\title{
RUREG--0540-101.19-10.8
}

NUREG-0540

Vol. 19, No. 8

Title List of Documents

Made Publicly Available

August 1-31, 1997

\author{
RECEIVFD \\ NOV $1 \cap 1997$ \\ OSTI
}

U.S. Nuclear Regulatory Commission

Ofrice of Information Resources Managemeat

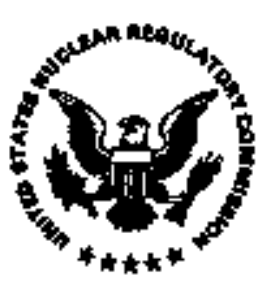




\section{AVAILABILTY MOTICE}

\section{Avatubitity of Referonce Materials Cited in NFC Publications}

Most documents ctied in NRC publications will be avalable from one of the following sources:

1. The NRC Public Document Room, $2120 \mathrm{~L}$ Street, NW., Lower Level, Washington, DC 20555-0001

2. The Superintendent of Documents, U.S. Government Printing Office, P. O. Box 37082, Washington, DC 20402-9328

3. The Nationel Technicel tinformation Service, Springfield, VA 2216i-0002

Although the listing that follows represents the majorty of cocuments cited in NRC publications, it is not intended to be exheustive.

Referenced documents avaluable for inspection and copying for a fee from the NRC Public Document Room include NAC comesponderce and interntal NFC memoranda: NAC bullutins, circulers, information notices, inspection and investigetion notices; licensee event reports: vendor reports and correspondence; Commission papers: and applicant and licensee documents and correspondence.

The following cocuments in the NUREG series are availeble for purchase from the Govertiment Printing Office: formbl NRC stalf and contractor reports, NAC-sponsorod conference pro. caedings, international sgresment reports, grantes reports, and NHC booklets and brochures. Atso available tre regulatory guxides, NFC regulations in the Codte of Federal Reguldtions. and Nuciear Regulatory Commisgion iasuanoss.

Documents availeble from the Nationel Technted Information Sarvice include NUFEG-8eries reports and tecknical reports prepared by ather Federal epencies and reports prepared by the Atortic Energy Commisston, forerunner agency to the Nuclear Repulatory Commission.

Documents avallable from public and opecial technical llbrarles include all open literature items, such as books, journal articles, and transactions. Fedoral Register notlces, Federal and Slate lepislation, and congressioned reperts can usually be obtained from these libraries.

Documents such as thesps, dissertetions, foreign reports and transiations, and non-NRC conference proceedings are available for purchase from the organization sponsoring the publice. tion cited.

Single copies of NRC draft reports are available tres, to the extent of suppty, upon written requeat to the Office of Adrninkstration, Distribution and Mail Services Section, U.S. Nuclear Regulatory Commisaion, Washington, DC 20565-0001.

Copies of industry codes and stendards used in a substantive manner in the NaC requlatory process are maintained at the NAC Library, Two White Fint North, 11545 Rockvile Pike, Fiockville, MD 20852-2739. for wa by the public. Codes and standards are usually copyrighted and mey be purchased trom the originating organization or, if they are American Nationaf Stendards, from the American Netional Standards Institute, 1430 Broadway. New York, NY 10018-3308.

A year's subscription of this report consists of 12 monthy issues. 
NUREG-0540

Vol. 19, No, 8

\section{Title List of Documents \\ Made Publicly Available}

August 1-31, 1997

Date Published: October 1997

E. B. Morris, NRC Projed Manager

Ofince of Information Resources Manazement

U.S. Nuclear Regulatory Commission

Washington, DC 20555-0001

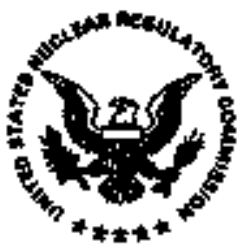




\section{DISCLAMIER}

This report was pitepted as as acconth of woxk sponsored by an agensy of the Uaited States Gowernment. Netihst the Uniled Staiss Gowernmest nof any tapecy

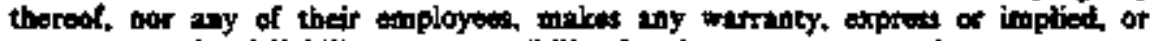

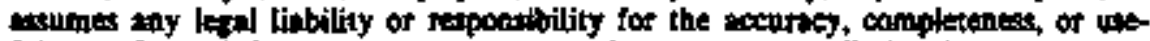
fuliness of any information, spparatus product, or process discloued, or repressonts that ihs wo would not infriase privetety owned rights. Referance herein to eny specifje commetciel product, procoss, of service by trade aame, tradernark, manufac.

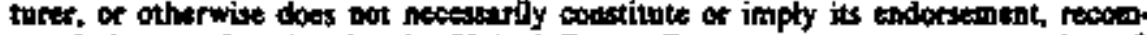
mendation, or farering by the Uaited States Govertment of any agtency thereof.

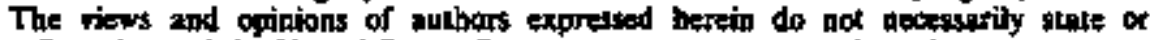
refiect those of the United Stales Govermment or any apency thersor. 


\section{DISCLAIMER}

Portions of this document may be illegible electronic image products. Images are produced from the best available original document. 


\section{CONTENTS}

Preface $\ldots \ldots \ldots \ldots \ldots \ldots \ldots \ldots \ldots \ldots \ldots \ldots \ldots \ldots \ldots \ldots \ldots \ldots \ldots, \ldots \ldots \ldots$

Atrangement of Items by Docket Number $\ldots \ldots \ldots \ldots \ldots \ldots \ldots \ldots \ldots \ldots \ldots$ xi

Alphabetic Arrangement of Docket Items by Facility Name $\ldots \ldots \ldots \ldots \ldots \ldots \ldots$ xvii

Docketed ftems $\ldots \ldots \ldots \ldots \ldots \ldots \ldots \ldots \ldots \ldots \ldots \ldots \ldots \ldots \ldots \ldots, \ldots \ldots \ldots$

Low-Level Radwaste Disposal Sites — Docket $27 \ldots \ldots \ldots \ldots \ldots \ldots \ldots \ldots$

Domestic Licensing of Source Material — Docket $40 \ldots \ldots \ldots \ldots \ldots \ldots \ldots$ I

Domestic Licensing of Production and Utilization Facilities -Docket $50 \ldots \ldots \ldots \ldots 8$

Standardized Nuclear Power Plant Design - Docket $52 \ldots \ldots \ldots \ldots \ldots \ldots \ldots \ldots 6$

Domestic Licensing of Special Nuclear Material —Docket $70 \ldots \ldots \ldots \ldots \ldots \ldots 167$

Packaging of Radioactive Material for Transport and Transportation of Radioactive

Material Under Certain Conditions - Docket $71 \ldots \ldots \ldots \ldots \ldots \ldots \ldots \ldots \ldots$

Licensing of Independent Spent Fuel Storage - Docket $72 \ldots \ldots \ldots \ldots \ldots \ldots \ldots 183$

Nondocketed Items $\ldots \ldots \ldots \ldots \ldots \ldots \ldots \ldots \ldots \ldots \ldots \ldots \ldots \ldots \ldots \ldots \ldots . \ldots \ldots$

10 CFR - Commission Meeting Transcripts and Reports . . . . . . . . . . . 187

ACNW - Advisory Comunittec on Nuclear Waste $\ldots \ldots \ldots \ldots \ldots \ldots \ldots \ldots \ldots \ldots 7$

ACRS - Advisory Committee on Reactor Safeguards Documents . . . . . . . . . 187

ADVCM - NRC Advisory Committees $\ldots \ldots \ldots \ldots \ldots \ldots \ldots \ldots \ldots \ldots \ldots \ldots$

COMMISSION - NRC Commissioner Correspondence $\ldots \ldots \ldots \ldots \ldots \ldots \ldots \ldots 188$

CONTRACT - NRC Contracts and Grants . . . . . . . . . . . . . . . 190

FOIA - Freedom of Information Act Requests and Appeals $\ldots \ldots \ldots \ldots \ldots \ldots \ldots, 193$

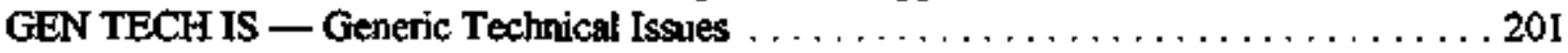

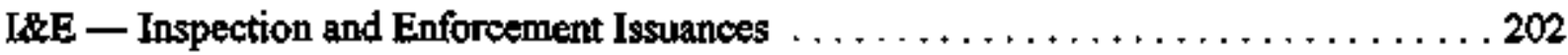

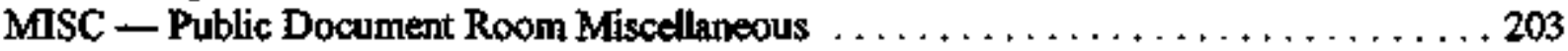

NUREG — NUREG Reports and Related Correspondence $\ldots \ldots \ldots \ldots \ldots \ldots \ldots 204$

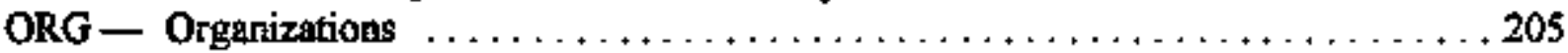

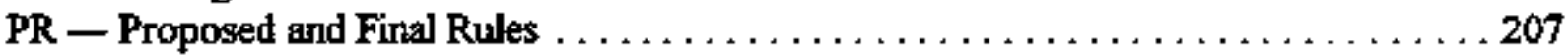

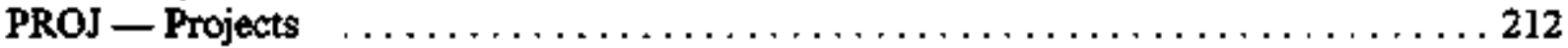

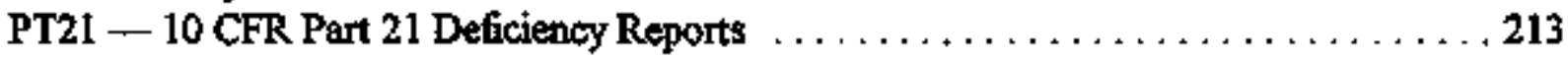

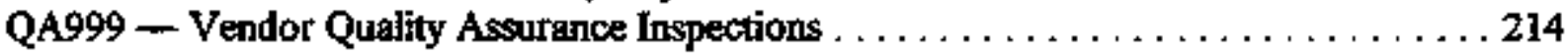

REG CERT - Registration of Certificates for Sealed Sources and Devices . . . . . . . 214

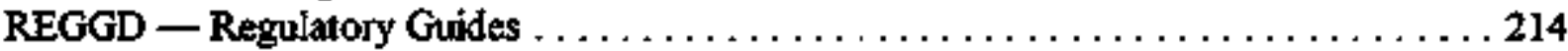

REVIEW GRP - Review Groups $\ldots \ldots \ldots \ldots \ldots \ldots \ldots \ldots \ldots \ldots \ldots \ldots \ldots \ldots \ldots \ldots \ldots, 215$

SECY — Staff Position Papers Prepared for the Commission $\ldots \ldots \ldots \ldots \ldots \ldots \ldots \ldots 216$

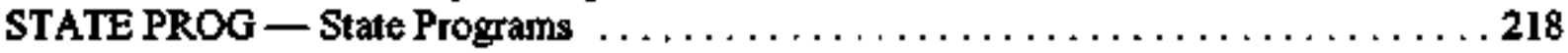

TOPREP - Topical Reports and Related Correspondence $\ldots \ldots \ldots \ldots \ldots \ldots \ldots, 222$

WASTE MGMT — Waste Management Documents $\ldots \ldots \ldots \ldots \ldots \ldots \ldots \ldots \ldots \ldots \ldots$ 


\section{CONTENTS}

Page

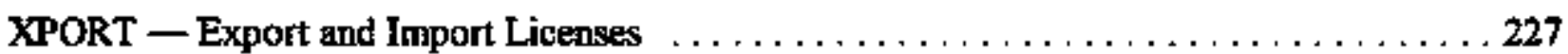

Personal Author Index . . . . . . . . . . . . . . . . . . . . . . . . . . . . 229

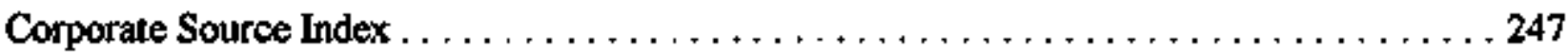

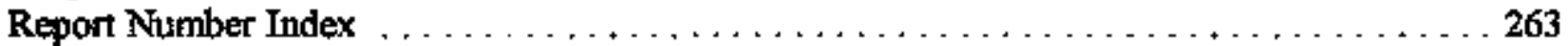

Cross Reference of Enclosures to Principal Documents $\ldots \ldots \ldots \ldots \ldots \ldots \ldots \ldots \ldots$

Appendix A - Definitions of Docket 50 Categories $\ldots \ldots \ldots \ldots \ldots \ldots \ldots \ldots \ldots$ A 1

Appendix B - Definitions of Nondocketed Categories ............... B - 1 


\section{PREFACE}

The Title List of Documents Made Publicly Avaitable is a monthly publication. It describes the information recejved and published by the U.S. Nuclear Regulatory Commission (NRC). This information inciudes (1) docketed material associated with civilían nuclear power plants and other uses of radioactive materials ard (2) nondocketed material received and pubilished by NRC pertinent to its role as a regulatory agency. As used here, docketed does not refer to Court dockets; it refers to the system by which NRC maintains its regulalory records. This series of documents is indexed by a Personal Anthor Index, a Corporate Source Index, and a Report Number Index.

The docketed information in the Tutle List inchudes the information formerly issued through the Department of Energy pubjication Power Reactor Docket Infomation, last published in January 1979. NRC docuntents that are publicly available may be examined without charge at the NRC Public Document Room (PDR). Duplicate copies in paper, microtiche, or (selectively) diskette, may be obtained for a fee.
Standing orders for certain categories of documents are also available. Clients may search for and order desired titles through the PDR computerized Bibliographic Retrieval System (BRS), whick is accessible both at the PDR and remotely. BRS also has some types of documents in full text. Other recent, selected documents, as well as information about the PDR, may be viewed/downloaded from an electronic bulletin board. The PDR is staffed by professional technical librarians, who provide reference assistance to users. See NOTES at the end of the preface for information about reaching the PDR. Microfiche of the docketed information listed in the Title List is available for sale on a subscription basis from the National Technical Information Service (NTIS). See NOTES at the end of the Preface for the complete NTIS address.

We encourage your comments, criticisms, and suggestions. In particular, if there are title descriptions that are not meaningful, please let us know so that we can correct the database. Insofar as possible, we would like this document to be a valuable part of your reference material.

\section{DOCKETED ITEMS}

Each utility or institution engaged in a nuclear activity licensed and regulated by the U.S. Nuclear Regulatory Commission (NRC) is assigned a docket number. As used by NRC, docket numbers refer to the particular system by which NRC maintains its regulatory records, not to law court dockets. The first two digits of a docket number identify the type of nuclear activity that NRC regulates. These docket categories are as follows:

\section{7 - Low-Leve] Radioactive Waste Disposa]} Sites

30 - Byproduct Material Licenses

40 -Nuclear Source Material Facilities

50-Nuclear Reactors and Futel Reprocessing PJants

52 -Standard Design Certification, Early Site Permit, and Combined Licenses for Nuclear Power Plants

$70-$ Special Nuclear Materials 


\section{DOCKETED ITEMS (cont.)}

71 -Design of Packages for Transport of Radioactive Material and Quality Assurance Programs

Except for Docket 27, these numbers correspond to the parts of Title 10 in the Code of Federal Regulations (10 CFR) that cover regulations pertaining to those types of licenses. The
72 -Independent Spent Fuel Storage Instaltations

remaining digits of a docket number uniquely identify a specific facility or institution. For example, Docket $50-317$ identifies the Calvert Cliffs Nuclear Power Plant, Unit 1.

(1) P. Operating license stage documents \& correspondence

(2)

(3)

8907070172 Commends util for exemplary hospitality extended to visiting Sotiet technical working group on 890608 .

(4)

(5)

(6)

DENTON, H.R.Office of Governmental \& Pubfic Affairs (Post 870413). 89/46/27<smiles>O</smiles>

CLARK, PR. 50442:181-50442:182
(8)

General Public Utilities Corp-GPU Service Corp. 2 pp.

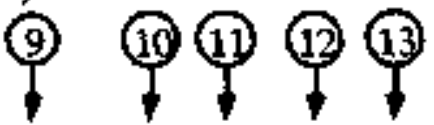

1. NRC/PDR Document category (see Appen$\operatorname{dix} A)$.

2. NRC/PDR Accession Number (year, month, day, and sequence number of iten).

3. Description of information contained in the doctument.

4. Personal author(s). An asterisk in this position indicates that there is no personal author.

5. Author affiliation (NRC, corporate, agency, or nonindividual source).

6. Date of document described.

7. Recipient of the document.

8. Recipient affiliation (NRC or corporate).

9. Pages in this document.

10. This five-digit number is the NRC $48 \mathrm{X}$ trictofiche designation.
11. The three-digit portion of this entry is the number of the frame on which this document starts.

12. This five-digit number is the microfiche on which this document ends.

13. The three-digit portion of this entry is the number of the frame on which this document ends.

The Docketed Iteros list is arranged sequentially by Docket Number, category, and then by date of principal iterns. Entries indented and pre-

ceded by a dash are items that were submitted as enclosures to principal itens. In general, indented items are not in accession number sequence. If the docketed document has a contract (job code) number of a formal report number, it with appear in the last line of the item following entry 5 . 


\section{NONDOCKETED ITEMS}

The nondocketed items are divided into the cate. gories used for filing and searching in the NRC Public Document Room. These categories and their symbols are as follows:

10 CFR 9.7-Commission Meeting Transcripts and Reports

ACRS-Advisory Committee on Reactor Safeguards Documents

ADVCM-NRC Advisory Committees

COMMISSION-NRC CommissioneI

Comespondence

CONTRACT -NRC Contracts and Grants

FOIA-Freedom of Information Act

Requests and Appeals

GEN TECH IS-Generic Technical Issues

I\&E-Inșpection and Enforcement Issuances

I\&E Manual-NRC lospection Manual

I\&E MISC-Draft Generic

Communications

MISC-Public Document Room

Miscellaneous

NUREG-NRC Staff and Contractor

Formal Reports, Conference Proceedirigs,

Brochures, International Agreement

Reports, and Related Correspondence

ORG-Organizations

PR-Proposed and Final Rules

PRESS-Press Releases

PRM-Petitions for Rulemaking

PROJ-Projects
PT 21-10 CFR Part 21 Deficiency Reports

QA 999-Vendor Quality Assurance

Inspections

RCSSD-Registration of Certificates for

Sealed Sources and Devices

REGGD-Regulatory Guides

RES-Research and Technical Assistance

Reports (1974 through 1988)

KEVIEW GRP-Review Groups

RM-Rulemakings

SAI - Strategic Assessment lssue Papers

SECY - Staff Position Papers Prepared for the Commission

SPCH-Speeches

STATE PROG-State Programs

TOPREP-Topical Reports and Related Correspondence

WASH-AEC-Issued Formal Reports and

Related Correspondence

WM-Waste Management Documents

WMRES - Waste Management Contract Research Documents

XPORTLIC-Export and Import Licenses

Appendix B lists these categories and describes the material available in each. Omission of a category in the listing for a docket indicates that no documents in that category were filed in the time period of the report.

The principal elements of the nondocketed itenis are- 


\section{NONDOCKETED ITEMS (cont.)}

(1) NUREG-NRC Staff and Contractor Formal Reports, Conference Proceedings, Brochures, International Agreement Reports, and Related Correspondence

(2)

(3)

(4)

8910100307 NUREG/CR-5340 DATA SUMMARY RFPORT FOR FISSION PRODUCT RELEASE TEST VI-2

(5)

(6)

OSBORNE, M.F, COLLINS, J.L., LORENZ, RA. Oak Ridge National Laboratory.

(7)

(8)

(9)

(19)

(1)

FIN B-0127. ORNLTM-11105. 89/09/30. Division of Systems Research (Post 880717). 69 pp.

(12) (13) (14) (15)

51409:065-51409:134.

1. Nondocket category.

2. NRC/PDR Accession Number (year, month, day, and sequence number of item).

3. NRC Report Number.

4. Title and description of information contained in the document.

5. Personal author(s). An asterisk in this position indicates that there is no personal author.

6. Corporate, agency, or other nonindividual source.

7. Confract or financial identification number source.

8. Secondary report number, if any.

9. Date of document described
10. NRC organizational recipient (or sponsor).

11. Pages in this document.

12. This five-digit number is the NRC $48 \mathrm{X}$ microfiche desigration.

13. The three-digit portion of this entry is the number of the frame on which this document starts.

15. The three-digit portion of this entry is the number of the frame on which this document ends.

The Nondocketed Items list is arranged sequentially by Accession Number within category. Indented items preceded by a dash are enclosures to unindented jtems.
Ordering and Viewing Documents:

1. The address for $\mathrm{GPO}$ is:

Superintendent of Documents U.S. Government Printing Office

P.O. Box 37082

Washington, DC 20402-9328
To place orders, send a check or money order payable to the Superintendent of Documents. GPO Deposit Account holders may charge their orders by calling 202-512-2249. 


\section{NONDOCKETED ITEMS (cont.)}

2. The address for NTIS is:

National Technical Information Service 5285 Port Royal Road

Springfield, Virginia 22161

To place orders for or to obtain price information about NRC publications at NTIS, either write to NTIS or call the Sales Desk at 703-487-4650. Specify the NRC report number and title when you place your order. You may charge publications to American Express, Visa, or Mastercard credit cards; supply the charge card number when placing your order.

3. The location of the PDR is:

2120 L Street, NW.

Washington, DC

The mailing address is:

U.S. Nuclear Regulatory Commission

Public Document Room

Mail Stop LL -6

Washington, DC 20555-0001
Telefacsimile

$202-634-3343$

Telephone

$202-634-3273$ or $1-800-397-4209$

Internet $\mathbf{e}-m$ at

PDR@NRC.GOV

For more information about PDR services, for reference assistance, to place an order for documents, or to arrange for access to the PDR computer retrieval system, write, e-mail, fax, or phone. When ordering documents listed in this publication, please identify the Document Accession Number for each citation. Reproduction charges and method of payment, based on the current reproduction contract, may be abtained from the PDR.

To access the PDR electronic bulletin board on FedWorld, contact the FedWorld Help Desk at $703-487-4086$. 
Manis

OCONEE NUCLEAR STATION, UNIT 2

OCONEE NYCLEAR STATION, UNIT 3

OHIO STATE WNIV. RESEAACH REACTOR

OREGON STATE WNY. RESEAFCH

FEACTOA

OYSTEA CREEK NUCLEAR POWER PLANT

PAUSADES MUELEAF PLANT

PALO VERDE NUCLEAR STATION, UNIT 1 STN-50.528

PALO VERDE NUCLEAR STA KON, UNIT 2 STN-60-529

PALO VERDE MUCLEAR STATIDL UNIT 3 STN-SO-530

PEACH BOTTOH ATOMIC POWER STATION, 60-171 UNIT 1

PEACH BOTTOM ATOMIC POWER STATION, $\quad 50.277 \quad 47$ UNIT 2

PEACH BOTTOM ATOMIC POWER STATHOM, 50-278 UNIT 3

PEMNSYLVANIA STATE UNV. RESEARCH FEAGTOR

PERRY NLICLEAR POWER PLANT, INIT 1

PERRY NUCLEAR POWER PLANT, UNIT 2

PULGFIM NUCLEAR POWER STATON, UNIT 1

PLUM EROOK FESEARCH REACTOR FACALTY

POINT BEACH NUCLEAR PLANT, LNITT 1

POINT EEACH NUCLEAR FLANT, UNIT 2

PAAIRIE ISLAND MUCLEAF STATION, UMTT 1

PAAIRIE ISLAND MJICLEAF STATION, UNIT

2

PURDUE UNN. RESEARCH REACTOR

QUAD-CMES STATION, UNIT 1

QUAD-CMES STATION, UNIT 2

RANCHO SECO NUCLEAF GENERATING STATION

PHOOE ISLAMD A PRONIOENCE PLANTATIONS AEC

FNER EEND STATION, UNIT 1

ROBEAT EMNKT GINNA NUCLEAA PLANT, UNIT 1

RPPI CFITICAL EXPERIMENT

SALEM NUCLEAP GENER/TING STATION, INT 1

SALEN NUCLEAA GEVIERATING STATION, UNit 2

SAN ONOFRE NUQLEAF STATION, UNIT 1

SAN ONOFRE MUCLEAF STATION, UNIT 2

SAN ONOFBL NUCLEAR STATKON, UNIT 3

SAXTON MUCLEAF EXPERIMENTAL

FAcALIT

SEABFOOK NUCLEAA STATION, UNT 1

(1)

\section{Docket Pape \\ Nime}

50-270 $\quad 41$

50-287 54

$50-150 \quad 12$

50-243 21

$50-219 \quad 17$

$50-255 \quad 30$

160

162

res

14

47

$50-5 \quad 9$

50.440 139

50-41 140

$50-253$, 57

$50-50$

50-286

$50-301$

50.282

$50-3096$

$504182 \quad 14$

60-254

$50-205$

$50-312$

50-193

$00-45$

50.244

$50-225$

50-272

$50-311$

50-206

$50-361$

$50-362 \quad 108$

$50-146 \quad 12$

$50-449 \quad 141$ 3
SEABROOK NUCLEAR STATON, UNIT 2

SEQUOYAH NUCLEAF PLANT. UNAT 1

SEQUOYAH NUCLEAP PLANT, UNIT 2

SHEARON HARFIS NUCA,EAF POWER PLANT, UNTT 1

SHOREHAN NUGLEAR POWER STATION

SOUTH TEXAS PROEECT, UNIT 1

SOUTH TEXAS PAONECT, UNIT 2

ST. LUGE PLANT, UNIT 1

ST. LUCEE PLANT, UNIT 2

SUFAY POWER STATION, ONAET 1

SUAAY POWER STATKN, UNT 2

SUSOUEHANNA STEAM ELECTIFIC

STATION, UNIT $Y$

SUSOUEHANNASTEAM ELECTFIC

STATION, U:T 2

TEXAS ABHYUNV. RESEAFCH REACTOA

THREE MULE ISLAND NUCLEAA STATION, UNIT

THREE MLE ISLAND NUCLEAP STATION, पxiा 2

TROIAN NUCLEAR PLANT

TURKEY POINT PLANT, UNT 3

TURKEY PONT FLANT, UNET 4

UNIV, Of RLOAIOA TRAINWNG REAGTOR

UNNY. OF ILLINOIS RESEAFCH REACTCF

UNIV. OF ILLINOIS RESEAFCH REACTOR

UNW. OF MARYLAND RESEARCH REACTOA

UNIV. OF MSSOUIRI RESEARCH AEACTORCOLMuBik

UNIV, OF VIRGINUA GAVALIEA REAGTOR

UNIV. OF VIAGINA RESEAPCH REACTOR

UNNY. OF WISLOONSIN RESEARCH

AEACTOR

UNIVERSTY OF WASSACEUSETTS,

LOWELL RESEANCH REACTOR

UNIVERSITY $O F$ MISSOURA FESEARCH

REACTOA, ROUL, WYO

VIAKONT YANKEE NUQ⿱一龰EAP POWER

VRGL C. SUNAER NUCLEA STATION, UNIT 1

WASHINGTON STATE UNN, REBEARCH PEACTOR (TRIGA)

WATERFOFD GENEFATING STATKNN, UNIT

WATTS BAF NLCLEAR FLANT, INTT

WATTS BAF NLCLEAR PLANT, LNIT 2

WESTINGHOUSE TEST REACTOR
Docket

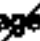

42

$50-3478$

$50,328 \quad 69$

50-400 127

$50-322$

STN-50-498 168

STN-50-49. 158

$50356 \quad 94$

50-369 121

$50-280 \quad 48$

50-281 49

$50-397 \quad 719$

$50.388 \quad 120$

50-128 12

$50-209$

$50-320$

50-344 100

$50-250 \quad 27$

50-251 25

$50-83 \quad 11$

$50-151 \quad 12$

60-356 105

50-166 14

$50-1883$

$50-396 \quad 125$

50-6e 11

$50-156 \quad 13$

$50-228 \quad 20$

$50-128 \quad 12$

$50-271 \quad 49$

$50-095 \quad 124$

$50-27 \quad 10$

50-382 118

50-390 128

50-391 128

$50-2210$ 


\begin{tabular}{|c|c|c|c|c|}
\hline Docket & Nane & Pagen & Docket & Mome \\
\hline $50-89$ & GENERAL ATCWMLS & 11 & $50-249$ & OAEGON STATE UNIV. RESEARCH \\
\hline $50-97$ & $\begin{array}{l}\text { COANELL UNIV. ZERO POWEF } \\
\text { AEACTOR }\end{array}$ & 11 & $50-244$ & $\begin{array}{l}\text { REACTOR } \\
\text { ROEERT EMMET GININA NUCLEAF } \\
\text { FLANT, UNIT } 1\end{array}$ \\
\hline 60-116 & $\begin{array}{l}\text { KOWA STATE UNIV, RESEARCH } \\
\text { AEACTOR }\end{array}$ & 14 & $50-245$ & $\begin{array}{l}\text { MALSTONE NUCAEAR POWER } \\
\text { STATHON, UNIT I }\end{array}$ \\
\hline $50-123$ & $\begin{array}{l}\text { UNVERSITY OF MISSOLAI FESEAFCH } \\
\text { REACTOF, ROLLA, MO }\end{array}$ & 12 & $50-247$ & INDIAN POWT STATICN, INIT 2 \\
\hline $50-128$ & $\begin{array}{l}\text { TEXAS ARM UNV. FESEAFCH } \\
\text { REACTOR }\end{array}$ & 12 & $50-249$ & $\begin{array}{l}\text { DAESDEN NUCLEAR POWEA STATION, } \\
\text { UNIT } 3\end{array}$ \\
\hline $50-133$ & HUMBOUT BAY POWER PLANT, VNMT 3 & 12 & $50-250$ & TURKEY PONT PLANT, UNIT 3 \\
\hline $50-134$ & $\begin{array}{l}\text { WORCESTEF POLYTECHNIC NSTIUUTE } \\
\text { HESEARCH REACTOR }\end{array}$ & 12 & $\begin{array}{l}50-251 \\
50-254\end{array}$ & $\begin{array}{l}\text { IUFXEY POINT PLANT, UNIT } 4 \\
\text { OUADCATIES STATION. UNIT } 1\end{array}$ \\
\hline $50-140$ & $\begin{array}{l}\text { SAXTON NUCAEAR EXPERIMENTAL. } \\
\text { FACLTY }\end{array}$ & 12 & $50-255$ & PALISADES NUGLEAR PEANT \\
\hline $50-150$ & $\begin{array}{l}\text { OHO STATE UNN, RESEARCH } \\
\text { REACTOF }\end{array}$ & 12 & $60-259$ & $\begin{array}{l}\text { BAOWNS FEARY NUCLEAR POWER } \\
\text { STATON, UNTT } 1\end{array}$ \\
\hline $50-151$ & $\begin{array}{l}\text { WNN. OF ILLNOIS RESEARCH } \\
\text { REACTOR }\end{array}$ & 12 & $50-260$ & $\begin{array}{l}\text { EROWNS FEPAY NUCLEAR POWEA } \\
\text { STATION, UNT } 2\end{array}$ \\
\hline $50-155$ & BIG ROCK PCINT NUCAEAR PLANT & 13 & $50-261$ & H.B. PDBNSON PLANT, UNIT 2 \\
\hline $50-158$ & $\begin{array}{l}\text { LNIY. OF WISCONGIN RESEARCH } \\
\text { REACTOR }\end{array}$ & 13 & $60-263$ & $\begin{array}{l}\text { MONTIGELLO NUGLAR GENERATWNG } \\
\text { PLANT }\end{array}$ \\
\hline $50-157$ & CORNELL UNIV. RESEAROH AEACTOA & 14 & $50-264$ & DOW GHEMICAL CO. \\
\hline $50-160$ & $\begin{array}{l}\text { GEOROIA NSTITUTE OF TECHNOLOGY } \\
\text { RESEARCH REACTOR }\end{array}$ & 14 & $\begin{array}{l}50-265 \\
50-266\end{array}$ & $\begin{array}{l}\text { OUADACATES STATION, UNIT } 2 \\
\text { PONT BEACH NUCLEAR PLNNT, UNTT } 1\end{array}$ \\
\hline $50-169$ & GENERAL ATOMICS & 14 & 50.267 & $\begin{array}{l}\text { FORT ST. VRAN NUCLEAR } \\
\text { GENERATING STATION }\end{array}$ \\
\hline $50-168$ & $\begin{array}{l}\text { UNIV. OF MAPYLAND RESEARCH } \\
\text { FEACTOA }\end{array}$ & 14 & $50-269$ & OCONEE NUCLEAR STATION, UNIT I \\
\hline $50-170$ & $\begin{array}{l}\text { AFAED FOACES RADIOBIOLOGY } \\
\text { AESEARGH INSTITUTE }\end{array}$ & 14 & $50-270$ & $\begin{array}{l}\text { OCONEE NUCLEAR STATION, UNIT } 2 \\
\text { VEFHONT YANKEE NUCAEAR POMER }\end{array}$ \\
\hline $50-171$ & $\begin{array}{l}\text { PEACH BOTTOH ATOMIC POWER } \\
\text { STATKW, UNIT } 1\end{array}$ & 14 & $=0-972$ & \\
\hline $50-182$ & PURDUE UNIV, FESEAFCH REACTOF & 14 & Nere & STATKON, UNAT I \\
\hline $50-184$ & $\begin{array}{l}\text { NATIONAL INSTITUTE OF STANDARCS } \\
\text { \& TECHNOLOCY REACTOF }\end{array}$ & 15 & $50-275$ & $\begin{array}{l}\text { D|ABLO GANYON NUCLEAA POWER } \\
\text { PLANT, UNT } 1\end{array}$ \\
\hline $50-188$ & $\begin{array}{l}\text { UNIV. OF HISSOURI RESEARCH } \\
\text { FEACTOROOUUABA }\end{array}$ & 15 & $50-277$ & $\begin{array}{l}\text { PEACH BOTTON ATOMLC POWEA } \\
\text { STATION, UNIT } 2\end{array}$ \\
\hline $50-198$ & $\begin{array}{l}\text { AHOOE SLAND \& PAOVIDENCE } \\
\text { PLANTATIONS AEC }\end{array}$ & 15 & 50-278 & $\begin{array}{l}\text { PEACH BOTTOM ATOMIC FOWER } \\
\text { STATION, UNIT } 3\end{array}$ \\
\hline $50+199$ & $\begin{array}{l}\text { WHWATTAN COLLEGE RESEARGH } \\
\text { AEAGTOR }\end{array}$ & 15 & $\begin{array}{l}50-280 \\
50-291\end{array}$ & $\begin{array}{l}\text { SURRY POWEP STATLON, LANTT } 1 \\
\text { SURAY POWER STATKON, UNT } 2\end{array}$ \\
\hline $50-205$ & SAN ONOFRE NUCLEAR STATION, UNIT & 15 & $50-292$ & $\begin{array}{l}\text { PRARAE ISLAND NUCLEAF STATION, } \\
\text { UNIT } 1\end{array}$ \\
\hline $50-213$ & HADDAM NECK PLANT & 16 & $50-284$ & $\begin{array}{l}\text { IDAHO STATE UNIY. FESEEAFACH } \\
\text { FEACIOR }\end{array}$ \\
\hline $50-219$ & $\begin{array}{l}\text { OYSTER CAEEK NUCLEAF POWEA } \\
\text { PLANT }\end{array}$ & 17 & $50-285$ & FOAT CALHOUN STATION, UNT 1 \\
\hline $50-220$ & $\begin{array}{l}\text { NUNE MILE POINT NUCLEAR STATION, } \\
\text { UNIT T }\end{array}$ & 18 & $50-286$ & INDAAN POINT STATION, UNIT S \\
\hline $50-223$ & $\begin{array}{l}\text { UNNERSTY OF MASSACHUSETTS, } \\
\text { LOWEL RESEARCH AEACTOA } \\
\text { AFI CRTIGAL EXFERUMET }\end{array}$ & 20 & $50-200$ & $\begin{array}{l}\text { THREE MLE ISLAND NUCLEAR } \\
\text { STATION, UNIT } \uparrow\end{array}$ \\
\hline $50-297$ & $\begin{array}{l}\text { OFESDEN MUCAEAR POWER STATION, } \\
\text { UNTT } 2\end{array}$ & 20 & $50-293$ & $\begin{array}{l}\text { PILGRIM NUCLEAR POWER STATION, } \\
\text { UNIT } 1\end{array}$ \\
\hline $50-238$ & NUCLEAF SHIP SAVANNAH & 21 & $50-205$ & ZION NUCLEAR FOWER STATION, UNT \\
\hline
\end{tabular}


bocke

50-290

$50-297$

50-298

$50-501$

50-302

$50-304$

$50-305$

50-306

50-309

$50-311$

$50-312$

50-313

50-315

$50-316$

$50-317$

50-318

$60-320$

$50-321$

50-522

$50-325$

$50-324$

$50-325$

50-327

50-398

50-329

50-330

50-381

50-333

$50+304$

$50-305$

$50-359$

50-3ss

50-339
Nama

BROMNS FEARY NUCLEAR POWER STATKON, UNT 3

NOFTH CAROLINA STATE UNIY.

PLLSTAR REACTOR

COOPEA NUCIEAR STATWON

POINT BEACH NUCLEAR PLANT, UNIT 2

CRYSTAL RIVER NICLEAR PLANT, UNIT

ZION MUCLEAR POWER STATION, UNIT 2

KENAJNAE NUCLEAF POWEF PLANT

PRAIRIE ISLAND NUCAEAR STATION. UNAT 2

MANE YANGEE ATOMTC POWER PLANT

SALEH HUCLEAR GENEFATWO STATKON, UNIT 2

PANCHO SECO NUCLEAR GENERATING STATION

ARKAMSAS NUCLEAR ONE, UNIT I

DONALD C. COOK MUCLEAP FOWER

PLANT, LNIT 1

DONALD C. COOK NUCLEAR FOWER PLANT, UNIT 2

CALVERT CUFFS NUCLEAR POWER PLANT, UNT I

CALVEAT CLFFS NUCAEAR DOWER PLANT, UNIT 2

THFEE MUE ISLAND NUCLEAR STATKON, UNT 2

EOWIN L HATCH NUCLEAP PLANT, LNT

SHORF⿻彐一AA NUCLEAR PONER STATTON

DUALO CANYON NUCLEAR POWEA PLANT, LNIT 2

BAUKSWCK STEAN ELECTRKG PLANT, UNT 2

BAUNSWCK STEAH ELECTRKC PLANT, UNAT 1

SEOUOYAH NUCAEAR PLANT. UNIT 1

SEQUOYAH NUCLEAF PLANT, UNIT 2

MIDLAND PLANT, UNIT 1

MDLAND FLANT, UNIT ?

DUANE AFNOLO ENERGY CENTEA

IANES A FIZPATRICK NUCUEAR POWEA PLANT

BEAVER VALLEY POWEA STATION, UNIT 1

ST. LUCAE PLANT, UNIT 1

MLLSTONE NUCLEAR POWER

STATION. UNIT 2

NORTH ANENA POHER STATION UNIT 1

NOPTH ANNA POWER STATION, UNTT 2
Docket rime

Page

$$
\text { UNIT } 2
$$

$50-34$

TAOJAN NUCLEAR PLANT

99

50-345

OEZMAU NUCLEAA STATION, UNIT 2

$50-346$

DAVISBESSE NUCLEAR POWEA STATKON, UNIT I

$50-348$

JOSEPH M. FARLEY NUCLEAR PLANT. UNIT 1

$50-352$ LHEFICK GENERATING STATLON, INNIT

$50-359$

$50-354$

LHIEFICK GENERATHNG STATION, UNIT 2

HOPE CFEEK NUCLEAR STATION, UNIT

(10)

50-350

UNV. OF ILLNONS FESEARCH AEACTOR

$50-361$

SAN ONOFAE NUCLEAR STATION UNIT 2

$50-362$

SAN ONOFAE NUCLEAR STATION, UNIT 3

$50-344$

JOSEPH M FAFLEY NUCLEAR PLANT, UNIT 2

50-396

$50-369$

$50-369$

$50-370$

EDWIN I. HATCH MUCAEAR FLANT, UNIT

ARKANSAS NUCLEAR ONE, UNIT 2

WILLAAM B. MCEUIFE NUCLEAF STATION, UNT 1

WILUAH B. MCEUIRE MUCLEAF STATION, UNT 2

50-37s

LASAUE COUNTY STATION, UNIT 1

300

101

101

102

109

104

105

106

105

108

100

110

111

LASALLE COUNTY STATIOA, UNIT 2

117

$50-382$

$\$ 0-397$

WATEAFORD GENERATTNG STATION, UNIT 3

SLISOUEHANWA STEAN ELECTRAC STATION, UNIT 1

$50-388$

SUSOUEHANNA STEAM ELECTFIC STATDN, UNT ?

50-389

ST. UUCGE PLANT, UNT 2

$50-990$

WATTS BAF NUCLEAR PLANT, UNT 1

WATTS BAF MUCLEAF PLANT, UNIT 2

VIFCIL C. SUMMER NUICLEAR STATION.

$50-995$

$50-396$

50-997

LNNT, OF VAGINIA CAVALER REACTOR

5

WPPSS MUCLEAR PAOVCT, INIT 2

50.400

$50-409$

SHEARON HARRIS NUCLEAF POWER PLANT, UNIT 1

$50-410$

LA CROSSE BOILING WATER REACTOR

128

NANE MRE POINT MUCLEAA STATION, UNIF 2

$50-412$

BEAVER VALLEY POWEA STATION, UNIT 2

$50-413$

CATAWBA NUTCLEAT STATON, UNIT 1

$50-414$ 


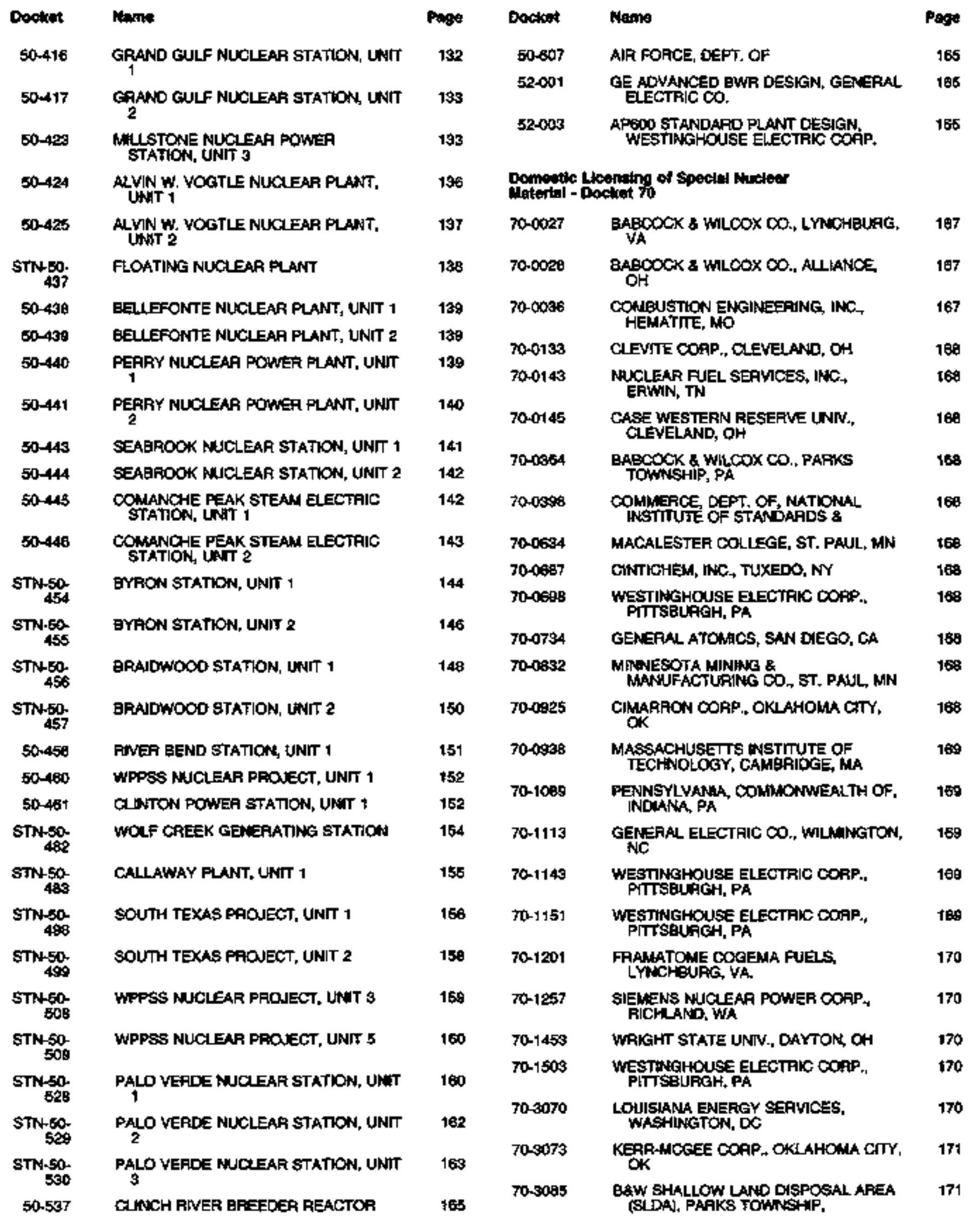




\begin{tabular}{|c|c|c|c|c|c|}
\hline Doeket & Name & Pag* & Docket & mam & Pege \\
\hline 70.3089 & $\begin{array}{l}\text { U.S. ENR|CFGAENT COFP. (AVLS } \\
\text { ENRICHENT PLANT) }\end{array}$ & 171 & $71-0228$ & GAPITAL X-RAY SEFVICE & 174 \\
\hline $70.700 t$ & US. ENRICHMENT CORP (PADUCAY & 171 & $71-0236$ & AMERIGAN ALALINES. INC. & 174 \\
\hline & GASEOUS DIFFUSHSN PLART) & & $79+0250$ & TRANSNUCLEAF, INC. & 174 \\
\hline 707002 & $\begin{array}{l}\text { U.S. ENRIOHWENT COFP. } \\
\text { (PORTSHOUTH GASEOUS DFFUSION }\end{array}$ & 172 & $71-0252$ & DAYTON X.RAY COD & 175 \\
\hline DOCKET & UNKNOWN & 3 & $71-0260$ & ASTPOTEGH, & 175 \\
\hline & & 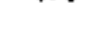 & 71.0269 & LEHIGH TESTNG LABORATORIES, ING. & 175 \\
\hline Packenging & 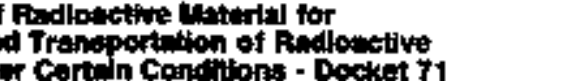 & & $71-0300$ & $\begin{array}{l}\text { INDEPENDENT TESTING } \\
\text { LABOOFATORIES }\end{array}$ & 175 \\
\hline 71.0011 & ARAOW TANK \& ENGWEERHG $C O$. & & 71001 & CTL ENGINEERING, INC. & 175 \\
\hline $71-0015$ & & - & $71-0308$ & ALASKA INDUSTRIAL X-RAY & 175 \\
\hline $71-0018$ & NORTTMEST ALFL NES, INC. & 173 & $71-0916$ & $\begin{array}{l}\text { MASON \& HANGER-SULAS MASON } \infty \text {. } \\
\text { INC. }\end{array}$ & 175 \\
\hline 310026 & EASTEPAN TESTING \& INSPECTION & 173 & 71.0319 & BENERAL TESTING \& INSPECTION CO. & 176 \\
\hline $71-0036$ & GRAMEU $\perp$ CORP. & 173 & $71-0326$ & $\begin{array}{l}\text { WOUSTRLAL NDT SERYCES OUY. } \\
\text { (INDUISTRIAL HEAT TREATING }\end{array}$ & 175 \\
\hline $71-0040$ & AMERSHAN CORP. & 173 & $71-0330$ & ALLIED INSPECTION SERVICES, INC. & 175 \\
\hline $71-0051$ & HAFEISON STEEL_ CASTINGS $C O$. & 173 & $71-0397$ & CALMET TESTING SETVICES, INC. & 176 \\
\hline $11-0000$ & HA. WNSPECTON SERVCE & 173 & 71.0345 & CAROLINA POWER \& LKGHT CO. & 175 \\
\hline $71-0066$ & $\begin{array}{l}\text { CERTIFIED TESTING LABORATOFIES, } \\
\text { WNG. }\end{array}$ & 173 & $71-0346$ & PUBLIC SEAVICE $\infty$. OF COLORADO & 175 \\
\hline $71-0075$ & $\begin{array}{l}\text { NOFFOUK SHIPBUILING \& DFYDOCK } \\
\text { CORP. }\end{array}$ & 173 & $71-0852$ & NlLES STEEL TANK 00 . & 175 \\
\hline $71-0079$ & BABCOCK \& WILCOX $\infty$ & 173 & Sor & 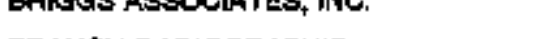 & 775 \\
\hline 71.0093 & H.JNTENGTON TESTING & 173 & $71-0308$ & $\begin{array}{l}\text { BFANCGARADIOISAAPHIC } \\
\text { LABOAATORIES, WNC. }\end{array}$ & 175 \\
\hline $71-0006$ & EG\&G FLOFIDA, INC. & 173 & 71.0992 & BAKER TESTING SERVICES, NNC. & 175 \\
\hline $71-0097$ & $\begin{array}{l}\text { NONOESTRUCTIVE INSPECTION } \\
\text { SERVKEE }\end{array}$ & 173 & $71-0404$ & CTI, INC. & 175 \\
\hline $71-0098$ & NEWPOAT NEWS SHIPBUILDUNG \& DFY & 173 & $71-0408$ & NASSOURI STEEL CASTINGS CO. & 175 \\
\hline & Lo & & 71.0417 & CAFIBE SHELL \& TUBE, ING. & 175 \\
\hline 7,0098 & FOSTIER WHEELLEA ENERTY COAP. & 174 & $71-0422$ & OKLAHOMA STEFI CASTINGS CO. & 976 \\
\hline $71-0102$ & $\begin{array}{l}\text { 5OUFCE PAODUCTICN S EQUIPMENT } \\
\infty ., \text { INC. }\end{array}$ & 174 & $71-0428$ & HQH X-AAY SERVICES, BNC. & 176 \\
\hline $71 \cdot 0103$ & TWIN CITY TESTINE CORP. & 174 & 71.0420 & BALL MILLEA, INC. & $17 \mathrm{~B}$ \\
\hline $71-0150$ & BUCKEYE STEEL CASTINBS & 174 & $71-0487$ & AOVEX CORP. & 176 \\
\hline 71.0111 & $\begin{array}{l}\text { MATEPIAL TESTING LBORATOAY OF } \\
\text { MFRIINIA, WNC. }\end{array}$ & 174 & $71-0438$ & GENERAL NOTORS CORP. & 176 \\
\hline $71-0113$ & NOOTER COAP. & 174 & & CONSULTANTS, INC. & 160 \\
\hline $7+-0114$ & COLBY-THIELMEIEA TESTING CO. & $\$ 74$ & $71-0460$ & UNIV. OF VIRGGINIA & 176 \\
\hline $71-0146$ & GLOBE X-FAY SEFVICES, ING. & 174 & $71+0468$ & EL PASO NATURAL GAS, ING. & 176 \\
\hline 71.0155 & BATH IRON WOFKS COAP. & 174 & $71 \cdot 0472$ & AEC TESTING & 176 \\
\hline $71 \cdot 0157$ & FROEHLFAY \& ROBERTSON, INC. & 174 & $71-0476$ & MIDWEST INSFECTION SERVICES, NKC. & 176 \\
\hline $71-0171$ & GUTSCAH FIED SEAVICESINOE, ING. & 174 & 71.0479 & $\begin{array}{l}\text { SOUTH GAROUNA ELECTHIC \& GAS } \\
\infty .\end{array}$ & 176 \\
\hline $71 \cdot 0181$ & CENTUAY INSFECTION, INC. & 274 & 71.0482 & UNV. OF MASSACHUSETTS & 176 \\
\hline $71-0186$ & HGA STEEL STAUCTURES, INC. & 174 & $71-0487$ & APINY, DEPT, OF & 176 \\
\hline 71.0201 & DURALLOY ELAW-KNOX, HWC. & 774 & $71-0494$ & MONTANA X-PAY, INC. & 176 \\
\hline $71-0205$ & PAYOR FOUNDRY, INC. & 174 & $71-0503$ & PENN NSPECTION CO. & 176 \\
\hline $\begin{array}{l}7.0219 \\
71.0218\end{array}$ & $\begin{array}{l}\text { INIERHOUNTANN TESTING CO. } \\
\text { CONAM INSPECTION, INC. }\end{array}$ & 174 & $71-0508$ & $\begin{array}{l}\text { MET-CHEM ENGGNEFEFING } \\
\text { LABORATORIES, INC. }\end{array}$ & 176 \\
\hline $71-0220$ & GENERAL DYNAMIOS CORP. & 174 & 710539 & ARAOW NDE CO., INC. & 176 \\
\hline
\end{tabular}




\begin{tabular}{|c|c|c|c|c|c|}
\hline Docket & Nime & Pago & Docket & Name & Pege \\
\hline $71-0542$ & MATTINGLY TESTNG SERVICES, IAC. & 176 & $71-0775$ & B \& G IN\$PECTION & 178 \\
\hline $71-0559$ & $\begin{array}{l}\text { HGH WOUNTAAN NSPECTION } \\
\text { SERVICES, NC. }\end{array}$ & 176 & $71-0770$ & DERBY GTY INSPECTION & 178 \\
\hline $71-0555$ & ALLEGMENY LABORATORIES & 176 & $71-0782$ & $\begin{array}{l}\text { INTERNATIONAL RADWOGRAPHY A } \\
\text { INSSECTION SEAVICES }\end{array}$ & $17 \mathrm{~b}$ \\
\hline $7:-0558$ & NON DESTRUCTIVE TESTING CORP. & 176 & $71-0783$ & $\begin{array}{l}\text { NOFTHWWEST INSPECTION \& TESTING } \\
\text { SERVCES }\end{array}$ & 178 \\
\hline $71-0560$ & EOWAPDS PIPELINE TESTING, INC. & 176 & & & \\
\hline 71.0583 & $\begin{array}{l}\text { MNNESOTA VALLY ENGTEERING, } \\
\text { INC. }\end{array}$ & 176 & $\begin{array}{l}71-0787 \\
71.00801\end{array}$ & $\begin{array}{l}\text { LAFAYETTE TESTING SERVICES } \\
\text { MCNDT LEASING, JNC. }\end{array}$ & $\begin{array}{l}178 \\
178\end{array}$ \\
\hline $71-0585$ & MOS MSPECTION, INC. & 177 & $71-0802$ & NORGAL TESTING, ING. & 179 \\
\hline 710592 & BFAUN ENGINEERING TESTING, INC. & 177 & 71.0007 & MAFYLAND Q C. LABOPATORES & 179 \\
\hline $71-0505$ & AKAON NOUSTRLAL SERYICES, INKC. & 177 & $71-0800$ & DEETA AIR LINES & 179 \\
\hline $71-0803$ & $\begin{array}{l}\text { JAN XFAY SERVICES, WKC. } \\
\text { INDUSTRIAL NOT CO }\end{array}$ & 17 & $71-0830$ & TI INSPECTKON-TECH & 179 \\
\hline $\begin{array}{l}71-0,911 \\
71-0,015\end{array}$ & $\begin{array}{l}\text { INDUSTALAL NOT CO. } \\
\text { ALONSO \& CAFUS IFON WORKS, INC. }\end{array}$ & $\begin{array}{l}177 \\
177\end{array}$ & $71-0062$ & $\begin{array}{l}\text { UNITED STATES ENAKCHMENT } \\
\text { COAFORATION }\end{array}$ & 179 \\
\hline $\begin{array}{l}71+0627 \\
71-0885\end{array}$ & $\begin{array}{l}\text { DONSOLIDATED NOE, NC. } \\
\text { H \& G INSPECTKON CO., INC. }\end{array}$ & 177 & $71-08868$ & $\begin{array}{l}\text { MANYAR ELECTROALLOYS } \\
\text { COAPOFATION }\end{array}$ & 179 \\
\hline $71-0537$ & WESTERN STRESS, INC. & 177 & $71-0899$ & DECASNE TESTING. INCC. & 179 \\
\hline 7100838 & WESTINGHOUSE ELECTRIC CORP. & 177 & $71-0840$ & $\begin{array}{l}\text { INOUSTRIAL MARINE TESTING } \\
\text { LABOAATORY }\end{array}$ & 179 \\
\hline $71-0890$ & ATOAIC INSFECTION 1 AES & 17 & 71-0855 & 日ЛTE INSPECTKON SERVICE OO., ING. & 179 \\
\hline $71-0862$ & BARNETT INDUSTRIAL XAAY & $\begin{array}{l}177 \\
177\end{array}$ & $71-0883$ & INSPECMCN SERVICE, INC. & 170 \\
\hline $71-0668$ & COWO TEGH, INC. & 177 & $71-0864$ & ADAMS NCUSTPIAL SERVCES, INC. & 179 \\
\hline $71-0072$ & LUCUS PIIKAN, BNC. & 177 & $71-0065$ & WTECRATED TECHNOLOOOIES, INC. & 179 \\
\hline $71-0667$ & $\begin{array}{l}\text { INDEPENDENT INSPECTION CORP. } \\
\text { PAE INC PPIEELWE NGPEGTON \& }\end{array}$ & 177 & $71-0870$ & MOLTEN METAL TECHNOLOGY & 179 \\
\hline $71-0684$ & $\begin{array}{l}\text { PIE INC (PIFELINE NSSECTKNA } \\
\text { ENGGINEEFING] }\end{array}$ & 177 & $71-0572$ & CONNORS PIPE SYSTEMS, INC. & 179 \\
\hline $71-0701$ & NOT SPECAALISTS, NAC. & 177 & $71-0878$ & ADFIAN COLLEGE & 179 \\
\hline $71-0705$ & APFUED TECHWUCAL SERMICES, INC. & 177 & $71-0879$ & EASTERN MKGHKAN UNNERSITY & 179 \\
\hline 71.0707 & CARONDELET FOUNCAY 0. & 177 & $71-00800$ & NOAFOUK STATE UNAVEASTY & 179 \\
\hline $71-0708$ & WESTINGHOUSE ELECTRIC COAP. & 177 & 71.3010 & $\begin{array}{l}\text { MODEL: NT-IX, TRANSPOFTATION } \\
\text { OEPT. OF }\end{array}$ & 179 \\
\hline $71-0716$ & $\begin{array}{l}\text { AFCTIC SLOPE WISFECTION SEFVICES, } \\
\text { WIC. } \\
\text { METALS FVAU WTON R TESTING INS }\end{array}$ & 178 & $71-3024$ & MODEL; 2842A, TFANSPORTATION, & 179 \\
\hline $\begin{array}{l}71-0734 \\
71-0739\end{array}$ & $\begin{array}{l}\text { METALS EVALUATKON \& TESTING, ING. } \\
\text { MACNA CHEK, INC. }\end{array}$ & $\begin{array}{l}178 \\
178\end{array}$ & $7 t-3025$ & MODEL: TN 7.2 GEFMAN CASK & 170 \\
\hline $71-0797$ & SERGENT, HAUSKANS \& BECKWITH & $\begin{array}{l}178 \\
178\end{array}$ & 713035 & $\begin{array}{l}\text { MODEL JPC-8OY-20T (JAPANESE) } \\
\text { CASK, TRANSFATION, }\end{array}$ & 180 \\
\hline $71-0741$ & EIX TESTING LABORATOFAES & 178 & $71-3036$ & $\begin{array}{l}\text { WOEG: JAF-GOY-9SOK, } \\
\text { TRANSPOATATION, DEPT OF }\end{array}$ & 180 \\
\hline $71-0750$ & BUANLYY TEOANOLOGY, WNC. & 178 & & 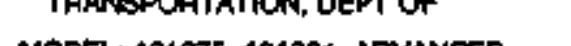 & \\
\hline 71.0753 & MOBILE-LAB, ING. & 178 & 71.5796 & 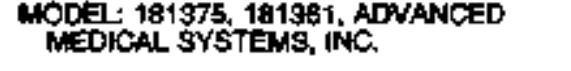 & 180 \\
\hline $71-0754$ & CODE SERVICES, WV. & 178 & 71.5939 & MODEL: 1500, GENEPAL ELECTRAC $C O$. & 180 \\
\hline $71+0758$ & NATIONWHDE TESTING SEAVICES, INC. & 178 & $71-5957$ & MODEL: BHF-1, CHNTKHEM, INC. & 180 \\
\hline $71-0761$ & KOONEY X-AAY, ING. & 178 & $71 \cdot 0400$ & $\begin{array}{l}\text { MODEL: 6400, WESTWEHOUSE } \\
\text { ELECTRIC COAP. }\end{array}$ & 180 \\
\hline $71-0762$ & KC INSPEGTION, INC. & 178 & & & \\
\hline $71-0764$ & DIAMOND H TESTING 00 . & 178 & 71.6568 & 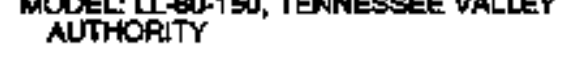 & 180 \\
\hline $\begin{array}{l}71-0768 \\
71.0769\end{array}$ & $\begin{array}{l}\text { DESERT WOLSTRIAE X-RAY, INC. } \\
\text { GFEAT LAKES TESTING, INC. }\end{array}$ & $\begin{array}{l}178 \\
178\end{array}$ & $71-6581$ & $\begin{array}{l}\text { MOCEL \$1O12:1 \& } 51032-1 A \text { A } \\
\text { ADVANICED NUCLEAP FUELS CORP. }\end{array}$ & 180 \\
\hline $71-0771$ & $\begin{array}{l}\text { LONGMEW INSPECTION - GULF DOAST } \\
\text { GAOUP, INC. }\end{array}$ & 178 & $71-9001$ & $\begin{array}{l}\text { MOOEL IF-300, VECTRA } \\
\text { TECHNOLOGIES, INC. }\end{array}$ & 180 \\
\hline 71.0774 & MHOWEST INOUSTRIAL XAAY & 178 & $71-9010$ & MOOEL: NLL1/2, NAC NTERNATIONAL & 180 \\
\hline \multicolumn{6}{|c|}{ xyi } \\
\hline
\end{tabular}




\begin{tabular}{|c|c|c|c|c|}
\hline Dackel & Nano & Page & Dosket & Nane \\
\hline $71-90099$ & WODEL 576 \& 676E, AMERSHAM COAP. & 180 & $71-9258$ & MOOEL F-294, NOFDKN \\
\hline 71.0093 & $\begin{array}{l}\text { WODELL: } 990, \text { BEOA, G60AE, 6608, 6608E, } \\
\text { OED, AMERSHAH }\end{array}$ & 180 & 71.0261 & $\begin{array}{l}\text { MOOEL: HLSTAR 100, HOLTEC } \\
\text { INTERMATIONAL }\end{array}$ \\
\hline $71-8067$ & $\begin{array}{l}\text { MOOEL: BCL-3, BATTELLE COLUMBUS } \\
\text { LABOAATORY }\end{array}$ & 190 & $71-9253$ & $\begin{array}{l}\text { WOOEL BPEC-150, SOUACE } \\
\text { PAOOUCTON \& EOUP. CO. }\end{array}$ \\
\hline 71.9079 & $\begin{array}{l}\text { MOPEL NUPAC 140-2.0, NUPAC } \\
\text { SEAVICE, INC. }\end{array}$ & 180 & $71-\$ 268$ & $\begin{array}{l}\text { MOOEL TRANSTOR SIEFRA MUKLEAA } \\
\text { COHP. }\end{array}$ \\
\hline $71-9081$ & $\begin{array}{l}\text { MODSL: CNS 1-19C, CHEMANHCLEAR } \\
\text { SYSTEMS, INC. }\end{array}$ & 190 & $71-84 \$ 9$ & MODEL: $660 \mathrm{~L}$ \\
\hline 71.9094 & $\begin{array}{l}\text { MOOEL: GNS 14-195-H, CHEM-NUCLEAF } \\
\text { SYSTEMS. WH. }\end{array}$ & 181 & $\begin{array}{l}71-9271 \\
71-9277\end{array}$ & $\begin{array}{l}\text { MODEL: TAOJAN AEAGTOR VESSEL } \\
\text { MOCEL: FSV-7, UNT } 3\end{array}$ \\
\hline $71-6096$ & $\begin{array}{l}\text { MODEL: CNS Z1,300, GHEM-NUUCLEAR } \\
\text { SYSTEMS, WVE. }\end{array}$ & 181 & $71-927 \%$ & MODEL- HALFPACK \\
\hline $71-9146$ & $\begin{array}{l}\text { MOOEL: NUPAC 50-1.5L, NUPAC 50-2.5L, } \\
\text { ET AL NUPAC }\end{array}$ & 181 & $\begin{array}{l}71-9511 \\
71-9964\end{array}$ & $\begin{array}{l}\text { MOOEL: R-1, ENERGY, DEPT. OF } \\
\text { MODELL UC-609, ENEAGY, DEPT. OF }\end{array}$ \\
\hline $71-9148$ & MOOEL: 7TO, AMERSHAM COFP. & 181 & \multirow{2}{*}{\multicolumn{2}{|c|}{$\begin{array}{l}\text { Leanding of molependint spent Fuel sterage } \\
\text { - Dockit } 72\end{array}$}} \\
\hline $71-9156$ & $\begin{array}{l}\text { MOOEL IR-SO, JNDUSTIHIAL NUCLEAR } \\
00 .\end{array}$ & 181 & & \\
\hline 71.9159 & $\begin{array}{l}\text { MODEL: NUPAC 14/199H, L \& M, LN.14. } \\
\text { 17OH, L \& M, MUPAC }\end{array}$ & 181 & $\begin{array}{l}72-0002 \\
72-0004\end{array}$ & $\begin{array}{l}\text { SURAY ISFSI 50-280 \& 50-281 } \\
\text { OCON4EE ISF\$, 50-269, 50-270 \& 50-297 }\end{array}$ \\
\hline $71-9576$ & $\begin{array}{l}\text { MOOEL: NUPAC 14/2 } \\
\text { NUPAC SERVICES, INC. L. ET AL. }\end{array}$ & 191 & $72-0005$ & $\begin{array}{l}\text { PONT BEACH ISFSI GEN LIC, S0-263 \& } \\
\text { SO-301 }\end{array}$ \\
\hline $71-9177$ & $\begin{array}{l}\text { MODEL- NUPAC 10/140 \& LN 10-135A, } \\
\text { NUPAC SEFVBCE, INC. }\end{array}$ & 181 & $\begin{array}{l}72-0007 \\
72-0008\end{array}$ & $\begin{array}{l}\text { PAUSADES ISFSI GEN UC, 50-265 } \\
\text { CALVEAT CLIFFS ISFSI، 50-317 \& E0-318 }\end{array}$ \\
\hline $71-9178$ & $\begin{array}{l}\text { MODEL: NUPAC 7/TO0 \& LN 7-100. } \\
\text { NUPAC SEAVICES, INC. }\end{array}$ & 181 & $72-0009$ & FOFT ST, VRAWN ISFSA, 50-267 \\
\hline $71-9179$ & 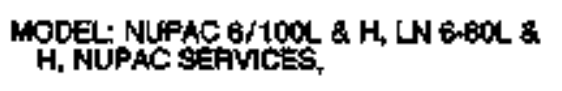 & 181 & 720010 & $\begin{array}{l}\text { PRAIAIE ISLAND ISFSI, SO-2B2 \& 50-306 } \\
\text { ARKANEAS NUCLEAA ONE, ISFSI GEN }\end{array}$ \\
\hline $71-9183$ & MODEL: NAC-1, NAC INTEANATXONAL. & 181 & & \\
\hline 71.9184 & $\begin{array}{l}\text { MODEL: PAS-1, NUCUEAF PACKAENA, } \\
\text { ING. }\end{array}$ & 181 & $\begin{array}{l}72-0014 \\
72-0016\end{array}$ & $\begin{array}{l}\text { DAVIS BESSE ISFSI GEN UC, 50-345 } \\
\text { NORTH ANNA ISFS - 50-398 \& 50-9398 }\end{array}$ \\
\hline $71-9202$ & MODEL: TNGFP, ENERGY, DEPT, OF & 181 & $72-0017$ & TRONAN ISF\$S, $50-344$ \\
\hline $71-9200$ & MODEL: TNFEG, ENEFGY, DEPT. OF & 191 & $72-0018$ & GOODHUE COUNTY ISFS \\
\hline 71.9208 & MODEL: 10.142, NUPAC SERVICES, INC. & 191 & $72-0020$ & INEL TMH-2 I\$FSI \\
\hline 71.0026 & $\begin{array}{l}\text { MODE: NACLWT, NAC } \\
\text { INTERTATIONAL } \\
\text { MODEL: GA-4, BENERAL ATOMICS }\end{array}$ & 181 & $\begin{array}{l}72-0021 \\
72-0022\end{array}$ & $\begin{array}{l}\text { CENTAAL WTERIM STORAGE ISFSI } \\
\text { MOOEL: PARATE FUEL STOGAGE } \\
\text { FACILITY }\end{array}$ \\
\hline $\begin{array}{l}71.9228 \\
71 . \cos 8\end{array}$ & MODEL $200 \%$, GENERAL ELECTRIC CO. & $1 \mathrm{B1}$ & $72-1001$ & 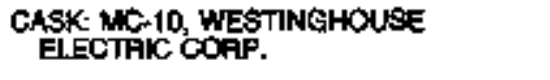 \\
\hline $71-\operatorname{gec} 34$ & 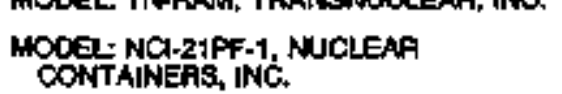 & 182 & 72.1004 & $\begin{array}{l}\text { CASK NUHOMS 24P/52B, VECTRA } \\
\text { TECHNOLOCES, WC. }\end{array}$ \\
\hline $71-9235$ & MODEL: NACSTT, NAC INTEANATIONAL & 182 & & CASK VSC-24, SIERAA NUGLEAR, INC. \\
\hline $71-9299$ & $\begin{array}{l}\text { MOOEL: MOC-S, WESTINGHOUSE } \\
\text { ELECTRUC COFP. }\end{array}$ & 192 & 72.1008 & $\begin{array}{l}\text { CAS*: HSTAA 100, HOLTEC } \\
\text { INTER }\end{array}$ \\
\hline 71-.9249 & $\begin{array}{l}\text { MODEL:CNS 14170, SERIES III, CHEM- } \\
\text { NUCAEAT SYSTEMS. }\end{array}$ & 182 & $72-1014$ & $\begin{array}{l}\text { CASK: W-STOAH 100, HOLTEC } \\
\text { INTEANATONAL }\end{array}$ \\
\hline $71-925:$ & MODEL: BW-2901, B\$W FUEL $\infty O$. & 182 & $72.102 \dagger$ & $\begin{array}{l}\text { OASK: THLO2 ORY STOAAGE GASK, } \\
\text { TRANISNUCLEAR, ING. }\end{array}$ \\
\hline 71.9255 & $\begin{array}{l}\text { MODELL MP197, VECTHA } \\
\text { TECHNOLOEIES }\end{array}$ & 182 & $72-1023$ & $\begin{array}{l}\text { CASK: TRANSTOA GASK, SIEFRA } \\
\text { NUCLEAR GORP. }\end{array}$ \\
\hline
\end{tabular}




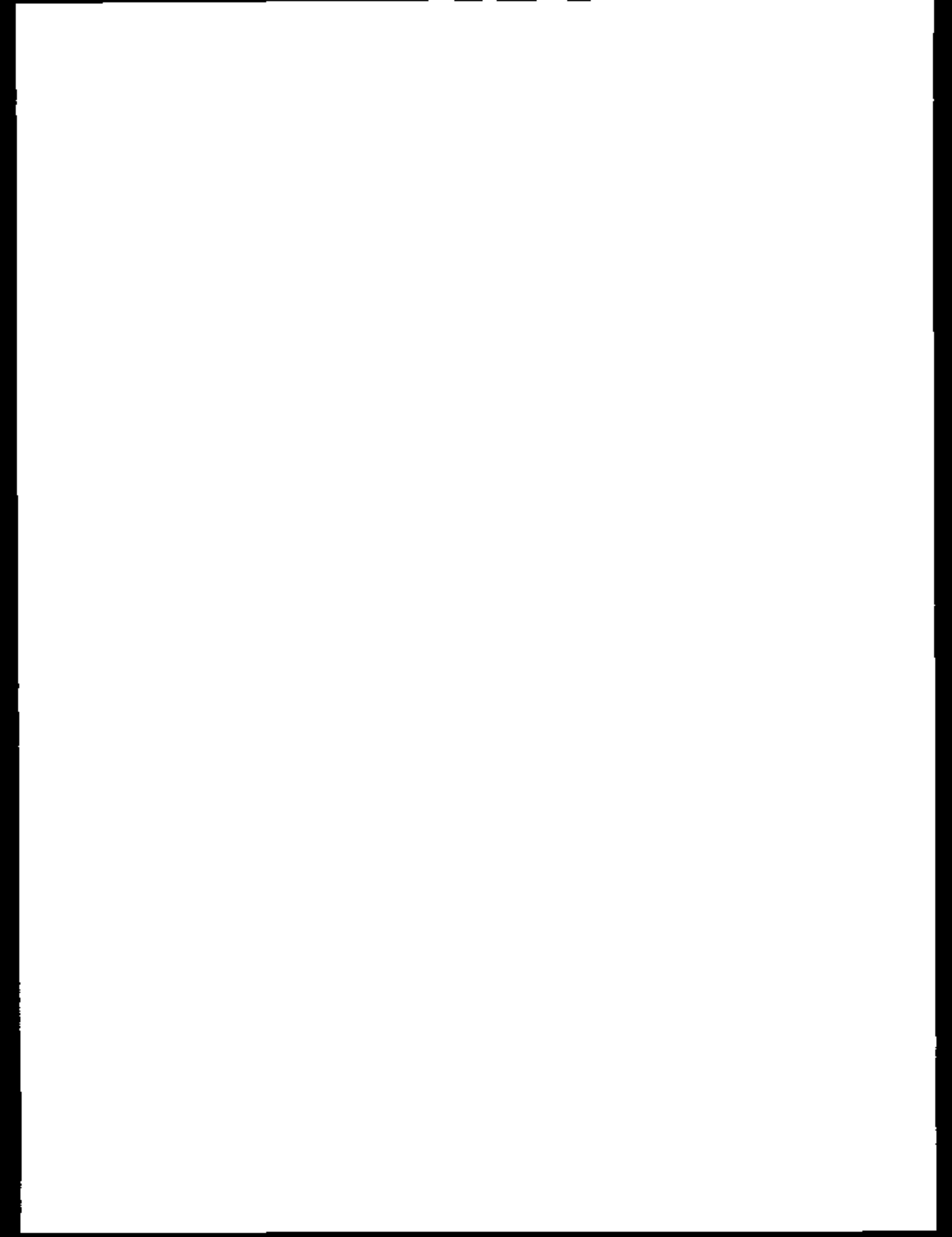




\section{ALPHABETIC ARRANGEMENT OF DOCKET ITEMS BY FACILITY NAME}

Aneme

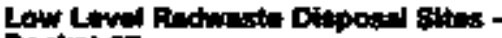
Docket 27

LOUISVILLE, KY, U.S. ECOLOBY. INC.

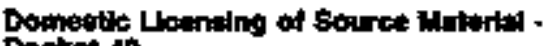
Dooket 40

AUED-SIGNAL, INC., METROFOLIS, IL

ANINE ARUNDE COUNTY/CUATIS BAY, BALTIMORE, WD

ATLANTIC RICHFIELD CO., GAANTS, NH

ATLAS CORP., DENVER, $C O$

BAECOCK \& WLOOX DO., ALLANCE, OH

GEAP CREEK URANIUM CO, CASPER, WY

AP CHEMICALS AMERICA, HC., LMM, OH

CHENETRON CORP., PAOMDENOE, RI

CINCMNNATI, UNIV. OF, GINGNNATL, OH

COLOAADO VENTURES, INC., DENVER, CO

DOW OHEMICAL U.S.A. \&UNTT OF DOW

CHEMICAL $\infty$ ). ENVIROCARE OF UTAL, INC, SALT LAKE
GTY, UT

FANSTEEL, INGG NOATH CHEAGO, IL

FEFRET EXPLORATION CO. OF NEBRASKA, DENVER, CO

FAONE INVESTMENT CO. (EROOKS \&

PERKINS DOAP, )

HAFTUYY HATTLEY LANDFILL (SCA), BAY COUNTY, MI

HOMESTAKE MHNING COn GRANTS, NW

HONEYWEL, WC, HOPKIAS, MN

HYOPO FESOUACES, INC, DALLAS, TX

INTERNATIONAL URANIUM USA CORP.

KENNECOTT URANFM CO., RAWLNS, Wr

MARTIN MAFIETTA ALUMWMU BALES, INC.. MLANA, TN

MICWIGAN CHEMKAL COAP. ST. LOUIS, M

MINNESOTA MINING \& MANUFACTURING

CO, 57. PAUL, MN

PATHFINDEA MINES COAP, WUS, WY

PATHFINDEI NINES COAP., ANERTON, WV

PATHFINDEA NINES OOPP, SHIFLEY BASIN, WY

PETROTOMCS CO. SHIPLEY BASW, WY

PLATEAU RESOUFCES, LTD, LAKE POWNELL, UT

POWEA RESOURCES, INK., CLENAOCK, Wr

OUIVIRA MINING $\infty$., ERANTS, NH

ATO ALGOM MANUNG COPP, MOAB, UT
Docket pag

$27-049$

40-3998

40-034t

40-9902

$40-3458$

40-8474

$\$ 0-8452$

40-7604

$40-8724$

40-2676

40-7271

$40-0017$

$40-8969$

$40-7560$

$40-8943$

$40-0235$

40-8022

$40-8903$

40-7982

40-80s8

40-8691

40-B584

$40-809$

40-6284

10.1020

40-481

40-2059

40-1602

40-\$650

40-8eg9?

40-8957

$40-8005$

40-8igina
Neme

RIO ALEOM MENINE COAP. OKLAHONA
CITY, OK

FHA CO, ASHTABLLA, OH

SHELDALOY OOFP, NEWFELD, NU

SHIELPAUOY METALLUAGICAL COFP. NEWFIELO NH

SOHD WESTERN MINANG CO., SALT LAKE GTY, UT

TOTAL MINEFALS CORP, CASPER, WY

U.S. ENERKYY COAP, FNERTON, WY

UNC MINING \& MILUNG ION. OF UNATED NUCLEAR COAP.).

WESTEFN NUCLEAR, NC., LAKEWOOD, CO

WESTINGHOUSE ELCTRIC CORP., PITSBURGH、 PA

Domentito Liotnoling of Protuction and Uit retion factinte - Deekst 50

AMP FOACE, DEPT. OF

$50-607 \quad 165$

ALVIN W. VOGTLE MJCLEAR PLANT, UNIT 1

$50-424 \quad 136$

ALVIN W, VOGTLE NUCLEAR PLANT, UNIT 2

50-425 137

ARKANSAS NUCLEAR ONE, UNTT 1

$50-313 \quad 76$

E0-3es 111

so-17t 14

AFN.CO FORCES RADIOGOLOGY

RESEARCH WNSTITUTE

BEAVER VALLEY POWER STATION, UNTT 1

$50-334 \quad 93$

BEAVER VALLEY FOWEA STATION, UNIT 2

$50-412 \quad 129$

$50-439$

$50-439 \quad 130$

$50-345101$

50-155 18

STN-60-456 148

STN-50-457 150

50-259 31

$50-280$

S0- 200

50-325 86

$50-324 \quad 85$

$50-57 \quad 11$

BUFFALO MATERIALSS RESEARCH CENTER

BYRON STATION. UNIT 1

STN-50-454 144

STN-50-455 146

STN-50-40: 155

xix 
Hims

CALVEFT CLIFFS NUCAEAR POWEA PLANT, UNT 1

CALVERT CUFFS NUCLEAM FOWER PLANT. UNIT 2

CATAWBA WUCLEAA STATION, UNT 1

CATAWFA NUCLEAR STATION, LANT 2

CANTICHEM

CLINCH RNEA BAEEDER REACTOR

CUNTON POWER STATION, UNIT 1

COMANGHE PEAK STEAM ELEOTRIC STATHON, UNTT I

COMANCHE PEAK STEAM ELECTR|C STATKON UNT 2

COOFEF NULLEAR STATION

CORNELL UNN, RESEARCH REACTOR

COFNEL UNY, ZERO POWEA REACTOR

CRYSTAL RIYEA NUCLEAP PLANT, UNIT 3

DAVS-BESSE NUCLEAF POMER STATION, UNT 1

DUAGO CANYON NUCLEAR POWER PLANT, UNT 1

CIABLO CANYON NUCLEAR PONER FLANT. UNT 2

DOHALD G. COOK NUCLEAF POWEA PLANT, UNIT 1

DONAIO C. COOK NUCLEAF POWEF PLANT, UNIT 2

DOW CHENICAL $O$.

DRESDEN MUCLEAA POWER STATION, UNIT 1

DRESOEN NUCLEAR POWER STATION, ENTT 2

OFESOEN NUCLEAR POWER STATION. UNAT 3

DUANE ARNOLD ENERBY GENTES

EDWIN I. HATCH NUCLEAR PLANT, UNTT 1

EDWN I. HATCH NUCLEAP PLANT, UNHT 2

ENRICO FERMI ATOMIC POWER PLANT, UNIT 1

ENRICO FERN ATOMK POWER PLANT, UNIT 2

FLOATING NUCLEAR PLANT

FOAD NUCLEAA REACTOR

FOFT CALHOUN STATION, LNIT 1

FOAT ST. VFAIN NUCLEAR GENERATING STATKON

FOSTER WHEELER COAP.

GENERAL, ATOWICS

GENERAL ATONWCS

GEOPGLA INSTITUTE OF TECHNOLOGY RESEARCH REACTOA
Dooknt Pein Mams

GAAND GULF NUCLLEAP STATLON, UNIT ;

bocket Page

$50-317 \quad 79$

$50-318 \quad 80$

$50-413 \quad 130$

60-414 131

$50-54 \quad \$ 1$

$50-557 \quad 165$

$50-461 \quad 152$

$60-445 \quad 142$

$80-446 \quad 143$

50-25g 61

50-157 14

$50-67 \quad 11$

50-902 64

$50-346 \quad 101$

$50-275 \quad 46$

50.32806

$50-315 \quad 76$

$50-316 \quad 76$

50-264 35

60-10 9

$\$ 0-237 \quad 20$

$50-249 \quad 28$

50-331 9t

$50-321 \quad 8 p$

50-300 110

50-10 10

$50-341 \quad 69$

STN-50-497 139

50-2 B

50-285 52

$50-267 \quad 30$

50-51 11

50-163 14

$50-89 \quad 11$

$50-160 \quad 14$

GRAND GULF NUCLEAF STATKON, UNIT 2

H.B. ROBINSON PLANT, UNIT 2

HADOAM NECK PLANT

HOFE CAEEK NUCLEAR STATKON, UNIT 1

HUNBOLDT BAY PONEA FLANT, UNAT 3

IDAHO STATE UNIY. RESEARCH REACTOR

INOAN PONNT STATION, UNT 1

MUIAN POINT STATION, UNIT 2

INDAAN PONT STATION, UNIT 3

CONA STATE UNW, RESEAPCH REACTOA

JAMES A, FTZPATFIOK NLCLEAF POWER PLANT

JOSEPH M. FAFLEY MUCLEAR PLANT, UNIT 1

NOSEFH W. FARLEY NULEAR PLANT, UNIT 2

$50-416 \quad 132$

$50-417 \quad 135$

50.261 30

50-213 16

$50-354 \quad 105$

$50-133 \quad 12$

$50.284 \quad 52$

5003

50-247 25

$50-286 \quad 54$

50-116 11

$50-933 \quad 92$

$50+348 \quad 108$

$50-364 \quad 100$

KEWAUNEE NUCLEAR POWER PLANT

LA CROSSE BOLLNG WATEA REACTOR

LASAШE COUNTY STATION, LAVT 1

LASAUE COUNTY STATION, UNT 2

LIAEFOK GENEAATING STATION, UNI 1

LINERGCK GENERATING STATION, UINIT 2

MANE YANKEE ATOWIG POWER PLANT

MANHATTAN COUEECE RESEAFCH REACTOR

MASSACHUSETTS INSTITUTE OF TECHNOLOGY RESEARCH REACTOR

MOLAND PLANT, UNTT 1

MADLAND PLANT, UNT 2

MULSTONE NUCAEAP POWER STATION, UNFIT 1 MILLSTONE NUCLEAP PONER STATION,

MHLLSTONE NUCUEAR PONEA STATION, UNTT 3

MONTCELLO NUCLEAR GENERATWG PLANT

NATONAL WSTMUTE OF STANDAPDS : TECHNOLOGY REACTOR

NANE WILE PONT NUCEAA STATION, UNIT 1

NINE MILE POINT NUCLEAR STATION, UNIT 2

NORTH ANNA POWEA STATION, UNIT 1

NORTH ANNA POWER STAKION, UNIT 2

NORTH CAROLNA STATE UNNIV. PULSTAR REACTOR

NUCLEAR SHIP SAVANNAK

OCONEX NUCAEAR STATION, UNIT 1
$50-305 \quad 69$

$50-409 \quad 128$

$50-373 \quad 115$

E0.374 117

50-352 100

50-359 104

$50-909 \quad 71$

50-199 †5

$50-20 \quad 10$

50-329 91

$50-930$ 81

50-245 22

$50-3368$

$50-423 \quad 130$

50-263 $\quad 34$

$50-184 \quad 15$

$50-220 \quad 18$

$50-410 \quad 128$

50-339 97

$50-3398$

50-297 61

$50.238 \quad 21$

$50-269 \quad 40$ 
OCONEE MUGLAA STATON, UNF 2

$\begin{array}{lll}50-270 & 41 & \text { SEABROOK NUCLEAR STATION, UNIT } 2 \\ 50-287 & 54 & \text { SECUOYAH NUCLEAR PLANT, UNIT } 1 \\ 50-150 & 12 & \text { SEQUOYAH NUCLEAR PLANT, UNIT } 2 \\ 50-243 & 21 & \text { SHEAFON HARRIS NUCLEAR POWEA } \\ & & \text { PLANT, UNIT } 1\end{array}$

$50-444 \quad 142$

OCONEF MUOLEA STATION, UNIT 3

56-218 17

SHOAEHAM MUCLEAR FOWEA STATION

$50-327 \quad 88$

OHO STATE UNIV, RESEAROH REACTOA

$50-255$

pALISADES NuCAEAR PLANT

SOUTH TEXAS PROJEGT, LINT 1

50-320

89

$50400 \quad 127$

PALO VEADE NUCLENR STATION, UNII 1 STN-80-528

PALO VERDE WUCEAR STATION, UNIT 2 STN50-520

PALO VERDE NUCLEAR STATION, UNNT 3 STN-50-590

PEACH EOTTOM ATOWK POWEA STATION,

$50-171$ UNIT 1

PEACH GOTTON ATOMIC POWER STATION, UALIT 2

$60-277 \quad 47$

SOUTH TEXAS PRONECT, UNT 2

$50-322$

B3

ST. LUCIE PLANT, UNTT 1

$5 T+50-498 \quad 166$

ST. LUCAE PLANT, UNT 2

SUARY POWER STATION, UPNT 1

STNE0-499 158

$50-335 \quad 94$

$50-389 \quad 121$

SURAY POWEA STAKKW, UNIT 2

$50-280 \quad 48$

50-281 48

SUSOUEHANNA STEAN ELFCTAKC

$50-387$

119

PEACH BOTTOM ATONC POWEA STATION,

$50-278 \quad 47$

UNit 3
PENNSYLVANMA STATE UNIY. RESEARCH REACTOA

$50-59$

$50-440 \quad 139$

FERTY NUCLEAA POWER PLANT, UNT 1

PEARY MUCGEAT POWEA PLANT, UNIT 2

PRGPAW NIKLEAF POWEA STATION UNT

1

B0-441 140

$50-293$

PLUM BPOOK RESEAFCH REAGTOR FACILITY

POINT BEAOH MUGLAR PLANT, UNIT 1

POINT BEACA NUCEEAR PLANT, UNIT 2

PRAFIE ISLAND NUCLEAP STATON, UNIT 1

PRAFIE LSLANO NUCLEAR STATION, UNIT 2

PIROUE UNIN. RESEARCH REACTOR

OUAD-GIIES STATION, UNIT I

OUAD-CITIES STATION, UNTT 2

RANCHO GECO NUCLEAR CIENEAATING STATION

RHODE ISLAND \& PAONIDENCE

PLANTATIONS AEC

RIVER BEND STAIION, UNIT 1

ROEEAT EMMET GINNA NUCLEAR PLANT,

FPI CFTICAL EXPERIMENT

SALEM MUCLEAR GENERATWM STATHN, UNIT I

SALEA NUCLEAR EENERATNG STATION, UNIT 2

SAN ONOFFE NUCLSAR STATHON UNIT 1

SAN ONOFRE NUCLEAP STATION, UNIT 2

SNN OHOFRE NUCLEAP STATION, LNIT 3

SAXTON NUCLEAF EXPERMNENTAL

FACALITY

50-50 11

60-285 36

50-301 62

$50-29250$

$50406 \quad 69$

60-182 14

50-254 28

50-265 35

$50-312 \quad 74$

$50-193 \quad 15$

E0-456 151

E0-244 21

$50-225 \quad 20$

50.272 44

\$0-208 $\quad 15$

$50-301 \quad 106$

$50362 \quad 108$

$60-148 \quad 12$

$50-443 \quad 14 \uparrow$

SEABROOK NKCLEAR STATION, UNIT I
50-311 72

STATION, UNIT 1

SUSOUEHANNA STEAN ELECTRK STATION, UNIT 2

TEXAS AEN UNIV. RESEARCH REACTOR

$50-386$

120

THFEE MUE ISLAND NLICAEAR STATION, UNIT 1

THFEE MUE ISLAND ADUGEAR STATION, UNTT 2

TROLAN MUGLEAH PLANT

TUFKEY PONT FLANT, UNIT I

TUFKEY PONT PLANT, UNIT 4

UNN. OF FLCFIDA TRAINING REACTOR

UNN, OF ILLINOES RESEAFCH REACTOR

UNN, OF ILUNOHS RESEAFCH REAGTOR

UNN, OF MARYLAND RESEAFCH FEACTOA

UNYY. OF MISSOURI RESEAACH REACTORCOLUMBAR

UNN. OF VIRGINDA CAVALIER REACTOF

UNIV. OF VIRGINA RESEAFCH REACTOR

UNIV, OF WISCONSIN RESEARCH AEAGTOR

UNIYERSITY OF MASSACHUSEITS, LOWEUL FESEARCH HEACTOA

UNIVEASTY OF MESOURI RESEAFCH

PERCTOR, ROUA, MO

VEFWONT YANKEE NUCLEAT POWER STATHON

VIAGH C. SUMMER MUCLEAA STATKN. UNT 1

WASHINGTON STATE UNIV. RESEARCH AEAGTOR (TRIGA)

WATERFORD GENEAATINE STATION, UNIT 3

WATTS BAA NUCLEAA FLANT, LNAT 1

WATTS BAF MUCLEAF FLANT, UNUT 2

\section{0-128 12 \\ $50-298 \quad 56$ \\ $50-320 \quad 82$}

$50-344 \quad t 00$

$50-250 \quad 27$

$50-251 \quad 28$

50-63 11

50-151 12

$50-356 \quad 106$

50-188 14

$50-188 \quad 15$

$50-396 \quad 125$

$50.62 \quad 11$

$50-156$ t3

50-223 20

$50-123 \quad 12$

$50-271 \quad 49$

$50-395 \quad 124$

$50-27 \quad 10$

30-382 118

50-390 122

50-391 123

$50-22 \quad 10$ 
Dookint Pags

WILUAN G. MCEUIFE MUCLEAR STATION,

$50-369 \quad 112$ VIT 1

WILIAN . MCOUHE MUCLEAR STATION, UNIT 2

WOLF CFEEK GENERATING STATION

WORCESTEA POLYTECHNLC NSTTTUTE FESEARCAH REACTOR

WPPSS NUCAEAR FROJECT, UNIT 1

WPPSS NUCLEAR PROLECT, LNT 2

WPFSS NUCLEAR PROVECT, UNTT

WPPSS AUCLEAR PROECCT, LHAT 5

$30-370$ itd

STNSO-452 154

$50.494 \quad 12$

$50.460 \quad 152$

$50-397$

STN-BO-508

STN $50-509$

YANKEE-POWE NUNCLEAR FOWER STATION

$50-29$

ZKN NLCLEAR POWER STATION, UNIT 1

$50-295$

ZION NUCUEAR FOWER STATION, UNGT 2

APEOO STANDARD PLANT DESIGN

WESTINATOUSE ELECTRIO CORP.

GE ACWANCED BWA DESWGN, GENERML ELFCTRLC CO.

Domentle Lbanting of Epectal Nuctuar

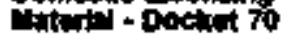

BEW SHALLOW LAND DISPOSAL AREA (SLDA), PARKS TOWNSHIP,

BABCOCK \& WILOOX CO., ALLLANICE, OH BABCOCK \& MILCOX CO., LYACHBURO, VA BABCOCK \& WLCOX CO., PAFKS TOWNSHIP, PA

GASE WESTERN RESERVE UNN, CLEVELAND, OH

CIMAFAON COAP., OKLAHOWA CITY, OK

CWTICHEM, IN, TUXEDO, NY

CUEVTE DOAP, CUEVIUAND, OH

COMEUSTON: ENGINEERANG, ING. HEAATITE, MO

CONMERCE, OEFT. OF, NATIONAL INSTITUTE OF STANOAFOS \&

FRAMATOME COGEMA FUELS. IVNCHAURG, VA.

GENERAL ATOMICS, SAN DIECO, CA

GENERAL ELECTFKC CO. WMUMGTON, NC KEARHCGEE DOFP, OKLAHOWA CITY, OK

LOLASLANA ENEAGY SERVICES, WASHINGTON, DC

MACALESTER COUEGE ST, PAUL, MAN

MASSACHUSETTS WSTITUTE OF

TECH MOLOCY, CAMBAIDEE, MA

MINNESOTA MINHE \& MANUFACTURING Co. ST. PAUL MN

MUCLEAR FUEL SERYICES, ING., EFWIN, TN

PENWSYLVANIA, COHMWONWEALTH OF, NDIANIA, PA

$50-504 \quad 67$

$52.003 \quad 165$

$62-001 \quad 168$

$70-3095 \quad 171$

$70-0028 \quad 167$

$70-0027 \quad 167$

$70-0364 \quad 168$

$70-0145 \quad 168$

$70-0925 \quad 168$

$70-0697 \quad 168$

$70-0133 \quad 168$

$70-00056167$

$70-0399 \quad 168$

$70-1201 \quad 170$

$70-0734 \quad 188$

$70-1113 \quad 168$

$70-8073 \quad 171$

$70.6070 \quad 170$

$70-0634 \quad 16$

$70-0999 \quad 169$

$70-0892 \quad 188$

20.0149 180

$70-10 \% 96$
Nome

Docket

SIENENS NUCLEAR POWEA CORP. FKOHLAD, WA

$70-1257$

Page

U.S. ENPICHNENT CORP. (AVUS ENRICHNENT PLANT)

U.S. ENAICMISTT COAF. PADUCAH GASEOUS DIFFUSTON PLANT]

U.S. ENAICHNENT CORP. (PORTSNOUTH GASEOUS PIFFUSHON

WESTINCHOUSE ELECTAIC COAP., PIT SSUROH, PA

WESTIMEHOUSE EIECTRIC CORP., PITTSSURGH, PA

WESTIMCHOUSE ELECTRIC CORP., PITTSBURGH, PA

WESTINGHOUSE ELFCTFAC CORP., FाT SBLPAH, PA

WRIGLIT STATE LNWV, DAYTON, OH

$70-3099$

$70-7001$

$70-7002$

172

Packinging of fodlowothe theterted for

Trineport end Trentaporiation of

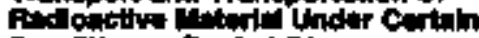

Coildtuin - Docket 71

ABC TESTING
ADAMS INDUSTRIAL SERVICES, WN.

ADRIAN COLLEGE

ADVEX CORP.

AKRON INDUSTRIAL SEFVICES, INC.

ALASKA NOESTRIAL X-FAY

AWEGHENY LABOAATOAIES

ALIED INSPECTION SERVICES, DHC.

ALONSO \& CARUS RON WORKS, INC.

AMERICAN AURLINES, INE.

AMtREHAN CORP.

APPLED TECHNICAL SERVKES, NC.

AFGTIC SLOPE INSPECTION SERVICES, INC.

ARATY, DEPT, OF

ARROW NOE CO., HA.

ARACW TANK \& ENGINEERING $C O$.

ASTROTECH, WC.

ATONIC INSPECTXON LABS

B \& G INSPECTION

BABCOCK \& WILCOX $C O$.

BAKER TESTING SEFVICES, ING.

BAFNETT INDUSTFAL X-FAY

BATH IRON WORKS COAP.

BILL MILLEF, INC.

BIX TESTING LABORATOAIES

BRANCH RADIOQRAPHIC LAEORATOFIES, inc.
$70-1151 \quad 169$

$70-0698$

168

$70-1503$

170

$70-1149$

169

$70-1458$

170

$71-0472$

178

$71-0064 \quad 179$

$71-0978 \quad 179$

$71-0497 \quad 176$

$71-0595 \quad 177$

$71-0008 \quad 175$

71 -0oss 176

$71-0580 \quad 175$

$7+-06+5 \quad 177$

7)-0es6 174

$71-0040 \quad 173$

$71.0706 \quad 177$

$71.0719 \quad 178$

$71.0487 \quad 176$

$71-0059 \quad 176$

71-00t5 173

$71.0280 \quad 175$

$74.0060 \quad 177$

$710775 \quad 178$

$710079 \quad 175$

71-0392 175

71-0062 177

$71-0155 \quad 174$

$71-0498 \quad 176$

$71-0741$

$71-0 \mathrm{ses} \quad \$ 75$ 
BRAUN ENGWNEERING TESTINAC INC.

BRIGGS ASSOCAATES, INC.

BUCKEYE STEEL GASTINES

BUANLEY TECHNOLOGY, INC.

CALUMET TESTING SERMCES, INC.

CAPITAL X-FAY SERVICE

CARTEE SHELL \& TUEE, ING.

CAROLNA POWEF \& UGHT $C O$.

CARGMNELET FOUNOAY CO.

CEI INDUSTRIES, INC.

GENTURY WSPECTION, WC.

CERTIFIED TESTTNG LABORATORIES, INK.

CODE SERVICES, INC.

COLGX.THIELMEIER TESTING $\infty$.

COMO TECH, INC.

CONAMN INSPEETTON, INC.

CONNOFS PWE SYSTENS, INC

CONSOLIDATED NDE, NC.

CT, NC.

CTL ENEANEERING, INC.

OAYTON X-AAY $C O$.

DEGISNE TESTING, WNG

DELTA ANA LINES

DERBY CTYY INSPECTION

DESERT INDUSTFAAL X-RAY, UNC.

DIAMOND H TESTING $\infty O$.

DUFALLLO BLAWHCNOX, INC.

EASTEFN MLCHGAN UNIVEFESTTY

EASTEAN TESTING \& INSPECTION

ECWAFDS FIFELINE TESTING, INC.

EGEG FLOFIDA, WNC.

EL PASO NATUAAL GAS, ING.

EUTE INSPECTION SERVICE $\infty O$, wC.

FOSTER WHEELER ENERGY CORP.

FROEHUNG \& ROBERTSON, INC.

GENERAL DYNANACS CORF.

GENERAL MOTOAS $O$ OAP.

GENERAL TESTING \& INSPECTION CO.

GLTSCH FIELD SEAVICES/NDE, WC.

GLOPE X-RAY SERVICES, WKC.

GREAT LAKES TESTING, INC.

GPINIEZLL COAF.

H \& G INSPECTION CO., INC.

HSH X-RAY SERVICES, INC.

\begin{tabular}{|c|c|}
\hline $71-0592$ & 177 \\
\hline 71.0368 & 175 \\
\hline $71-0+10$ & 174 \\
\hline $71-0750$ & 178 \\
\hline $71-08587$ & 175 \\
\hline $71-0220$ & 174 \\
\hline $71-0417$ & 175 \\
\hline $71+00345$ & $\$ 75$ \\
\hline 7100707 & 17 \\
\hline $71-0016$ & 173 \\
\hline 770181 & 174 \\
\hline $71-0065$ & 173 \\
\hline $71-0754$ & 178 \\
\hline $71+0114$ & 174 \\
\hline $71-0689$ & 177 \\
\hline $71+0216$ & 174 \\
\hline $71-0672$ & 179 \\
\hline $71-0627$ & $\$ 77$ \\
\hline $71-0404$ & 175 \\
\hline $71-0501$ & 175 \\
\hline $71-0252$ & 175 \\
\hline 710839 & 179 \\
\hline 71-0800 & 170 \\
\hline $71-0778$ & 178 \\
\hline $71-0768$ & 178 \\
\hline $7+0704$ & 178 \\
\hline $71-0201$ & 174 \\
\hline $71-0679$ & 179 \\
\hline $7 t-0026$ & 173 \\
\hline $71-0568$ & 176 \\
\hline 71.0096 & 173 \\
\hline $71-0488$ & 176 \\
\hline $71-0855$ & 179 \\
\hline $71-0099$ & 174 \\
\hline $71-0157$ & 174 \\
\hline $71-0220$ & 174 \\
\hline 710439 & 176 \\
\hline $71-0319$ & 175 \\
\hline 710171 & 174 \\
\hline $71-0146$ & 174 \\
\hline $71-0768$ & 178 \\
\hline $71-0003$ & 173 \\
\hline $7 t-0605$ & 177 \\
\hline 710429 & \\
\hline
\end{tabular}

H.R. WEPECTION SERVICE

hafaison STEEL CASTINGS CO.

HIGH MOUNTAN RSPECTION SERVICES, INC.

HIGH STEEL STRUCTUAES, INC.

HWNTINGTON TESTING

INDEPENDENT INSPECTION CORP.

INDEPENDENT TESTING LABOAATOAIES

INDUSTTRAAL MAFINE TESTING LABORATORY

INDUSTRIAL NDT 00 .

INDUSTRTAL NDT SERVICES DIV.

(BNDUSTRAAL HEAT TREATING

INSPECTHON SERUCE, INC.

INTEGRATED TECHNOLOGES, INC.

INTERMOUNTAIN FESTING CO.

INTERMATIONAL AADLORAPHY \& INSPECTION SEAVICES

IT INSPECTLON-TECH TECHNCIANS, INC.

JAN X-RAY SERVICES, ING.

KC NSPECTHNL, INC.

KOONEY X-RAY, INC.

LAFAYETTE TESTING SEAVICES

LERICH TESTING LABORATORIES, INC.

LONBVIEW INSPECTION - GULF COAST GAOUP, INC.

LUCOUS PITKIN, ING.

MABNA CHEK, WNC.

MANOIF ELEICTROALOYS COAPORATION

MARYLANOO C. LABCRATOFIES

MASON \& HANGER-SHLAS MASON CO, INC.

MATEALAL TESTING LABOFATOFY OF VIAGINIA, INC.

MATTWGLY TESTING SERVICES, INC.

MCNDT LEASINGS, INC. MET-GHEM ENGINEEAING LAEOPATOHES,

METALS EVALLATION \& TESTING, INC.

MIDWEST INDUSTRAAL XPAY

MIDWEST WLSFECTION SERVICES, INC.

MINWESOTA VALLEY ENGINEEFANG, NAC.

MISSOURI STEE CASTRESS $\infty$.

MOBILELAB, INC.

MODEL: 10-142, WUPAC SEFVKCES, WN.

MODE: 1500. GeNERAL E.EGTFUC $C O$.

MODEL: 181375, 1913S1, AOVANCED

MEOKCAL Srstens, ING

MOOEL: 2000, GENERAL ELECTRKC $\infty$.
Donitot

$71-0065$

$71-0051$

$71-0555$

$71-0186$

$71-0098$

$71-060$ ?

71.0000

71-0840

71-0611

$71-0928$

71.0863

71-0865

71-0213

71-0782

$71-0850$

710000

71-0762

71-0781

71-0787

71.0269

71-071

$71-0672$

71-0738

$71-0836$

71-10007

71-0816

71.0111

$71-0542$

$71-6901$

$71-0508$

$71-0734$

71-0774

71-0476

$71-068$

$71-0408$

$71-0753$

$71-0008$

$71-5039$

$71-570$

71-5028
173

173

176

174

173

177

175

179

17

175

179

179

174

178

179

137

178

178

178

175

178

17

178

170

179

175

174

176

178

178

178

173

176

176

175

178

181

t80

180

781 


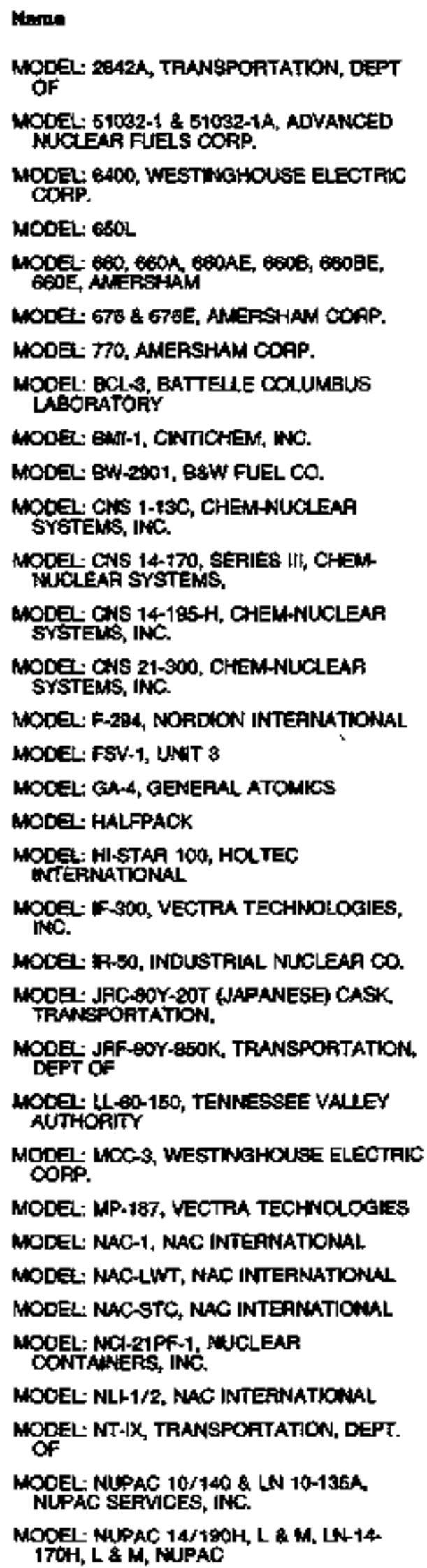

\begin{tabular}{|c|c|}
\hline Docktt & Pagu \\
\hline 71.3024 & 170 \\
\hline 716581 & 180 \\
\hline $71-8400$ & 180 \\
\hline $71-0$ ast & 182 \\
\hline $71-9033$ & 180 \\
\hline $71-0029$ & 180 \\
\hline 71.9148 & 181 \\
\hline 71.9067 & 180 \\
\hline $71-5057$ & 180 \\
\hline $7 t-9251$ & 182 \\
\hline $7 t-9081$ & 180 \\
\hline 71.9249 & 182 \\
\hline 7t-9094 & 181 \\
\hline $71-9006$ & 181 \\
\hline 71.9258 & 182 \\
\hline $7+5277$ & 1 diz \\
\hline $71-8220$ & 191 \\
\hline $71-9270$ & 183 \\
\hline $71-3<61$ & 182 \\
\hline $71-0001$ & 100 \\
\hline 71.9156 & 181 \\
\hline 712005 & 180 \\
\hline 71-3086 & 180 \\
\hline 716568 & 180 \\
\hline $71-9039$ & 182 \\
\hline $71-9 \cos 6$ & 182 \\
\hline $71-9783$ & $18 t$ \\
\hline $71-9225$ & 281 \\
\hline $71-9235$ & 182 \\
\hline $71.92 x 4$ & 182 \\
\hline $71-8014$ & 180 \\
\hline 715010 & 179 \\
\hline $71-9177$ & 187 \\
\hline $71-8150$ & 181 \\
\hline
\end{tabular}

Nime

Dockent

Parat

MODEL: NUPAC 14/219H \& L, ET AL NUPAC 71-9176 18 SEAVWCES, HWC.

MODEL: NUPAC 140-20, NUPAC SERVICES, 79-9070 190 INC.

NODEL: NUPAC 50-1.5L, NUPAC 50.2.54 ET 71-9145 AL, NUPAC

NOOEL: NUPAC 6/100H, \& H, LN 6-8OL \& H, MUPAC SEPVCES.

MODEL: NUPAC 7/100 \& LN 7-100, NUPAC 71-9178 181 SEPVICES, NC.

MODEL: PAS-1, NUCLEAR PACKAGING, ING- 719984 181

MODEL: 9-1, ENEAGY, DEPT. OF

71-9519 189

MODEL SPEC-150, SOURCE PAODUCTION 71-92:3 182 $\triangle$ EOUP. CO.

MODEL TN 7-2 GERMAN CASK

$71-3025 \quad 179$

MODELL: TNGRP, ENEFGSY, DEPT, OF

WODEL- TNAAM, THANSNUCUEAR, INC.

$71-9202 \quad 181$

71-9233 102

$71-9206 \cdot 181$

MODEL: TNAEG. ENERGY, OEPT. OF

MODEL: TRANSTTOR SUERRA NUCLEAR CRP.

MODEL: TROUAN REACTOA VESSEL

MODEL UC-609, ENERGY, DEPT. OF

MOLTEN METAL TECHWOLOGY

MONTANA X-AAY, INGC.

MOS INSPECTION, NC.

NATIONAL NSPECTION \& CONSULTANTS, INC.

NATIONWIDE JESTING SERVICES, INC.

NDT SPECIAUSTS, NC.

NEWPOFT NEWS SHIPEUILONG \& DRY DOCK 00.

NILES STEEL TANK $\infty$.

NON DESTRACTIE TESTING COAP.

NONDESTRUCTWE INSPECTION SERVICE NOOTEA CORP.

NCFCAL TESTINE, NC.

NOAFOLK SHFEUILDING a DAYDOCK CORP.

MOAFOLK STATE UNIVERSITY

NOFTHWEST AIPL:NES, INK.

NOFTHWEST MSPECTION \& TESTING SERVICES

OKLAHOMA STEFl GASTINGS CO.

PENN INSPECTION COD.

PE NC. (PIPEUNE INSPECTDON \& ENGINERING)

PAYOR FOUNDAY, NC.

REALC SEPVICE CO. OF COLOPADO

SEROENT, HAUSKINS $a$ EECKWITH
$71-9988 \quad 189$

$71-9271 \quad+82$

$71-9932 \quad 183$

71-00870 179

$71.0494 \quad 176$

$71-0595 \quad 177$

$71.0444 \quad 176$

$71.0759 \quad 178$

$710701 \quad 17$

$710008 \quad 179$

$71.0952 \quad 175$

$71+0559 \quad 176$

$79-00037 \quad 175$

$71-0113 \quad 174$

$75-0602 \quad 179$

$74-0075 \quad 175$

$71.0680 \quad 179$

$71-0016 \quad 178$

$710703 \quad 178$

$71-0422 \quad 176$

$71-0500 \quad 176$

71-0694 177

$71-0209 \quad 174$

$71-0946 \quad 176$

$71-0737 \quad 178$ 


\begin{tabular}{|c|c|c|c|c|}
\hline Dociket & Pape & Hent & Dockinat & Paco \\
\hline-0102 & 174 & $\begin{array}{l}\text { CASK NIJHOMS 24P/ERE, VECTRA } \\
\text { TECHNOLOGIES, INC. }\end{array}$ & $72-1004$ & 185 \\
\hline-0.79 & 176 & $\begin{array}{l}\text { CASK TN-SE DRY STORABE CASK, } \\
\text { TRANSNUCLEAR, INC. }\end{array}$ & $72 \cdot 1021$ & 186 \\
\hline-0103 & $\begin{array}{l}174 \\
174\end{array}$ & $\begin{array}{l}\text { CASK: TFANSTOA CASK, SIERAA NUCLEAR } \\
\text { CORP. }\end{array}$ & $72 \cdot 1023$ & 186 \\
\hline-0932 & 179 & CASK: VSC-24, SERAA NUCLEAG, NNC. & $72-1007$ & 185 \\
\hline & & CENIAAL INTEFIM STORAGE ISFSI & $72-0021$ & 185 \\
\hline 492 & 178 & DAVS BESSE ISF\$ GEN LIC, 50-346 & $72-0014$ & 184 \\
\hline$\$ 480$ & 178 & FORT ST. VRAIN ISF\$I, 50-267 & $72-0008$ & 184 \\
\hline - & 177 & GOOCHUE COUNTY ISF\$ & 720018 & 164 \\
\hline-0700 & 177 & INEL TMIR ISFS & 720000 & 184 \\
\hline$-0 \$ 98$ & 177 & MODEL: PANATE FUEL STORAGE FACIUTY & 720022 & 186 \\
\hline & & NOFTH ANNA ISFSI - 50-338 \& 50-339 & $72-0016$ & 184 \\
\hline & & OCDNEE NSSI, 50-299, 50-270 \& 50-287 & $72-0004$ & 160 \\
\hline-0013 & 184 & PALISADES ISFSI GEN LXC, 50-256 & $72-0007$ & 123 \\
\hline 00008 & 163 & PONT BEACH ISFSI GEN LIC, SO-266 \& 60 . & $72-0005$ & 185 \\
\hline-1006 & 185 & PAAIRE ISLAND ISFSI, 50-2B2 \& $50-306$ & 720010 & 164 \\
\hline & & SURAY ISFS1 50-260 \& 50-281 & 720002 & 163 \\
\hline 014 & 186 & TROJAN ISFSL, S0-344 & $72-0017$ & 184 \\
\hline$\infty 01$ & 185 & UNKNOWN & DOCKET & 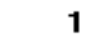 \\
\hline & & UNKNOWW & DOXKET & 170 \\
\hline
\end{tabular}




\section{DOCKETED ITEMS}

\section{LOW LEVEL RADWASTE DISPOSAL STES}

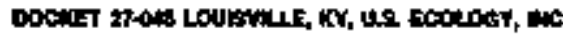

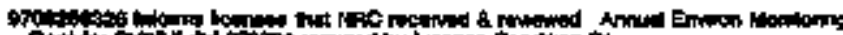

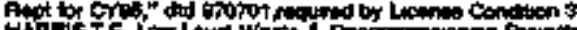

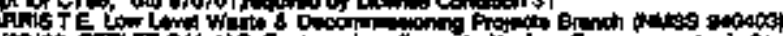

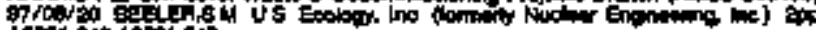

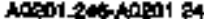

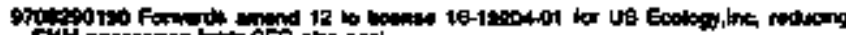
E⿰讠十

Fip

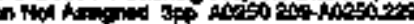

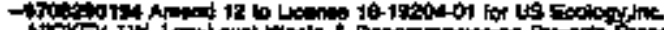

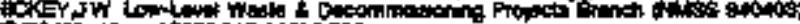

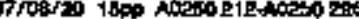

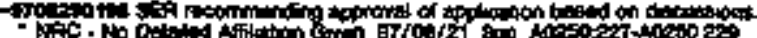

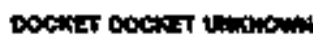

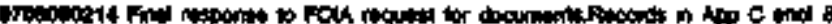

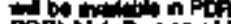

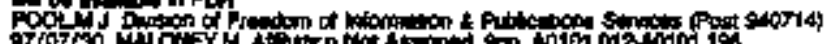

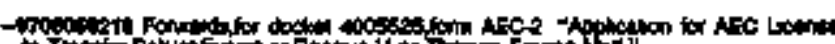

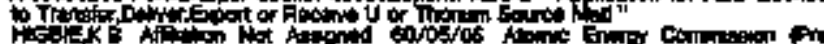

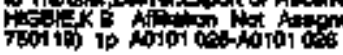

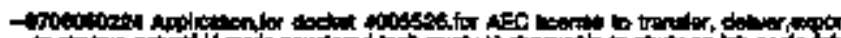

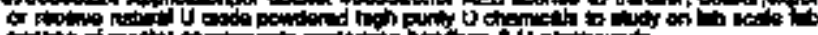

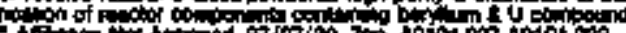

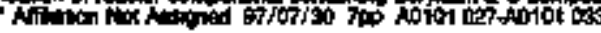

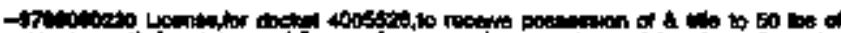

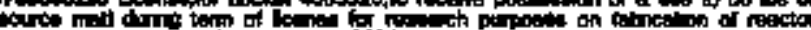

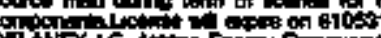

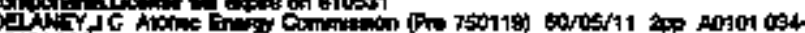
ANo1 tos

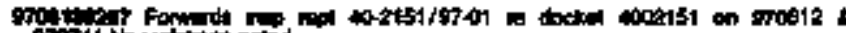

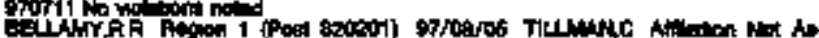

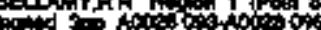

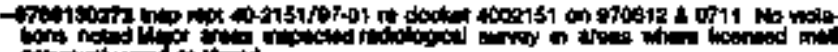

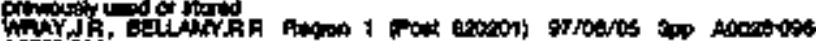
atom

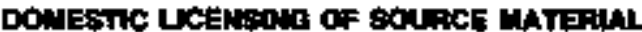

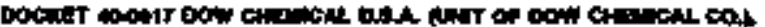

97 G

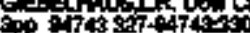

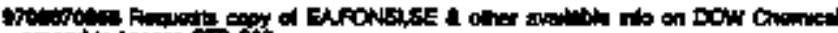
mond to lecte fir

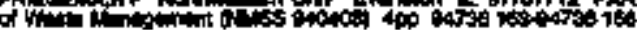

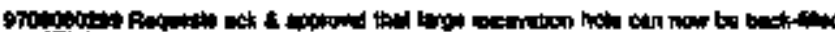

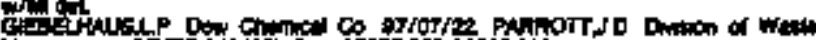

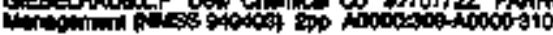

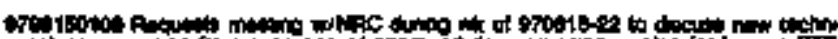

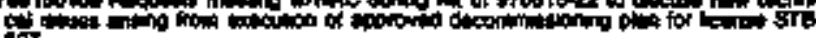

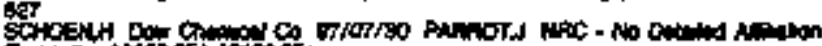

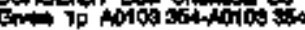

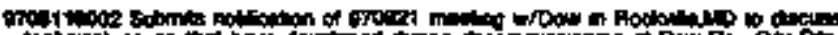

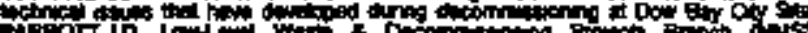

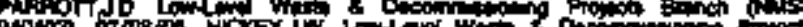

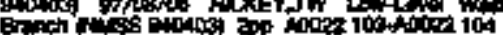

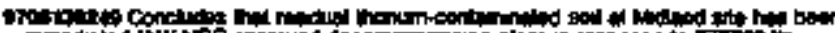

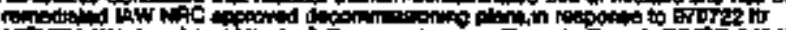

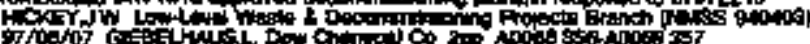

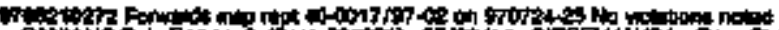

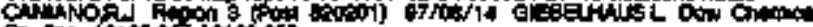

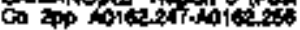

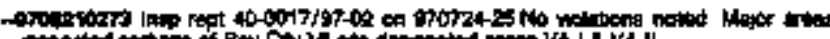

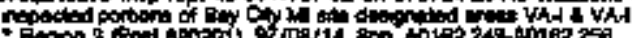

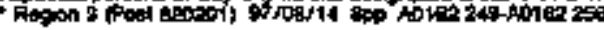

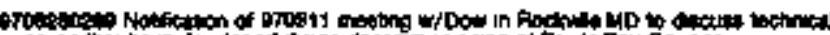

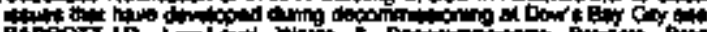

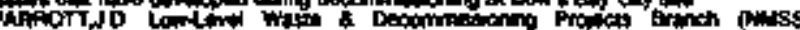

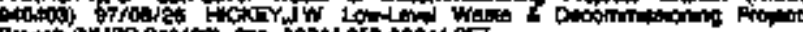

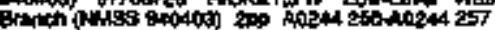

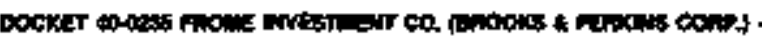

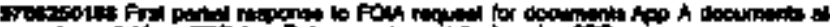

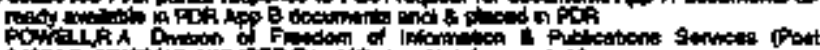

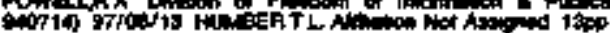

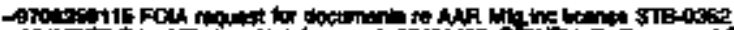
H J I

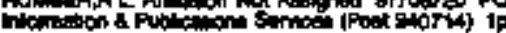

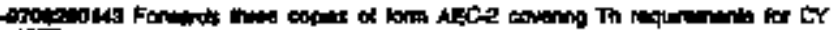
103

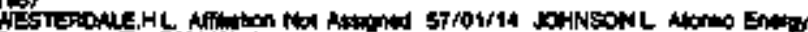

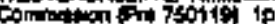

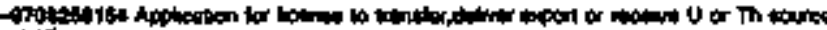
Pi

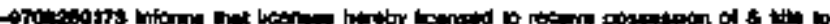

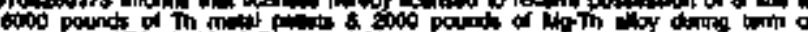

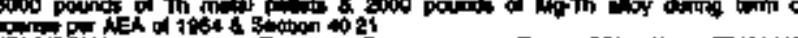

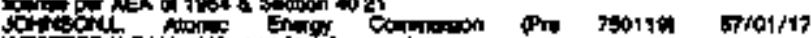

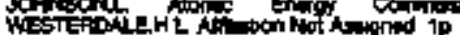

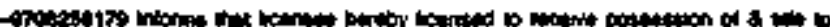

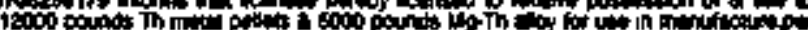
1. of of PAt

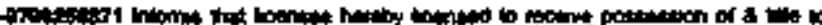

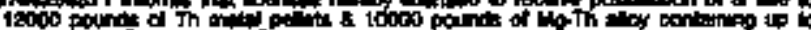

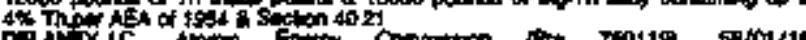

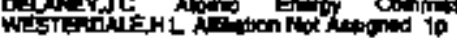

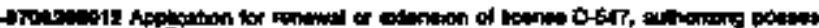
the of 6 600 the thatal poition to

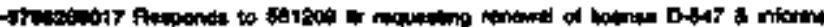

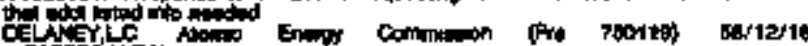
in

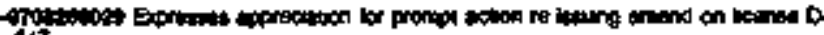

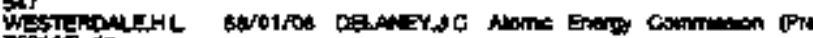
$760197 \mathrm{p}$

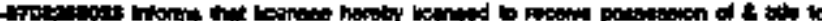

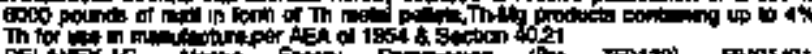

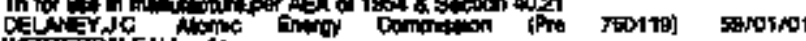

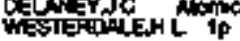

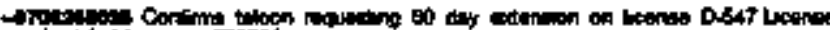

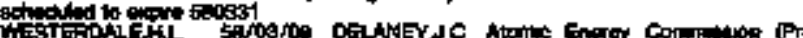

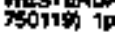

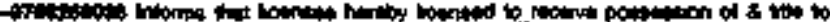

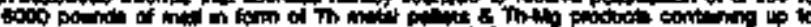

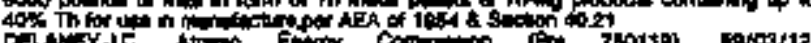

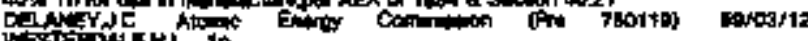

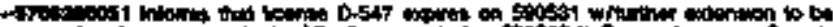

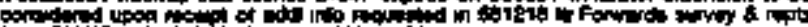

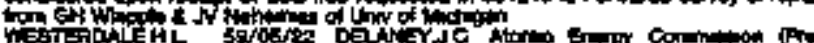

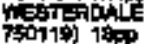

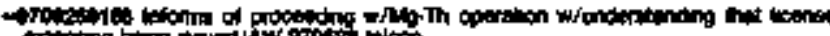
Ar 750 16: 10

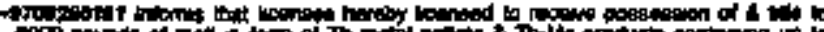

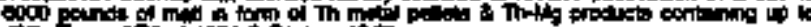

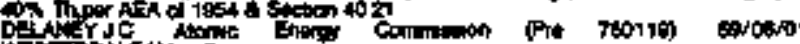

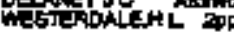




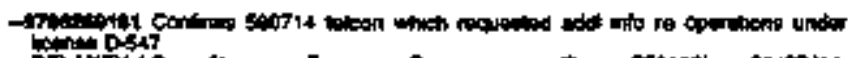

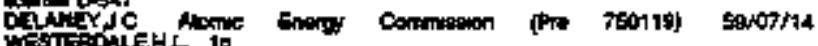

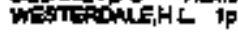

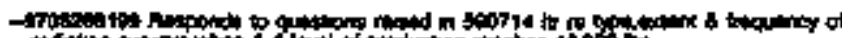

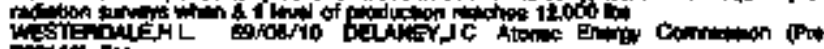
Totit in 70

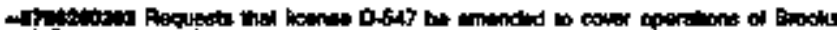

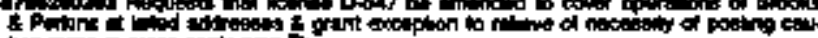
bens. TEOA 19

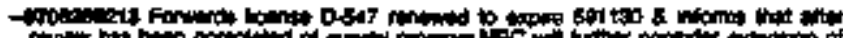

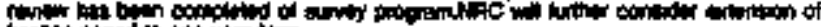

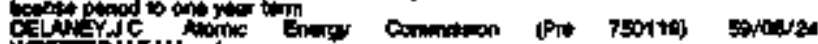

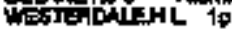

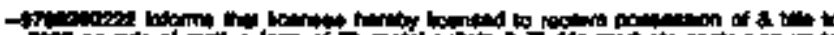

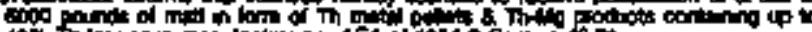

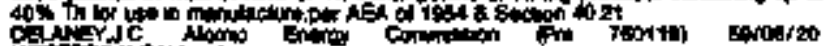

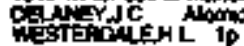

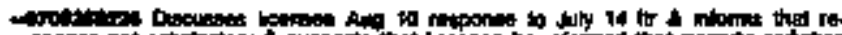

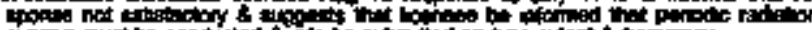

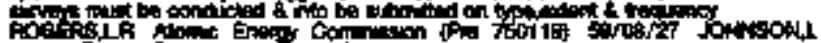

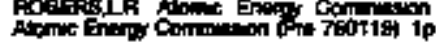

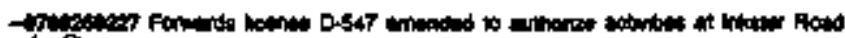

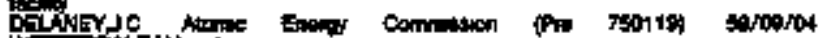

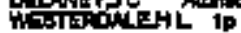

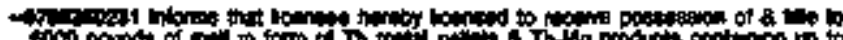

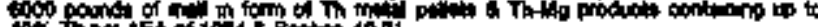

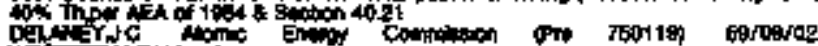

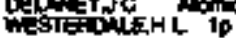

$\rightarrow$ Fots

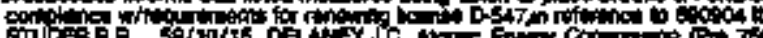

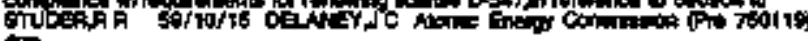
4

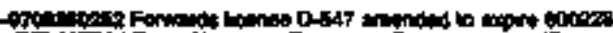

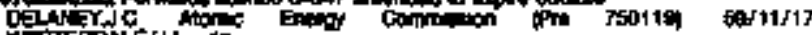
(1)

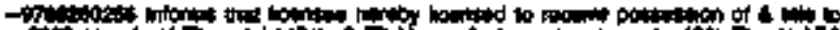

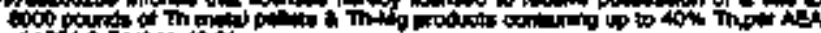

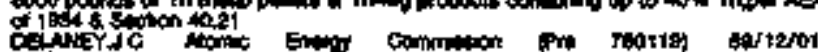
WITHE

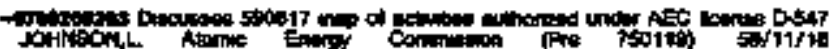

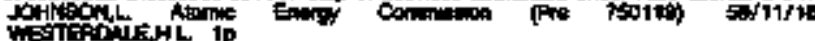

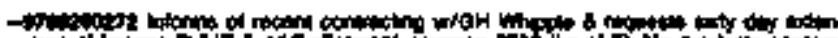

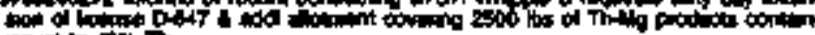
Nity

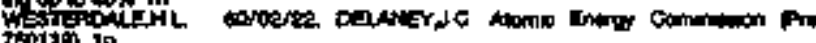
rowion ip

*7.

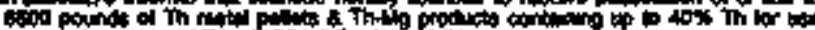

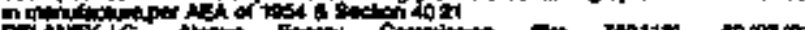

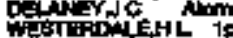

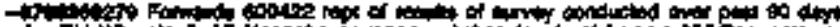

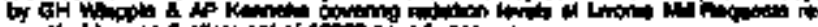

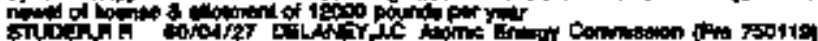
100

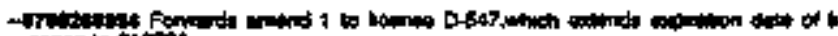
cenat to tor

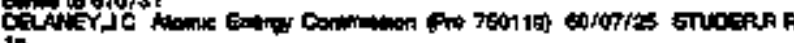
10

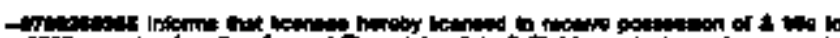

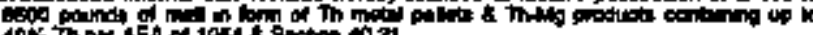

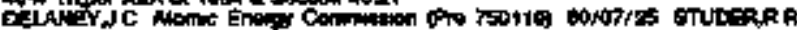
10

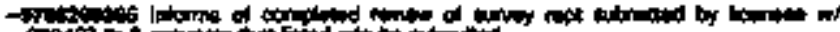

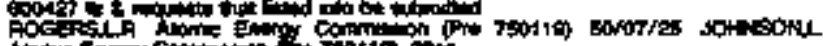

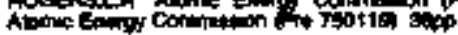

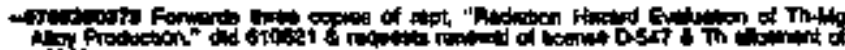

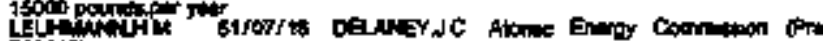
75011에 ip

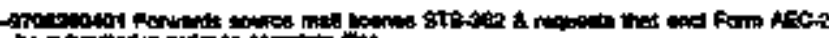

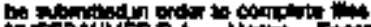

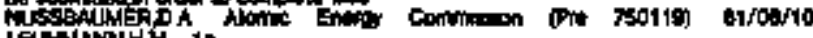
LETHANOAH ip

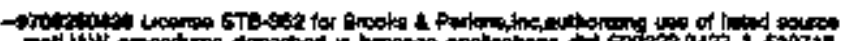

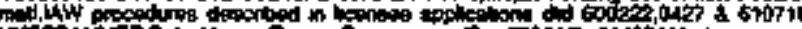

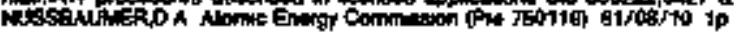

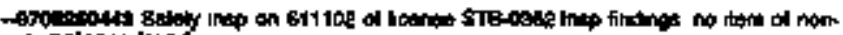

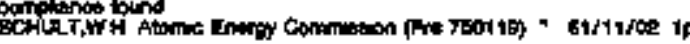

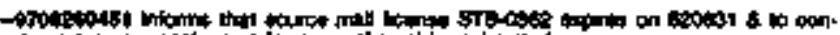
Ald

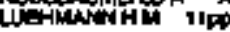

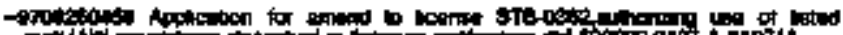

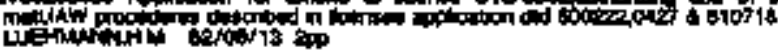

How

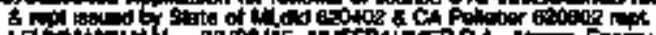

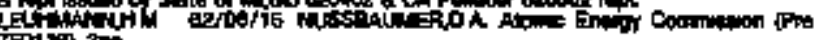
(1001 18) 37

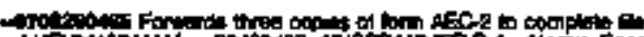

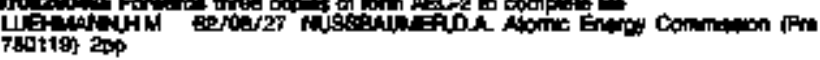

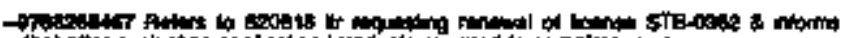
Hit

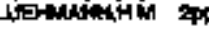

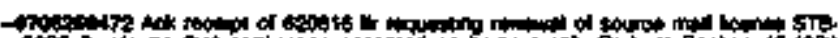

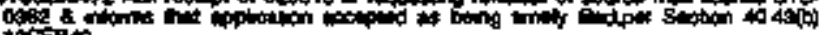

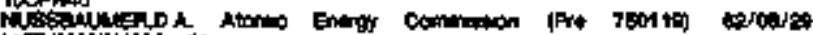

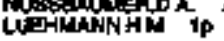

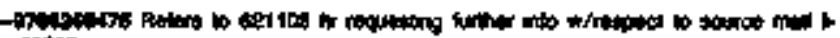

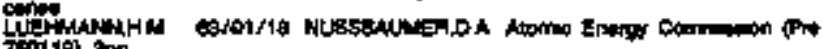
70011 ) 300

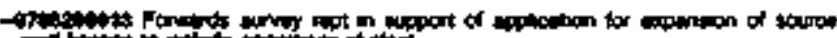

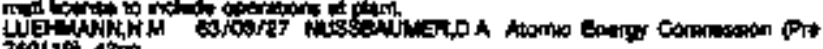
75011934

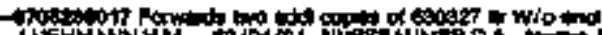

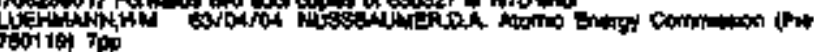

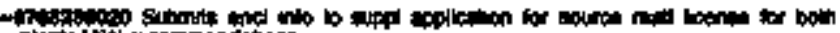
bu J Tot 19) 20

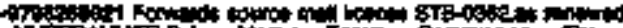

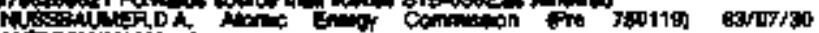

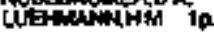

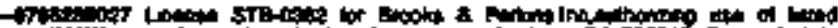

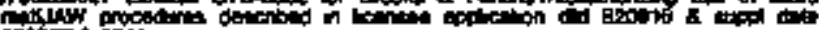
60002 \& 6011

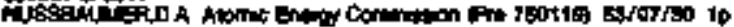

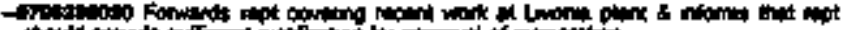

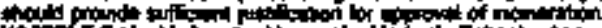

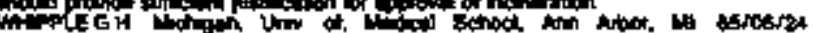
sore ineY

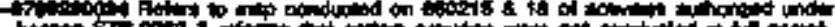

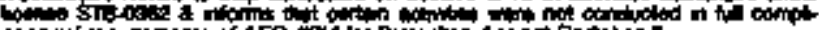

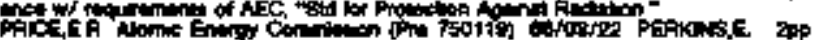

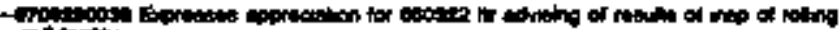

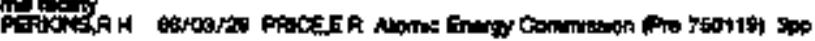

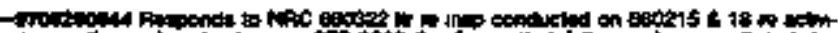

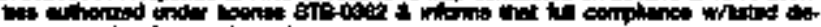

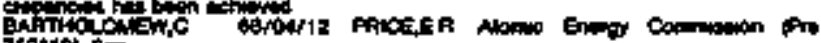
istifion sar

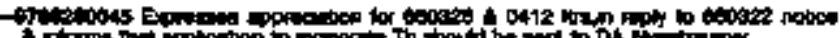

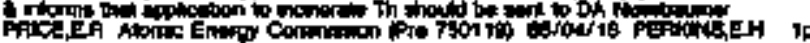

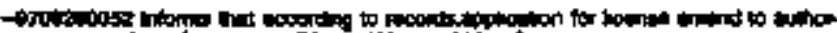

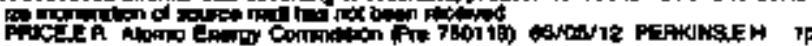

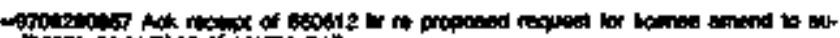

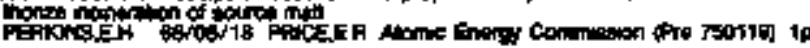

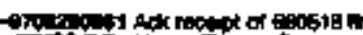

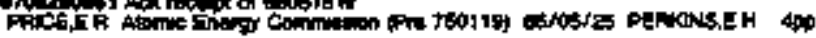

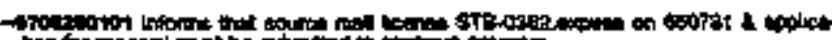
bop for non PEAkist H ip 


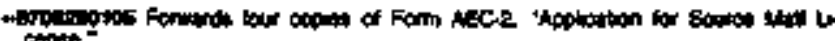

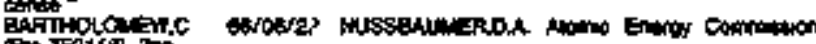
Tru rodin 20

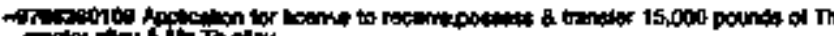

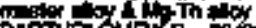

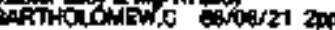

- If

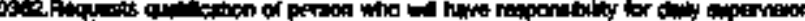

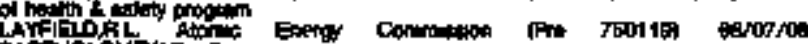
ENRTHOLOHAW, $2 p$

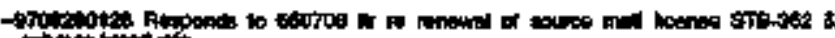

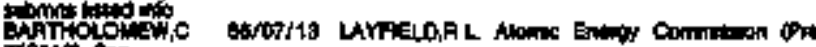
T3011s 3pp

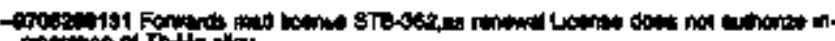

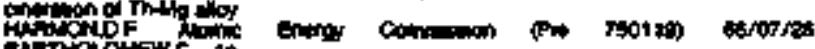
Anithoungw,

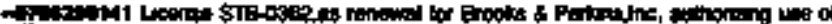

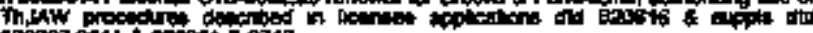

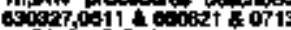

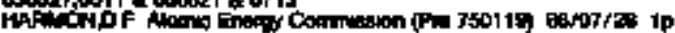

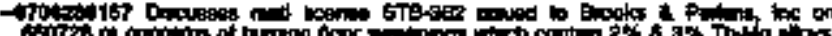

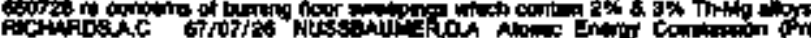

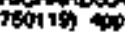

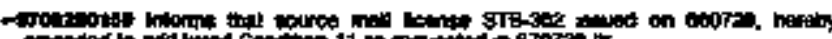

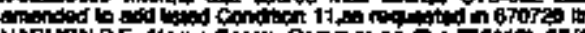

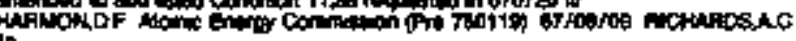
ip

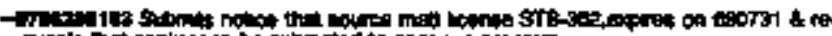

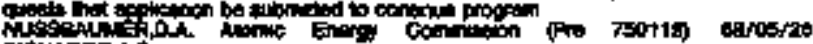

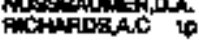

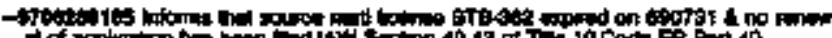

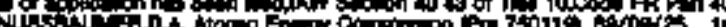

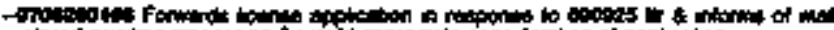

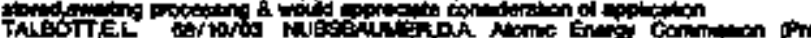
Fo11日]

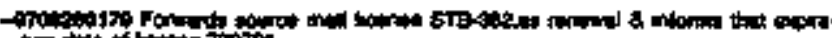

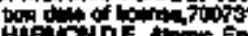

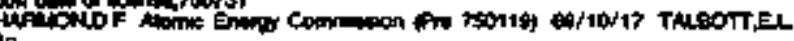
in

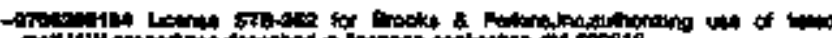

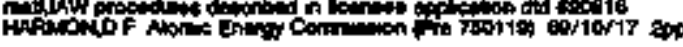

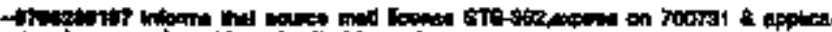

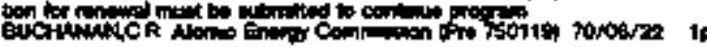

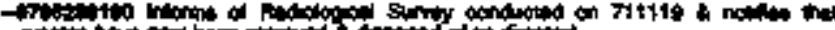

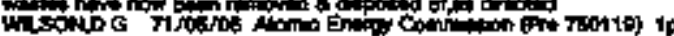

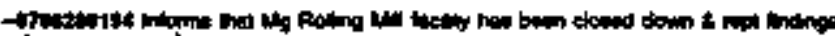
Wit

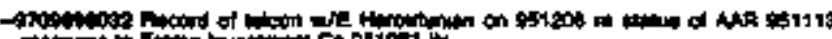

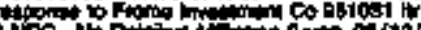

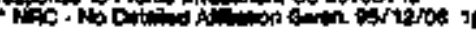

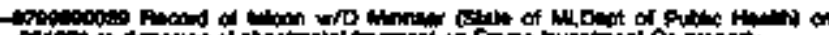

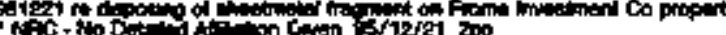

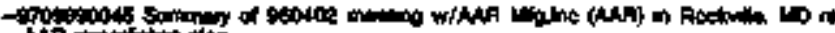

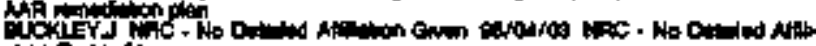
cont Eman ip

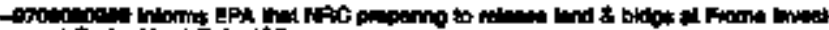

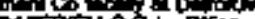

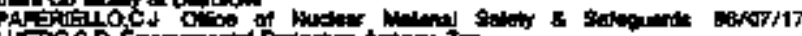

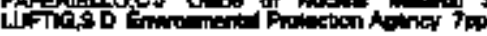

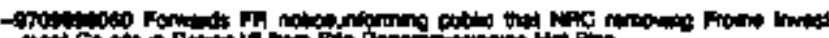

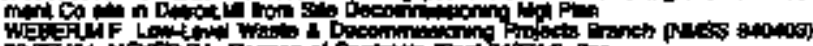

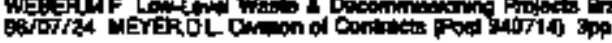

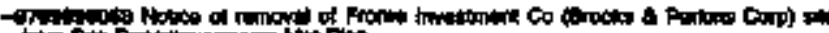

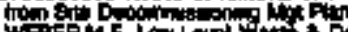
ofotion

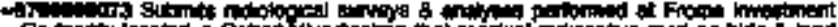

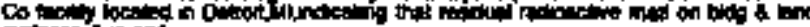

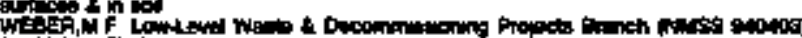

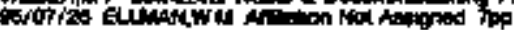

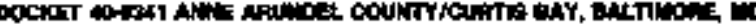

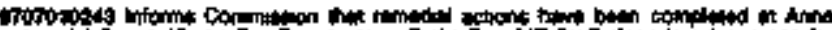

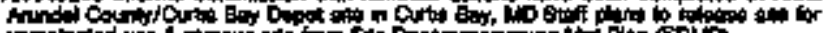

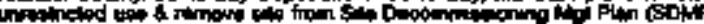

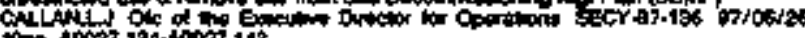

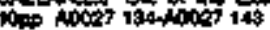

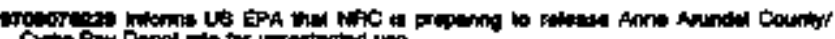

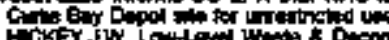

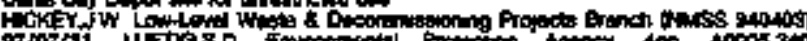

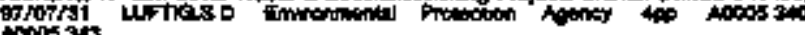
Nonos 34s

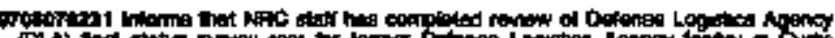

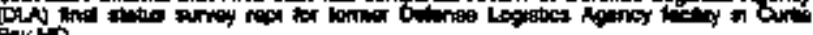

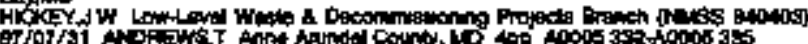
烈

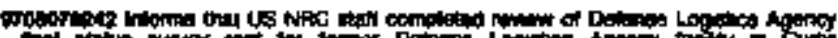

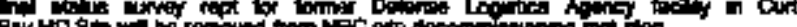
X

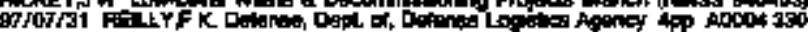
1000430

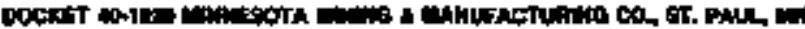

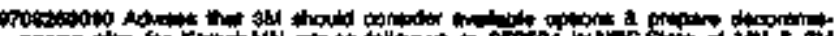

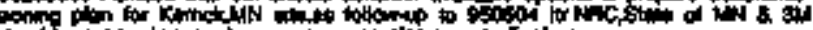

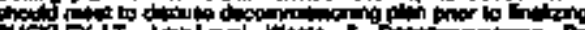

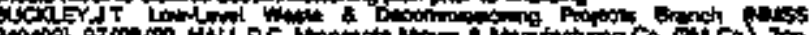
(a)

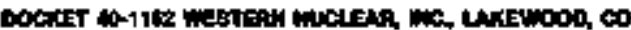

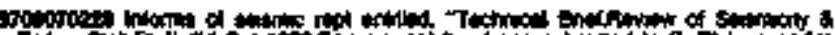

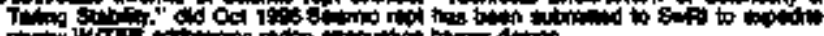

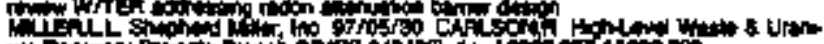

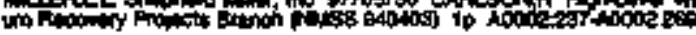

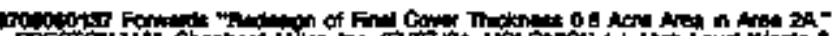

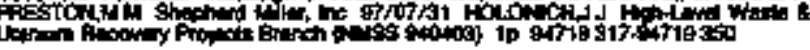

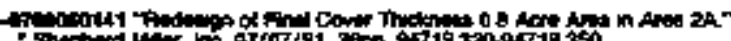

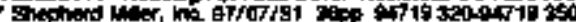

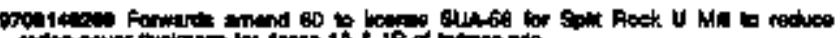

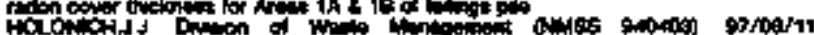

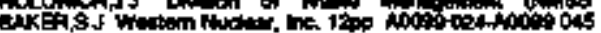

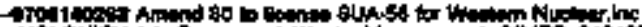

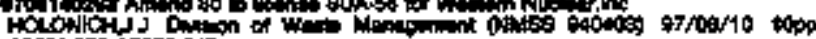

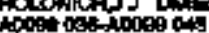

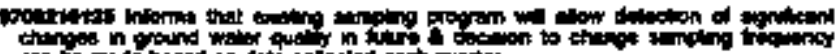

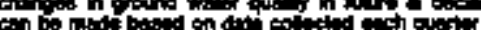

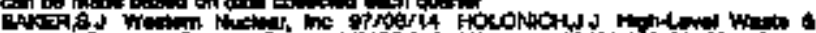

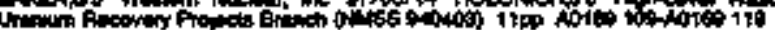

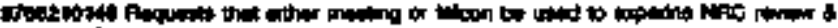

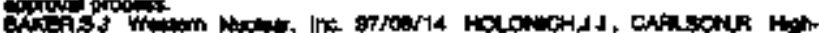
1 Nolष 30

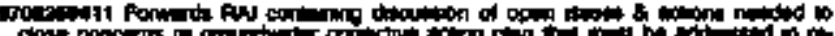

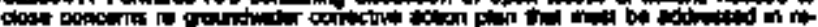

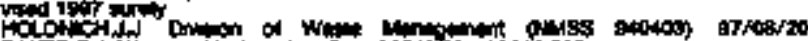

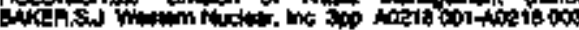

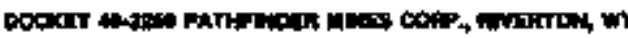

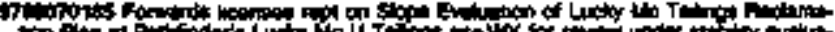

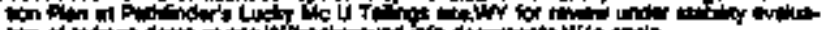

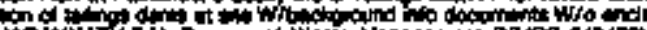

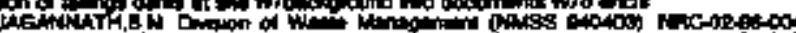

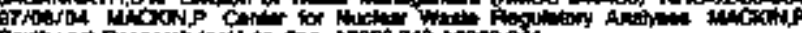

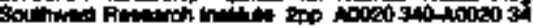

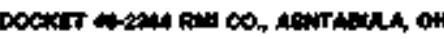

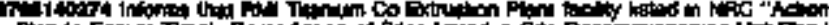

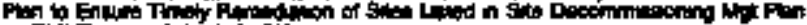
4 Alin The

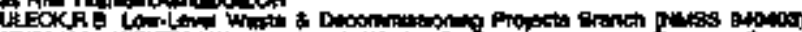

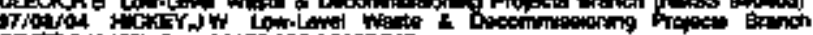

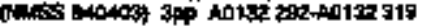

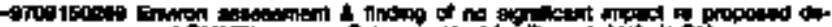

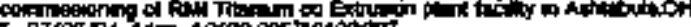

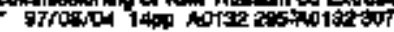




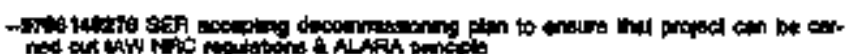

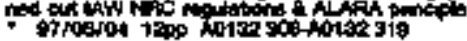

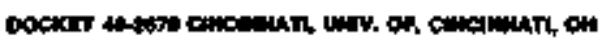

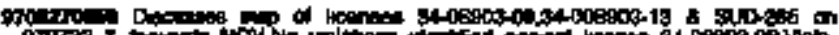

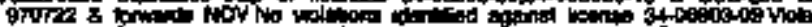

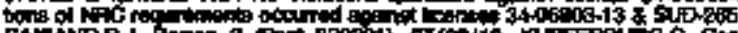

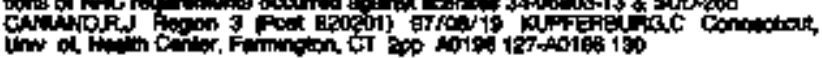

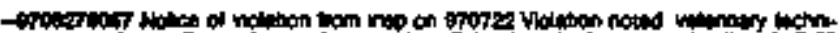

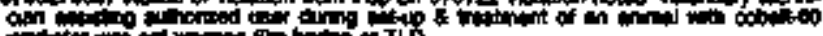

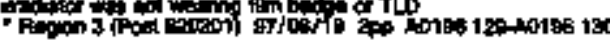

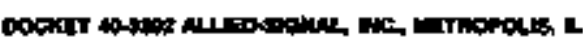

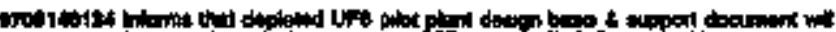

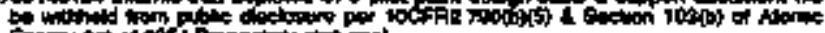

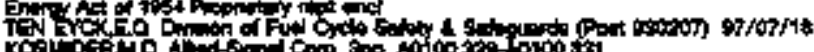

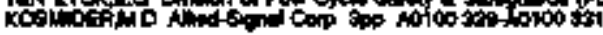

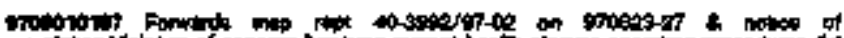

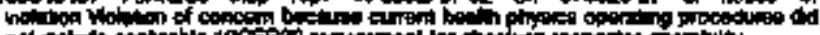

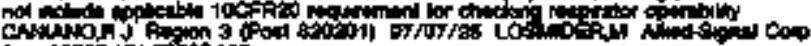

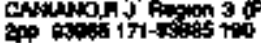

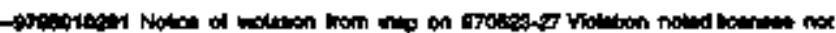

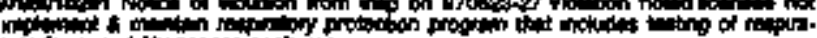

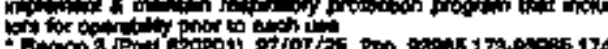

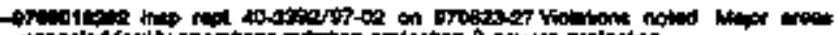

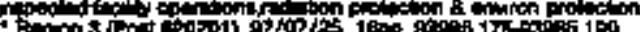

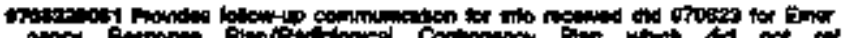

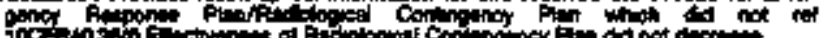

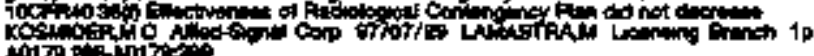

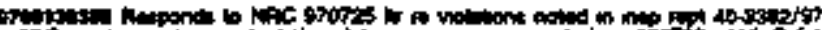

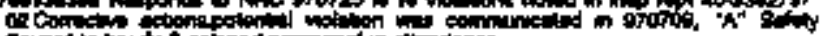

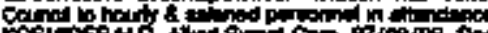

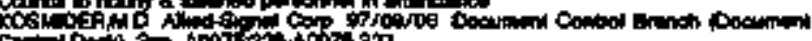

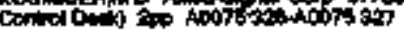

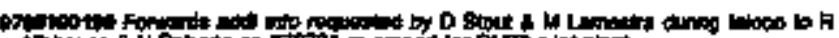

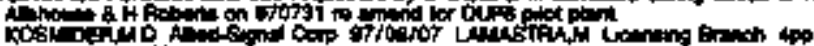

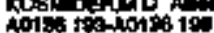

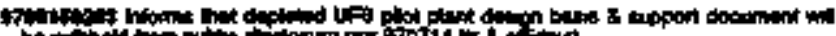

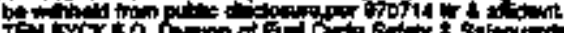

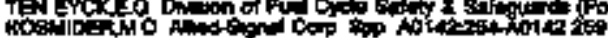

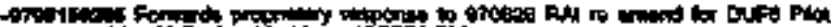

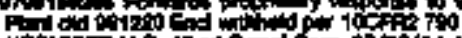

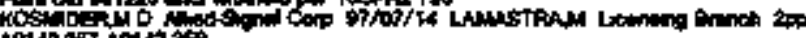
40142 $267+0142 \%$

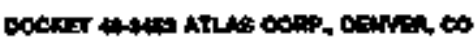

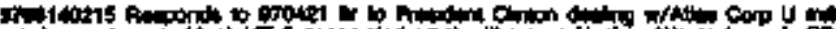

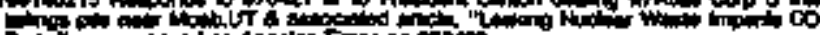

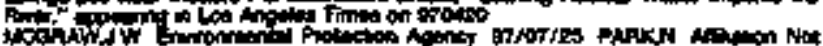

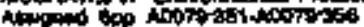

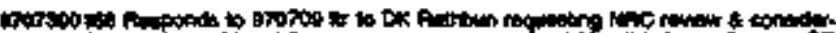

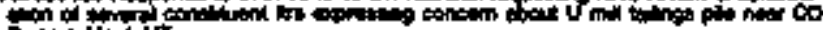

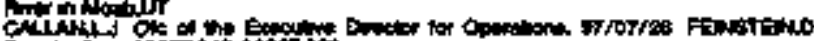

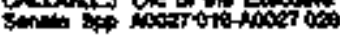

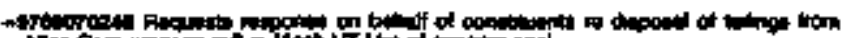

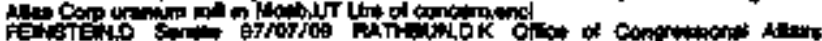

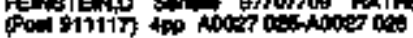

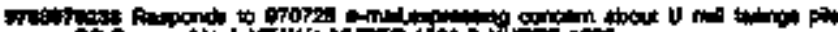

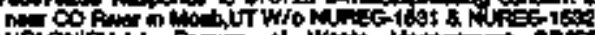

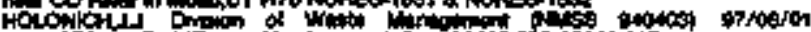

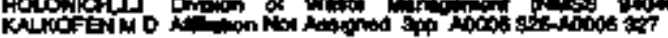

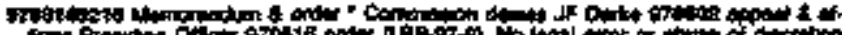

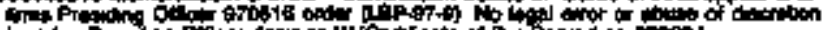

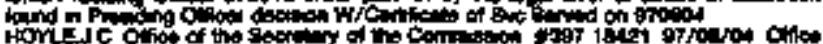

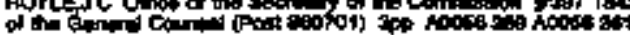

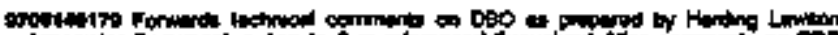

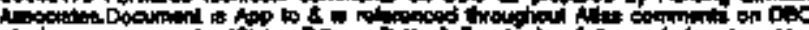

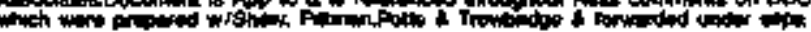

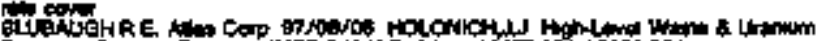

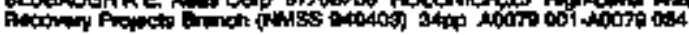

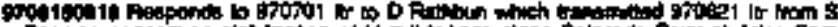

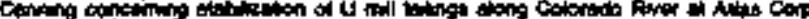

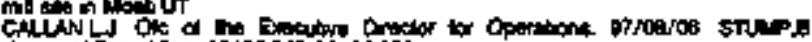

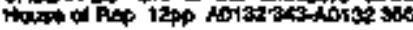

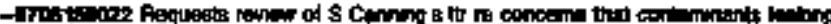

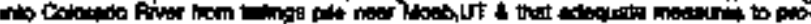

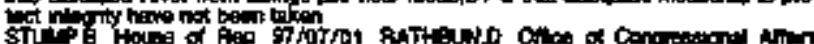

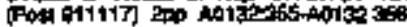

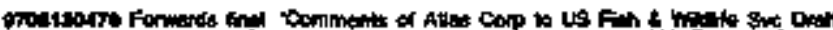

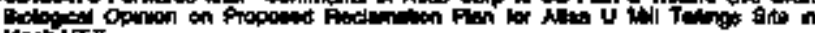

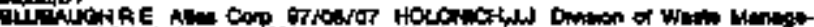
ment (0)

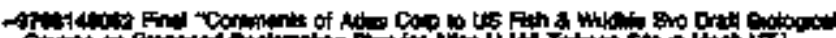

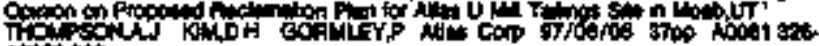
Alom

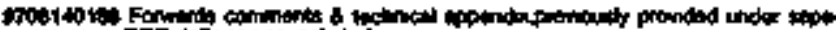

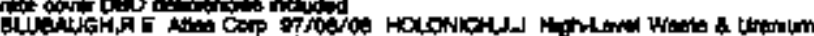

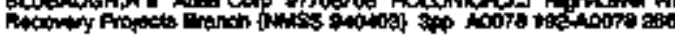

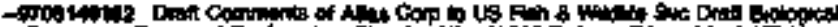

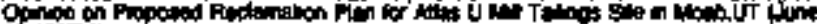

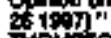

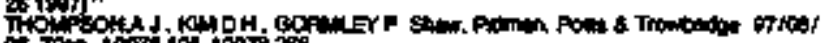

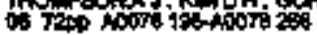

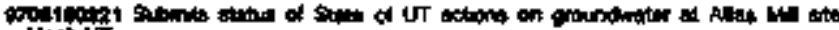

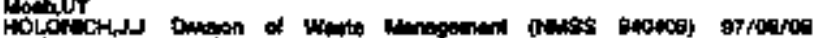

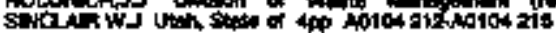

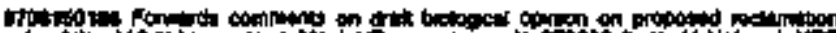

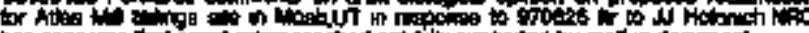

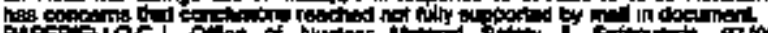

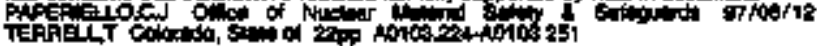

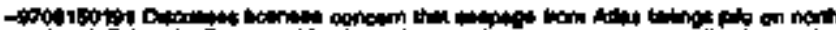

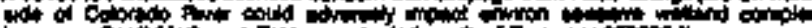

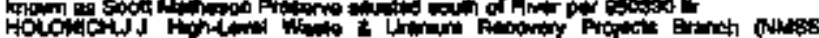

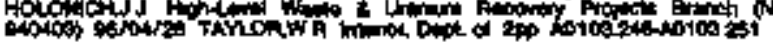

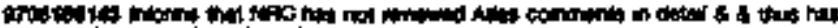

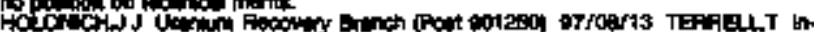

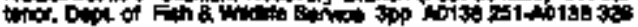

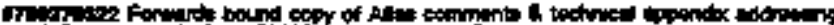

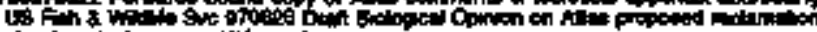

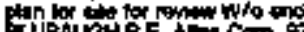

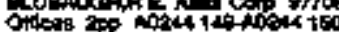

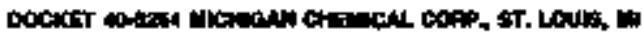

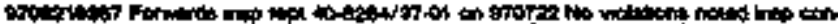

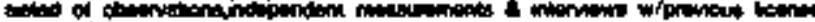

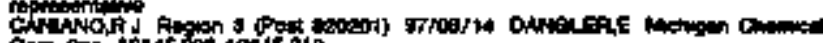
Corp 200 inds

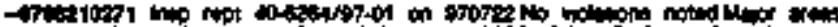
The

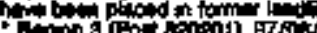

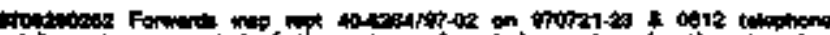

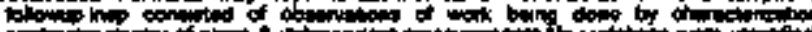

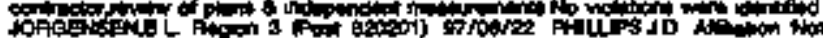

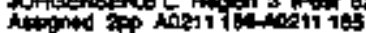

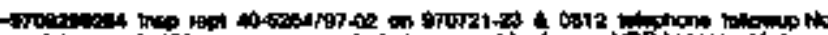

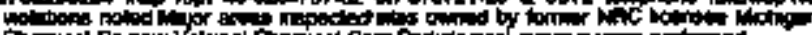

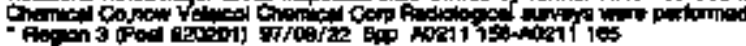

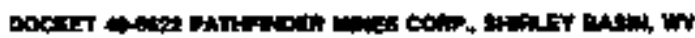

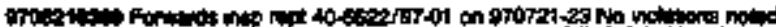

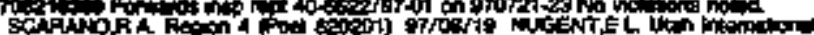

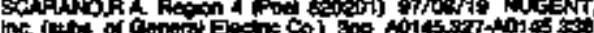

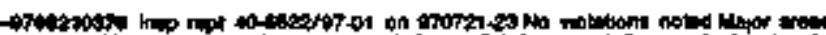

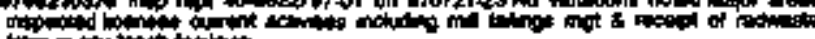

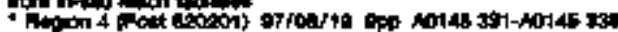




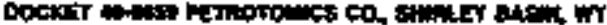

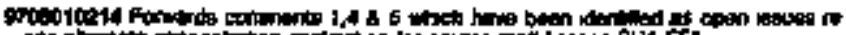

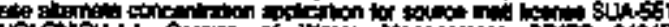

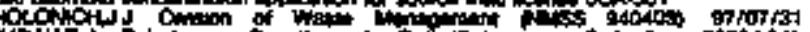

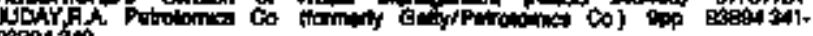

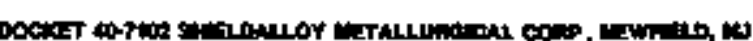

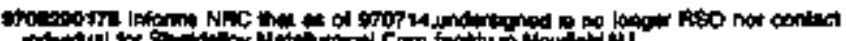

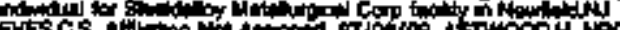

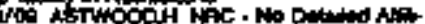

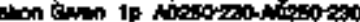

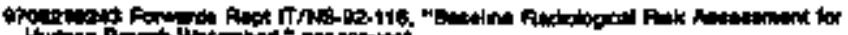

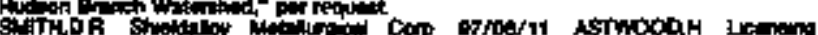

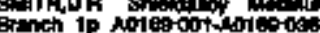

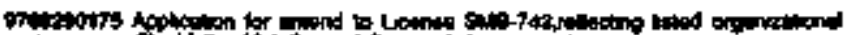

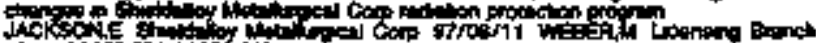

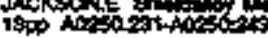

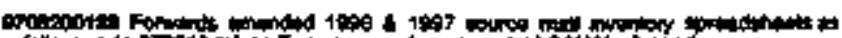

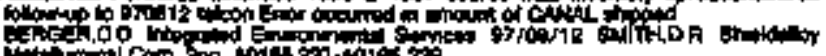

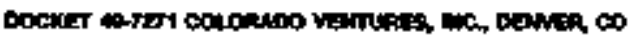

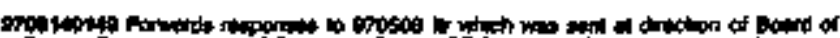

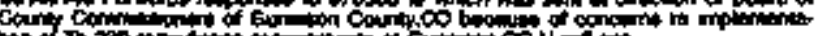

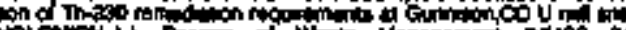

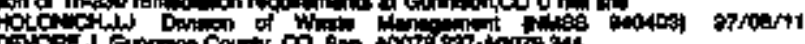

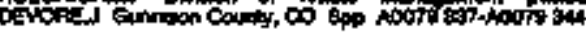

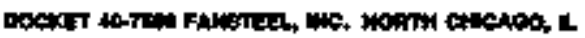

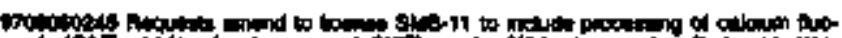

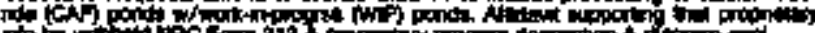

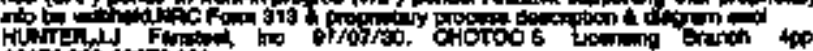

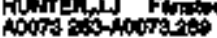

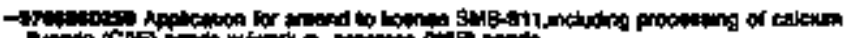

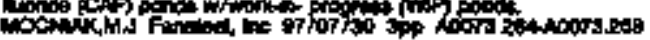

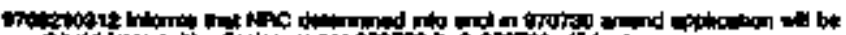

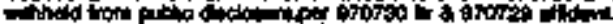

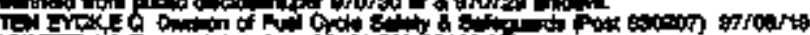

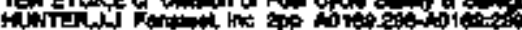

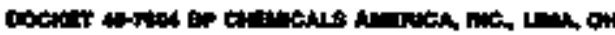

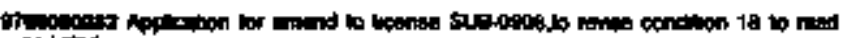

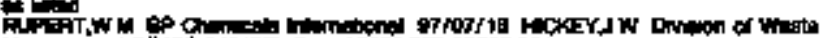

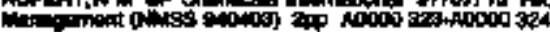

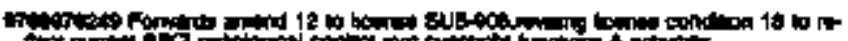

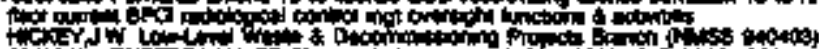

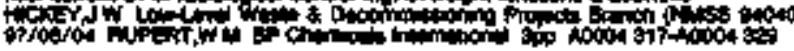

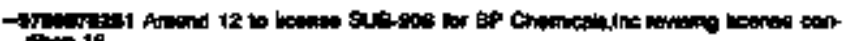
in 10

7F

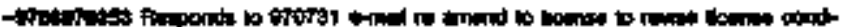

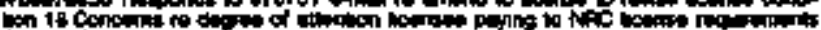

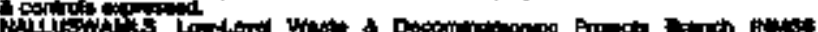

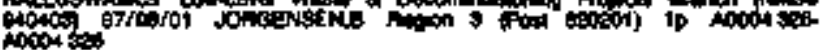

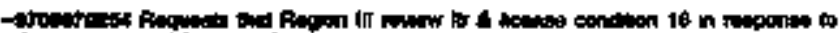

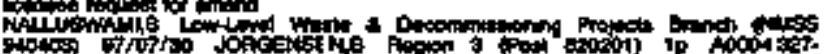

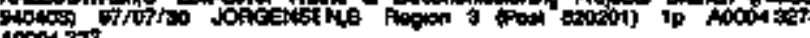
10004377

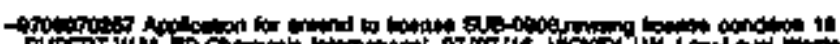

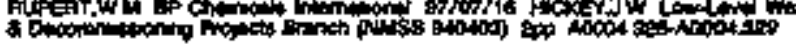

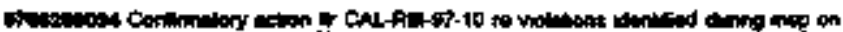

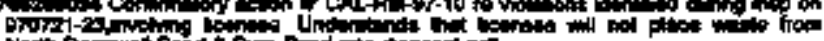
Worth

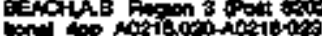

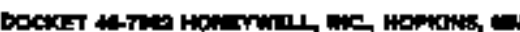

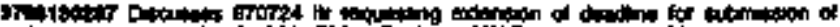

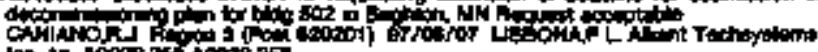
the if

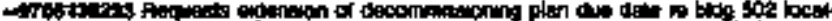

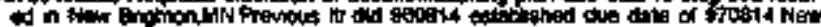

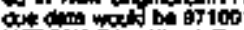

USBOH

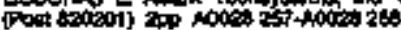

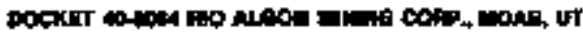

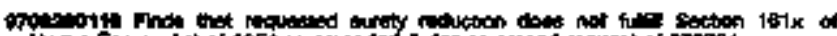

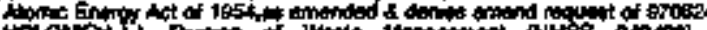

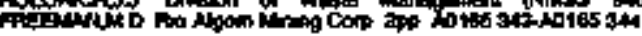

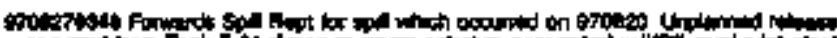

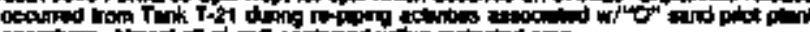

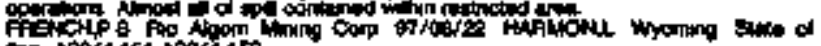

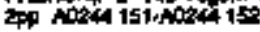

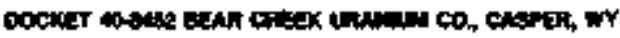

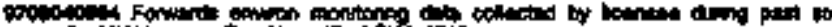

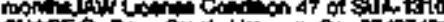

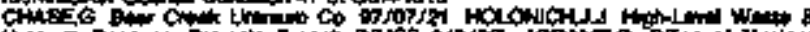

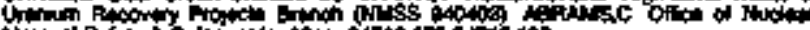

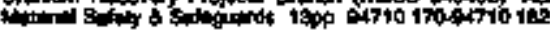

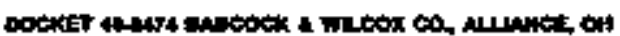

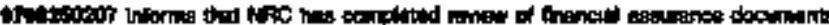

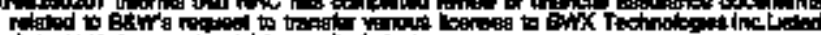

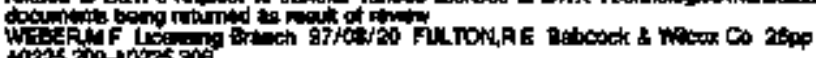

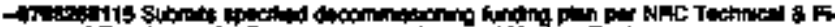

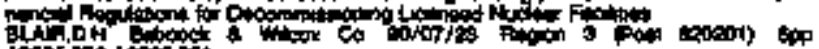

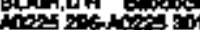

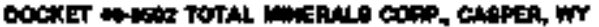

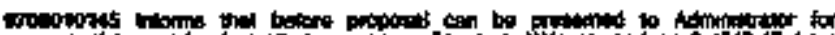

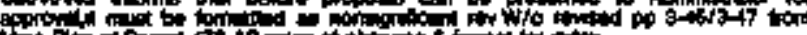

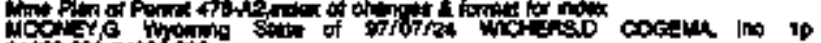
Hotos

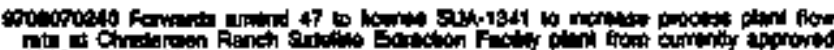

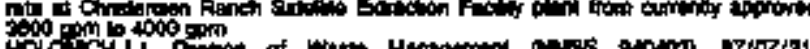

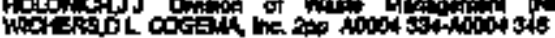

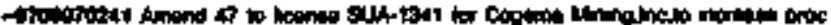

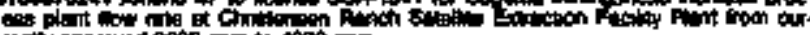

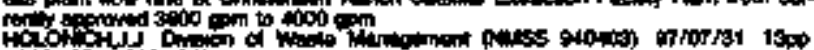
ingor of

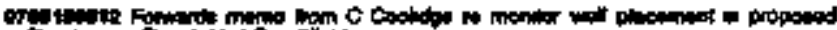

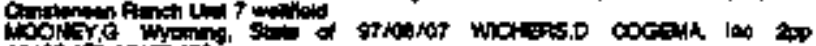

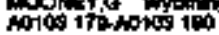

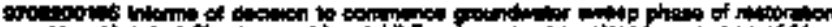

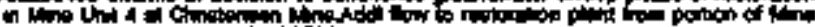
WTd

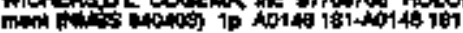

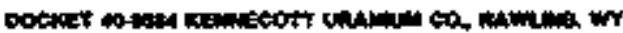

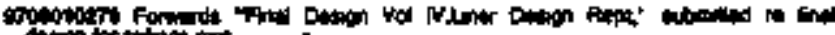

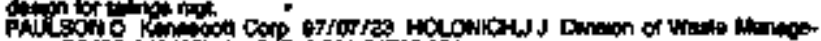

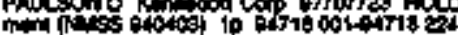

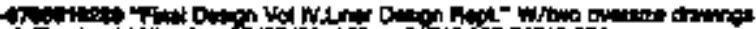

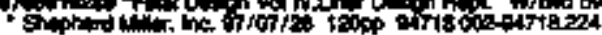

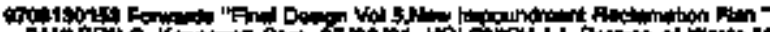

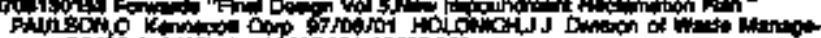

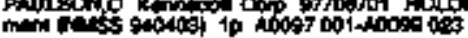

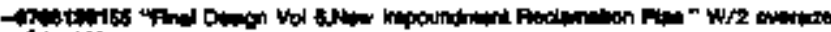

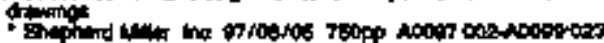

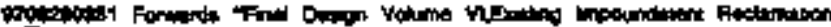

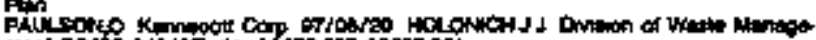
mand (Ret:

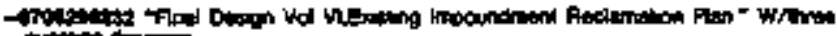

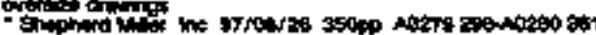




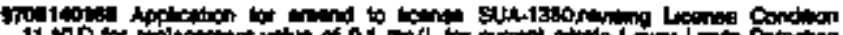

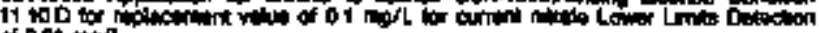
of $01 \mathrm{~mol}$

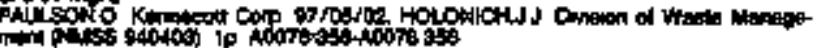

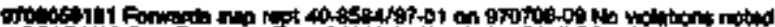

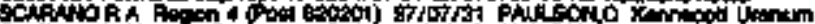

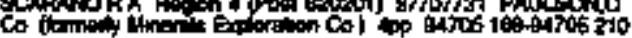

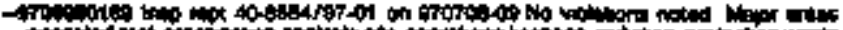

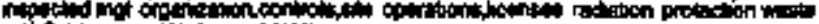

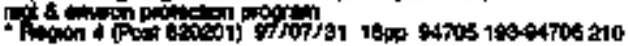

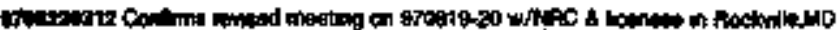

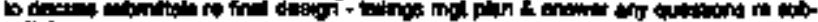

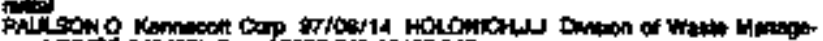

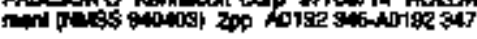

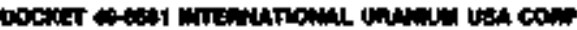

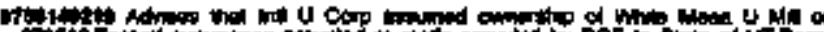

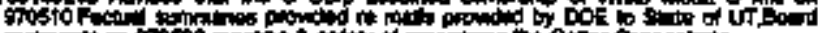

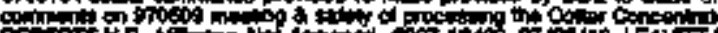

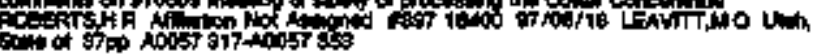

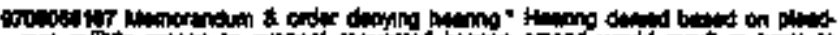

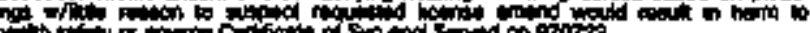
hitrit the

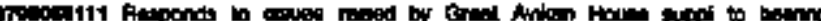

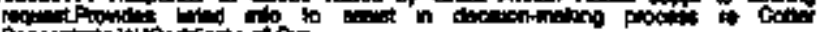

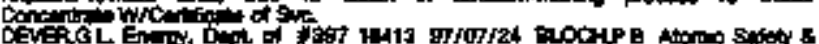

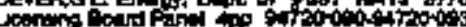

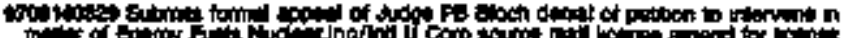

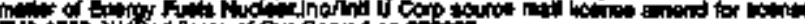

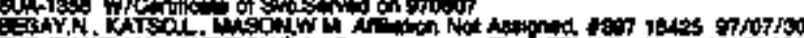

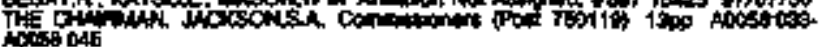

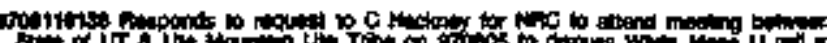

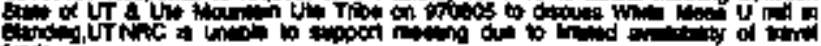

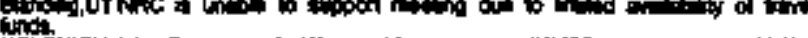

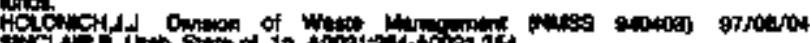

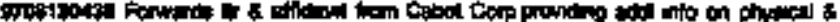

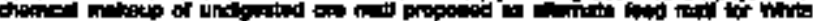

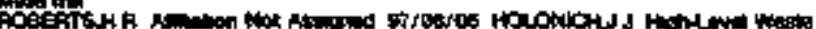

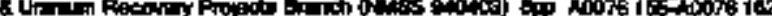

If

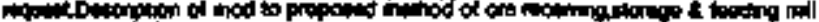

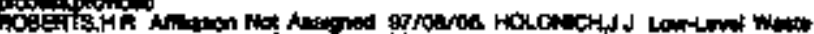

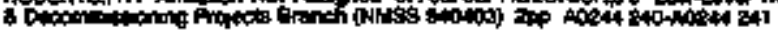

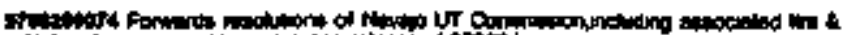

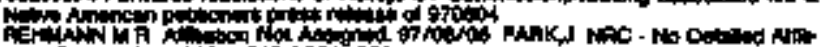

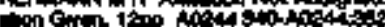

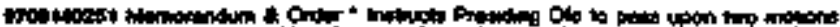

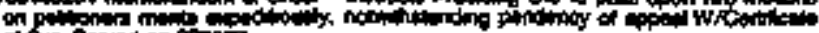

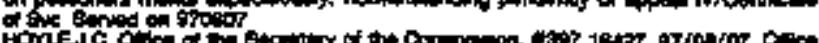

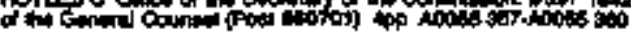

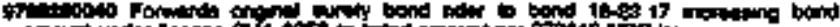

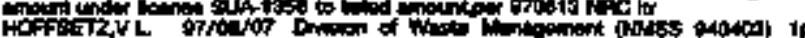

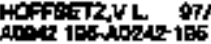

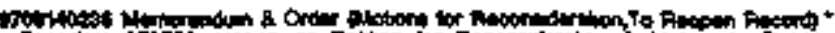

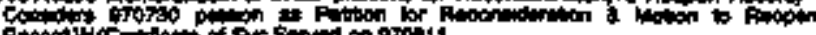

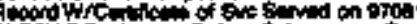

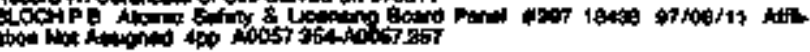

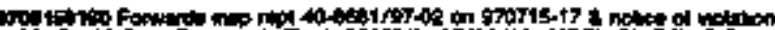

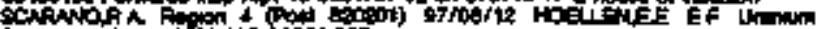

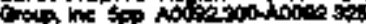

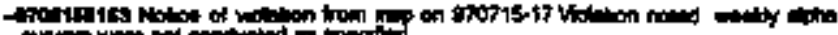

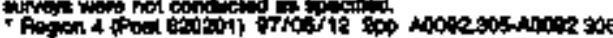

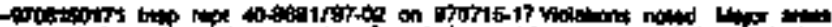

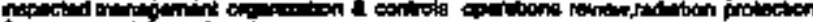

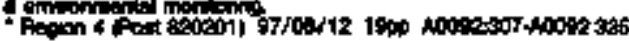

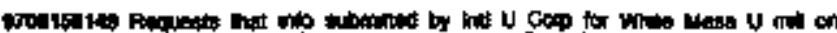

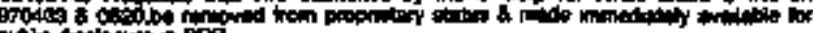

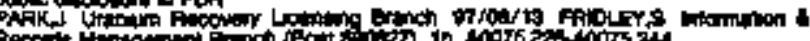

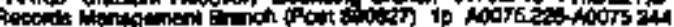

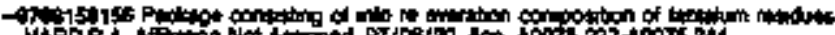

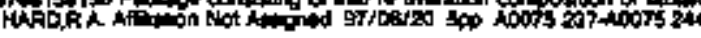

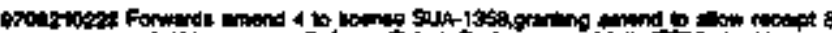

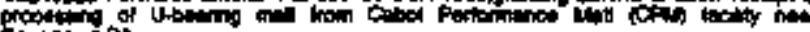

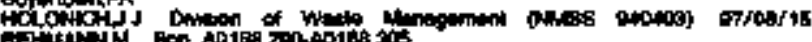

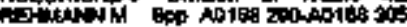

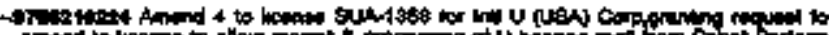

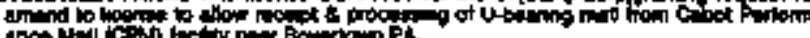

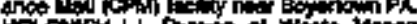

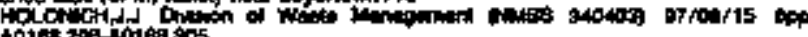

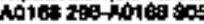

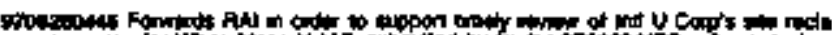

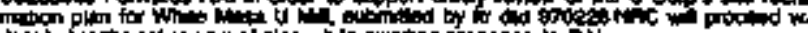

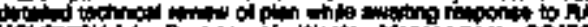

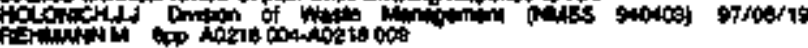

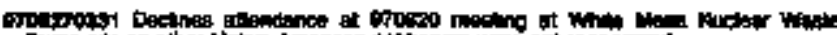

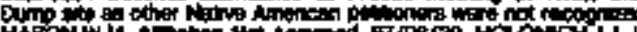

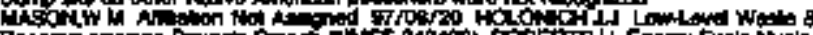

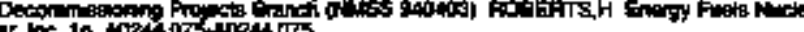

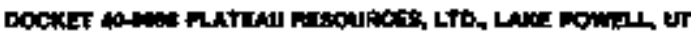

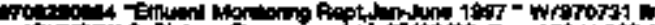

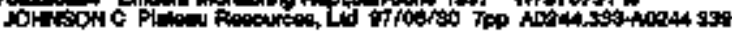

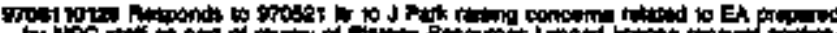

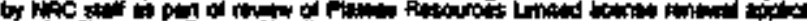

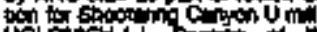

HOLC

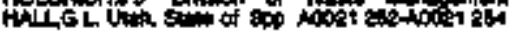

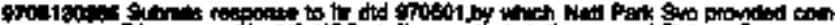

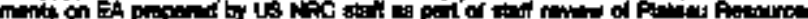

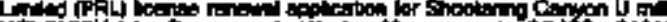

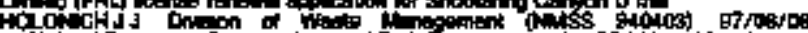

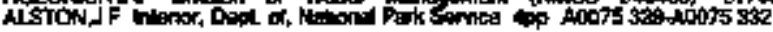

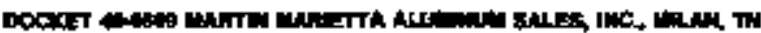

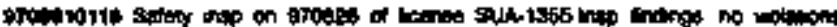

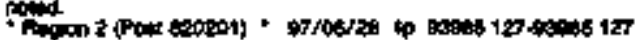

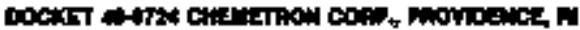

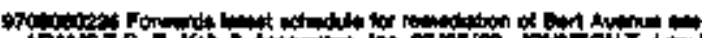

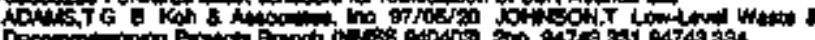

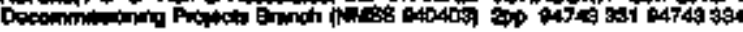

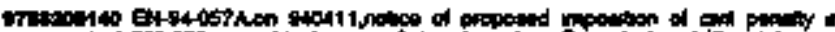

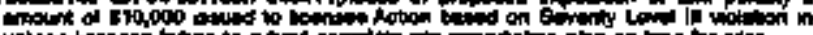

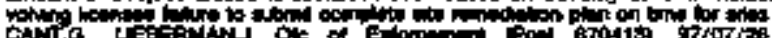

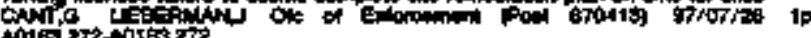

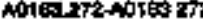

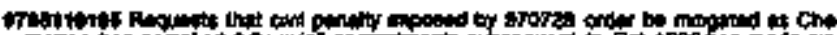

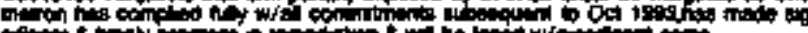

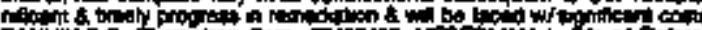

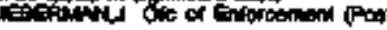

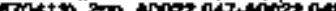

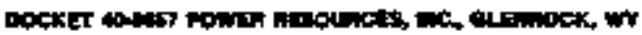

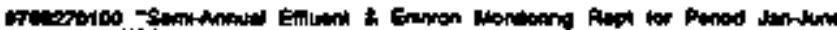

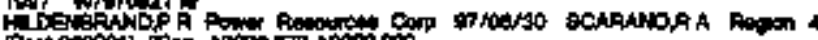

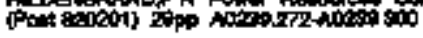

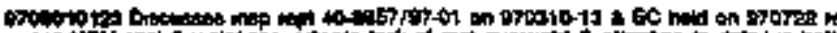

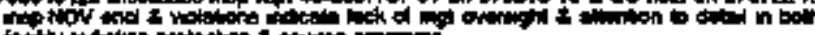
f.

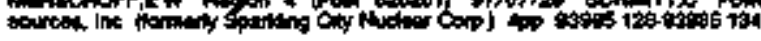

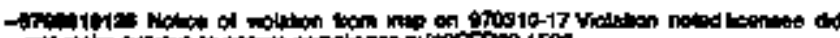

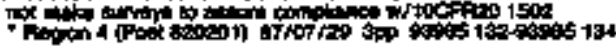

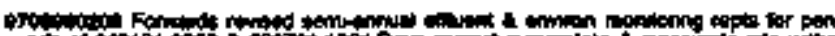

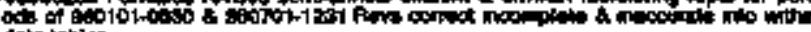
Antations

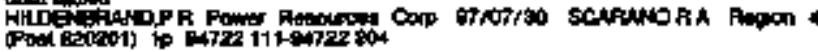

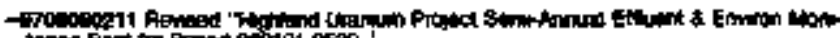

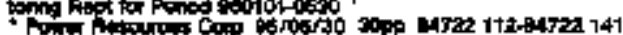

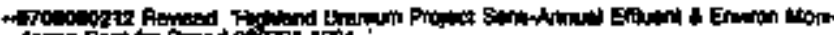

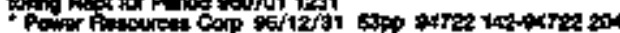




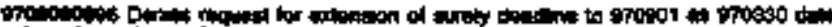

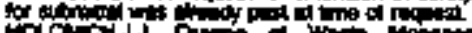

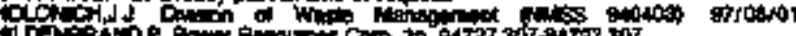
等

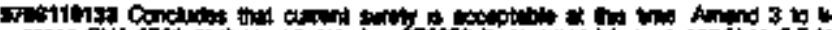

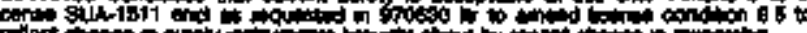

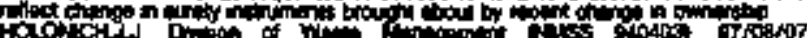

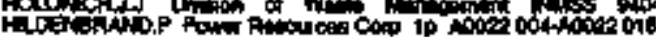

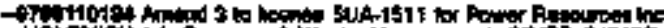
Hotaliah

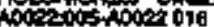

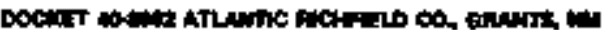

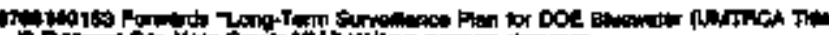

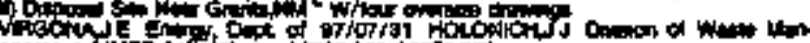

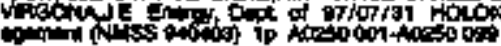

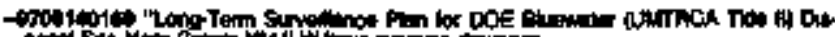

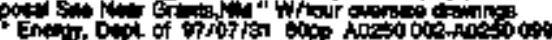

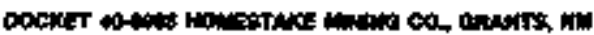

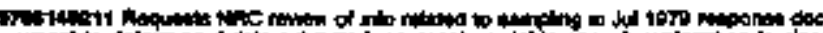

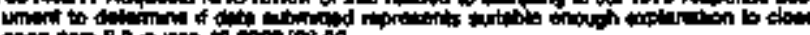
coen

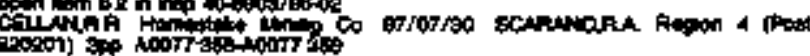

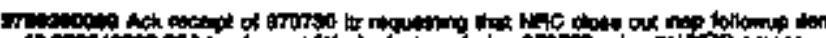

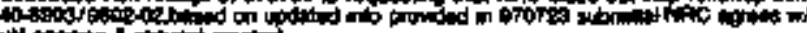
tot

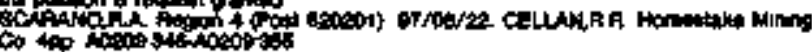

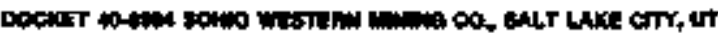

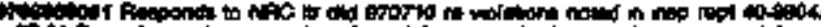

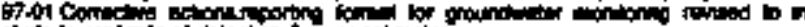

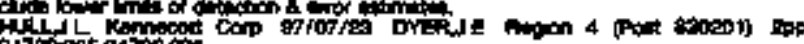

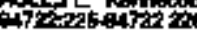

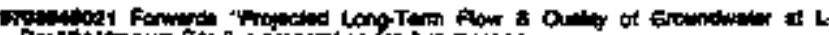

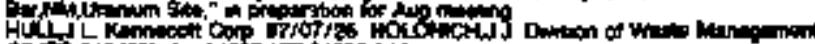

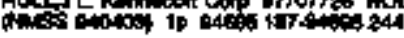

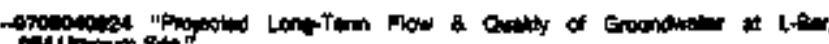

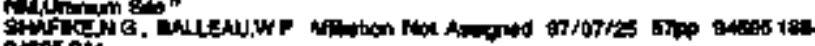
ontexs

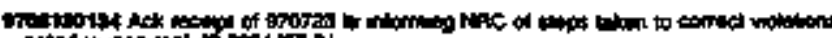

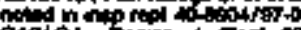

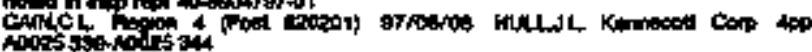

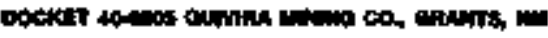

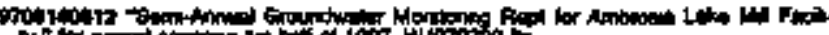

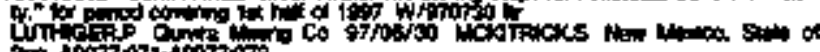

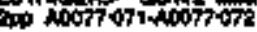

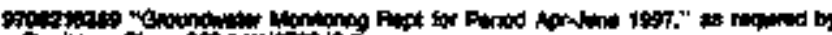

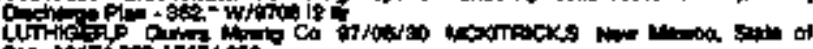

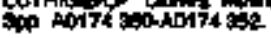

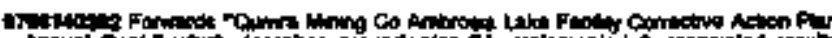

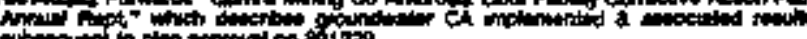
whon

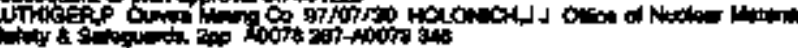

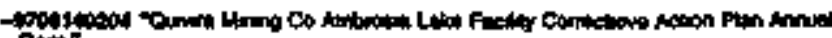

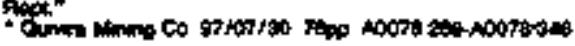

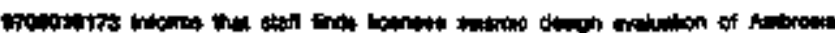

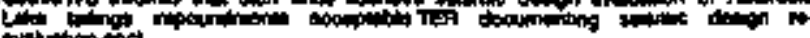

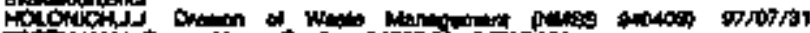
of

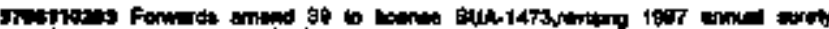

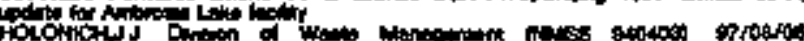

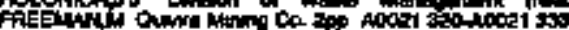

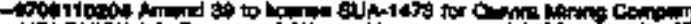

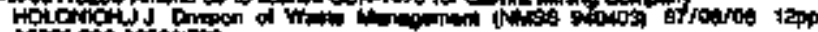
notrey owating

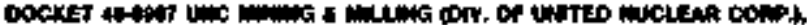

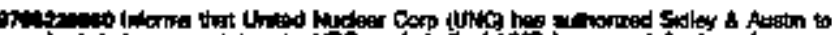

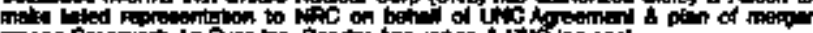

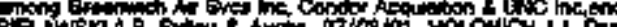

Drem Man

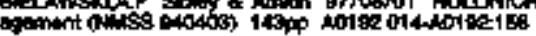

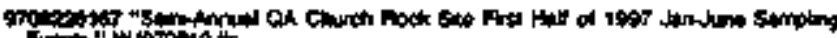

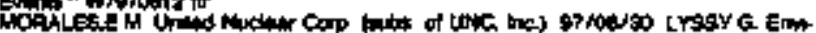
mond

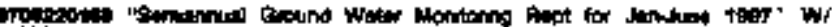
ond1

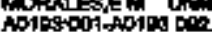

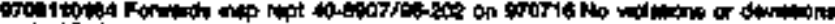

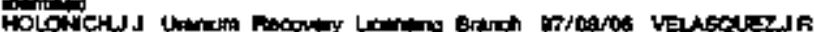

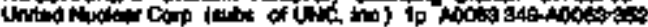

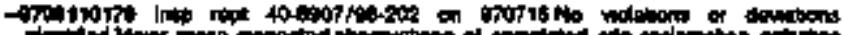

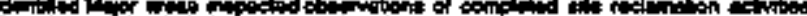

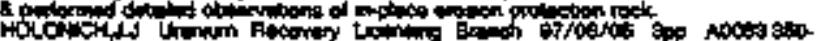
Hotom

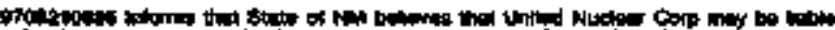

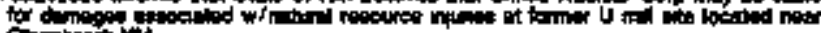

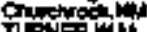

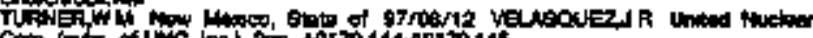

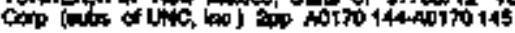

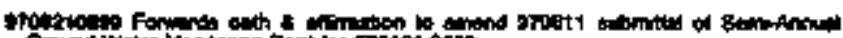

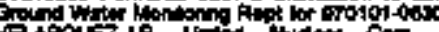

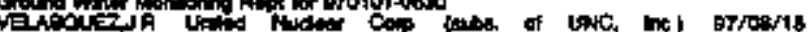

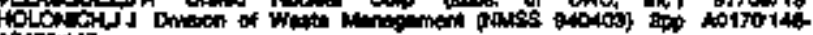

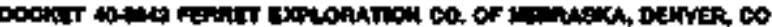

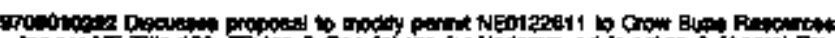
1.

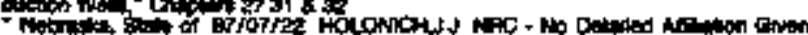

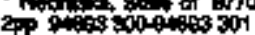

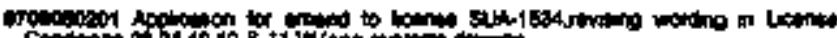

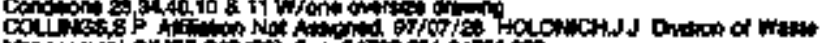

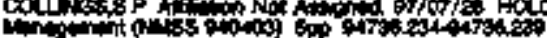

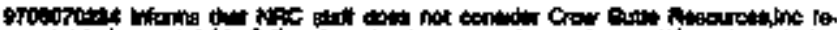

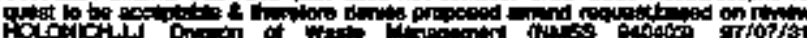

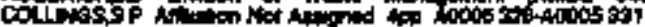

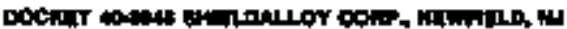

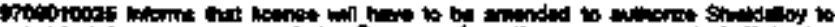

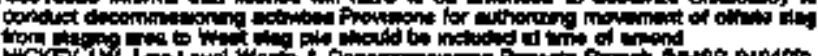

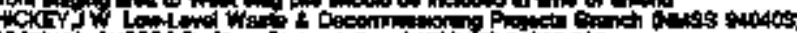

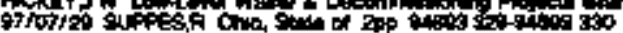

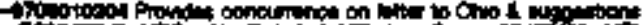

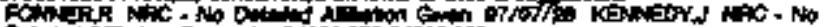

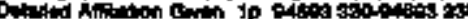

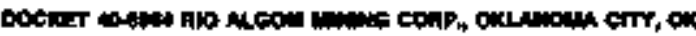

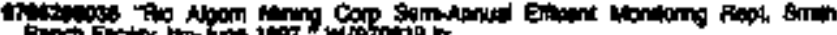

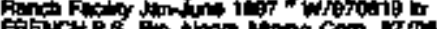

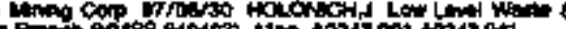

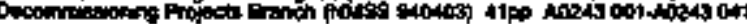

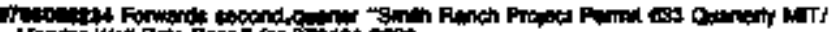

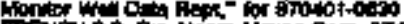

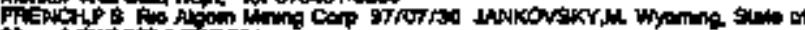

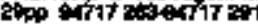

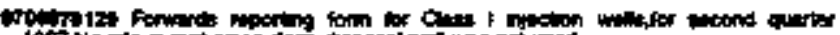

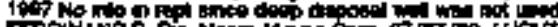

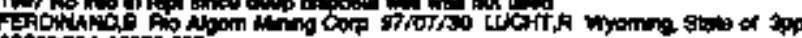

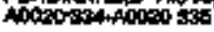

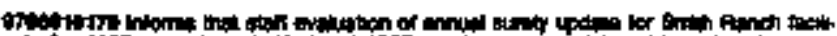

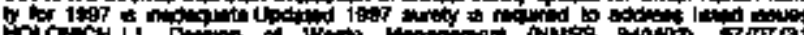

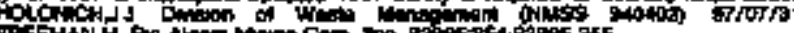

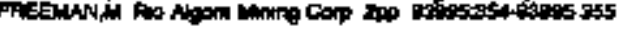

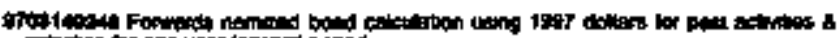

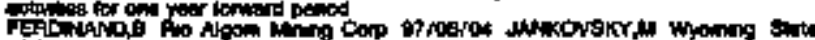

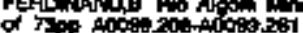

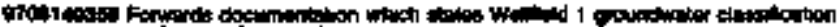

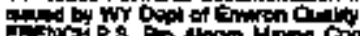

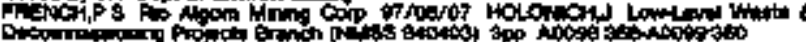




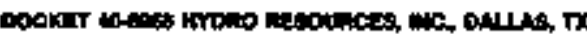

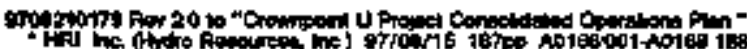

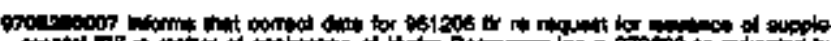

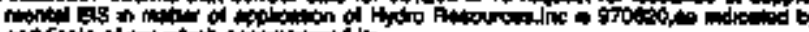

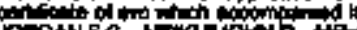

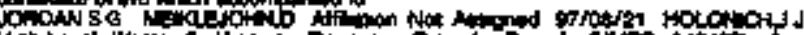

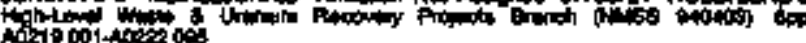

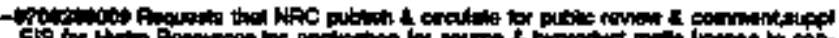

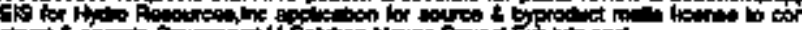

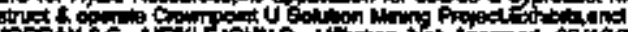

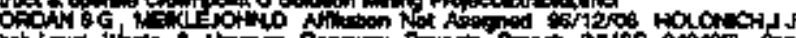

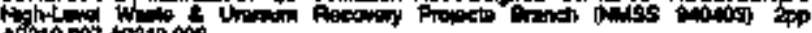

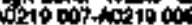

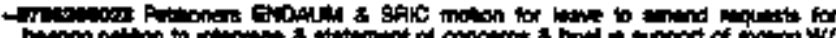

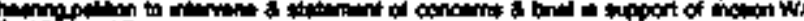
Corlifict of ste

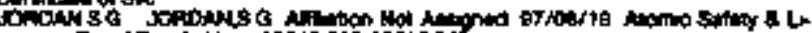

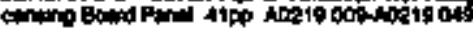

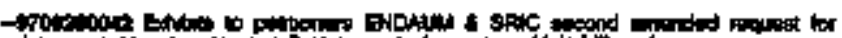

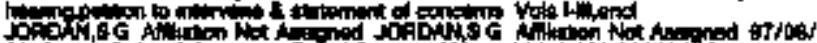

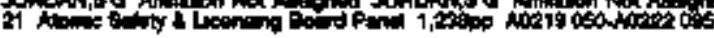

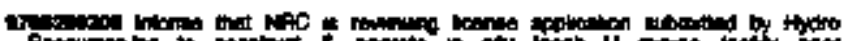

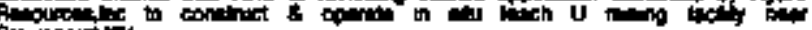

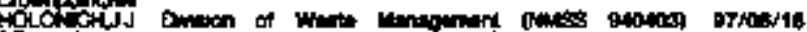

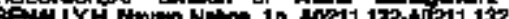

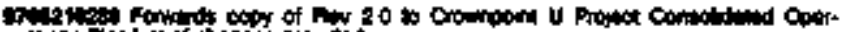

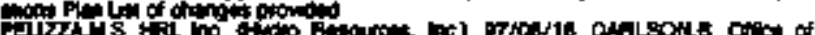

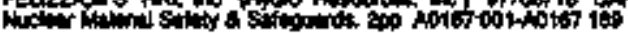

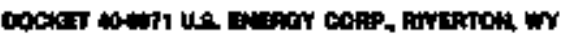

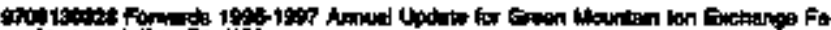

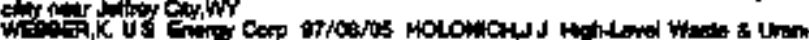

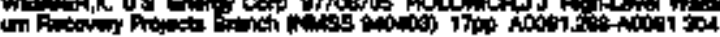

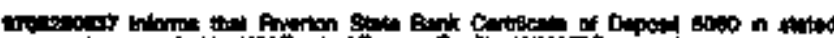

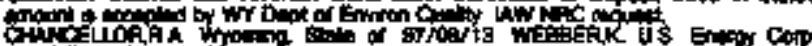

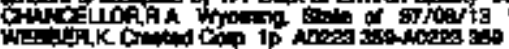

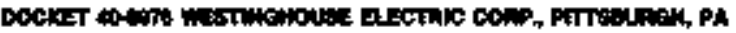

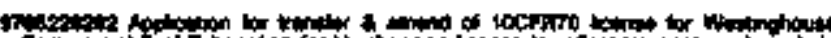

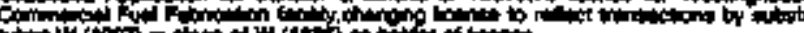

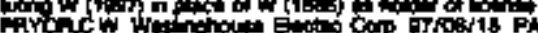

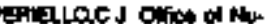

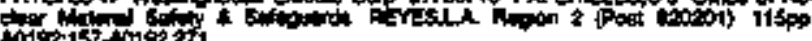

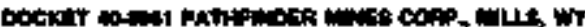

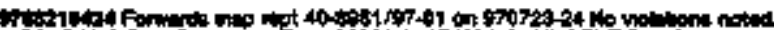

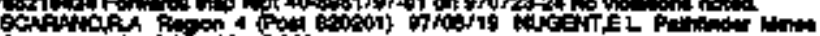

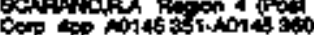

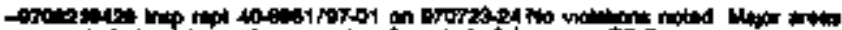

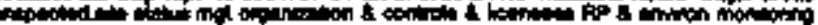

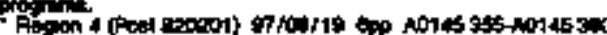

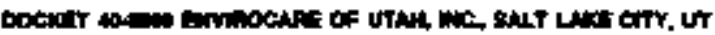

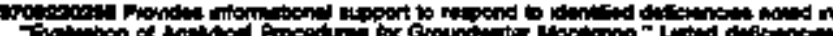

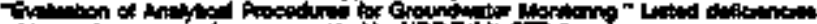

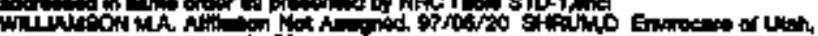

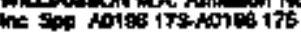

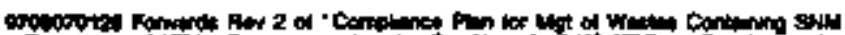

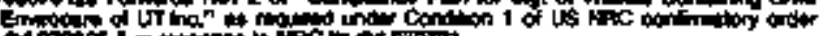

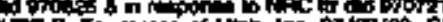

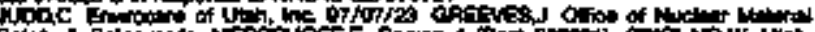

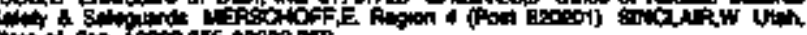

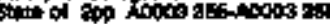

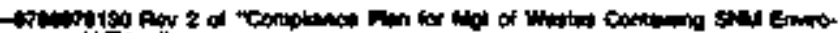

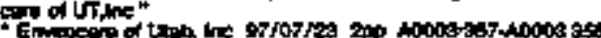

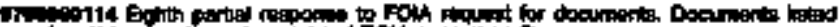

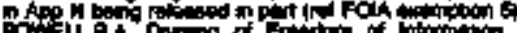

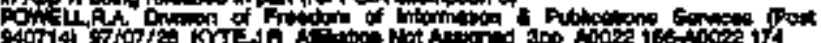

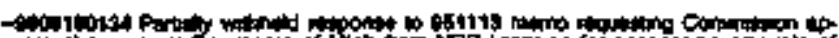

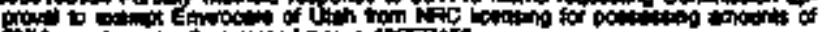

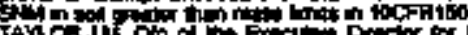

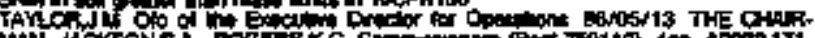

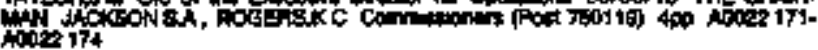

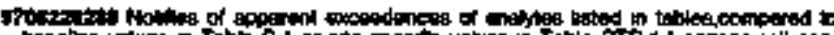

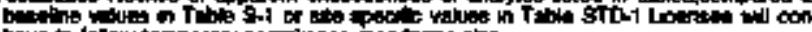

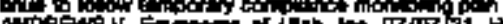

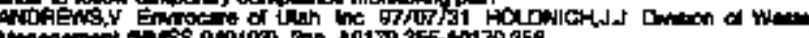

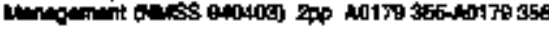

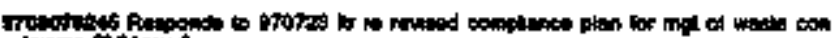

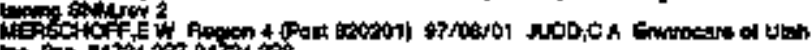

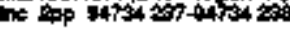

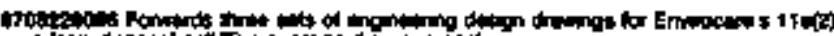

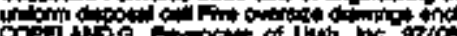

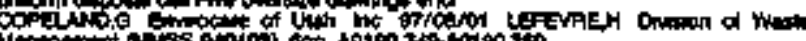

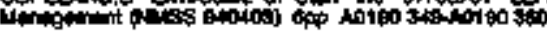

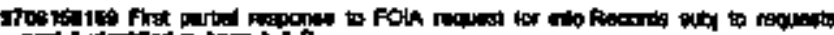

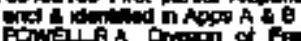

Ponf

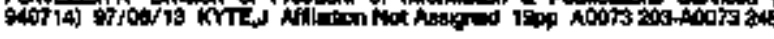

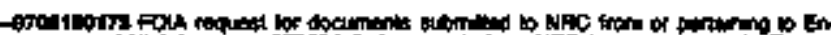

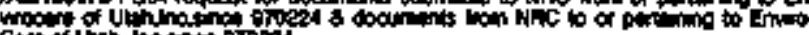

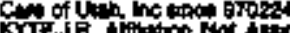

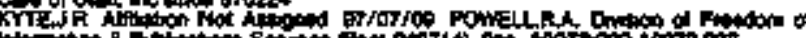

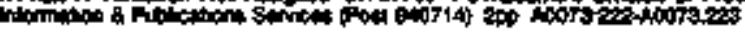

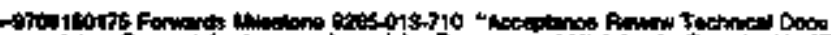

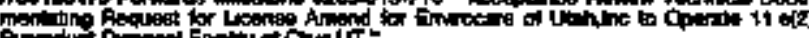

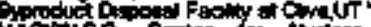

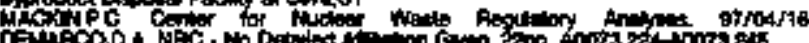

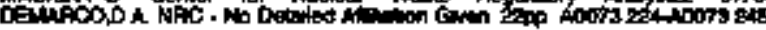

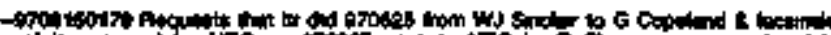

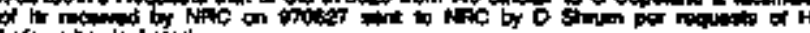

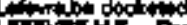

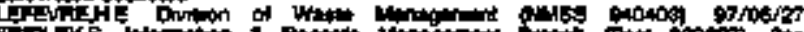

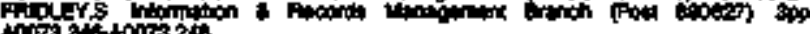

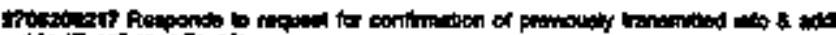

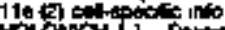

F

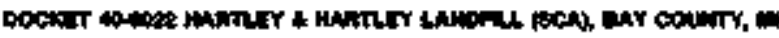

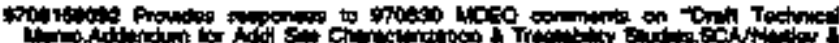

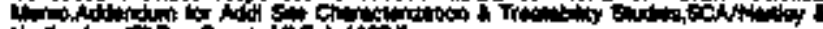

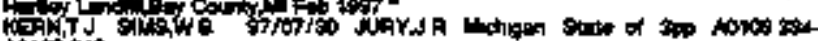

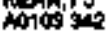

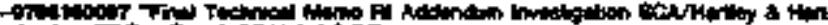

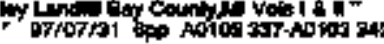

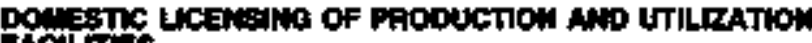

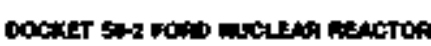

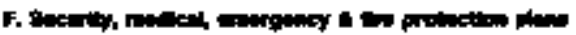

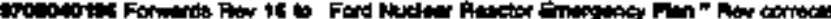

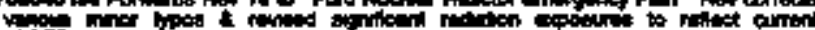

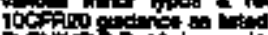

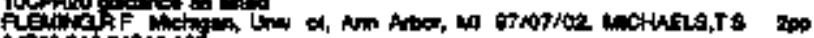
arto to

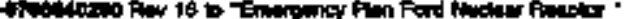

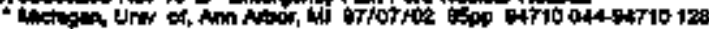

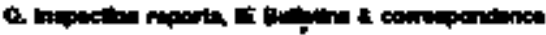

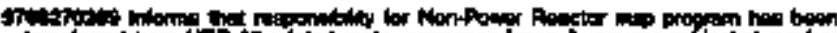

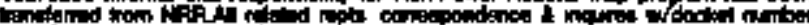

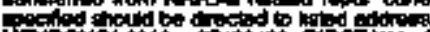

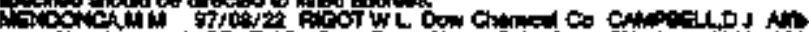

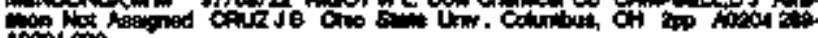

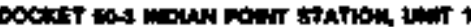

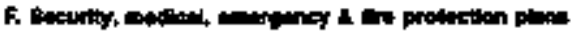

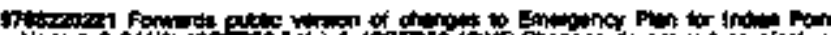

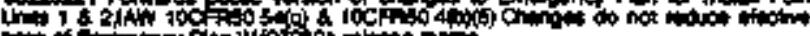

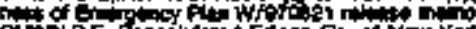

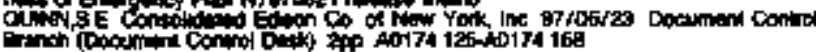

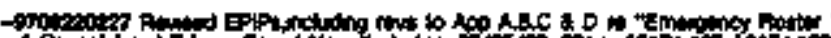
- Contitided Edeon co of 


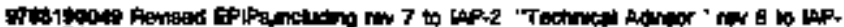

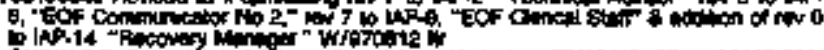

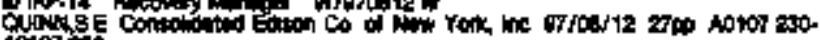
norot 200

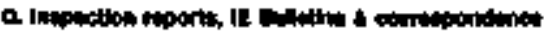

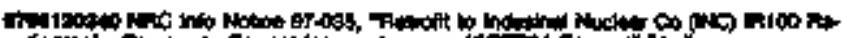

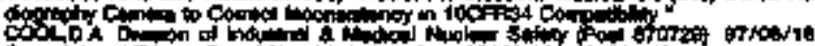

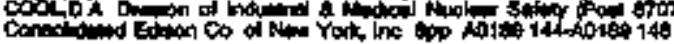

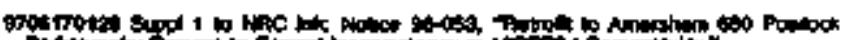

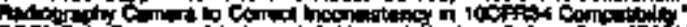

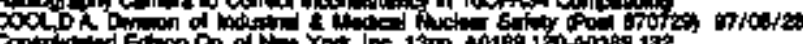

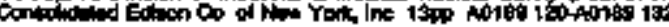

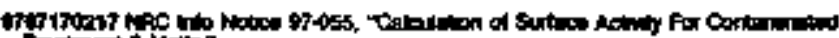

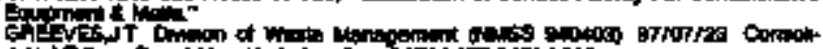

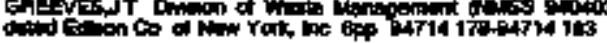

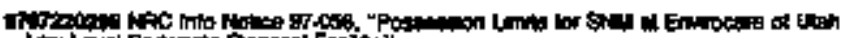

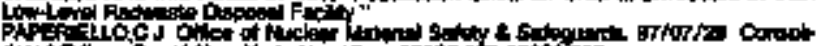

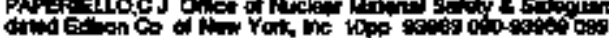

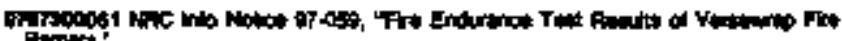

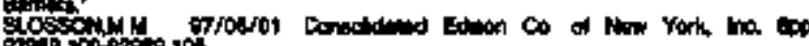

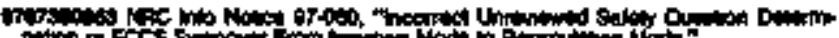
gLoos of

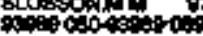

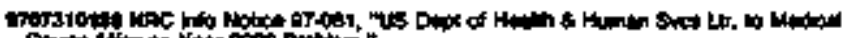

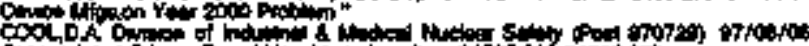

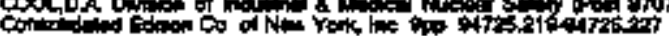

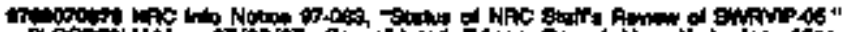

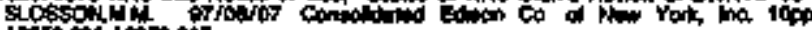

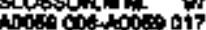

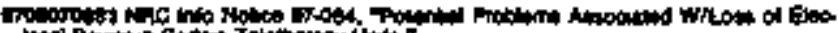

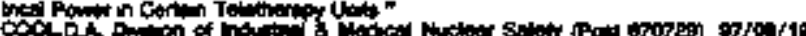

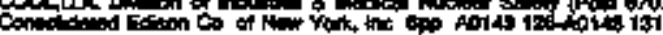

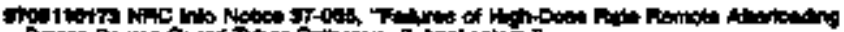

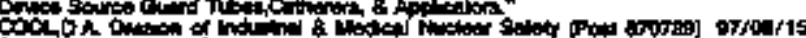

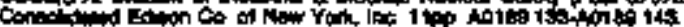

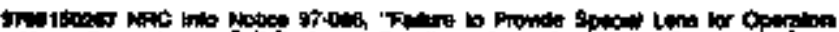

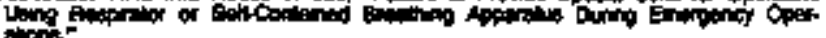

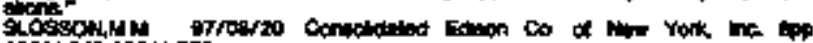

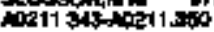

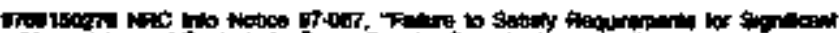

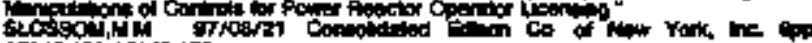

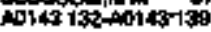

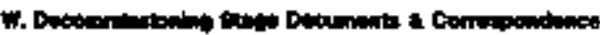

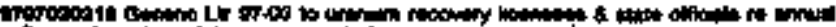

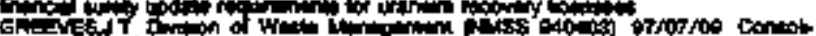

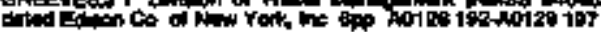

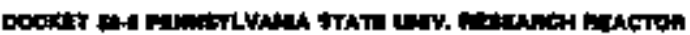

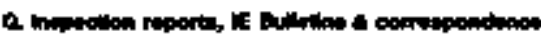

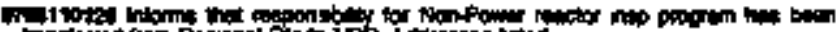

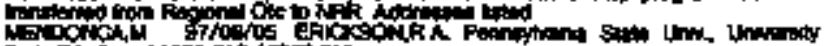

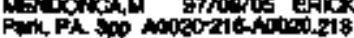

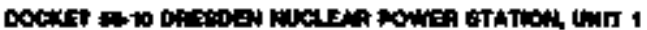

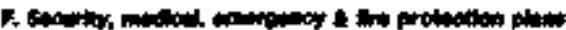

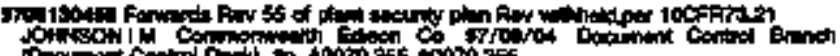

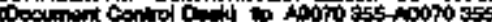

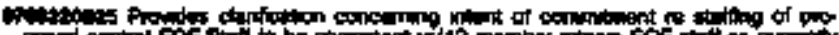

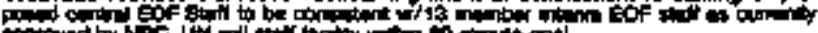

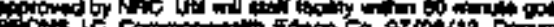

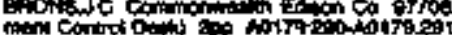

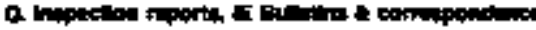

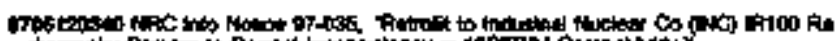

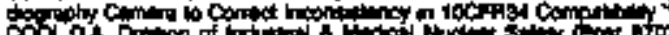

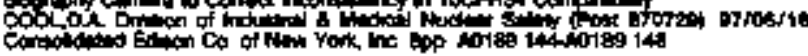

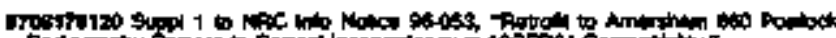

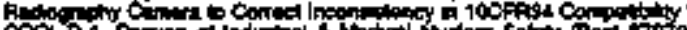

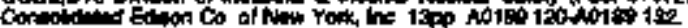

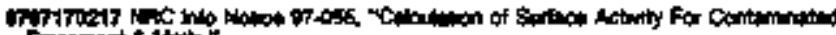

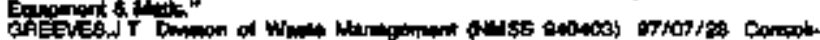

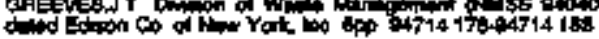

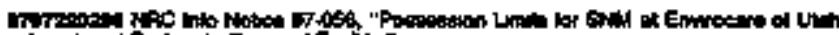
PAf B

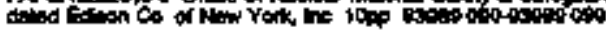

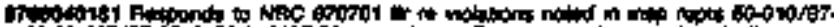

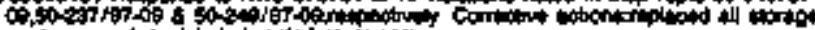

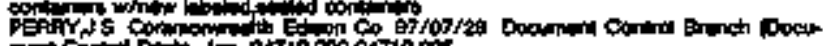

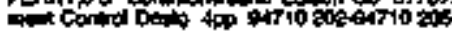

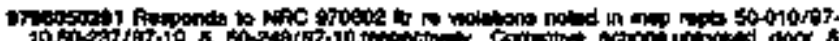
10 B0\% J

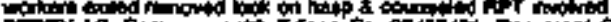

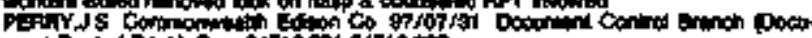

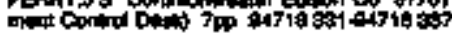

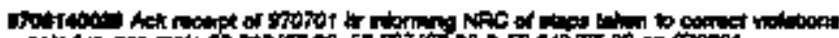

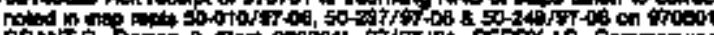

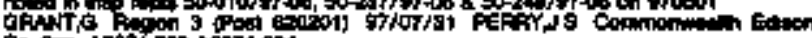

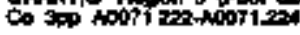

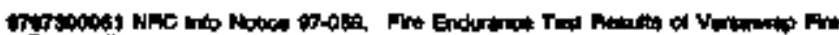

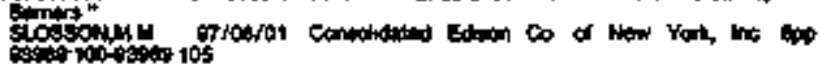

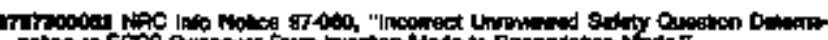

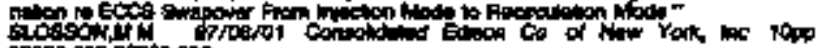

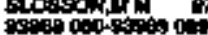

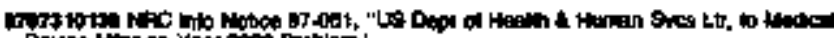

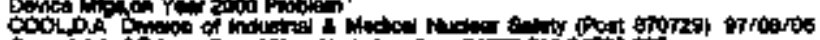

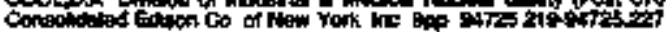

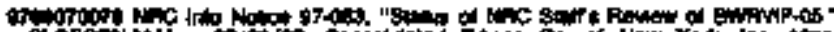

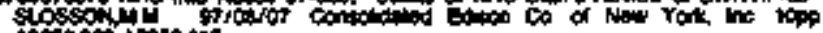

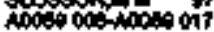

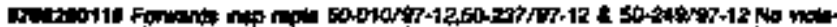

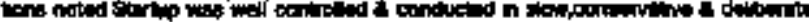

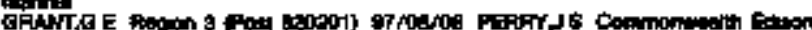

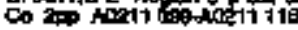

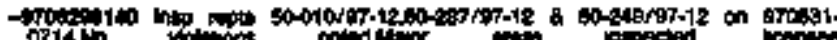
Sowations 3 (1)

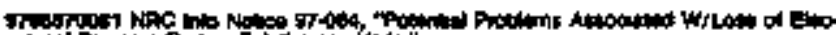

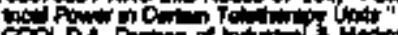

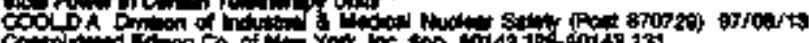

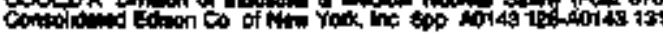

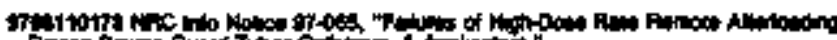

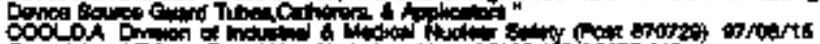

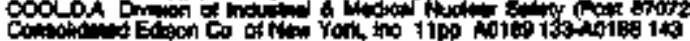

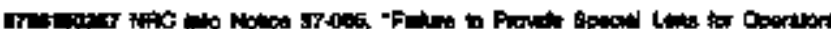

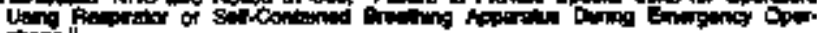

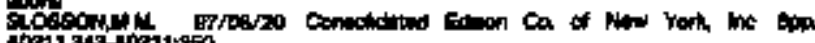

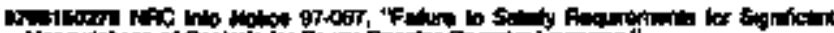

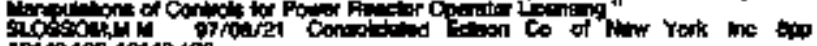
A.

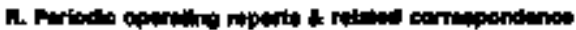

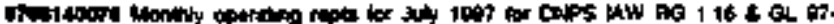

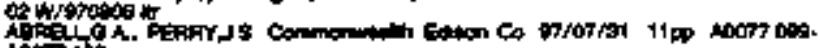
ADontion

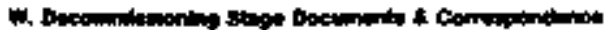

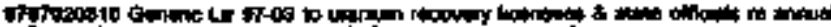

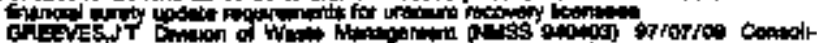

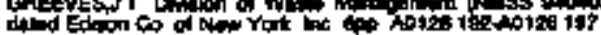




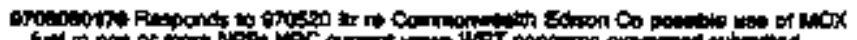

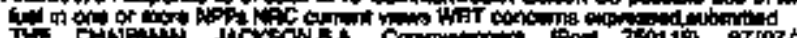

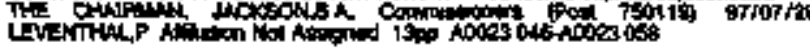

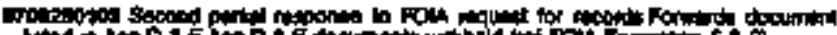

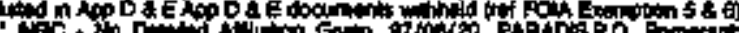

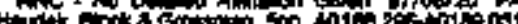

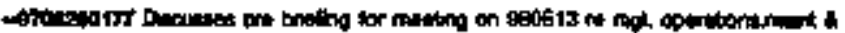

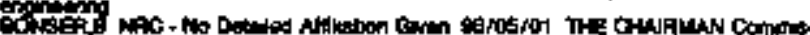
pones ford J

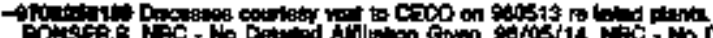

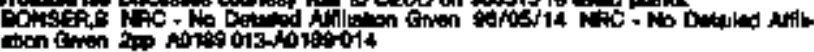

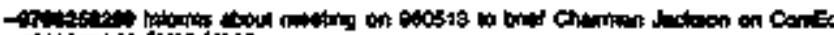

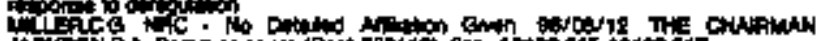

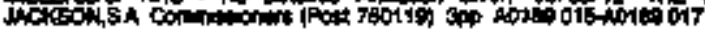

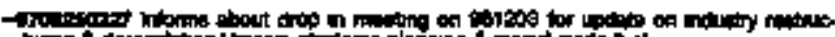

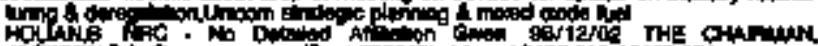

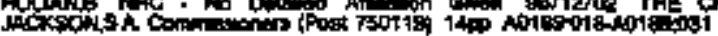

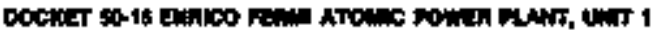

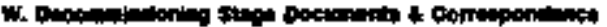

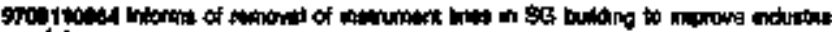

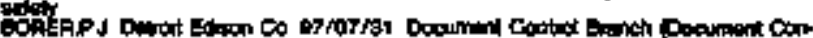

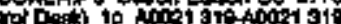

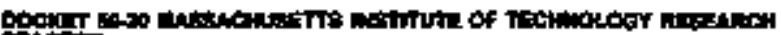
iftion

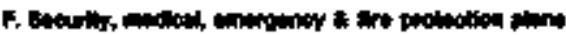

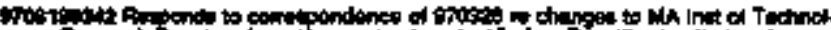

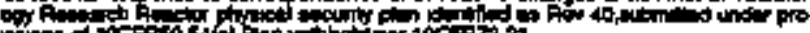

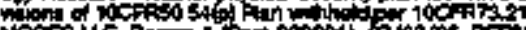

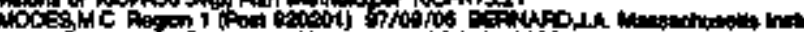

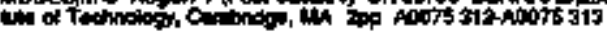

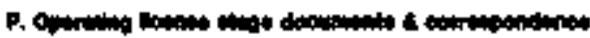

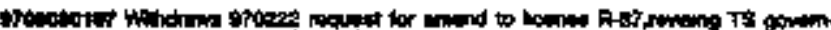

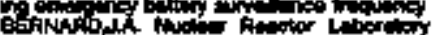

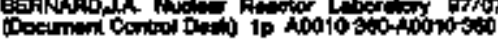

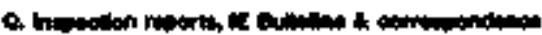

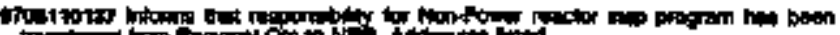

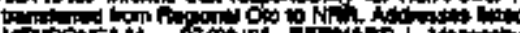

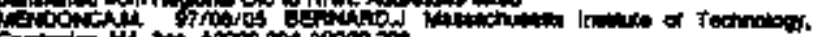

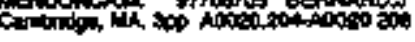

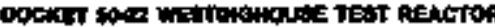

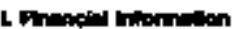

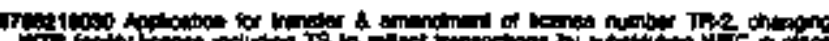

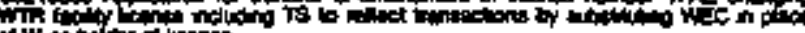

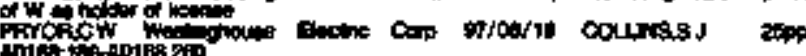

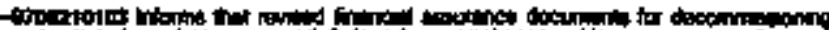

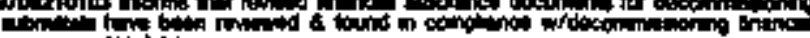

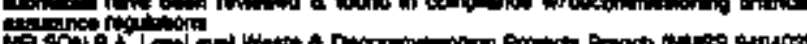

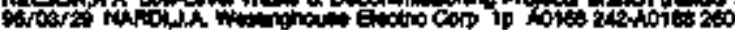

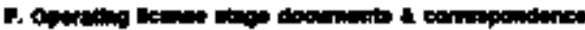

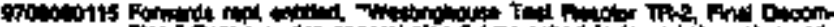

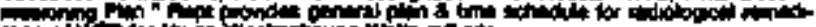

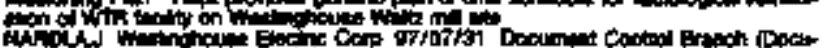

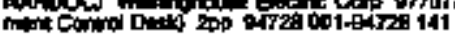

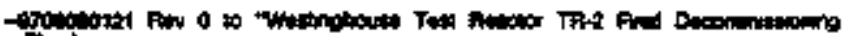<smiles>[Te]</smiles>

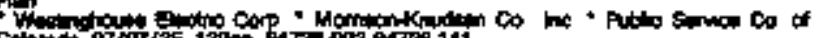

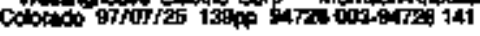

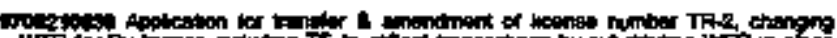

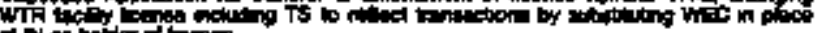

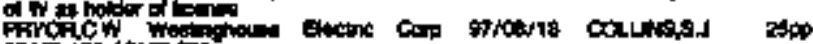

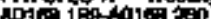

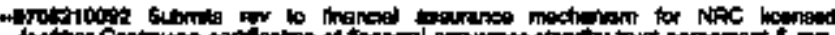

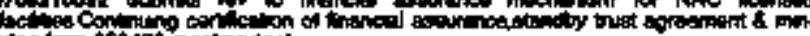

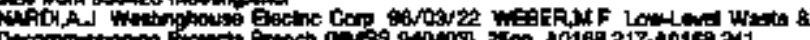

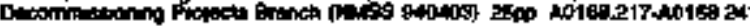

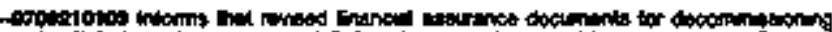

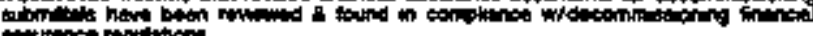

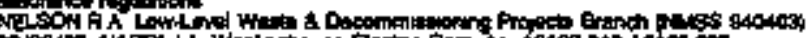

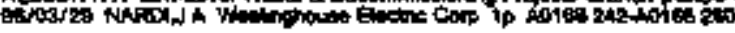

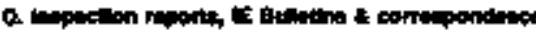

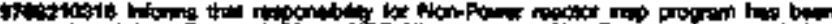

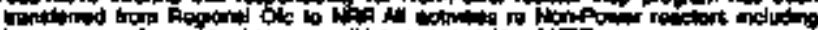

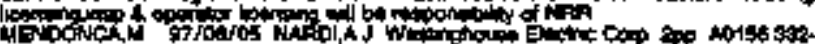
10150 39

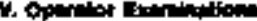

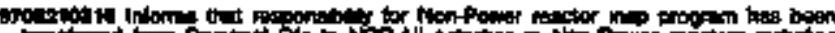

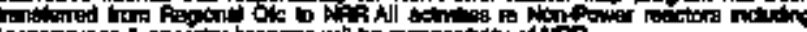

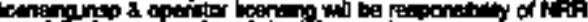

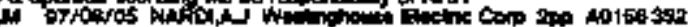

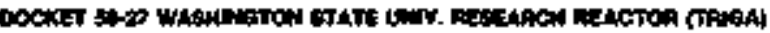

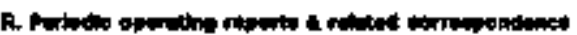

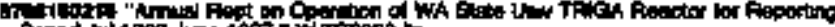

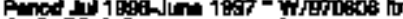

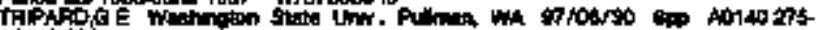
AOMW

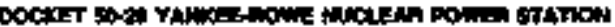

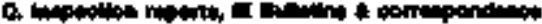

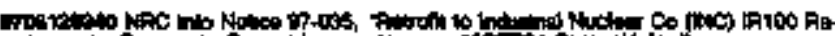

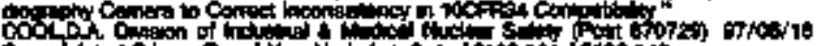

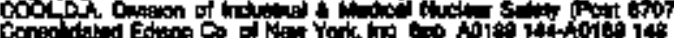

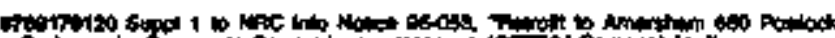

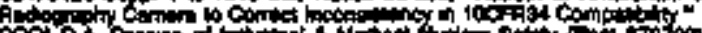

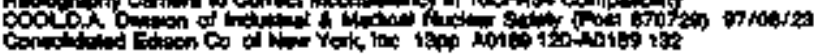

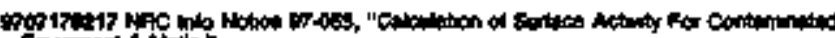

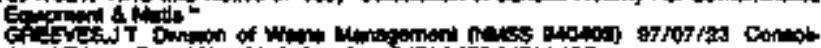

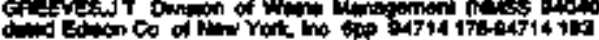

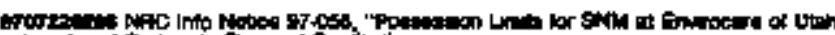

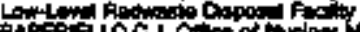

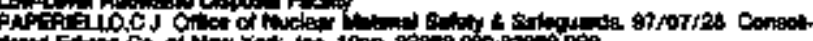

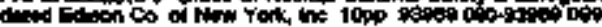

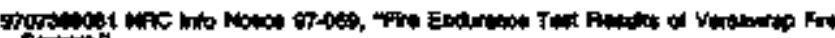
fichos

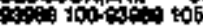

wersond slogn $3 x^{3}$

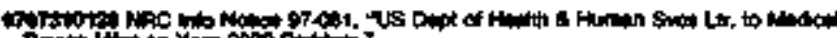

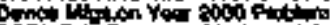

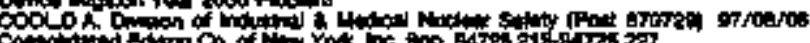

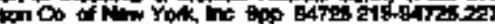

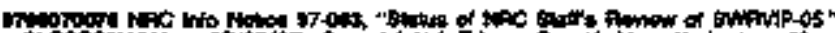

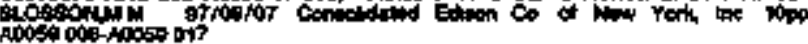

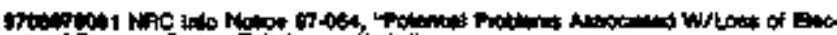

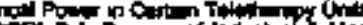

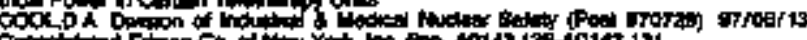

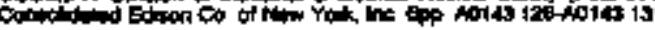

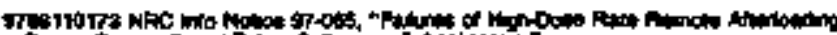

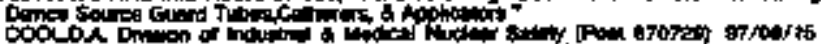

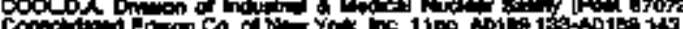

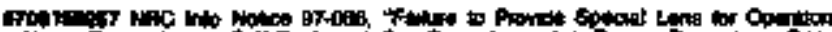

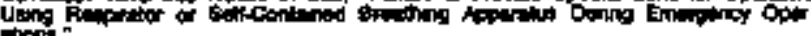

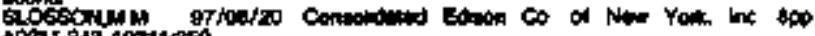

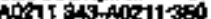




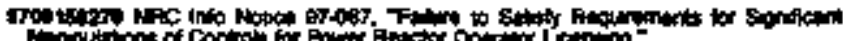

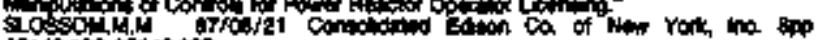

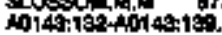

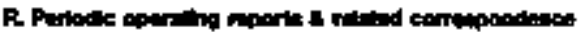

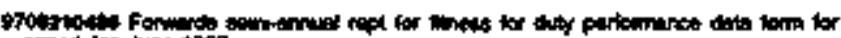

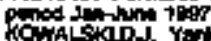

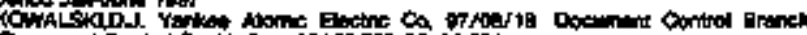

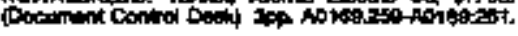

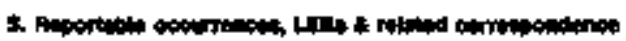

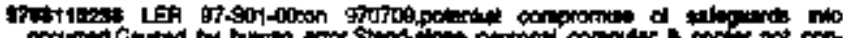

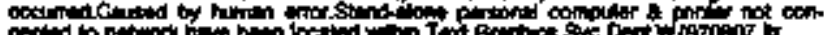

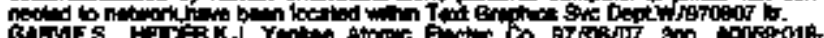

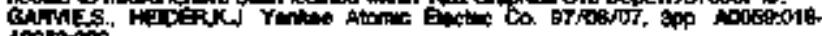
antiotion

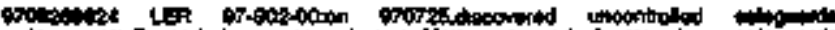

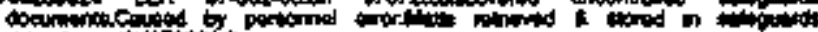

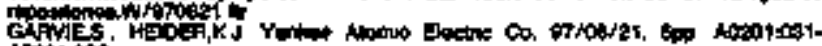
A02015006.

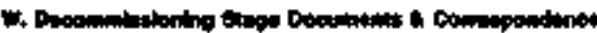

काgre:

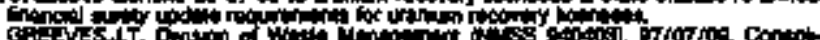

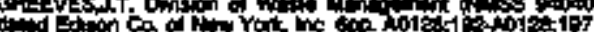

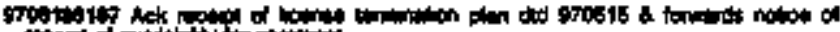

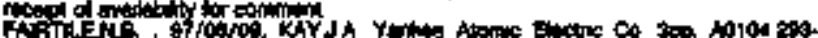
mitop

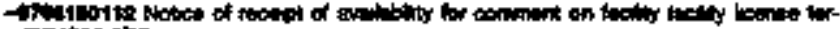

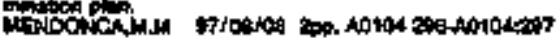

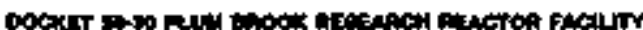

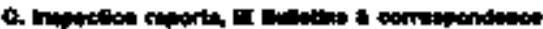

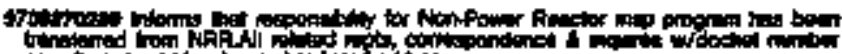

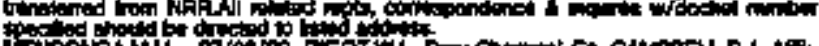

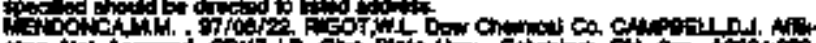
ton

occker bast nosith wimeg cosp.

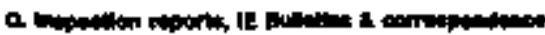

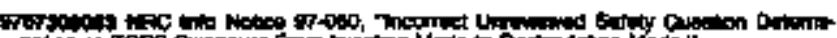

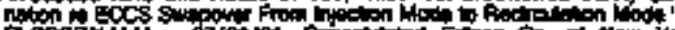

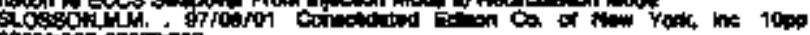

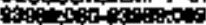

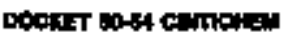

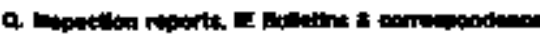

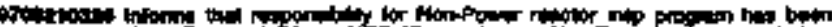

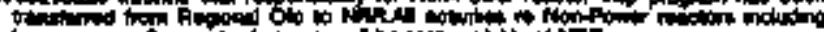

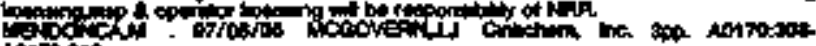

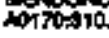

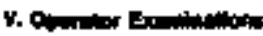

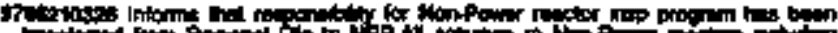
1.

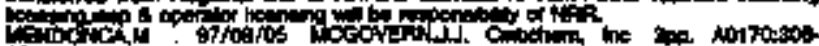
Aotrosio

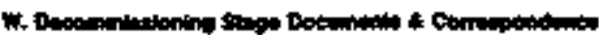

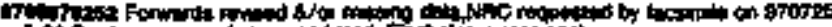

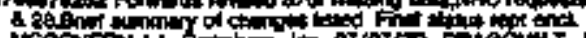

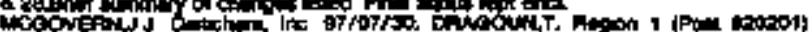

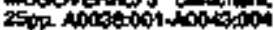

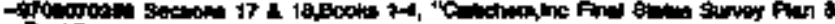
Prite

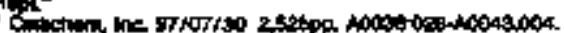

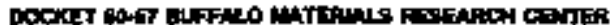

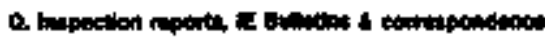

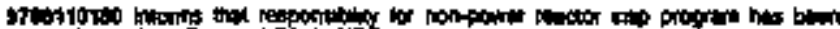

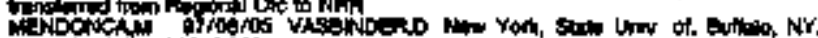

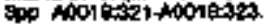

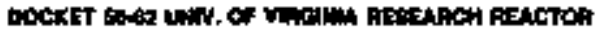

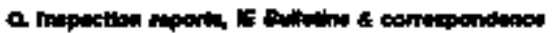

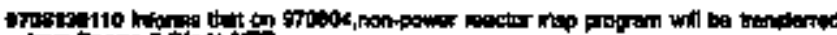

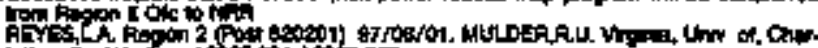

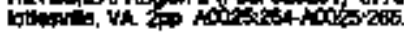

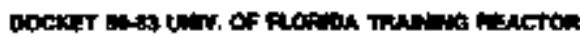

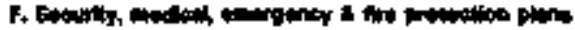

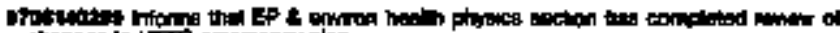

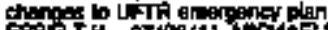

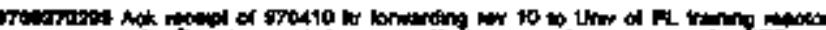

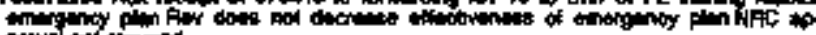

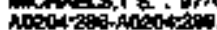

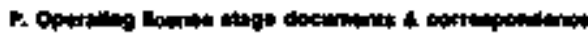

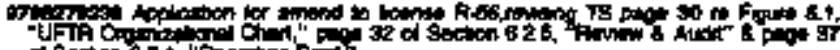

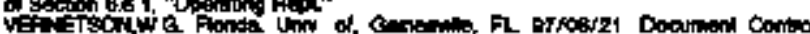

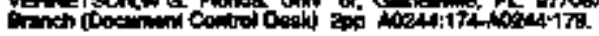

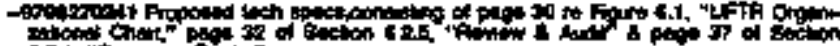

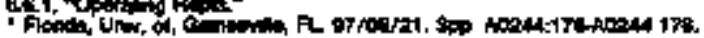

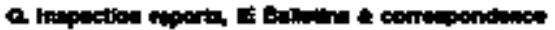

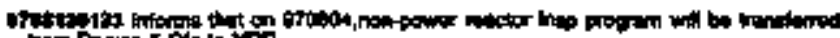

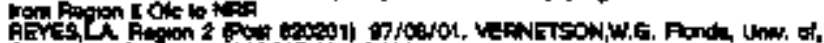

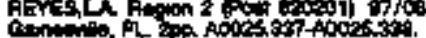

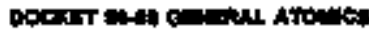

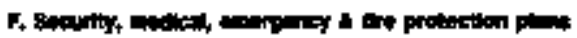

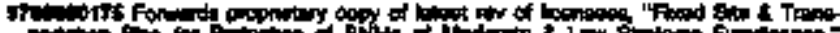

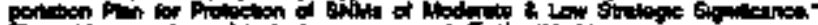

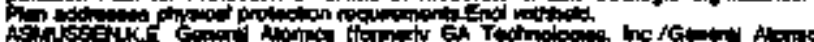

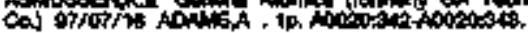

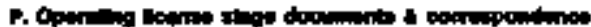

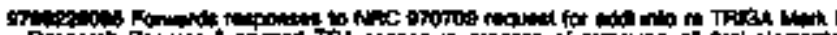

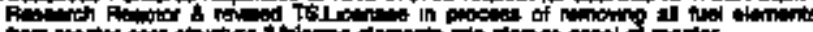

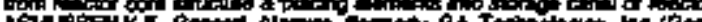

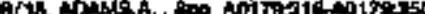

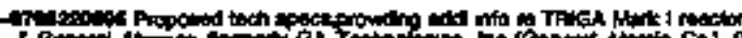

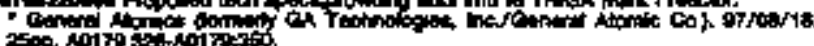

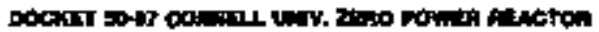

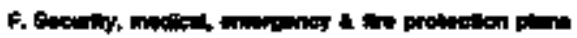

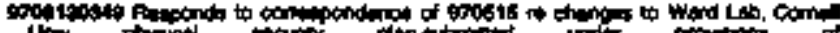
Pock

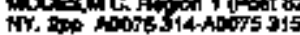

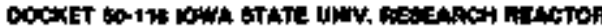




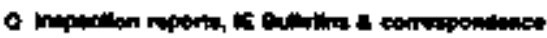

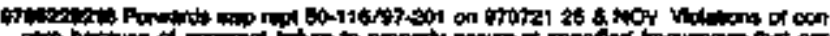

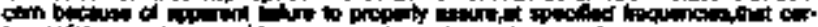

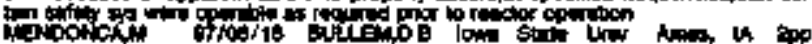

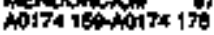

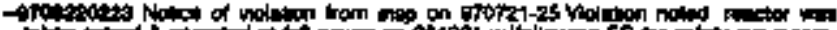

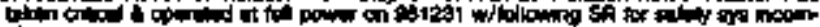

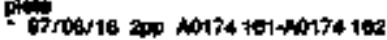

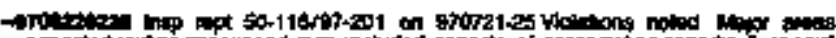

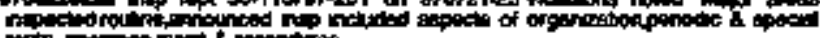

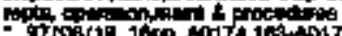

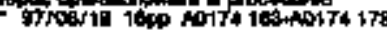

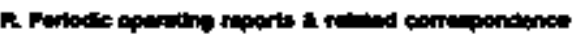

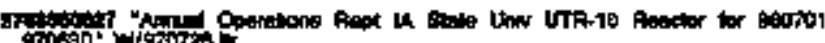

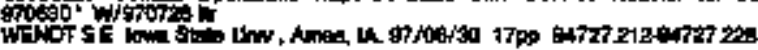

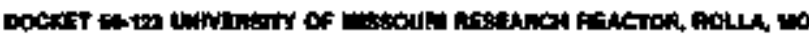

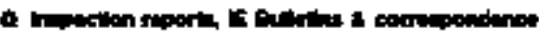

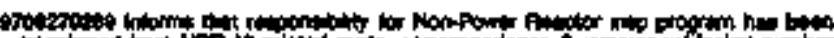

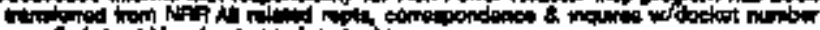

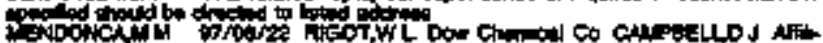

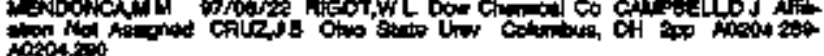

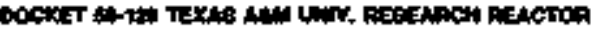

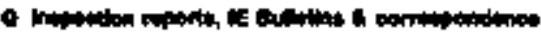

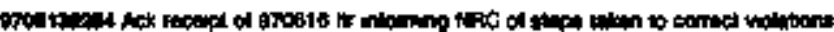

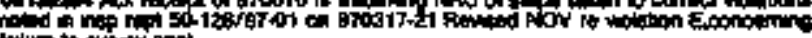
onction

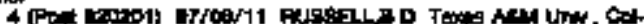

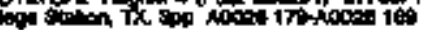

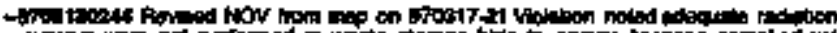

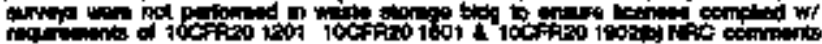
risingent

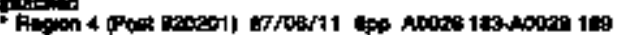

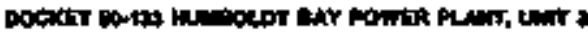

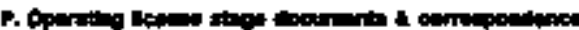

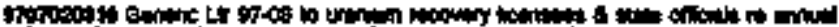

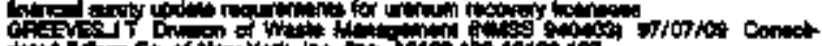

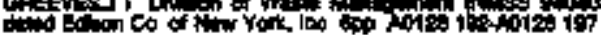

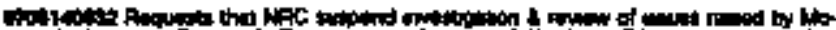

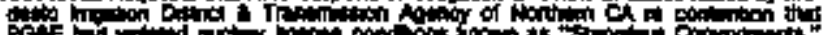

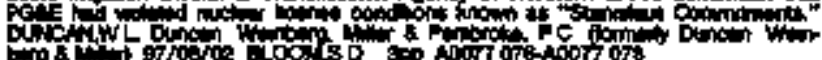

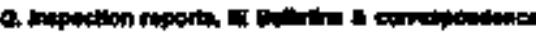

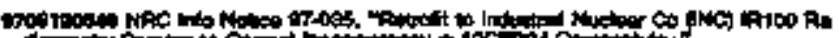

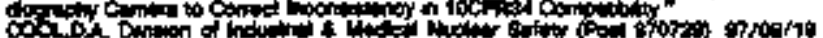

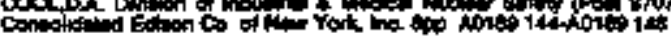

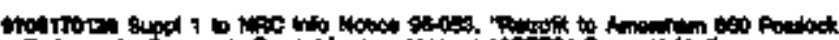

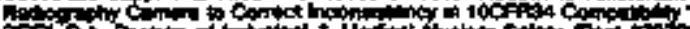

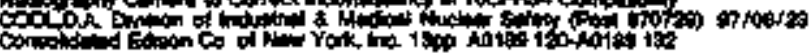

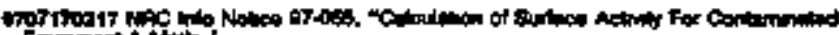

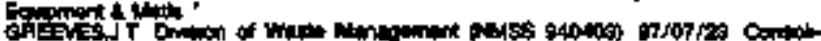

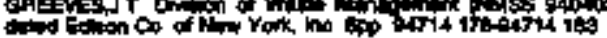

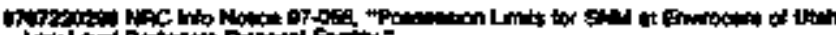

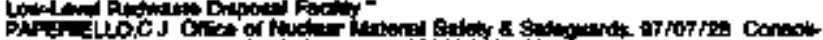

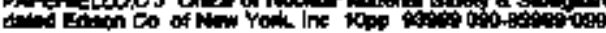

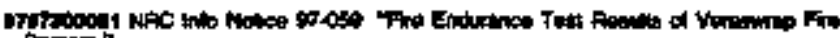

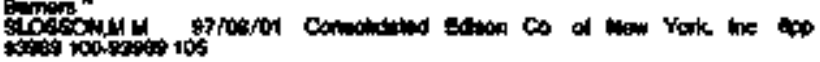

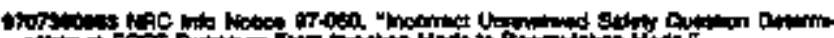

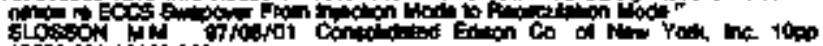
sion

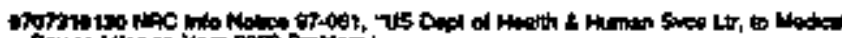

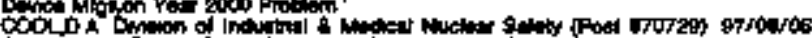

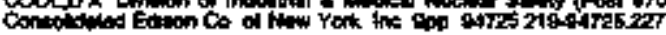

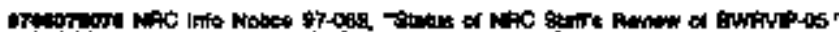

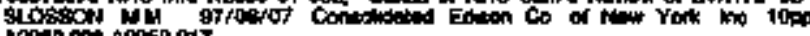
nowe cotations on

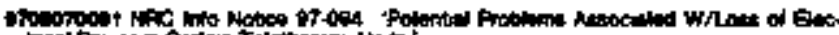

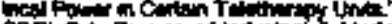

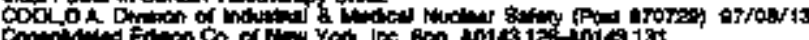

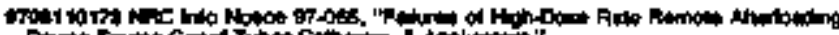

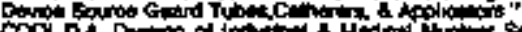

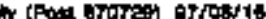

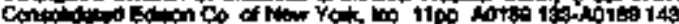

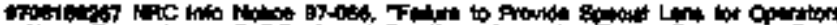

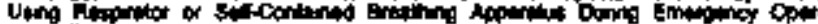

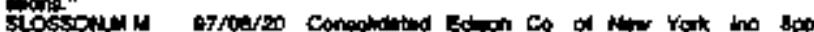

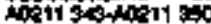

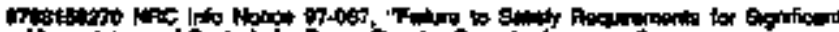

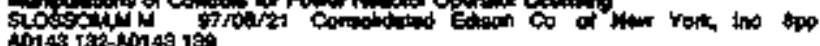

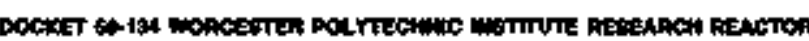

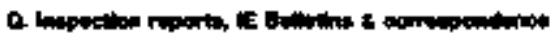

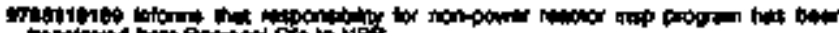

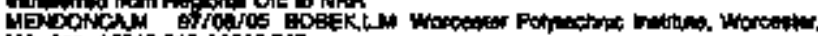

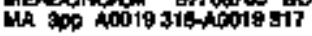

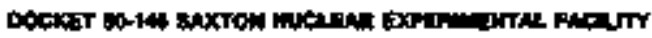

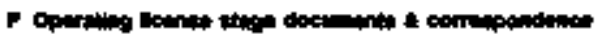

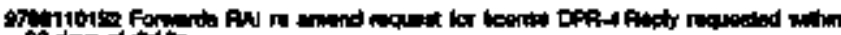

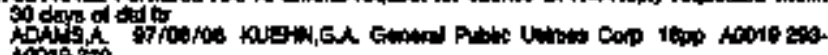
tots 30

Q70

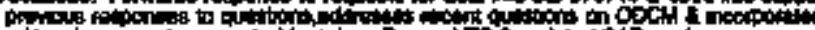
Kúj

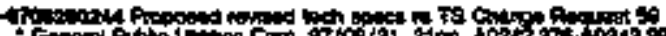

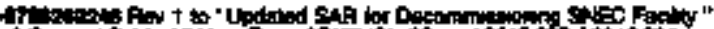

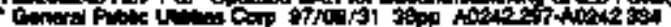

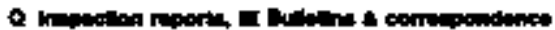

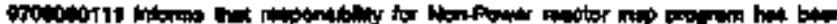

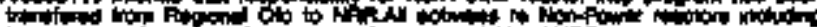

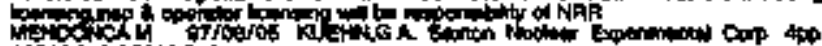
10012 Q1340012 018

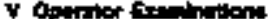

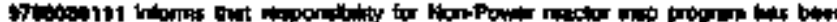

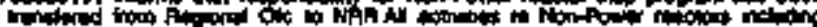

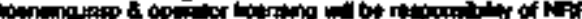

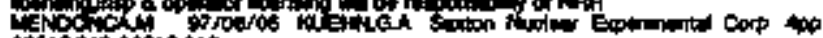
A0012013010012 016

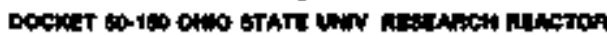

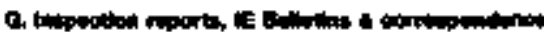

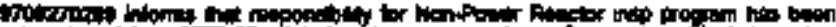

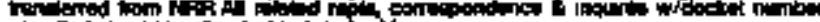

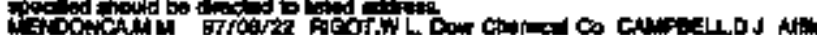

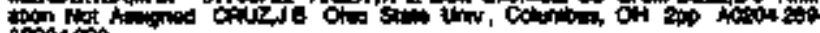

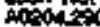

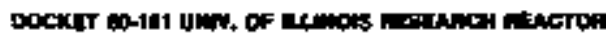

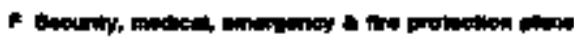

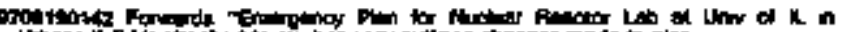

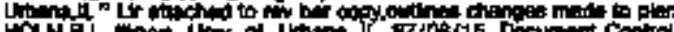

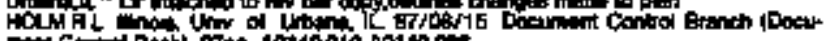

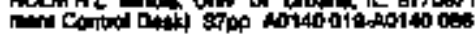




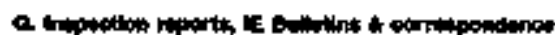

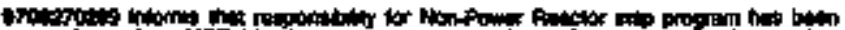

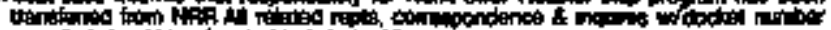

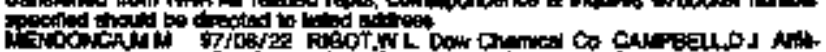

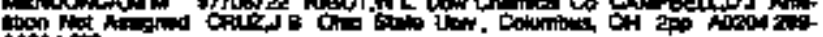
$\cos 040$

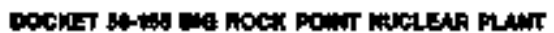

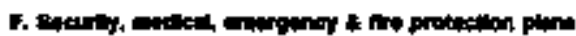

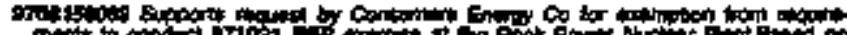

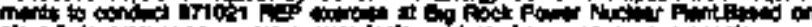

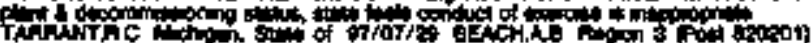

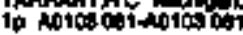

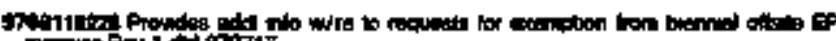

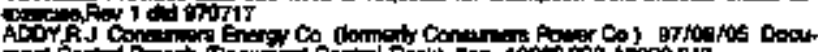

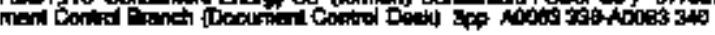

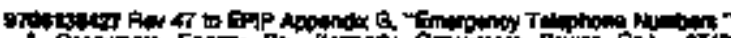

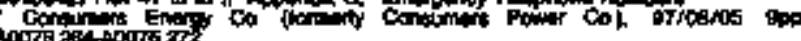

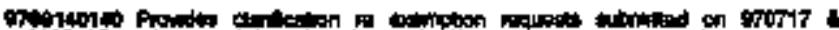

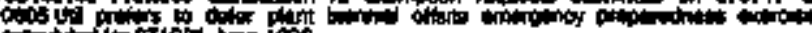

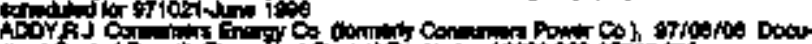

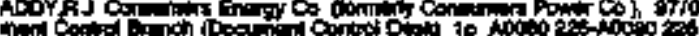

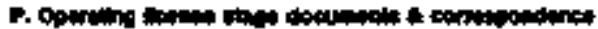

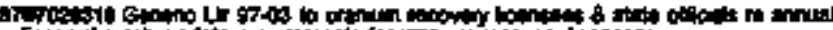

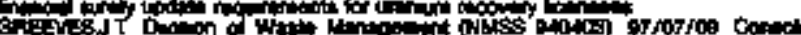

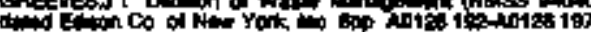

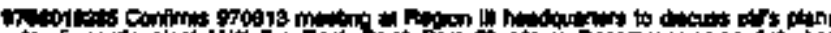

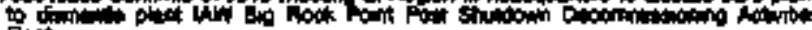

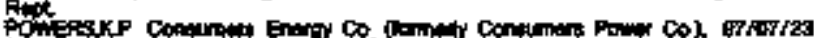

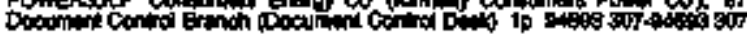

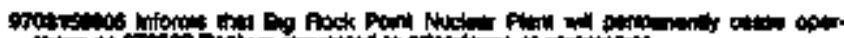
otos of of

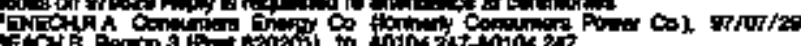

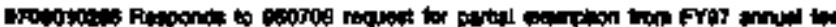

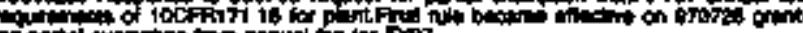

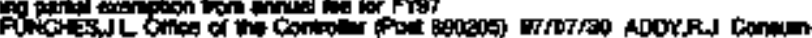

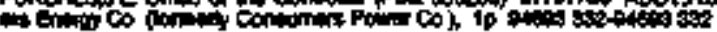

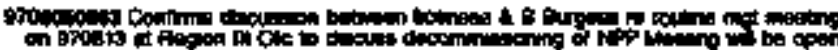
oping

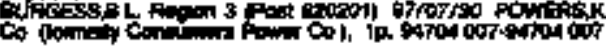

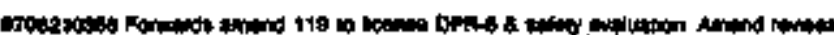

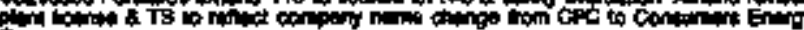

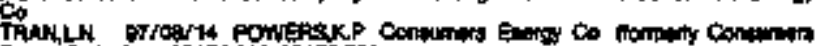

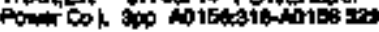

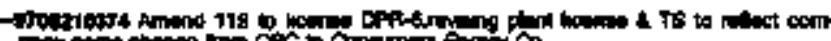

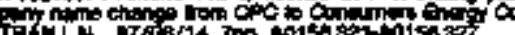

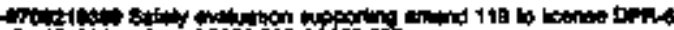

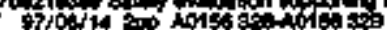

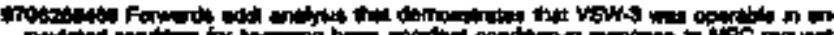

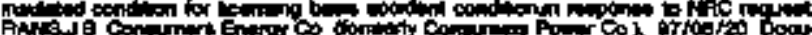

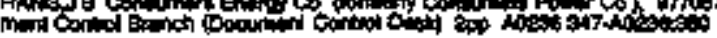

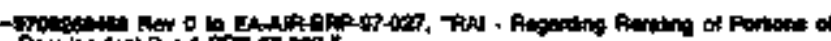

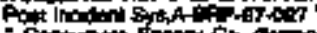

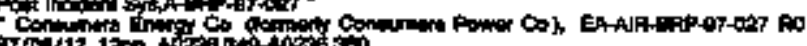

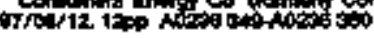

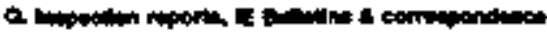

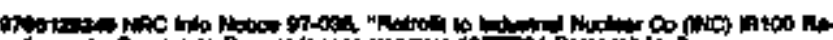

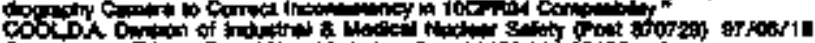

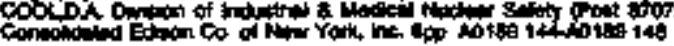

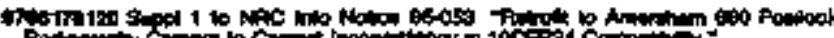

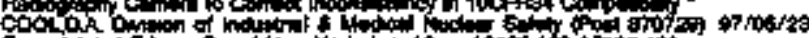

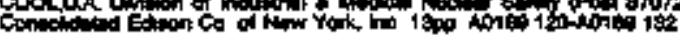

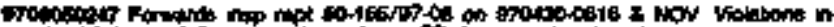

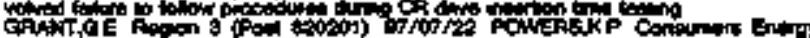

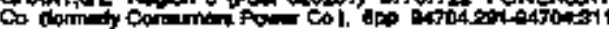

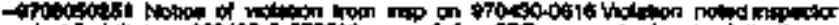

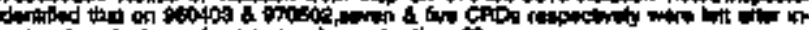

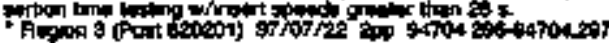

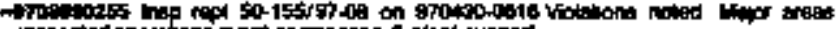

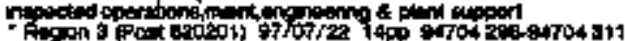

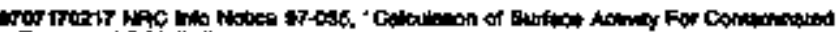

CQPi

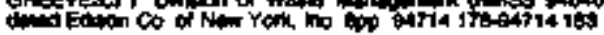

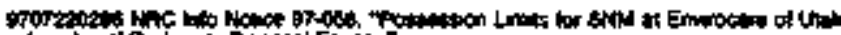

PA

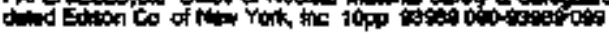

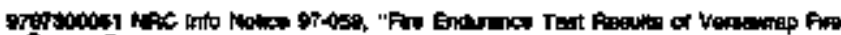

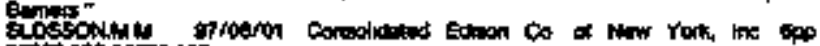

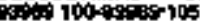

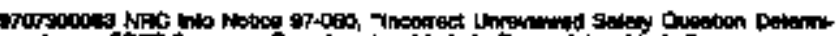

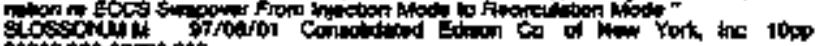

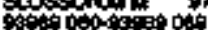

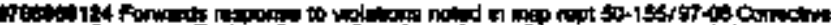

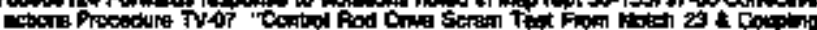

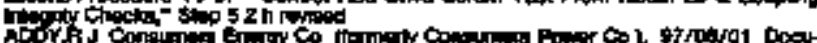

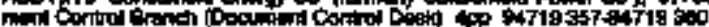

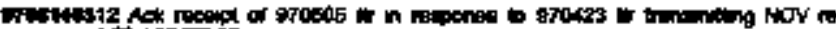

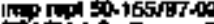

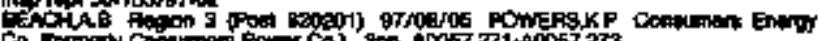

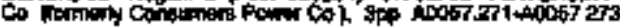

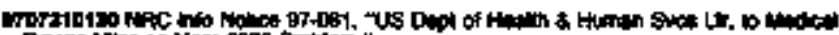

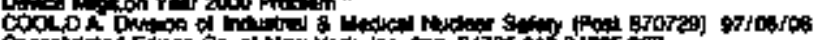

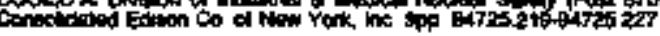

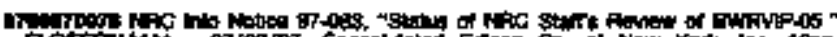

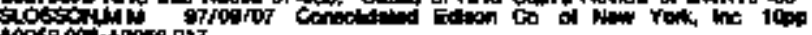

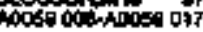

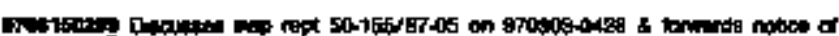

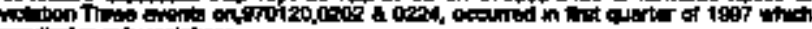

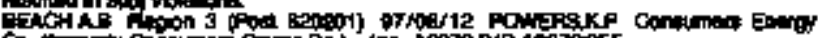

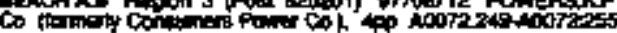

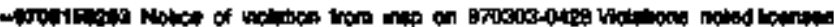

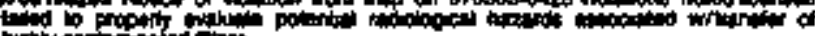

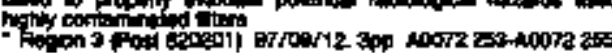

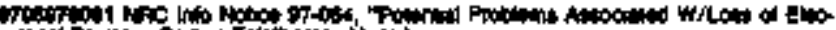

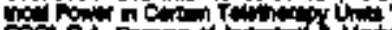

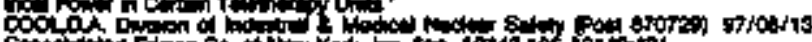

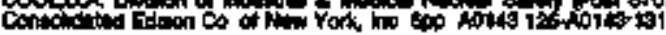

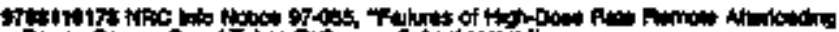

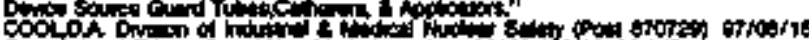

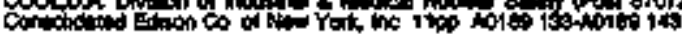

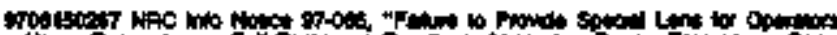

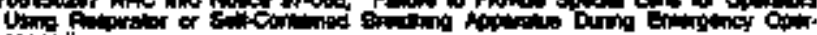

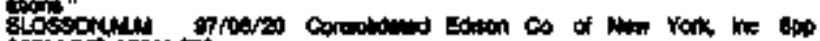
A021 1 sक

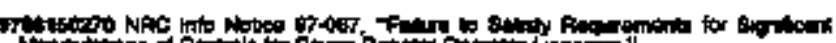

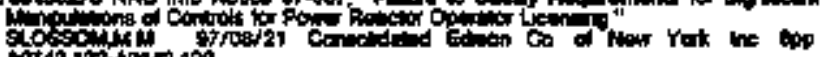

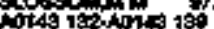

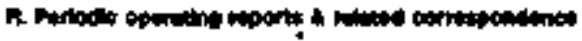

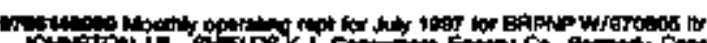

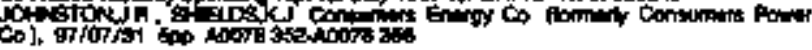

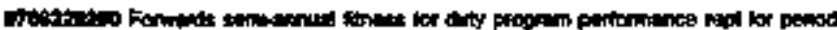

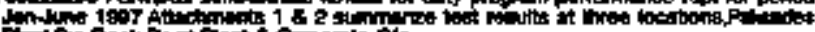

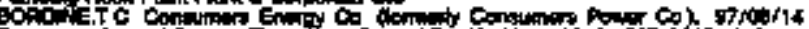

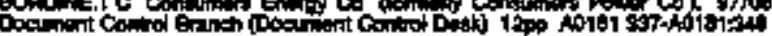

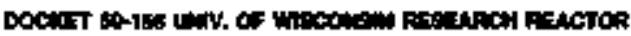

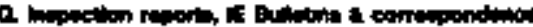

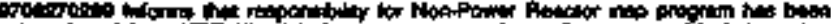

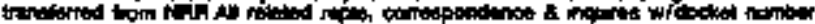

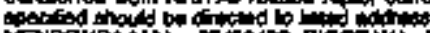

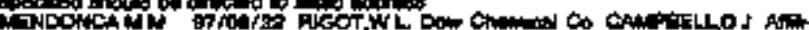

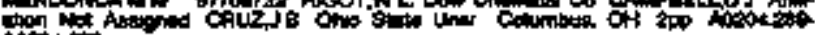
tom tom 


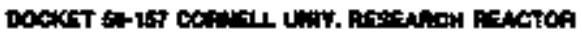

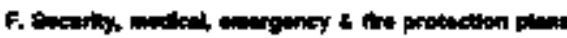

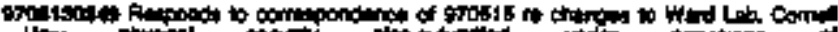

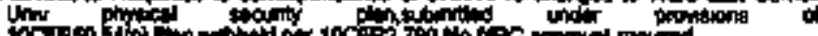

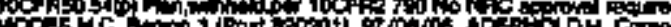

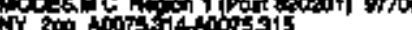

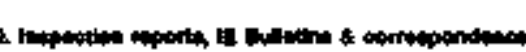

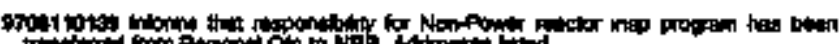

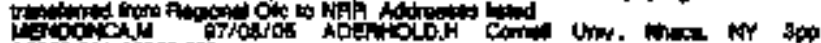
noto at 1000020

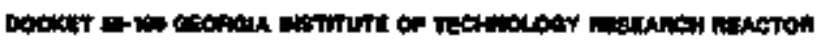

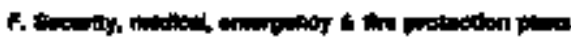

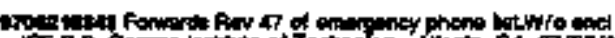

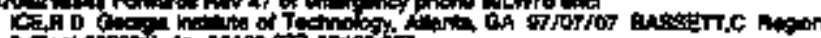

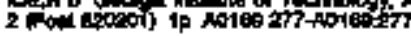

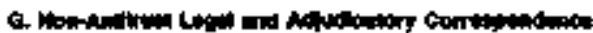

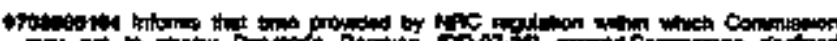

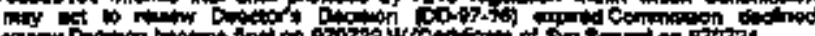

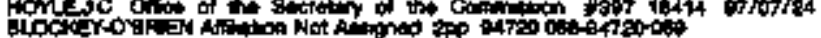

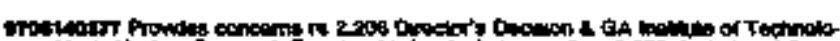

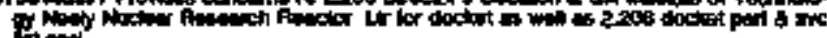

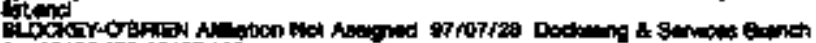
ip AO102 100

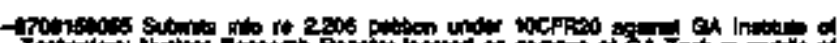

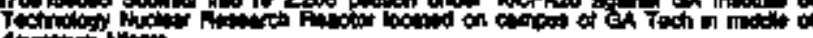

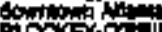

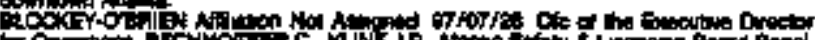

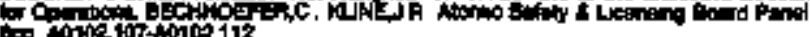

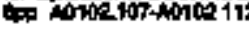

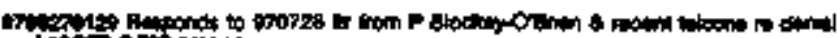

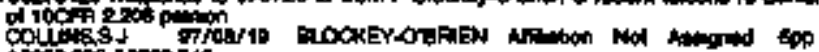

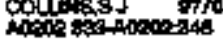

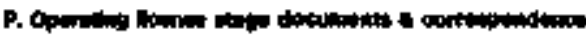

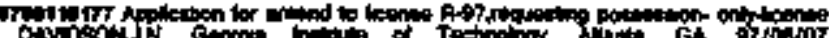

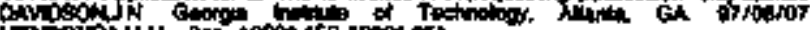

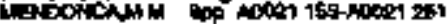

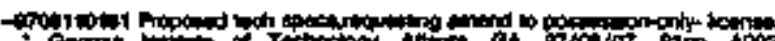

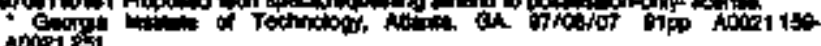

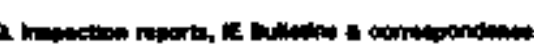

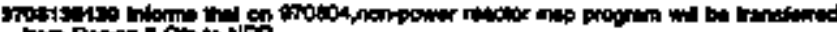

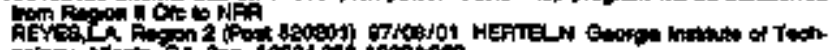

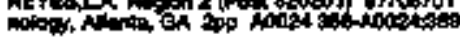

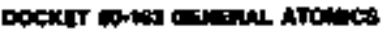

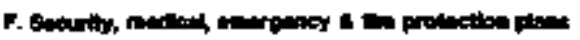

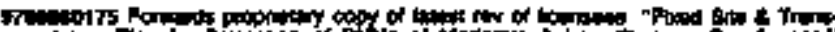

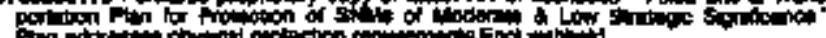

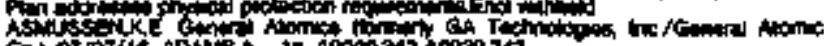

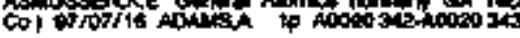

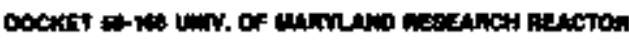

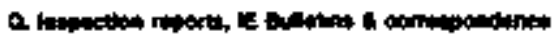

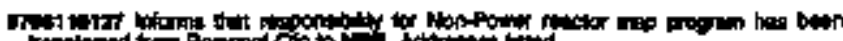

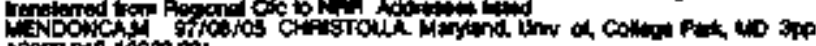

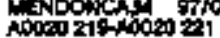

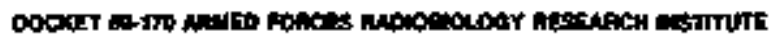

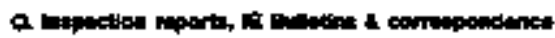

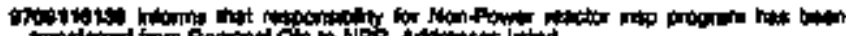

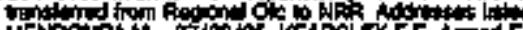

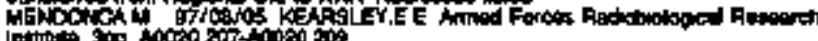

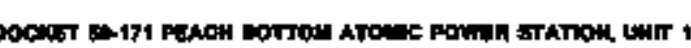

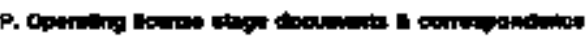

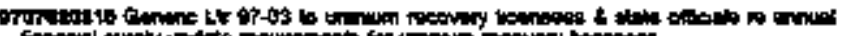

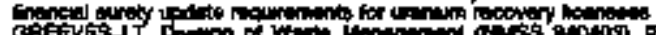

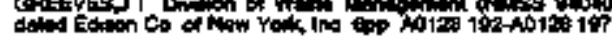

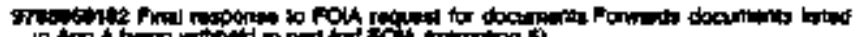

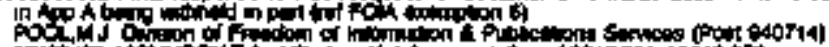

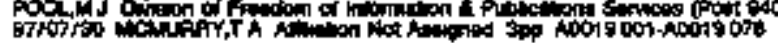

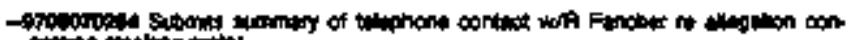

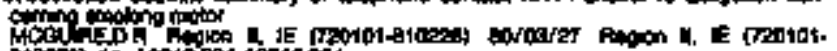

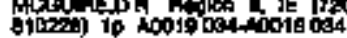

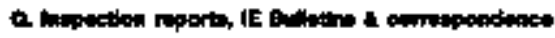

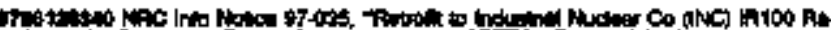

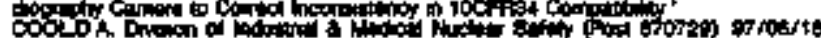

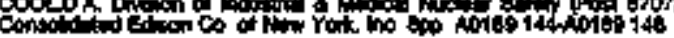

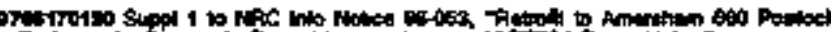

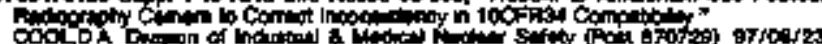
con

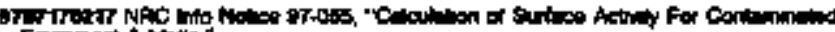

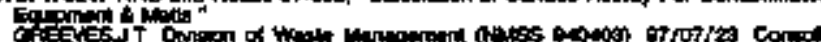

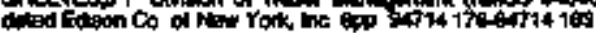

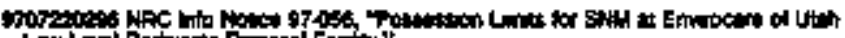

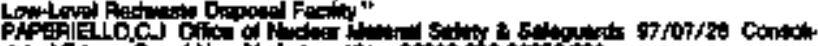

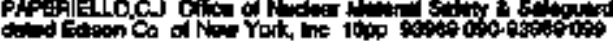

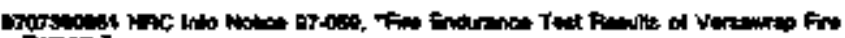

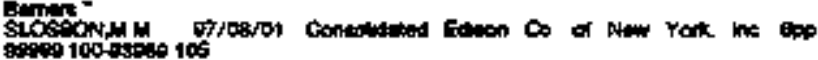

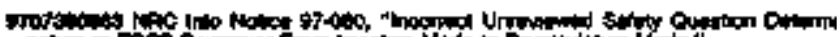

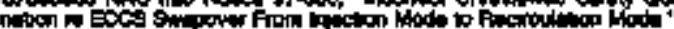

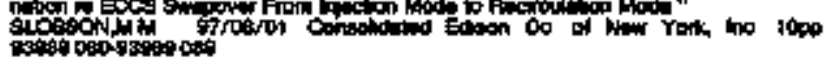

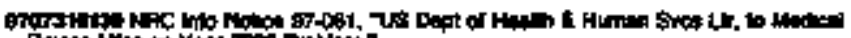

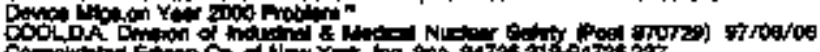

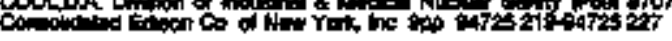

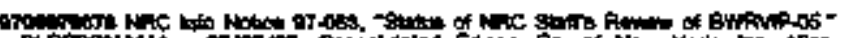

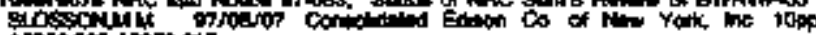

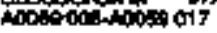

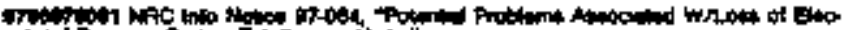

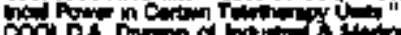

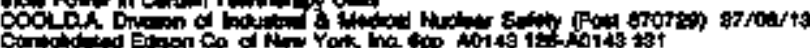

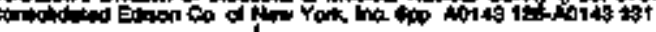

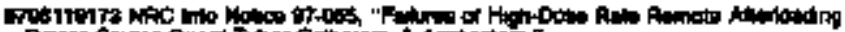

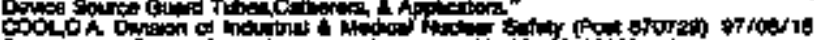

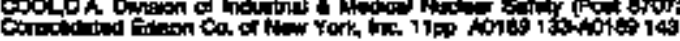

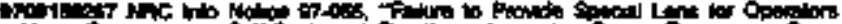

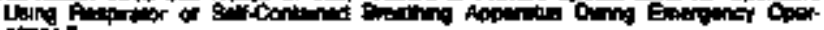

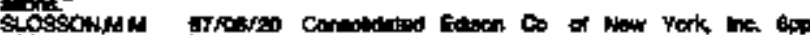

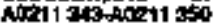

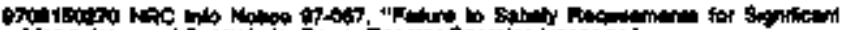

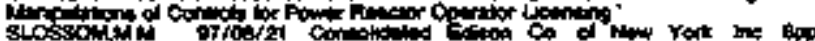

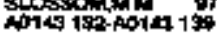

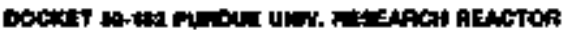

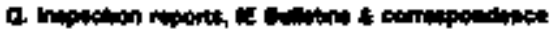




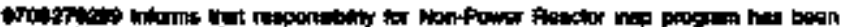

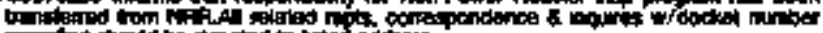
in

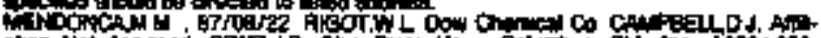

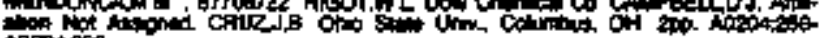
nom 202

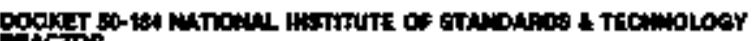

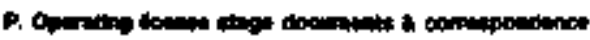

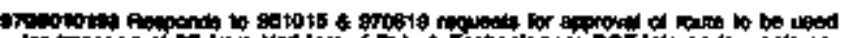

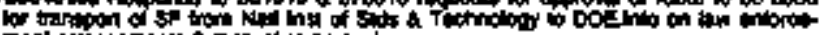

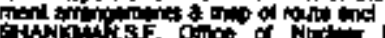

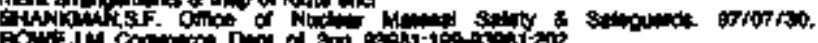
A

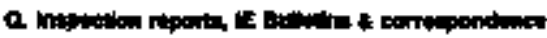

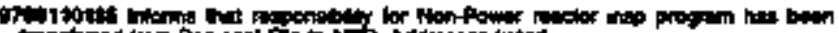

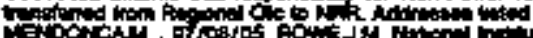

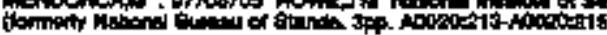

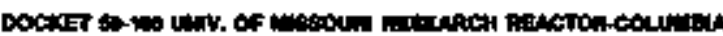

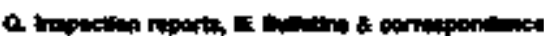

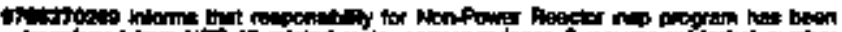

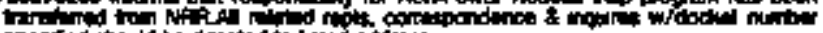

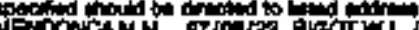

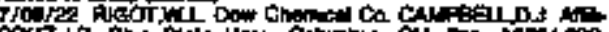

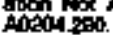

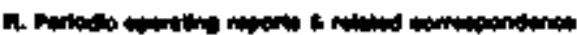

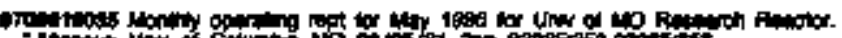

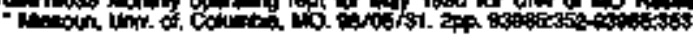

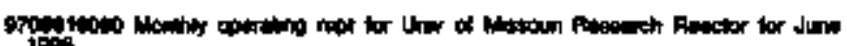
1etis.

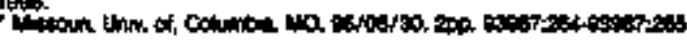

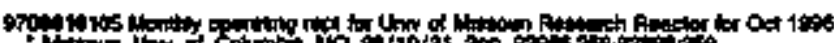

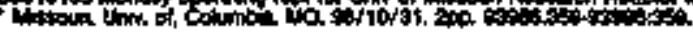

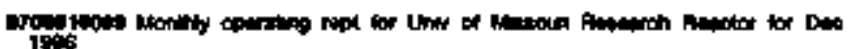

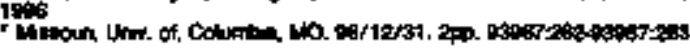

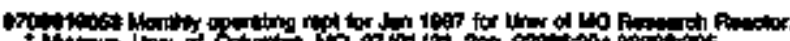

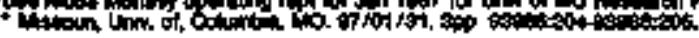

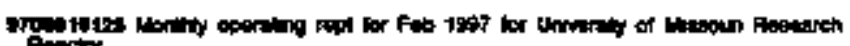

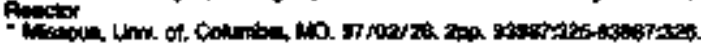

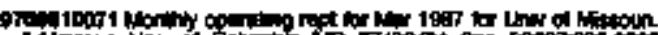

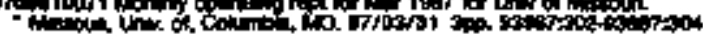

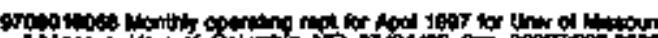

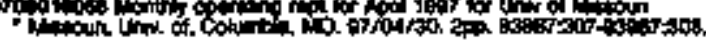

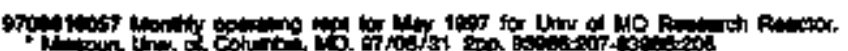

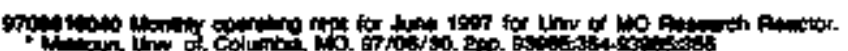

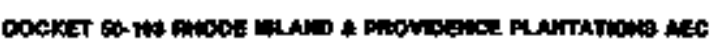

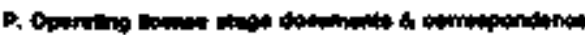

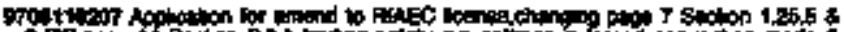

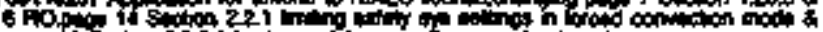

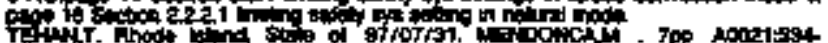
Ainctis:

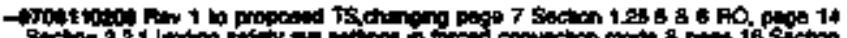

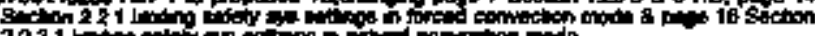

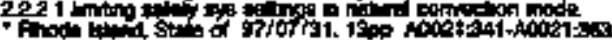

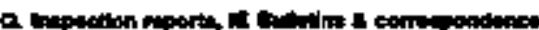

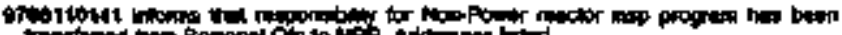

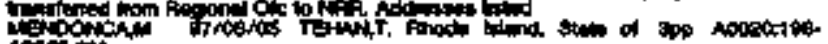
inosico

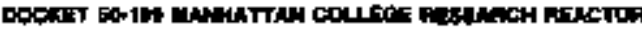

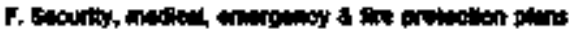

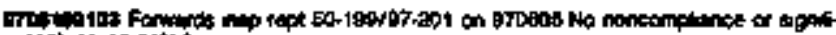

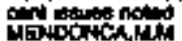
Aoristath-40134 134

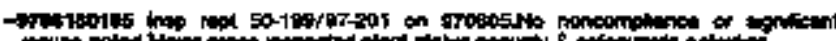

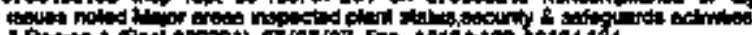

Progen 1 oroed

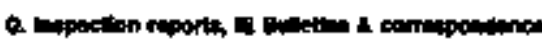

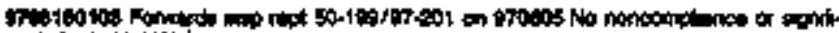

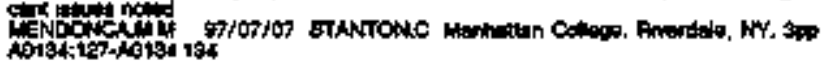

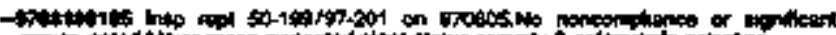

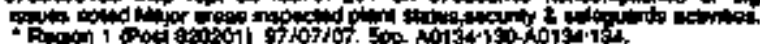

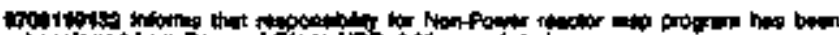

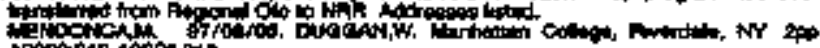
$\cos 2010-\sin 20212$

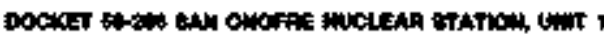

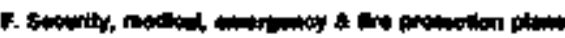

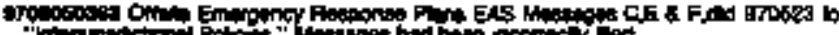

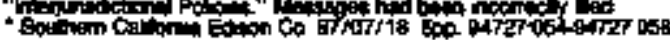

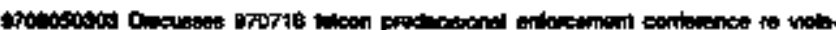

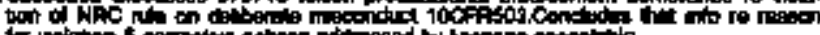

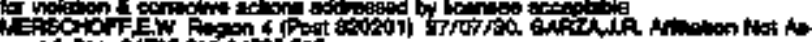

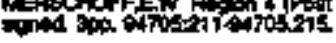

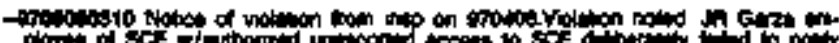

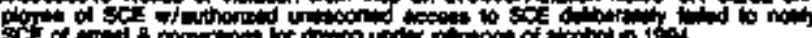

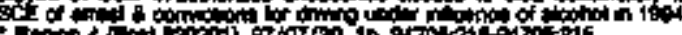

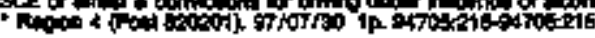

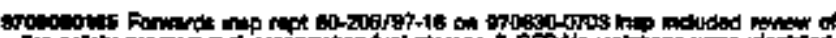

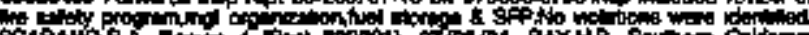

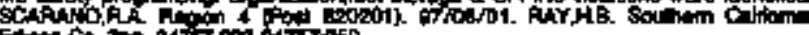

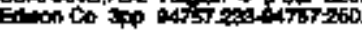

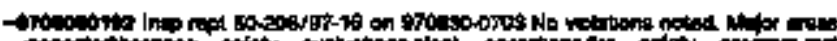

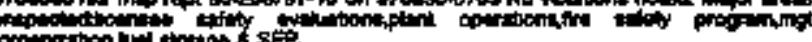

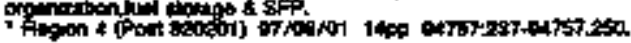

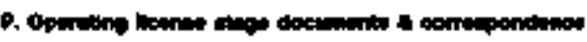

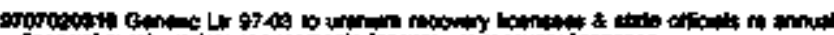

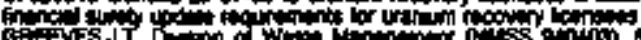
of

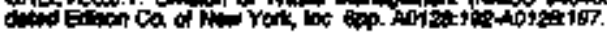

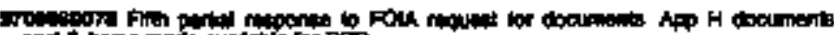

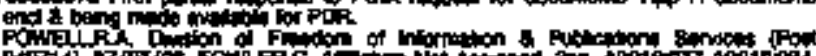

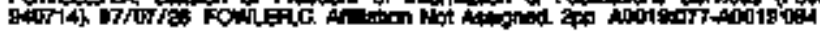

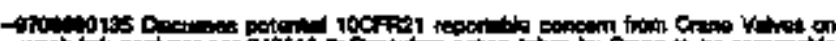

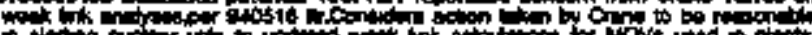

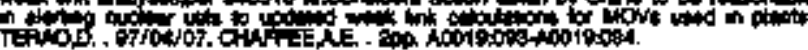

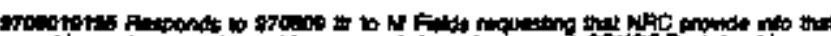

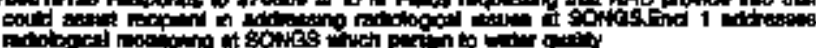

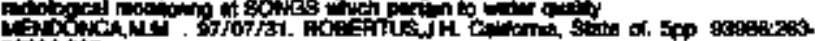

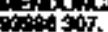

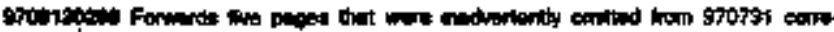

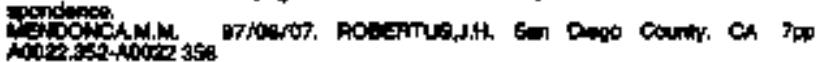

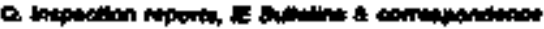

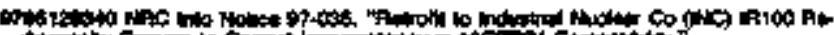

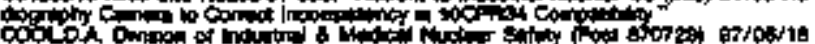
Co

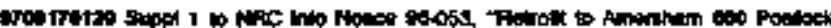

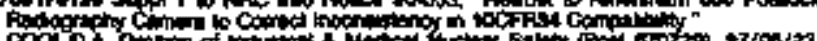

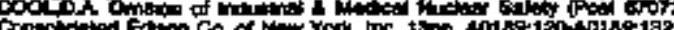




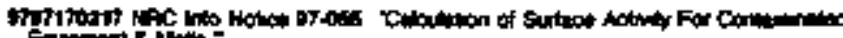

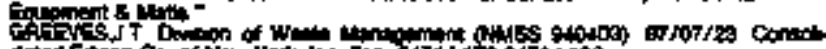

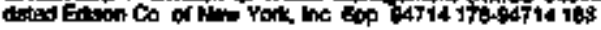

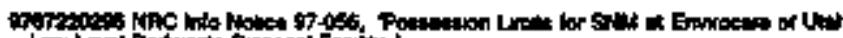
DH

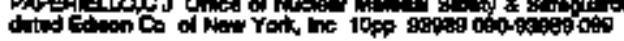

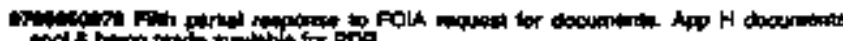

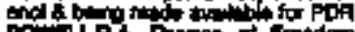

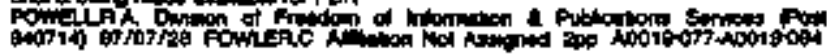

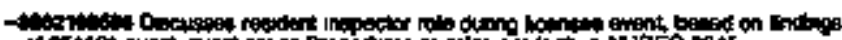

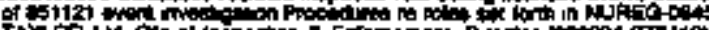

Thy

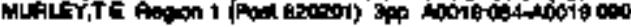

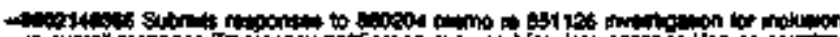

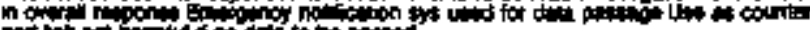

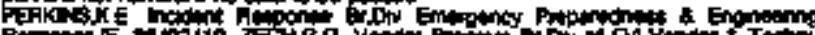

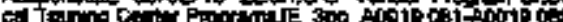

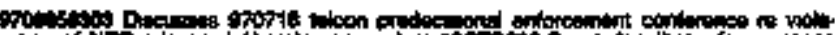

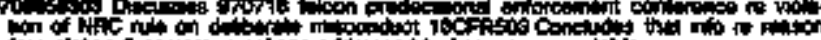

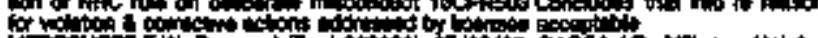

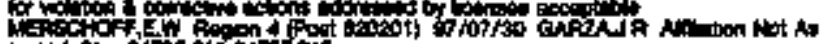

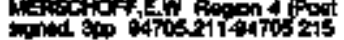

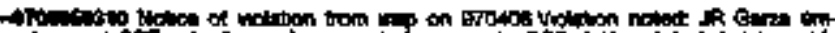

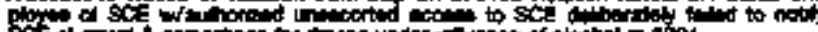

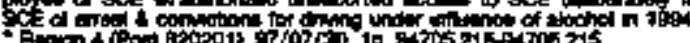

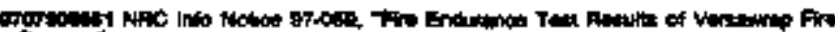

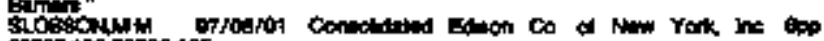

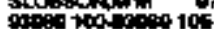

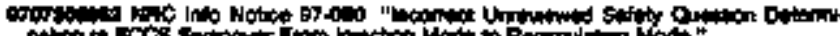

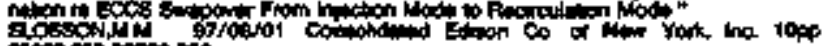

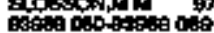

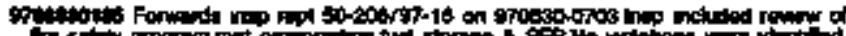
\&

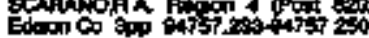

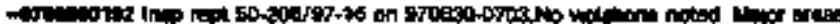

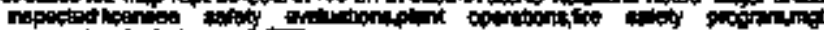

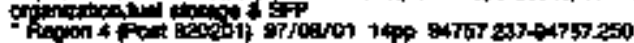

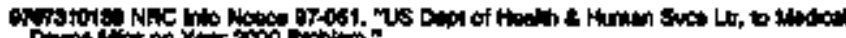

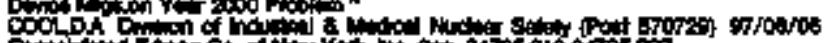

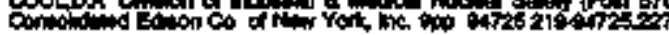

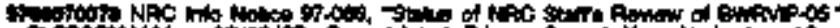

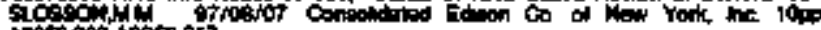

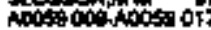

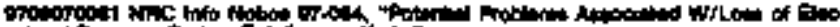

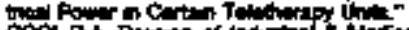

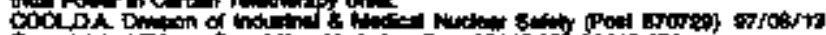

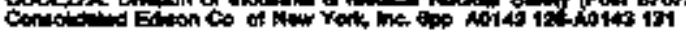

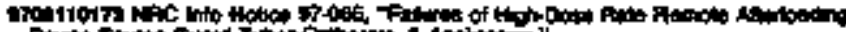

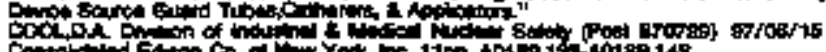

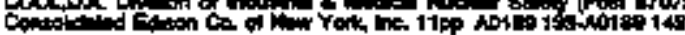

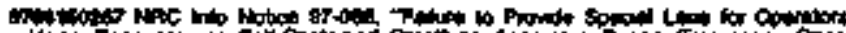

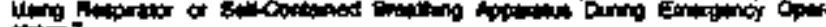

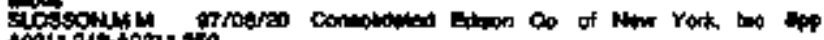

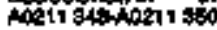

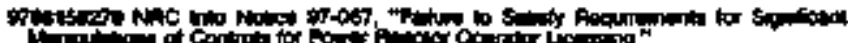

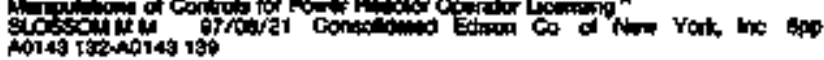

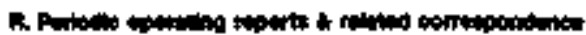

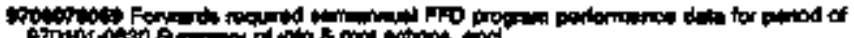

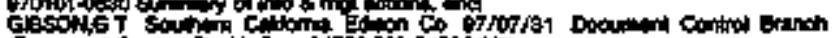

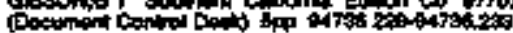

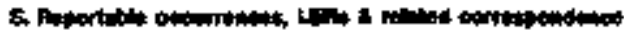

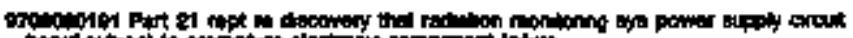

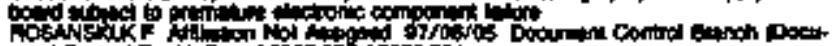

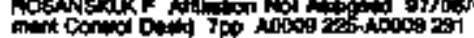

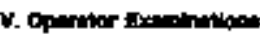

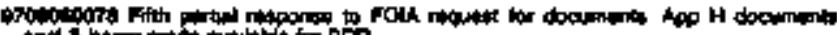

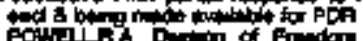

Fond

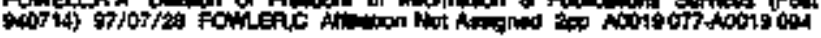

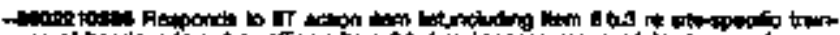

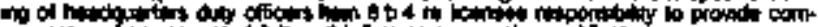

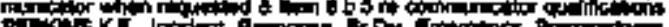

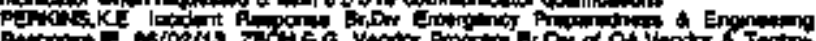
A

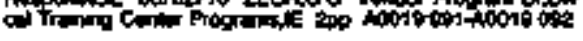

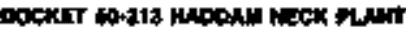

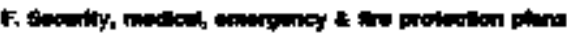

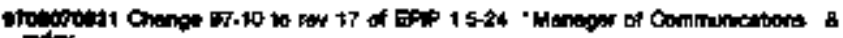
tox

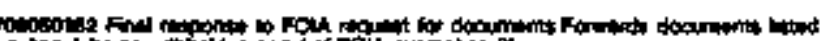

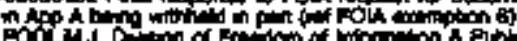

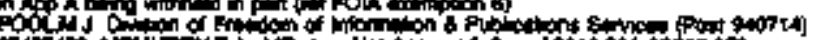

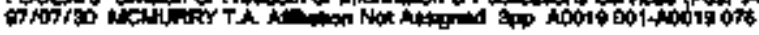

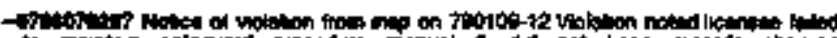

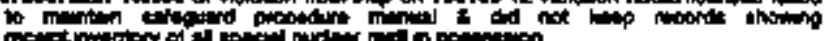

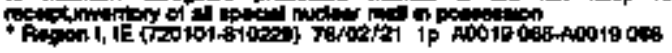

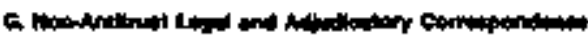

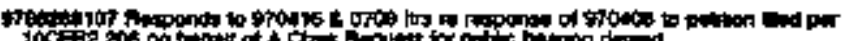

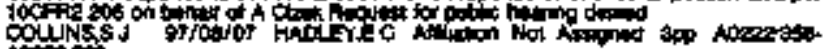
intexis

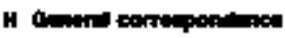

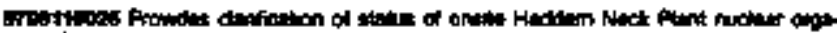

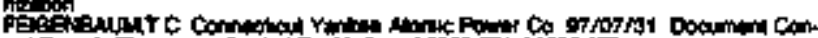

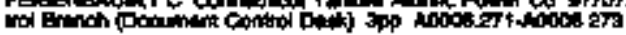

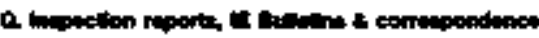

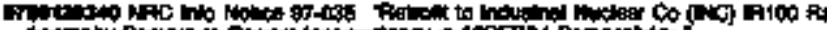
CO

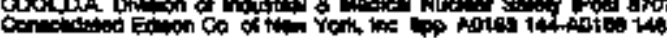

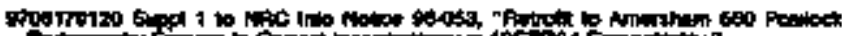

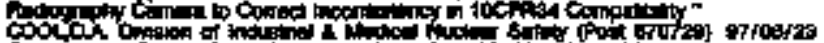

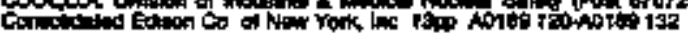

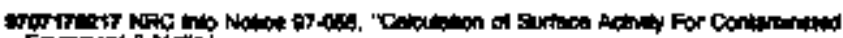

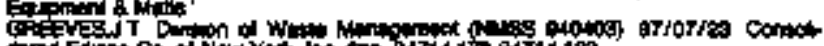

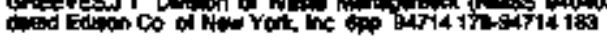

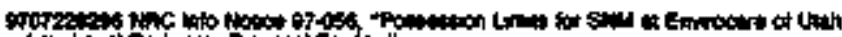

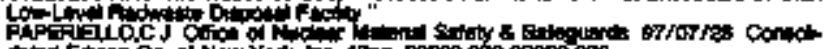

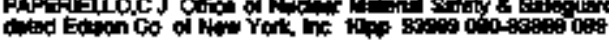

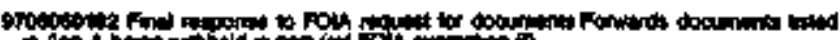

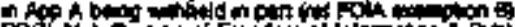

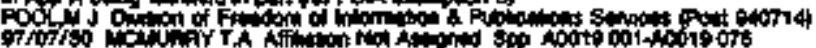

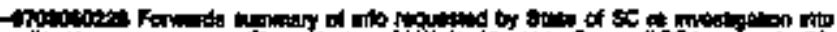

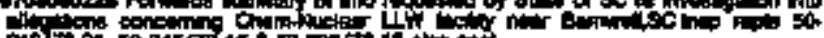

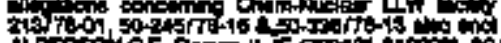

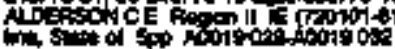

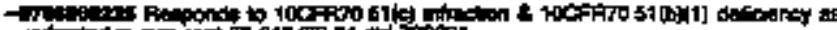

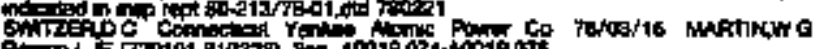

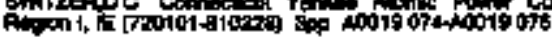

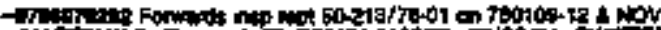

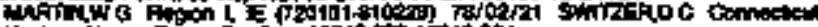

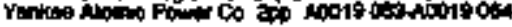

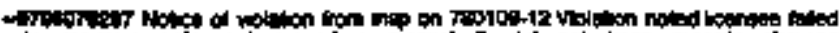

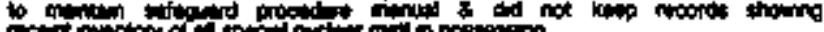

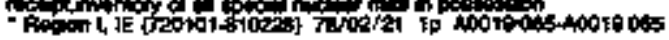

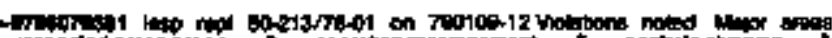

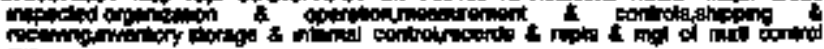

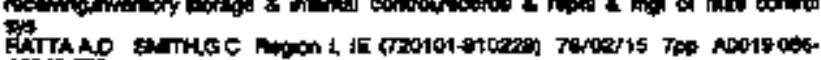
A0010002 


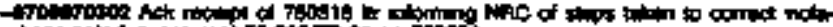

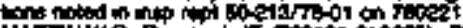

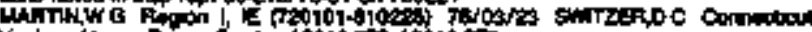

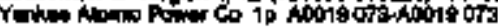

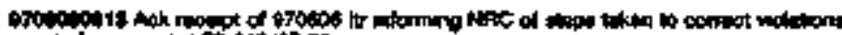

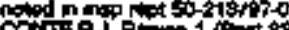

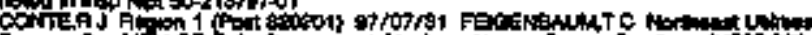

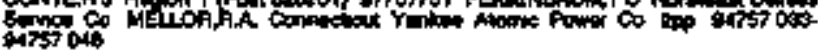

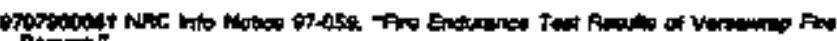

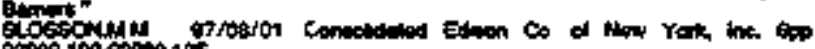

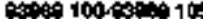

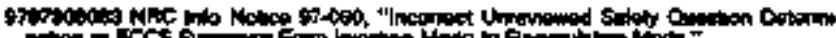

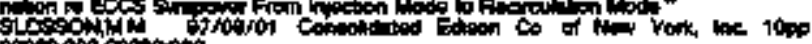

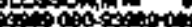

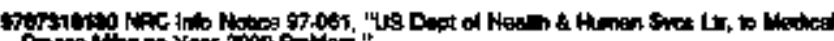

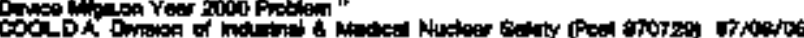

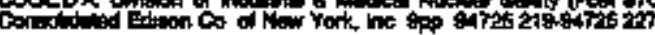

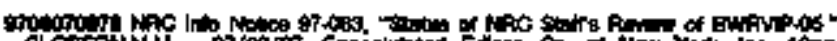

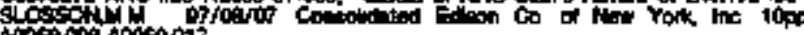

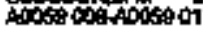

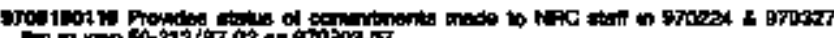

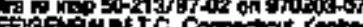

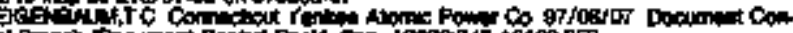

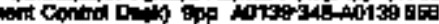

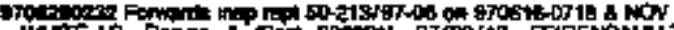

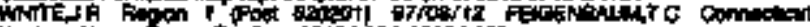

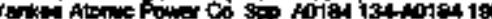

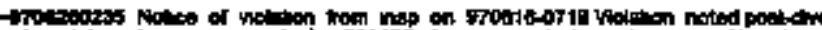

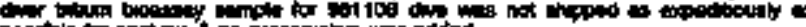

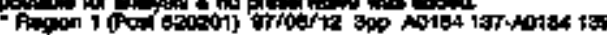

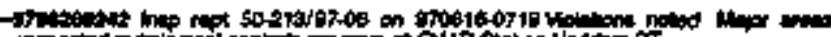

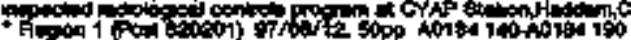

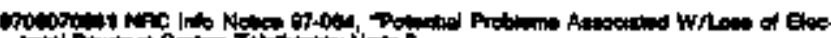

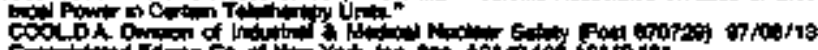

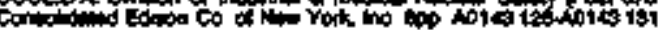

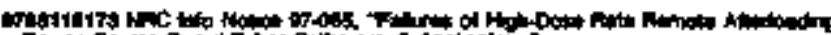

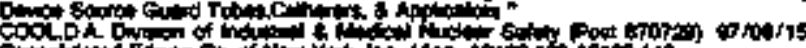

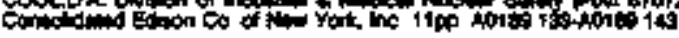

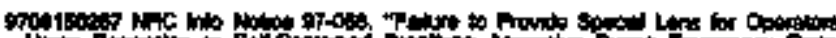
U.

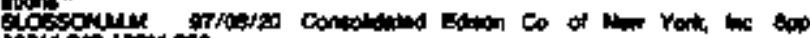

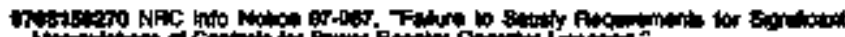

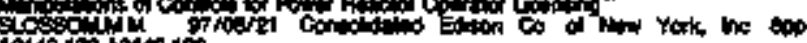

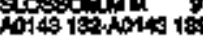

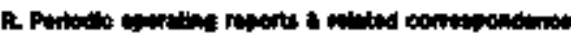

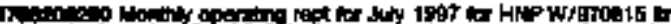

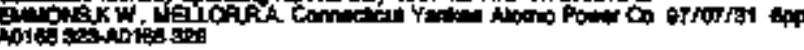

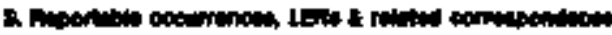

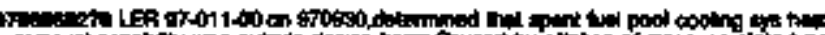

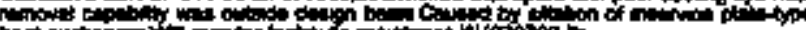

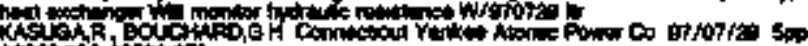

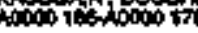

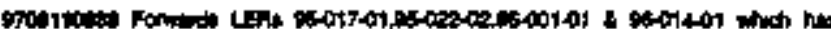

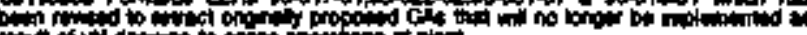

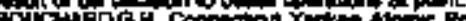

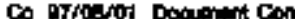

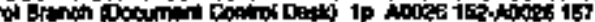

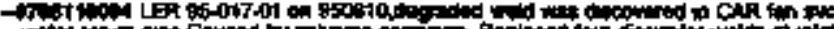
1

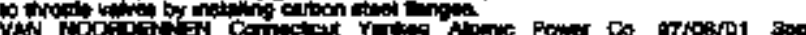
Vis

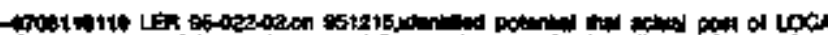

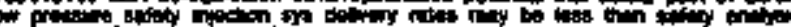

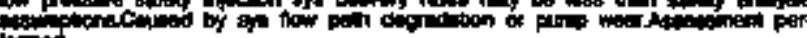

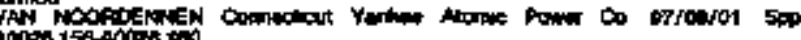

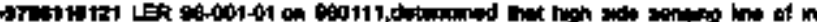

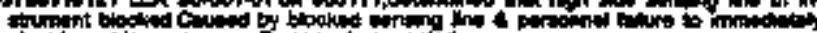

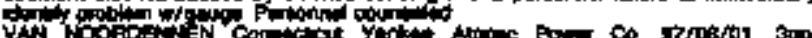

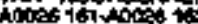

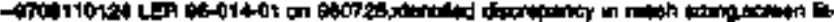

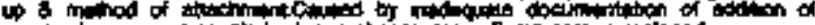

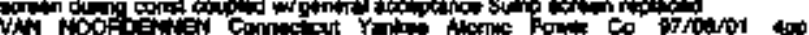

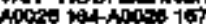

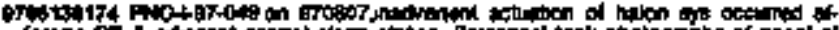

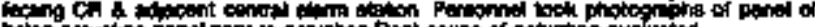

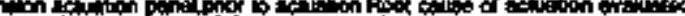

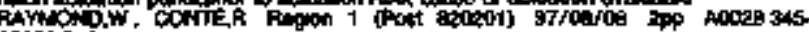
Metat 34:

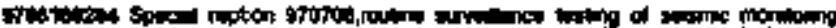

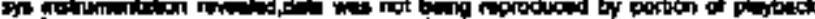

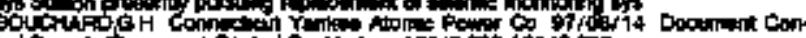

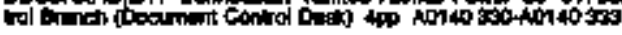

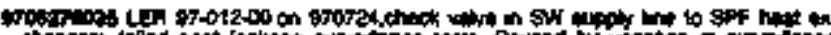

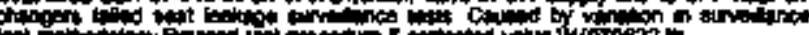

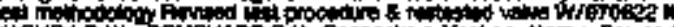

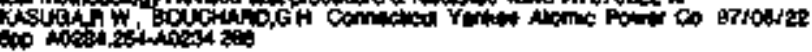

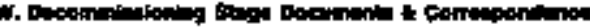

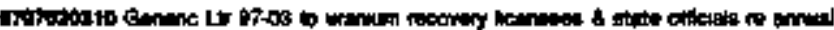

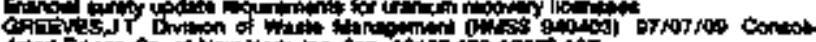

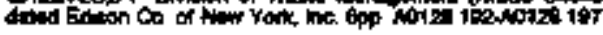

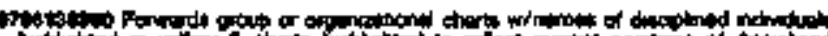

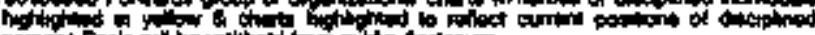

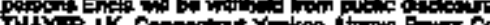

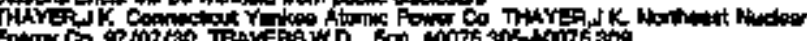

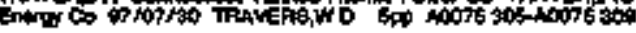

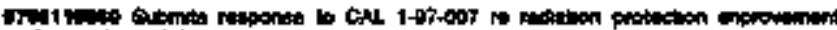

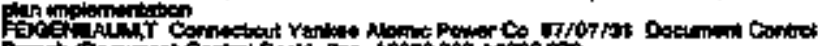

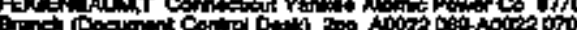

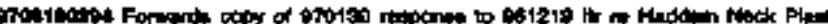

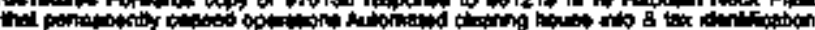

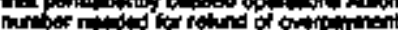

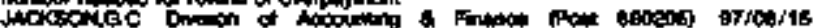

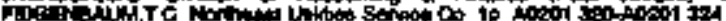

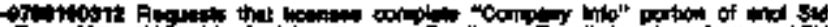

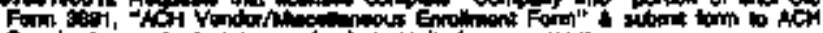

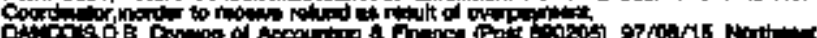

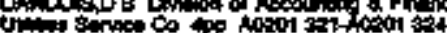

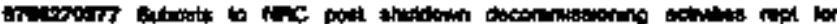

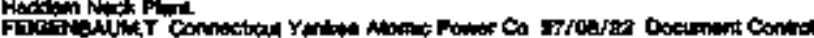

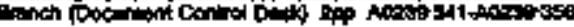

- Hay 10

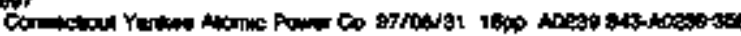

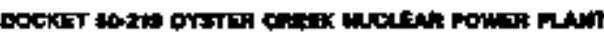

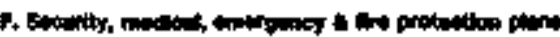

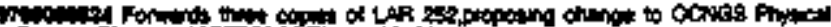

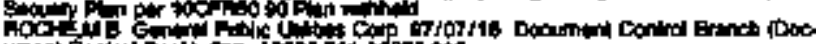

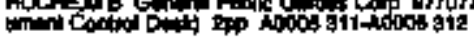

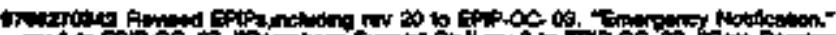

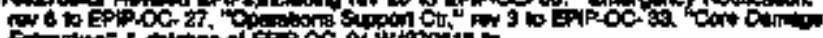

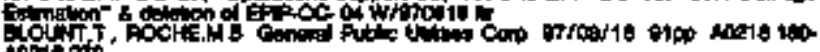

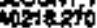

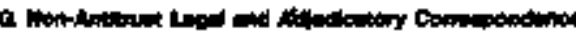

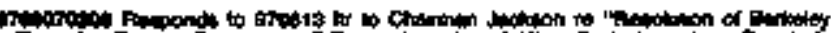

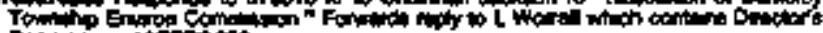

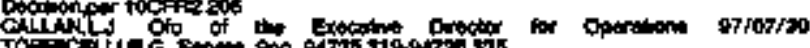

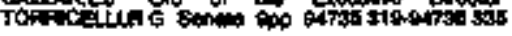

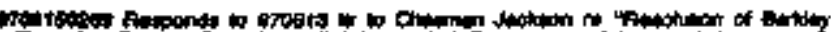
To

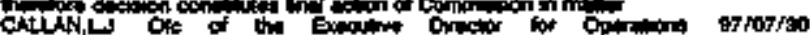
Golly

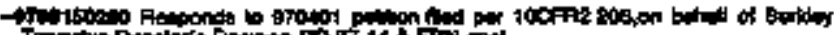

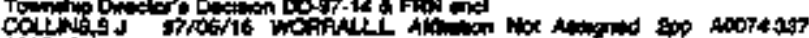
10034 3

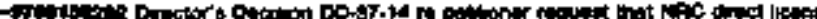

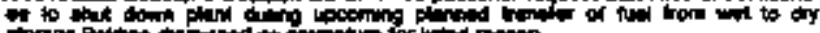

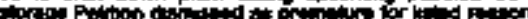

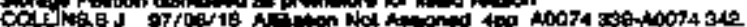




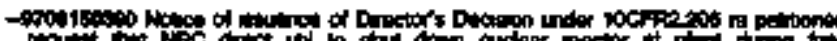

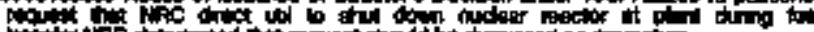

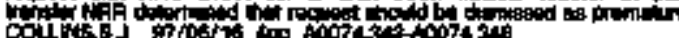

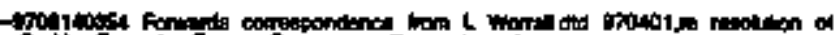

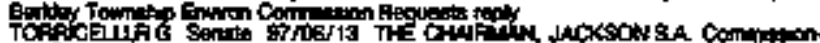

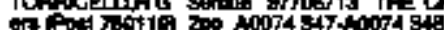

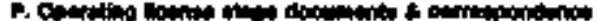

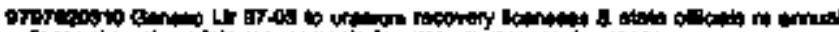

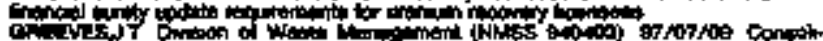

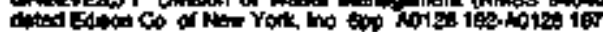

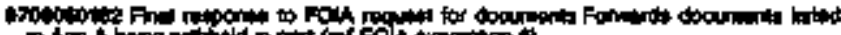

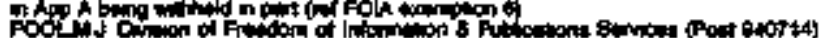

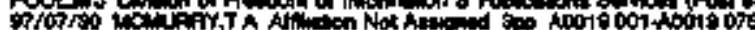

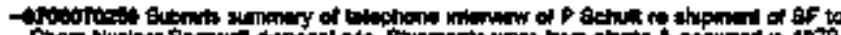

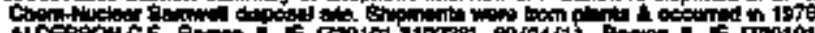

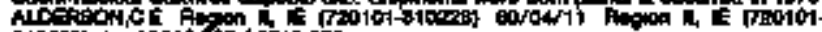

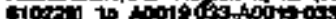

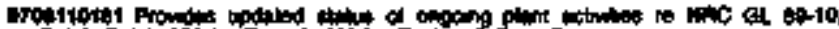

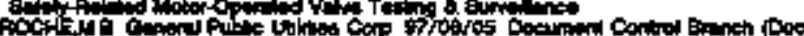

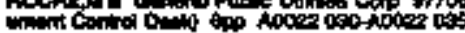

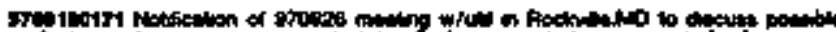

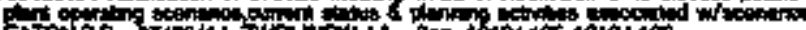

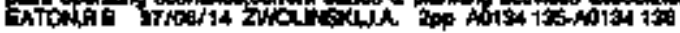

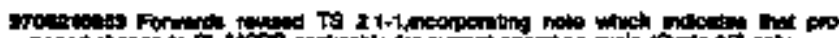

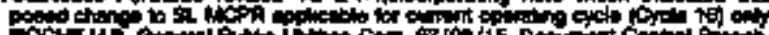

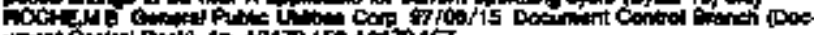

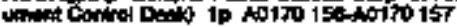

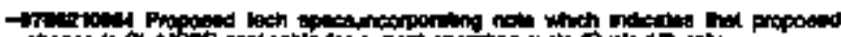

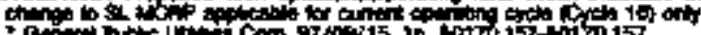

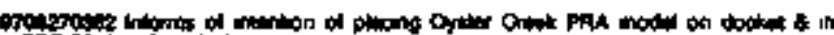

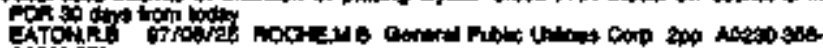
intolin

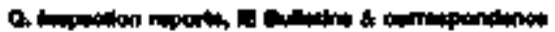

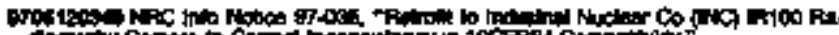

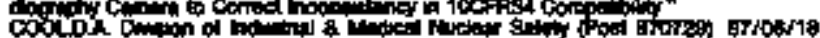

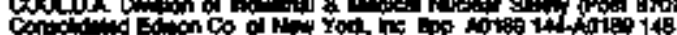

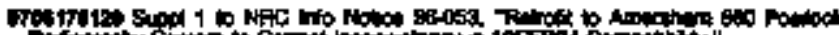

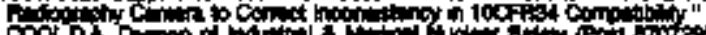

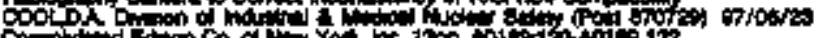

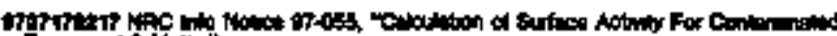
cion:

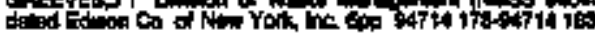

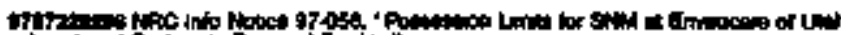

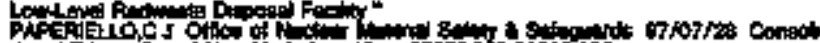

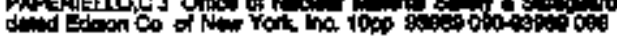

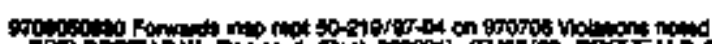

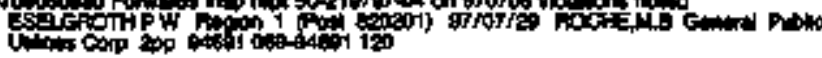

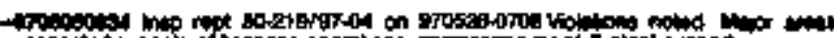

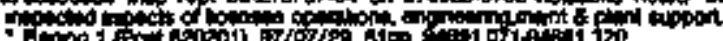

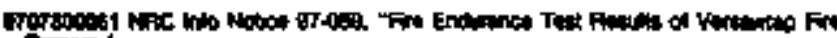

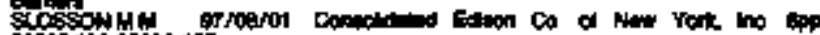

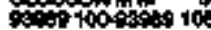

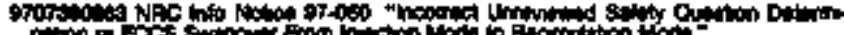

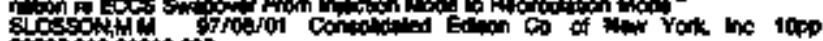
\%.

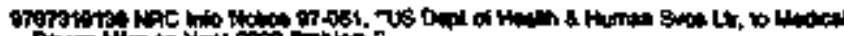

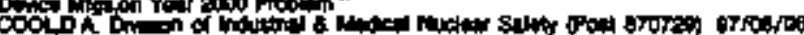

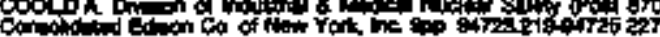

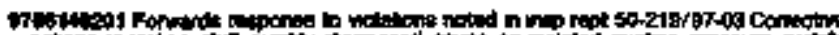

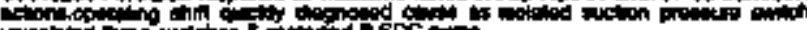

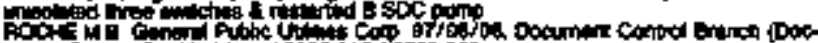

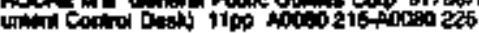

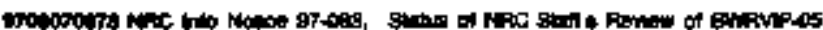

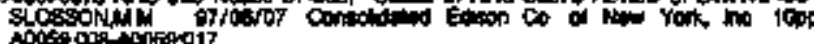

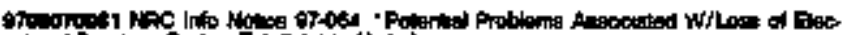

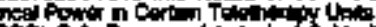

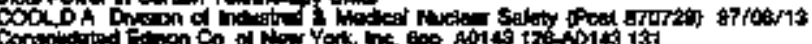

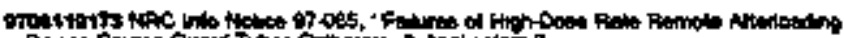

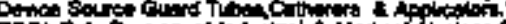

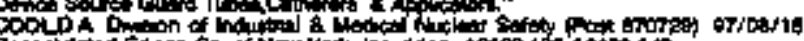

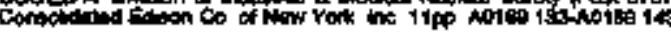

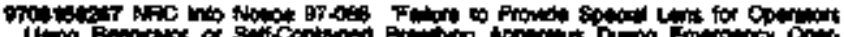

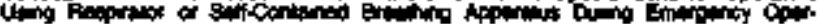

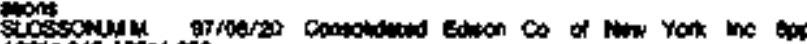

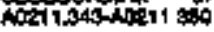

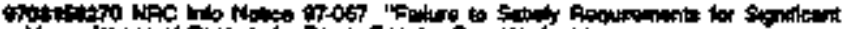

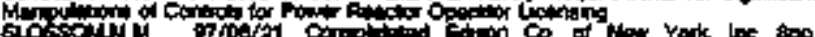

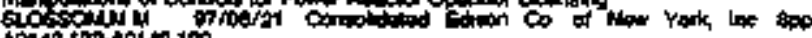

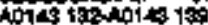

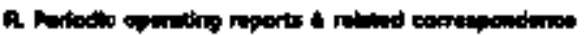

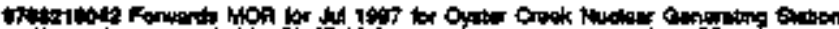

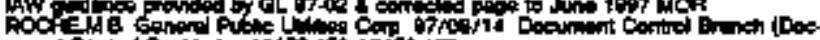

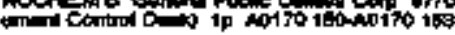

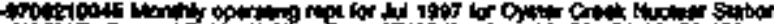

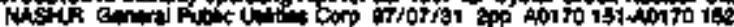

- mona

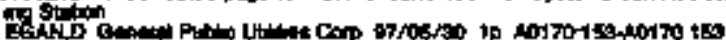

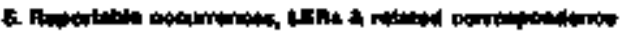

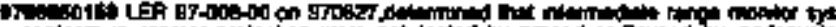

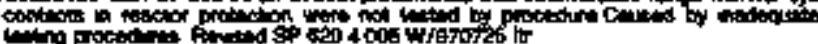

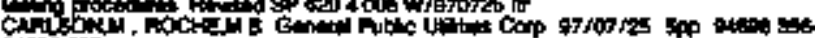
exposen

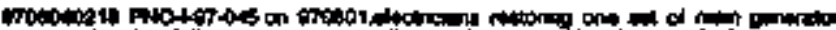

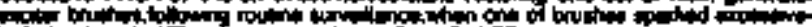

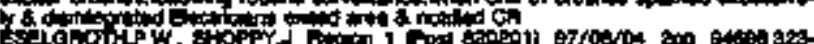
oforite

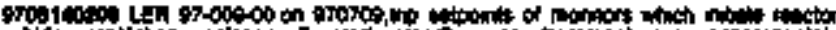
H.

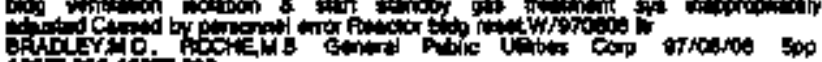
Mon

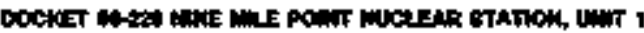

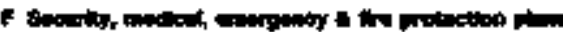

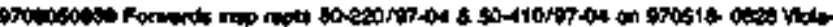
of

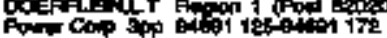

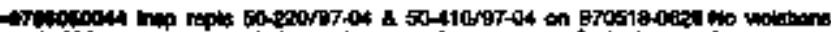

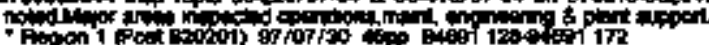

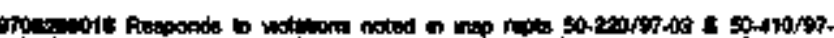

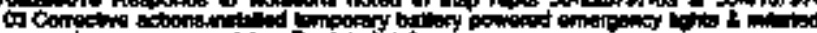

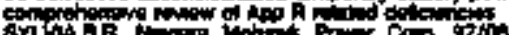

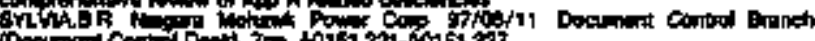

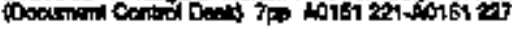

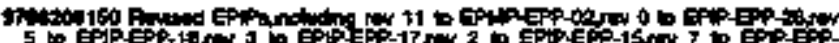
5 te to

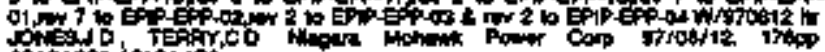

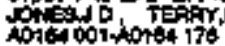

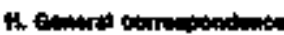

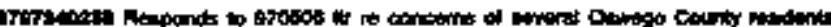

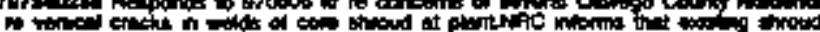

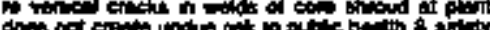

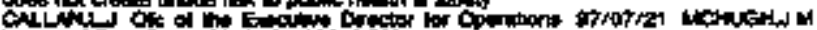

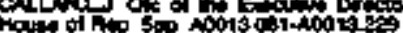

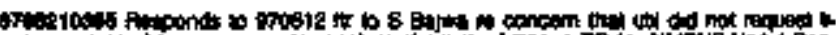

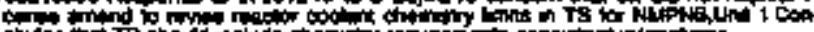

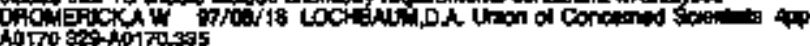




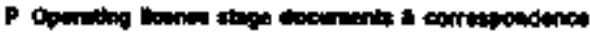

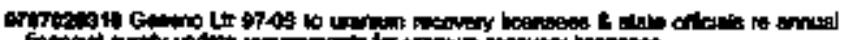

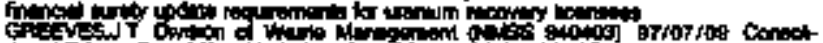
the

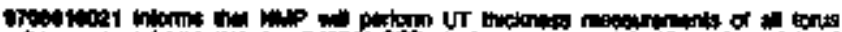

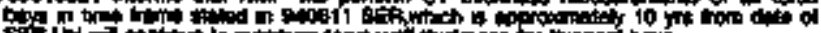

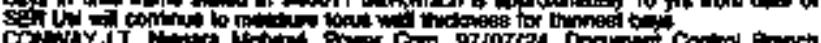
Cothly J

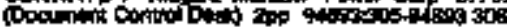

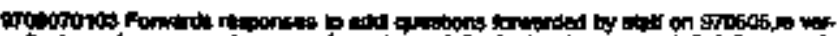

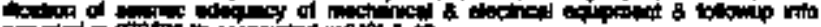

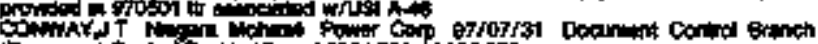

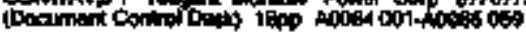

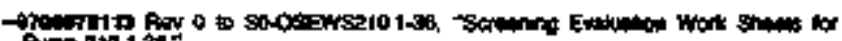
Anp pro 1s.

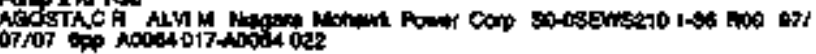

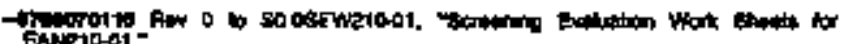
(a)

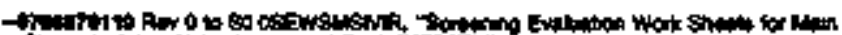

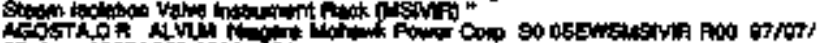

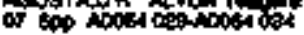

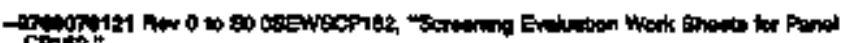

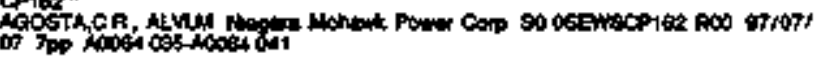

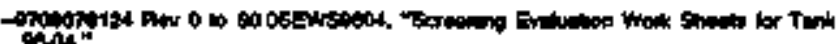

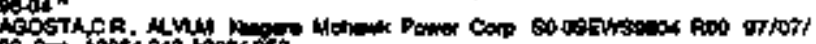

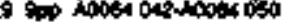

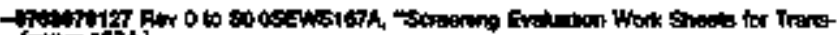

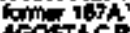

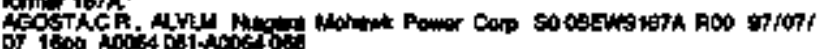

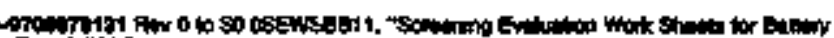

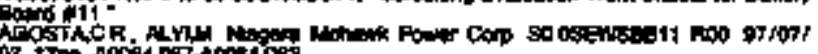

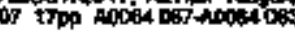

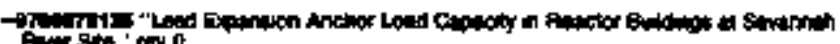

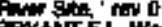

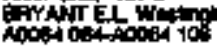

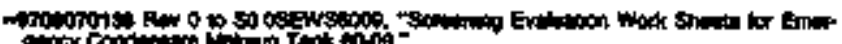

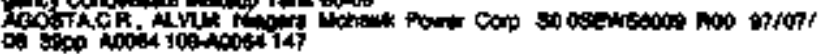

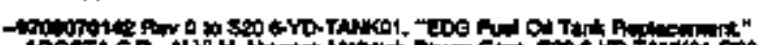

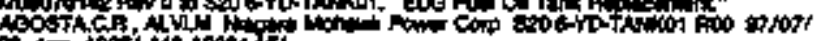

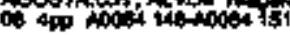

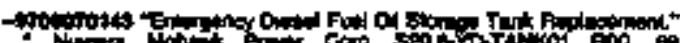

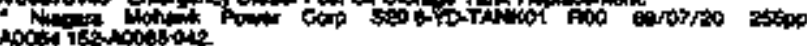

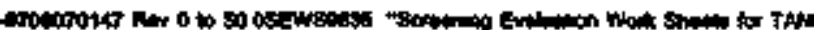

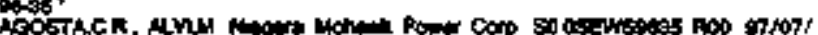

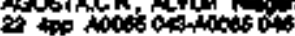

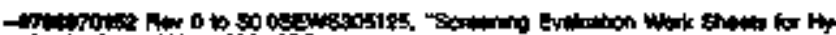

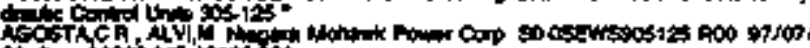

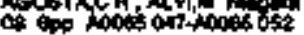

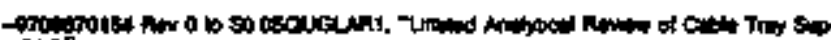

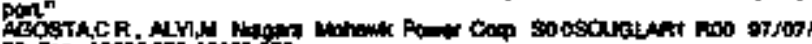

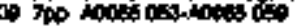

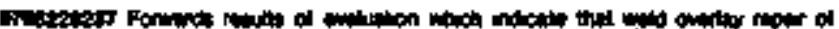

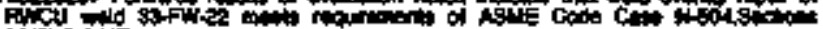

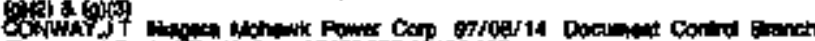

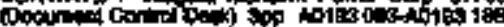

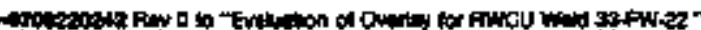

SCMC

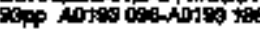

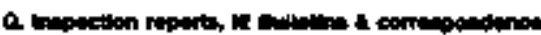

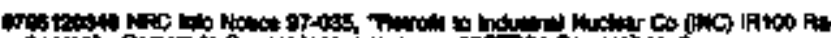

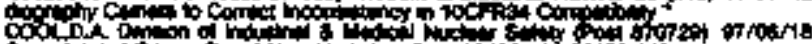

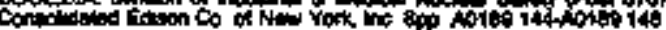

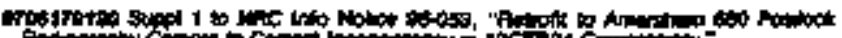

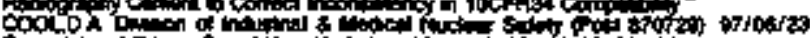

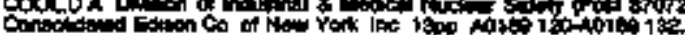

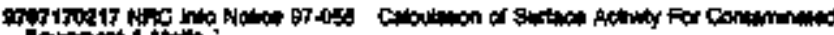

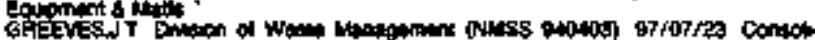

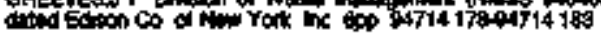

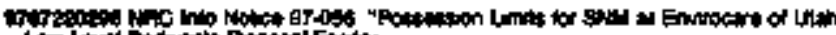

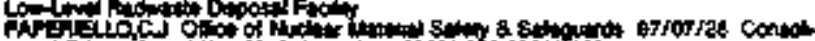

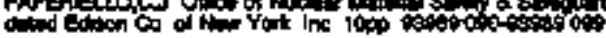

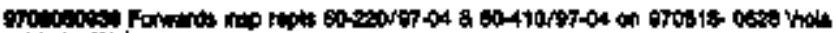

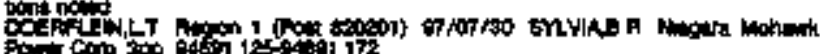

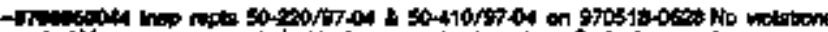

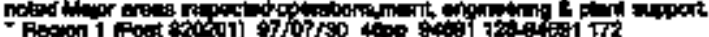

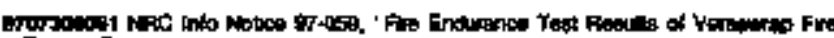

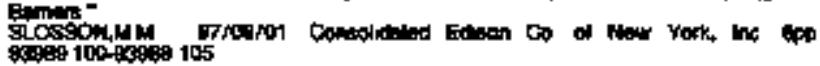

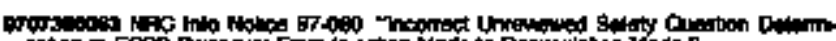

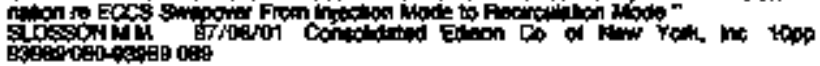

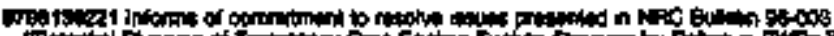

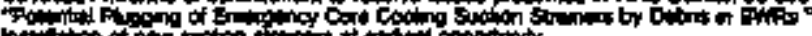

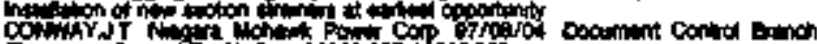

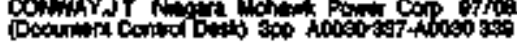

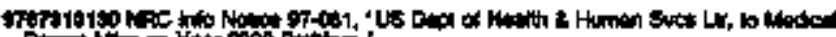

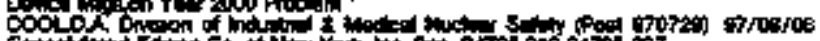

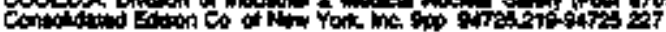

ITh:

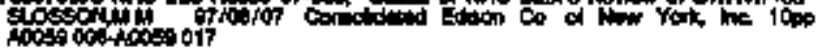

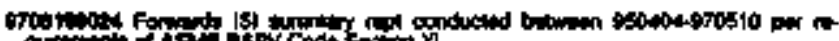

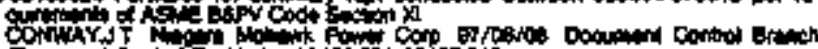

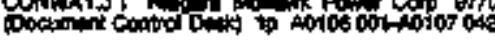

- MD

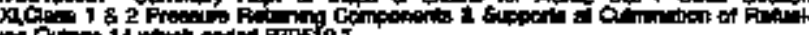

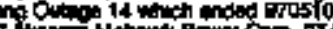

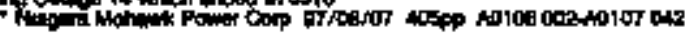

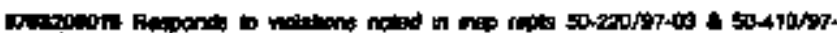

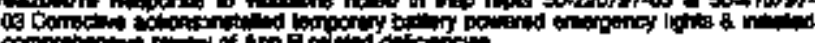
gemproin on

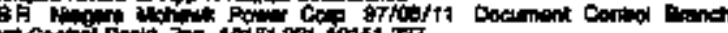

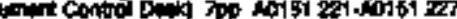

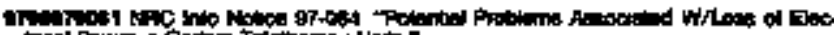

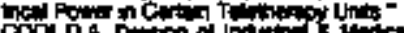

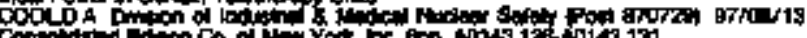

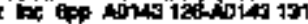

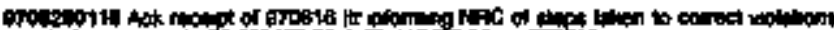

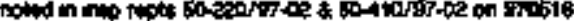

Do Pone

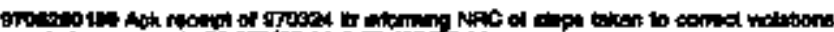

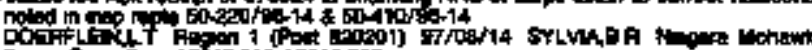

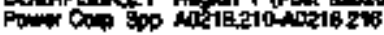

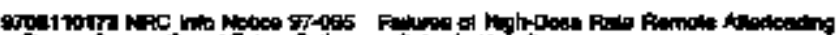

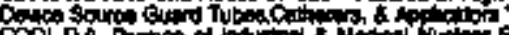

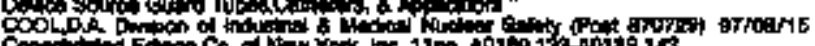

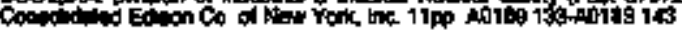

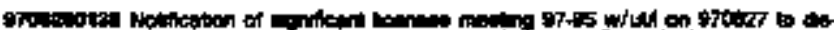

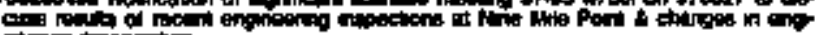
Dodn Hitos

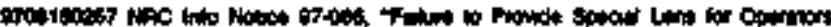

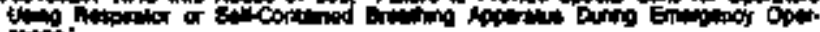

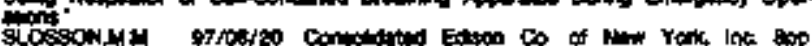
Aat1 843-40et1 30

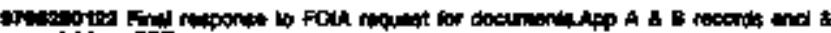

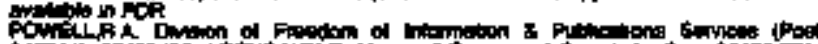

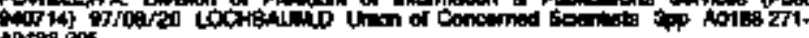
ADtiges

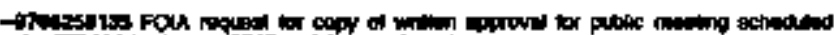

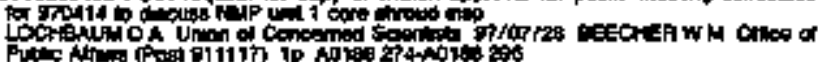

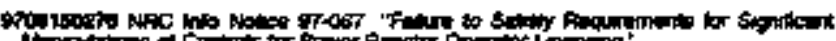

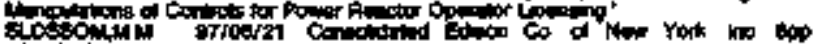

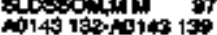




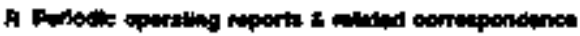

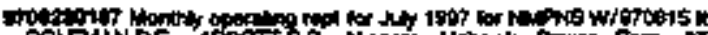

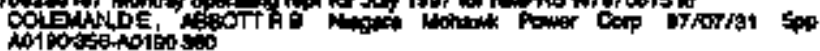

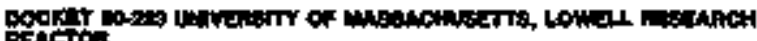
pection

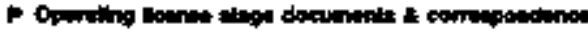

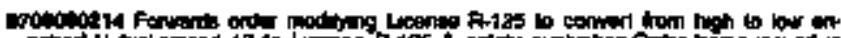

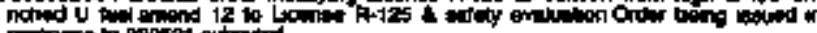

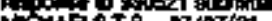

How Une of $17+\cos 2 \pi 00$

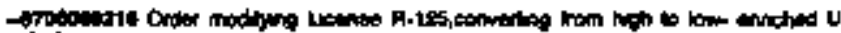

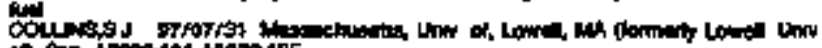
of

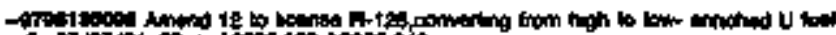

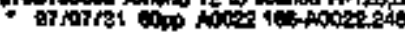

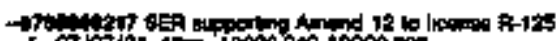

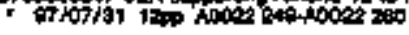

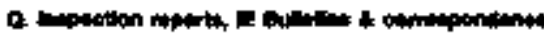

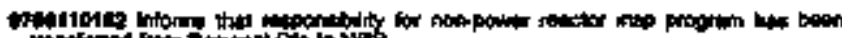

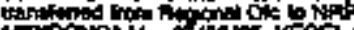

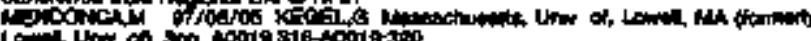

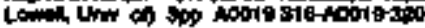

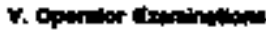

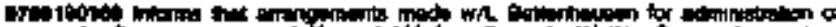

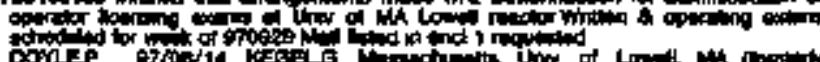

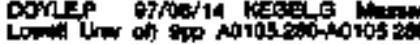

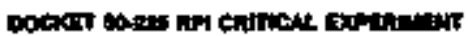

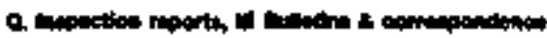

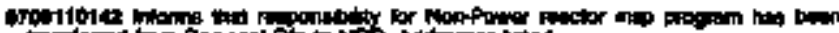

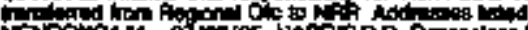

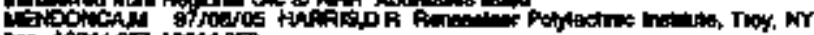

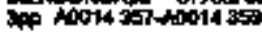

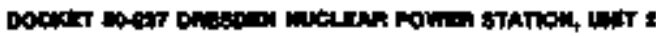

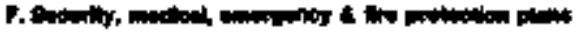

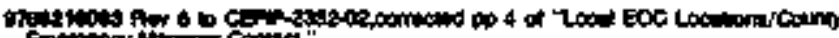
Ecomor

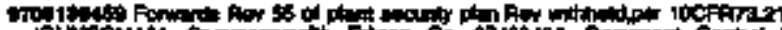

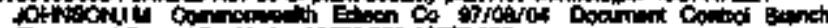

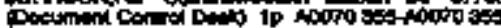

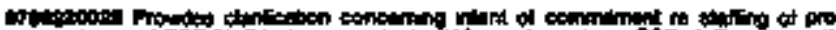

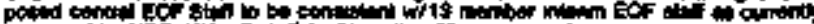

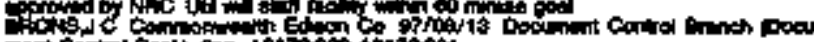

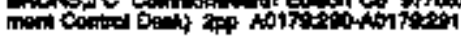

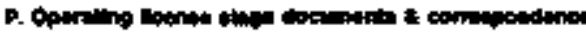

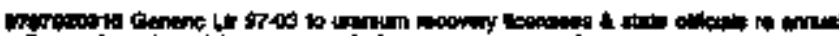

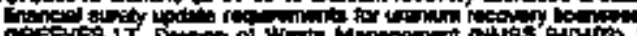

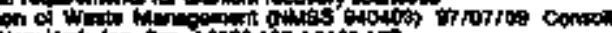

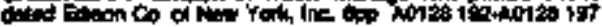

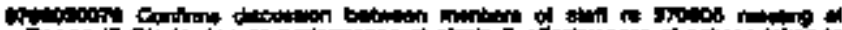

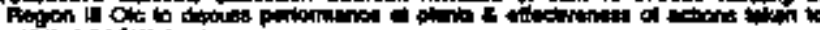

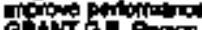

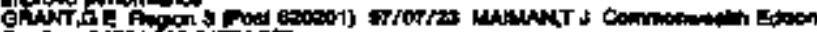

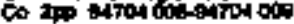

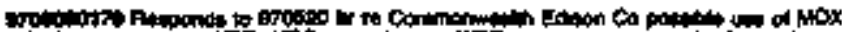

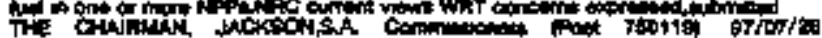

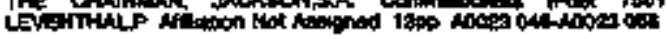

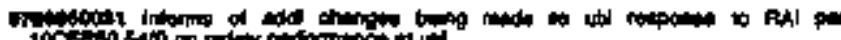

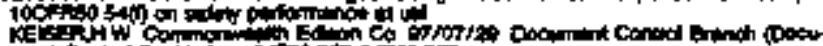

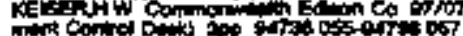

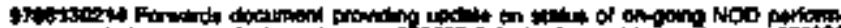

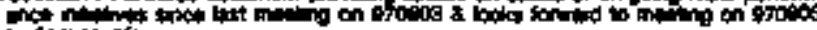
odegrin

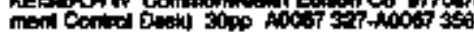

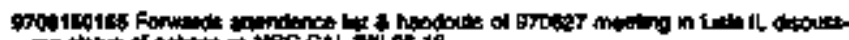

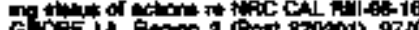

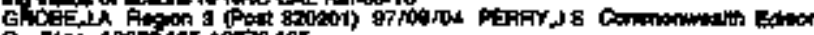

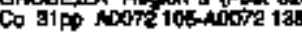

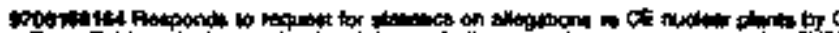

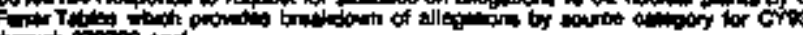

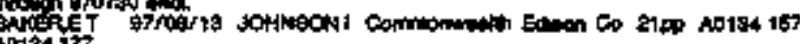
ANHâ it?

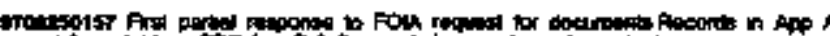

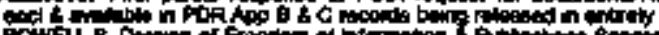

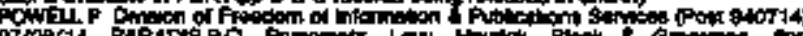

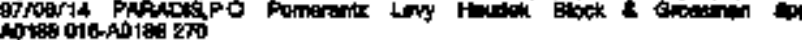

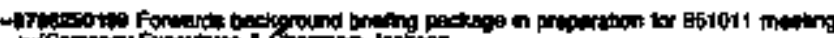

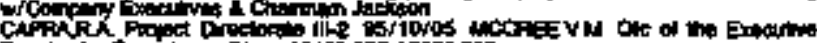

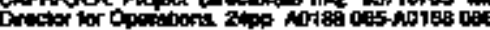

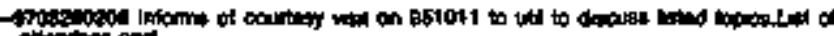

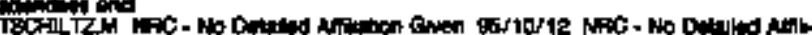

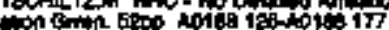

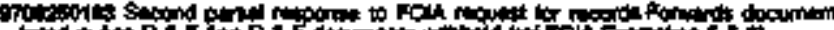

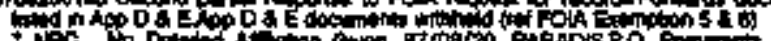

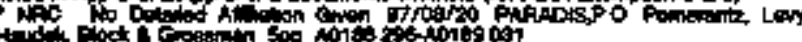

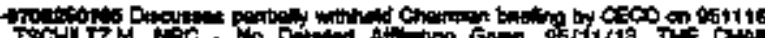

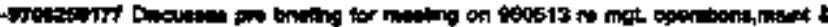

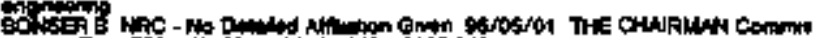

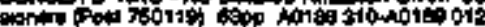

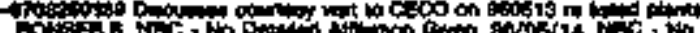

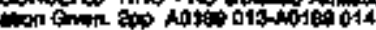

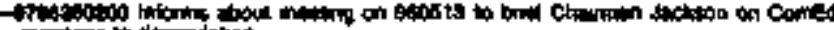
Hinc

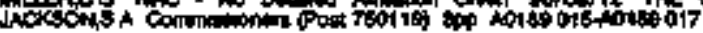

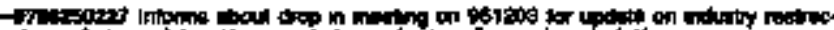
Hoin

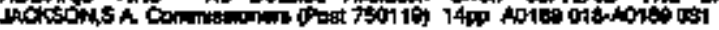

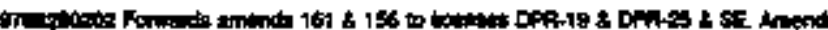

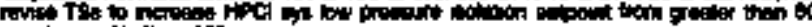

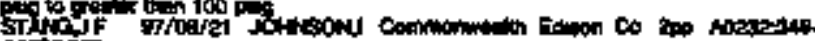
AORAOS

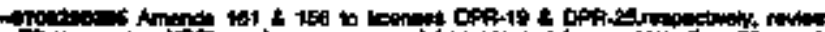

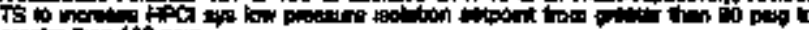

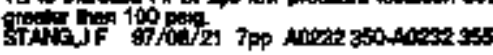

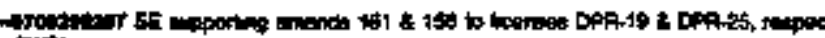
trin

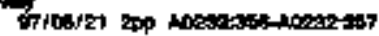

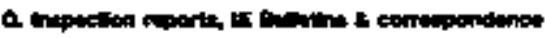

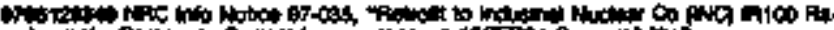

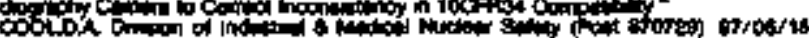

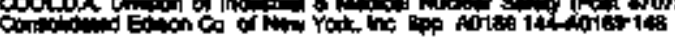

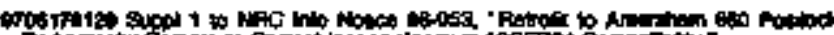

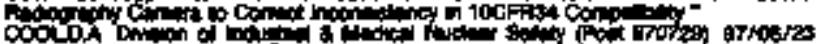

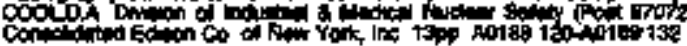

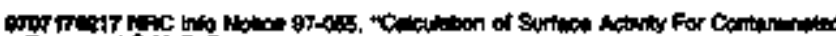

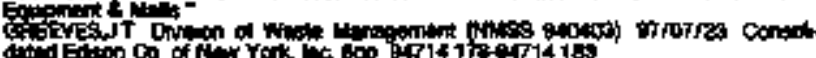

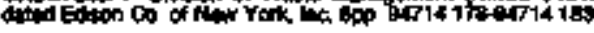

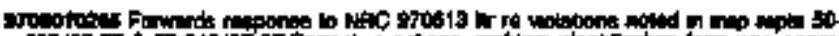

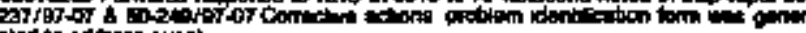

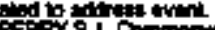

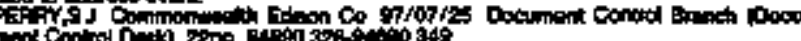

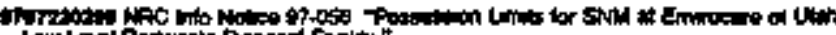

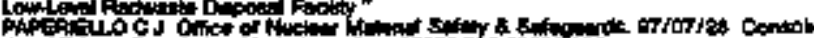

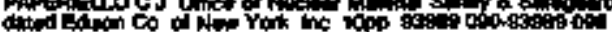

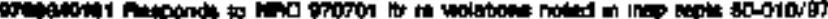

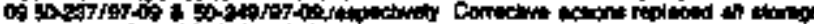

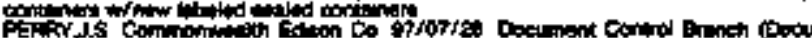

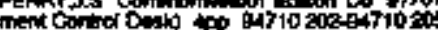




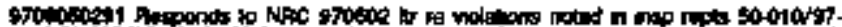

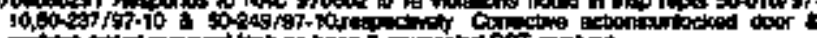
10.7.

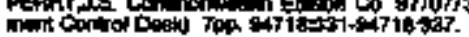

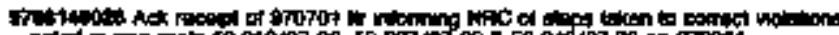

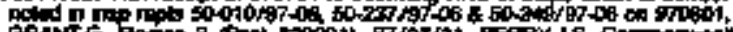

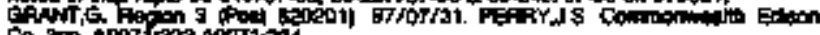

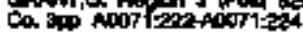

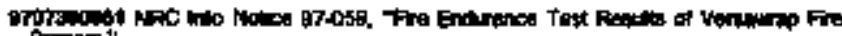

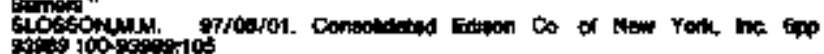

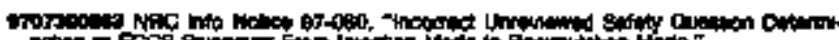

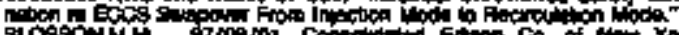

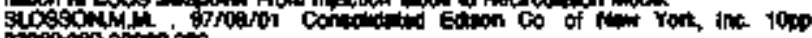

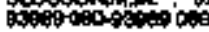

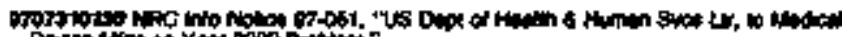

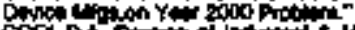

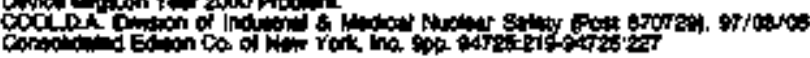

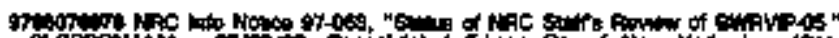

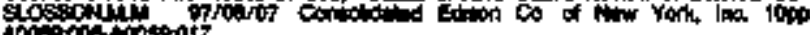

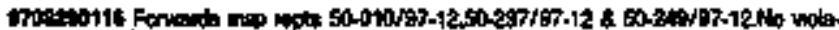

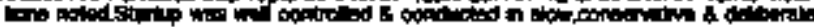

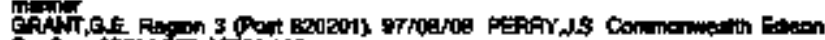

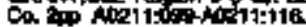

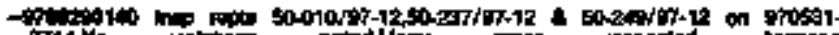

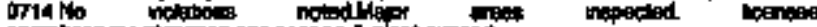

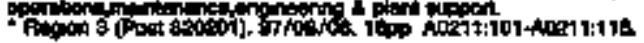

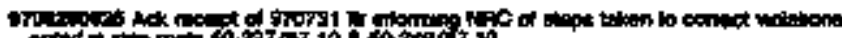

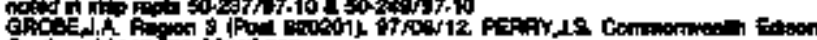

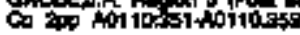

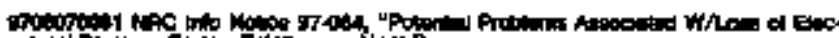

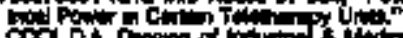

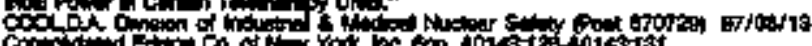

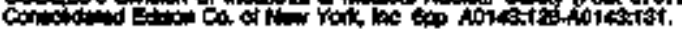

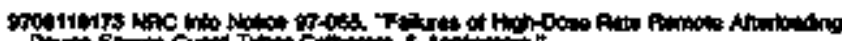

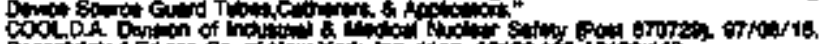

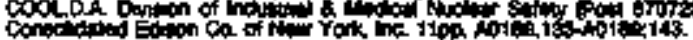

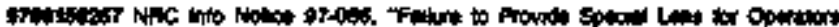

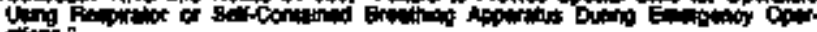

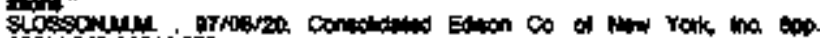

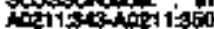

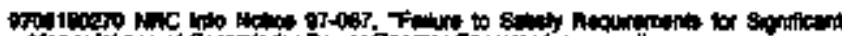

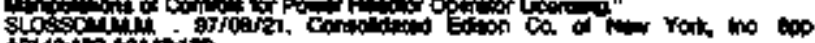

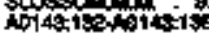

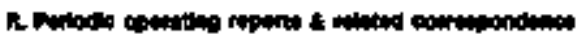

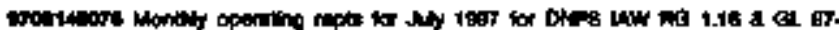
Cew Frowe lit. ADOTr:100.

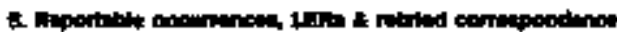

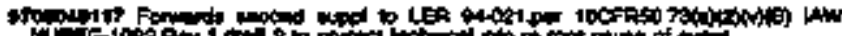

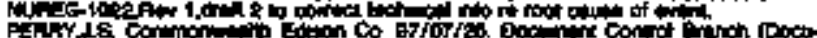

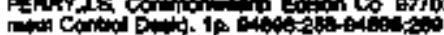

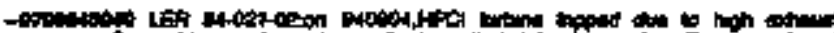

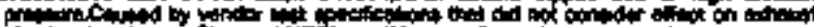

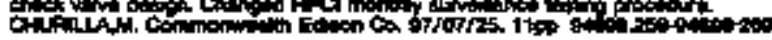

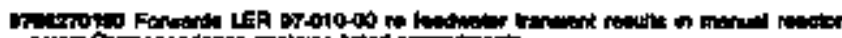

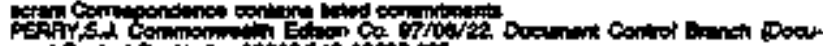

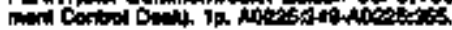

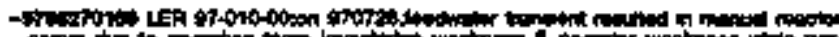

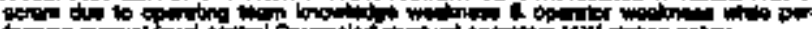

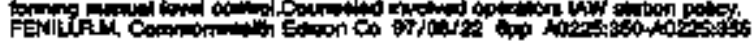

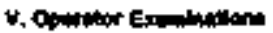

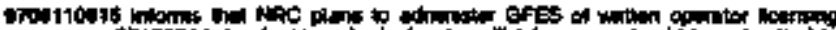

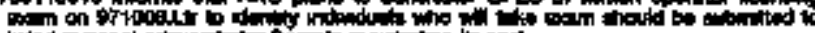

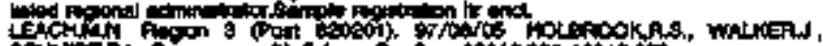

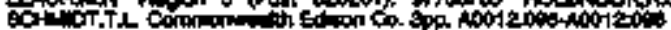

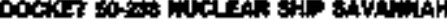

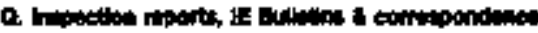

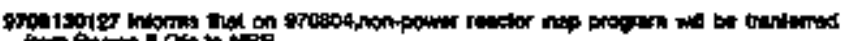

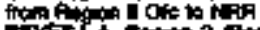

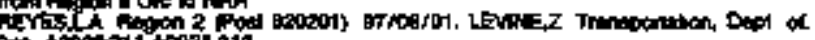
\& A

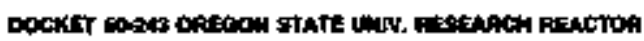

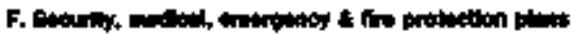

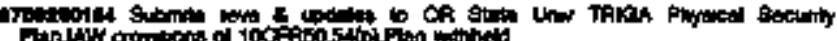

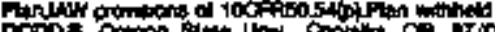

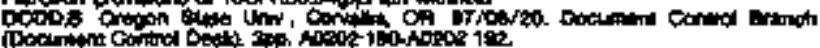

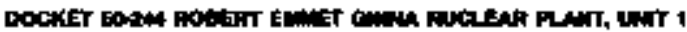

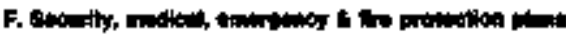

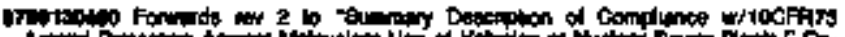

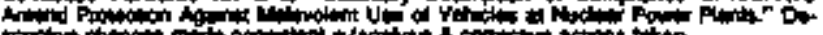

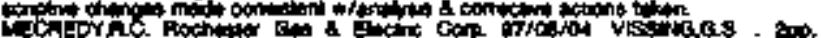

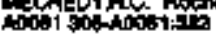

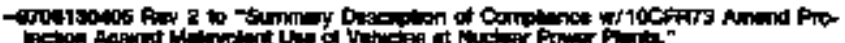

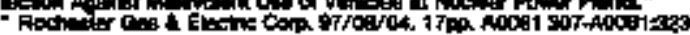

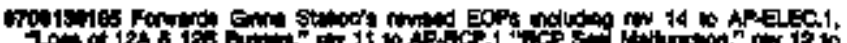

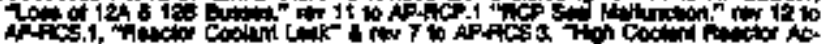

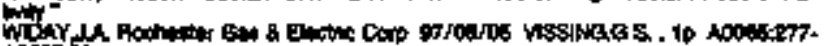
tootsis

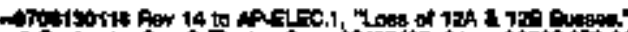

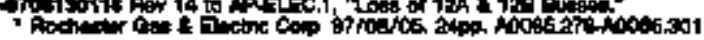

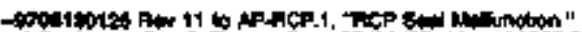

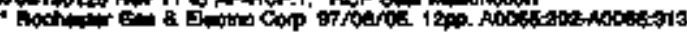

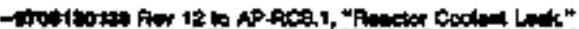

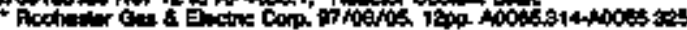

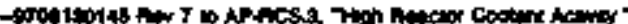

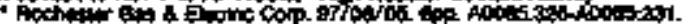

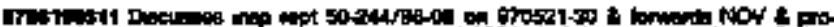

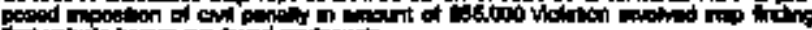

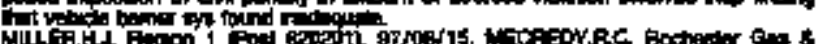

Tococt

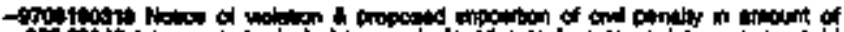

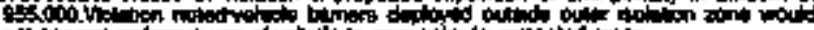

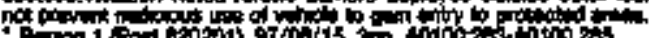

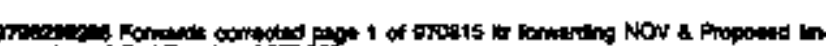

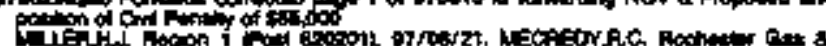

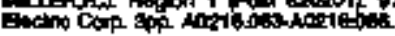

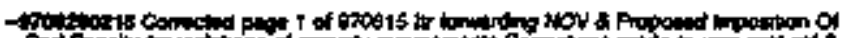

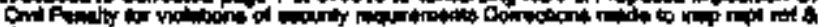

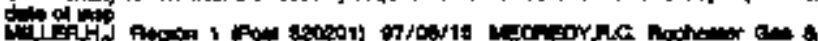

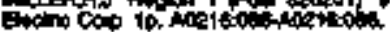

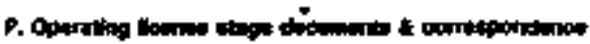

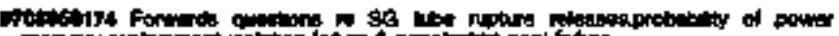

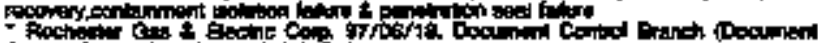

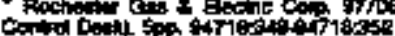

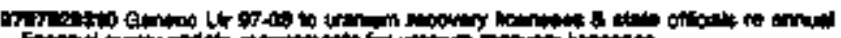

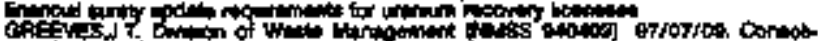

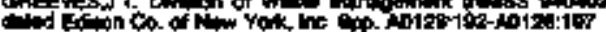

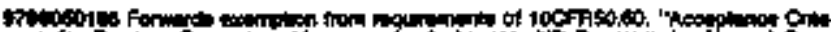

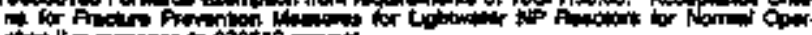
Mista $40000.117+0000$ id

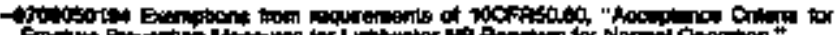
OOIN

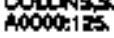




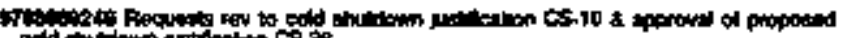

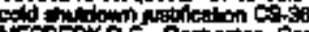

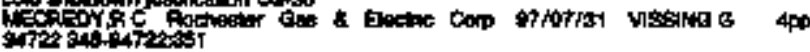

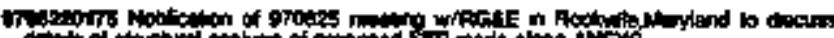

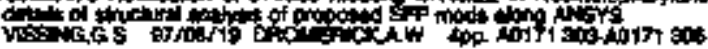

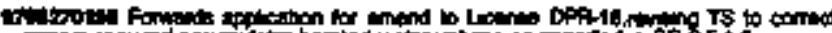

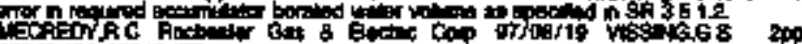

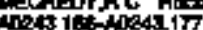

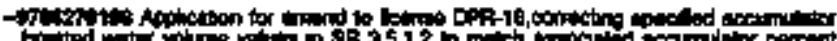

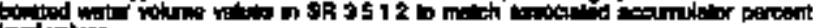

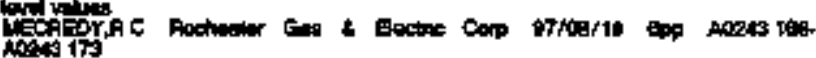

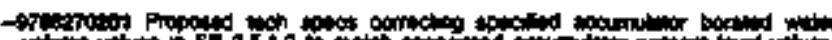

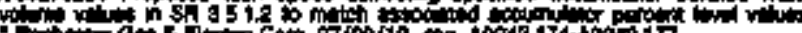

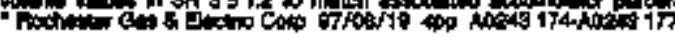

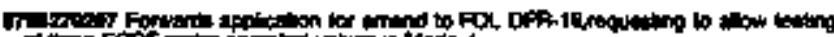
of torition.

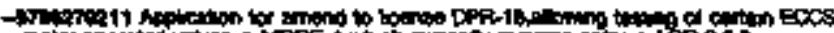

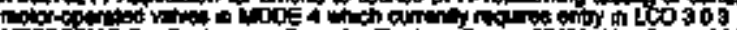

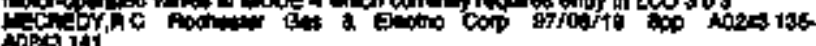

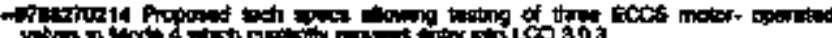

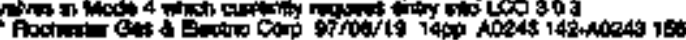

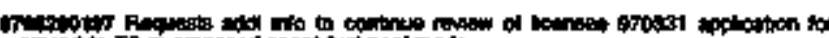

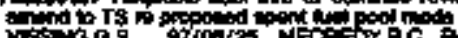

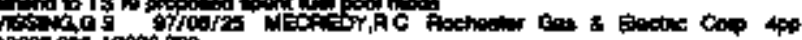

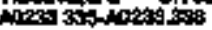

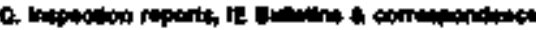

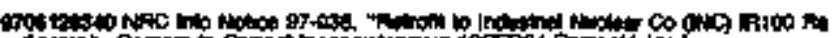

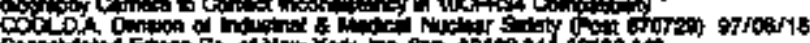

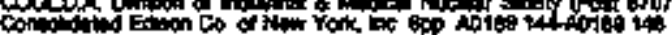

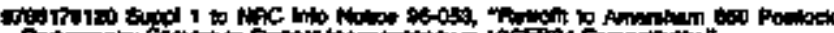

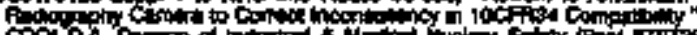

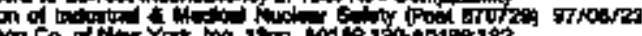

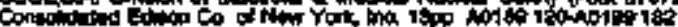

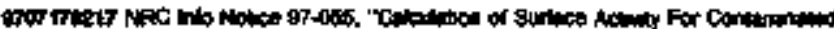

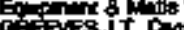

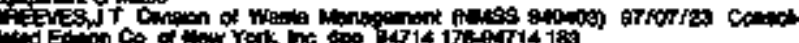

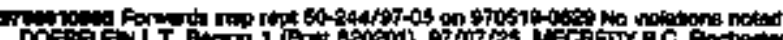

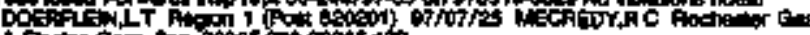

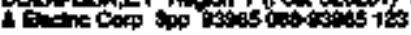

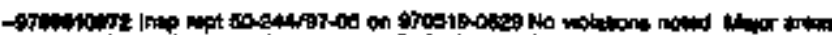

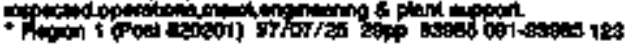

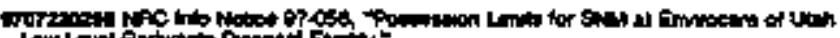

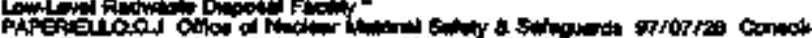

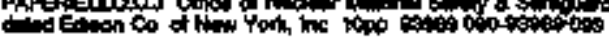

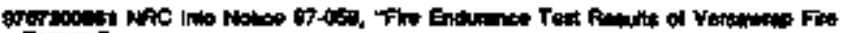

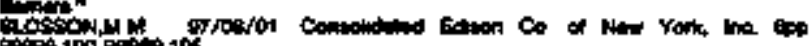
100000

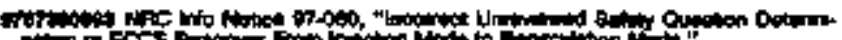

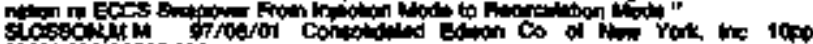
定

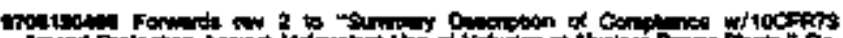

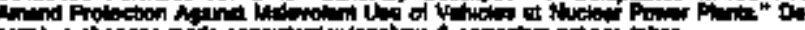

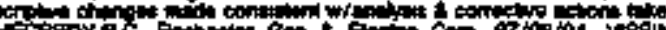

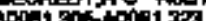

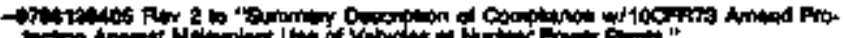

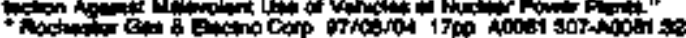

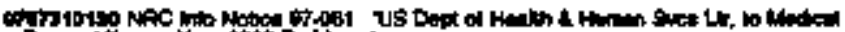

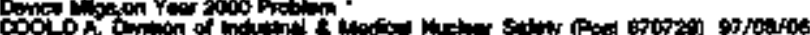

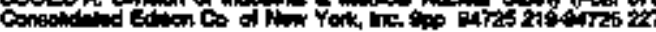

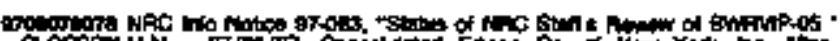

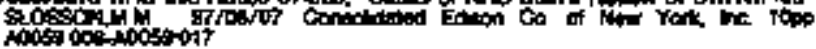

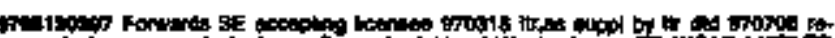

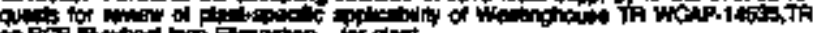

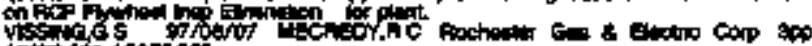

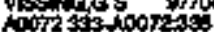

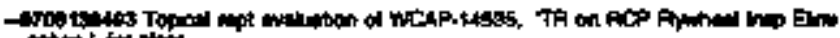

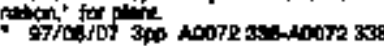

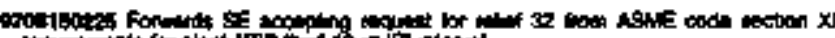

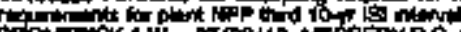

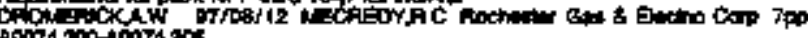

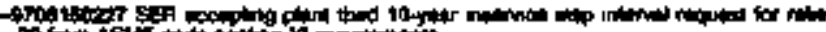

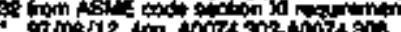

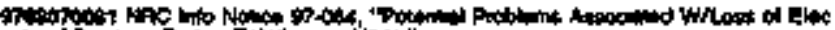

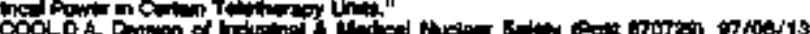

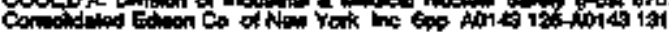

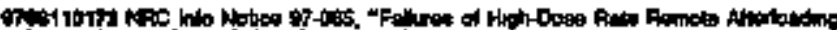

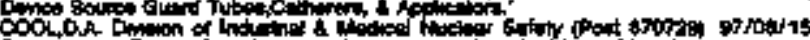

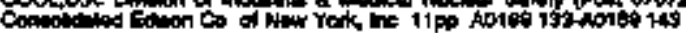

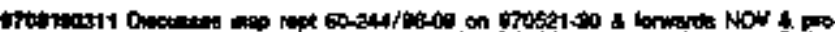

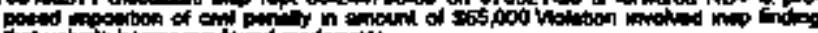

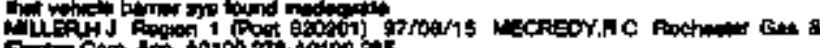

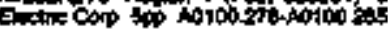

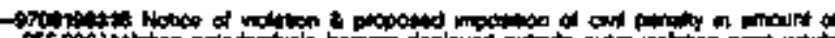

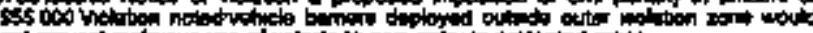

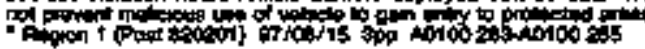

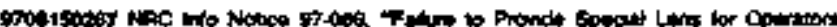

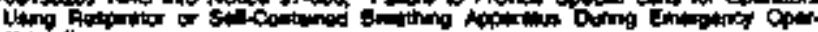

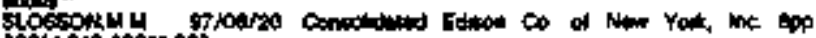

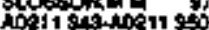

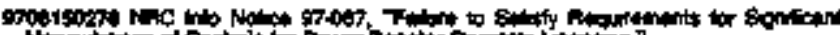

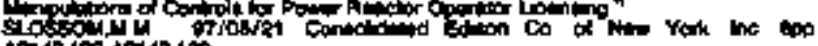
1004351301496

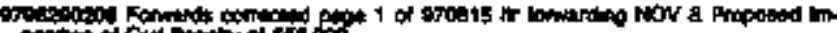

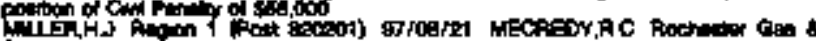

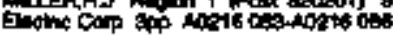

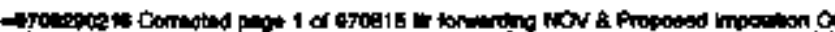

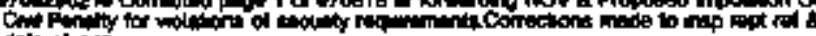

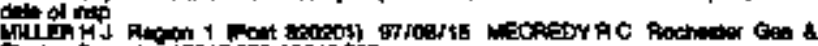

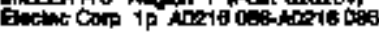

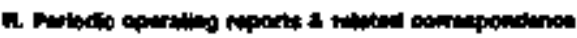

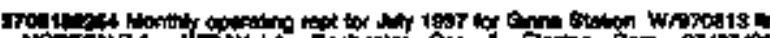

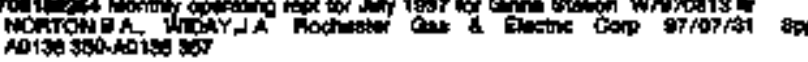

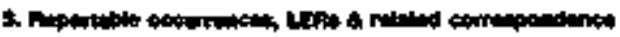

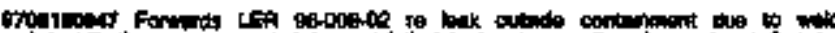

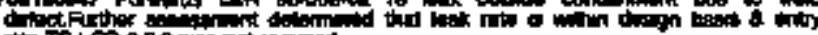

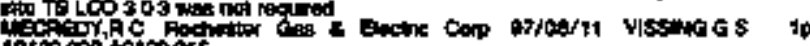

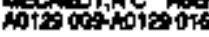

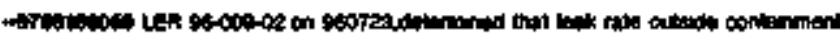

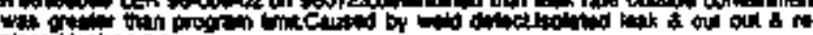

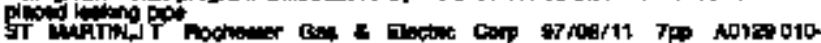
intorito

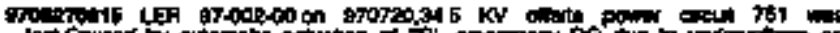

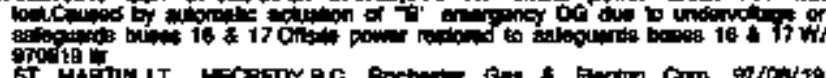

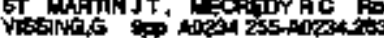

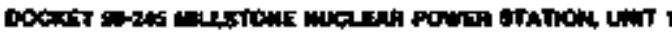

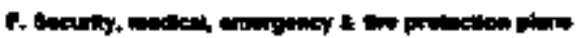

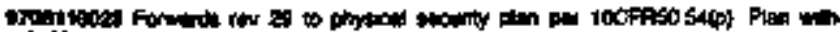

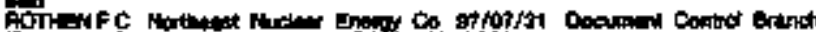

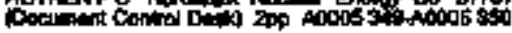

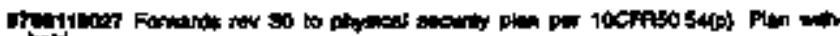

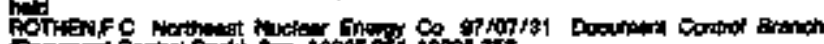

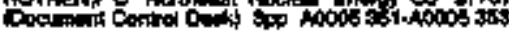

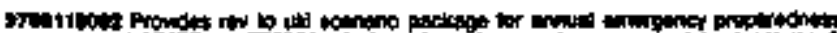

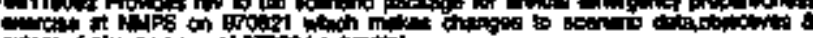

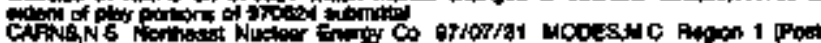

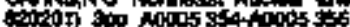




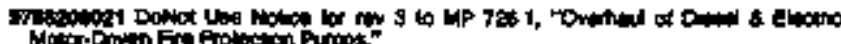

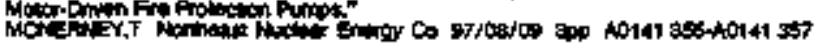

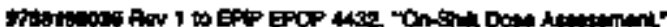

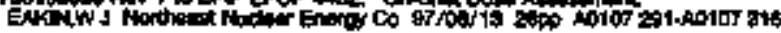

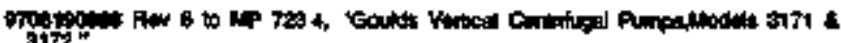

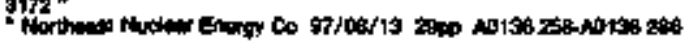

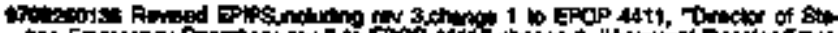
wan Entrency Ogor:

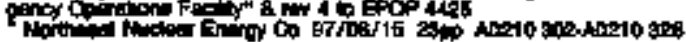

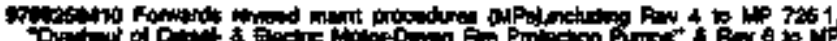

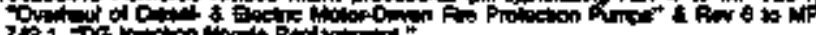

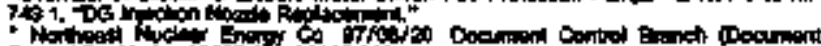

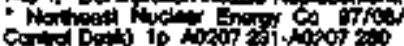

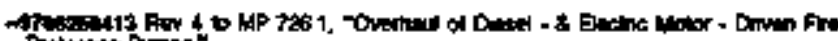

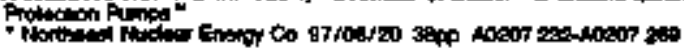

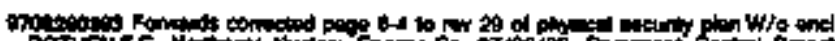

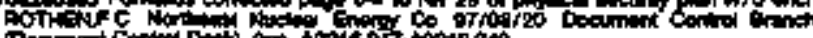

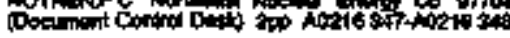

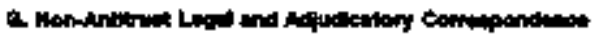

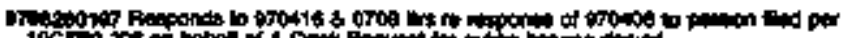

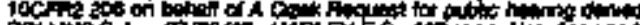

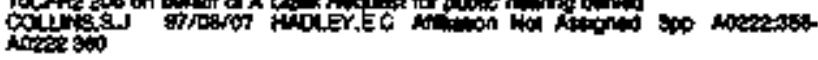

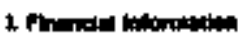

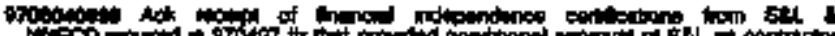

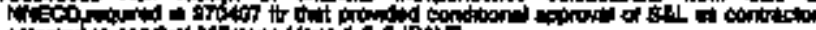

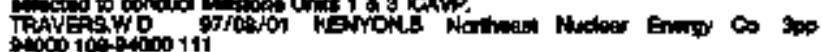

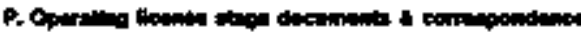

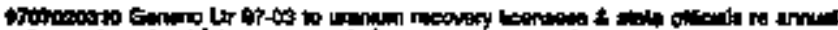

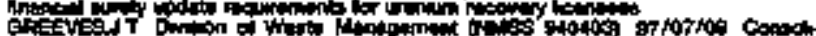

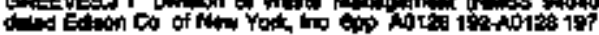

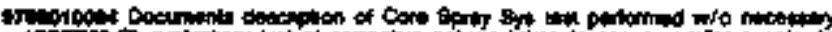

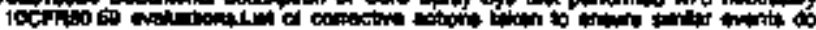

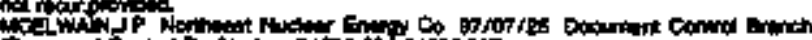

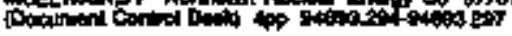

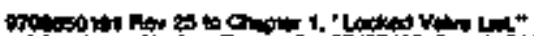

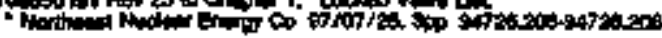

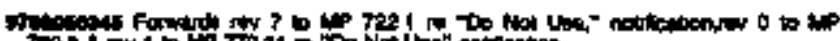
cond

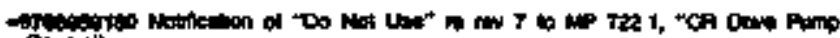

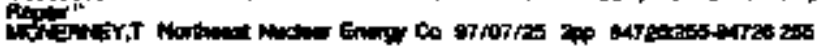

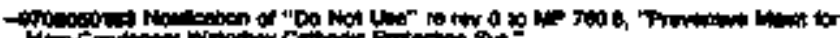

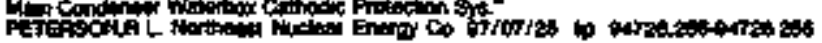

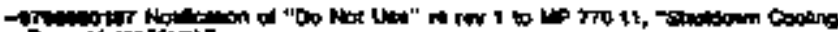

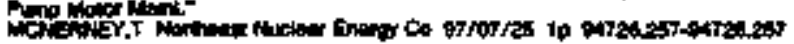

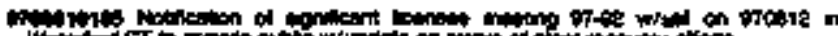

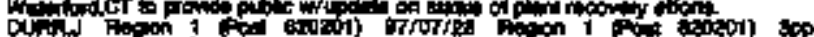

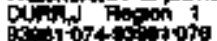

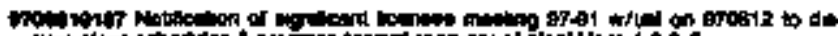

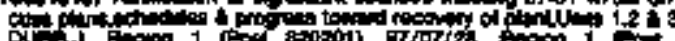

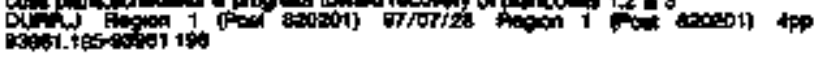

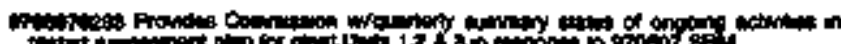

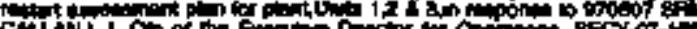

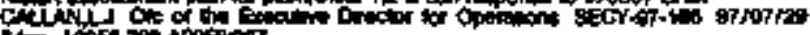

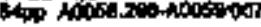

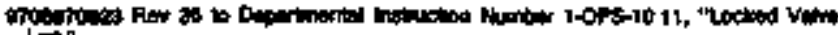

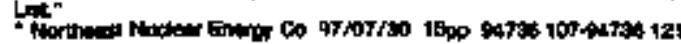

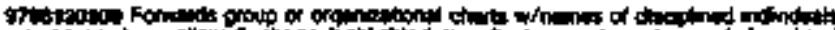

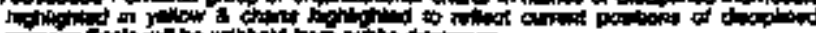

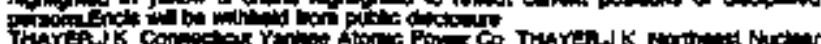

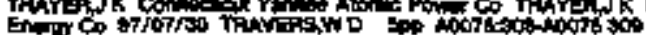

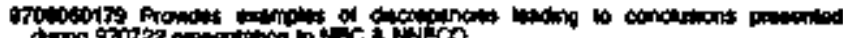

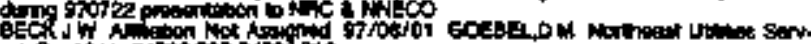

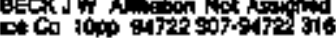

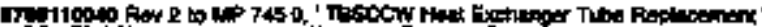

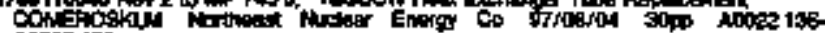
Aon? tes

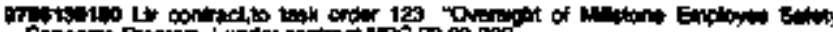

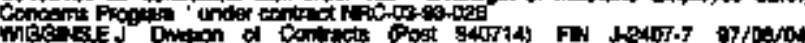

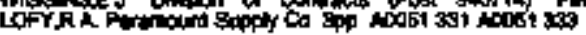

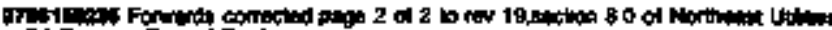

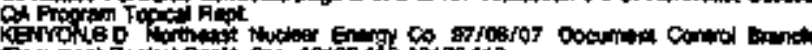

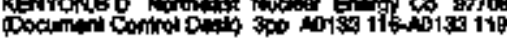

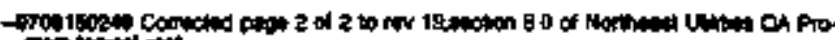

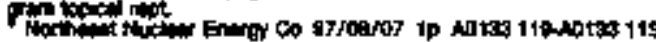

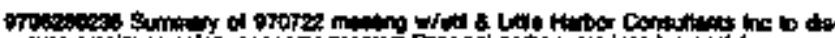

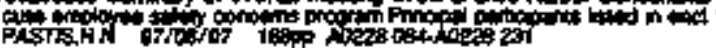

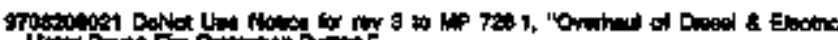

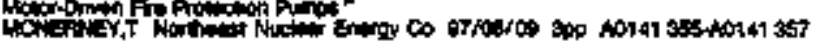

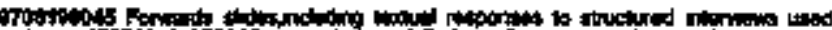

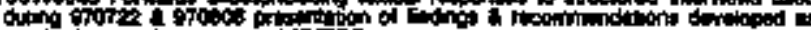
Hot of

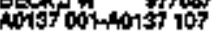

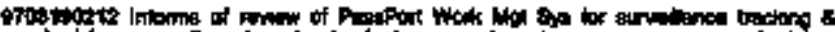

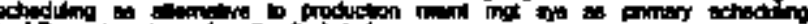

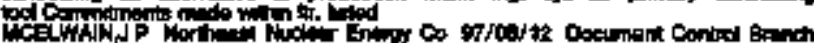

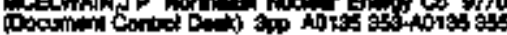

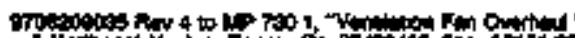

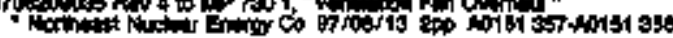

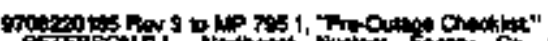

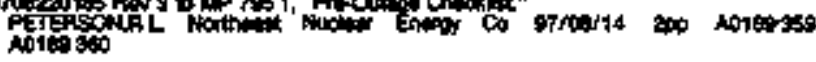

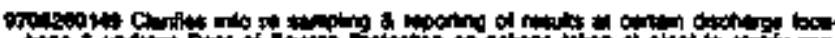

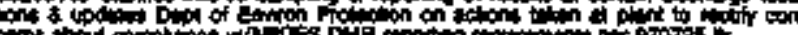

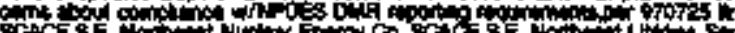
由Jis

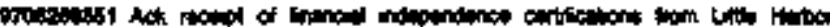

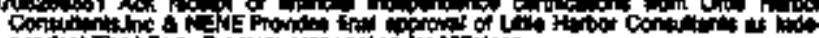

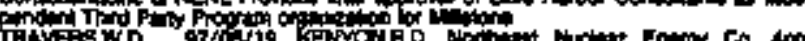

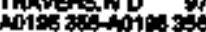

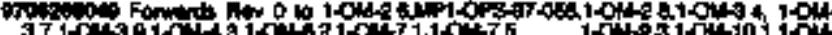

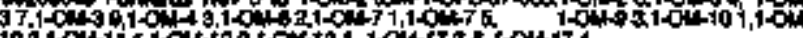

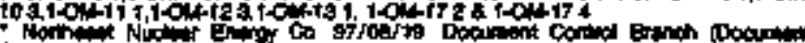

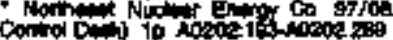

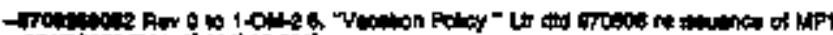

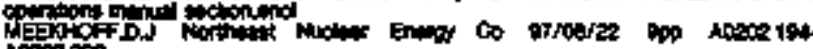
Alowe

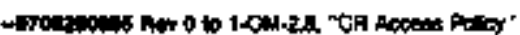

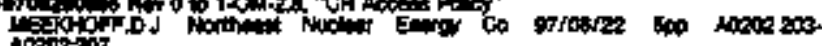

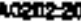

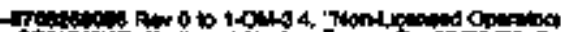

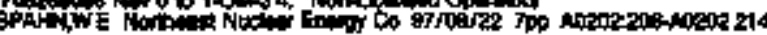

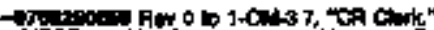

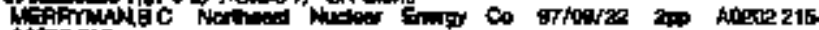
40.02 216

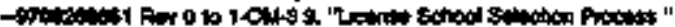

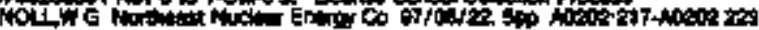

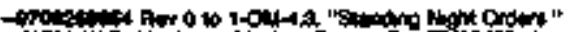

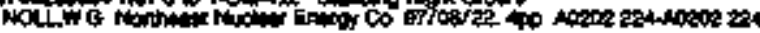

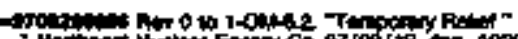

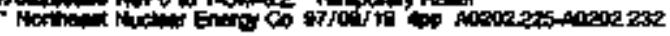

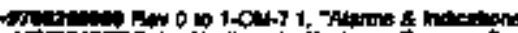

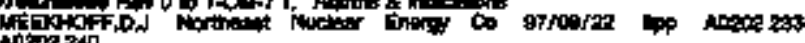
Maspent

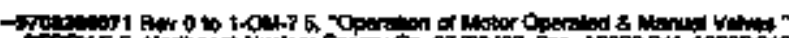

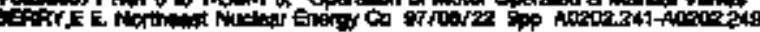

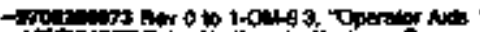

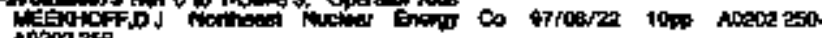

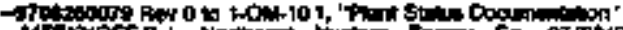
A A 


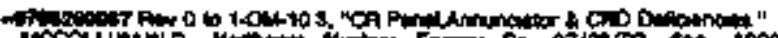

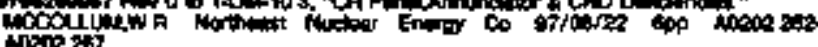

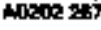

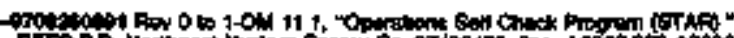

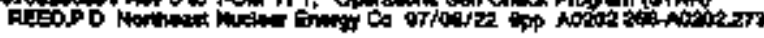

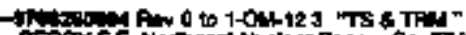

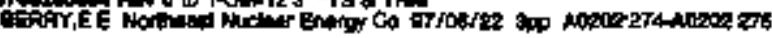

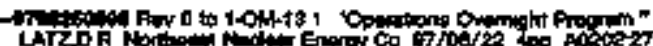

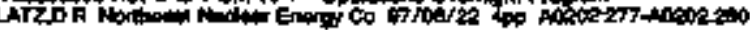

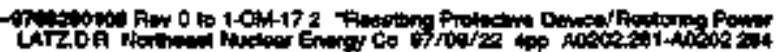

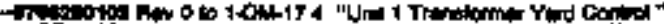

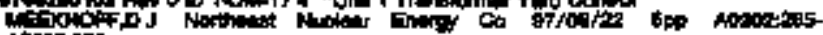
100030

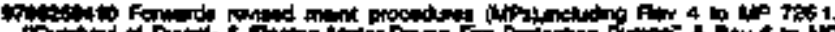

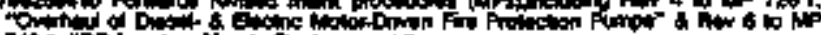

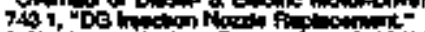

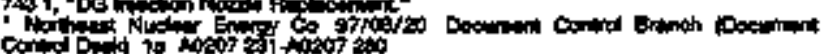

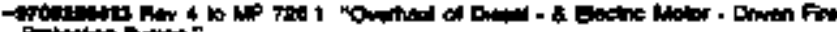

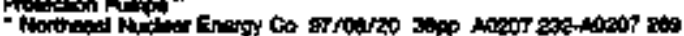

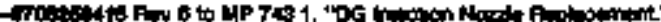

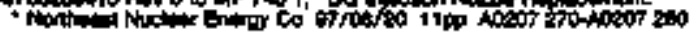

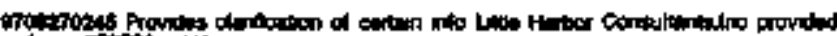

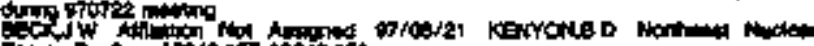

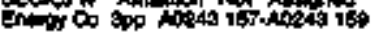

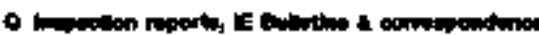

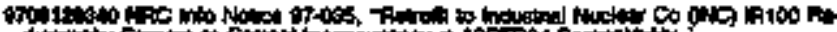

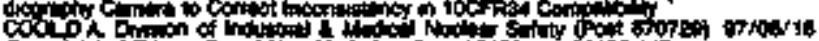

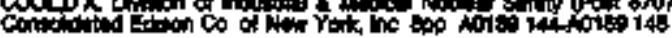

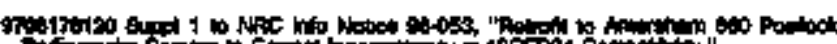

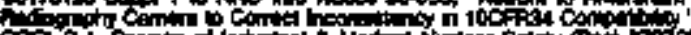

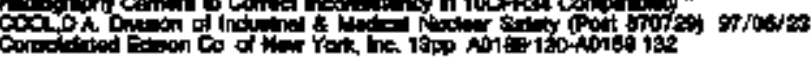

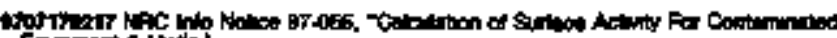

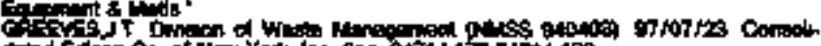

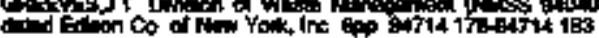

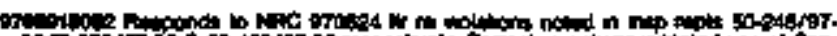

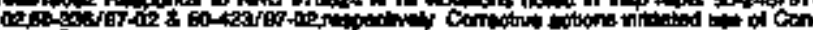

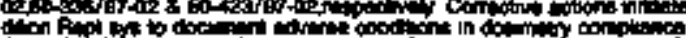

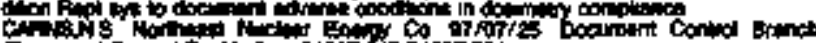

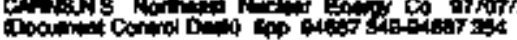

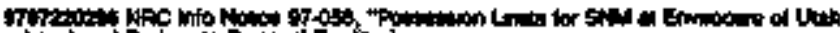

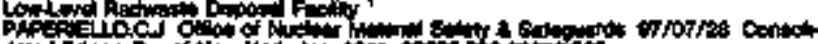

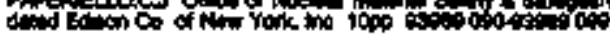

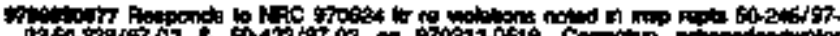

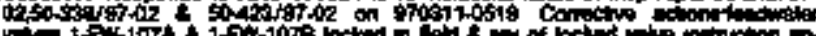

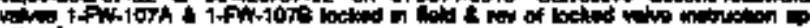
otons

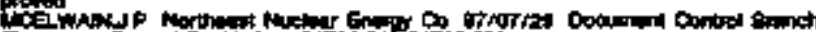

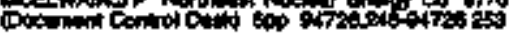

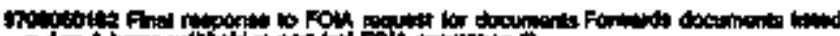

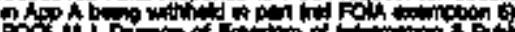

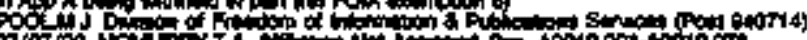

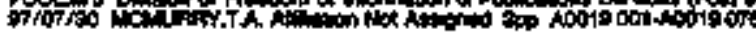

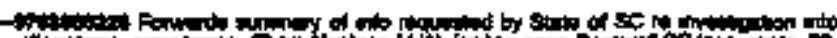

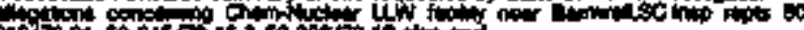

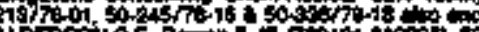

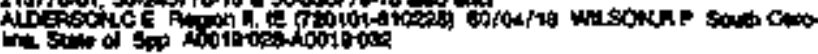

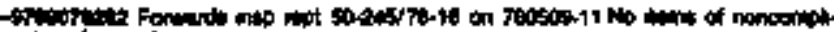
WA

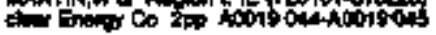

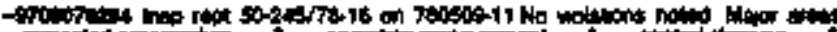

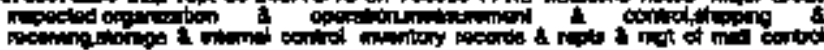

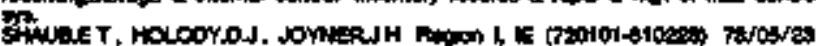

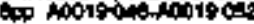

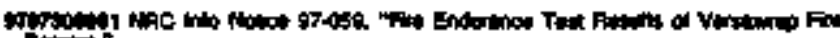

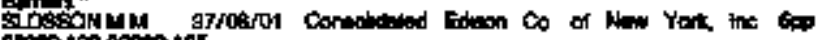

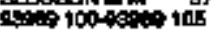

Dow

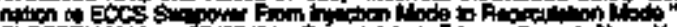

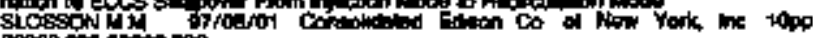

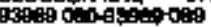

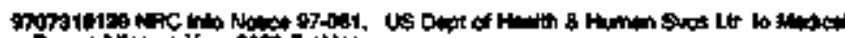

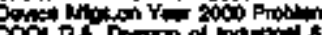

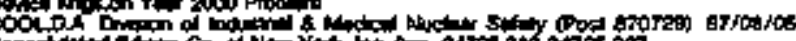

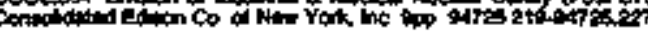

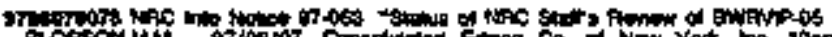

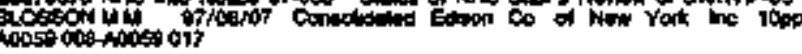

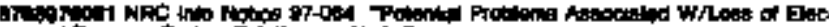

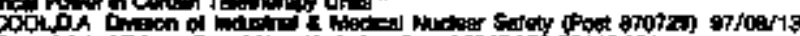

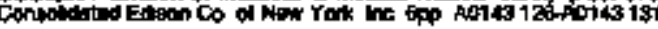

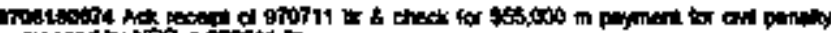

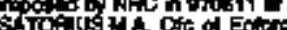

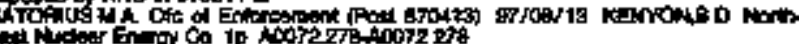

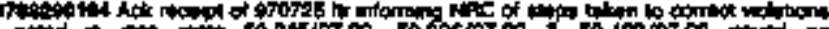

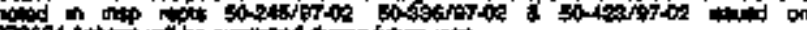

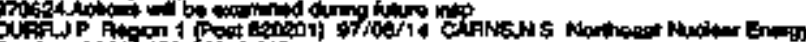

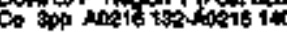

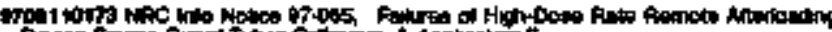

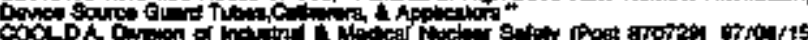

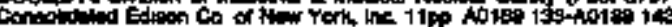

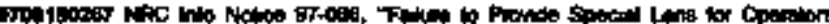

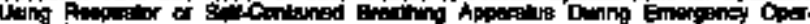

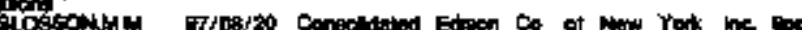

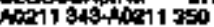

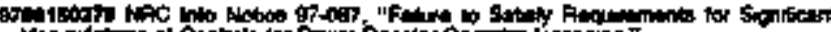

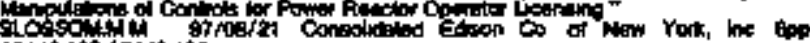

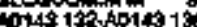

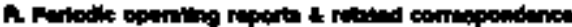

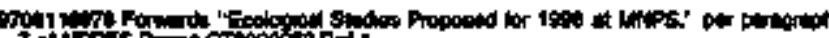

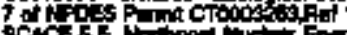
SdC Hal

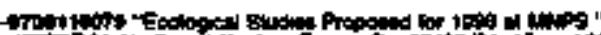

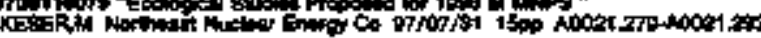

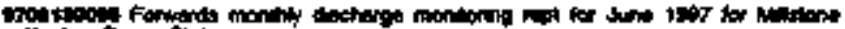

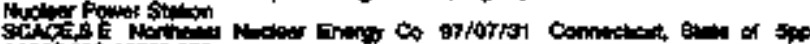

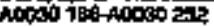

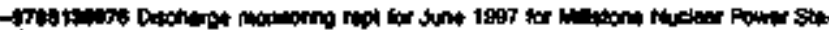

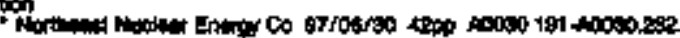

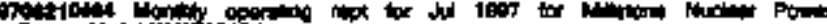

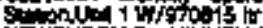

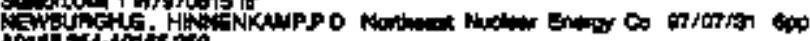

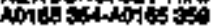

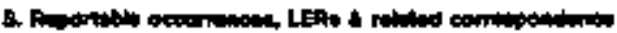

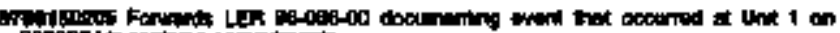

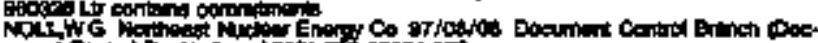

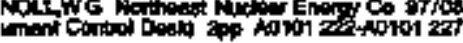

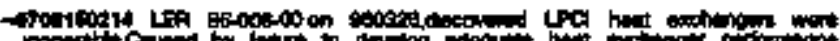

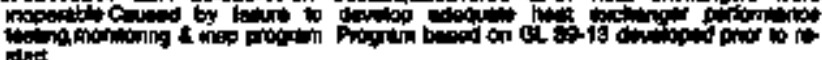

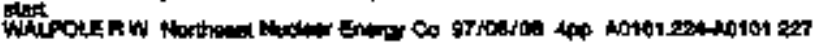

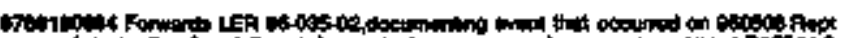

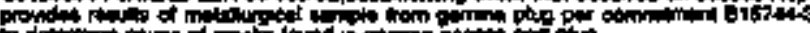

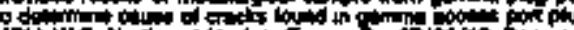

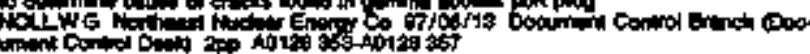

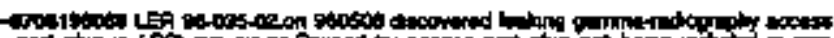

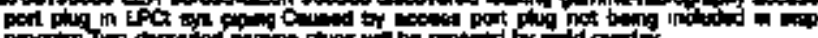

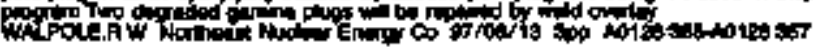

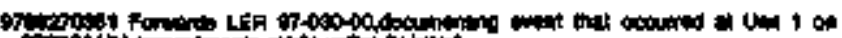

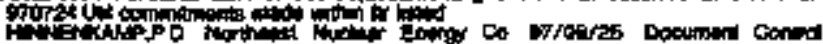

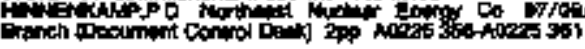

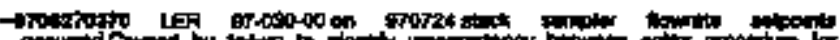

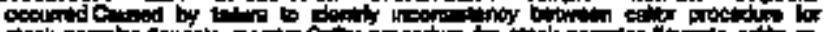

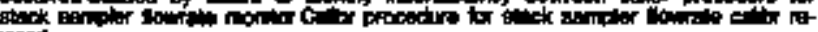

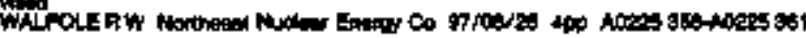




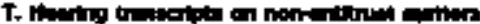

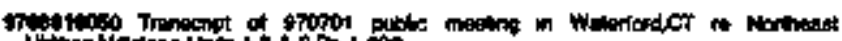

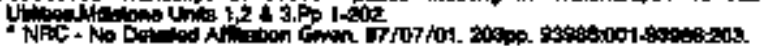

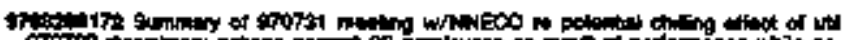

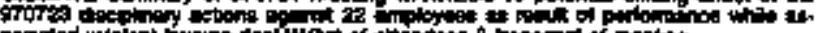

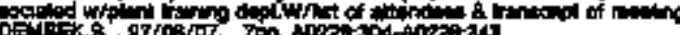

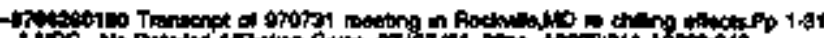

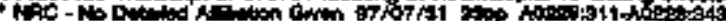

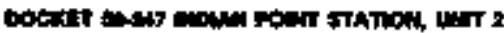

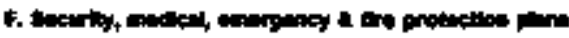

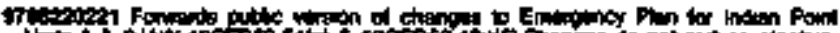

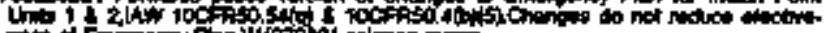

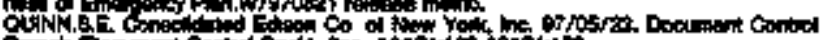

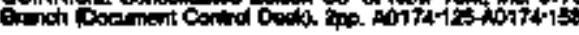

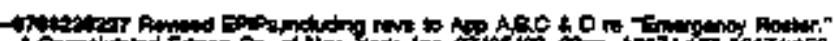

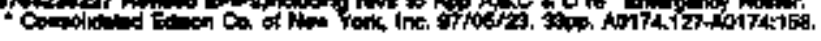

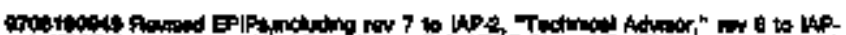

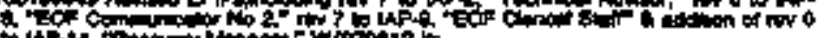

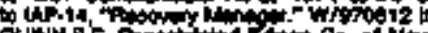

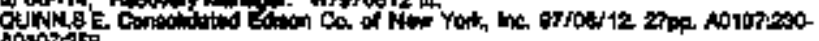
Antorse

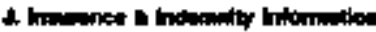

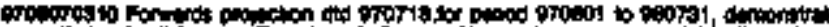

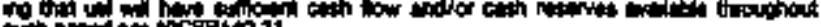

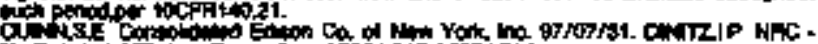

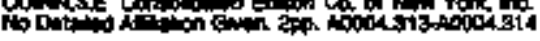

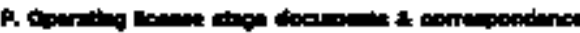

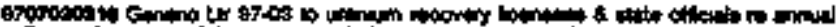

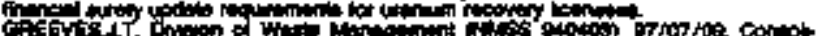

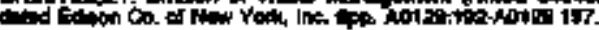

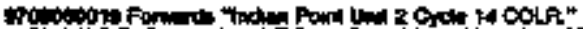

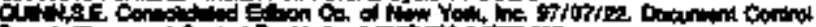

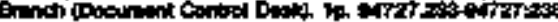

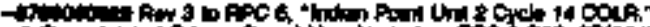

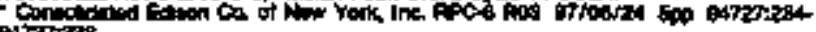

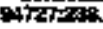

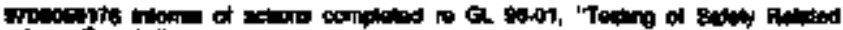

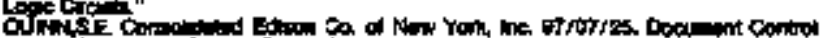

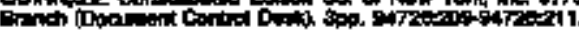

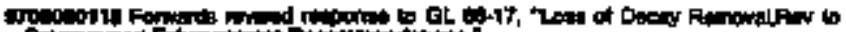

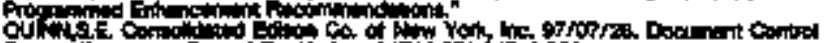

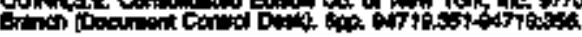

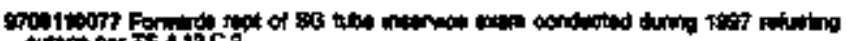

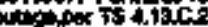

Ot if

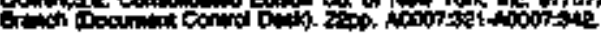

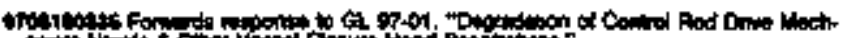

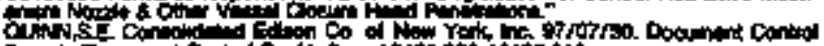

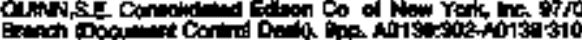

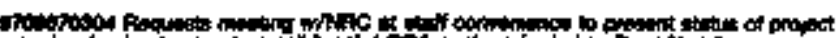

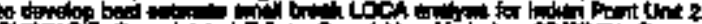

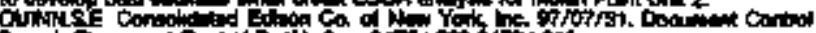

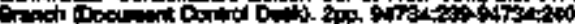

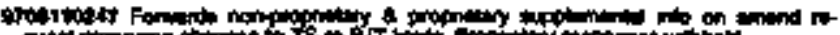

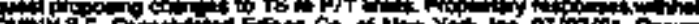

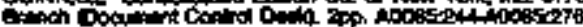

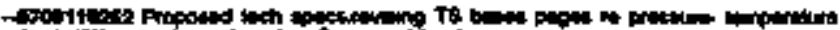

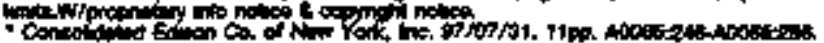

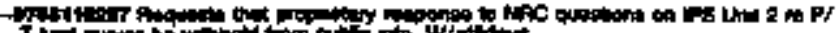

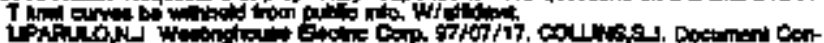

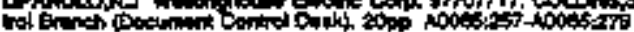

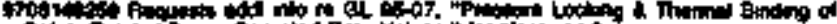

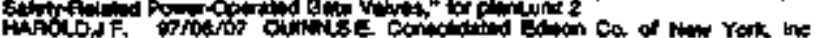

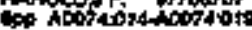

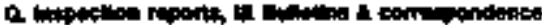

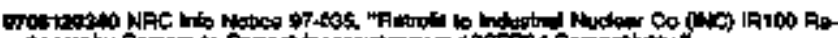

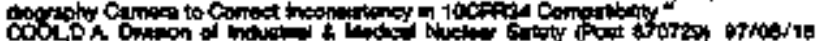

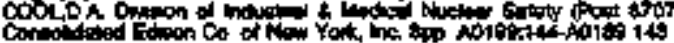

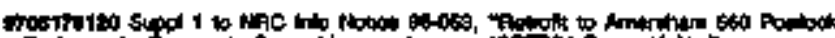

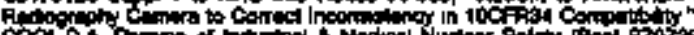

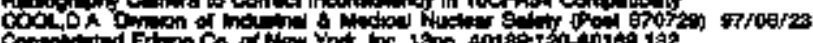

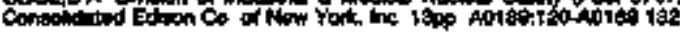

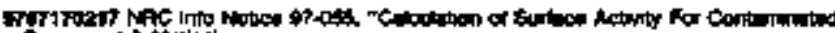

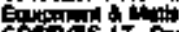

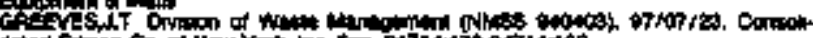

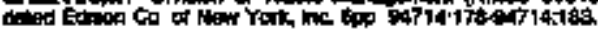

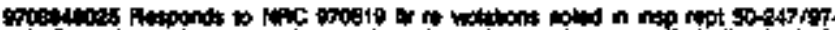

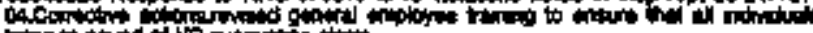

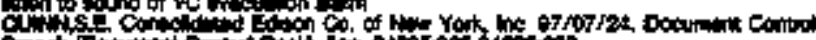

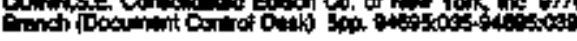

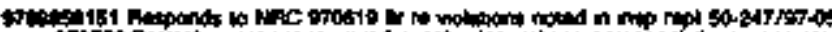

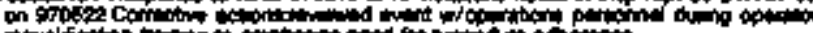

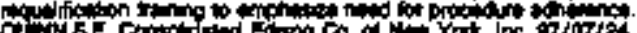

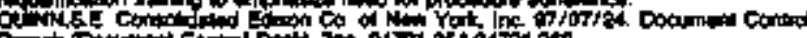

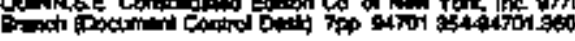

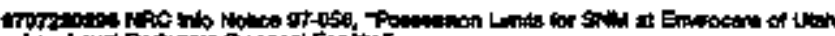
PAf

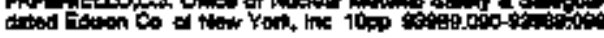

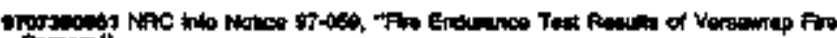

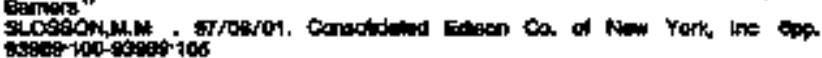

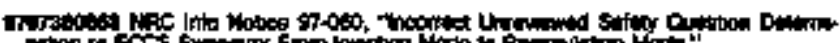

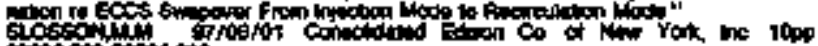

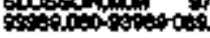

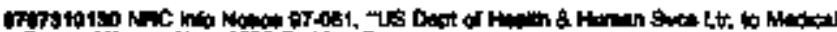

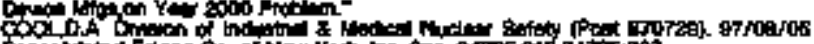

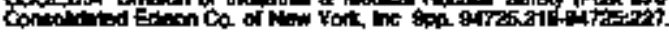

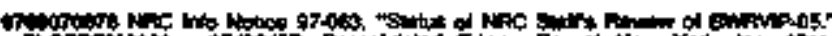

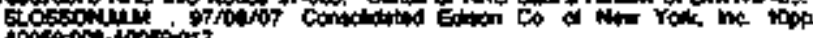

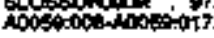

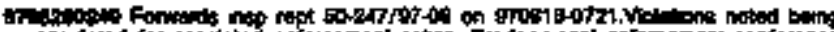

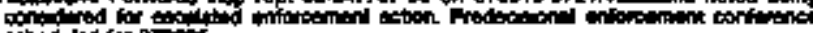

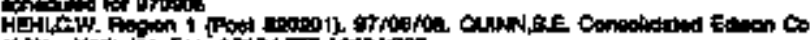

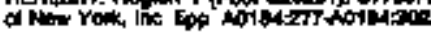

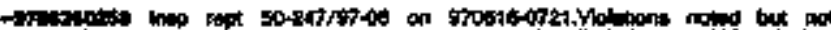

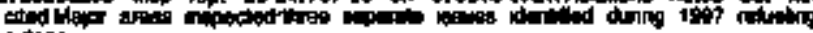

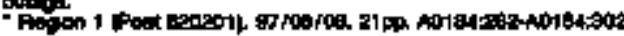

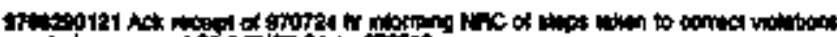
node

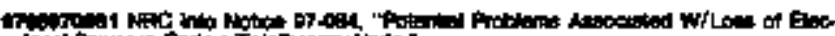

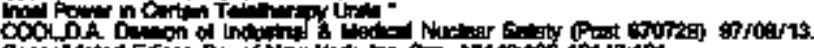
Contalinted Ef

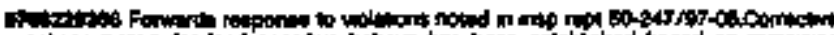

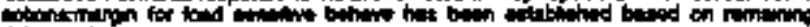

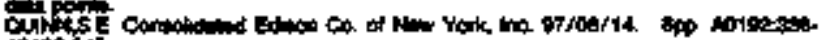
Antios 3 s.

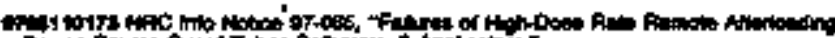

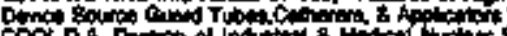

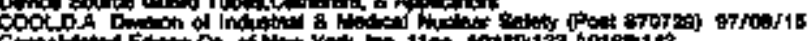

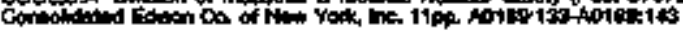

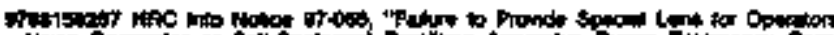

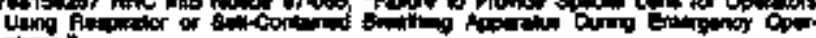

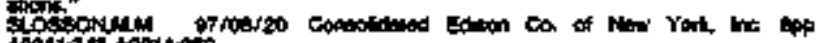

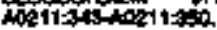

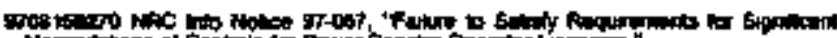

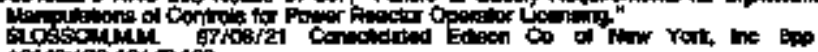

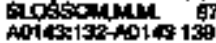

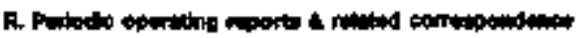

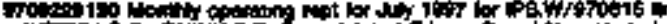

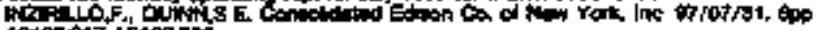

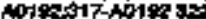




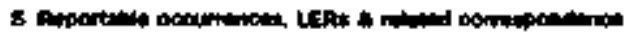

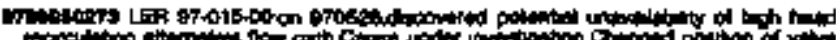

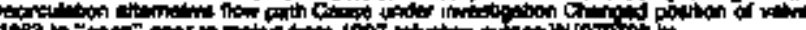

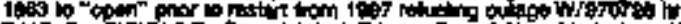

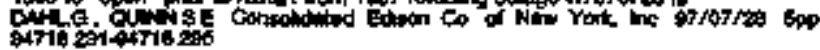

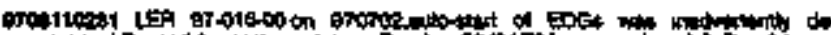

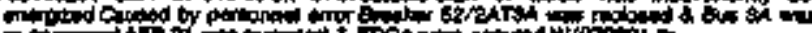

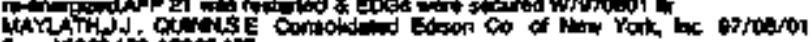

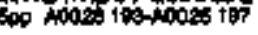

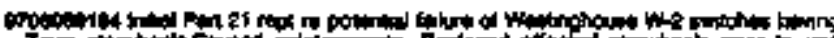

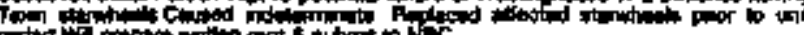

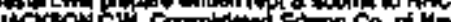

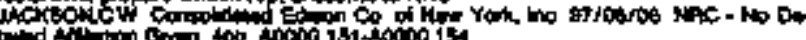

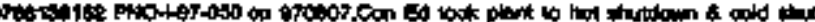

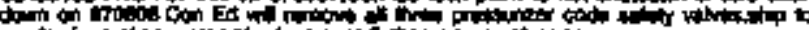

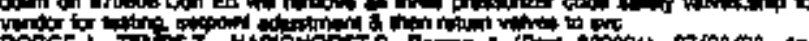

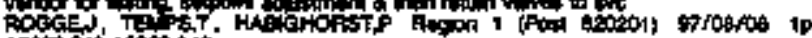

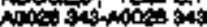

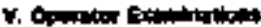

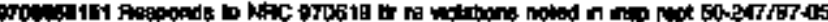

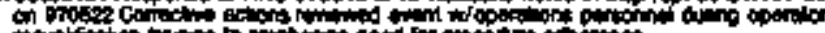

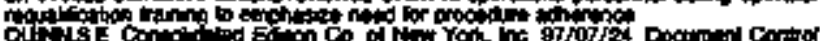

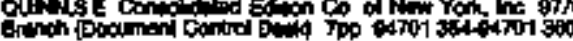

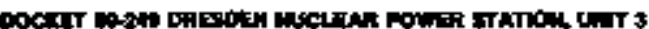

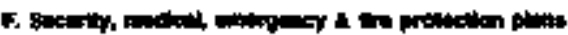

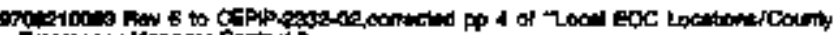

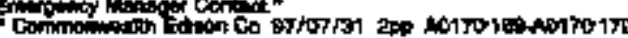

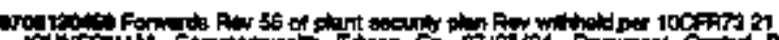

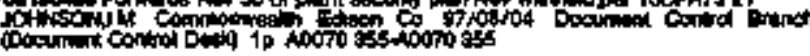

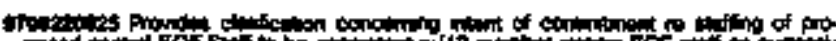

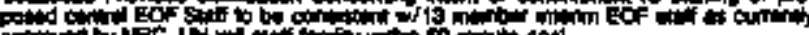

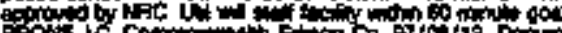

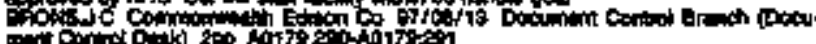

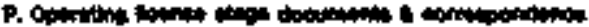

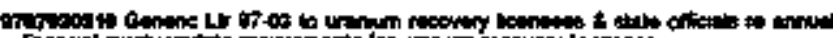

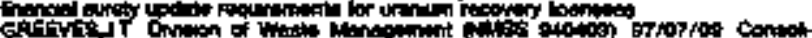

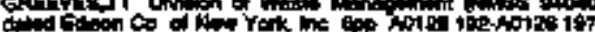

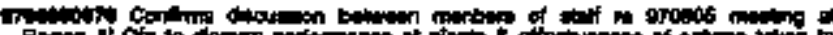

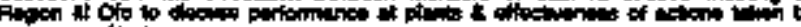

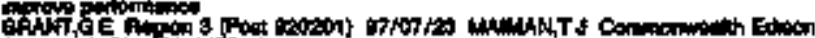
Co 200 antod of

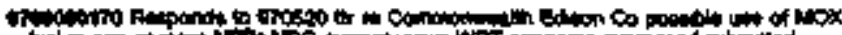

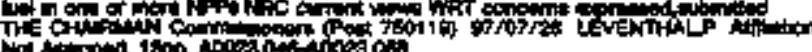

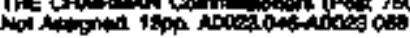

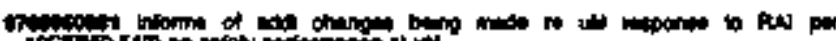

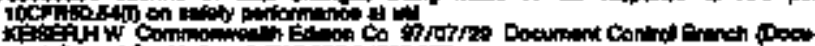

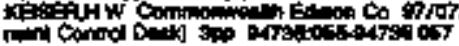

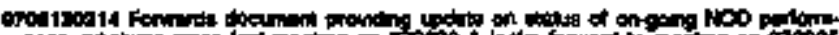

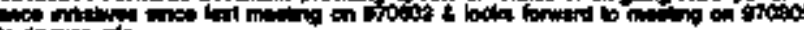

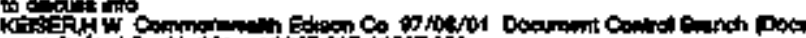

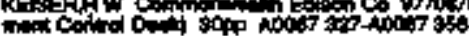

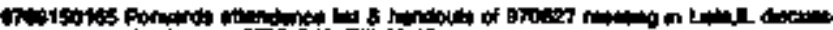

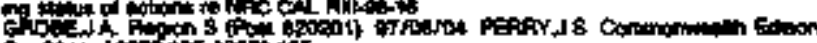

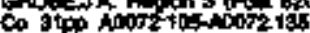

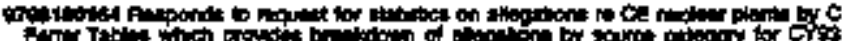

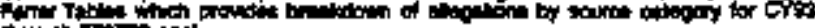

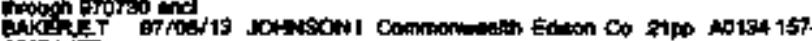
indal is

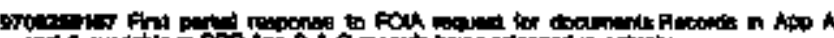

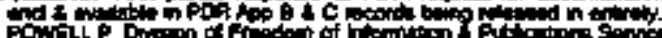

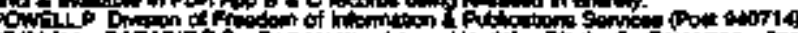

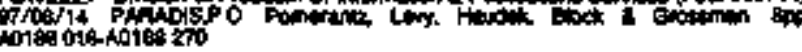

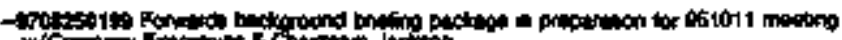

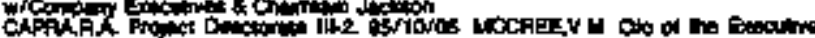

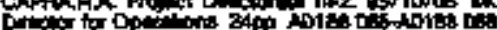

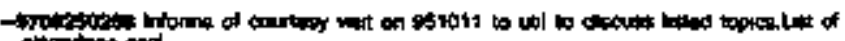
is

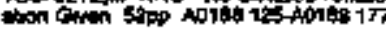

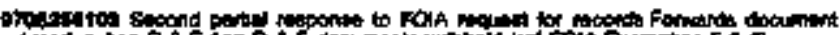

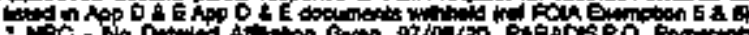

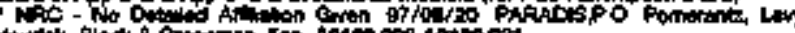

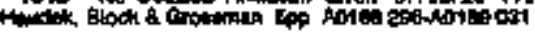

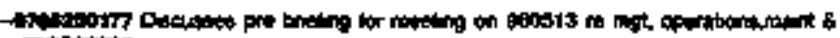

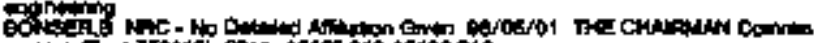

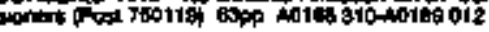

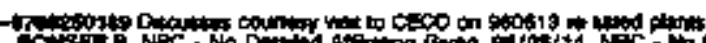

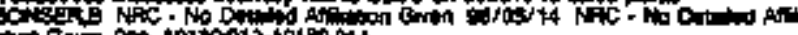

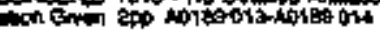

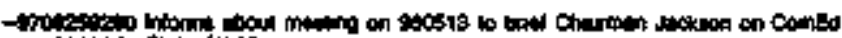

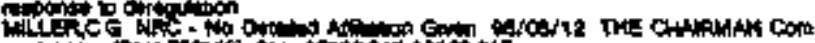
nutions

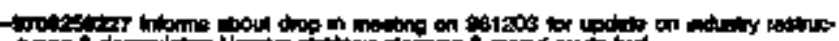

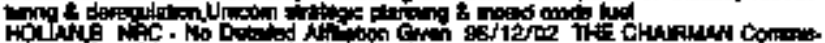

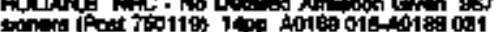

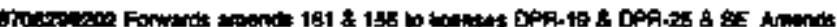
Hined T⿱宀⿻三丨口

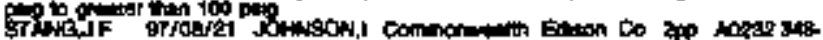
incestis

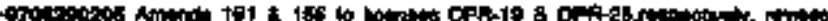

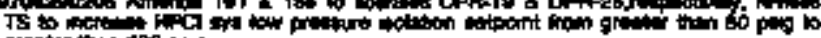

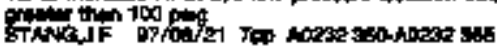

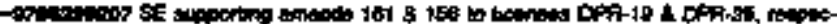
mots

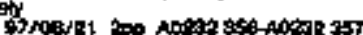

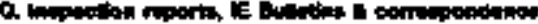

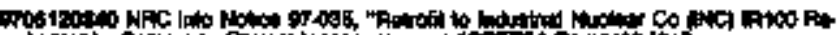

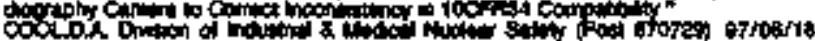

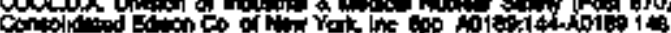

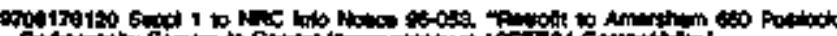

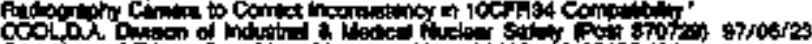

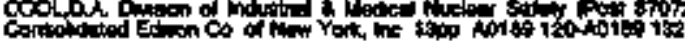

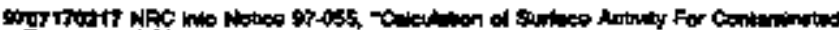

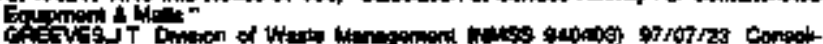

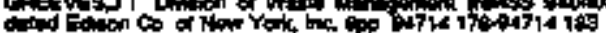

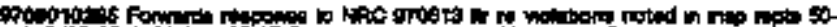

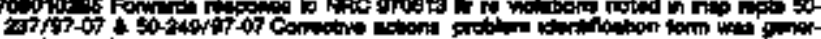

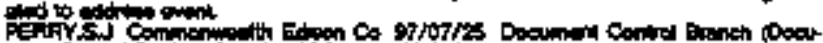

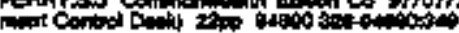

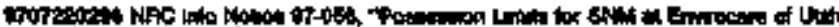
PA

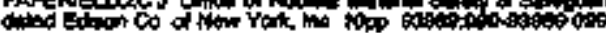

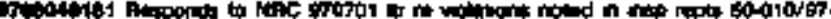

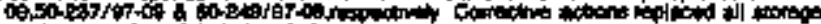

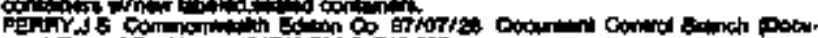

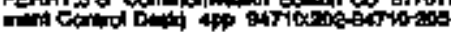

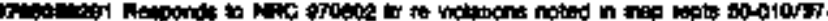

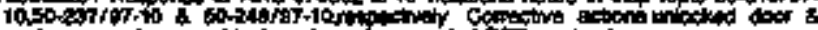

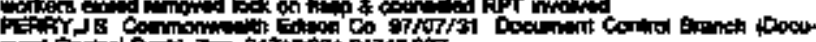

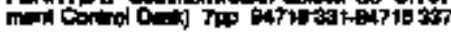

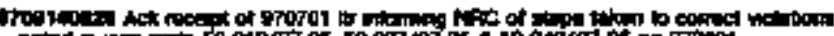

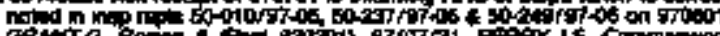

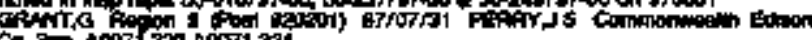

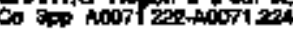

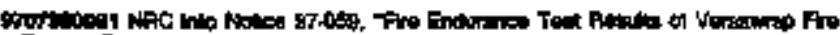

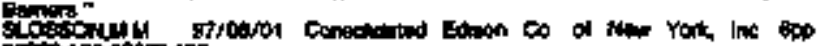
sinto 10000 105

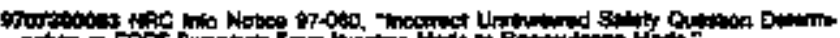

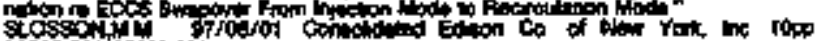
sitson

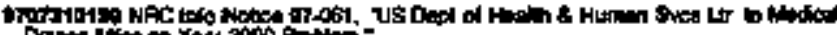

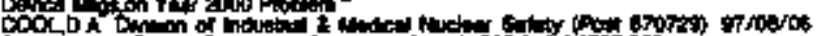

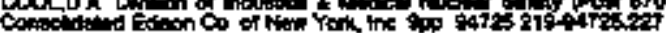




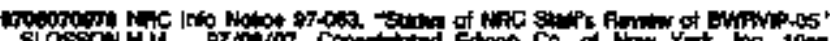

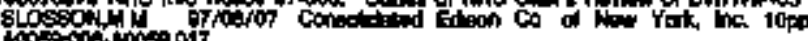

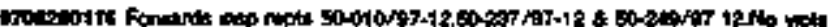

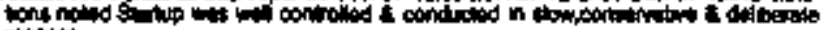

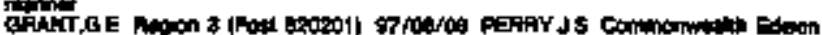

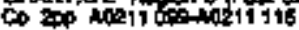

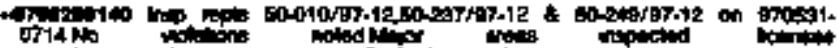

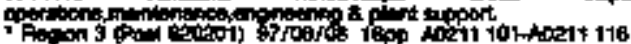

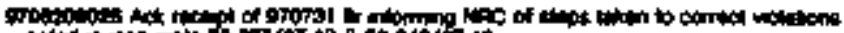
ny

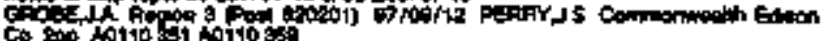

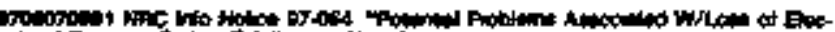
Co

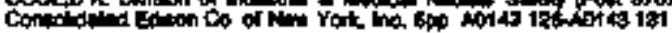

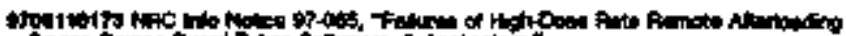

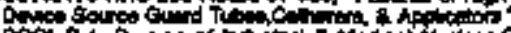

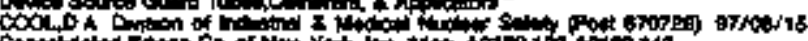

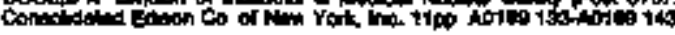

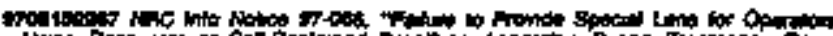

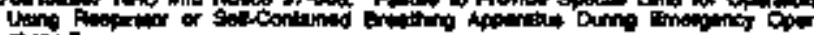

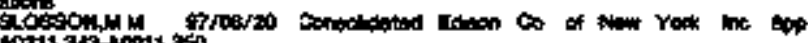

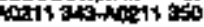

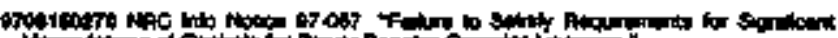

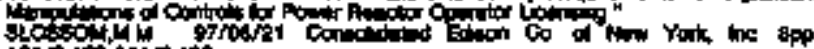

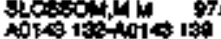

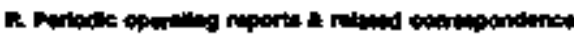

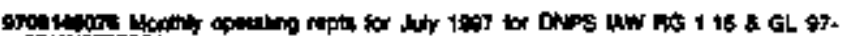

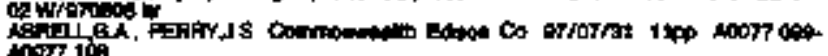

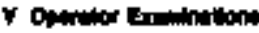

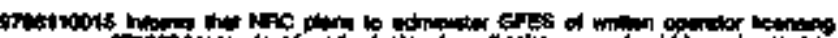

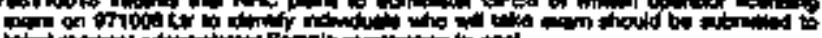

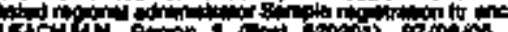

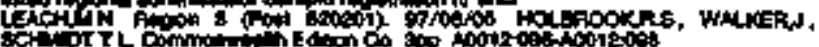

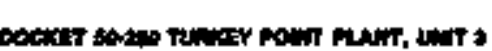

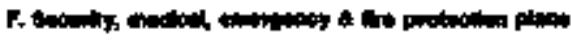

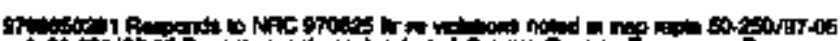

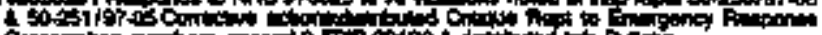

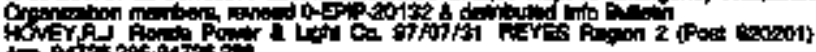

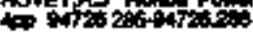

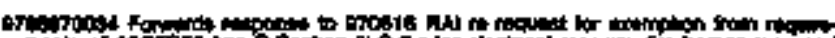

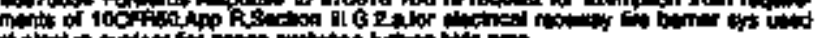
萑

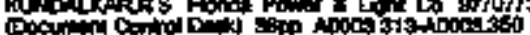

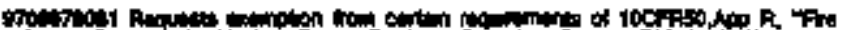

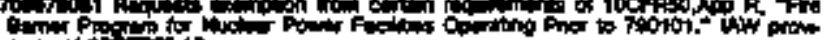
楠

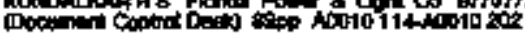

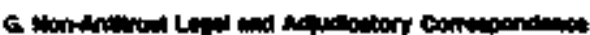

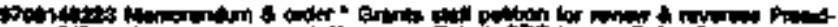

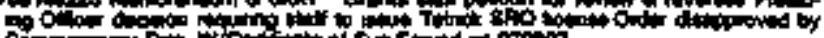

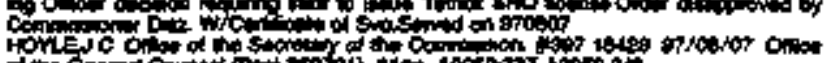

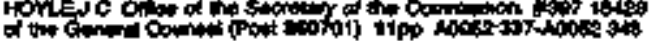

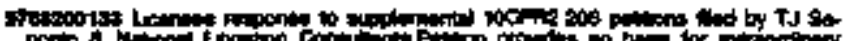

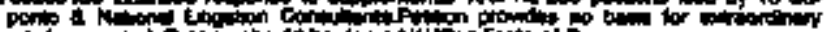

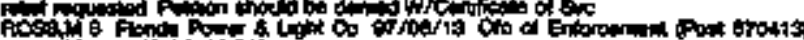

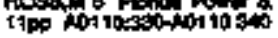

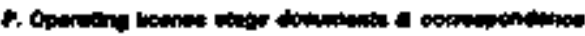

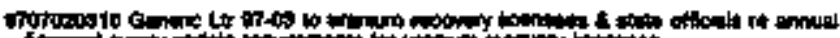

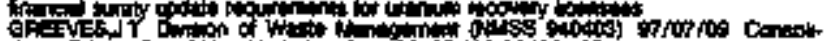

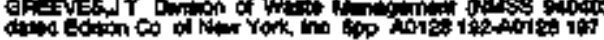

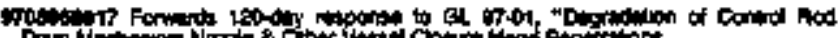

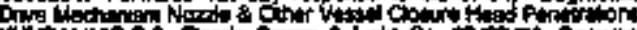

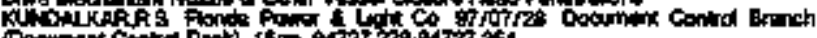

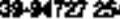

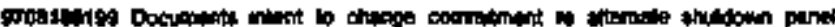

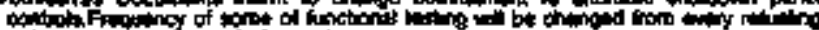

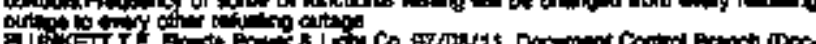

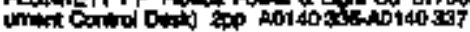

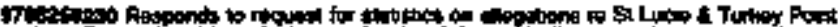

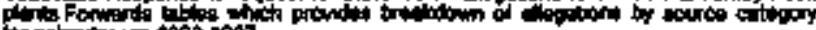

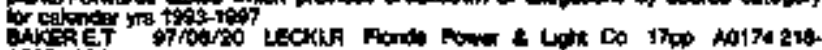
aitin the

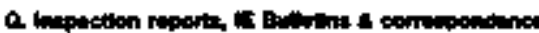

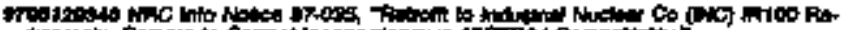

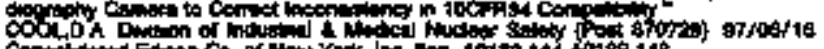

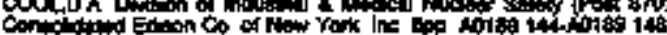

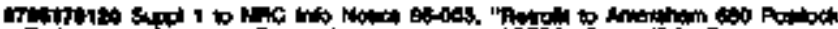

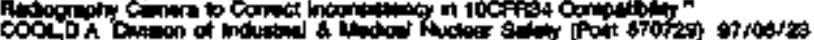

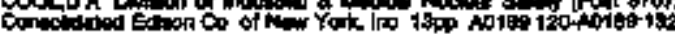

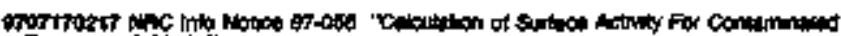

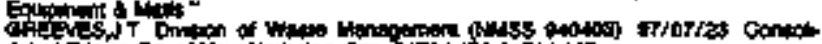

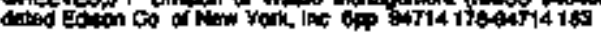

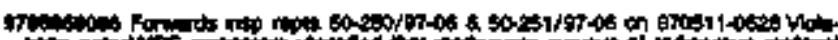

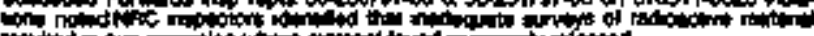

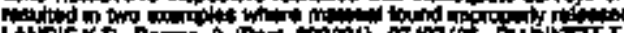

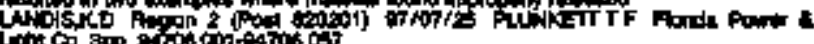

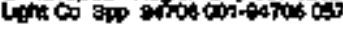

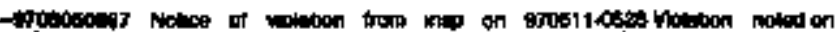

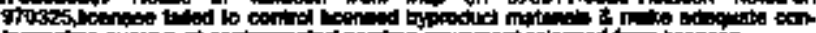

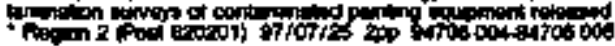

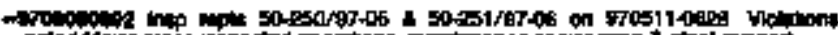

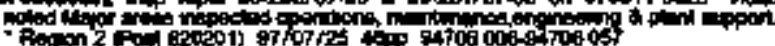

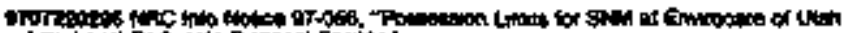

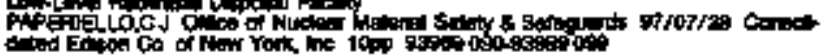

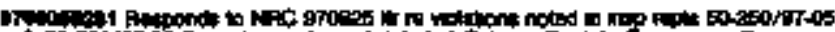

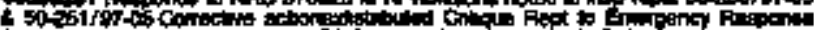

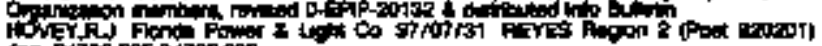

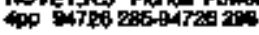

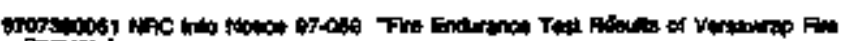

Fiosoly

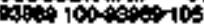

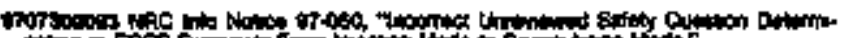

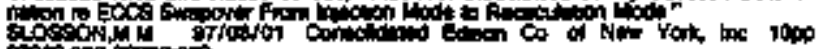

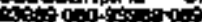

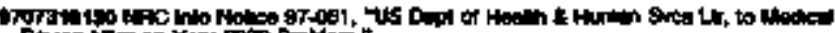

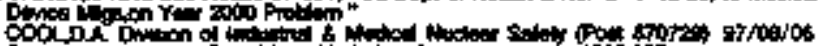

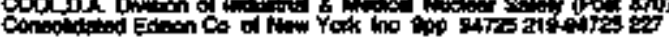

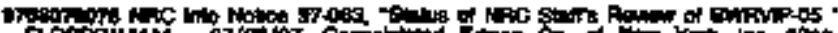

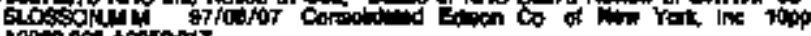

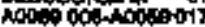

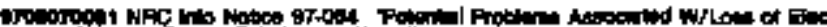

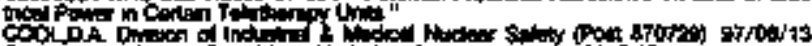

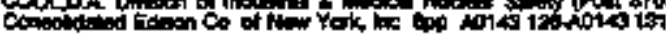

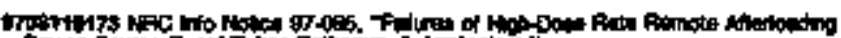

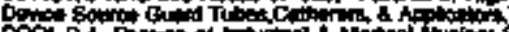

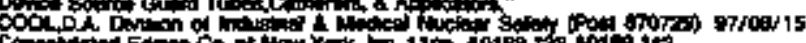

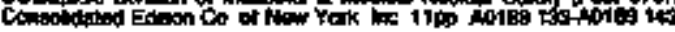

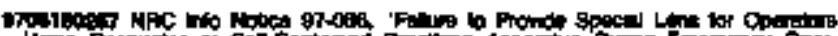

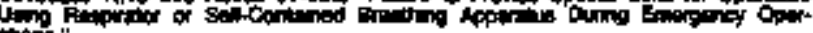

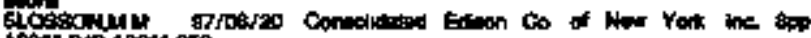
then 11 34 11 250

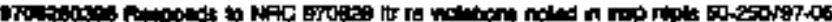
50051 f

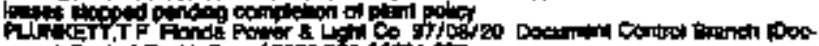

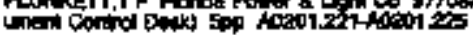

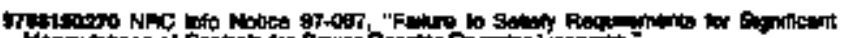

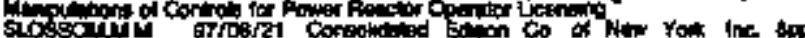

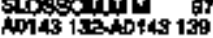




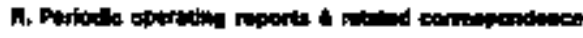

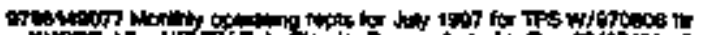

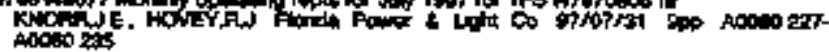

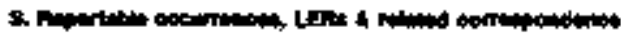

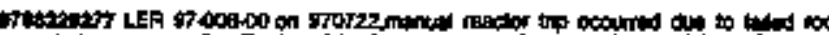

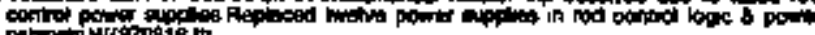

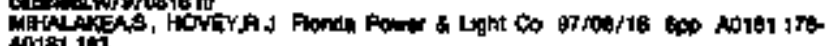
Nat8 10:

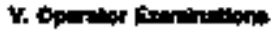

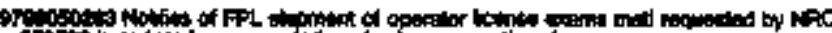

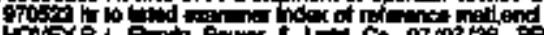

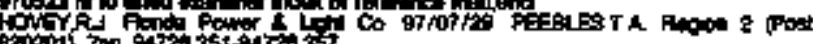

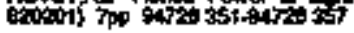

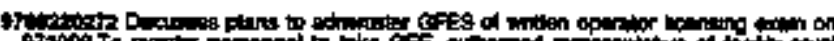

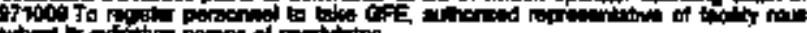

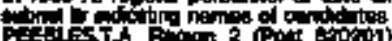

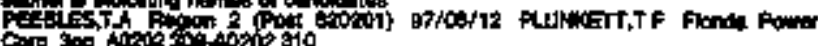

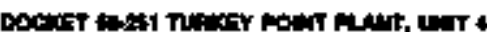

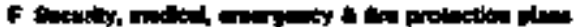

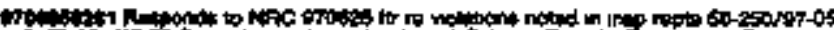

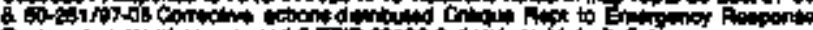

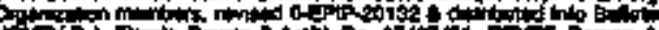
4 .

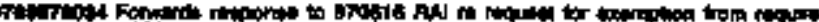

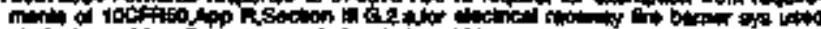

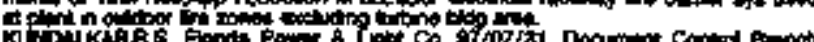

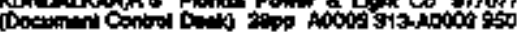

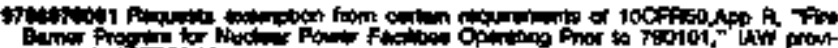

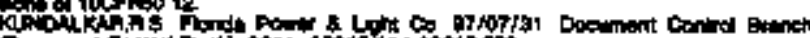

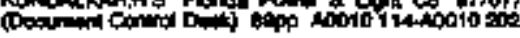

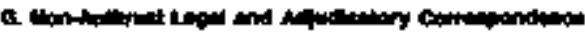

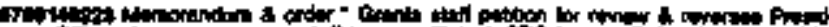

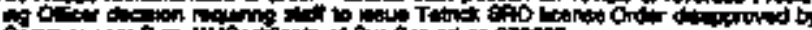

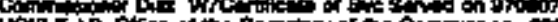

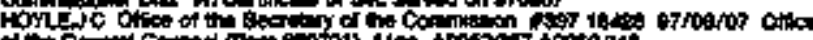

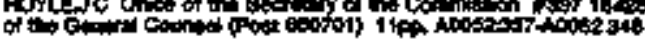

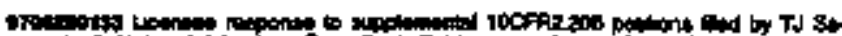

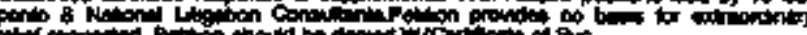

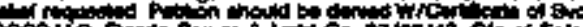

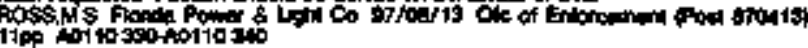

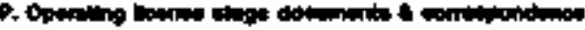

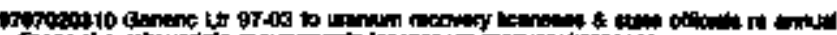

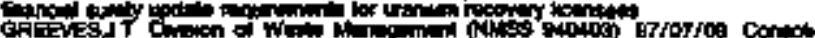

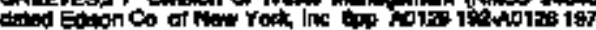

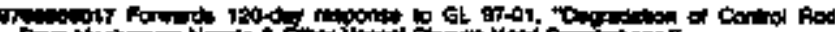

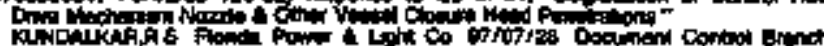

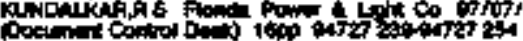

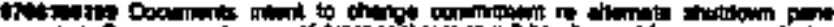

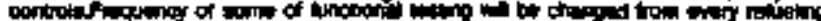

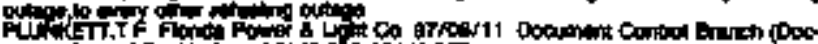

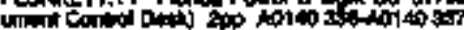

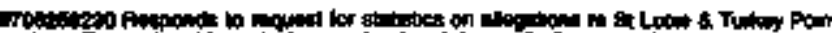

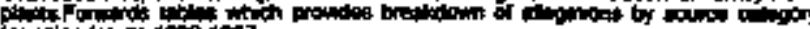

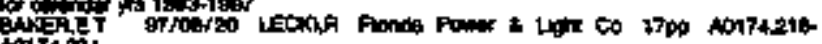
antion

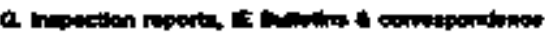

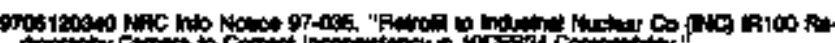

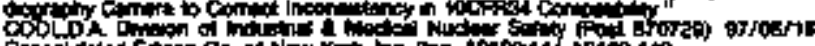

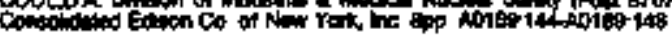

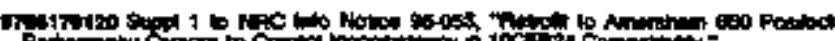

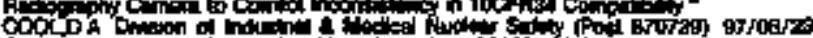

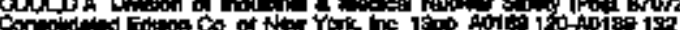

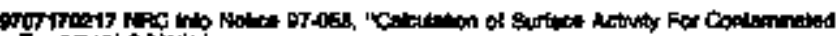

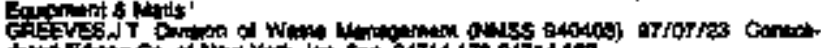

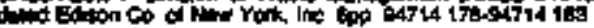

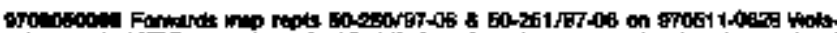

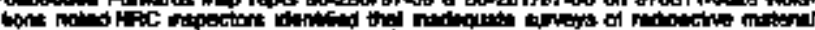

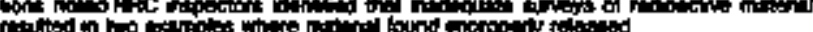

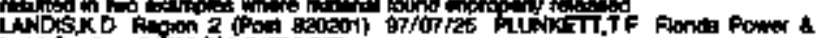

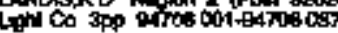

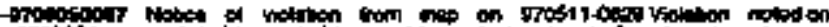

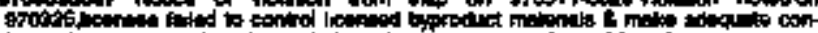

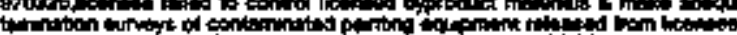

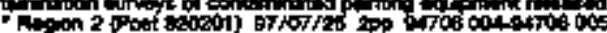

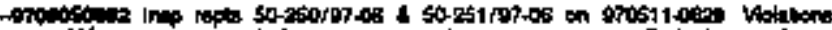

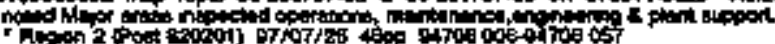

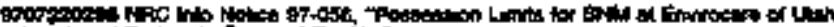

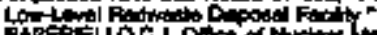

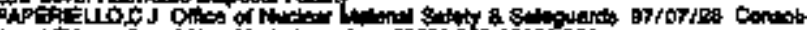

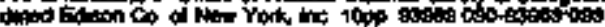

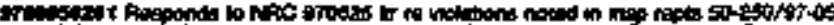

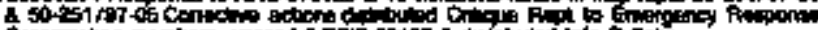

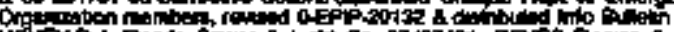

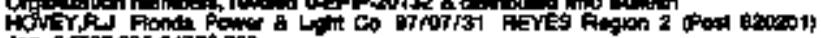

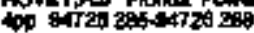

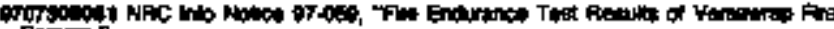

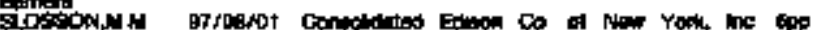

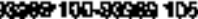

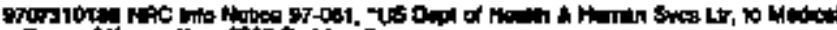
DOH

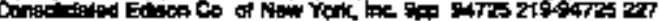

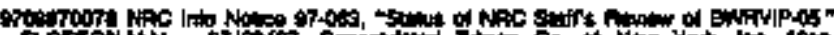

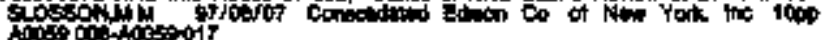

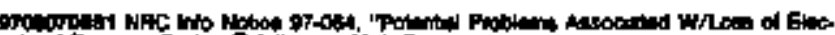

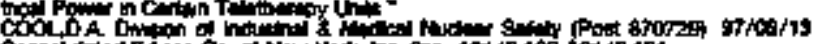

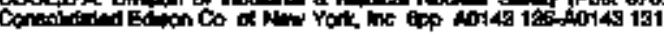

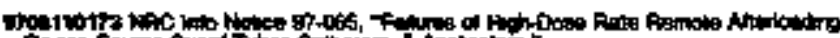

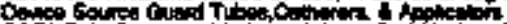

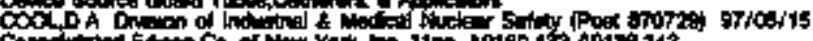

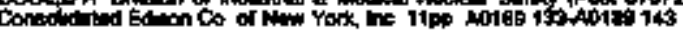

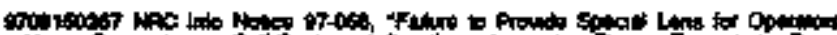

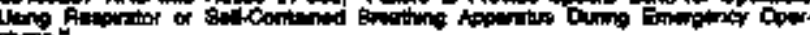

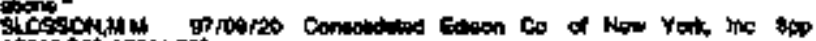

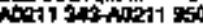

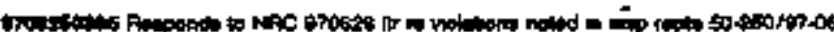

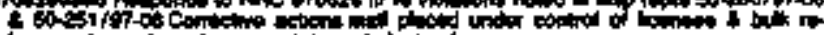
pLid

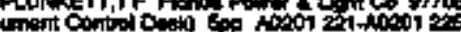

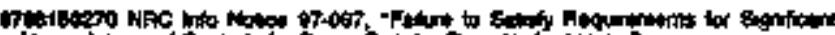

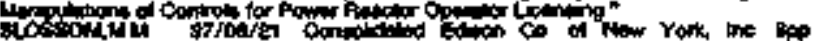

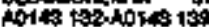

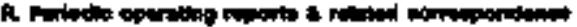

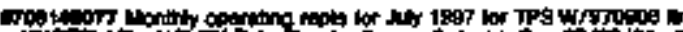

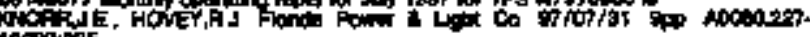
1000020

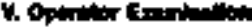

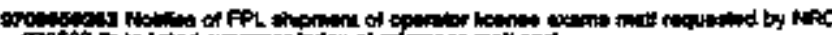

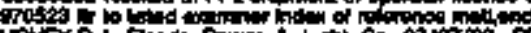

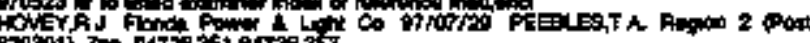

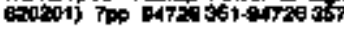

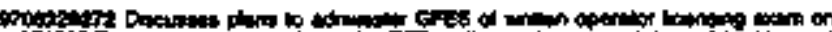

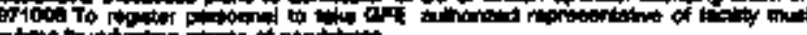

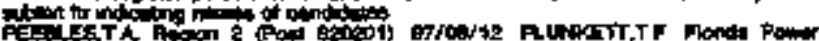

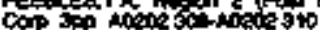

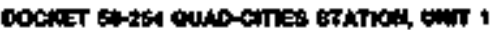

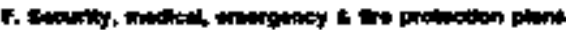

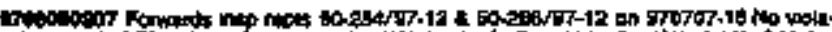

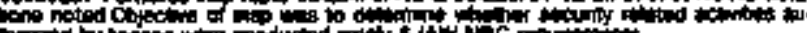

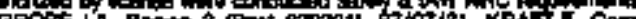

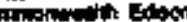
c sop strot $246-9404=0$ 


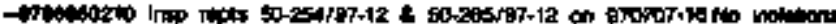

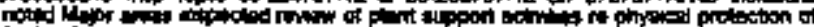
(1)

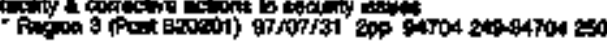

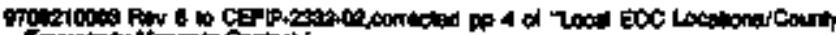

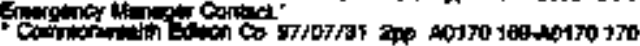

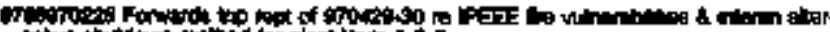

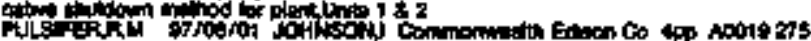

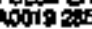

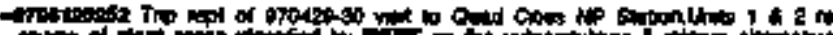

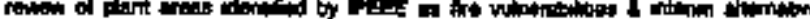

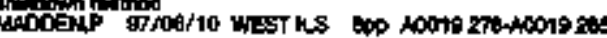

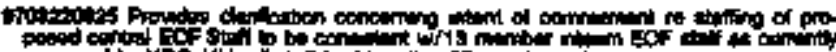

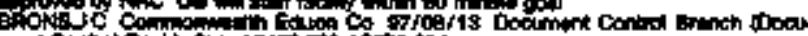

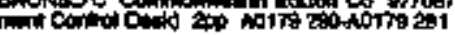

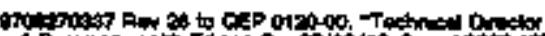

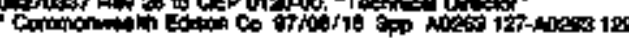

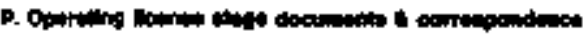

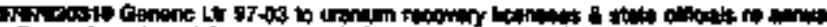

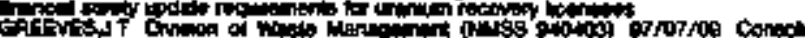

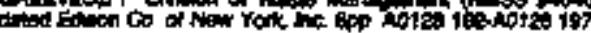

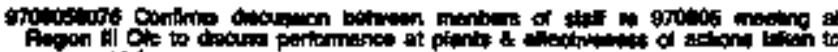
Gitin,

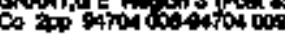

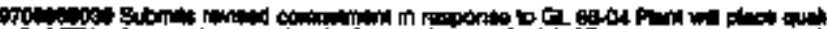

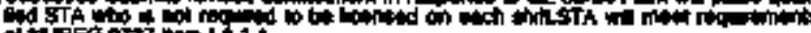
on

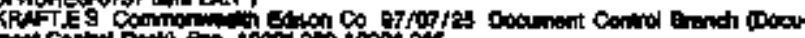

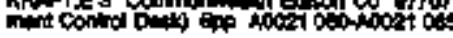

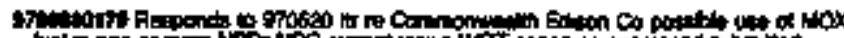

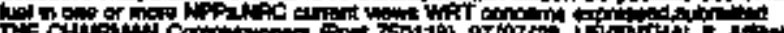

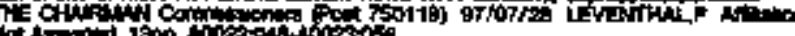

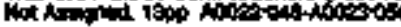

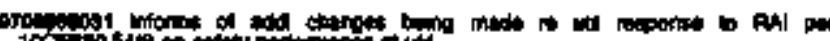

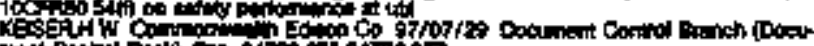

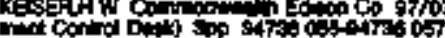

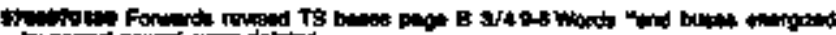

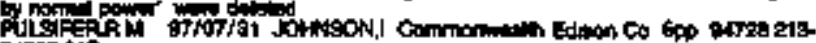
garas?

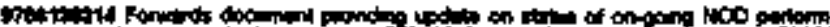

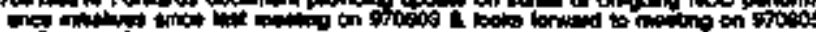

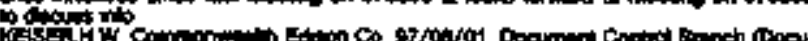

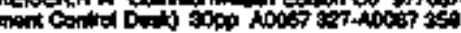

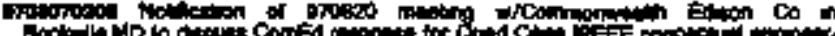

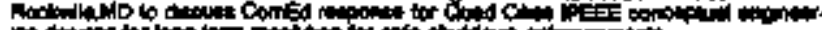

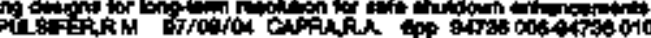

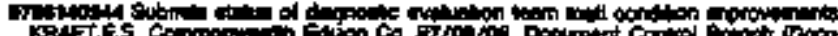

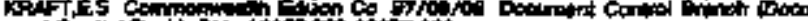

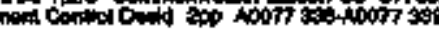

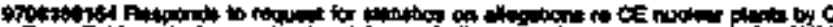

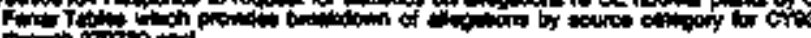

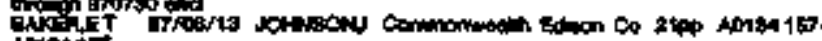
Wortsint

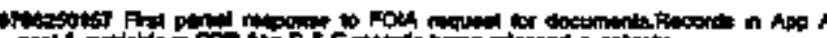

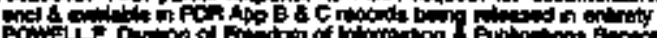

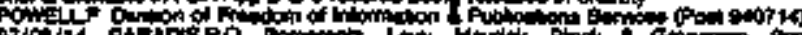

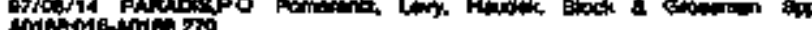

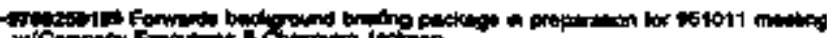

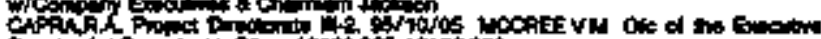

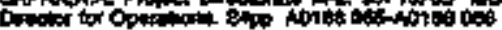

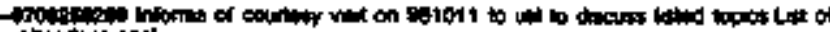

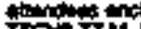

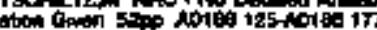

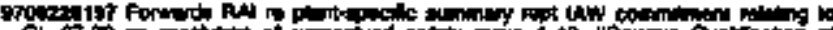
GL 6 Put

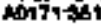

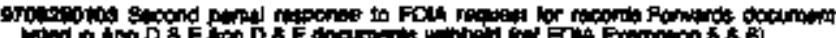

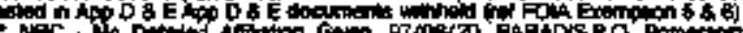

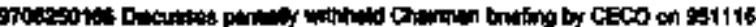

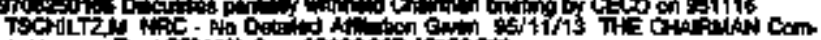

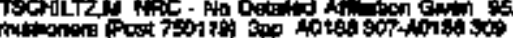

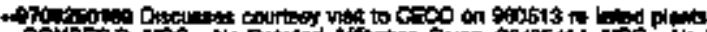

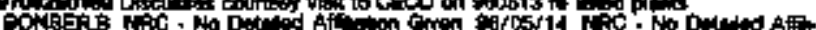

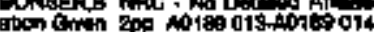

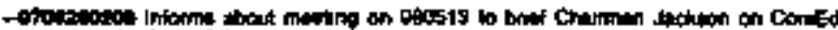

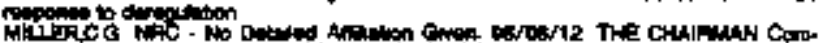

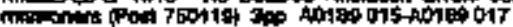

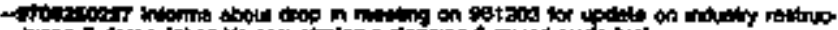

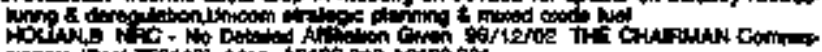

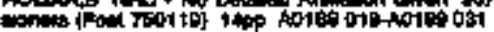

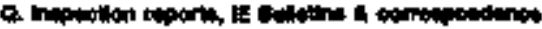

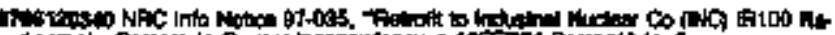

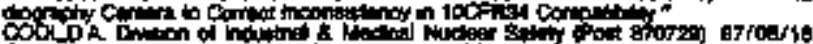

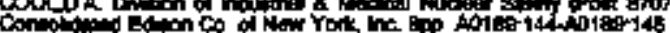

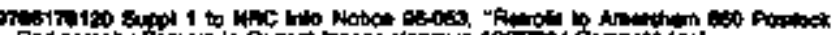

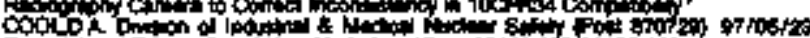

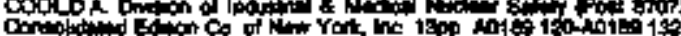

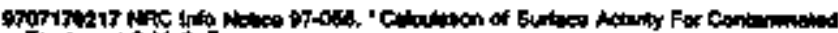

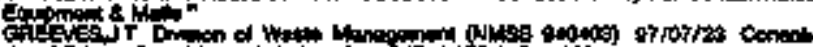

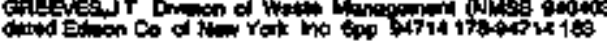

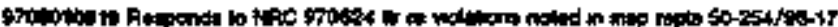

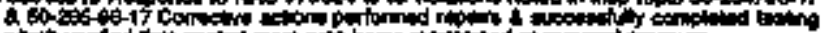

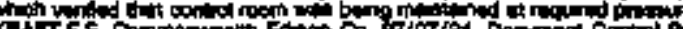

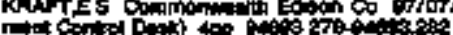

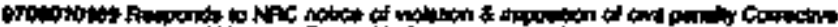
K. $\operatorname{lip}_{0 \rightarrow 0}$

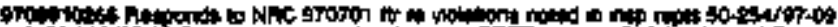

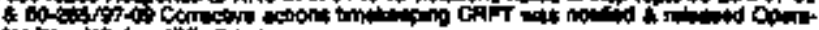

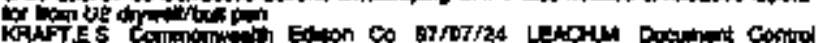

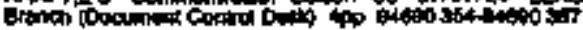

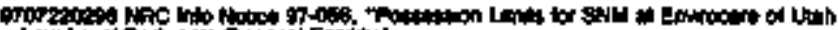

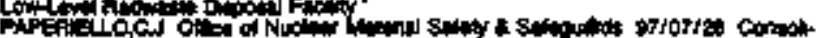

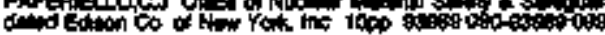

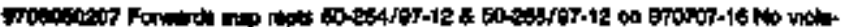

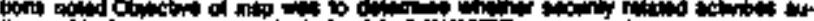

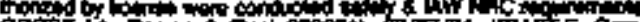

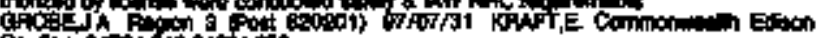

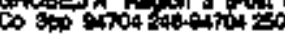

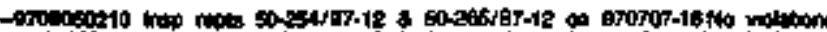

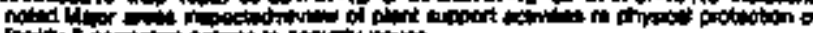

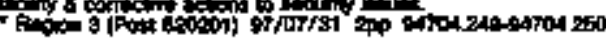

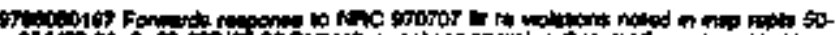

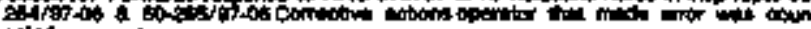

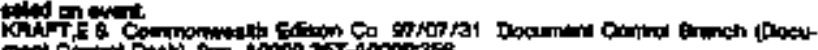

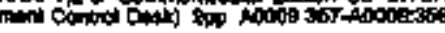

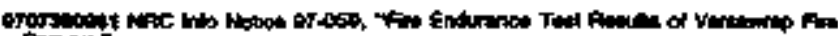

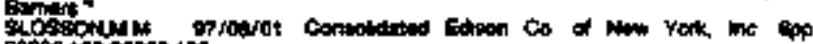

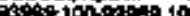

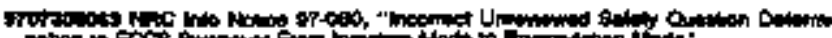

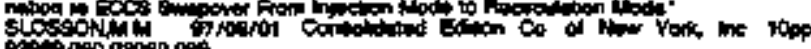
(1)

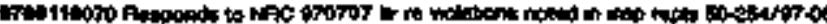

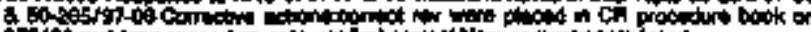

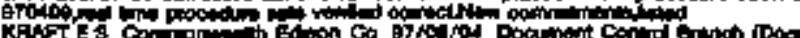

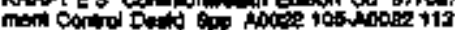

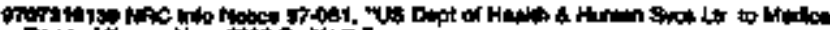

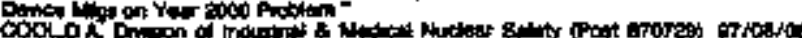

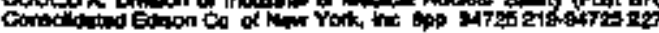

of

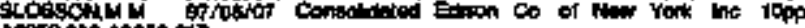
Aocoso ochention on 
rimis

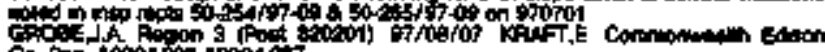

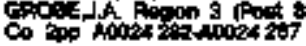

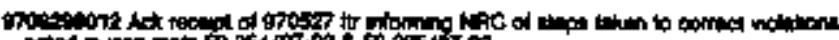

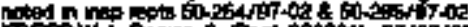

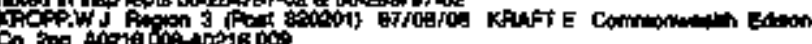

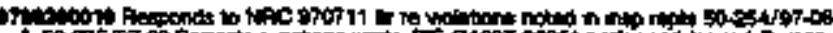

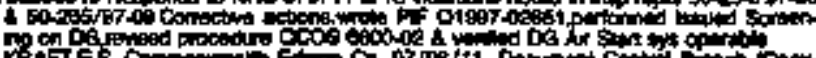

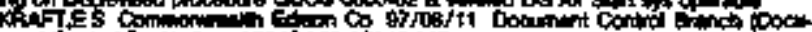

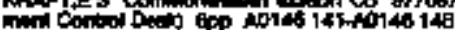

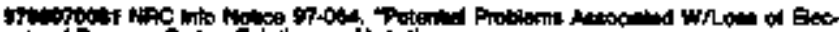

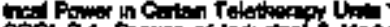

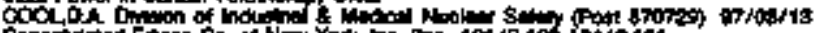

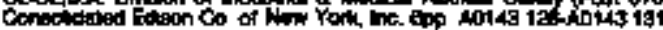

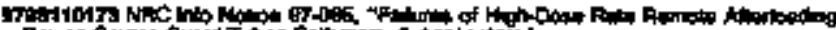

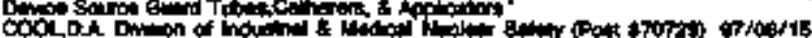

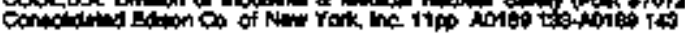

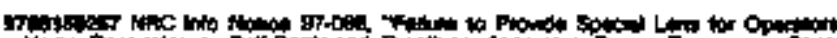

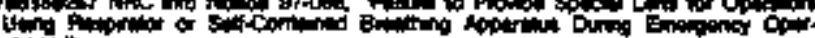

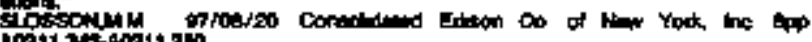

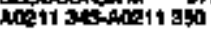

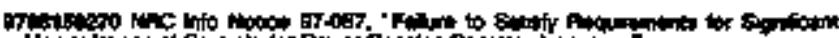

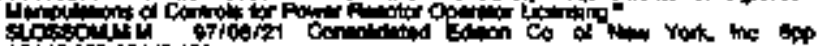

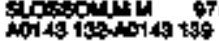

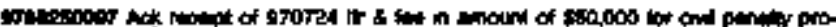

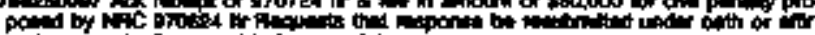

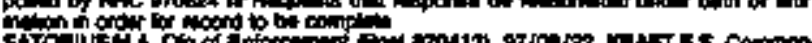
surpent

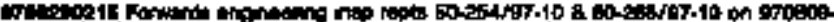

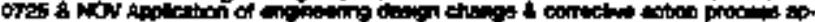

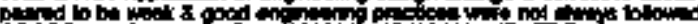

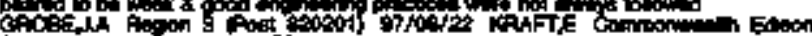

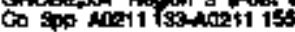

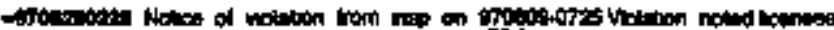

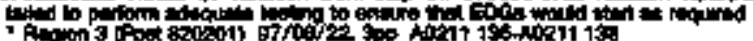

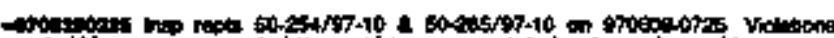

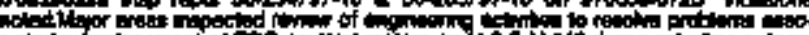

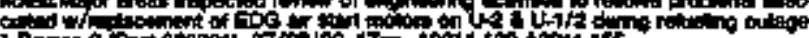

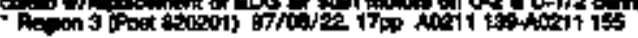

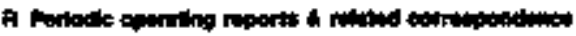

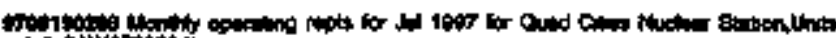

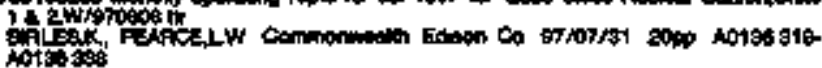

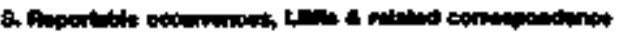

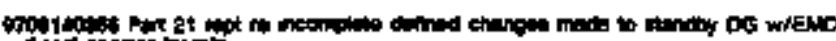

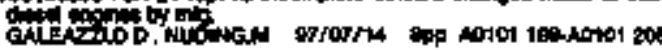

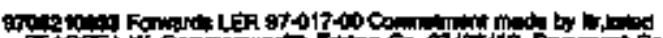

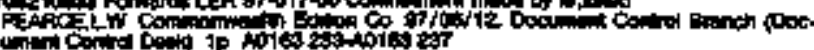

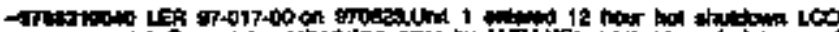

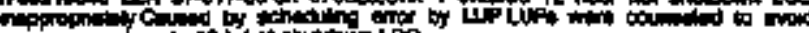
fot

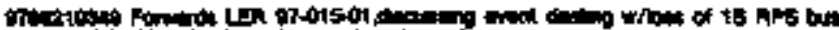

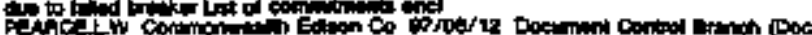

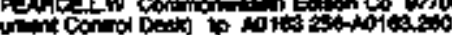

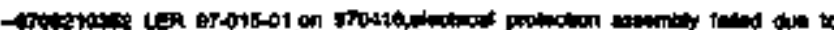

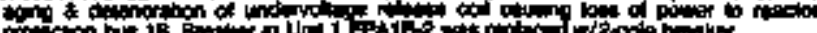

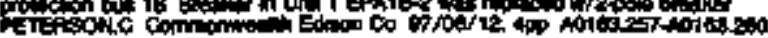

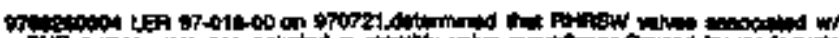

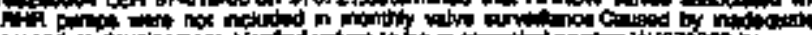

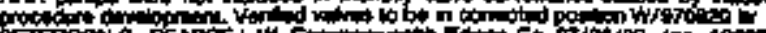

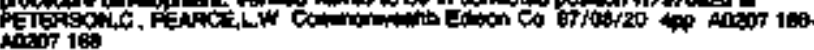

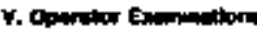

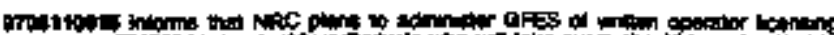

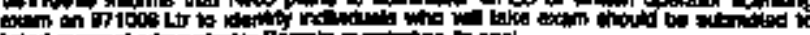

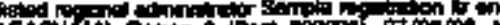

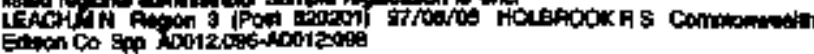

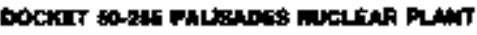

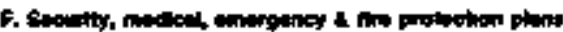

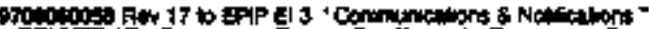

gititus ian of

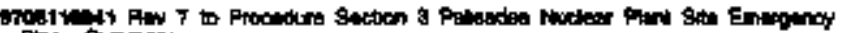

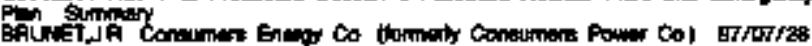

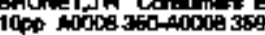

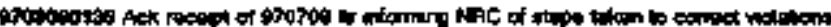

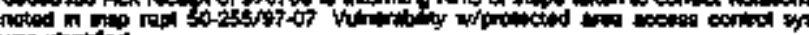

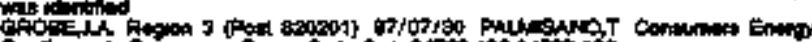

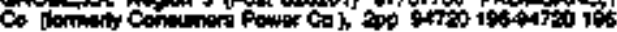

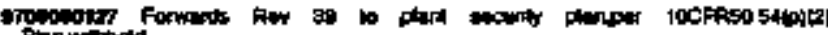

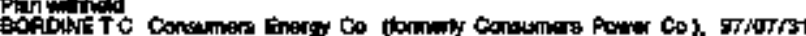

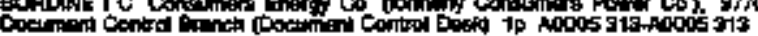

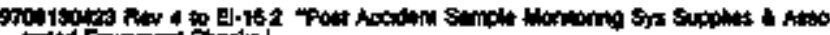

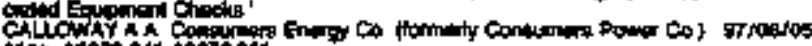

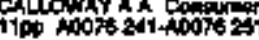

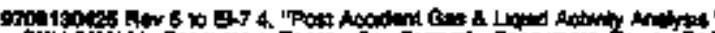

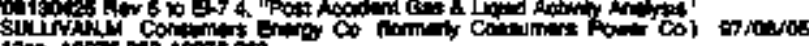

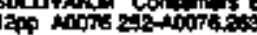

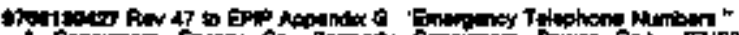

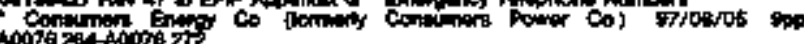

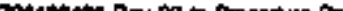

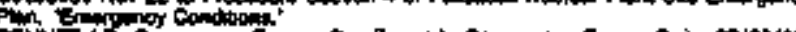

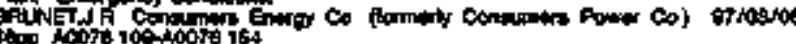

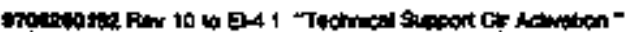
14.9p

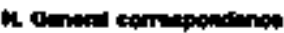

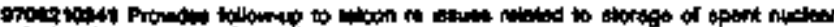
fud 101703:

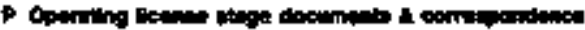

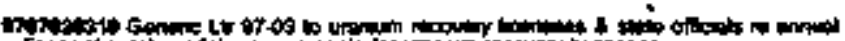

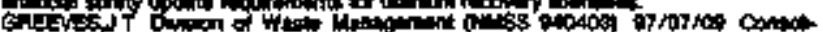

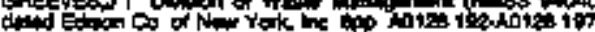

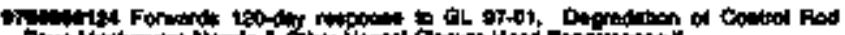

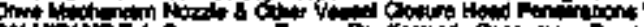

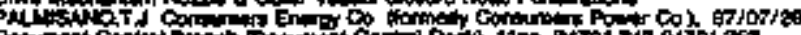

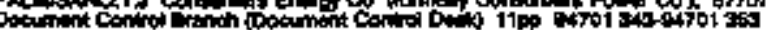

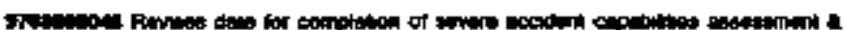

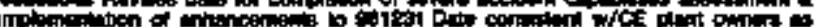

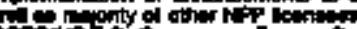

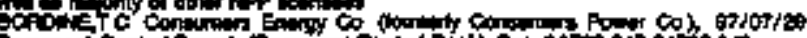

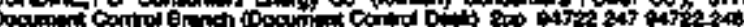

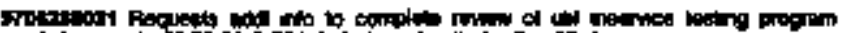

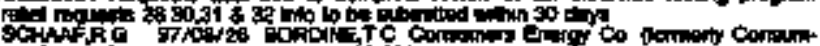

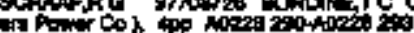

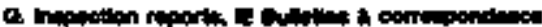

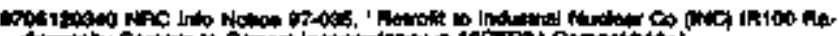

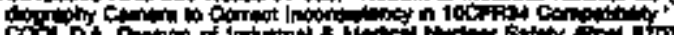

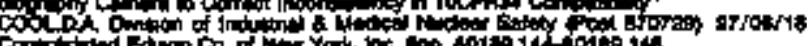

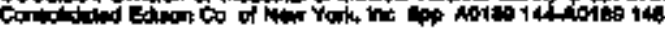

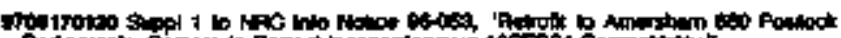

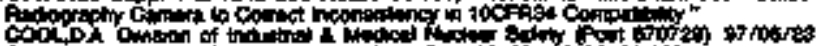

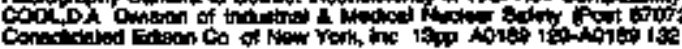

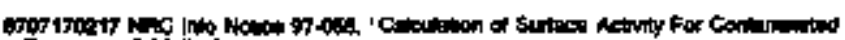
Equping \&

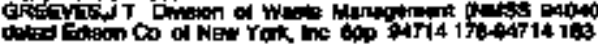

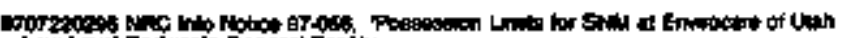

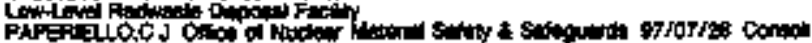

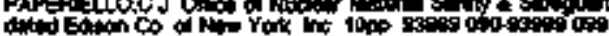




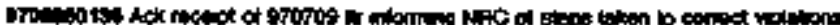

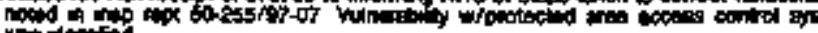
(1)

Go

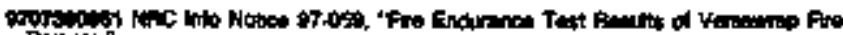

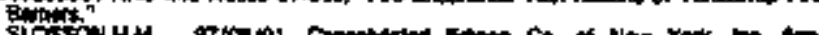

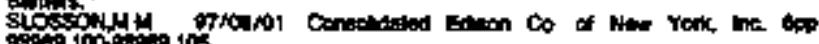

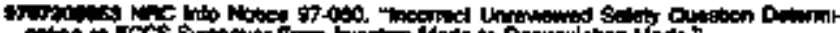

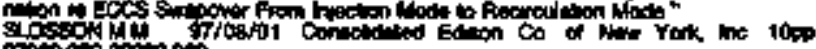

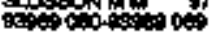

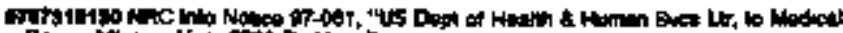
Do

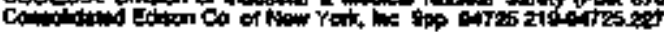

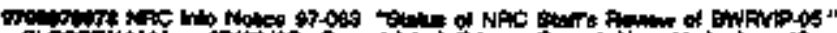

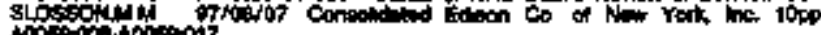

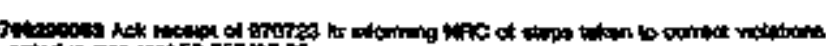

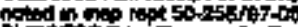

at

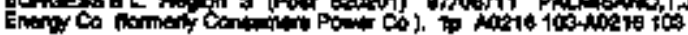

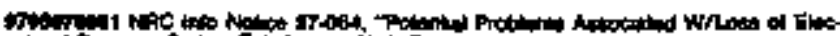

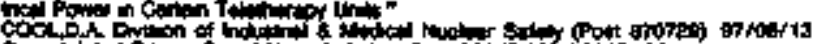

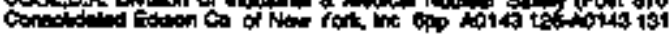

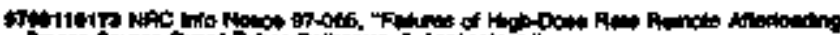

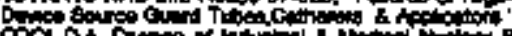

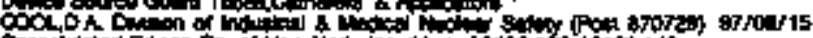

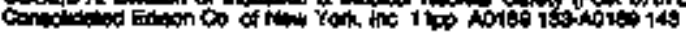

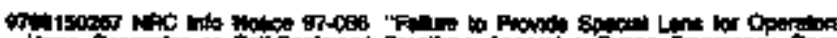

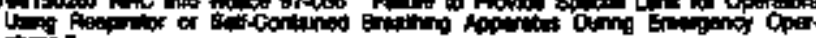

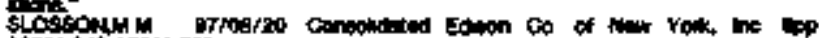

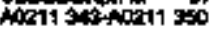

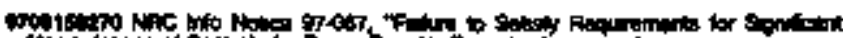

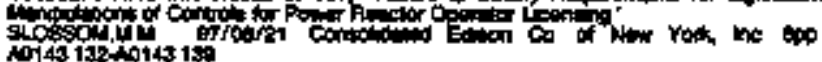

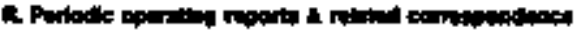

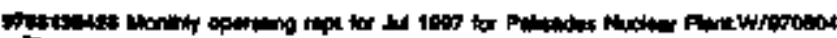

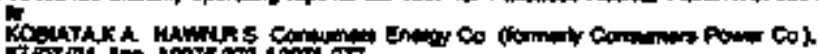

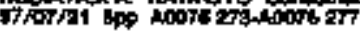

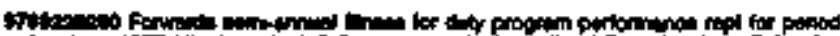

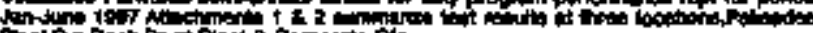

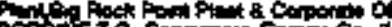

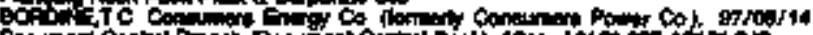

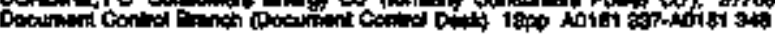

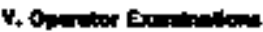

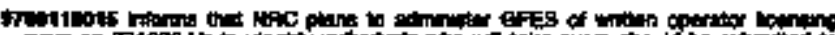

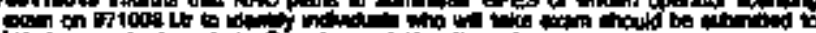

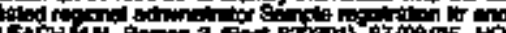

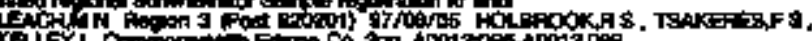

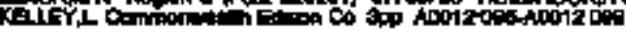

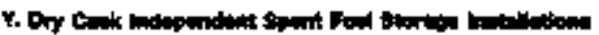

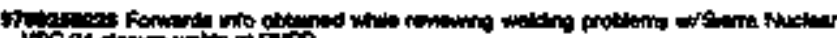

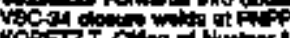

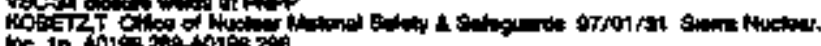
ine. ip 70 rision

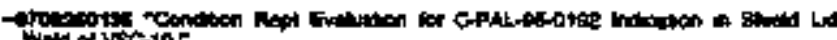
Waid of is $10^{-2}$

Ku

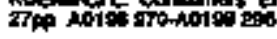

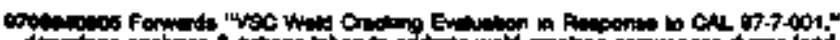

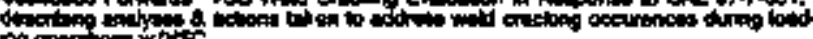

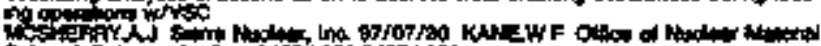

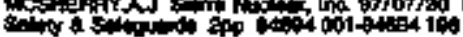

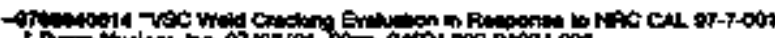

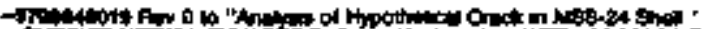

Gidot-

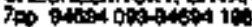

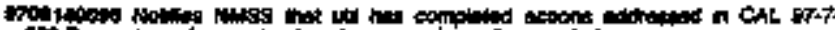

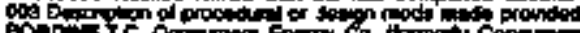

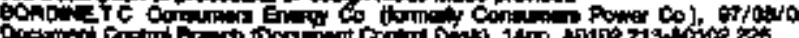

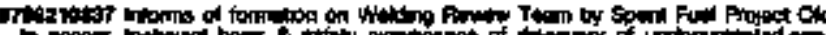

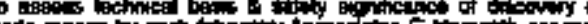

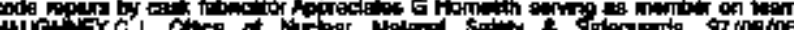

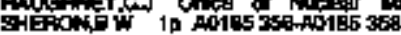

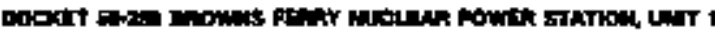

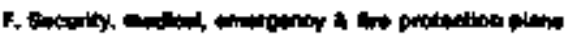

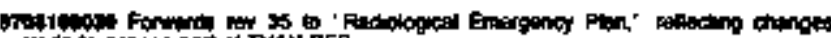

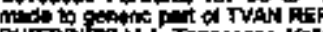

PURK

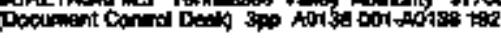

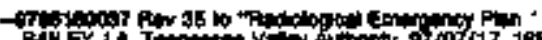

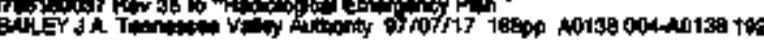

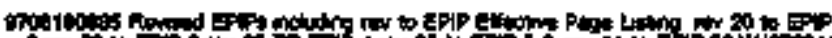

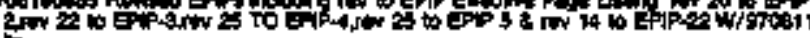

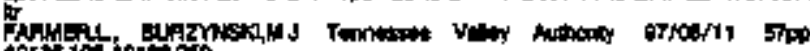

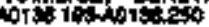

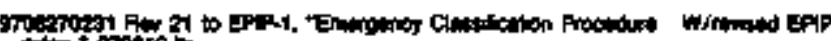

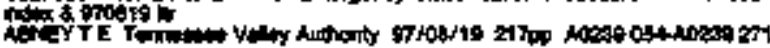

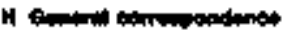

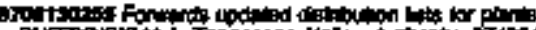

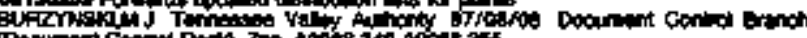

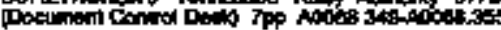

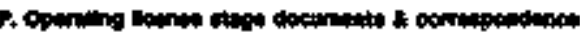

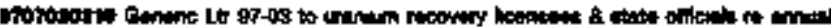

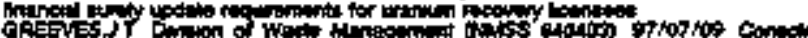

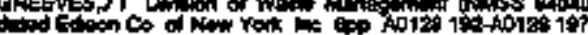

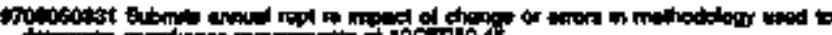

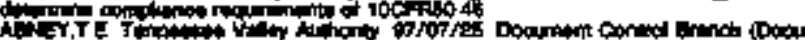

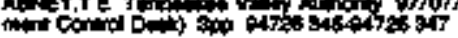

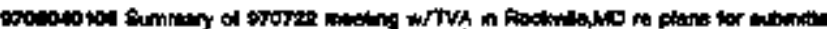

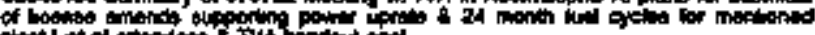

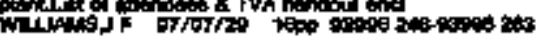

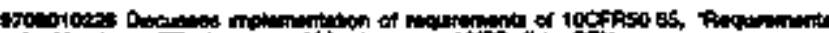

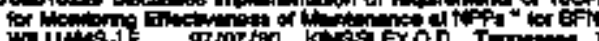

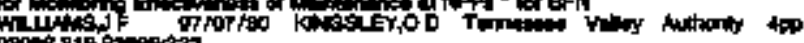

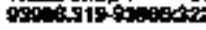

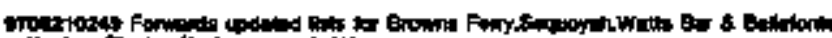

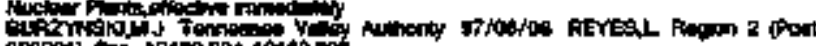

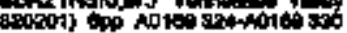

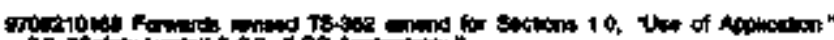
20 , "

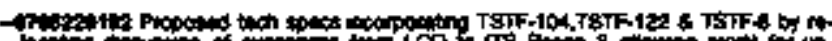

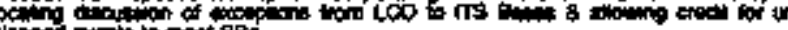
(1)

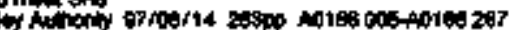

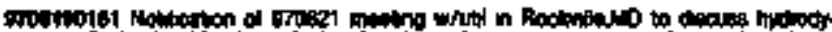

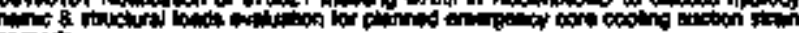

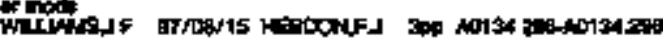

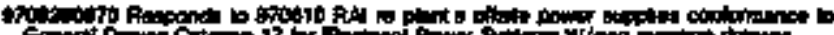

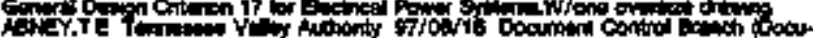

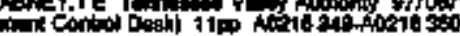

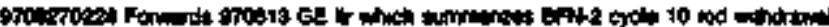

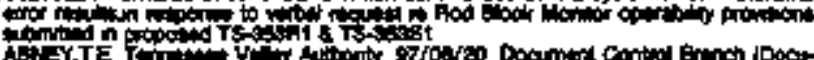

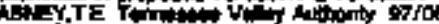

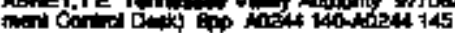

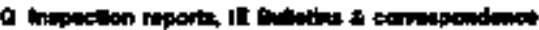

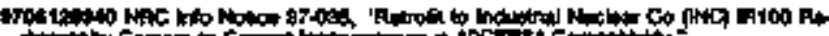

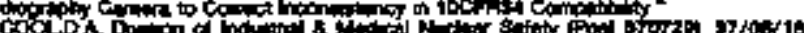
Gon

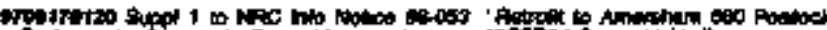

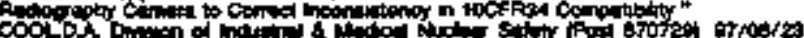

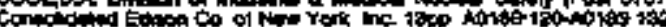




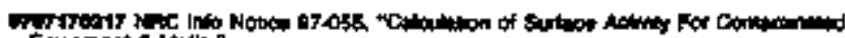

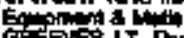

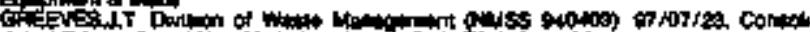

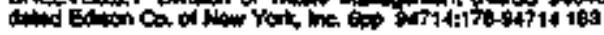

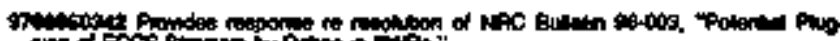

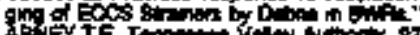

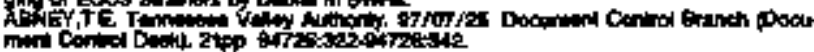

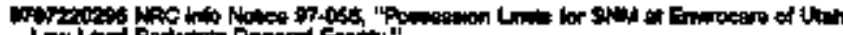

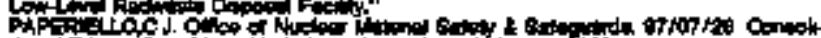

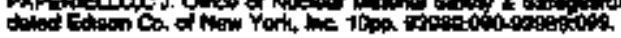

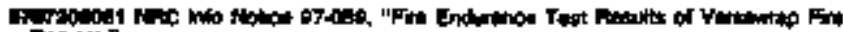

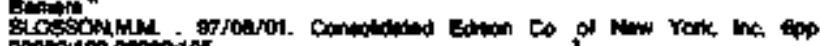

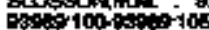

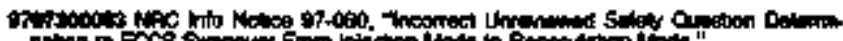

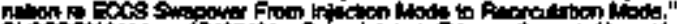

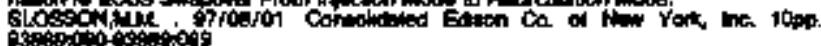

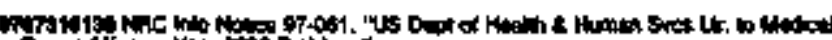

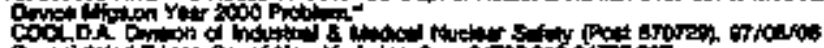

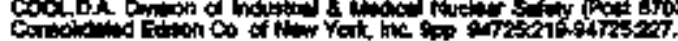

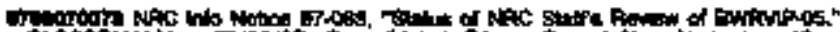

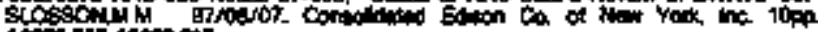

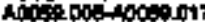

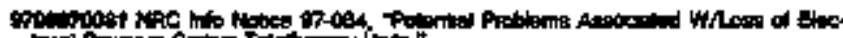

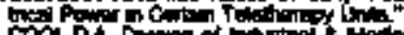

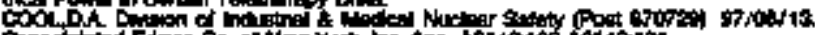

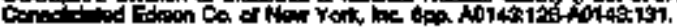

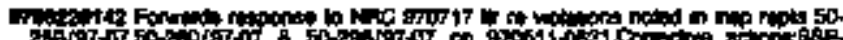

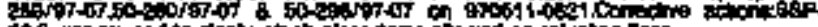

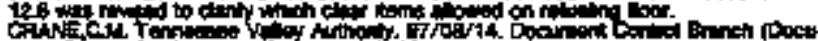

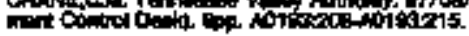

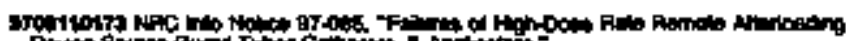

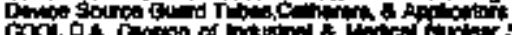

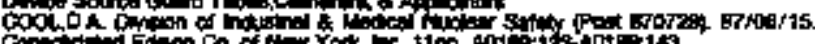

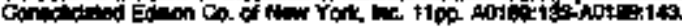

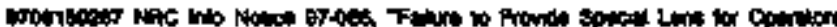

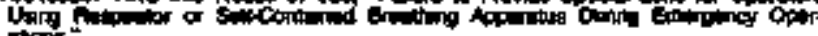

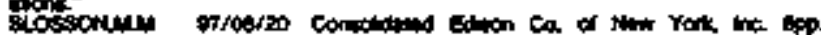
$40211543 \operatorname{lng} 1$ 1:30

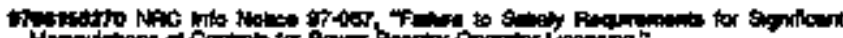

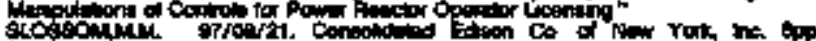

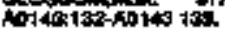

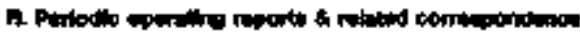

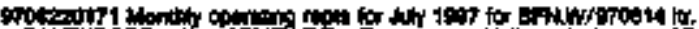

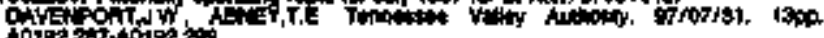

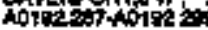

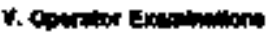

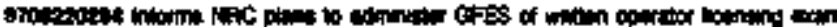

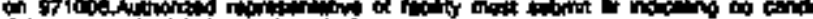

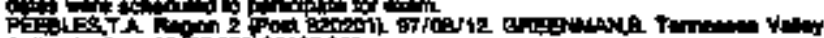

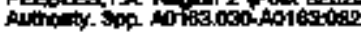

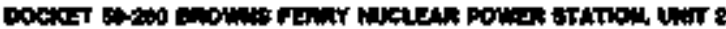

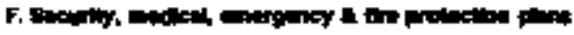

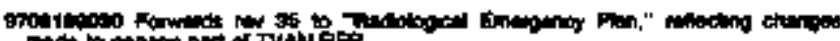

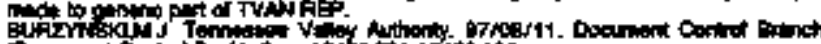

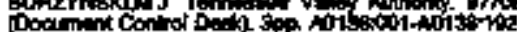

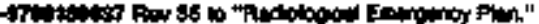

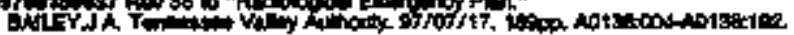

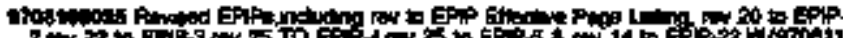

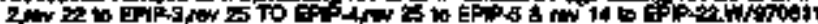

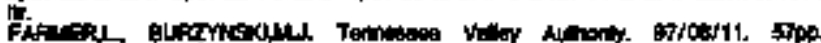

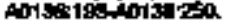

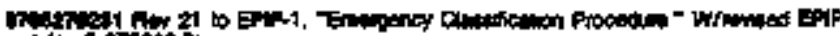

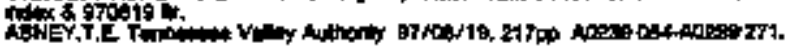

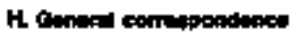

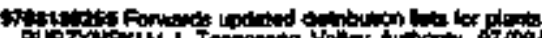

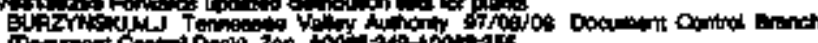

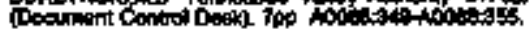

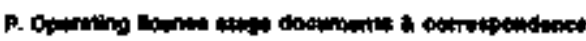

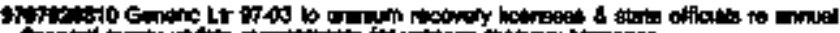

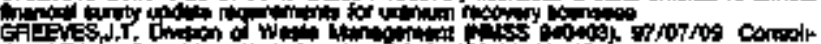

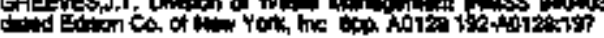

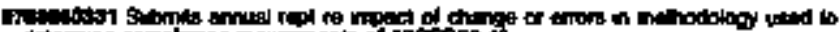

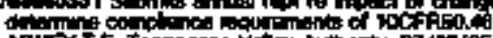

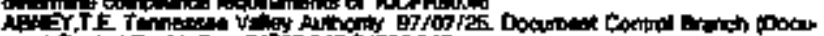

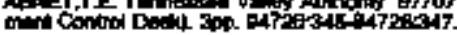

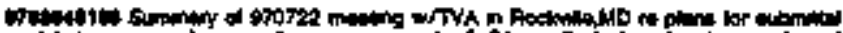

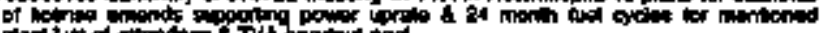

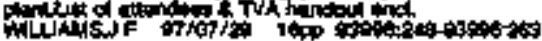

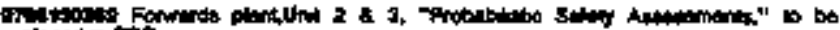

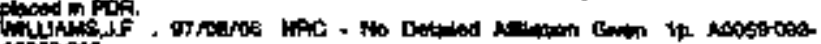

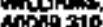

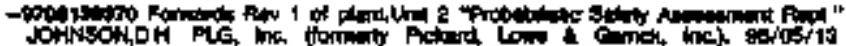

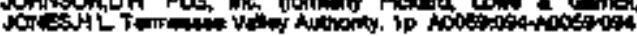

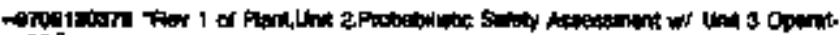

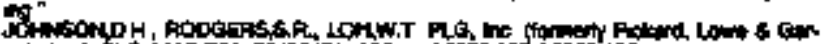

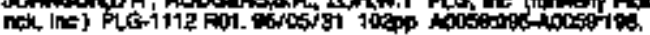

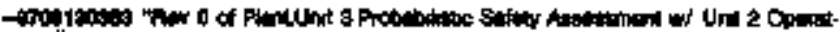

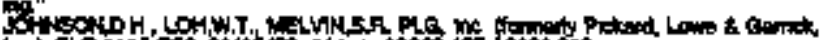

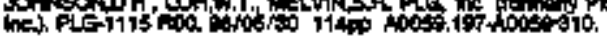

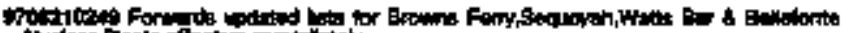

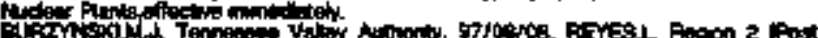

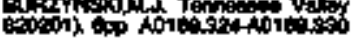

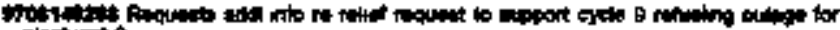

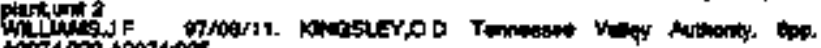

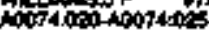

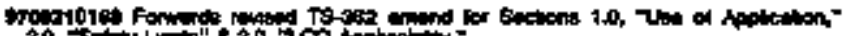

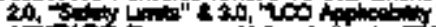

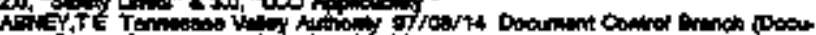

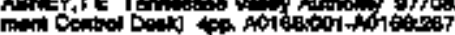

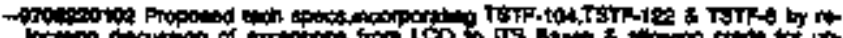

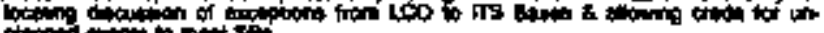

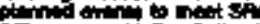

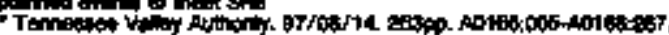

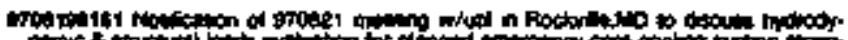

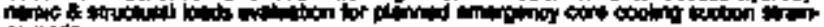

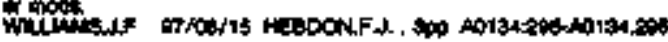

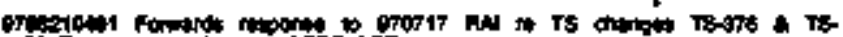

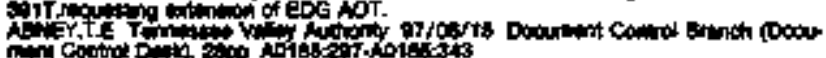

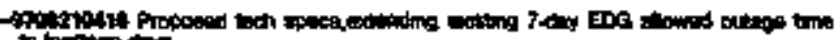
Tomand ot

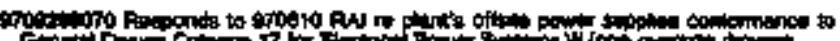

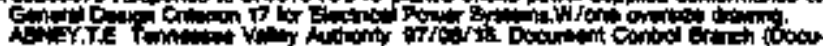

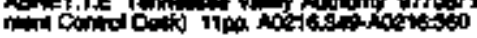

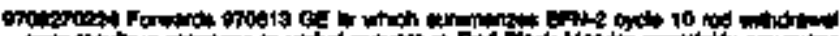

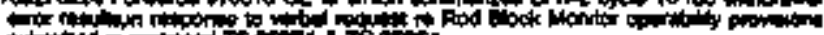

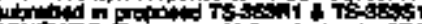

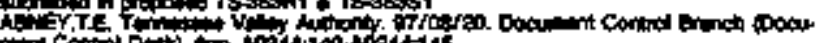

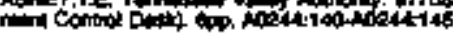

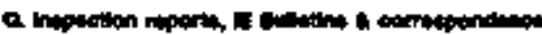

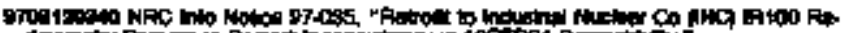

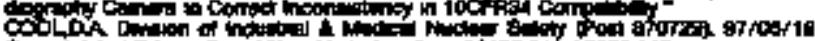

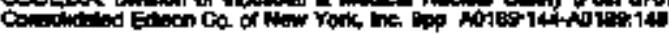

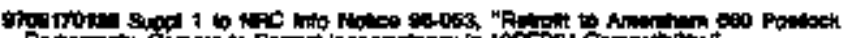

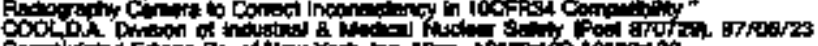

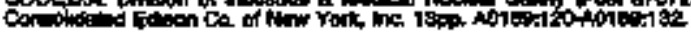

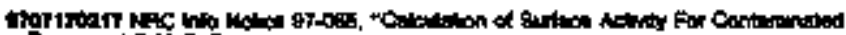

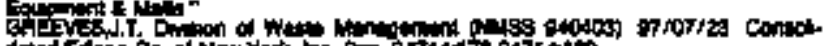

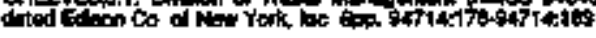

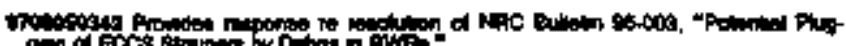

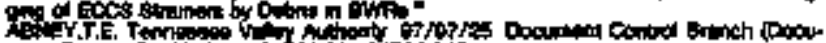

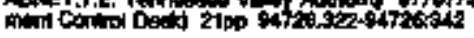




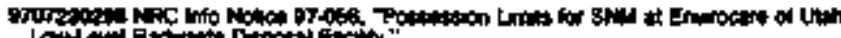

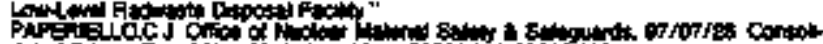

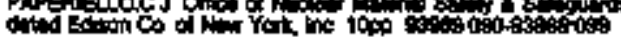

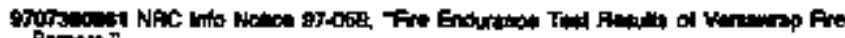

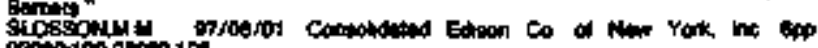

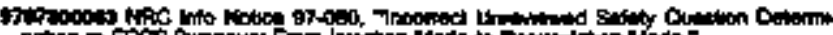

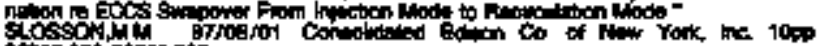

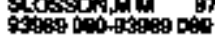

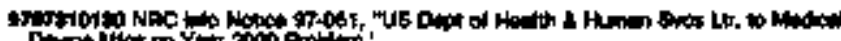

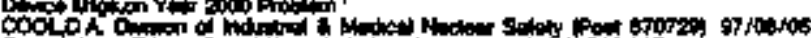

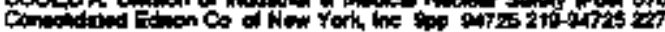

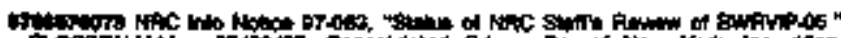

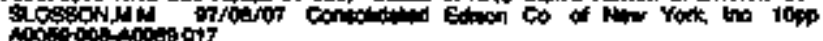

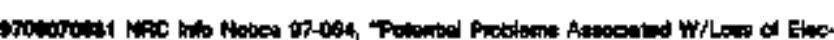
I

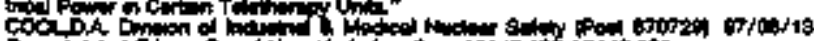

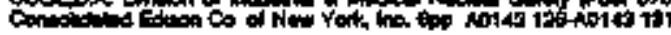

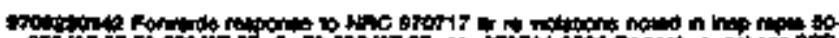

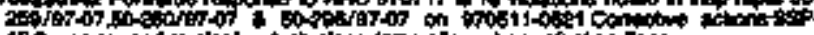

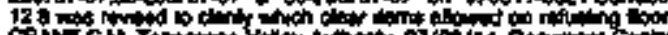

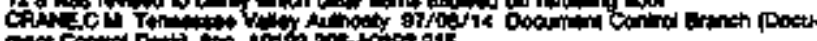

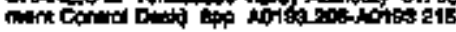

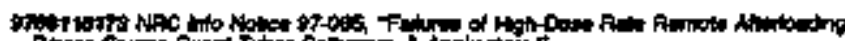

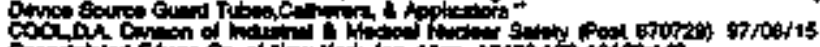

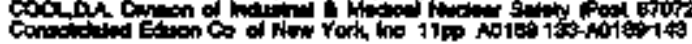

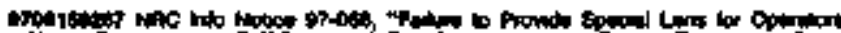

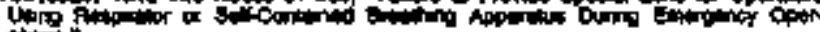

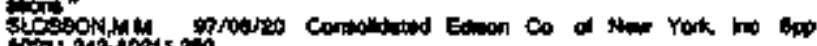

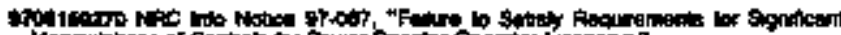

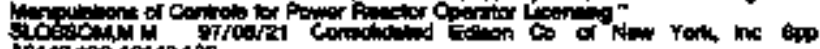

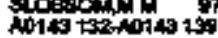

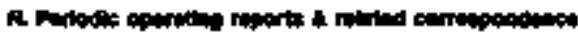

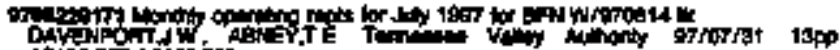

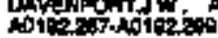

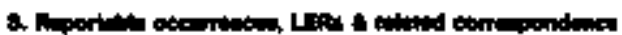

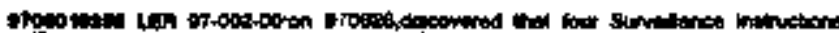
(Ot)

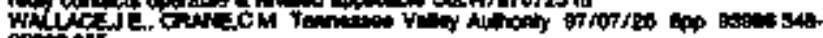

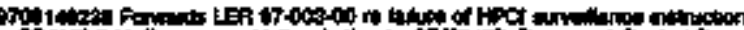

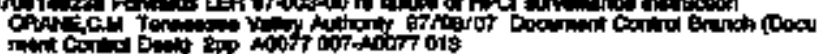

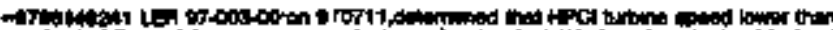

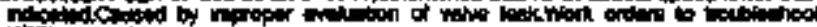
Hich trition

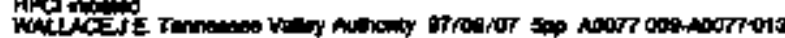

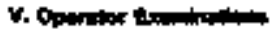

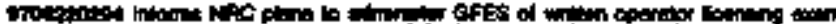

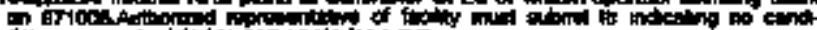

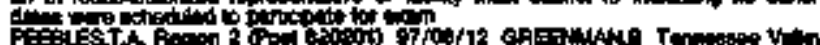

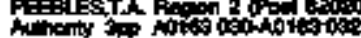

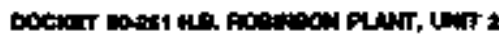

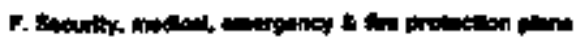

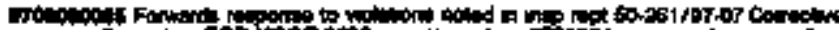

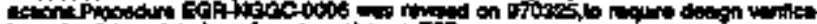

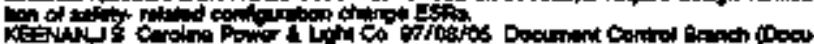

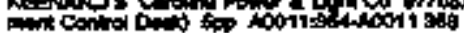

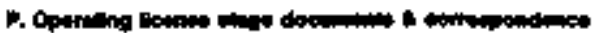

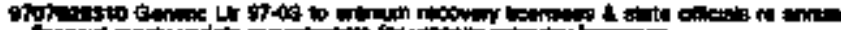

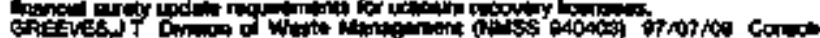

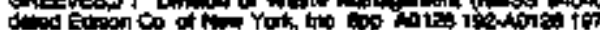

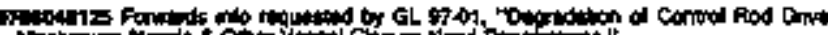

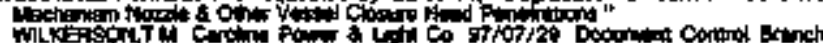

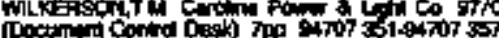

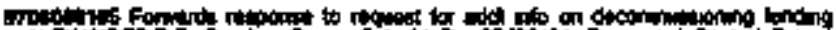

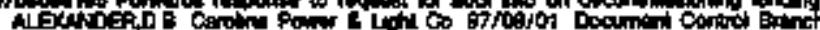

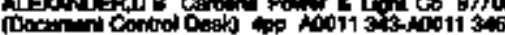

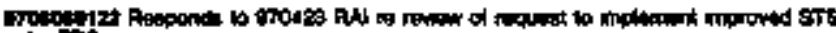

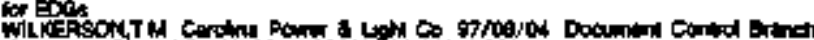

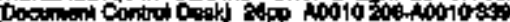

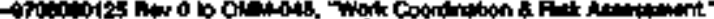

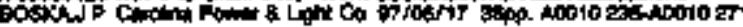

- 970

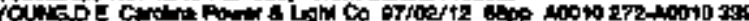

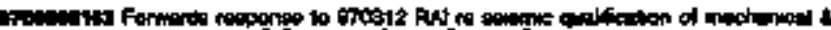

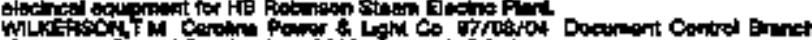

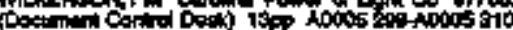

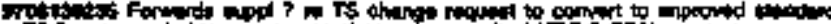

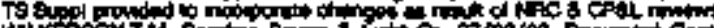

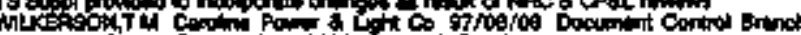

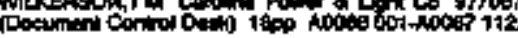

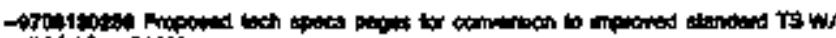

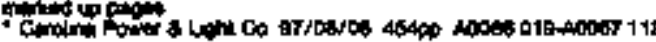

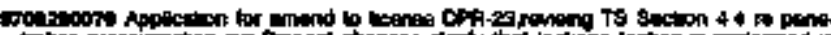

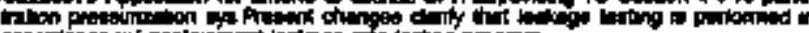

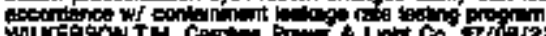

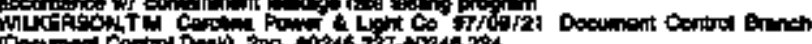

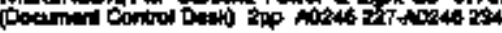

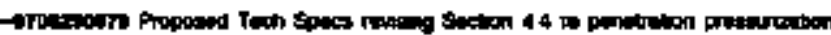

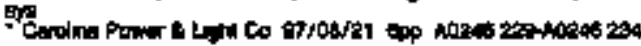

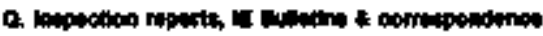

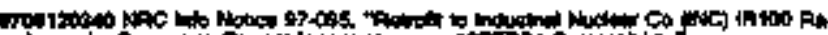

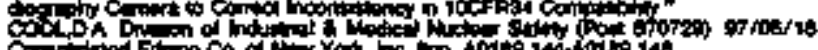

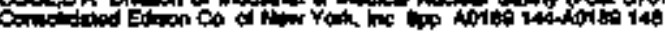

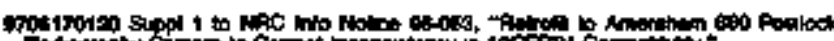

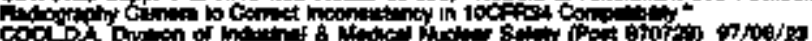

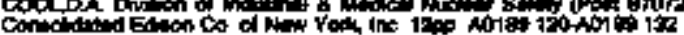

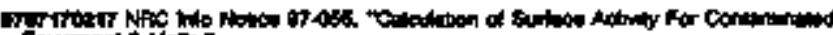

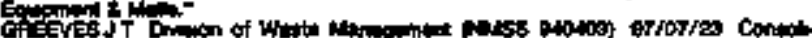

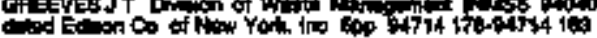

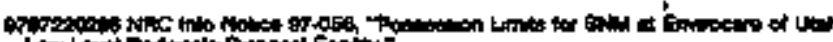

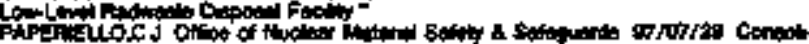

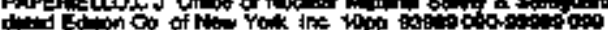

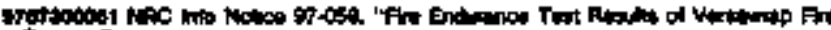

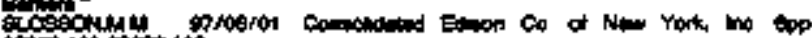

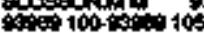

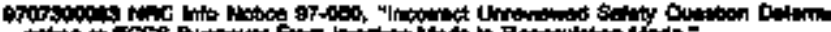

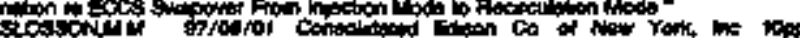
Q

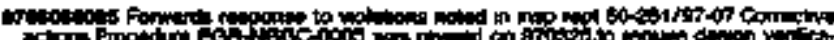

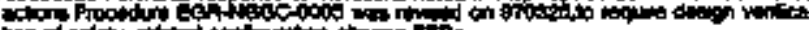

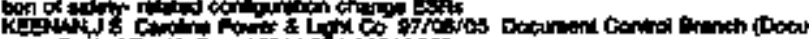

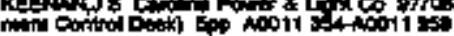

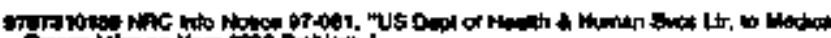

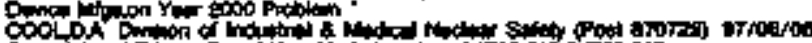

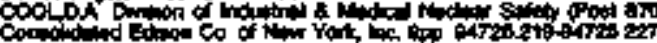

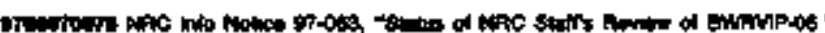
tod

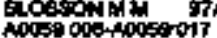

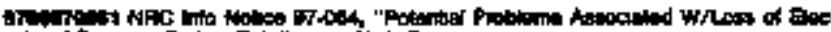

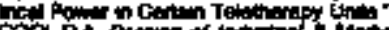

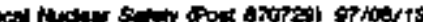

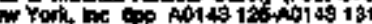

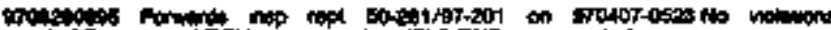

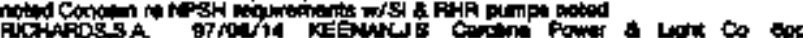

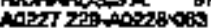

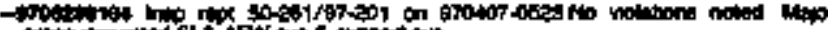

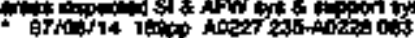




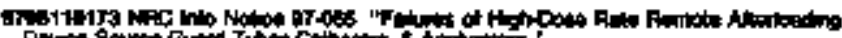

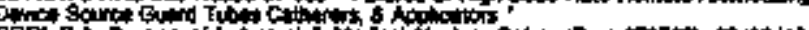

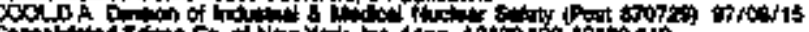

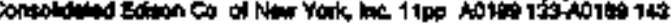

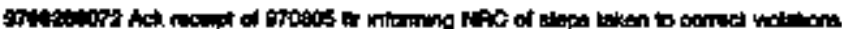

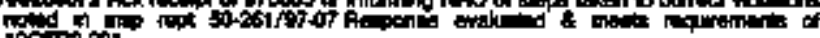

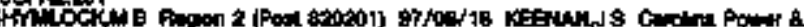

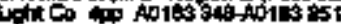

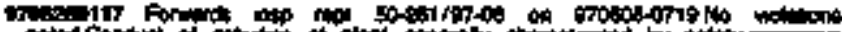

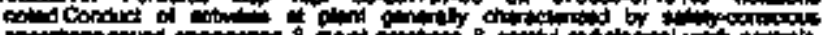

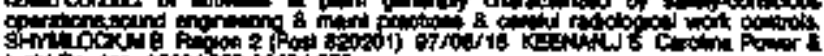

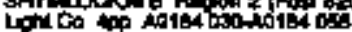

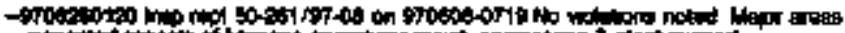

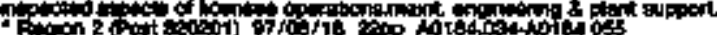

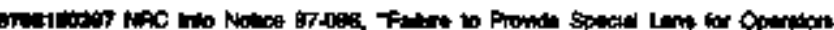

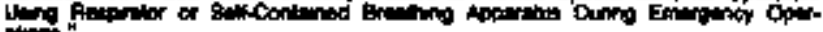

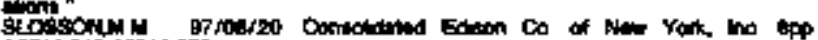

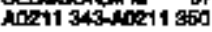

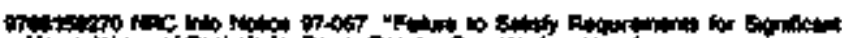

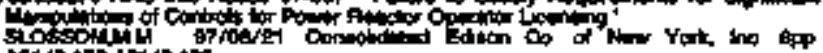

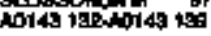

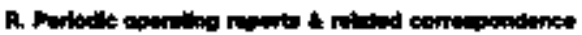

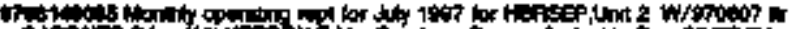

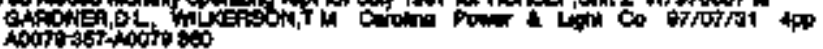

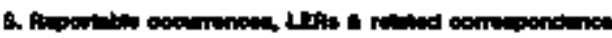

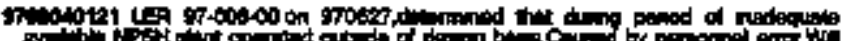

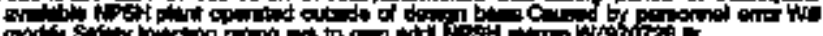

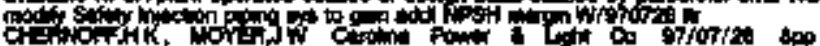

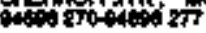

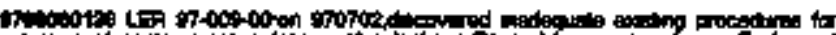

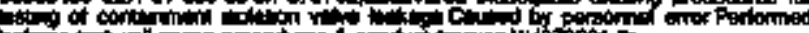

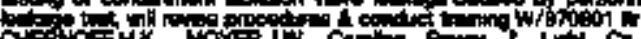

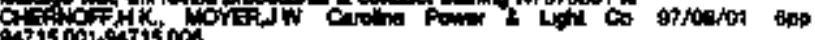
a47 is $001-94715006$

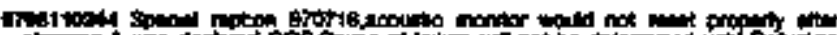

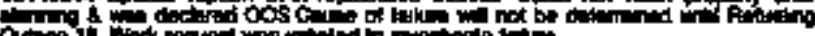

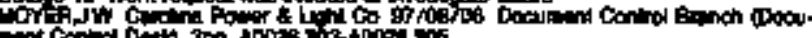

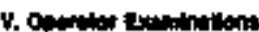

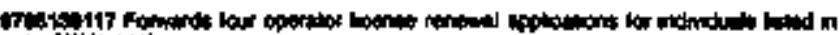

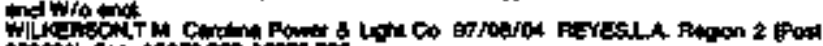

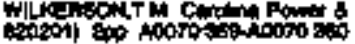

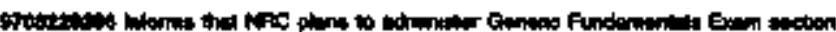

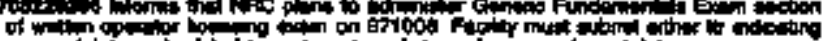

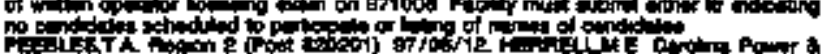

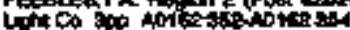

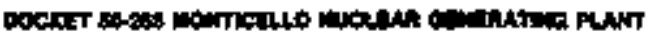

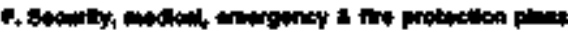

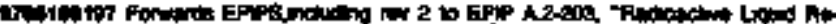

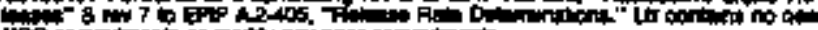

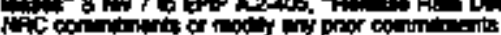

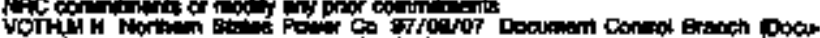

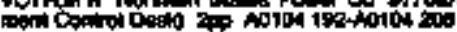

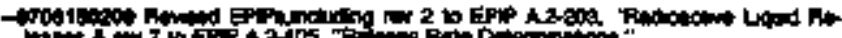

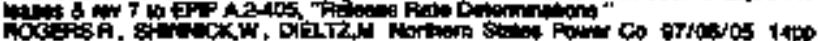

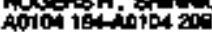

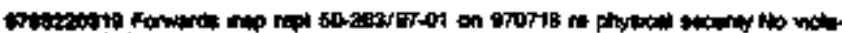

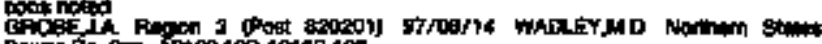

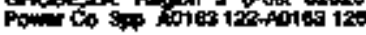

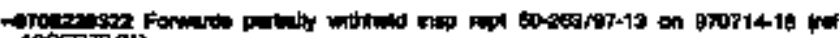

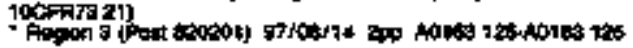

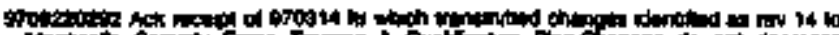

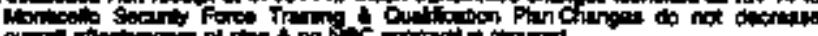
chet of Co 301010101010

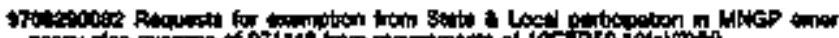

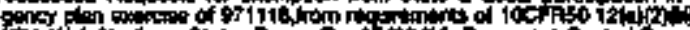

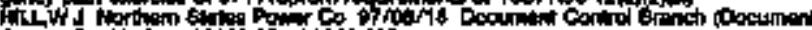

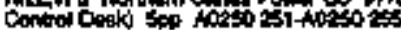

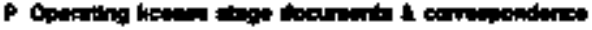

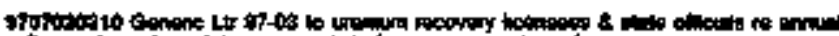

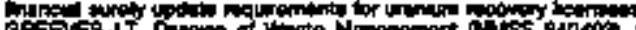

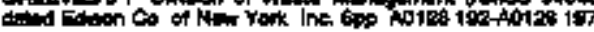

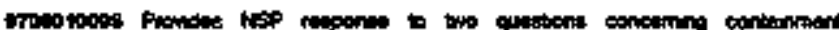

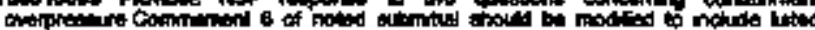
into

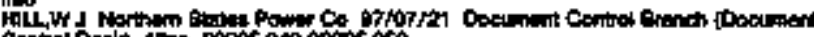

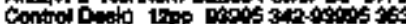

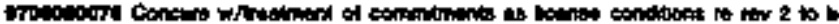

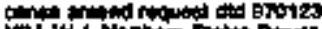

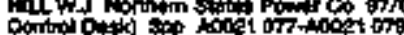

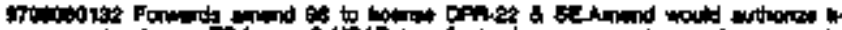

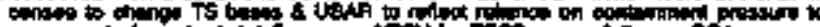

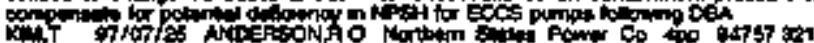
atist as:

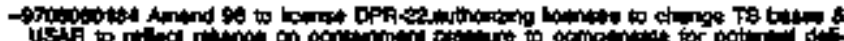

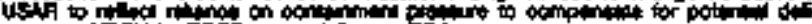

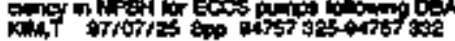

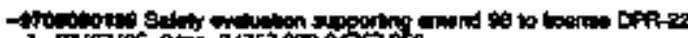

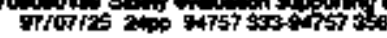

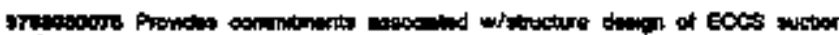

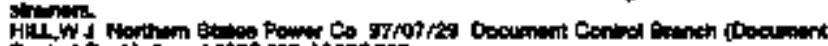

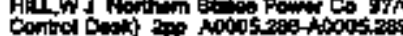

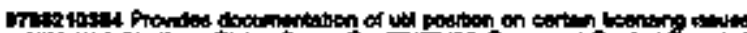

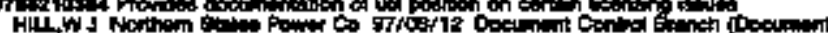

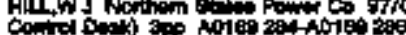

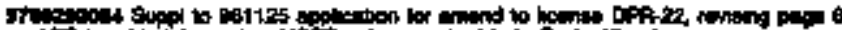

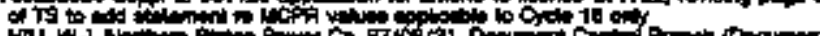

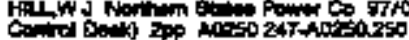

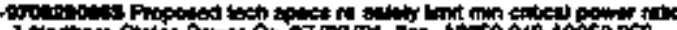

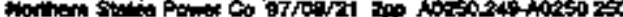

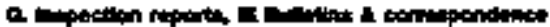

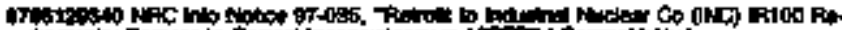

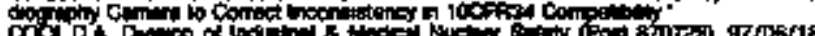

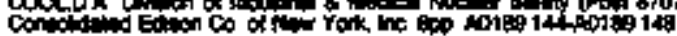

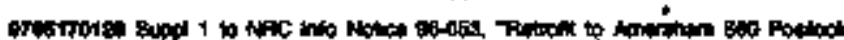

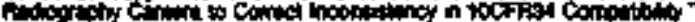

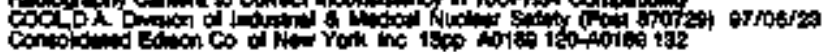

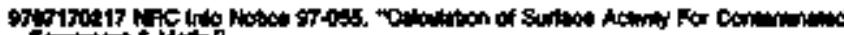

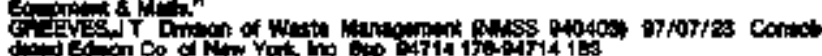

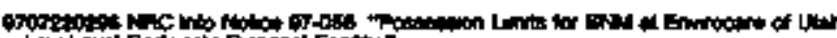
Loti

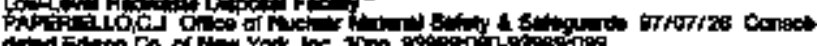

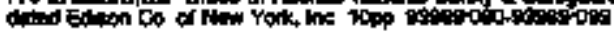

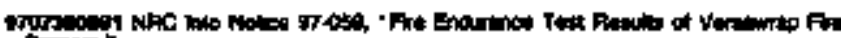

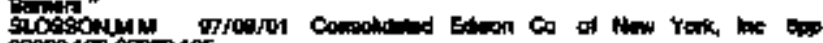
105

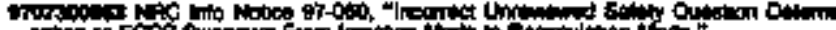

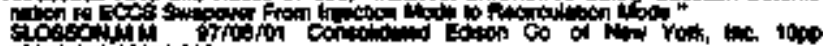

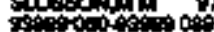

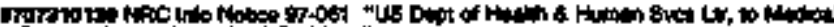

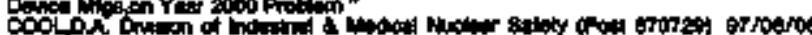

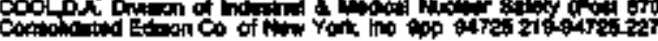

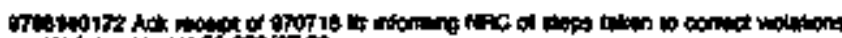

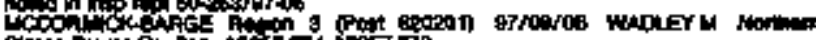

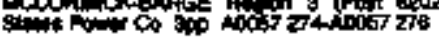

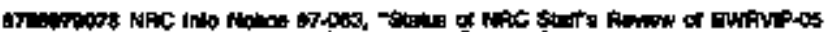

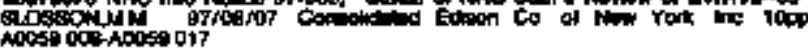

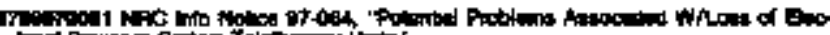

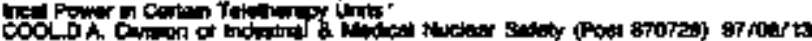

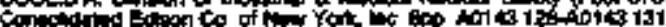




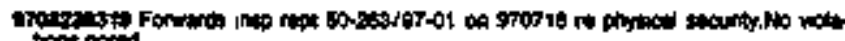

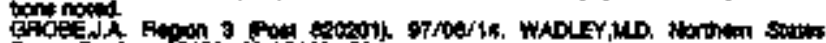

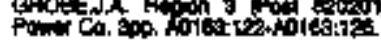

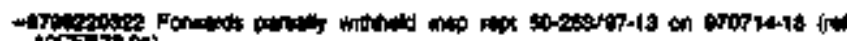

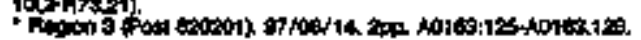

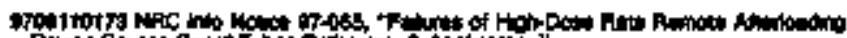

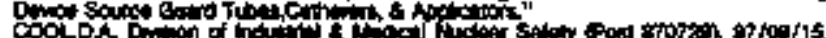

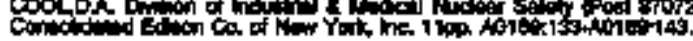

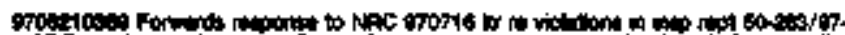

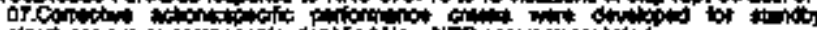

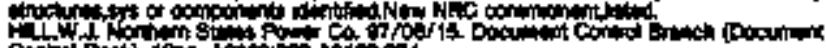

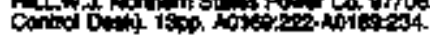

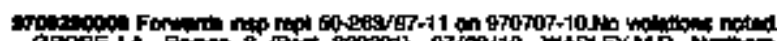

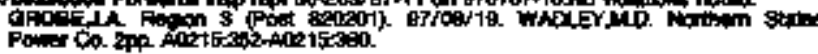

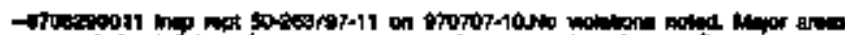

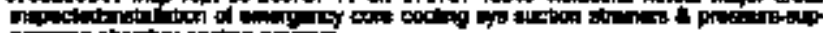

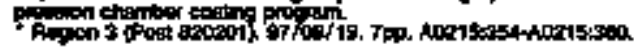

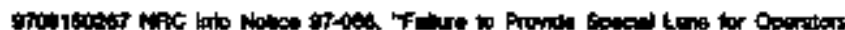

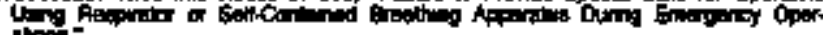

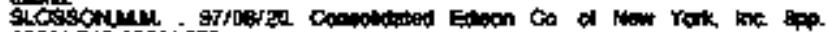
10217: $543-10211360$

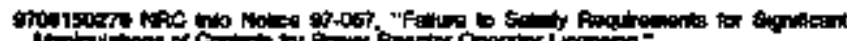

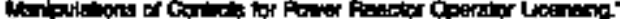

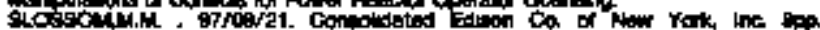
intis: $13200145+39$

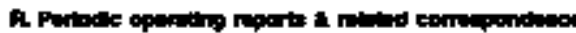

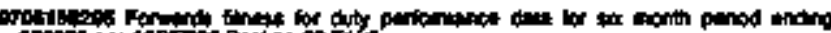

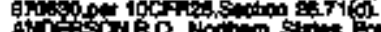

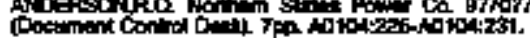

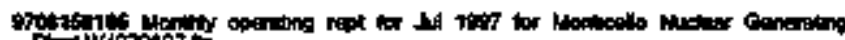

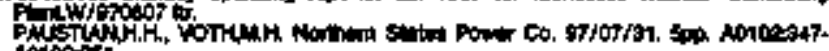
A

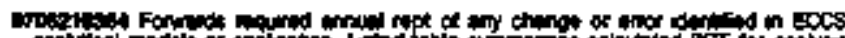

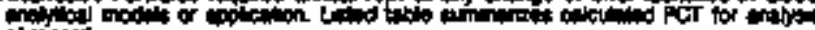

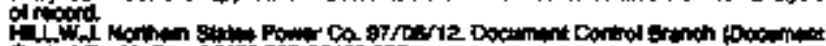

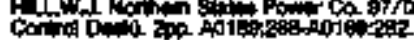

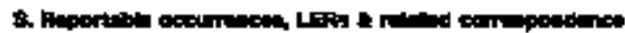

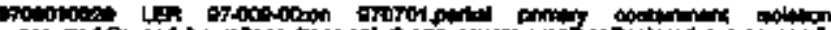

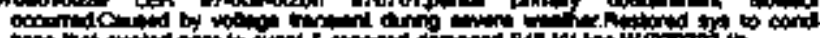

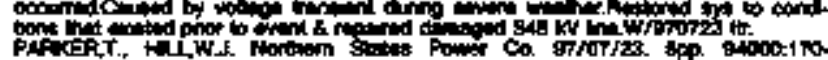
pandist

\section{Openar firamianione}

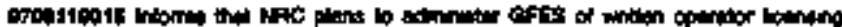

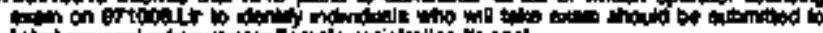

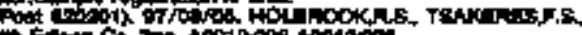

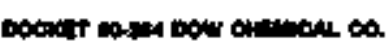

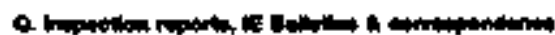

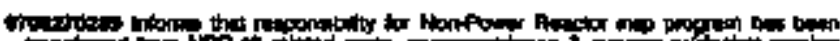

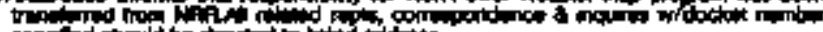

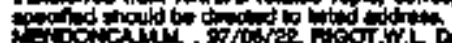

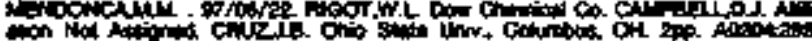
intion.

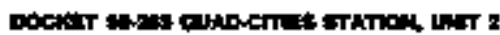

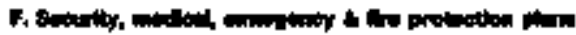

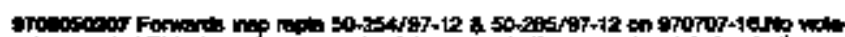

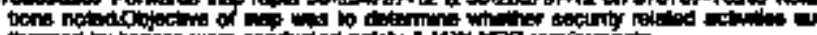

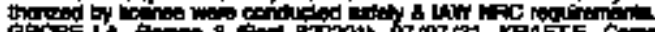

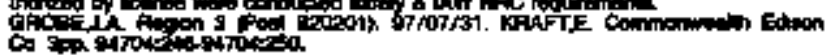

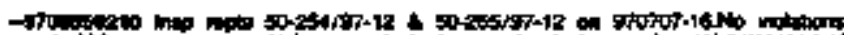

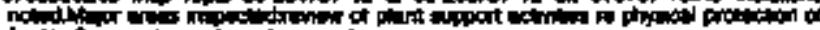

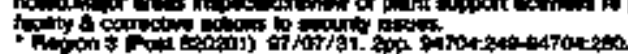

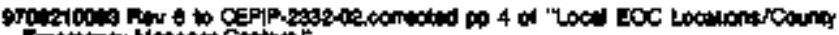

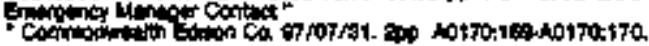

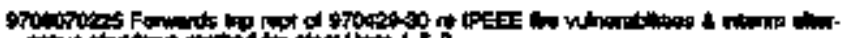

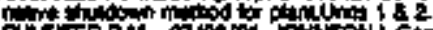

Atotion

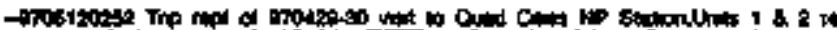

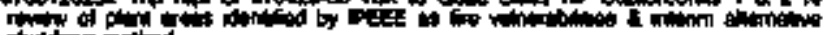

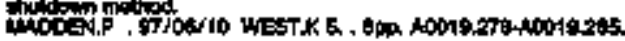

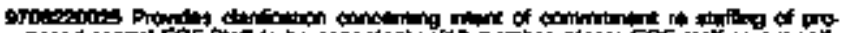

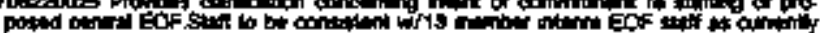

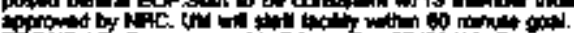

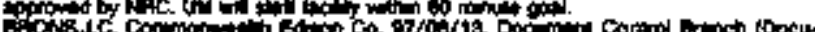

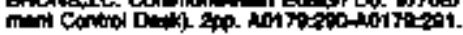

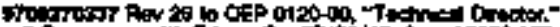

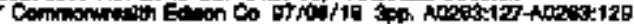

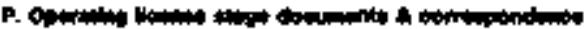

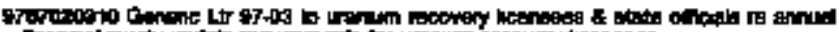

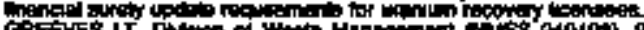

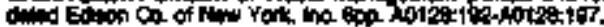

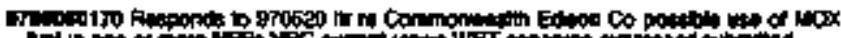

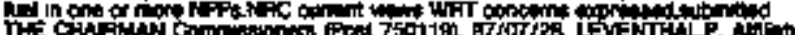

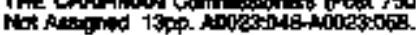

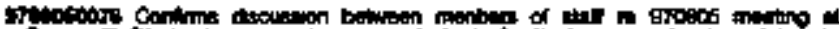

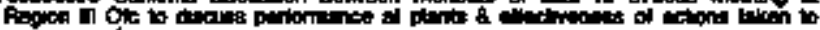

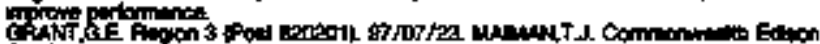

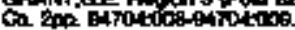

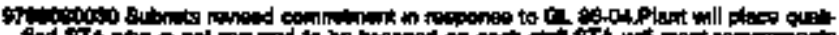

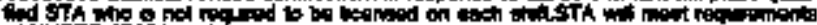
of WRER

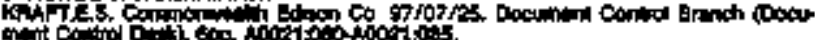

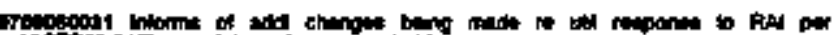

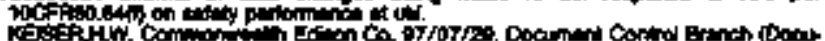

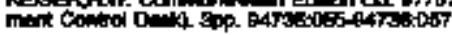

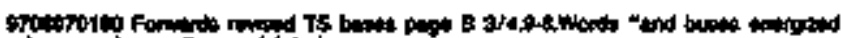

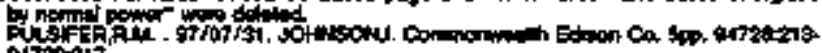
ortis:

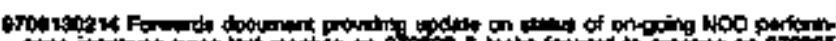

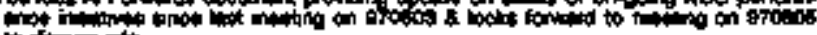

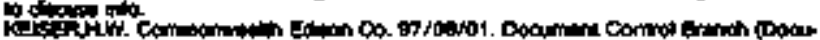

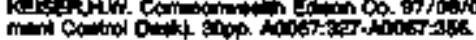

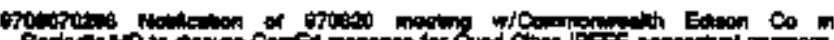

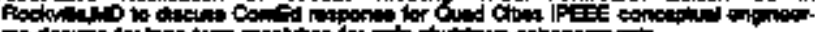

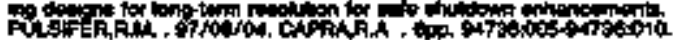

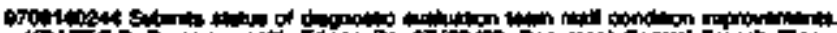

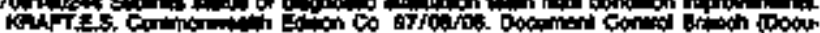

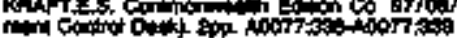

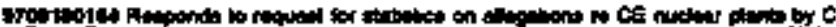

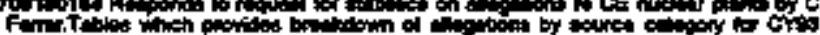

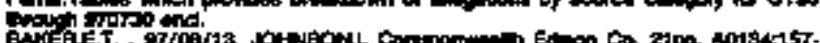
otion.

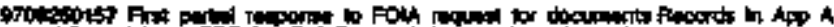

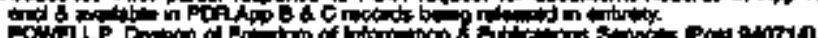

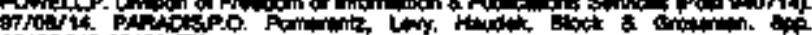

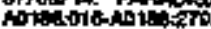

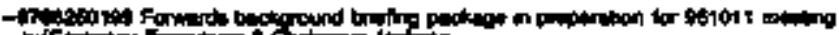

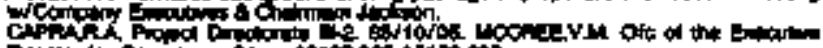

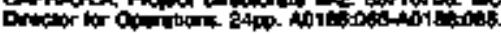

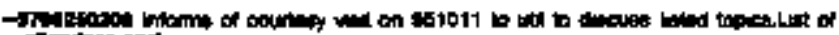
ToCh

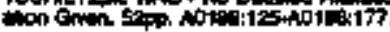

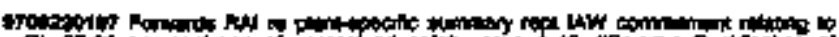

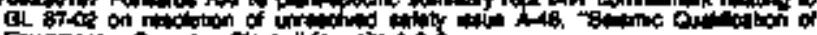

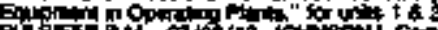
Pustrith

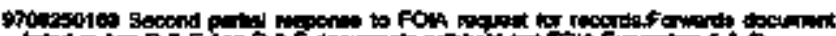

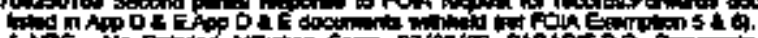

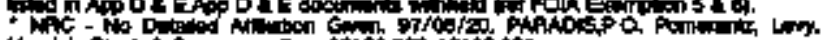

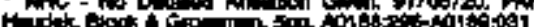




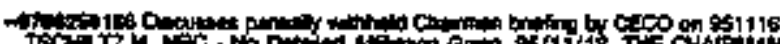

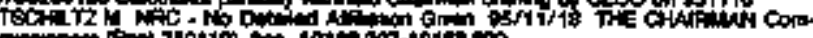

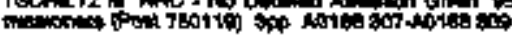

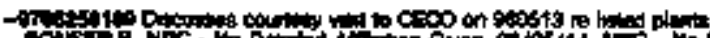

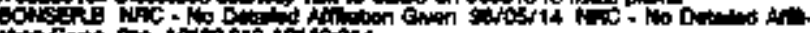

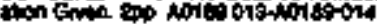

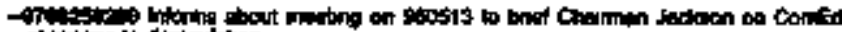

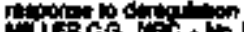

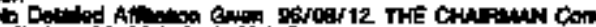
等

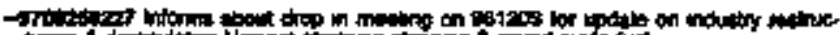

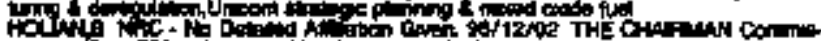

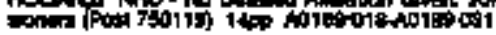

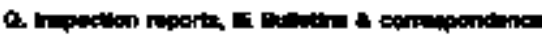

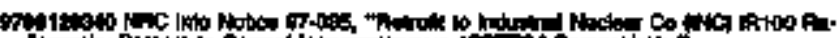

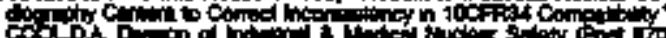

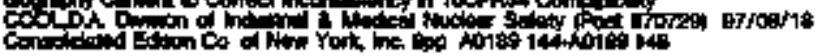

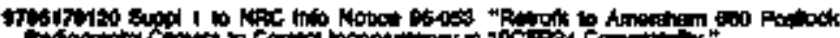
Co,

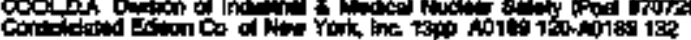

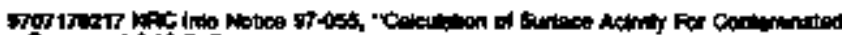

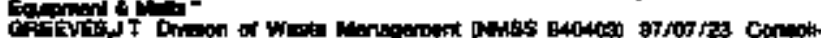

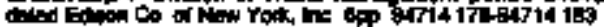

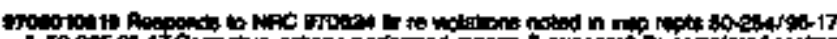

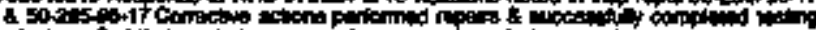

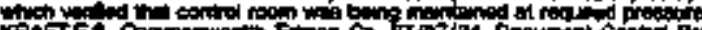

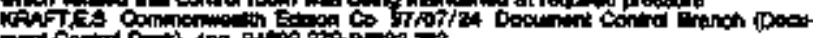

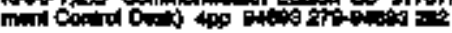

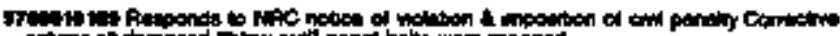
KF

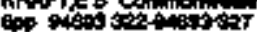

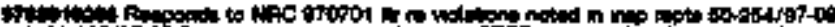

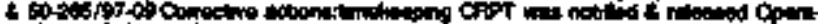

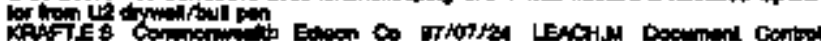

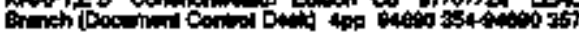

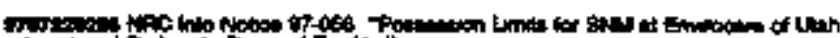

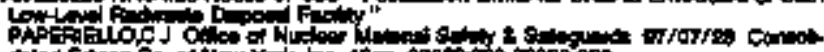

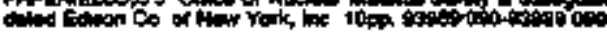

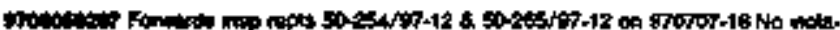

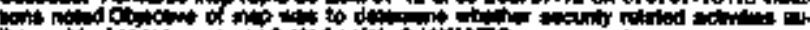

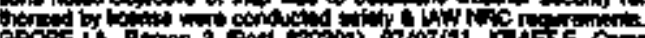

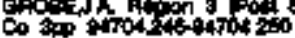

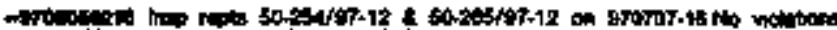

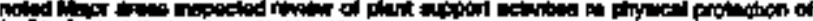

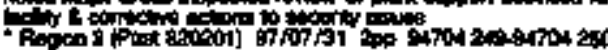

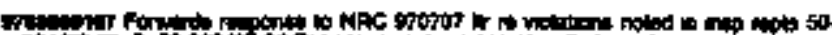

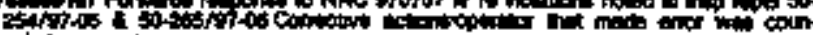

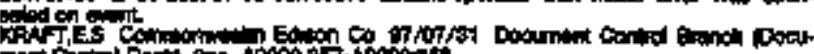

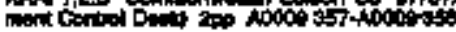

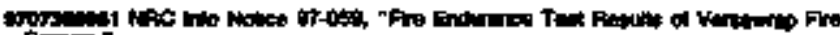

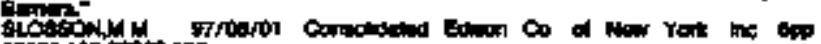
sicoson, 105

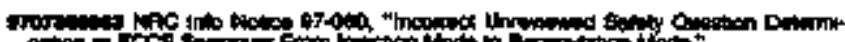

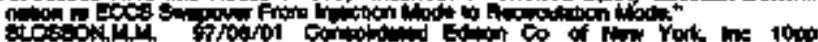
舟

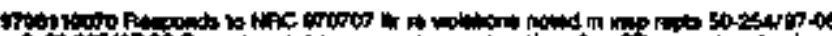

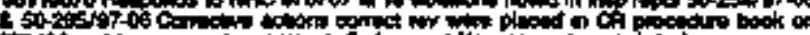

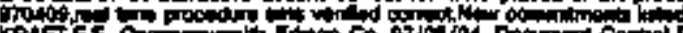

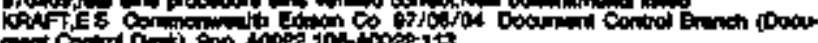

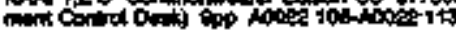

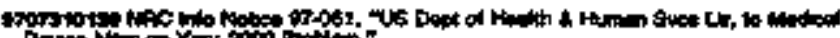

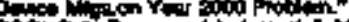

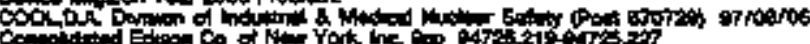

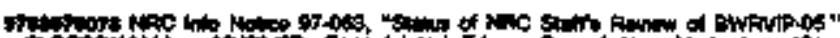

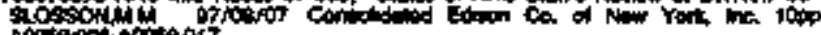

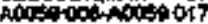

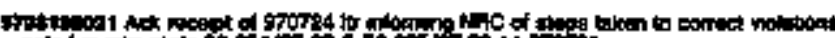
cich

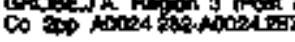

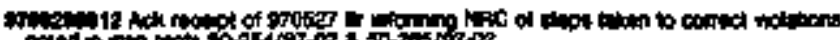

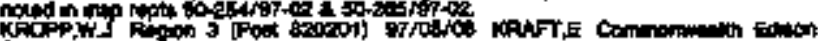

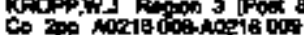

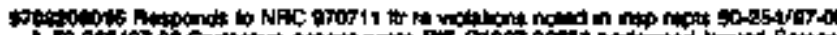

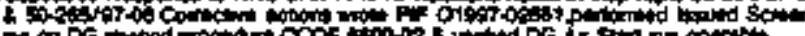

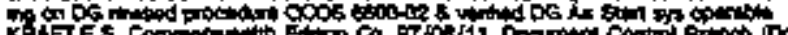

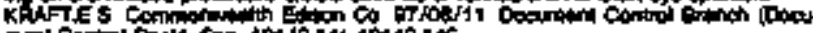

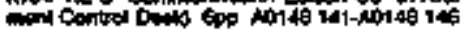

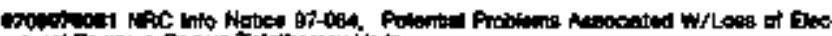

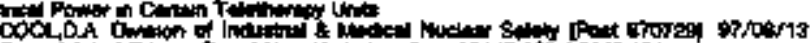

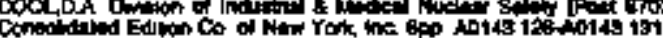

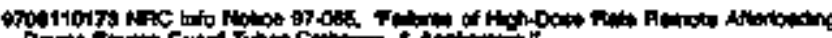

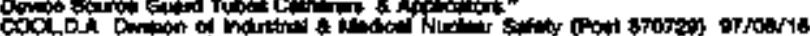

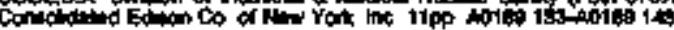

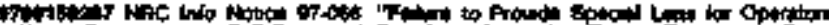

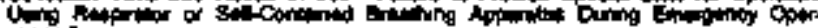

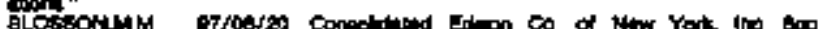
Axer1 3434021130

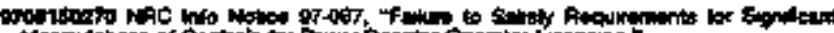
th ADIsis

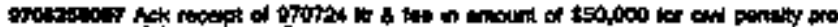

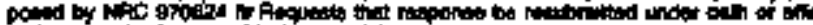

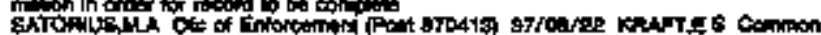

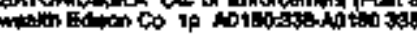

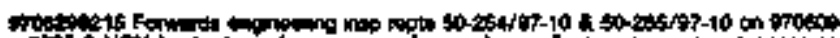

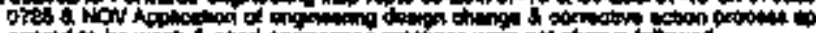

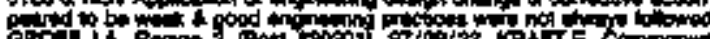

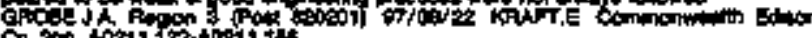

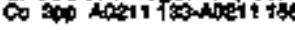

How

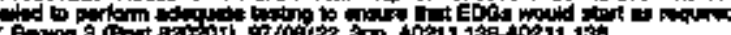

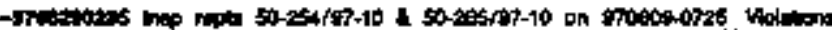

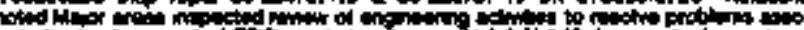

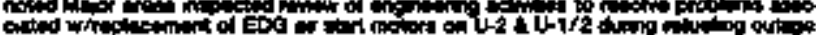

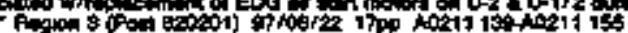

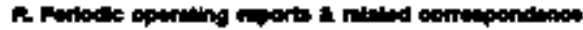

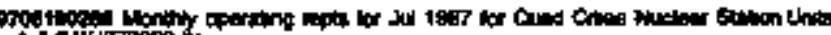

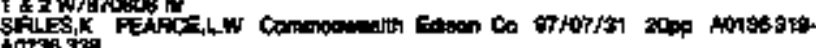

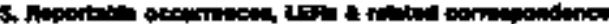

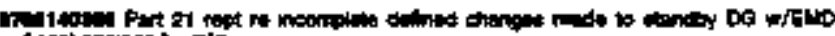

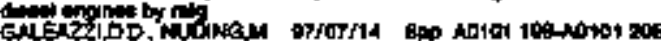

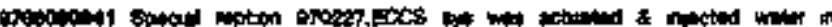

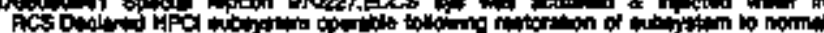

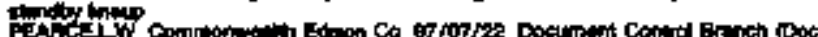

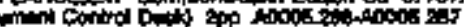

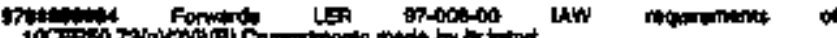

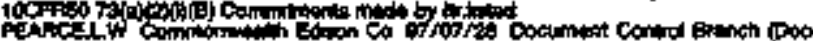

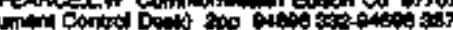

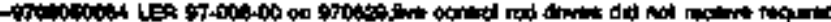

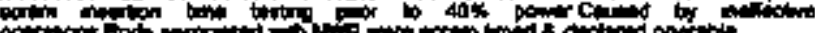

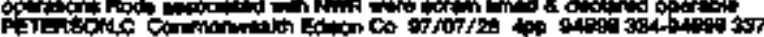

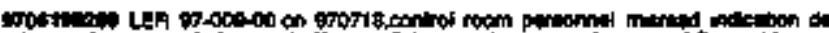

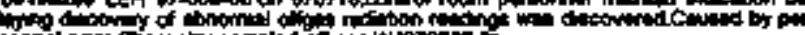
ont crot on

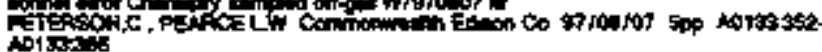

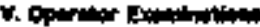

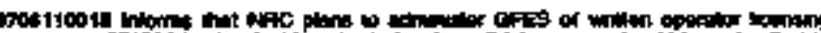

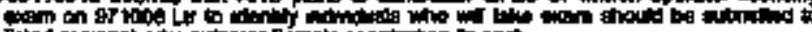

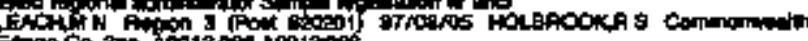

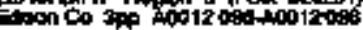

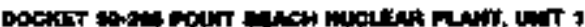

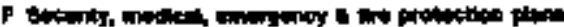

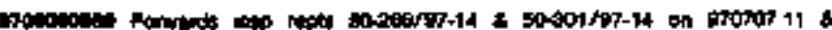

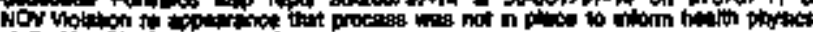

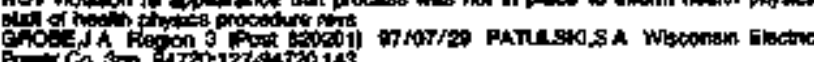




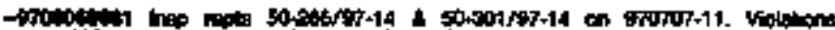

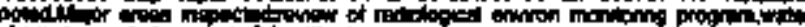

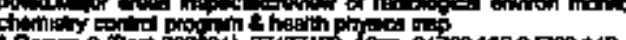

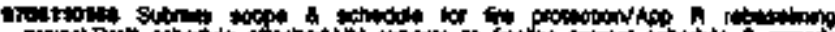

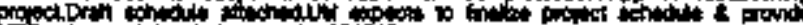

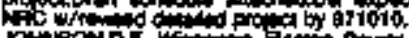

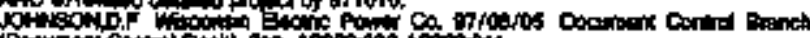

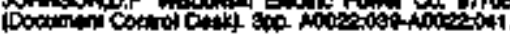

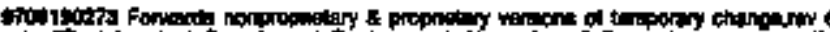

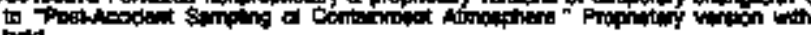

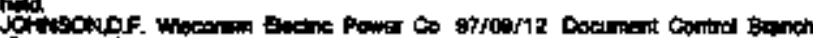

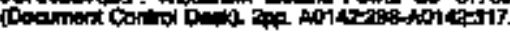

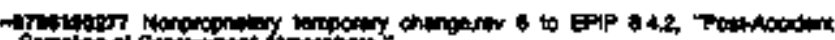

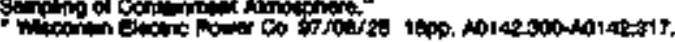

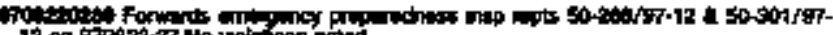

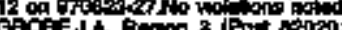

Pane

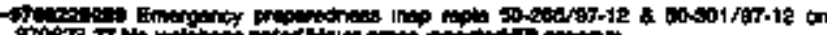

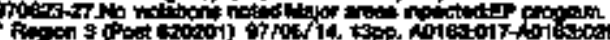

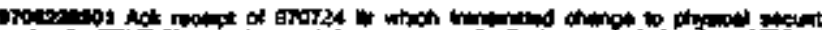

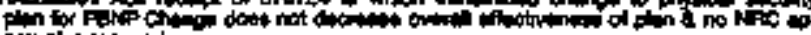

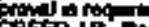

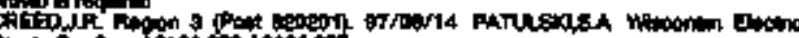

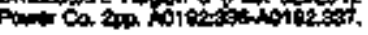

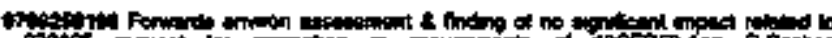

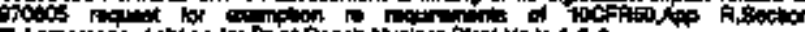

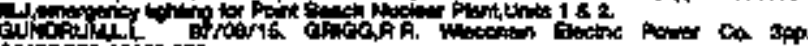

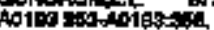

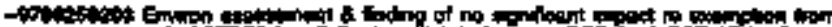

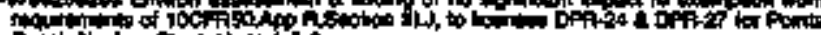

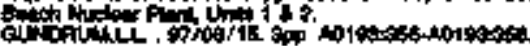

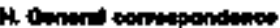

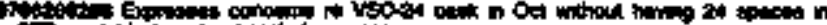

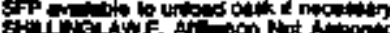

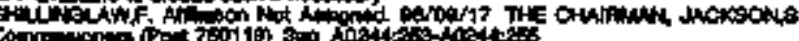

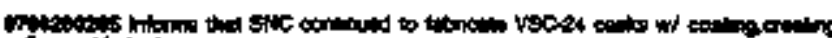

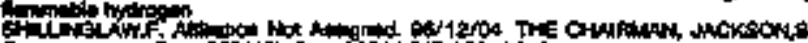

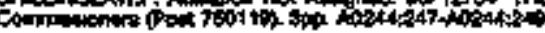

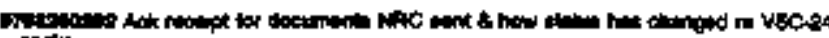

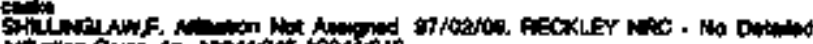

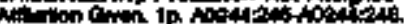

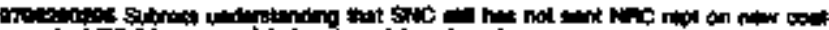

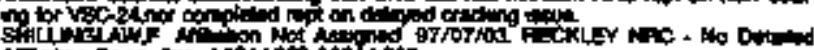

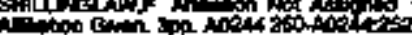

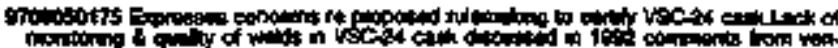

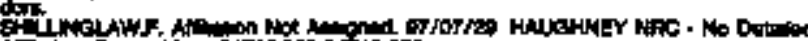

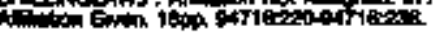

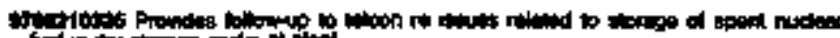

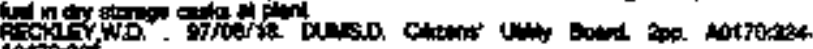
atrosit.

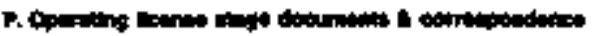

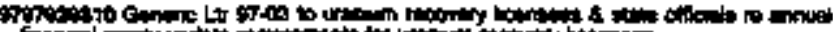

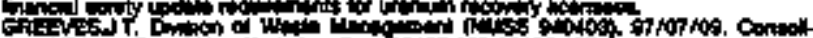

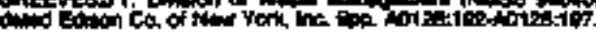

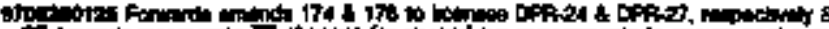

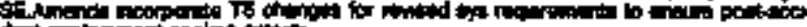

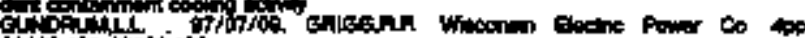

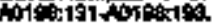

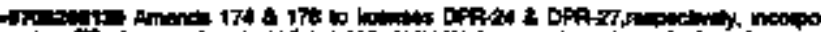

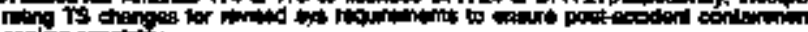
coling

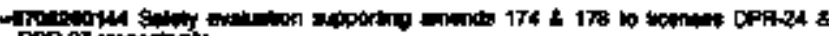

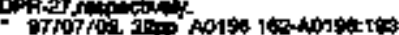

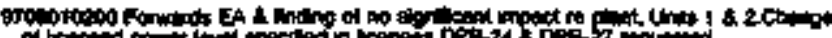

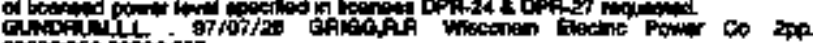

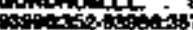

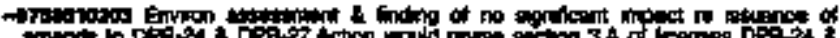

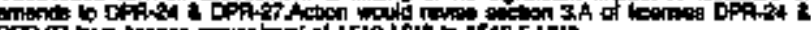

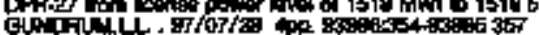

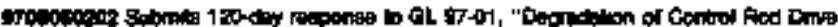

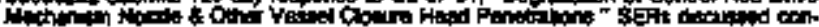

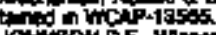

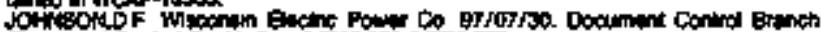

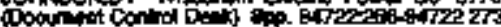

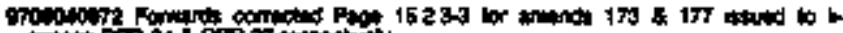

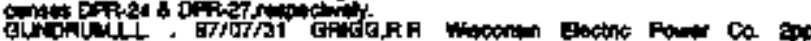

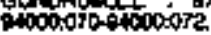

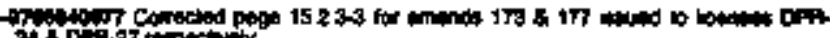

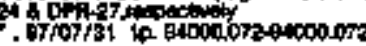

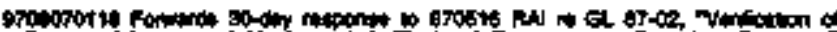

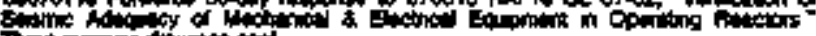

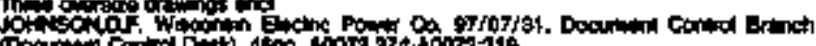

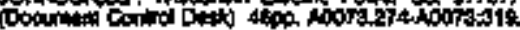

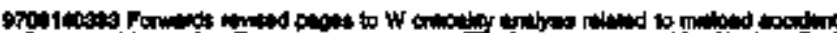

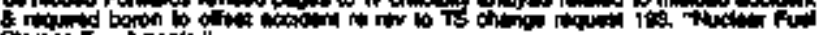

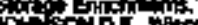

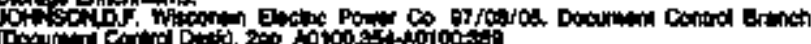
然

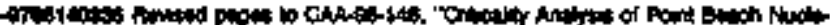

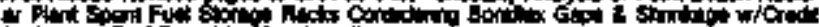

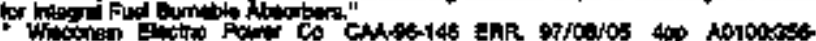
Nototices

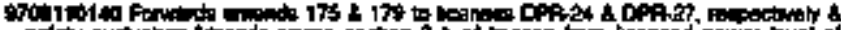

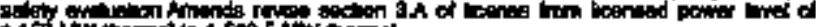

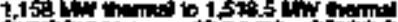

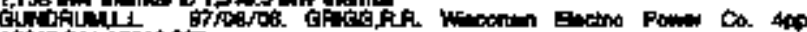

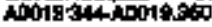

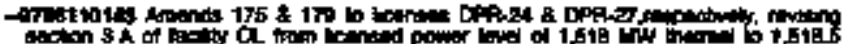

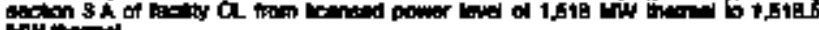

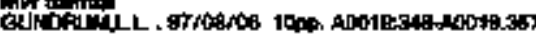

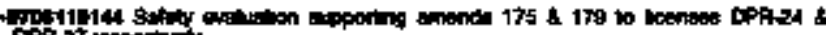

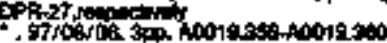

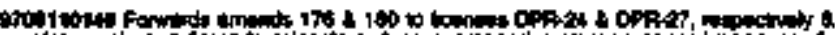

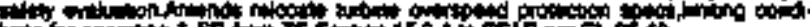

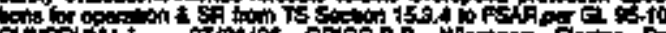
Sit

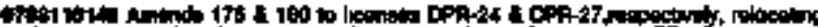

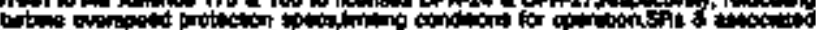

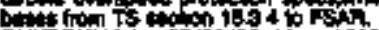

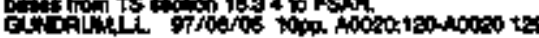

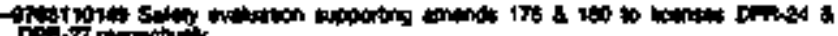

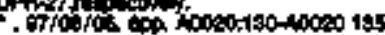

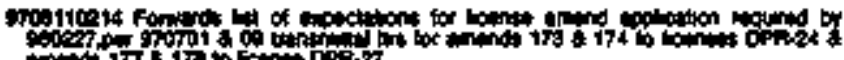

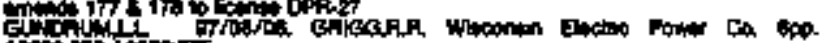

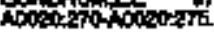

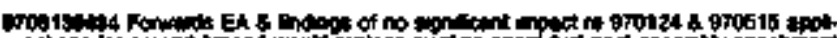

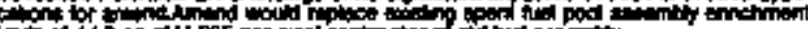

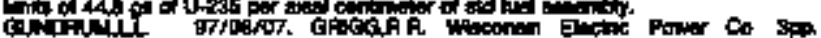

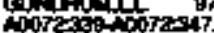

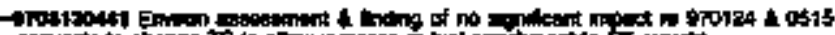

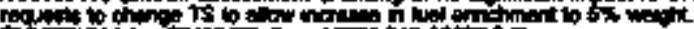

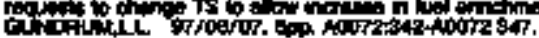

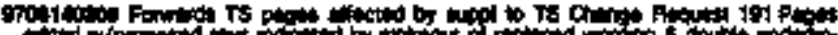

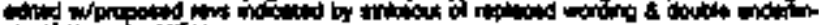
of of

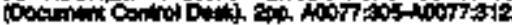

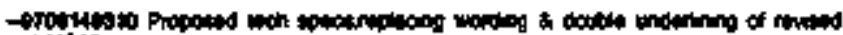

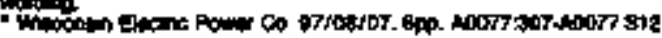

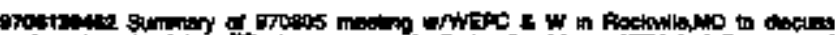

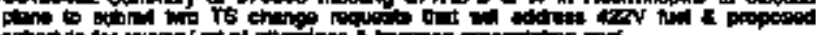

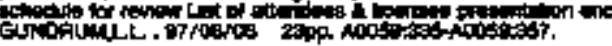

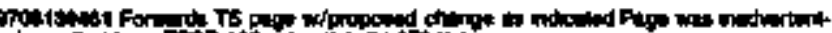

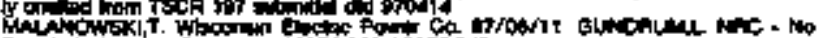

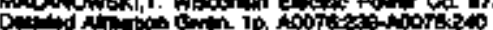

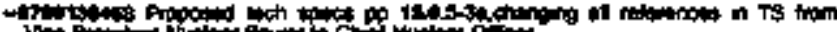

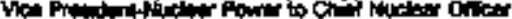
1 


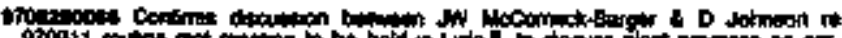

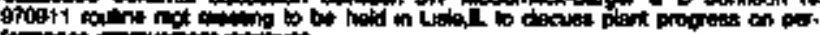

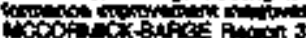

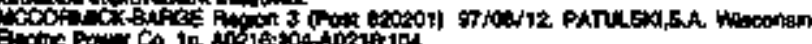

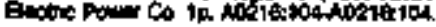

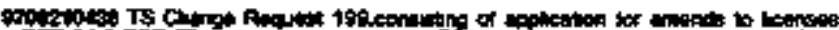

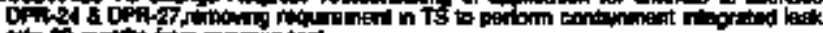

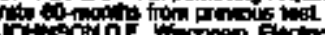

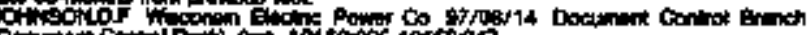

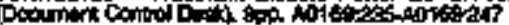

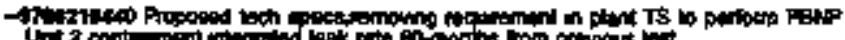

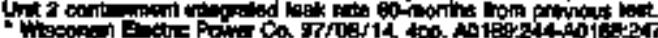

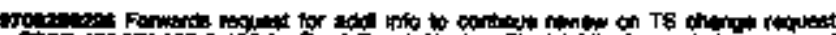

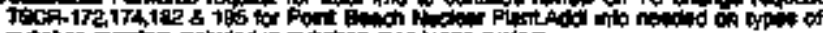

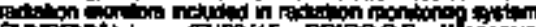

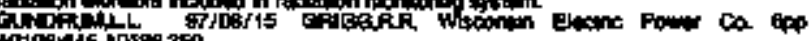

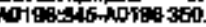

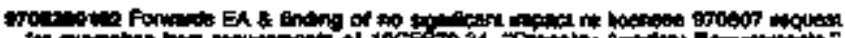

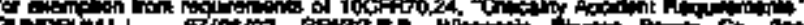

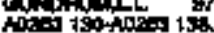

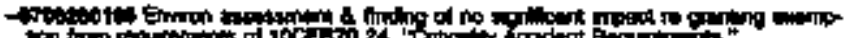

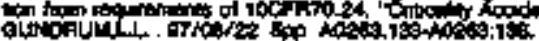

if

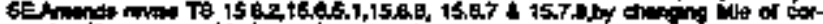

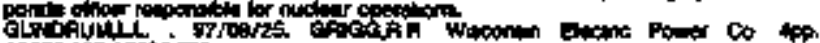

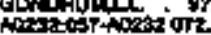

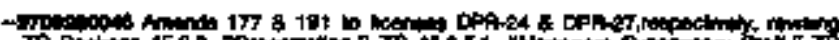

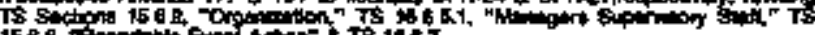

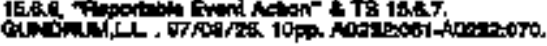

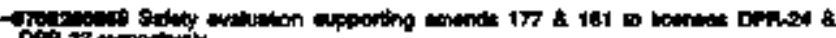

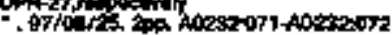

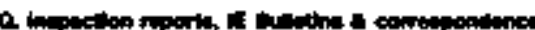

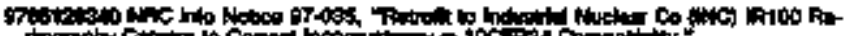

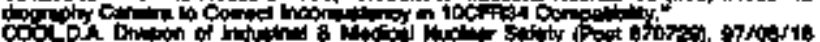
Con

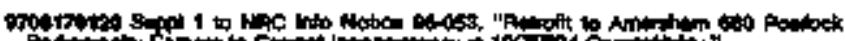

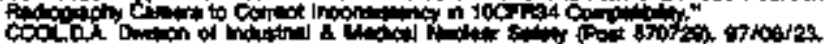

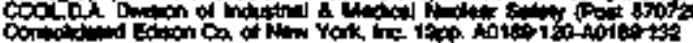

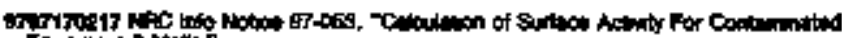

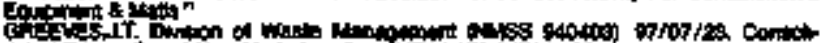

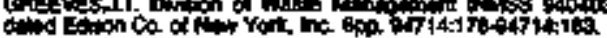

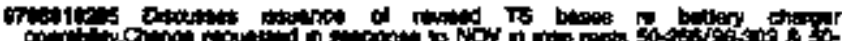

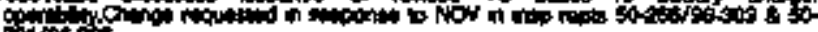

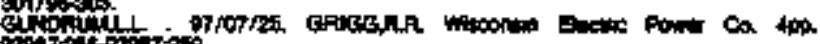
a

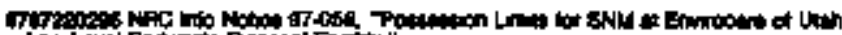

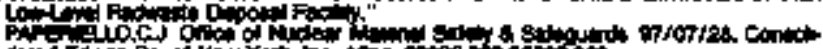

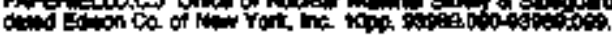

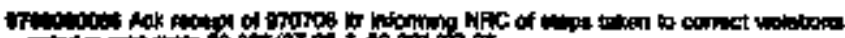

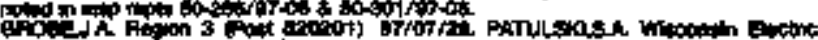

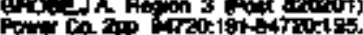

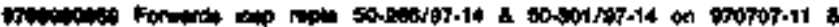

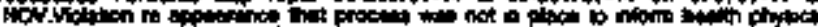

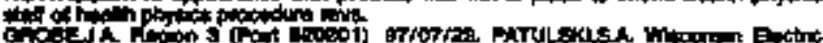

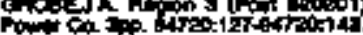

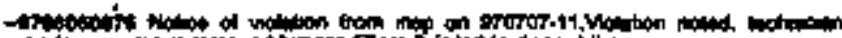

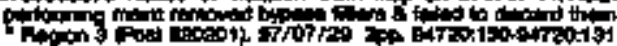

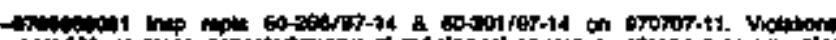

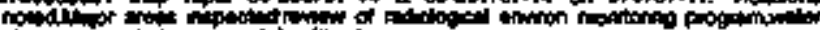

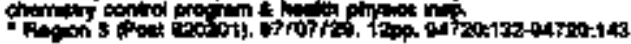

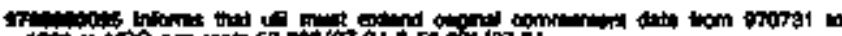

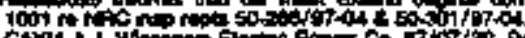

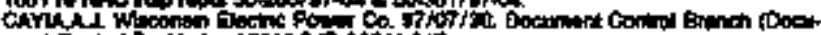

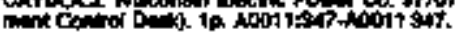

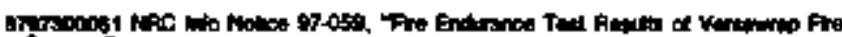

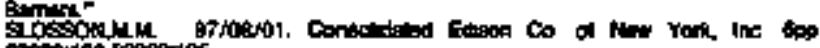

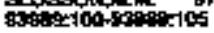

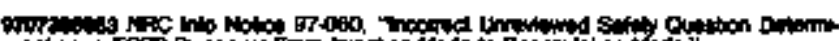
stosso GLos

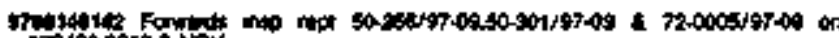

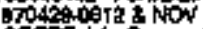

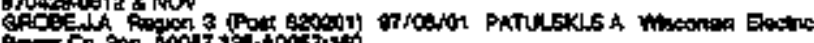

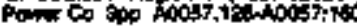

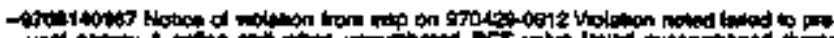

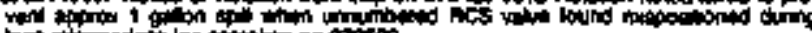

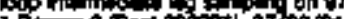

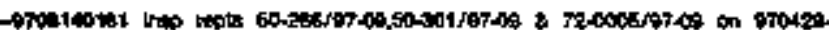

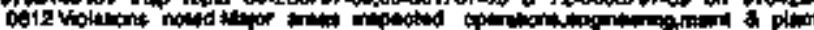
Peppot.

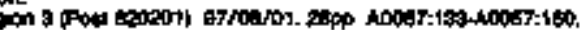

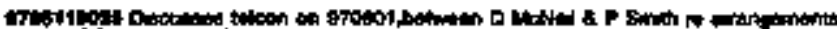

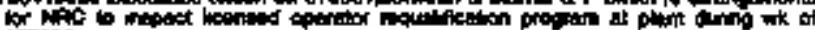

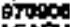

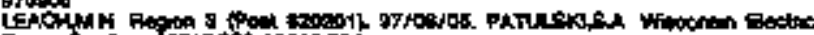

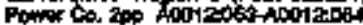

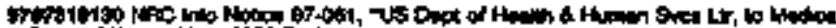

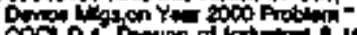

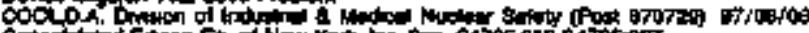

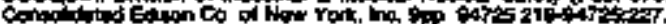

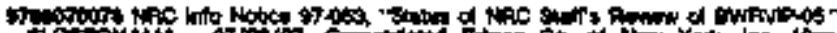
Losonint in

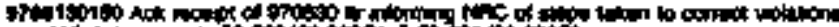

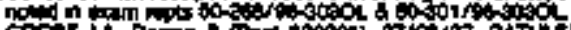

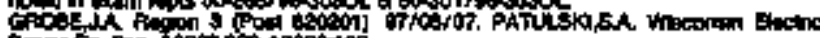

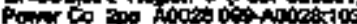

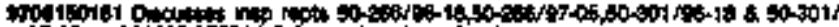

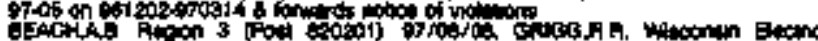

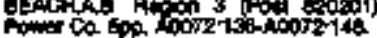

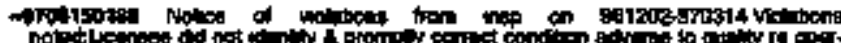

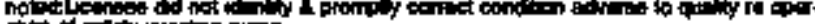

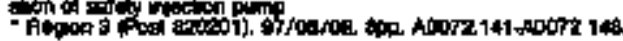

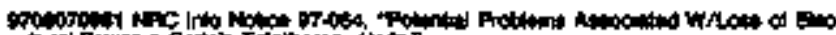

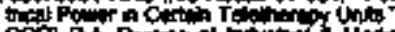

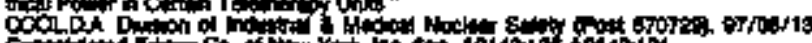

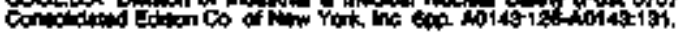

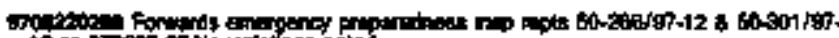

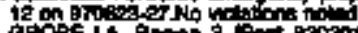

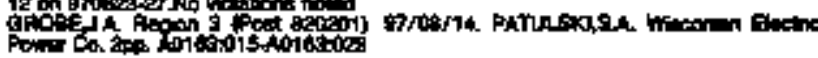

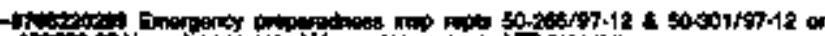

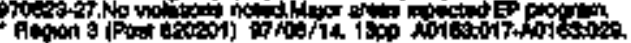

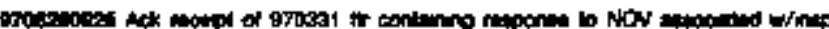

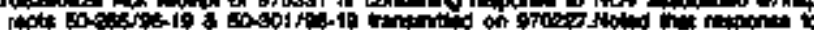

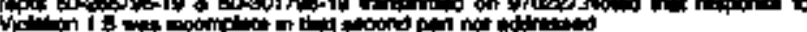

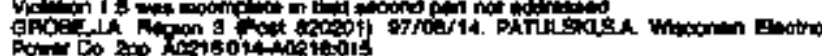

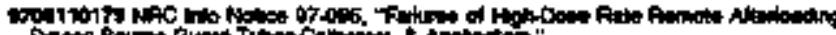

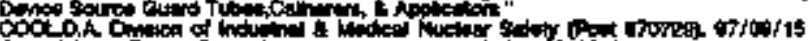

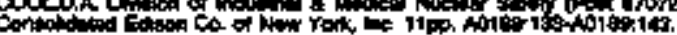

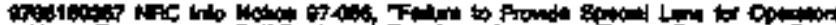

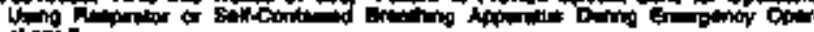

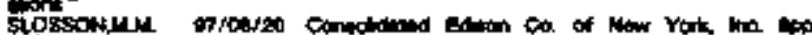
Aocis 1)

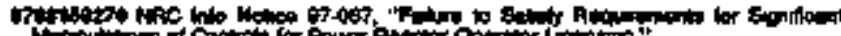

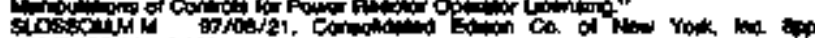

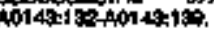

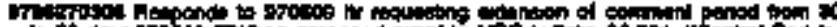

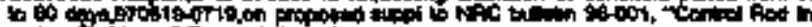

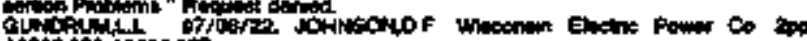

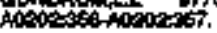

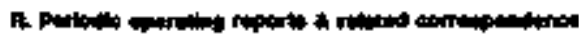

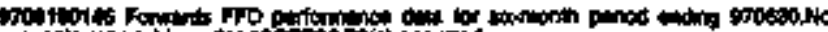
cold

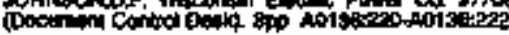

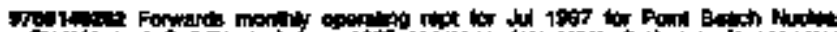

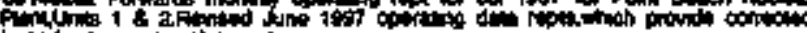

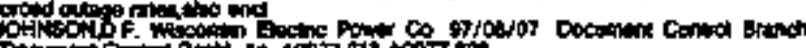

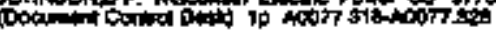

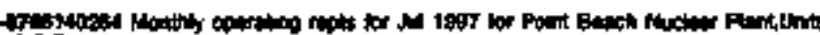

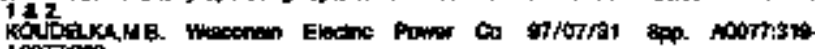
notrąas 


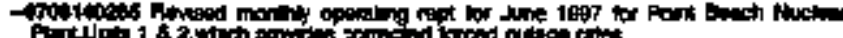
noto hom 3 at

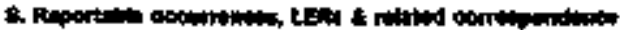

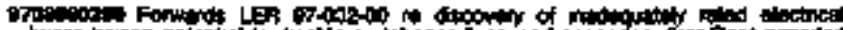

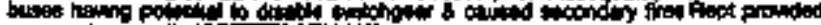

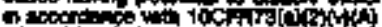

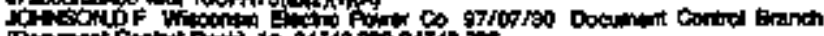

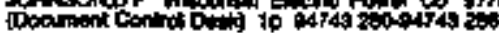

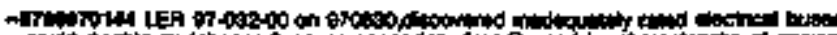

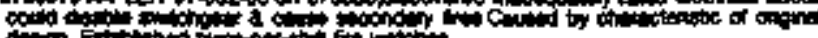

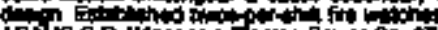

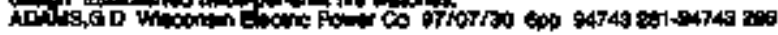

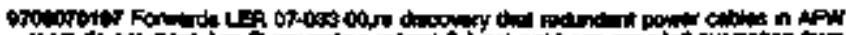

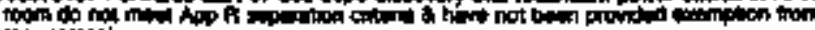
trom

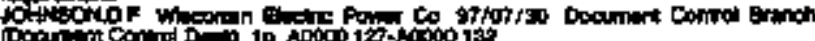
(1000 132

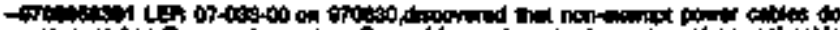

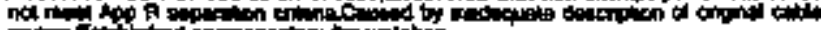

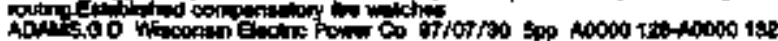

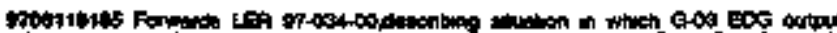

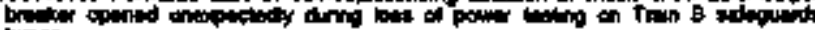

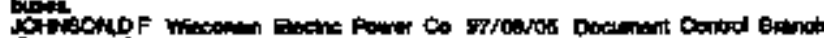

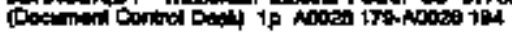

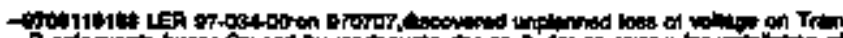

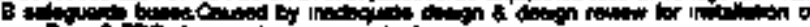

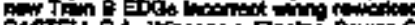

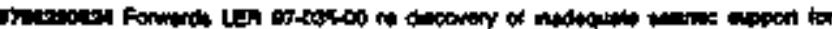

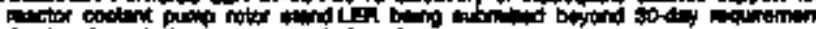

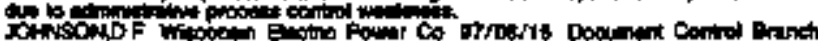

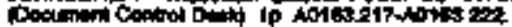

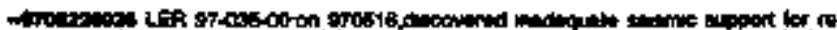

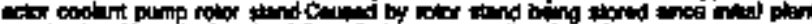

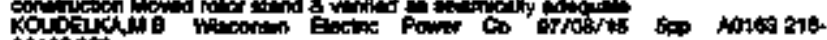
A0tiones

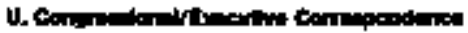

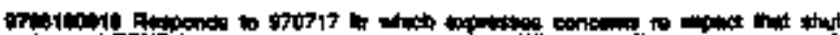

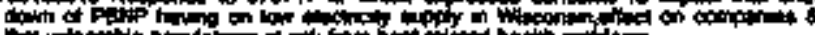

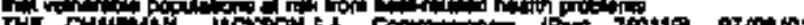

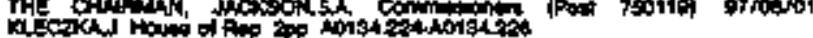

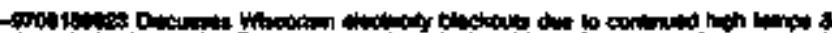

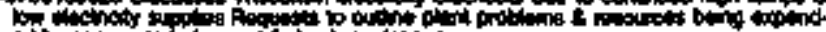

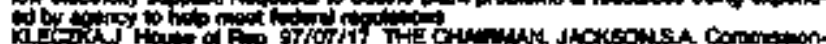

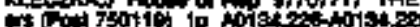

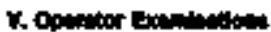

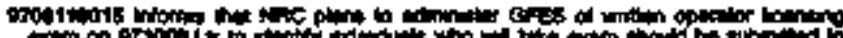

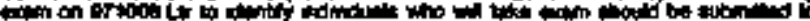

U.

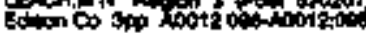

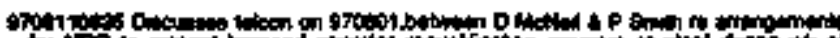

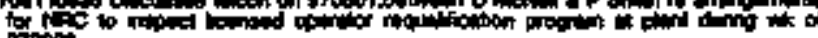

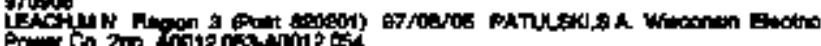

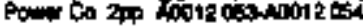

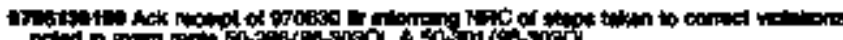

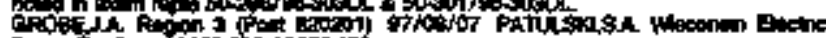

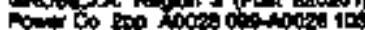

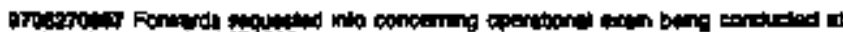

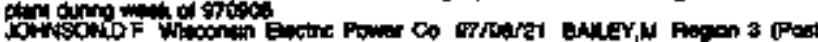

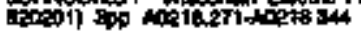

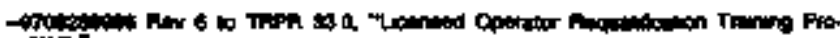

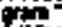

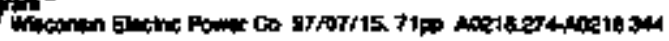

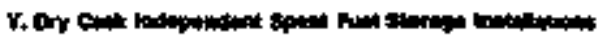

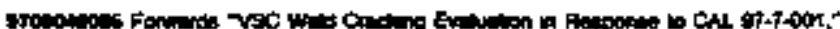

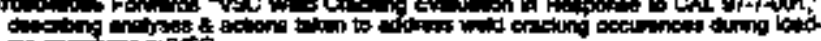

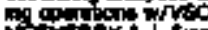

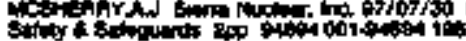

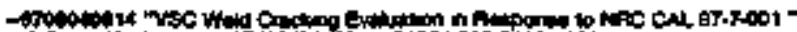

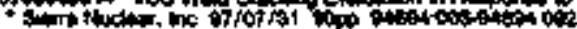

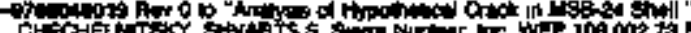

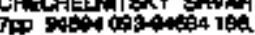

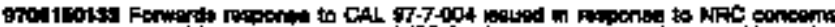

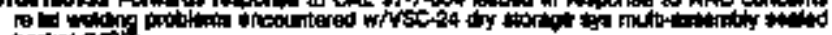
toin

管

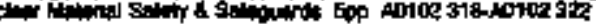

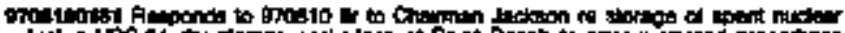

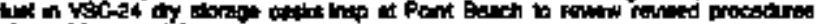

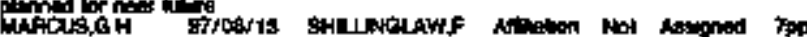

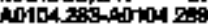

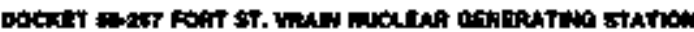

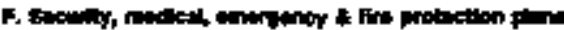

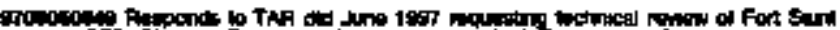

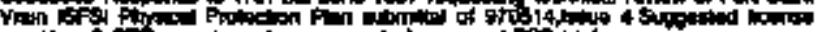

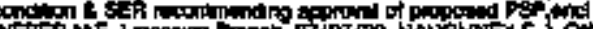

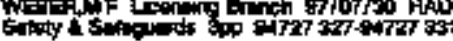

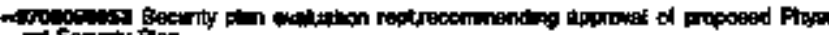

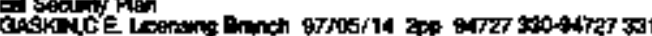

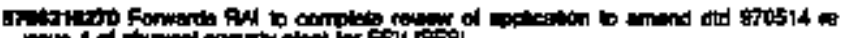

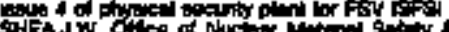

Phe

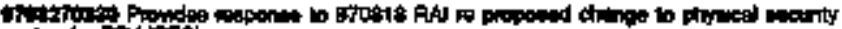

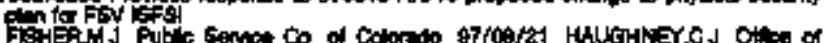

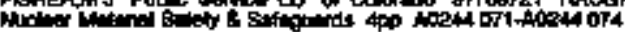

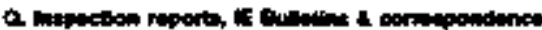

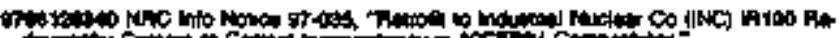

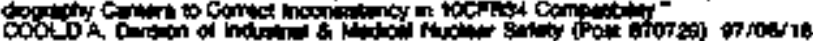

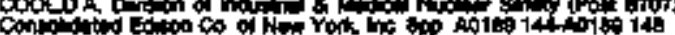

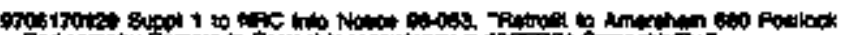

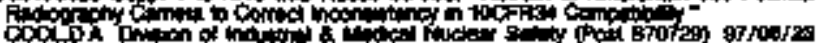

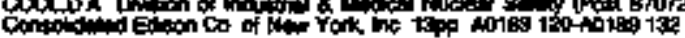

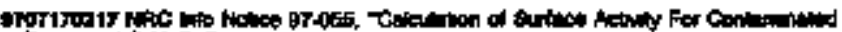

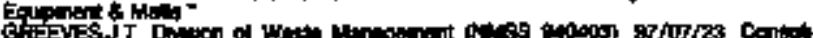

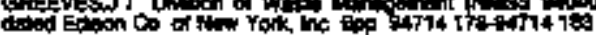

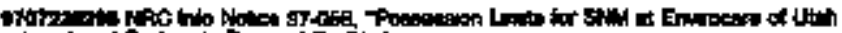

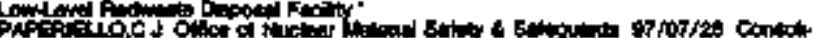

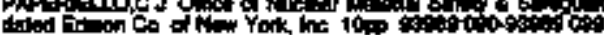

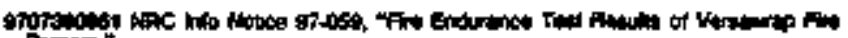

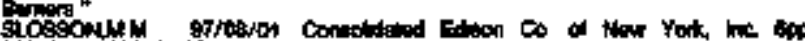
thos 100 toting 106

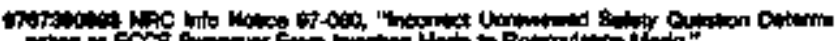

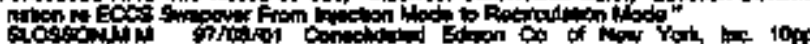

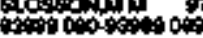

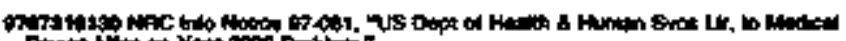

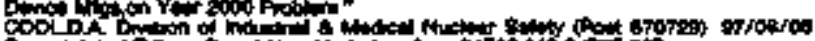

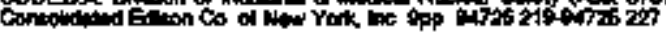

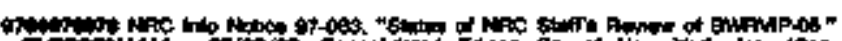

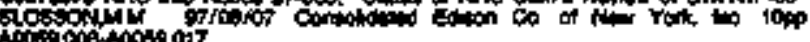

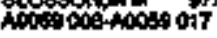

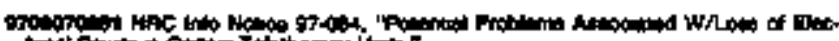

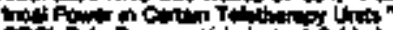

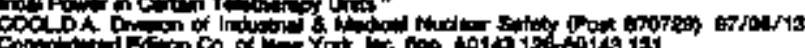

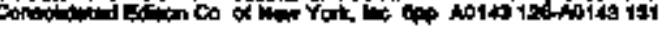

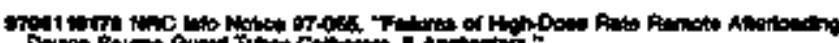

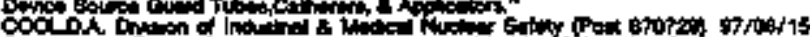

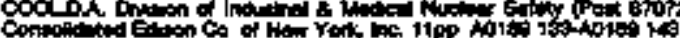

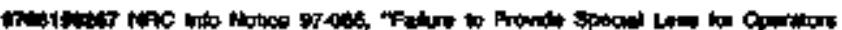

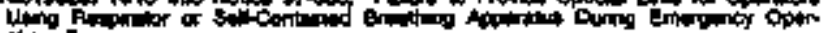

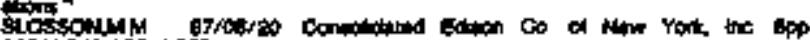

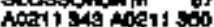

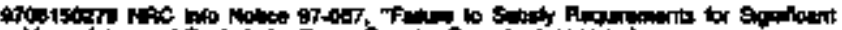

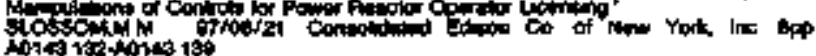




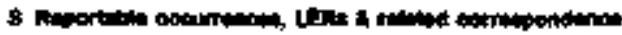

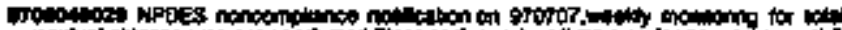

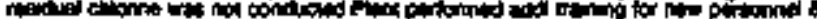

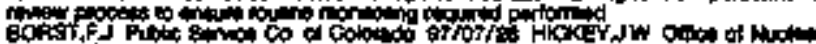

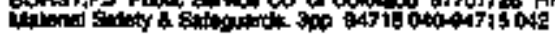

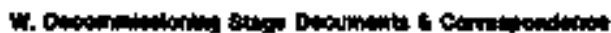

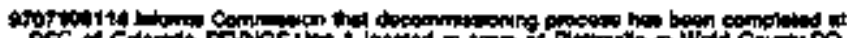

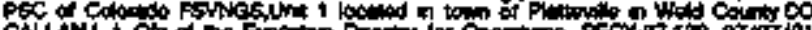

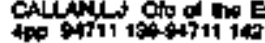

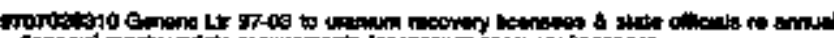

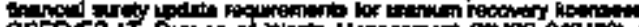
incorion conot

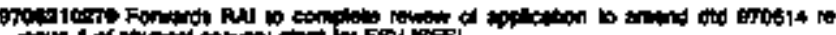

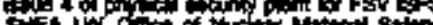

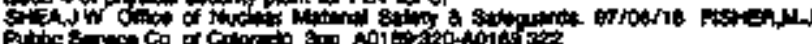

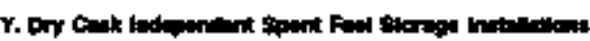

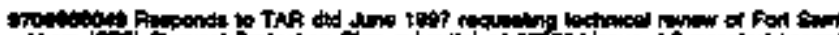

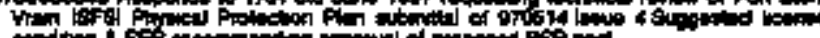

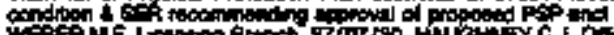

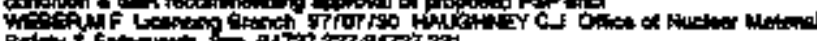

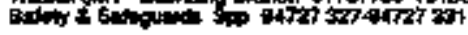

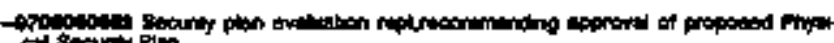

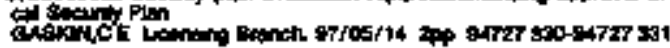

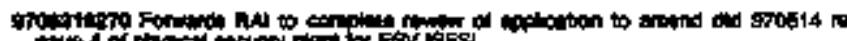

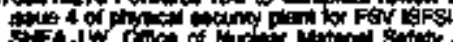

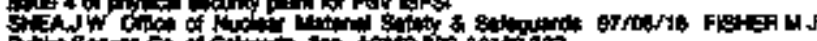

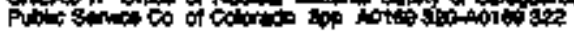

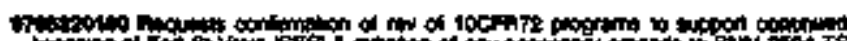

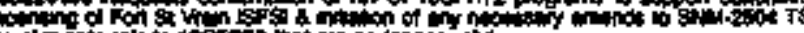

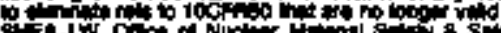

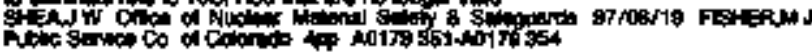

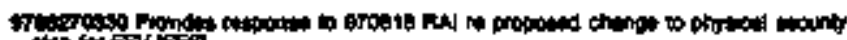

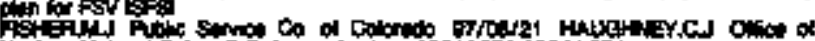

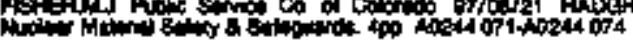

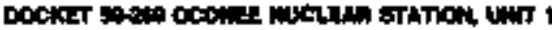

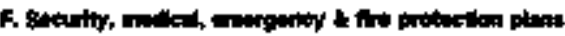

3hection

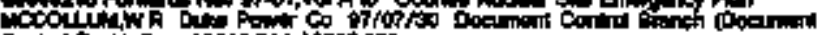

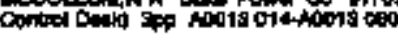

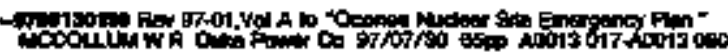

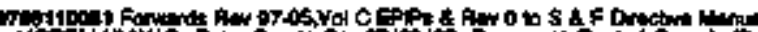

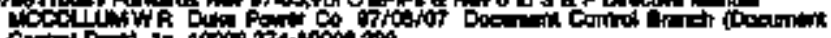

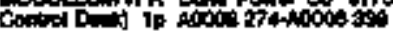

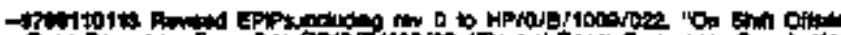

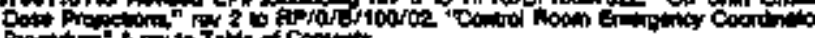

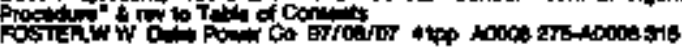

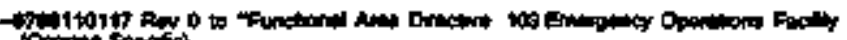

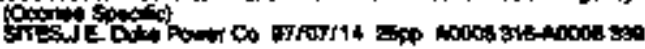

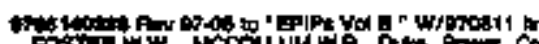
Pond

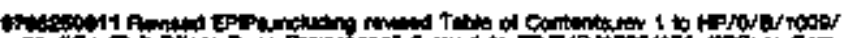

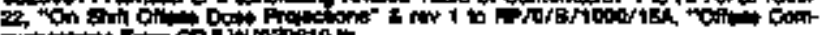

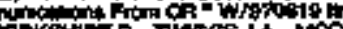

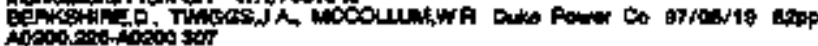

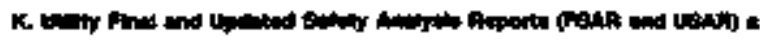

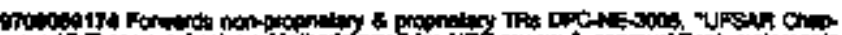

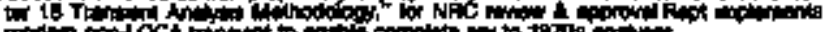
Tuctin Nows $001-40001$ 25:

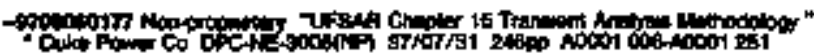

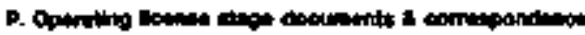

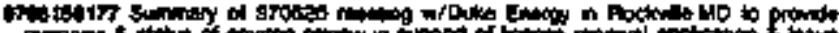

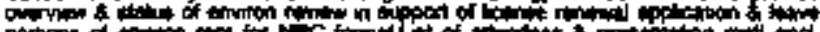

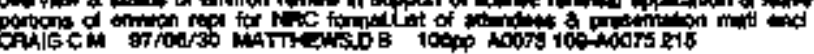

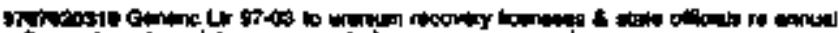

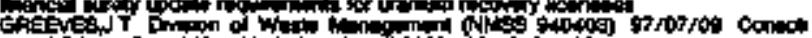

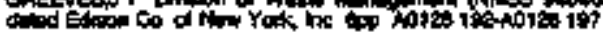

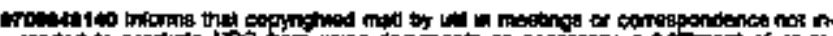

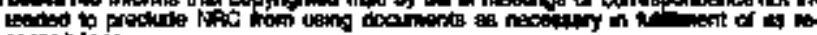

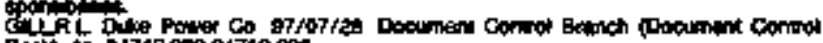
Detip ip $1470200-96710200$

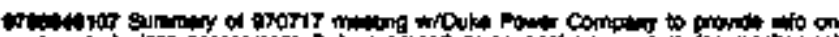

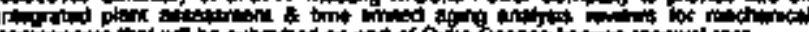

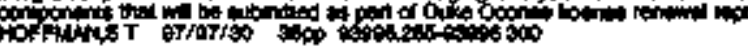

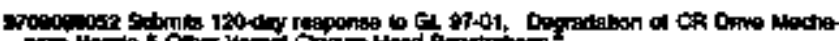

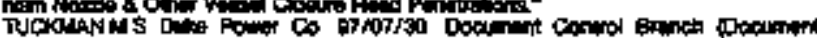

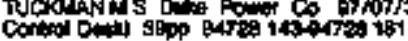

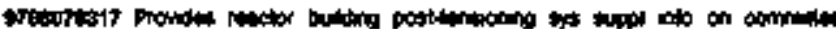

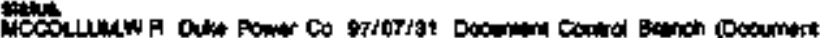

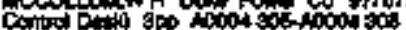

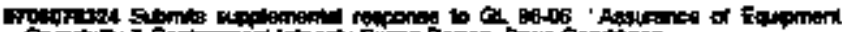
Path

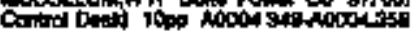

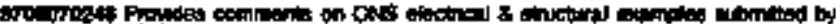

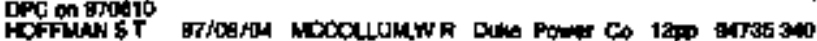
90753 31

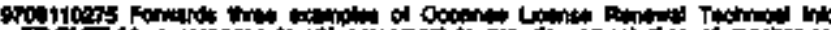
In

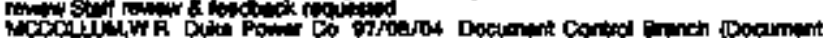

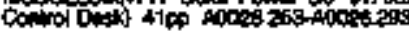

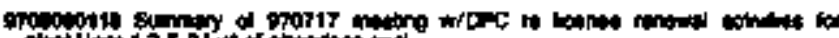

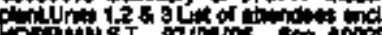

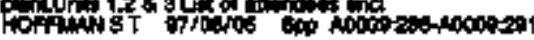

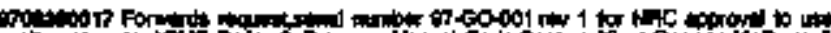

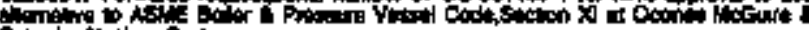

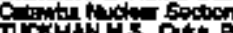

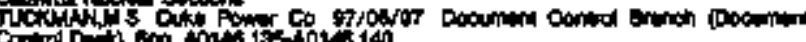

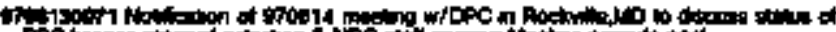

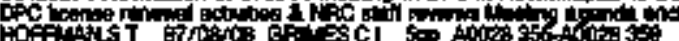

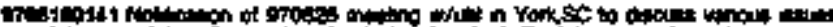

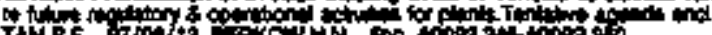

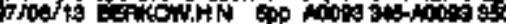

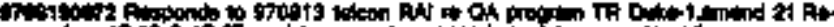

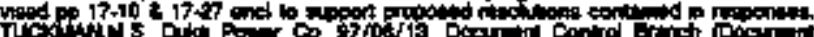

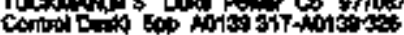

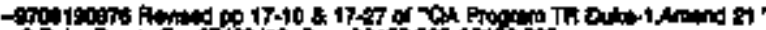

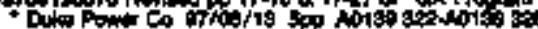

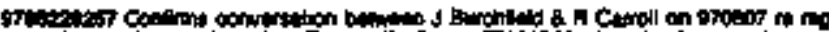

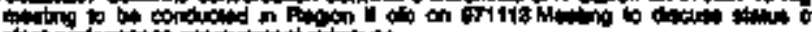

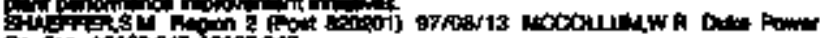

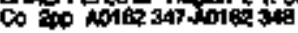

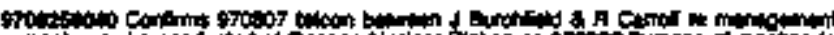

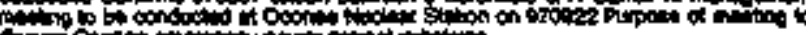

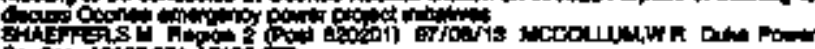
Co

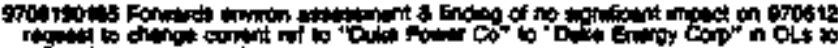

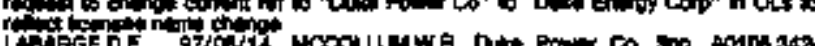
Antos

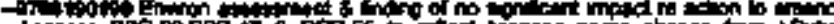

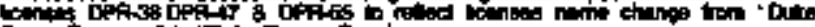

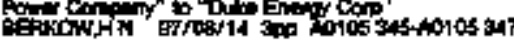

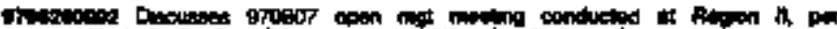
Boven Co 100p Notesorithoisto

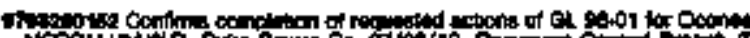

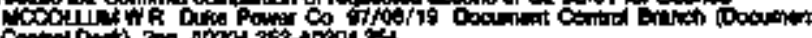
controd Deito 


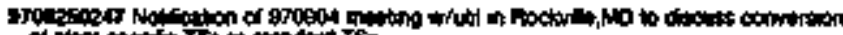
if

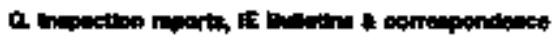

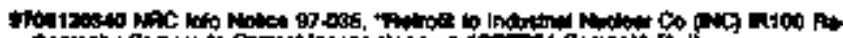

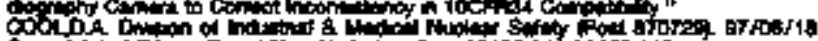

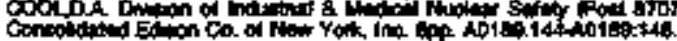

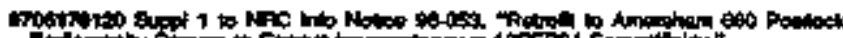

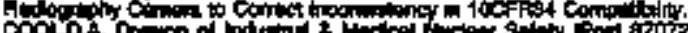

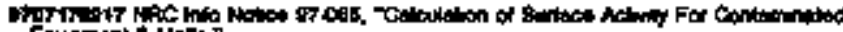

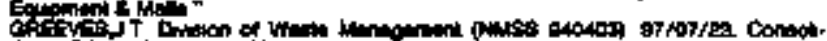

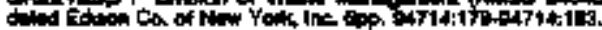

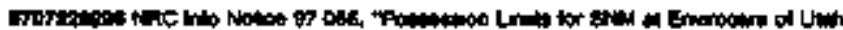

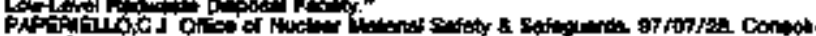

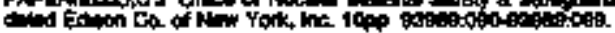

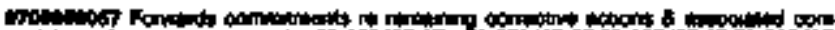

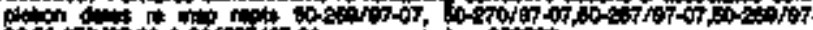

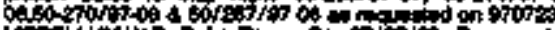

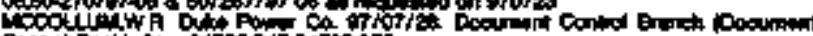

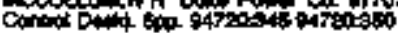

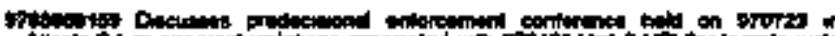

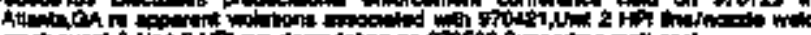

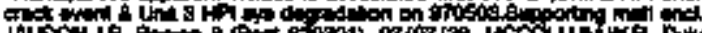

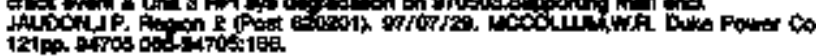

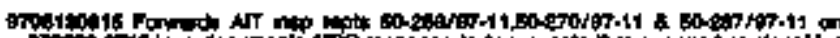

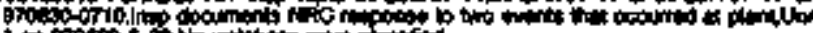
1 in Fis.

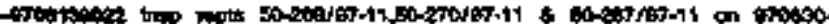

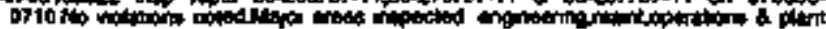

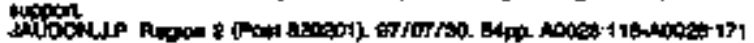

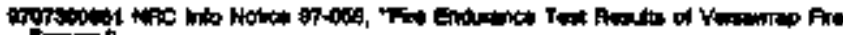

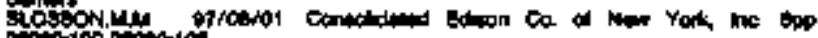
B.

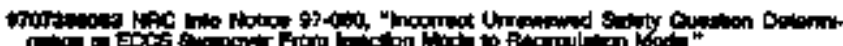

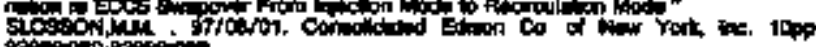

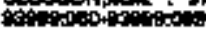

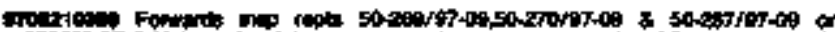

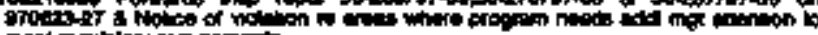

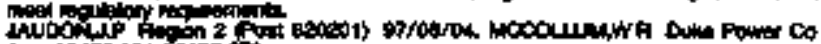

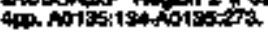

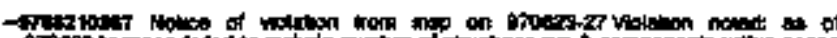

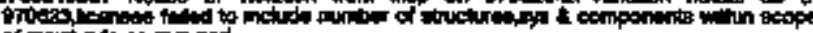

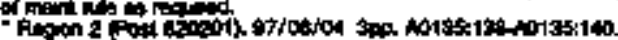

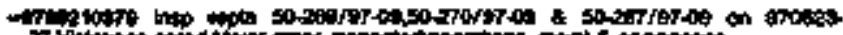

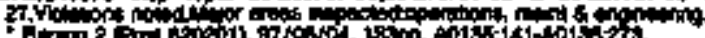

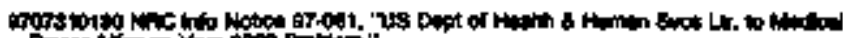

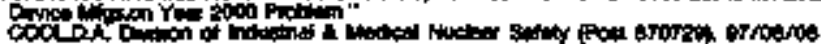

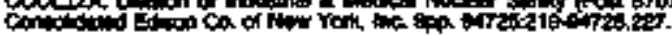

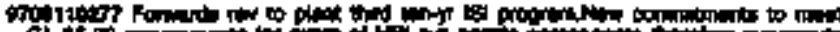

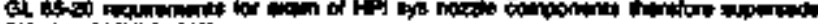

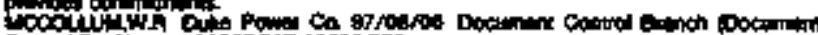

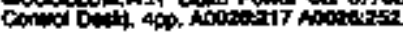

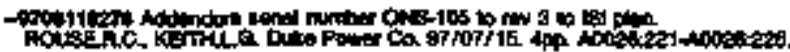

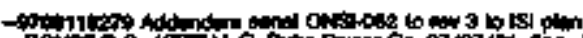

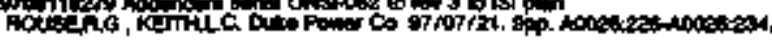

- Q7.

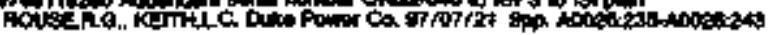

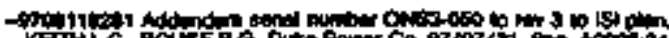

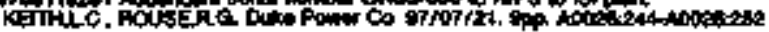

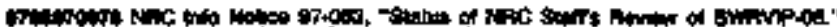

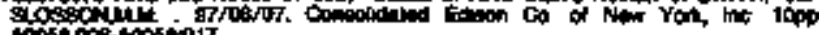

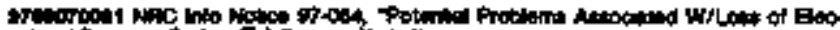

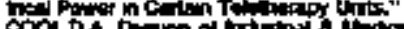

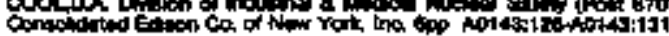

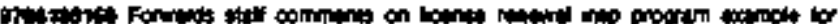

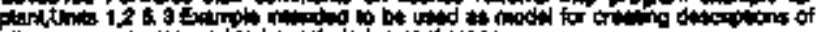

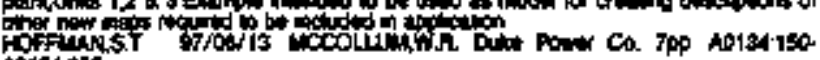

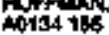

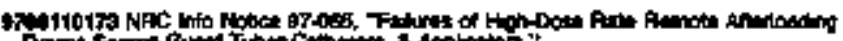

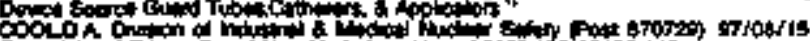

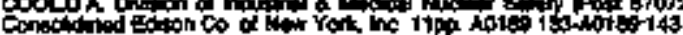

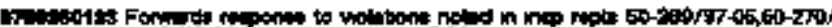

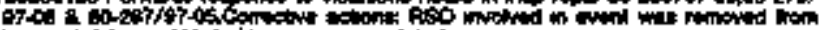

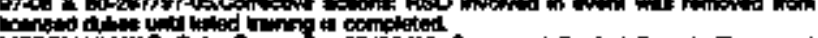

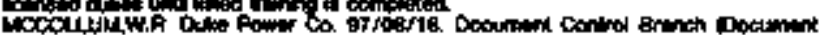

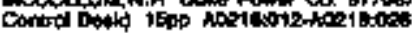

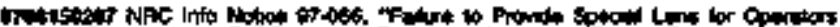

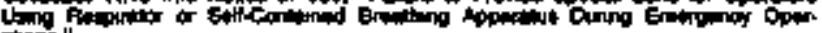

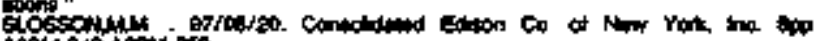

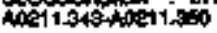

17.

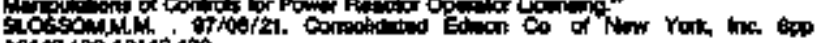

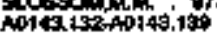

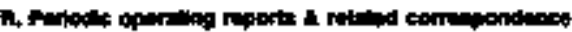

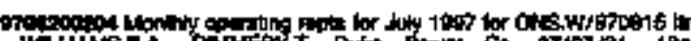

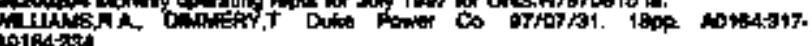

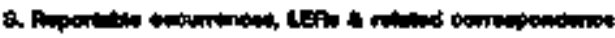

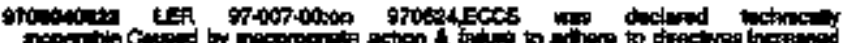

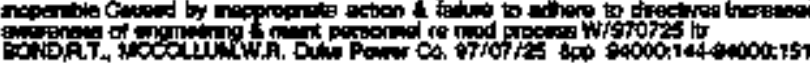

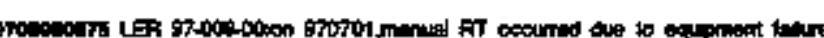

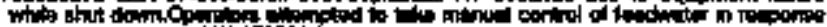

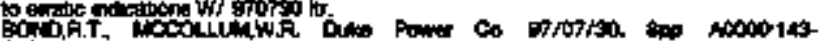

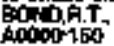

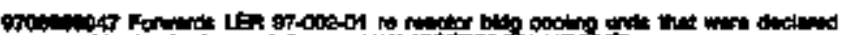

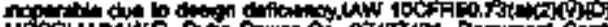

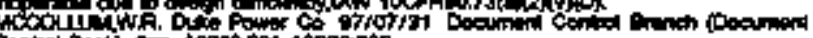

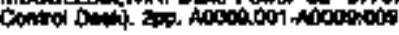

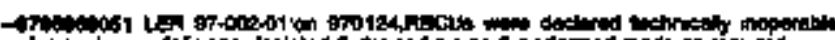

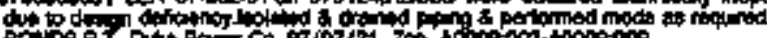

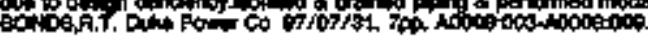

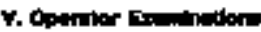

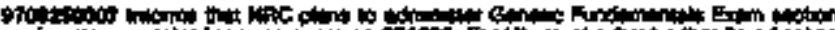

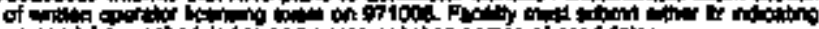

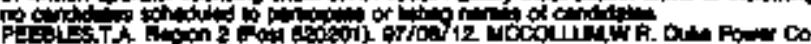
200.

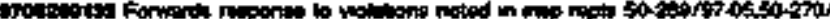

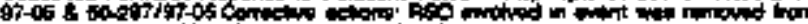

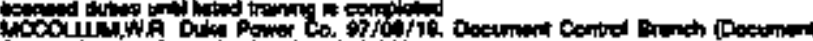

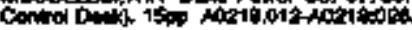

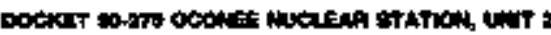

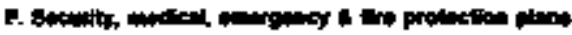

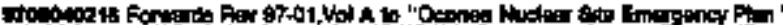

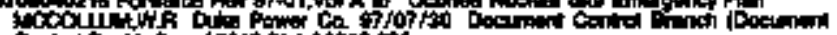

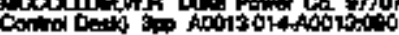

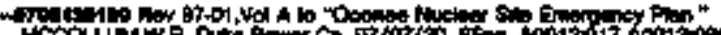

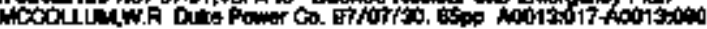

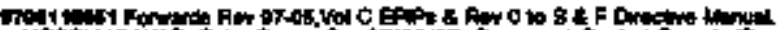

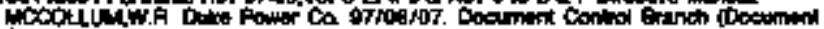

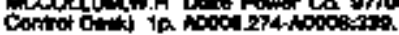

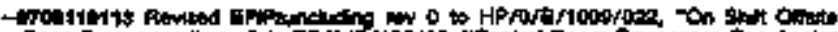

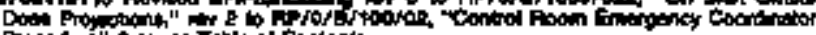

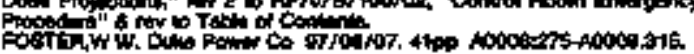

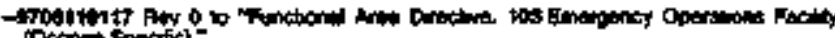

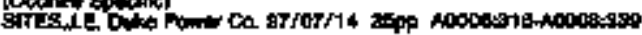

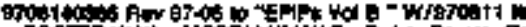

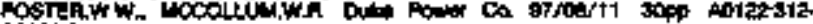
1012ะ2011 


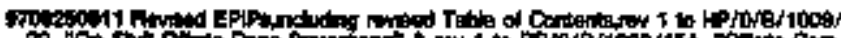

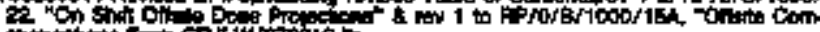
onchom orifor

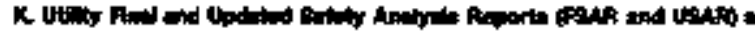

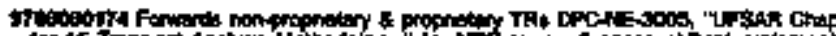

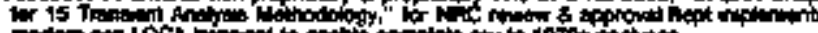

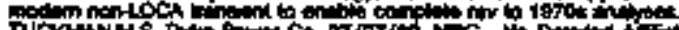

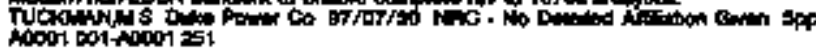

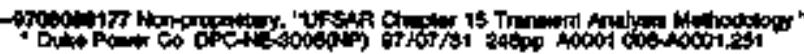

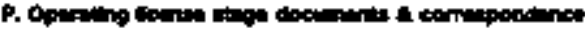

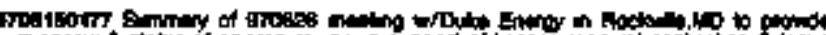

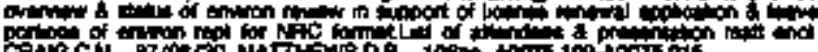

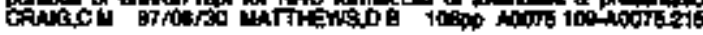

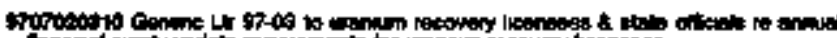

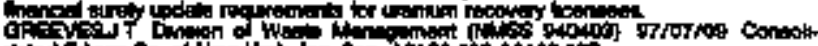

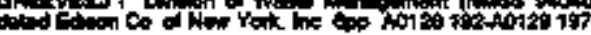

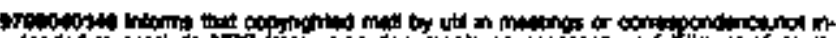

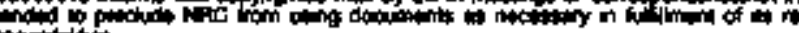

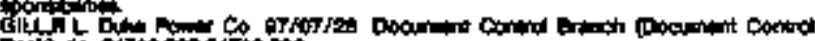
Dip th

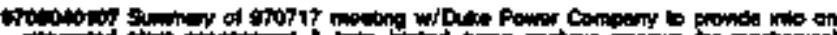

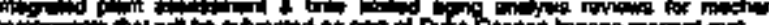

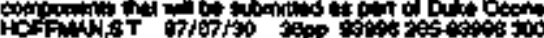

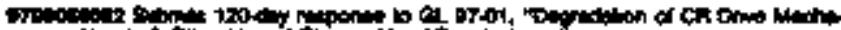

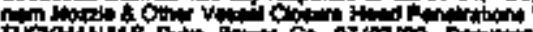

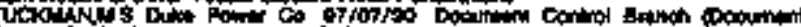

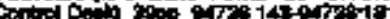

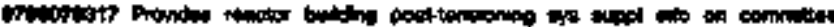

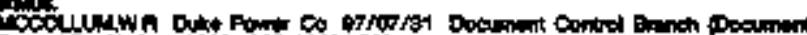

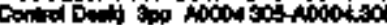

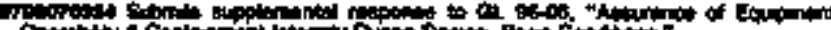

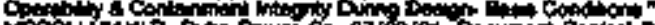

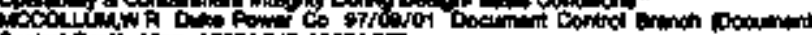

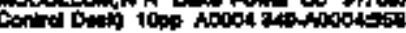

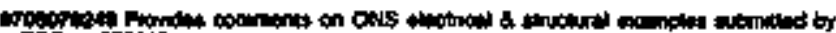

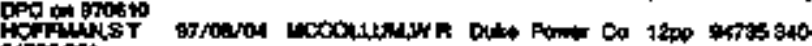
6itiont

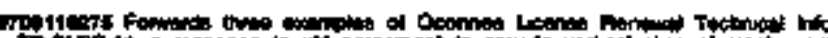

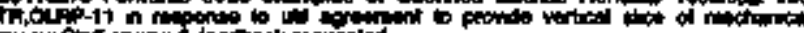

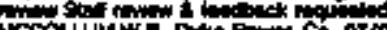

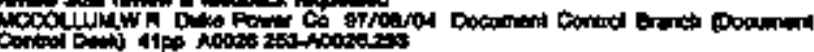

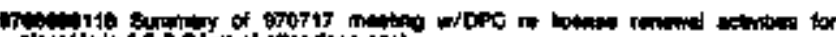

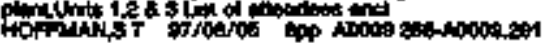

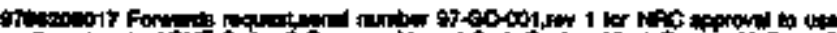

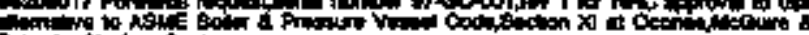

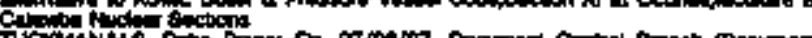

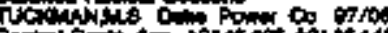

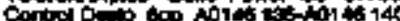

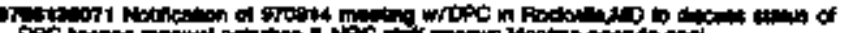

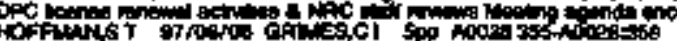

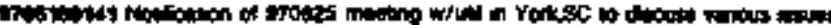

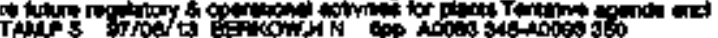

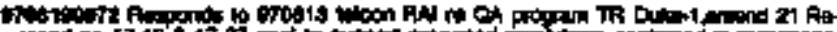

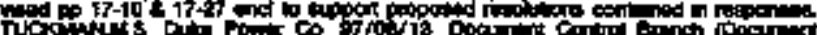

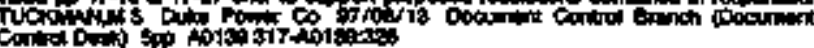

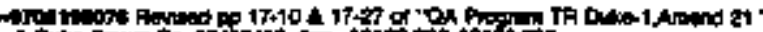

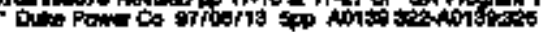

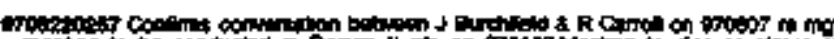

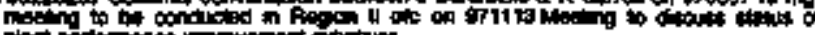

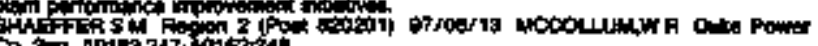

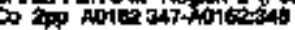

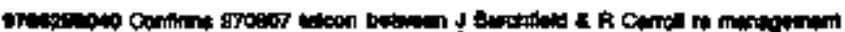

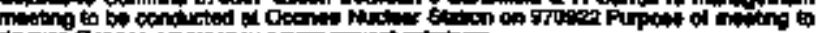

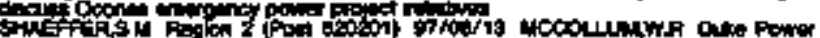

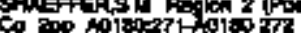

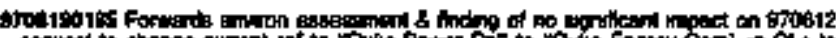

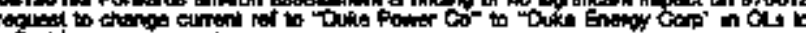

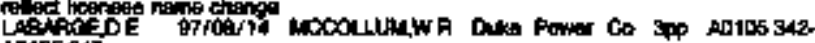
An106 347

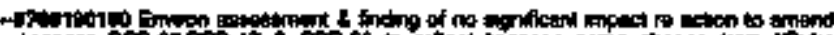

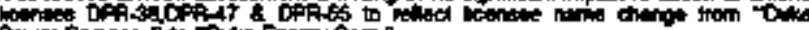

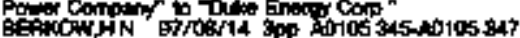

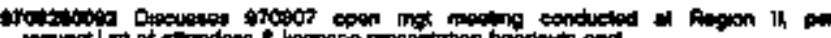

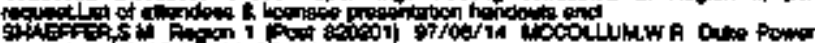

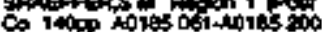

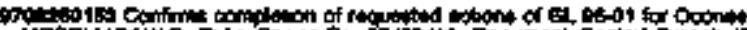

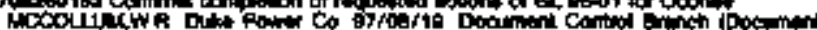

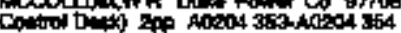

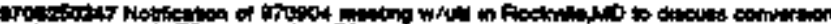

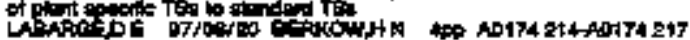

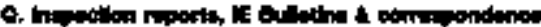

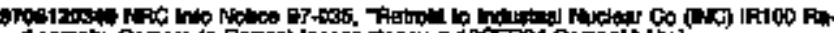

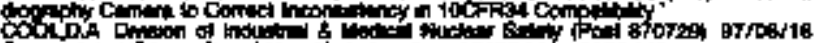

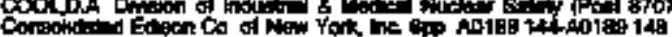

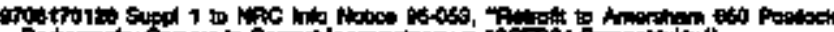

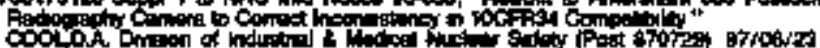

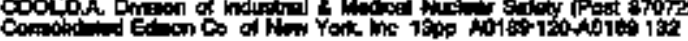

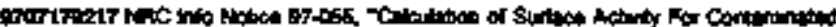

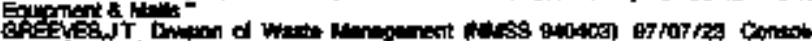

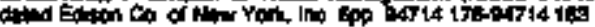

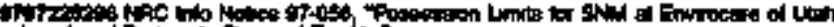

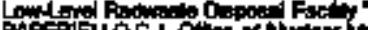

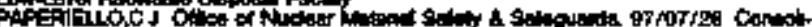

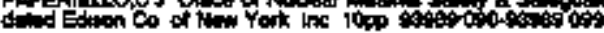

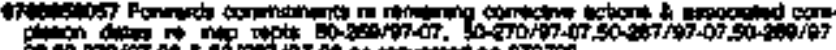

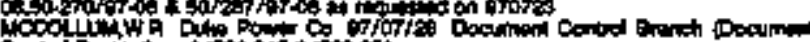

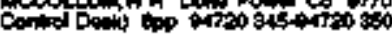

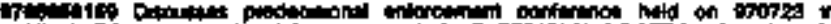

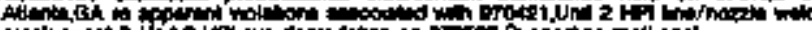

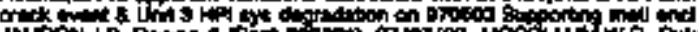

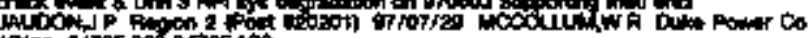

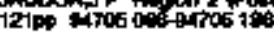

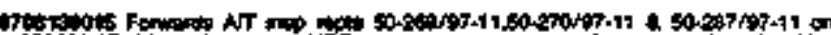

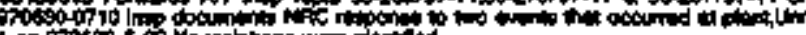

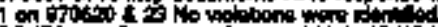

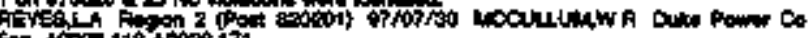

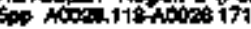

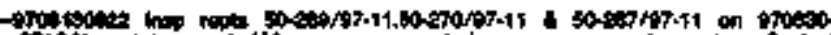

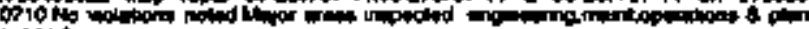

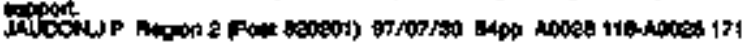

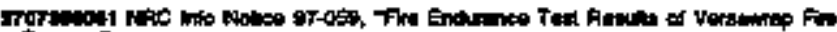

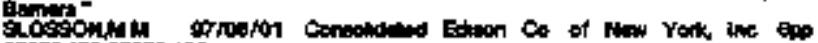

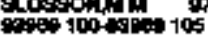

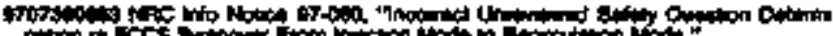
itcin of

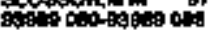

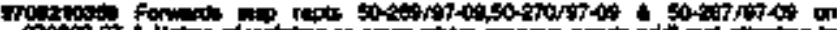

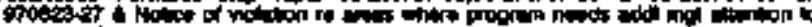

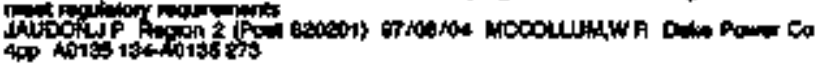

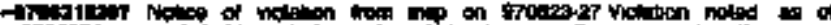

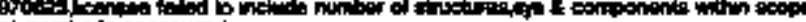

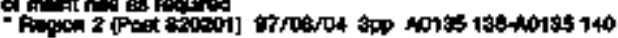

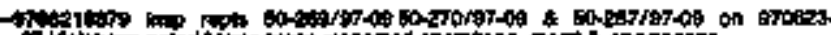
2.

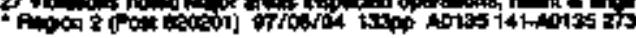

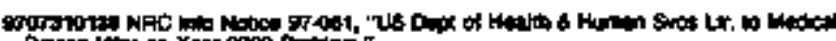

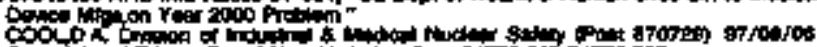

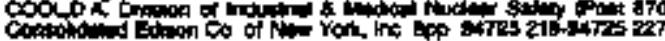

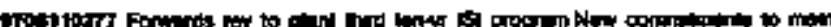

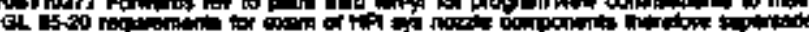

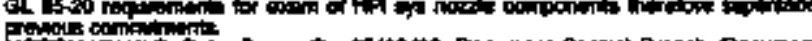

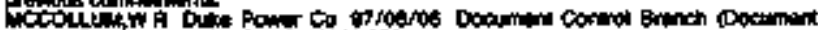
coldom 


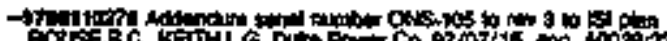

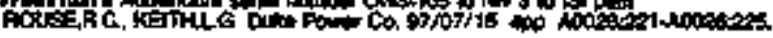

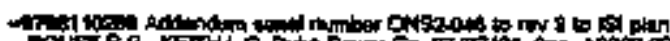

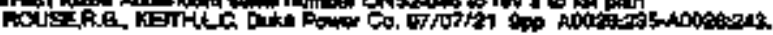

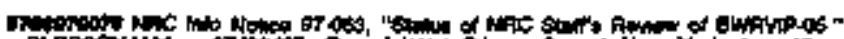

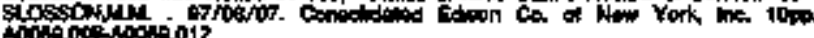

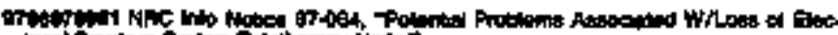

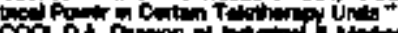

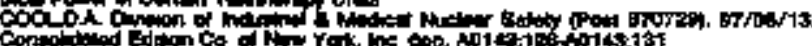

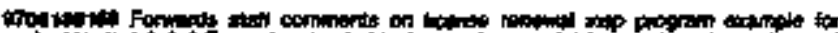

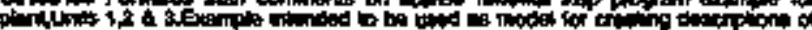

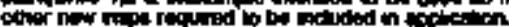

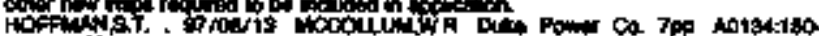
notistitio

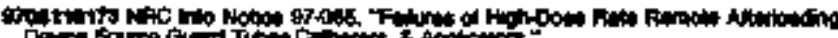

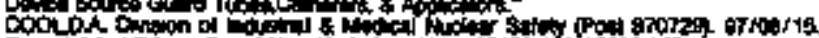

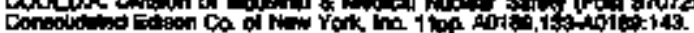

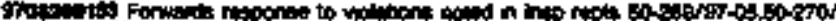

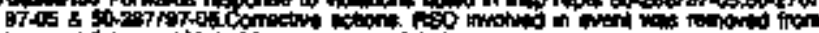

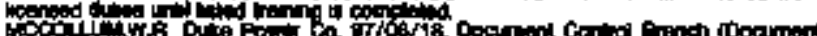

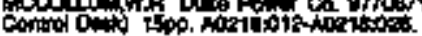

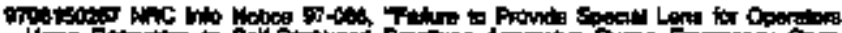

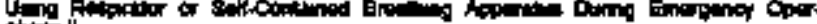

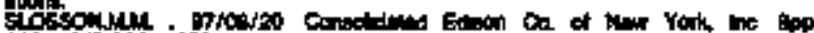

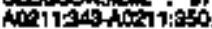

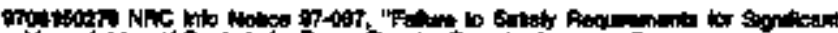

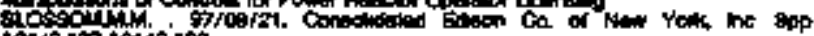

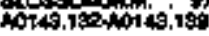

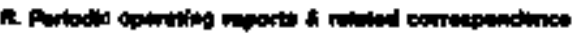

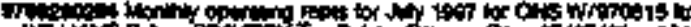

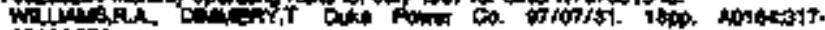
aibusin

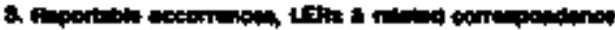

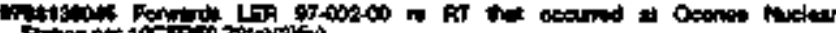

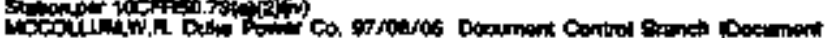

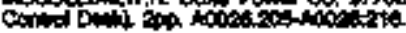

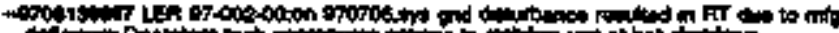

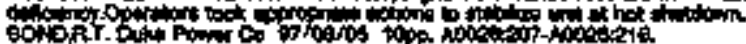

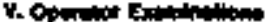

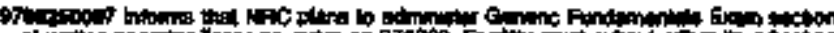

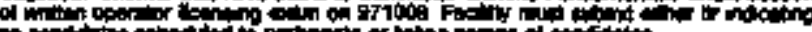

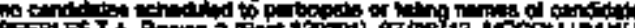

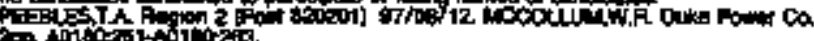

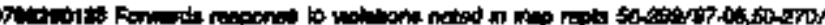

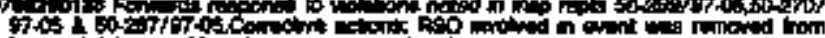

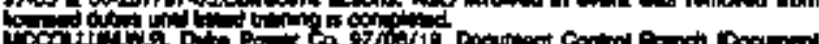
Cocon

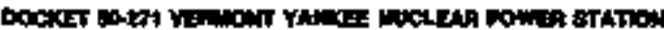

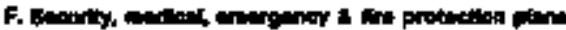

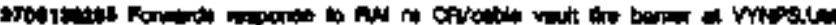

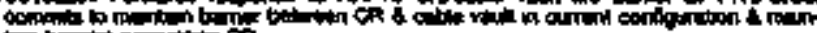

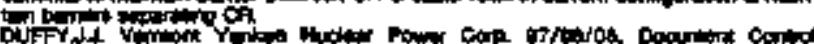

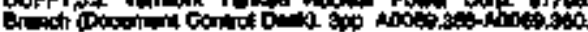

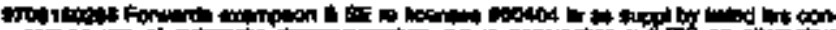

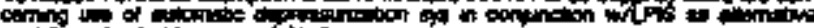

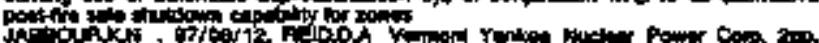

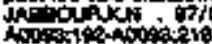

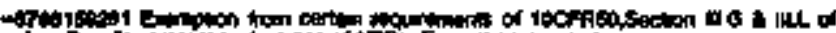

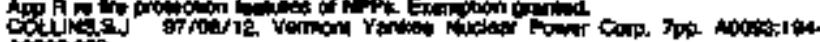
Alotion

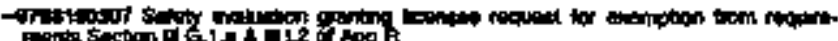

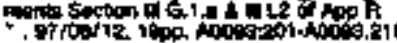

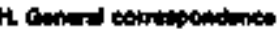

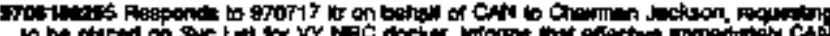

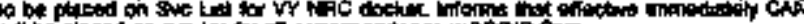

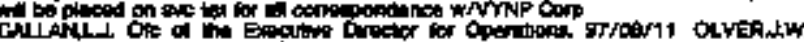

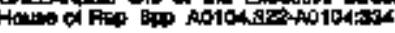

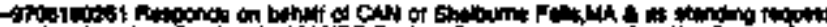

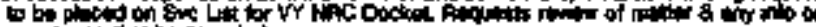

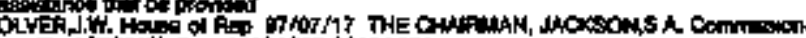
or pool

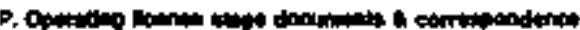

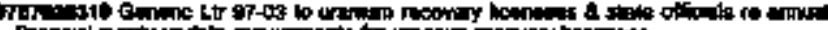

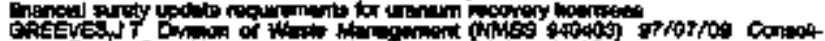

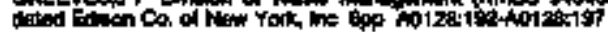

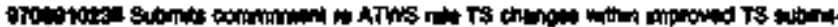

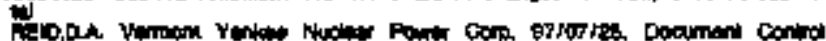

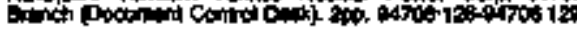

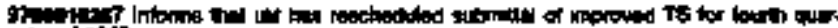
ior 品 100 .

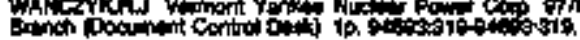

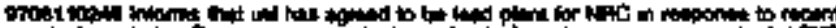

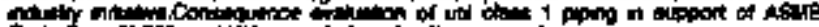

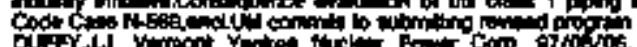

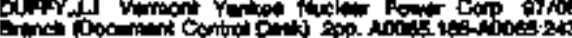

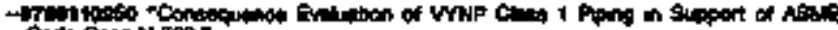

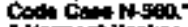

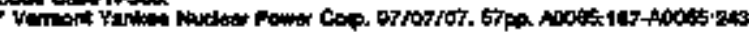

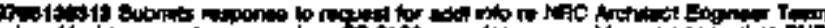
krep Y

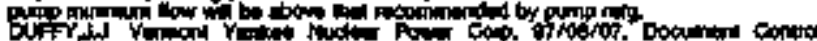

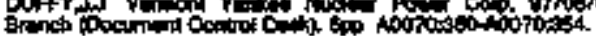

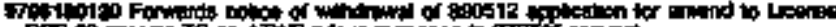

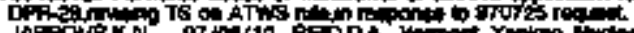

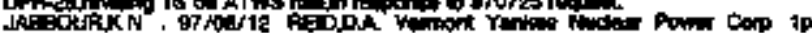
A0134100 AOTS172

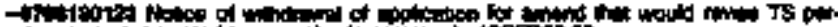
IN.

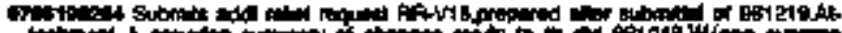

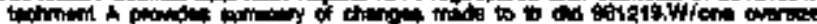

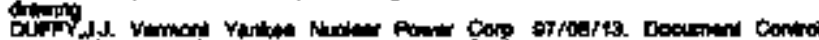

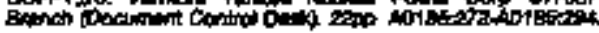

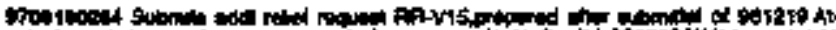

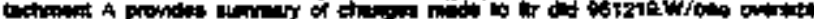

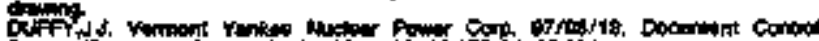

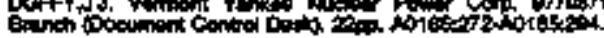

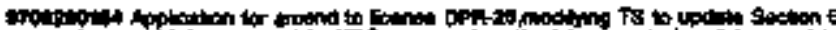

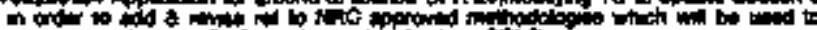
is

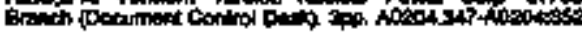

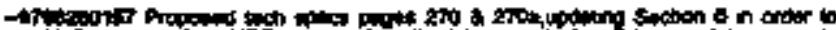

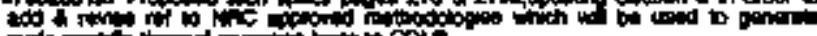

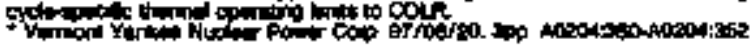

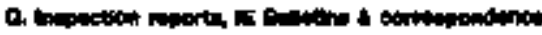

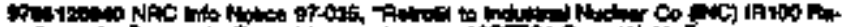

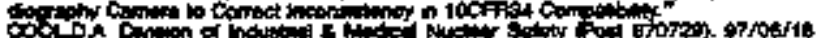

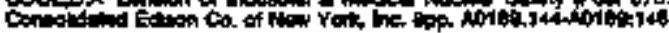

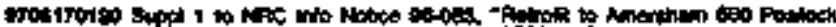

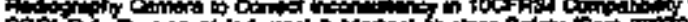

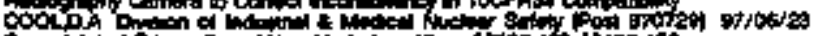

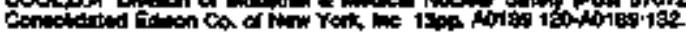

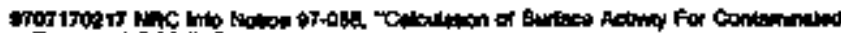

Cot

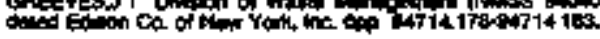

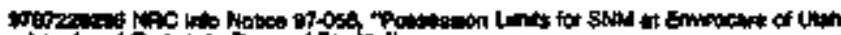
PAP

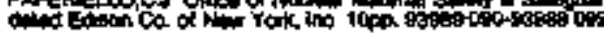




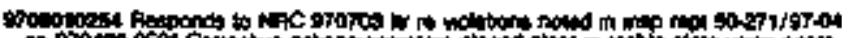

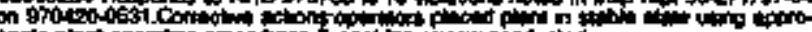

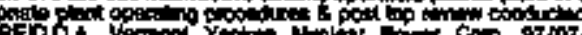

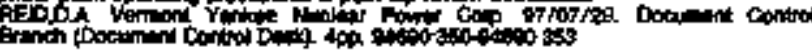

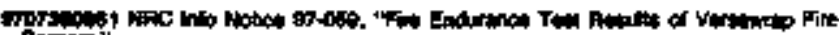

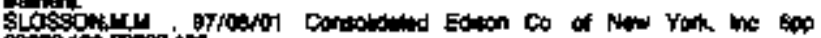
os.

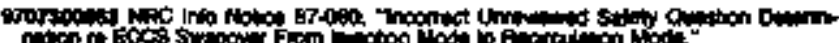

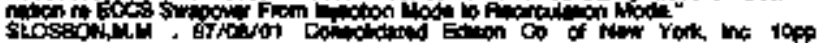

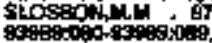

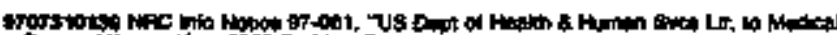

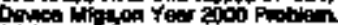

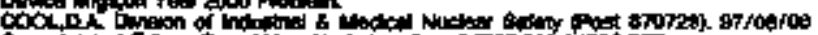

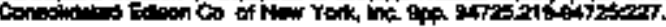

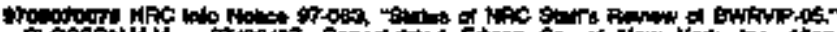

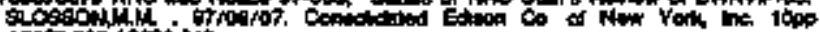

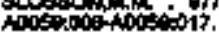

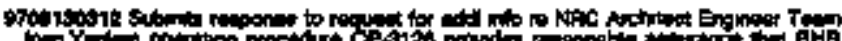

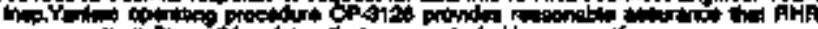

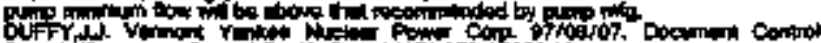

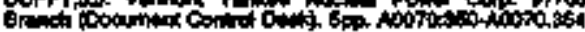

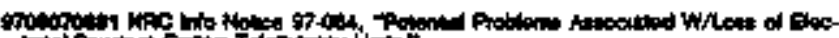

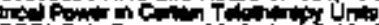

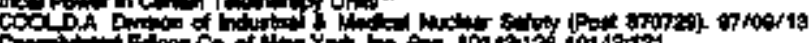

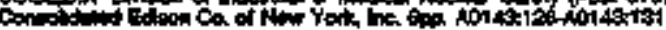

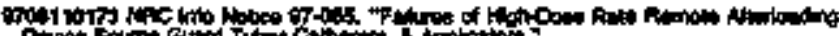

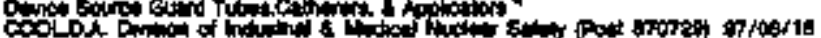

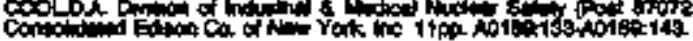

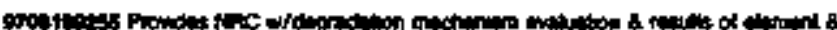

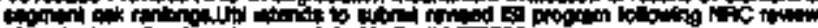

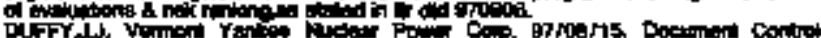

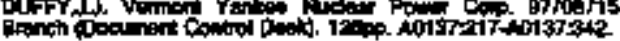

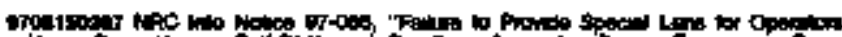

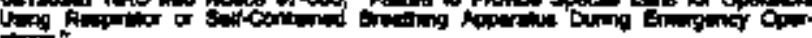

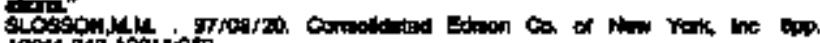

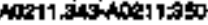

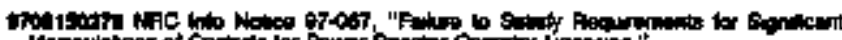

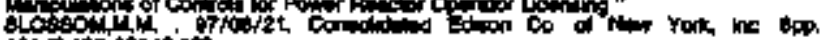
Horationation

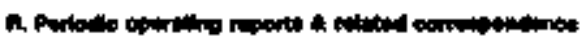

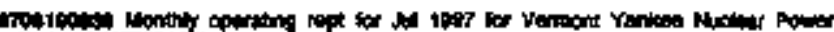

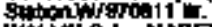

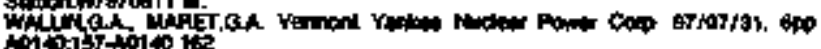

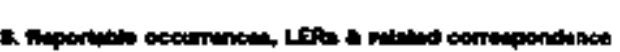

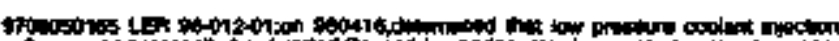

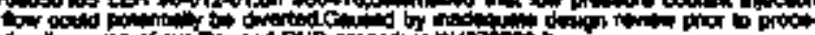

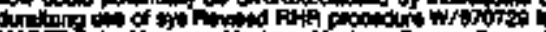

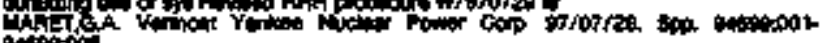
antotot

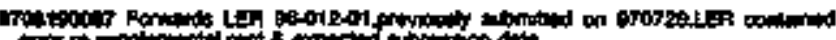

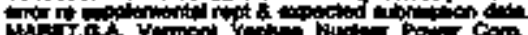

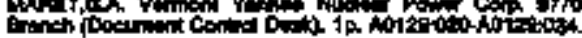

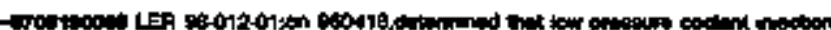

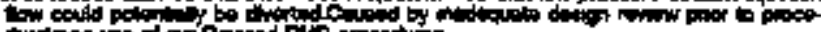

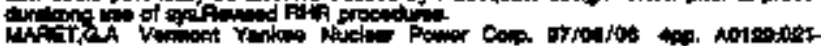
Antzoned

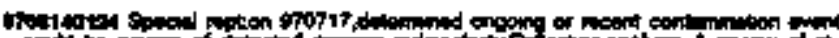

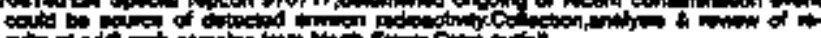

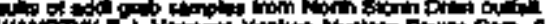

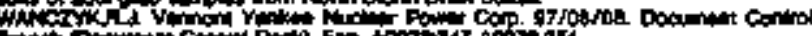
Ding

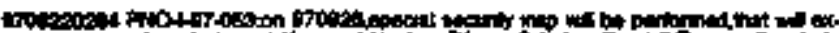

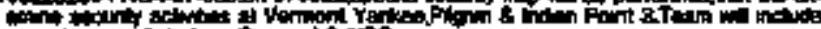

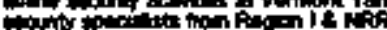

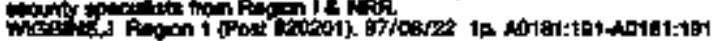

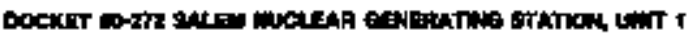

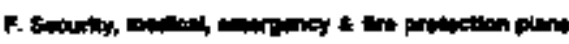

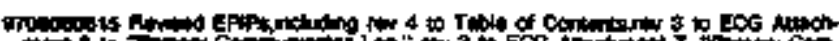

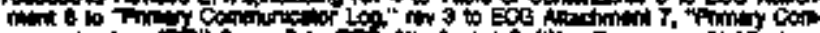

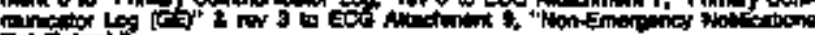

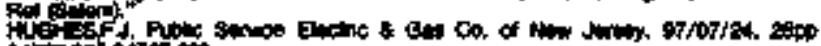

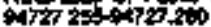

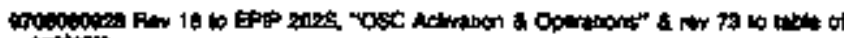

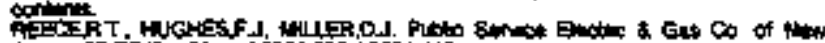

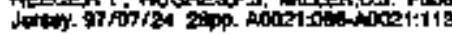

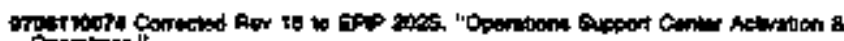

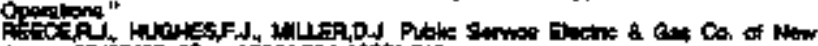

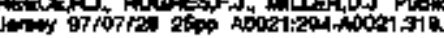

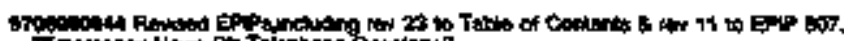

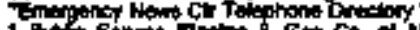

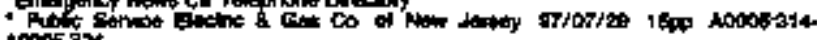
40005 2 3

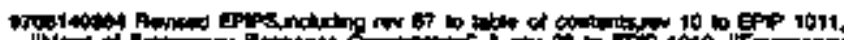

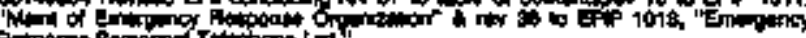

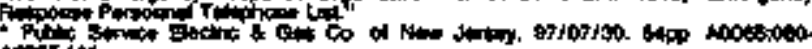
atowiti

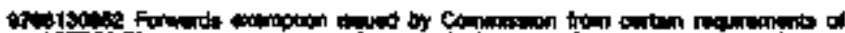

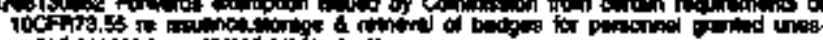

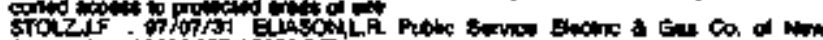

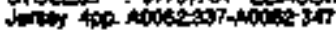

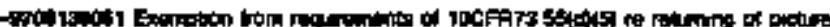

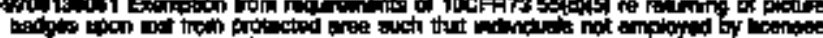

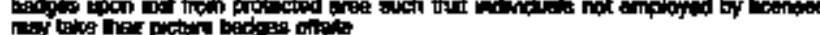

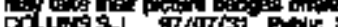

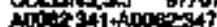

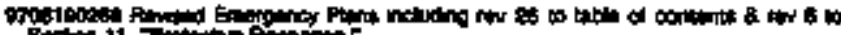

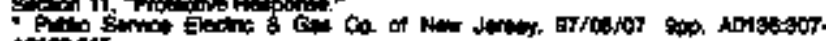
noist 315

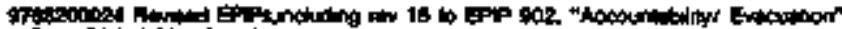

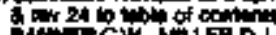

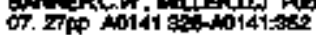

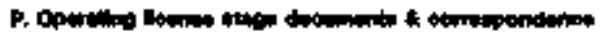

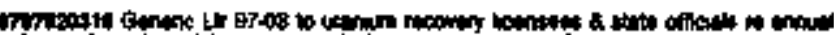

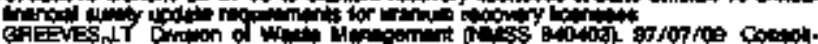

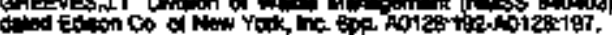

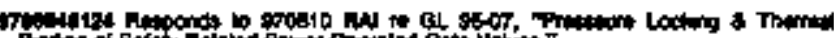

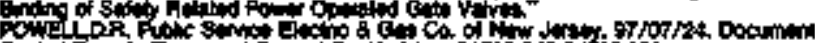

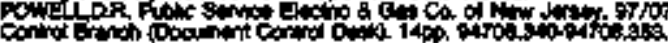

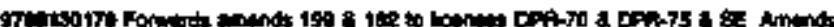

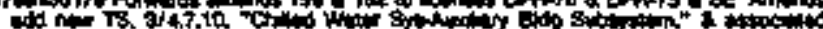

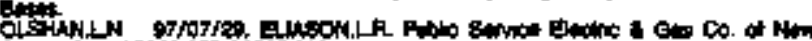
Dising

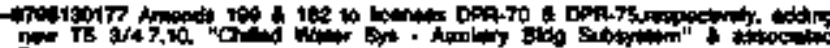

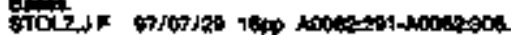

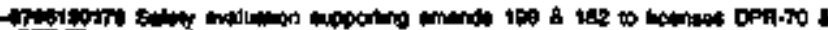

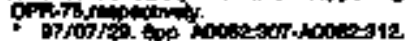

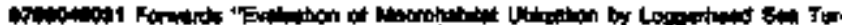

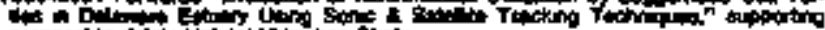

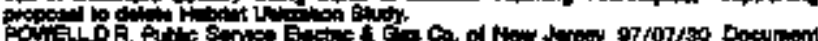

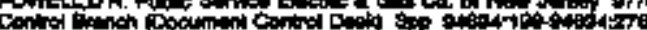

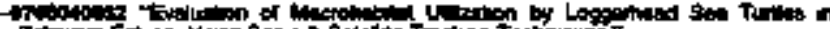

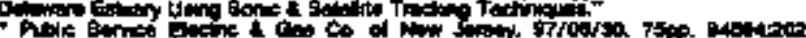
sing 2 th

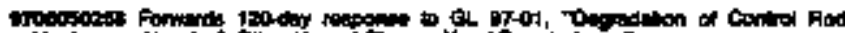

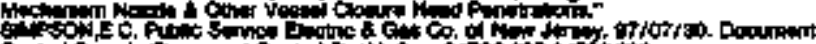

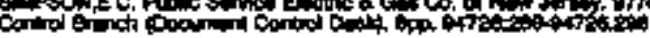

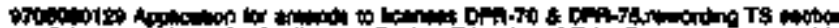

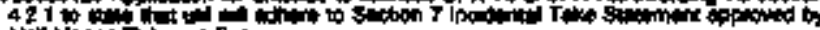

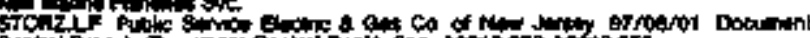

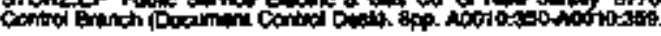

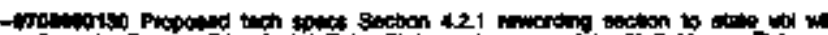

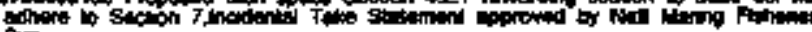

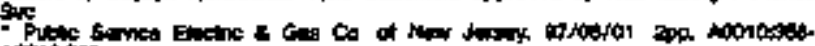
Notolos:

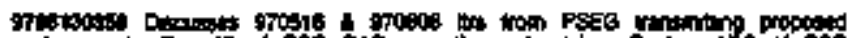

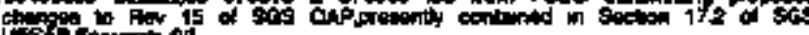

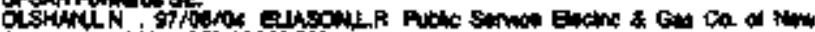

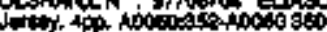




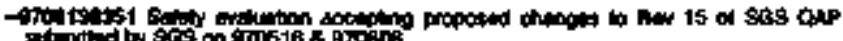

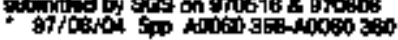

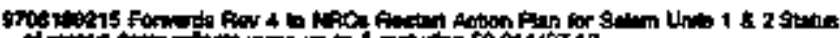

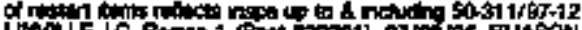

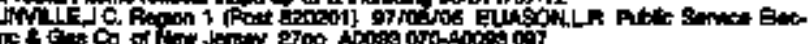

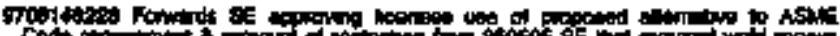

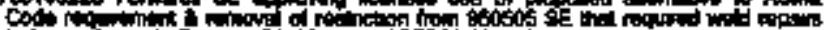

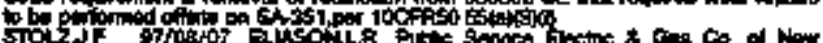
Jition

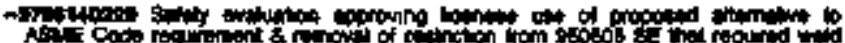

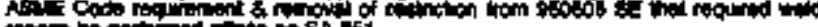

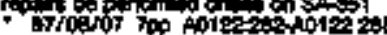

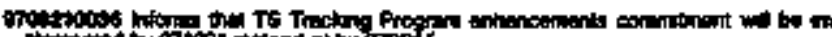

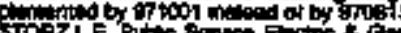

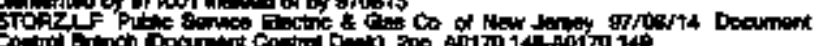

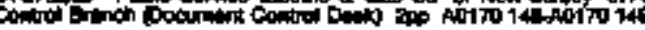

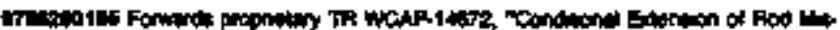

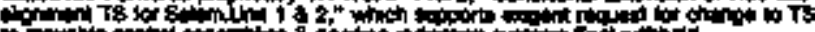

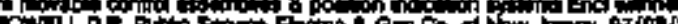

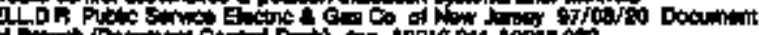

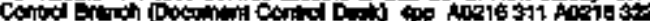

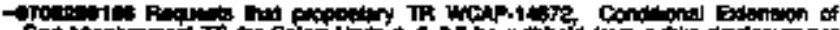

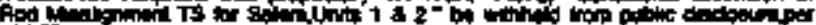

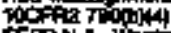

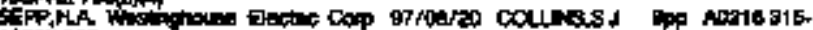
$10210-302$

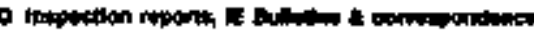

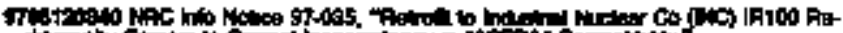

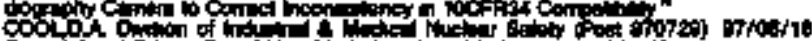

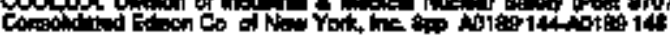

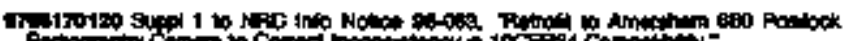

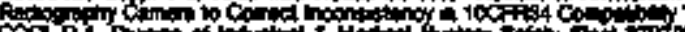

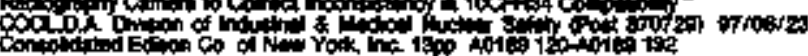

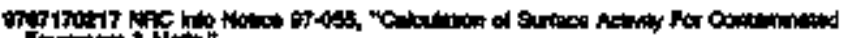

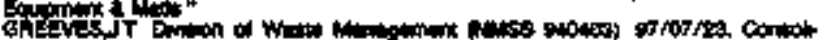
ot

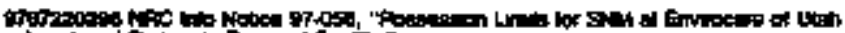

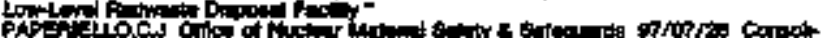

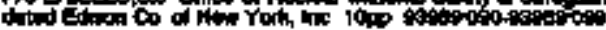

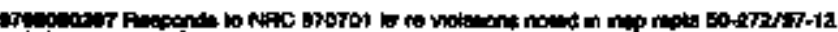

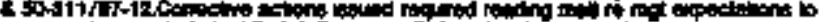

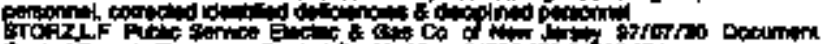

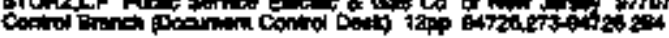

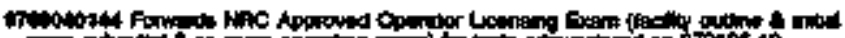

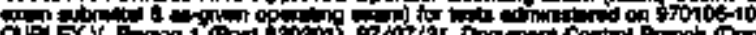

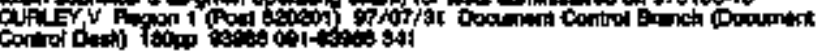

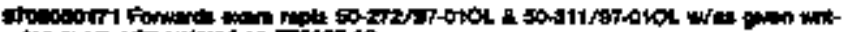

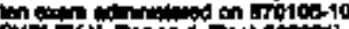

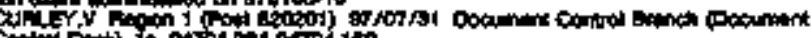

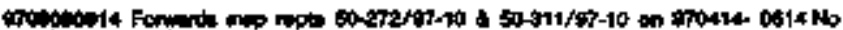

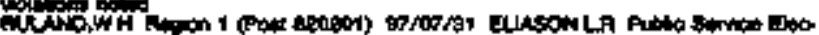

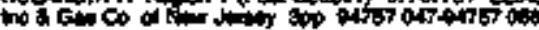

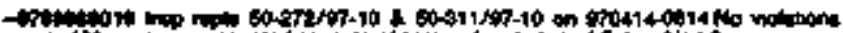

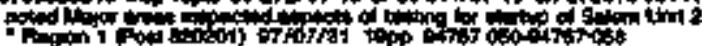

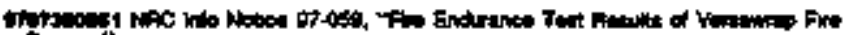

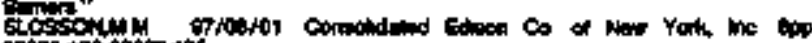

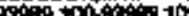

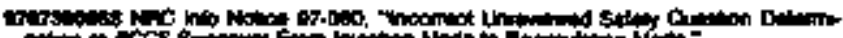

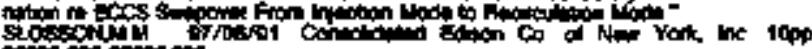
rosing

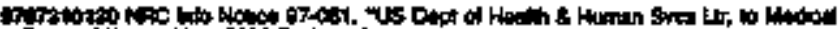

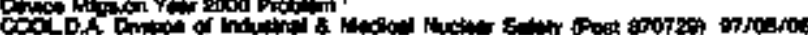

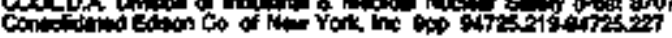

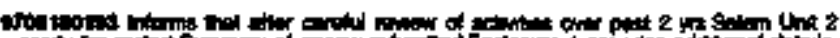

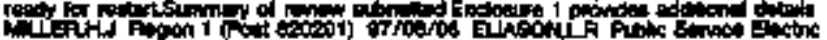

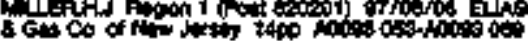

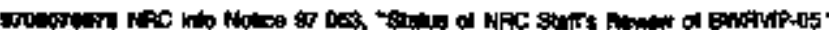

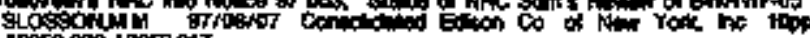

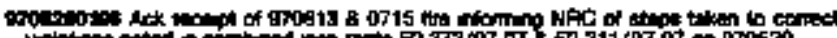

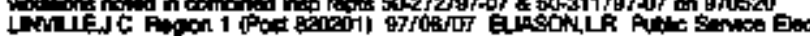

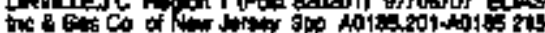

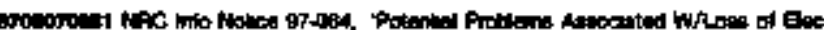

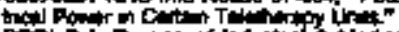

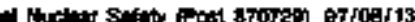

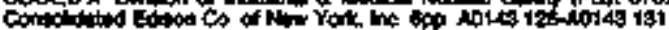

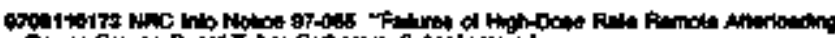

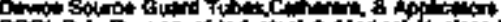

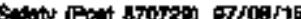

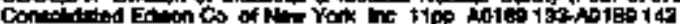

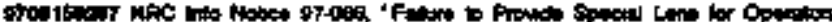

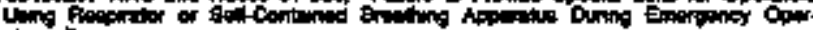

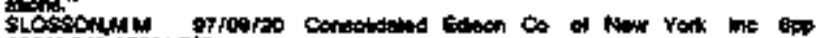
10211 240

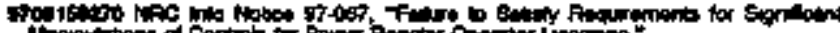

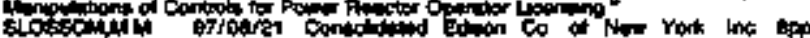
1019132101431

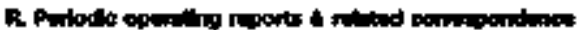

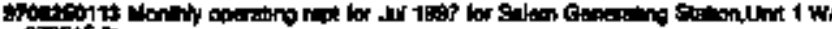

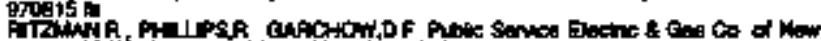

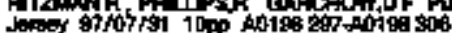

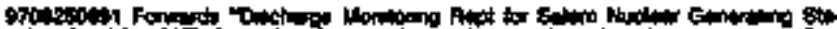

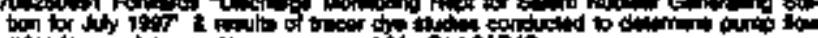

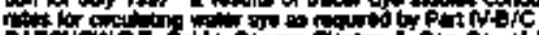

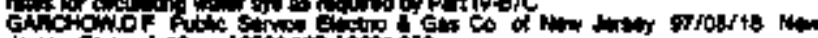

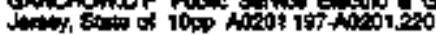

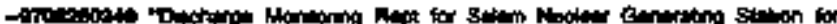

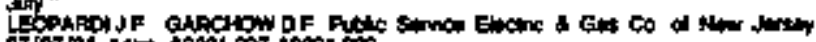

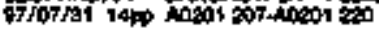

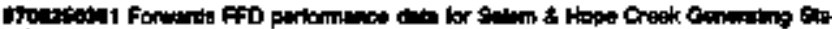

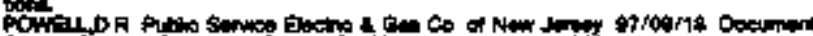

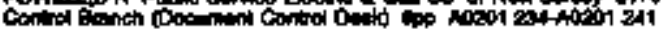

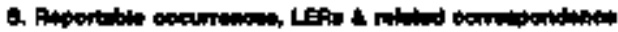

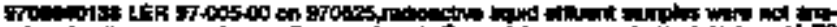

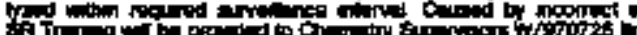

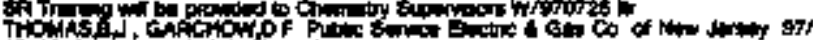

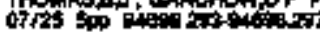

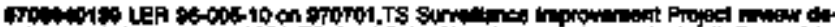

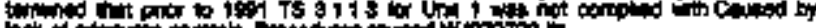

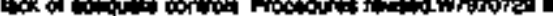

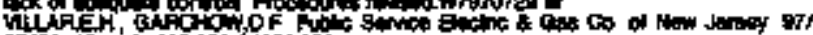

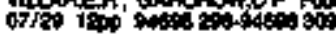

Trow

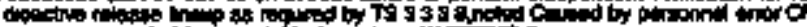

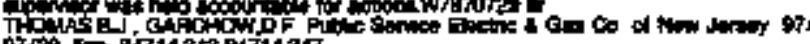
th

970)

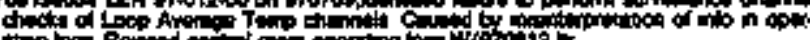

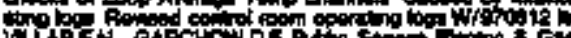

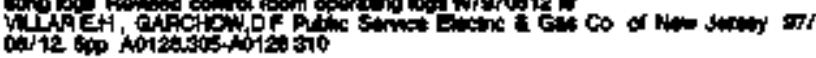

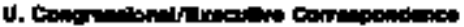

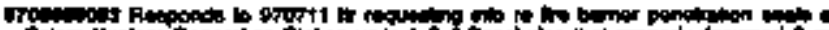

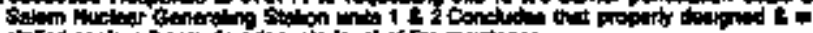

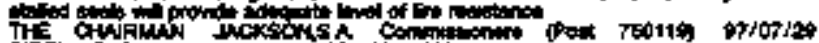

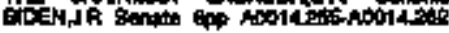

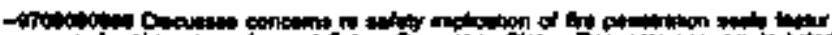

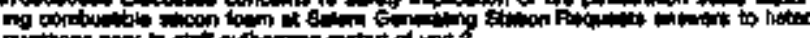

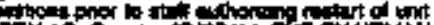

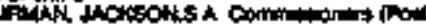

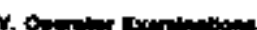

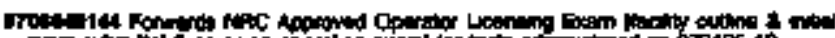

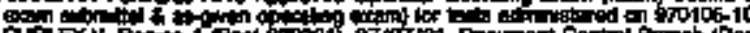

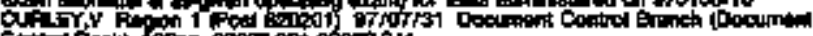

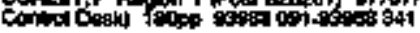

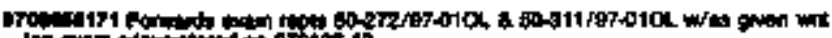

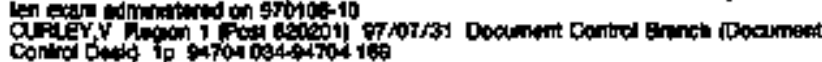




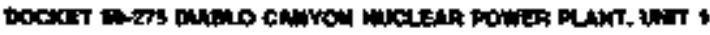

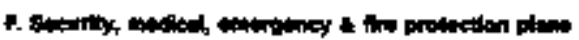

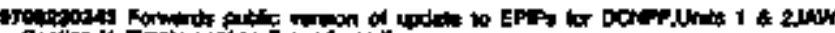

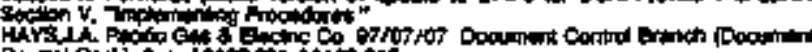

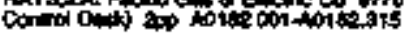

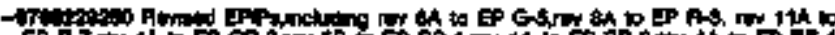

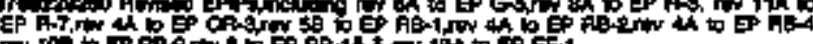

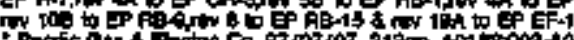

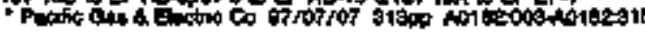

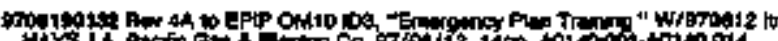

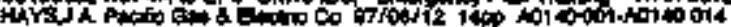

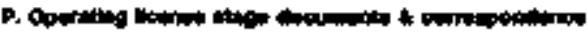

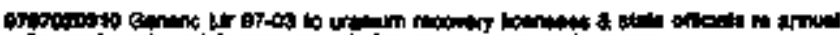

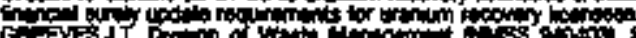

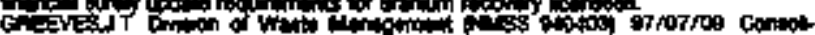

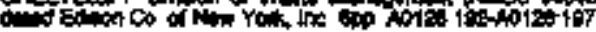

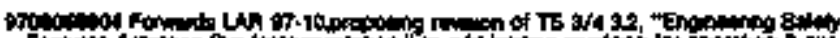

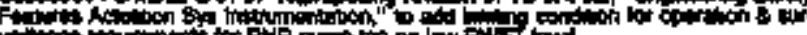

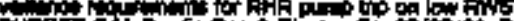

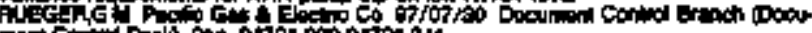

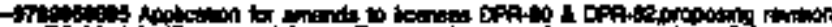

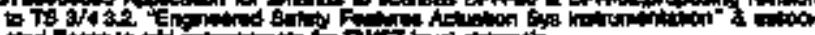

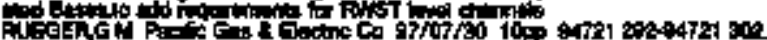

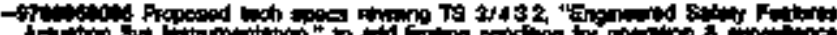

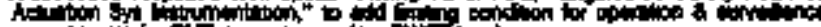

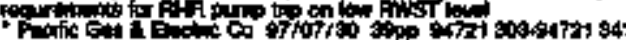

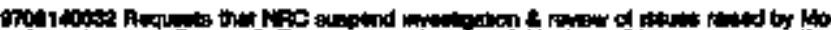
doto lat

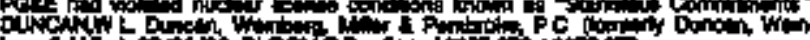

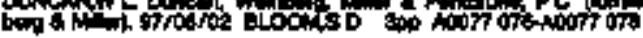

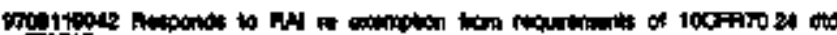

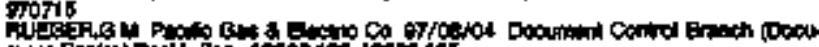

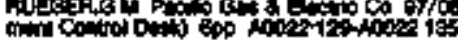

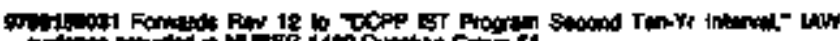

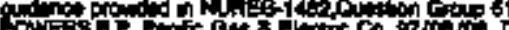

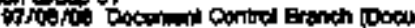

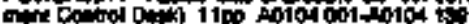

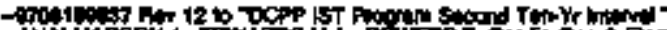

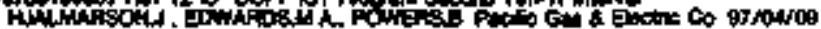
Hace alof of

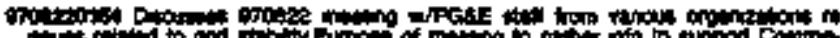

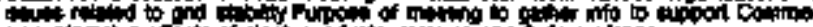

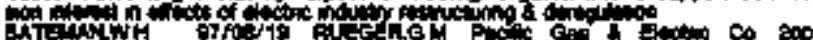
A017 30\%4017\% 300

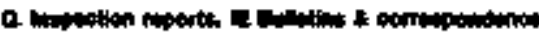

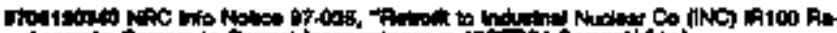

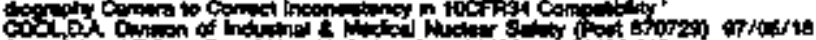

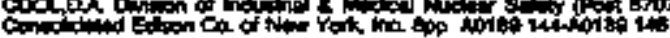

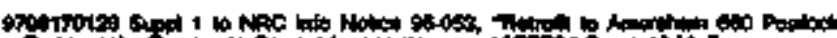
A

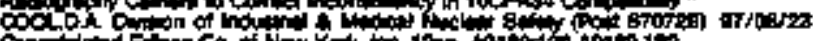

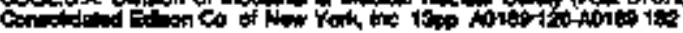

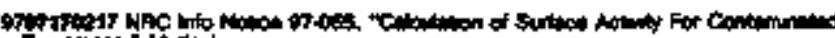

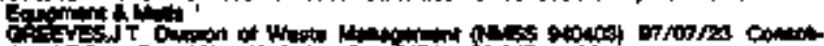

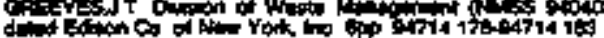

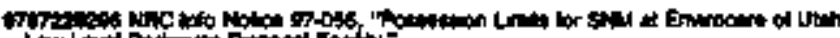

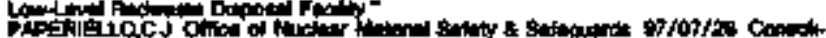

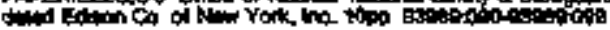

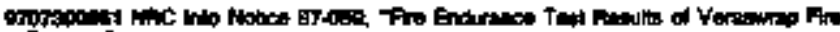

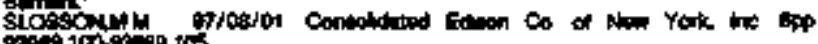

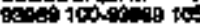

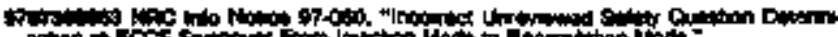

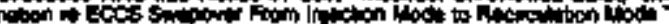

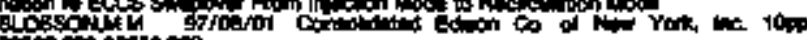

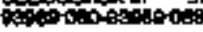

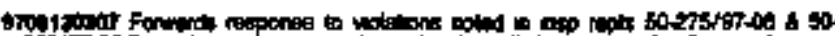

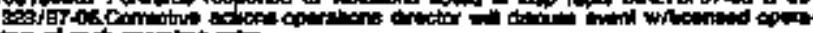

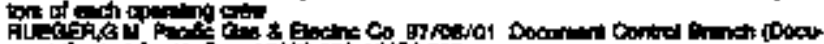

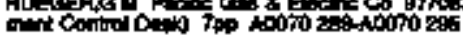

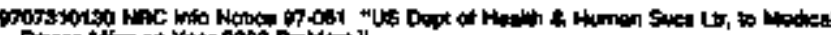

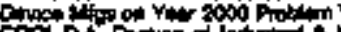

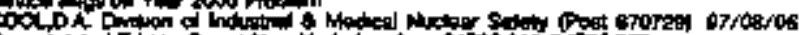

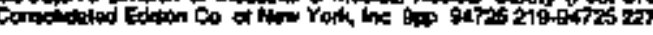

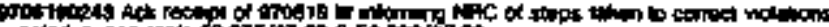

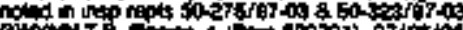

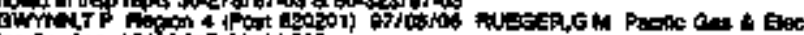

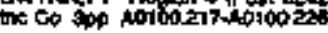

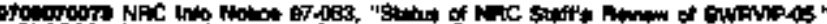

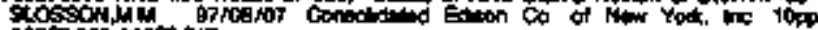

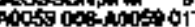

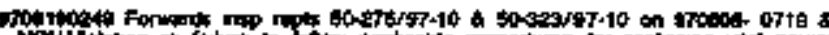

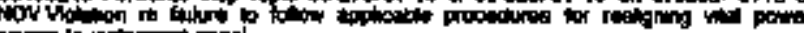

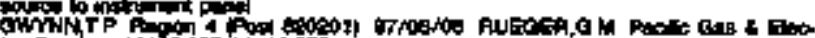

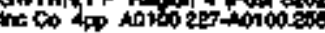

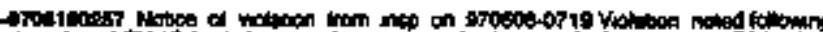

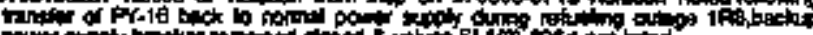

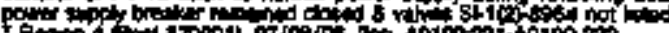

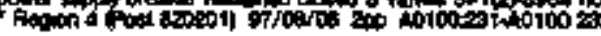

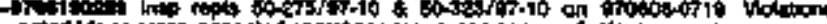

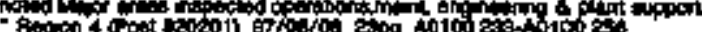

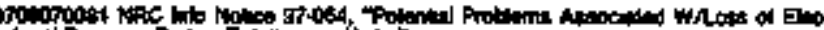

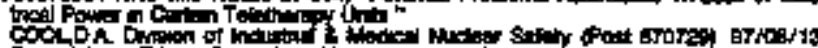

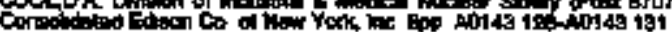

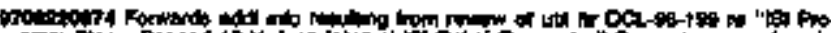

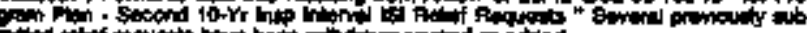

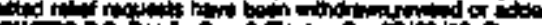

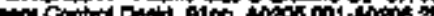

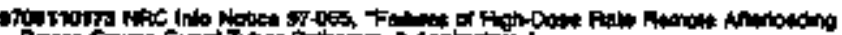

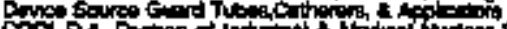

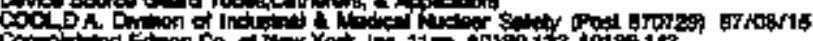

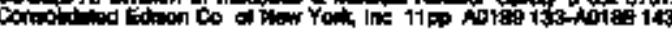

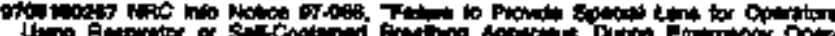

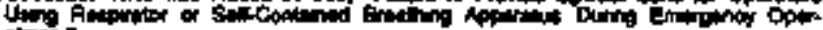

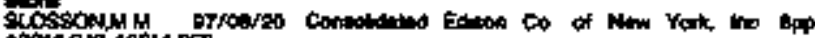

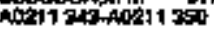

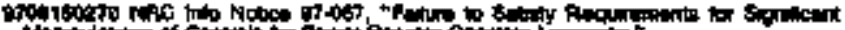

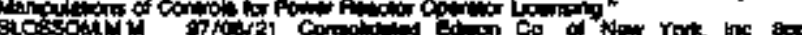
a

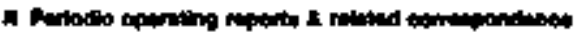

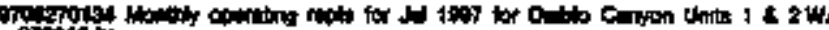

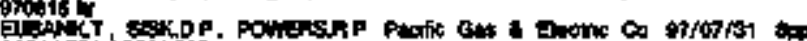

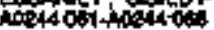

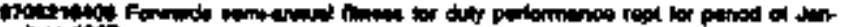

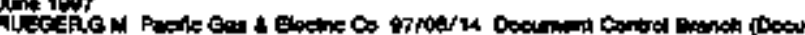

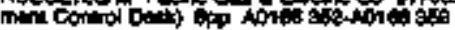

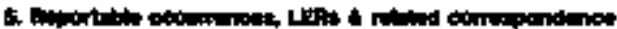

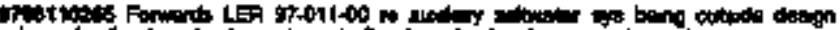

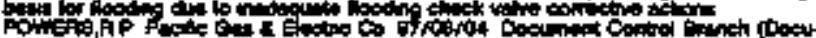

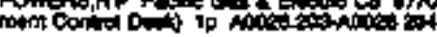

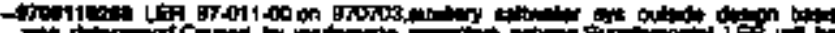

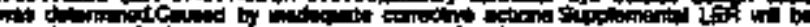

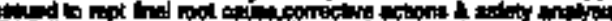

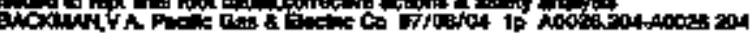

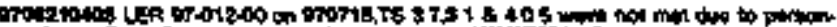

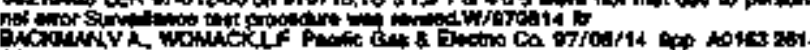
ionosion

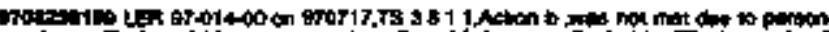

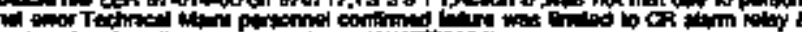

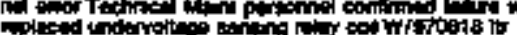

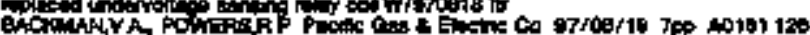
40161 132

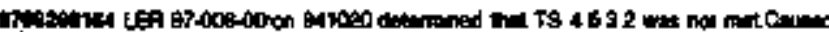

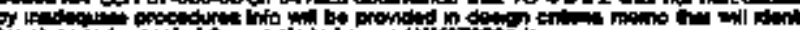

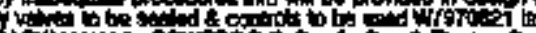

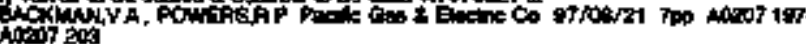




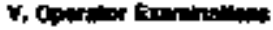

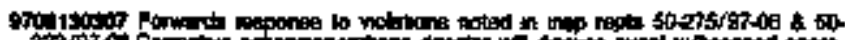

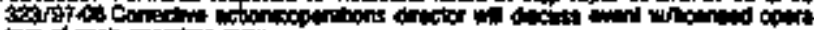

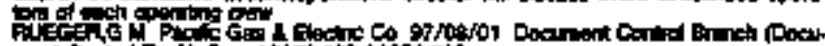
minl Gental D:

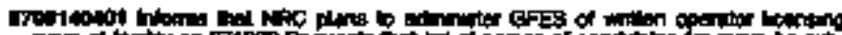

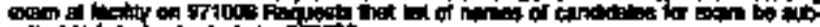

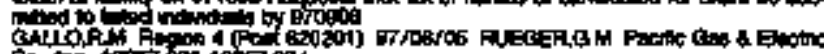

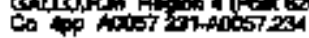

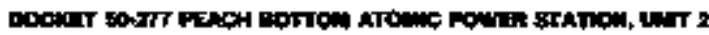

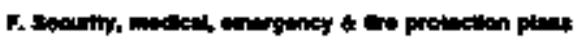

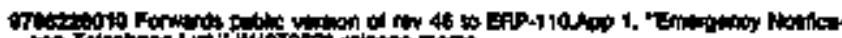

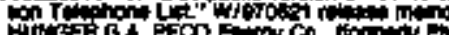

Nyte

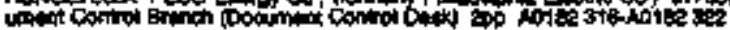

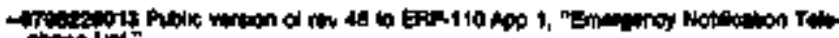

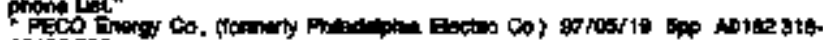
aldien

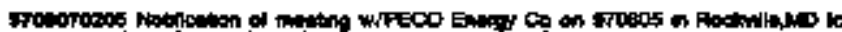

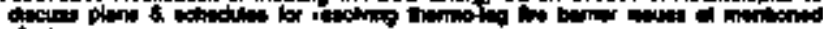

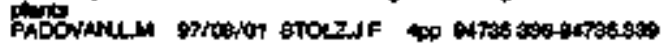

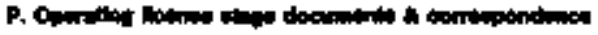

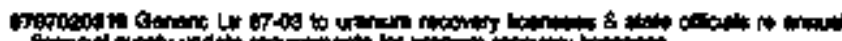

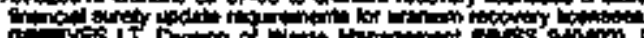

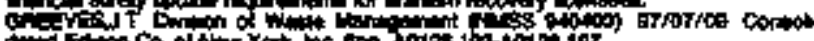

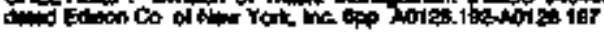

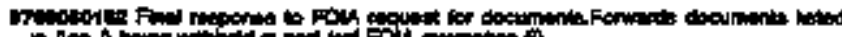

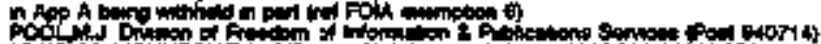

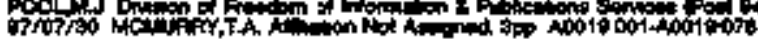

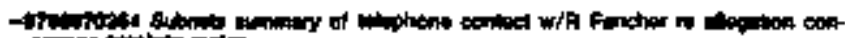

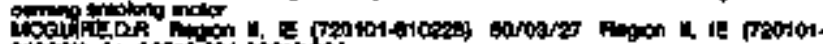

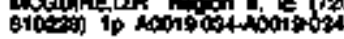

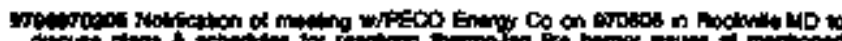

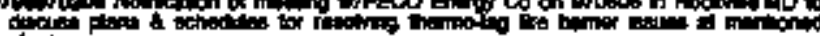

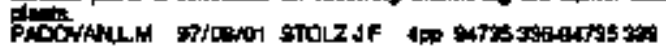

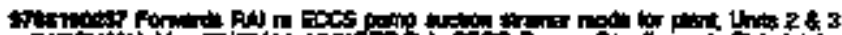

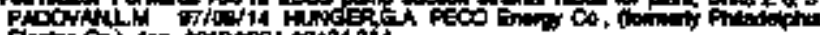

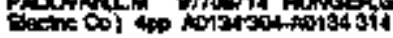

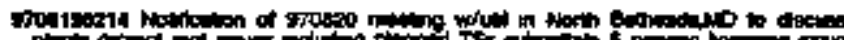

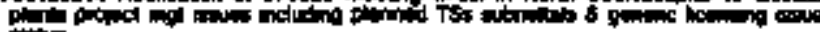

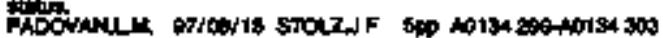

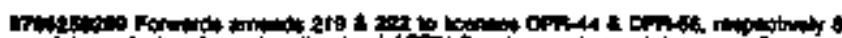

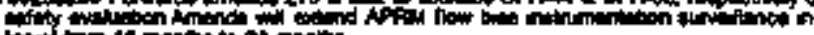

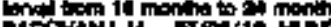

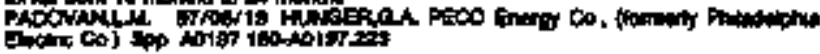

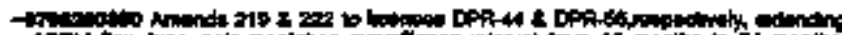

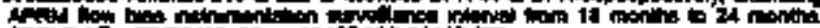

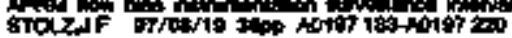

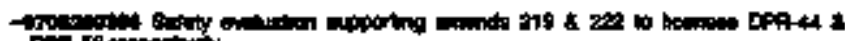

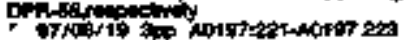

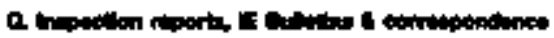

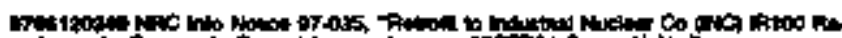

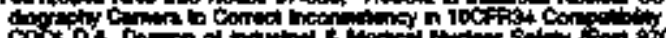

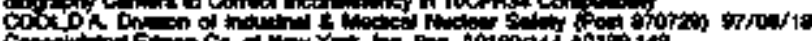

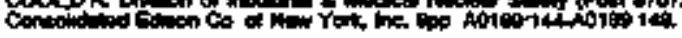

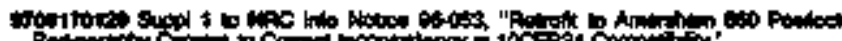

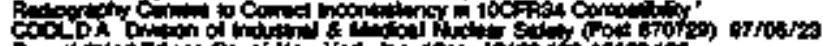

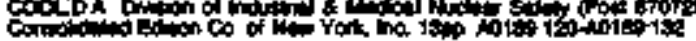

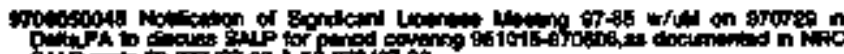

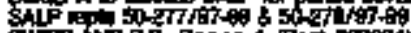

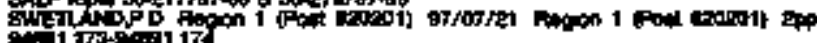

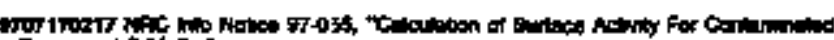

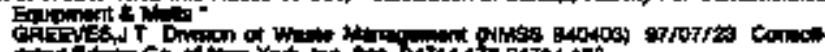

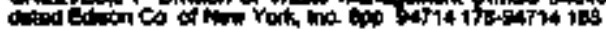

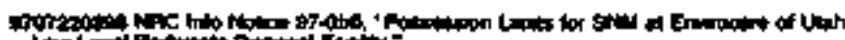

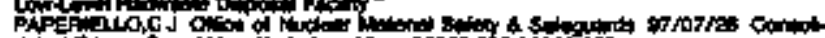

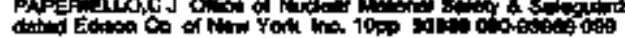

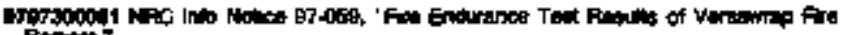

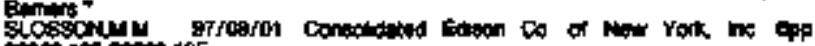

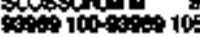

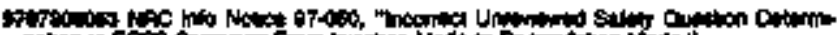

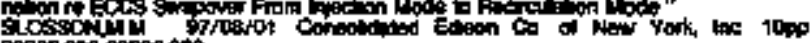

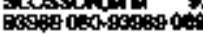

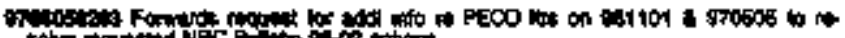

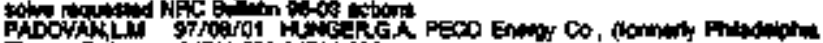

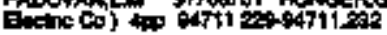

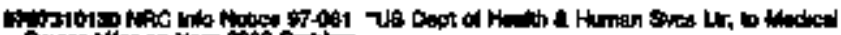

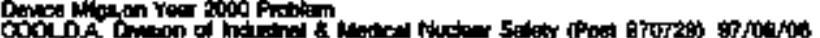

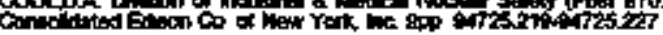

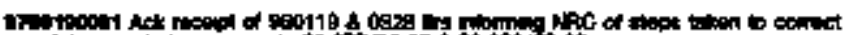

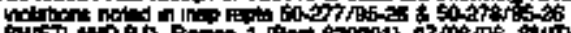

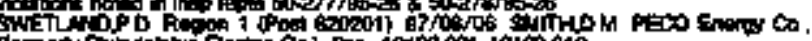

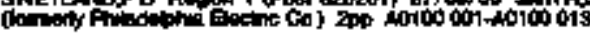

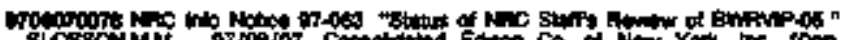

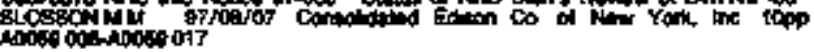

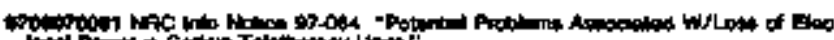

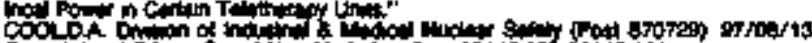

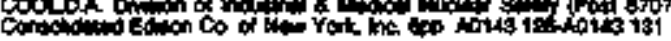

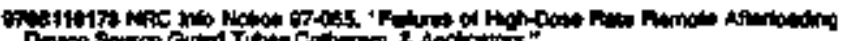

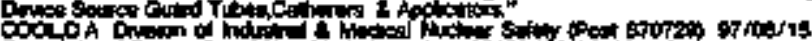

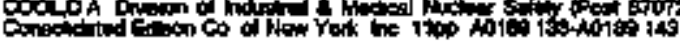

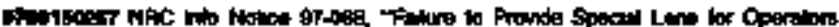

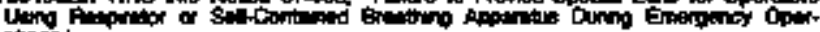

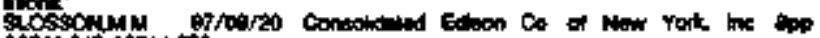

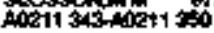

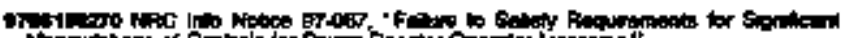

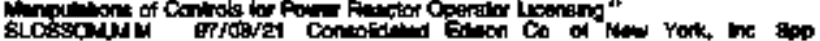
Mids 13everks 139

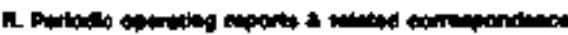

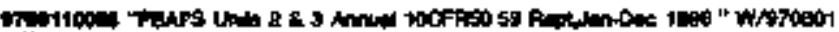

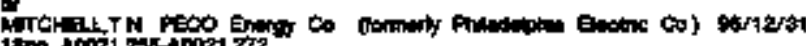

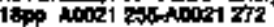

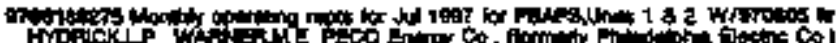

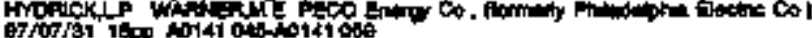

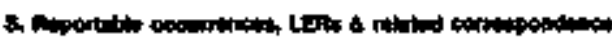

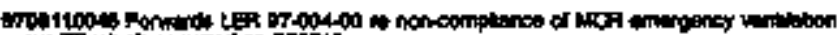

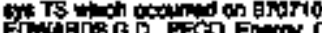

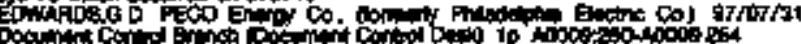

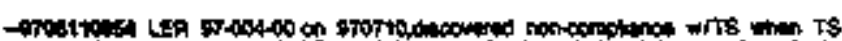

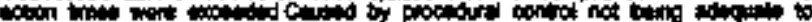

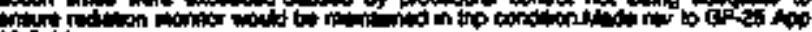

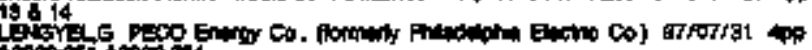

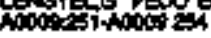

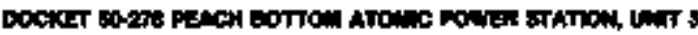

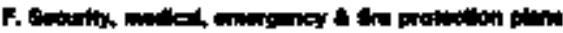

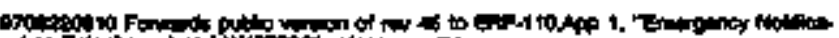

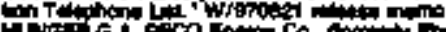
HIN

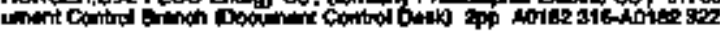

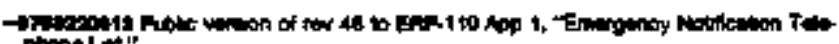
Phone Let"

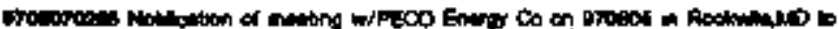

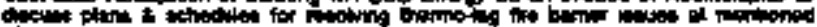

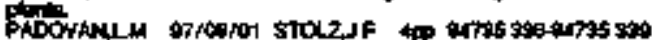

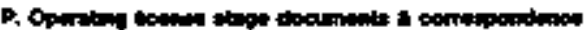

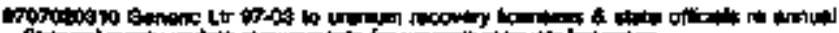

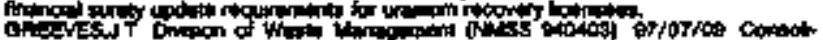

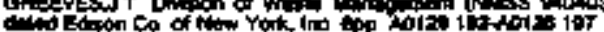




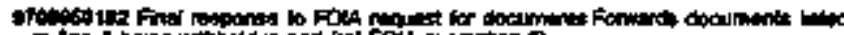

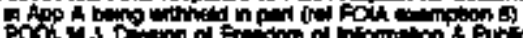

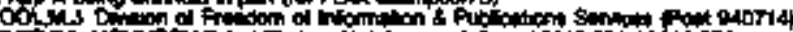

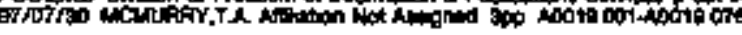

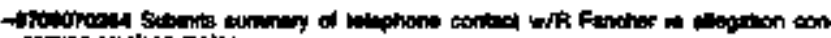

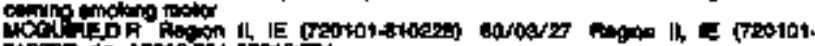

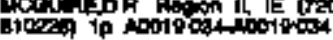

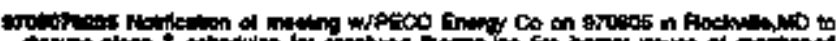

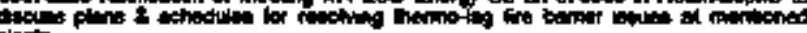

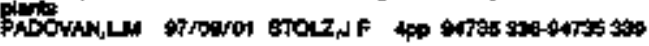

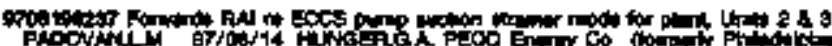

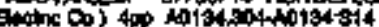

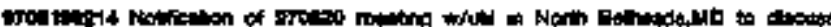

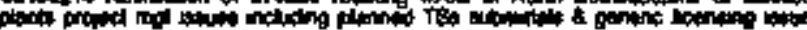

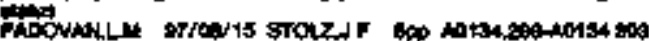

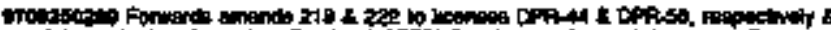

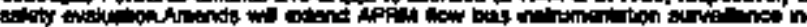

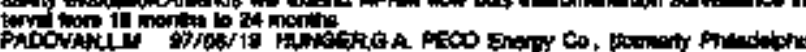

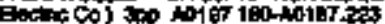

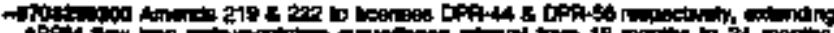

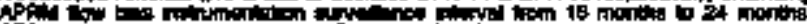

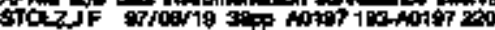

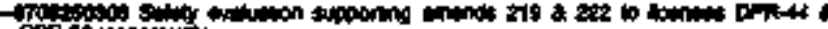

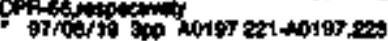

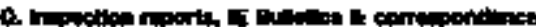

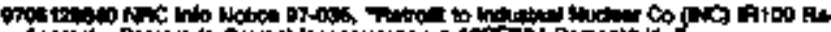

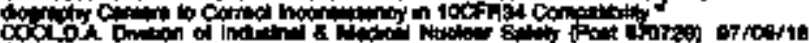

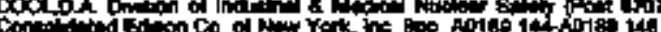

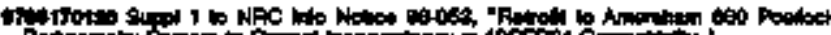

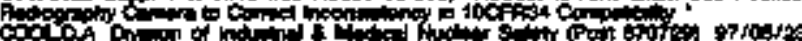

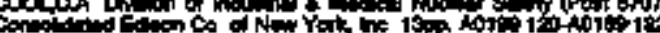

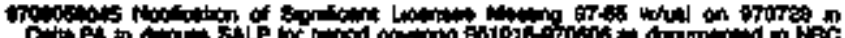

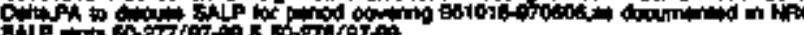

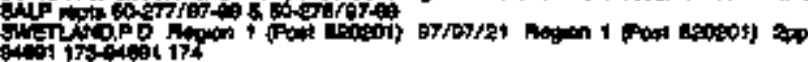

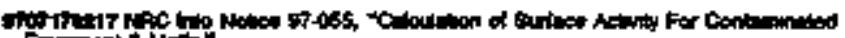

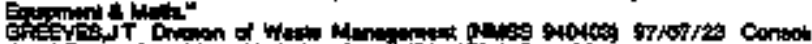

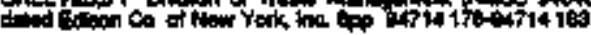

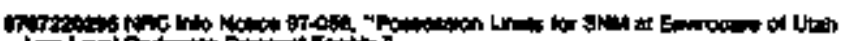

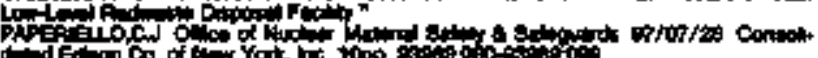

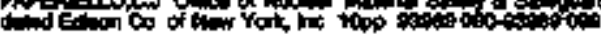

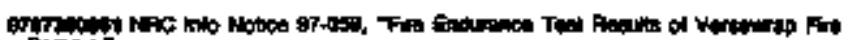

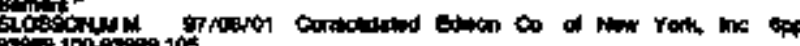
100 acoso 10

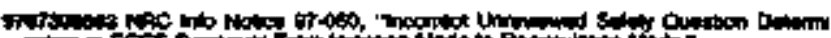

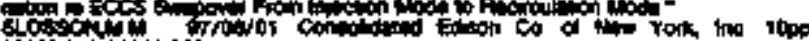

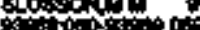

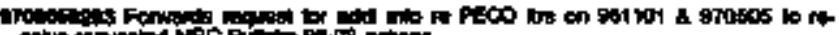

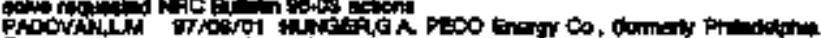

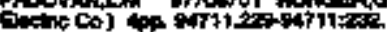

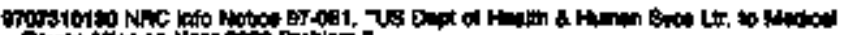

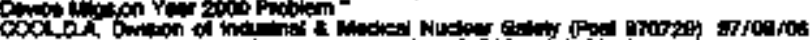

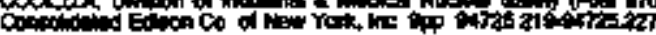

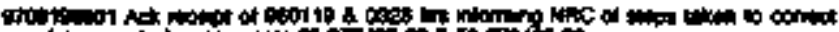

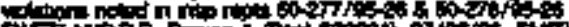

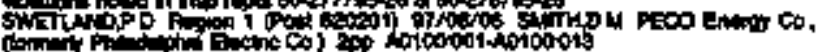

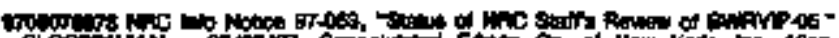

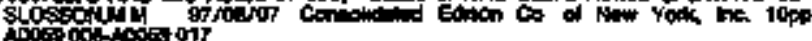

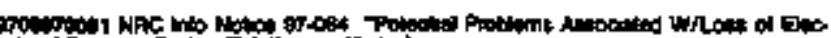

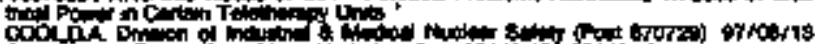

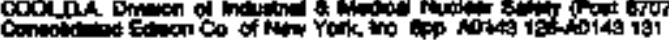

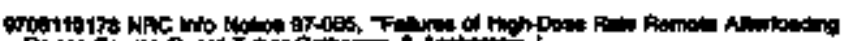

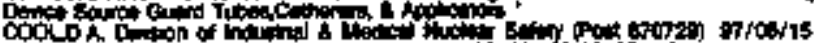

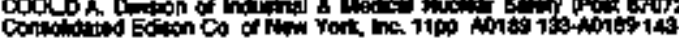

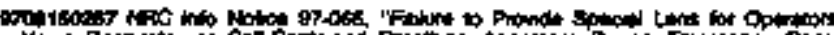

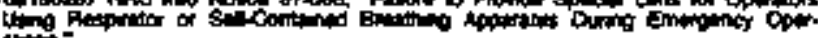

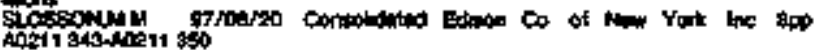

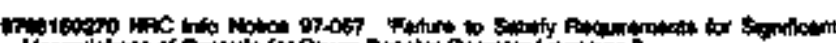

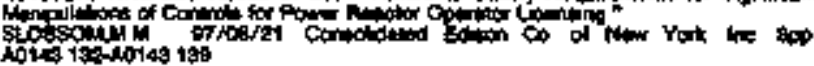

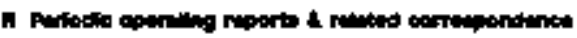

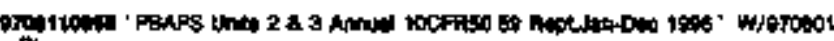

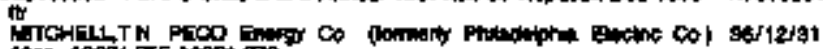

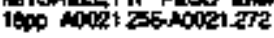

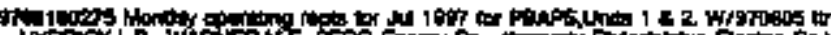

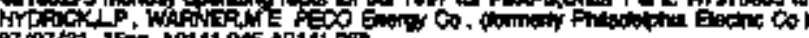

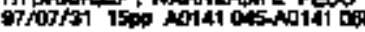

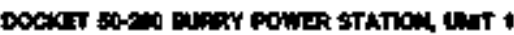

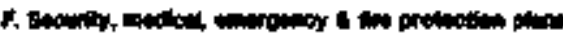

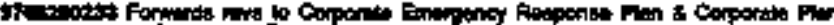

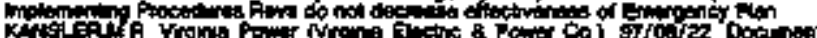

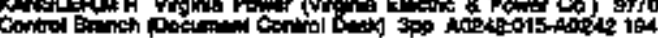

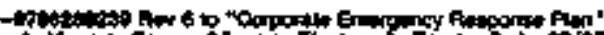

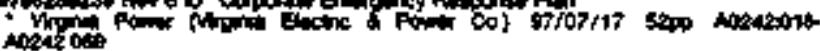

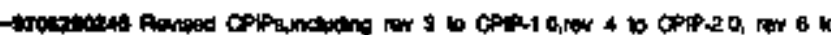

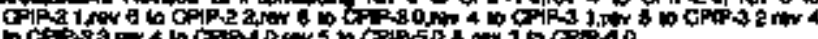

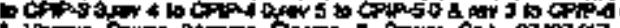

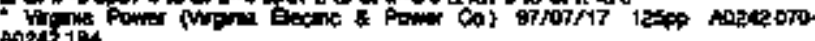

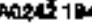

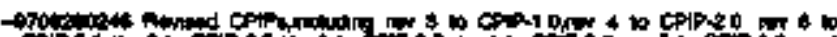

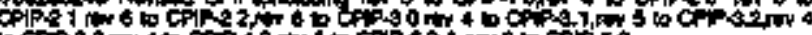

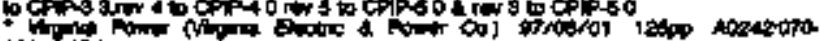
$00242 \tan$

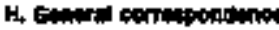

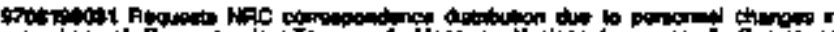

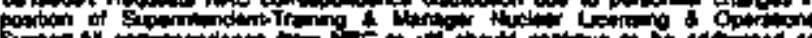

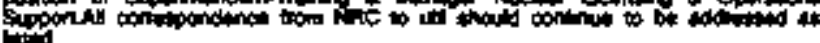

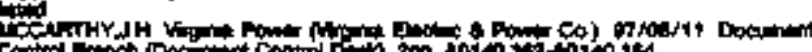

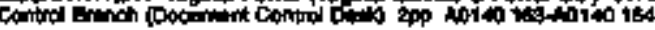

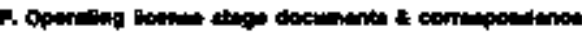

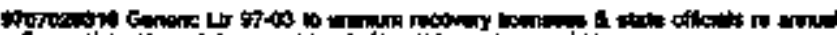
Ge

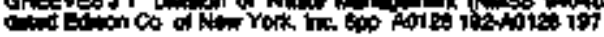

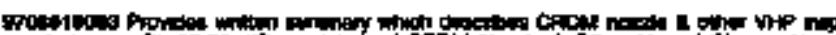

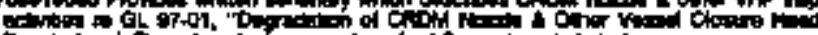

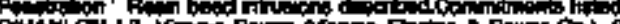

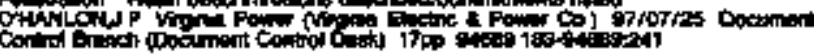

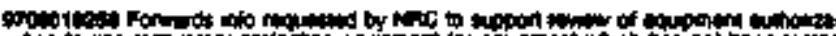

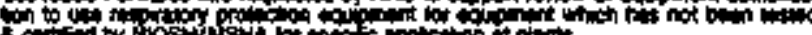

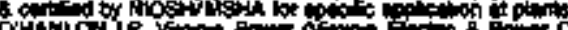

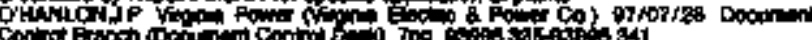

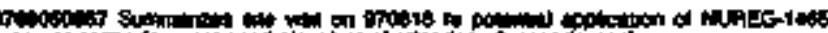

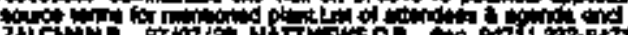

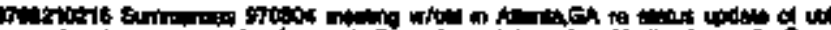

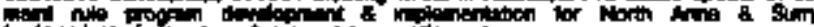

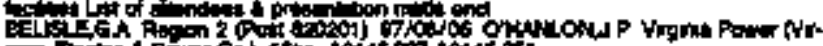

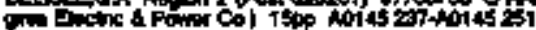

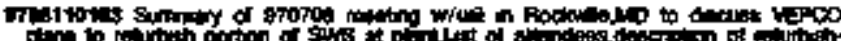

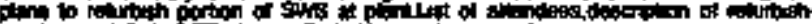

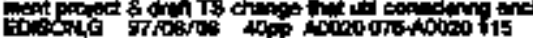

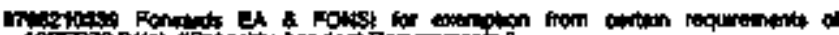

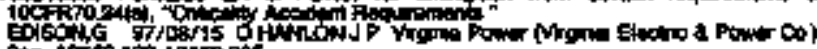

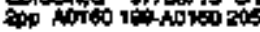

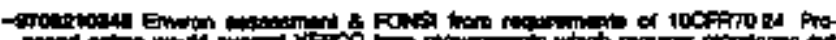

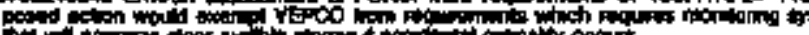

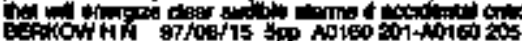




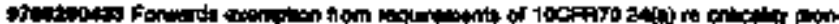

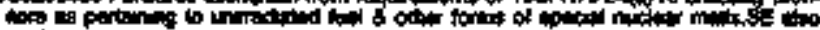

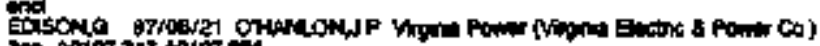

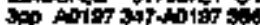

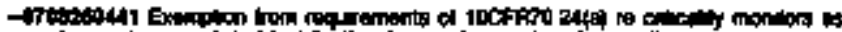

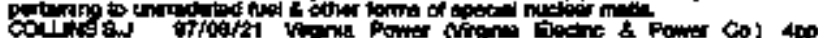

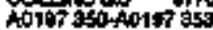

-

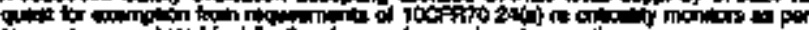

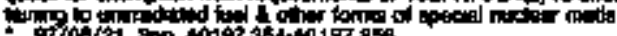

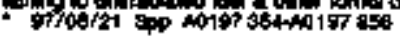

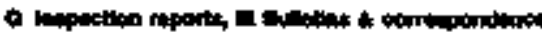

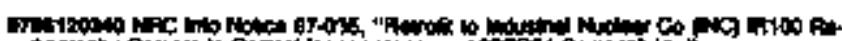

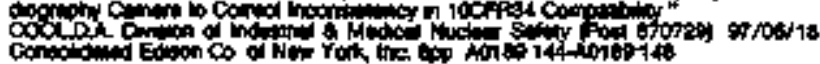

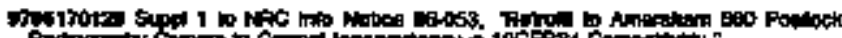

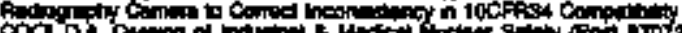

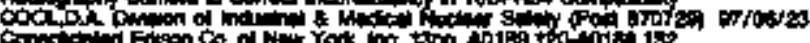

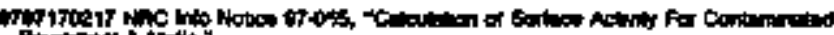

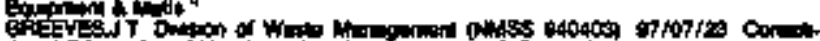

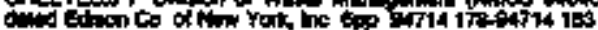

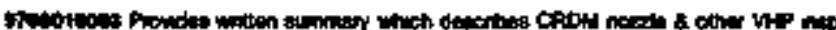

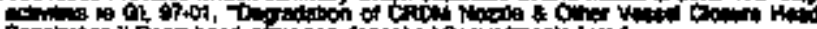

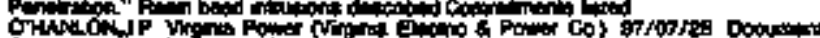

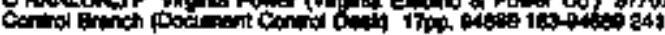

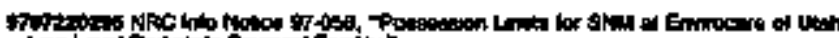
Lor

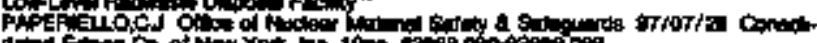

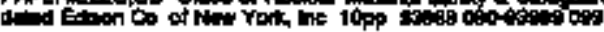

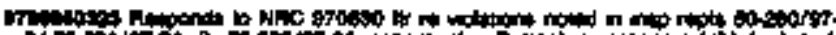

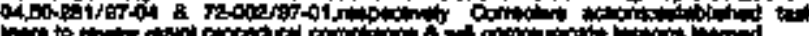

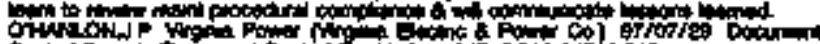

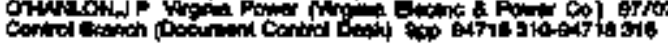

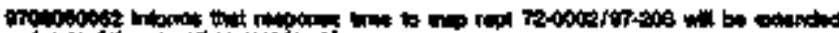

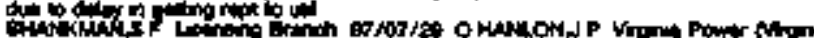

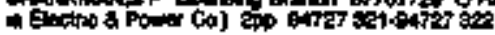

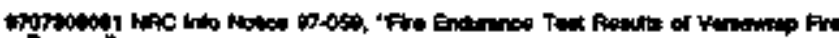
留

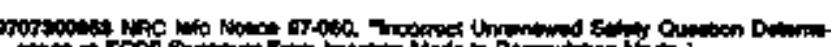

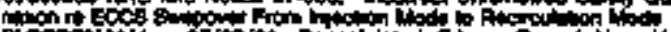

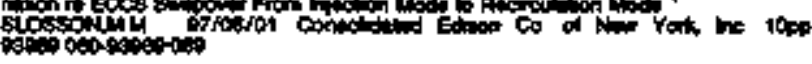

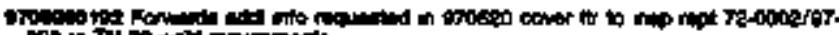

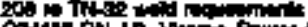

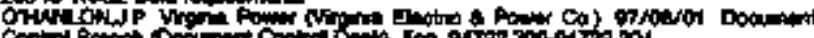

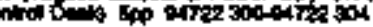

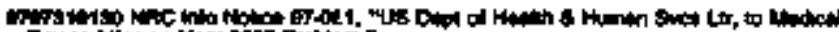

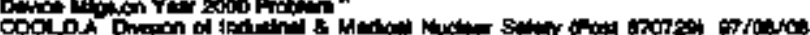

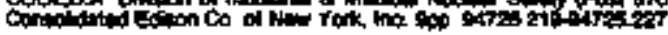

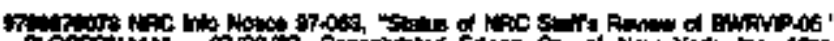

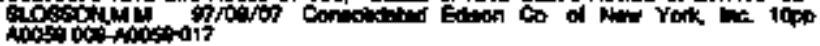

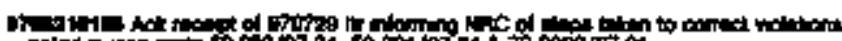

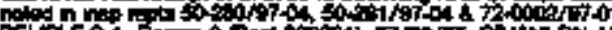

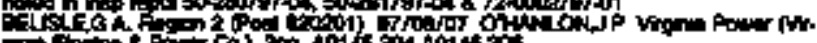

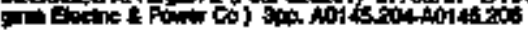

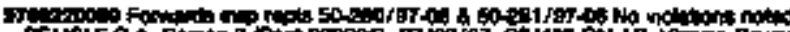

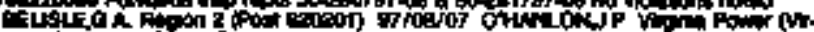

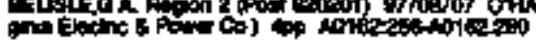

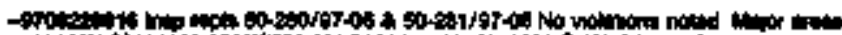
H.

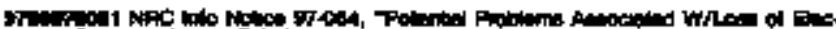

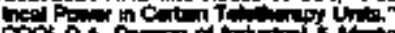

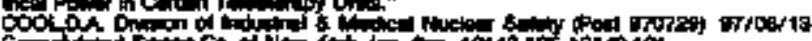

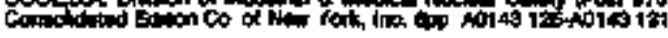

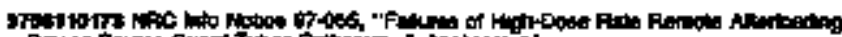

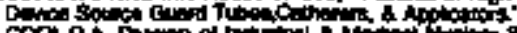

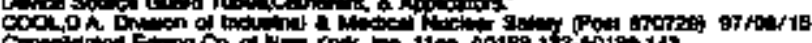

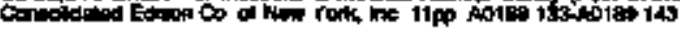

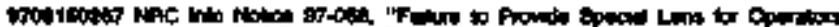

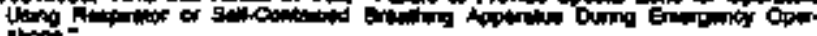

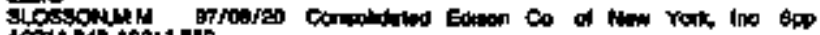

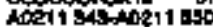

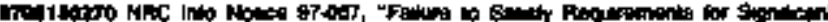

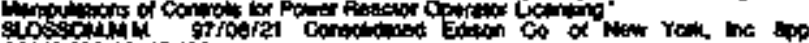

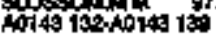

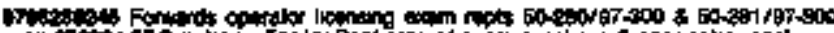

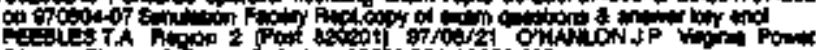

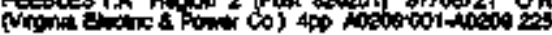

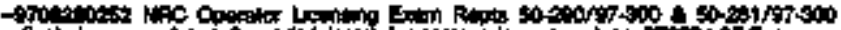

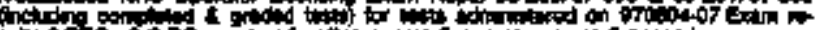

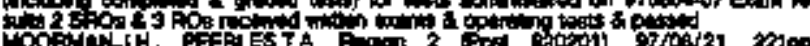
nodotosition

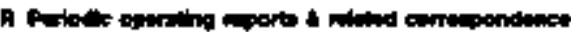

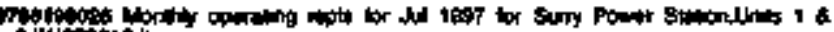

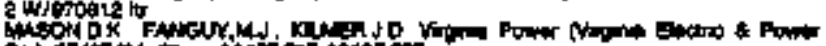

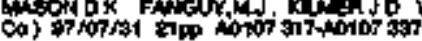

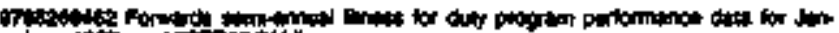

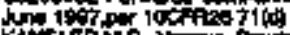

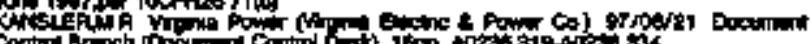

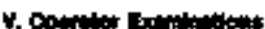

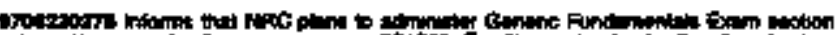

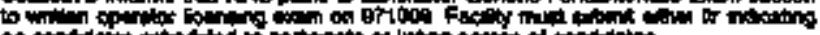

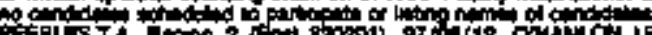

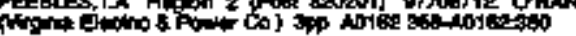

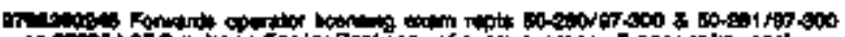

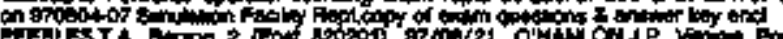

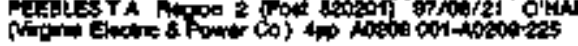

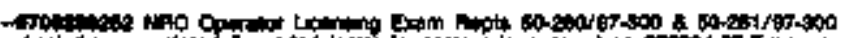
(1)

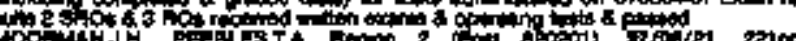

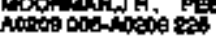

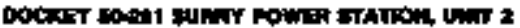

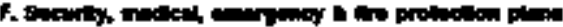

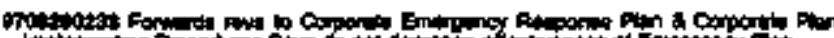

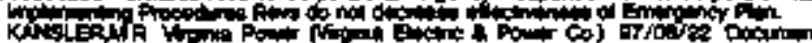

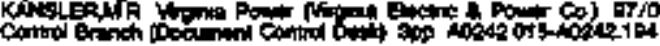

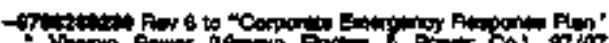

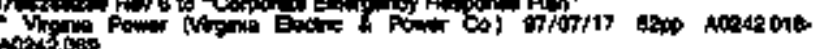

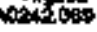

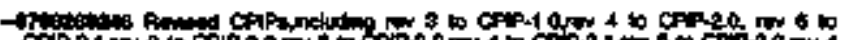

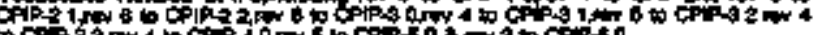

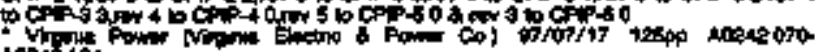
now 104

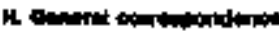

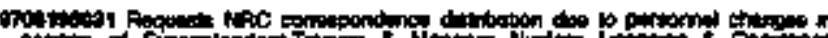

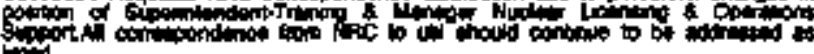

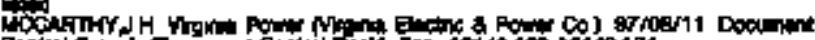

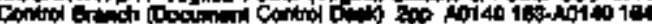

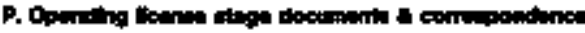

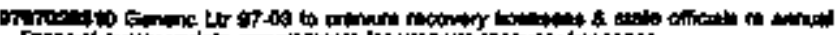

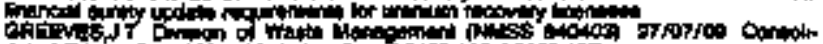

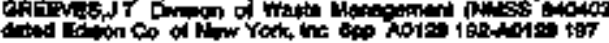

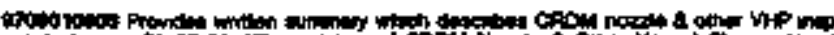

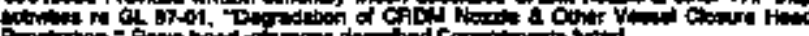

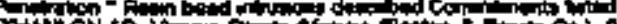

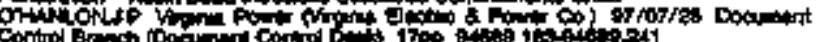

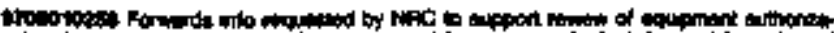

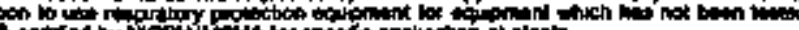

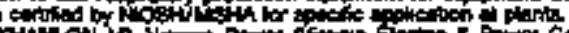

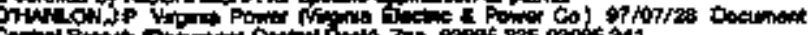

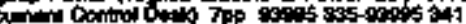

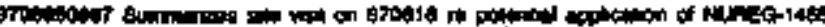

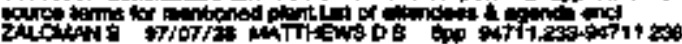




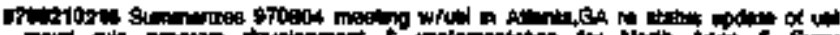

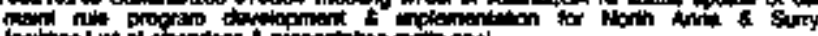

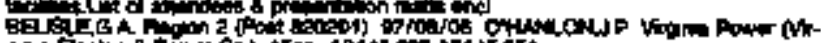

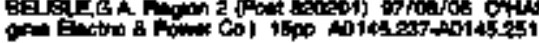

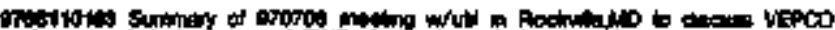

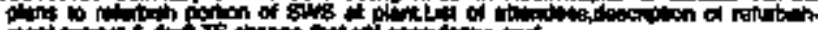

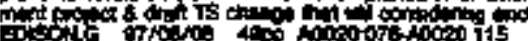

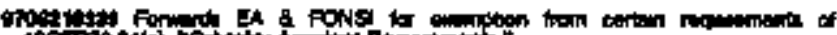

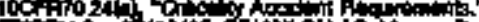

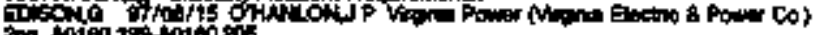

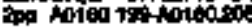

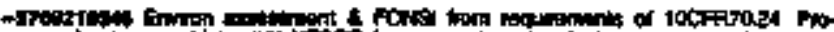

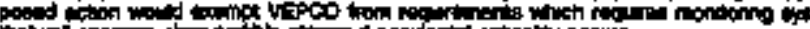

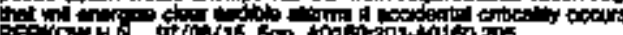

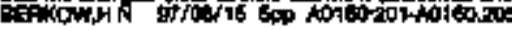

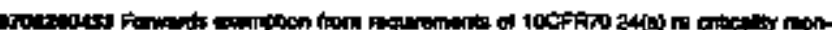

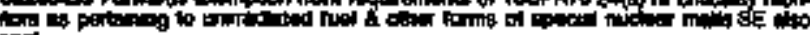

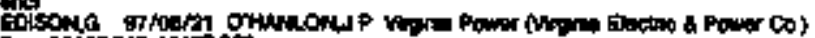

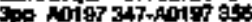

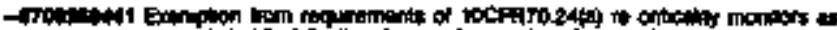

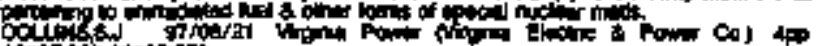

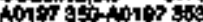

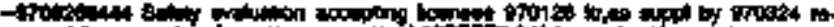

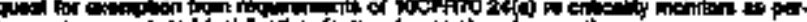

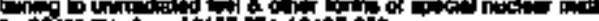

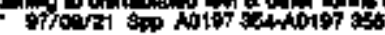

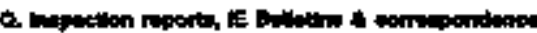

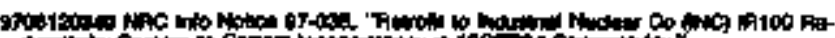

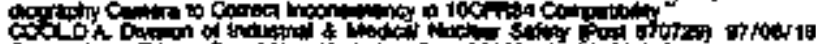

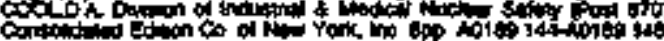

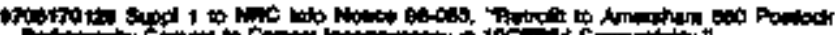

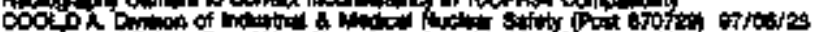

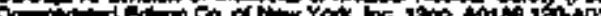

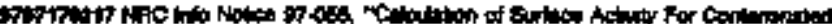

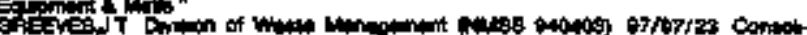

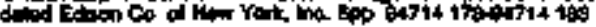

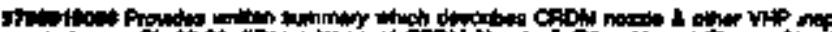

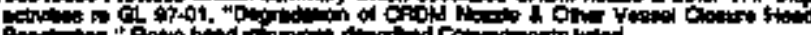

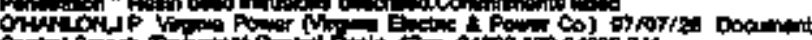

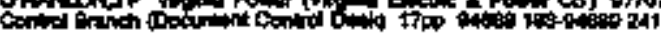

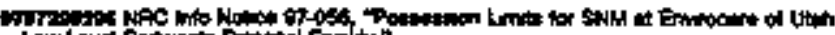

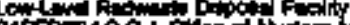

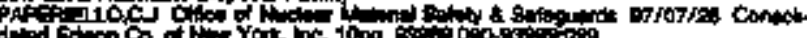

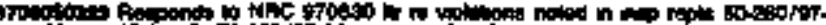

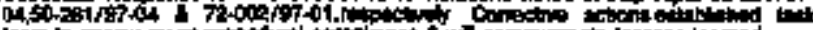

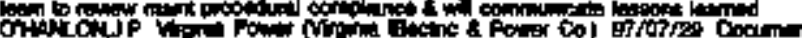

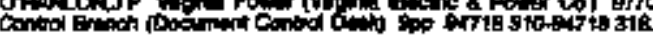

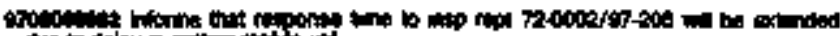

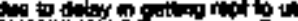

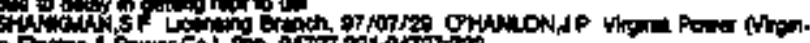

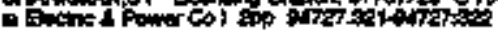

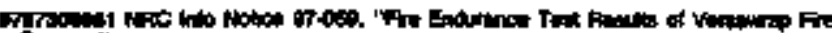

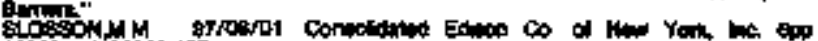

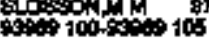

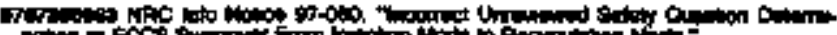

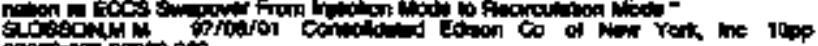

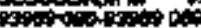

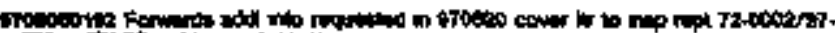

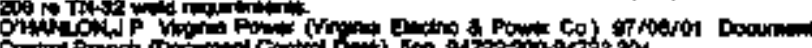

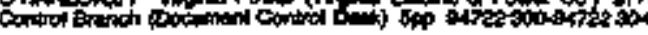

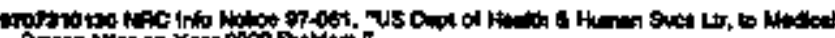

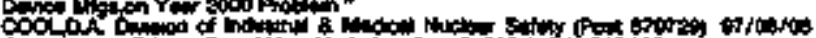

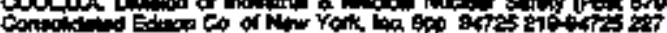

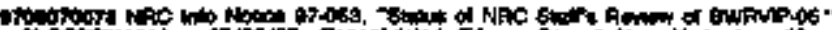

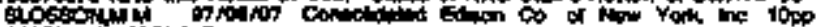

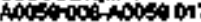

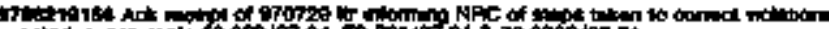

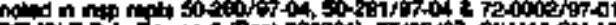

E-Fis

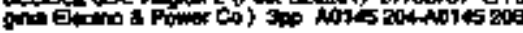

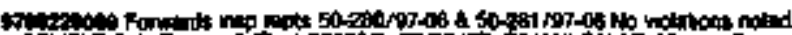

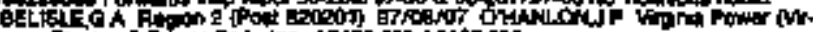

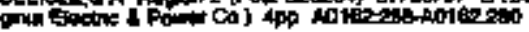

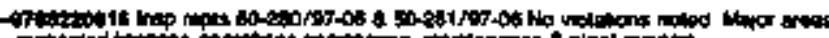

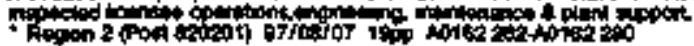

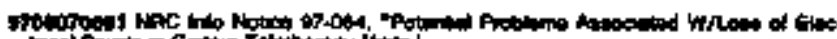

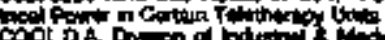

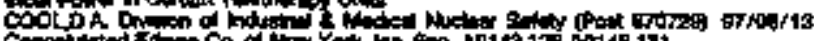

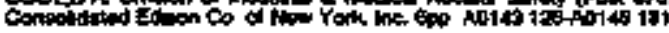

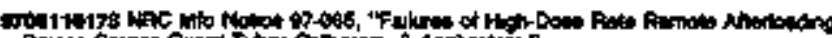

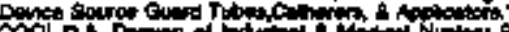

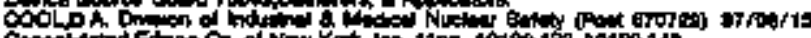

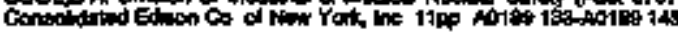

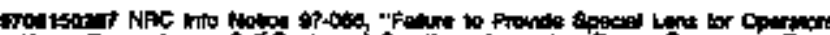

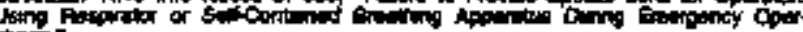

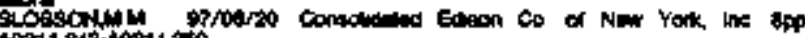
Nor 1 S43 1130

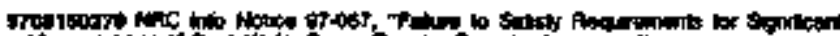

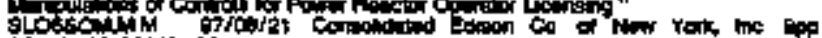

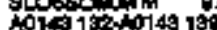

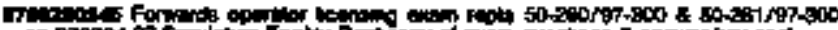

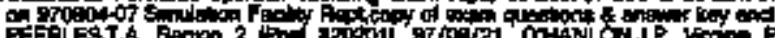

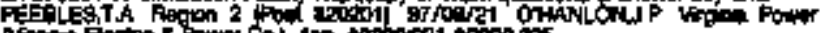

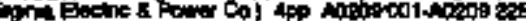

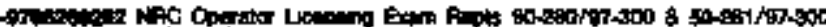

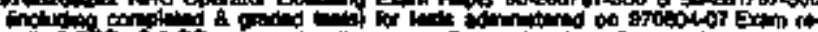

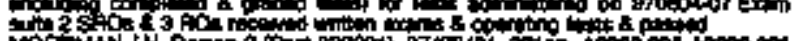
IOD

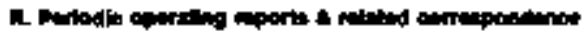

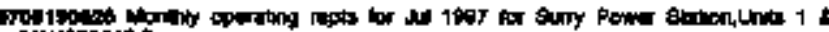

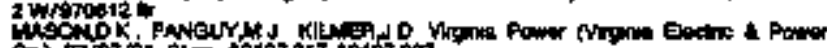

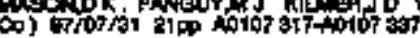

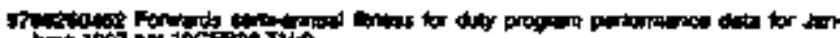

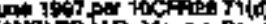

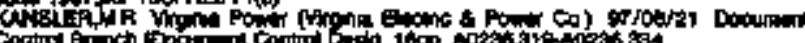

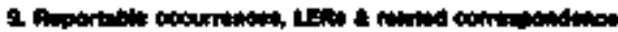

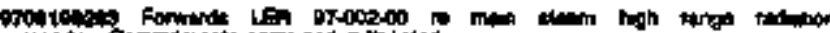

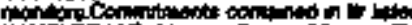

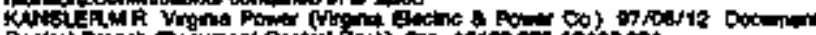

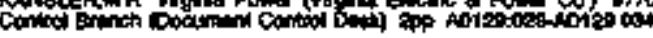

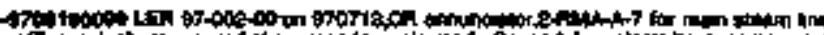

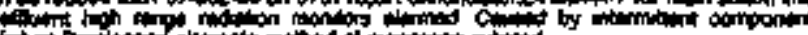

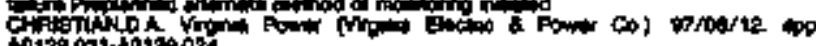

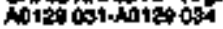

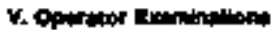

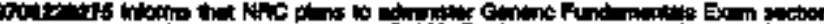

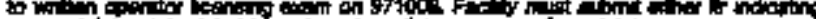

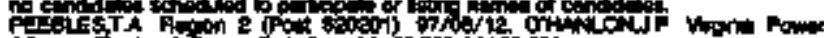
(V.

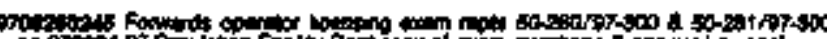

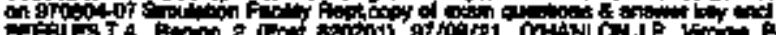

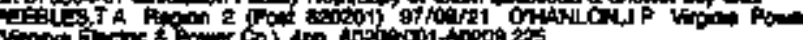

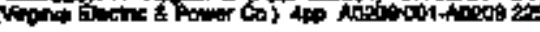

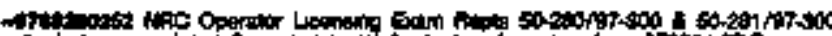

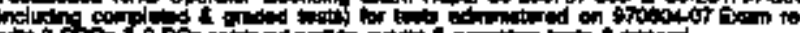

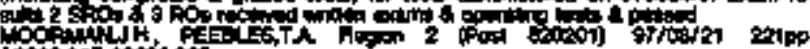

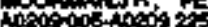

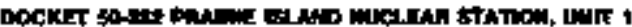

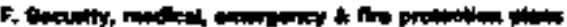

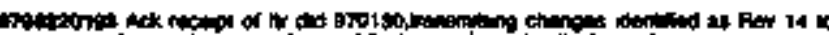

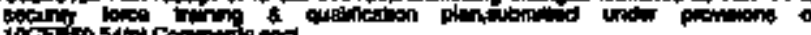

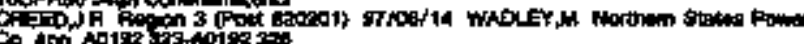

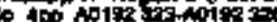

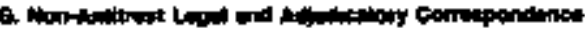

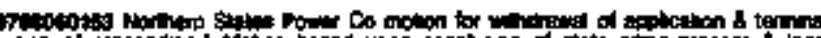

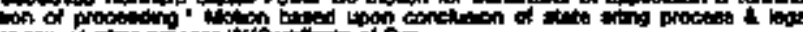

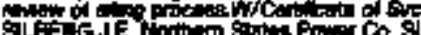

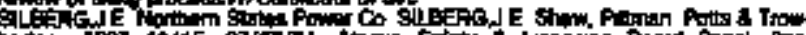

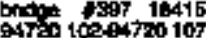




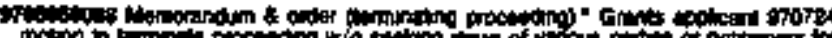

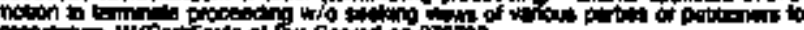

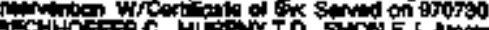

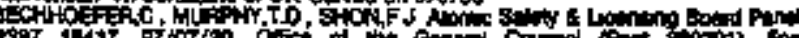

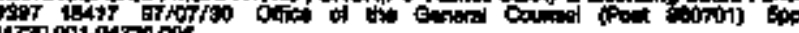

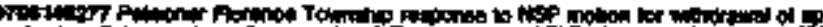

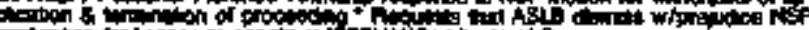

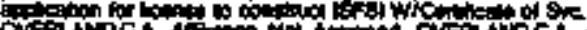

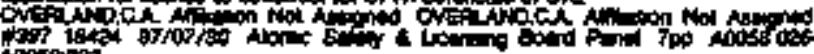
$40008 \times 130$

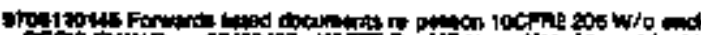

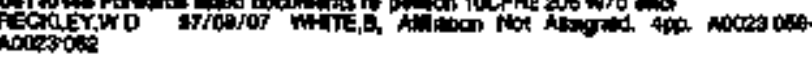

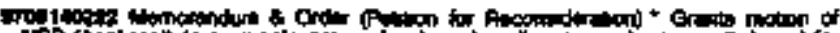

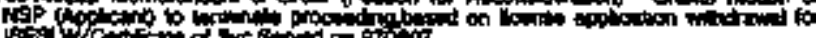
=cinto

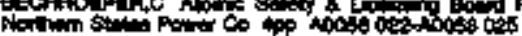

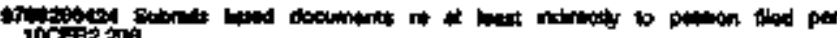

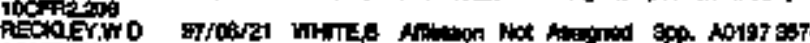

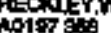

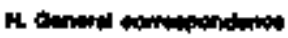

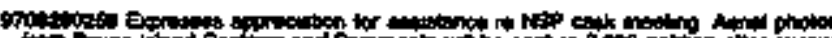

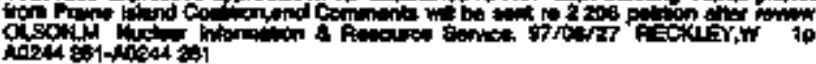

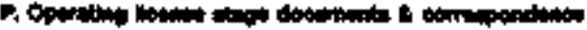

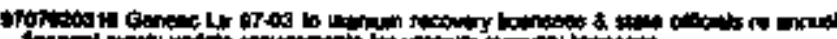

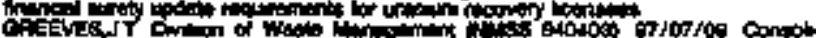

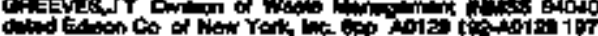

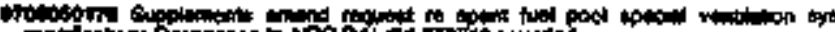

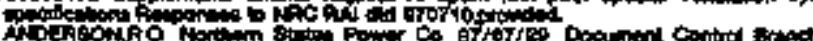

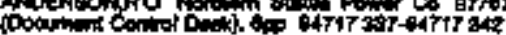

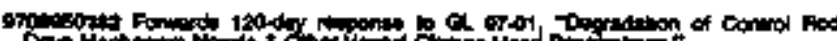

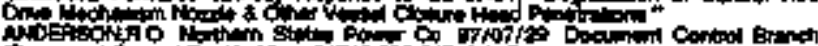

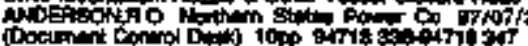

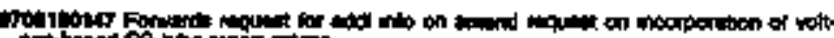

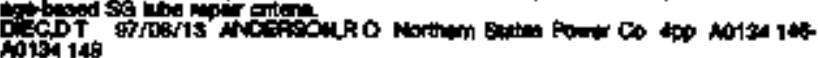

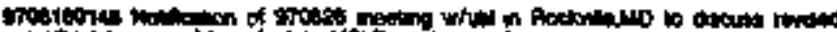

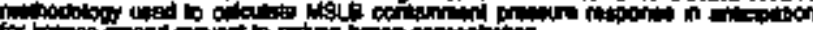
of

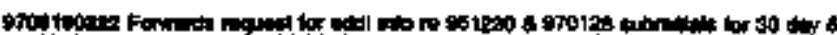

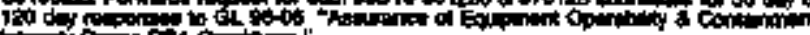

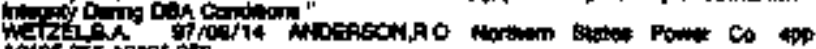

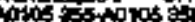

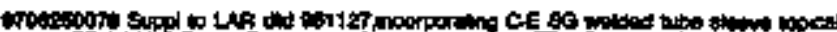
W C C

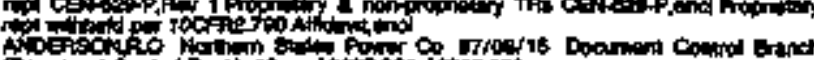

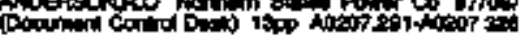

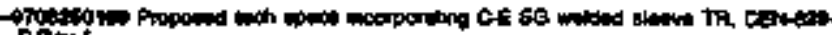
Prit

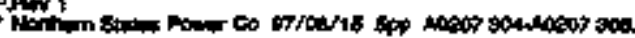

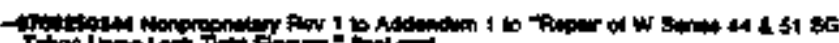

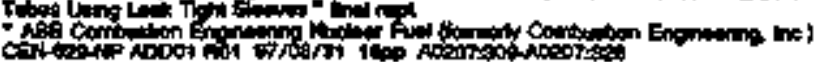

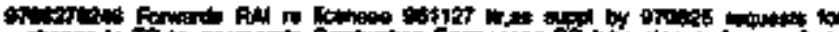

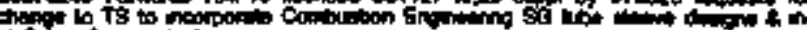
OC ADoros

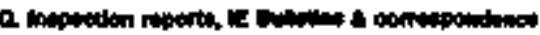

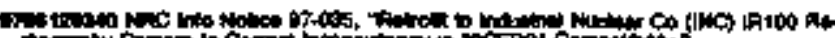

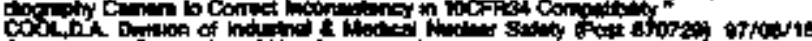

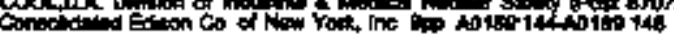

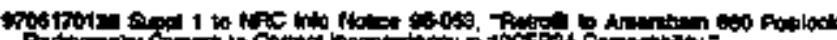

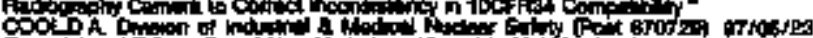

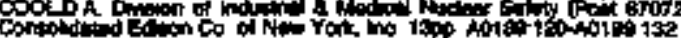

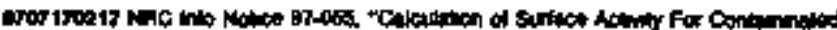

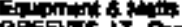

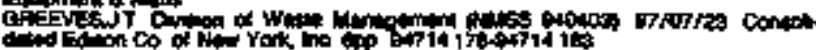

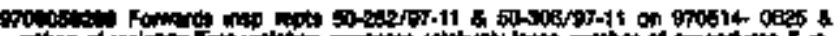
noteo of wol:

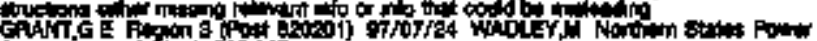

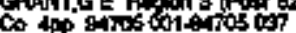

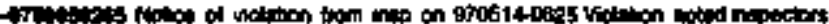

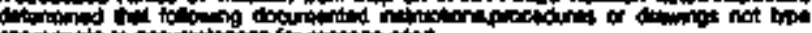

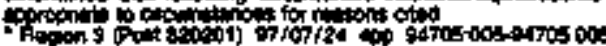

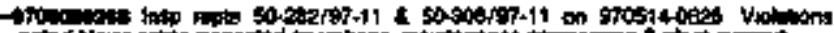

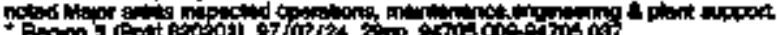

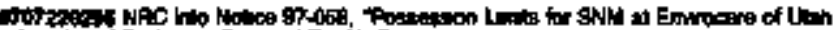

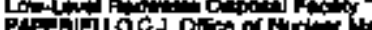

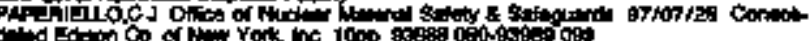

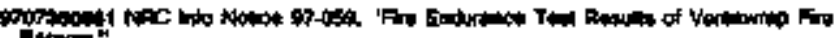

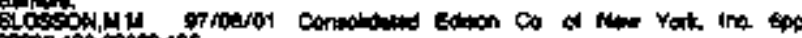
100 istos $10 \%$

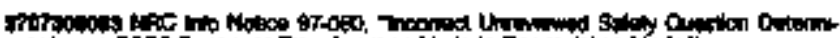

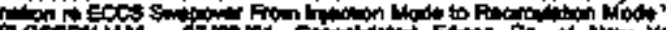

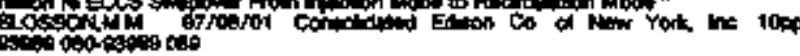

17r3101

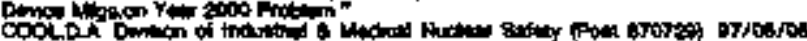

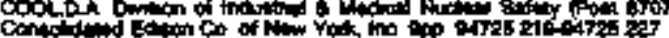

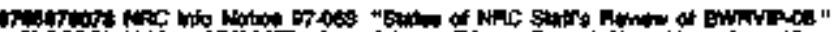

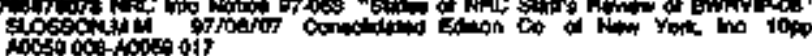

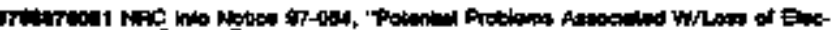

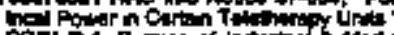

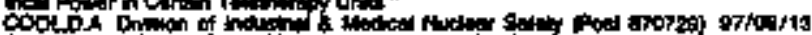

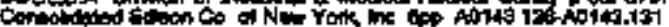

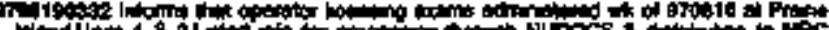

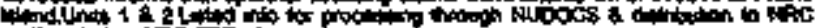

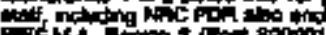

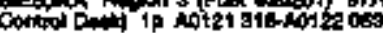

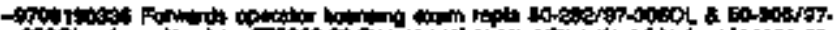

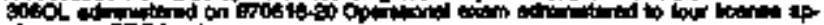

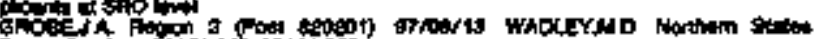

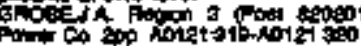

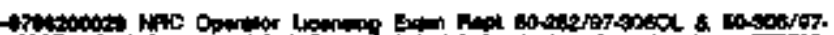

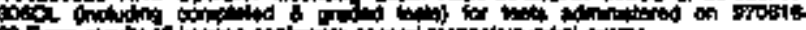

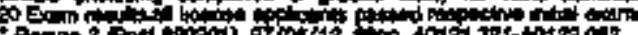

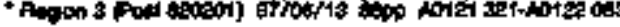

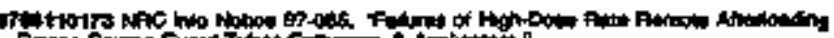

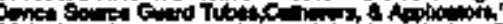

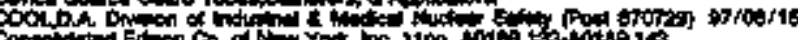

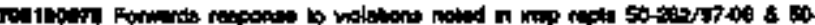

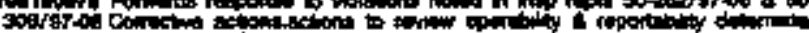

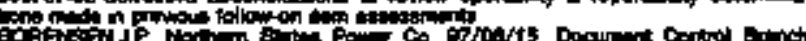

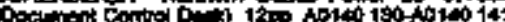

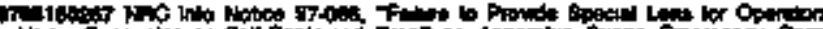

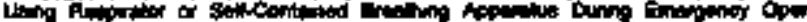

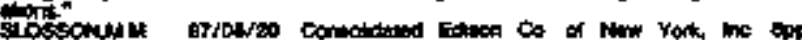

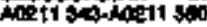

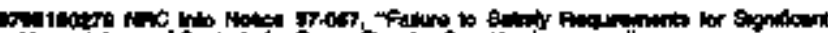

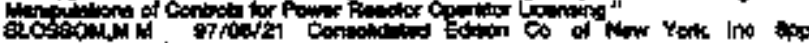

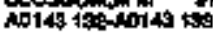

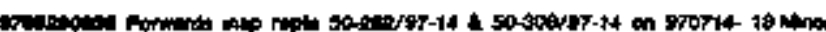

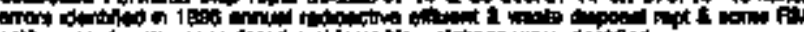

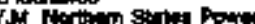

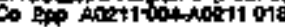

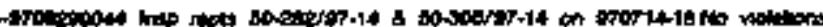

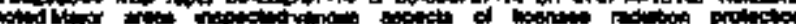

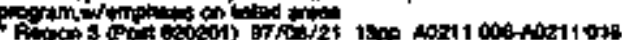

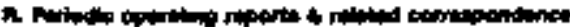

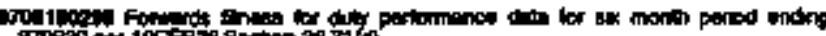

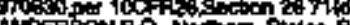

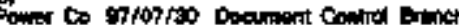

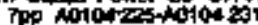

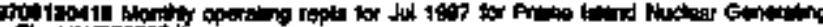

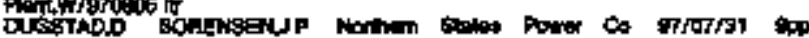

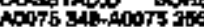


It)

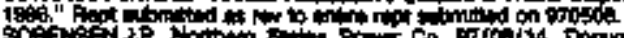

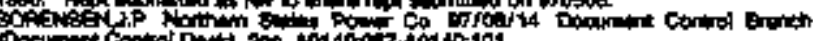

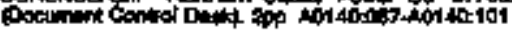

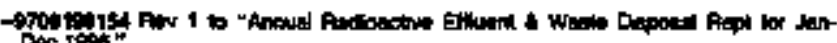

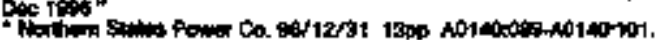

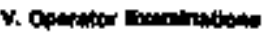

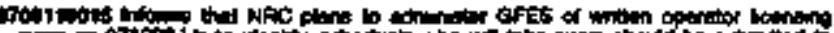

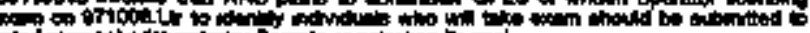

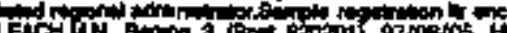

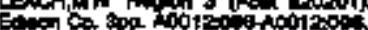

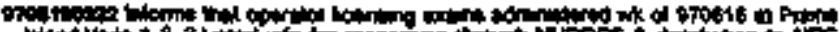

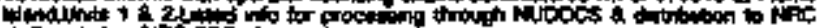

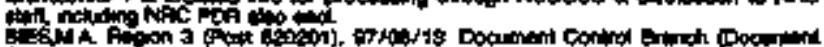

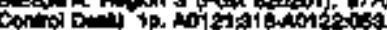

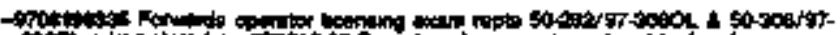

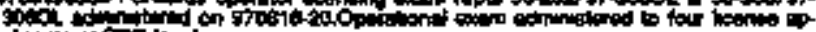

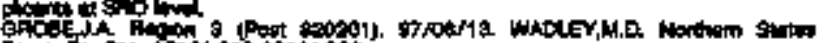

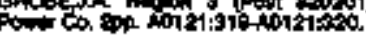

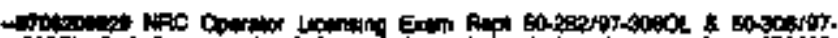

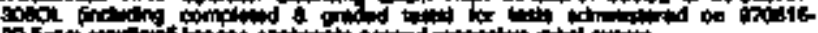

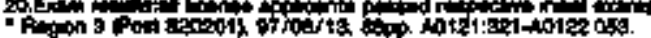

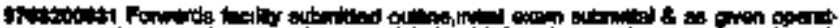

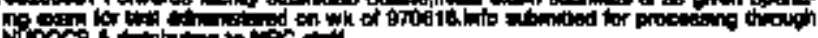

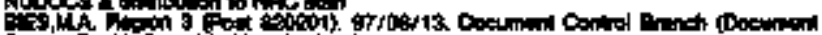

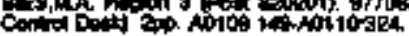

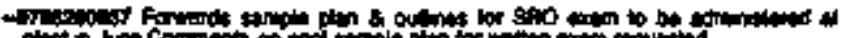

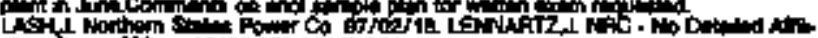

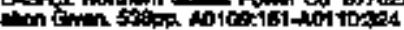

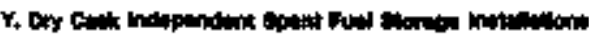

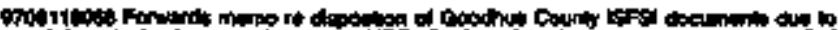

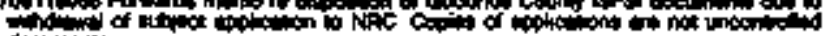
|

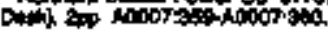

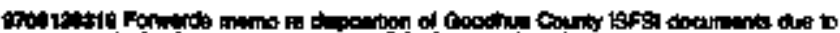

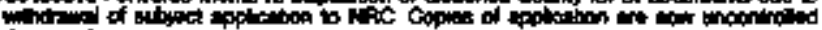

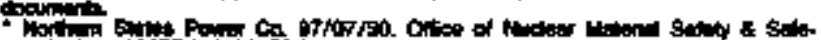

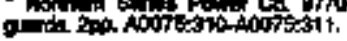

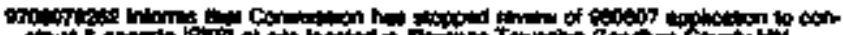

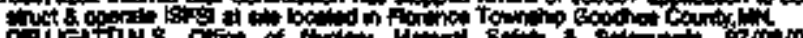

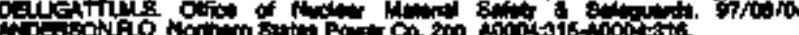

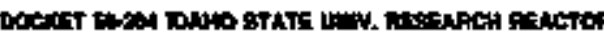

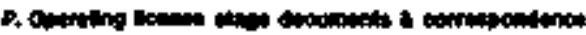

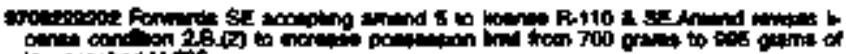

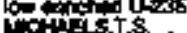

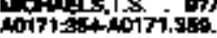

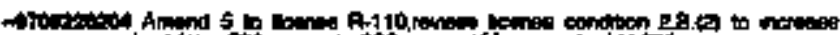

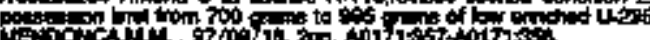

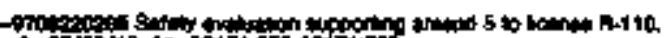

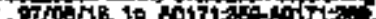

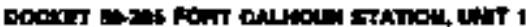

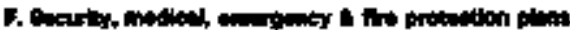

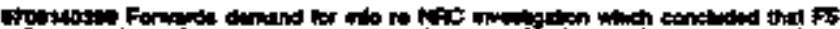

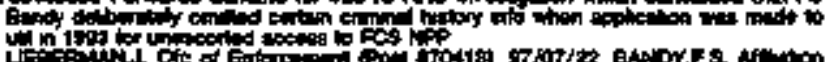

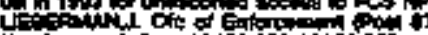

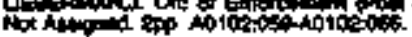

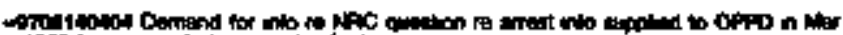

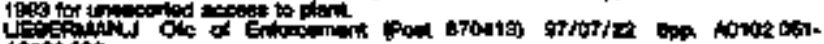

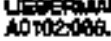

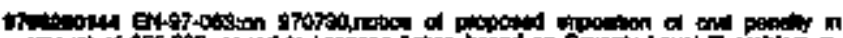

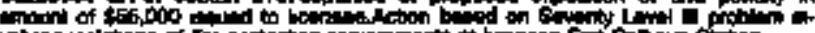

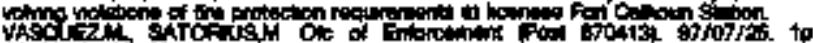

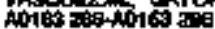

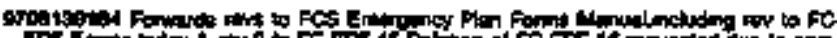

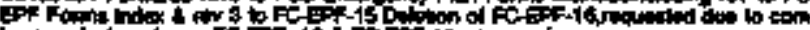

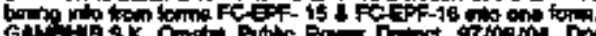

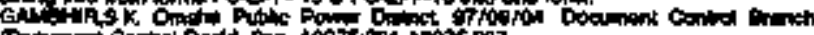

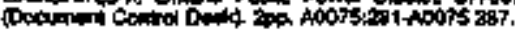

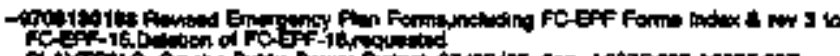

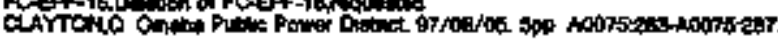

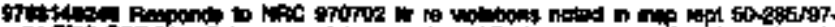

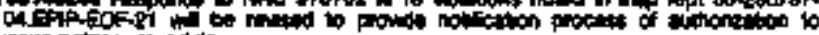

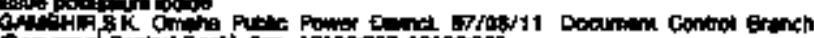

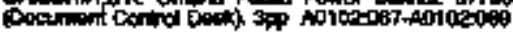

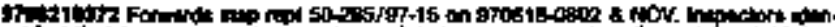

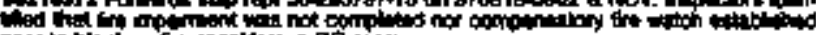

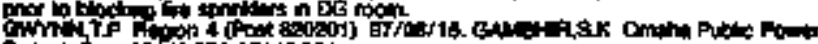

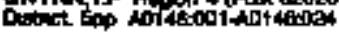

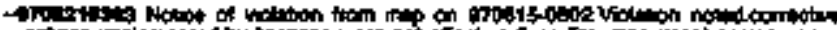

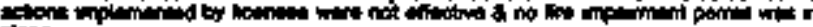
Piace

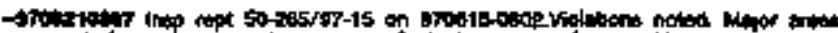

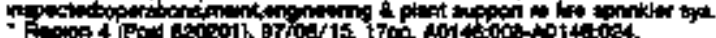

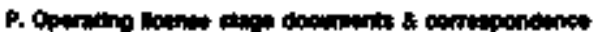

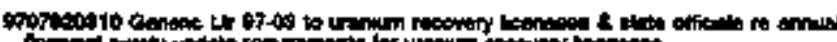
G

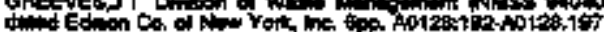

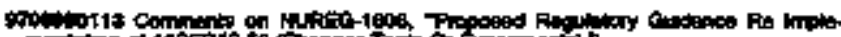

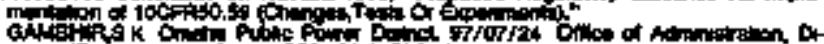

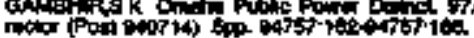

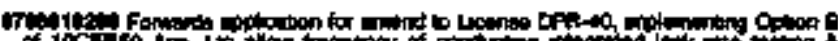

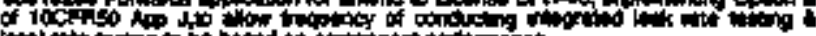

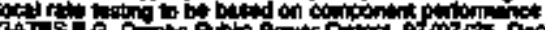
ches $Q$

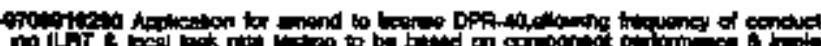

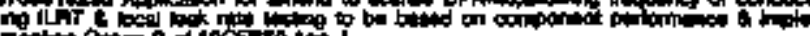

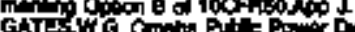

烈

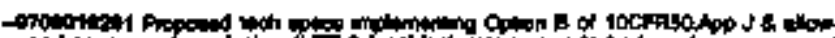

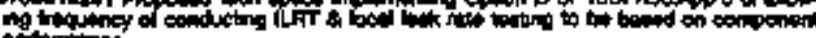

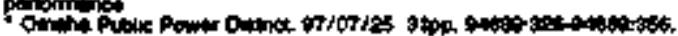

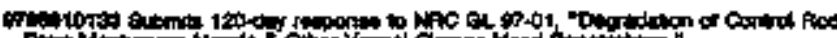

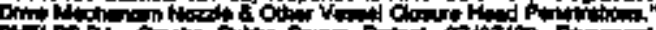

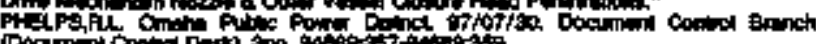

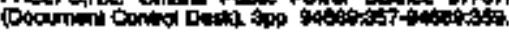

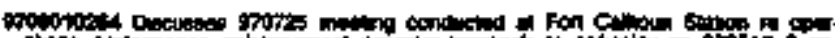

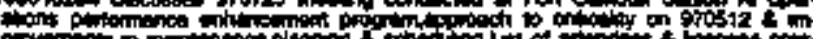

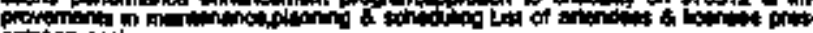

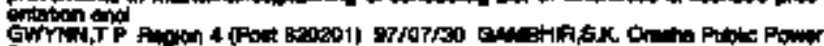

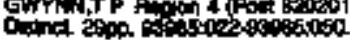

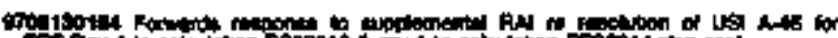

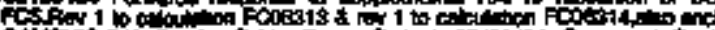

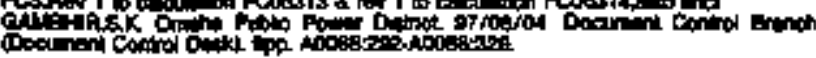

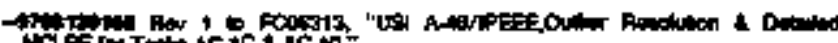

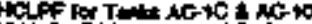

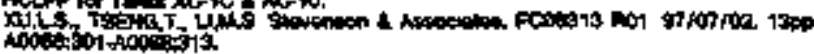

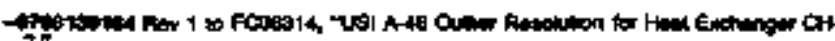

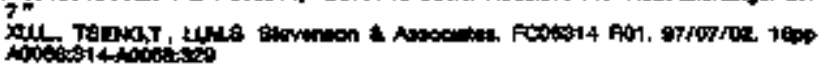

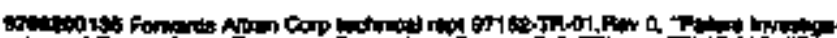

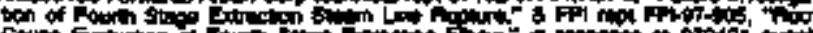

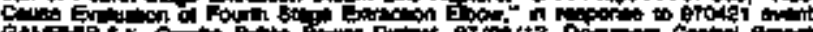

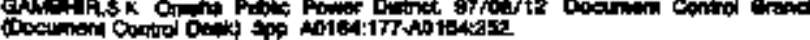

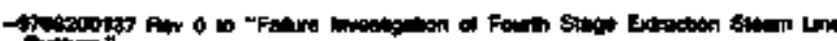

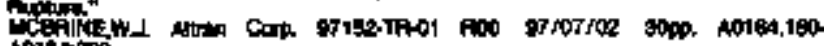
Moritezos

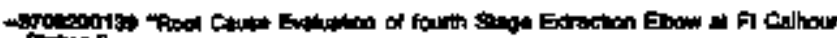

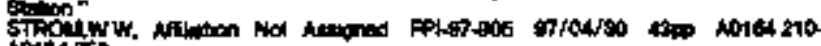
noted a 


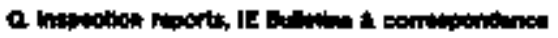

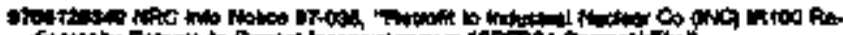

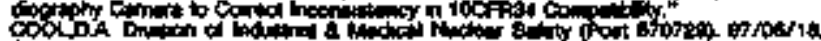

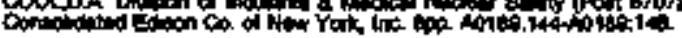

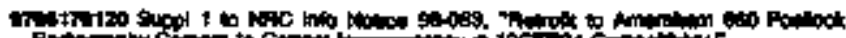

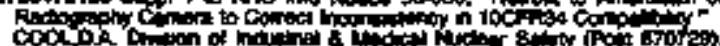

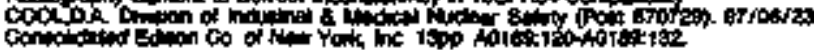

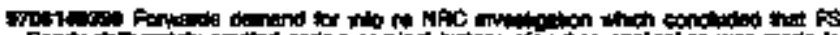

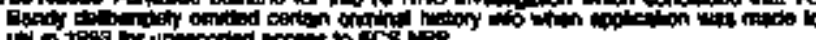

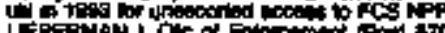

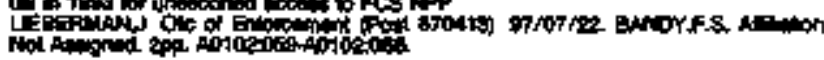

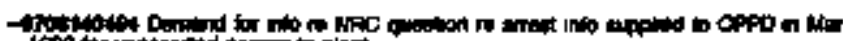

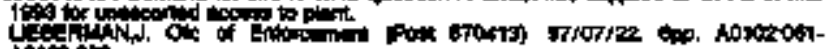
Notion

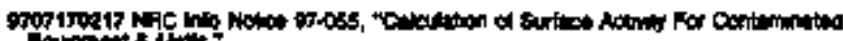

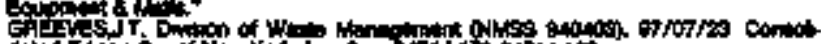

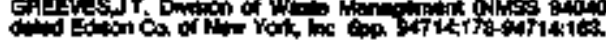

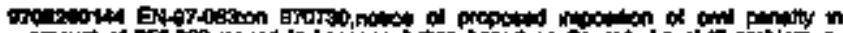

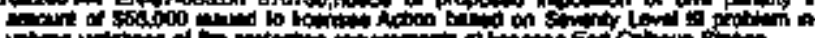

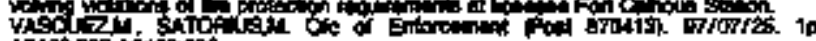

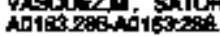

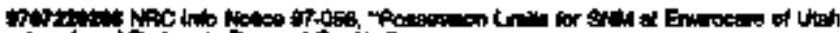
Lof

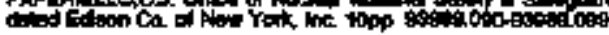

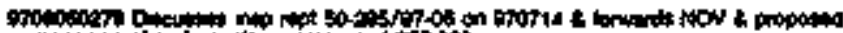

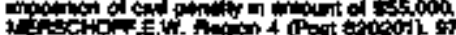

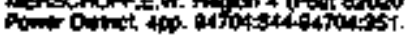

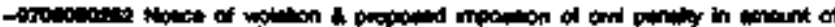

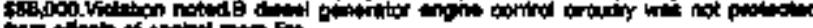

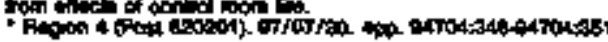

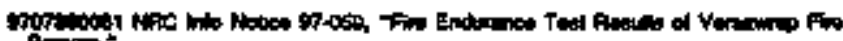

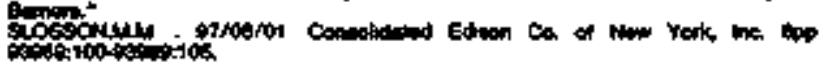

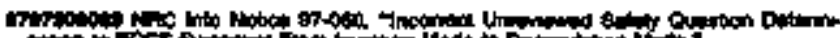

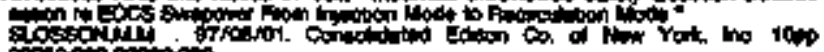

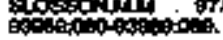

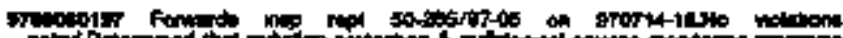

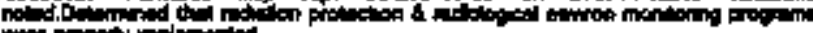

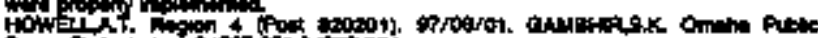

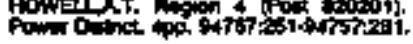

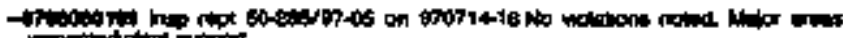

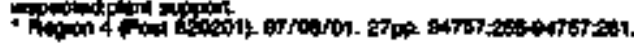

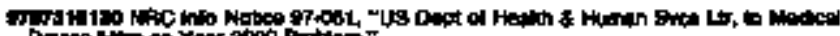

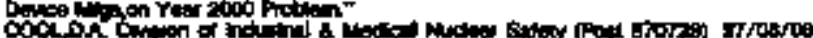

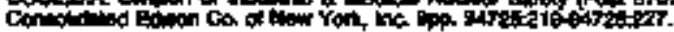

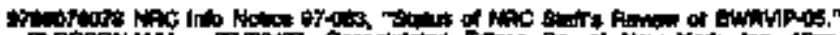

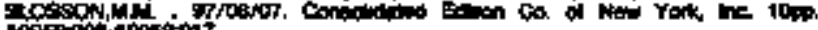

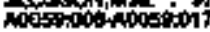

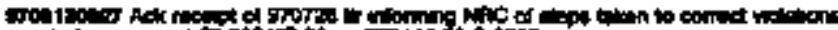

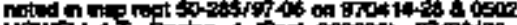

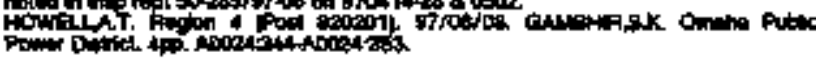

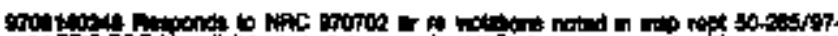

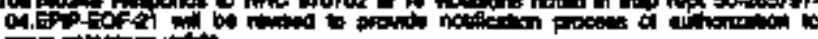

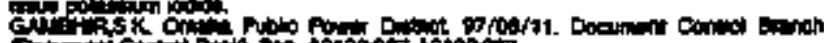

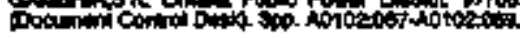

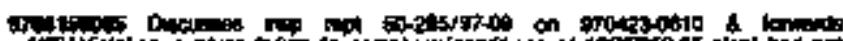

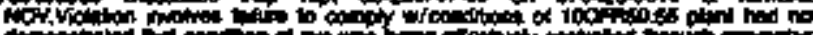

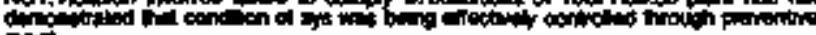

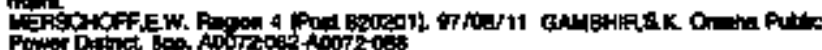

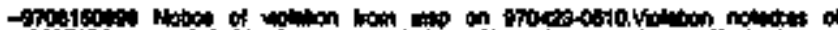

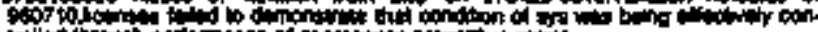

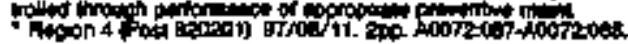

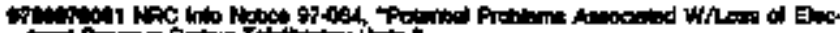
led

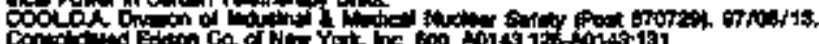

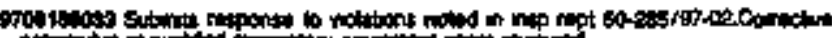
Gow

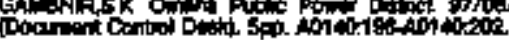

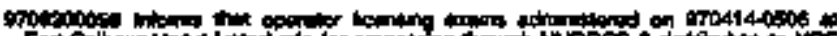

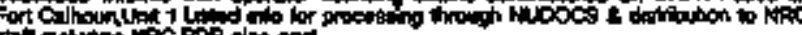

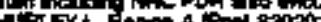

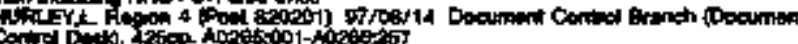

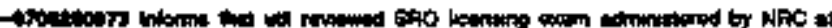

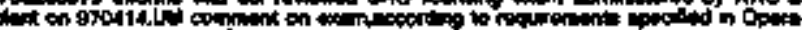

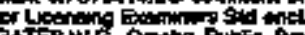

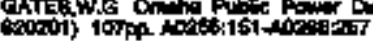

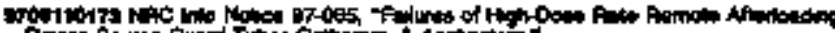

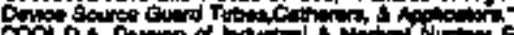

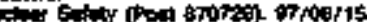

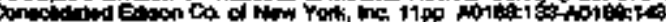

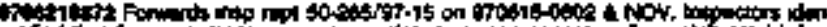

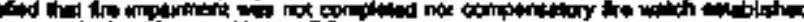

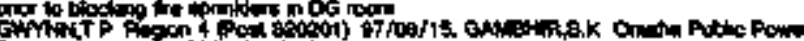

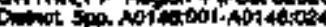

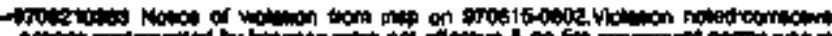

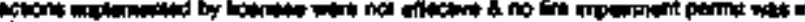

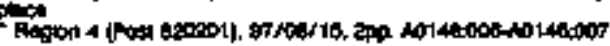

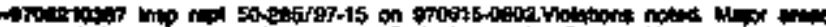

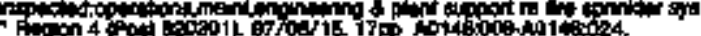

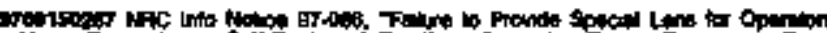

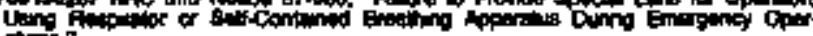

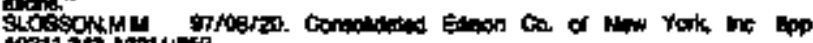

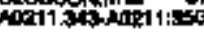

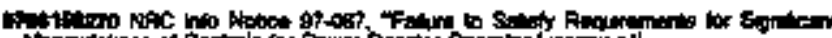

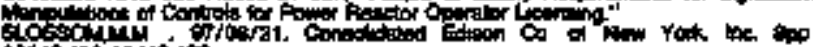
Bostacting

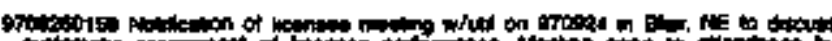

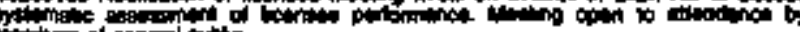

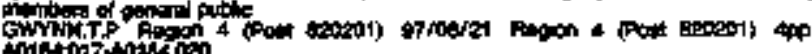

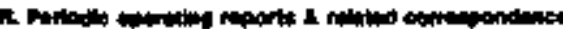

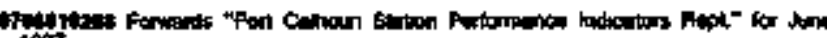
19t?

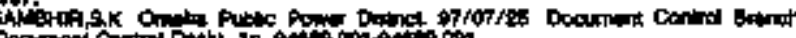

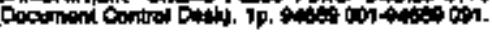

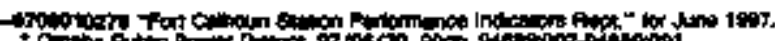

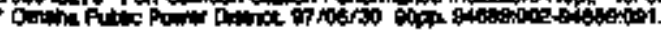

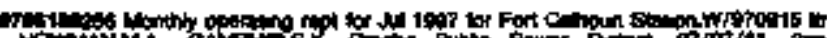

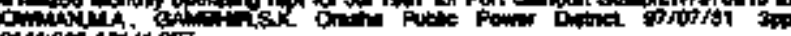

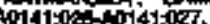

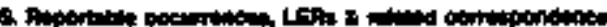

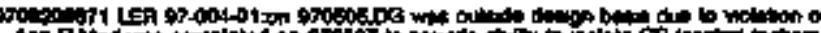

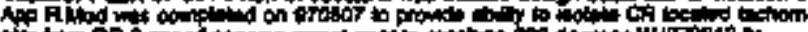

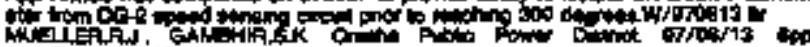

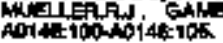

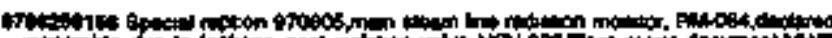

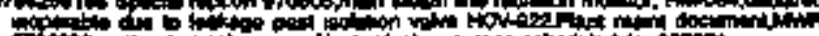

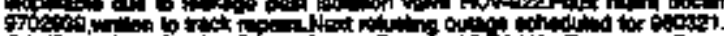

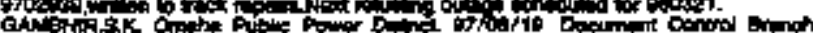

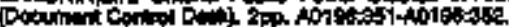

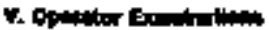

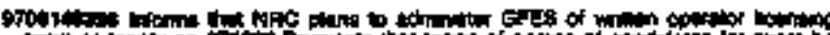

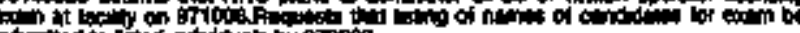

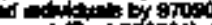

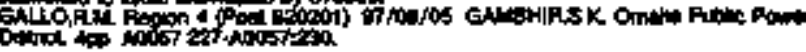

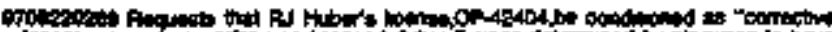

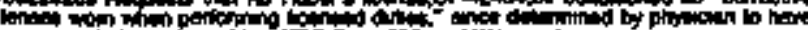

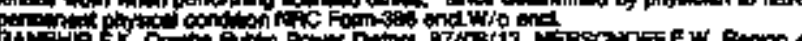

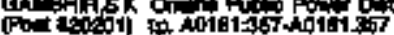

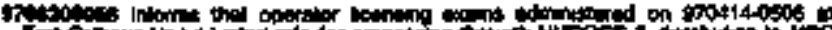

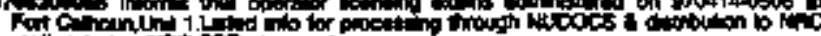

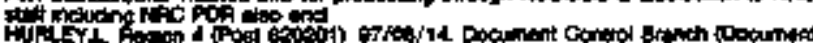

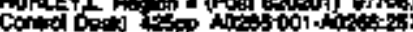




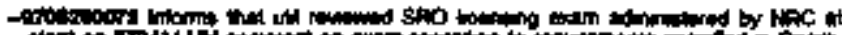

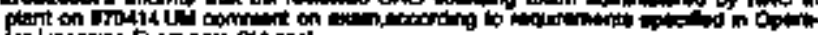

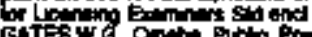

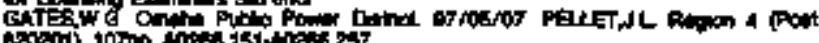

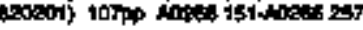

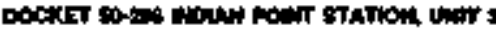

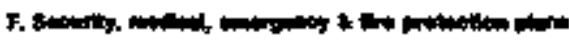

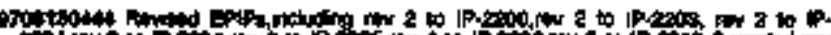
zad, 1 1. IPespld

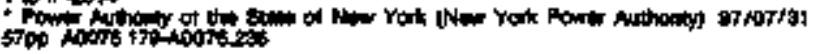

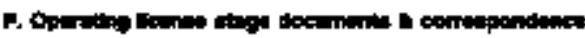

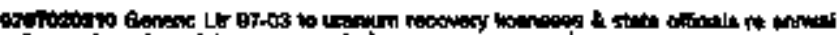

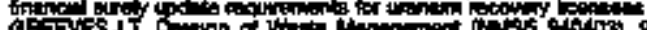

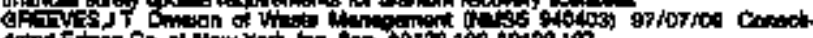

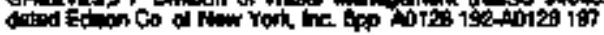

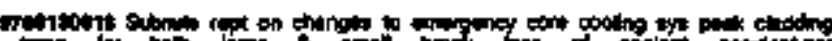

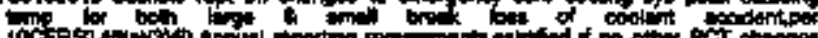

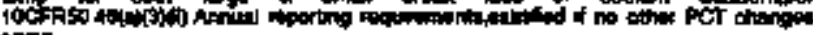

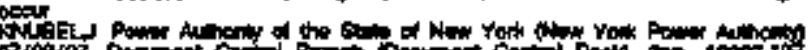

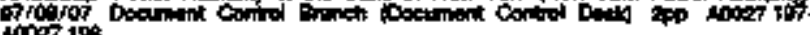
10027 19:

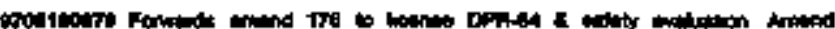

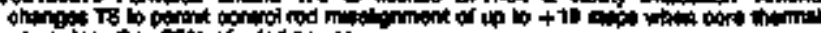

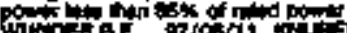

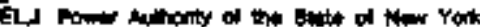

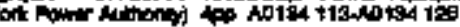

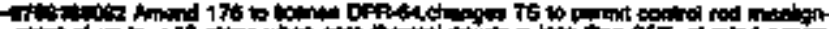

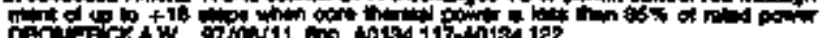

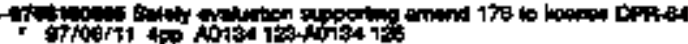

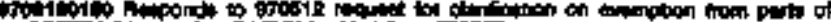

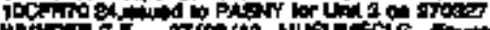

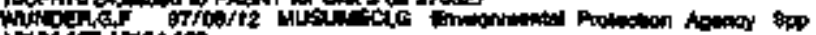

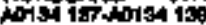

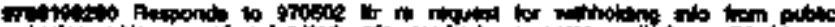

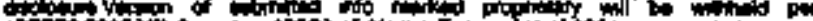

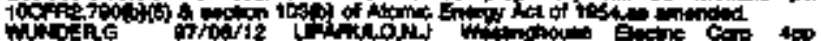
winos

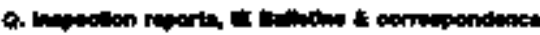

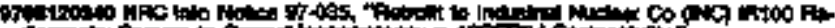

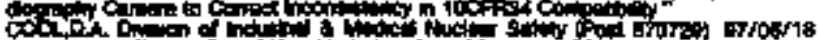

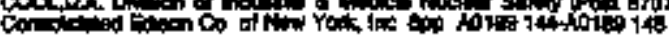

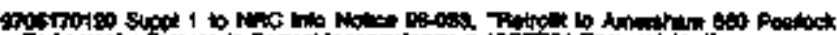

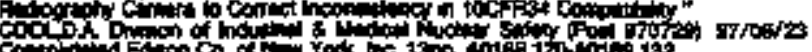

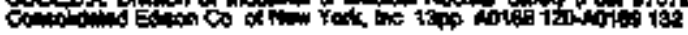

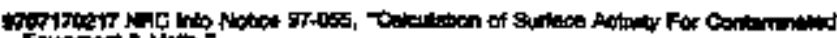

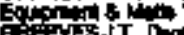

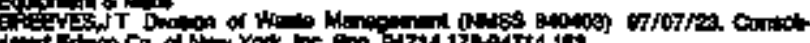

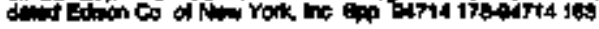

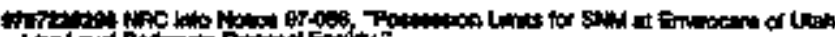
PAP

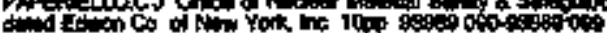

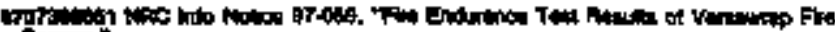

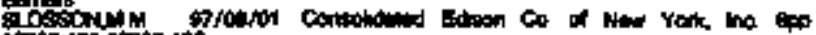

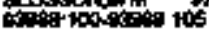

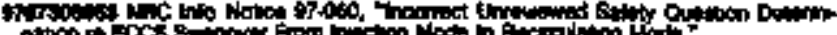

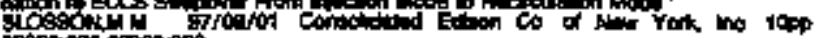

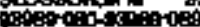

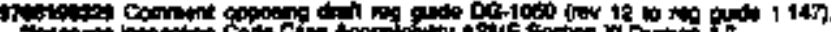

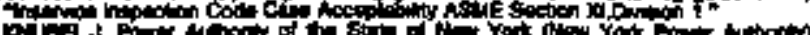

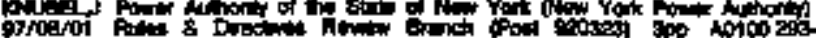
Notio: 286

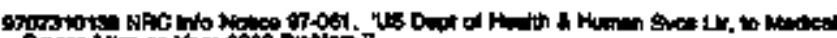

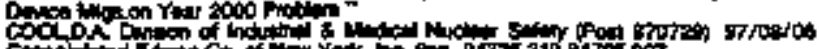

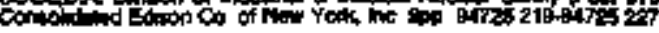

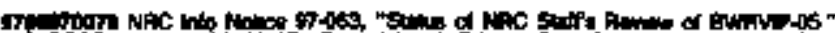
常

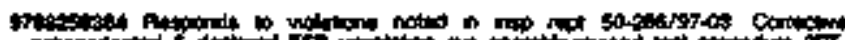

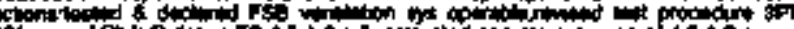

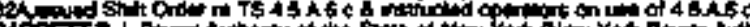

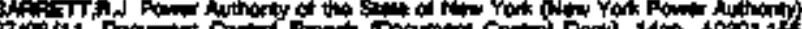

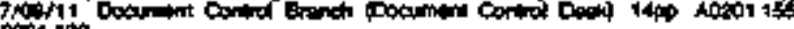

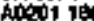

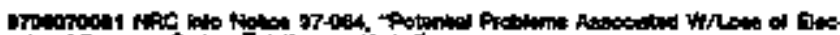

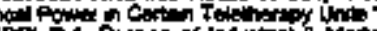

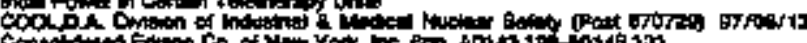

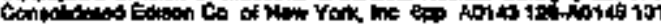

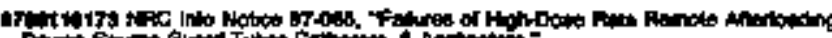

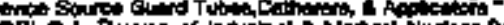

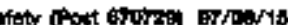

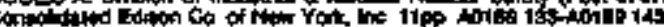

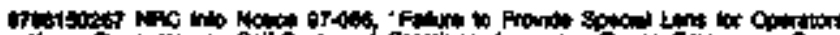

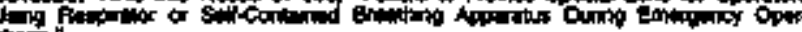

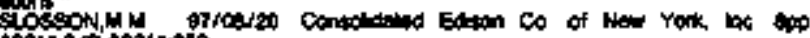

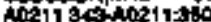

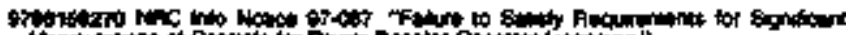

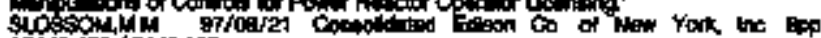
int 40 is 40140

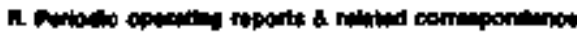

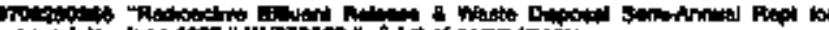

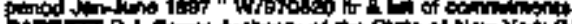

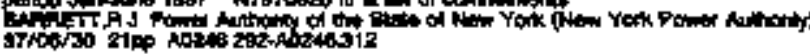

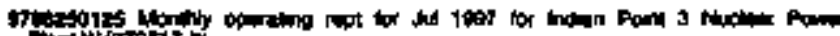

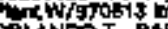

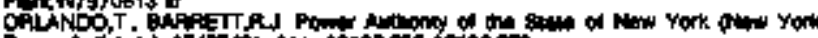

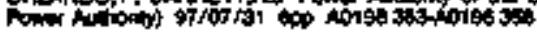

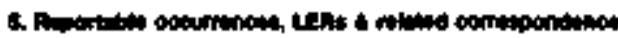

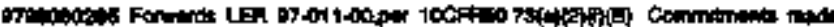

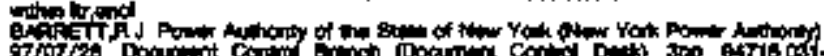
6471603

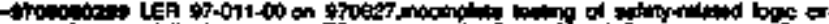

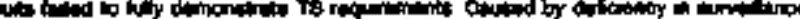

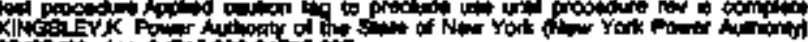

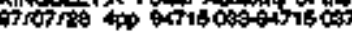

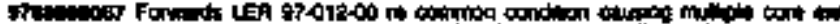

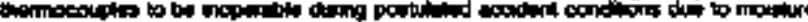

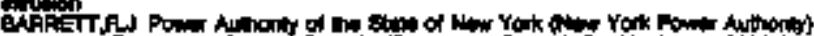

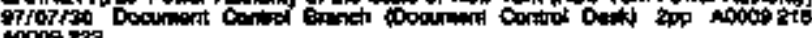
1000020

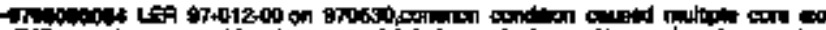

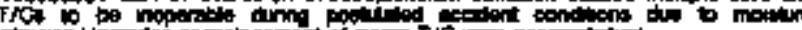
B

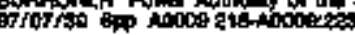

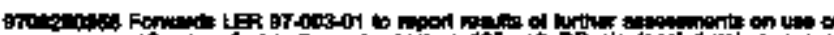

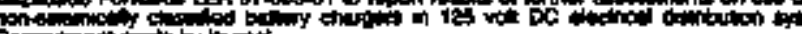

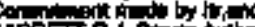

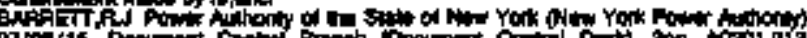

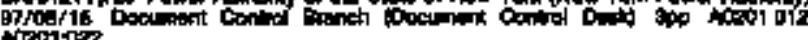

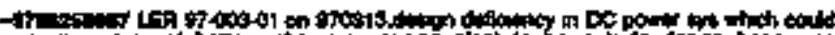
cout a $\mathrm{k}$.

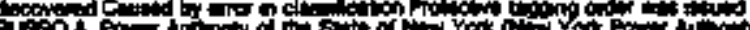

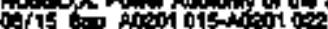

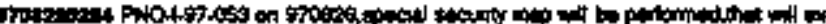

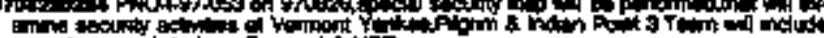

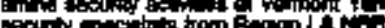

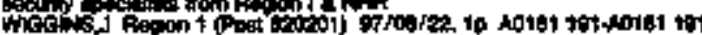

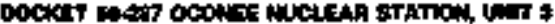

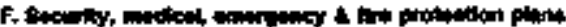

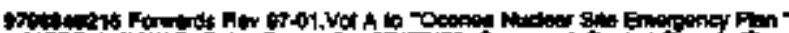

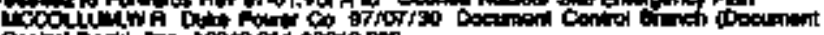

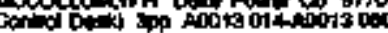

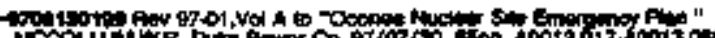

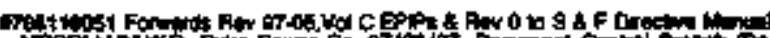

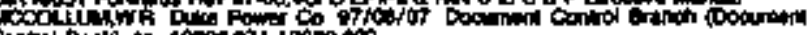

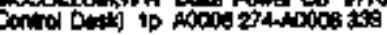




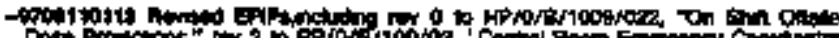

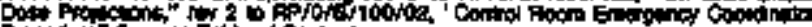

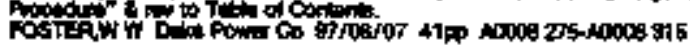

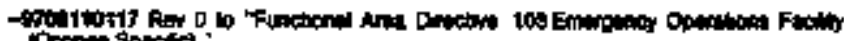

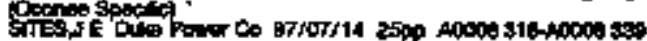

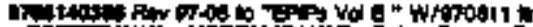

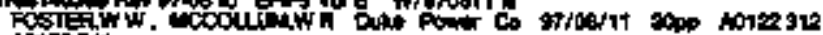
wionsin

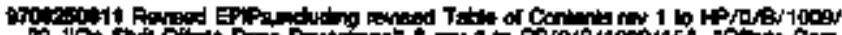

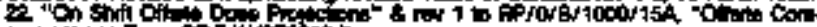

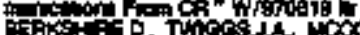

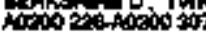

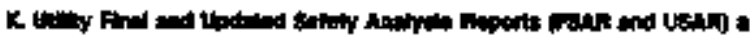

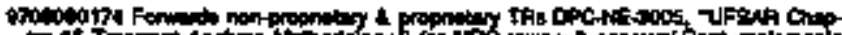

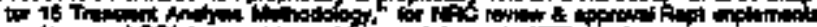

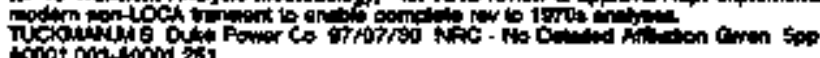

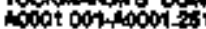

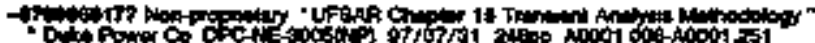

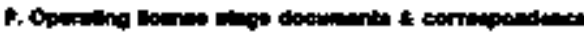

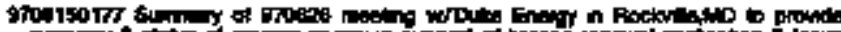

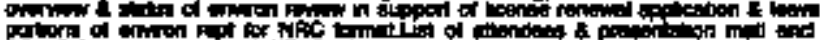

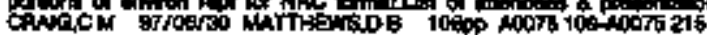

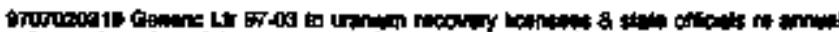

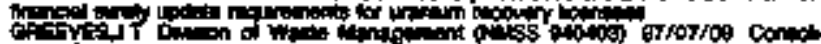

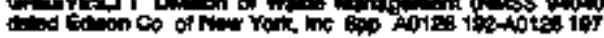

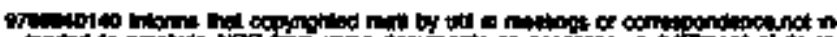

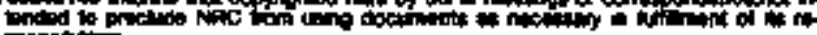

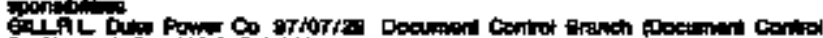

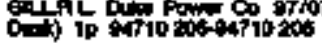

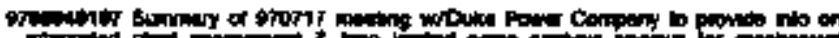

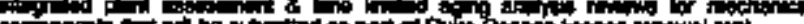

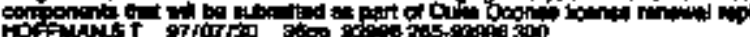

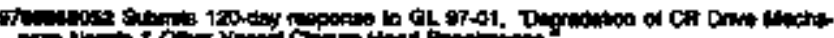
TUCK

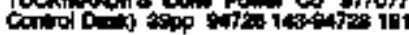

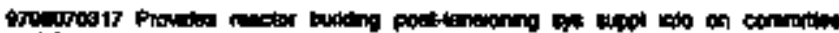

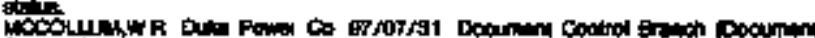

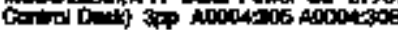

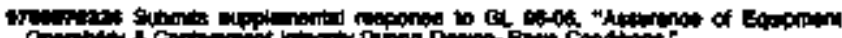

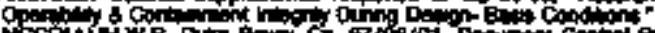

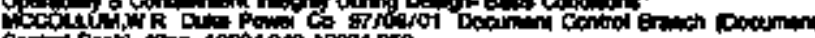

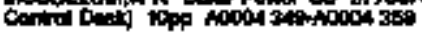

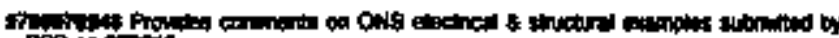

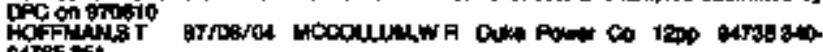
ofjos

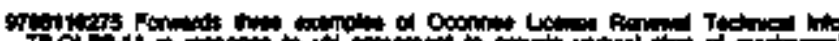

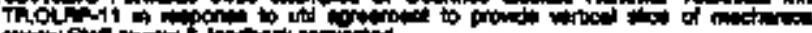

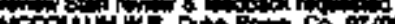

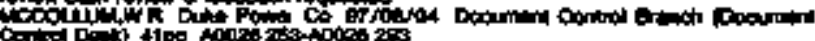

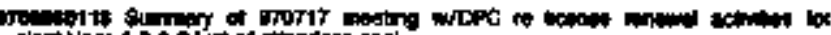

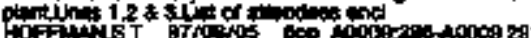

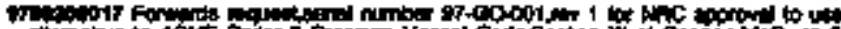

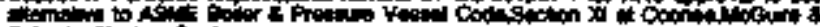

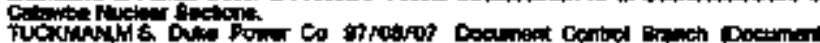

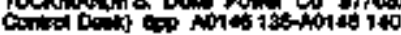

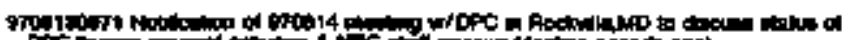
HCF

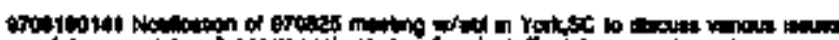
$\rightarrow$ hit

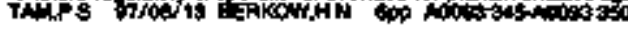

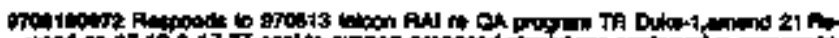

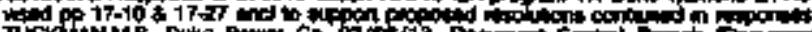

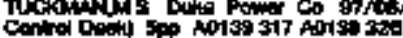

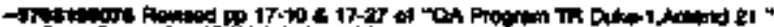

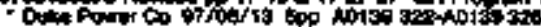

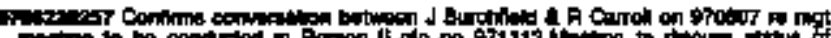

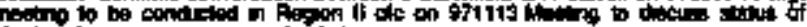

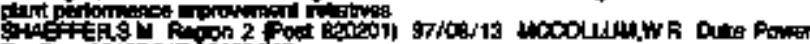

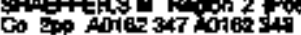

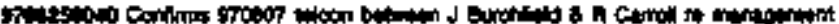

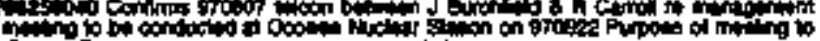

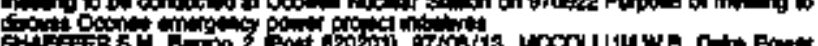

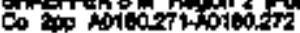

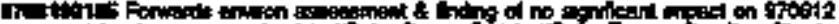

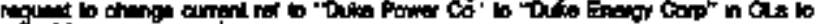

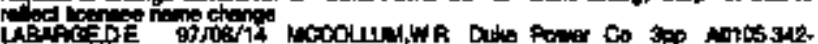
Antos $\$ 47$

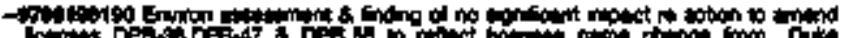

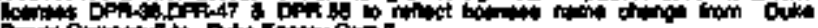

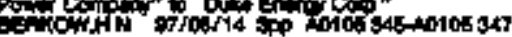

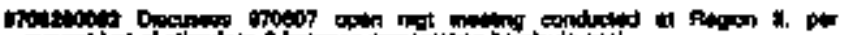

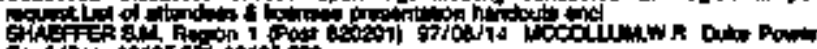

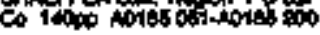

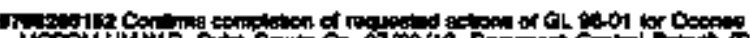

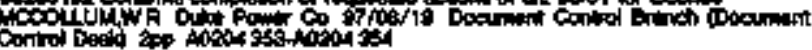

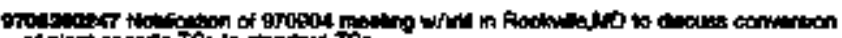

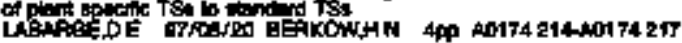

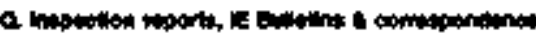

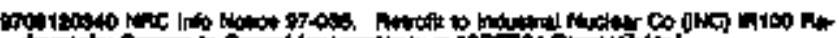

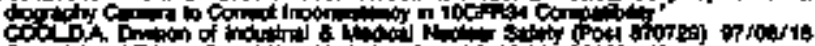

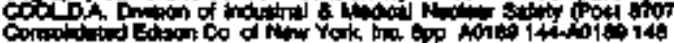

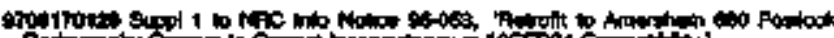

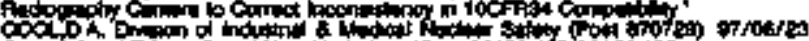

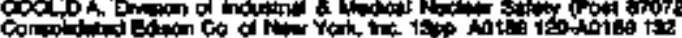

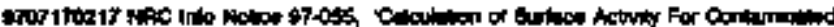

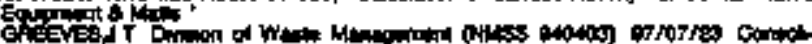

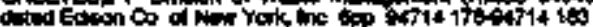

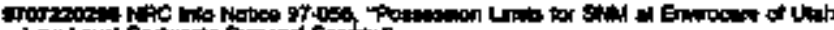

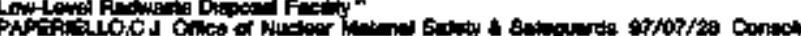

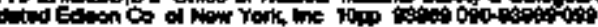

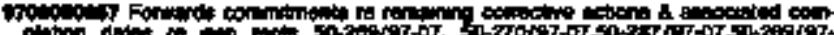

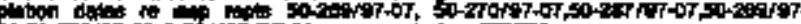

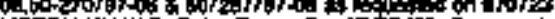
Cox

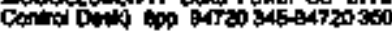

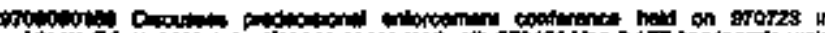

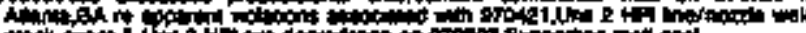

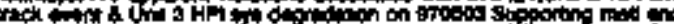

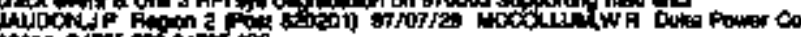

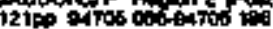

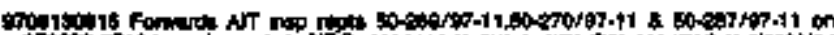

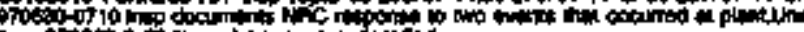

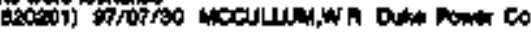
$8113+40028$ i7t

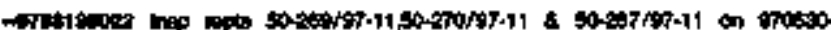

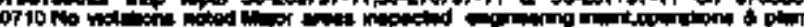

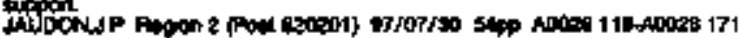

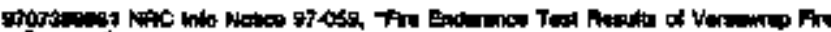

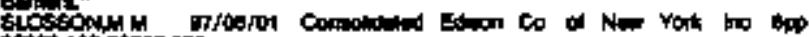

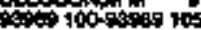

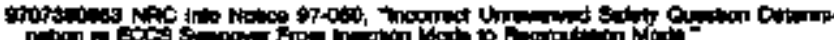

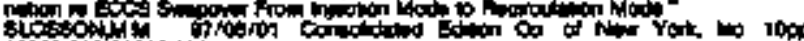
10.0\%

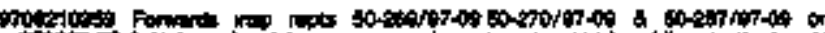

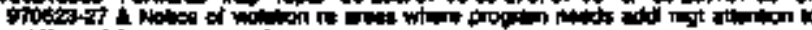

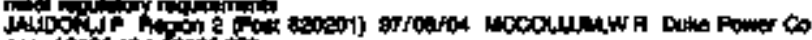

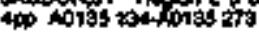

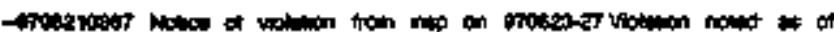

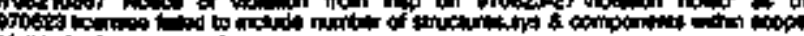

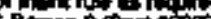

管

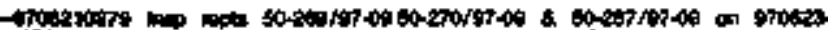

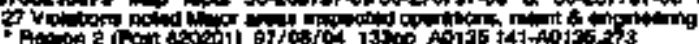




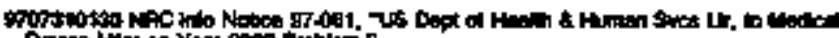

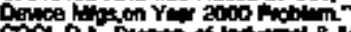

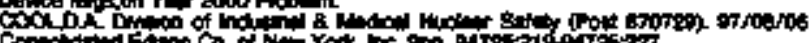

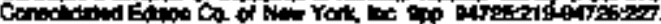

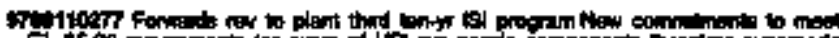

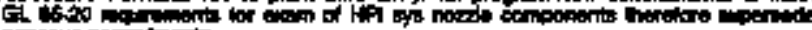

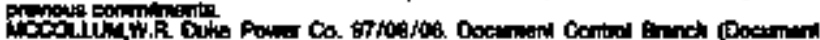

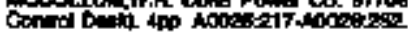

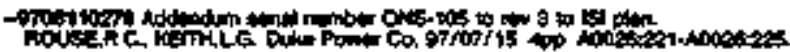

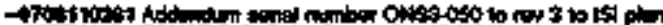

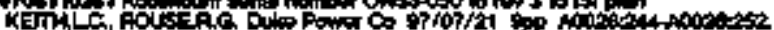

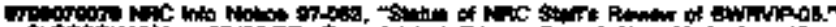

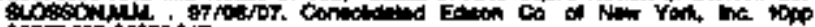

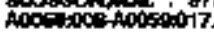

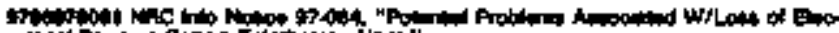

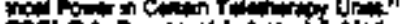

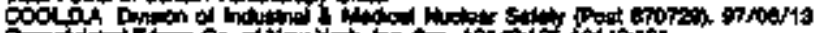
Cond

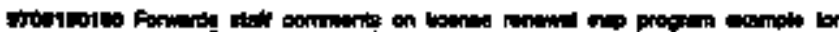

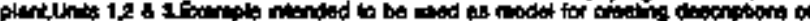

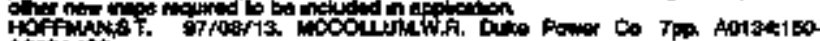
Actisio

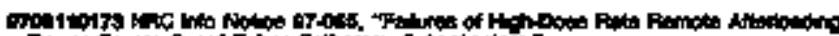

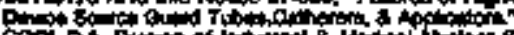

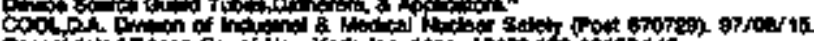

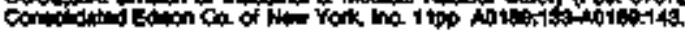

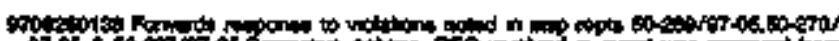

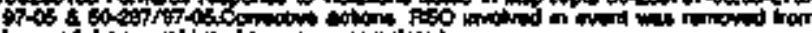

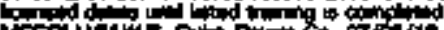

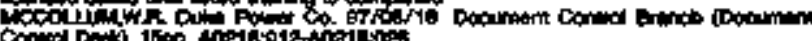

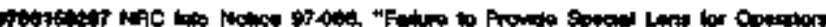

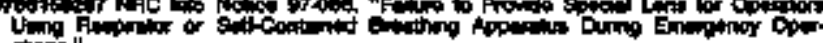

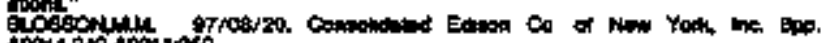

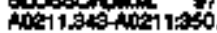

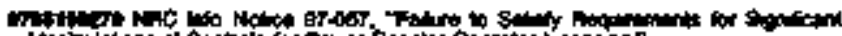

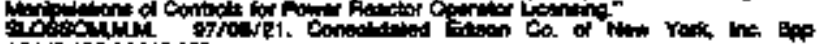

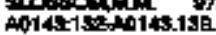

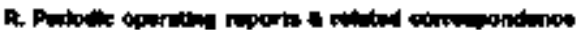

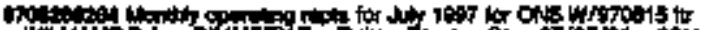

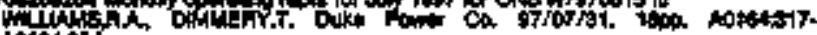
Noted 32

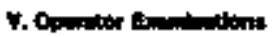

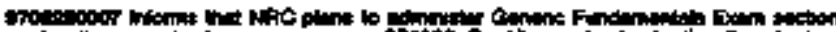

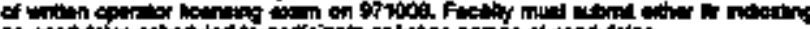

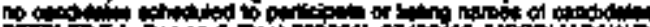

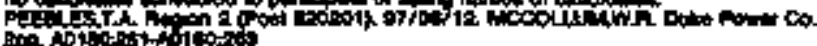

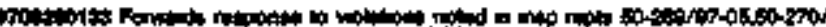

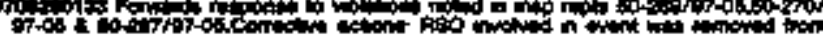
cenced che

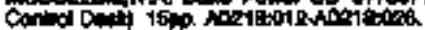

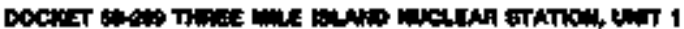

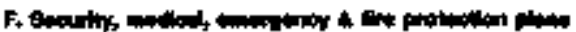

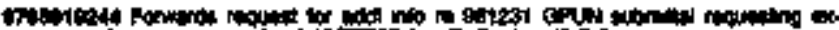

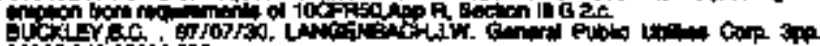
oxistion

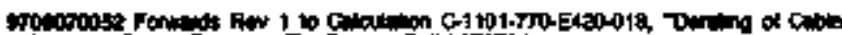

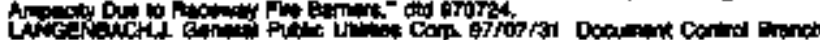

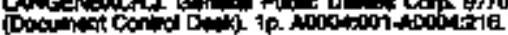

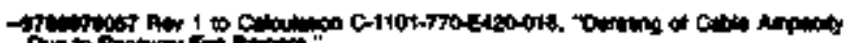

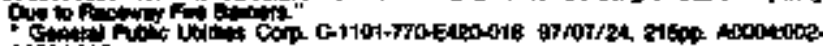
nototits

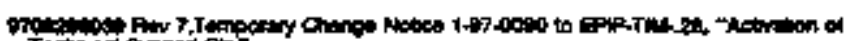

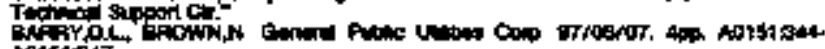
notsisut

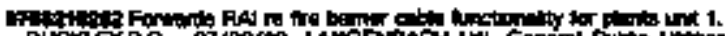

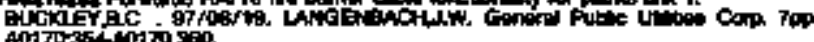

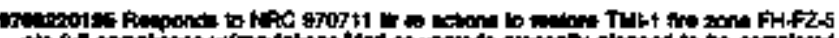

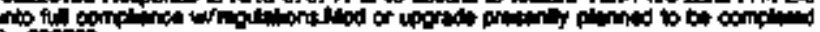
90000 .

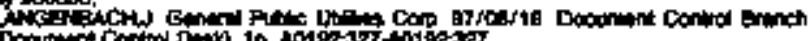

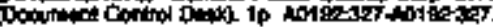

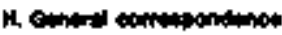

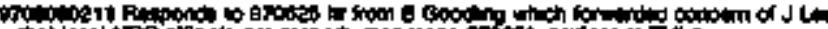

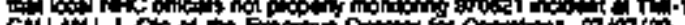

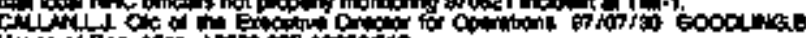

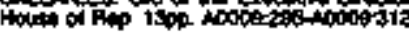

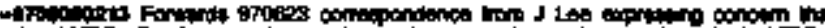

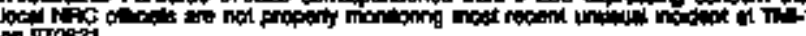

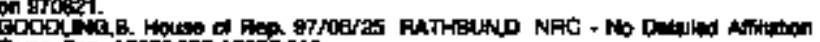

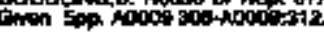

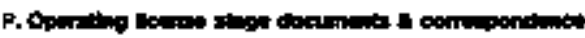

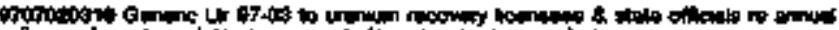

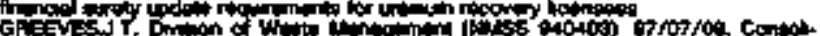

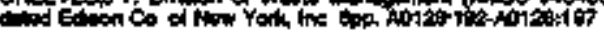

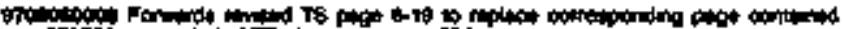

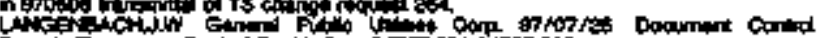

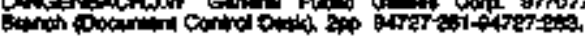

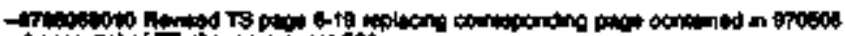

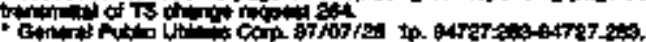

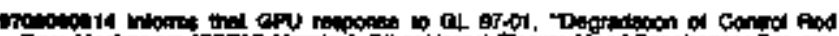

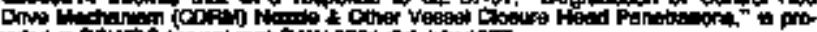

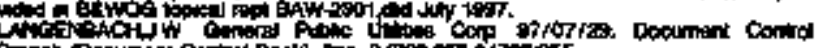

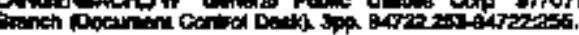

of

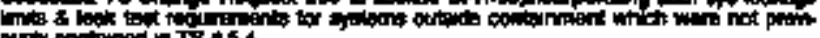
a:

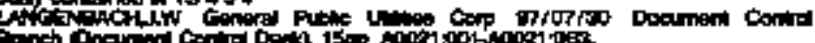

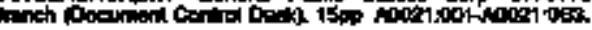

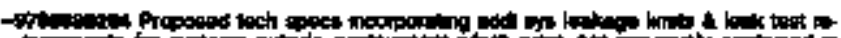
fon.

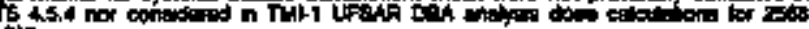
trite

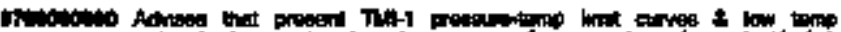

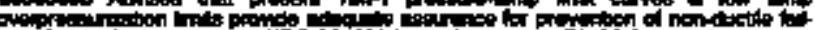

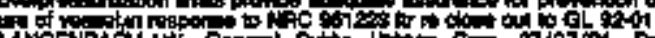

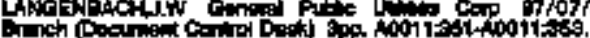

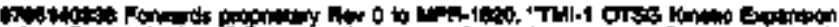

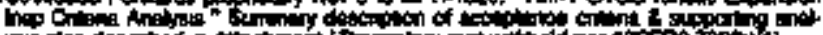

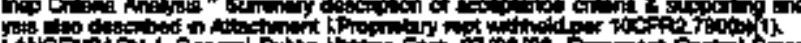

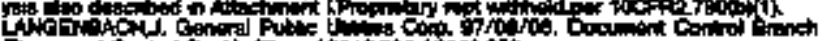

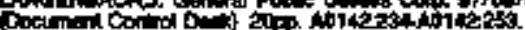

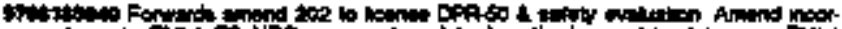

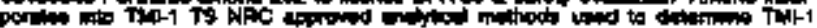

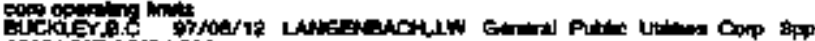

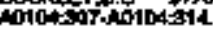

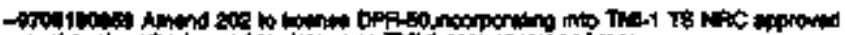
(1)

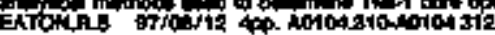

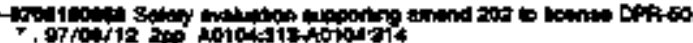

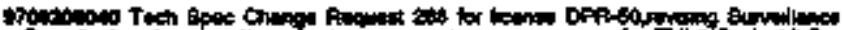

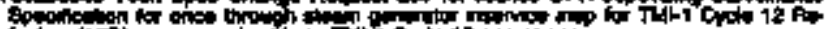

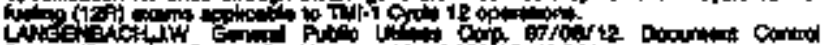

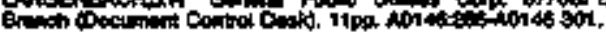

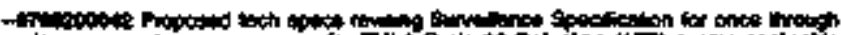

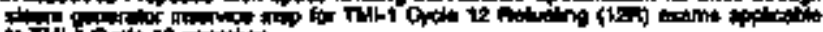

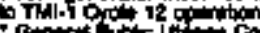

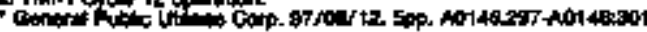

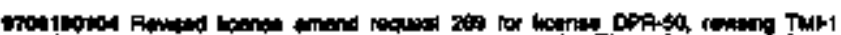

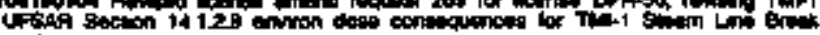

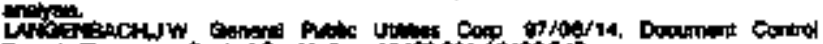

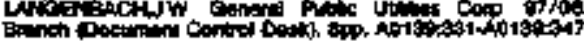

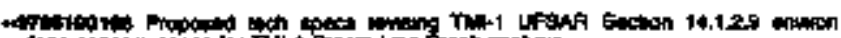

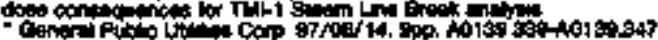

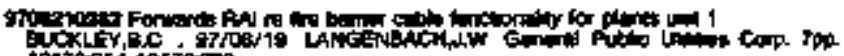

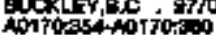




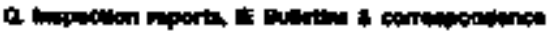

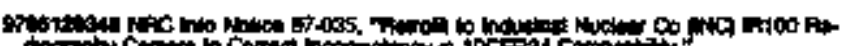

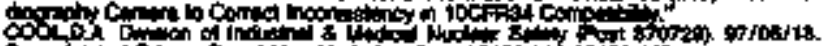

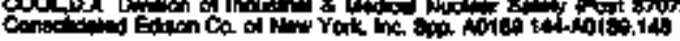

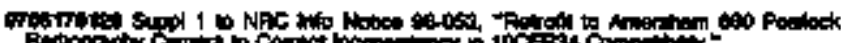

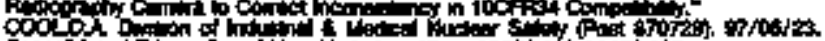

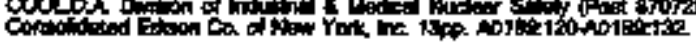

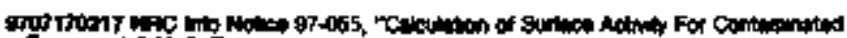

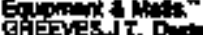

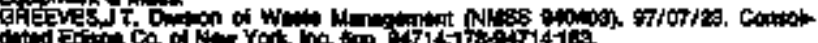

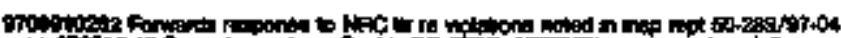
cht

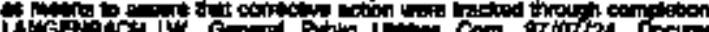

LWG

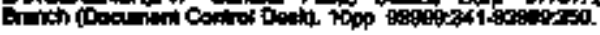

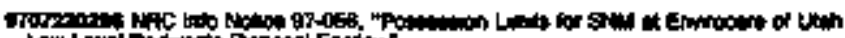

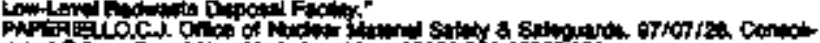

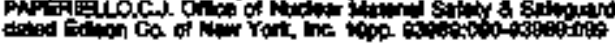

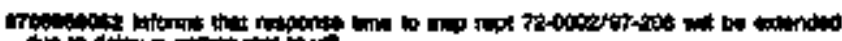

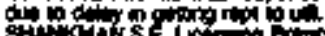

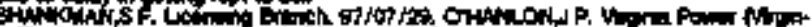

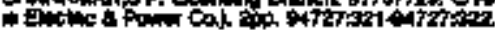

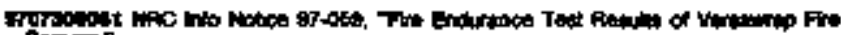

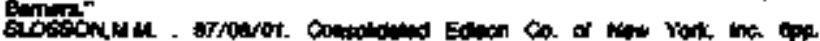

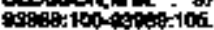

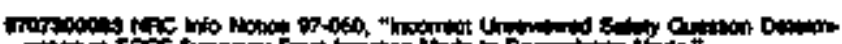

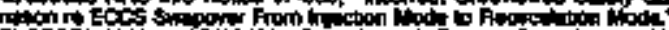

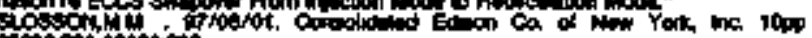

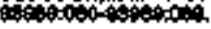

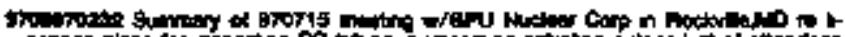

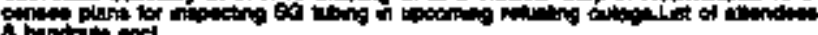

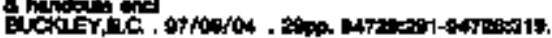

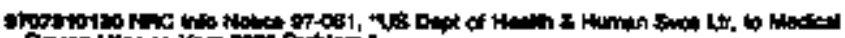

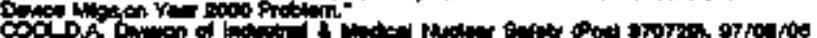

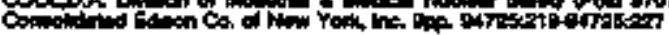

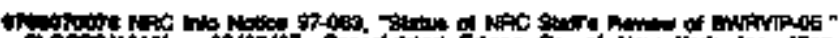

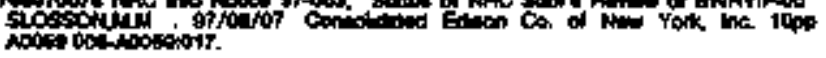

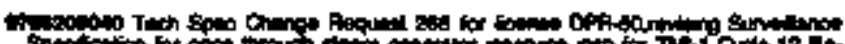

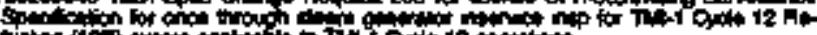

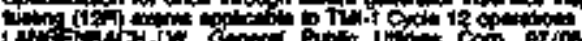

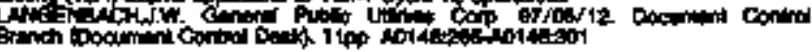

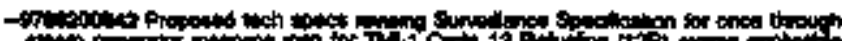
H.t.

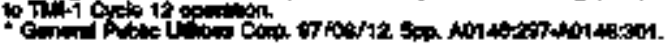

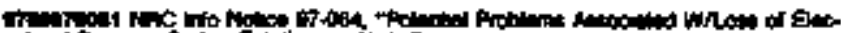

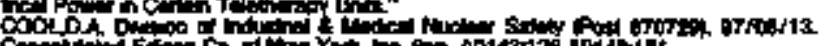

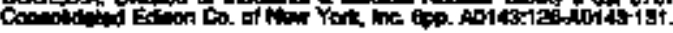

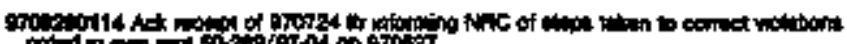

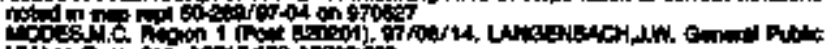

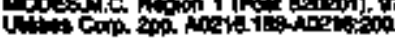

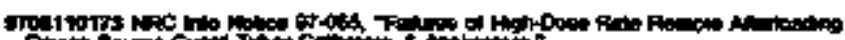

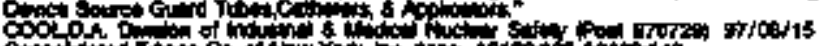

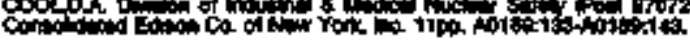

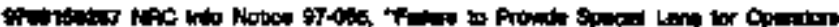

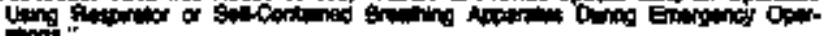

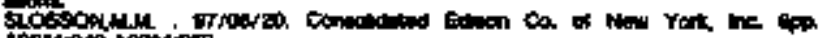

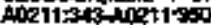

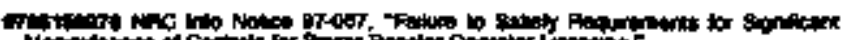

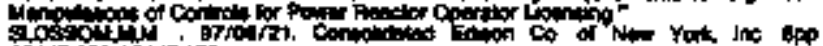
40 $45: 132-401431139$

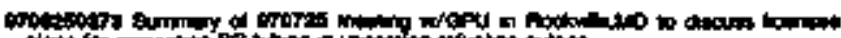

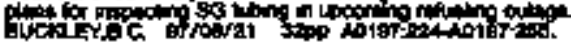

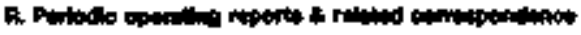

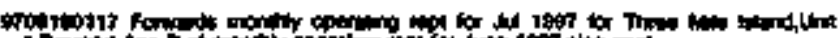

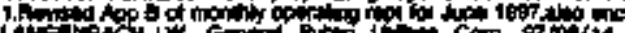

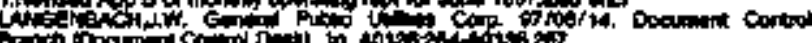

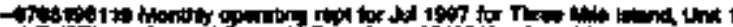

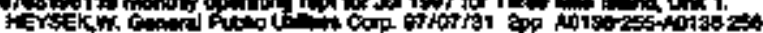

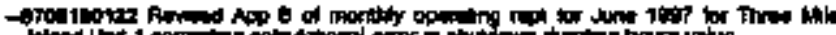

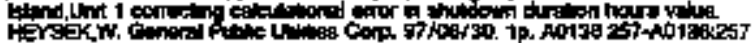

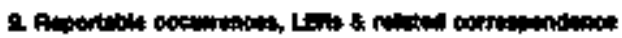

Fow

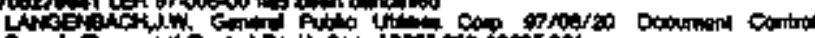

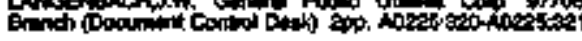

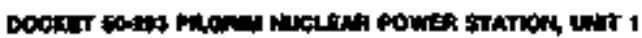

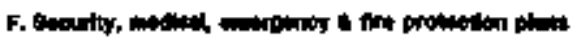

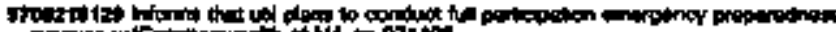

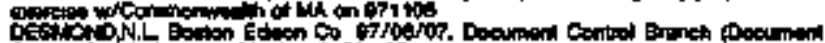

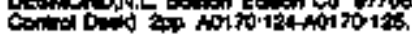

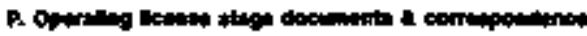

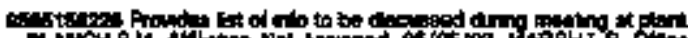

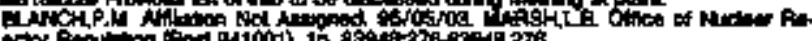

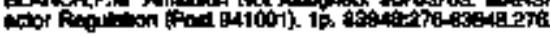

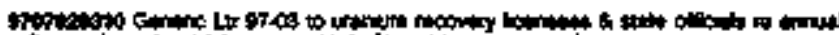

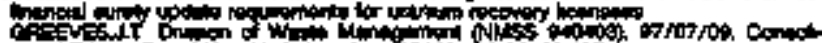

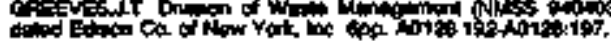

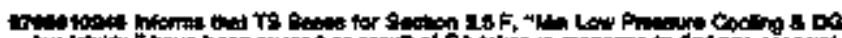

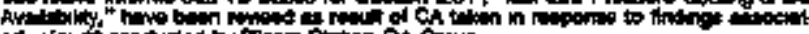

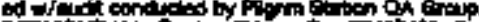

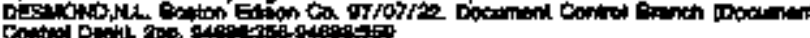

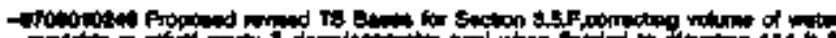

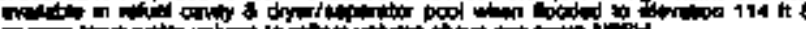

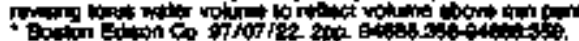

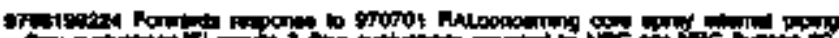

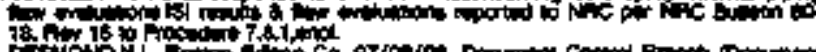

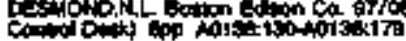

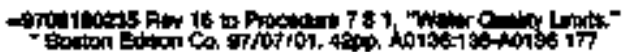

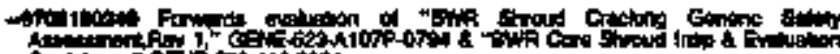

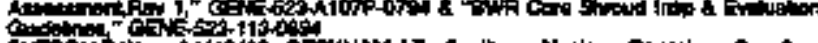

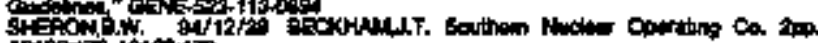
Anstion

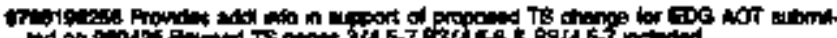

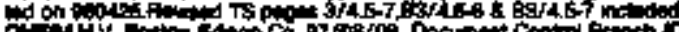

i

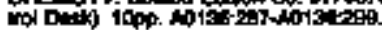

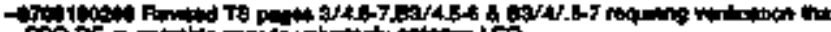

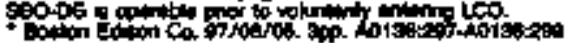

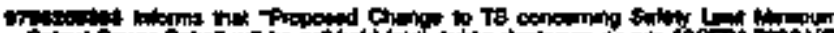

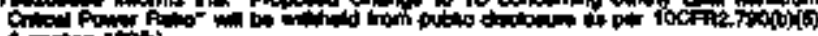

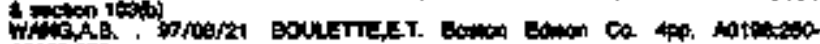
서연도.

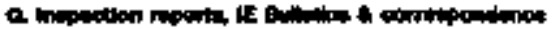

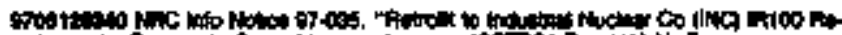

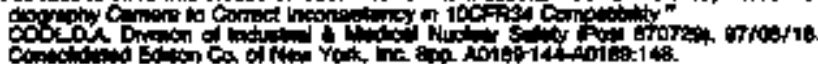

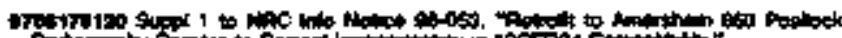

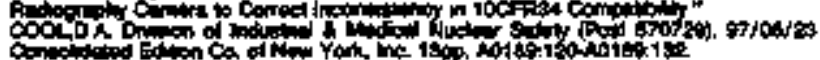

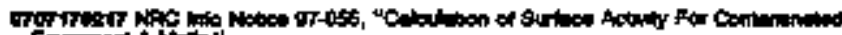

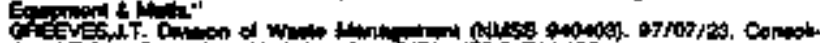

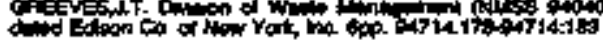

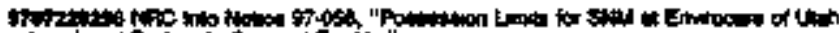

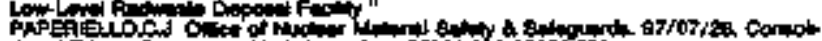

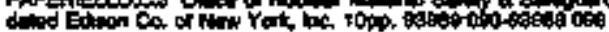

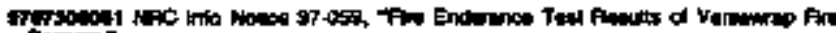

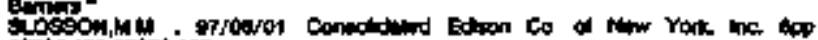
sion. 100 - 


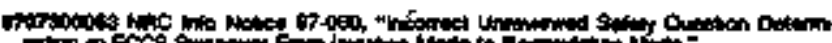

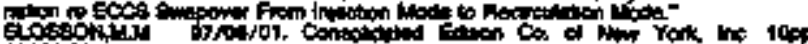

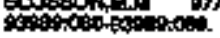

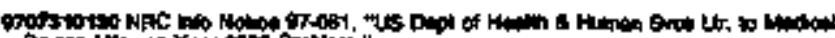

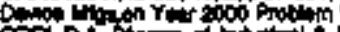

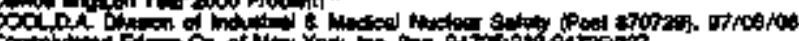

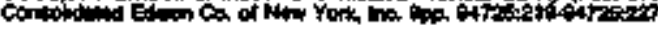

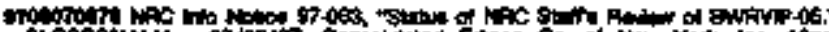

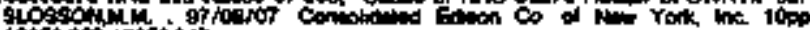

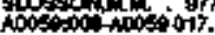

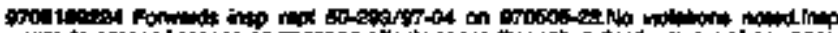

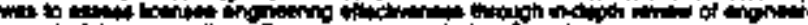

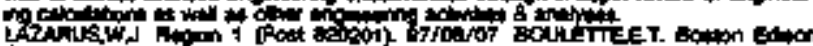

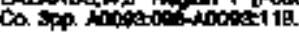

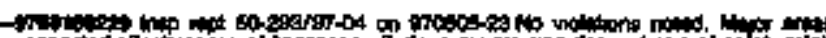

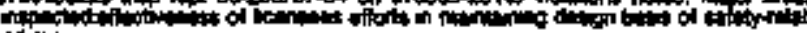

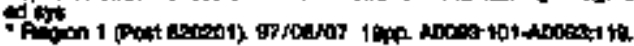

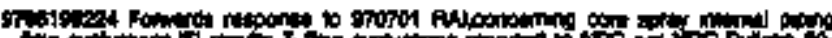

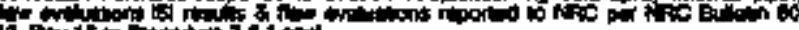

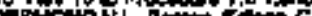

Oontat De

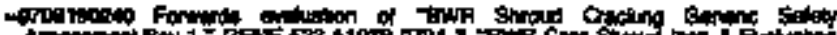

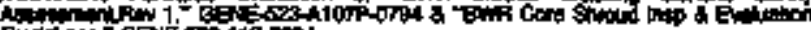

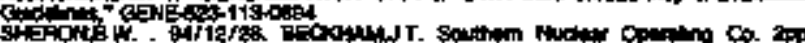
A

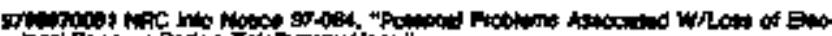

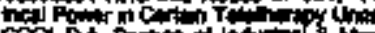

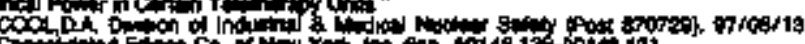

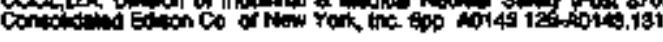

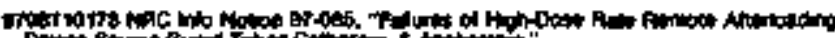
cool of

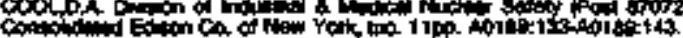

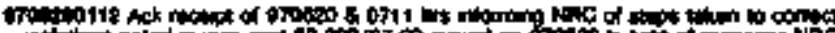

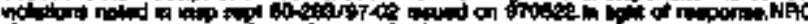

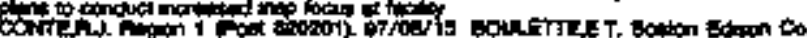

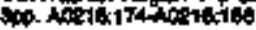

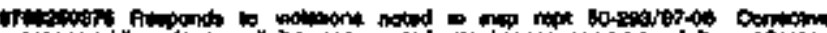

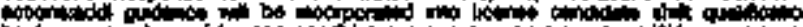

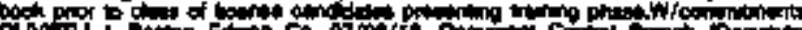

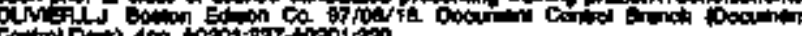

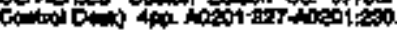

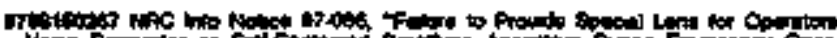

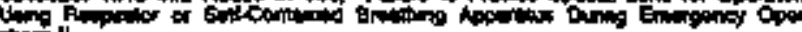

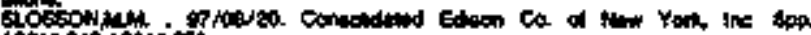

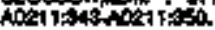

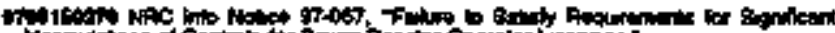

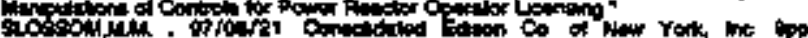
AN

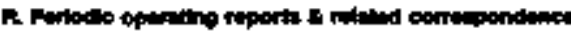

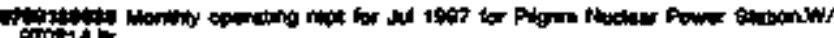

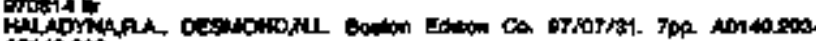
and $40+210$.

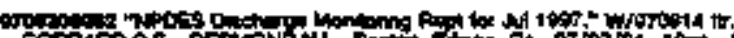

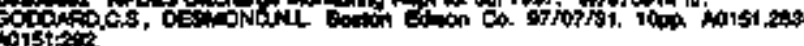

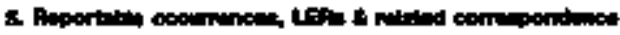

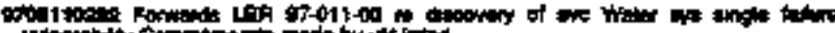

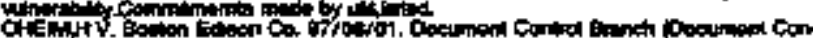

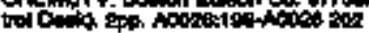

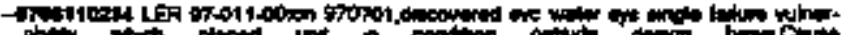

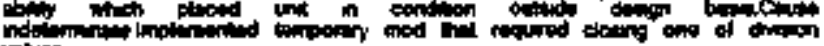

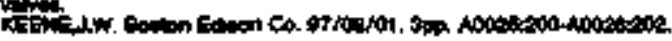

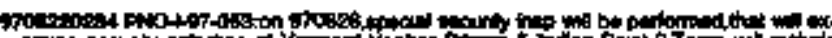

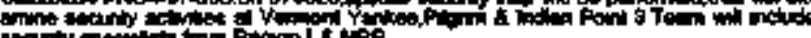

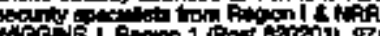

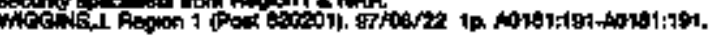

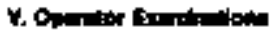

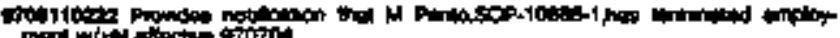

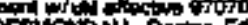

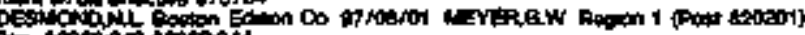
Th

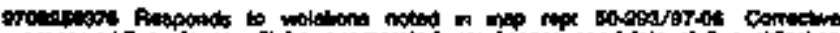

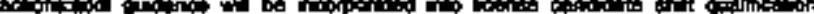

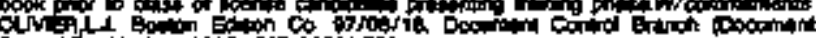

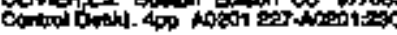

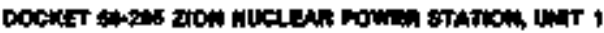

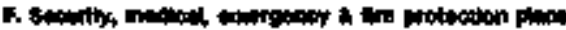

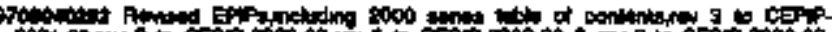

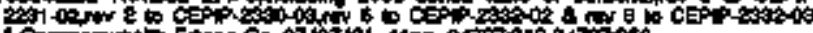

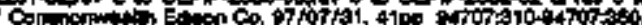

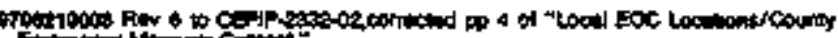

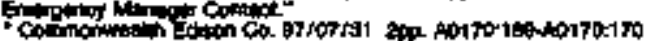

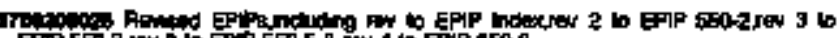

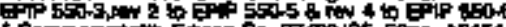

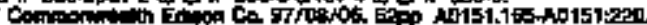

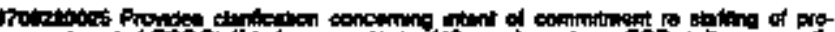

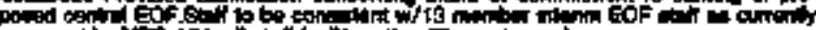

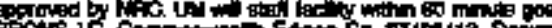

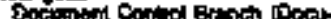

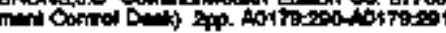

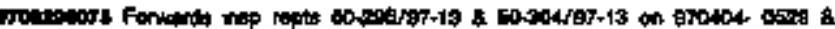

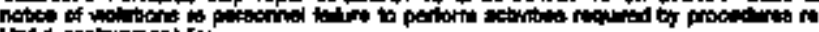
(1)

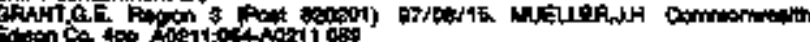

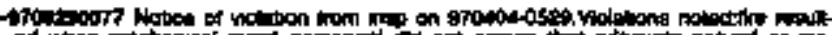

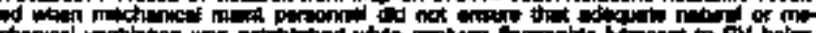

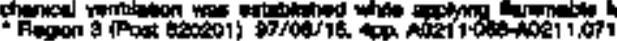

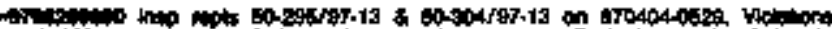

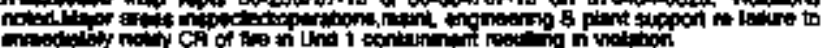

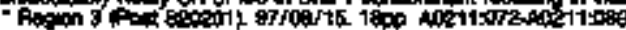

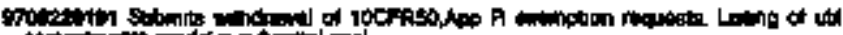

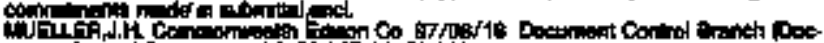

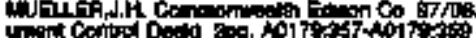

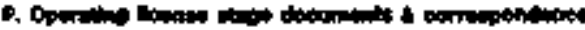

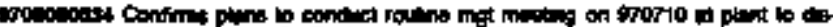

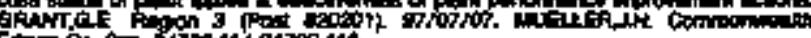

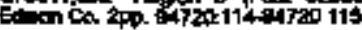

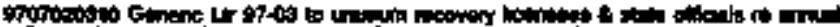

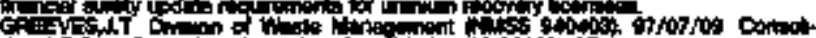

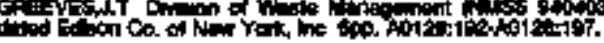

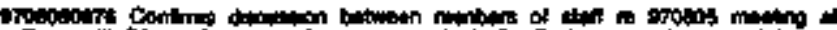

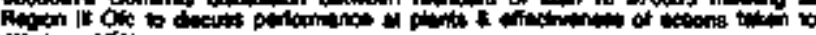

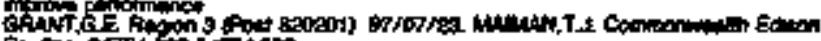

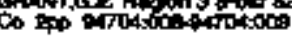

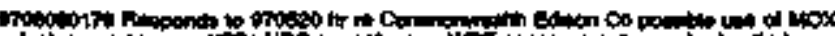

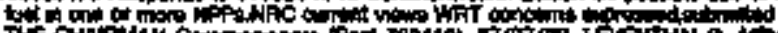

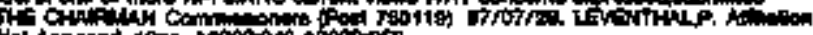

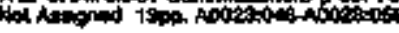

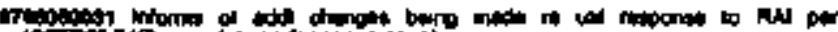

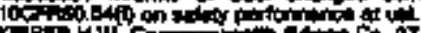

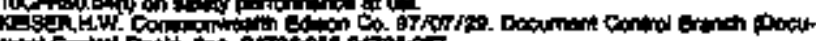

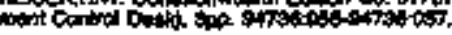

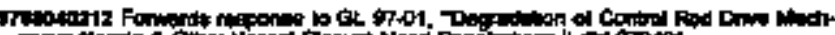

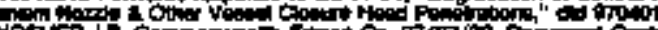

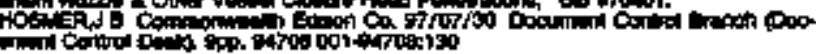

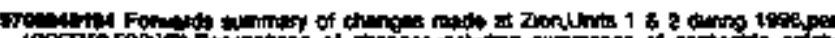

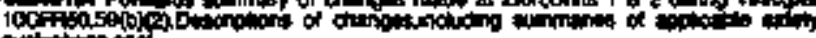

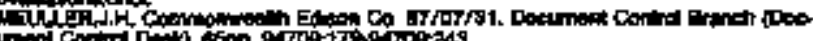

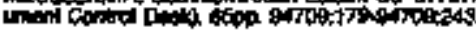

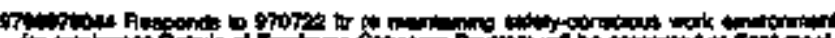

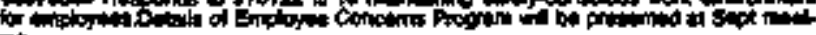

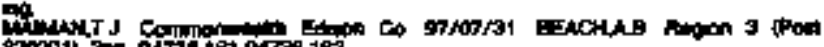

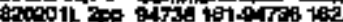

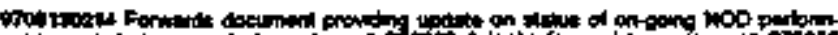

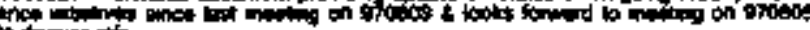

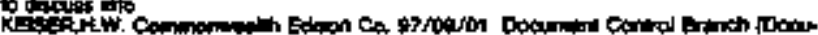

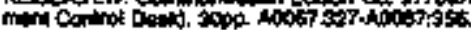




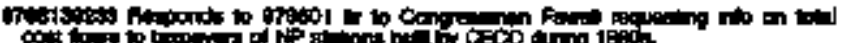

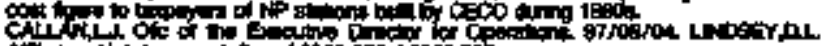

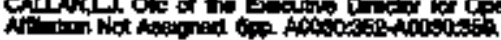

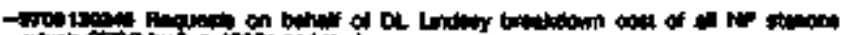

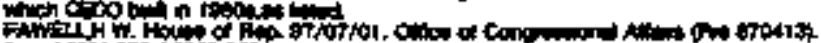

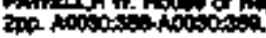

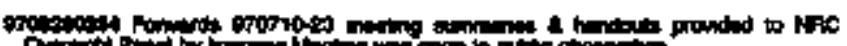

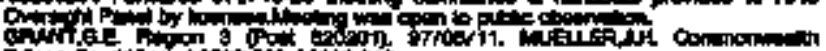

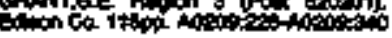

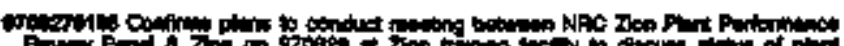

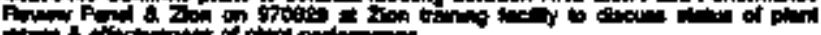
Coritat

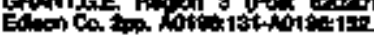

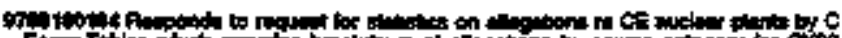

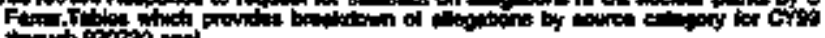

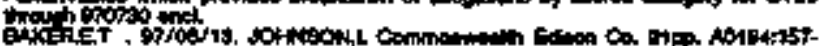
atsit7.

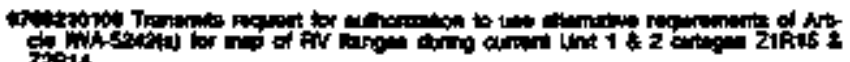

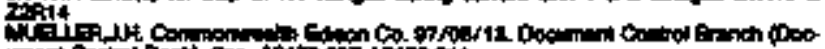

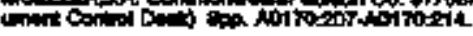

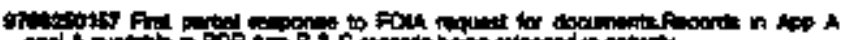

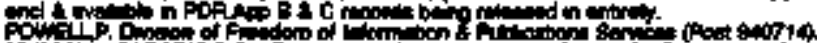

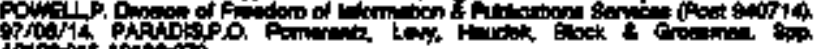

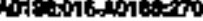

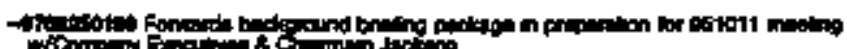

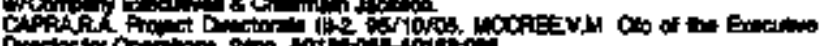

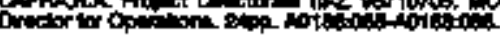

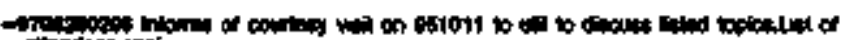

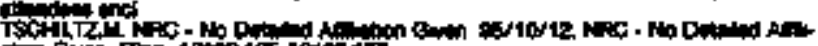

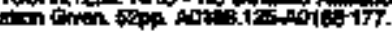

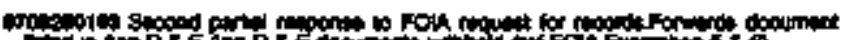

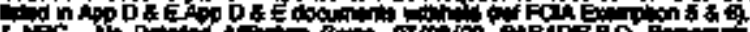

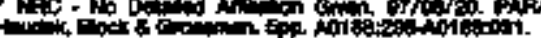

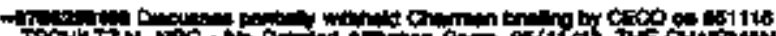
Foc

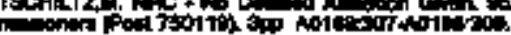

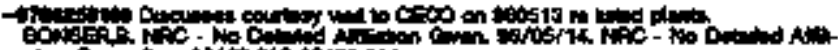

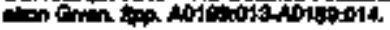

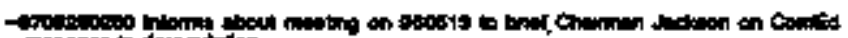

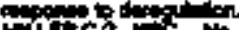

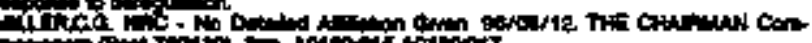

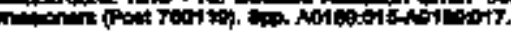

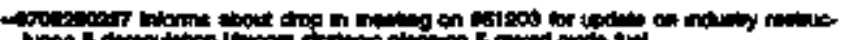

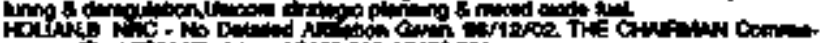

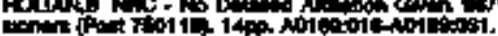

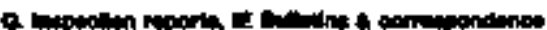

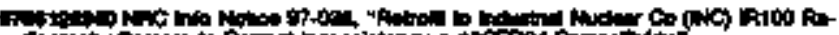

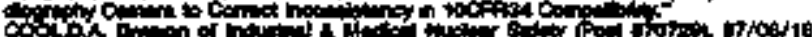

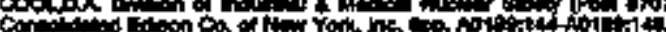

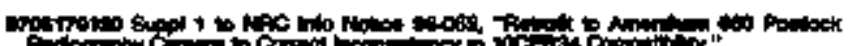

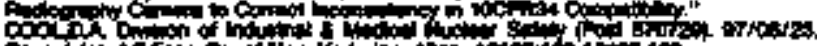

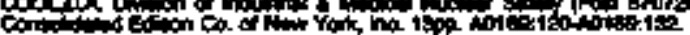

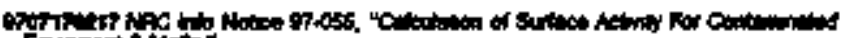

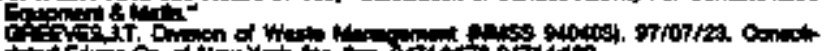

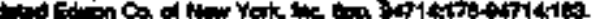

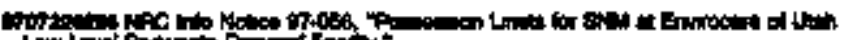
PA

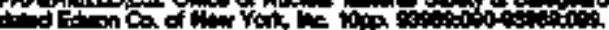

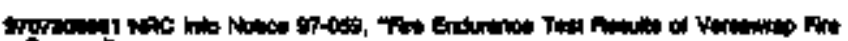

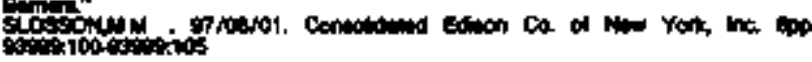

For

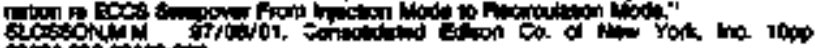
Wom

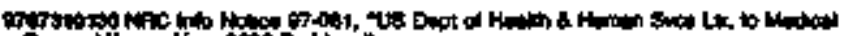

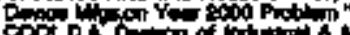

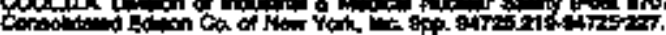

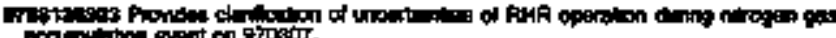

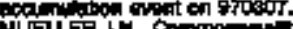

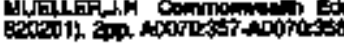

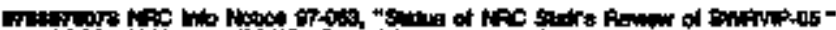

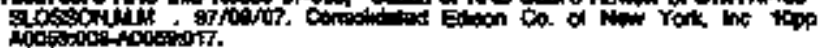

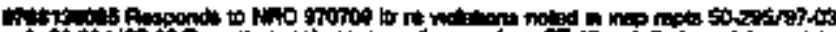

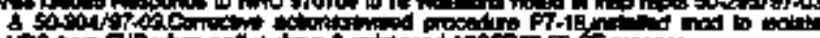

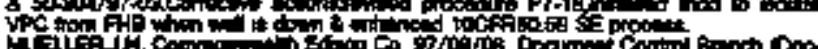

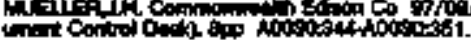

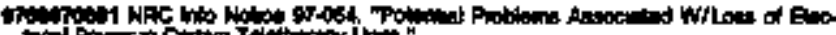

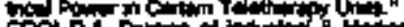

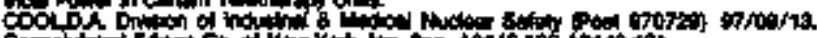

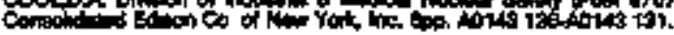

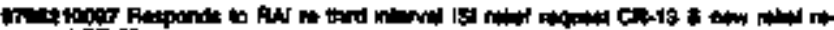

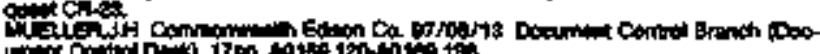
untion

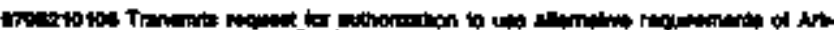

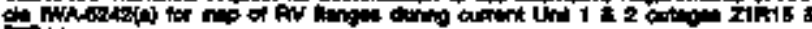

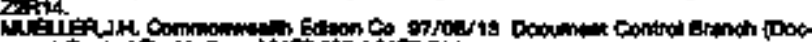

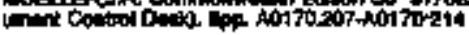

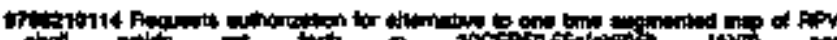

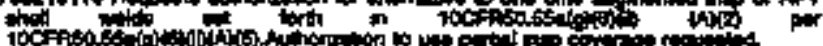

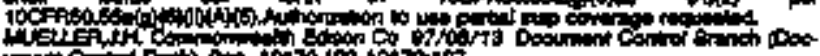

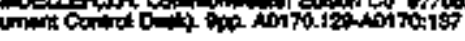

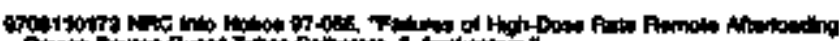

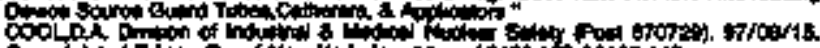

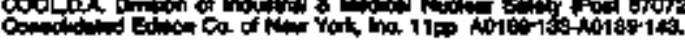

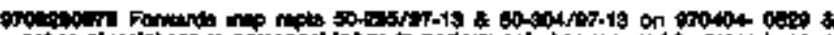

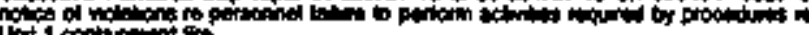

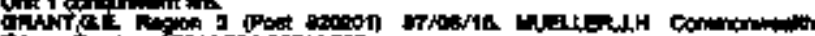

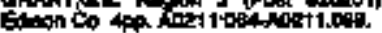

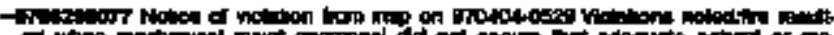

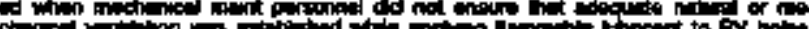

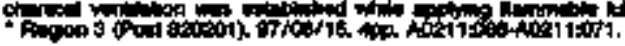

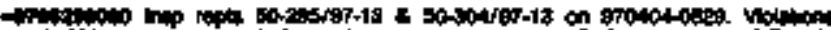

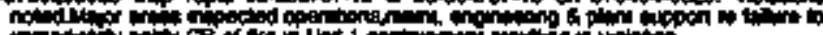

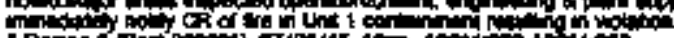

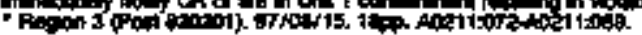

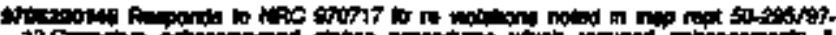

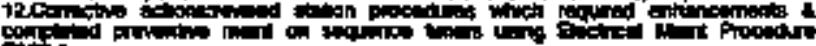
cotitis

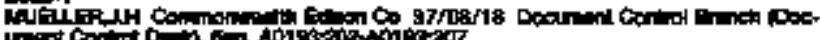

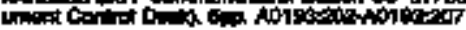

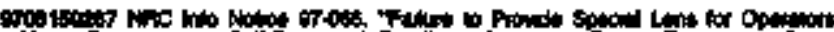

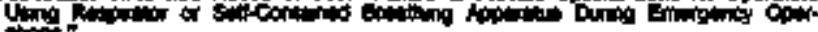

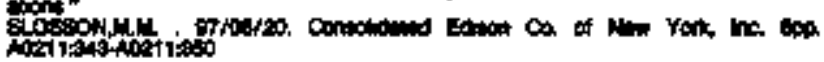

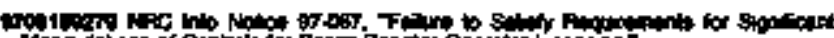

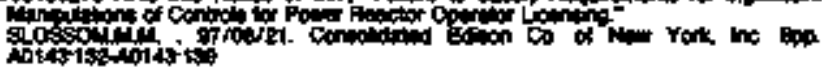

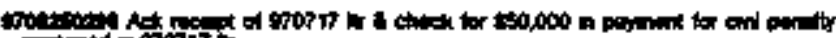

Hof

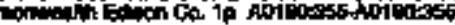

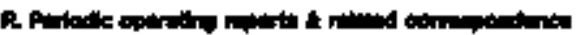

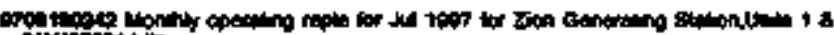

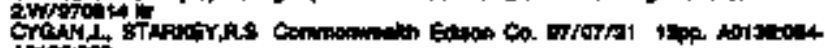
aiston.

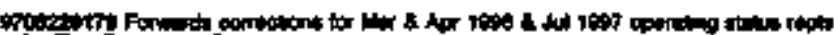

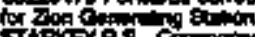

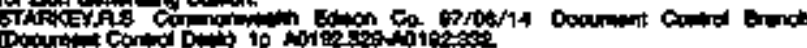

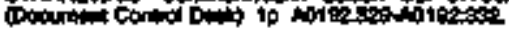

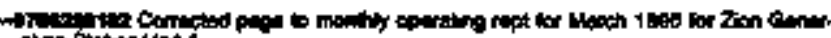

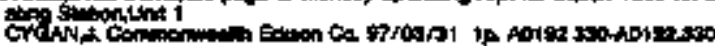

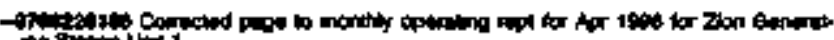

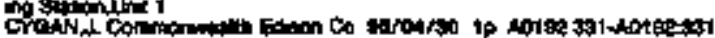

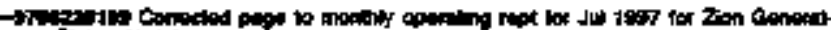
CP 


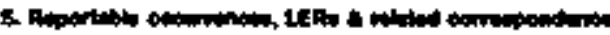

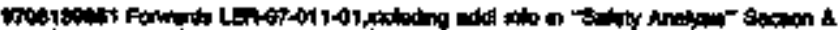

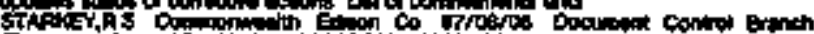

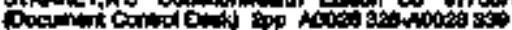

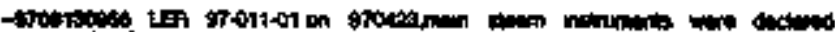

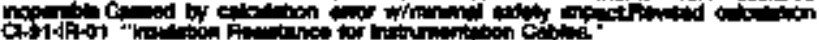

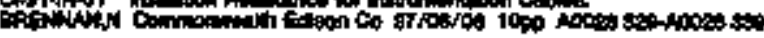

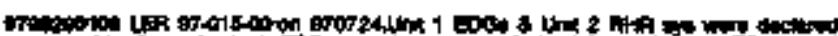

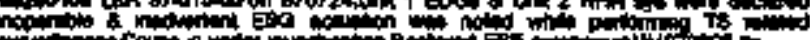

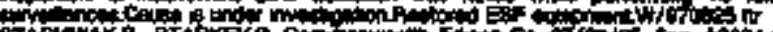

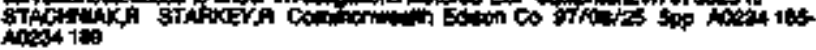

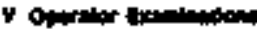

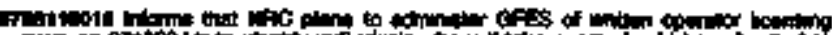

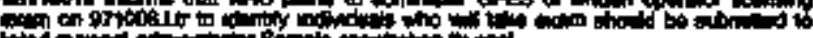

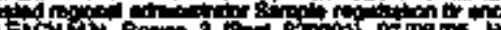

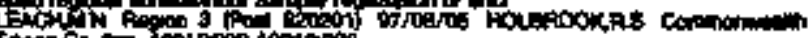

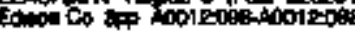

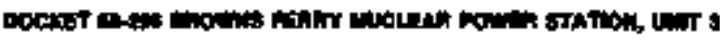

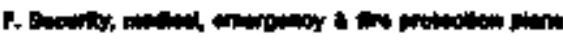

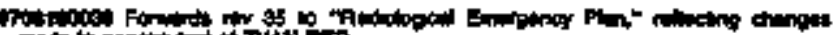

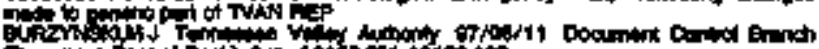

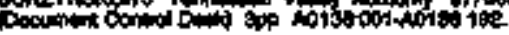

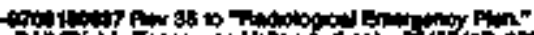

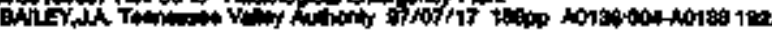

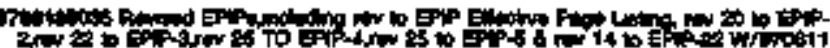

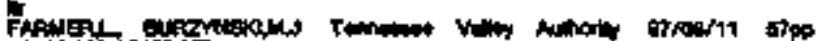
Aortsotion

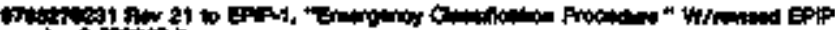

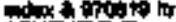

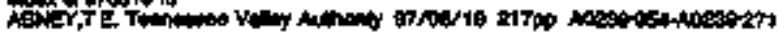

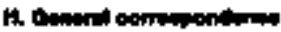

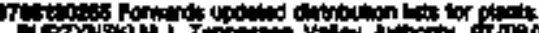

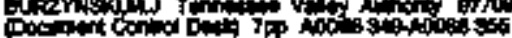

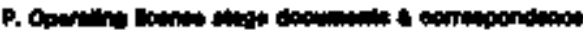

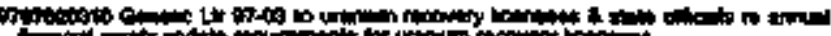
che

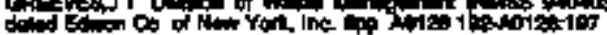

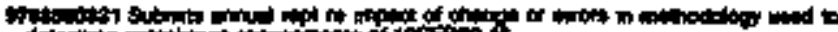

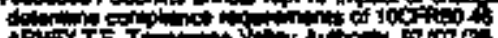

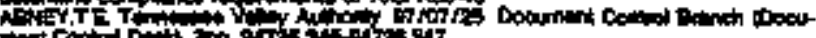

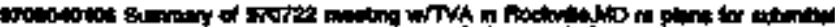

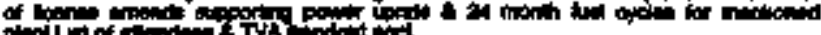

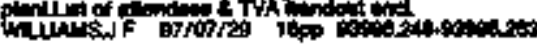

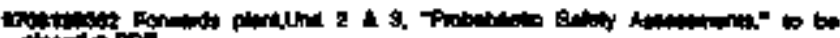

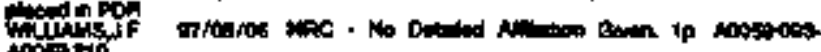
100 क्ष

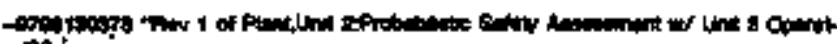

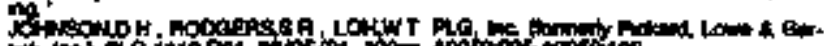

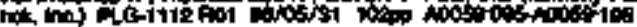

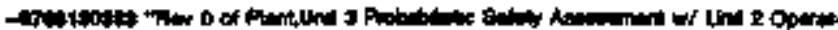

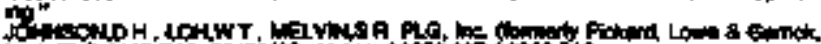

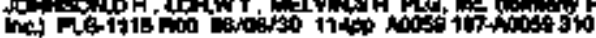

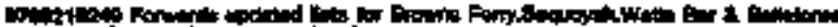

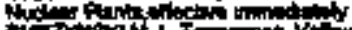

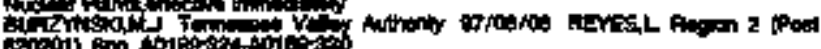

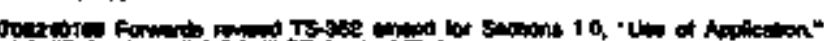
in

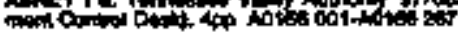

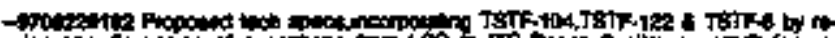

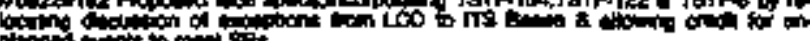

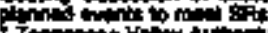

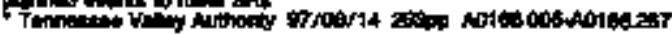

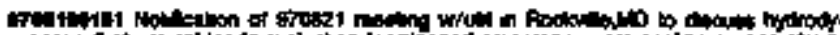

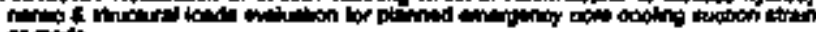

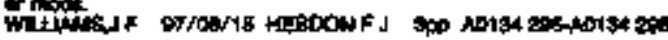

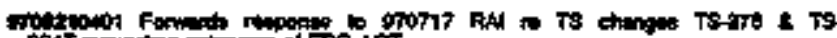

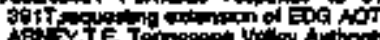

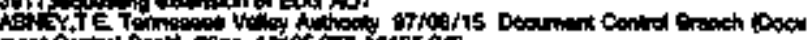

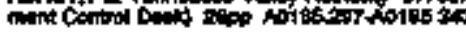

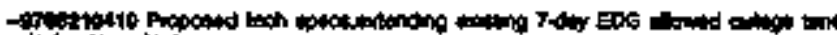

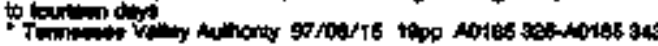

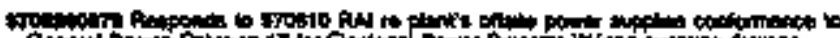

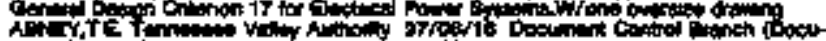

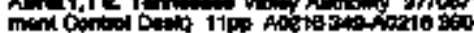

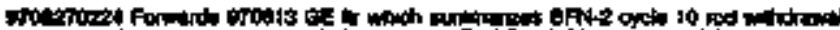

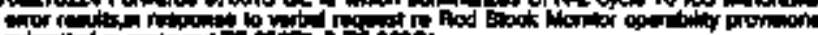

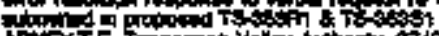

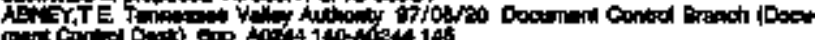

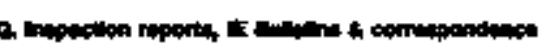

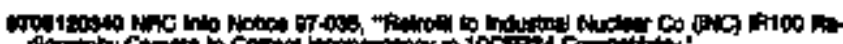

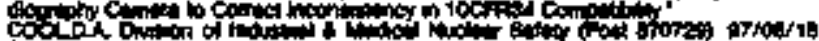

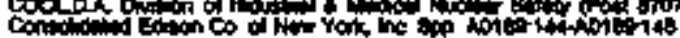

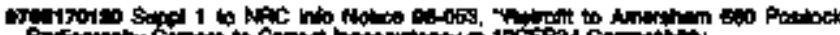

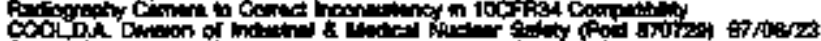

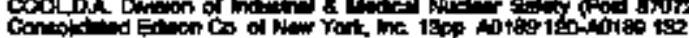

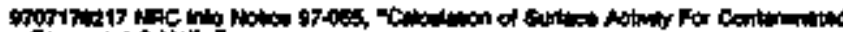

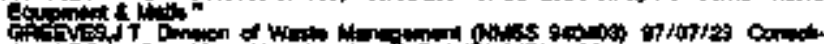

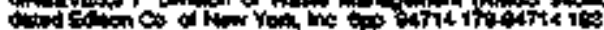

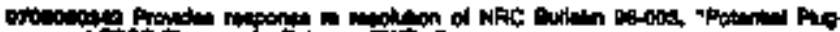

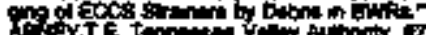

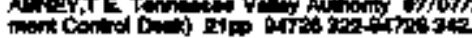

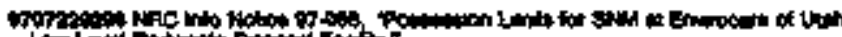
Pain

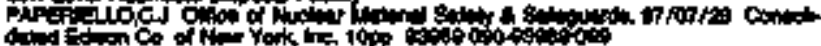

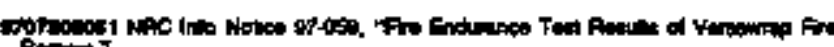

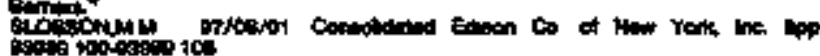

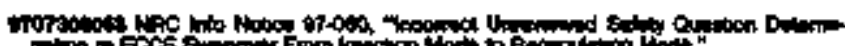

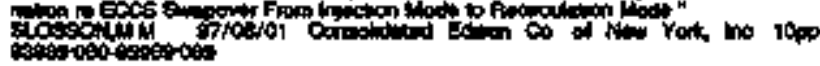

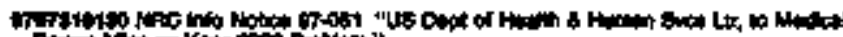

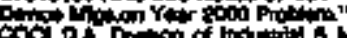

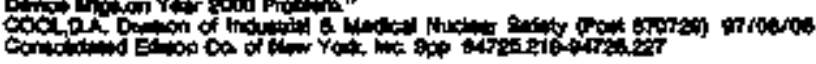

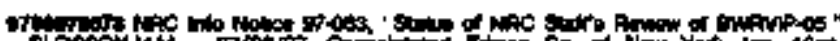

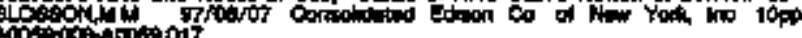

Now

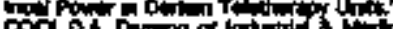

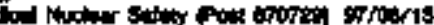

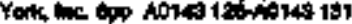

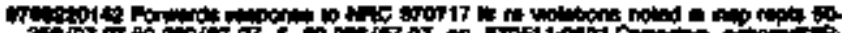
2..

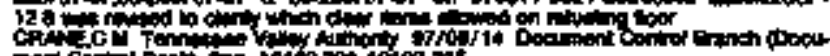

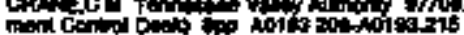

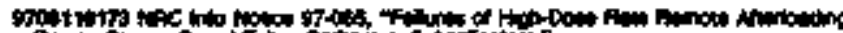

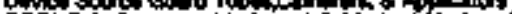

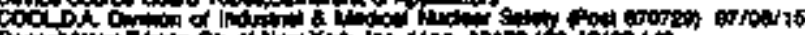

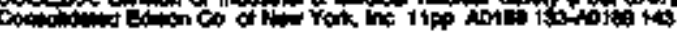

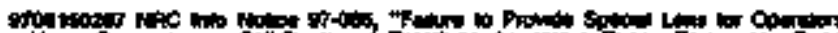

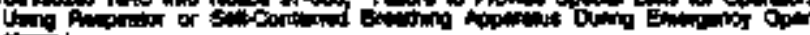

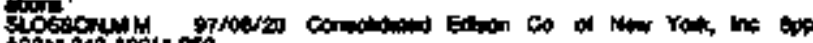

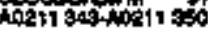

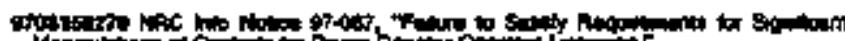
Fos

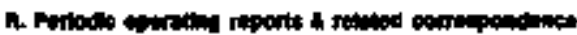

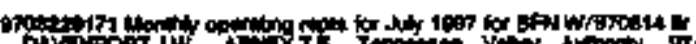
nichorit 


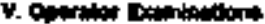

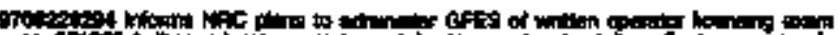

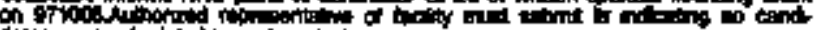

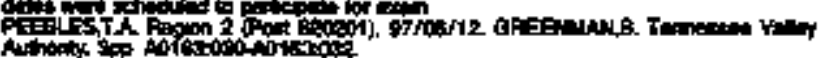

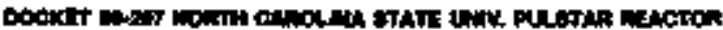

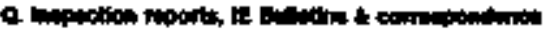

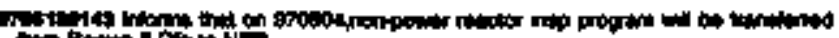

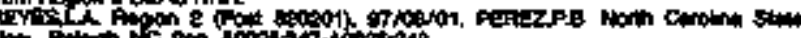

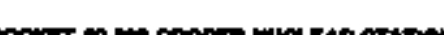

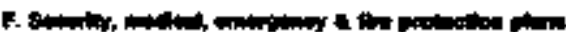

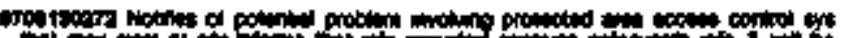

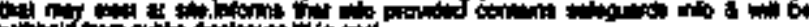

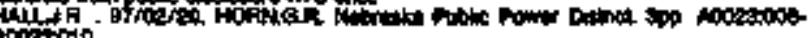

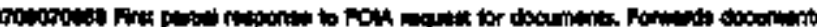

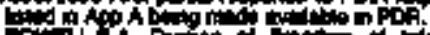

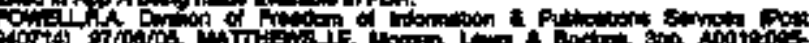
(1)

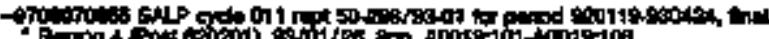

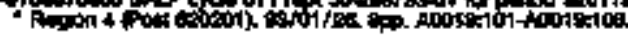

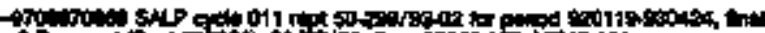

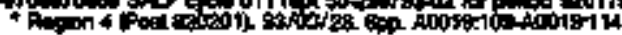

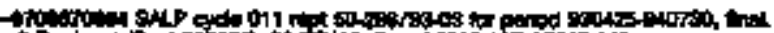

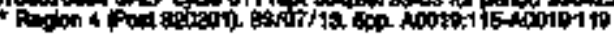

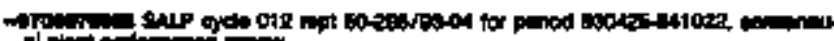

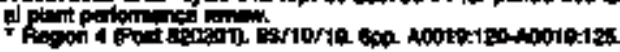

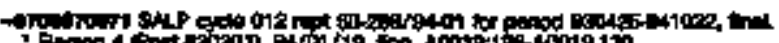

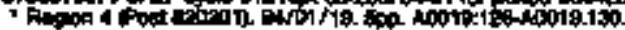

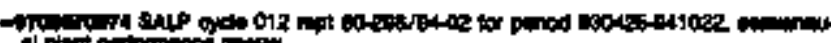

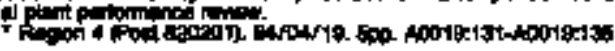

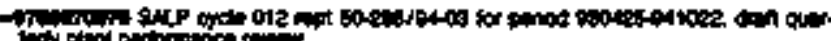

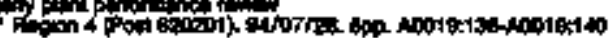

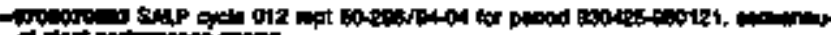

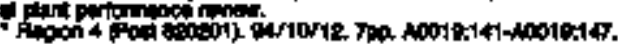

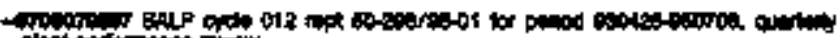

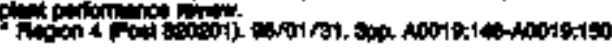

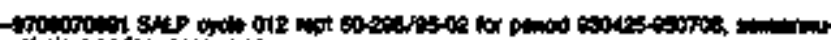

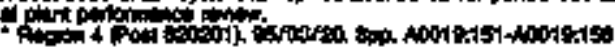

-

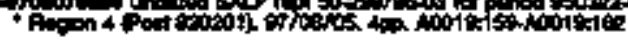

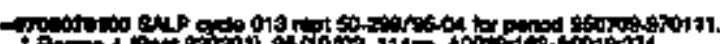

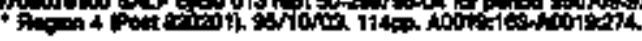

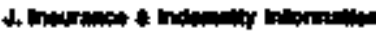

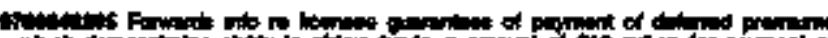

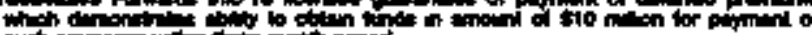

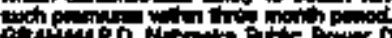

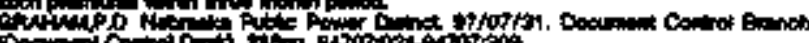

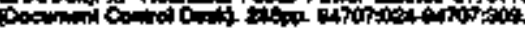

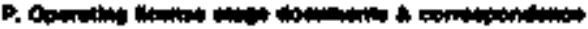

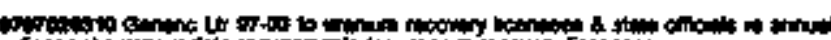

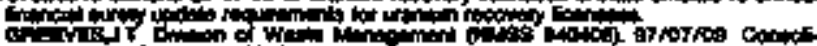

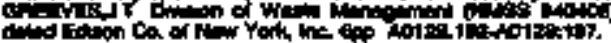

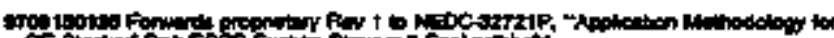

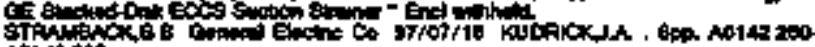

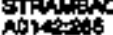

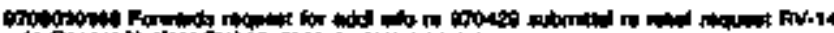

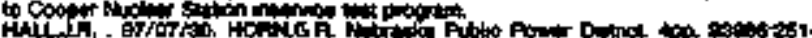
intis

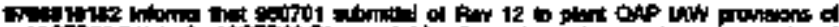
hochas

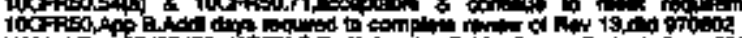

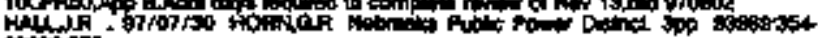
istimatis

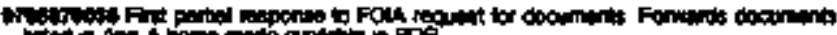

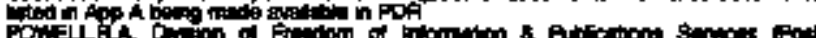

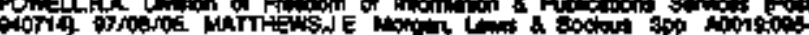
atosiat

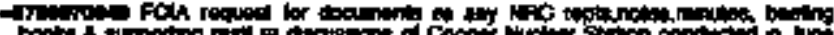

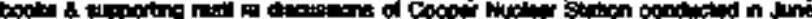

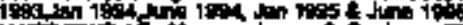

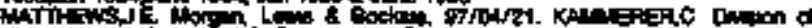

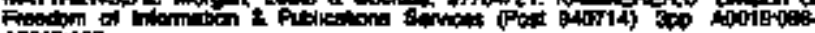
Anotitiod

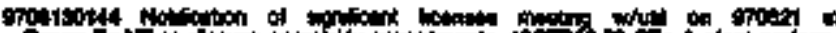

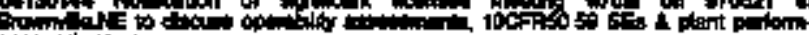

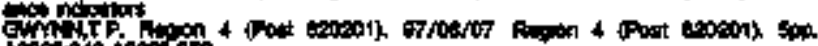
Alochations

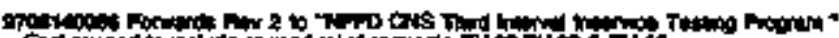

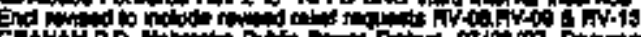

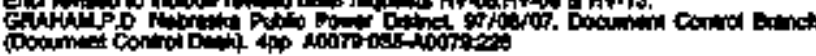

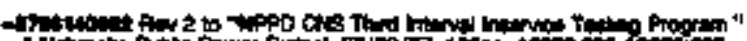

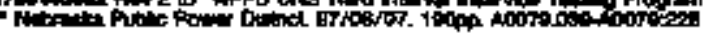

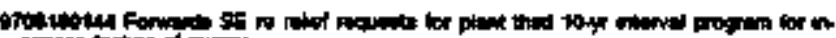

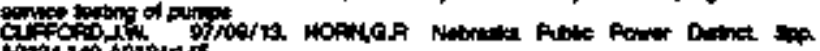

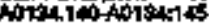

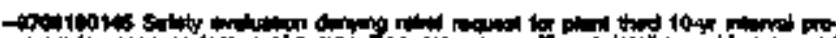
管

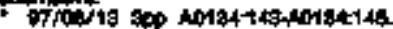

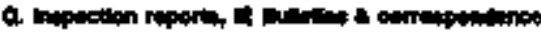

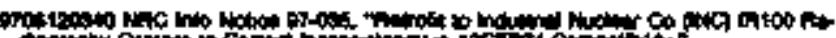
Wo

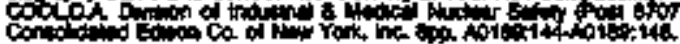

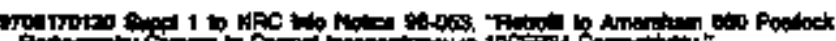
Condy

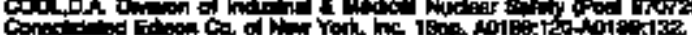

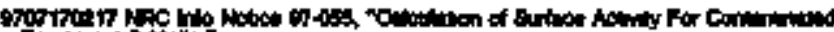
cyly

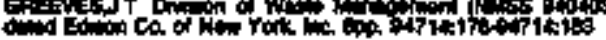

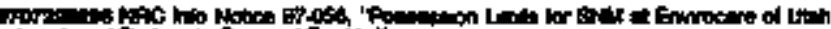

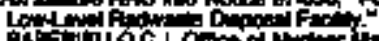

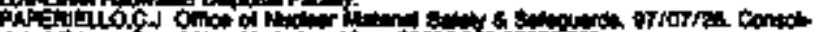

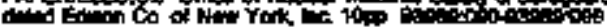

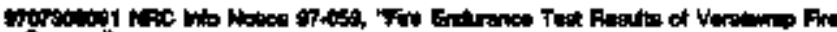

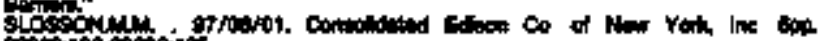

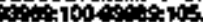

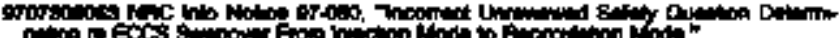

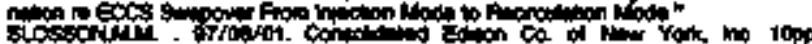

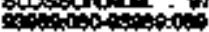

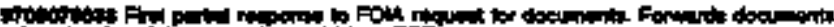

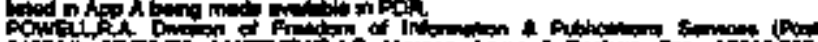

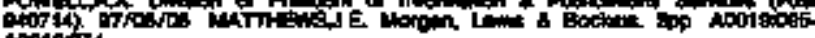
mation

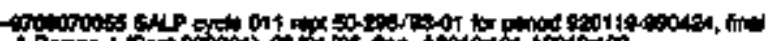

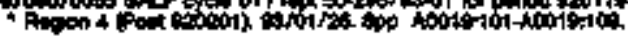

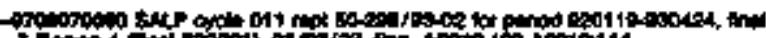

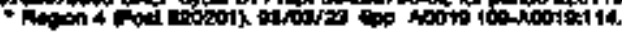

-4

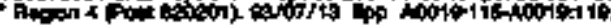

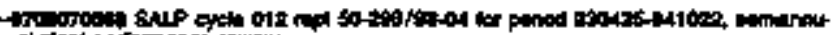

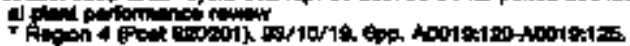

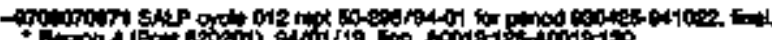

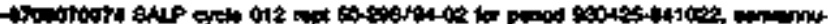

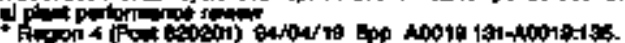

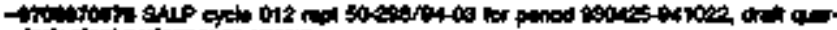

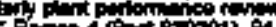

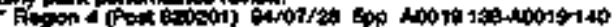




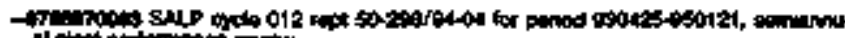

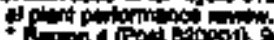

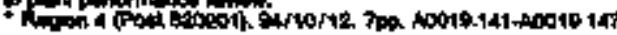

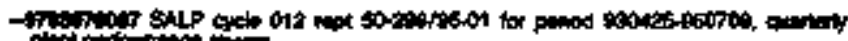

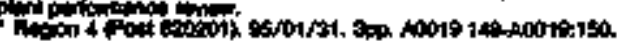

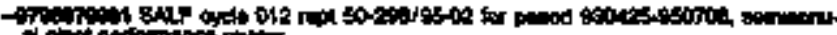

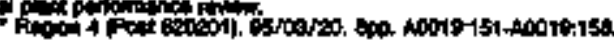

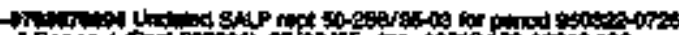

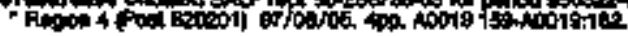

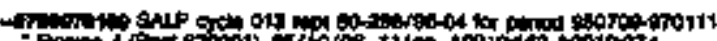

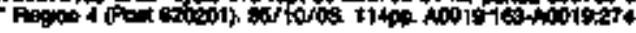

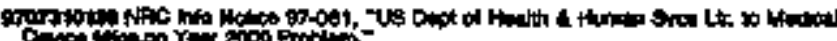

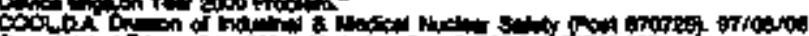

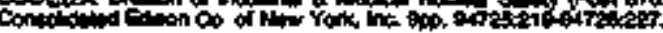

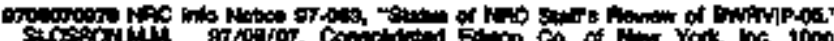

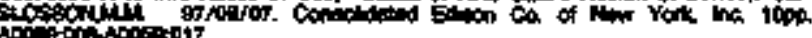

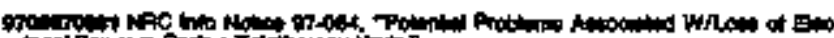

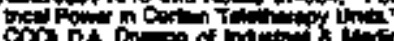

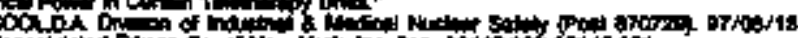

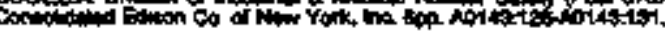

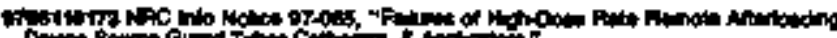

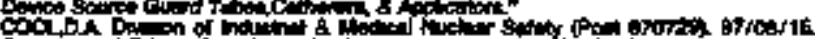

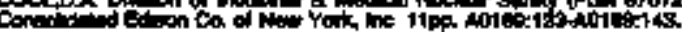

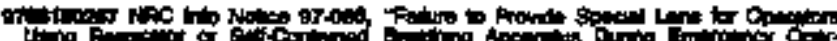

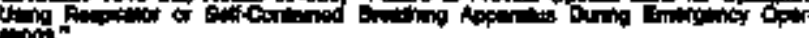

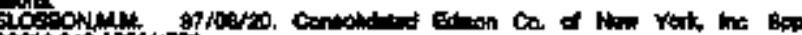

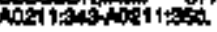

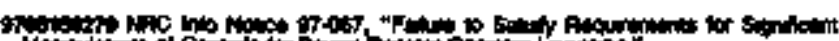

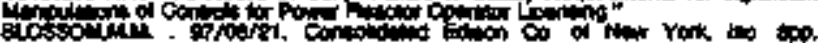

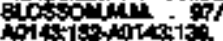

of

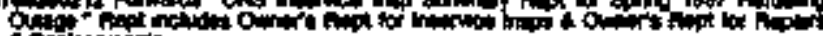

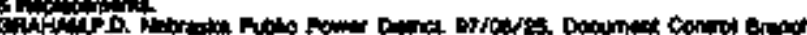

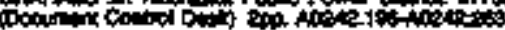

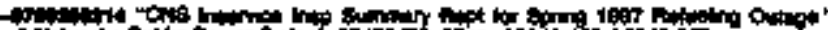

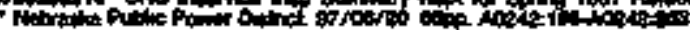

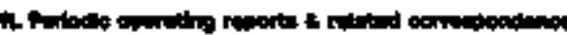

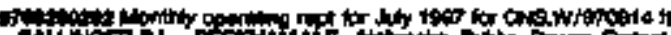

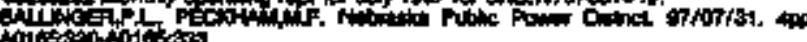

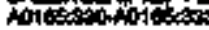

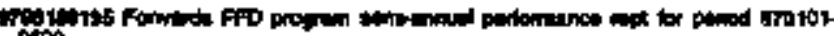

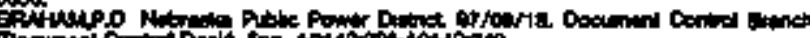

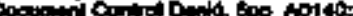

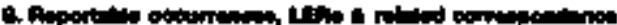

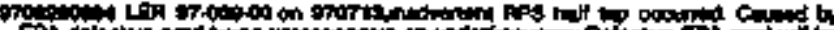

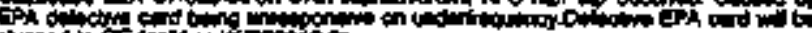

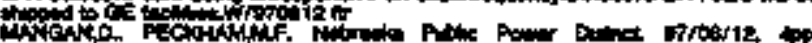

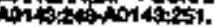

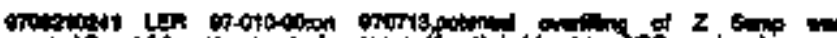

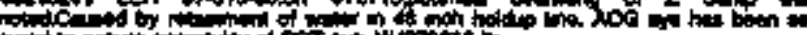
fot

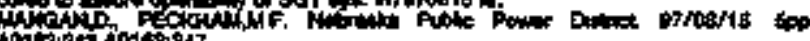

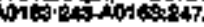

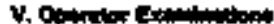

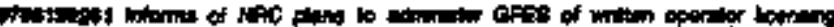

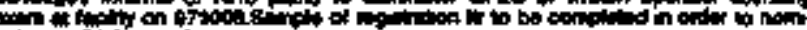
(a)

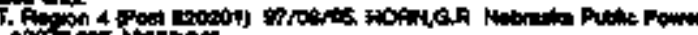

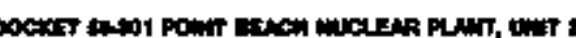

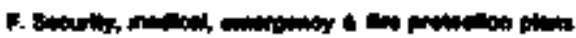

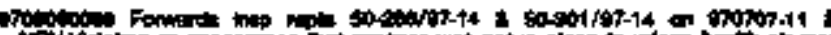

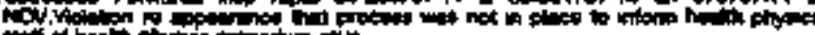

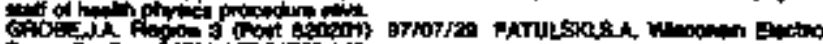

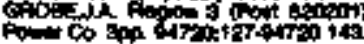

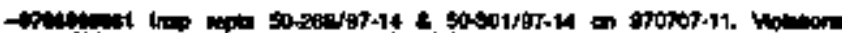

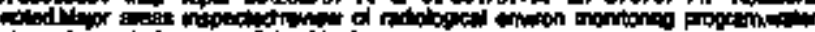

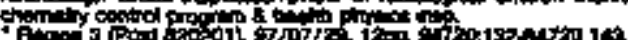

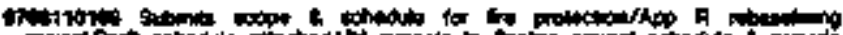

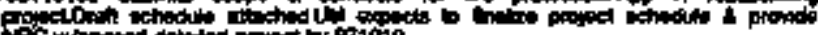

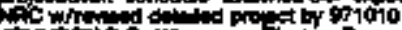

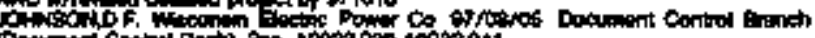

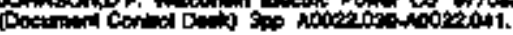

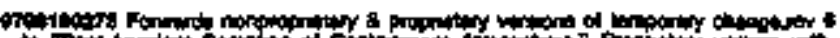

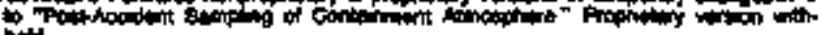

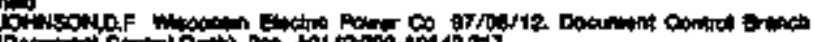

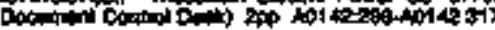

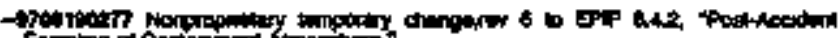

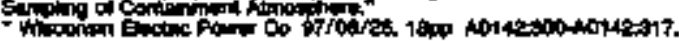

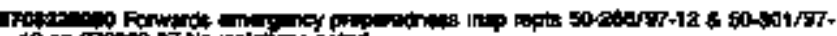

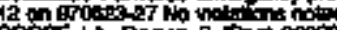

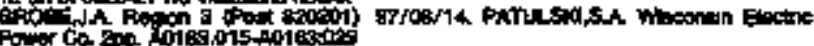

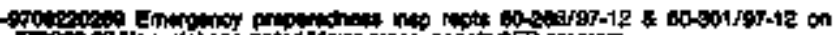

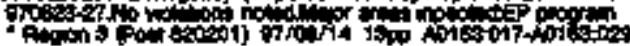

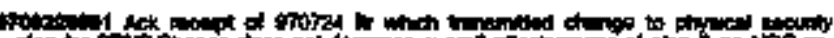

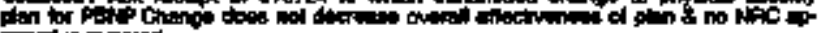

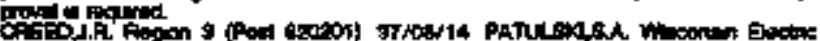

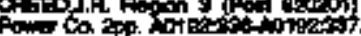

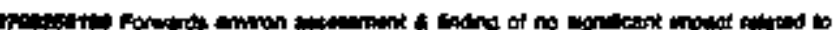

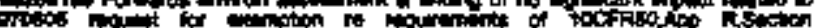

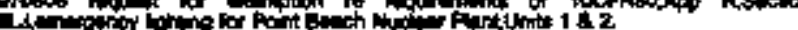

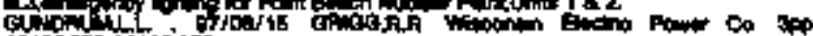

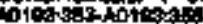

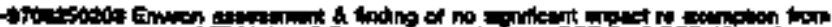

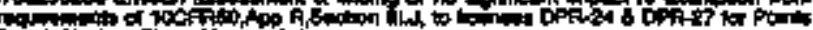
The

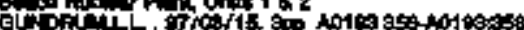

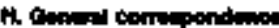

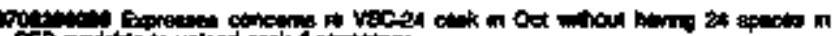

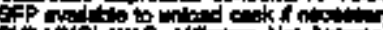

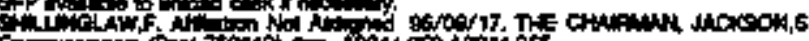

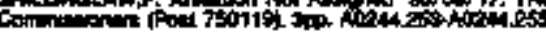

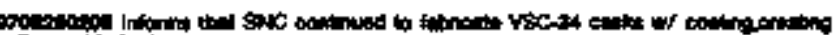

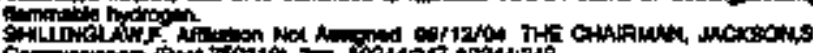

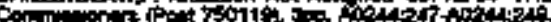

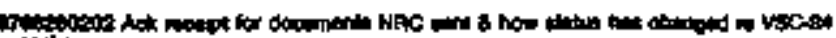

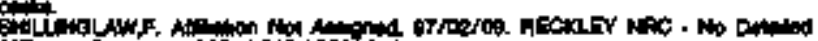

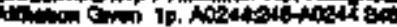

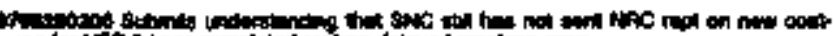

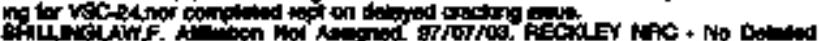

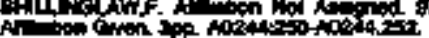

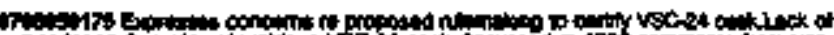

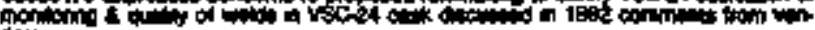

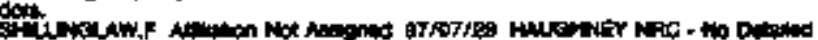

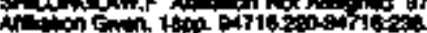

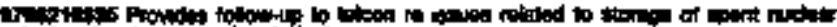

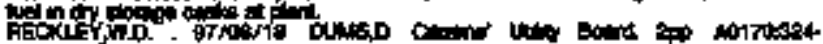
Apisus:

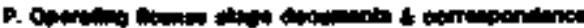

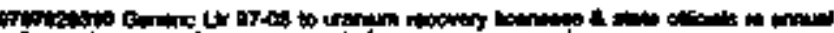

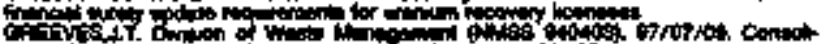

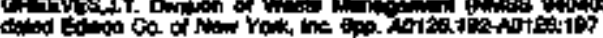

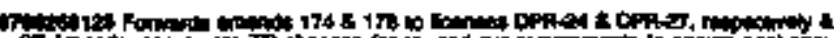

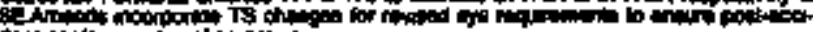

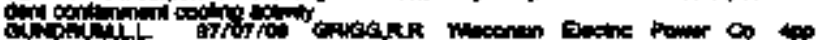

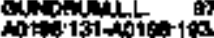

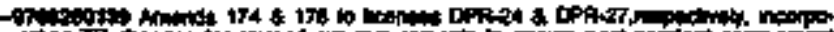

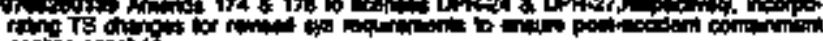

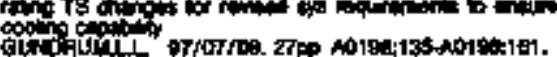

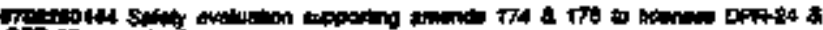

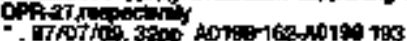

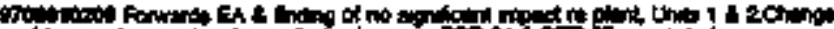

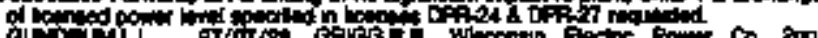

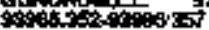




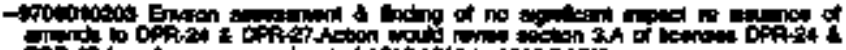

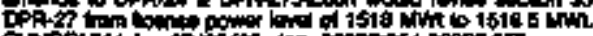

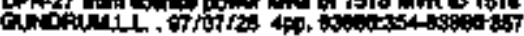

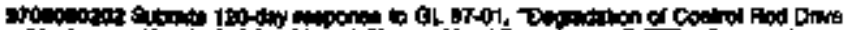

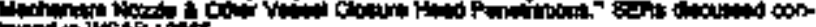
ined in wation.

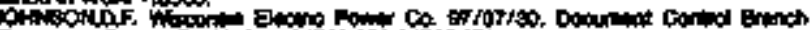

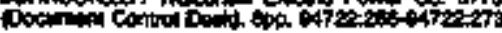

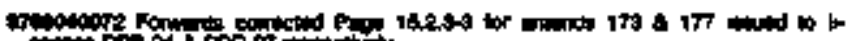

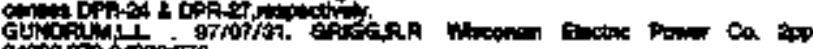
siloto of

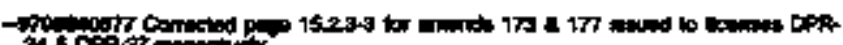

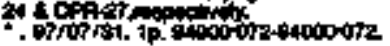

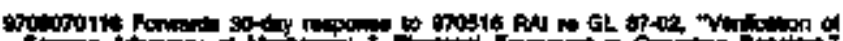

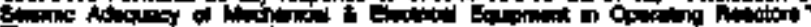

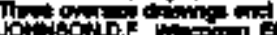

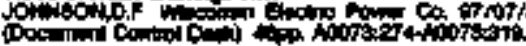

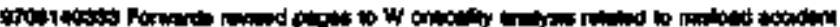

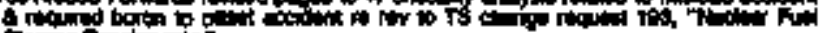

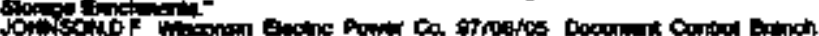

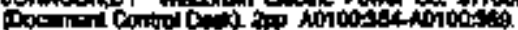

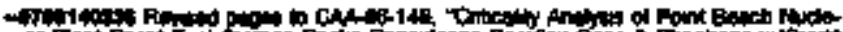

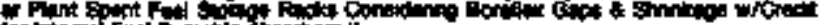

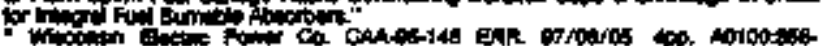
atomits

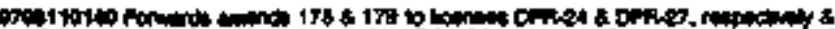

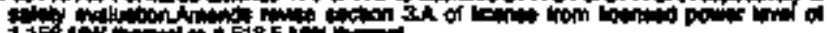

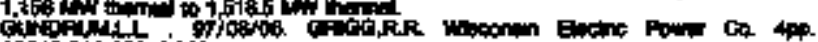
Acol 934 -

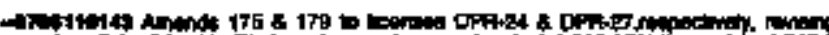

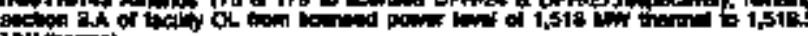

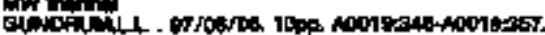

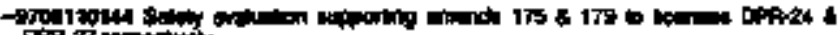

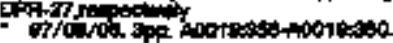

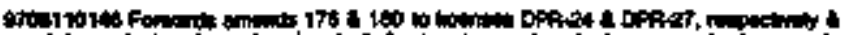

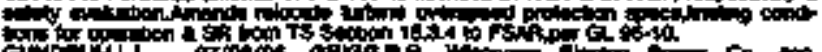

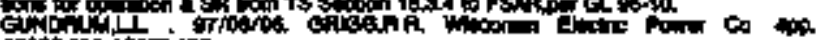

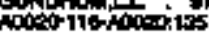

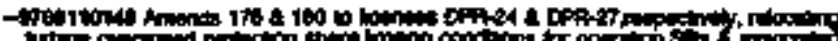
ut

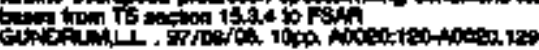

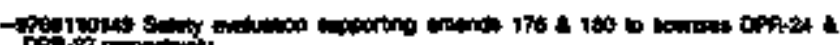

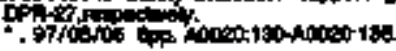

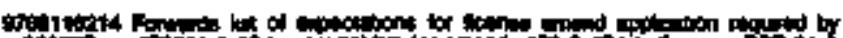

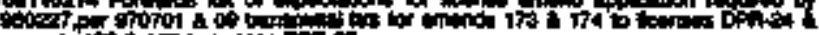

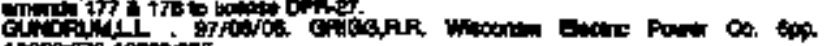
worotratoogens.

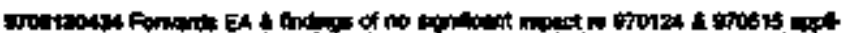
calom to

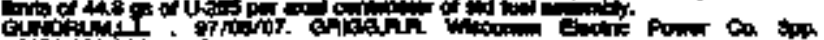

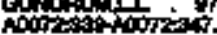

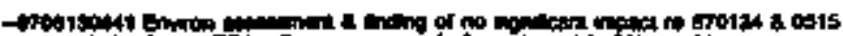

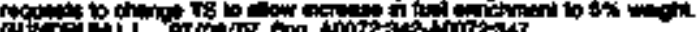

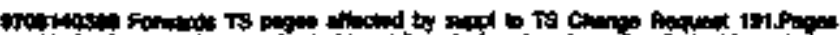

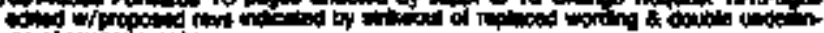

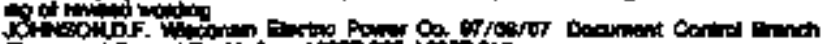

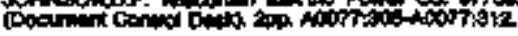

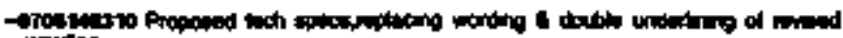
ind

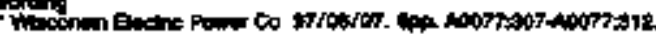

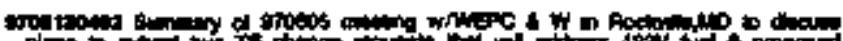

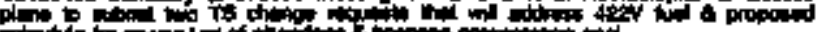

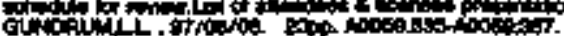

of

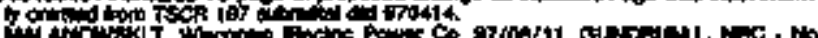

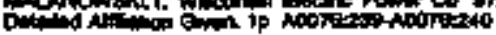

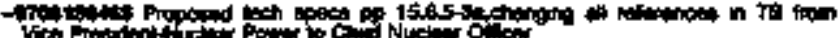

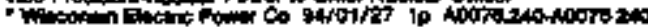

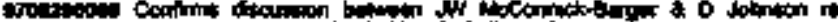

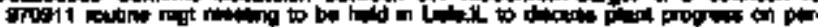

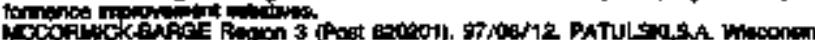

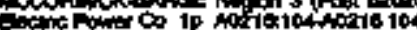

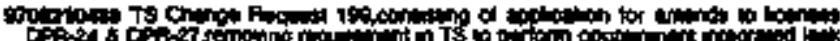

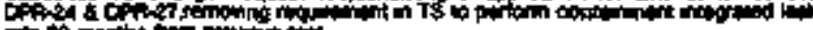

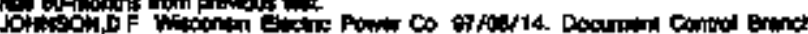

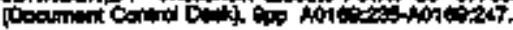

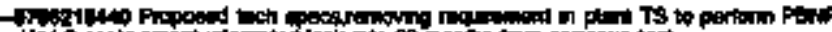

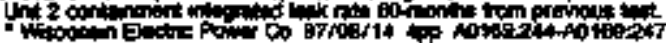

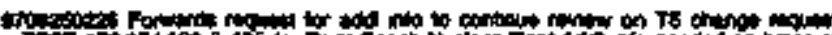

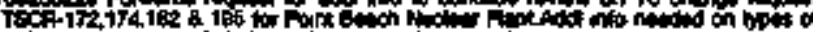

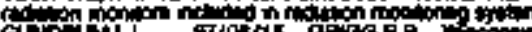
G

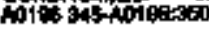

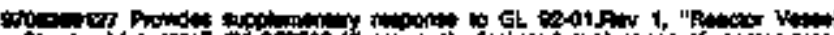

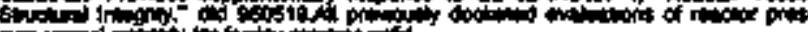
24.

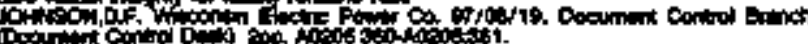

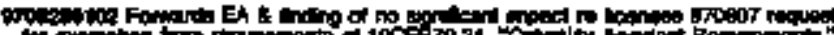

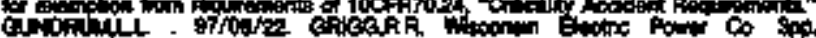

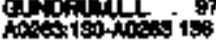

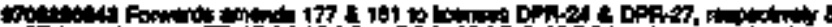

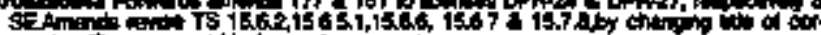
peris of

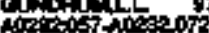

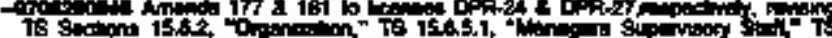

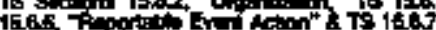

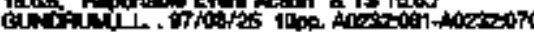

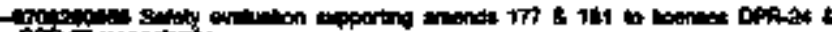

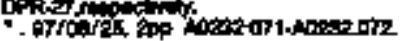

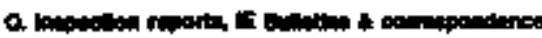

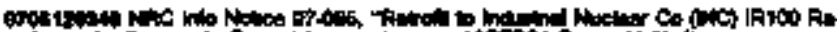

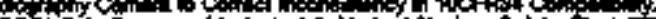

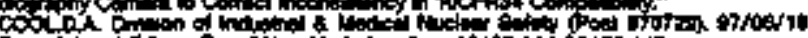

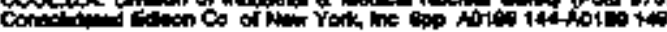

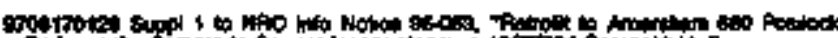

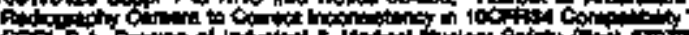
Cordor.

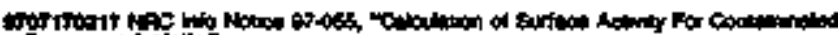

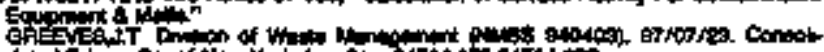

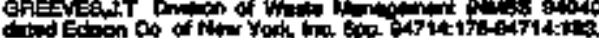

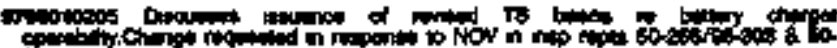

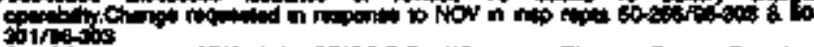
CSTH

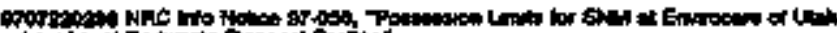

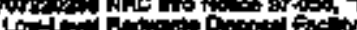

Lot

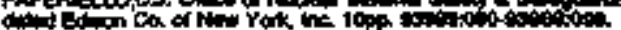

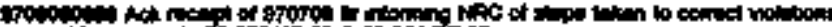

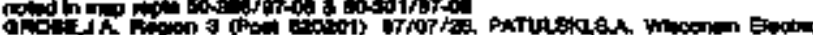

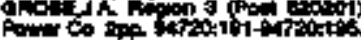

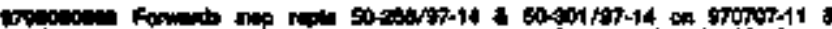

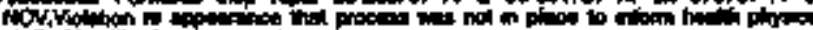

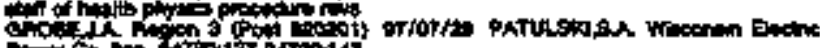

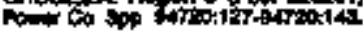

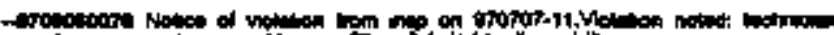

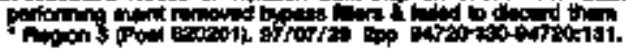

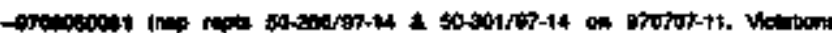

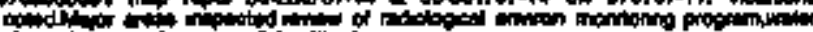

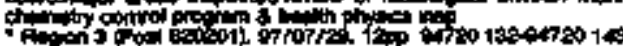

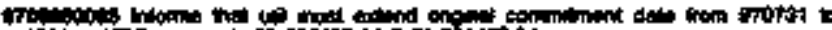

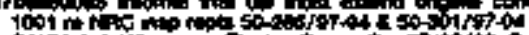

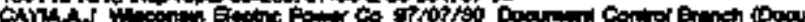

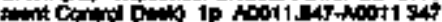

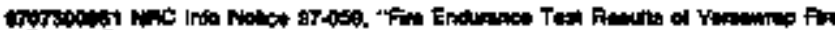

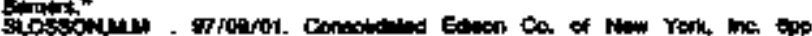

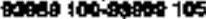




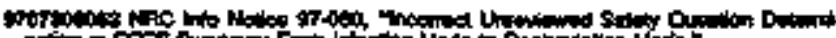

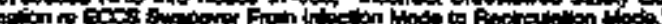

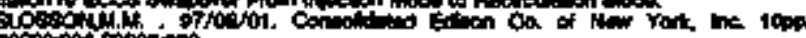

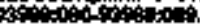

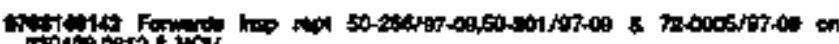

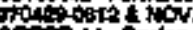

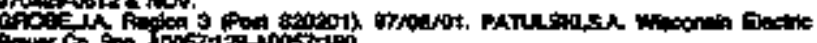

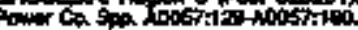

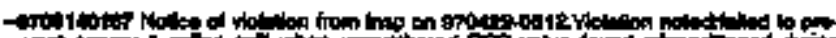

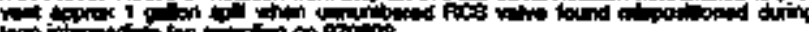

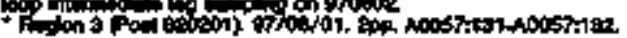

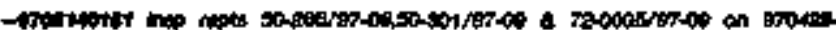

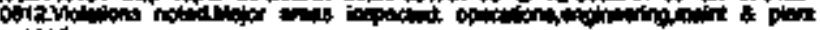

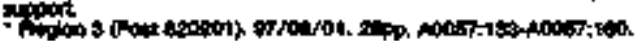

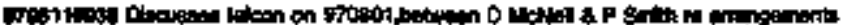

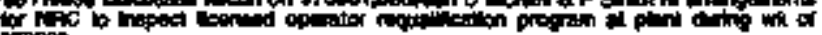

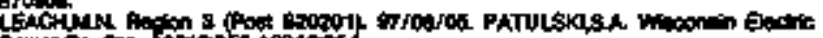

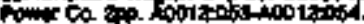

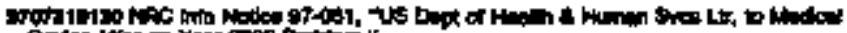

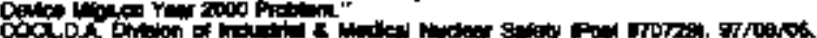

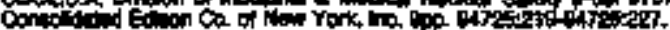

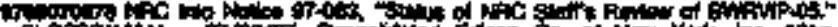

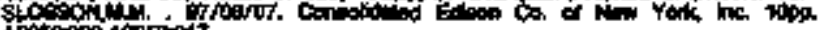

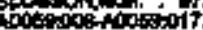

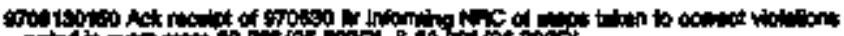

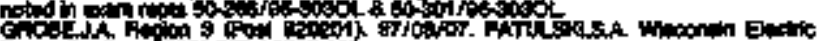

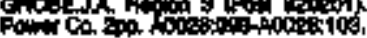

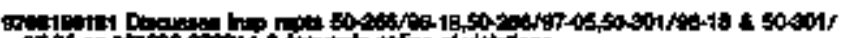

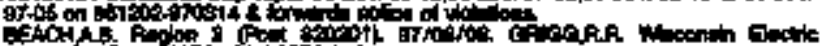

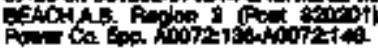

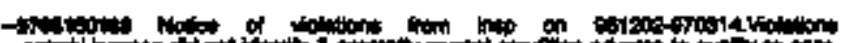

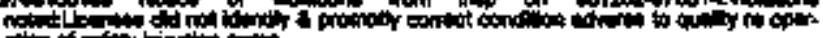

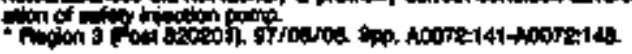

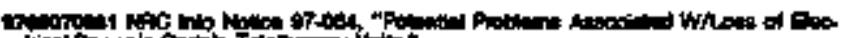

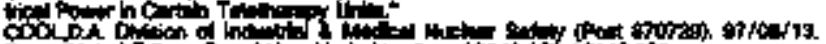

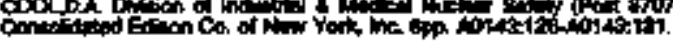

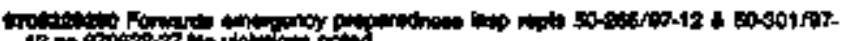

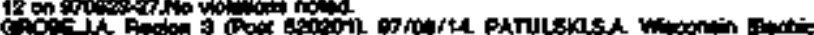

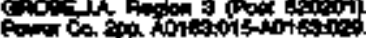

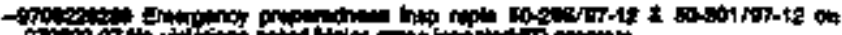

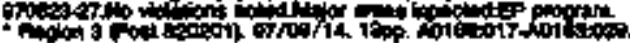

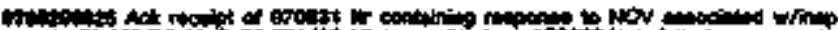

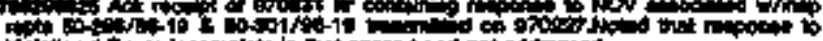

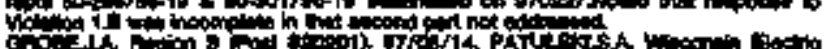
Cond ot

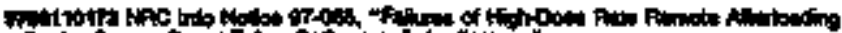

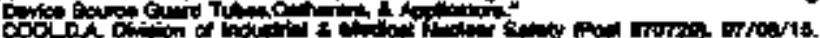

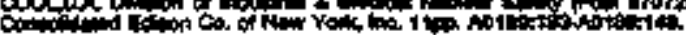

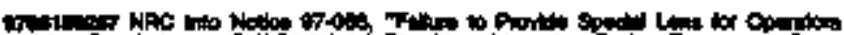

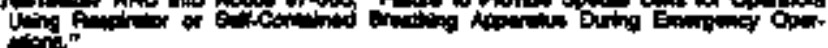

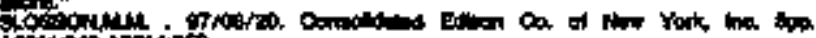

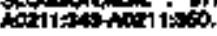

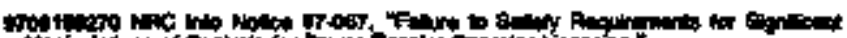

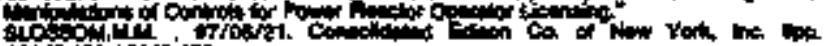

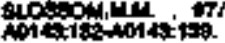

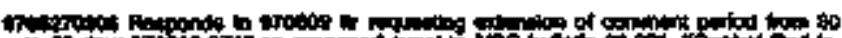

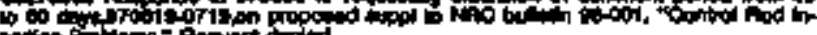

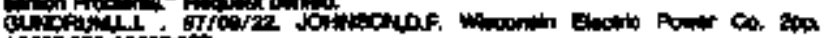
Anom

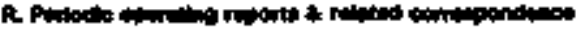

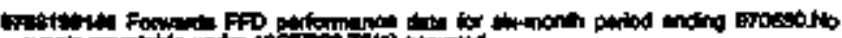

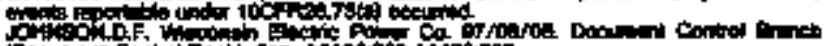

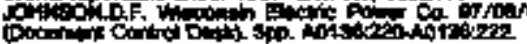

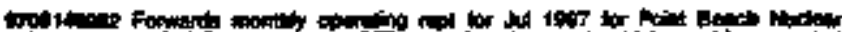

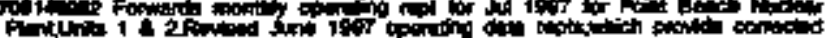

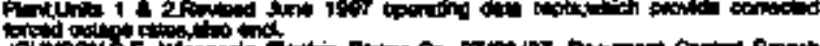

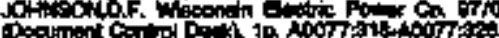

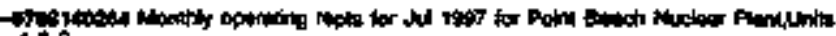

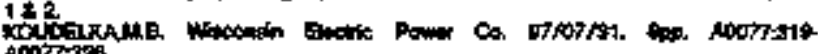
A00\%7286.

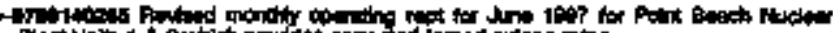

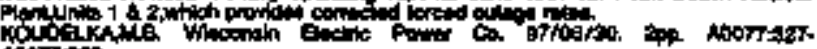
nopryatis.

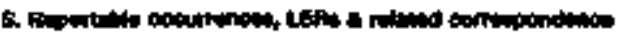

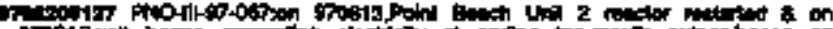

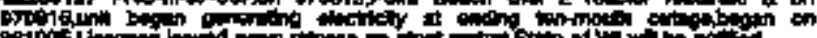

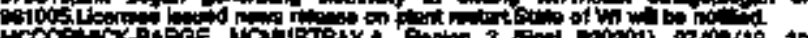

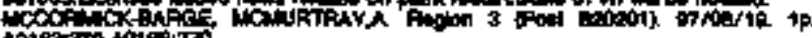

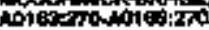

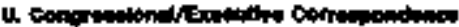

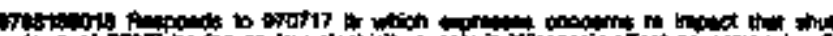

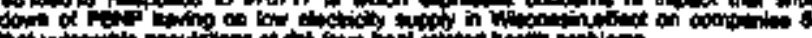

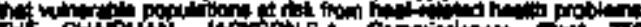

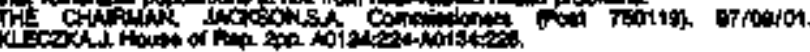

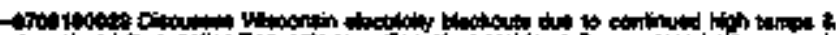

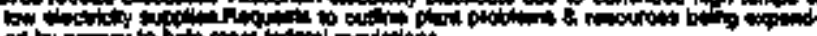

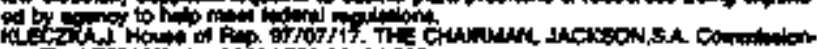

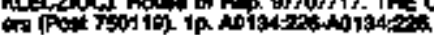

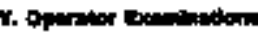

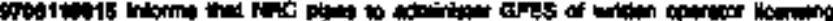

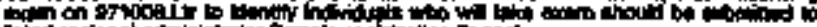

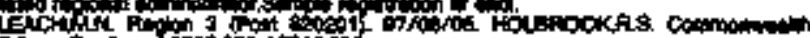

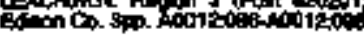

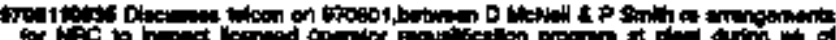

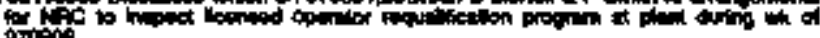

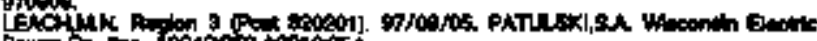

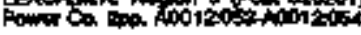

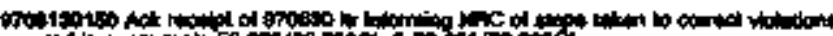

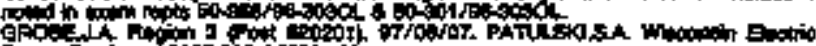

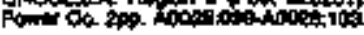

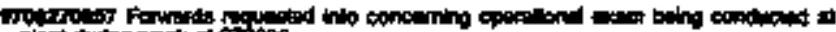

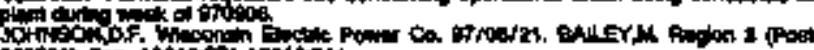

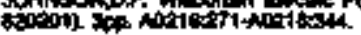

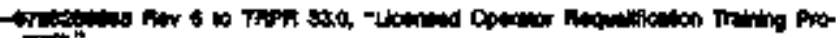

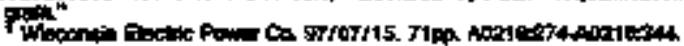

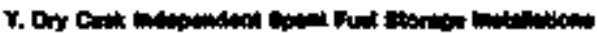

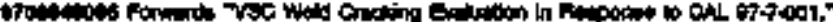

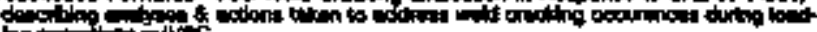

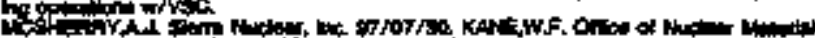

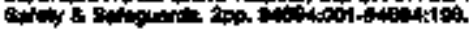

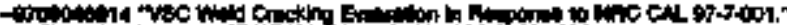

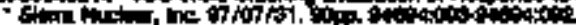

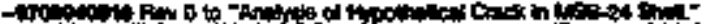
C,

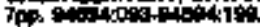

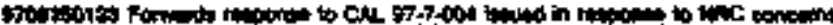

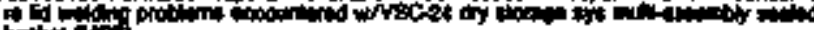

PAnt

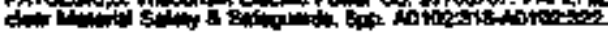

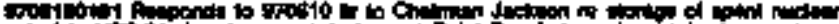

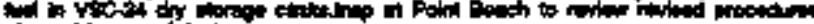

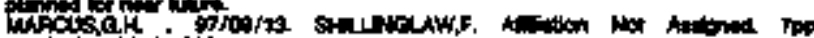
Apious sistopistos.

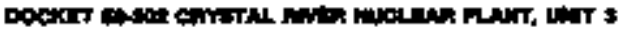

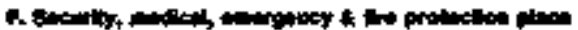

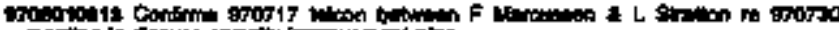

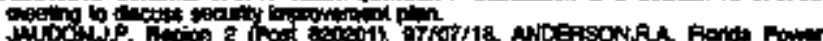

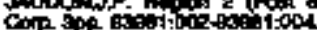




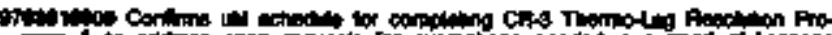

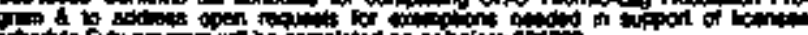

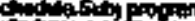

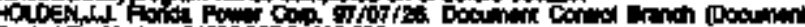

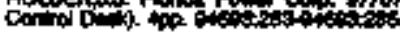

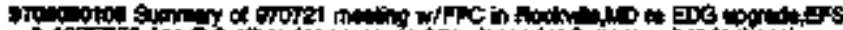

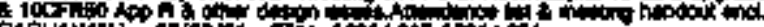

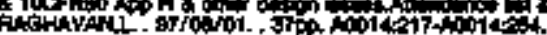

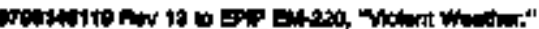

81-

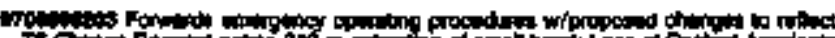

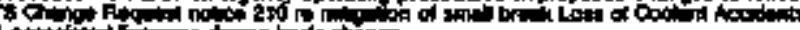

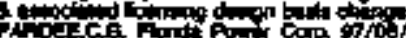

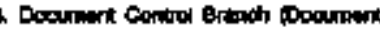

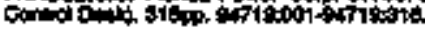

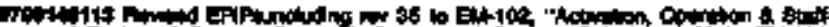

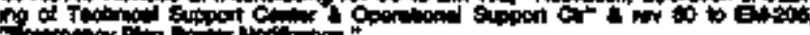

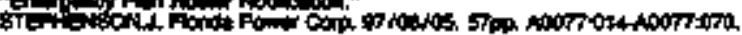

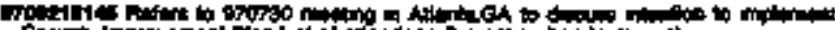

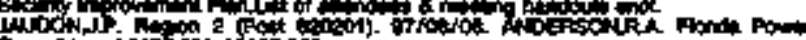

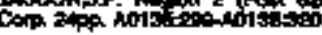

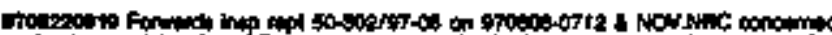

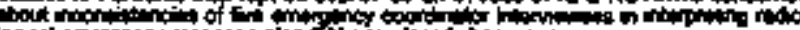

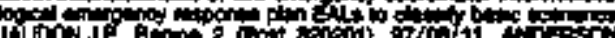

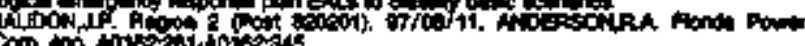

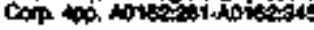

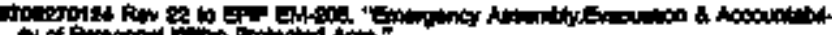

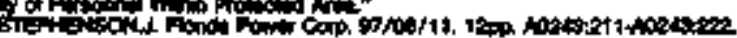

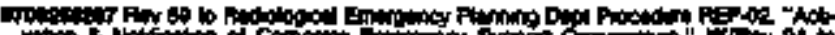

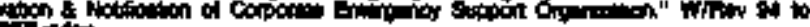

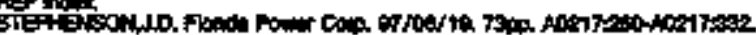

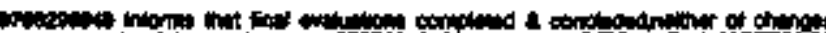

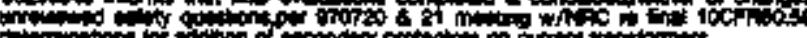

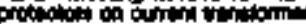

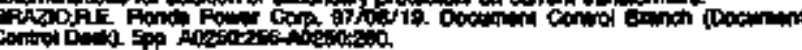

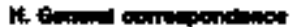

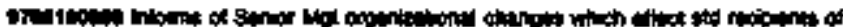

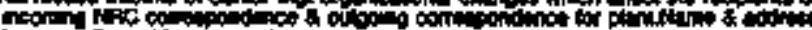

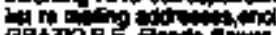

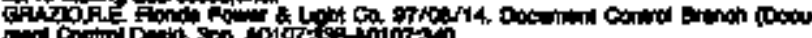

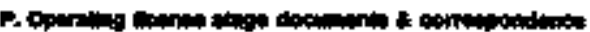

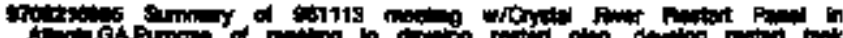

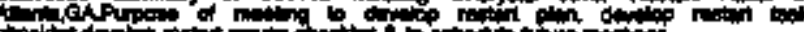
chectid Altistiolition.

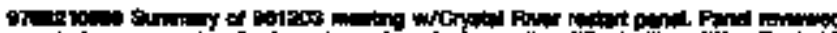

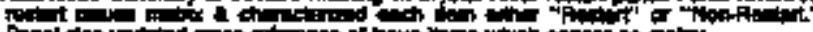

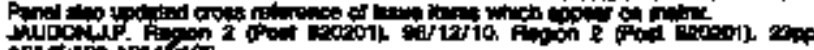
Nolosionionation

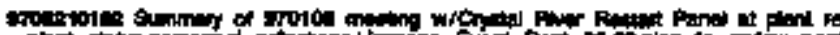

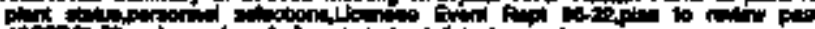
10FPRo

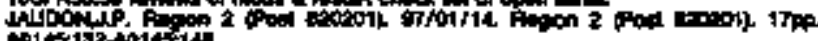

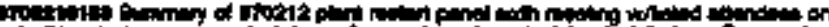

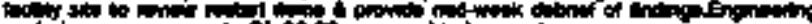

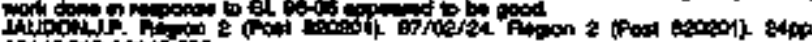

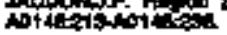

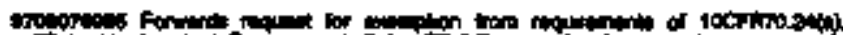

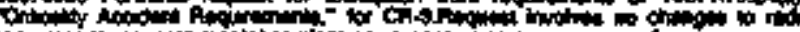

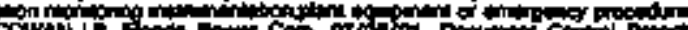

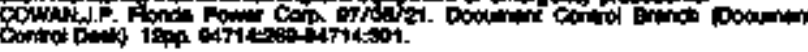

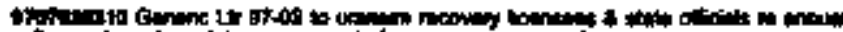

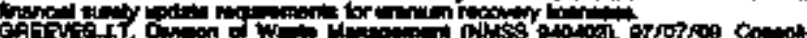

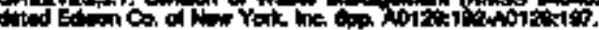

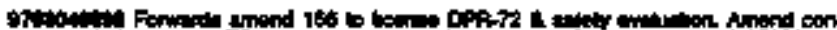

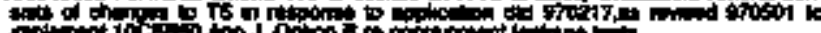

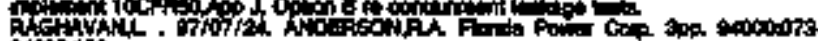
A400:

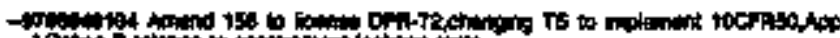

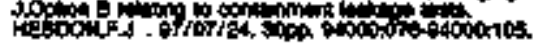

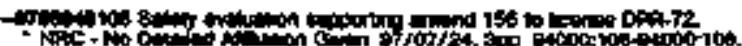

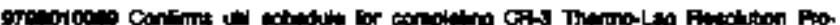

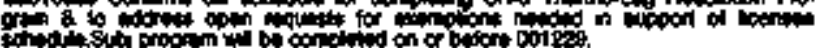

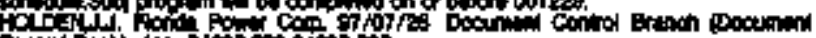

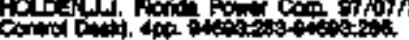

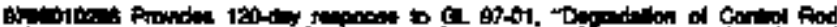

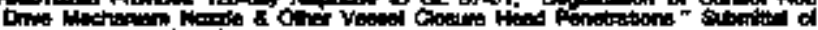

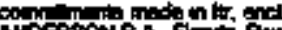

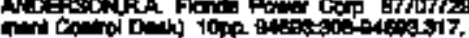

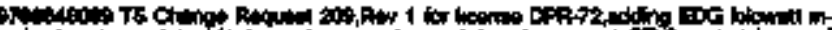

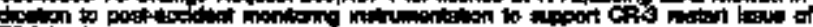

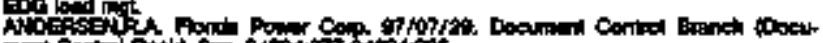

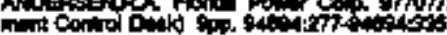

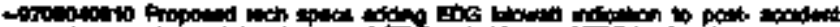

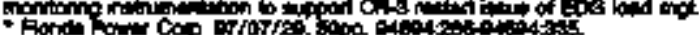

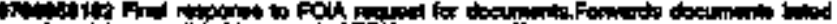

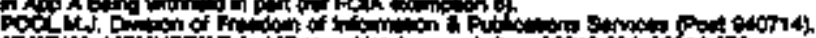

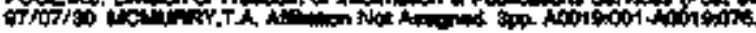

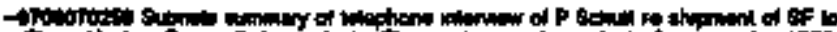

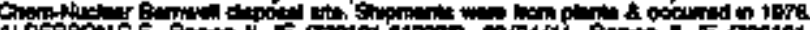

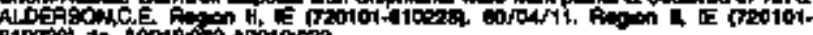

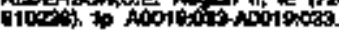

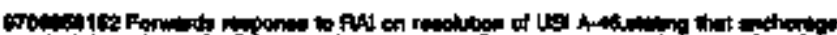

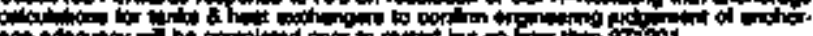

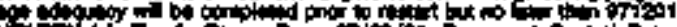

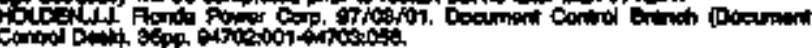

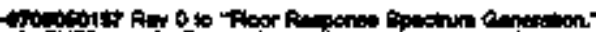

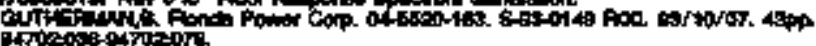

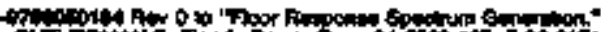

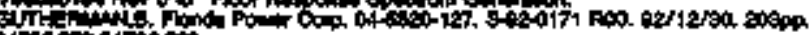

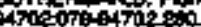

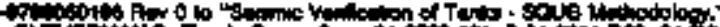

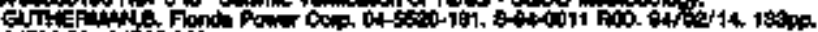

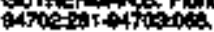

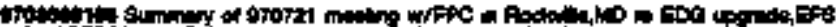

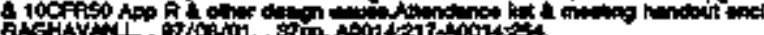

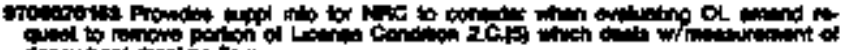

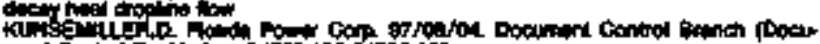

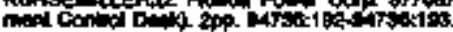

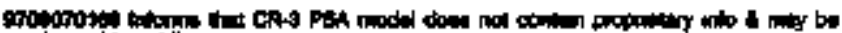

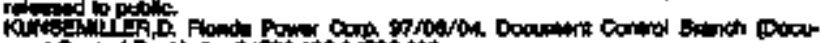

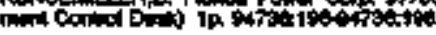

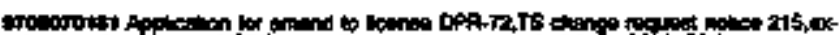

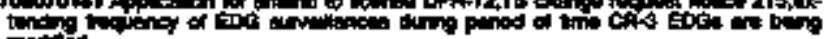

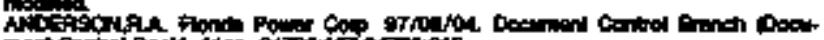

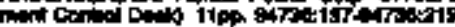

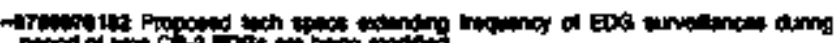

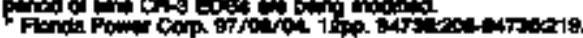

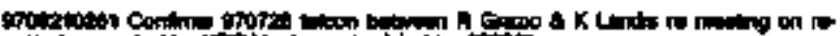

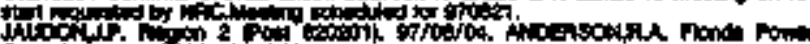

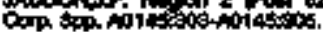

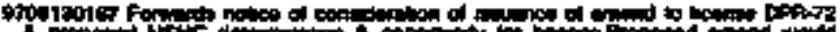

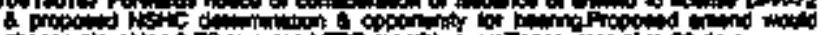

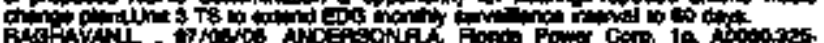
mocosos

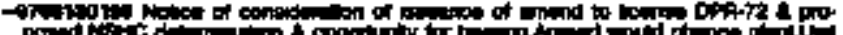

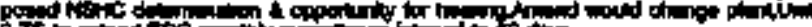
3 To 0 .

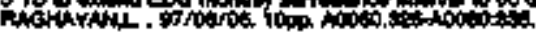

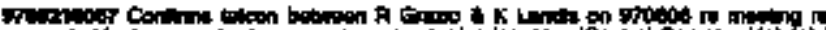

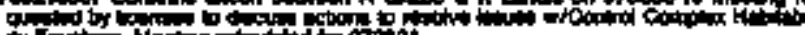

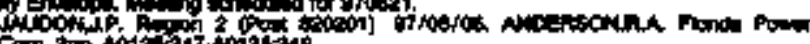

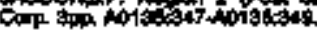

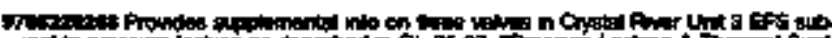

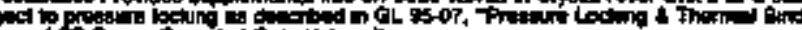

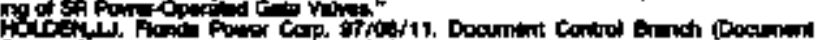

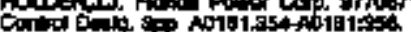

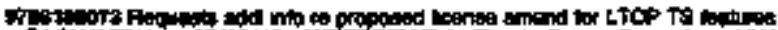

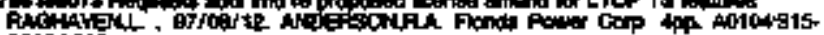

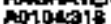




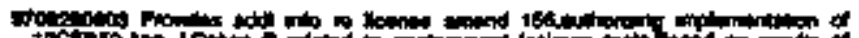

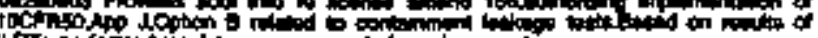

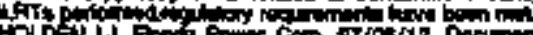

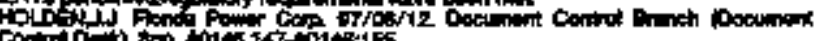

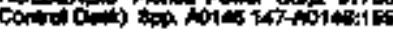

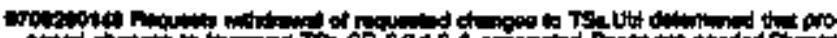

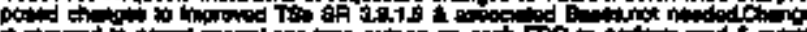

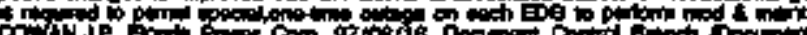

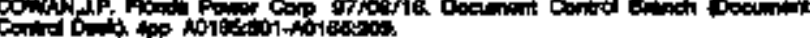

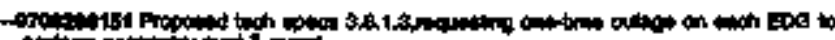

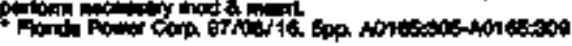

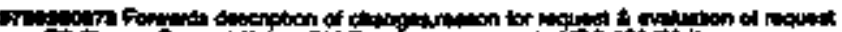

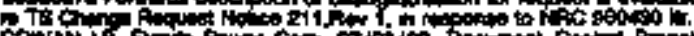

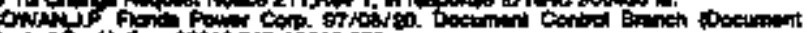

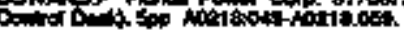

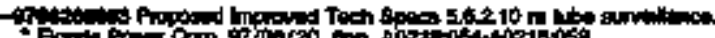

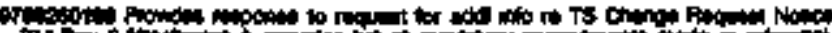

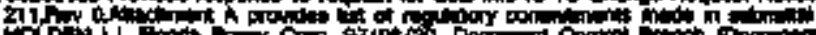

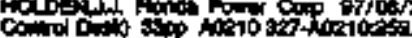

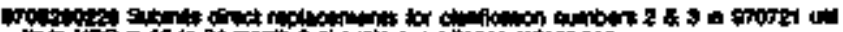

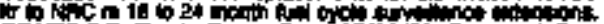

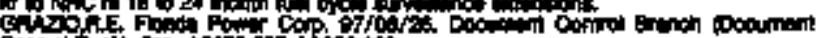

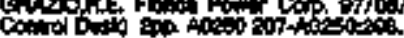

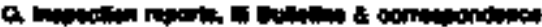

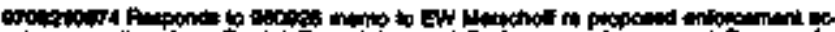

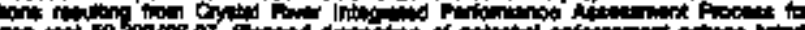

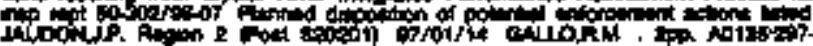
Noristion

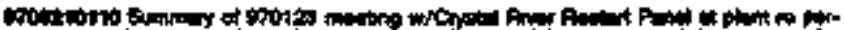

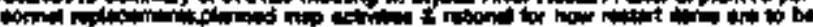

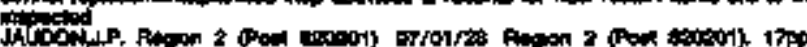

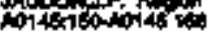

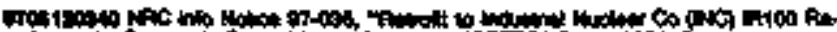

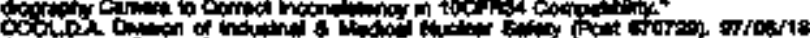

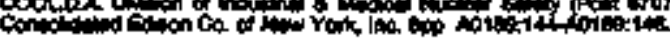

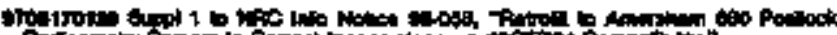

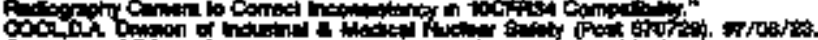

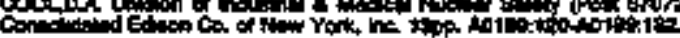

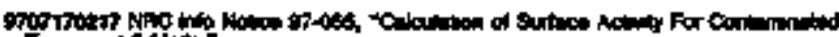

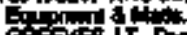

C. 3 if

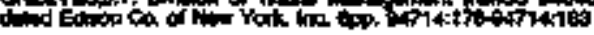

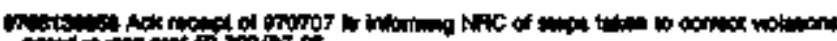
sinity

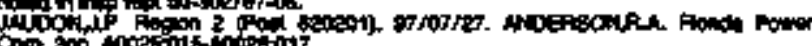

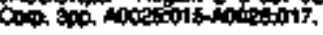

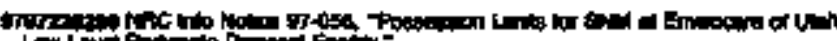

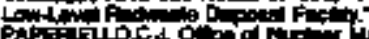

Af Fi

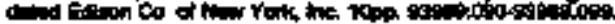

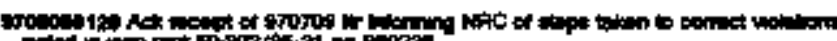

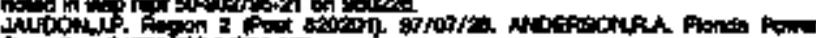

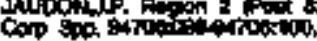

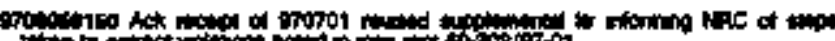

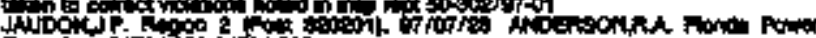

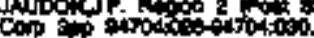

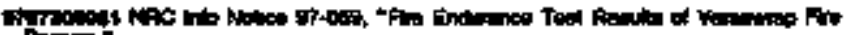
int

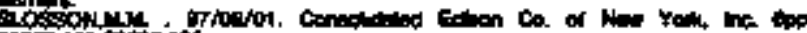

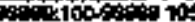

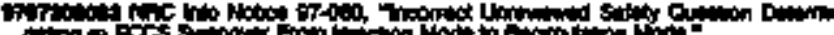

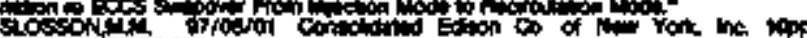

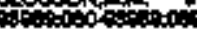

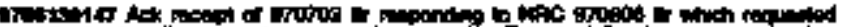

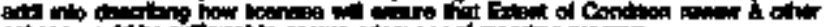

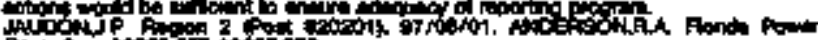

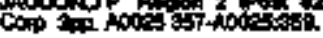

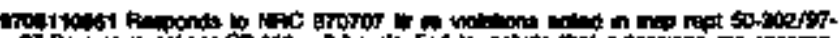

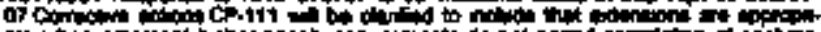

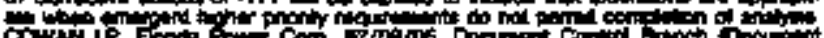

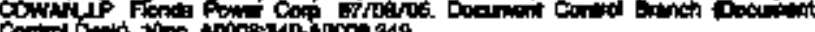

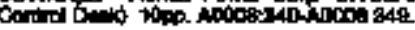

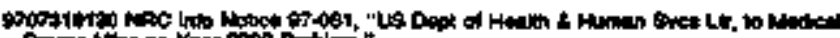

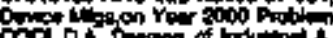

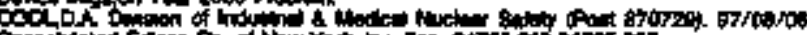

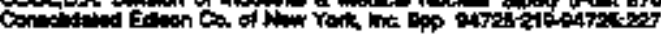

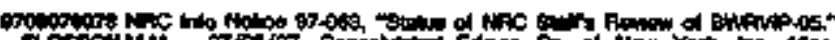

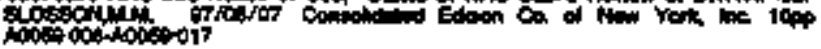

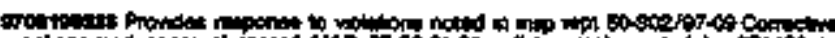

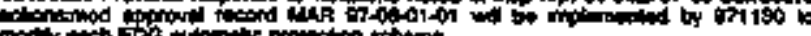

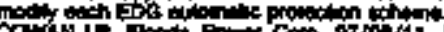

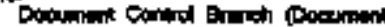

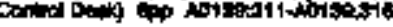

thes

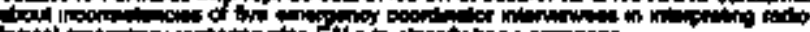

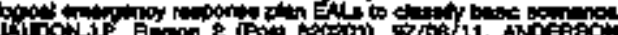

Com top and

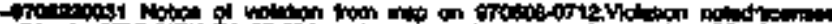

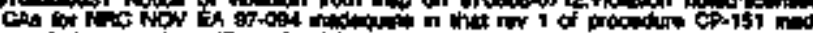

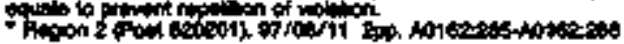

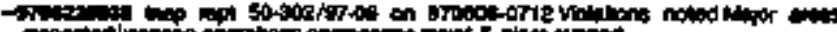

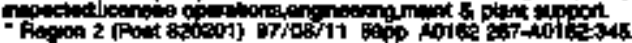

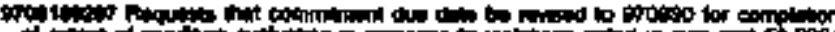

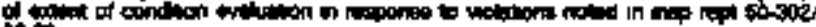
\%

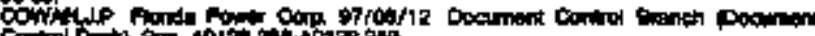

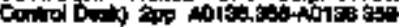

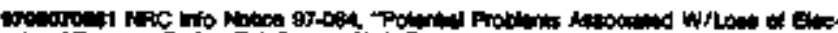

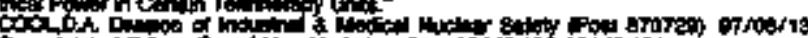

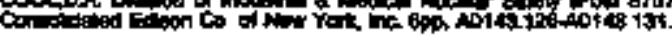

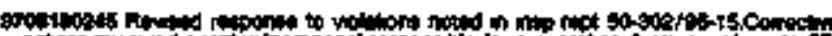

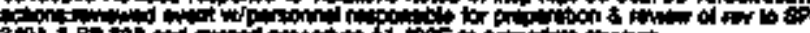

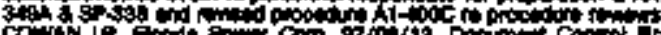

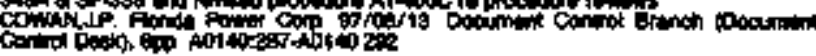

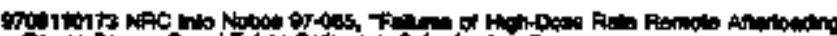

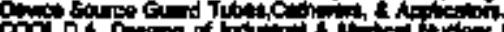

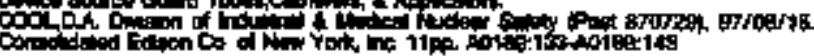

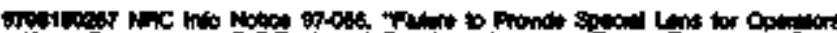

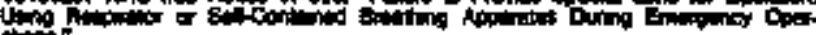

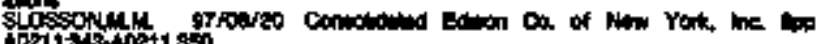

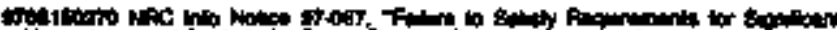

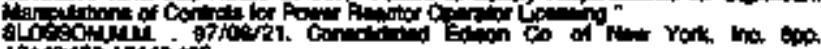

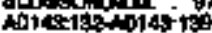

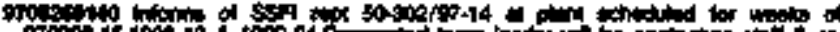

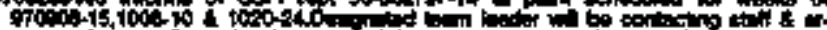

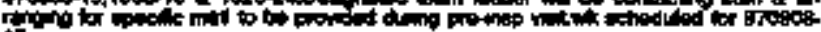

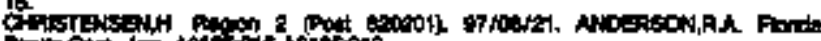

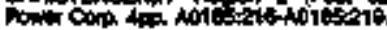

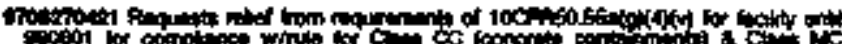

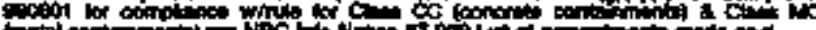

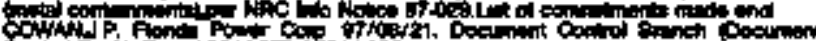

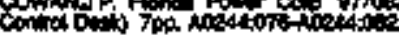

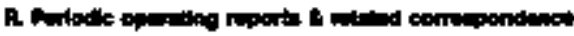

Fond

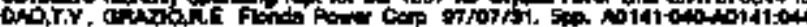

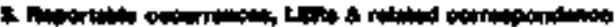

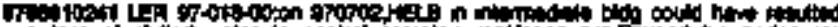

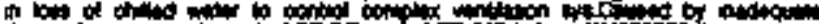

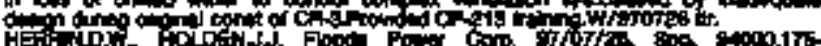
Dicos: 100

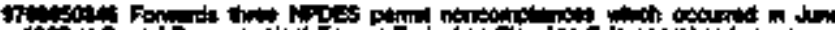

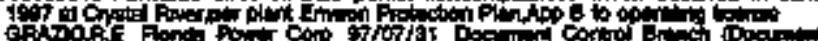

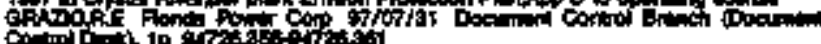
(1)

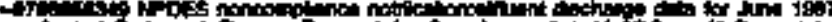

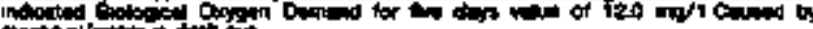

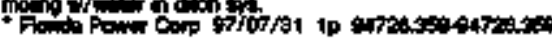

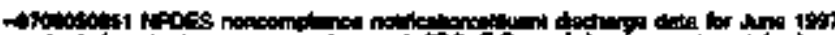

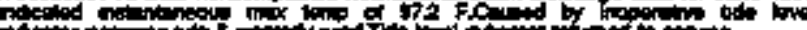

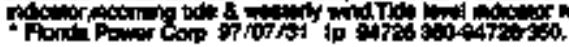




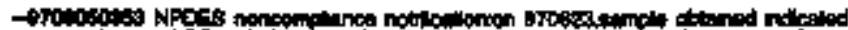

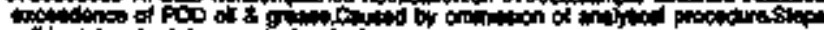

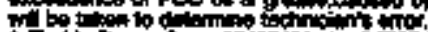

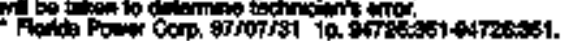

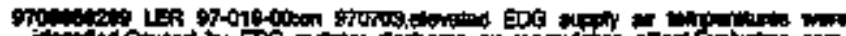

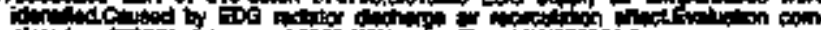

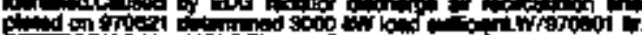

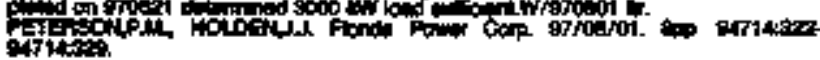

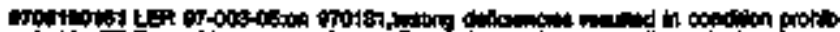

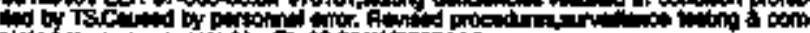

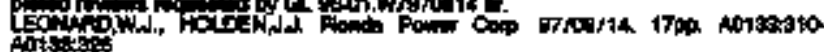

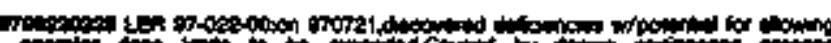

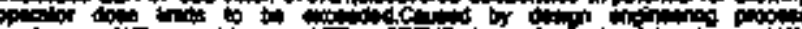

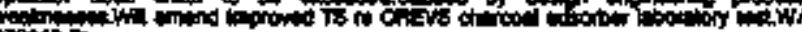
9rositis.

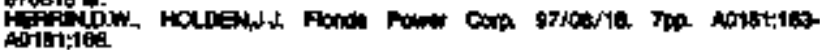

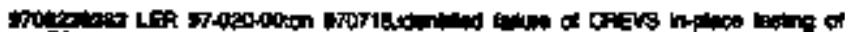

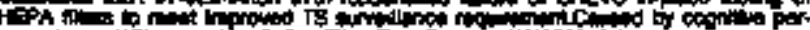

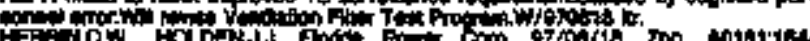
Aitiotion

Py

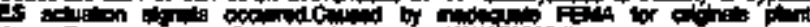

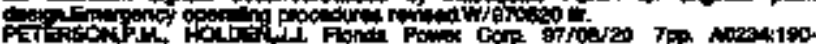
Aoratis

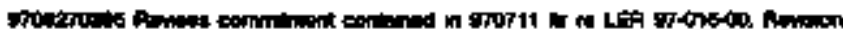

HoLb

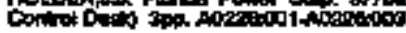

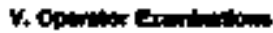

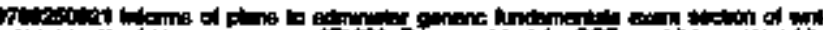

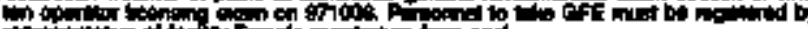

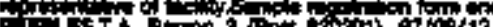

di.

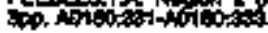

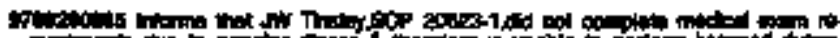

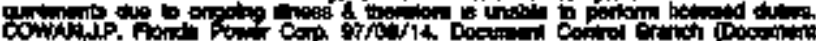

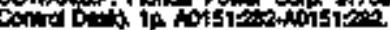

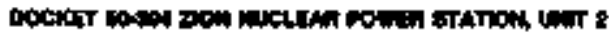

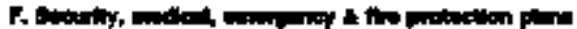

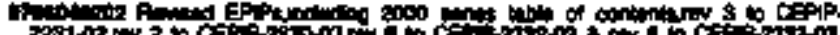

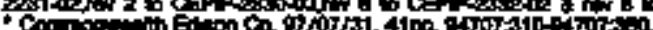

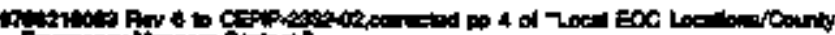

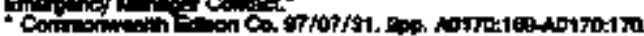

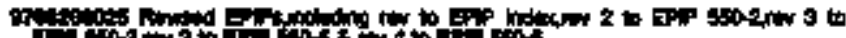
to

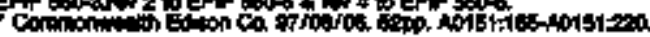

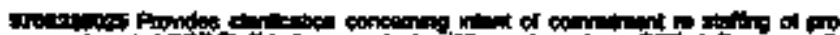

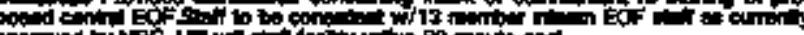

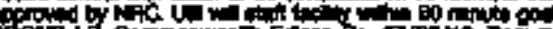

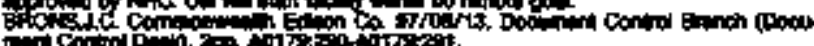

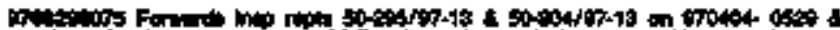

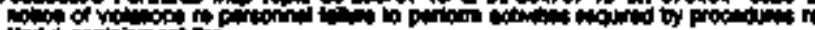
Lh

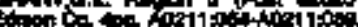

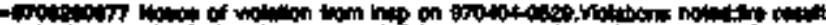

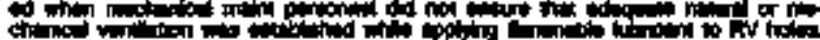

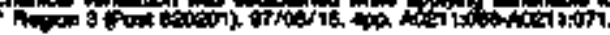

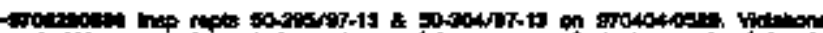

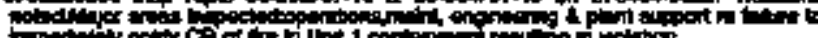

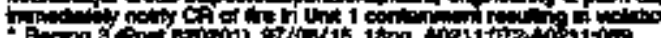

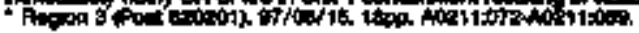

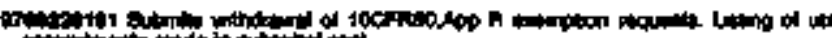

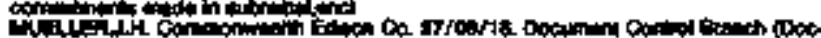

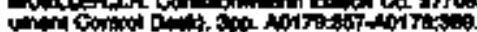

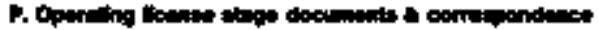

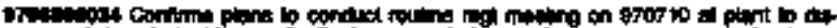

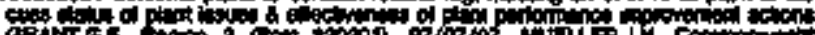

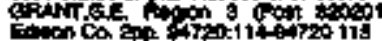

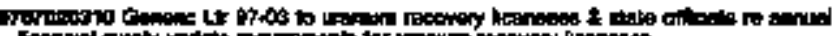

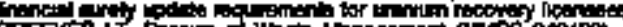

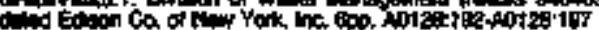

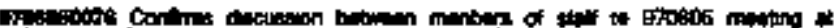

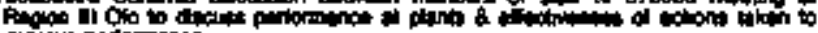

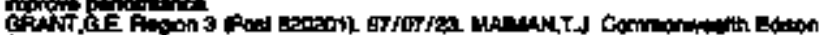

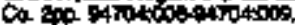

7. The Cind of

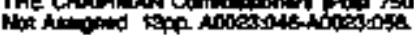

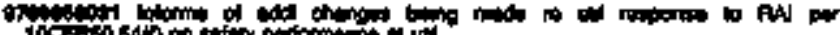

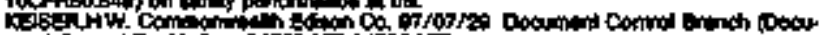

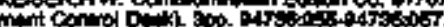

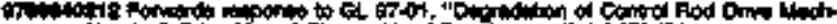

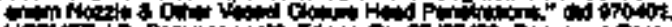

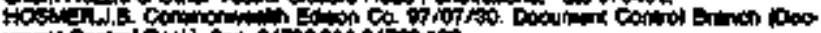

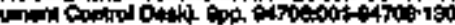

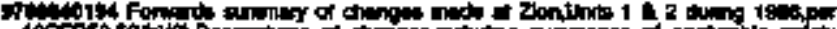

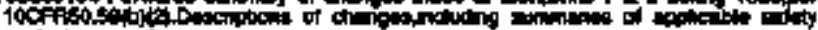

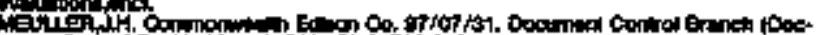

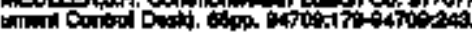

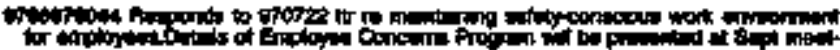

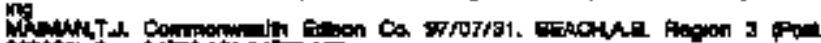

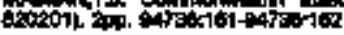

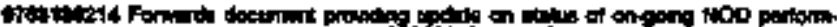

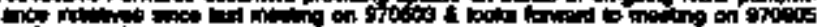

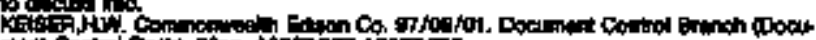

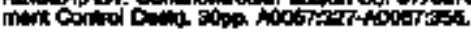

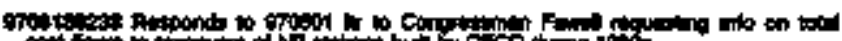

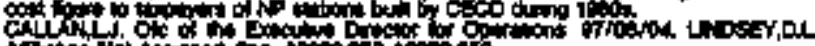

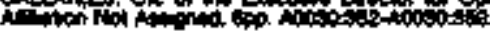

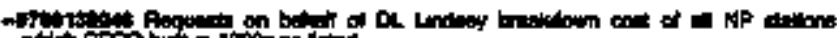
Phin

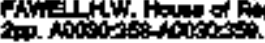

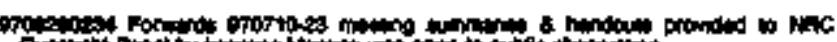

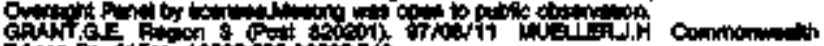

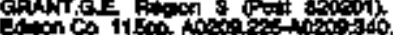

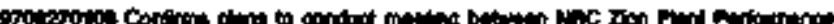

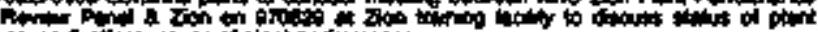

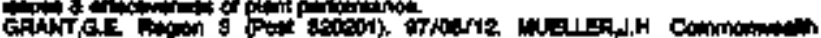
Fand da son

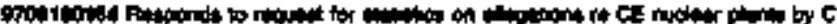

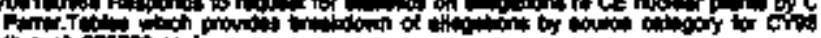

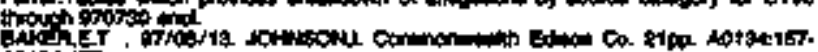
absutis.

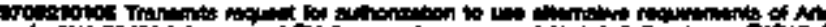

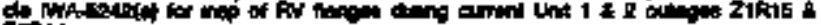
20714.

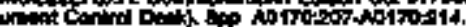

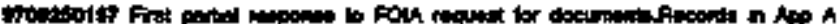

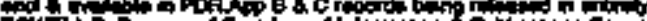

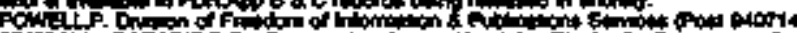

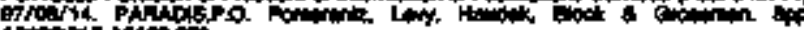

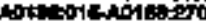

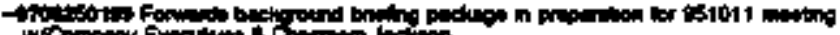

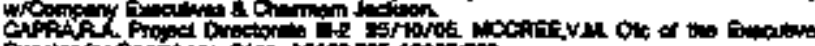

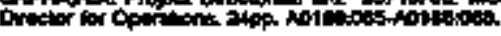

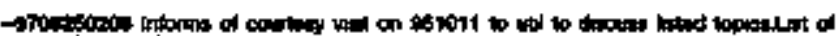

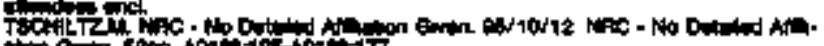

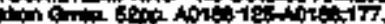

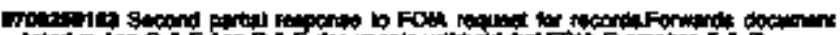

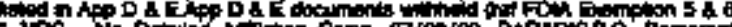

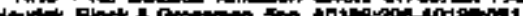

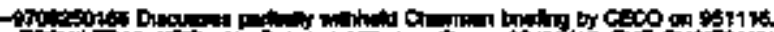

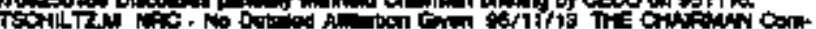

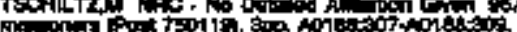

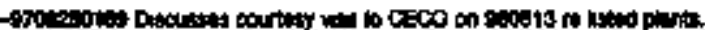

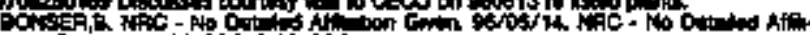

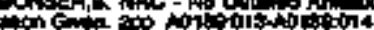




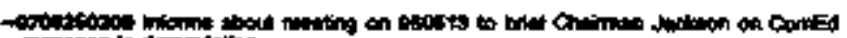

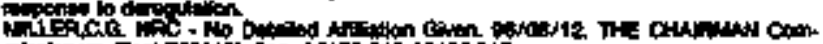

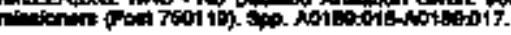

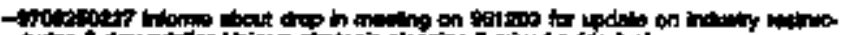

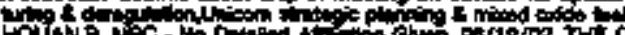

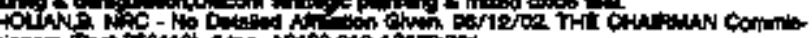

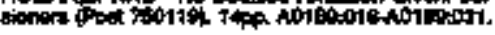

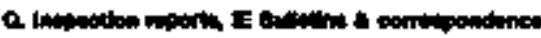

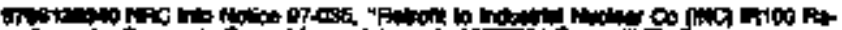

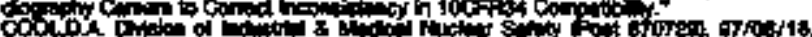

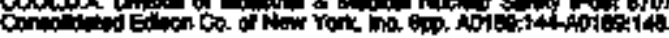

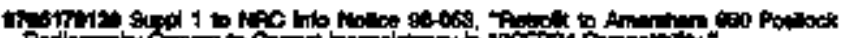

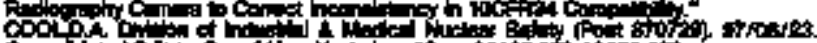

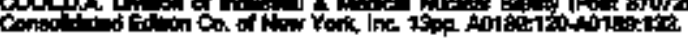

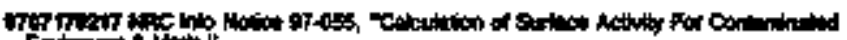

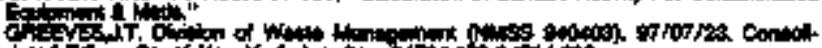

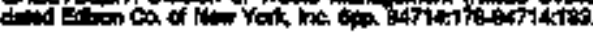

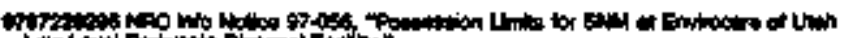

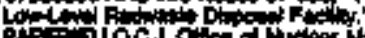

PH

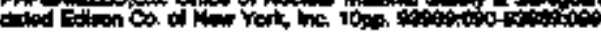

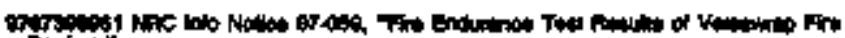

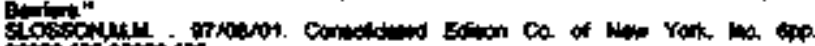

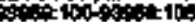

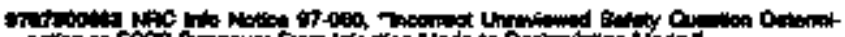

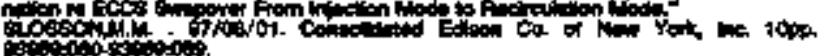

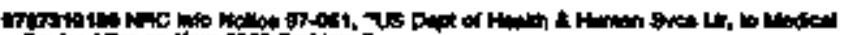

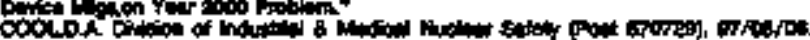

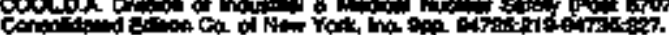

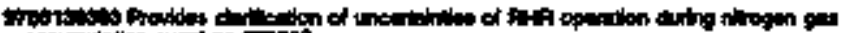

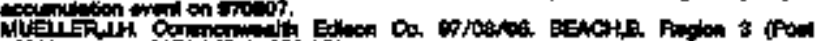

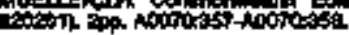

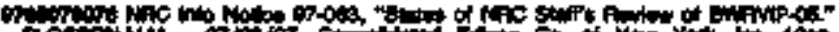

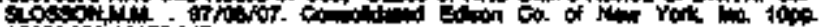

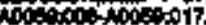

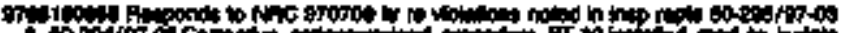

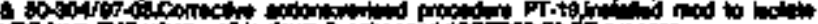

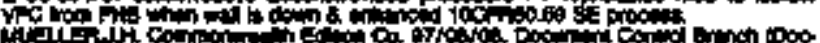

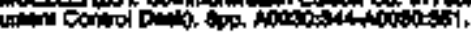

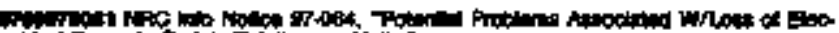

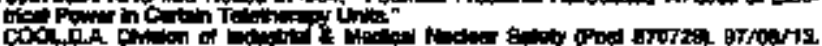

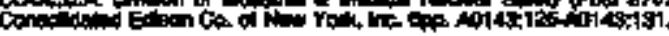

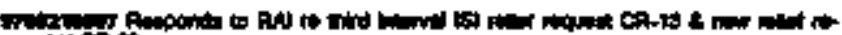

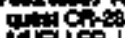
T:

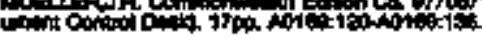

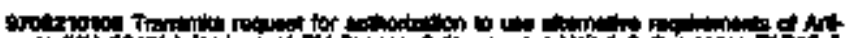

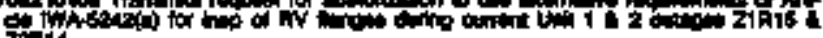
2.

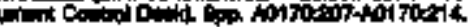

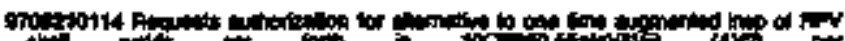

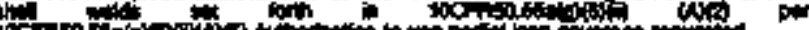
$16 \mathrm{C}$ if

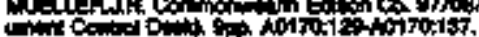

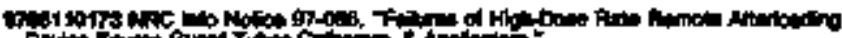

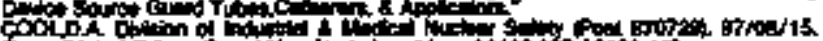

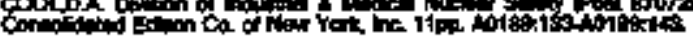

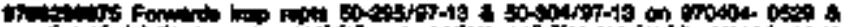

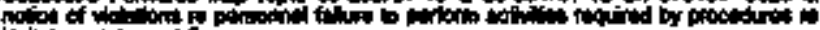

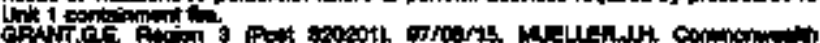

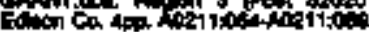

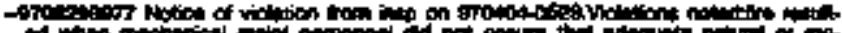

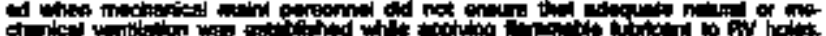

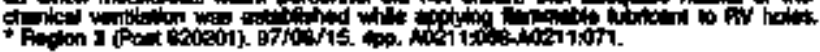

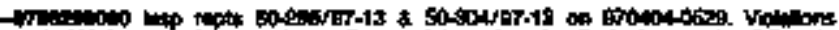

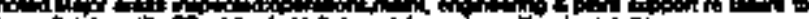

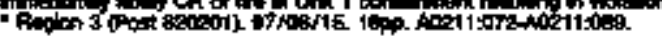

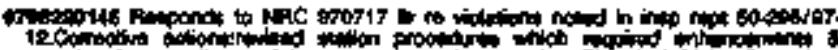

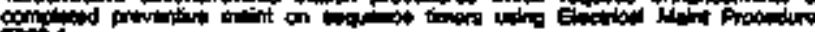

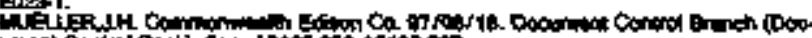

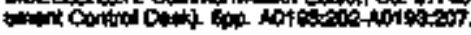

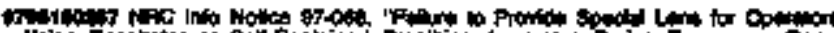
low

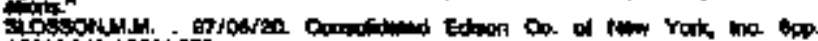

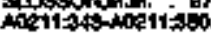

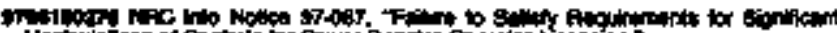

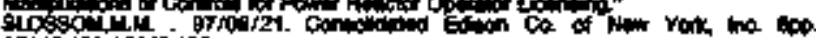

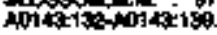

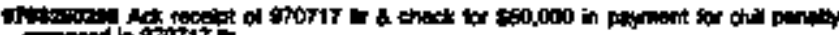

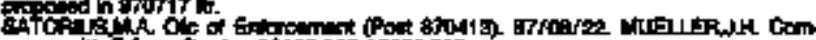

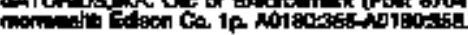

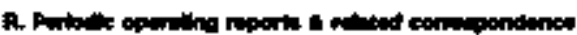

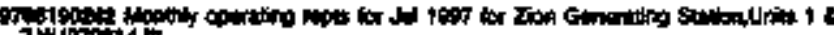

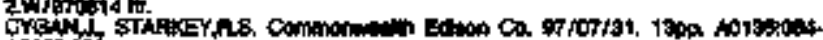
infistion

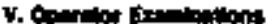

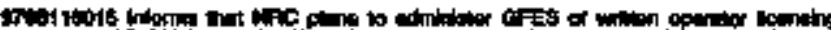

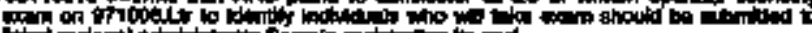

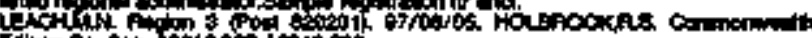

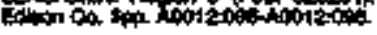

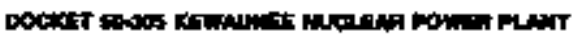

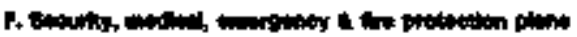

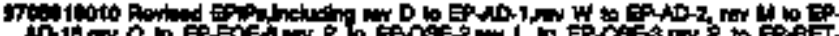
mol inter.

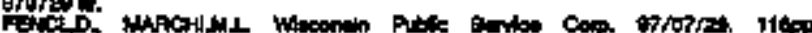

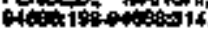

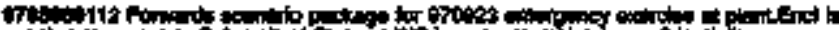

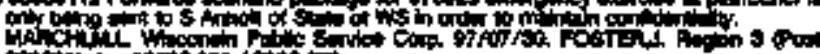
totion

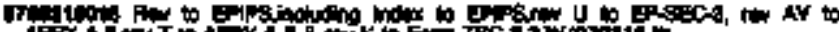

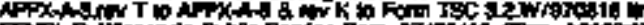

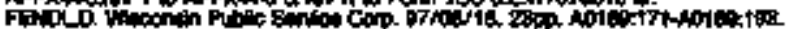

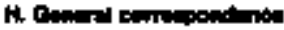

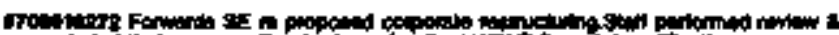

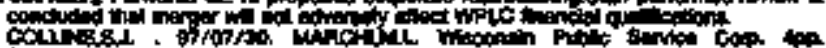
Cxings, 1 , 1 in

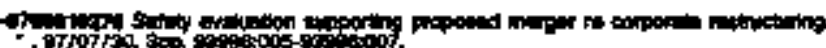

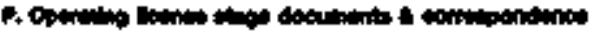

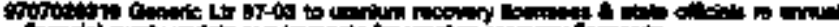

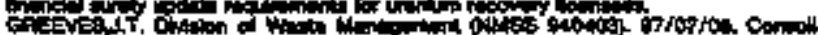

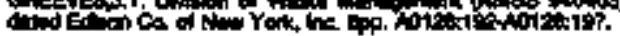

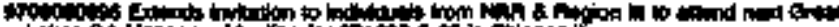

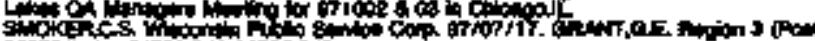

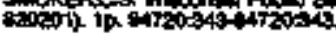

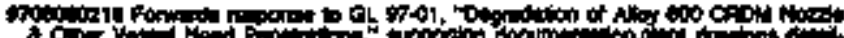

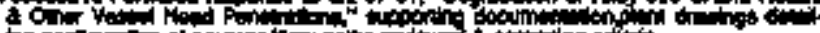

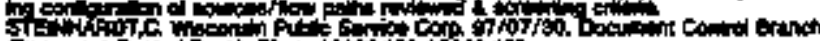

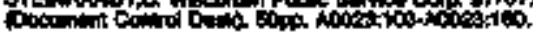

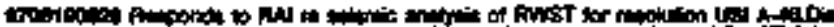

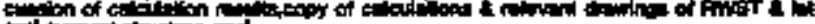

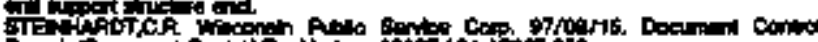

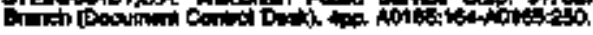

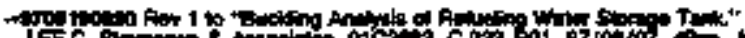

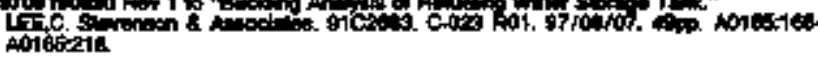




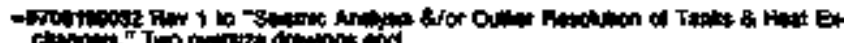

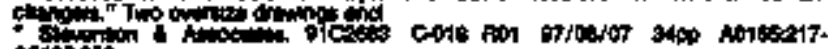
Aitestion

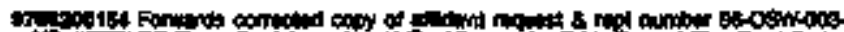
H. "J

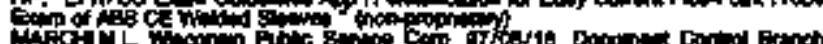

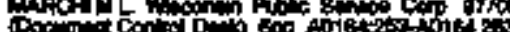

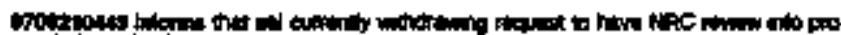

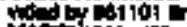

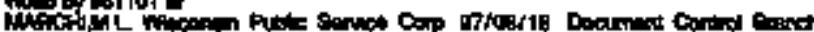

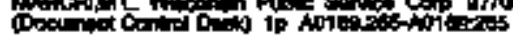

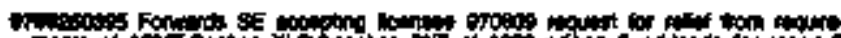
ind of Ast

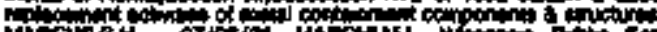
tolotion of

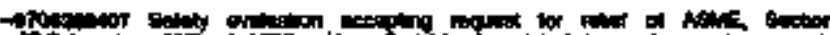

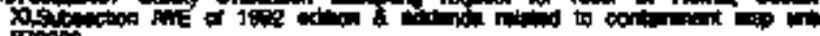

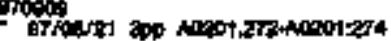

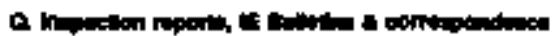

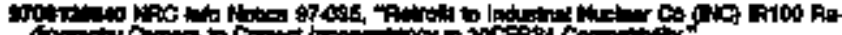
COth

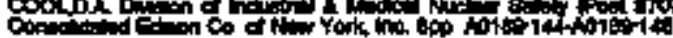

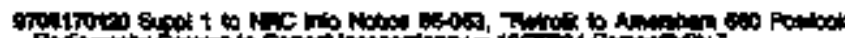

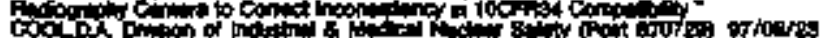

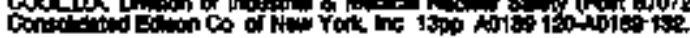

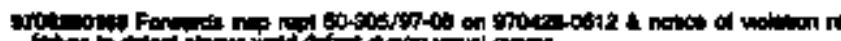

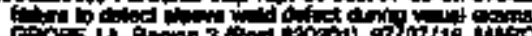

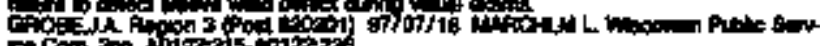

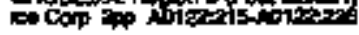

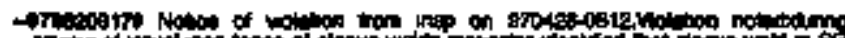

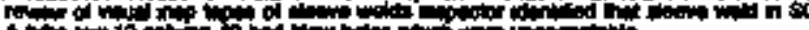

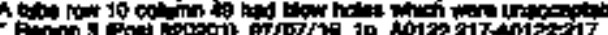

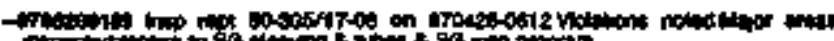

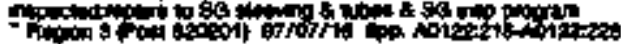

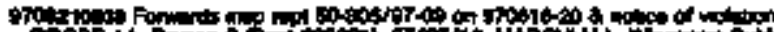

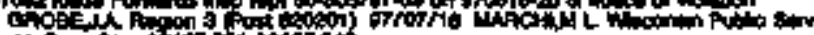

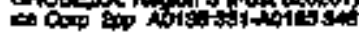

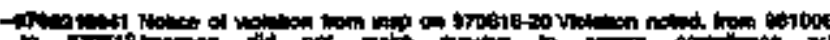

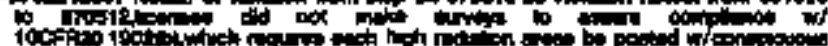

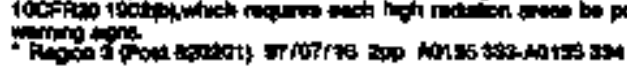

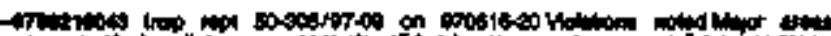

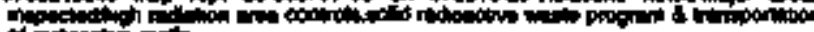

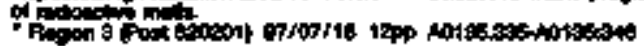

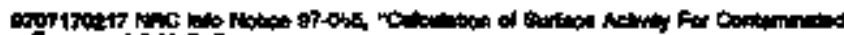

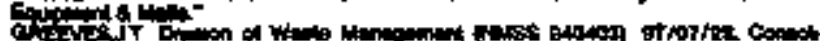

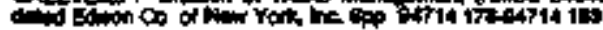

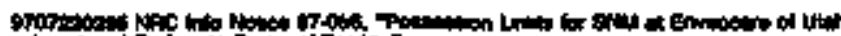

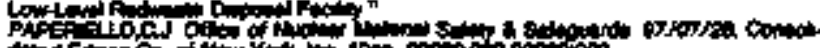

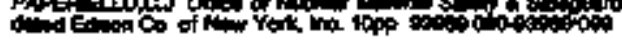

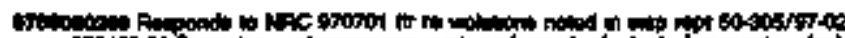

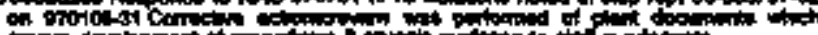

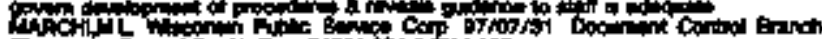

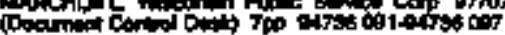

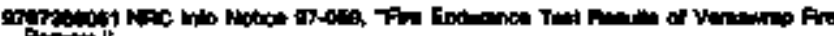

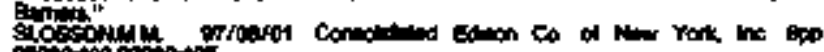

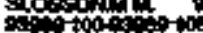

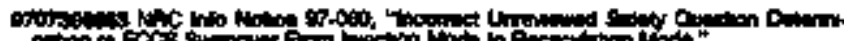

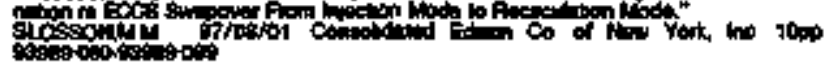

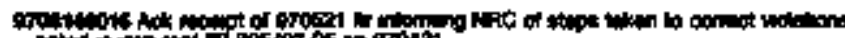

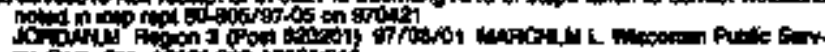

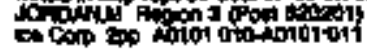

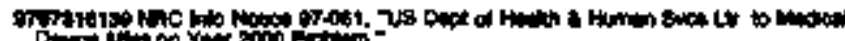

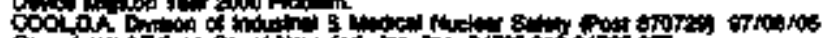

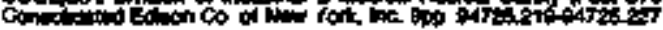

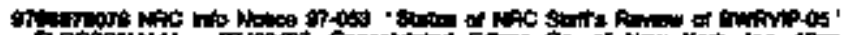

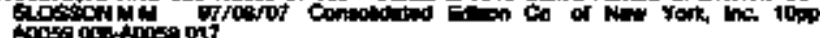

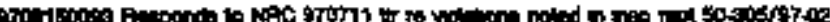

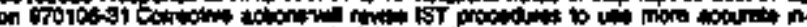
on \$1 THF

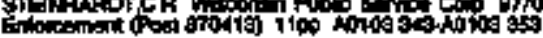

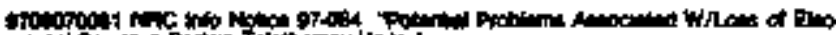

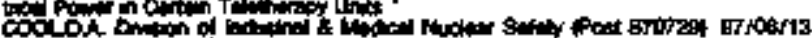

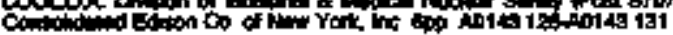

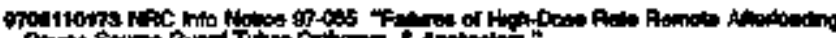

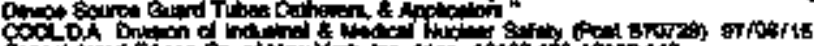

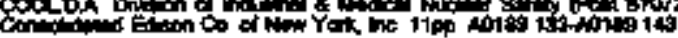

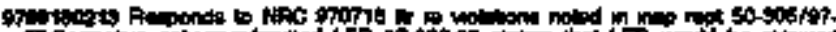

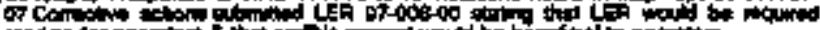

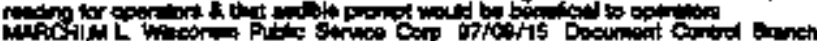

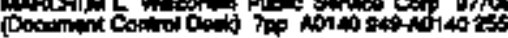

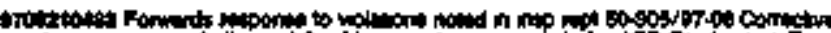

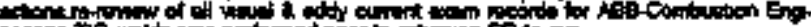

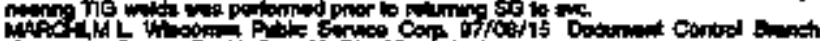

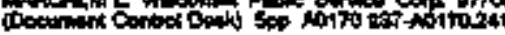

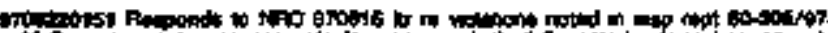

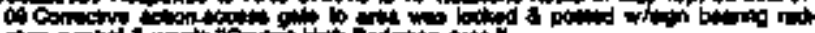

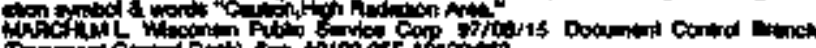

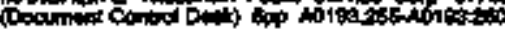

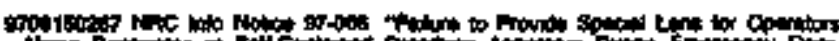

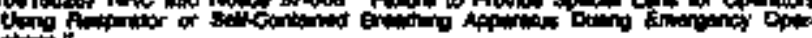

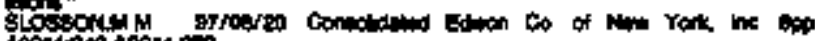

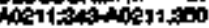

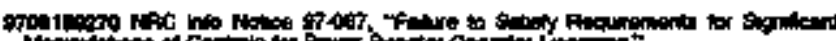

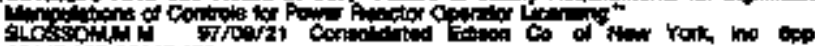

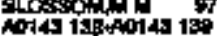

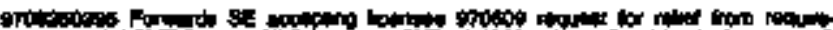

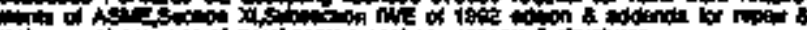

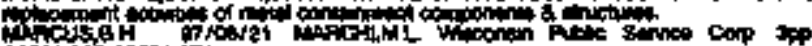
ritol L

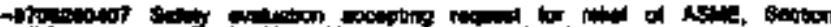

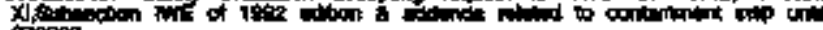
spoos

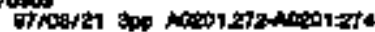

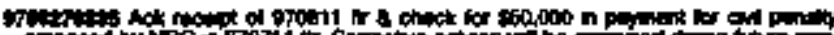

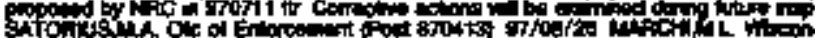

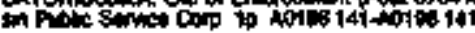

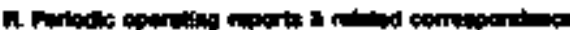

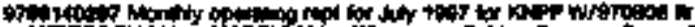

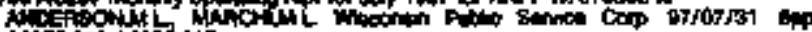
Anot arstion? 317

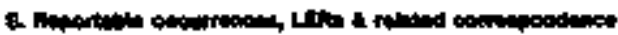

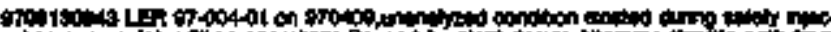

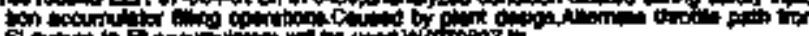

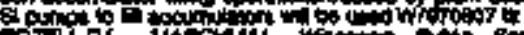

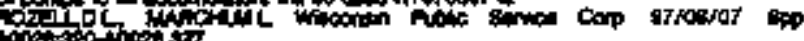

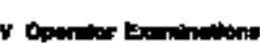

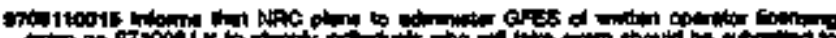

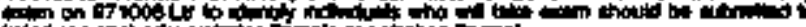

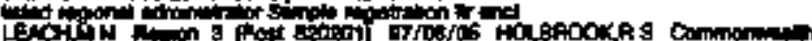

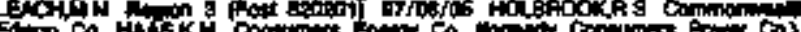

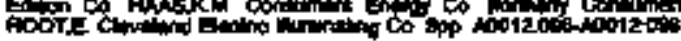

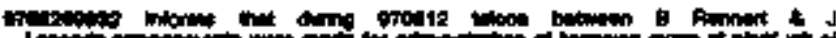

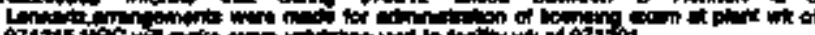
of 121

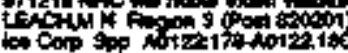

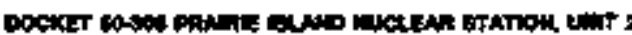

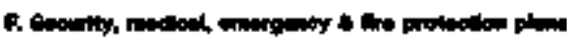

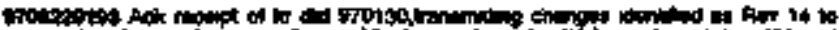

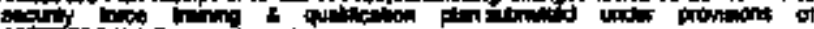

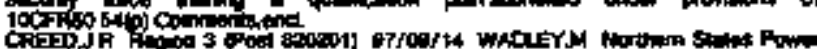

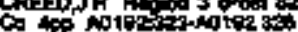




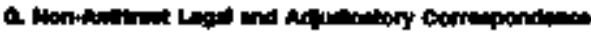

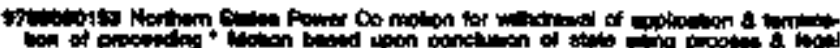

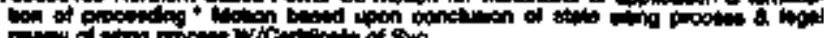

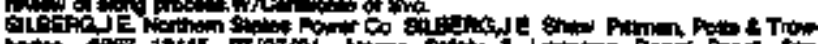

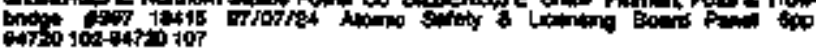

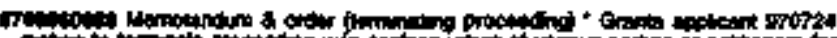

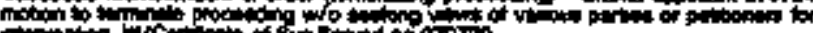

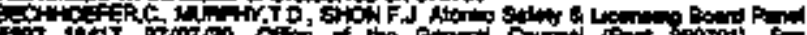

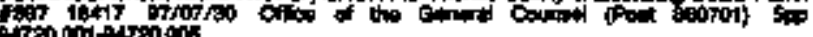

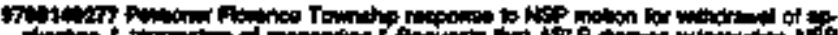

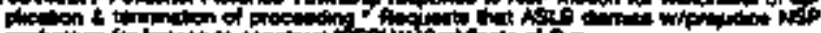

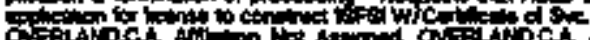

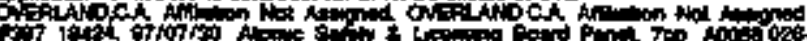
tiotsos

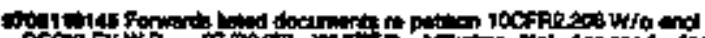

PACM1YY W $\sin 000$

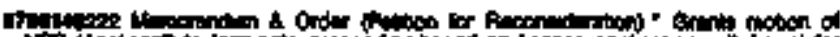

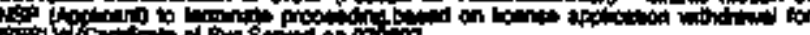
-

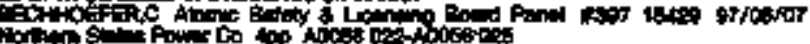

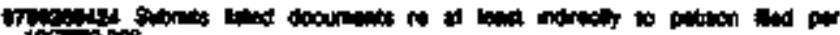

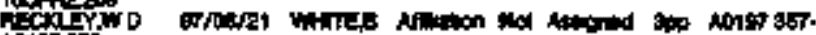
Aकाषे

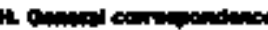

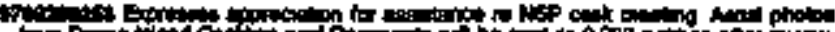

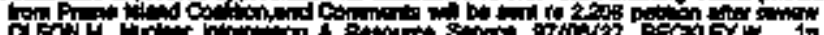

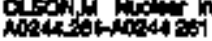

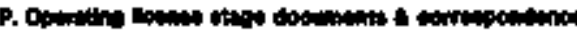

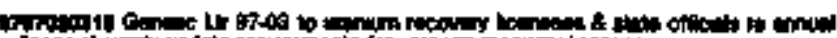

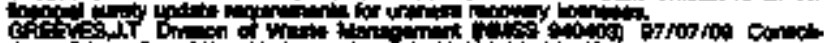

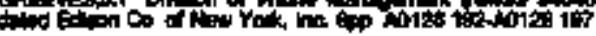

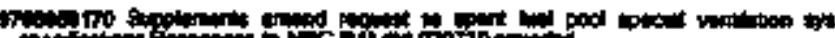

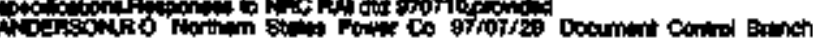

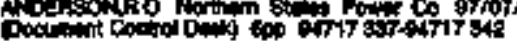

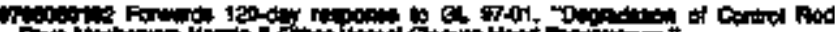

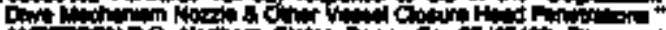

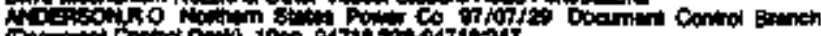

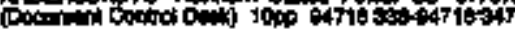

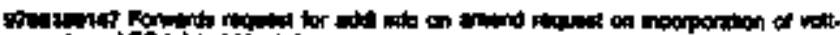
Ö. ATing

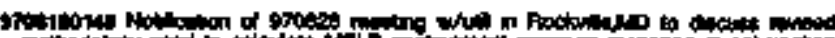

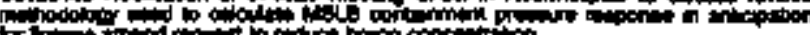

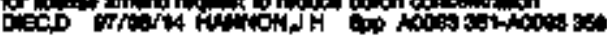

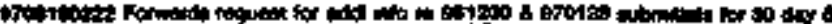

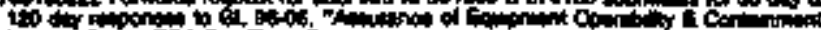

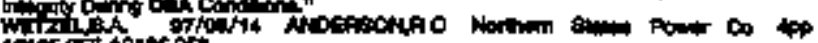
Notos isinoros os.

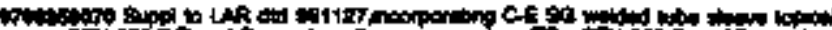

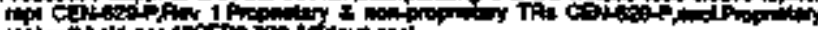

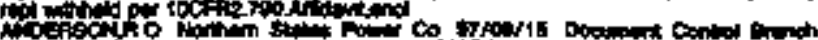

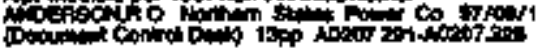

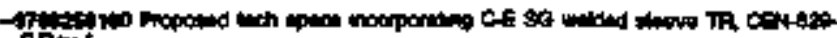

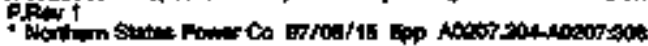

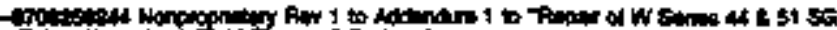

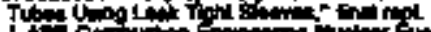

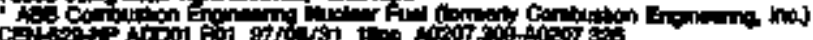

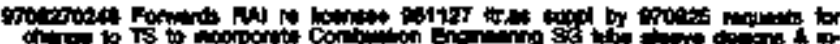

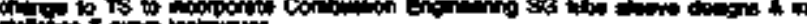

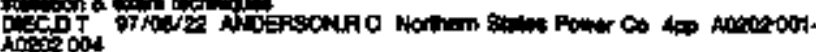

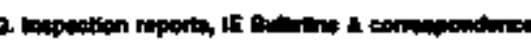

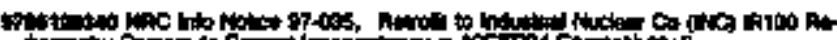

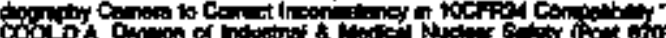

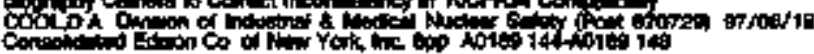

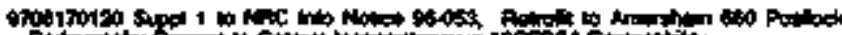

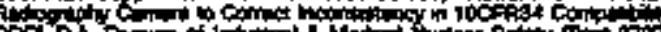

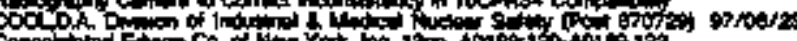

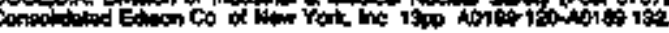

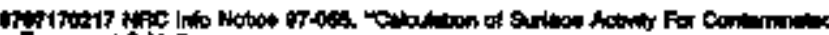

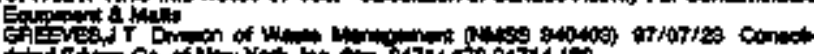

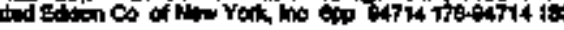

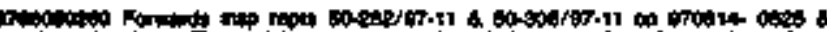

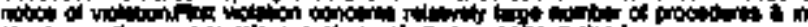

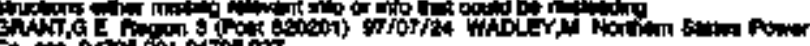

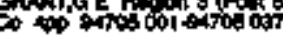

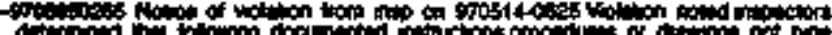

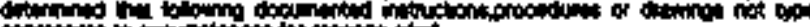

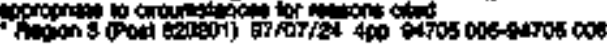

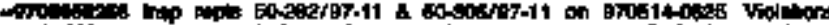

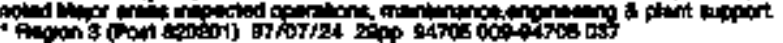

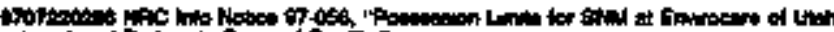

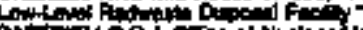

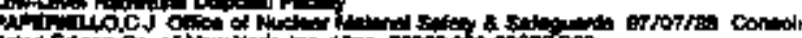

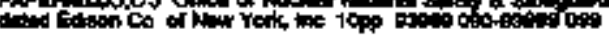

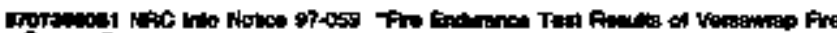

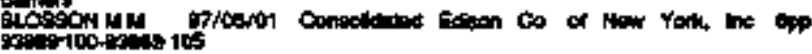

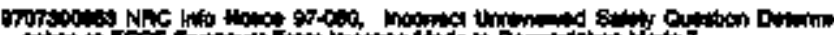

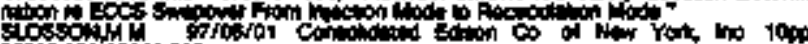

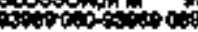

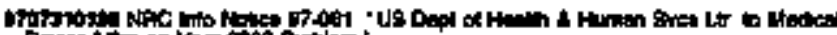
Oa

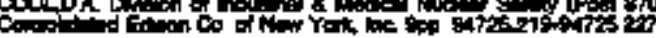

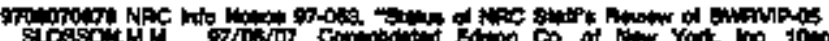

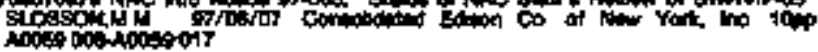

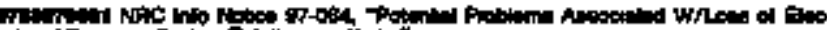

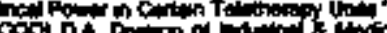

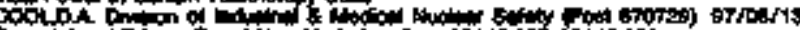

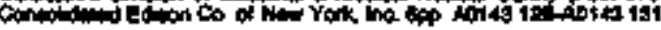

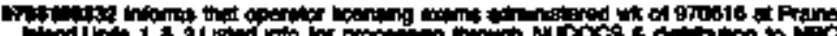

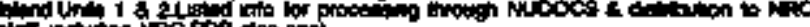

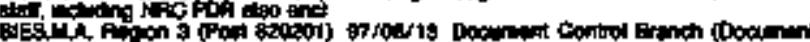

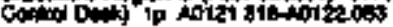

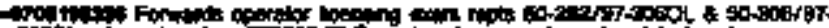

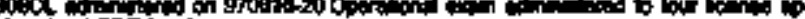

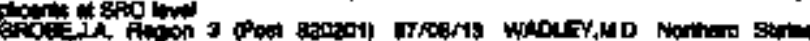

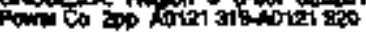

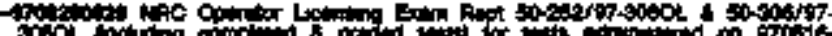

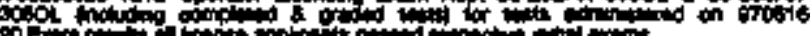
\% -

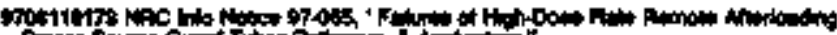

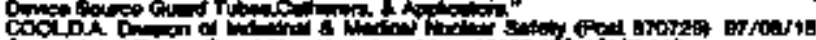

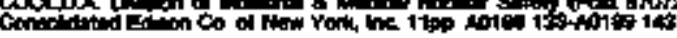

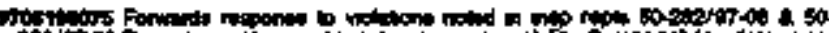

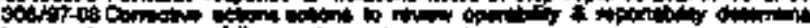

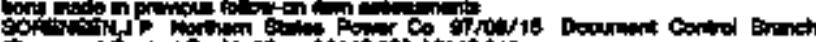

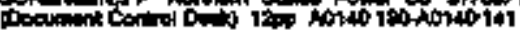

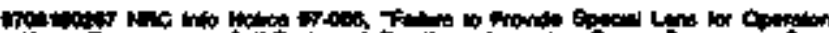
Ute

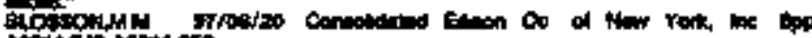

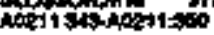

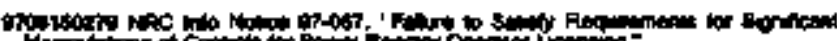

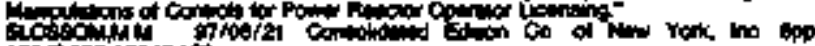
1014 18240140 13

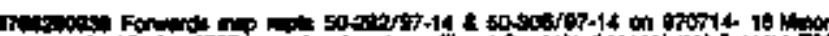

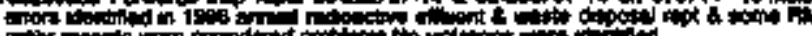

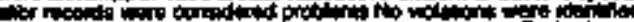
(T)

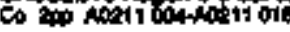

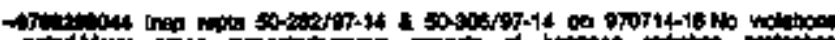

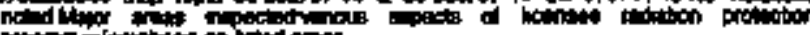

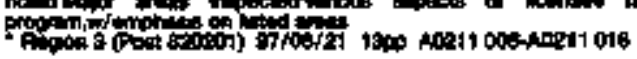




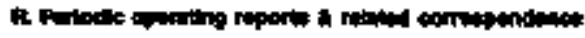

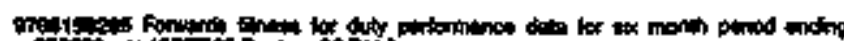

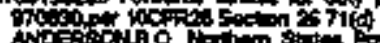

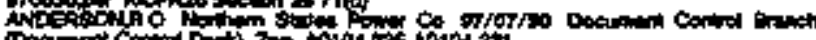

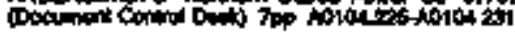

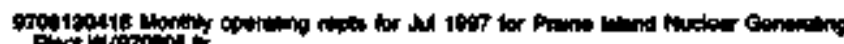

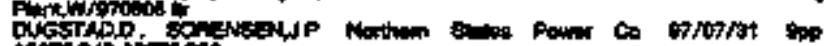

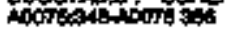

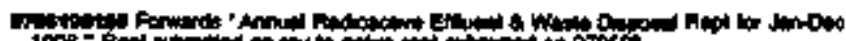

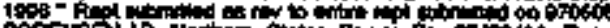

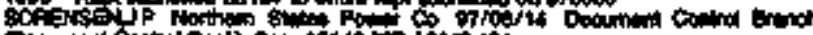

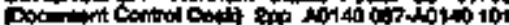

-

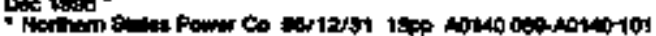

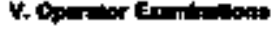

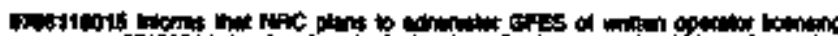

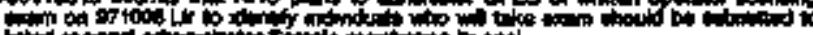

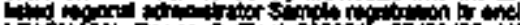

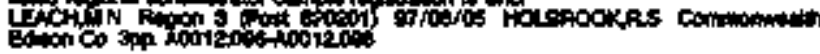

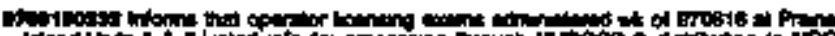

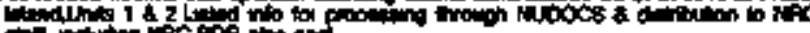

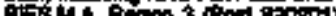

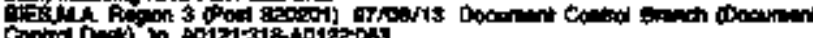

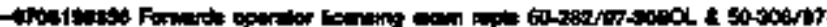

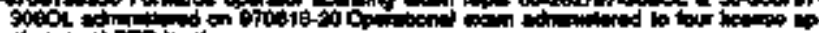

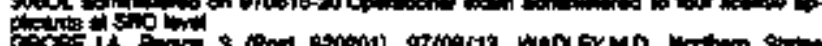

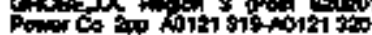

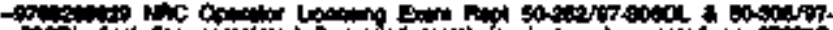

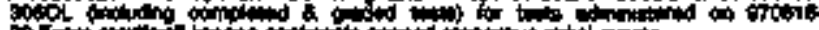

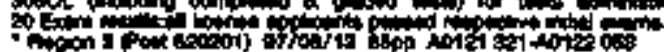

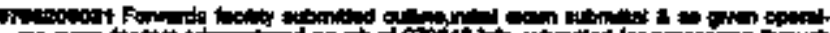

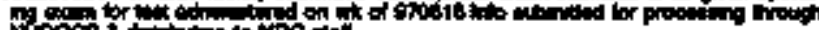

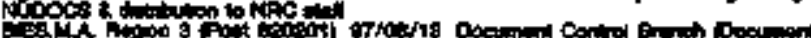

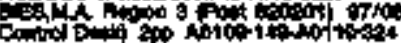

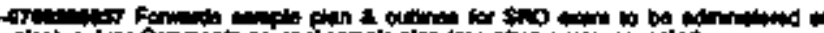

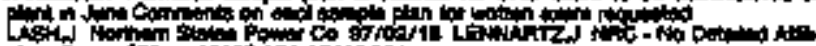

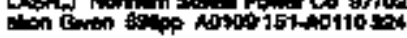

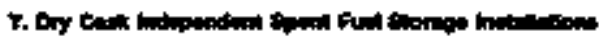

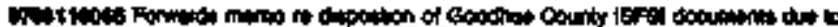

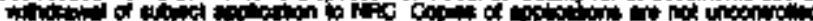

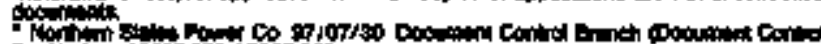
Ding

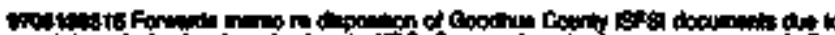

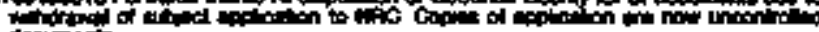

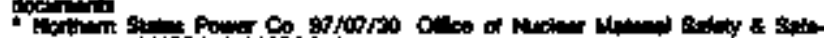

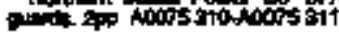

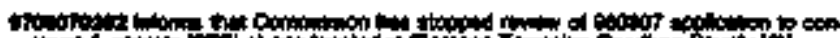

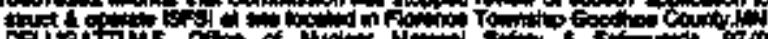

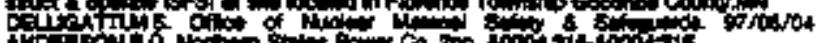

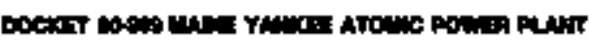

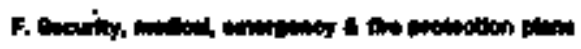

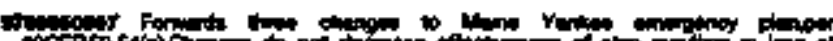

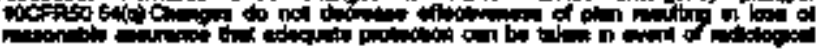

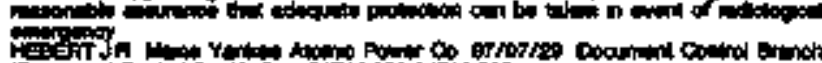

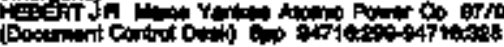

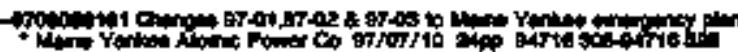

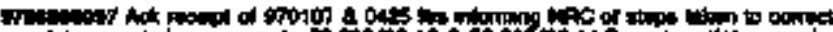

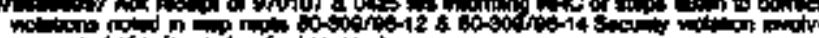

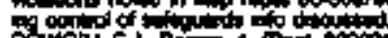

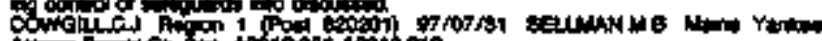

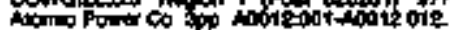

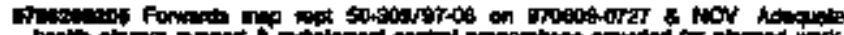

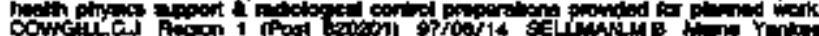

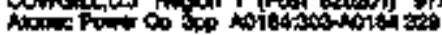

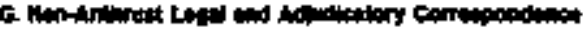

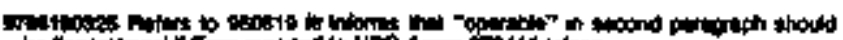

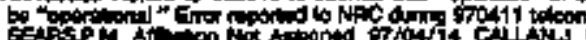

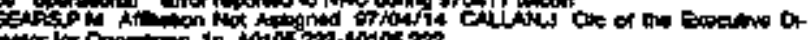

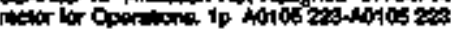

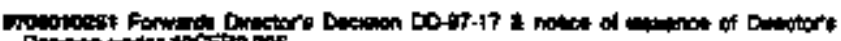

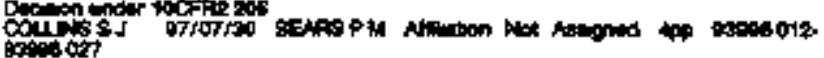

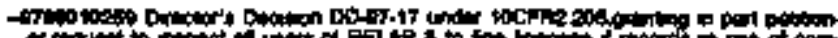

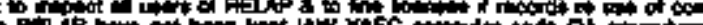

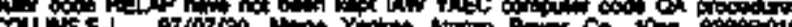
coun

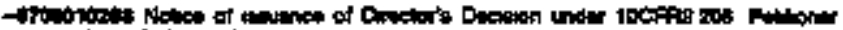

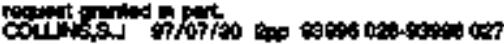

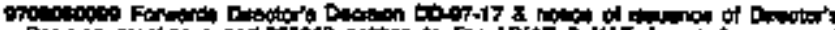

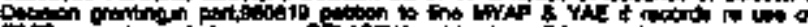

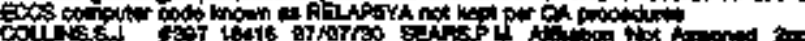

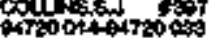

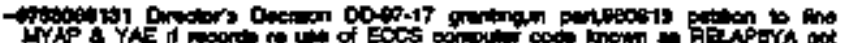

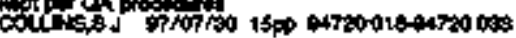

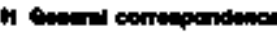

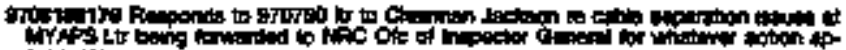

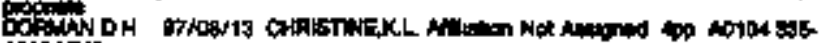
Antod 30

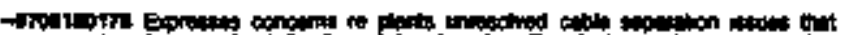

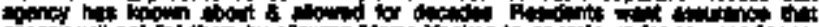

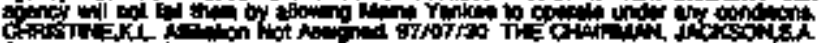

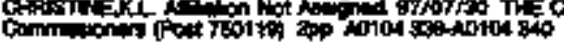

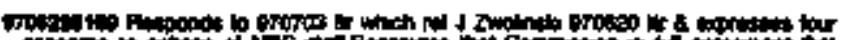

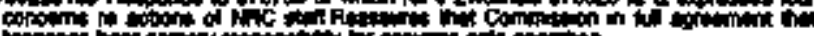

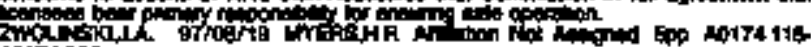
ior? 124

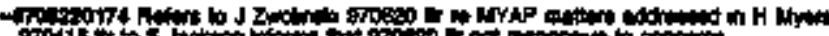

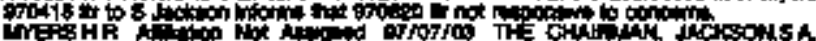

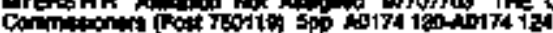

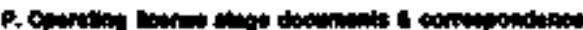

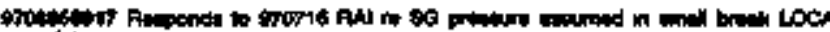

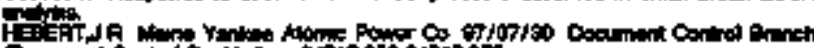

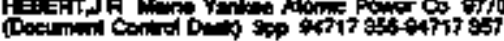

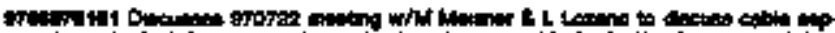

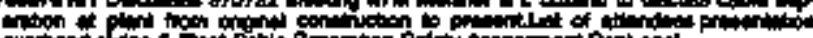
over

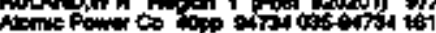

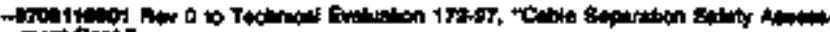

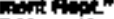

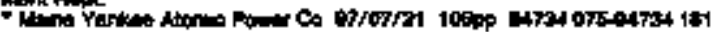

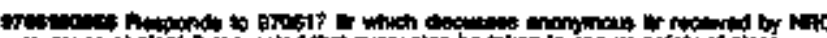

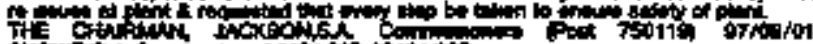

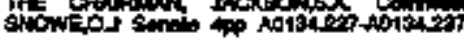

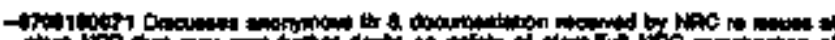

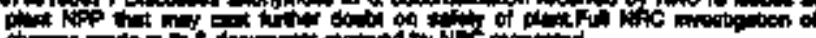

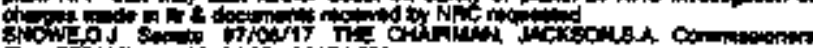

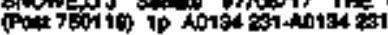

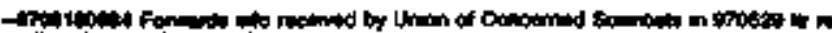

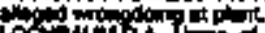

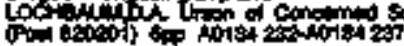

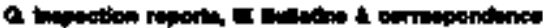

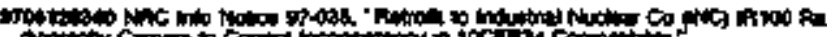

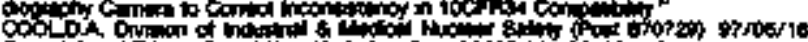

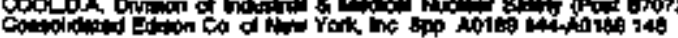

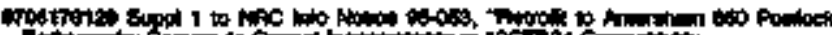

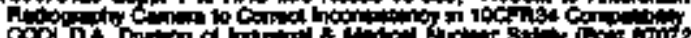

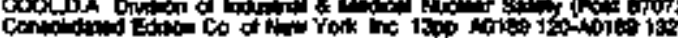




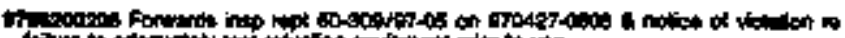

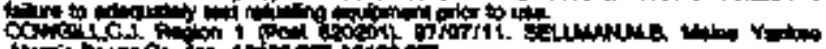

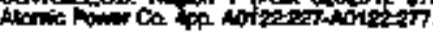

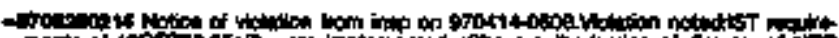

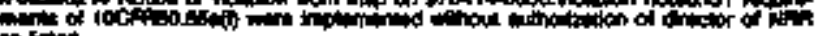

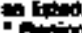

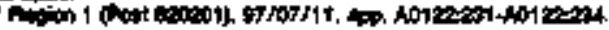

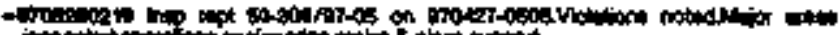

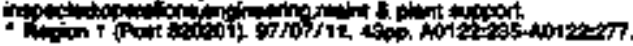

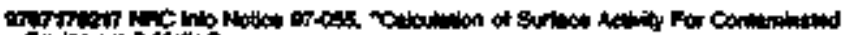

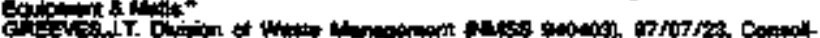

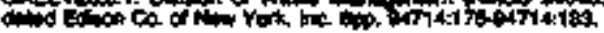

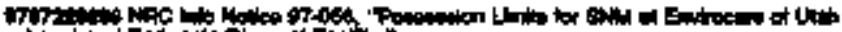

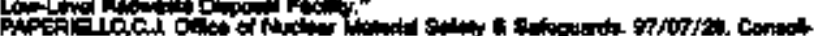

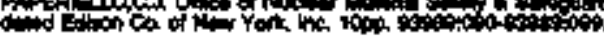

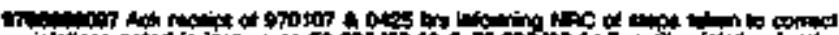

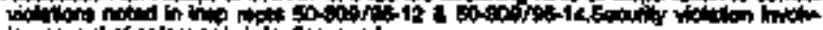

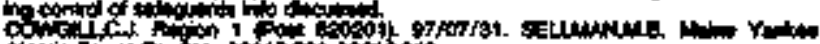
Atong

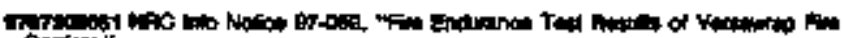
Shosoly a

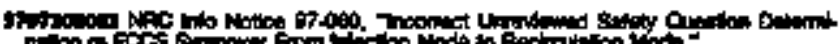

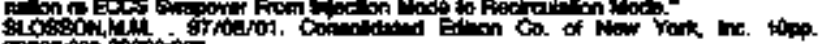

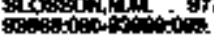

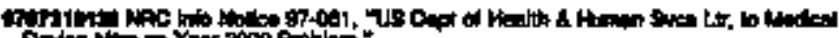

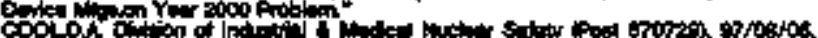

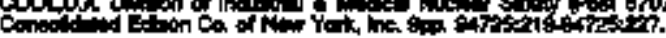

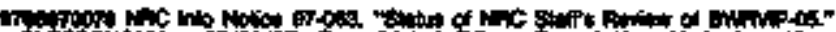

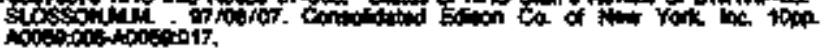

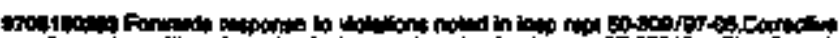

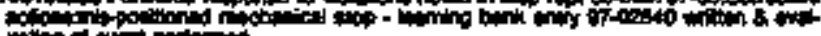

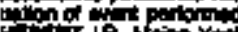

Fon

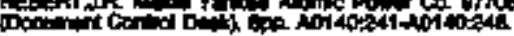

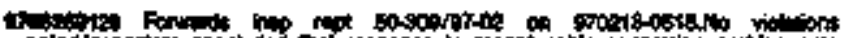

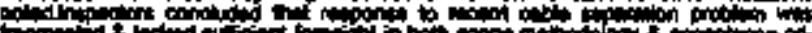

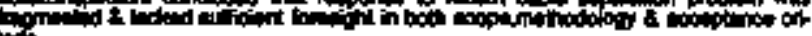

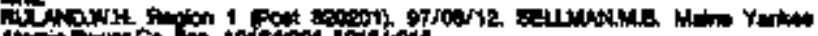

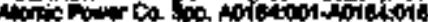

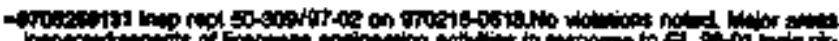

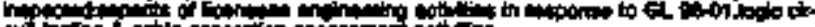

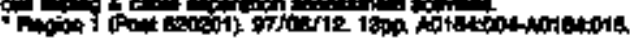

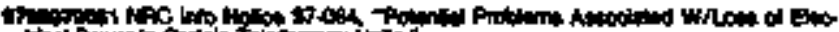

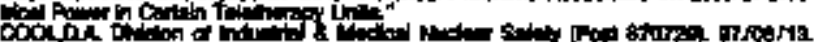

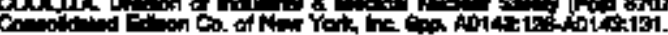

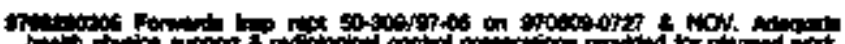

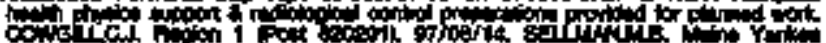

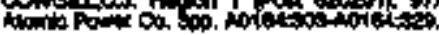

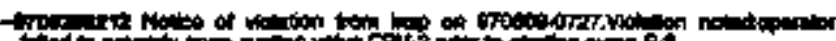

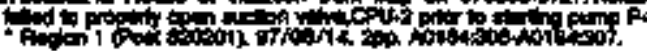

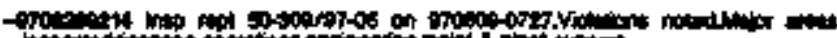

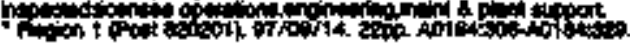

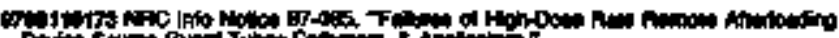

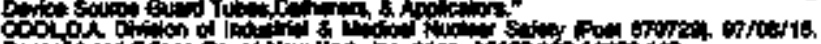

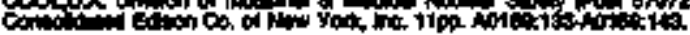

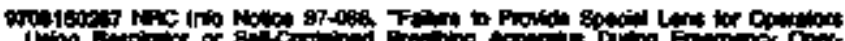

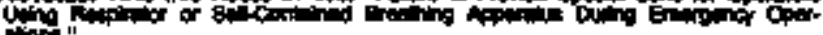

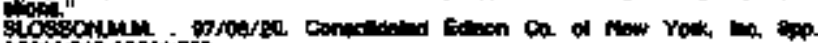

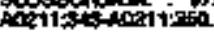

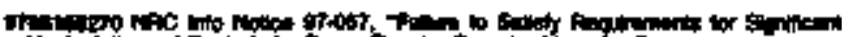

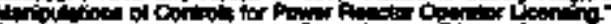

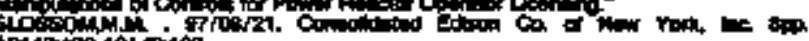

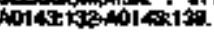

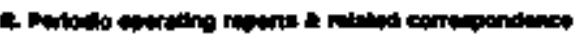

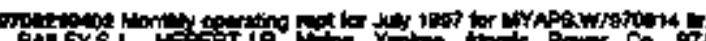

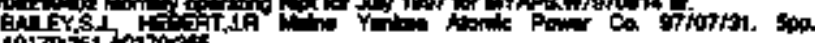

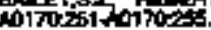

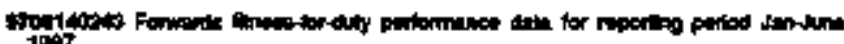
1007.

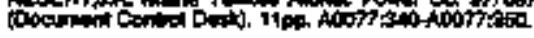

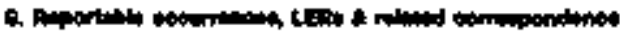

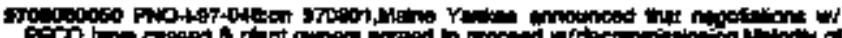

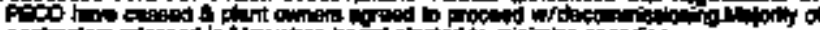

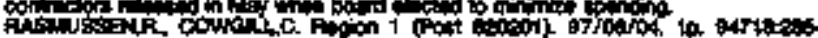
$94710 \mathrm{xas}$

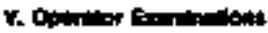

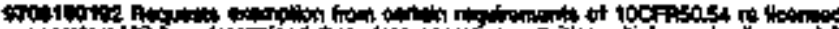

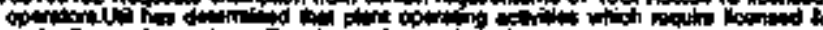

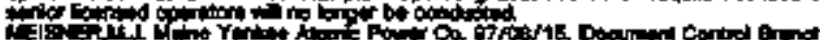

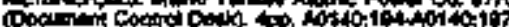

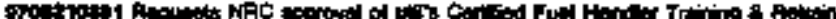

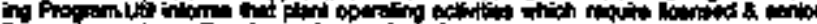

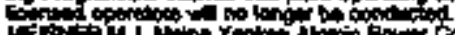

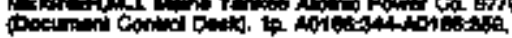

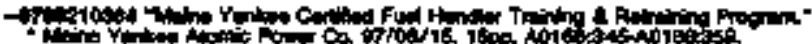

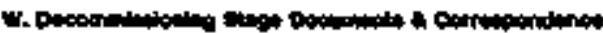

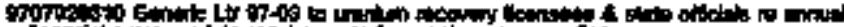

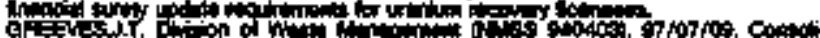

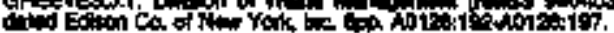

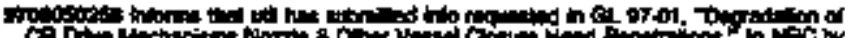

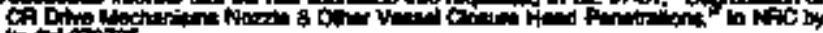

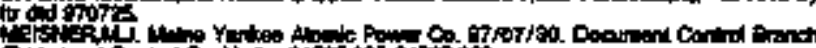

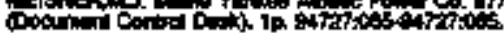

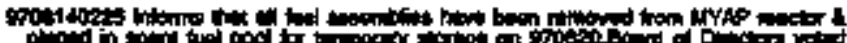

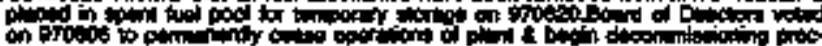

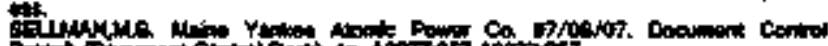

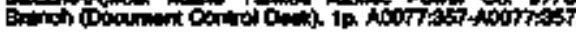

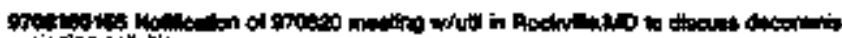

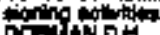

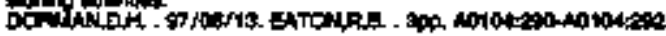

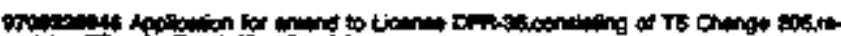

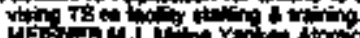

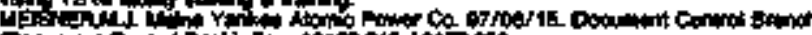

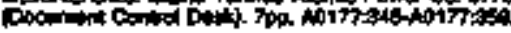

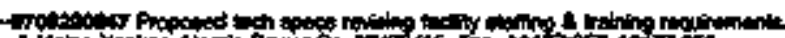

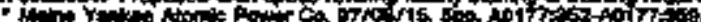

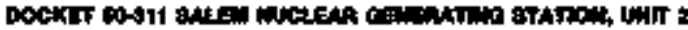

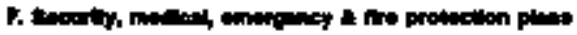

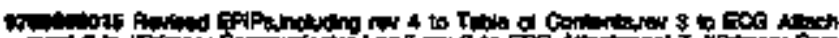

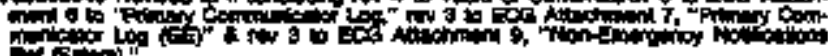

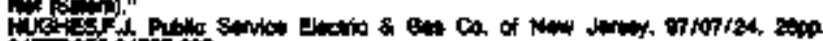

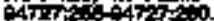

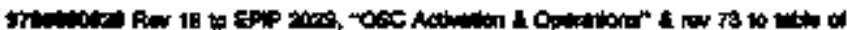

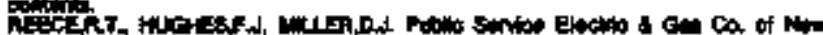

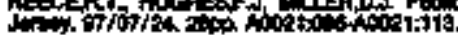

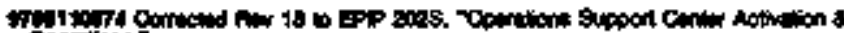

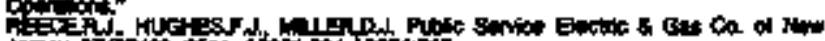

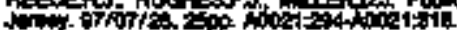

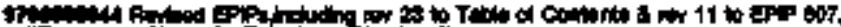

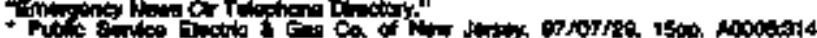
nocosisa,

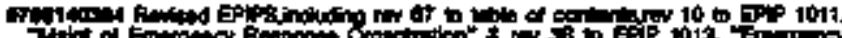

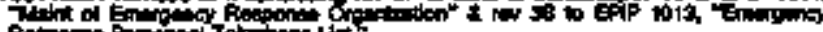

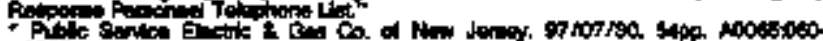
Aoptiti.

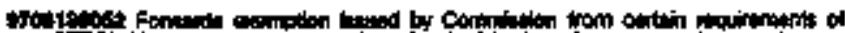

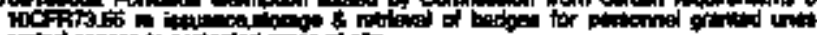

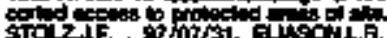

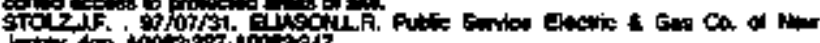

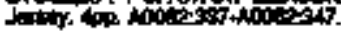




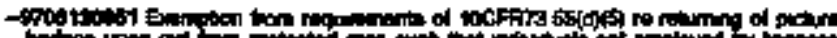

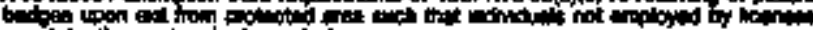

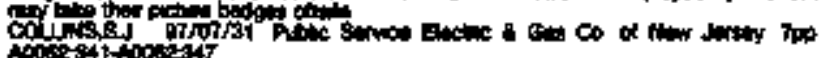

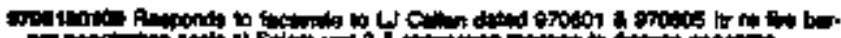

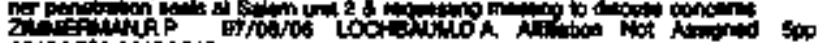

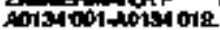

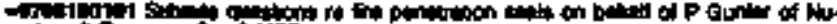

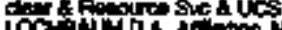

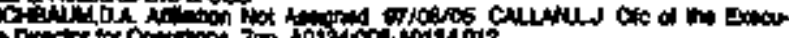

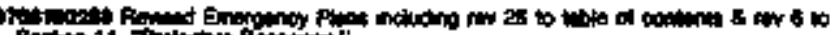

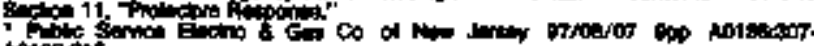
Aotsions

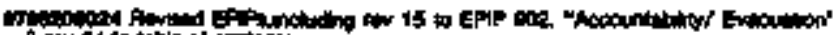

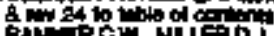

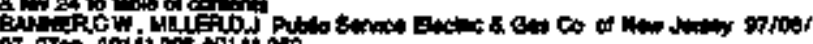

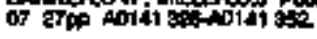

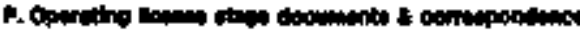

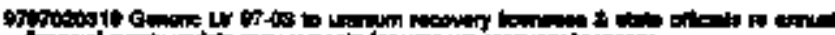

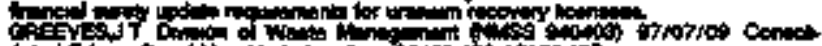

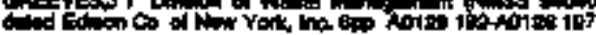

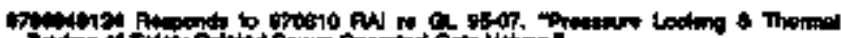

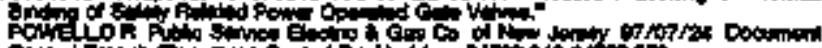
contion

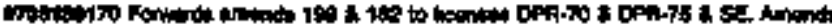

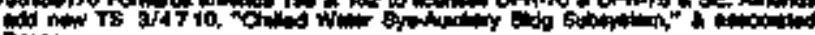

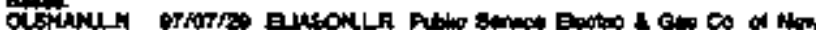

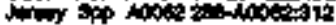

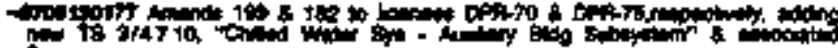

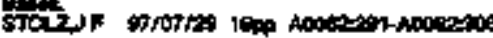

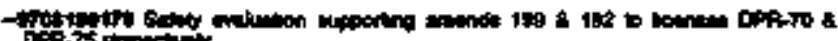

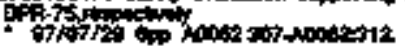

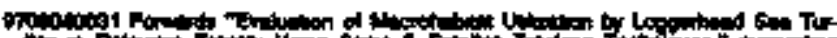

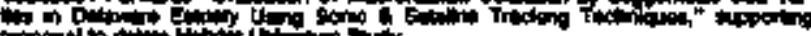

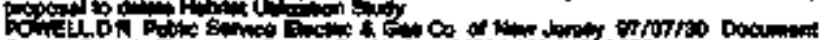
Con

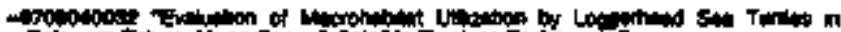

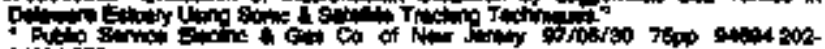
anciotis

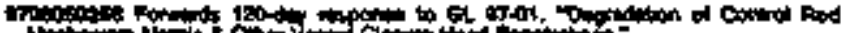

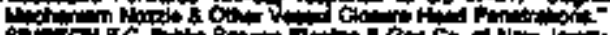

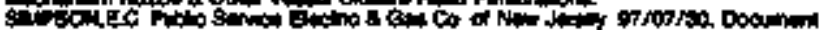

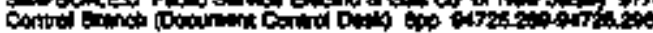

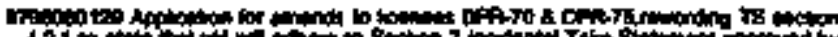

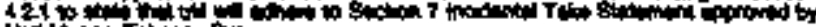

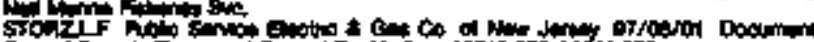

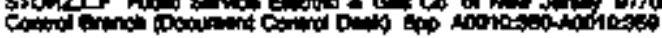

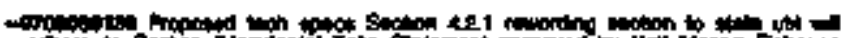

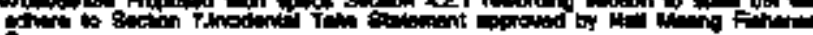

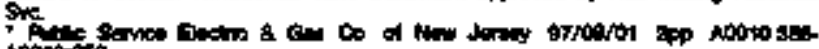
$\cos 0-30$

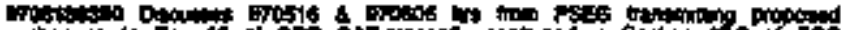

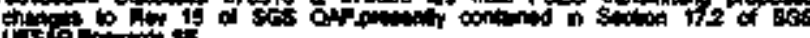

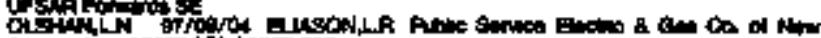

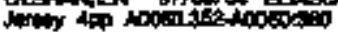

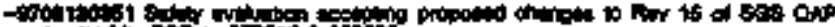

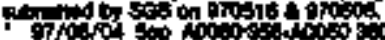

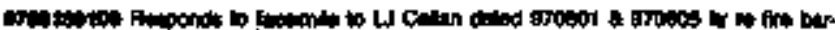
if Aol34001-4014012

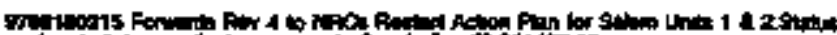

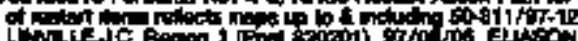

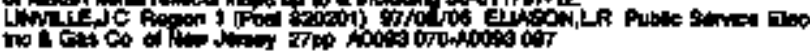

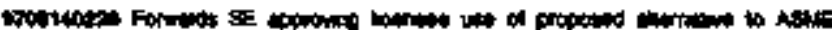

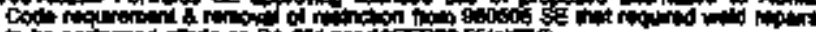

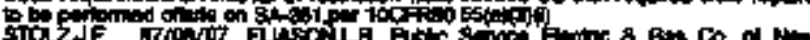

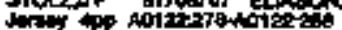

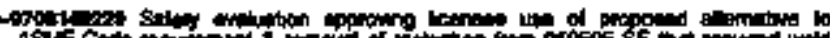

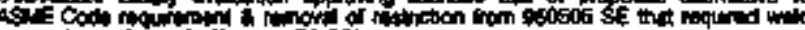
का

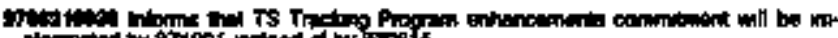

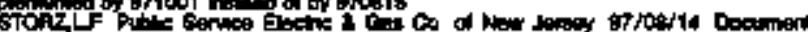

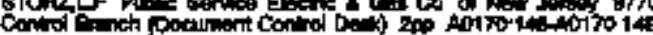

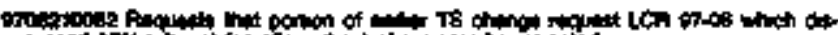

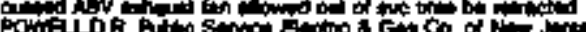

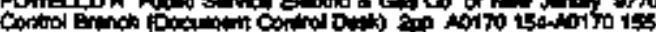

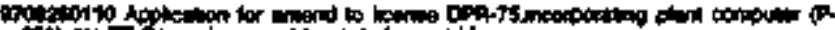

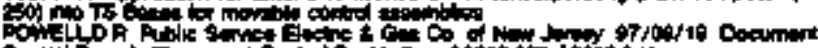

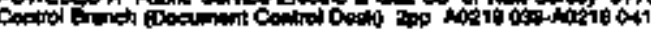

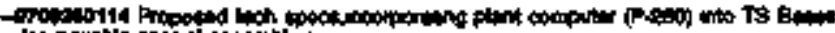

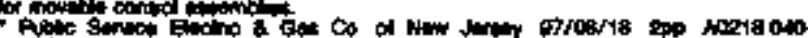

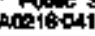

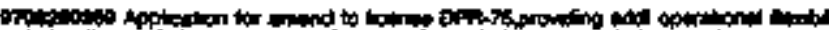

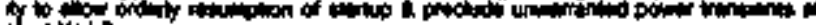
Tht lint 2

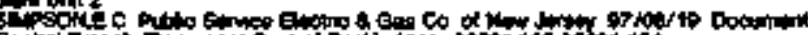

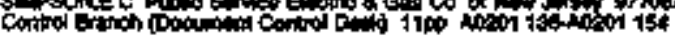

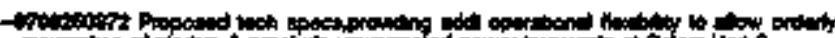

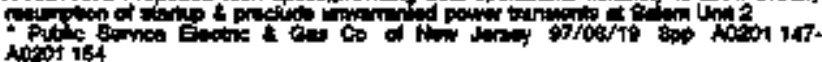

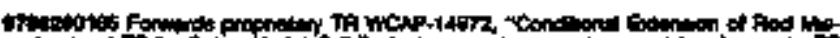

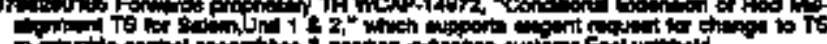

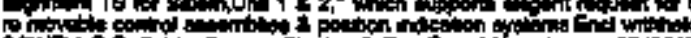
C

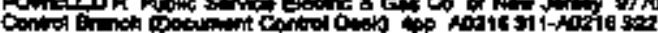

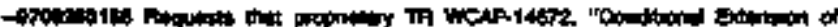

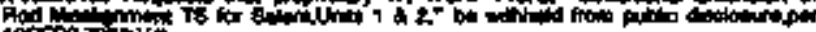

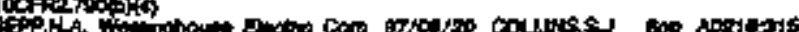
Acisis

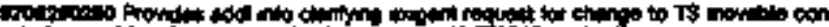

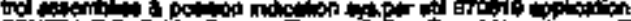

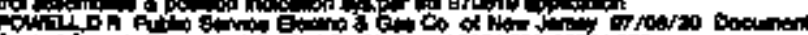

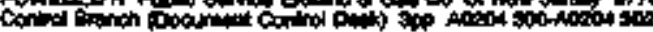

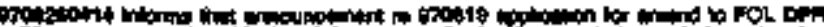

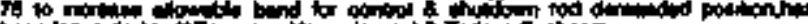

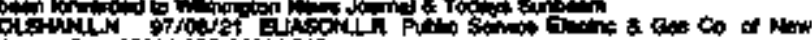

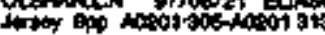

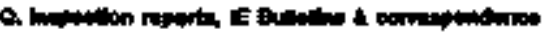

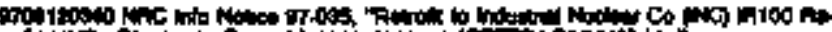

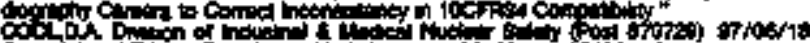

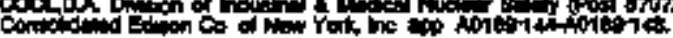

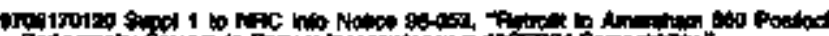

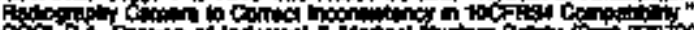

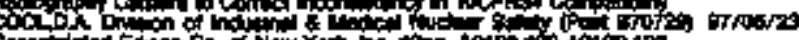

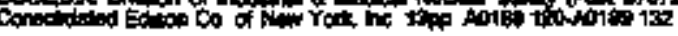

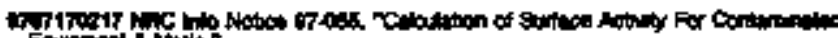

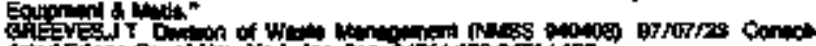

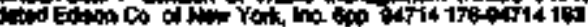

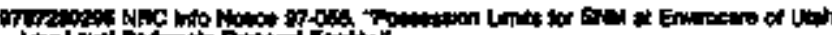

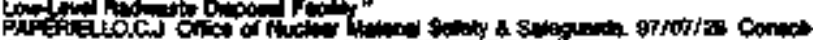

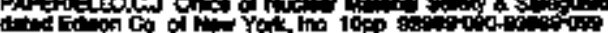

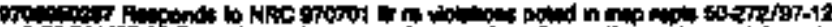

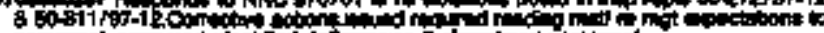

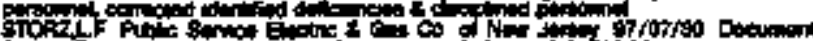

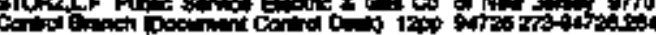

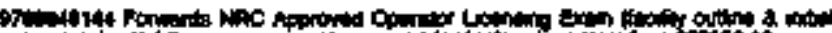

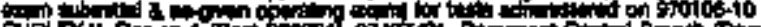

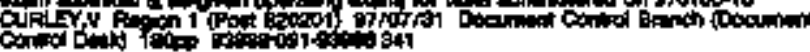

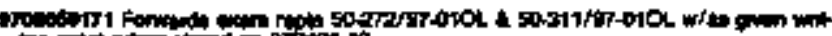

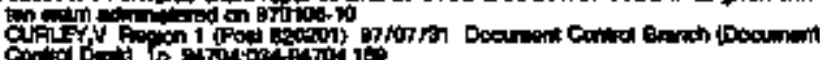

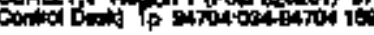

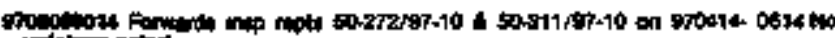

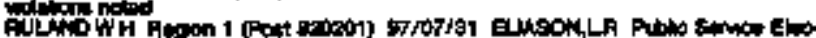

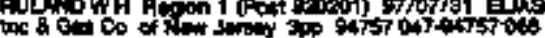

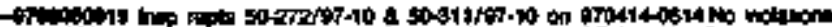

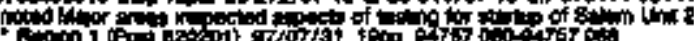




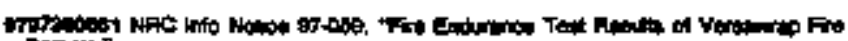

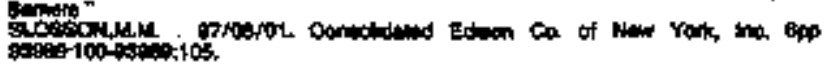

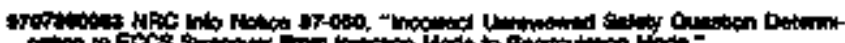

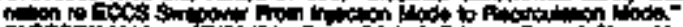

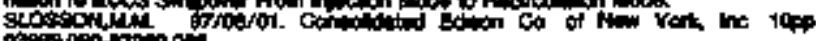

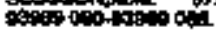

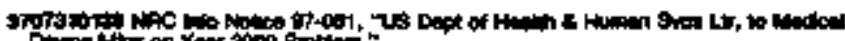

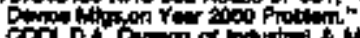

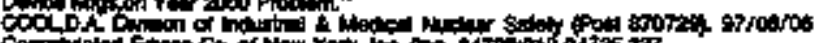

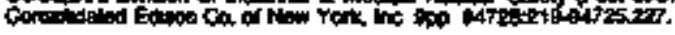

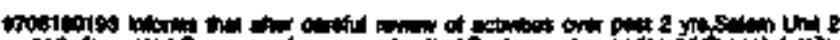

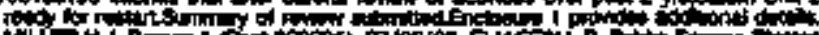

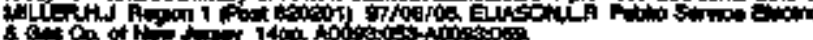

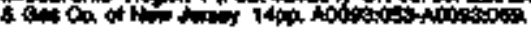

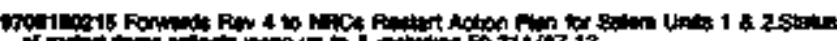

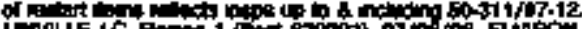

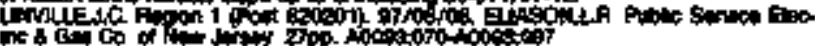

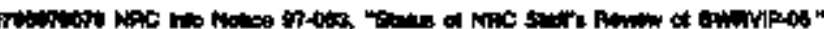

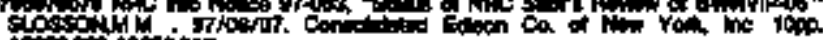

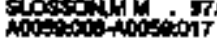

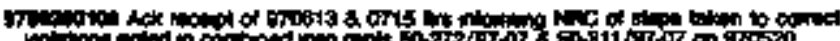
I-ivite

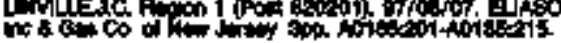

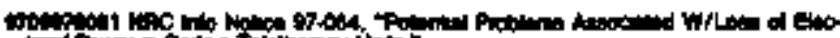

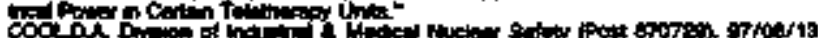

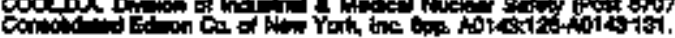

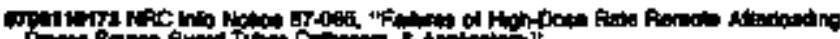

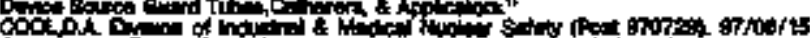

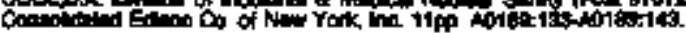

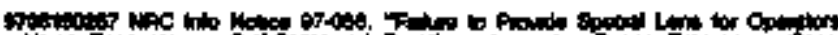

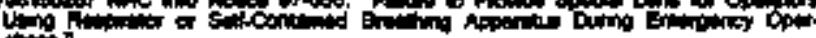

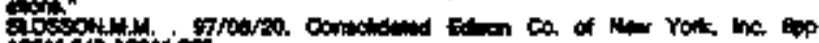

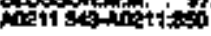

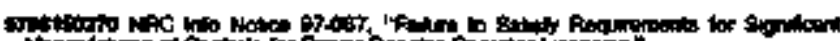

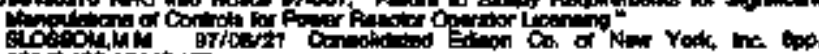

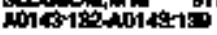

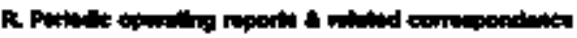

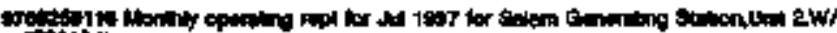

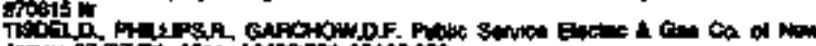

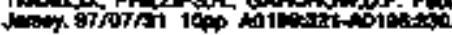

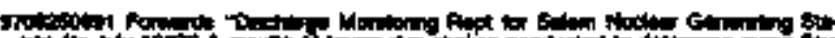

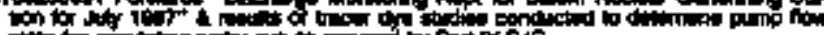

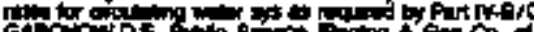

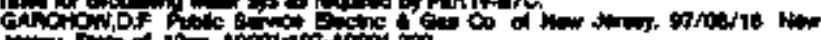

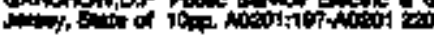

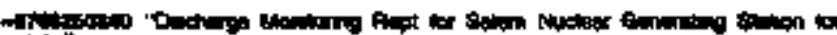

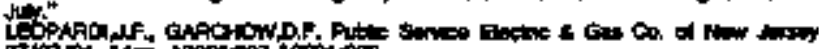

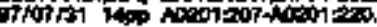

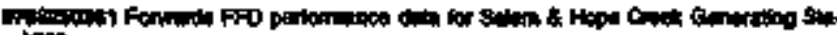
pan

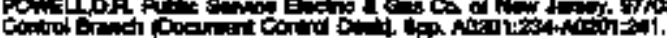

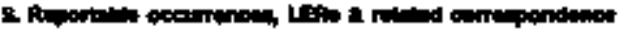

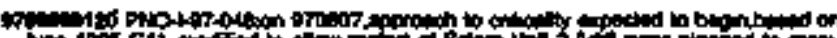

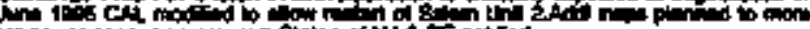

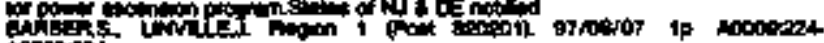

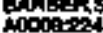

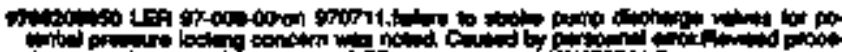

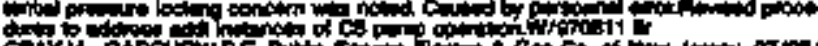

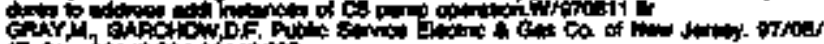
ir. top 1001 .

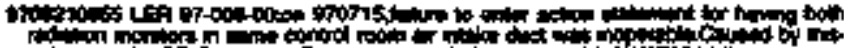

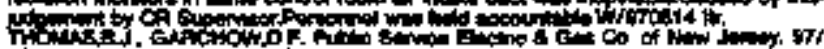

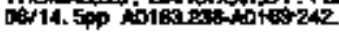

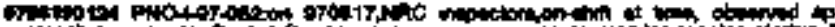

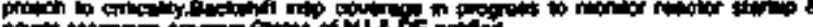

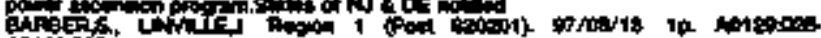
Dotionat

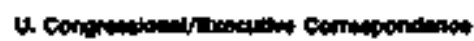

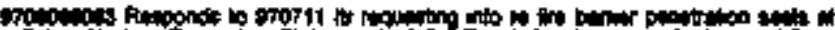

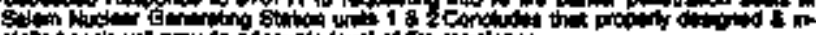

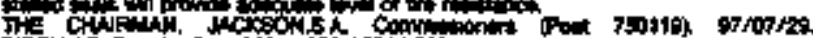

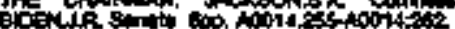

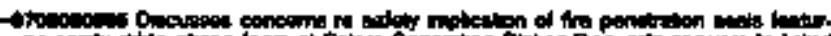

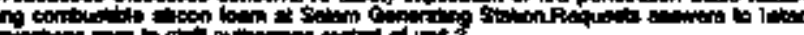

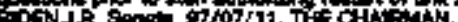

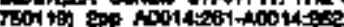

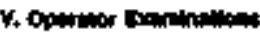

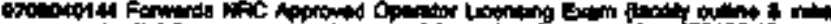

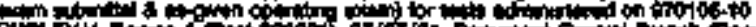

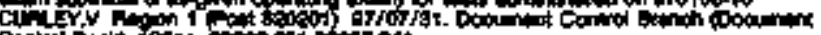

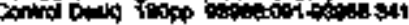

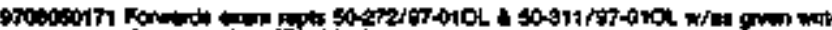

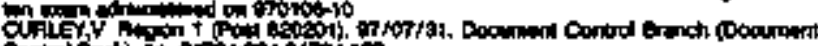

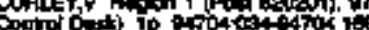

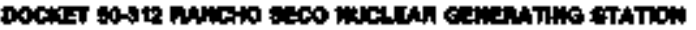

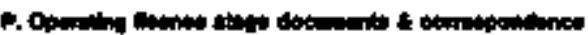

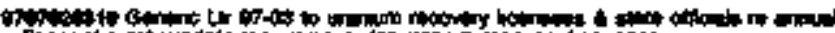
at

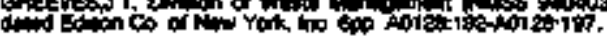

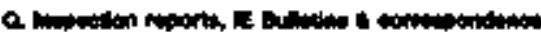

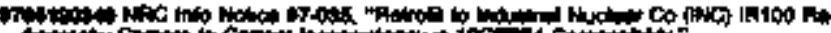

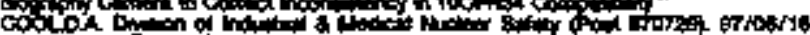

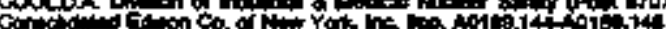

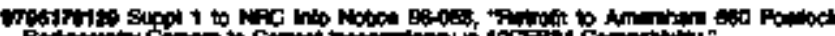

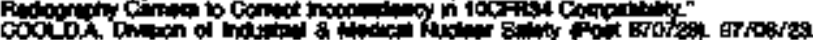

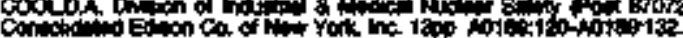

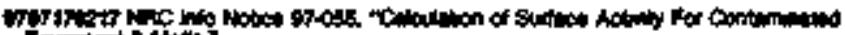

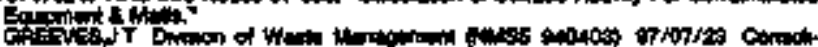

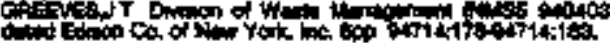

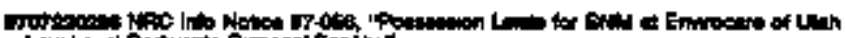

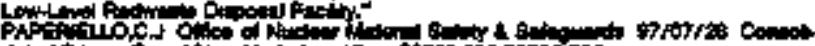

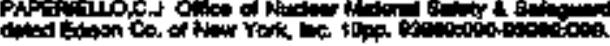

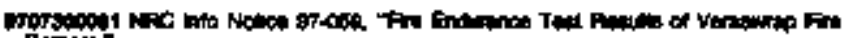
Bumper

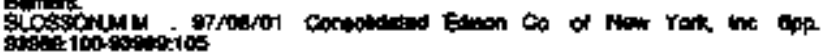

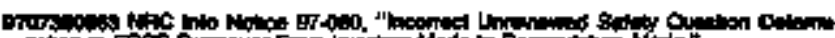

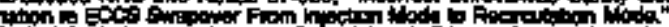

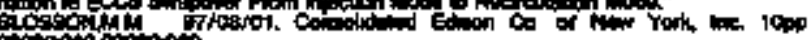
\$

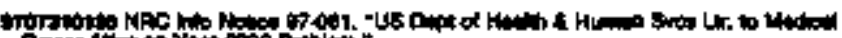

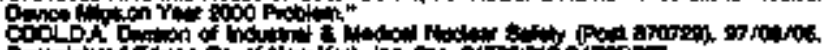

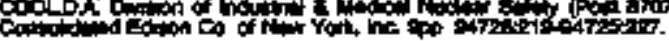

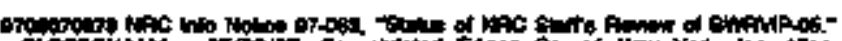

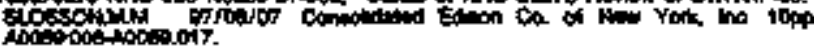

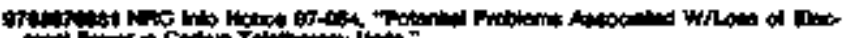

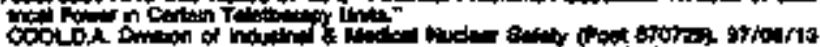

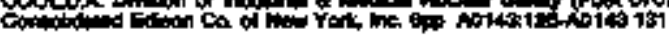

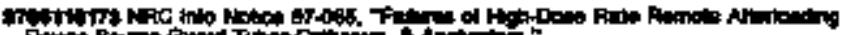

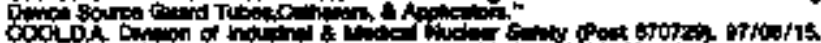

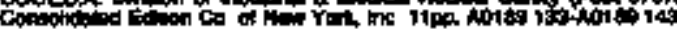

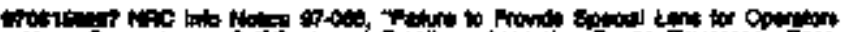

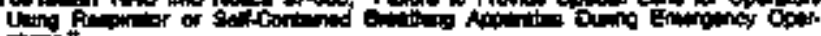

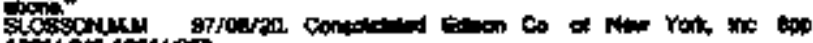

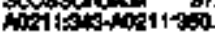

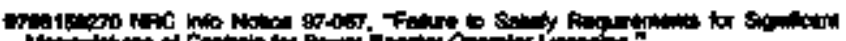

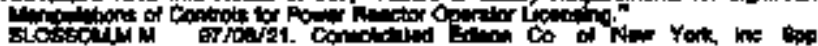
closecthy in wo 


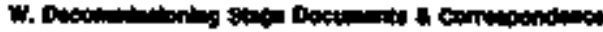

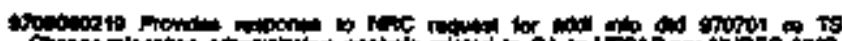

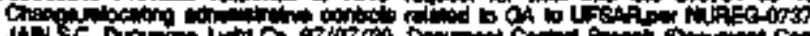

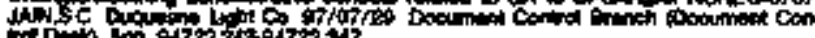

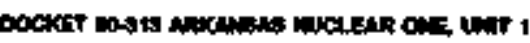

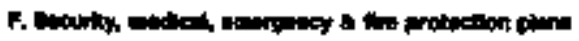

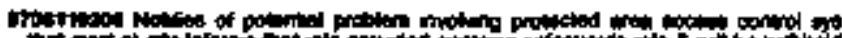

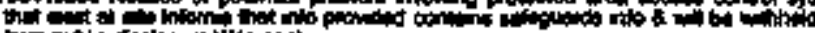

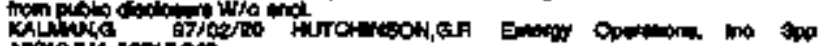

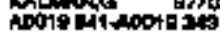

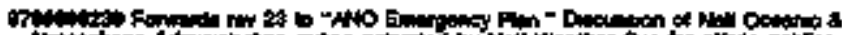

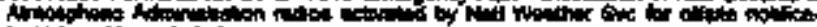

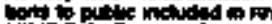

Con

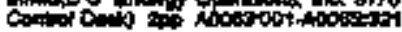

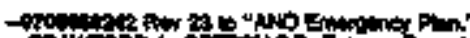

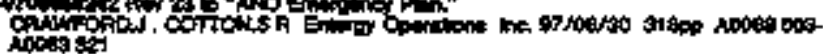

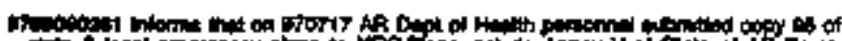

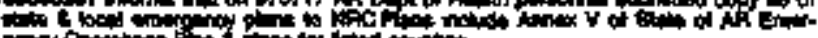

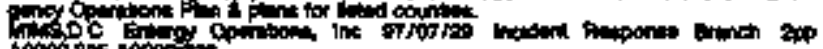
A0000 so:

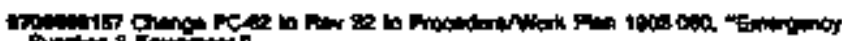

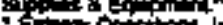

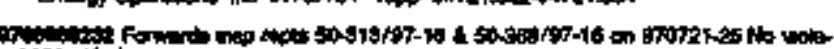

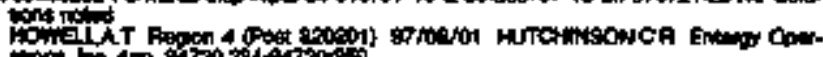

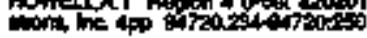

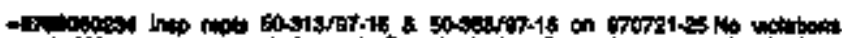

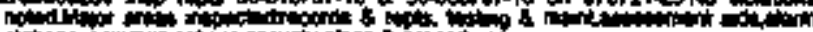

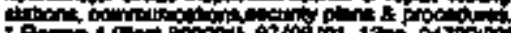

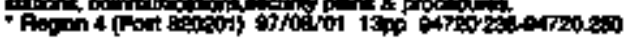

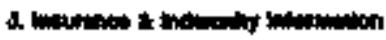

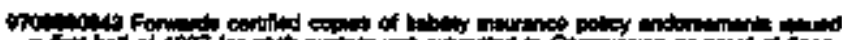

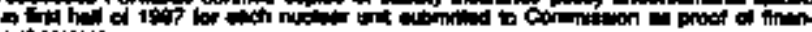

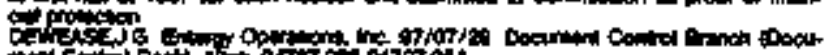

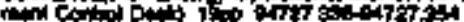

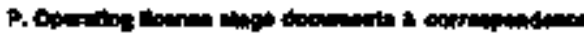

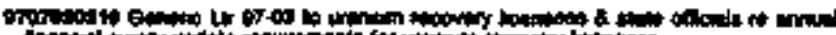

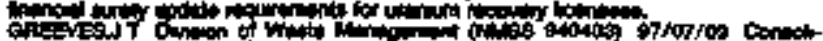

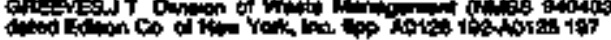

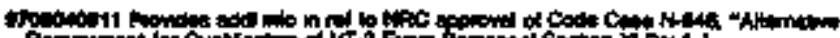

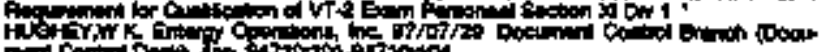

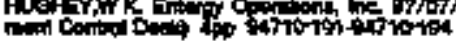

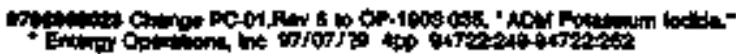

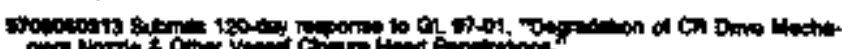

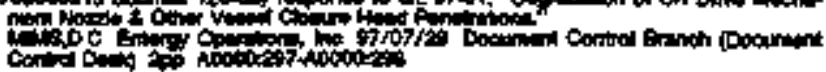

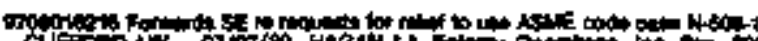

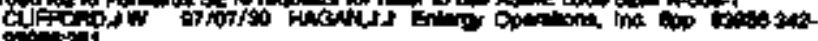

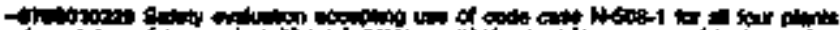

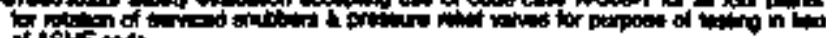
of $\sin$ god

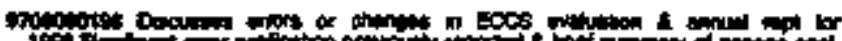

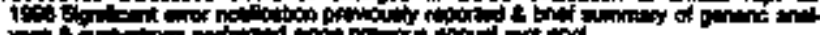

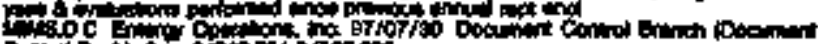

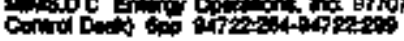

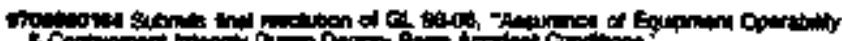

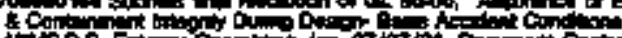

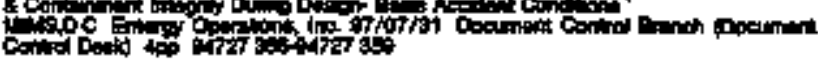

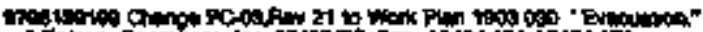

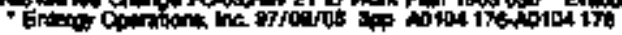

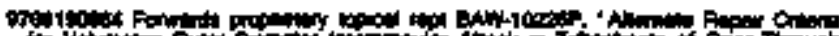

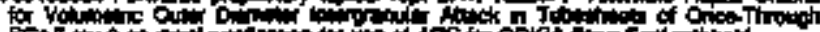

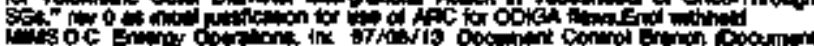

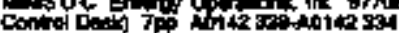

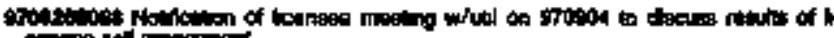

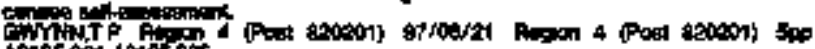
colós

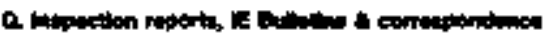

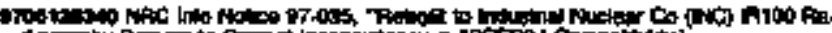

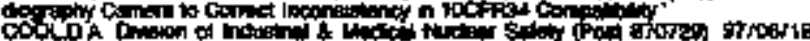

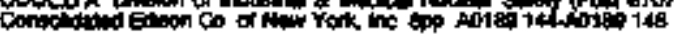

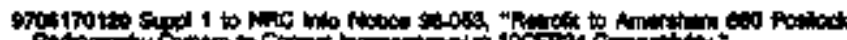

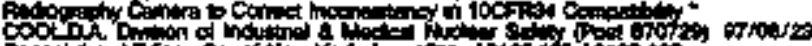

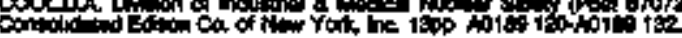

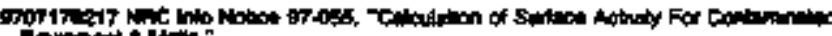

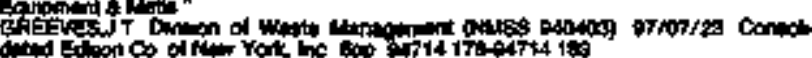

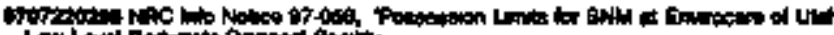

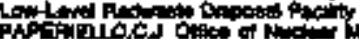

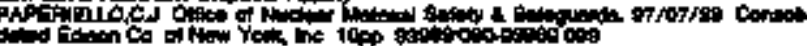

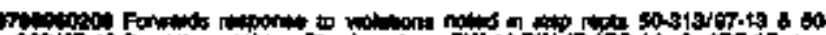

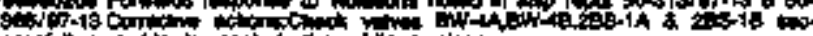

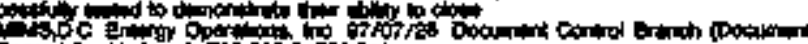

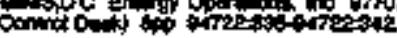

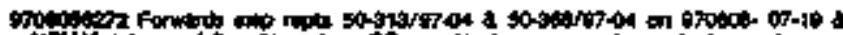

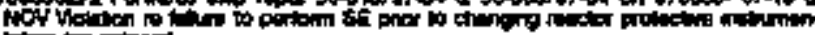
itim trition

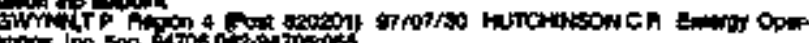

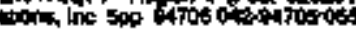

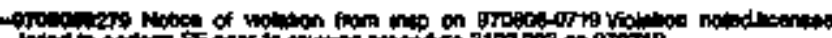

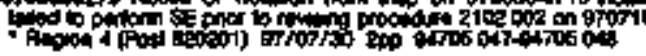

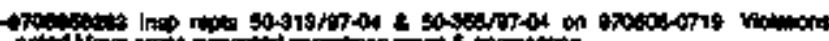

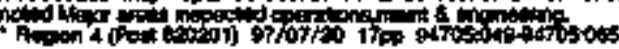

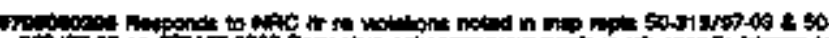

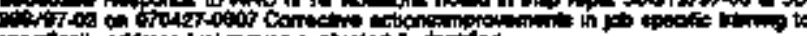

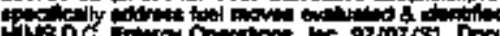

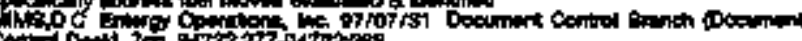

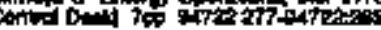

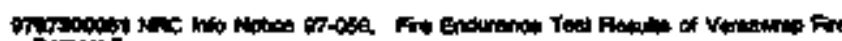

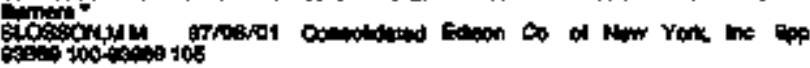

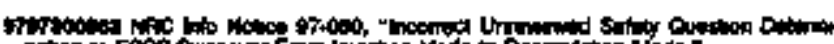

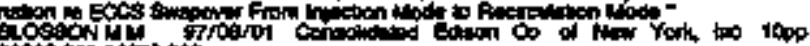
$\cos _{\text {ond }}$

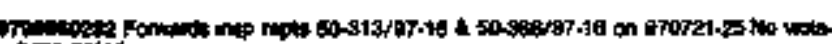

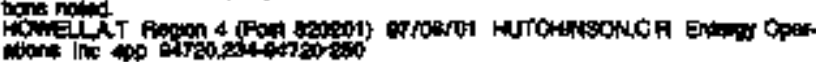

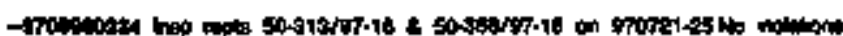

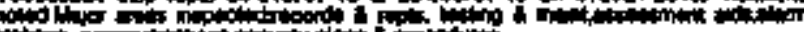

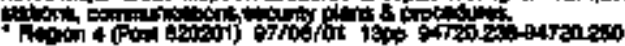

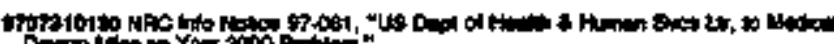

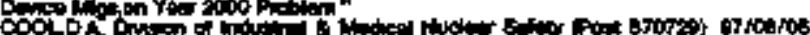

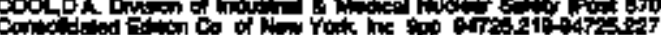

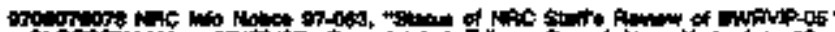

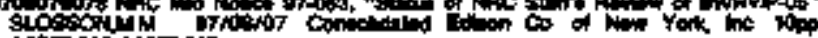

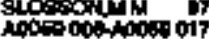

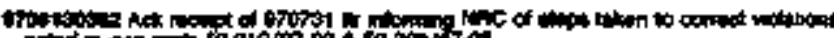

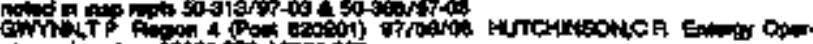

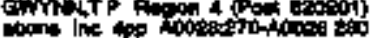

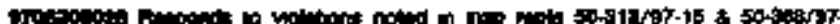

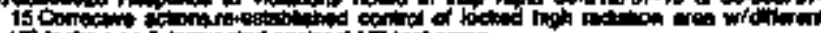

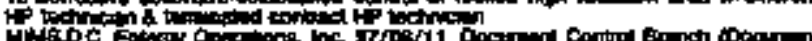

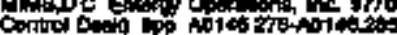

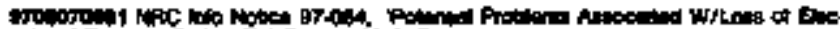

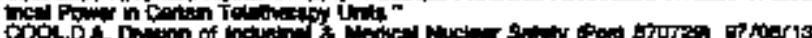

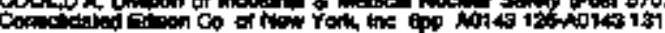

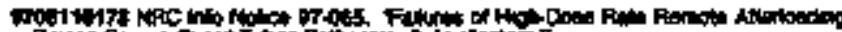

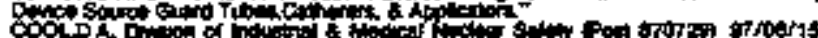

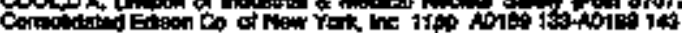




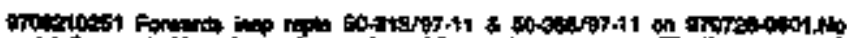

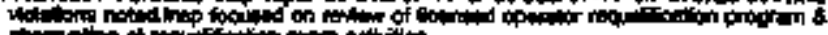
Fth

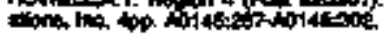

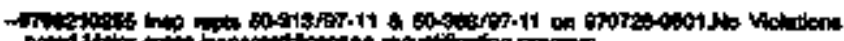

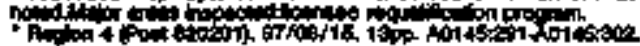

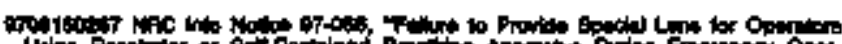

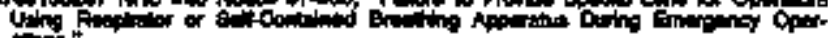

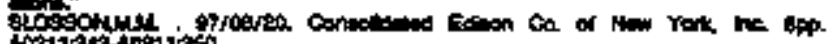
A

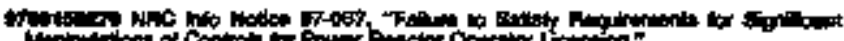

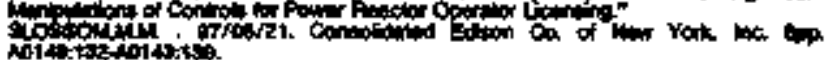

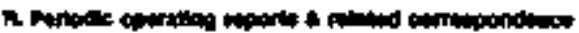

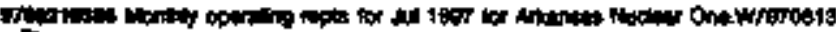

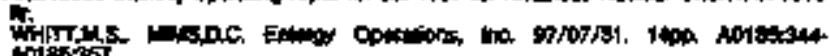

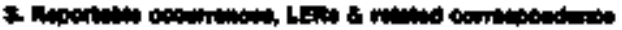

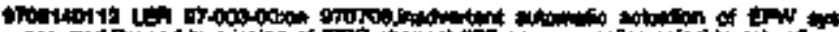

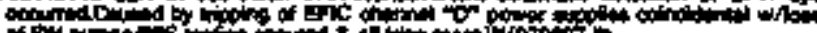

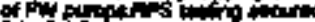

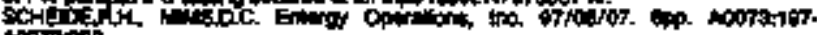
notion

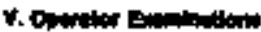

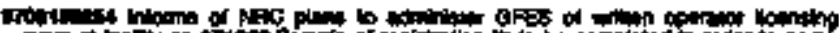

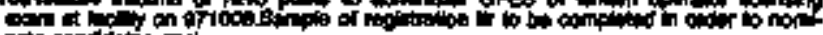

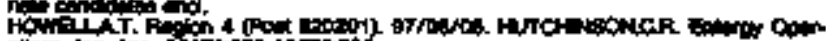

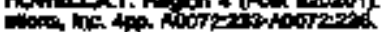

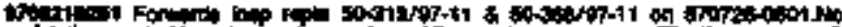

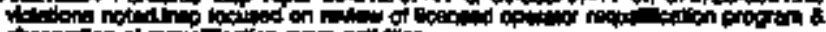

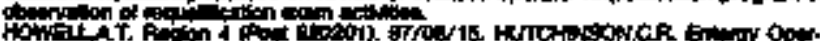

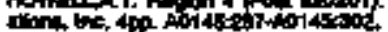

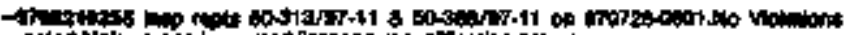

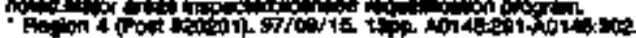

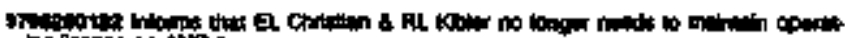

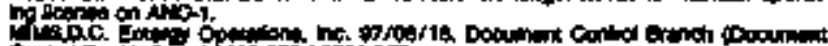

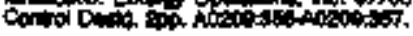

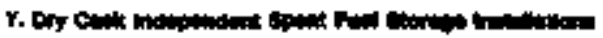

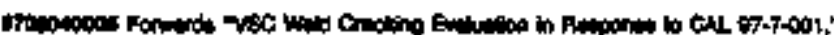

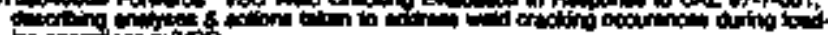

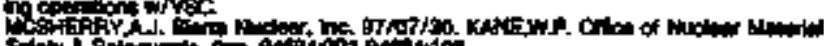

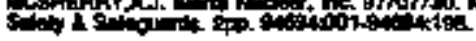

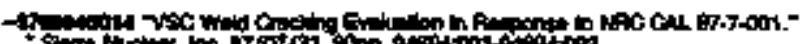

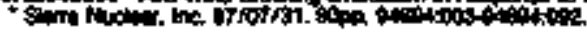

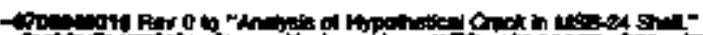

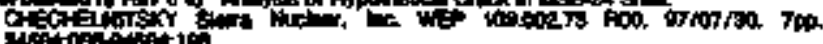

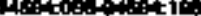

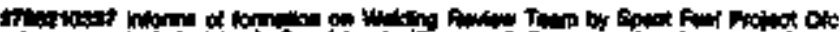

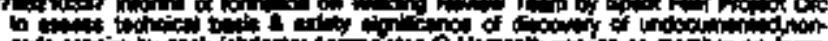

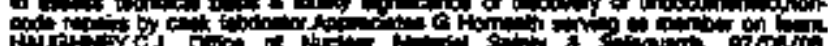

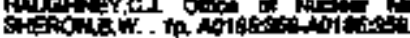

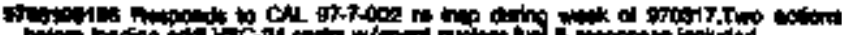

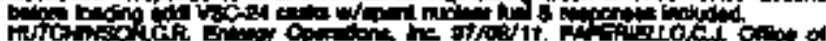

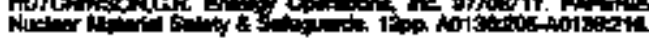

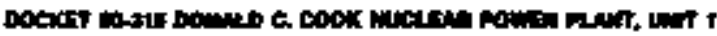

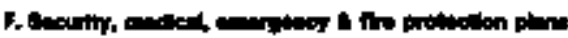

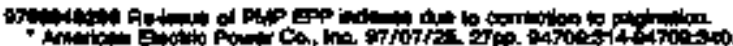

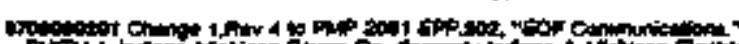

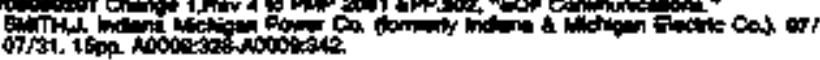

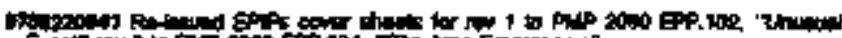

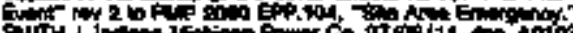

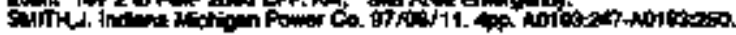

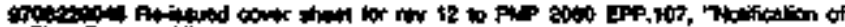

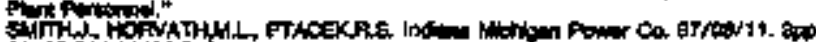
not

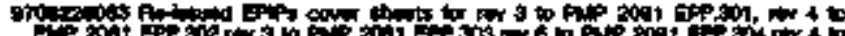
Fir 200\%

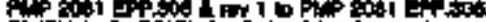

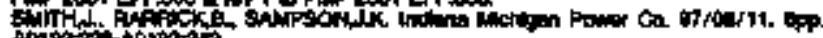
Antos

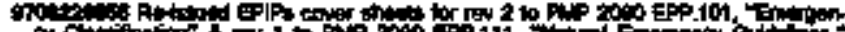

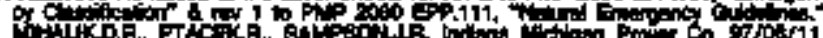

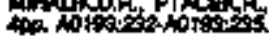

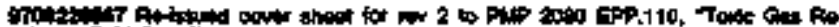

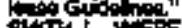

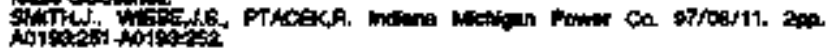

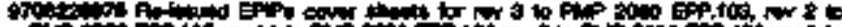

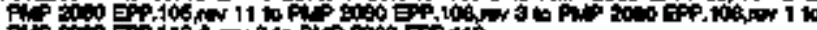

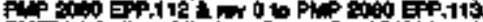

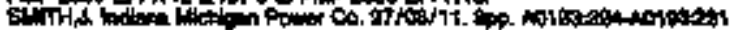

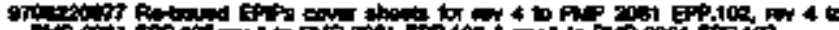

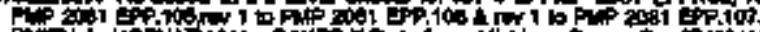

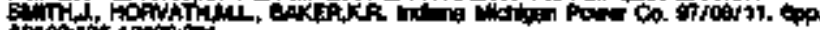

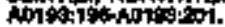

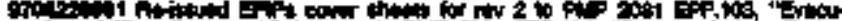

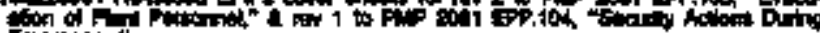

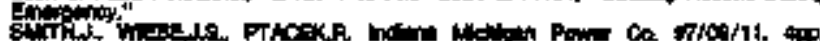
Aorition

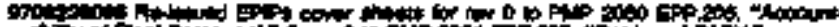

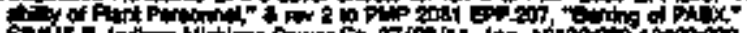

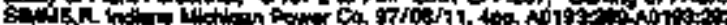

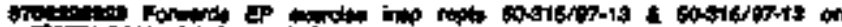

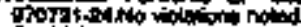

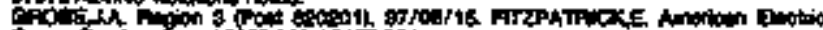

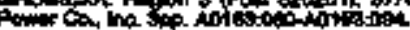

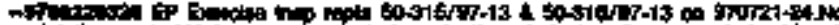

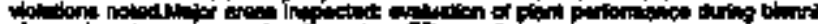

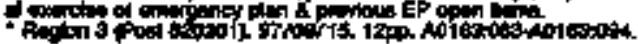

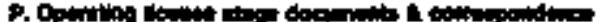

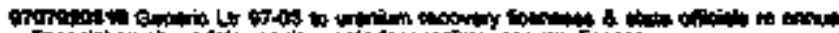

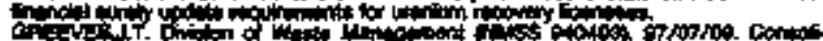

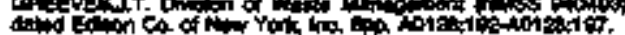

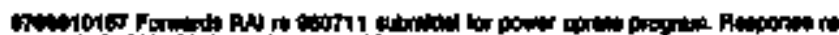

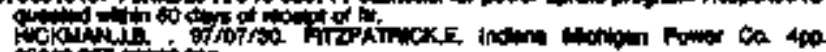

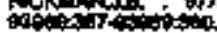

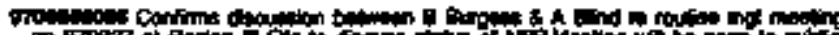

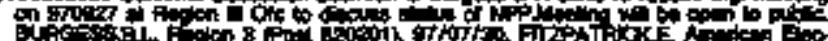

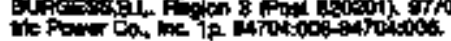

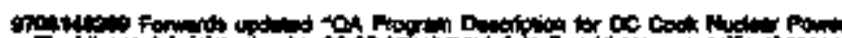

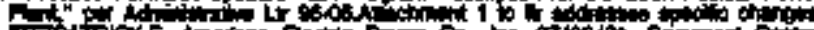

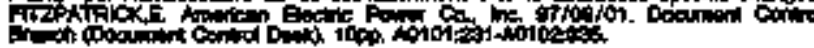

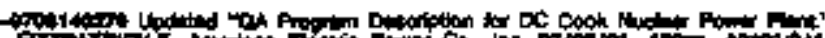

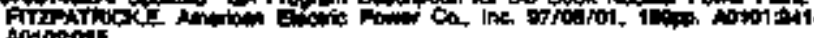

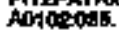

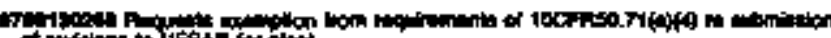

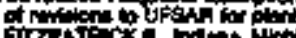

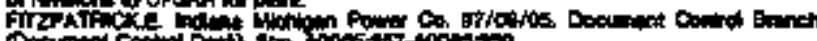

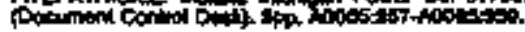

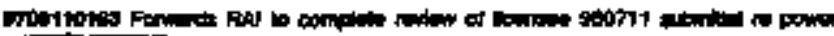

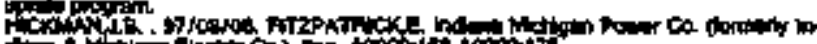

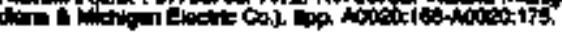

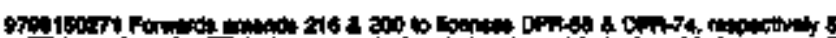
g.

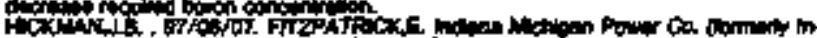

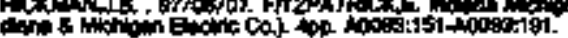

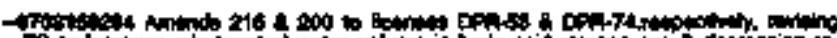

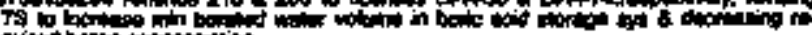

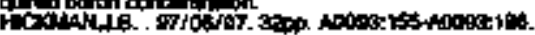

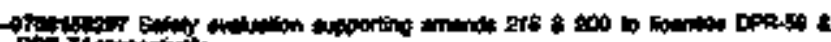

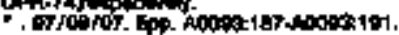




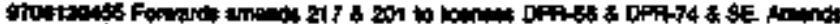

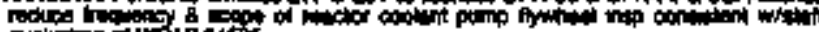

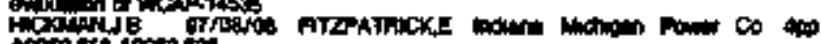

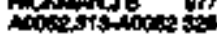

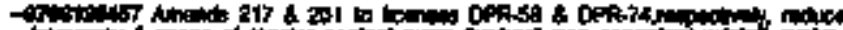

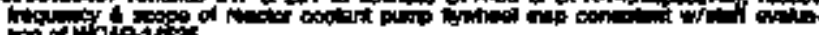

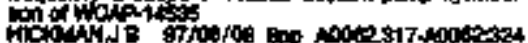

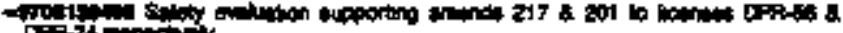

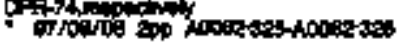

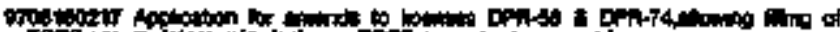

Cocs

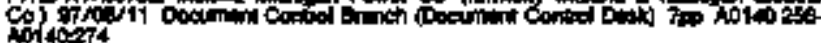

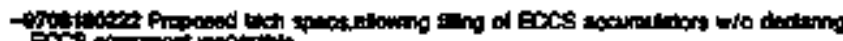

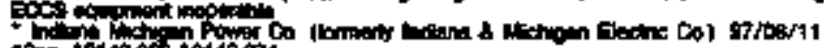

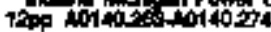

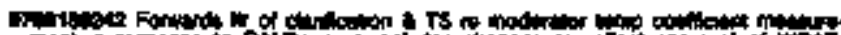

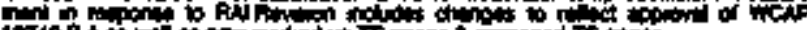

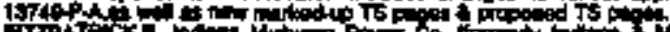

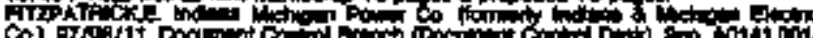
Col of 10.41

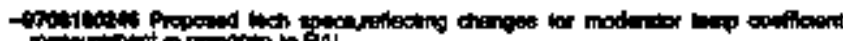

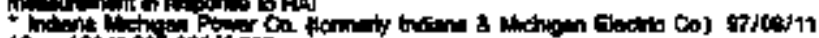

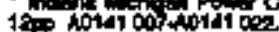

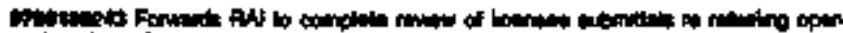

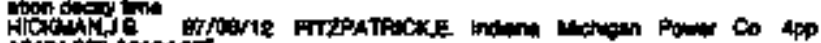

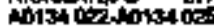

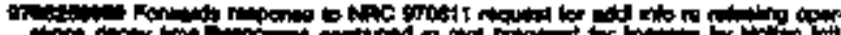

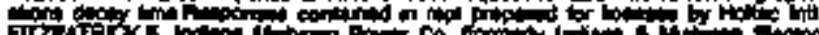

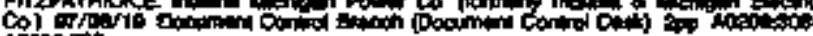
Col woons

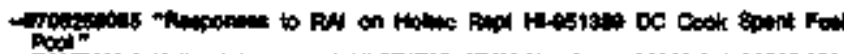

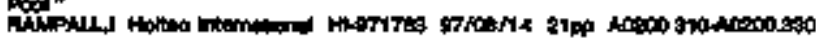

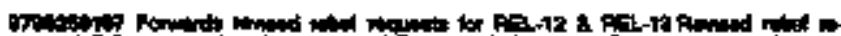

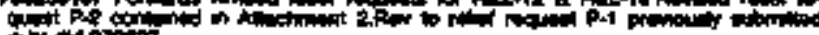

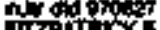

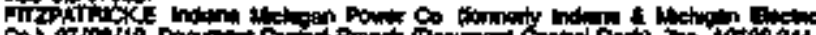

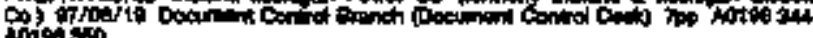

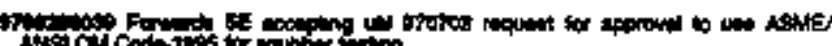

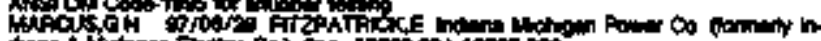

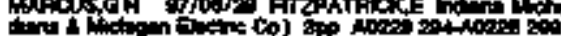

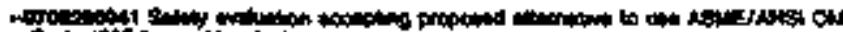

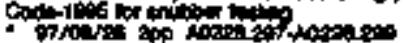

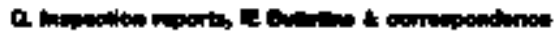

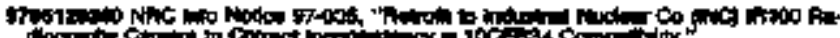

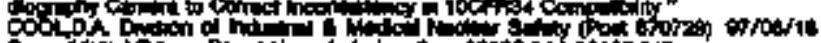

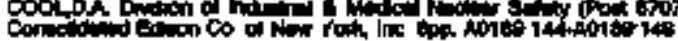

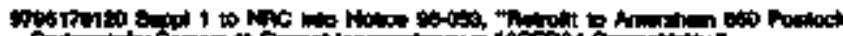

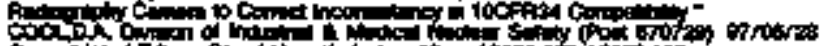

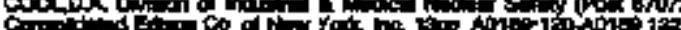

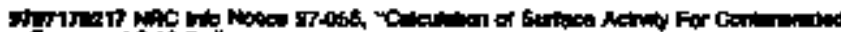

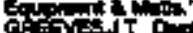

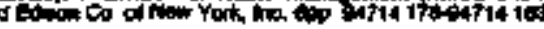

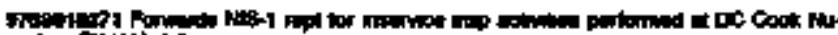

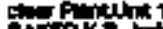

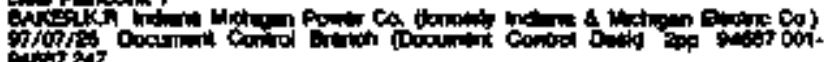

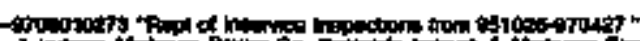

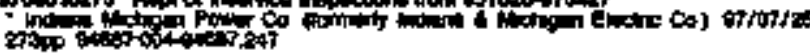

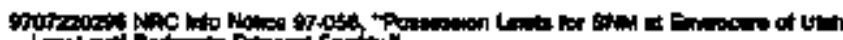

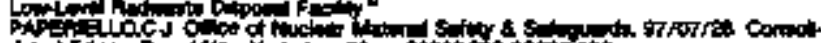

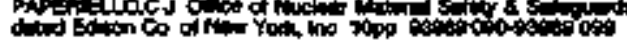

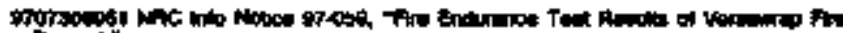

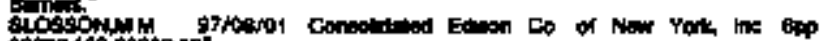

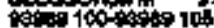

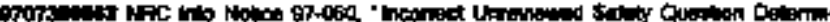

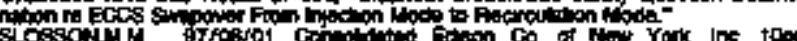

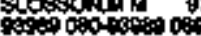

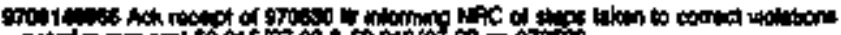

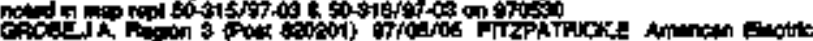

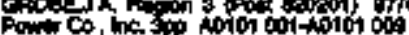

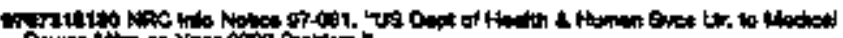

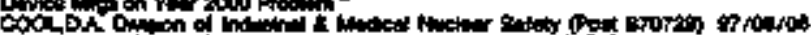

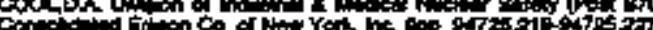

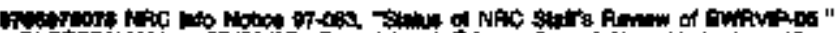

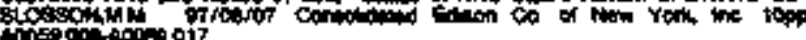

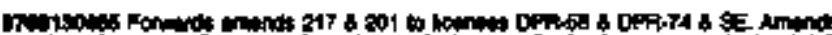

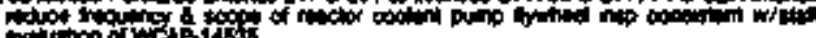
Hof

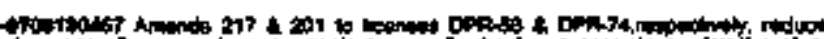

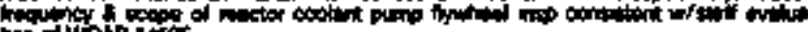

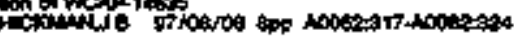

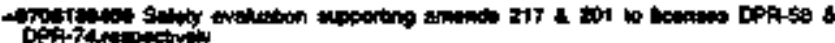
DF Hod

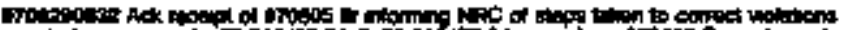

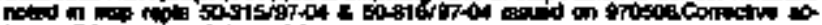

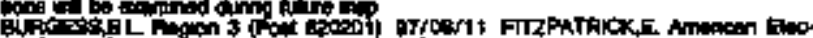

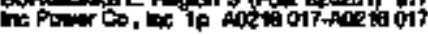

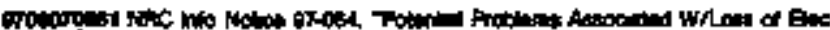

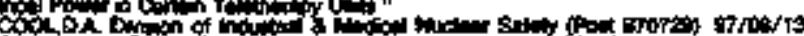

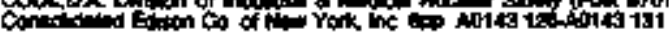

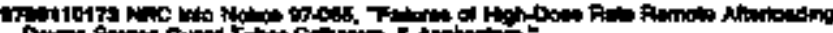

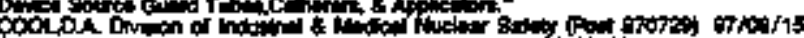

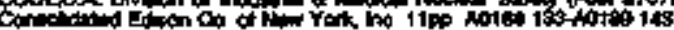

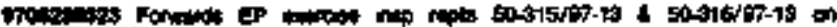

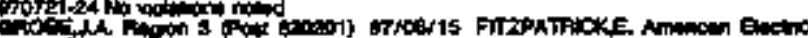

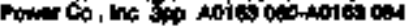

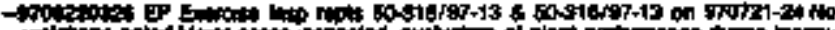

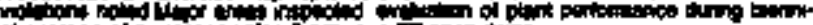

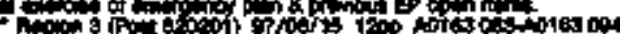

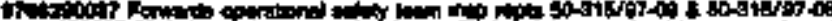

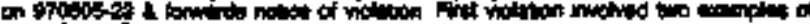
o

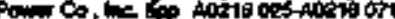

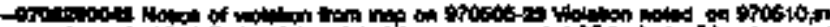

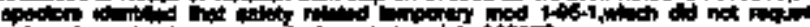

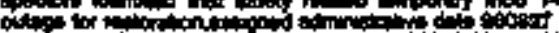

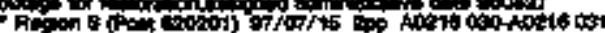

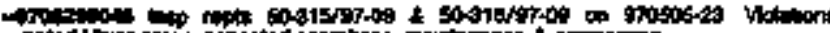

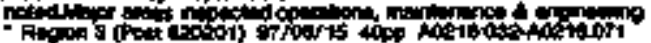

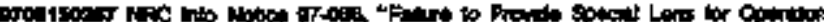

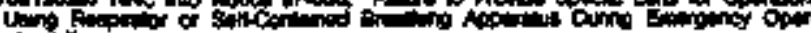

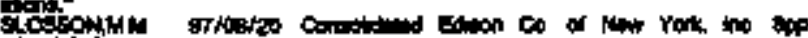

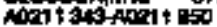

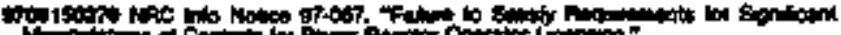

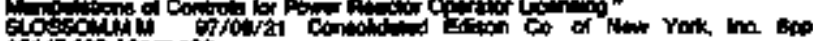

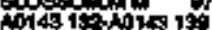

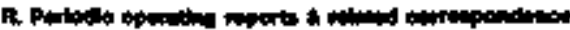

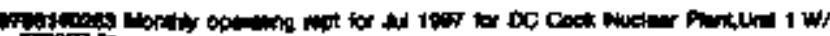

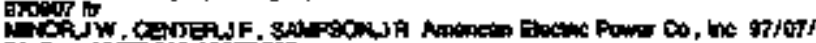

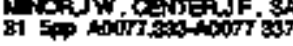

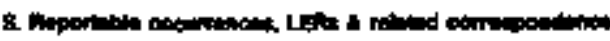

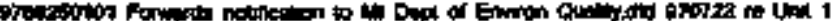

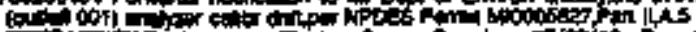
Fidsh

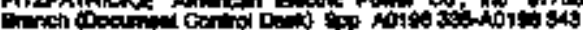




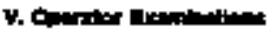

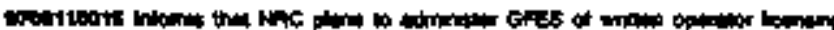

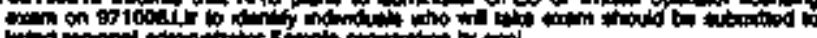

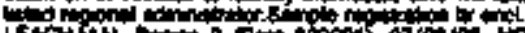

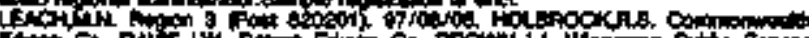

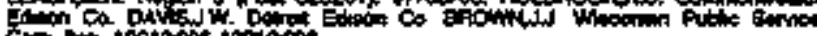

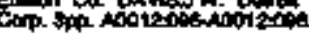

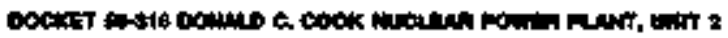

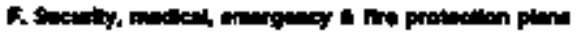

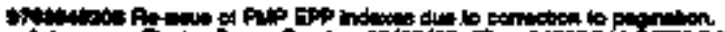

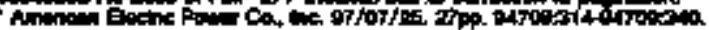

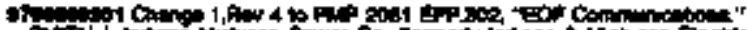

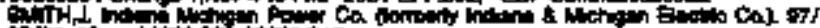

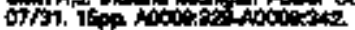

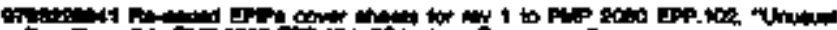

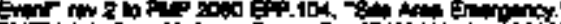

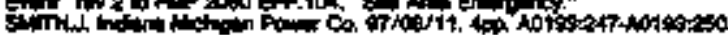

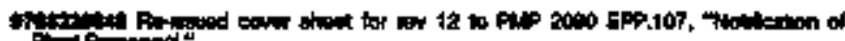
Pin Poroont.

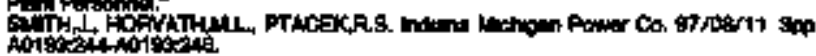

Fin

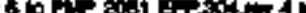

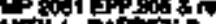

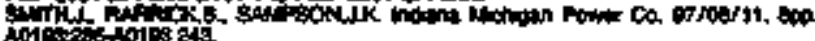

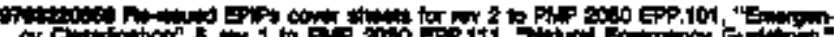

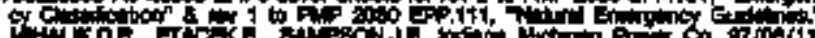

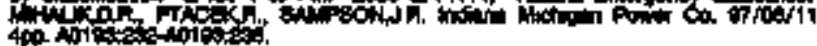

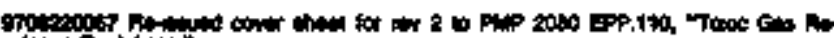

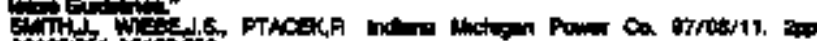

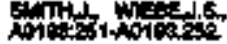

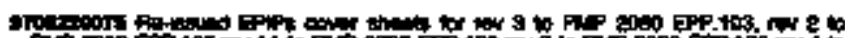

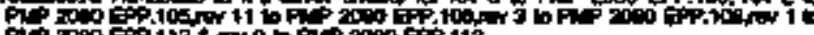

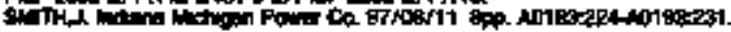

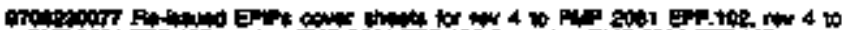

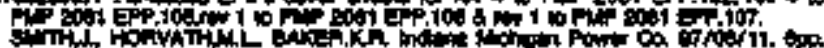

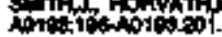

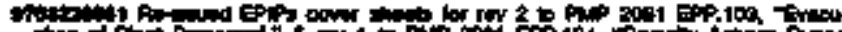

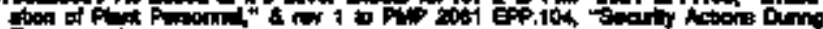

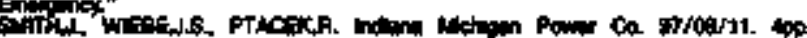

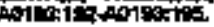

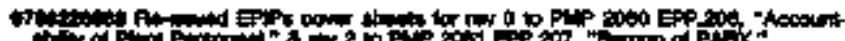

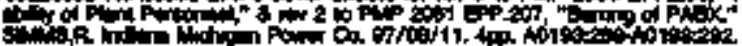

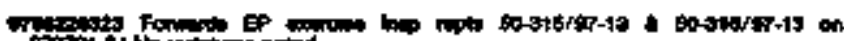

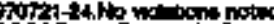

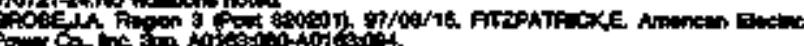

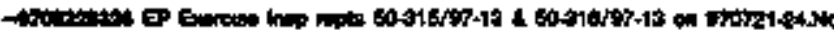

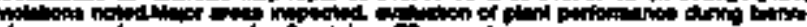

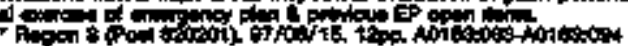

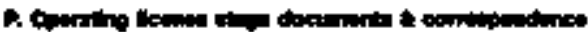

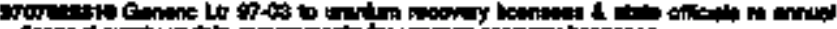

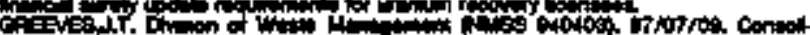

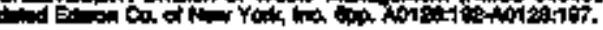

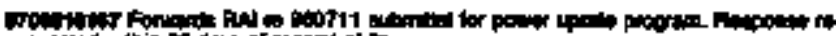

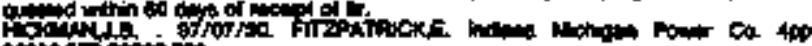

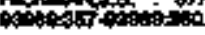

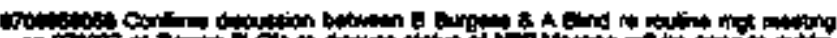

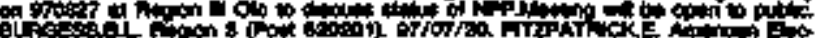

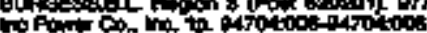

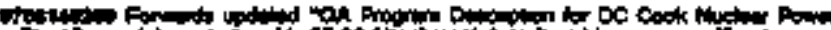

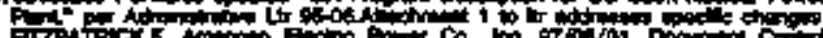

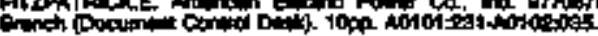

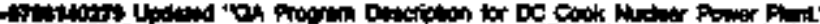

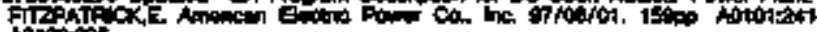
Nonescos.

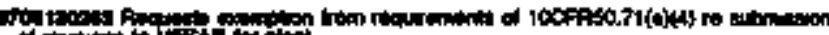

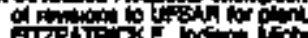

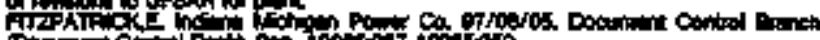

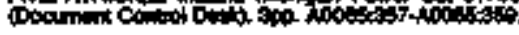

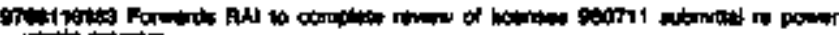

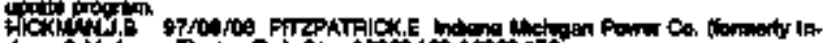

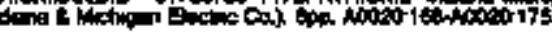

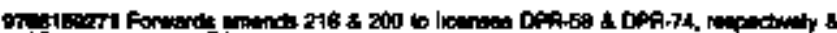

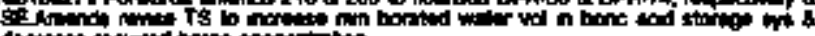

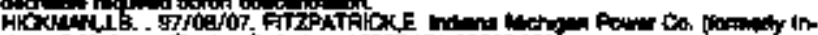

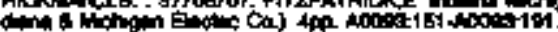

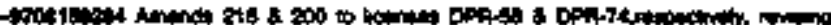

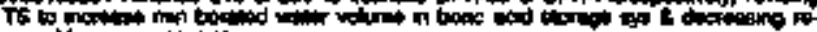

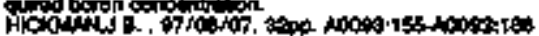

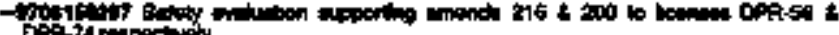

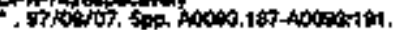

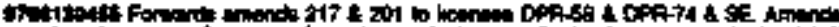

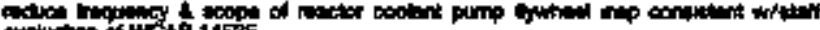

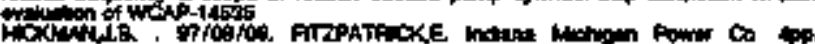

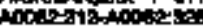

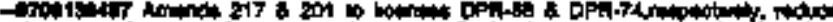

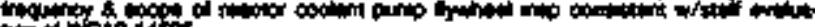

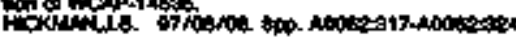

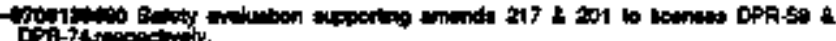

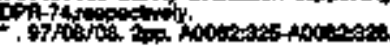

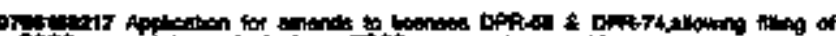

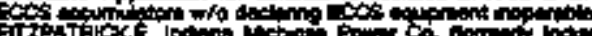

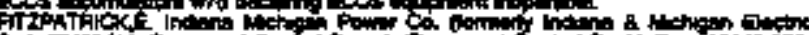

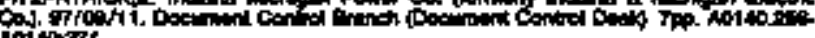

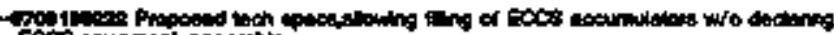

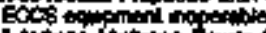

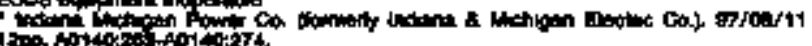

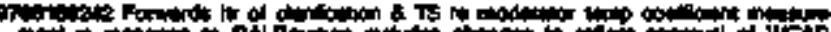

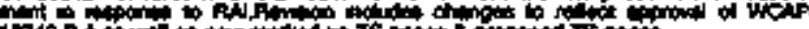

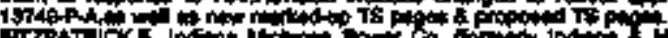

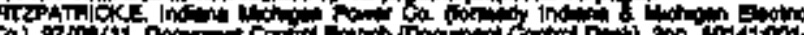
ca)

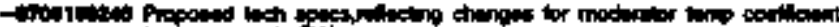

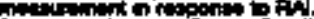

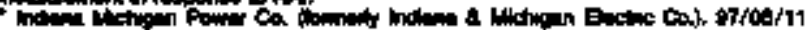

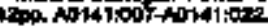

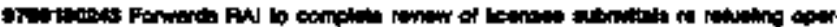

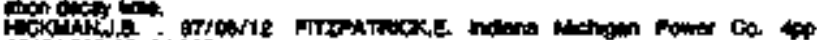

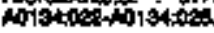

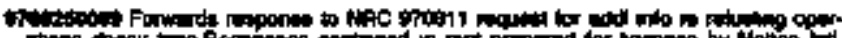

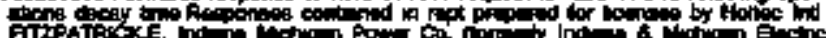

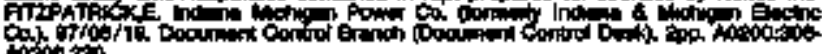
Nodistio.

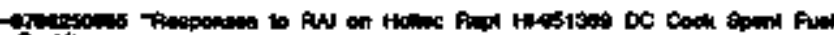

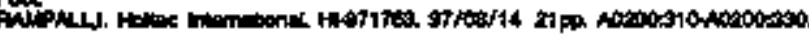

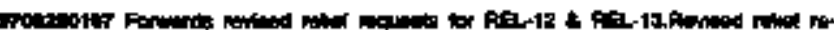

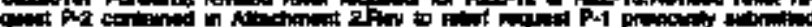

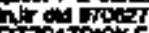

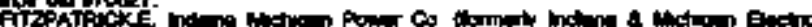

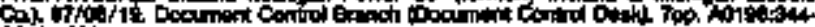
notion

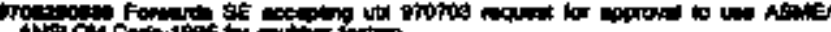

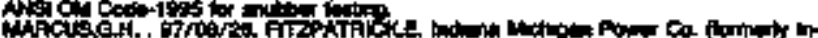

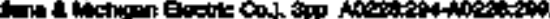

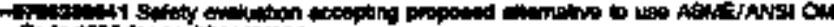

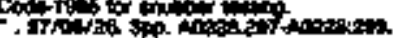

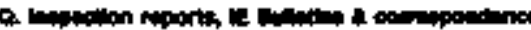

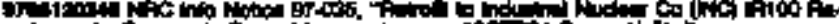

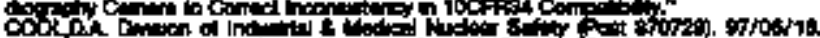

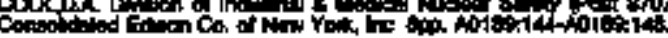

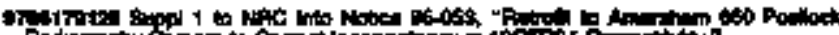

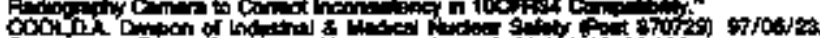

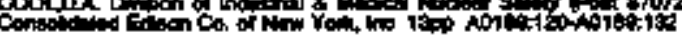

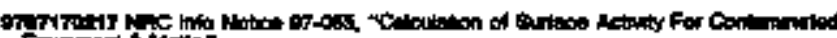

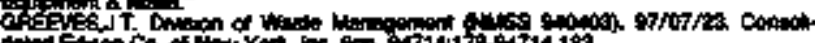

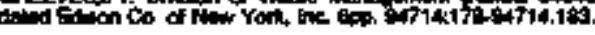




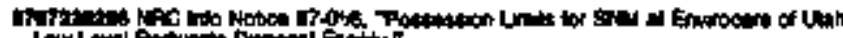

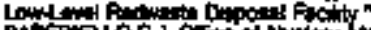

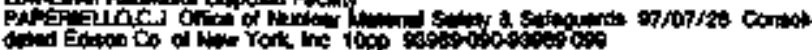

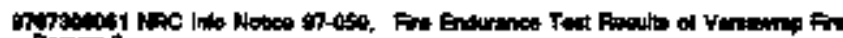

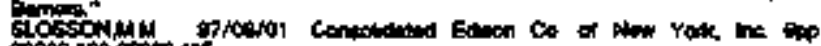
$100+165$

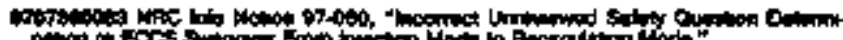

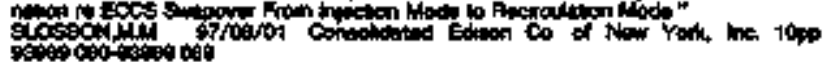

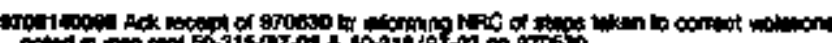

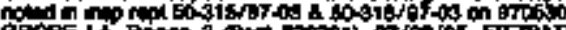

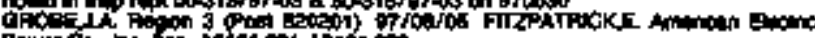

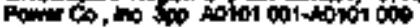

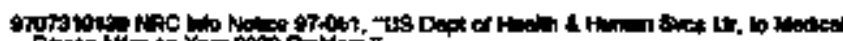
con

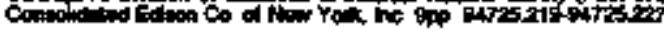

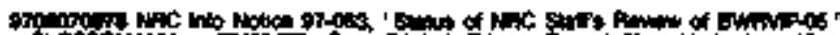

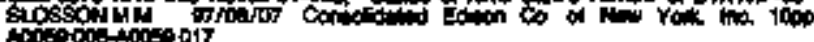

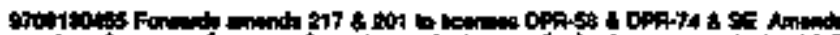

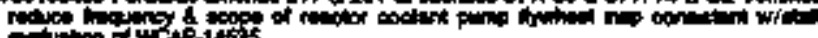
Hotition of

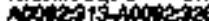

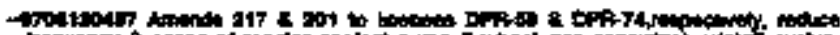

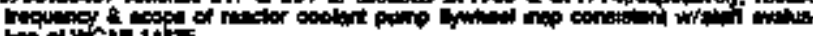

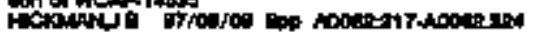

-

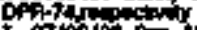

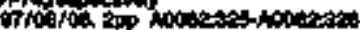

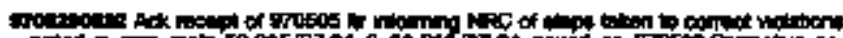

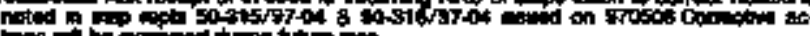

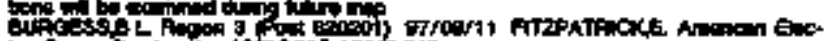

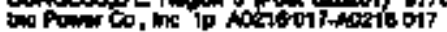

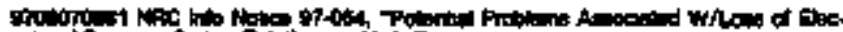

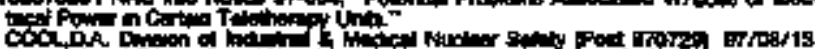

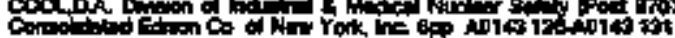

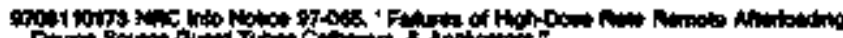

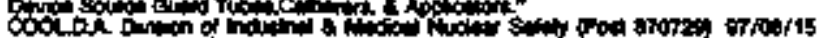

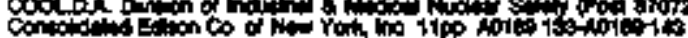

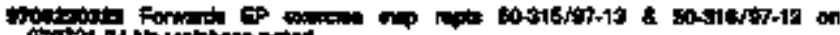

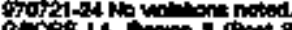

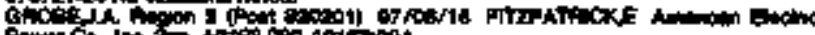
烈

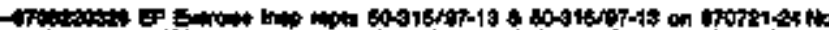

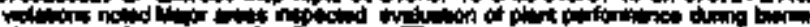

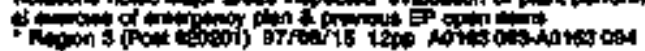

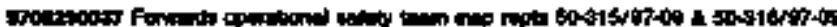

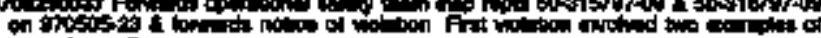

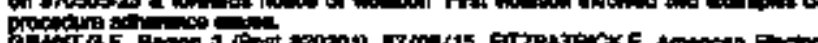

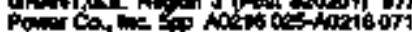

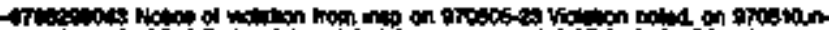

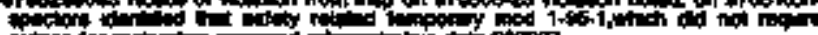

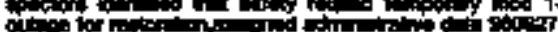

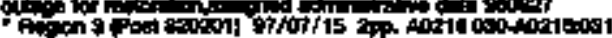

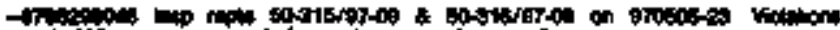
10.4.

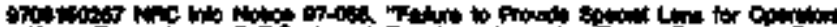
Lhe

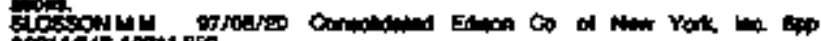

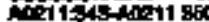

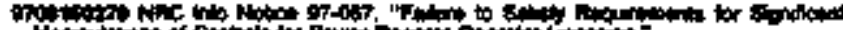

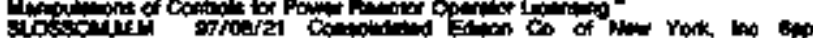

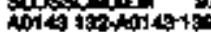

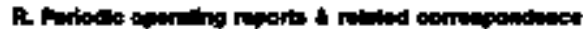

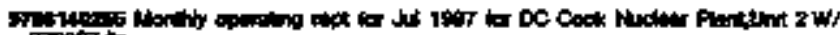

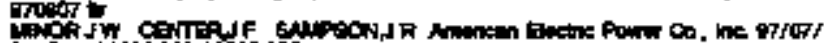
a

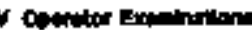

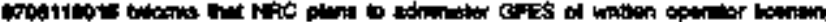

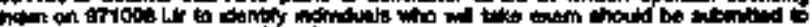

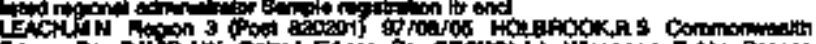

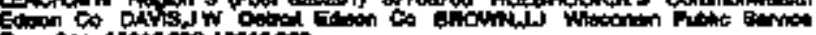

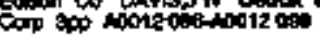

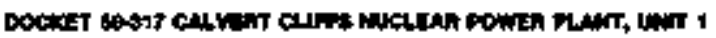

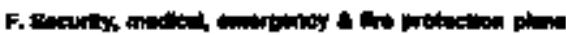

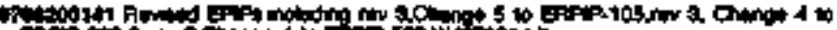

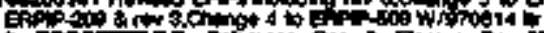

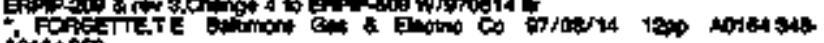
Andos

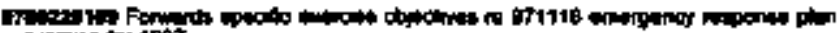

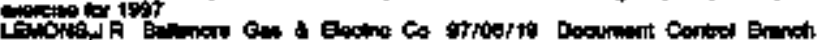

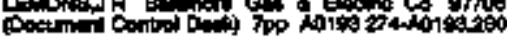

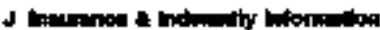

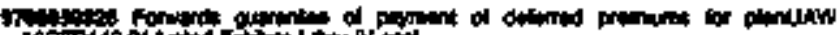

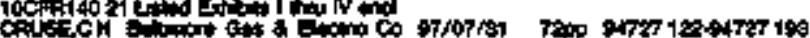

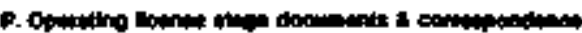

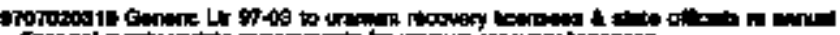

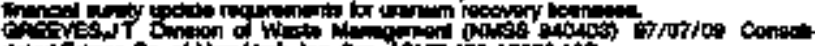

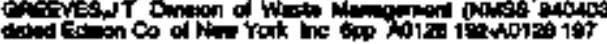

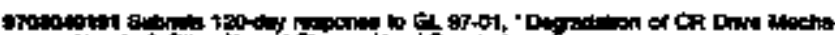

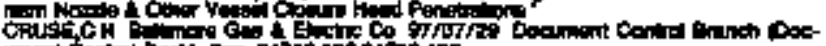

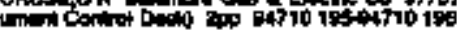

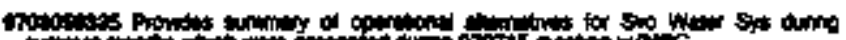
citiof

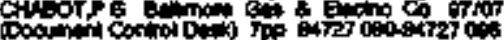

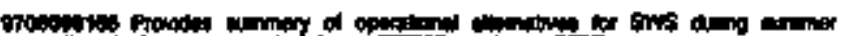

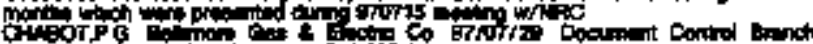
Dool

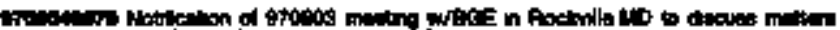

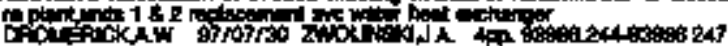

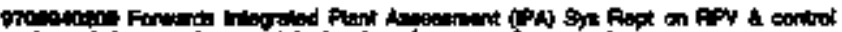

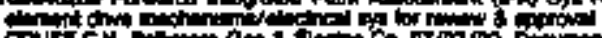

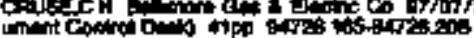

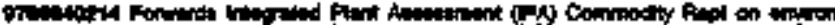

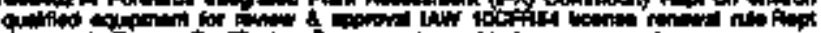

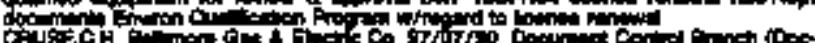

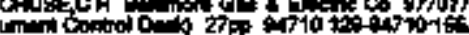

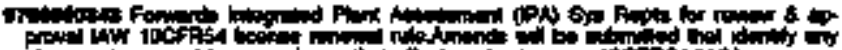

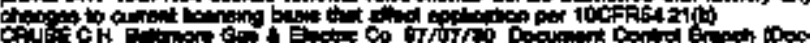

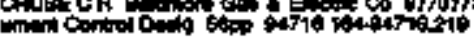

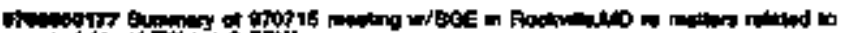

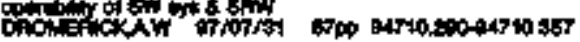

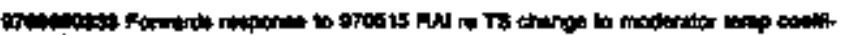

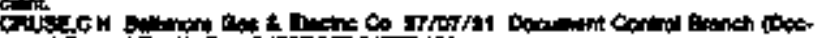

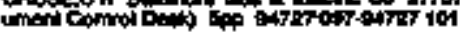

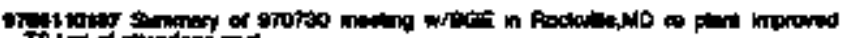

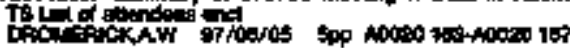

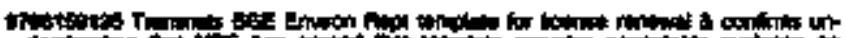

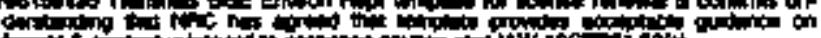

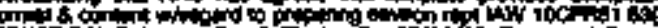

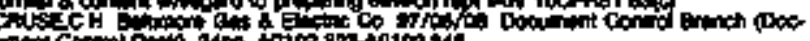

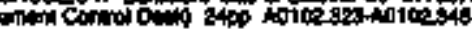

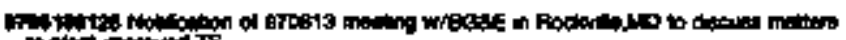

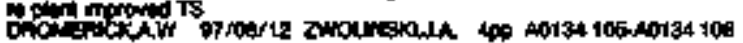

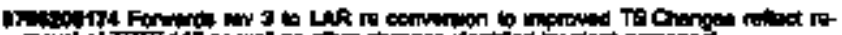

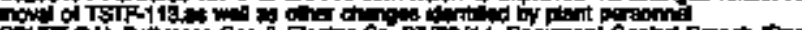

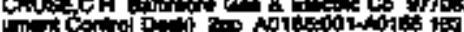




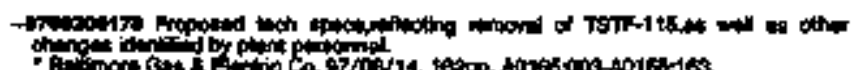

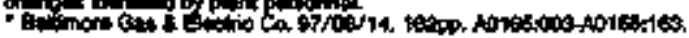

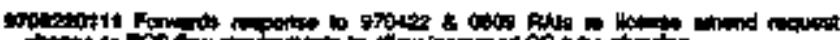

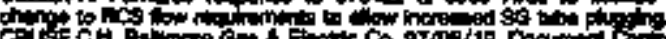

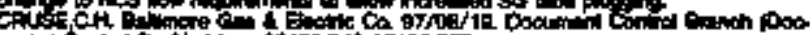

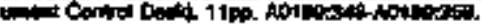

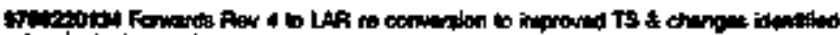

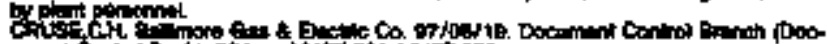

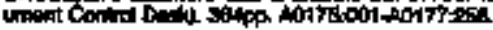

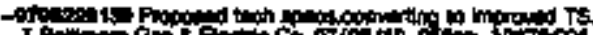

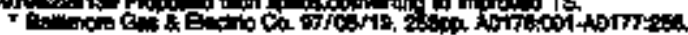

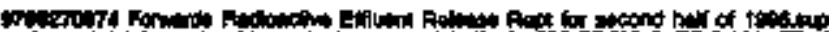

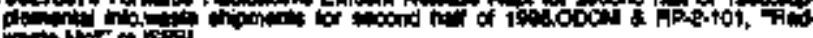

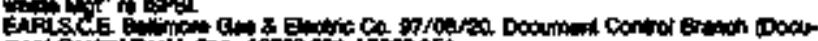

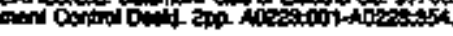

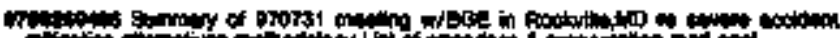

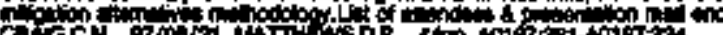

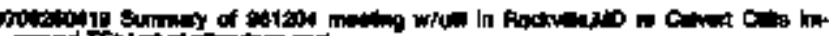

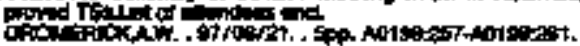

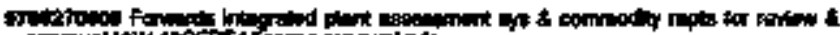

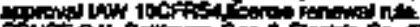

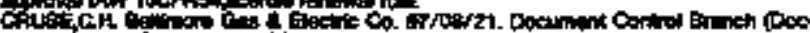

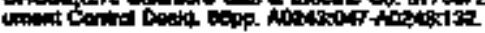

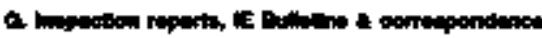

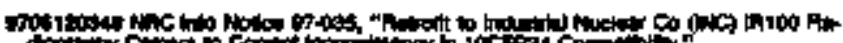

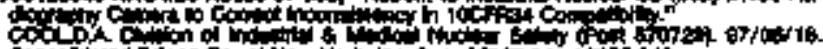

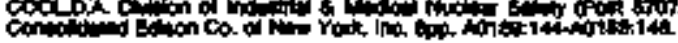

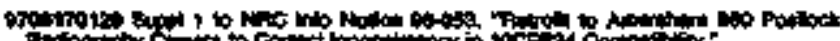

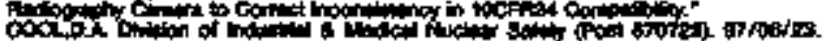

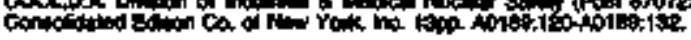

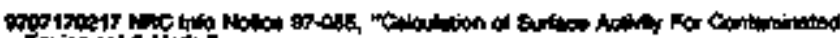

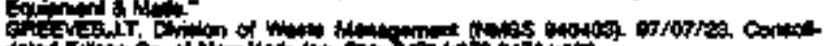

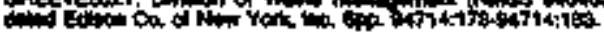

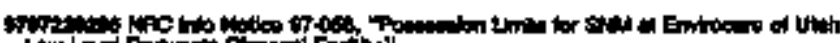
LApir

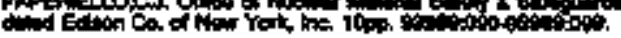

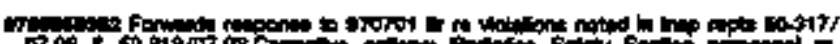

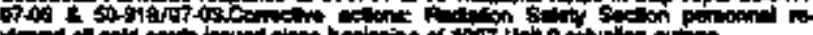

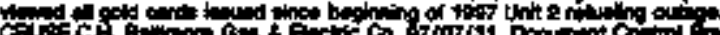
chin

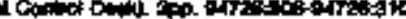

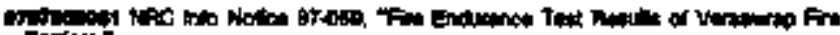

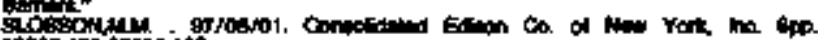

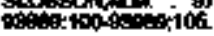

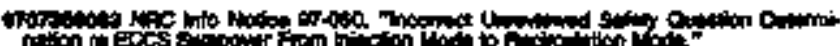

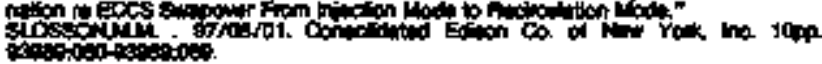

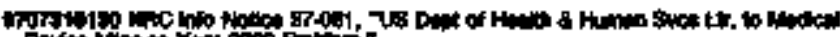

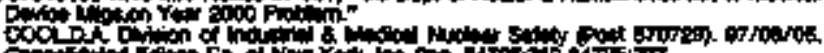

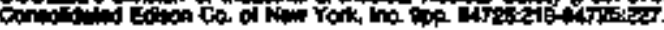

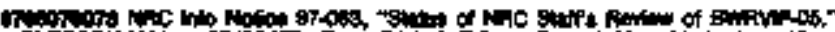

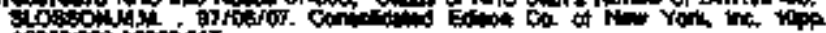

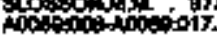

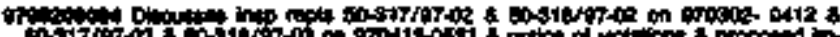

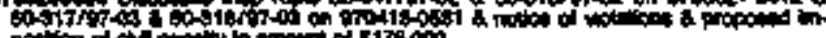

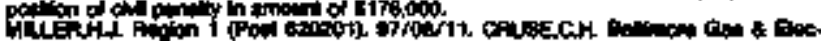

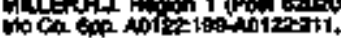

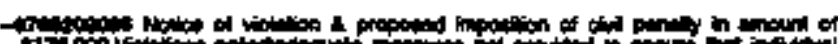

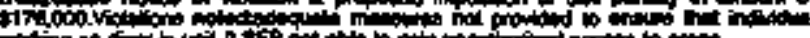

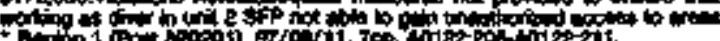

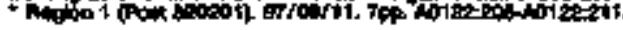

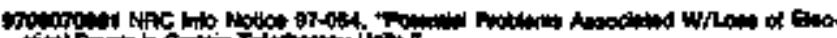

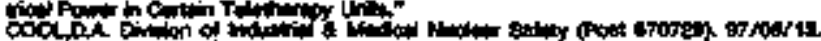

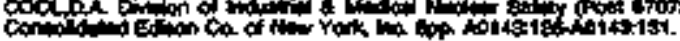

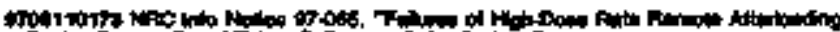

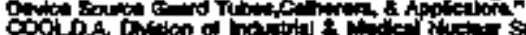

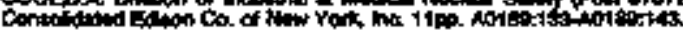

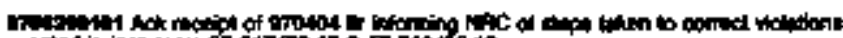
fond in in

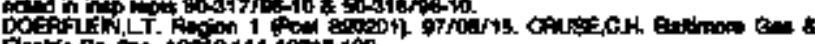

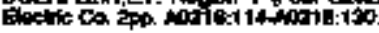

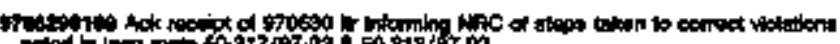
podpl in

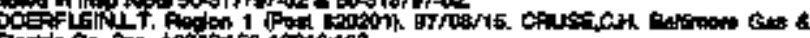

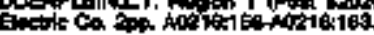

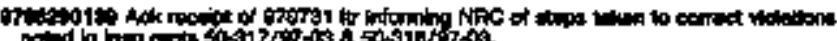

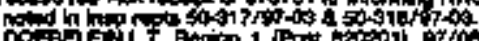

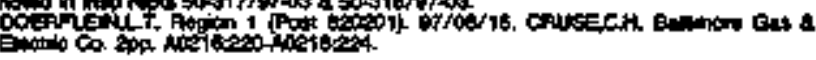

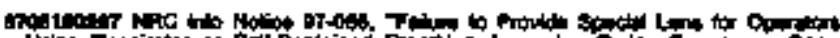

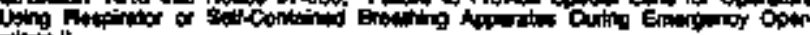

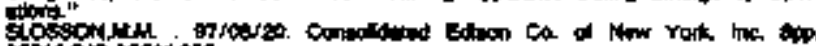

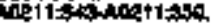

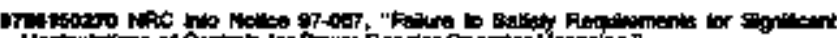

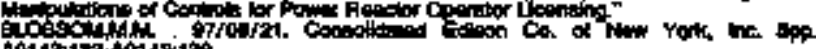
1014 132 - 4014010

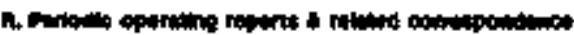

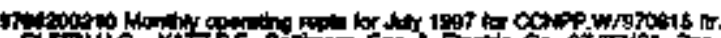

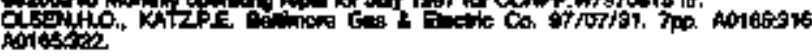

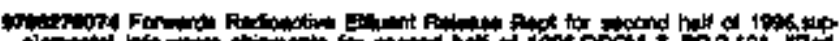
plemintion ins PAlis

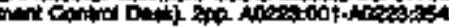

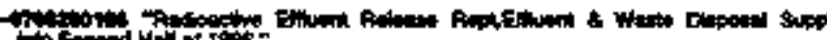

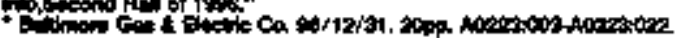

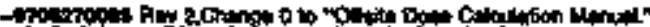

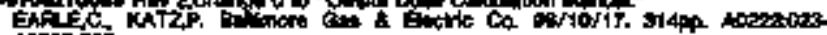
ADrasis.

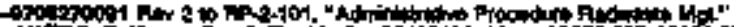

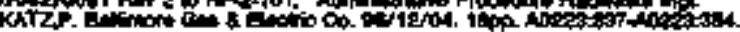

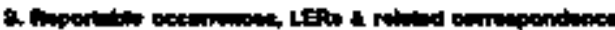

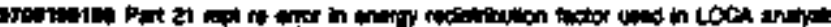

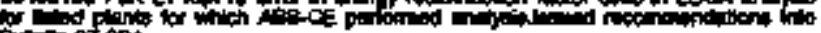

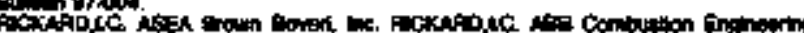

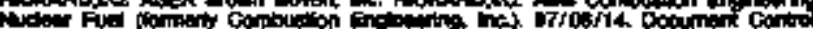

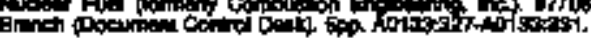

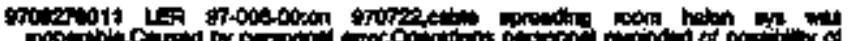

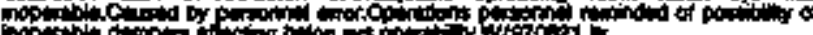

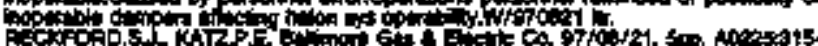
totom

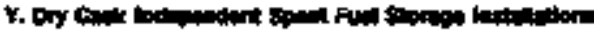

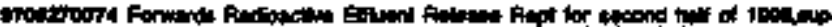

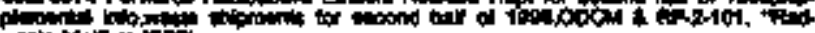

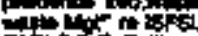

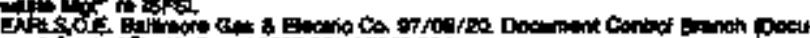

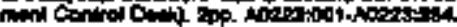

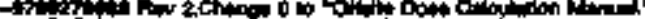

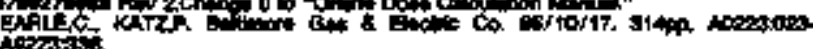

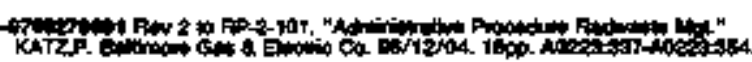

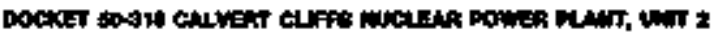

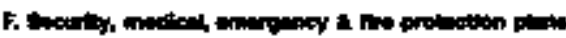

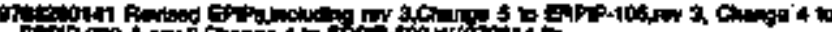
EHA

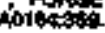

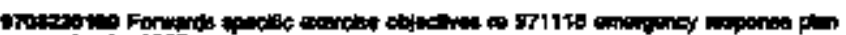

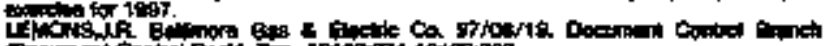

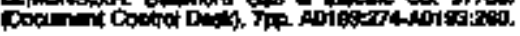

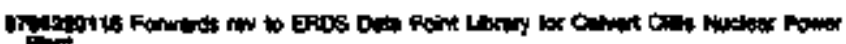

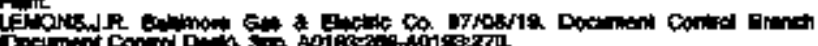
Doeimint Confol Do

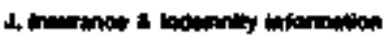

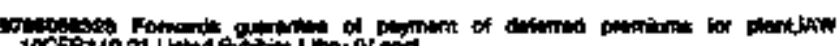

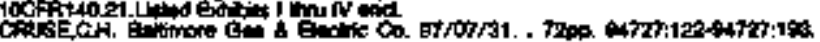




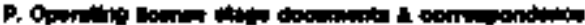

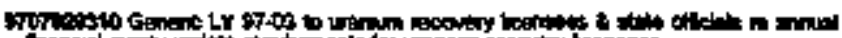

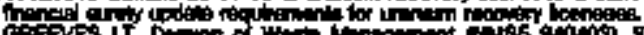

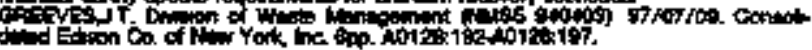

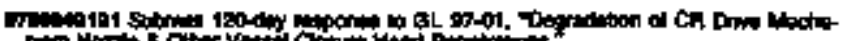

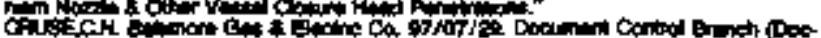

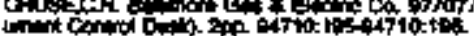

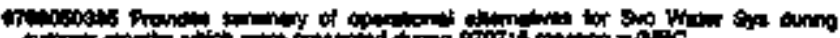

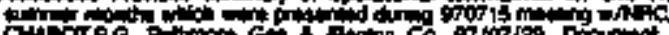

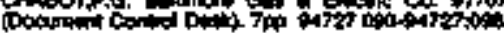

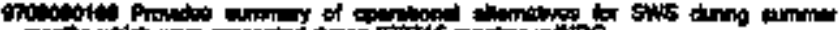

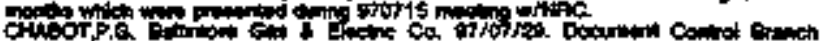

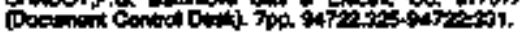

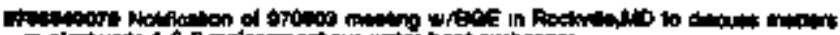

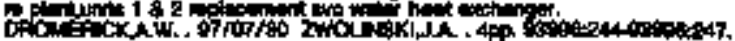

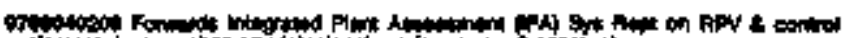

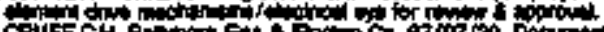

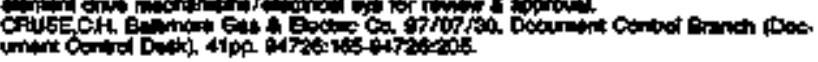

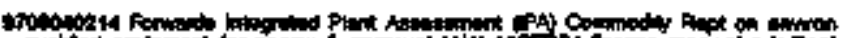

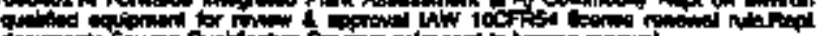

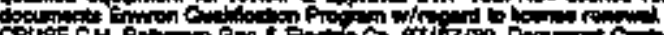

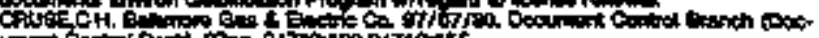

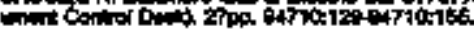

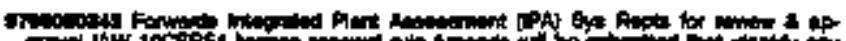

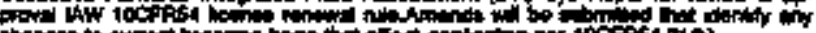

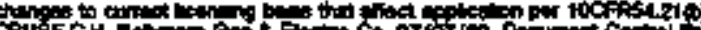

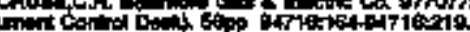

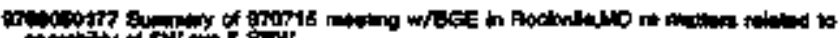

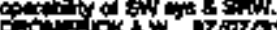

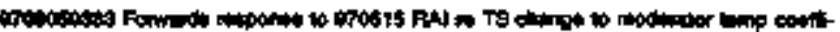

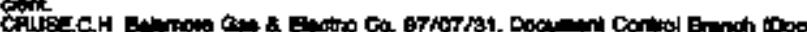

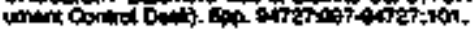

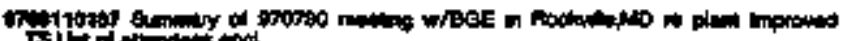

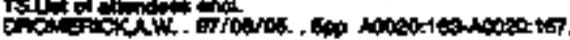

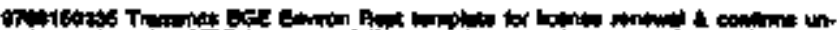

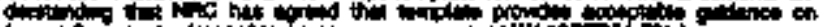

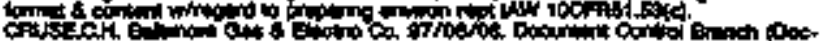

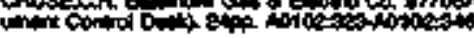

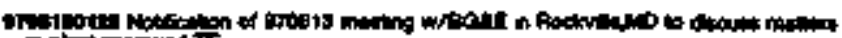

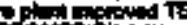

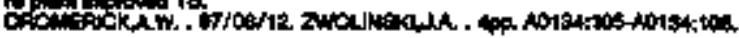

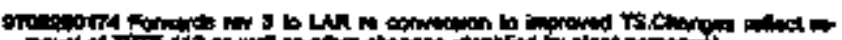

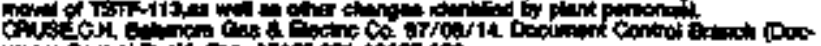

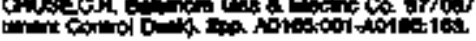

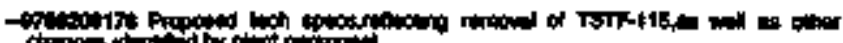

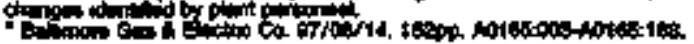

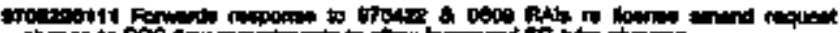

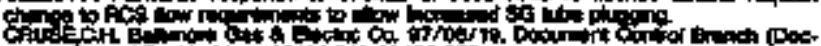

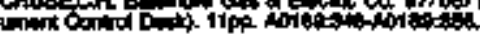

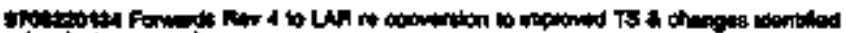

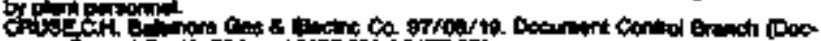

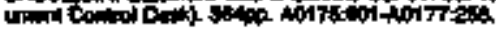

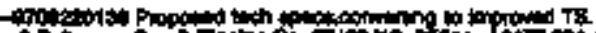

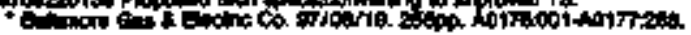

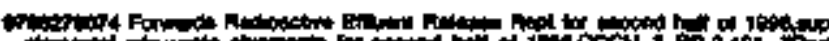

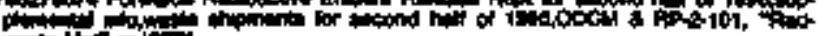
Jint if

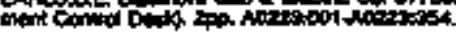

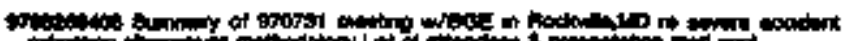

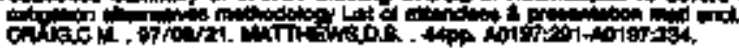

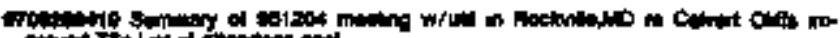

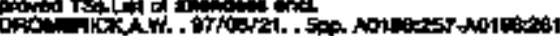

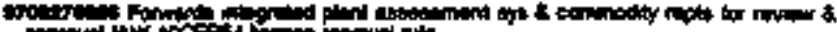

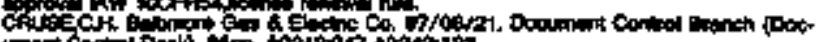

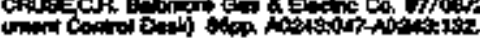

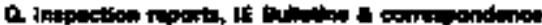

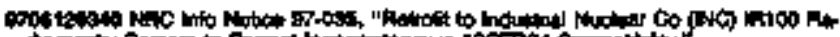

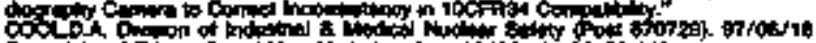

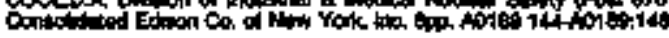

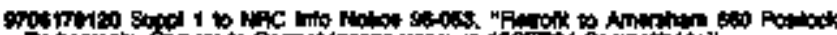

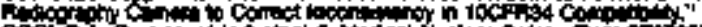

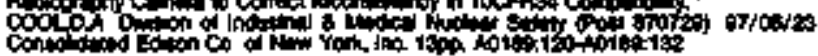

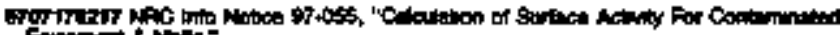

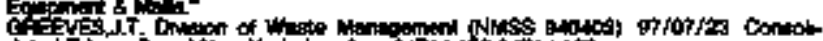

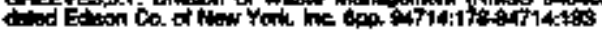

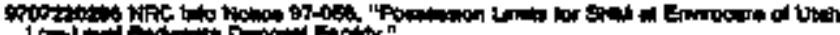
PAPE

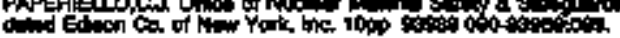

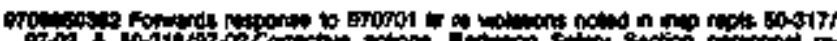

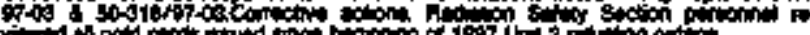

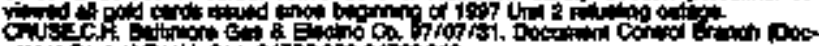

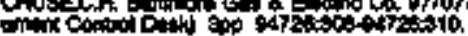

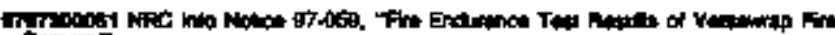

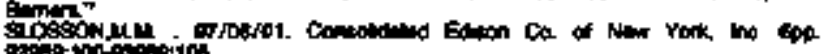

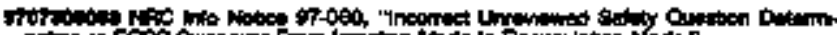

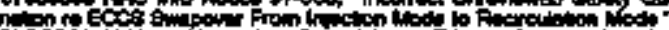

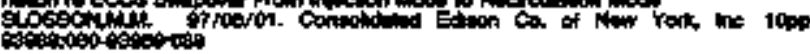

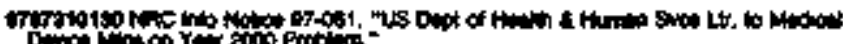

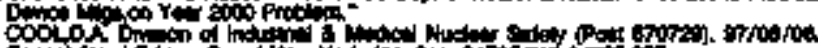

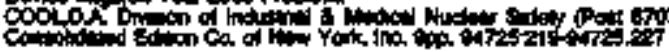

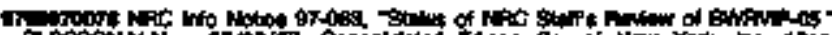

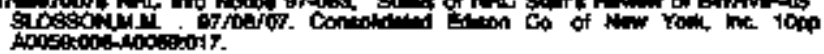

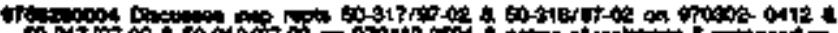

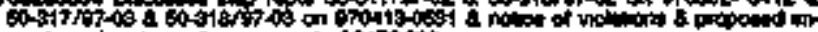

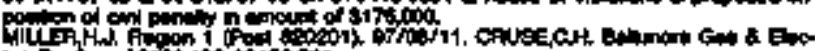

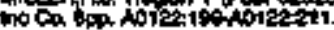

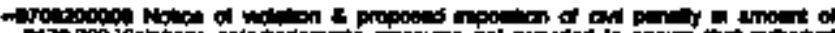

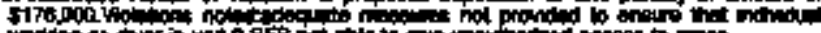

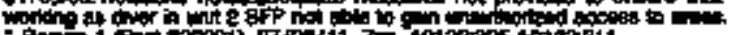

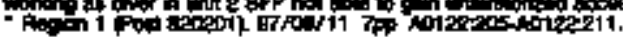

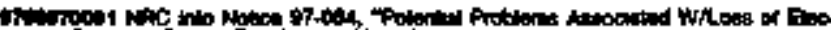

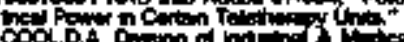

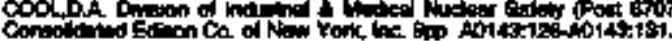

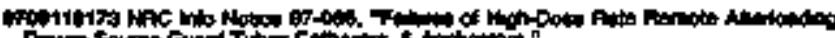

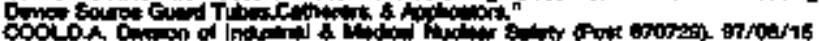

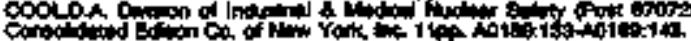

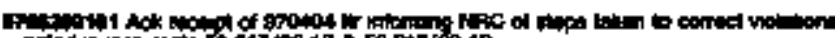

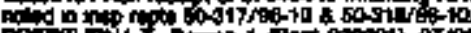
Dodn on

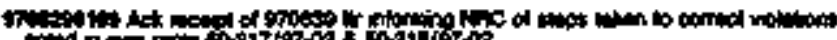

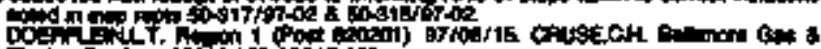

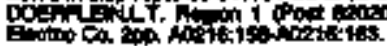

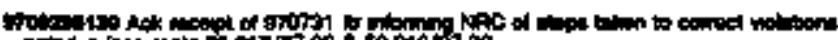

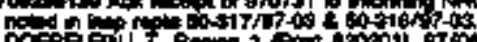

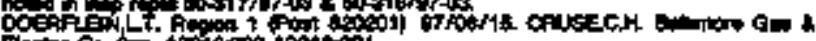

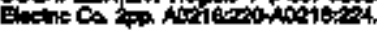

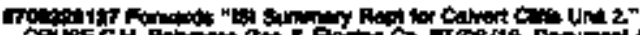

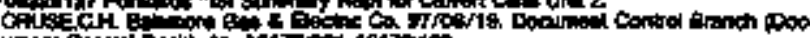

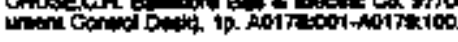

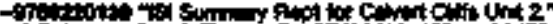

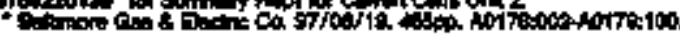

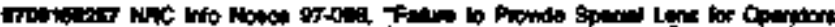

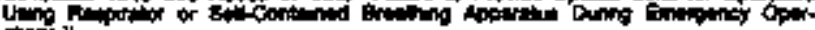

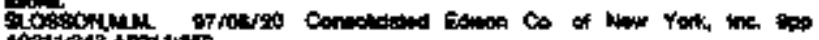
10211543 the 1400

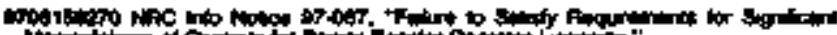

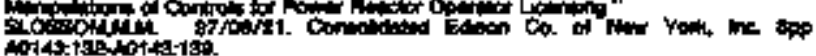




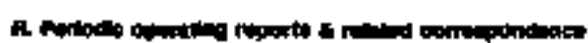

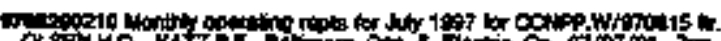

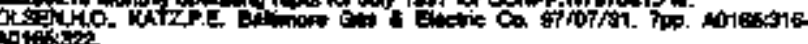

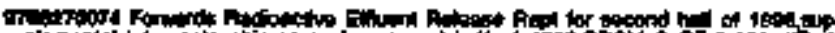

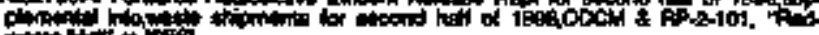
Con

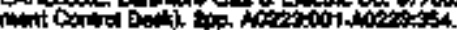

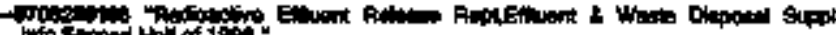

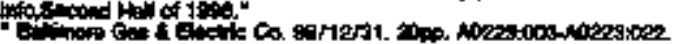

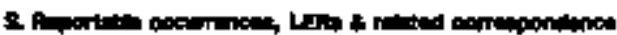

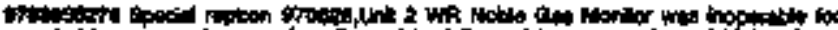

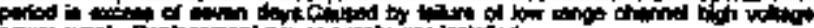

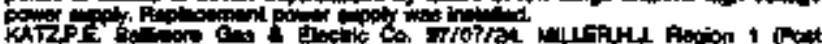

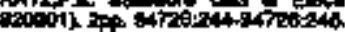

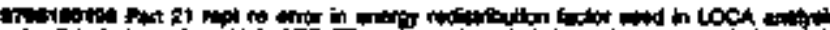

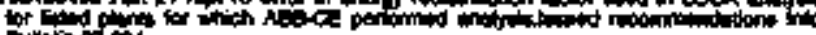

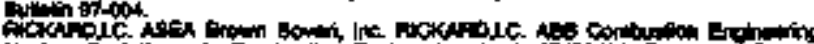

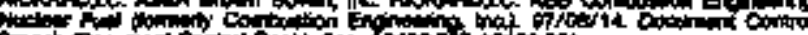

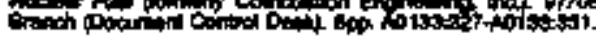

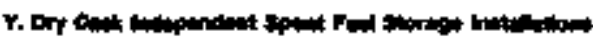

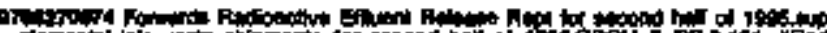

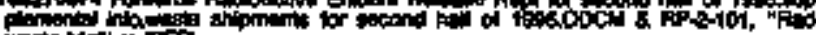

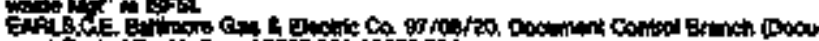

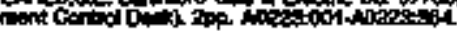

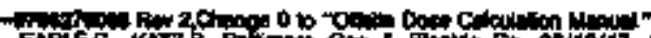

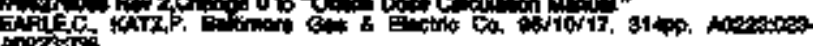

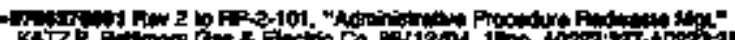

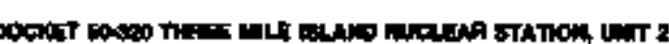

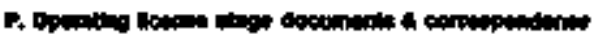

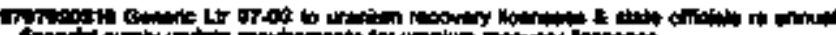

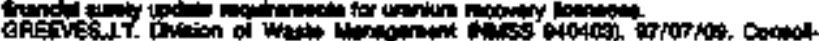

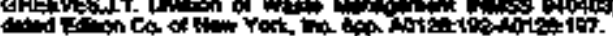

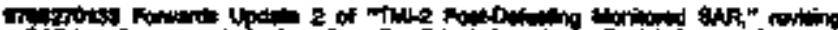

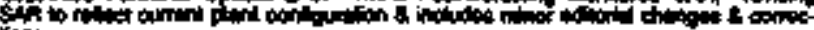

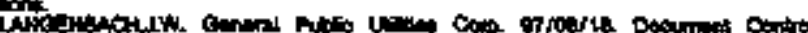

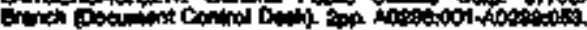

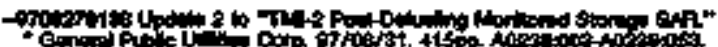

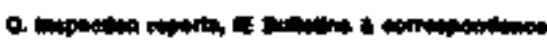

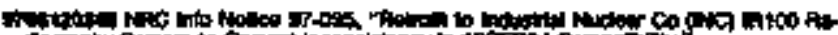

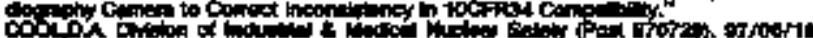

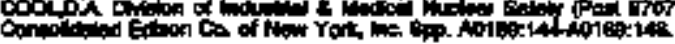

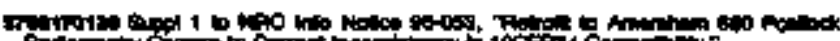
R.

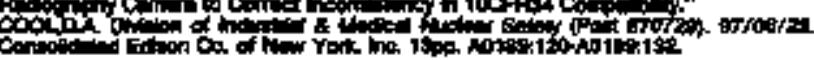

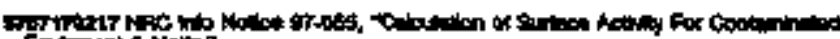

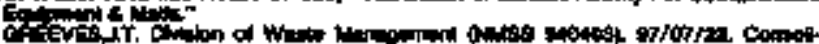

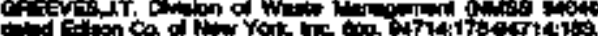

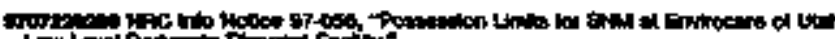
Lat

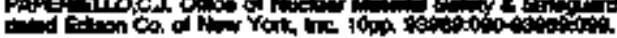

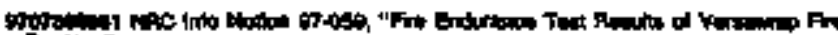

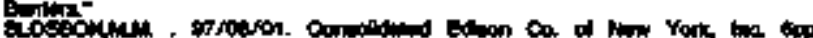

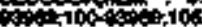

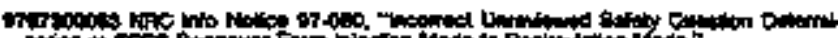

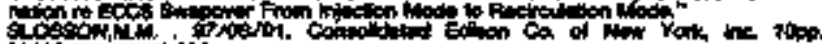

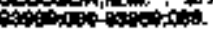

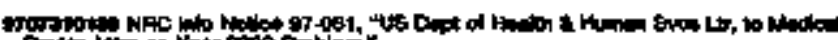
Cocolo

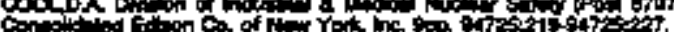

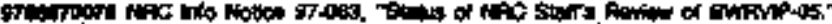

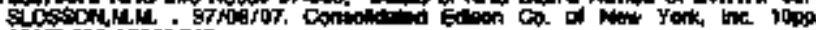
(1)

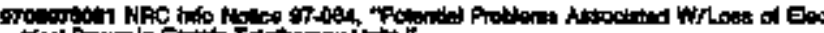

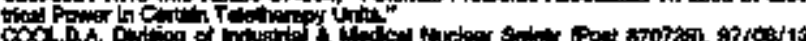

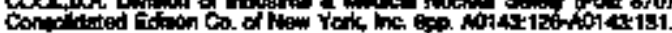

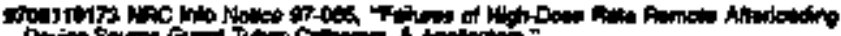

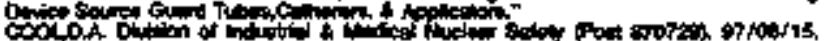

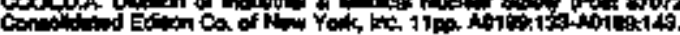

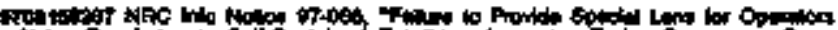

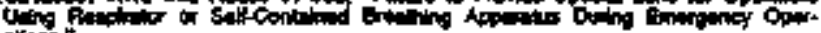

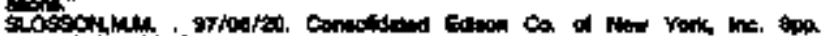
Al11

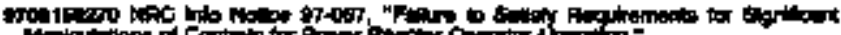

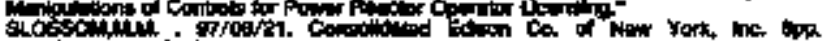

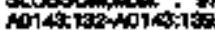

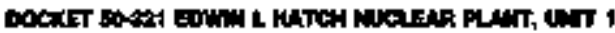

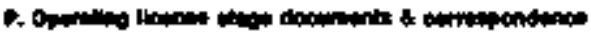

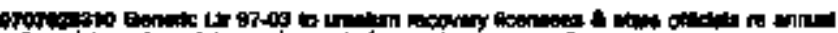

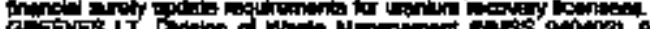

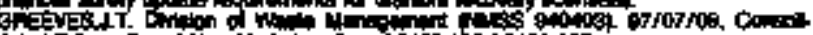

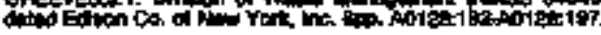

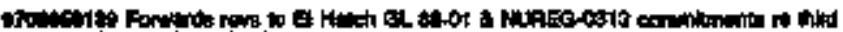

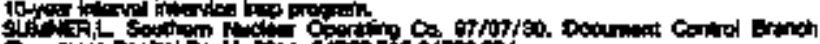

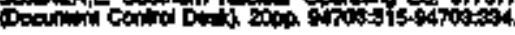

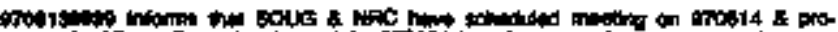

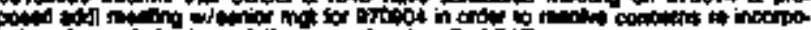

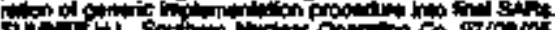

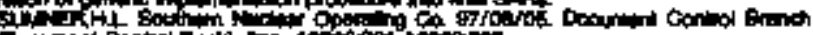

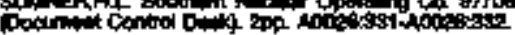

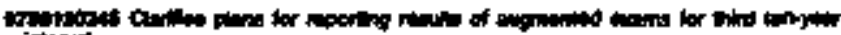

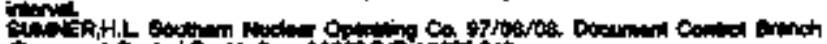

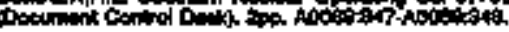

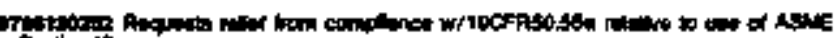
$\sin x$

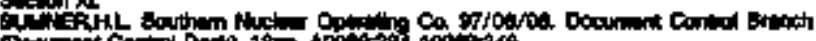

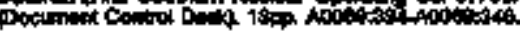

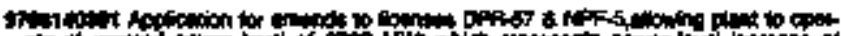
的售

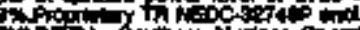

Sir

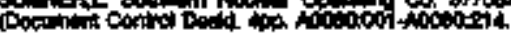

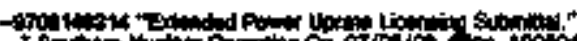

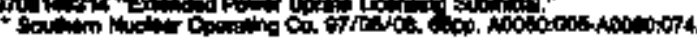

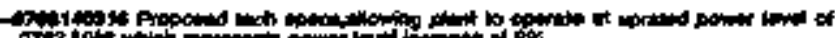

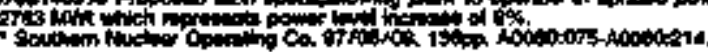

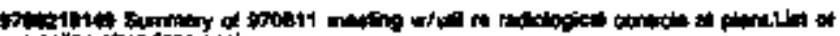

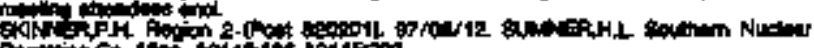

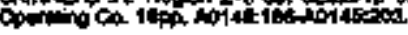

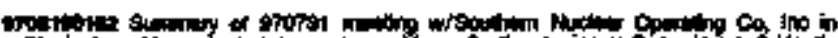

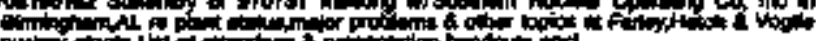

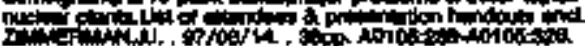

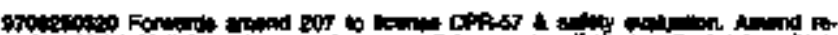

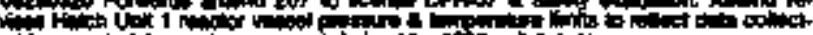
A Tom

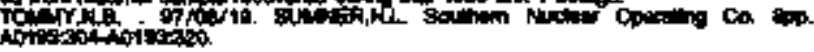

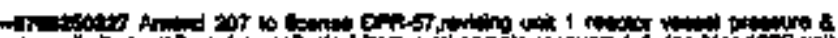

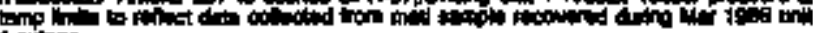

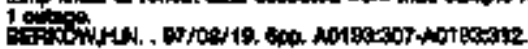

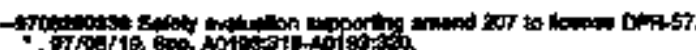

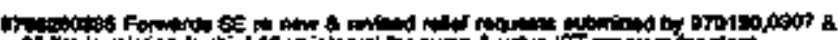

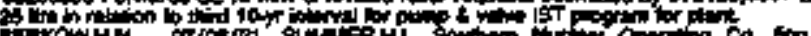

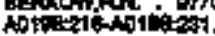

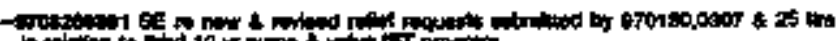

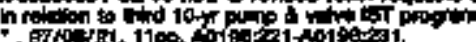




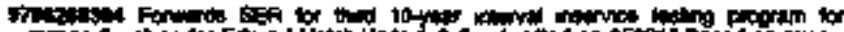

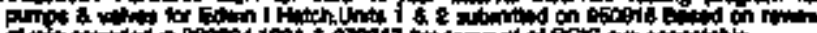

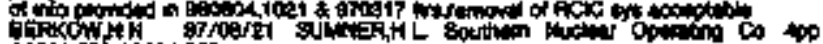

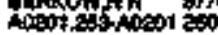

-

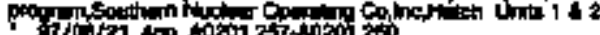

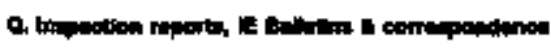

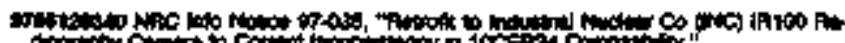

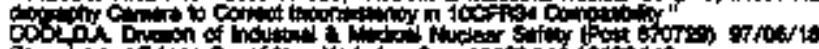

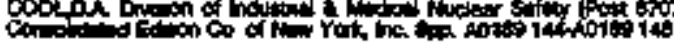

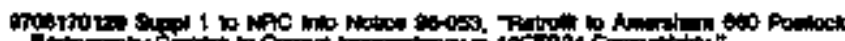

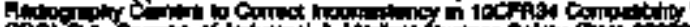

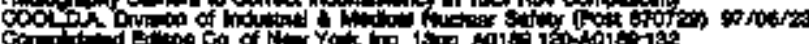

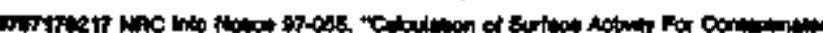
Sation

aris

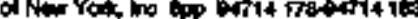

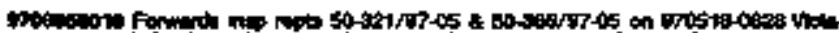

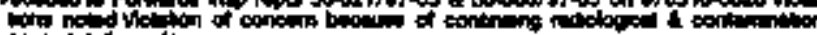

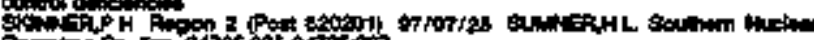

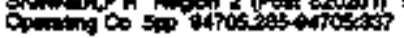

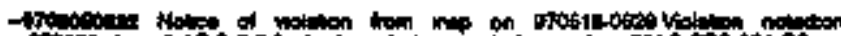

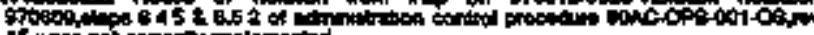

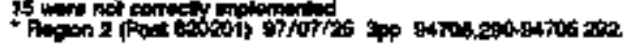

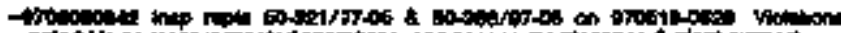

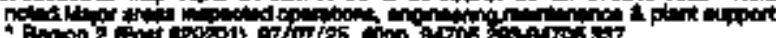

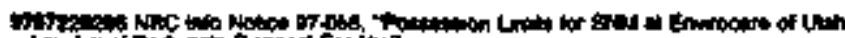

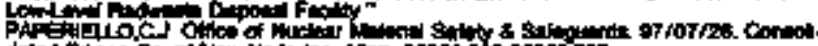

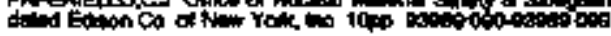

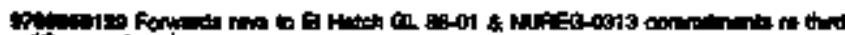

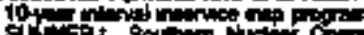

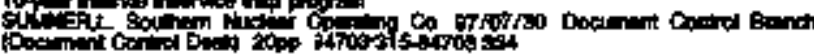

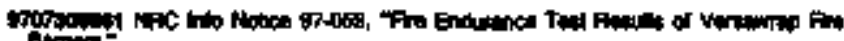

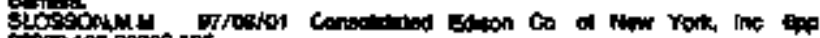

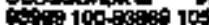

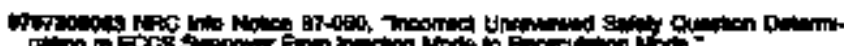

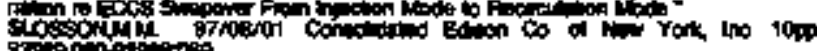

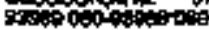

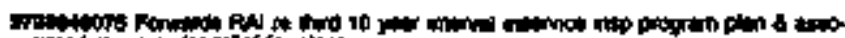

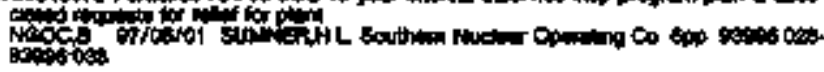

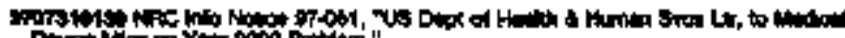

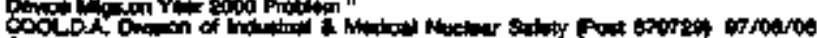

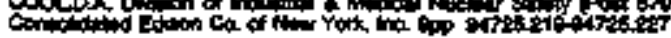

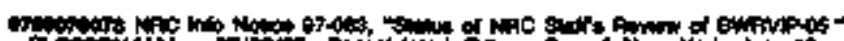

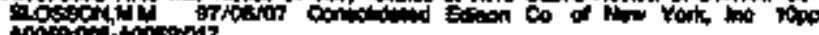

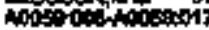

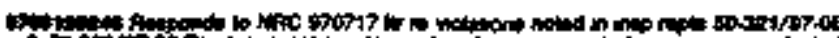

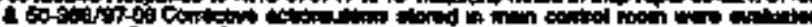

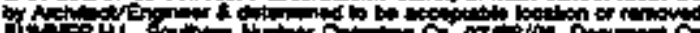

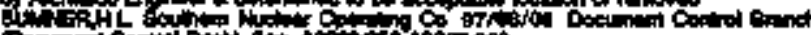

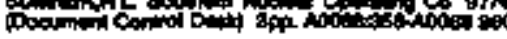

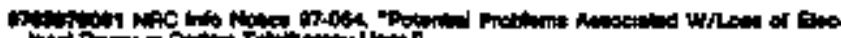

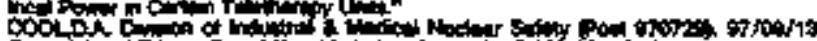

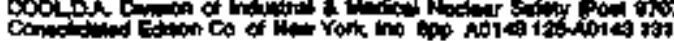

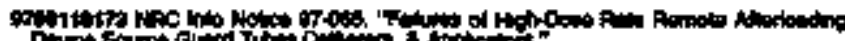

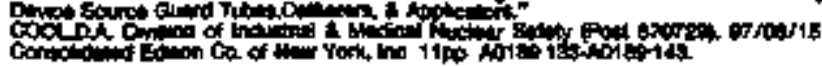

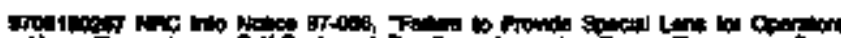

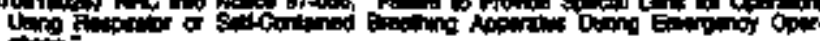

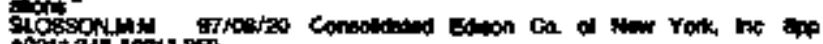

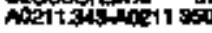

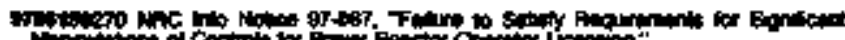

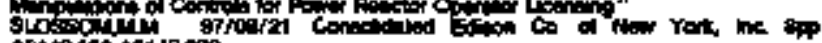
1016313240145 tr:

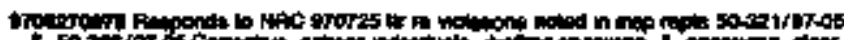

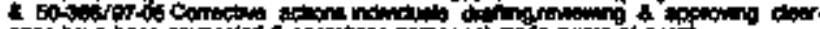

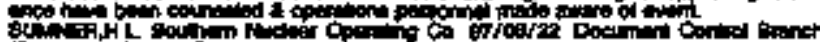

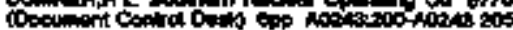

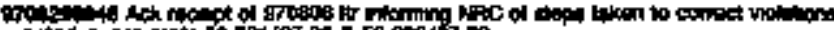

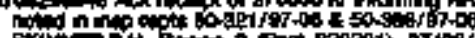

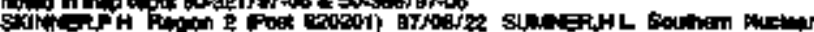

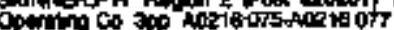

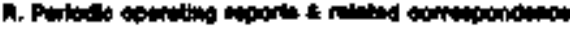

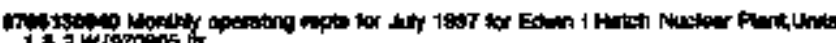

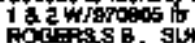

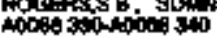

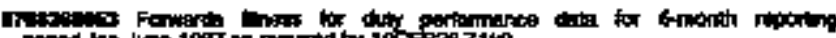

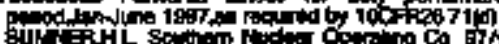

Uil

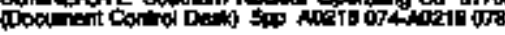

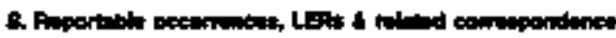

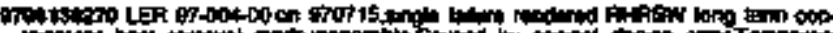

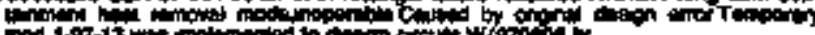

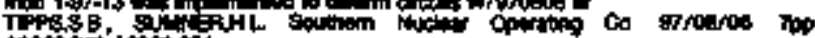
toostosined

\section{Opwator Exoninatim}

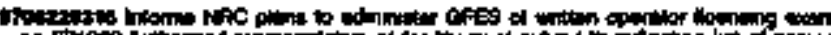

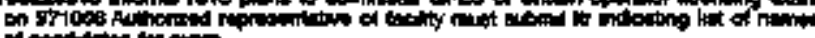

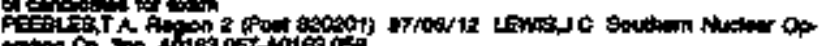
anding Co

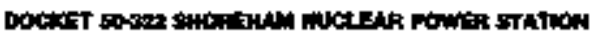

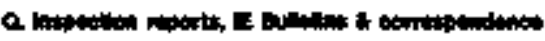

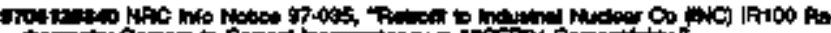

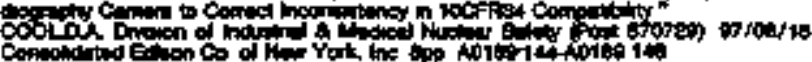

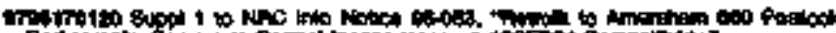

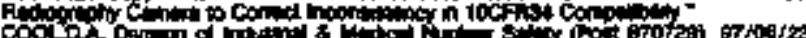

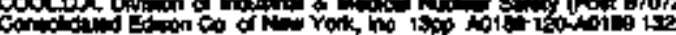

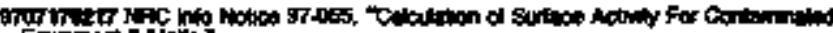

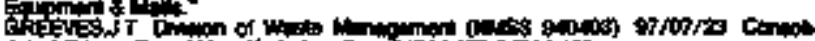

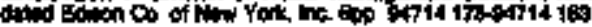

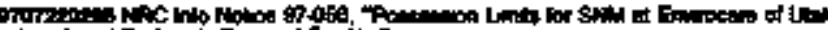

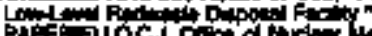

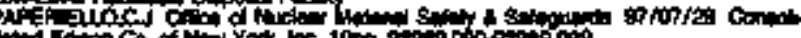

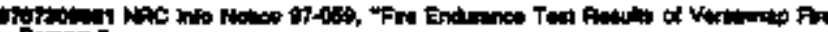

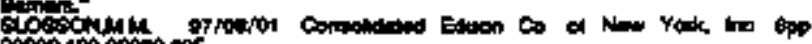
Q

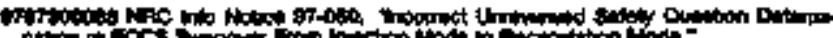

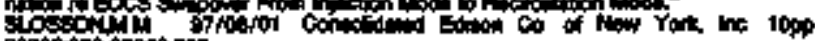

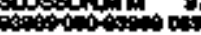

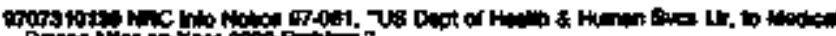
GCo

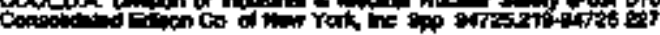

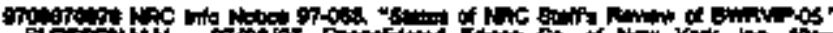

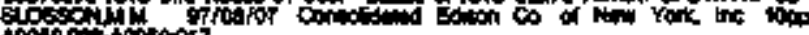

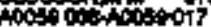

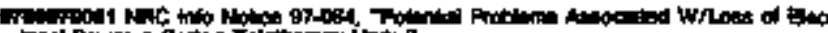

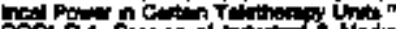

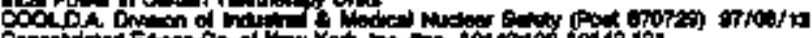

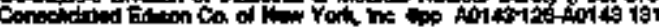

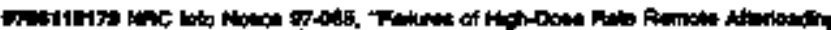

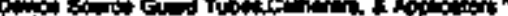

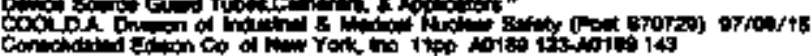

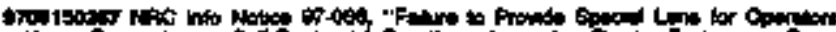

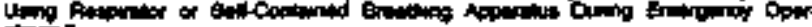

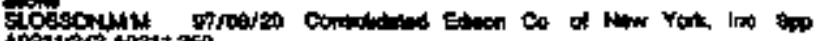
Anet1-30210 350

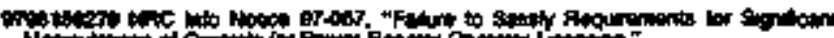

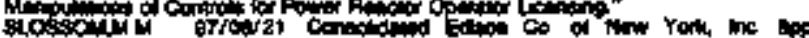

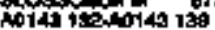




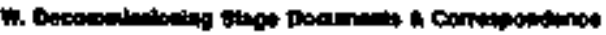

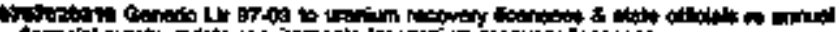

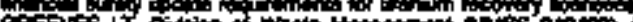

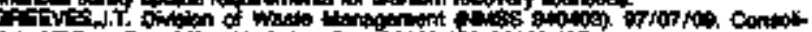

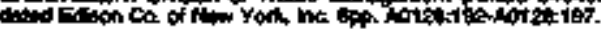

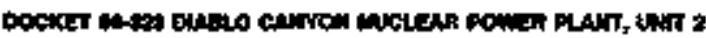

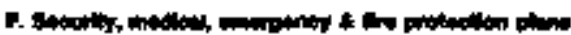

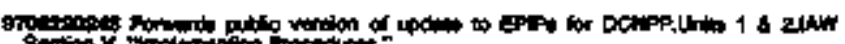

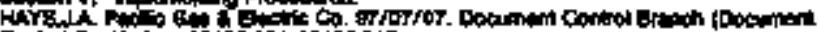

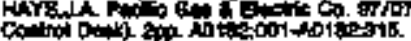

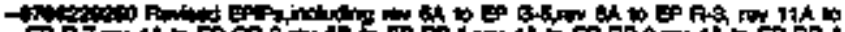
GR7 A Tot 7 .

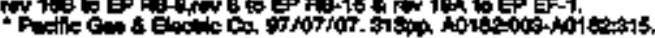

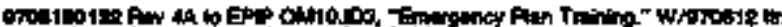

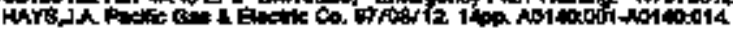

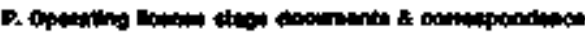

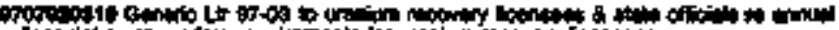

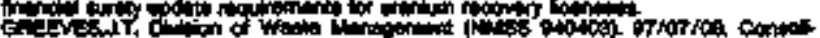

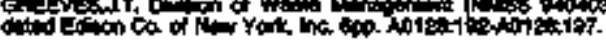

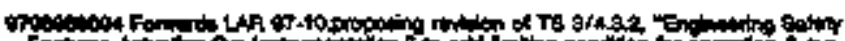

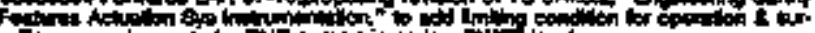
A

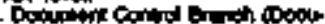

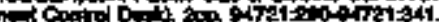

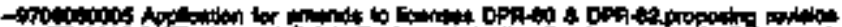

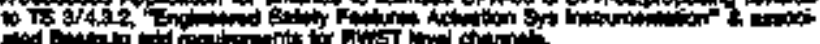

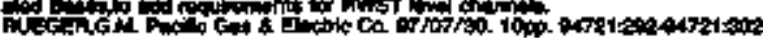

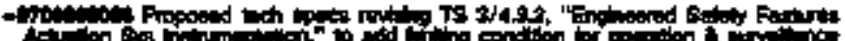

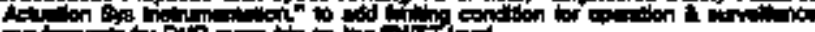

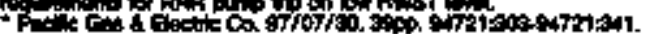

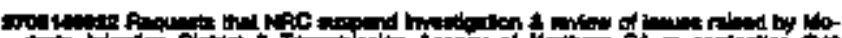

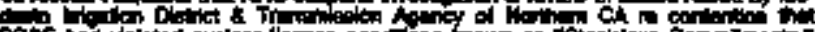

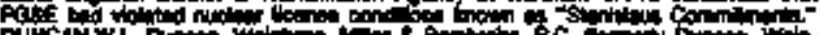

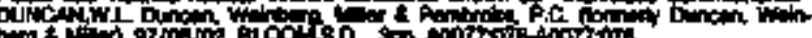

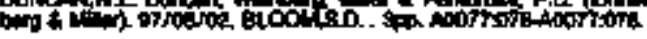

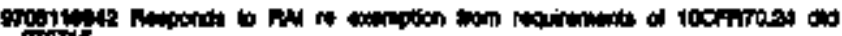
Prist.

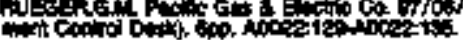

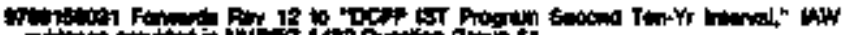

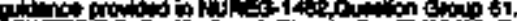

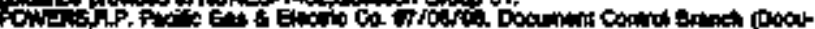

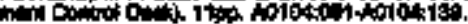

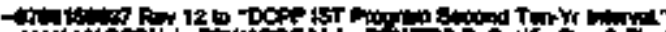

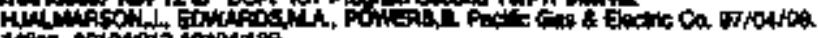

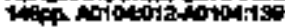

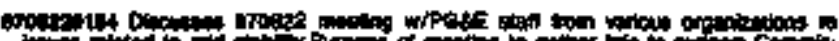

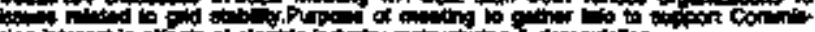

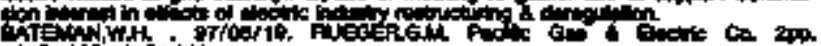

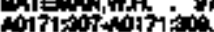

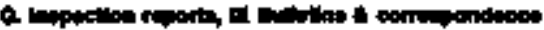

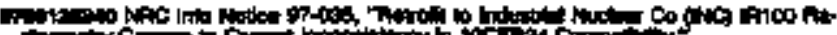

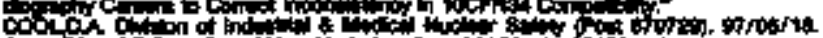

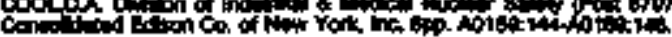

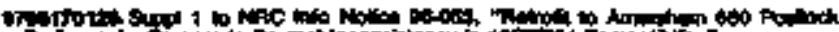

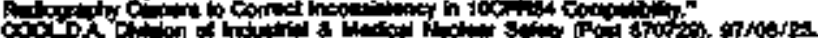

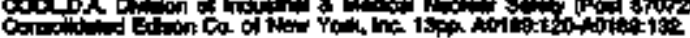

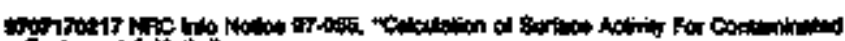

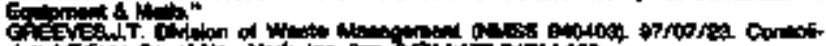

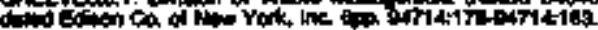

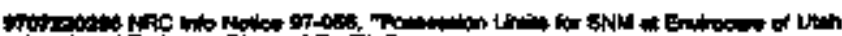

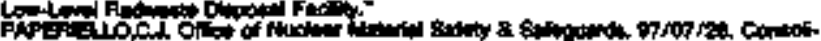

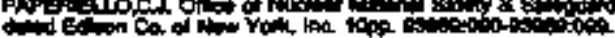

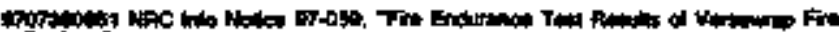

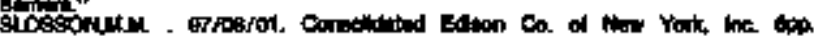

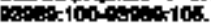

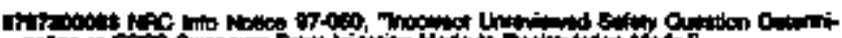

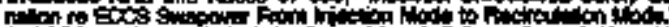

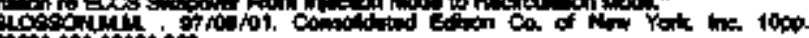

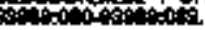

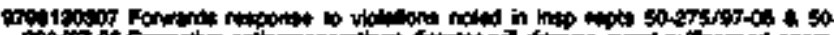

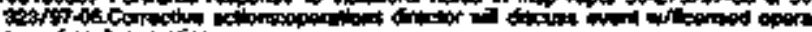

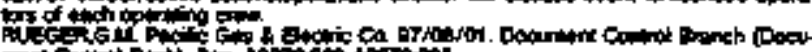

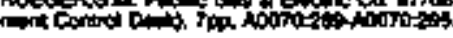

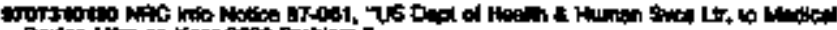

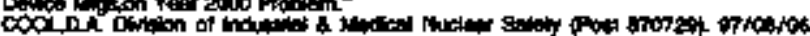

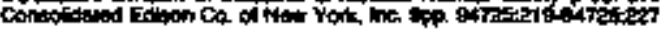

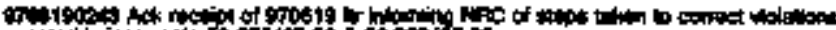

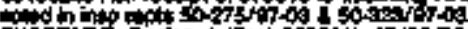

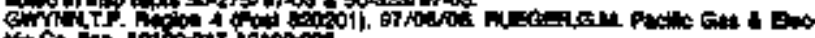

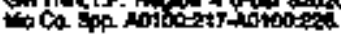

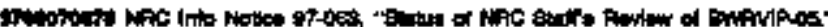

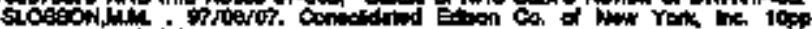

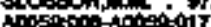

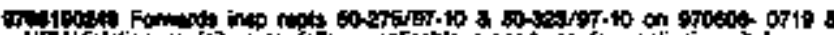

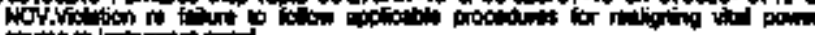

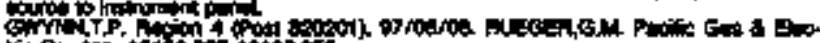

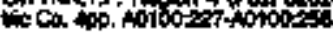

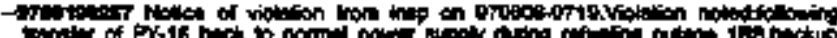

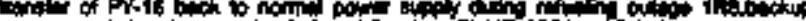

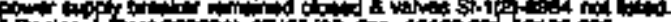

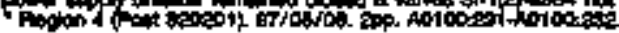

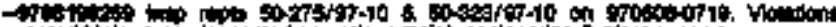

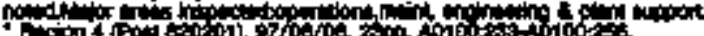

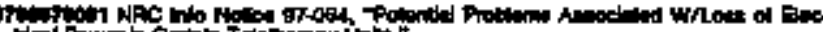

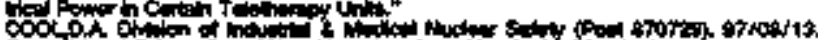

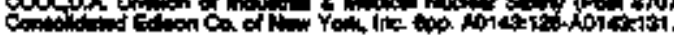

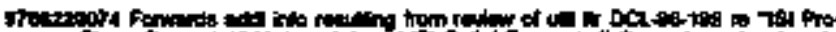

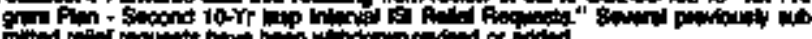

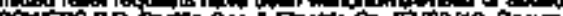
Min

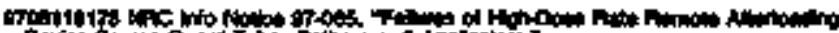

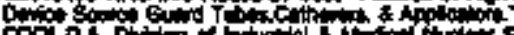

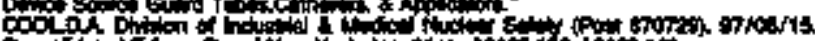

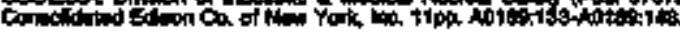

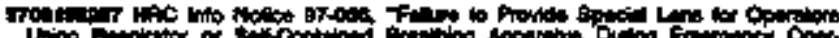

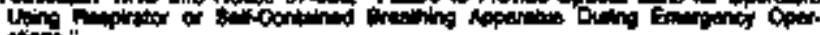

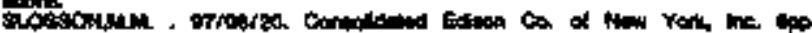
intisisumet istion.

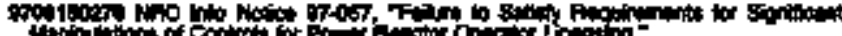

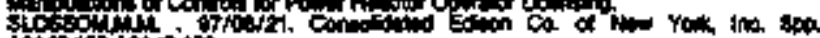

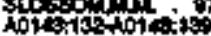

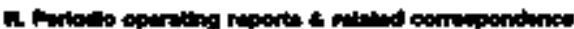

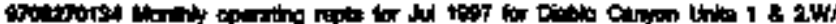

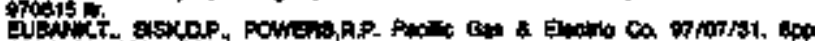

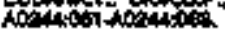

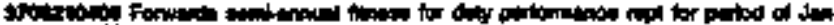

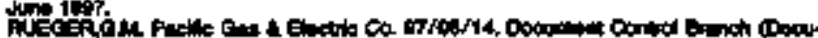

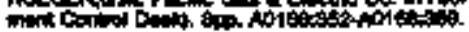

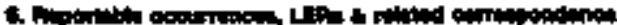

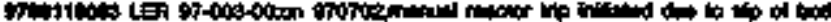

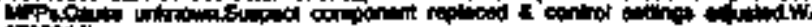
eropitit.

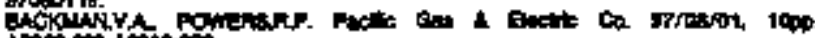

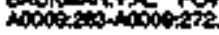

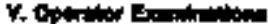

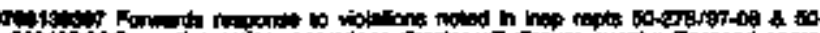

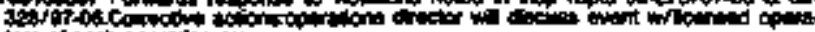

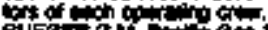

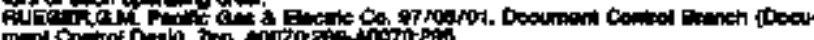

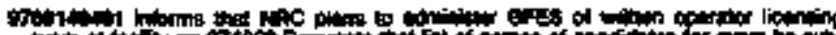

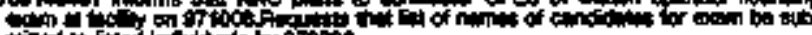

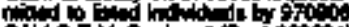

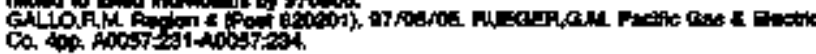




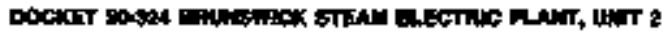

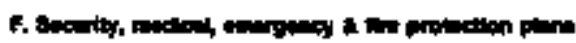

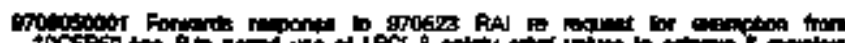

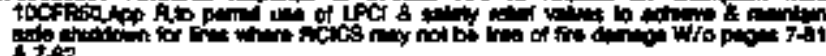
ition

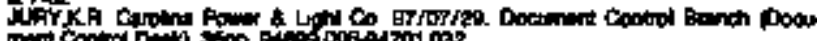

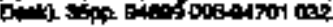

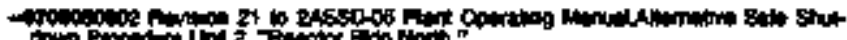

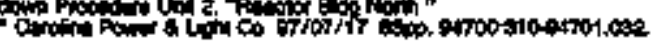

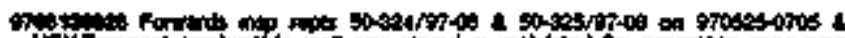

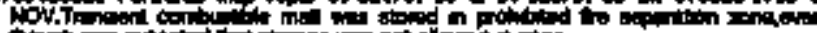

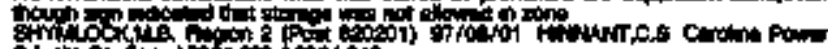
a

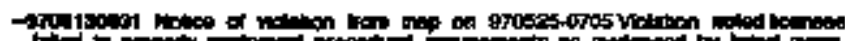

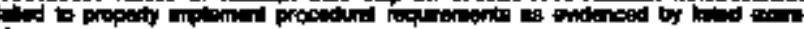

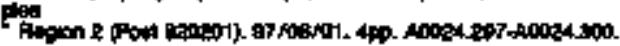

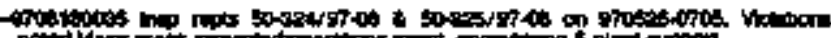

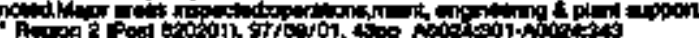

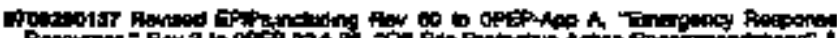

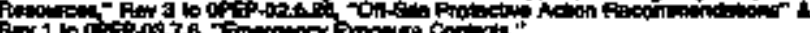

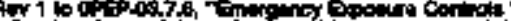

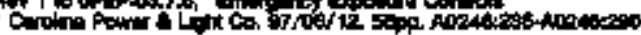

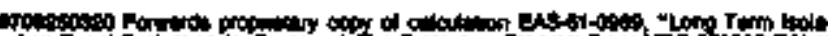

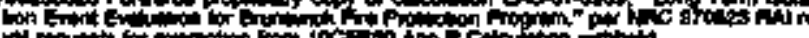

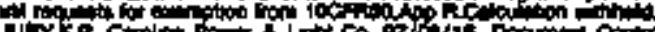

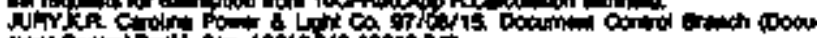

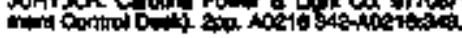

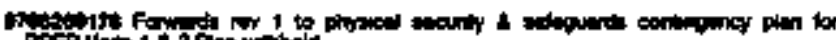

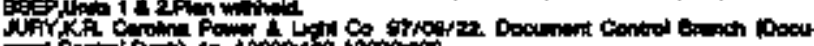

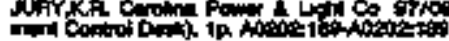

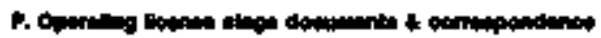

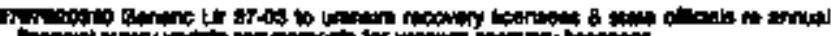

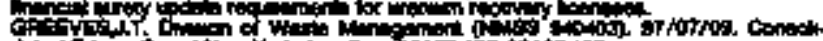

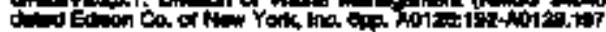

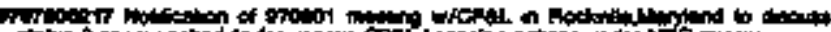

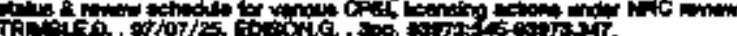

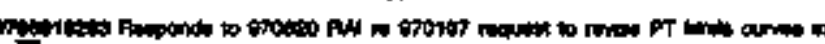

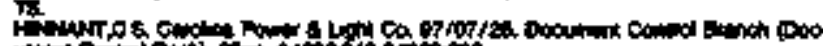

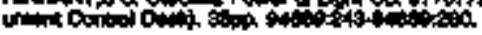

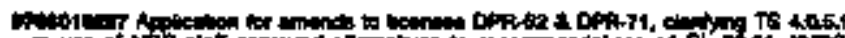

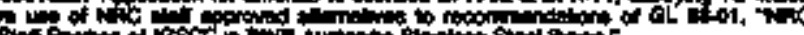

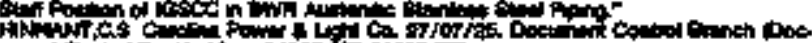

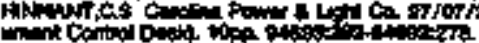

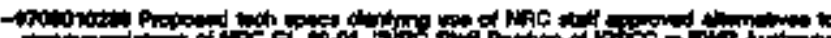

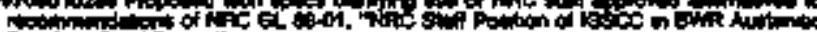

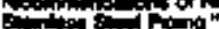

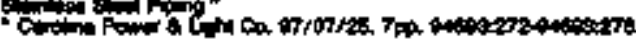

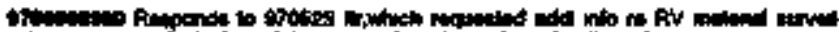

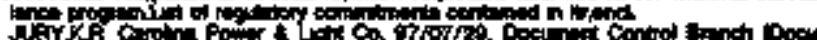

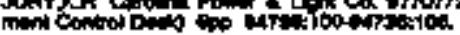

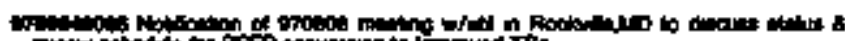

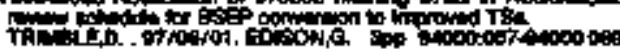

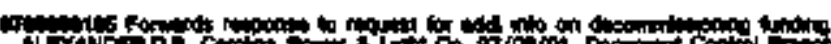

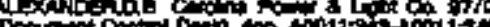

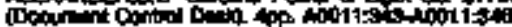

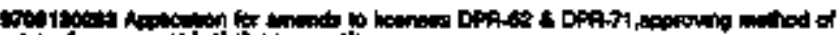

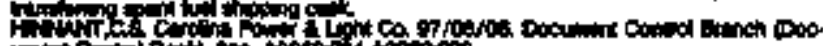
untion

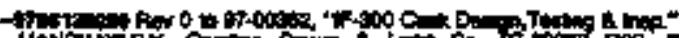

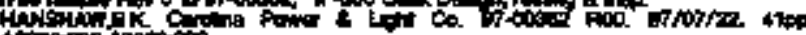

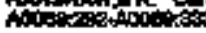

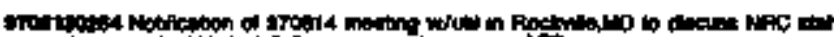

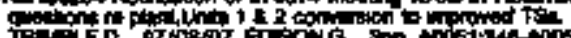

if:

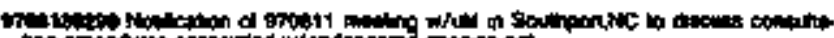

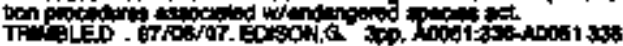

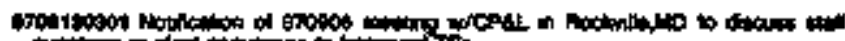

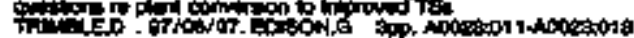

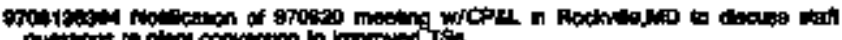

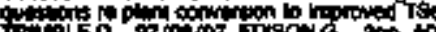

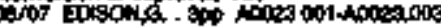

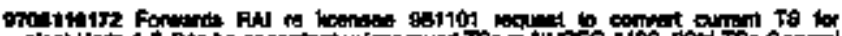

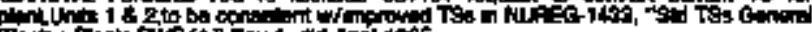

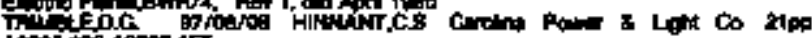

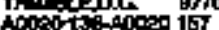

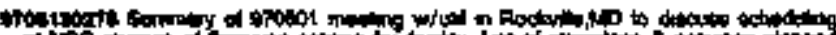

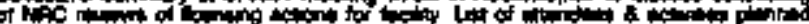

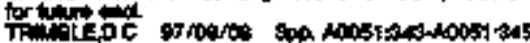

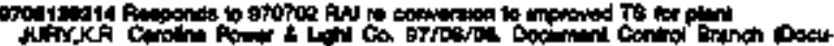

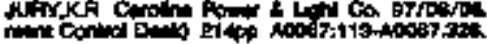

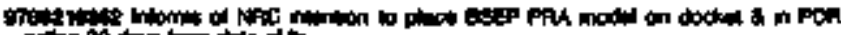

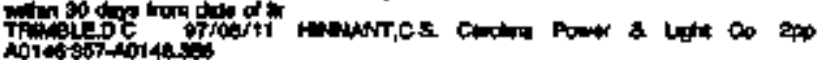

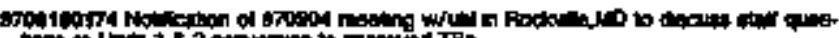

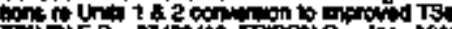

等

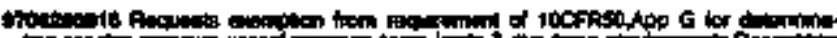

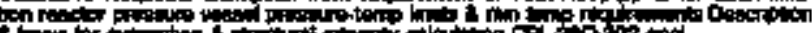

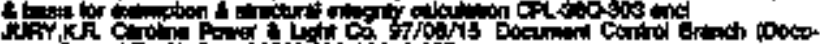

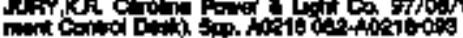

- PW

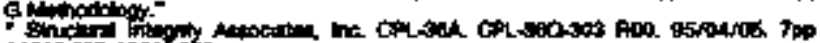

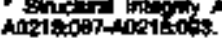

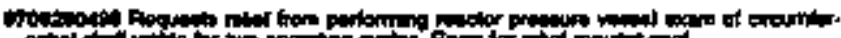

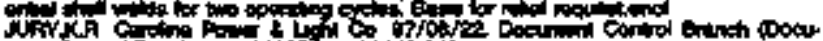

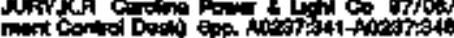

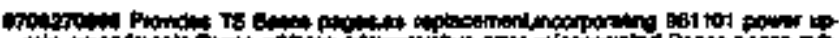

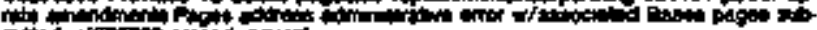

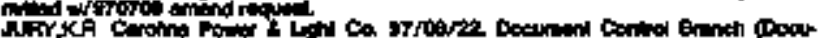

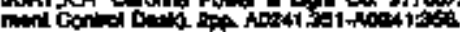

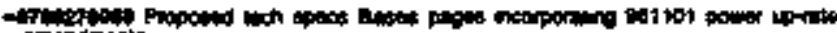

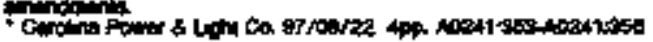

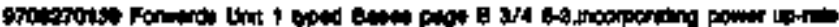

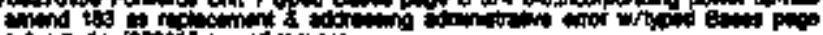

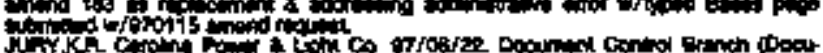

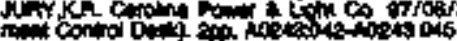

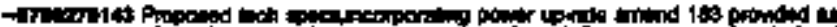

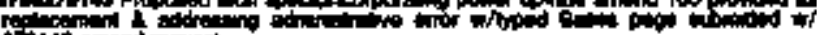
grostit anond aciet

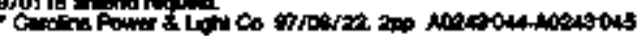

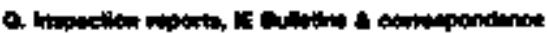

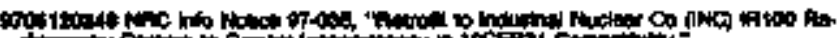

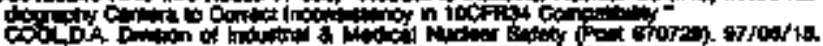

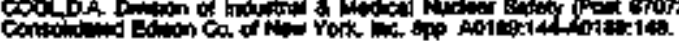

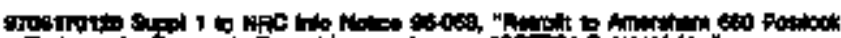

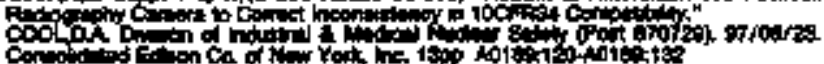

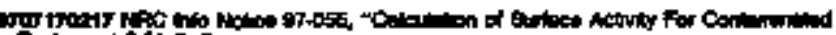

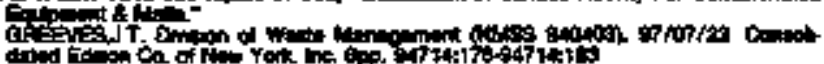

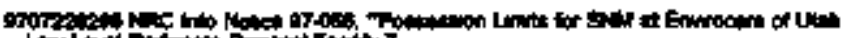

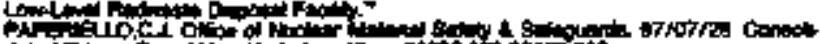

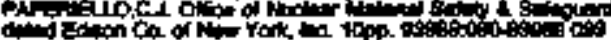

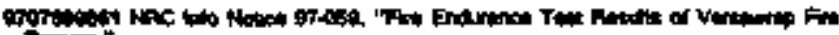

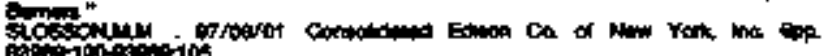

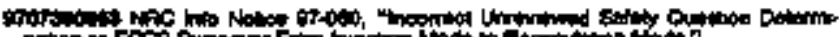

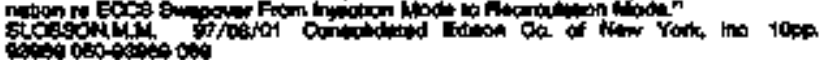

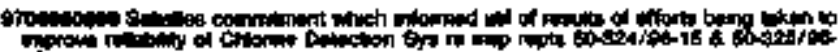

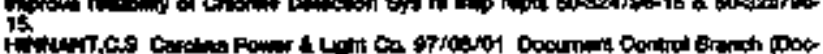

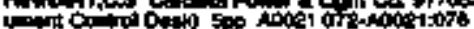




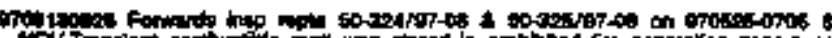

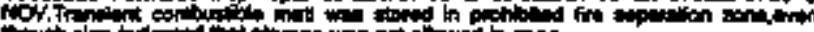

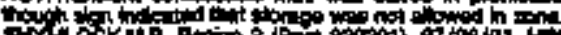

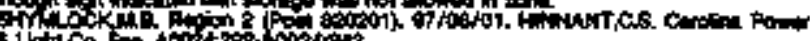

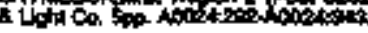

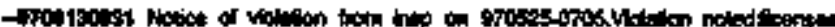

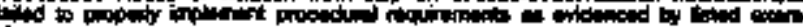

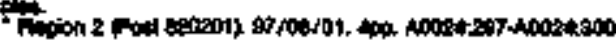

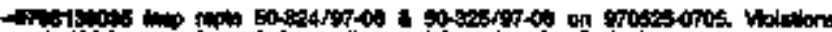

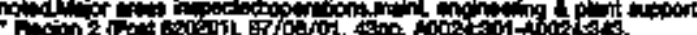

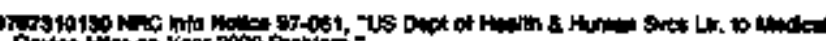

Coflot

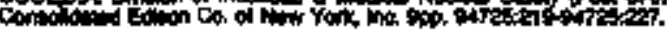

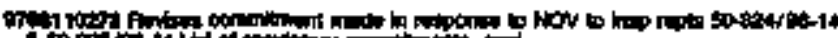

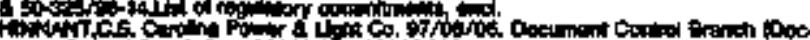

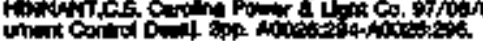

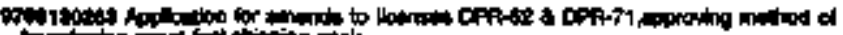

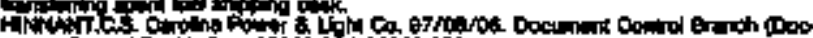

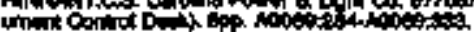

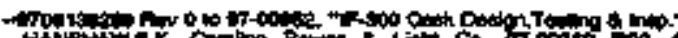

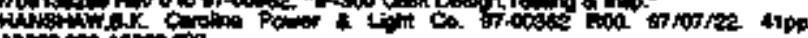

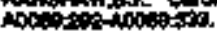

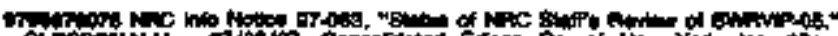

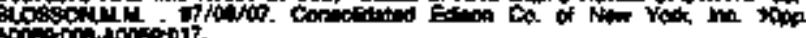

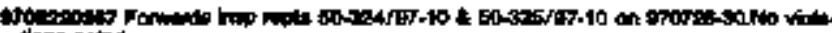

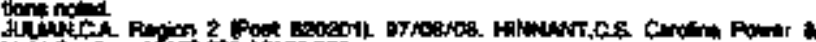

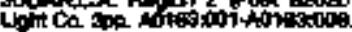

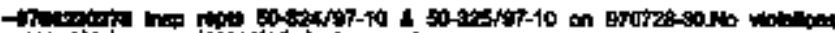

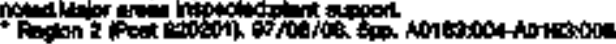

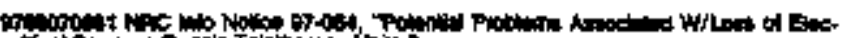

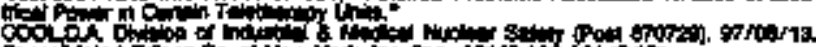

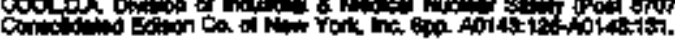

Wow

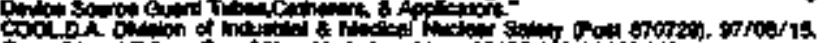

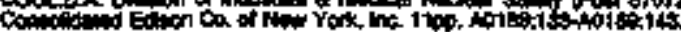

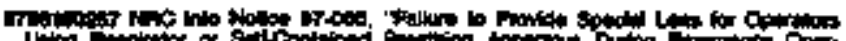

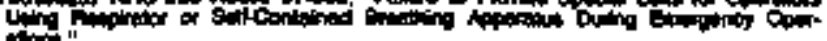

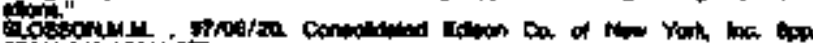

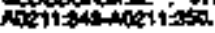

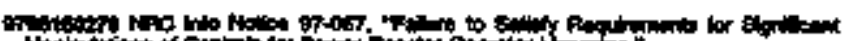

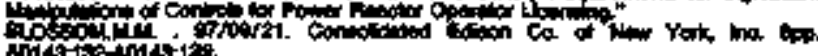

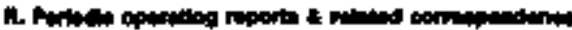

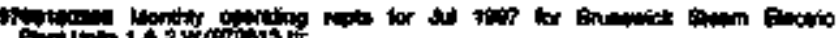

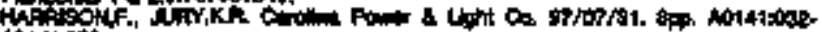
whatios

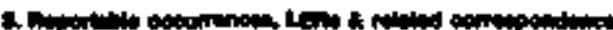

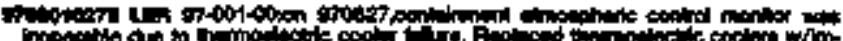

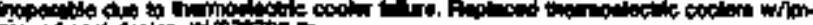

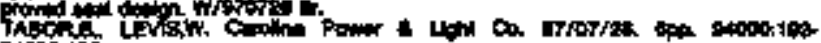
ontom

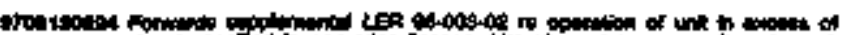

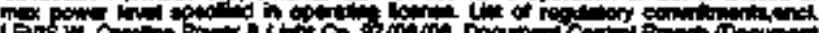

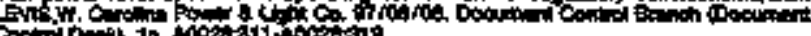

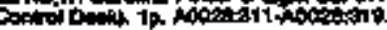

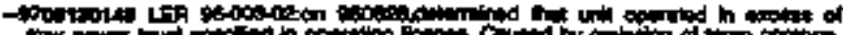

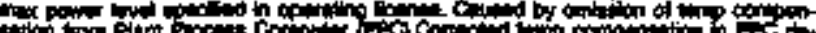
ation

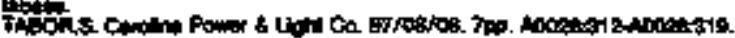

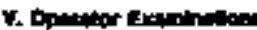

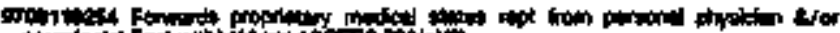

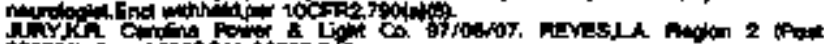

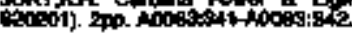

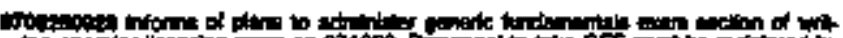

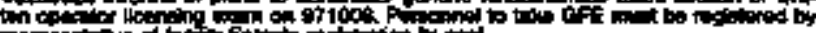

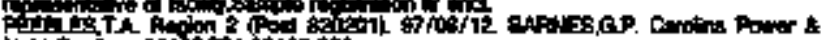

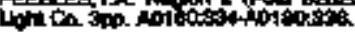

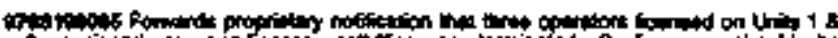

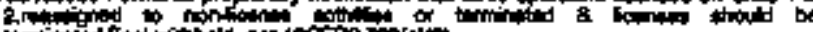

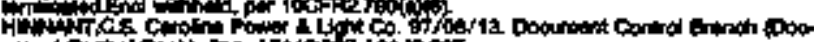

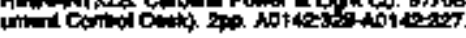

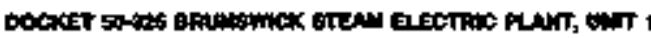

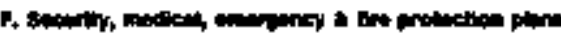

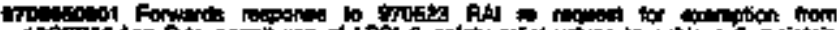

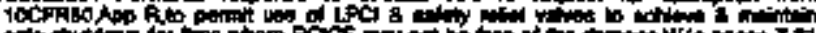

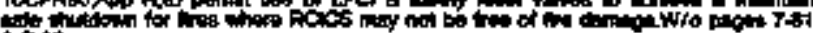

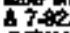

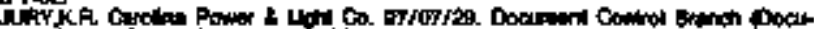

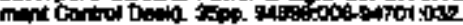

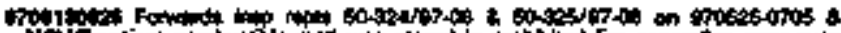

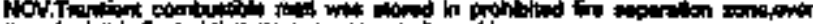

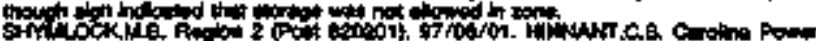

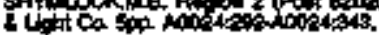

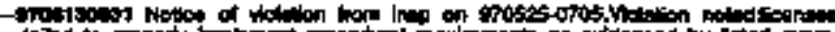
1. Paten

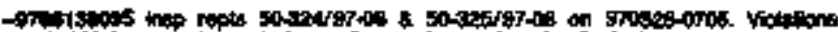

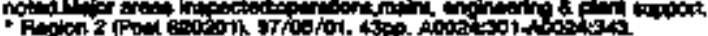

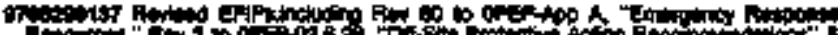

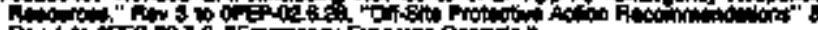

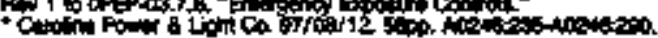

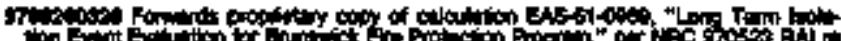

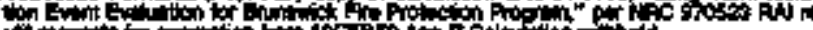

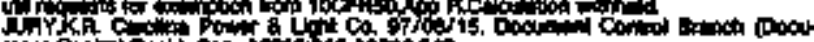

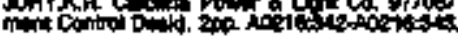

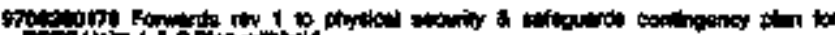

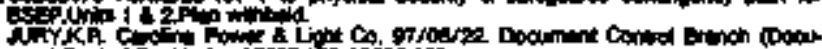

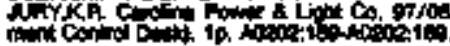

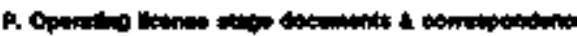

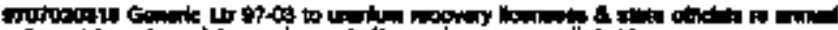

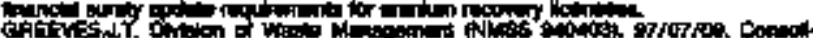

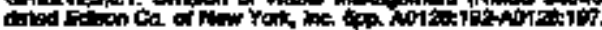

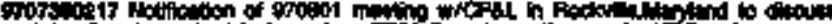

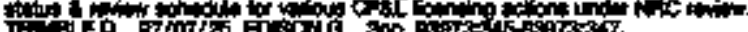

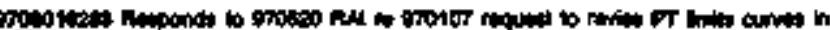

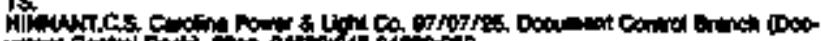

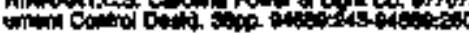

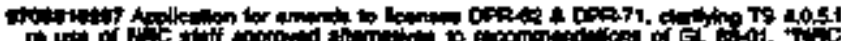
D u

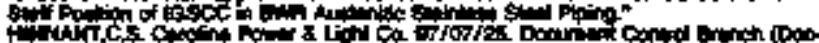
ind

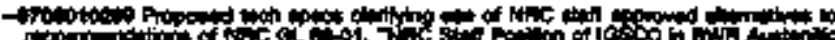

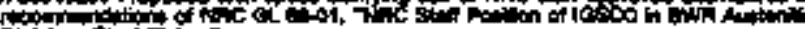

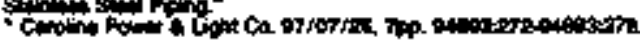

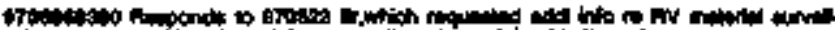

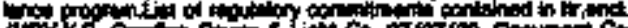

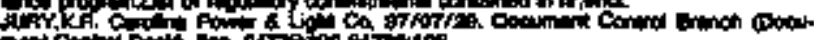

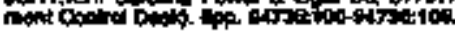

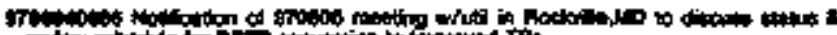

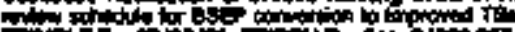

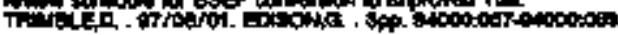

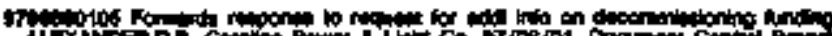

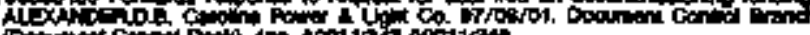

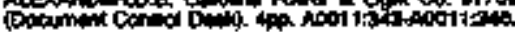

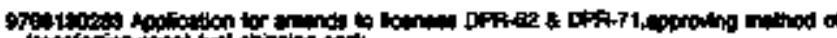

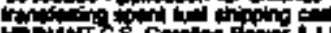

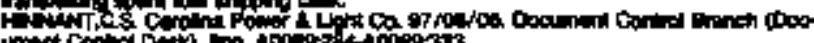

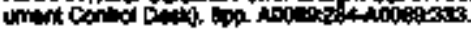

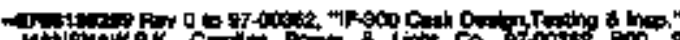

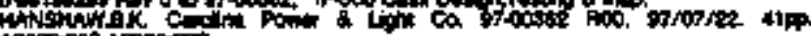

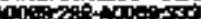

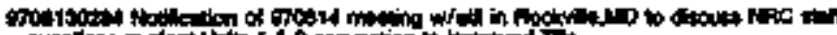

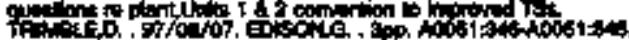




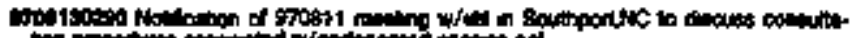

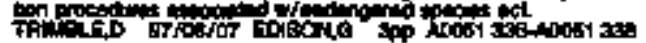

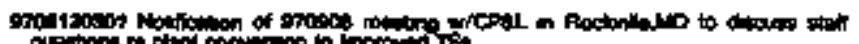

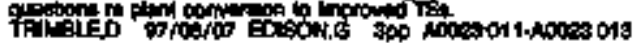

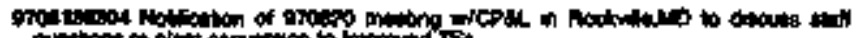

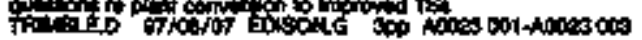

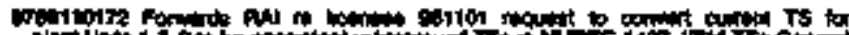

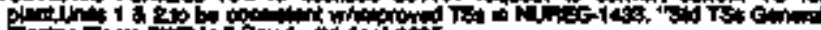

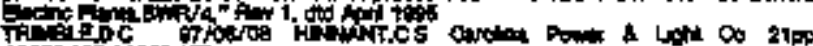

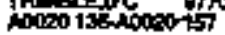

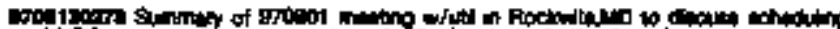

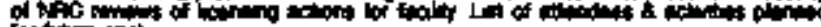

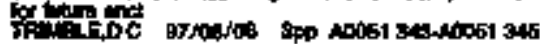

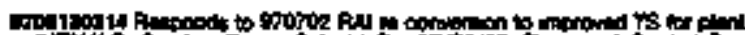

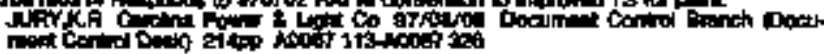

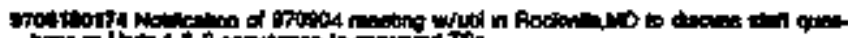

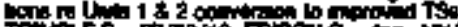

con

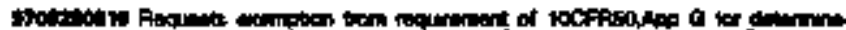

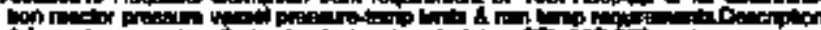

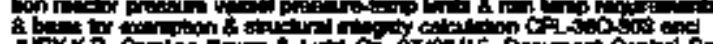

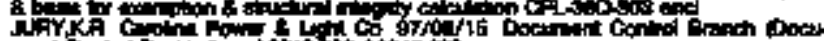

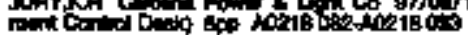

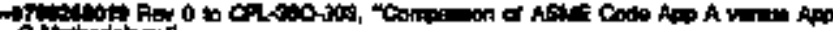
a thibodoty"

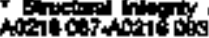

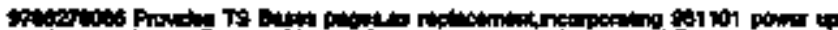

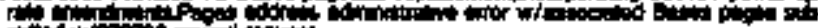

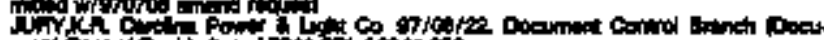
net Coint Dind

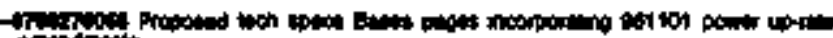

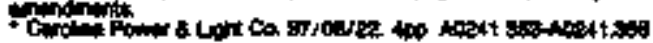

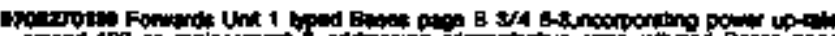

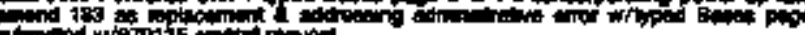

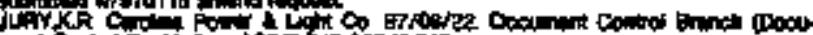

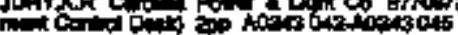

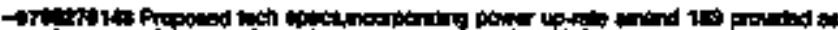

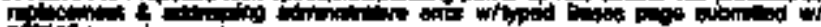

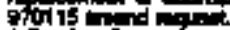

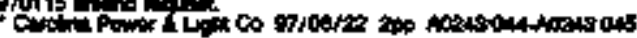

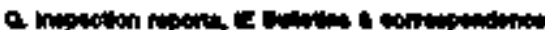

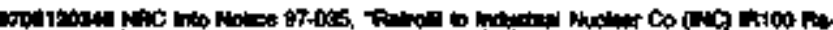

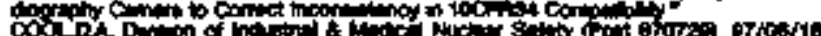

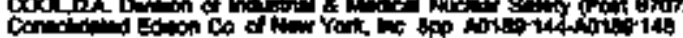

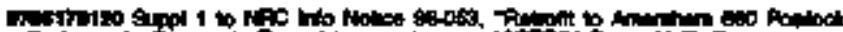

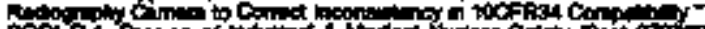

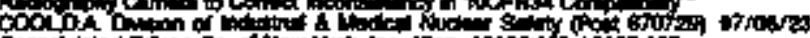

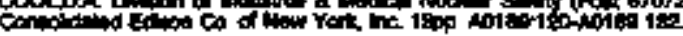

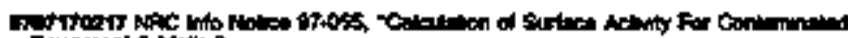

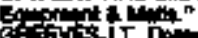

S.

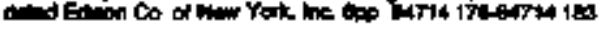

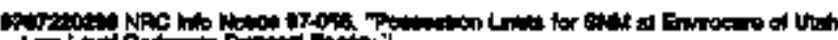

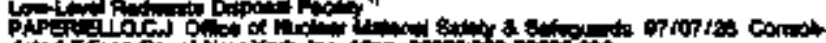

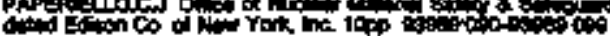

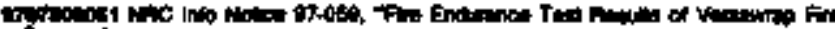

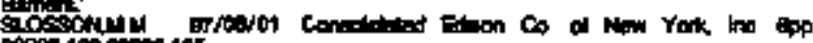

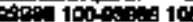

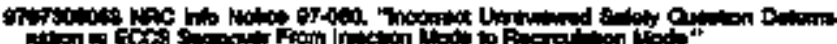

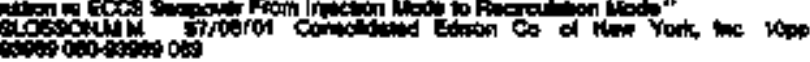

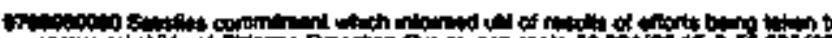

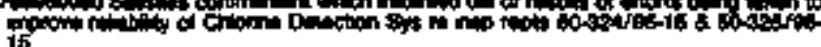

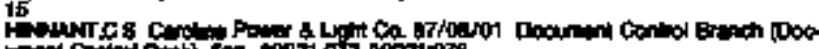

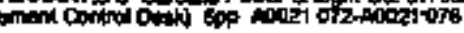

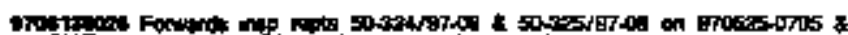

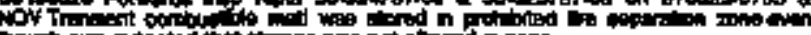

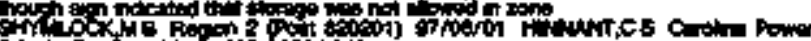

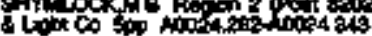

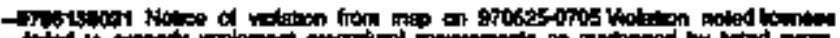

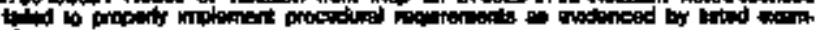

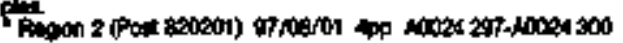

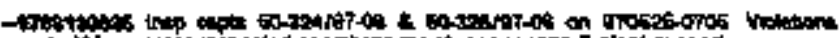

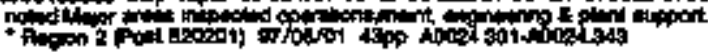

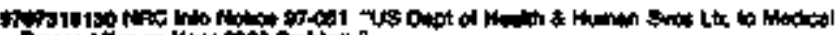

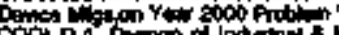

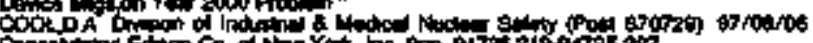

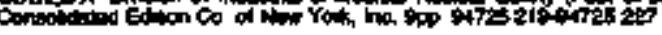

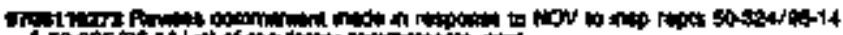

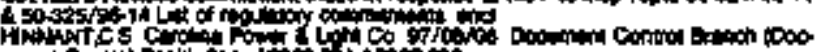

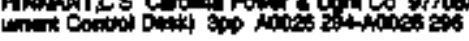

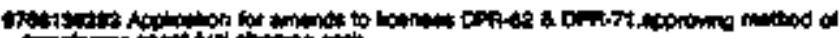

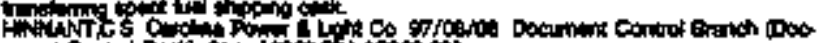

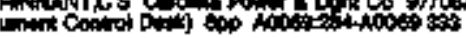

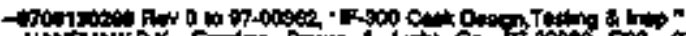

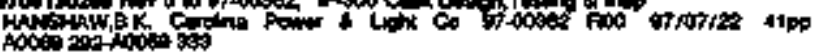

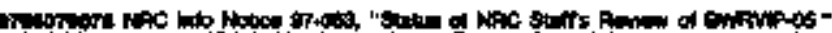

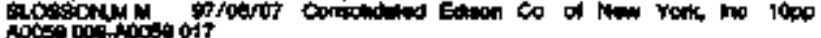

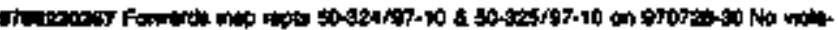

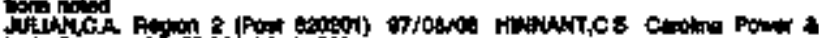

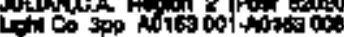

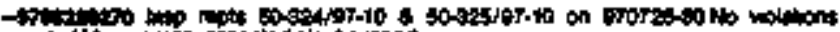

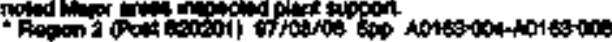

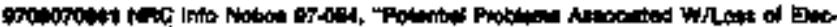

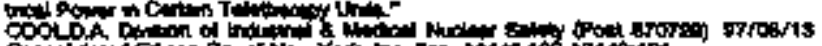

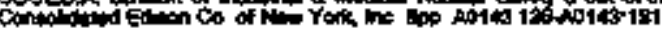

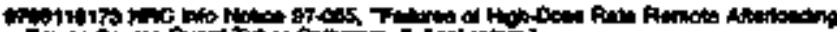

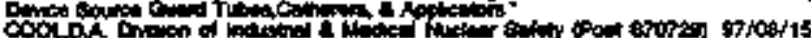

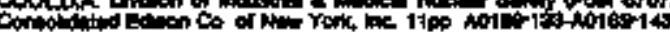

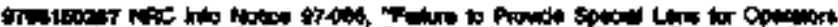

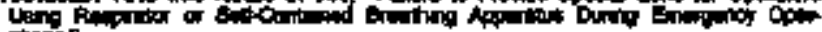

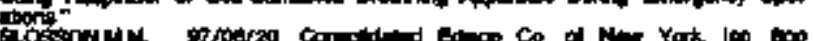

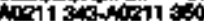

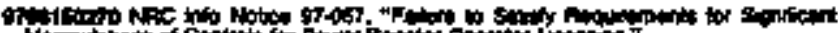

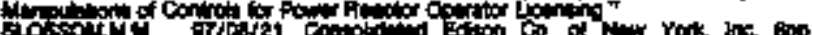

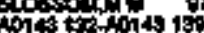

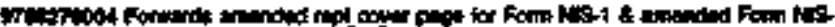

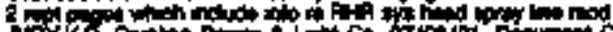

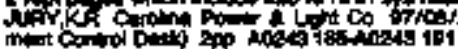

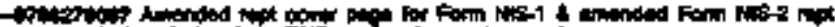

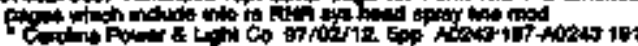

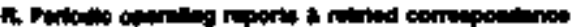

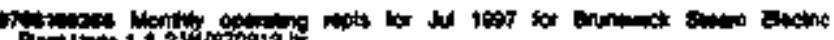

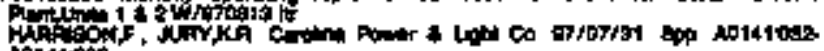
Novitos

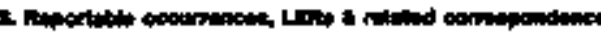

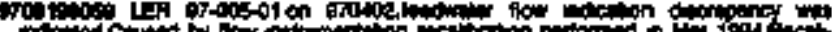

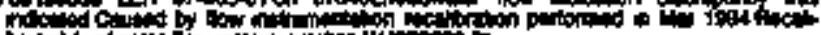

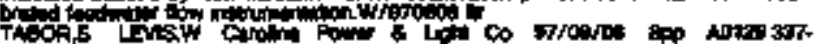
Antosis

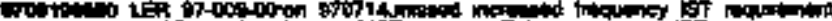

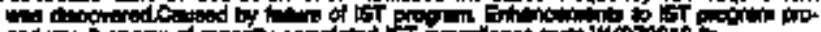

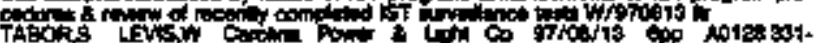
arizis

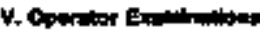

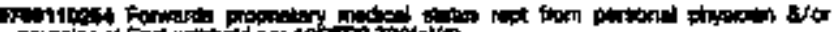

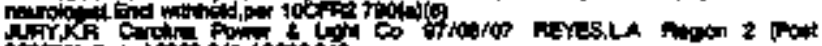

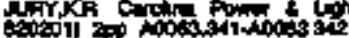




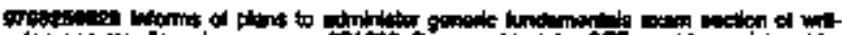

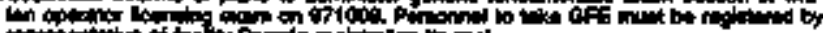

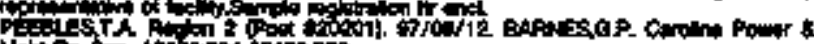

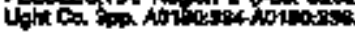

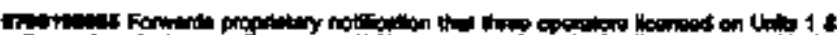

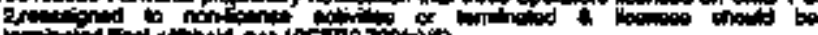

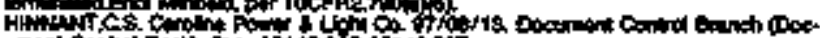

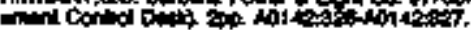

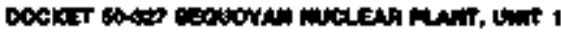

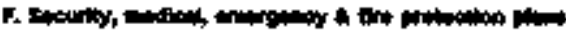

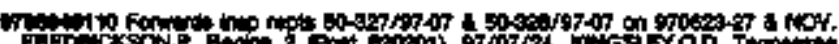

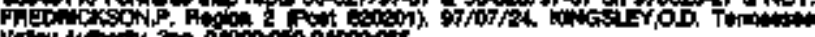

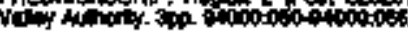

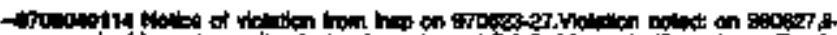

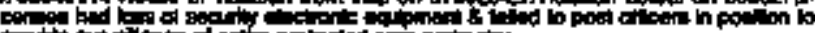

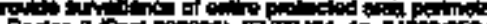

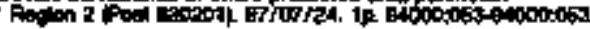

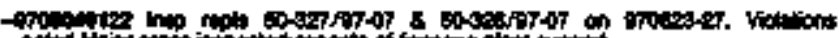

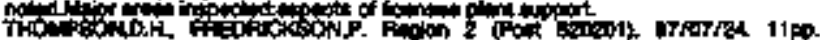

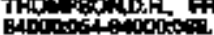

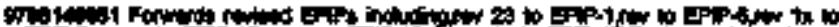

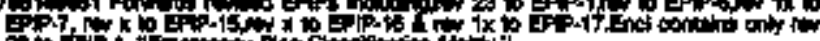
2 it

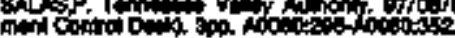

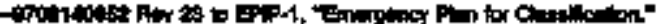

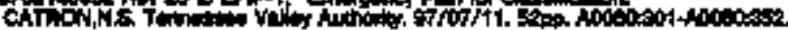

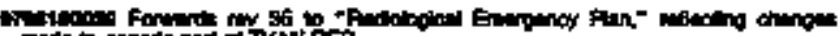

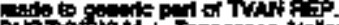

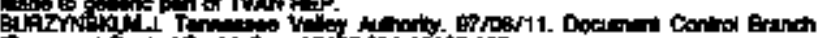

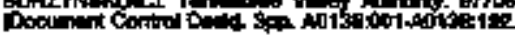

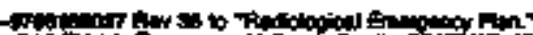

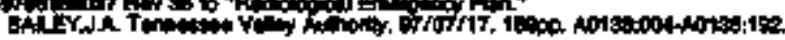

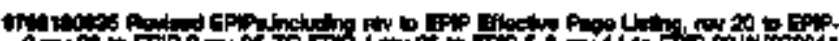

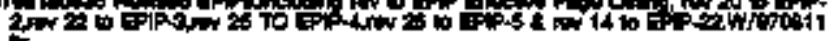

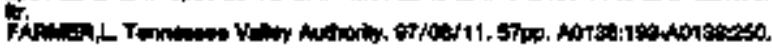

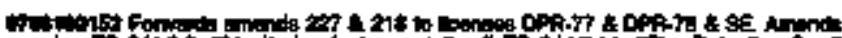

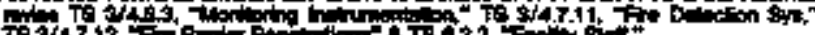

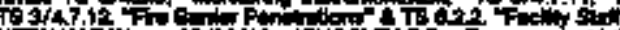

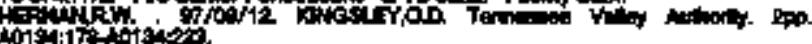

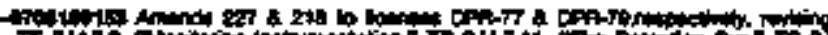

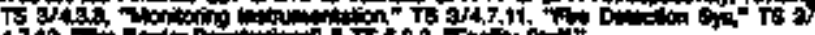

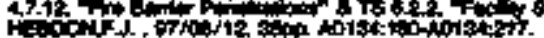

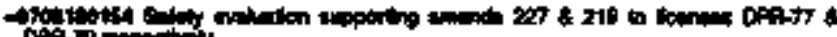

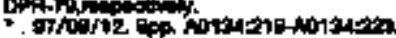

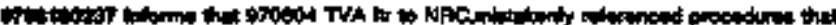

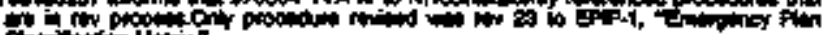

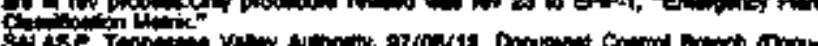

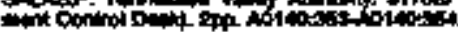

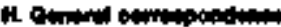

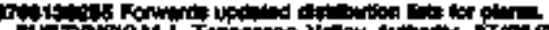
A c

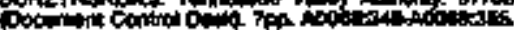

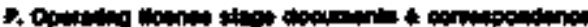

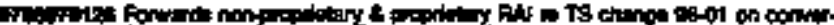

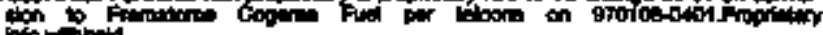
5.

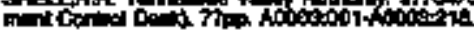

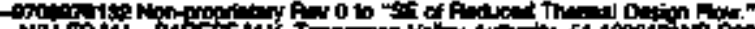

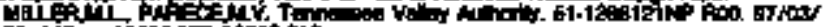

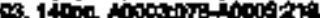

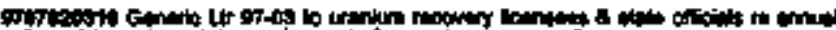

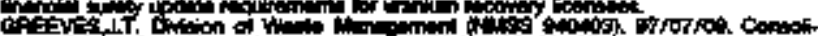

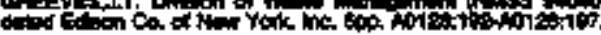

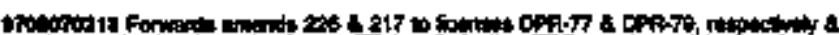

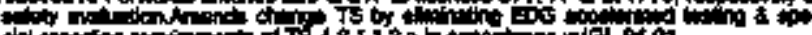

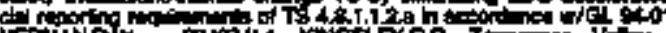

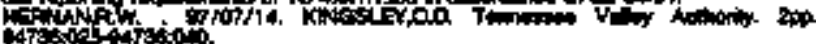

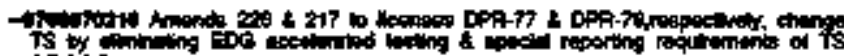
4.1.12.

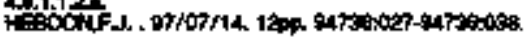

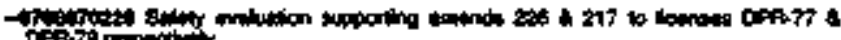

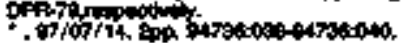

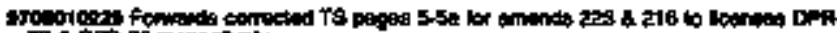

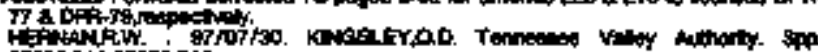

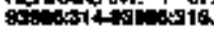

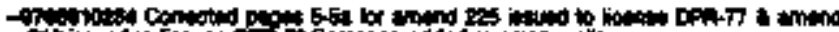

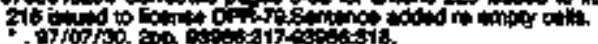

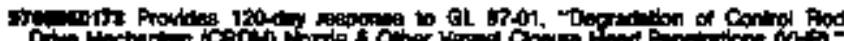

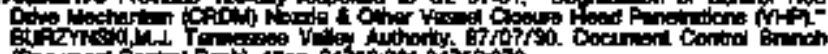

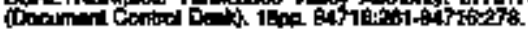

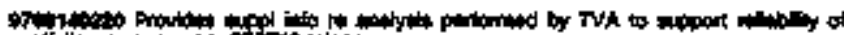

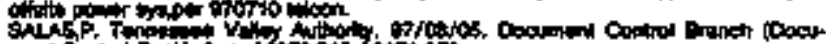

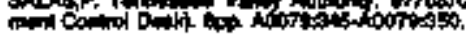

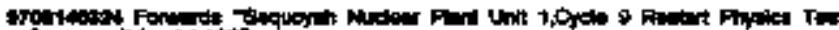

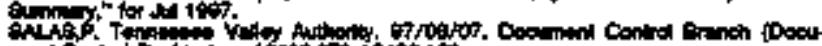

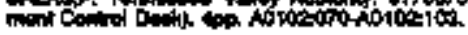

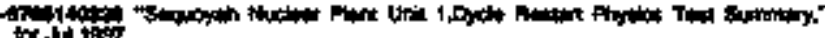
tor touts

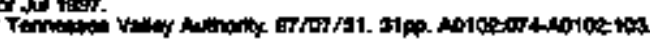

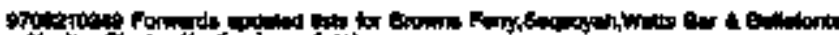

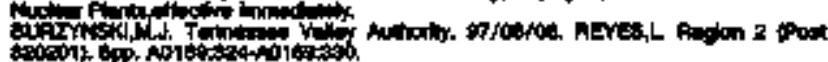

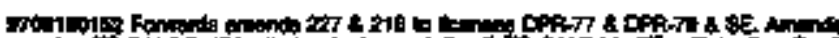

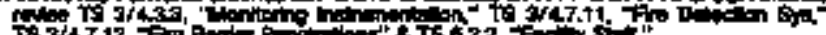

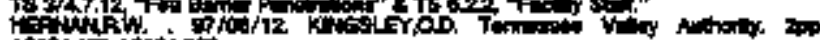

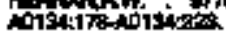

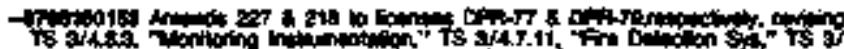

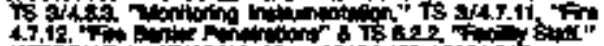

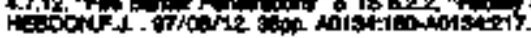

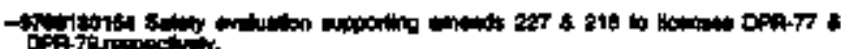

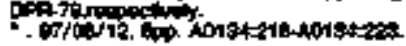

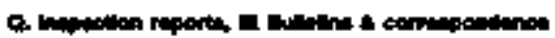

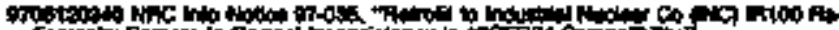

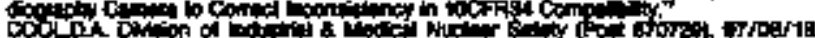

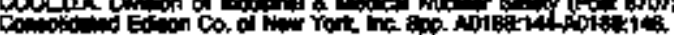

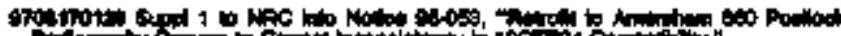

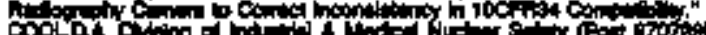

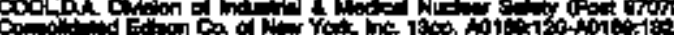

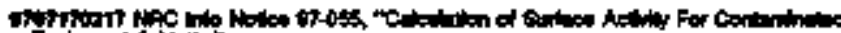

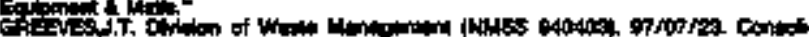

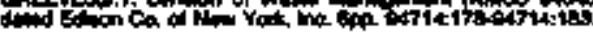

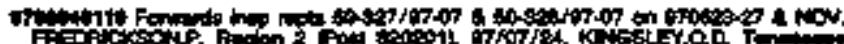

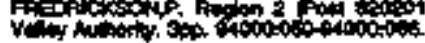

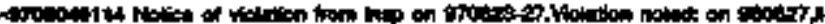

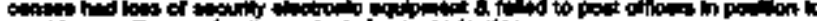

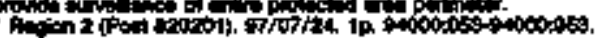

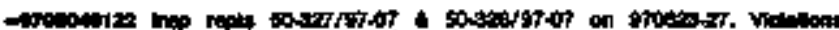

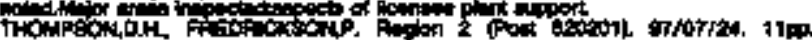

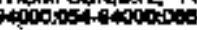

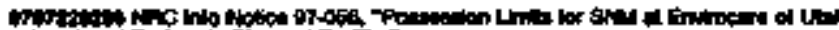

PAP T

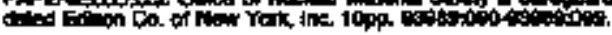

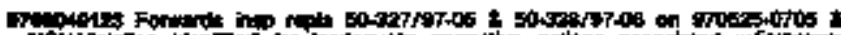

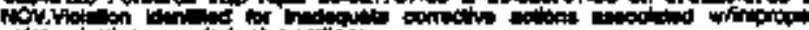

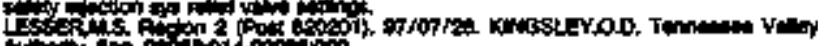

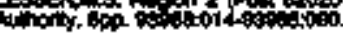

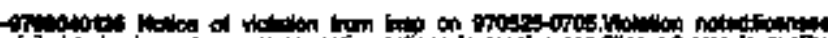

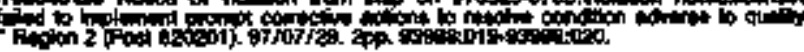




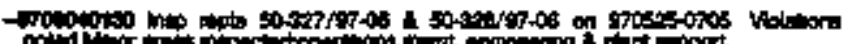

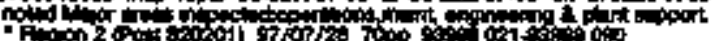

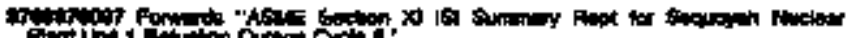

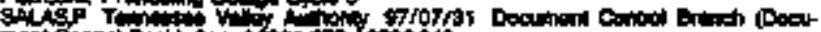

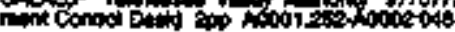

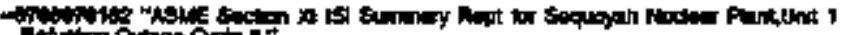

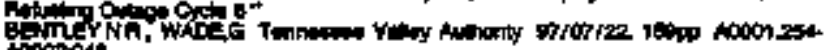
atopos:

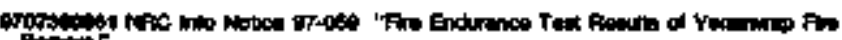

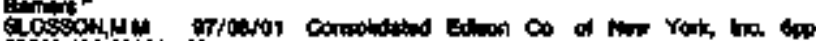

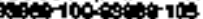

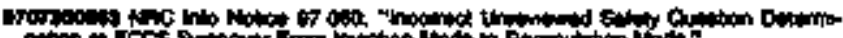

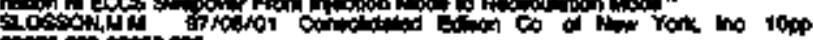

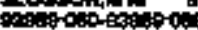

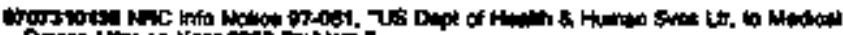

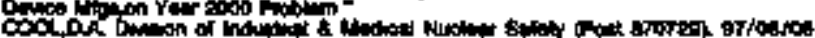

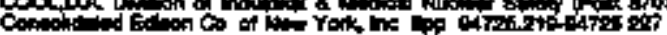

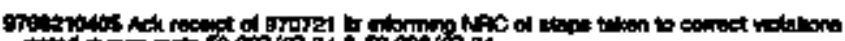

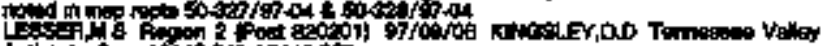

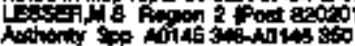

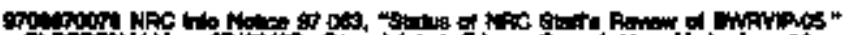
a iocosotionso of?

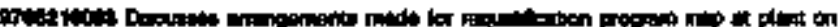

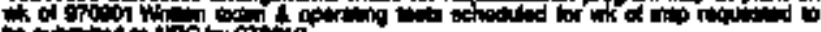

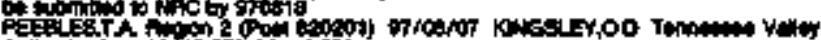

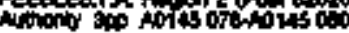

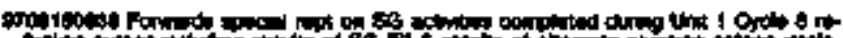

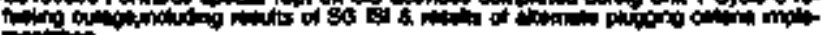

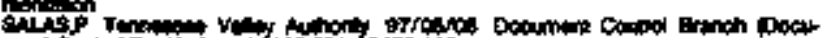

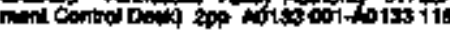

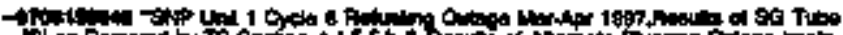

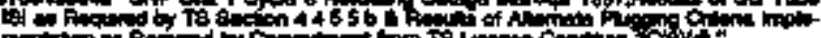

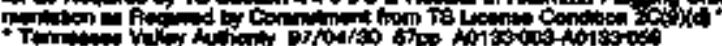

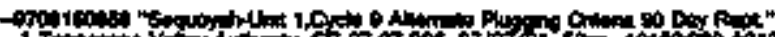

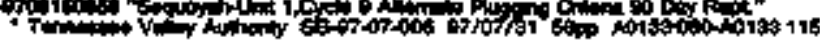

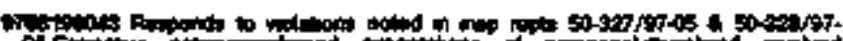

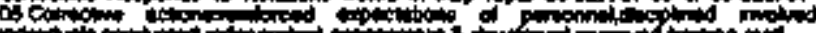

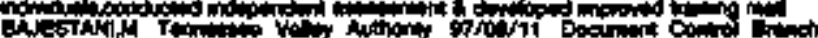
(i)

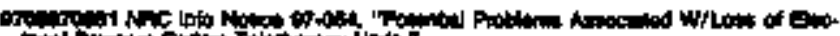

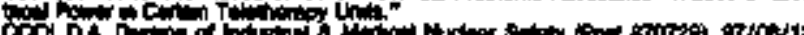

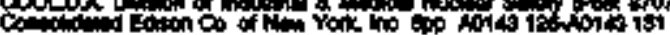

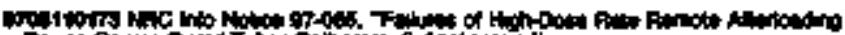

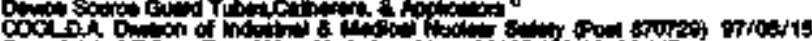

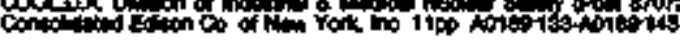

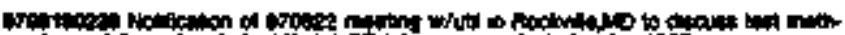

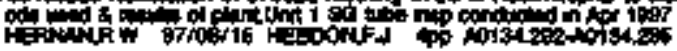

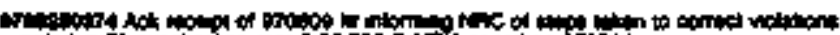

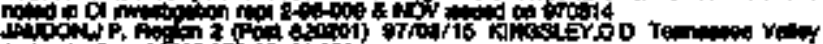

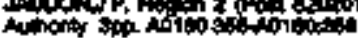

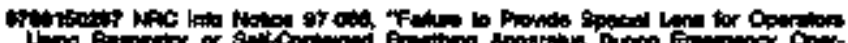

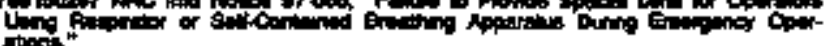

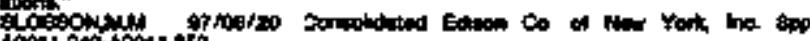

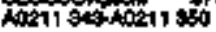

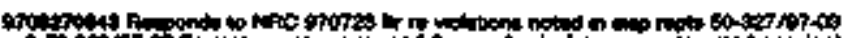

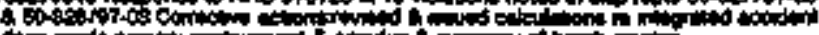

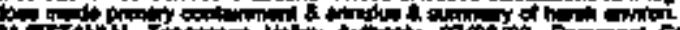

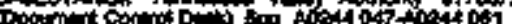

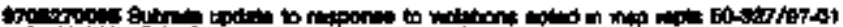

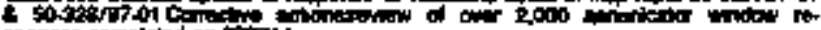

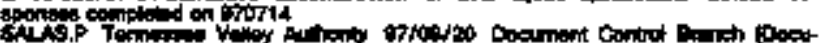

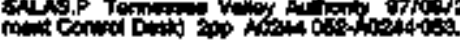

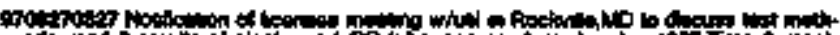

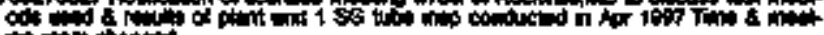

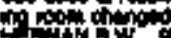

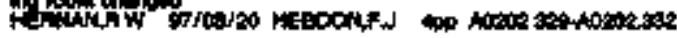

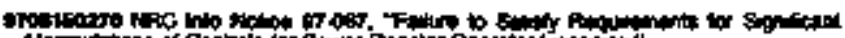

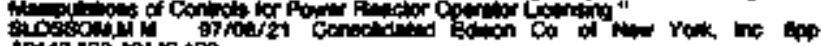

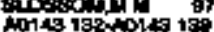

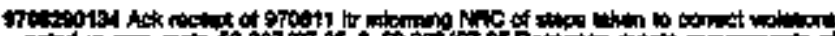

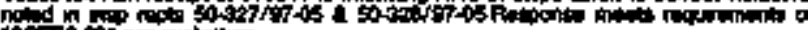

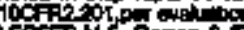

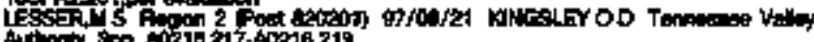

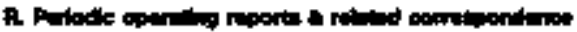

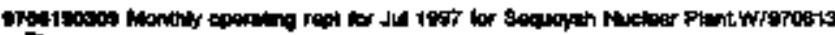

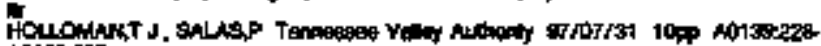
not3ato?

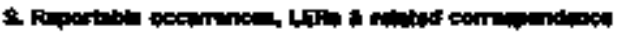

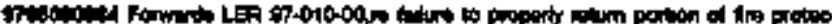

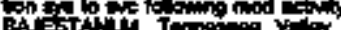

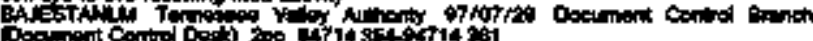

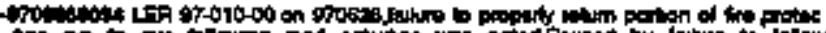

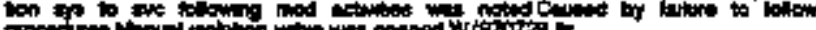

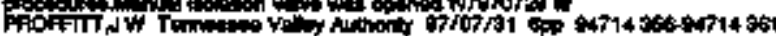

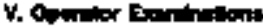

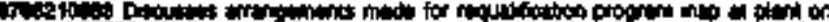
M of कropi if

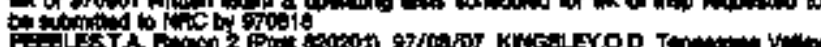
in

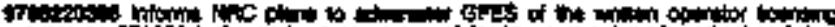

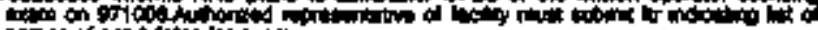

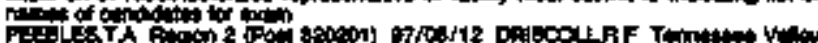

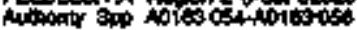

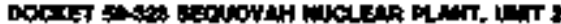

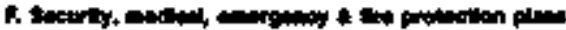

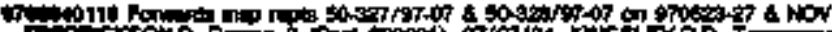
in

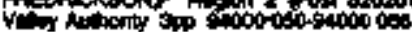

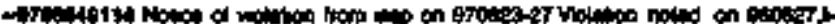

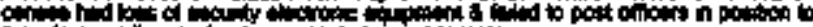

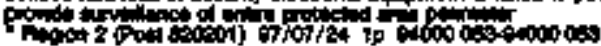

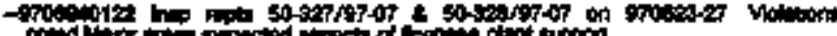

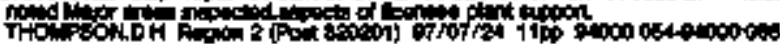

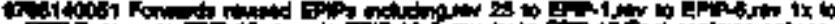

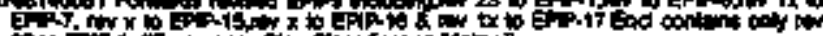

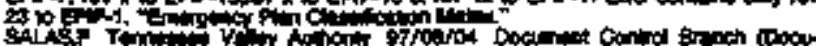

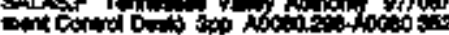

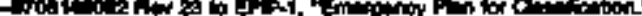

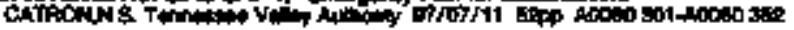

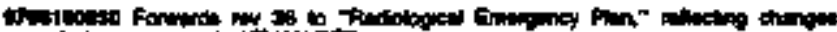

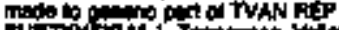

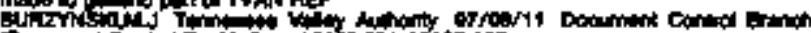

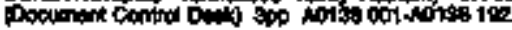

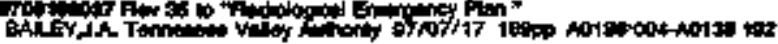

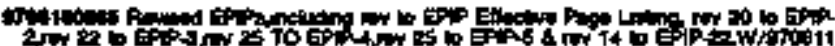

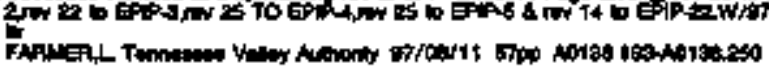

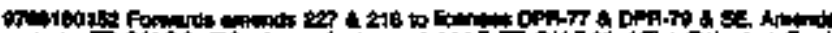

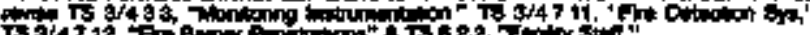

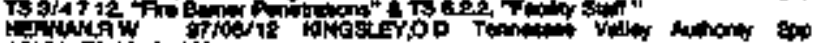

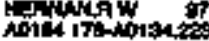

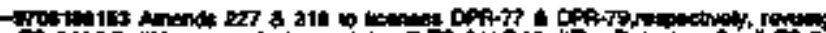

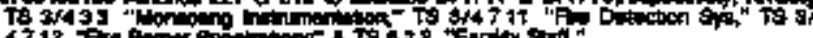

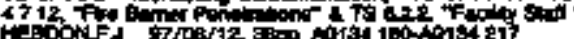

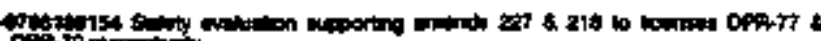

of

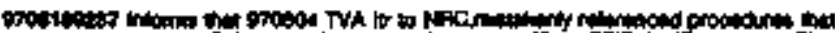

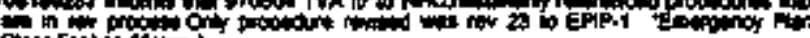

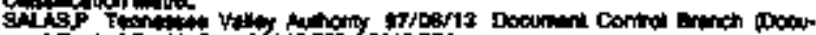

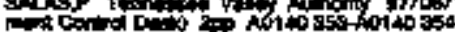




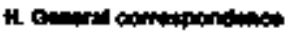

maxtrain Fon

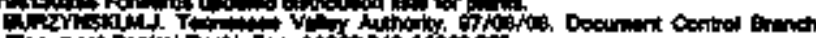

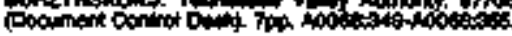

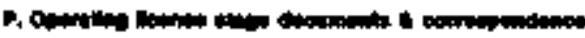

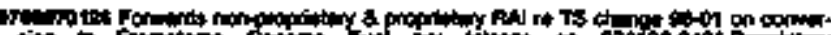

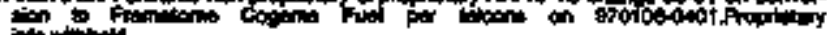

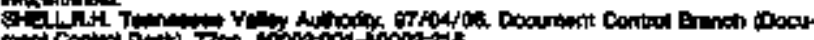

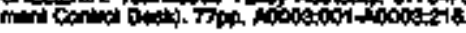

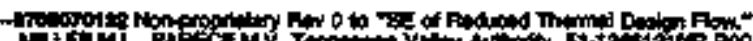

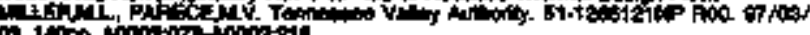

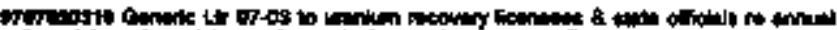

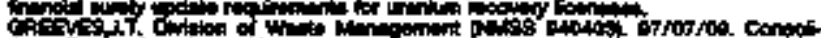

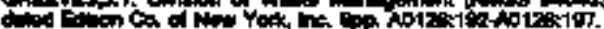

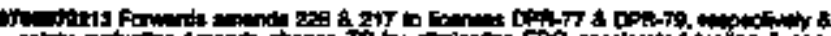

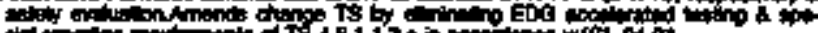

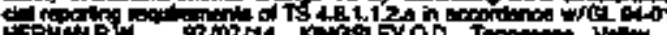

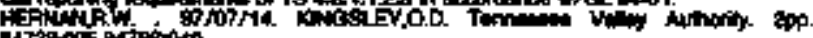

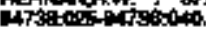

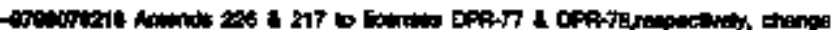
T5 by

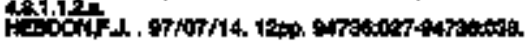

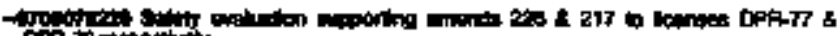

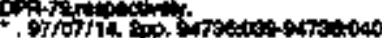

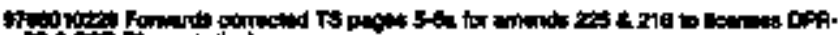

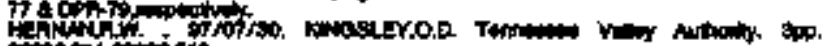

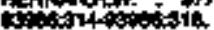

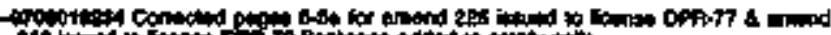
If

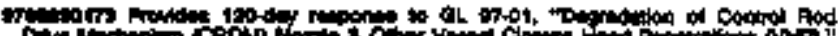

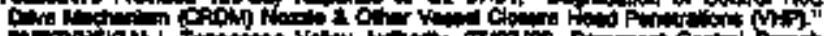

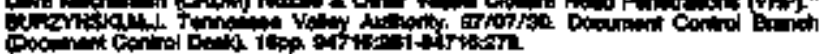

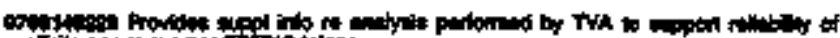

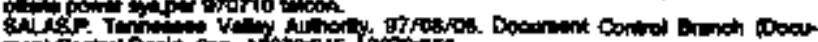

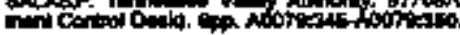

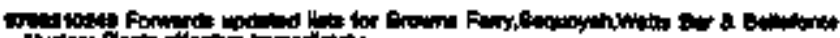

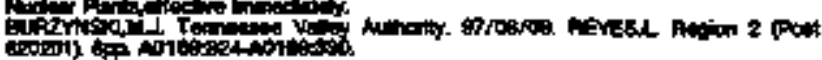

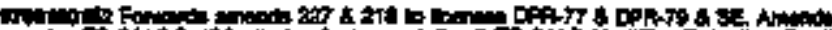

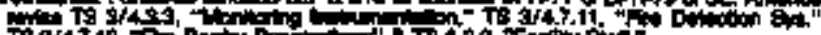

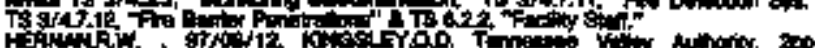

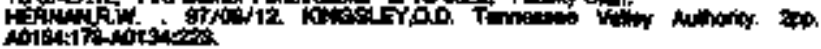

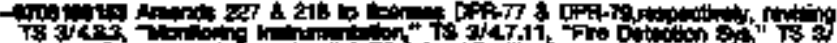

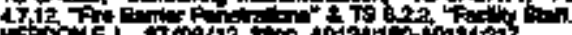

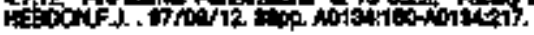

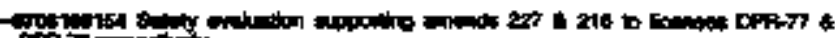

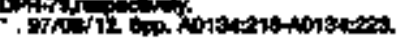

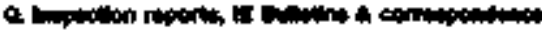

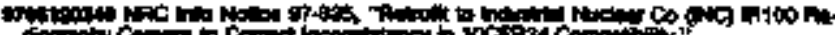

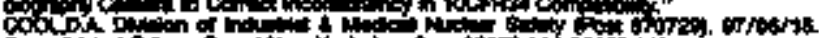

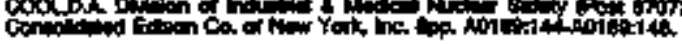

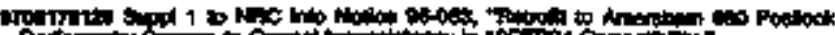

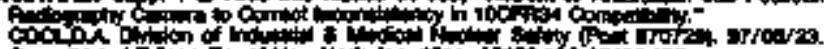

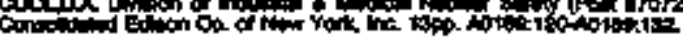

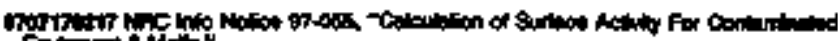

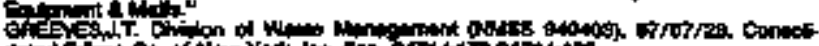

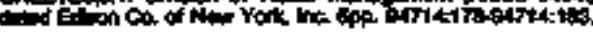

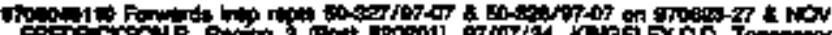

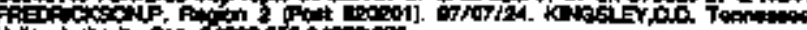

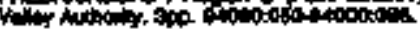

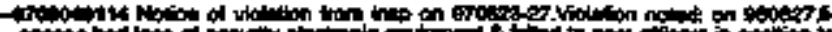

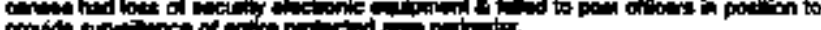

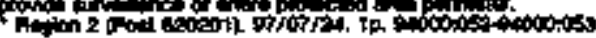

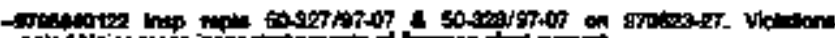

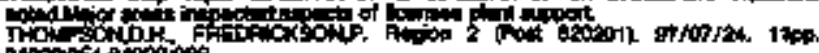

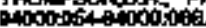

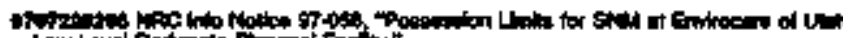
Low

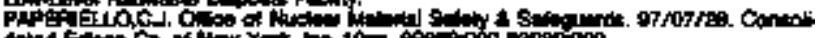

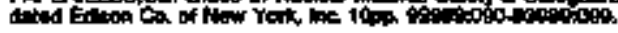

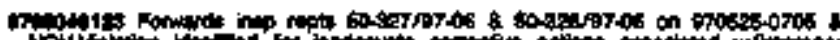

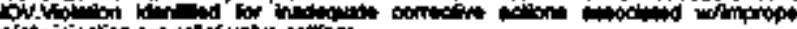

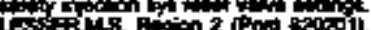

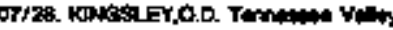

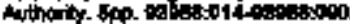

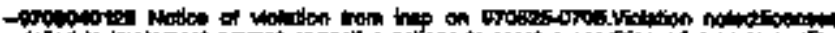

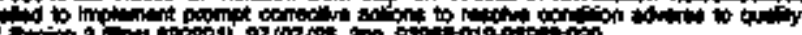

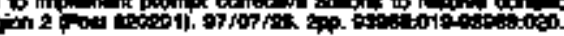

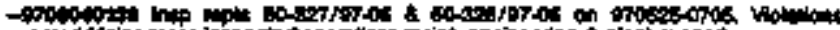

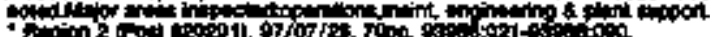

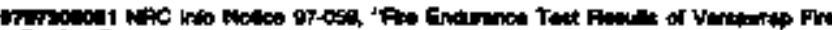
整

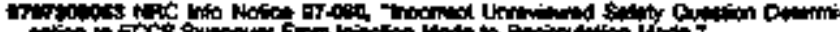

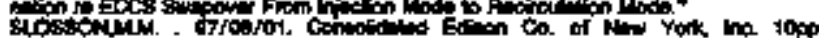

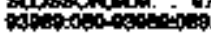

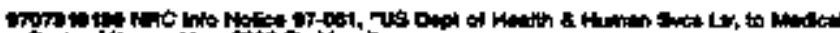

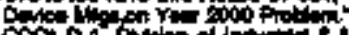

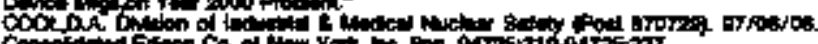

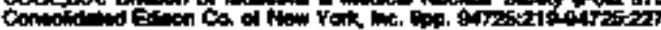

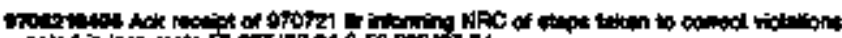

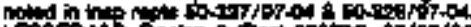

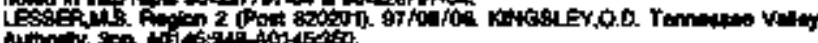

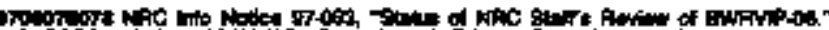

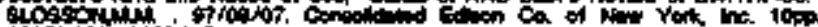

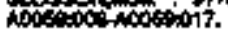

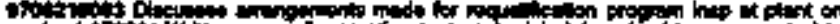

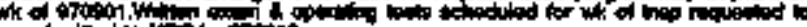

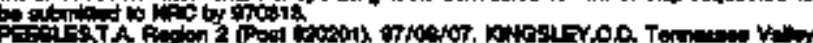
wowly.

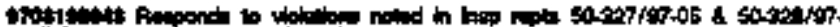

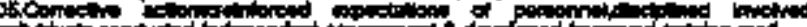

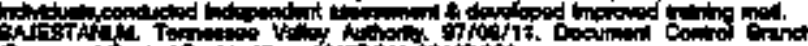

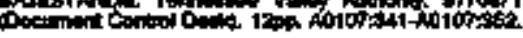

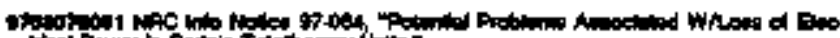

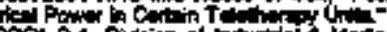

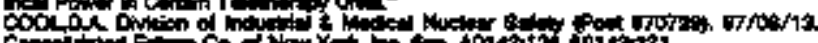

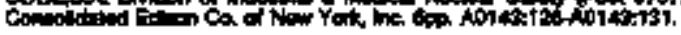

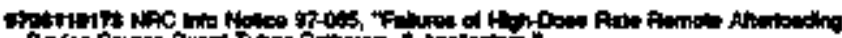

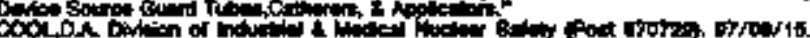

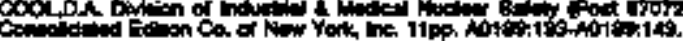

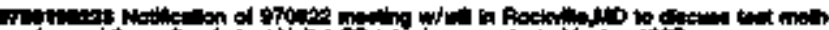

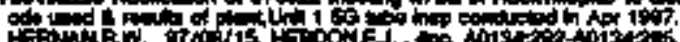

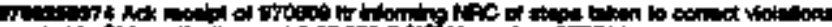

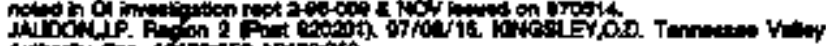

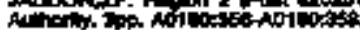

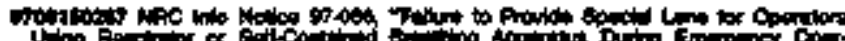

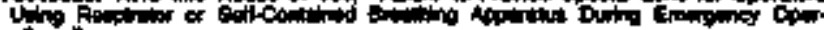

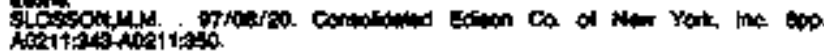

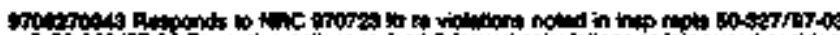

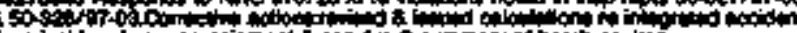

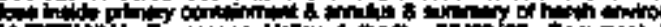

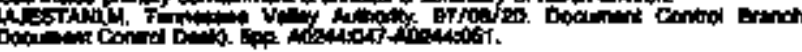

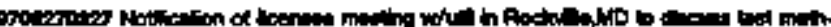

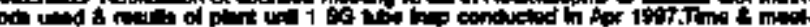

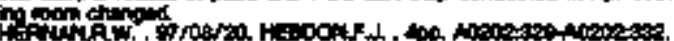

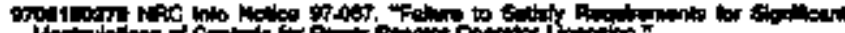

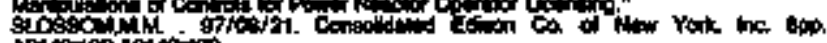

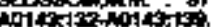

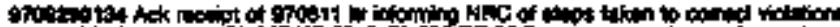

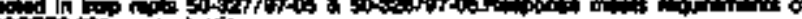
100,

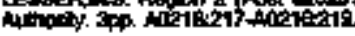

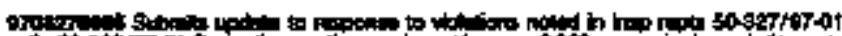

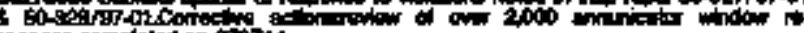

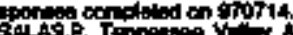

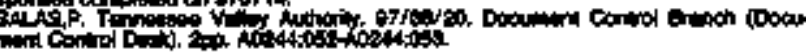




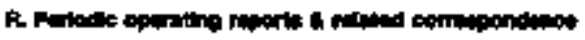

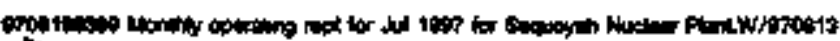
直

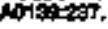

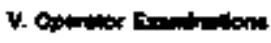

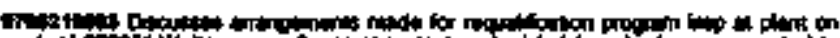

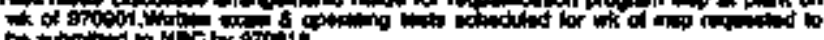

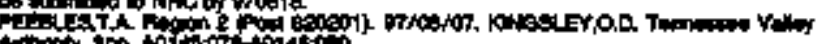

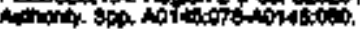

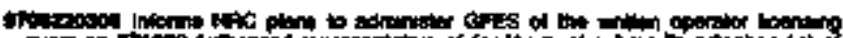

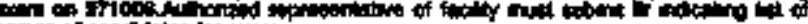

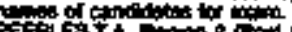

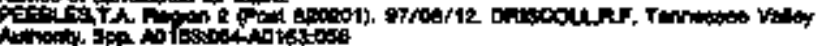

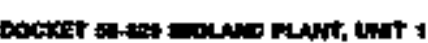

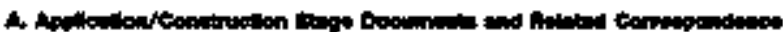

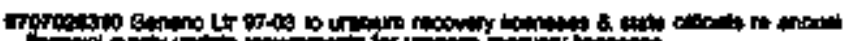

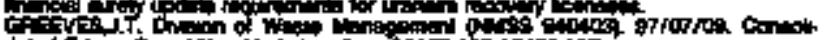

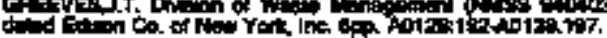

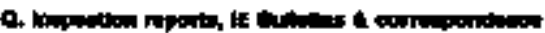

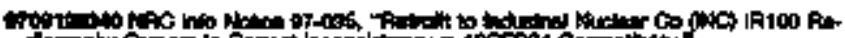

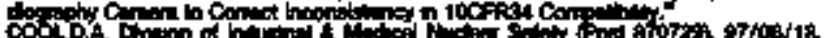

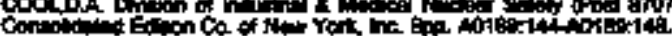

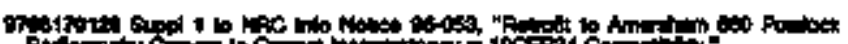

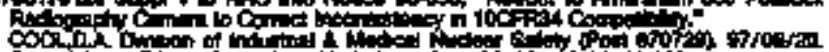

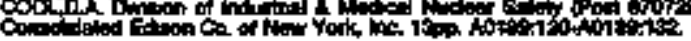

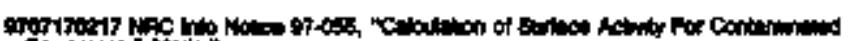

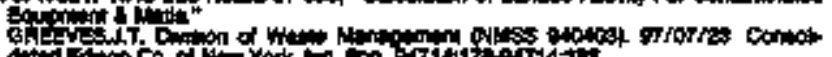

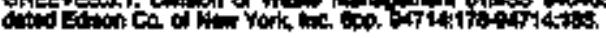

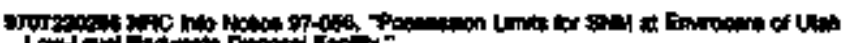

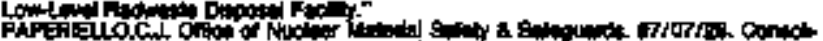
PAPE

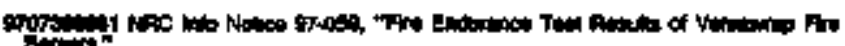

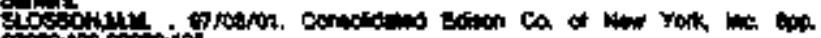

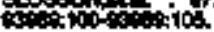

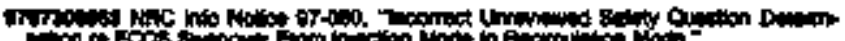

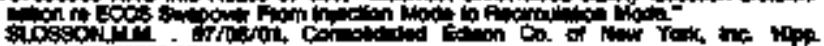
a

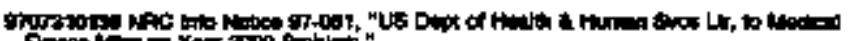

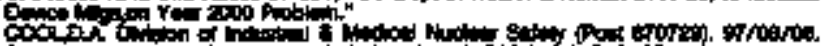

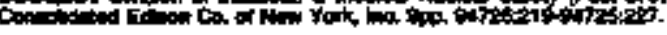

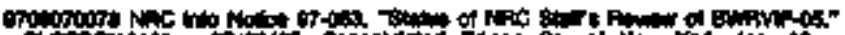

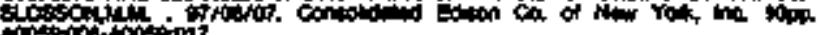

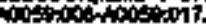

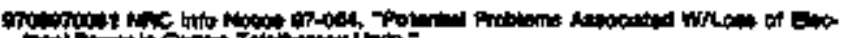
Con

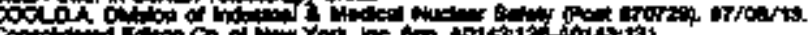

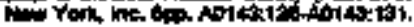

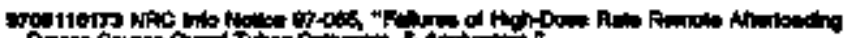

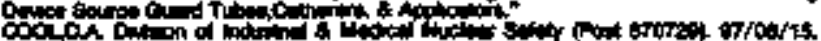

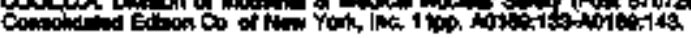

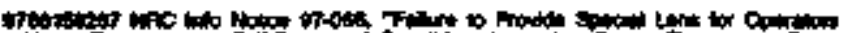

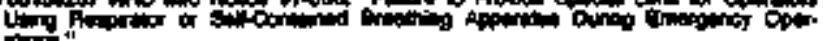

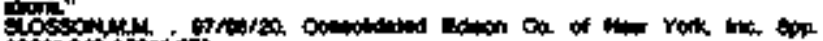

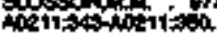

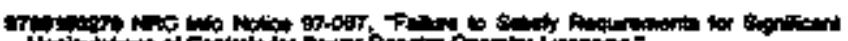

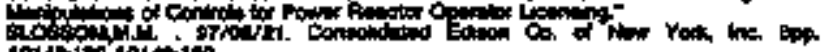

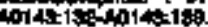

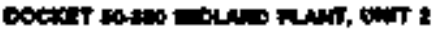

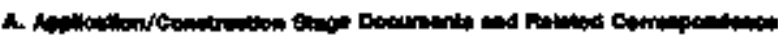

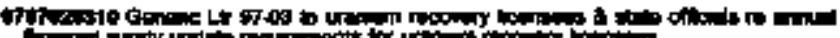

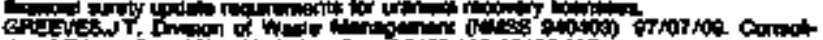

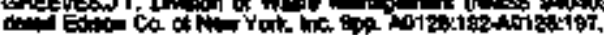

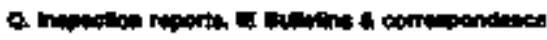

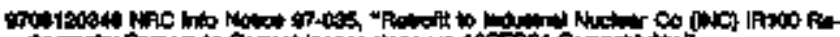

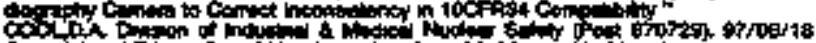

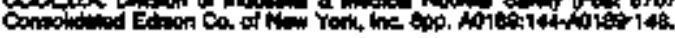

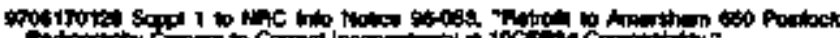

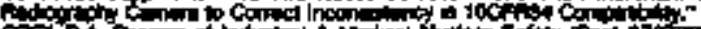

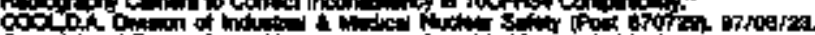

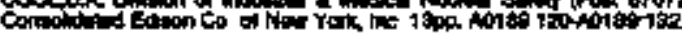

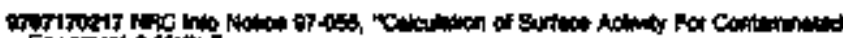
Equ intion

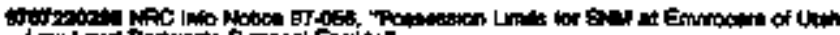

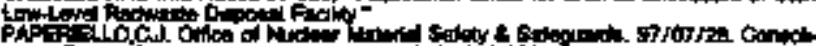

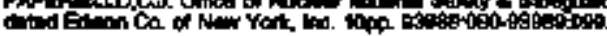

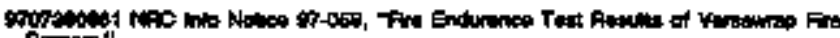

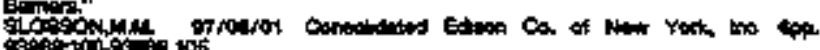
Sototo

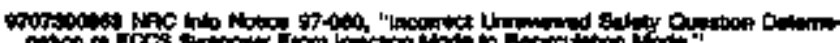

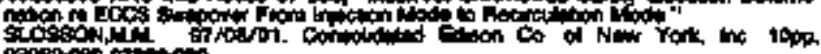

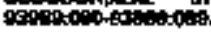

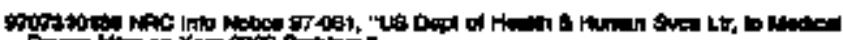

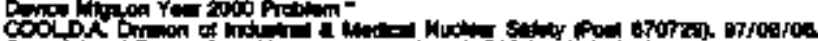

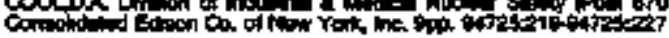

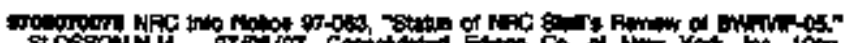

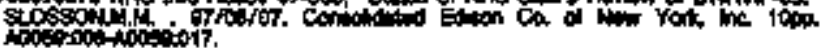

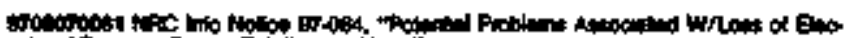

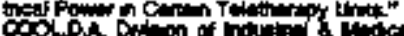

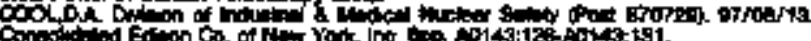

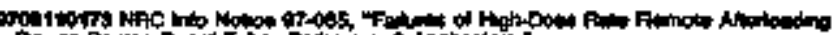

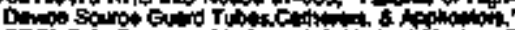

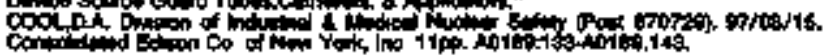

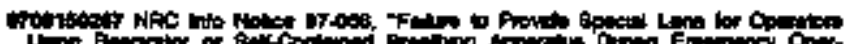

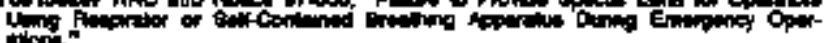

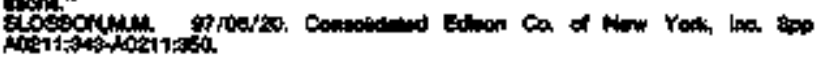

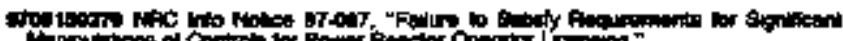

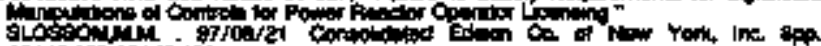

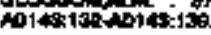

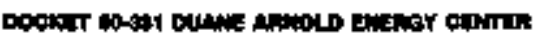

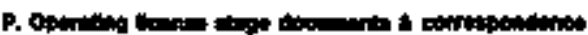

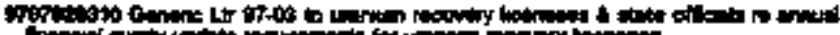

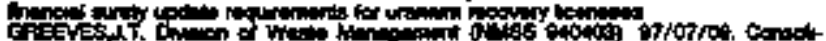

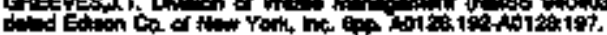

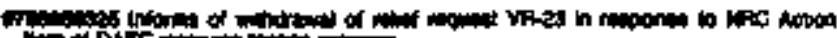

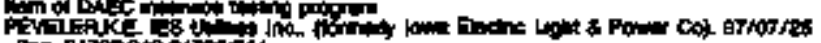

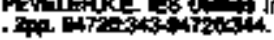

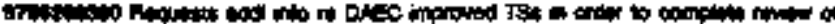

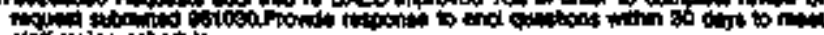
(t)

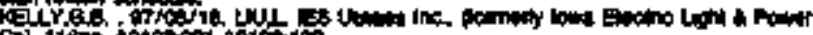

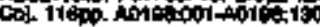

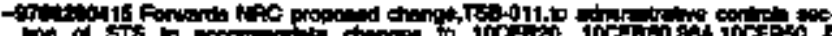

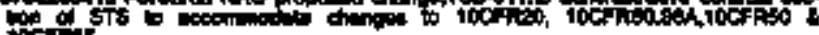

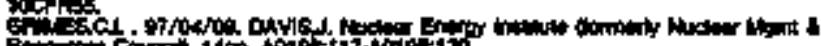

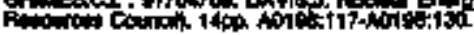

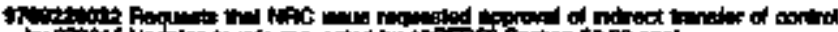

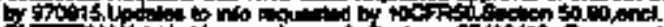

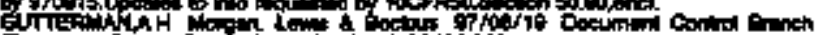

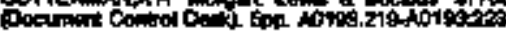

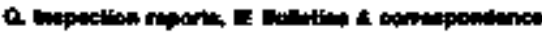

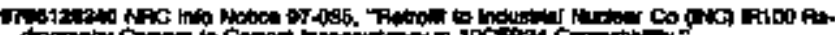

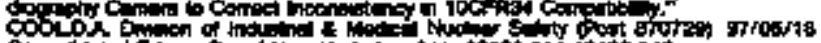

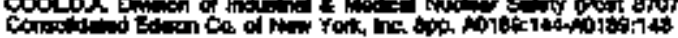




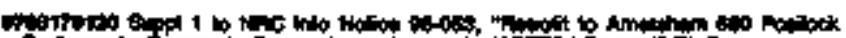

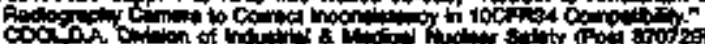

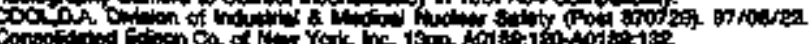

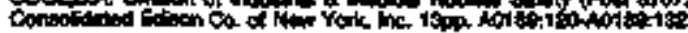

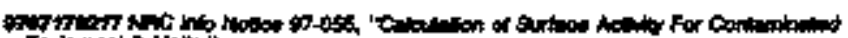

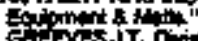

Pif

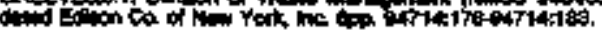

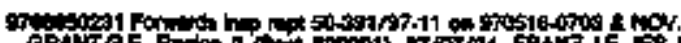

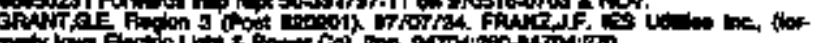

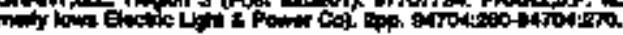

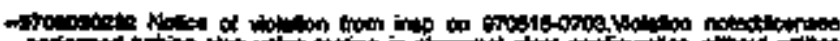

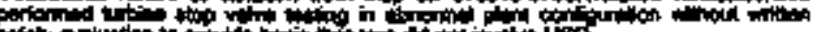

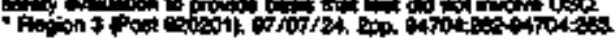

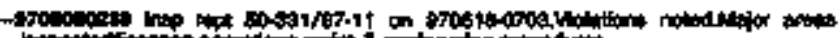

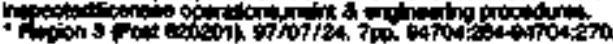

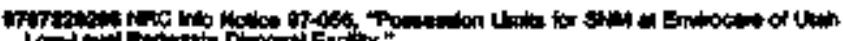

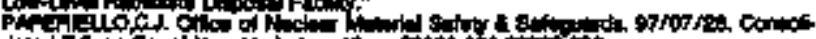

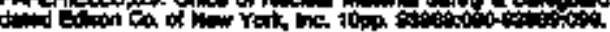

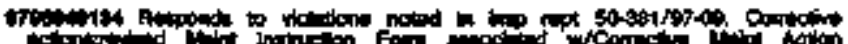

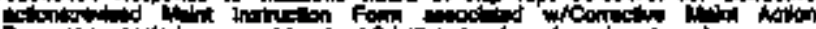

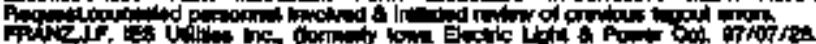

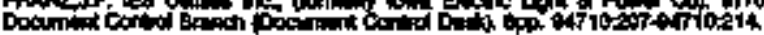

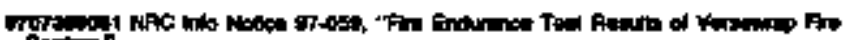

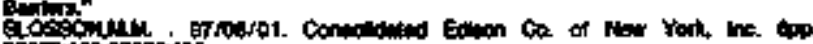

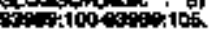

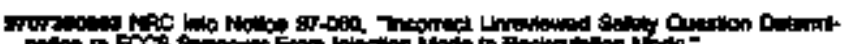

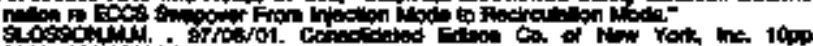
scosecting

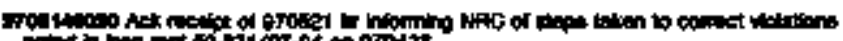

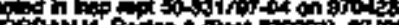

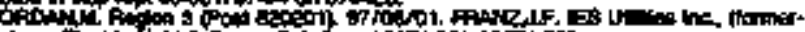
dite

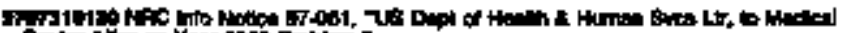

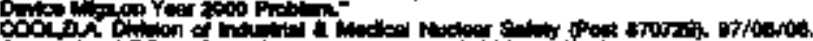

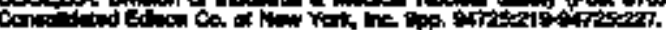

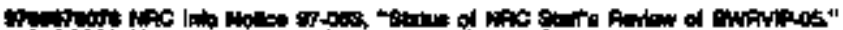

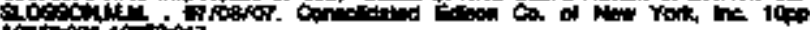

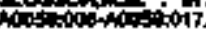

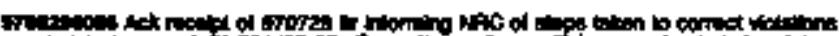

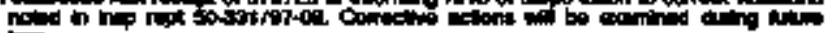

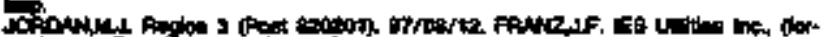

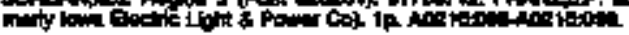

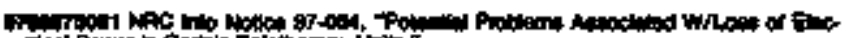
1.

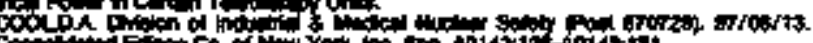
Con

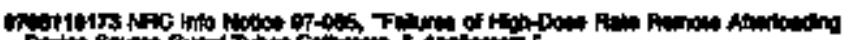

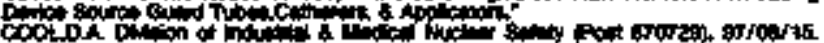

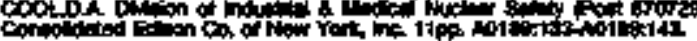

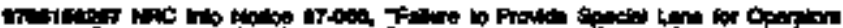

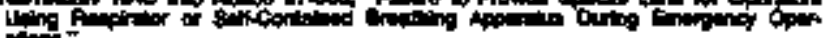

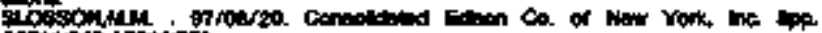

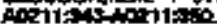

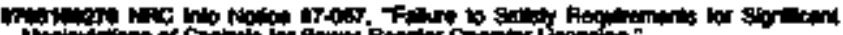

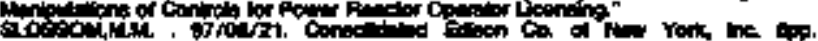

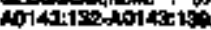

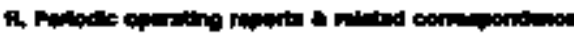

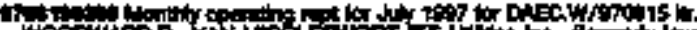

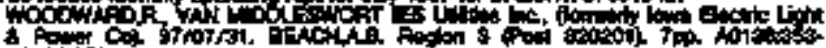
A01sion

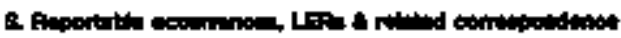

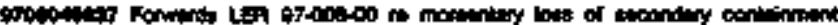

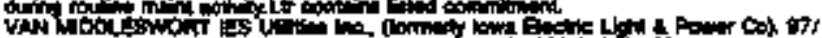

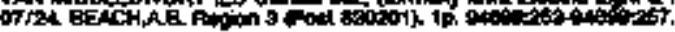

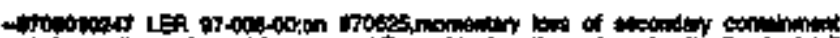

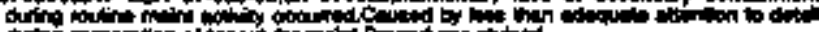

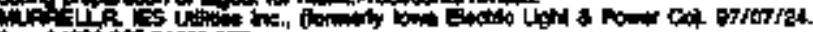

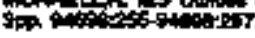

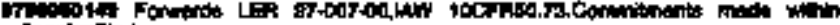
fil to

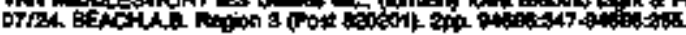

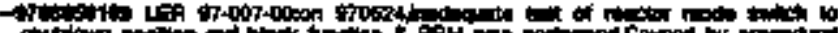

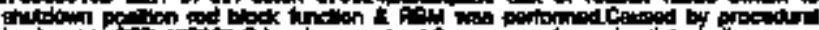

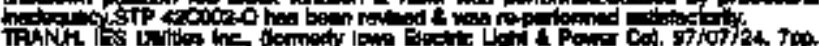
1.p.

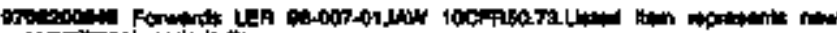

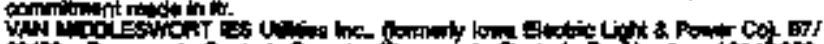

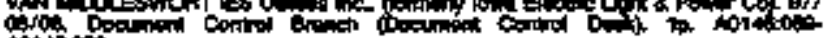
intion

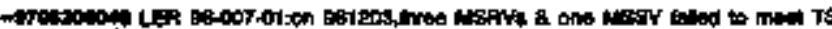

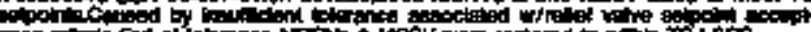

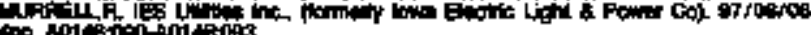

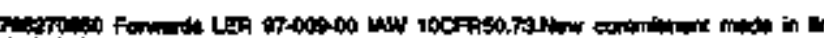

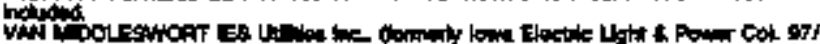

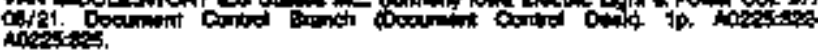

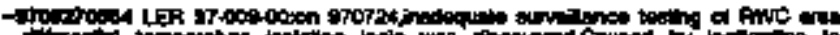

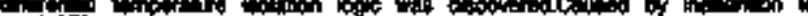

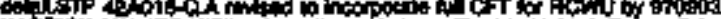

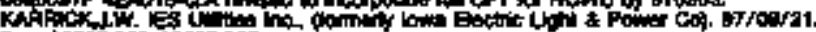

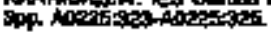

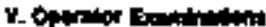

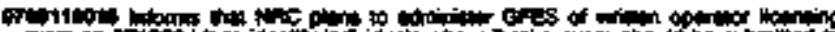

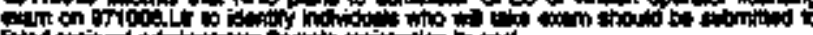

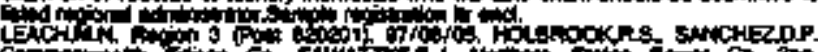

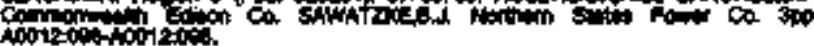

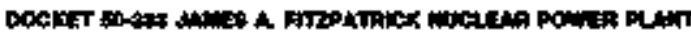

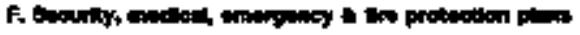

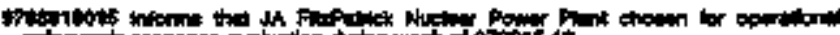
+

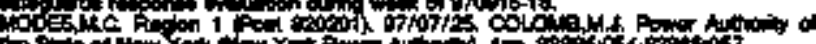

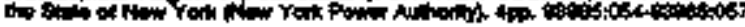

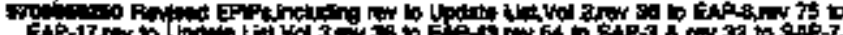

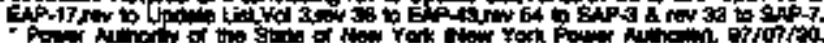

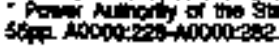

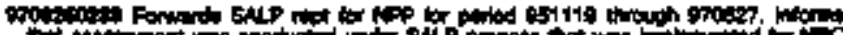

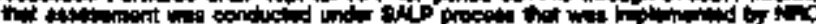

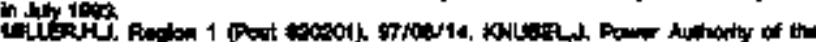

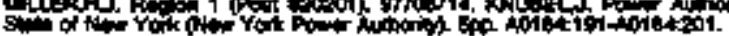

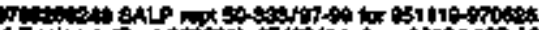

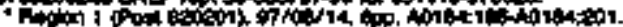

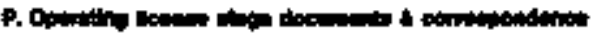

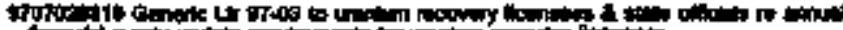
(1)

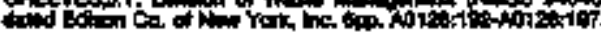

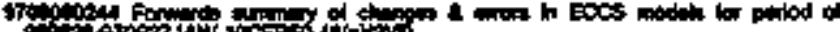

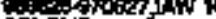

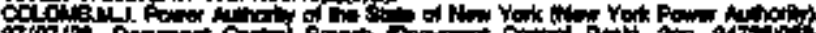
irotion

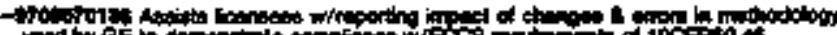

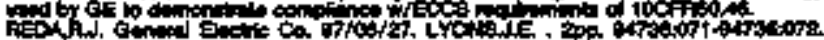

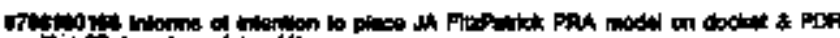

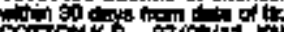

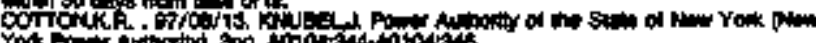

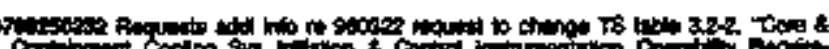

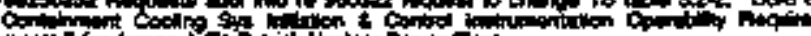

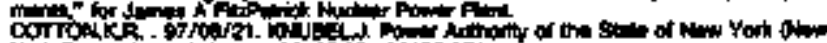

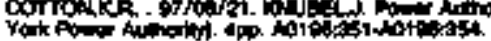




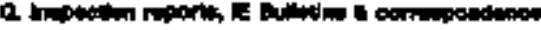

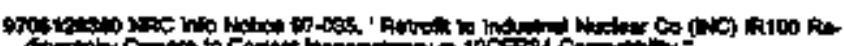

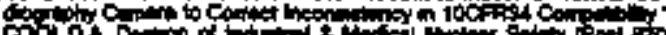

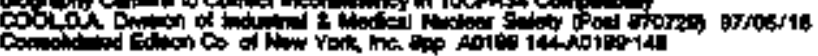

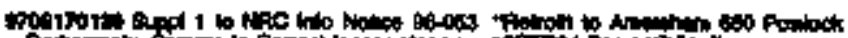

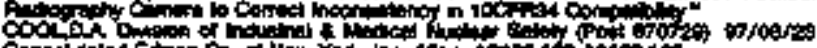

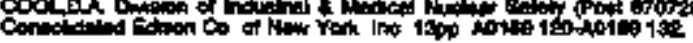

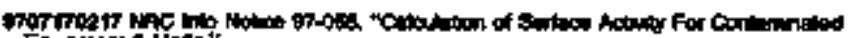

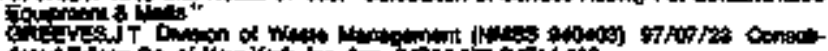

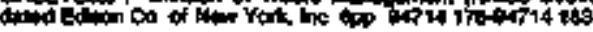

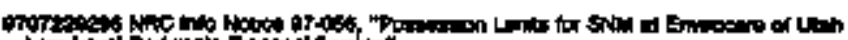

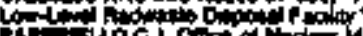

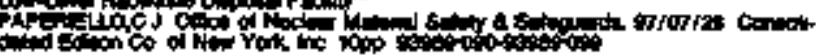

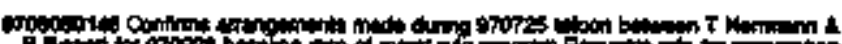
P E

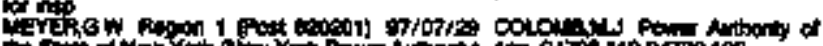

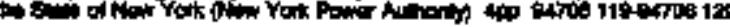

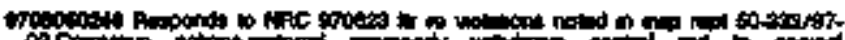

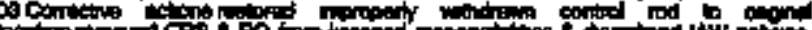

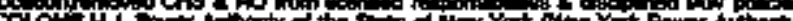

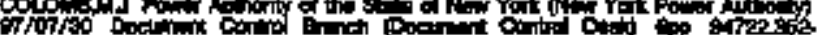
thes

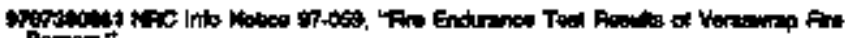

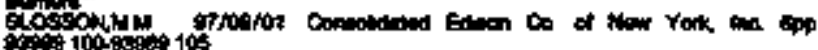

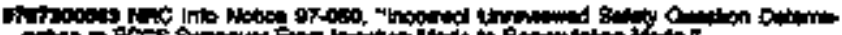

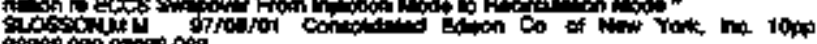

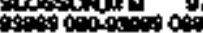

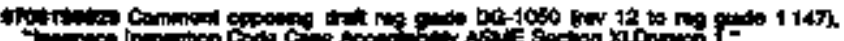
Not:

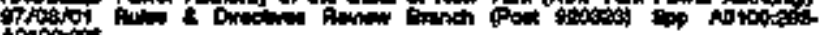
onot:

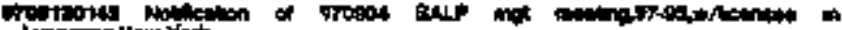

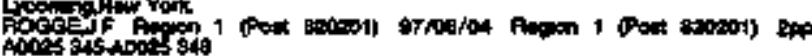

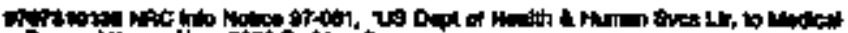

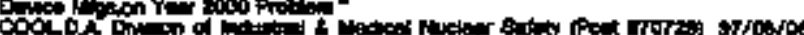

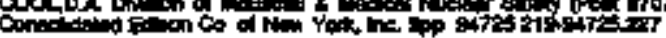

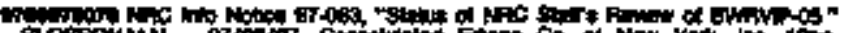

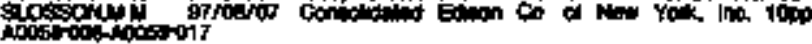

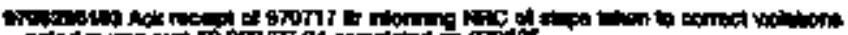

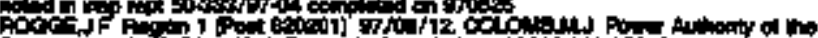

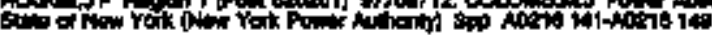

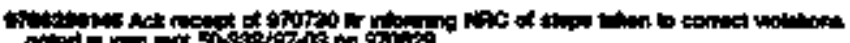

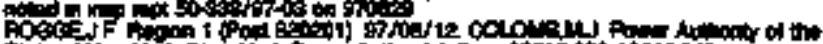

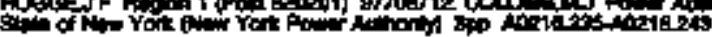

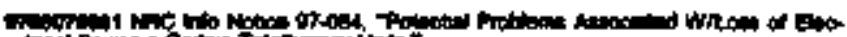

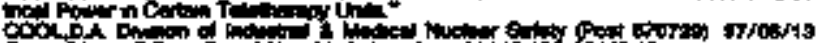
Conchinged

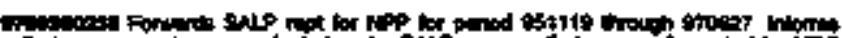

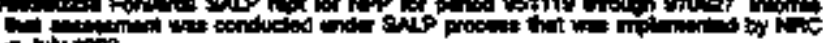
intions

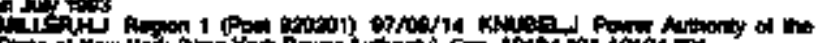

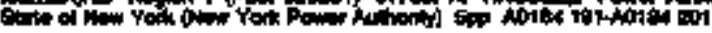

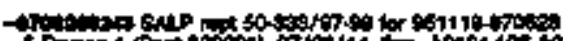

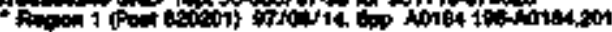

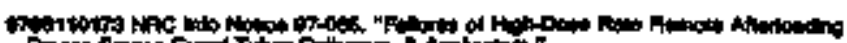

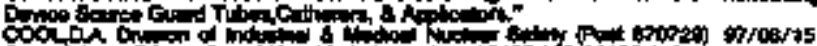

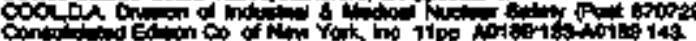

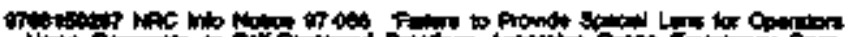

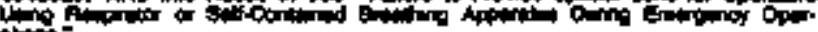

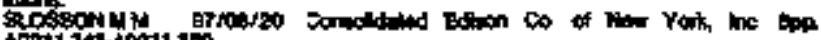

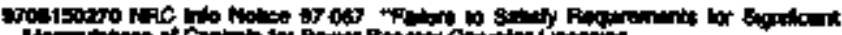

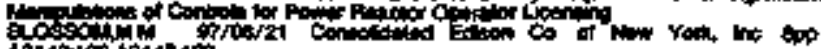

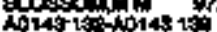

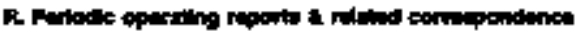

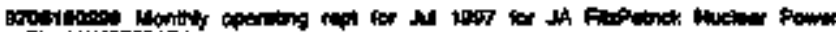

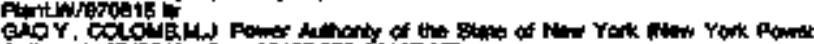
intuing on

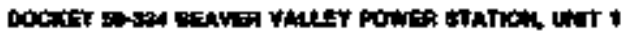

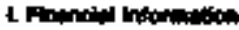

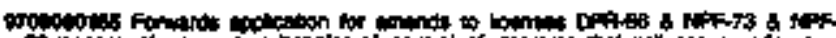

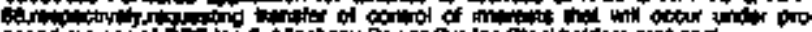

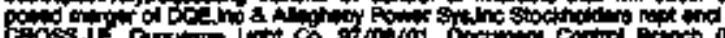

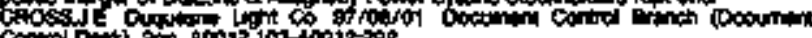

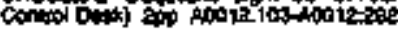

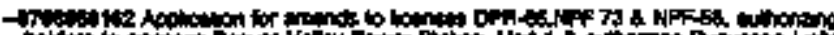

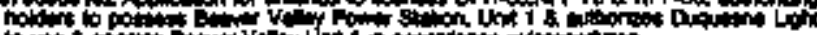

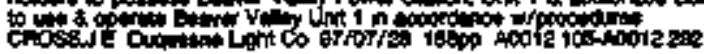

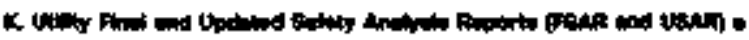

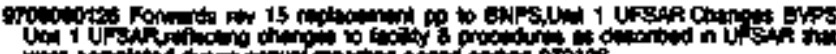

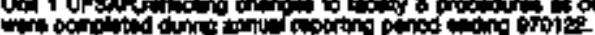

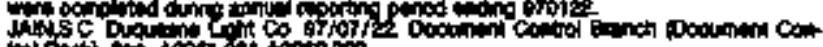

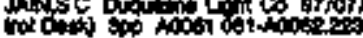

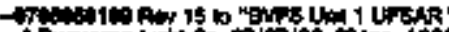

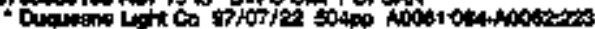

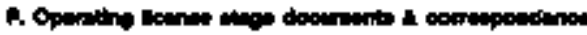

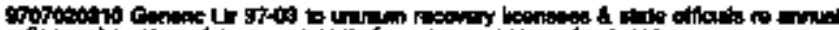

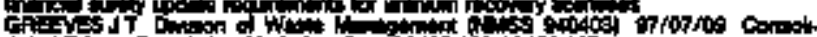

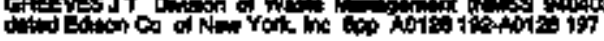

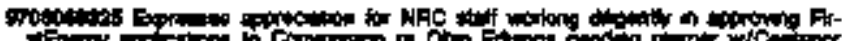

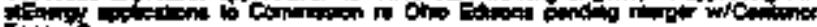

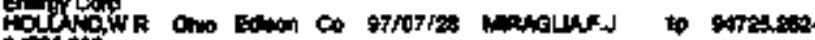
काt?

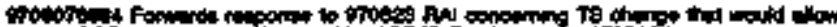

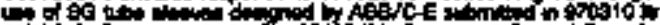

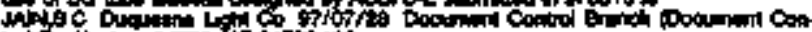

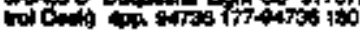

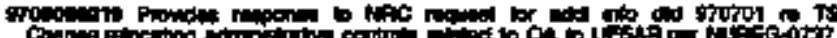

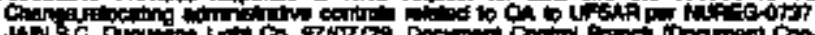

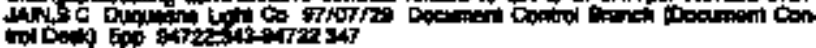

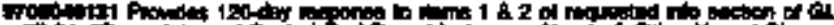

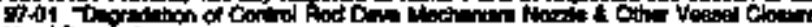

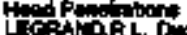

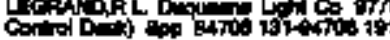

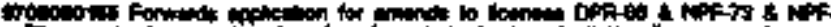

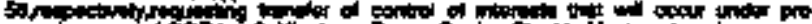

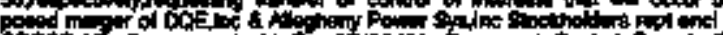

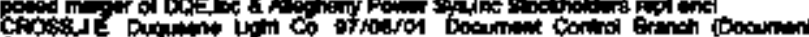

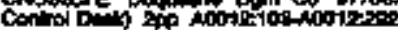

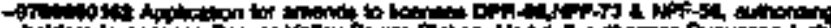

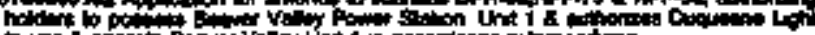

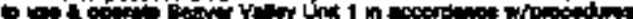

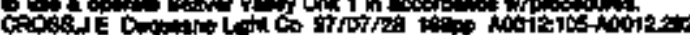

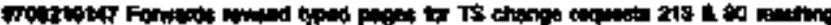

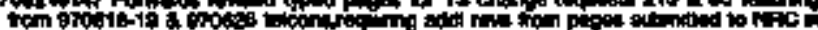
97009

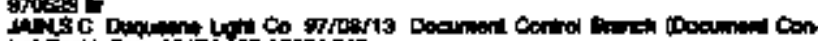

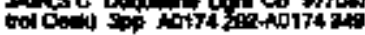

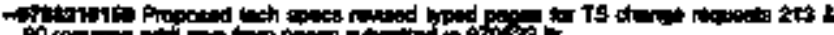

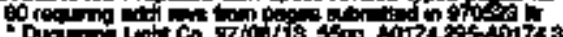

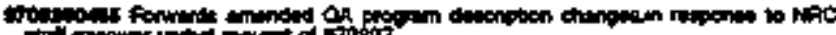

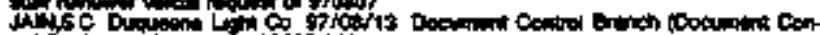

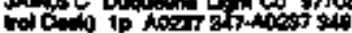

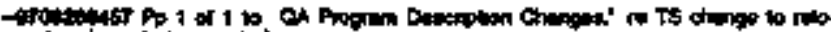

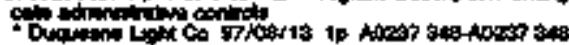

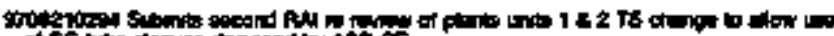

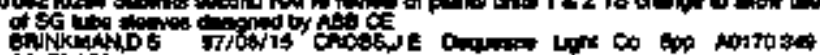
inl 70 sta 


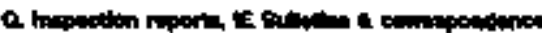

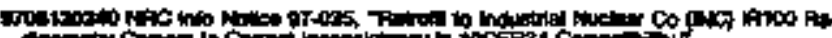

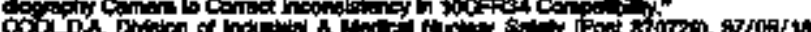
Con

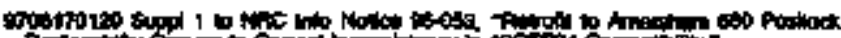

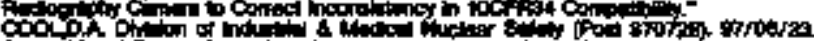

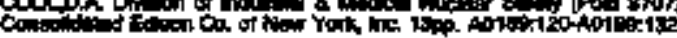

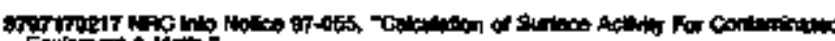
Cong

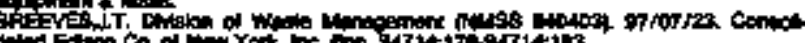

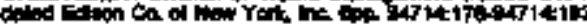

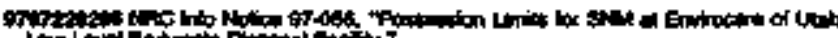

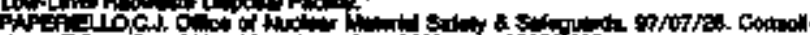

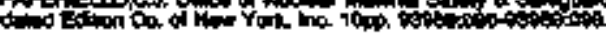

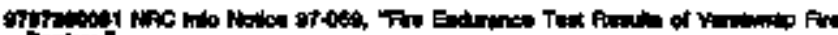

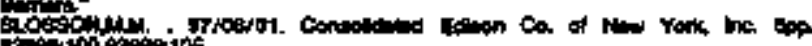

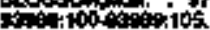

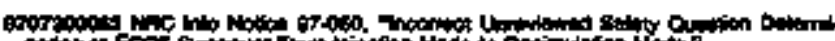

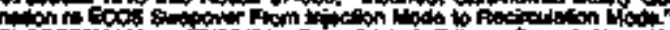

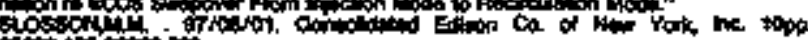

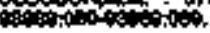

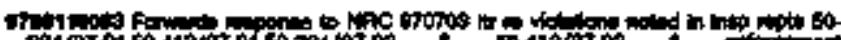

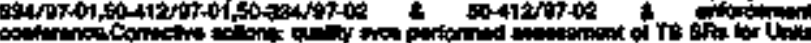

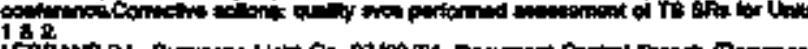

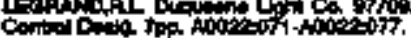

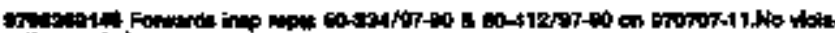

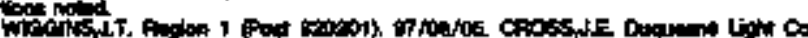

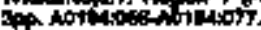

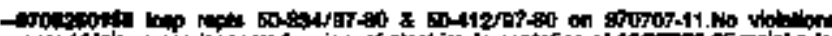

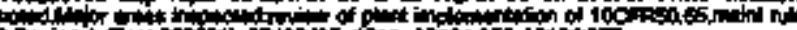

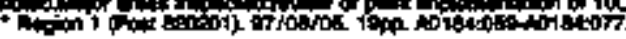

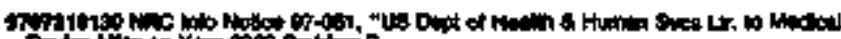
Dow Collind

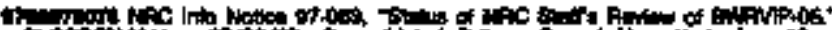
sos 100 .

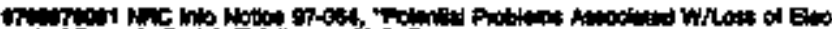

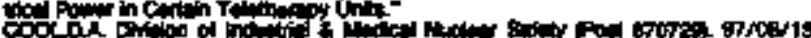

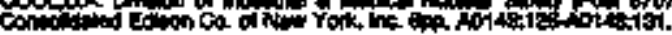

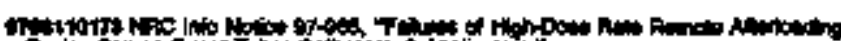

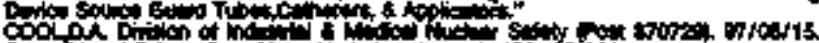

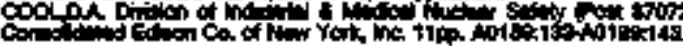

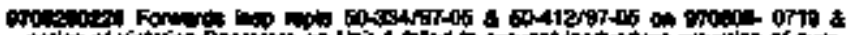

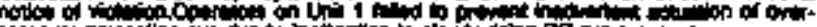

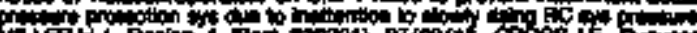

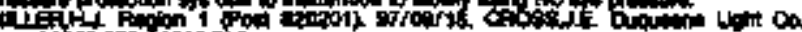

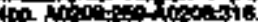

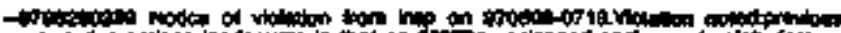

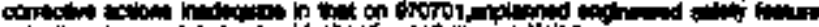

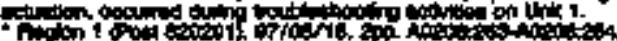

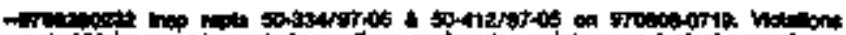

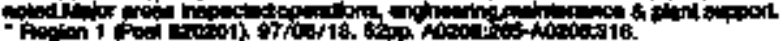

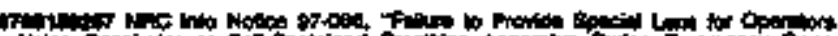

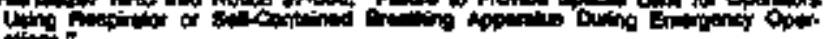

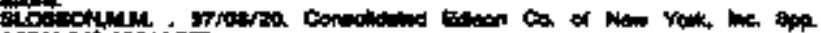

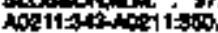

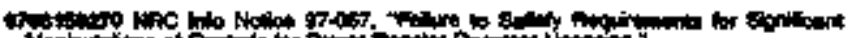

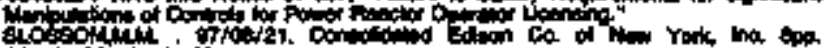
ito is

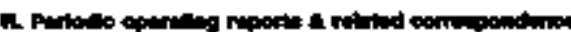

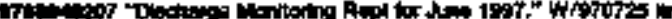

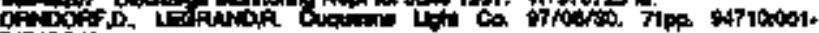
4710041 .

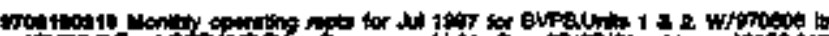

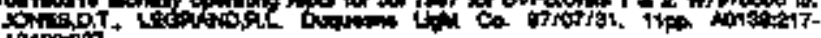

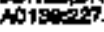

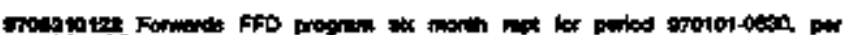
10Cimartion.

JANAC OU

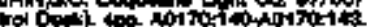

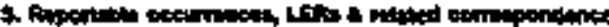

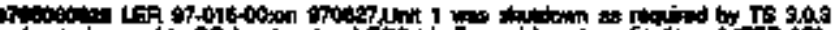

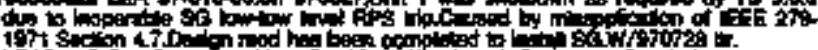

IEH

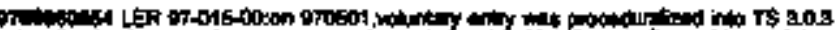

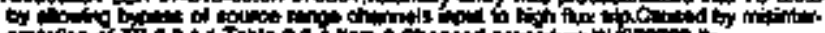

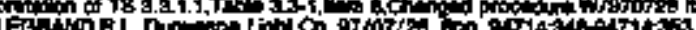

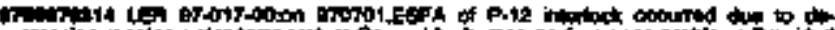

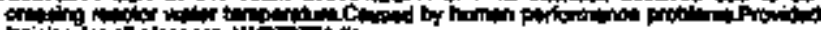

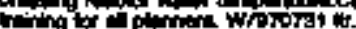

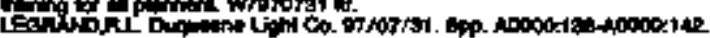

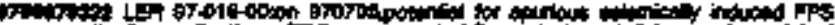

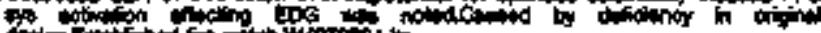
and

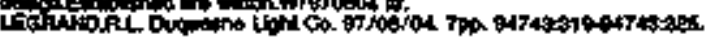

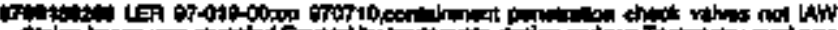

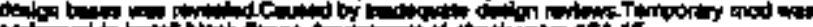

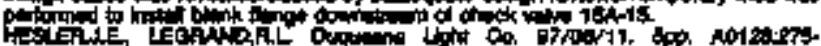

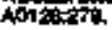

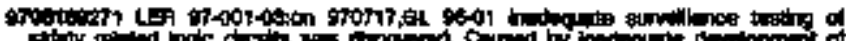

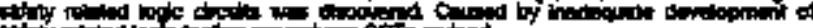

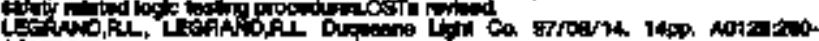

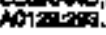

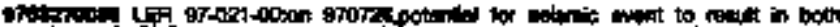

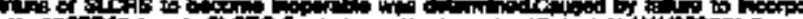

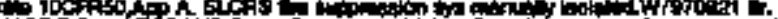

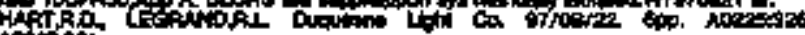
Aft:

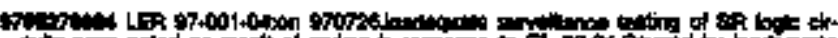

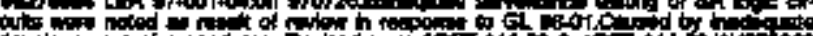

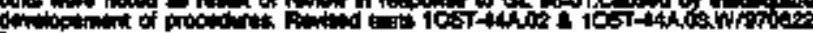

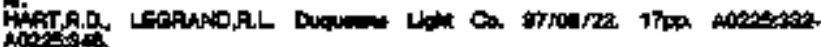

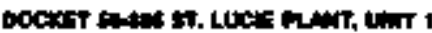

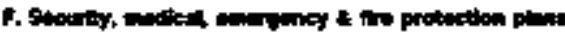

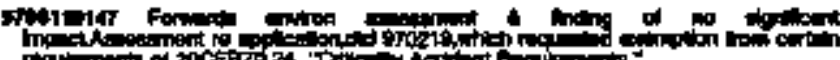

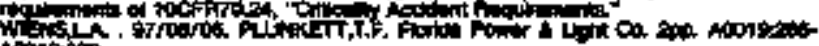

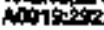

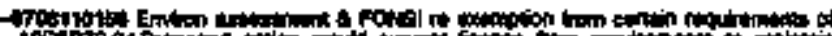

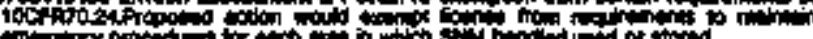

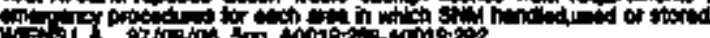

if

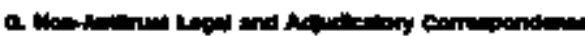

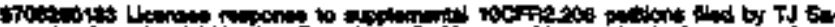

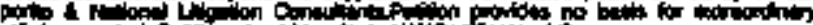

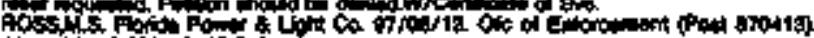

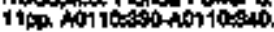

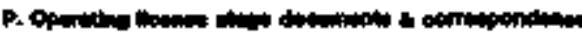

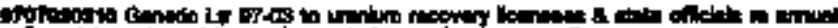

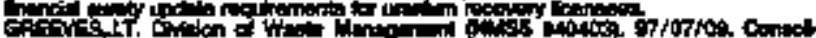

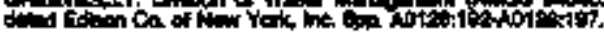

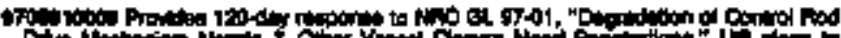

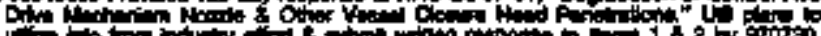

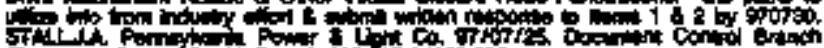

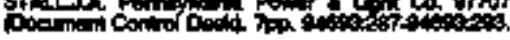

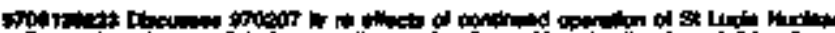

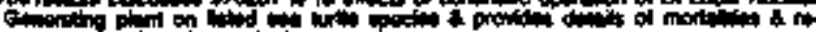

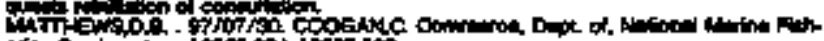

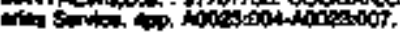

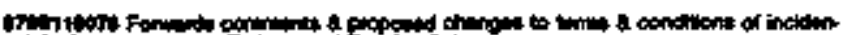

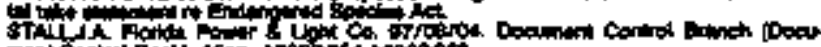

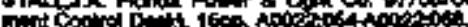

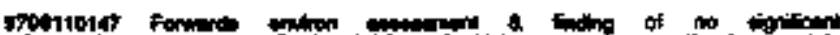

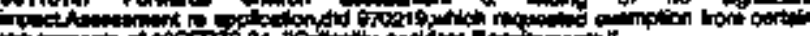

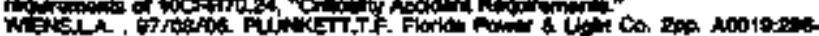
Nations 


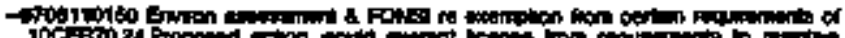

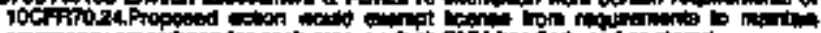

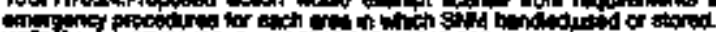

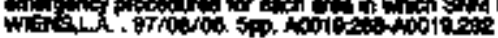

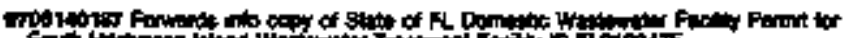

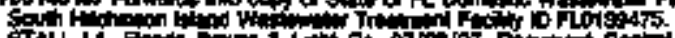

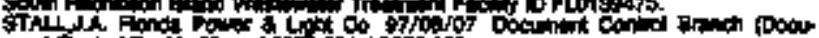

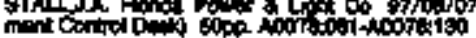

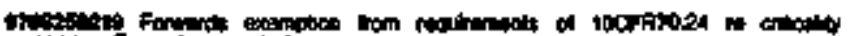

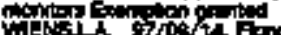

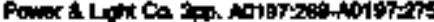

towistion

This:

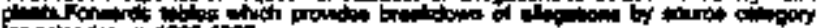

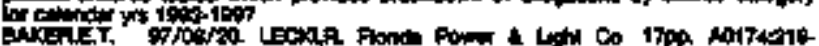

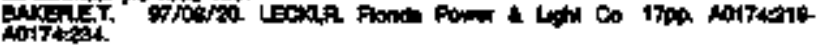

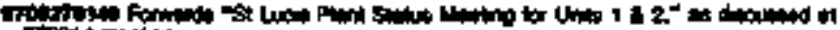

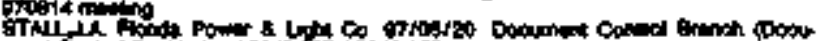

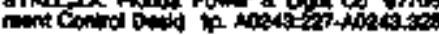

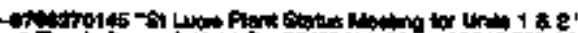

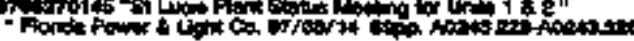

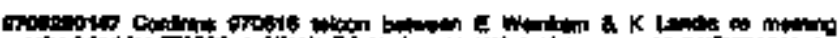

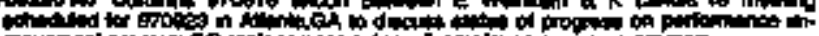

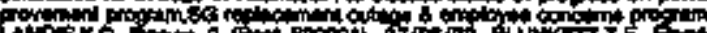

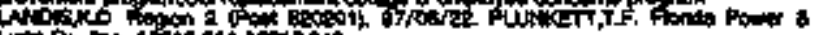

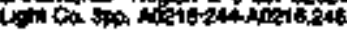

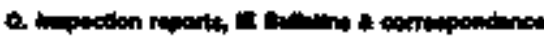

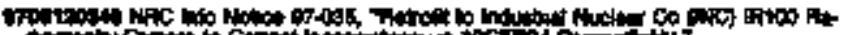

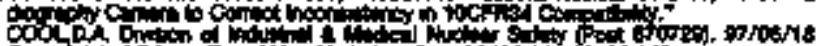
Son

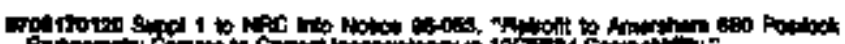

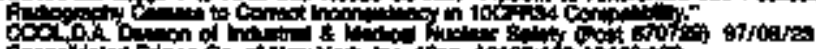

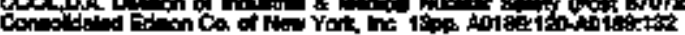

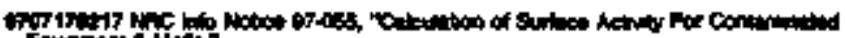

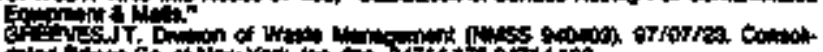

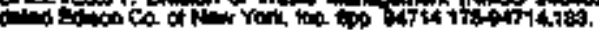

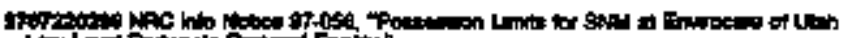
PAt

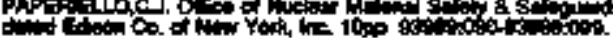

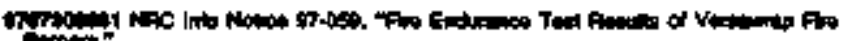

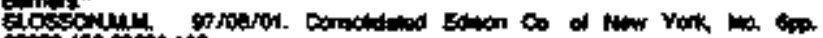

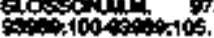

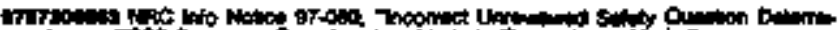

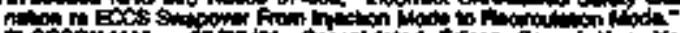

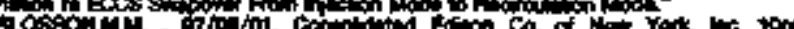

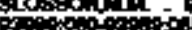

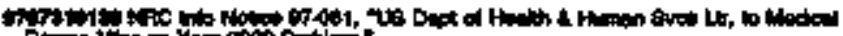

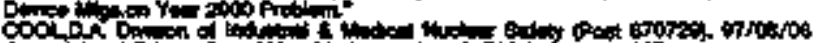

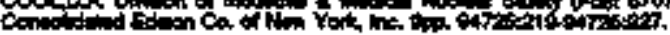

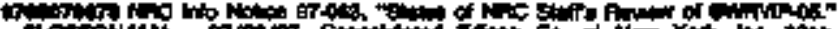

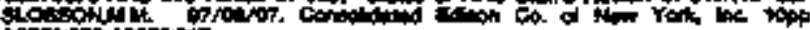

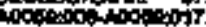

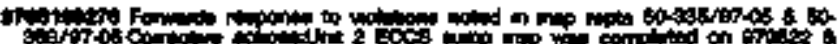

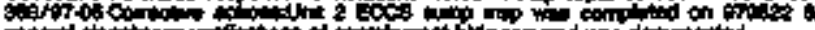

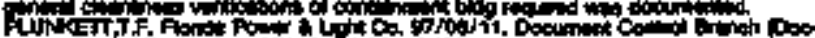

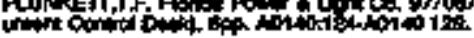

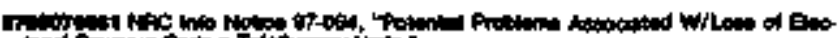

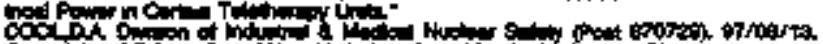

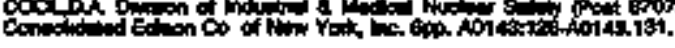

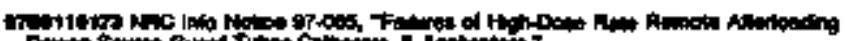

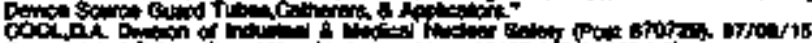

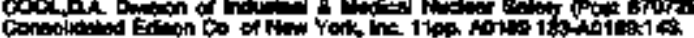

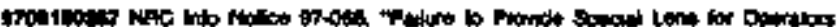

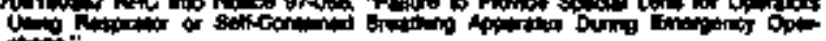

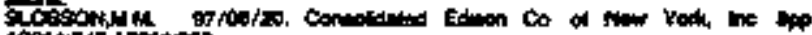

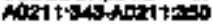

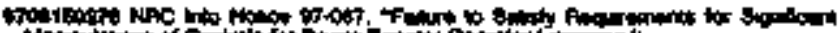

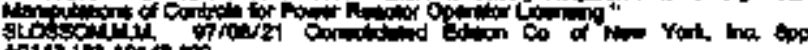

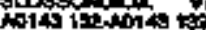

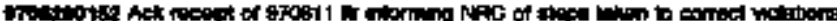

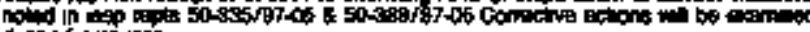

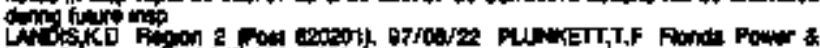

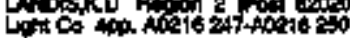

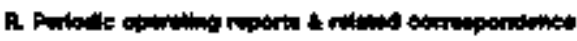

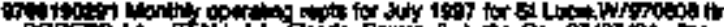

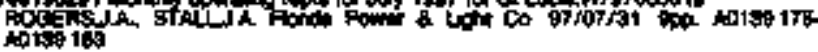

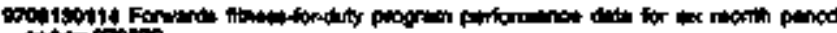
Gintoptosio.

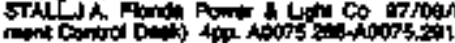

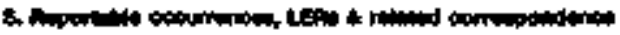

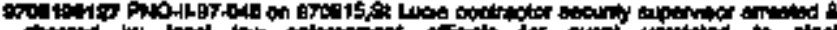

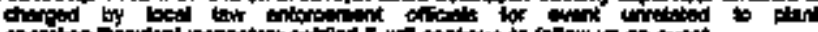

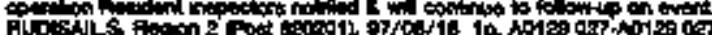

\section{Opintor Et ton:}

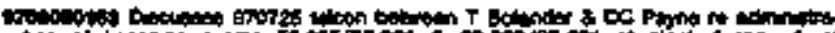

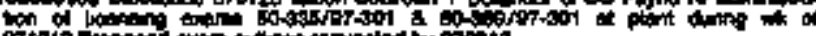

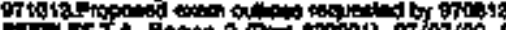

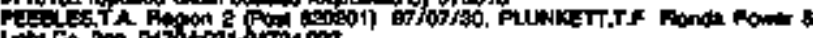

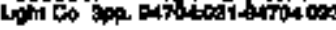

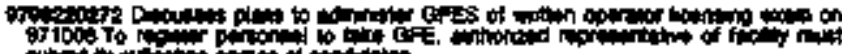

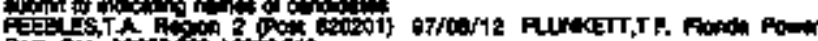
Con

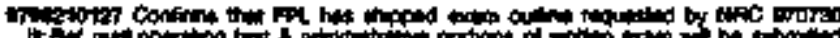

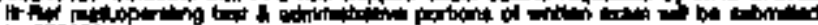

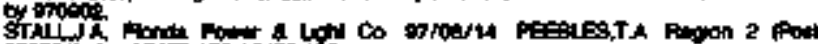

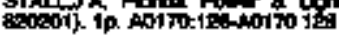

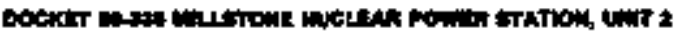

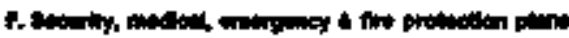

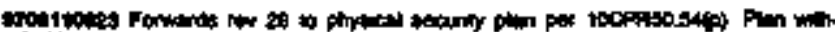

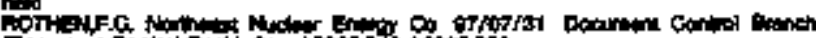

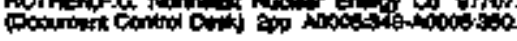

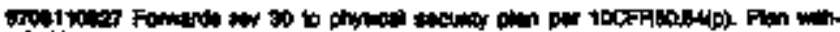

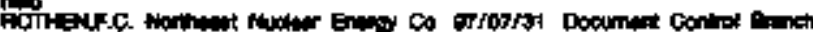

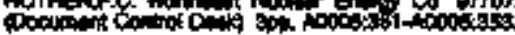

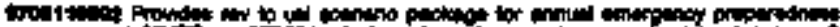

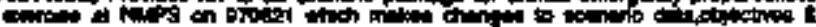

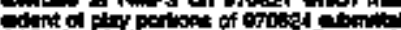

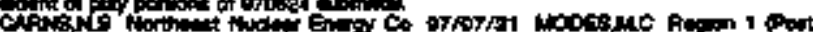
ctition ipo A

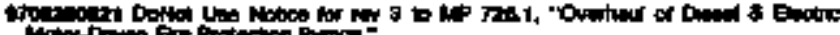

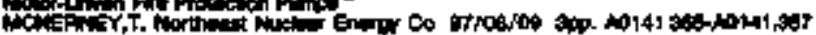

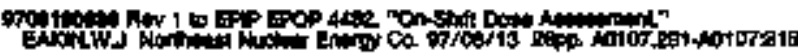

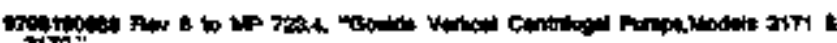

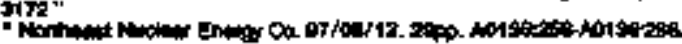

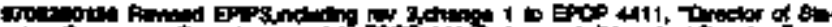

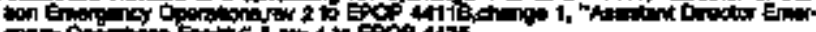

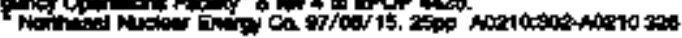

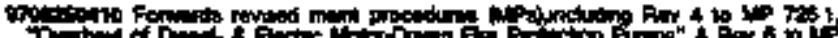

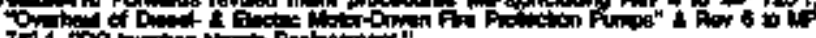

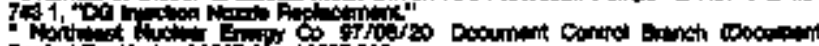

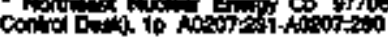

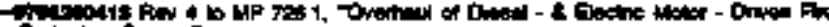

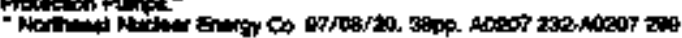

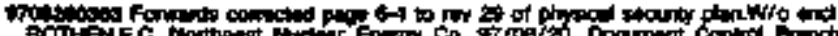

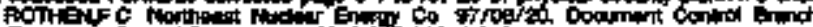

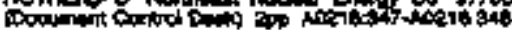




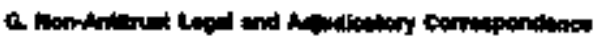

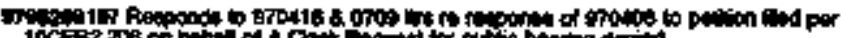

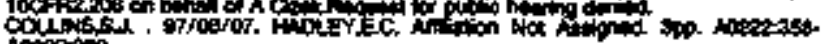
Aotosion.

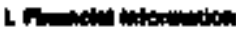

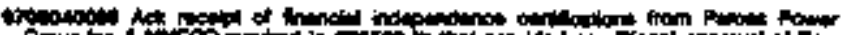

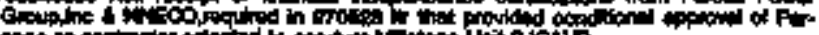

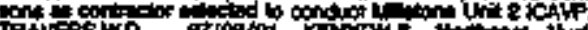

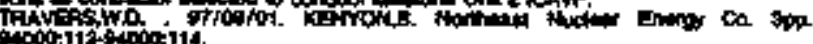

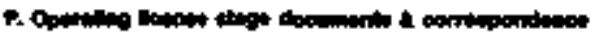

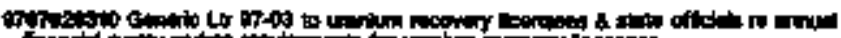

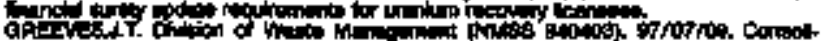

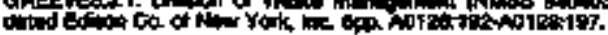

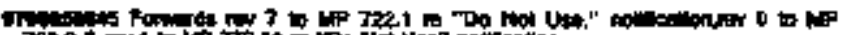

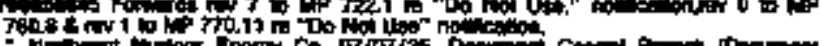

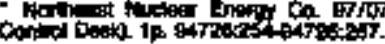

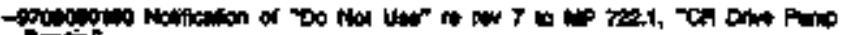

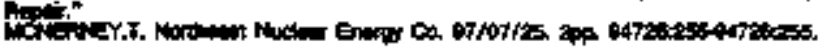

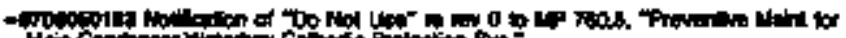

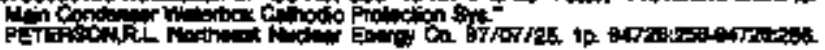

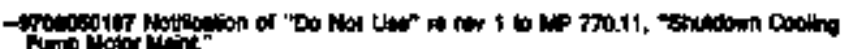

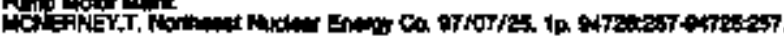

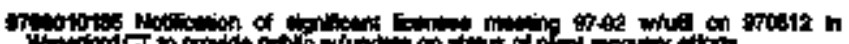
of

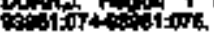

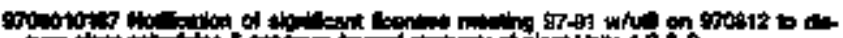
a.

Diph

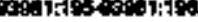

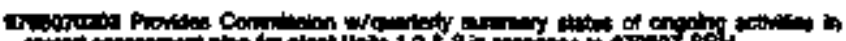

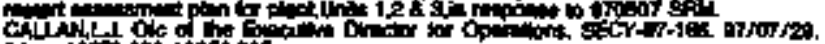

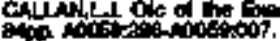

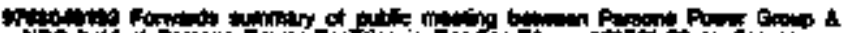

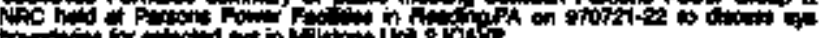

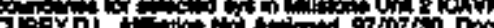

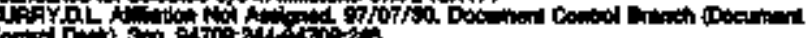

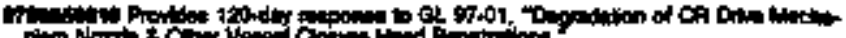

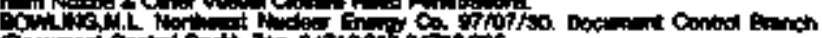

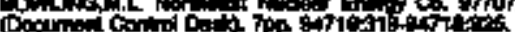

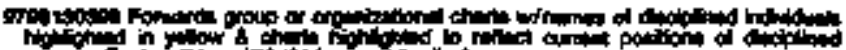

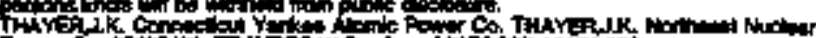

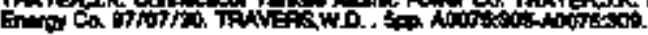

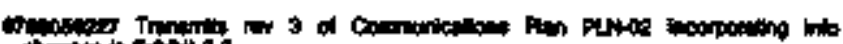

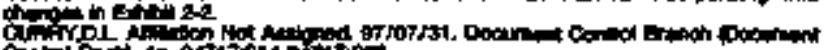

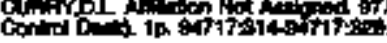

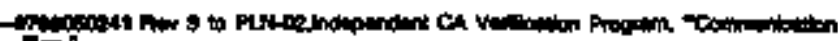

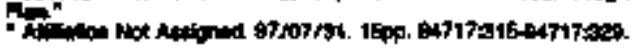

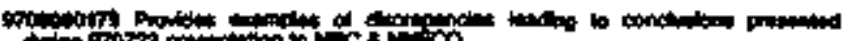

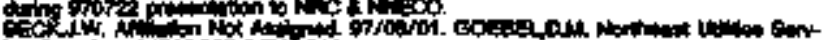

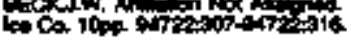

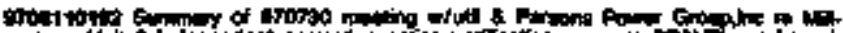

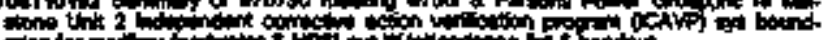

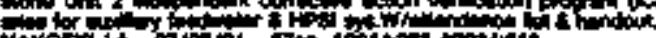

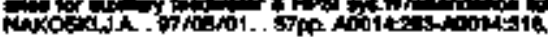

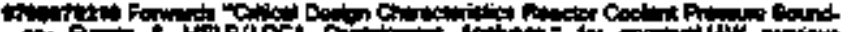

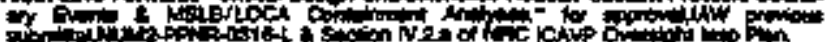

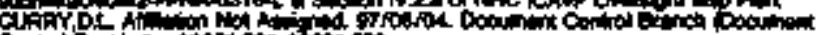

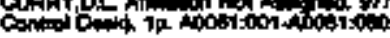

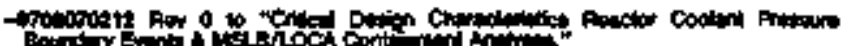

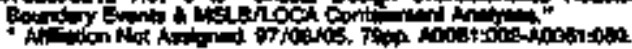

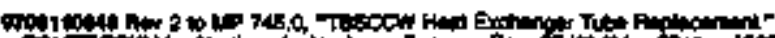

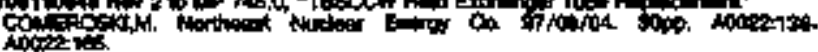

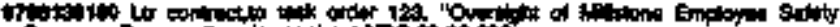

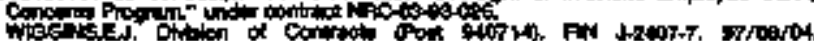

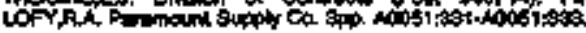

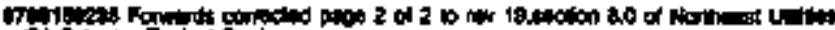

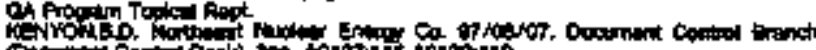

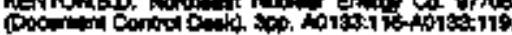

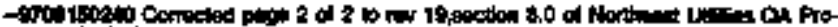

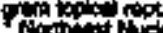

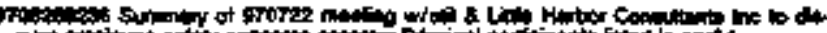

DA

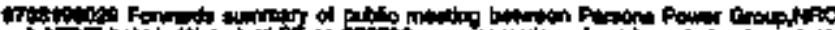

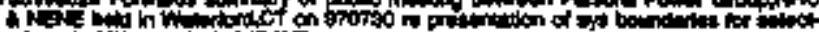

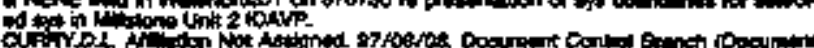

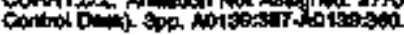

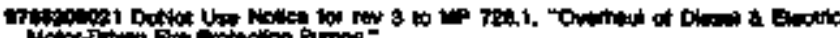

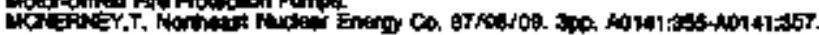

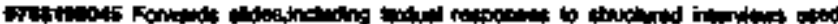

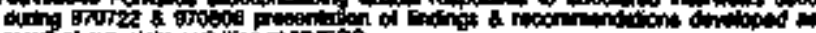

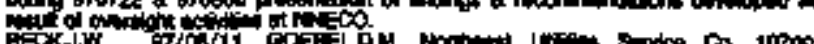

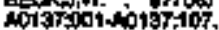

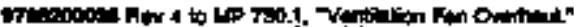

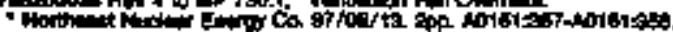

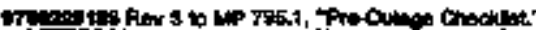

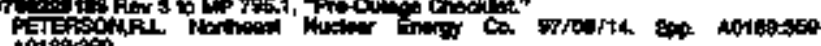
not

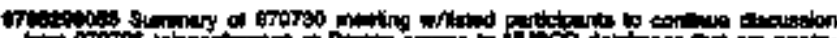

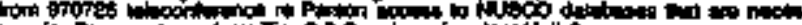

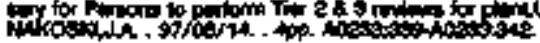

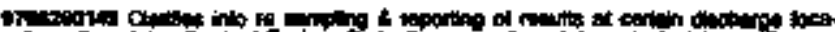

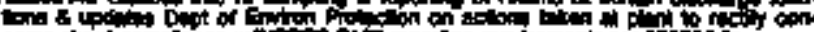

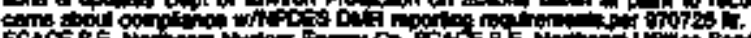

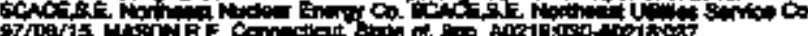

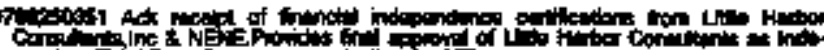

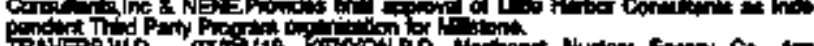
Aorstom

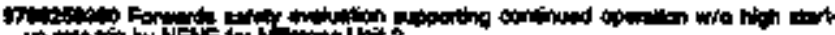

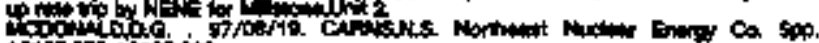

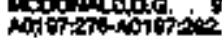

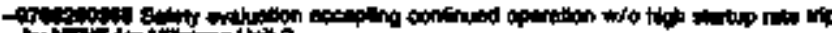

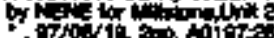

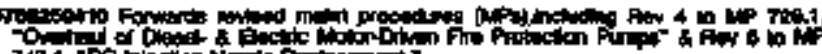

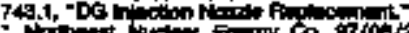

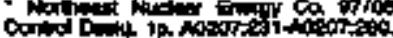

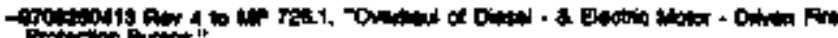

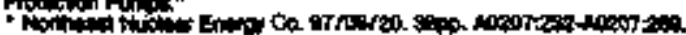

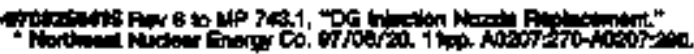

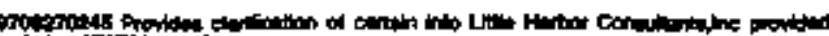

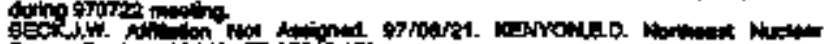

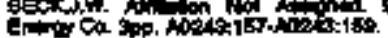

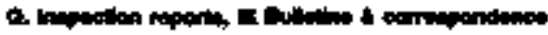

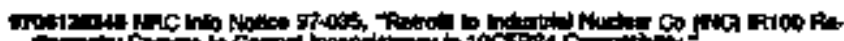

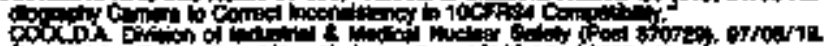

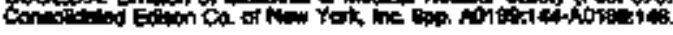

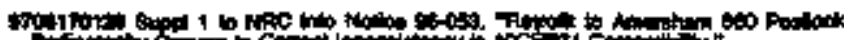

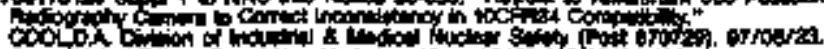

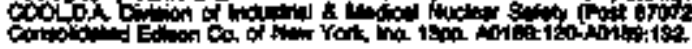

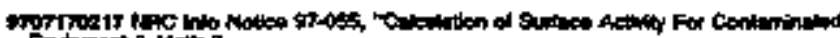

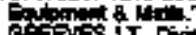

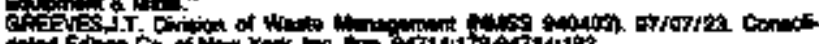

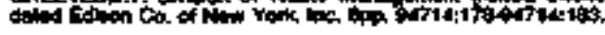




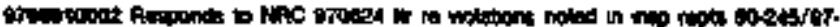

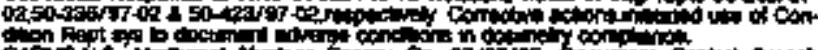
chatis

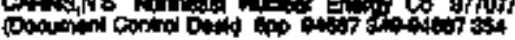

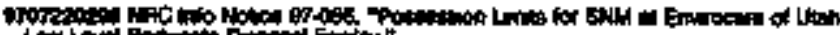

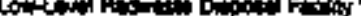

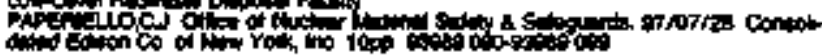

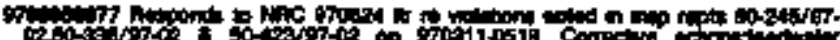
Whe 14 w

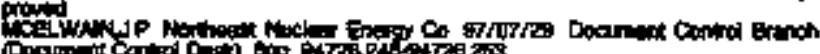

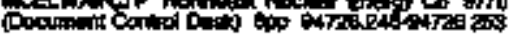

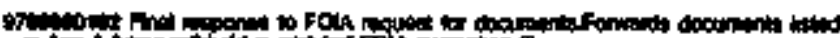

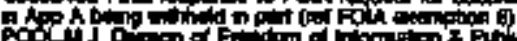

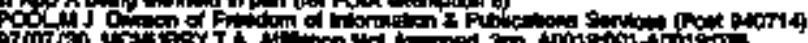

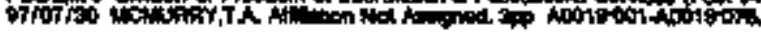

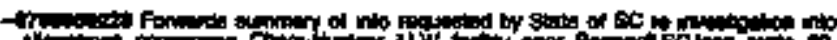

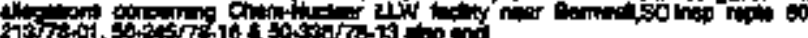

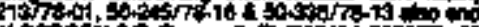

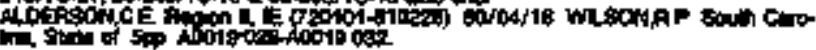

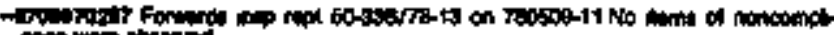

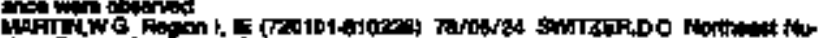

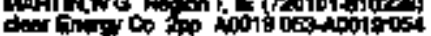

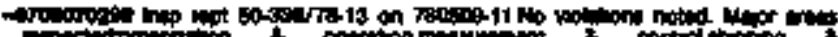

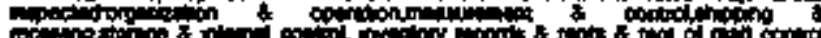
t.t.

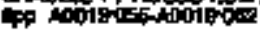

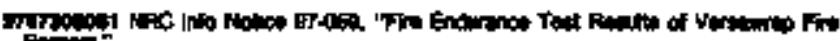

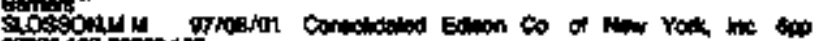
sion 100

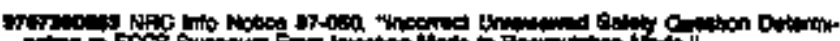

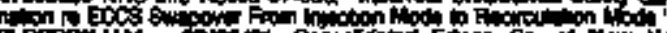

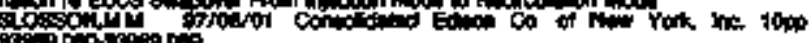

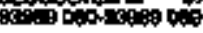

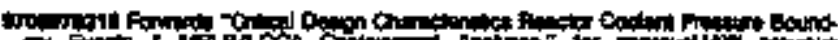

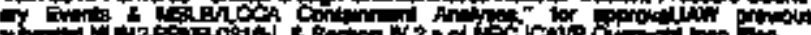

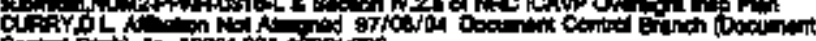

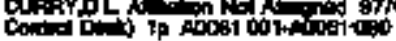

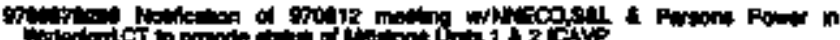

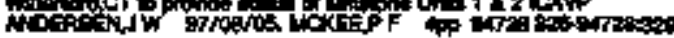

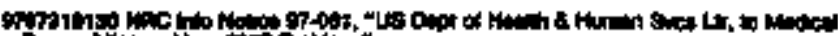

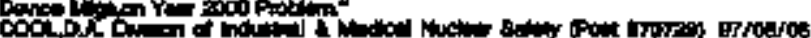
Coned d

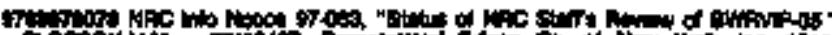

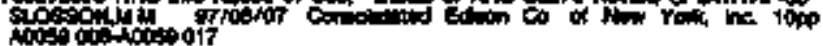

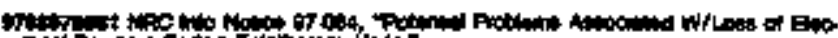

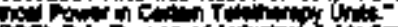

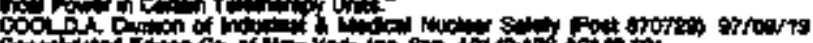

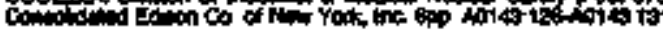

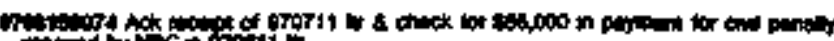

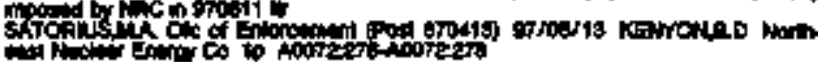

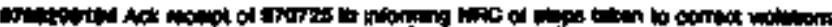

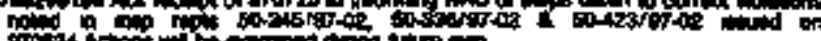

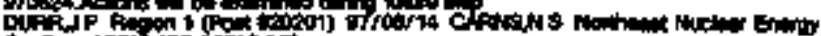

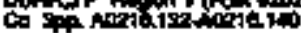

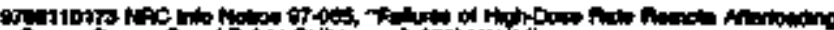

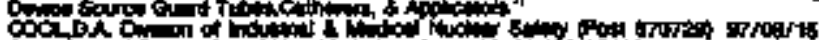

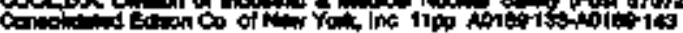

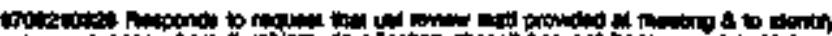

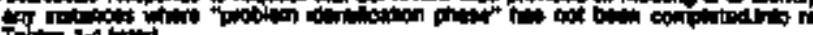

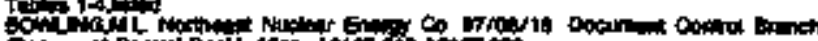

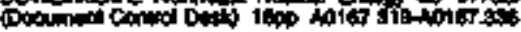

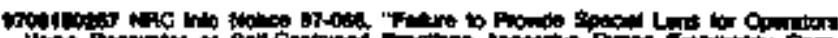

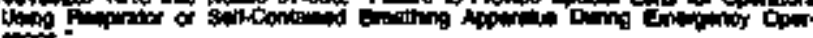

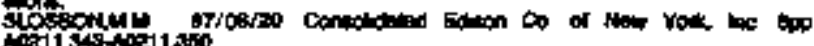

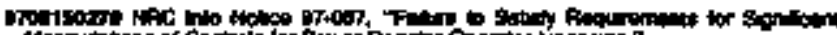

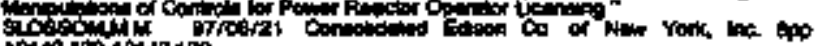
Antes is

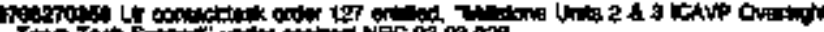

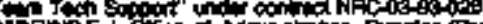

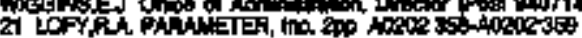

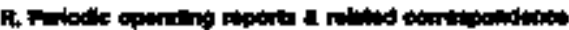

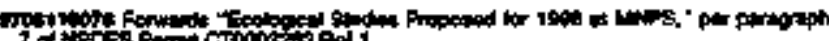

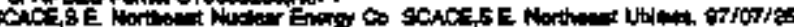

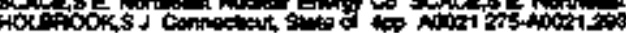

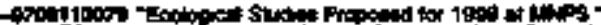

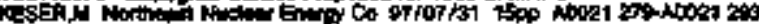

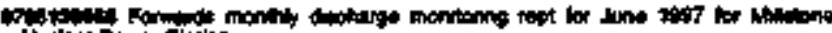

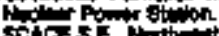

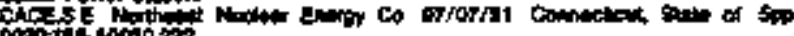

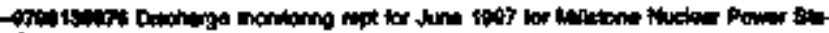

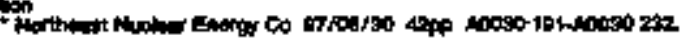

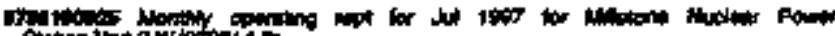

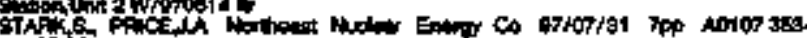
N107

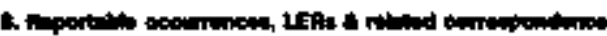

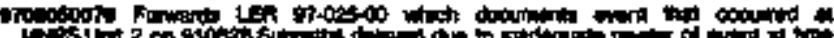

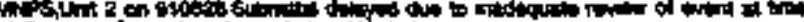

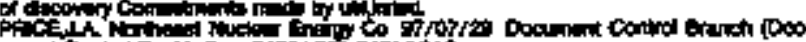

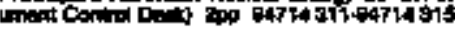

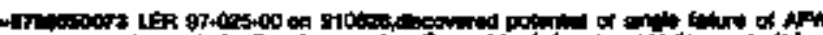

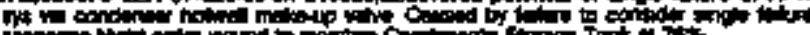

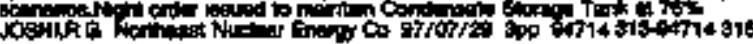

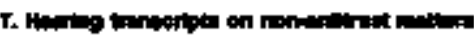

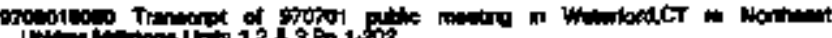

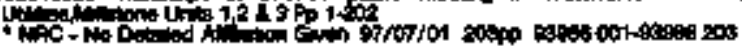

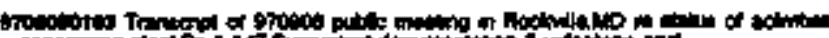

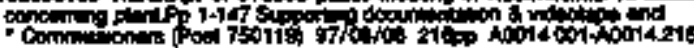

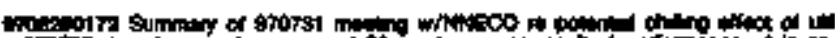

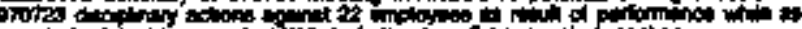

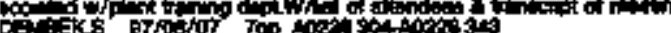

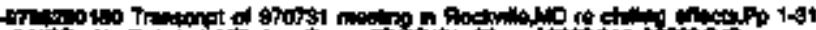

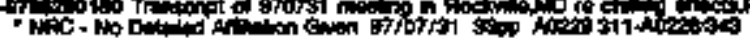

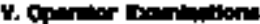

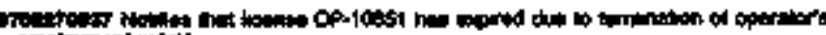

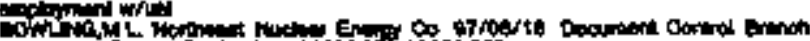

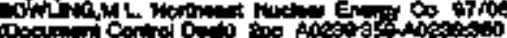

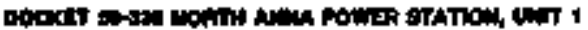

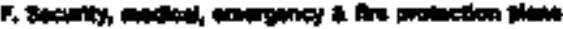

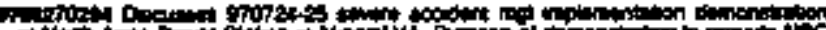

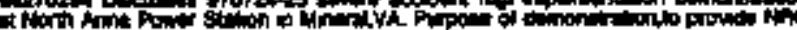

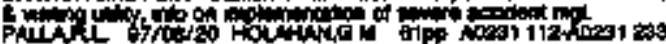

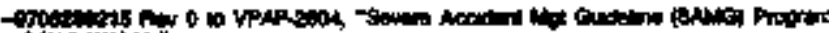

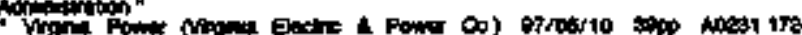
ingiotio

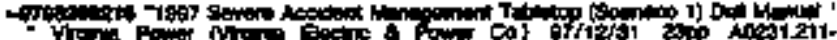

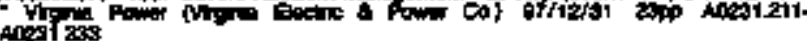

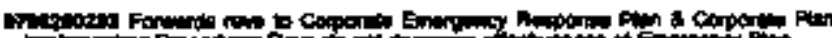

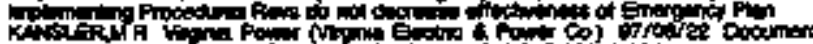

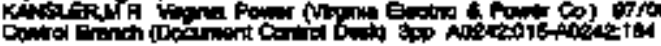

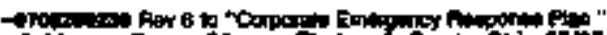

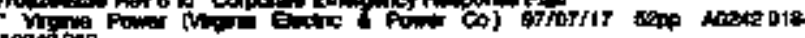

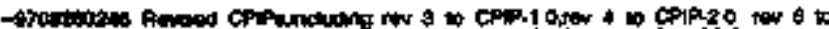

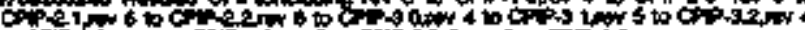

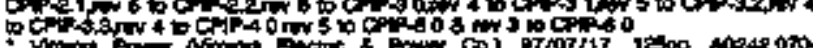
ineting 


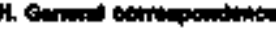

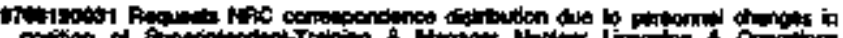

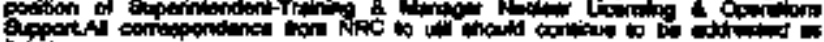
tion.

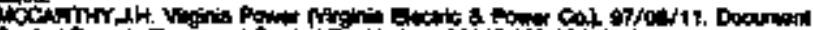

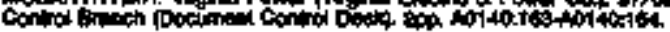

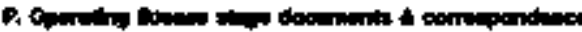

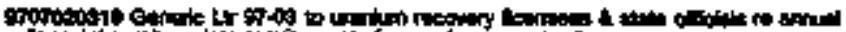
of.

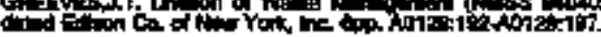

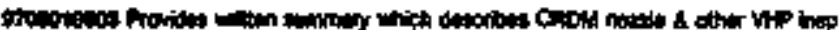

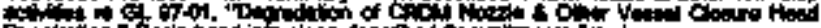

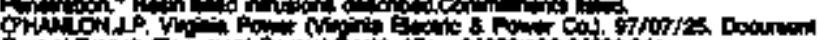

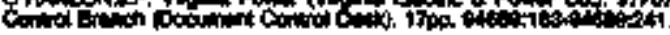

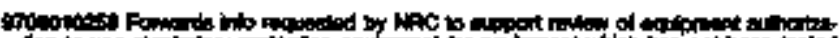

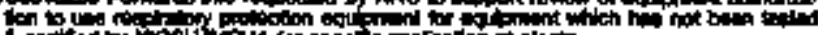

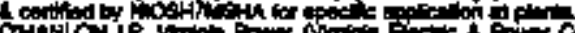

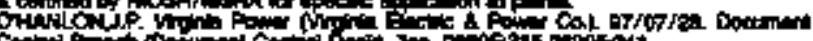

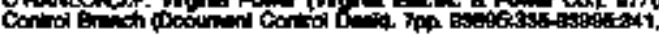

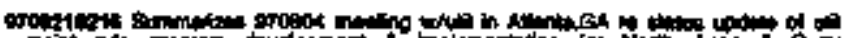

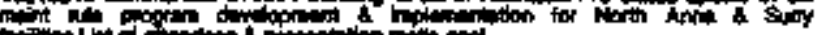

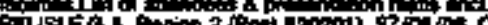

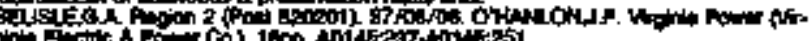

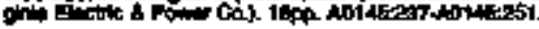

9rom: polod of ofo.

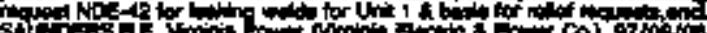

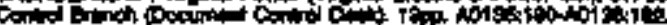

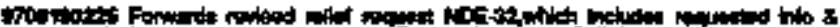

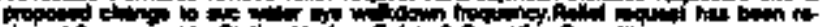

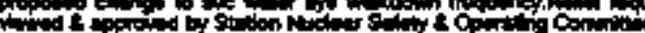

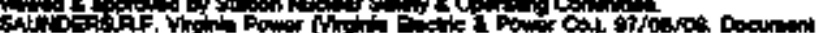

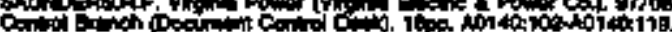

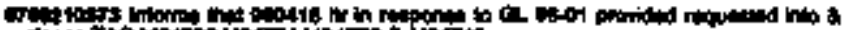

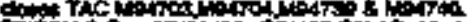

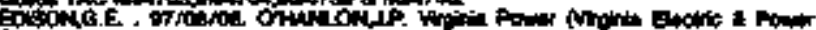

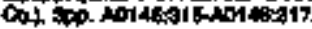

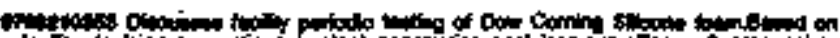

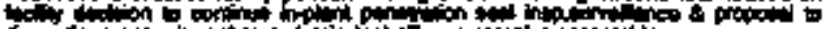

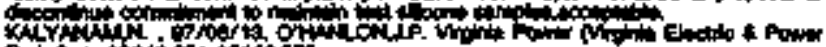

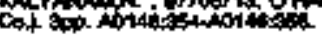

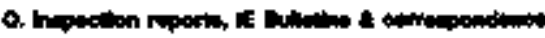

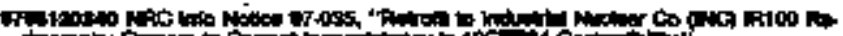

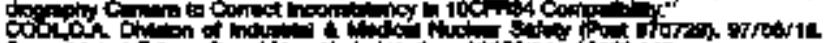

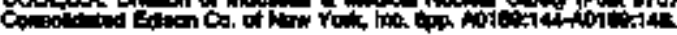

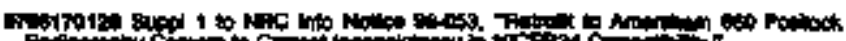

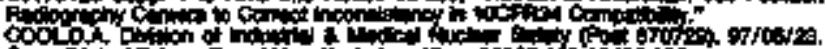

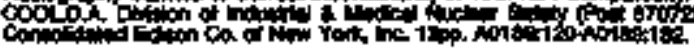

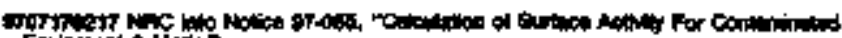

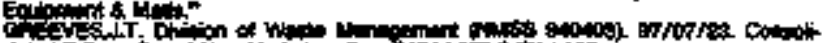

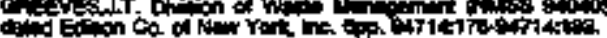

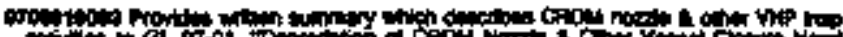

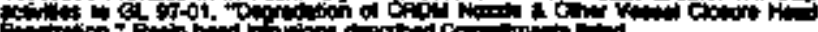

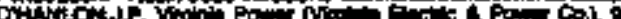

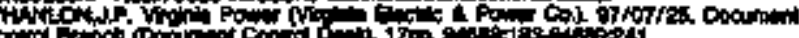

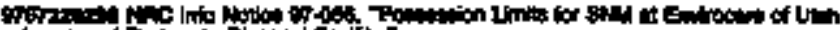
of

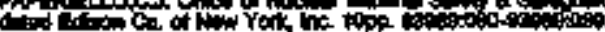

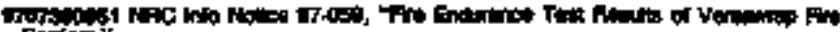

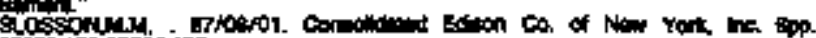

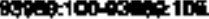

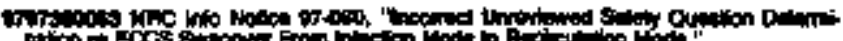

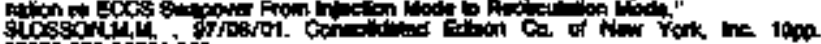

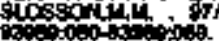

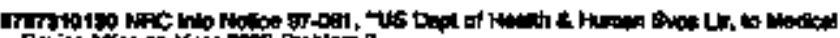

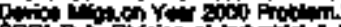

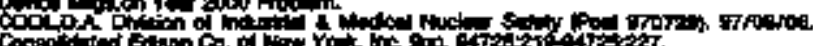

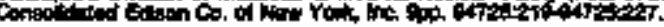

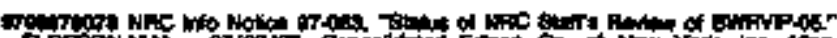

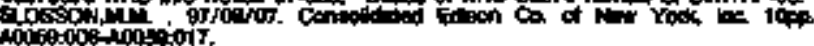

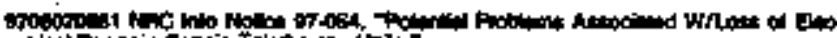

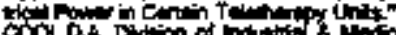

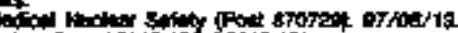

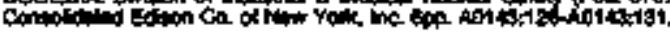

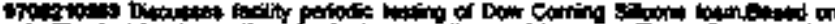

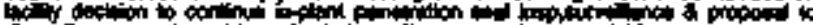

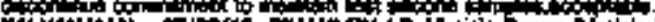

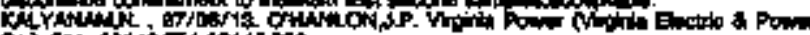

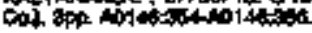

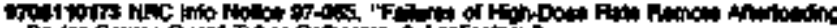

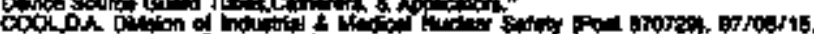

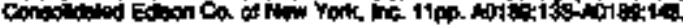

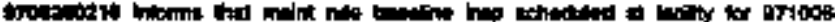

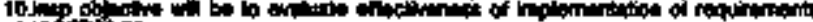
of $10 \times+4005$.

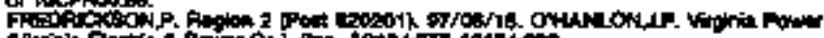

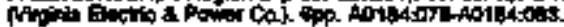

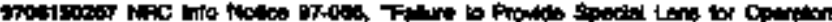

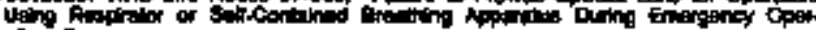

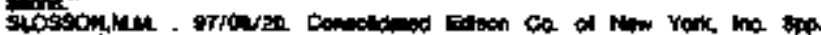

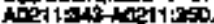

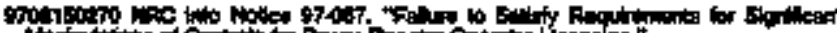

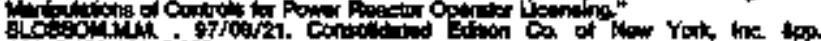

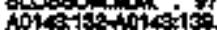

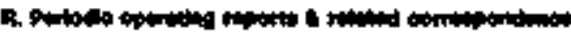

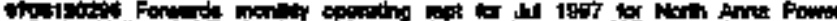
c.

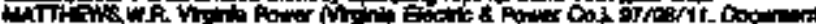

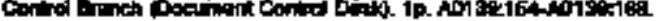

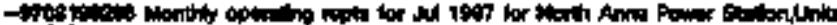
1t2

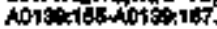

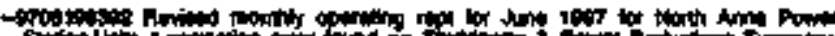

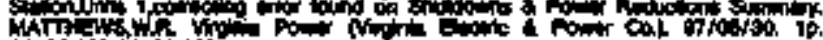

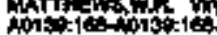

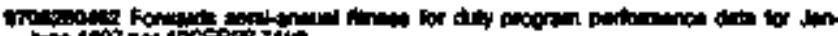

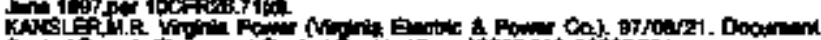

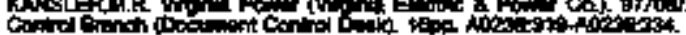

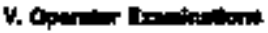

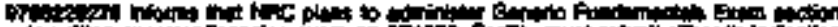

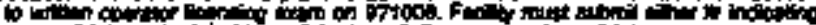

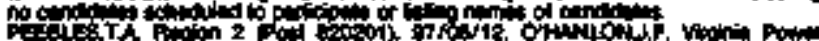

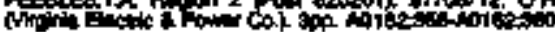

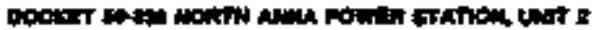

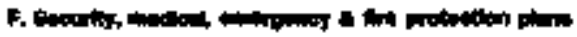

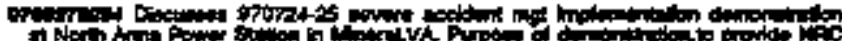

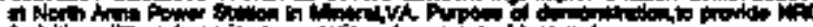

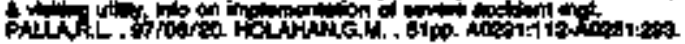

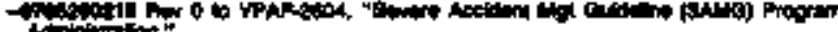

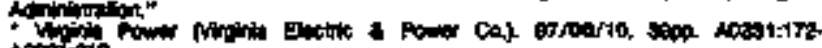
Notitio.

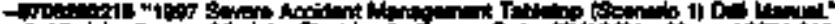

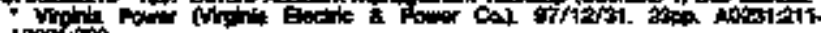
Nometson.

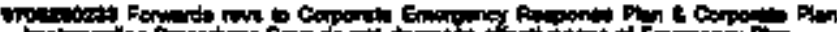

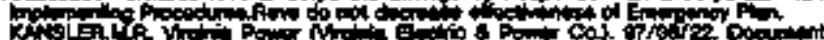

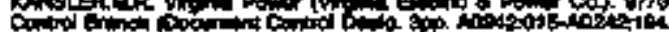

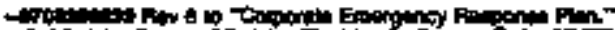

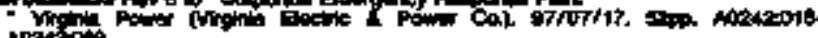
$\lim _{\rightarrow \infty}$

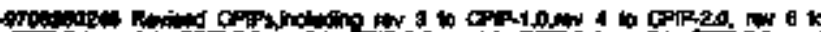
G

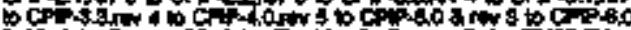

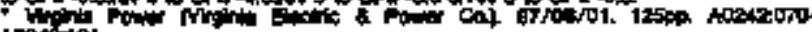
noper 194 . 


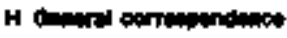

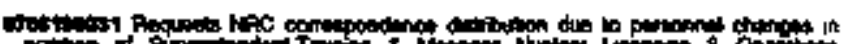

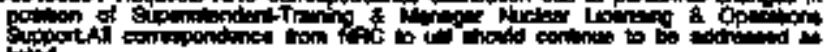

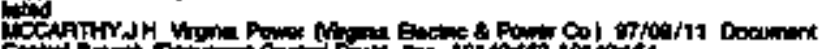

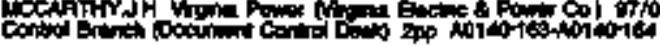

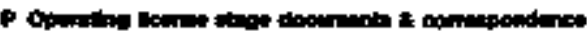

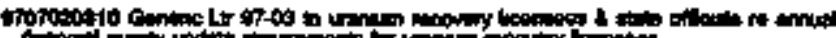
G

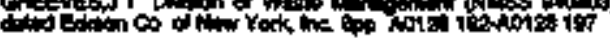

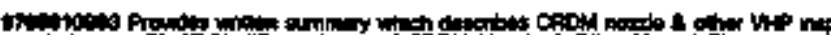

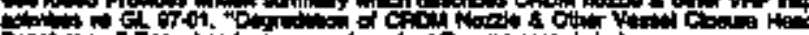

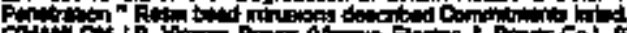

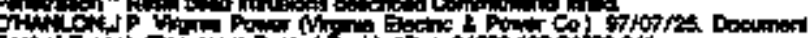

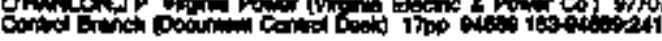

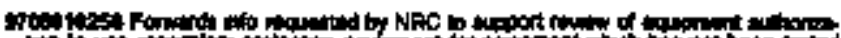

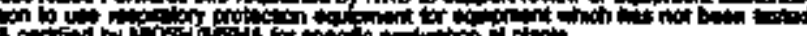

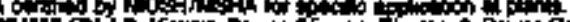

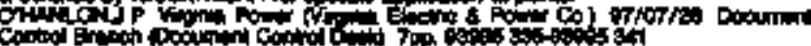

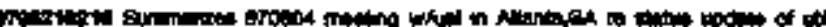

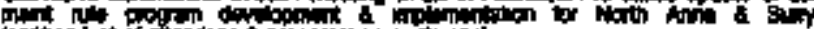
crich of

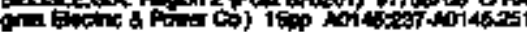

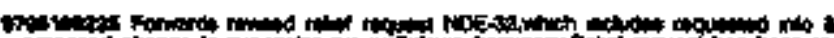

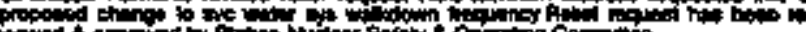

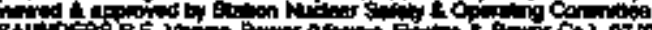

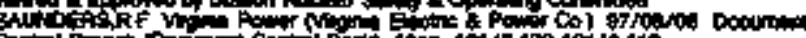

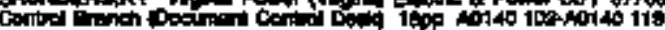

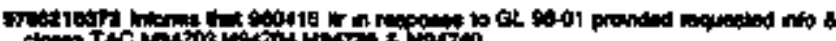

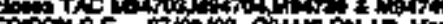

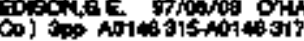

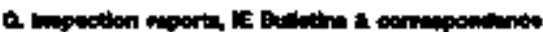

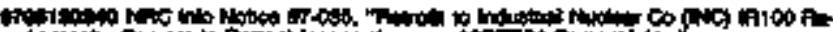

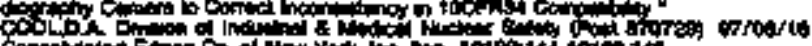

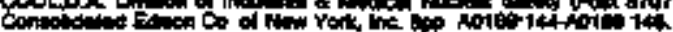

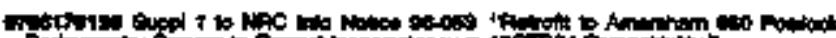

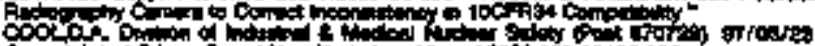

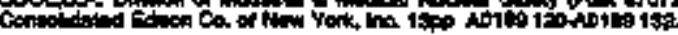

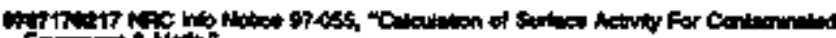
E F

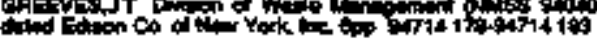

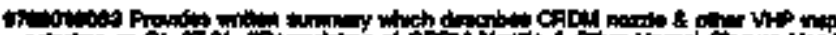

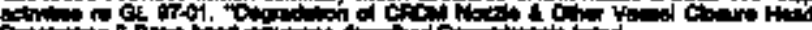

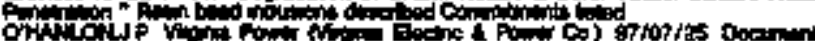

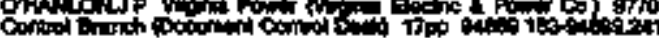

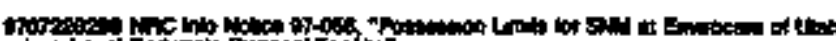

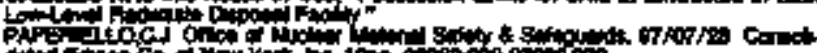

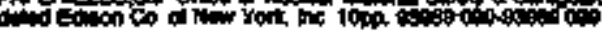

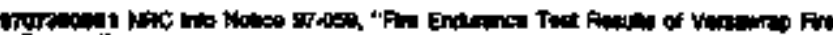

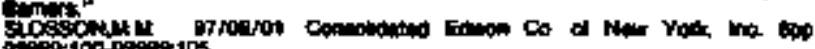

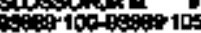

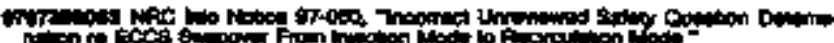

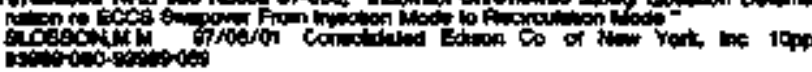

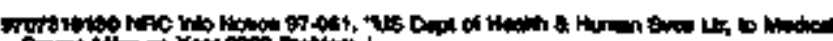

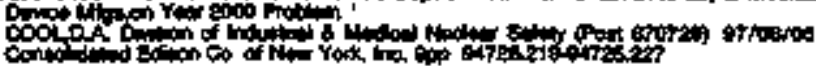

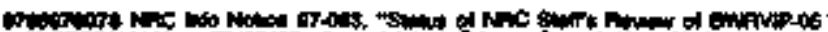

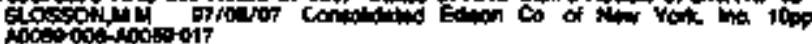

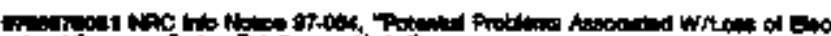

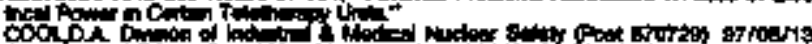

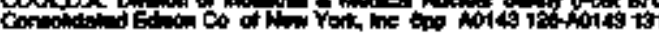

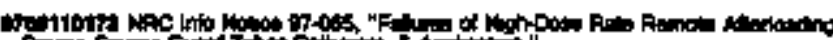
Coot Con

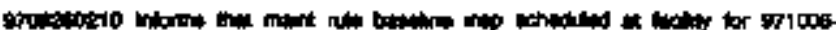
10 ine of

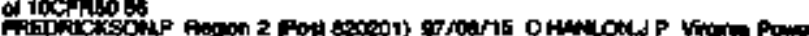

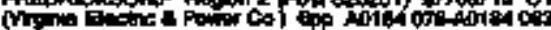

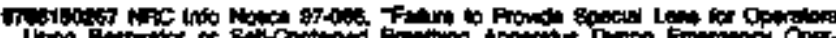

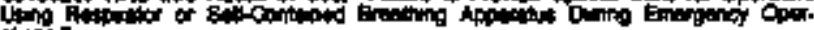

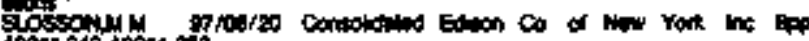

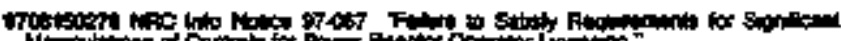

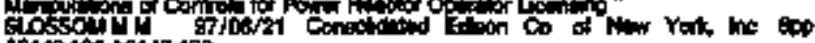
A143 132-40143 139

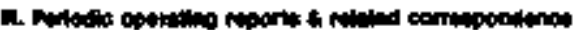

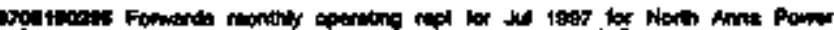

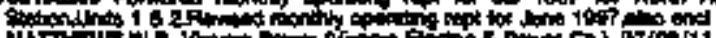

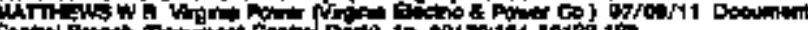

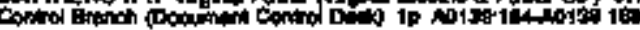

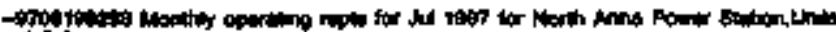

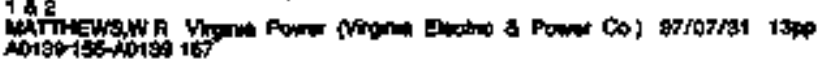

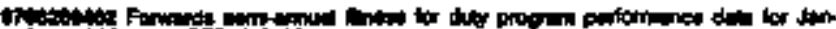

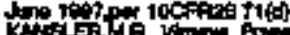

KHAB

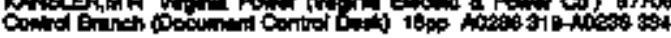

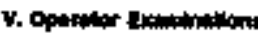

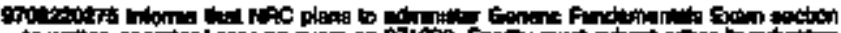

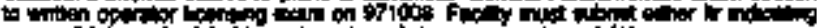
nocrita

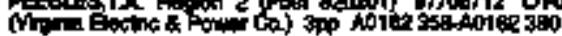

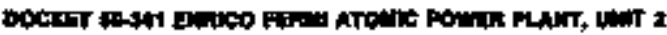

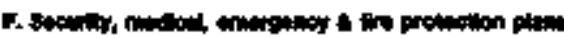

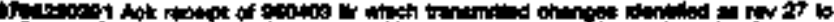

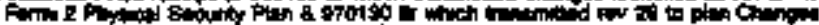

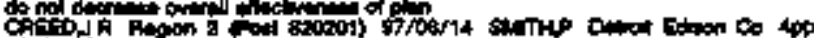

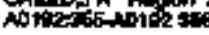

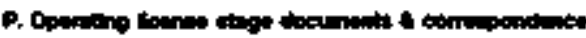

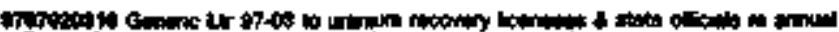

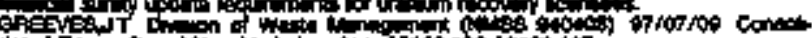

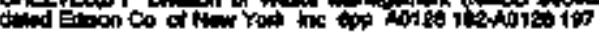

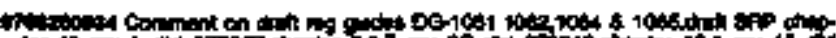

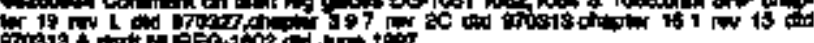

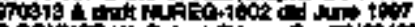

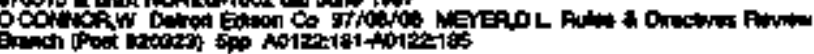

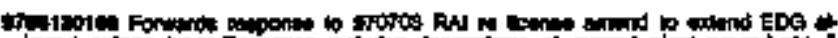

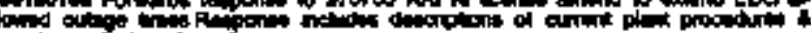

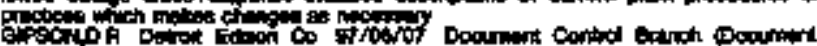

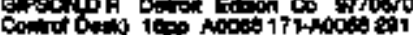

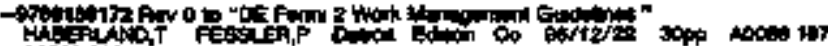
notiots

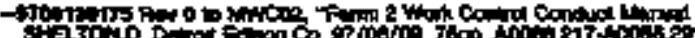

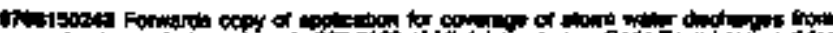

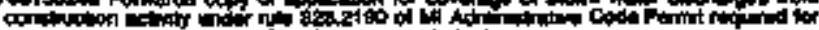

chold

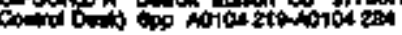

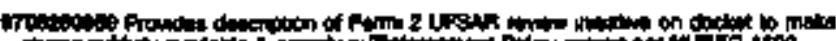

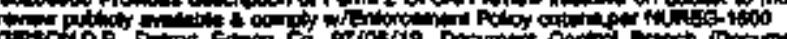

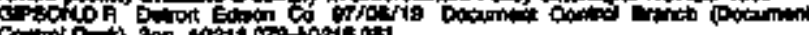

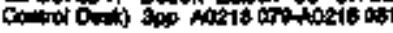

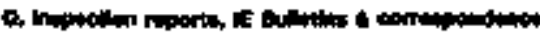

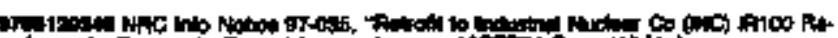

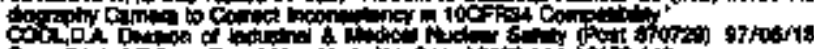

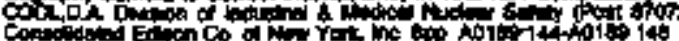

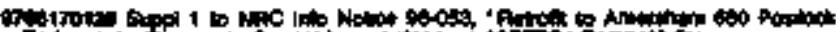
coorot

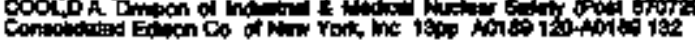

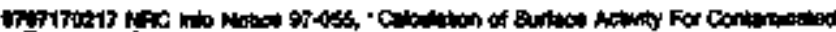

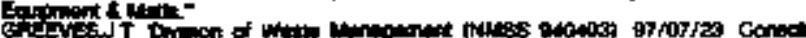

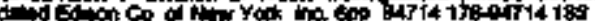




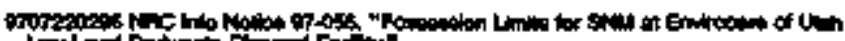

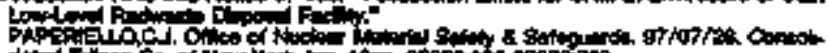
DAping

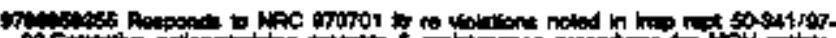

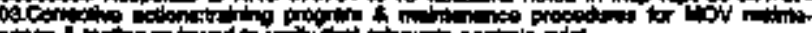

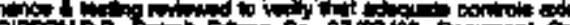

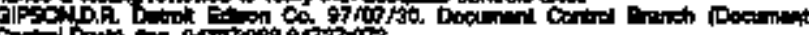
Trom

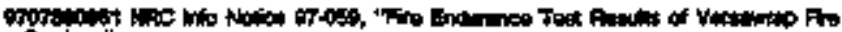

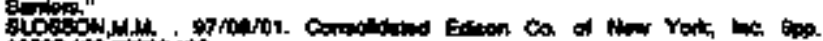
\%

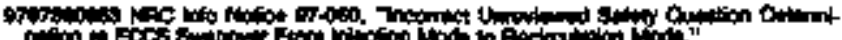

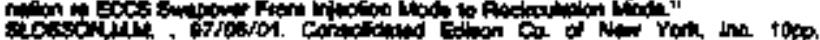

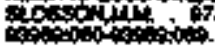

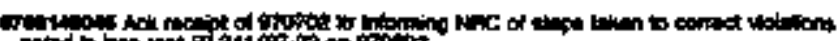

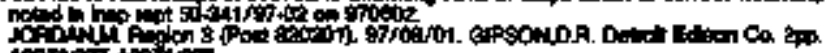

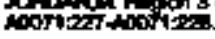

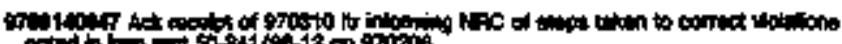

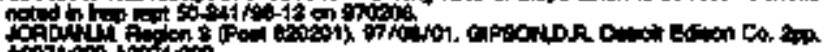
motition

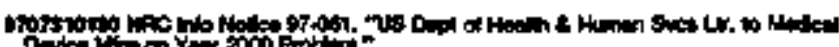

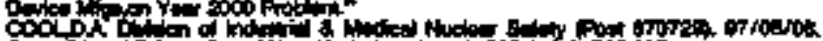

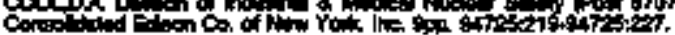

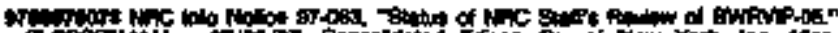

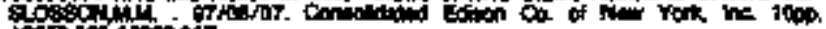

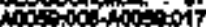

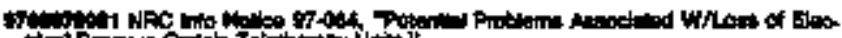

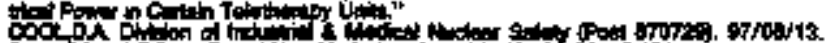

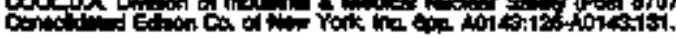

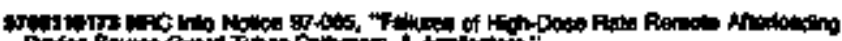

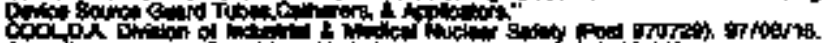

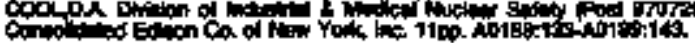

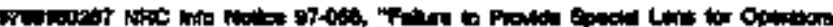

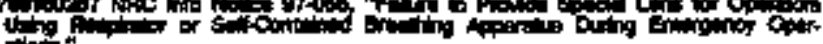

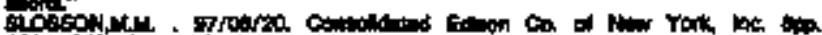

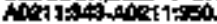

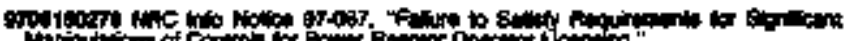

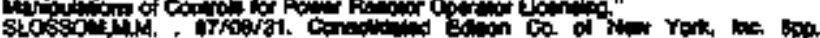

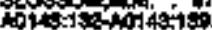

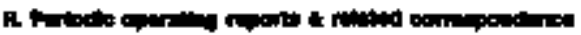

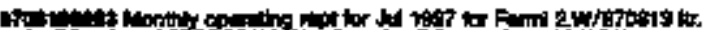

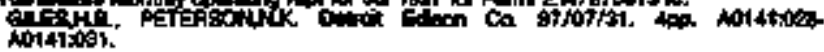

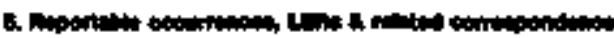

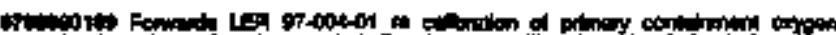

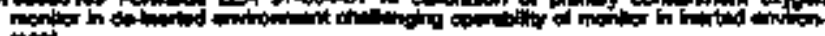

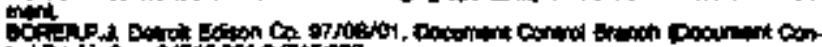

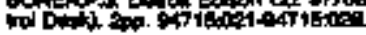

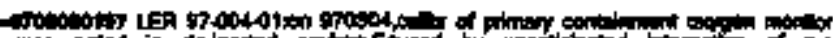

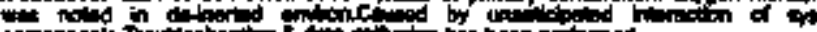

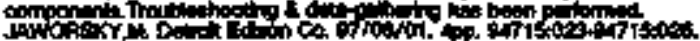

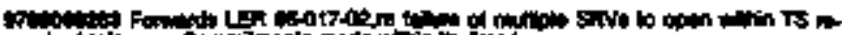

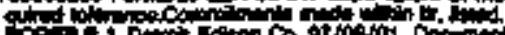

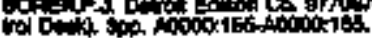

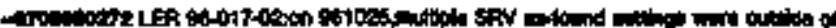

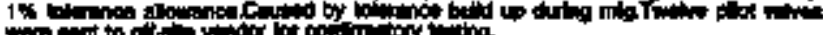

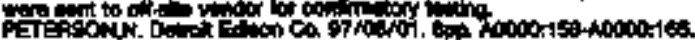

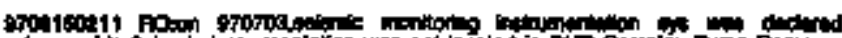

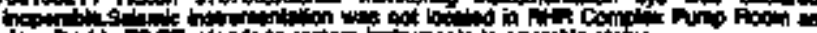

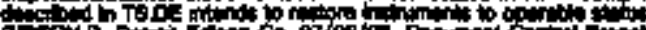

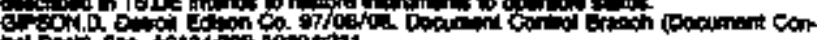

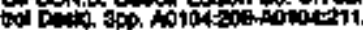

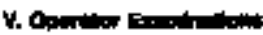

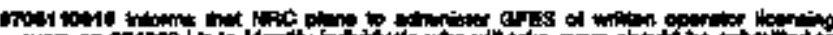

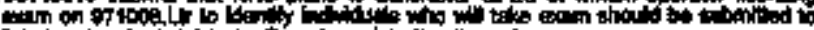

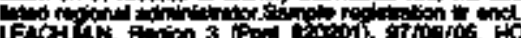

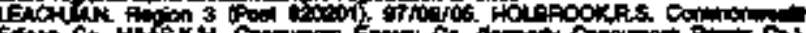

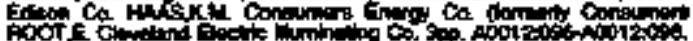

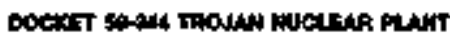

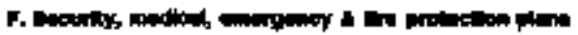

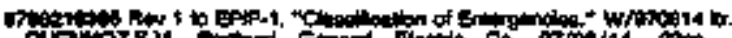

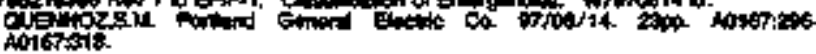

L Frated bionowion

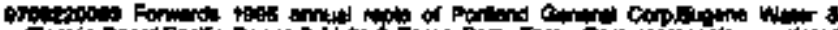

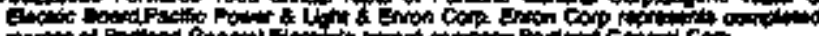

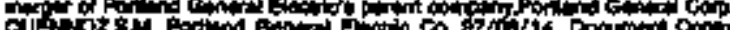

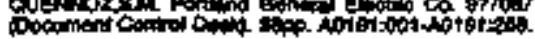

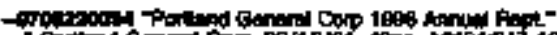

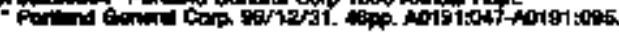

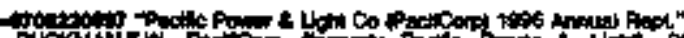

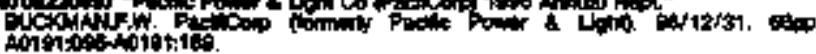

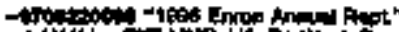

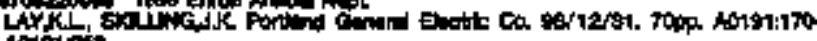
M.

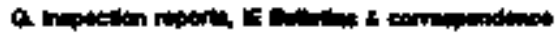

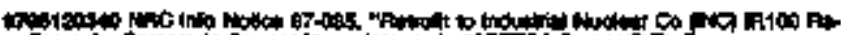

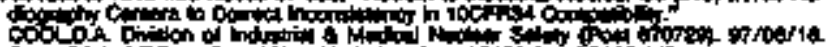

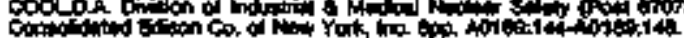

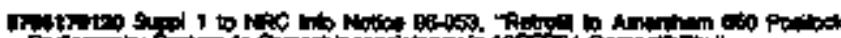

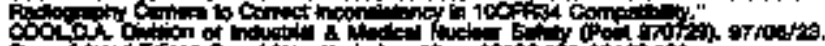

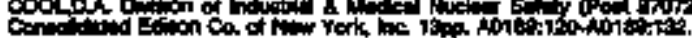

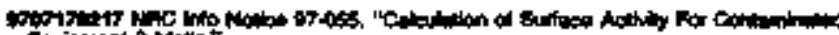

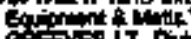

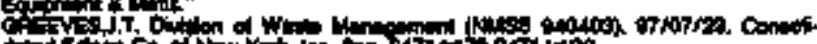

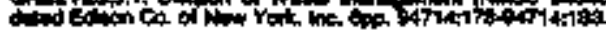

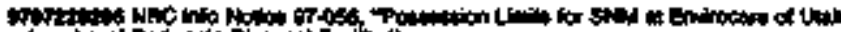
Po-

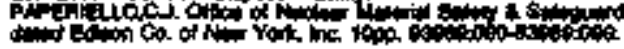

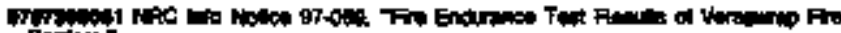

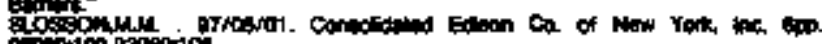

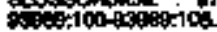

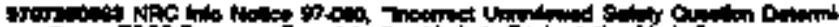

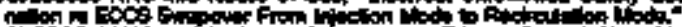

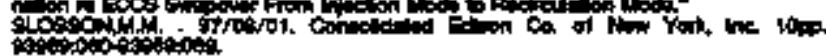

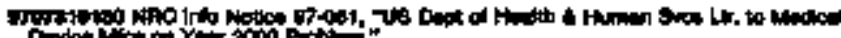

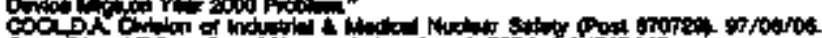

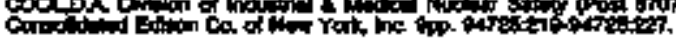

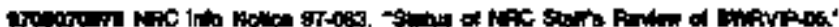

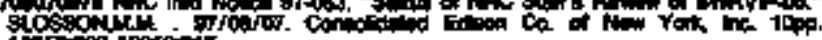

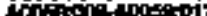

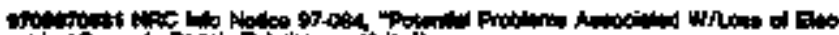

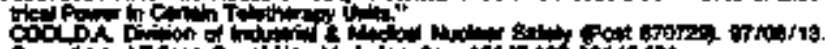

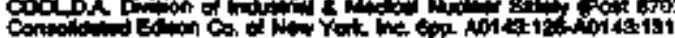

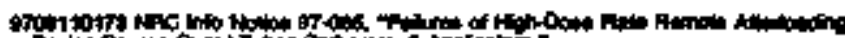

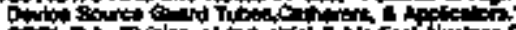

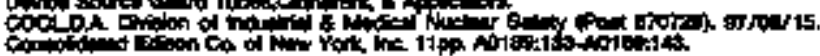

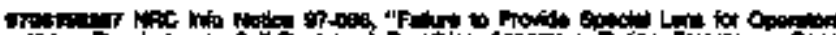

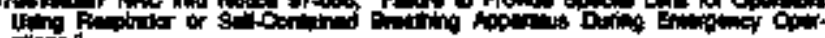

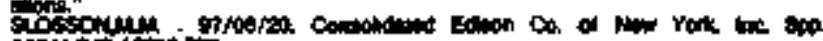

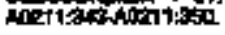

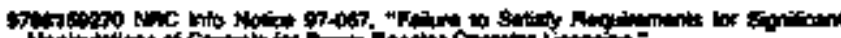

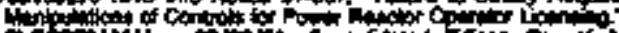

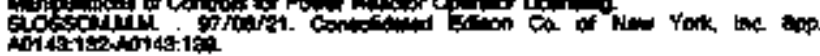

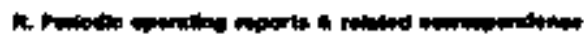

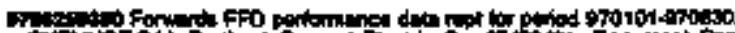

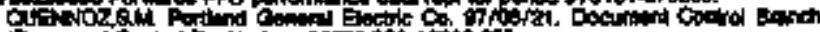

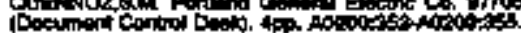




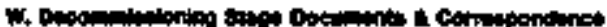

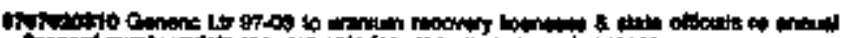

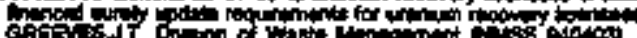

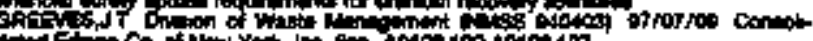

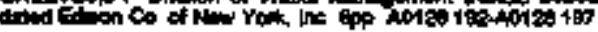

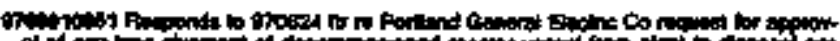

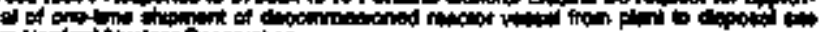

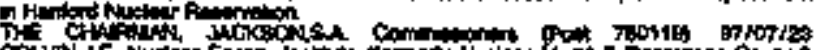

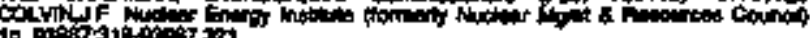

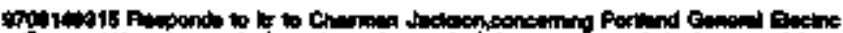
Oo nop

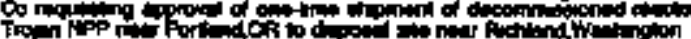

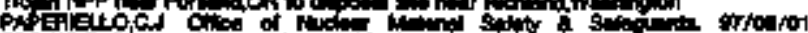

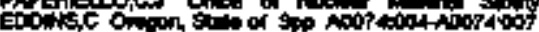

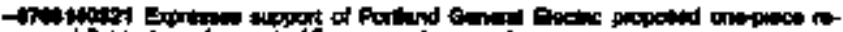

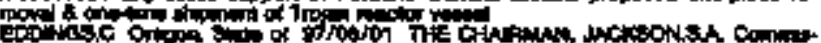

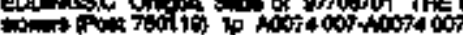

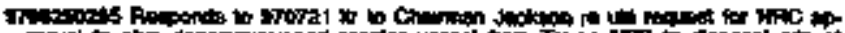

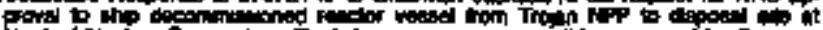

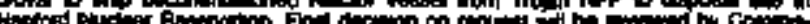

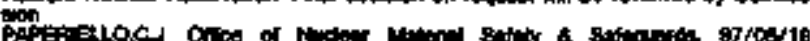

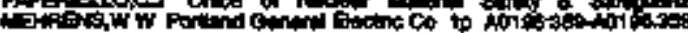

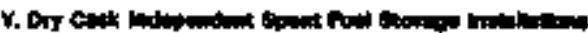

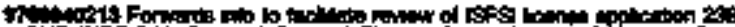

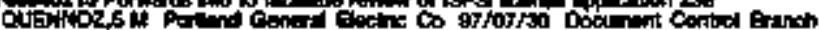

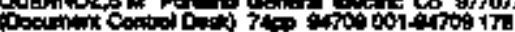

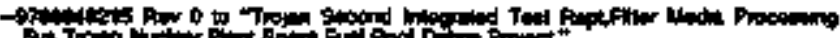
Q

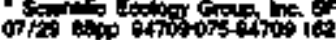

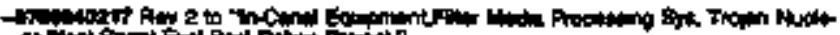
4 Find aito

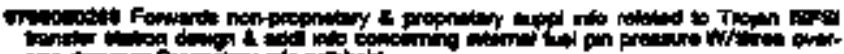
od

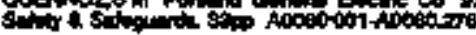

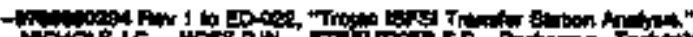

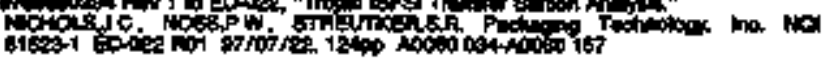

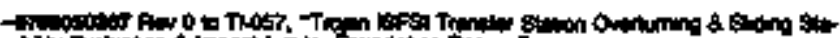

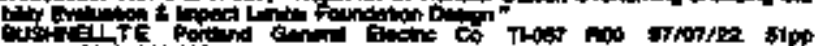
conotion

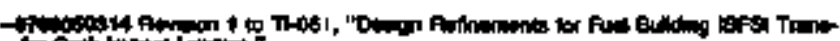

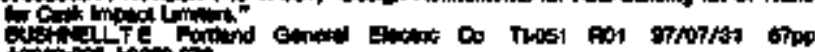
(t)

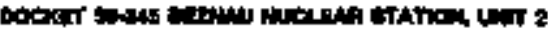

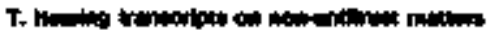

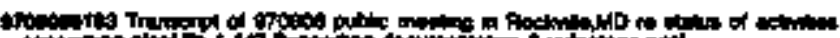
coming on

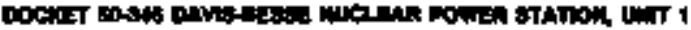

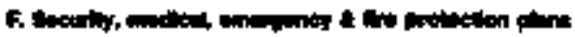

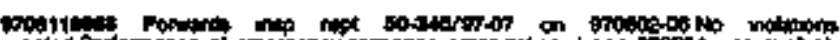

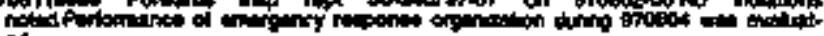
4

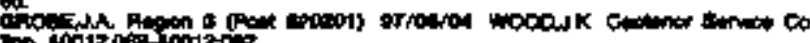

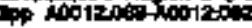

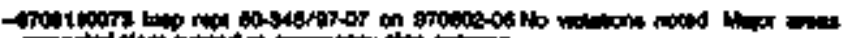

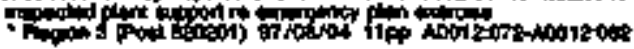

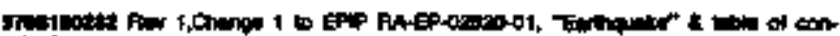

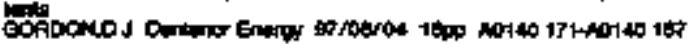

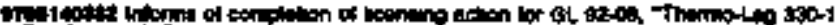

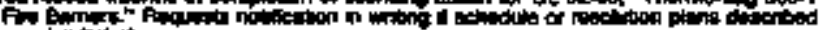

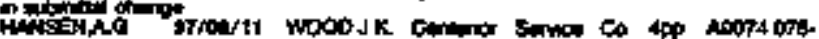
noturoso

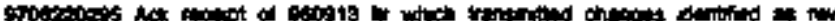

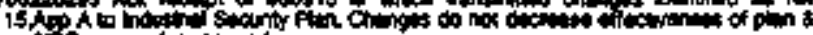

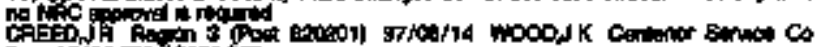

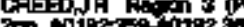

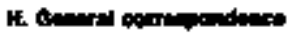

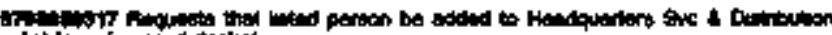

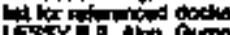

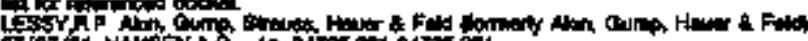

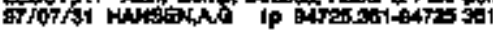

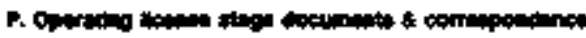

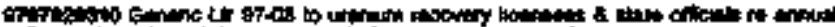

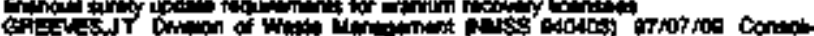

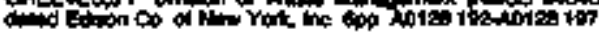

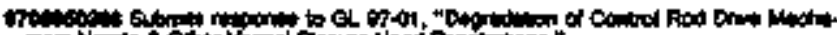

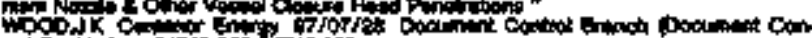

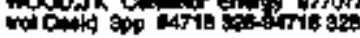

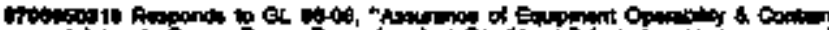

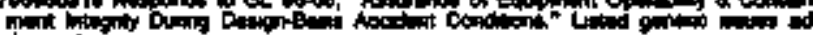

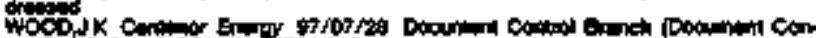

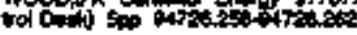

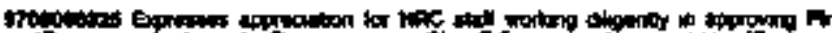

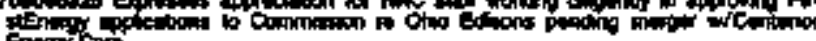

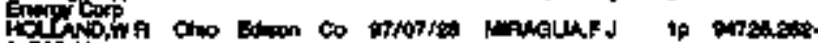
onters

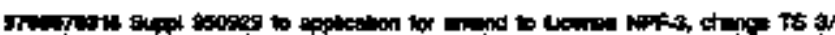

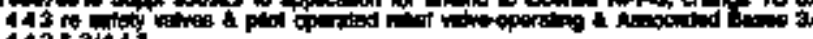
$42+344$

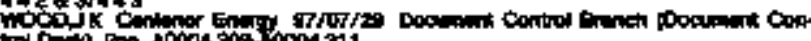

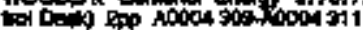

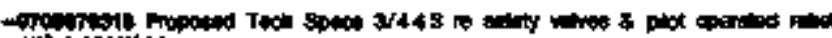

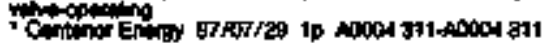

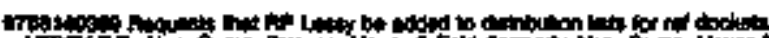

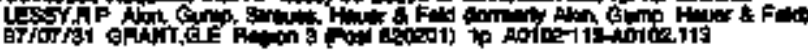

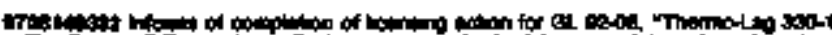

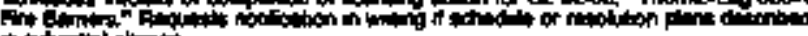

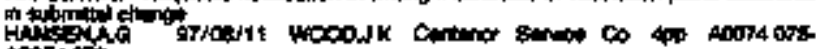
10074 orts

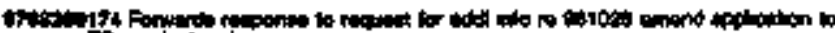

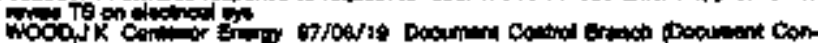

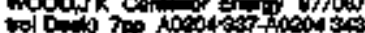

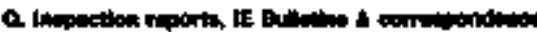

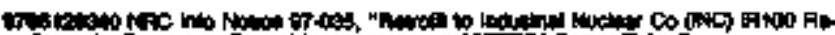

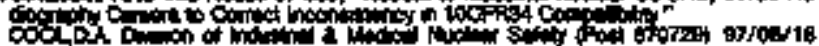

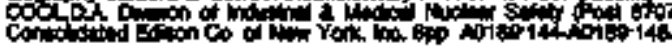

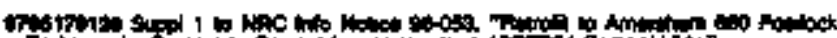

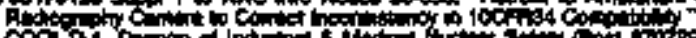

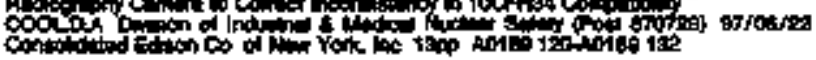

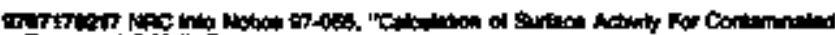

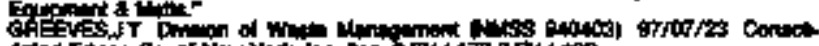

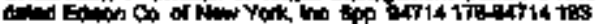

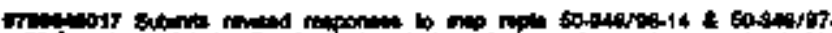

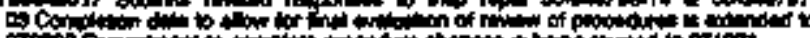

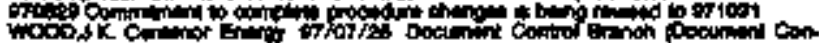

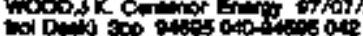

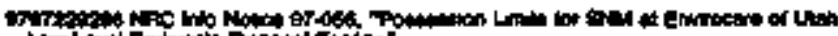

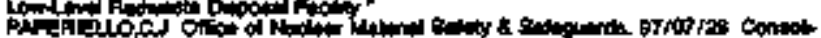

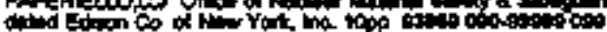

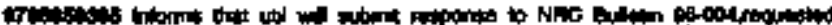

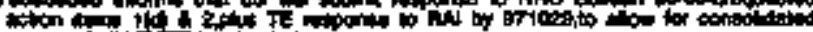

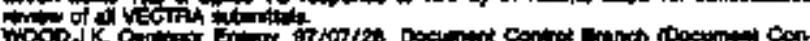

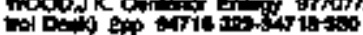

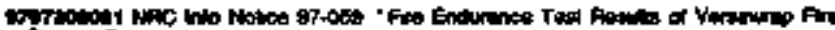

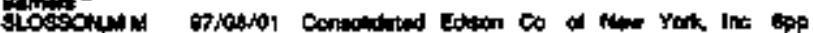
100- 100 


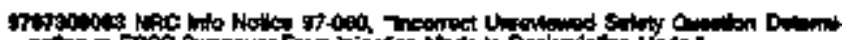

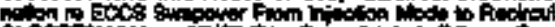

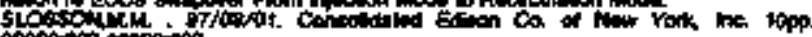

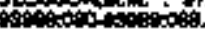

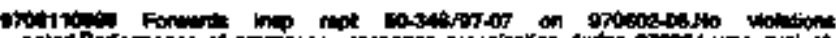

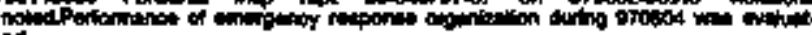

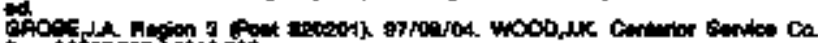

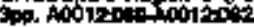

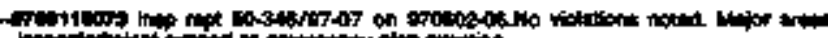

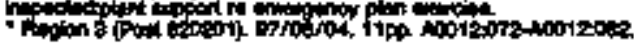

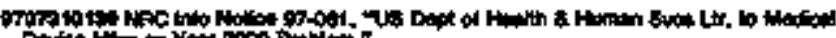

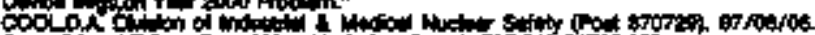

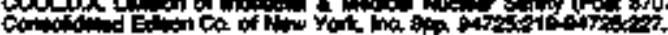

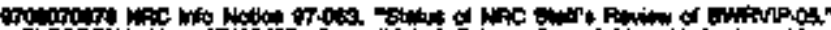
a

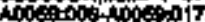

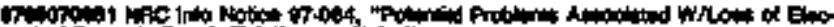

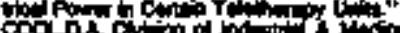

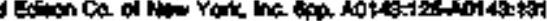

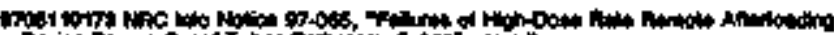

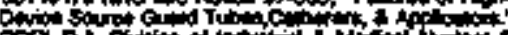

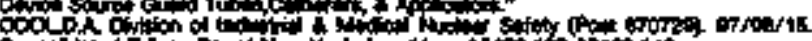

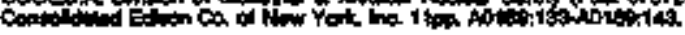

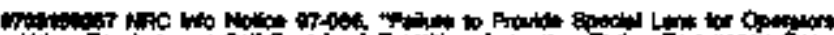

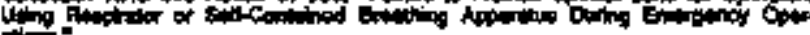

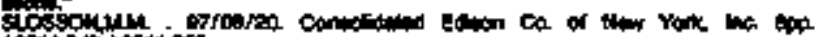

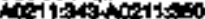

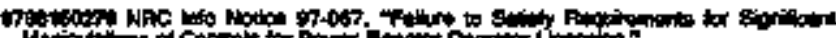

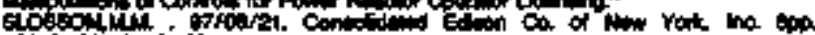

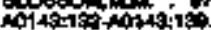

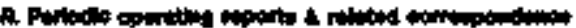

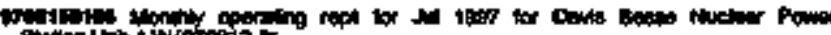

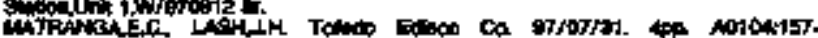
colotion.

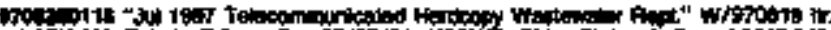

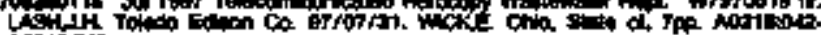
inction.

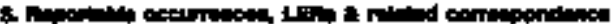

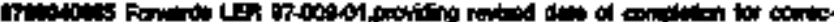

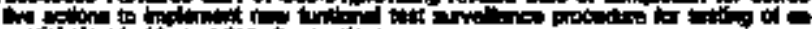

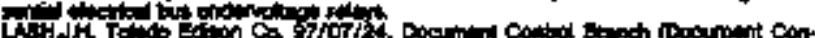

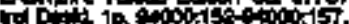

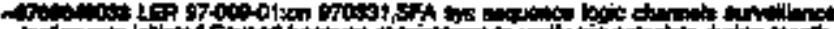

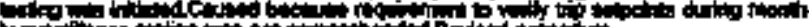

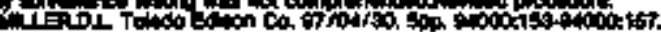

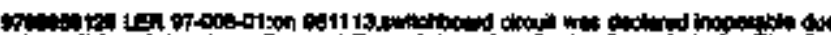

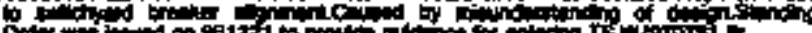

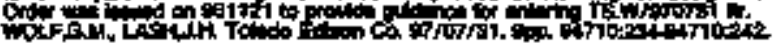

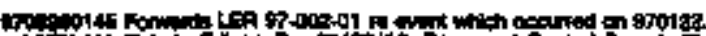

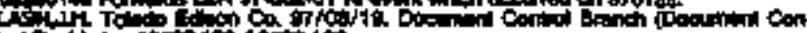

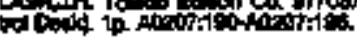

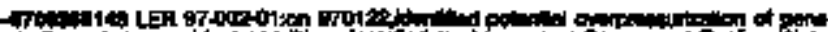

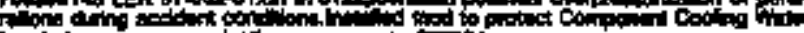

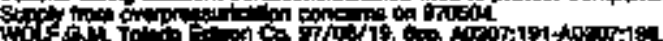

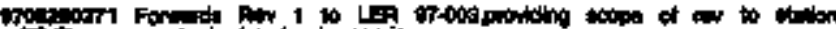

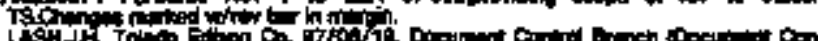

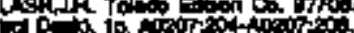

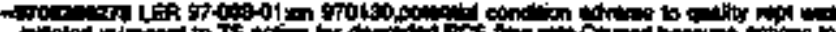

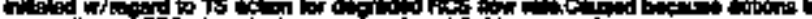

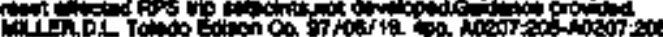

V. Opdor Extmanom:

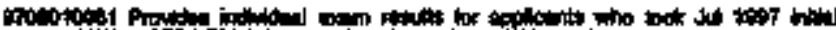

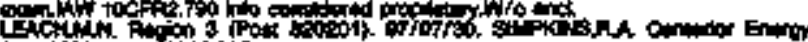

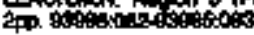

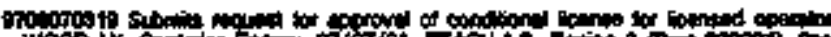

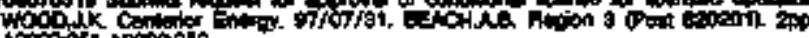
ition

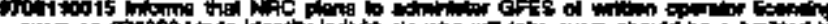

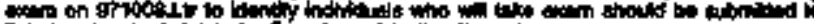

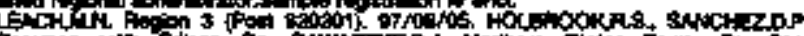

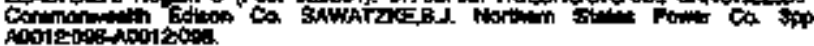

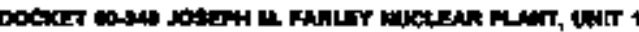

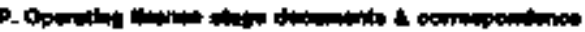

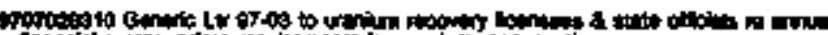
p

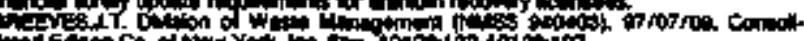

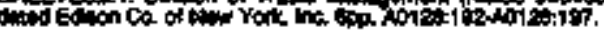

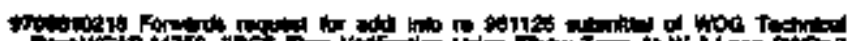

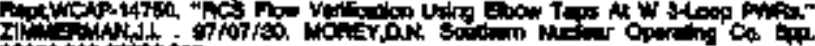

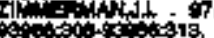

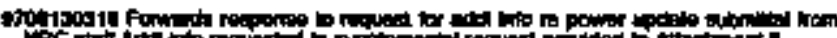

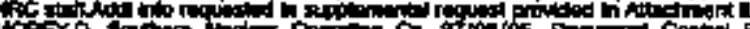

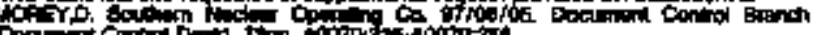

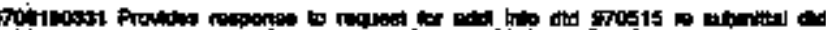

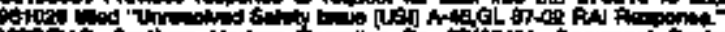

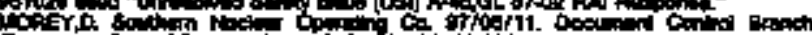

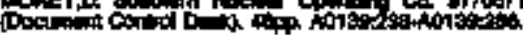

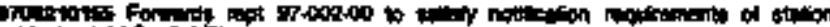

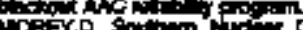

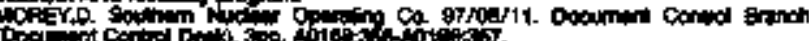

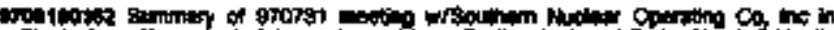

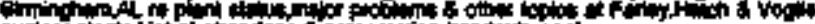

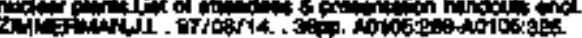

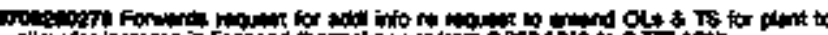

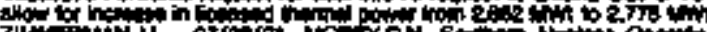

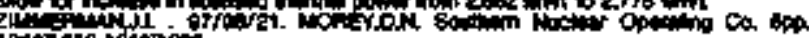

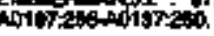

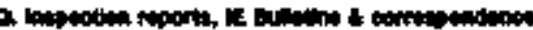

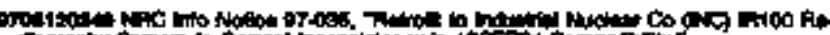

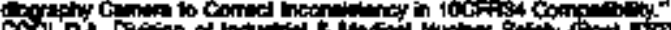

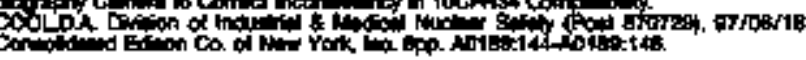

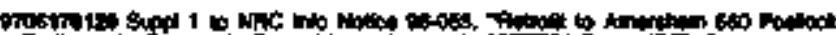

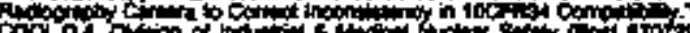

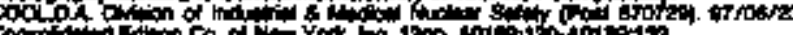

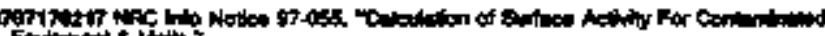

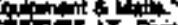

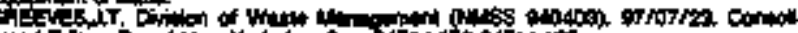

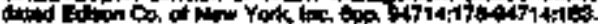

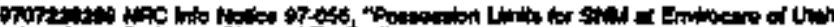

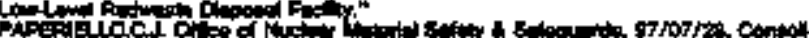

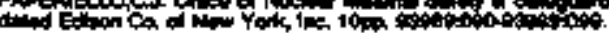

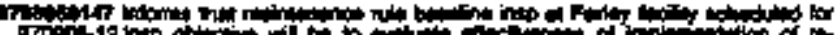

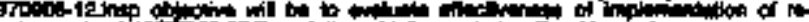

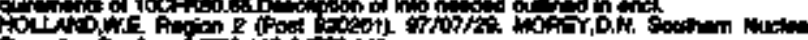

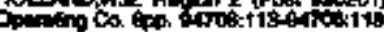

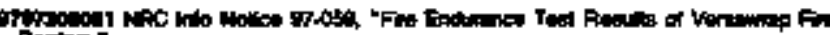

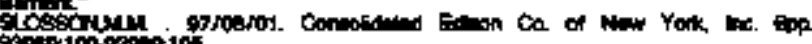

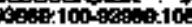

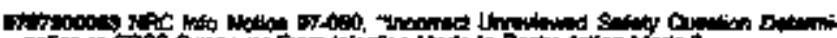
arcin ia

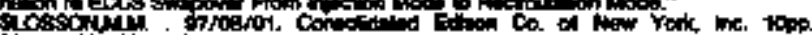

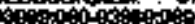

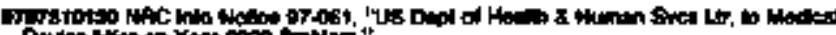

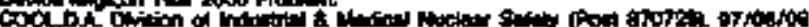

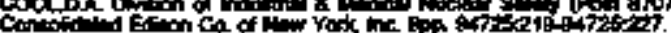

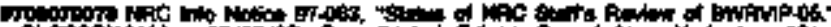

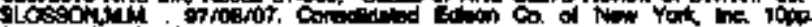

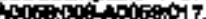

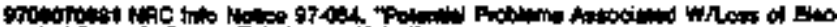

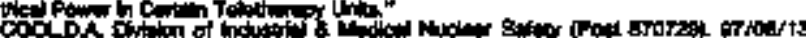

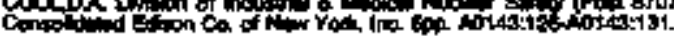

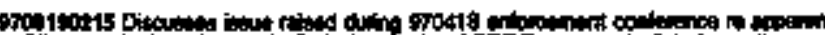

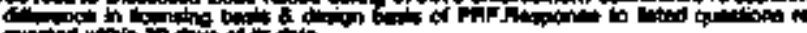

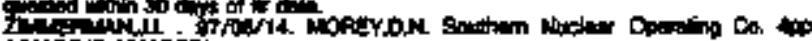

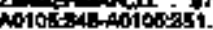




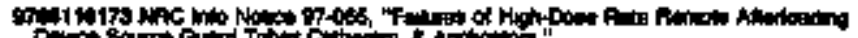

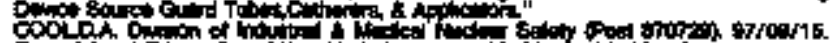

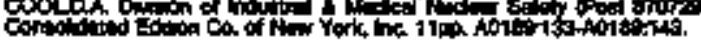

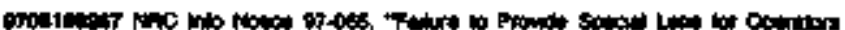

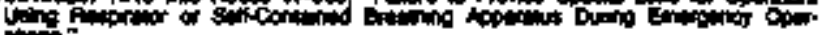

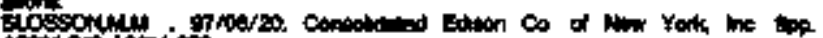

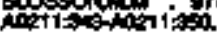

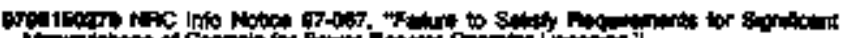

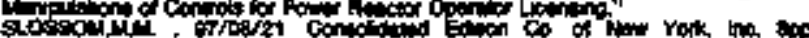

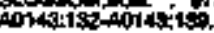

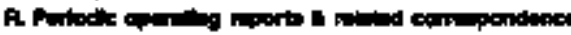

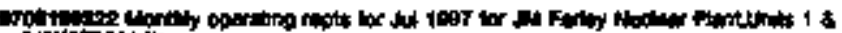

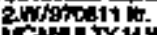

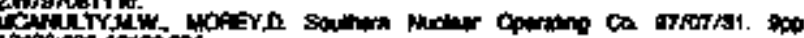
ato

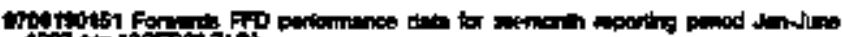

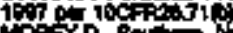

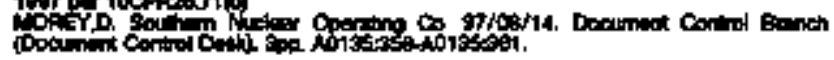

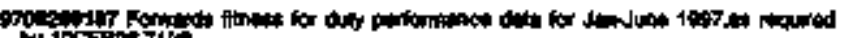

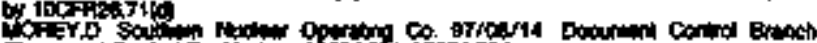

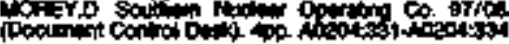

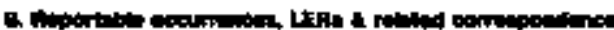

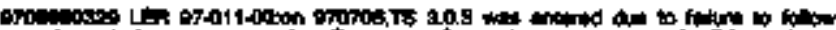

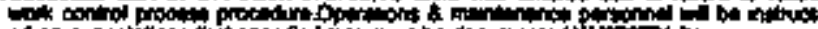

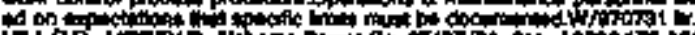

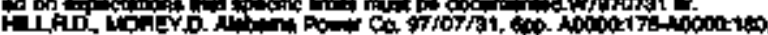

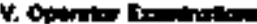

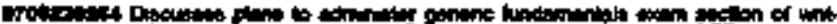

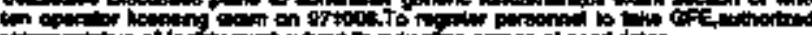

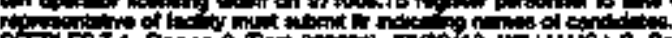

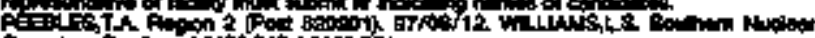
Orich

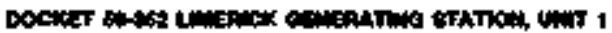

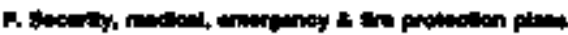

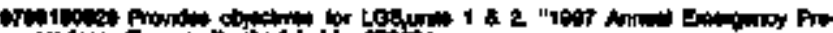

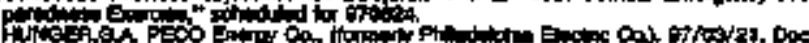

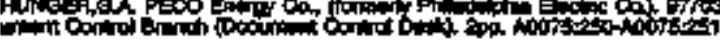

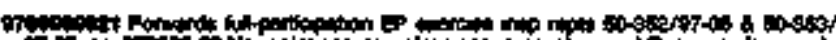

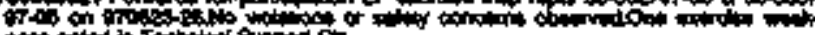

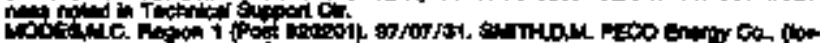

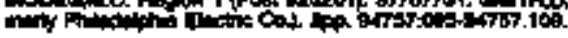

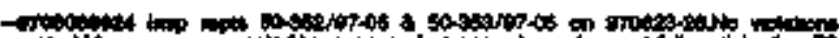
now d.

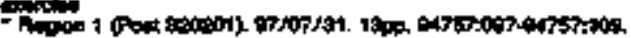

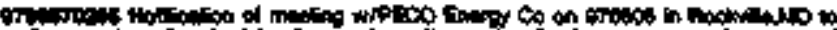

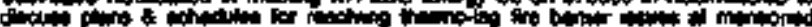

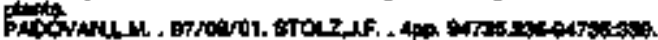

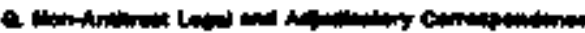

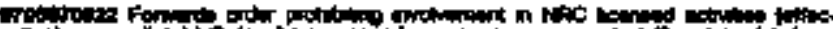

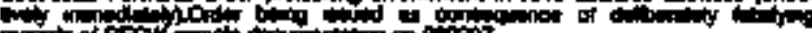

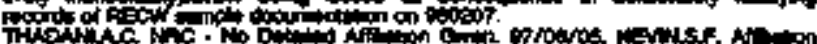

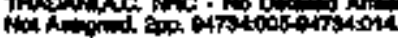

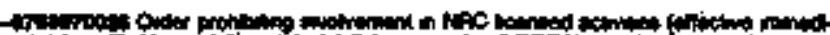

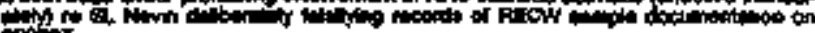

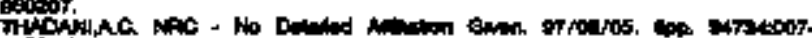

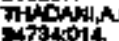

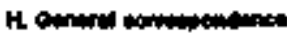

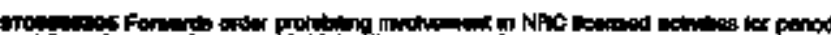

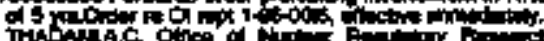

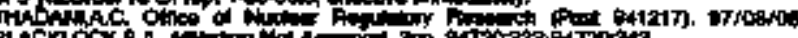

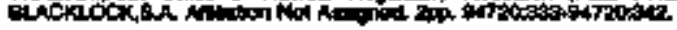

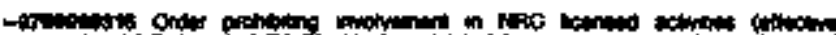

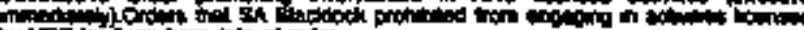

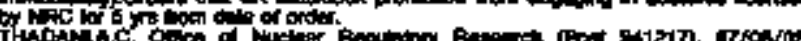

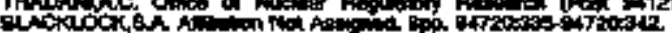

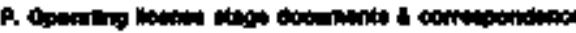

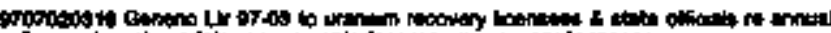
dy

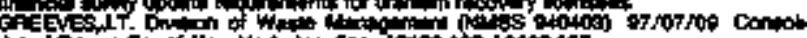

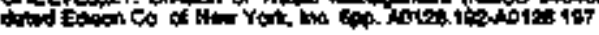

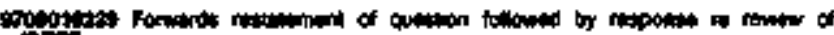

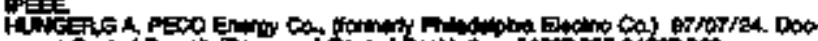

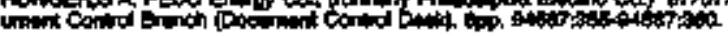

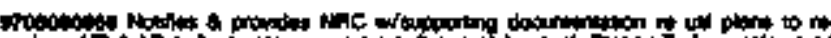

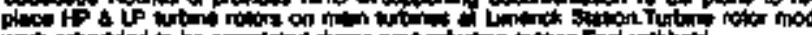

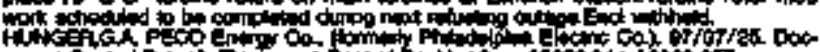

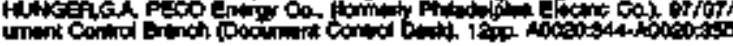

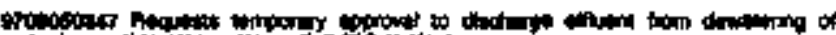

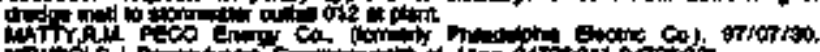

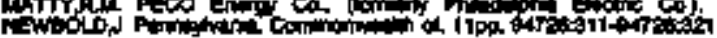

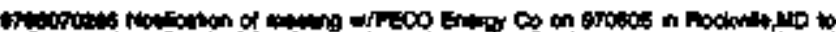

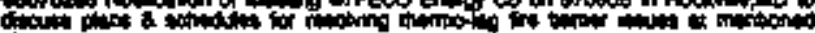

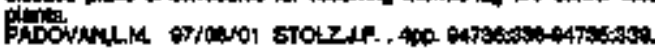

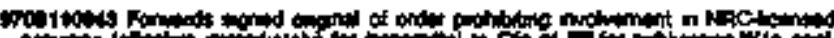

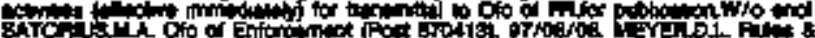

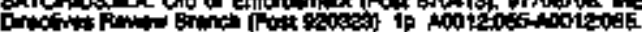

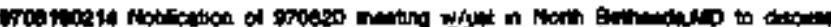

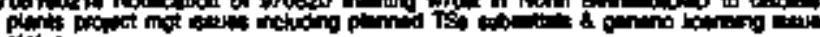

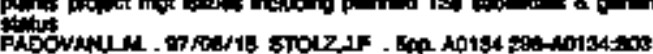

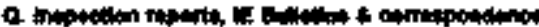

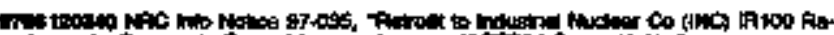

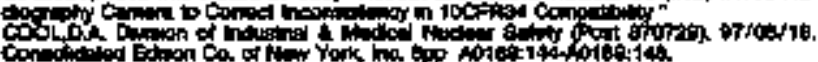

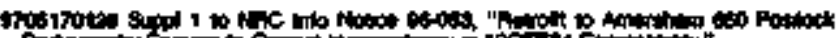

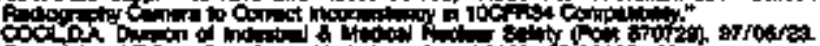

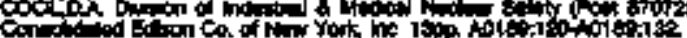

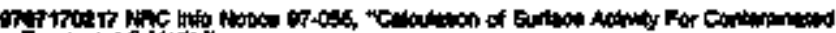
cdidy

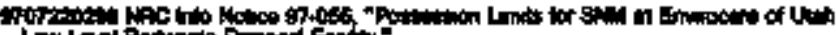

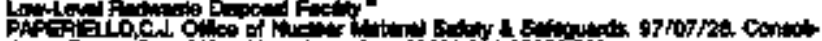

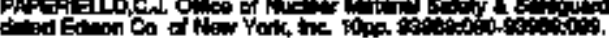

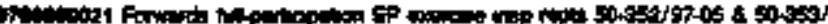

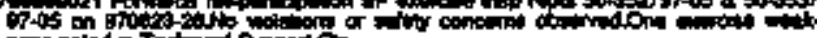

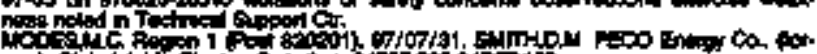

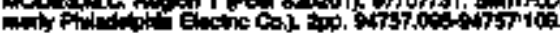

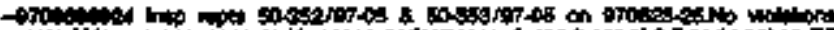

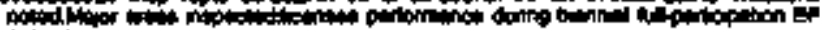

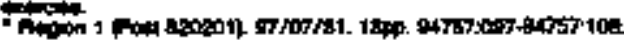

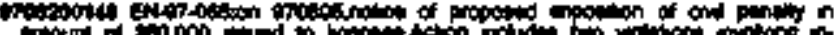

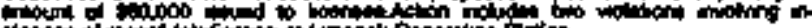
1.

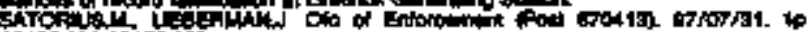

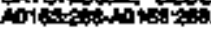

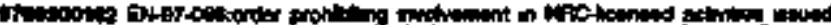

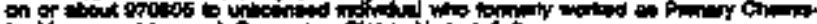

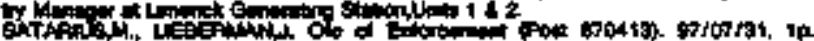

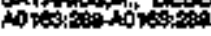

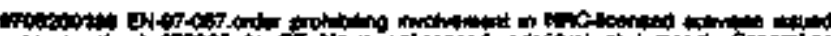

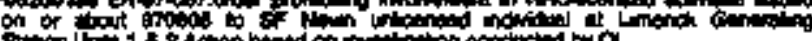

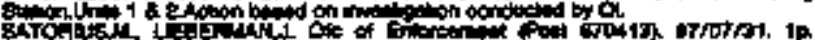
salo

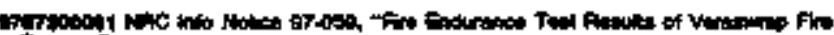

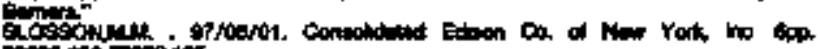

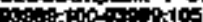

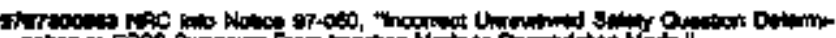

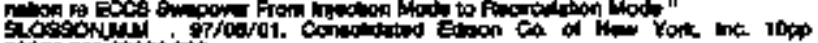
scosonuy, of?

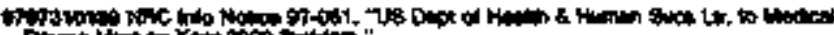

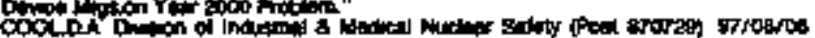

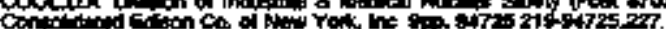




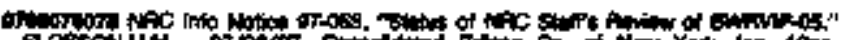
gosopin, of

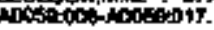

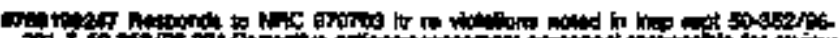

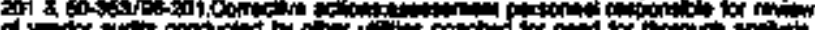
of

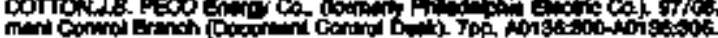

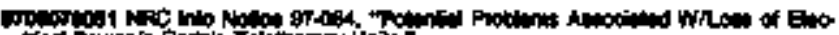

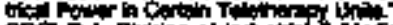

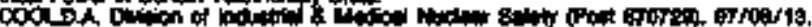

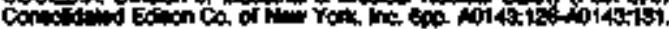

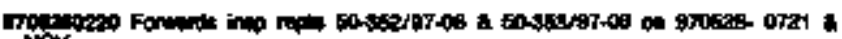

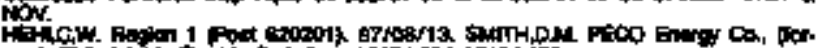

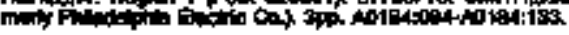

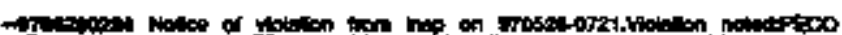

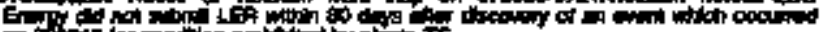

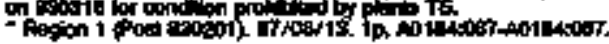

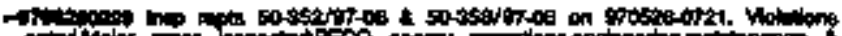

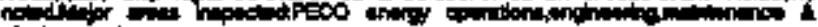

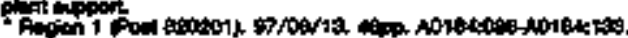

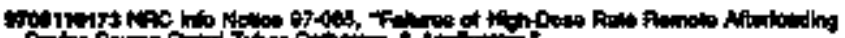

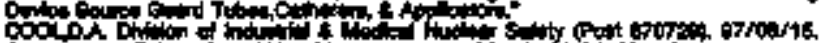

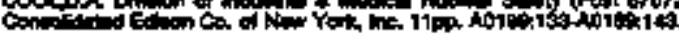

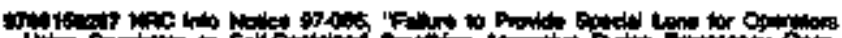

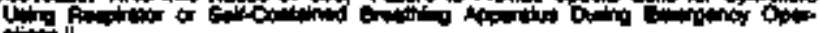

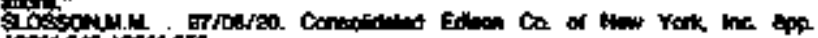

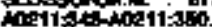

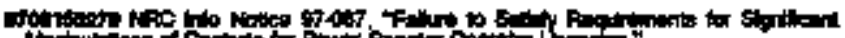

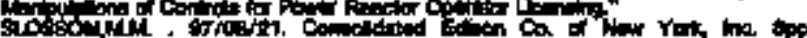

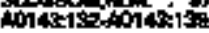

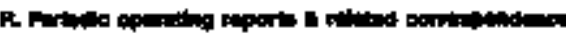

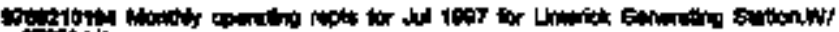

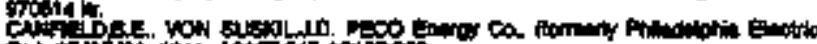
Ca)

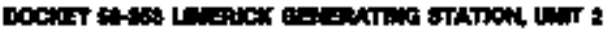

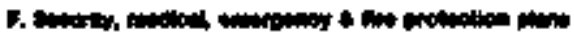

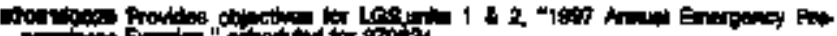

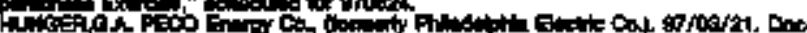

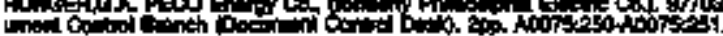

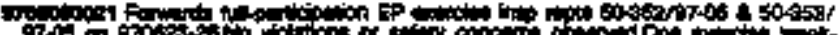

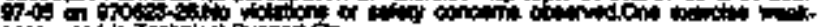

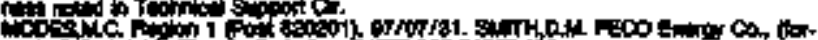

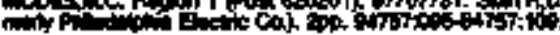

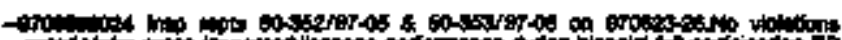

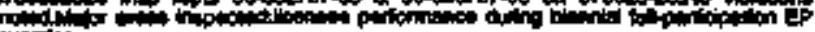

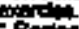

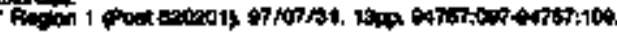

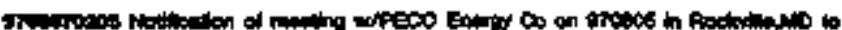

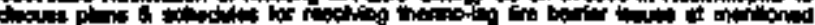

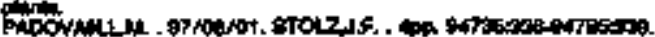

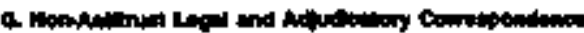

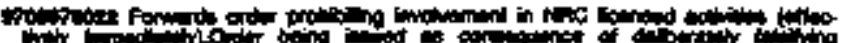

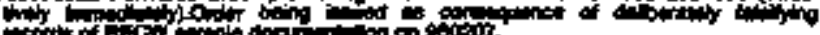

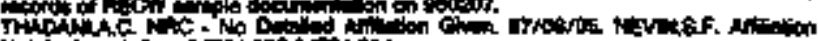

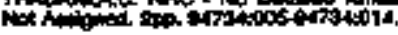

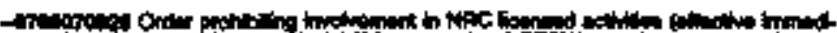

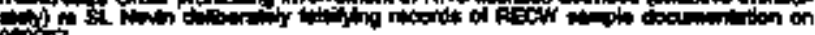
$\prod_{i \rightarrow 0}=0$

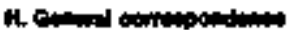

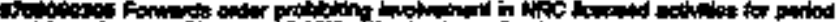

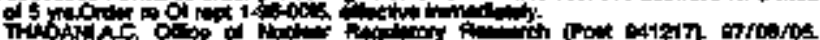

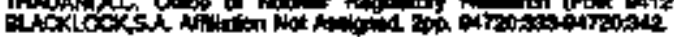

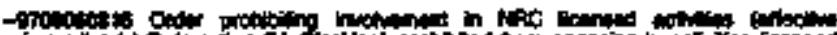

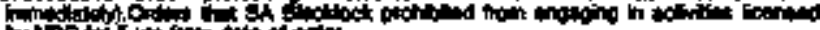

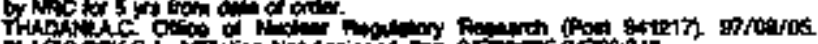

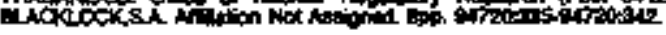

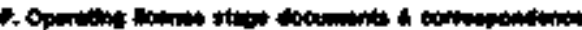

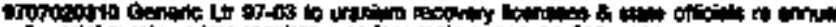

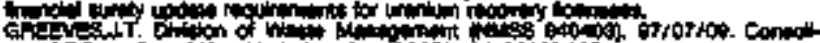

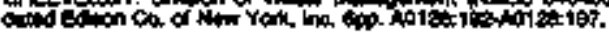

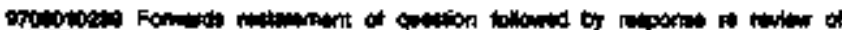

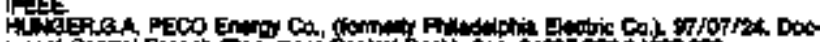

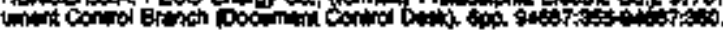

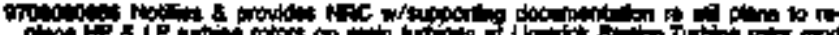

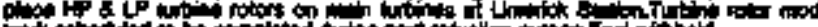

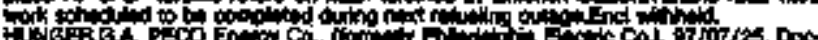

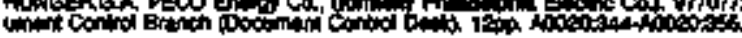

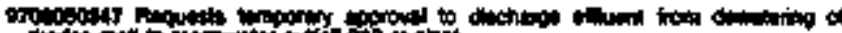

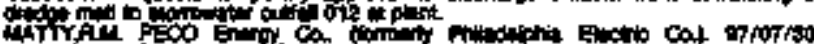

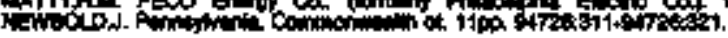

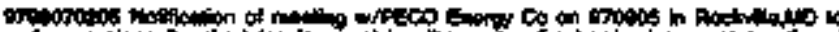

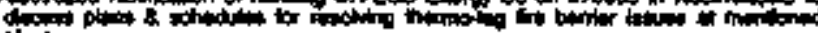

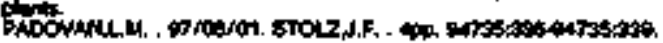

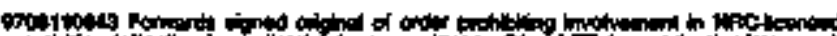
Sorop

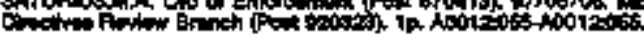

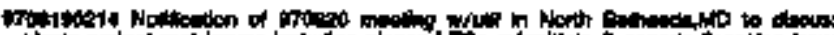

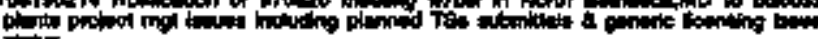

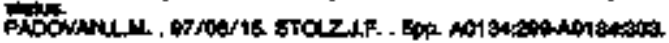

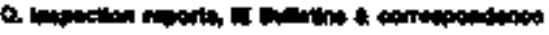

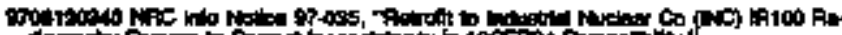

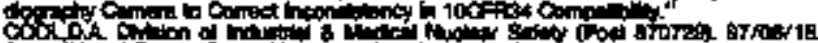

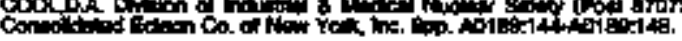

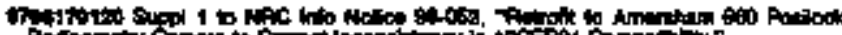

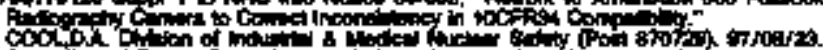

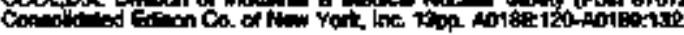

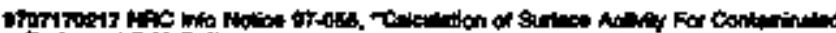

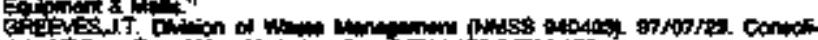

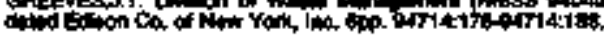

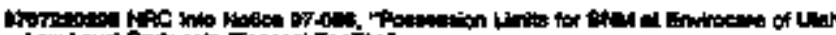
Low

PN

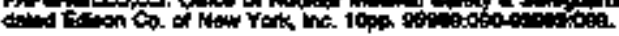

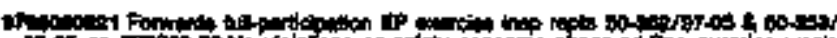

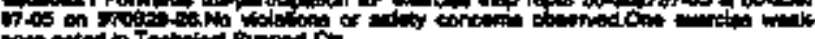

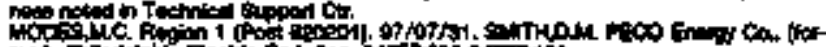

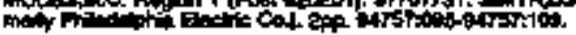

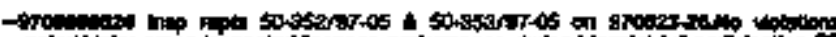

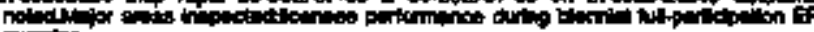

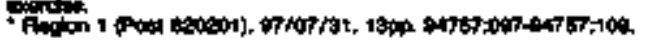

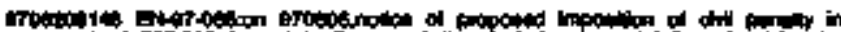

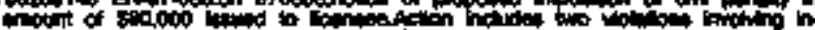

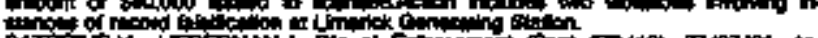

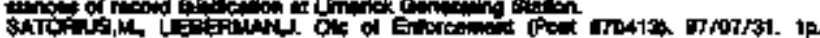
Anteintion

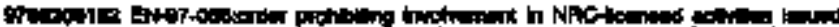

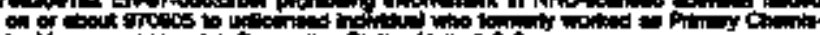

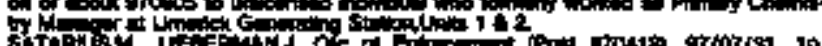
A0: 0 to 0 .

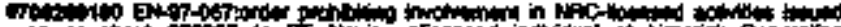

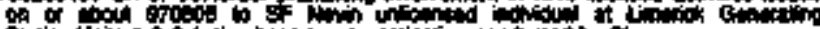

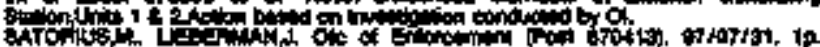

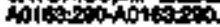

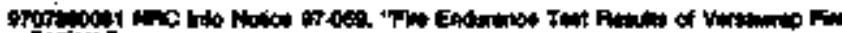

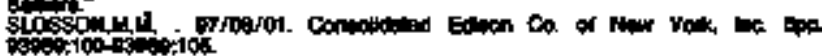

oros

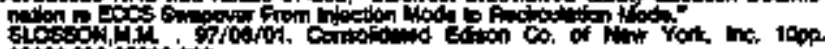
Hotosion

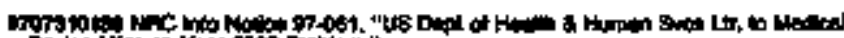

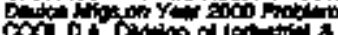

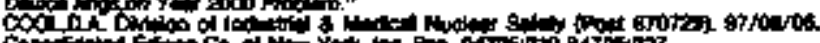

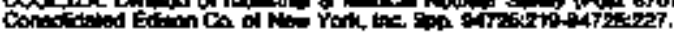




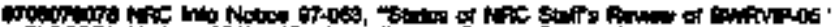

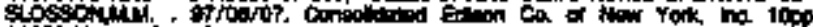
iosotimation?

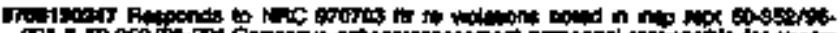

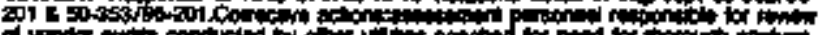

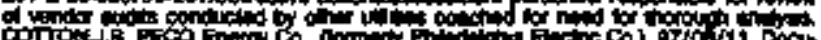

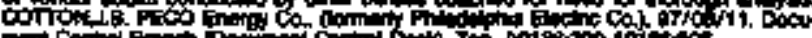

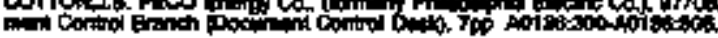

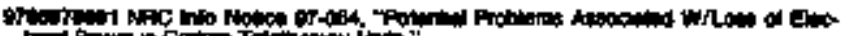

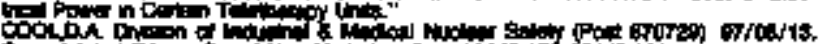

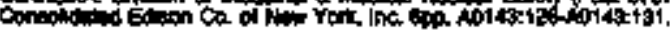

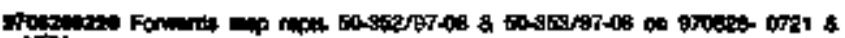

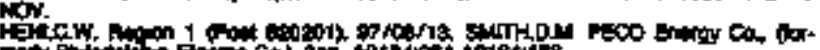

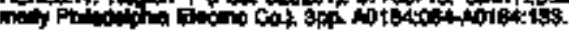

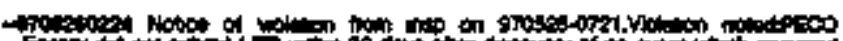

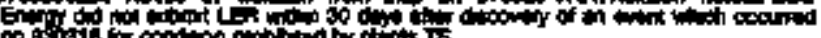

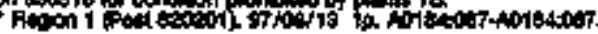

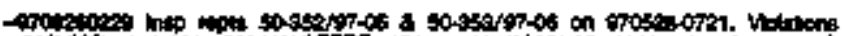

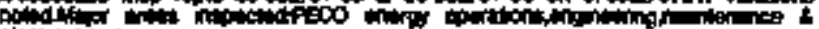

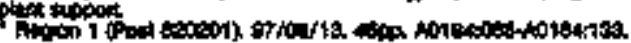

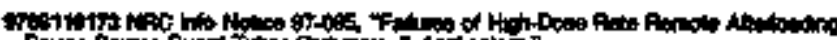

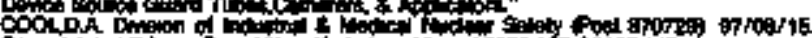

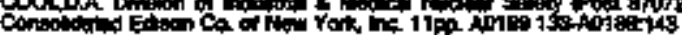

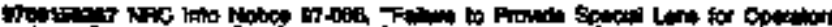

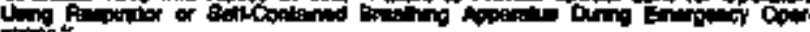

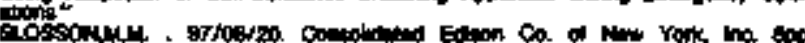

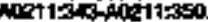

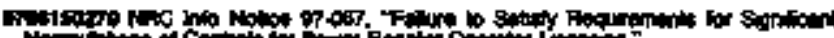

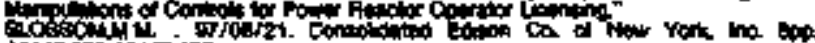

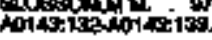

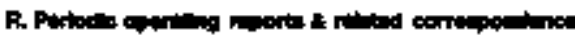

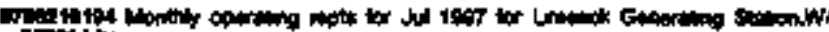
कinati

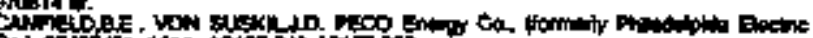

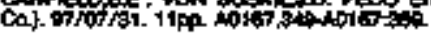

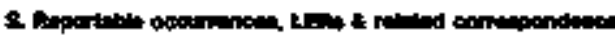

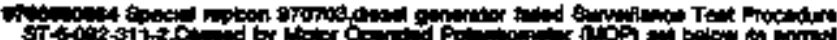

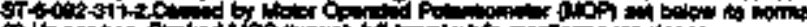

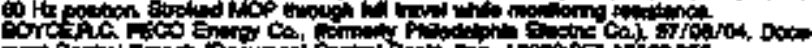

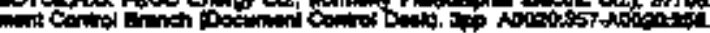

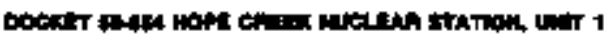

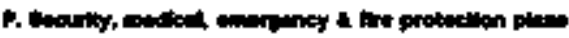

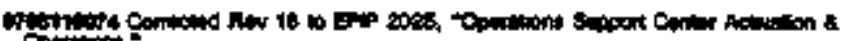

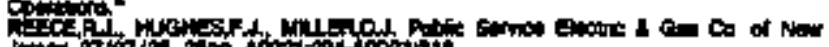

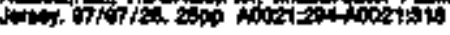

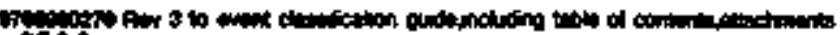

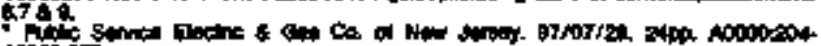
10000\%?

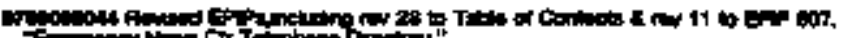

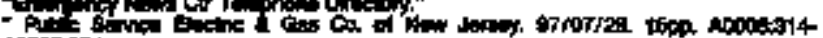
1000000

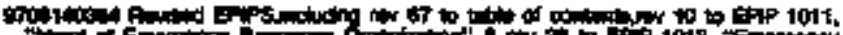

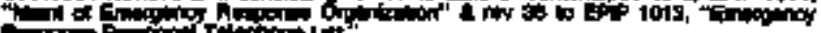

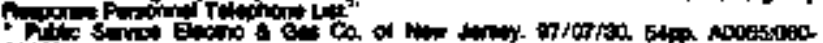
Nito $11 \%$.

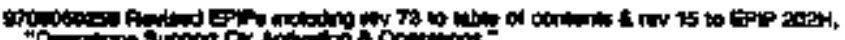

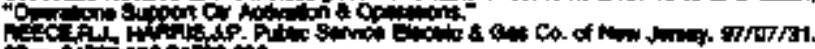

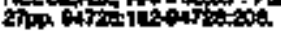

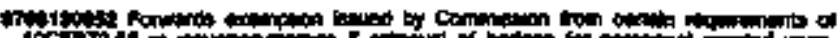

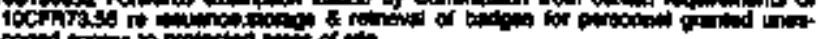

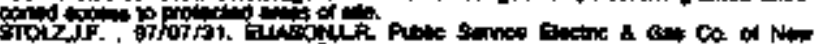

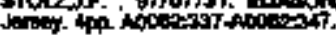

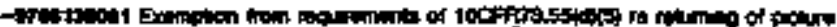

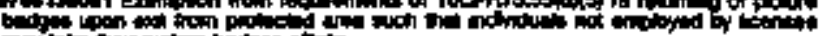

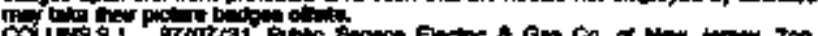

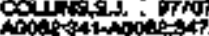

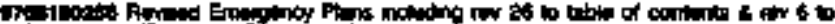

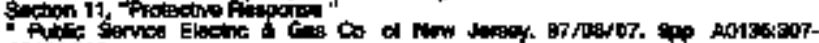
A013015.

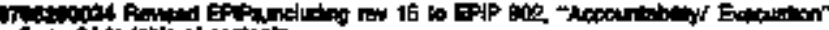

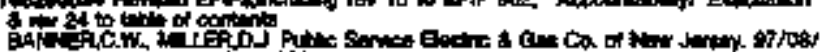

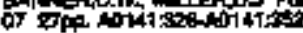

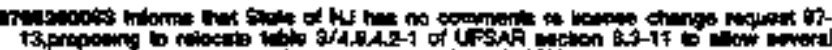

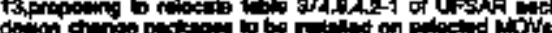

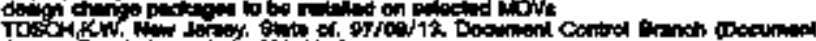

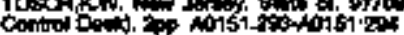

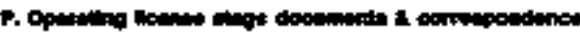

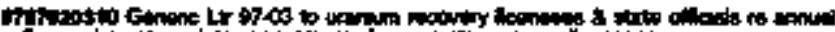

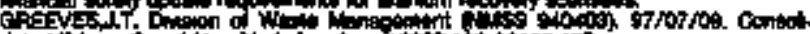

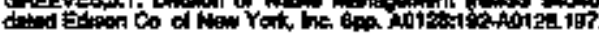

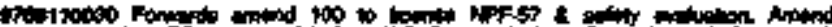

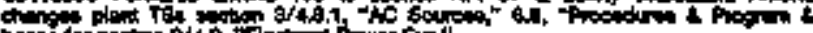
JAf tor

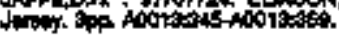

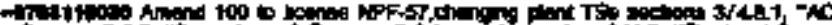

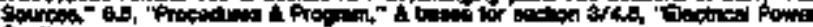

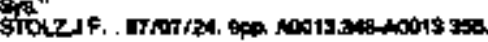

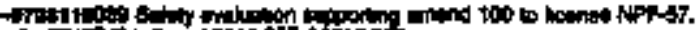

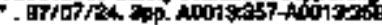

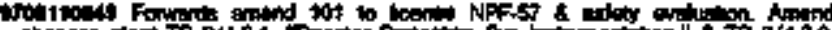

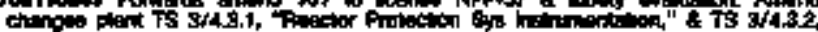

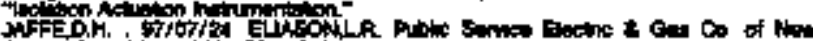

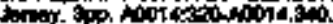

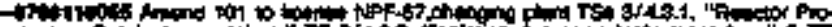

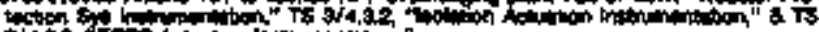

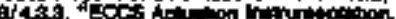

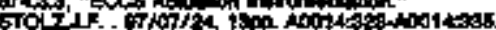

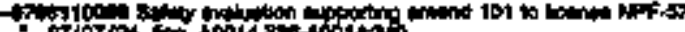

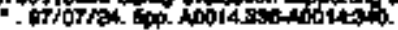

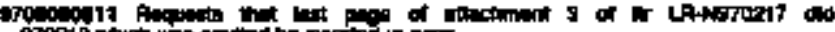

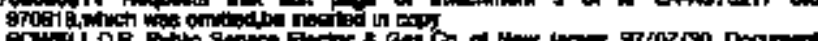

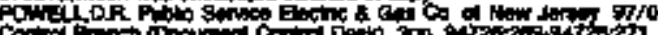

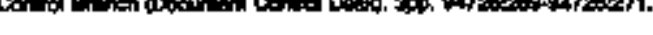

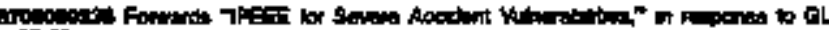

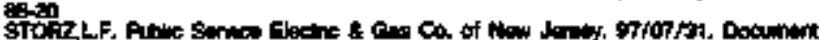

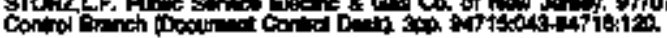

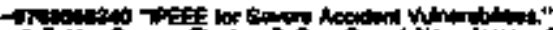

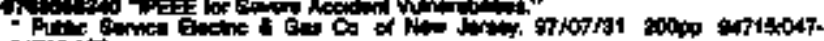
artexis.

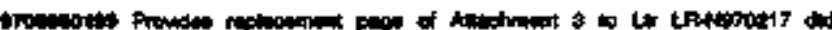

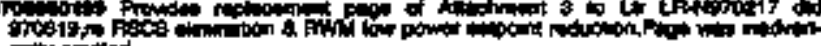

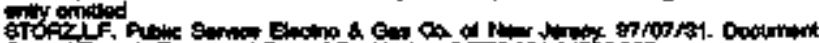

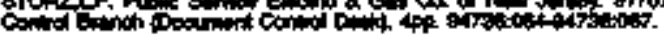

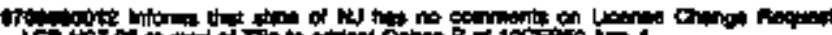
ICA Ho

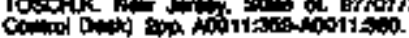

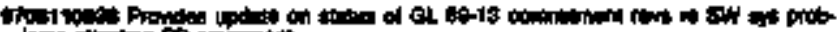

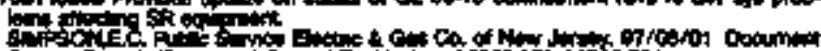

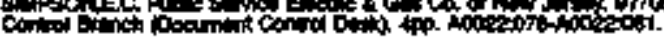

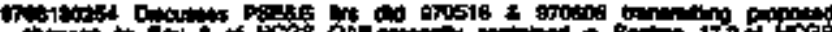

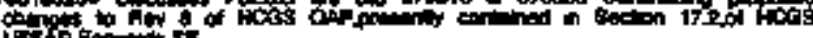

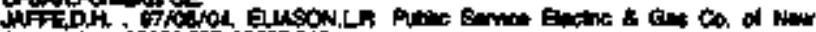

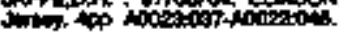

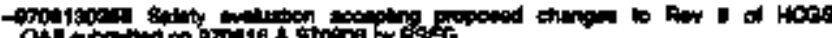

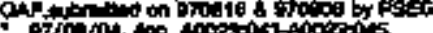

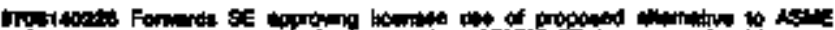

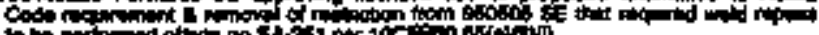

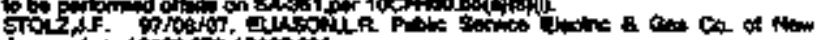

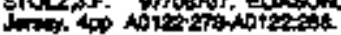

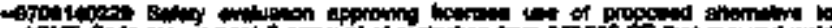

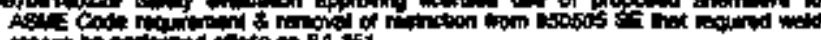

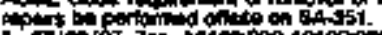




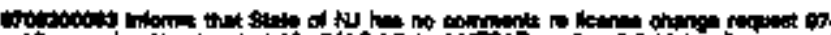

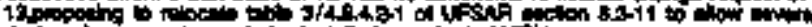

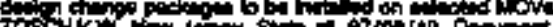

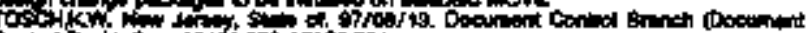

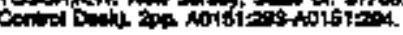

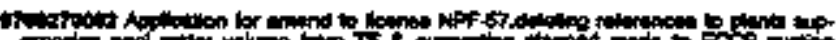

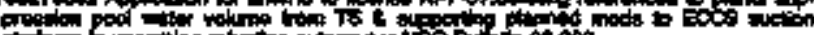

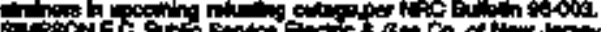

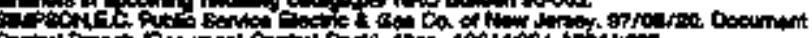

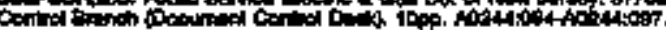

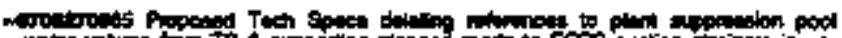

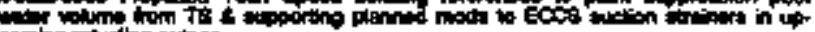

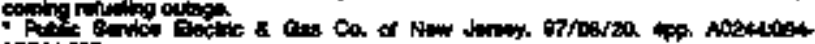
incoser.

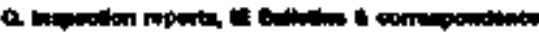

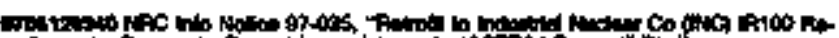

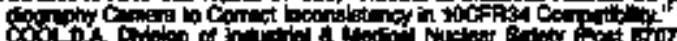

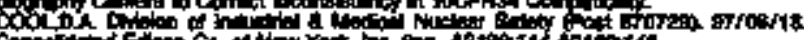

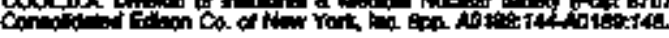

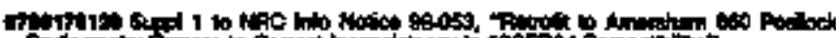

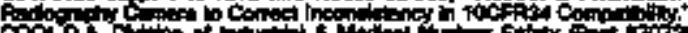

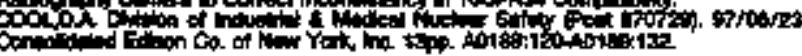

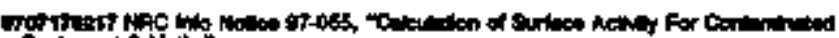

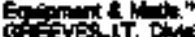

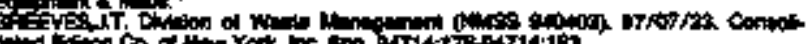

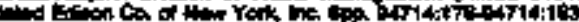

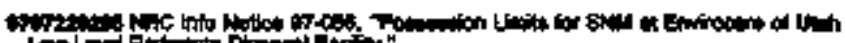

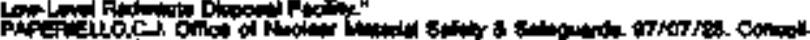

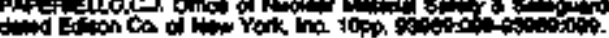

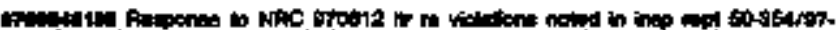

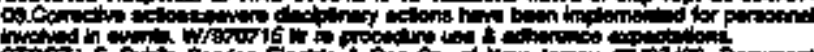

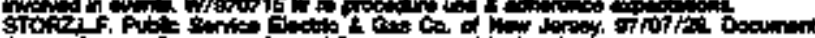

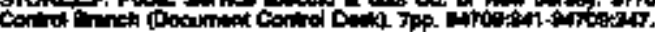

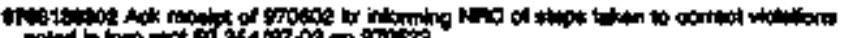

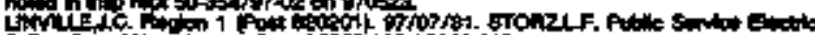

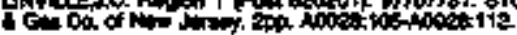

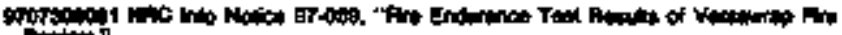

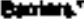

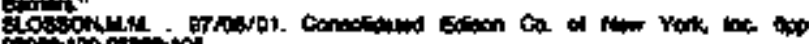

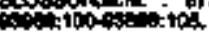

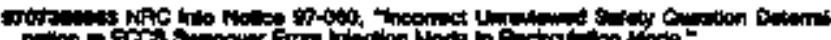

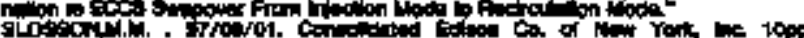

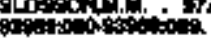

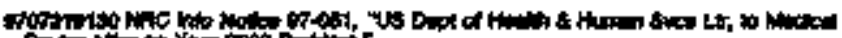

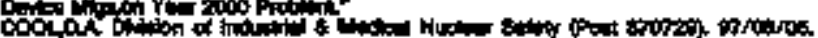

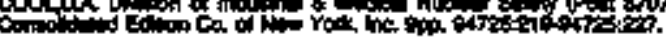

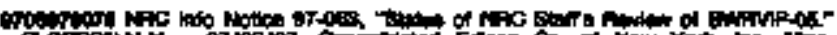

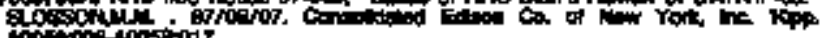

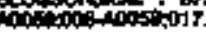

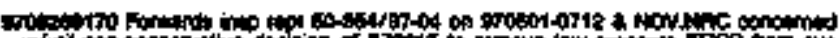

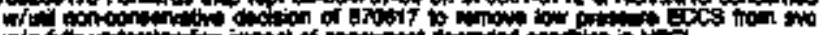

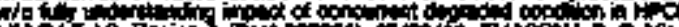
to

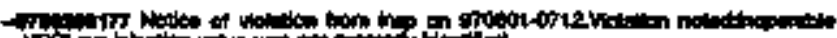

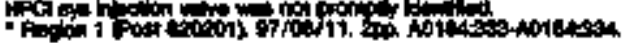

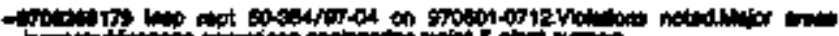

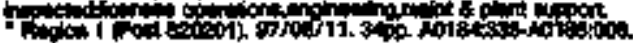

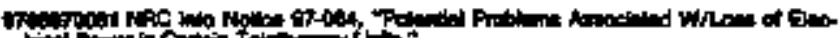

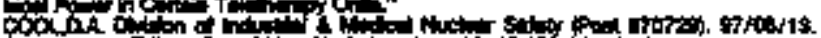

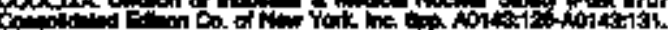

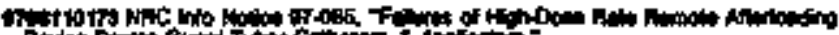

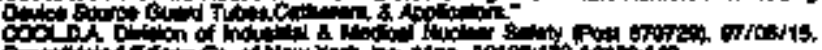

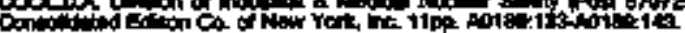

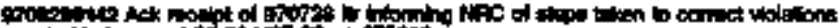

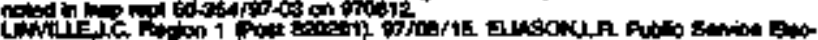

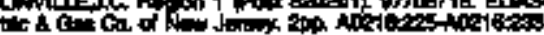

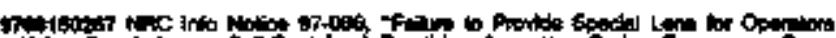

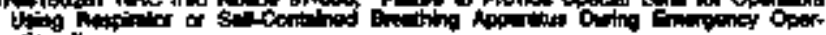

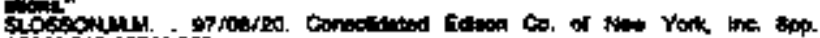

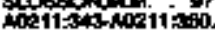

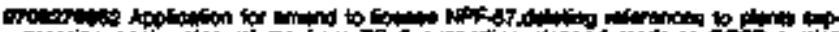

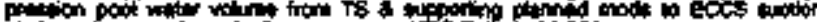

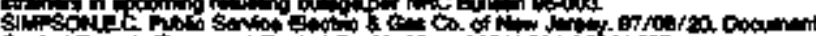

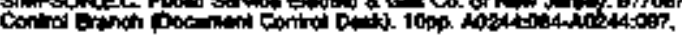

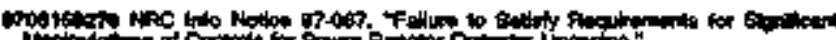

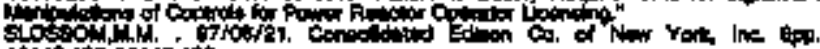

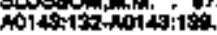

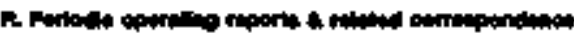

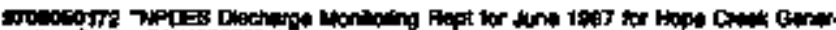

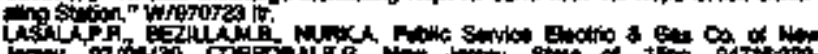

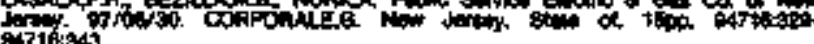

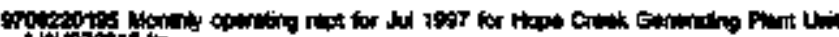
1,407015 it

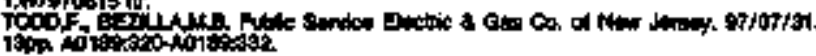

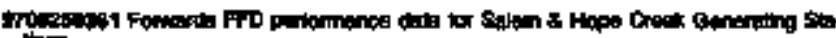

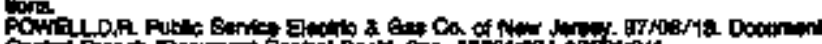

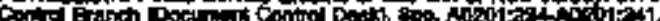

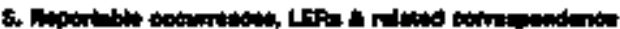

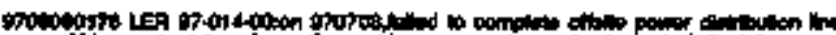

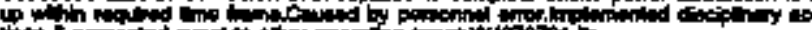

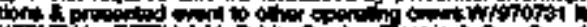
KFi

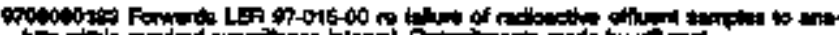

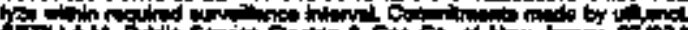

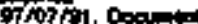

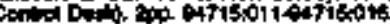

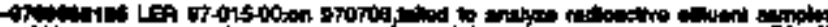

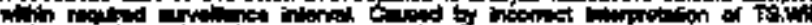
ring con

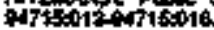

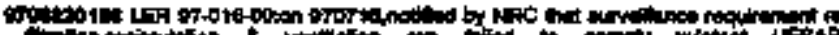

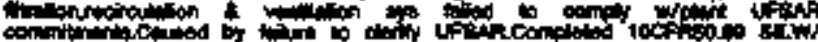

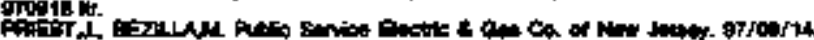

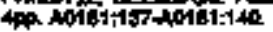

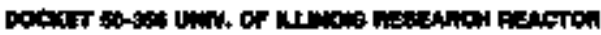

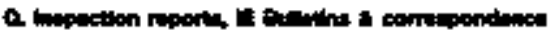

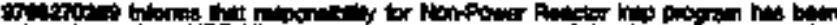

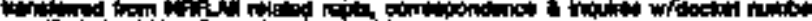

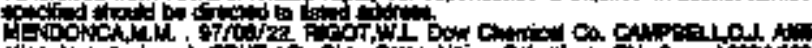

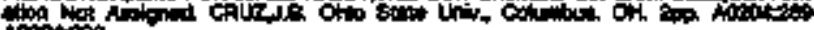
topetos

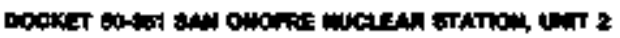

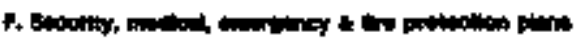

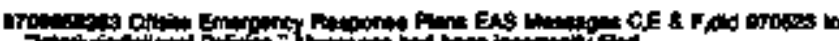

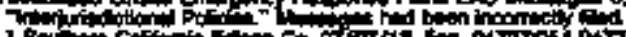

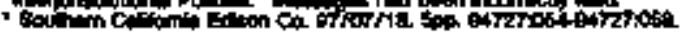

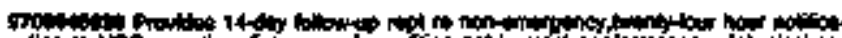

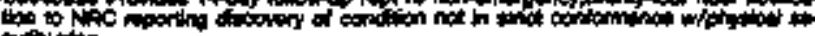
arit

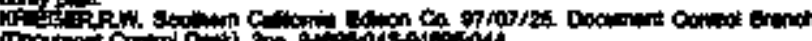

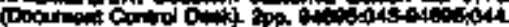

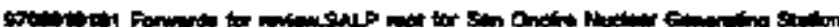

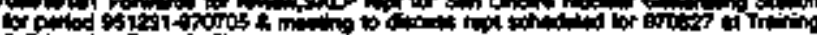

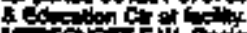

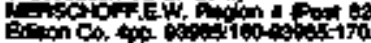

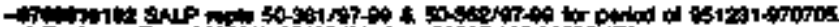

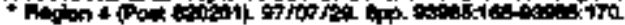

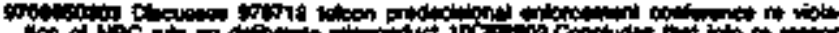

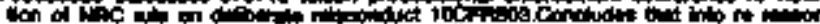

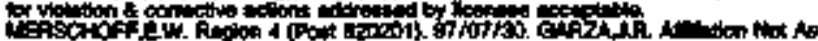
siped app of

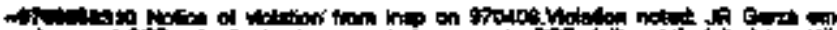

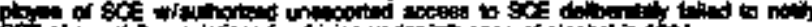

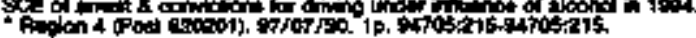




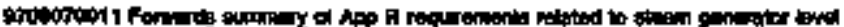

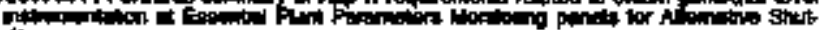

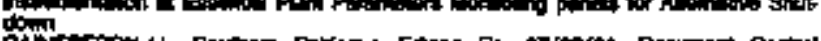

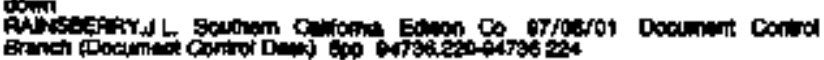

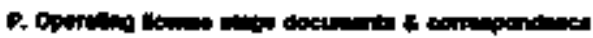

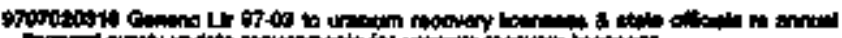

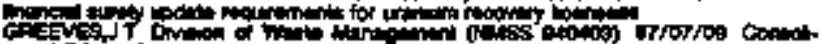

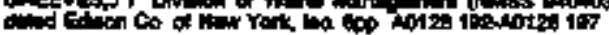

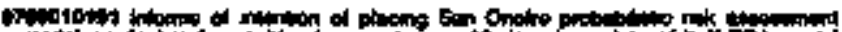

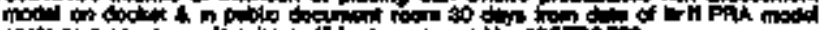

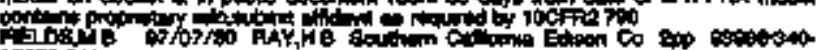
$\lim _{0.04}$

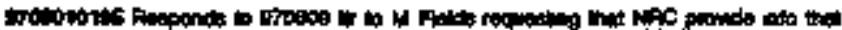

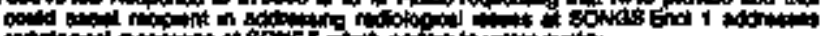

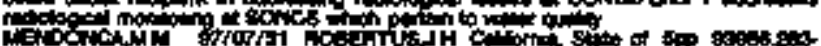
oneas 207

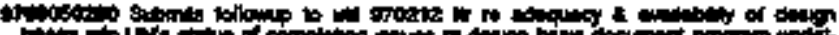

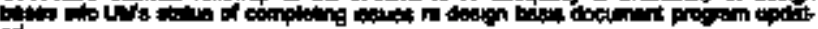

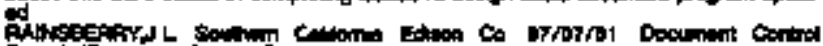

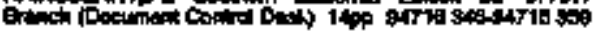

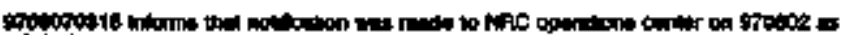

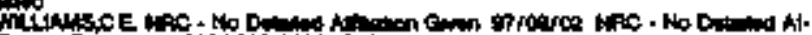

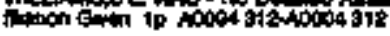

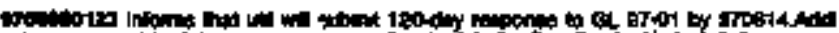
Ge ro PHN

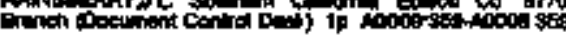

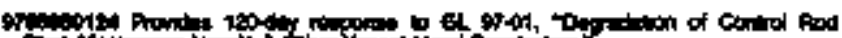

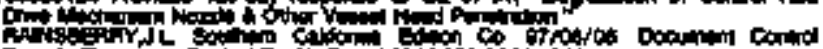

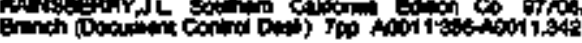

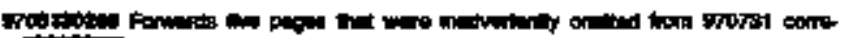

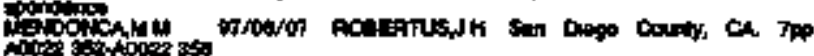

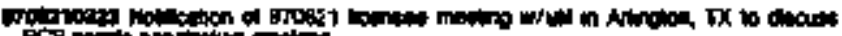

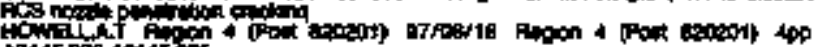

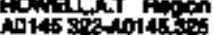

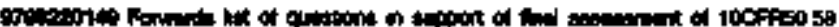

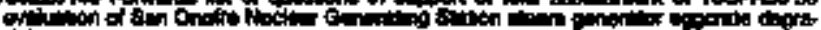
dection With of

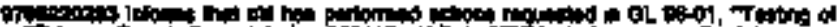

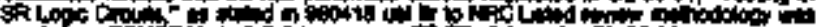
ve

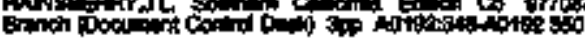

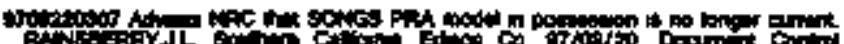

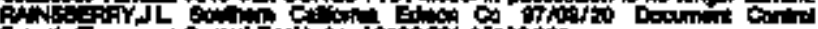

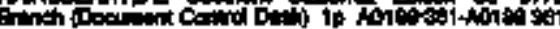

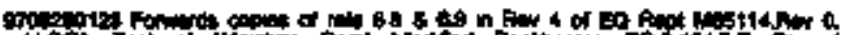

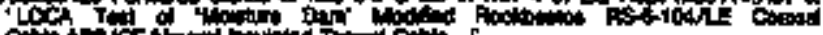

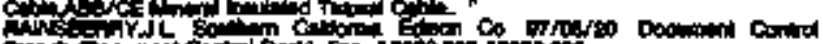

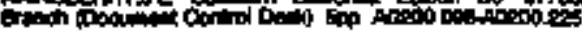

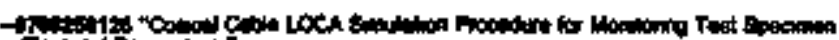

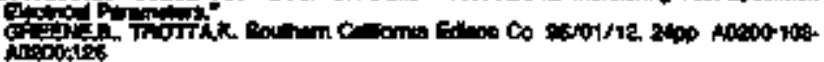

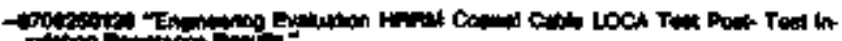

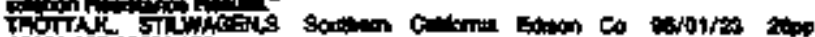
19012

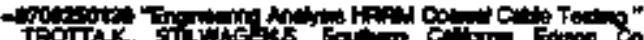

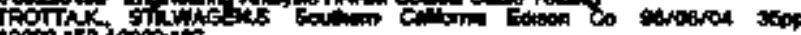
is 10.0010

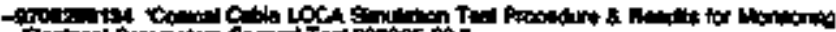

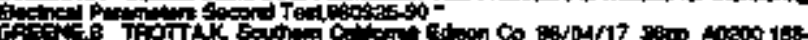
Chentions

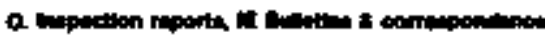

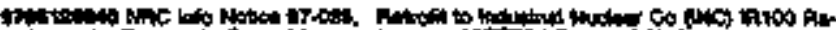

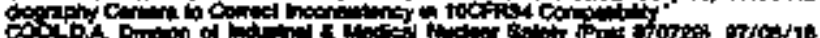

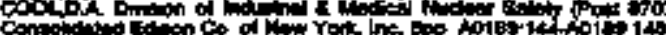

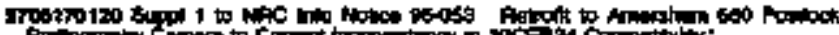

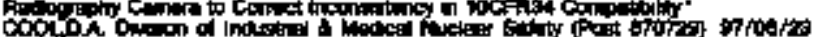

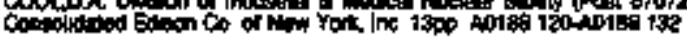

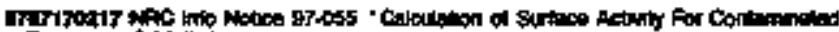

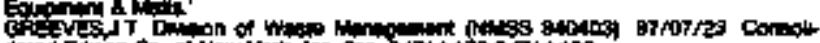

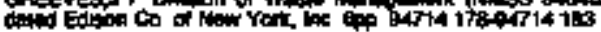

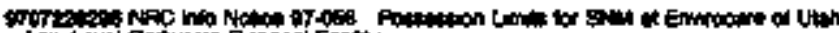

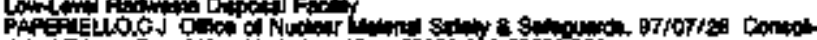

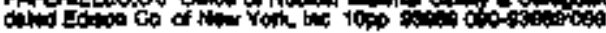

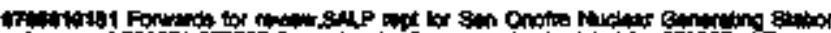

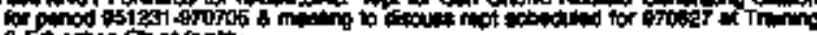

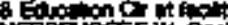

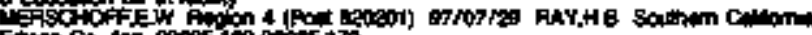

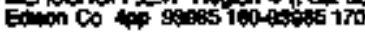

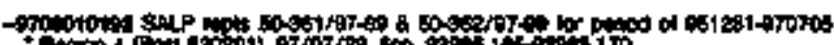

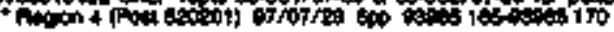

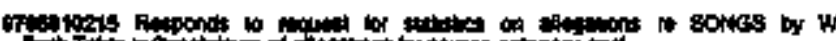

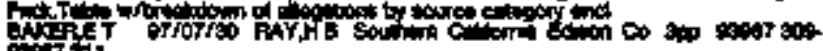
C.7 31

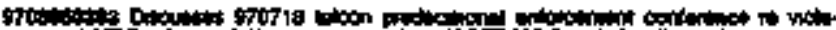

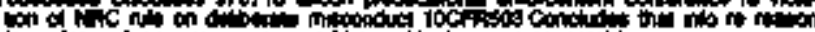

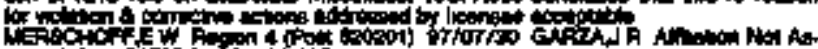

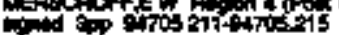

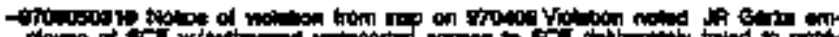

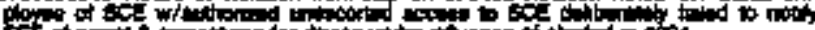
C.

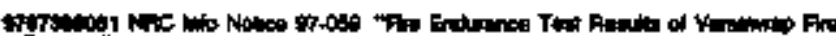

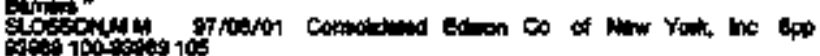

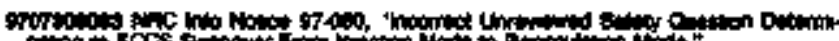

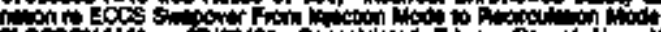

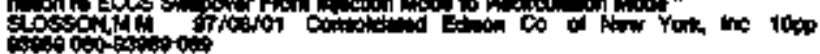

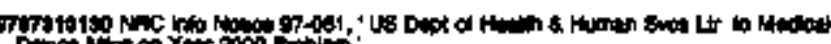

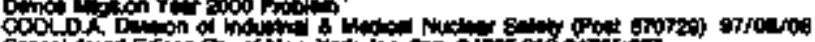

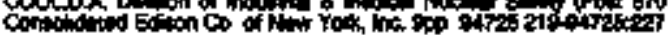

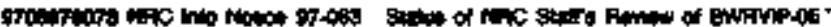

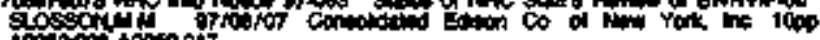

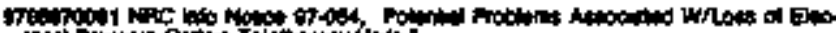

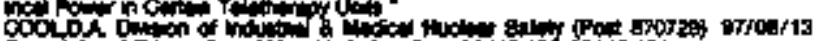

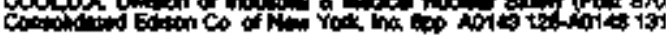

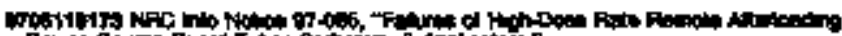

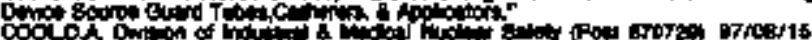

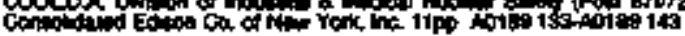

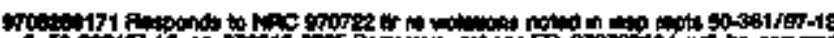

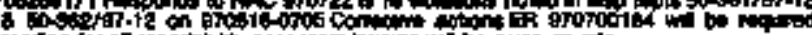

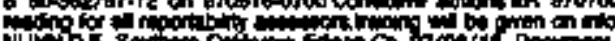

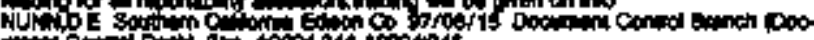

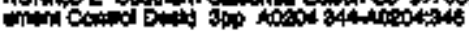

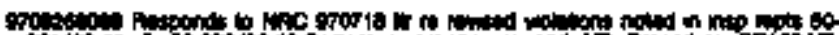

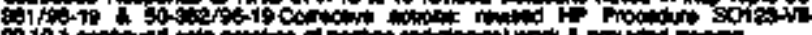

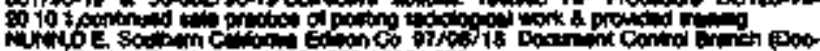
intul

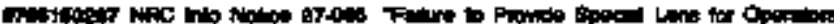

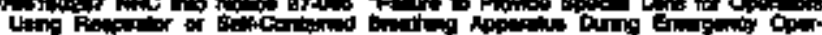
cong of

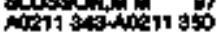

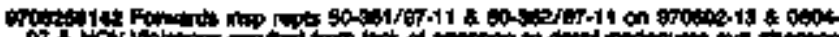

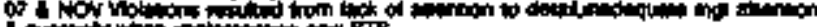

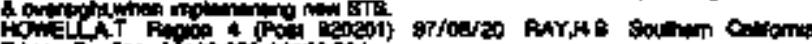

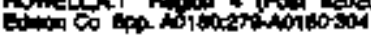

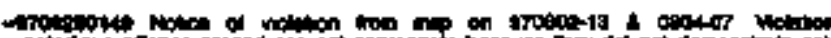

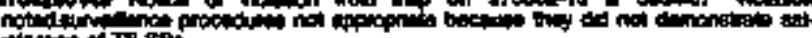

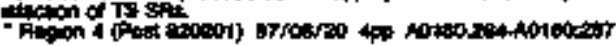

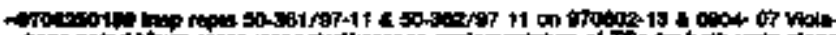

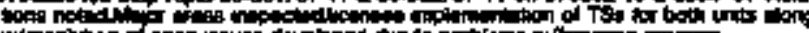

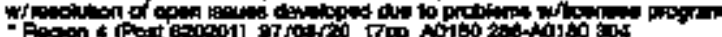

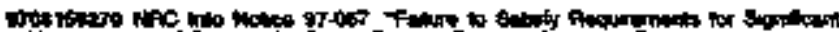

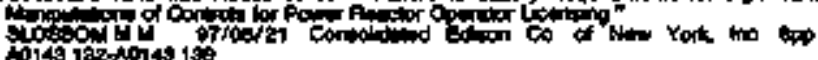




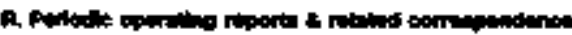

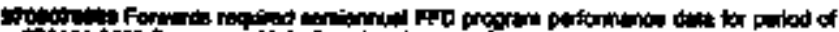

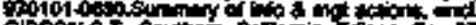

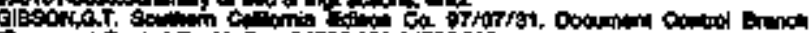

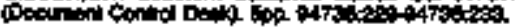

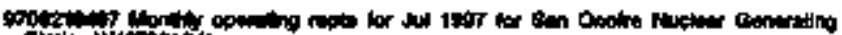

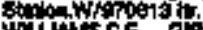

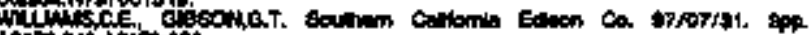

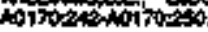

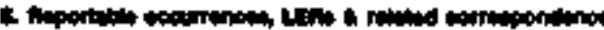

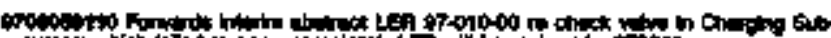

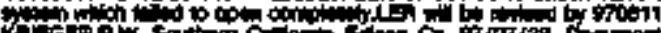

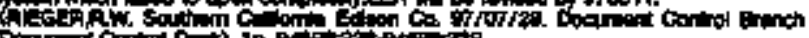

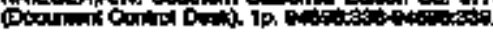

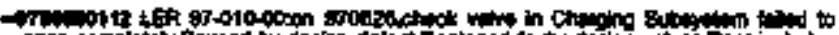

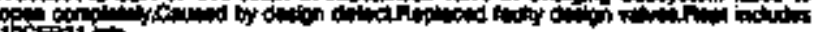

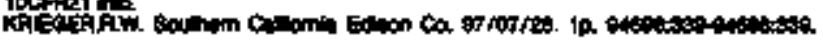

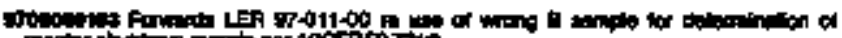

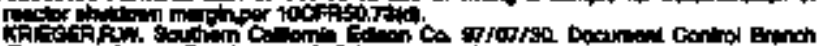

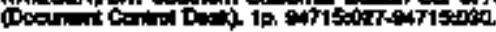

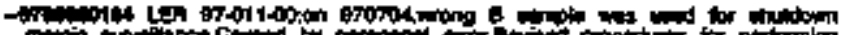

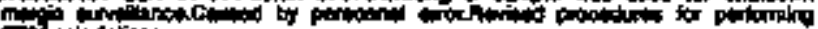

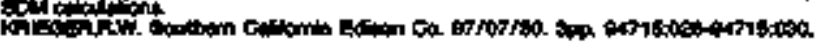

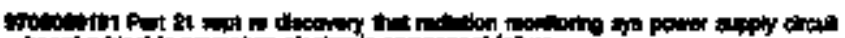

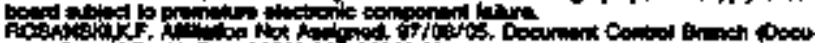

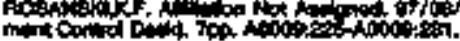

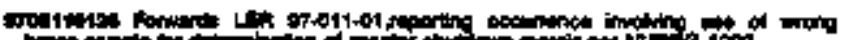

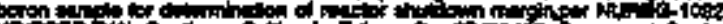

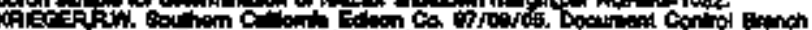

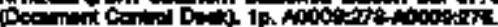

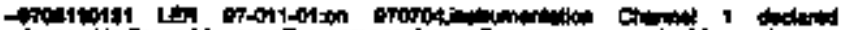

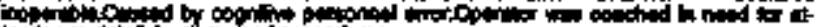

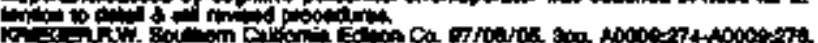

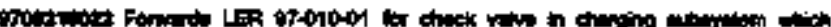

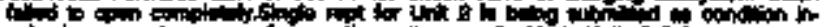

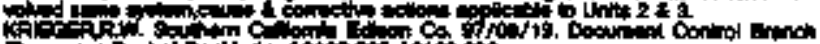

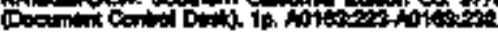

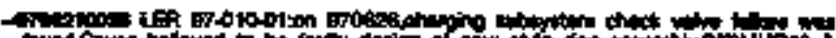

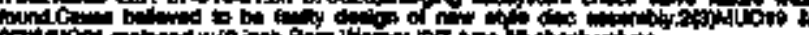

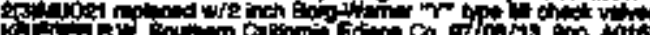

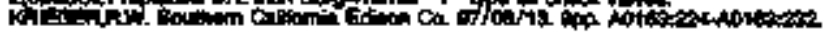

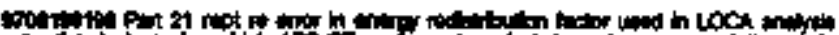

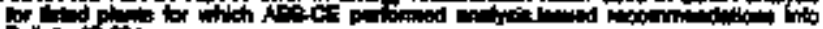

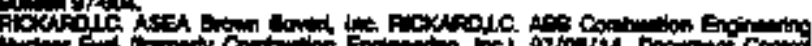

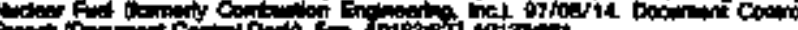

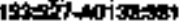

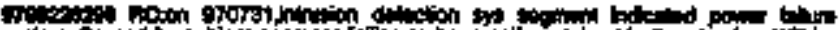

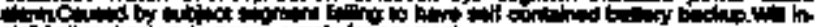

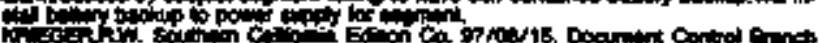

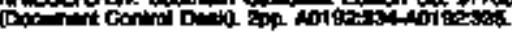

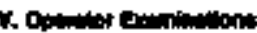

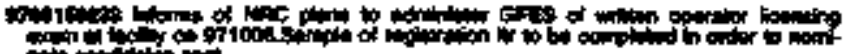

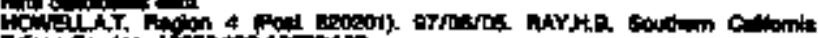

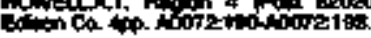

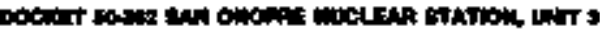

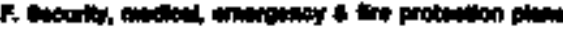

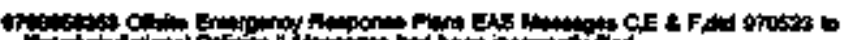

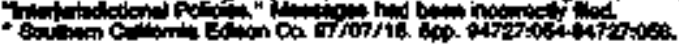

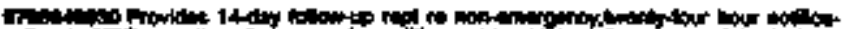

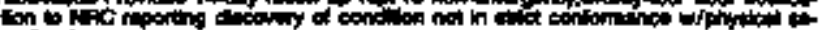

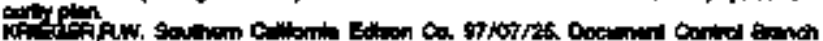

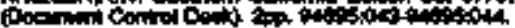

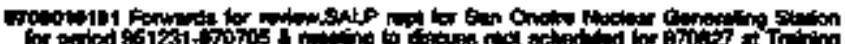

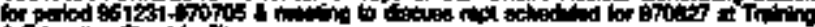

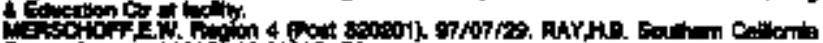

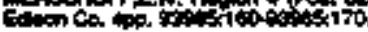

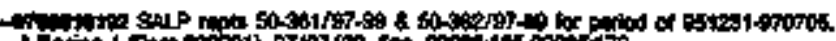

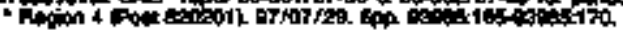

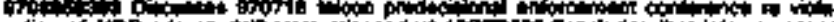

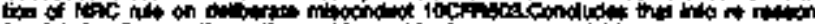

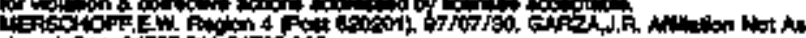

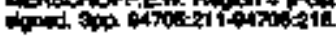

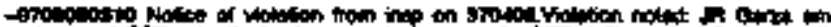

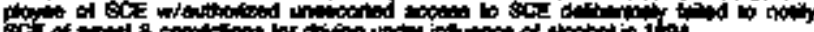

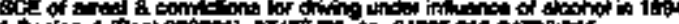

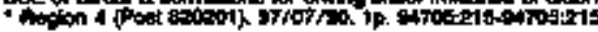

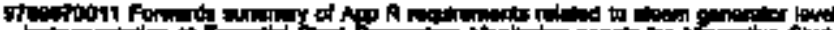

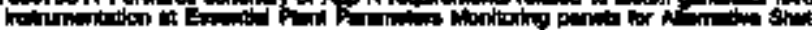

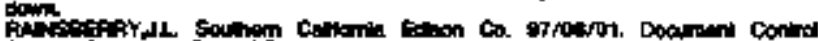

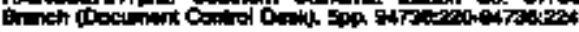

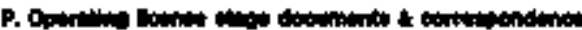

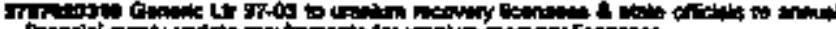

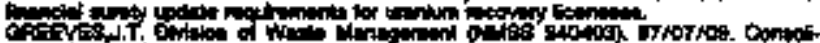

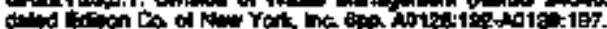

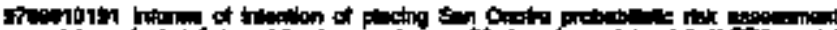

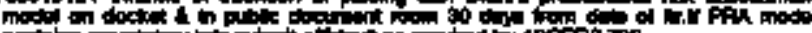

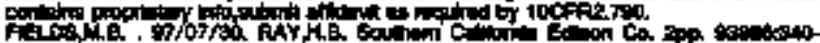
Pionitist.

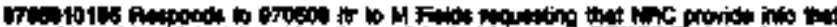

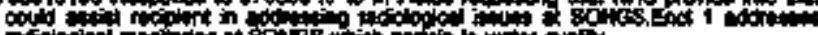

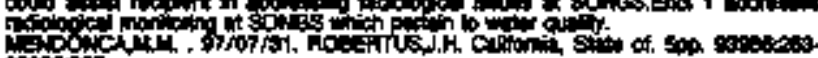
sing

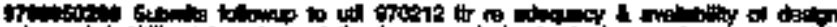

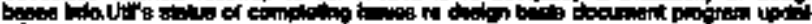

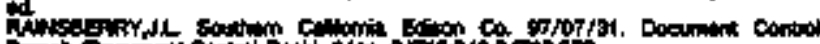

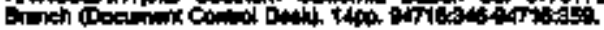

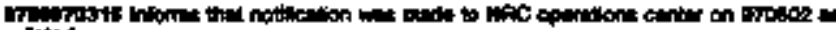

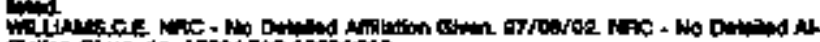

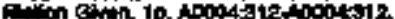

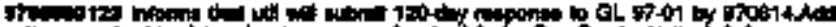
pit

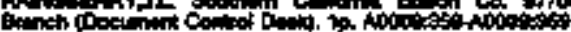

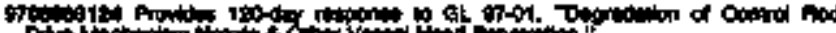

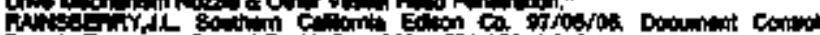

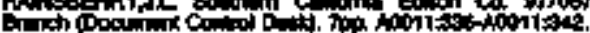

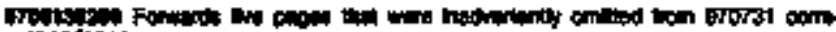

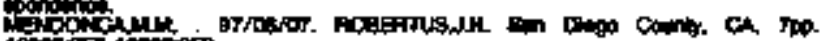

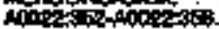

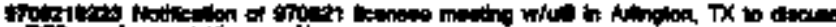

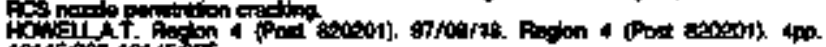
of 4 sin

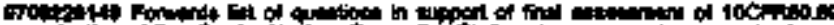

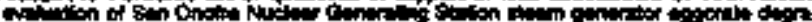

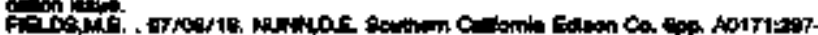

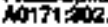

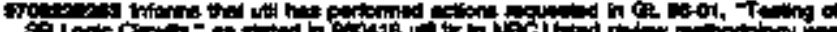

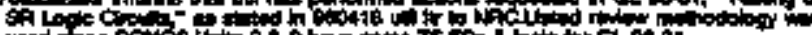

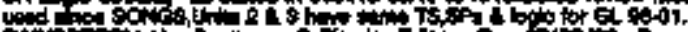

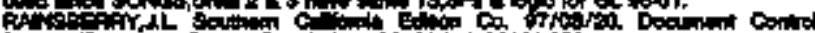

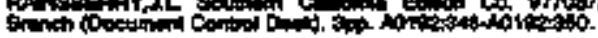

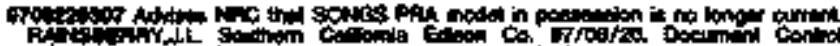

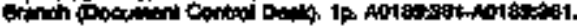

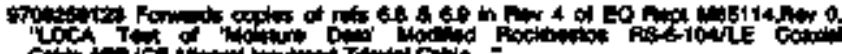

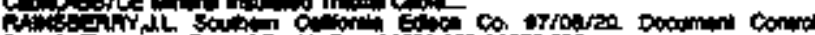

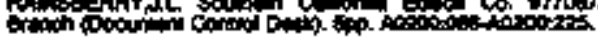

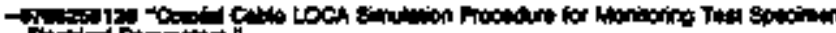

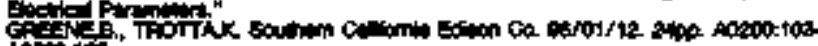
4000,

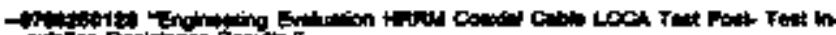

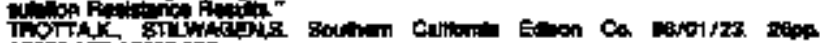

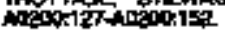

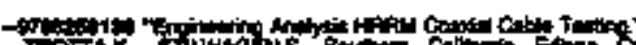

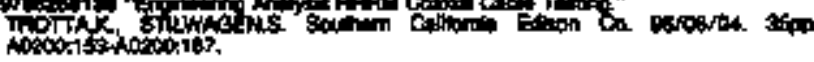




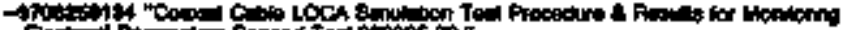

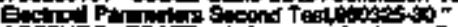
G atoos:

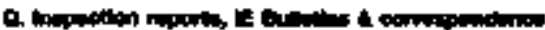

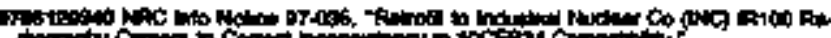

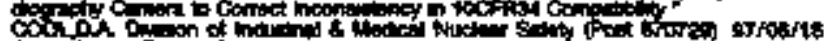

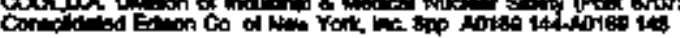

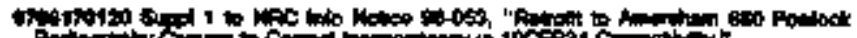

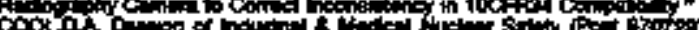

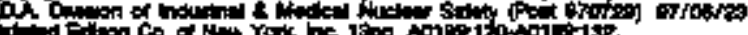

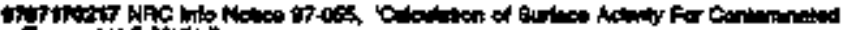

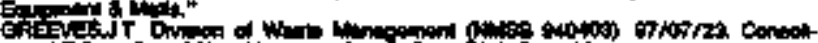

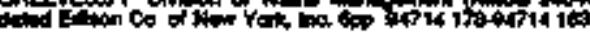

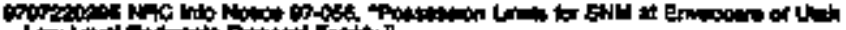

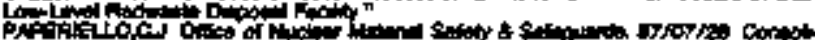

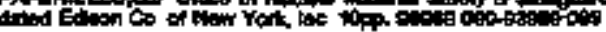

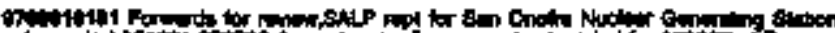

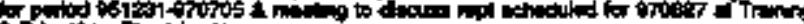

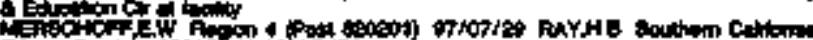

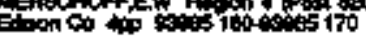

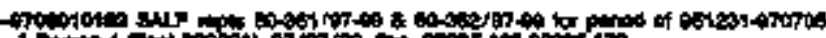

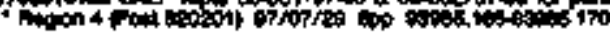

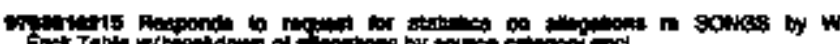

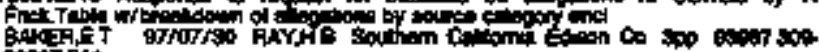
atesit

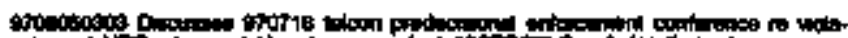

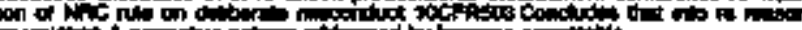
势

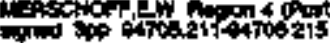

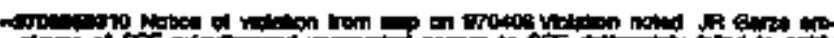

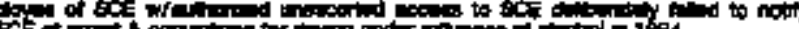

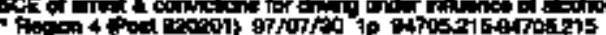

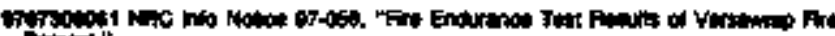

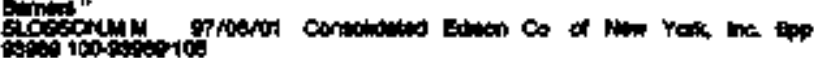

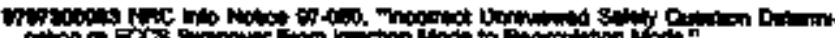

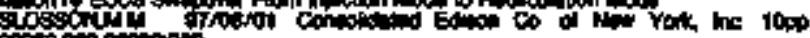

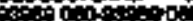

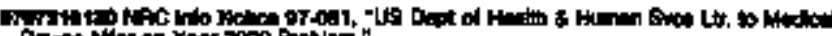

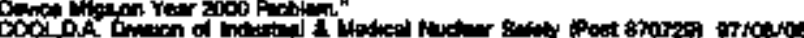

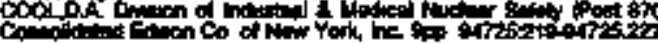

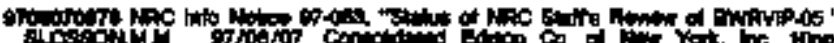

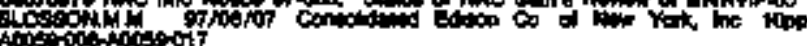

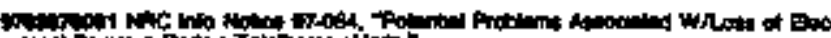

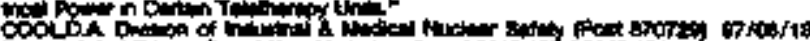

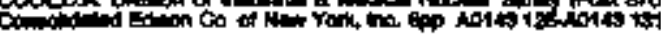

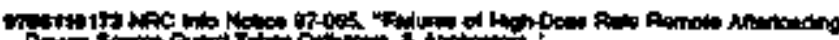

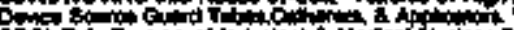

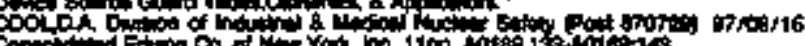

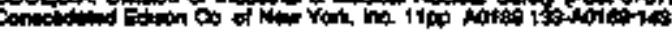

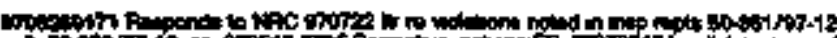
4 S0

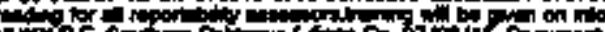

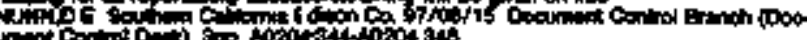

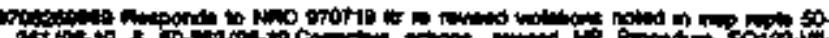

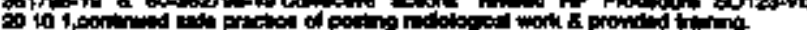

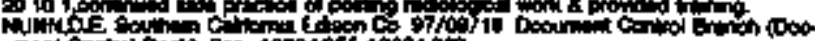

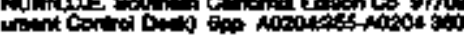

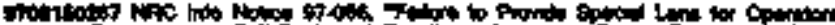

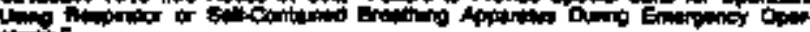

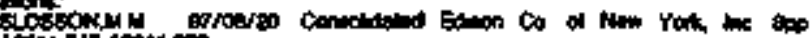

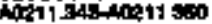

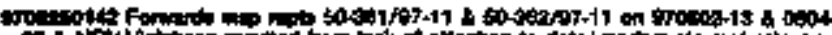

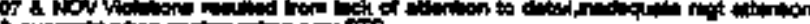

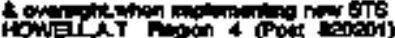

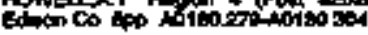

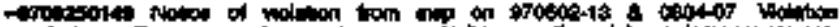

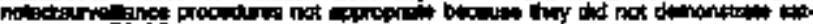
gtacion of Ts stit

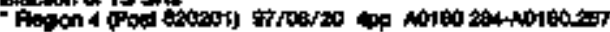

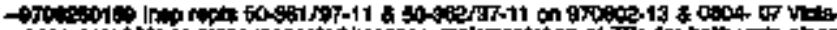

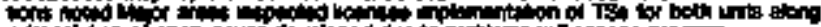

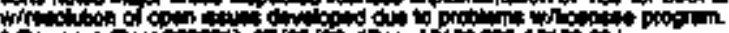

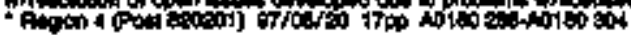

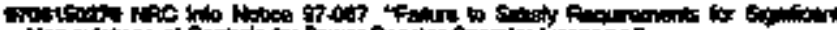

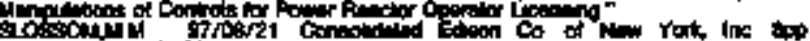

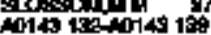

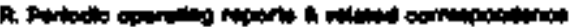

in

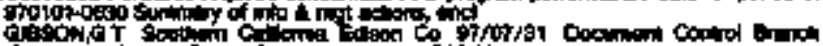

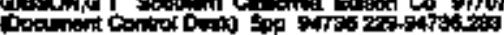

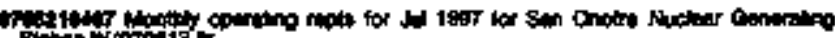

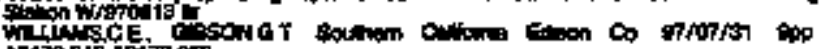
10130240170

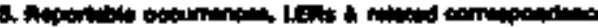

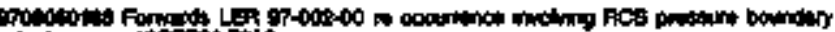

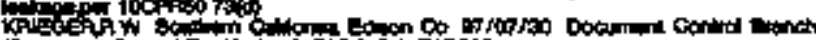

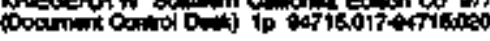

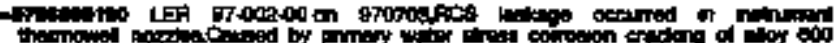

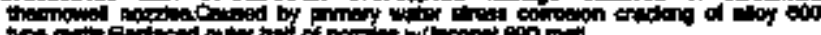
tof

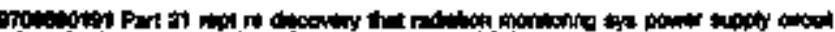

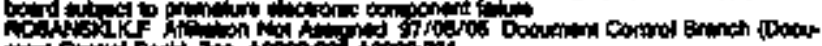

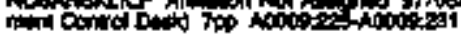

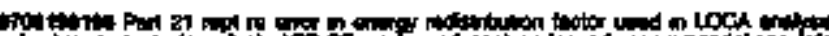

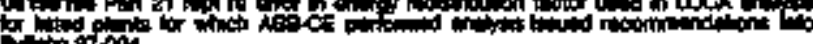
(n)

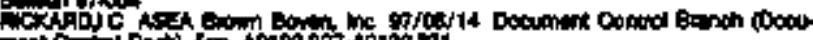

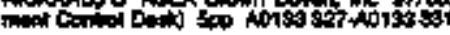

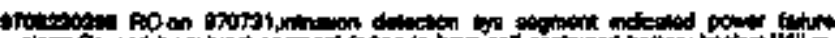

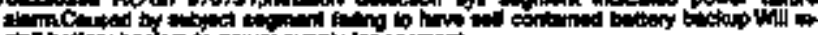

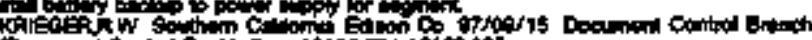

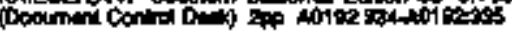

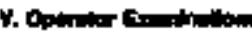

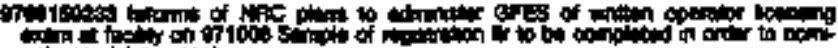

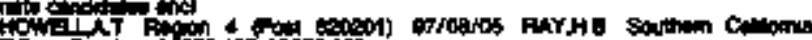

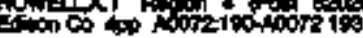

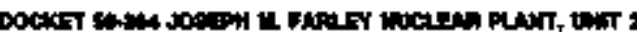

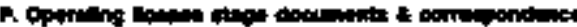

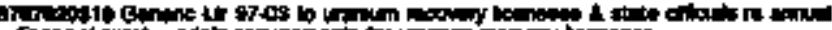

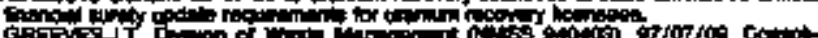

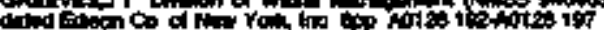

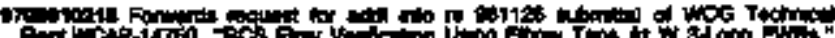

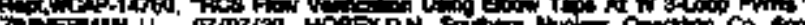

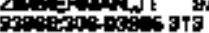

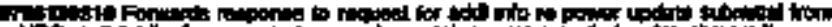

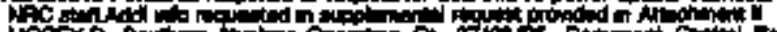

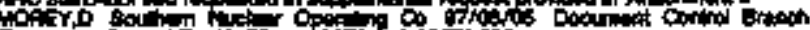

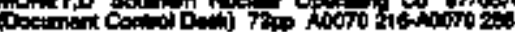

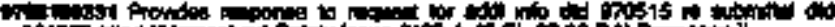

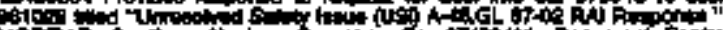

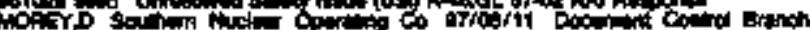

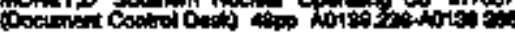

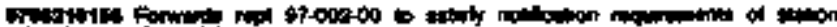

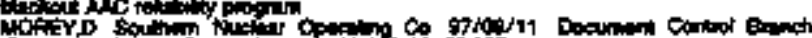

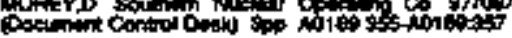

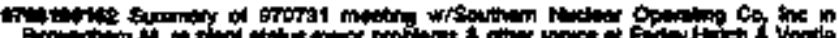

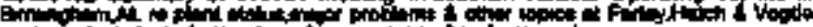

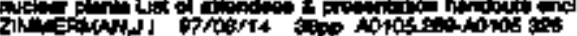




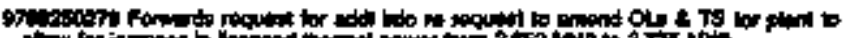

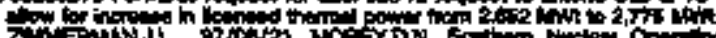

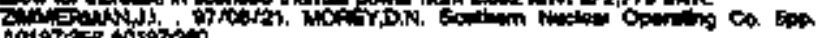

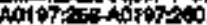

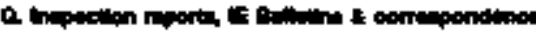

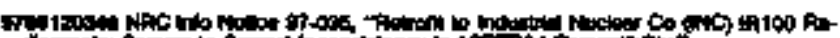

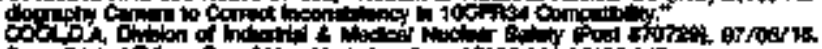

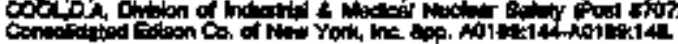

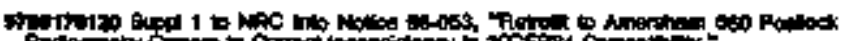

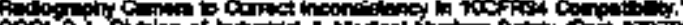

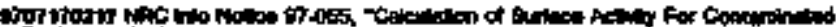

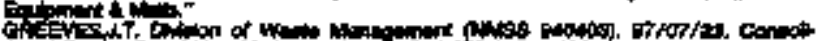

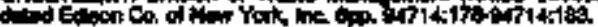

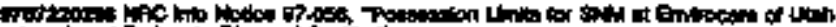

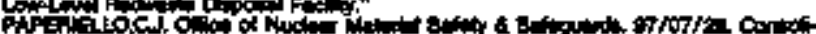

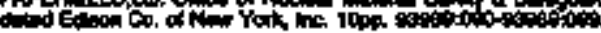

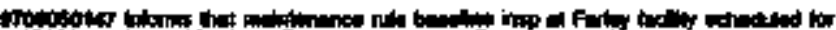

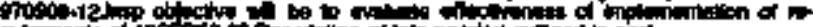

How

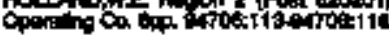

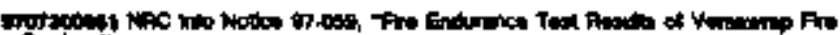

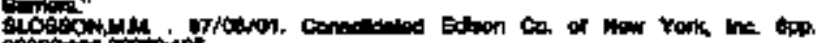

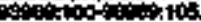

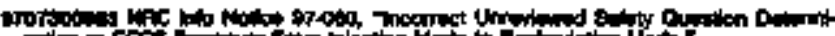

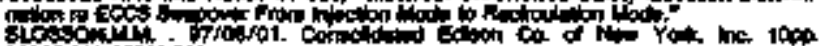

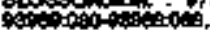

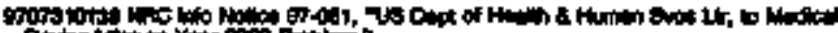

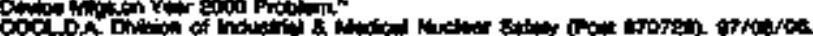

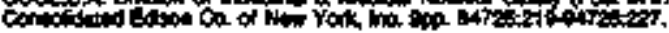

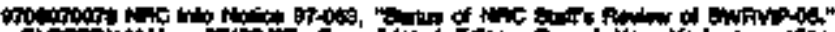

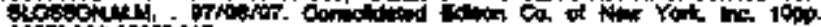
10000000017.

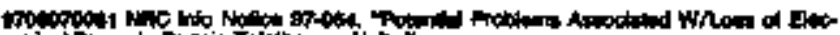

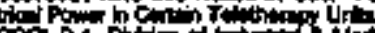

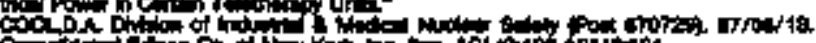

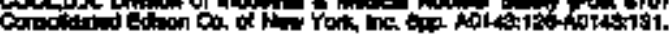

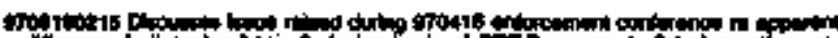

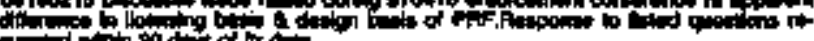
Tenn Nointo th

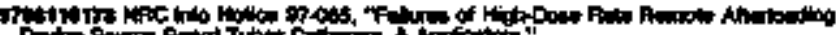

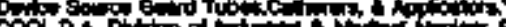

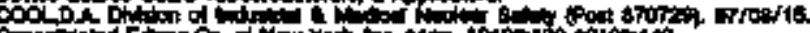

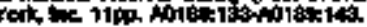

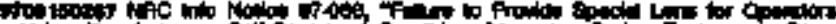

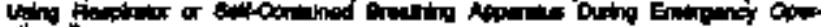

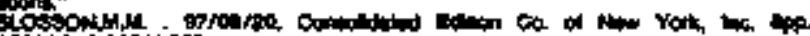

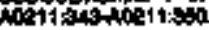

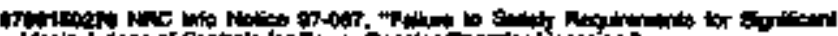

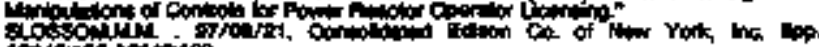

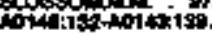

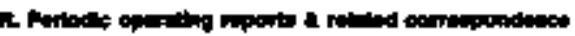

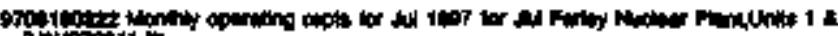

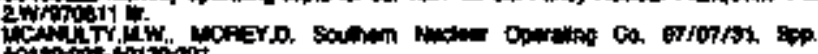

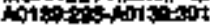

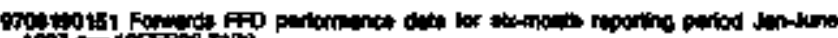

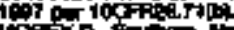

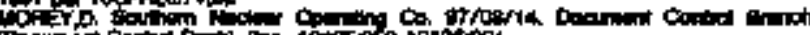

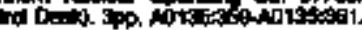

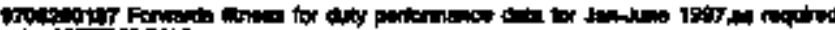
106izazilo

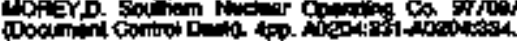

V. Oporider ti. mbloint

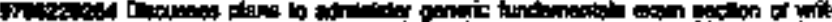

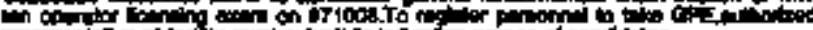

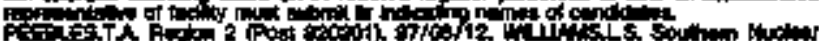

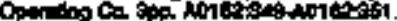

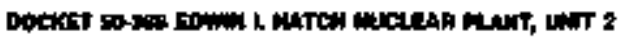

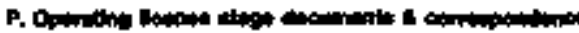

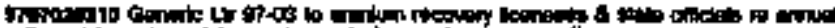

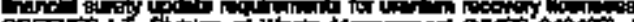

c:Fezy

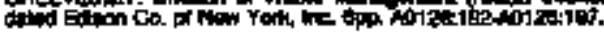

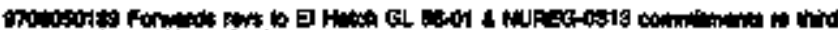

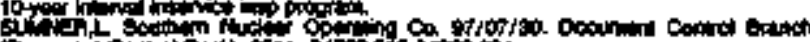

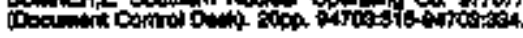

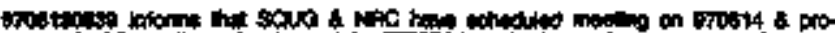

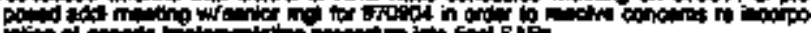

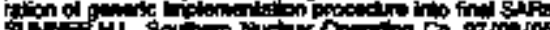

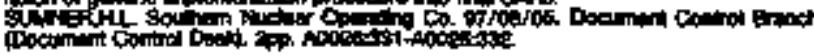

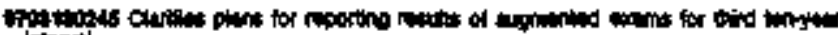

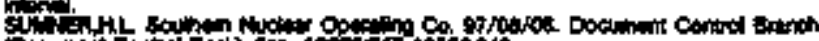

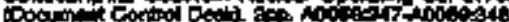

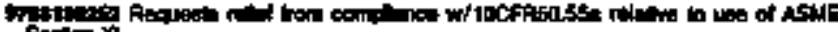
section $x$

IDech

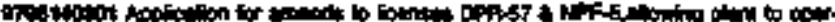

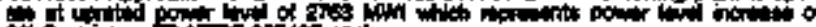

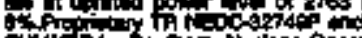

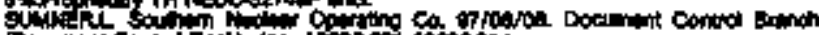

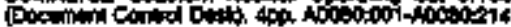

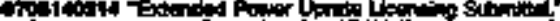

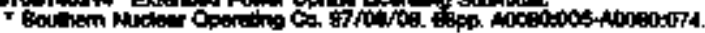

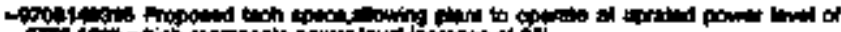

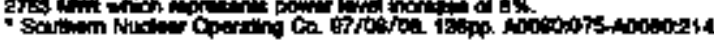

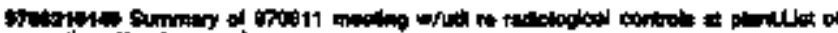

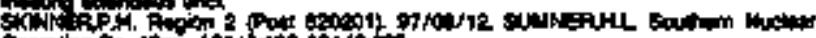

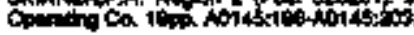

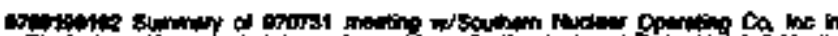

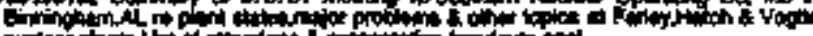

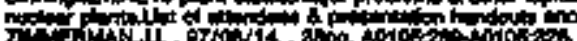

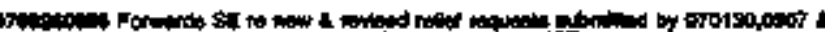

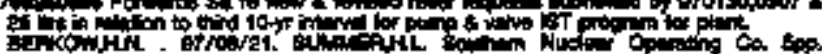

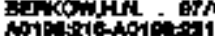

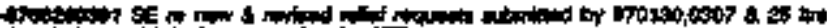

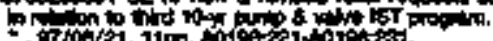

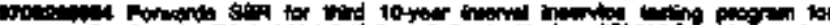

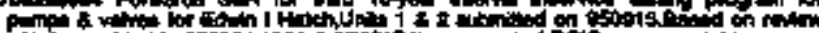

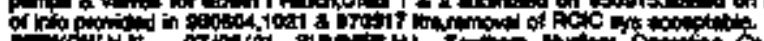

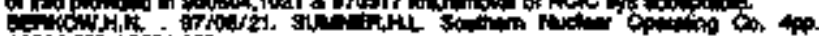

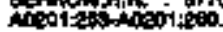

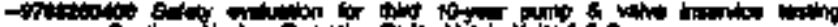

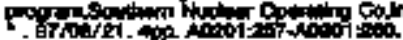

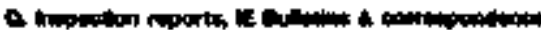

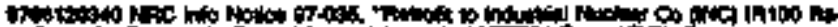

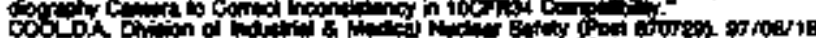
Contos

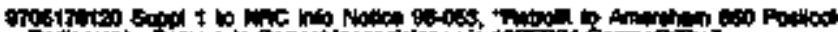

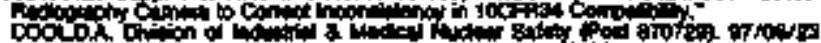

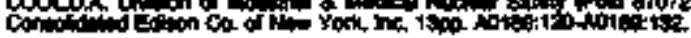

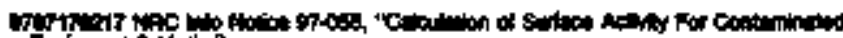
Eq

G

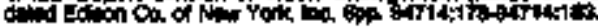

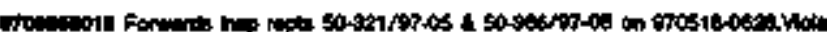

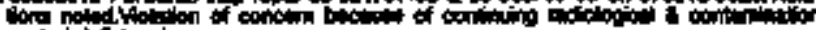

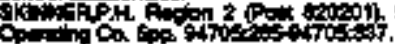

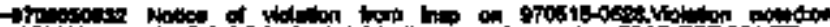

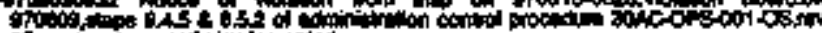

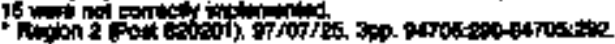

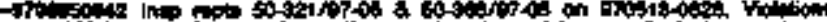

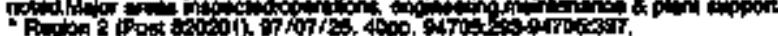




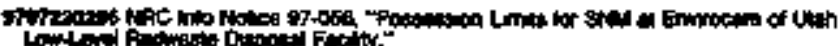

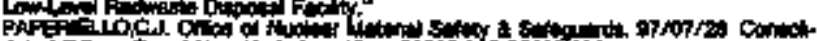

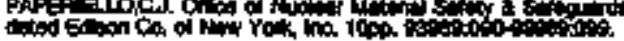

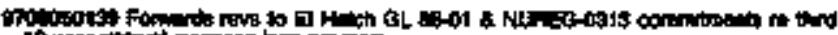

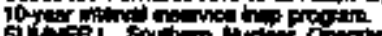

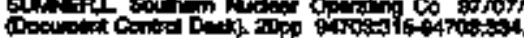

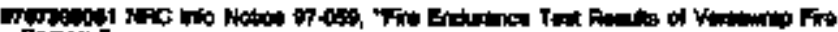

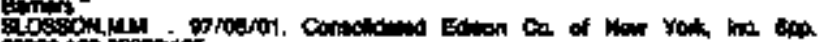

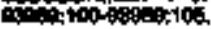

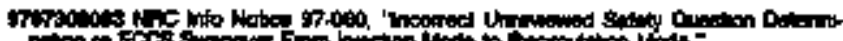

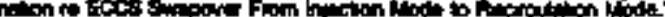

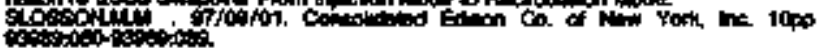

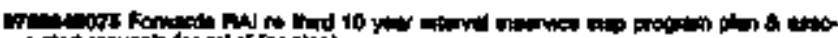

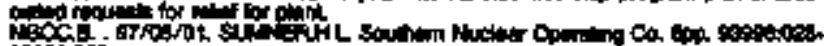
atios:

Find

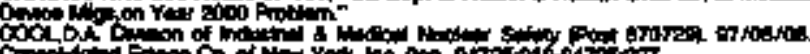

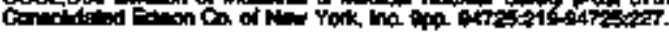

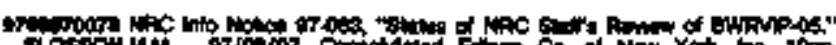

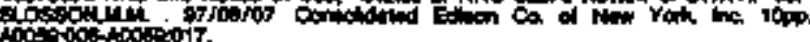

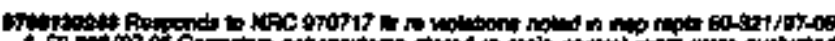

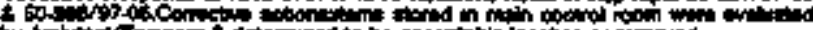

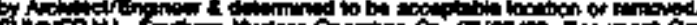

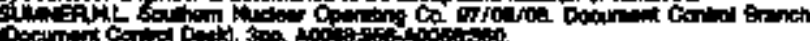

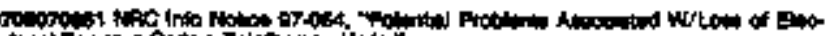

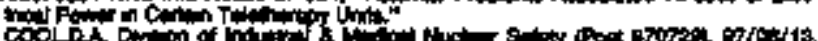

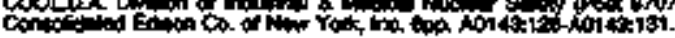

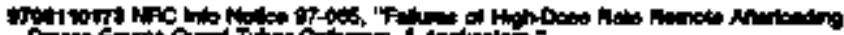

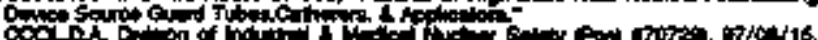

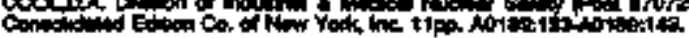

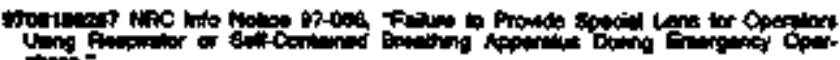

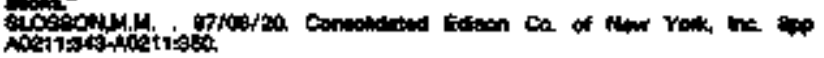

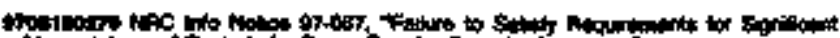
等

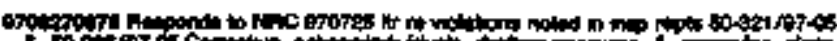

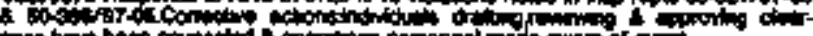

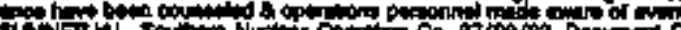

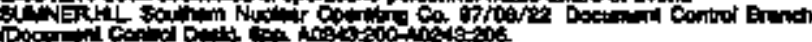

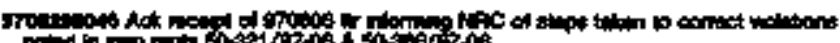

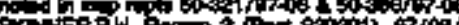

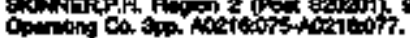

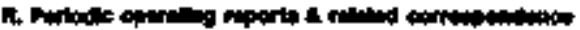

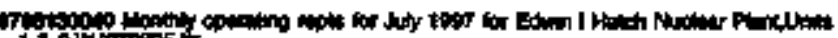

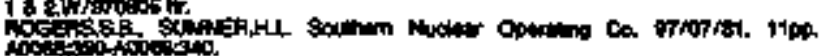

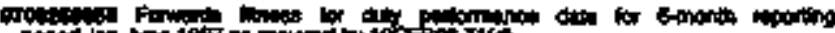

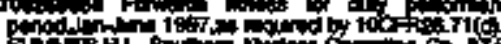

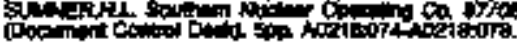

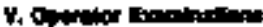

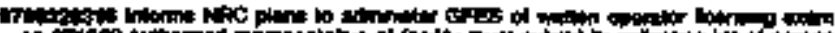

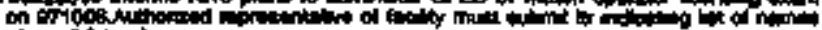

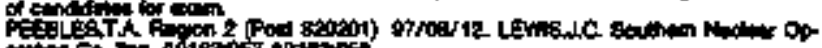

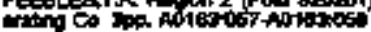

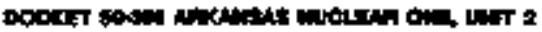

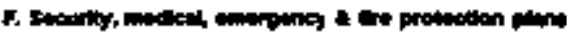

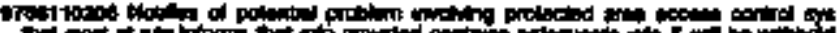

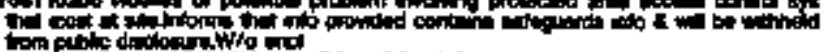

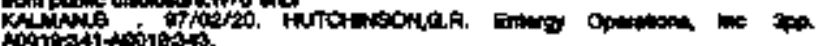

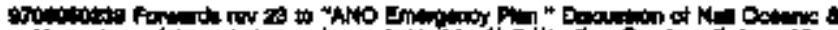

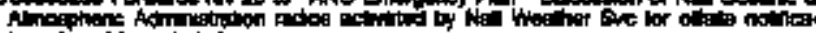
thens bo pere metuded in rev.

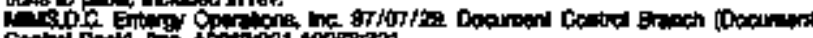

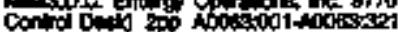

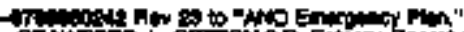

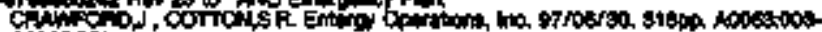
Atostist.

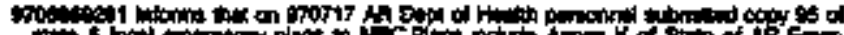

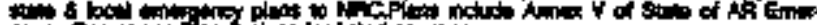

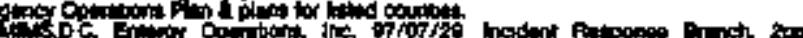
我

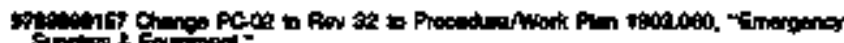

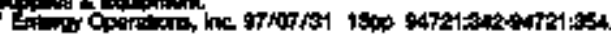

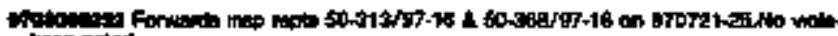

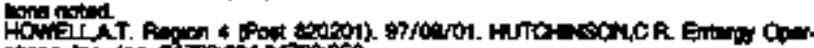

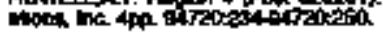

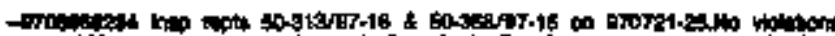

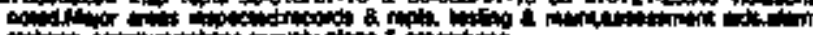

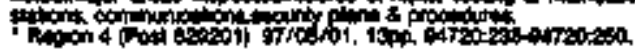

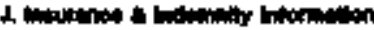

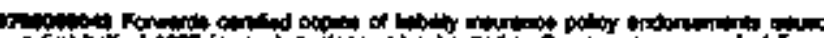

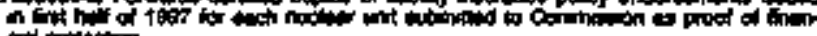
oid

Dow

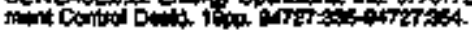

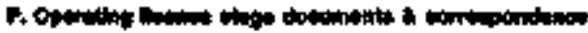

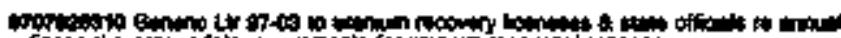

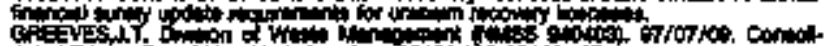

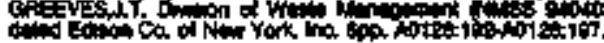

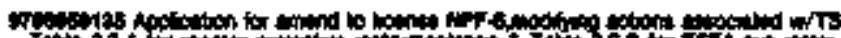

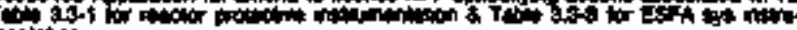

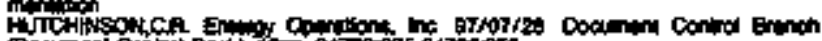

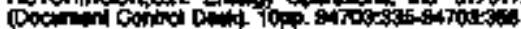

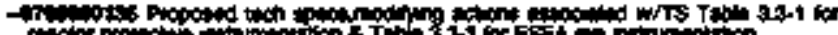

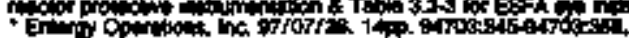

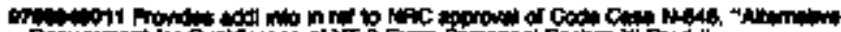

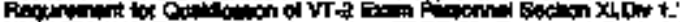

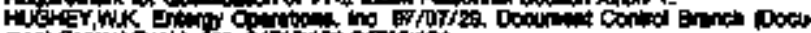

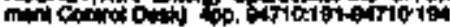

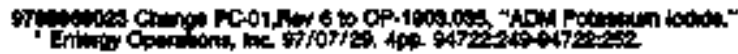

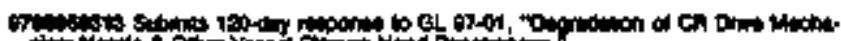

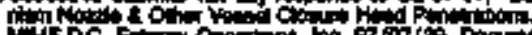

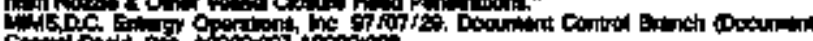

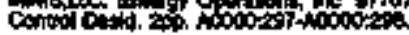

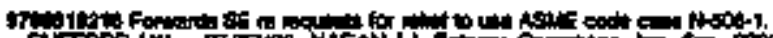

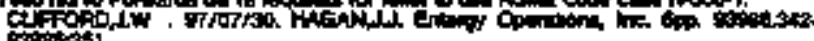
sions

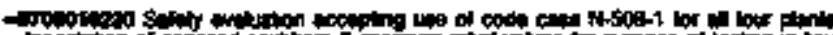

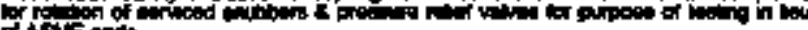
thot

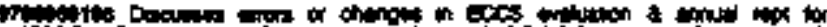

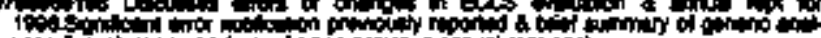

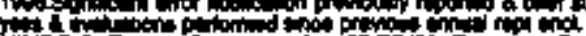

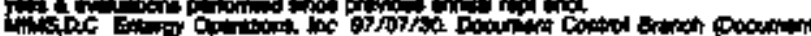

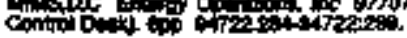

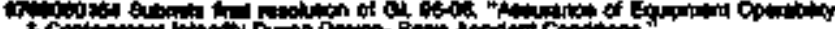

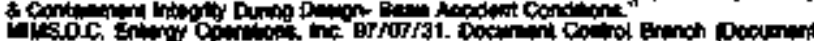

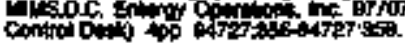

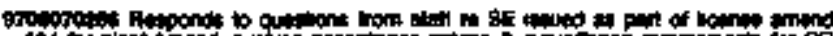

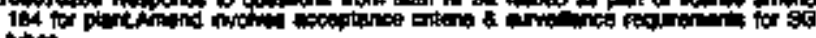

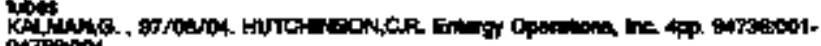

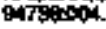

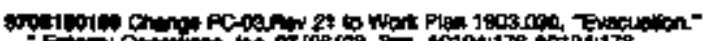

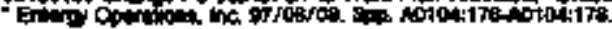

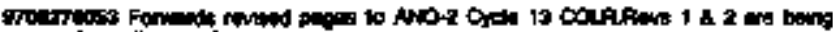

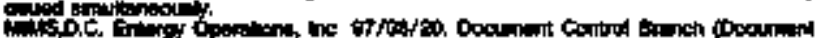

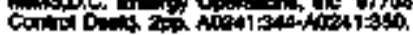

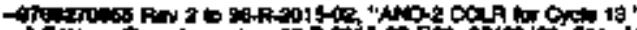

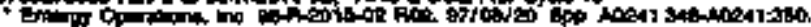




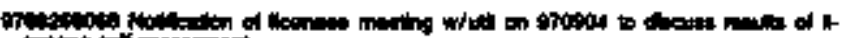

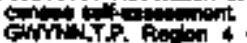

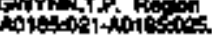

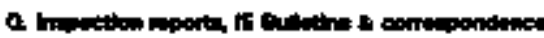

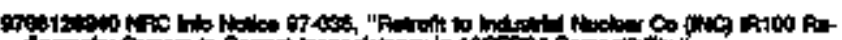

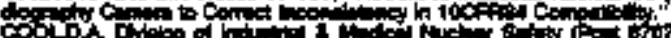

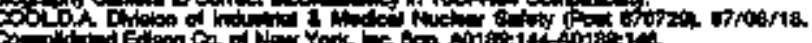

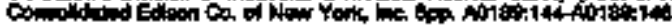

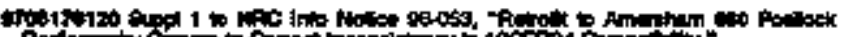

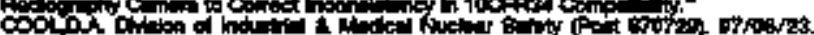

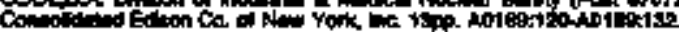

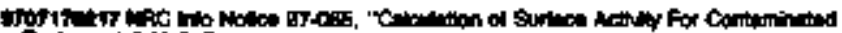

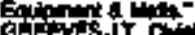

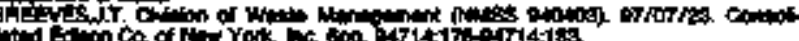

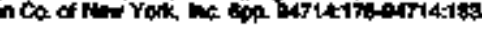

管

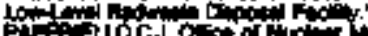

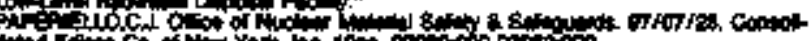

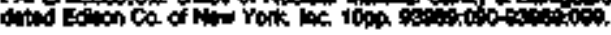

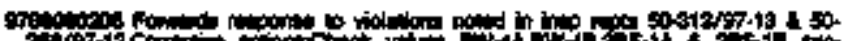

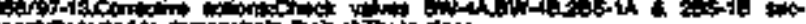

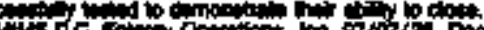

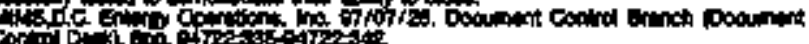

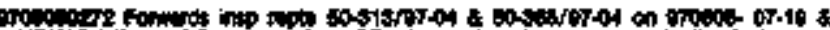

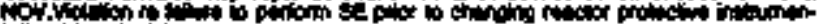

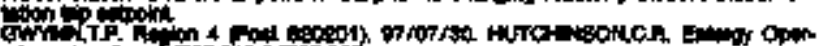

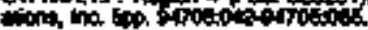

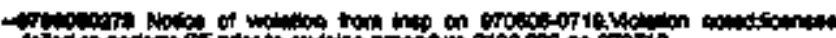

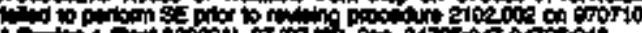

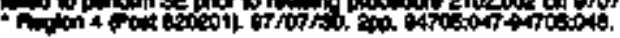

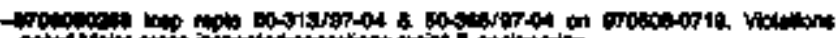

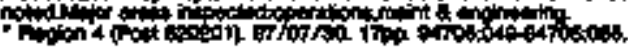

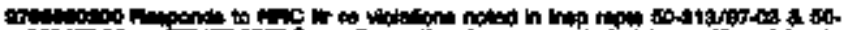

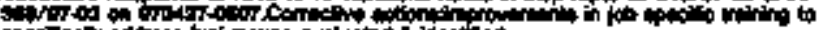
it

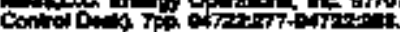

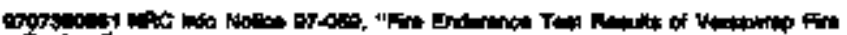

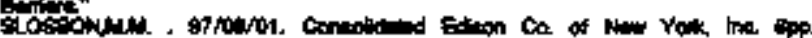

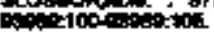

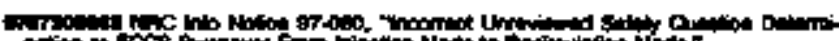

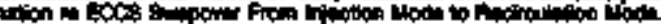

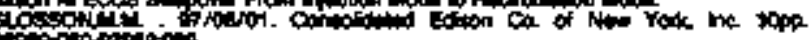

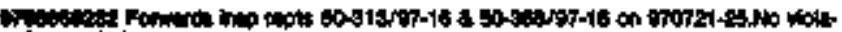
Hithotid.

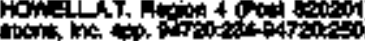

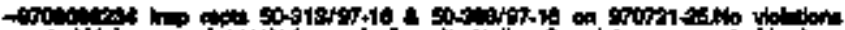

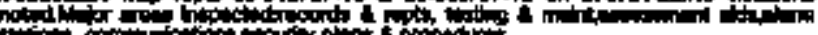

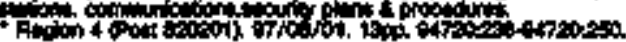

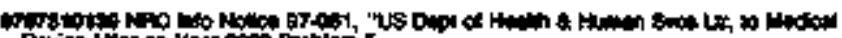

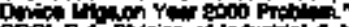

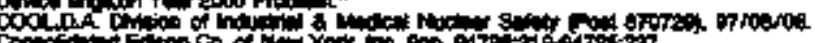

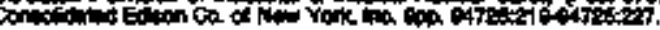

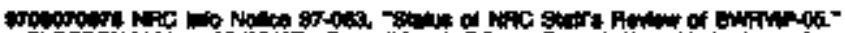

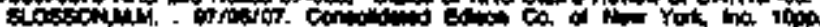

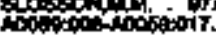

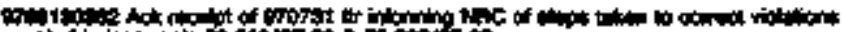

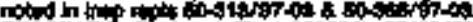

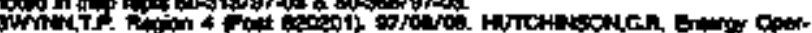

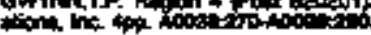

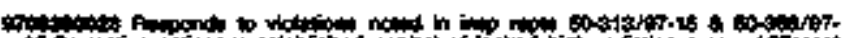

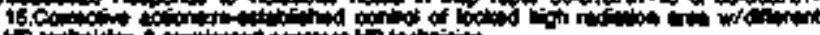

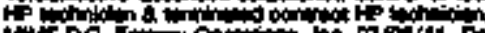

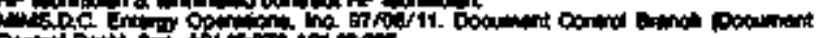

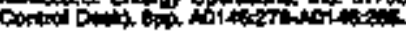

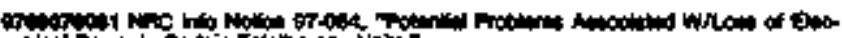

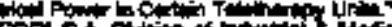

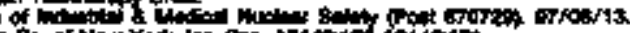

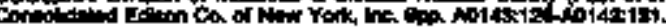

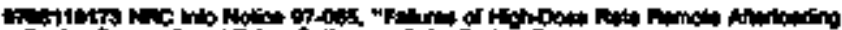

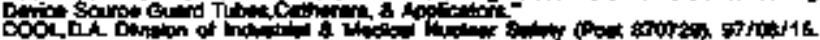

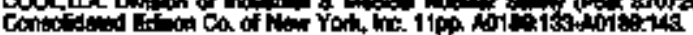

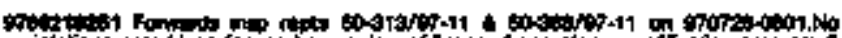

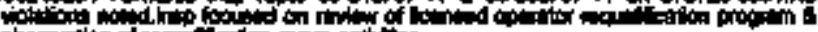

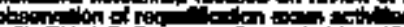

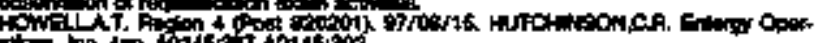

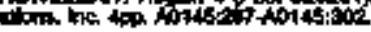

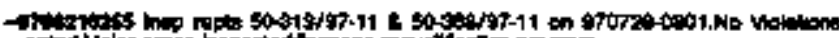

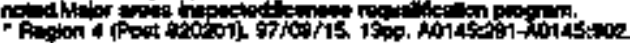

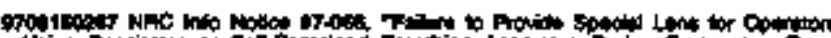

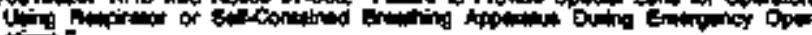

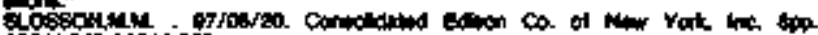

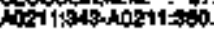

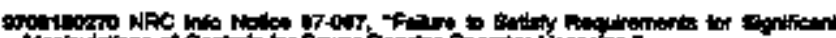

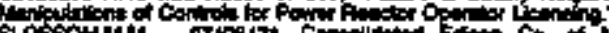

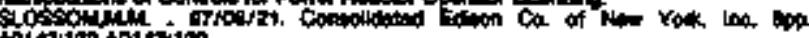

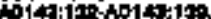

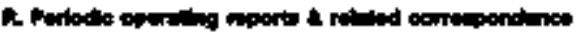

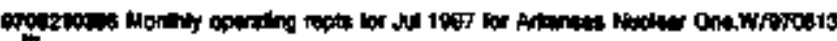

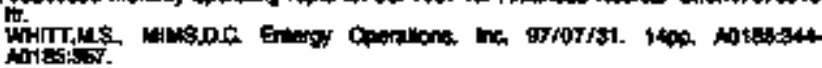

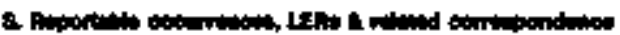

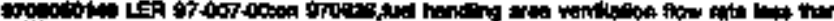

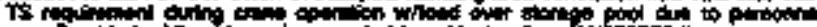

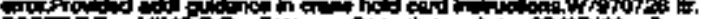

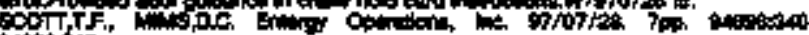
sinosiar.

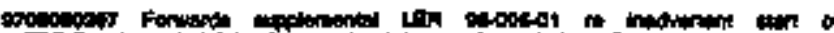

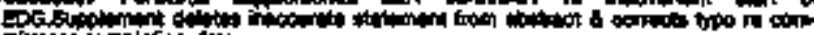

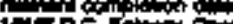

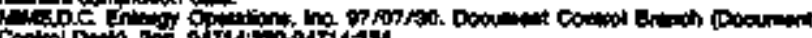

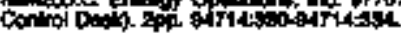

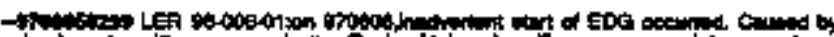

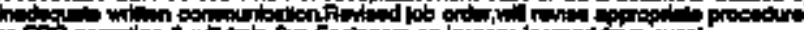

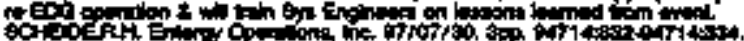

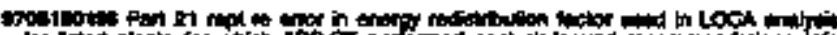

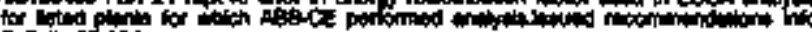
Bation grtood

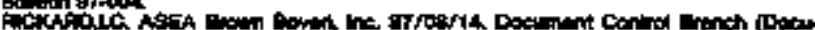
hime Con

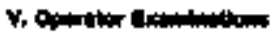

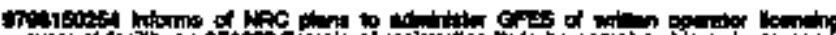

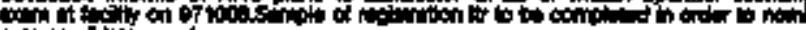

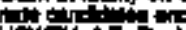

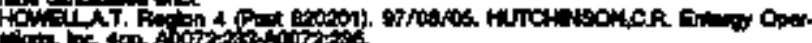

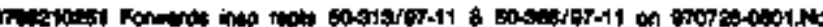

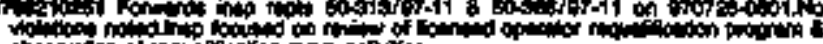

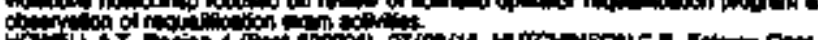

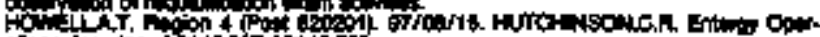

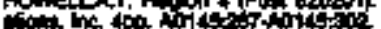

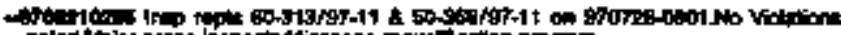

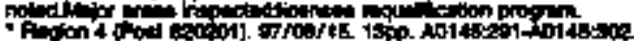

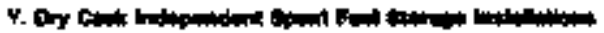

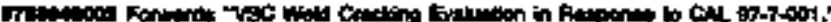

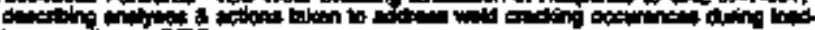
in opostint ting

CE

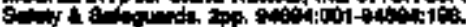

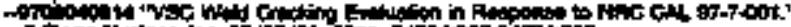

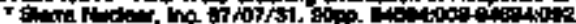

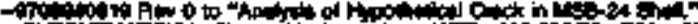
Ge

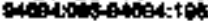

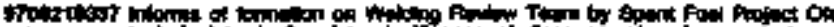
o

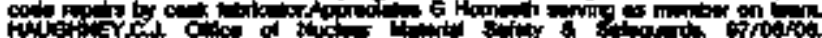

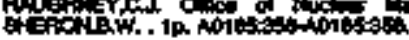

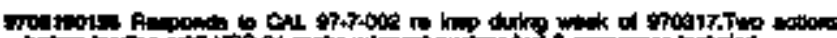

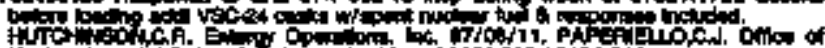

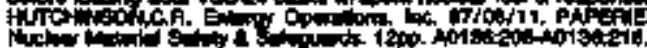

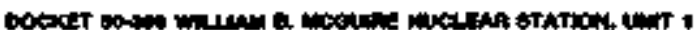

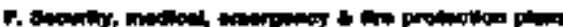

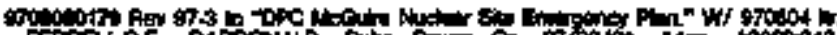

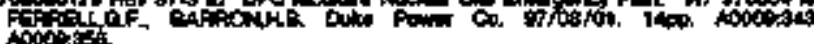




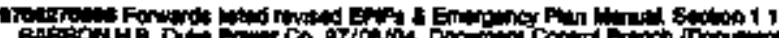

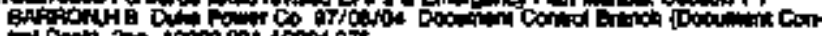

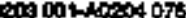

-

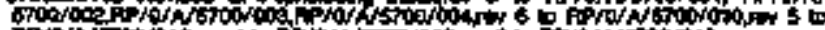

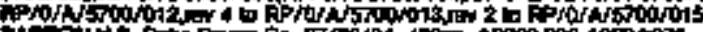

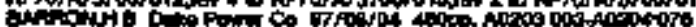

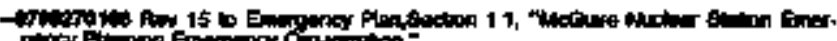

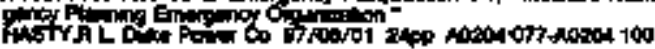

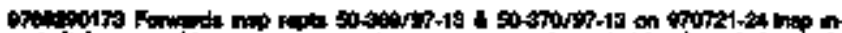

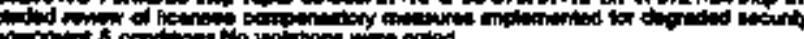

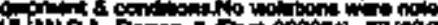

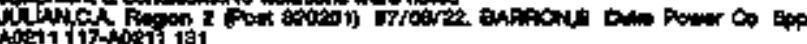

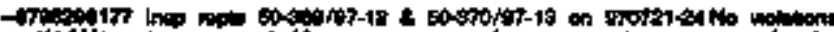

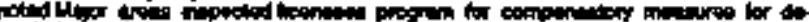

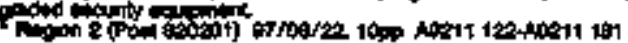

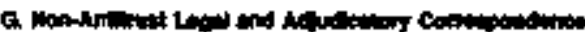

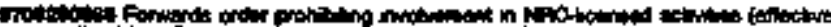

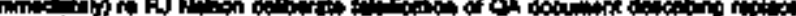

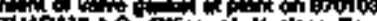

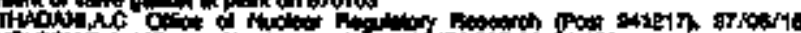

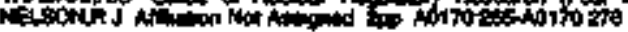

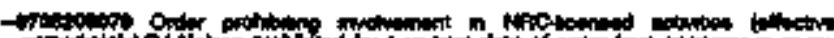

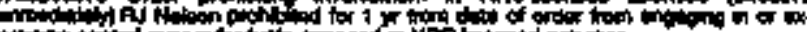

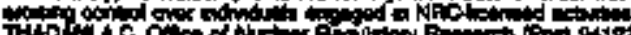

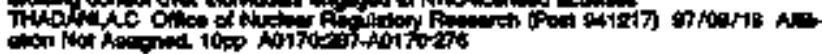

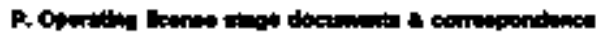

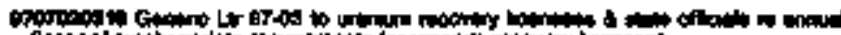

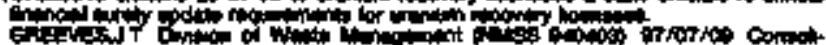

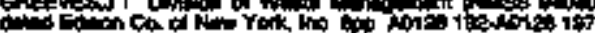

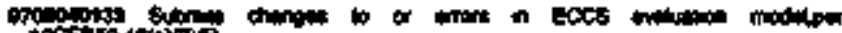

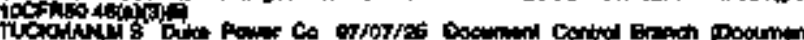

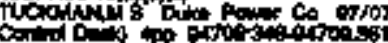

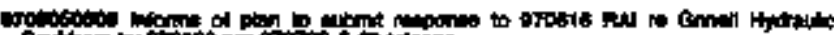

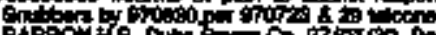

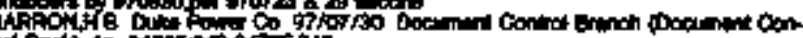

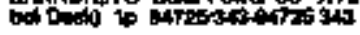

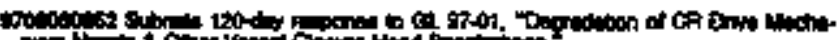

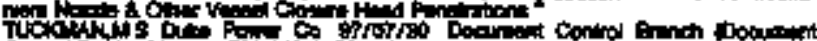

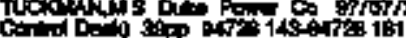

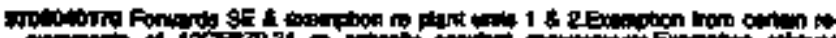

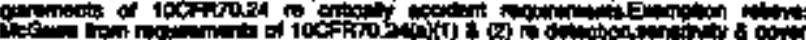

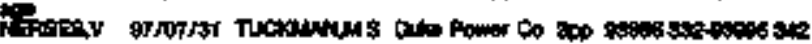

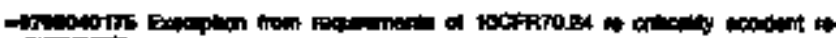

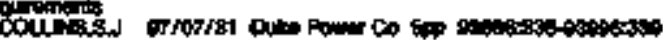

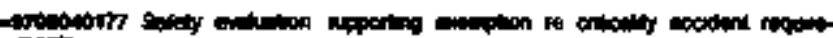

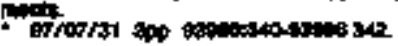

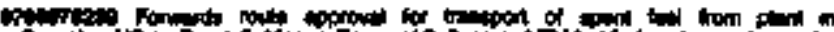

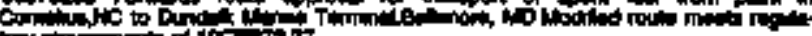

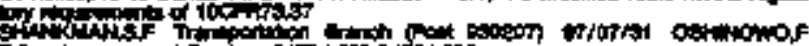

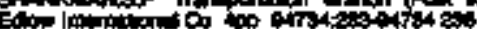

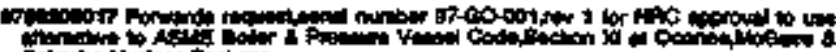

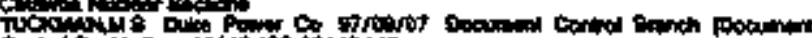

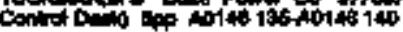

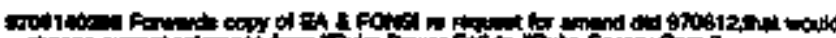

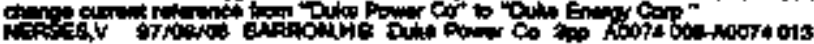

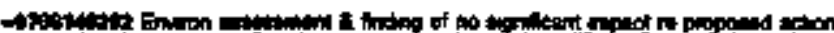

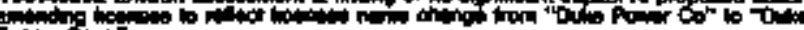

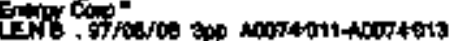

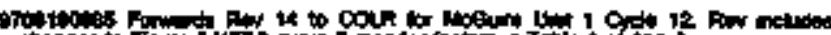

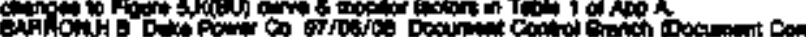

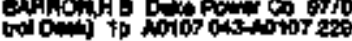

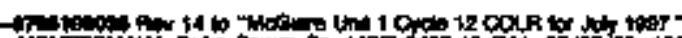

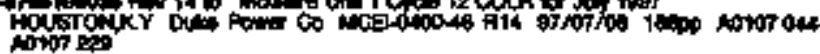

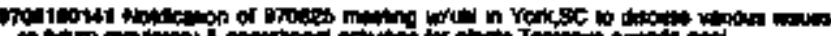

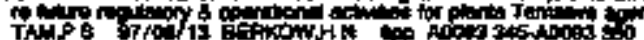

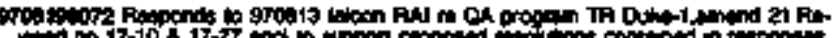

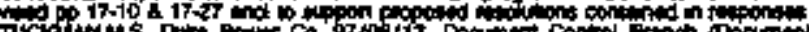

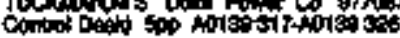

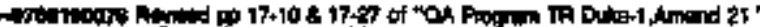

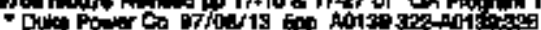

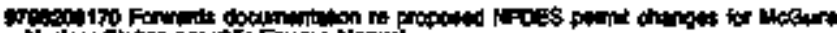

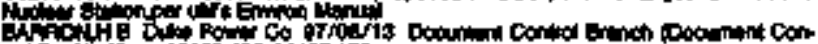

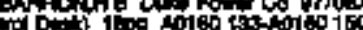

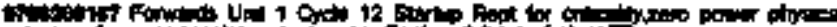

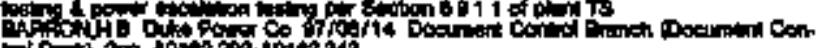

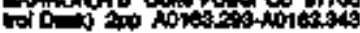

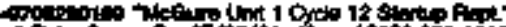

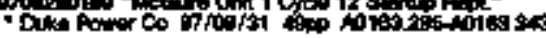

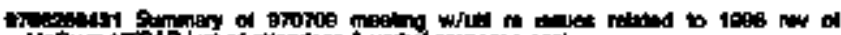

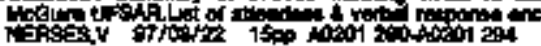

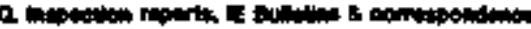

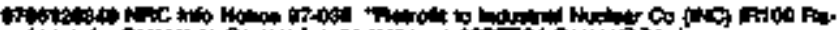

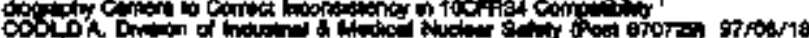

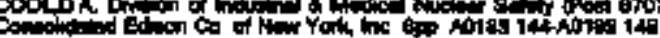

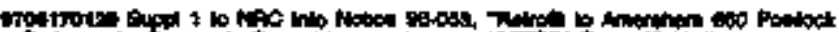

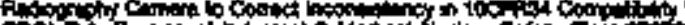

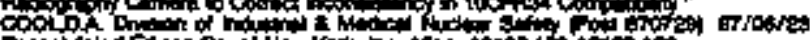

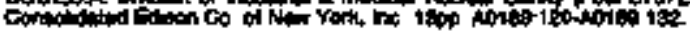

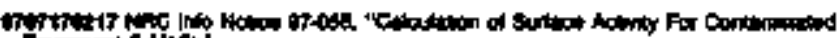

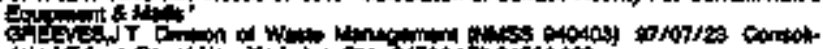

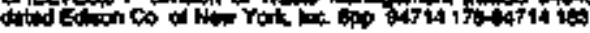

1

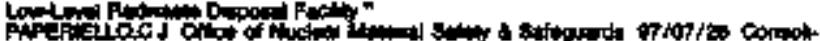

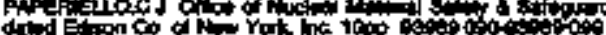

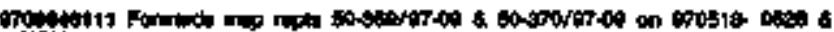
(1)

W

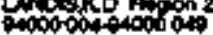

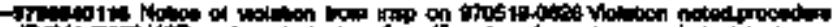

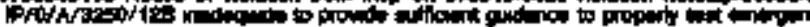

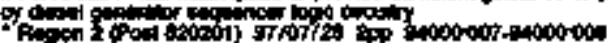

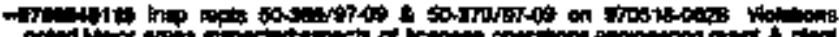

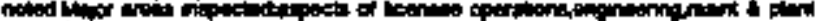

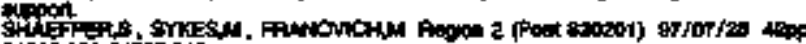
9000 of topocos

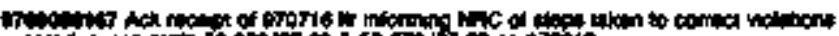

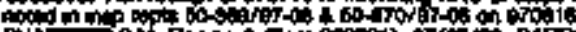

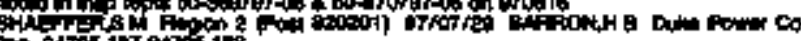

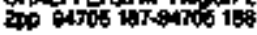

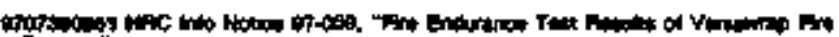

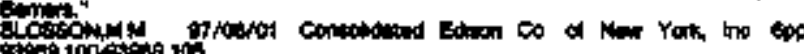

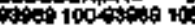

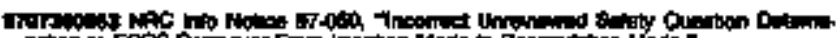

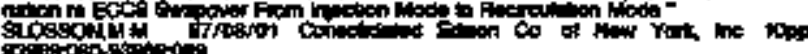

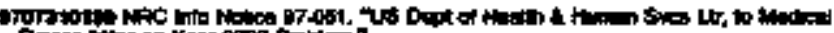

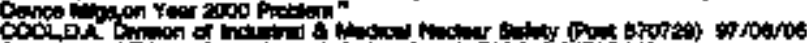

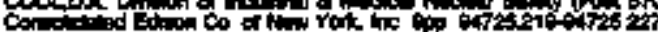

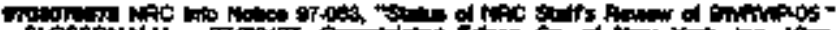

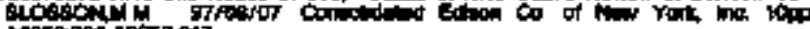

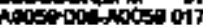

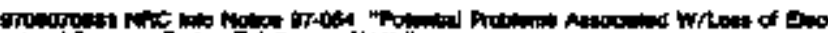

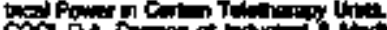

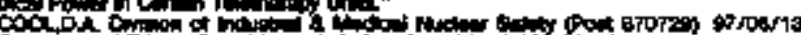

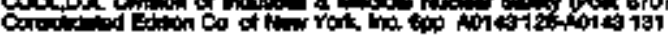

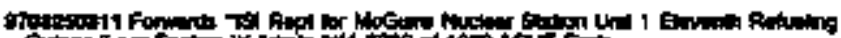

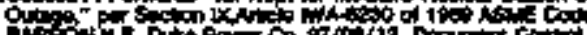

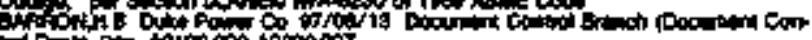

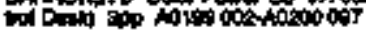

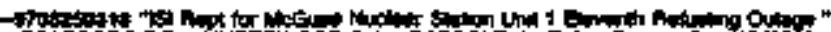

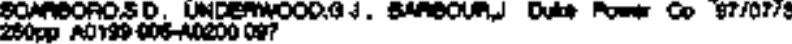

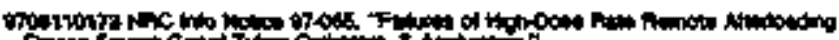

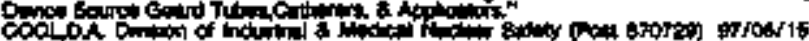

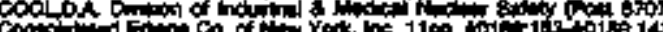




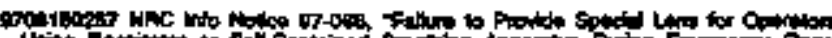

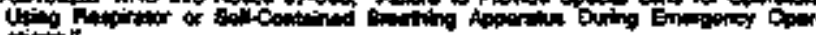

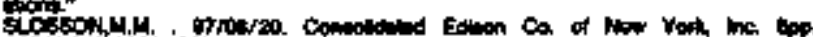

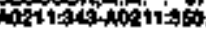

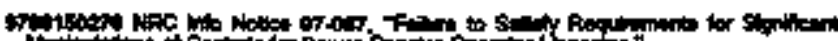

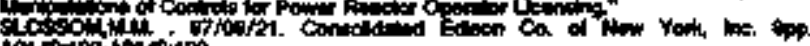

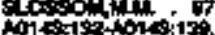

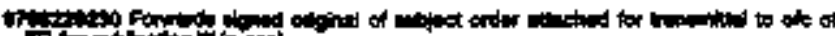

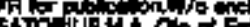

On

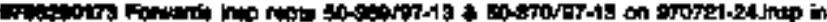

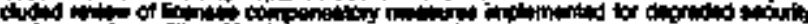

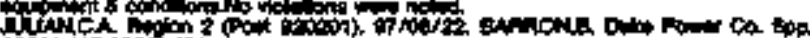

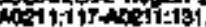

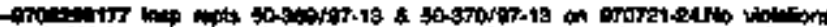

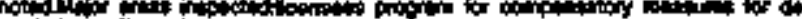

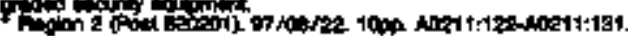

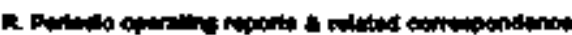

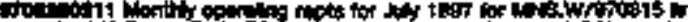

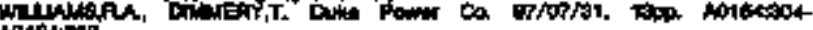
nowata

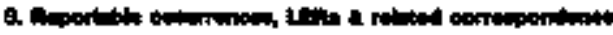

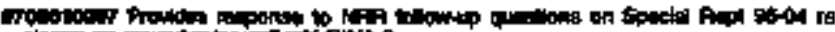

Eif

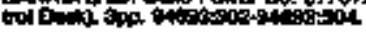

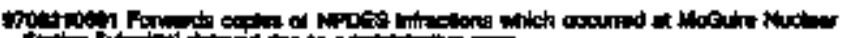

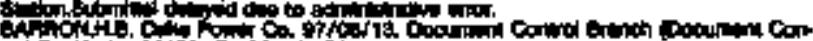

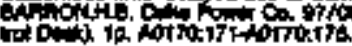

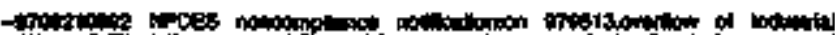

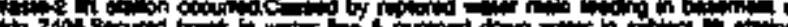
Ca

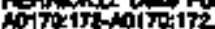

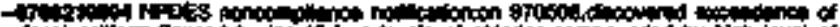

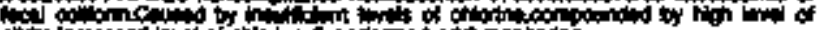

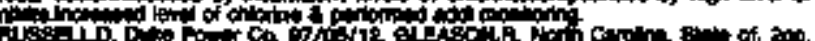

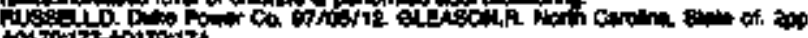

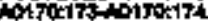

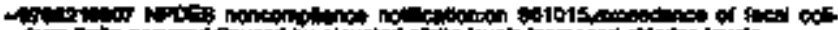

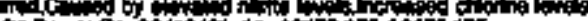

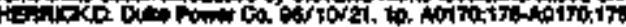

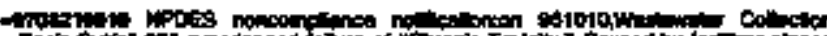

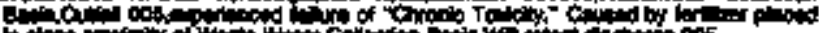

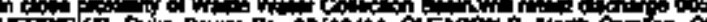

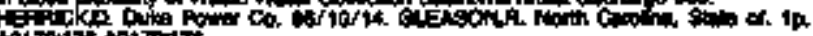
A 170 it

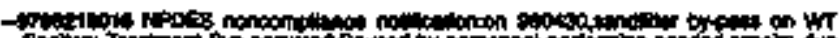

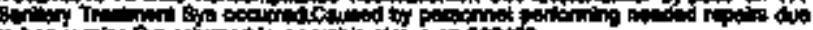

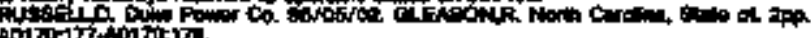

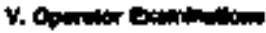

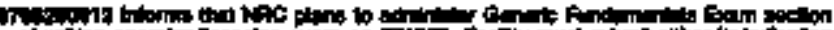

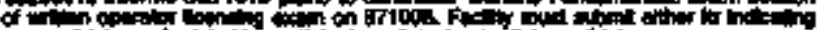

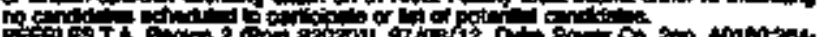
dorbetiot

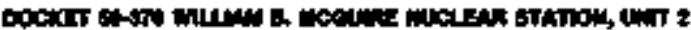

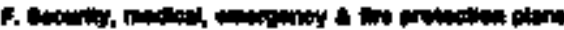

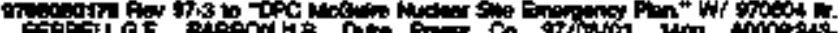

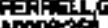

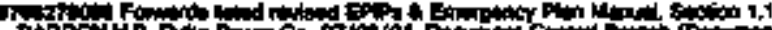

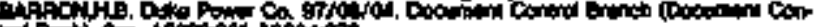

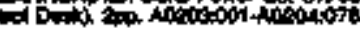

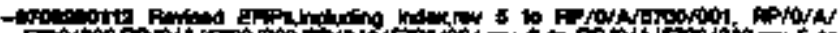

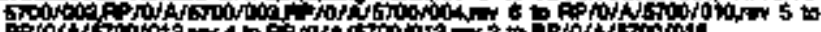

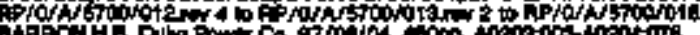

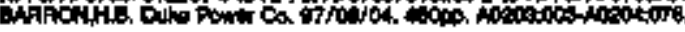

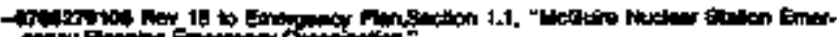

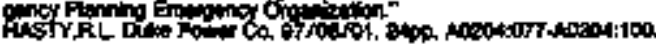

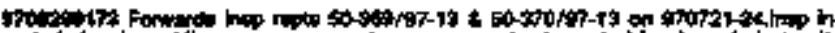

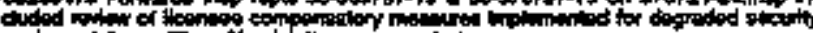

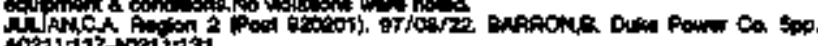

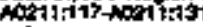

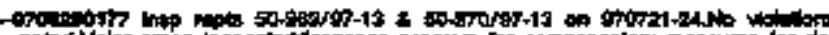

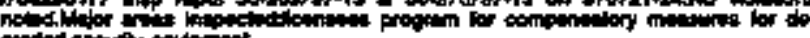

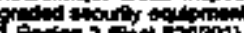

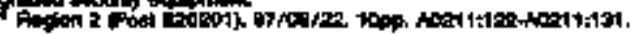

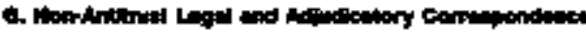

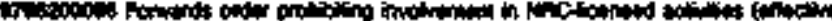

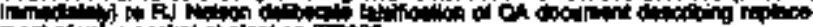

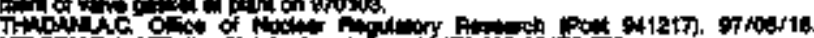

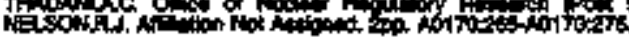

- 970

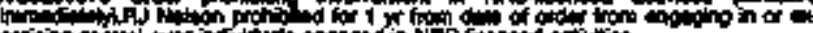

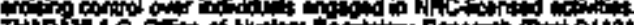

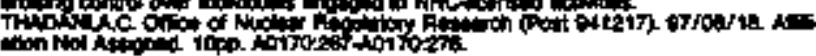

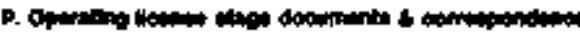

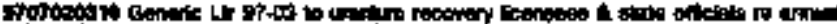
1.

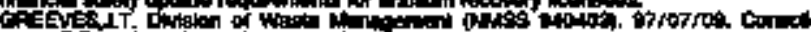

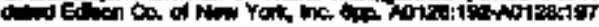

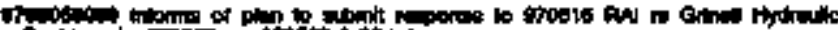

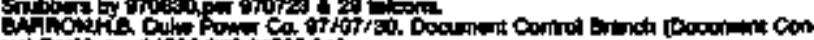

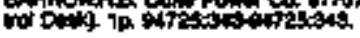

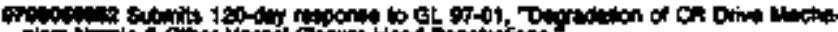

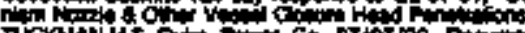

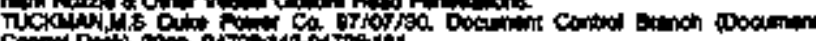

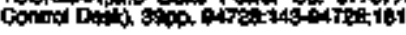

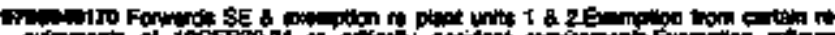

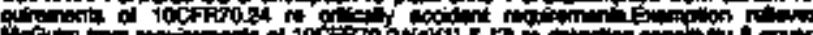

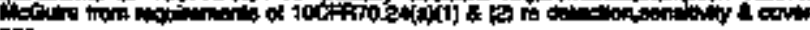

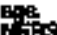

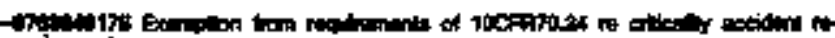

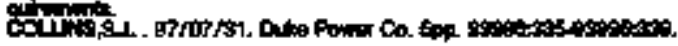

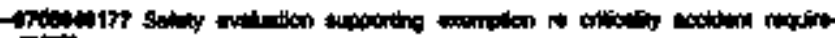

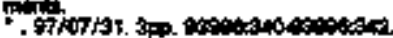

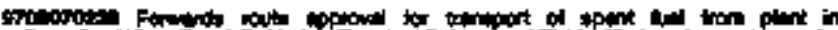

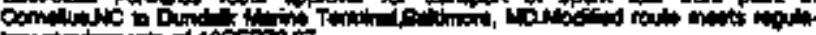

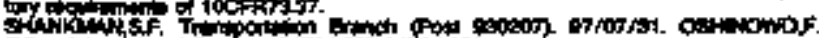

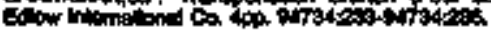

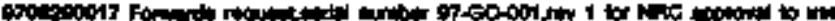

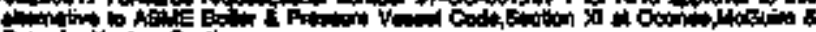

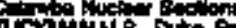

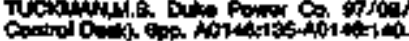

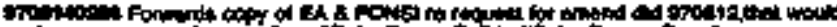

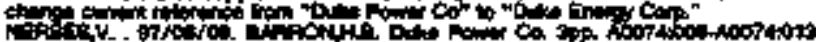

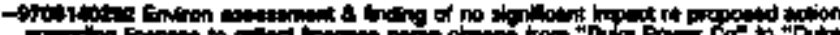

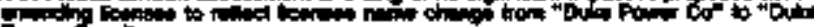

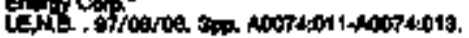

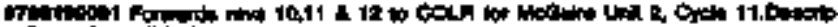

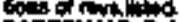

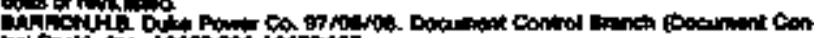

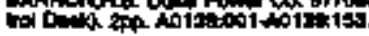

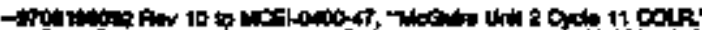

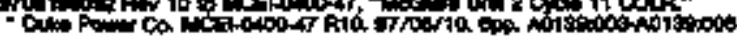

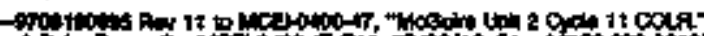

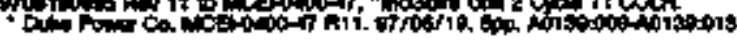

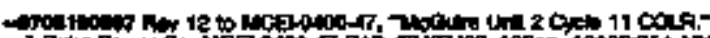

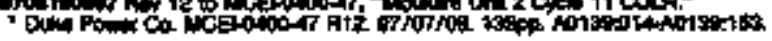

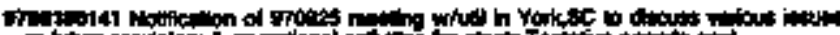

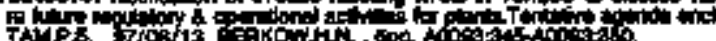

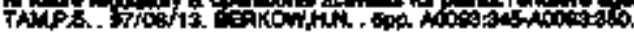

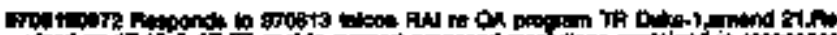

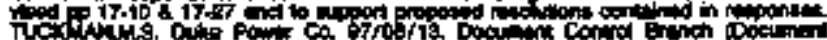
Colol Doma

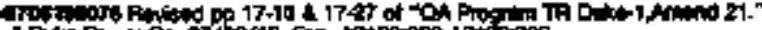

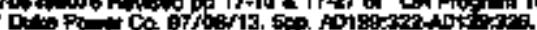




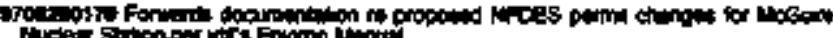

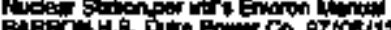

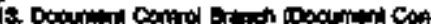

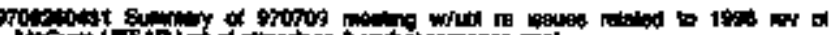

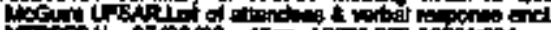

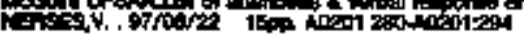

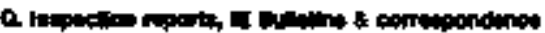

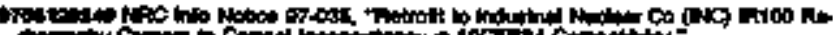

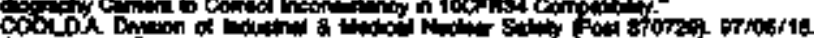

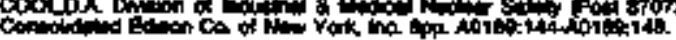

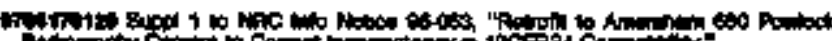

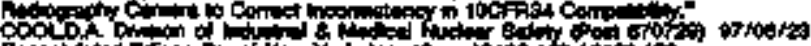

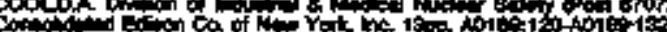

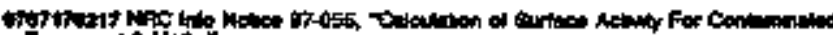
cising

Gid

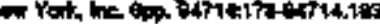

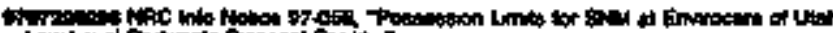

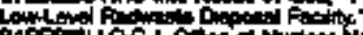

PAf -

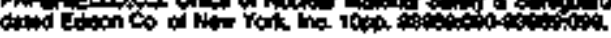

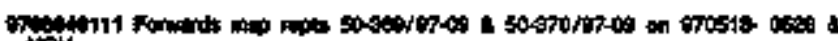

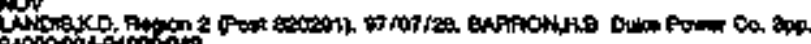
4000:001040060tis

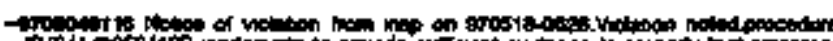

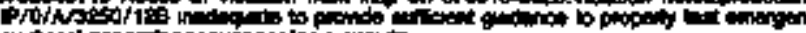

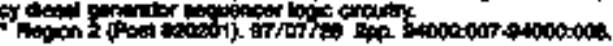

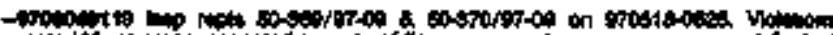

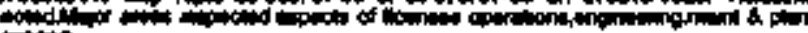

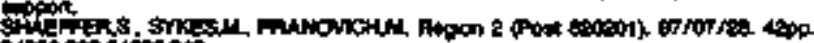

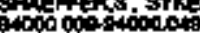

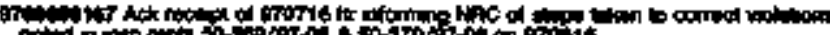

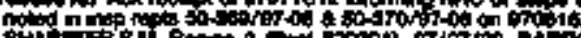

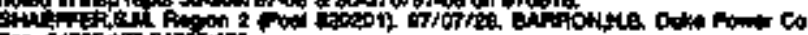

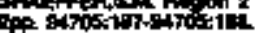

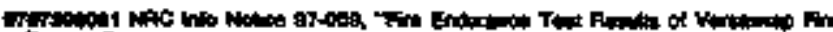
cimatis"

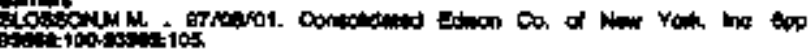

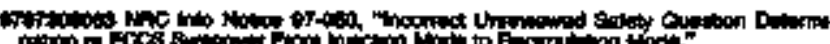

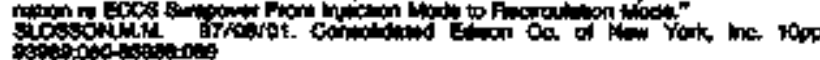

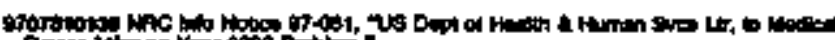

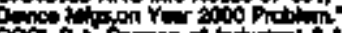

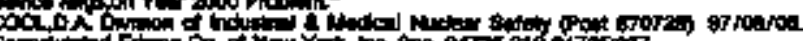

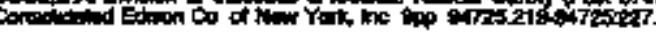

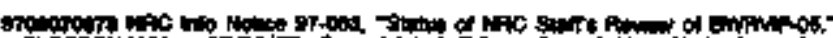

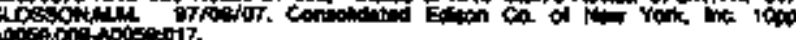

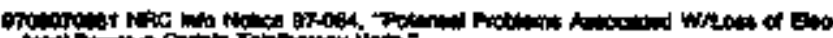

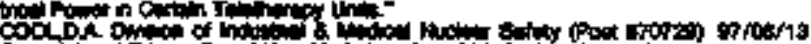

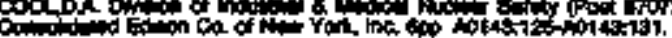

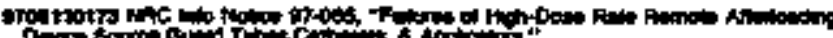

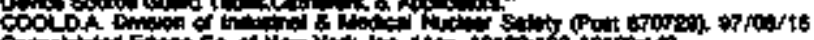

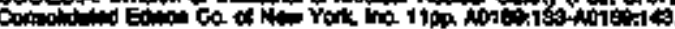

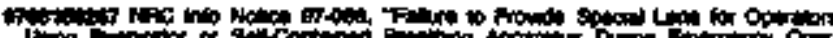

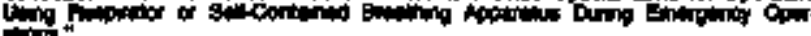

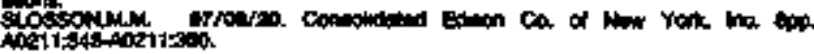

on

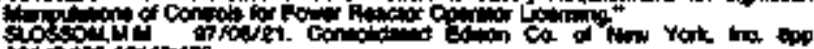
tortistaristis:

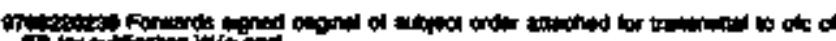

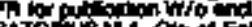

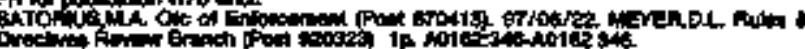

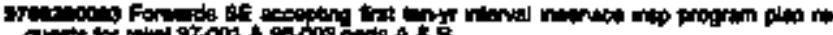

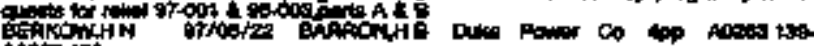
tetsicis.

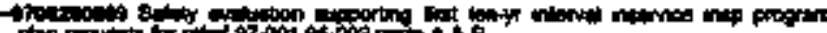

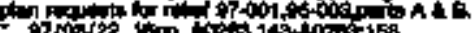

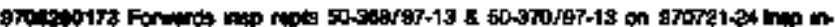

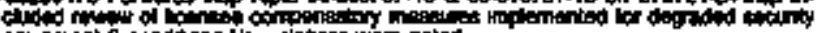
Jif AD: 1 -

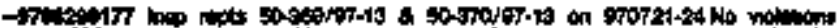

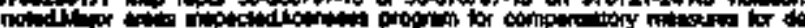

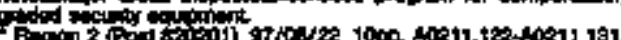

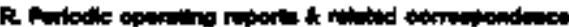

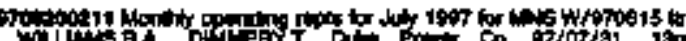

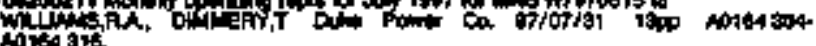

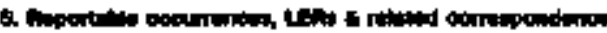

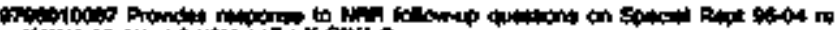

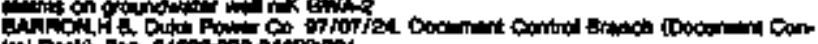

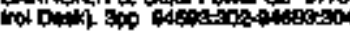

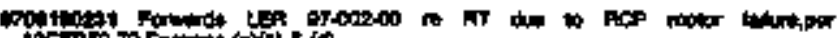

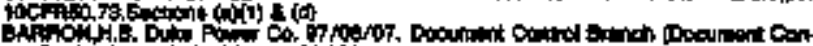

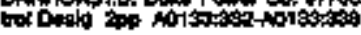

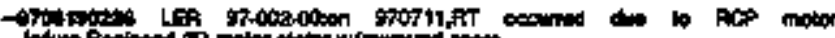

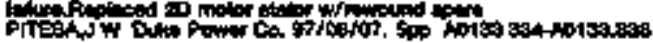

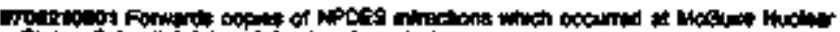

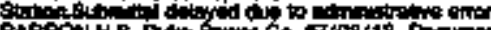

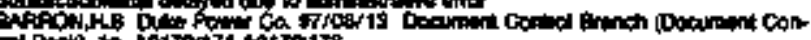

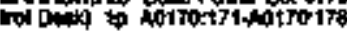

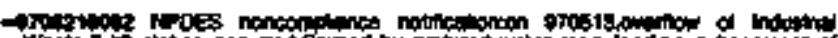

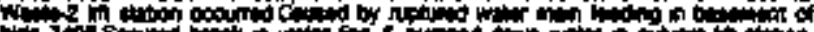

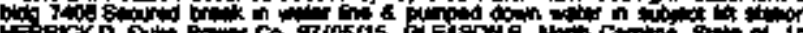

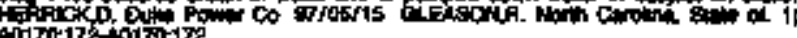

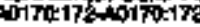

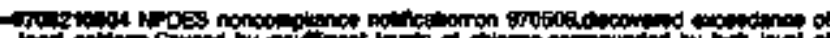

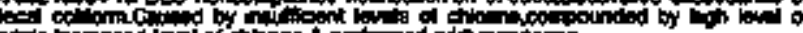

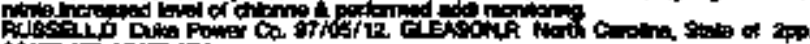
norkitiondink174

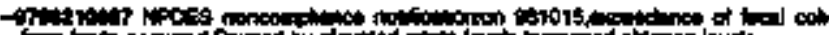

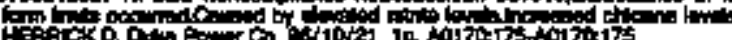

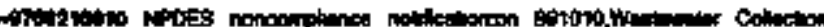

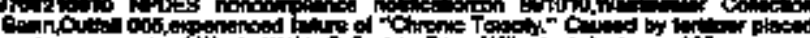

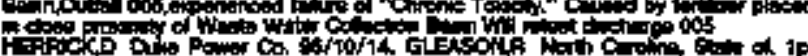

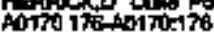

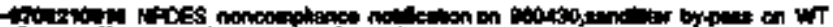

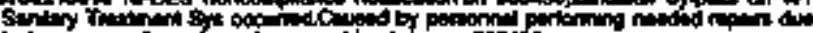

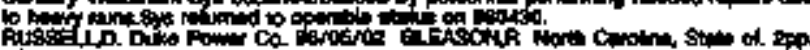

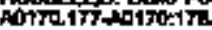

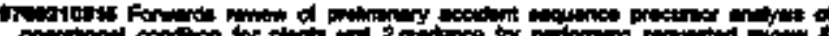

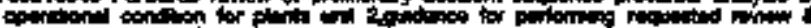

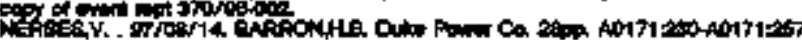

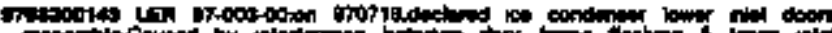

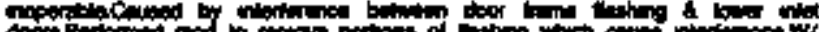

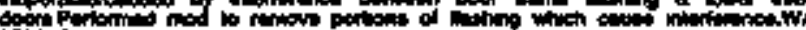

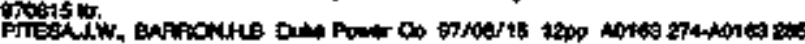

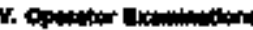

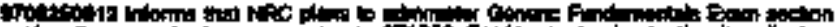

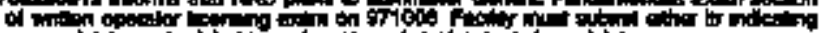

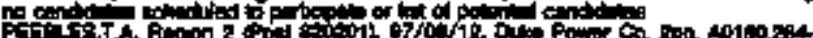
197.

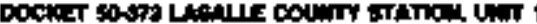

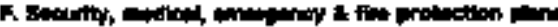

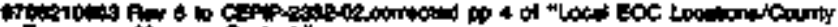

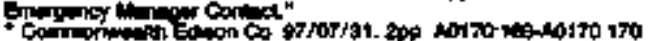

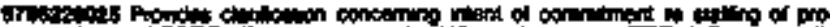

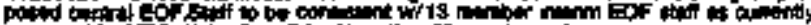

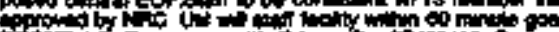

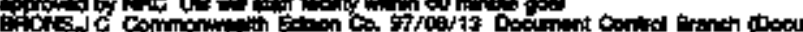

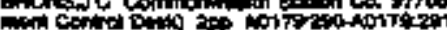




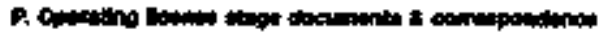

Fon

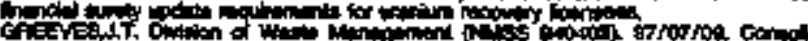

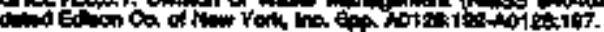

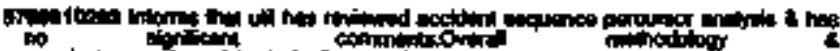

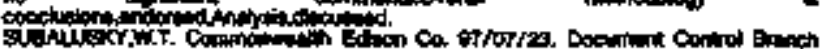

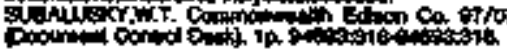

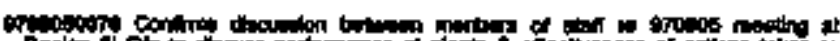

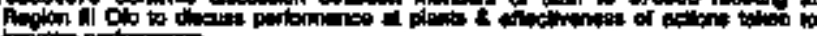

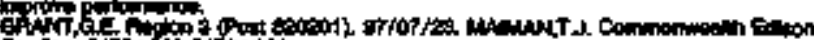

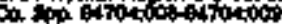

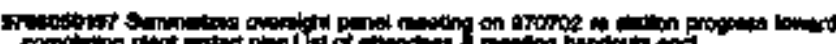

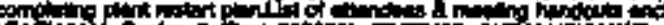

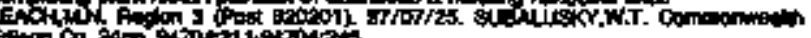

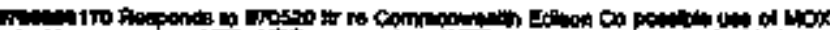

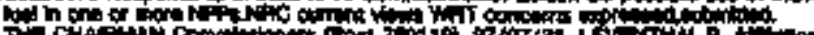

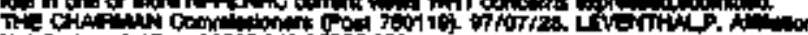

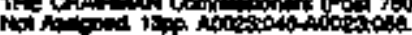

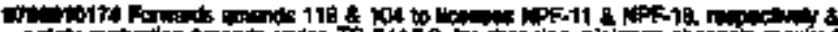

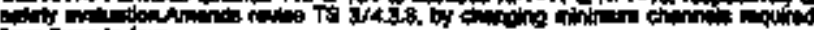

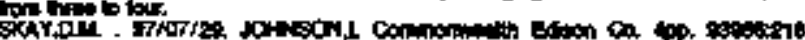

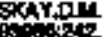

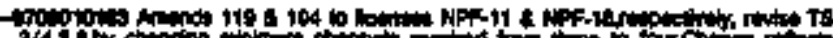

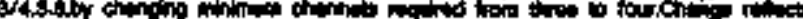

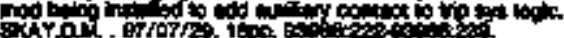

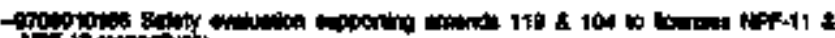

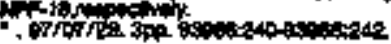

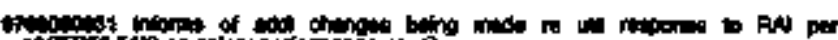

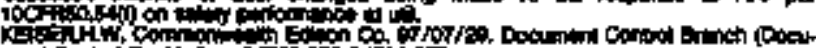

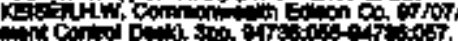

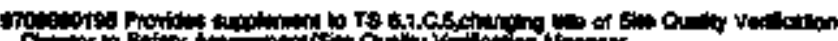

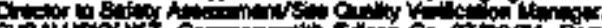

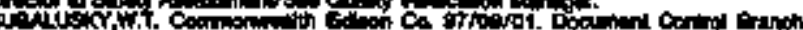

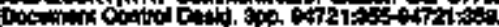

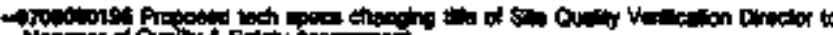

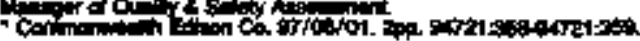

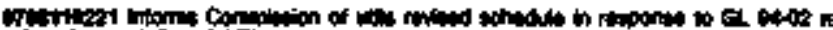
by

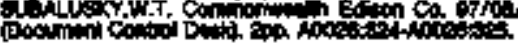

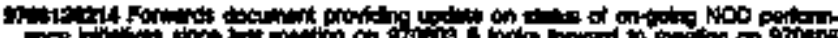

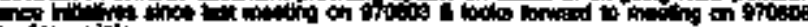

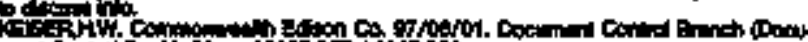

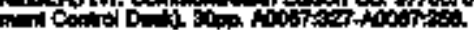

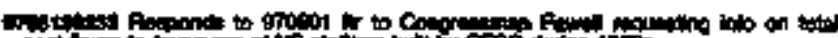

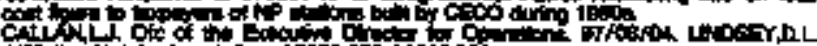

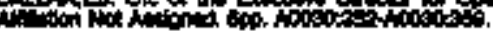

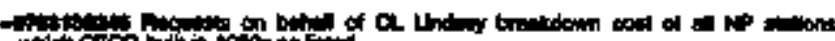

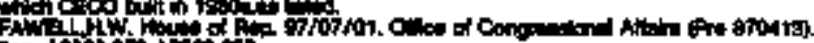
Bap nothos

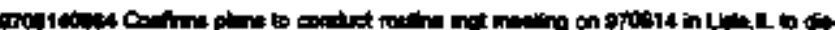

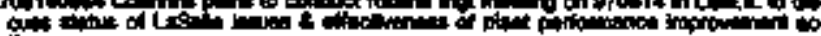

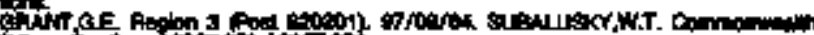

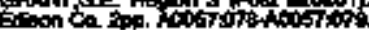

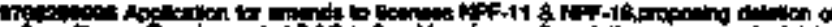

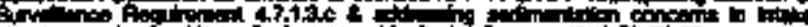

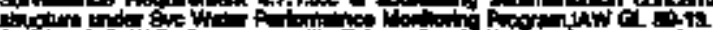
sids

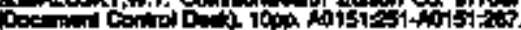

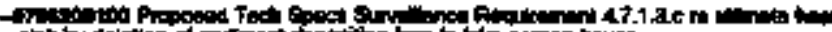

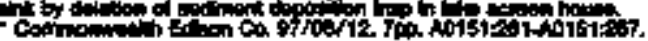

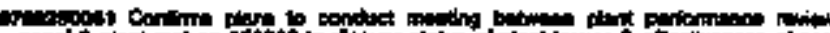

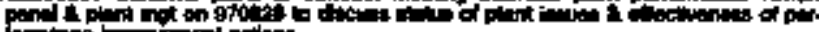

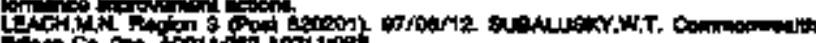

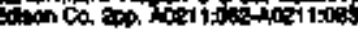

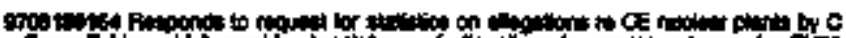

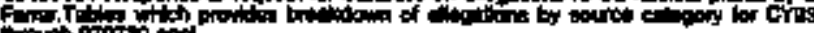

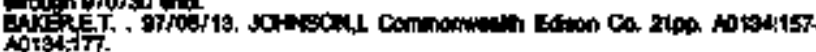

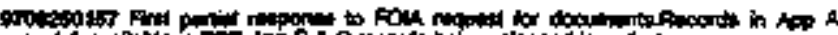

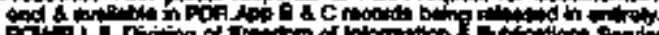

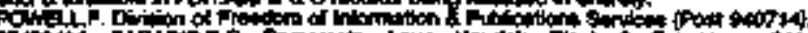

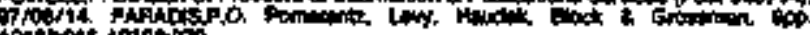

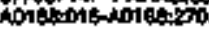

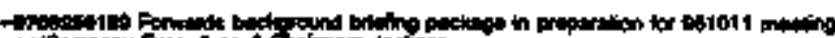

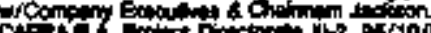

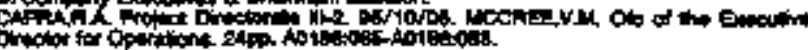

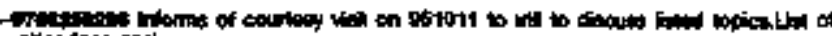

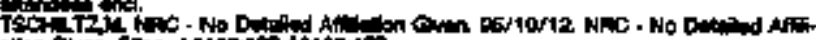

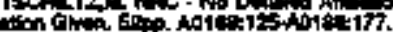

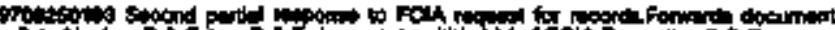

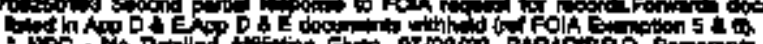
作

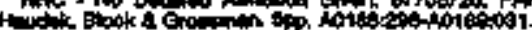

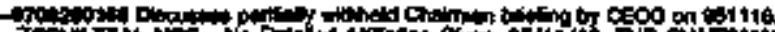

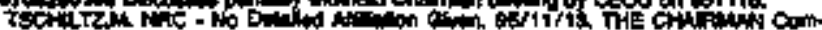

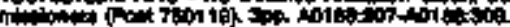

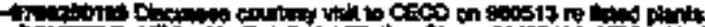
bon

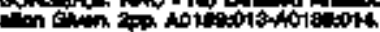

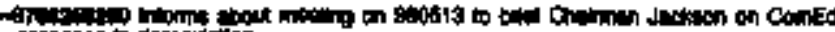

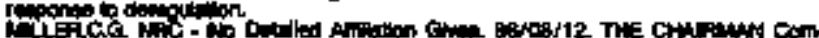

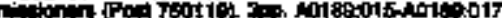

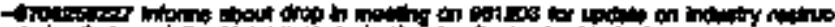

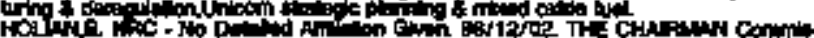

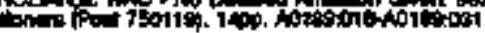

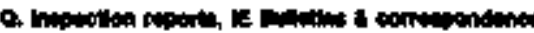

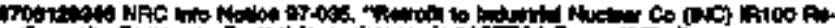

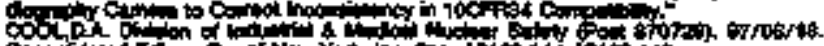
Conchl.

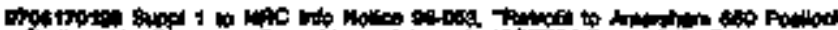

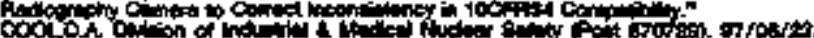

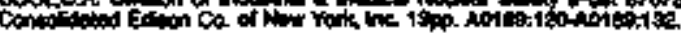

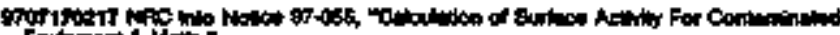

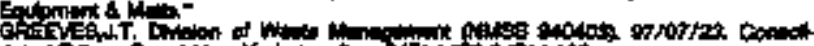

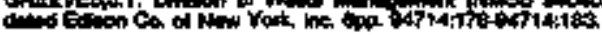

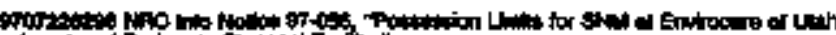
$L$ L

PAt

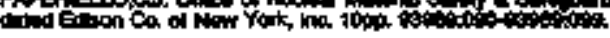

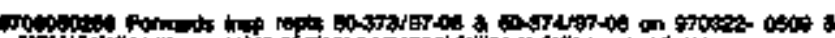
of

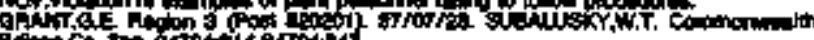

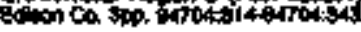

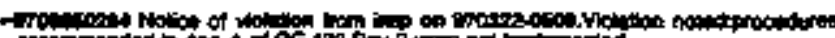

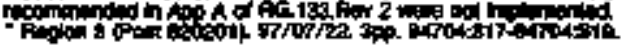

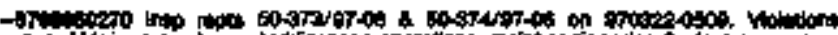

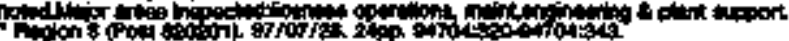

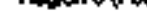

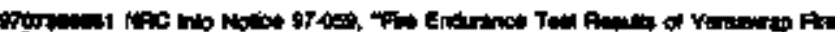

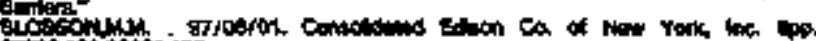

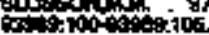

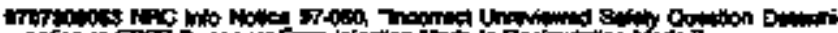

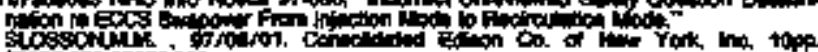

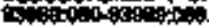

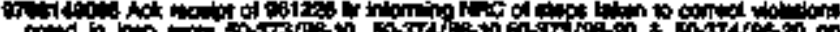

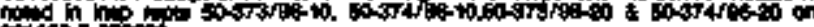

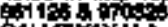

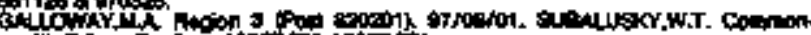

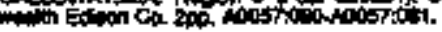

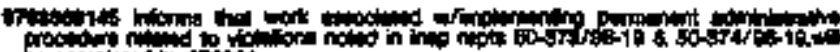

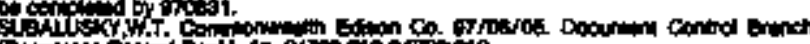

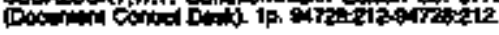

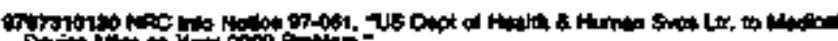

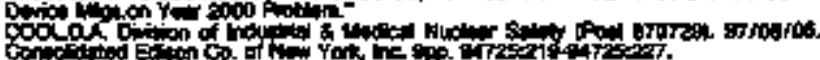

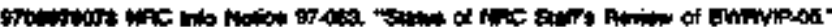

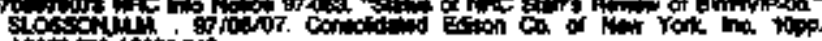

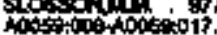




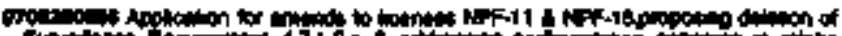

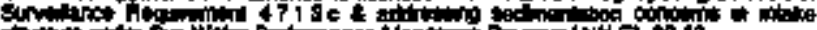

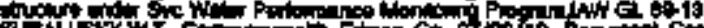

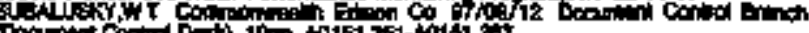

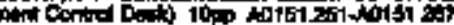

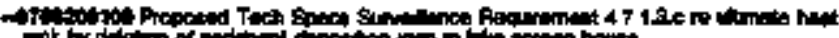

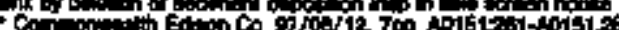

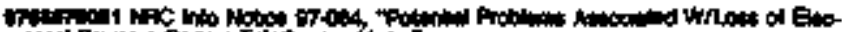

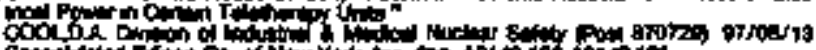

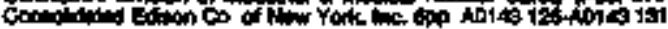

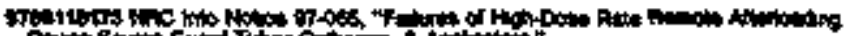

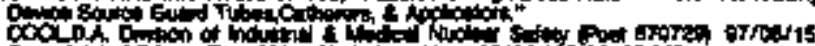

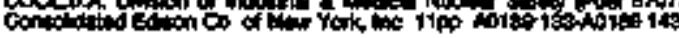

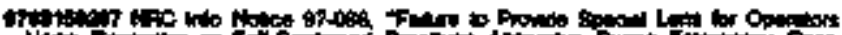

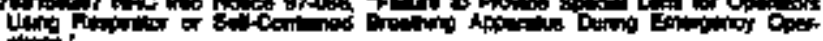

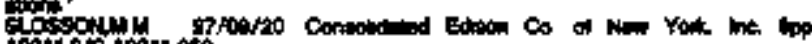

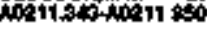

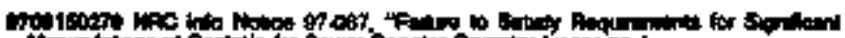

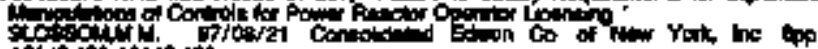

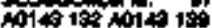

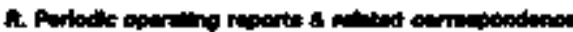

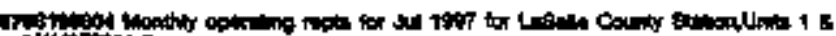

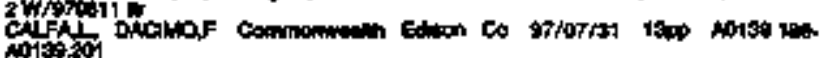

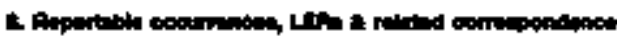

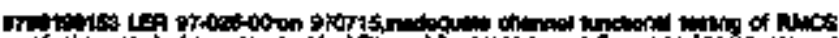

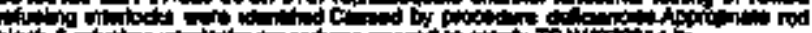

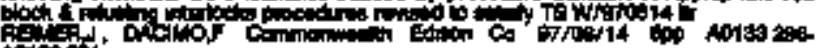
40

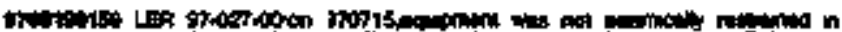

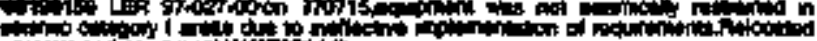

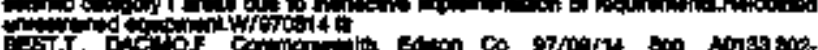
Afos sto

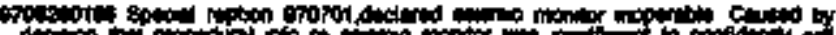

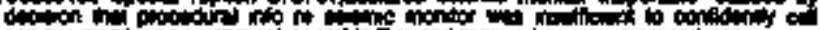

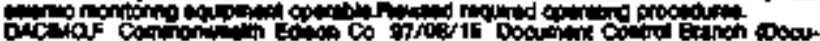

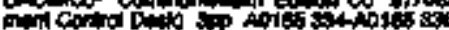

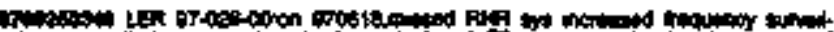

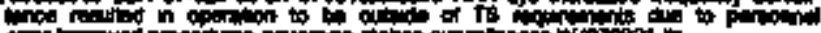

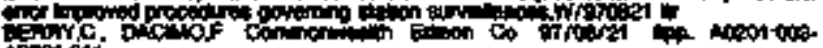
Ane01 011

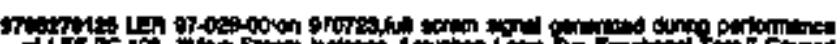

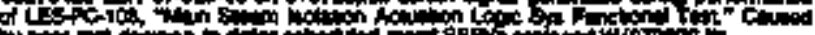

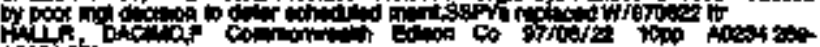
Atositis:

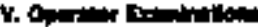

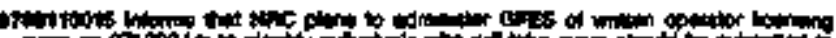
Pin on of

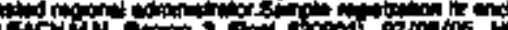

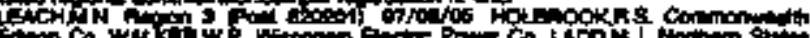

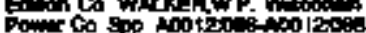

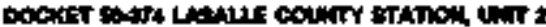

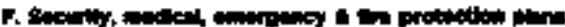

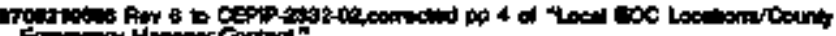

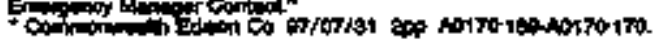

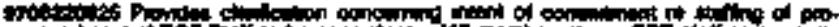

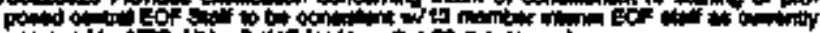

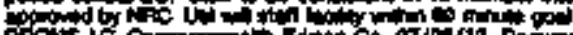

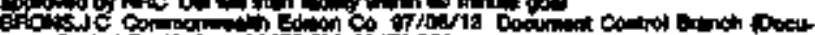

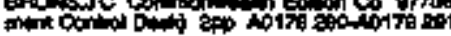

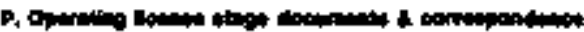

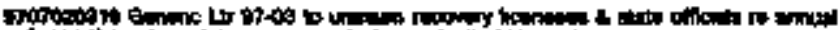

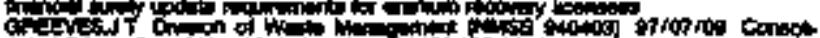

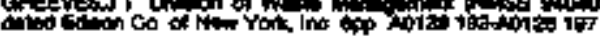

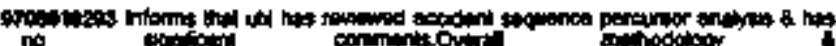
cition

a

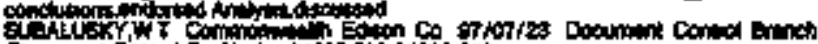

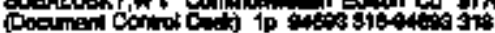

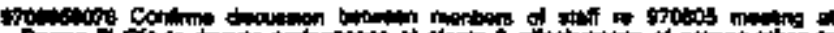

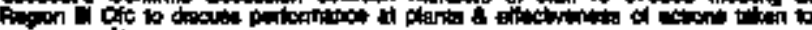

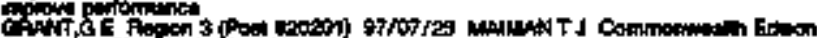

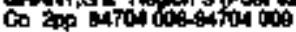

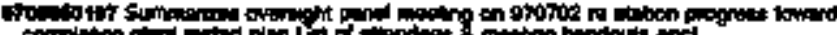

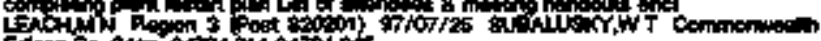

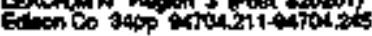

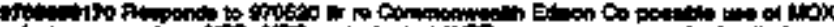

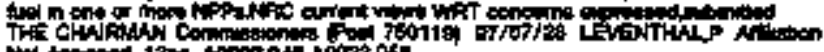

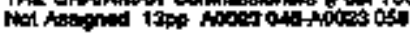

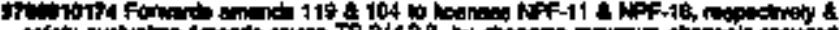

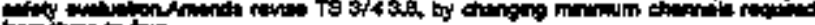

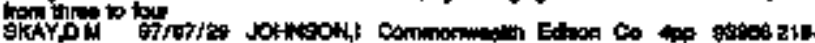
oisation

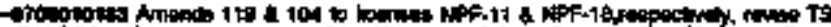

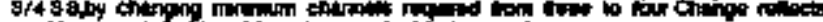

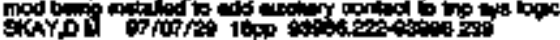

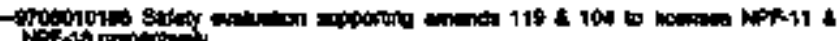

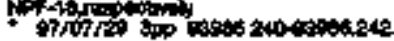

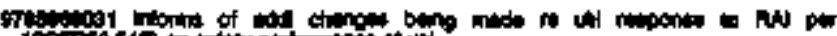
if

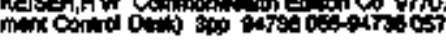

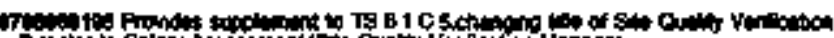

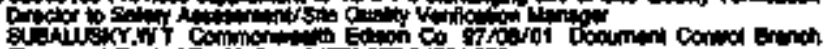

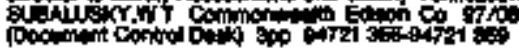

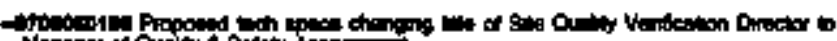

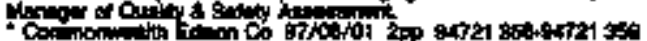

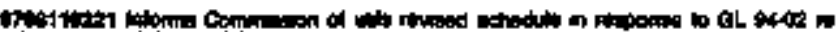

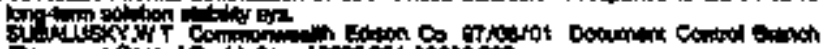

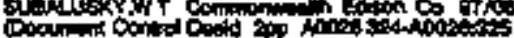

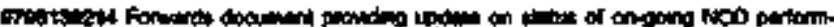

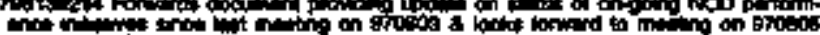

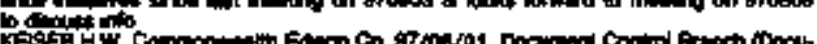

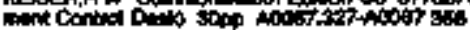

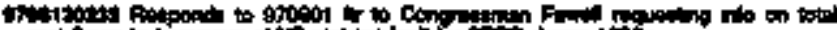

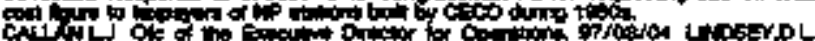

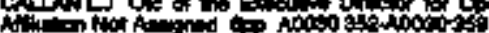

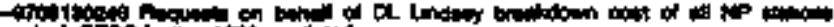

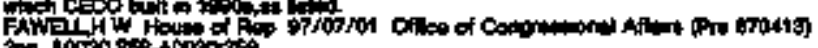

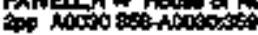

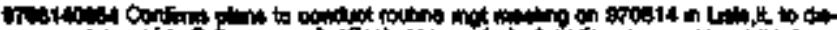

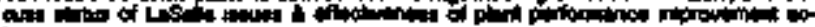

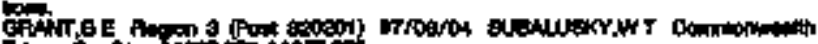

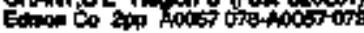

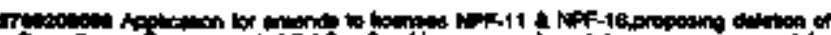

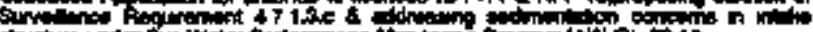

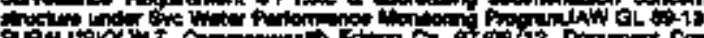

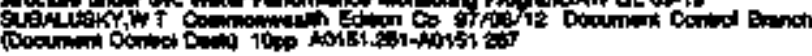

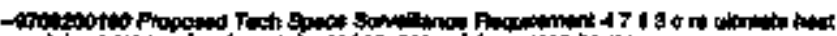

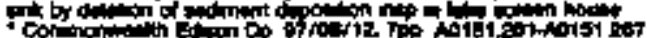

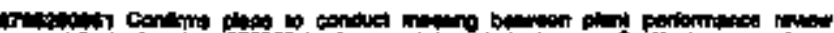

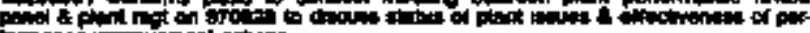

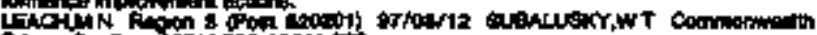

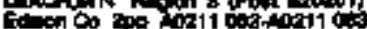

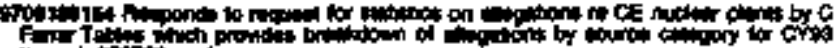

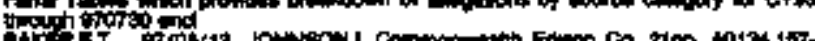

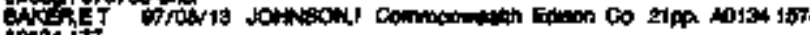
indist it

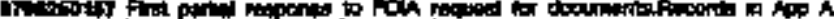
De

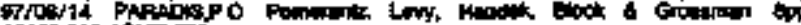

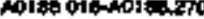




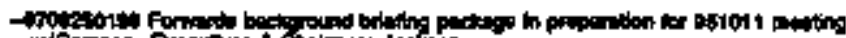

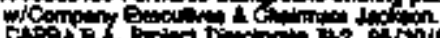

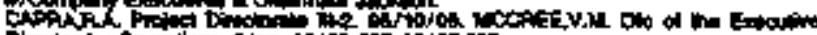

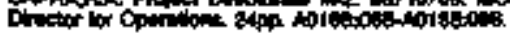

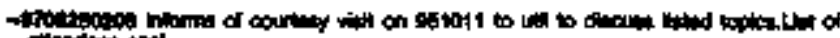

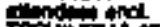

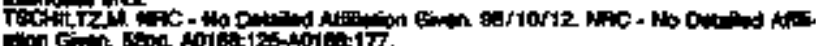
Tis.

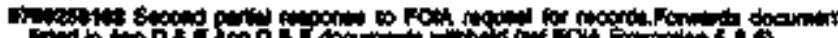

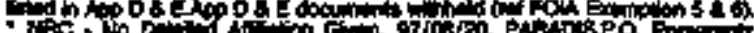

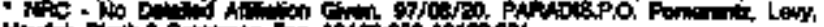

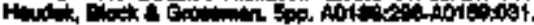

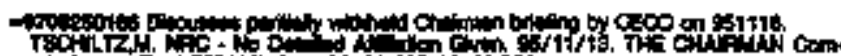

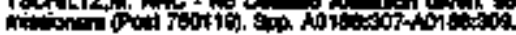

-

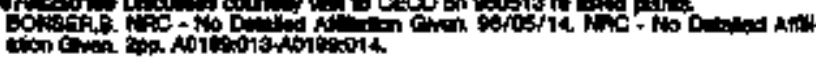

$\rightarrow$ H.

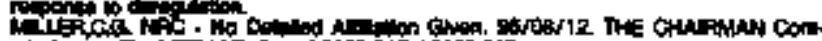

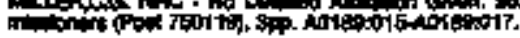

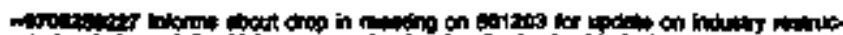

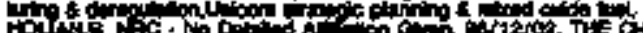

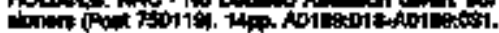

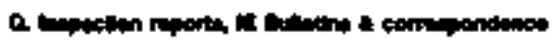

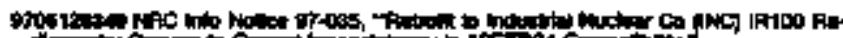
CODL

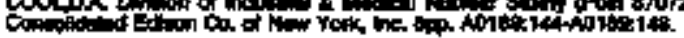

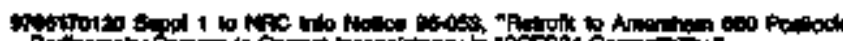

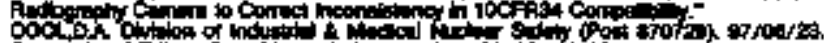

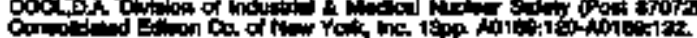

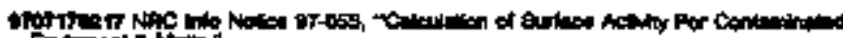

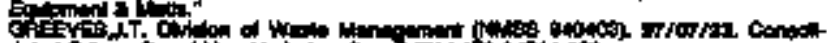

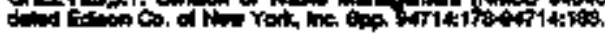

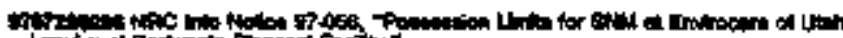

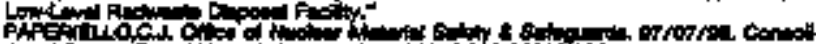

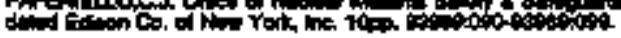

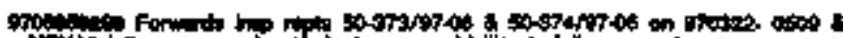

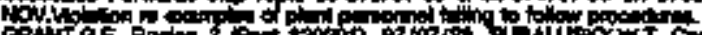

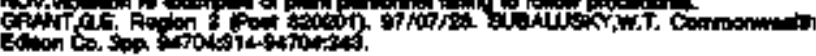

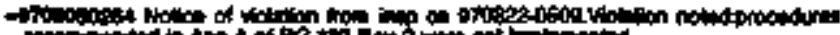

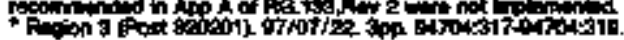

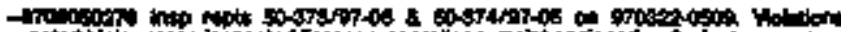

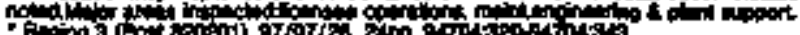

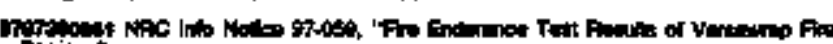

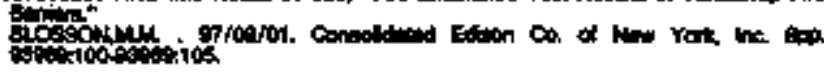

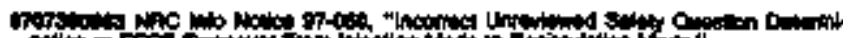

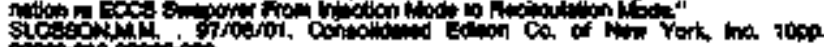
Q

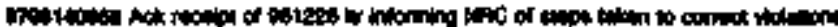

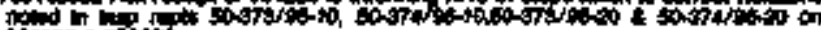

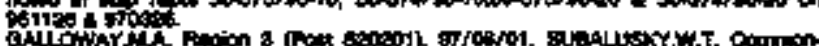

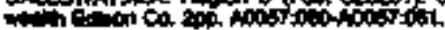

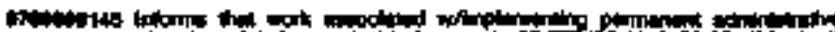

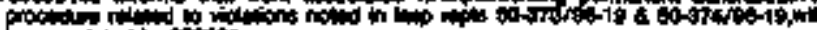

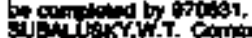

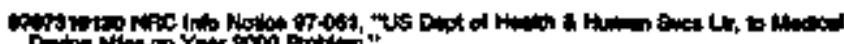

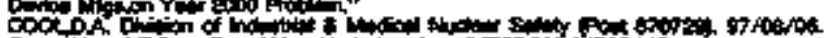
Cof

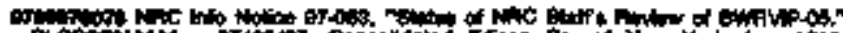

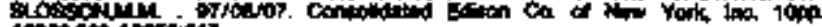

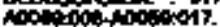

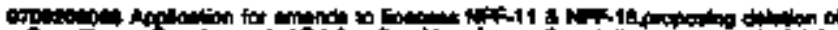

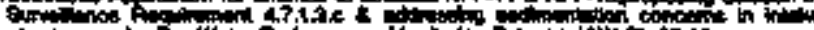

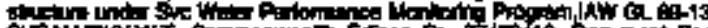

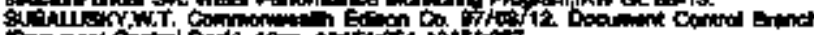

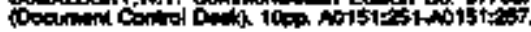

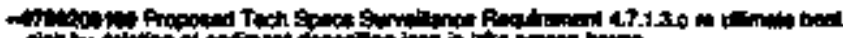

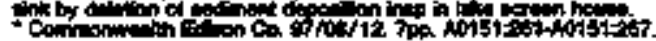

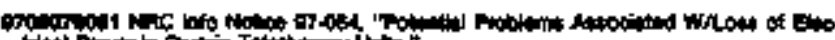

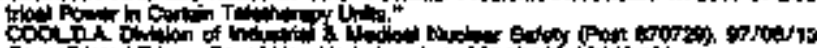

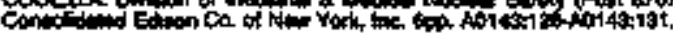

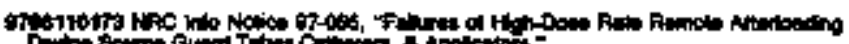

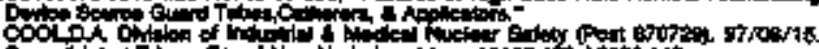

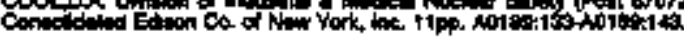

Frotion

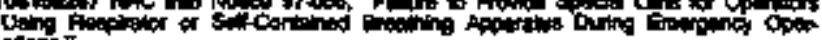

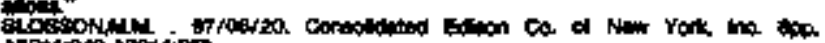

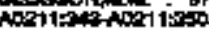

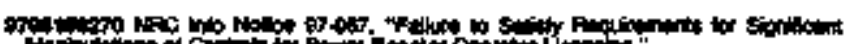

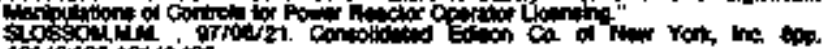

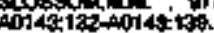

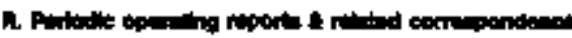

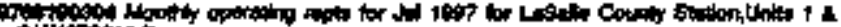

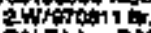

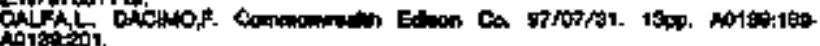

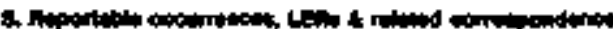

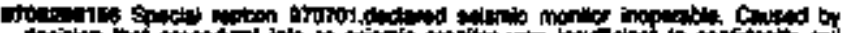

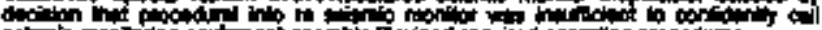

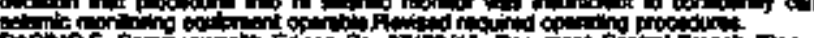

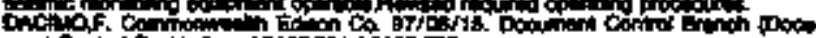

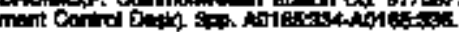

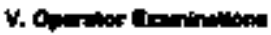

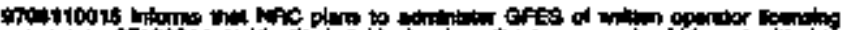

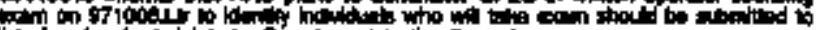

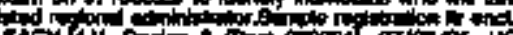

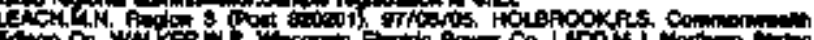

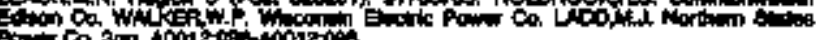

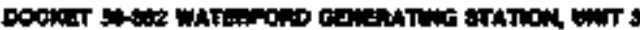

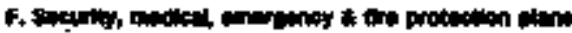

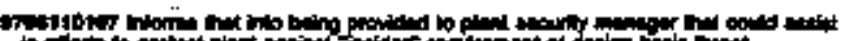

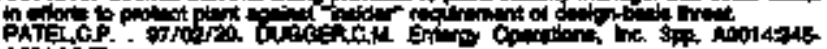
antitar.

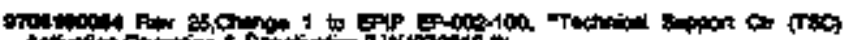

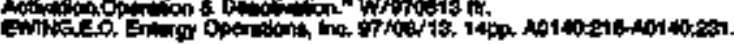

Frow

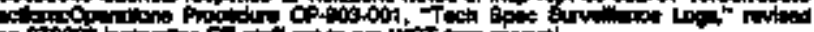

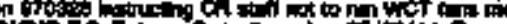

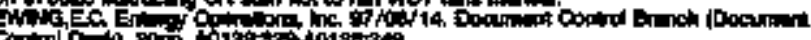

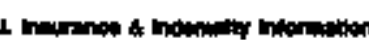

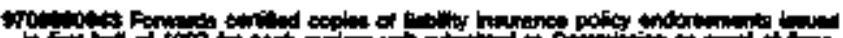

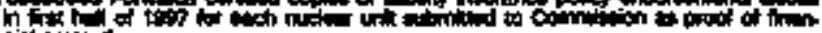

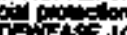

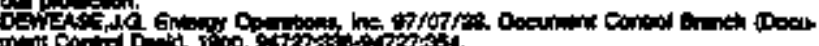

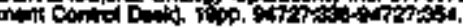

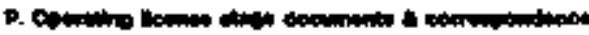

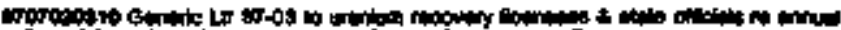

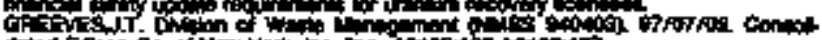

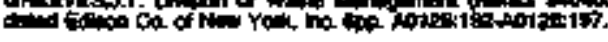

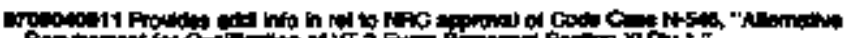

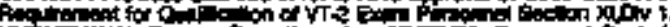

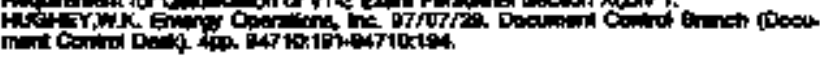

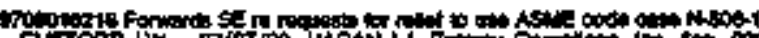

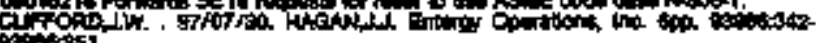
Kinctisi.

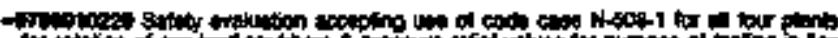

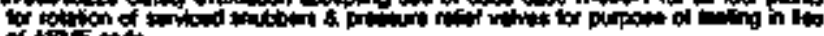

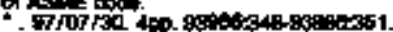

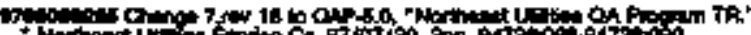




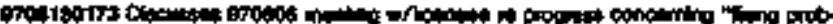

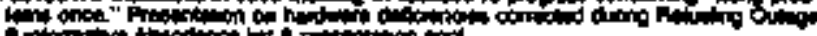

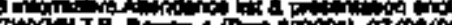

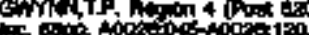

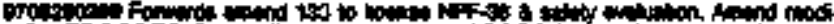

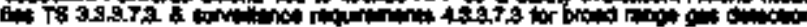

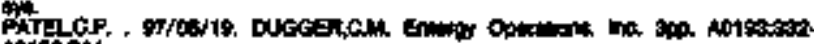
A01:30

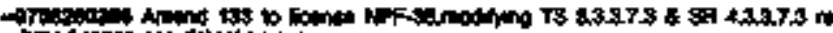

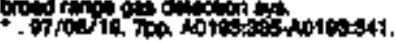

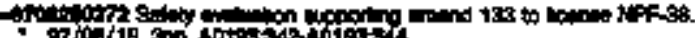

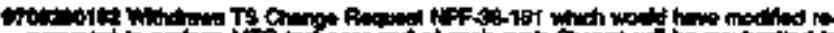

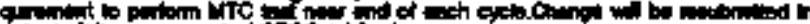

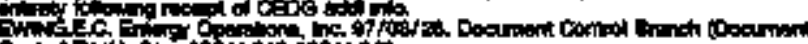

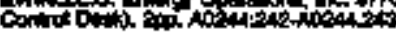

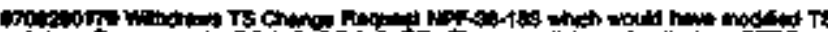

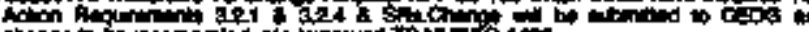

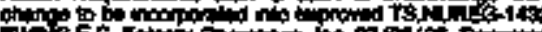

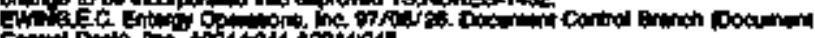

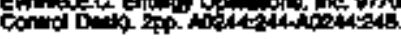

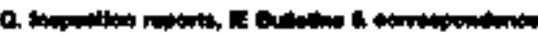

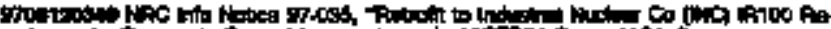

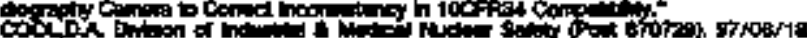

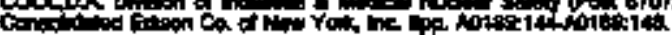

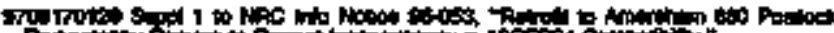

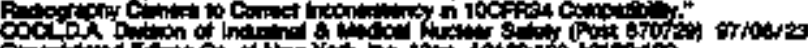

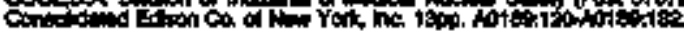

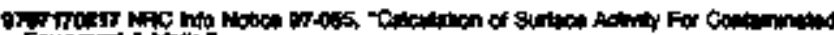

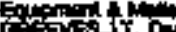

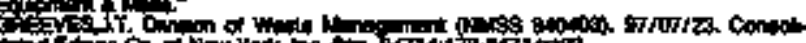

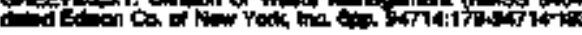

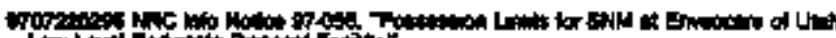

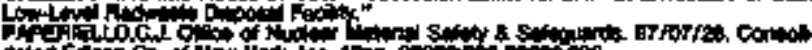
and

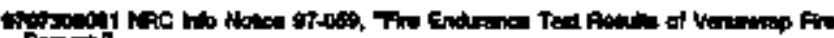

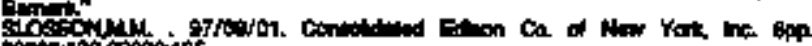

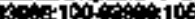

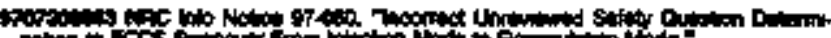

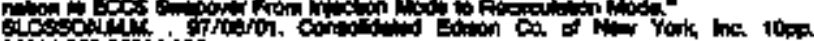

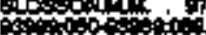

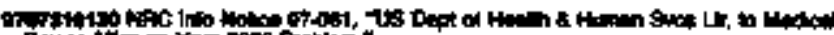

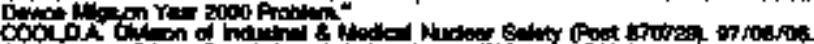

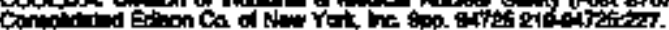

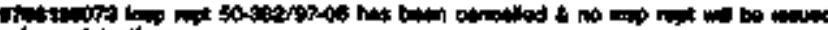

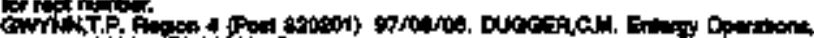

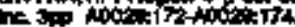

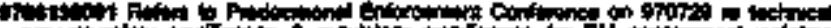

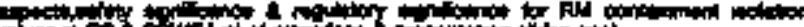

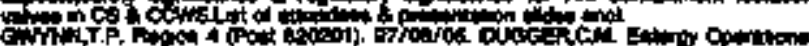

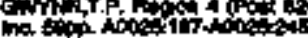

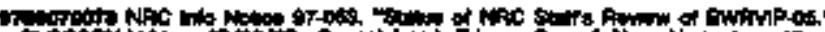

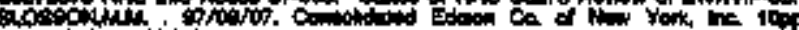

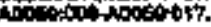

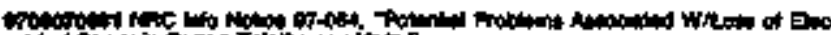

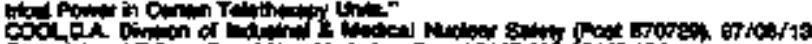

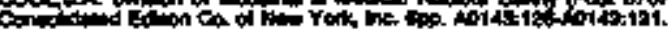

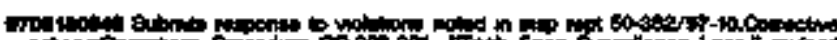

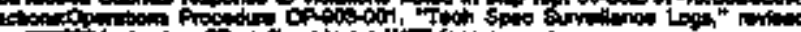

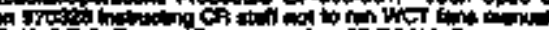

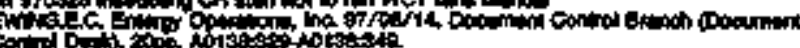

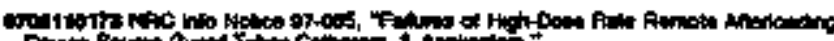

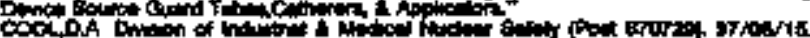

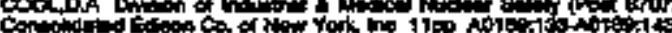

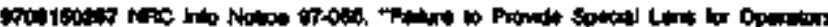

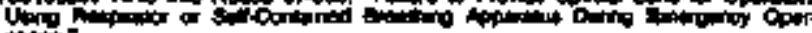

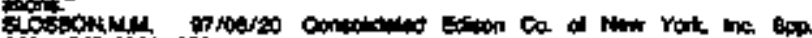

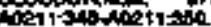

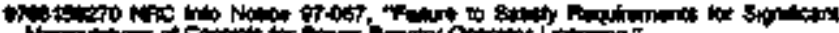

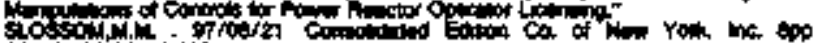

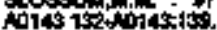

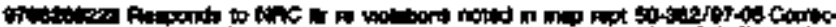

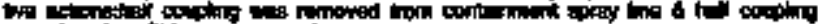

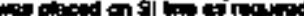

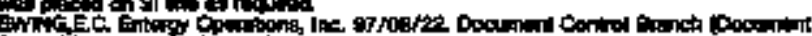

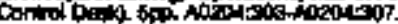

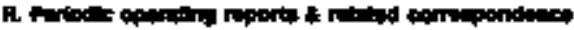

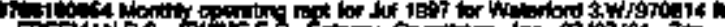
F

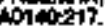

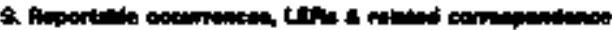

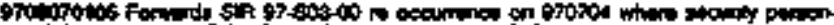

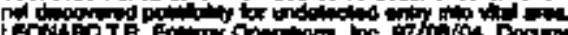

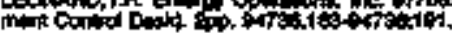

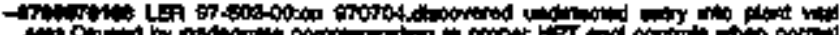

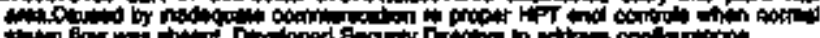

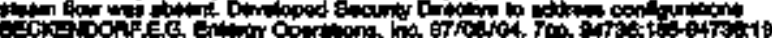

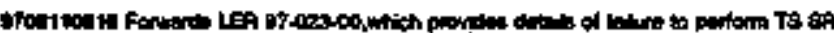

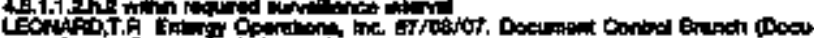

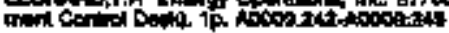

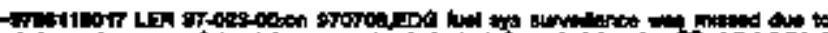

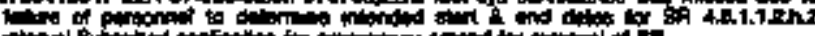

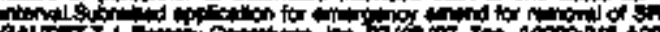

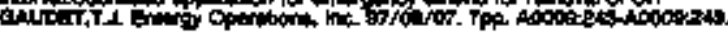

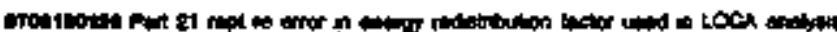

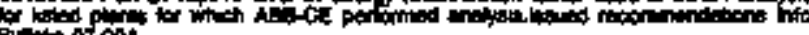

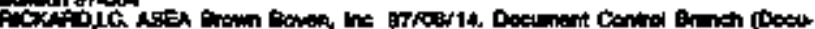

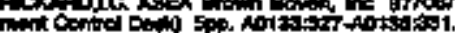

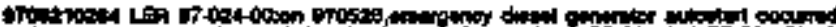

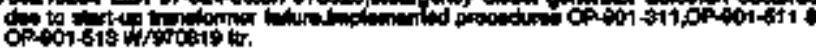

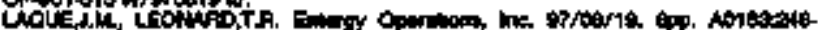
A1

\section{Opintor Escintion:}

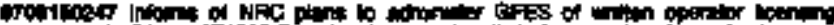

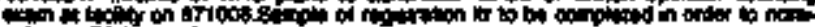

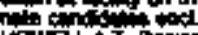

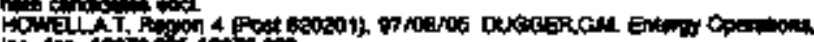

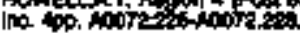

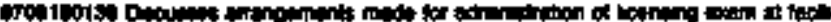

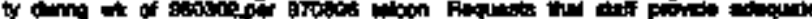

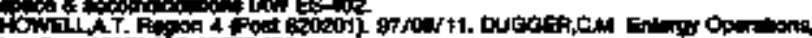
inc iop 100720 is 40072100

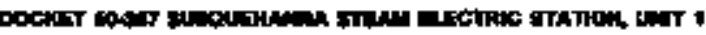

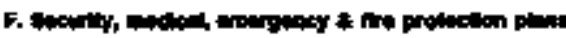

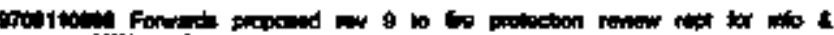
Gofothwio ind.

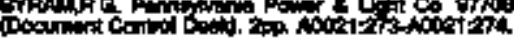

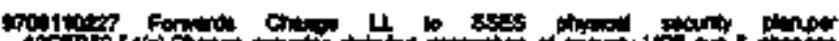

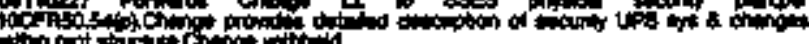

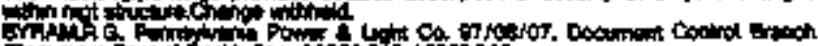

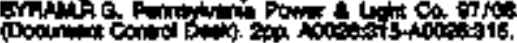

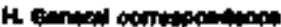

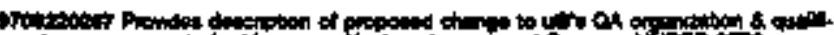

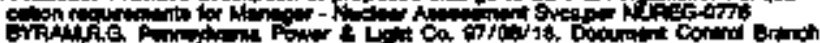

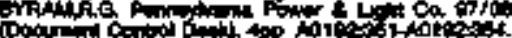

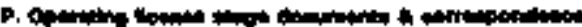

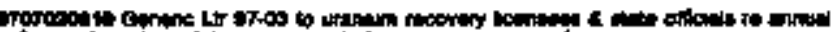

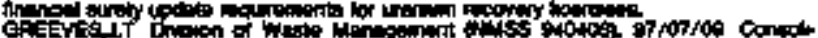

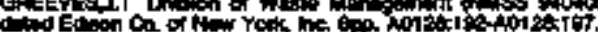

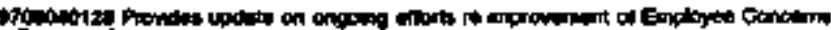

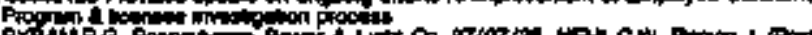
cor

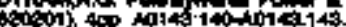




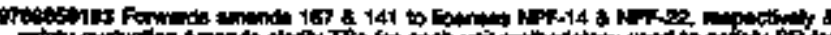

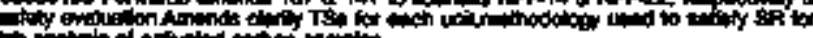

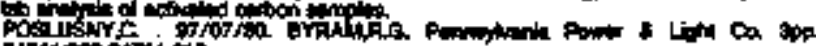

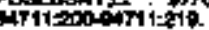

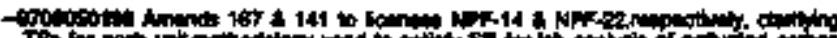

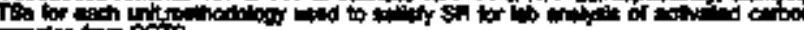

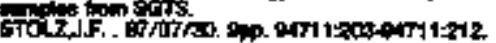

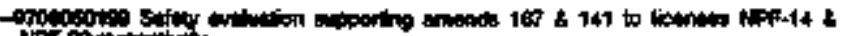

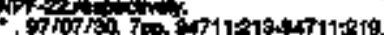

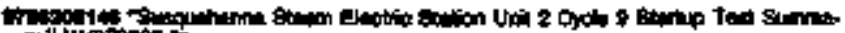

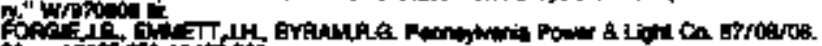

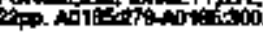

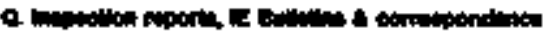

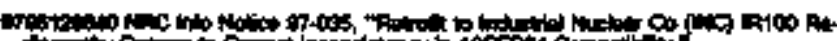

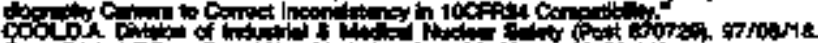

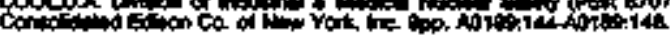

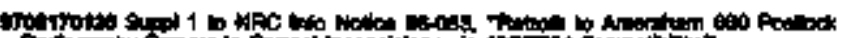

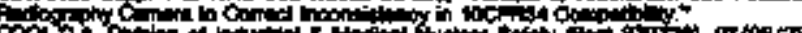

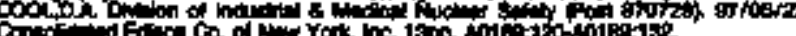

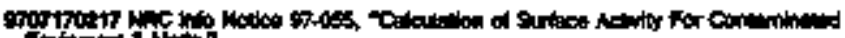

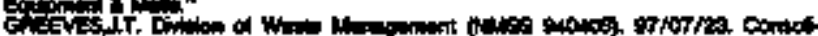

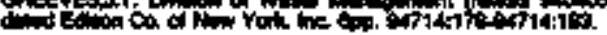

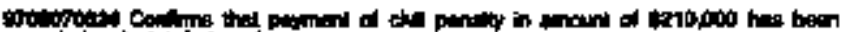

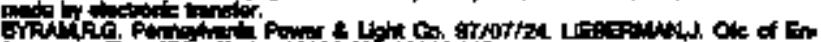

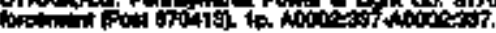

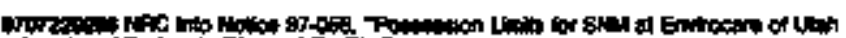
PH

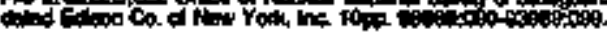

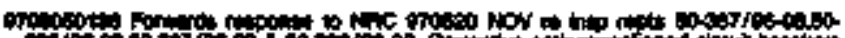

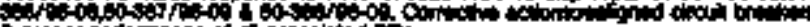

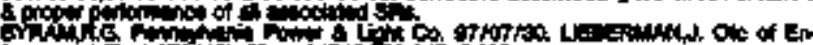

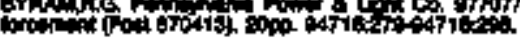

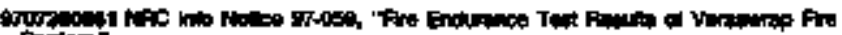

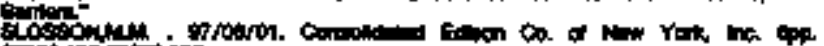
sistion

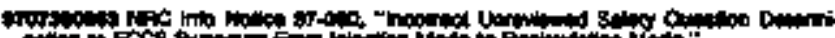

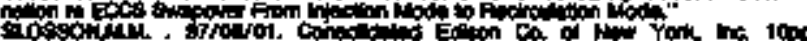

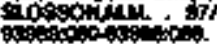

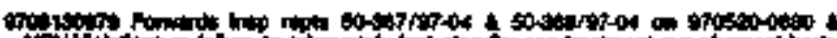
40.t.

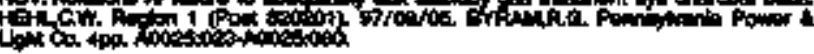

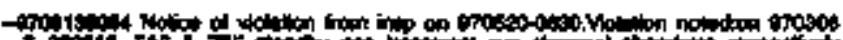

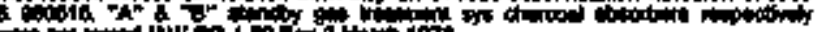

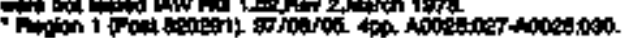

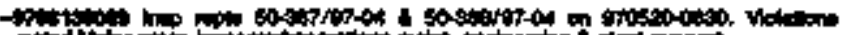

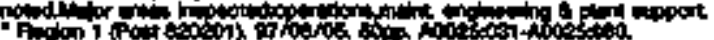

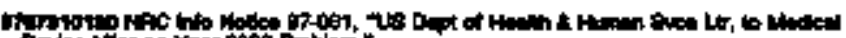

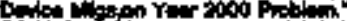

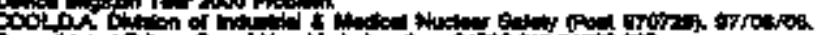

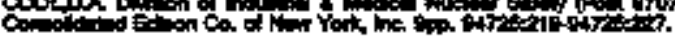

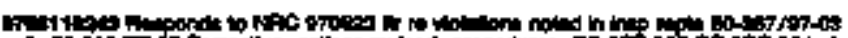

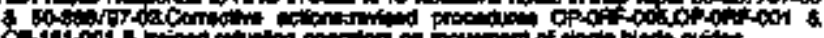

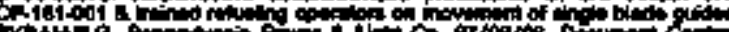

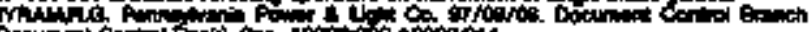

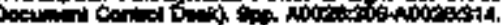

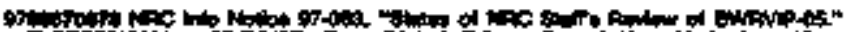

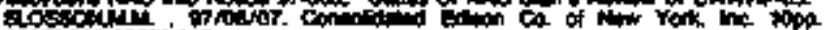

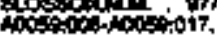

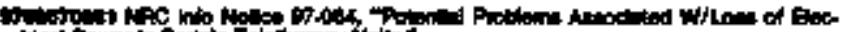

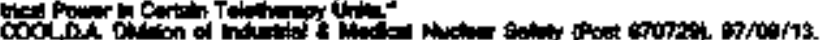

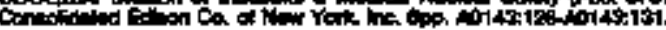

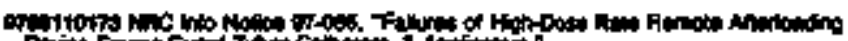

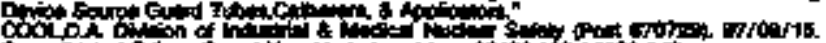

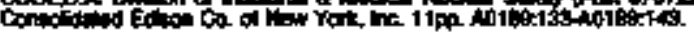

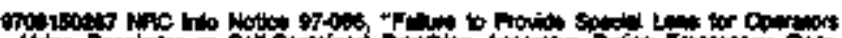

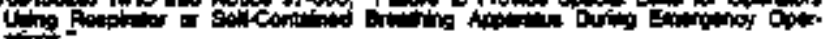

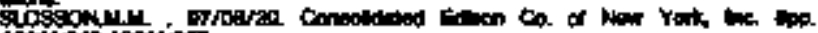

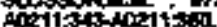

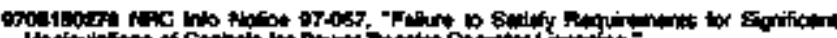

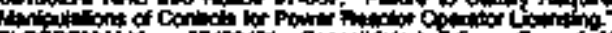

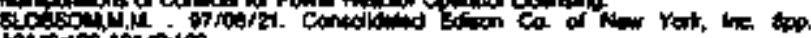

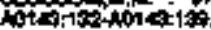

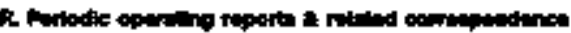

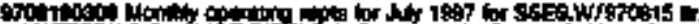

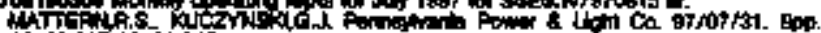

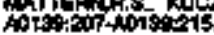

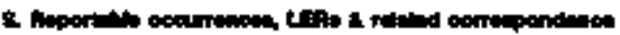

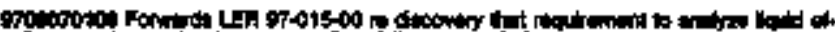

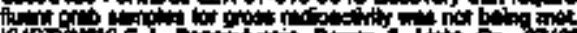
klo

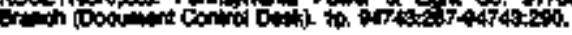

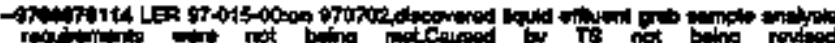

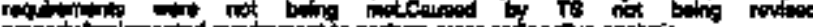

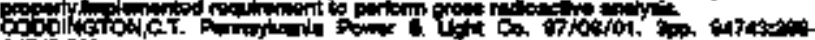

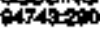

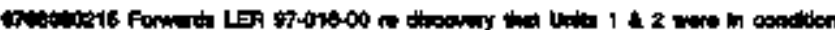

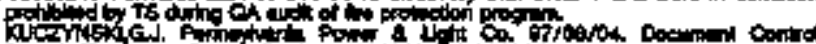

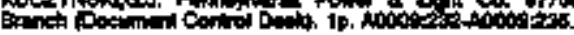

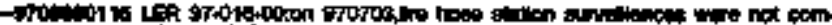

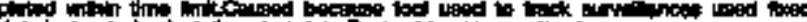

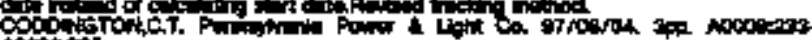
topotios

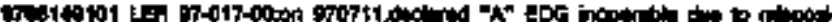

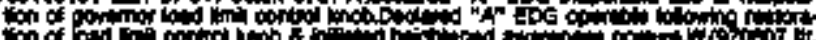

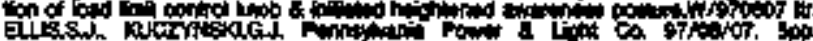

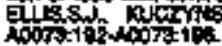

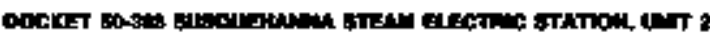

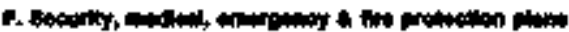

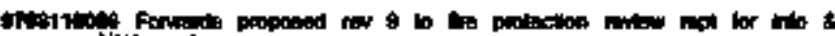

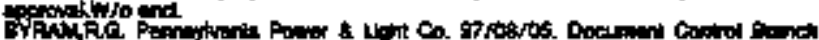

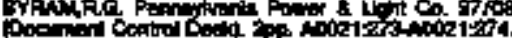

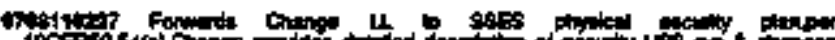

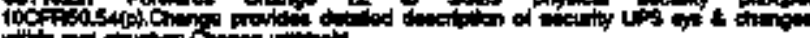

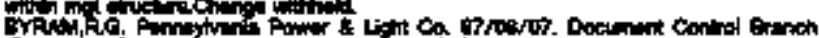

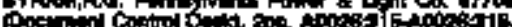

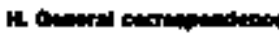

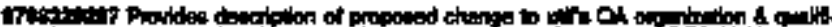

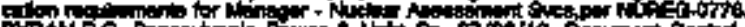

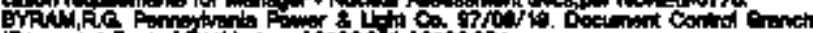

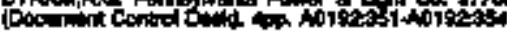

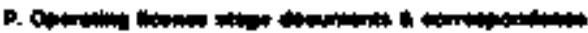

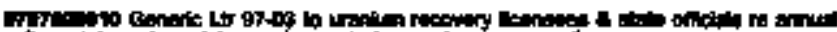

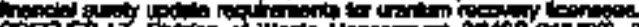

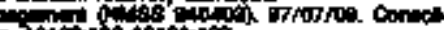

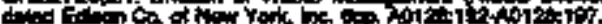

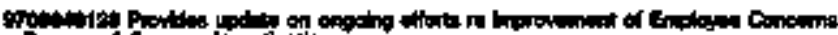

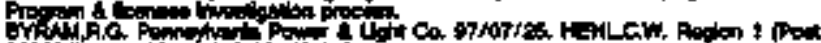

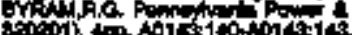

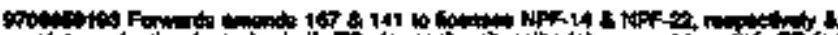

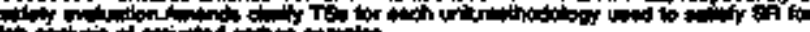

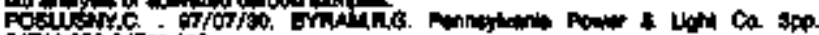

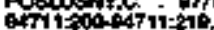

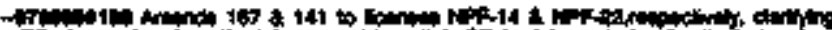

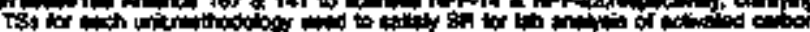
man

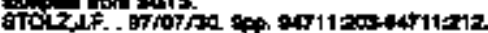

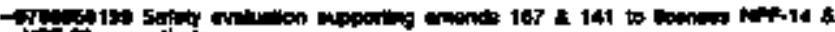

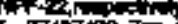

mot.

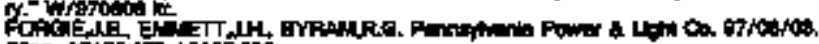

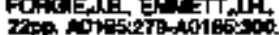




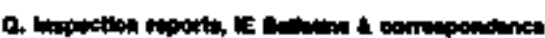

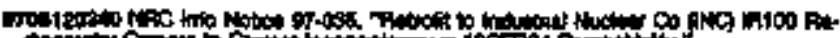

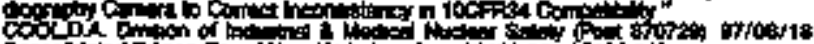

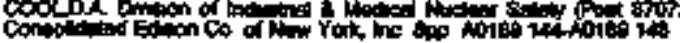

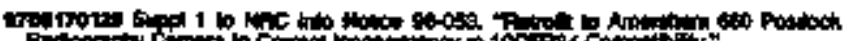

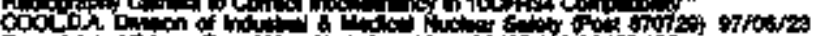

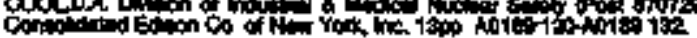

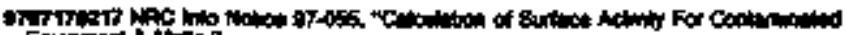

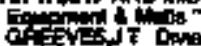

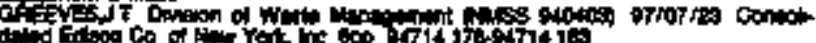

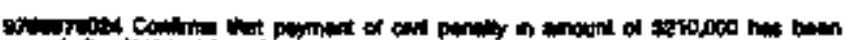

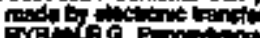

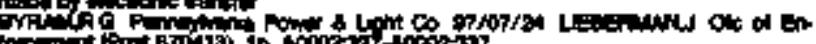

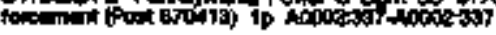

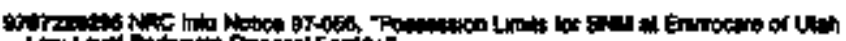
Pht L

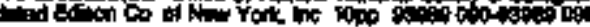

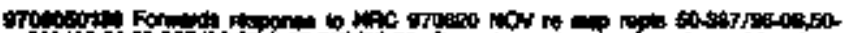

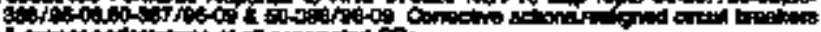

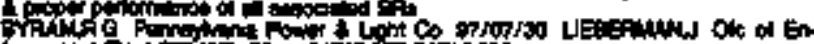

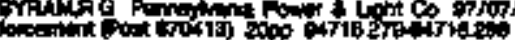

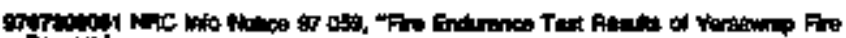

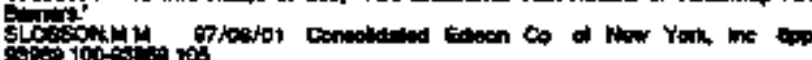

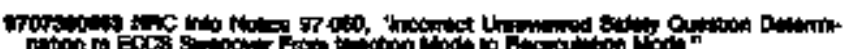

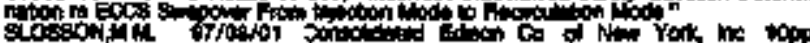

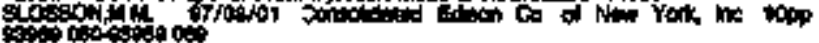

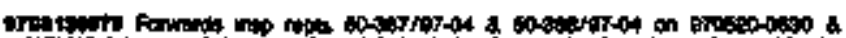

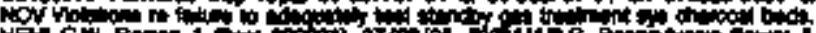

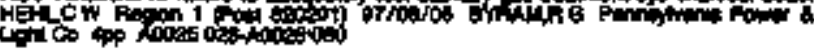

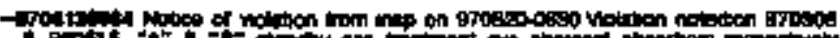

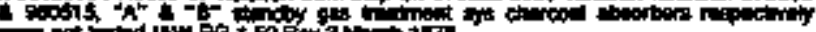

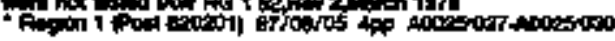

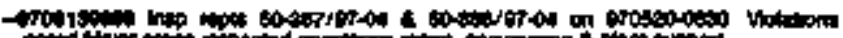

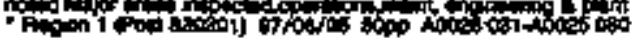

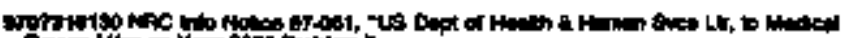

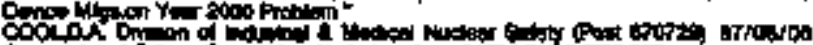

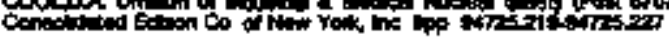

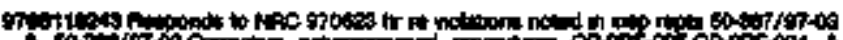

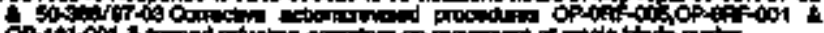

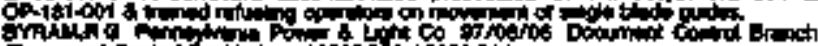

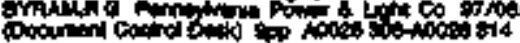

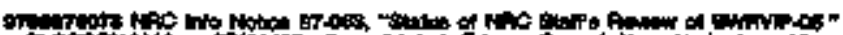

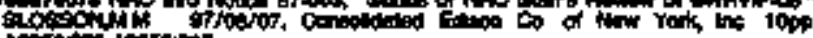

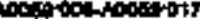

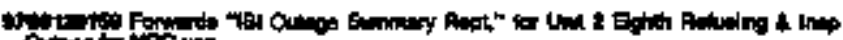

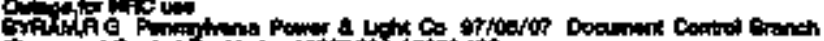

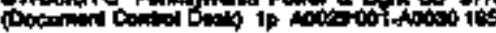

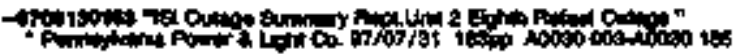

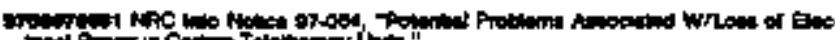

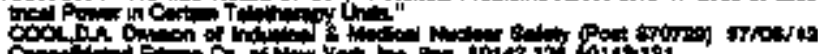

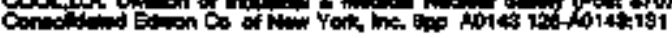

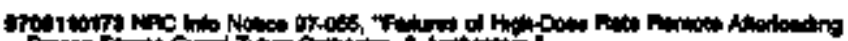

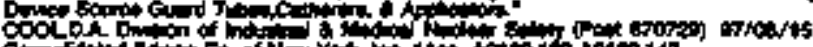

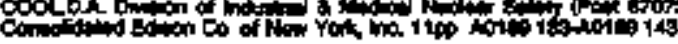

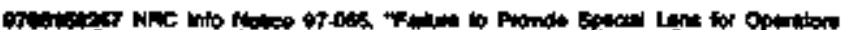

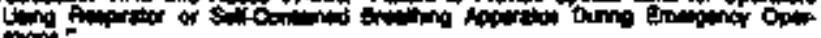

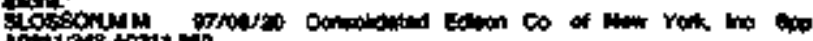

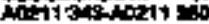

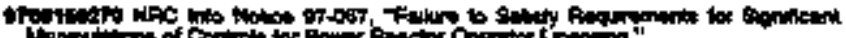

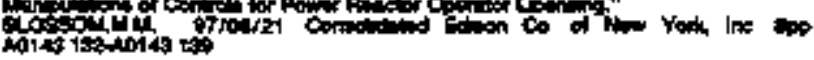

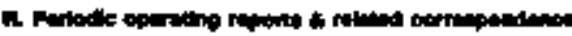

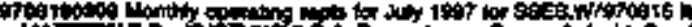

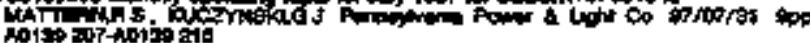

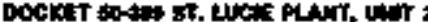

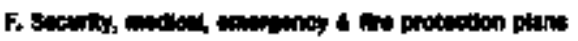

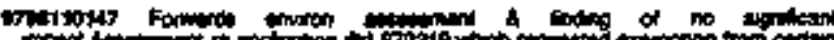

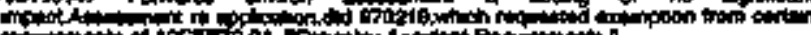
Hastion of Minotion

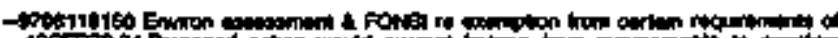

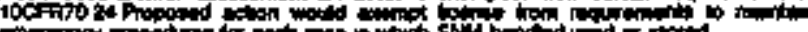

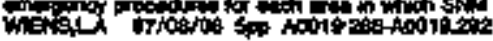

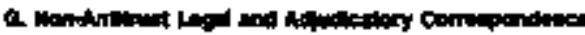

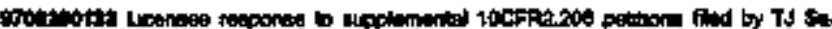

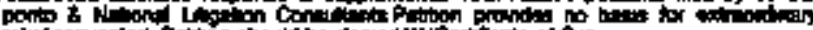

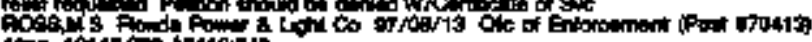

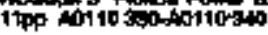

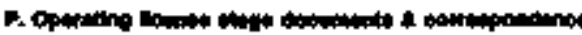

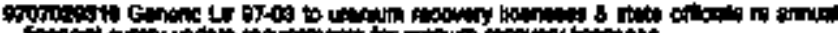

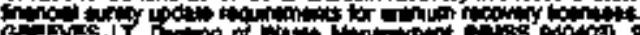

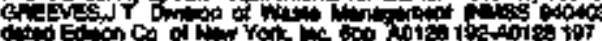

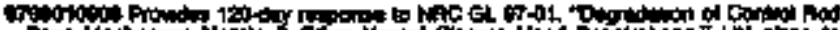

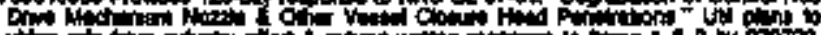

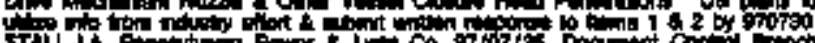

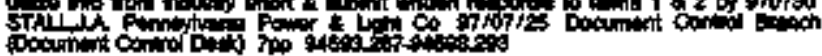

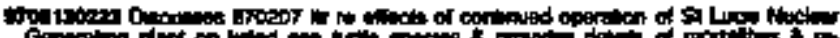

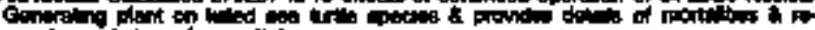
Pitions

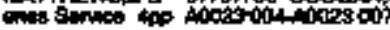

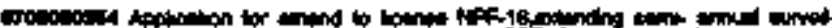

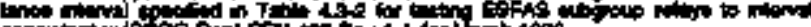

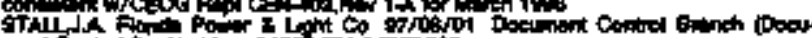

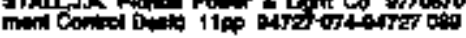

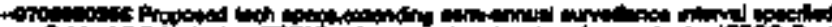

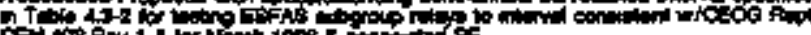

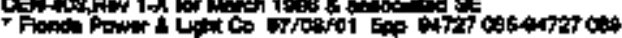

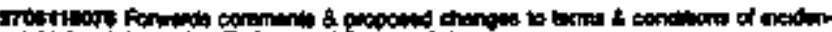
-

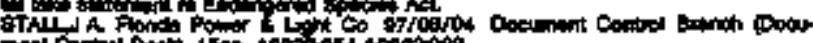

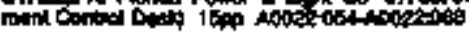

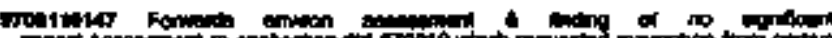

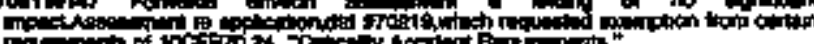

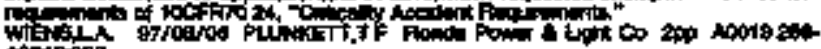
wotong

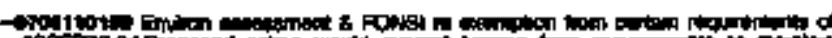

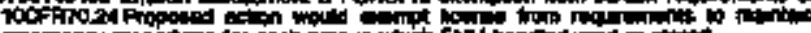

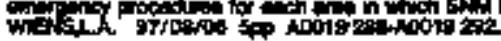

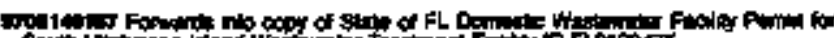

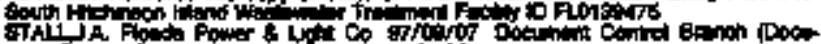

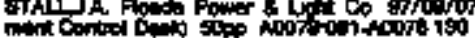

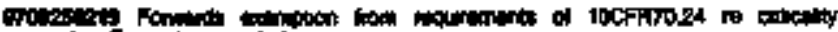

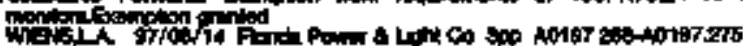

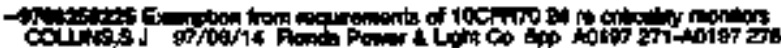

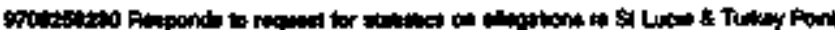

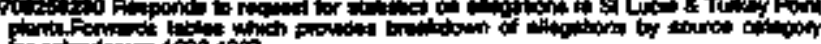

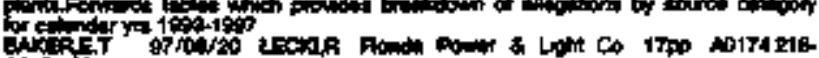
antis

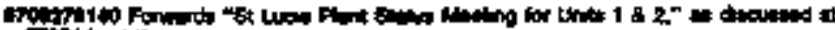

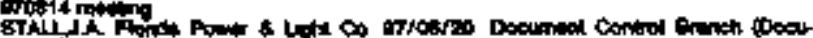

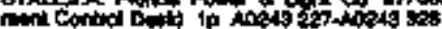

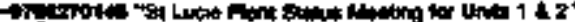

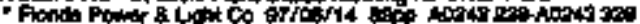

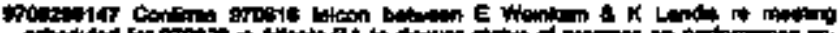

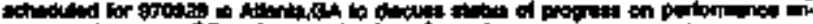

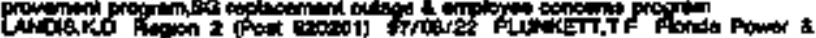

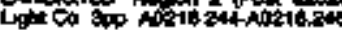




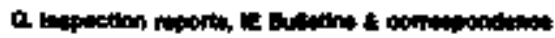

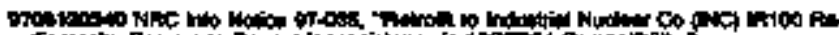

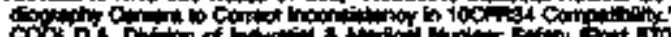

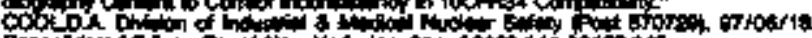

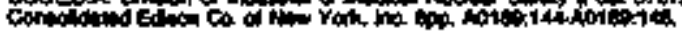

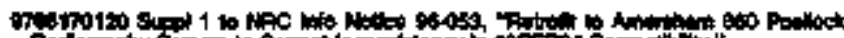

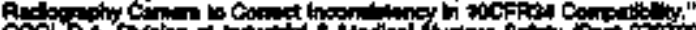

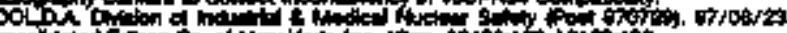

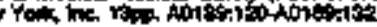

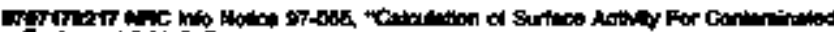

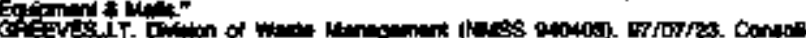

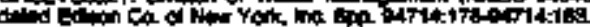

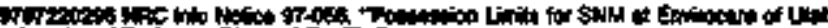

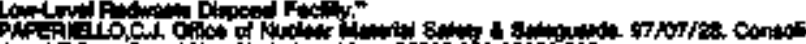

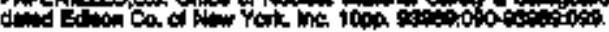

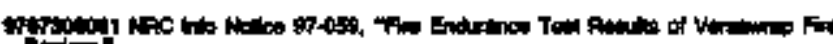

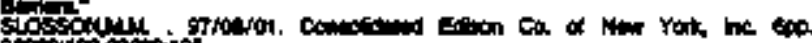
कि

Fir wow

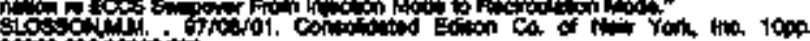

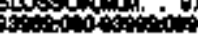

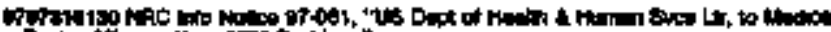

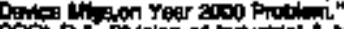

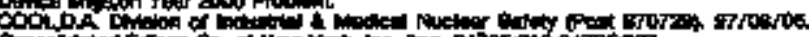

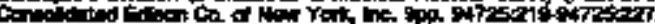

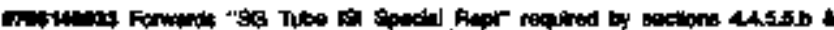

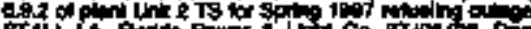

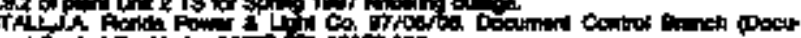

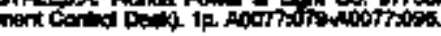

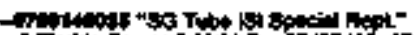

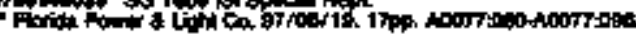

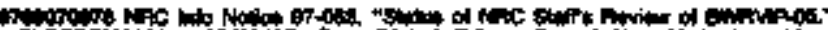

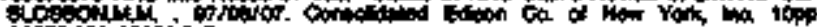

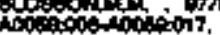

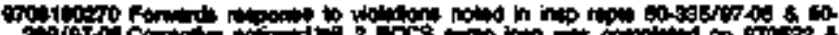

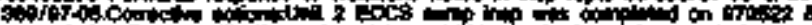

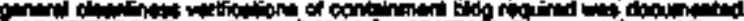

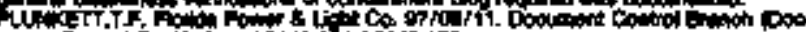

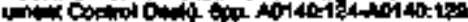

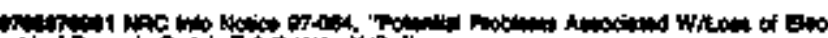

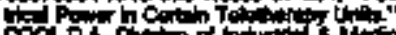

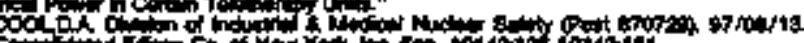
Con

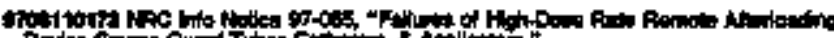

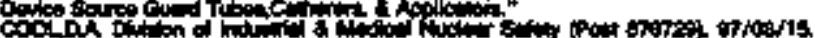

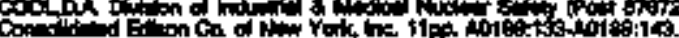

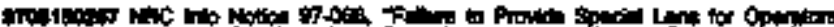

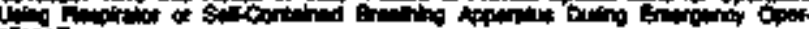

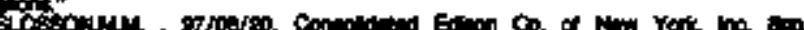

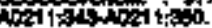

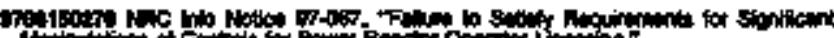
"Wo: AT:18

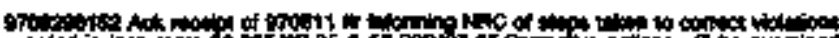

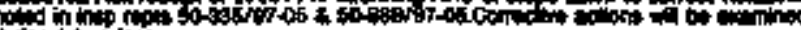
tint

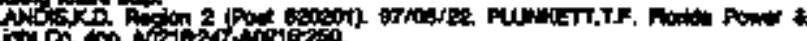

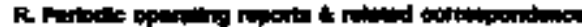

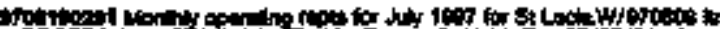

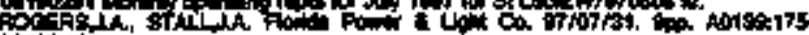

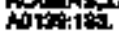

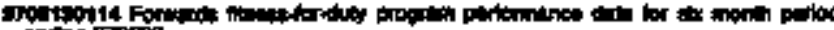

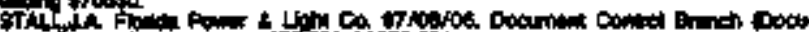

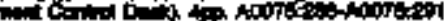

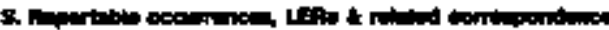

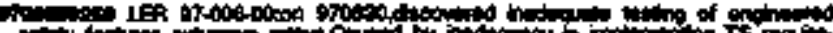

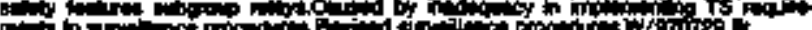

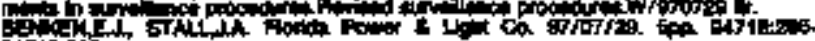
atration

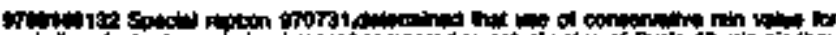

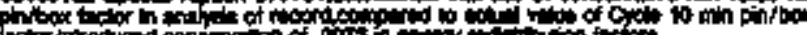

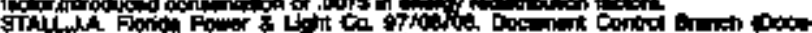

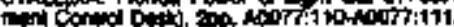

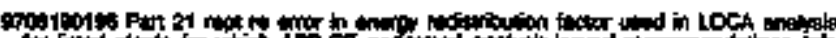

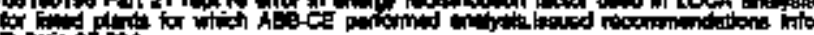

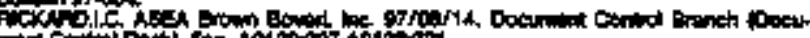

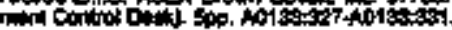

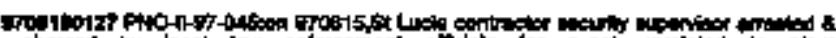

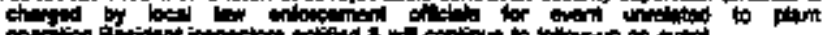

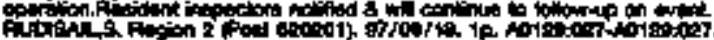

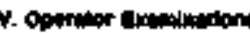

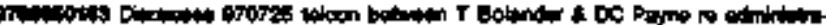

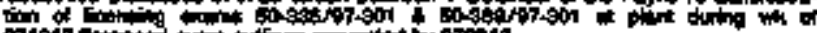

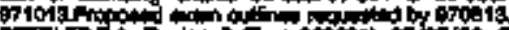

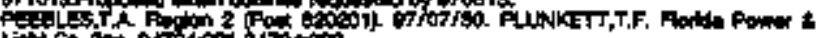

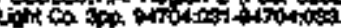

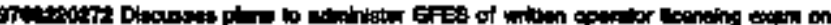

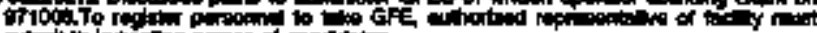

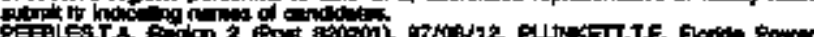

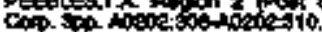

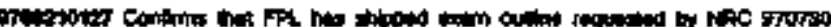

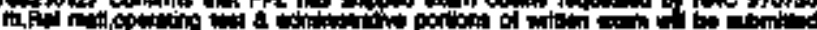

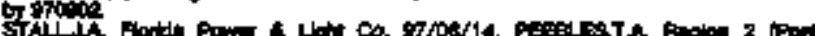

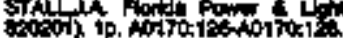

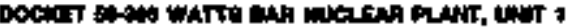

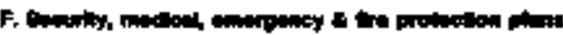

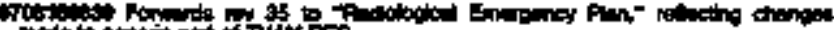

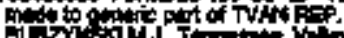

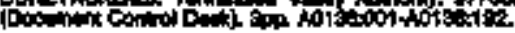

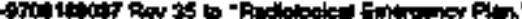

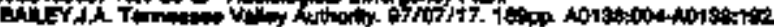

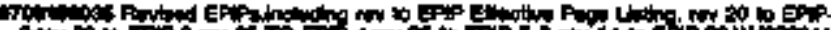

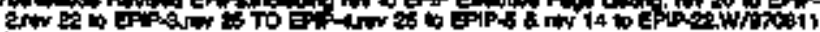

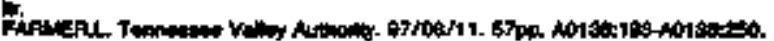

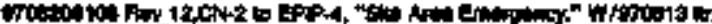

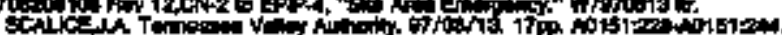

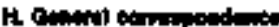

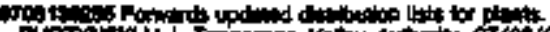

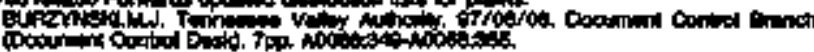

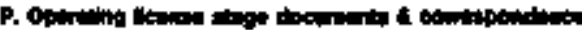

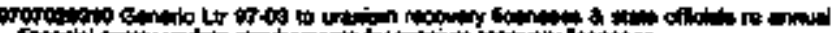

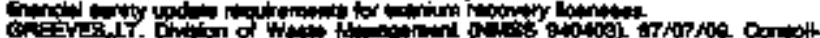

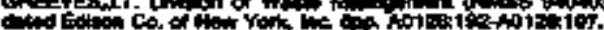

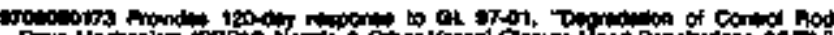

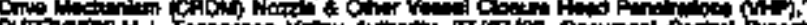

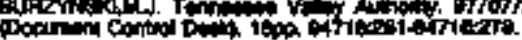

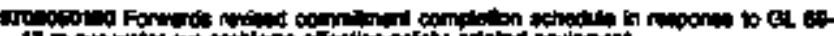

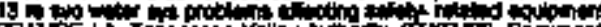
(1)

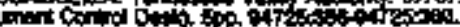

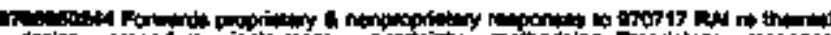

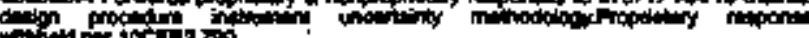

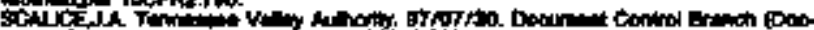

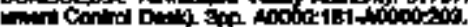

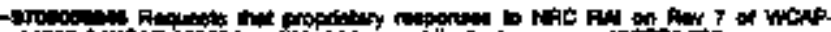

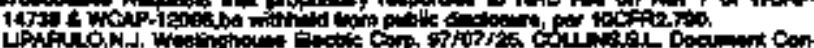

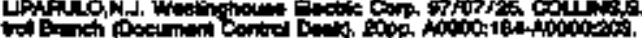

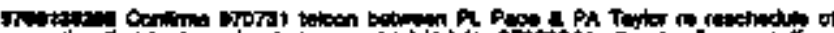

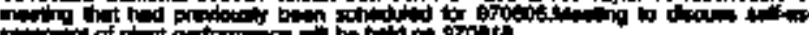
ís tor

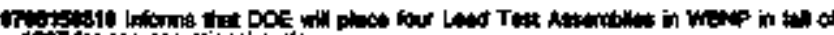

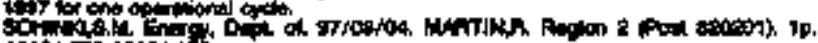

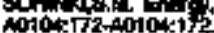




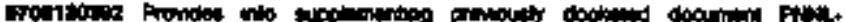

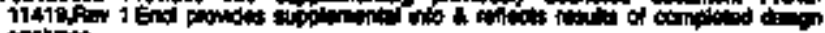

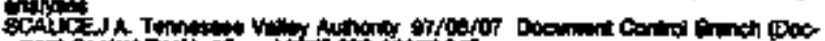

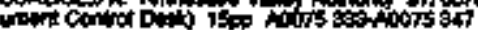

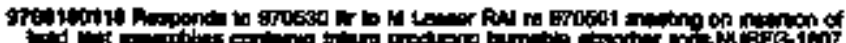

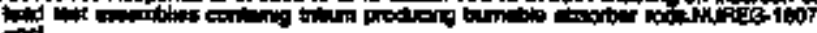

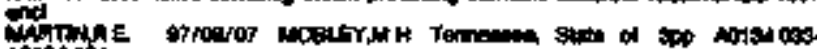
Anot 20

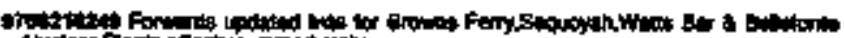

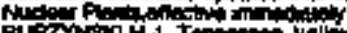

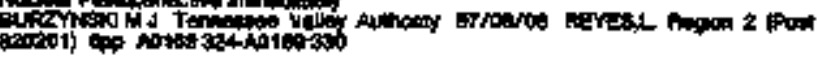

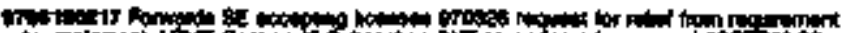

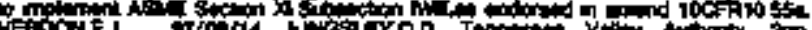

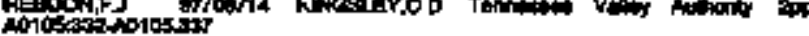

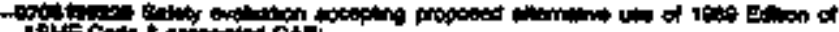

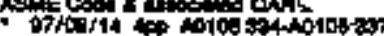

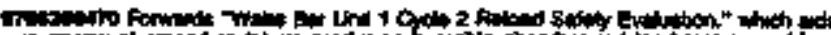

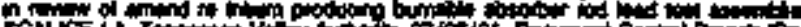

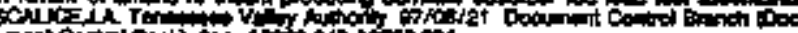

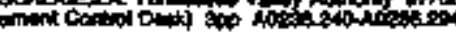

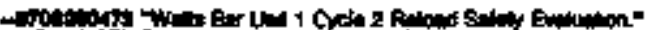

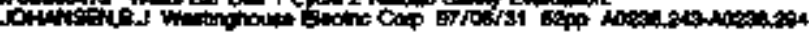

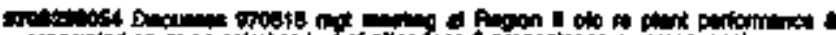

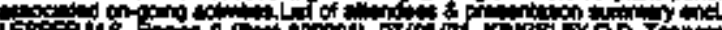

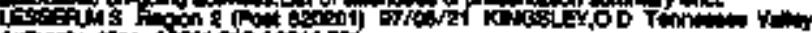

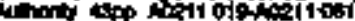

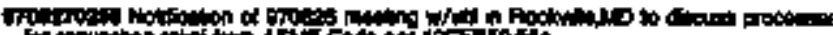

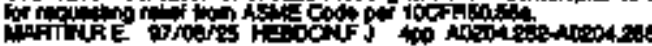

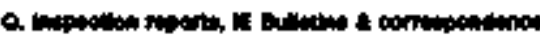

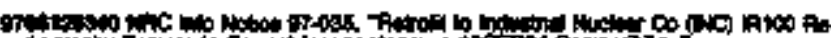

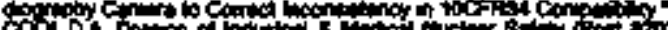

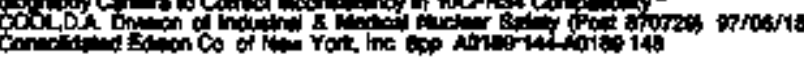

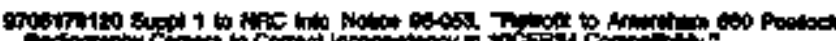

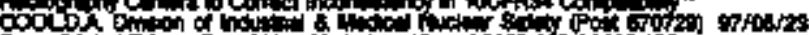

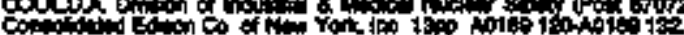

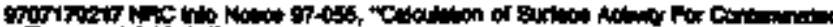

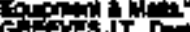

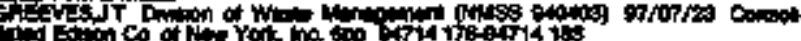

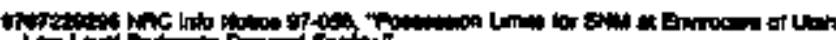
Pot

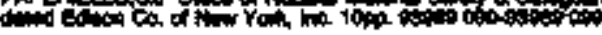

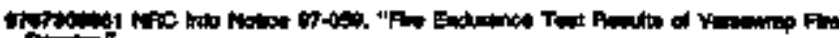

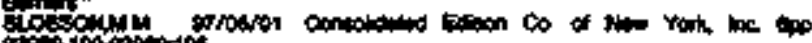

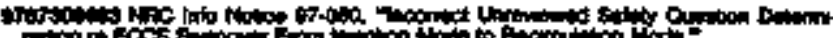

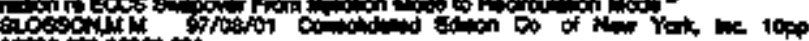
sto

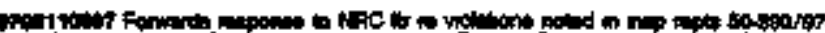

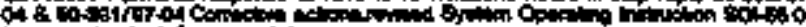

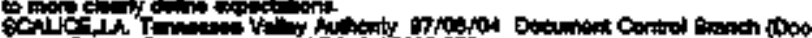

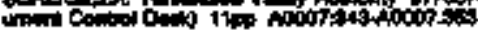

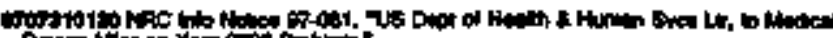

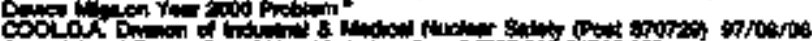

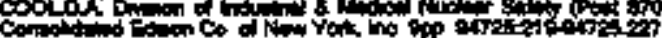

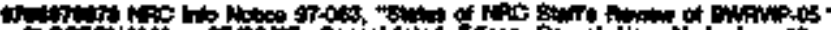

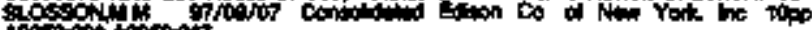

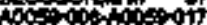

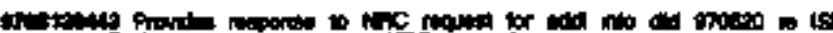
ogrth

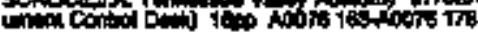

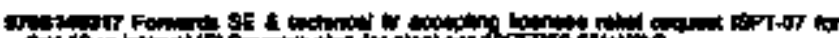

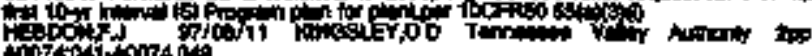

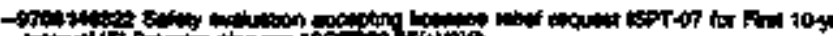

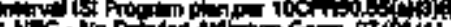

Ant

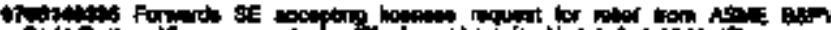

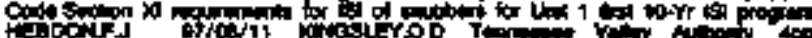

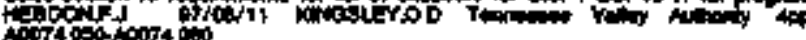

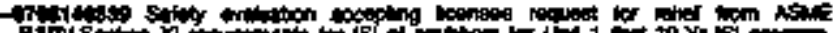

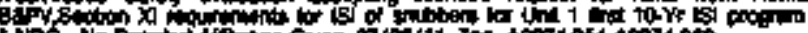

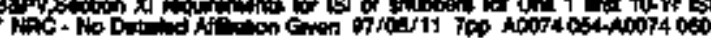

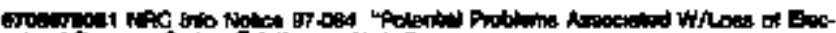

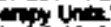

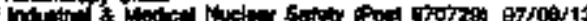

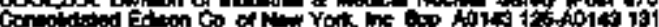

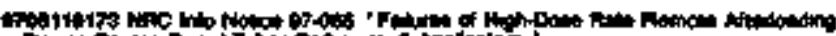

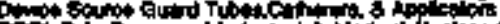

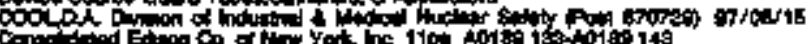

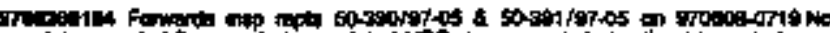

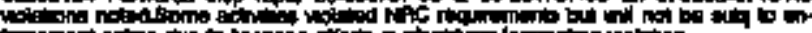

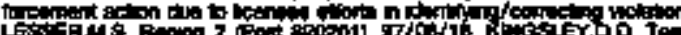

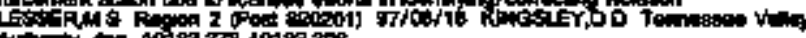

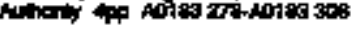

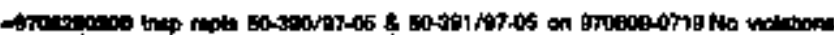

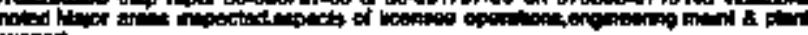
arpoct

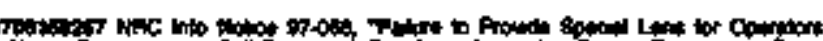

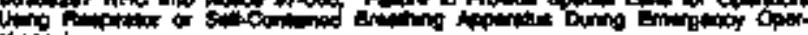

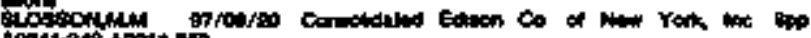
toe 11:3484ket1 30

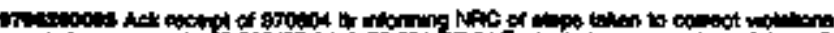

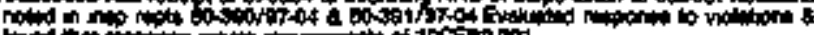

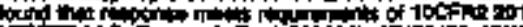

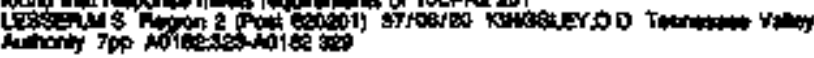

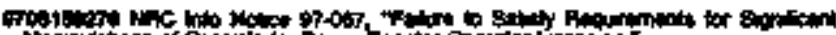

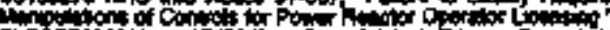

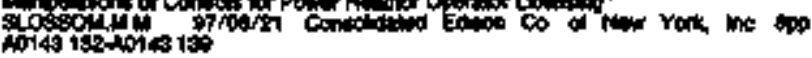

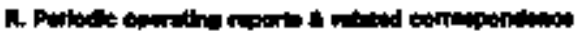

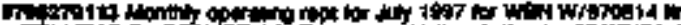

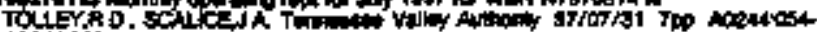
A

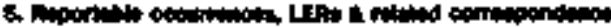

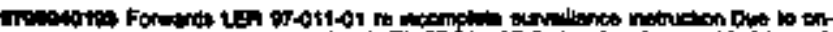

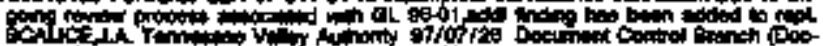

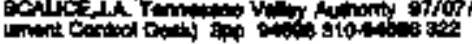

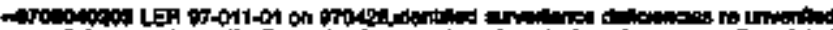

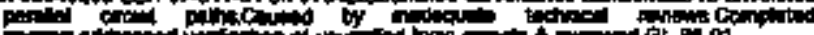

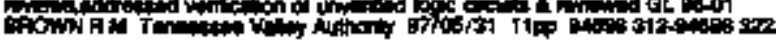

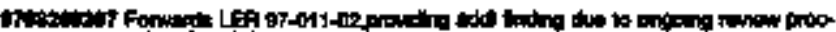

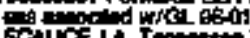

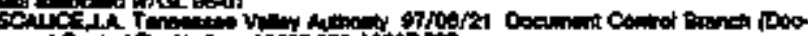

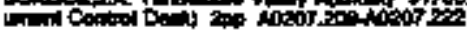

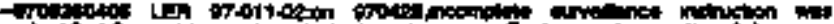

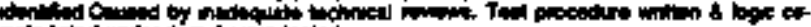

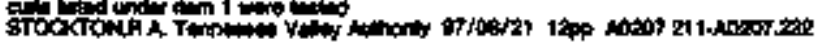

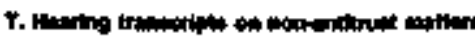

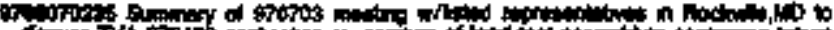

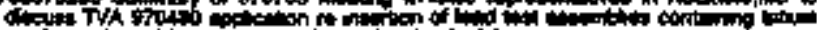

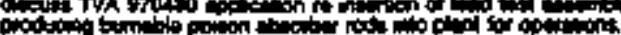

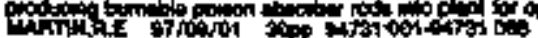

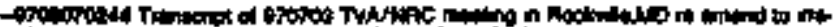

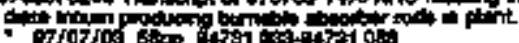

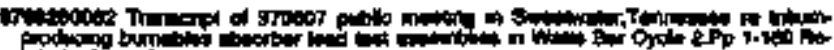
Fidering by

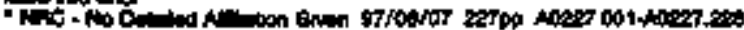

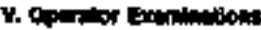

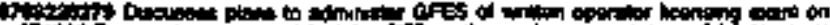

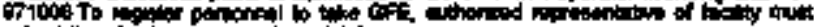

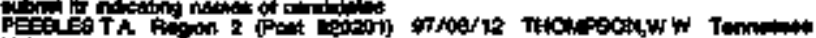

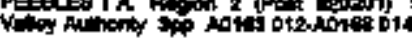

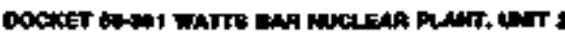




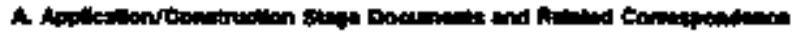

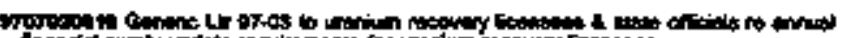

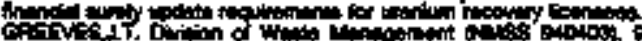

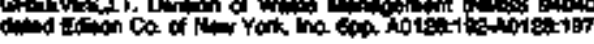

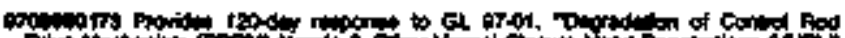

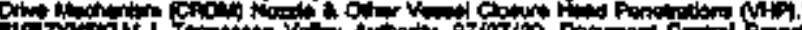

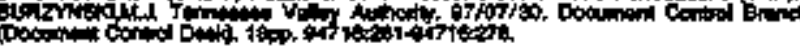

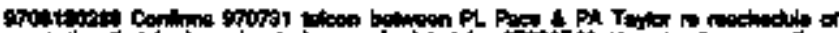

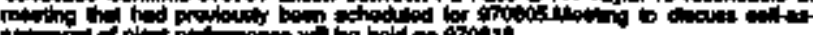
1

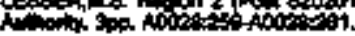

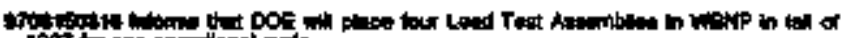

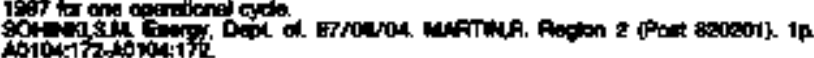

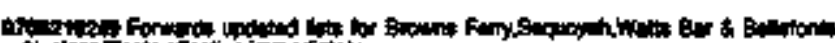

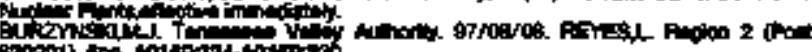

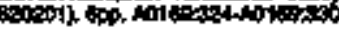

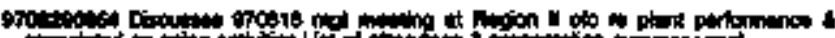
I

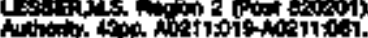

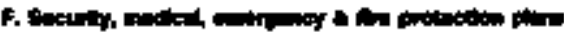

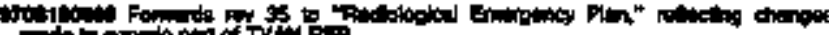

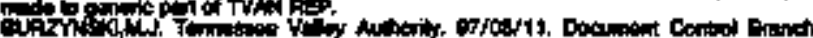

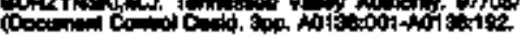

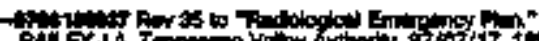

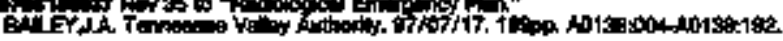

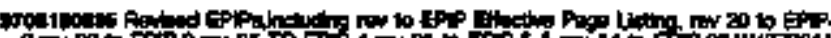

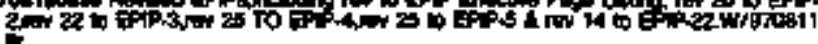

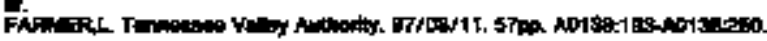

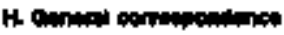

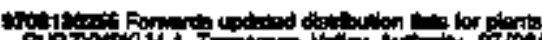

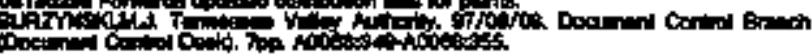

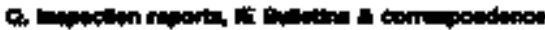

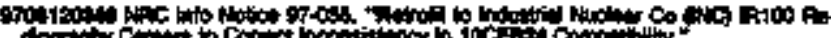

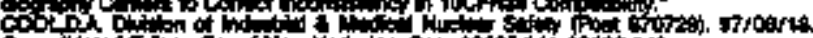

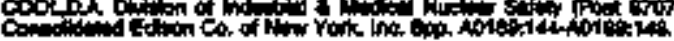

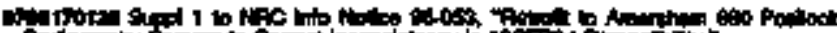

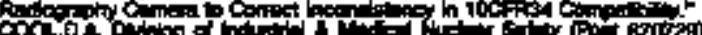

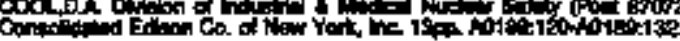

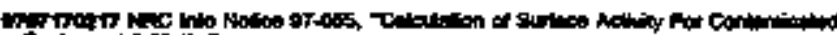

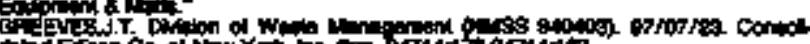

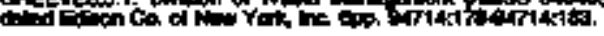

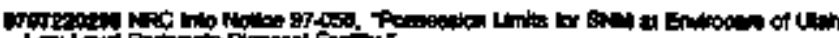
PAPG

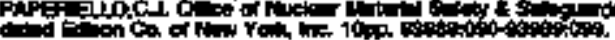

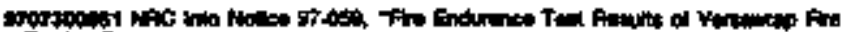

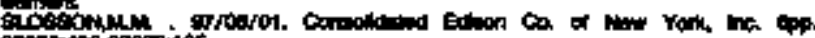

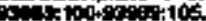

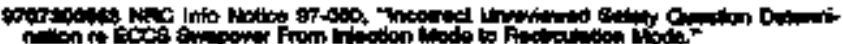

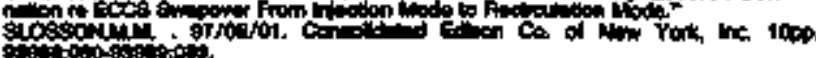

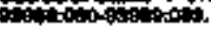

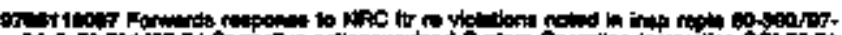

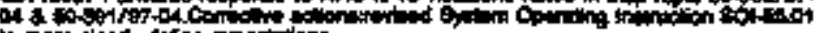

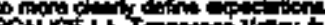

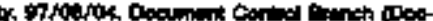
(1)

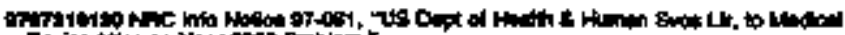

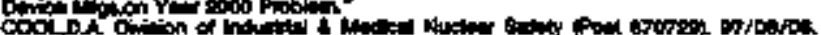

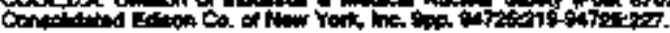

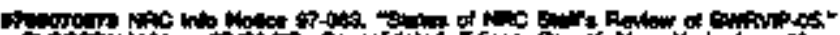
stopow

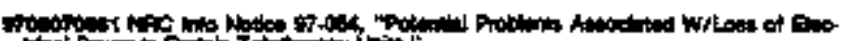

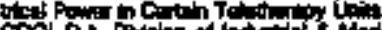

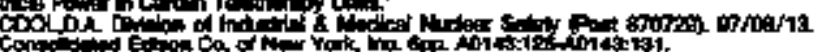

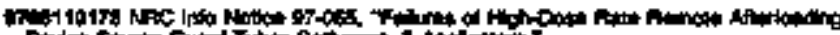

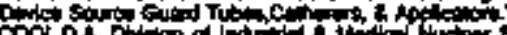

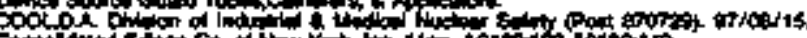

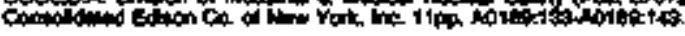

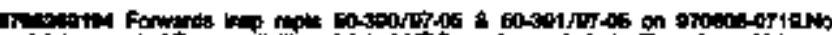

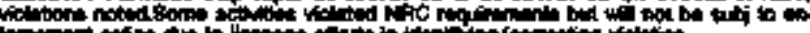

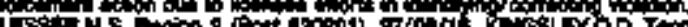

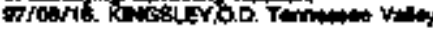

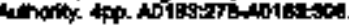

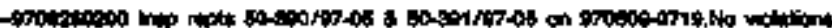

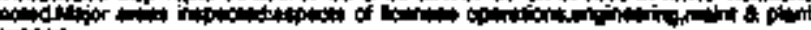

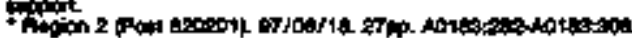

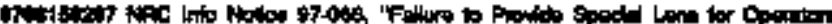

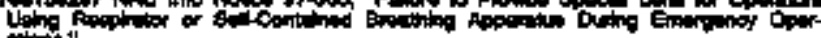

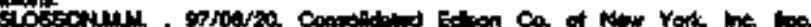

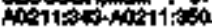

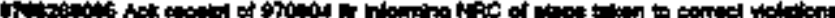

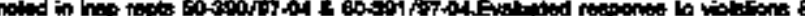

I

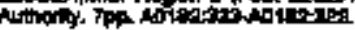

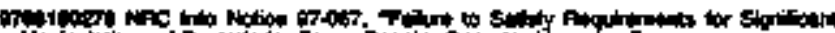

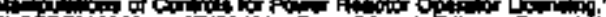

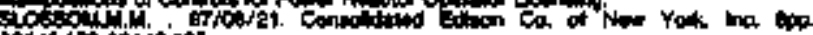

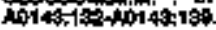

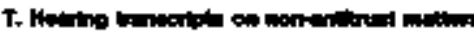

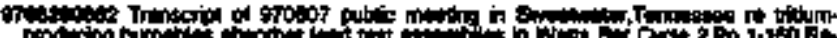

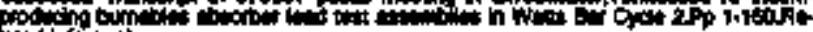

-

\section{Opuld Fration:}

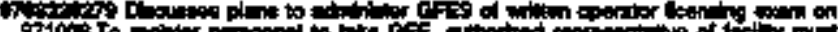

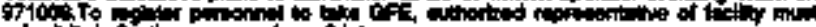

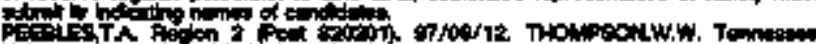

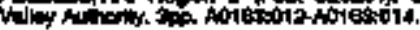

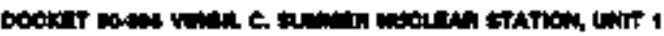

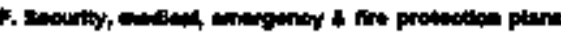

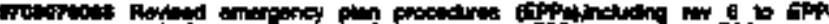

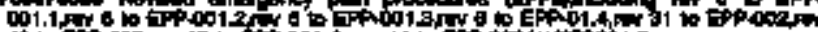

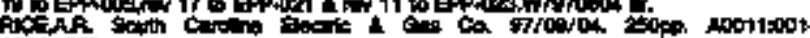
M0011250

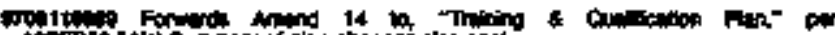

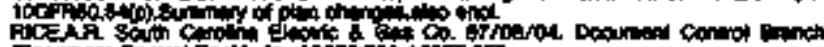

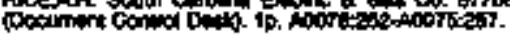

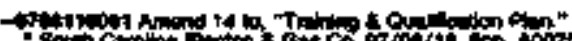

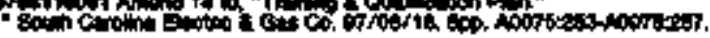

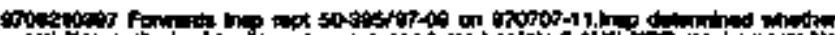

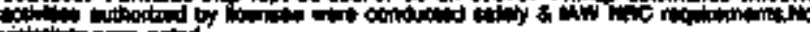

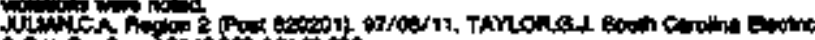

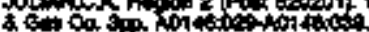

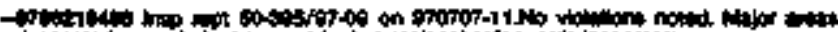

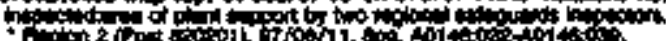

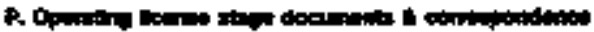

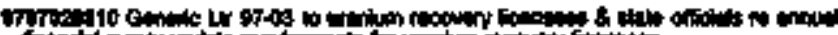

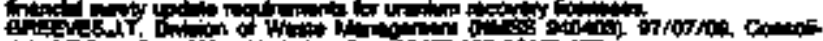

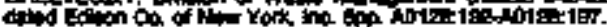

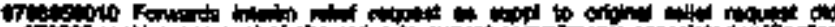

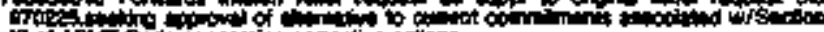

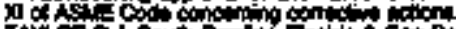
TArla

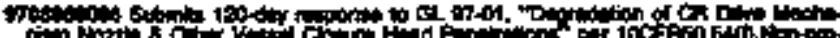

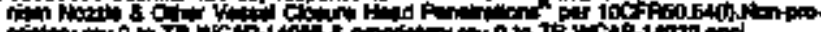

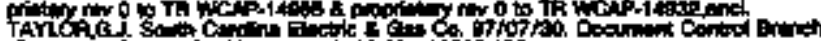

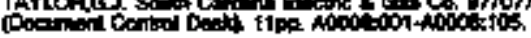

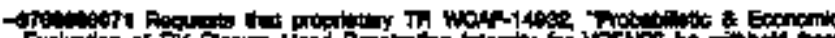

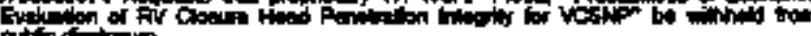
poritition

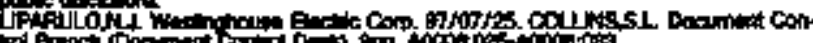

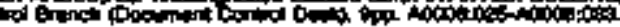




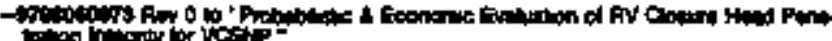

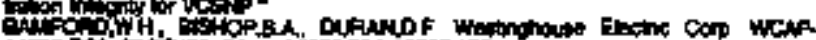

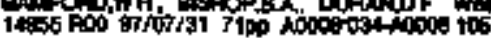

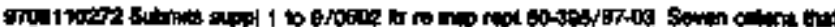

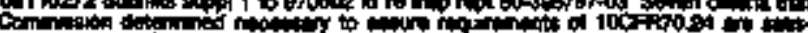
Cod 1 atotond

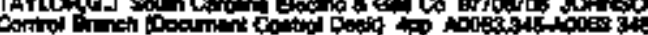

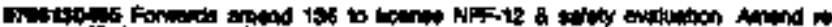

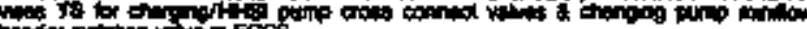

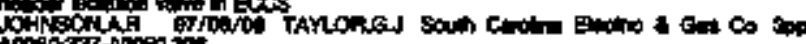

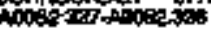

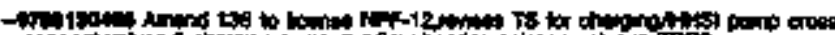

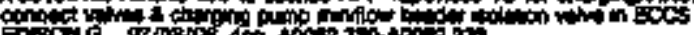

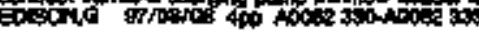

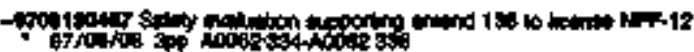

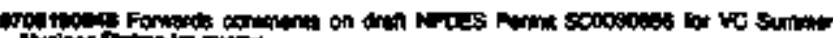

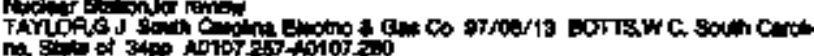

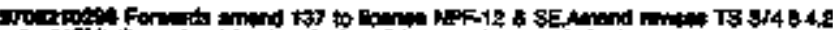

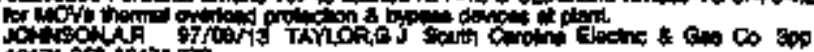

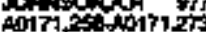

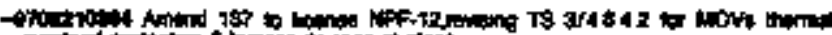

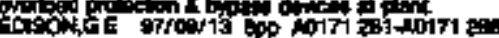

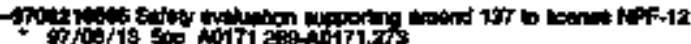

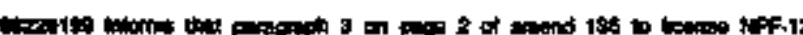

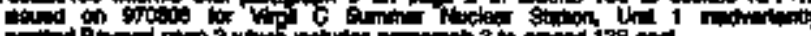

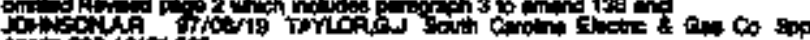

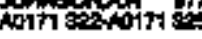

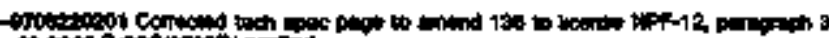

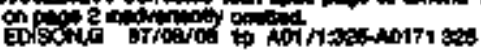

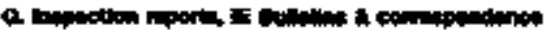

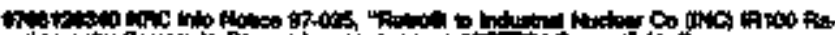
cortin

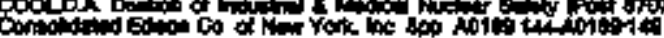

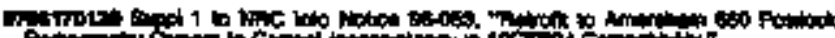

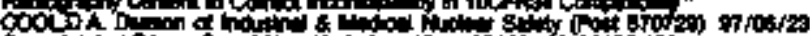
cool

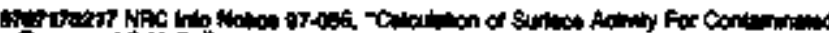

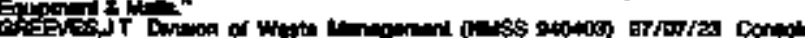

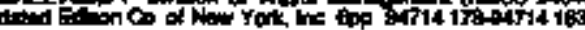

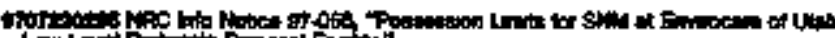

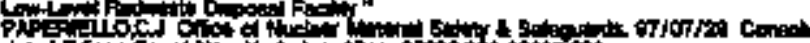

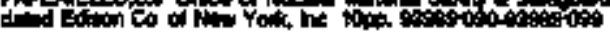

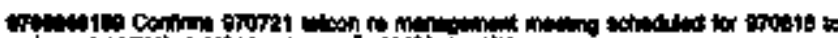

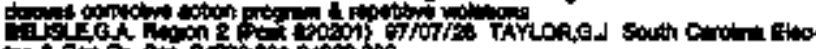

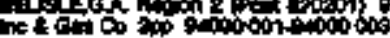

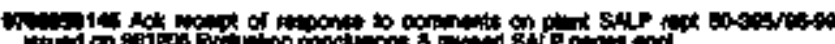

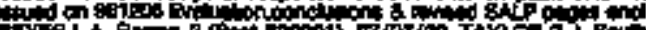

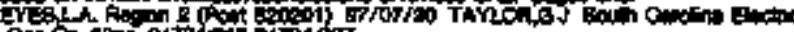

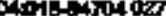

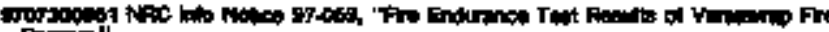

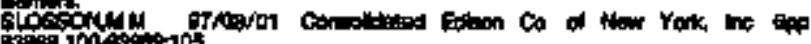

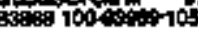

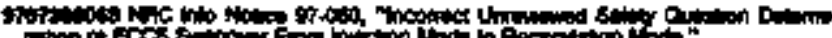

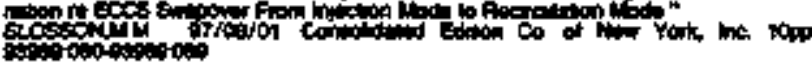

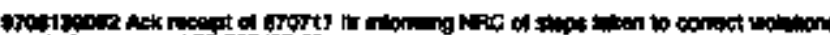

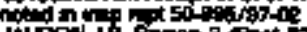

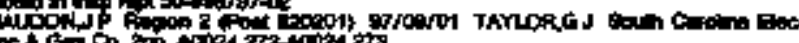

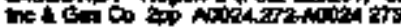

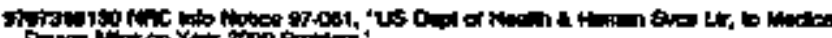
Co

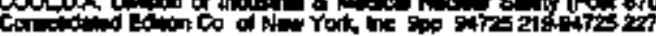

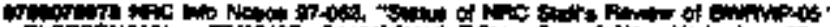

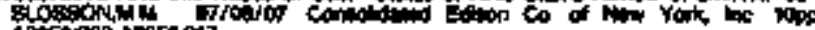

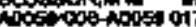

Wint

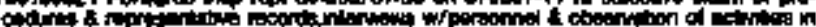

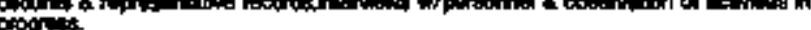

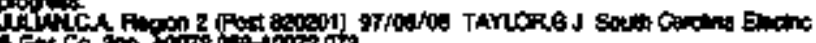

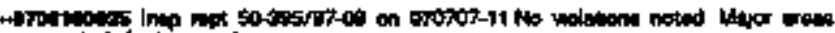

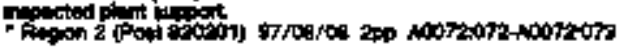

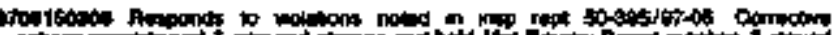

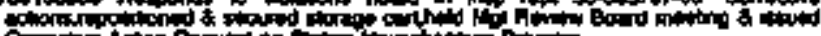

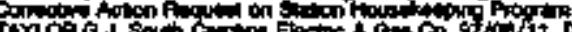

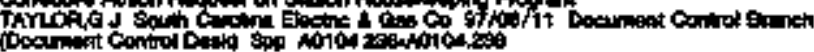

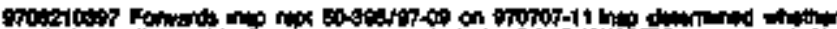

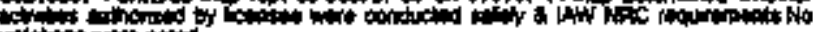

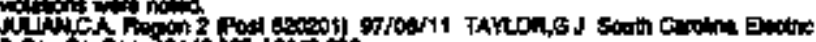

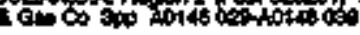

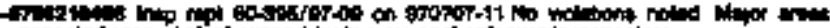

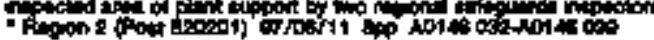

sfow

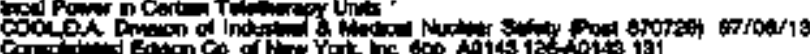

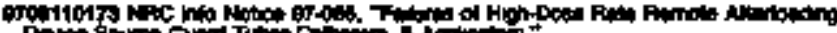

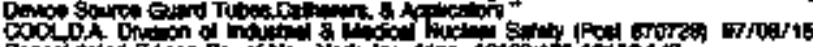

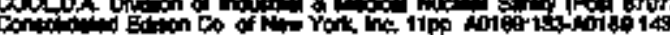

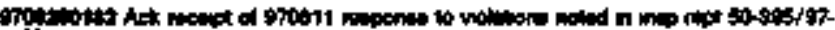

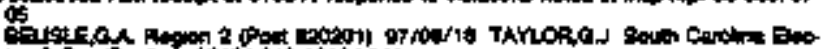

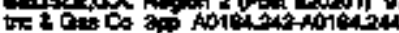

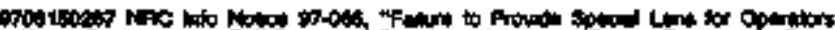

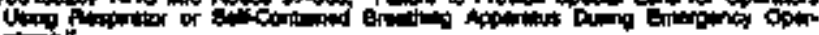

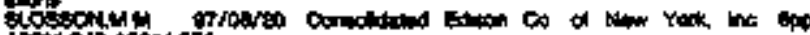

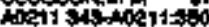

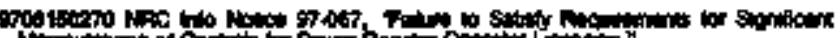

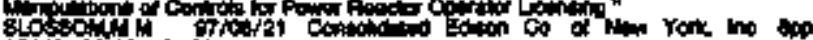
a

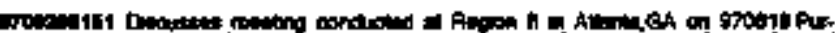

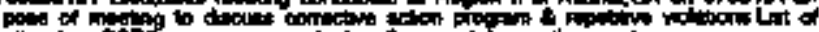

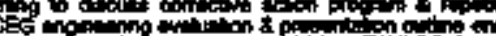

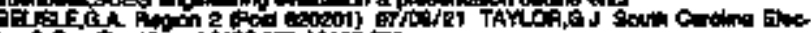

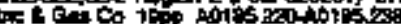

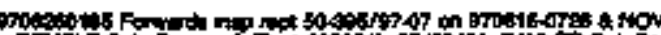

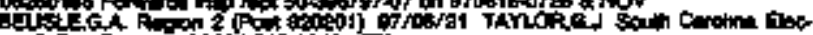

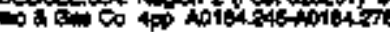

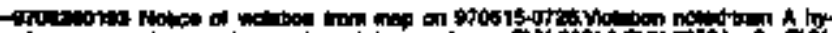

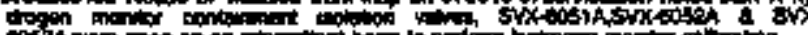

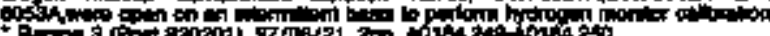

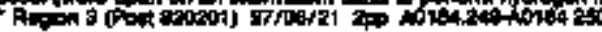

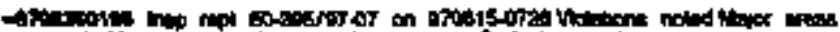

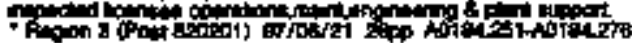

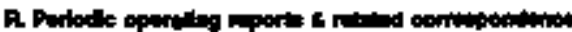

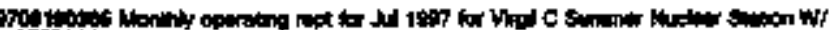

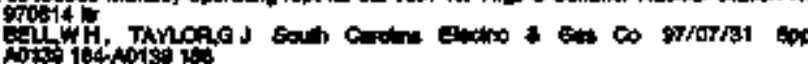

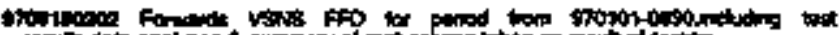

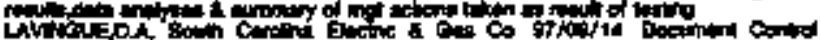

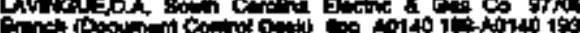

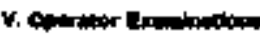

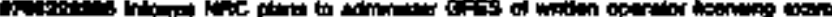

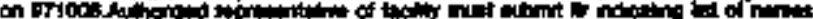
of

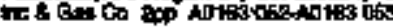

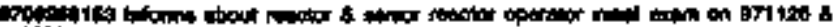

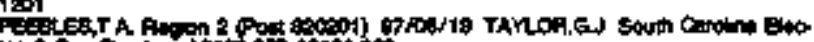

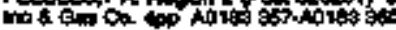

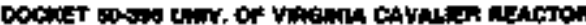




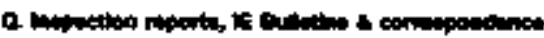

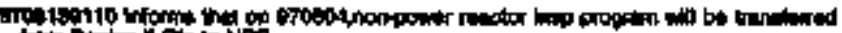

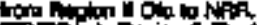

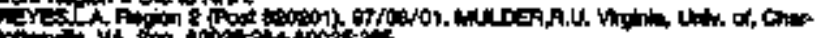

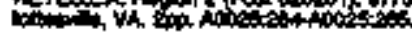

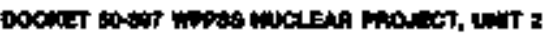

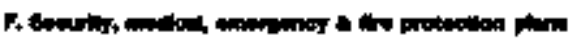

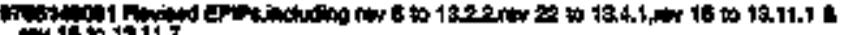

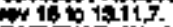

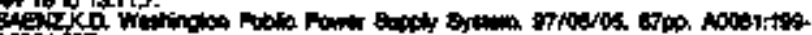

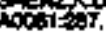

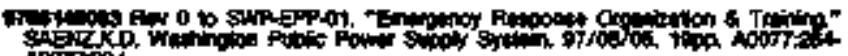

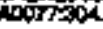

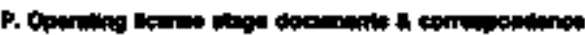

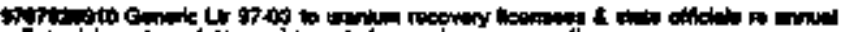

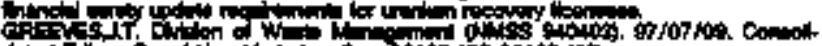

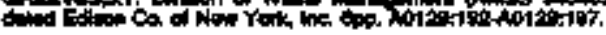

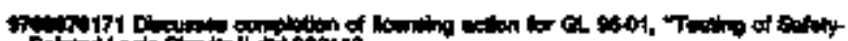

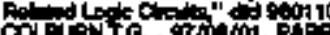

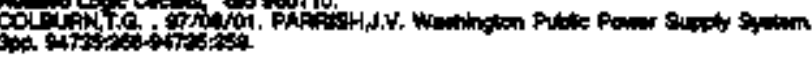

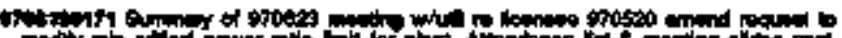

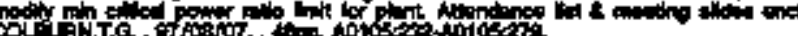

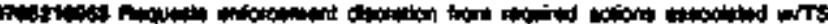

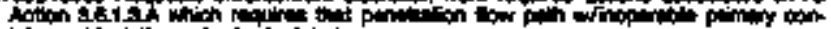

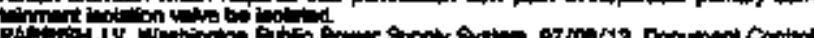

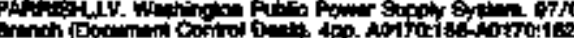

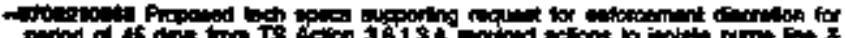

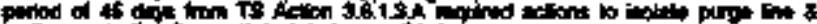

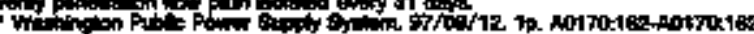

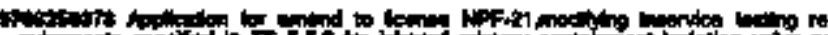

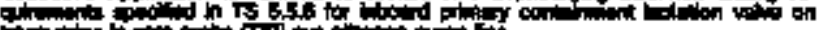

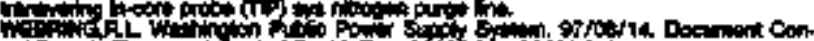

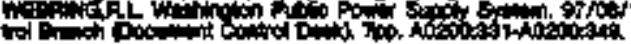

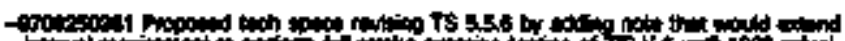

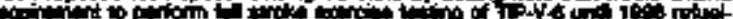

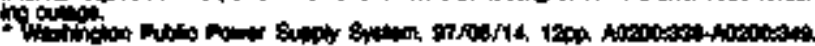

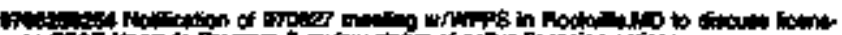

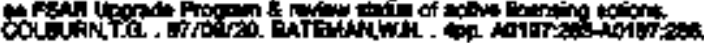

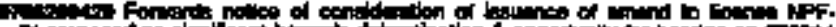

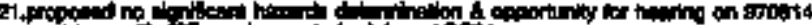

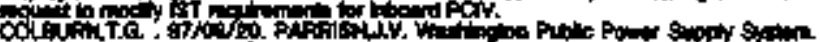

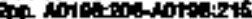

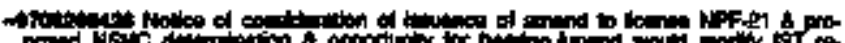
pond K10\%

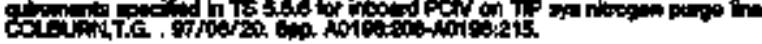

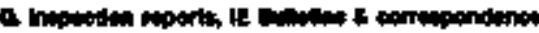

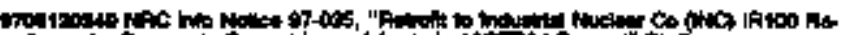

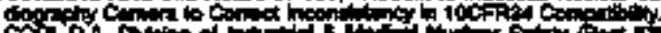

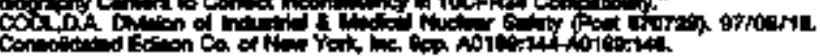

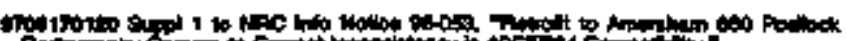

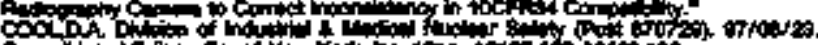

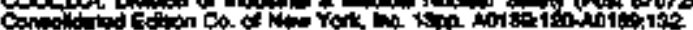

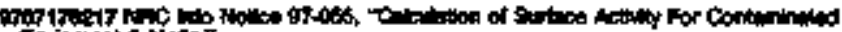

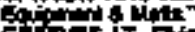

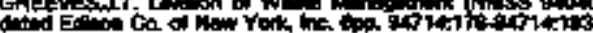

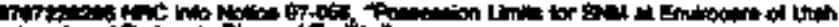

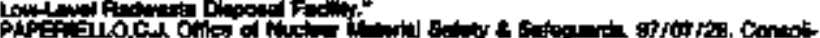

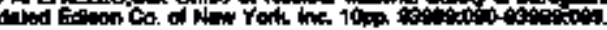

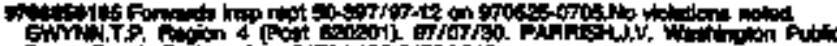

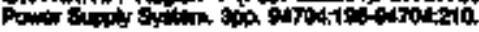

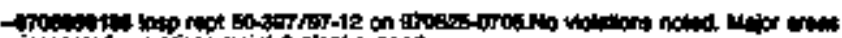

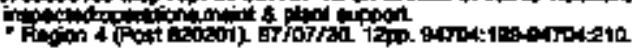

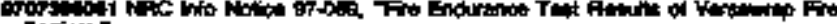

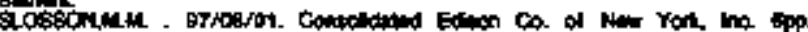

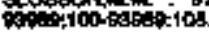

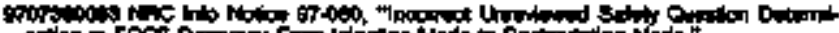

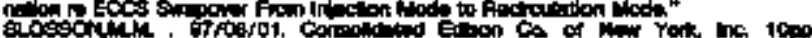

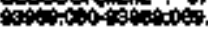

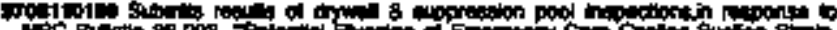

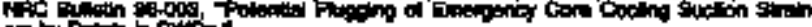

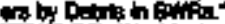

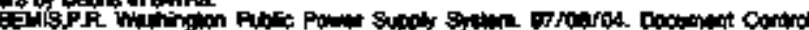

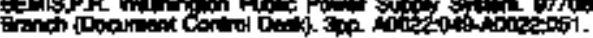

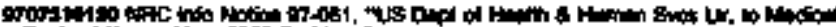

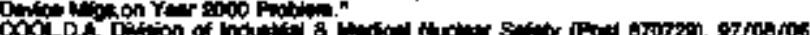

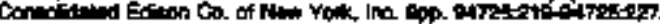

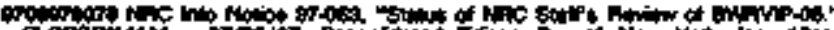

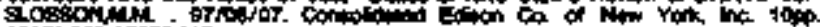
1006000

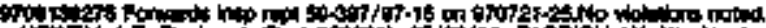

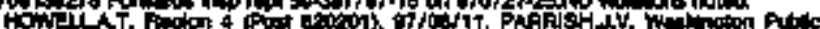

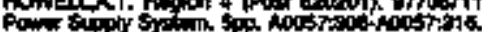

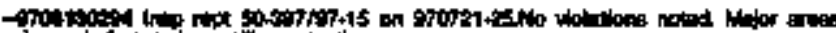

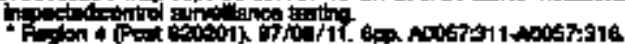

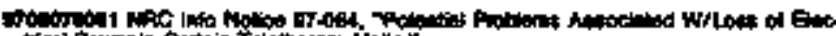

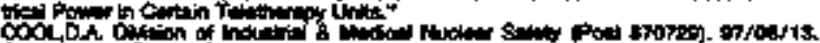

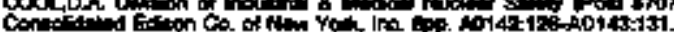

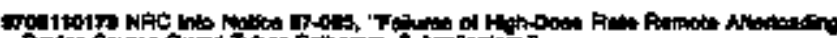

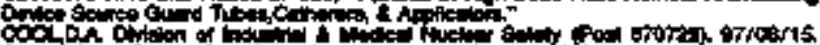

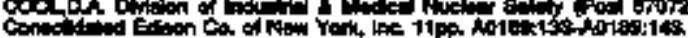

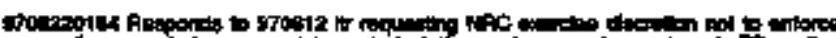

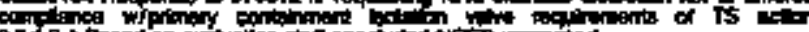

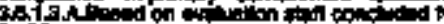

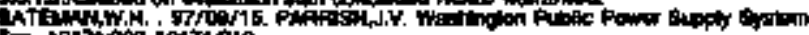

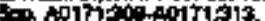

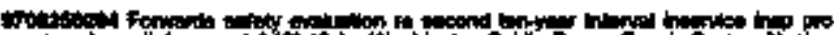

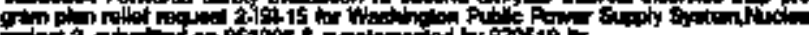

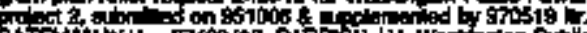

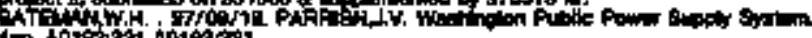

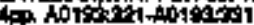

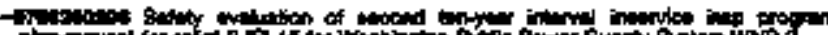

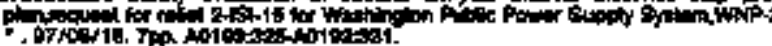

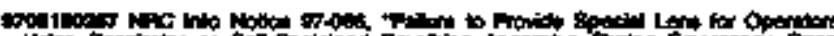

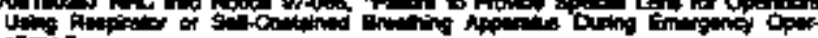

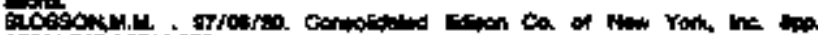

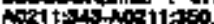

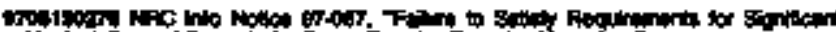

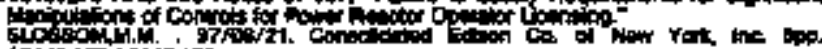

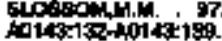

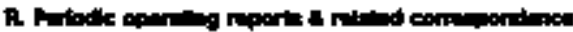

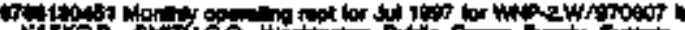

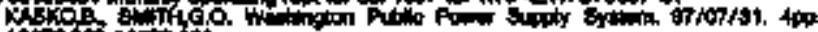

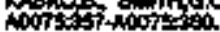

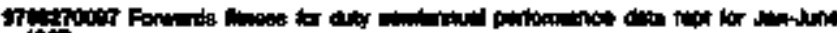
197.

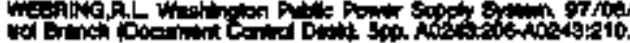

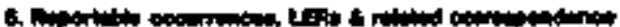

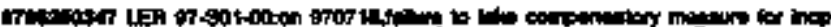

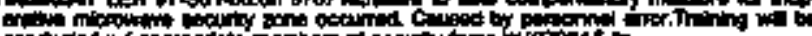

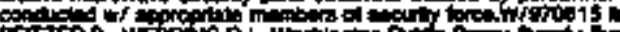

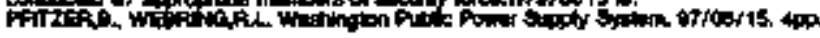

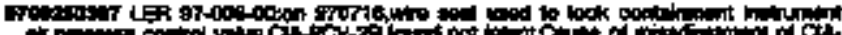

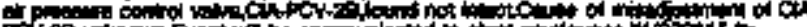

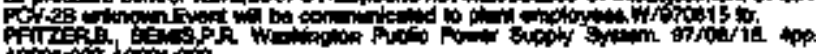

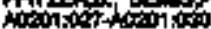

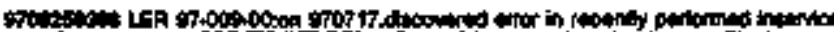

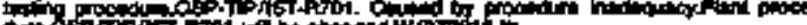
A 0 os

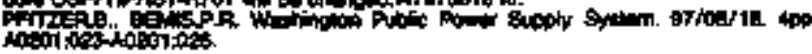




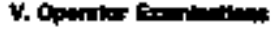

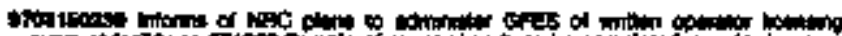

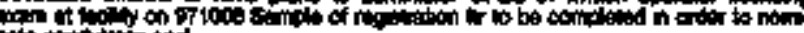

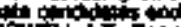

HCw

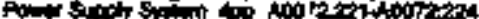

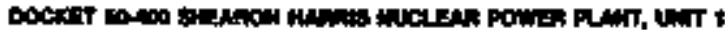

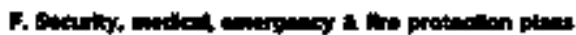

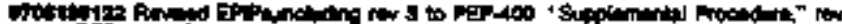

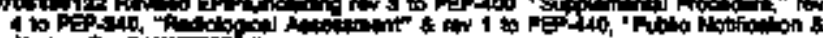
A ming

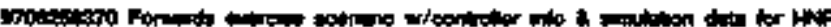

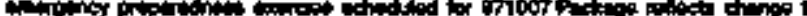

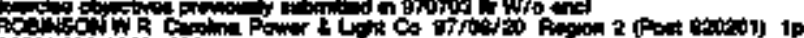

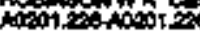

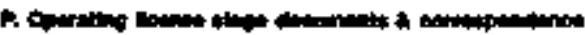

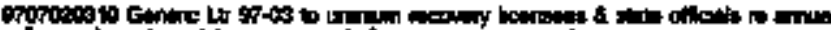

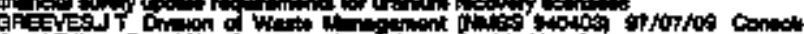

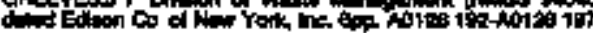

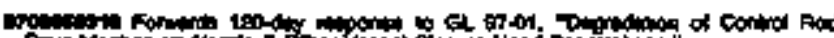

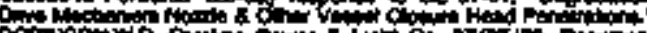

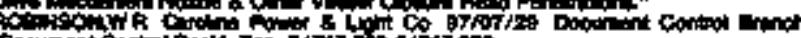

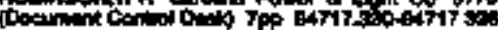

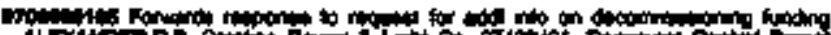
LOA A011 34.

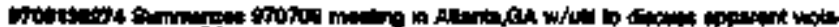

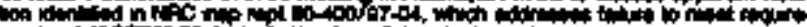

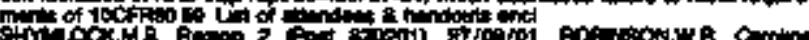

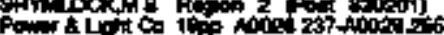

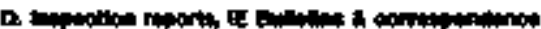

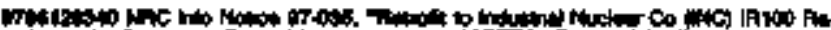

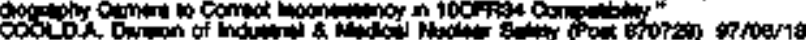

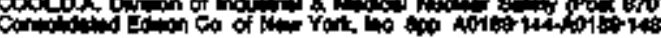

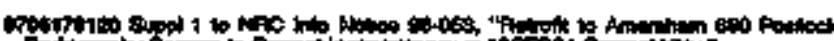

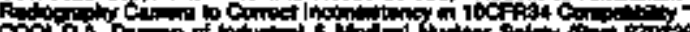

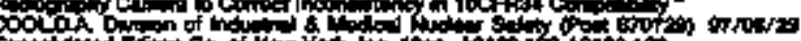

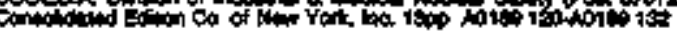

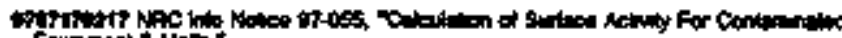
Eorich

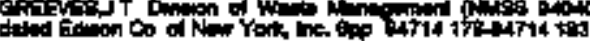

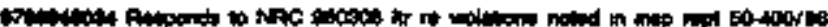

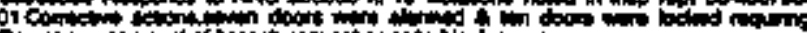

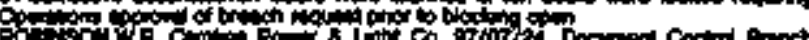

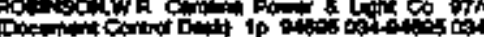

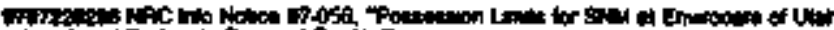

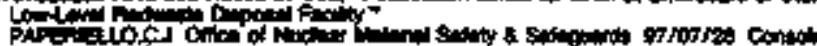

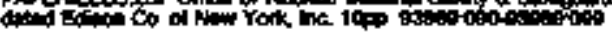

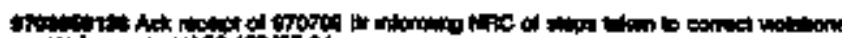

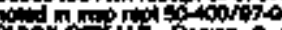

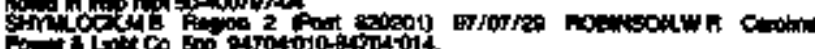

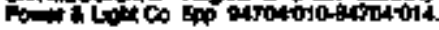

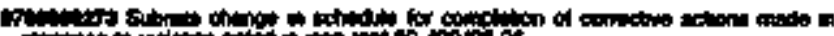
H.

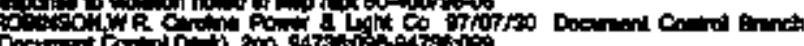

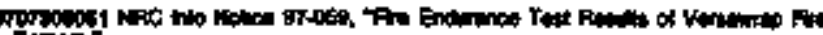

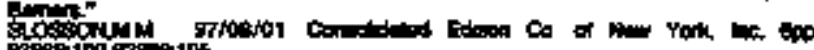

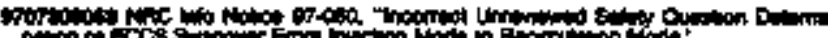

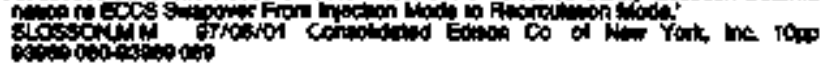

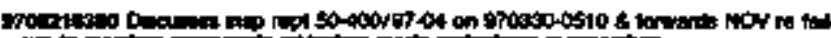

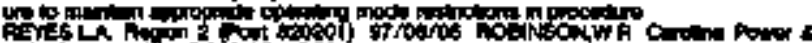

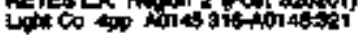

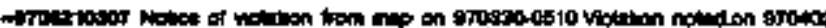

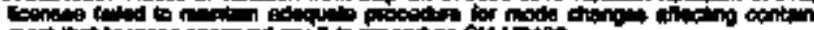

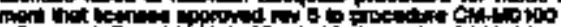

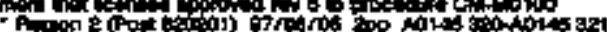

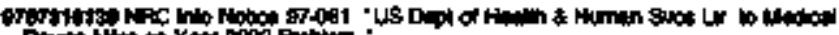

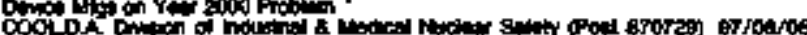

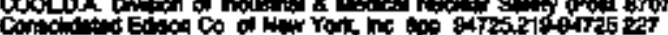

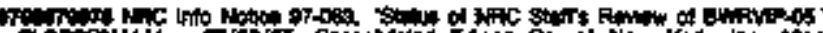

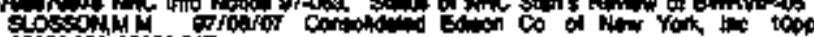

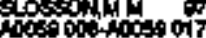

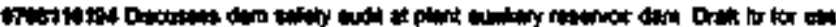

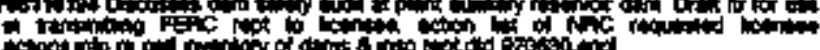

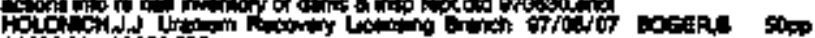

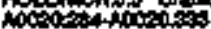

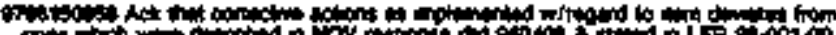

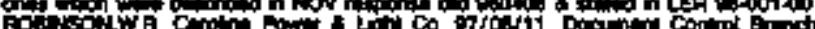

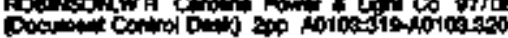

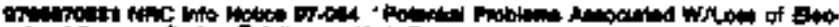

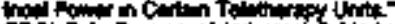

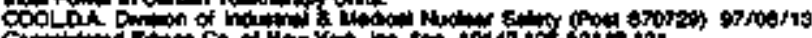

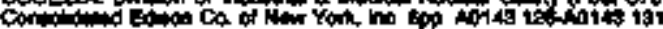

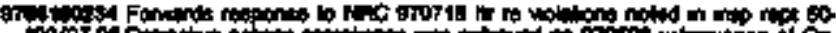
- oos

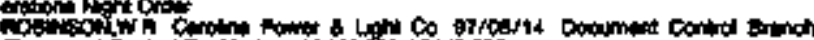

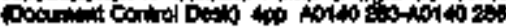

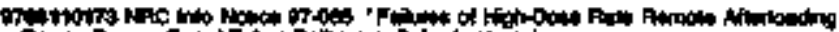

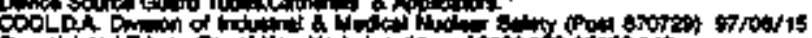

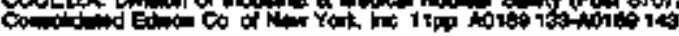

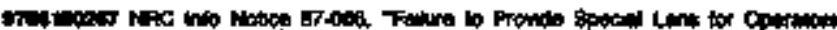

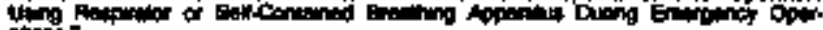

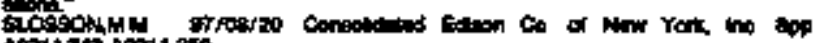

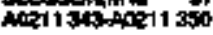

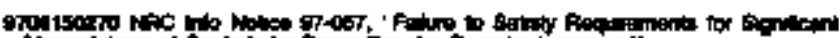

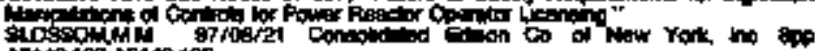

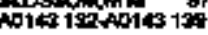

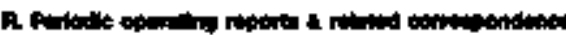

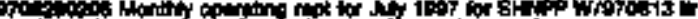

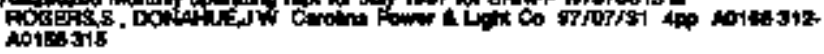

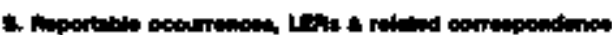

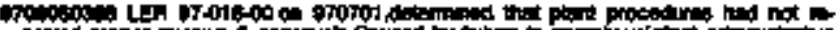

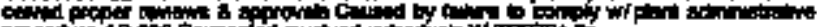

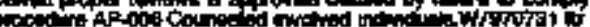
羊1

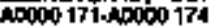

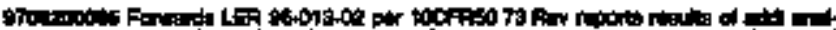

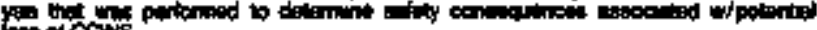

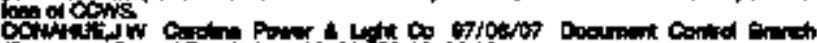

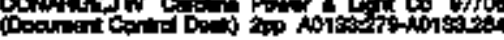

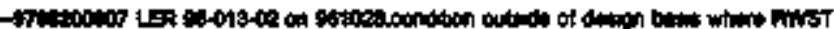

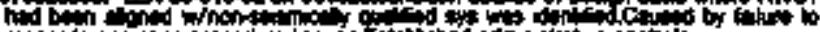

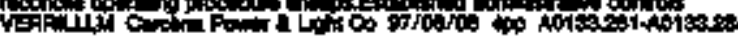

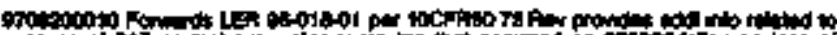

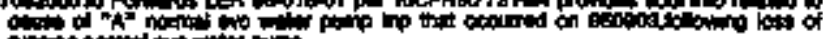

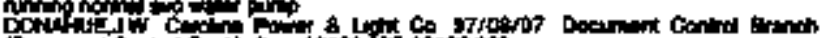

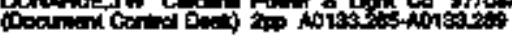

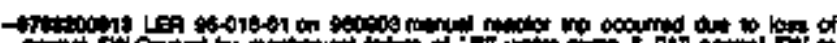

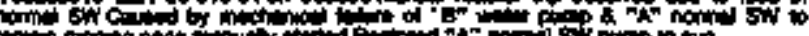

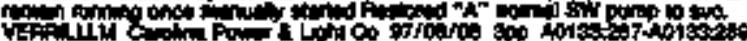

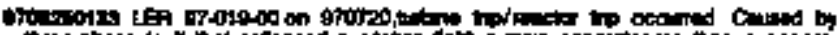

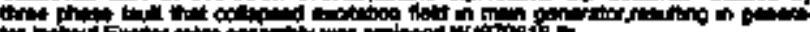

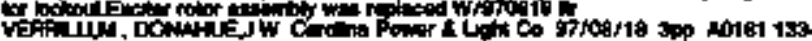
Avisi 106

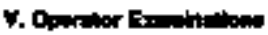

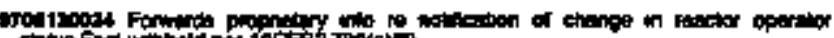

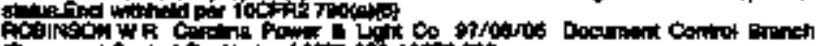

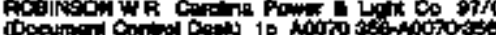

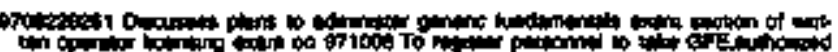

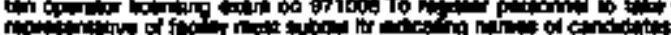

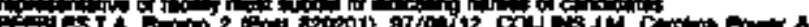

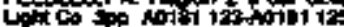




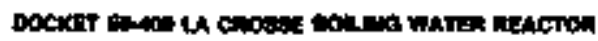

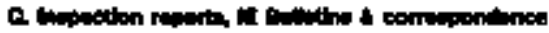

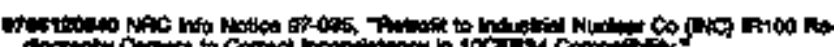

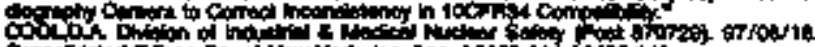

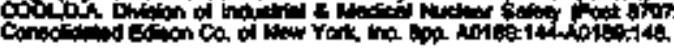

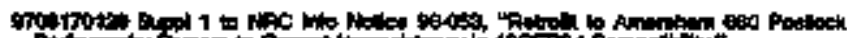

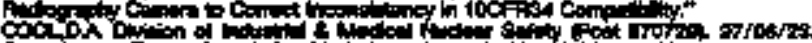

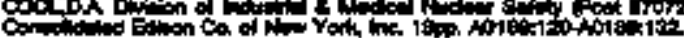

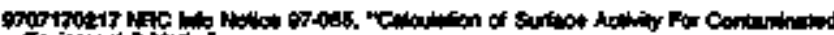

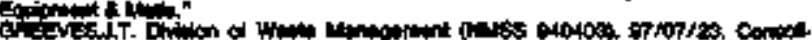

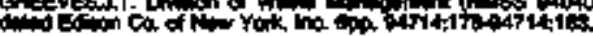

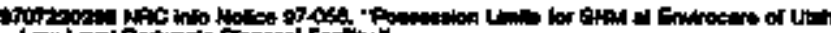

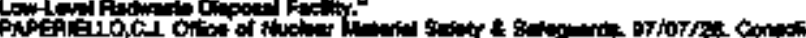

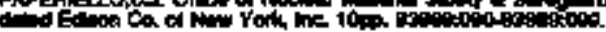

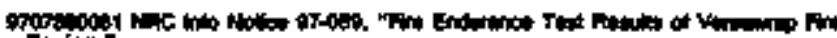

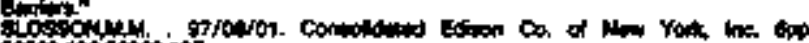

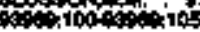

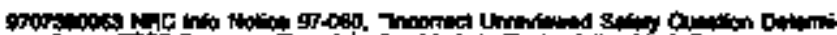

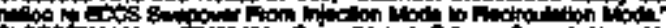

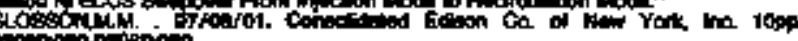

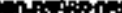

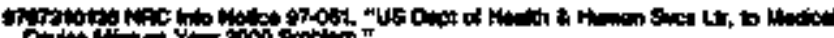

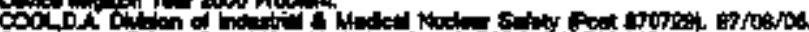

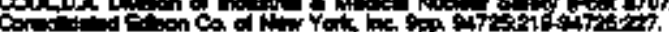

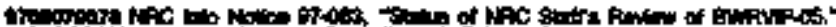

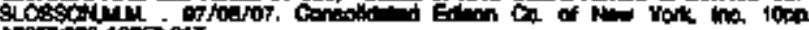

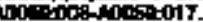

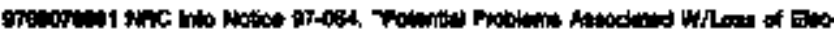

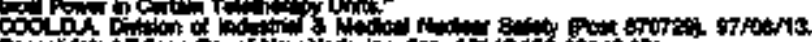

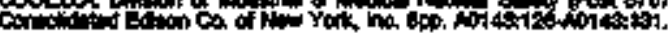

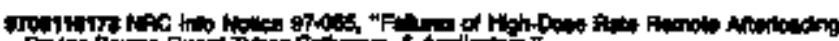

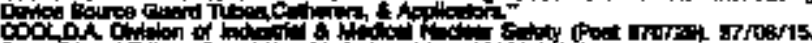

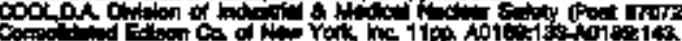

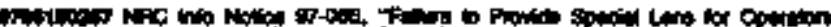

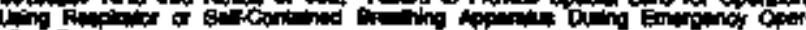

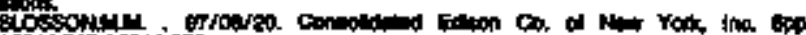

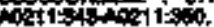

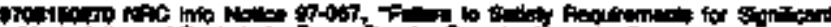

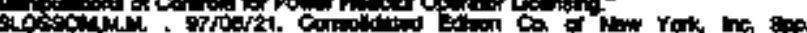

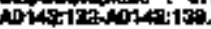

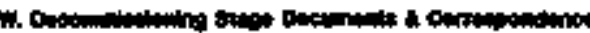

In

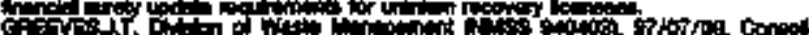

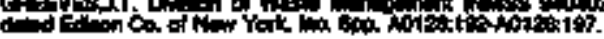

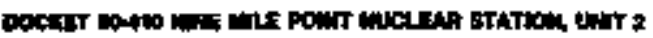

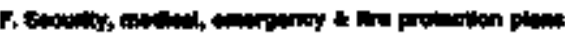

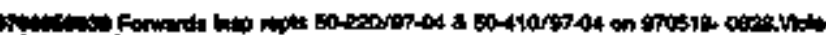
Fin

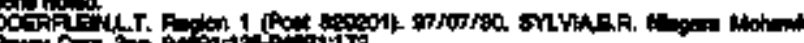

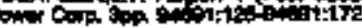

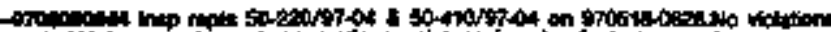

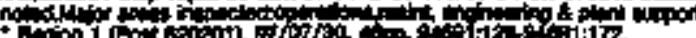

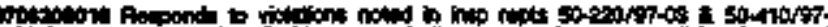

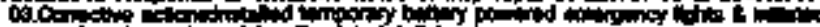
contith

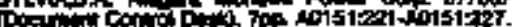

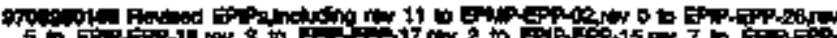

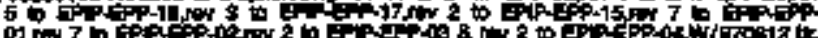

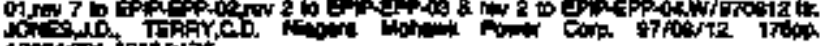

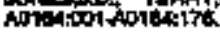

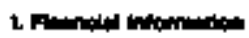

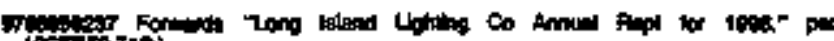

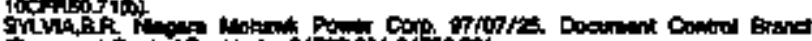

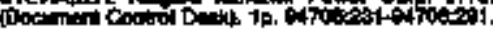

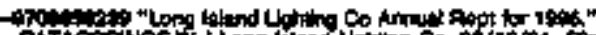

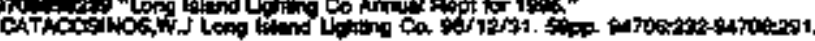

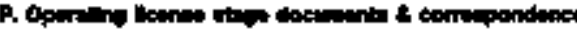

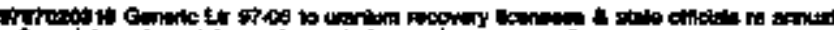

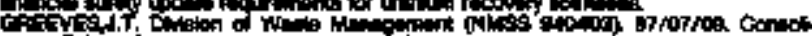

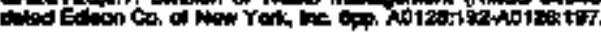

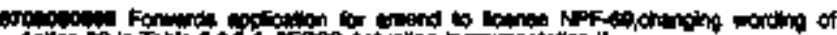

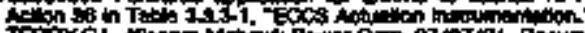

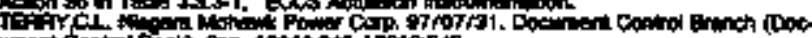

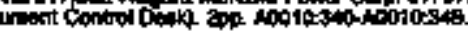

- Fon

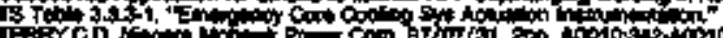

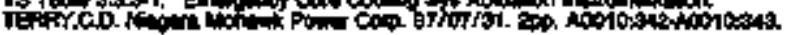

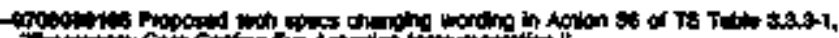

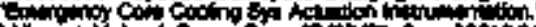

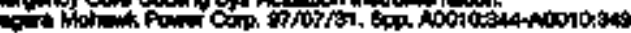

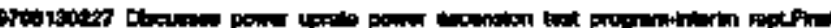

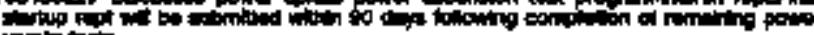

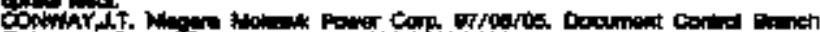

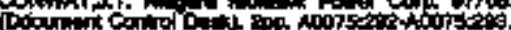

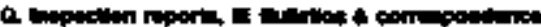

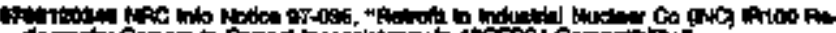
Cot

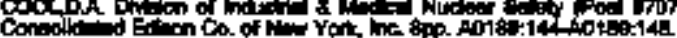

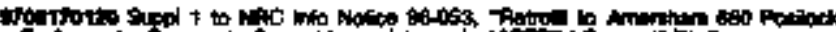
F.

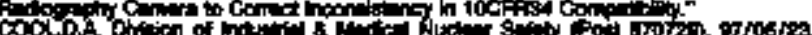

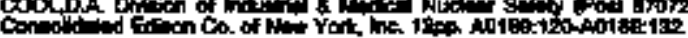

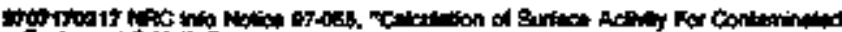

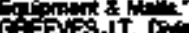

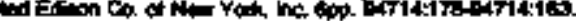

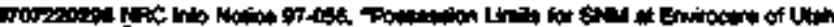

PAp G

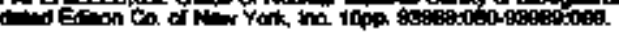

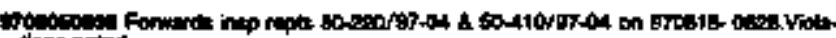
lont nomid.

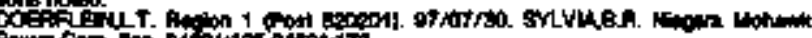

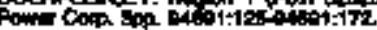

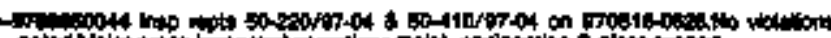

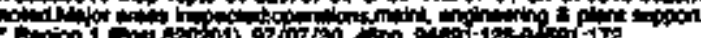

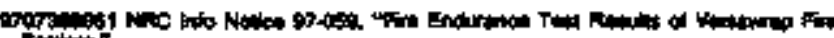

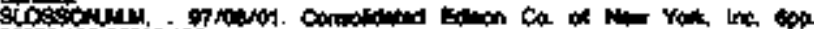
\&.tionosios.

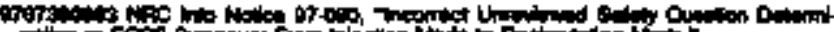

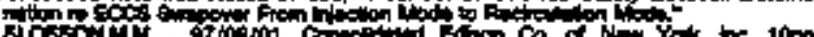

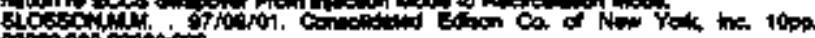
(1)

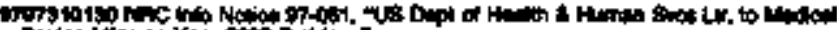

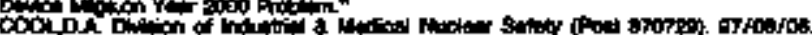

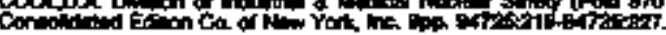

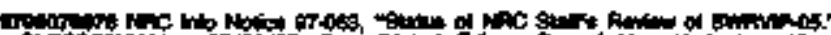

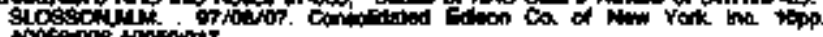

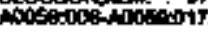

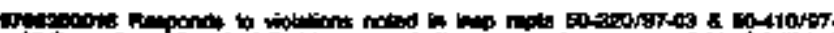

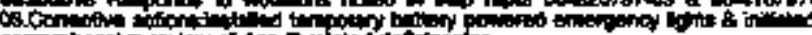

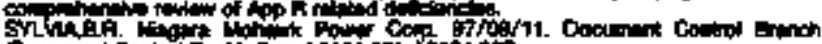

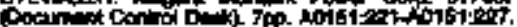

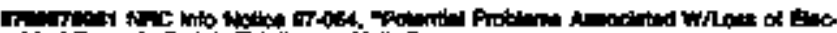

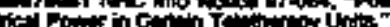

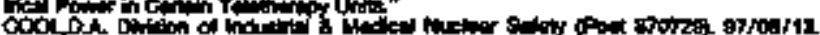

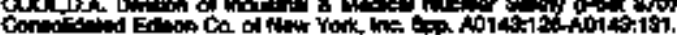

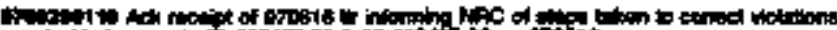

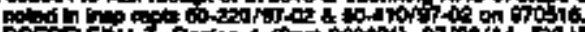

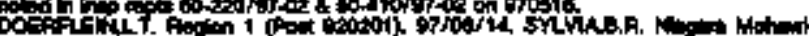

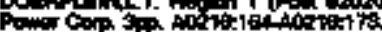

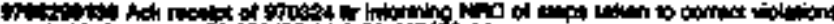

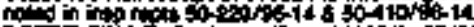

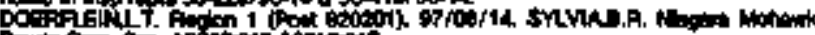

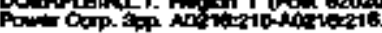

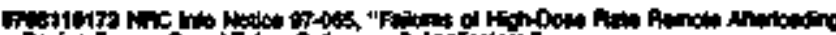

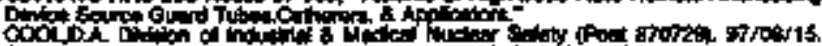

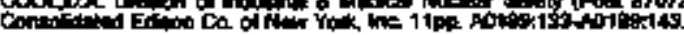




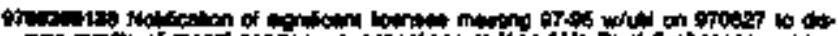

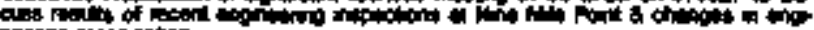

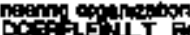

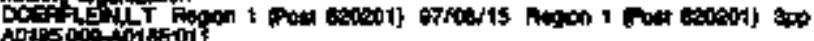

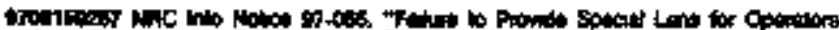

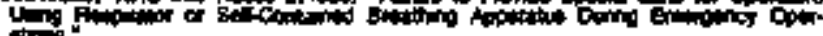

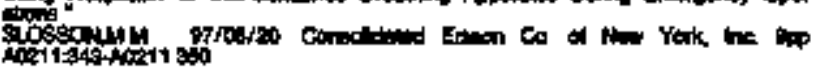

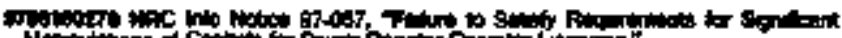

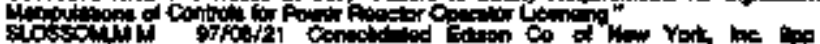

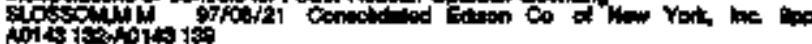

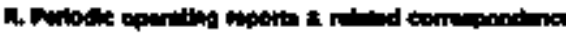

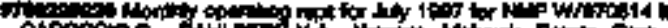

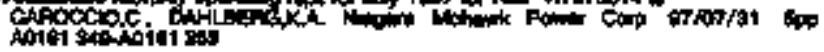

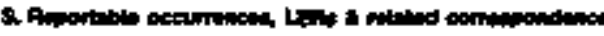

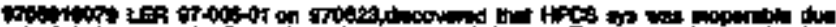

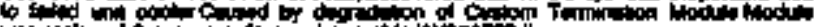

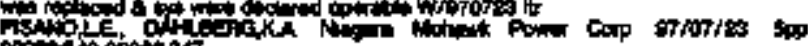

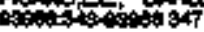

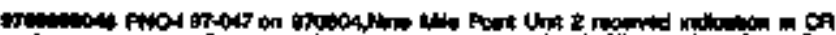

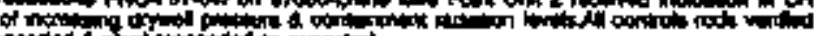

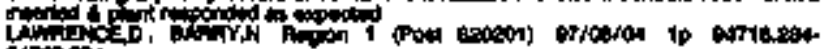

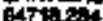

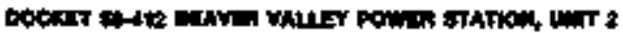

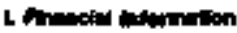

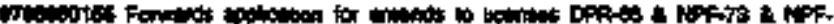

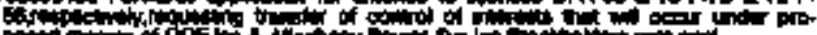

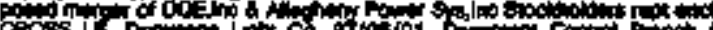

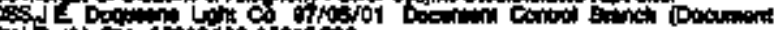

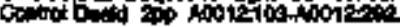

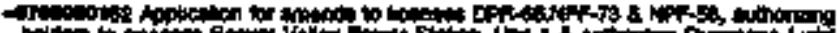

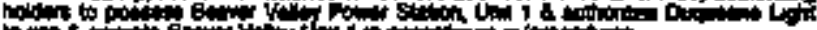

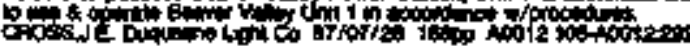

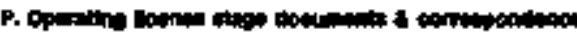

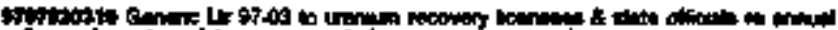
a

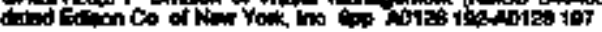

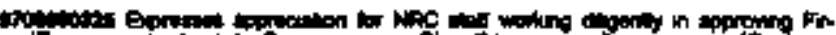

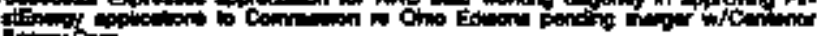

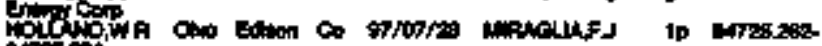
of

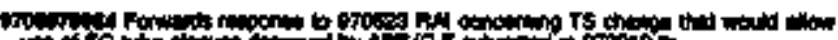

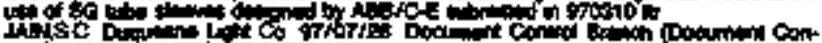

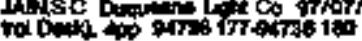

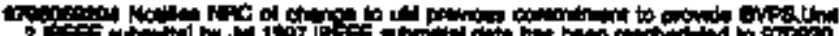

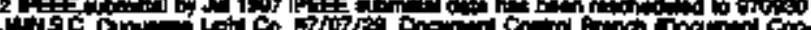

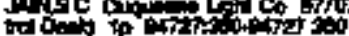

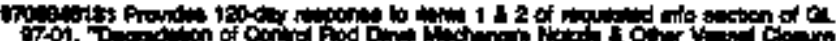

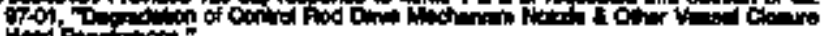
H

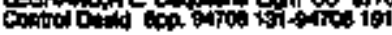

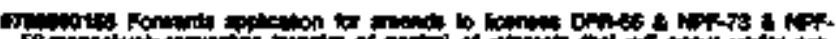

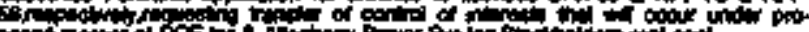

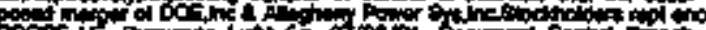

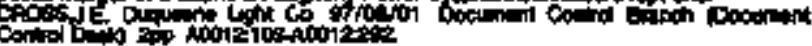

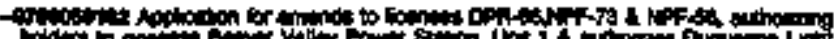

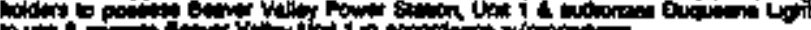

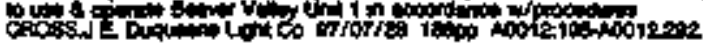

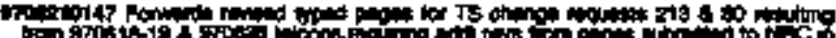

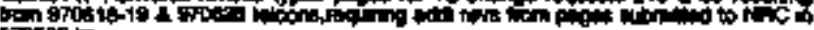
$\cos 6$

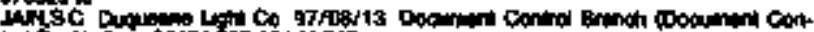

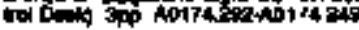

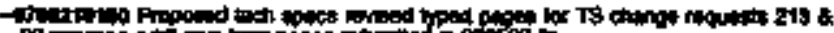

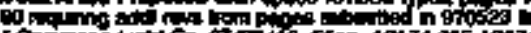

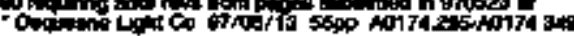

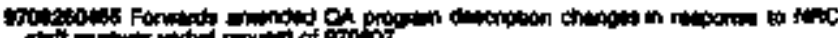

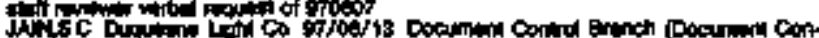

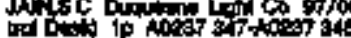

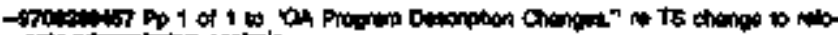

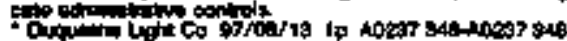

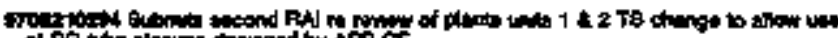

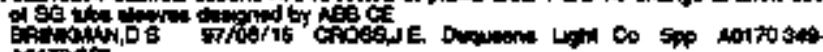
incirotes

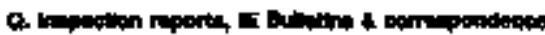

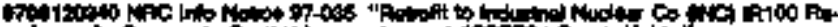

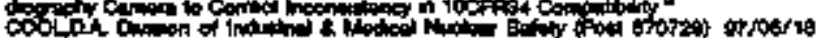

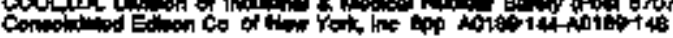

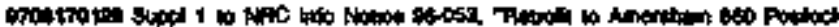

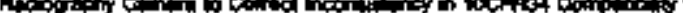

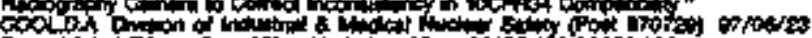

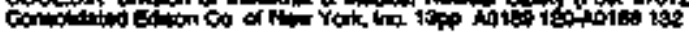

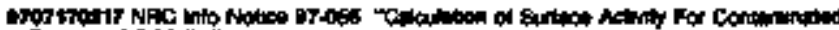

krive

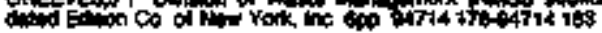

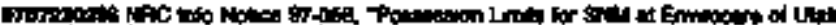

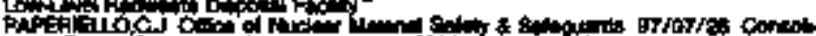

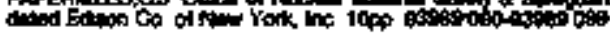

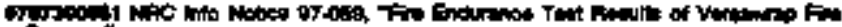

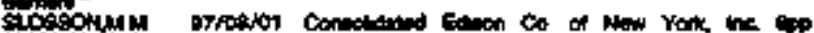

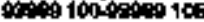

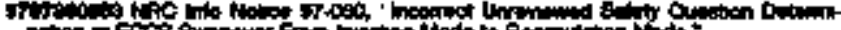

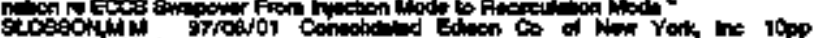
pion

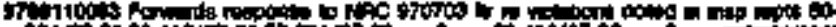
340

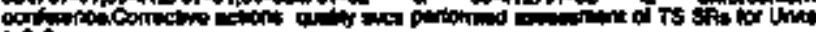
if

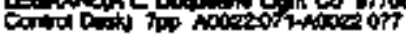

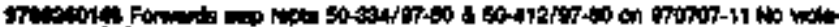

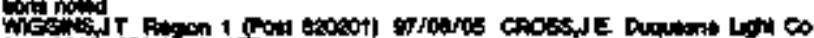

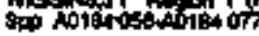

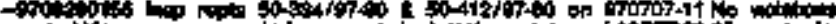

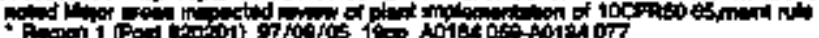

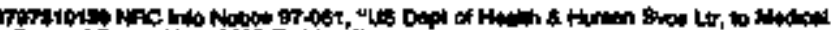

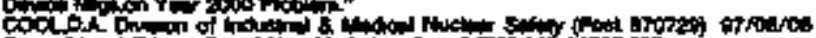

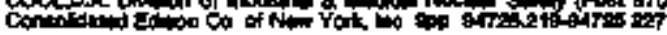

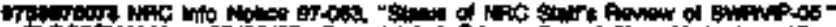

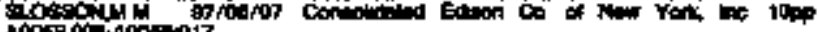

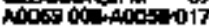

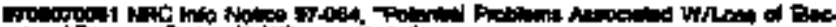

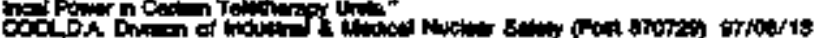

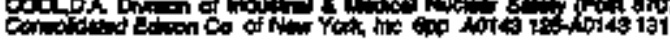

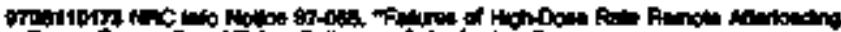

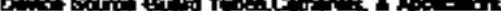

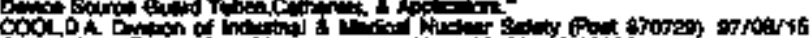

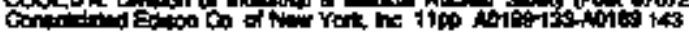

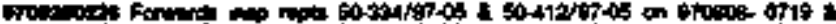

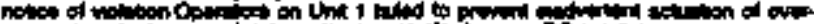

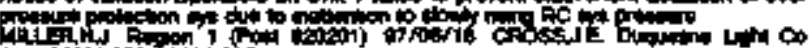

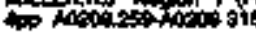

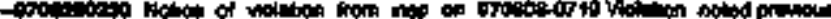

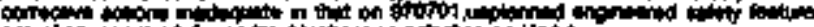

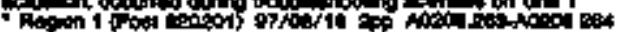

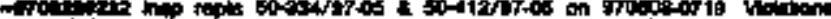

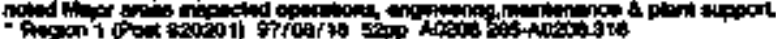

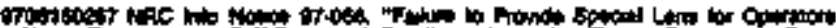

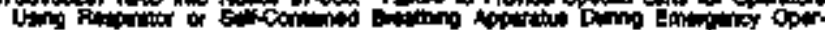

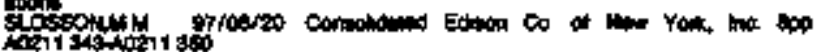




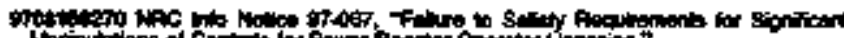

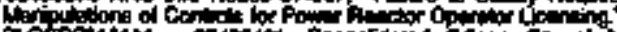

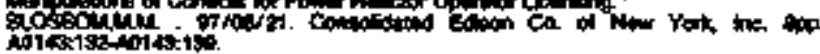

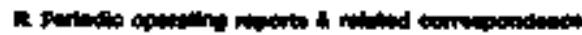

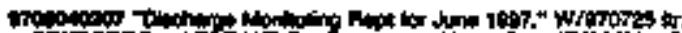

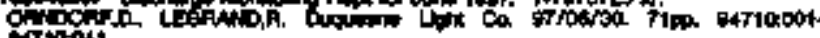
arviti.

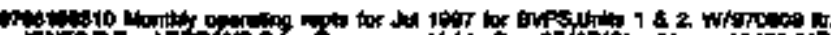

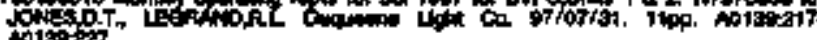
A13027.

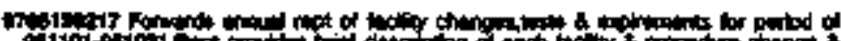

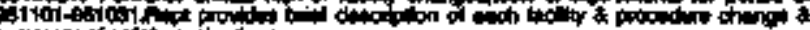

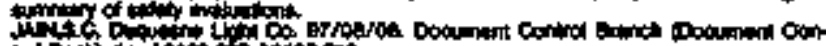

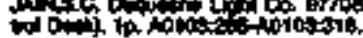

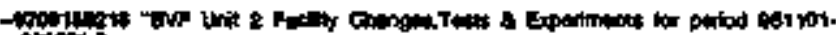
tionit"

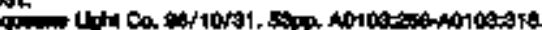

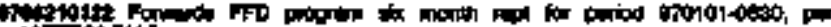

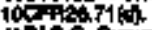
Whick of

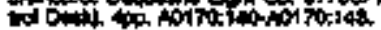

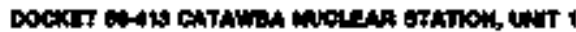

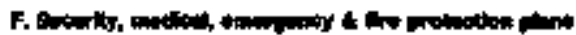

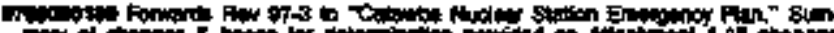

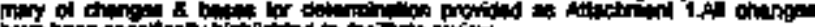

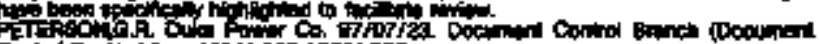

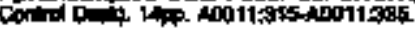

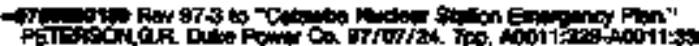

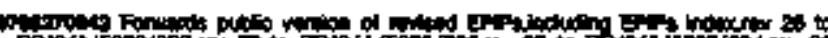

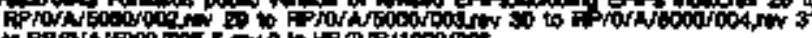

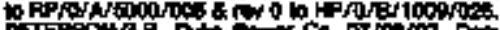

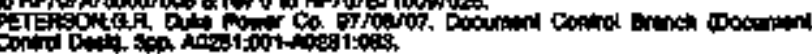

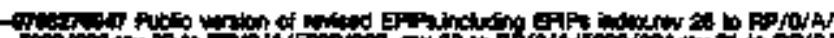
然

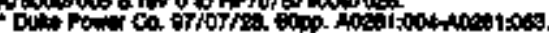

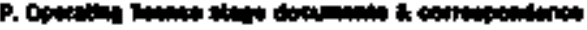

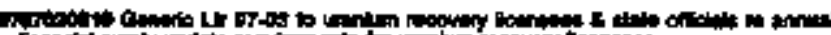

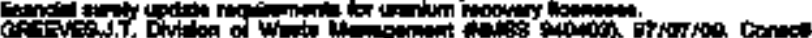

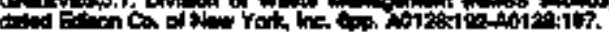

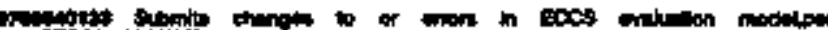

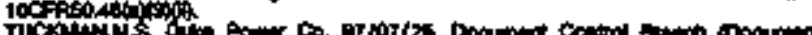

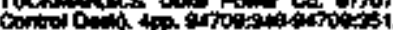

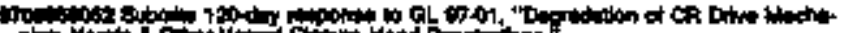

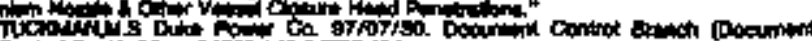

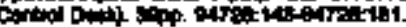

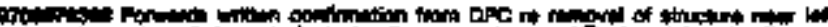

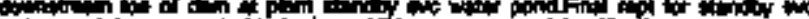

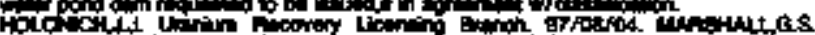

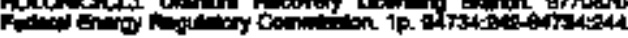

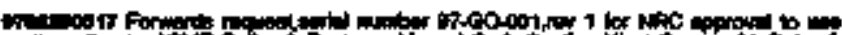

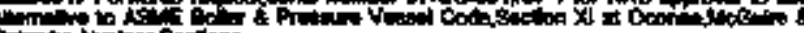

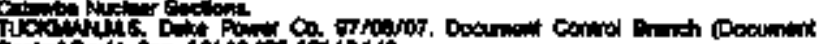

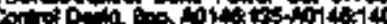

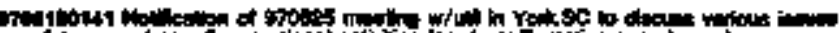

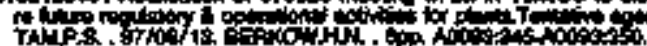

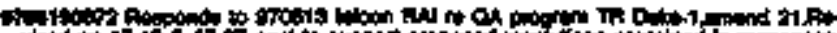

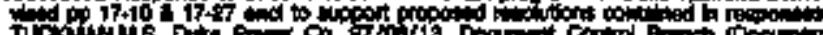

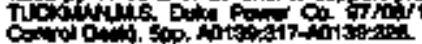

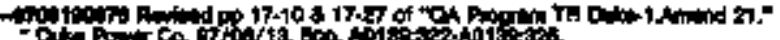

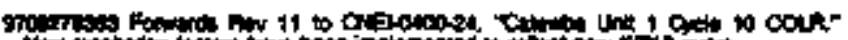

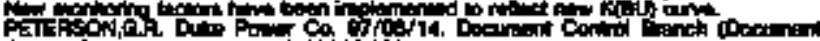

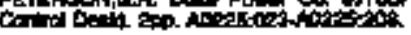

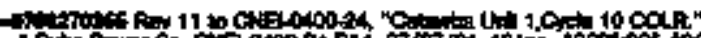

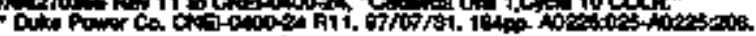

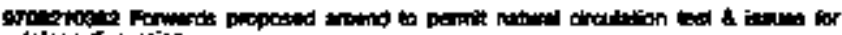

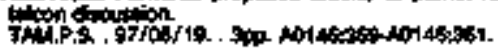

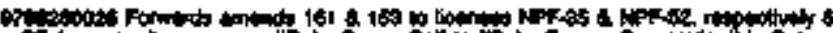

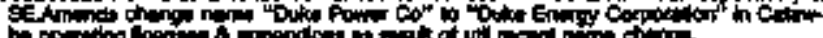
Th

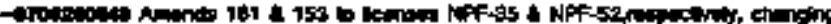

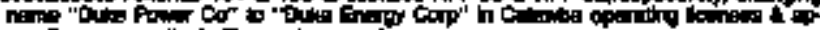

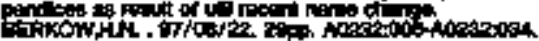

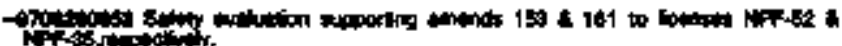

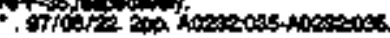

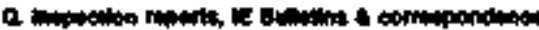

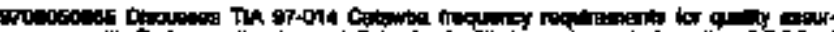

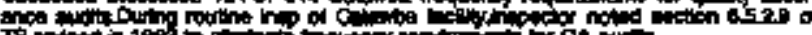

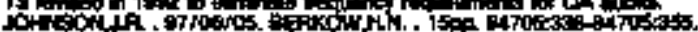

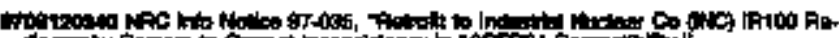

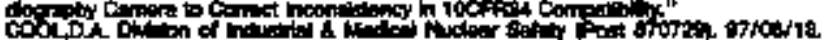

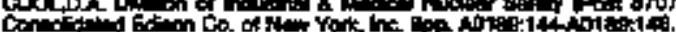

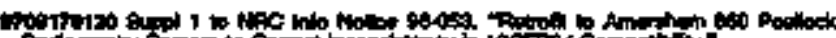

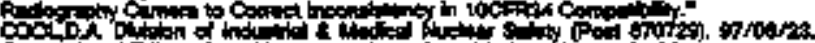

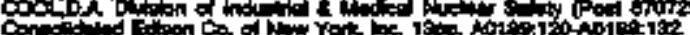

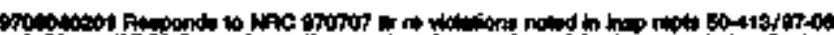

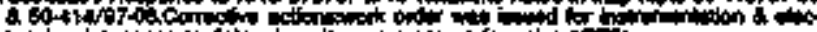

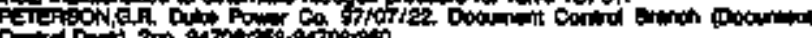

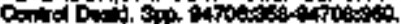

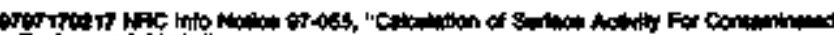
E

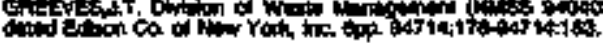

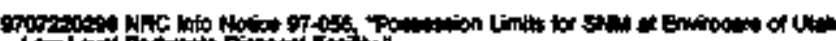

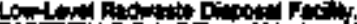

AP

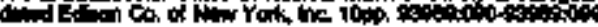

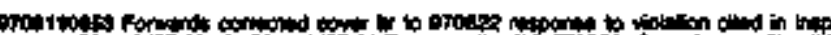

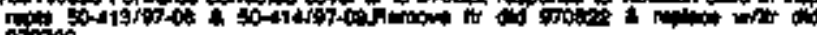

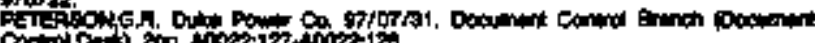

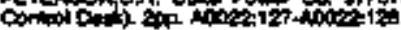

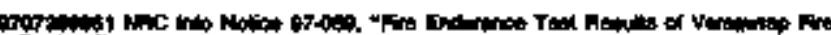

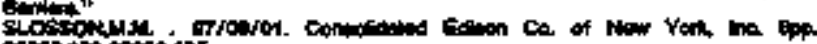

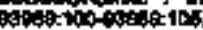

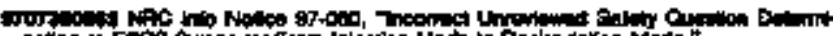

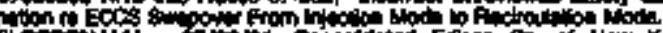

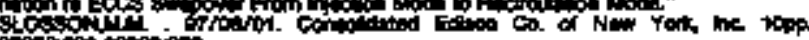
is:

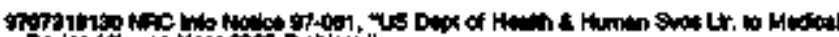

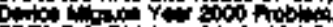

coo for Dulen of ind

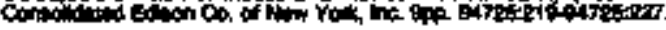

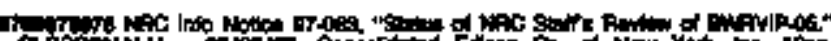

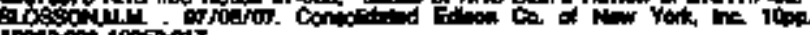

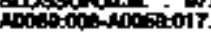

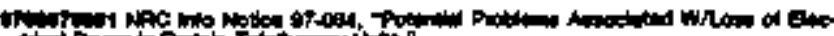

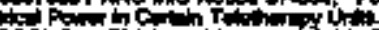

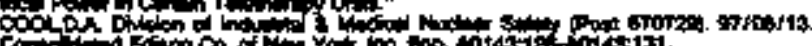

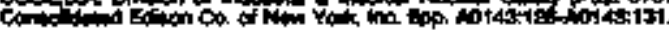

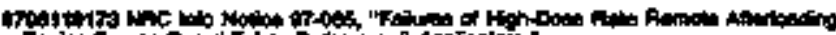

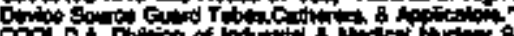

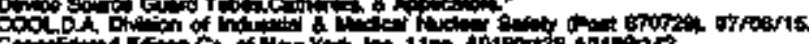

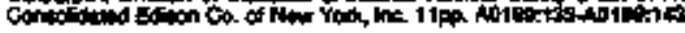

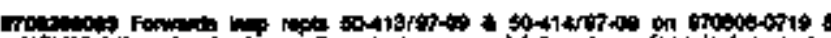

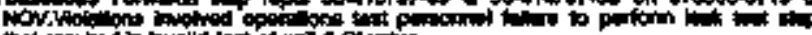

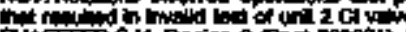

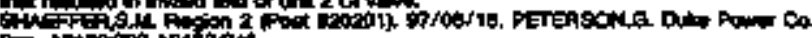

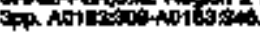

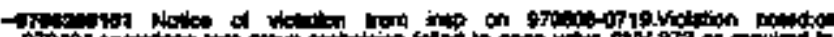

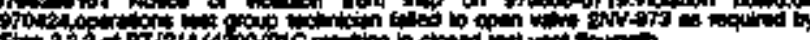
Sto 25 of $P / 2 / N 4$ SOF

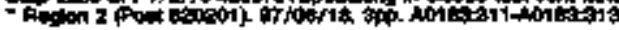

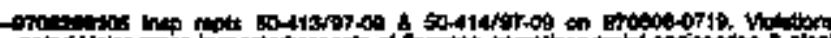

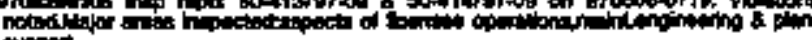

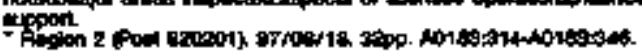




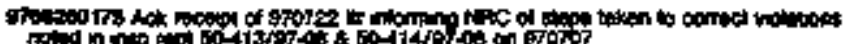

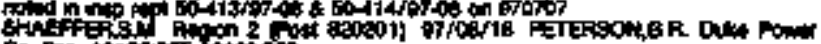
Ca 20 , not

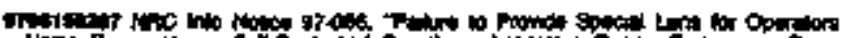

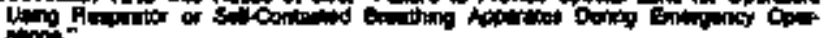

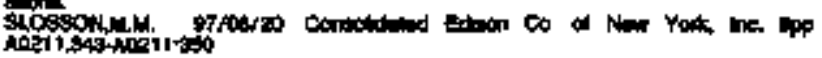

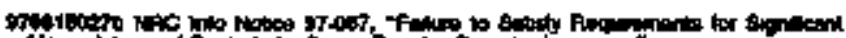

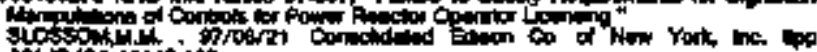

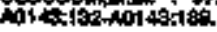

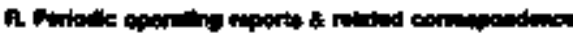

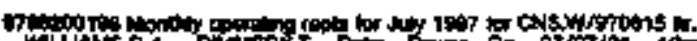
hol

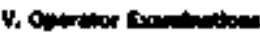

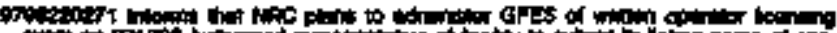

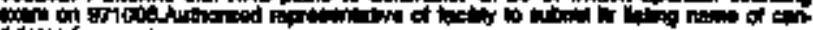

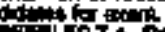

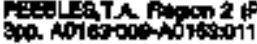

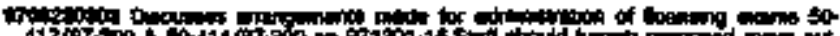

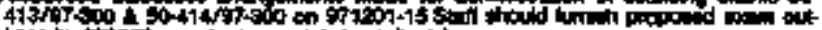
Preis

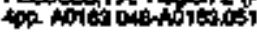

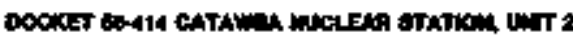

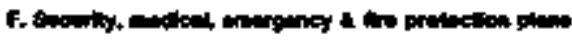

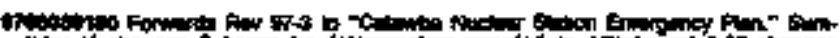

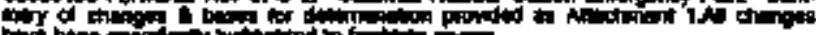

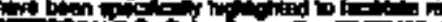

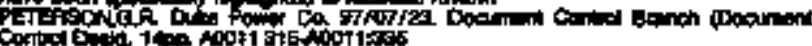

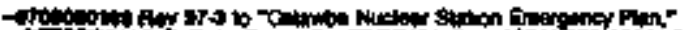

PETE

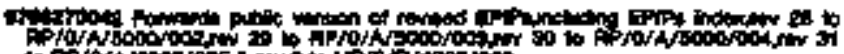

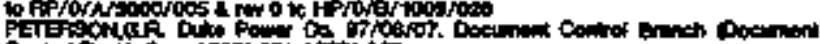

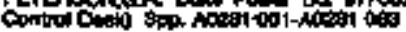

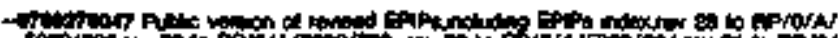

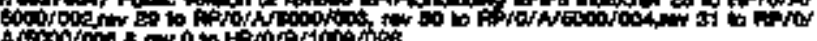

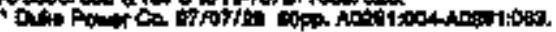

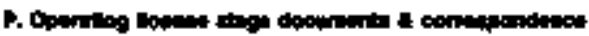

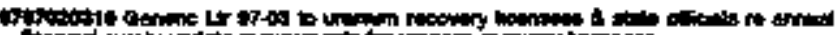

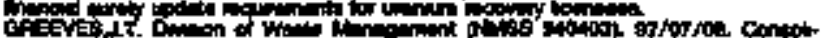

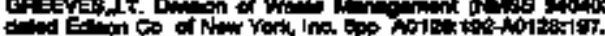

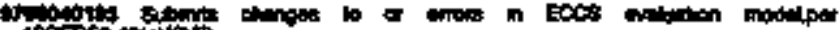

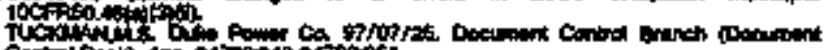

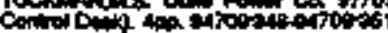

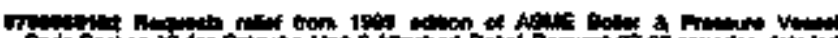

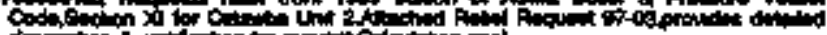
Feriton

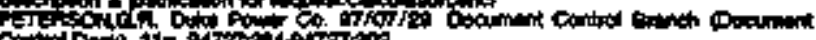

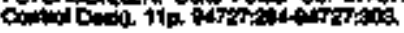

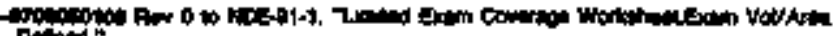
(1)

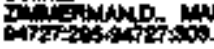

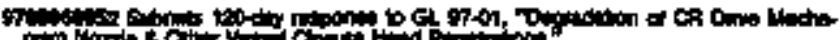

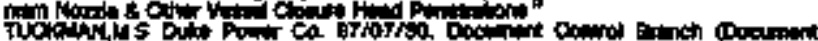

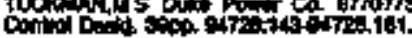

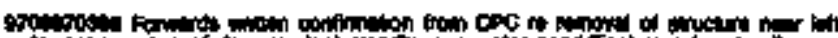

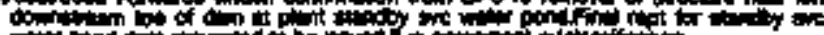

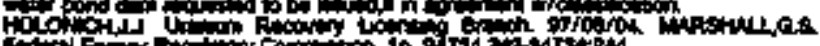

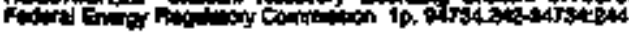

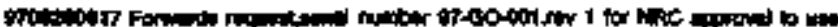

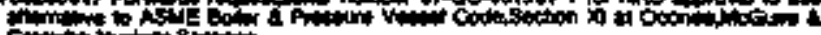

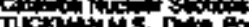

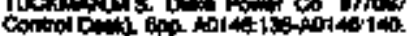

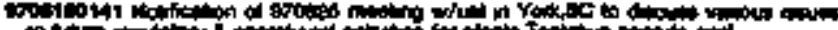

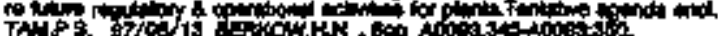

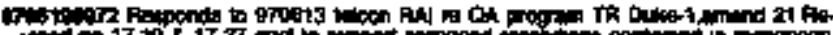

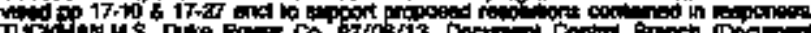

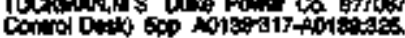

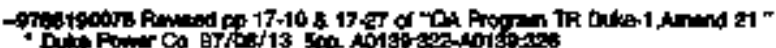

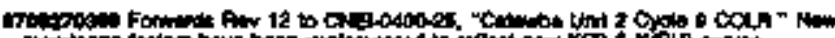

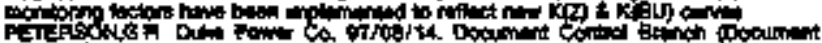

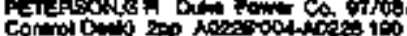

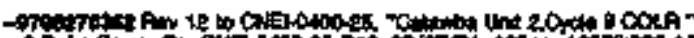

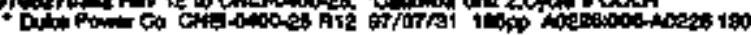

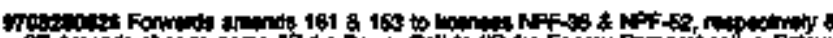
sectmints of

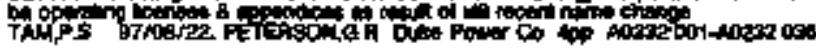

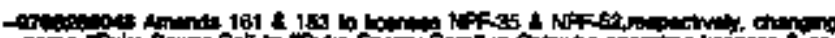

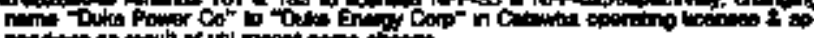

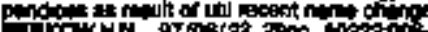

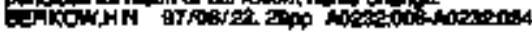

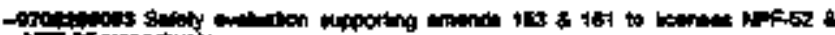

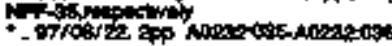

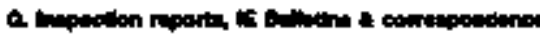

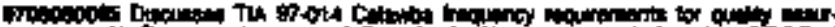

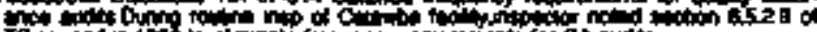
Tston

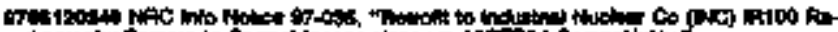

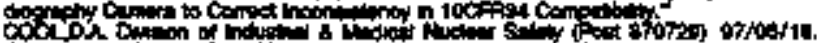

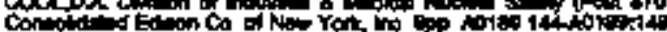

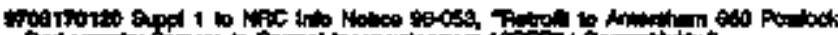

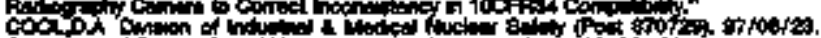

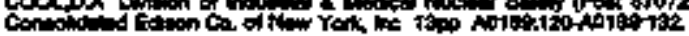

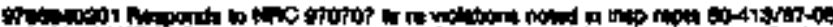
a so-d14 of a so-414 Hele

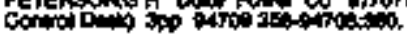

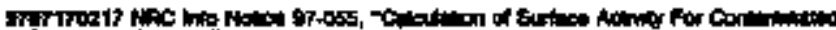

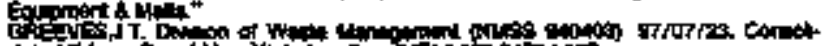

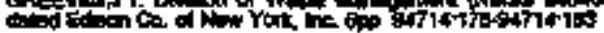

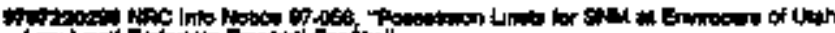
Pot in

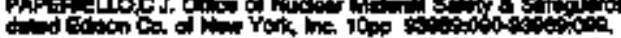

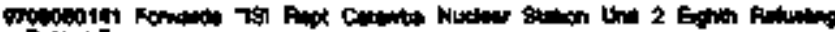

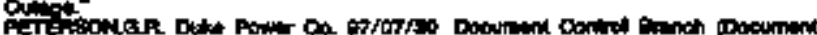

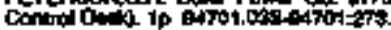

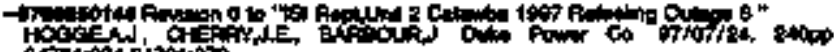

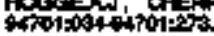

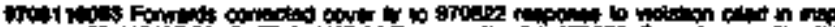

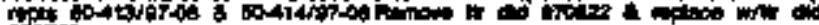

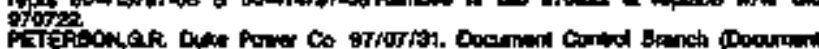

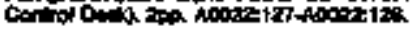

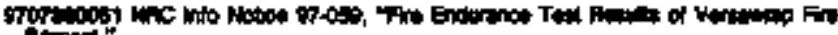

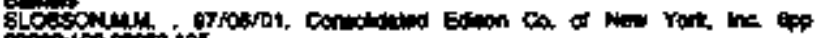
ofion

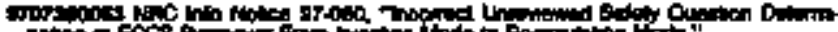

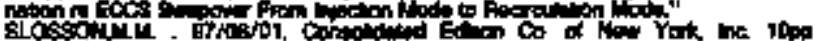

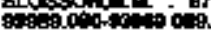

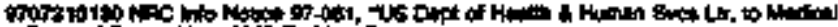

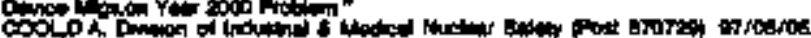

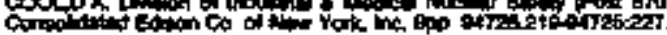

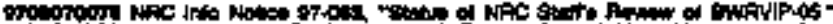
s.s.

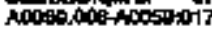

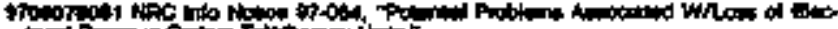

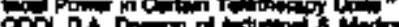

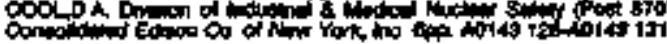




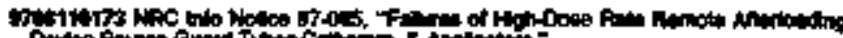

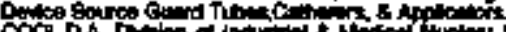

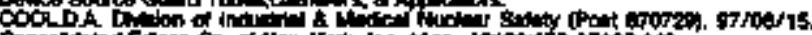

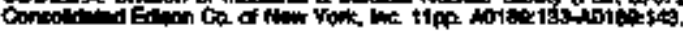

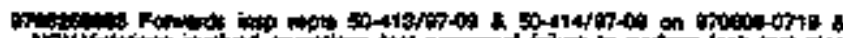

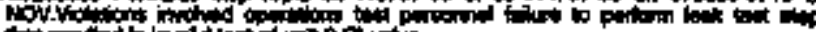

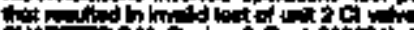

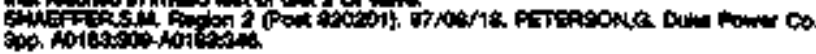

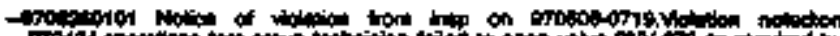

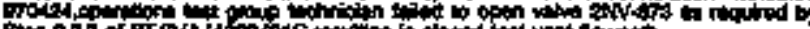

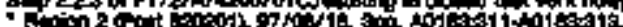

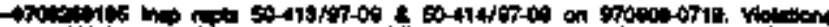

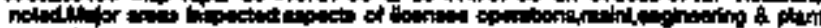

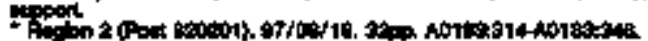

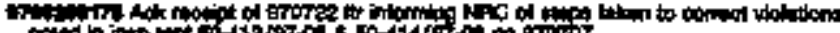

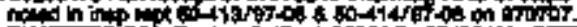

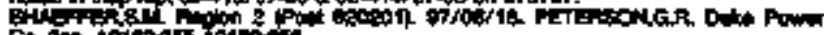

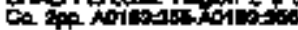

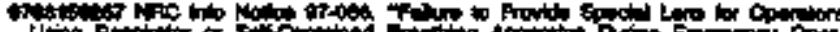

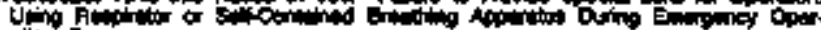

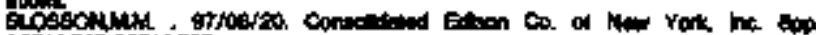

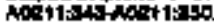

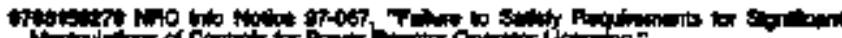

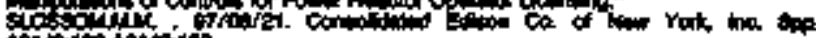

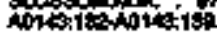

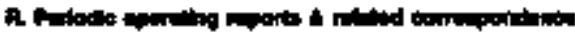

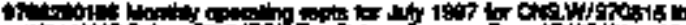

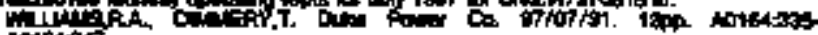
nothers?

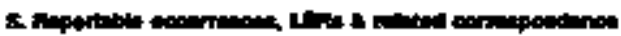

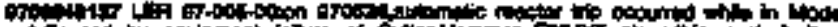

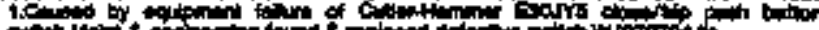

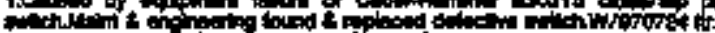

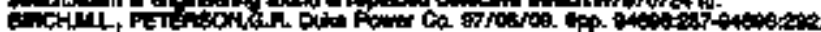

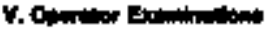

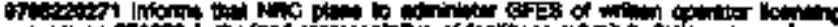

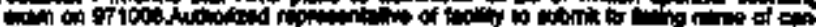

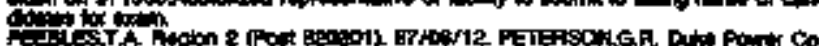

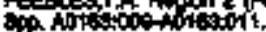

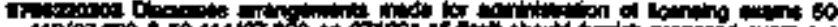

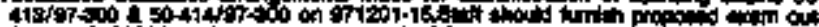

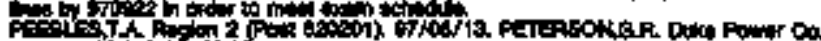

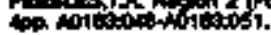

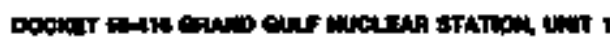

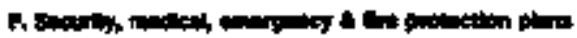

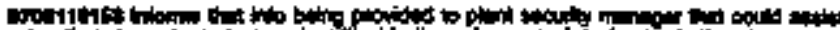

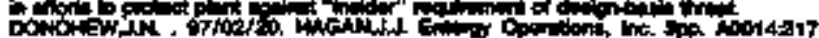
intistio

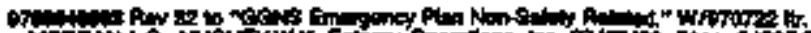

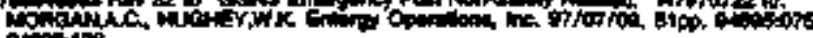
ofocitio

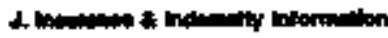

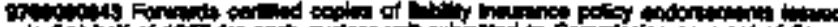

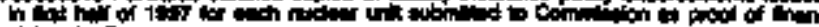

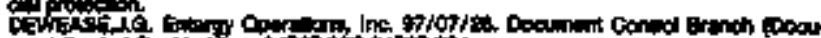

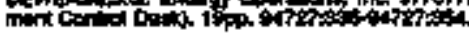

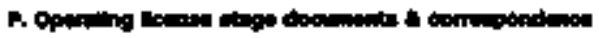

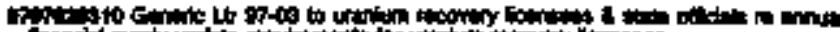

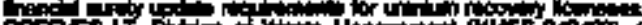

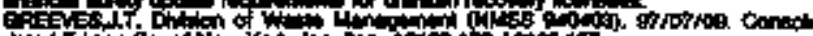

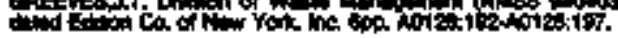

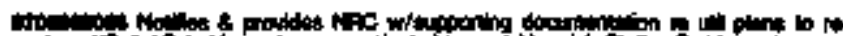

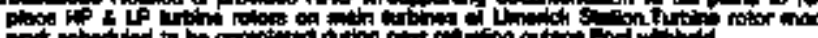

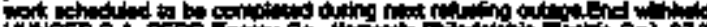
uning

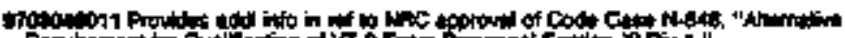

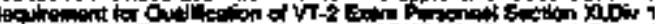

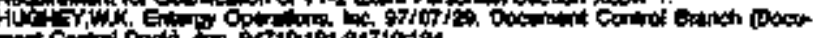

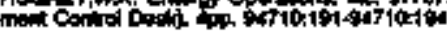

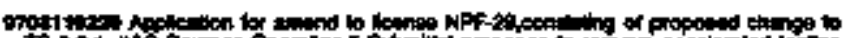

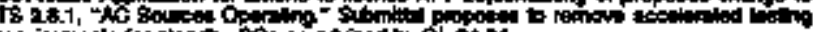

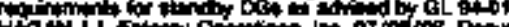

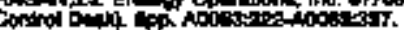

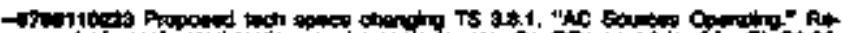

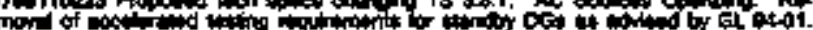

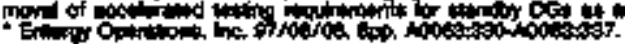

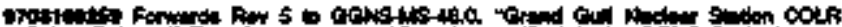

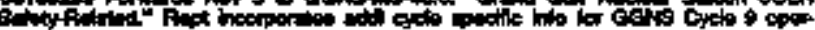

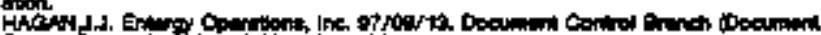

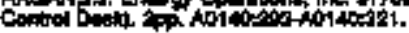

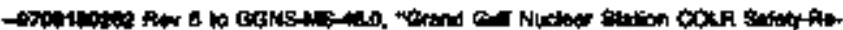

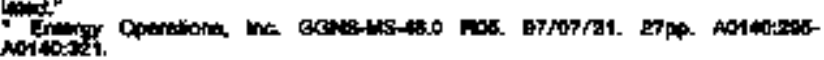

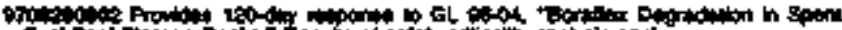

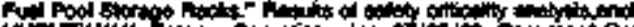

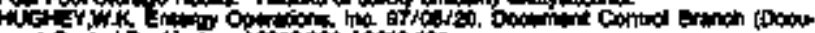

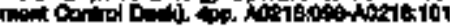

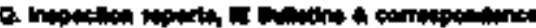

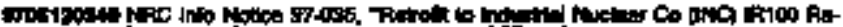

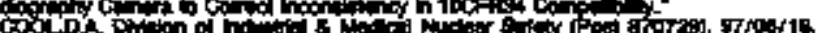

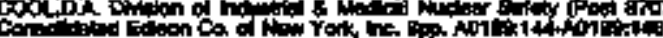

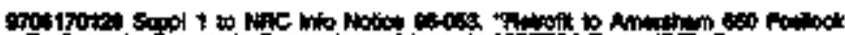

co

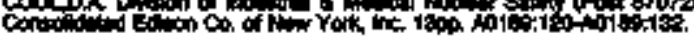

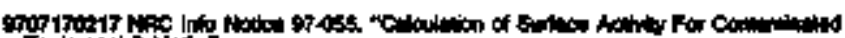
ch

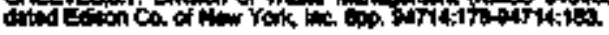

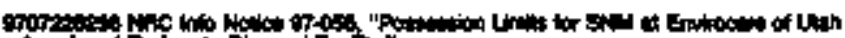

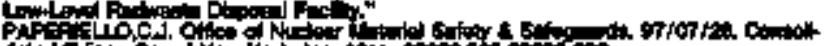

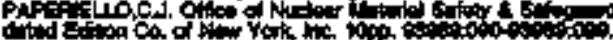

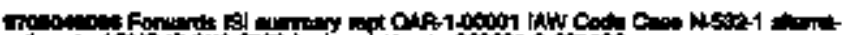

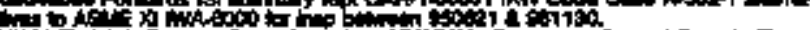

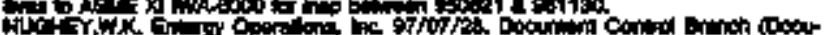

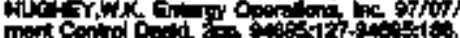

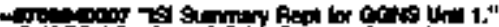

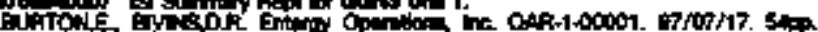

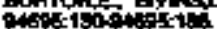

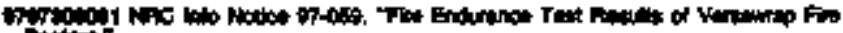

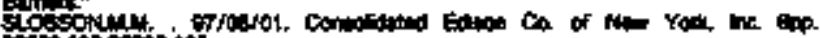
Hintoporing

SW

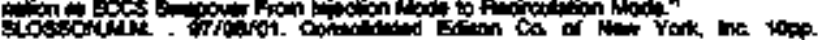

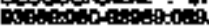

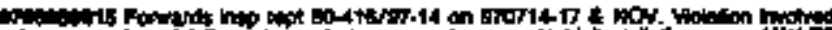

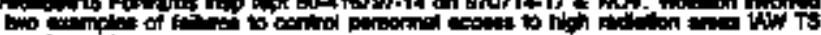

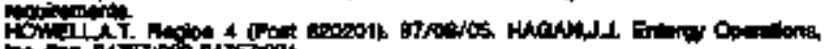

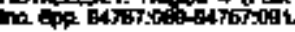

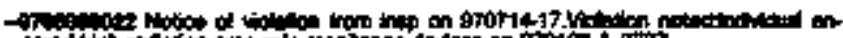

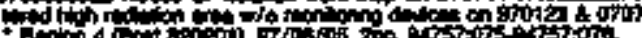

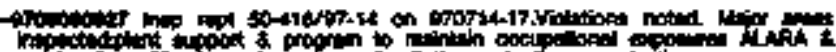

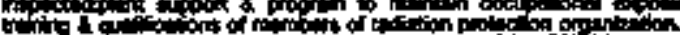

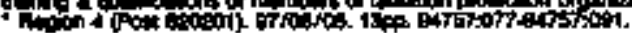

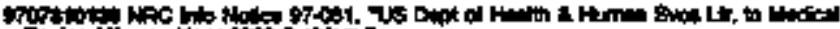

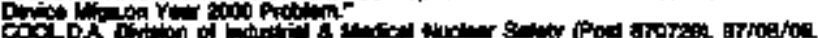

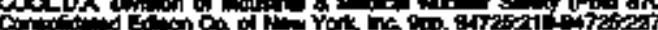

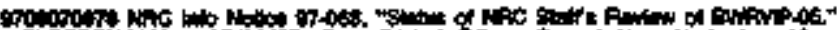

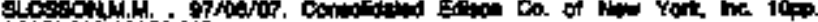

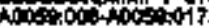

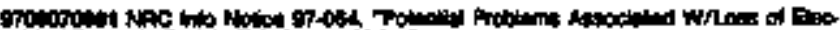

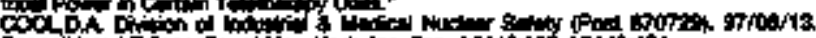

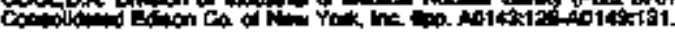

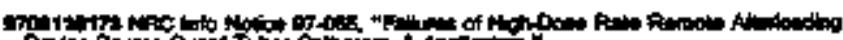

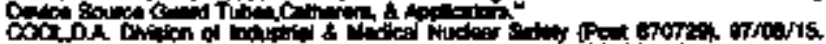

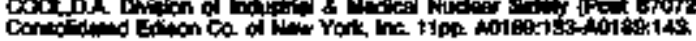




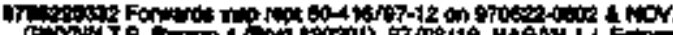

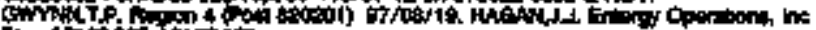

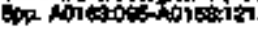

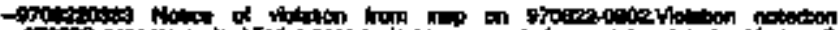

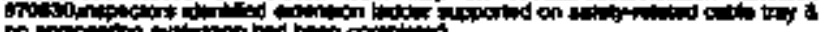

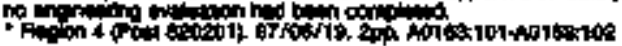

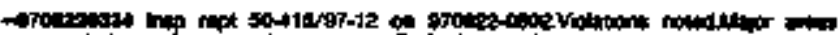

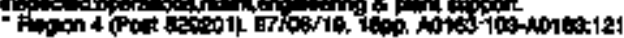

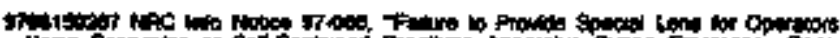

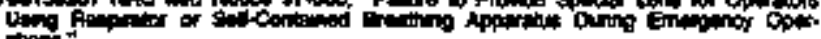

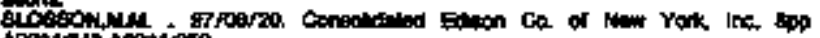

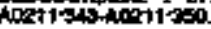

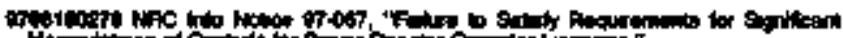

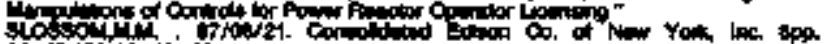

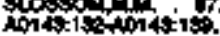

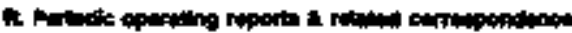

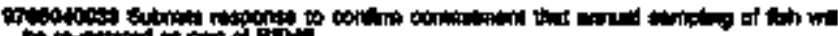

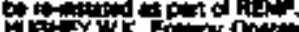
Maj-Y,

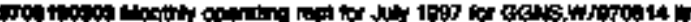

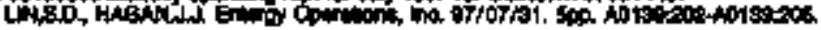

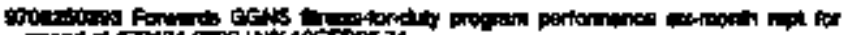

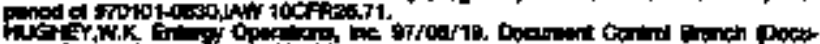

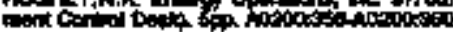

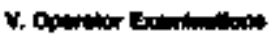

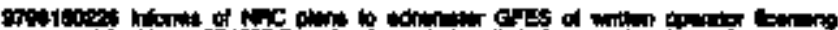

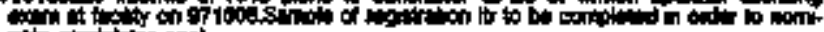

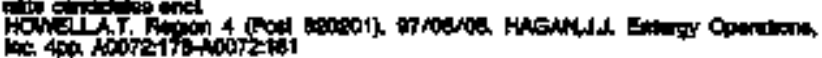

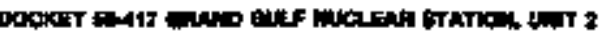

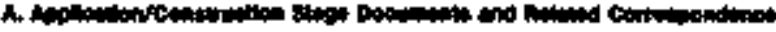

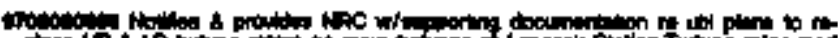

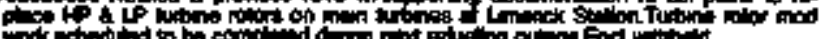

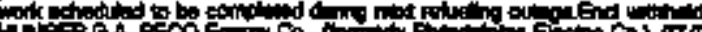

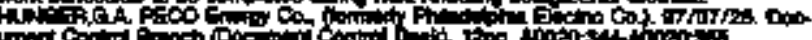

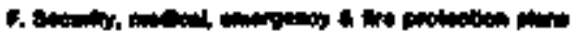

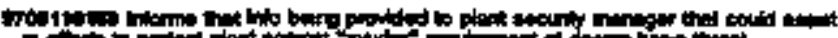

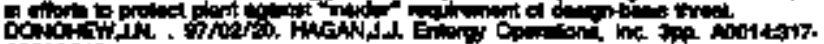
Aodititis

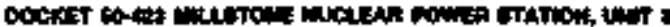

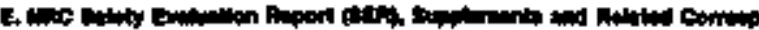

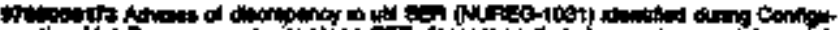

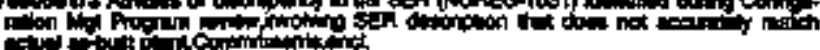

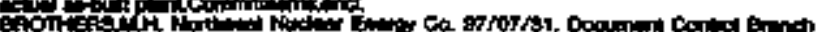
(10)

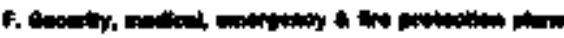

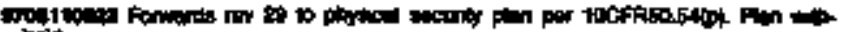

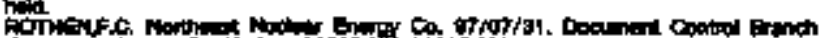

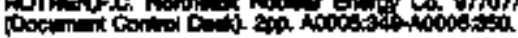

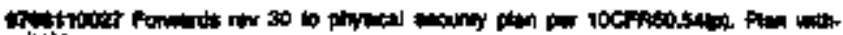

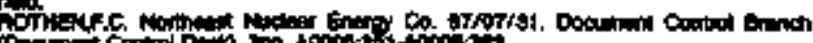

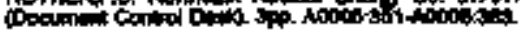

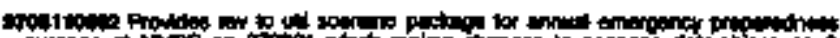

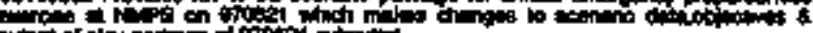

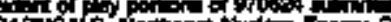

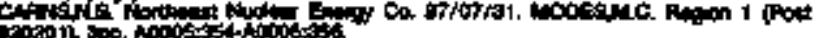

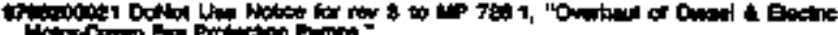

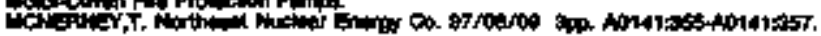

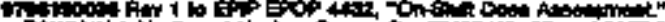

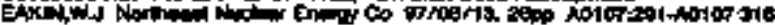

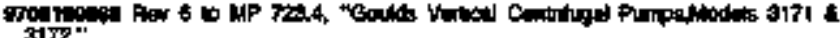

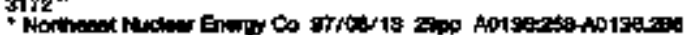

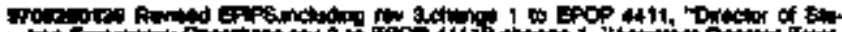

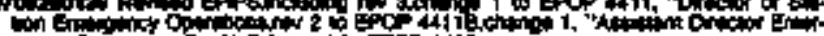

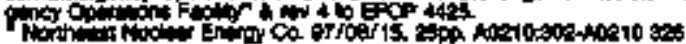

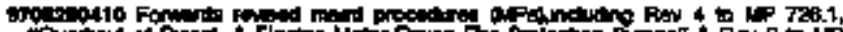

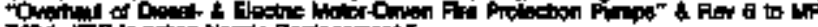

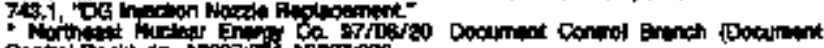

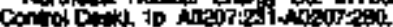

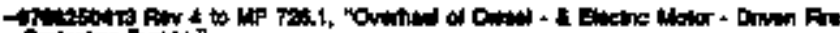

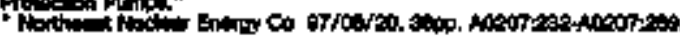

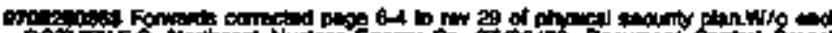

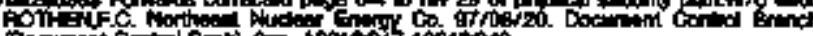

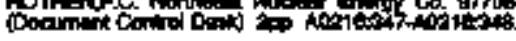

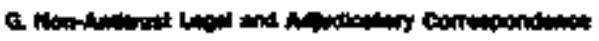

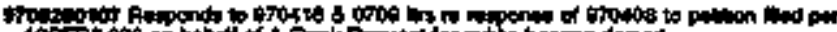

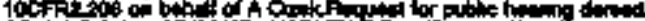

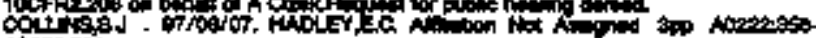
$1002 x=0.00$.

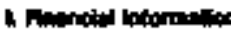

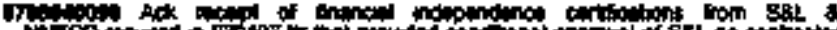

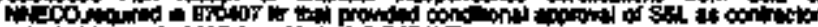

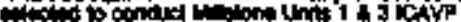

Thity

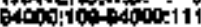

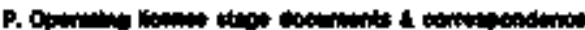

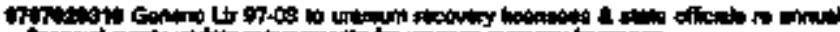

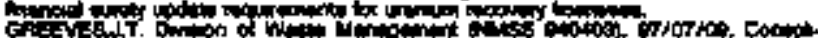

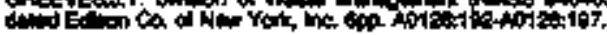

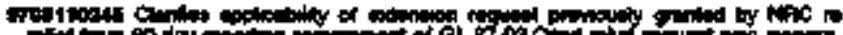

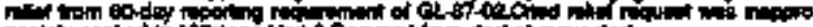

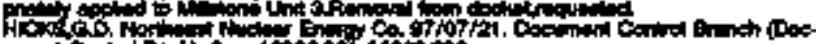

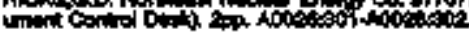

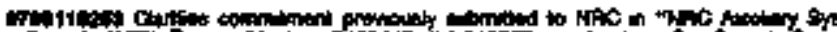

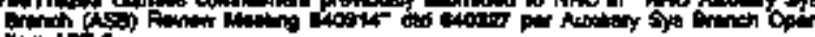

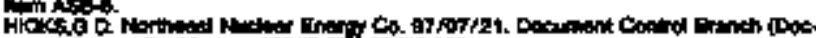

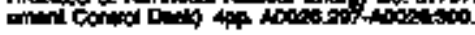

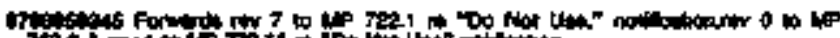

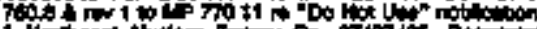

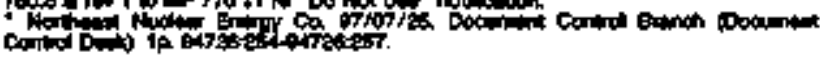

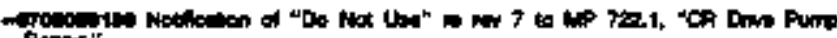

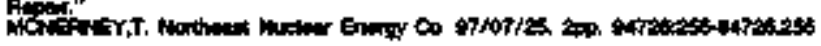

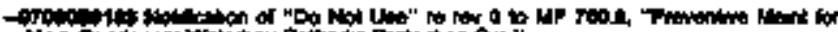

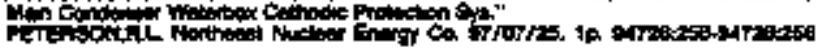

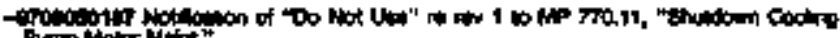

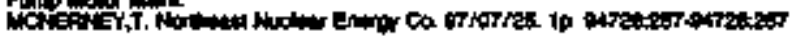

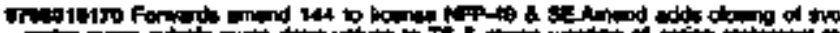

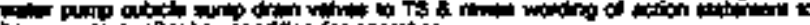

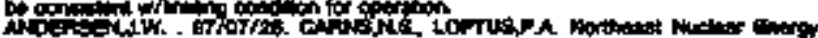

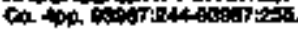

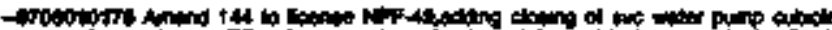

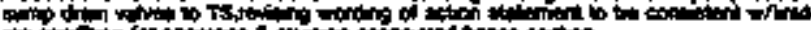

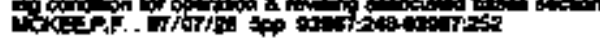

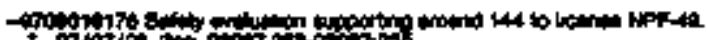

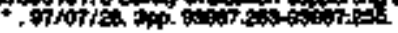

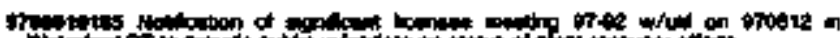

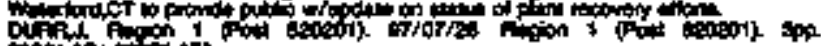
Dind

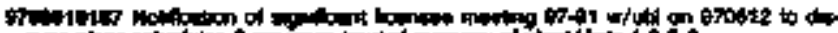

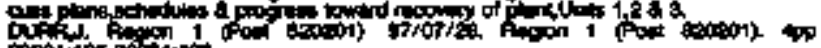

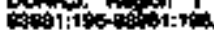

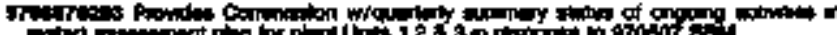

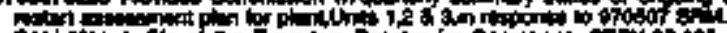

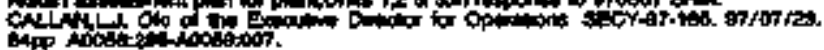




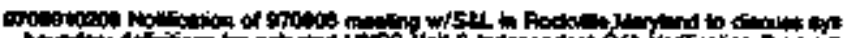

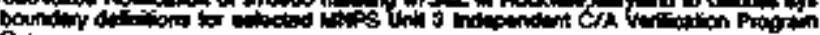

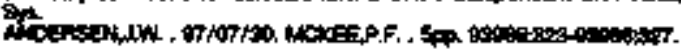

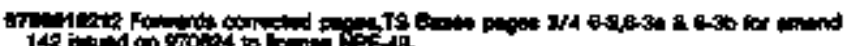

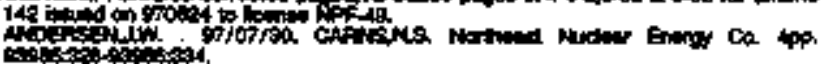

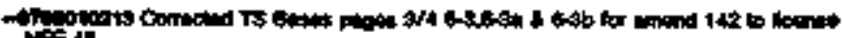

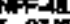

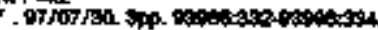

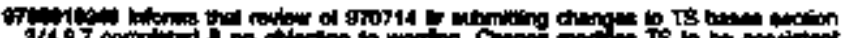

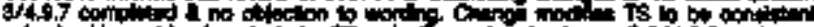

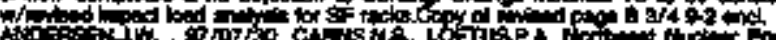

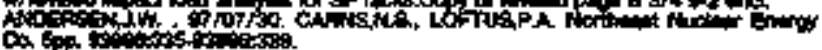

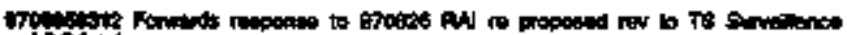

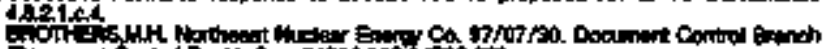

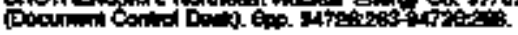

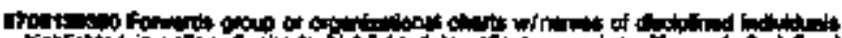

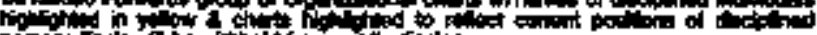

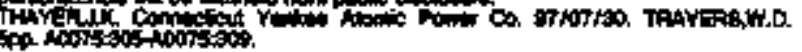

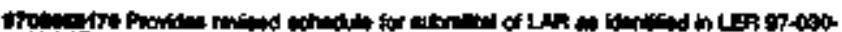

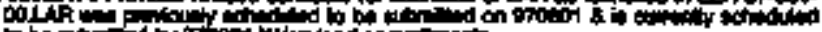

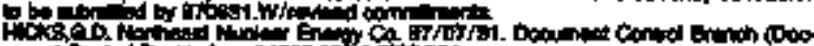

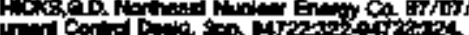

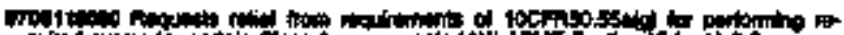
OH

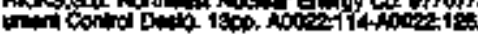

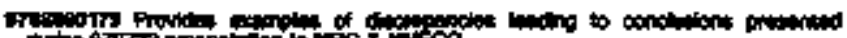
D.

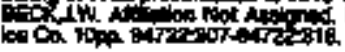

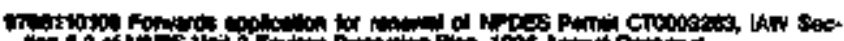

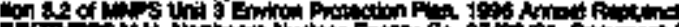

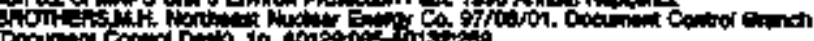
morstom

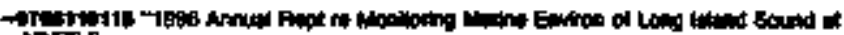
thes

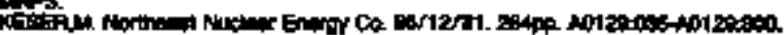

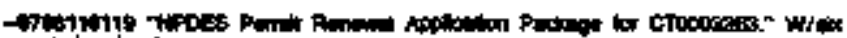

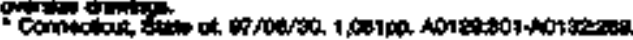

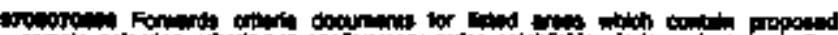

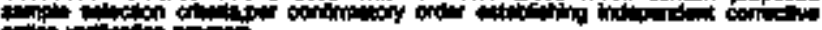

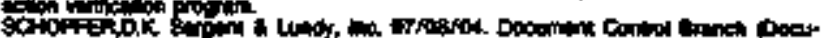

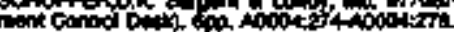

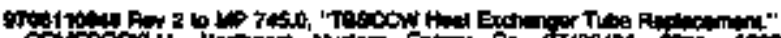

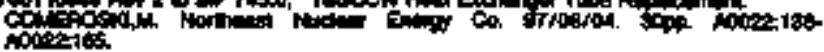

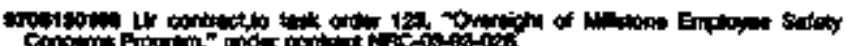

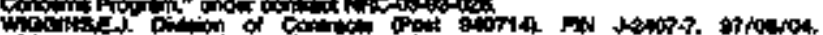

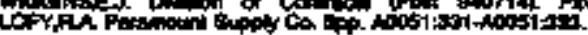

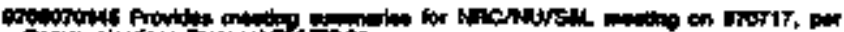

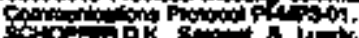

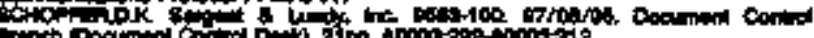

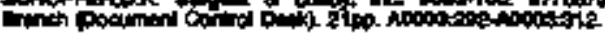

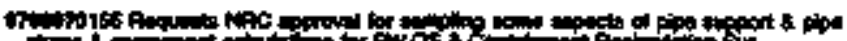

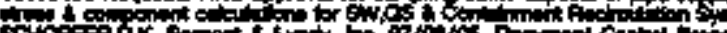

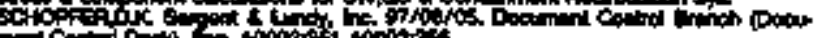

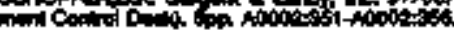

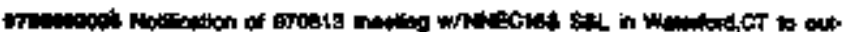

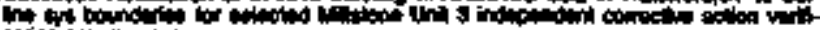

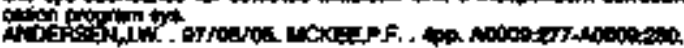

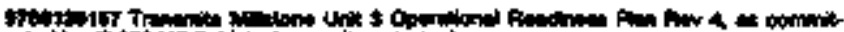

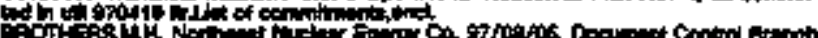

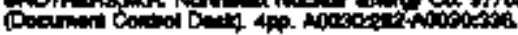

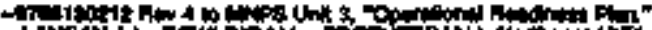

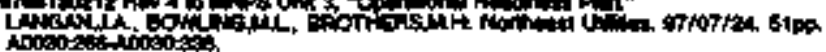

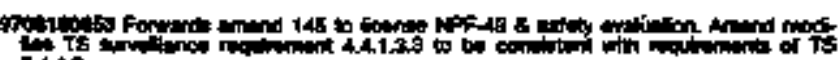
3.t.

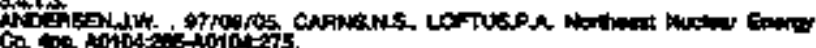

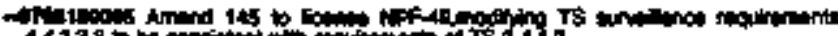

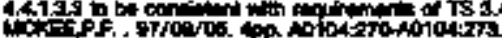

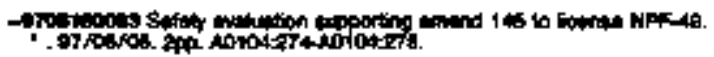

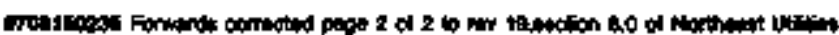

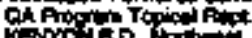

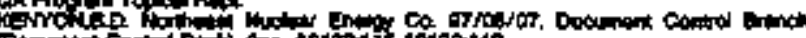

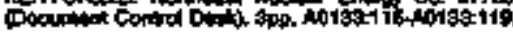

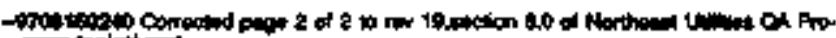

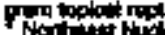

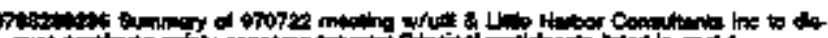

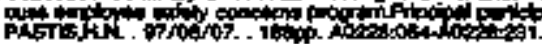

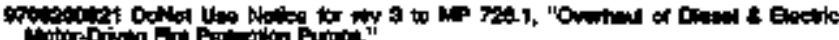

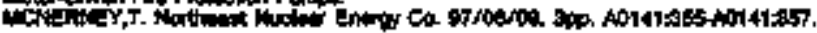

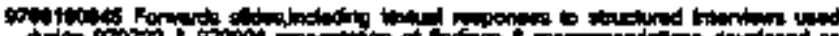

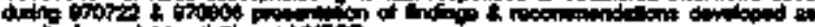

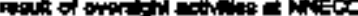

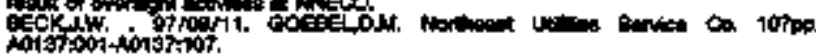

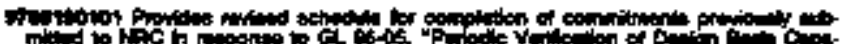

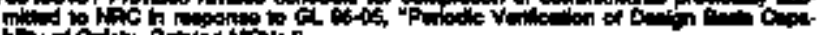

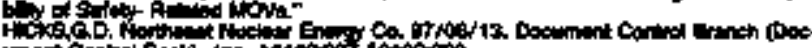

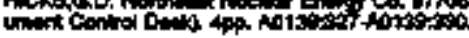

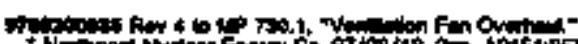

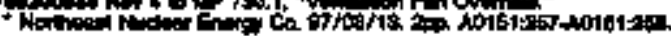

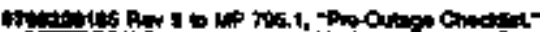

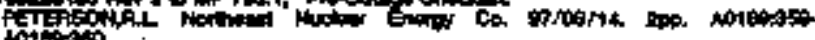

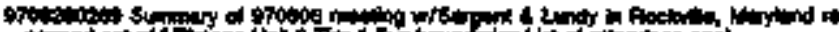

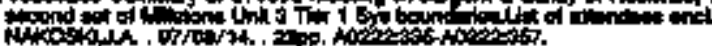

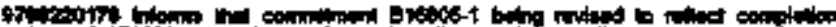

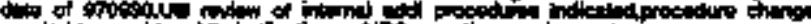

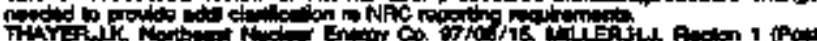

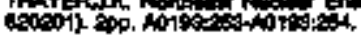

97w

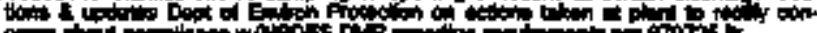

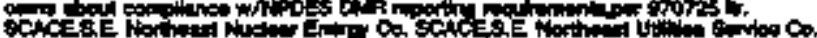

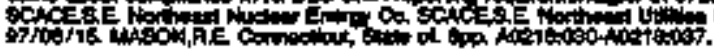

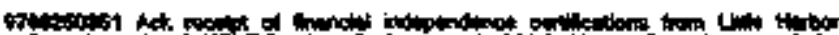

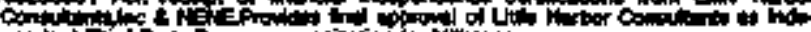

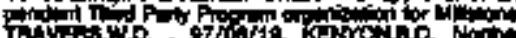

Thly

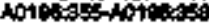

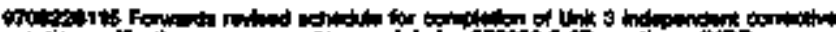

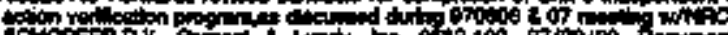

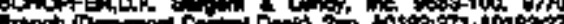

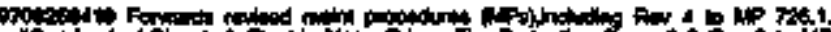

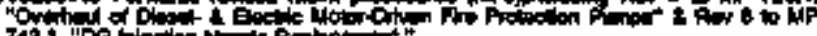

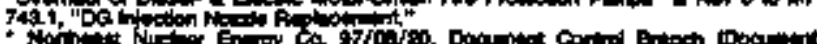

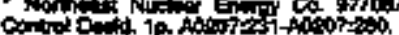

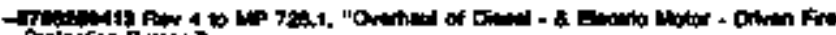

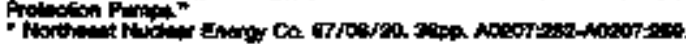

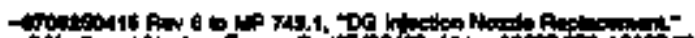

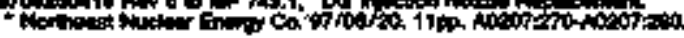

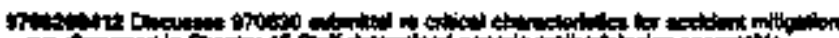

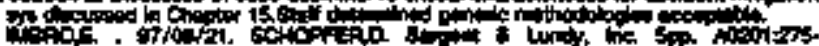

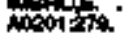

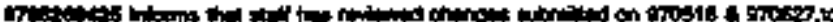

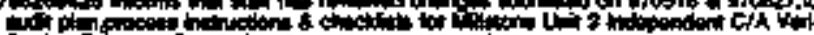

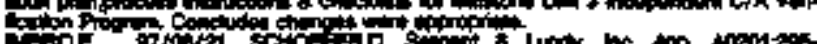
ingive

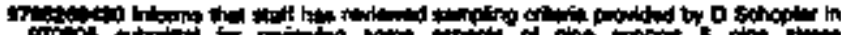

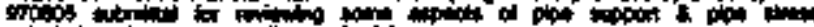

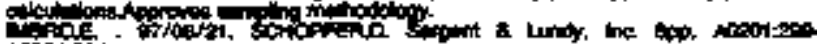
Anotidit.

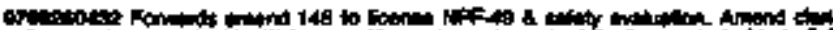

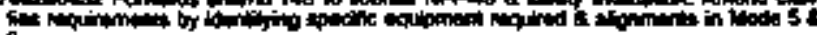
6.

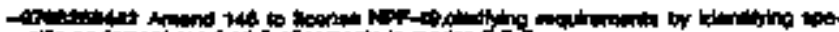

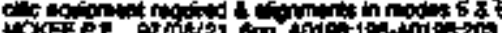

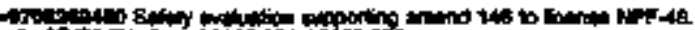




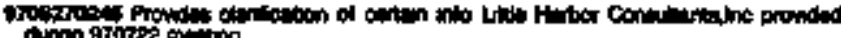

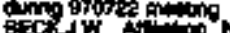

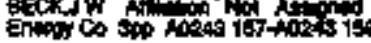

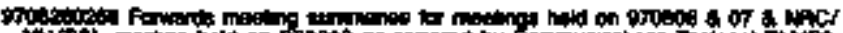

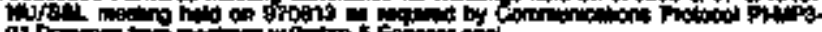

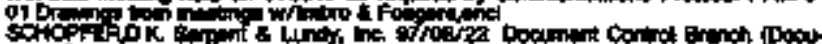

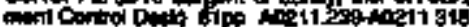

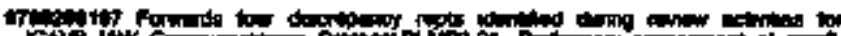

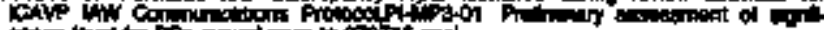

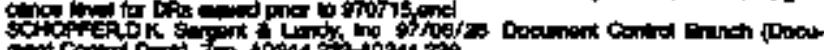

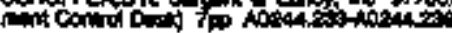

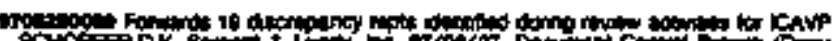

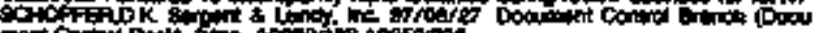

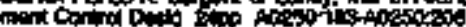

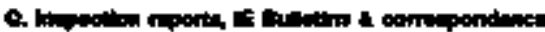

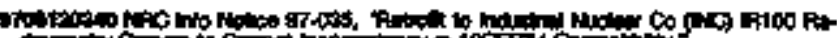
Cobifh

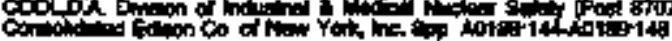

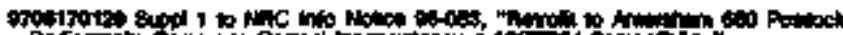

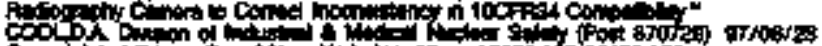

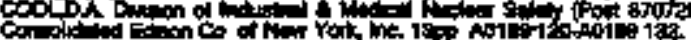

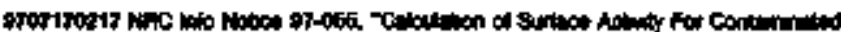

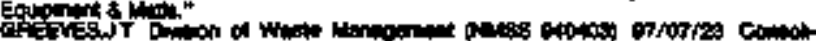

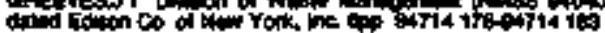

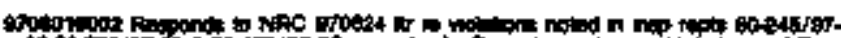

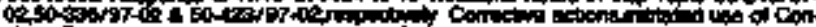

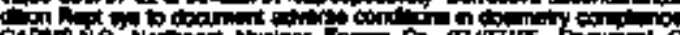

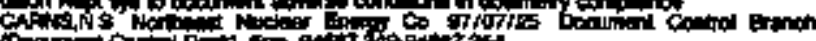

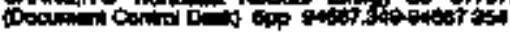

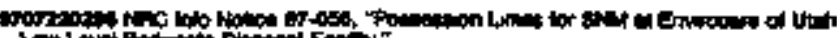
P. W

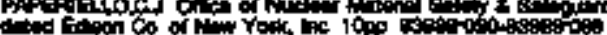

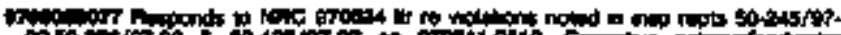

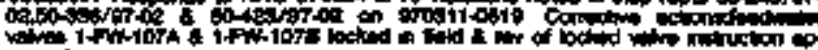

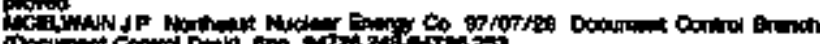

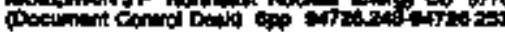

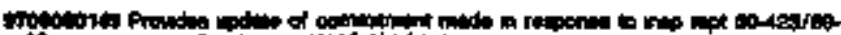
A

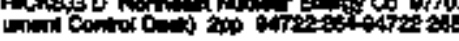

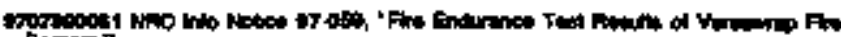

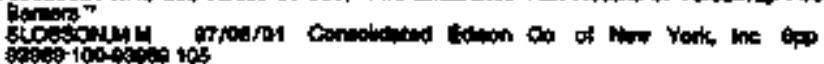

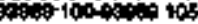

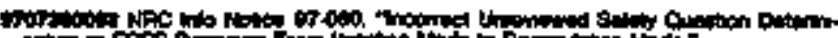

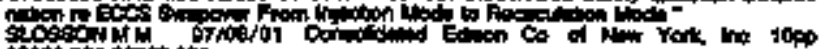

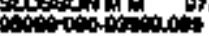

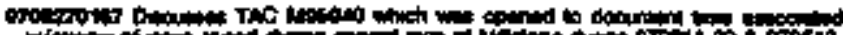

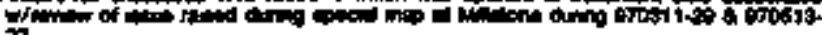

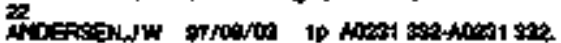

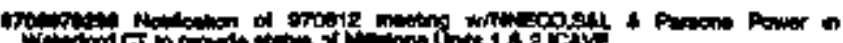

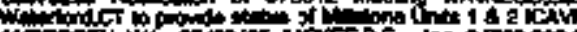

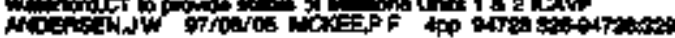

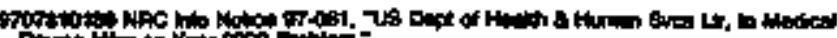

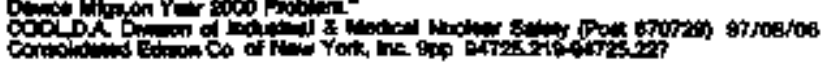

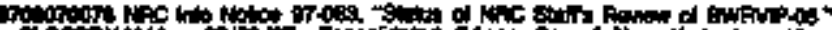

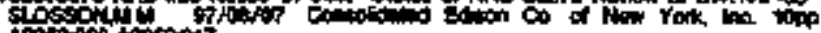

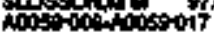

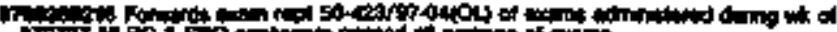

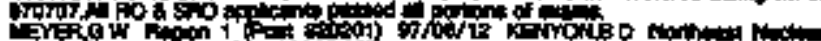

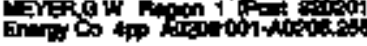

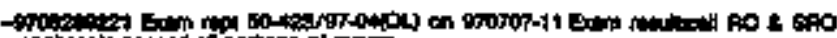

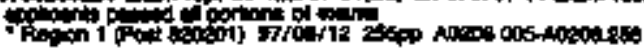

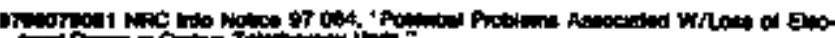

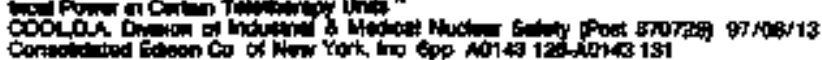

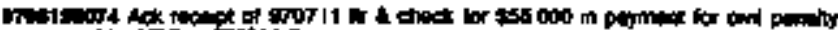

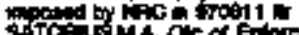
SATC

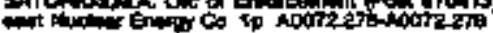

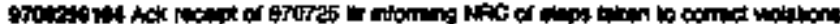

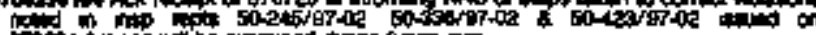

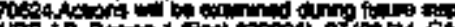

Co:

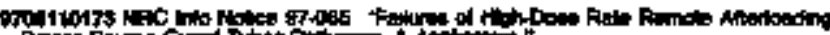

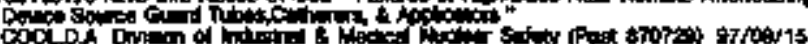

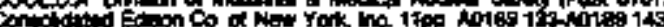

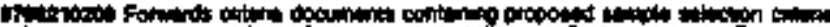

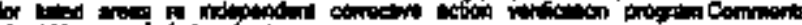

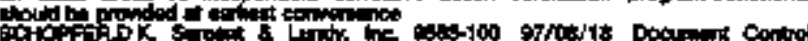

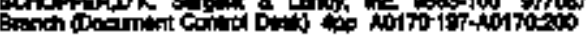

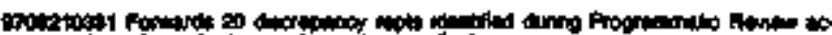

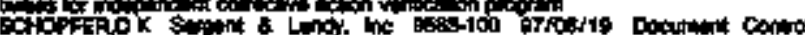

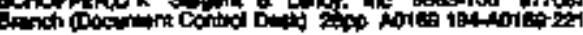

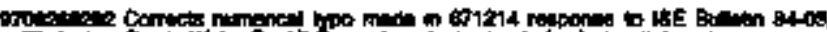

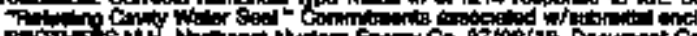

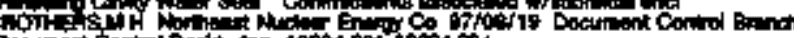

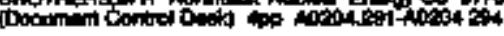

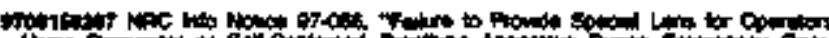

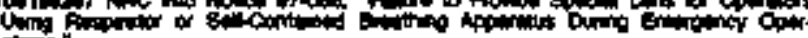

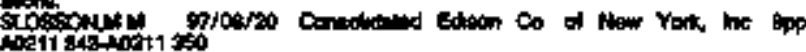

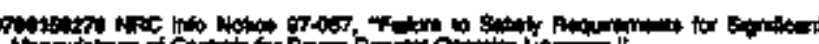

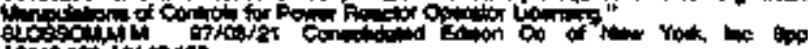

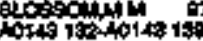

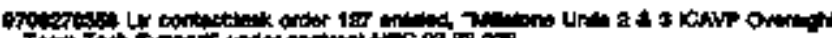

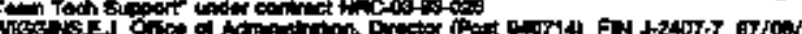

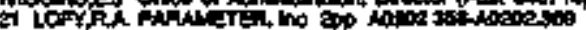

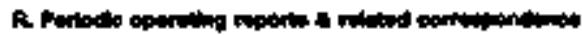

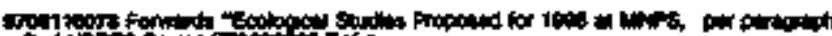

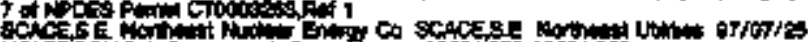

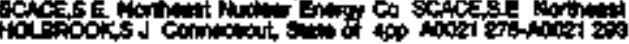

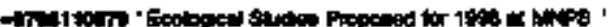

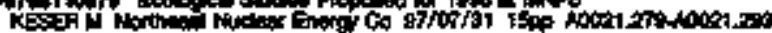

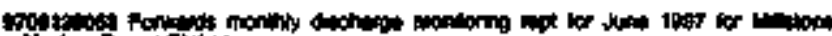

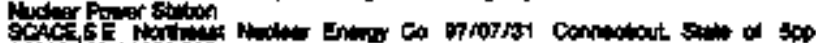
action

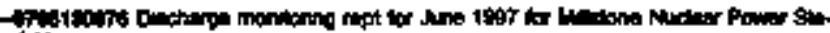

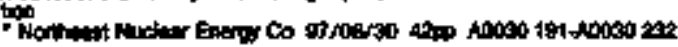

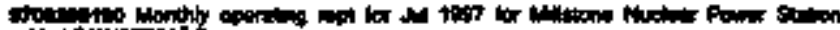
Unt 1 wrojosis

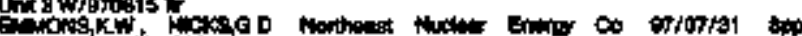

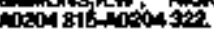

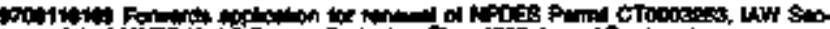

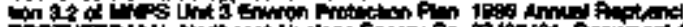

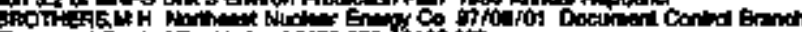

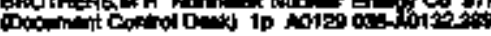

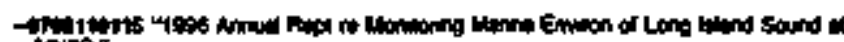

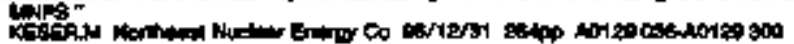

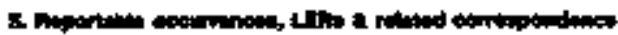

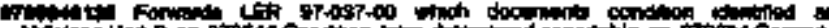

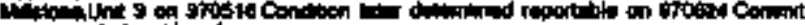

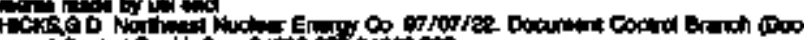

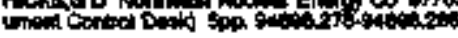

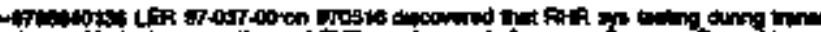

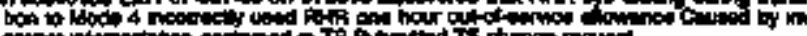

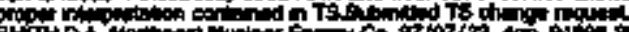

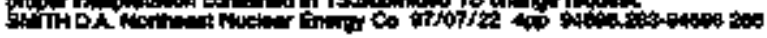

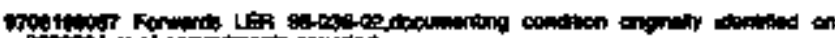

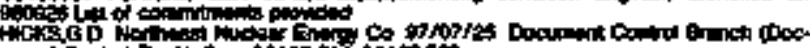

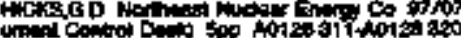

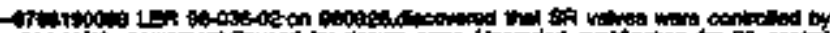

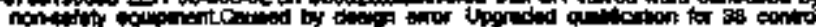

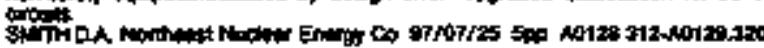

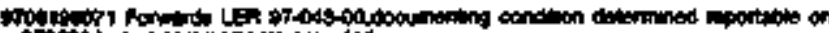

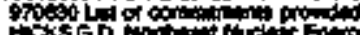

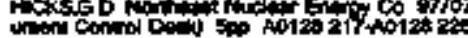




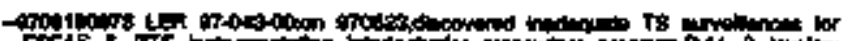

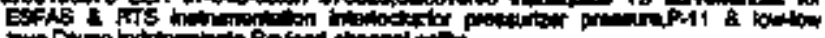

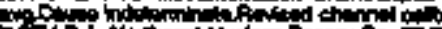

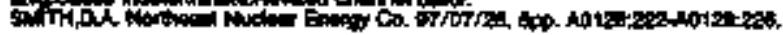

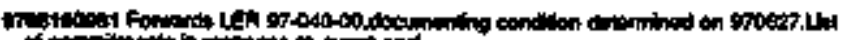

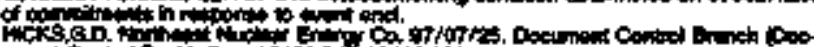

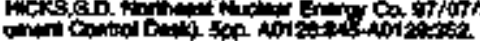

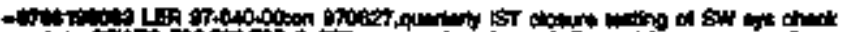

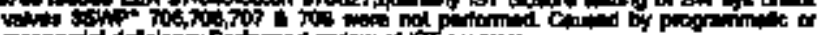

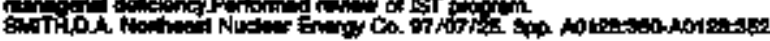

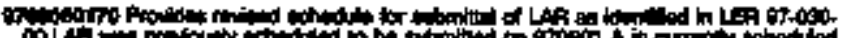

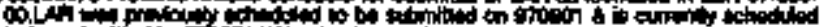

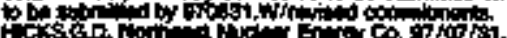

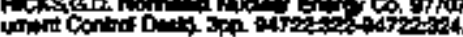

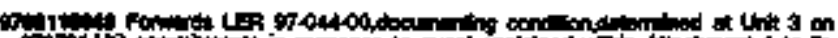

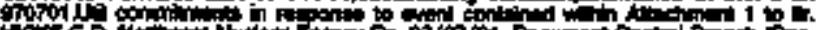

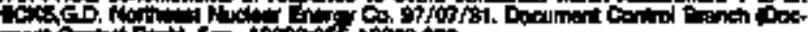

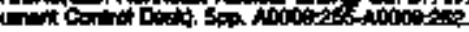

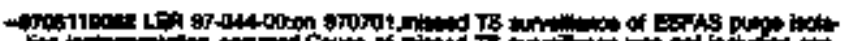

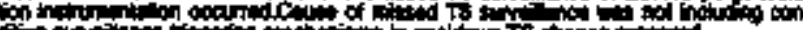
Sali

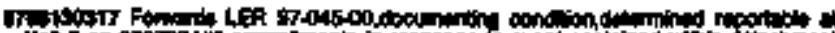

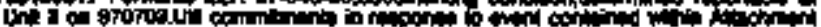
i to

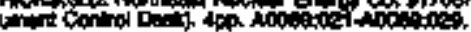

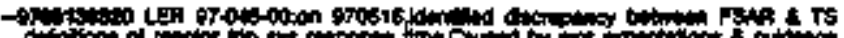

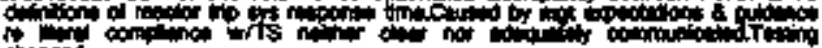

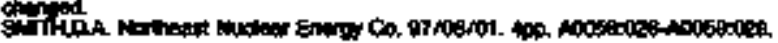

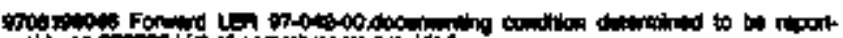

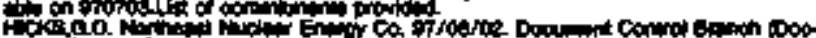

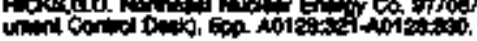

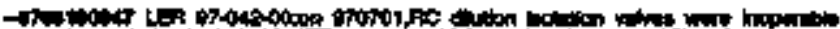

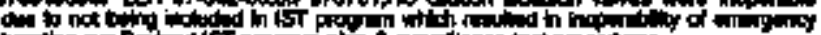

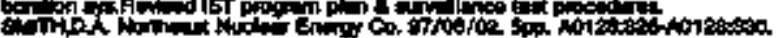

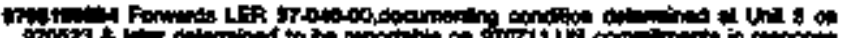

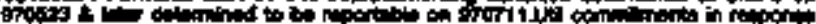

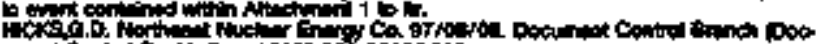

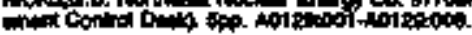

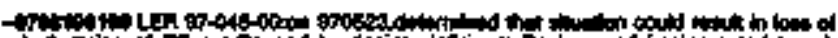

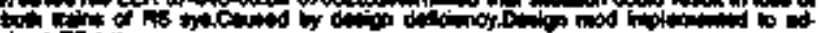

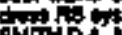

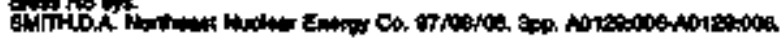

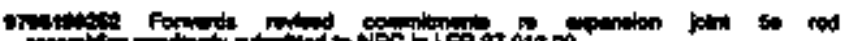

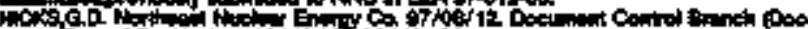

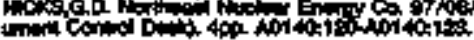

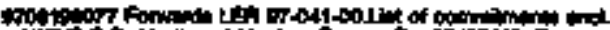

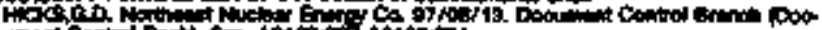

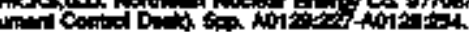

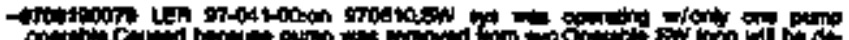

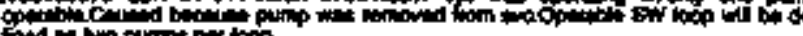

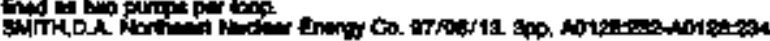

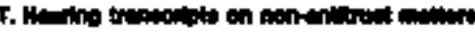

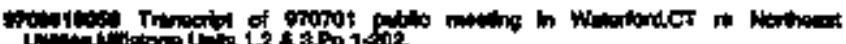

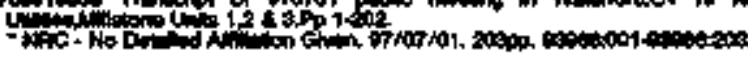

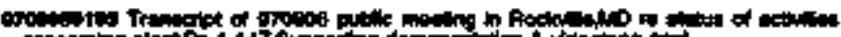

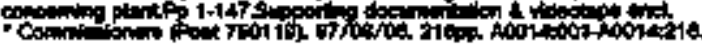

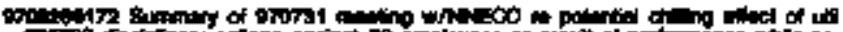

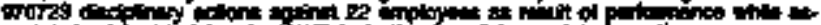

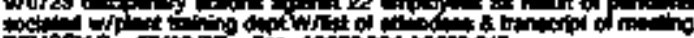

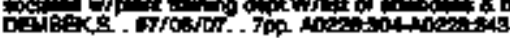

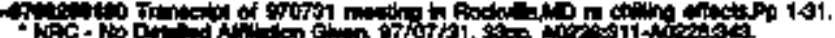

v. Operion Romine

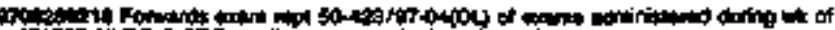

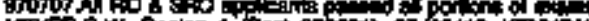

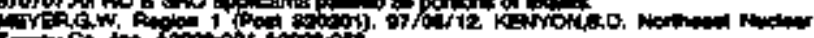

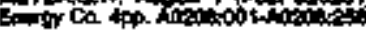

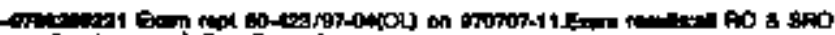

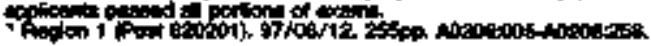

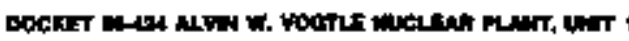

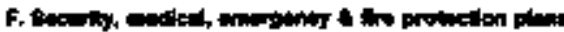

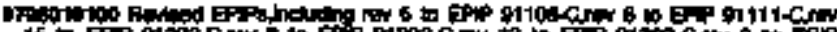
16 b EFP gizis

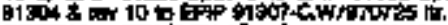

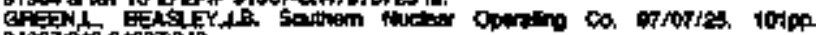
Bution.

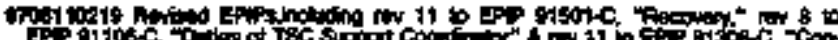

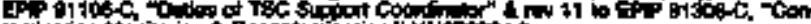

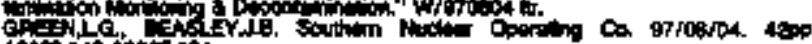

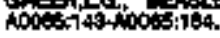

Iros

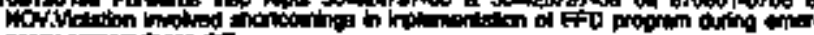

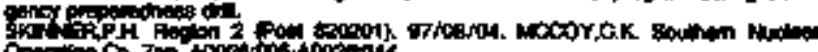

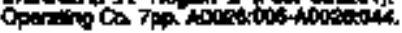

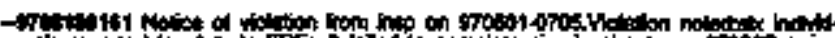

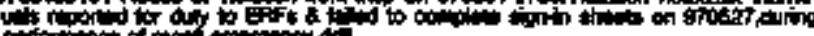

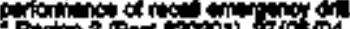

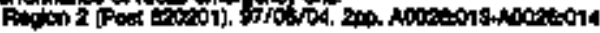

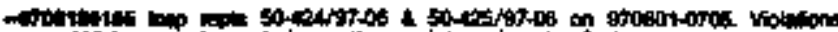

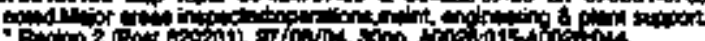

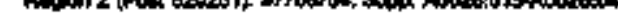

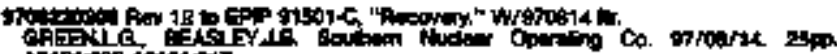

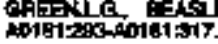

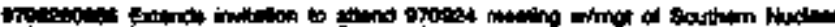

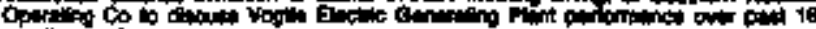

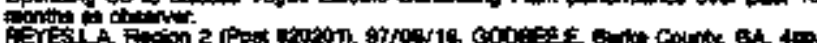

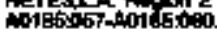

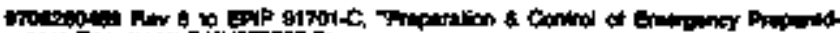

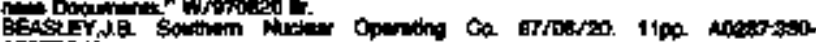
negrato.

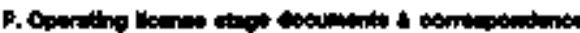

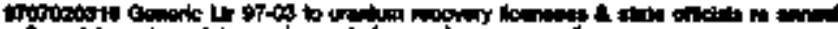

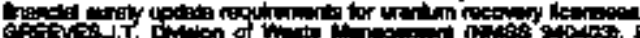

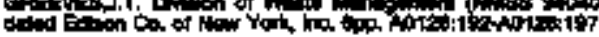

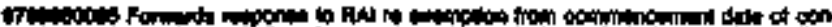

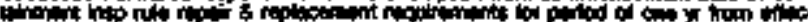
iction

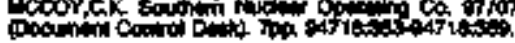

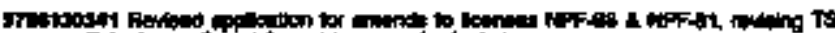

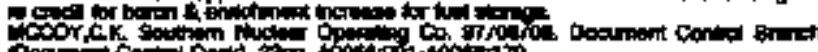

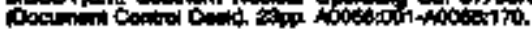

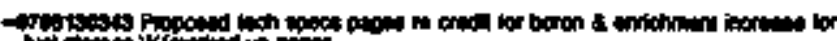

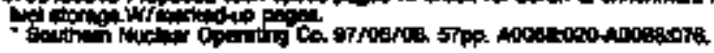

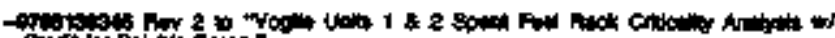

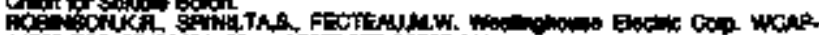

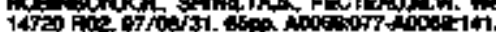

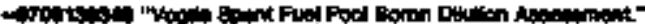

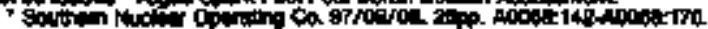

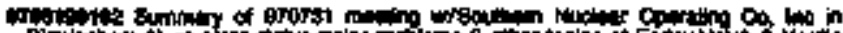

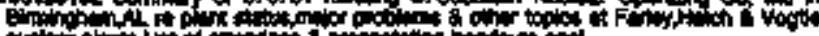

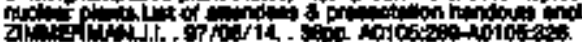

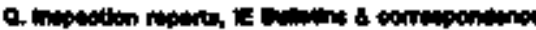

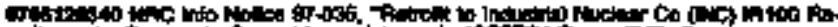

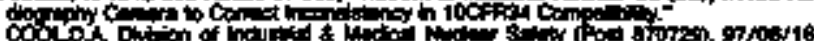

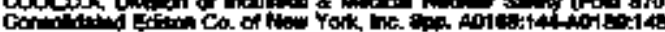

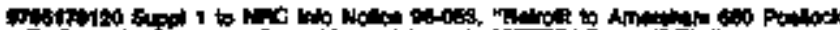

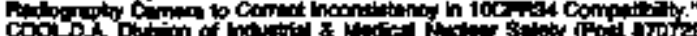
con

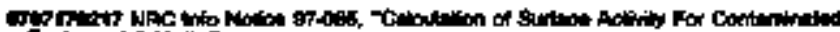

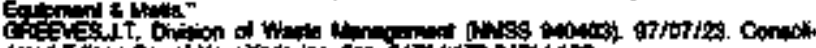

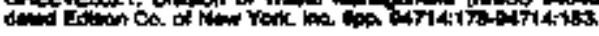

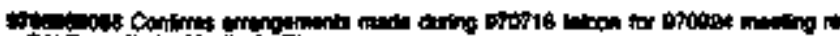
Step

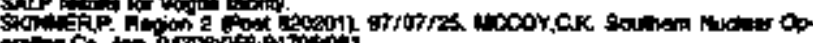

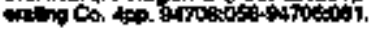




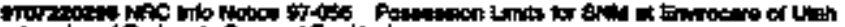

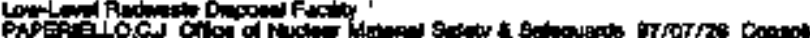

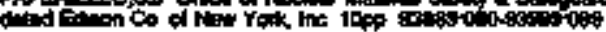

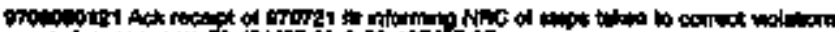

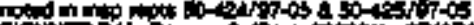

Hon Q

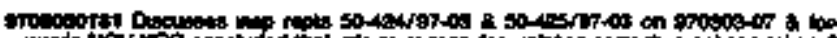

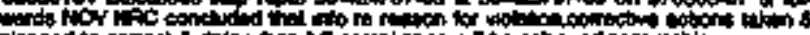

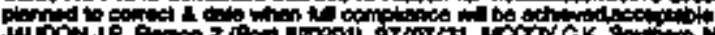

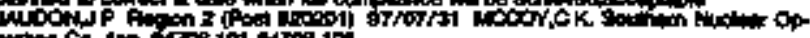

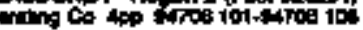

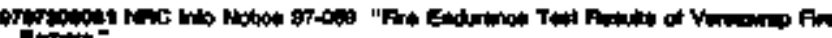

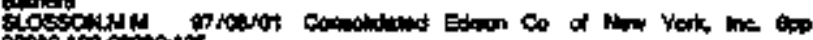

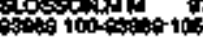

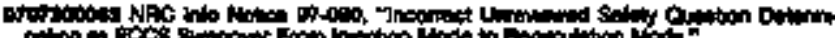

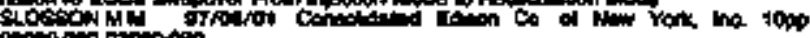

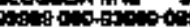

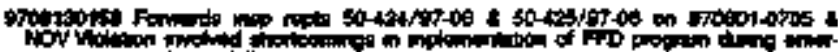

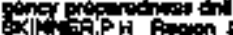

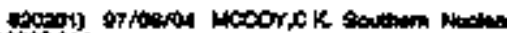

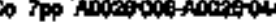

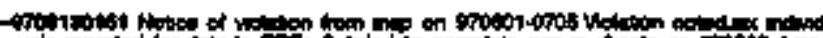

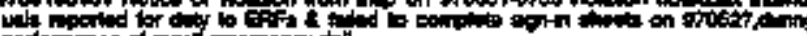

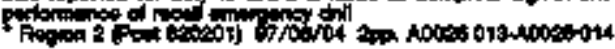

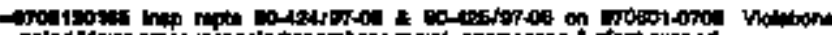

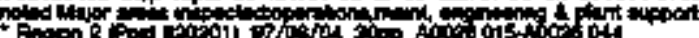

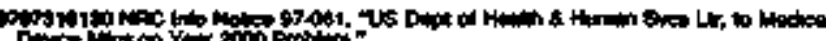

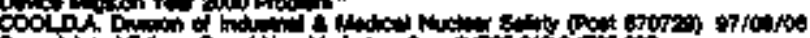

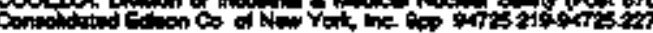

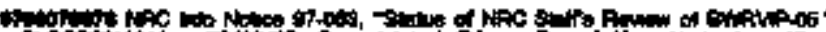

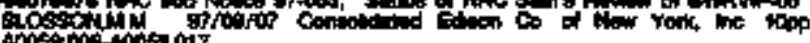
Aoos botidoto oir

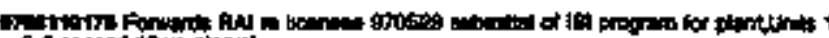

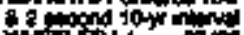

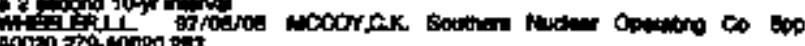

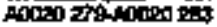

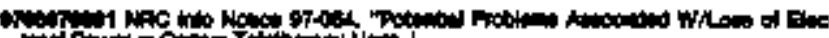

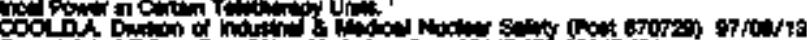

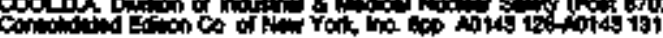

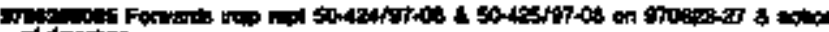

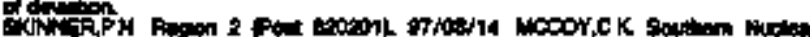

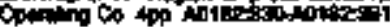

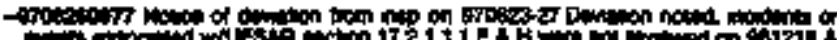

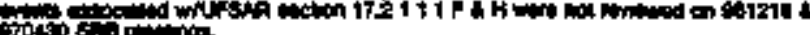

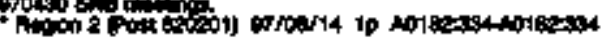

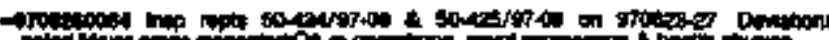

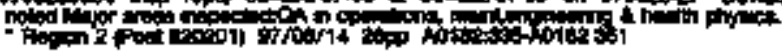

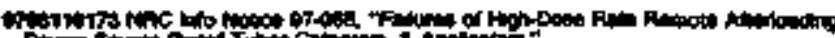

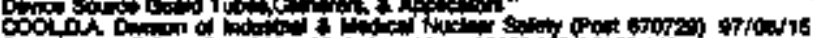

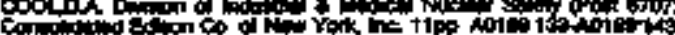

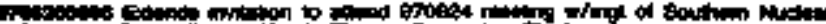

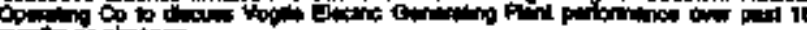

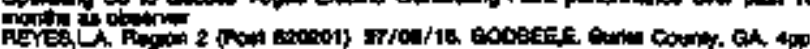

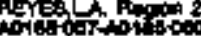

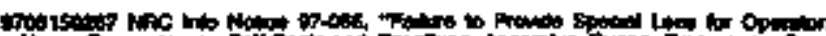

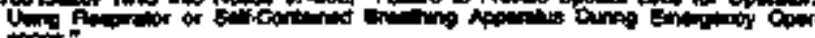

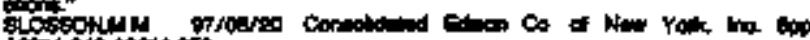

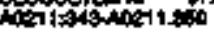

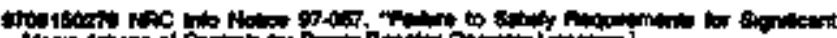

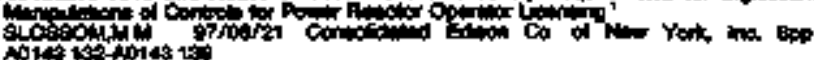

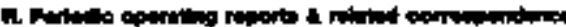

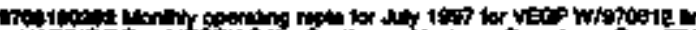

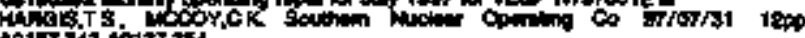

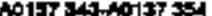

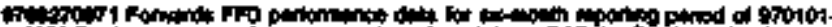

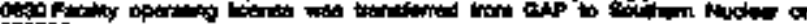
groner

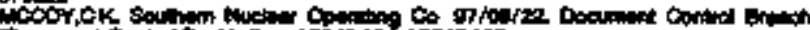

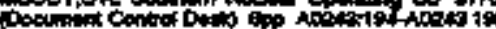

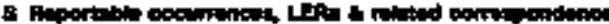

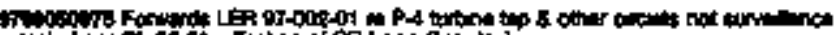

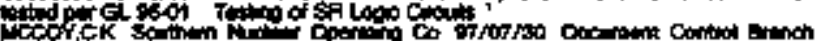

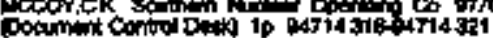

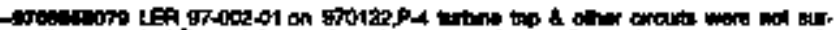
nate

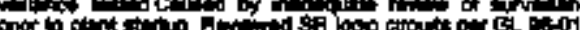

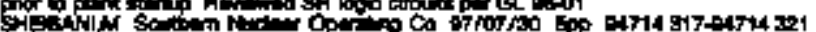

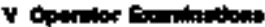

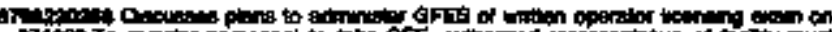

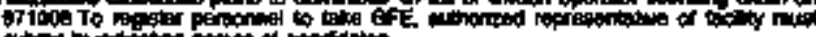

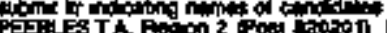

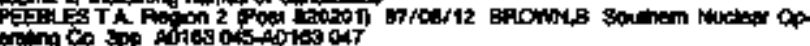

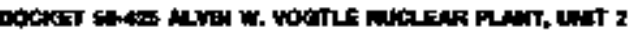

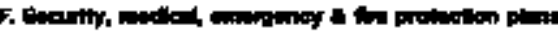

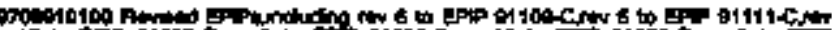

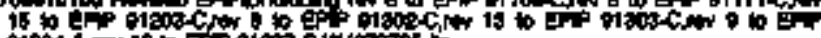

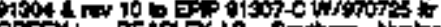
give

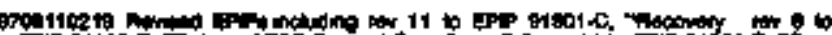

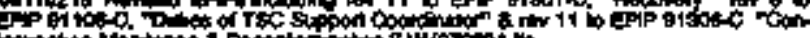

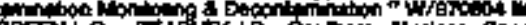

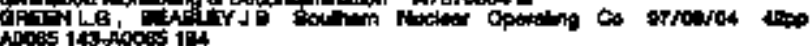

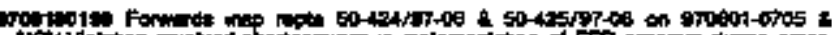

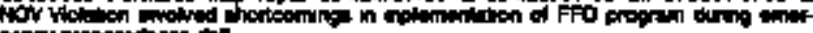

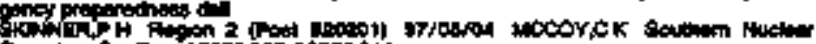

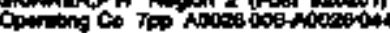

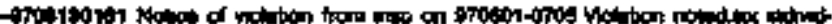

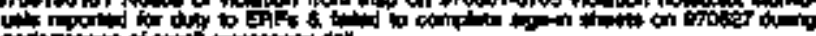

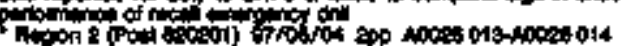

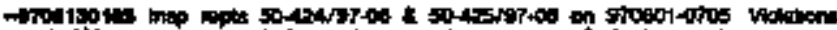

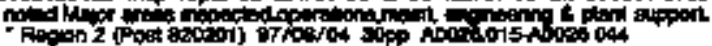

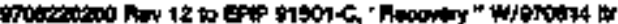

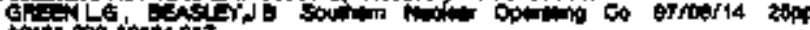

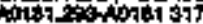

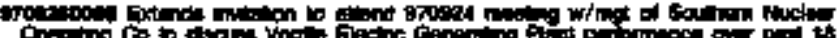

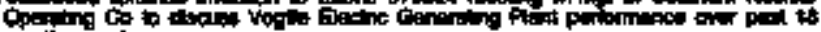

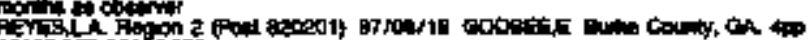

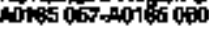

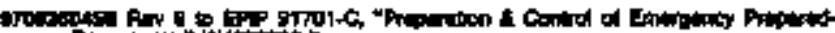

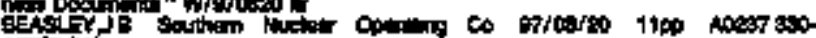
Antorion

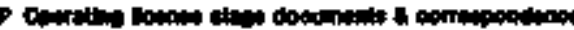

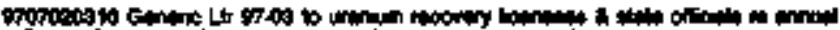
thow

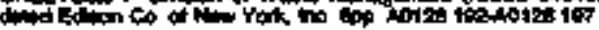

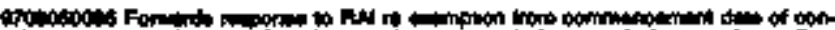

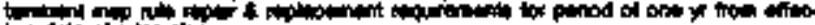

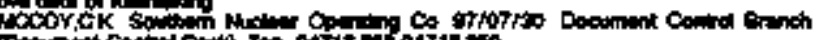

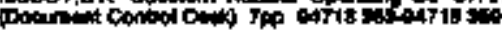

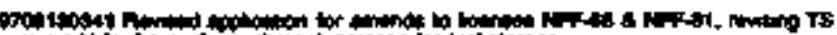

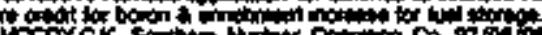

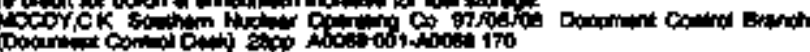

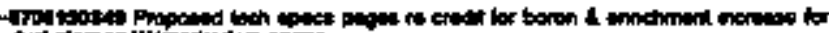

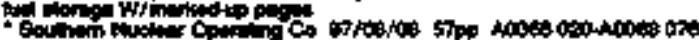

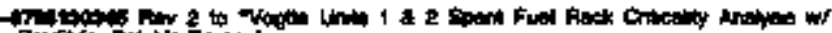

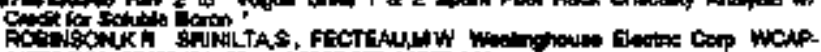

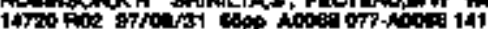

- 77.

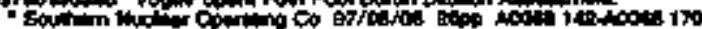

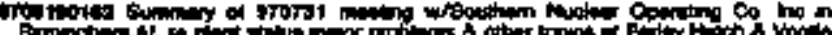

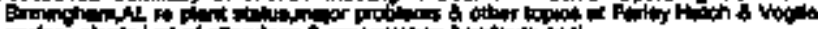

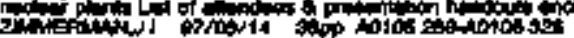




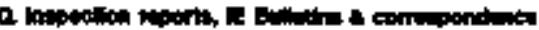

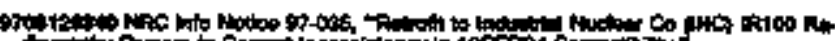

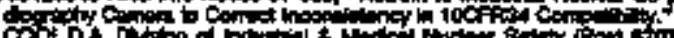

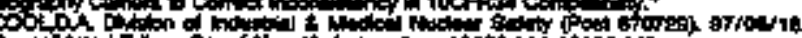

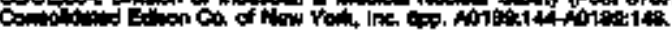

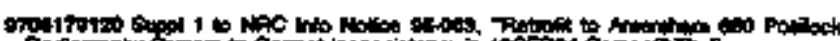

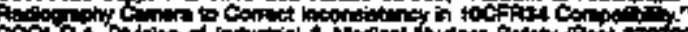

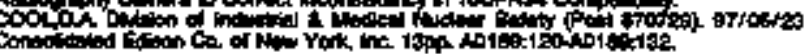

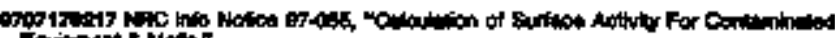

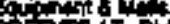

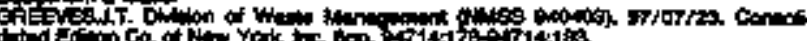

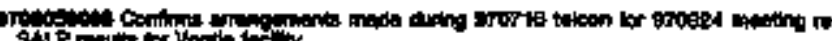

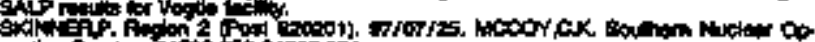

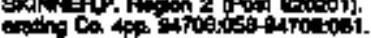

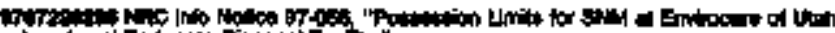

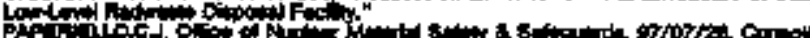

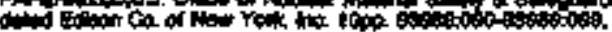

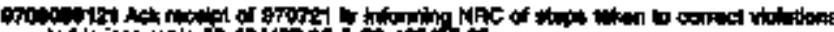

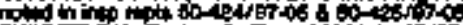

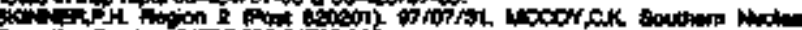

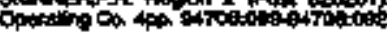

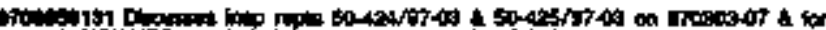

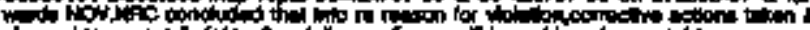

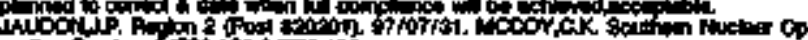

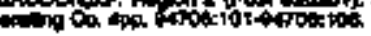

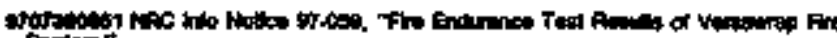
Priter.t.

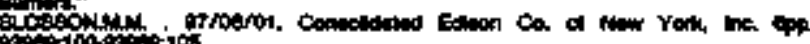

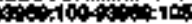

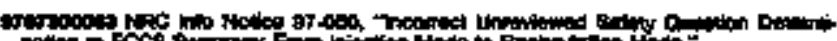

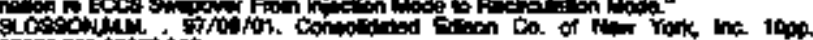
sicosing

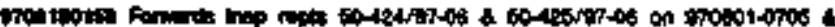

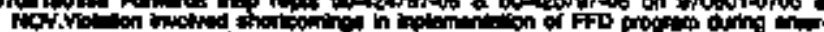

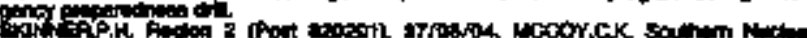

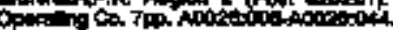

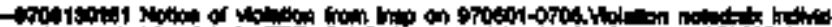

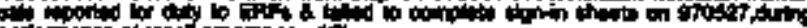

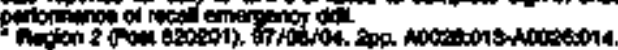

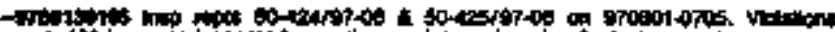

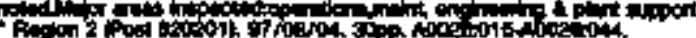

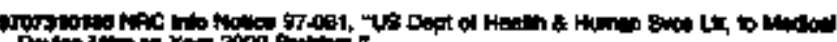

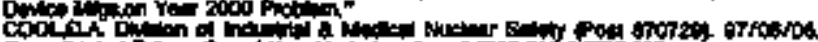

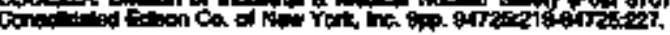

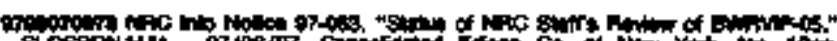

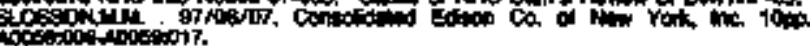

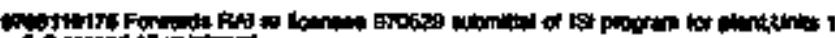

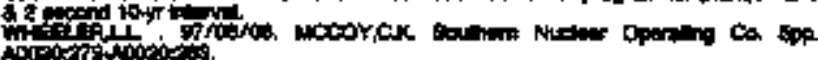

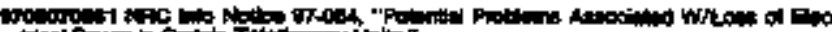

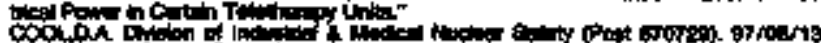

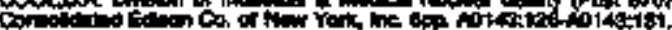

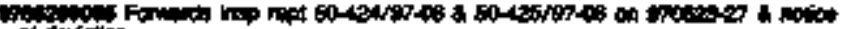

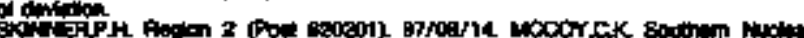
Qjos.

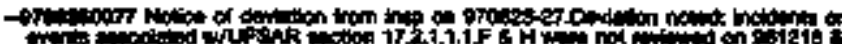

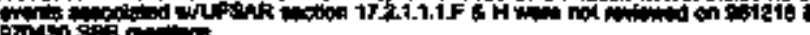

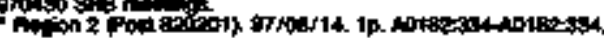

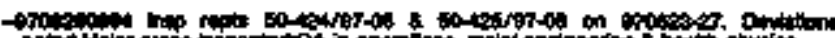

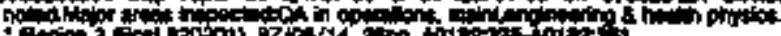

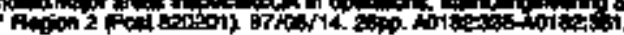

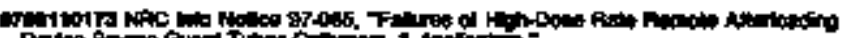

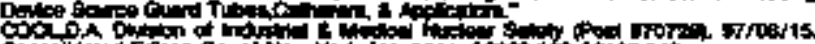

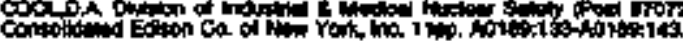

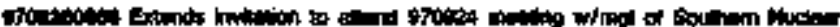

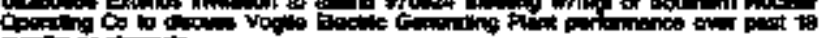

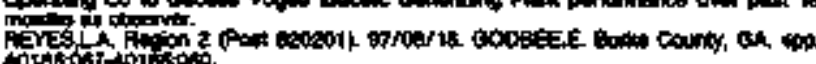

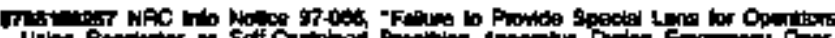

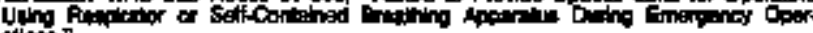

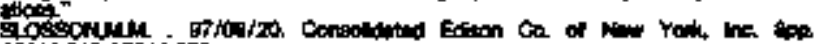

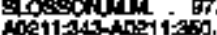

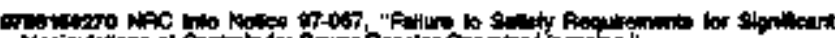

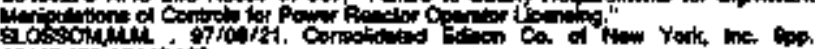

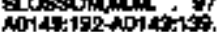

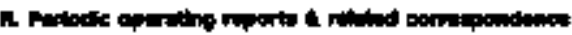

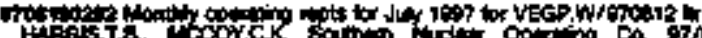
A013

tow

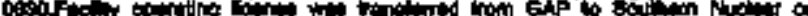

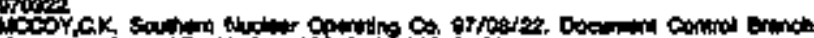

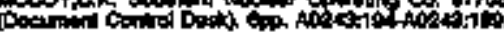

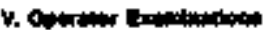

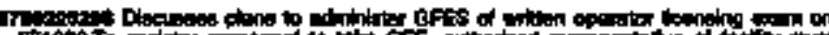

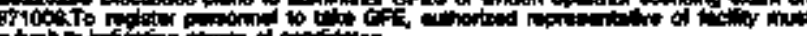

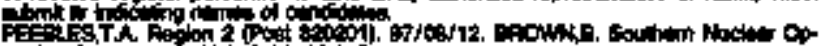

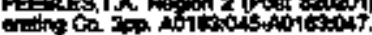

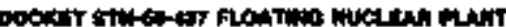

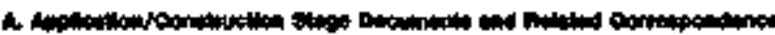

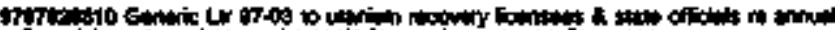

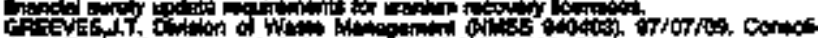

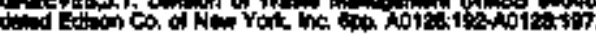

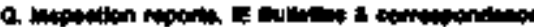

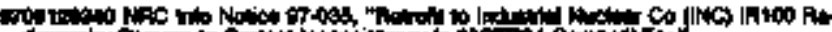
Co do

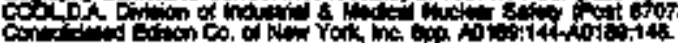

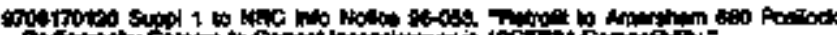

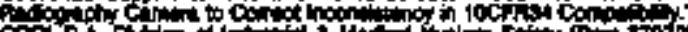

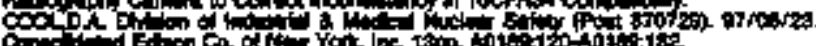

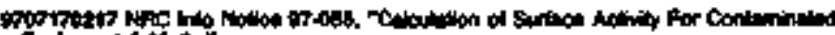

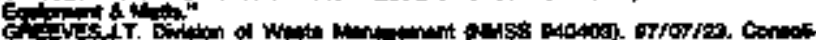

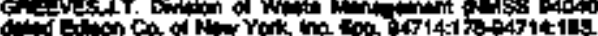

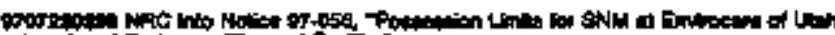

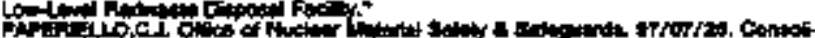

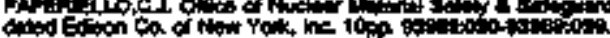

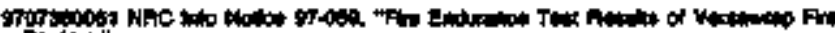

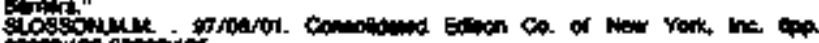

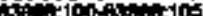

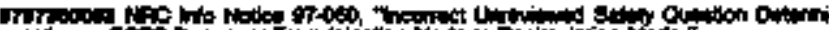

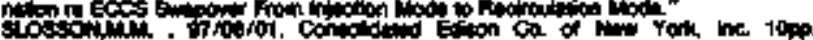

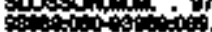

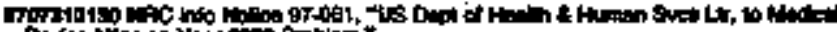

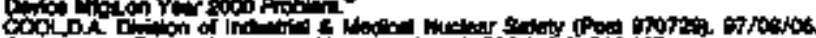

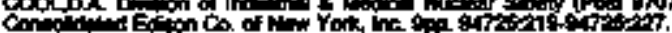

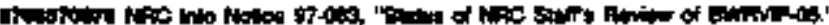

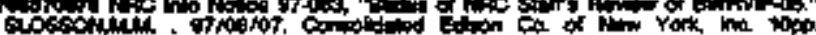

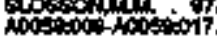

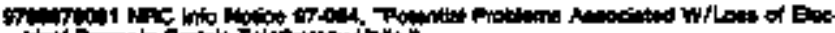

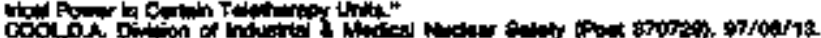

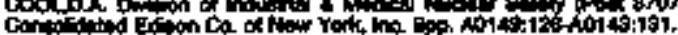

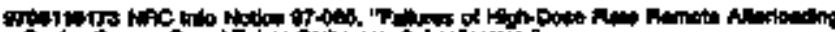

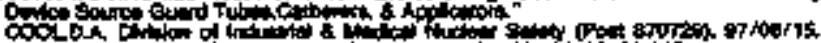

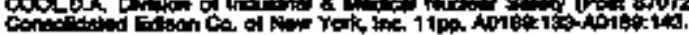

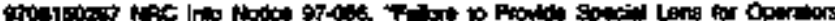

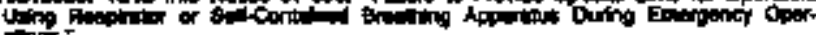

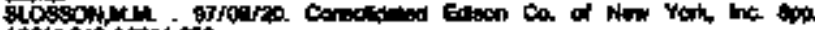

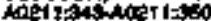




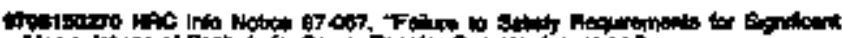

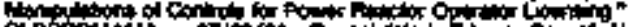

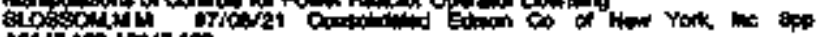
10143 13e-1040 139

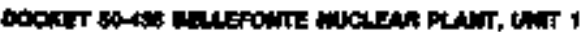

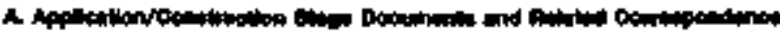

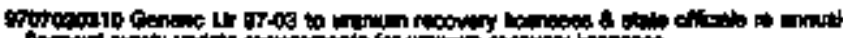
or

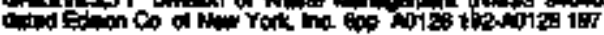

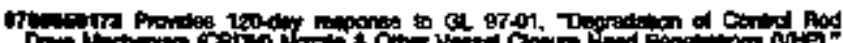

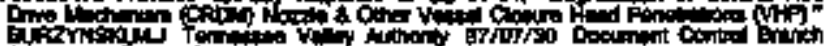

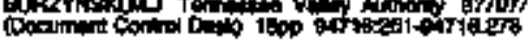

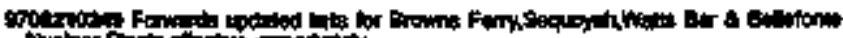

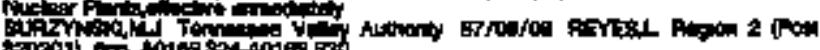

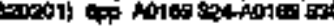

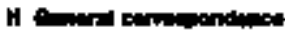

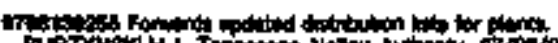

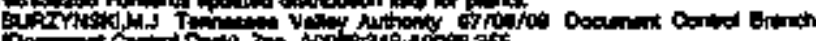

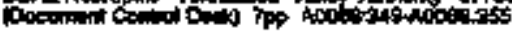

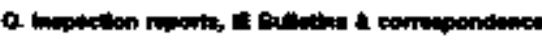

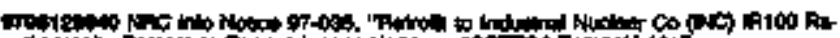

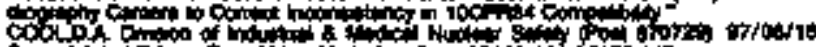

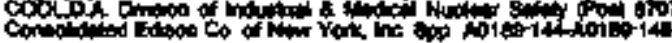

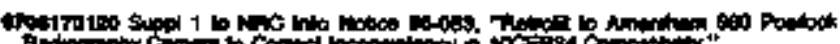
coofih

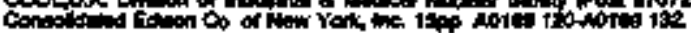

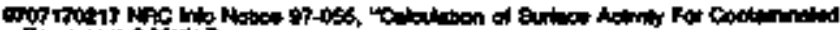

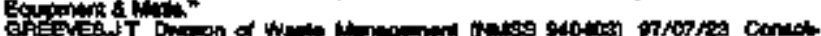

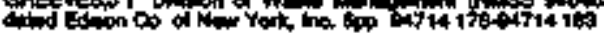

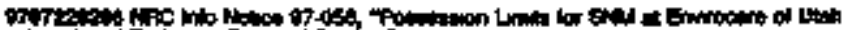

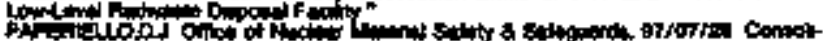

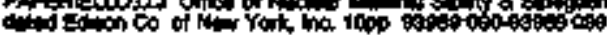

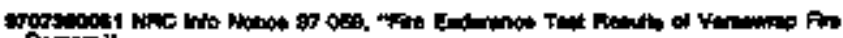

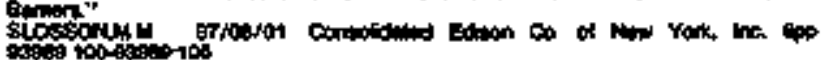

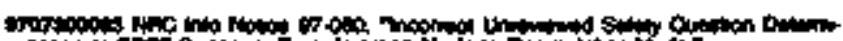

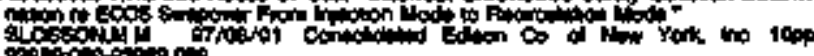

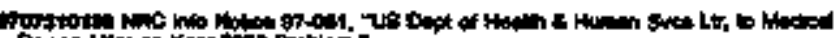

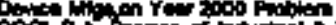

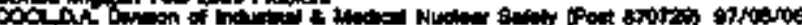

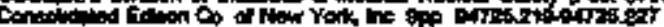

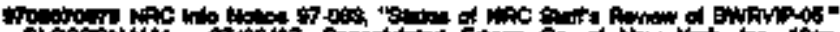

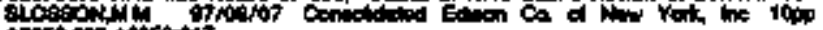

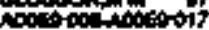

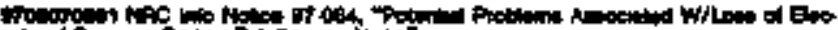

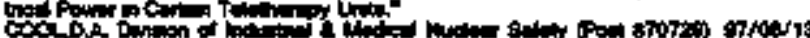

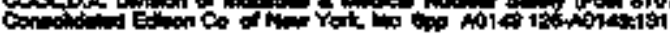

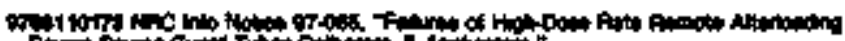

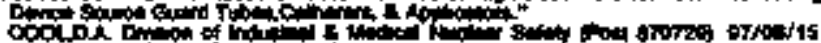

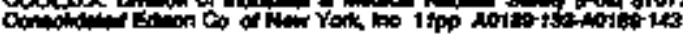

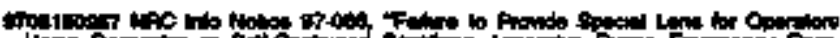

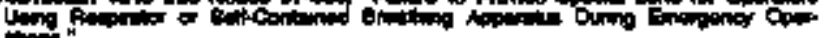

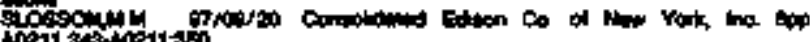

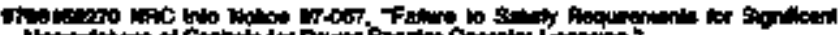

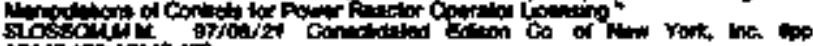

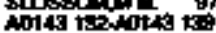

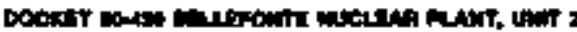

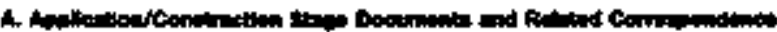

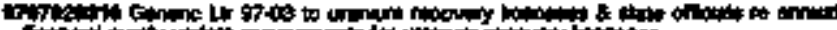

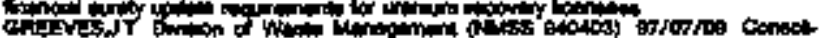

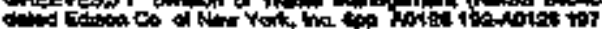

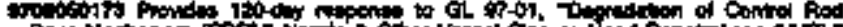

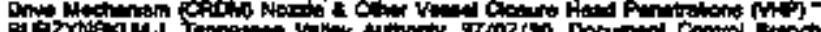

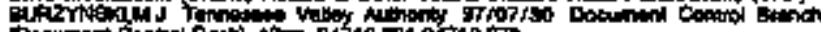

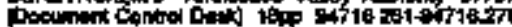

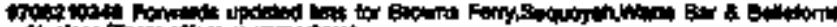

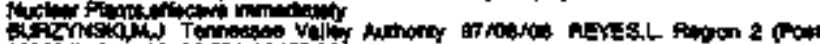

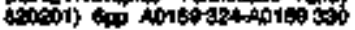

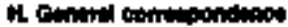

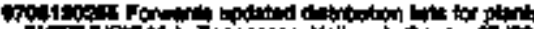

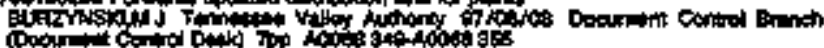

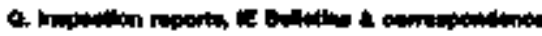

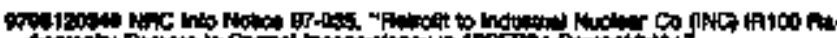

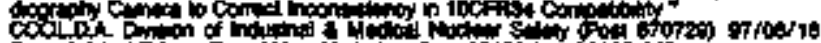

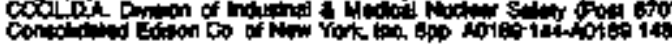

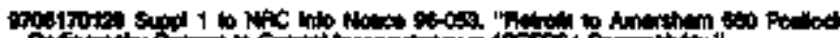
cothin

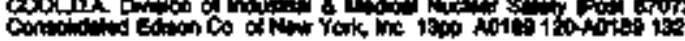

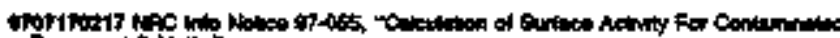
7.

CFli

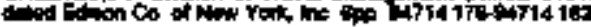

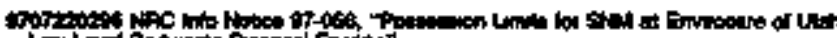

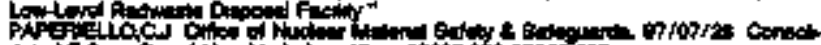

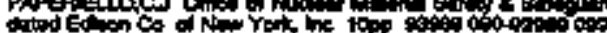

Hrof

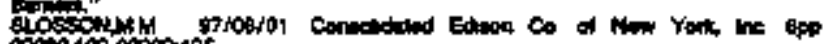

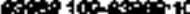

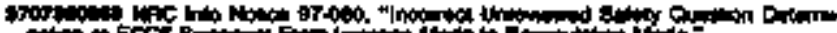

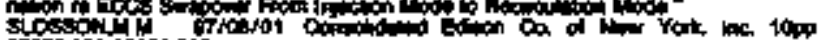
wits of on

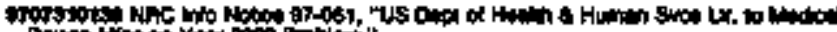

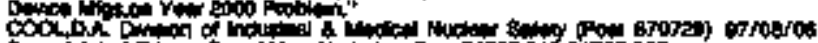

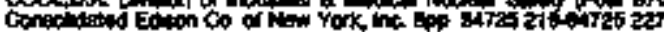

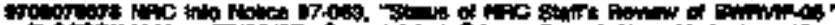

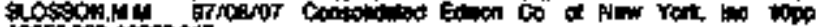

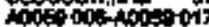

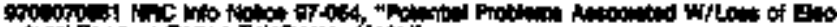

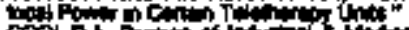

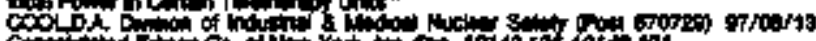

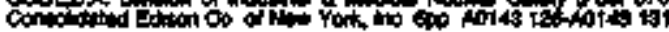

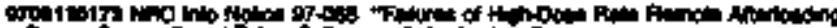

Co

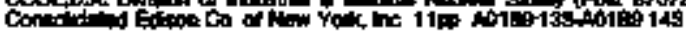

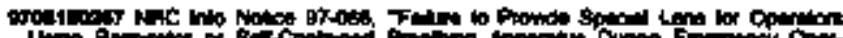

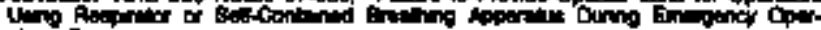

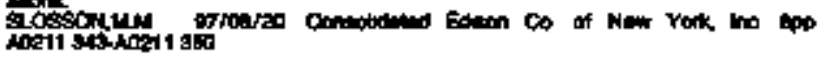

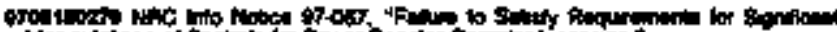

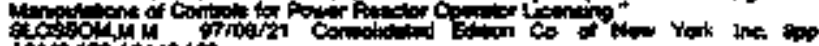

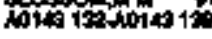

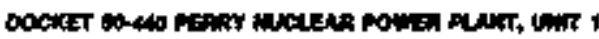

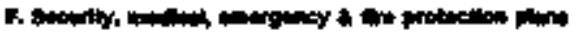

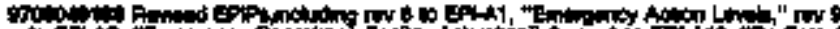

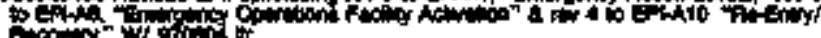

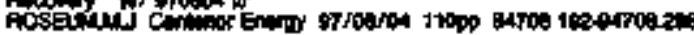

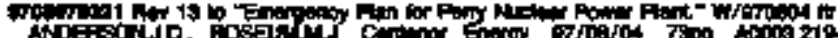

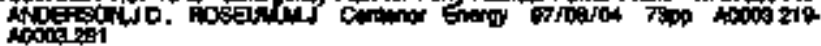

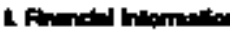

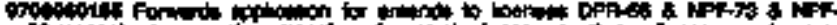

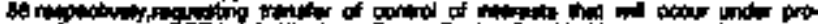

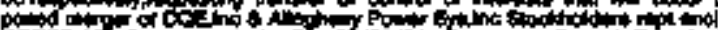

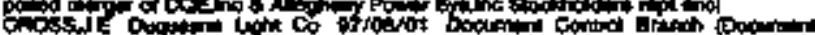

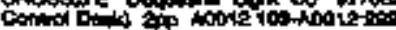




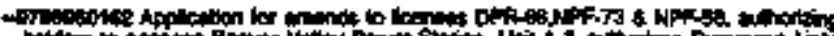

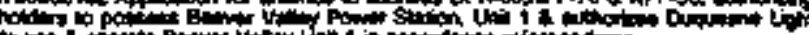

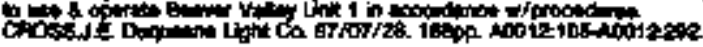

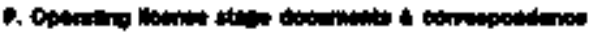

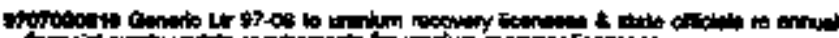

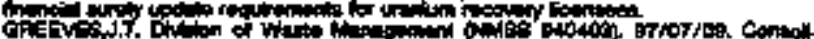
drad

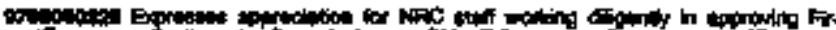

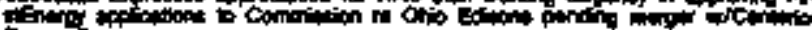

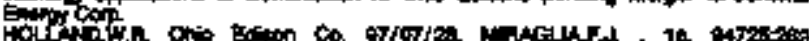
ortitat

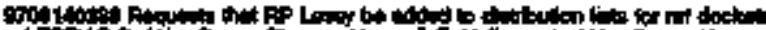

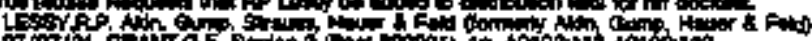

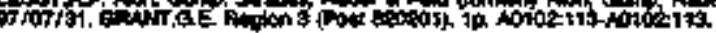

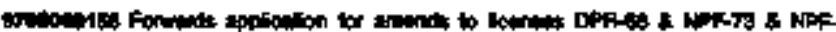

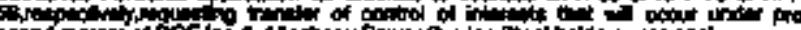

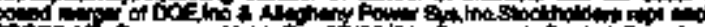

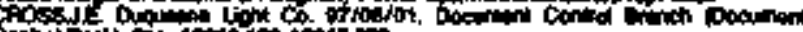

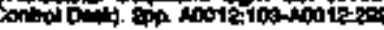

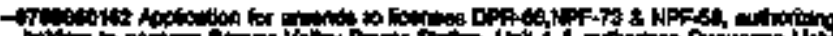

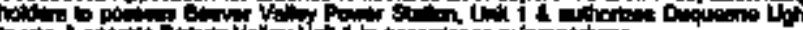

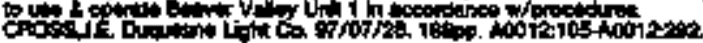

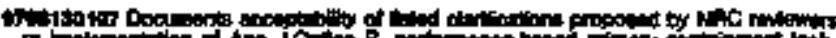

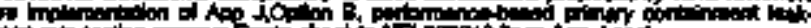

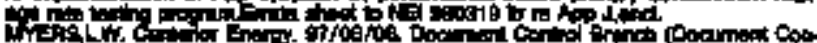

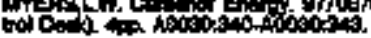

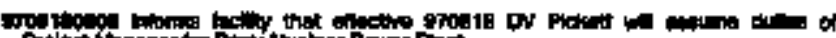

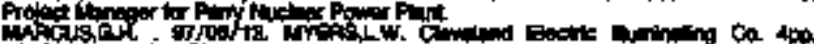

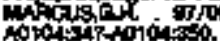

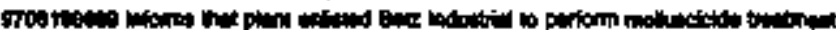

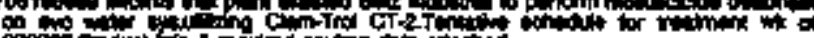

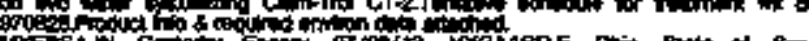

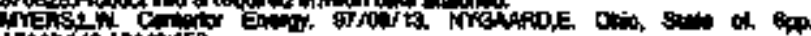

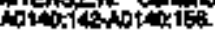

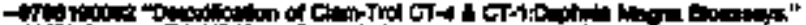

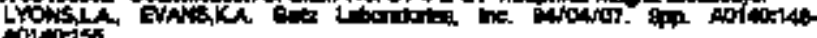
initios:

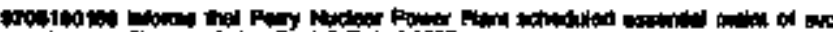

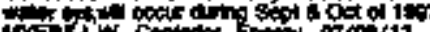

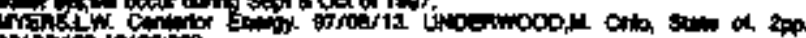

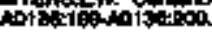

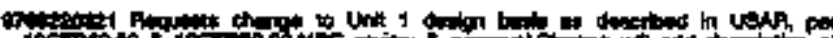

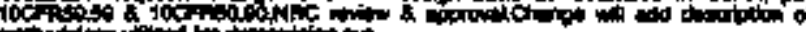

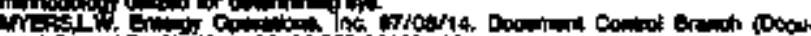

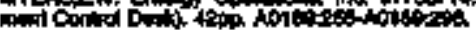

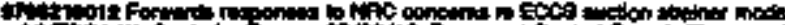

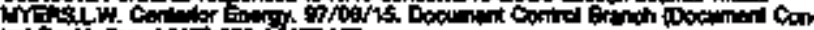

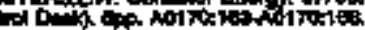

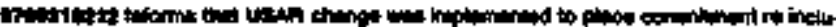

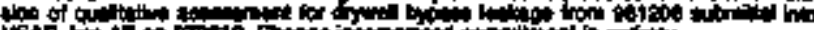

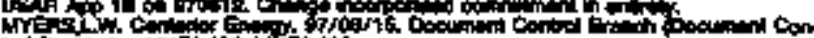

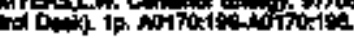

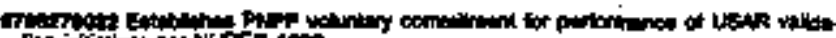
in

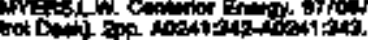

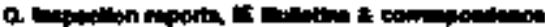

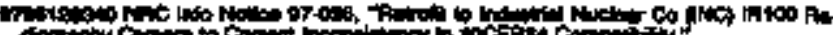

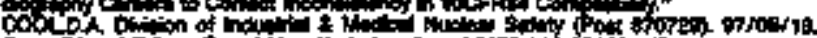

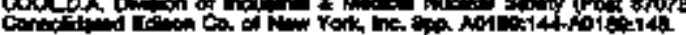

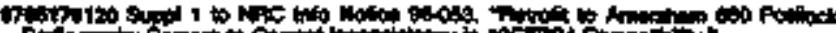

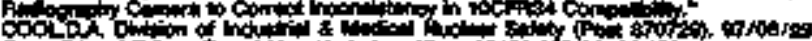

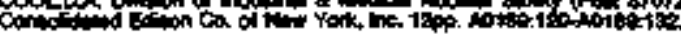

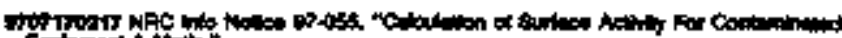

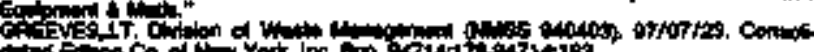

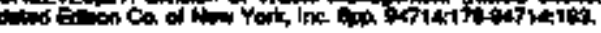

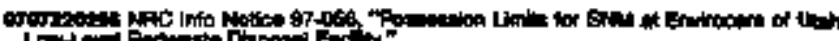

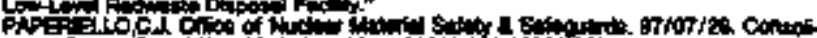

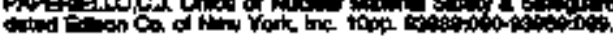

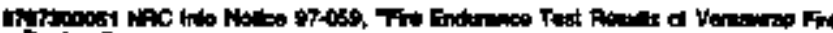

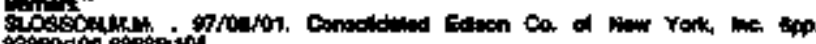

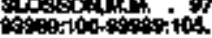

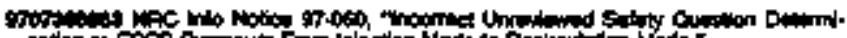

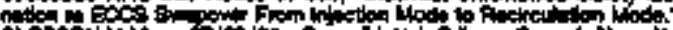

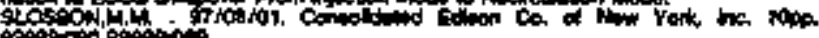

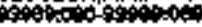

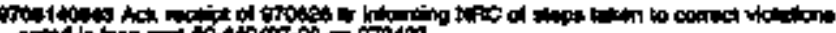

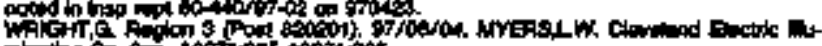

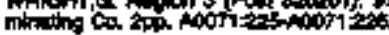

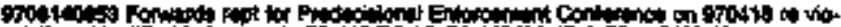

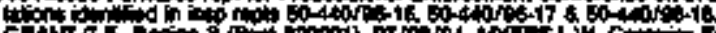
cipot,

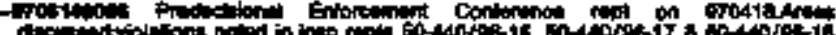

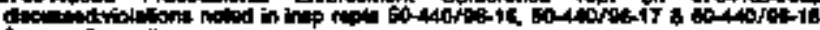

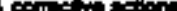

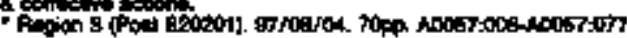

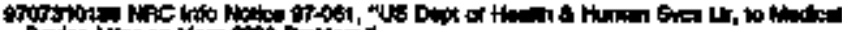

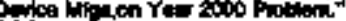

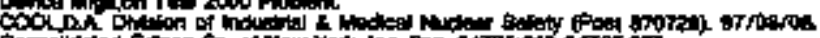

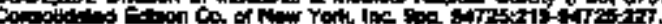

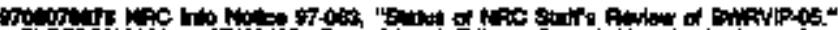

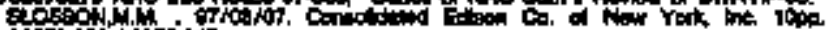

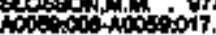

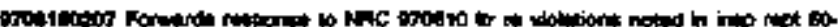

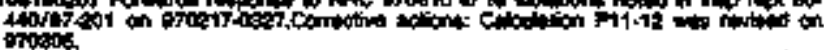

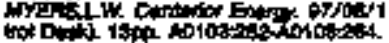

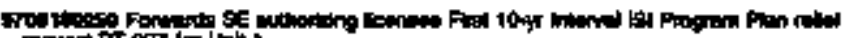

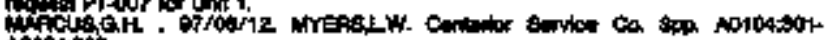
atoison.

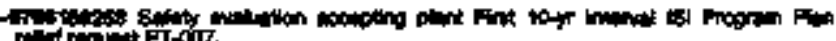

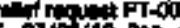

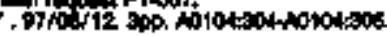

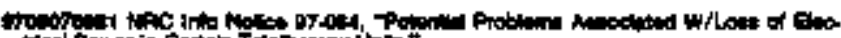
trid Powar in Centes To

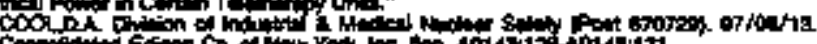

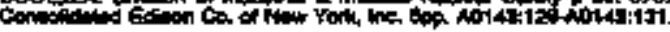

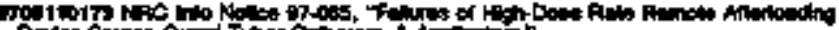

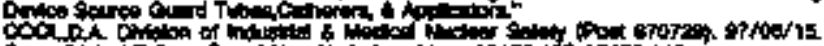

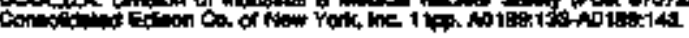

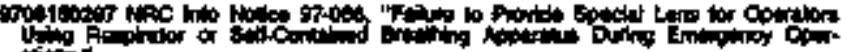

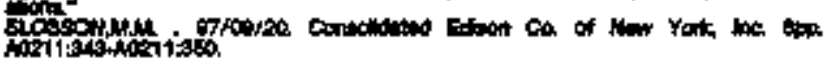

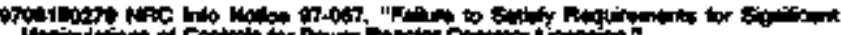

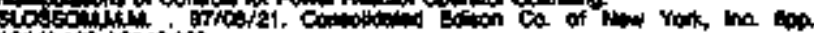
A143:13:A014\%13\%

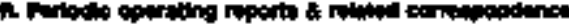

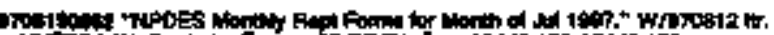

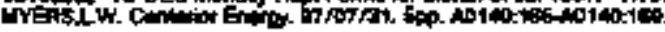

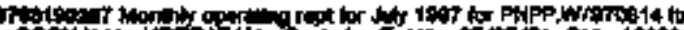

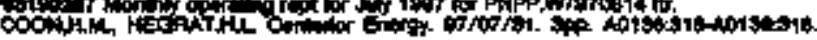

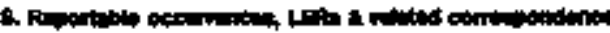

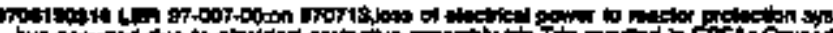

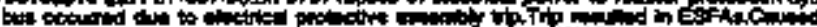

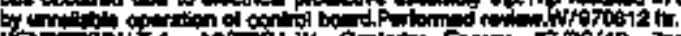
ionstsits.

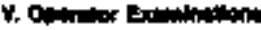

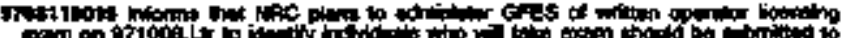

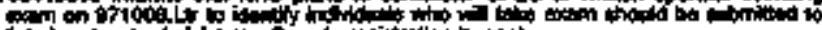

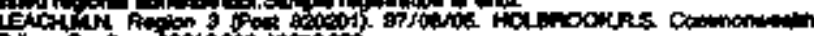

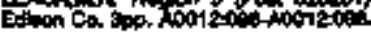

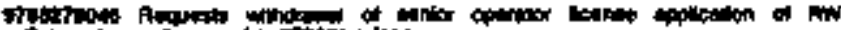

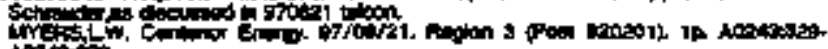

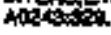

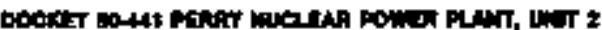




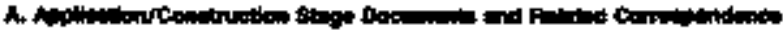

Pry

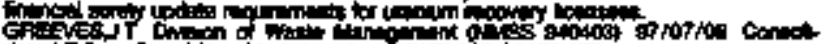

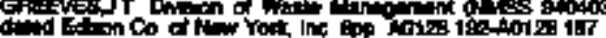

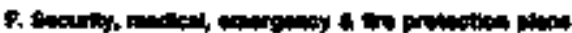

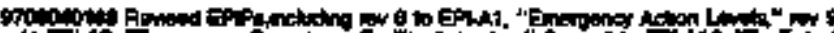

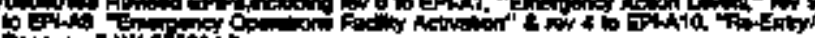

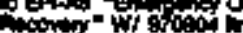

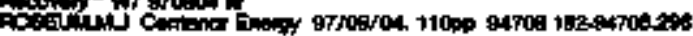

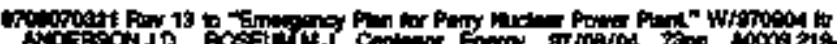

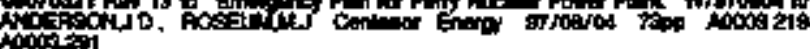

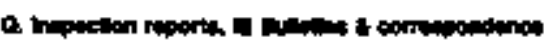

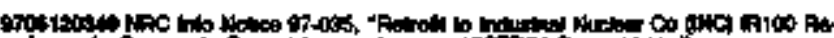

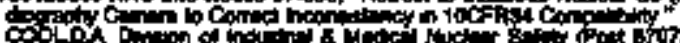

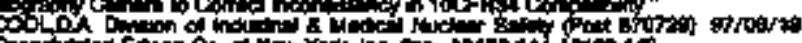

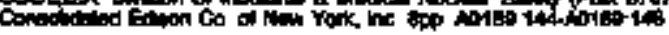

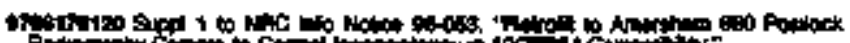

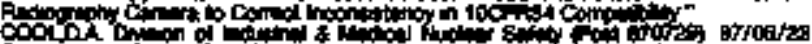

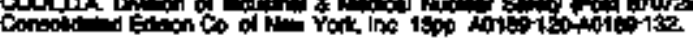

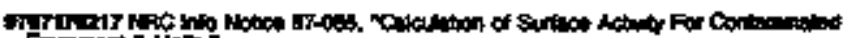

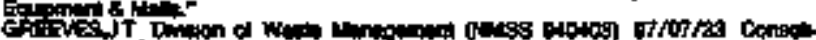

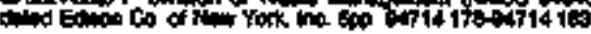

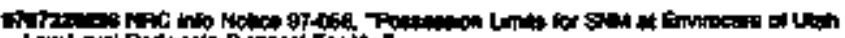

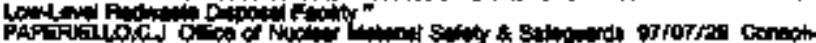

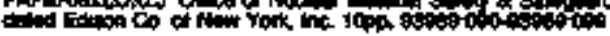

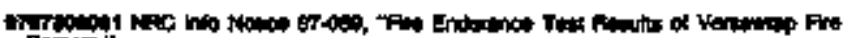

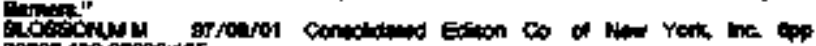

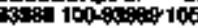

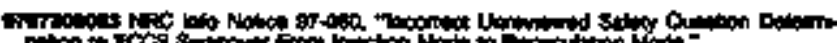

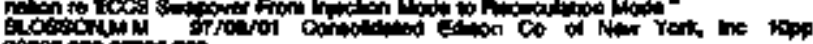

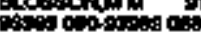

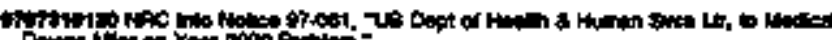

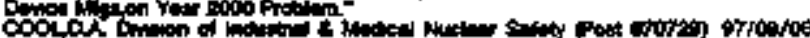

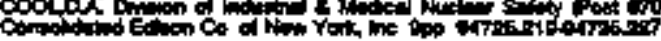

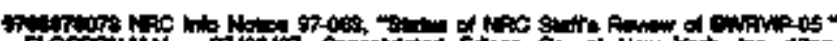

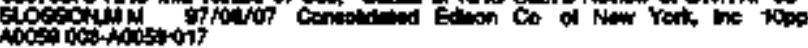

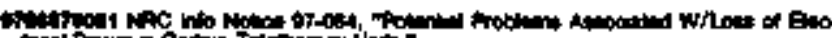

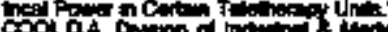

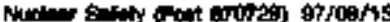

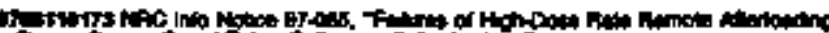

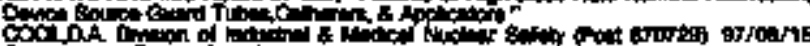

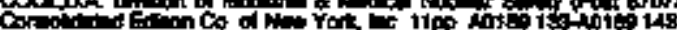

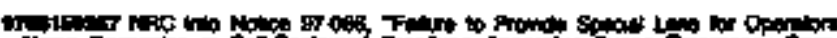

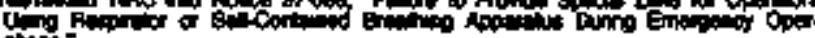

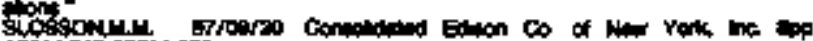

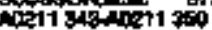

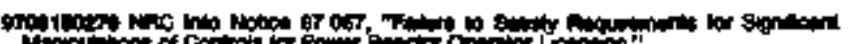

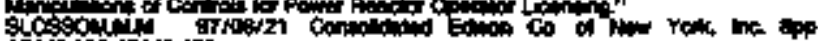

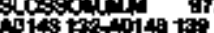

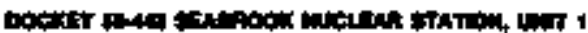

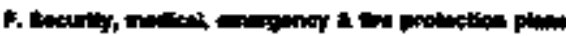

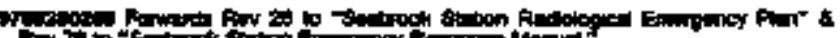

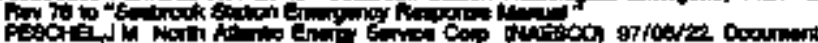

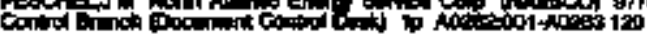

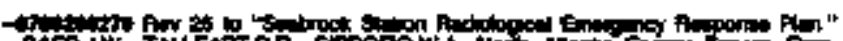
(140,

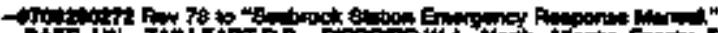

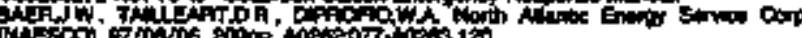

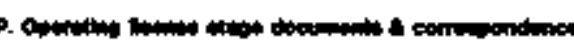

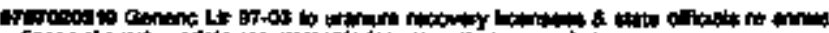

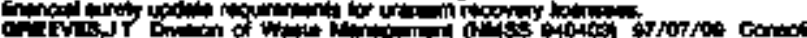

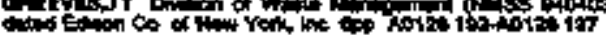

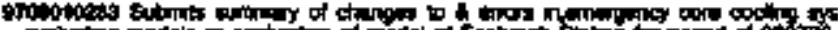

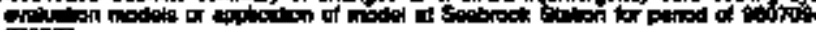
Trop?

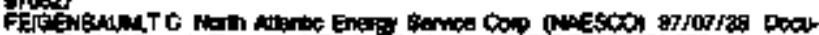

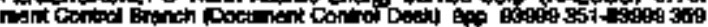

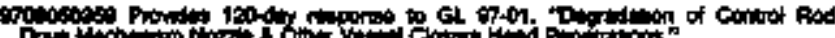

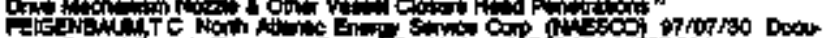

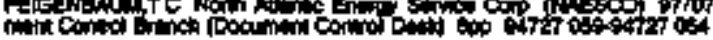

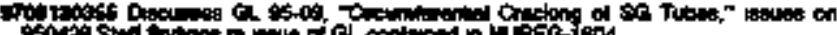

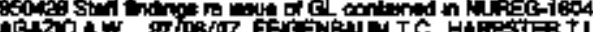
Sornce don on

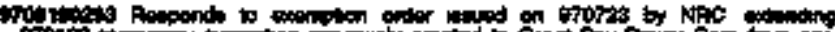

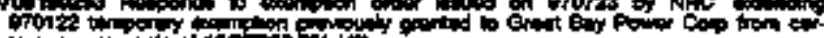

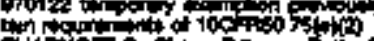

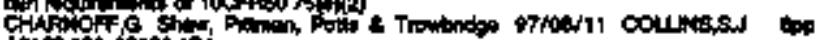

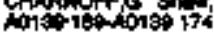

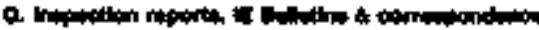

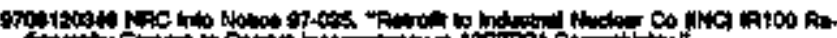

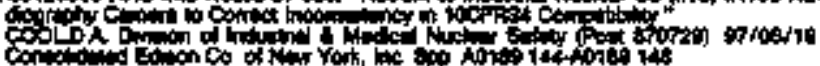

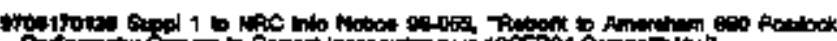

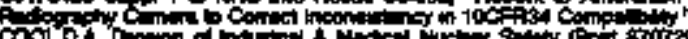

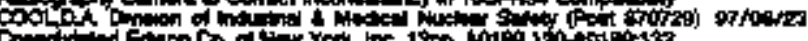

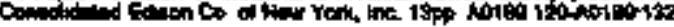

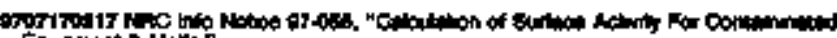

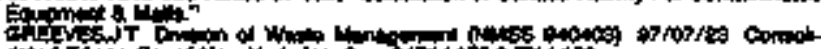

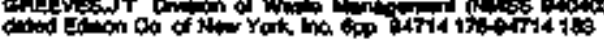

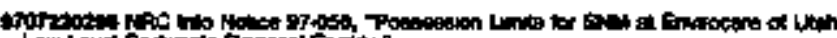

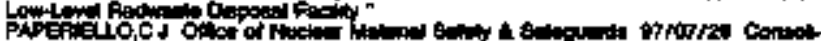

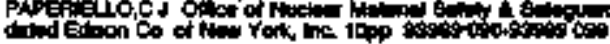

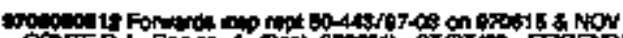

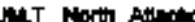

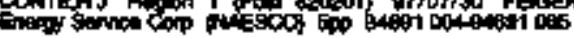

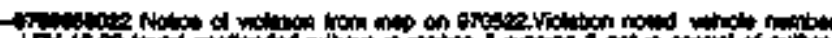

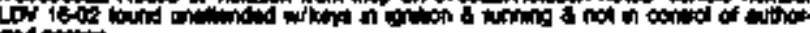

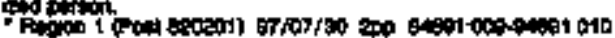

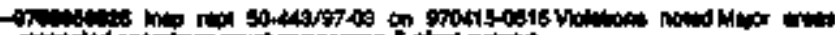

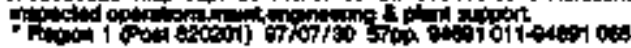

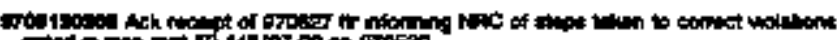

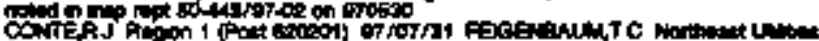

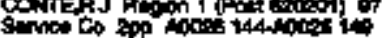

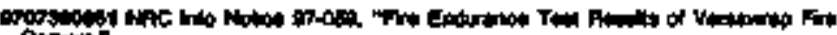

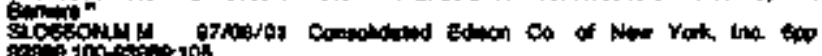

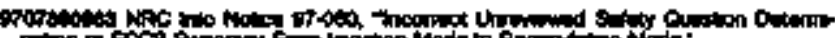

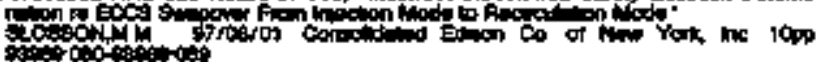

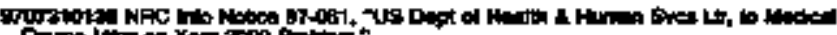

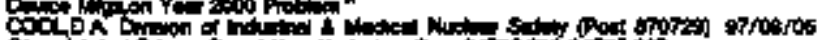
Con

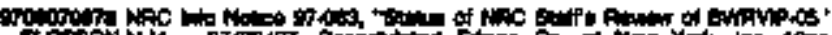
PO

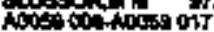

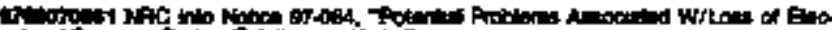

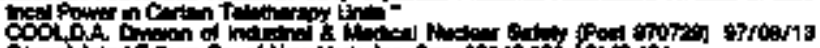

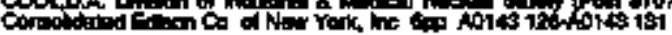

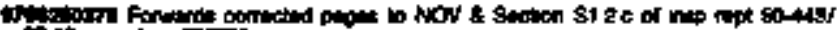

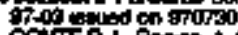

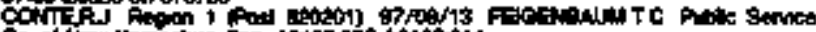

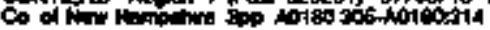

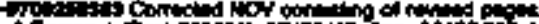

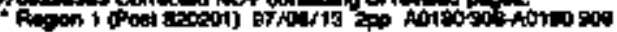

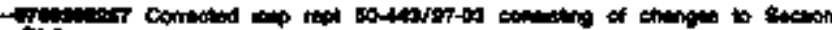

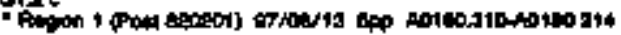

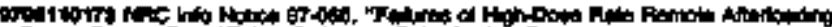

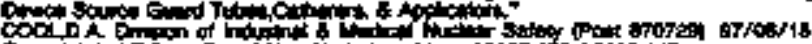

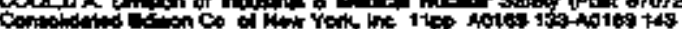




\section{DOCKETED ITENS}

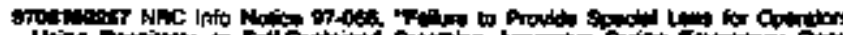

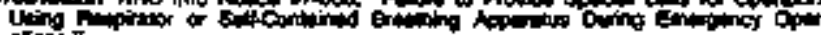

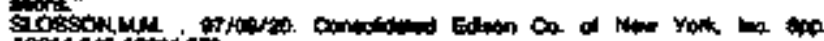

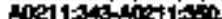

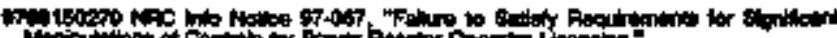

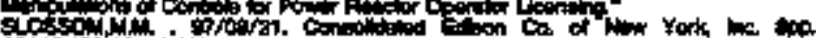
Aitation

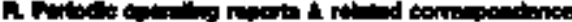

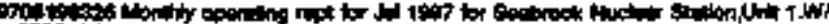

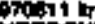

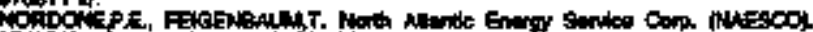

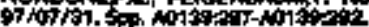

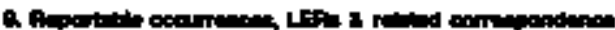

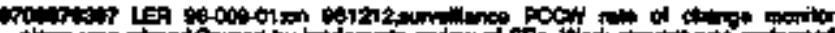

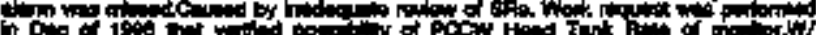
Then

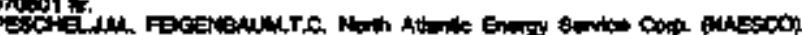

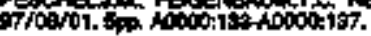

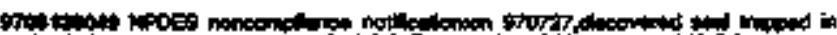

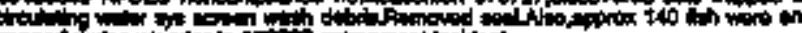

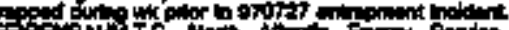

Fin

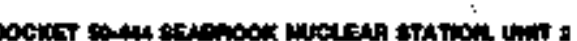

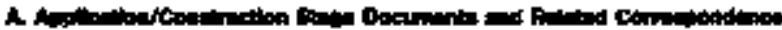

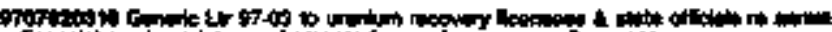

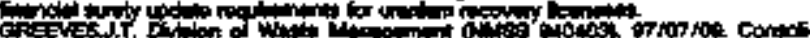

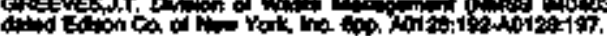

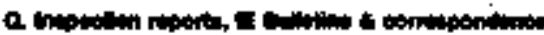

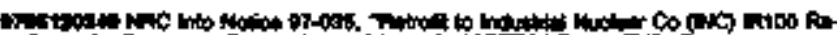

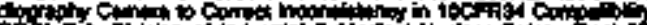

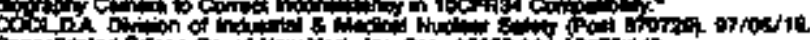

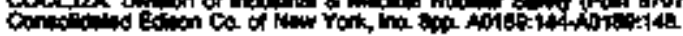

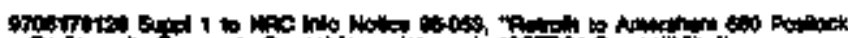

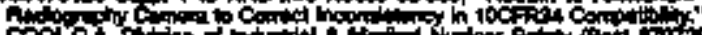

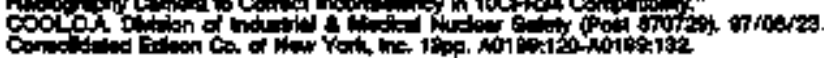

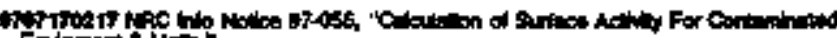

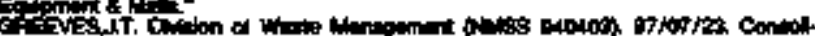

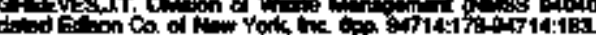

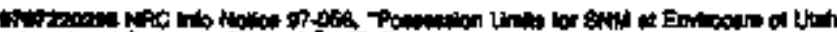

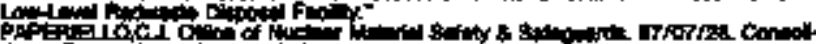

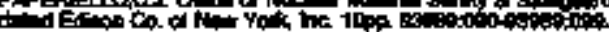

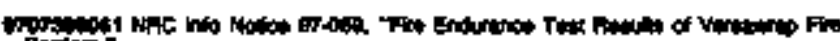

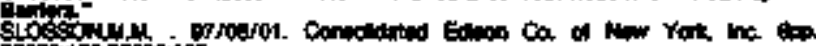

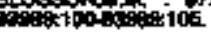

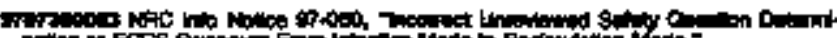

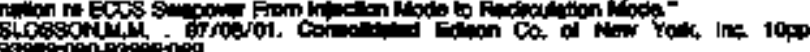

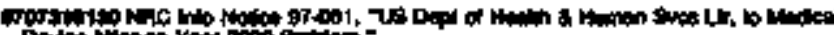

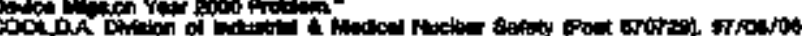

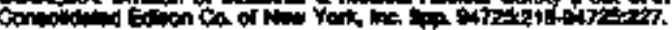

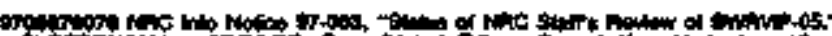

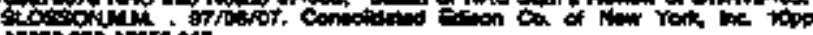

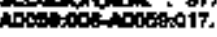

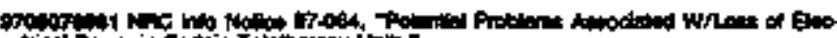

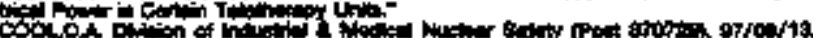

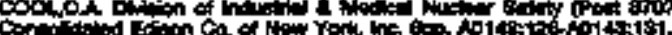

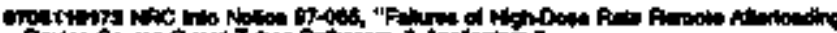

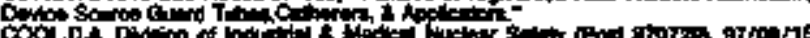

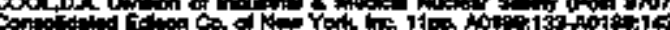

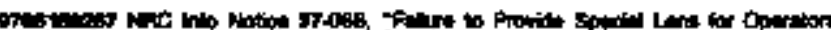

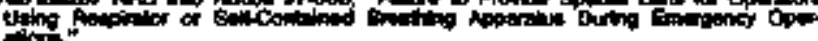

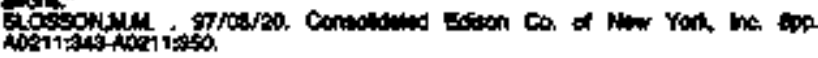

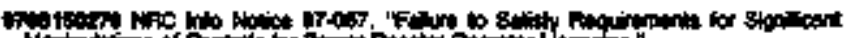

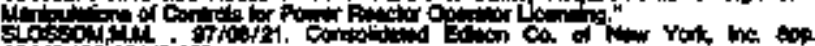
Adisting

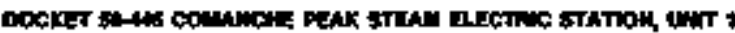

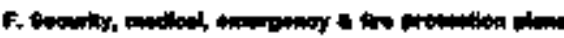

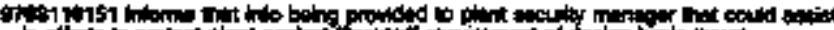

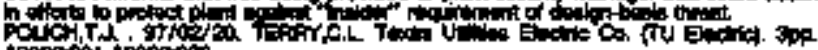

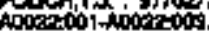

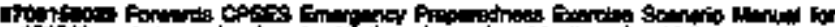

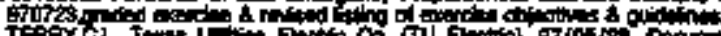

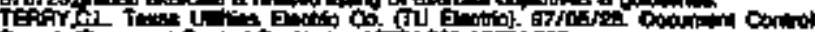

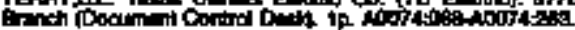

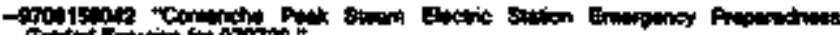

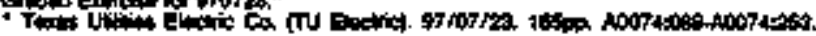

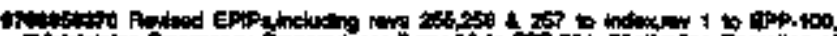

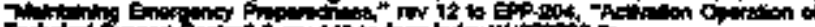

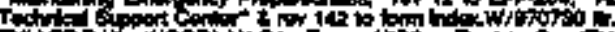

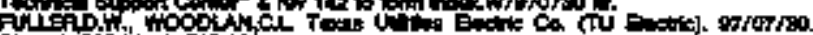

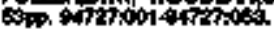

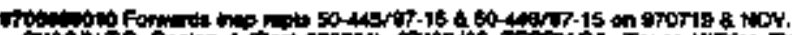

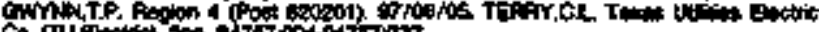

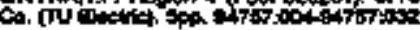

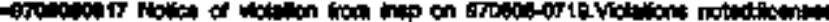

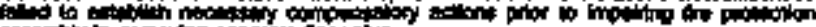

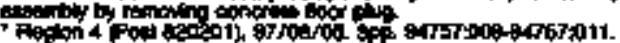

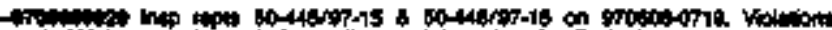

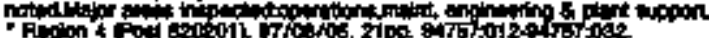

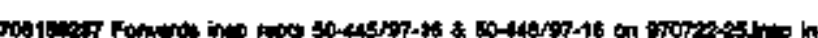

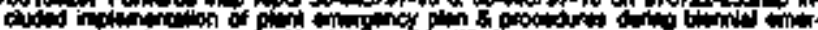

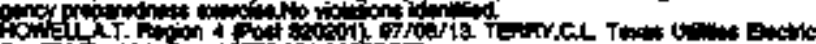

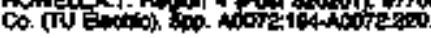

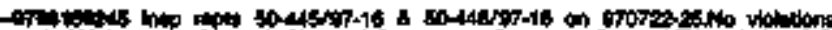

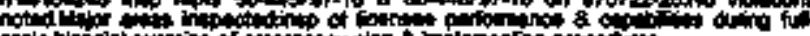

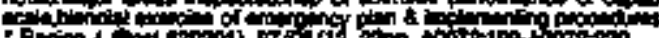

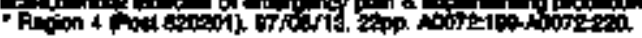

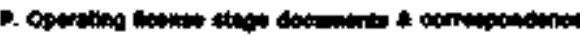

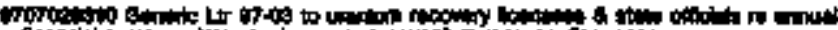

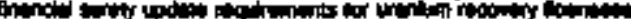

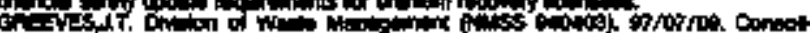

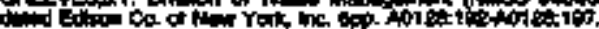

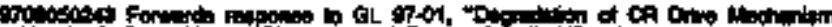

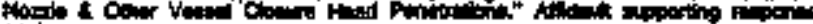

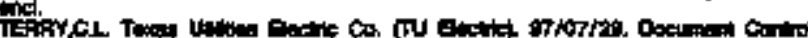

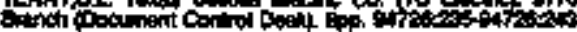

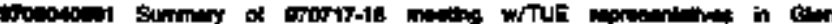

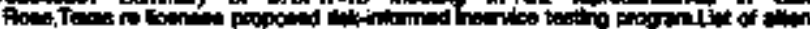

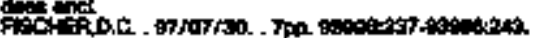

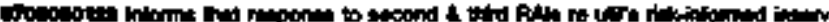

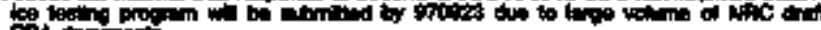

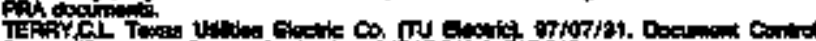

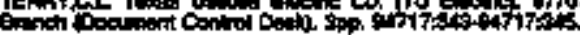

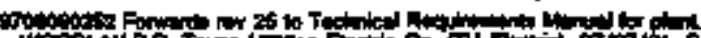

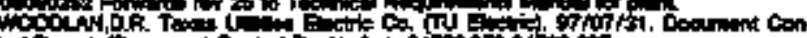

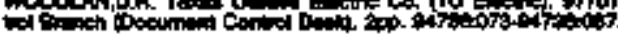

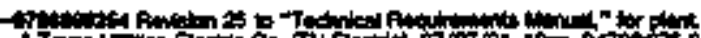

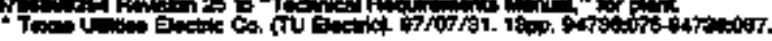

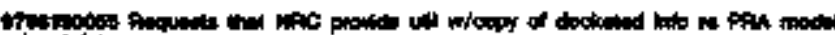

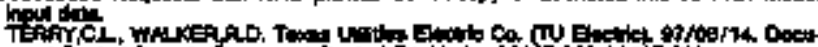

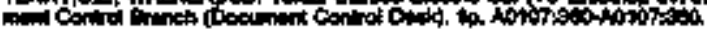

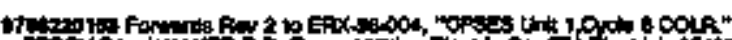

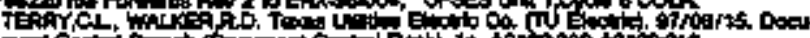

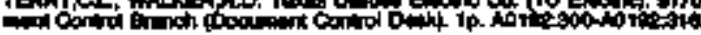

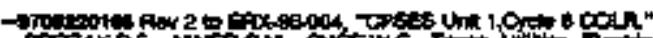
Exis

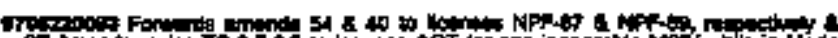

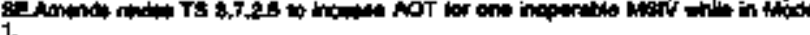

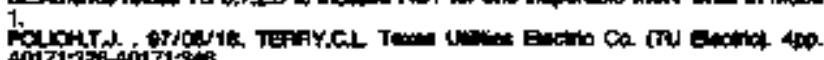

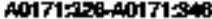

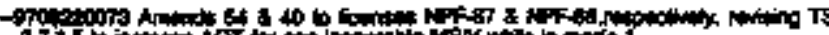

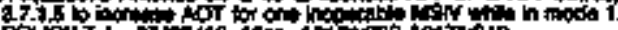

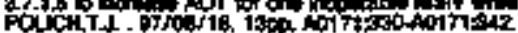




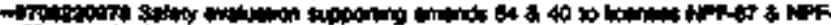

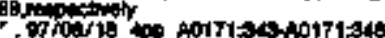

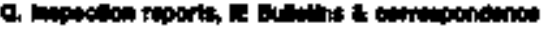

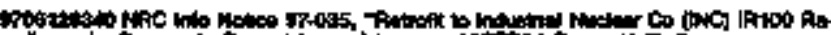

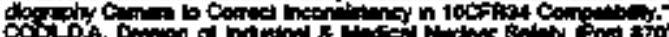

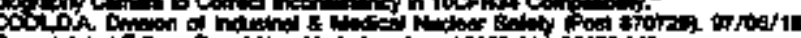

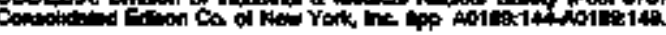

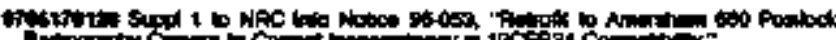

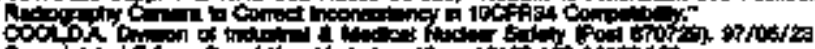

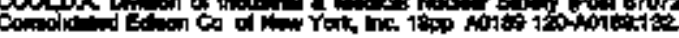

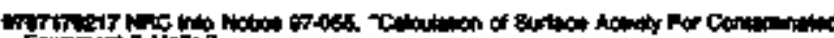

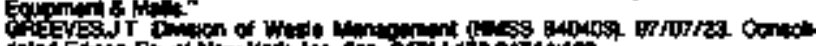

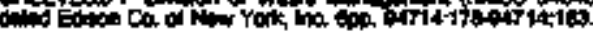

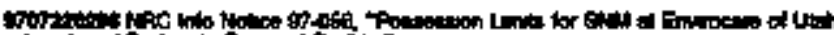

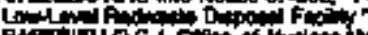

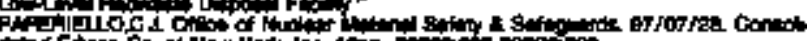

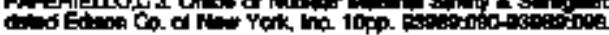

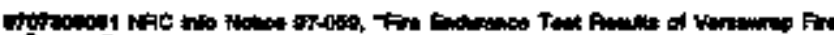

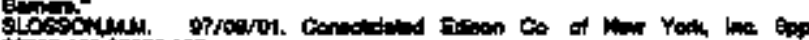

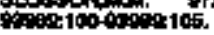

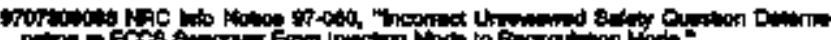
"

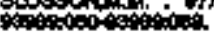

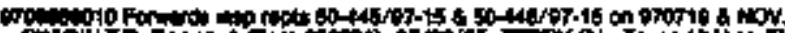

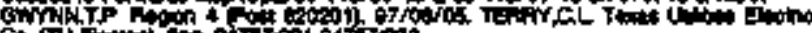

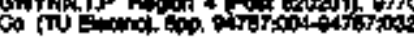

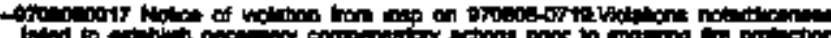

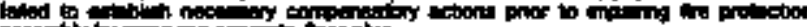

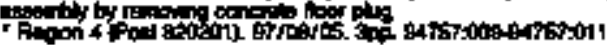

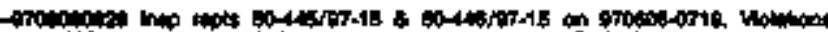

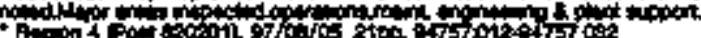

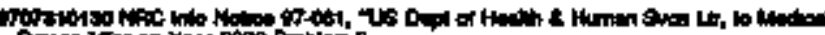

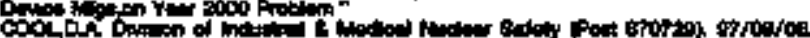

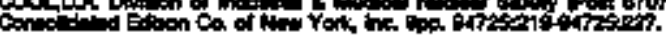

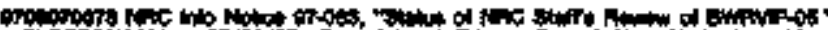

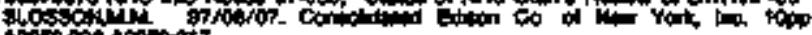

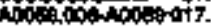

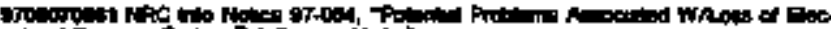

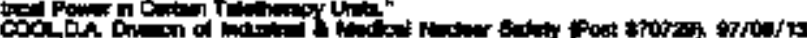

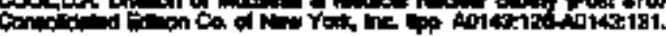

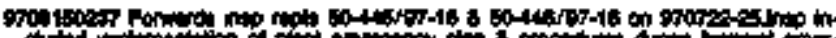

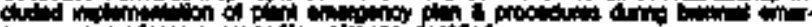

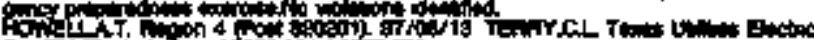

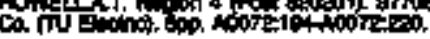

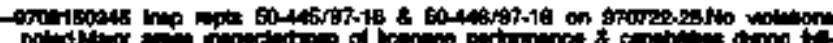

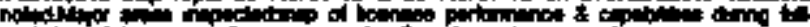

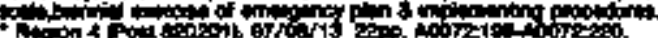

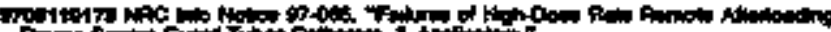

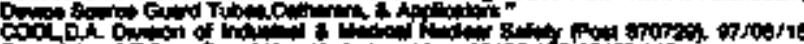

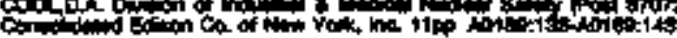

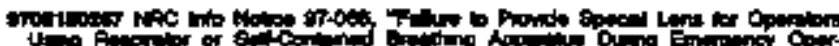

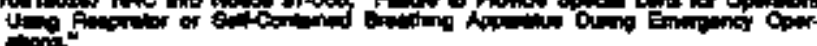

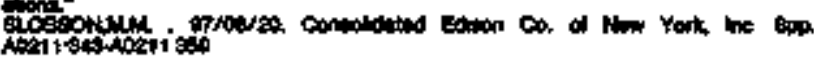

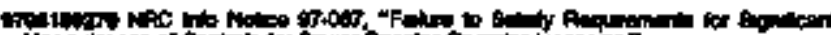

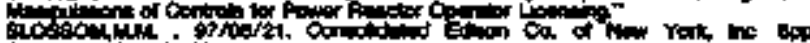

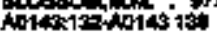

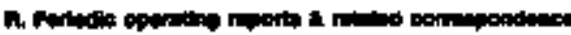

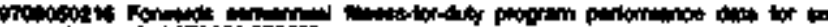

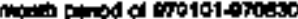

Min:

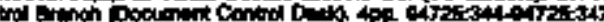

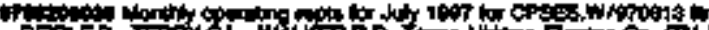

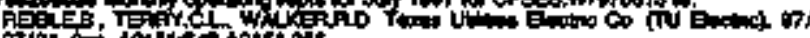

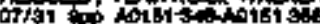

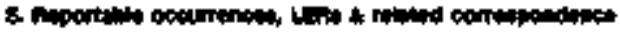

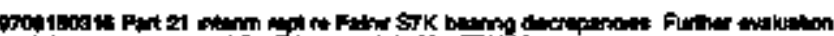

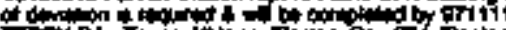

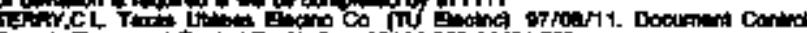

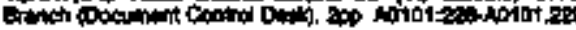

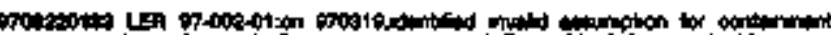

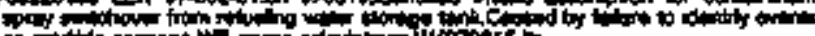

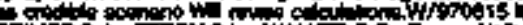

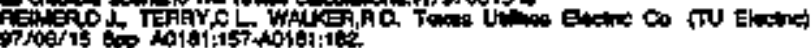

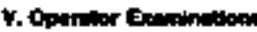

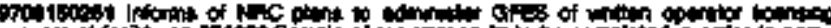

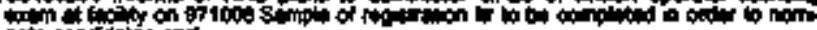

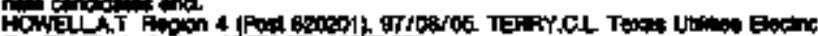

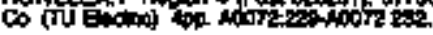

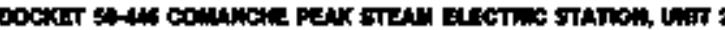

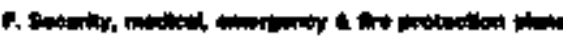

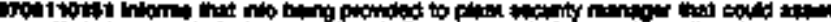
ROUCAT T. Nondion

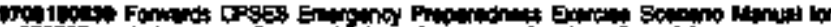

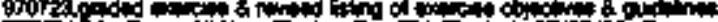

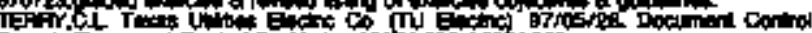
Branch poofy

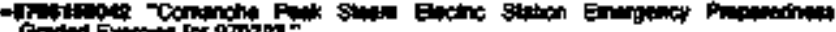

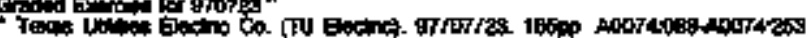

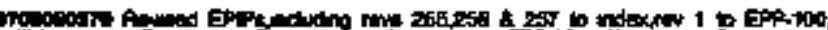

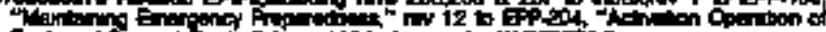

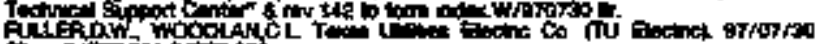

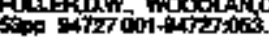

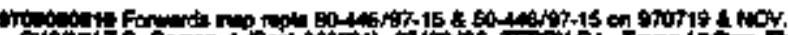
Givi T P. A

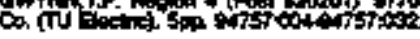

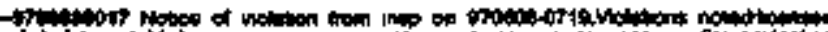

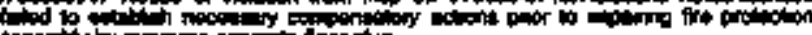

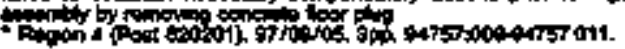

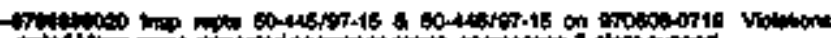

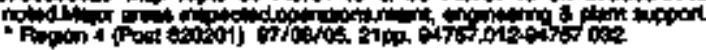

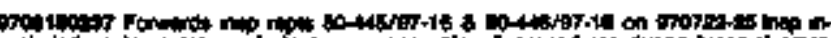

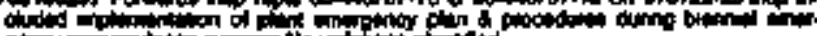

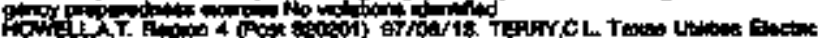

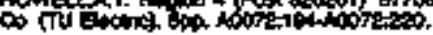

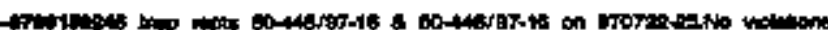

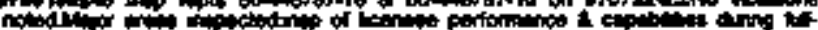

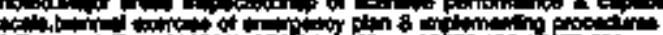

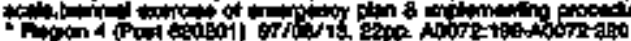

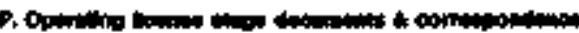

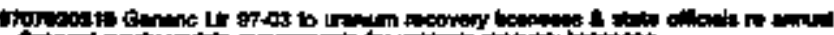

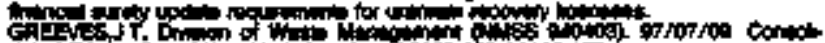

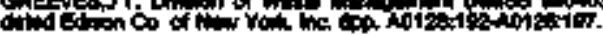

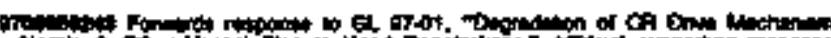

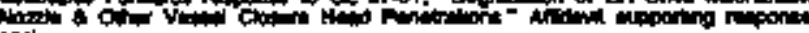

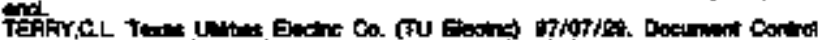

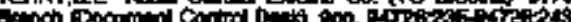

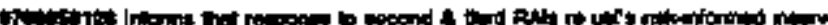

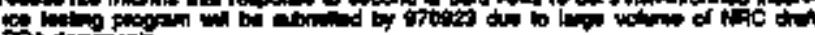

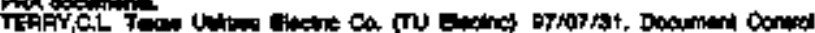

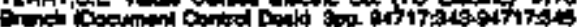

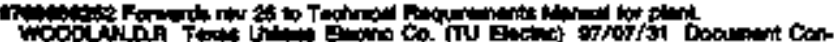

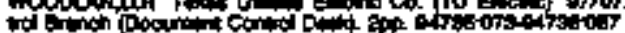

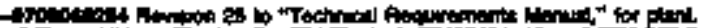

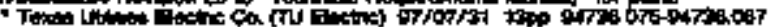

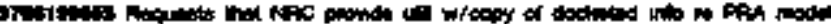

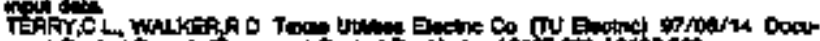

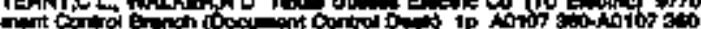




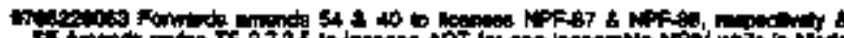

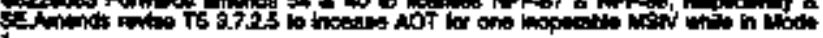

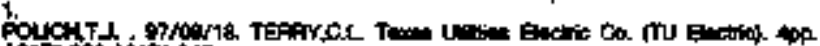
017า

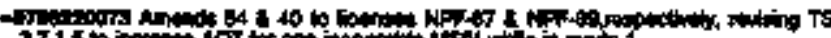
a7.1.

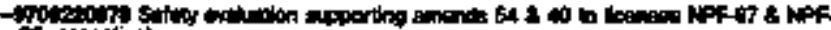

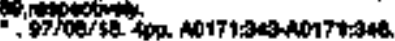

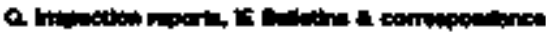

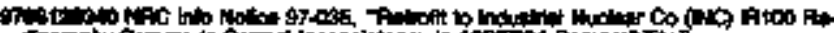

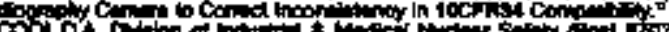

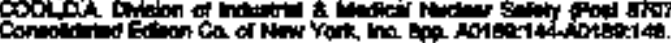

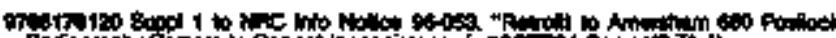

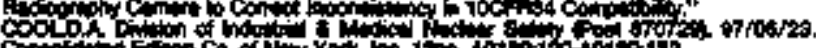

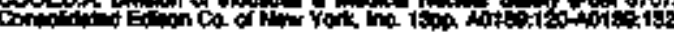

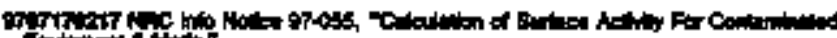

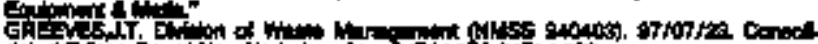

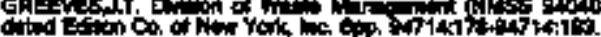

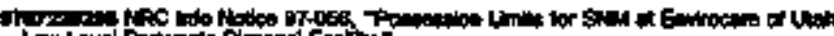

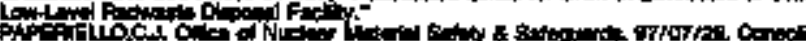

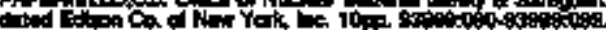

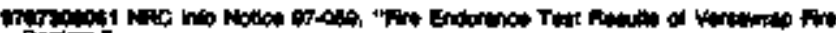

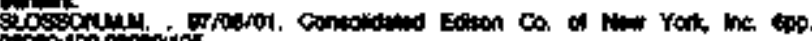

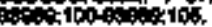

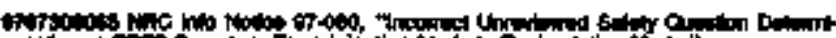

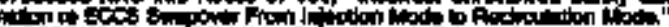

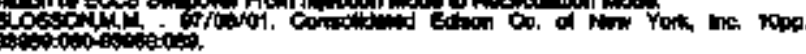

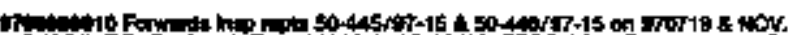

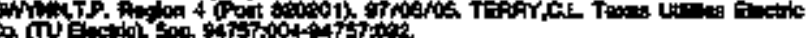

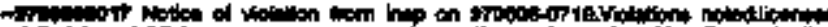

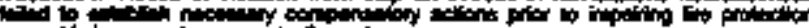

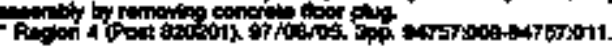

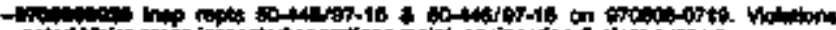

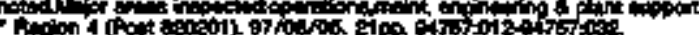

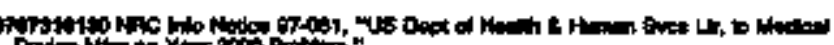

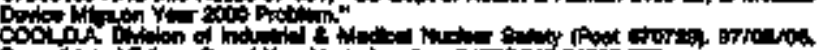

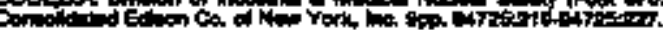

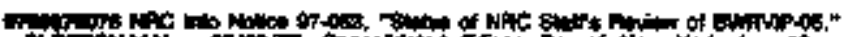

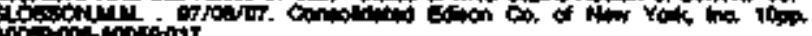

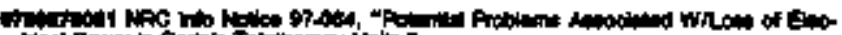

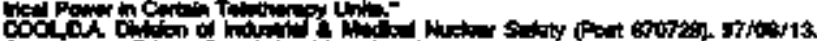

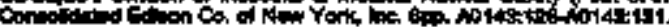

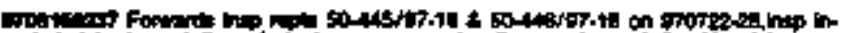

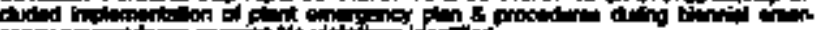
Fon

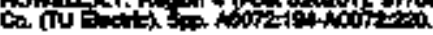

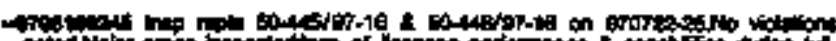

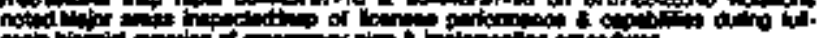

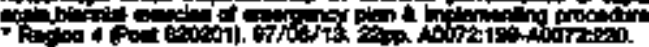

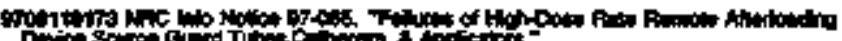

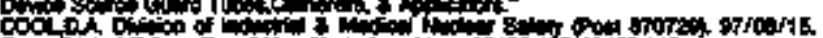

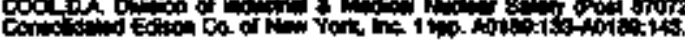

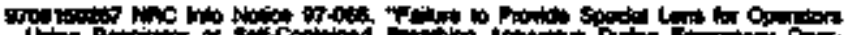

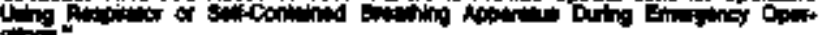

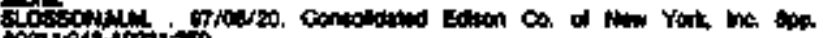

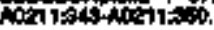

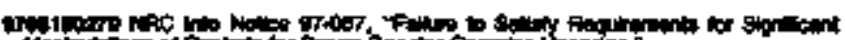
of

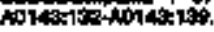

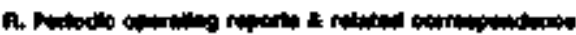

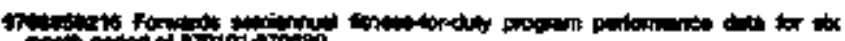

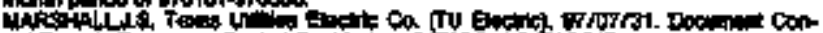

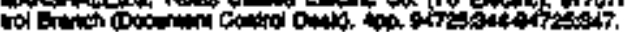

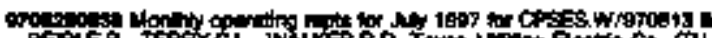

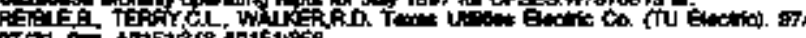

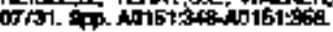

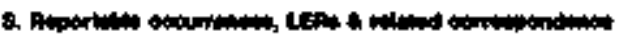

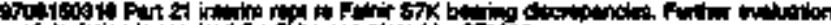

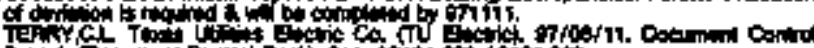

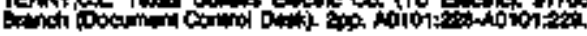

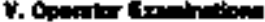

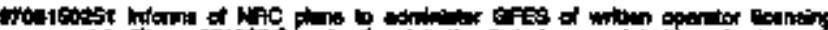

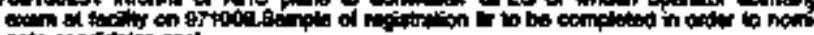

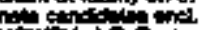

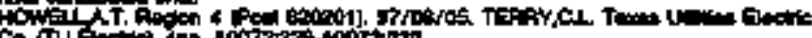

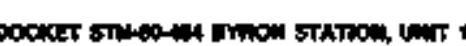

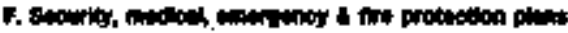

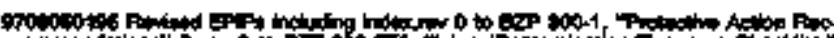

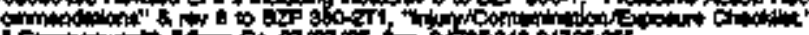

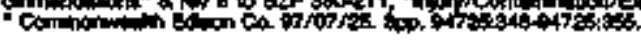

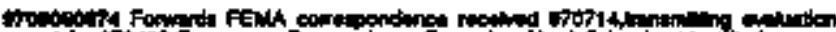

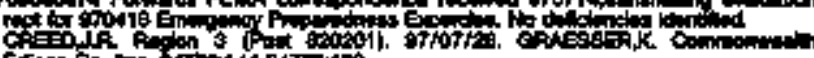

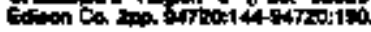

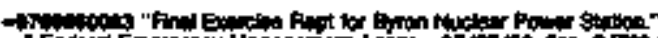

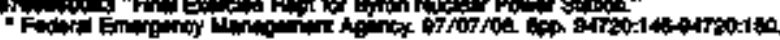

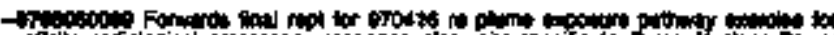

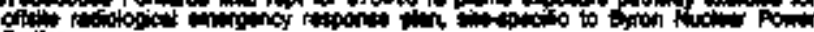

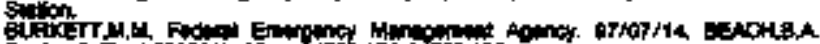

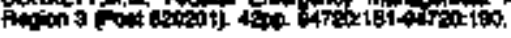

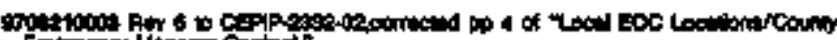

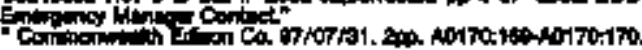

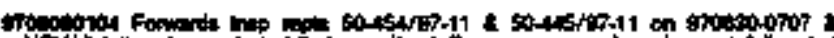

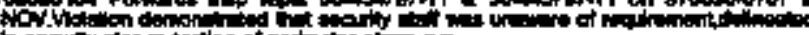

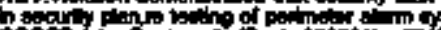

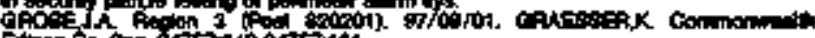

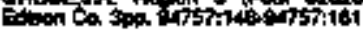

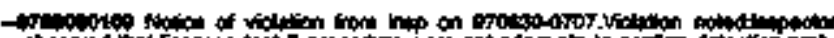

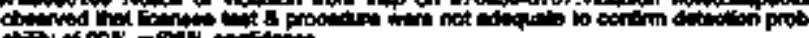

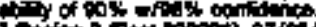

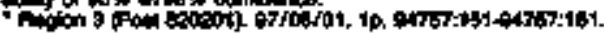

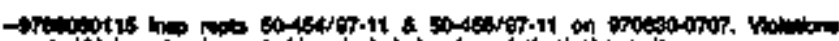

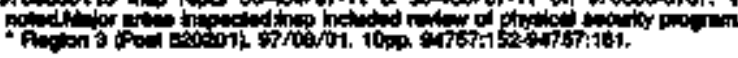

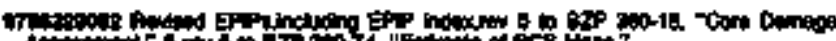

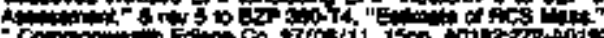

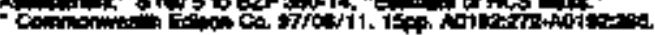

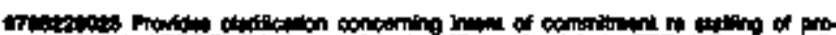

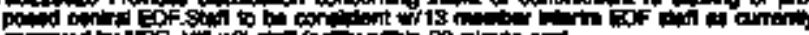

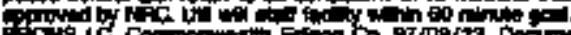

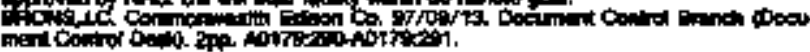

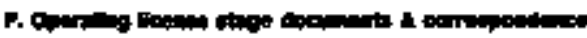

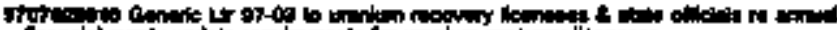
che

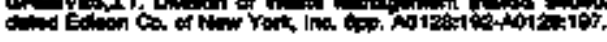

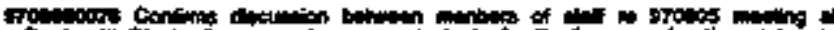

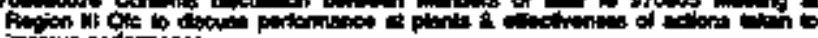

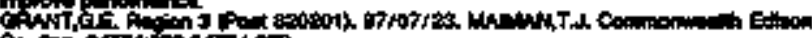
Co.

Toworos

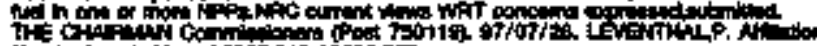

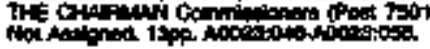

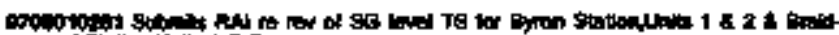
jood stiony

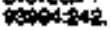

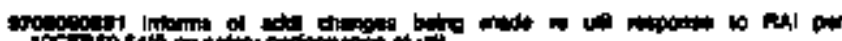

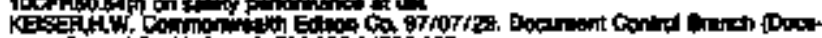

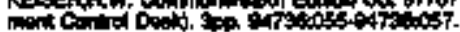




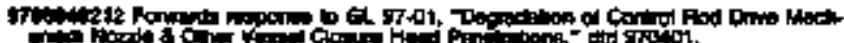

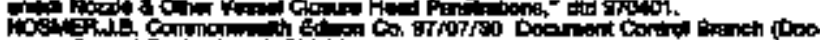

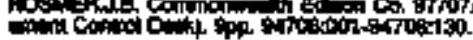

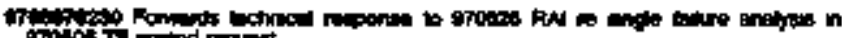

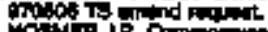

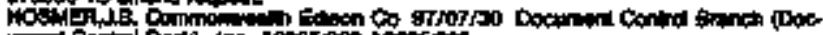

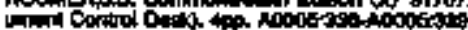

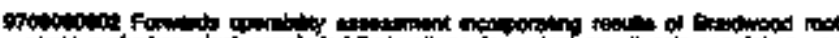

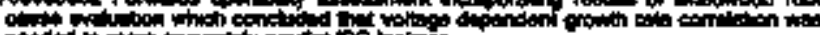

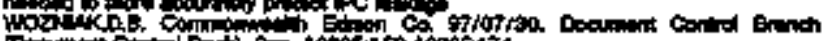

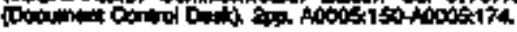

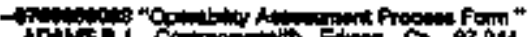

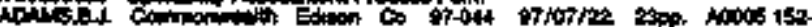
Aocalitit

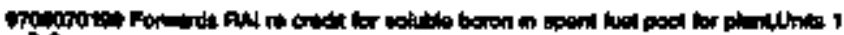

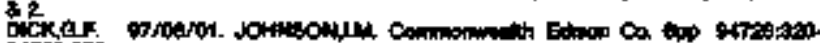
artacis

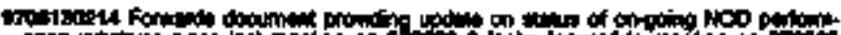

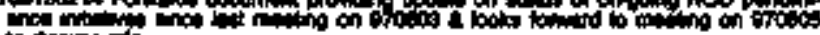

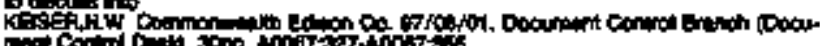

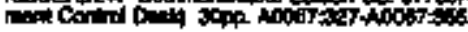

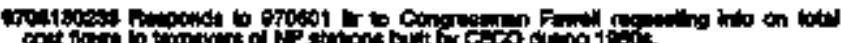

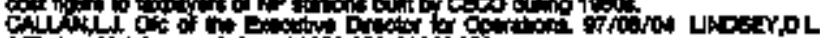

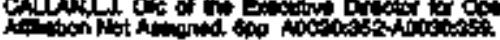

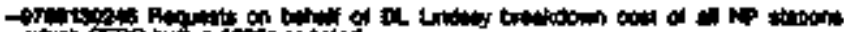

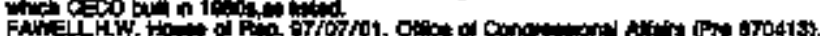
exp.

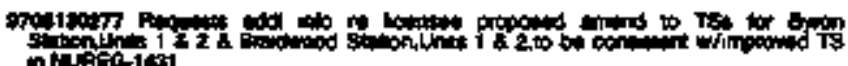
Al: $4002 \times 0 \times 5$

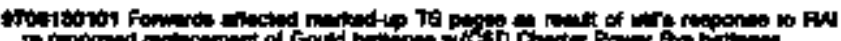

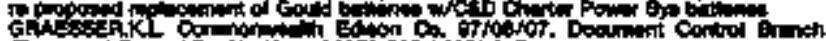

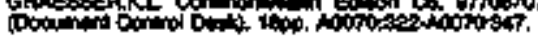

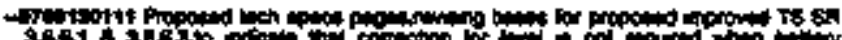

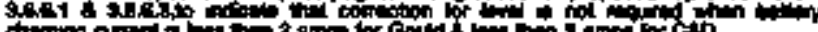

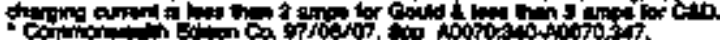

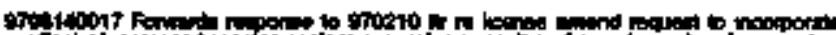

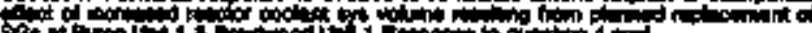

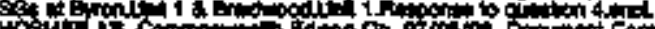

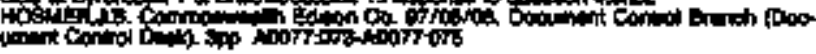

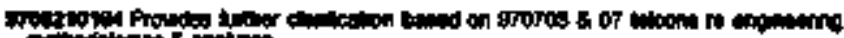

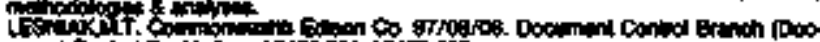

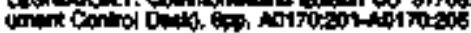

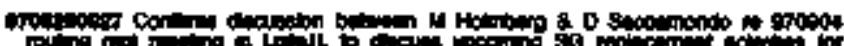

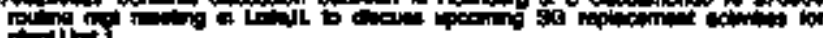

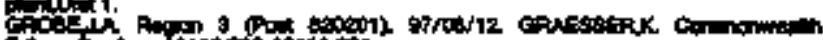

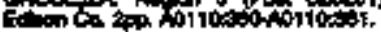

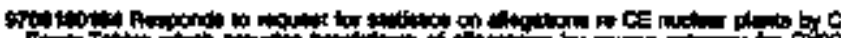
Pits T.

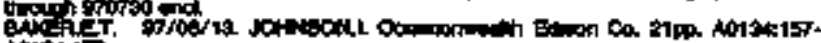
Not3in.

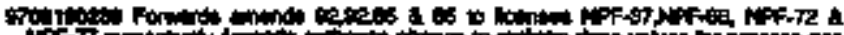
NAf

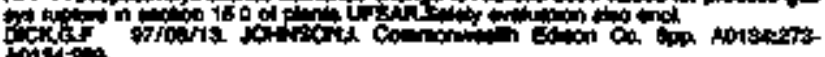
Antis:

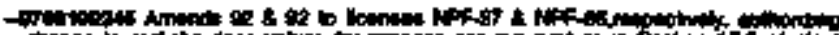

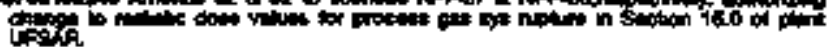

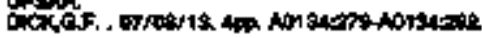

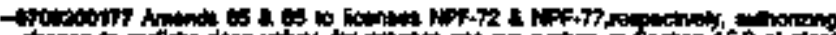

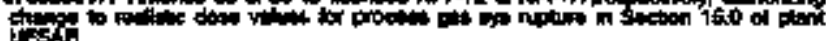

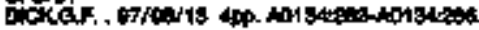

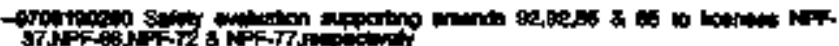

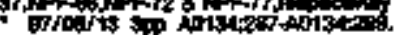

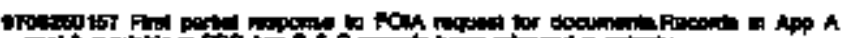

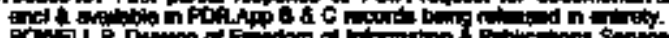

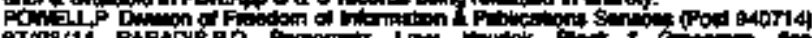

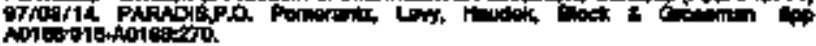

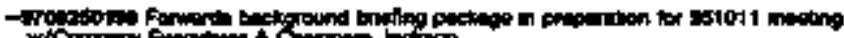

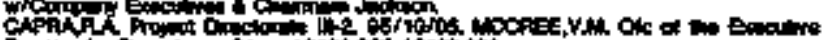

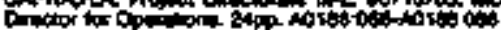

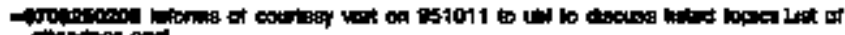

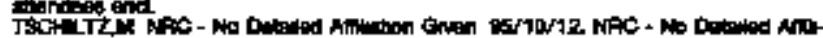

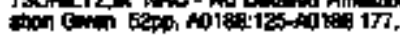

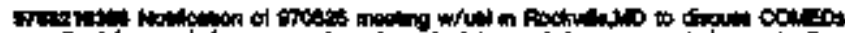

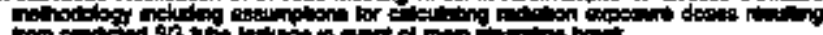
LYW

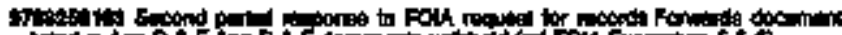

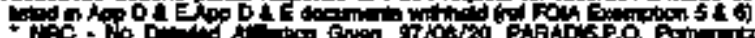

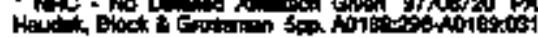

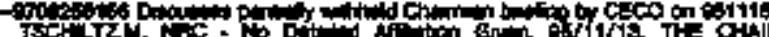

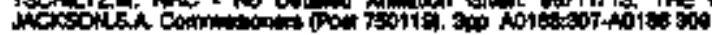

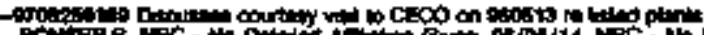

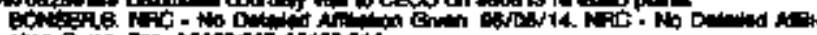

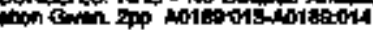

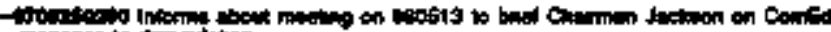
Hition

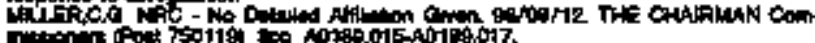

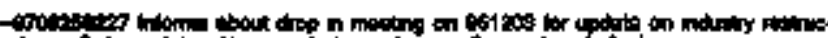

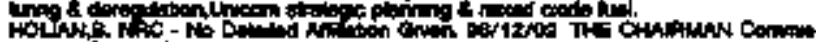

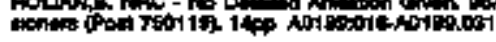

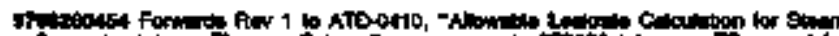

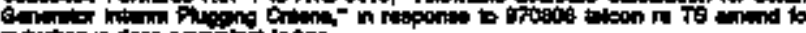

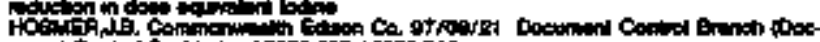

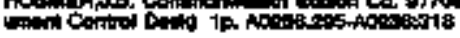

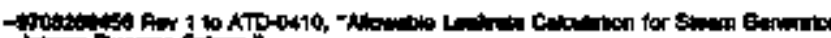

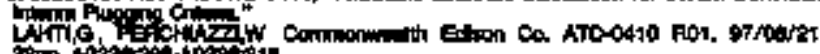

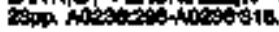

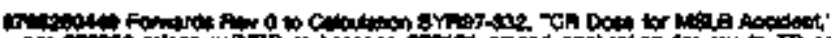

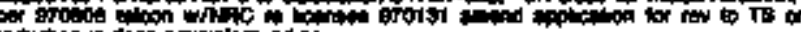

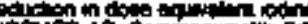

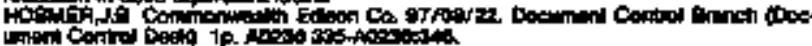

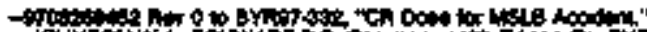

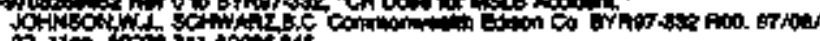

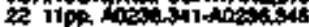

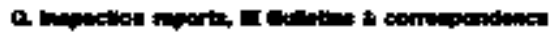

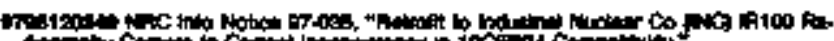

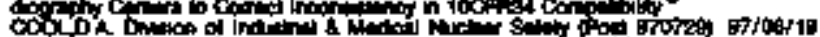

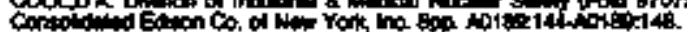

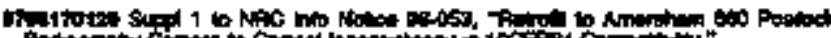

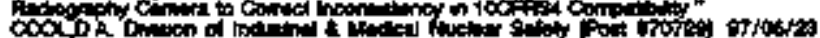

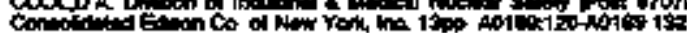

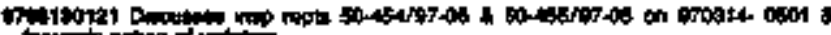

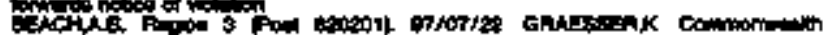

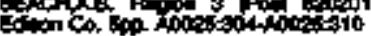

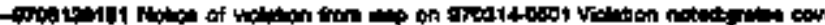

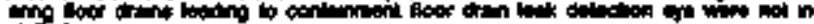

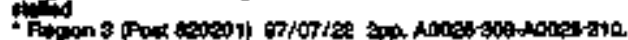

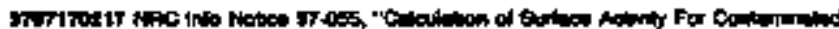

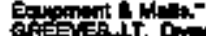
a

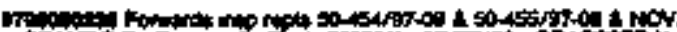

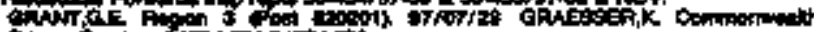

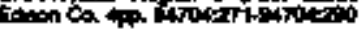

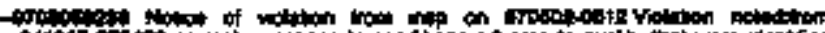

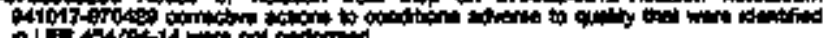

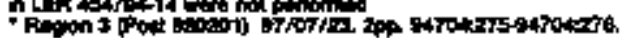

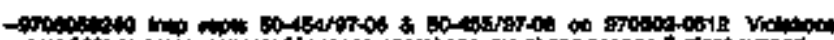

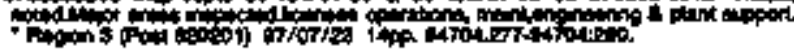

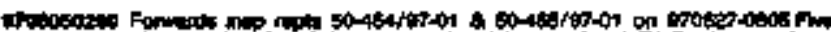

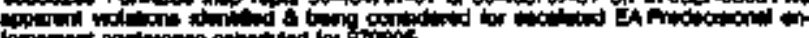

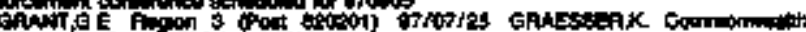

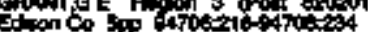

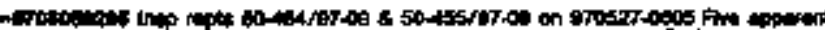

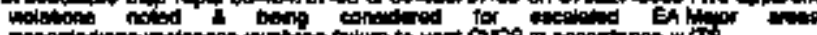

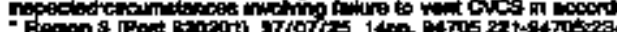




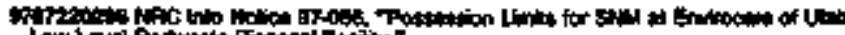

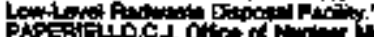

PAr

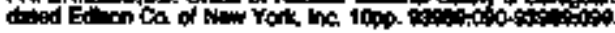

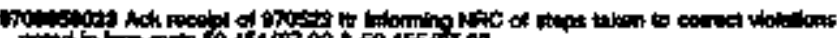

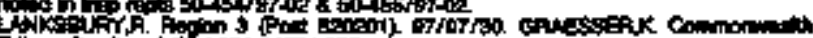

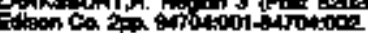

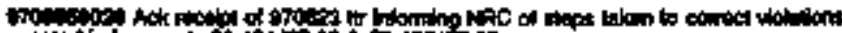
noly

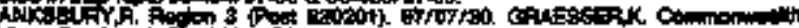

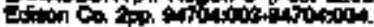

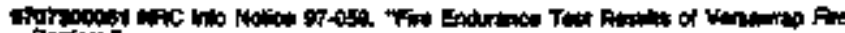

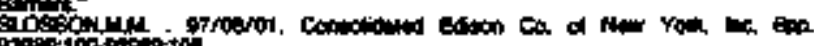

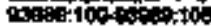

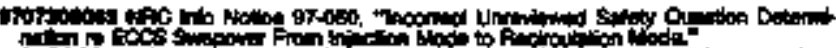

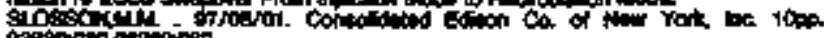

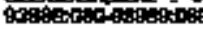

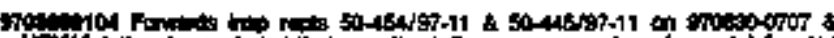

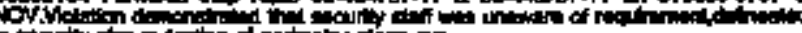

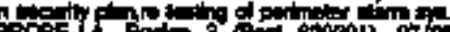

corcosak Componom

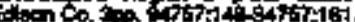

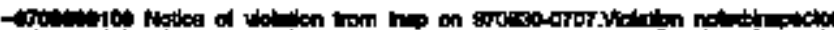

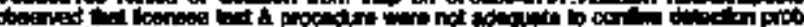

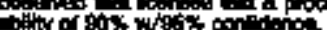

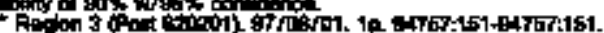

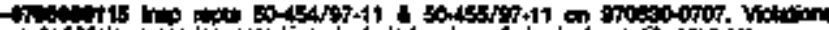

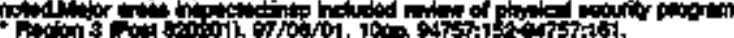

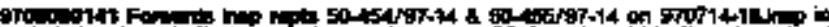

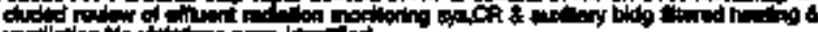

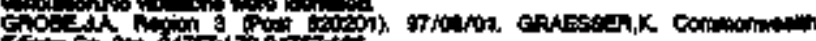

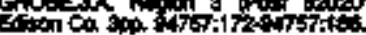

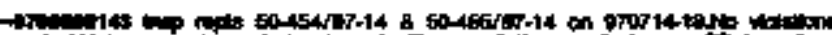

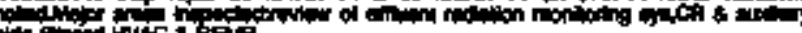
(1)

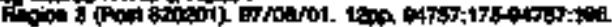

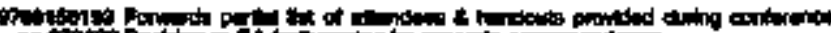

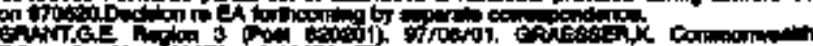

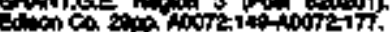

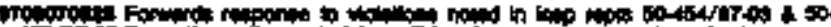

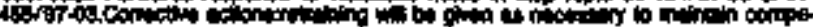

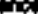

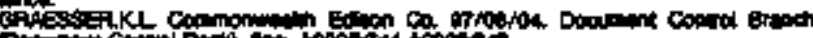

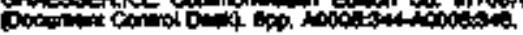

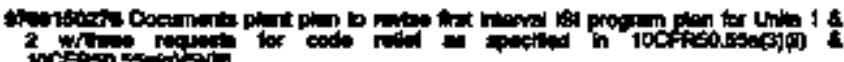

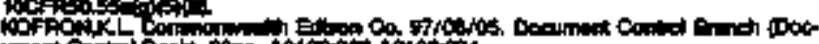

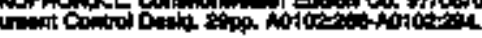

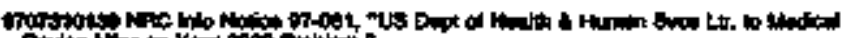

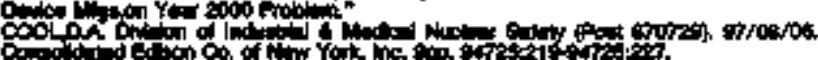

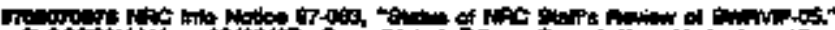

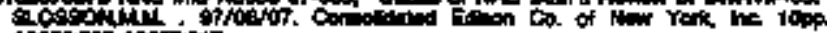

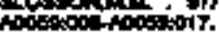

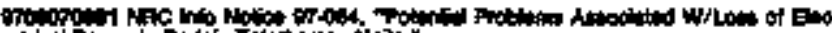
cint

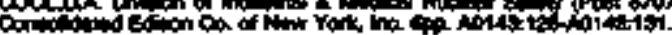

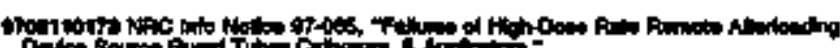

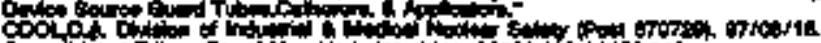

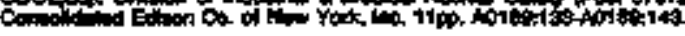

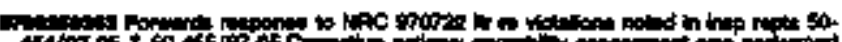

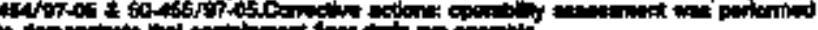

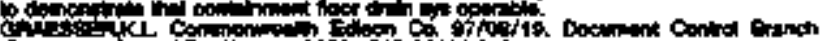

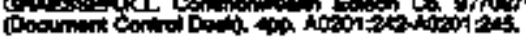

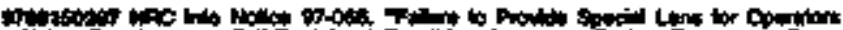

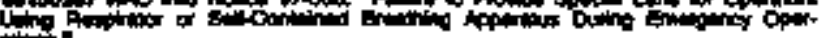

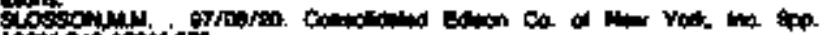

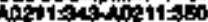

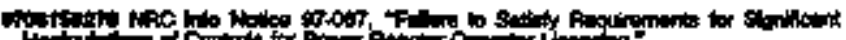

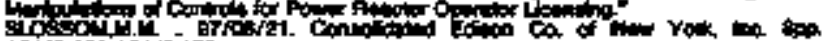

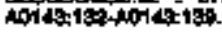

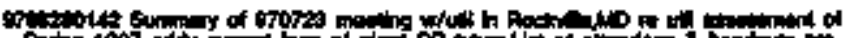

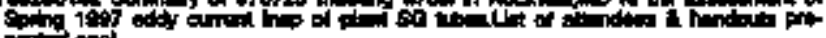

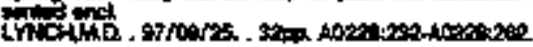

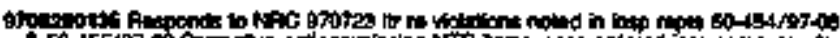

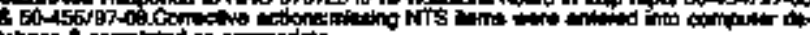

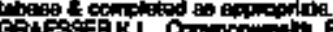

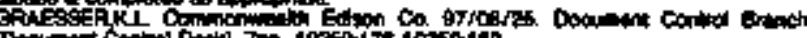

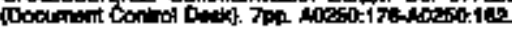

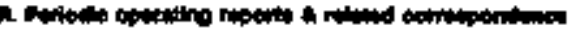

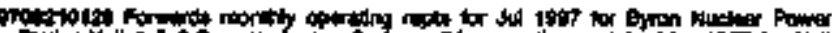

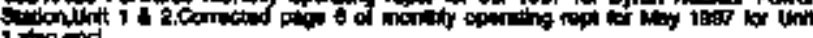

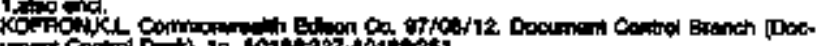

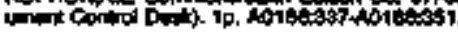

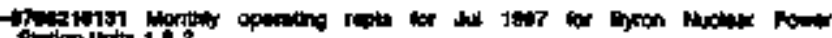

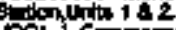

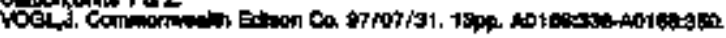

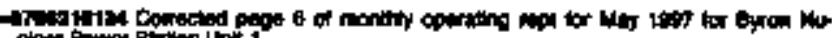

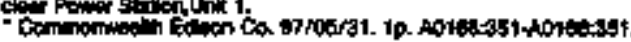

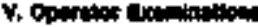

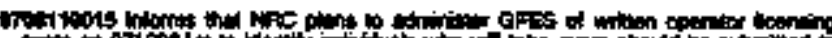

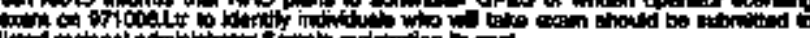

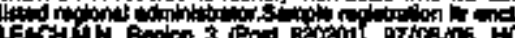

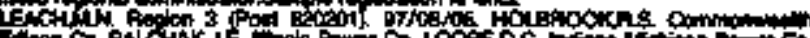

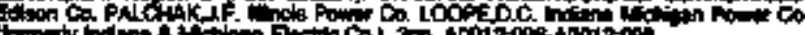

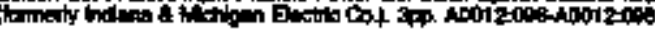

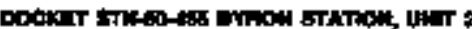

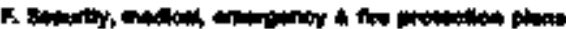

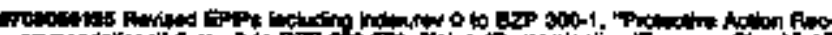

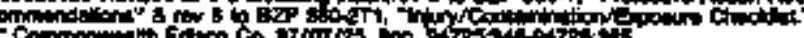

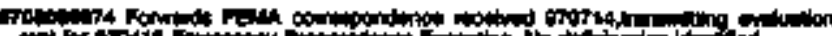

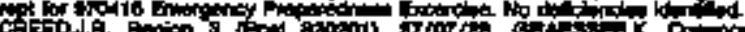

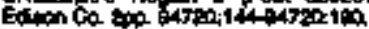

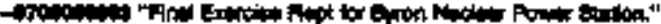

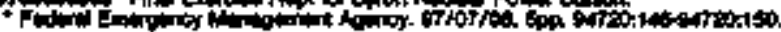

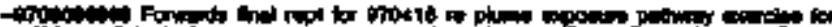

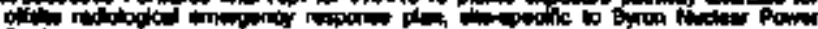

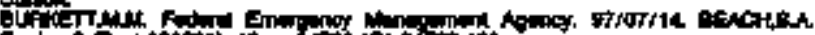

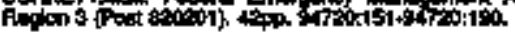

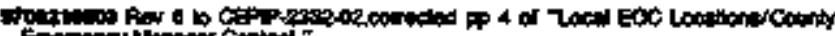

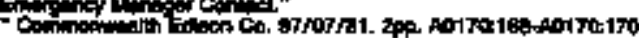

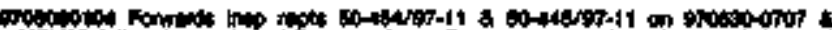

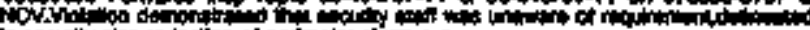

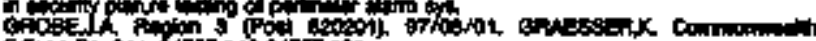

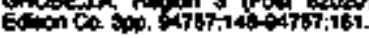

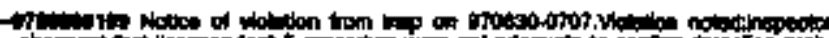

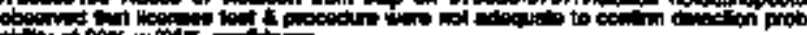

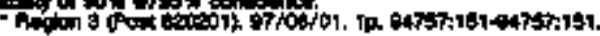

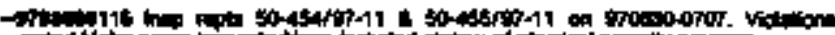

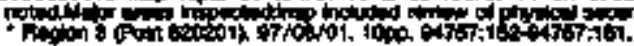

Fror.

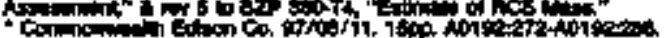

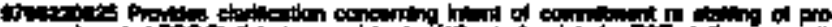

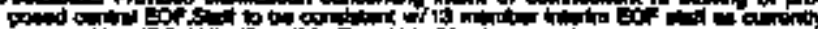

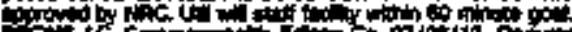

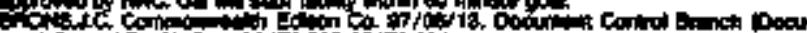

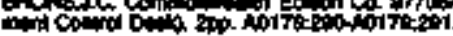

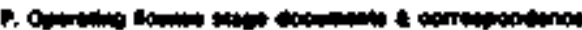

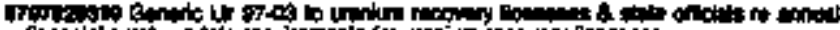

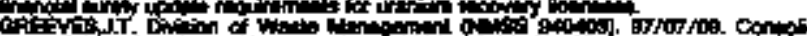

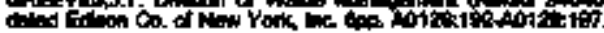

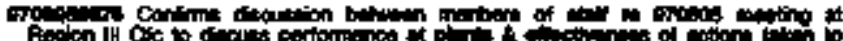

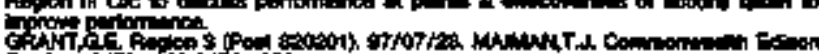

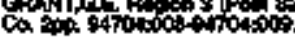

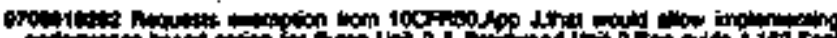

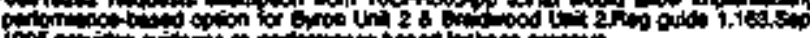

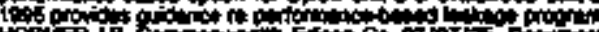

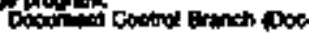

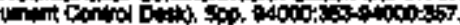




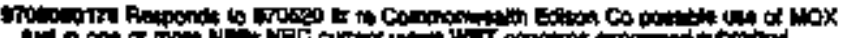

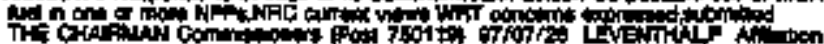

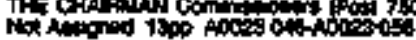

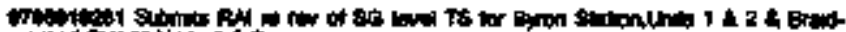

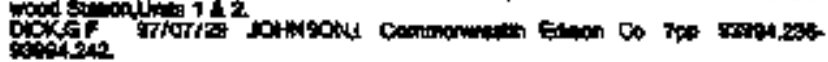

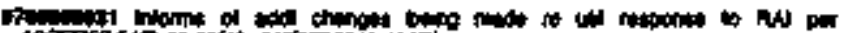

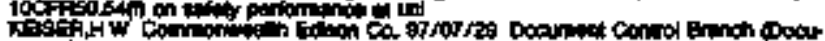

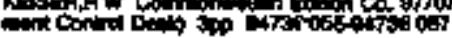

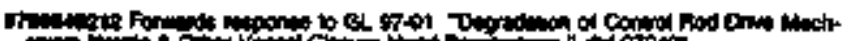

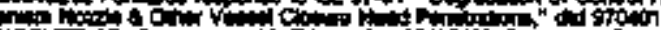

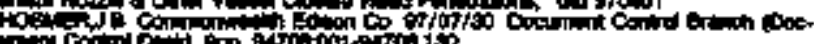

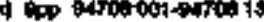

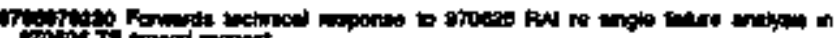

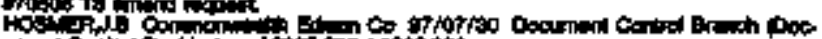

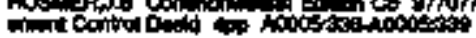

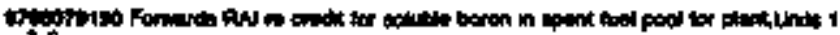

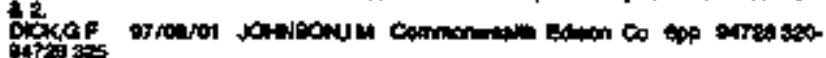

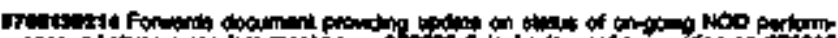

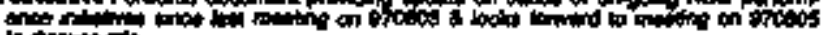

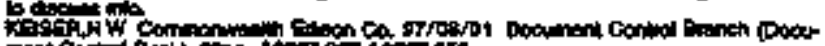

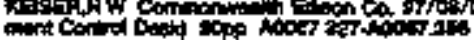

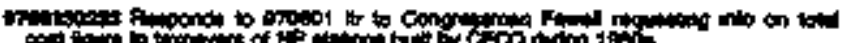
of A

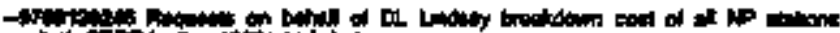

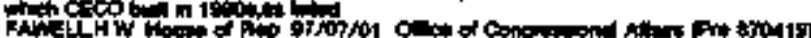

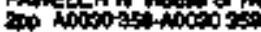

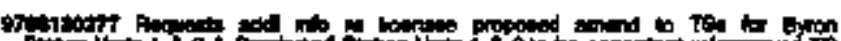

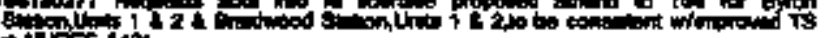

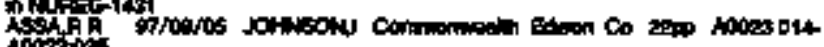
$\cot 0$ is

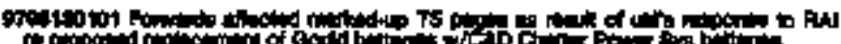
a the

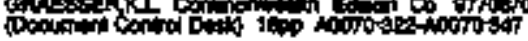

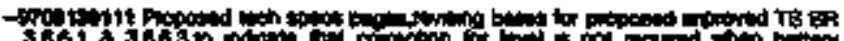

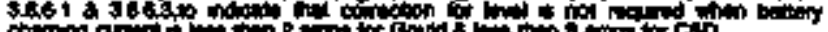

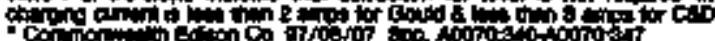

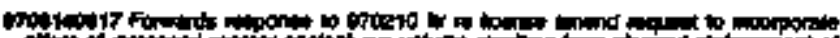

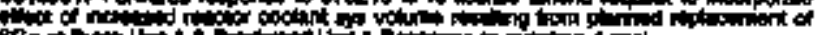

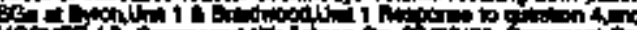

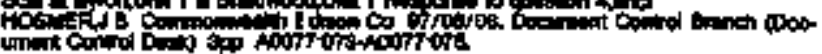

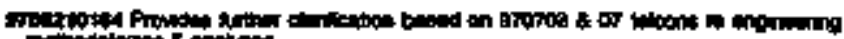

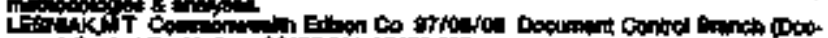

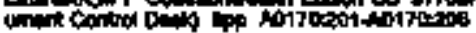

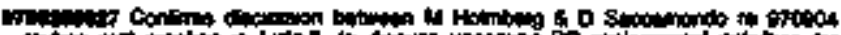

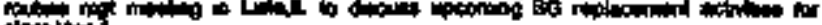

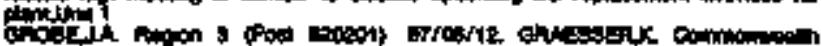
Edion 60 sop hotios

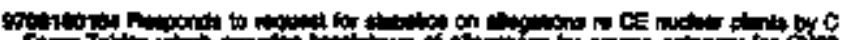

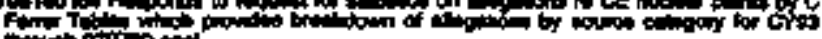

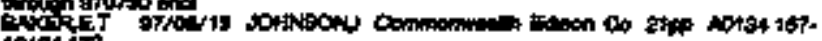
1017is is

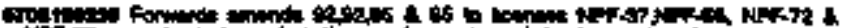

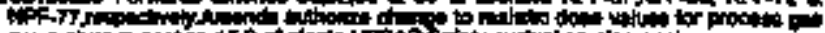

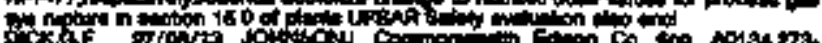
Aritsos

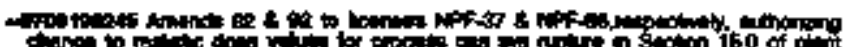

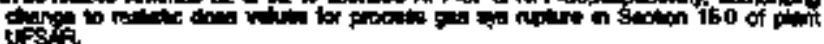

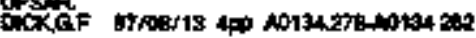

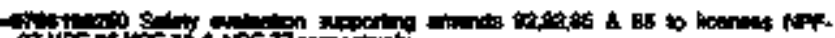

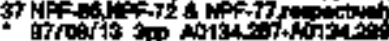

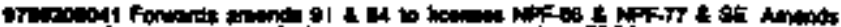

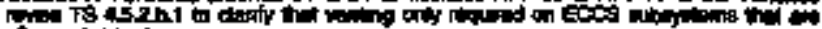

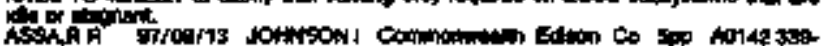
tortion

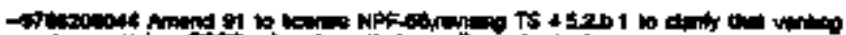

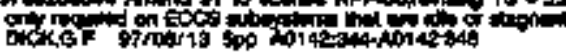

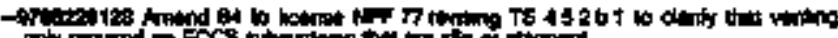

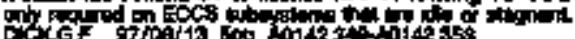

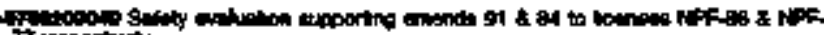

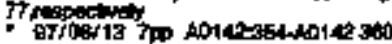

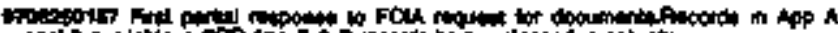

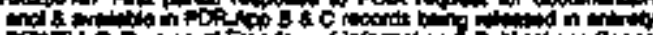

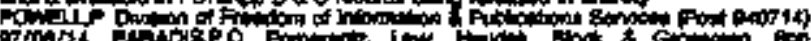

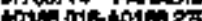

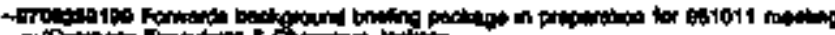

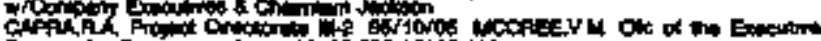

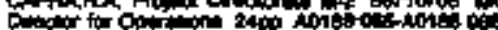

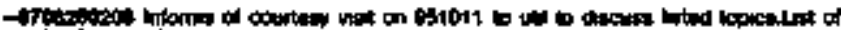

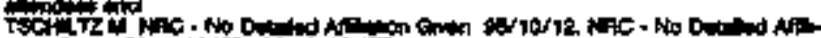

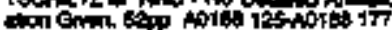

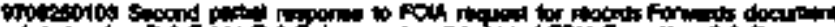

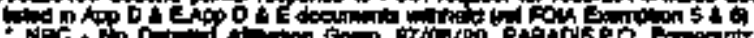

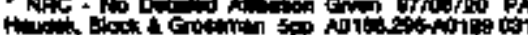

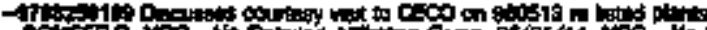

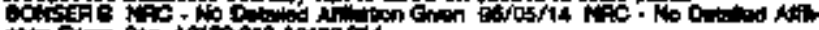

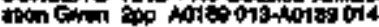

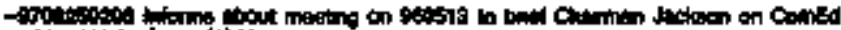

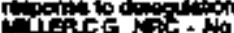

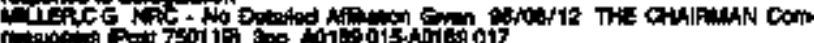

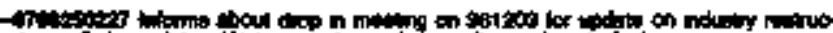
Hoth $\mathrm{B}$.

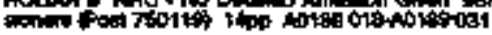

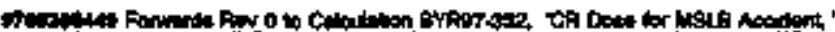

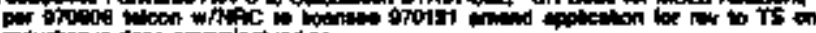

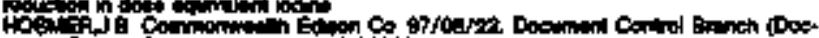

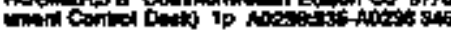

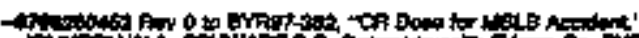
22 ilpo

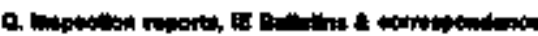

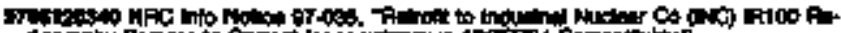

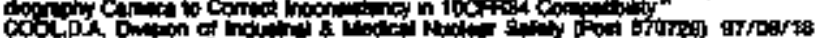
Cos

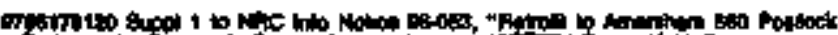

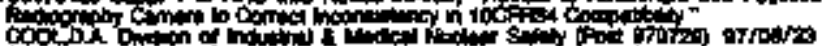

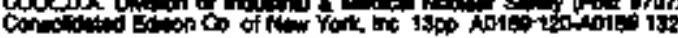

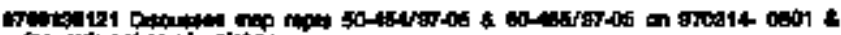

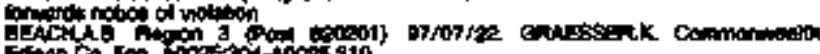

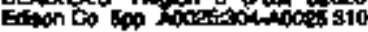

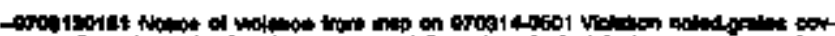

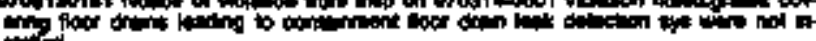

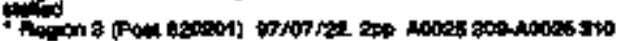

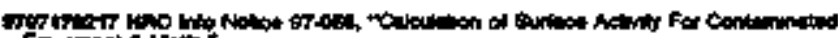
Cy G a :

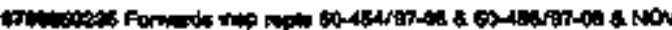

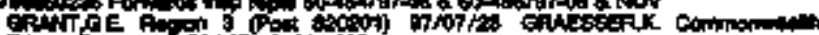

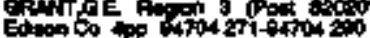

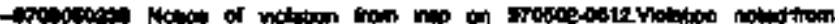

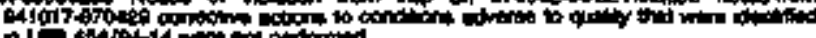

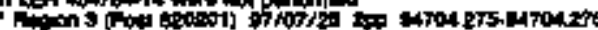

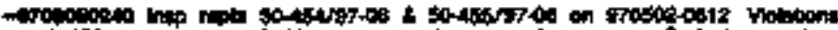

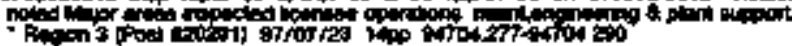

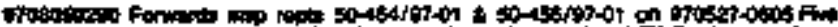

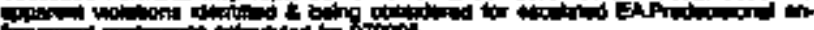

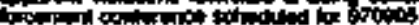

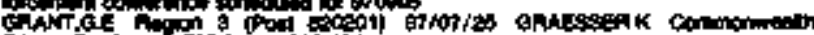

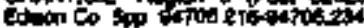

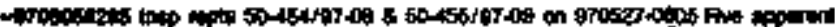

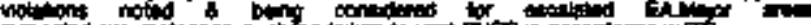

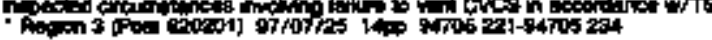




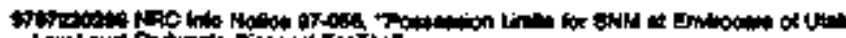

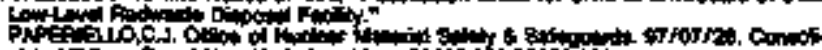

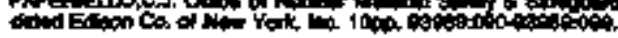

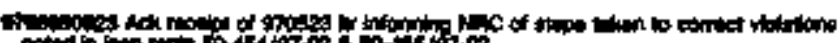

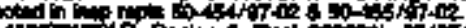

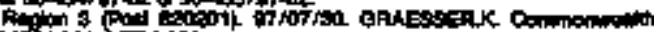

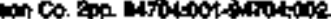

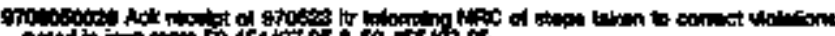

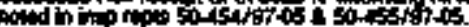

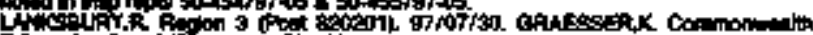

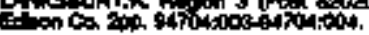

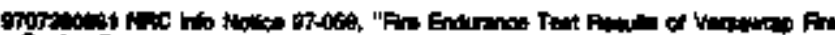
然然o sitionstion

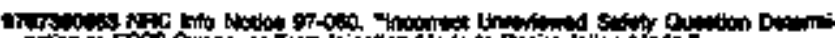

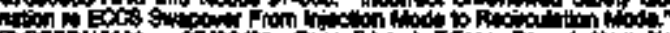

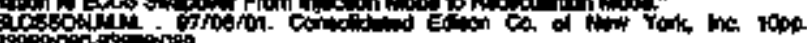

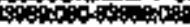

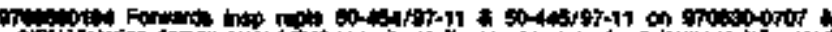

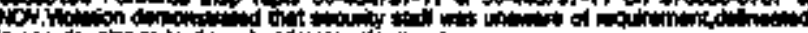

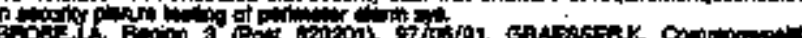

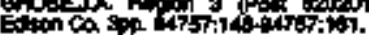

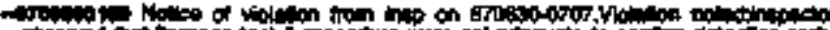

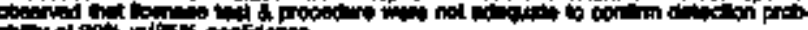

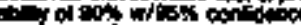

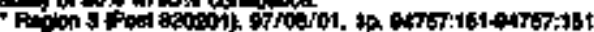

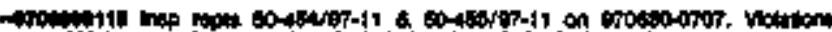

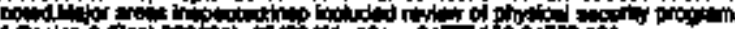

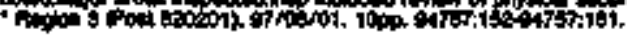

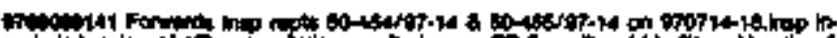

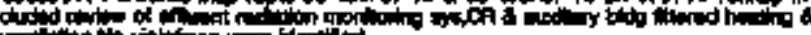

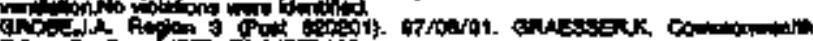

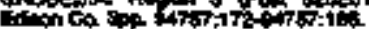

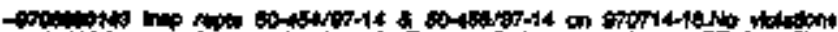

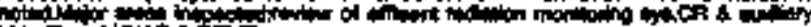

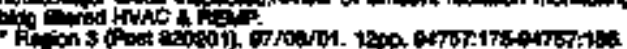

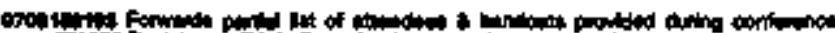

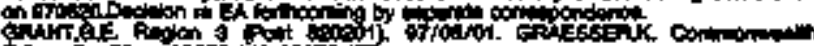

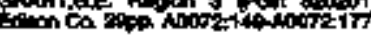

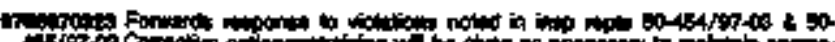

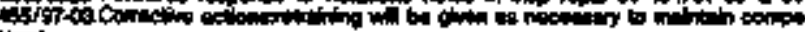

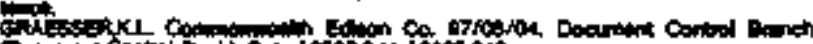

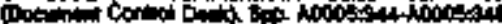

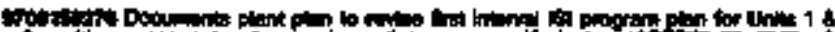

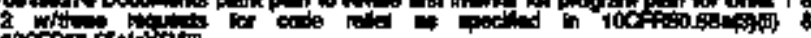

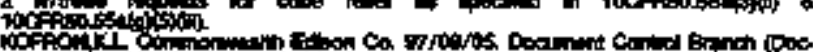

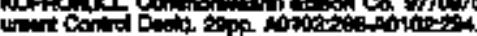

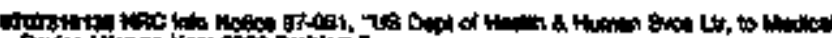

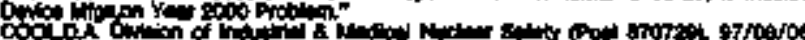

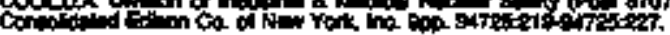

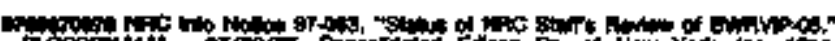

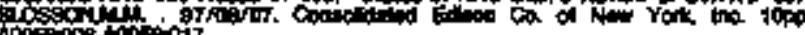
100000

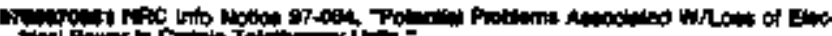

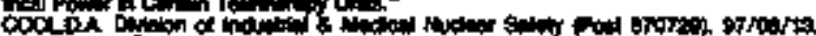

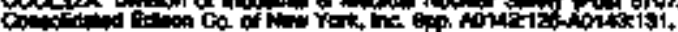

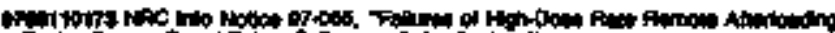

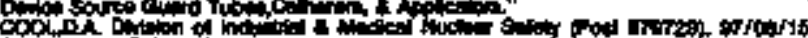

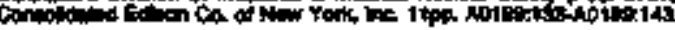

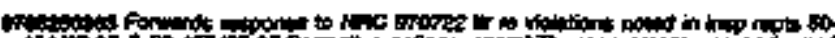

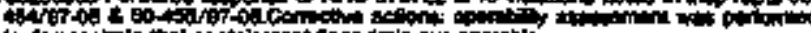

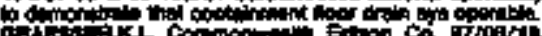

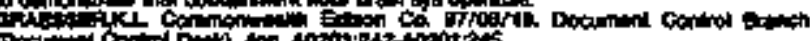

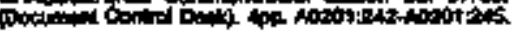

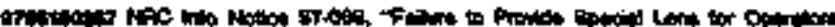

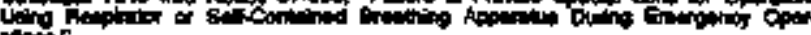

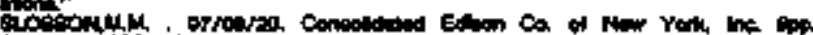

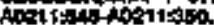

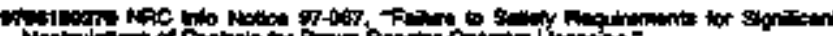

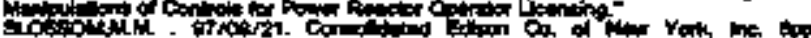

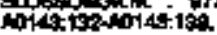

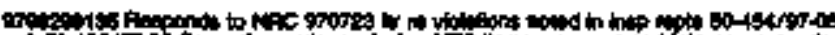

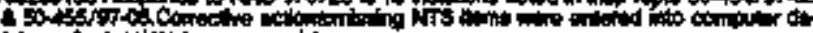

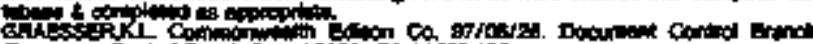

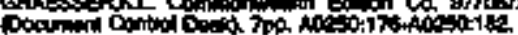

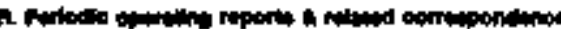

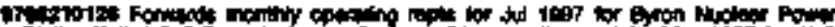

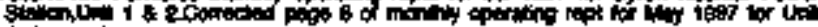

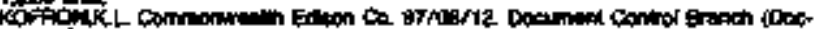

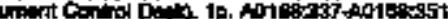

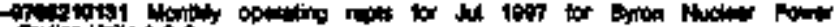

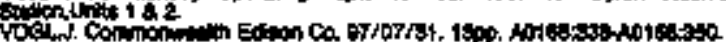

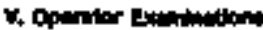

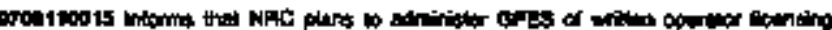

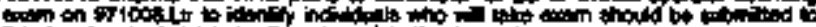

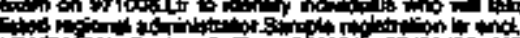

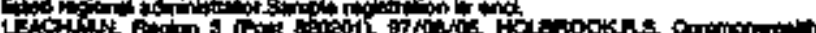

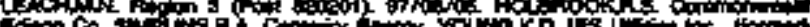

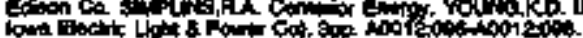

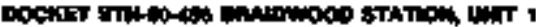

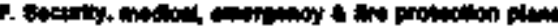

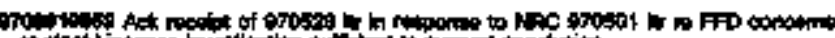

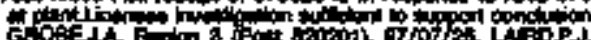

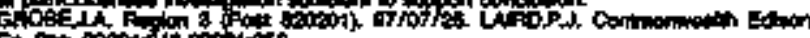

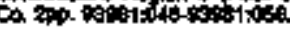

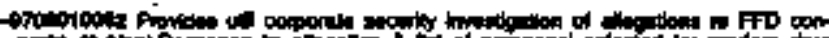

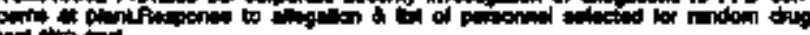
arting

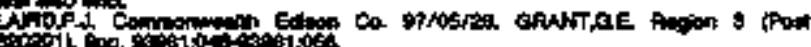

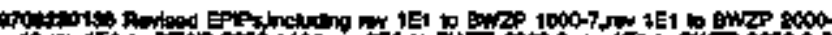

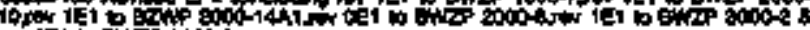
151 to

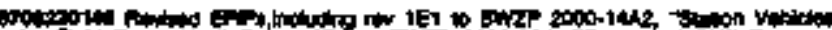

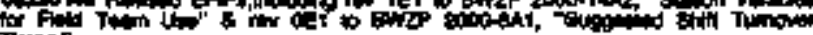

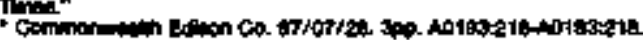

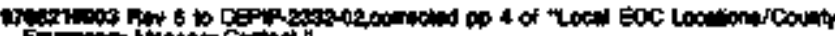

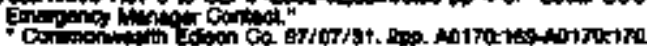

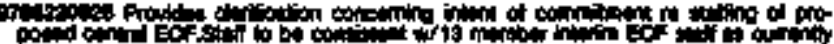

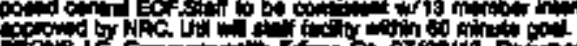

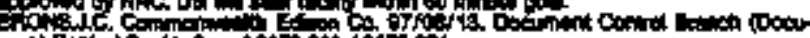

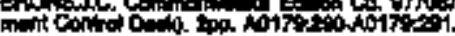

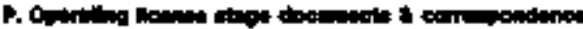

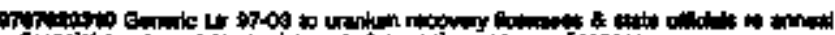

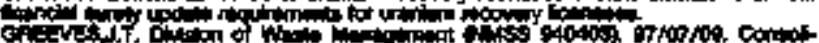

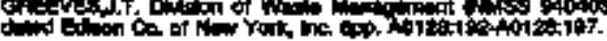

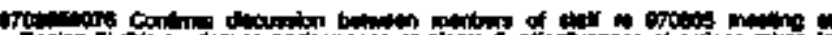

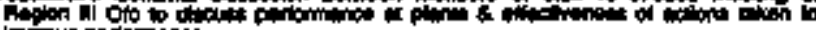

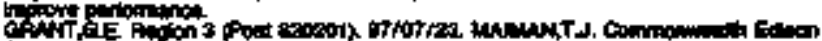

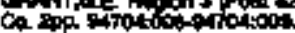

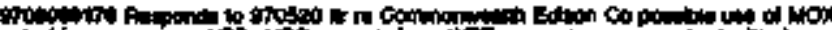
n

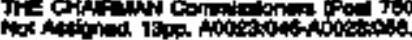

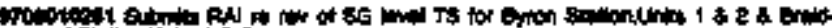

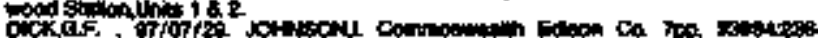
operter.

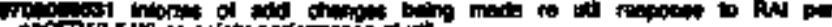

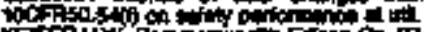
Wiek-

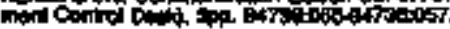

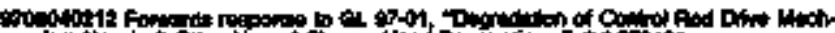
rin

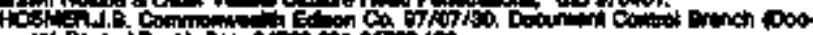

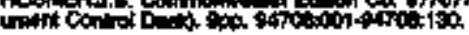

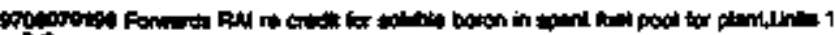

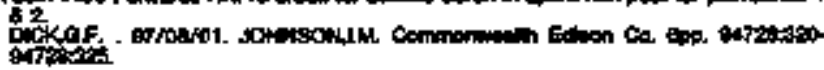

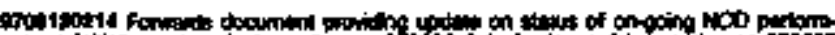

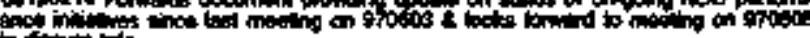
Ki

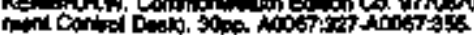




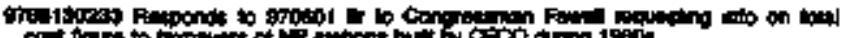

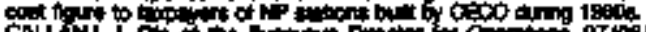
cail LiN

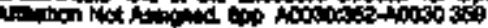

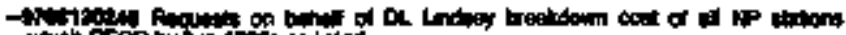

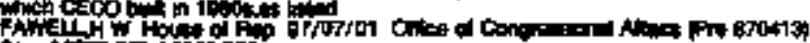
4 iñ

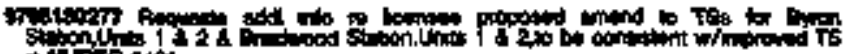
Af

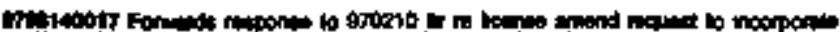

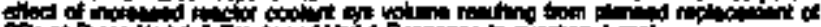
Host

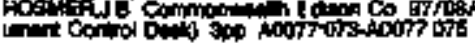

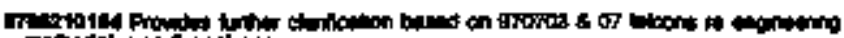

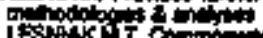

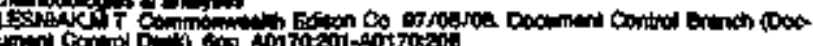

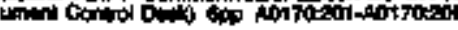

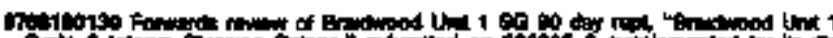

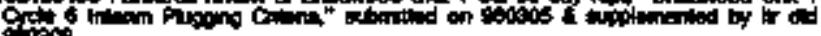

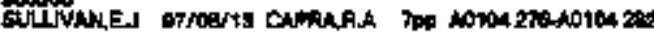

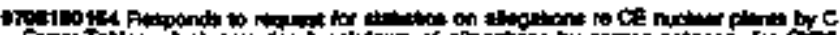

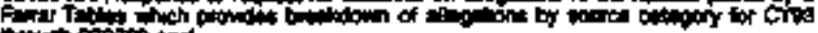

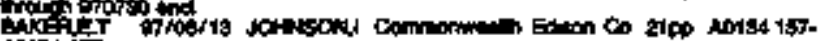
a일 it?

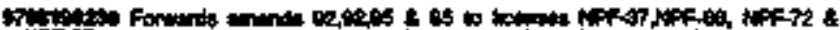

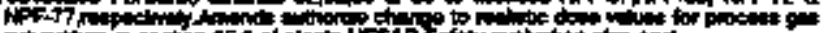
atrotion $n$ Aor

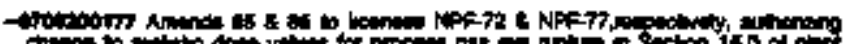

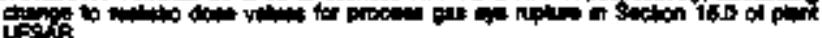

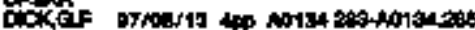

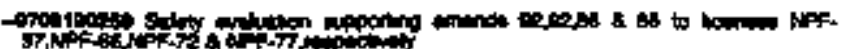

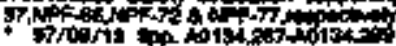

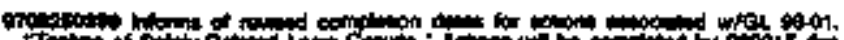

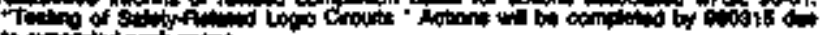

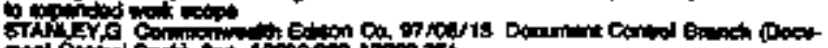

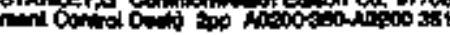

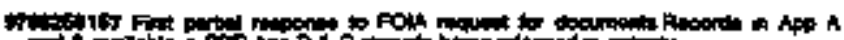

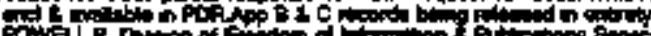

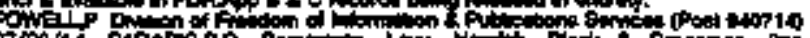

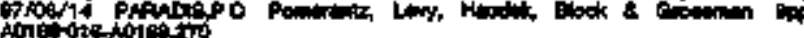

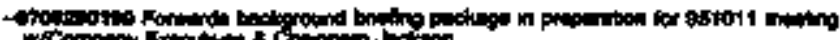

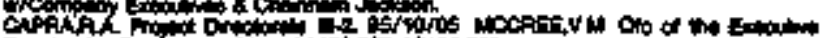

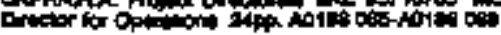

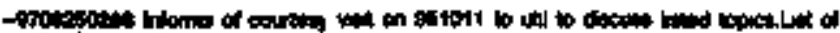

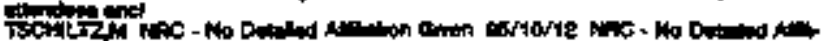

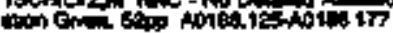

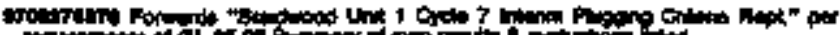

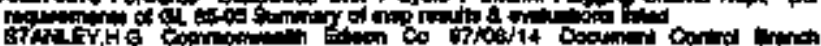

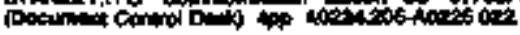

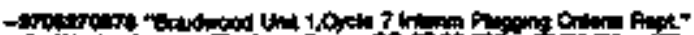

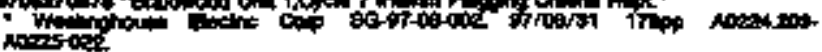

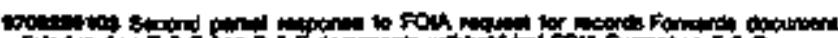

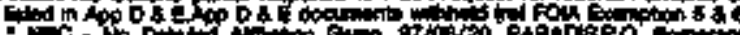

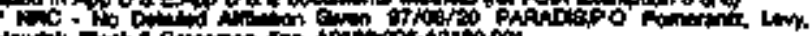

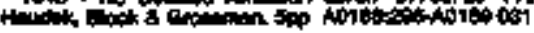

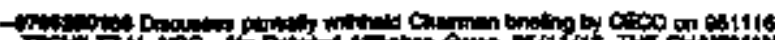

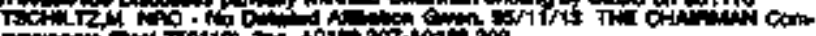

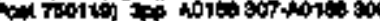

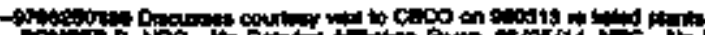

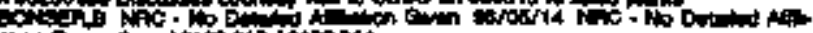
(1)

要

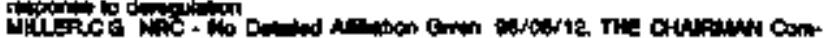
in

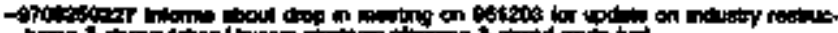

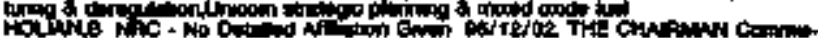

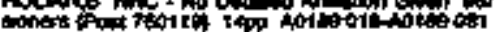

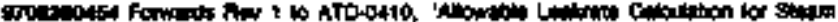

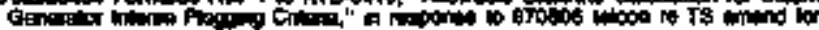

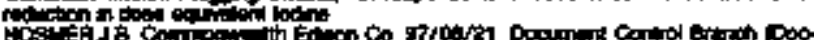

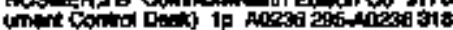

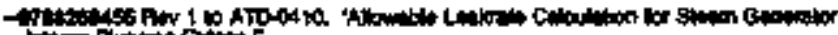
HaH Tine

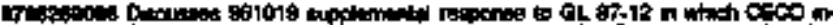

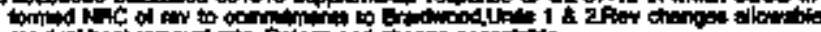

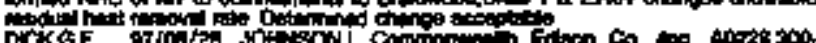
ancesis

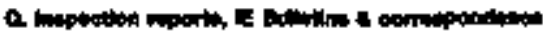

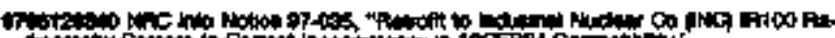

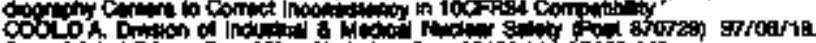

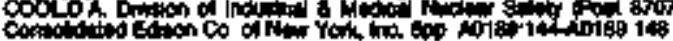

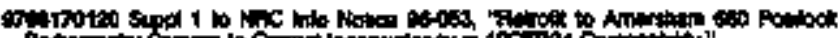
A $\alpha 0$ O

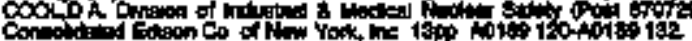

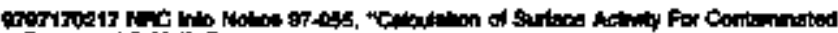

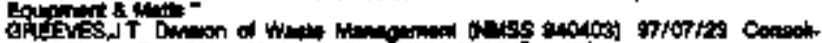

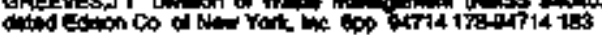

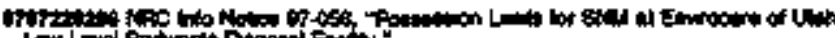
Low

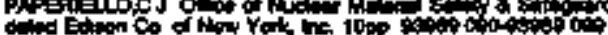

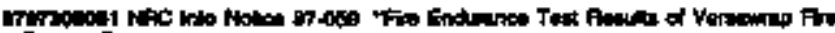

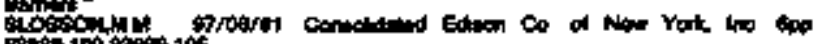
Disto $100-106$

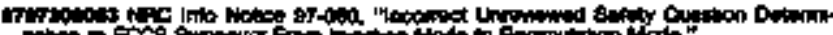

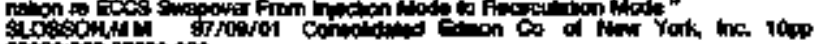

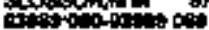

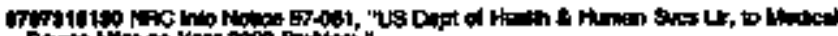

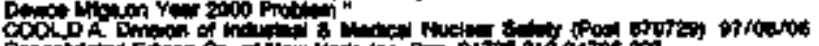

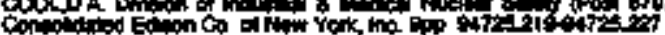

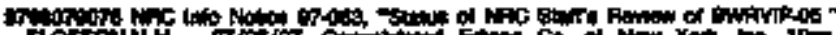

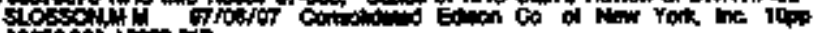
toos 0 ot

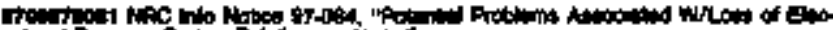

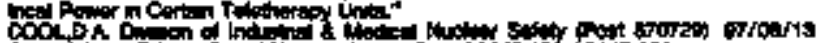
Conached

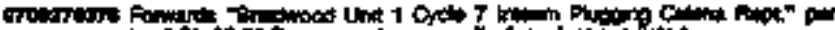
riv

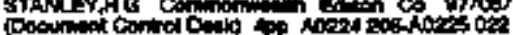

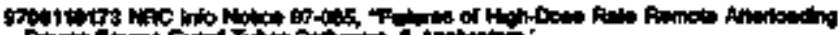

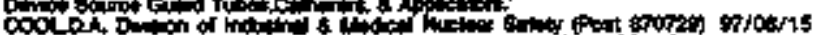

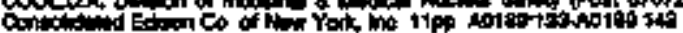

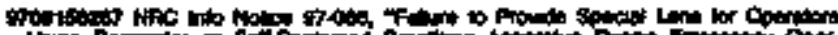

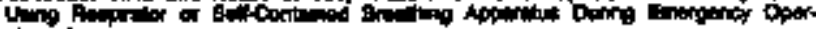

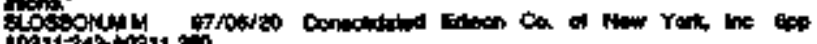

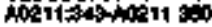

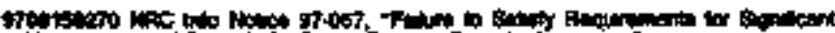

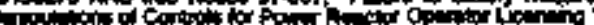

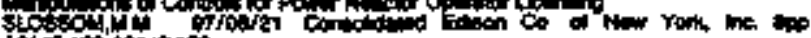

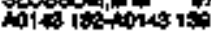

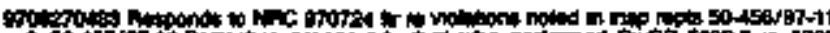

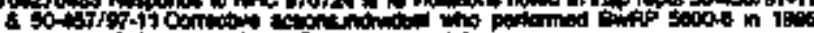

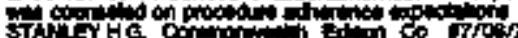

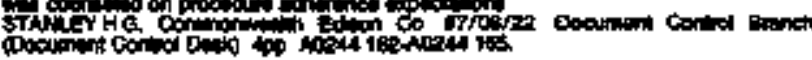

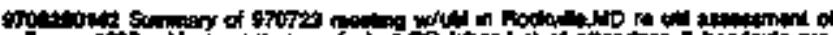

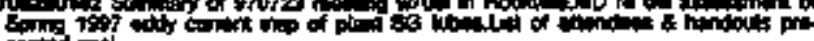

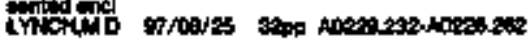

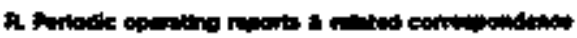

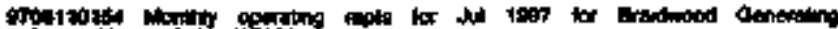

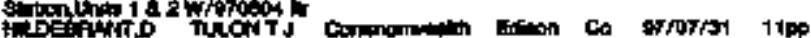

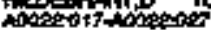




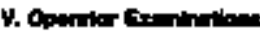

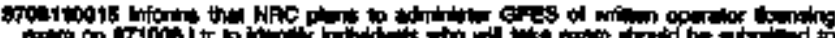

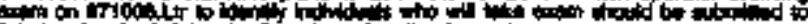

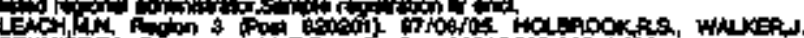

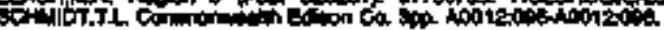

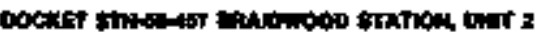

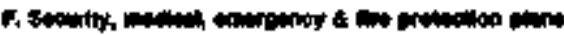

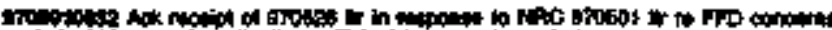
ation

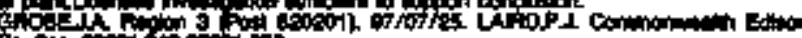

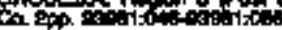

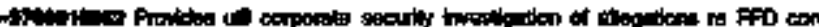

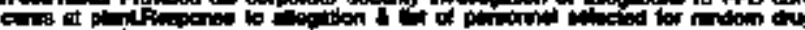
Hof Fine

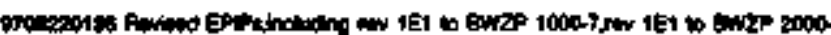

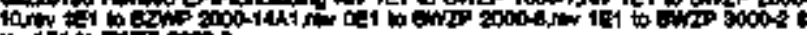

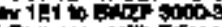

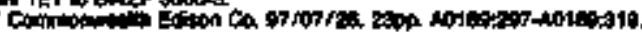

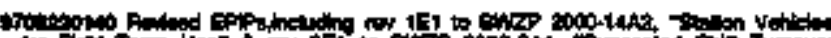

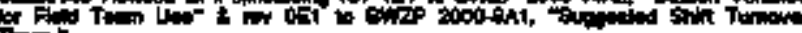

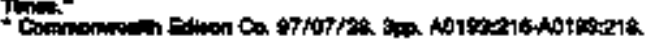

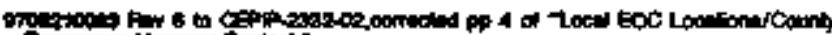

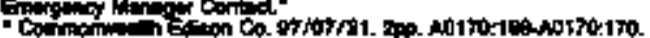

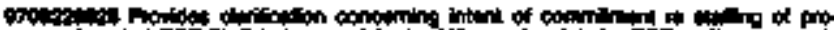

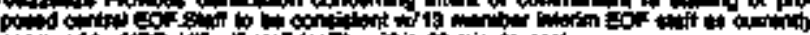

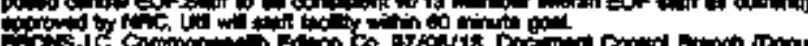

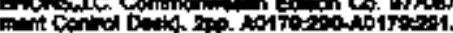

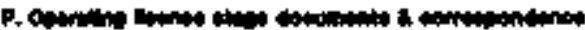

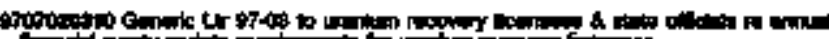

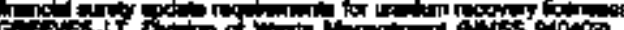

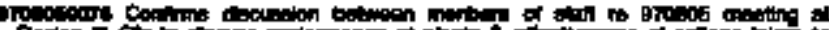

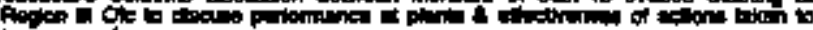

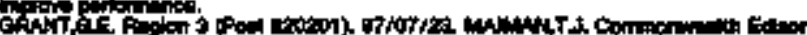

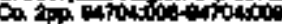

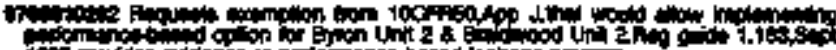

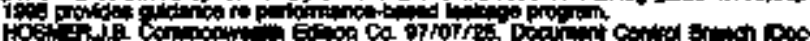

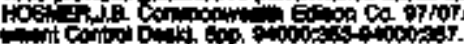

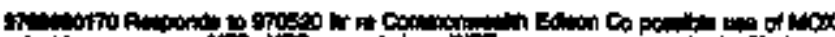

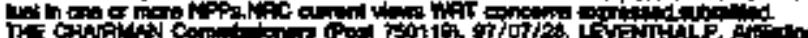

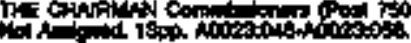

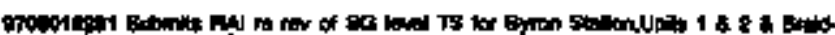

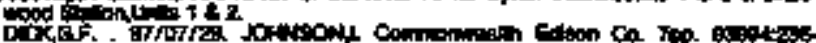
Dicis:-

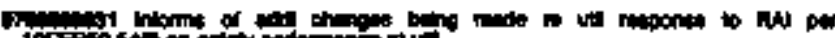

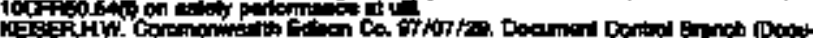

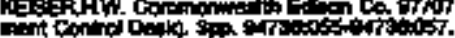

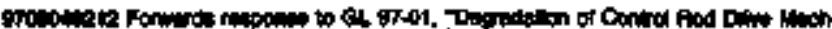
H.

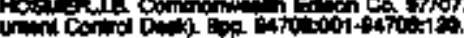

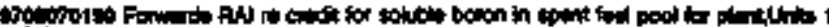

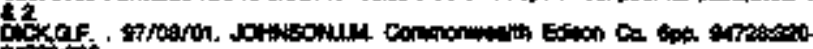
antis

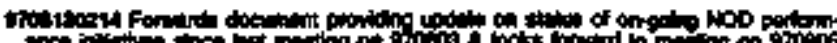

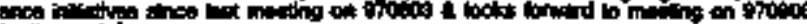

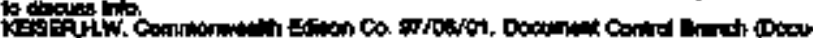

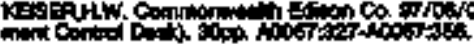

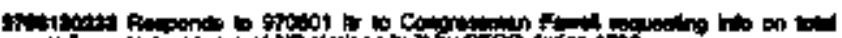

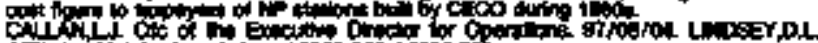

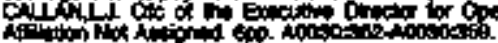

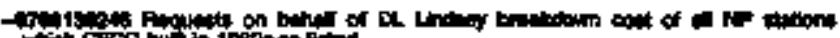

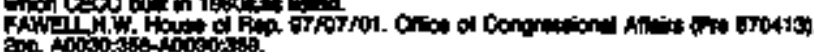

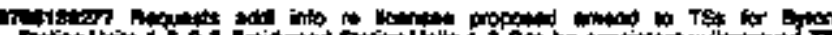

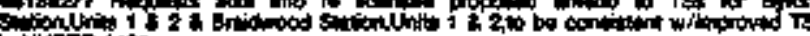
in

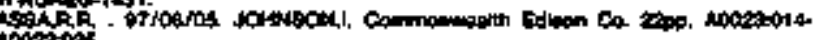
1002003

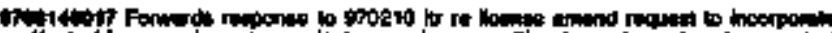

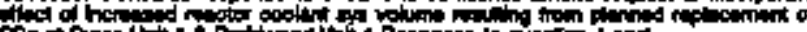

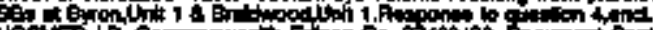
Host:

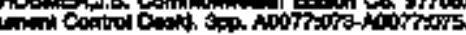

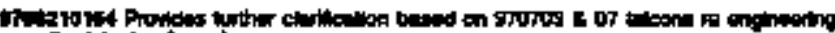

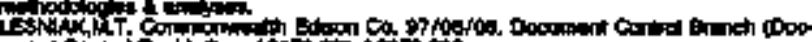
unil of

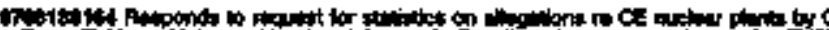

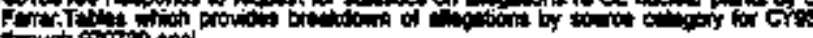

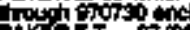

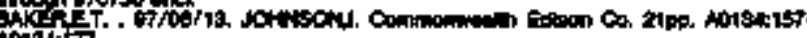
Amativ.

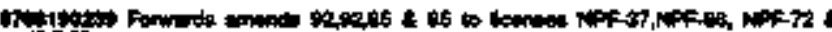

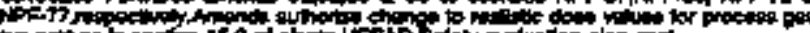

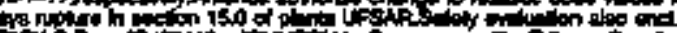

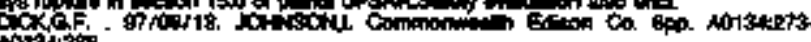
AOr

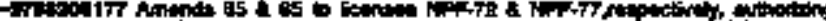

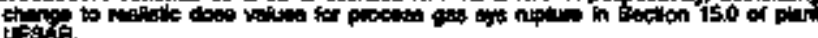

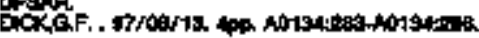

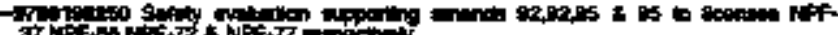

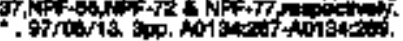

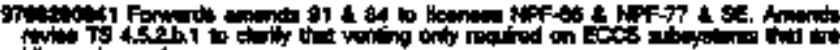

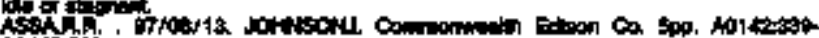
Aot towo

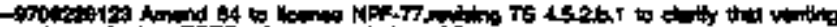

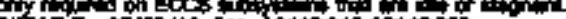

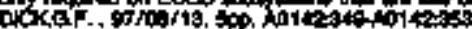

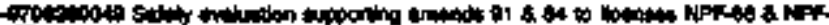

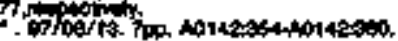

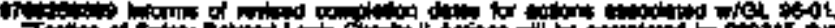

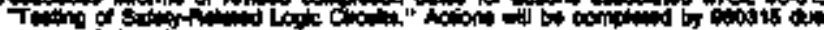

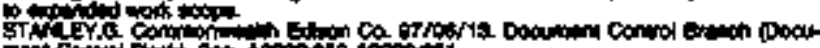

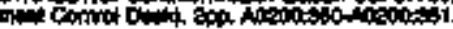

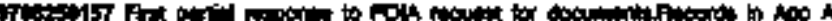

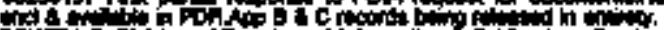

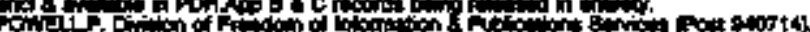

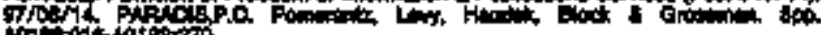

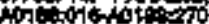

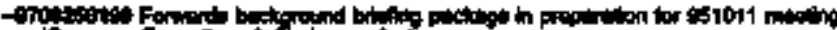

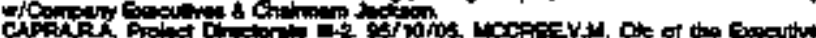

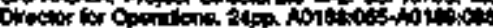

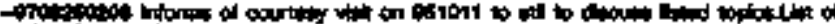

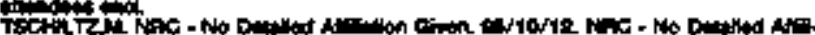

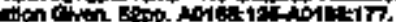

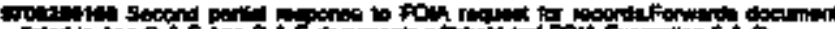

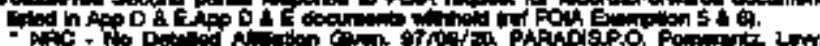

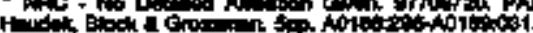

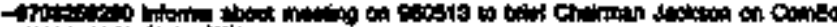
Hificion

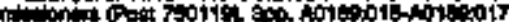

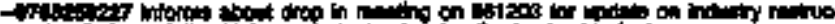
1.

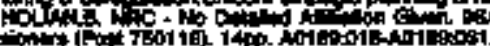

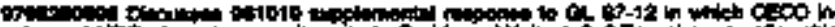
lormed N

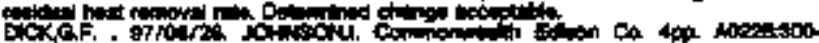
inosios.

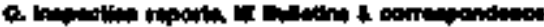

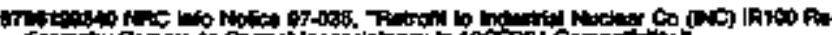

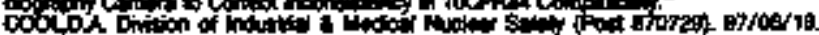
coont pingon of ind of 


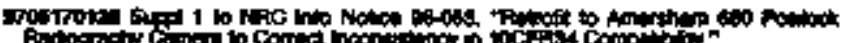

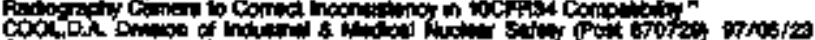
Con

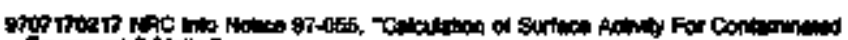

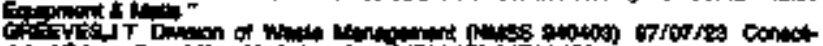

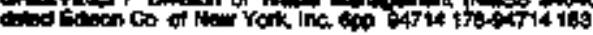

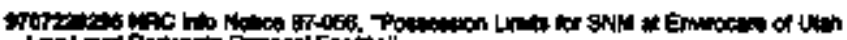

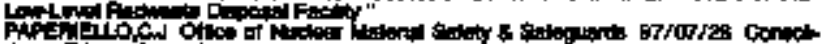

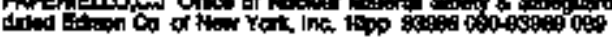

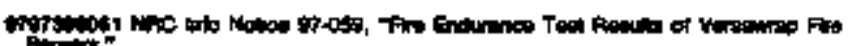

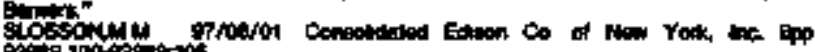

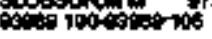

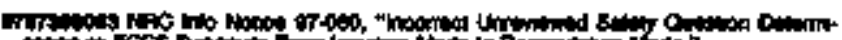

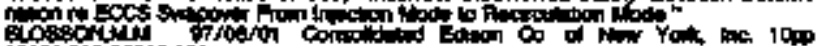
(x)

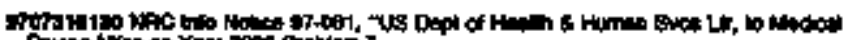

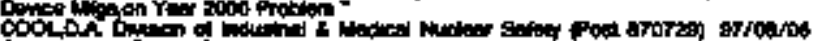

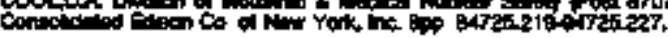

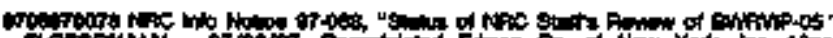

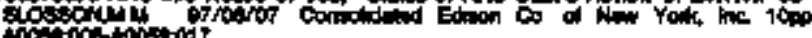

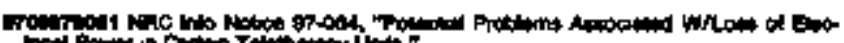

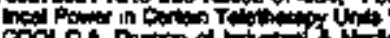

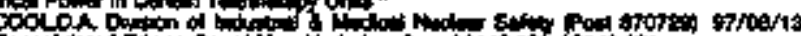

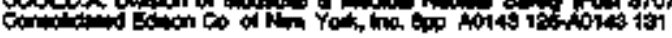

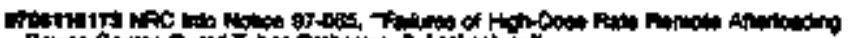

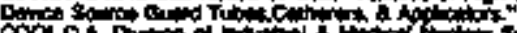

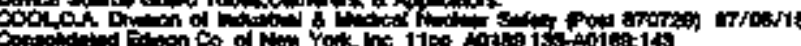

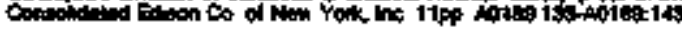

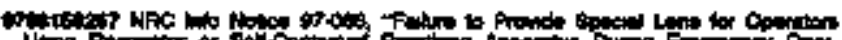

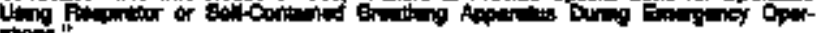

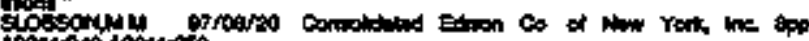

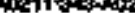

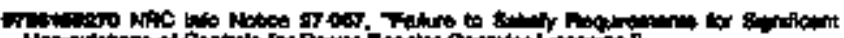

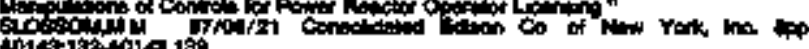

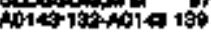

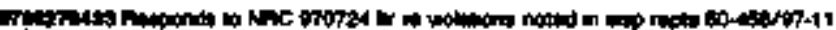

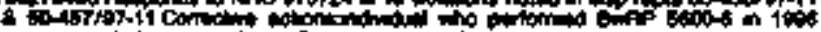

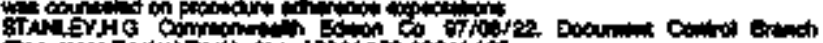

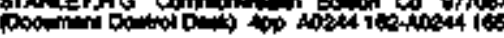

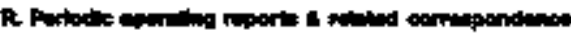

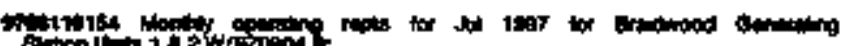

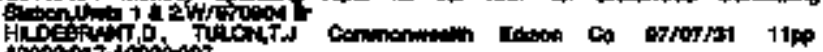

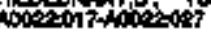

\section{V.}

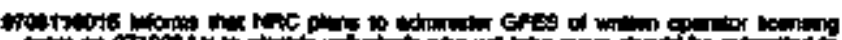

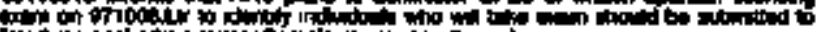

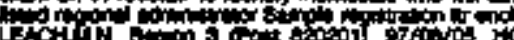

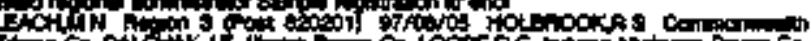

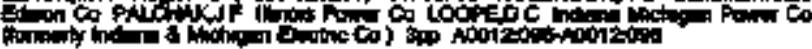

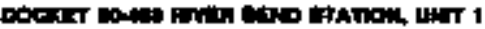

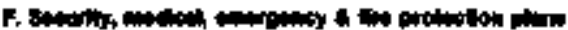

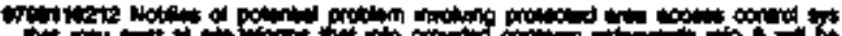

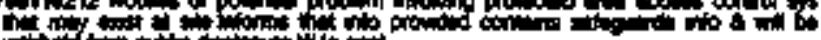

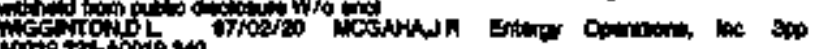

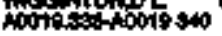

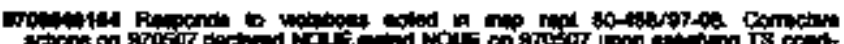

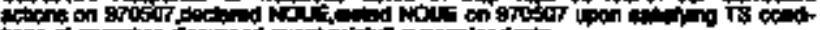

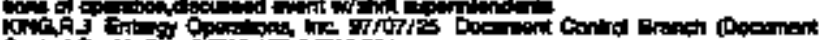

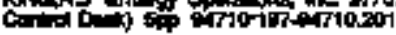

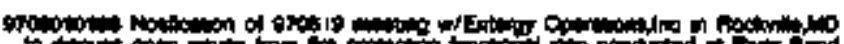

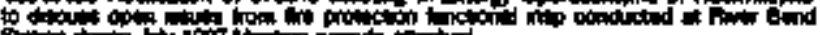

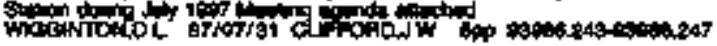

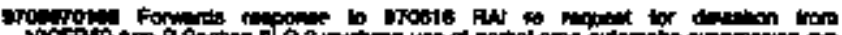

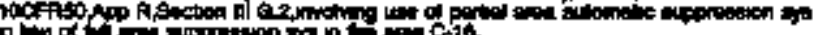

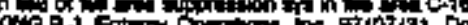

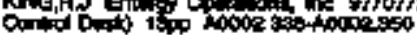

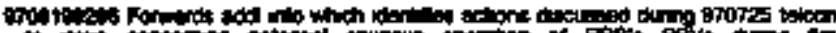

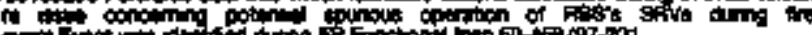

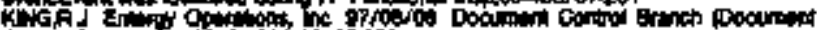

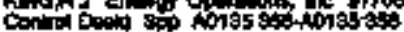

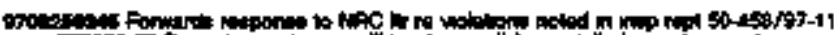

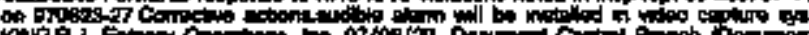

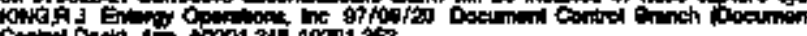

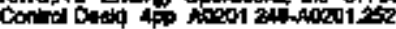

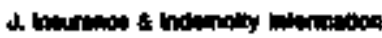

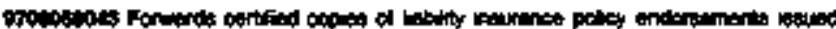

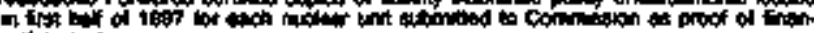

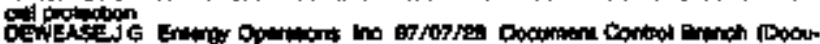

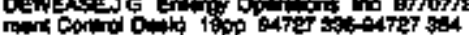

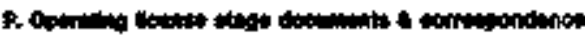

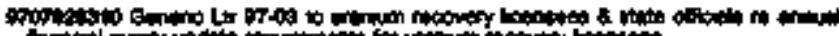

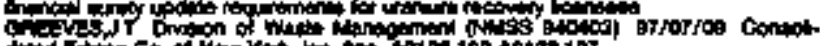

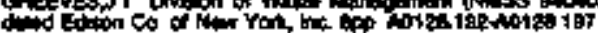

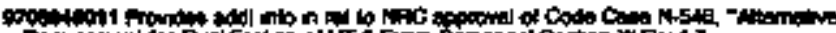

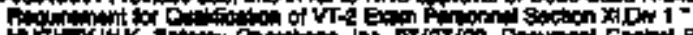

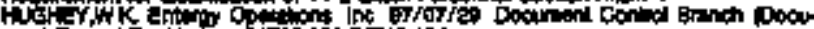

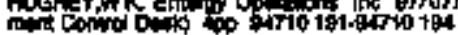

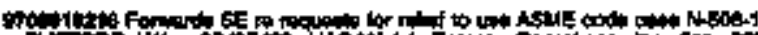

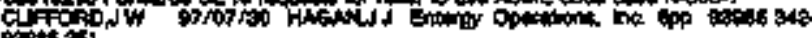

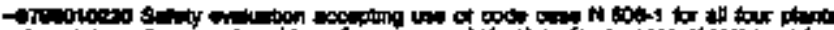

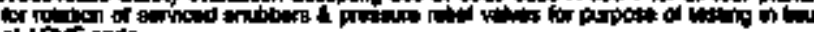

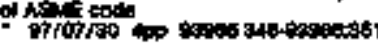

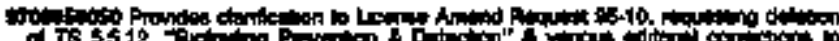

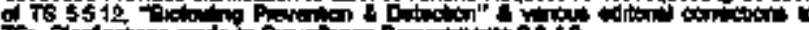

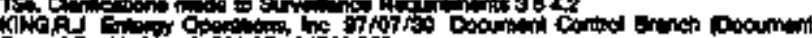

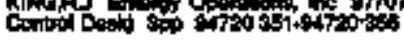

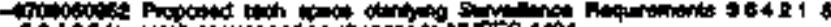

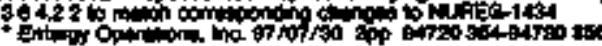

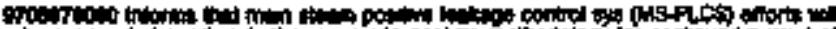

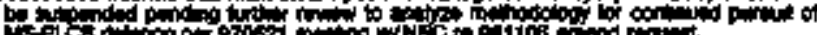

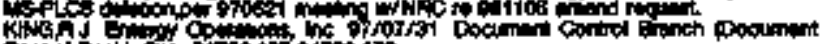

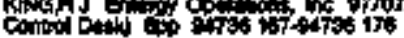

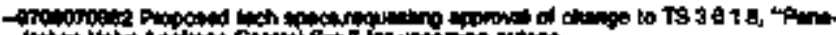

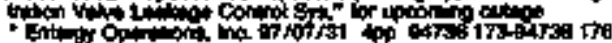

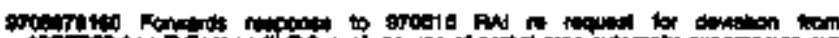

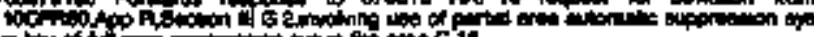

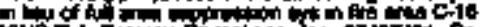

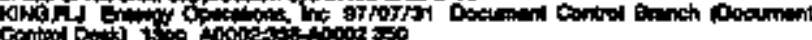

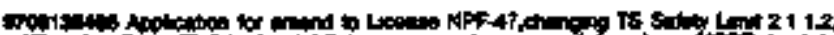

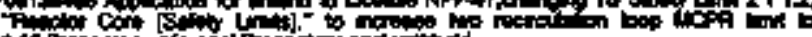

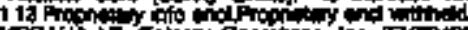

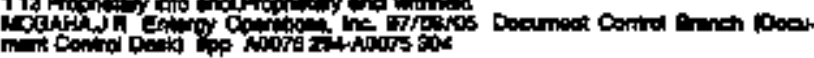

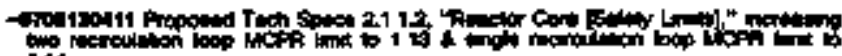

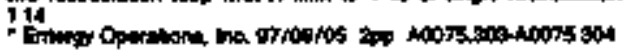

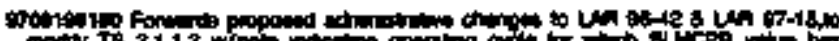

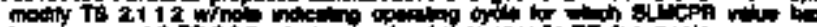

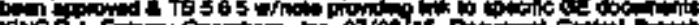

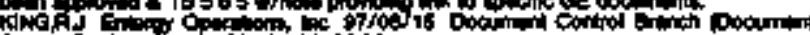

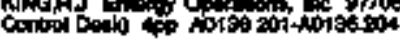

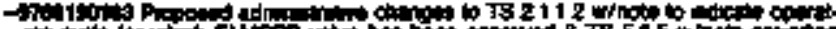

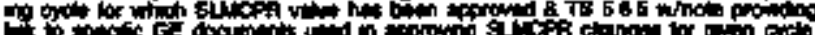

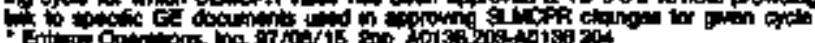

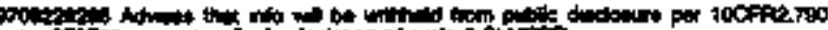

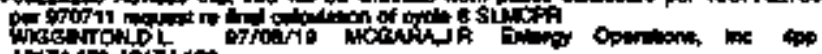
A0174 170,0174 yes

wow

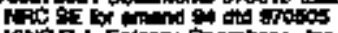

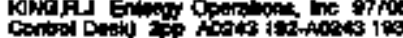




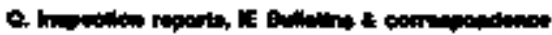

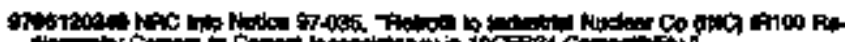

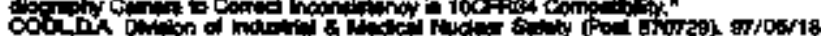

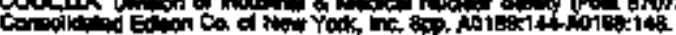

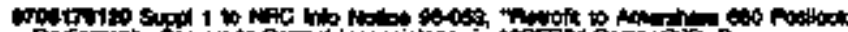
co

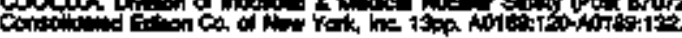

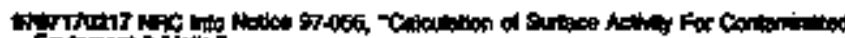

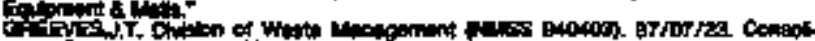

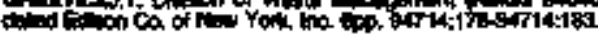

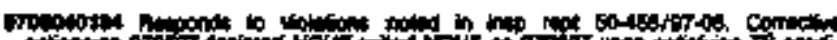

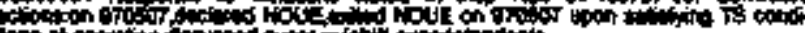

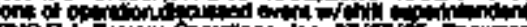

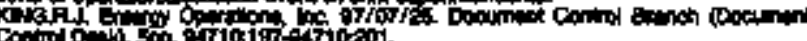

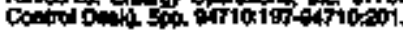

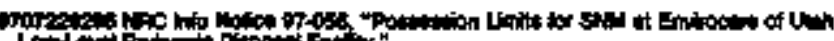

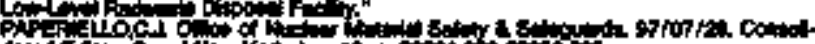

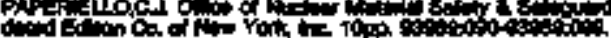

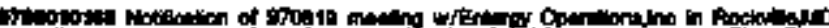

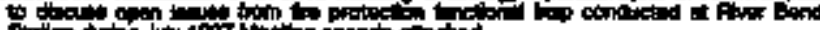

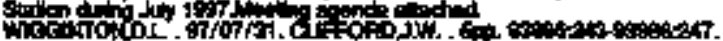

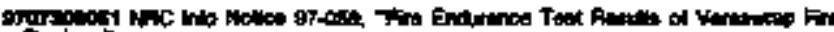

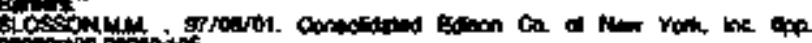

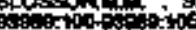

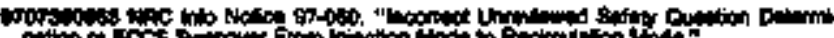

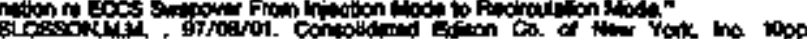

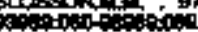

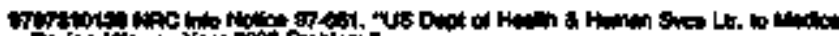

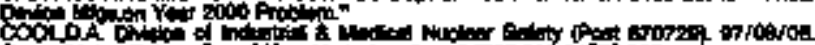

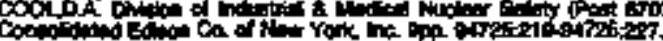

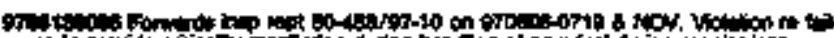

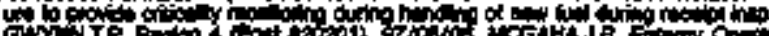

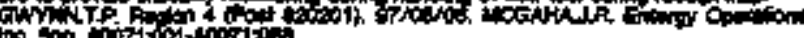

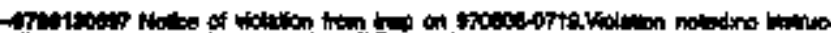

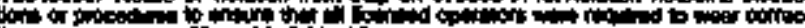

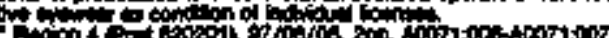

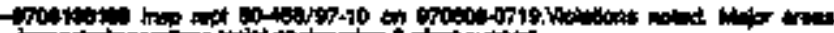

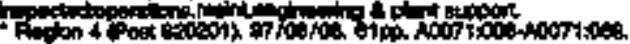

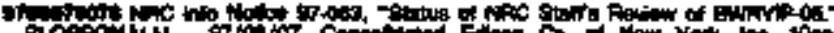

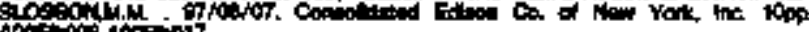

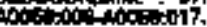

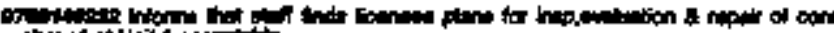

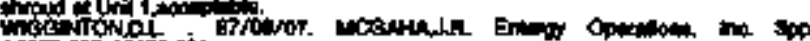

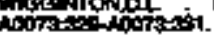

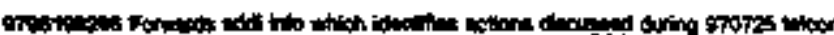

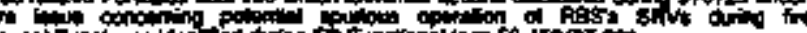

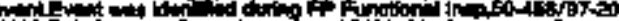

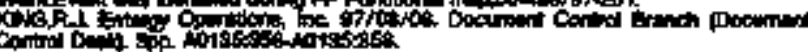

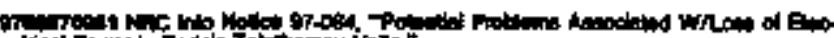

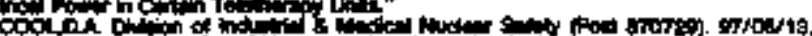

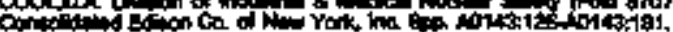

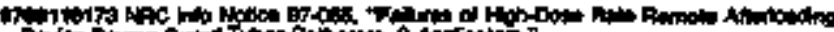

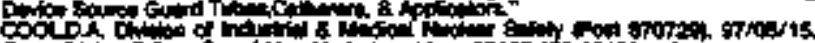

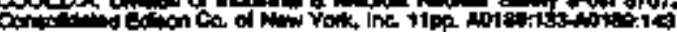

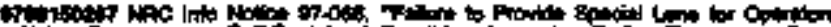
U.

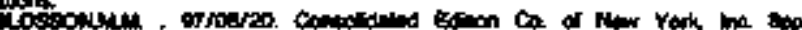

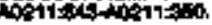

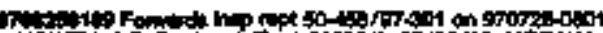

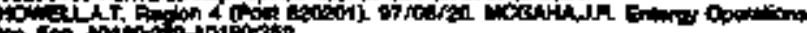

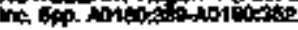

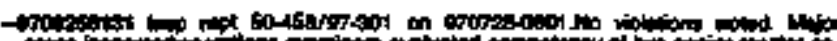

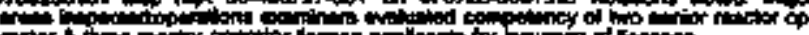

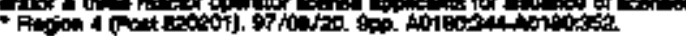

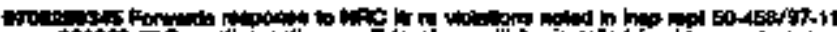

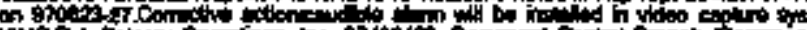

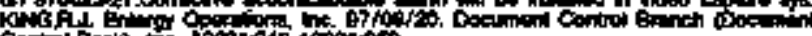

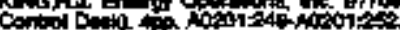

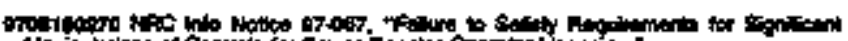

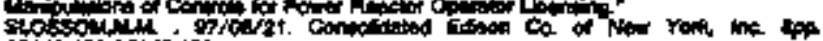

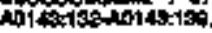

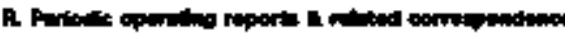

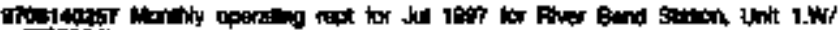

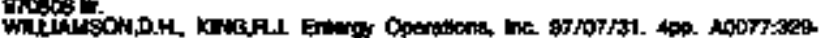
Non 192

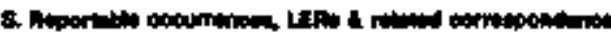

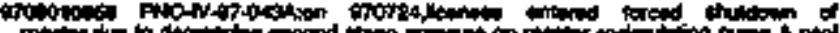

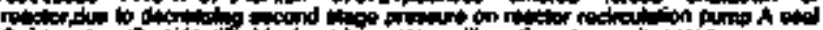

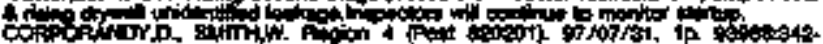

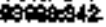

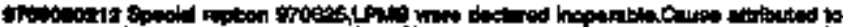

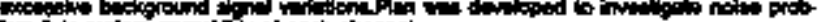

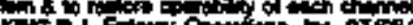

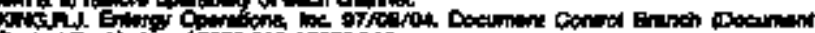

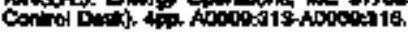

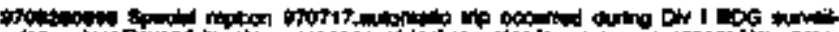

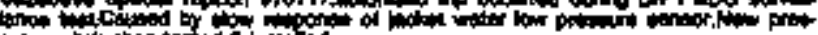

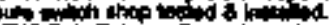

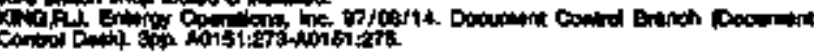

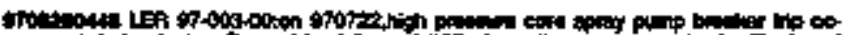

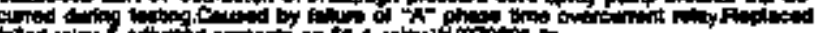

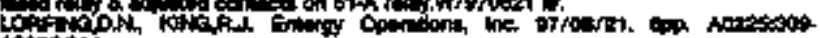
toresing

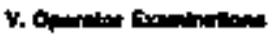

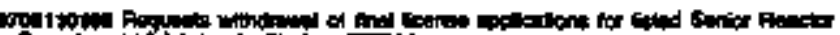
A

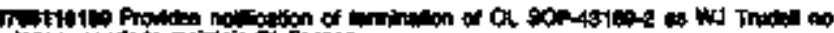

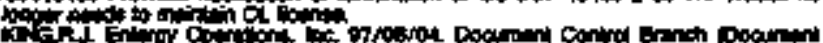

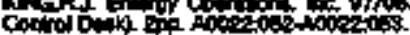

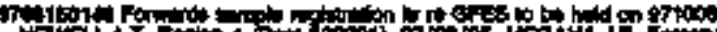

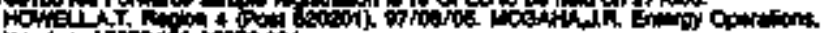

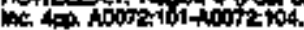

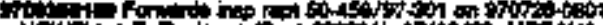

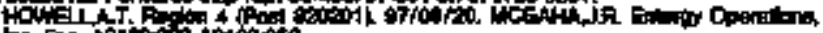

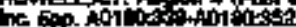

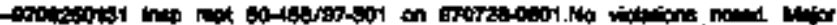

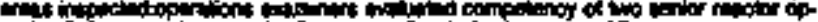

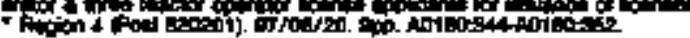

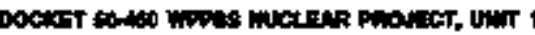

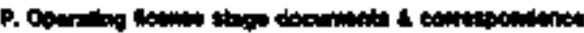

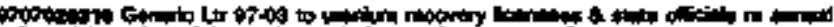

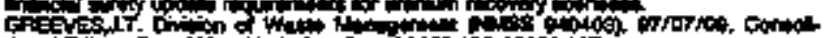

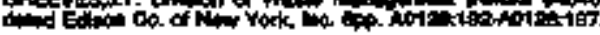

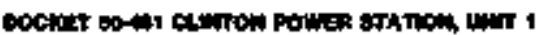

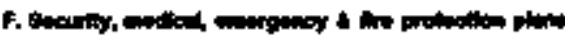

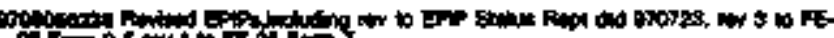

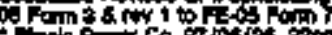

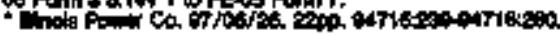

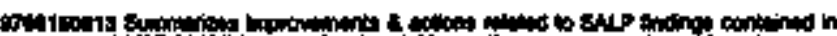

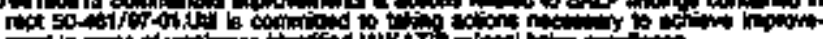

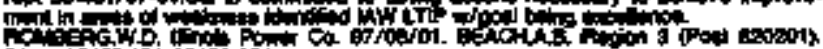

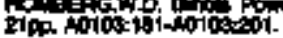

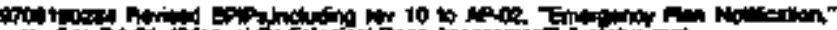

Prs

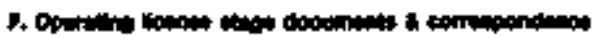

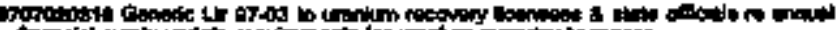

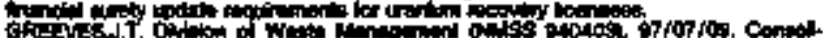

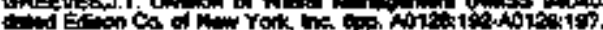




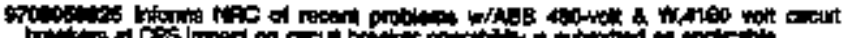
pot.

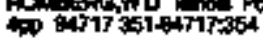

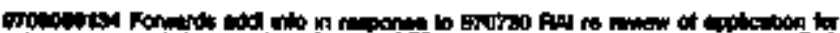

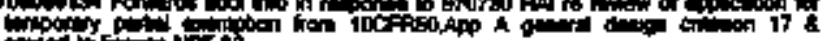

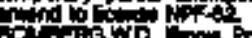

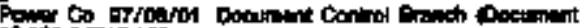
Conion D.

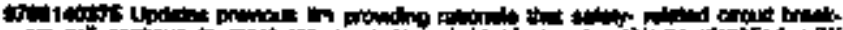

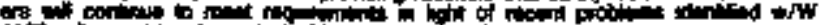
4 to POtso

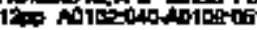

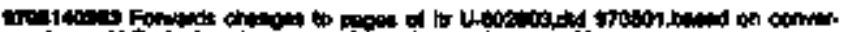

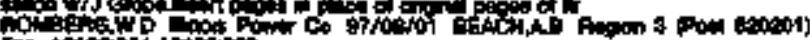

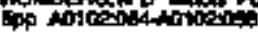

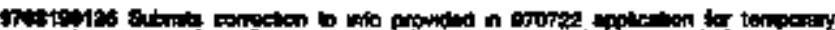

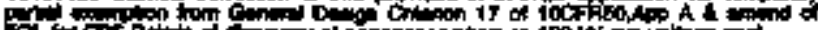

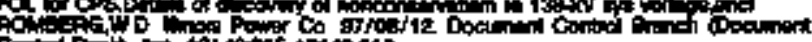

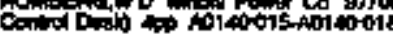

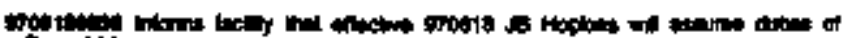

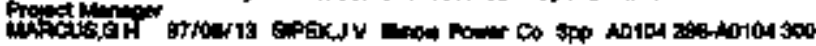

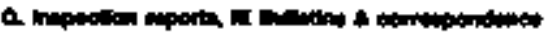

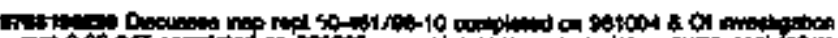

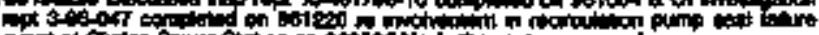

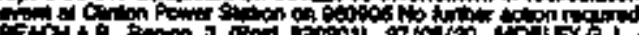

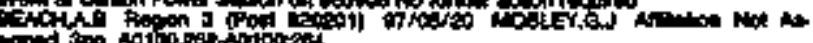

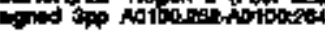

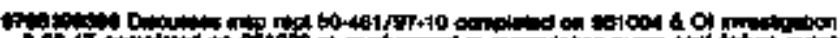

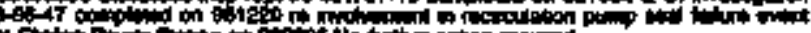

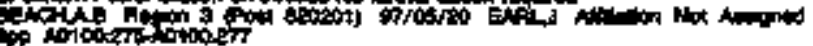

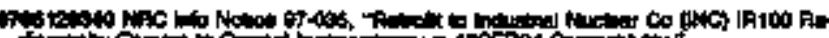

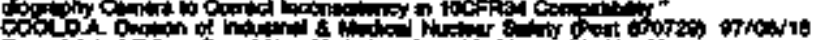

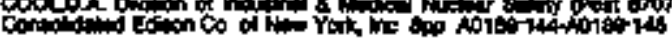

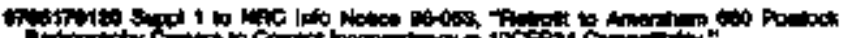

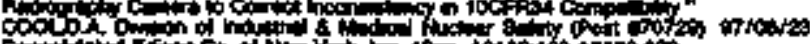
Com

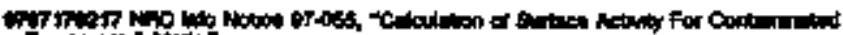
Grit

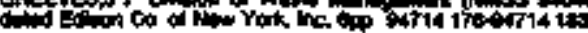

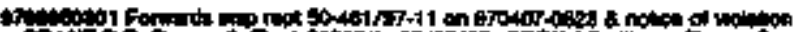

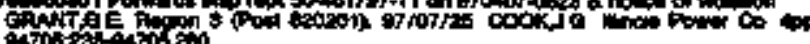

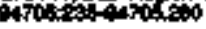

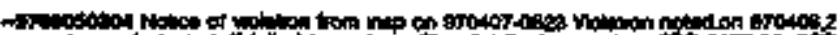

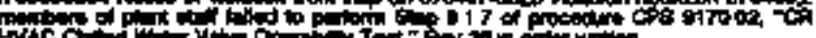

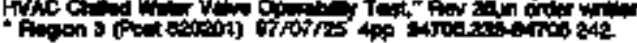

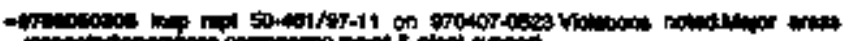

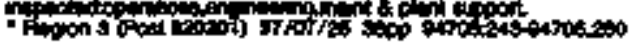

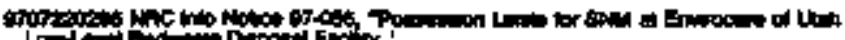

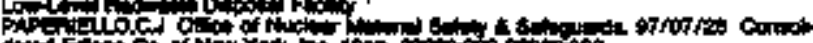

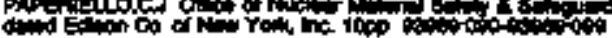

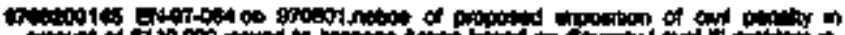

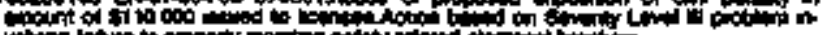

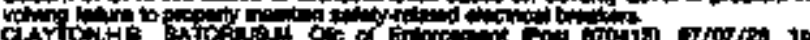

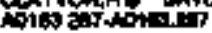

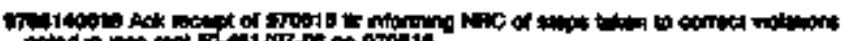
and if

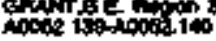

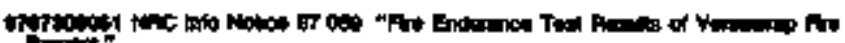

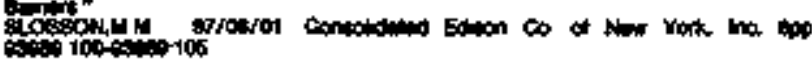

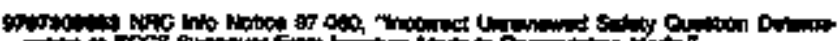

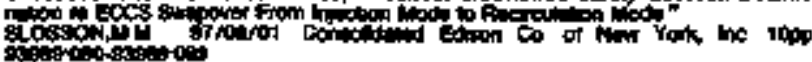

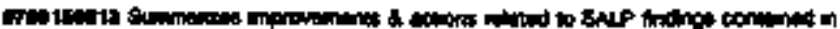

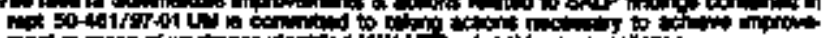

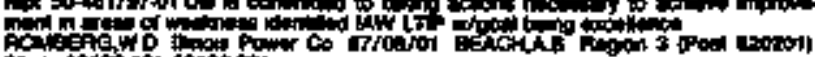

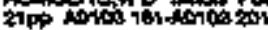

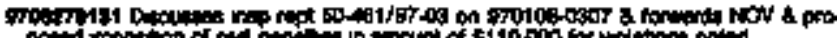

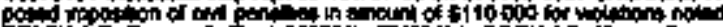

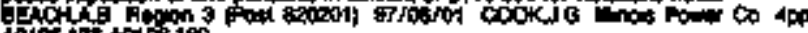
(180

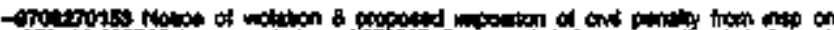

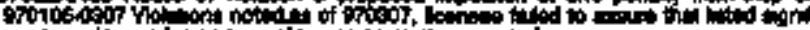

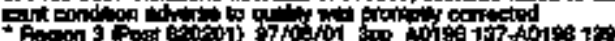

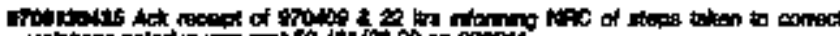

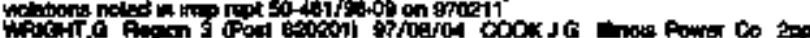

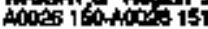

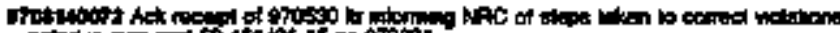

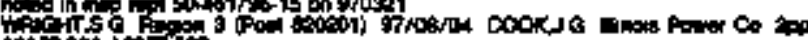

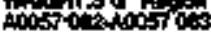

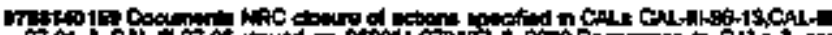

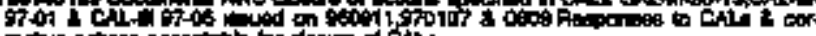

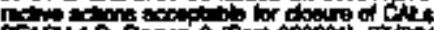

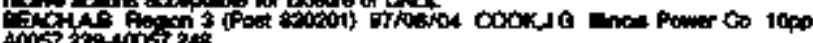
A00s

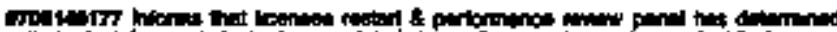

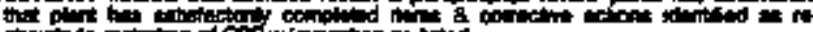

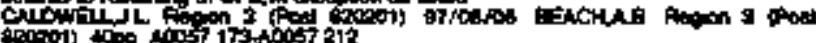

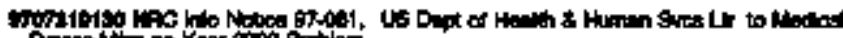

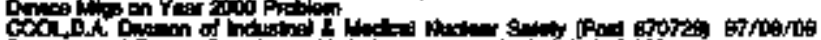

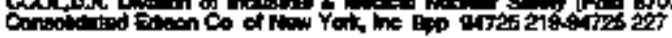

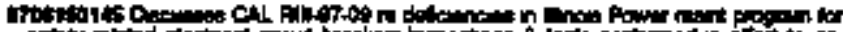

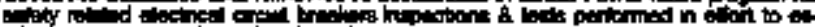

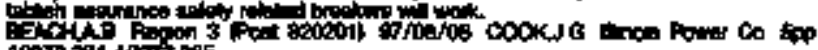

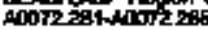

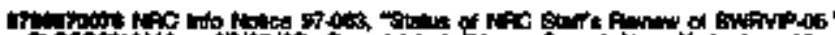

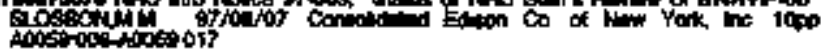

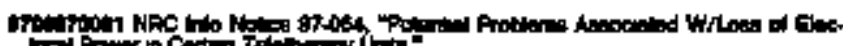

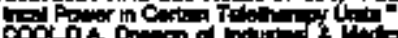

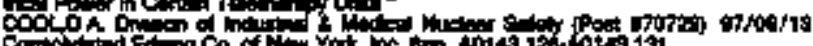

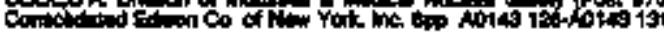

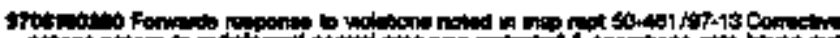

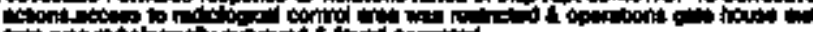

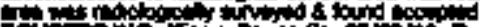

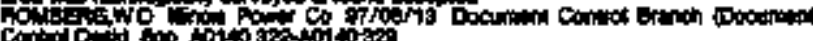

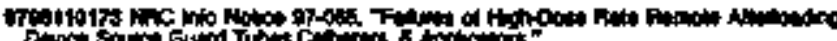

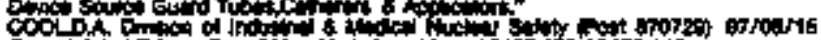

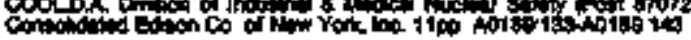

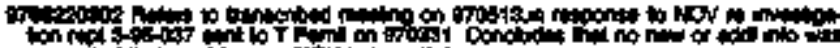

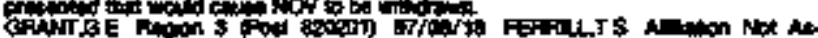

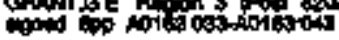

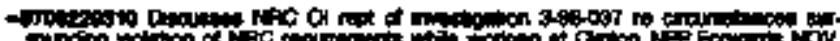

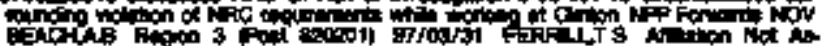

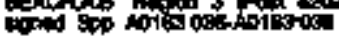

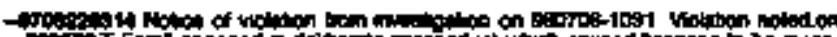

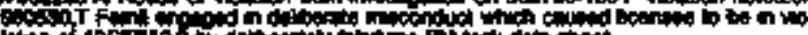

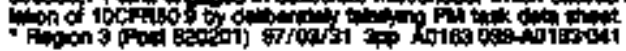

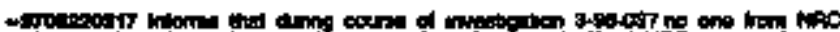

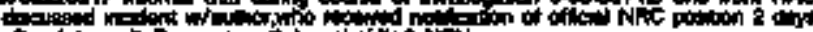

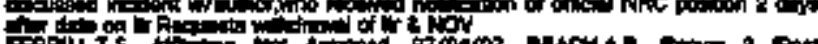

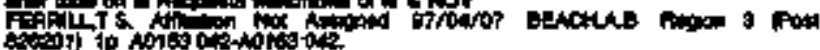

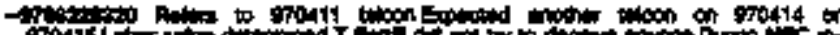
90415 L

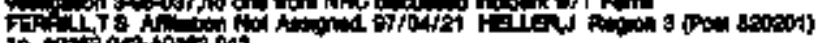

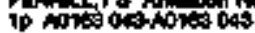

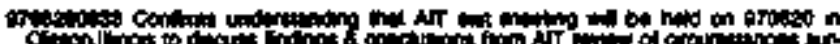

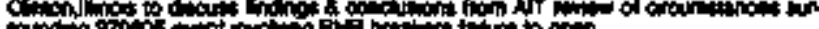
chini

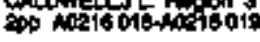

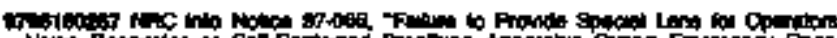

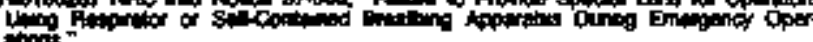

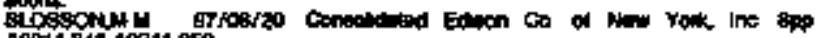
A0P' 1 \%4 10211 950

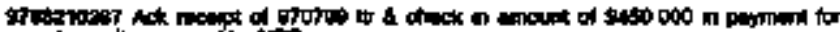

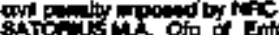

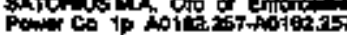




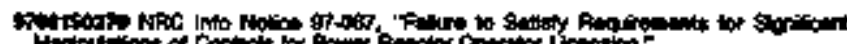

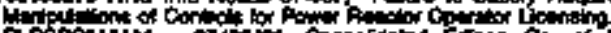

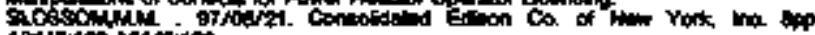

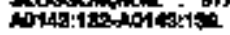

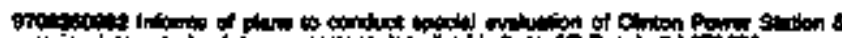

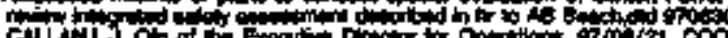

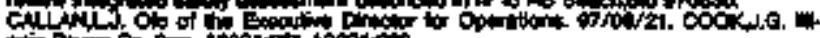

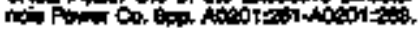

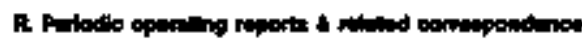

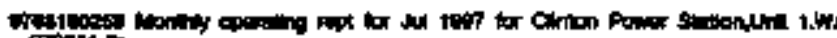

Sili11 :

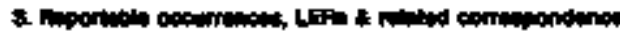

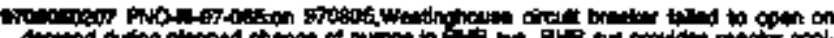

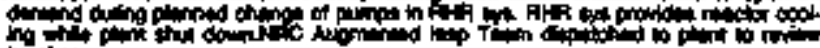

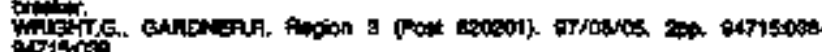

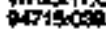

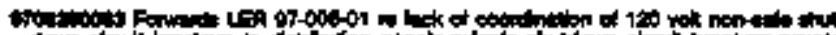

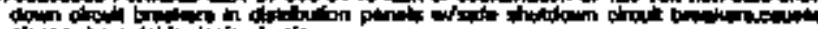

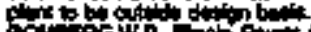

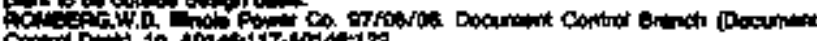

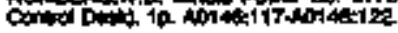

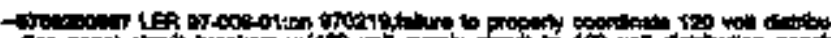

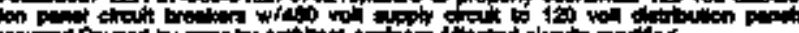

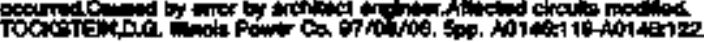

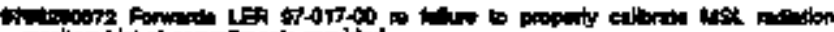

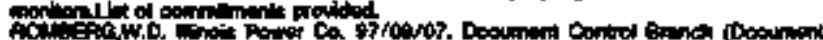

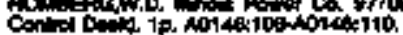

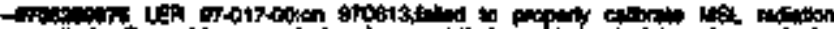

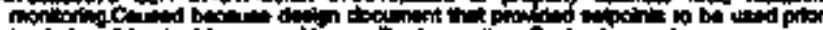

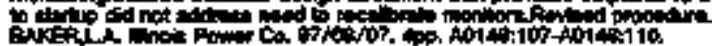

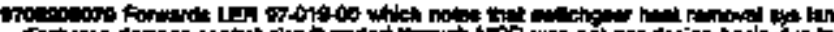

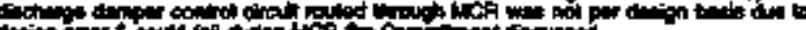

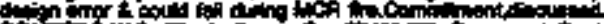

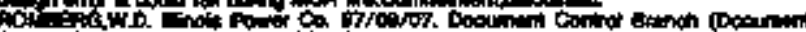

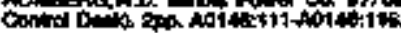

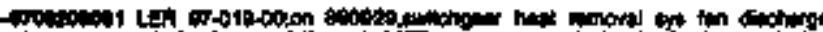

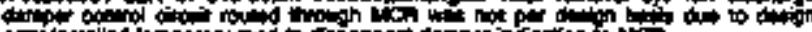

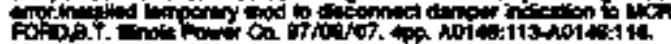

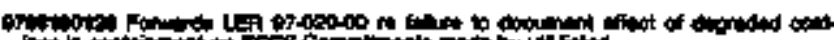

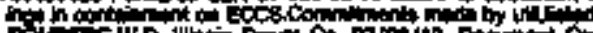

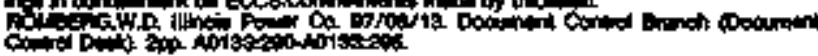

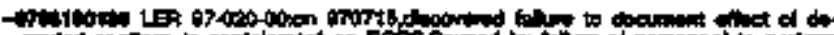

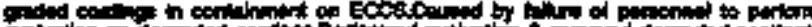

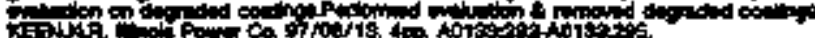

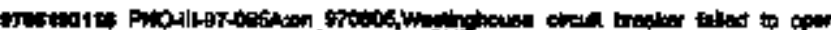

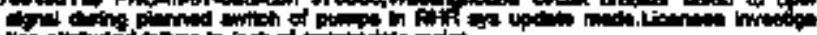

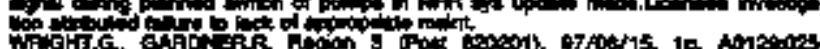
Wistis.

\section{Y. Orner Grointan}

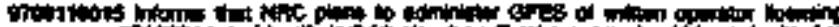
on m in

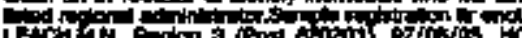

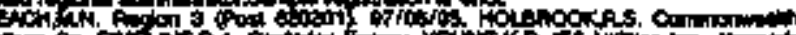

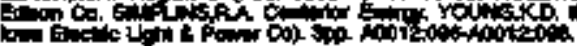

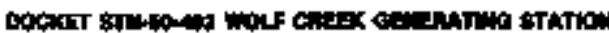

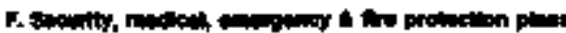

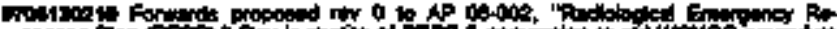
oponed fin of

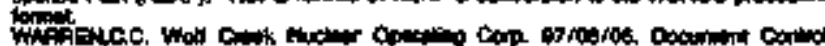

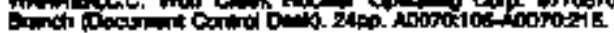

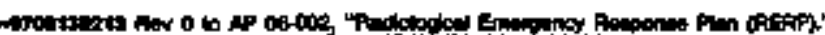

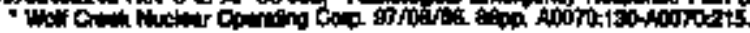

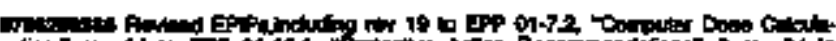

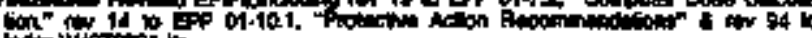

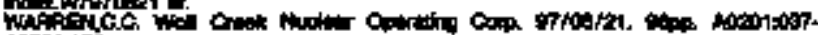
Anevi:tis

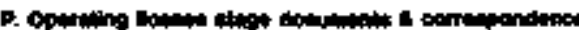

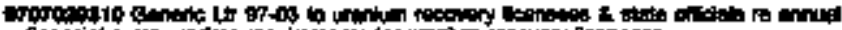

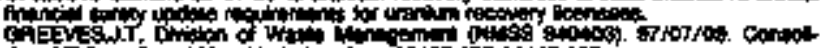

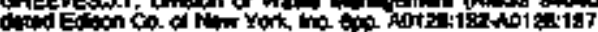

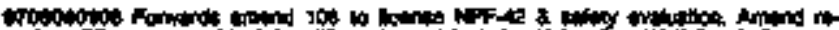

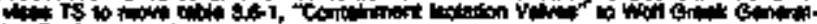

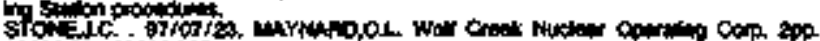

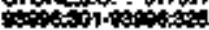

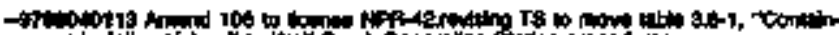

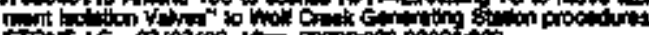

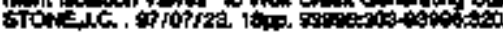

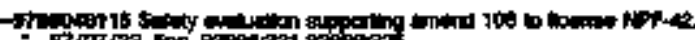

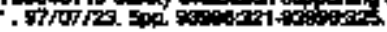

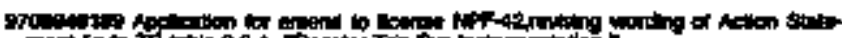

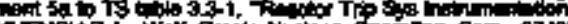

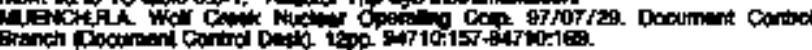

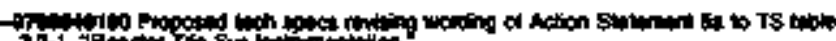

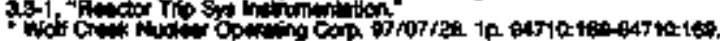

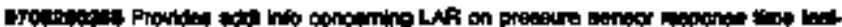

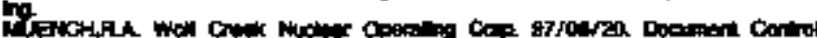

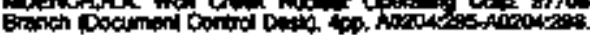

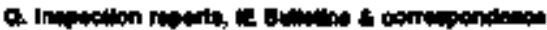

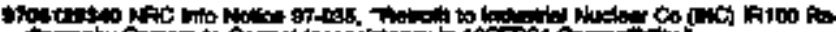

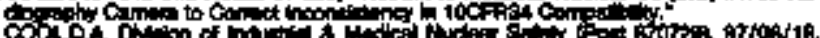

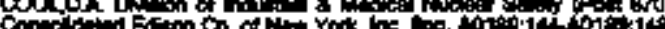

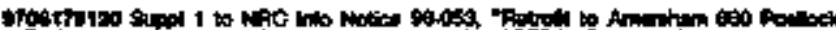

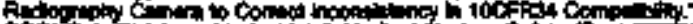

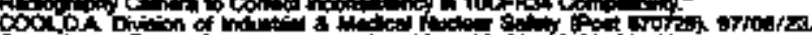

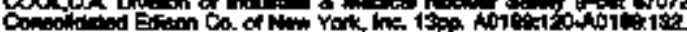

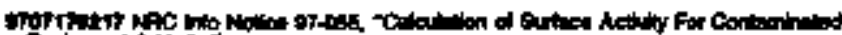

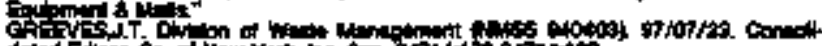

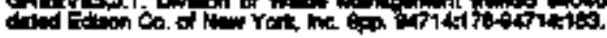

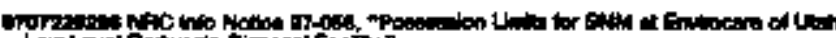

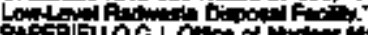

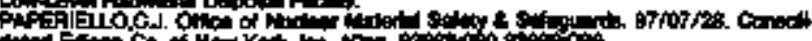

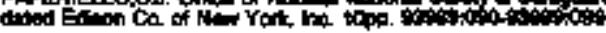

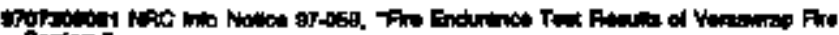

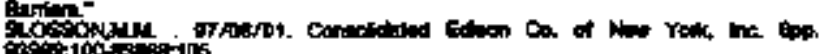

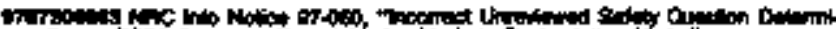

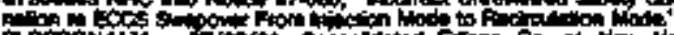

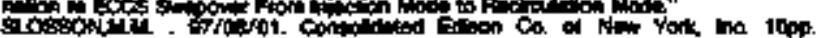

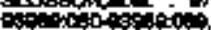

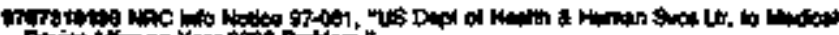

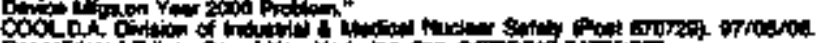
con

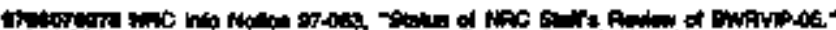

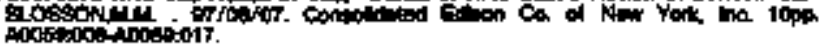

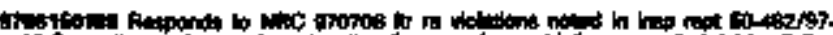

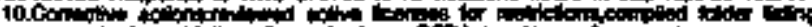

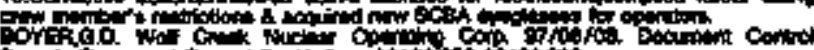

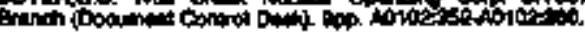

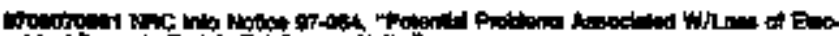

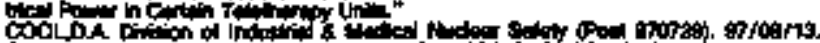

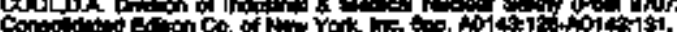

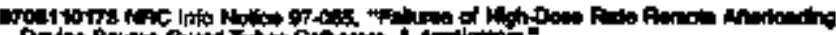

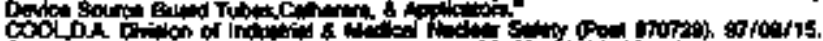

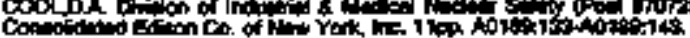

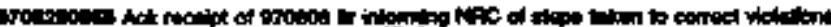

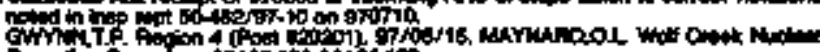

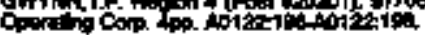

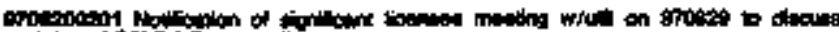

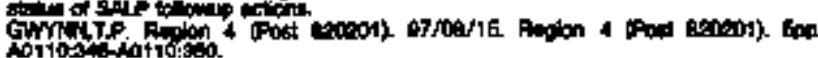

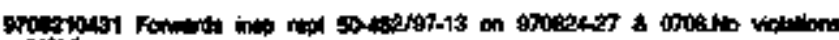

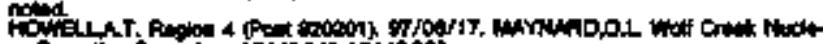

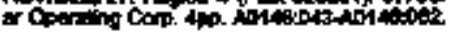




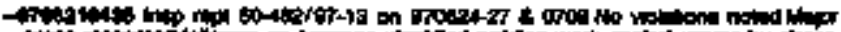

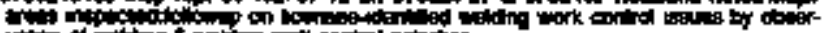

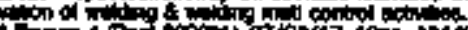

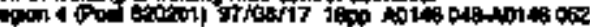

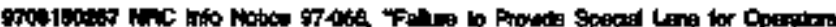

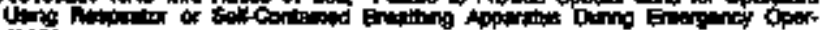

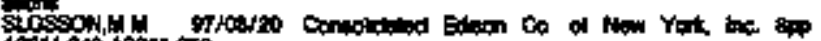
A011 34

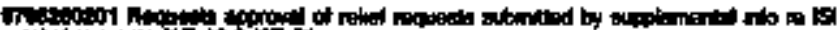
int

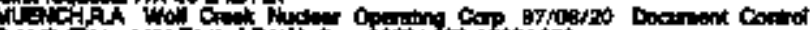

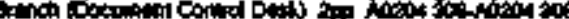

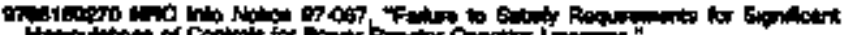

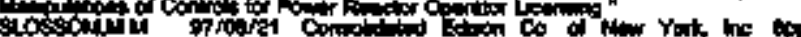

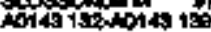

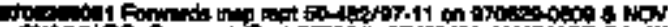

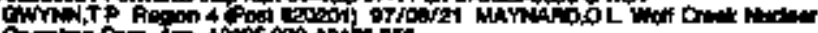

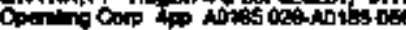

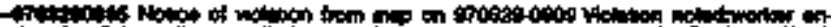

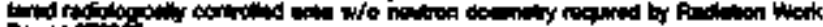
Peningog.

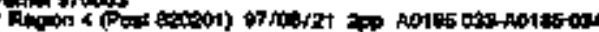

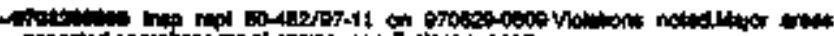

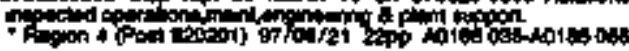

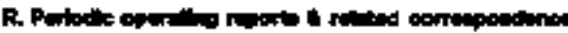

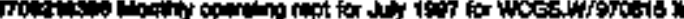

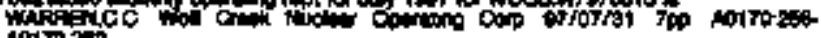

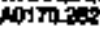

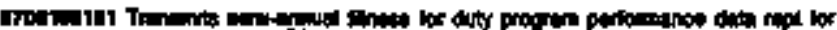

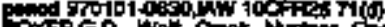

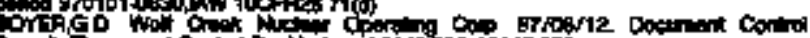

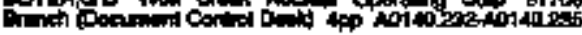

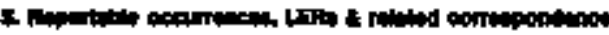

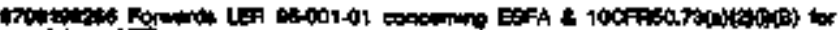

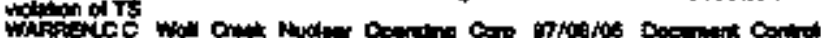

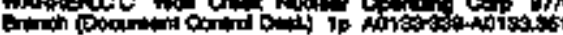

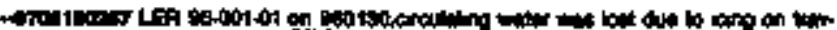

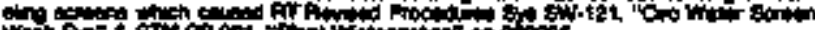

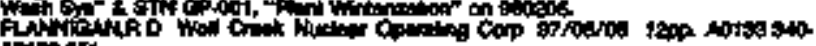

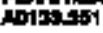

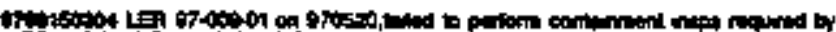

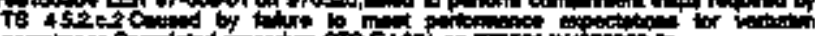

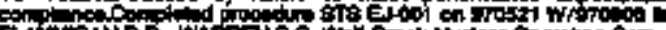

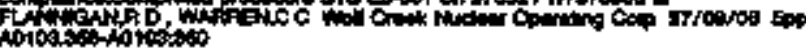

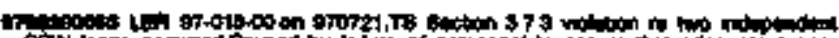

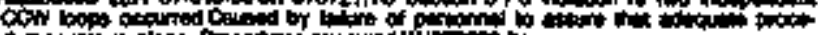

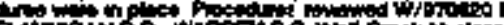

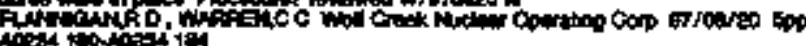

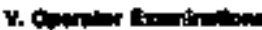

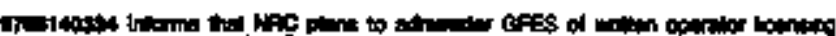

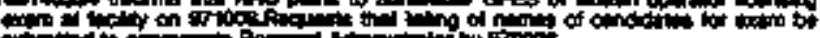

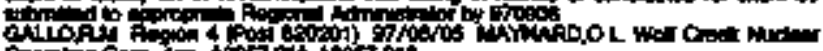

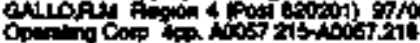

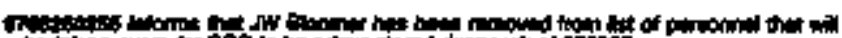

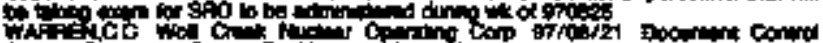

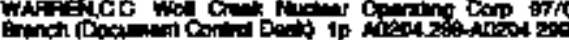

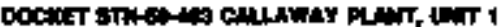

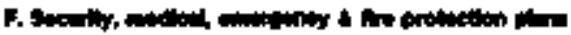

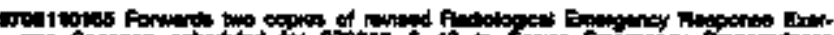

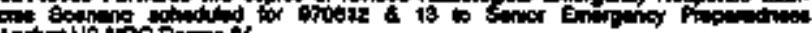
A

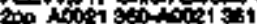

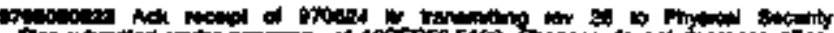

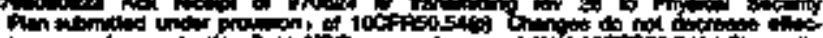

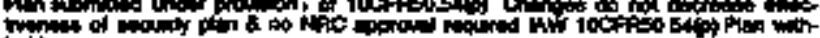
nild.

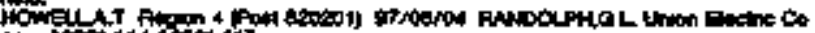
thp thotet 114 incet 19

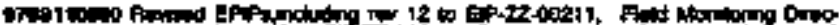

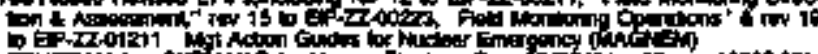

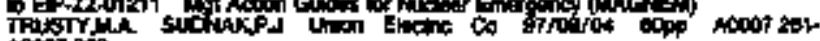
Alotion

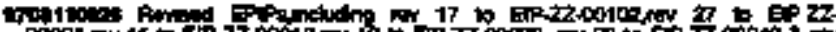

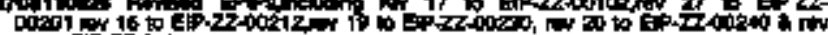
1970 to

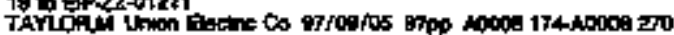

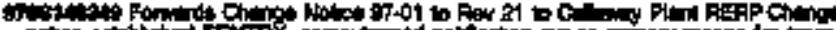

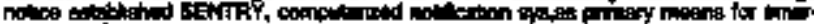

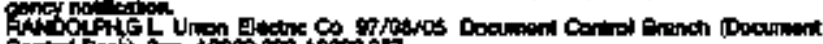

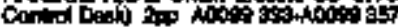

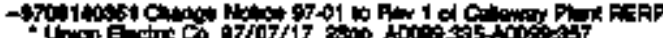

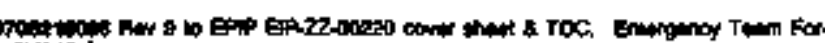

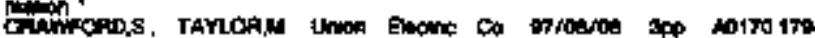
AOAT ist

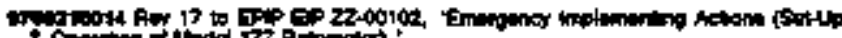

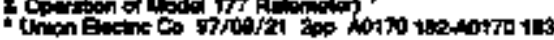

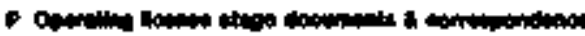

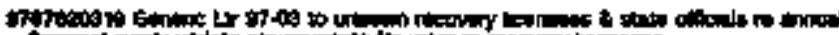

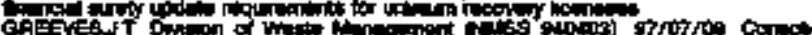
GAE

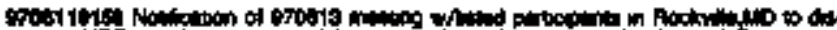

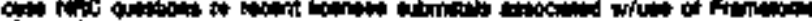

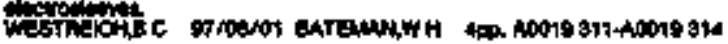

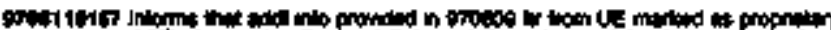

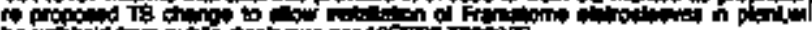

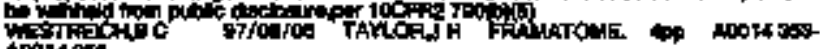
toold sos

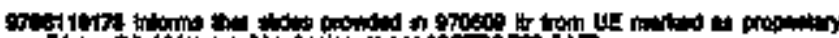
Whe $x_{1000} \rightarrow 00$

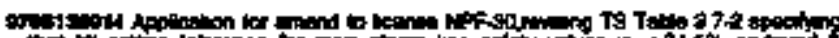

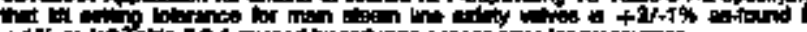

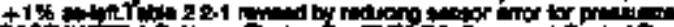
Conch

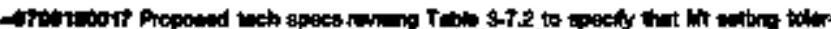

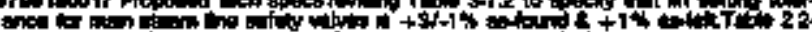

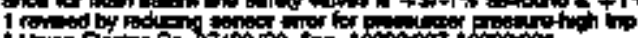

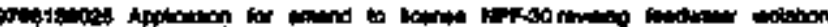

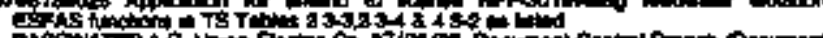

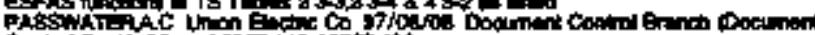

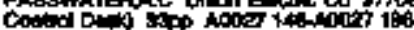

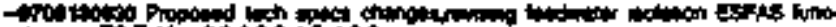

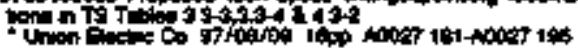

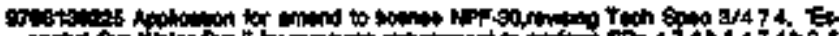

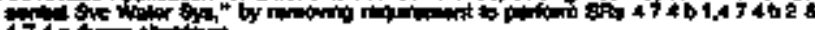
Pison

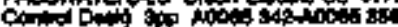

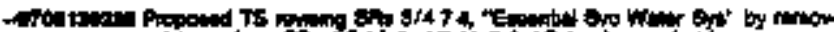

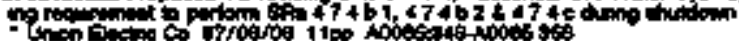

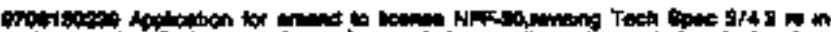

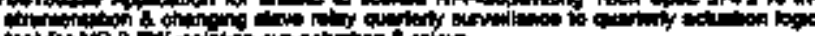

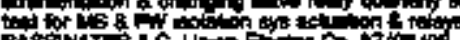

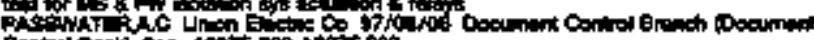

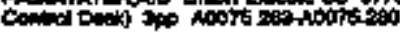

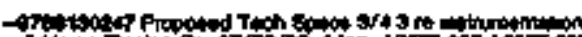

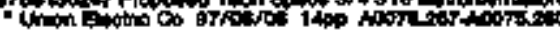

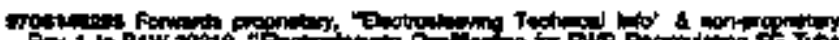

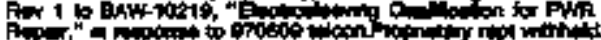

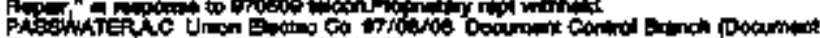

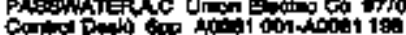

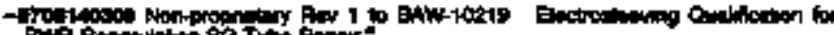

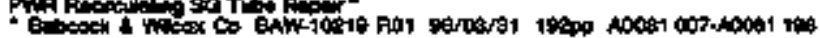

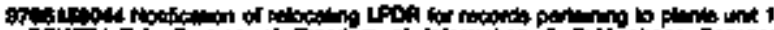

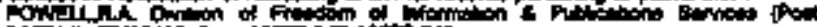

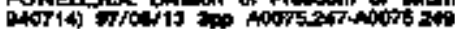




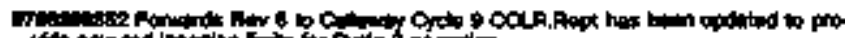

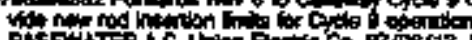

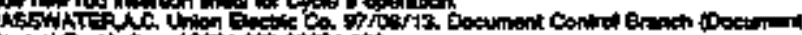

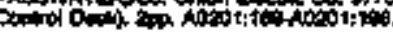

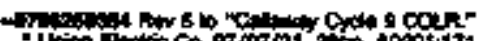

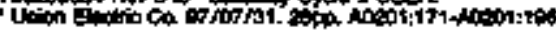

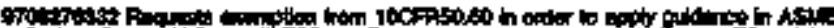

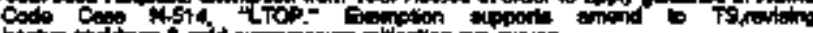
Pet Cond on 40 .

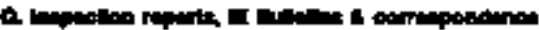

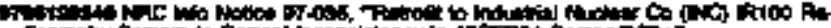

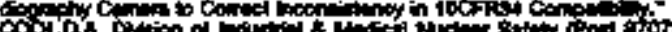

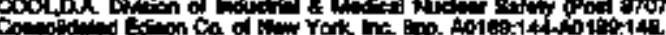

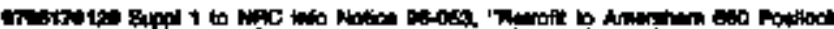

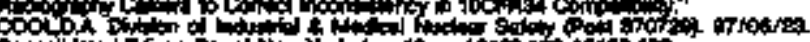
os

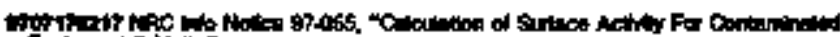

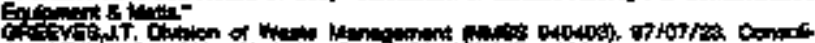

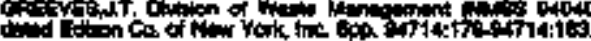

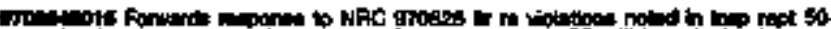

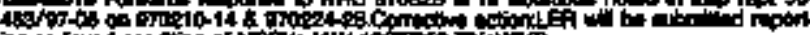

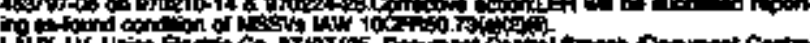

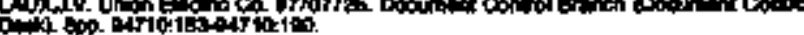

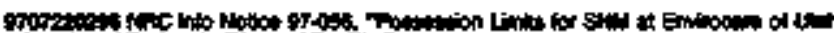

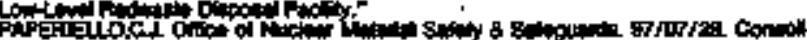

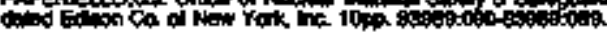

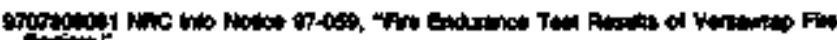

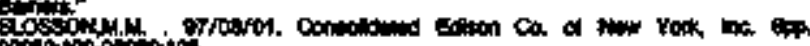

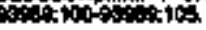

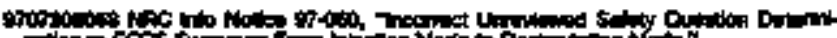

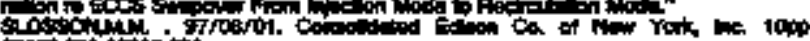
sing

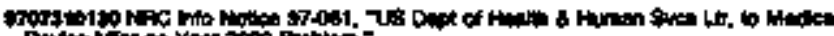

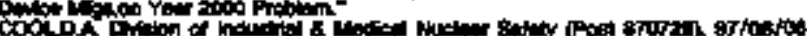

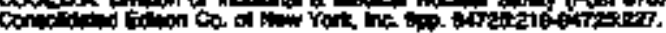

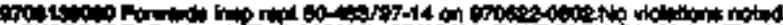

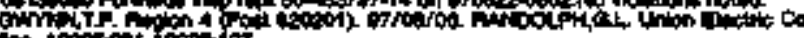

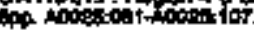

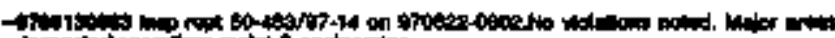

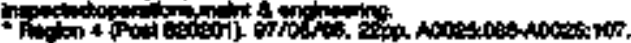

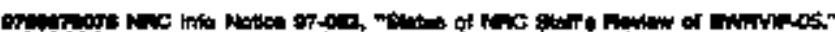

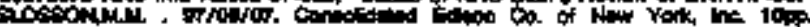

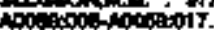

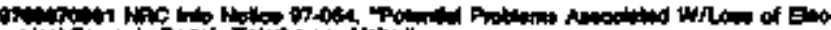

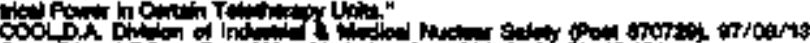

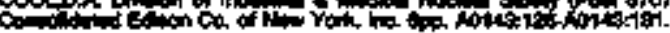

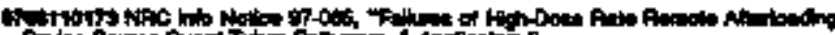

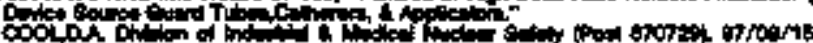

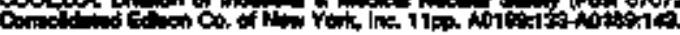

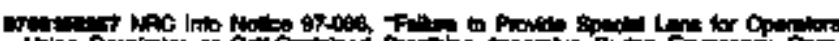

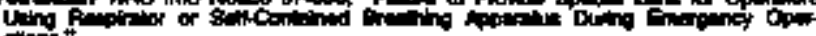

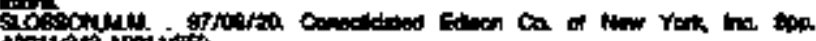

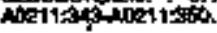

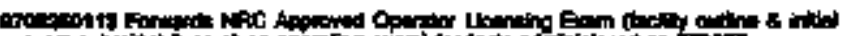

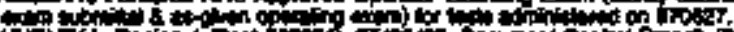

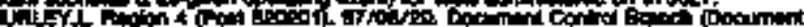

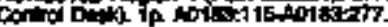

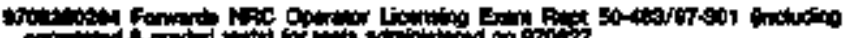

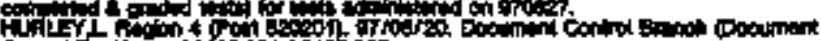

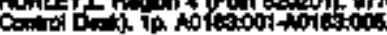

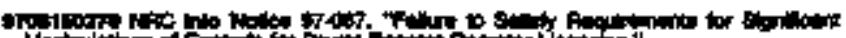

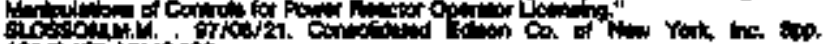

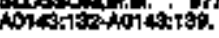

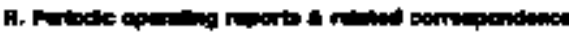

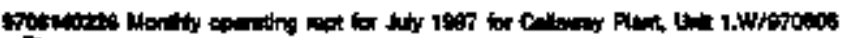

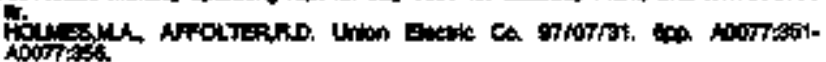

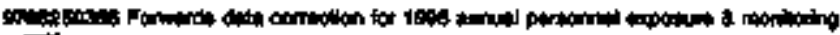

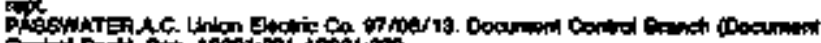

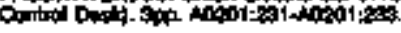

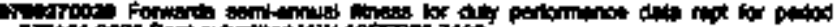

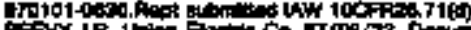

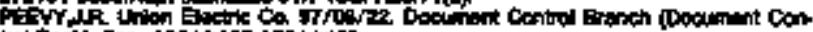

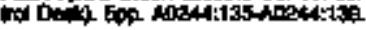

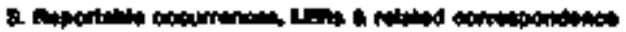

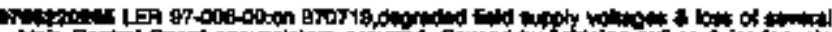

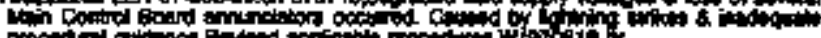

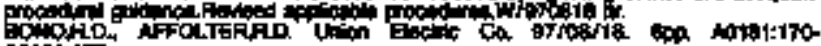

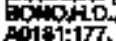

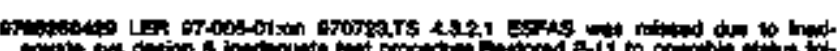

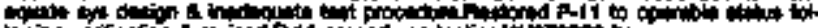

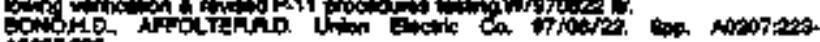
atorion.

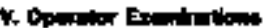

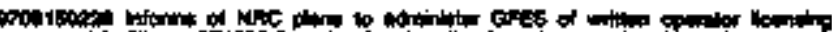

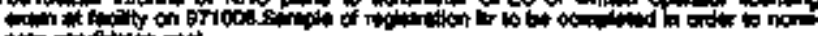

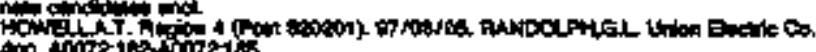

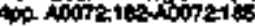

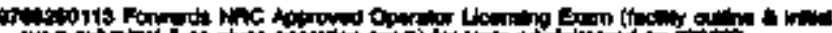

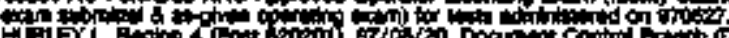

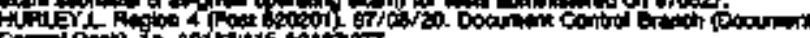

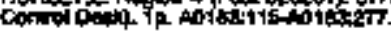

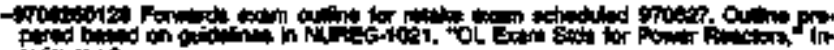

il 7 op

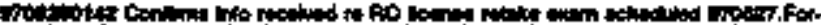

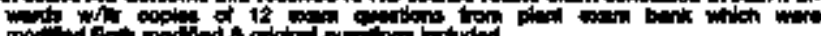

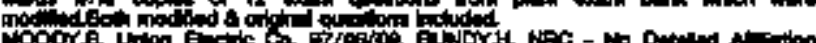

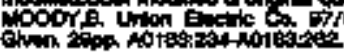

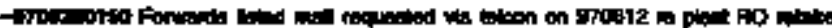

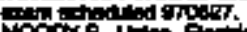

intion

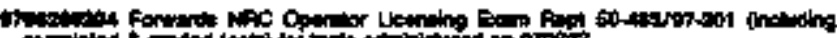

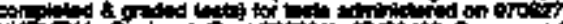

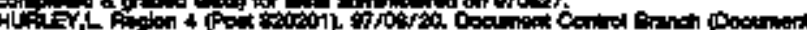

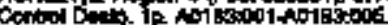

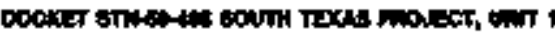

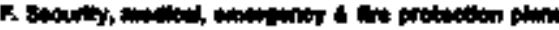

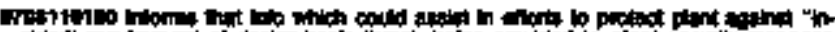

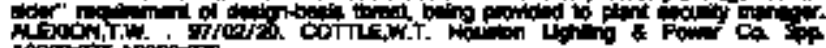

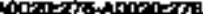

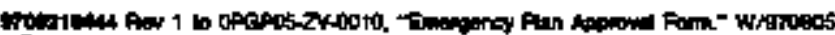

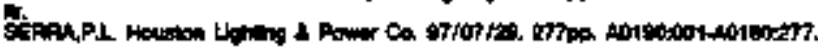

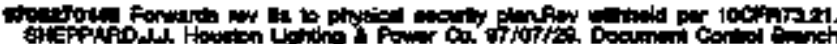

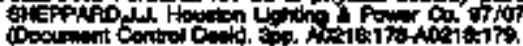

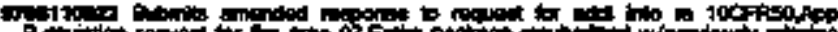

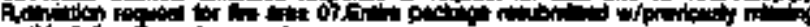

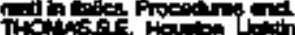

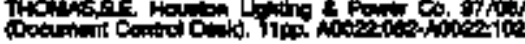

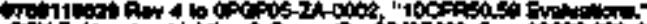

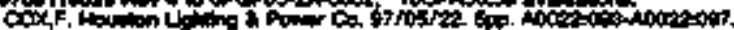

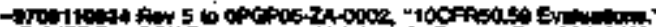

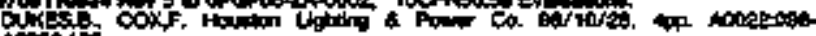
$1002=100$

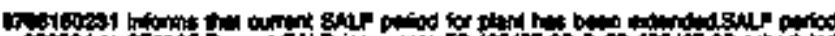

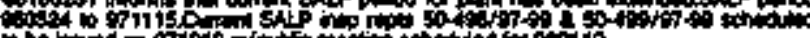

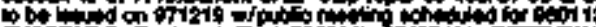

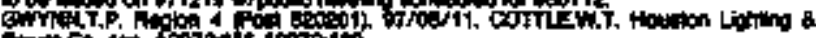

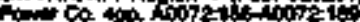




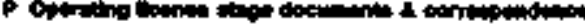

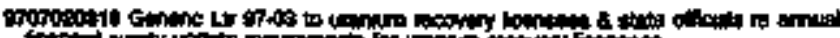

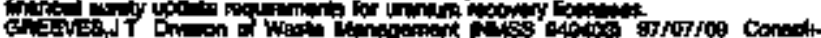

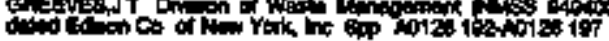

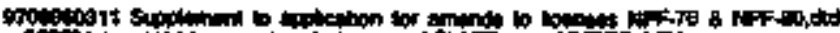

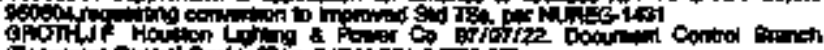

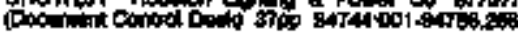

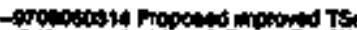

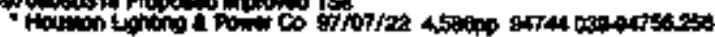

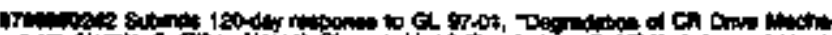

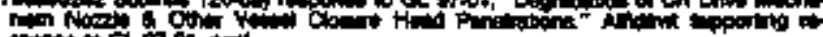
cont

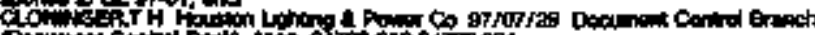

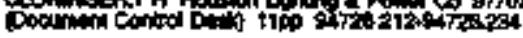

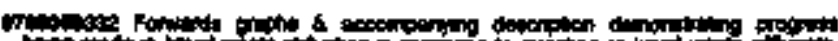

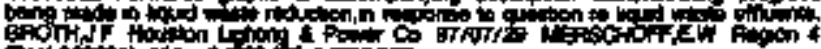

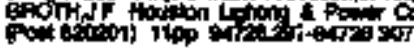

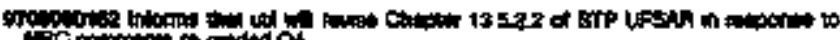

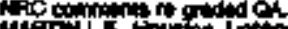

Monim

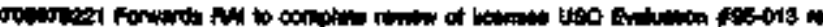

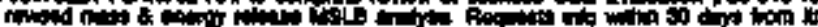

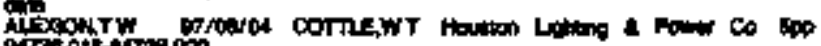
onsotiontion

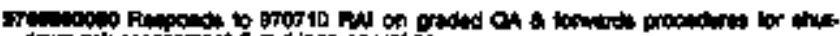

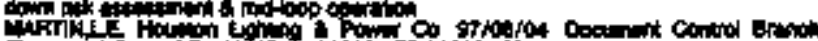

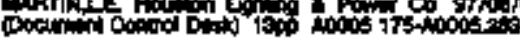

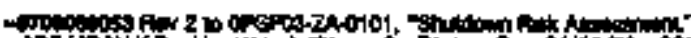

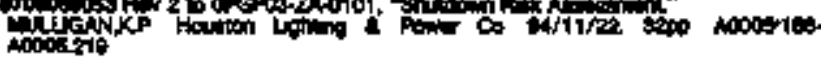

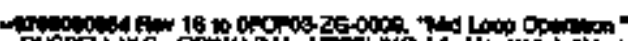
is on

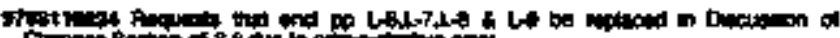

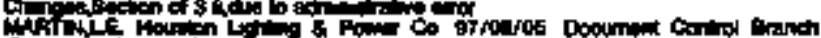

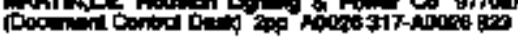

- OH

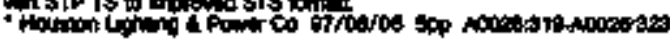

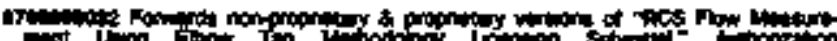

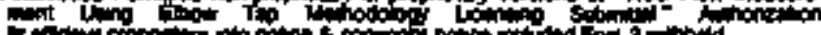

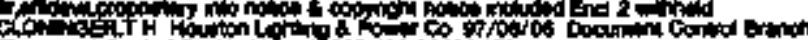

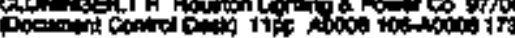

Cof

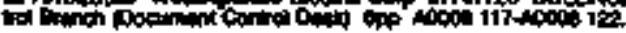

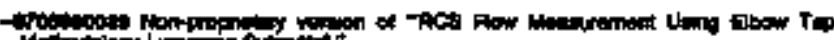

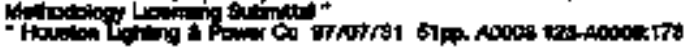

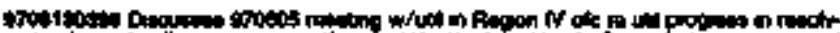

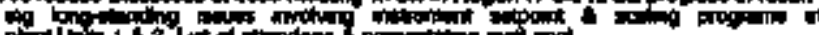

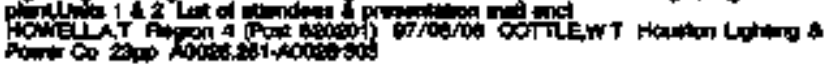

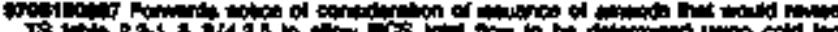

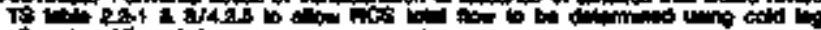

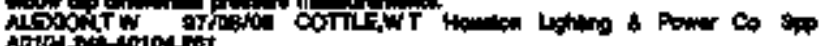

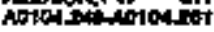

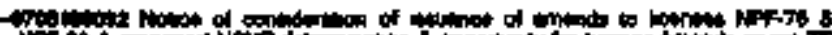

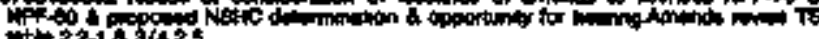

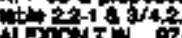

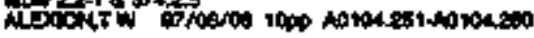

-

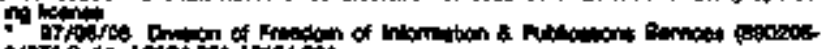

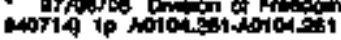

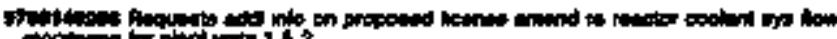

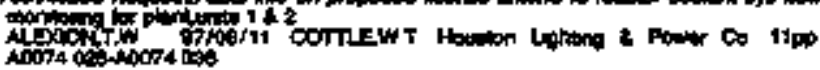

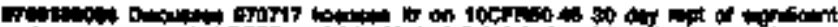
the

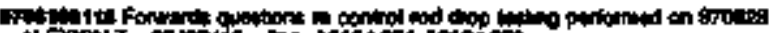

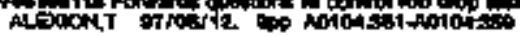

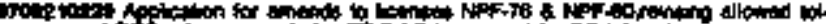

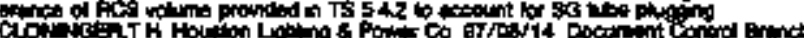

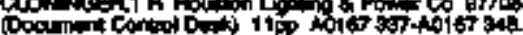

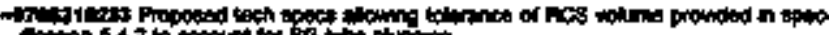

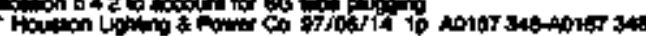

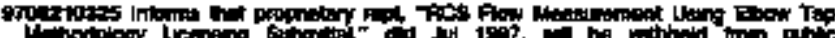

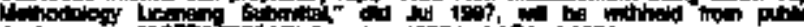

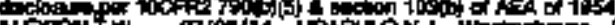

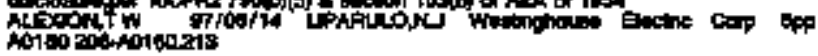

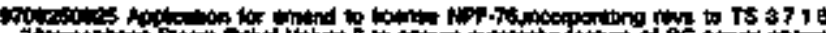

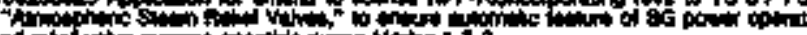
ed

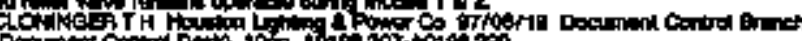

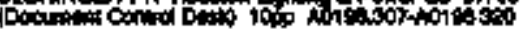

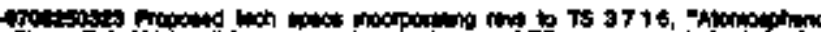

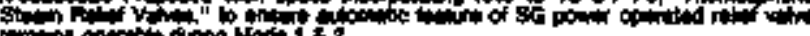

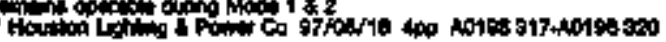

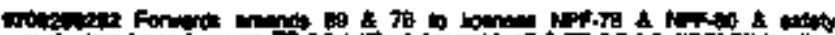

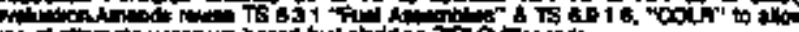

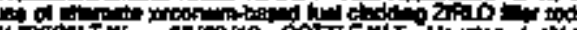

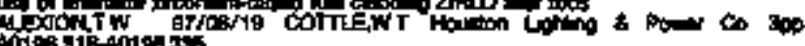

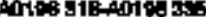

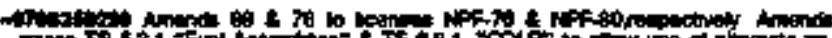

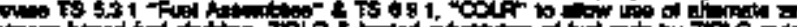

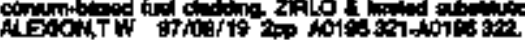

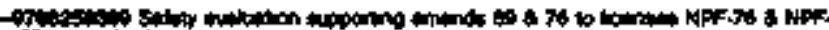

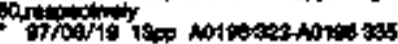

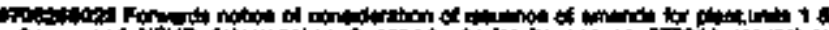

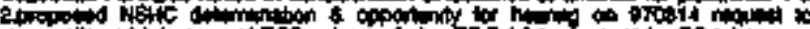

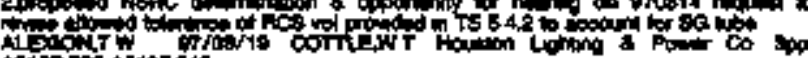

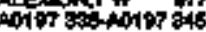

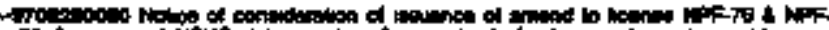

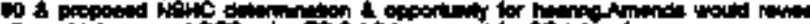

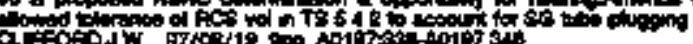

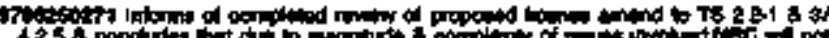

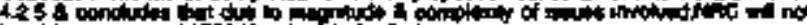

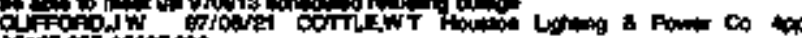

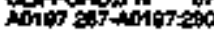

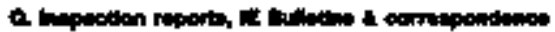

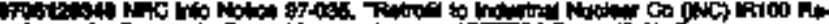

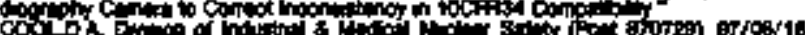

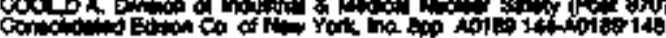

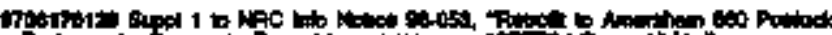

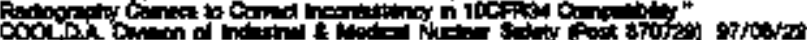

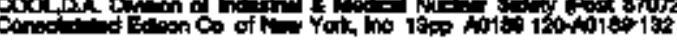

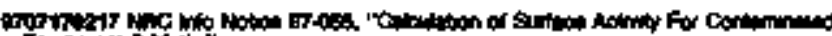

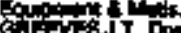
:

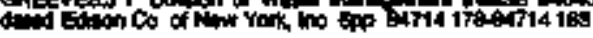

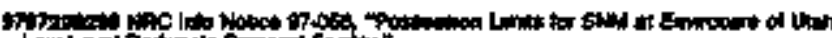
Low

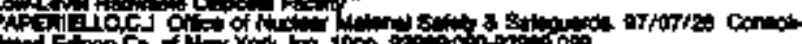

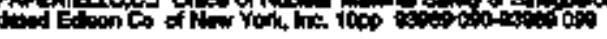

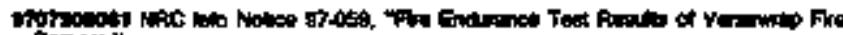
Antrat"

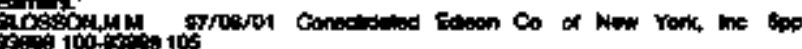

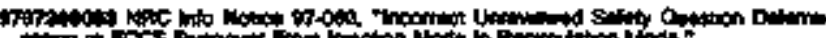

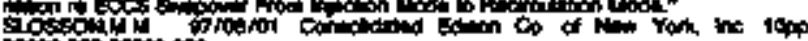

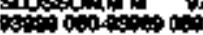

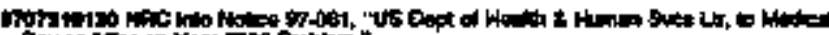

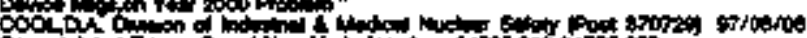

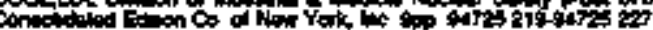

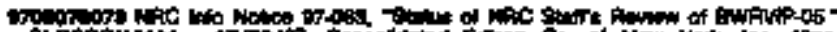

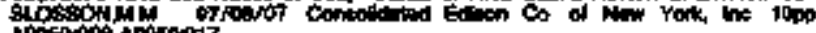
10060 000 A00600'?

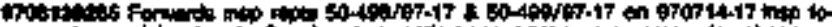

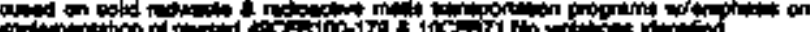

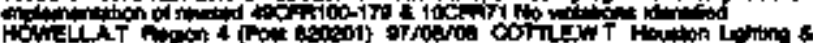

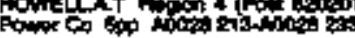




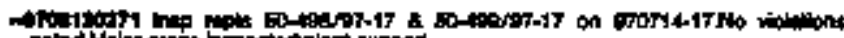

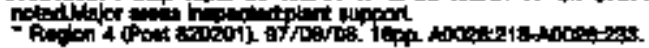

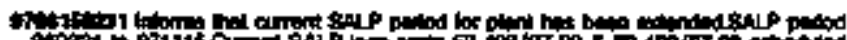

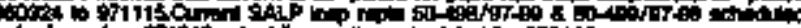

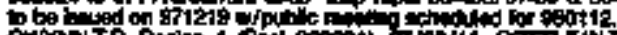

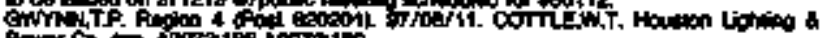

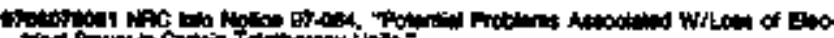

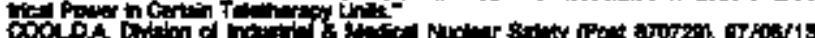

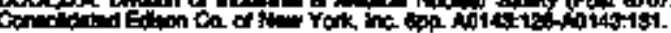

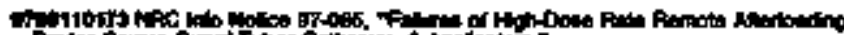

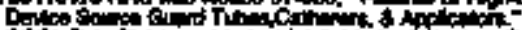

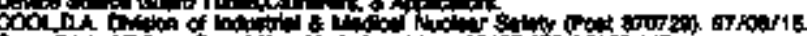

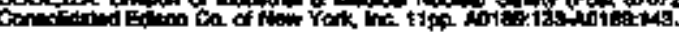

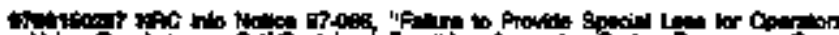

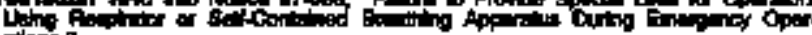

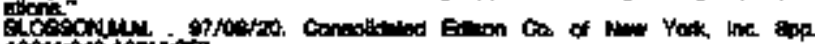
1011

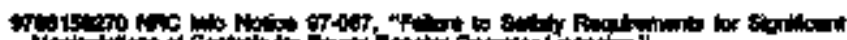

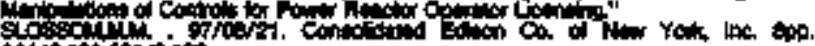

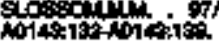

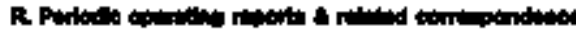

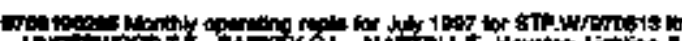

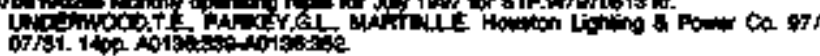

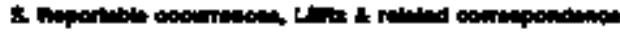

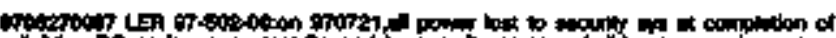

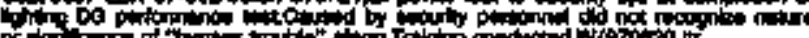

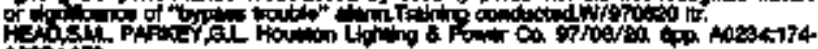
Atation

\section{Oparter Thematione}

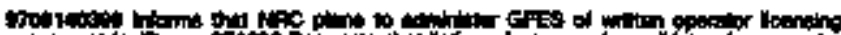

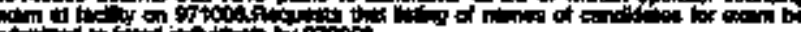

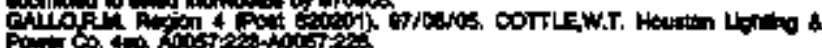

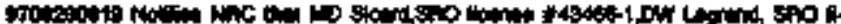

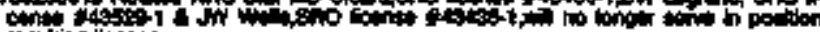

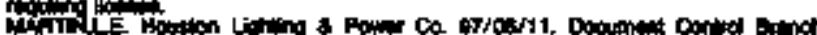

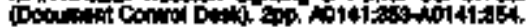

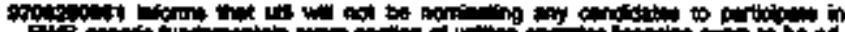

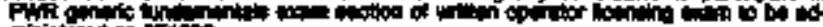

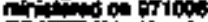

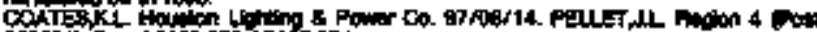

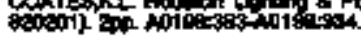

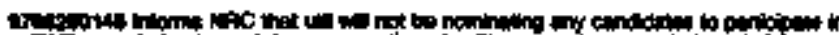

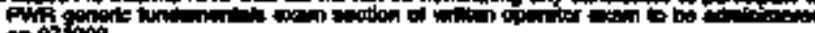

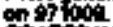

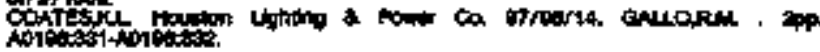

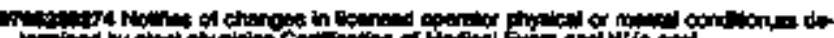
okif

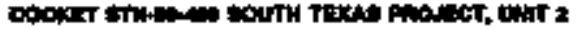

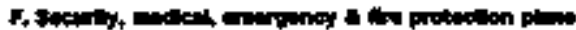

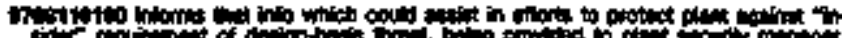

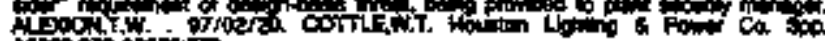

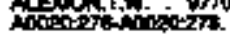

-

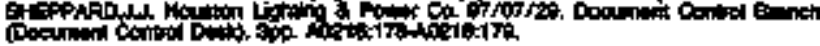

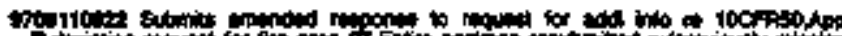
By

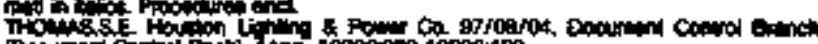

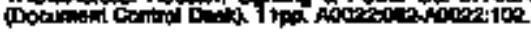

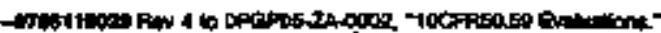

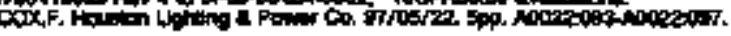

$\rightarrow$ DON Aoction

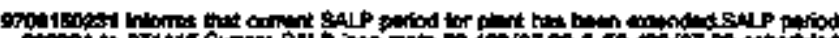

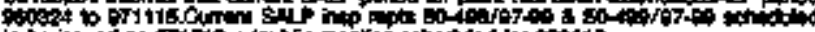

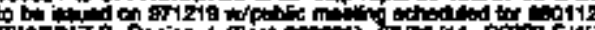

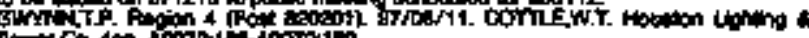

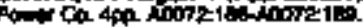

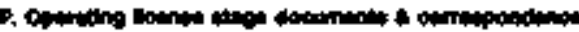

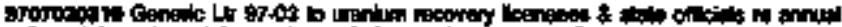

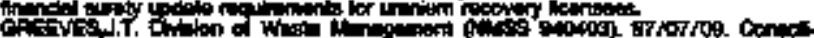

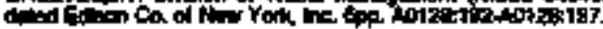

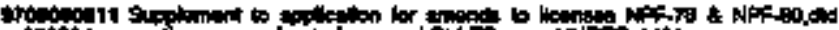

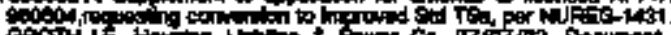

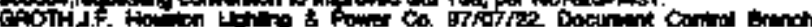

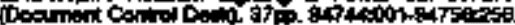

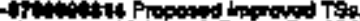

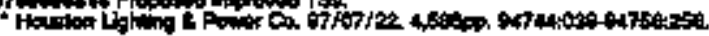

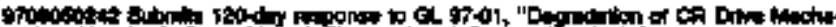

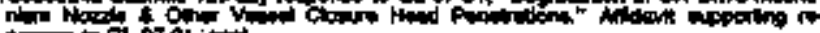
Now

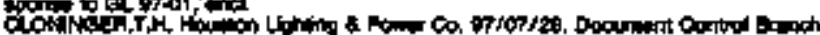

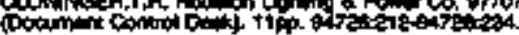

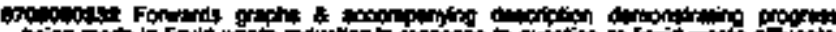

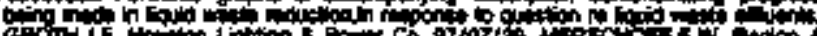

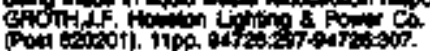

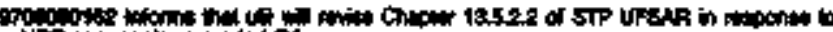
Nhe oction

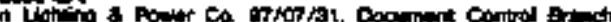

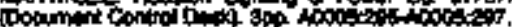

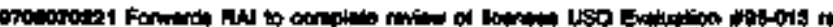

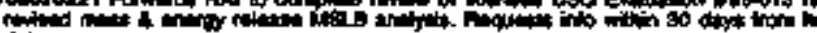

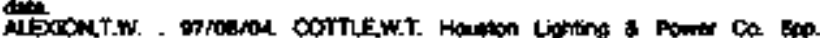

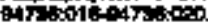

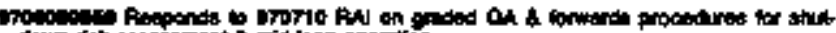

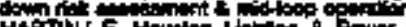

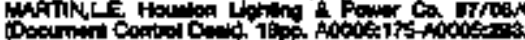

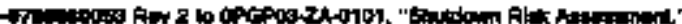

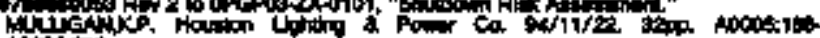
Notodis

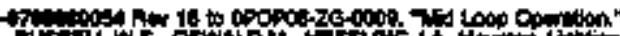

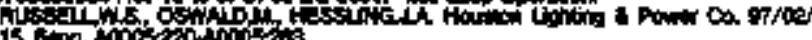

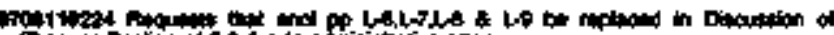
Oth th

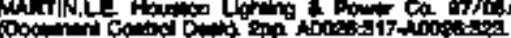

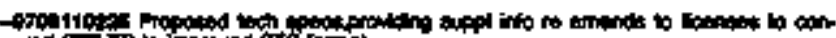

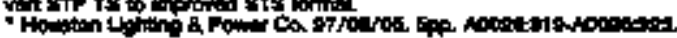

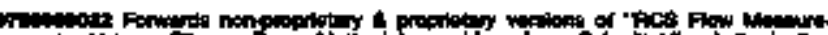

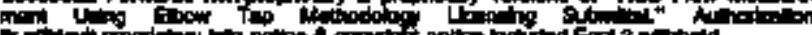

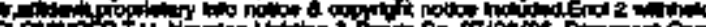

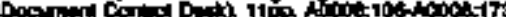

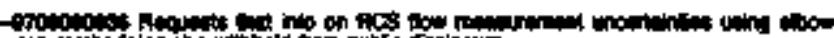

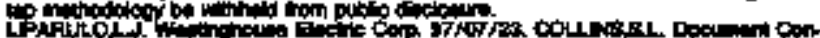

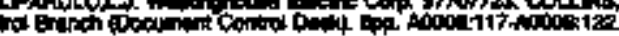

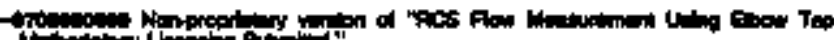

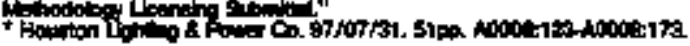

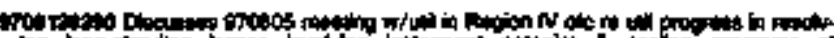

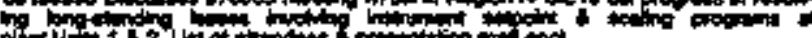
pom

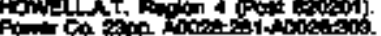

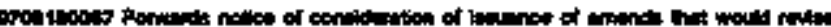

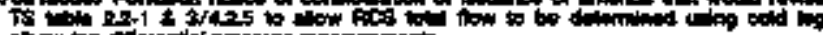

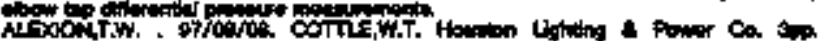
A

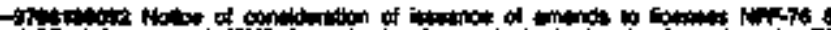

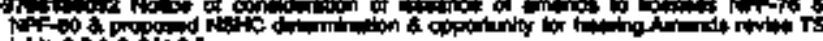

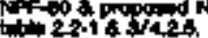

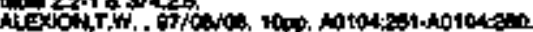

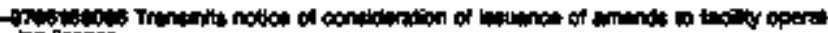

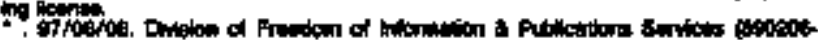

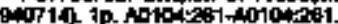




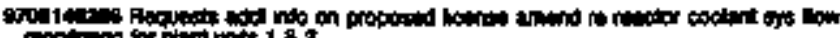

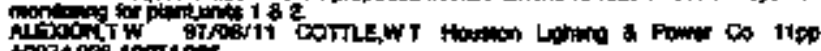
10074020 100 400

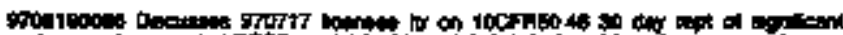

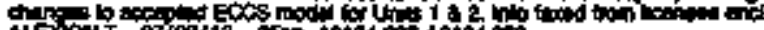
Alf

OM.

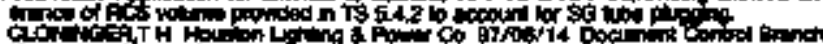

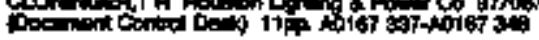

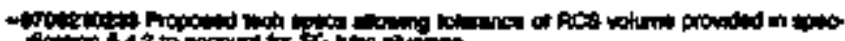

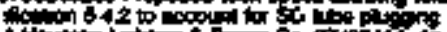

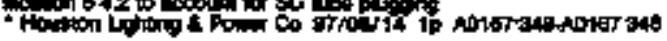

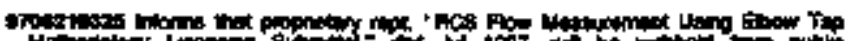

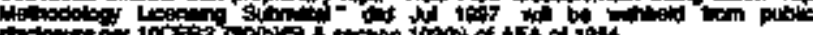

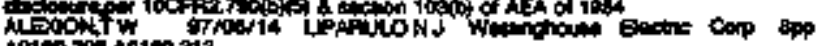

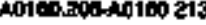

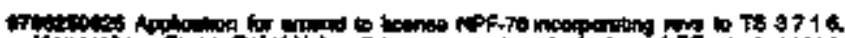

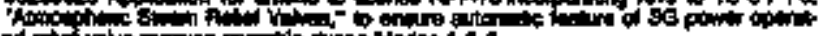
od

clow cefTH How (00h)

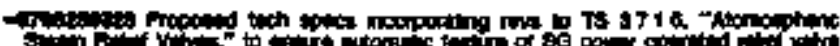

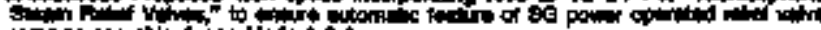

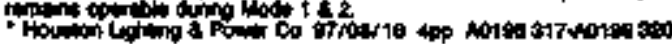

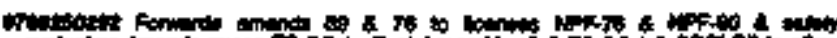

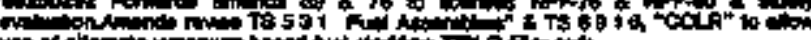

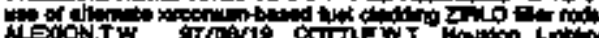

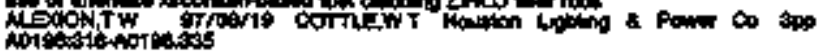

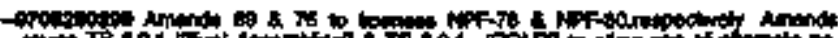

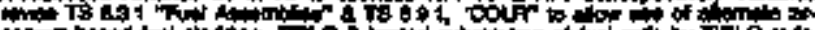

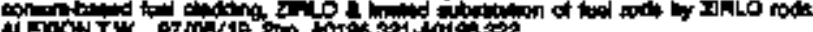

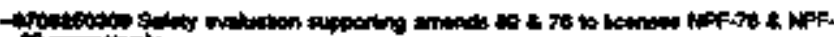

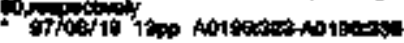

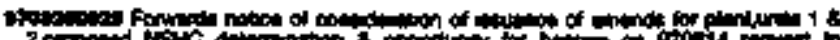

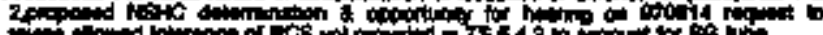

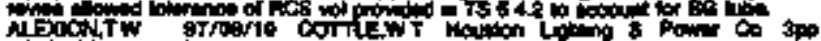

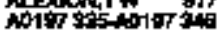

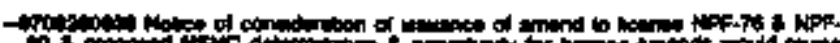

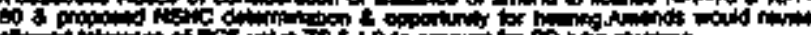

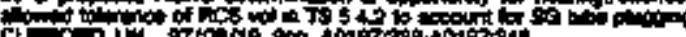

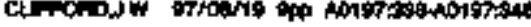

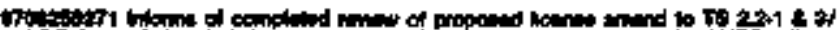

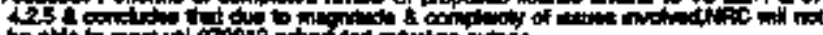

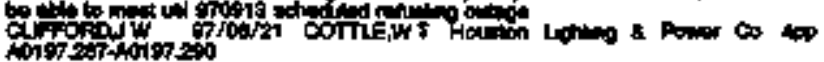

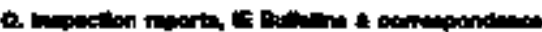

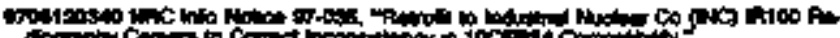

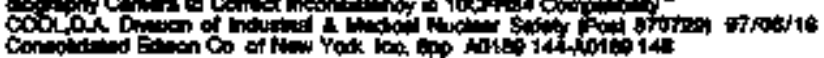

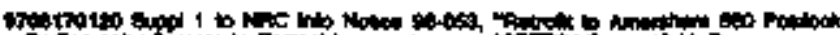

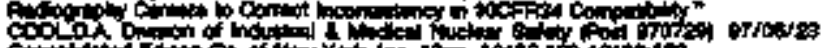

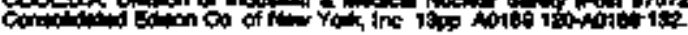

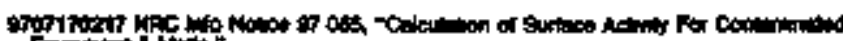

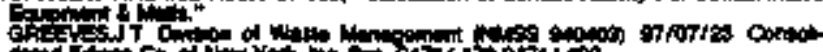

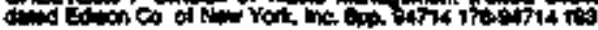

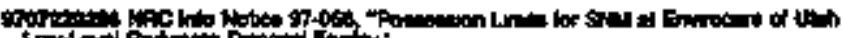

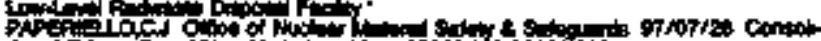

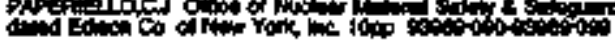

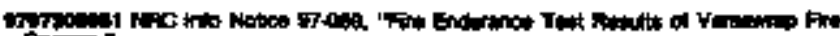

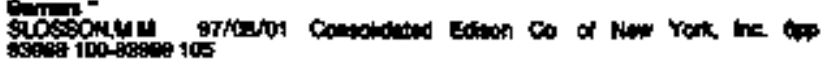

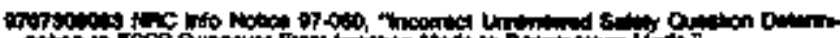

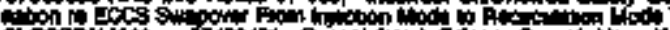

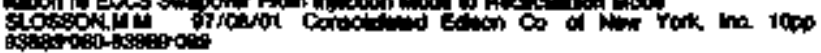

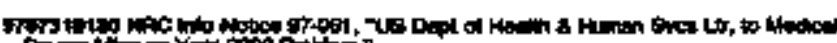

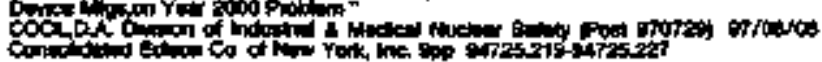

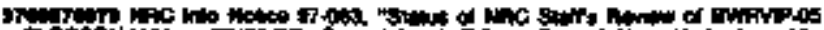

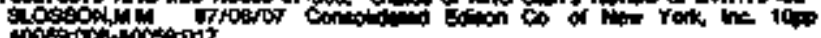

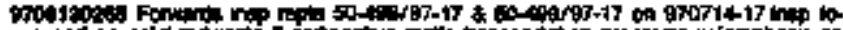

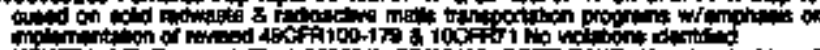

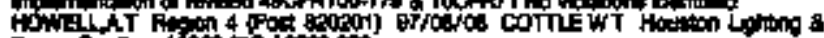

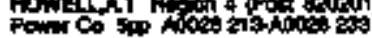

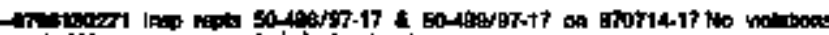

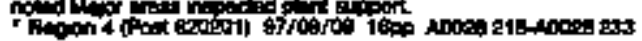

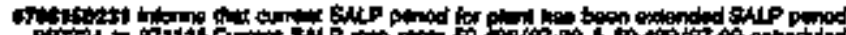

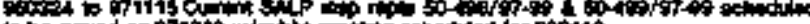

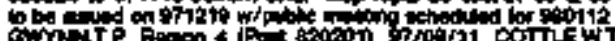

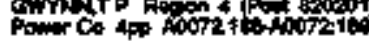

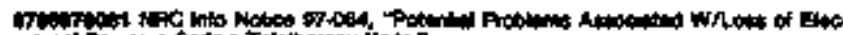

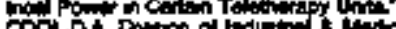

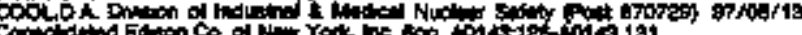

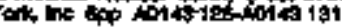

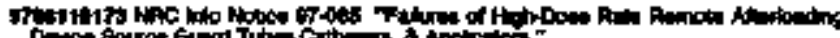

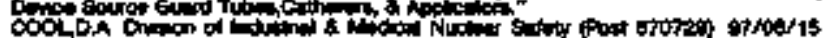

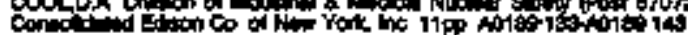

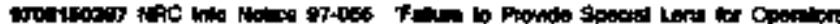

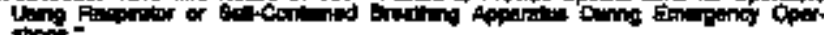

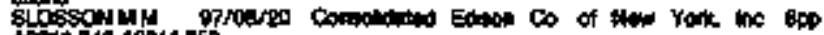

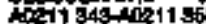

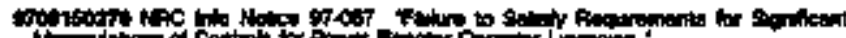

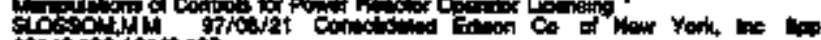

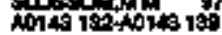

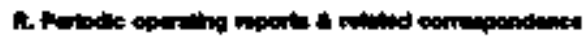

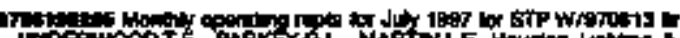

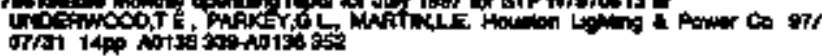

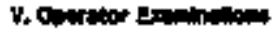

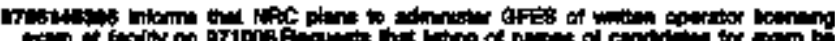

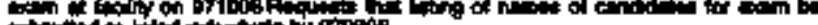

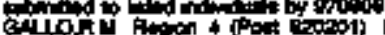

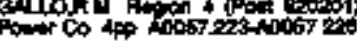

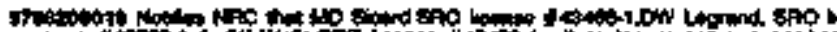
on

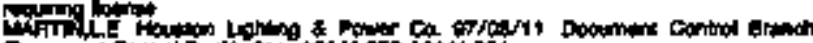

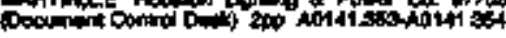

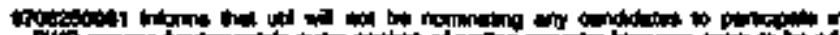

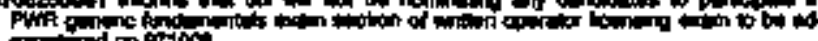

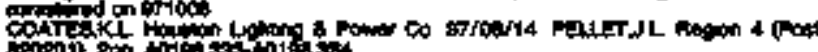

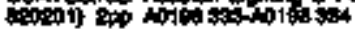

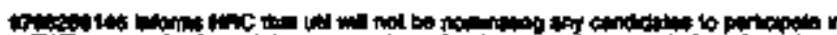
Pit ansiog.

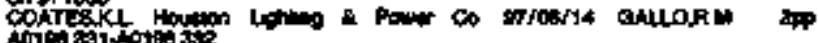

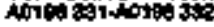

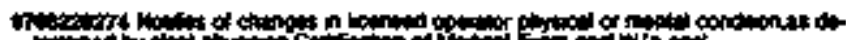

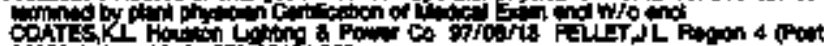

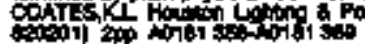

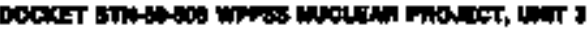

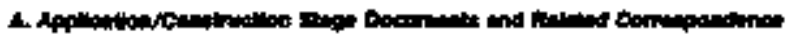

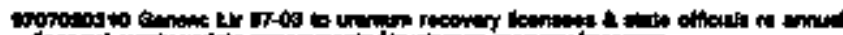
th

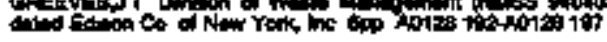

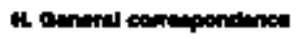

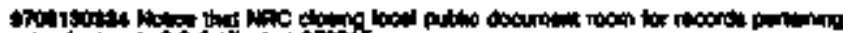

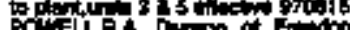

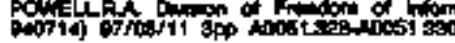

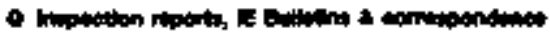

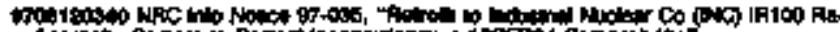

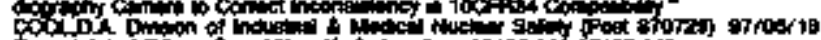

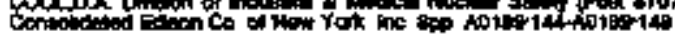




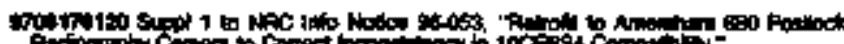

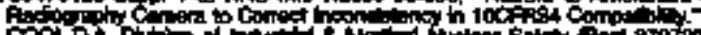

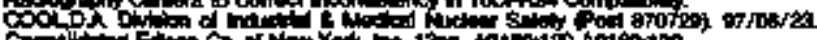

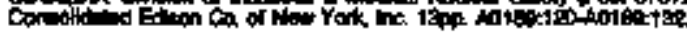

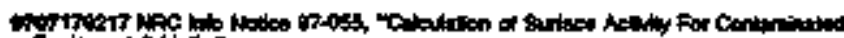

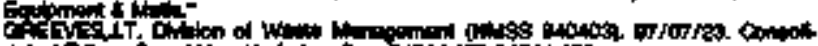

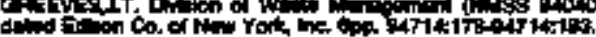

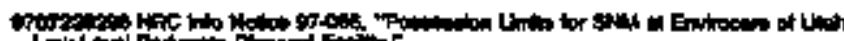

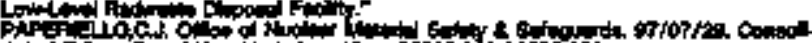

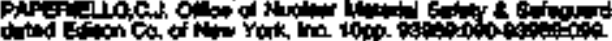

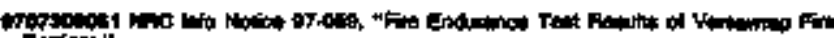

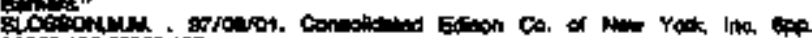

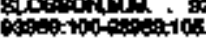

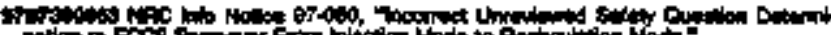

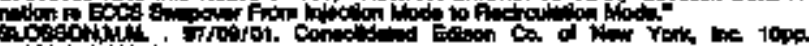
1.

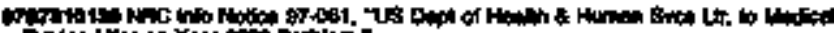

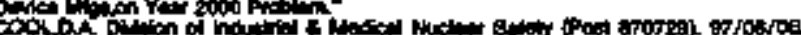

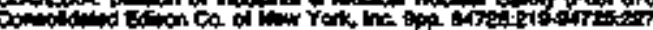

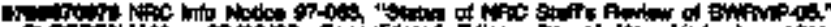

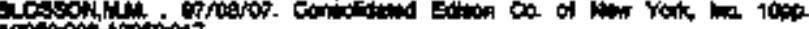

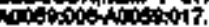

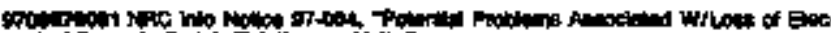

C

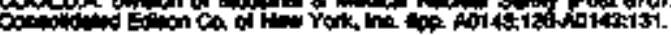

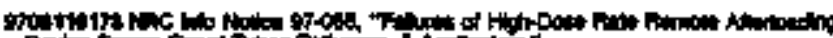

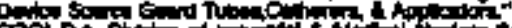

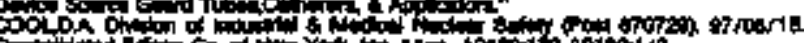

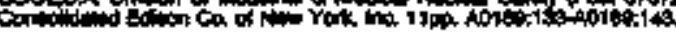

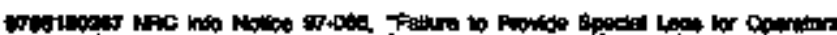

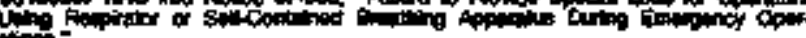

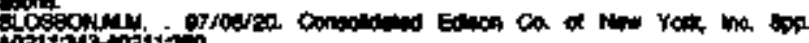

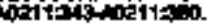

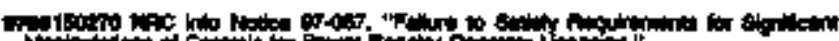
直

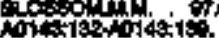

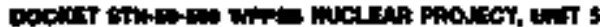

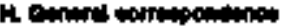

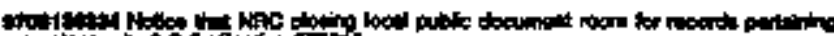

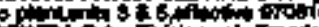

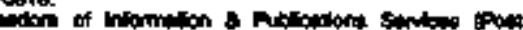

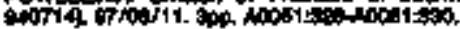

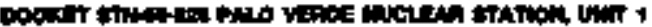

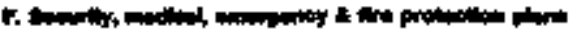

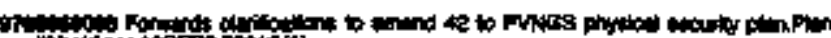

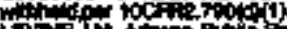

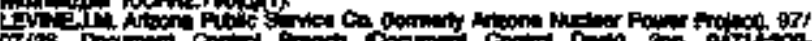

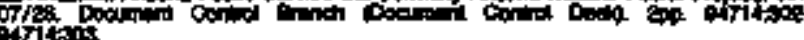

or of

It:

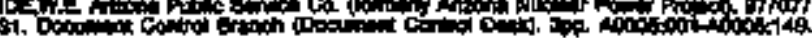

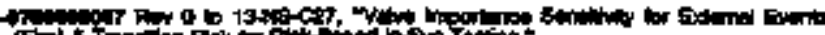

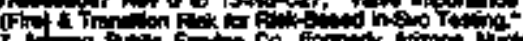

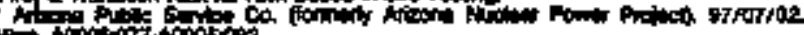

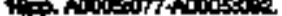

onj

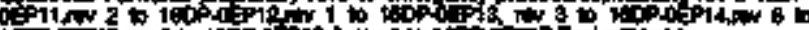

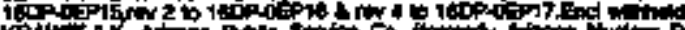

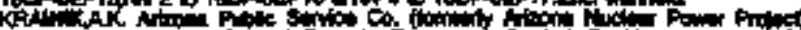

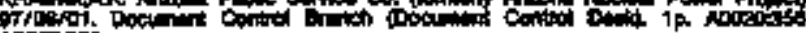
Anotise.

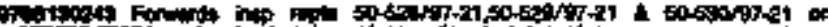

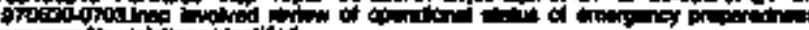

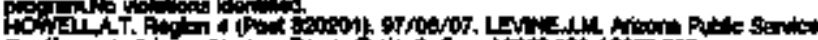

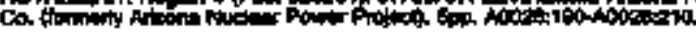

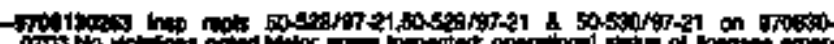

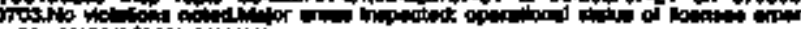

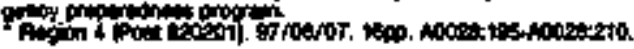

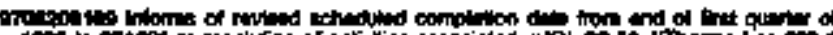

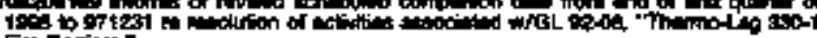

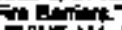

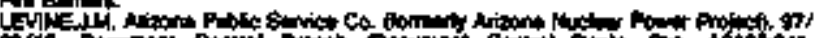

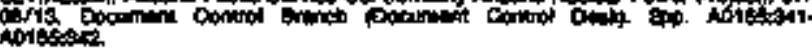

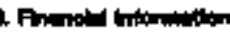

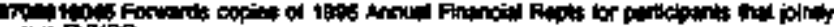
OH PNTBS.

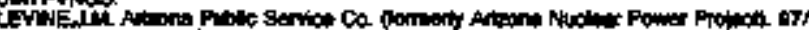
of $0+000$

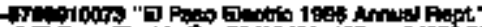

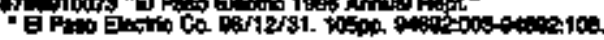

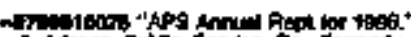

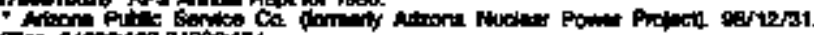

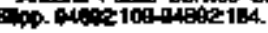

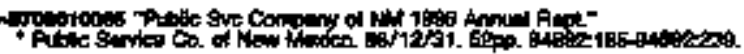

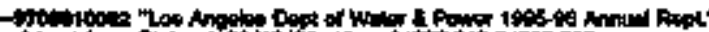

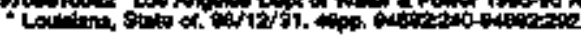

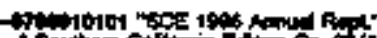

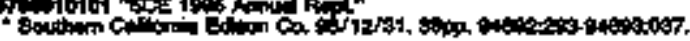

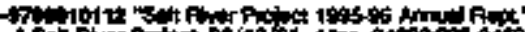

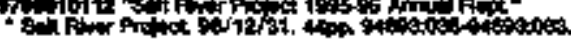

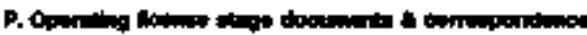

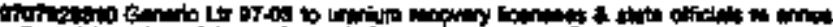

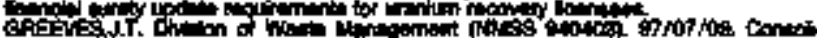

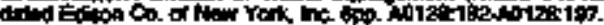

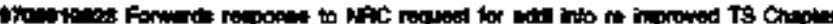

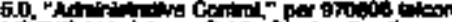

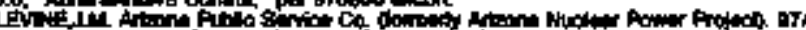

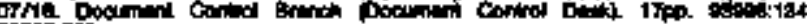
acosian.

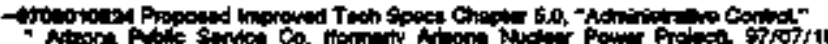

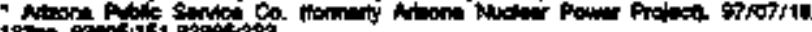

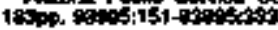

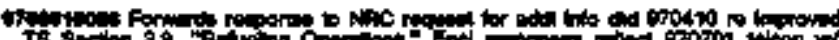

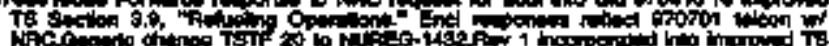
Nincers is

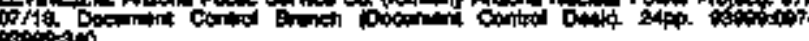
(a)

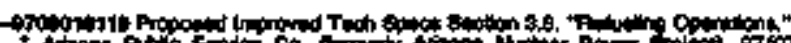

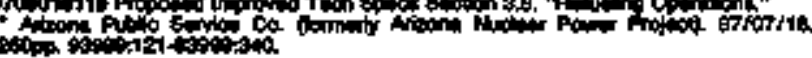

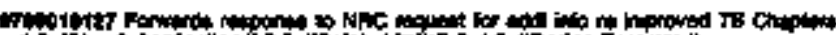

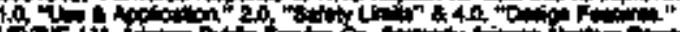

C.

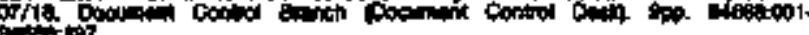

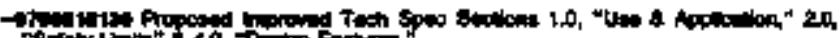

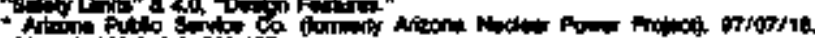
1.0.

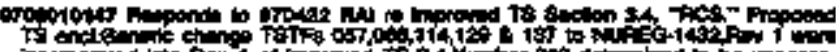

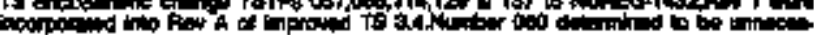

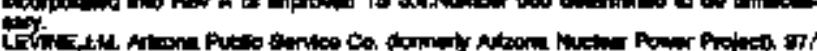

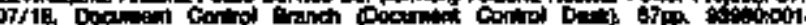
stistion.

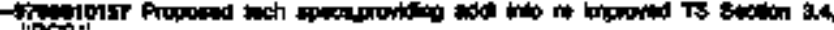
"Act:

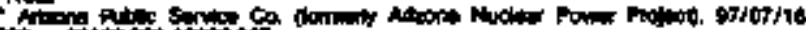
Jotin

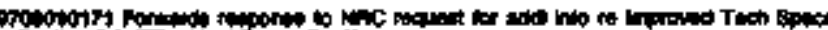

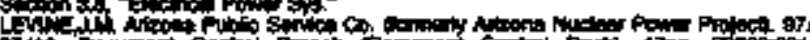

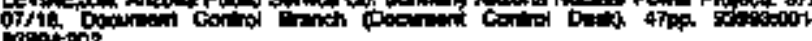

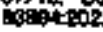

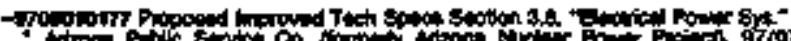

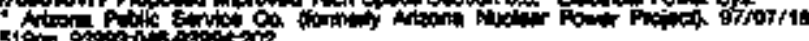
510 pistats

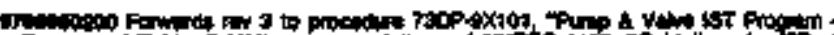

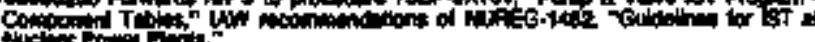

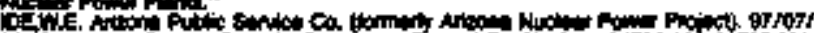

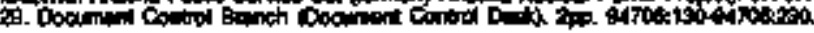




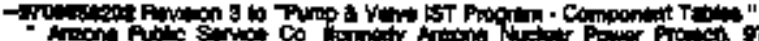

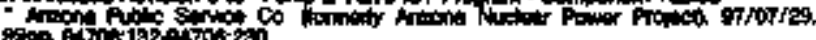

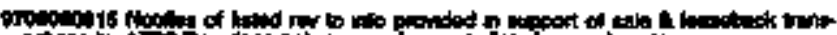
ofion th

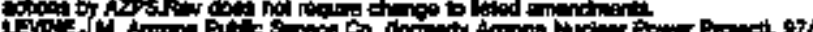

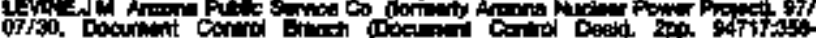
arisoso

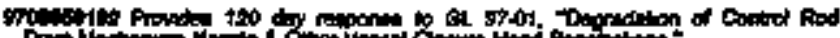

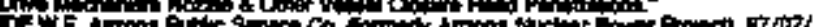

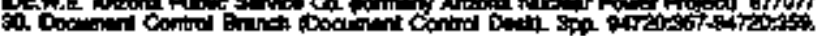

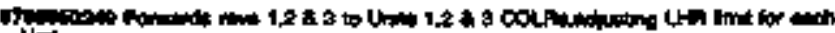

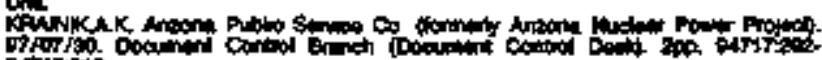
antionts

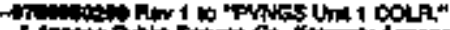

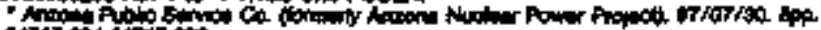

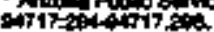

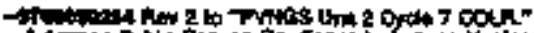

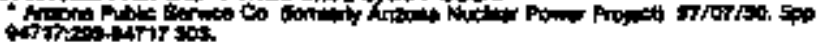

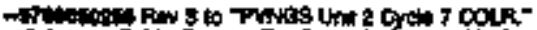

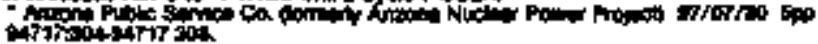

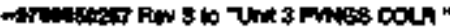

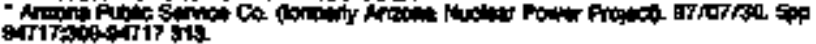

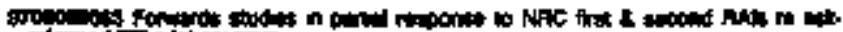

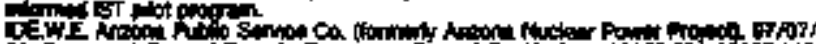

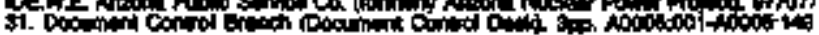

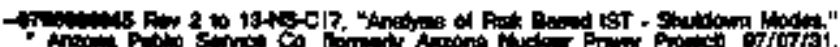

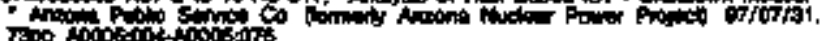

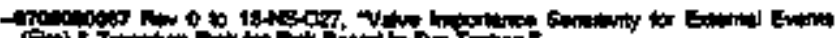

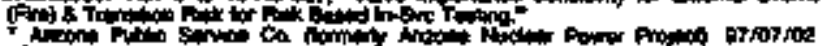

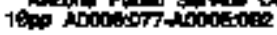

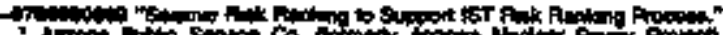

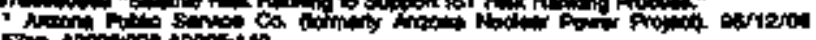

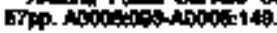

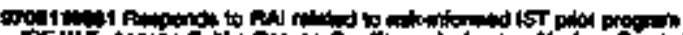

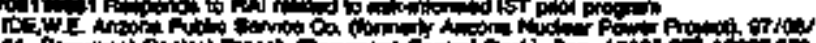

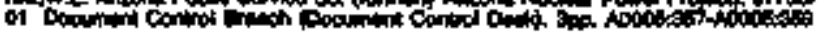

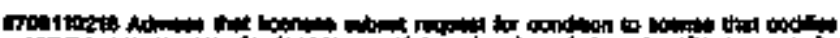

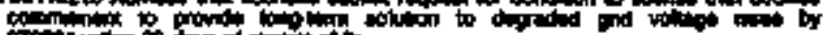

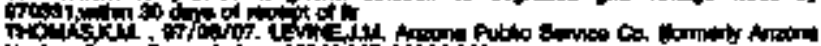

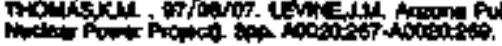

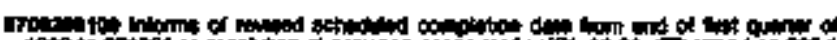

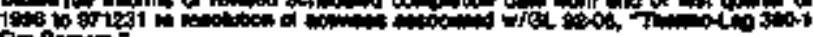

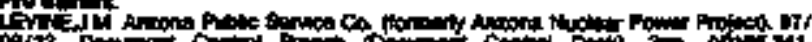

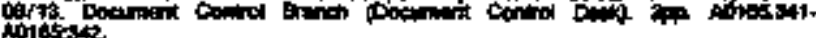

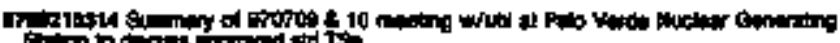

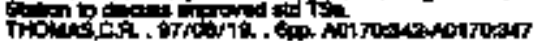

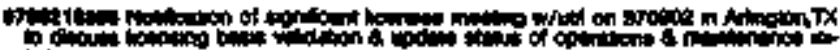

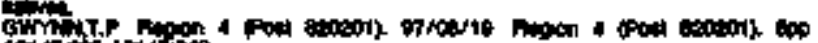
inditionition

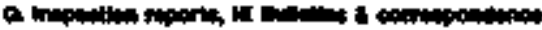

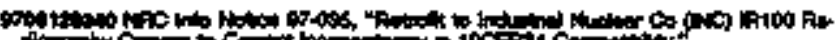

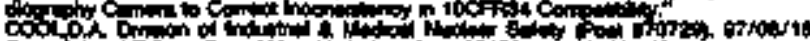

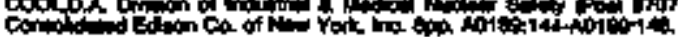

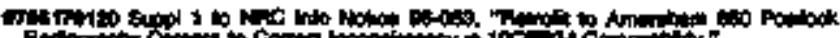

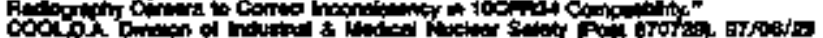

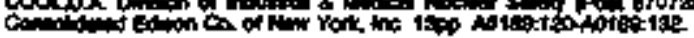

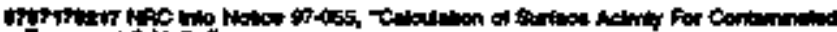
Egind

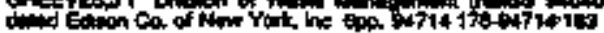

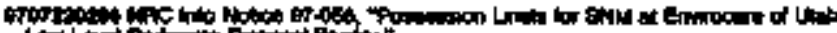

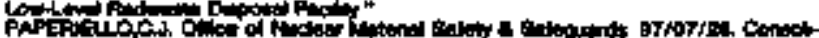

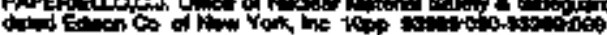

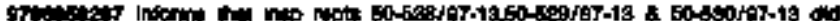

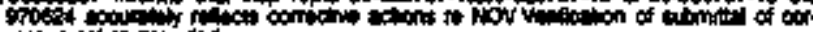

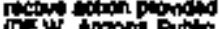

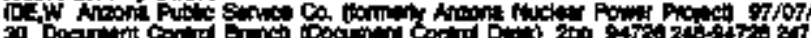

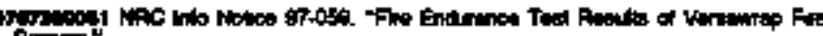

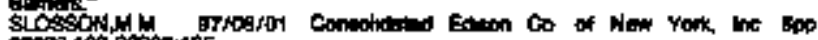

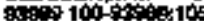

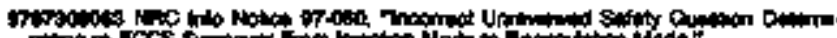

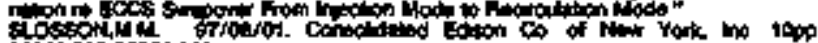
sos 000000000

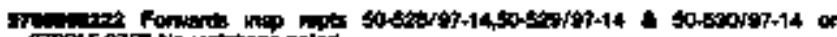

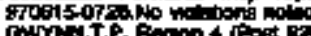

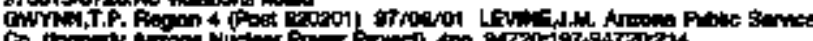

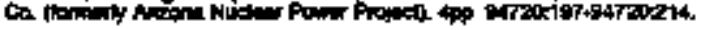

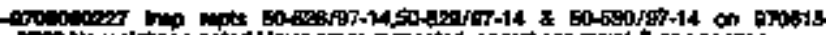

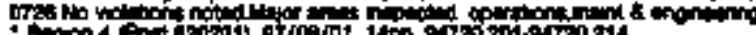

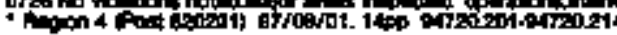

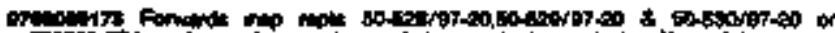

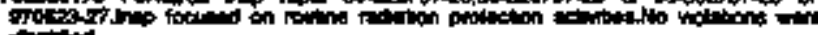

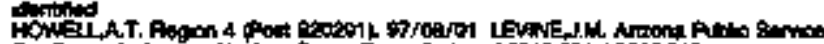

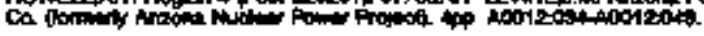

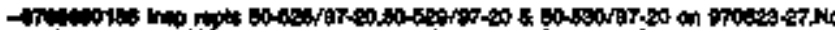

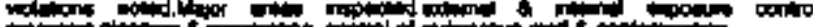

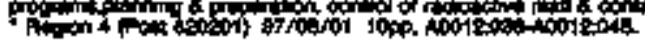

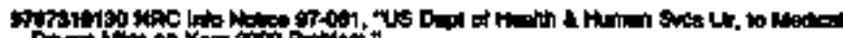

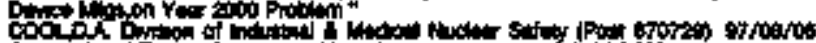

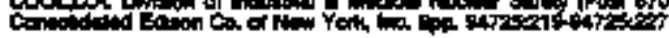

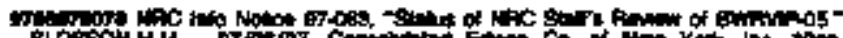

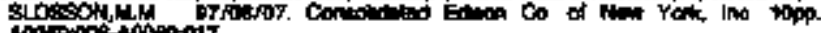

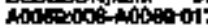

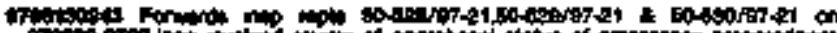

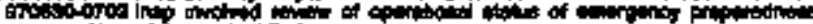

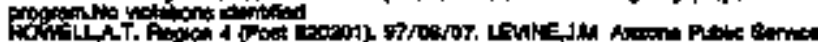

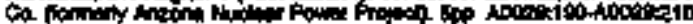

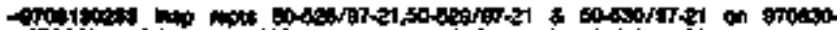

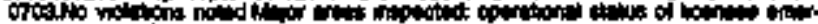

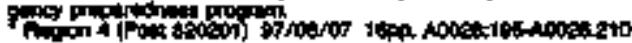

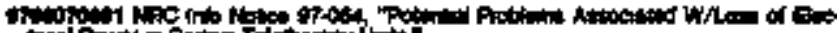

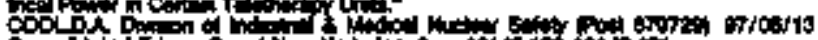

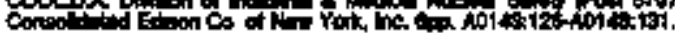

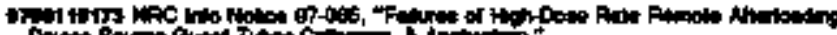

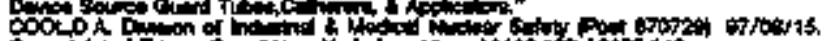

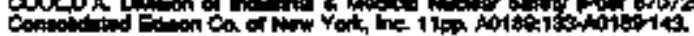

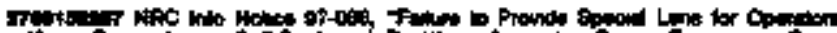

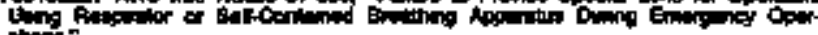

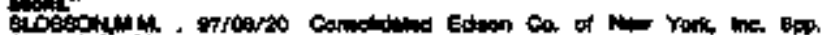

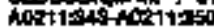

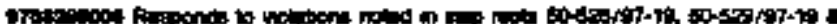

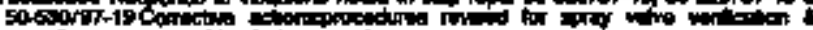

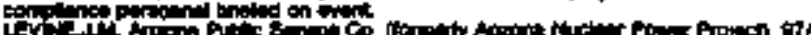

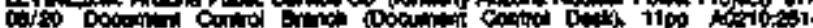
An⿴囗十) 269 .

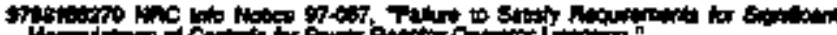

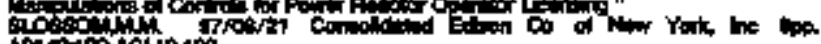

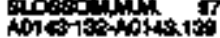

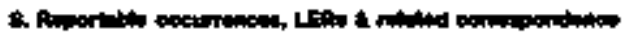

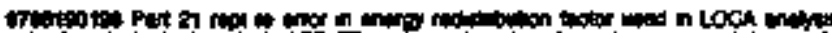

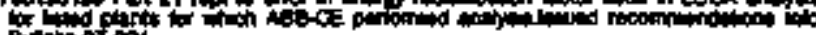

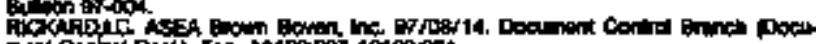

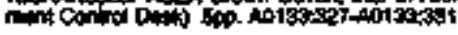

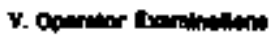

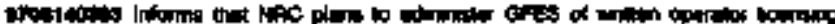

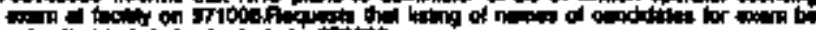

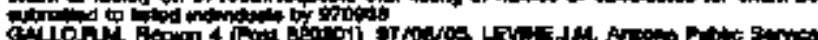

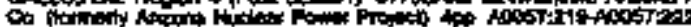




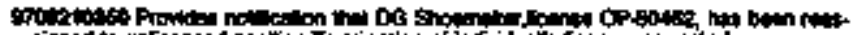

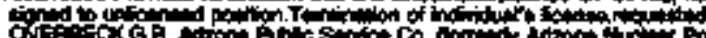

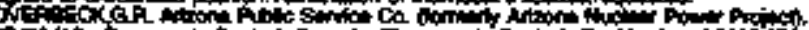

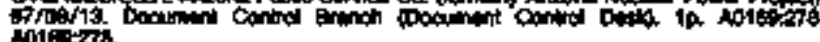

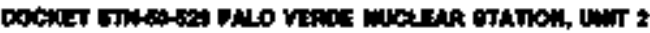

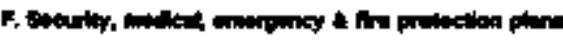

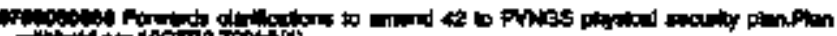

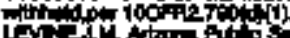

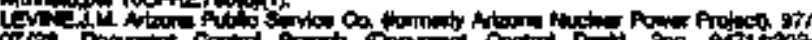

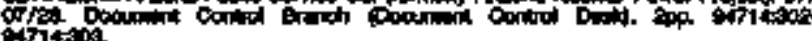

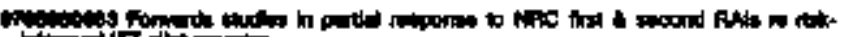
Cothe

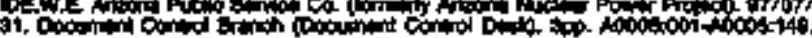

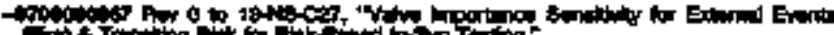

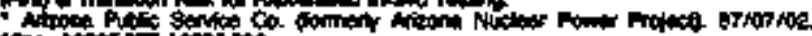

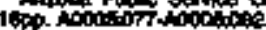

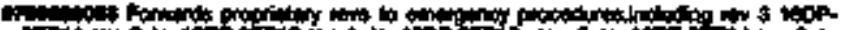

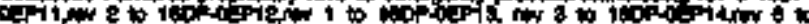

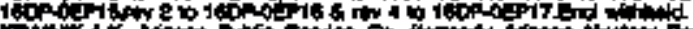

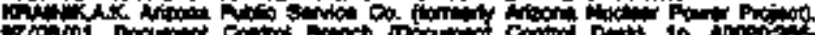

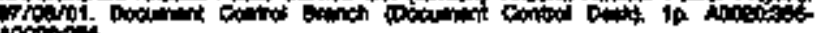
Aoctoser.

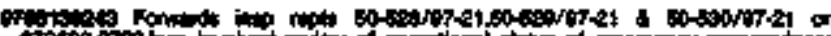

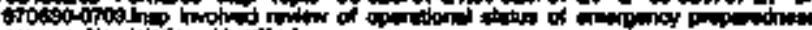

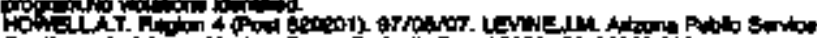

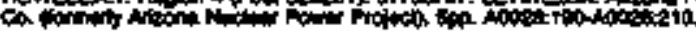

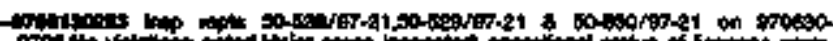

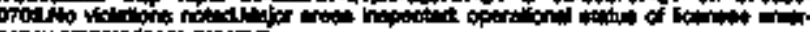

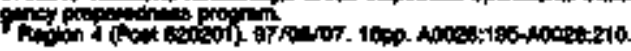

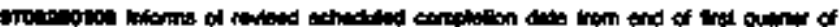

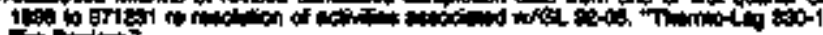
Th

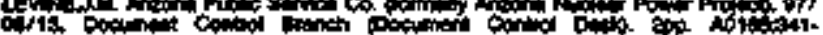
A010.034

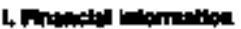

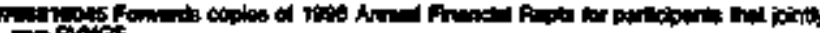
topprest

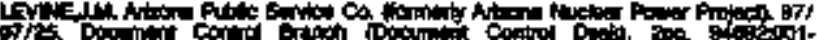

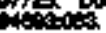

-

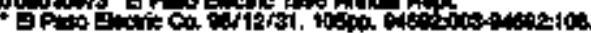

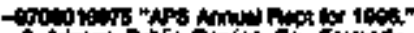

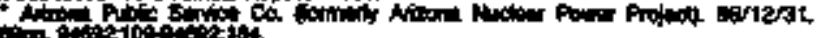

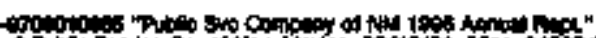

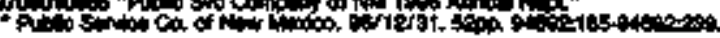

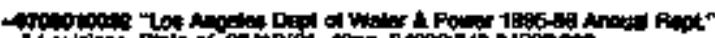

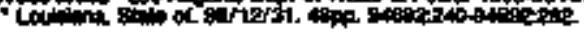

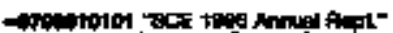

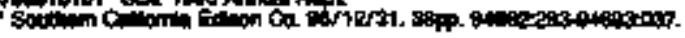

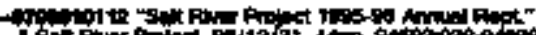

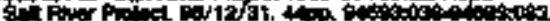

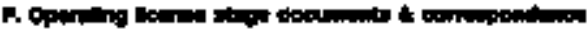

A

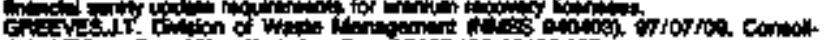

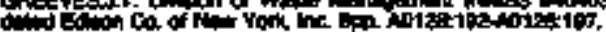

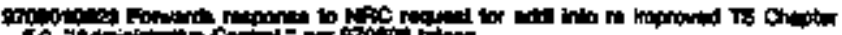

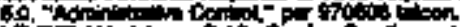

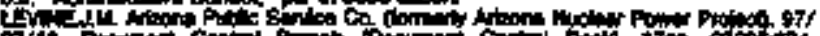

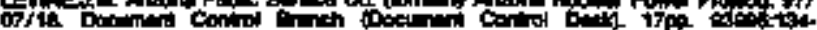
remesed

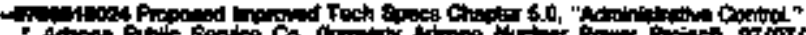

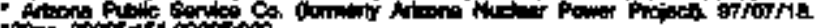

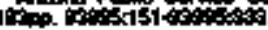

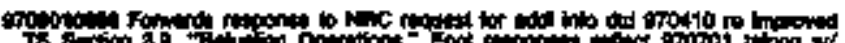

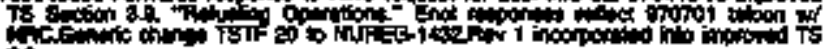
19.

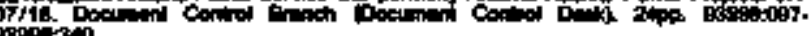

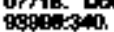

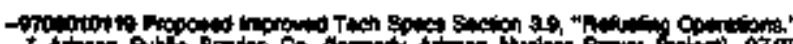

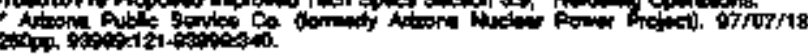

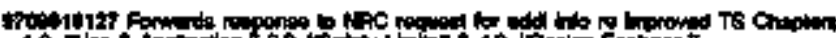

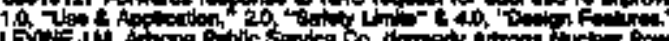

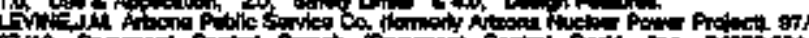

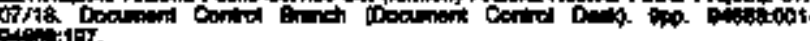

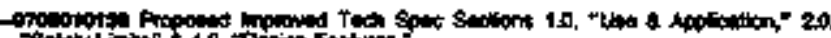

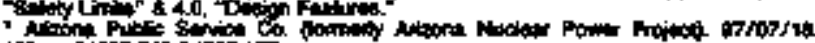

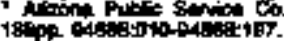

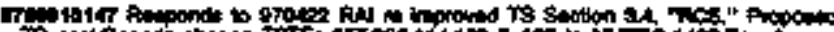

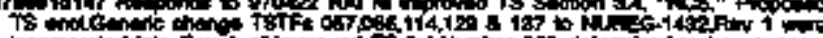

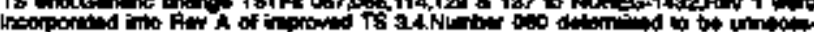
try.

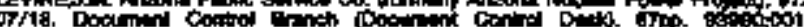
goraz:00T.

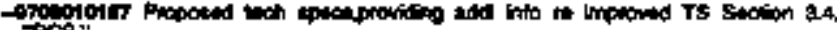

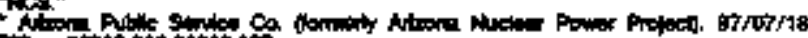

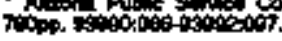

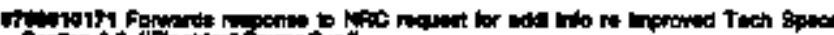

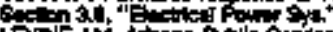

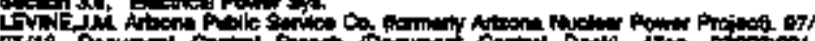

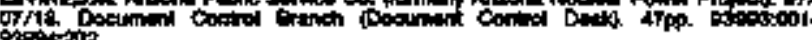

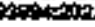

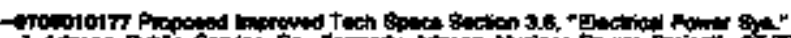

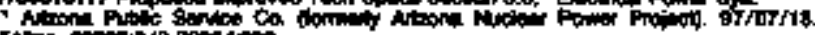

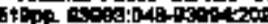

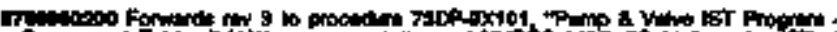

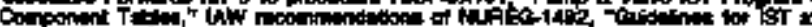

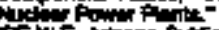

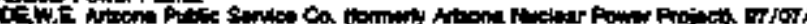

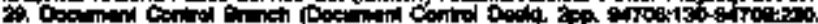

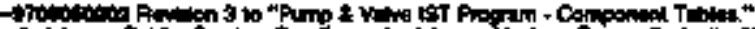

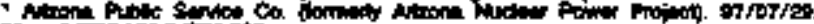

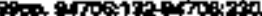

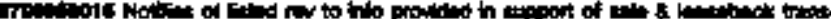

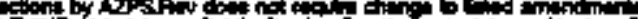

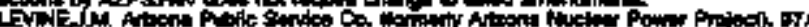

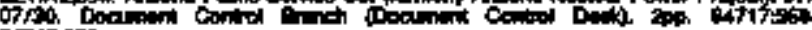
at717\%5.

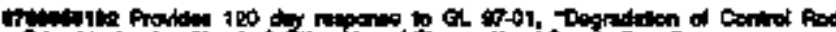

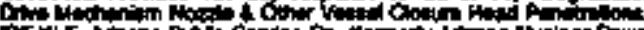

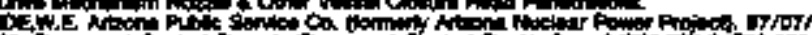

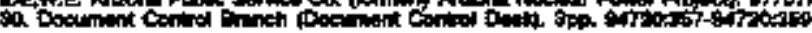

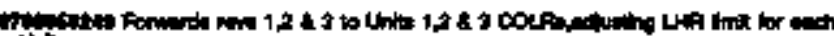
ut

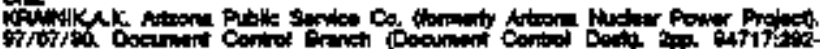
sition

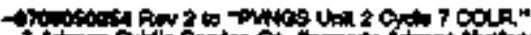

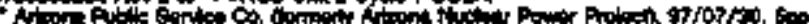

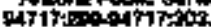

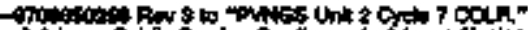

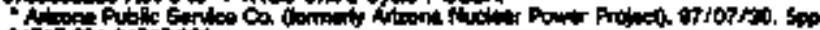

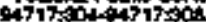

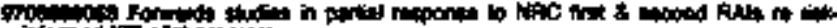

IO

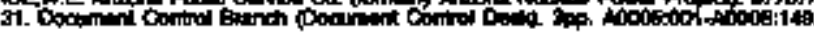

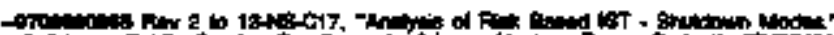

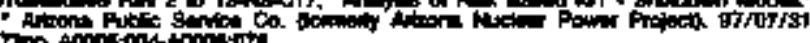

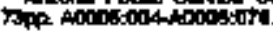

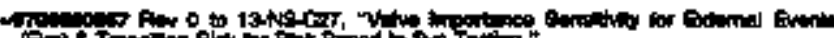

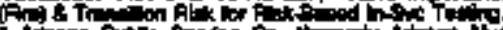

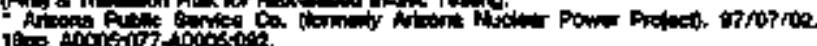

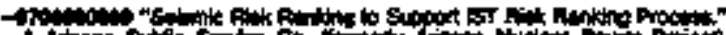

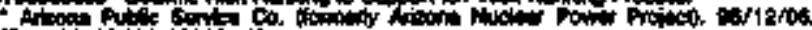

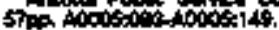

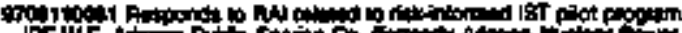

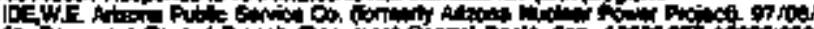

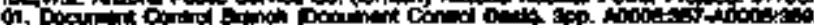

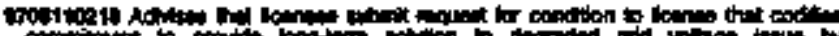

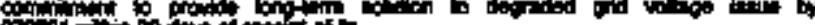
Fos

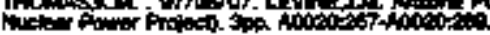

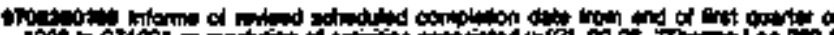

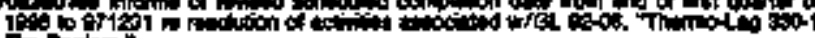

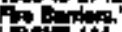

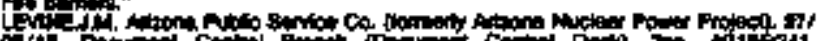

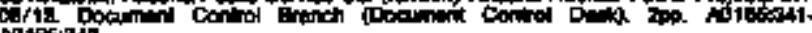
Antasio 


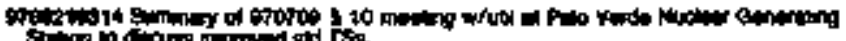

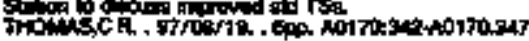

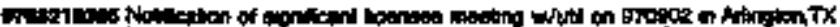

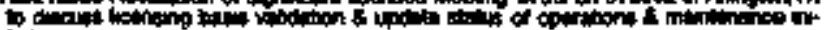

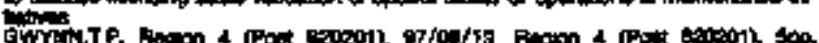

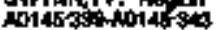

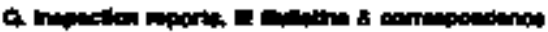

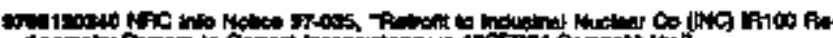

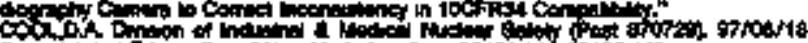

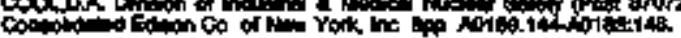

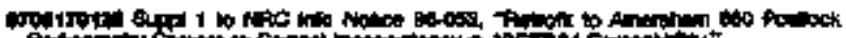

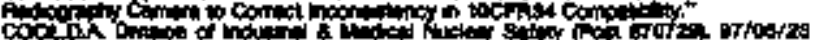

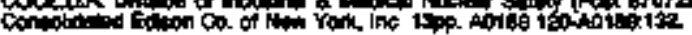

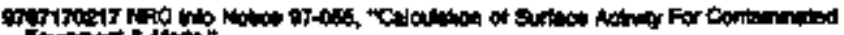

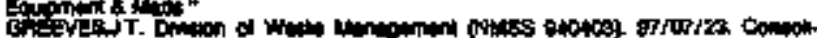

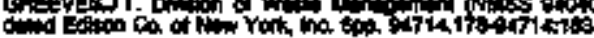

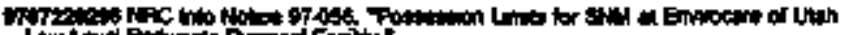

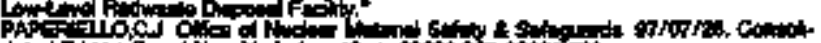

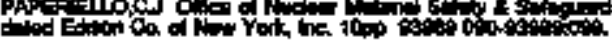

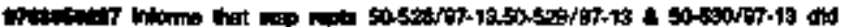

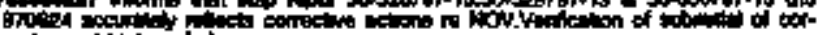

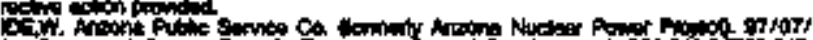

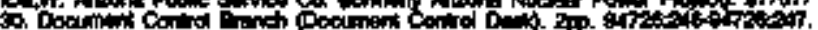

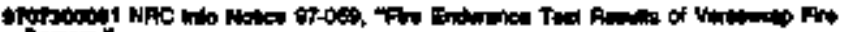

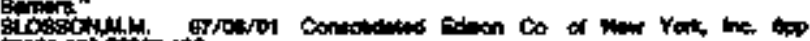

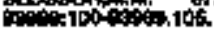

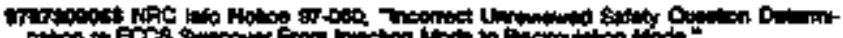

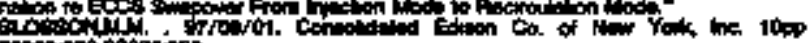

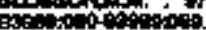

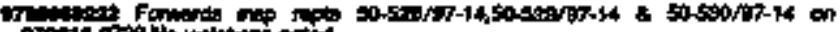

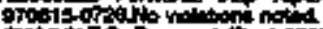

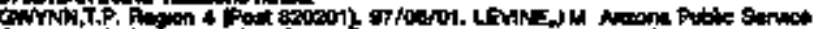

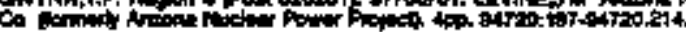

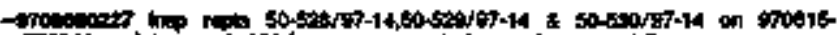

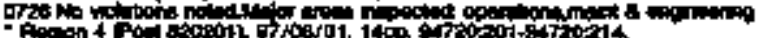

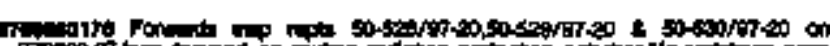

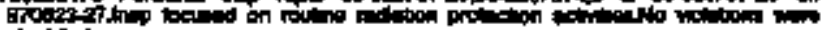

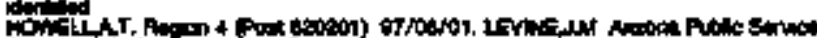

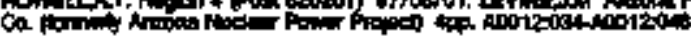

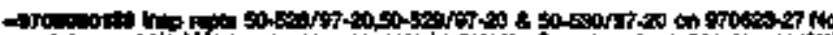

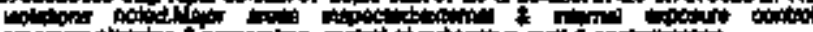

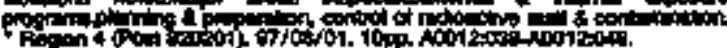

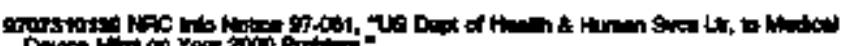

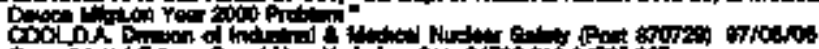

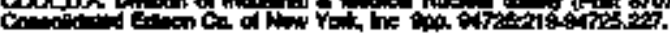

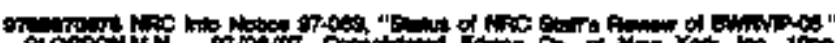

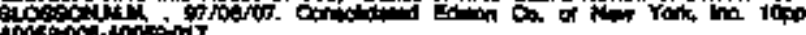

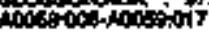

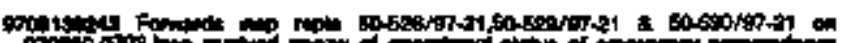

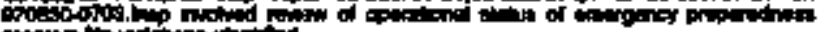

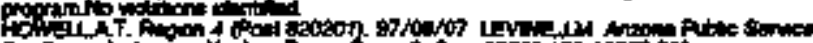

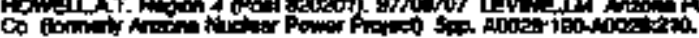

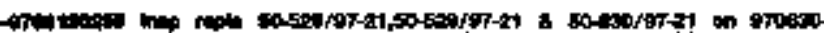

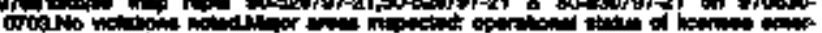

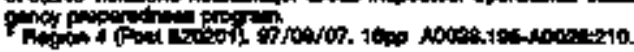

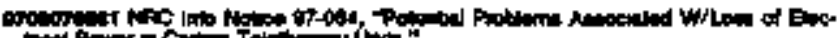

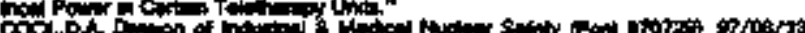

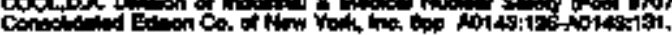

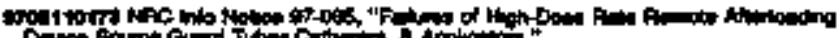
Cooloh

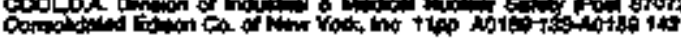

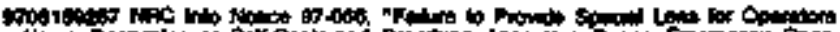

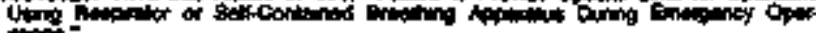

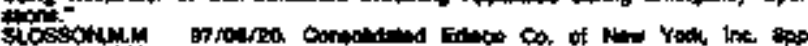

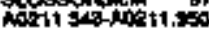

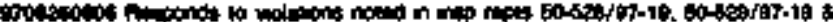
to sots

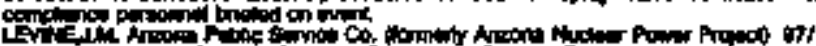

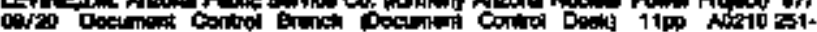
10210

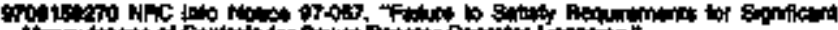

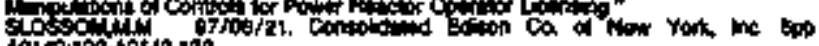

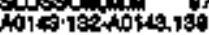

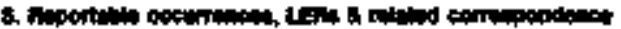

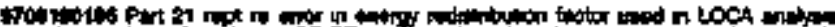

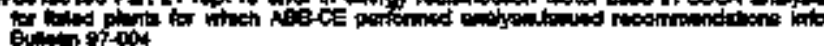

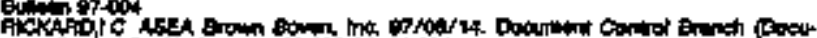

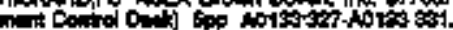

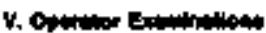

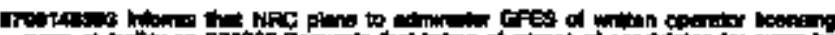

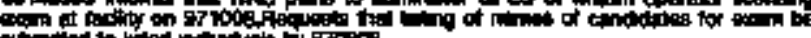

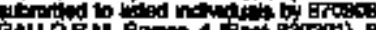

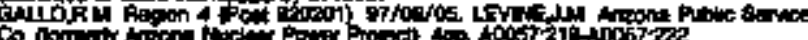

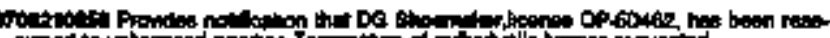

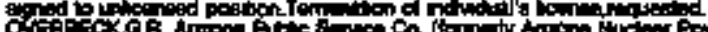

On side

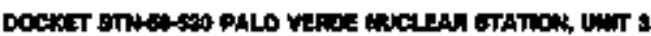

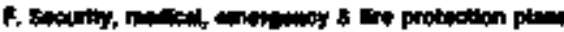

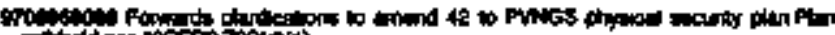

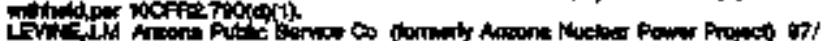

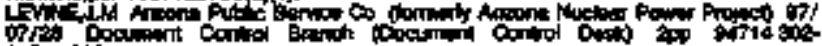
orintion.

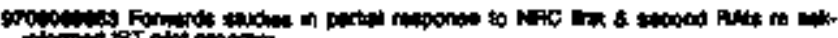

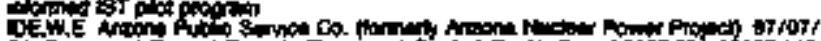

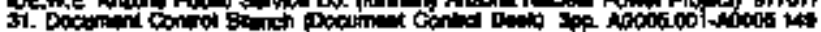

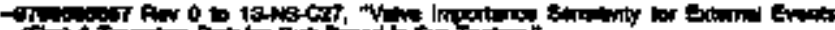

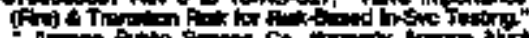

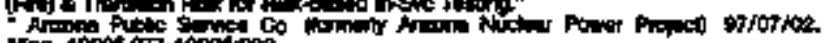

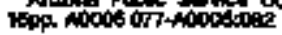

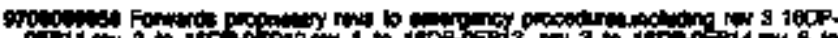

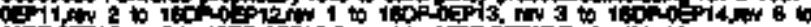

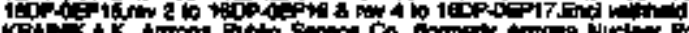

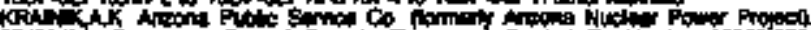

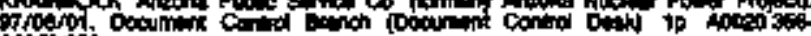


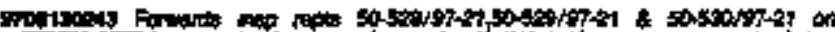

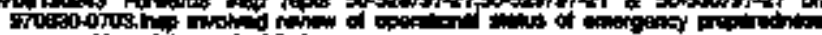

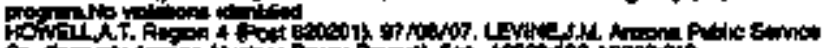

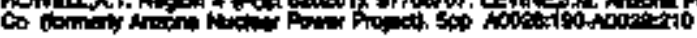

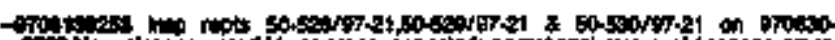

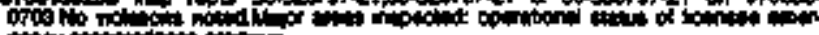

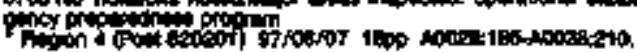

9ro:

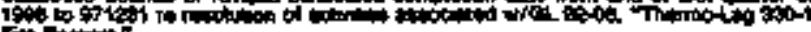

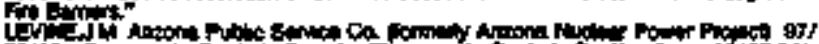

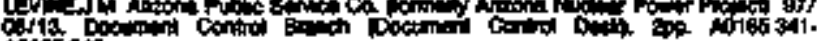
intion

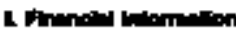

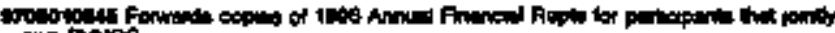
I

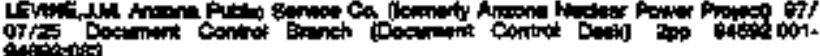

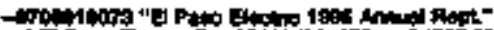

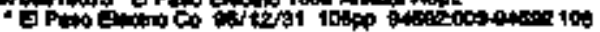

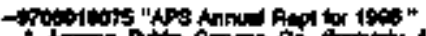

An

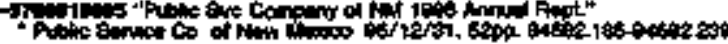

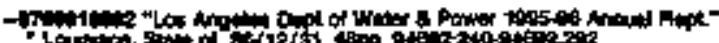




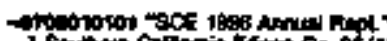

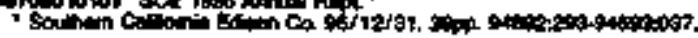

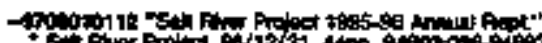

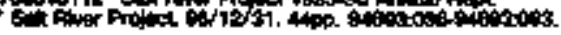

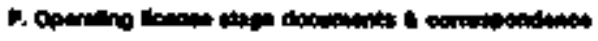

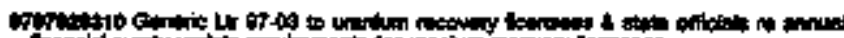

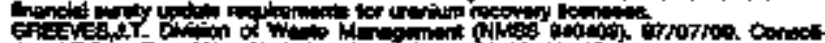

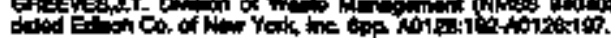

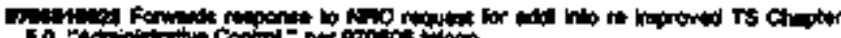

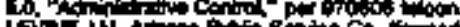

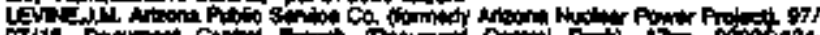

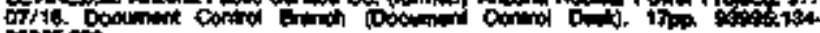
Exosises

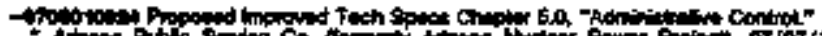

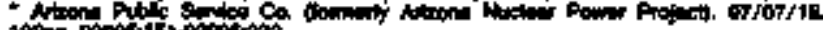

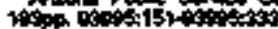

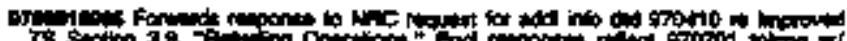

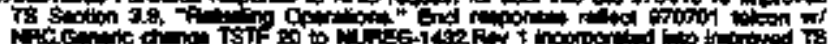

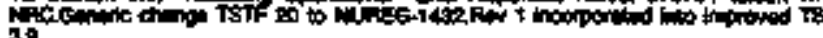
30.

L

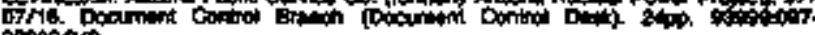
asposin

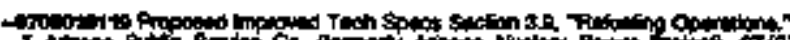

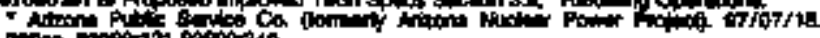
coupo

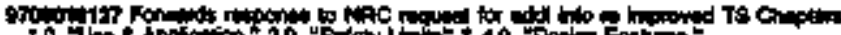
I.

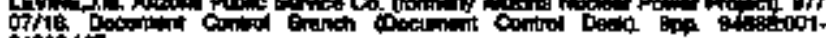
oftistis.

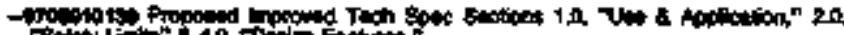

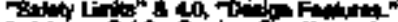

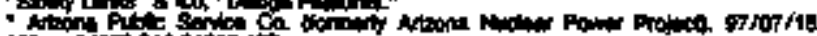
18:p. Ang

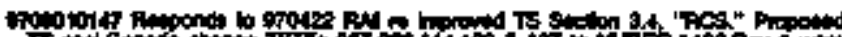

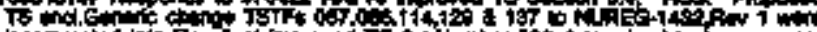

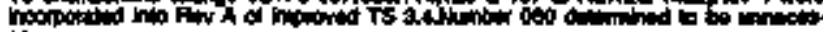

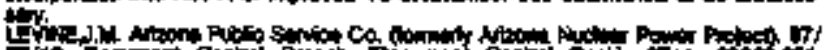

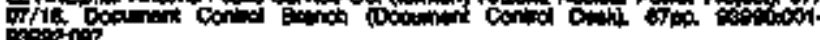
isocion

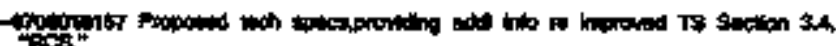

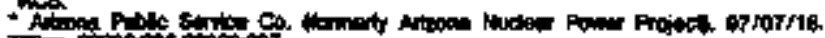
Fing

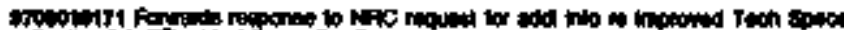

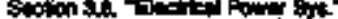

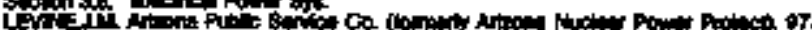

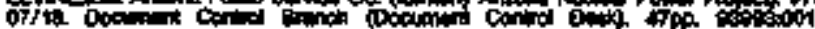
atimete

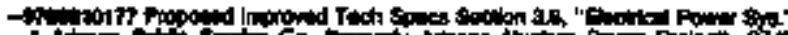

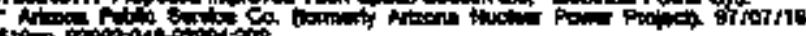

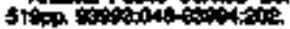

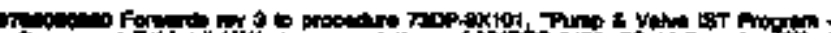

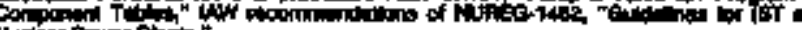

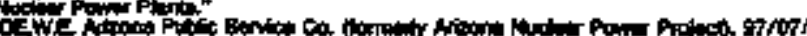

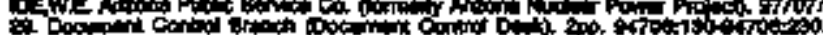

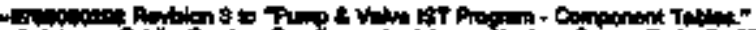

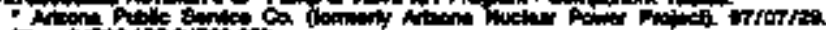

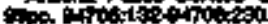

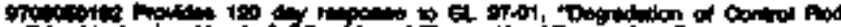

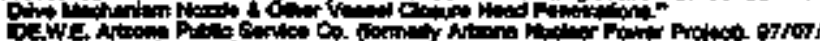

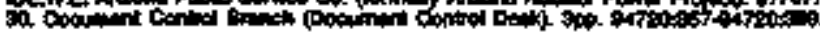

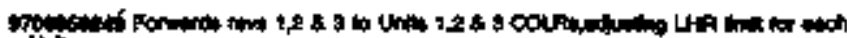

Kitw

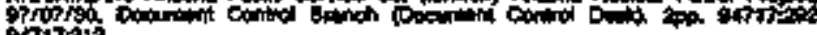
A717ais.

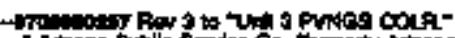

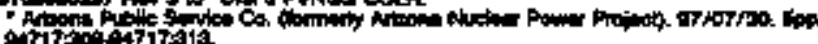

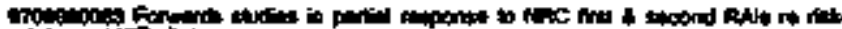

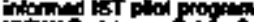

ID

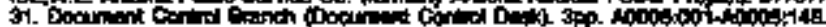

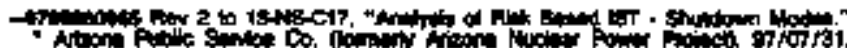

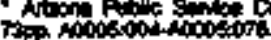

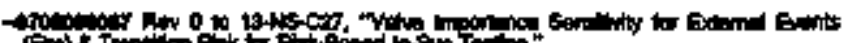

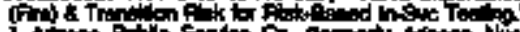

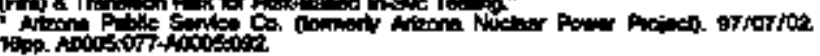

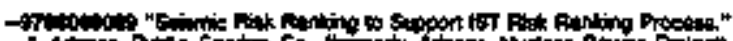

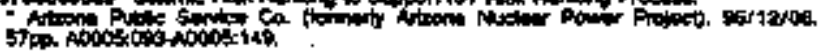

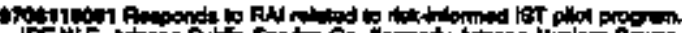

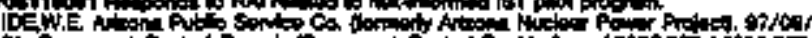

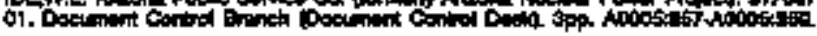

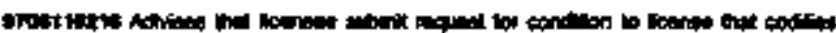

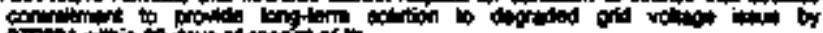

thow

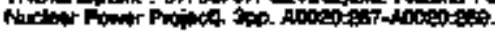

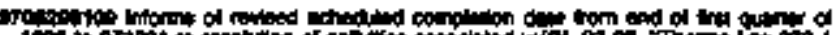

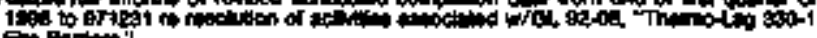

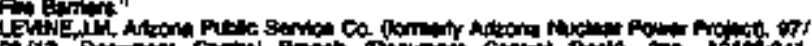

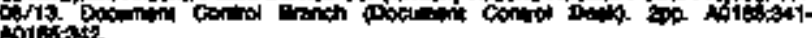

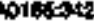

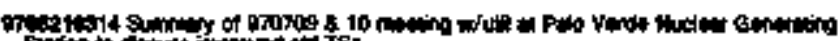

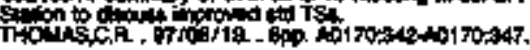

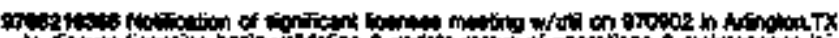

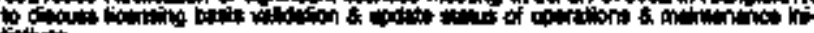

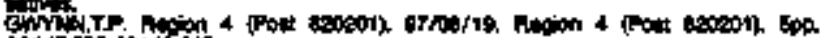

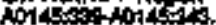

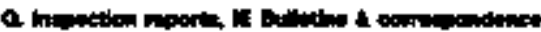

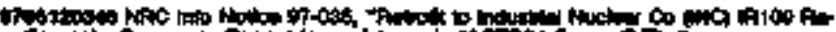

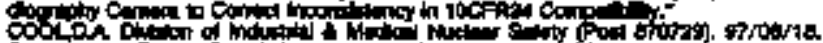

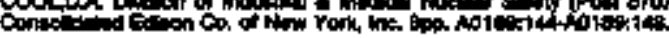

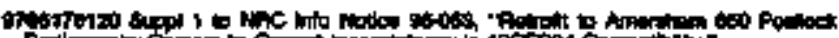

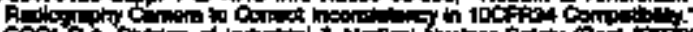

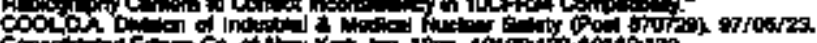

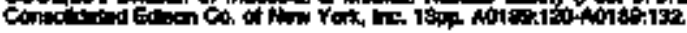

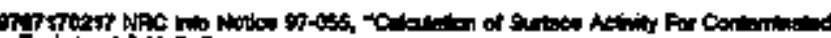

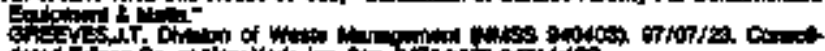

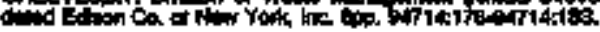

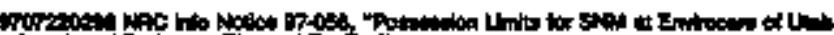

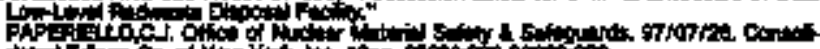

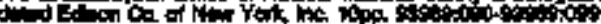

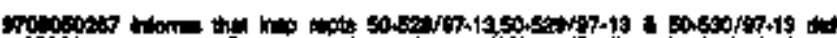

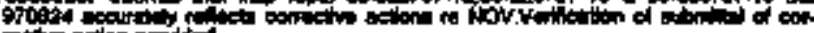

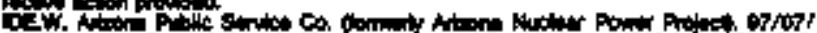

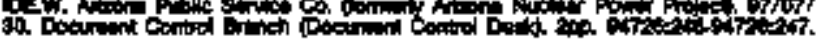

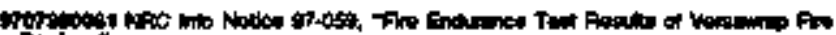

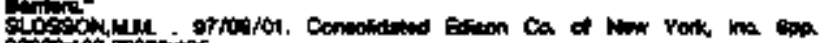

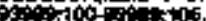

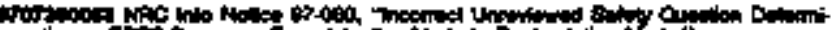

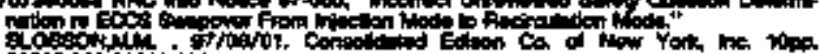
(a)

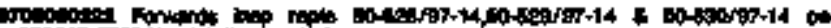

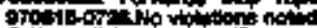

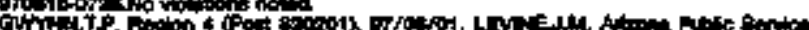

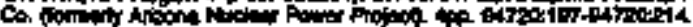

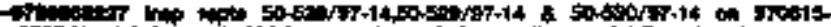

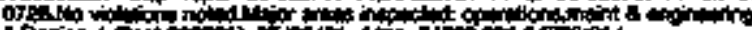
Presen 4 (1)

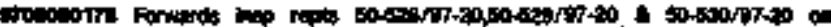

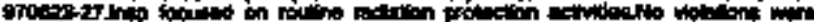

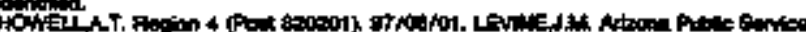

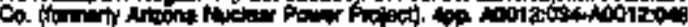

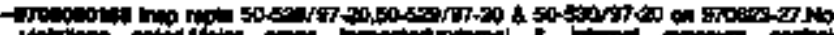
Voline now

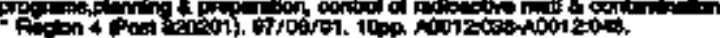

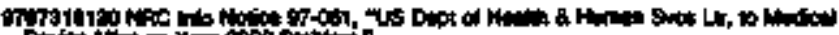

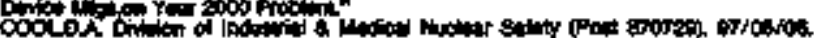

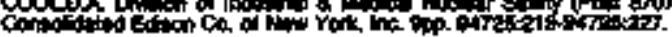

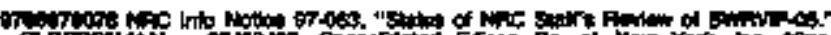

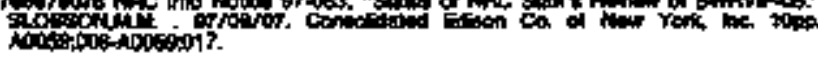

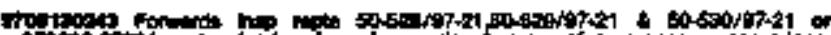

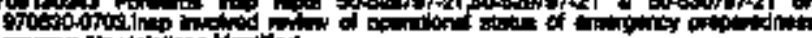

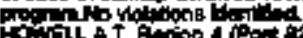

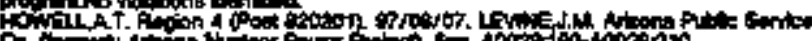

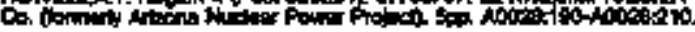




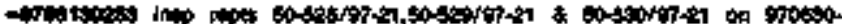

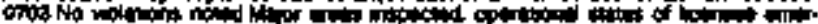

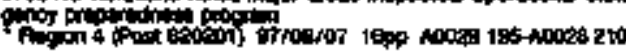

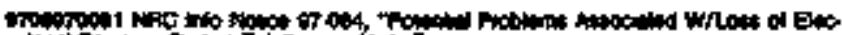

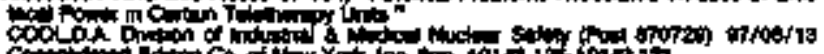

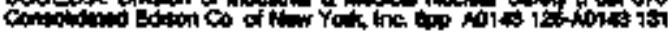

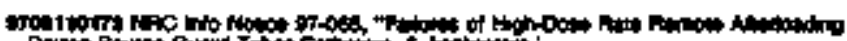

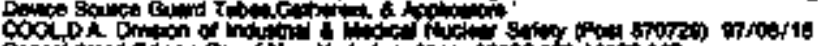

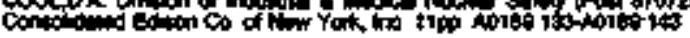

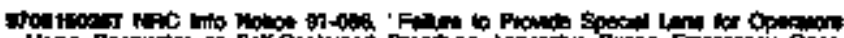

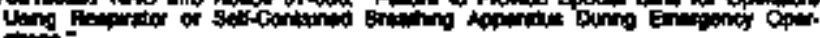

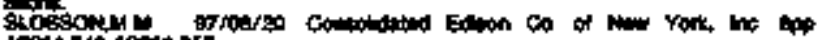

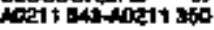

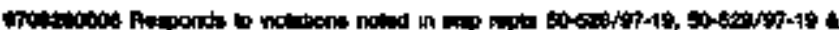

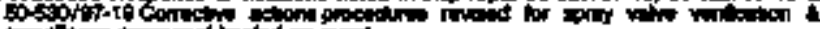

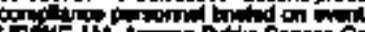

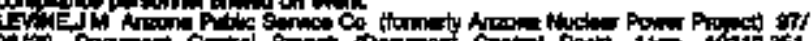
ingotion

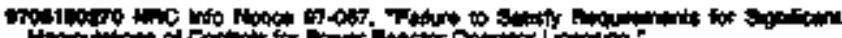

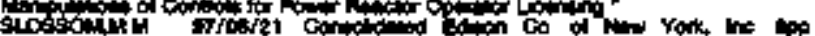

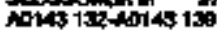

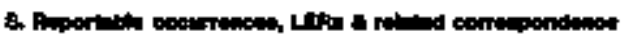

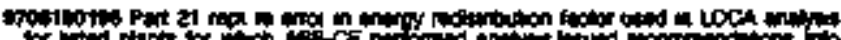

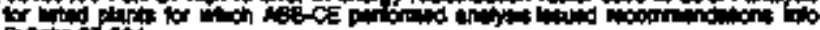
Batin 5004

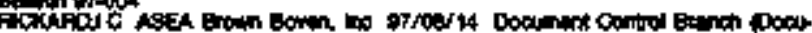

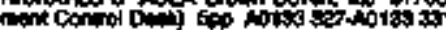

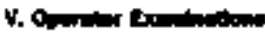

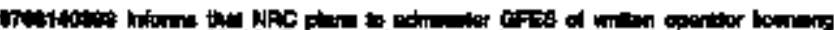

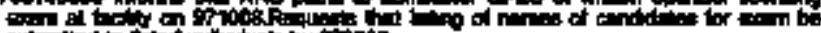

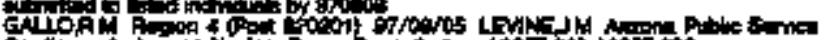

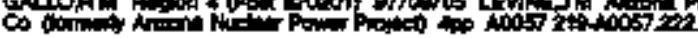

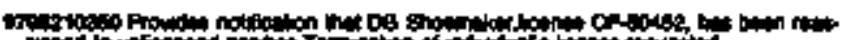

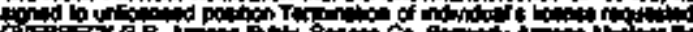

Fis

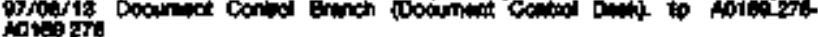

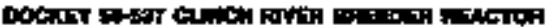

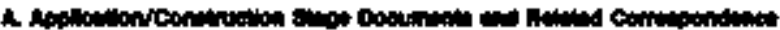

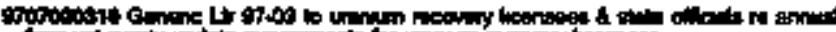

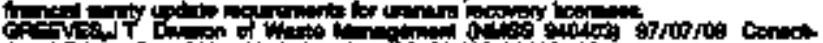

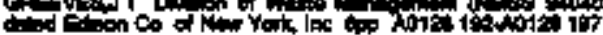

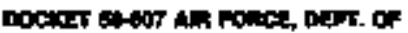

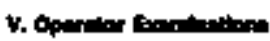

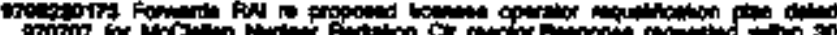

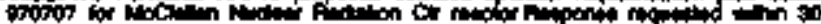

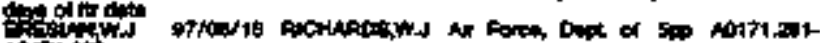
Artion

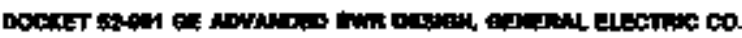

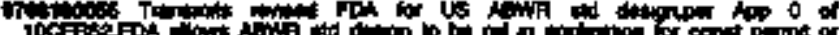

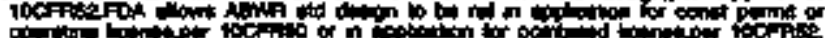

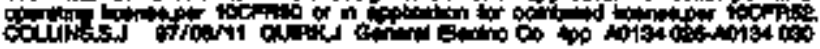

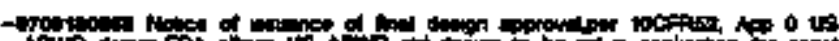

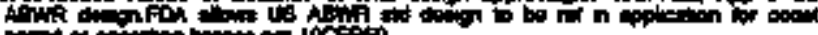

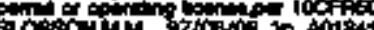

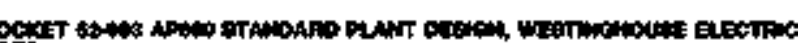

(a)

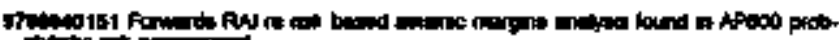

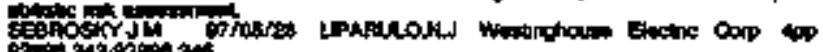

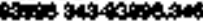

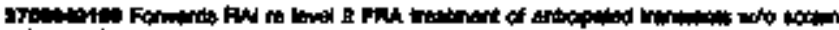

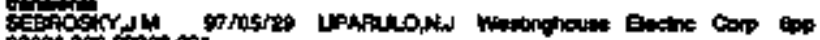

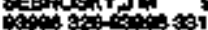

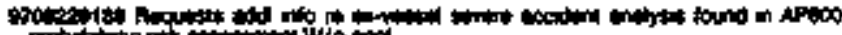
Pction E Aon?

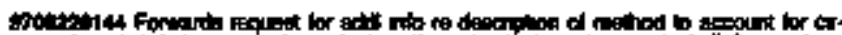

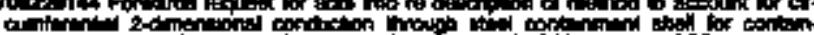

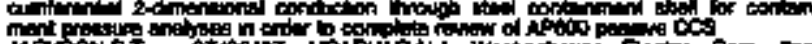

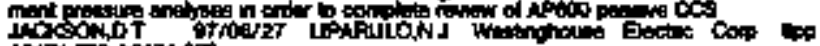

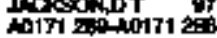

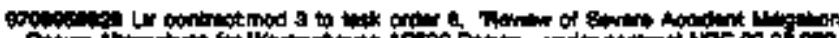

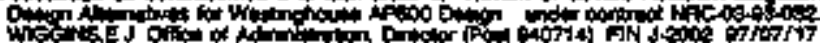

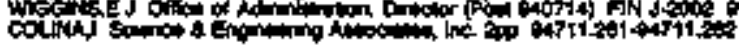

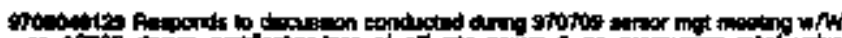

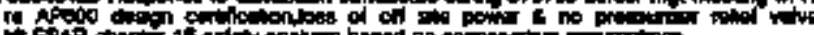

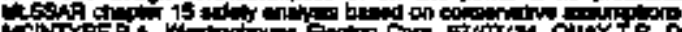

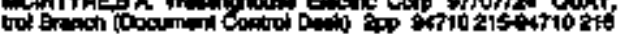

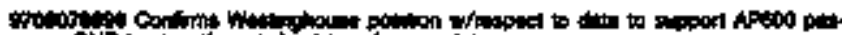

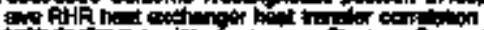

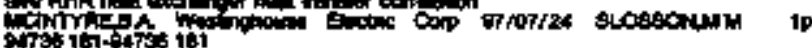

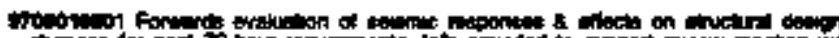

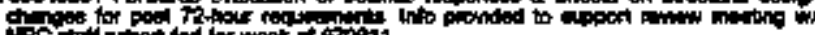

Hif

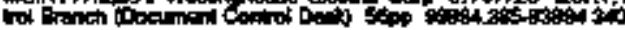

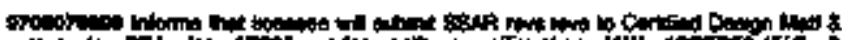

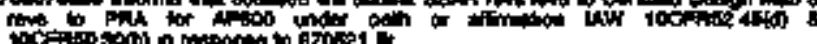
acintrito

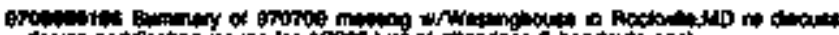

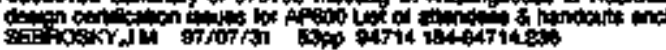

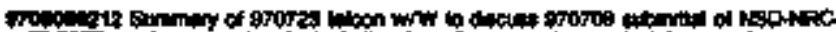

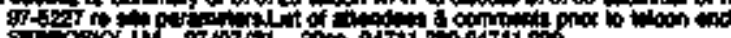

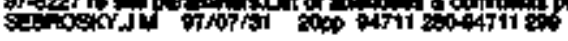

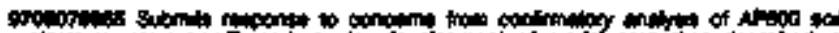

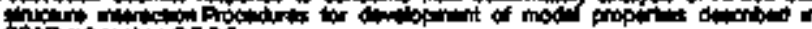

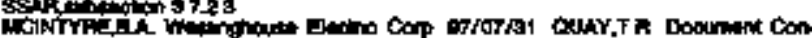

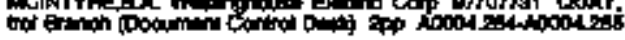

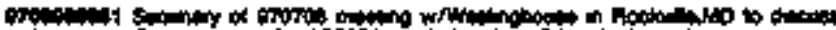

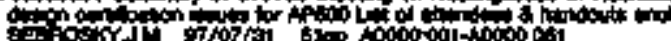

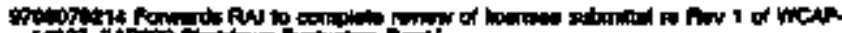
Fat.

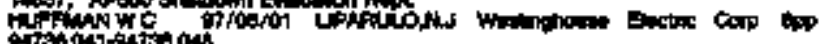

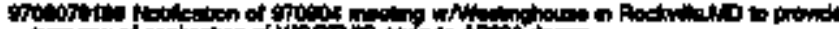

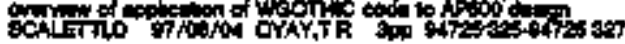

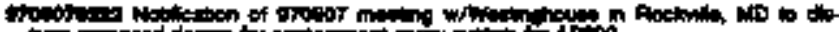

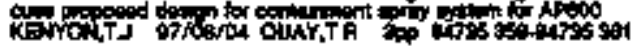

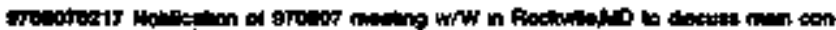

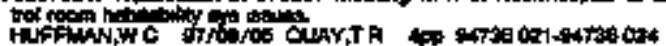

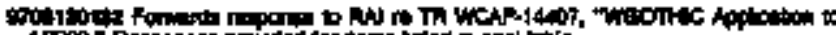

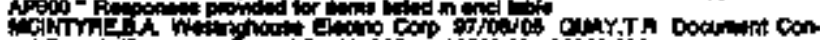

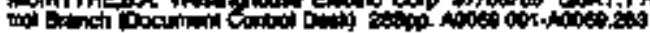

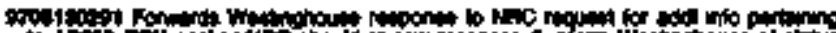

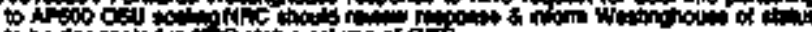

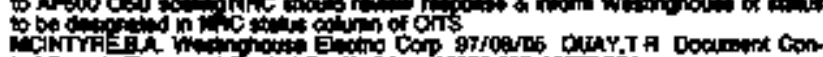

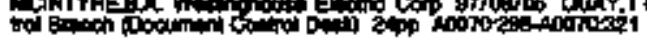

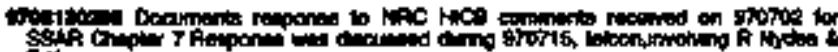

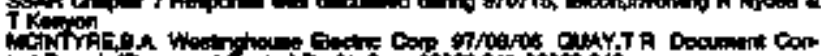

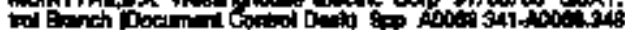

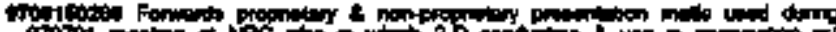

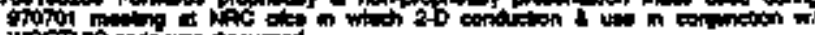

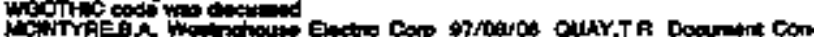

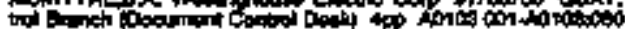

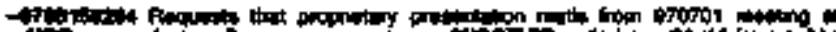

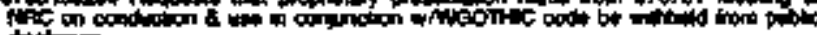

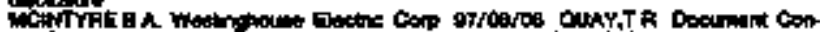

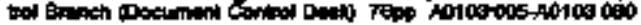




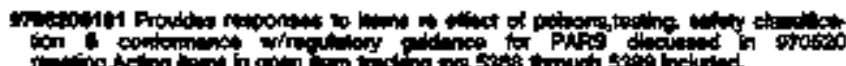

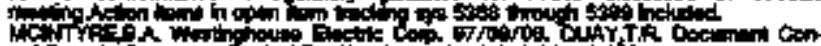

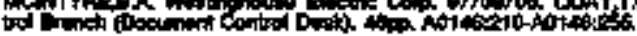

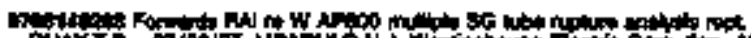

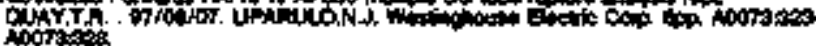

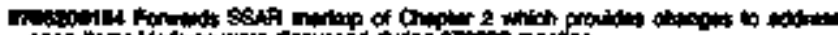

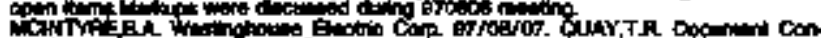

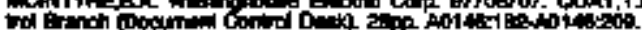

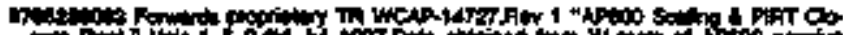

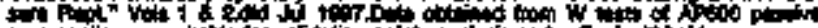

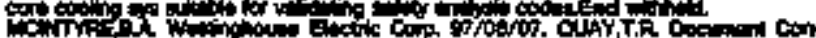

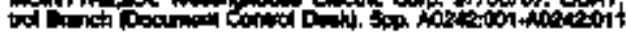

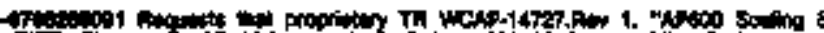

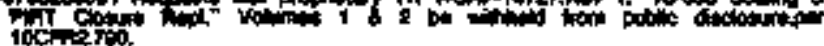

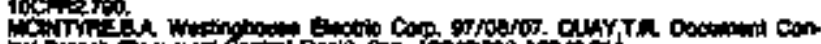

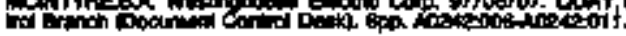

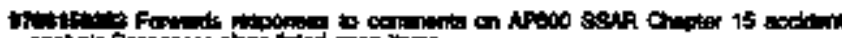

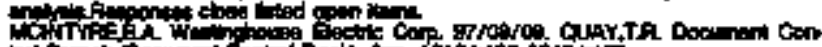

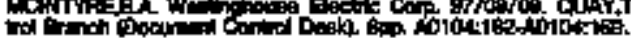

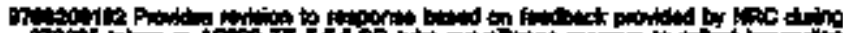
otots

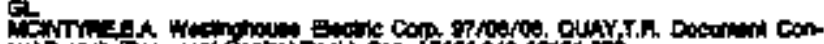

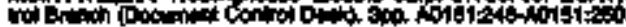

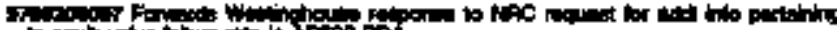

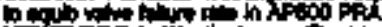

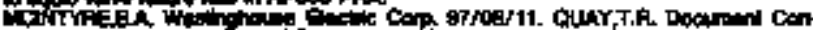

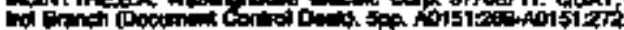

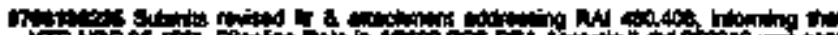

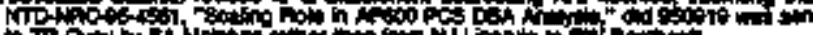

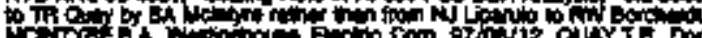
in

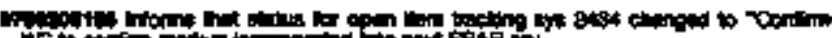

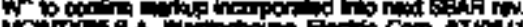

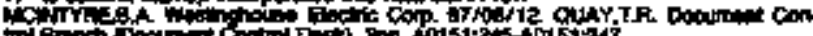

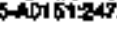

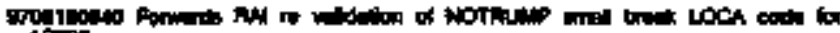

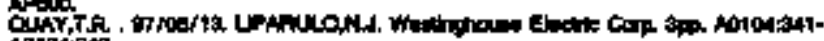
Alokist

\$70

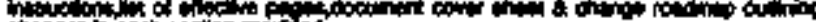

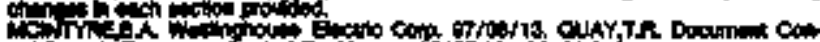

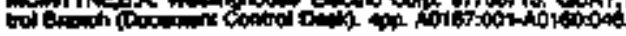

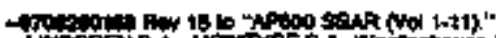

Uhe

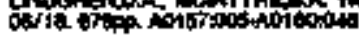

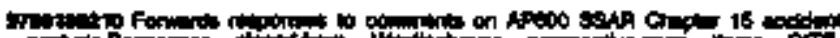

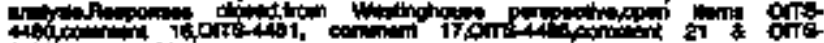

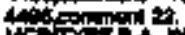

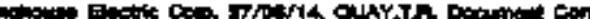

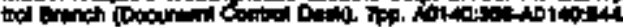

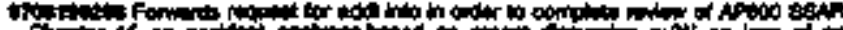

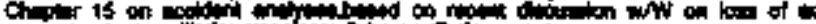

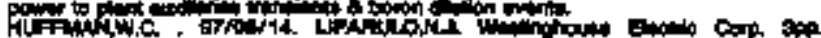

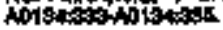

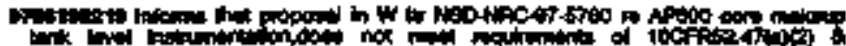

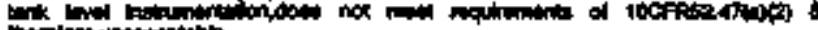

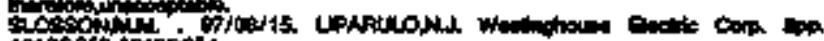

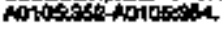

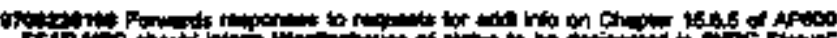

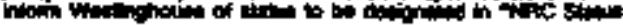
thing of

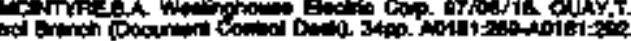

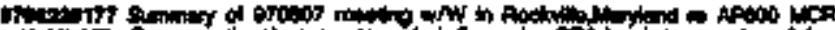

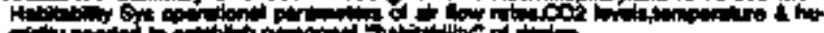

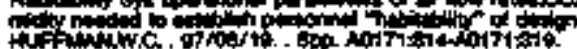

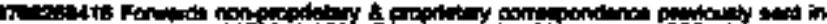

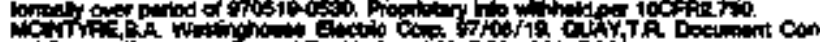

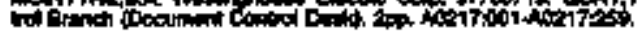

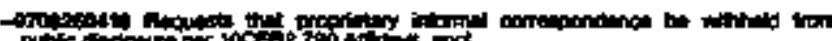
pow to

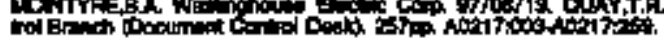

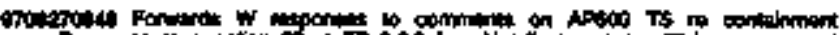

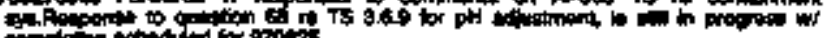

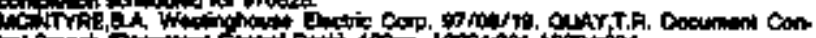

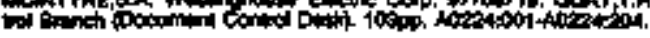

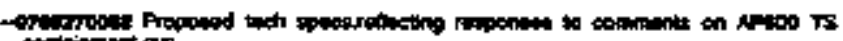

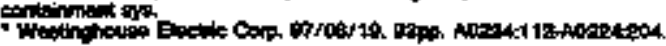

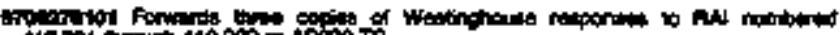

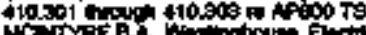

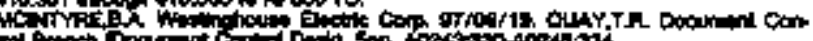

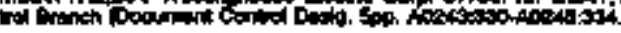

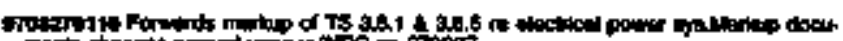

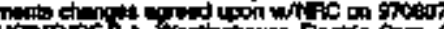

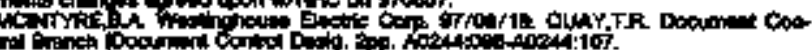

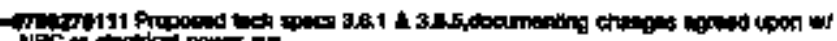

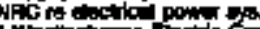

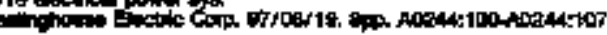

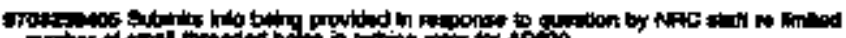

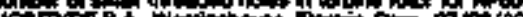
Fine

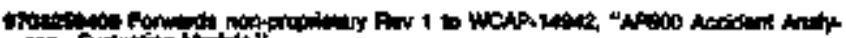

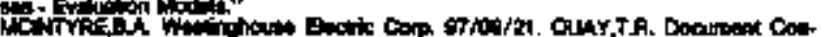

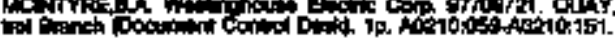

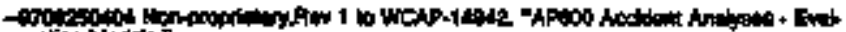

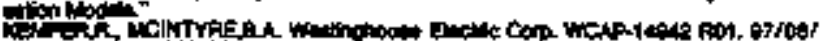

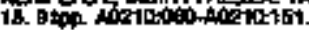

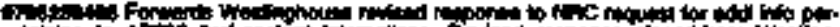

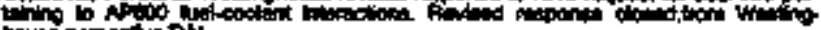

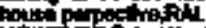

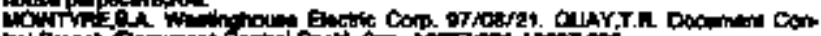

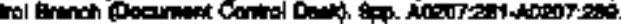

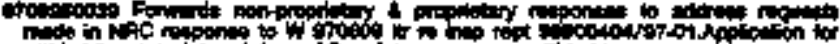

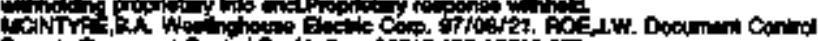

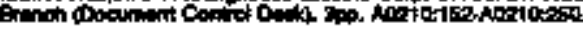

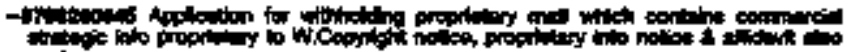

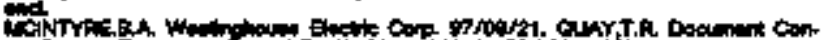

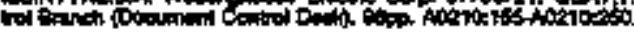

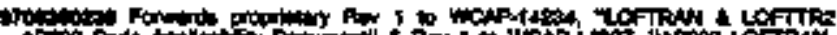

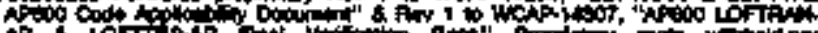

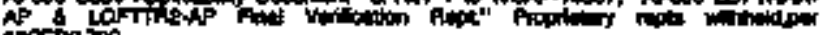

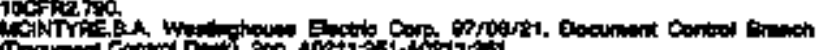

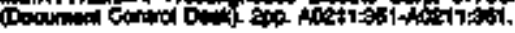

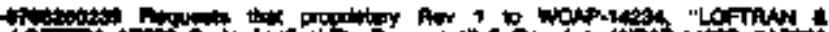

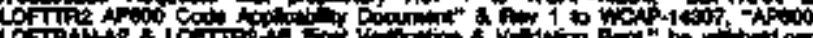

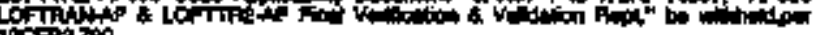
10

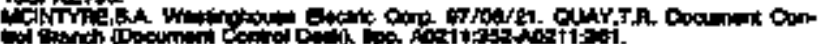

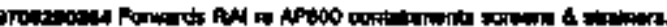

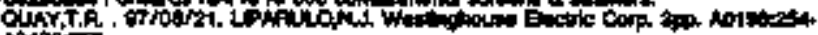
altons.

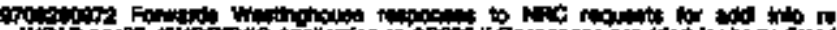

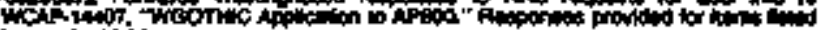

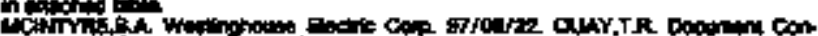

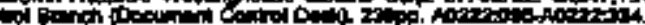

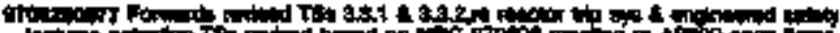

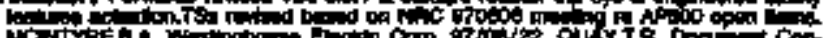

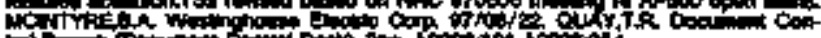

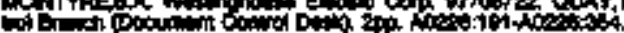

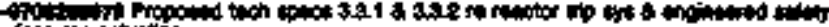

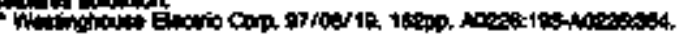

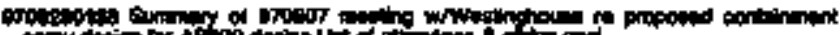

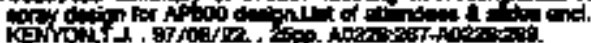

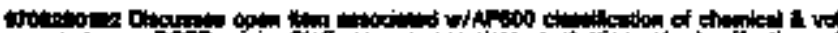

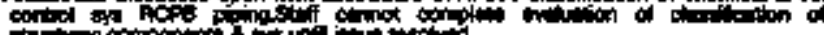
gith: Nosise

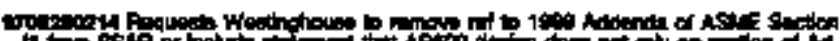

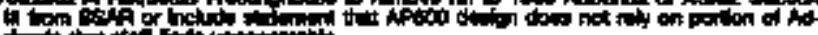

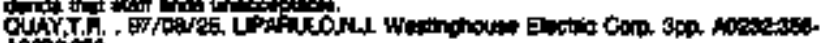
A 20200 


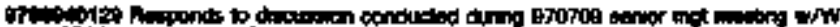

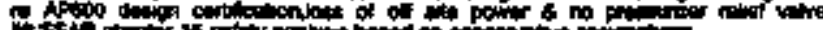

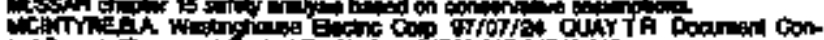

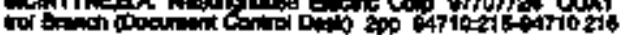

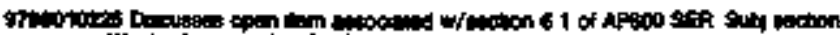

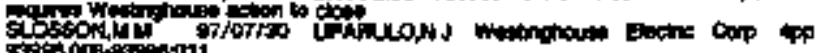

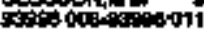

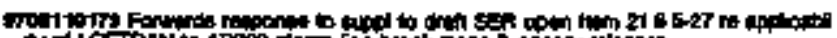

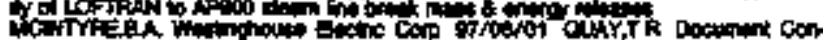

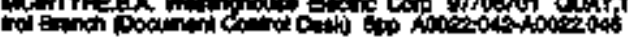

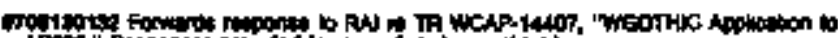

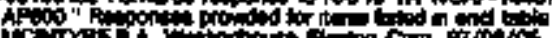

YC.MTE

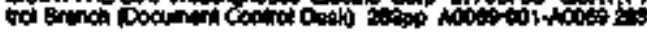

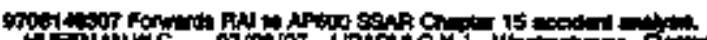

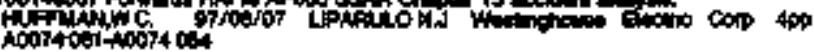

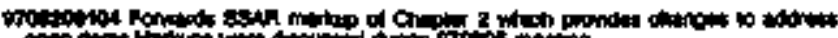

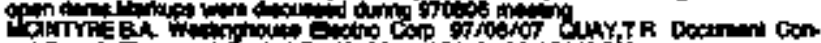

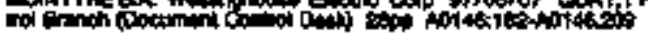

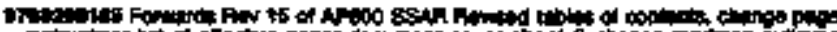

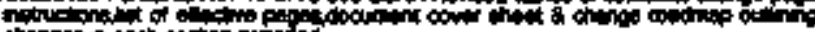

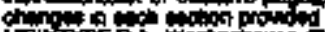

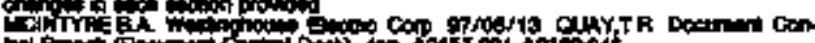

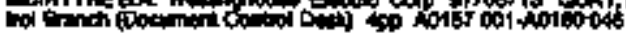

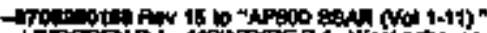

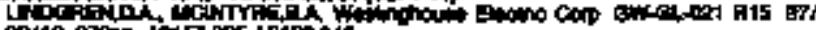

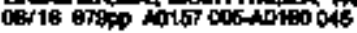

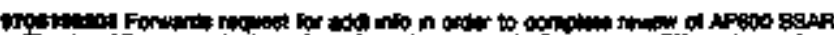

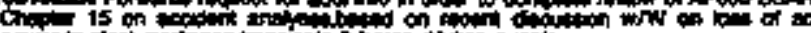

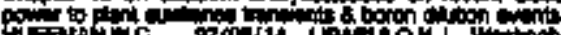

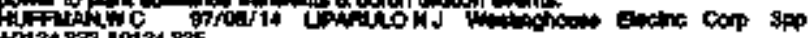

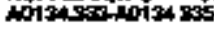

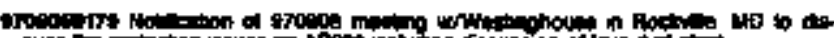

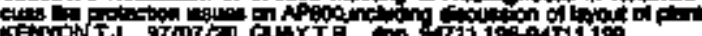

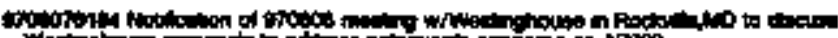

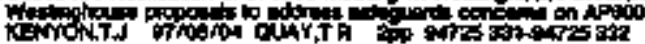

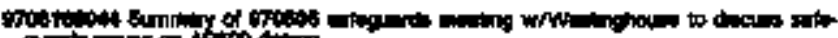

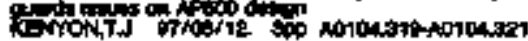

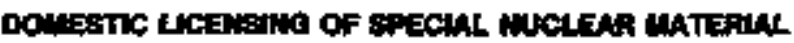

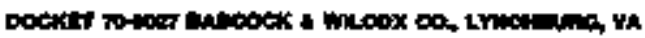

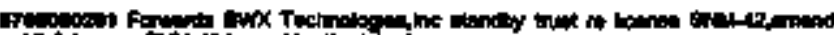

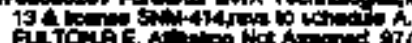

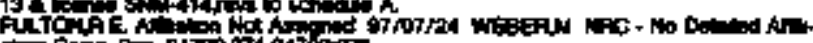
intion of

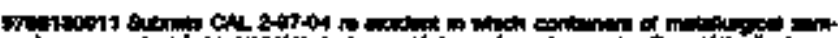

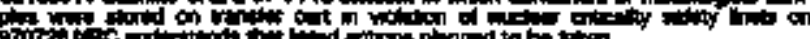

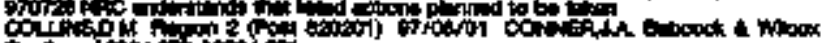
Co 300 not

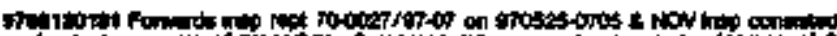

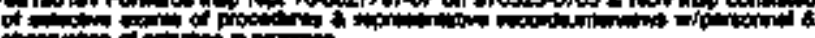

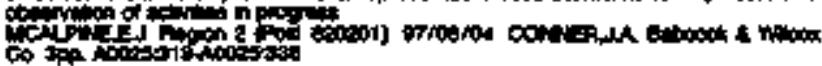

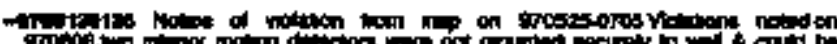

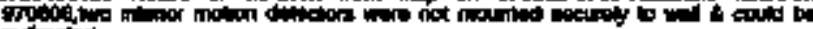

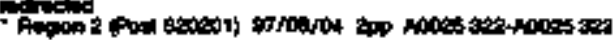

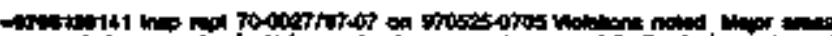

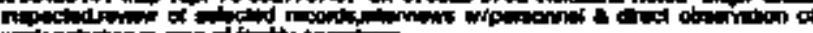

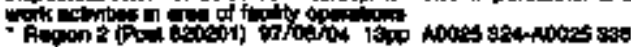

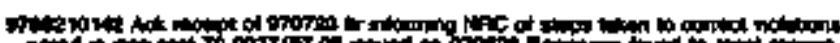

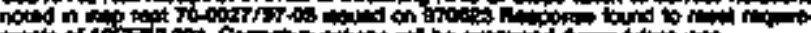

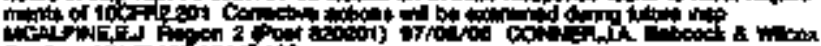

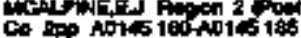

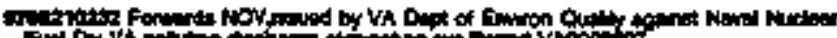

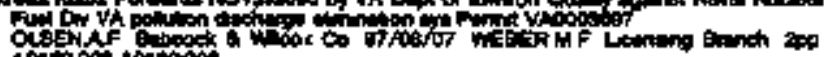
Ot:

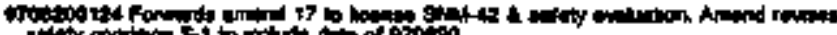

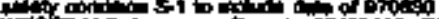
Notes as

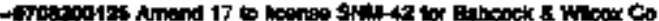

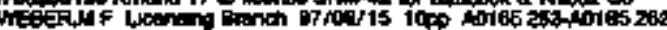

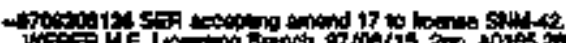

Wr: $\rightarrow$ MF I I

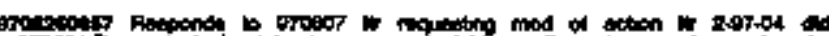

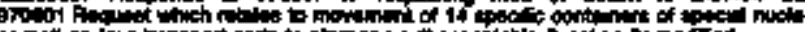

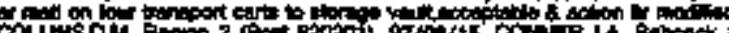

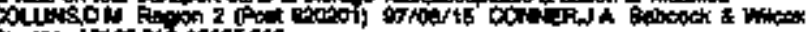

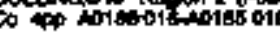

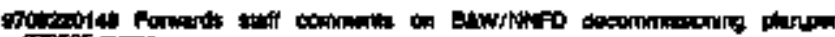

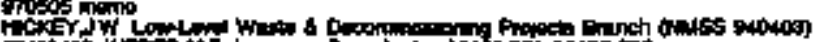

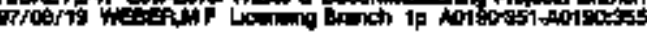

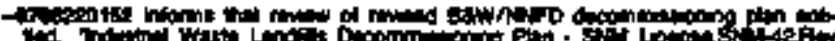

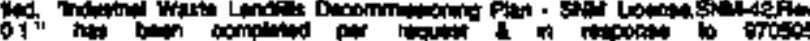

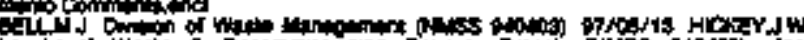
10.

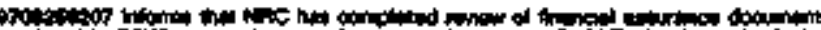

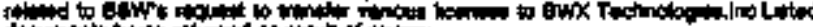

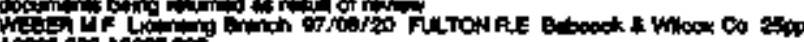

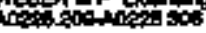

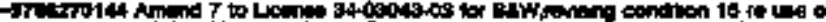

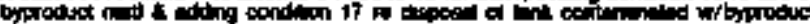

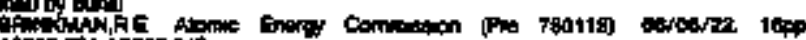

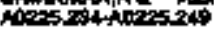

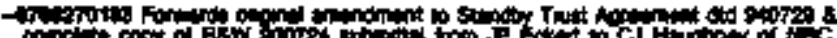

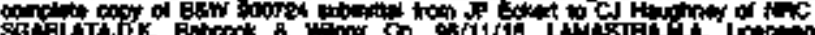

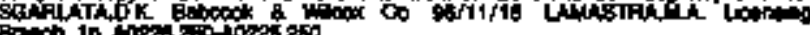

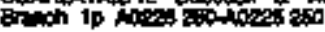

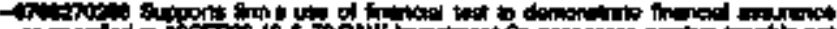

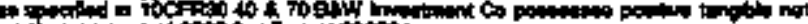
int ph toun to

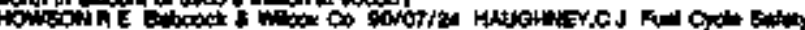

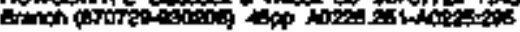

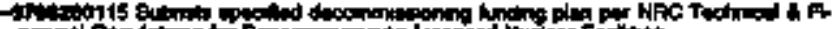

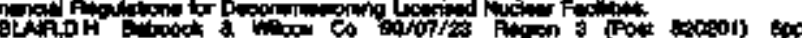

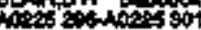

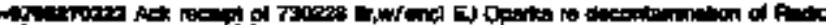

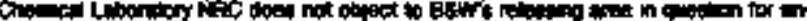

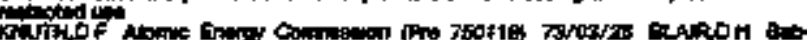

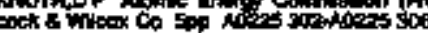

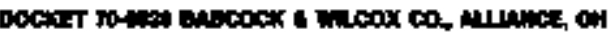

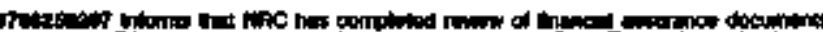

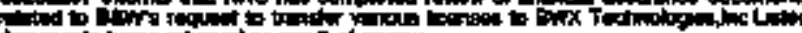

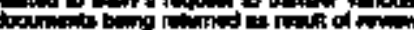

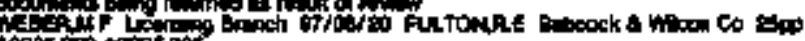

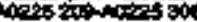

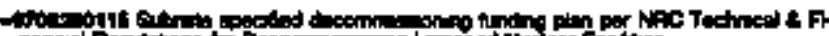

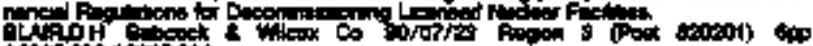

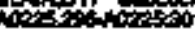

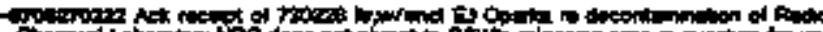

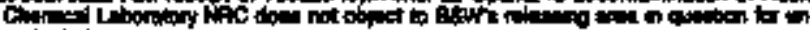

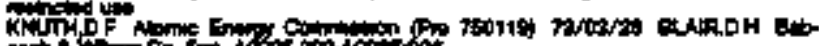
cocich

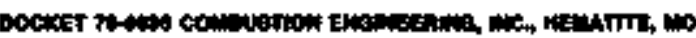

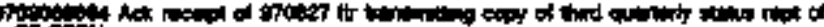

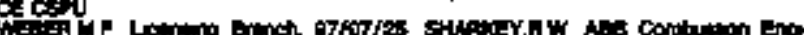
(n) otrotion

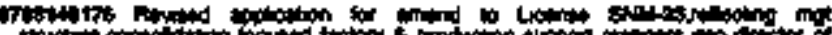

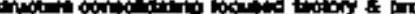

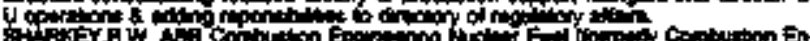

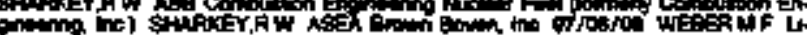

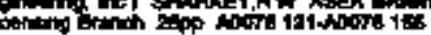

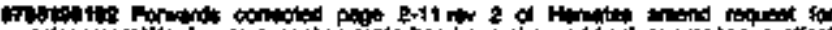

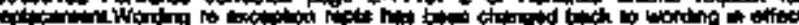

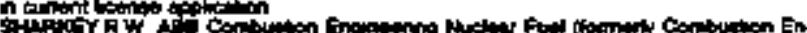

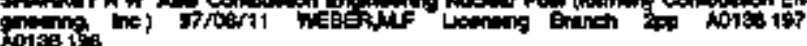




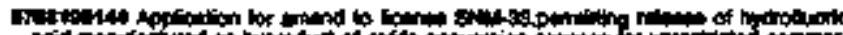

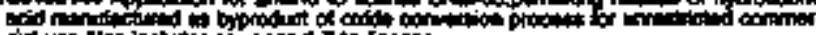

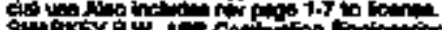

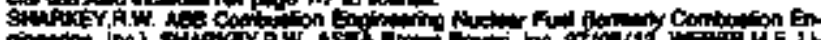

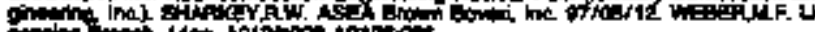

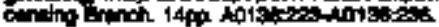

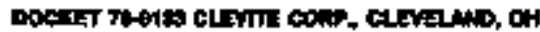

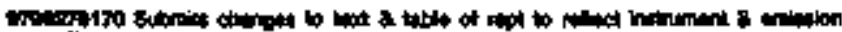

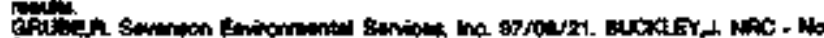

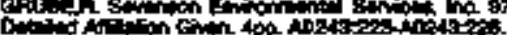

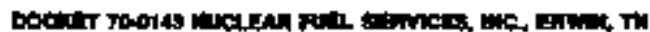

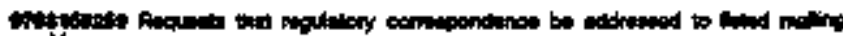

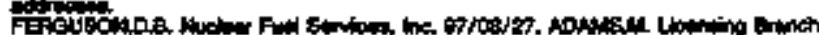

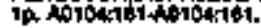

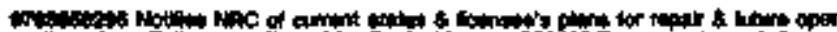

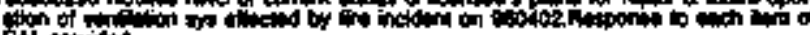

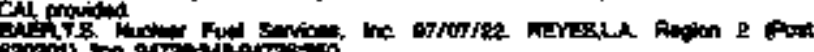
Pacoly ipp. on

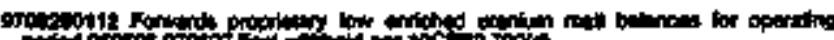

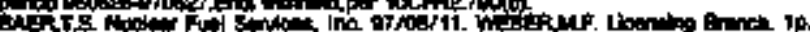

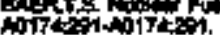

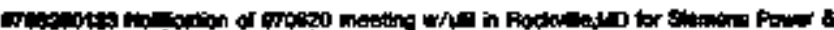

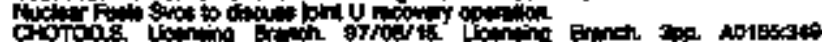
ciolis.

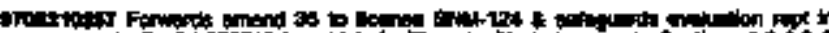

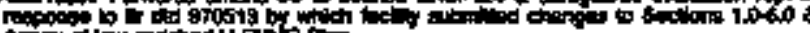

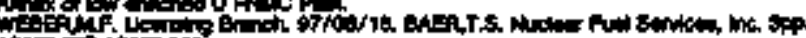

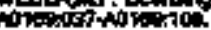

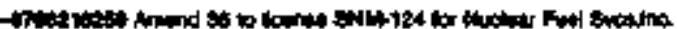

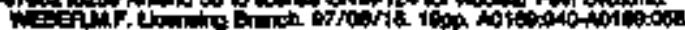

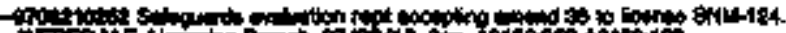

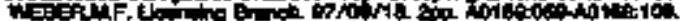

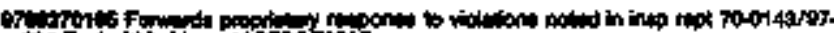

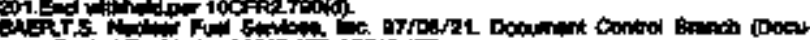

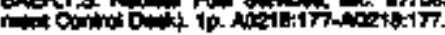

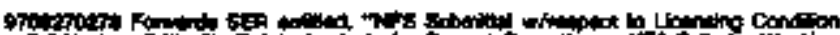
orth Py.

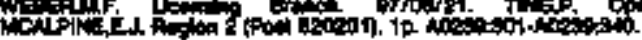

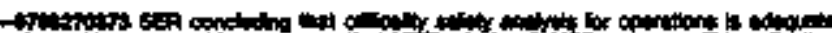

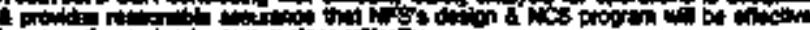
4

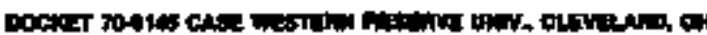

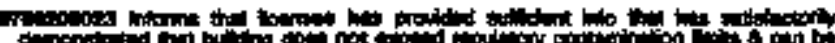

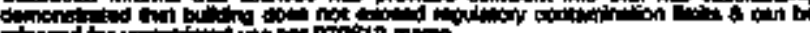

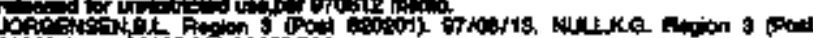

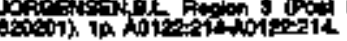

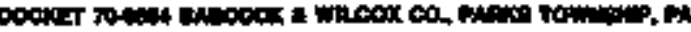

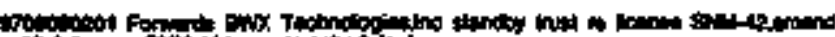

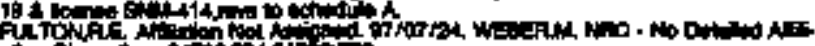

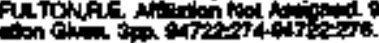

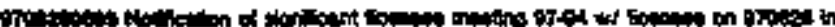

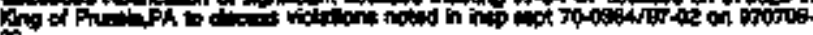
尊

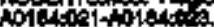

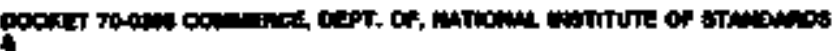

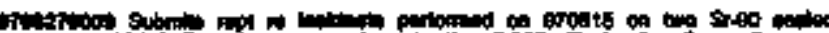

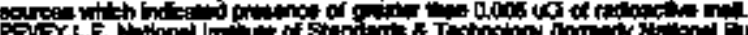

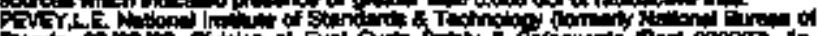

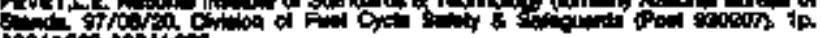

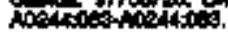

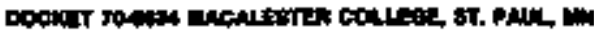

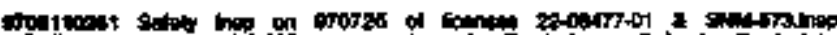

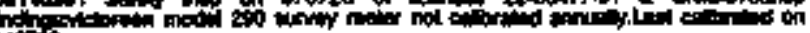

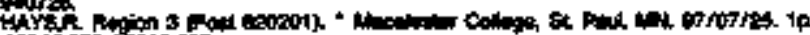

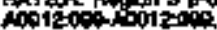

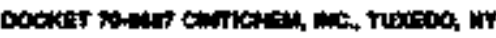

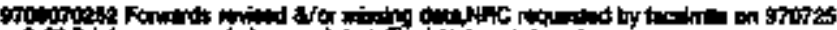

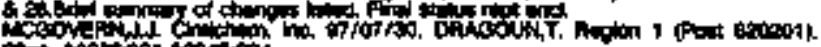

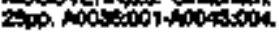

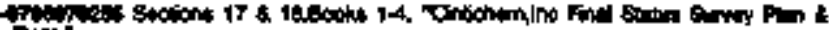

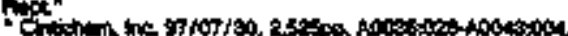

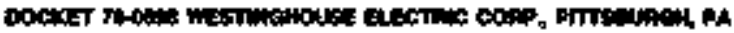

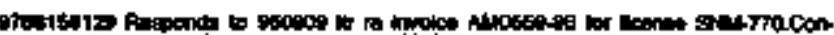

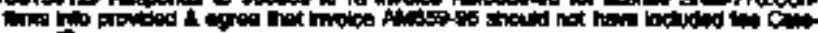
orts

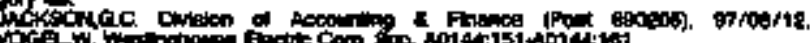

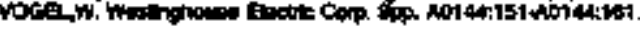

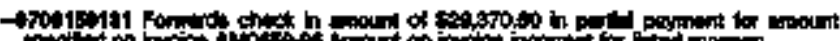

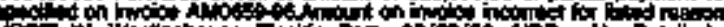

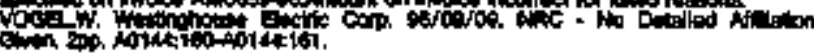

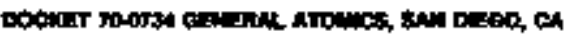

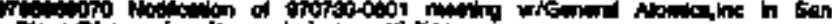

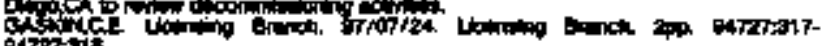
otrotita

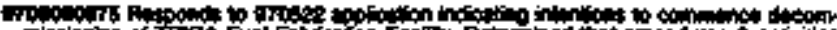

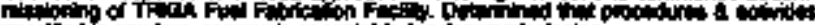

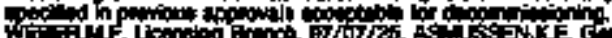

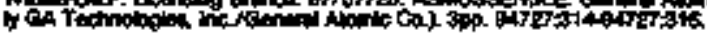

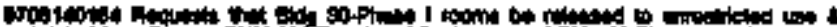

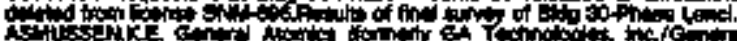

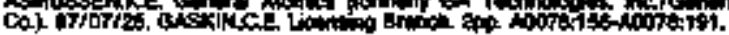

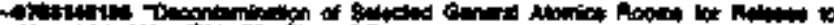

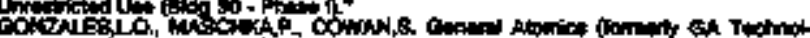

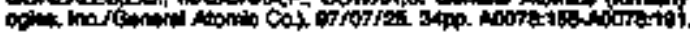

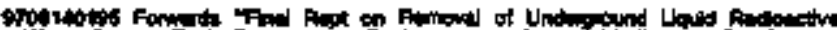

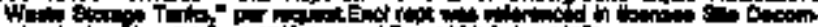

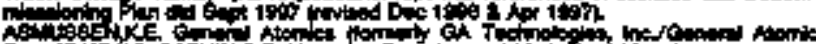

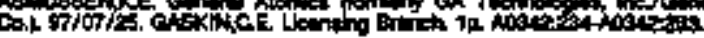

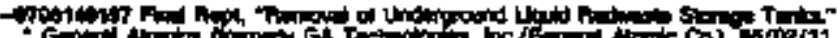

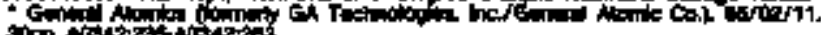

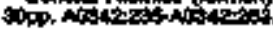

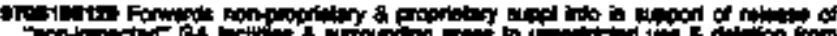

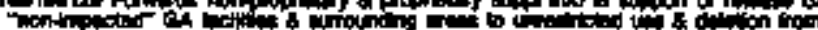

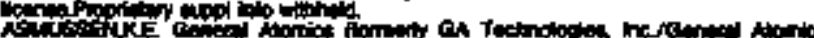

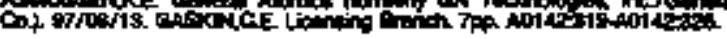

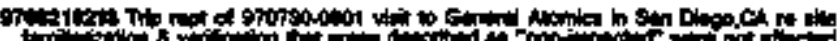

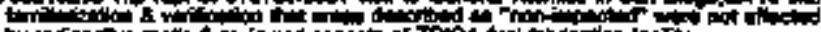

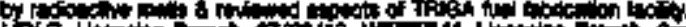

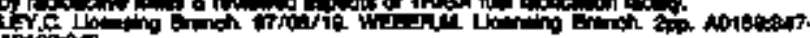
Anibog

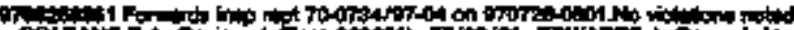

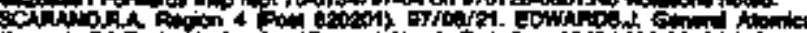

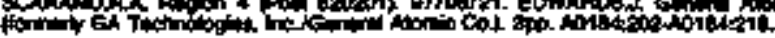

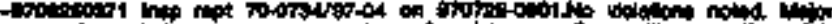

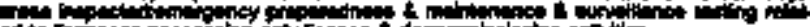

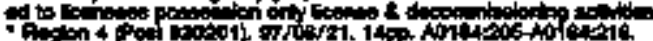

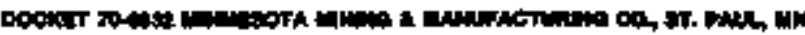

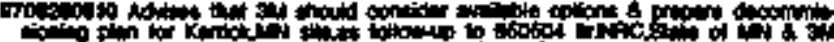

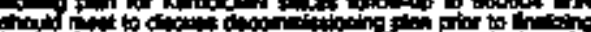

tut 90.0.

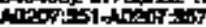

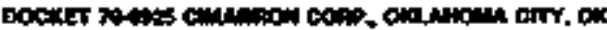

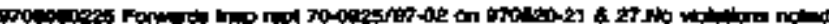

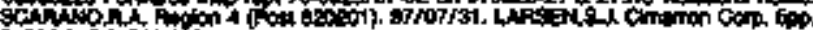
artist of

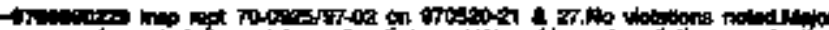

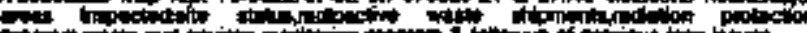

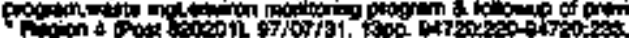




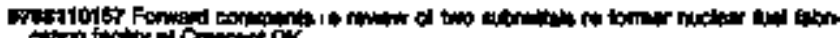

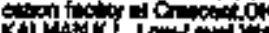

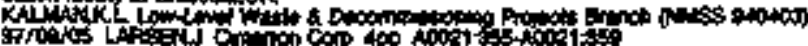
管

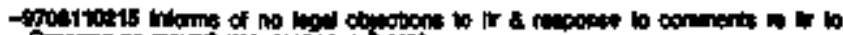

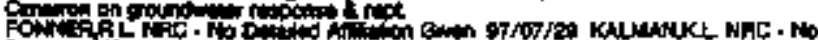

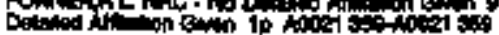

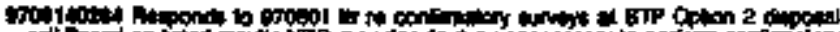

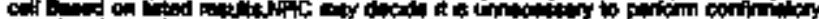
intion

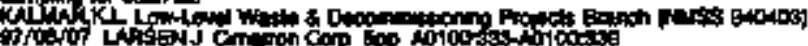

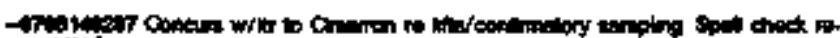

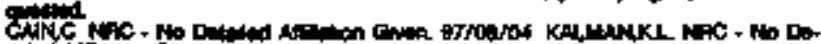

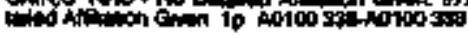

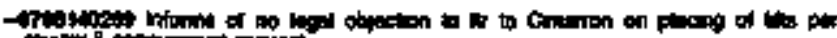

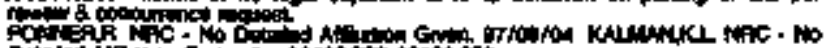

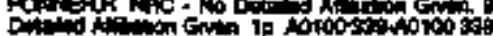

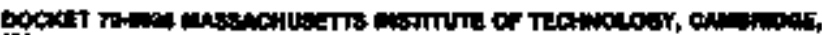
14.

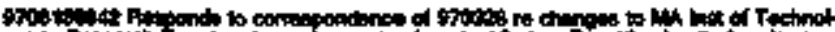

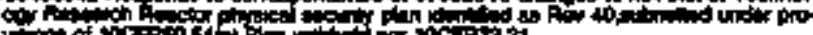
Hor

th Toche

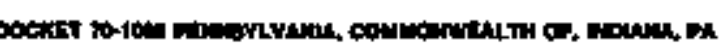

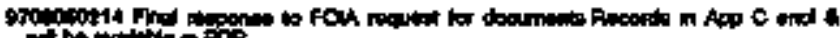

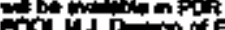

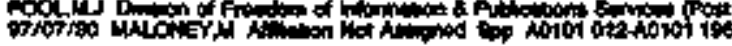

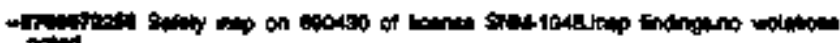

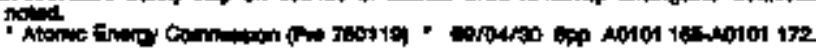

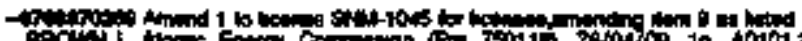

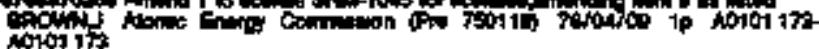

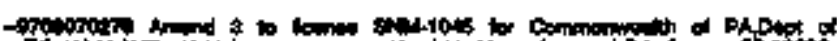

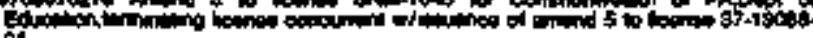

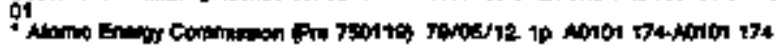

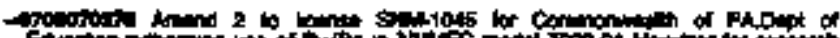

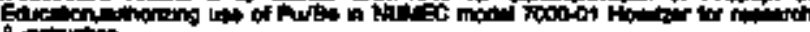

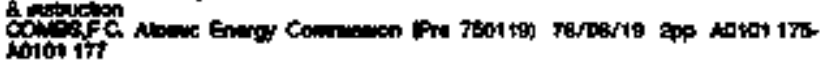

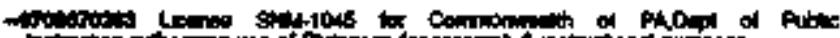

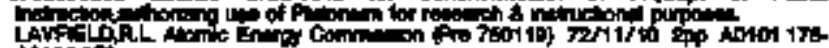
d0ㅇำ 170

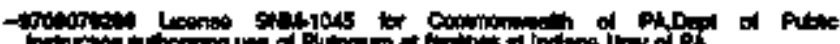

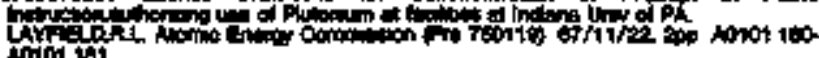
intoi to

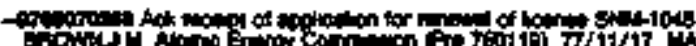

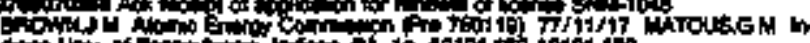

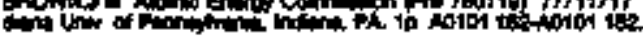

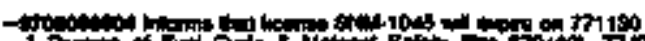
ochench of

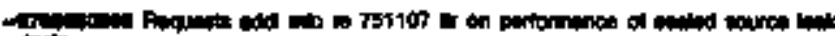

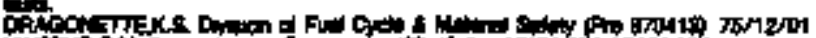

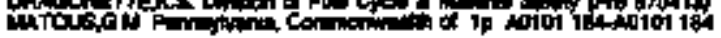

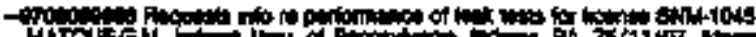

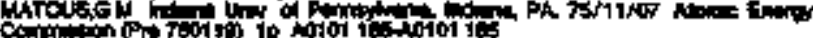

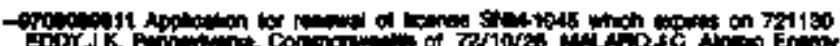

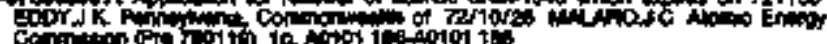

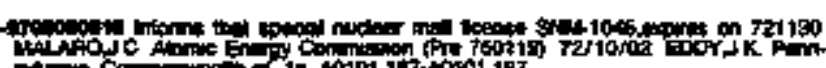

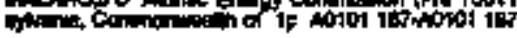

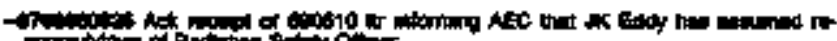

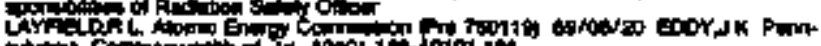

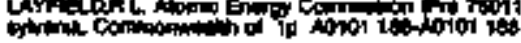

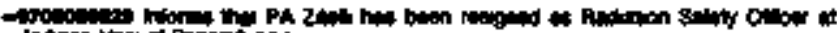

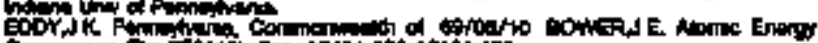

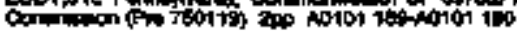

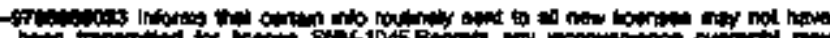

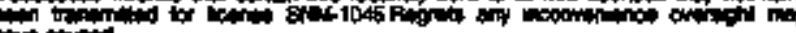

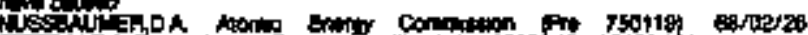

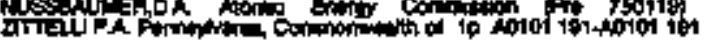

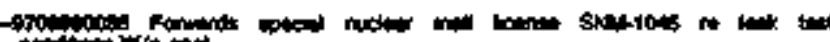

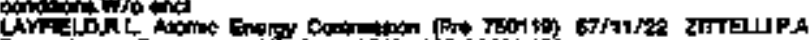

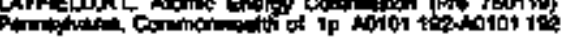

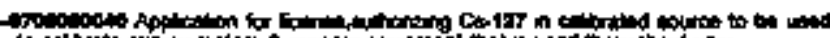

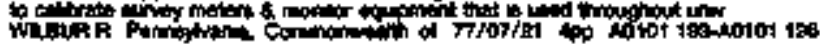

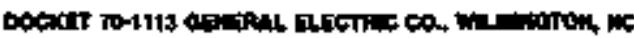

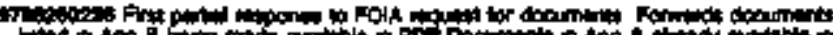

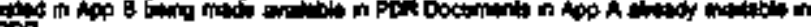
Dof

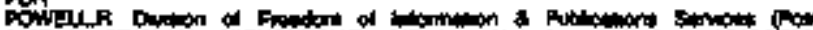

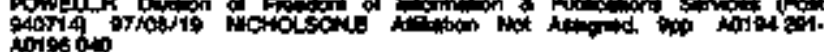

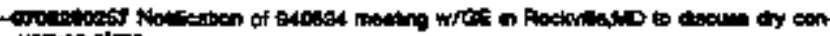

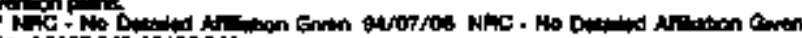

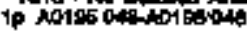

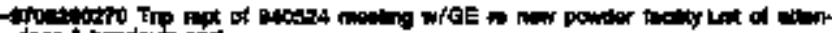

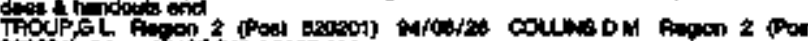

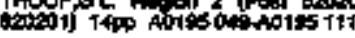

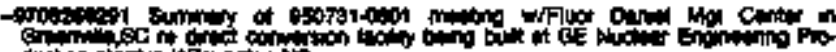

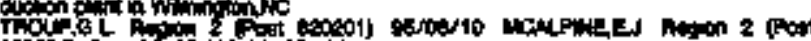
1.

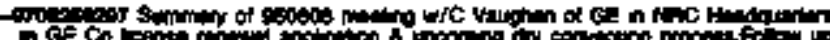

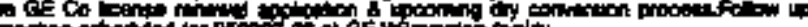
wction

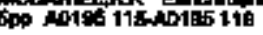

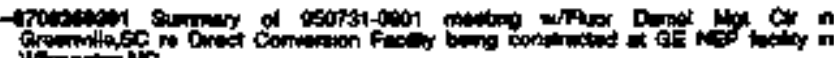

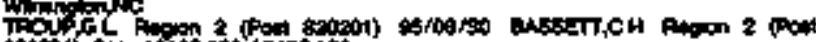

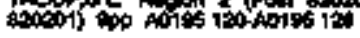

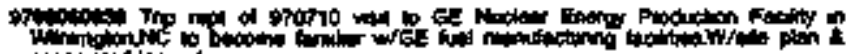

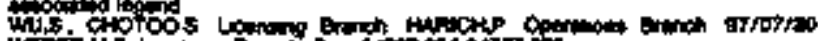

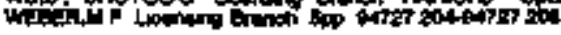

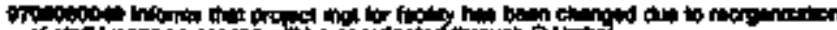

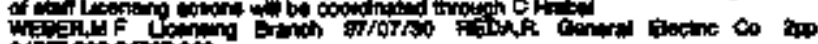

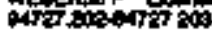

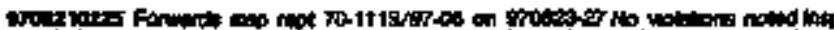

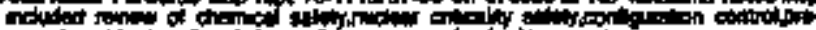

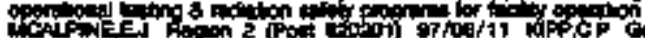

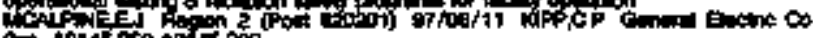

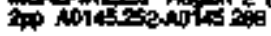

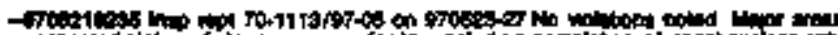

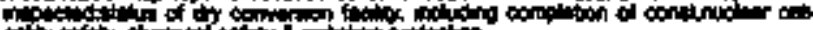

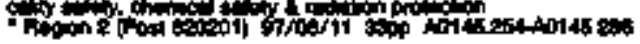

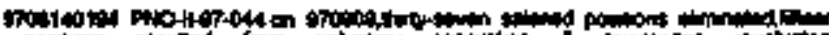

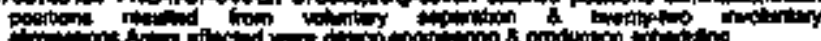

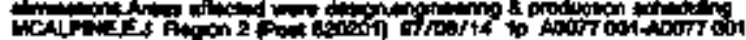

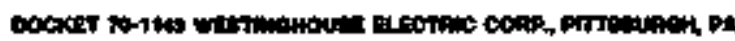

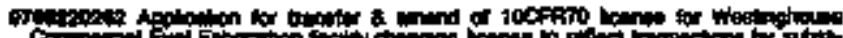

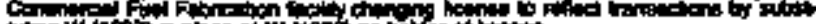

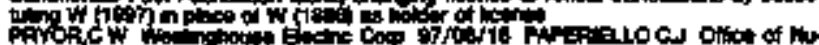

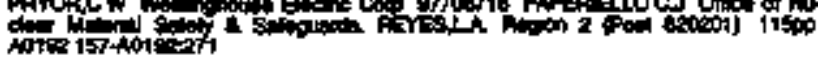

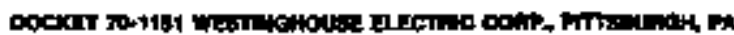

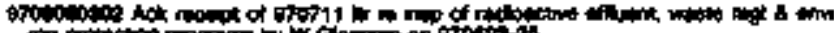
in

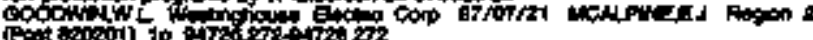

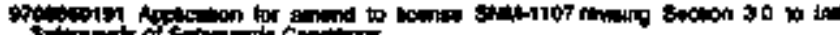

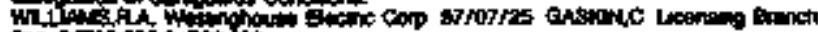

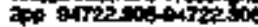




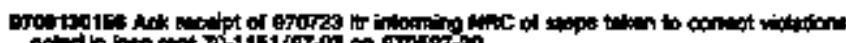

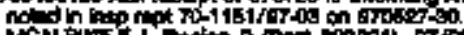

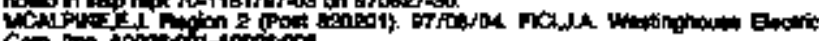

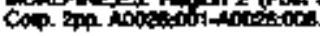

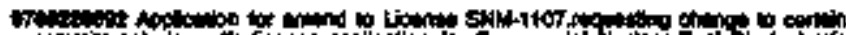

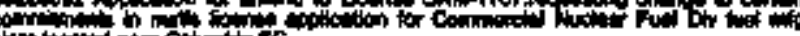
int

Nin wot

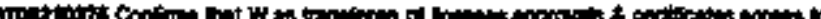

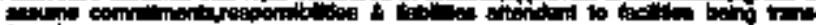
cle

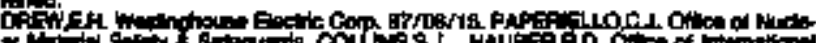

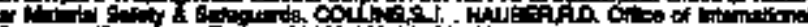

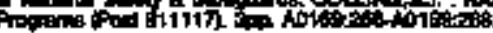

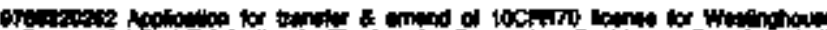

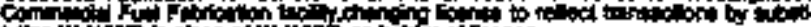

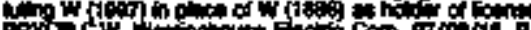

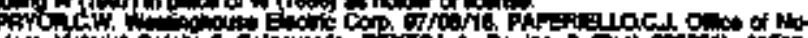

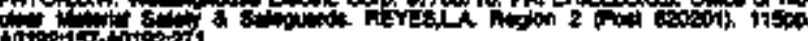

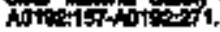

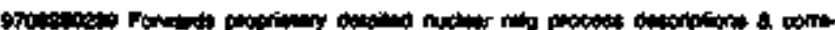

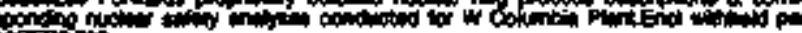

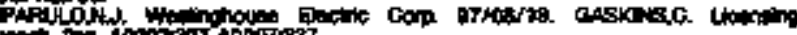
年

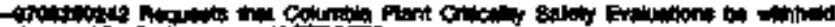

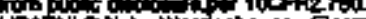

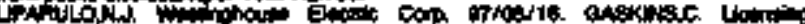

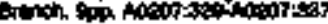

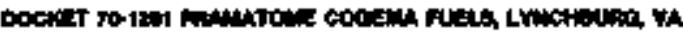

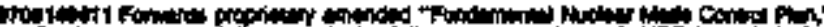

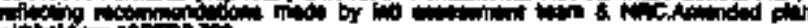

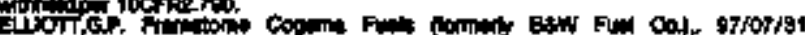

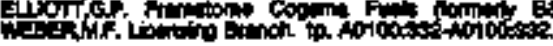

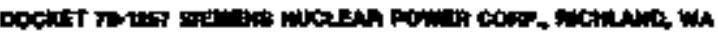

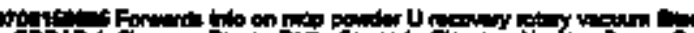

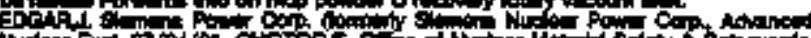

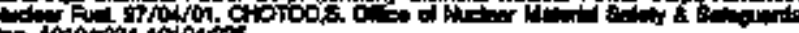

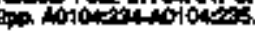

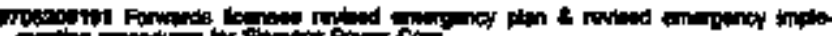

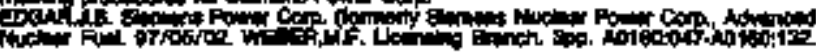

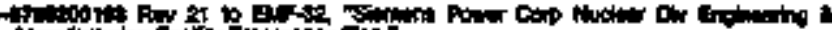
Wits

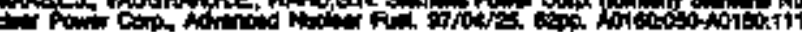

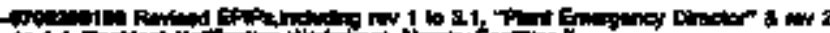

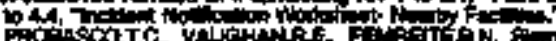

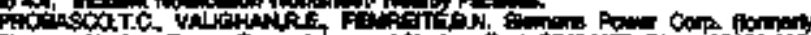

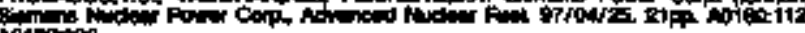
intook

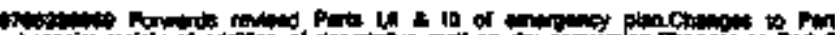

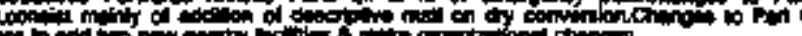

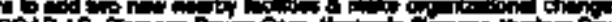

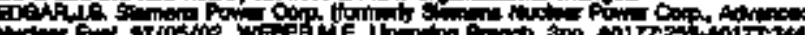

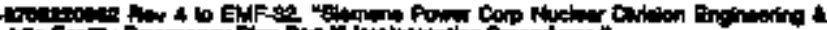

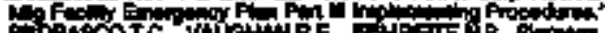

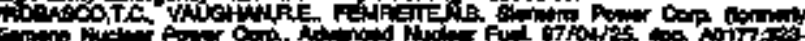
contingin.

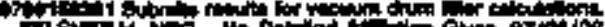

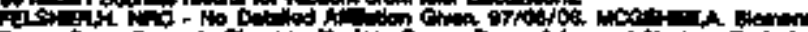

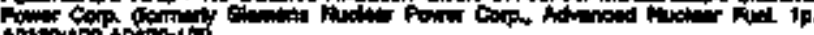

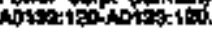

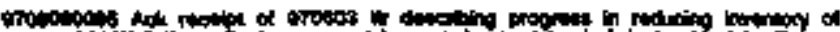

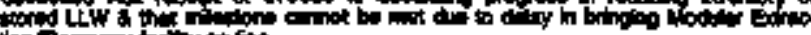

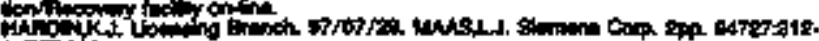
arzata

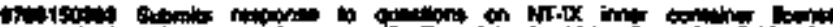

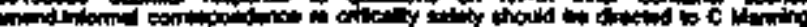

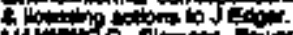

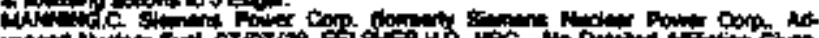

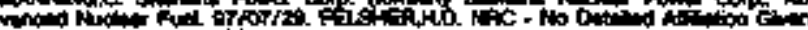
up Aorses:-

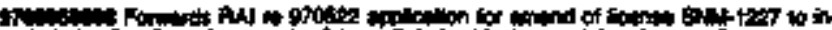

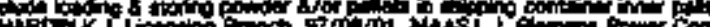

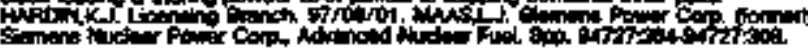

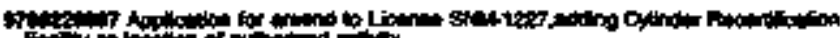

Eu bin

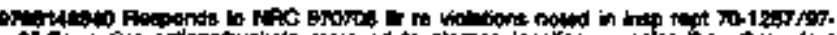

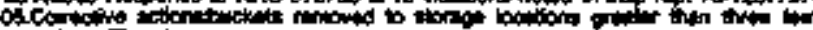

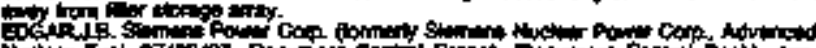

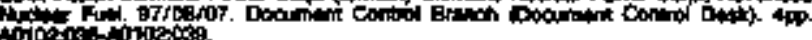

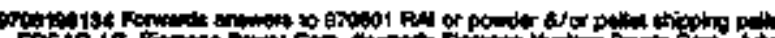

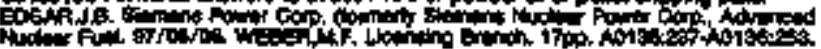

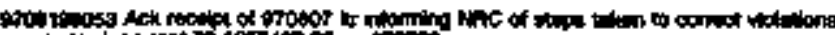

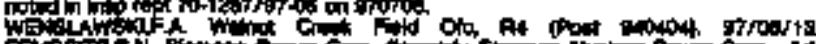

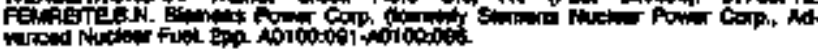

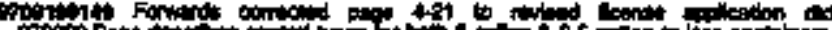

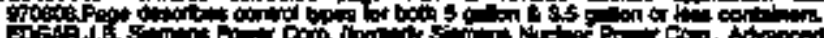

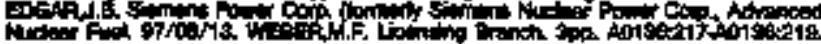

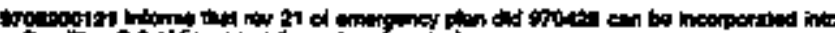

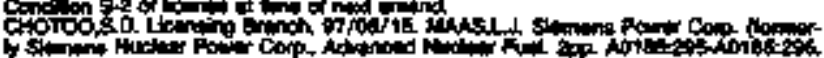

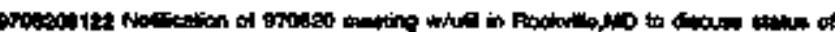

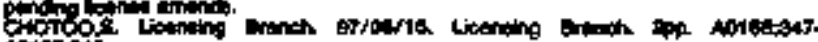
iplotion

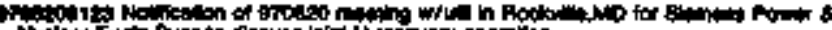

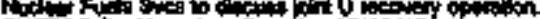

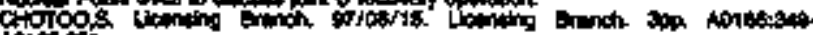
alcotit.

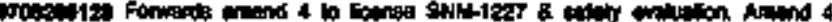

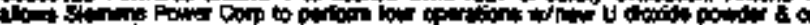

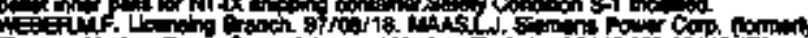

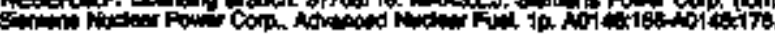

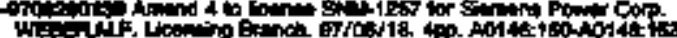

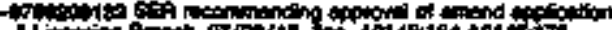

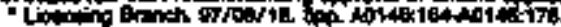

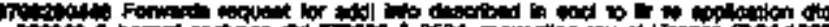

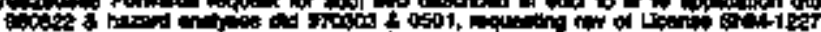

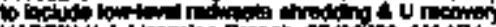

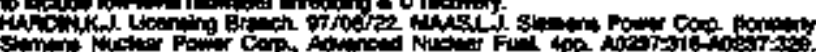

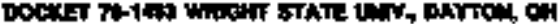

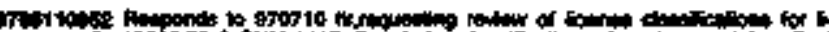

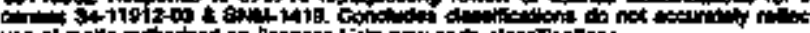

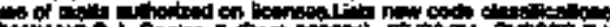

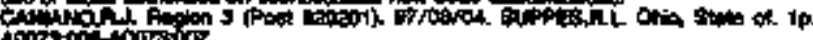

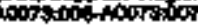

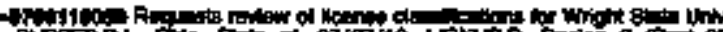

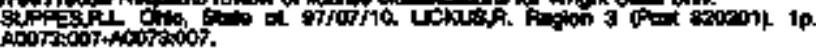

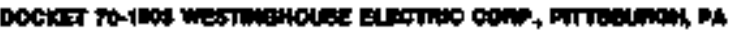

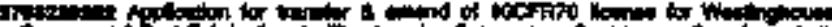
Com

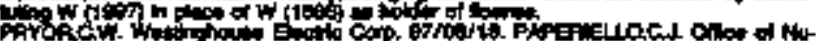

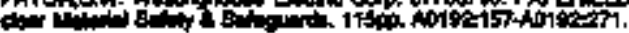

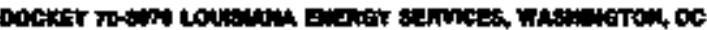

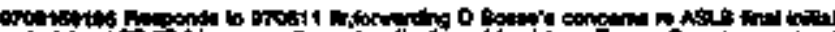

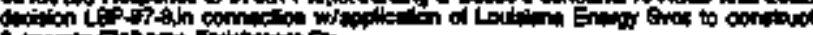

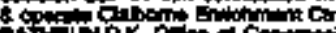

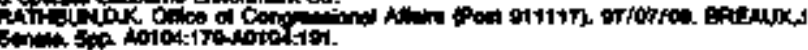

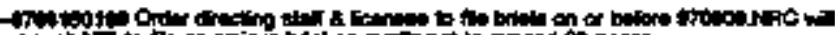

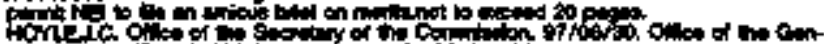

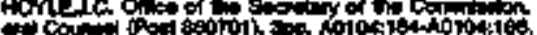

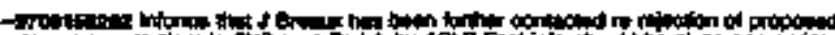

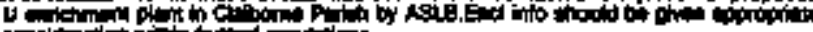

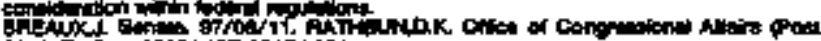

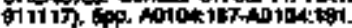

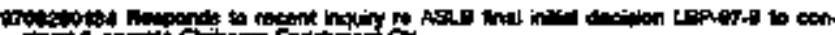

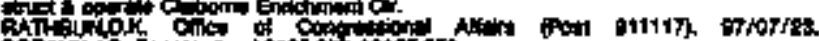

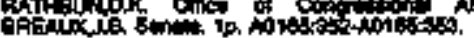




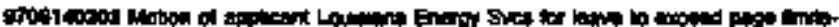

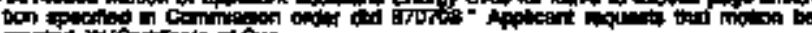

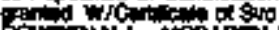

ANu totig

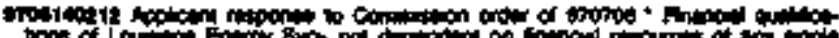

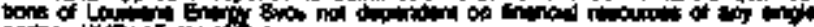

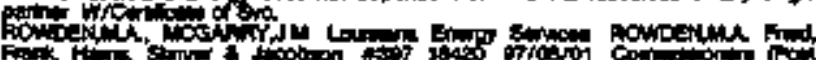

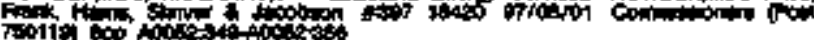

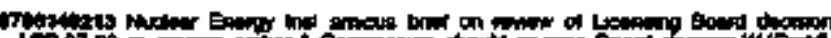

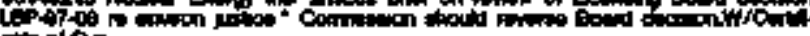

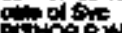

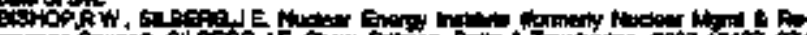

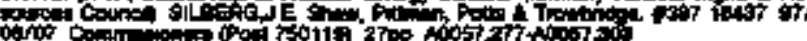

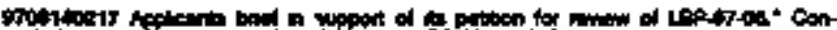

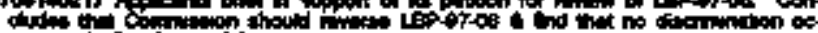

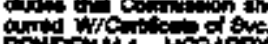

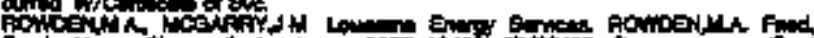

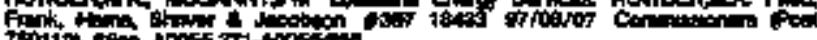

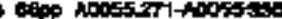

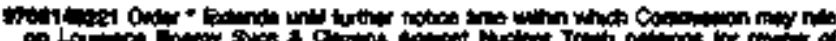

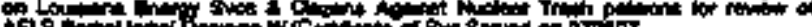

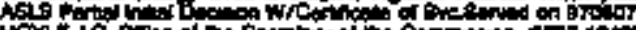

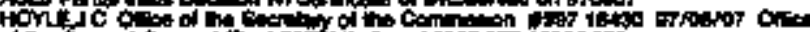

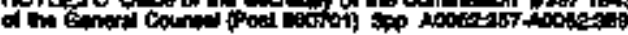

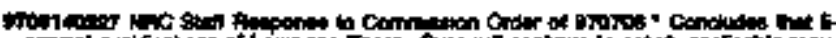

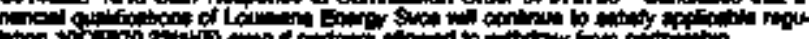

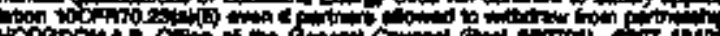

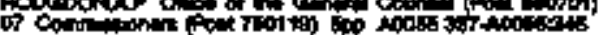

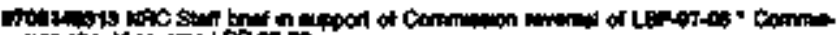

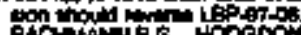

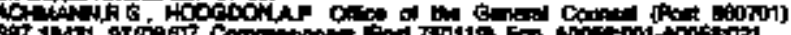

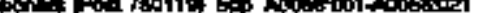

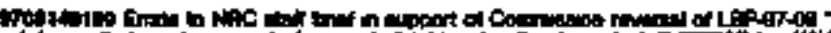

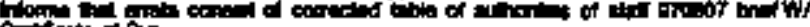
Con of Com

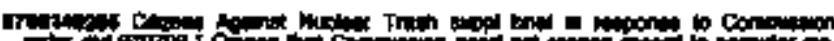

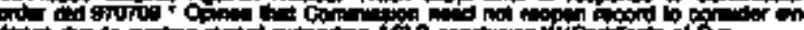

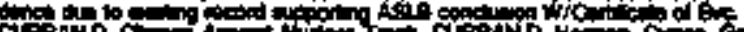

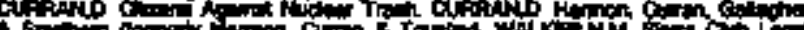

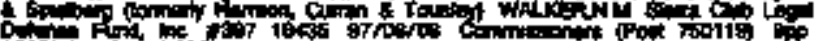

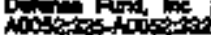

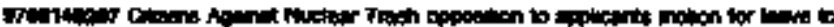

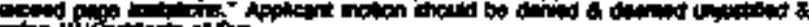

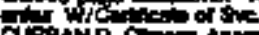

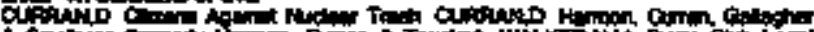

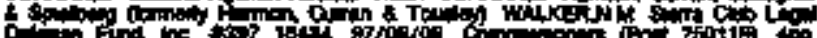

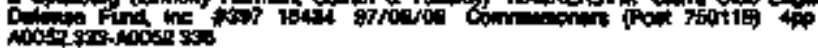

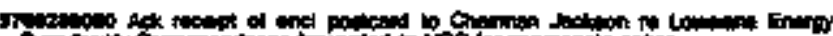

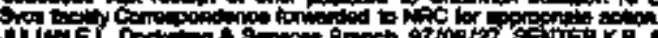

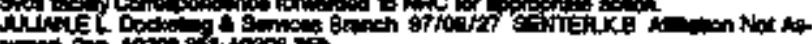

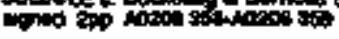

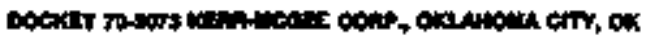

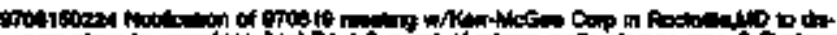
alo phent to

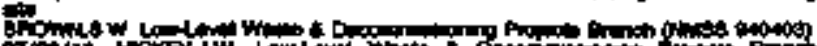
OH

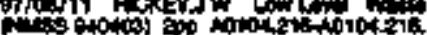

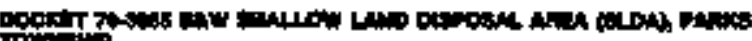

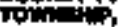

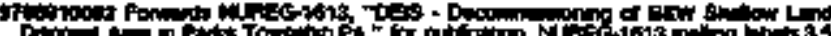

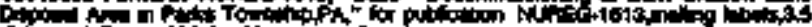

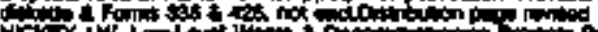

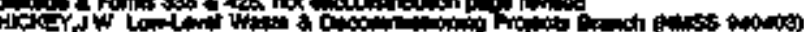

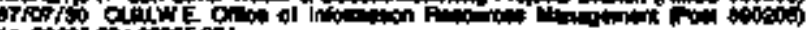

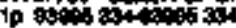

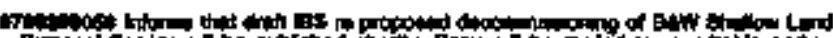

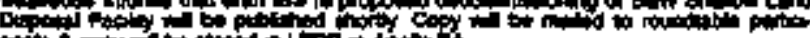

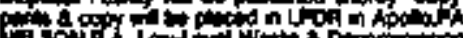

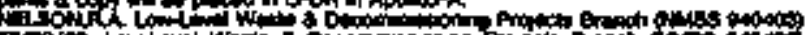

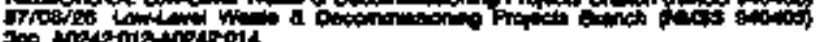

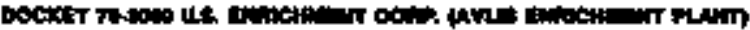

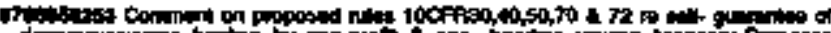

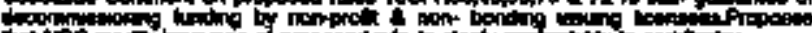

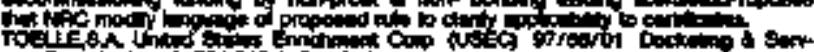

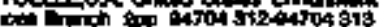

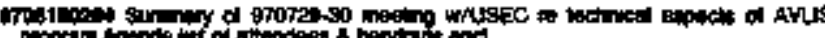

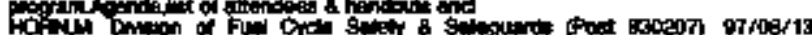

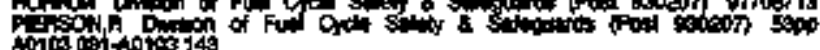

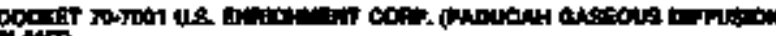
(1).17

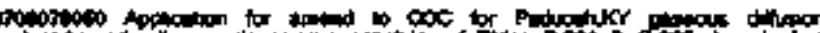

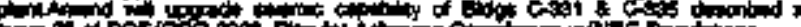
Tif

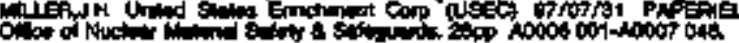

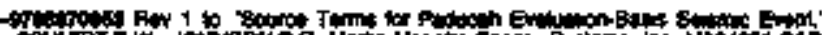

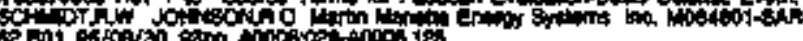

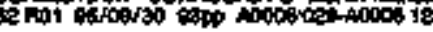

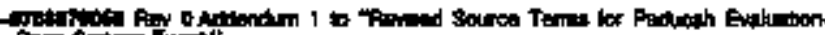

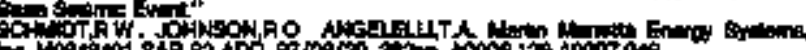

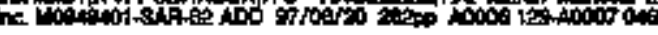

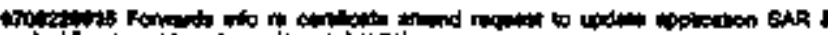

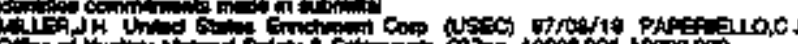

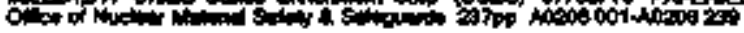

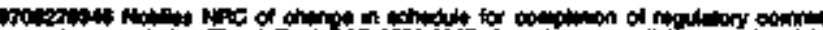

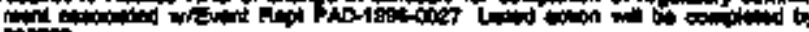

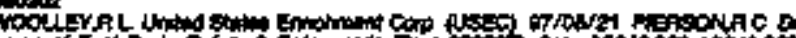

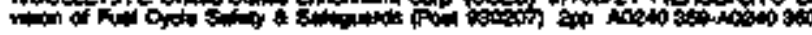

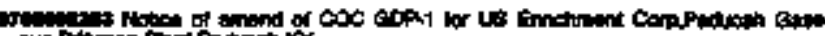
of

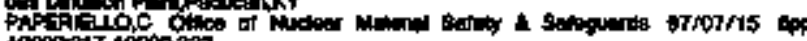
A0000-317-0000322

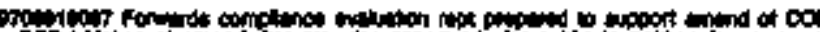

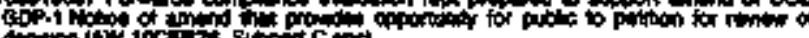

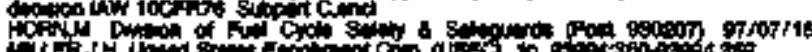

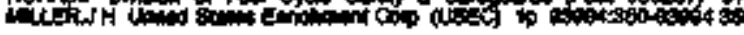

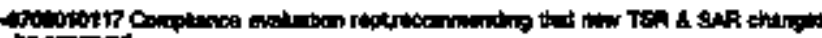
ba apponed

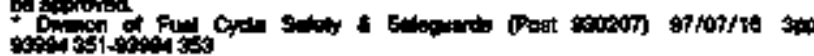

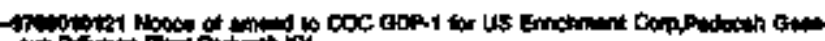

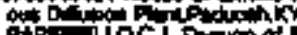
Anp in is of

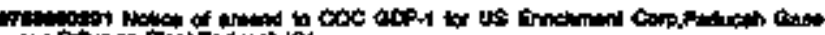
Not

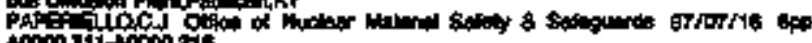

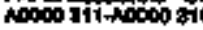

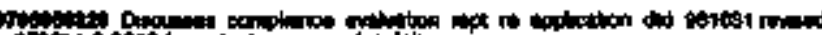

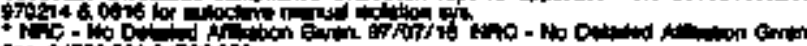

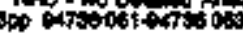

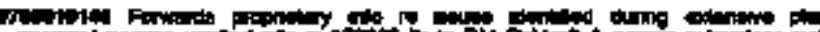

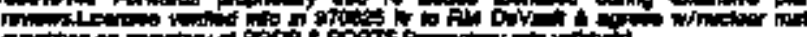

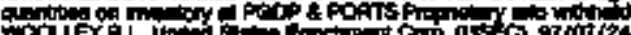

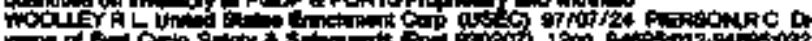

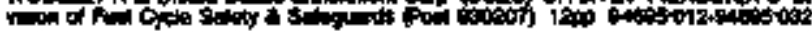

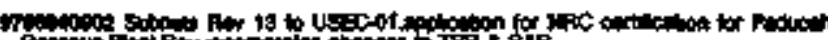

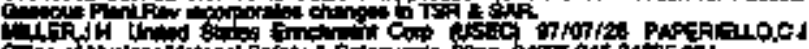

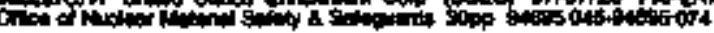

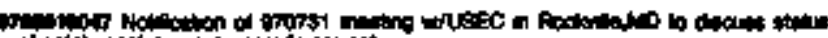

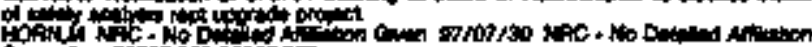
(and

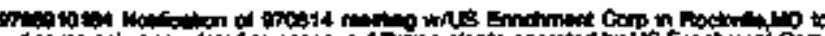

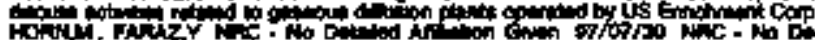

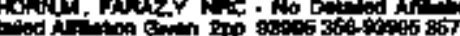

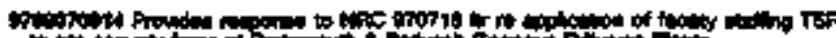

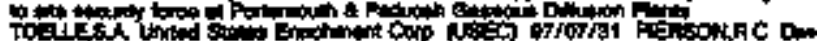
tond of

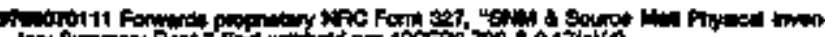
of

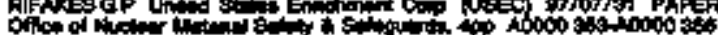

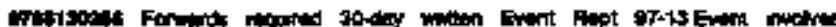

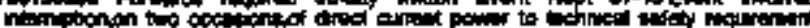

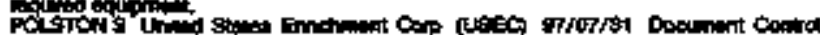

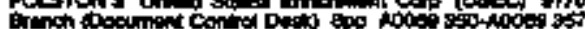




\section{DOCKETED ITEMS}

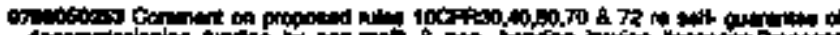

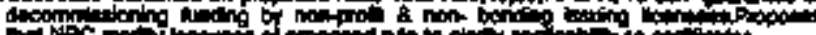

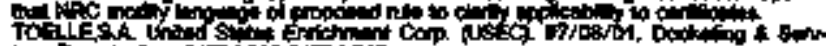

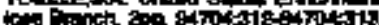

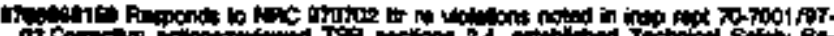
Q

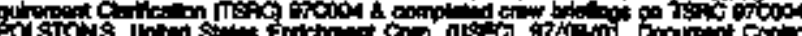

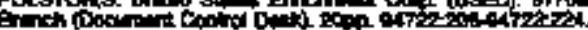

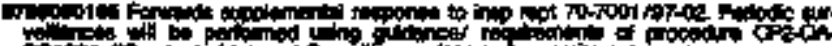

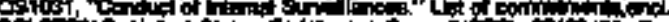

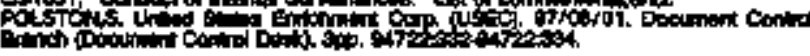

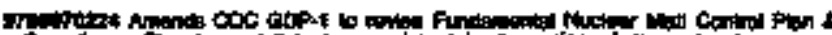

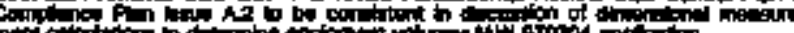

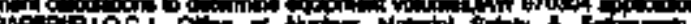
管 (4)

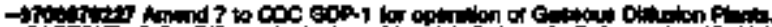

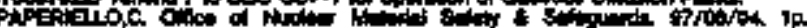

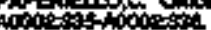

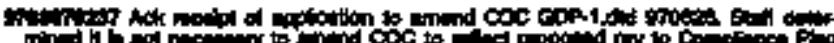

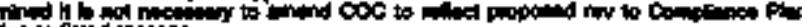

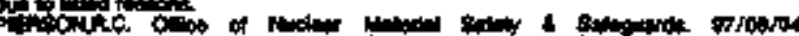

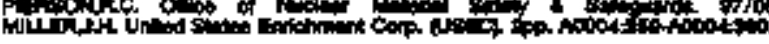

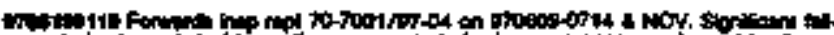

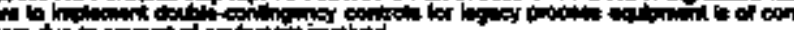

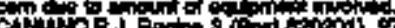

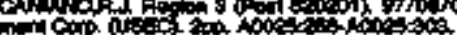

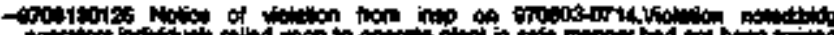

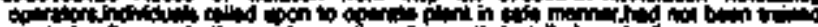

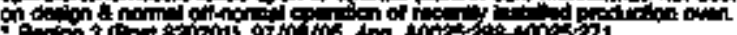

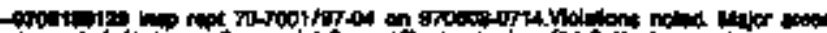

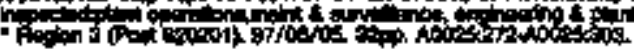

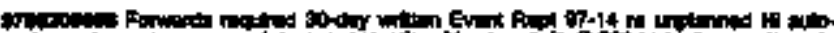

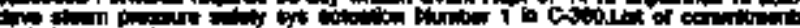

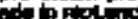

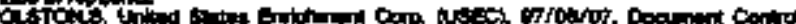

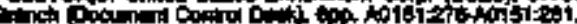

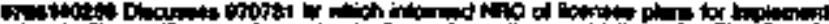

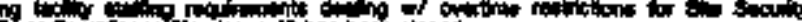

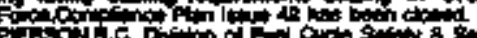
J

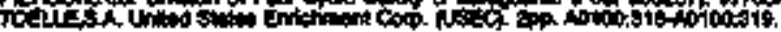

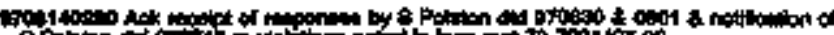

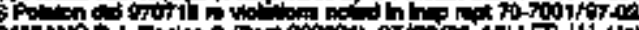

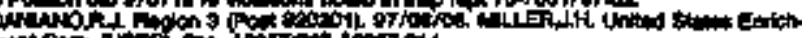

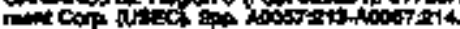

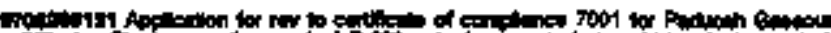
Cum fins

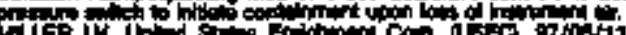
aprailo,a.t A

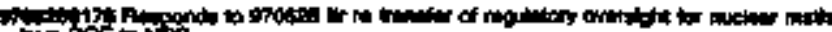

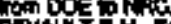

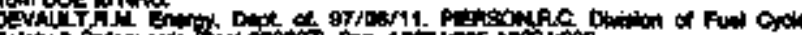

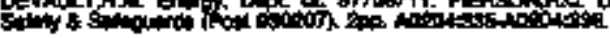

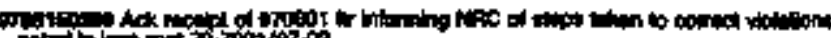

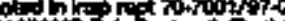

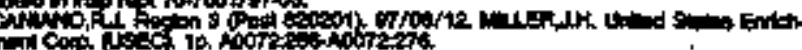

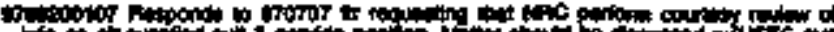

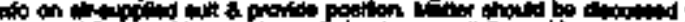

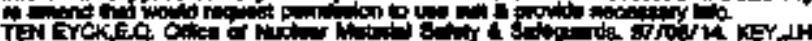

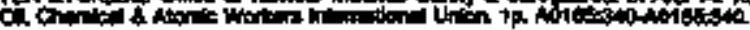

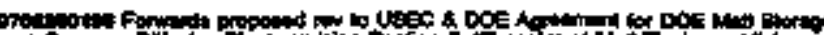

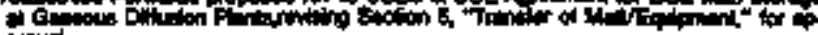

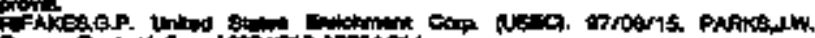

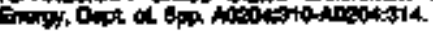

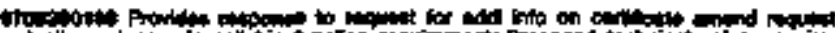

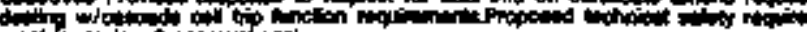
(a)

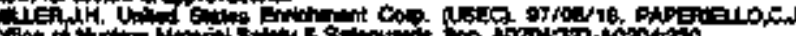

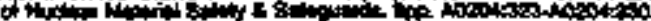

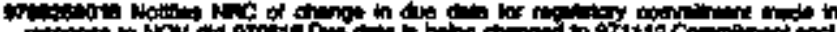

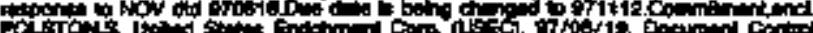

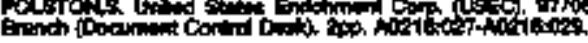

9roveron the

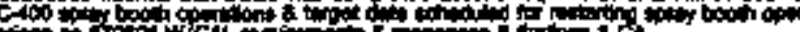

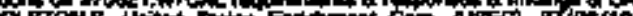
20201\% 1400 .

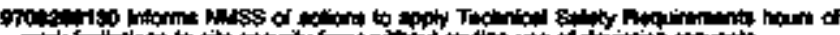

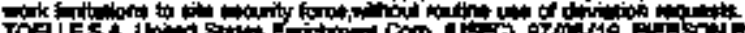

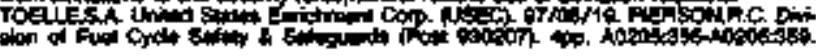

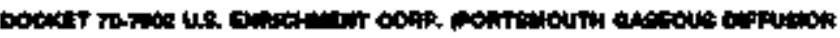

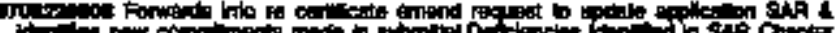

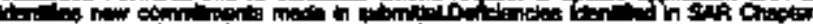

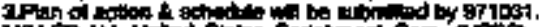

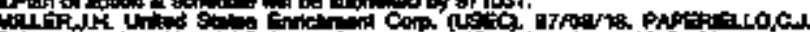

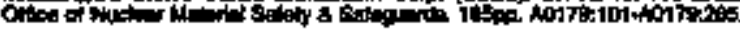

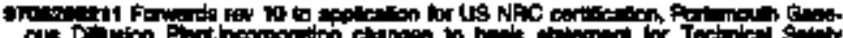

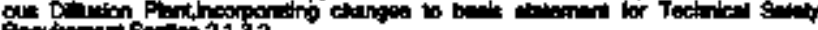

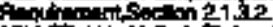

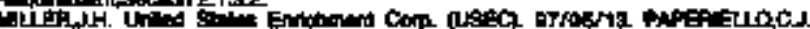

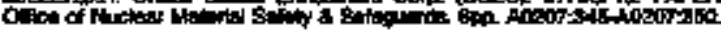

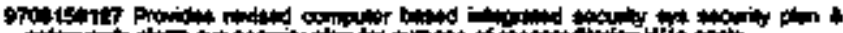

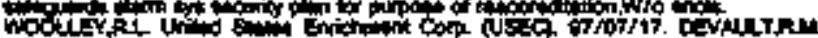

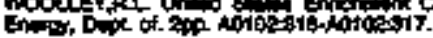

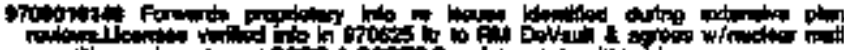

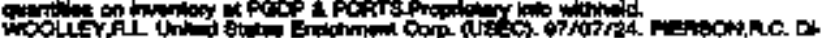

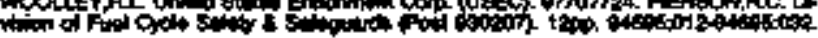

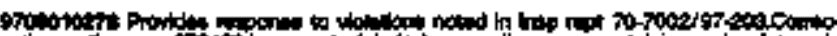

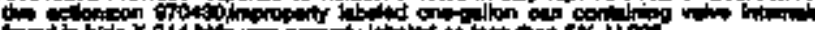

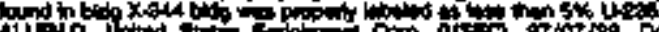

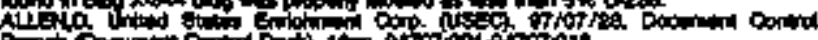

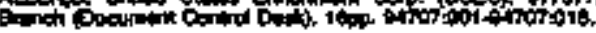

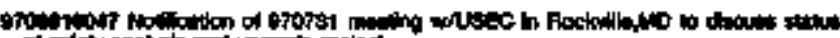
of

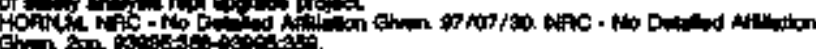

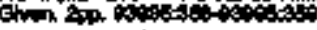

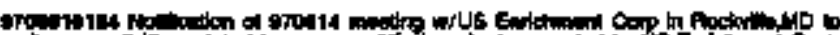

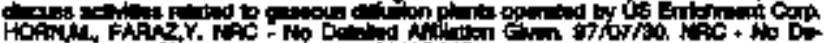

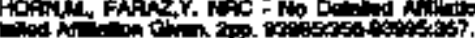

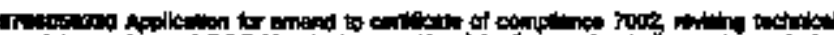

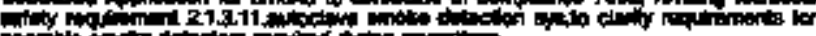

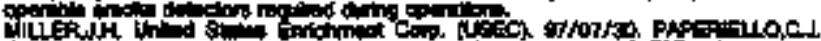

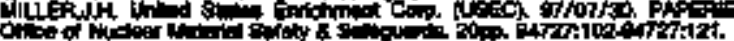

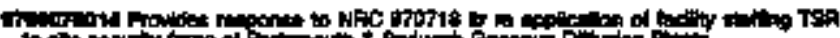

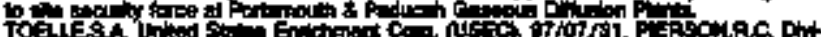

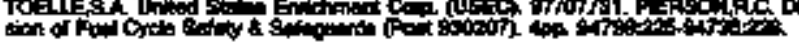

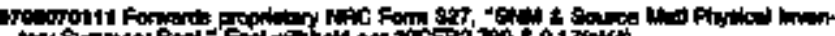

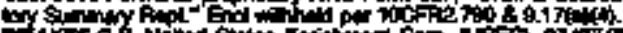

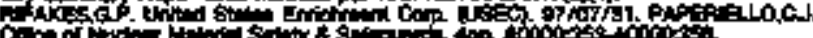

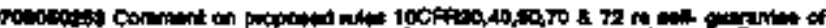

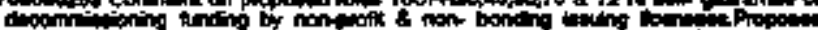

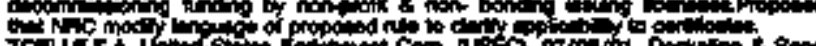

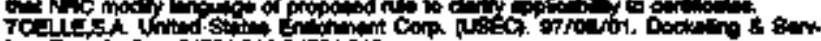

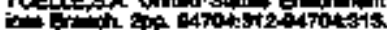

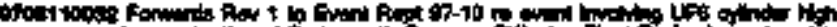

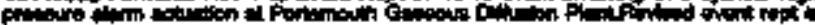

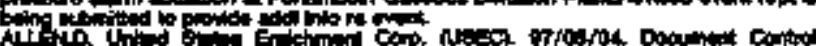

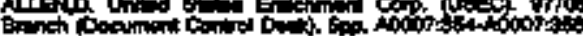

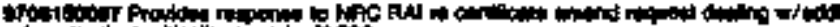

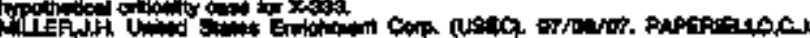

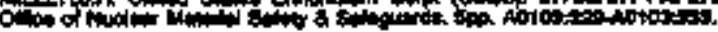

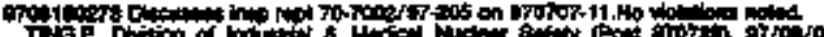

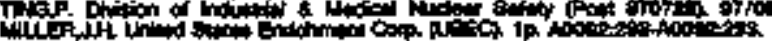

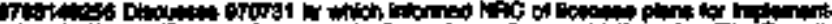

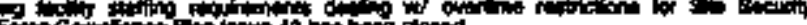

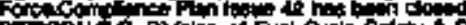

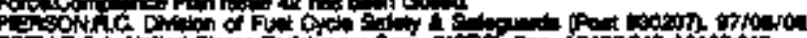

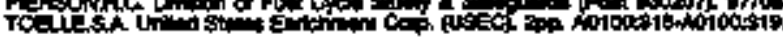

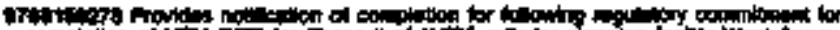

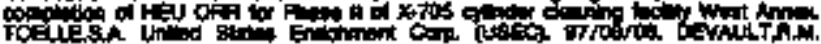

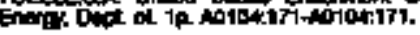

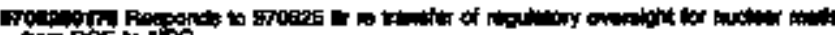

gey alt

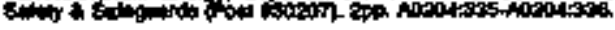

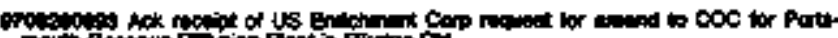

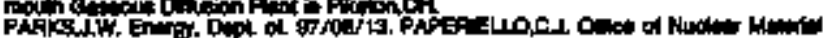

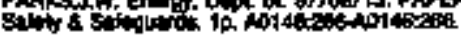




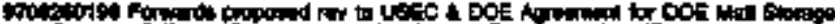

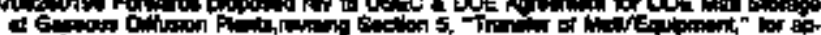
pront

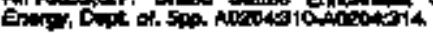

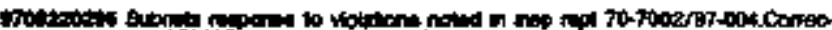

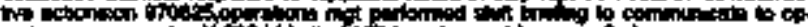

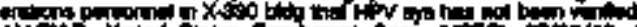

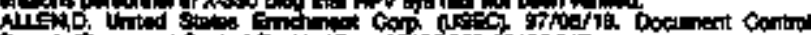

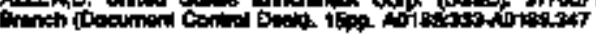

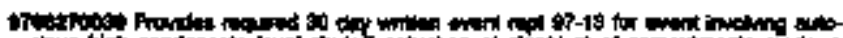

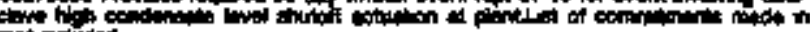

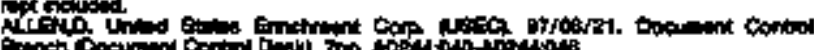

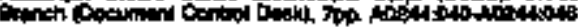

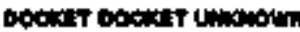

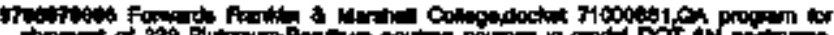

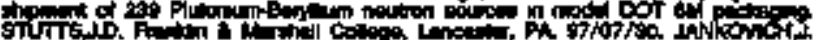

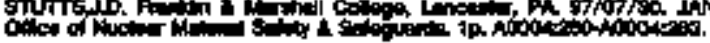

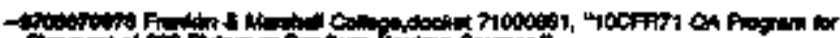

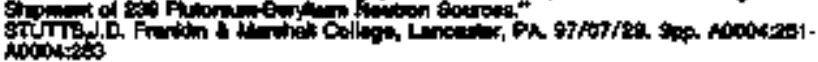

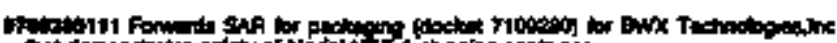

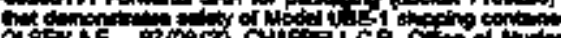

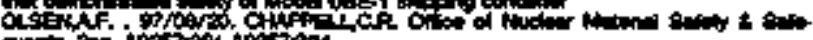

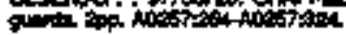

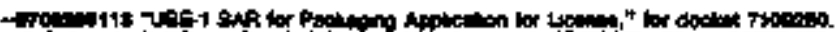

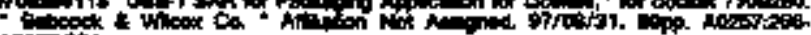
$\cos \cos ^{2}$

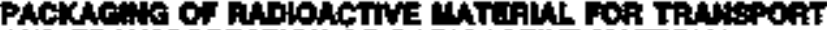 Ato TRA

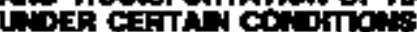

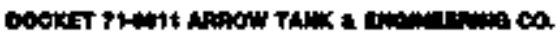

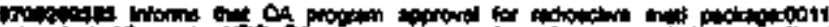

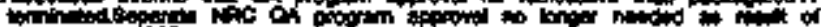

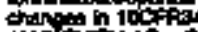

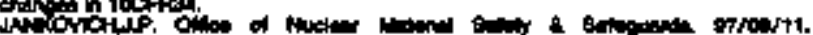

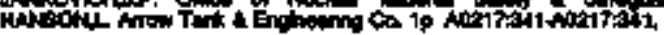

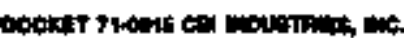

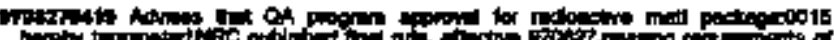

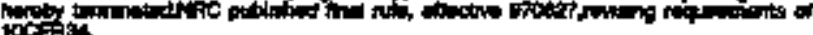

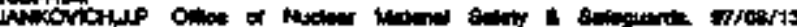

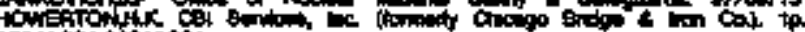

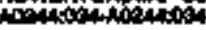

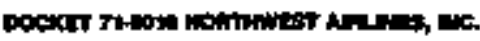

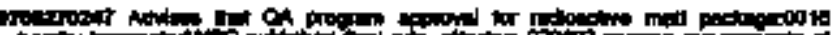
no

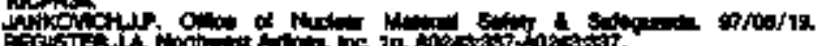

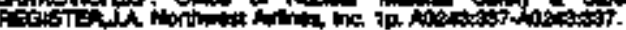

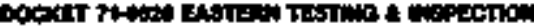

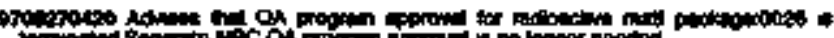

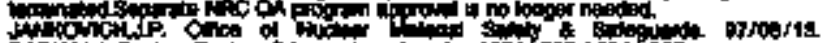

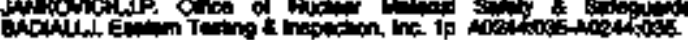

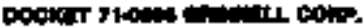

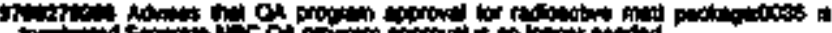
1.

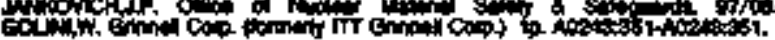

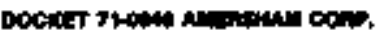

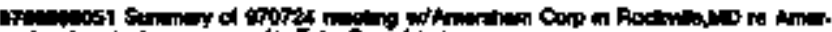

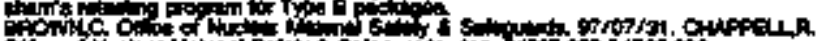

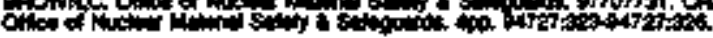

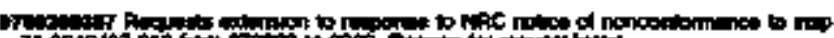
T1000\%

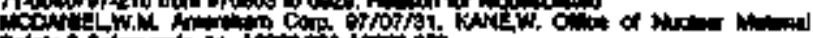

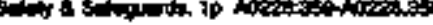

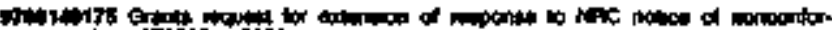

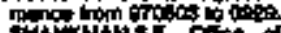

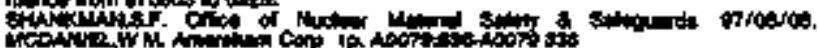

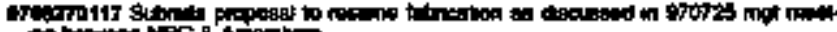

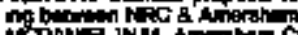

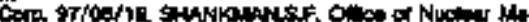

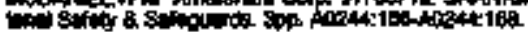

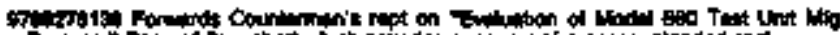

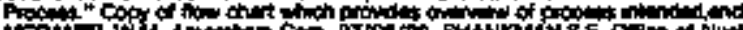

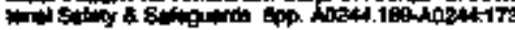

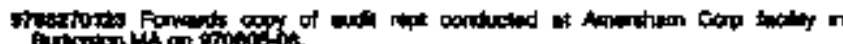

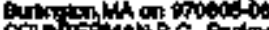

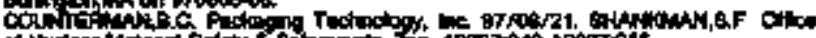

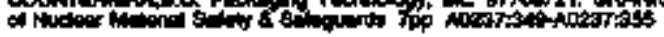

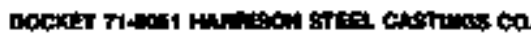

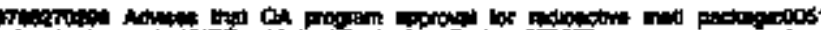

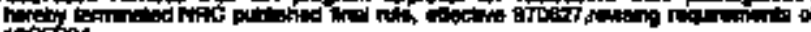

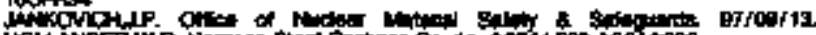

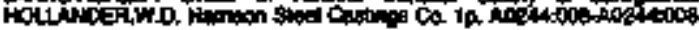

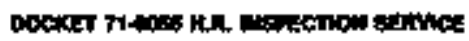

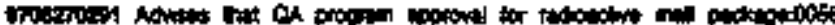

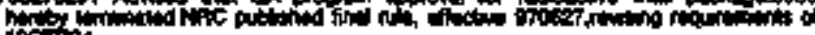

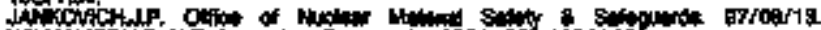

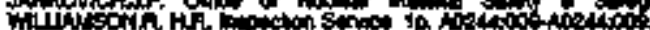

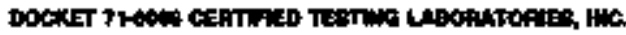

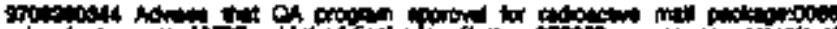

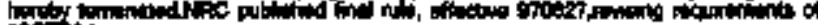
$10 \mathrm{C}$ 年

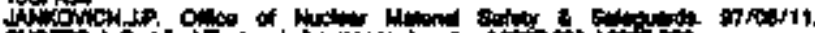

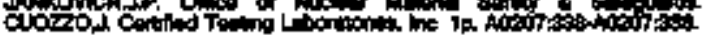

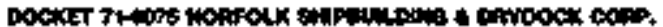

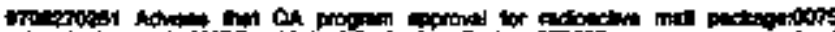

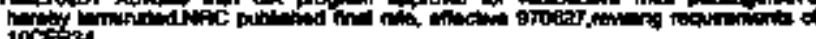

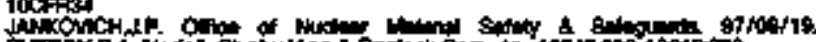

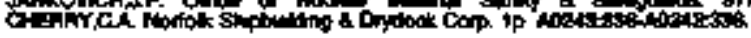

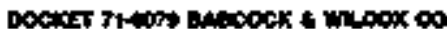

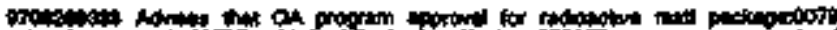

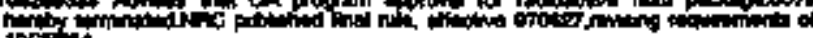

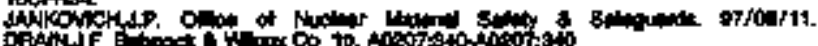

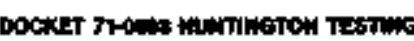

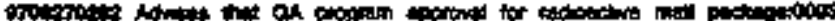

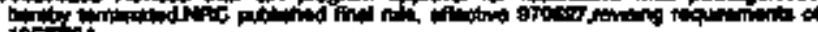

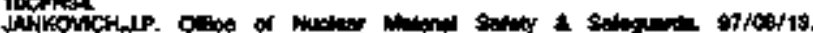

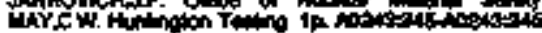

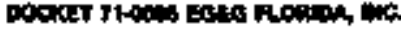

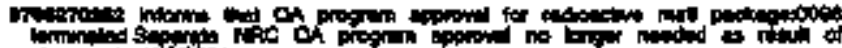

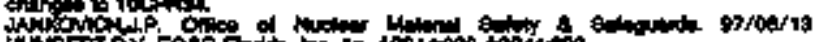

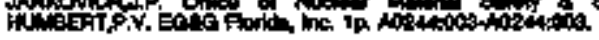

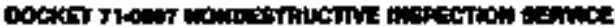

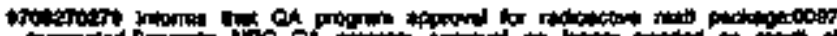

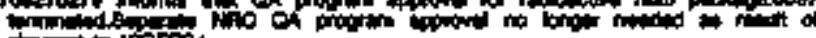

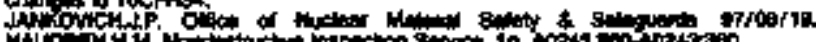

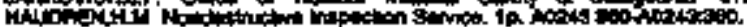

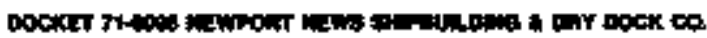

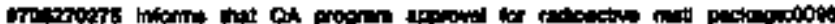

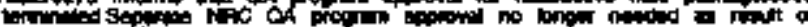

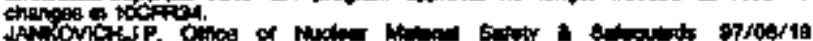

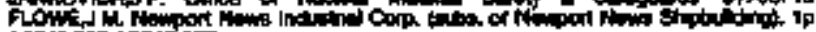
actor of 


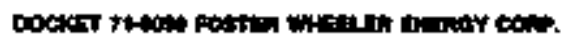

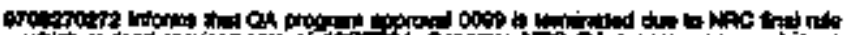

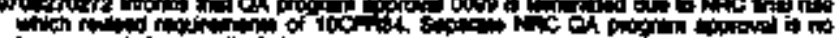

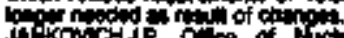

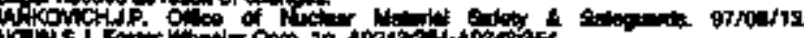

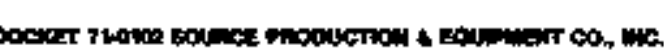

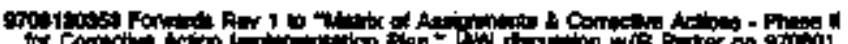

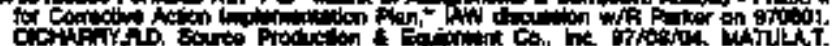
Cons of in

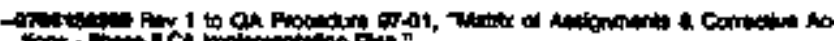

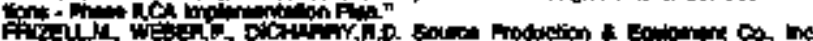

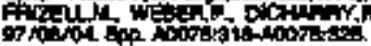

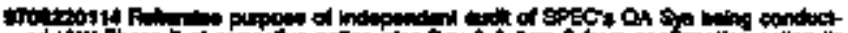

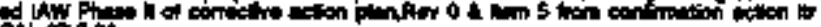
chis

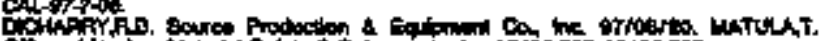

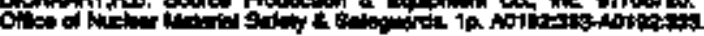

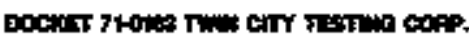

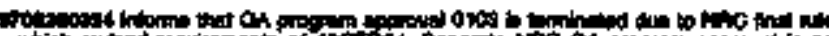

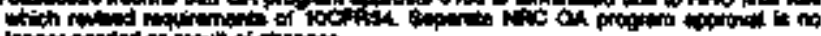

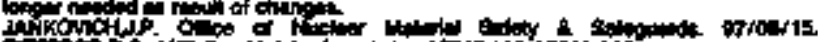

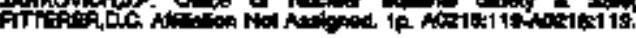

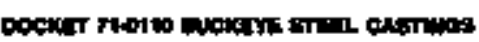

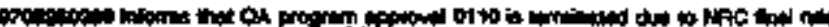

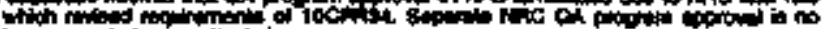

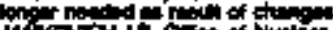

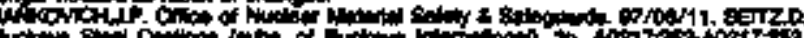

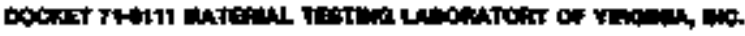

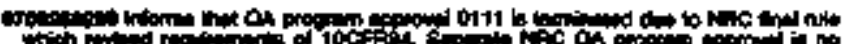

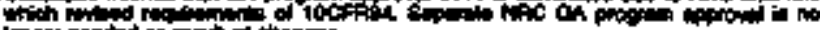

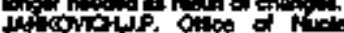

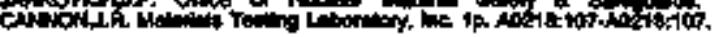

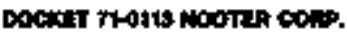

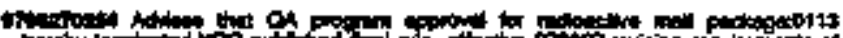

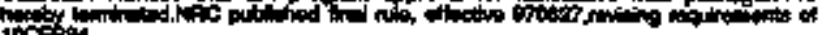

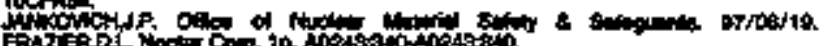

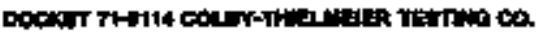

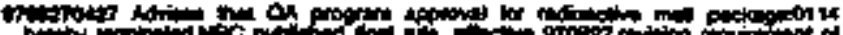

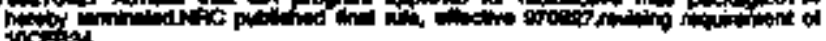

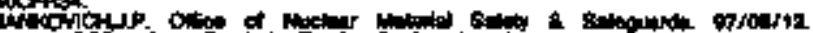
intide

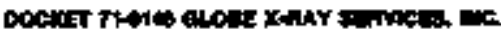

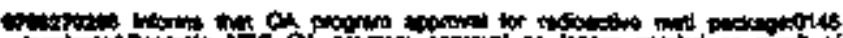

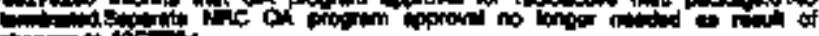

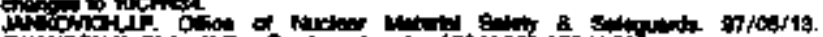

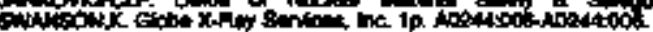

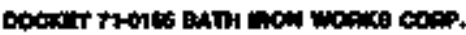

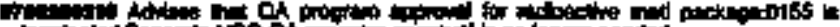

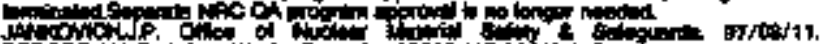

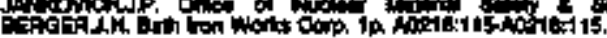

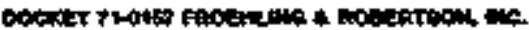

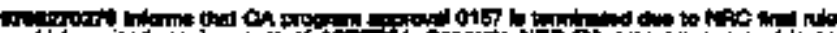

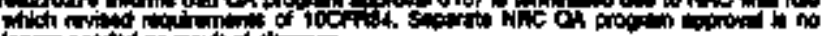

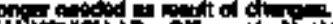

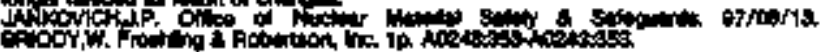

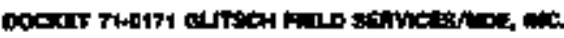

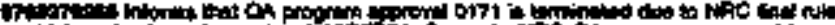

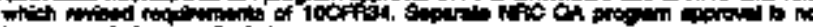

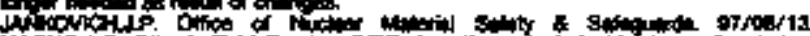

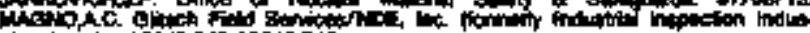

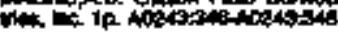

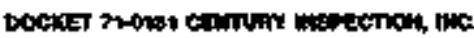

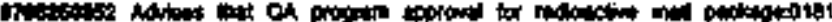

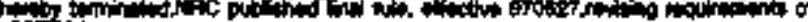
$10 \mathrm{criat}$

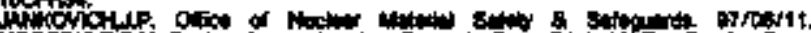

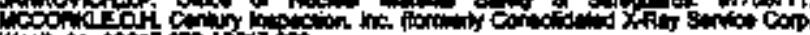

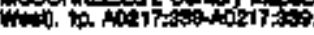

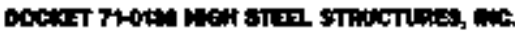

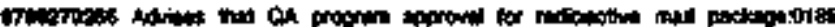

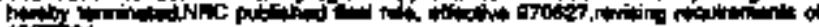

Pok

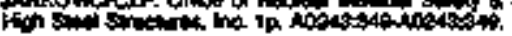

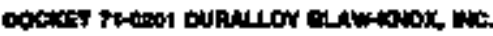

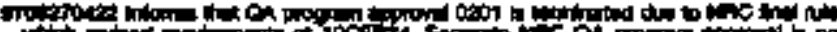

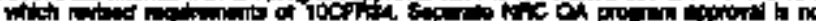

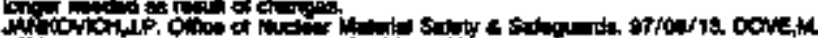

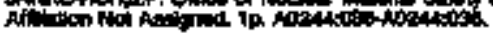

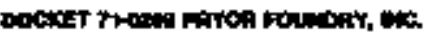

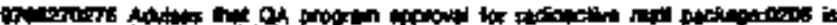

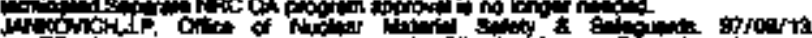

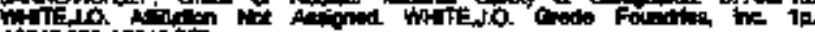

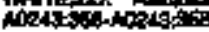

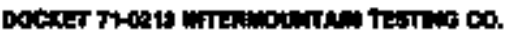

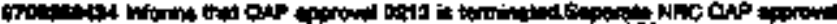

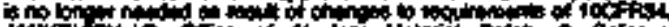

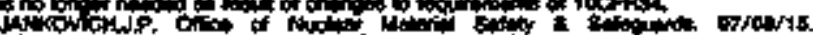

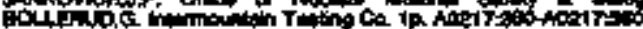

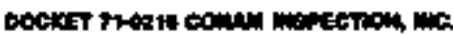

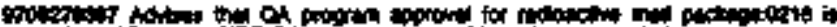
nitich

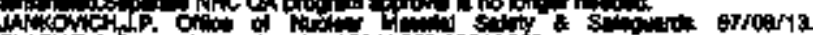

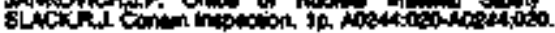

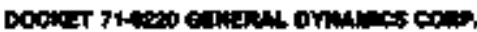

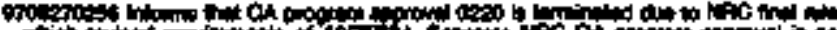

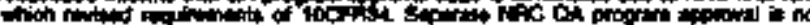

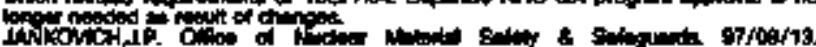

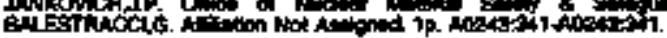

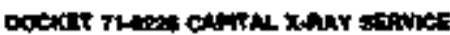

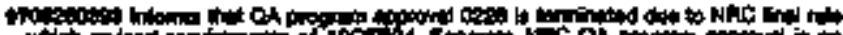

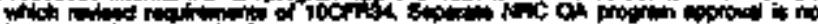

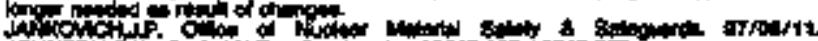

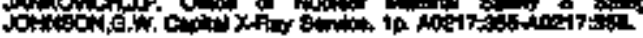

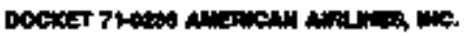

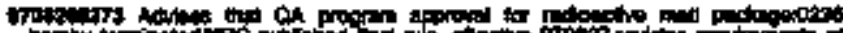

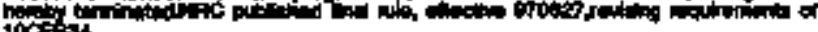

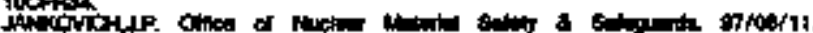

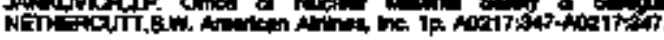

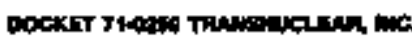

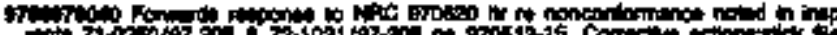

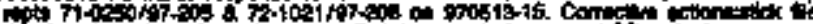

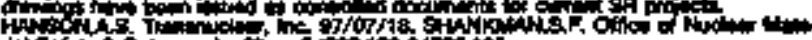

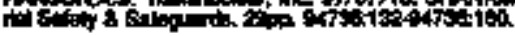

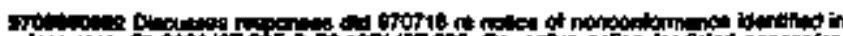

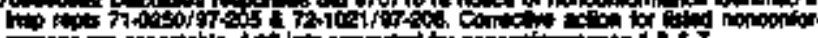

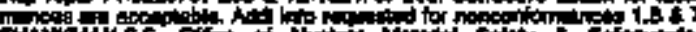

groprom

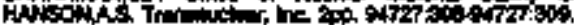




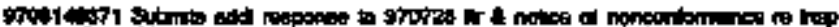

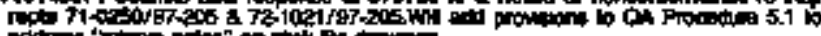
of

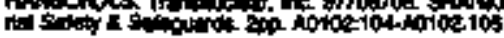

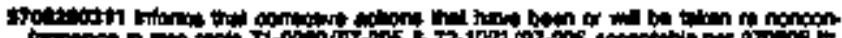
1 Hats

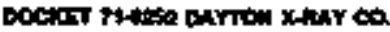

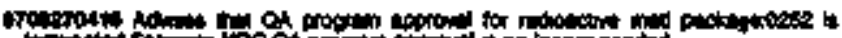

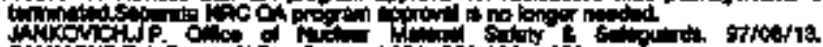

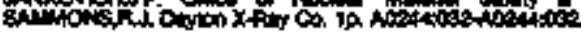

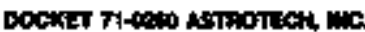

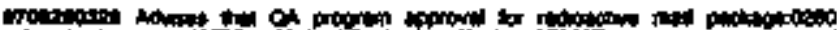

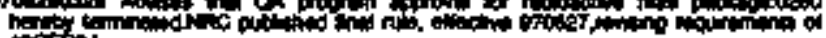

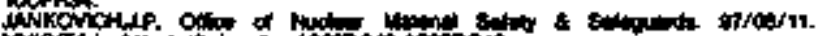

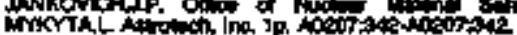

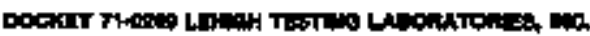

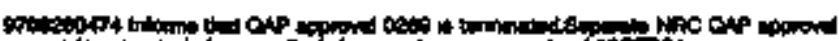

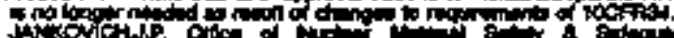
M)

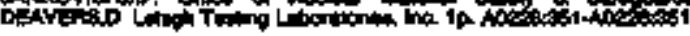

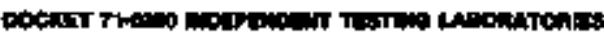

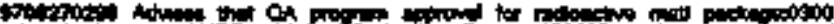

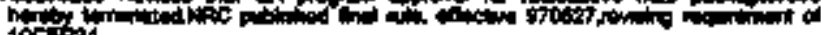

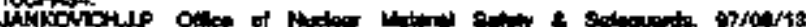

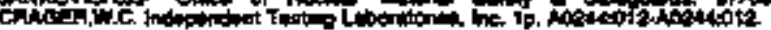

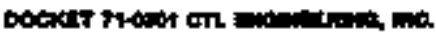

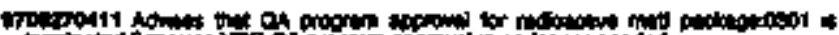

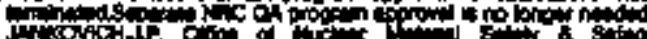

(1)

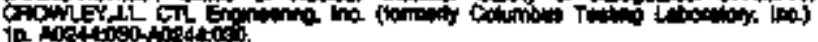

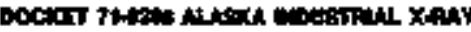

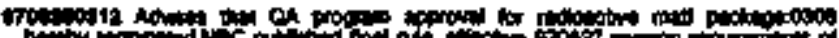

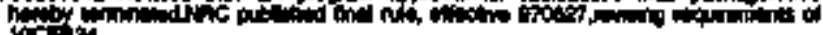

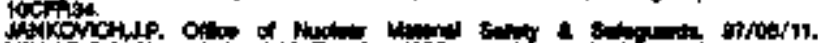

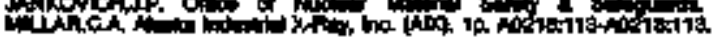

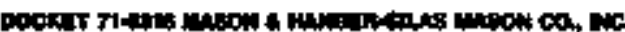

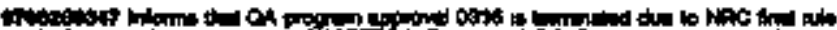

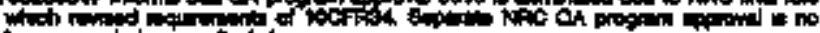

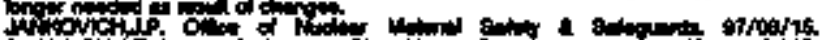

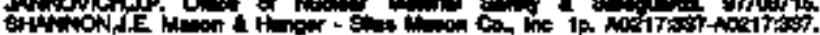

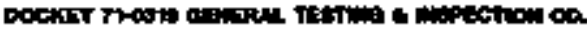

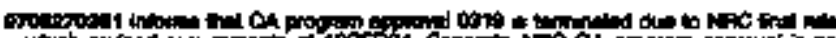

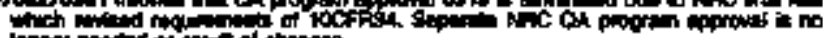

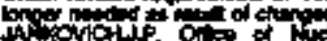

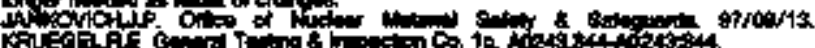

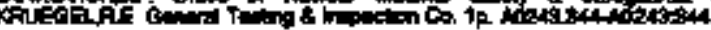

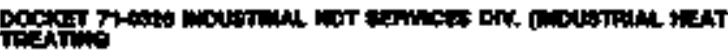

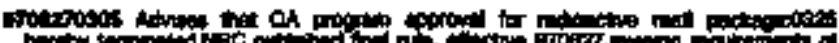

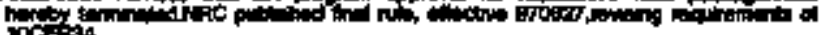
rocrisa.

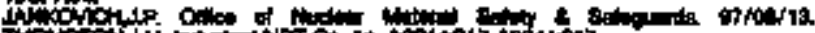

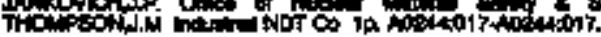

\section{Dodit ryote}

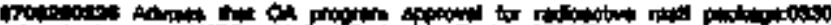

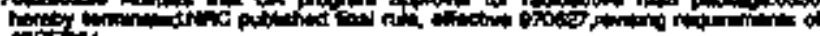

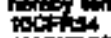

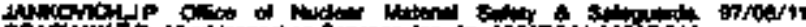

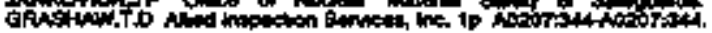

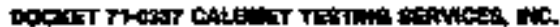

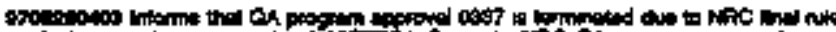

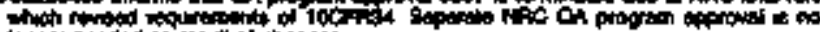

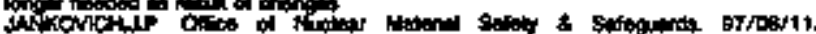

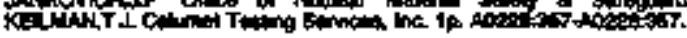

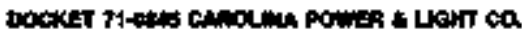

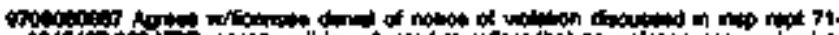

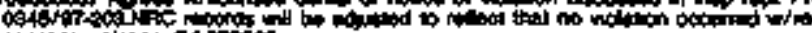
17s

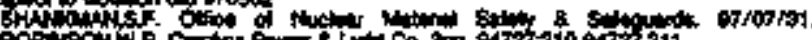

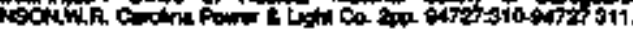

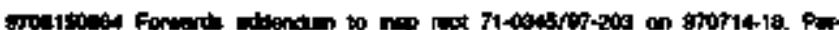

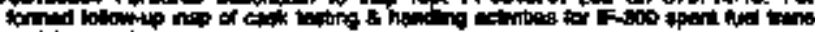

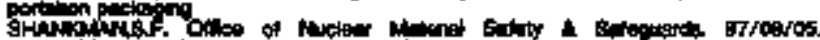

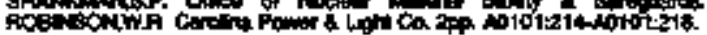

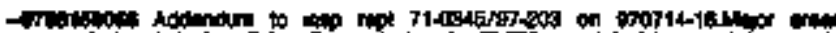

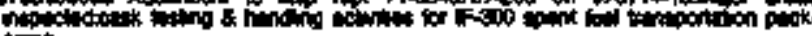
tant

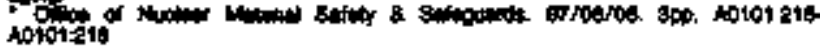

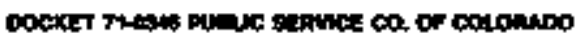

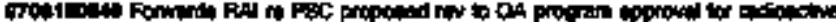

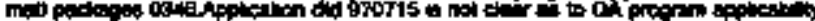

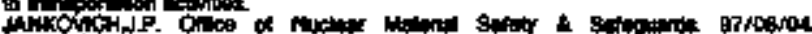

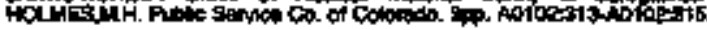

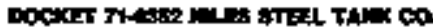

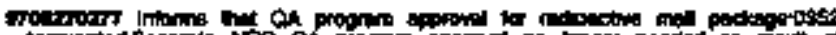

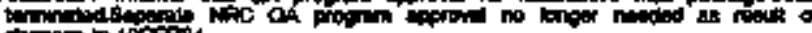

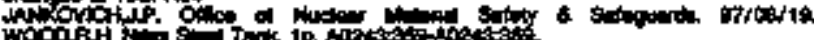

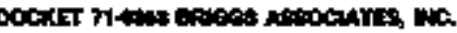

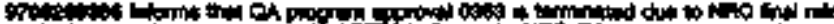

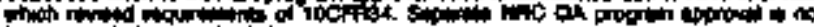

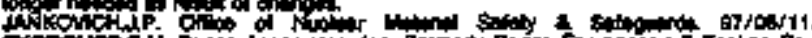

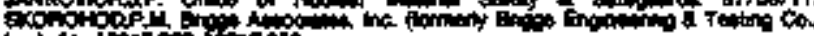

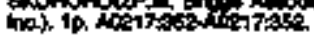

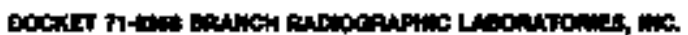

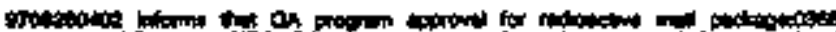

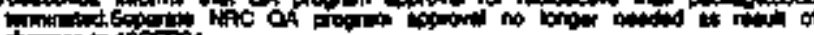

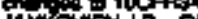

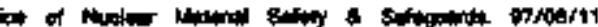

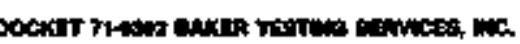

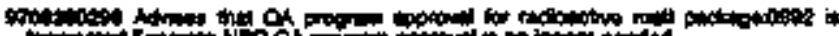

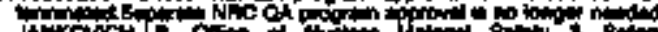

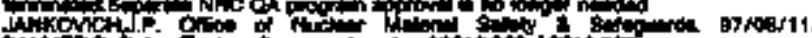

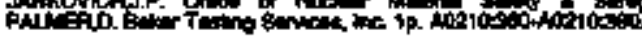

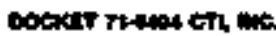

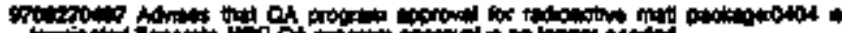

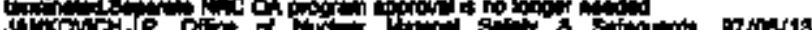

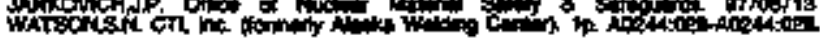

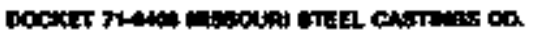

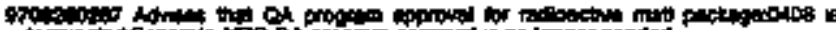

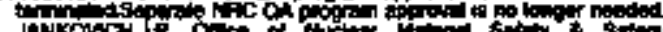

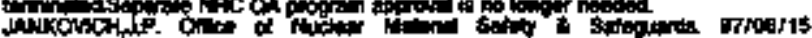

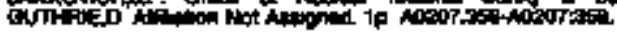

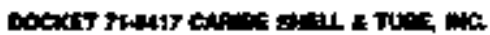

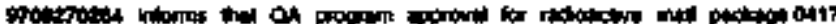

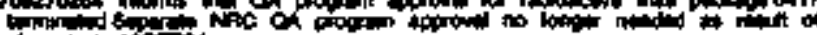

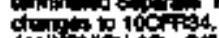

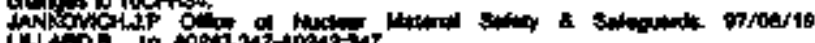




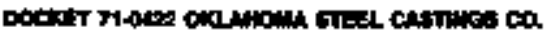

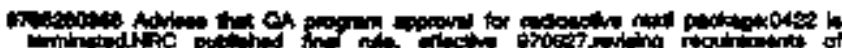
100 ind

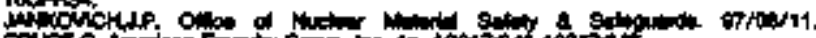

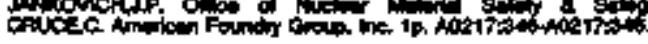

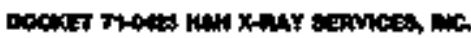

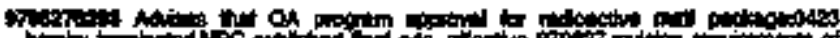

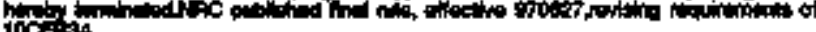
10 cist.

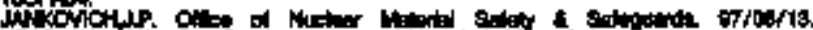

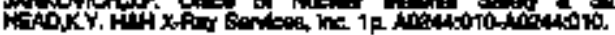

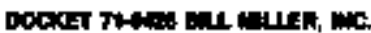

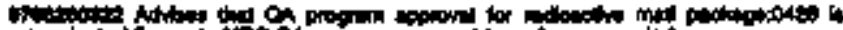

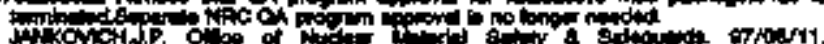

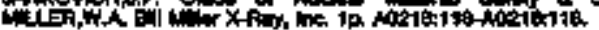

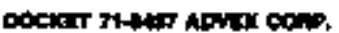

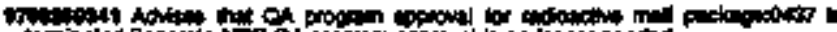
Tlint

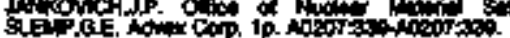

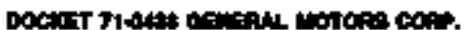

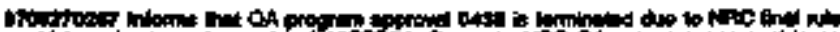

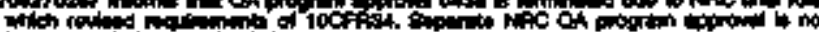

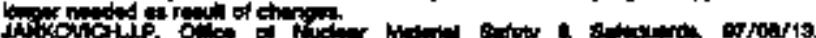

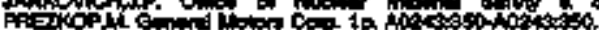

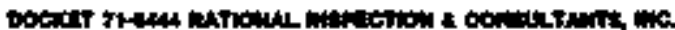

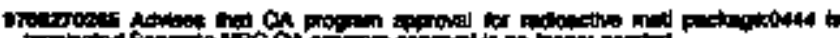

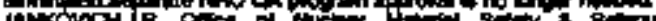

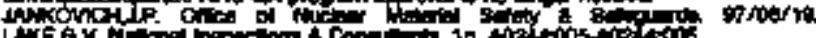

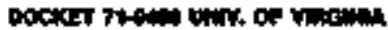

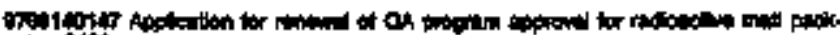

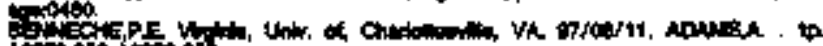
intoritas

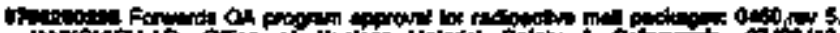
Bow

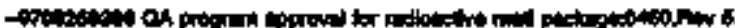

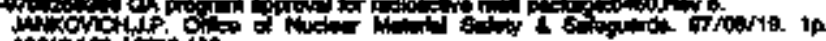

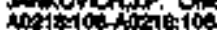

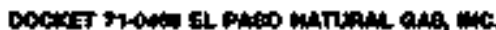

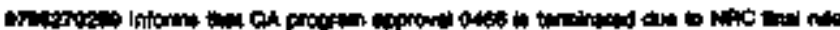

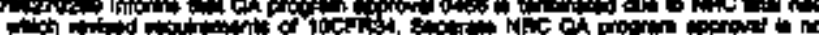

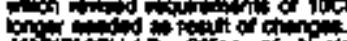

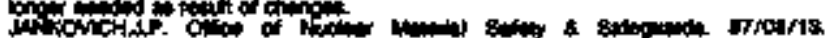

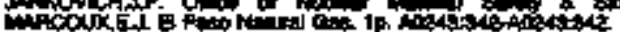

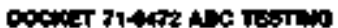

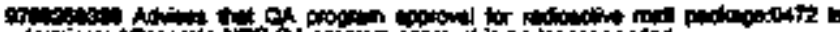

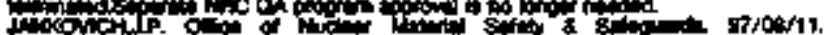

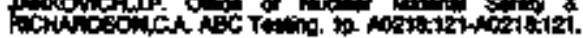

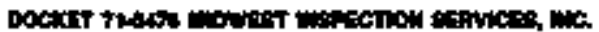

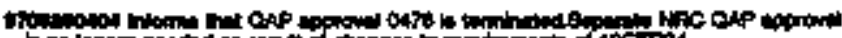

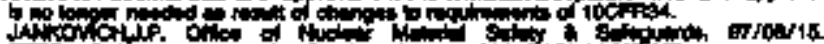

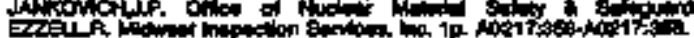

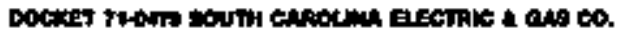

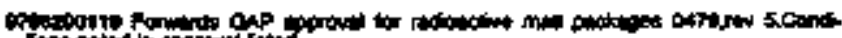

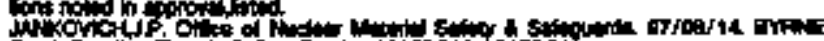

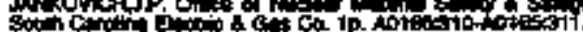

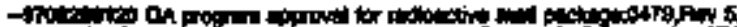

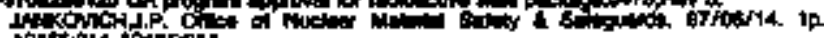

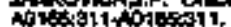

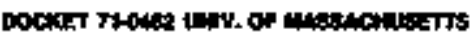

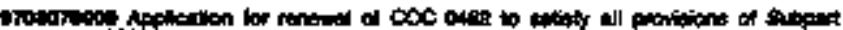

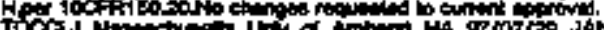

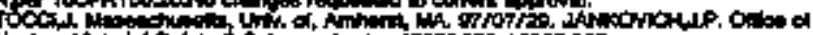

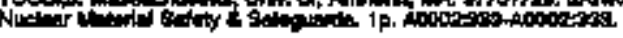

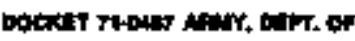

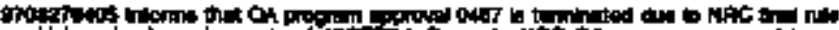

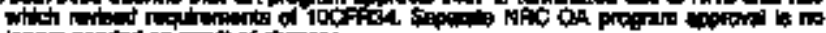

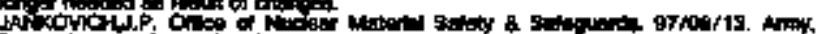

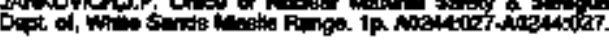

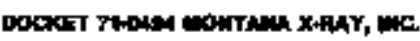

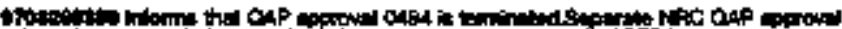

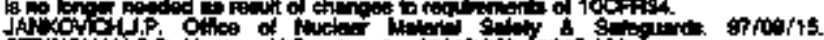

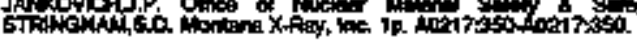

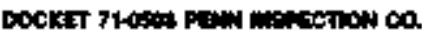

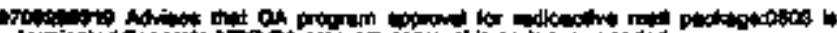

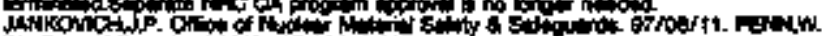

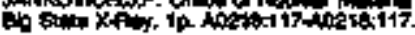

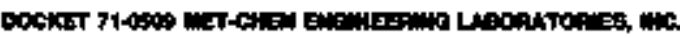

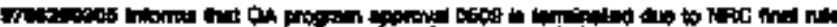

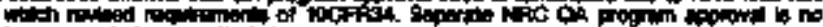

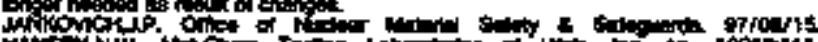

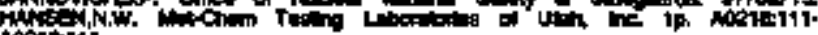
Mectivit.

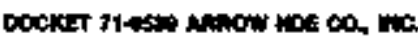

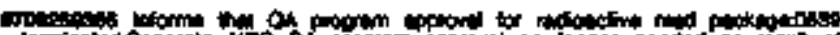

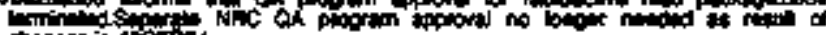

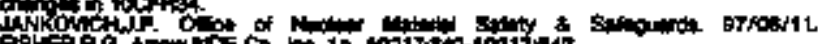

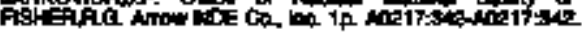

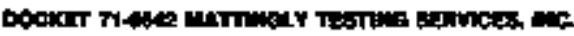

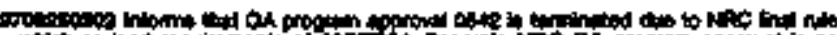

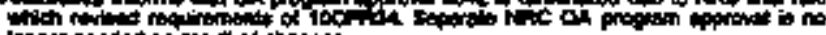

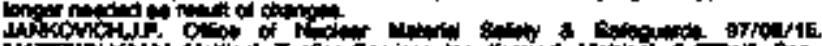

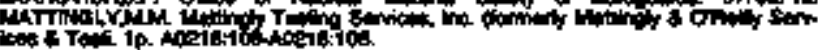

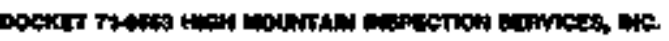

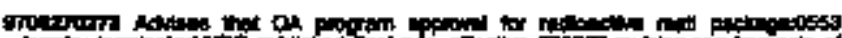

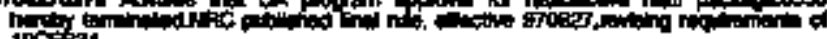

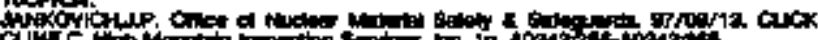

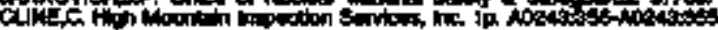

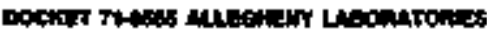

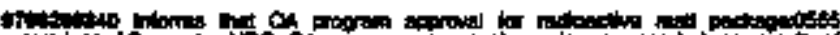

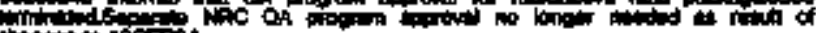

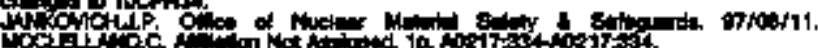

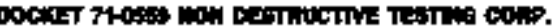

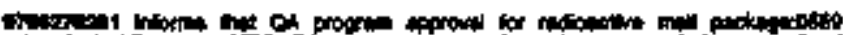
temineld

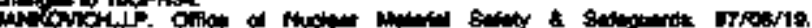

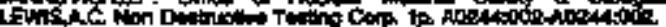

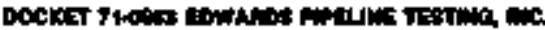

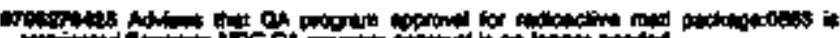

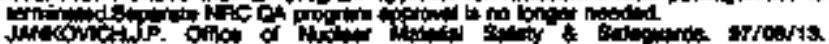

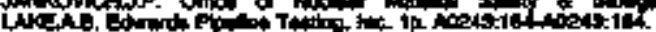

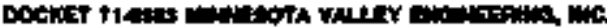

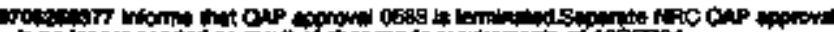

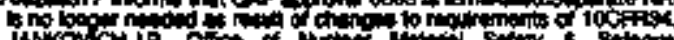
oproest5.

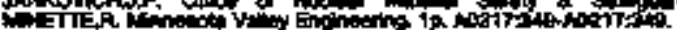




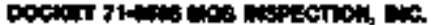

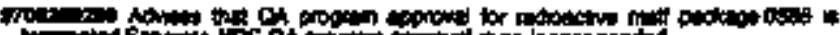

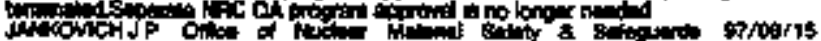

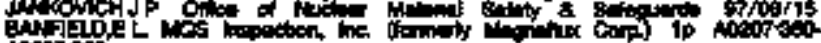
incol 300

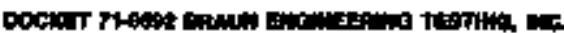

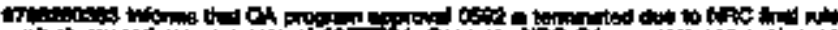

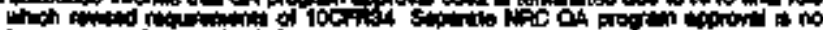

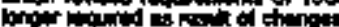

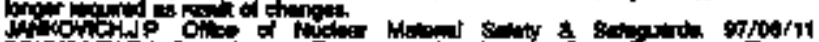

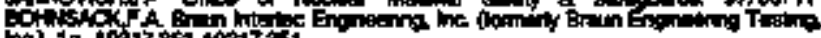

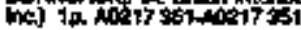

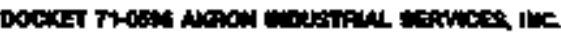

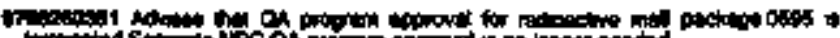
intide

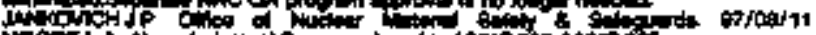

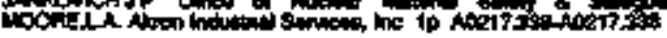

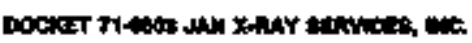

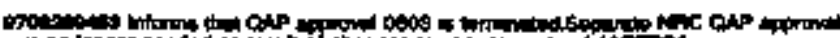

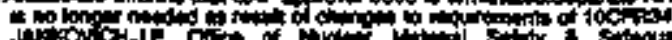

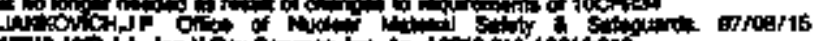

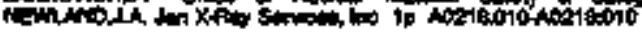

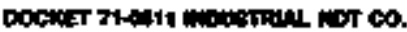

mos:

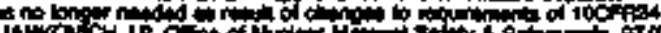

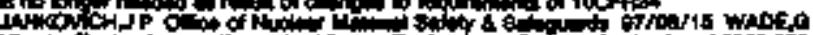

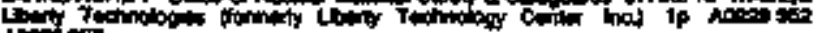

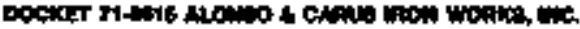

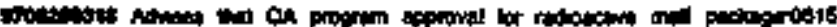

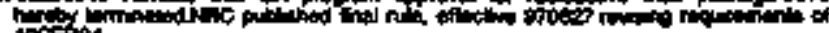

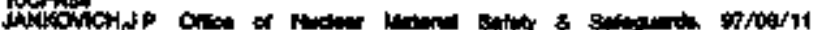

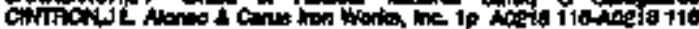

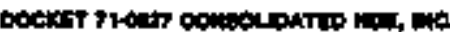

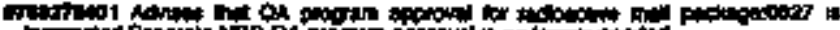

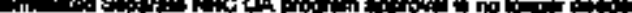

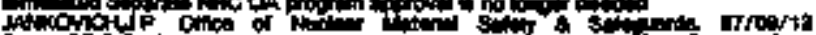

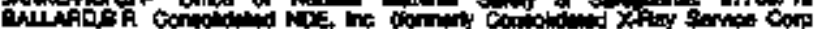

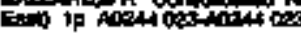

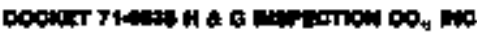

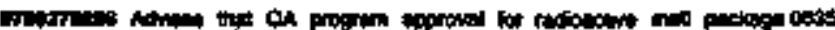

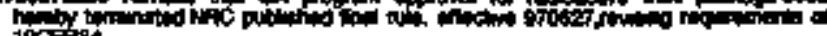

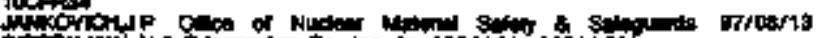
है।

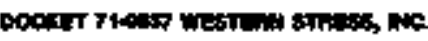

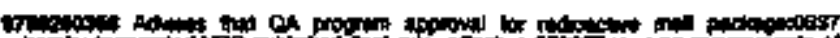

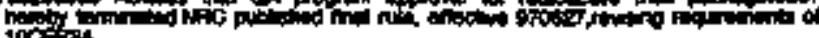
$100 \mathrm{pto}$

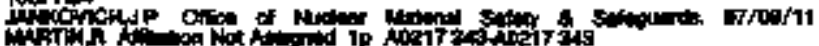

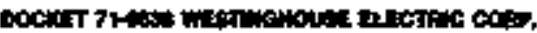

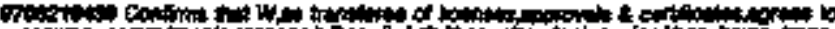

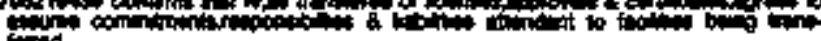

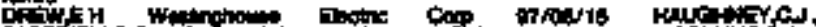

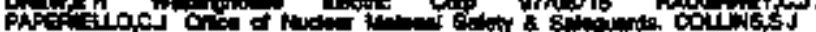

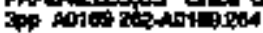

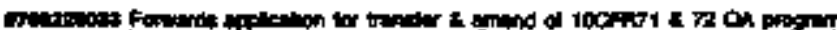

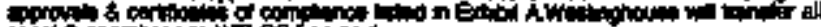

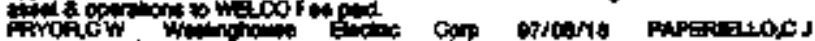

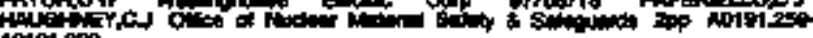

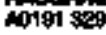

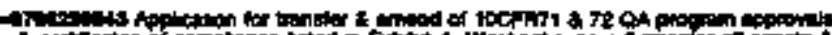

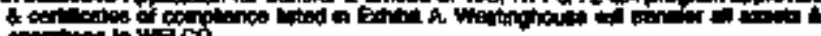

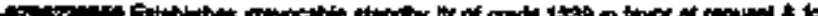

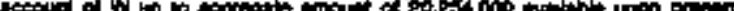

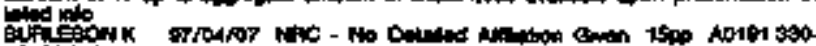
Anto

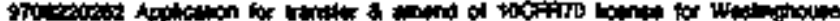

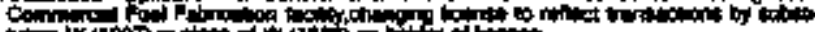

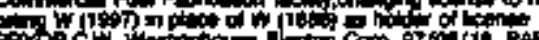

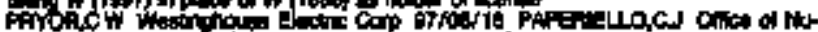

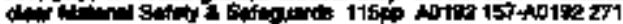

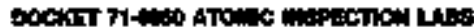

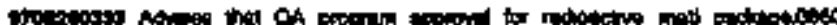

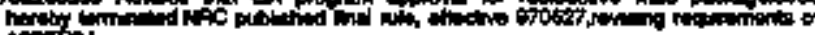

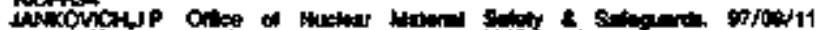

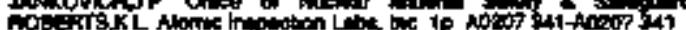

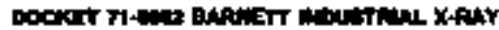

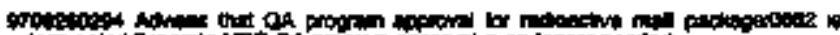
int orrogrtis ENiviT,

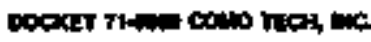

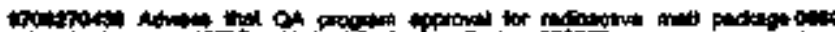

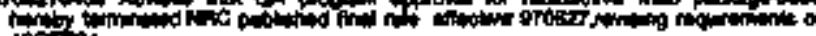

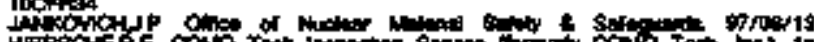

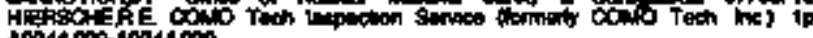

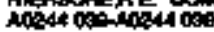

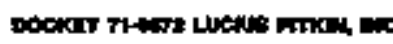

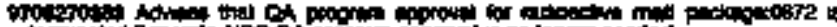

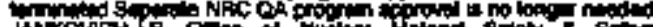

HW XWV

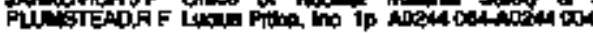

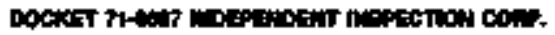

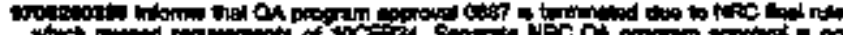

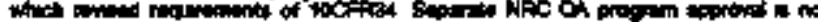

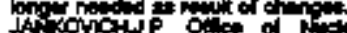

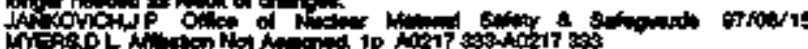

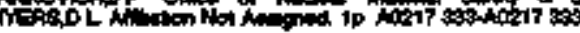

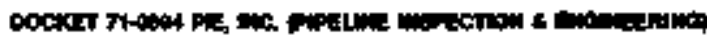

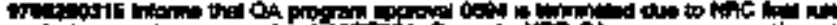

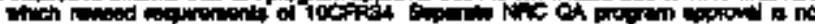

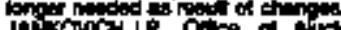

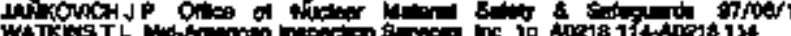

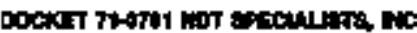

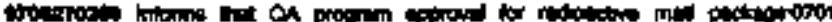

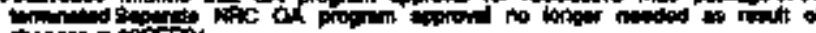
cintion

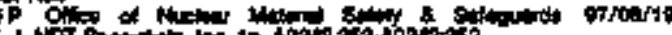

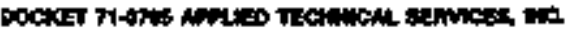

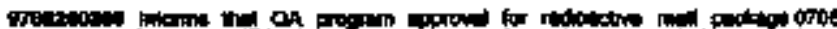

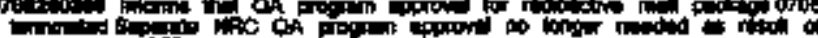

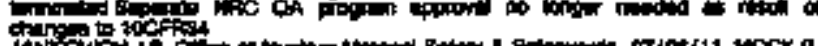

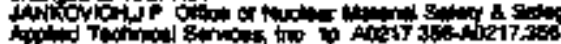

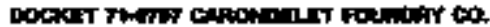

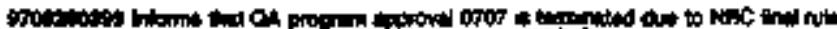

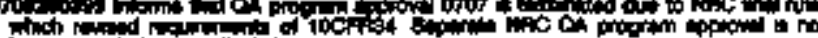

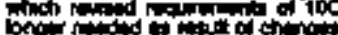

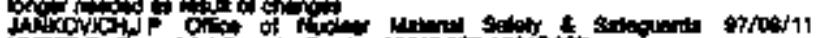
Fi:-RR 0 .

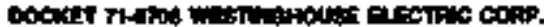

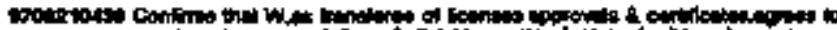

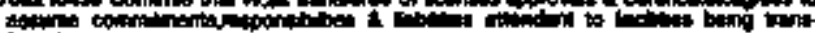

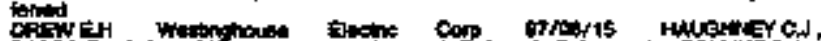

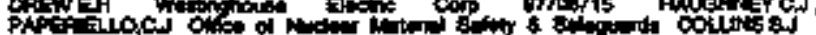

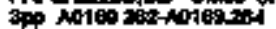

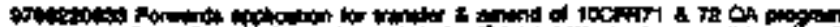

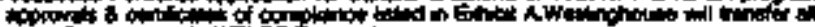

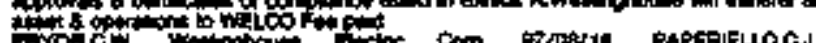
Huct Aots in 


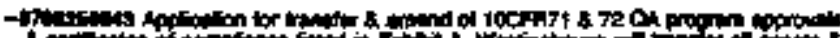

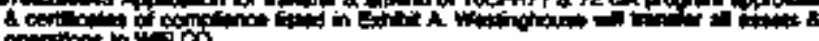

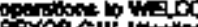

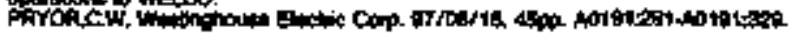

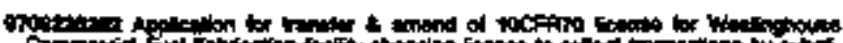

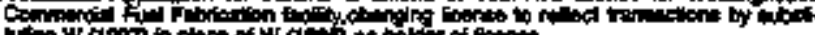

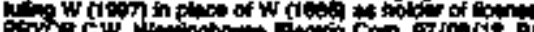

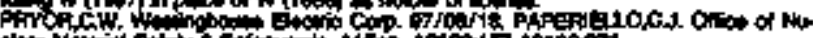

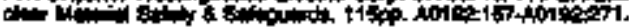

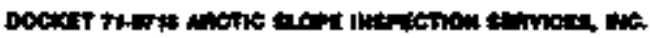

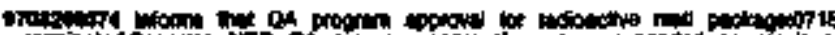

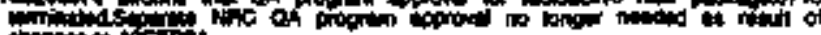

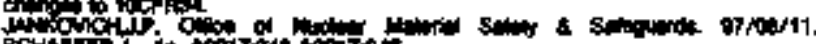

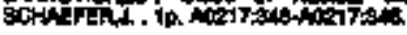

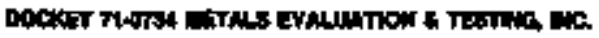

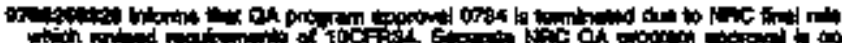

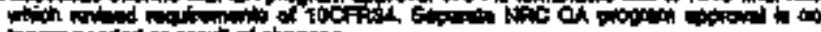

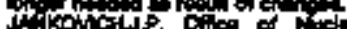

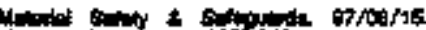

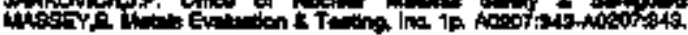

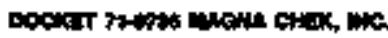

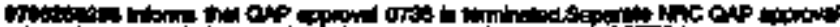

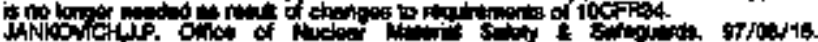

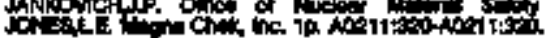

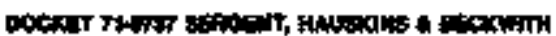

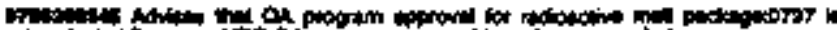

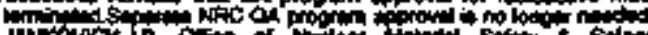

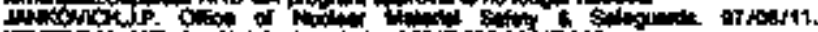

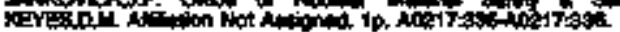

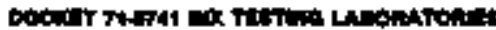

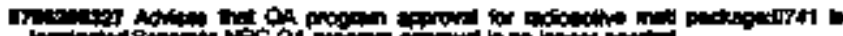

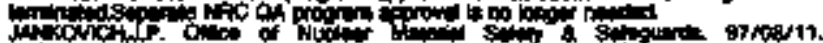

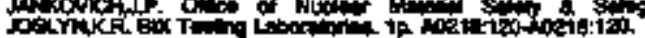

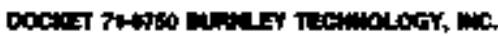

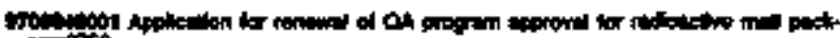
Pot

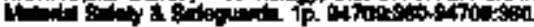

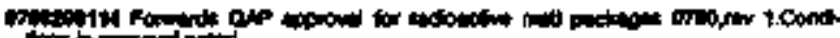

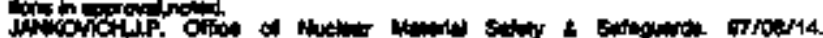

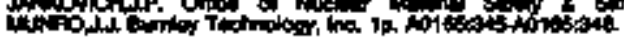

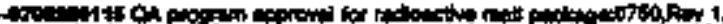

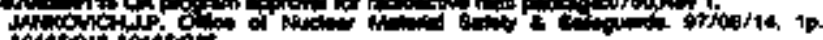

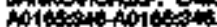

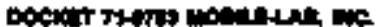

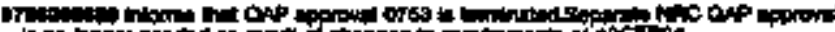

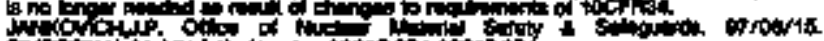
3.

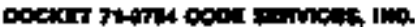

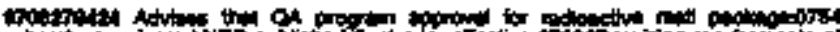

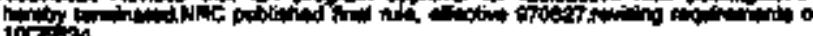

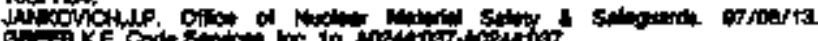

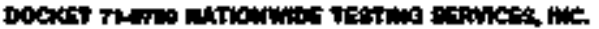

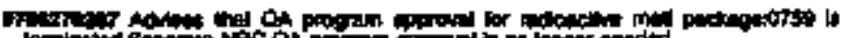

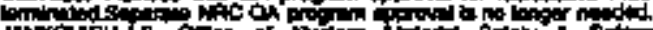

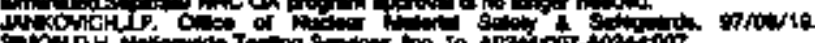

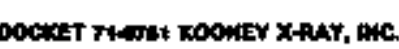

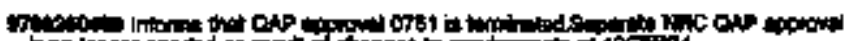

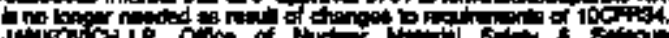

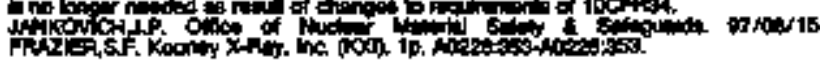

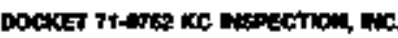

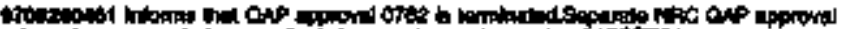

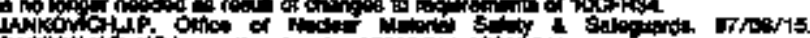

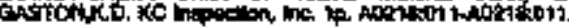

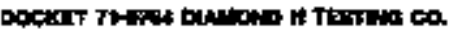

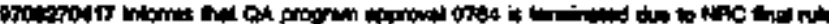

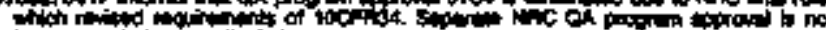

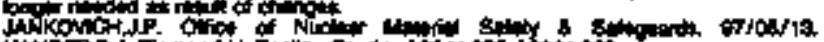

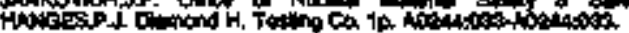

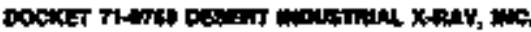

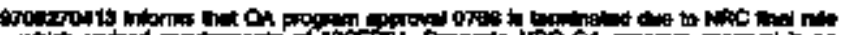

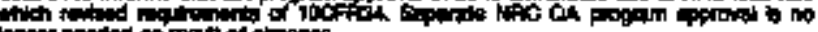

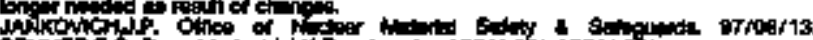
orv

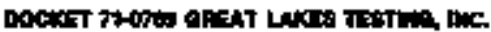

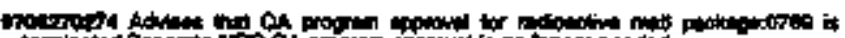

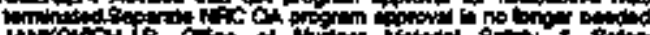

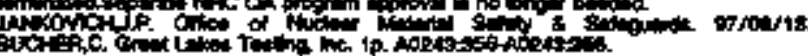

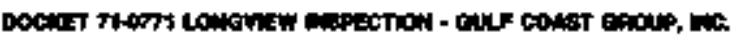

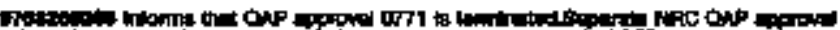

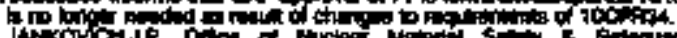

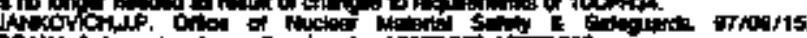

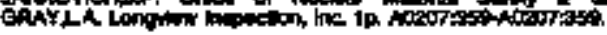

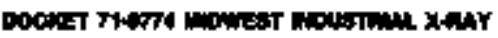

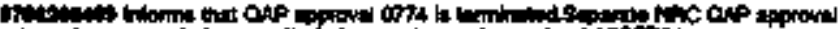

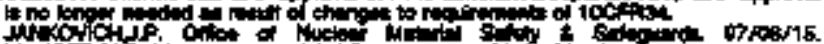

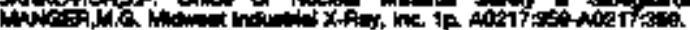

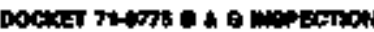

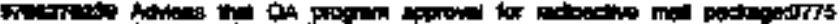

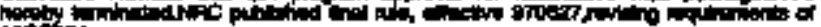

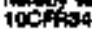

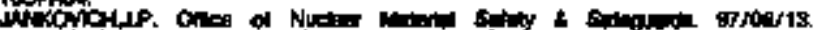

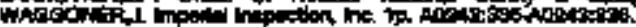

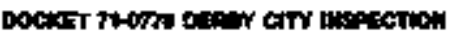

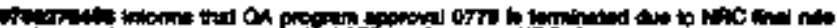

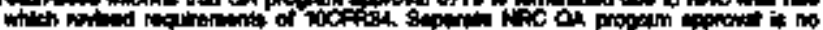

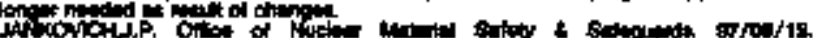

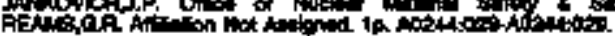

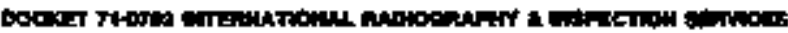

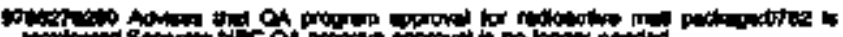

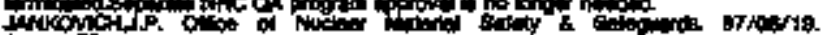

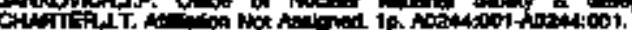

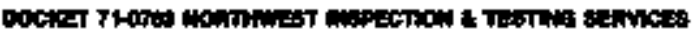

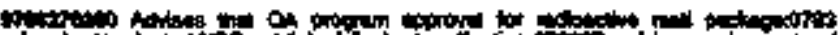

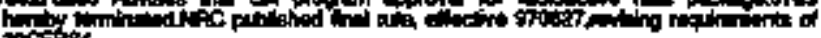

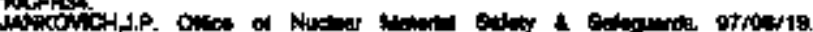

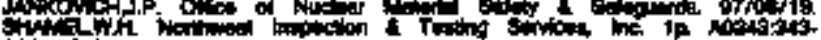
A

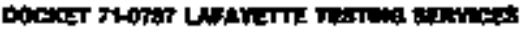

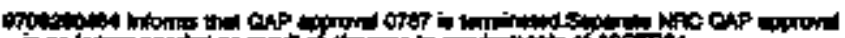

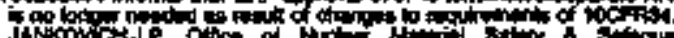

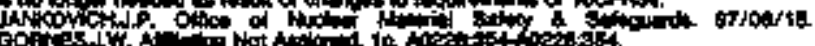

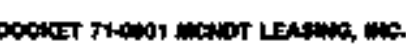

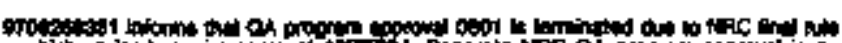

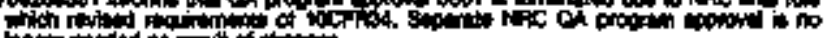

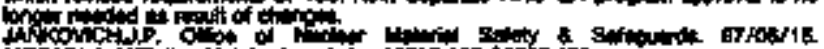

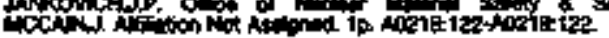




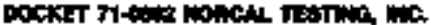

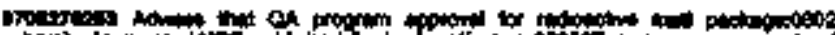

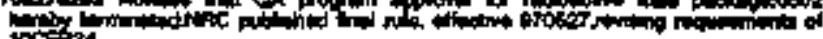

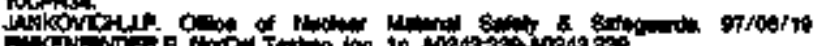

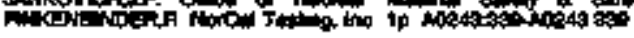

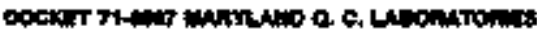

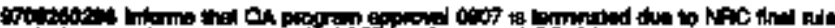

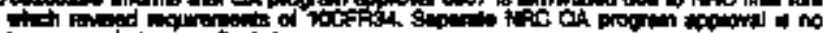

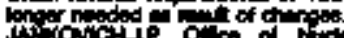

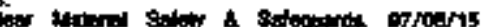

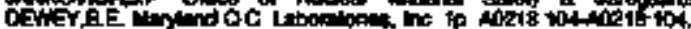

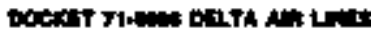

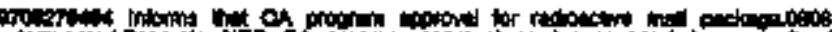

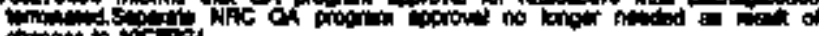

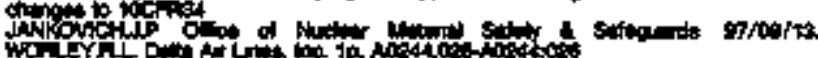

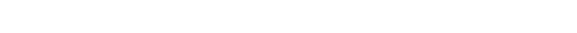

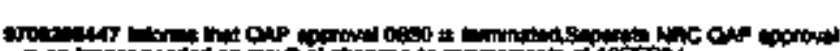

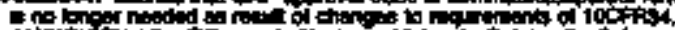

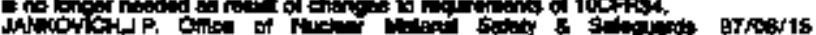

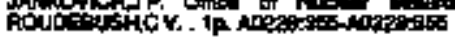

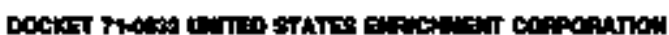

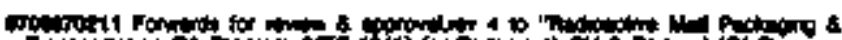

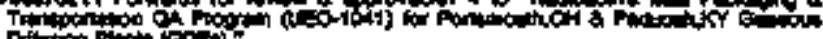

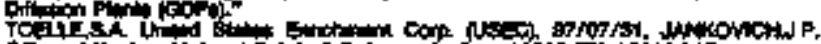

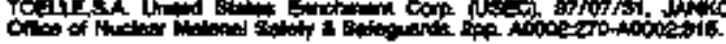

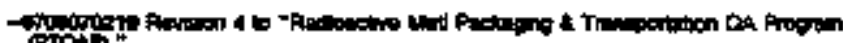

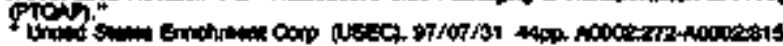

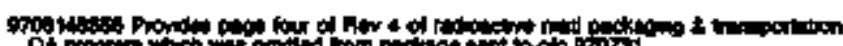

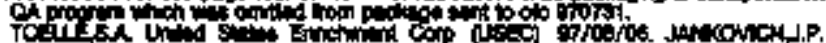

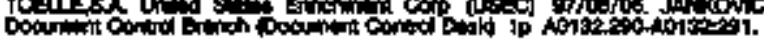

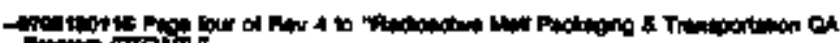

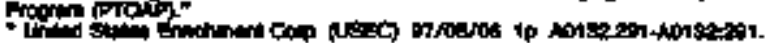

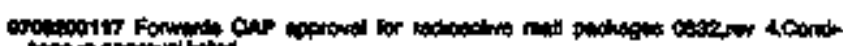

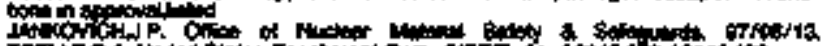

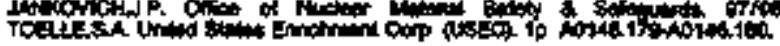

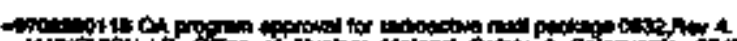

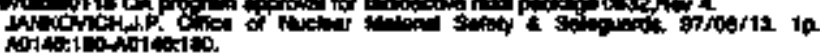

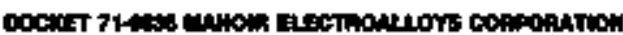

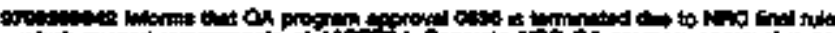

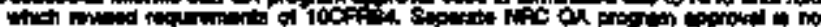

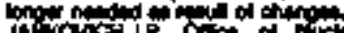

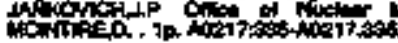

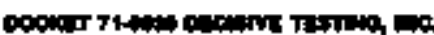

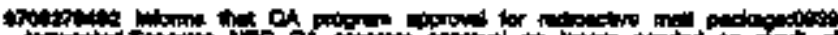

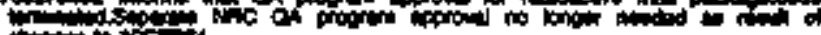
of the to to

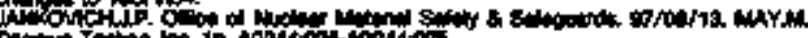

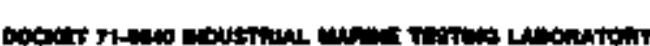

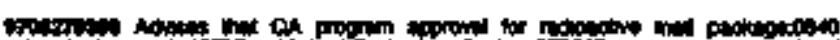

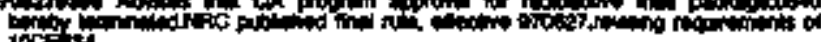

1GFVlis

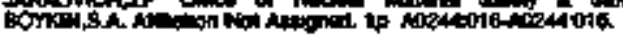

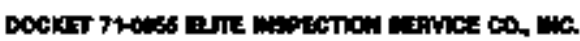

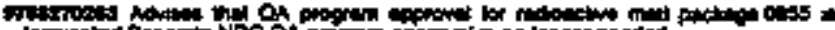

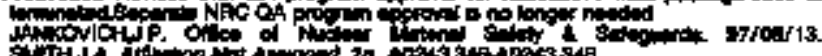

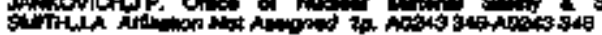

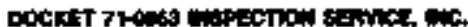

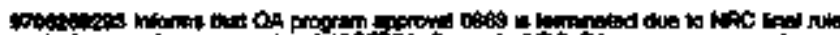

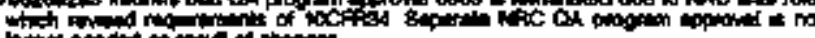

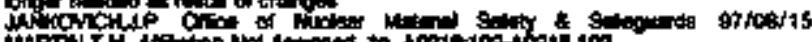

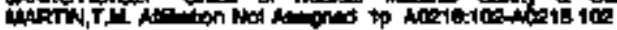

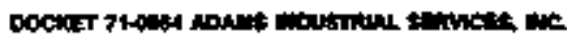

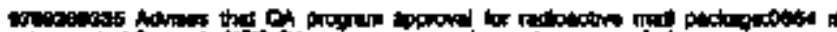

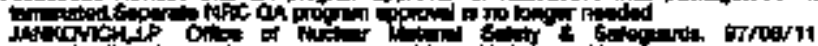

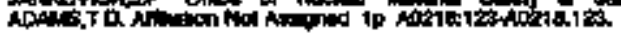

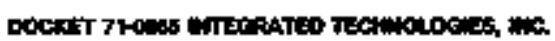

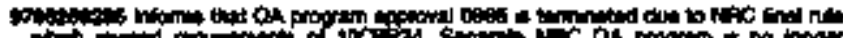

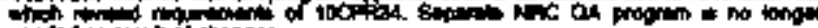

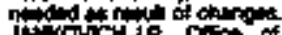

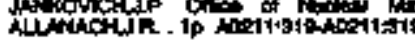

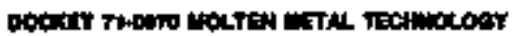

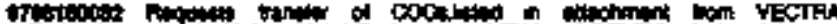

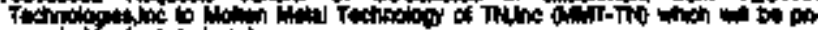

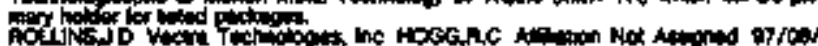

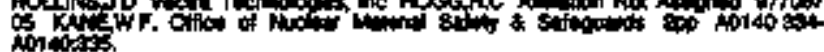

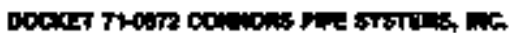

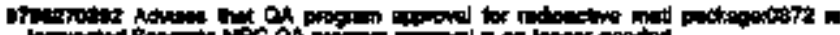
low

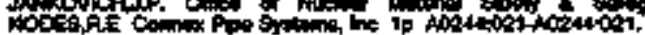

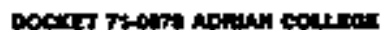

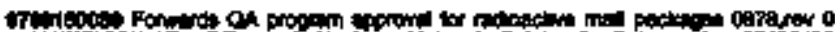

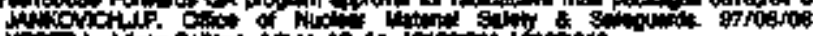

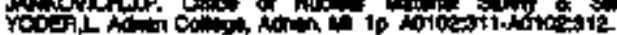

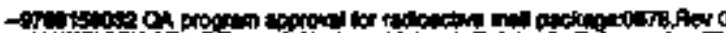
How solos 13 a

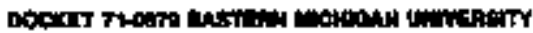

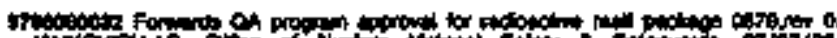

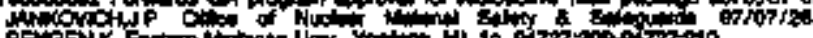

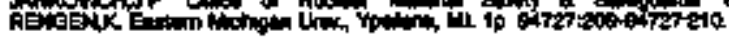

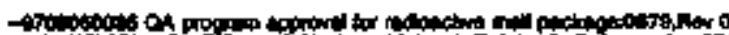

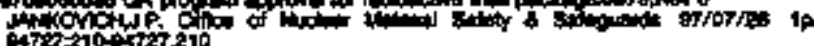

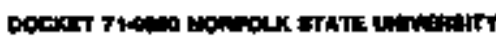

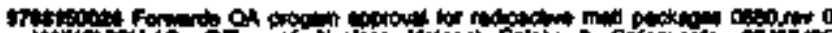

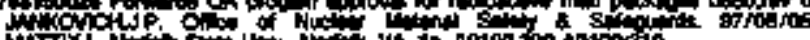
Hor X

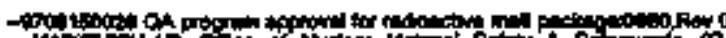

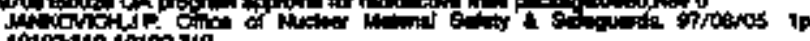
iotosstowioesia.

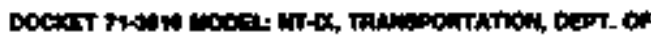

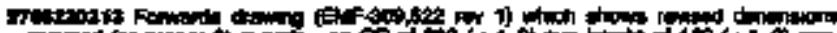

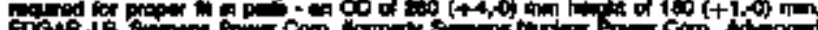
coevel notion Fuch

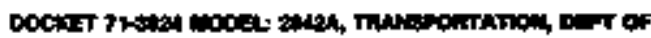

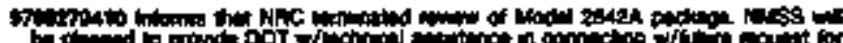

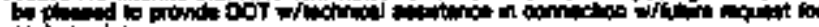
Cripite

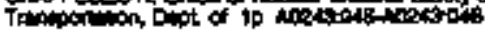

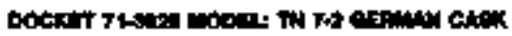

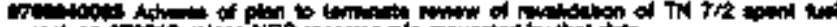

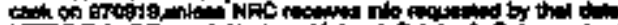

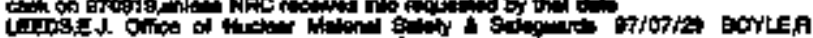

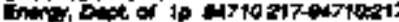




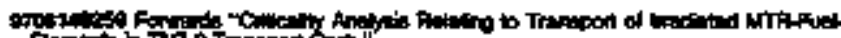

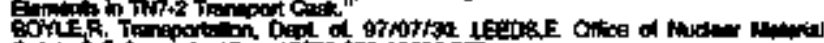

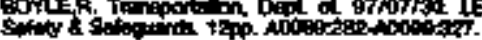

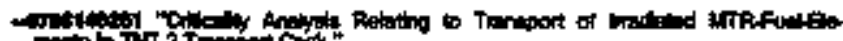
schol, sition.

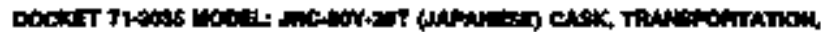

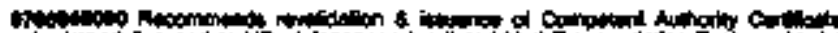

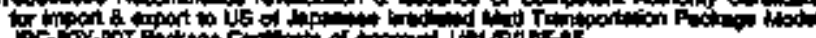

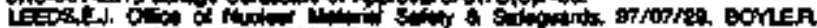

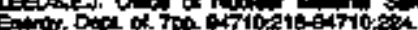

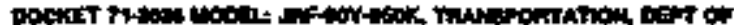

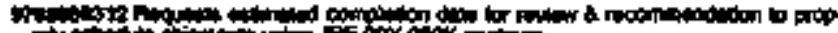

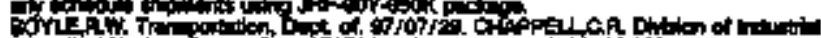

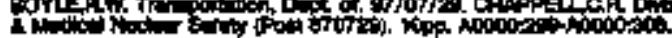

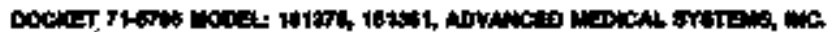

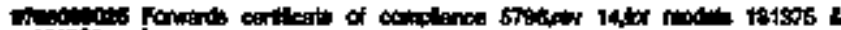

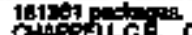

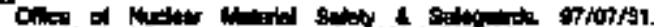
等

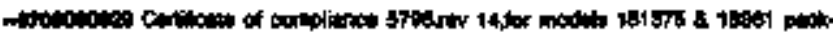

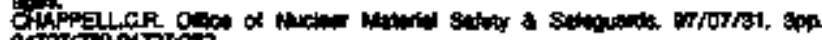

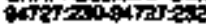

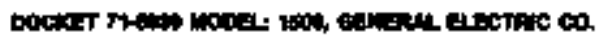

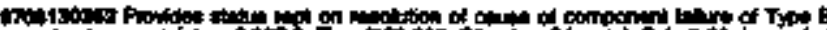

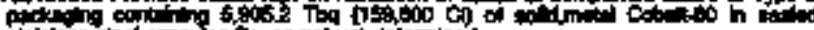

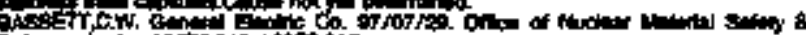

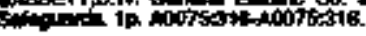

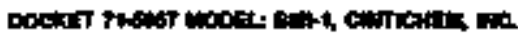

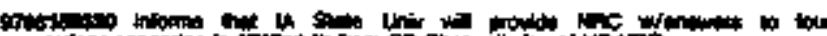

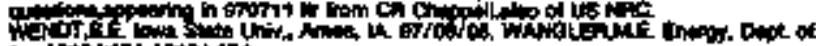

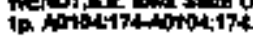

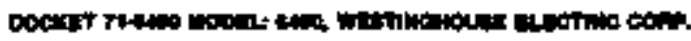

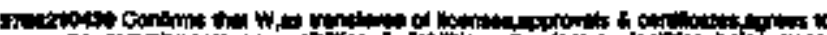

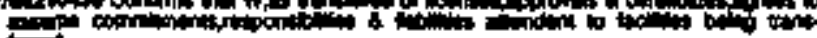

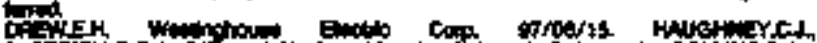

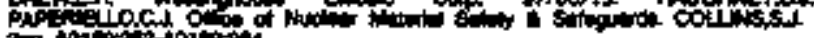

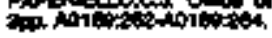

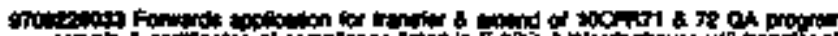

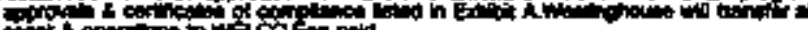

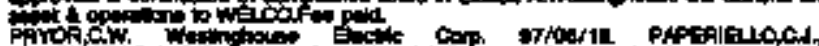
Hew Antist.

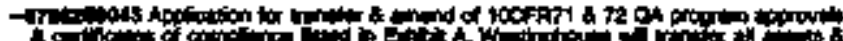

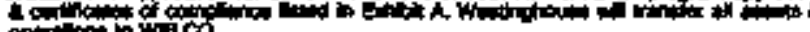

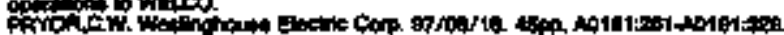

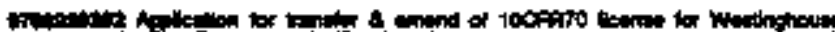

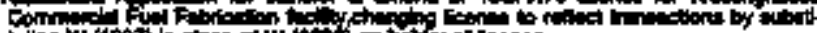

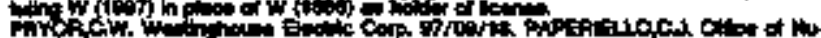

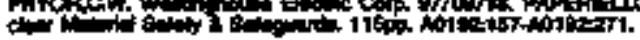

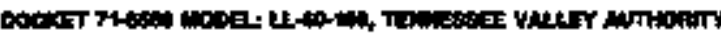

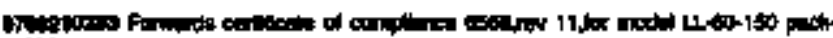

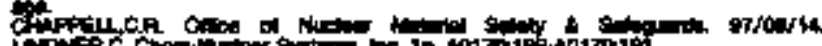

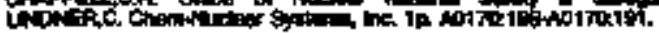

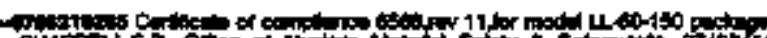

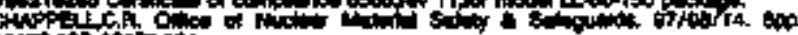

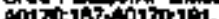

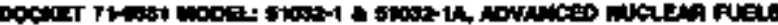
contis

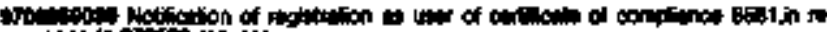
ation

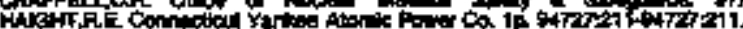

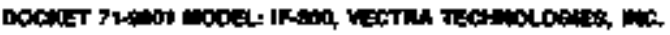

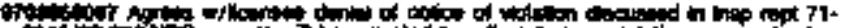

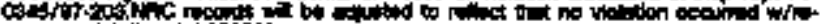

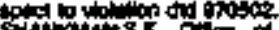

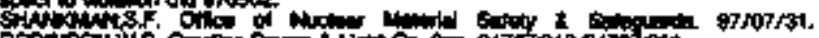

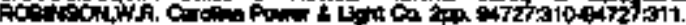

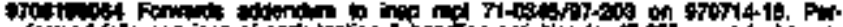

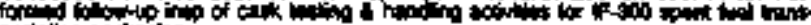

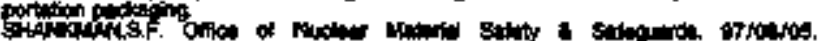

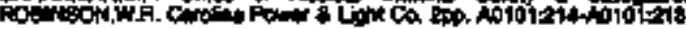

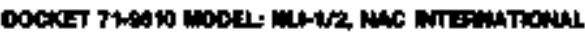

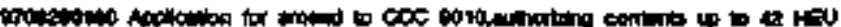

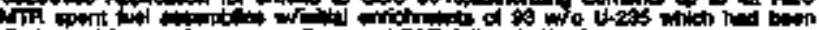

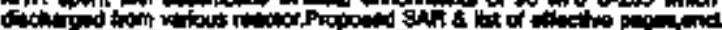

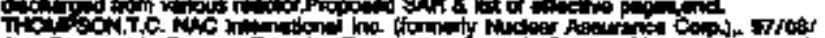

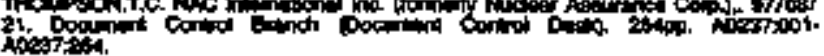

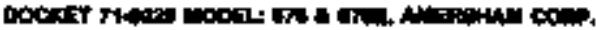

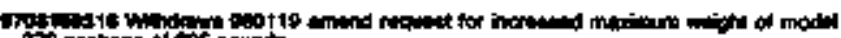
ox pietering of tess pound

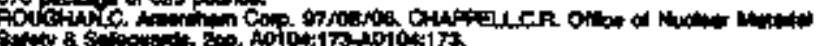

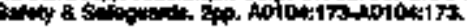

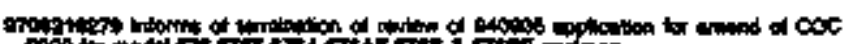

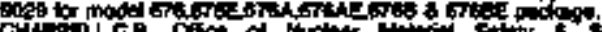

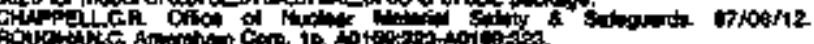

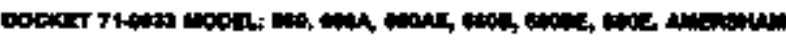

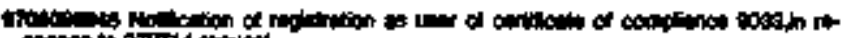

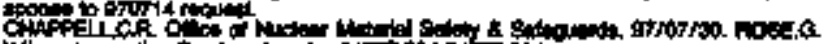

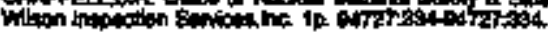

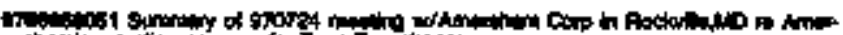

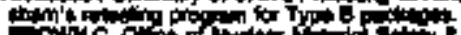

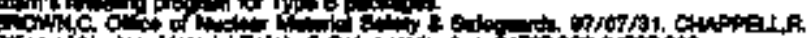
की of

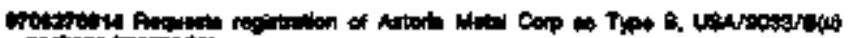

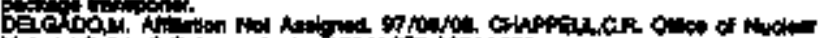

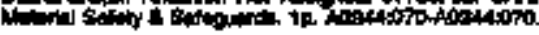

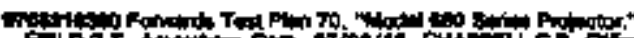

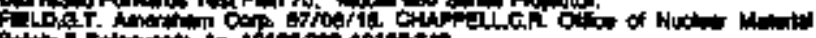
Hoto

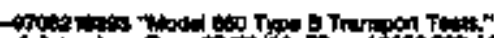

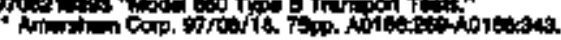

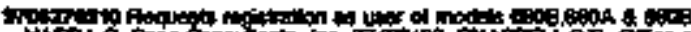

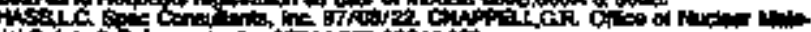

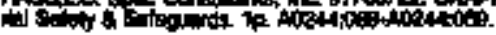

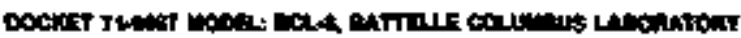

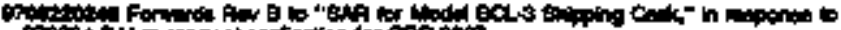

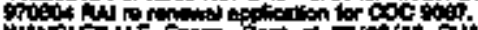

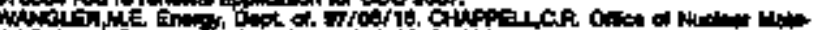

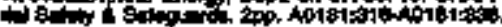

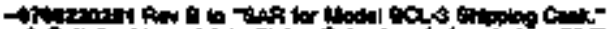

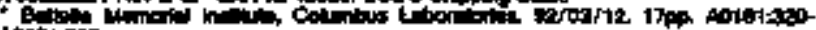
notisis:

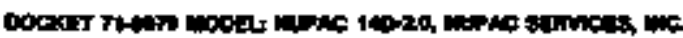

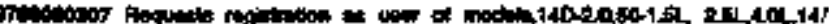

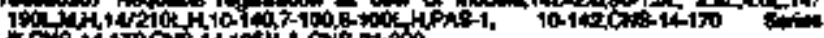
HOGG

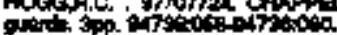

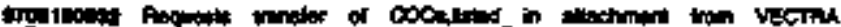

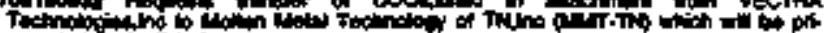

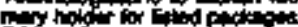

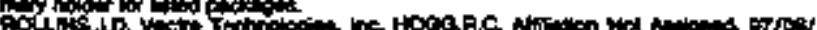

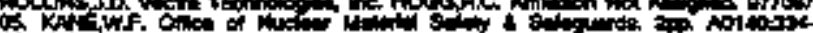

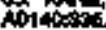

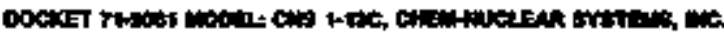

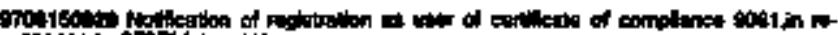

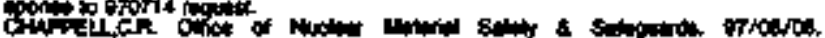

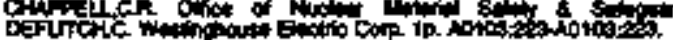




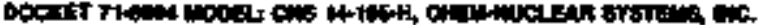

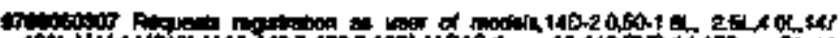

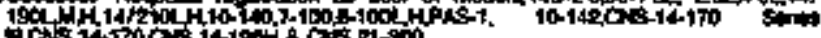
POCORC

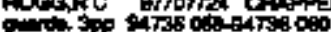

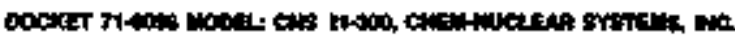

Fonow

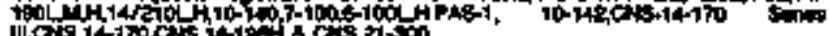

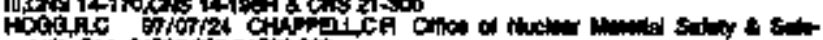

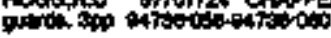

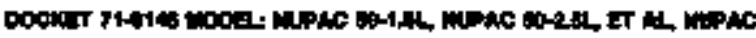

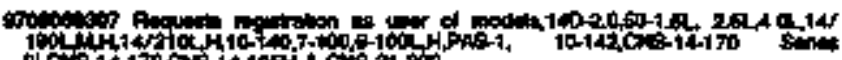

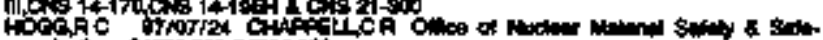

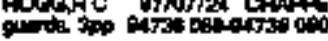

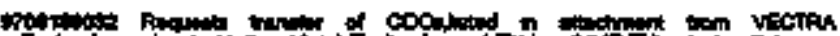

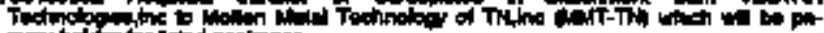

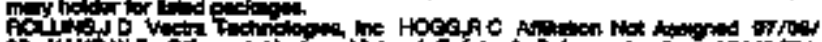

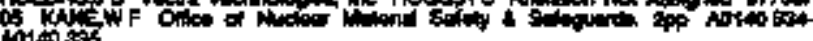
1014

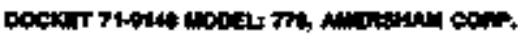

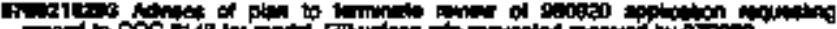

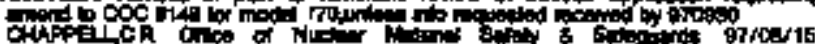

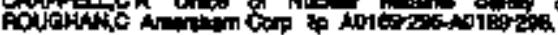

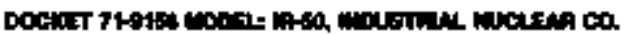

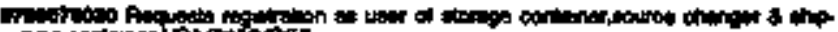

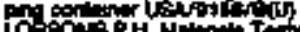

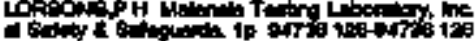

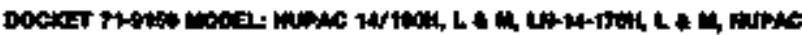

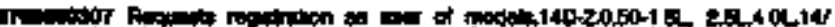
191, ROP H C

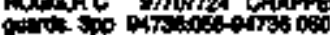

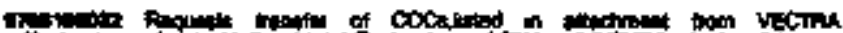

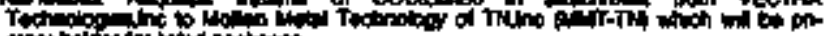

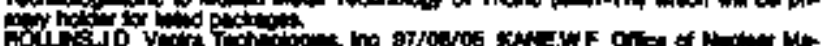

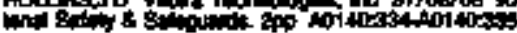

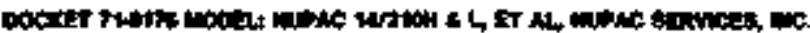

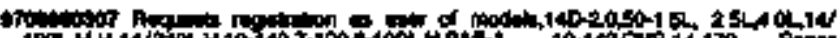

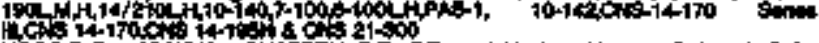

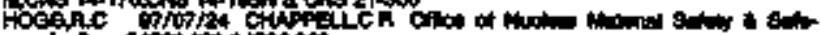
to

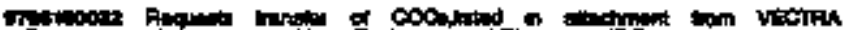

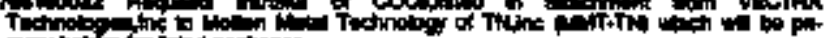

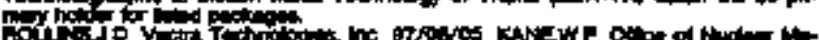

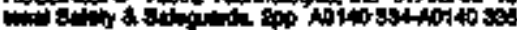

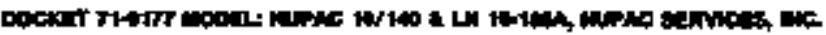

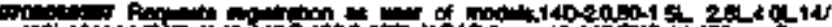
tol thit 14 .

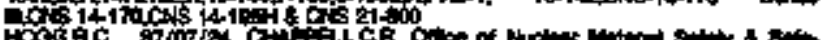

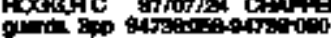

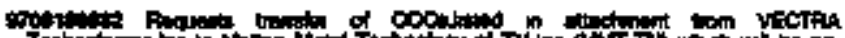

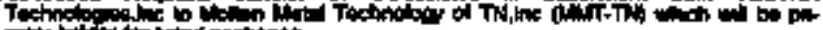

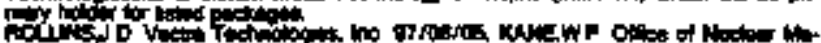
1.

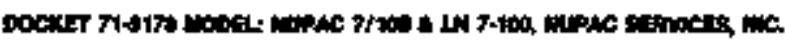

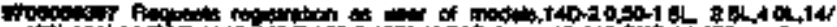

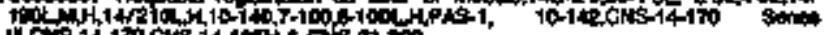

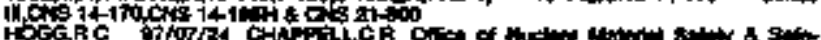

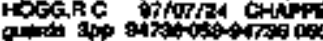

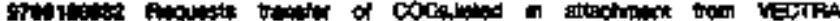

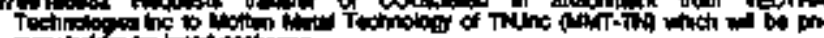

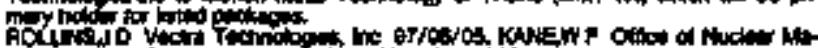

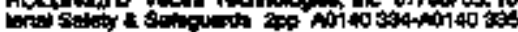

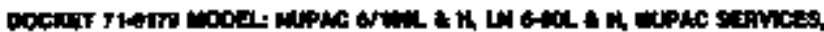

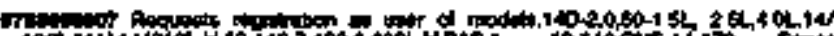

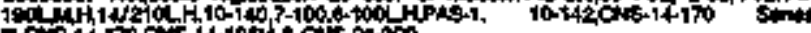
HOG

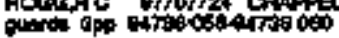

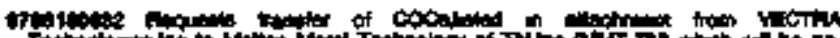

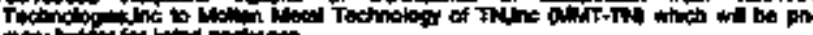

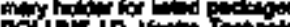

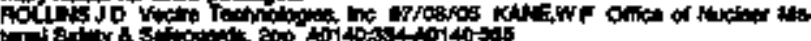

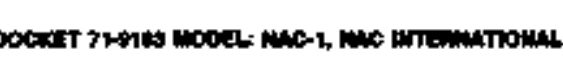

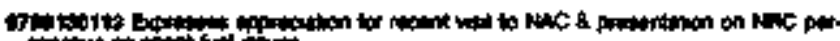

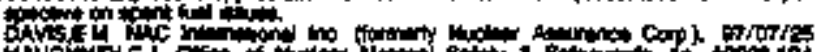
Hol of

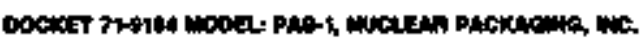

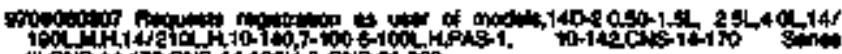

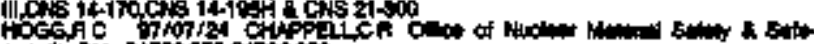

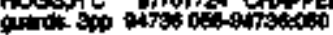

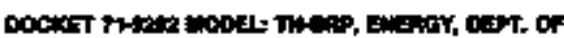

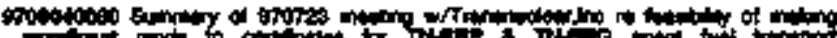

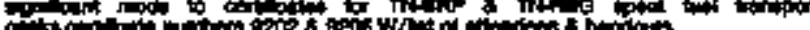

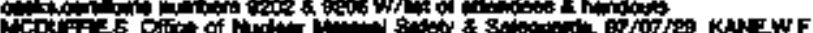
Of

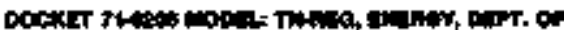

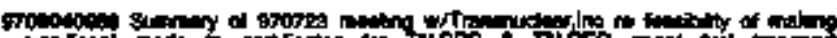

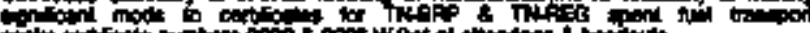

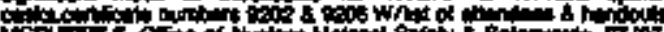

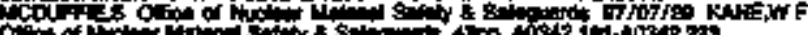

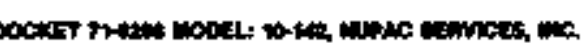

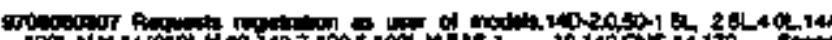

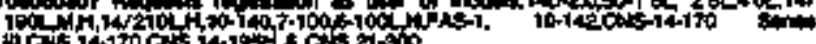

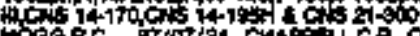

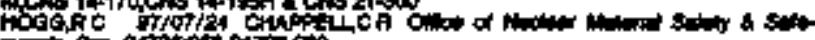

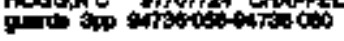

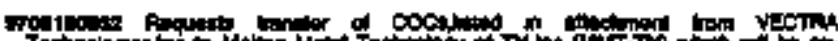

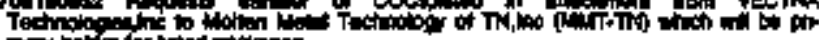

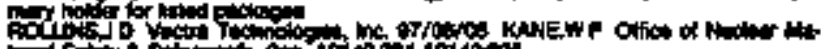

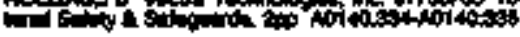

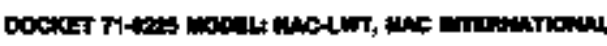

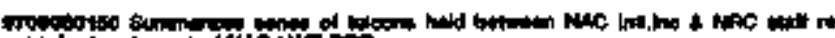

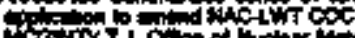

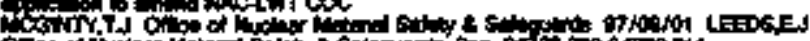

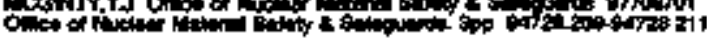

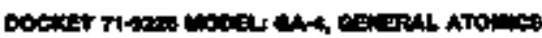

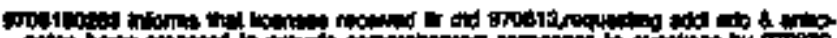

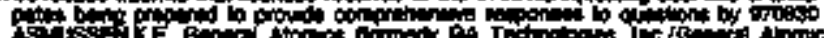

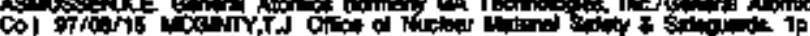
की

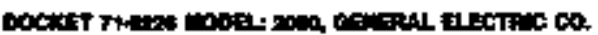

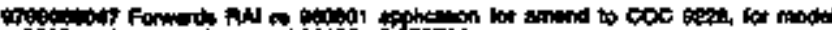

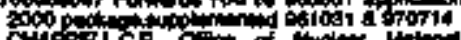

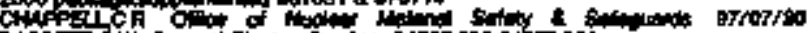

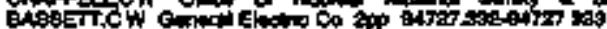

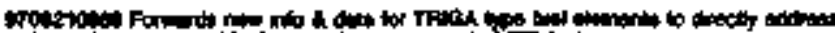

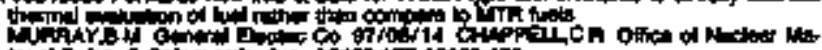

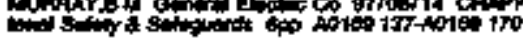




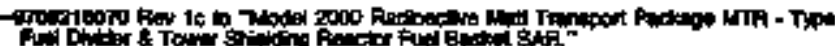

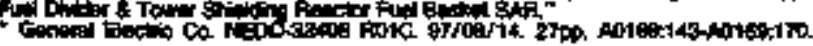

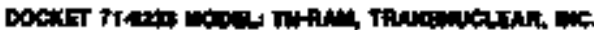

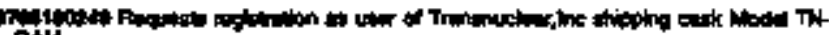

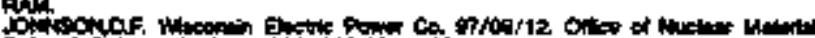

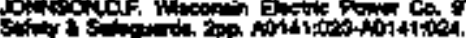

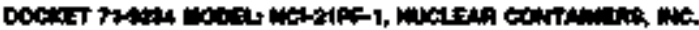

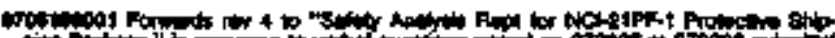

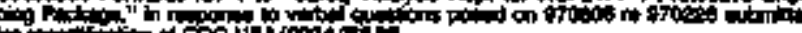

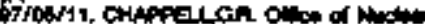

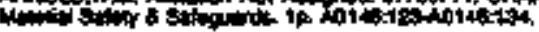

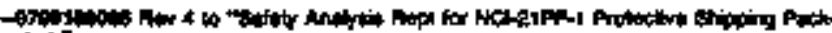

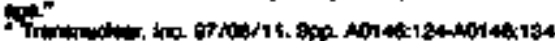

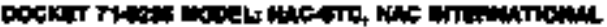

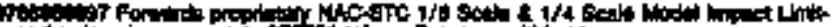

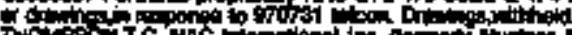

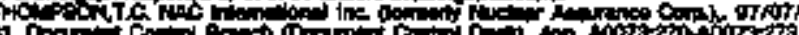

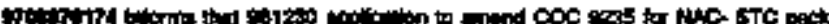

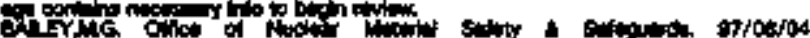
Now

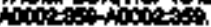

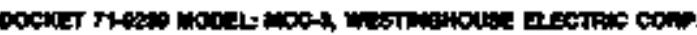

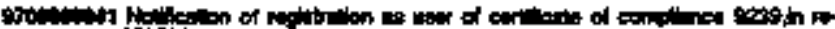

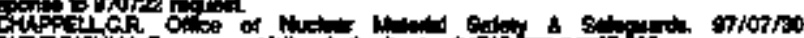

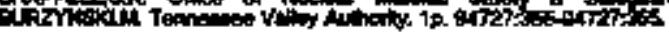

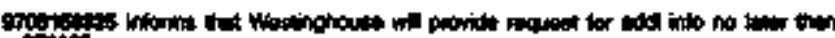
कito.

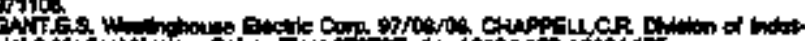

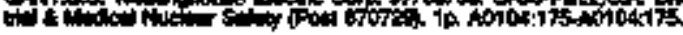

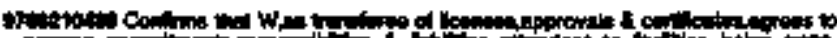

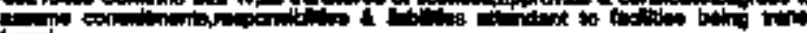

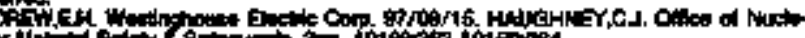

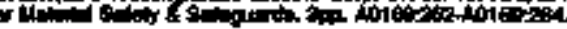

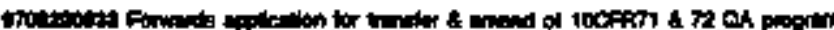

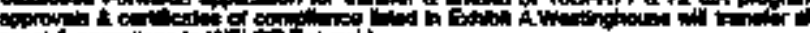

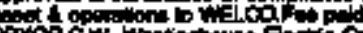

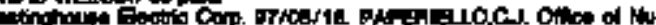

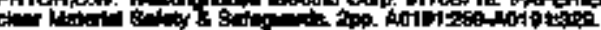

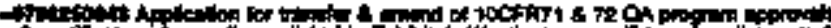

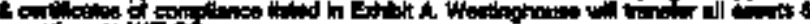

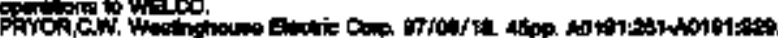

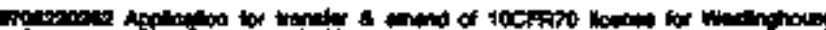

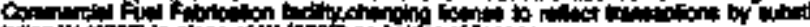

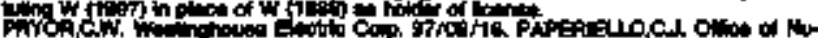

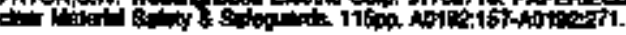

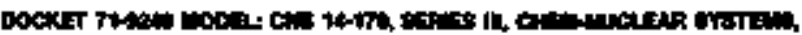

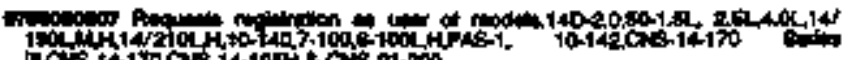

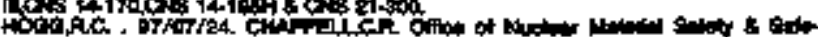

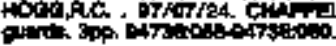

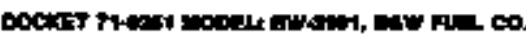

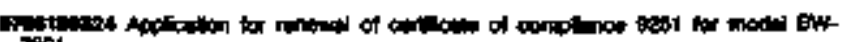
-

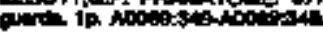

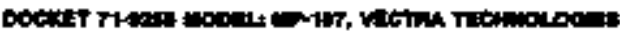

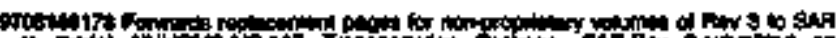

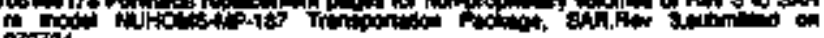

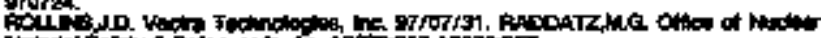

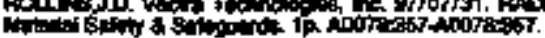

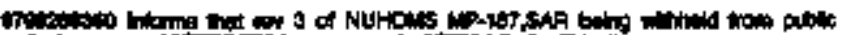

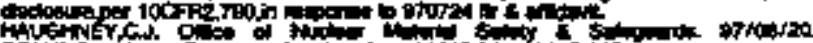

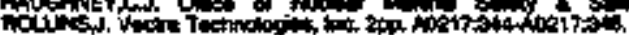

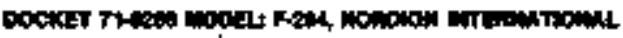

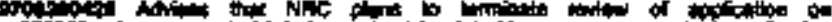

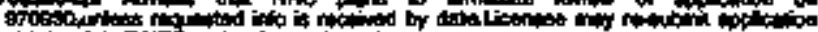

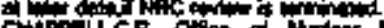

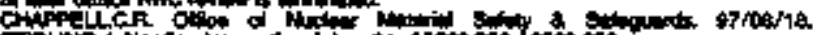

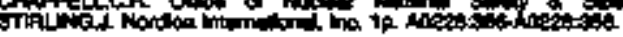

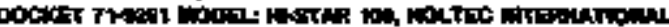

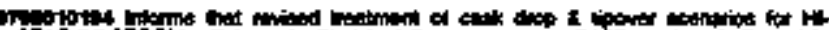

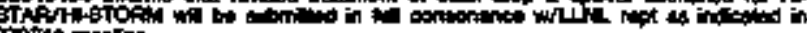

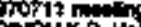

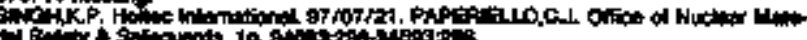

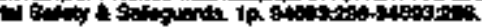

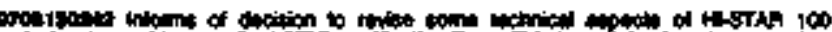

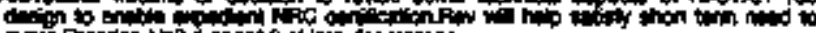

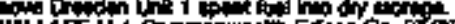

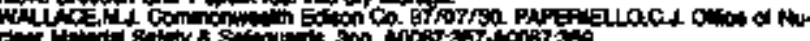

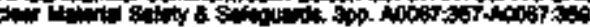

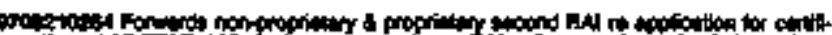

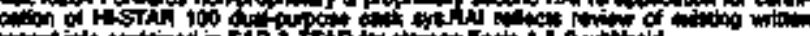

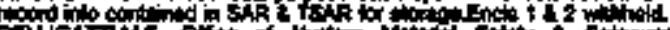

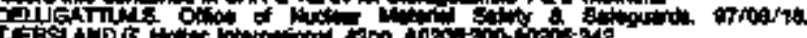

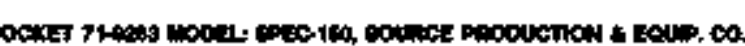

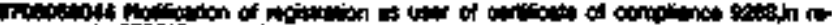

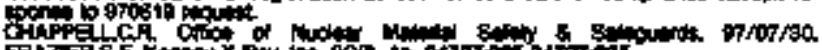

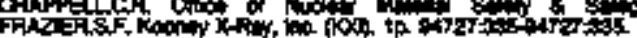

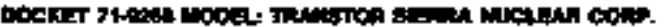

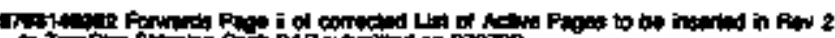

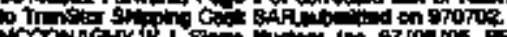

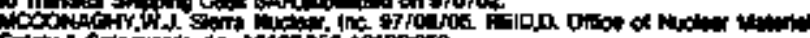

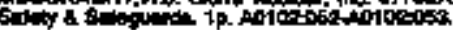

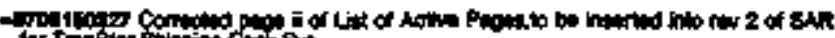

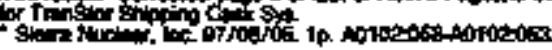

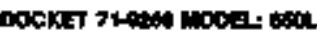

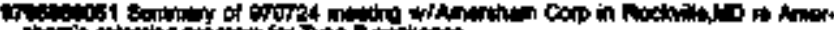

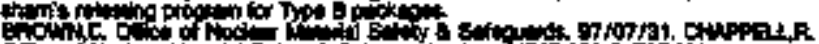

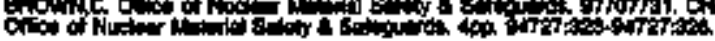

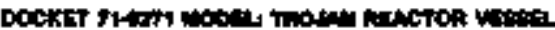
Orow PA

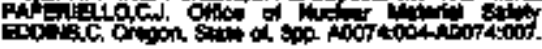

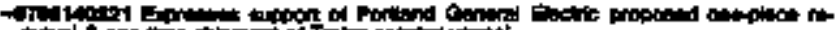

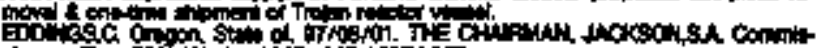

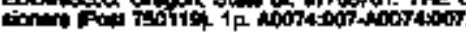

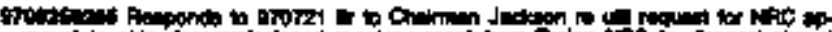

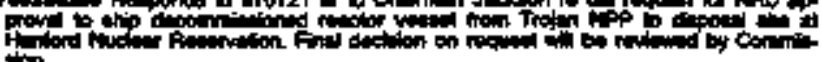

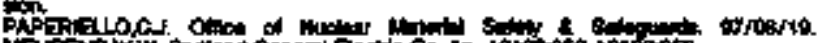

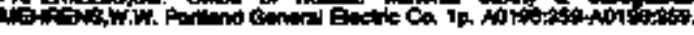

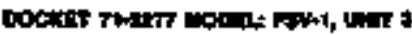

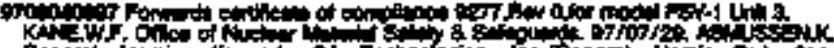

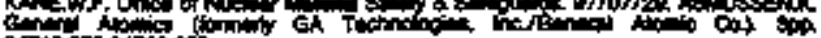

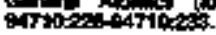

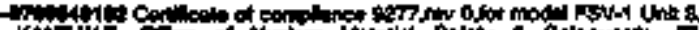

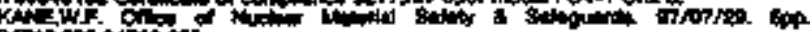

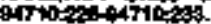

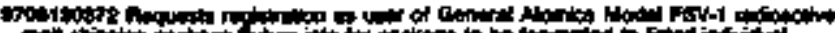

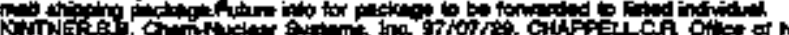

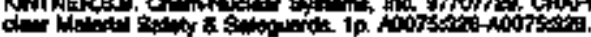

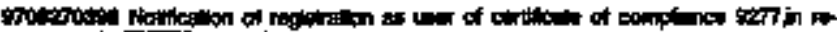

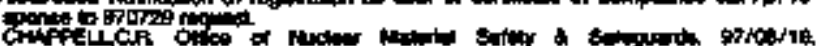

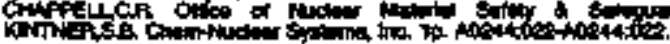




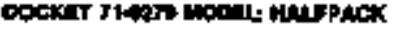

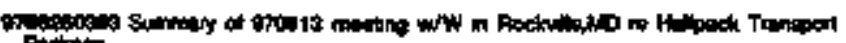
OSto

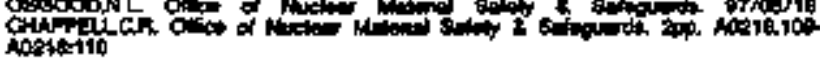

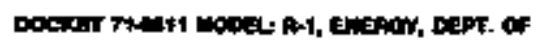

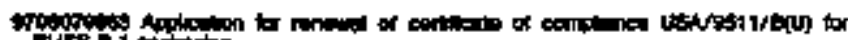

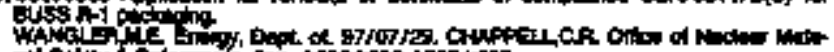

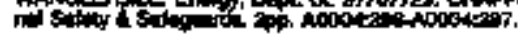

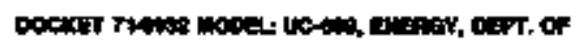

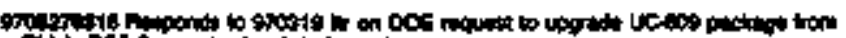

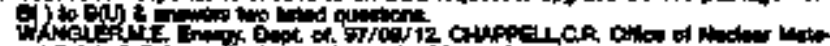

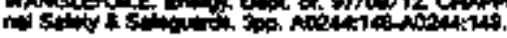

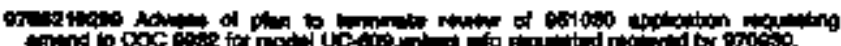

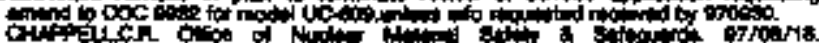

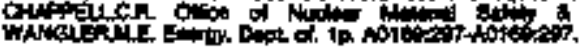

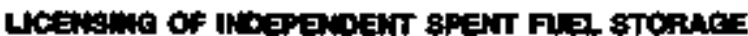

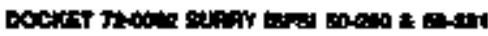

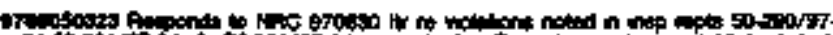

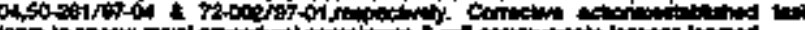

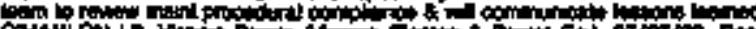

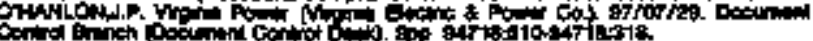

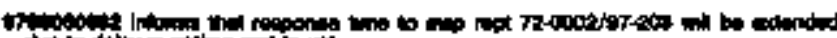

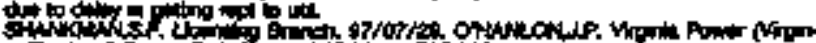

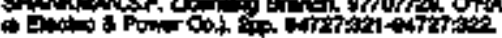

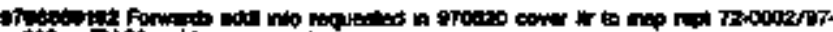

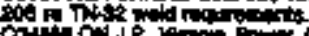

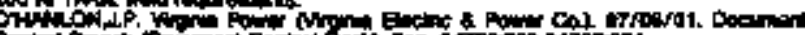

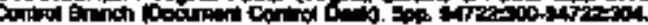

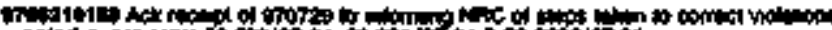

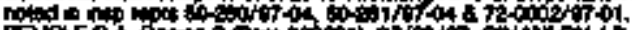

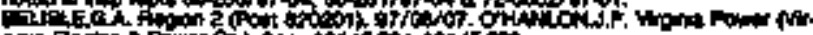

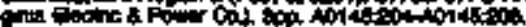

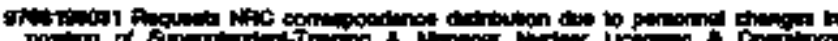

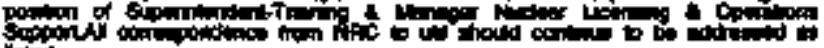

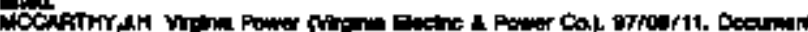

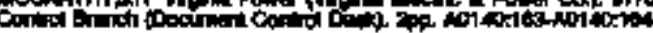

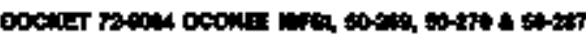

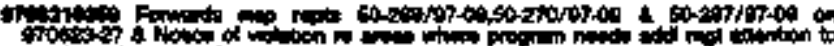

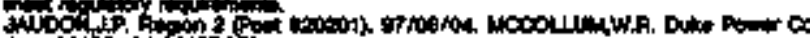
40 d0M

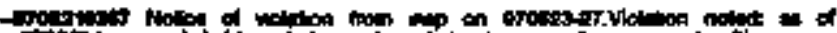

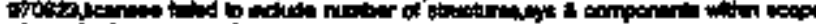

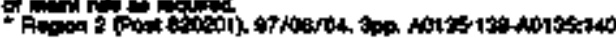

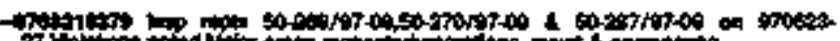

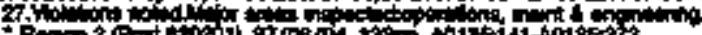

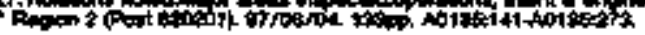

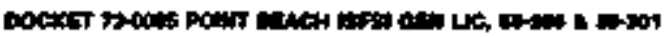

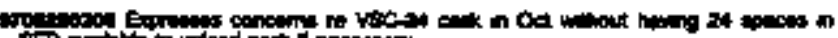

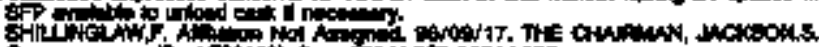

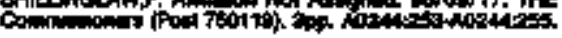

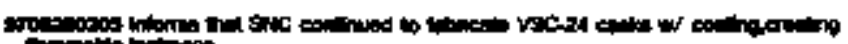

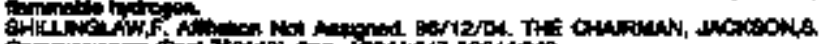

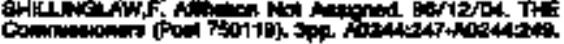

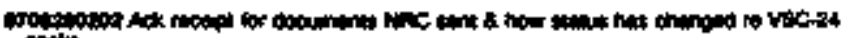

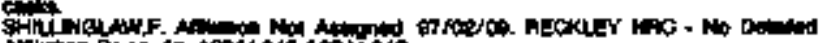

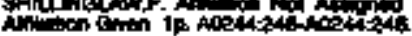

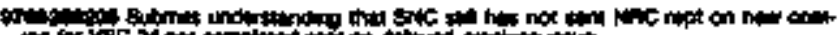

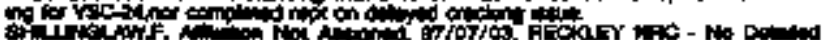

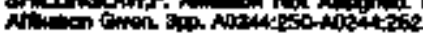

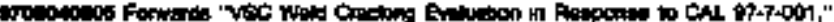

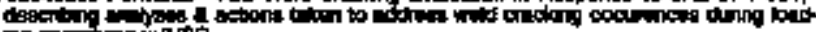

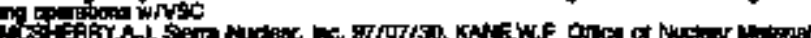

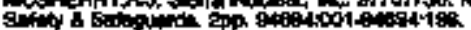

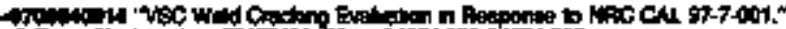

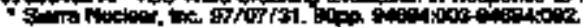

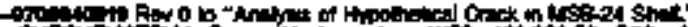

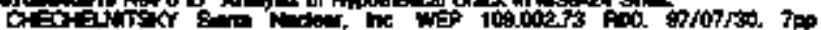

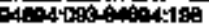

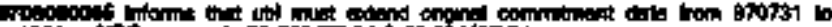

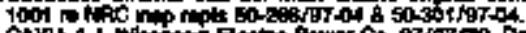

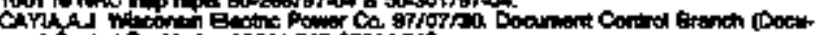

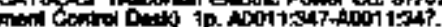

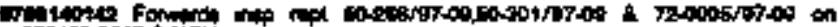
Droteo-012 \& NOW

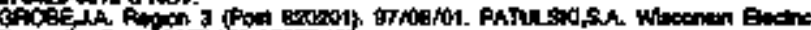

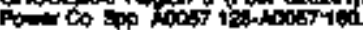

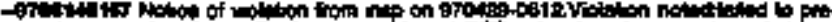

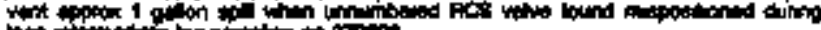

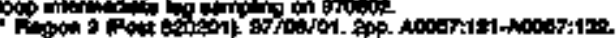

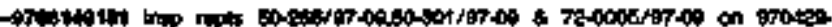

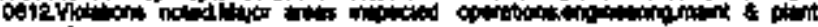

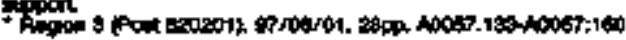

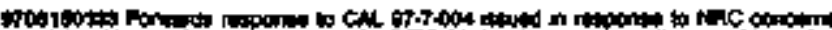

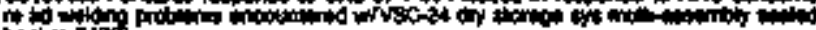

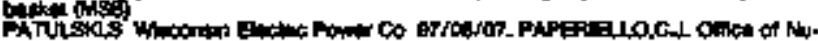

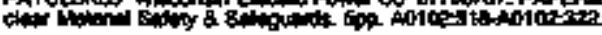

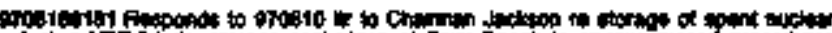

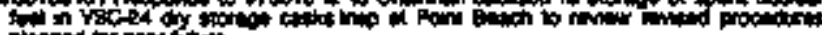

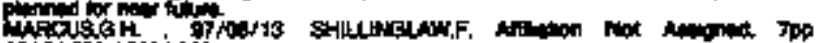
Nolon

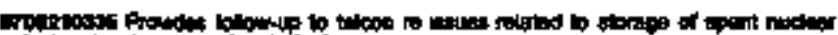

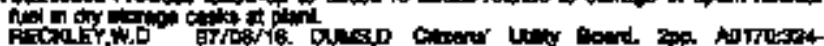
Rition

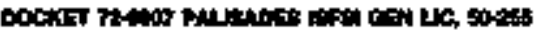

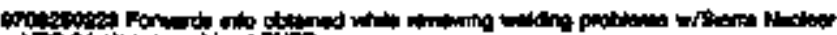
Y

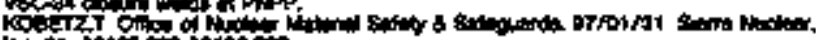

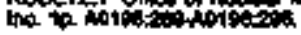

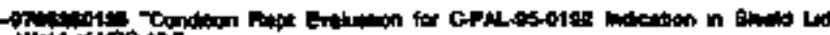
Win of $\cos ^{2}$

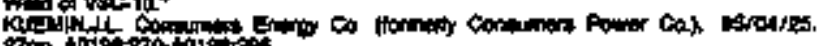

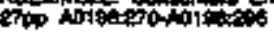

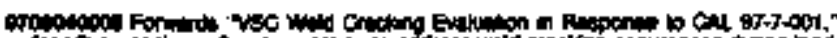

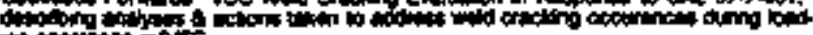
Pcoping

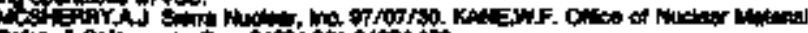

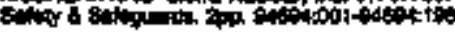

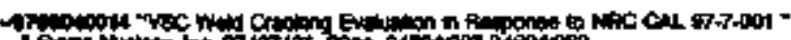

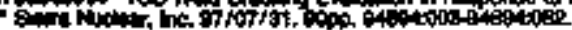

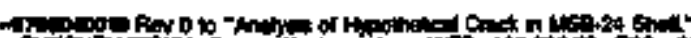

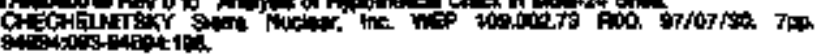

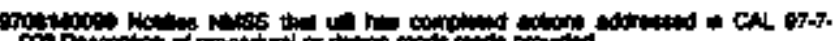

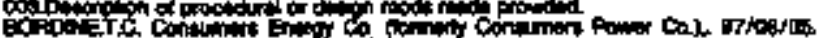

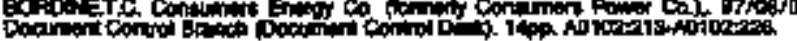

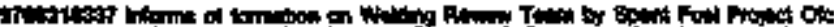

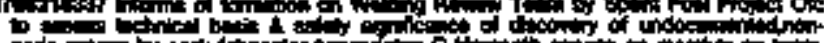

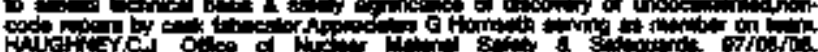

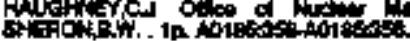

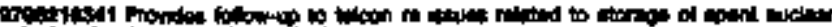

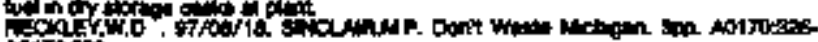
action.

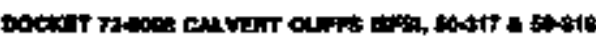

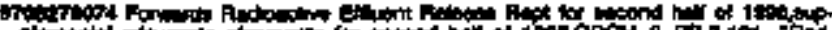

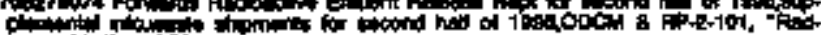
(1)

fint

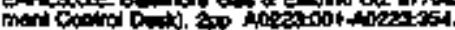

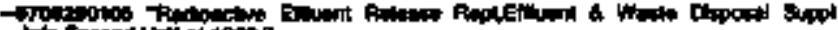

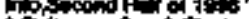

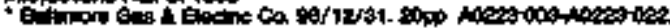




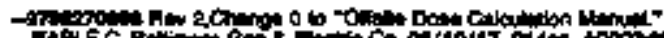

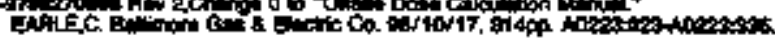

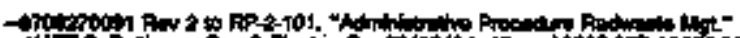

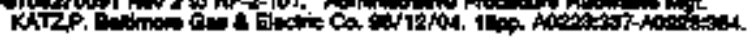

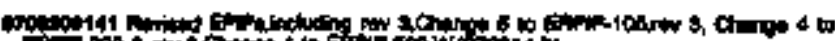

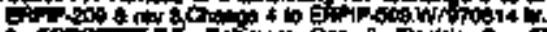

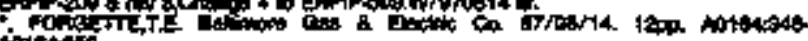
intomition

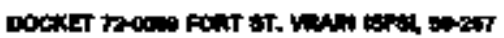

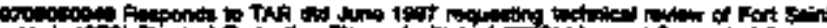
Wil to

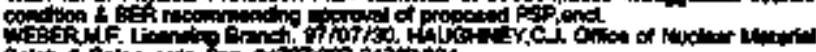

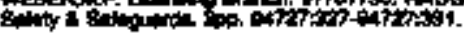

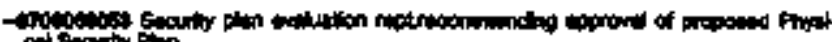
cal imation the

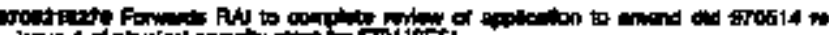

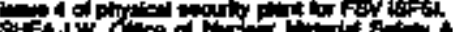

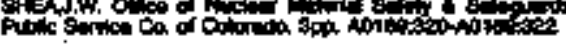

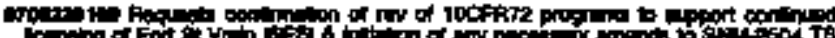

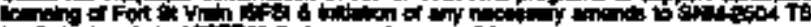

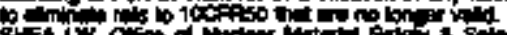

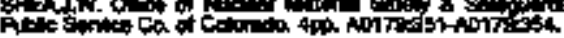

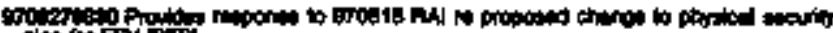

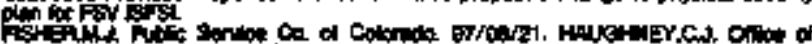

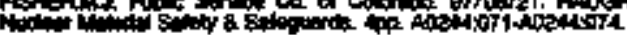

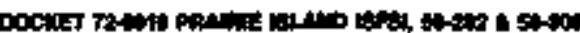

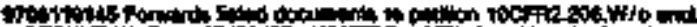

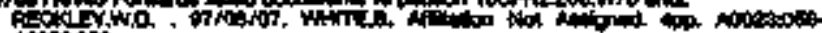
$\sin 0$

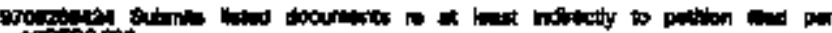

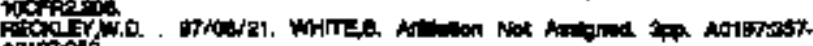
not

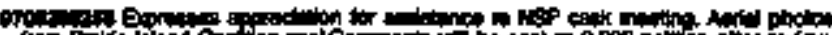

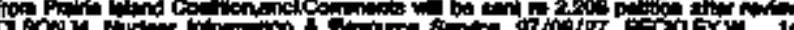

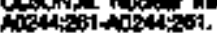

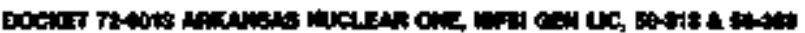

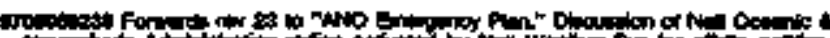

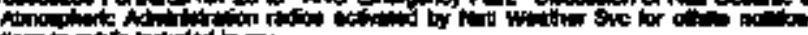

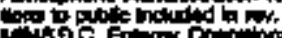

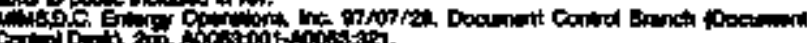

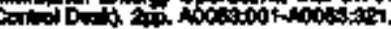

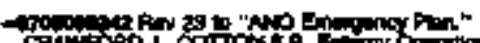

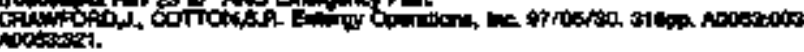

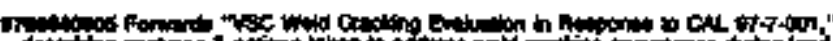

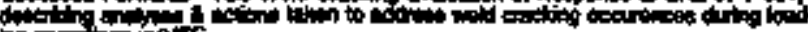

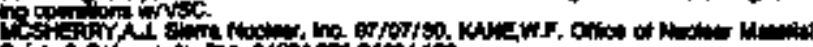

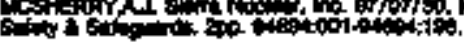

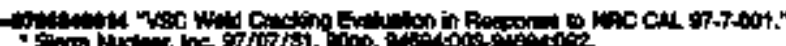

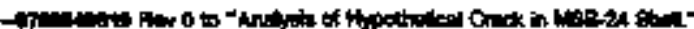
CHoch

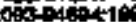

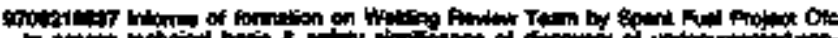

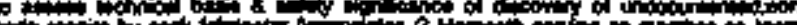

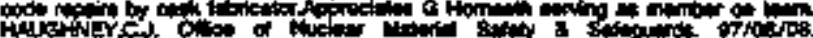

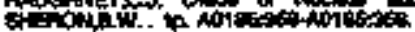

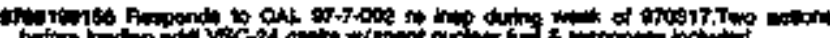

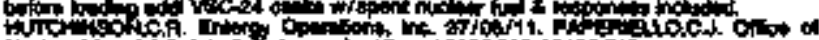

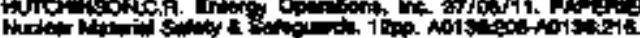

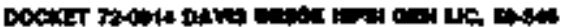

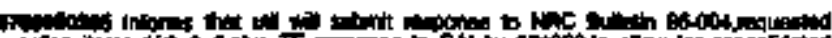

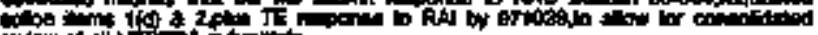

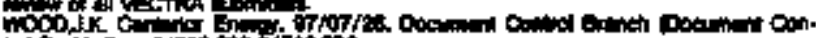

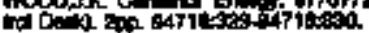

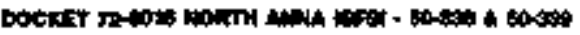

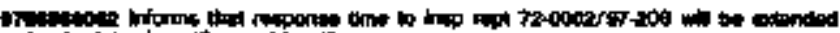

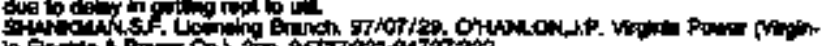

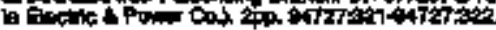

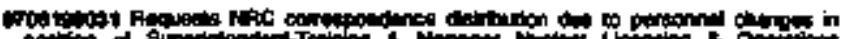

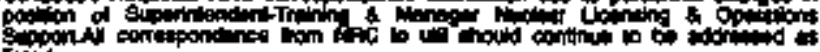

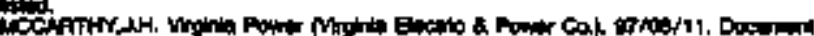

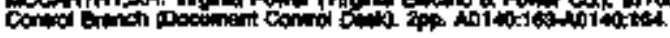

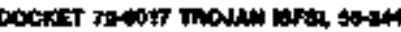

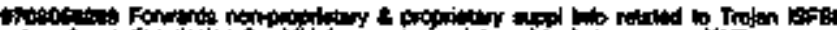

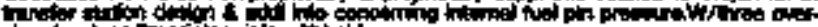

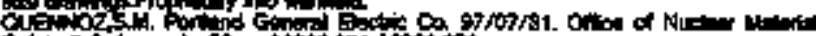

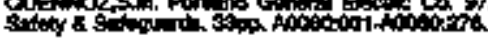

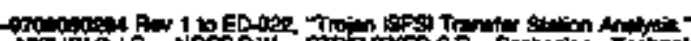

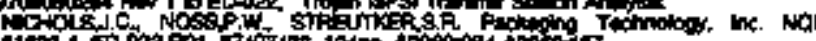

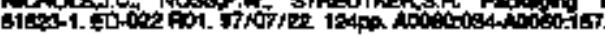

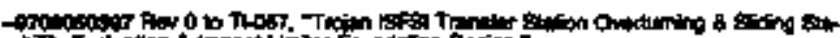

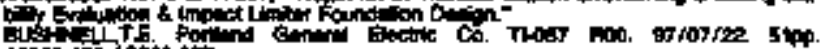

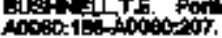

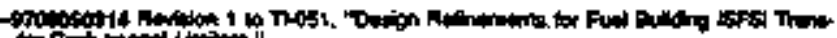

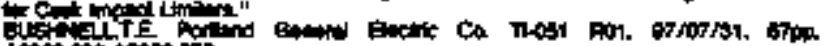

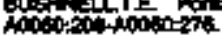

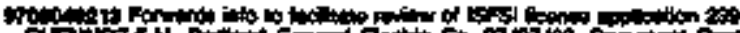

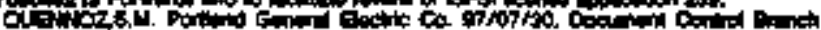

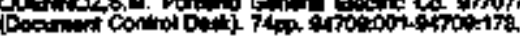

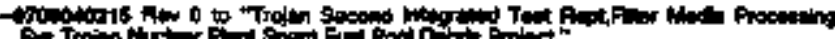

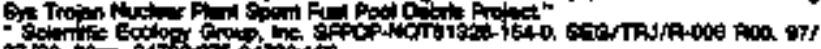

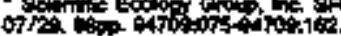

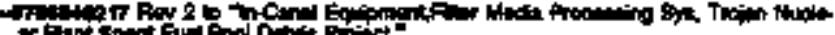

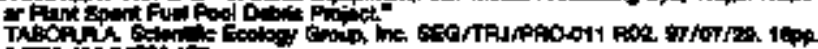

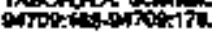

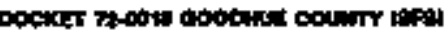

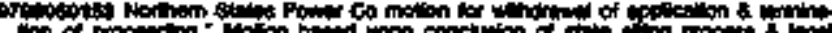

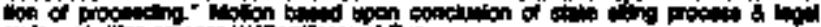

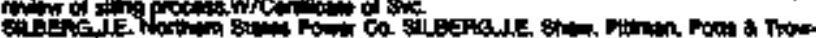

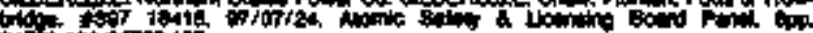

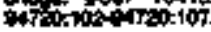

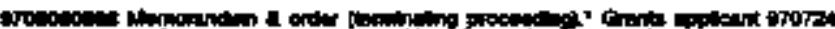

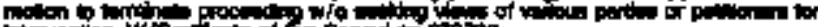

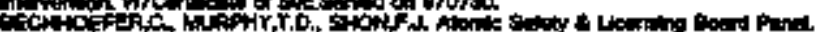

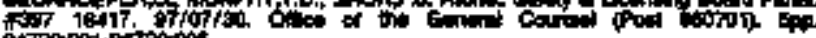

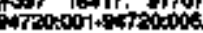

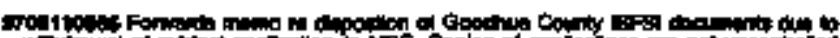

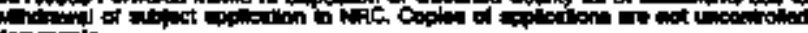

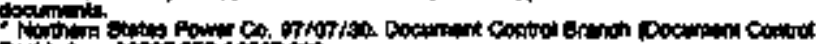

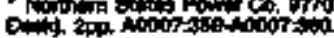

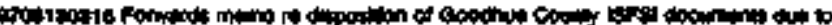

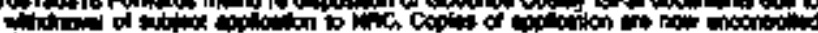

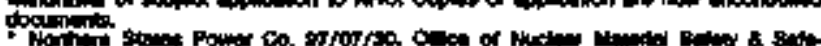

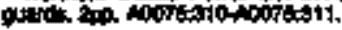

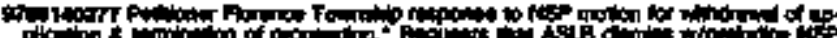

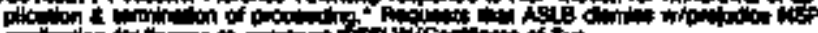

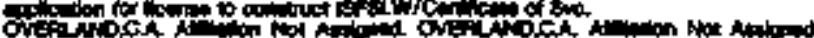

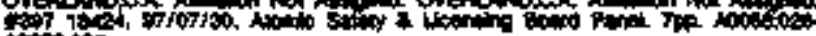
Afotos

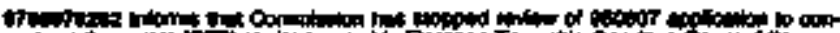
Fuct

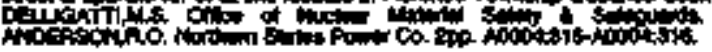

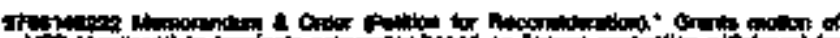
Le (4)

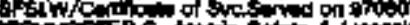

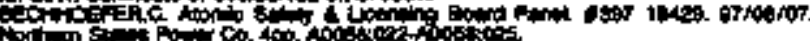

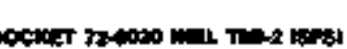

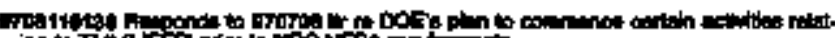

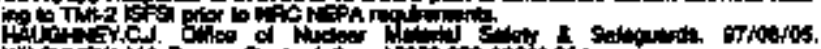

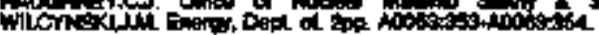




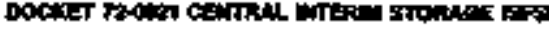

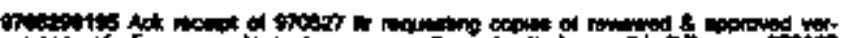

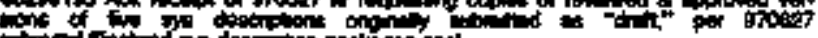

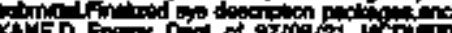

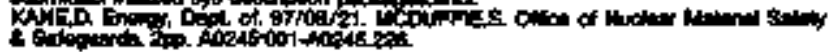

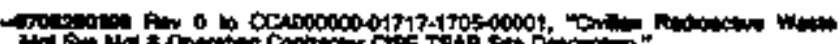

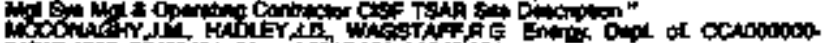

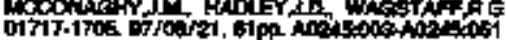

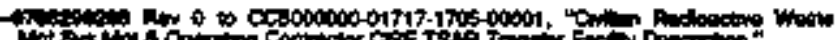

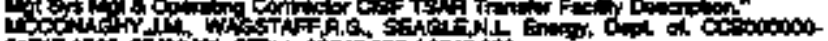

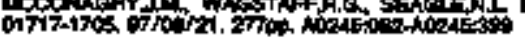

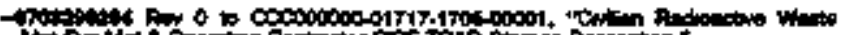

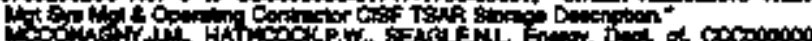

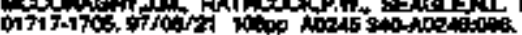

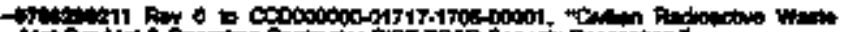

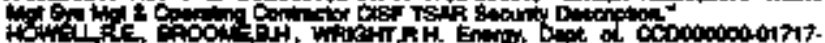

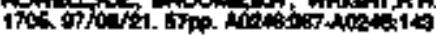

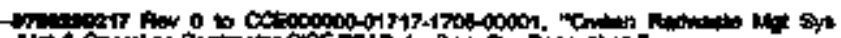

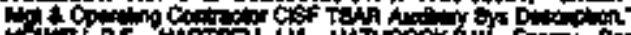

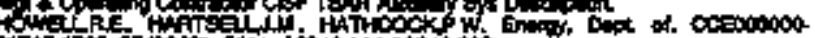

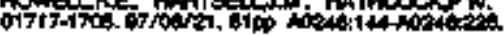

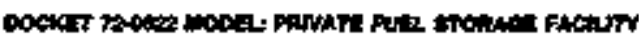

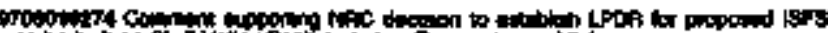

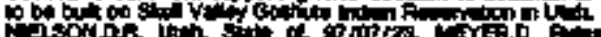

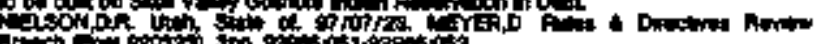

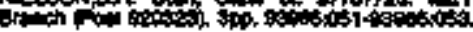

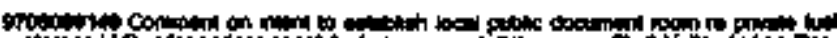

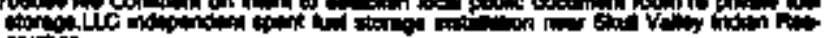

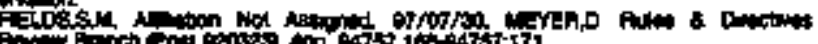

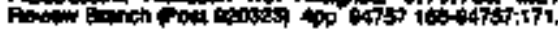

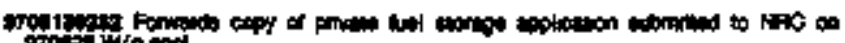
moticinto the

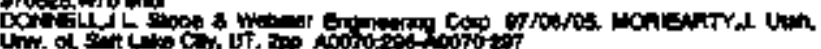

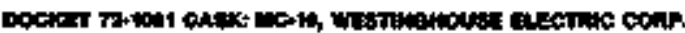

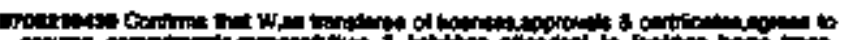

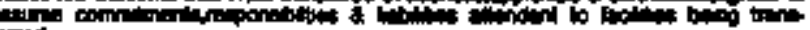

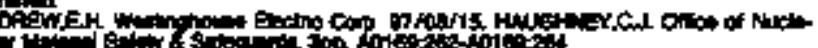

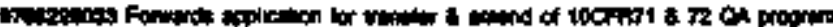

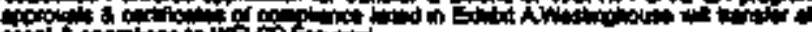

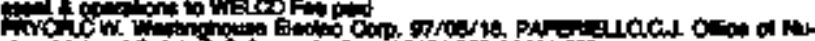

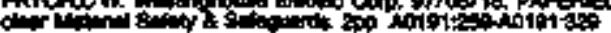

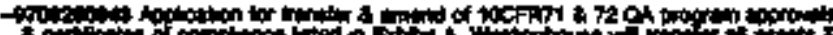

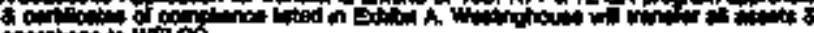

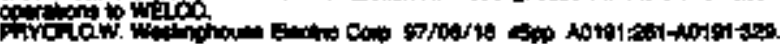

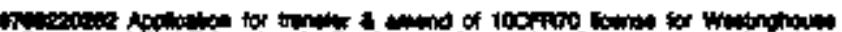

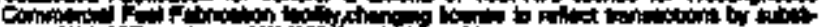

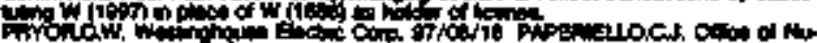

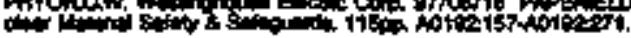

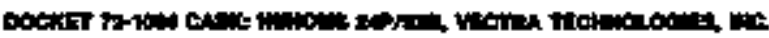

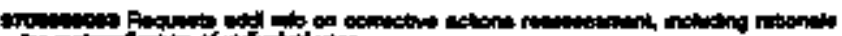

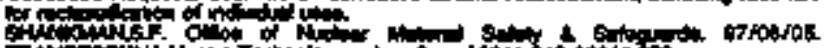

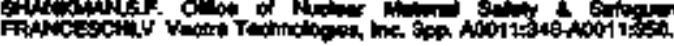

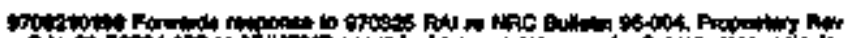
2 to

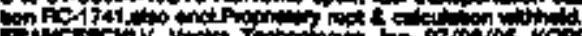

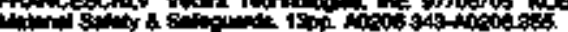

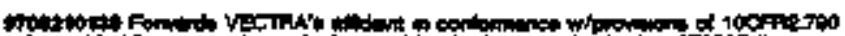

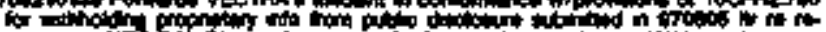

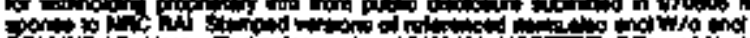

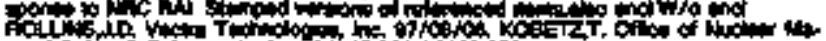

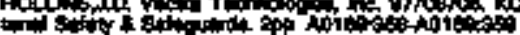

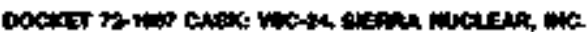

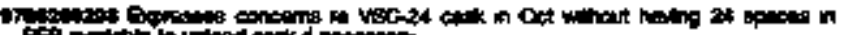

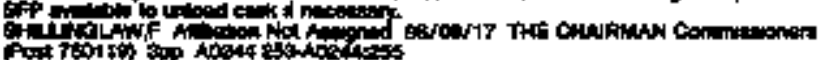

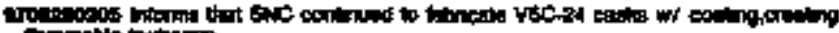

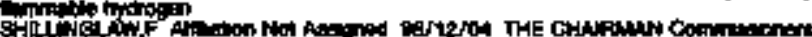

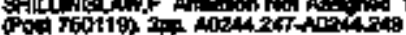

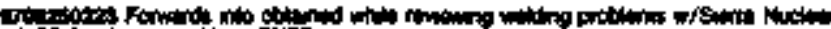

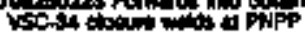

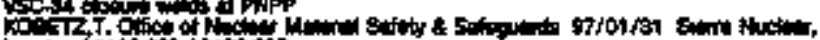

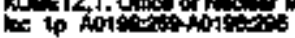

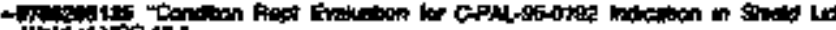

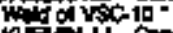

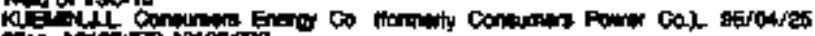

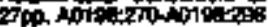

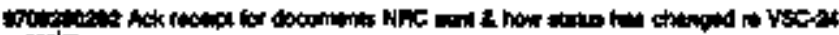

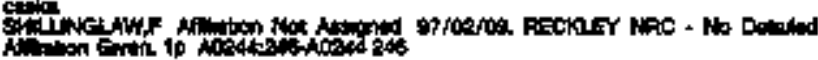

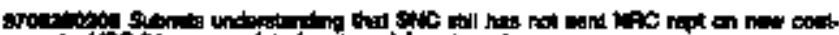

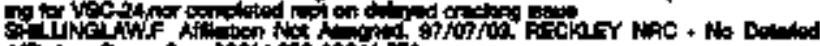

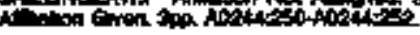

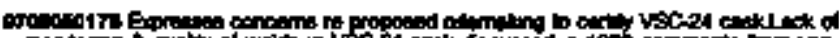

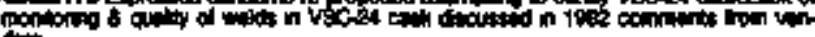
ditis

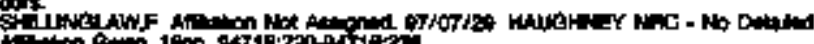

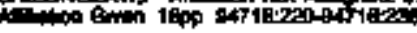

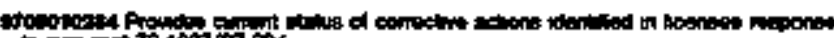

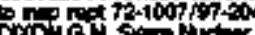

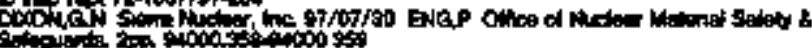

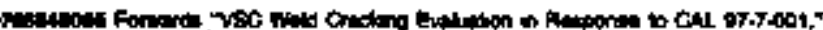

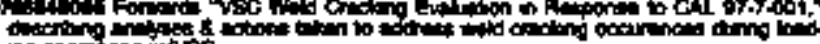

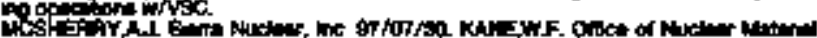

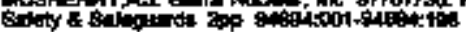

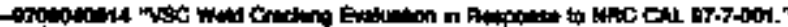

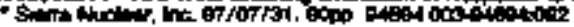

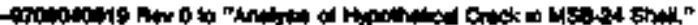

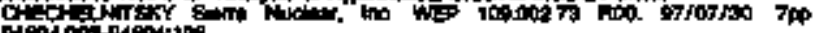

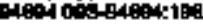

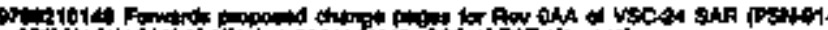

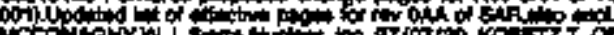

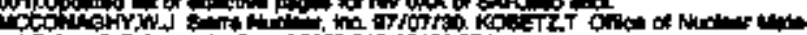

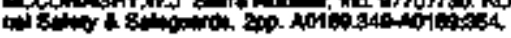

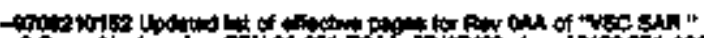

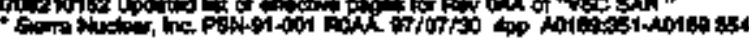

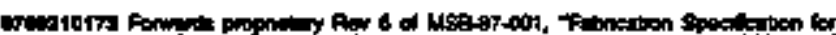

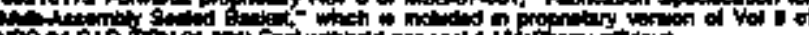

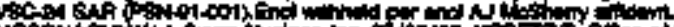

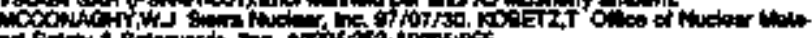

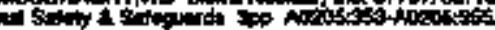

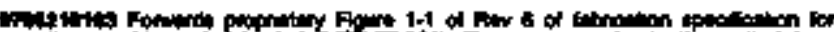

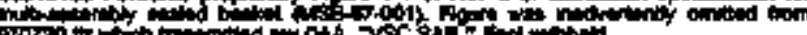

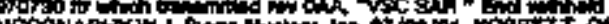

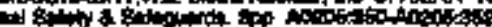

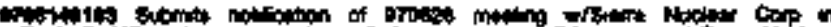

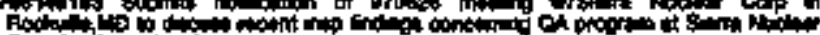

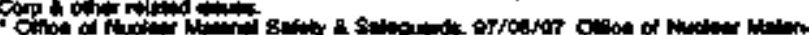

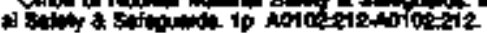

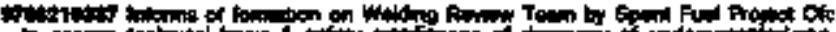

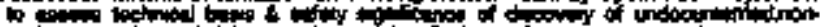

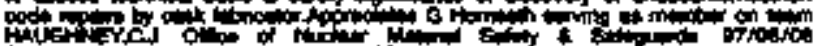

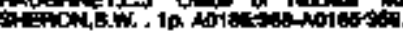

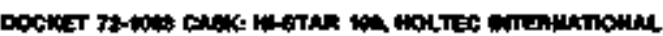

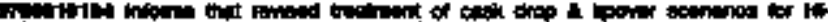

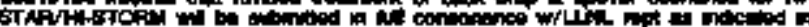

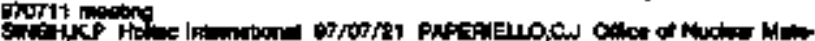

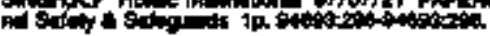

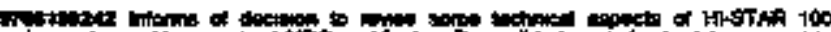

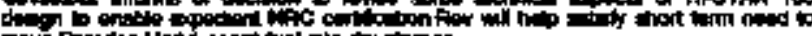

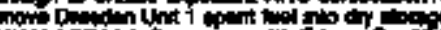

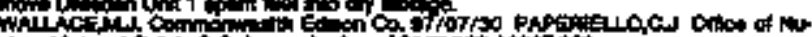

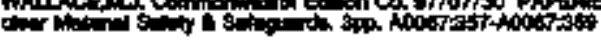

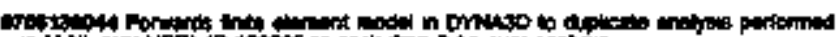

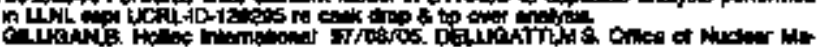

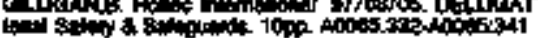

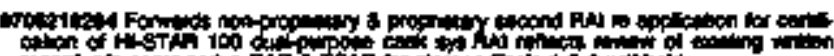

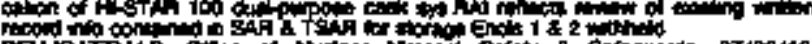

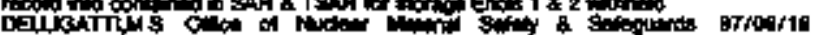

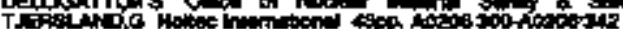





\section{NONDOCKETED ITEMS}

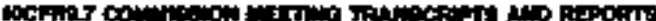

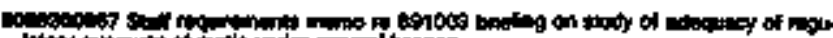

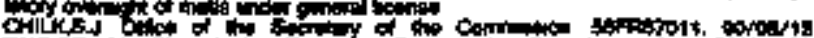

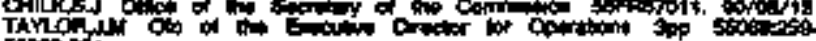

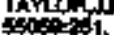

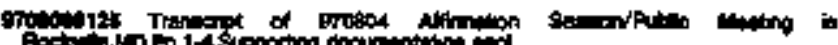

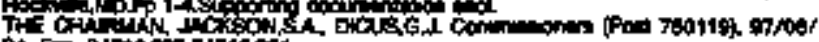

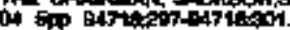

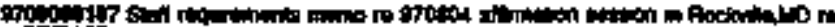

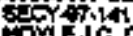

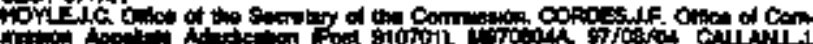

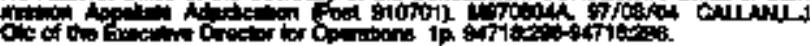

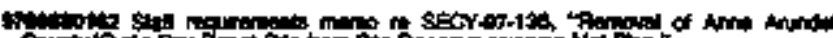

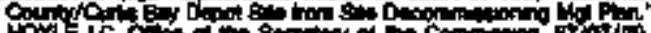

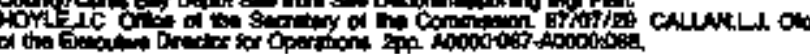

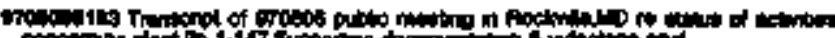

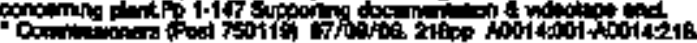

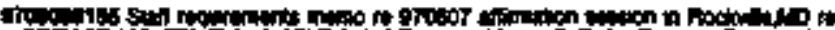
SEC

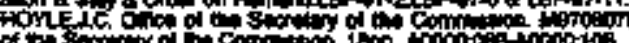

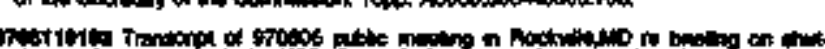

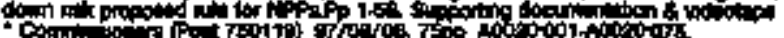

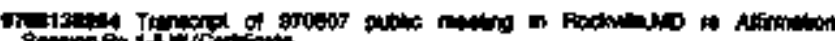

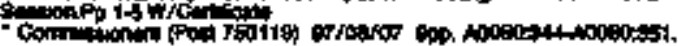

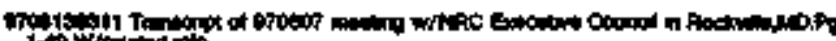

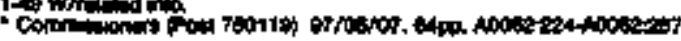

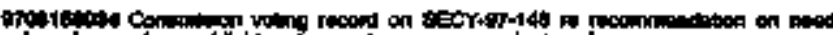

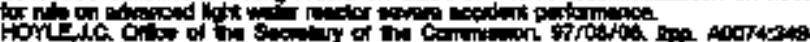
$x$

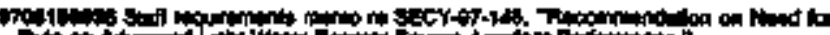

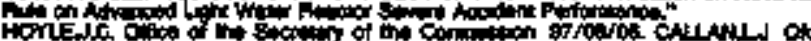

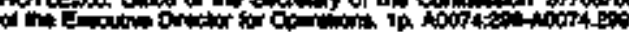

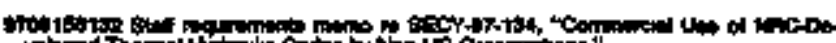

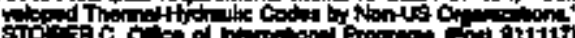

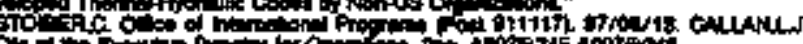

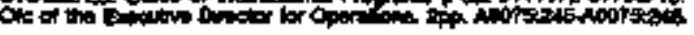

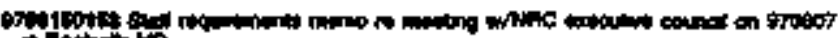

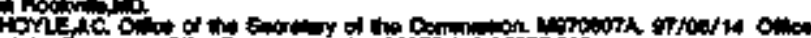

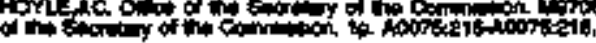

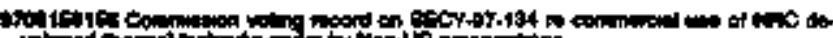
fort

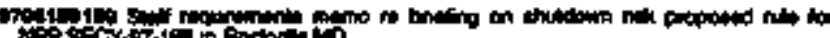

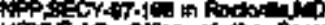

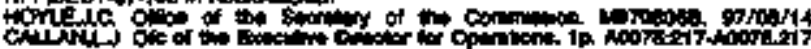

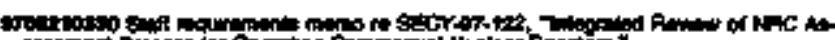

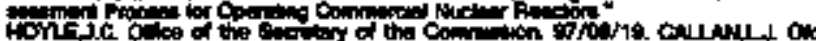

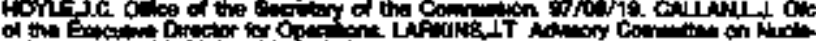

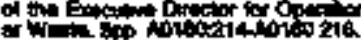

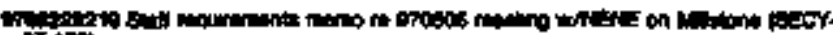
1.t+ot

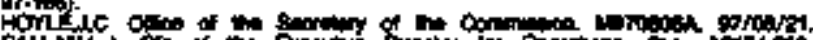

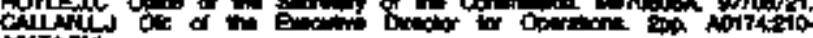
Amitazit.

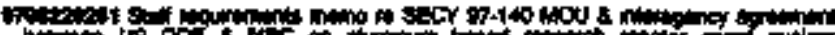

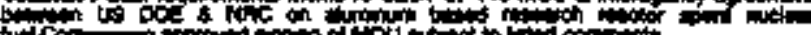

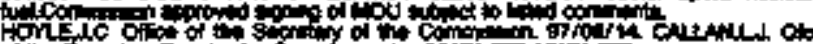

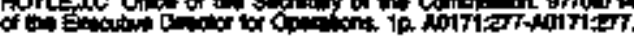

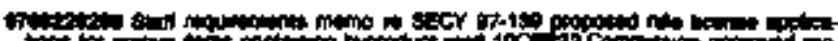

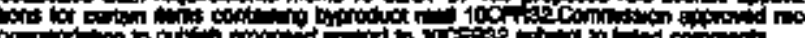

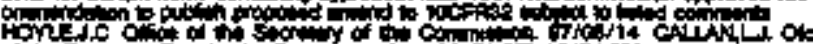

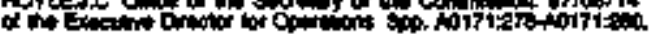

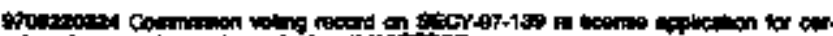

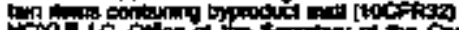

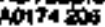

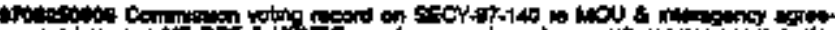

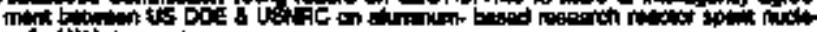

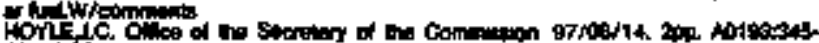
Aprod

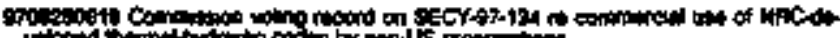

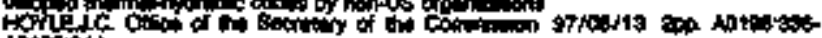
NDtosid

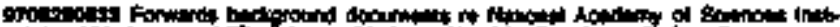

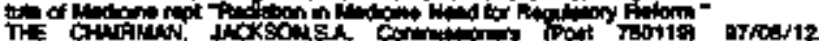

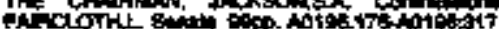

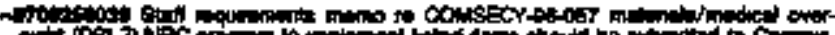

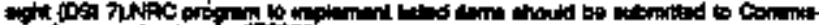

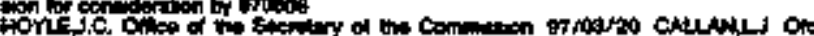

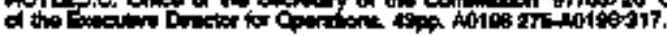

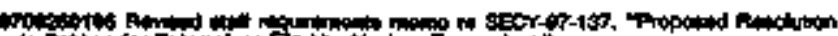

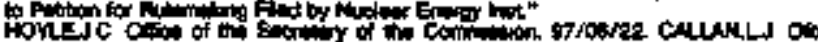

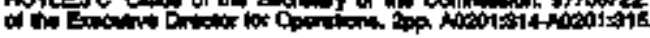

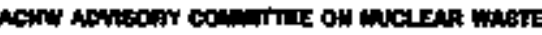

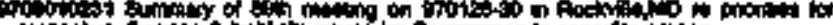

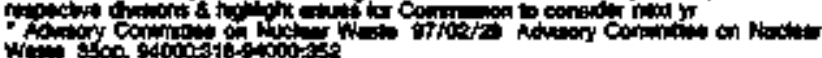

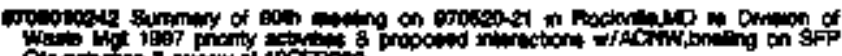

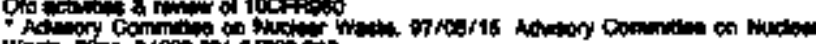
wint in of

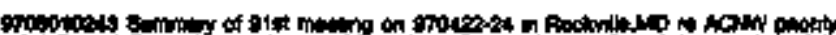

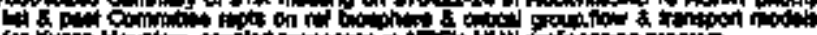
Whant a

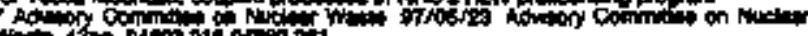

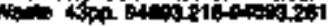

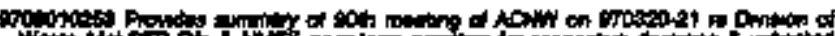

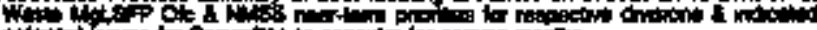

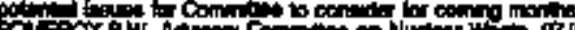

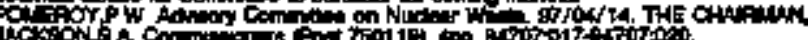

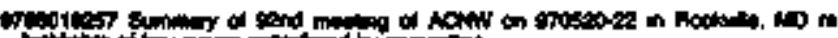

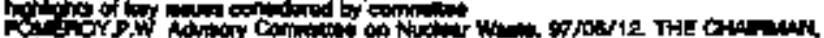

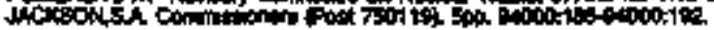

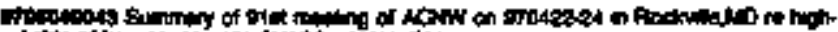

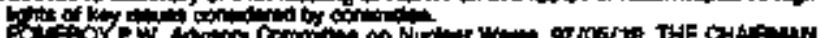

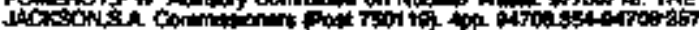

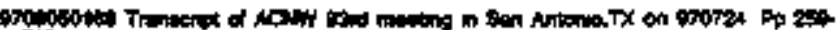
5

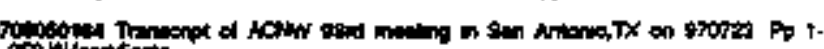
Sit W/ant

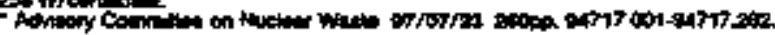

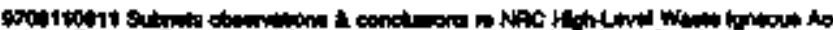

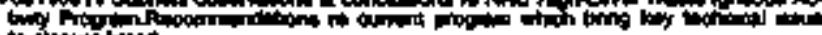

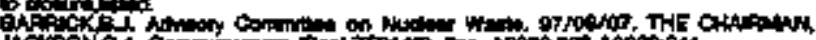

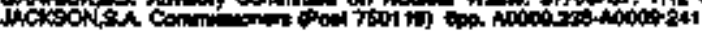

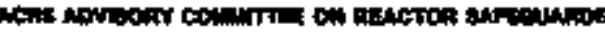

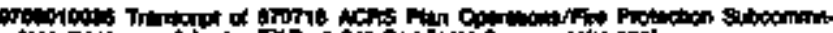

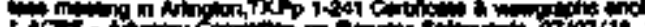

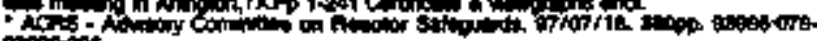
$\cos$

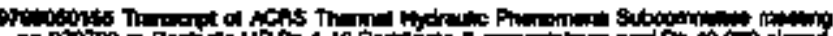

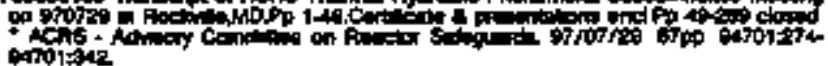

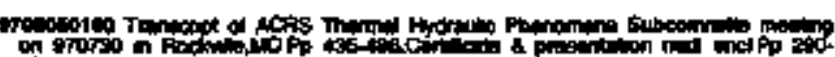

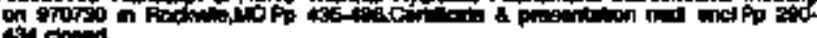

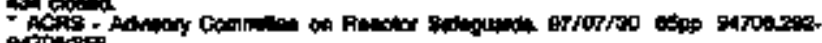
arioss

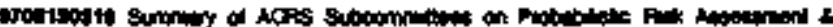

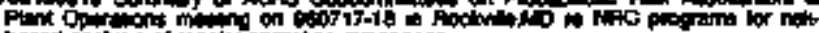

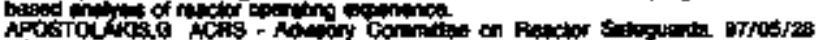

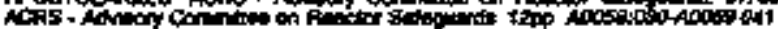




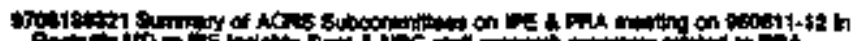

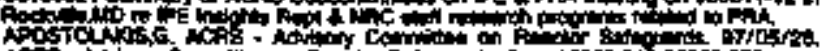

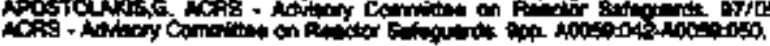

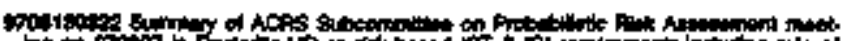

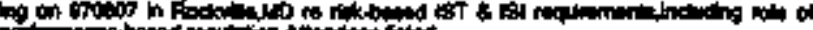

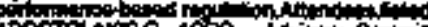

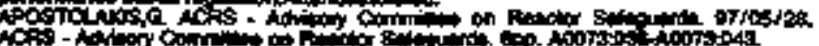

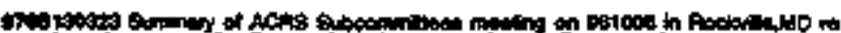

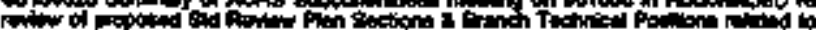

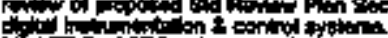

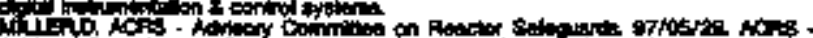

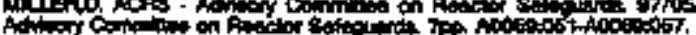

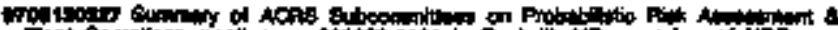

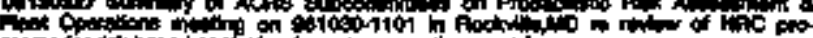

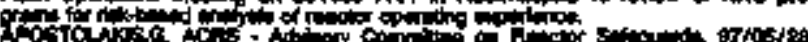

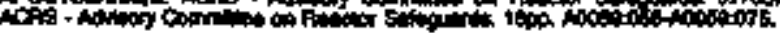

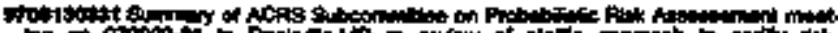

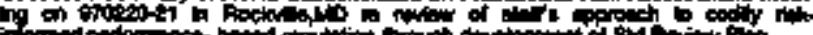

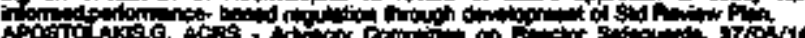

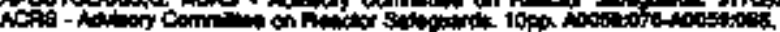

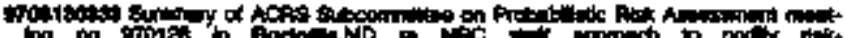

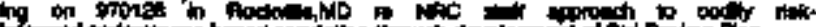

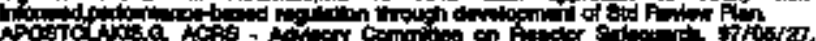

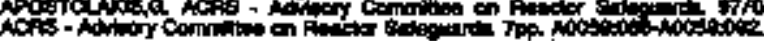

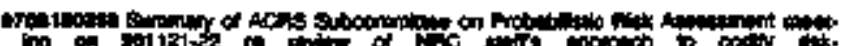

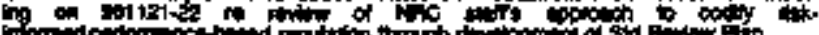

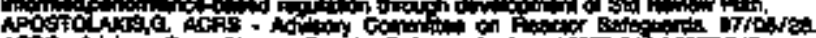

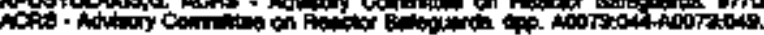

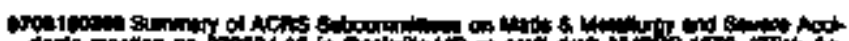

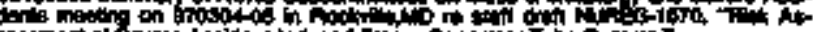

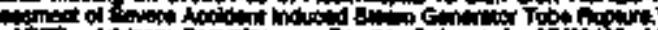

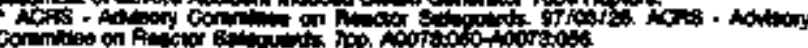

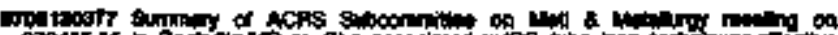

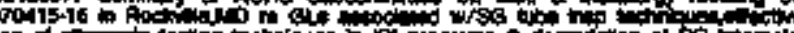

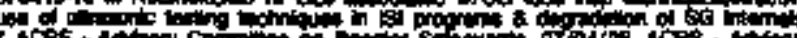

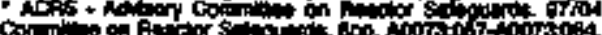

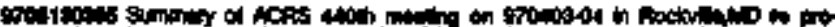

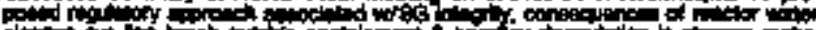

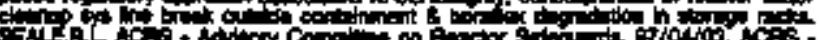

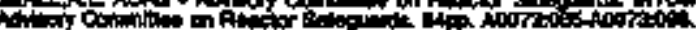

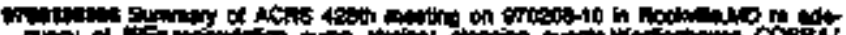

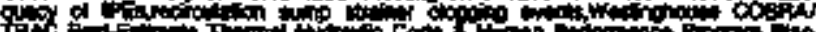
THA

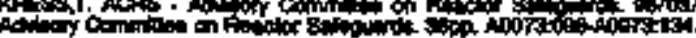

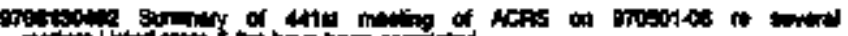

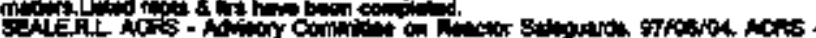

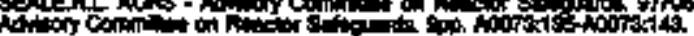

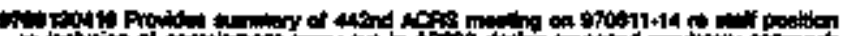

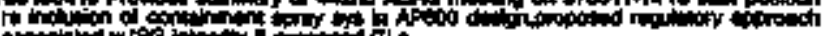

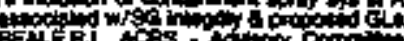

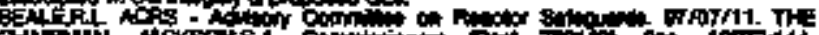

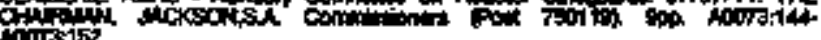

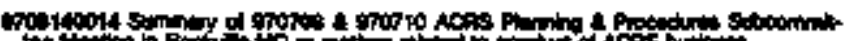

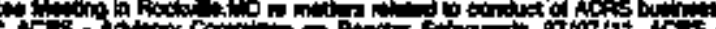

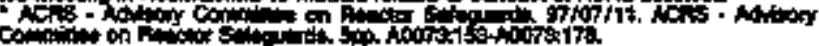

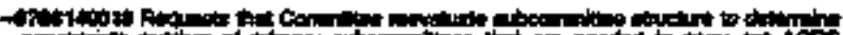

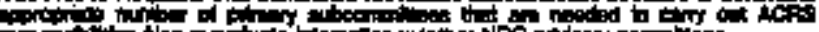

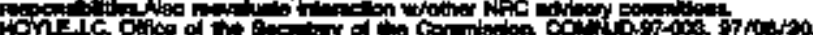

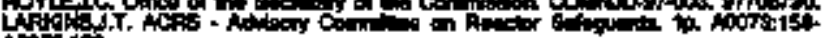
onts

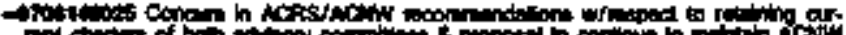

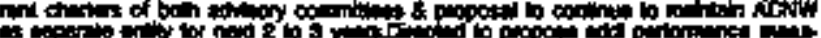

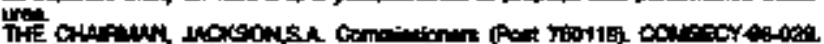

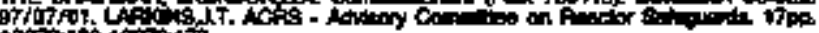

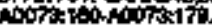

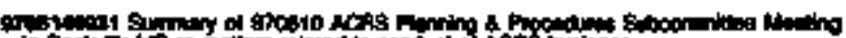

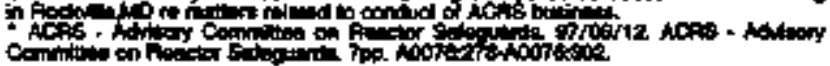

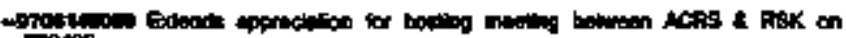

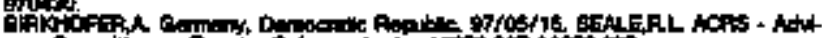

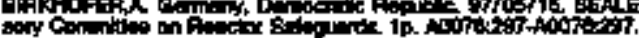

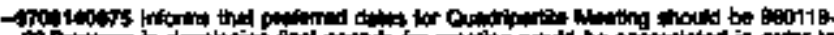

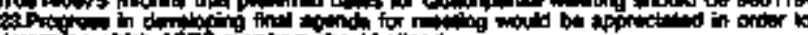

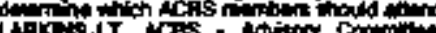

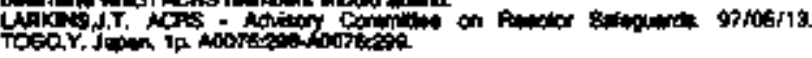

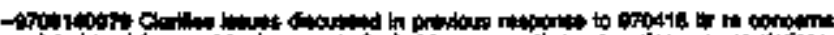

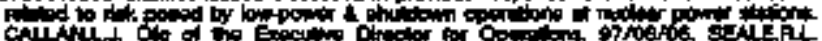

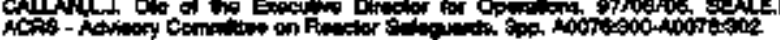

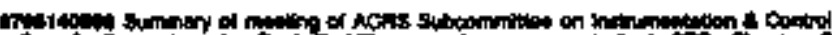

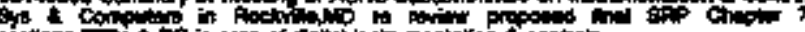

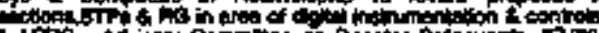

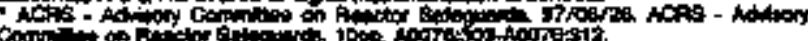

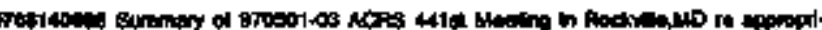

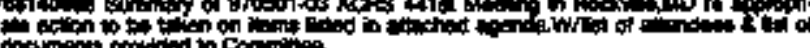

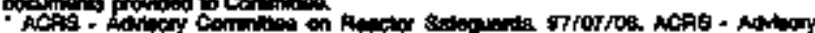

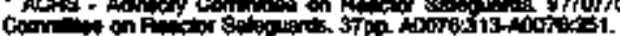

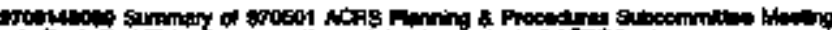

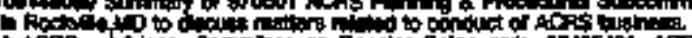

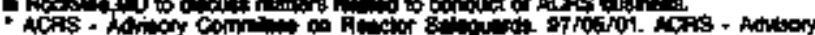

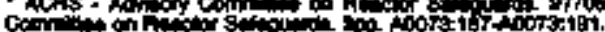

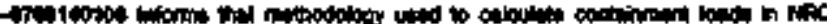

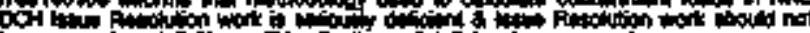

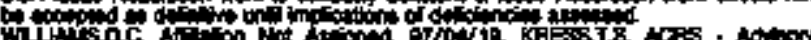

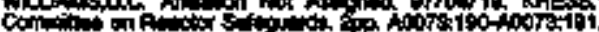

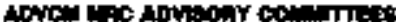

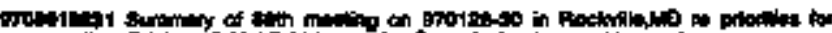

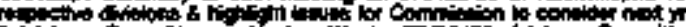

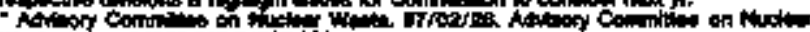

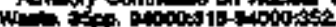

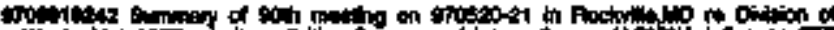

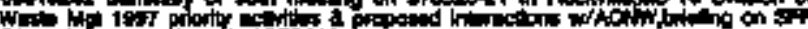
C.

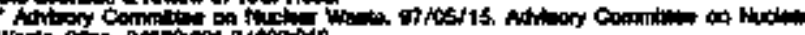

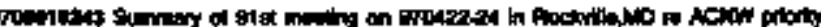

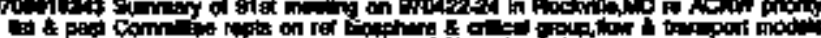

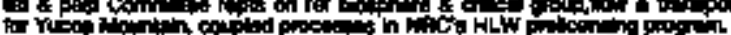

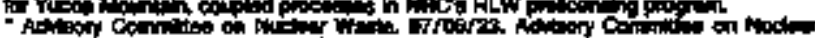

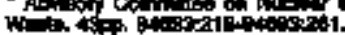

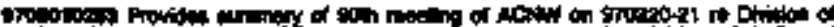

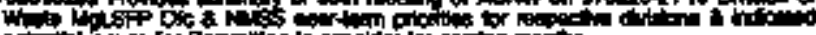

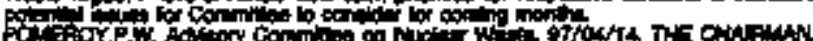

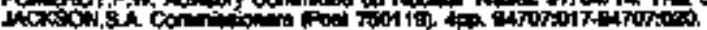

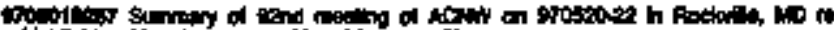

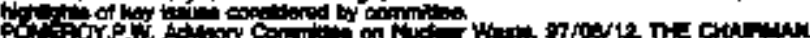

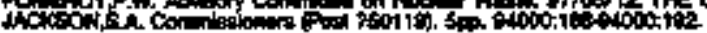

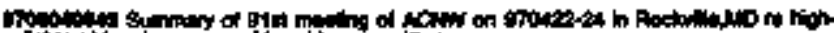

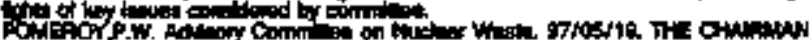

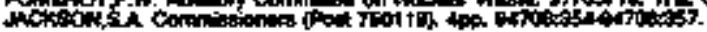

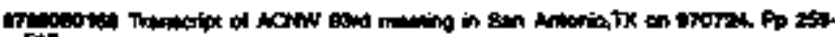

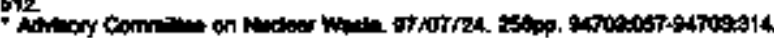

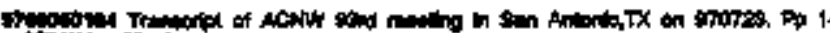

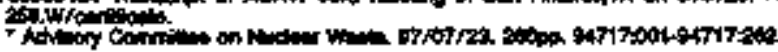

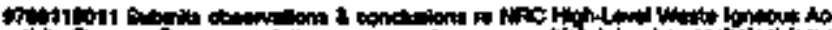

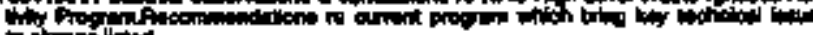
ons

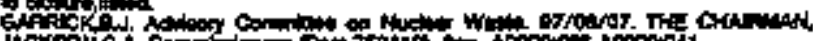
IAcxootioh Com

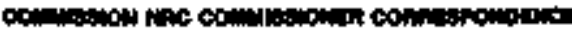

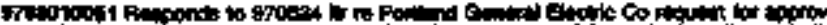

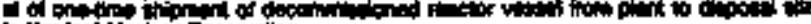

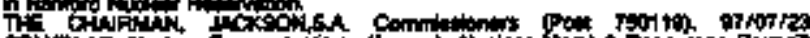

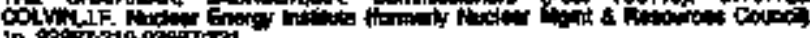

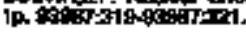

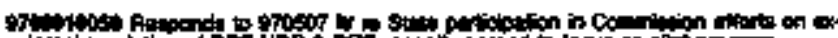

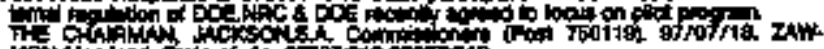

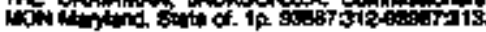

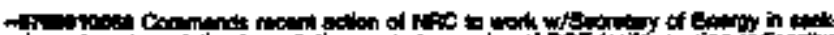

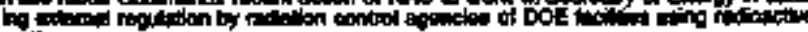

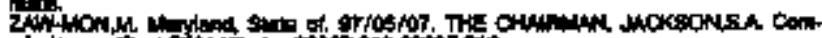

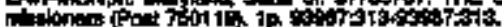




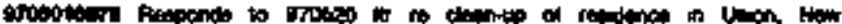

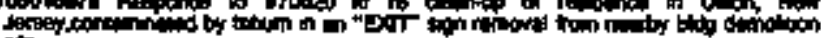

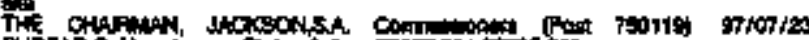

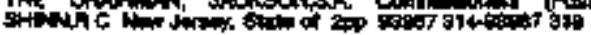

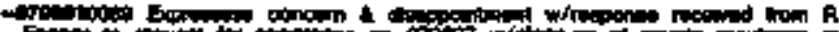

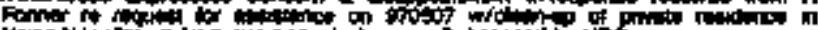

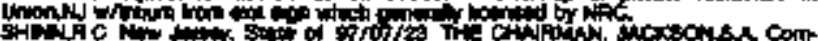

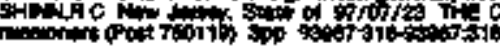

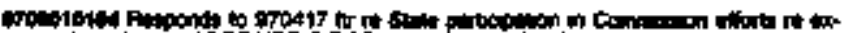

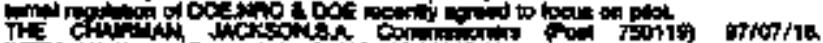

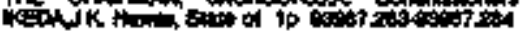

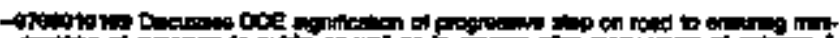

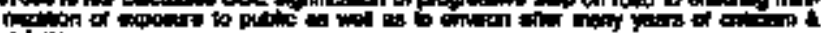

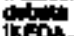

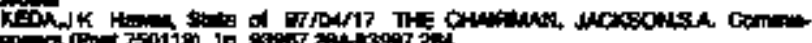

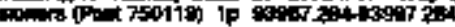

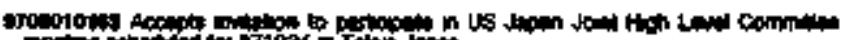

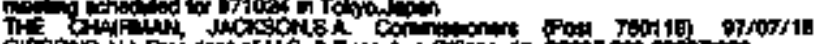
G

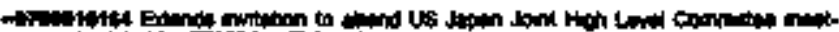

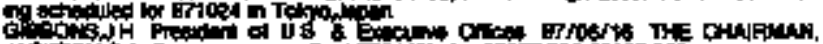

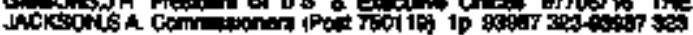

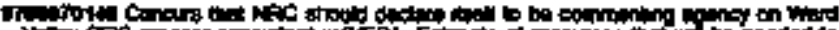

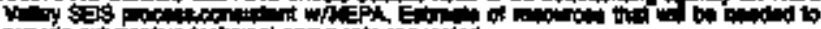

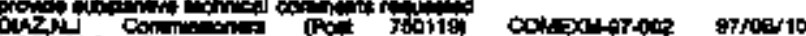

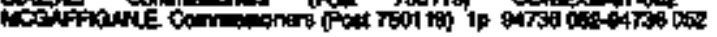

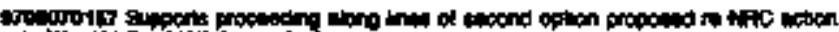
tory-d Vy

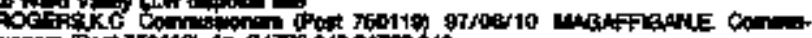

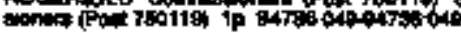

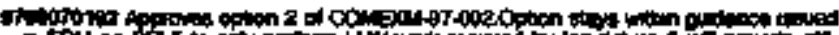

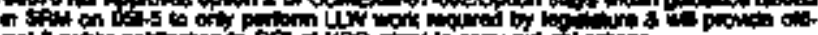

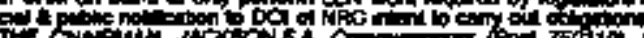

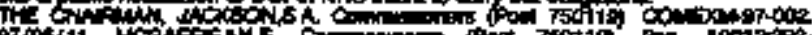

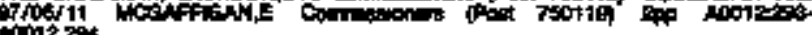

A

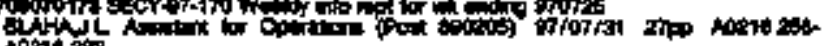
Notitiso

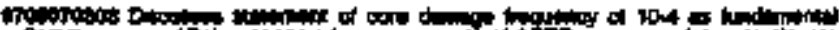

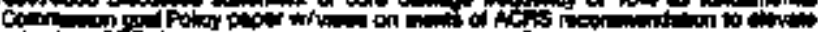

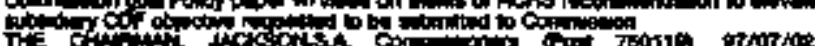

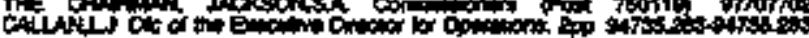

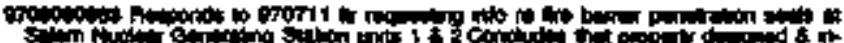

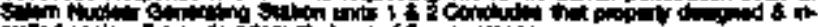

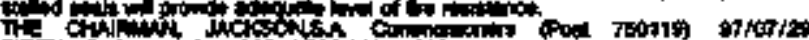
The of

-

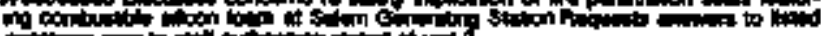

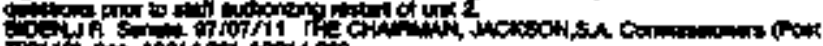

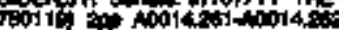

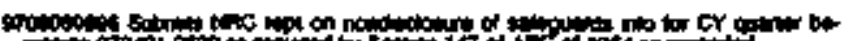
Find of

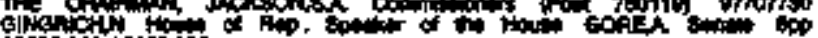

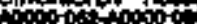

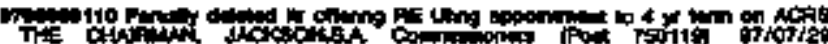

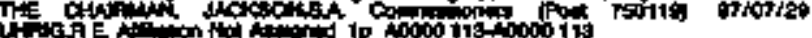

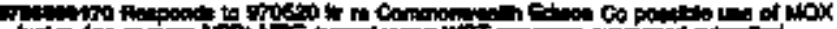

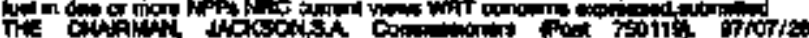

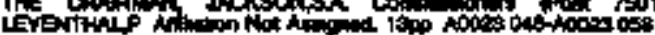

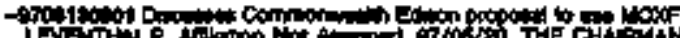

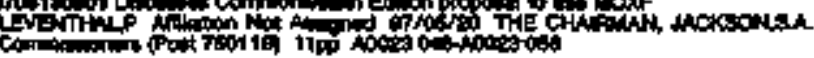

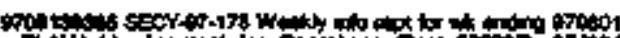
Diplition

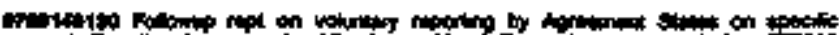

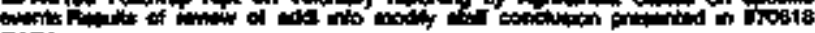

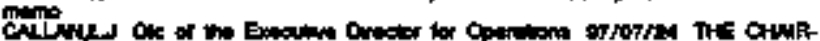

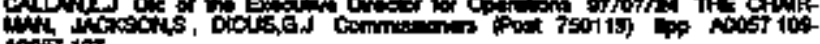
Ant 10.

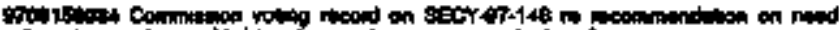

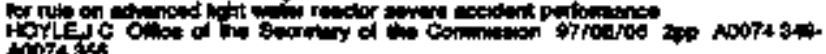
intists

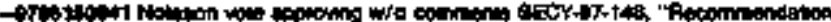

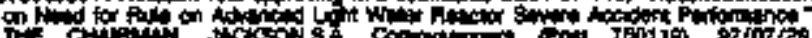

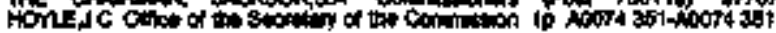

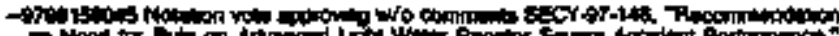

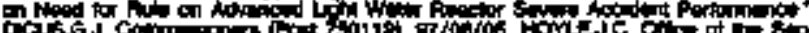

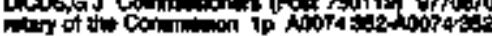

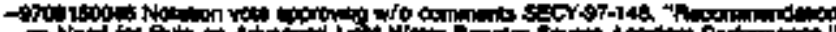

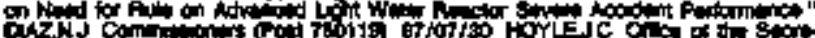
Caky J com

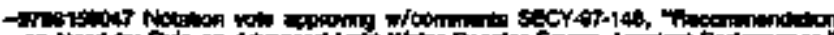

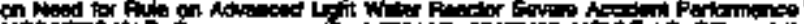

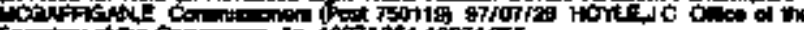

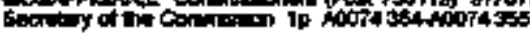

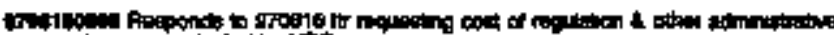

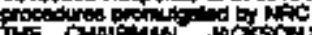

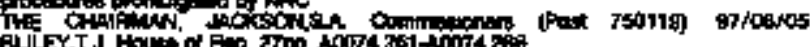

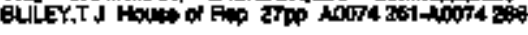

Prow

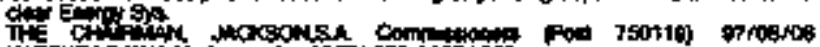

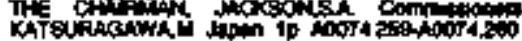

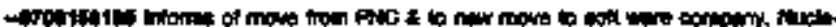

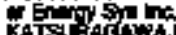

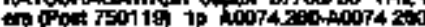

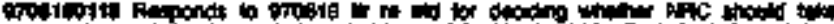

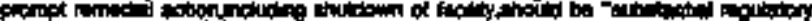

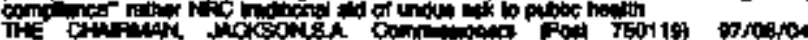

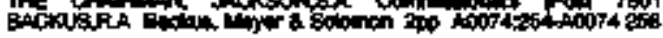

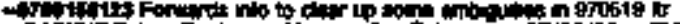

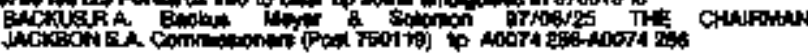

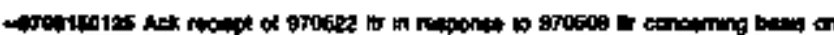

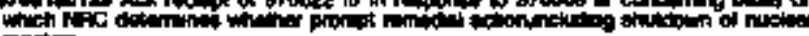

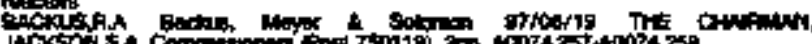

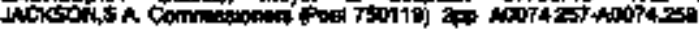

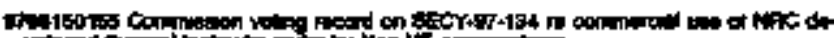

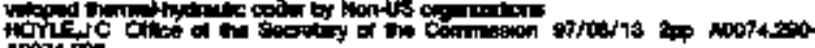
1007420

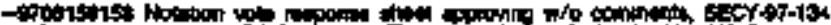

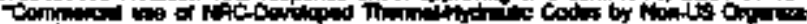

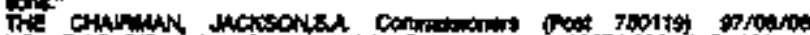

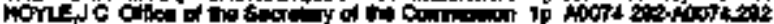

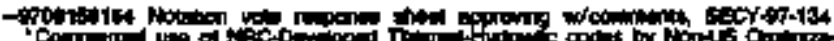

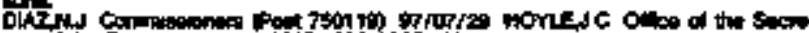

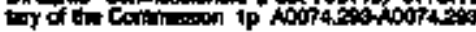

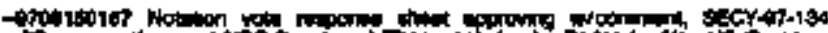

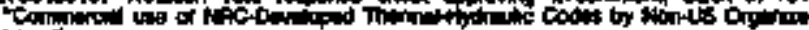

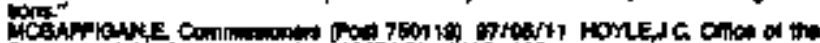

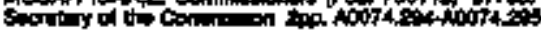

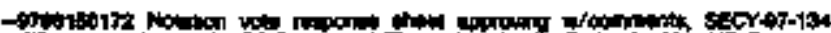
Tow

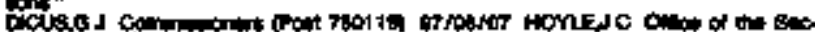

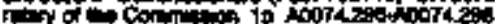

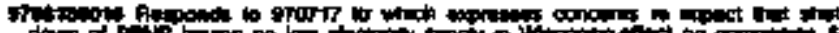

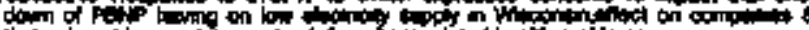

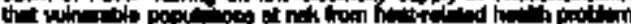

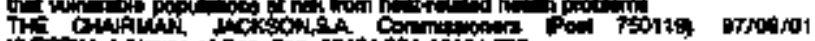

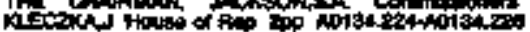

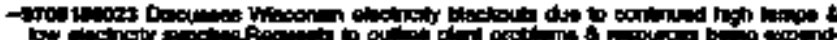

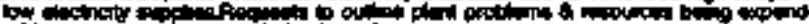

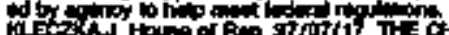

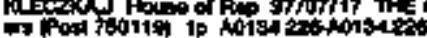

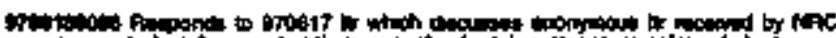

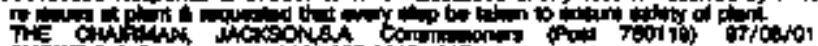

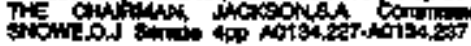

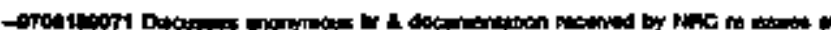

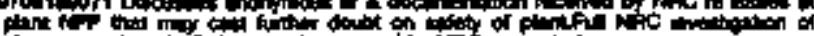

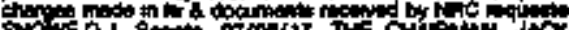

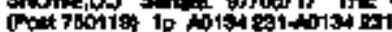

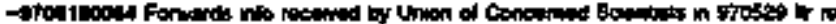

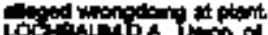

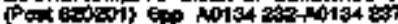




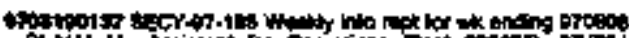

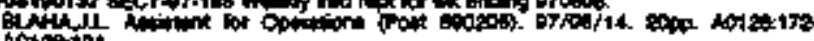
Aora:iar.

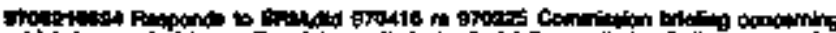

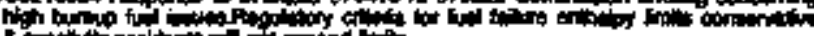

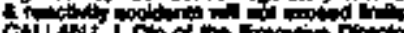

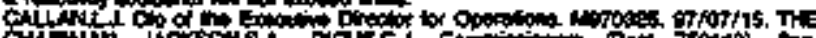

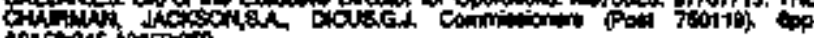

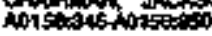

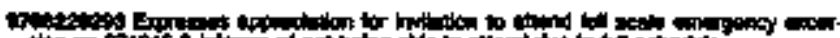

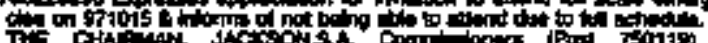

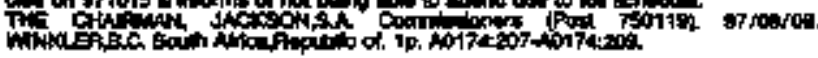

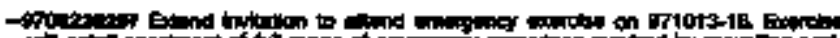

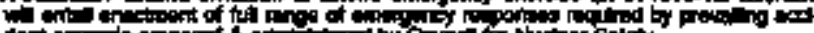

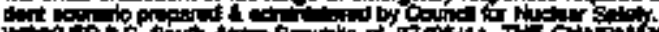

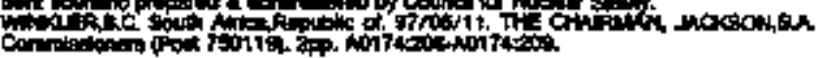

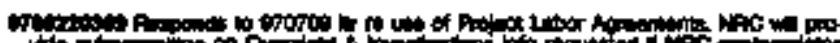

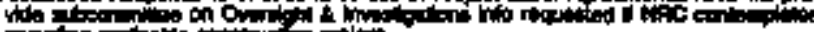
In Cif Hogks

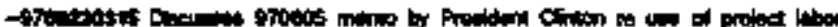

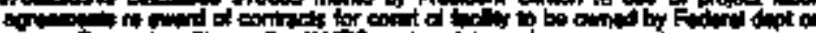

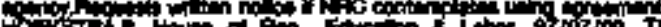

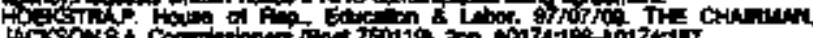

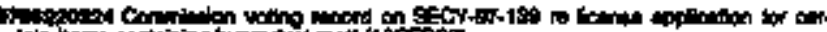

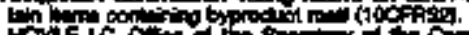

然 Aartion

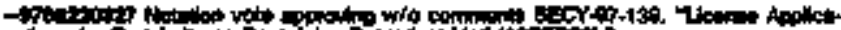

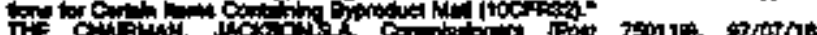

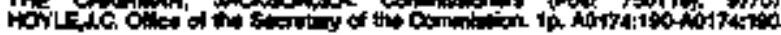

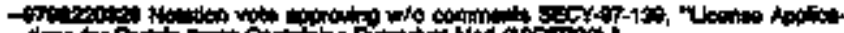

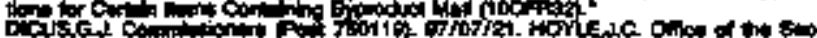

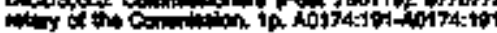

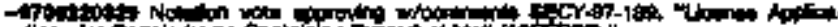

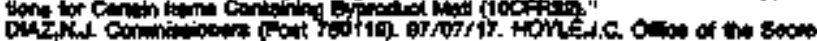

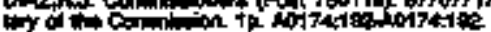

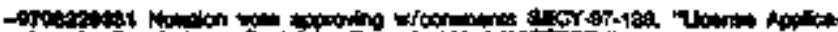

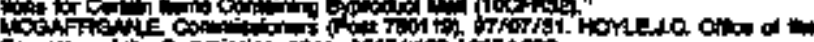

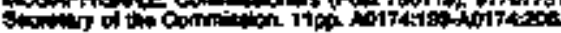

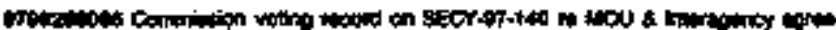

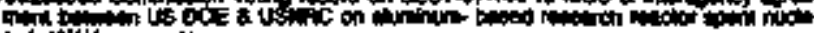

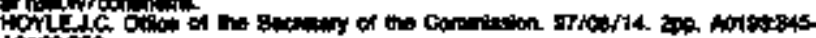
atos

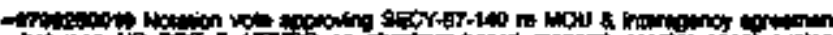

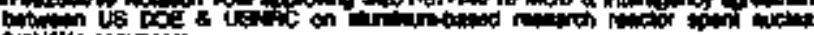
Tin

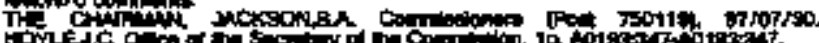

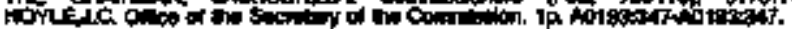

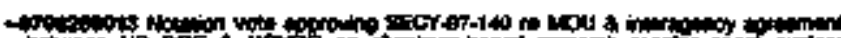

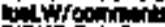

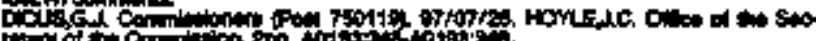

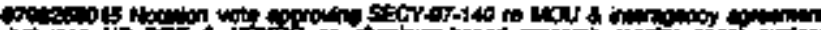

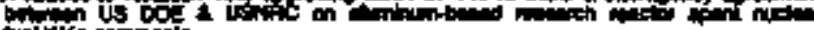

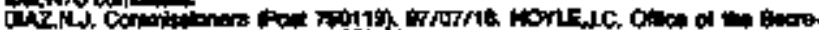

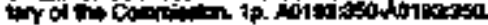

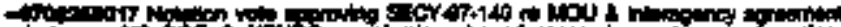

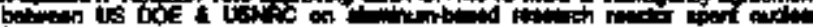

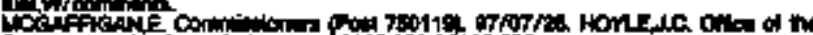

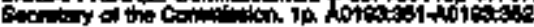

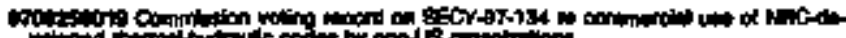

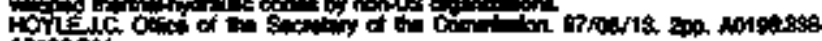
nitopit.

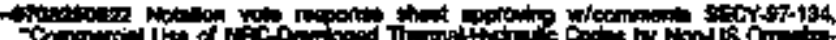

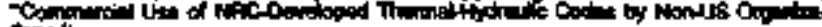

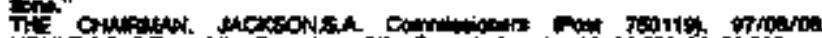

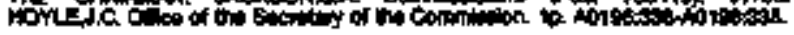

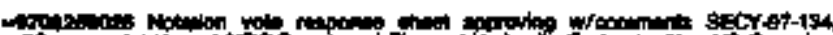

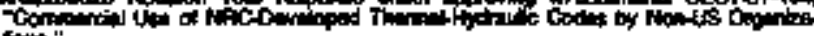

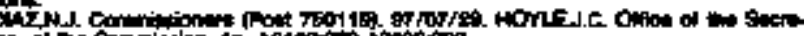

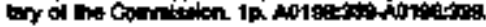

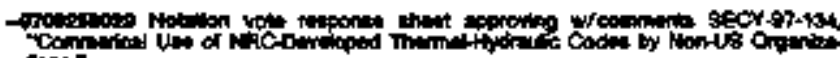

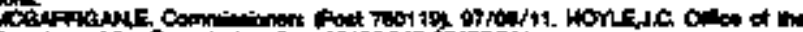

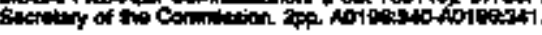

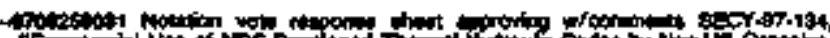

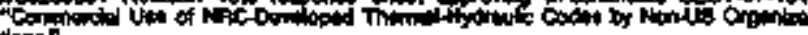

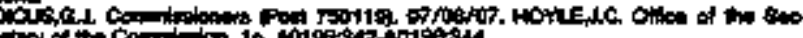

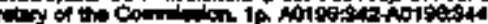

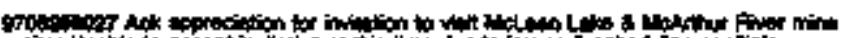

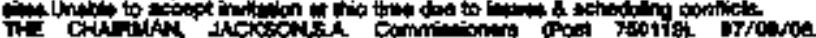

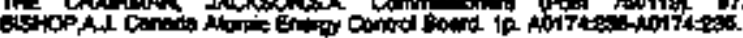

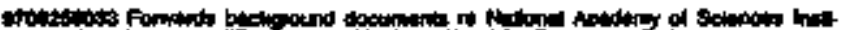

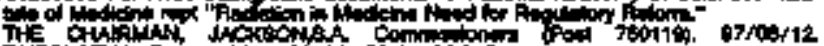

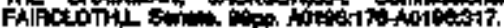

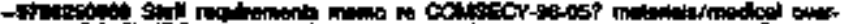

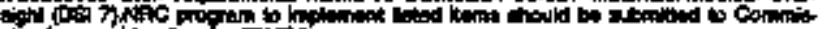

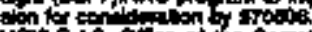

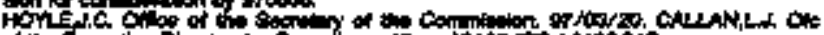

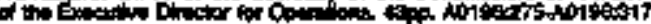

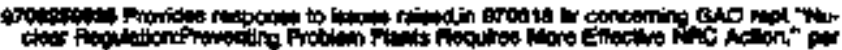

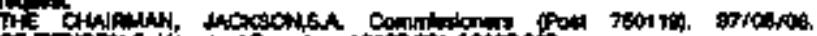

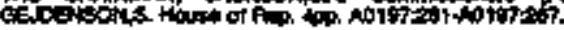

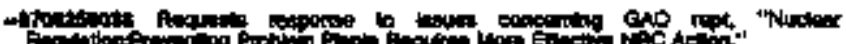

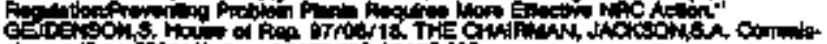

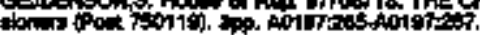

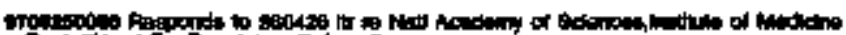

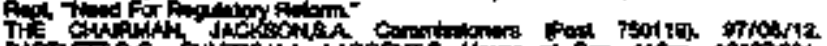

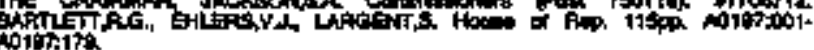

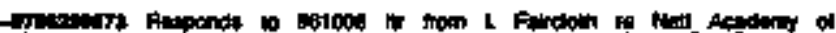

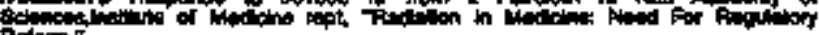

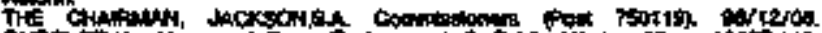
FA A019:17.

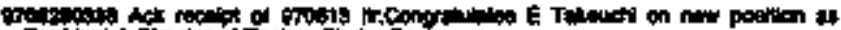

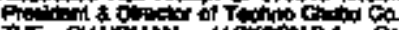

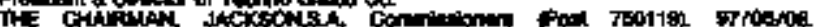
Thk

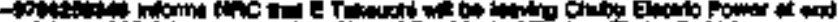

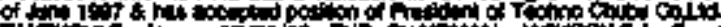

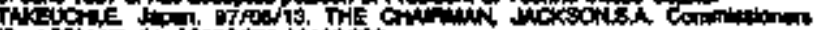

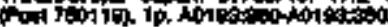

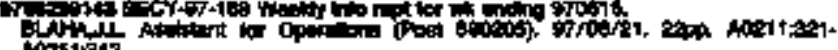
toptisit

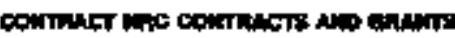

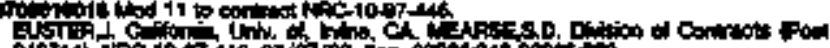

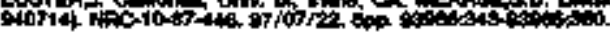

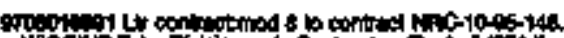
tor.t.

of

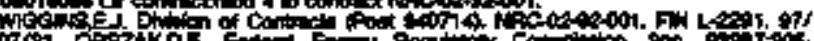

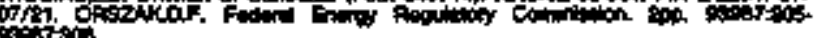

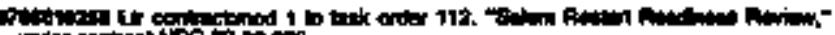

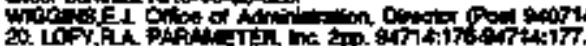

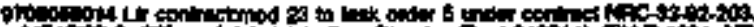

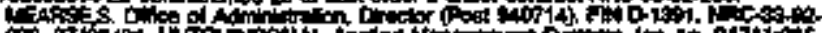

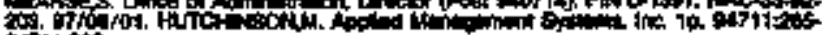
antites.

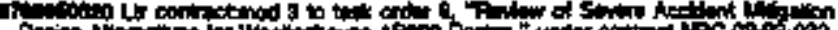
Pan ${ }^{2}$ N Whe tont1:202

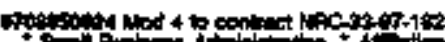

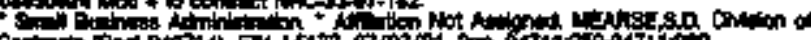

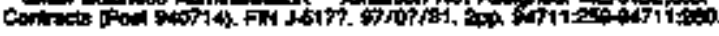

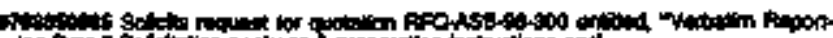

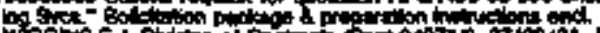

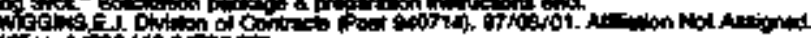

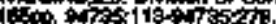




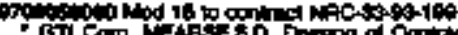

Gil

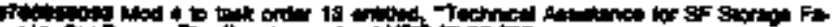

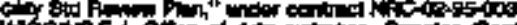

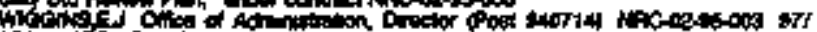

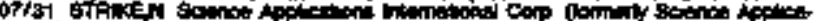

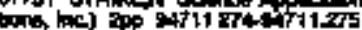

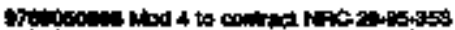

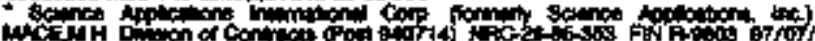

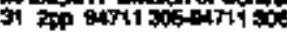

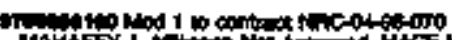

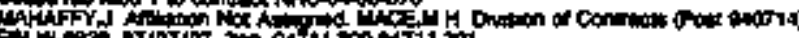

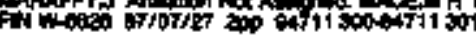

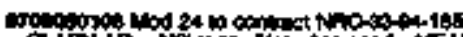

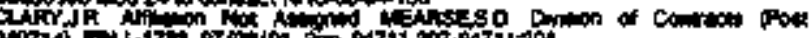

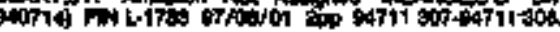

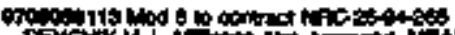

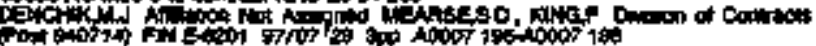

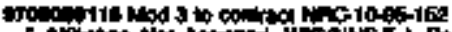

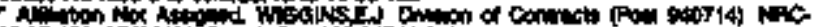
10-8\%

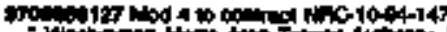

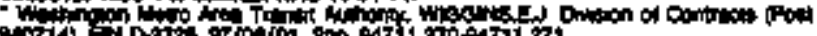

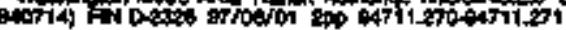

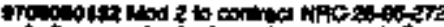

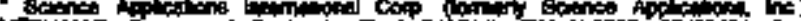

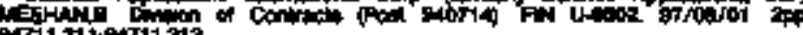

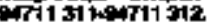

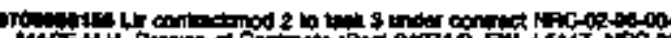

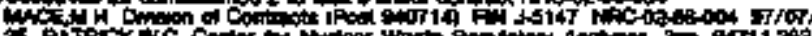

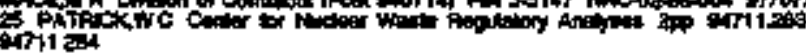

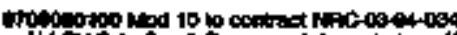

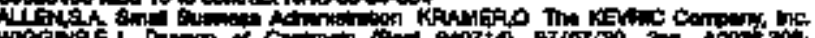

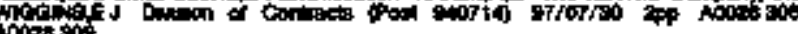

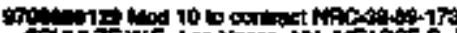

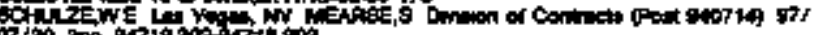
opton 290 ar?

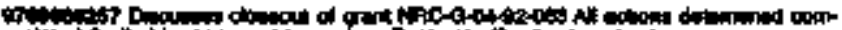

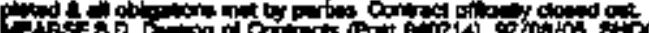

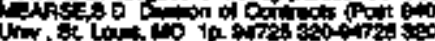

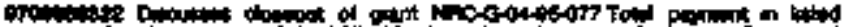

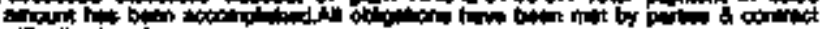

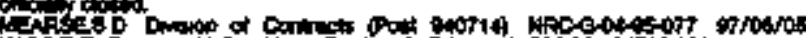

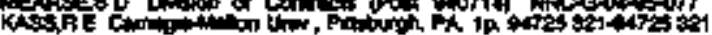

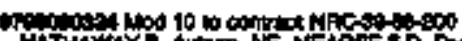

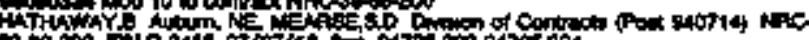

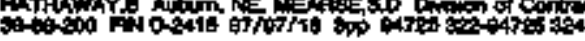

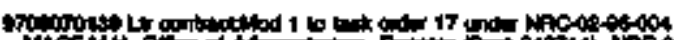

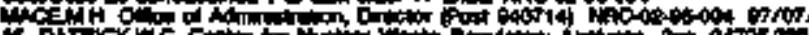

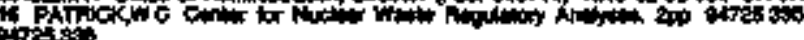

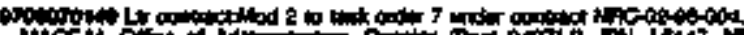

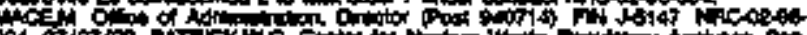

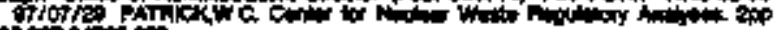

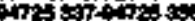

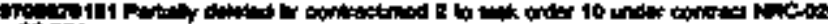

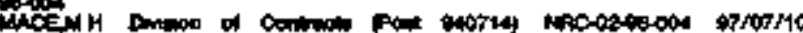

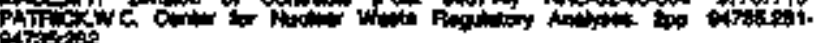

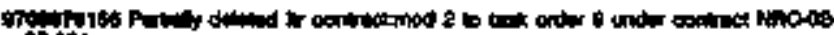
\%in

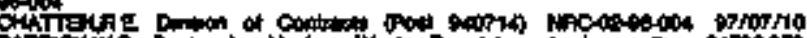

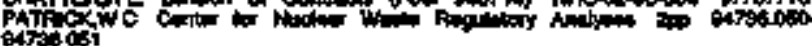

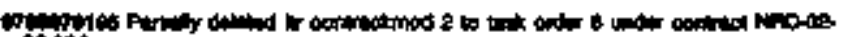
Gelof

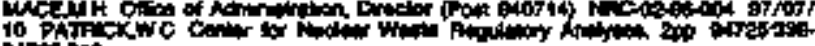
artion 340

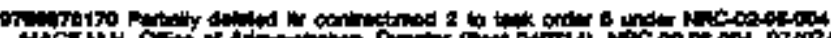

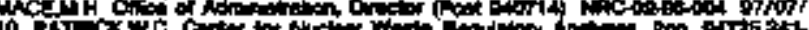
ires ith

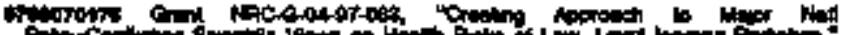

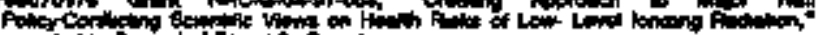

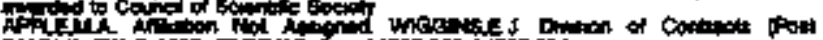

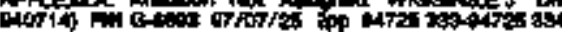

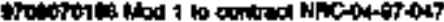

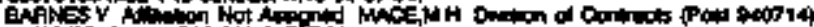

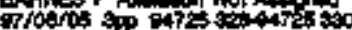

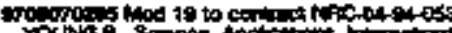

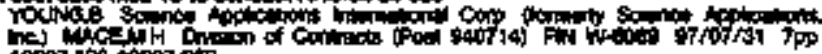
10007 10601000

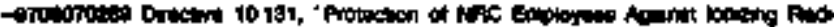

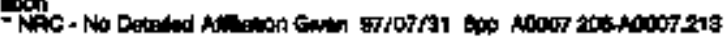

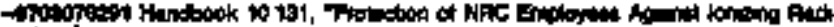

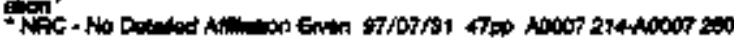

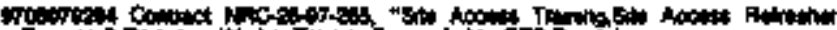

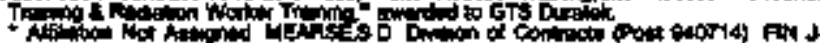

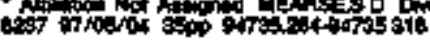

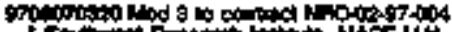

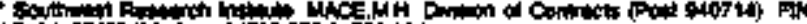
IST

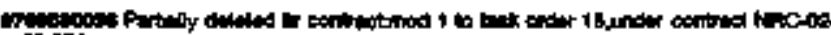

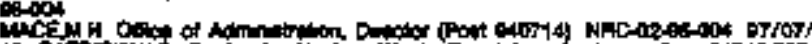

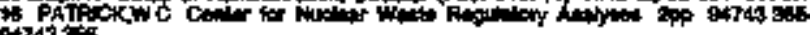

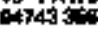

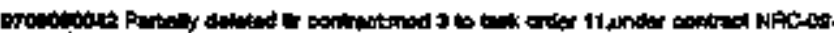

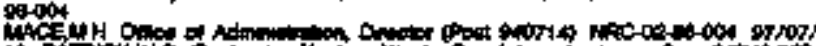

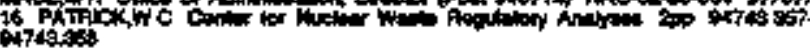

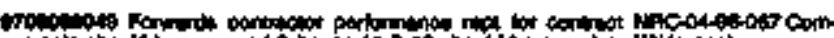

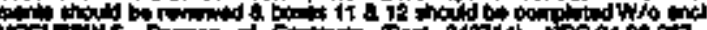

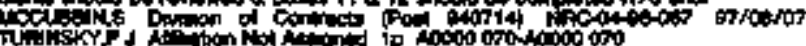

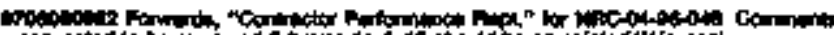

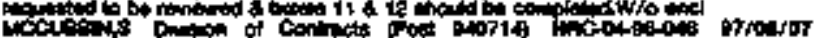

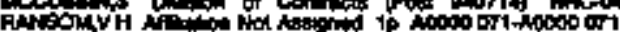

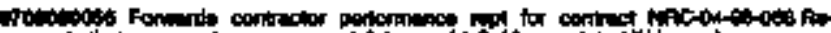

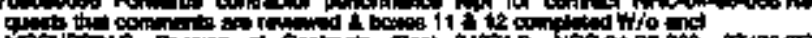
qut

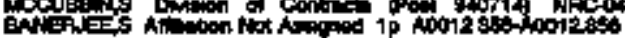

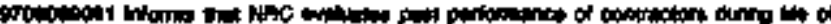

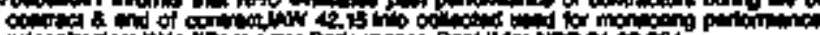

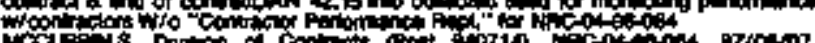

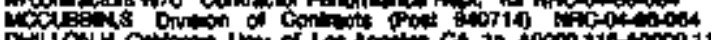
DH

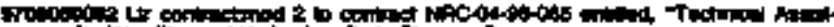

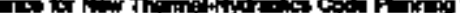

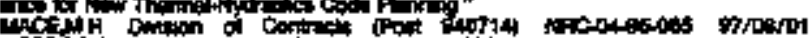
L

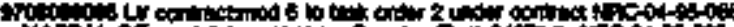

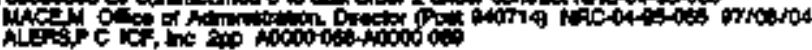

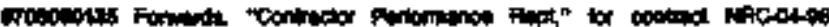

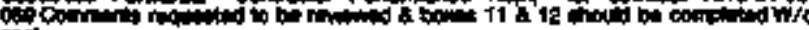

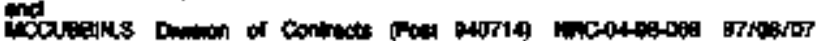

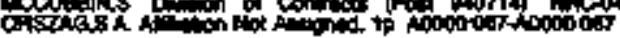

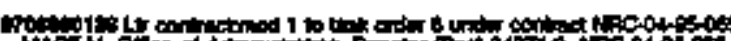

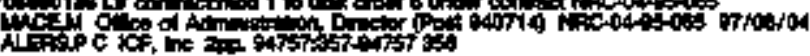

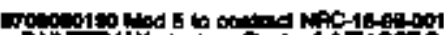

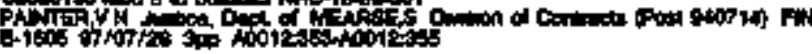

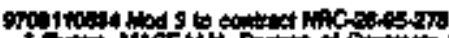

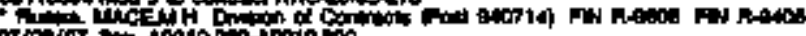

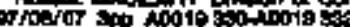

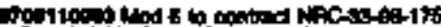
sultir,

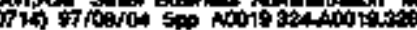

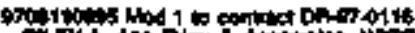

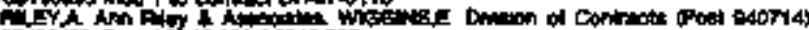

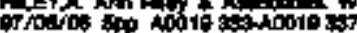

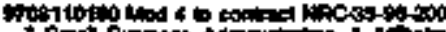

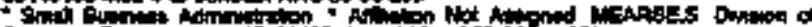

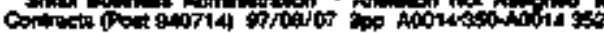

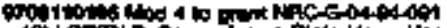

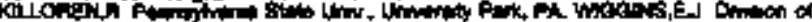

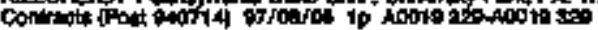

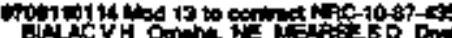

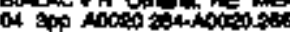




\section{MONDOCKETED ITEMS}

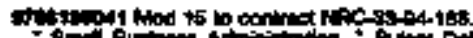

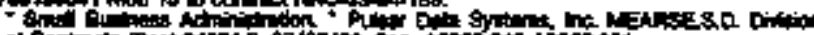

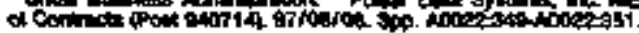

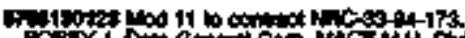

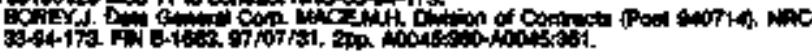

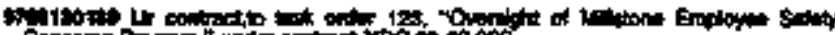

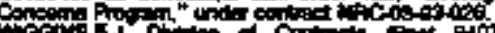

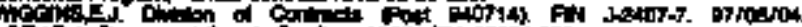

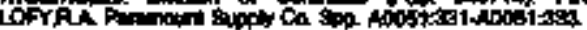

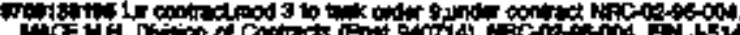

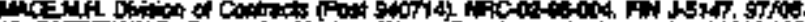

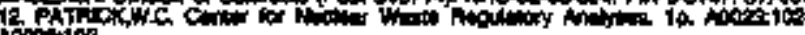
Anoction

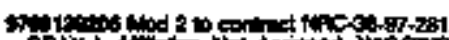
Fot

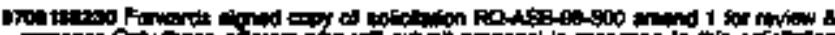

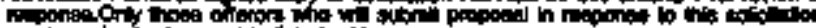

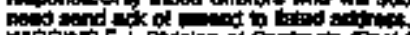

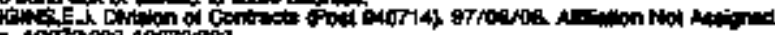

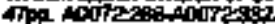

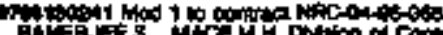

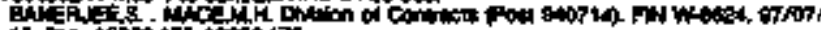

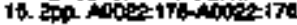

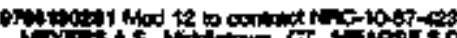

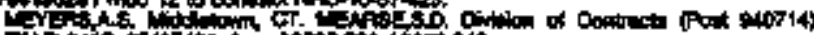

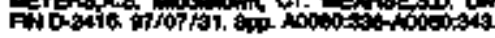

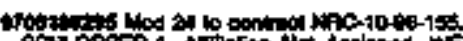

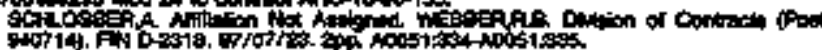

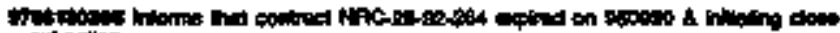

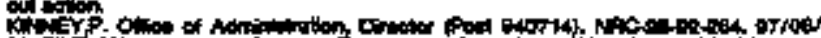

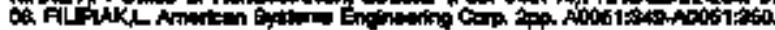

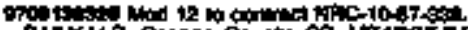

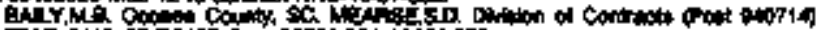

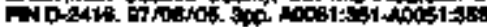

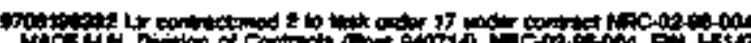

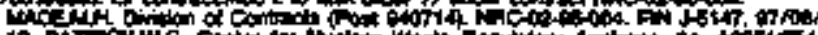

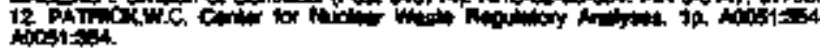

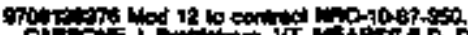
Chich

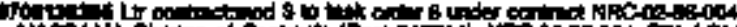

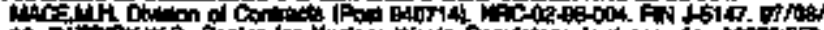
12 PKty

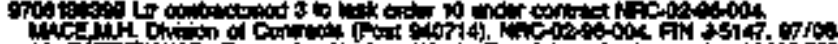

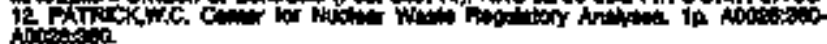

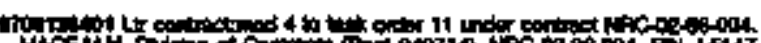

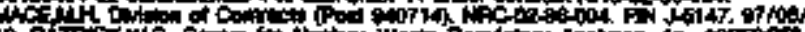

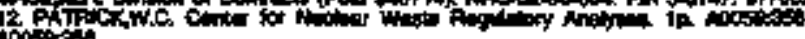

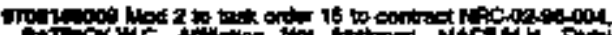

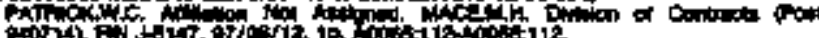

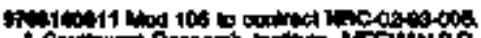

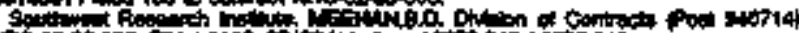

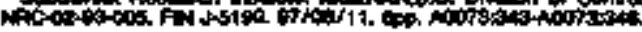

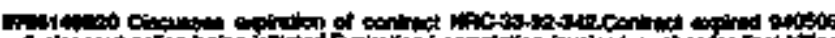

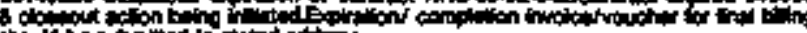

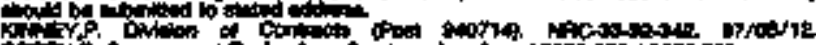

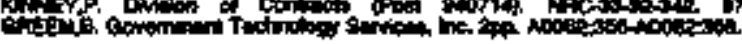

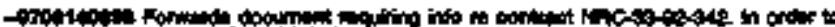
alo

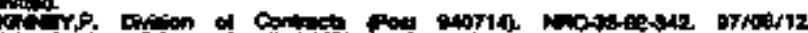

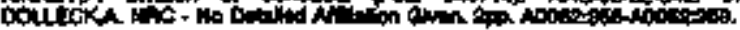

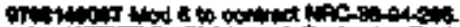

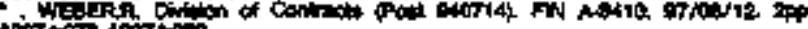

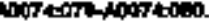

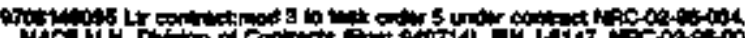

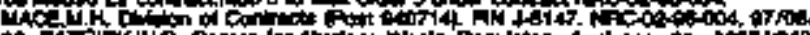

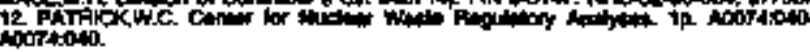

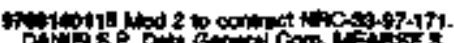

W

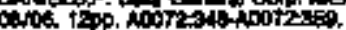

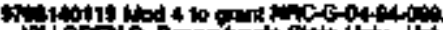

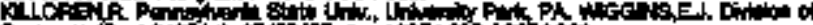

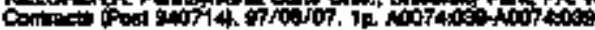

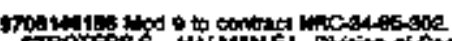

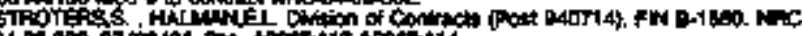

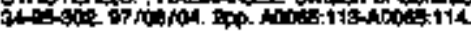

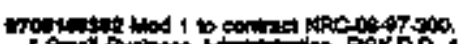

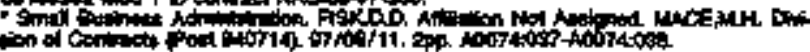

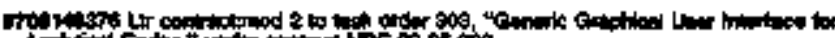

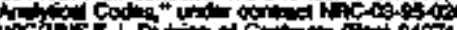

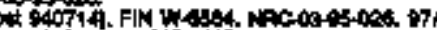

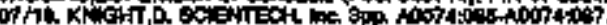

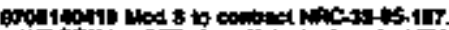

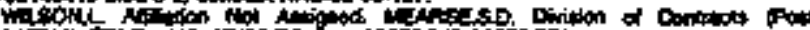

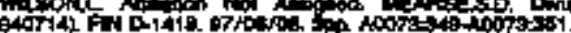

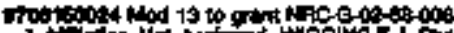

ina of

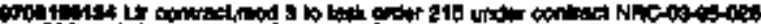
W

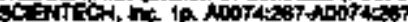

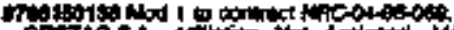

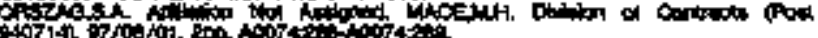

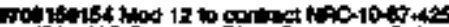

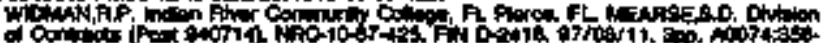
atomiti.

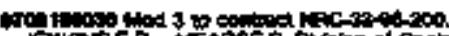

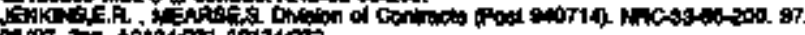

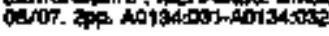

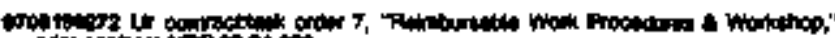

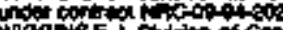

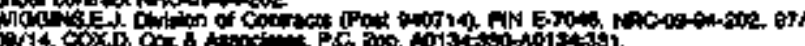

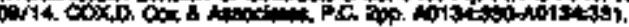

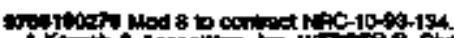

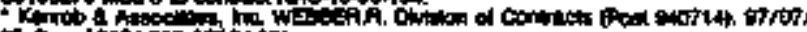

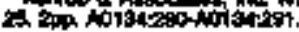

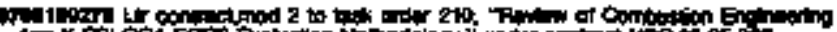
A.

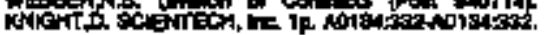

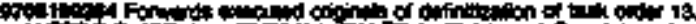
AI 941217]. 10, Aot

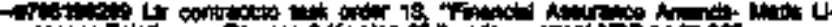

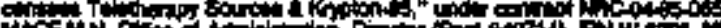

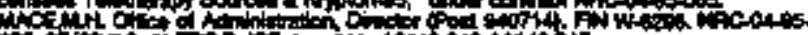

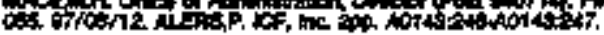

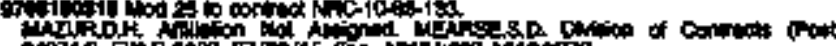

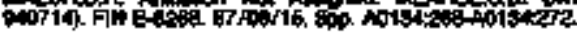

H

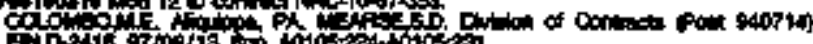

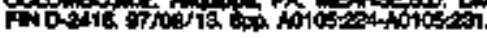

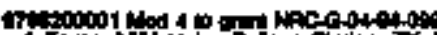

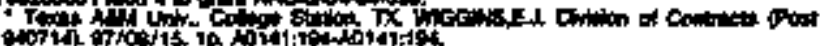

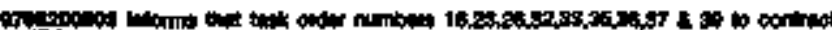

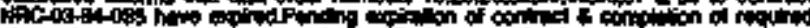

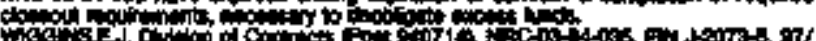

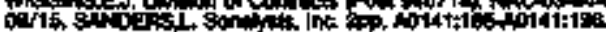

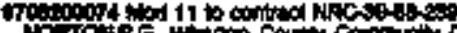

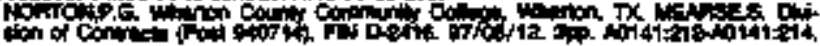

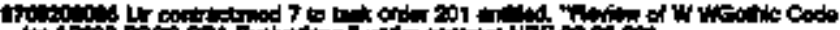

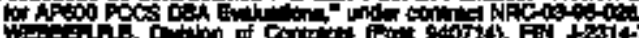

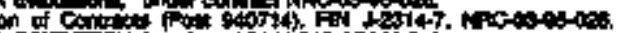

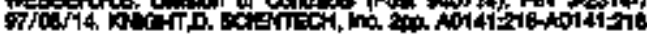

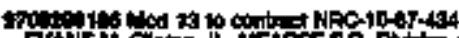

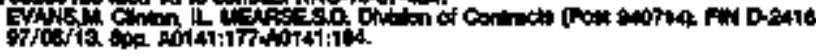

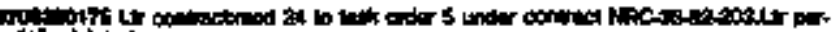

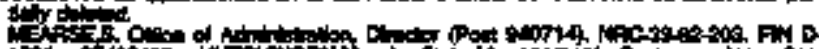

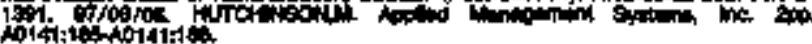

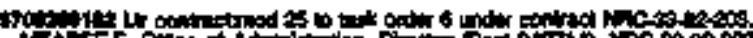
1 intorat:

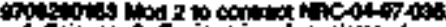

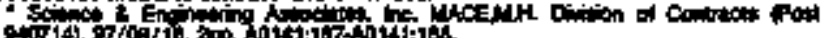




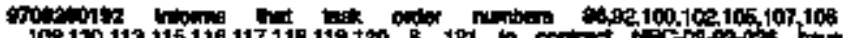

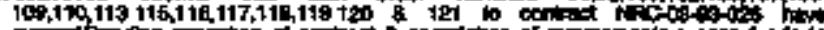
oq of

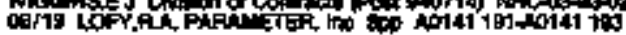

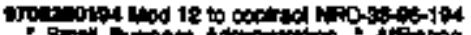

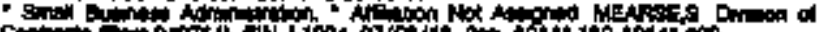

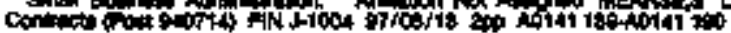

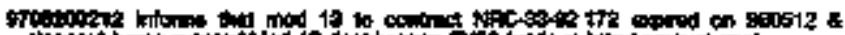

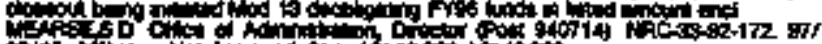

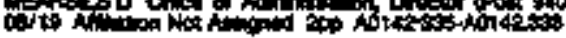

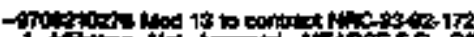

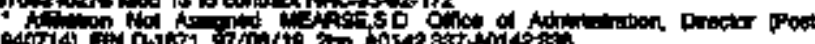

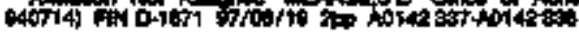

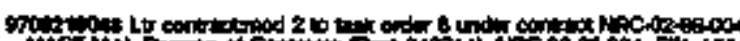

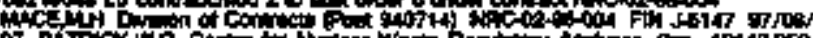

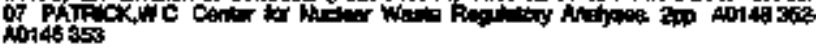

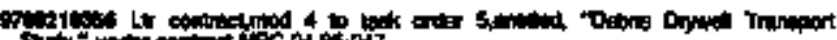

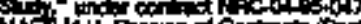

74 CSt

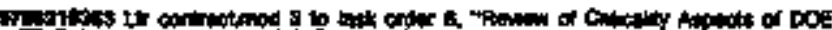

W

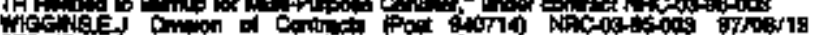

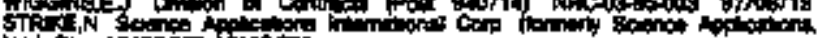

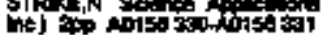

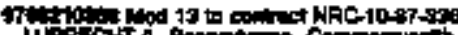
Woif

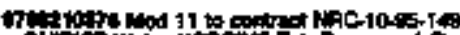

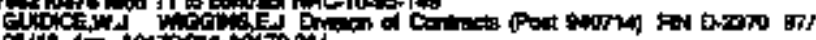
ath ip ro1

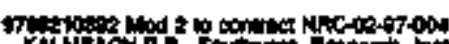

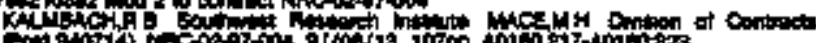

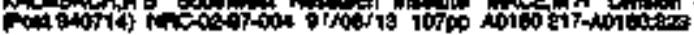

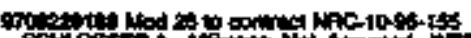

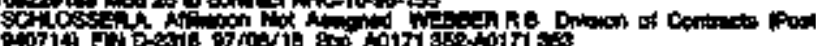

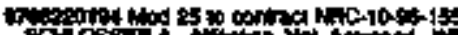

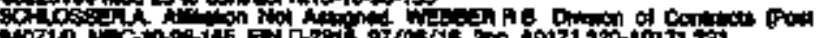

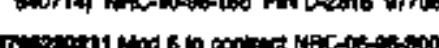

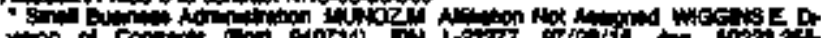

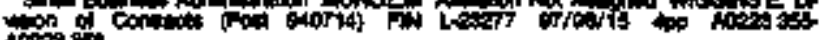

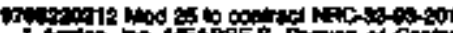

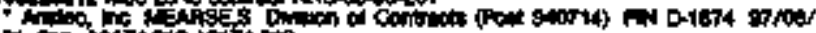

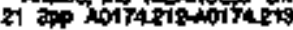

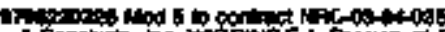

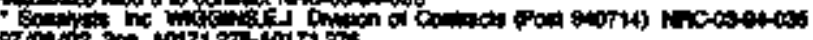

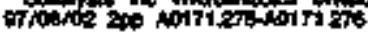

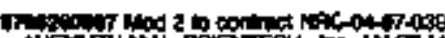

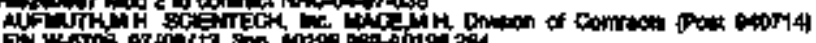

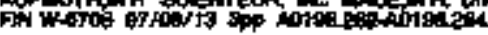

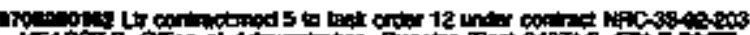

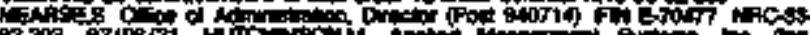

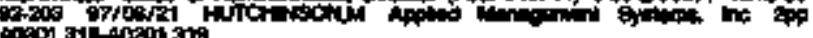

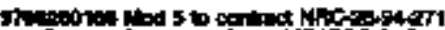

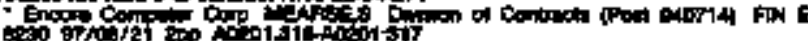

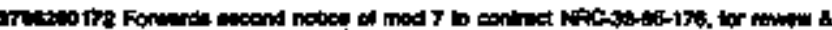

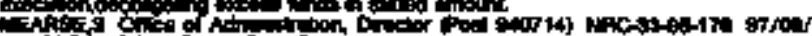

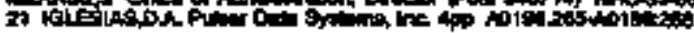

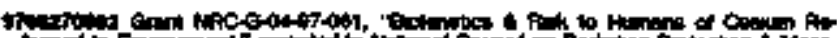

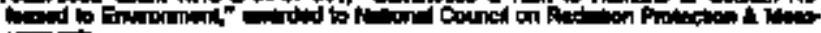

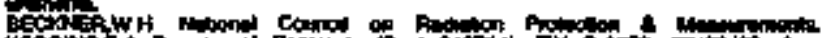

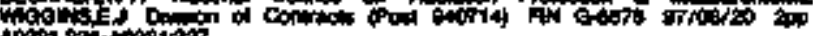
tow 1000

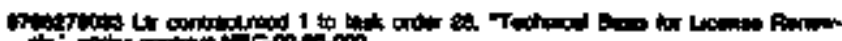

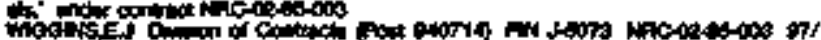
a

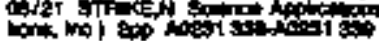

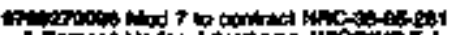

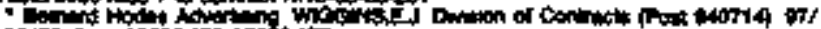

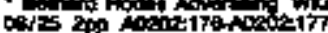

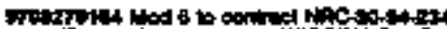

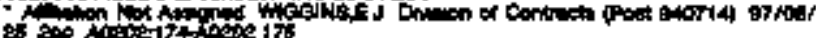

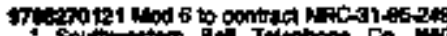

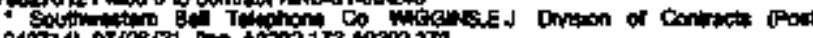

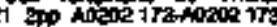

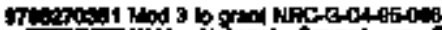

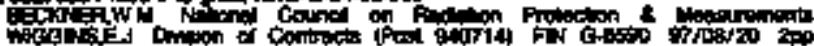

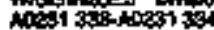

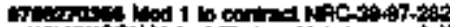

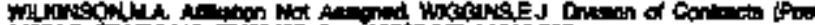

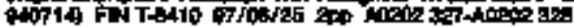

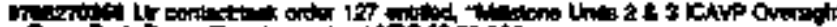

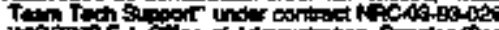

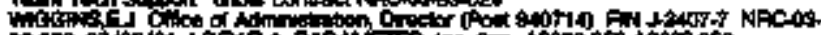

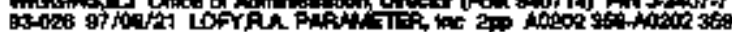

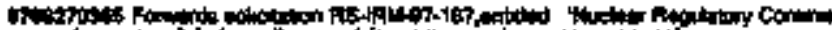

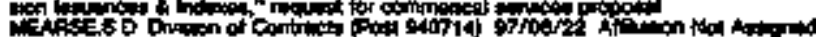

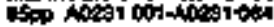

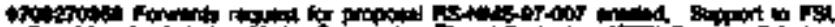

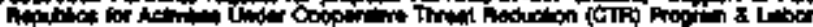

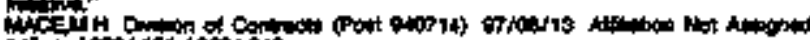

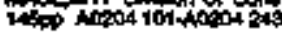

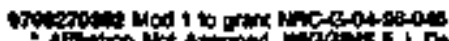

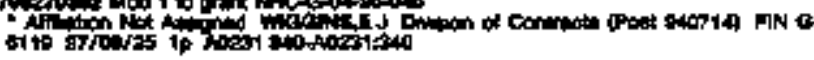

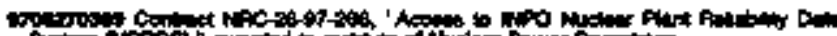
We Weta for

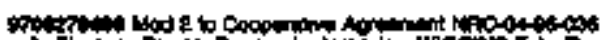

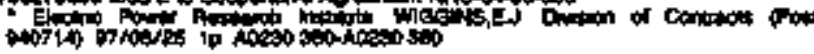

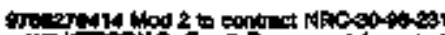

Whe

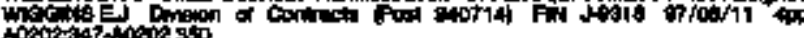

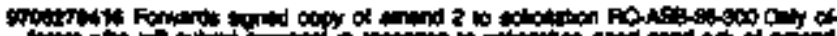

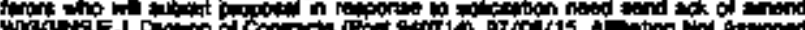

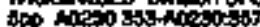

STOE:

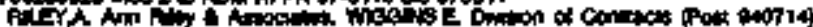

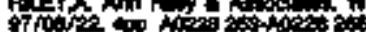

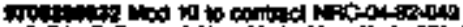

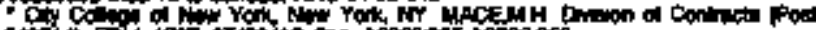

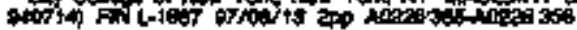

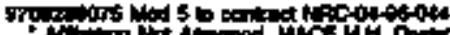

Allon Ha

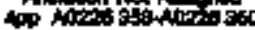

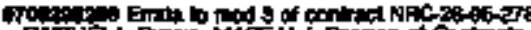

P.

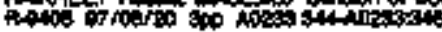

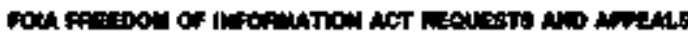

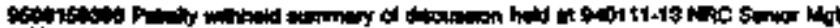

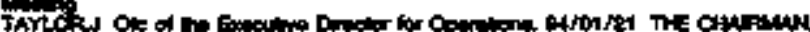

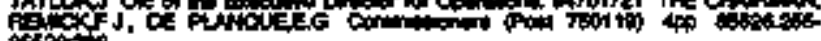

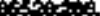

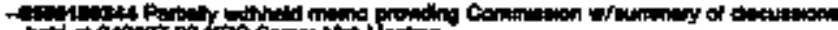
ho TAY and

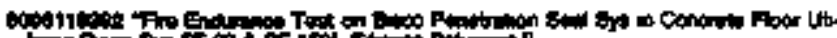

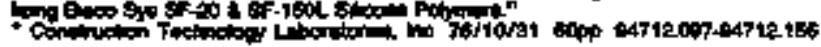

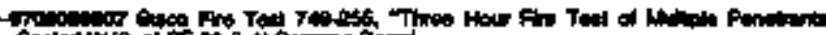

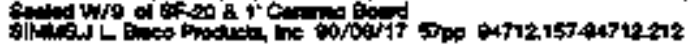

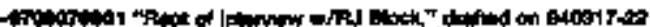

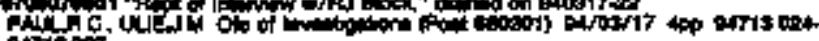

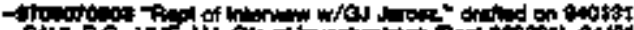

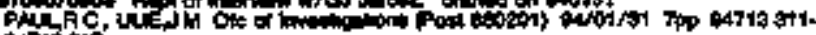
4713 औरत

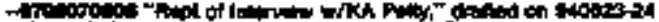

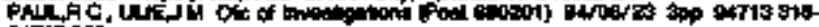
94T19 300

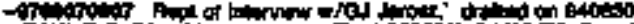

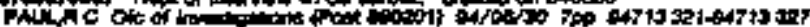




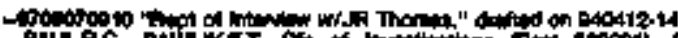

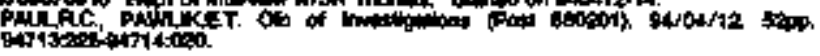

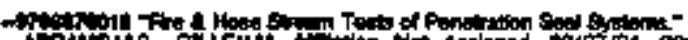

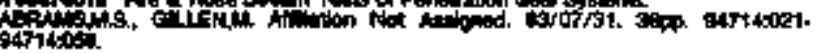

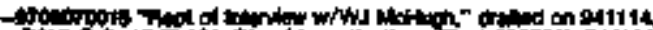

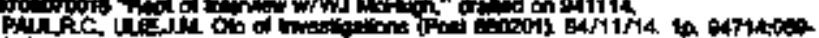
actringe

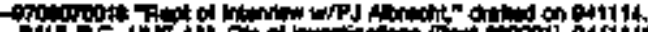

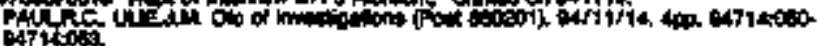

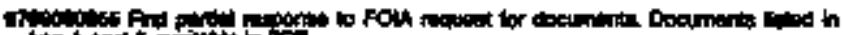
A

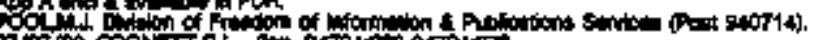

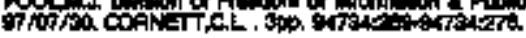

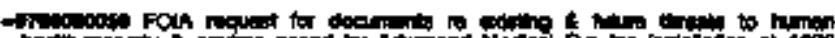

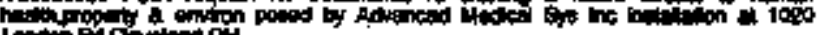

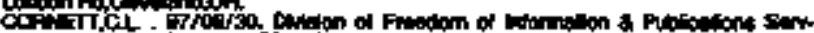

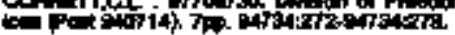

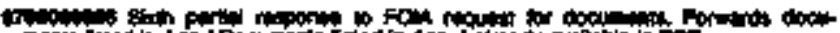

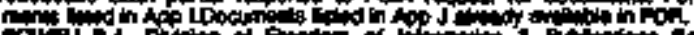

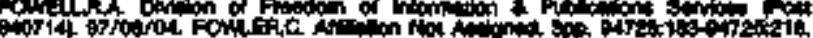

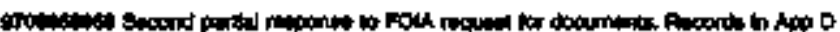

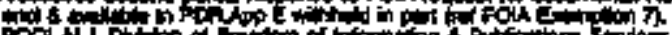

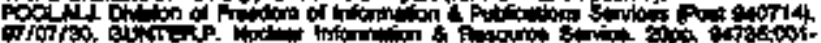

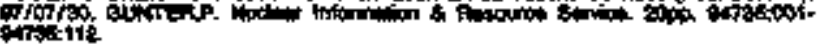

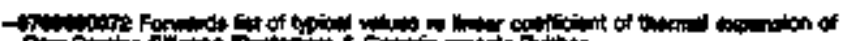

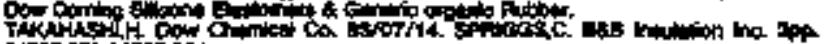
and

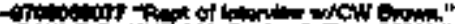

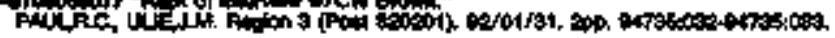

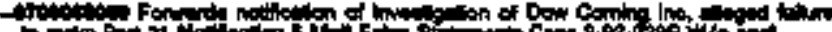

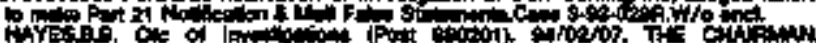

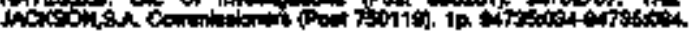

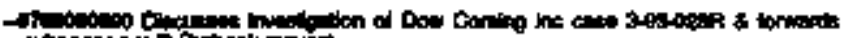

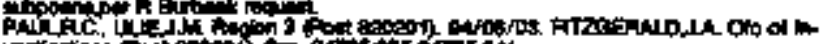

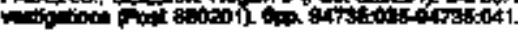

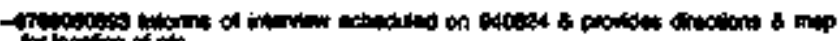

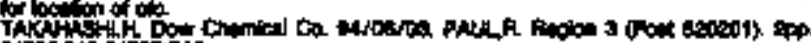
Thation

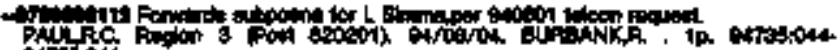
orition

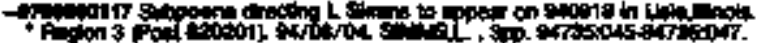

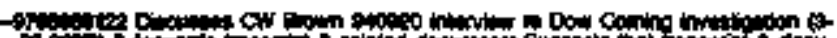

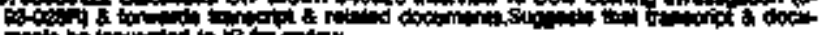

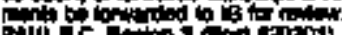

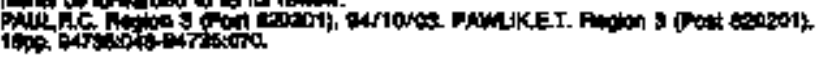

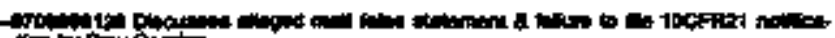

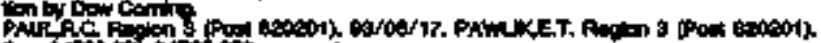

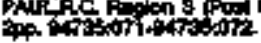

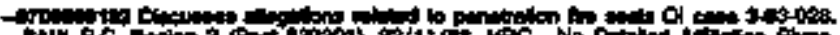

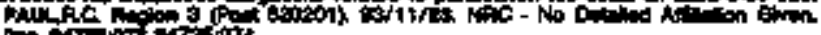

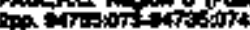

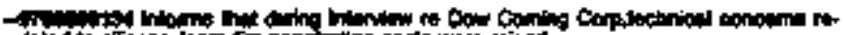

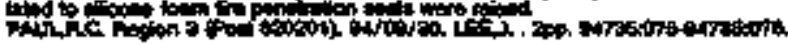

מ

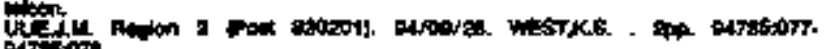

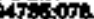

-

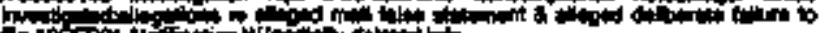

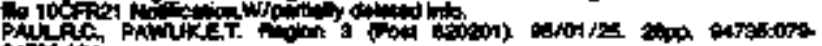
artsian

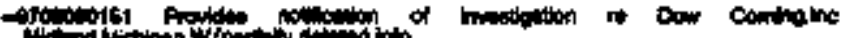

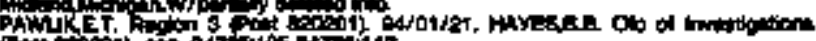

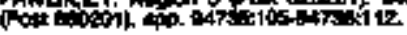

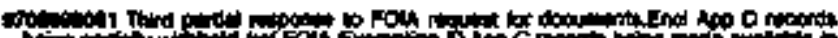
becion.

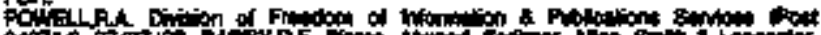
น 5 Jut

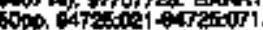

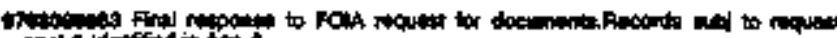

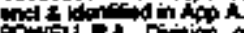

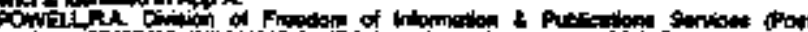

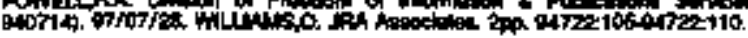

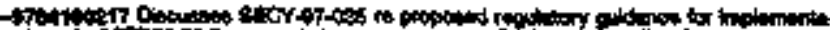

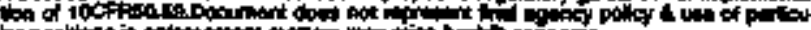

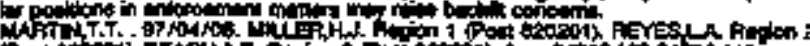

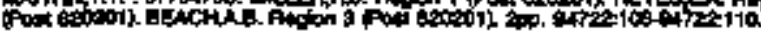

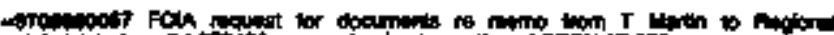

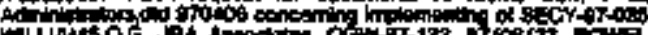

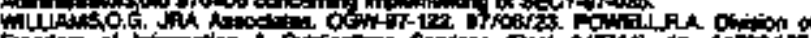

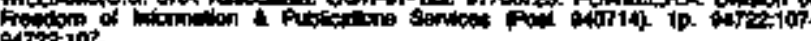

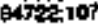

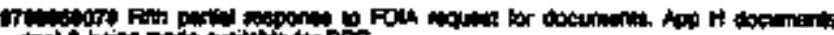

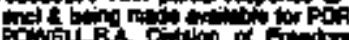

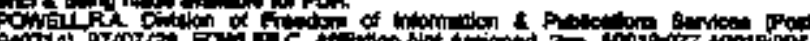

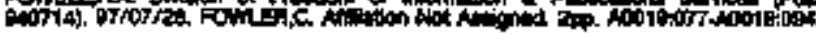

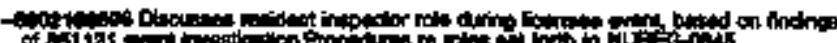

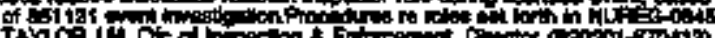

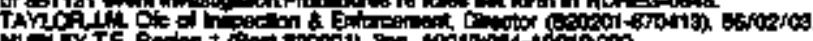

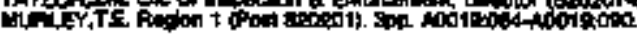

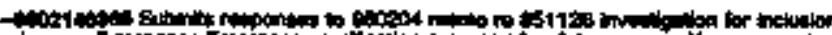

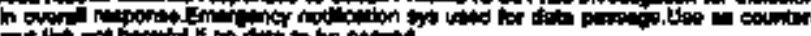

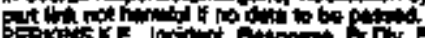

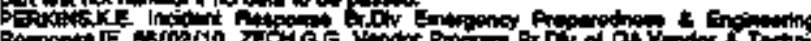

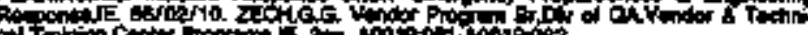

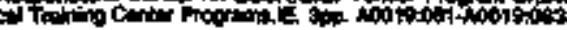

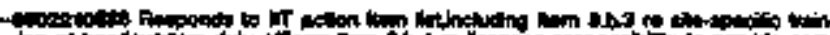

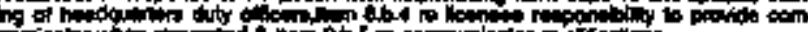

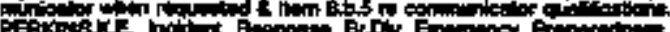

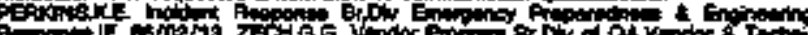

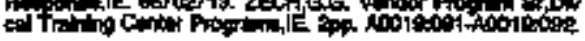

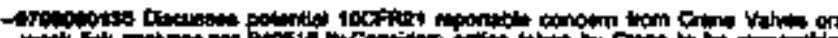

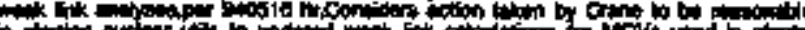

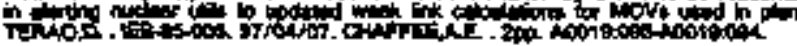

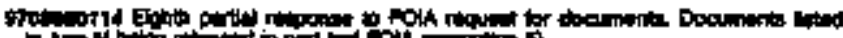

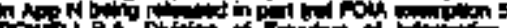

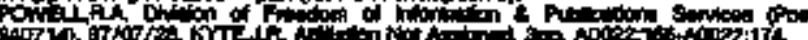

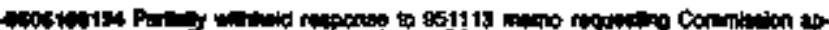

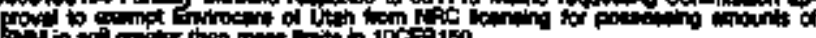

TAY Po f Apocelon.

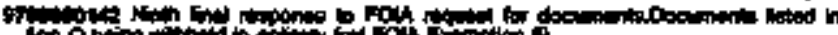

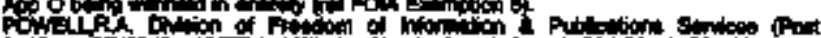

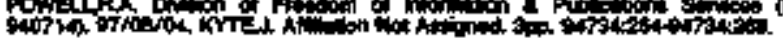

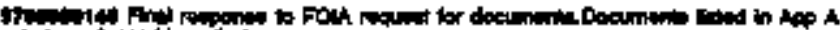

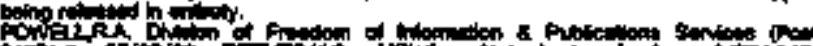

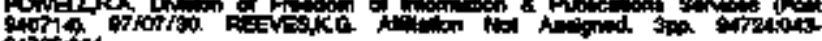
947

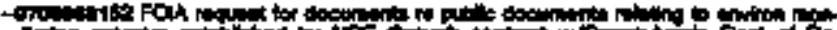

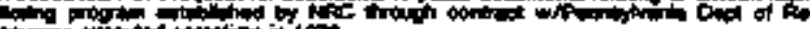

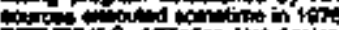

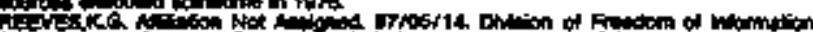

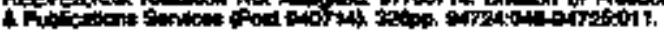

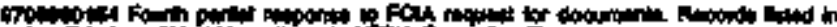

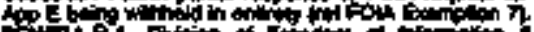

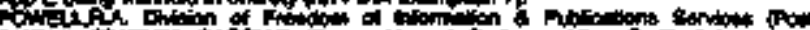

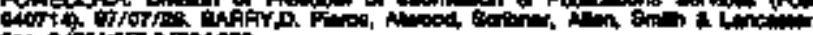

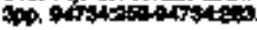

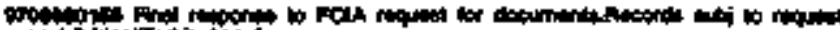

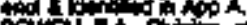

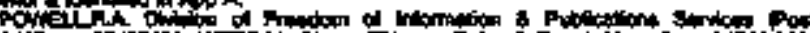

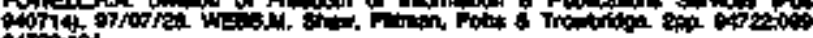
arion

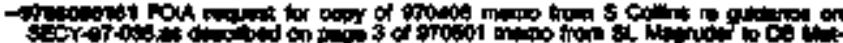

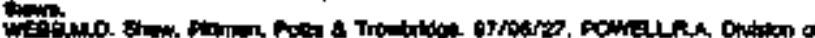

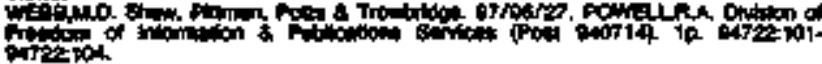

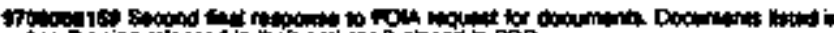

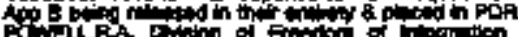
Tovit

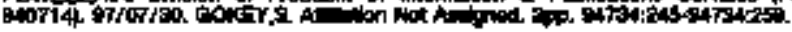

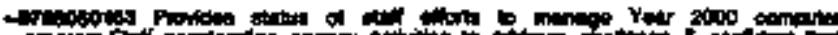

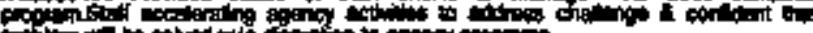

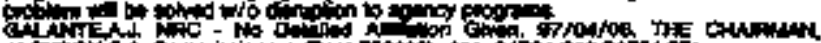

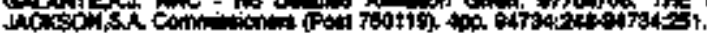




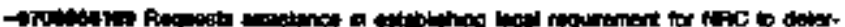

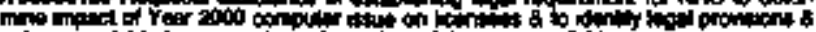
ator

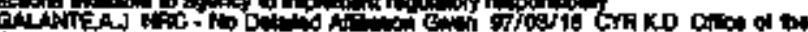

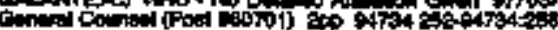

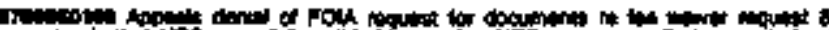

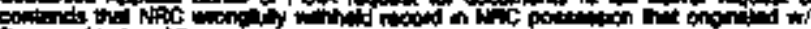

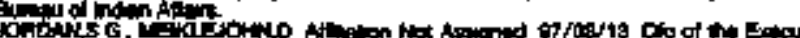

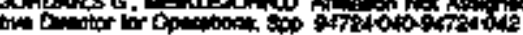

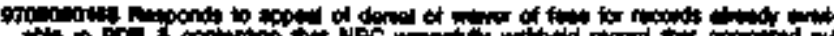

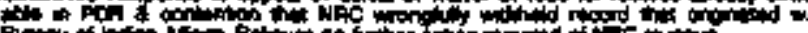

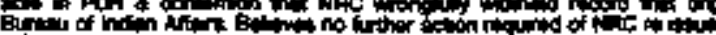

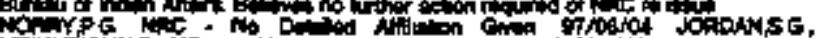

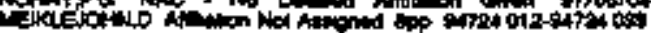

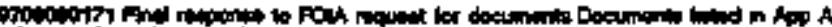

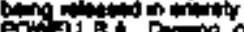

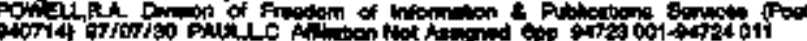

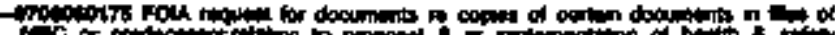

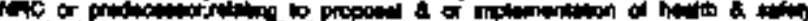

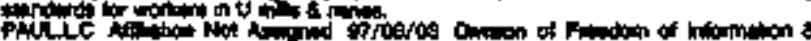

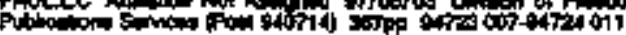

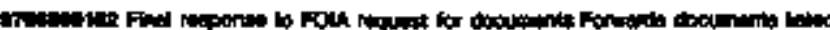

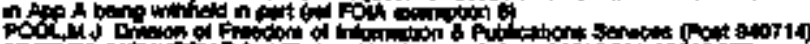

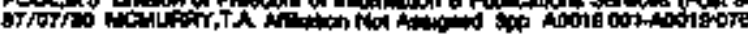

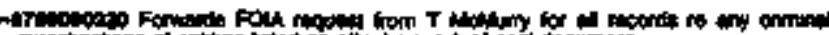

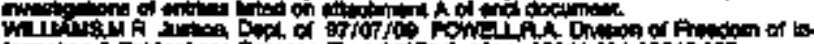

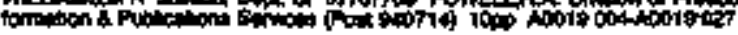

- In

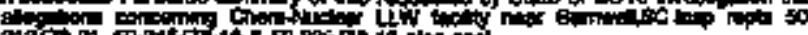

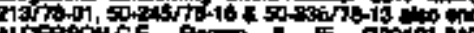

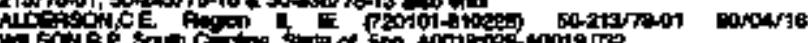

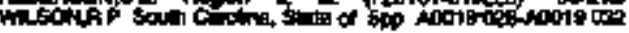

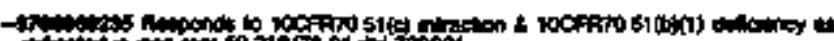

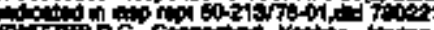
8ile

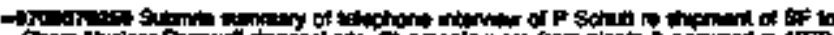

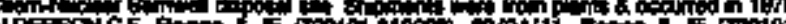
10

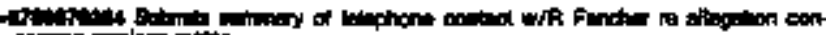

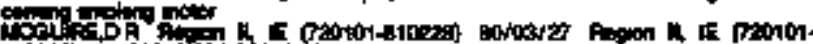

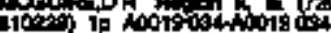

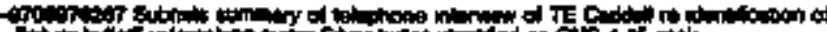
Do:

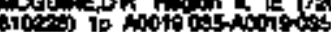

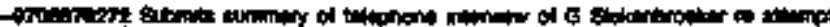

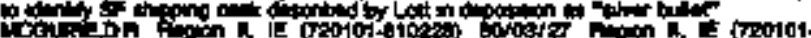

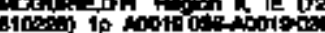

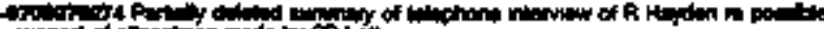

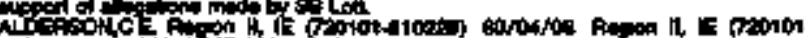

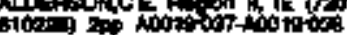

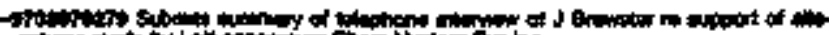

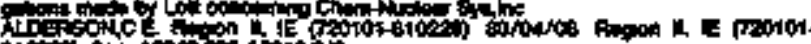
of

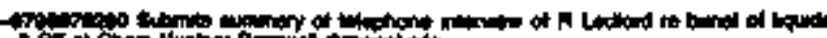

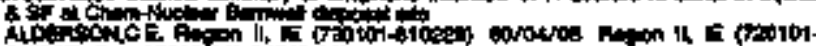

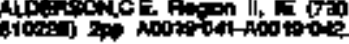

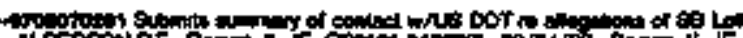

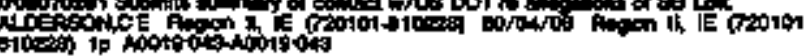

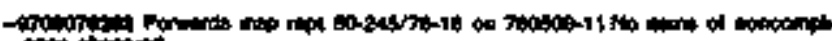

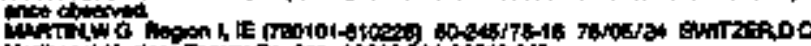

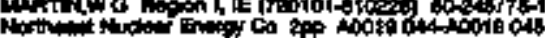

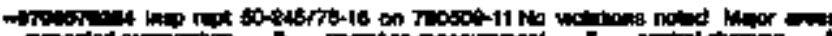

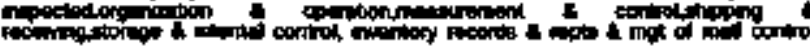

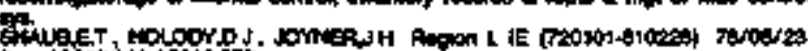

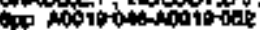

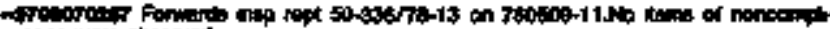

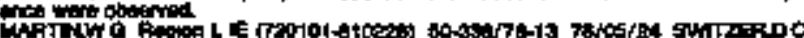

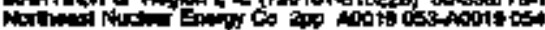

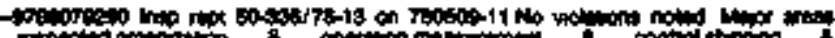

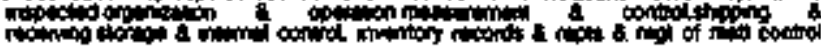

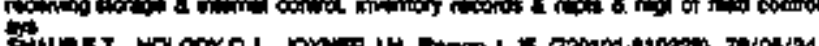
in

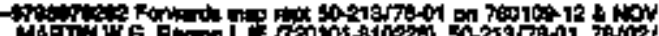

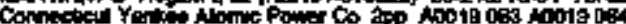

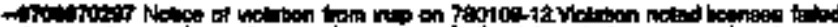

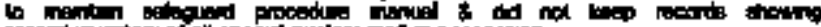

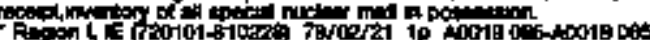

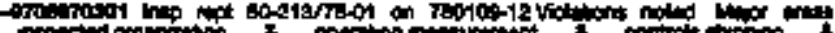

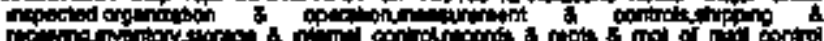

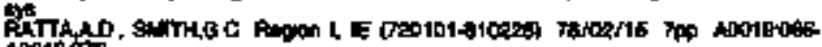
Antiotio

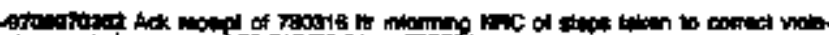

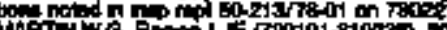

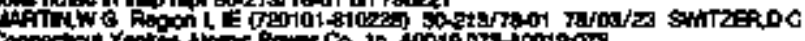

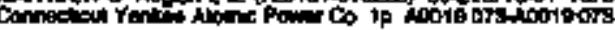

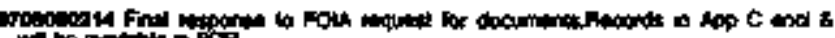

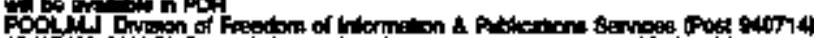
offot

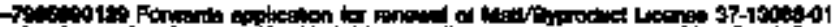

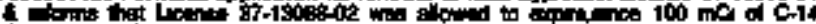

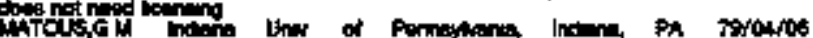

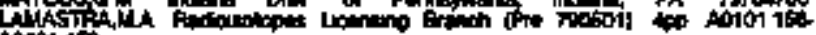
and 100

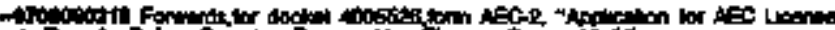
O Triti

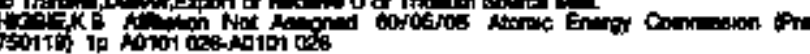

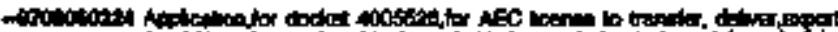

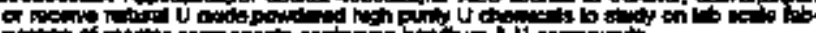

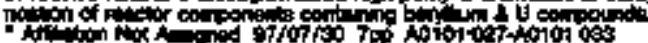

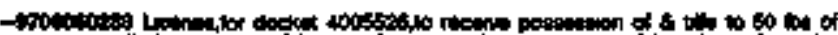

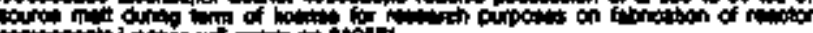

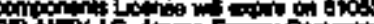

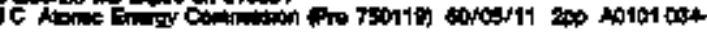

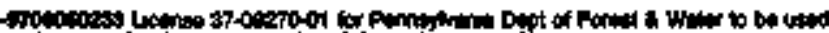
nWticic of

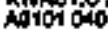

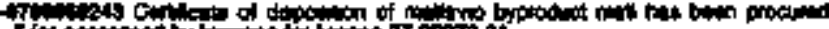

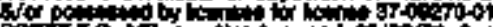

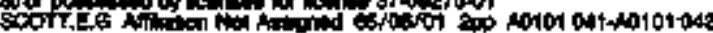

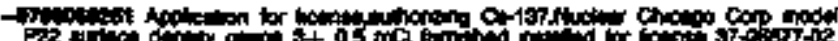

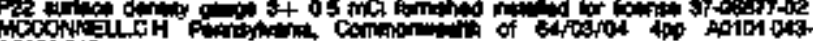
Actoi 048

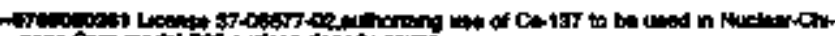

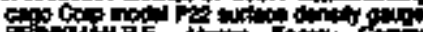

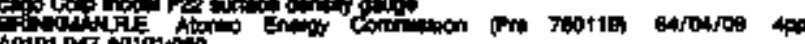

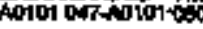

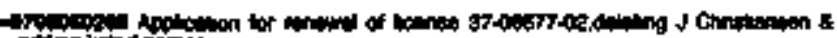

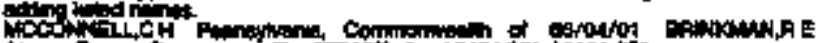

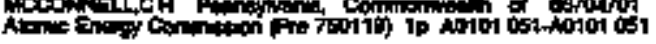

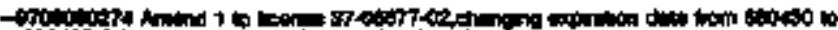

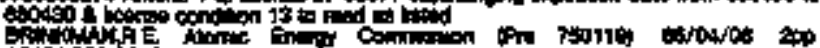

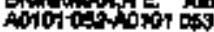

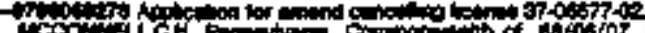

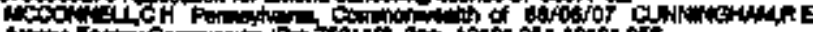

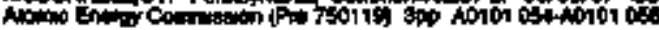

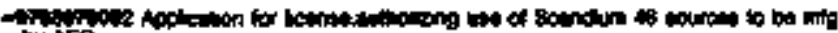

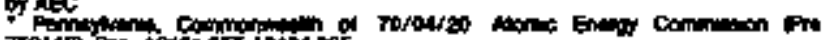

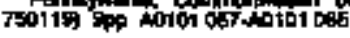

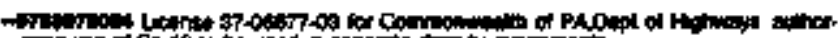

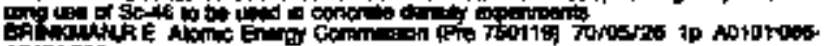
Aloi 060

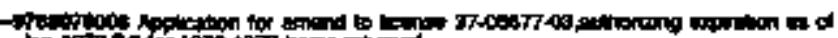

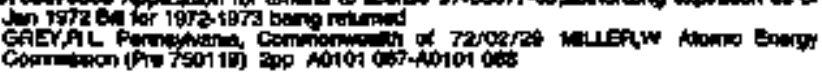

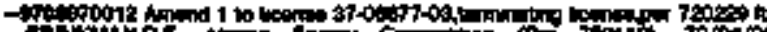

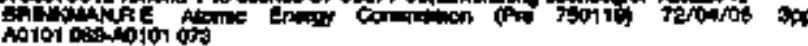




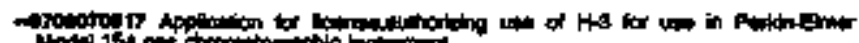

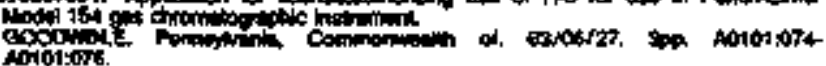

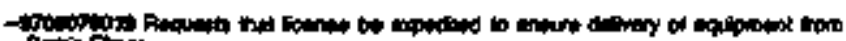

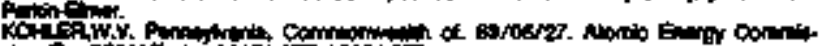

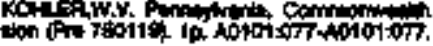

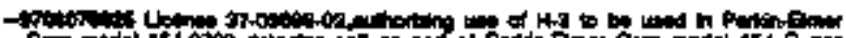

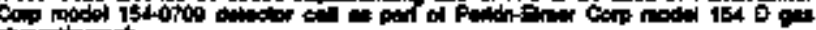

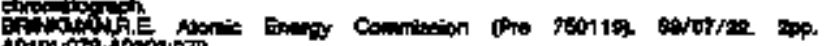

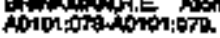

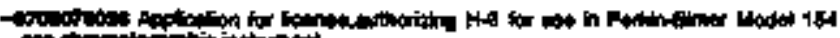

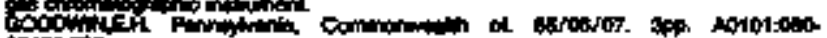
Aplonthe

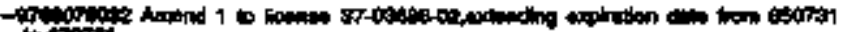
hotorst

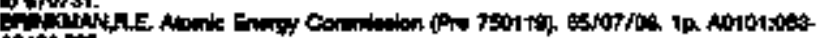
intotsolat.

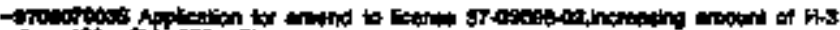

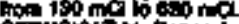

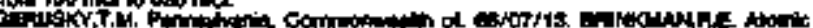

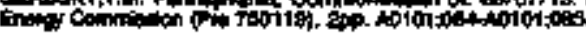

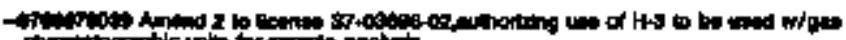

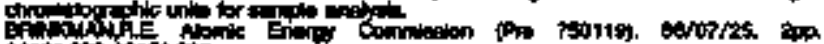

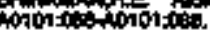

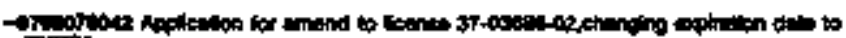
oureri.

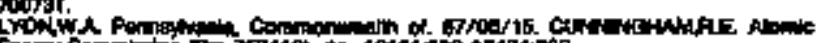

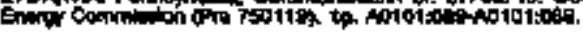

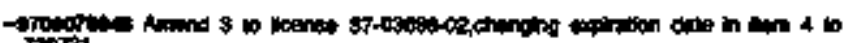
ragris

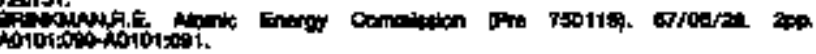

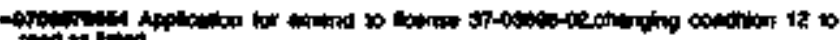
beflis

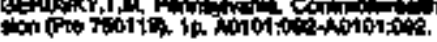

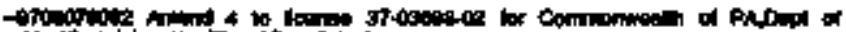

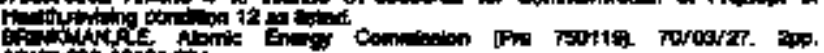

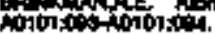

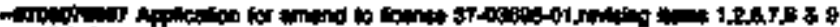
ertod

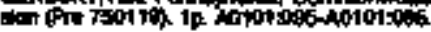

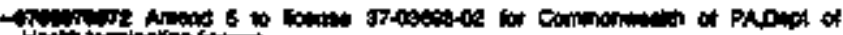

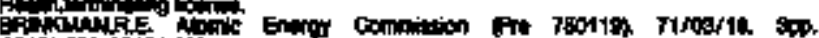

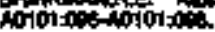

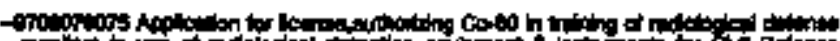

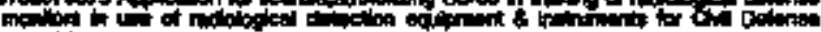

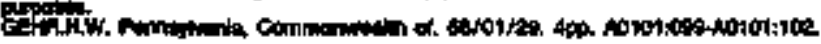

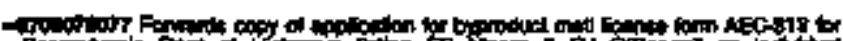

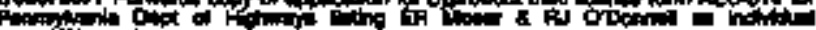

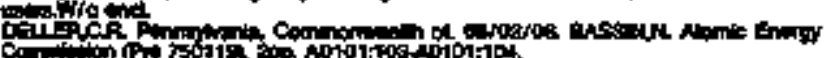

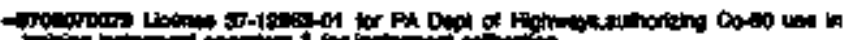

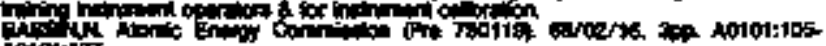
notoliver.

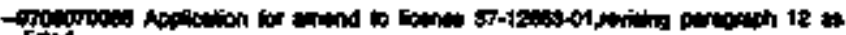

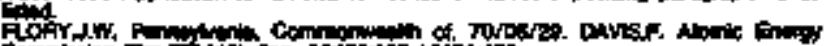
Combing (Pnt

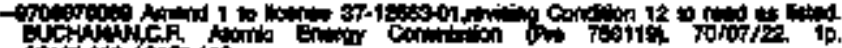

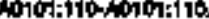

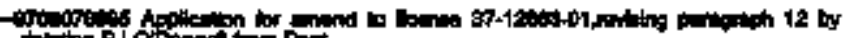

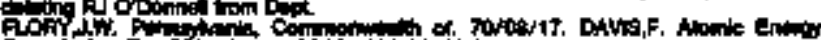

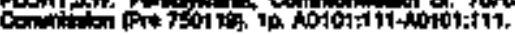

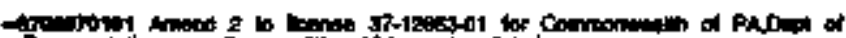

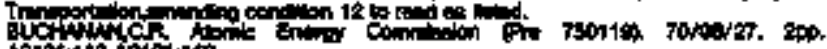
A00:1 12-A0ito: 1

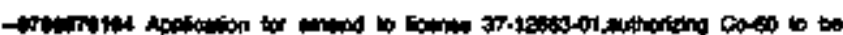

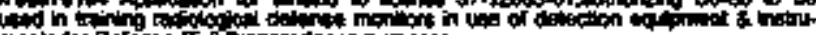

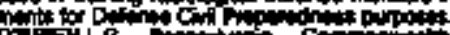
ợi:

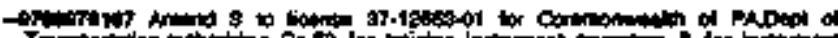

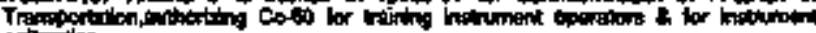

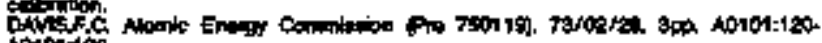
wion:

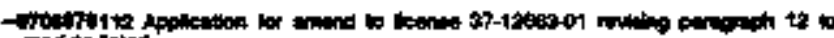

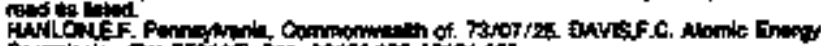

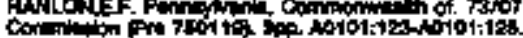

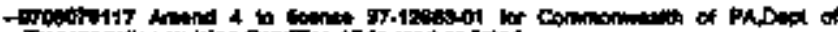

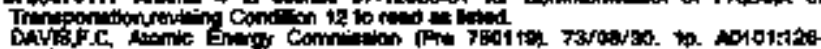
notition

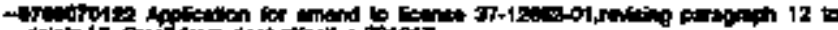

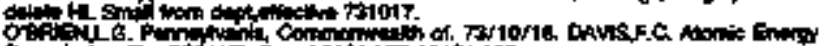

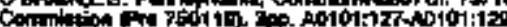

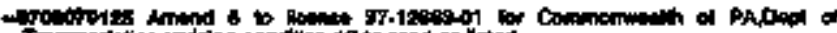

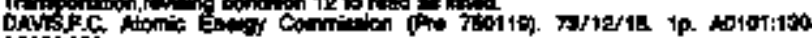

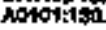

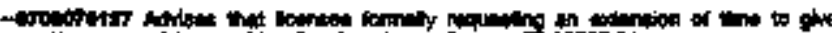

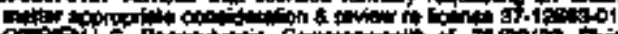

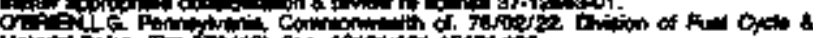

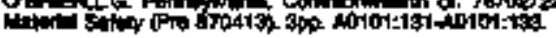

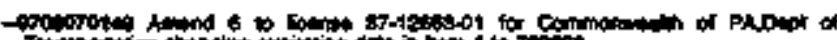

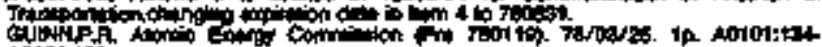
âdolition

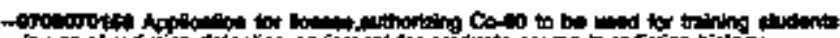

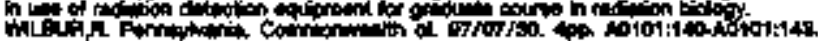

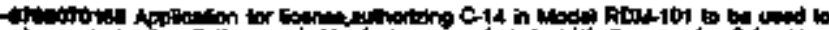

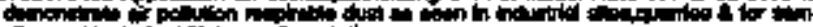

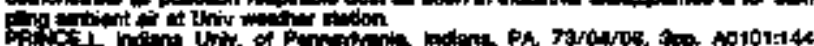

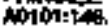

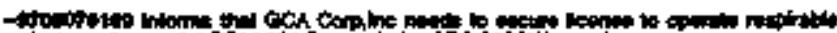

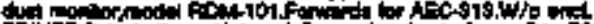

Find

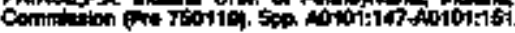

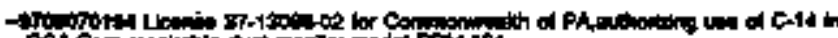

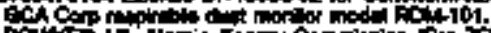

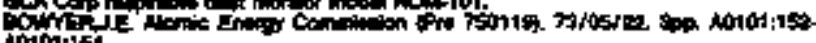
Aniton:16.

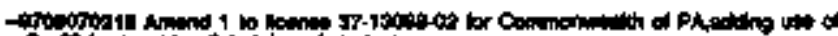

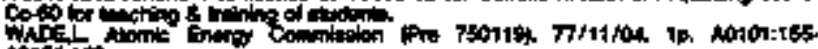
Hônitis.

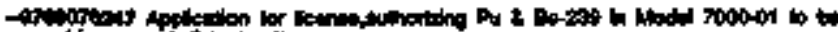

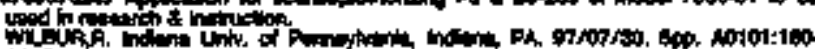
Noivitise.

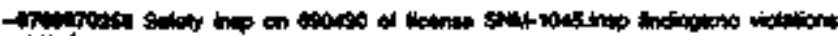

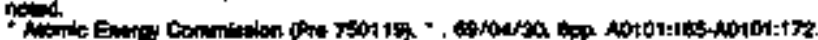

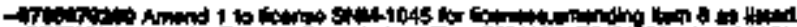

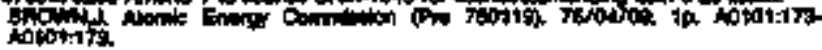

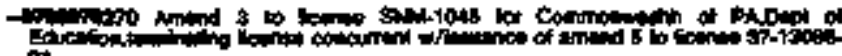

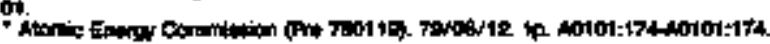

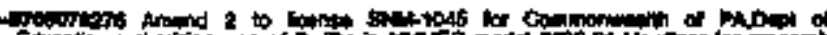

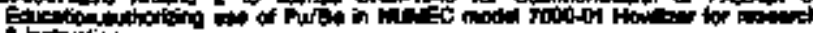

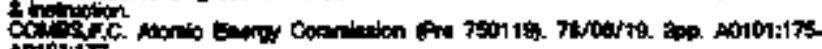
AOtonitr.

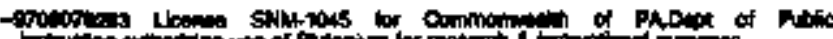

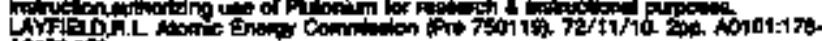
Ant:17n.

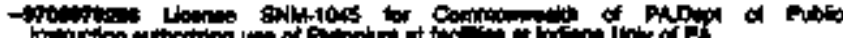

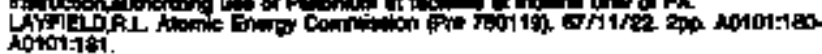

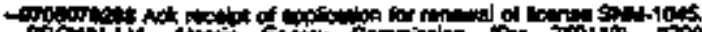

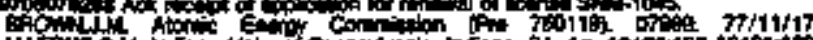

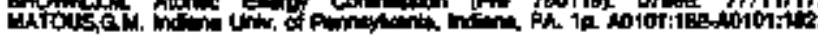

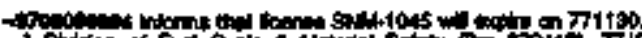

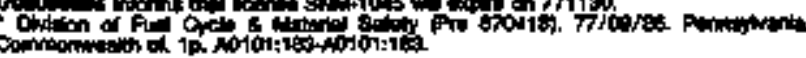

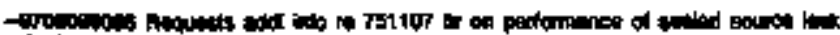

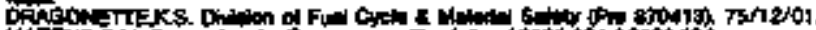

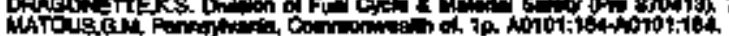




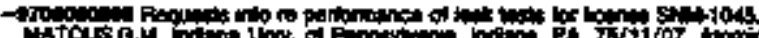

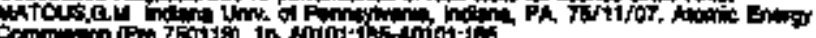

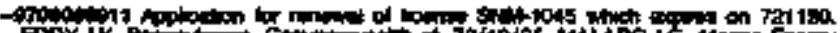

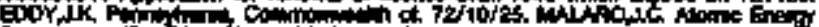

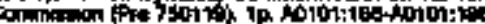

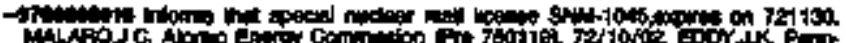

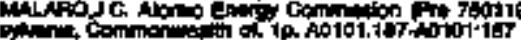

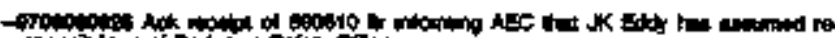

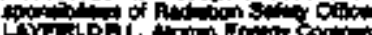

CYMLLD

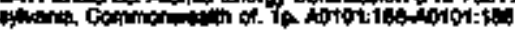

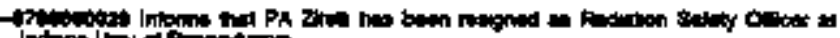

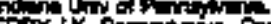

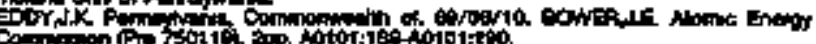

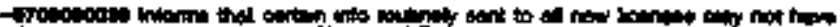

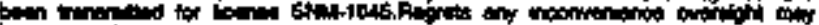

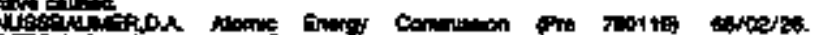

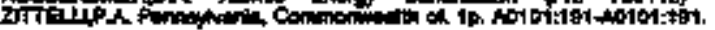

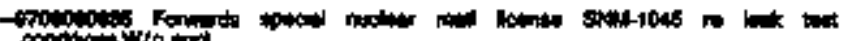

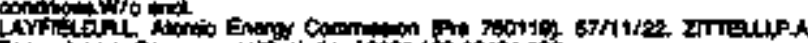

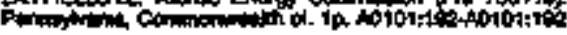

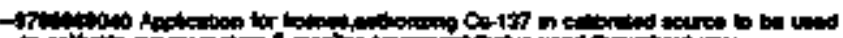

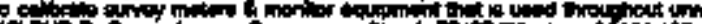

Wh-Th, Peng

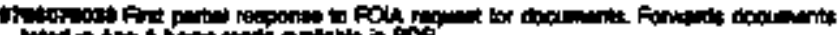

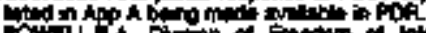

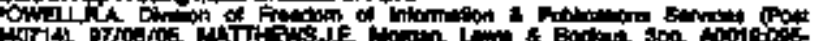
10014

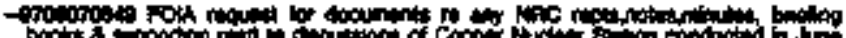

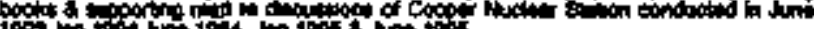
10 on

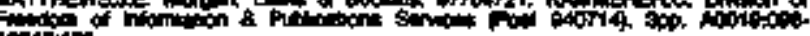
Andicion

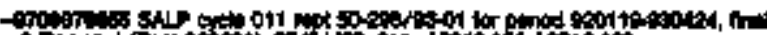

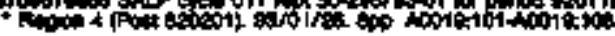

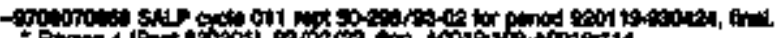

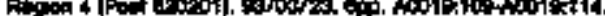

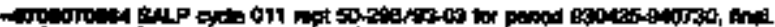

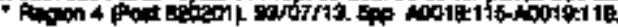

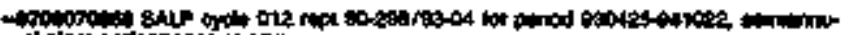

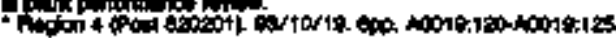

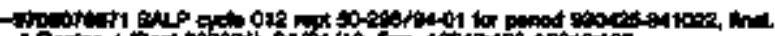

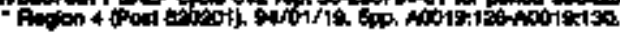

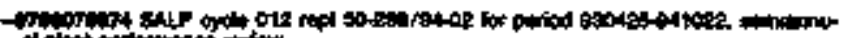

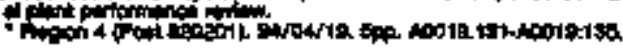

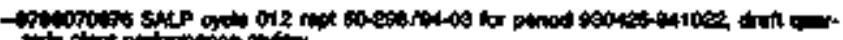

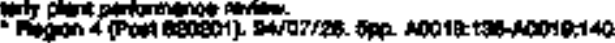

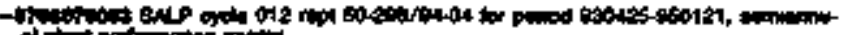

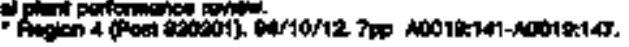

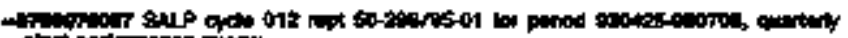

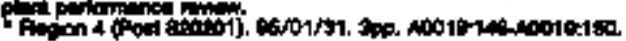

-

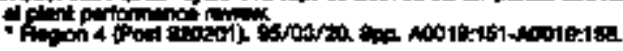

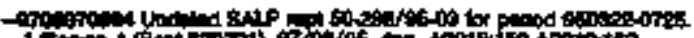

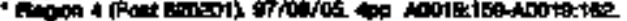

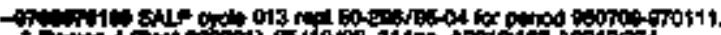

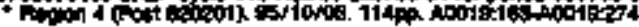

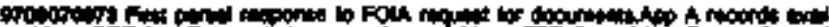

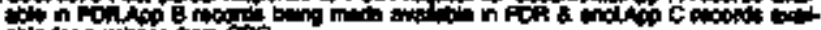

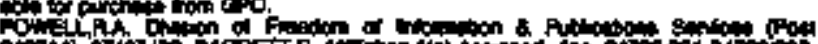

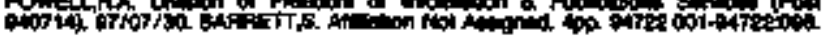

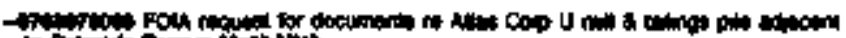

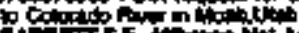

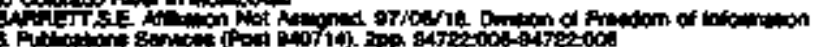

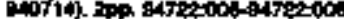

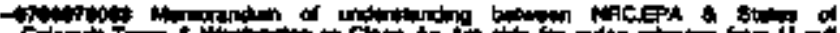

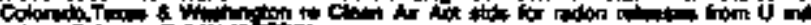

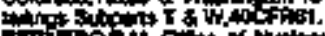

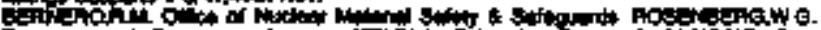

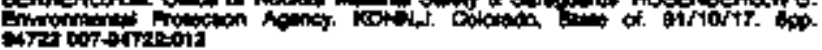

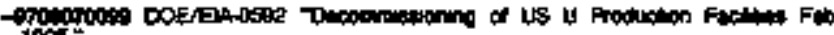
Iof

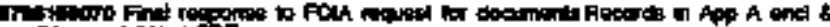

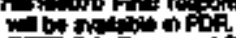

PE DOC

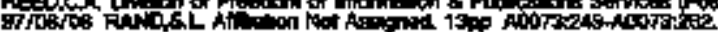

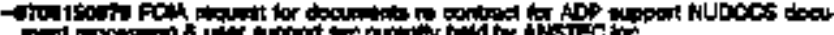

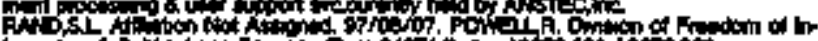

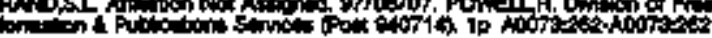

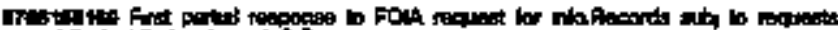

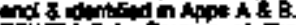

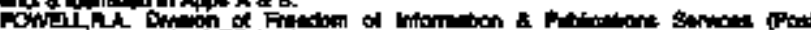

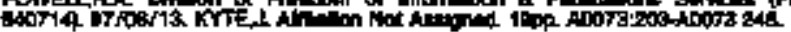

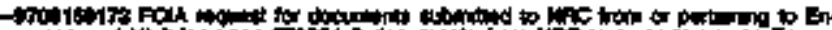

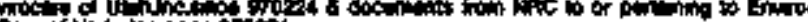

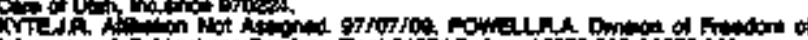

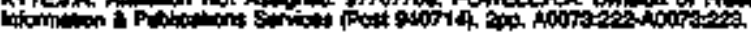

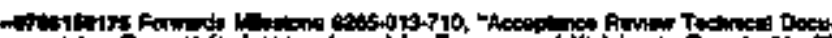

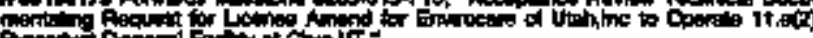

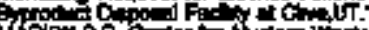

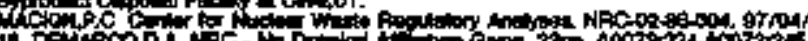

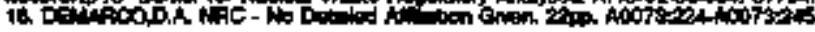

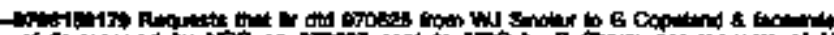

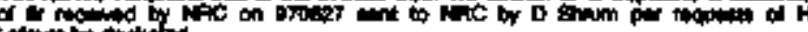

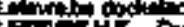

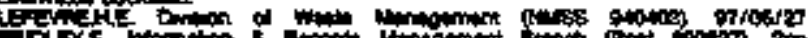

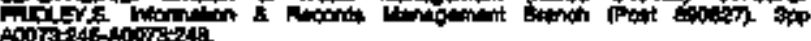

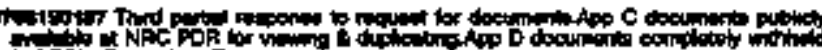
trool

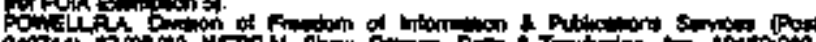

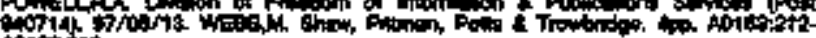
Altom

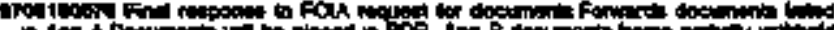

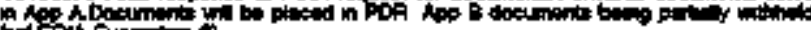
(t) on

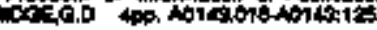

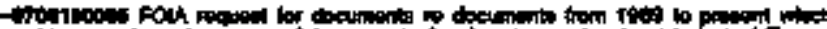

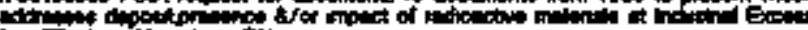

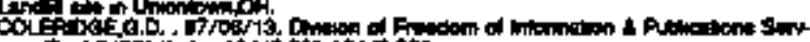
cen poel

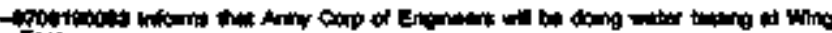

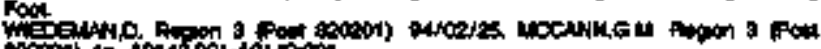

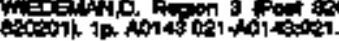

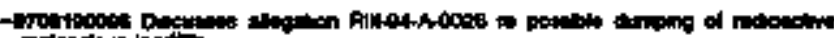

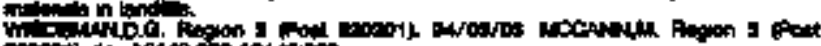

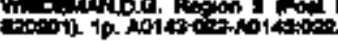

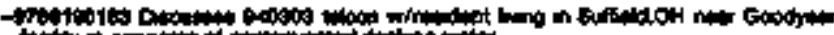

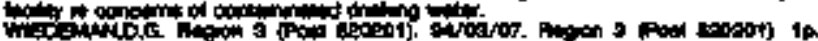
initios

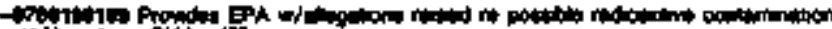

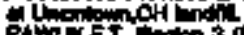

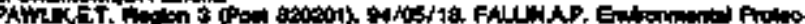

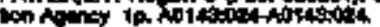

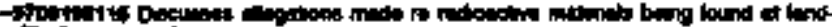
CA

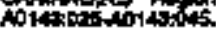

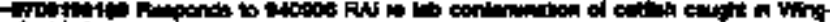

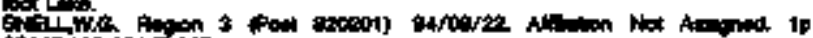

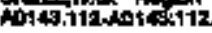

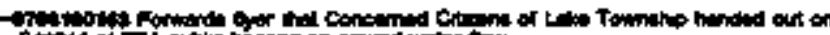

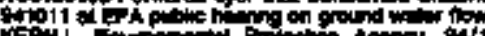
If

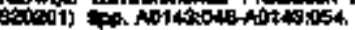

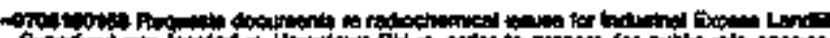

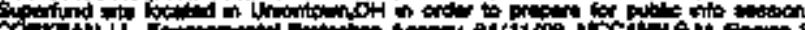

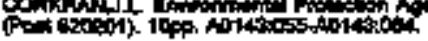

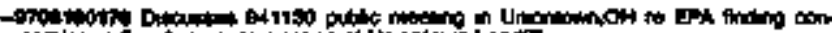

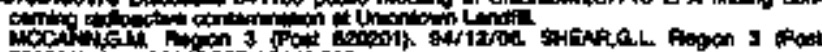

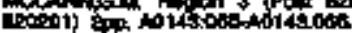

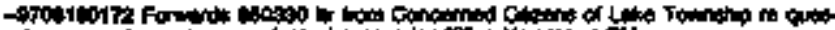

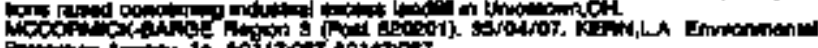

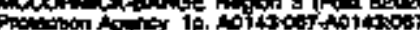




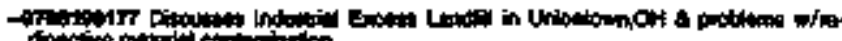

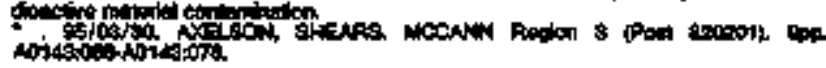

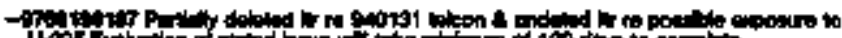
Fut ol

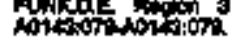

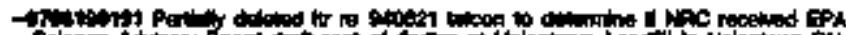

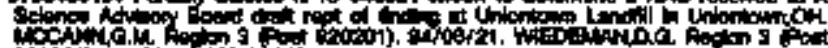

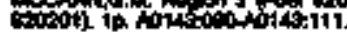

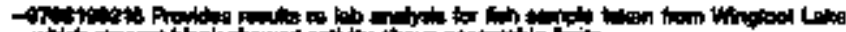

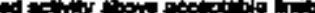

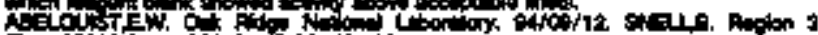

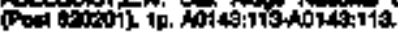

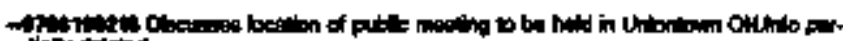

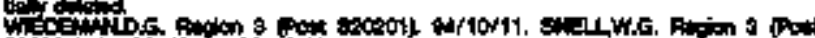

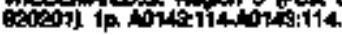

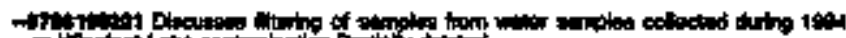

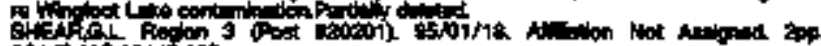

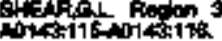

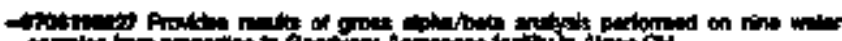

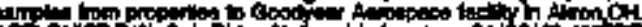

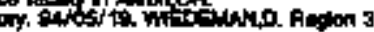

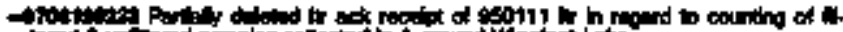

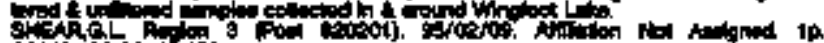

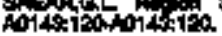

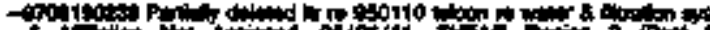

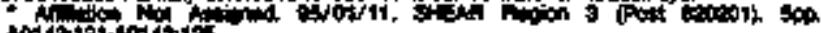

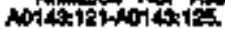

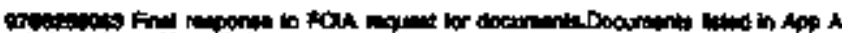

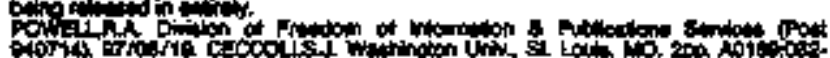
Alotits

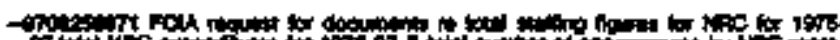
of

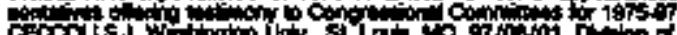

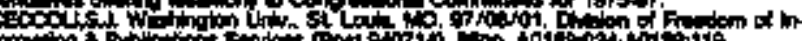

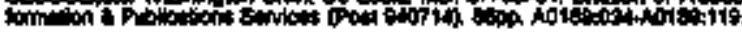

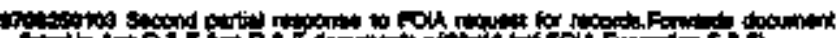

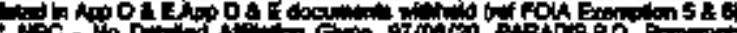

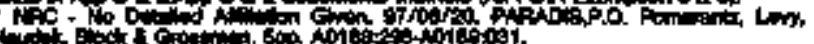

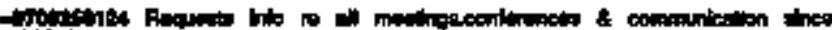

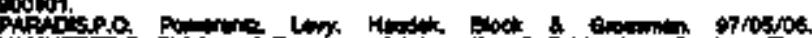
Kut antria 800 not

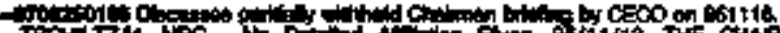

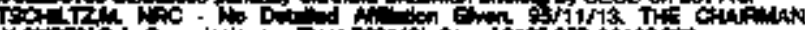
Ho.

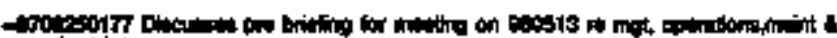

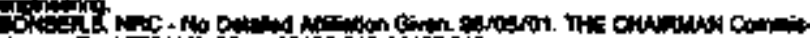

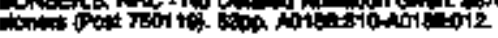

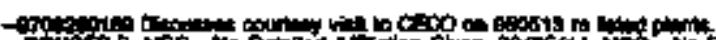

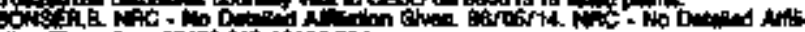

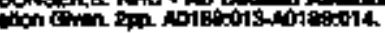

moger.

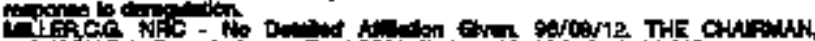

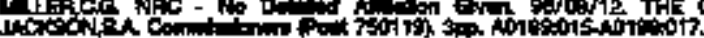

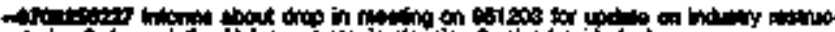

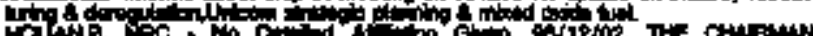

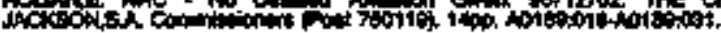

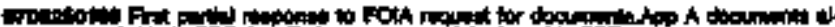
corit

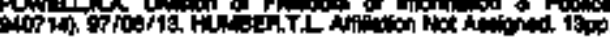

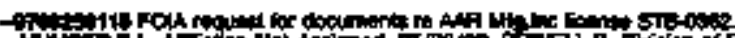

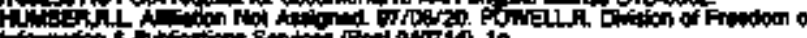

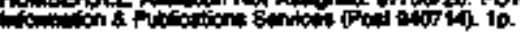

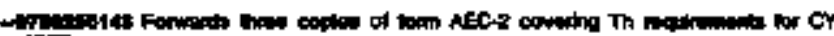
1757. 年

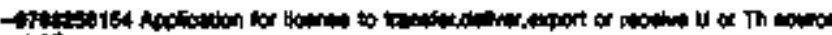

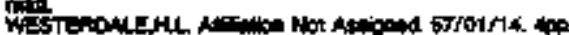

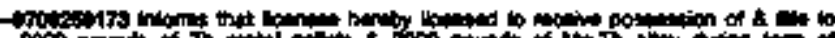

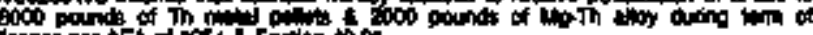

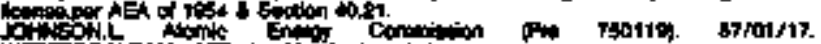

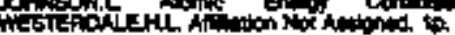

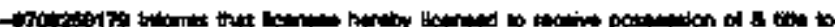

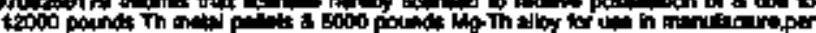

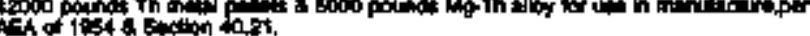
Oت

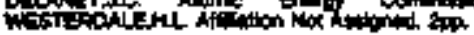

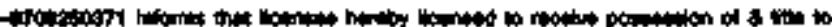

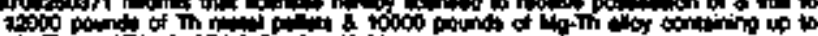

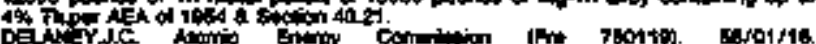

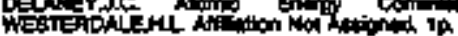

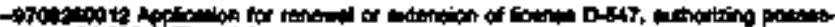
in of 1000 or thente

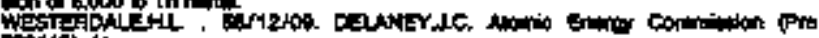
roption ip

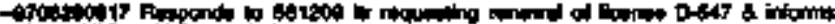

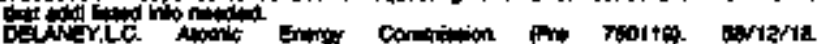

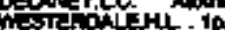

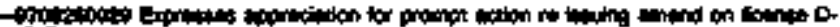
047. Piorig 拈

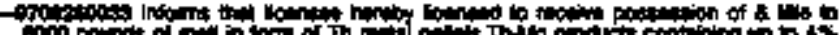

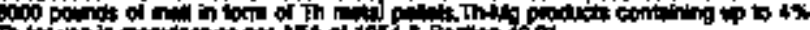

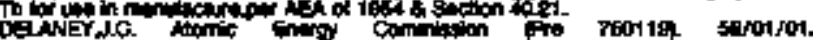
TBIPRDNEML - 19.

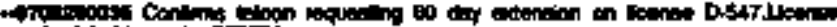

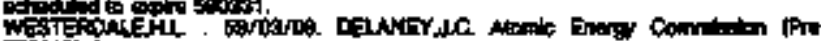
T:0110

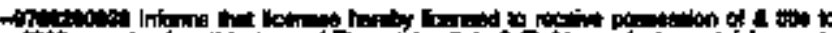

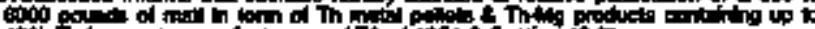
con

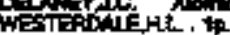

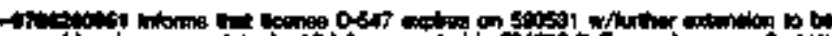

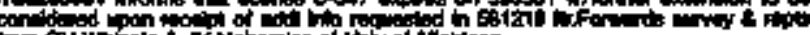

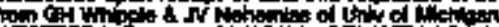

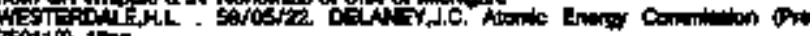

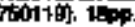

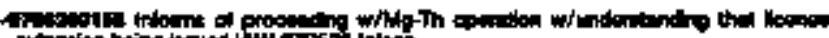

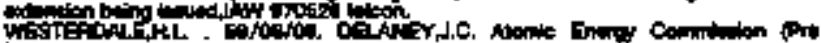
trotivi ip.

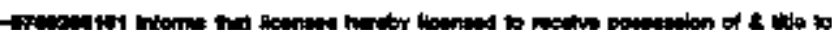

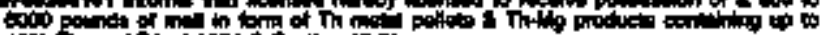
政

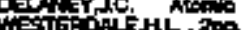

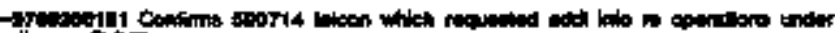

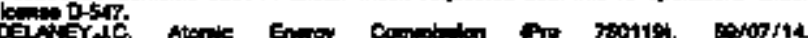

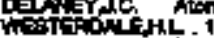

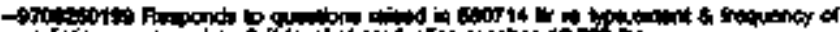

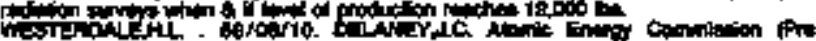

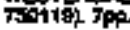

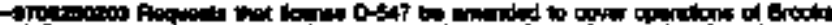

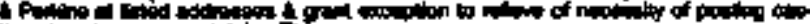

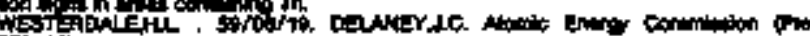
7 isich 10.

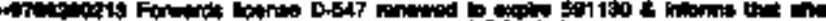

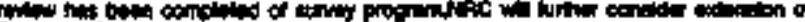

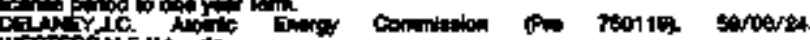
We

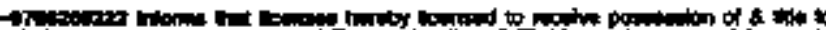

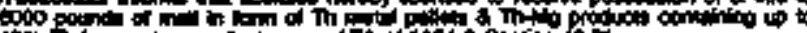

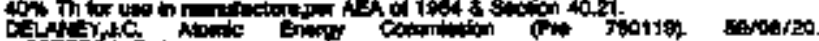

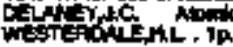

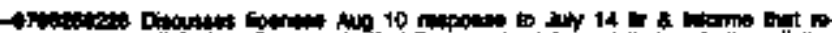

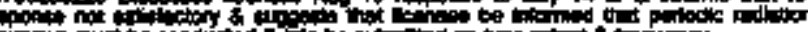

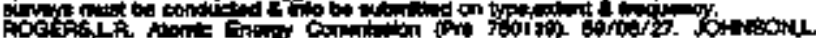

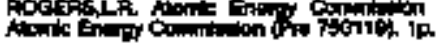

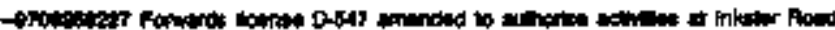

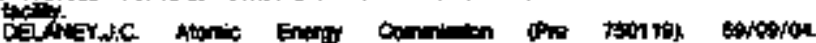

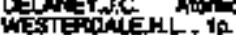




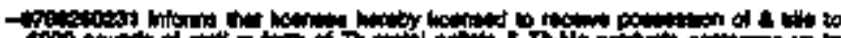

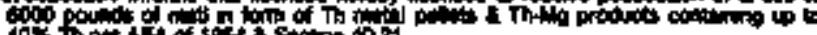

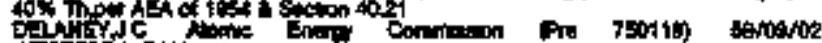

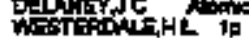

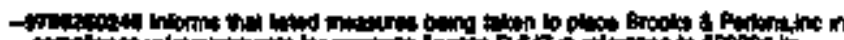

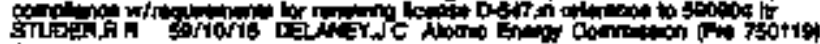
4av

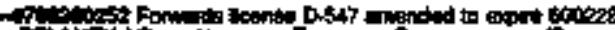

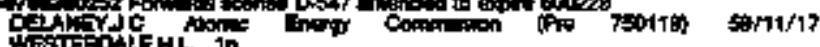

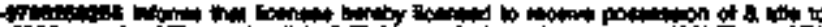

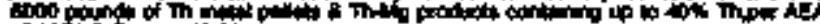

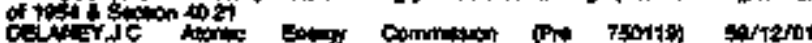
intrith

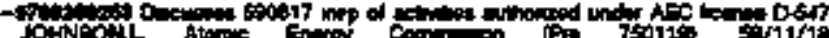

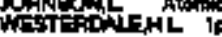

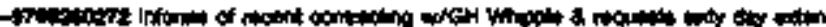

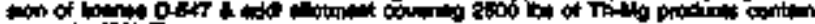

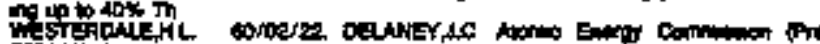

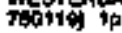

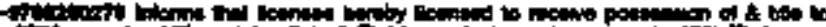

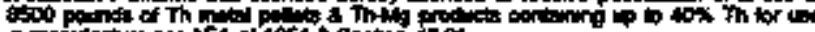

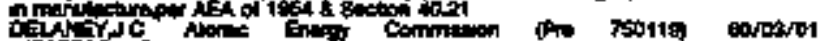

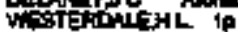

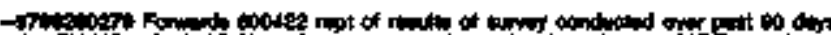

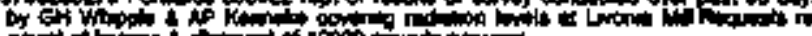

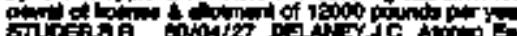

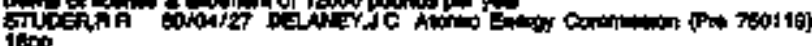
140.

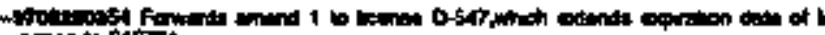

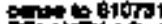

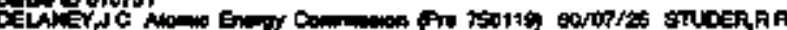
10

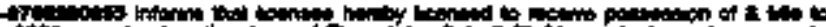

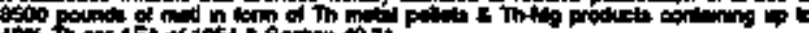

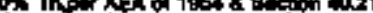
10

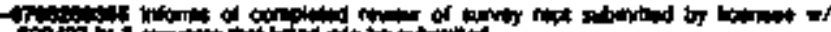

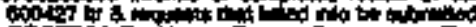

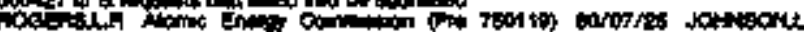

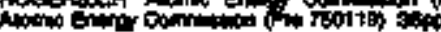

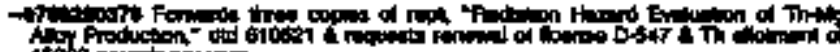

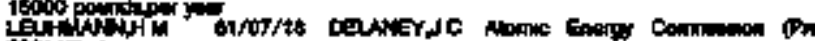

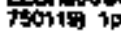

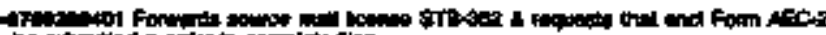

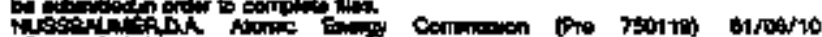

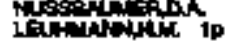

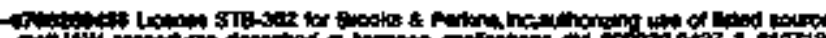

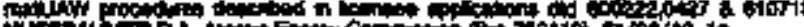

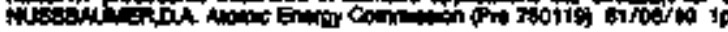

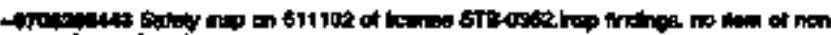

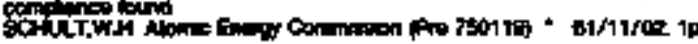

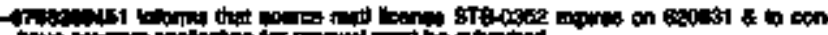

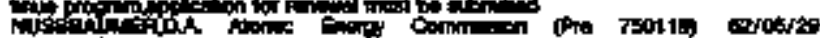
Hatrition 110

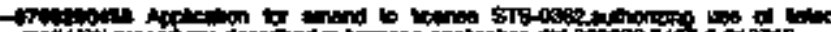

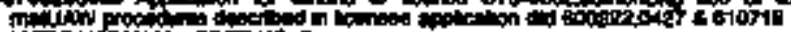

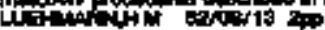

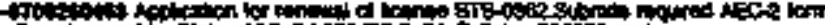

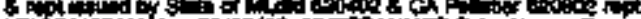

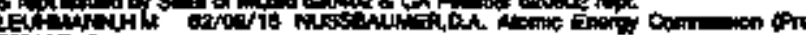

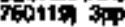

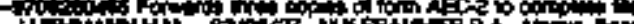
u 7sortis

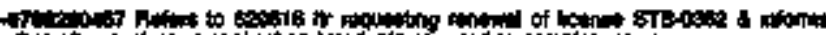

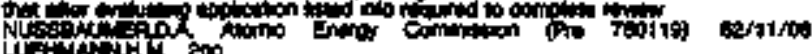

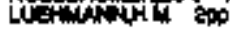

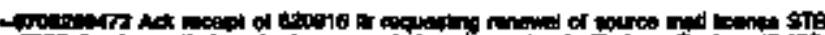

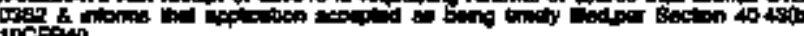

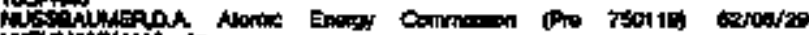
Lofintivent ip

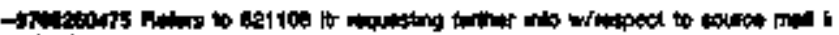

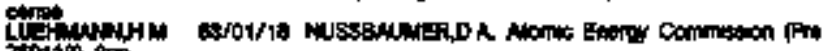
tortion

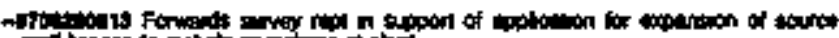

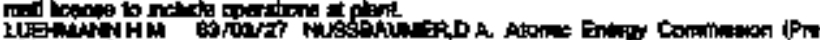
Tot

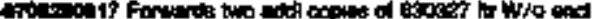

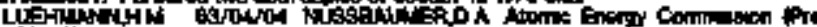

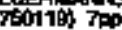

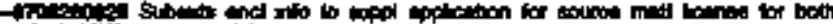

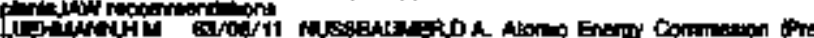
Thotito

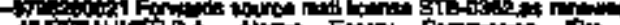

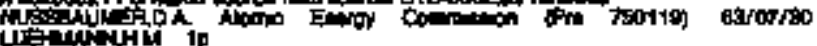

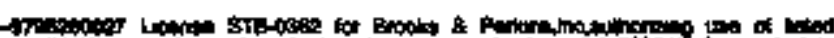

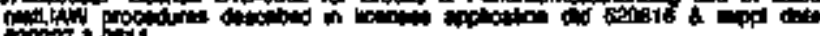

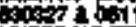

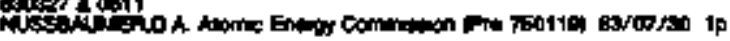

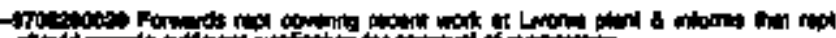

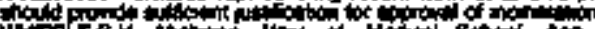

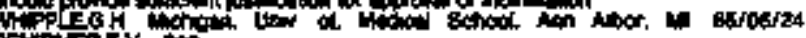

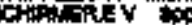

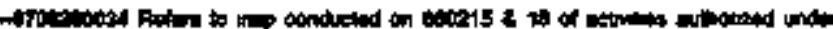

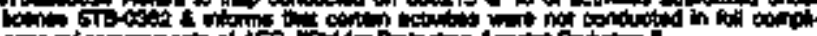

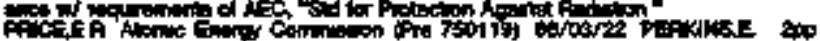

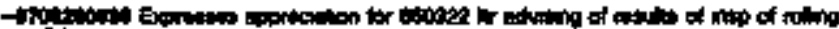

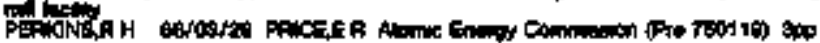

- Q70.

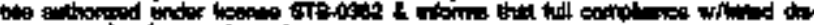

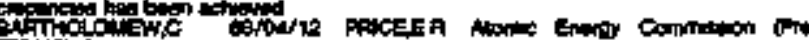
$7 \mathrm{Th} \tan$

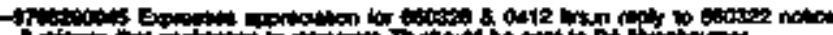

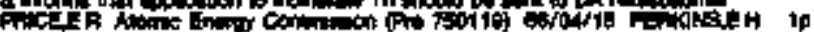

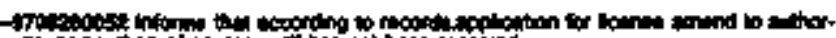

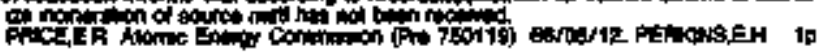

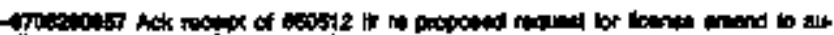

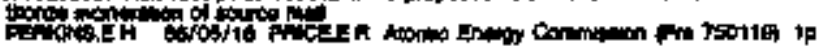

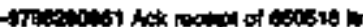

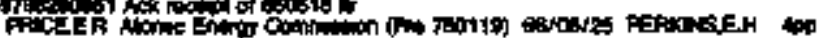

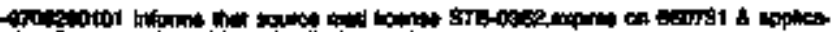

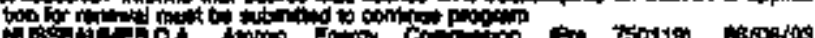

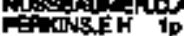

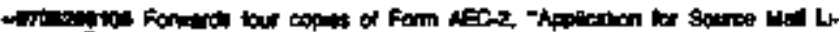

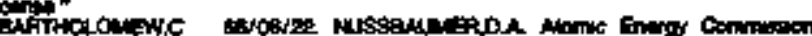
(P) To1 18) 200

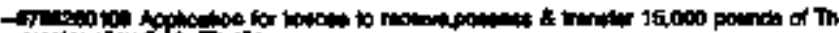

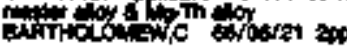

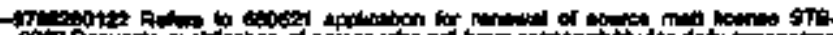

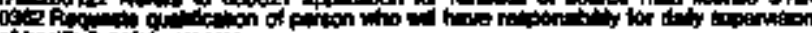
of BHFTHLLCTENA am

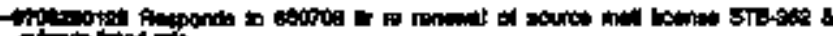

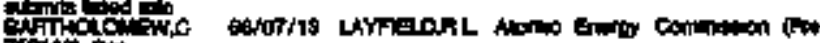
Toth th

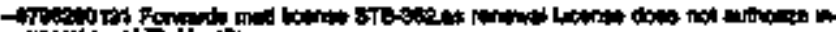

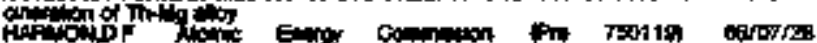
aritionare ip

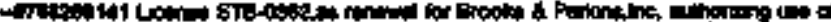

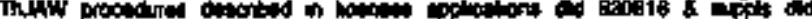

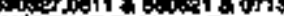

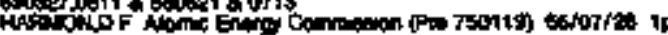

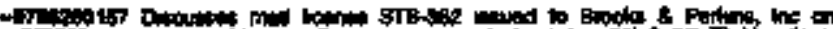

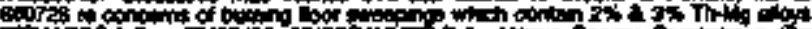

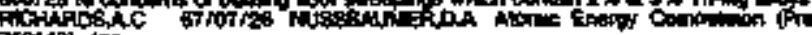
$750119) 400$

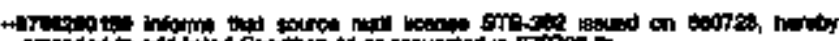

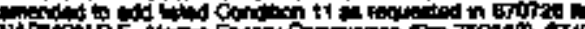

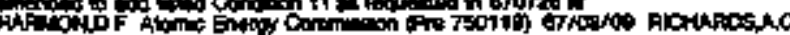
10 


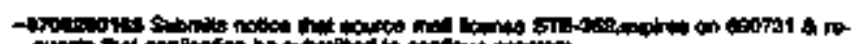

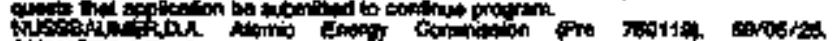
pitiofotic. ip

- 970 a

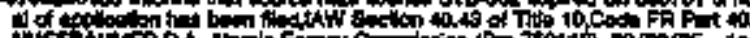

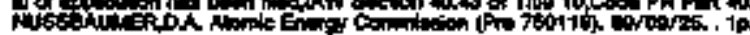

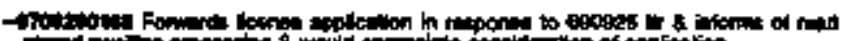

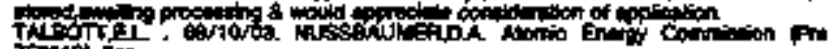
Tolis, apt

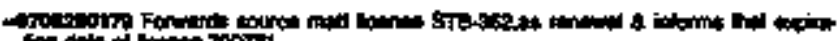

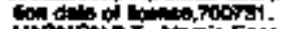

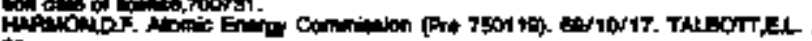
in

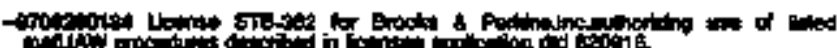

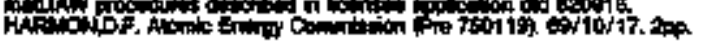

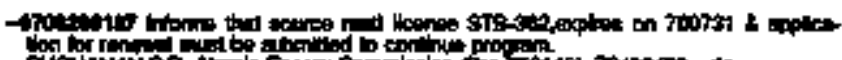

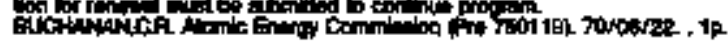

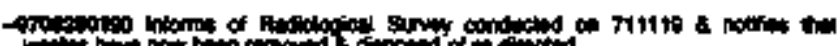

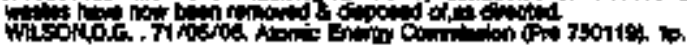

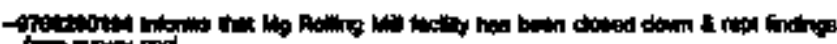

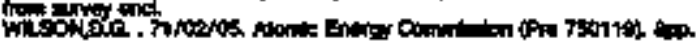

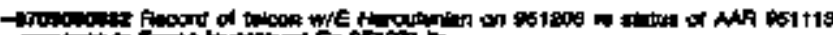
+

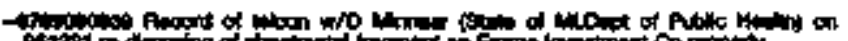

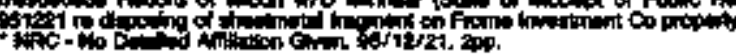

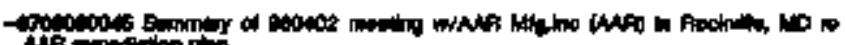

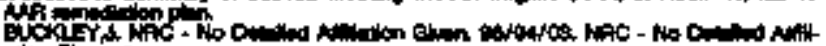

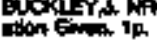

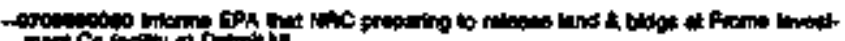

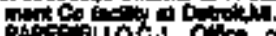

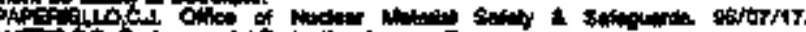

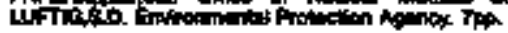

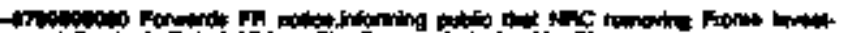

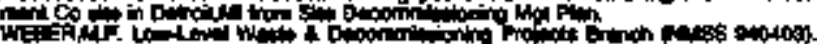

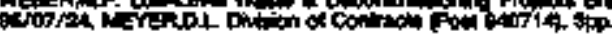

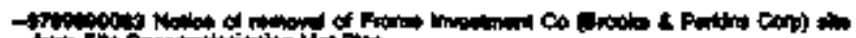

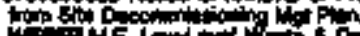

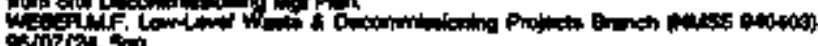
opotion son

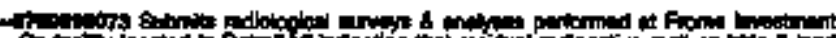

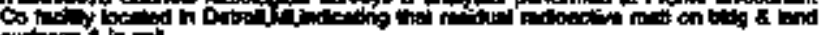

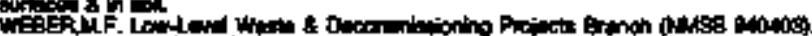

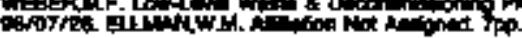

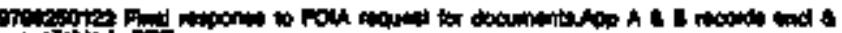

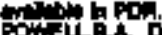

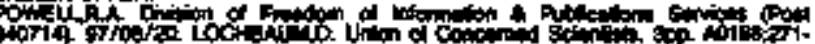
noteises.

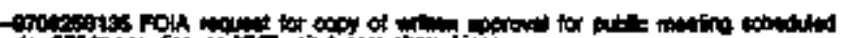

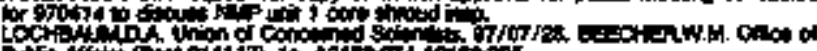

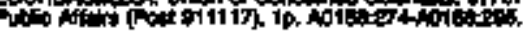

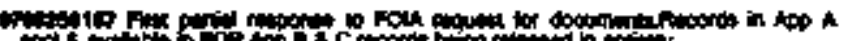

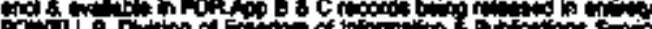

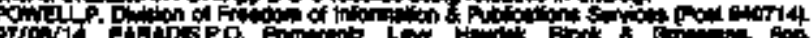

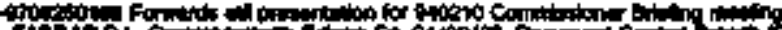

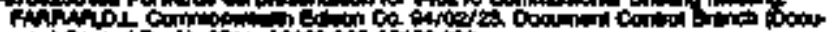

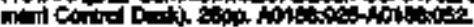

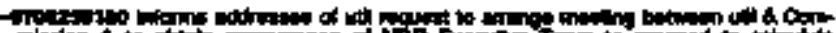

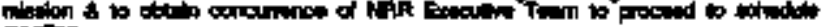

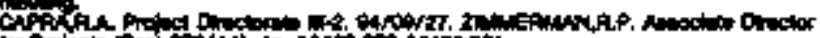

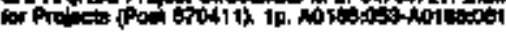

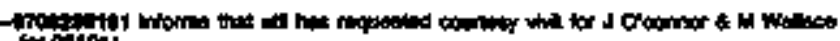

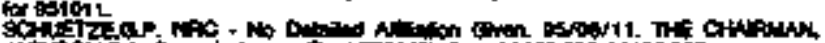

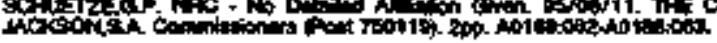

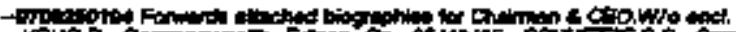

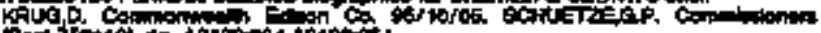

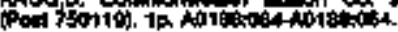

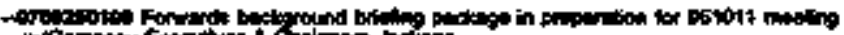

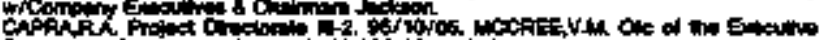

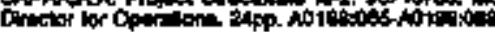
-

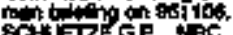

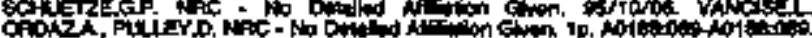

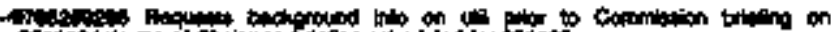

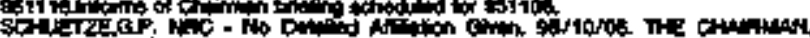

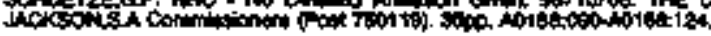

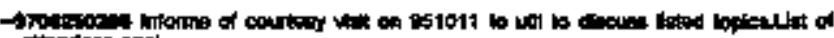

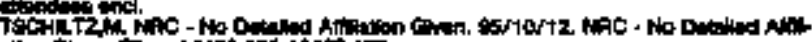

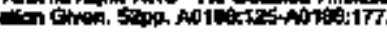

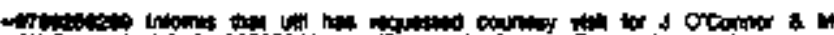

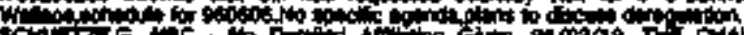

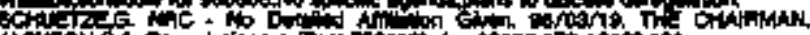

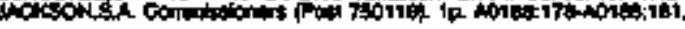

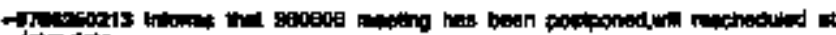

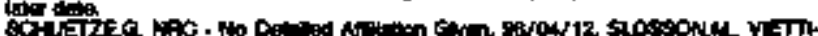

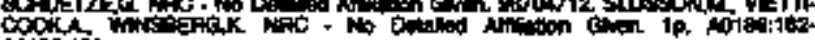
corotition

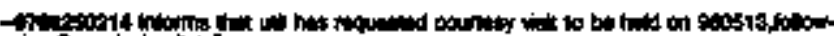

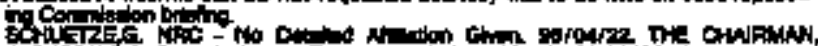

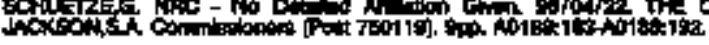

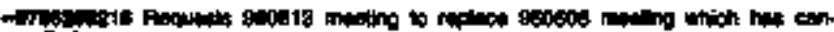
anted

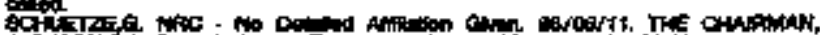

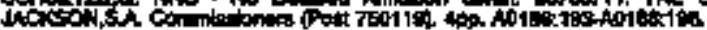

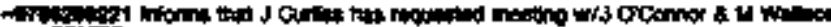
cn retion

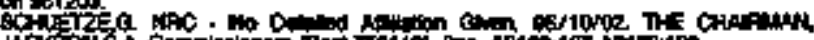

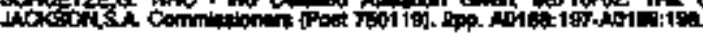

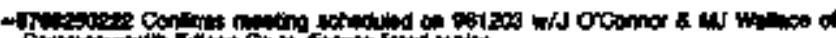
Q

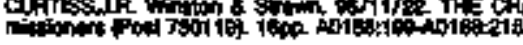

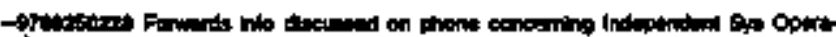

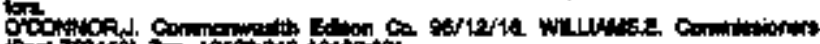

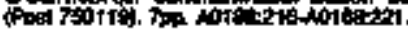

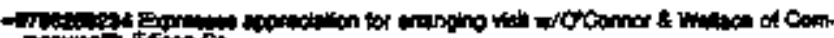

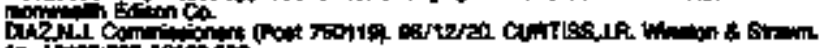

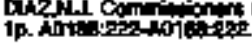

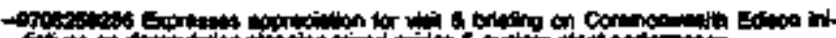

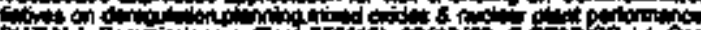
Eolon $\mathrm{C}_{0}$.

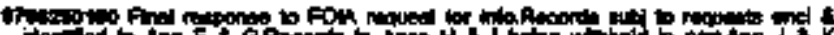

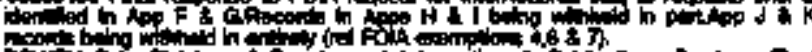

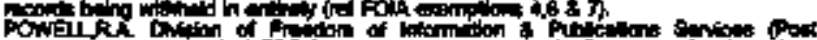

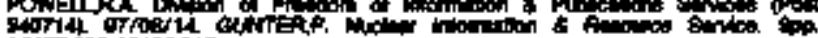

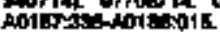

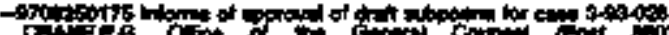

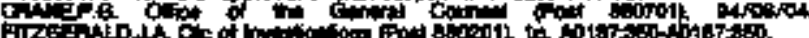

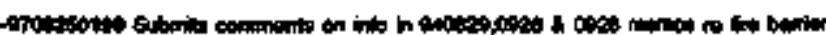

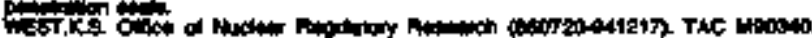

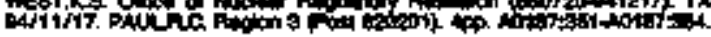

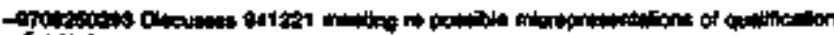

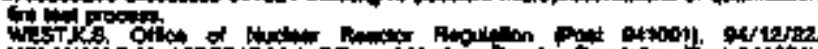
How 10. Antog

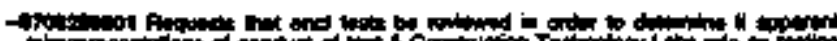

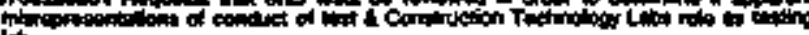

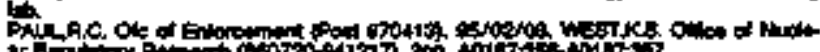

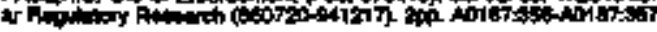

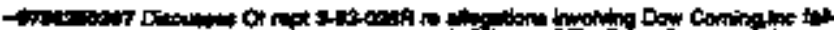

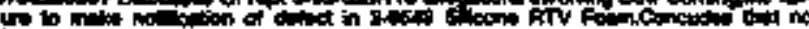

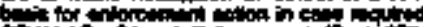

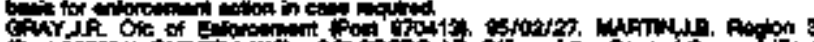

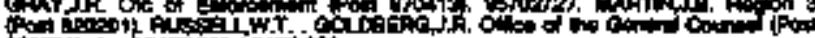

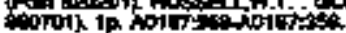

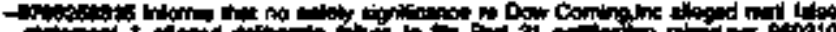

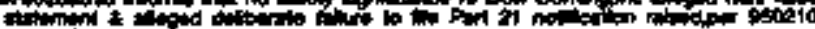

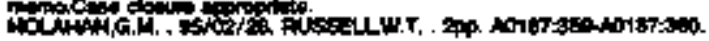

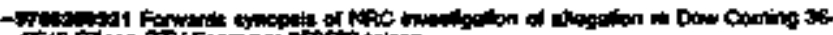

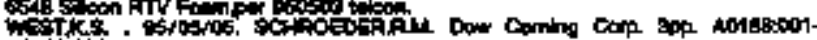
tois 


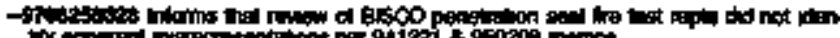

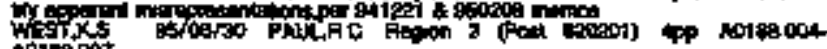
A 1000 of

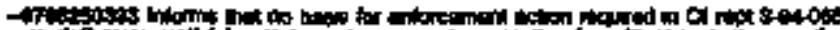

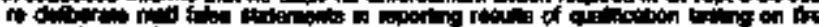

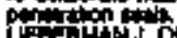

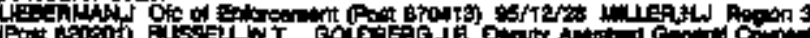

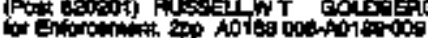

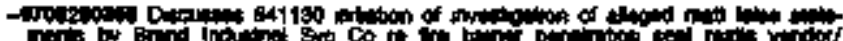

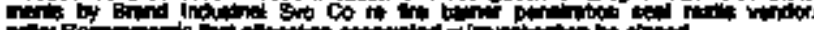

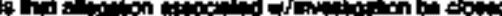

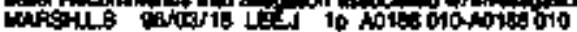

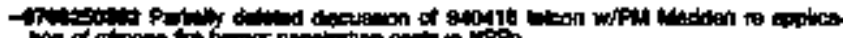

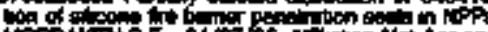

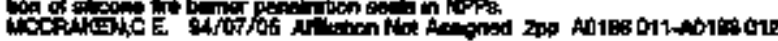

Iy.e.

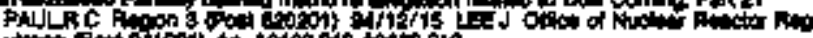

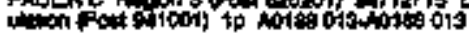

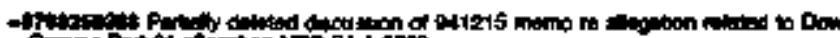
Cong

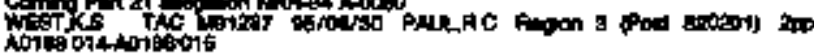

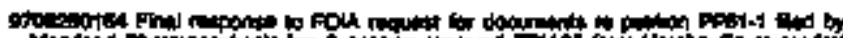

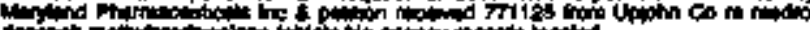

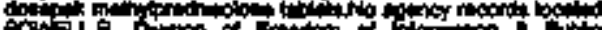

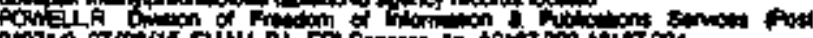

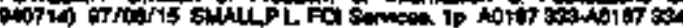

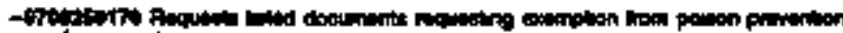

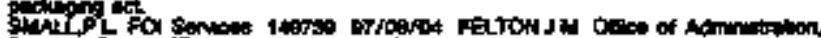

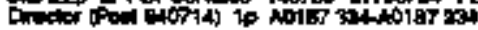

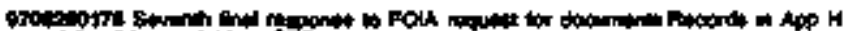

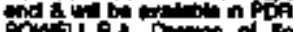

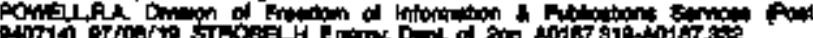

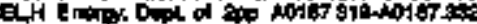

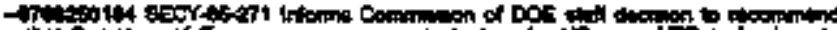

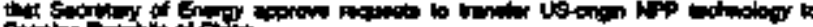

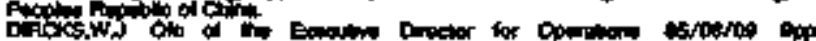
Norit की

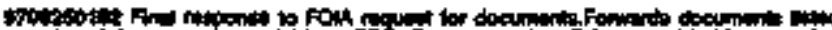

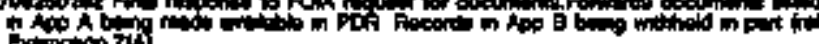

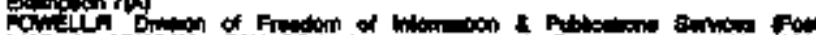

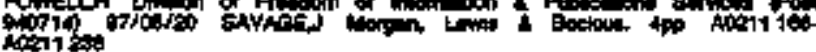

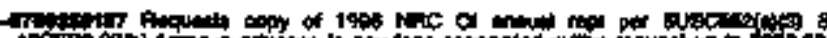

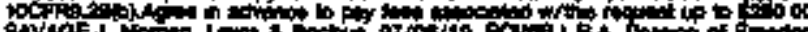

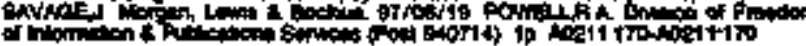

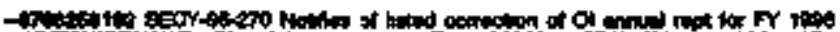

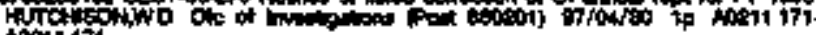

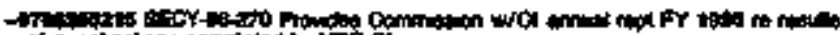

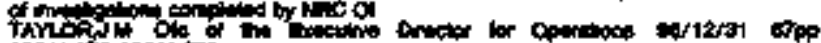
Not 11 th

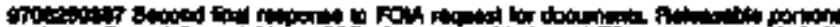

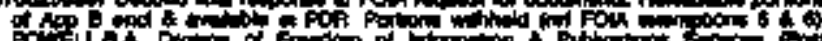

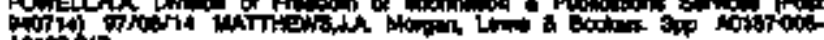
ningor

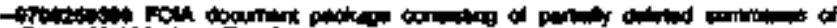

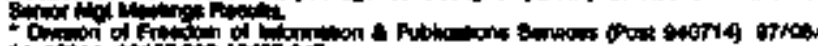

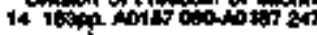

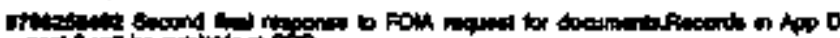

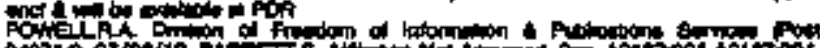

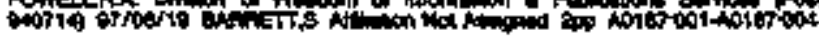

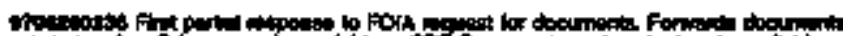

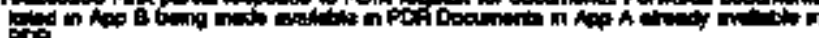

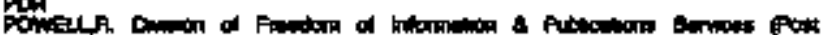

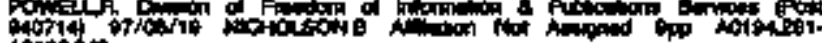
tontoto

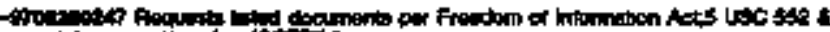

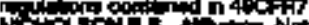

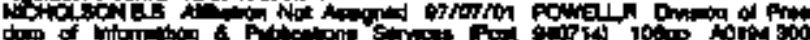

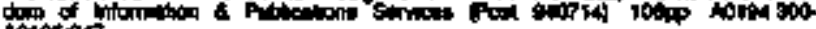

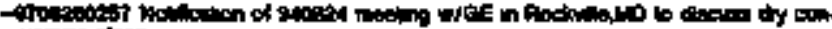

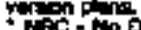

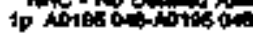

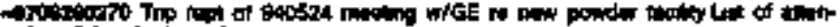

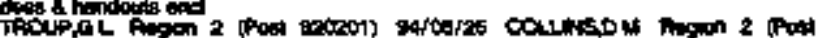

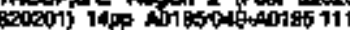

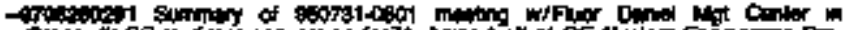

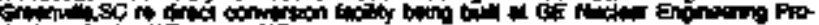

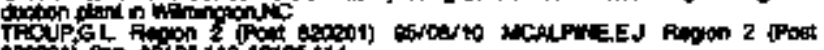

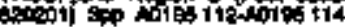

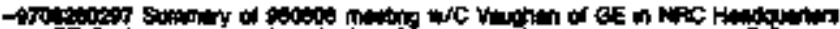

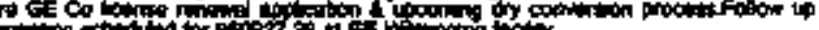

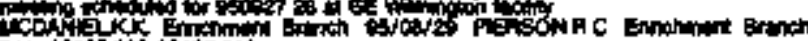

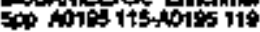

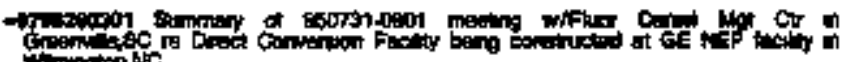

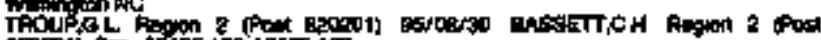

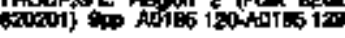

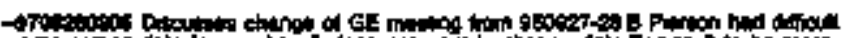

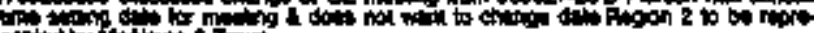
and

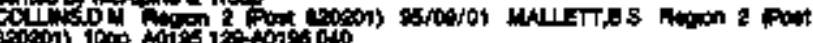

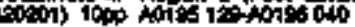

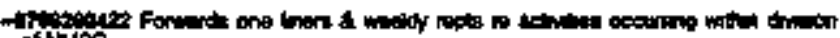

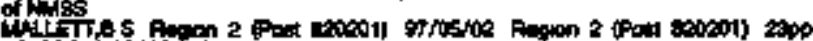

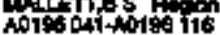

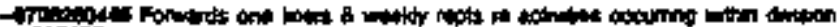

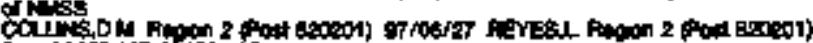

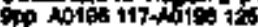

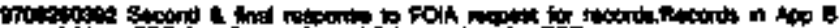

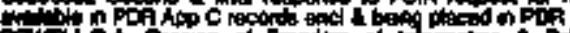

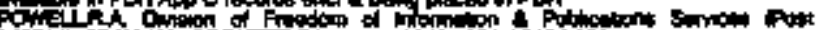

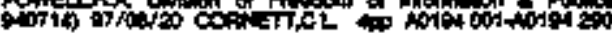

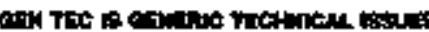

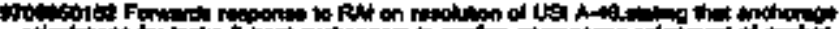

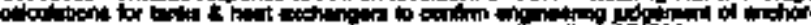

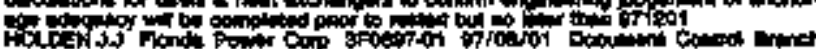

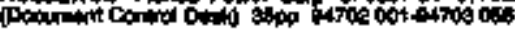

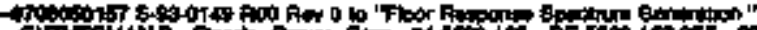

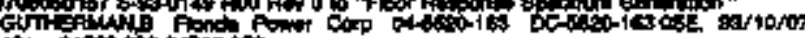

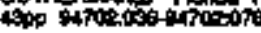

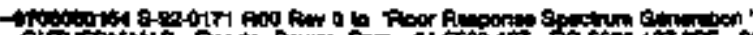

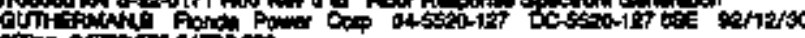

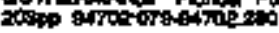

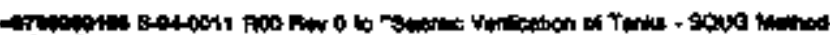

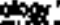

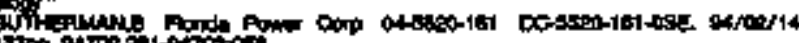

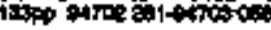

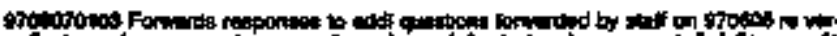

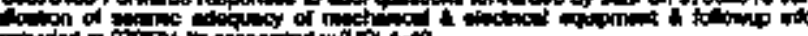

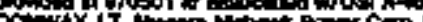

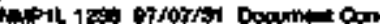

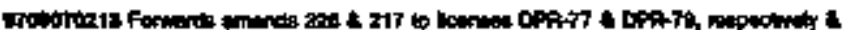

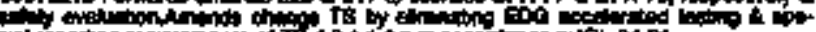

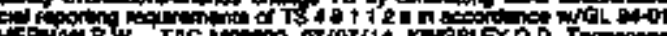

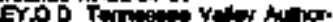

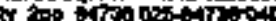

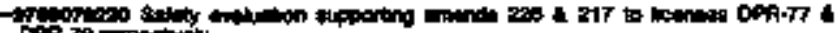

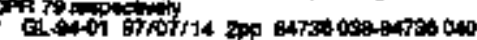

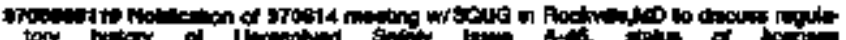

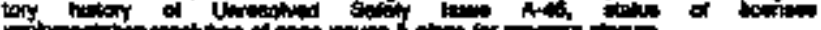

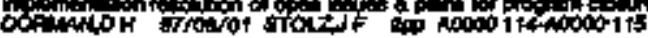

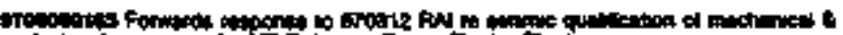

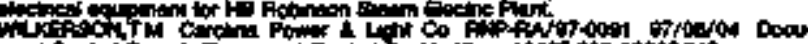

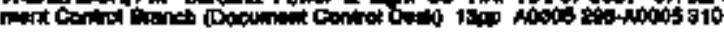

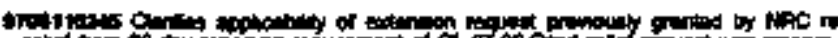

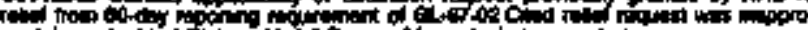

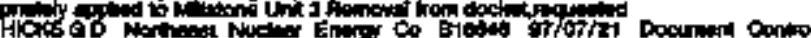

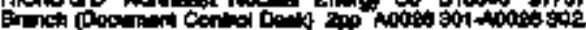

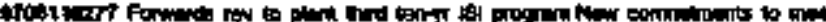

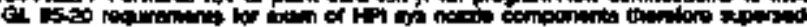

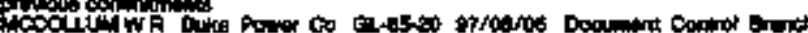

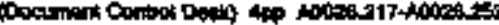

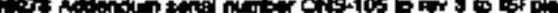

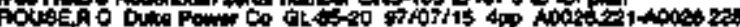




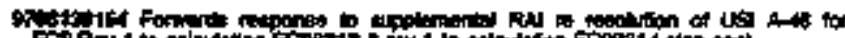

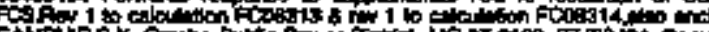

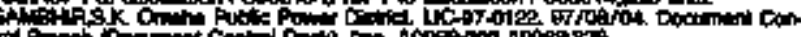

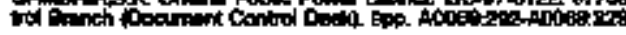

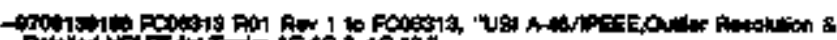

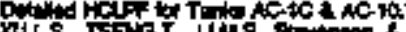

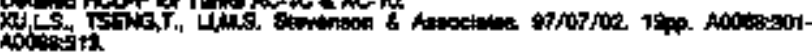

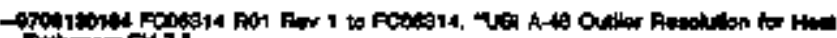
Oبll tot.

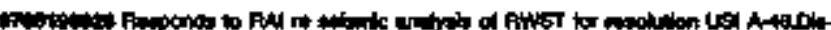

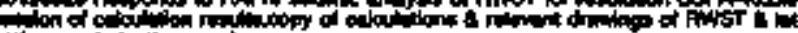

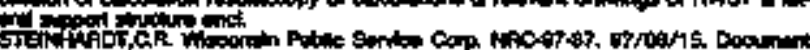

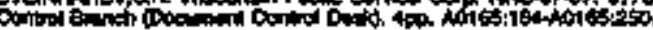

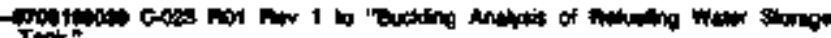

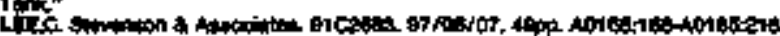

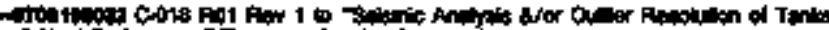

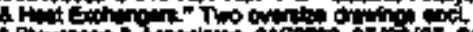

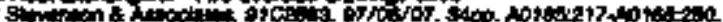

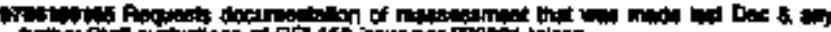

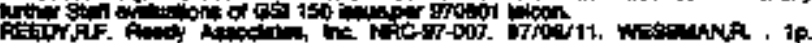
Mitos 31 ingtoses

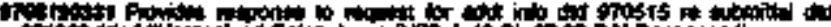

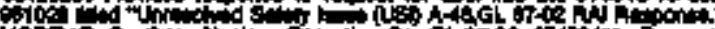

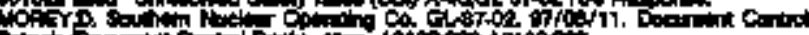

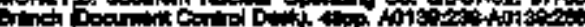

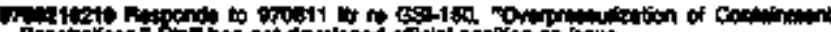

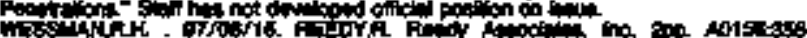
A01030.7.

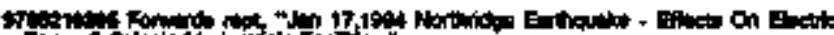
Por

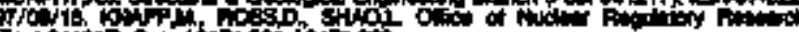

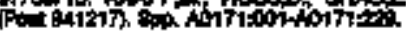

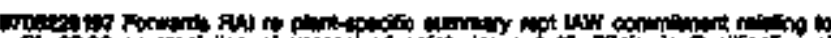

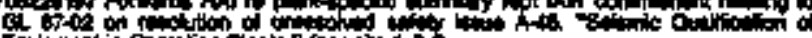

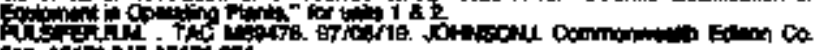

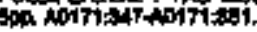

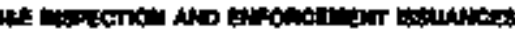

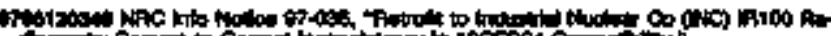

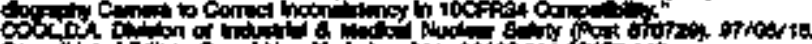

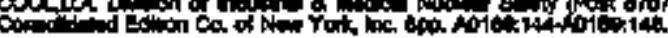

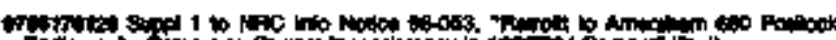

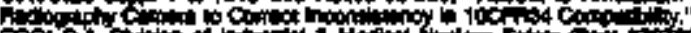
cootox ow of in

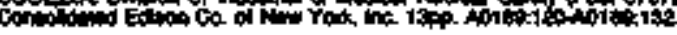

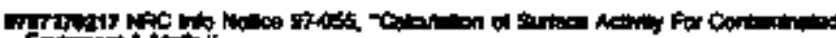

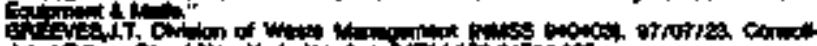

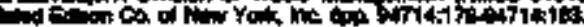

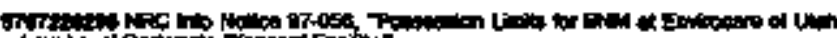

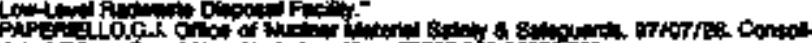

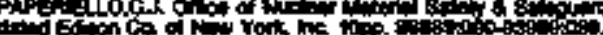

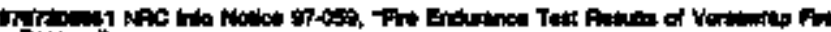

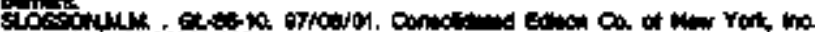

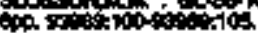

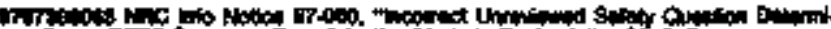

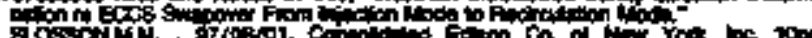
gosochin (1)

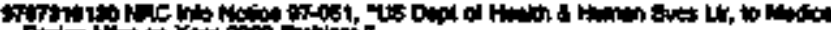

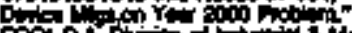

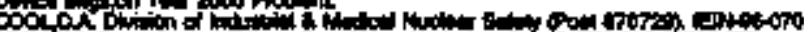

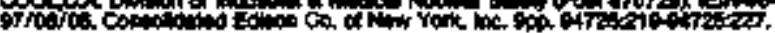

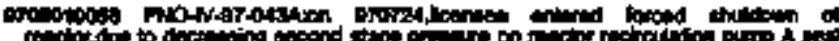

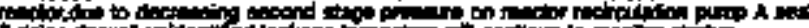

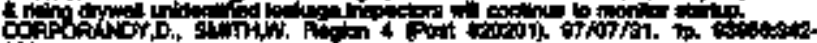
sistive

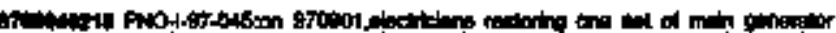

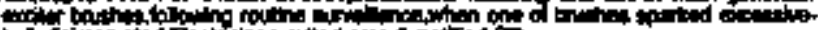

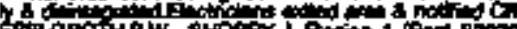

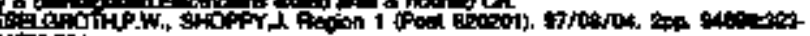
antiotiot.

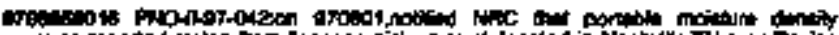

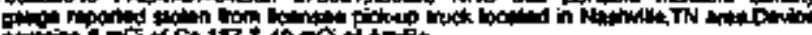

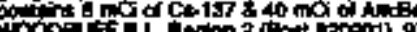

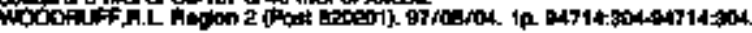

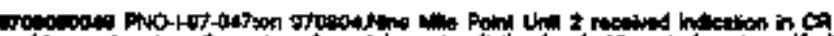

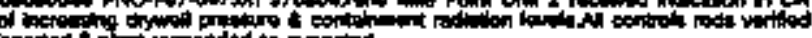

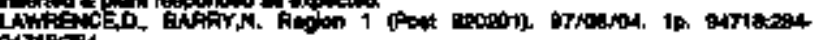

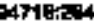

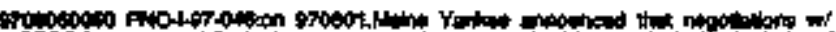

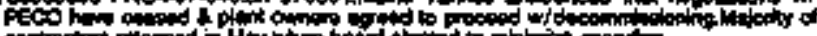

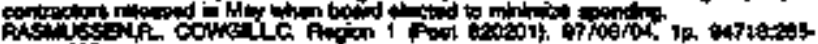
Dipition:

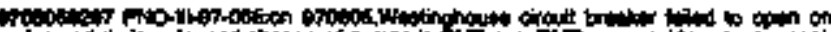

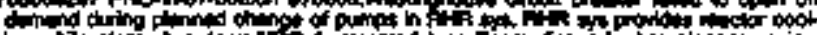

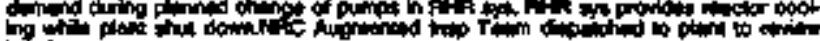

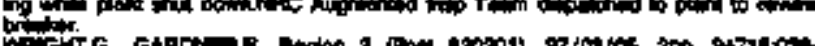
o4tistios.

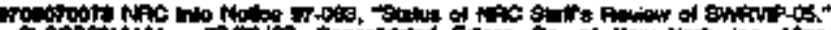

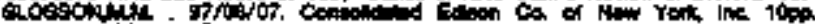

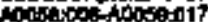

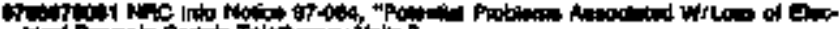

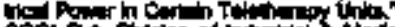

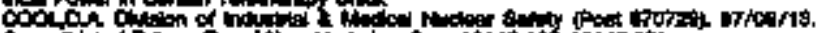

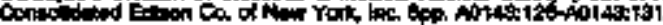

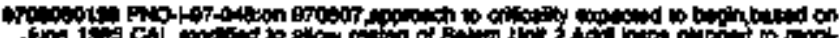

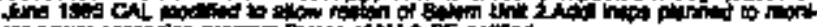

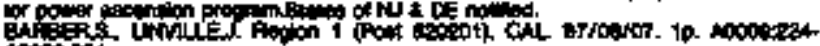
antoing

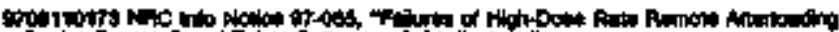

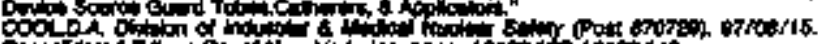

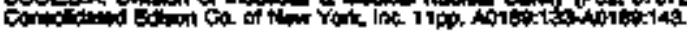

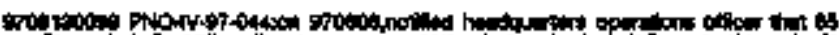

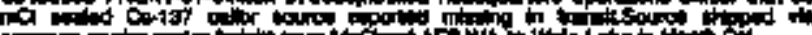

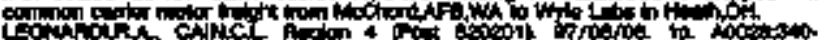

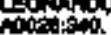

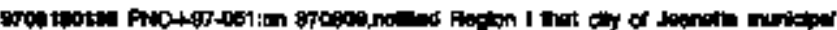

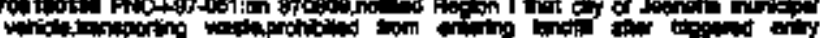

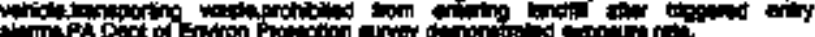

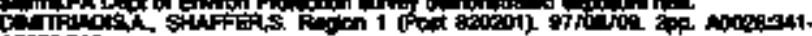
Anozesiat

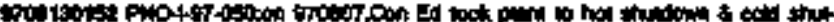

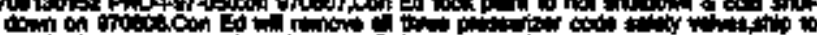

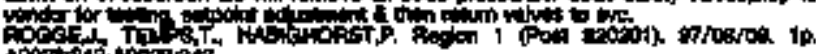

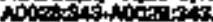

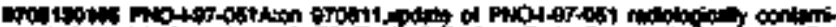

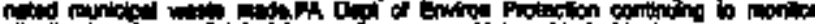
Alin.

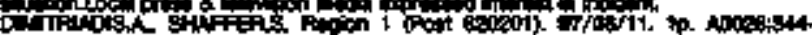
Anowistis

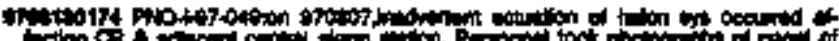

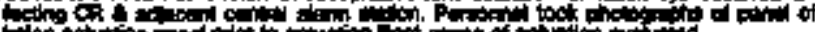

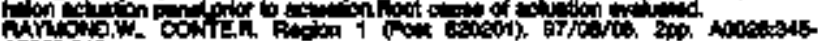

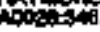

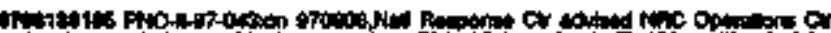

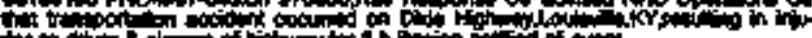

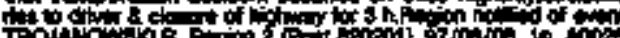

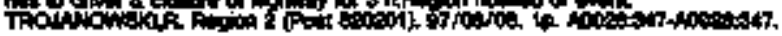

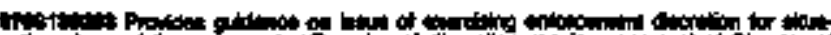

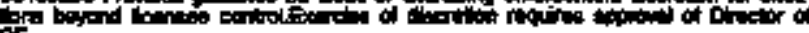
Uf

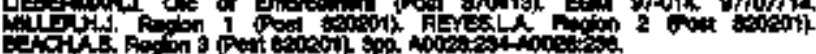

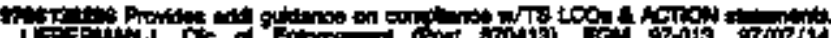

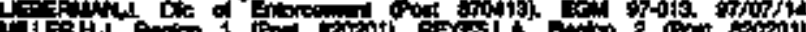

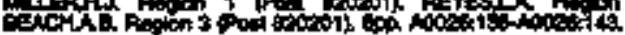

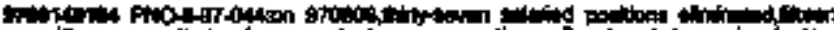

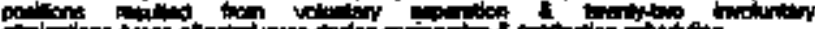

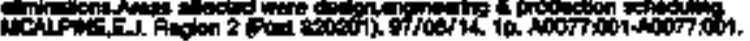

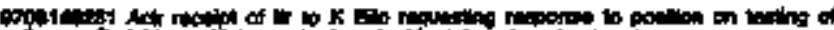

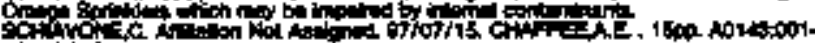
Actistis.

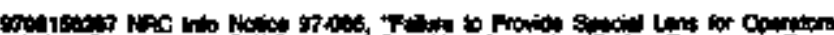

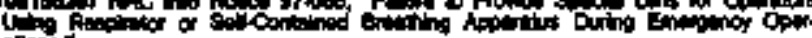

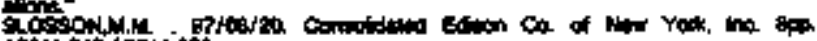

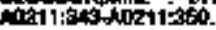




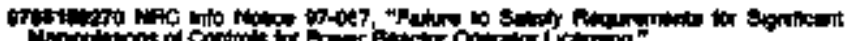

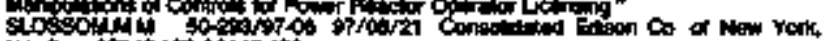

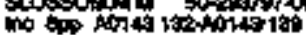

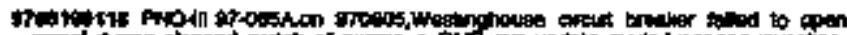

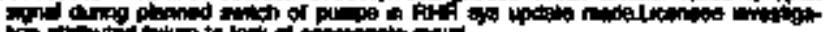

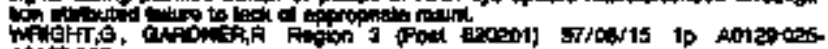
AOH:

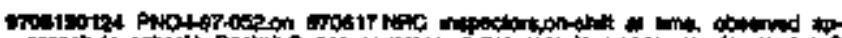

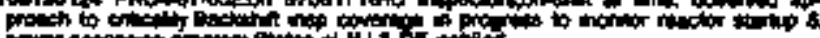

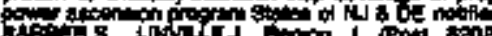

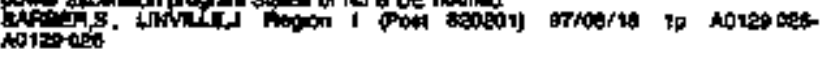

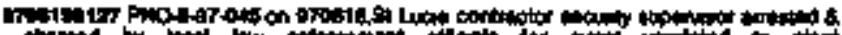

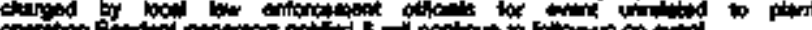

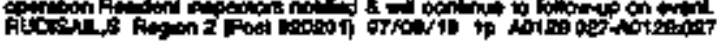

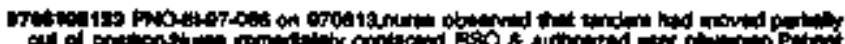

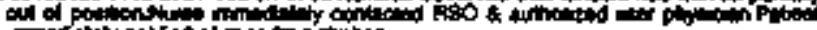

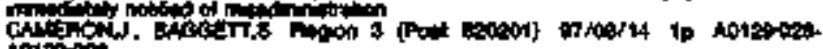
intor 0

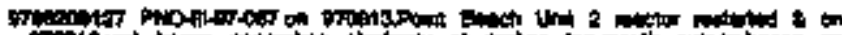

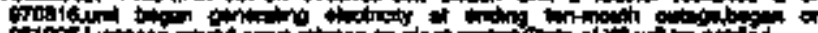

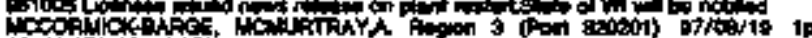

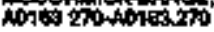

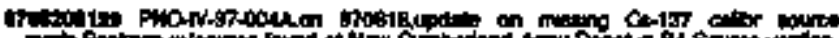

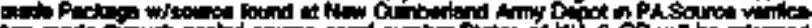

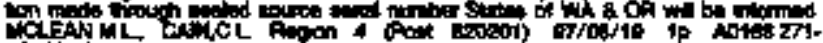

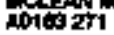

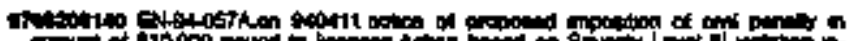

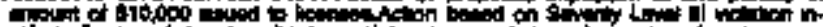

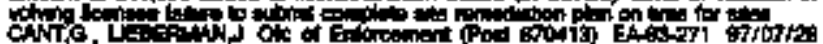

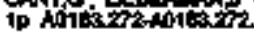

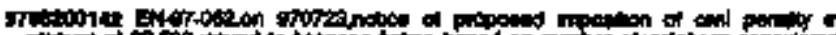

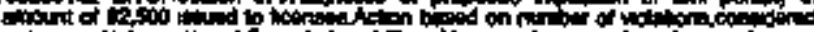
Dein ficot

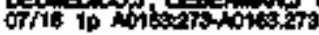

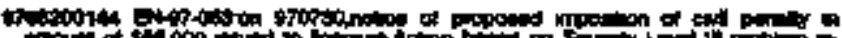

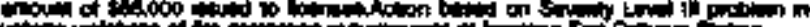

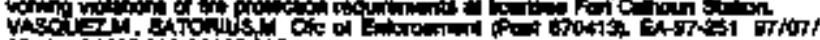
a ip Aols:

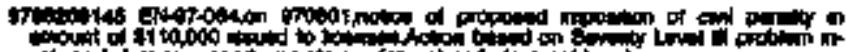

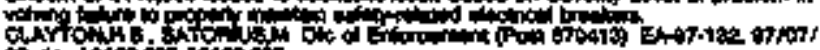
Th ip At

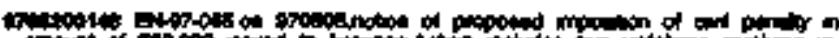

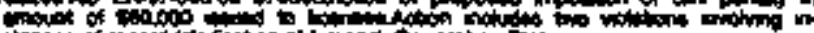

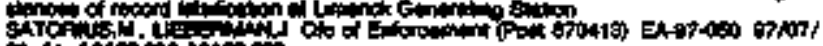
S1 ip tis

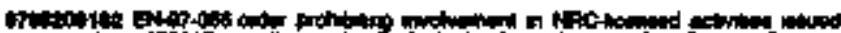

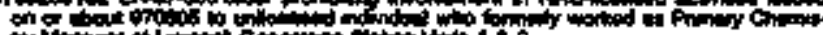

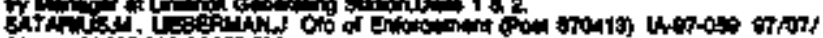

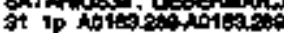

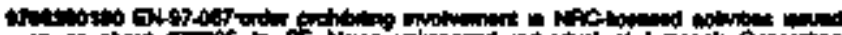

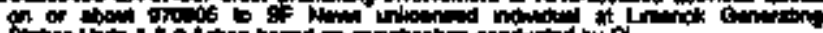

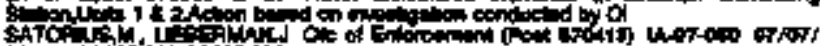

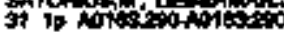

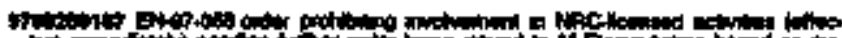

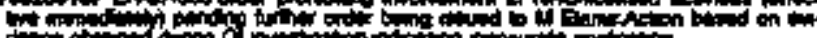

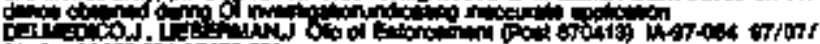
J1 10 1016.

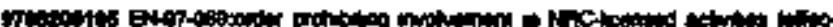

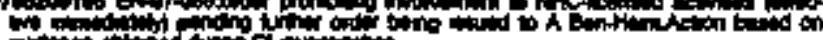
DAt Gi 19 A01,

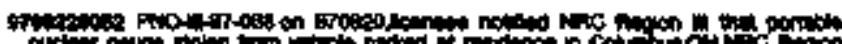

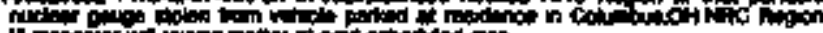
PATike A0tot 1s:

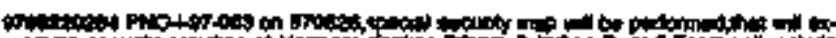

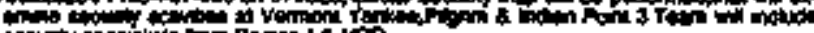

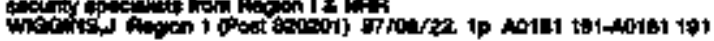

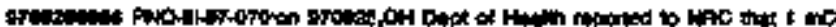

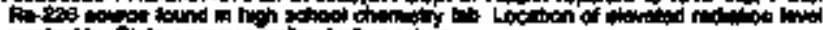

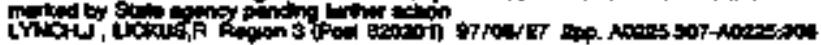

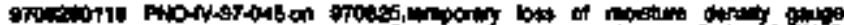

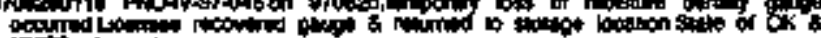

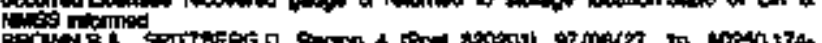
Monisis

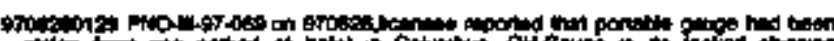

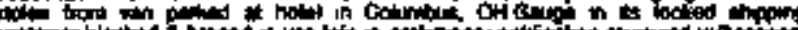

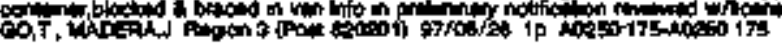

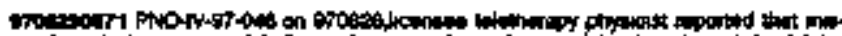

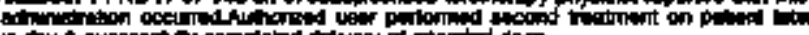

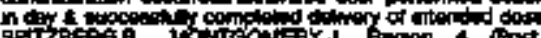
M0250 100

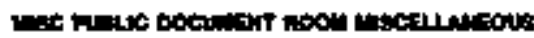

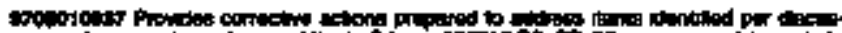

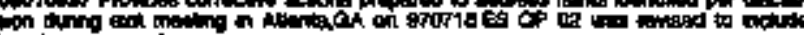

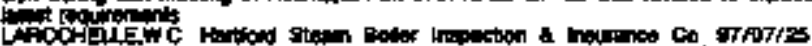

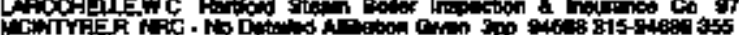

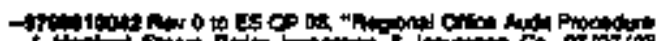

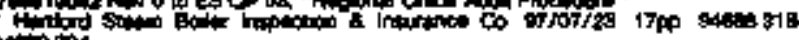

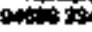

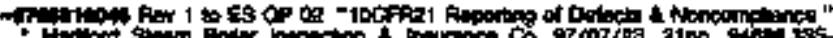

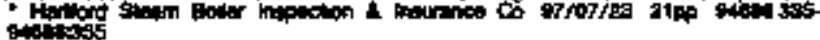

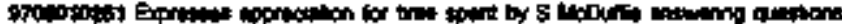

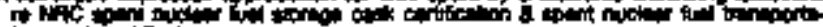
wath

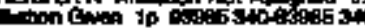

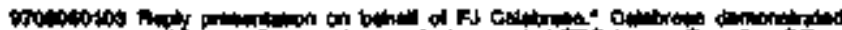

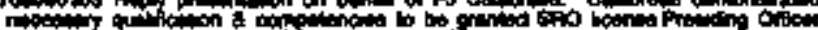

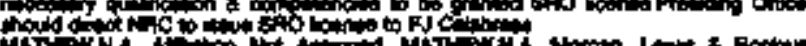

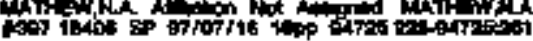

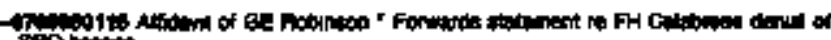

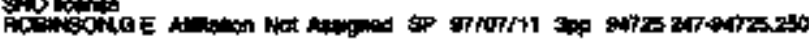

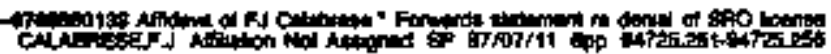

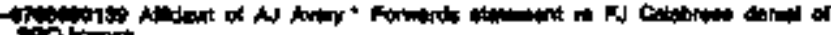

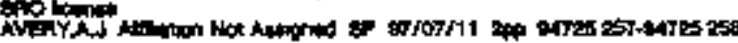

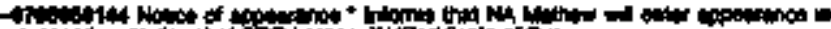

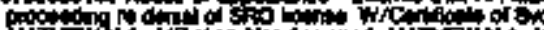

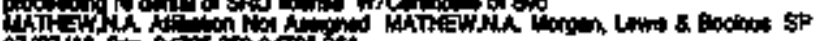

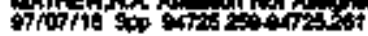

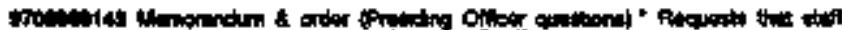

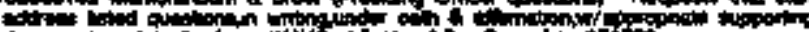

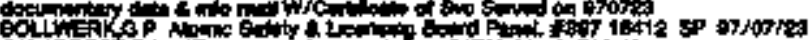

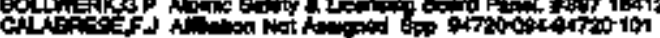

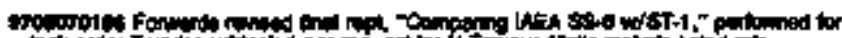

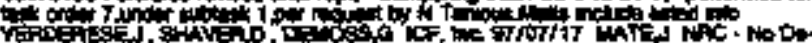

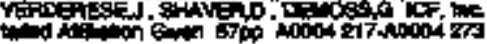

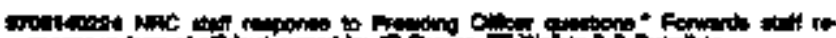

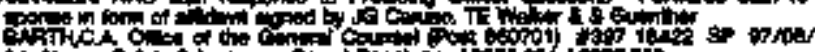

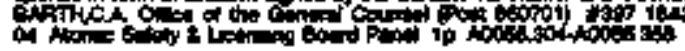

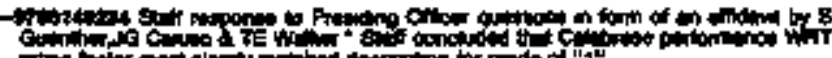

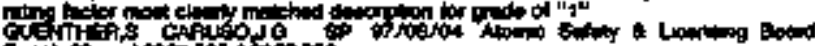

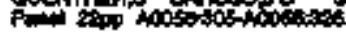

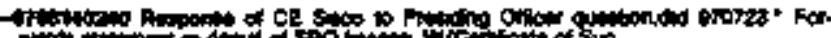

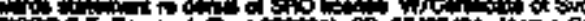
Gate

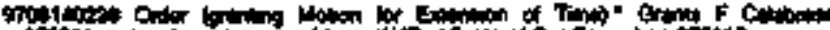

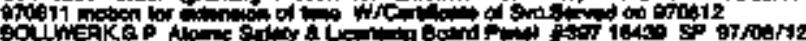

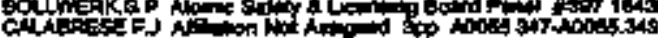

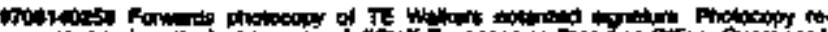

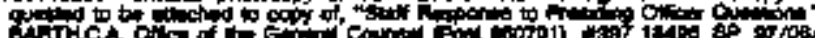

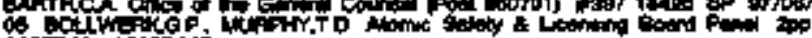
6. 


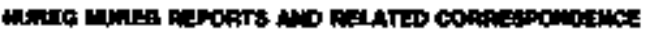

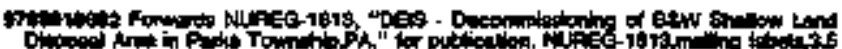

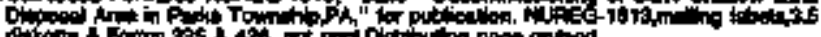

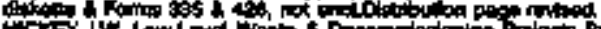

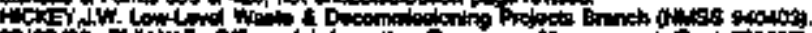
9710

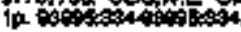

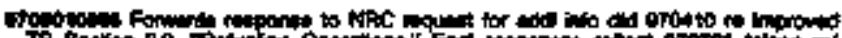

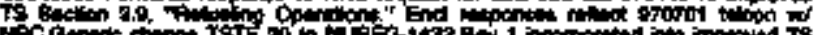
in.

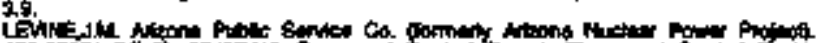

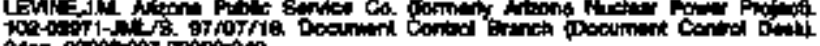

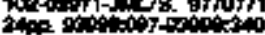

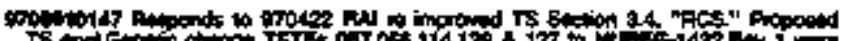
TS and la

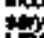

Ui 620 \%

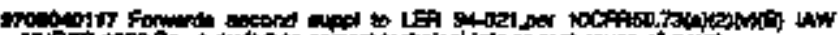

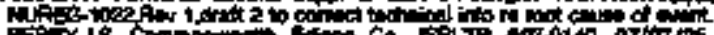

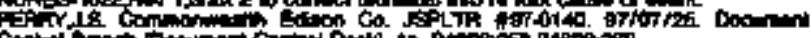

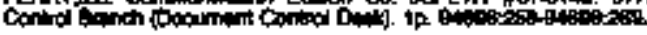

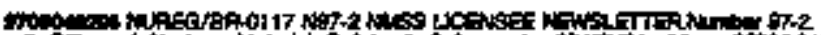

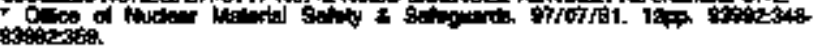

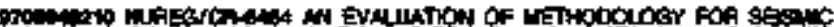

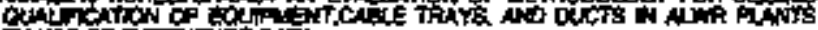
or to of ox in

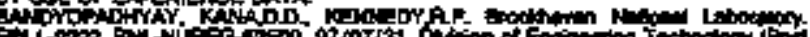

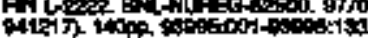

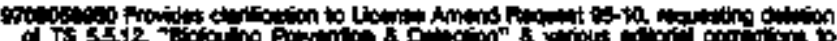

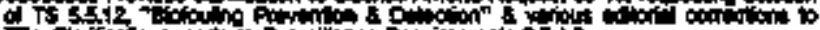

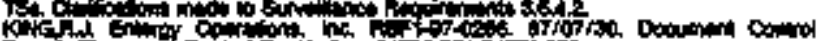

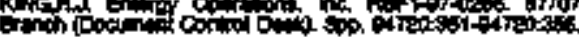

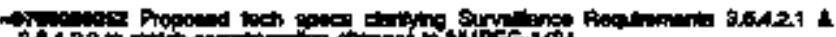

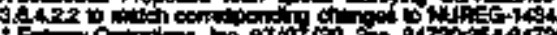

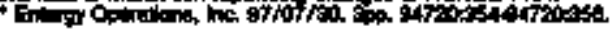

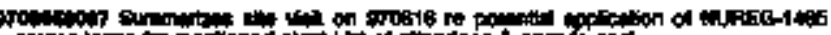

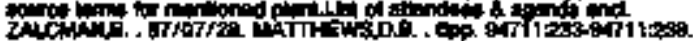

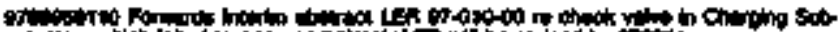

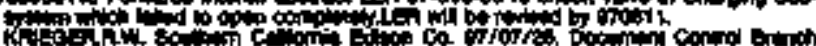

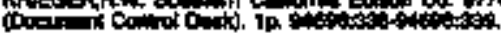

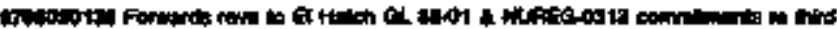
$10 \%$ W

Wint in

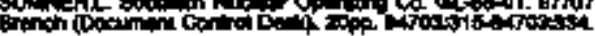

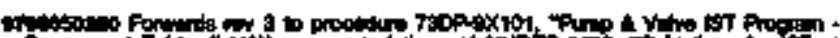

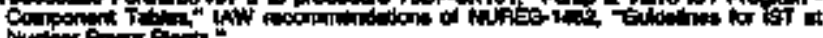

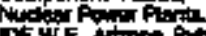

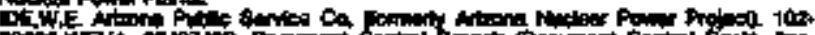

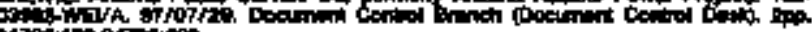

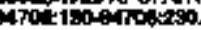

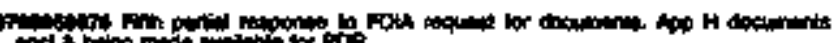

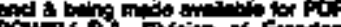

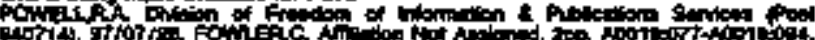

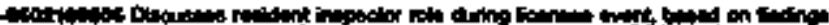

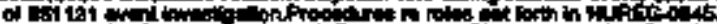

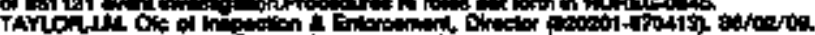

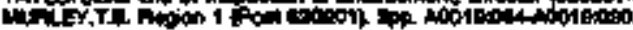

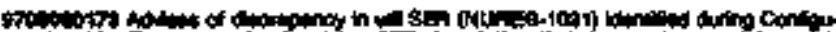

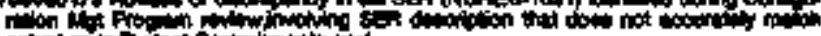

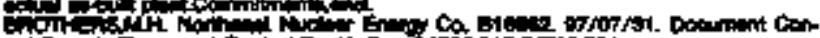

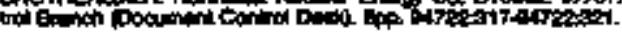

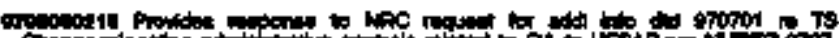
In

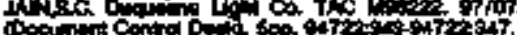

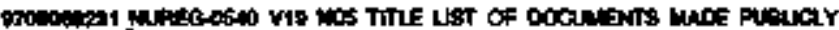

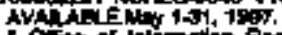

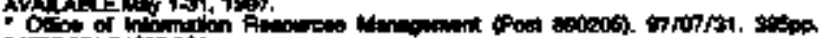

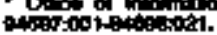

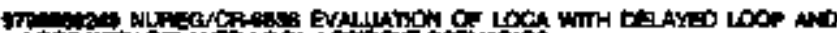

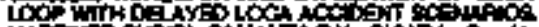

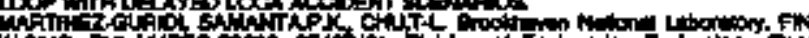

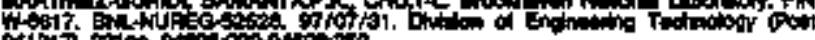

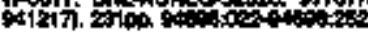

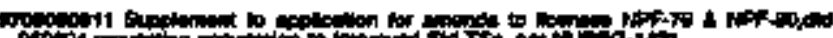

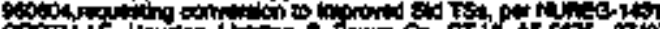

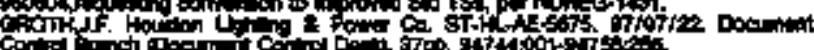

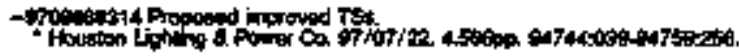

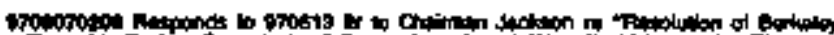

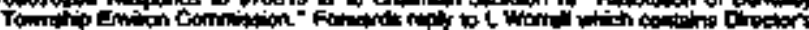
10CFine ono.

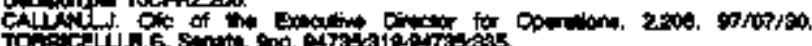

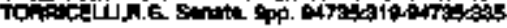

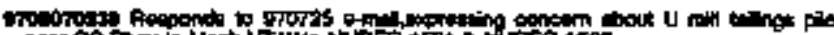

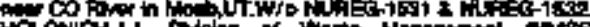

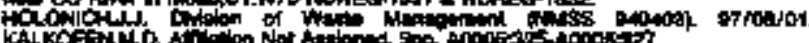

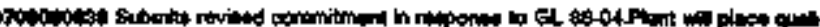

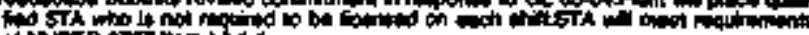

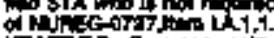

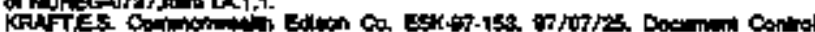

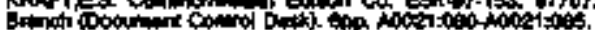

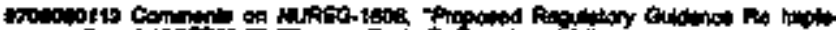

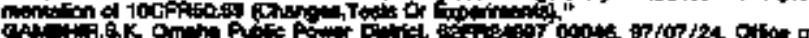

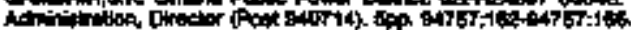

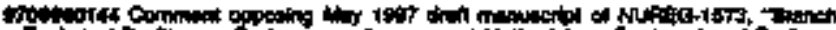

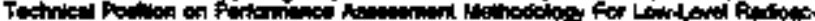

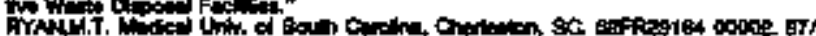

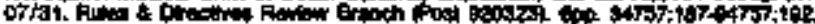

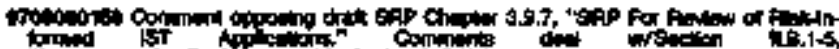

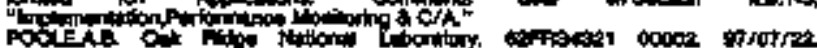

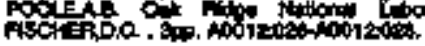

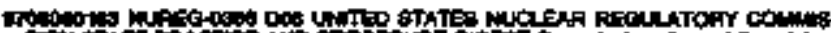

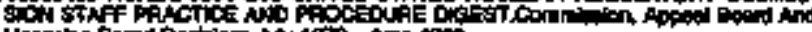

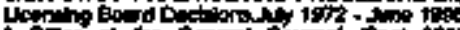

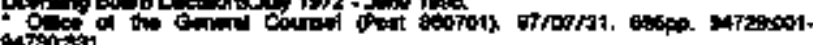
Arsotisy.

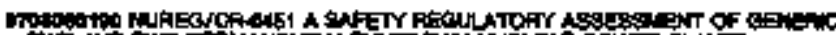

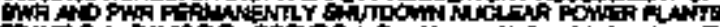

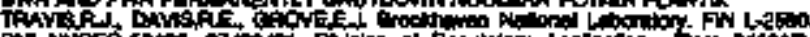

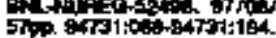

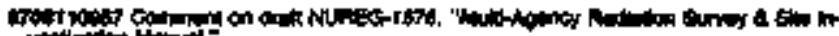

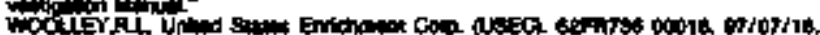

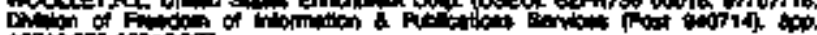

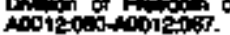

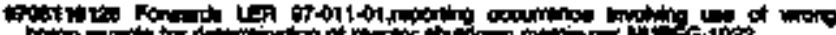

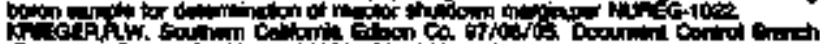

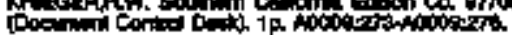

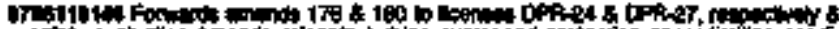

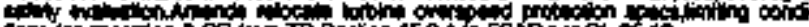

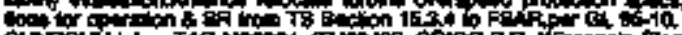

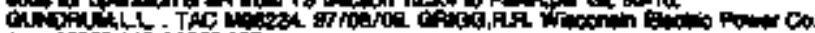

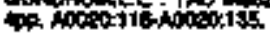

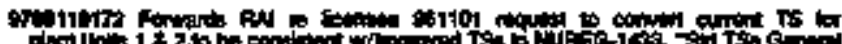

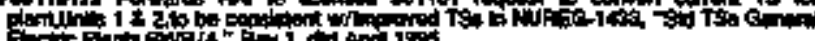

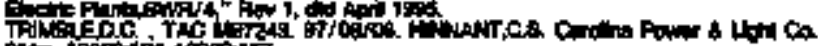
210

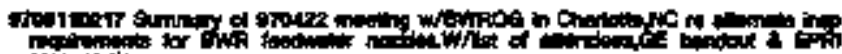

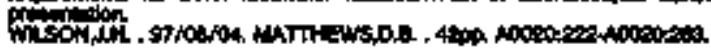

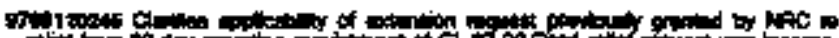

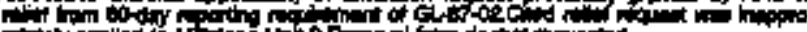

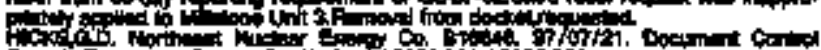

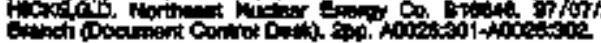

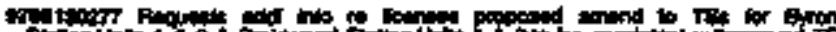

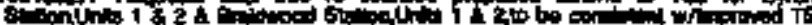
At A

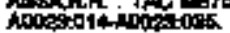

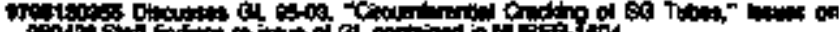

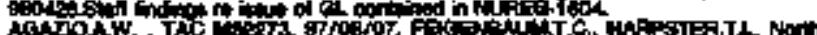

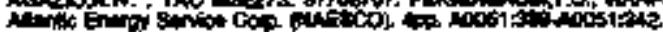

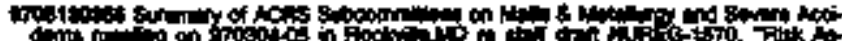

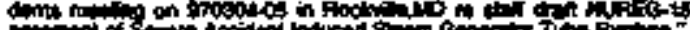

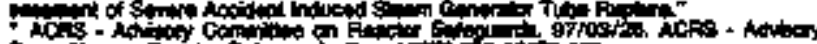

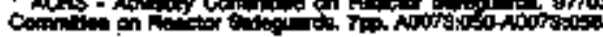

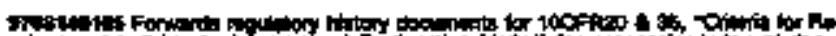

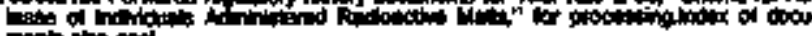
sefer

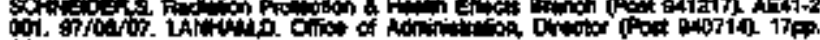

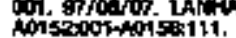




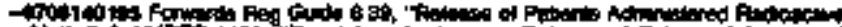

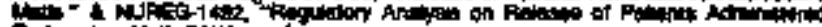

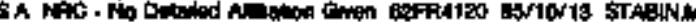

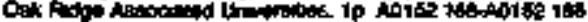

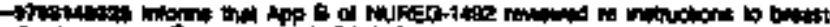

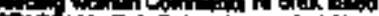

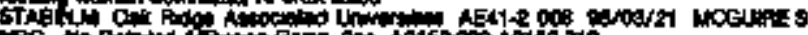

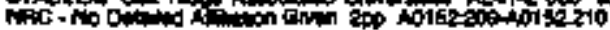

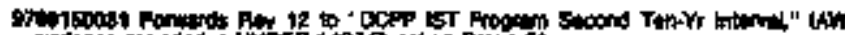

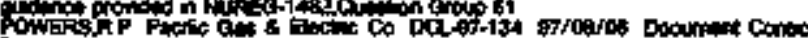

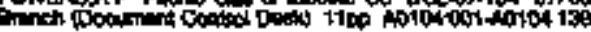

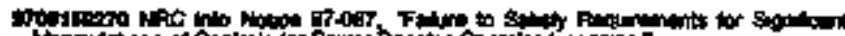

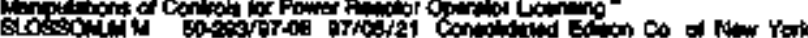

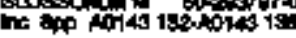

Fob

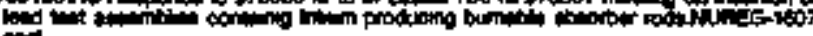

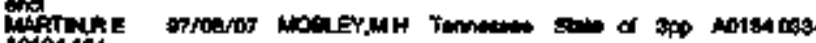
notis 104

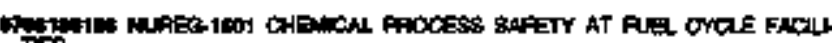

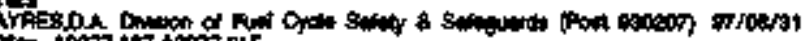

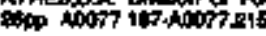

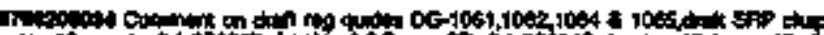

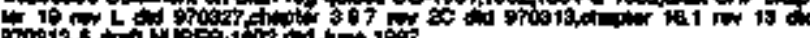

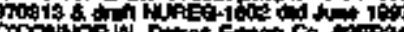

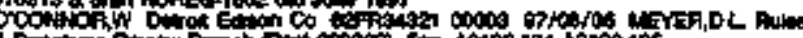

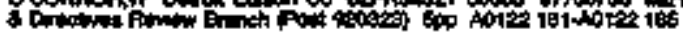

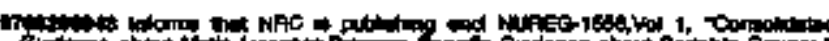

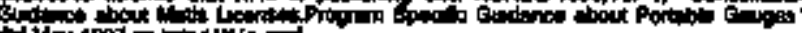

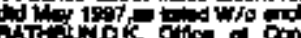

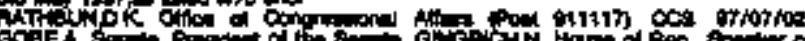

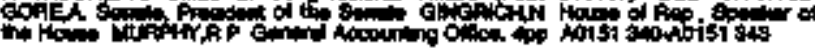

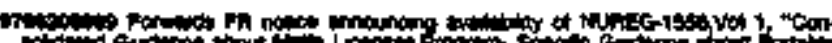

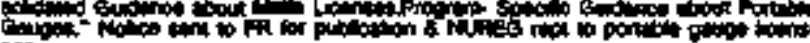

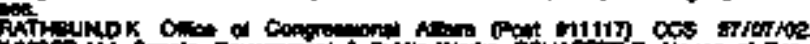

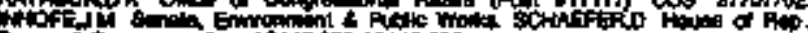

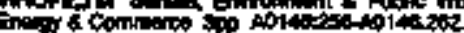

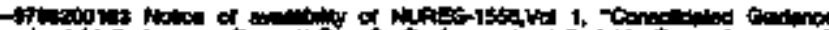

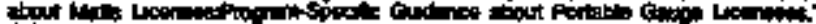
dition

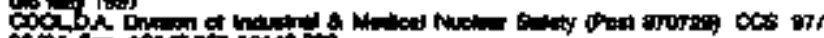

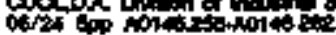

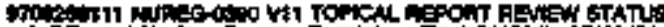

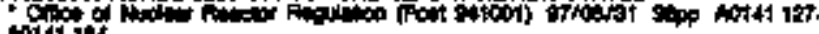
mist 10

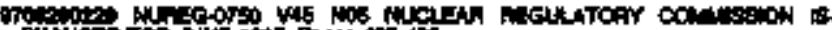

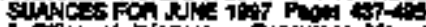

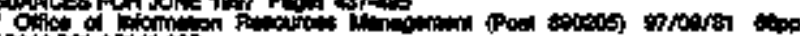

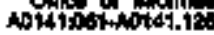

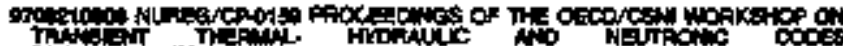

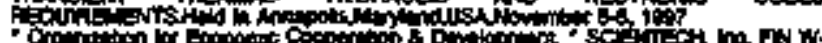

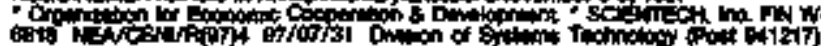

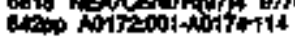

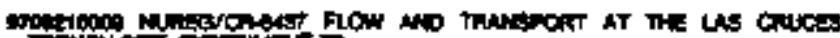

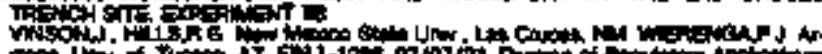

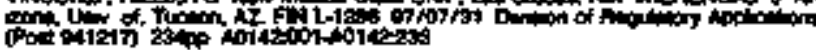

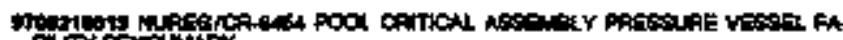

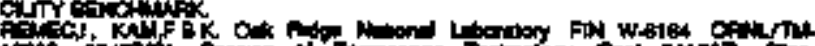

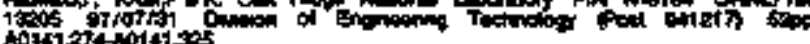

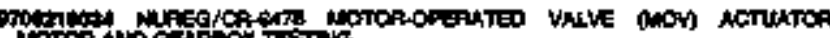

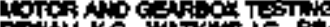

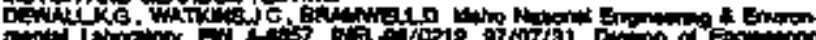

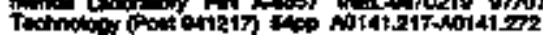

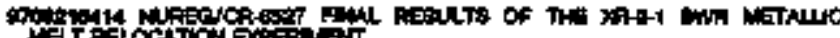

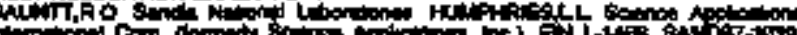

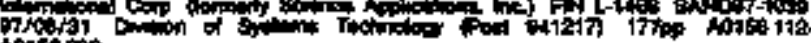
intion

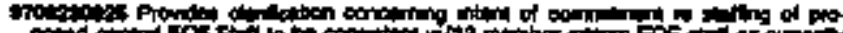

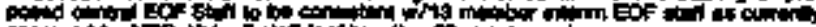

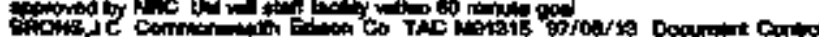

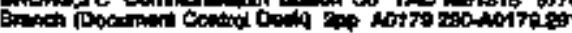

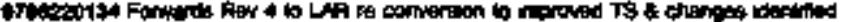

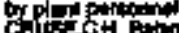

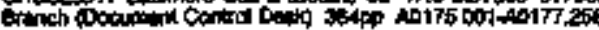

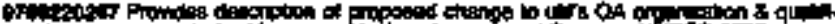

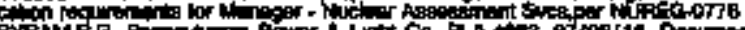

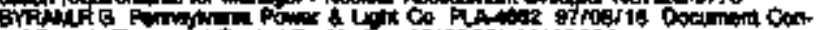

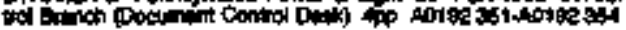

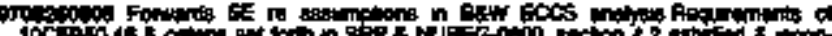

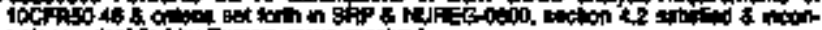

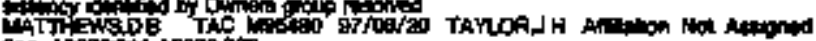

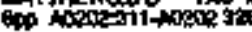

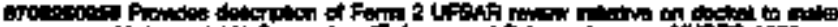

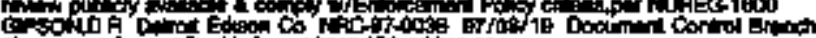

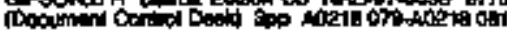

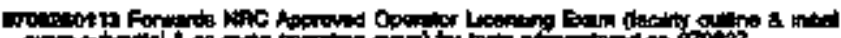

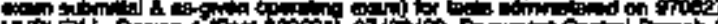

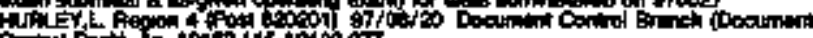

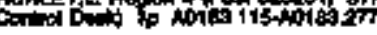

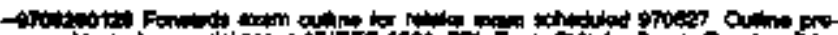

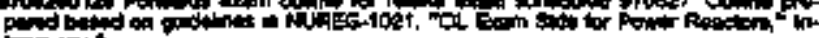

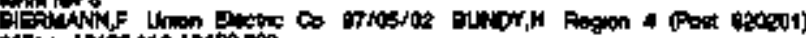

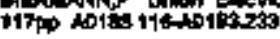

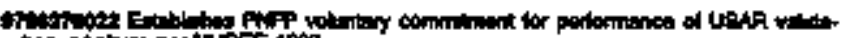

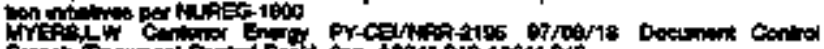

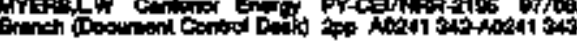

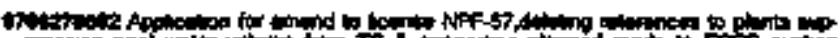

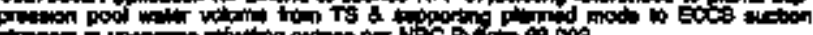

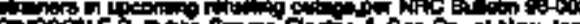

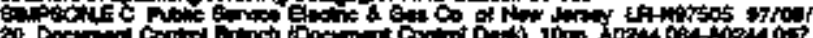

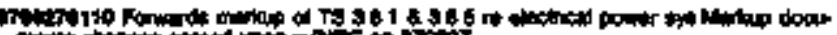

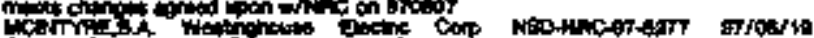

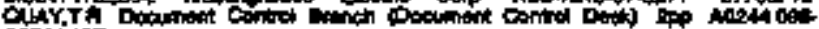
Nopis ior

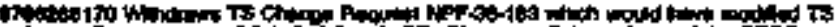

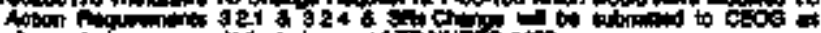

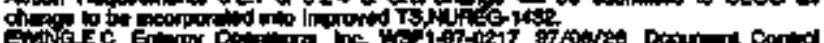

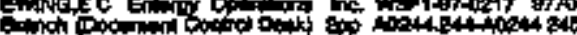

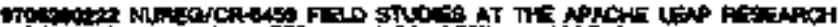
Gi ci

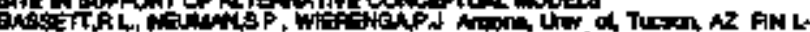

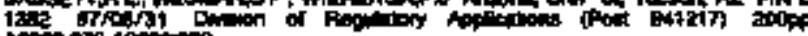

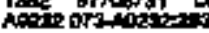

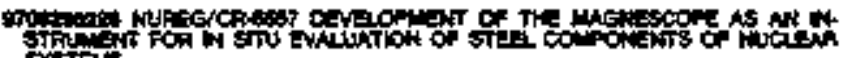

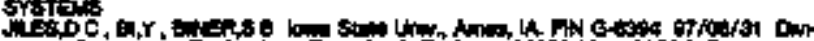

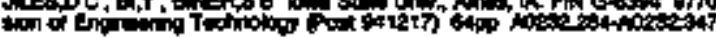

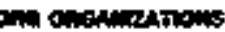

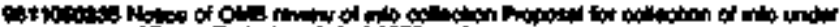

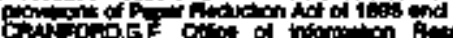

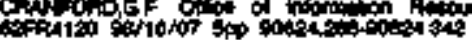

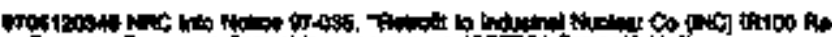

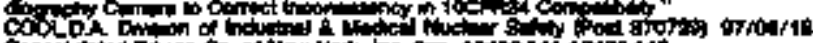

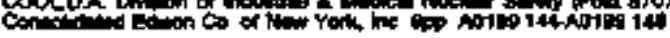

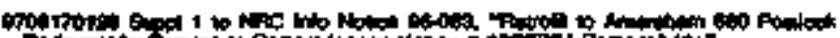

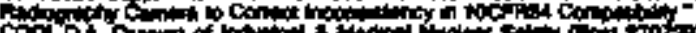

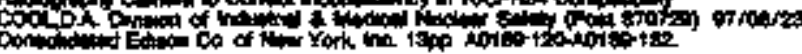

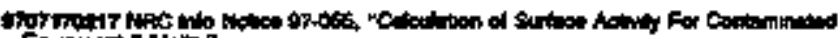

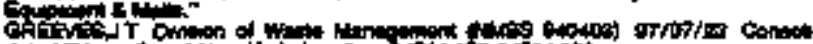

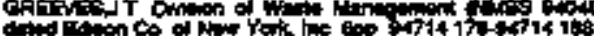

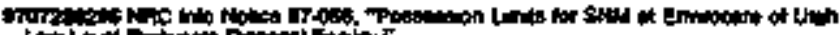

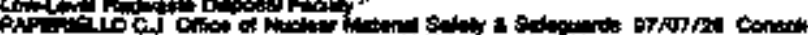

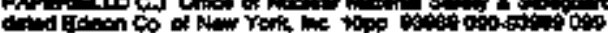

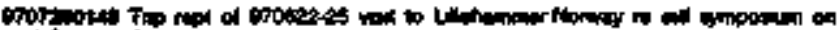

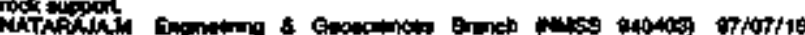
TAfl siation.

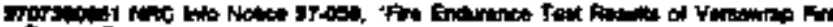

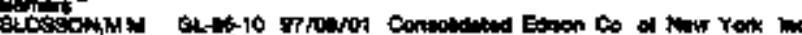

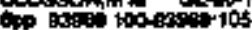




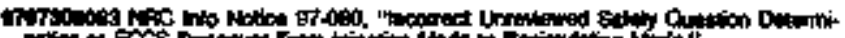

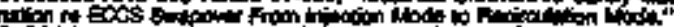

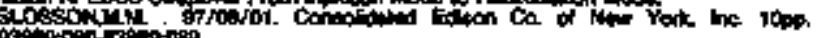
1036079

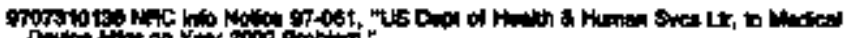
Co

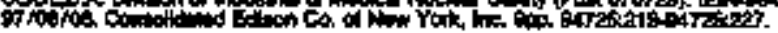

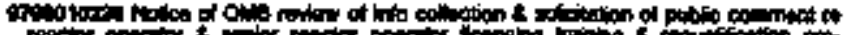

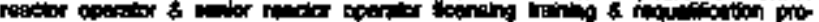
fi

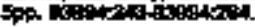

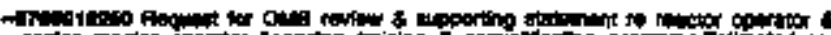

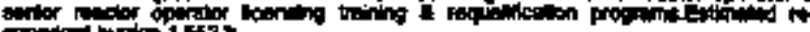

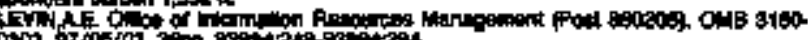

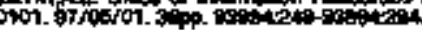

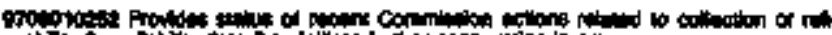

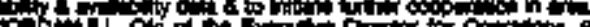

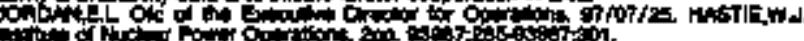

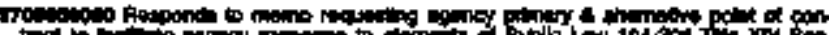

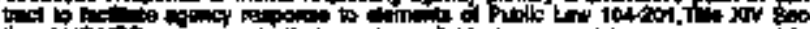

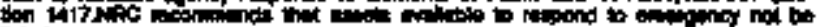
ROS

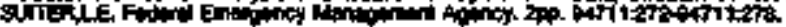

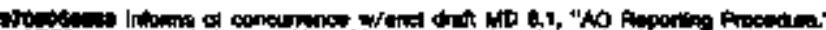

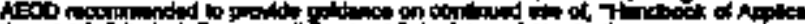

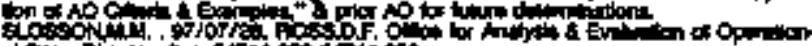

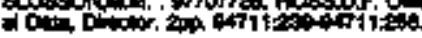

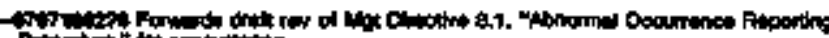

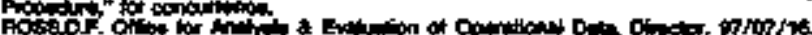

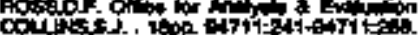

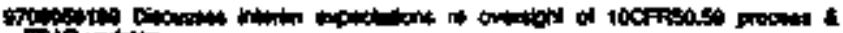

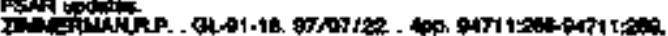

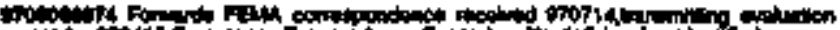

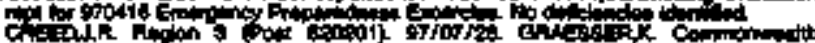

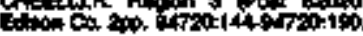

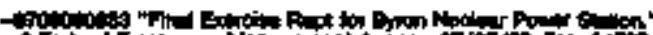

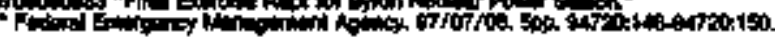

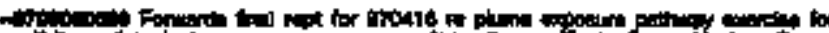

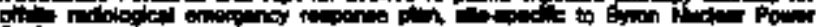

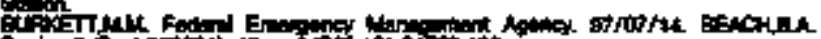

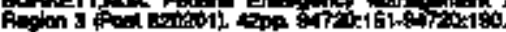

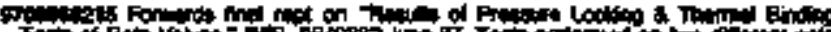

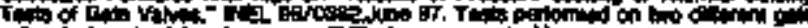

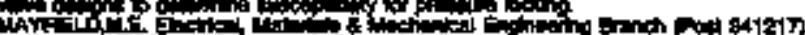

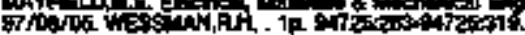

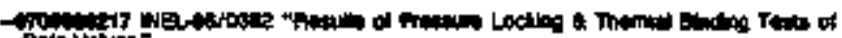

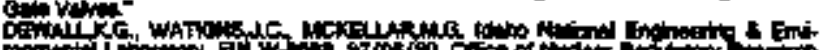

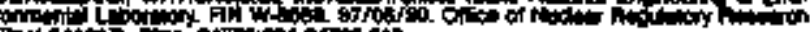

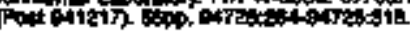

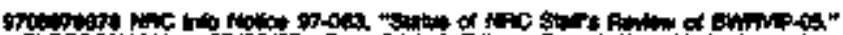
\$100\%

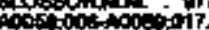

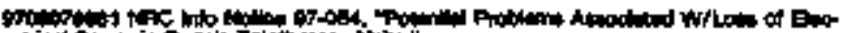

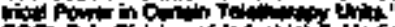

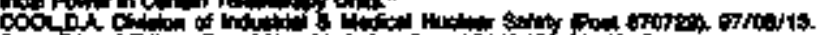

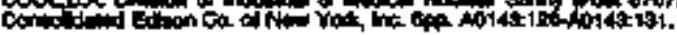

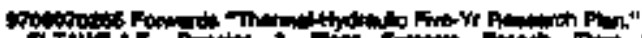

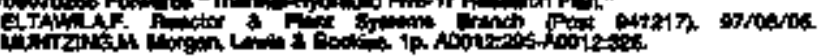

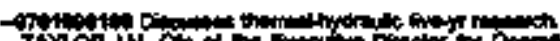

A r or

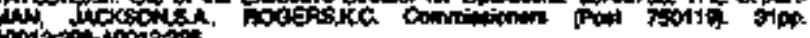
tron

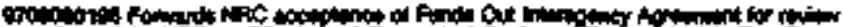

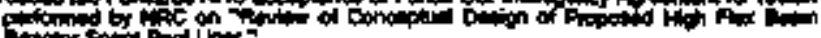

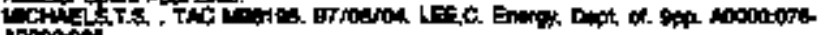
Anotoses.

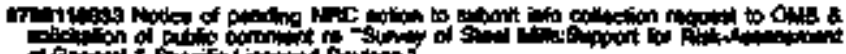

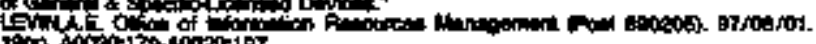

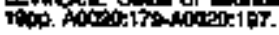

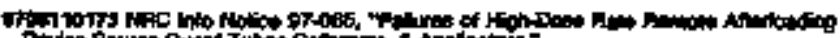

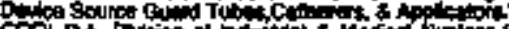

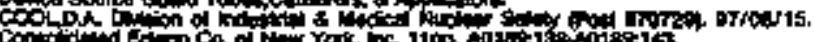

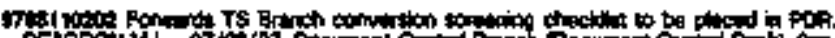

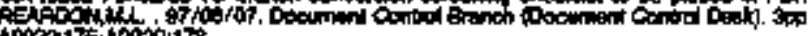
1000017640000,178

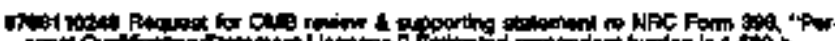

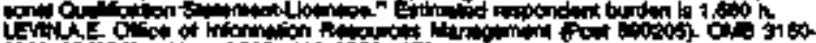

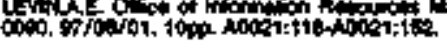

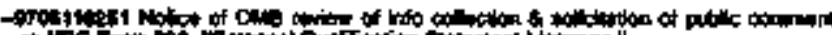

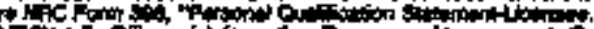

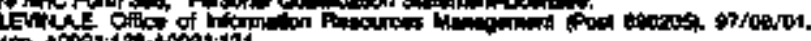

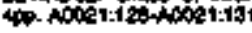

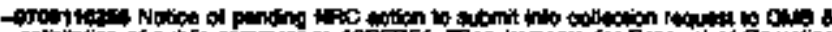

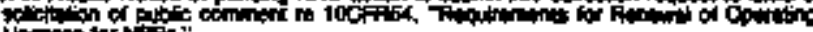

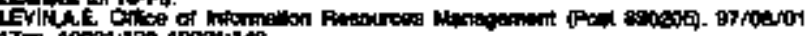
I700 A0021:19240021:14:

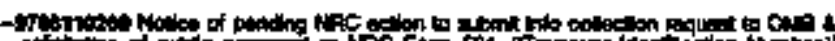

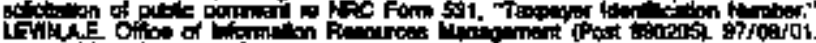

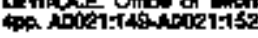

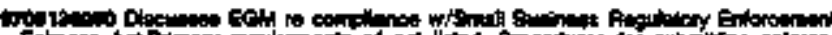

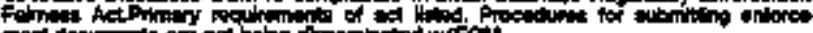

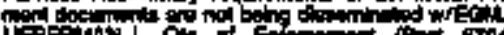

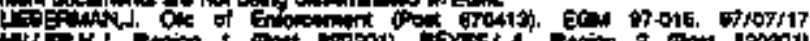

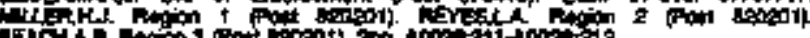

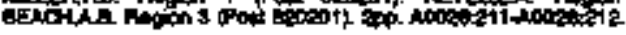

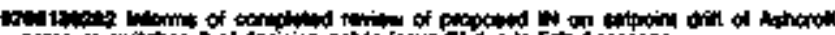

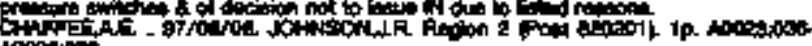
Anopias:

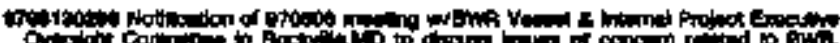

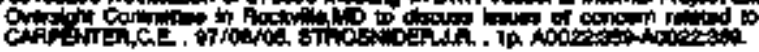

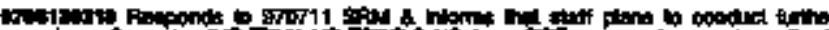

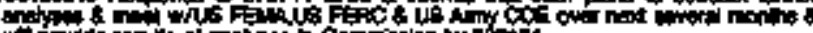

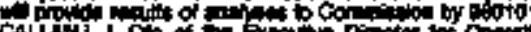

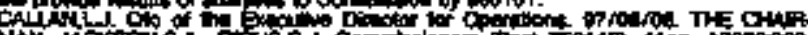

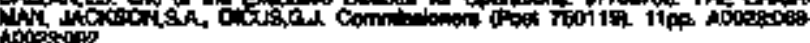
tonesson: 17.

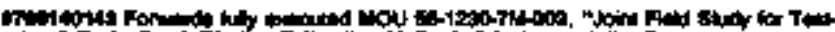

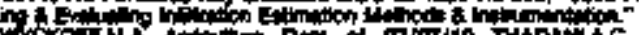

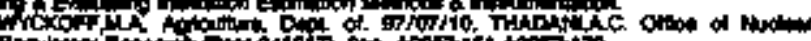

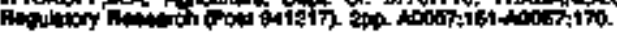

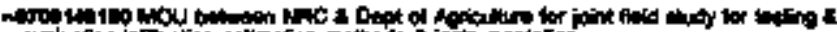

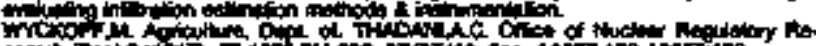

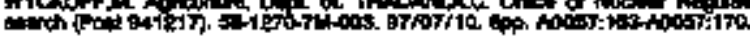

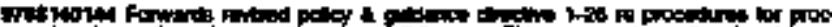

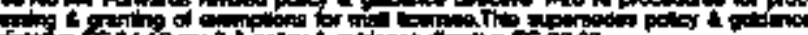

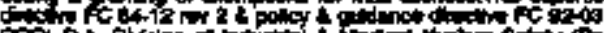

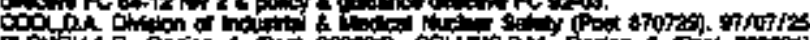

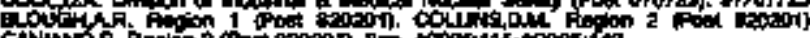

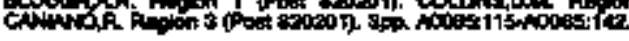

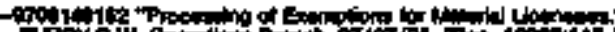

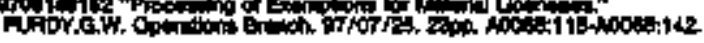

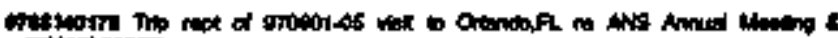

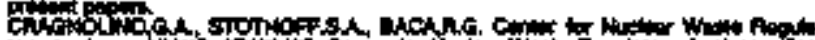

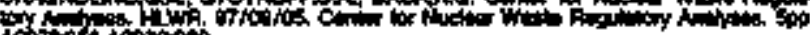

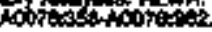

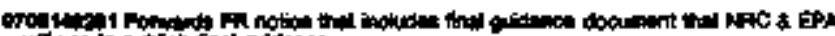

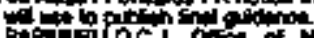

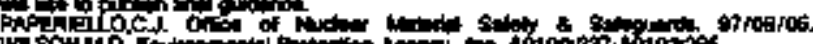

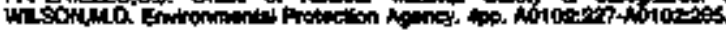

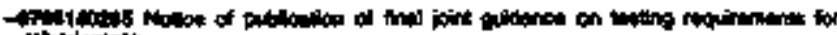

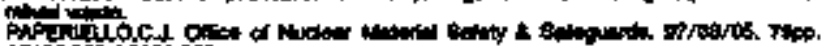

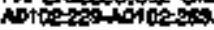

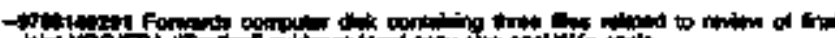
pill

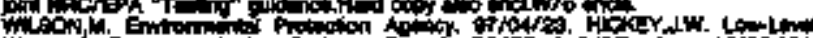

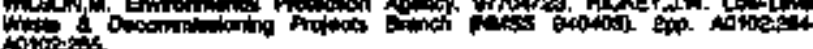

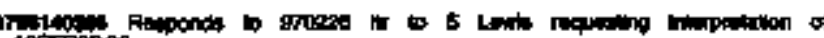

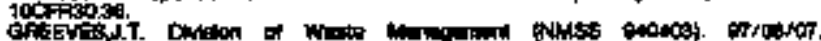

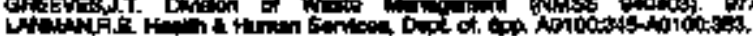

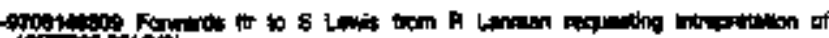

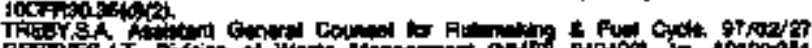
erpyos. 


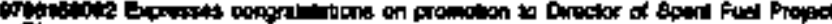

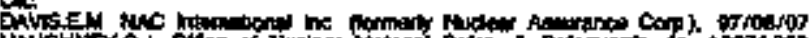

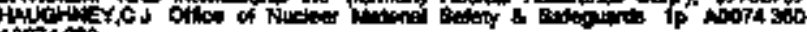
Anja 300

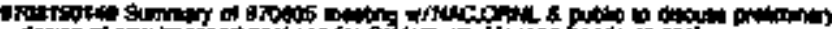

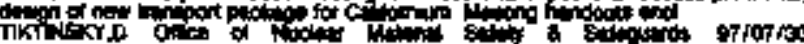

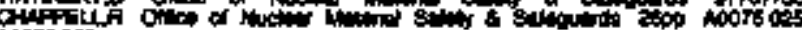
atomsoso

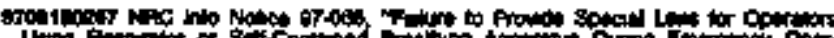

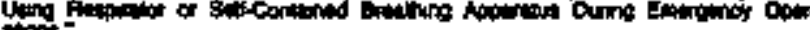

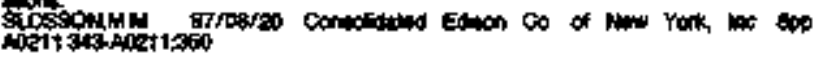

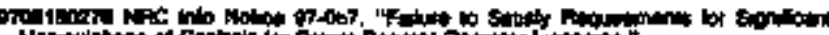

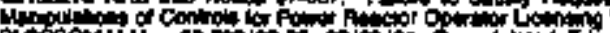

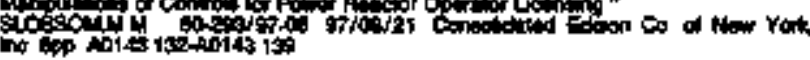

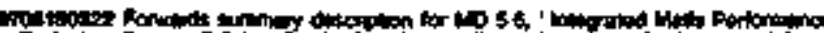

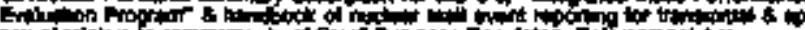

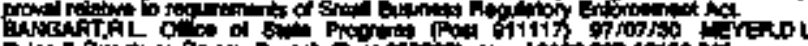

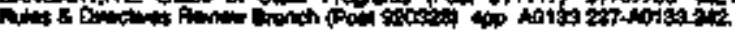

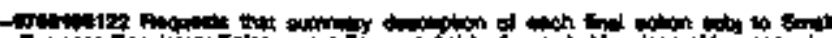

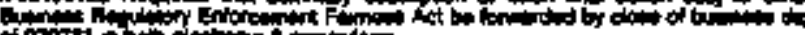

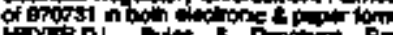

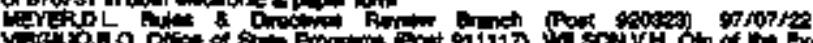

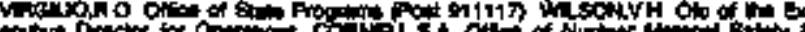

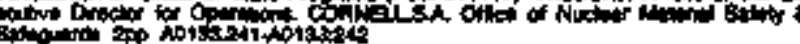

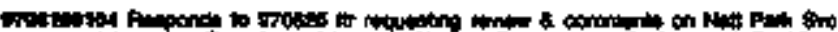

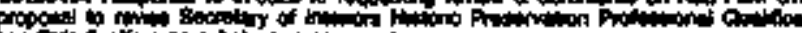

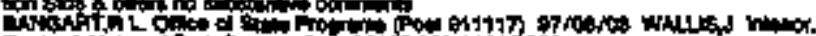

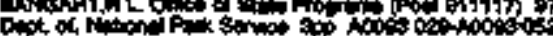

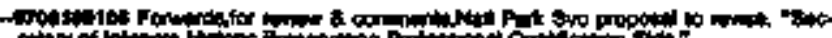

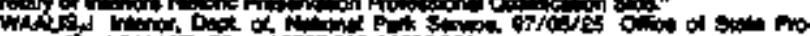

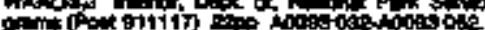

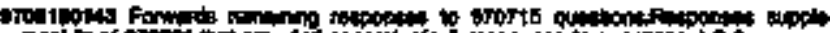

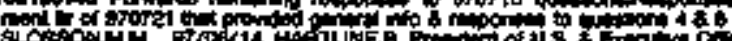
AO134347013450

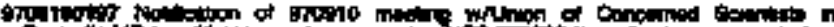

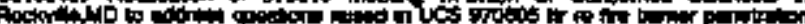

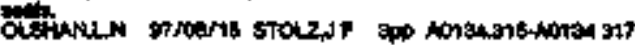

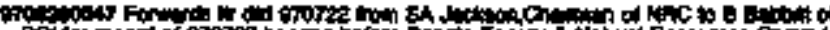

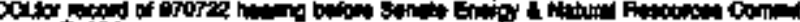
ton

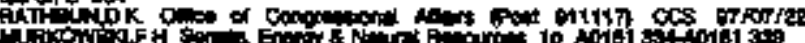

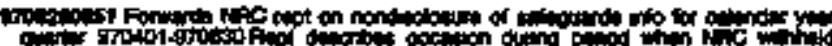

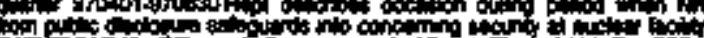

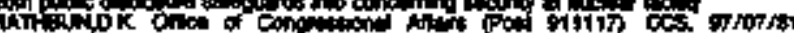
DH

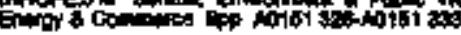

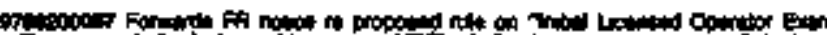

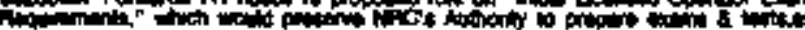

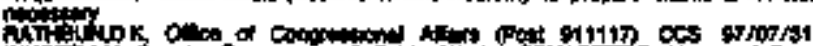

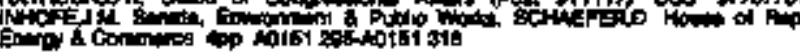

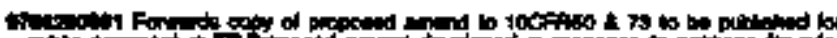

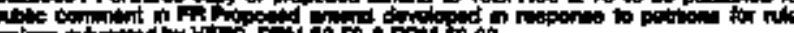

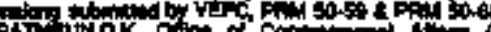

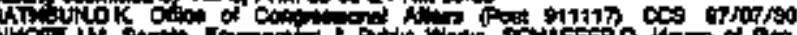

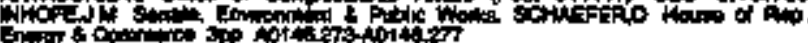

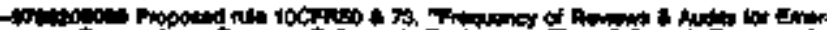

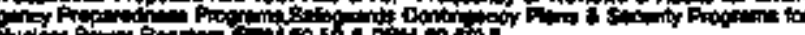

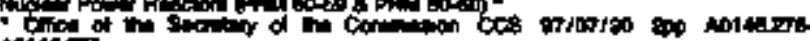
Aoition

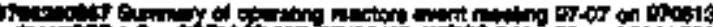

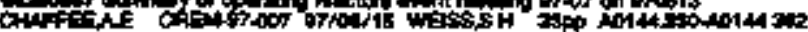

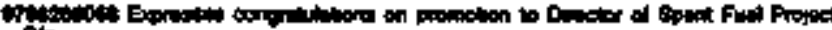

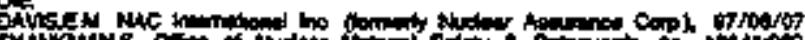

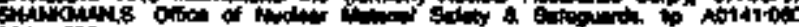
4atd1 o0d

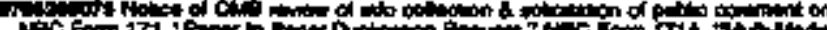

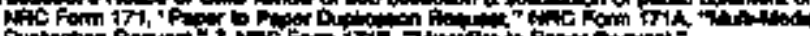

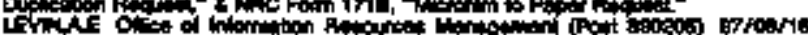
400 Not 48 300

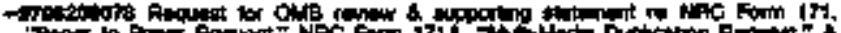

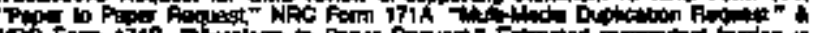

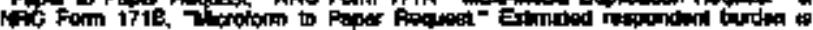

L

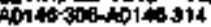

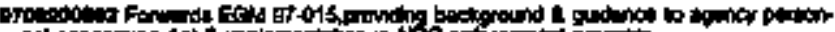

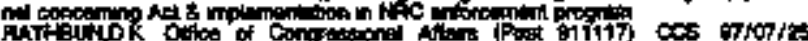
wanch

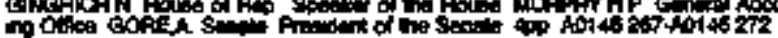

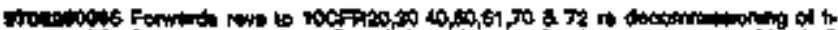

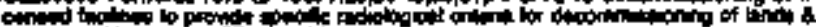

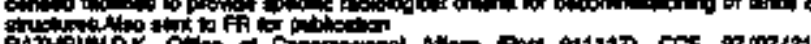

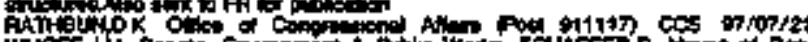

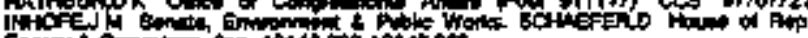

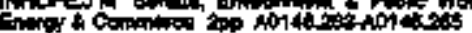

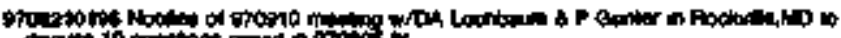

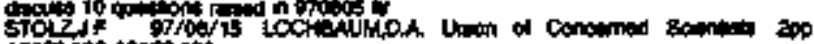

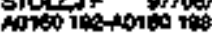

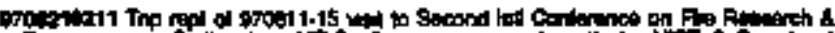

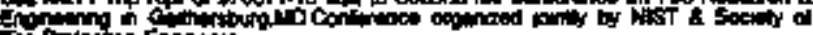

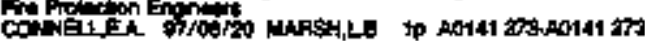

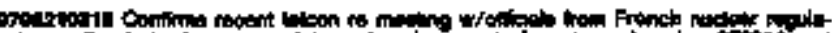

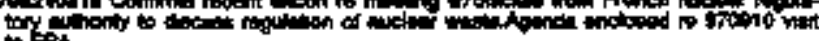

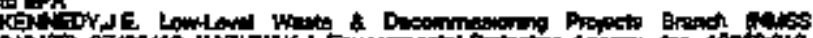

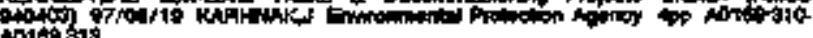

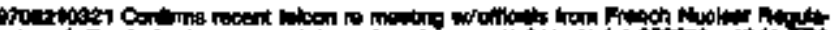

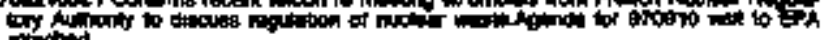

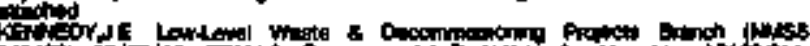

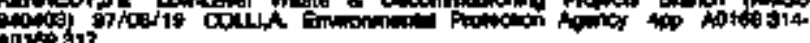

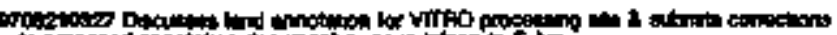

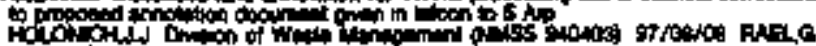

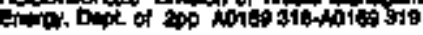

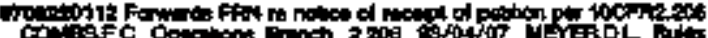

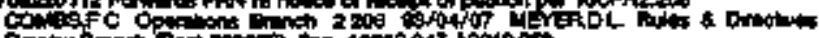

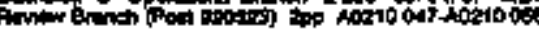

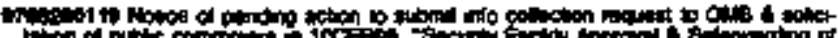

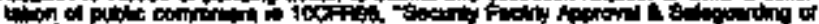

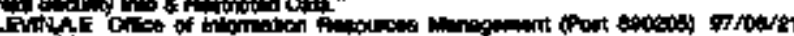

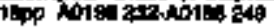

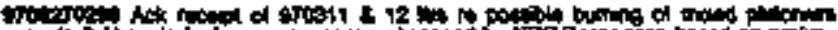

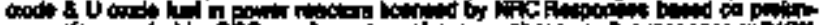

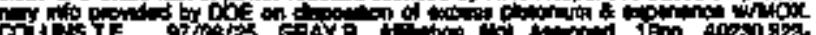
Atron 341

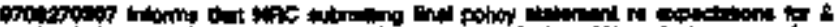

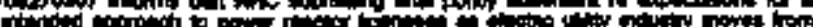

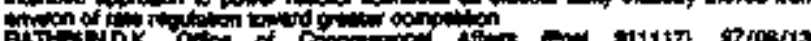

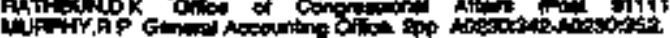

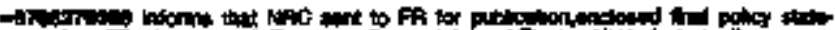

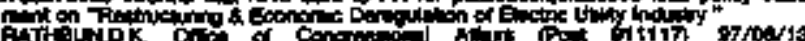

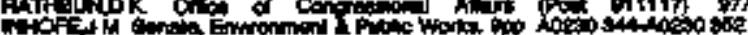

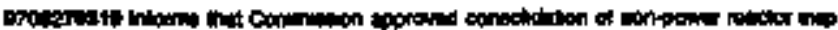

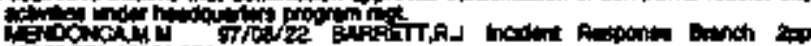

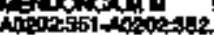

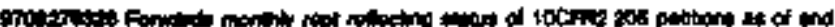

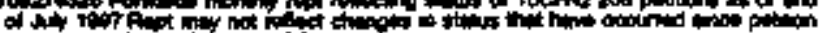

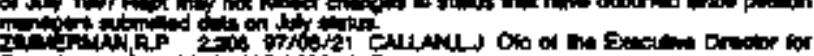
Cominion in

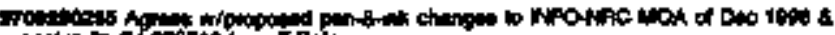

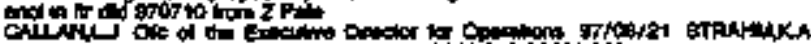

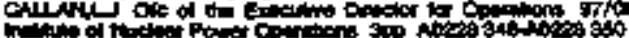

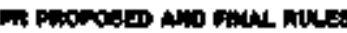

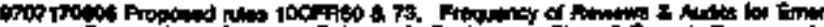

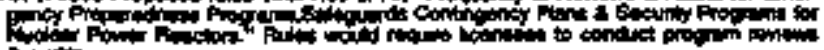

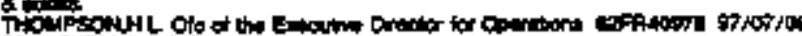

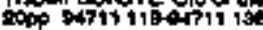




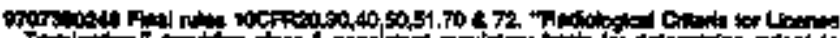

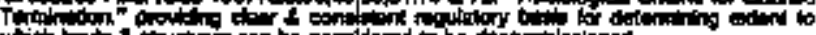

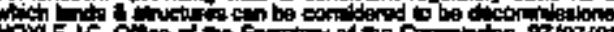

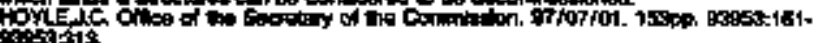

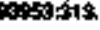

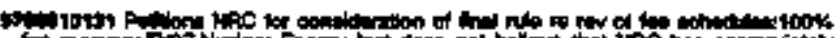

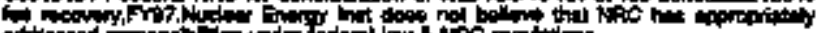

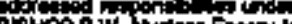

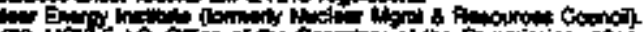
की

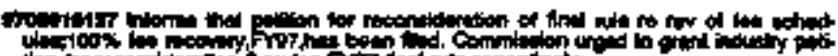

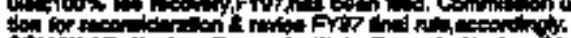

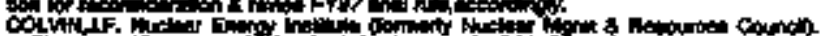

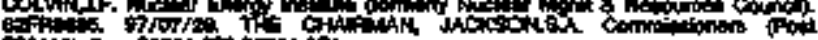

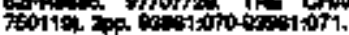

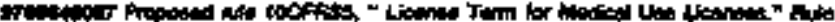

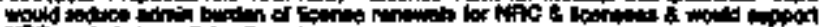
Hef

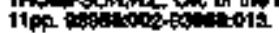

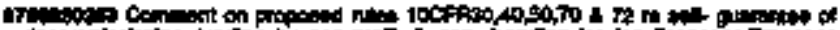

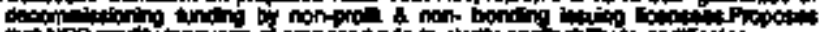

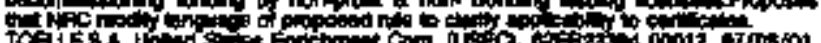

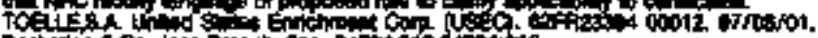

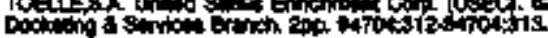

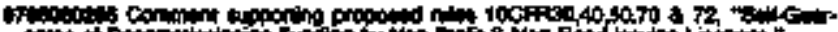

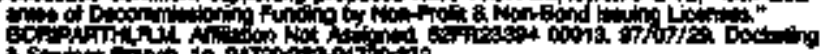

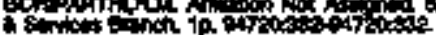

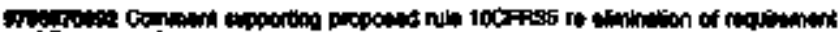

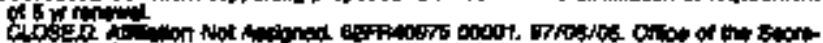

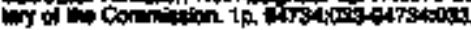

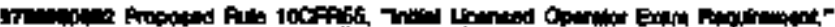

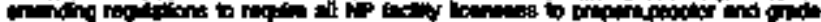

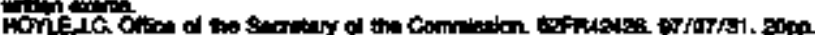

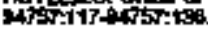

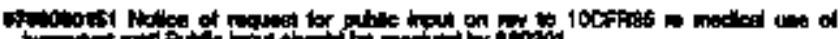

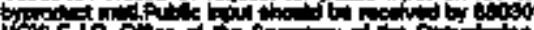

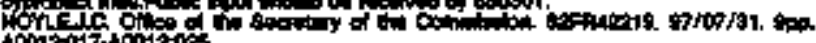

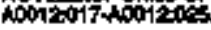

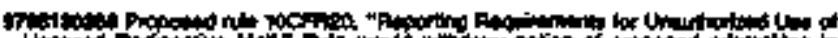

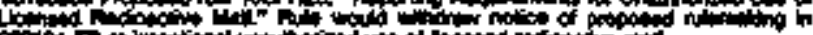

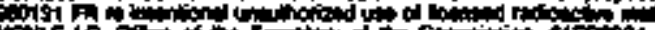

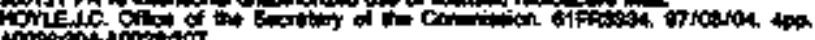

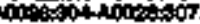

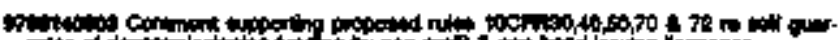

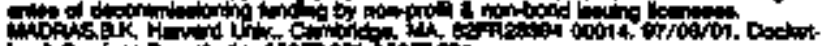

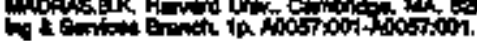

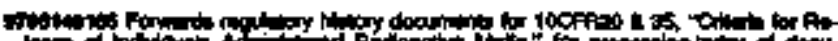

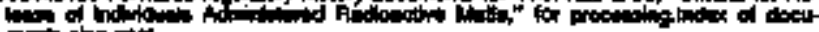
action

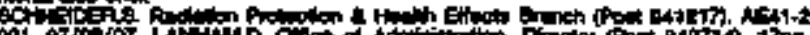

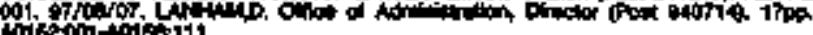

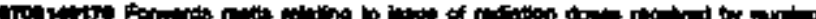

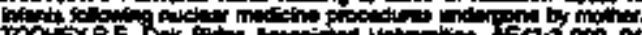

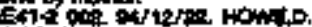

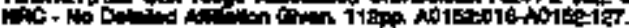

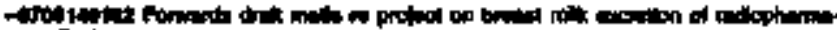

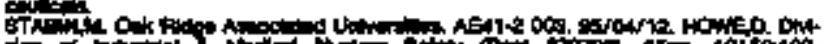

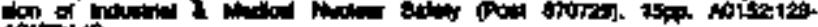
Aitritur

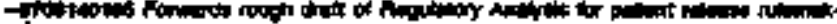

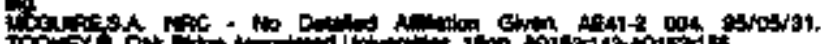

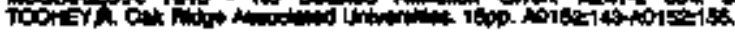

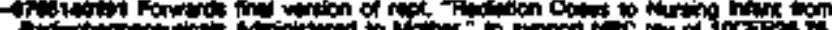

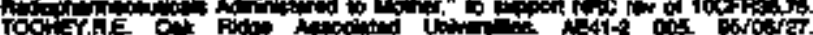

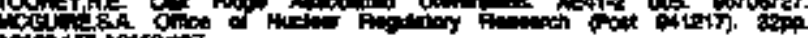

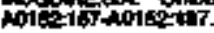

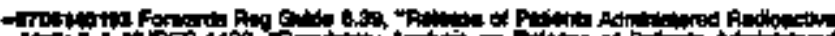

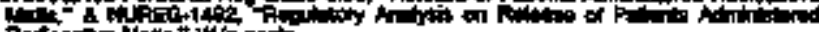

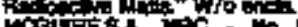

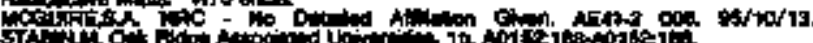
STh:

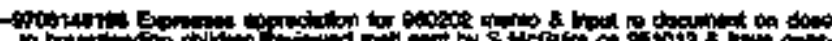
b bot

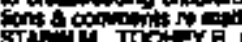

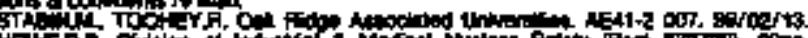

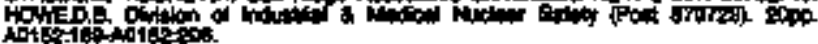

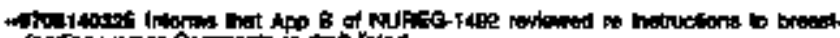

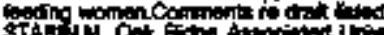

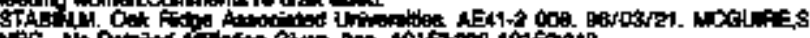

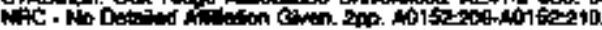

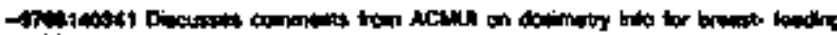

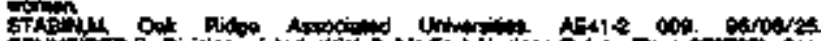
SC 10:

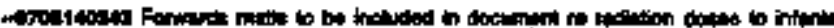

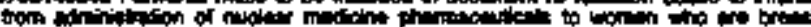

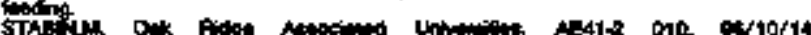

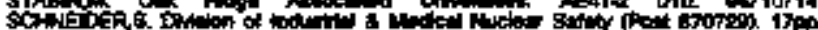
No1

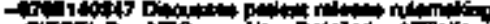

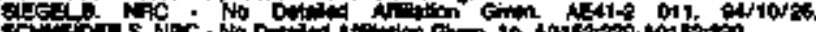

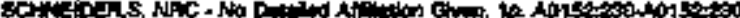

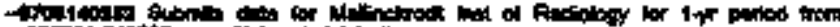

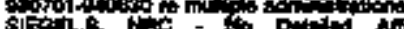

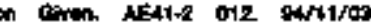

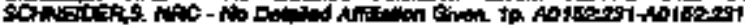

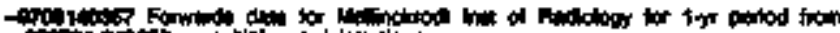

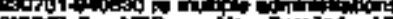

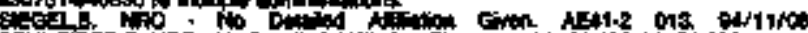

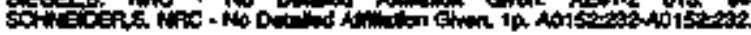

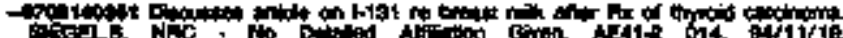

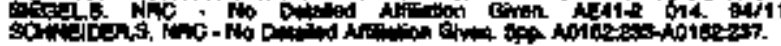

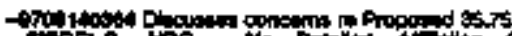

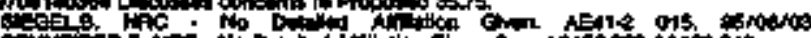

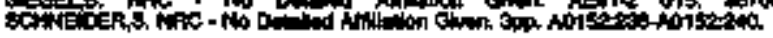

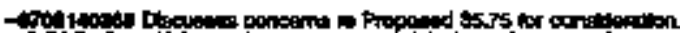

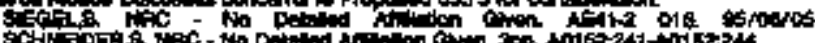

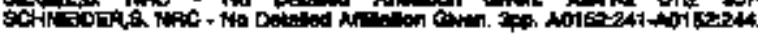

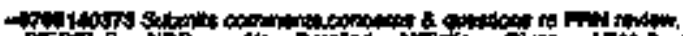

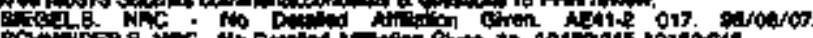

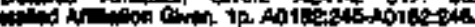

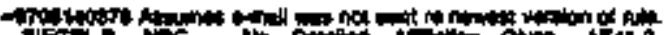

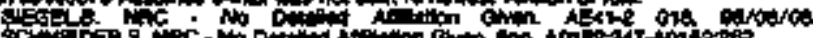

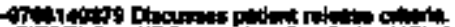

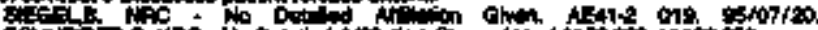

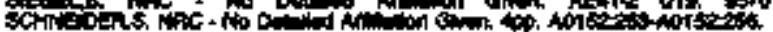

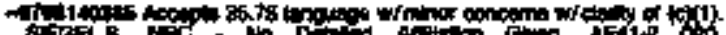

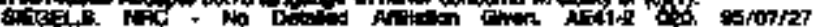

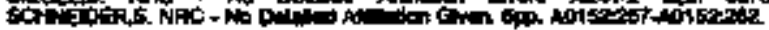

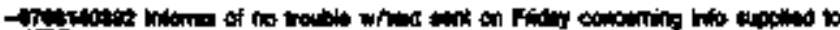

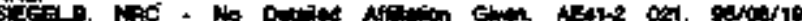

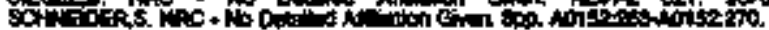

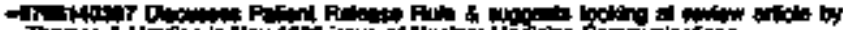

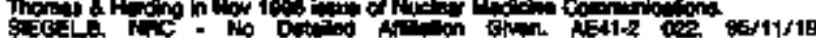

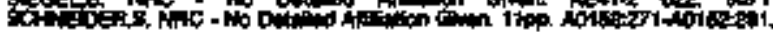

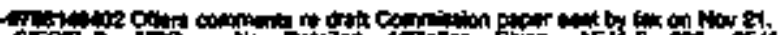

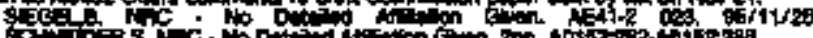

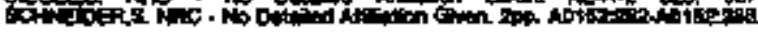

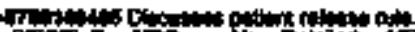

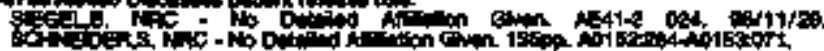

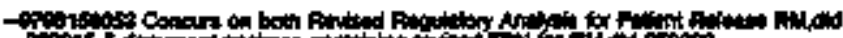

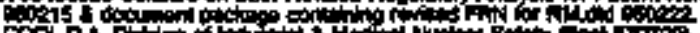

$600 \mathrm{DA}$ D.

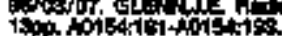

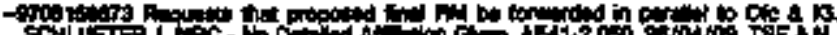

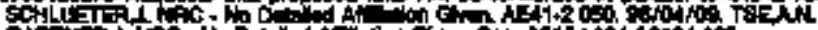

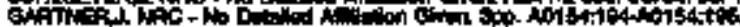

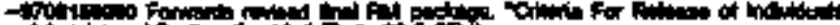
A.

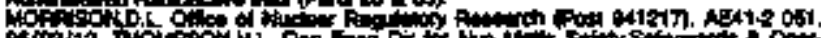

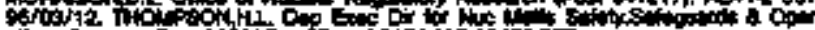

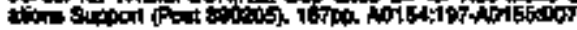

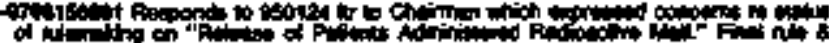

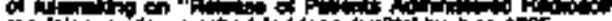

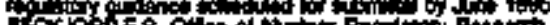

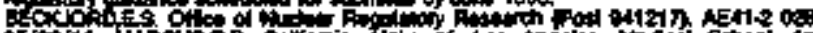

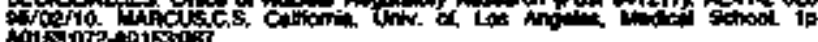
A01

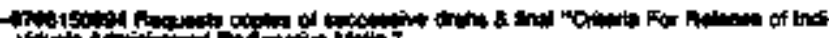

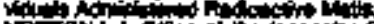

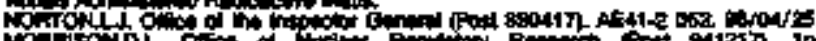

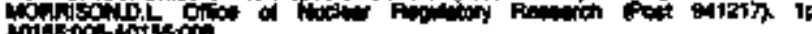




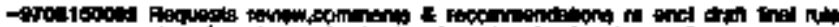

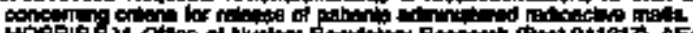

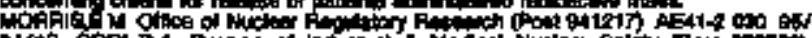

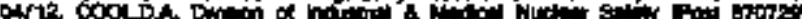

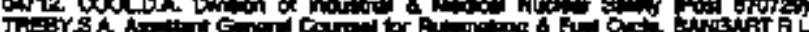

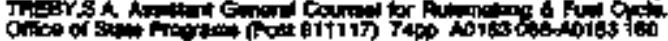

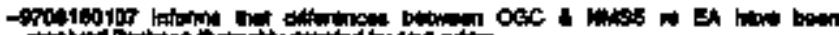

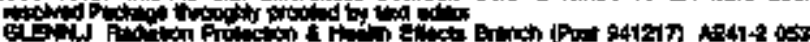

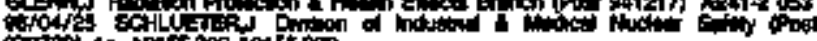

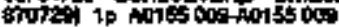

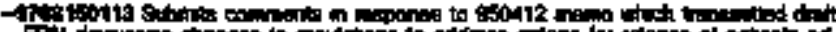
Fill doar.

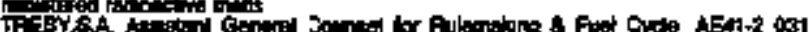

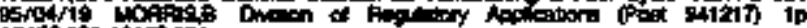
जIड़

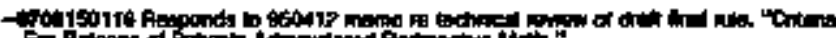

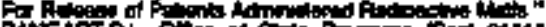

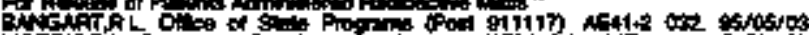

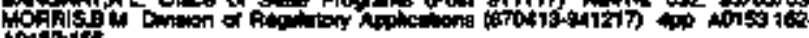
Horas $\rightarrow 10$

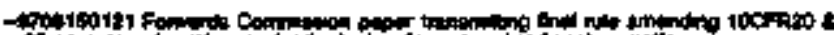

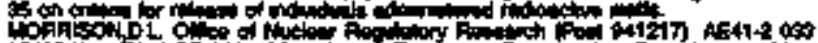

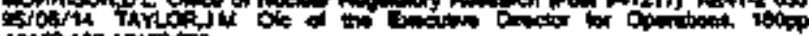

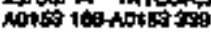

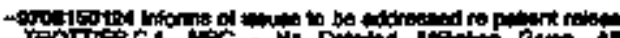

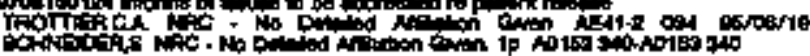

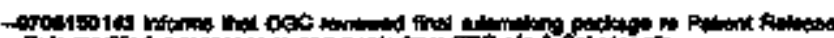

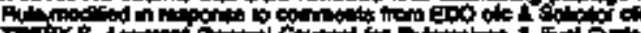

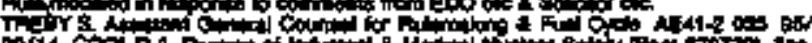

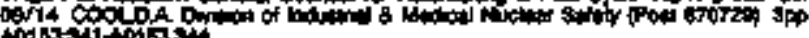

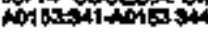

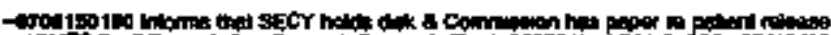
W

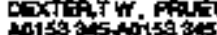

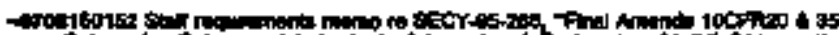

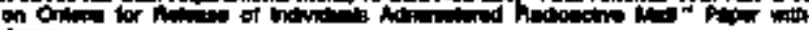

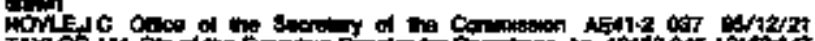

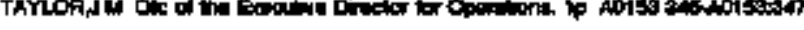

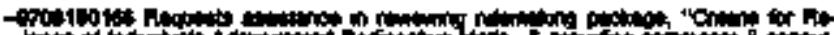

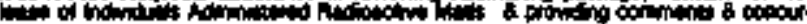

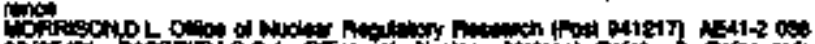

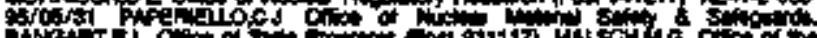

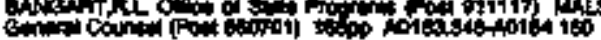

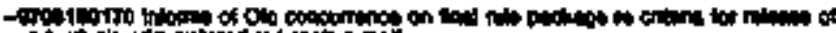

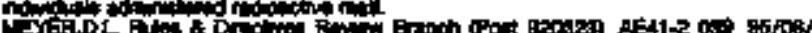

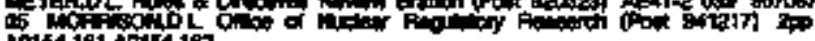

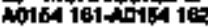

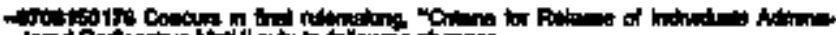

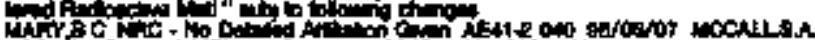
Nic. Wu D.

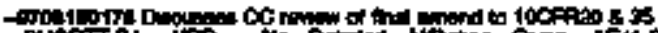
stopisL

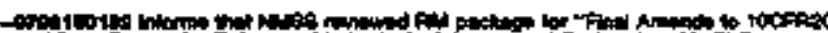

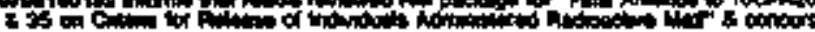
Pf

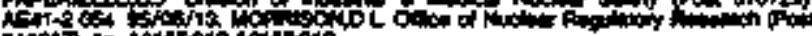

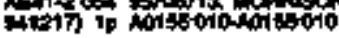

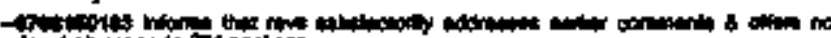
Hot of

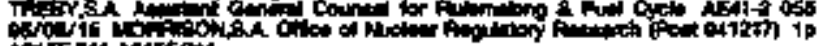

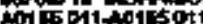

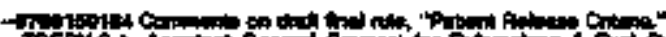

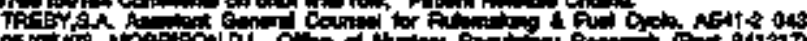

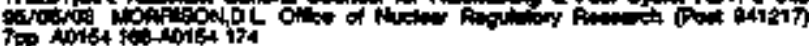

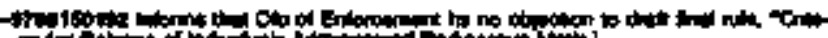

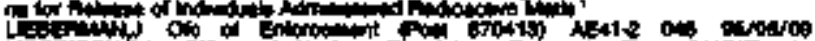

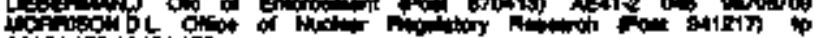

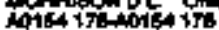

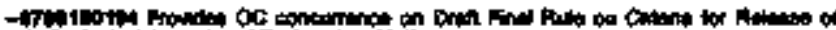

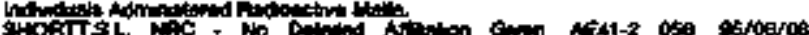
schis 10 A015 dis Ant

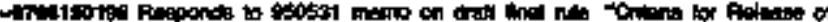

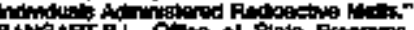

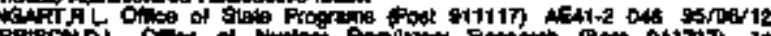

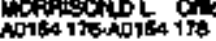

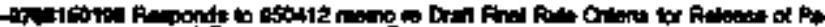

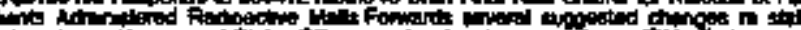

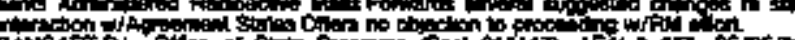

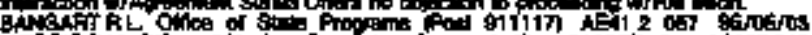

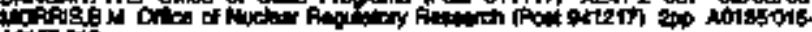

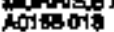

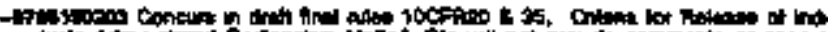

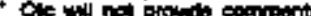

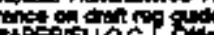

at

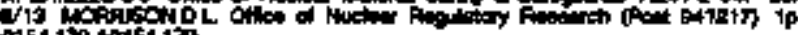

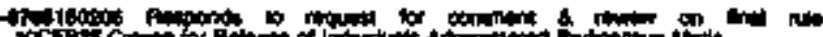

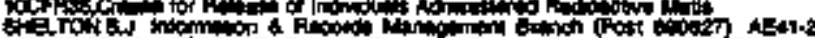

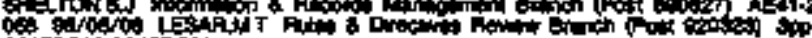
कon

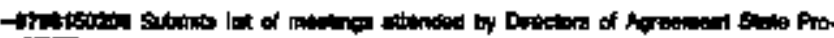

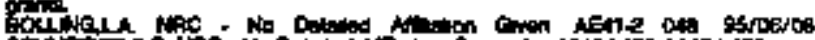

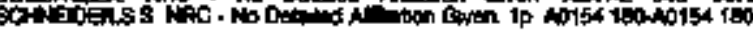

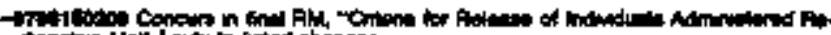

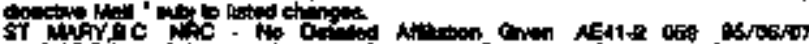

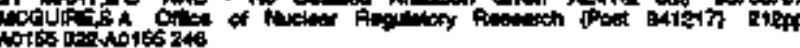

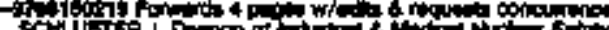

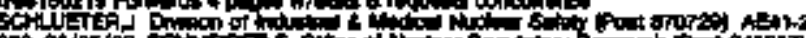

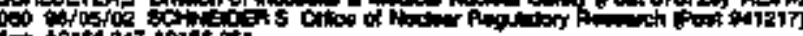

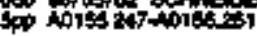

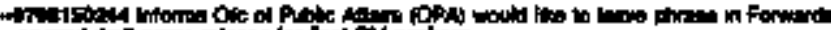

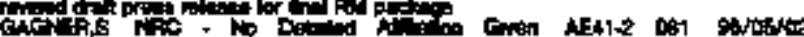

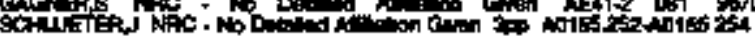

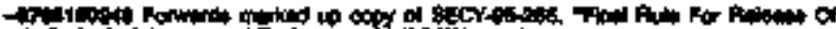

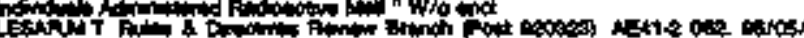

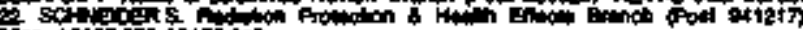

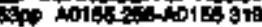

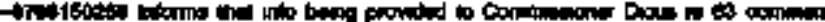

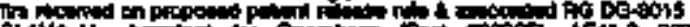

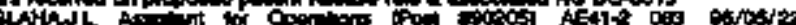

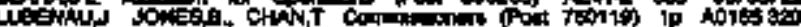
Ant5 300

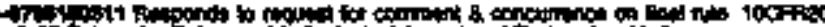

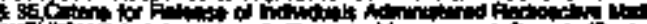

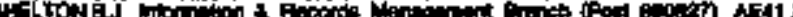

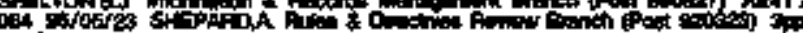

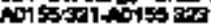

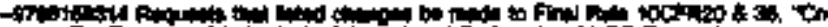

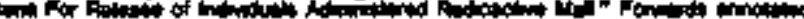

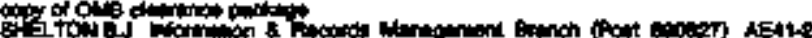

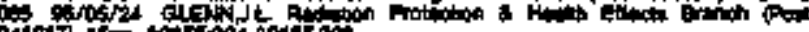

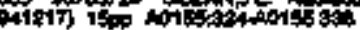

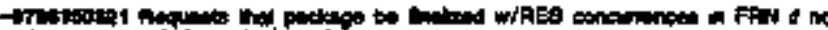

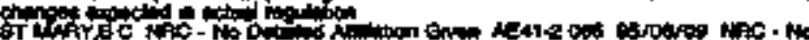

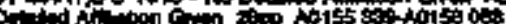

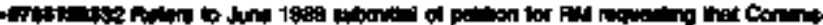

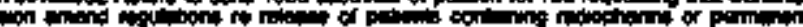

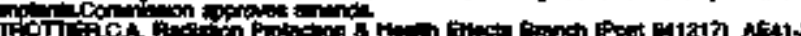

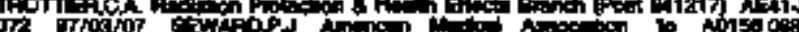
antston

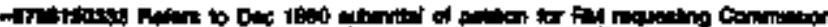

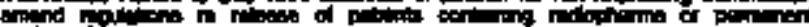

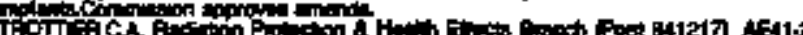

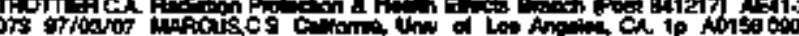
Hotson

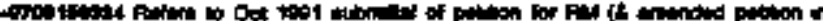

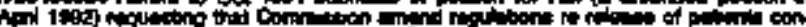
(1)

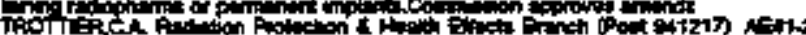

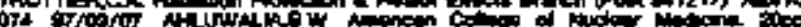

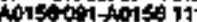

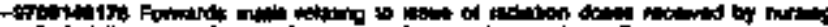

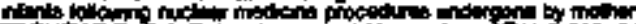

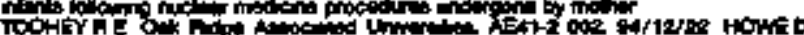

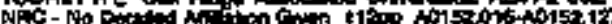




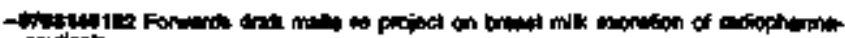

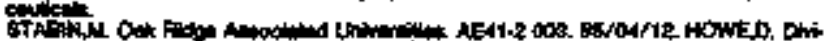

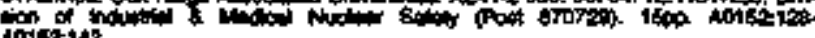

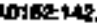

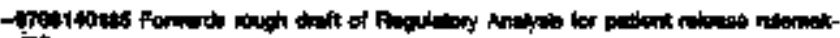

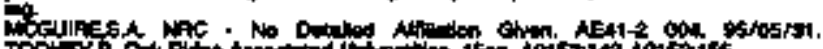

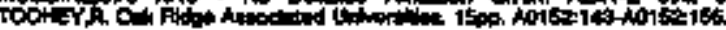

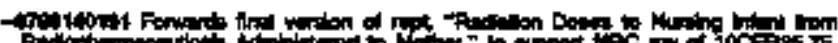

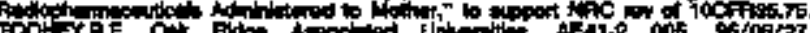

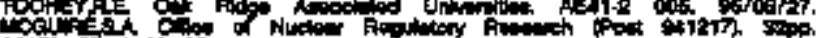

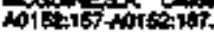

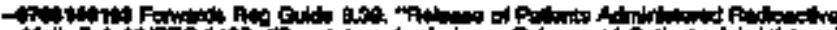

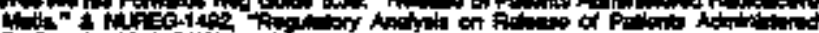

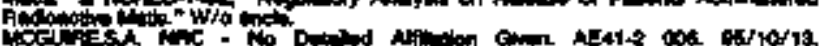

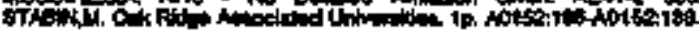

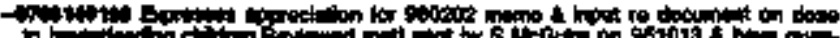

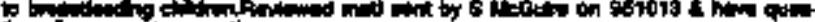

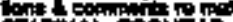

FT:

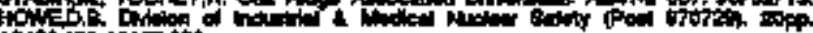

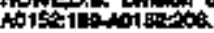

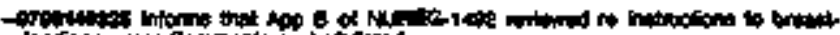

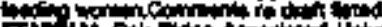

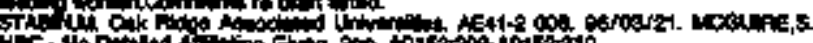

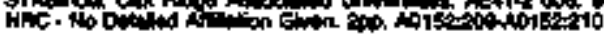

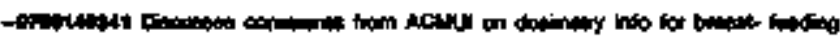

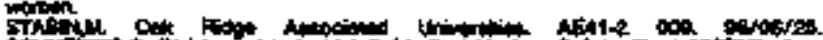
ICt

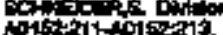

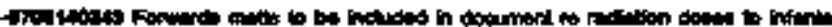

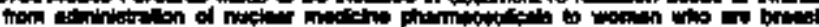

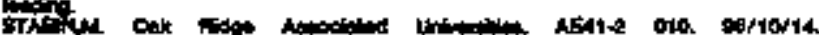

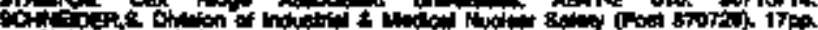
Aots

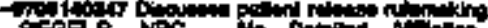

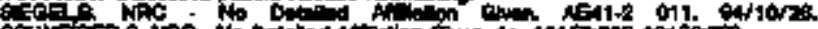

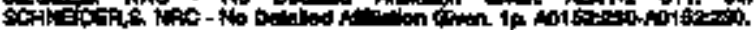

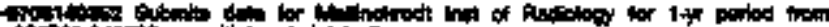

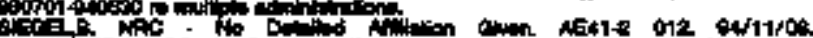

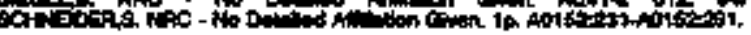

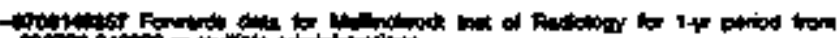

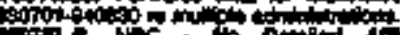

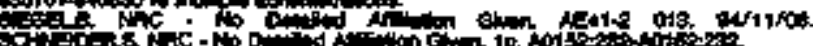

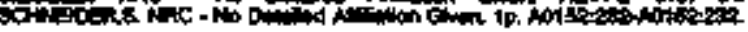

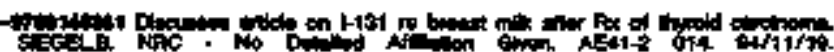

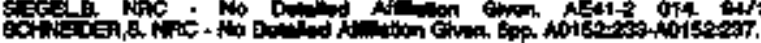

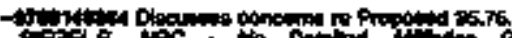

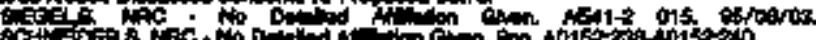

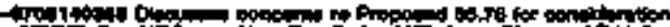

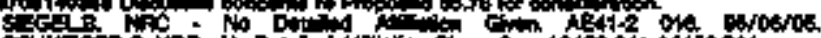

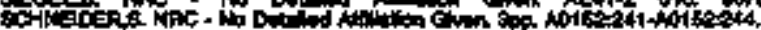

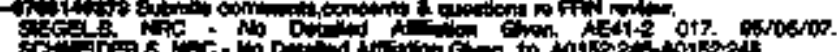

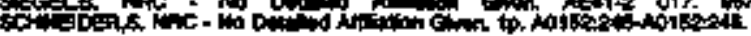

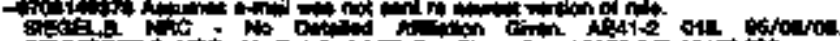

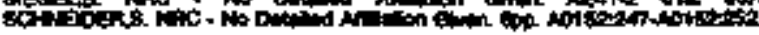

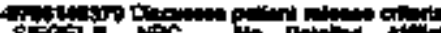

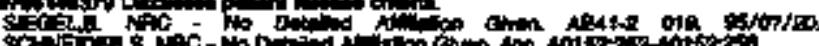

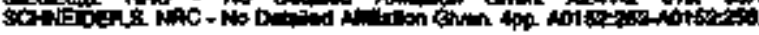

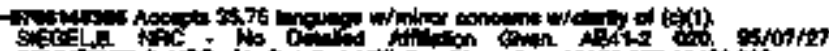

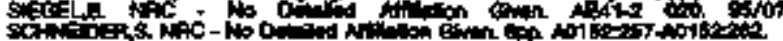

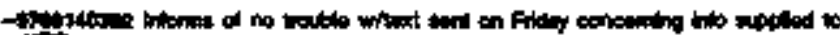

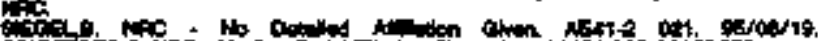

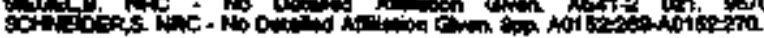

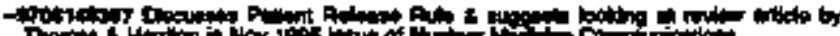

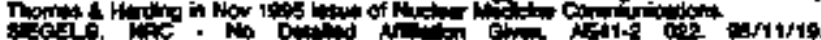

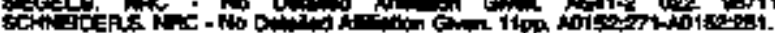

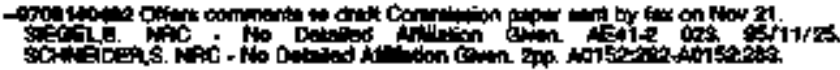

r tow

\&

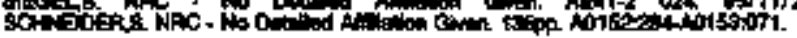

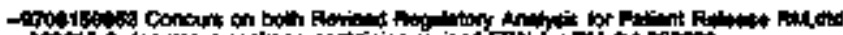

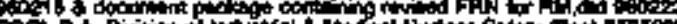

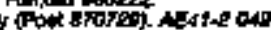

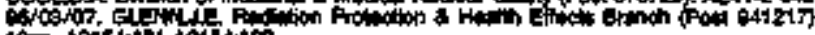

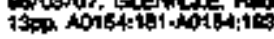

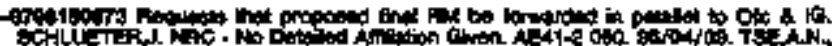

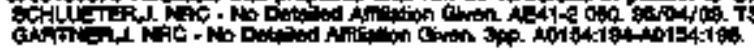

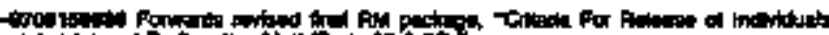

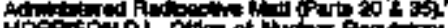

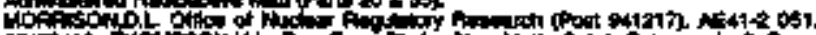

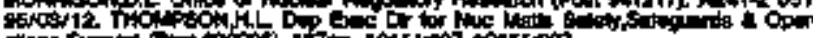

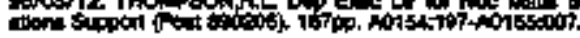

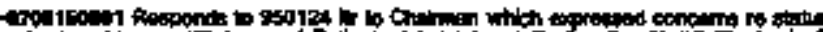

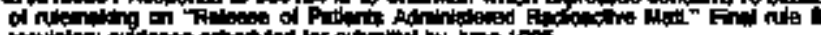

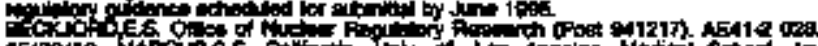

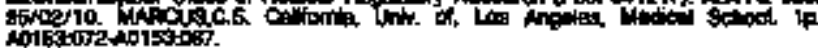

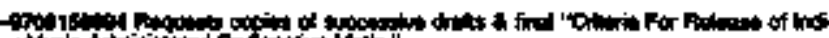

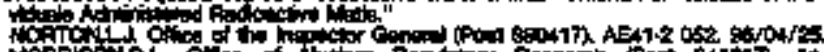

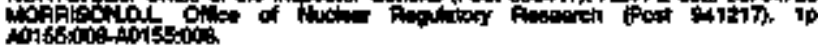

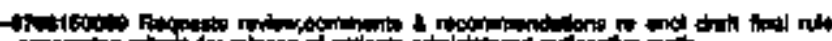

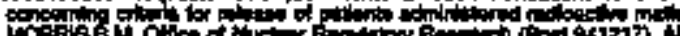

WOF

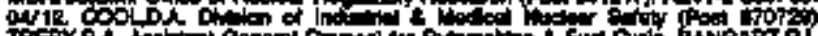

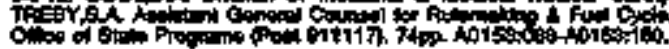

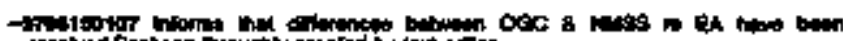

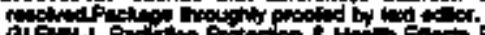

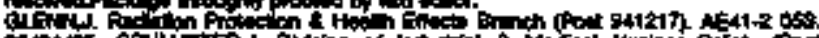

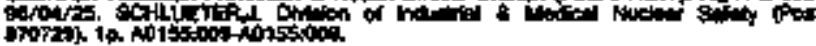

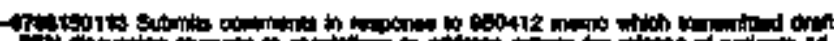

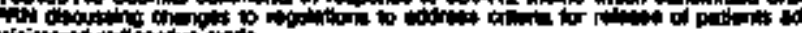
(1)

Tire

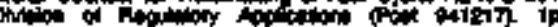

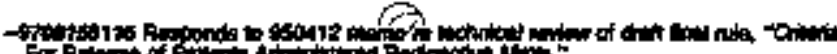

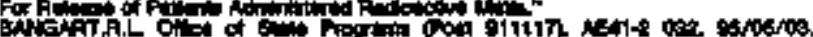

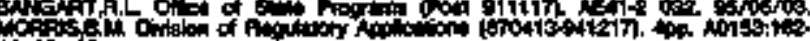
toition

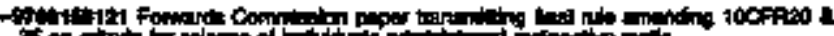

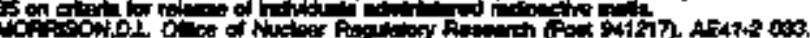

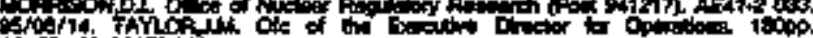

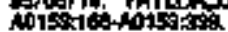

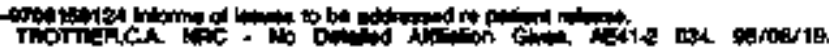

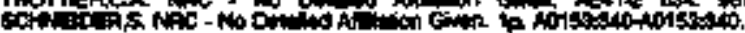

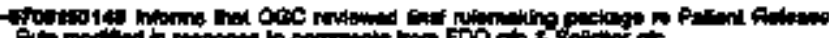

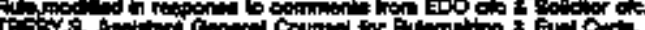

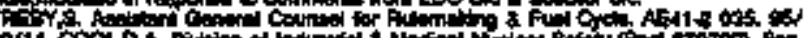

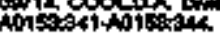

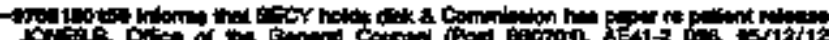

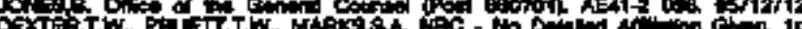

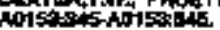

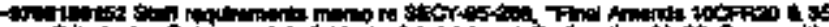

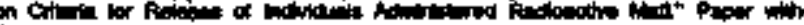
HWint

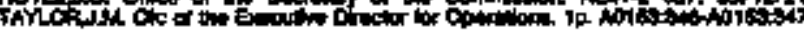

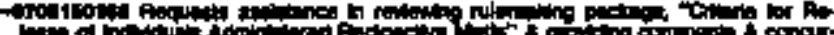

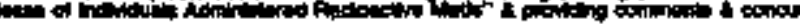

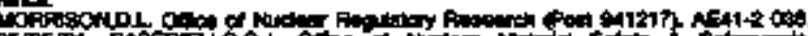

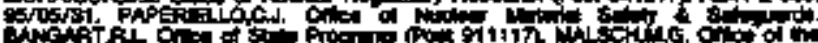

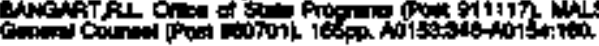

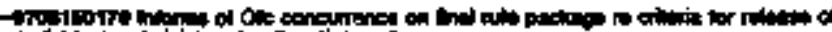

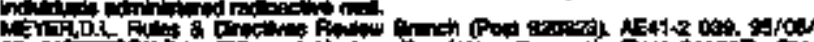
of We

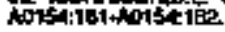

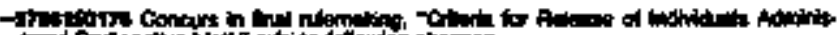

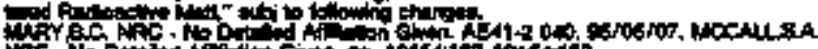

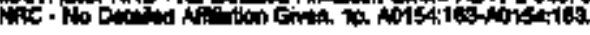

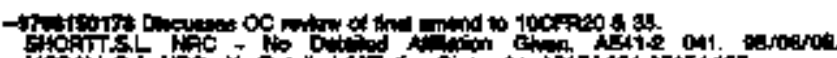

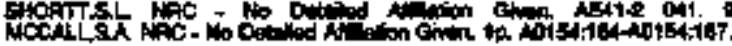




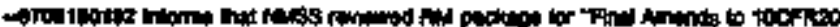

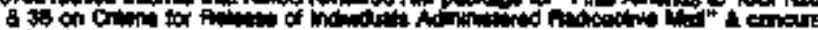
Prection

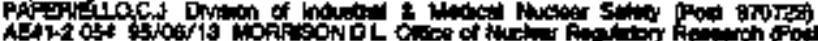

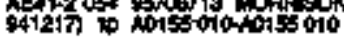

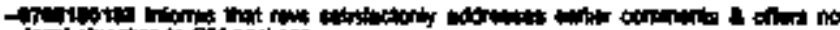

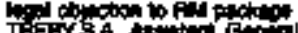

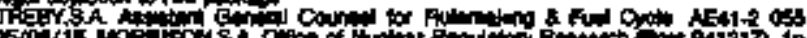

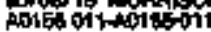

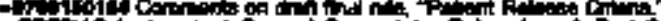

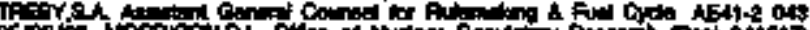

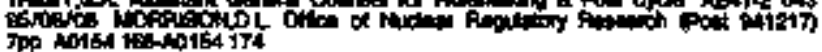

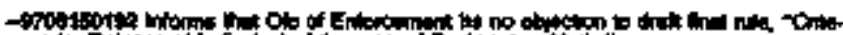

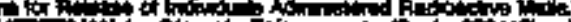

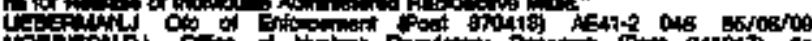

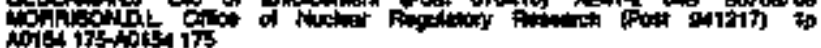

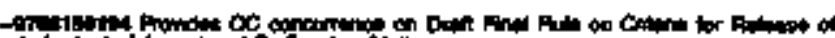

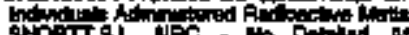

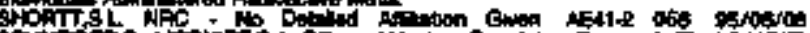

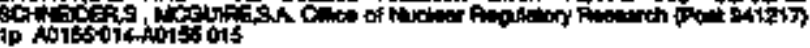

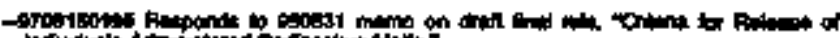

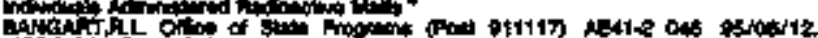

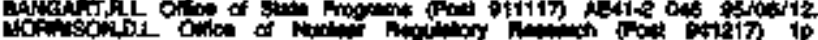

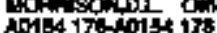

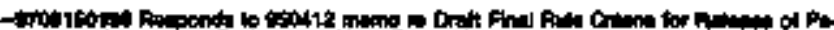

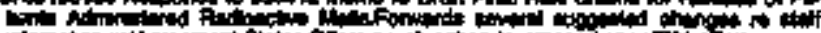

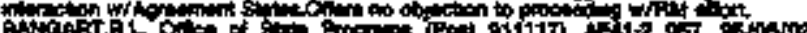

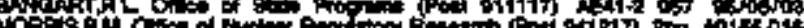
ADitio 618

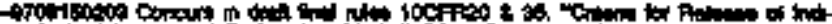

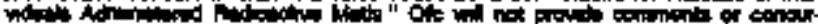

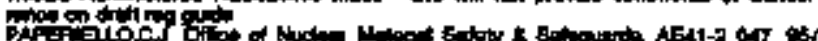

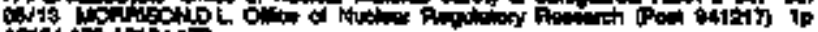
iond towatis is

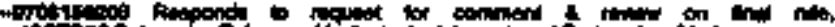

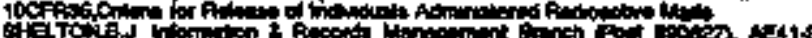

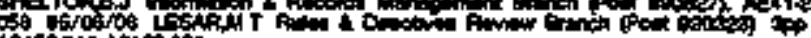

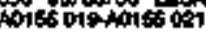

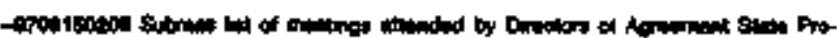

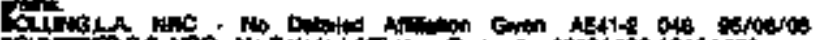

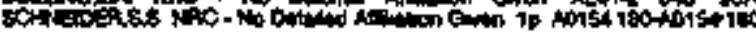

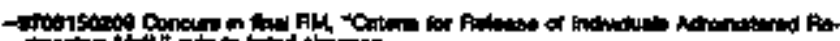

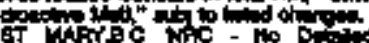

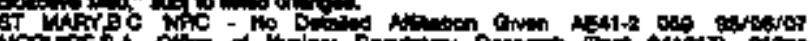

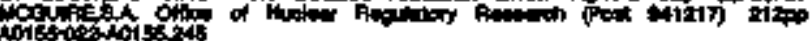

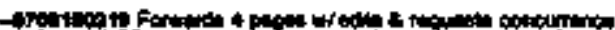

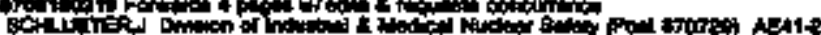

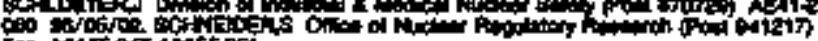
top now

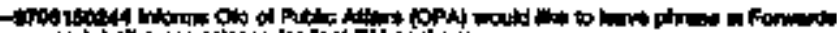

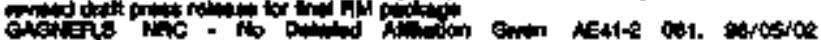

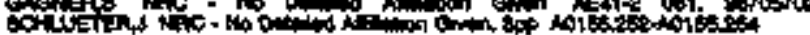

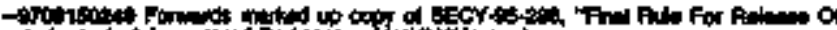

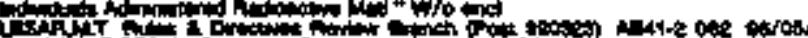

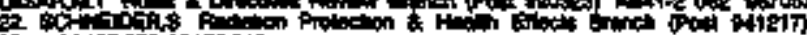

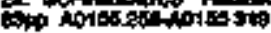

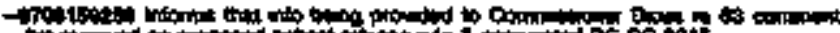

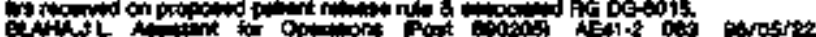

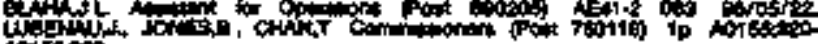

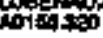

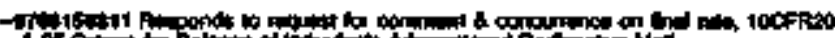

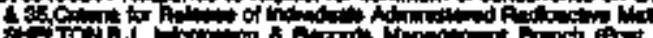

\%

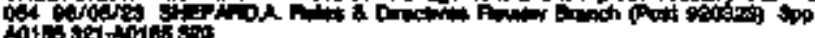

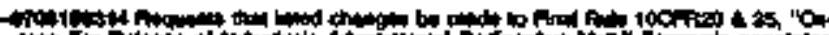

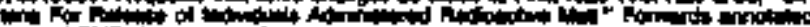

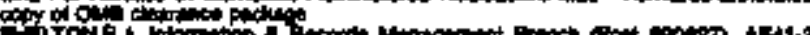
on a 1217 in

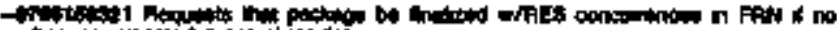

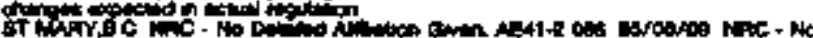

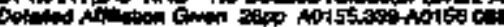

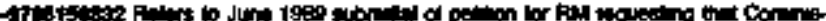

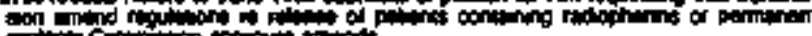

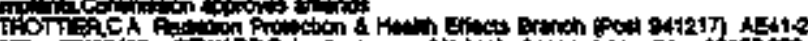

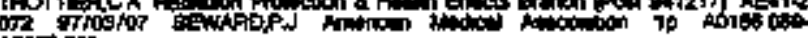
orstos

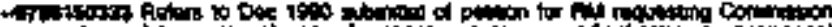

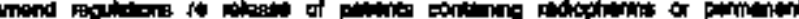

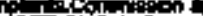

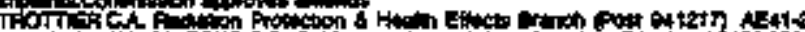
Ors orios THEB DQ90

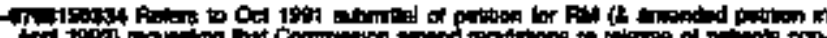

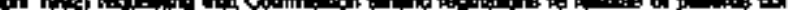

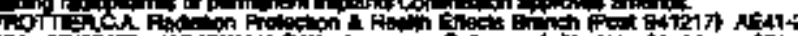

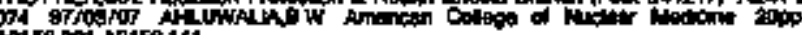
tors toith158 if

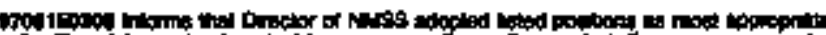

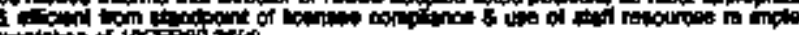

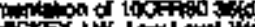

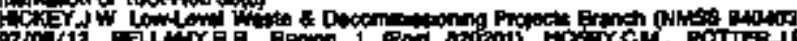

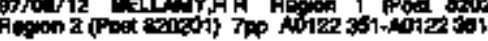

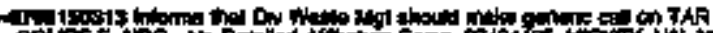

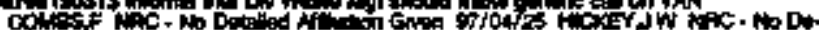

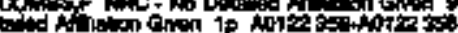

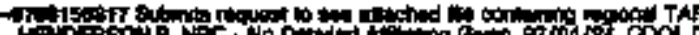

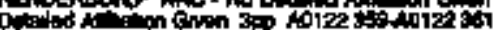

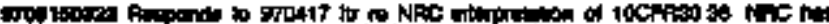
mecent com

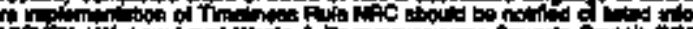
然

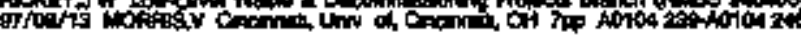

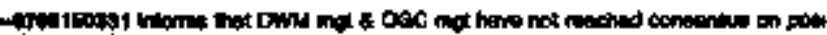

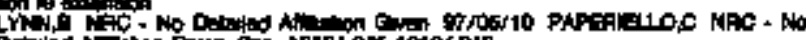

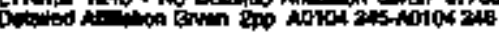

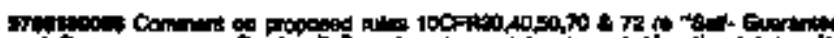

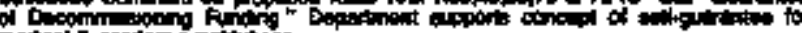

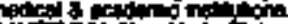

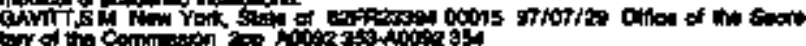

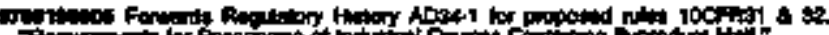

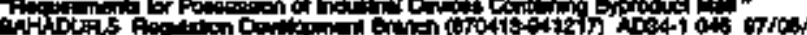

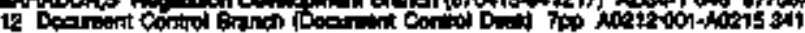

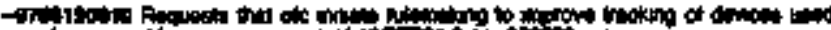

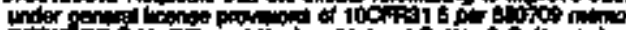

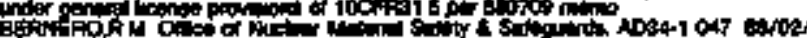

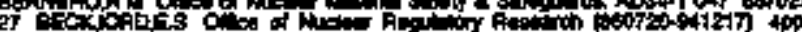
Meziz 194thriarios

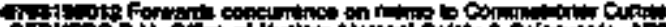

A

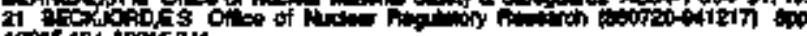
Mrets istorts 241

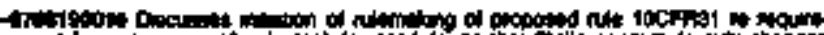

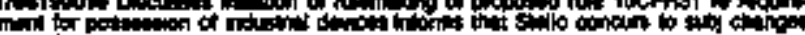

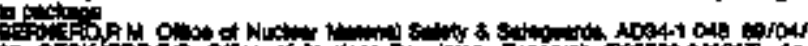

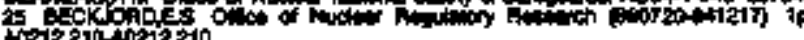

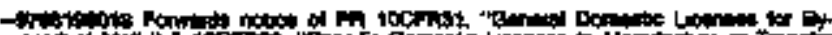

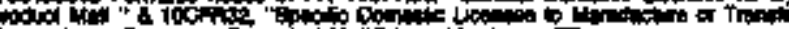

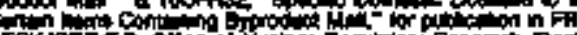

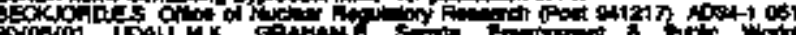

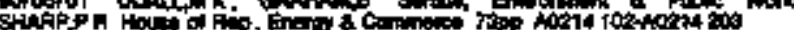

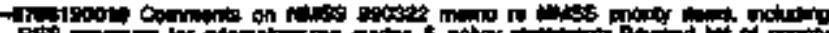

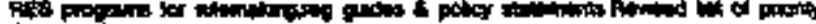

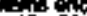

然

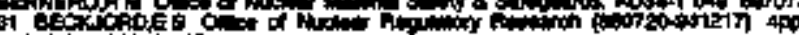

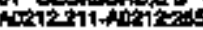

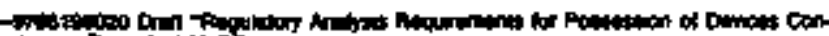

all

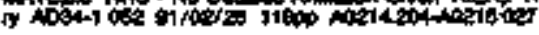

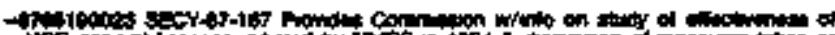

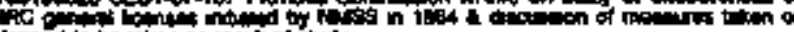

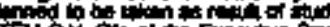

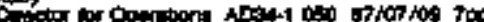

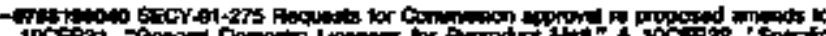

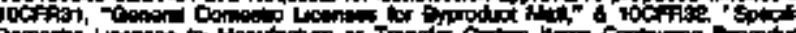

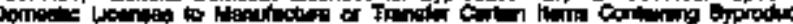

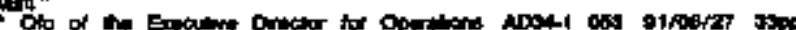

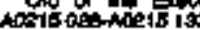




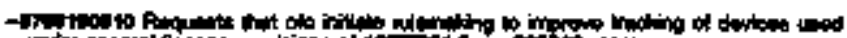

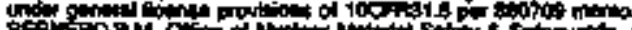
befic.

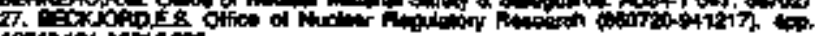

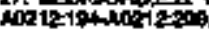

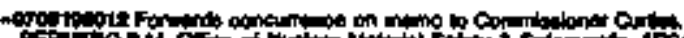

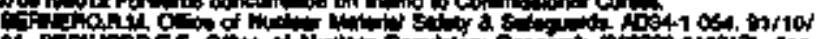

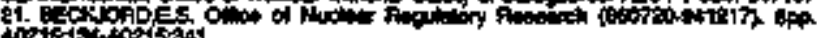

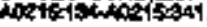

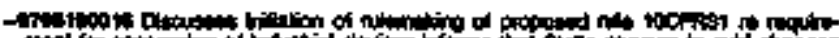

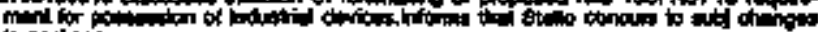

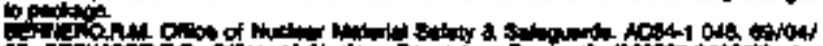

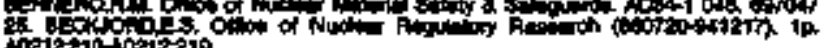

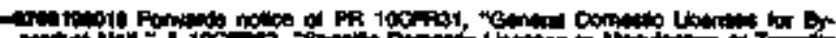

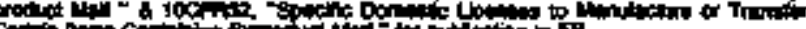

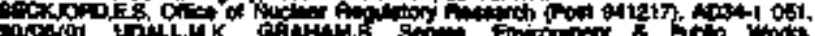

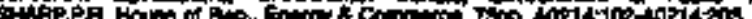

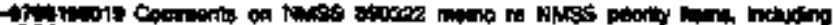

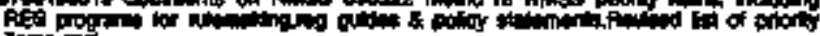

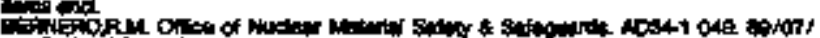

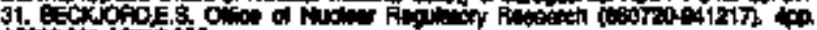

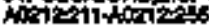

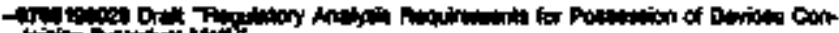
ith

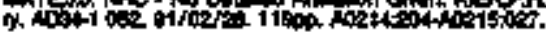

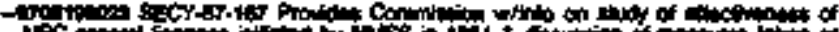

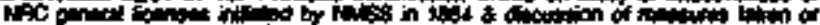

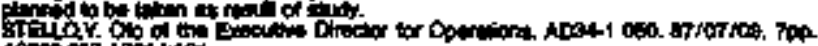

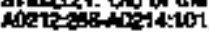

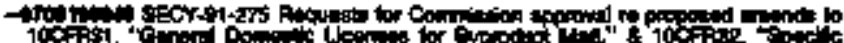
Q

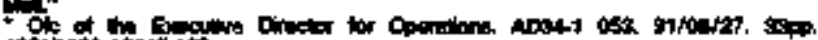

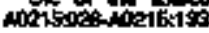

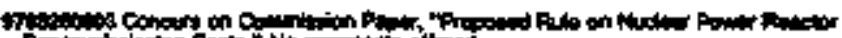

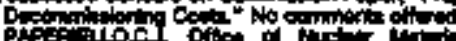

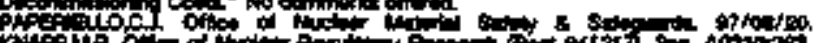

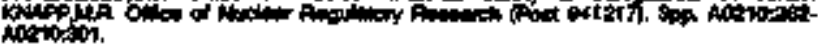

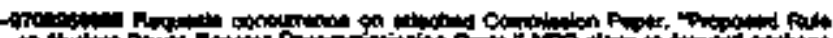

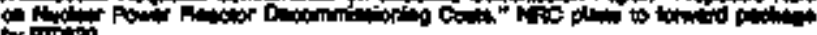

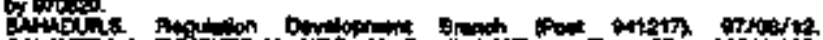

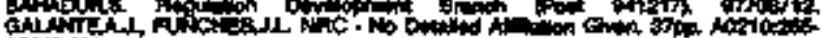

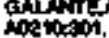

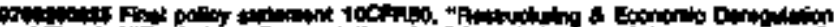

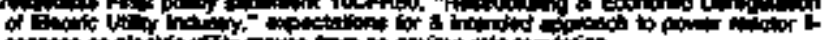

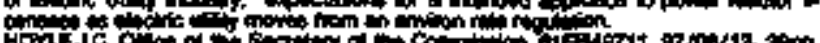
40

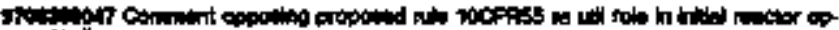

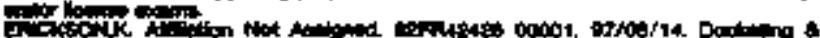

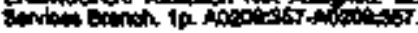

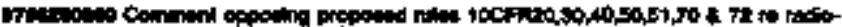

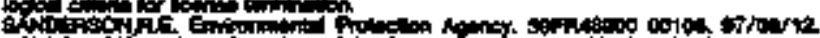

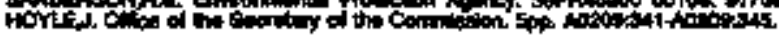

\section{Mov maverom}

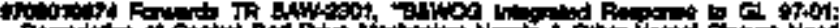

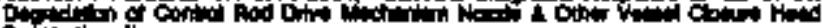

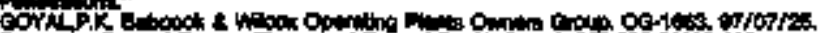

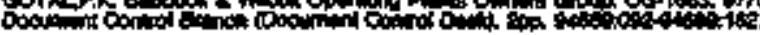

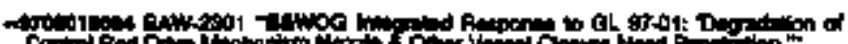

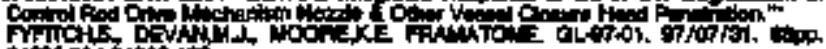

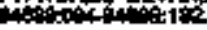

97w

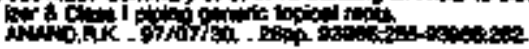

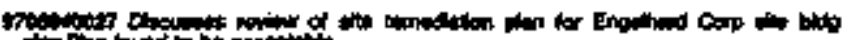

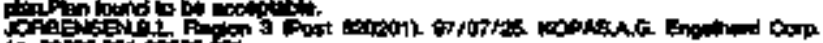
ia

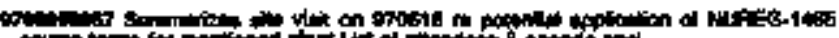

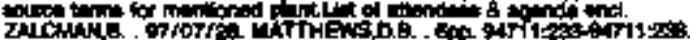

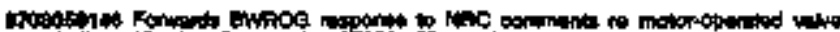

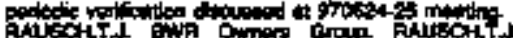

Alus

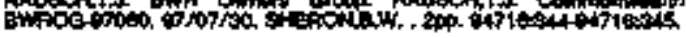

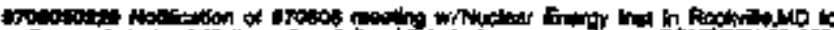

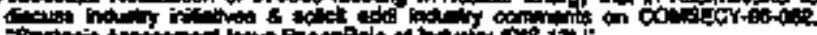

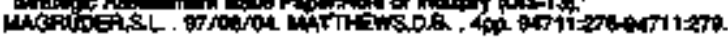

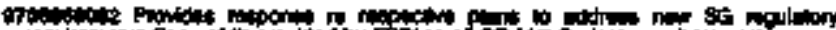

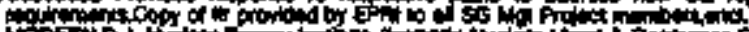

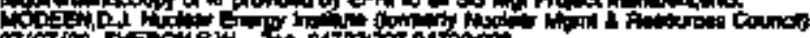

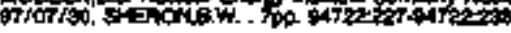

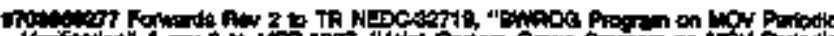

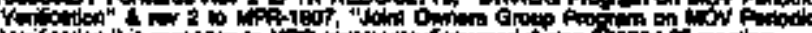

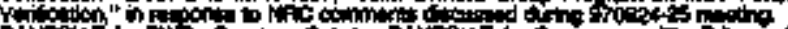

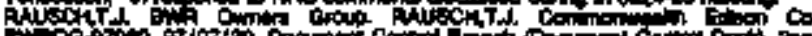

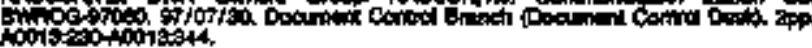

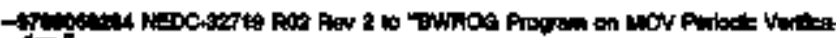

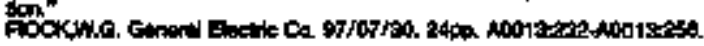

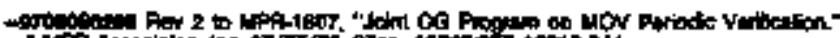

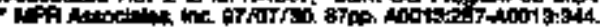

o

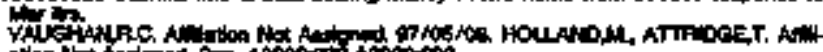

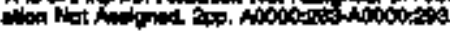

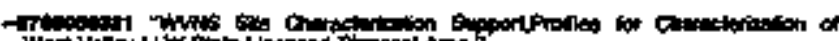

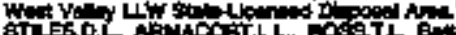

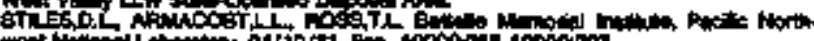

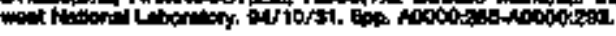

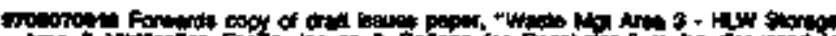

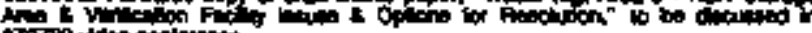

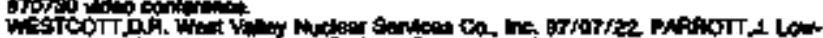

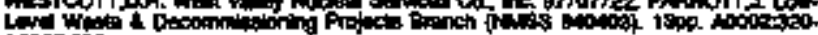
Anogotite

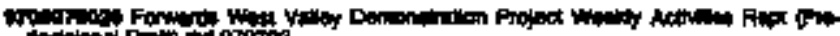

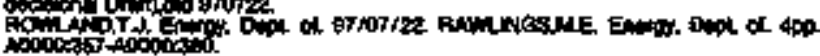

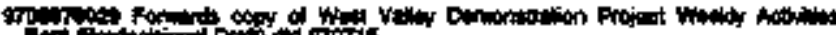

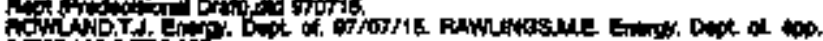

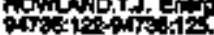

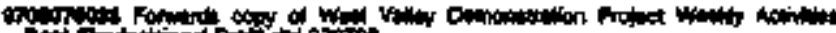

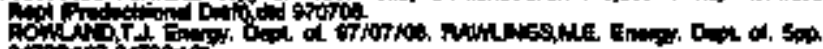
Oimation

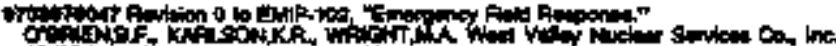

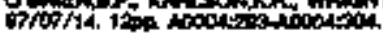

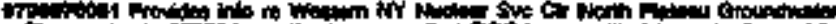

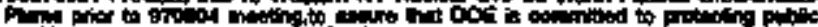

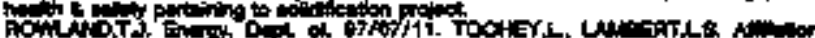

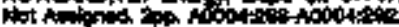

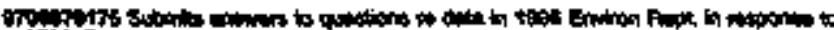
\%015 15

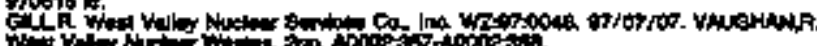

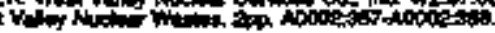

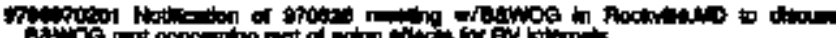

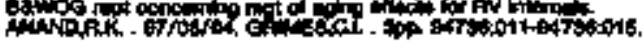

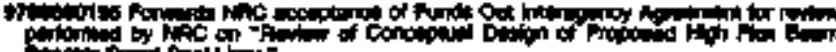

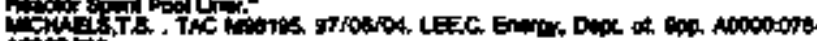

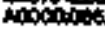

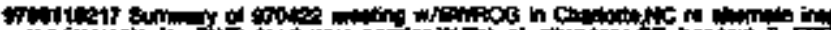

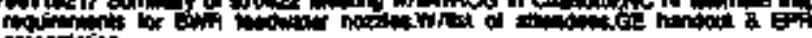

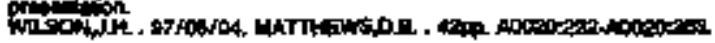

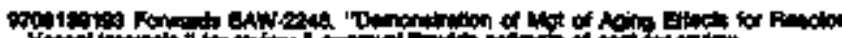

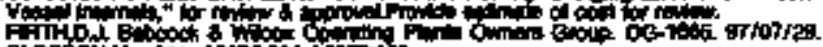

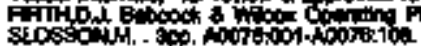

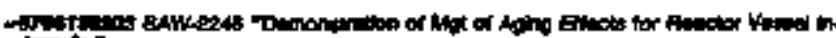

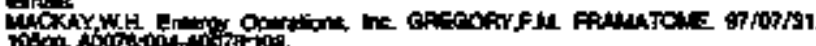

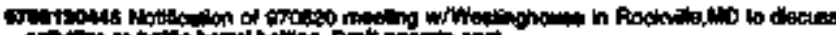

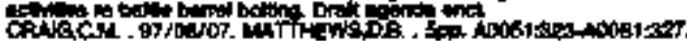




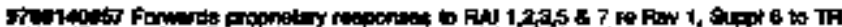

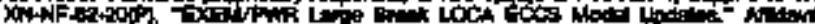

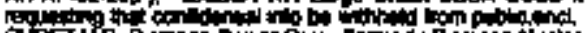

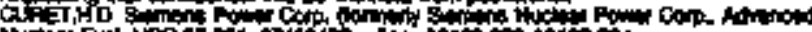

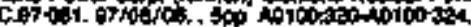

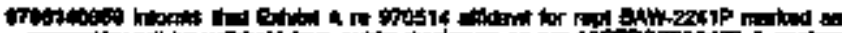

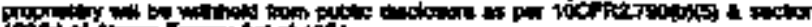

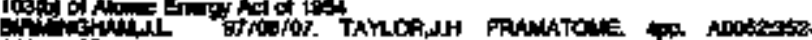
Anocists

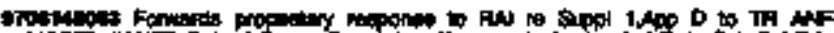

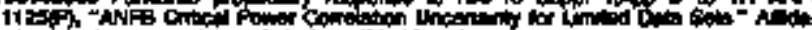

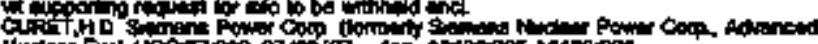

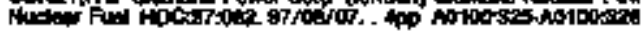

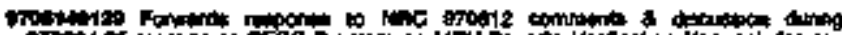

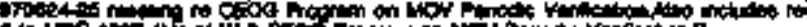

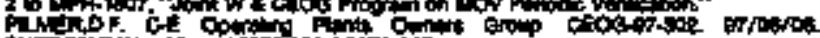

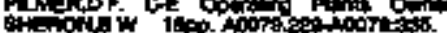

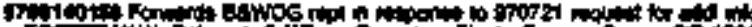

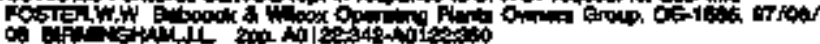

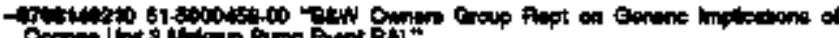

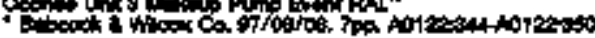

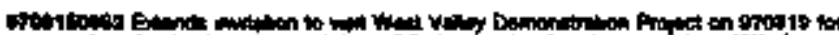

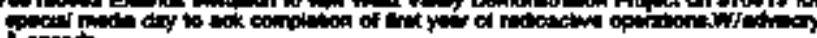

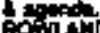

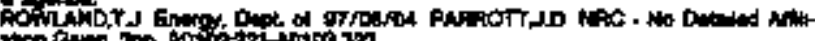

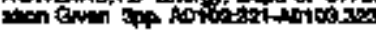

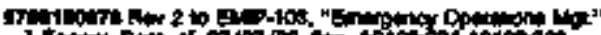

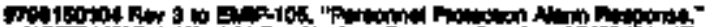

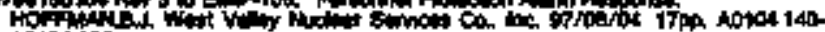
Aotodites

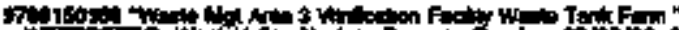

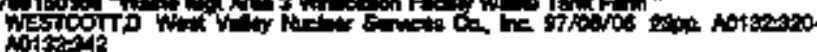

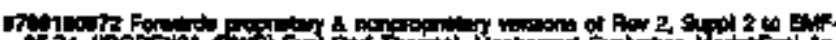

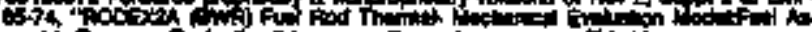

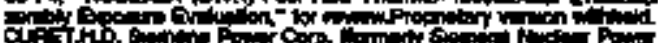

cirith

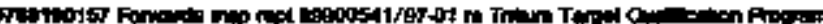

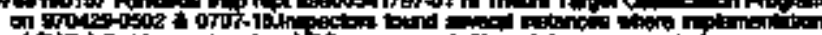
A

Horits

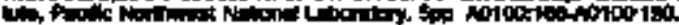

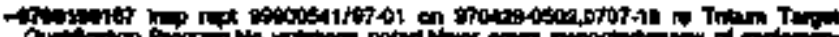

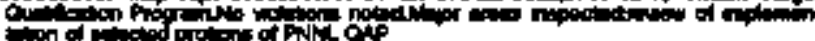

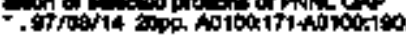

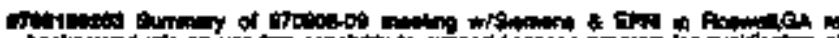

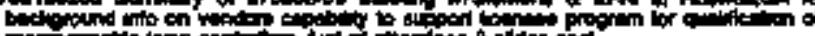

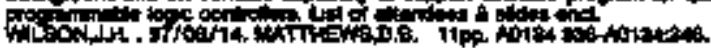

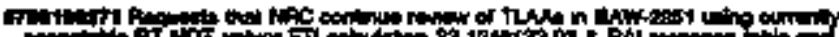

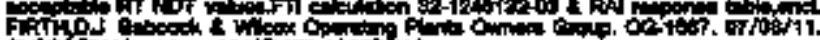
Pos

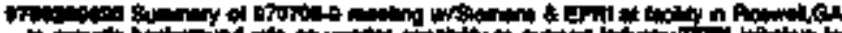

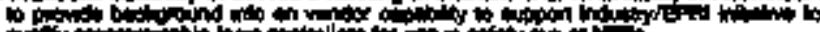
wis of

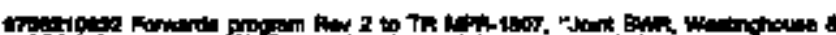

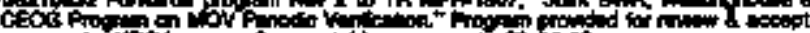

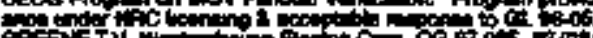

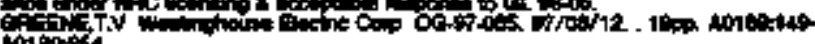
instats

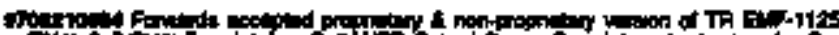

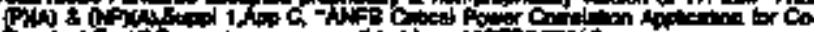

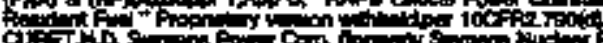

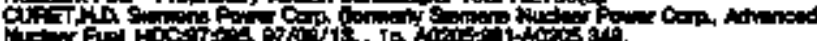

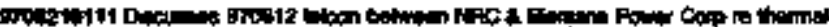

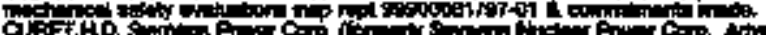

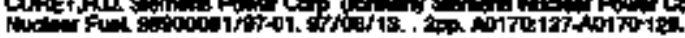

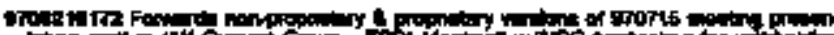

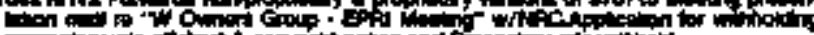

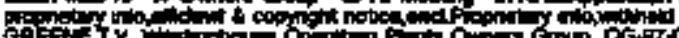

QA

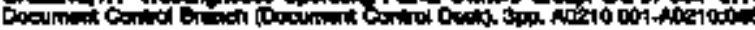

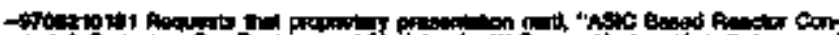

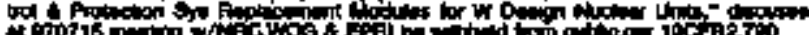

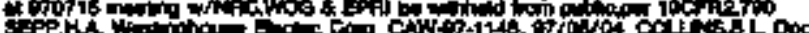
WP

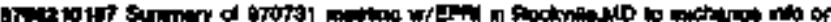

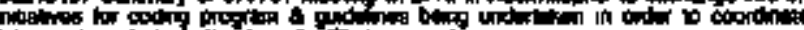

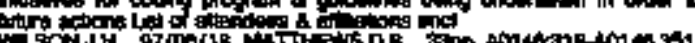

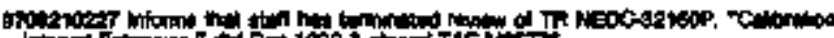

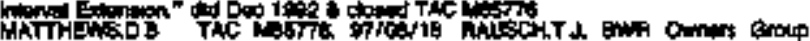

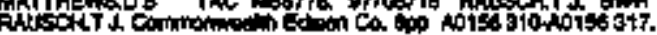

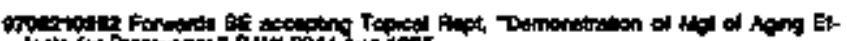

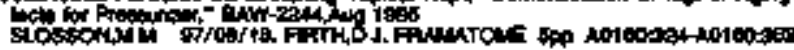

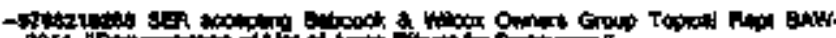

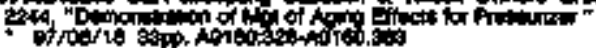

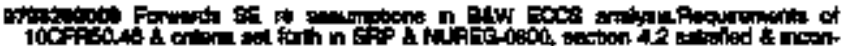

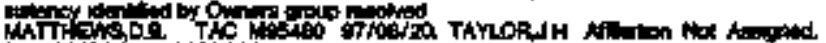
ep A A

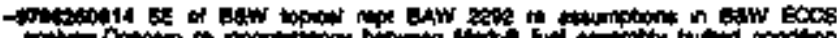

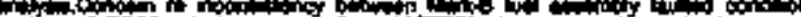

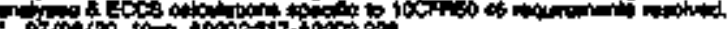

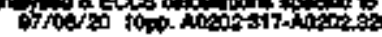

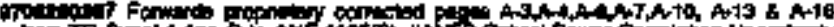

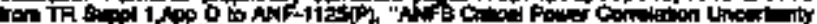

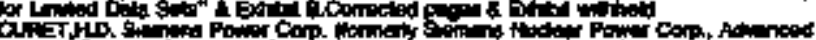

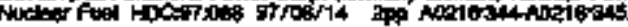

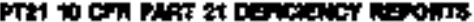

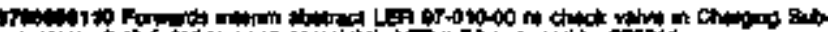

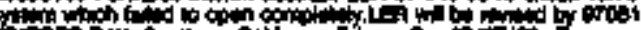

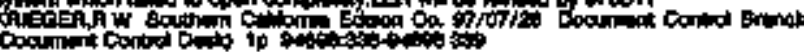

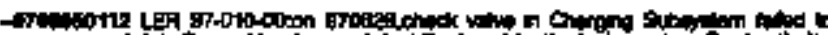

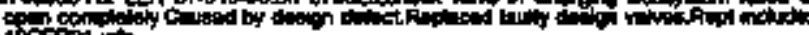

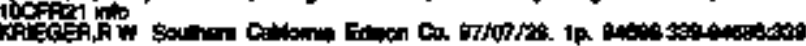

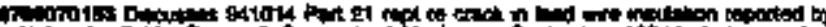

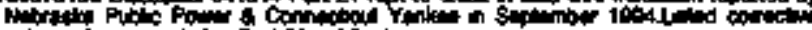
if

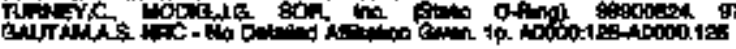

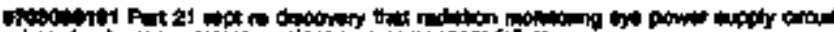
华

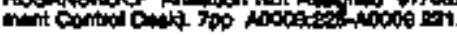
Fiver

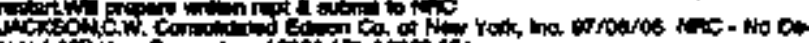

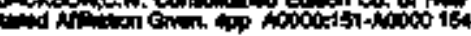

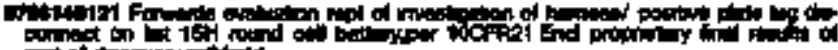

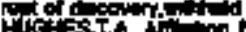

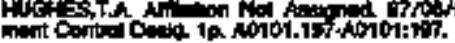

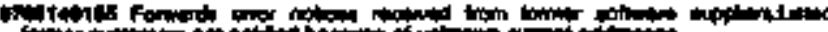

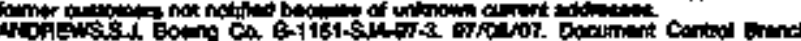

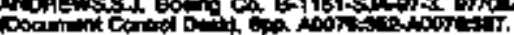

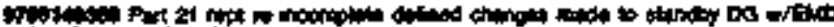

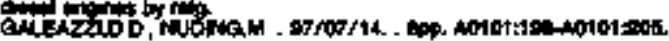

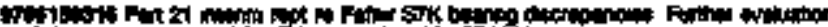

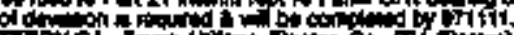

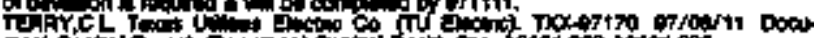

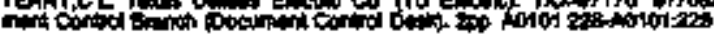

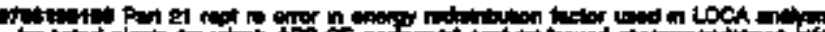

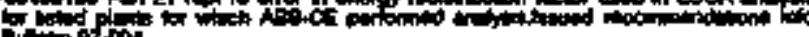

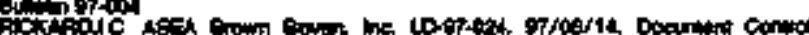

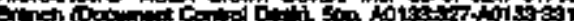

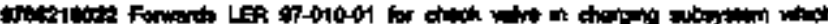

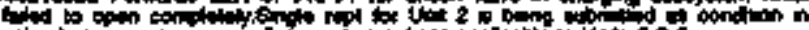

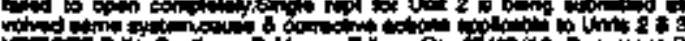

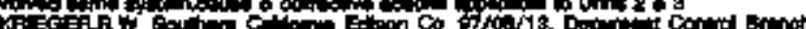

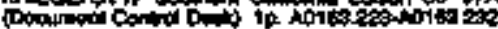

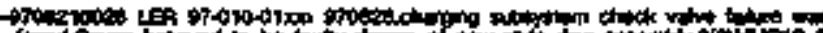

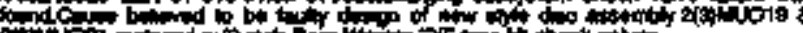

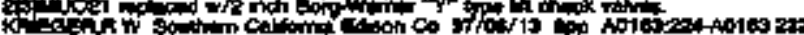




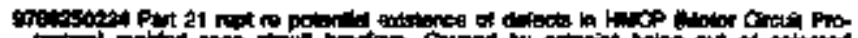

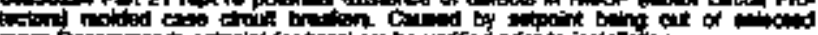

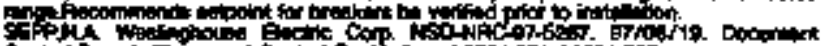

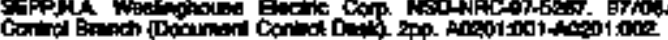

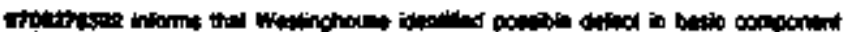

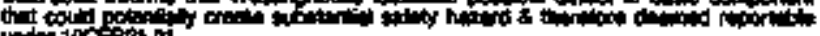

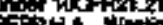

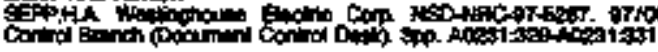

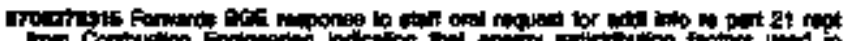

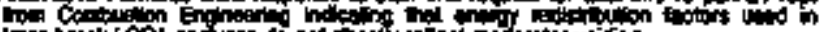
"

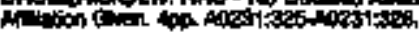

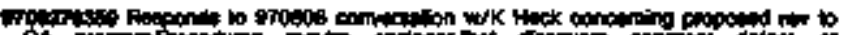

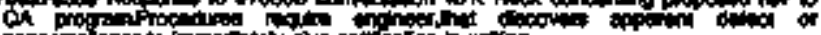

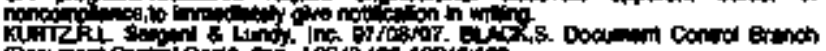

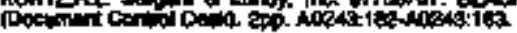

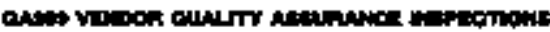

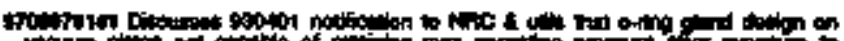

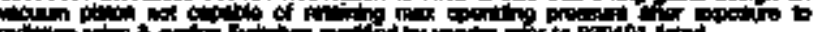

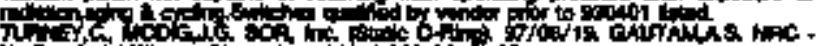
No Don

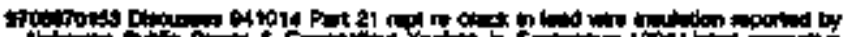

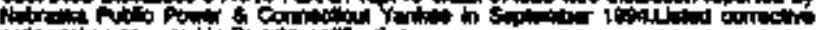

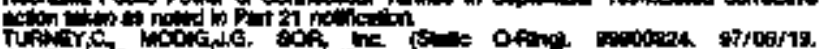

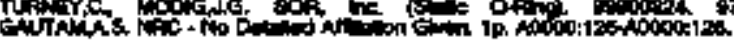

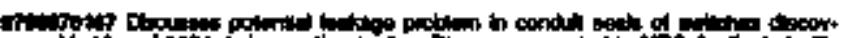

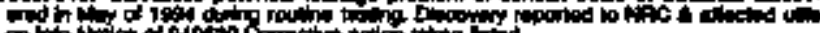

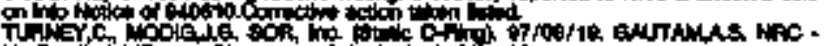

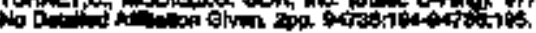

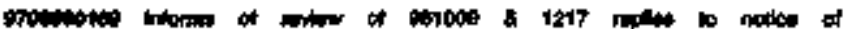

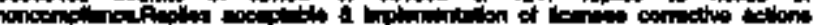
然

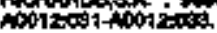

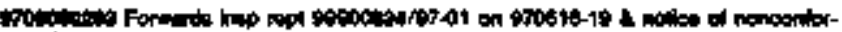

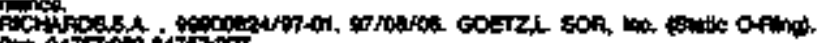

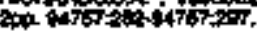

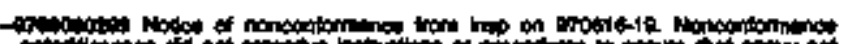

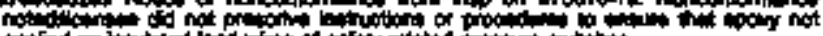

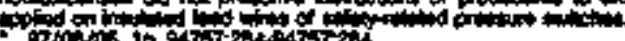

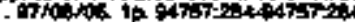

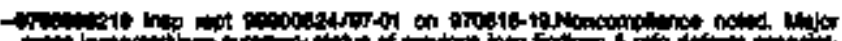
Ger 4 of

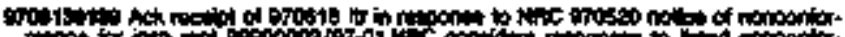

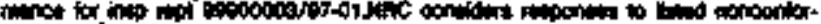

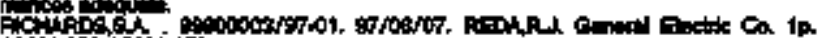

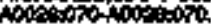

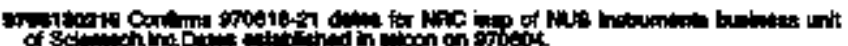
of ateatis

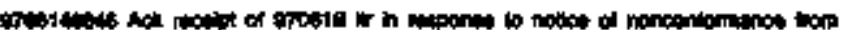

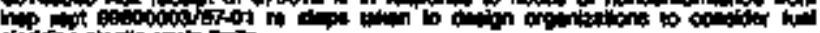

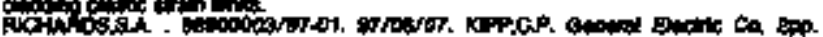

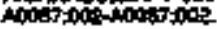

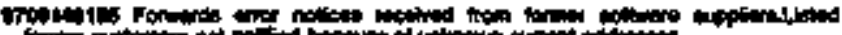

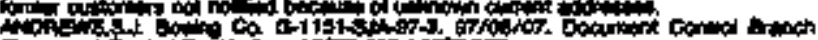

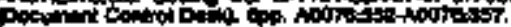

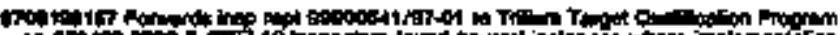

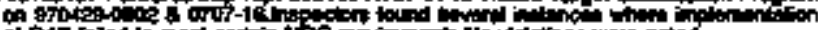

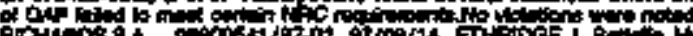

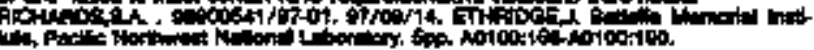

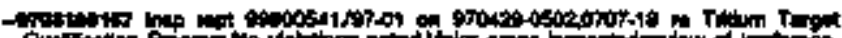

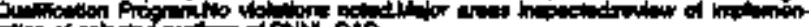

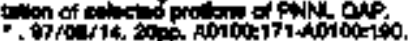

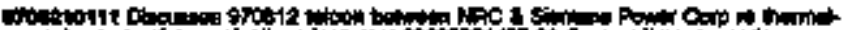

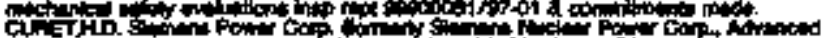

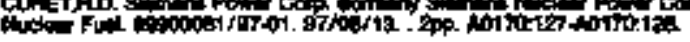

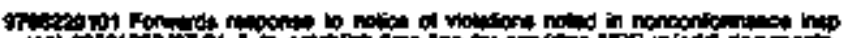

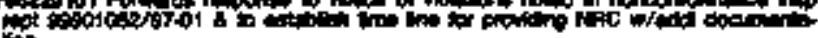
in. PII

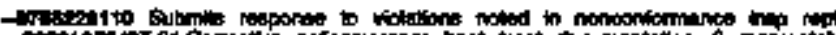

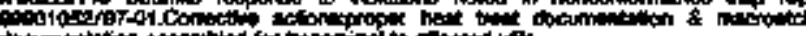

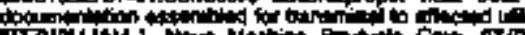

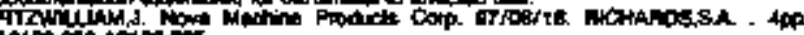

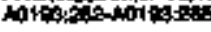

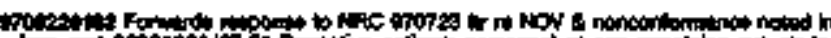

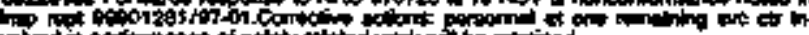

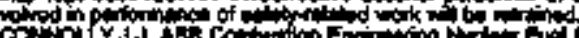

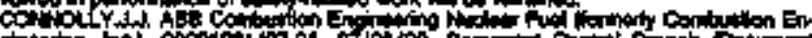

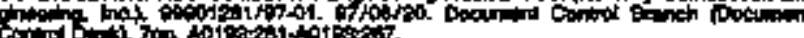

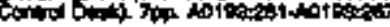

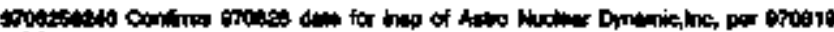

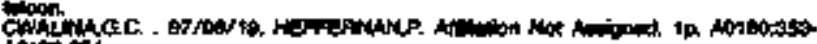
M1:003.

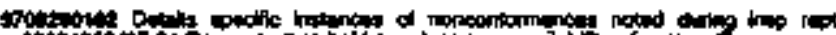

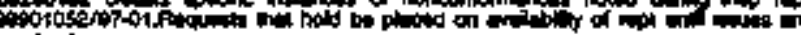

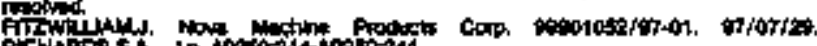

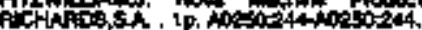

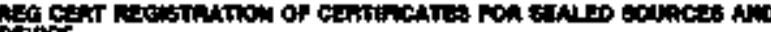

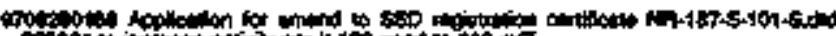

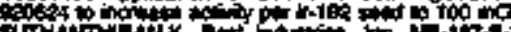

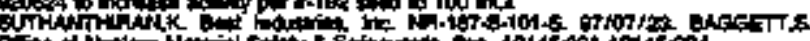

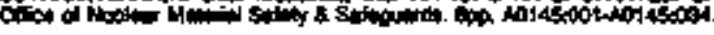

\section{FECGO RESULATOFY GUDES}

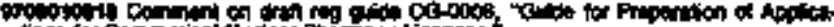

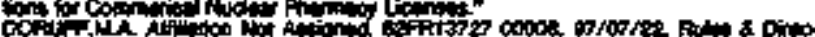

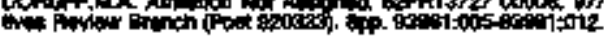

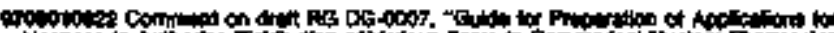

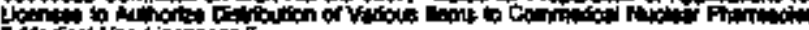

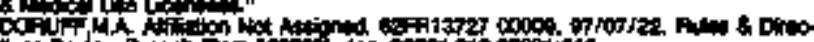

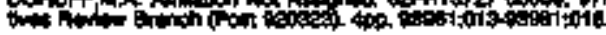

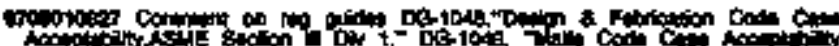
Now int."

HWE

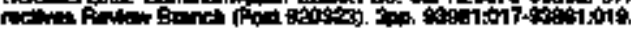

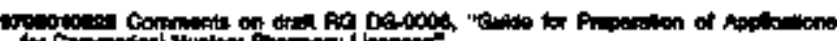

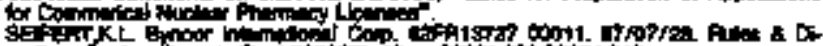
SE

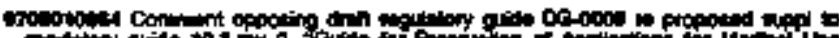

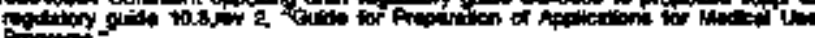

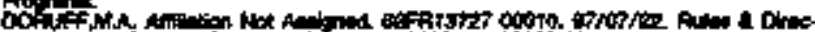

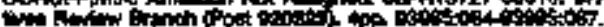

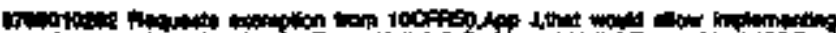

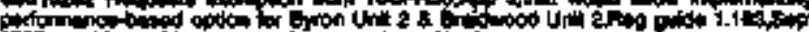

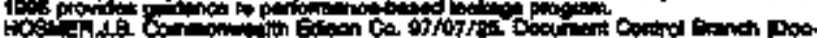

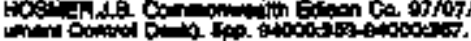

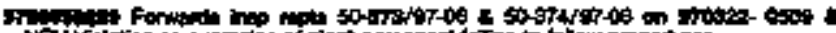

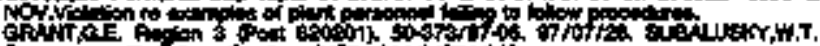

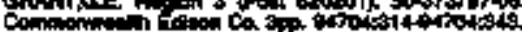

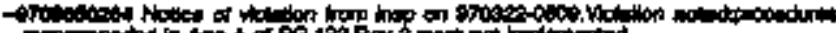

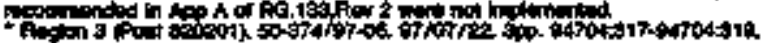

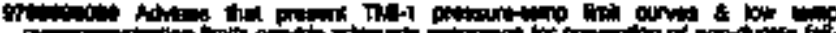

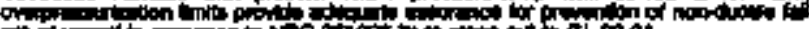

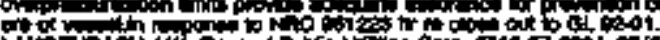

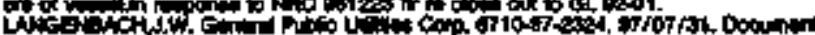

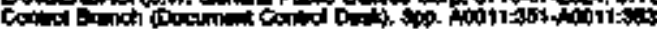

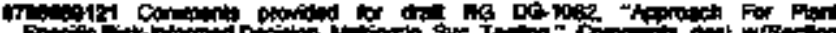

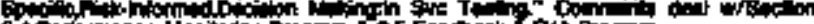

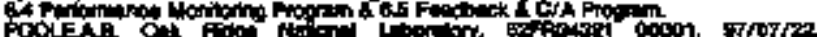

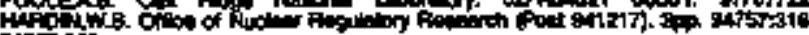
sing

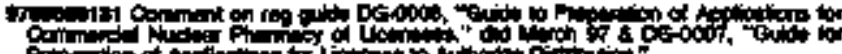

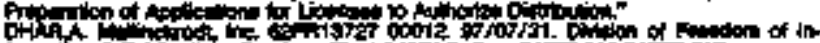

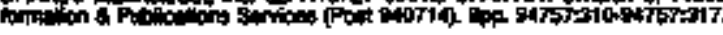

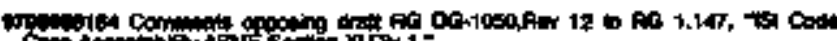

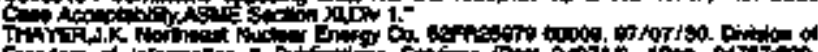

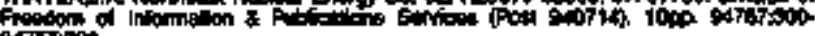
sirros:on. 


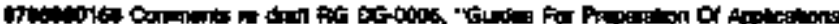

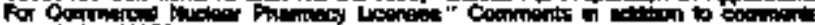
Gond

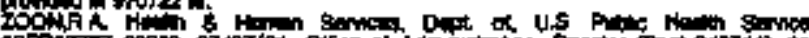

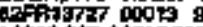

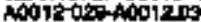

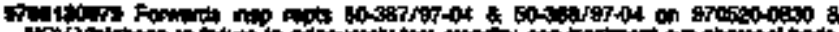

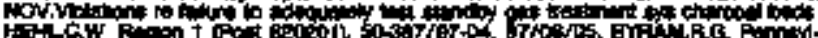

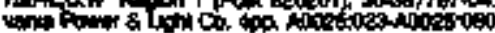

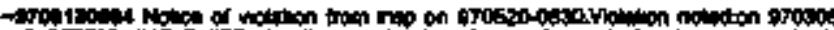

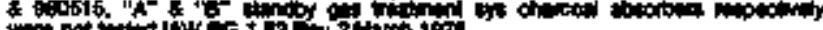

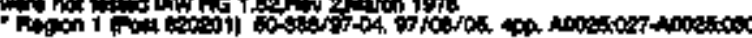

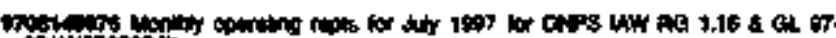
chation.

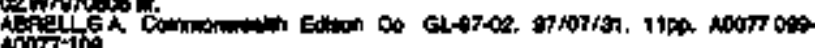
ADOT:10

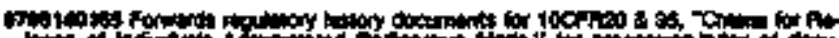

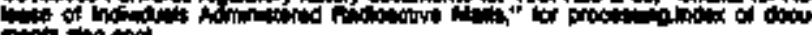

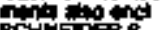

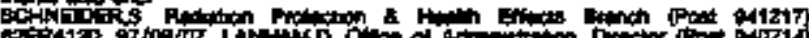

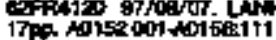

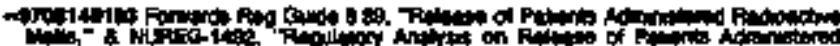

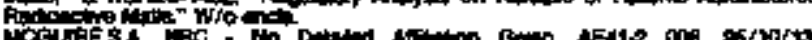

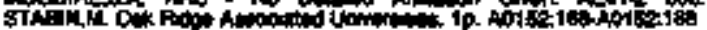

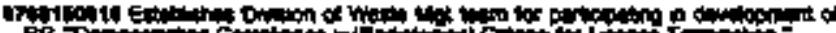

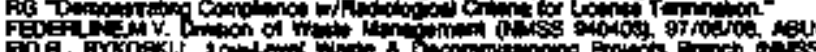
Fof, 由rk0\%

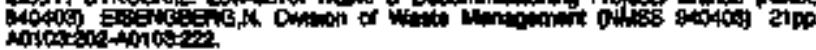

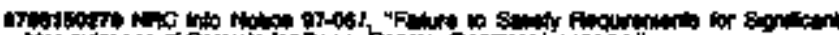

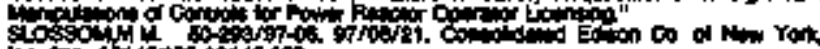

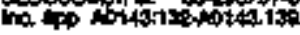

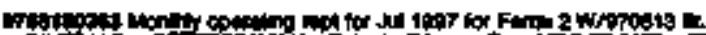

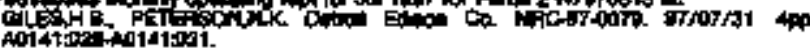

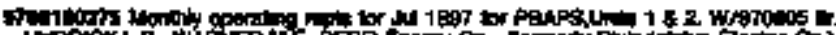

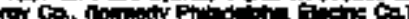

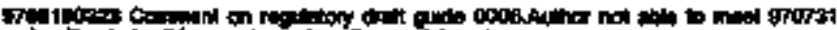

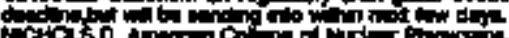

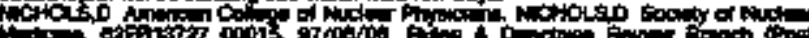

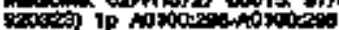

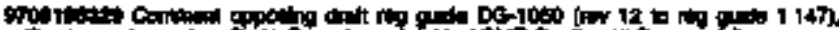

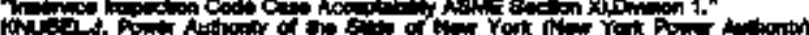

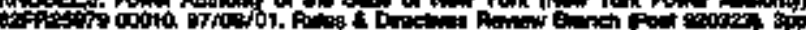

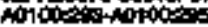

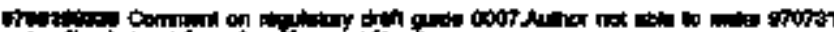
4.

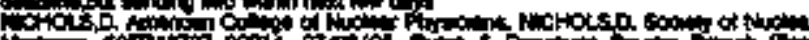

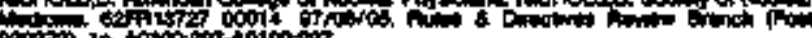

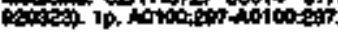

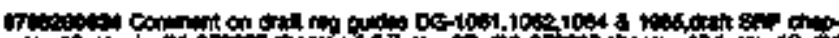

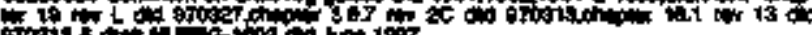
(1)

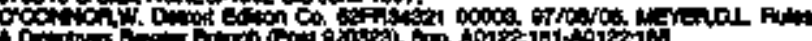

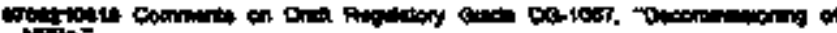

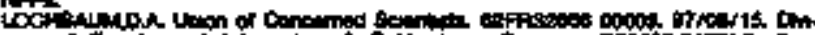

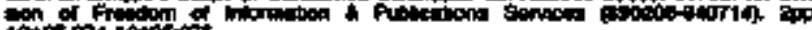

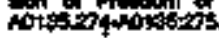

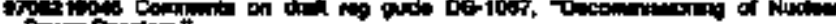

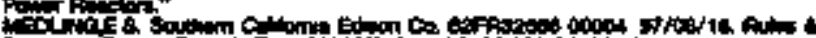

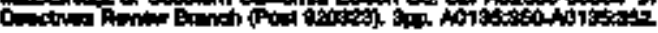

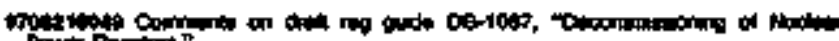
Arich Put

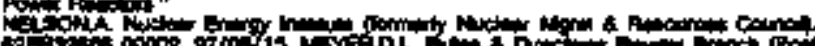

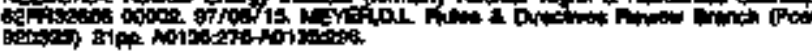

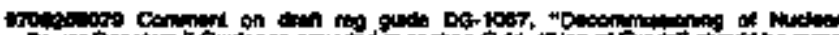

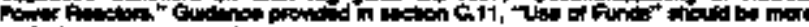

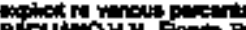

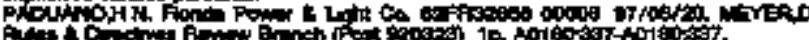

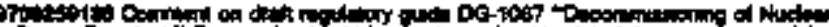

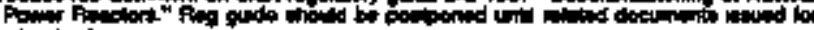

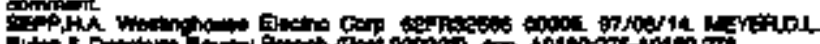
Arte 8 D.

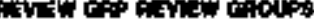

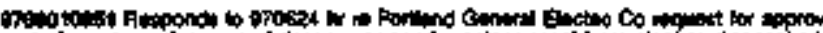

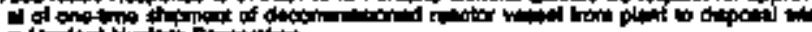

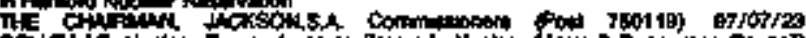

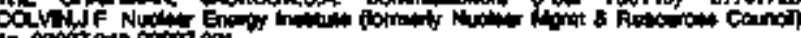

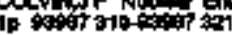

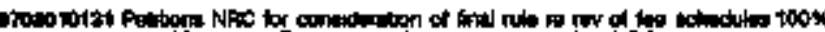

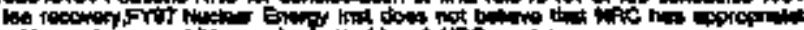

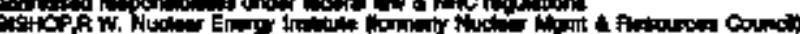

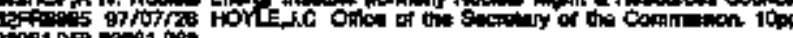

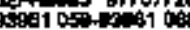

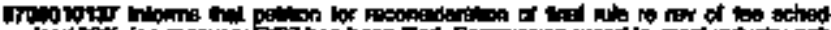

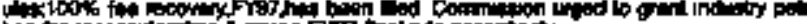

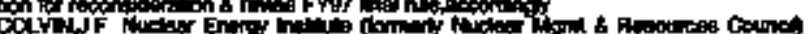

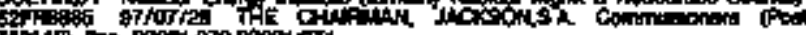

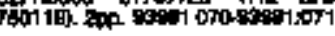

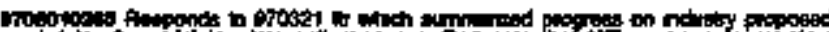

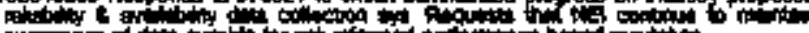

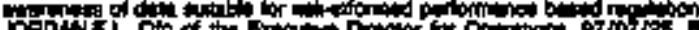

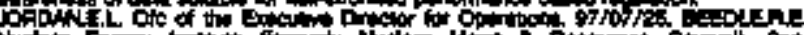

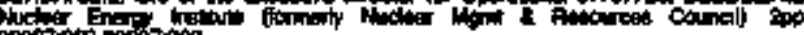

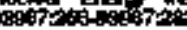

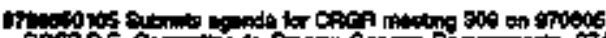

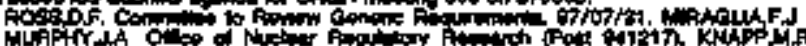

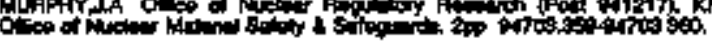

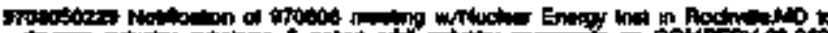

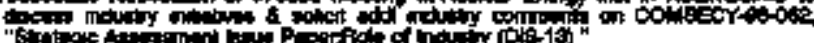

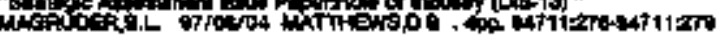

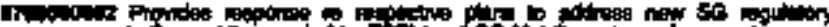

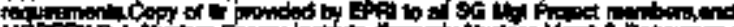

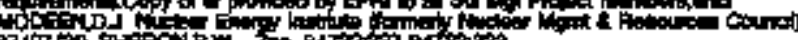
sirotiso ste

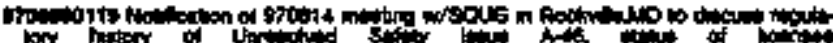

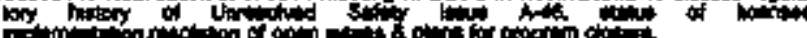

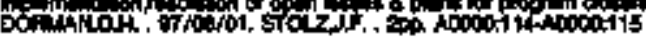

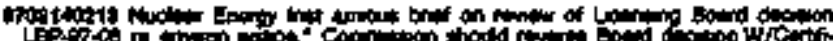

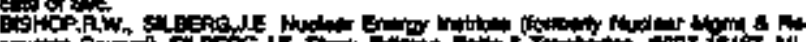

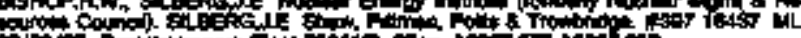

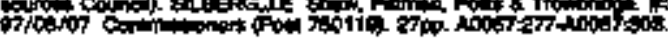

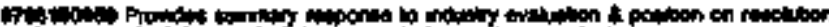

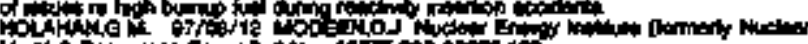

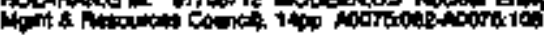

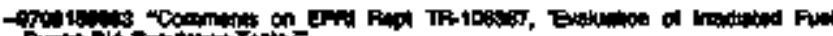

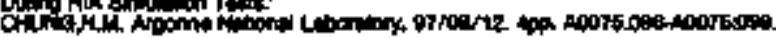

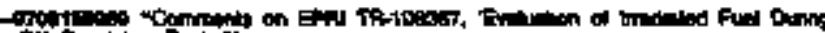

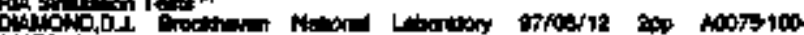
AOAT:

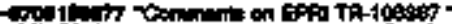

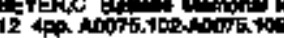

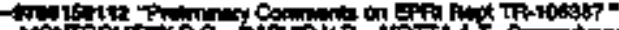

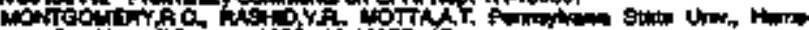

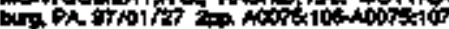

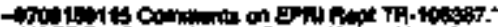
topis

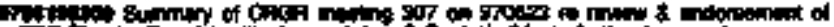

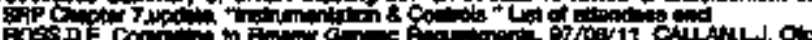

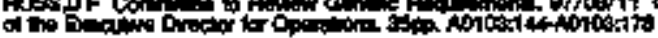

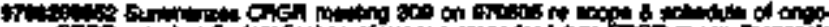

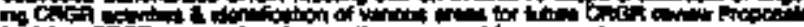

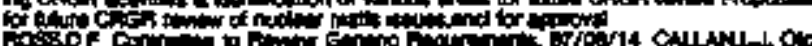

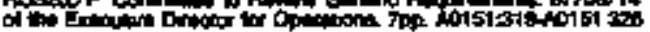

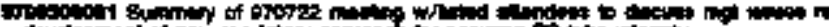

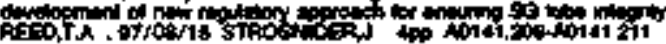

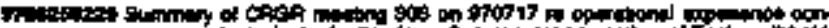

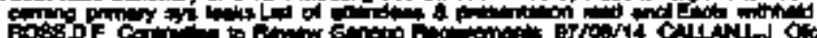

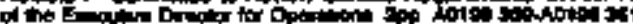




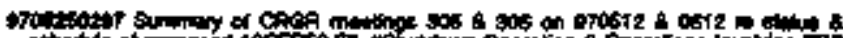

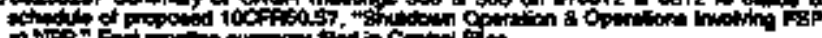

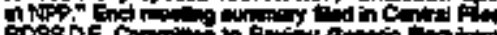

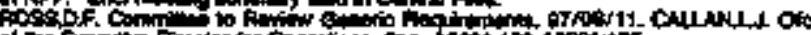

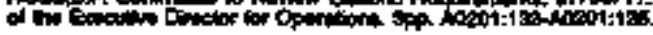

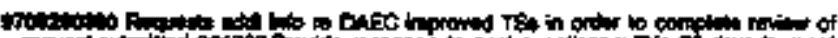

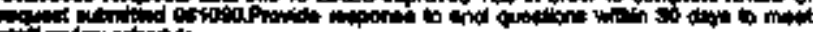

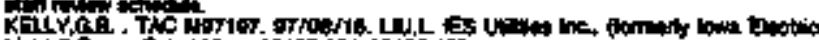

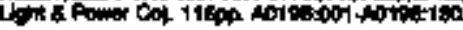

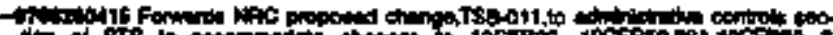

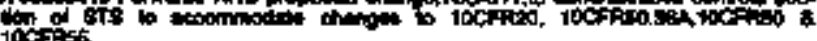

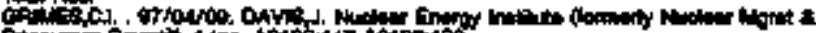

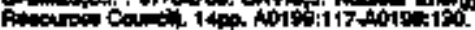

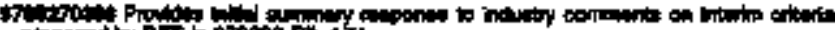

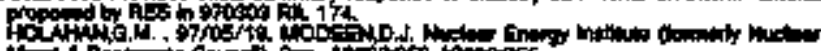

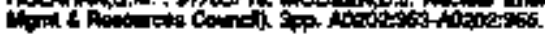

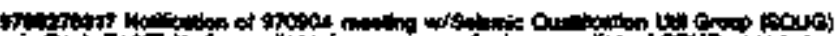

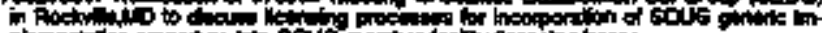

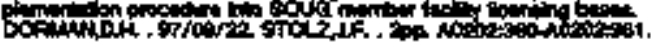

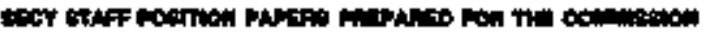

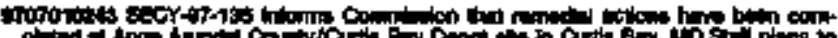

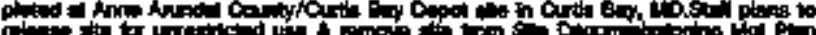

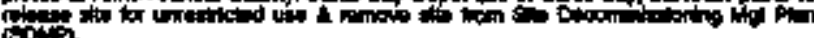
towis.

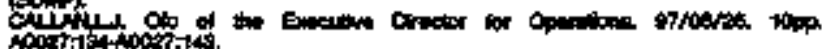

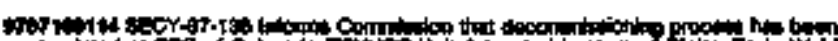

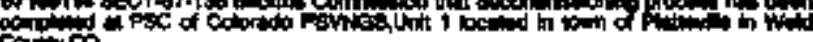

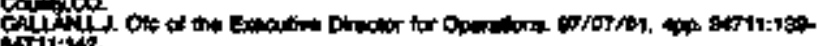

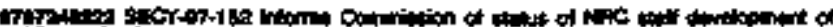

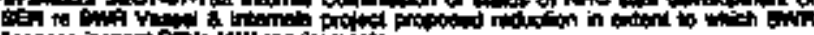

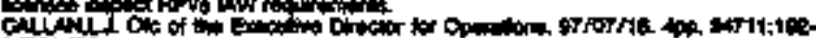
crivitit

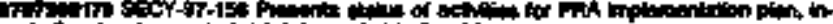

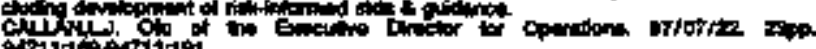

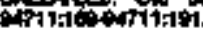

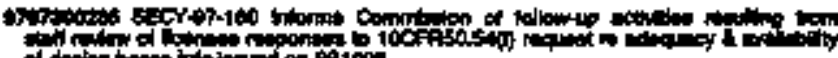

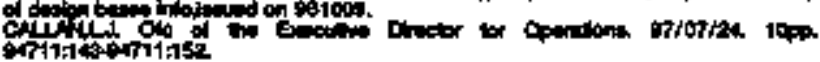

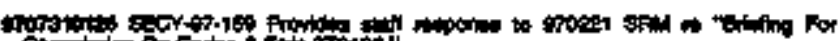

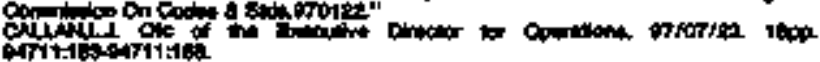

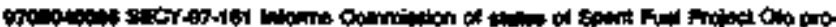

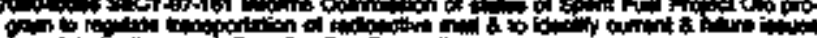

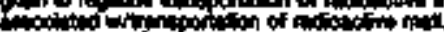

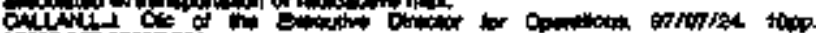

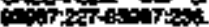

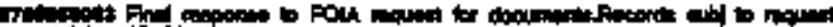

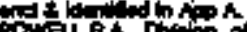

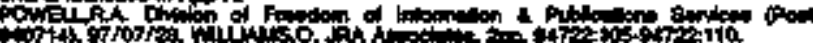

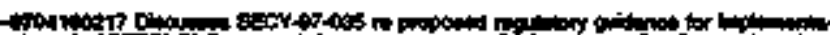
an $210 \mathrm{c}$.

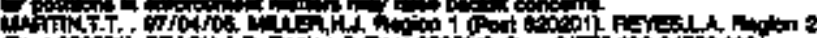

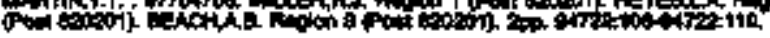

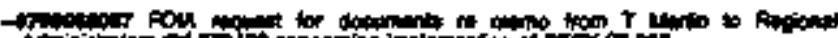

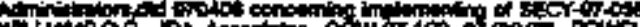

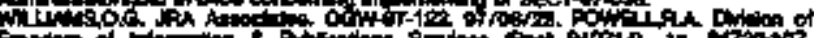

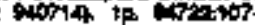

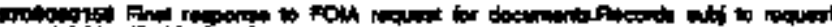
odel if

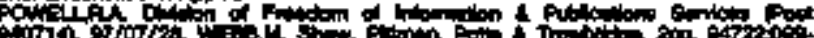
thithos

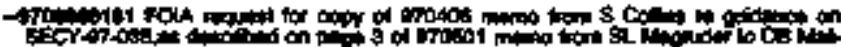

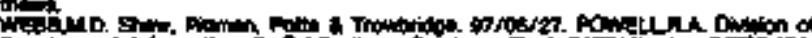

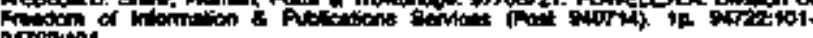
Borptod

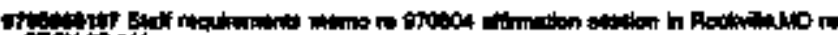
s.t.tit

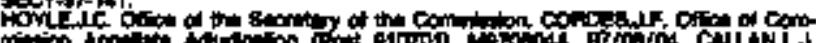

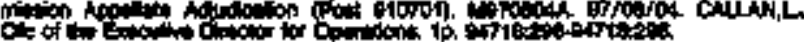

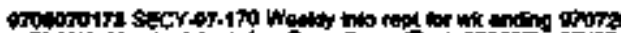

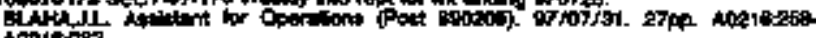
napisers.

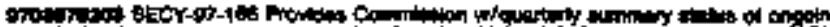

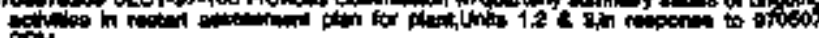

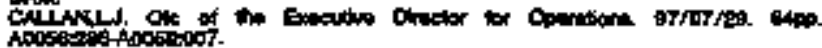

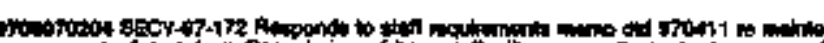

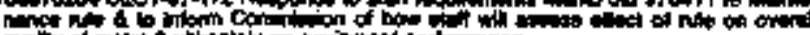

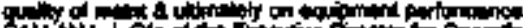

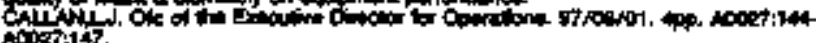

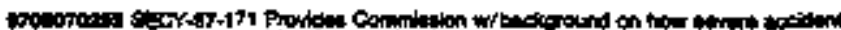

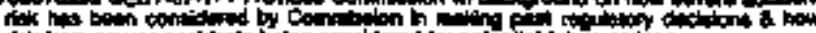

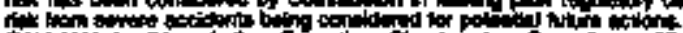

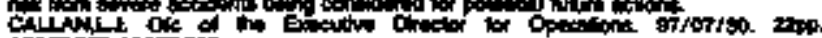
Notrotom

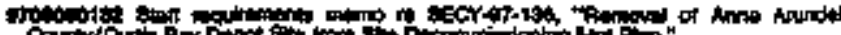

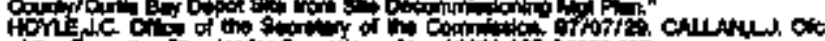

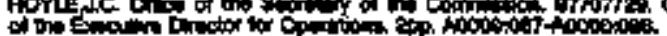

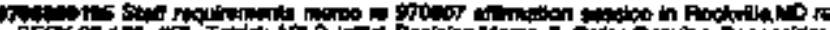

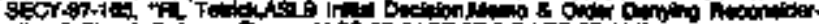

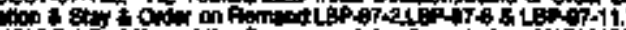

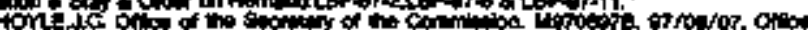

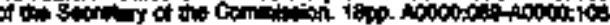

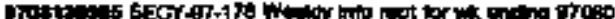

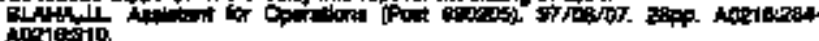

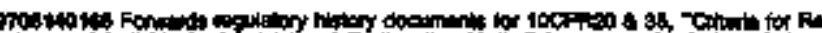

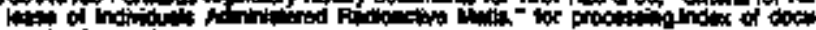
Bictions

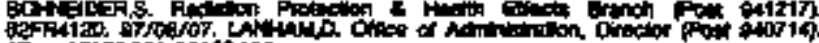

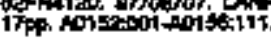

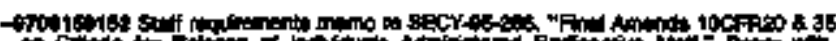

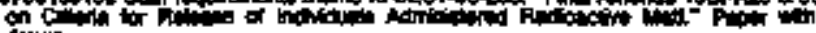
Artin.

Thy

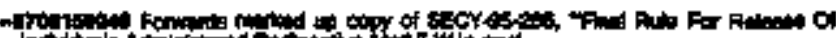

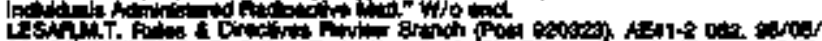
L

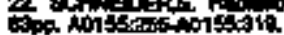

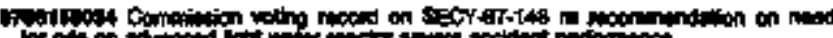

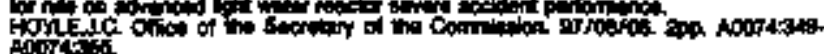

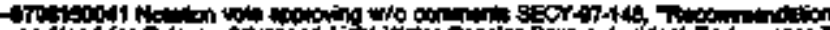

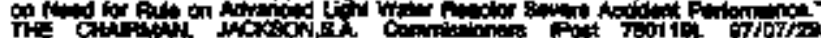

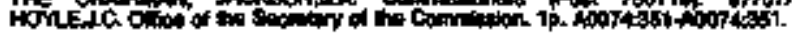

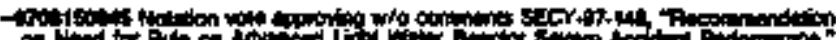

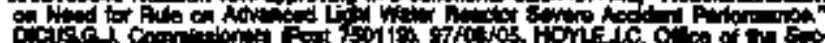

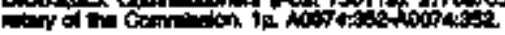

1.

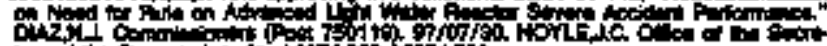

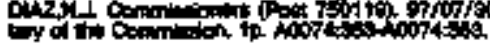

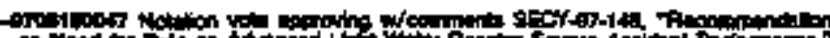

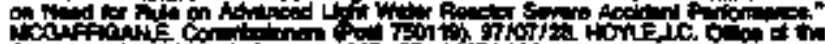

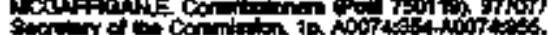

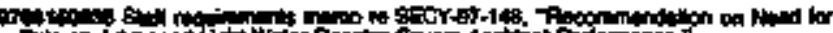

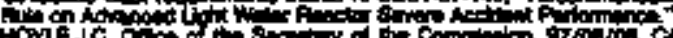

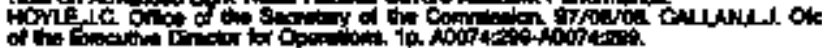

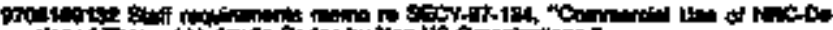

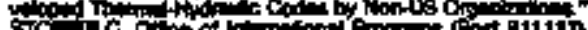

Gic d

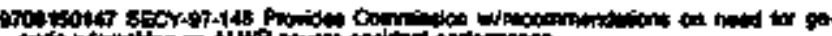

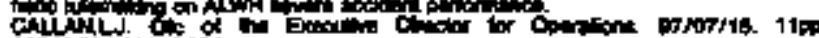

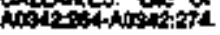

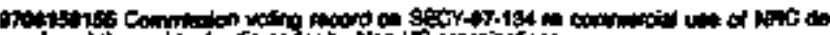

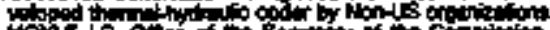
forif

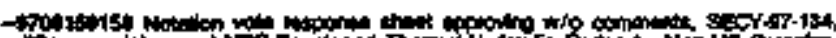

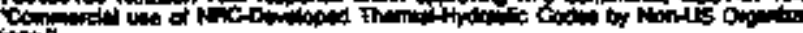

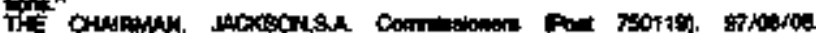

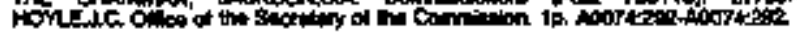




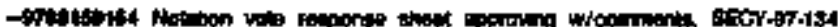

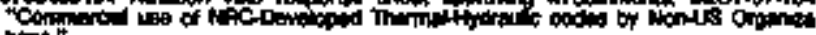

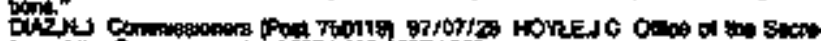

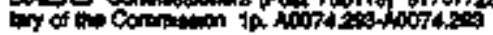

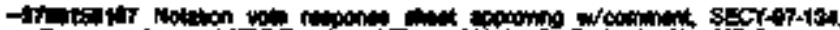

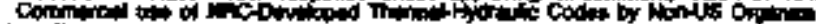

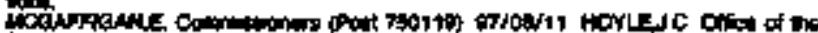

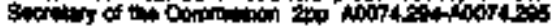

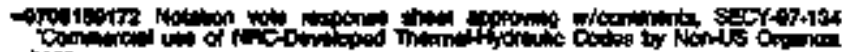

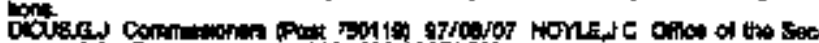

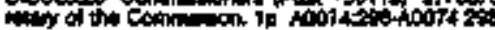

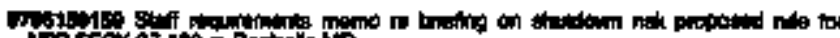

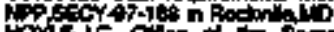

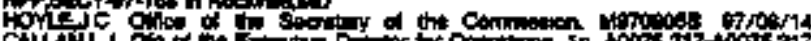

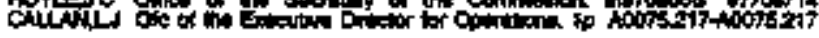

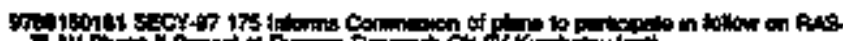

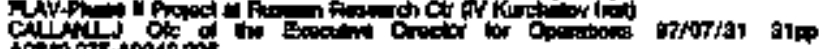

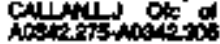

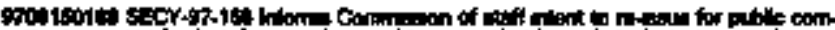

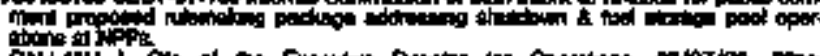

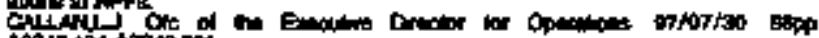

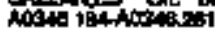

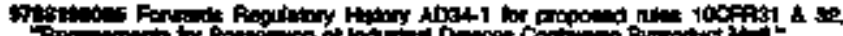

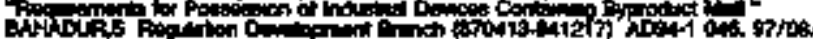

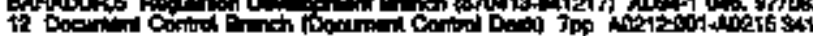

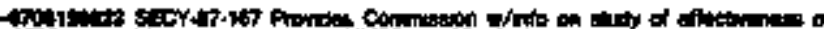

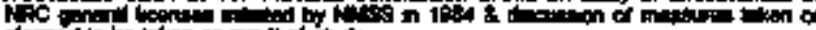

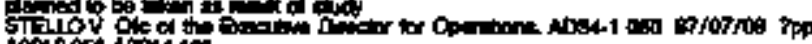

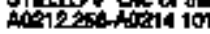

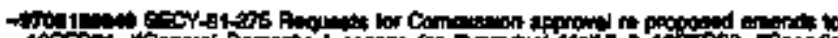

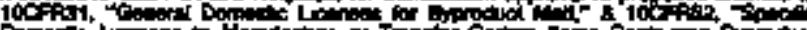

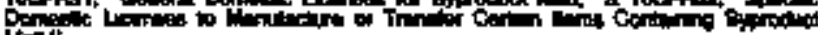

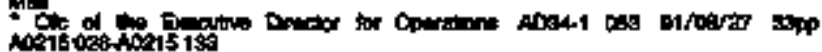

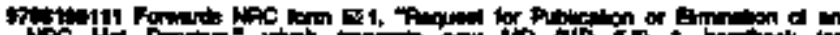

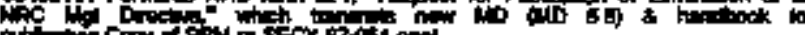

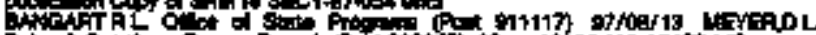

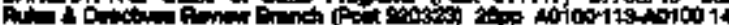

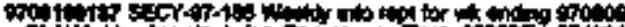

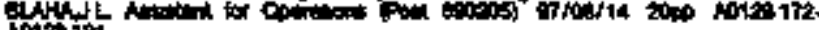
120

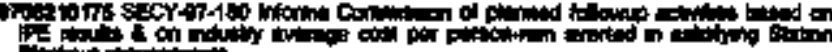

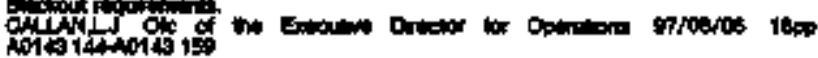

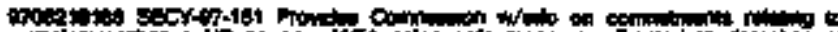

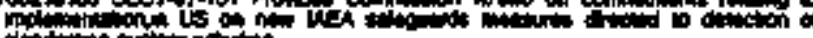

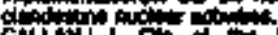

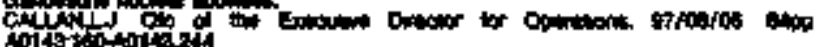

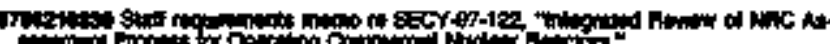

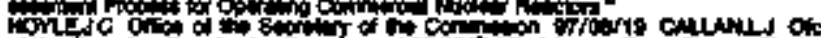

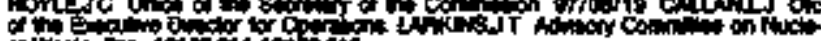

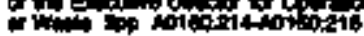

Pro D.to

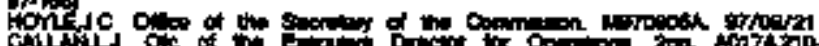

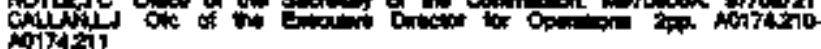

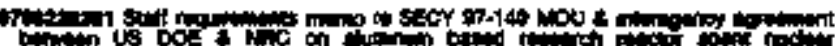

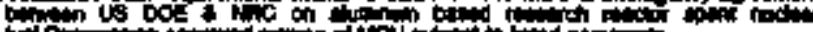

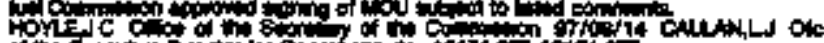
cith te

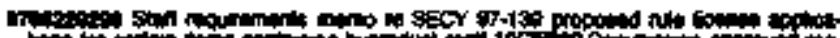

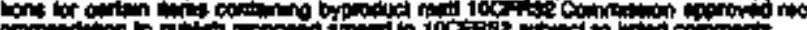

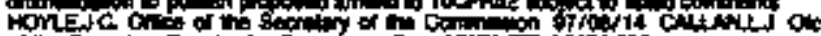

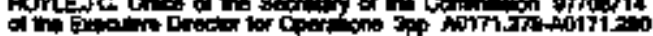

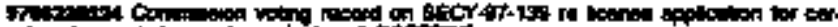

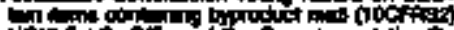

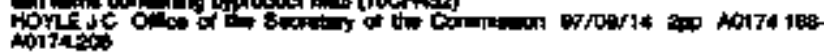

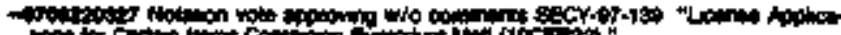

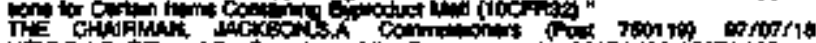

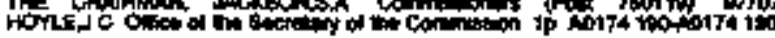

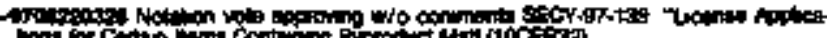

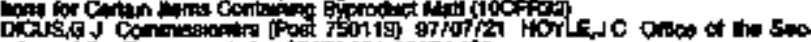

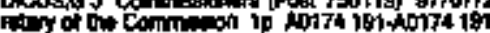

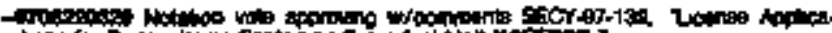

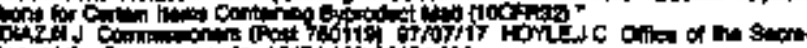

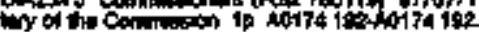

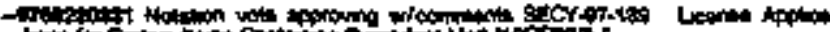

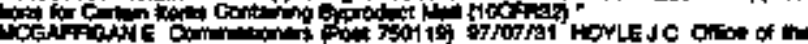

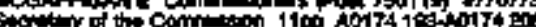

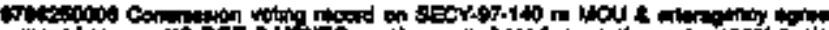

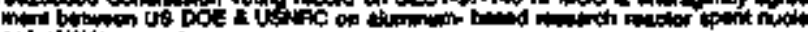

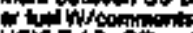

Hoy A

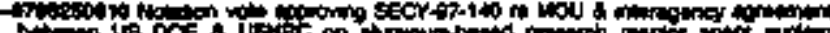

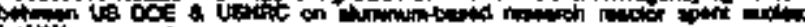

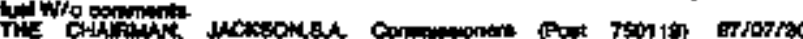

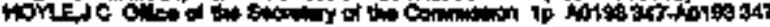

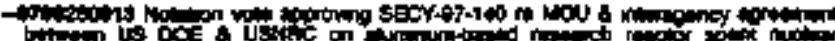

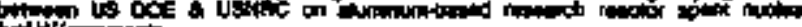
Hithomments

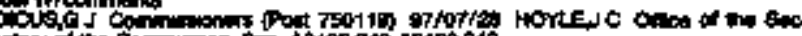

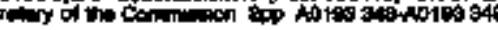

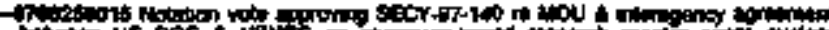

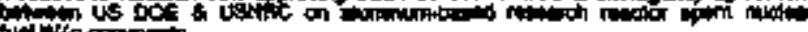

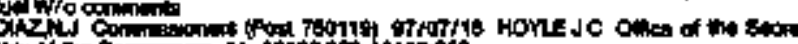

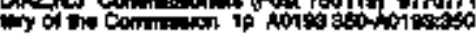

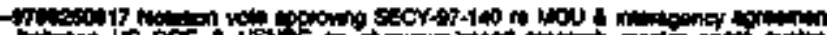

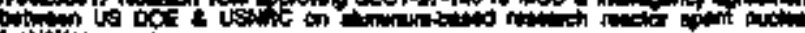

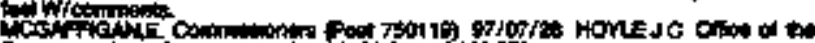

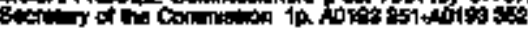

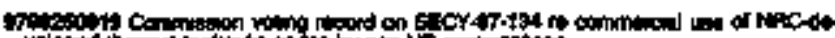

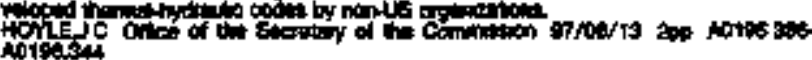

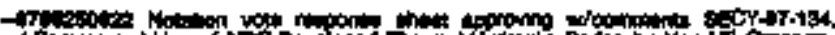

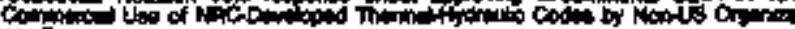

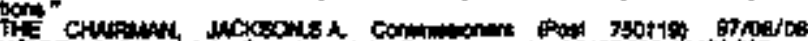

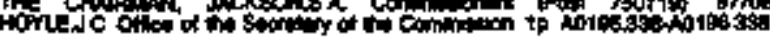

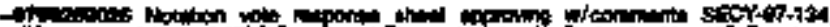

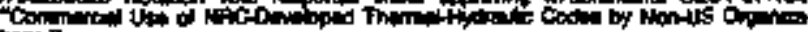

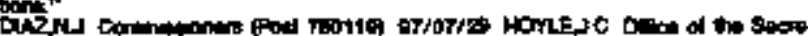

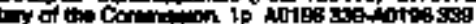

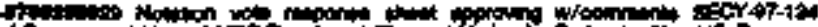

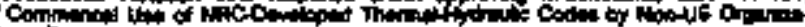

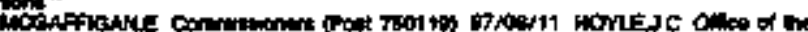

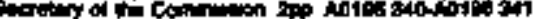

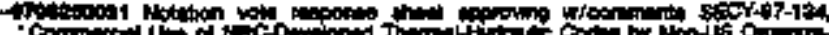

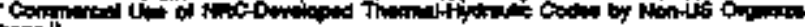

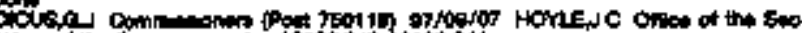

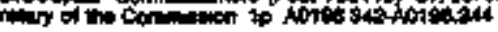

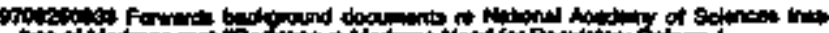

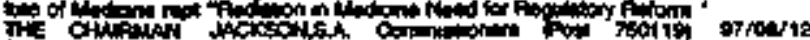

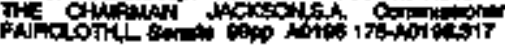

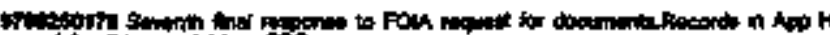
Pot in

OHa

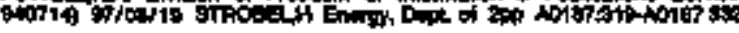

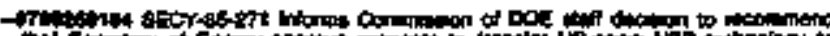

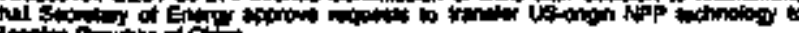

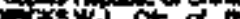

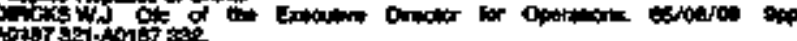

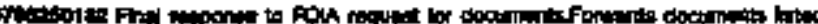

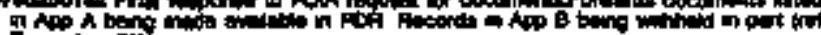
Erivion 7 h

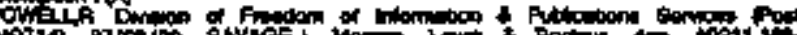
90719.

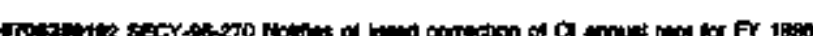

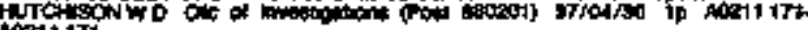
WP11 1T

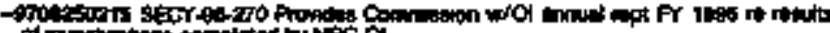

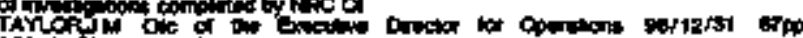

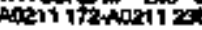




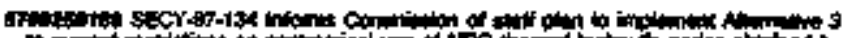

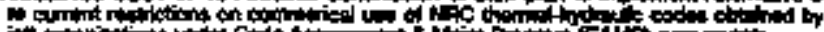

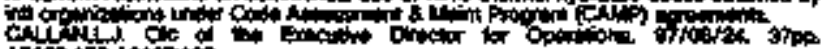

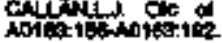

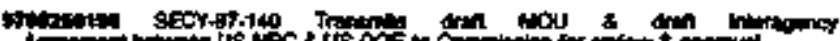

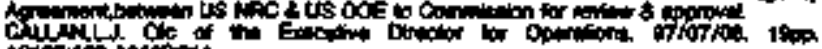

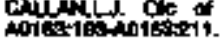

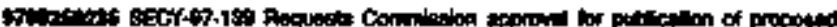

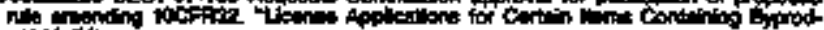

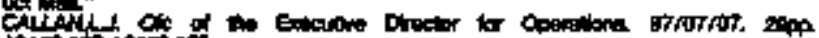

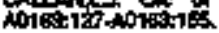

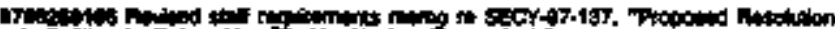

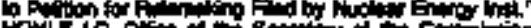

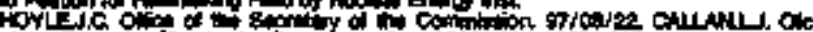

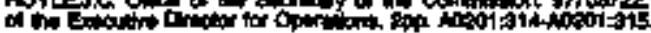

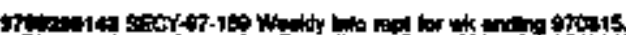

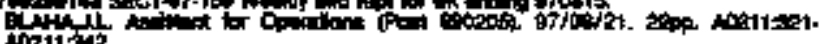
toz11 11 at?

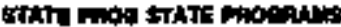

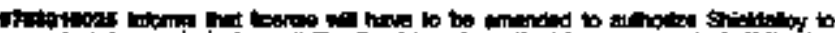

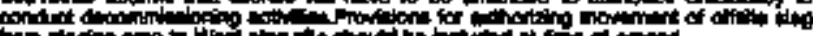

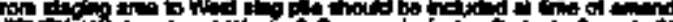

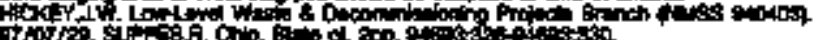

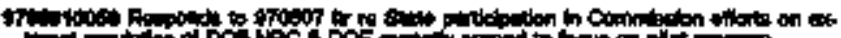

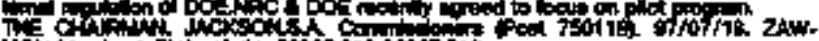

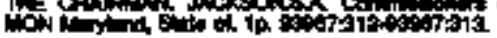

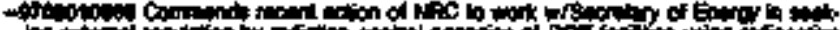

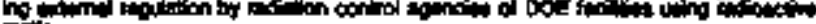

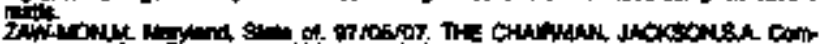

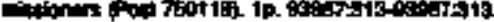

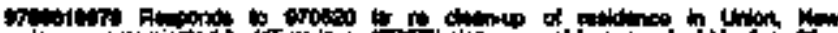

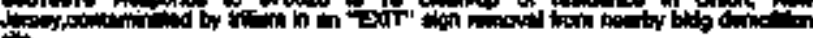

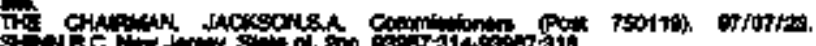

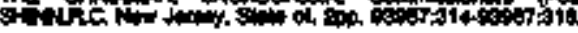

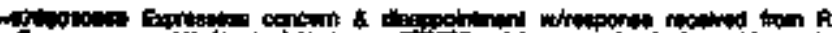

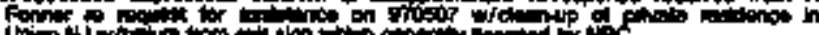

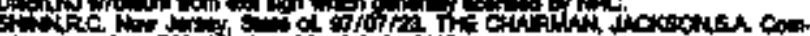

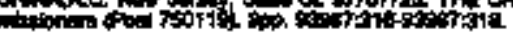

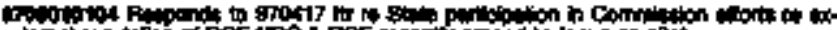

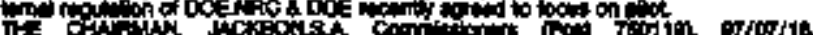
IF

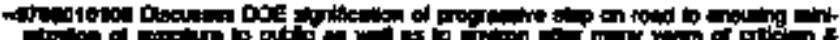

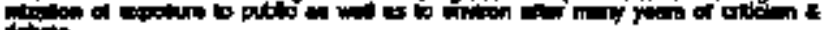

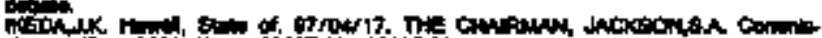

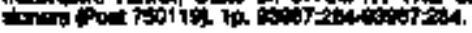

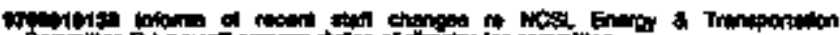
oq

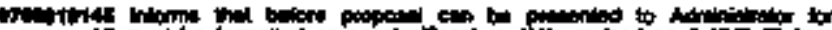

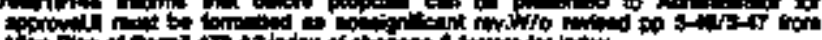

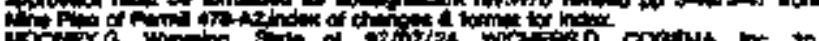

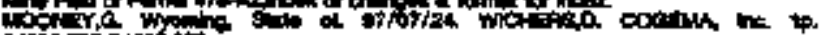

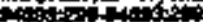

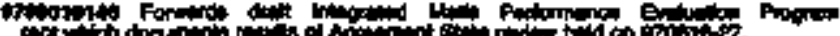

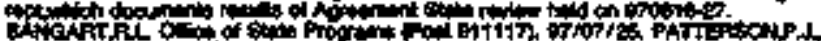

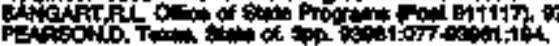

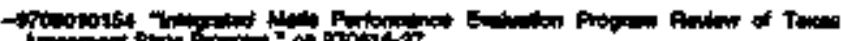

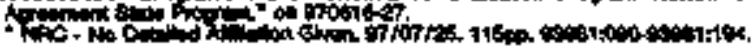

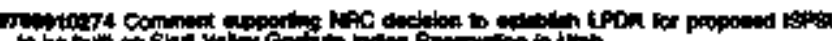

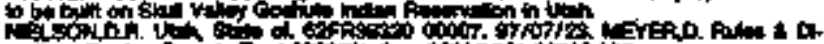
A

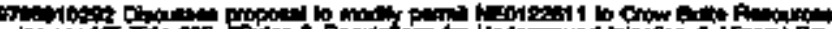

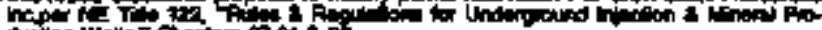

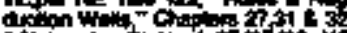

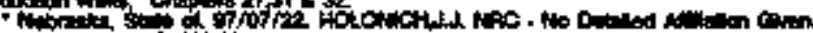

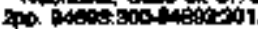

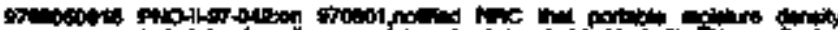

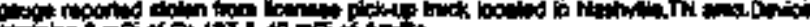

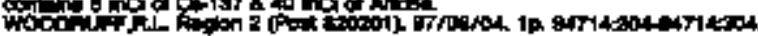

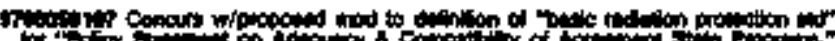

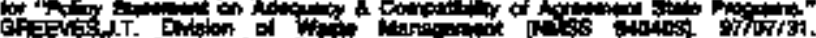

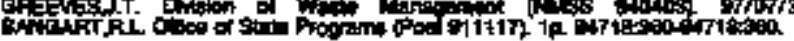

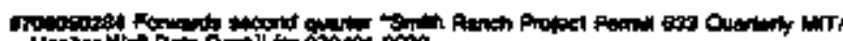

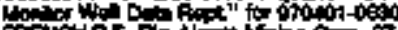

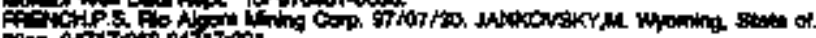
20po git?

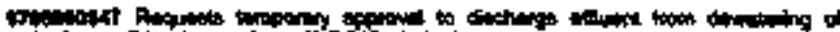

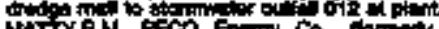

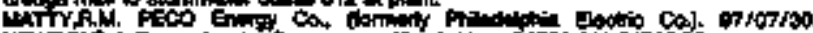

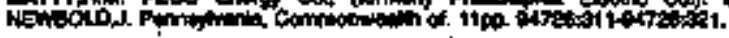

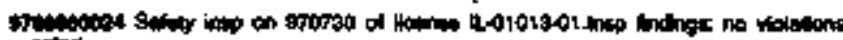
Pinging

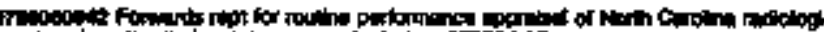

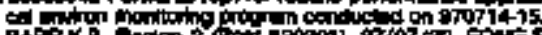

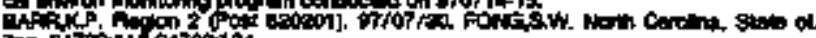
Tpe ofto:11004720124,

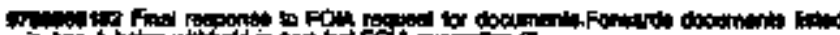

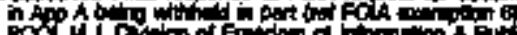

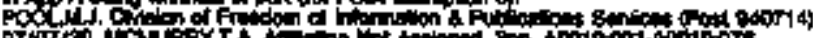

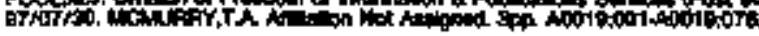

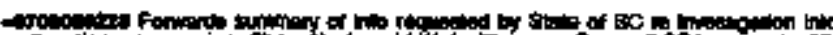

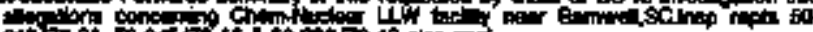

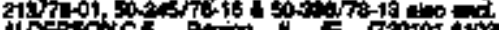

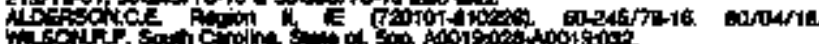

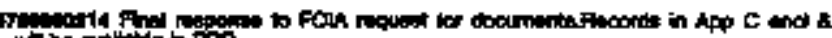

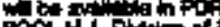

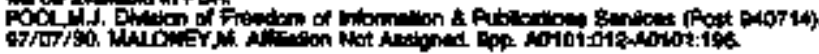

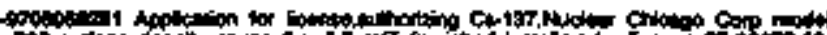
P2

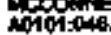

A

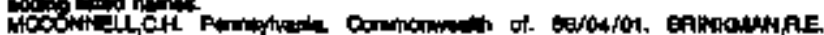

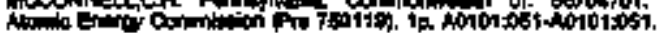

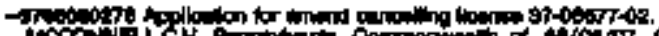

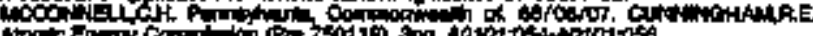
型

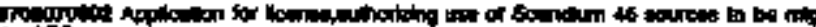
by tec.

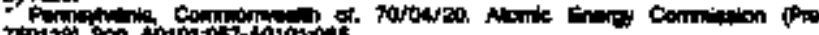

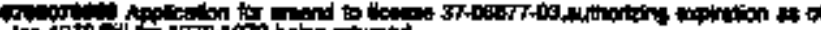
an 1 fot

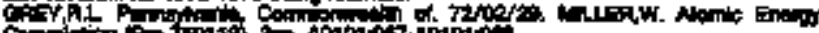

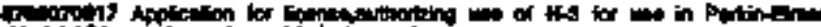

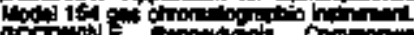
Arotions

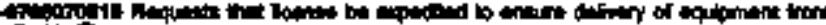

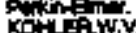

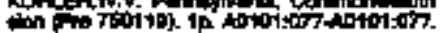

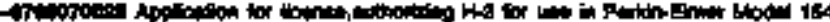

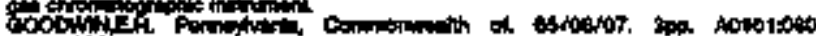
nototion

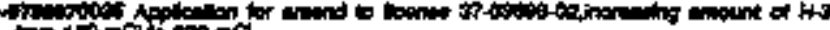
ton 130 mo to

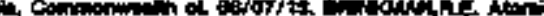

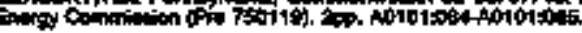

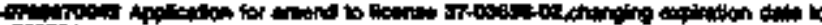

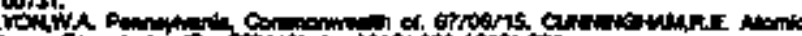

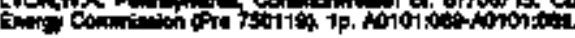

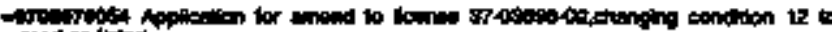

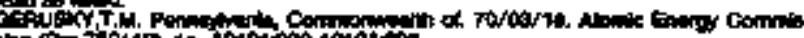

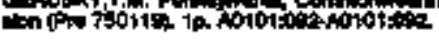

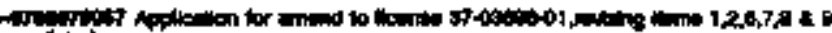
E.

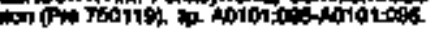

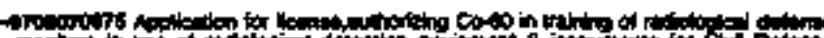

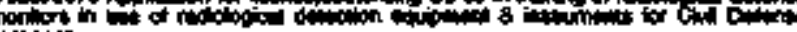

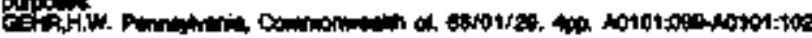

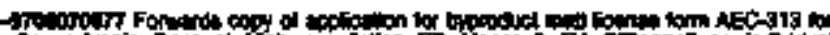

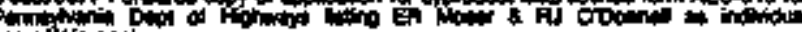
toing

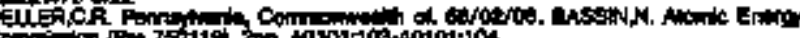

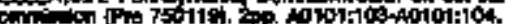




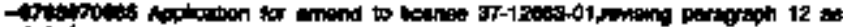

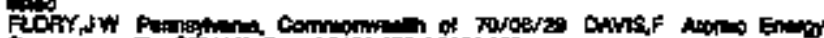

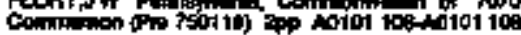

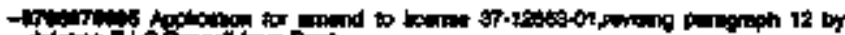

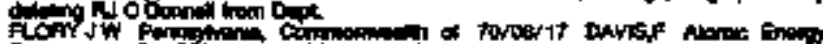

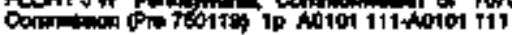

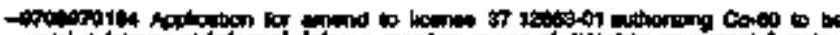

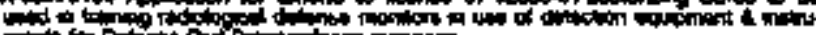

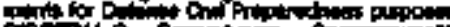

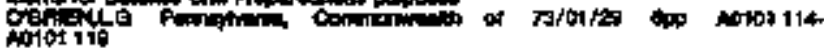

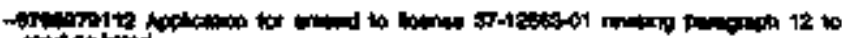

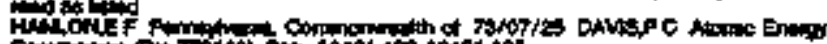

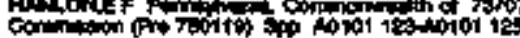

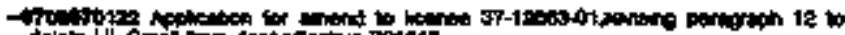
dever HL

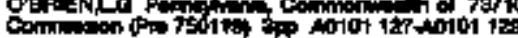

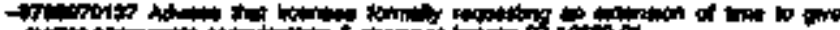

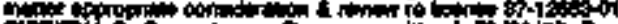

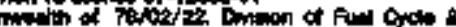

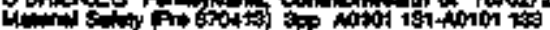

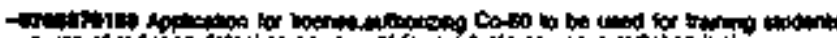

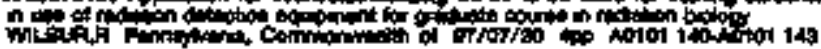

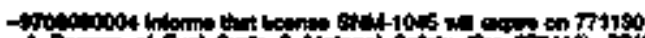

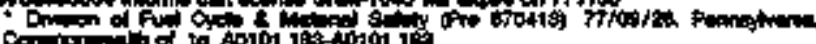

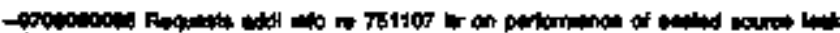

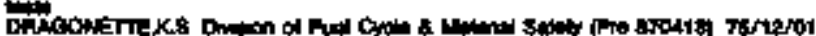

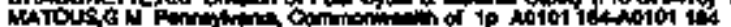

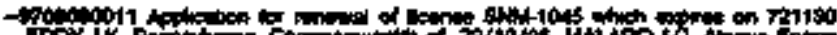

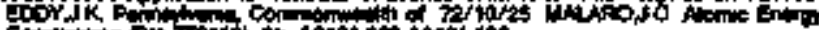

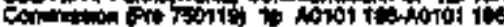

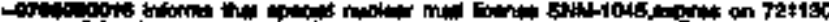
Waroj J

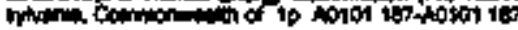

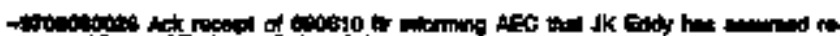

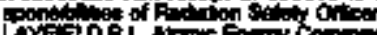

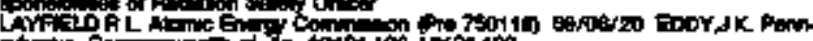

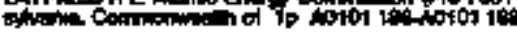

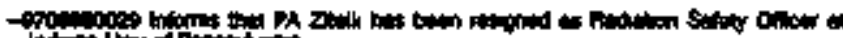

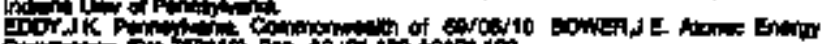

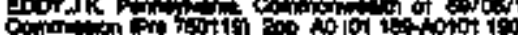

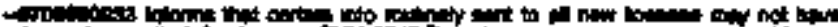

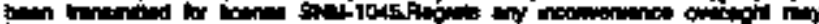

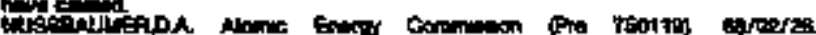

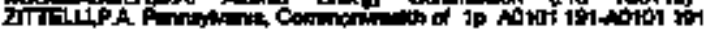

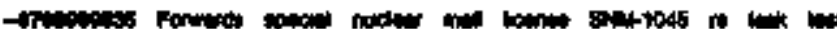

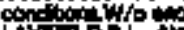

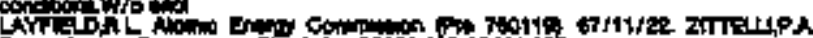

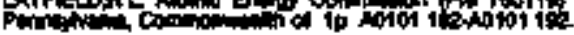

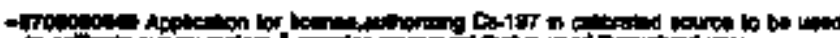
be

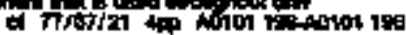

की (0)

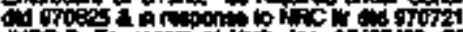

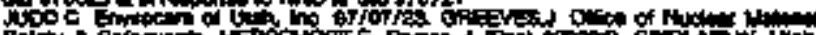

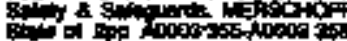

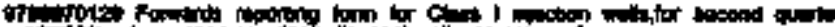

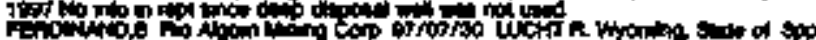

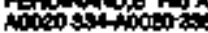

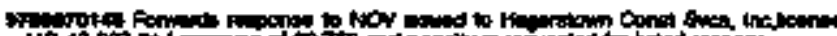

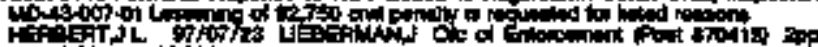
Aco.t.

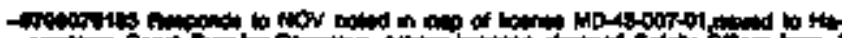

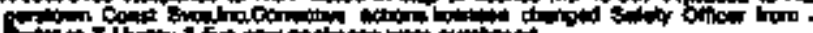

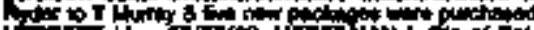

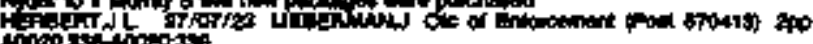

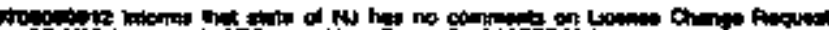
UAH

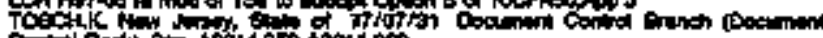

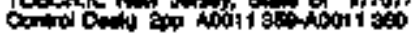

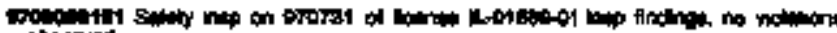

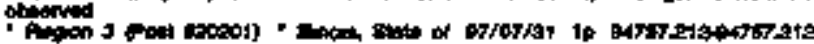

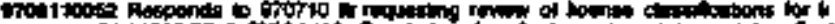

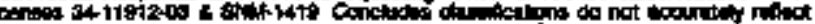

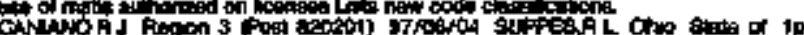
inor3otedororoor

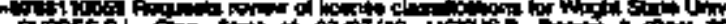

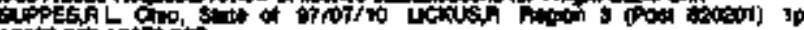

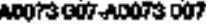

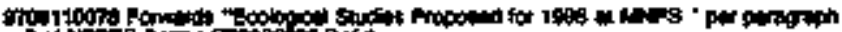

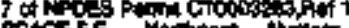

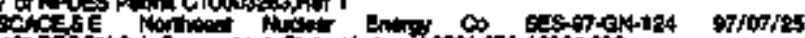

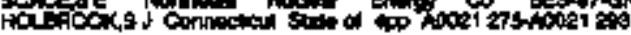

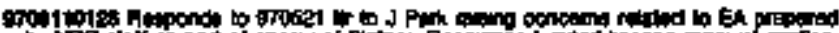

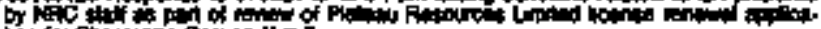

folo H L

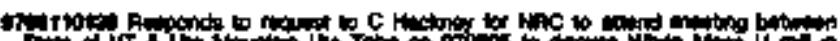

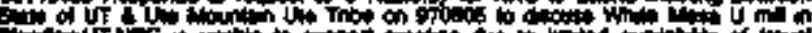

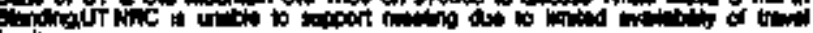

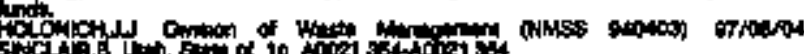

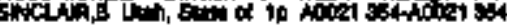

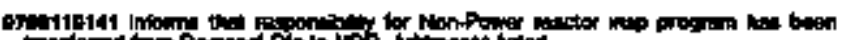

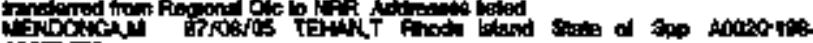

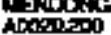

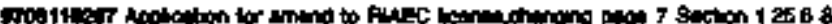

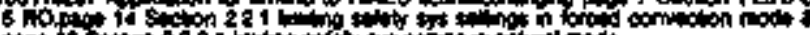

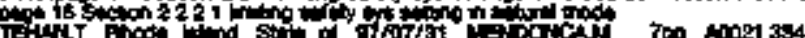
Alozit 3

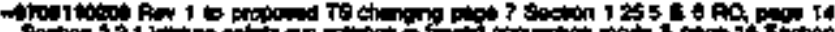

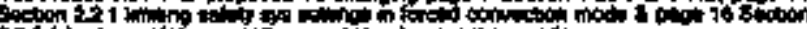

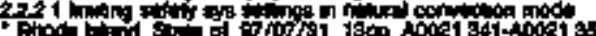

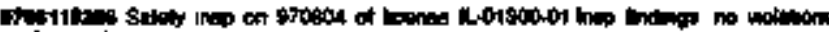

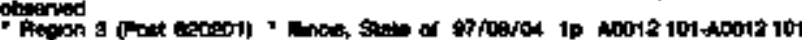

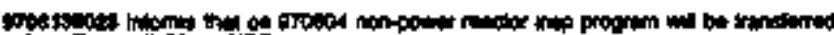

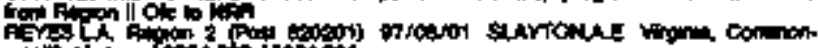

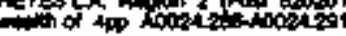

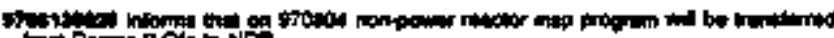

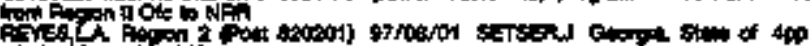
ionstions

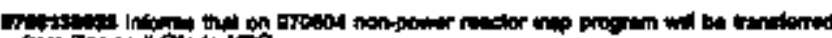

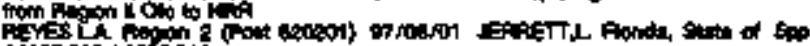

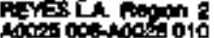

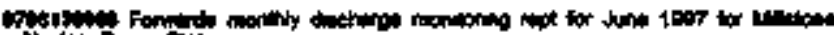

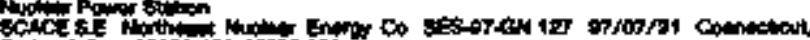

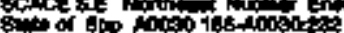

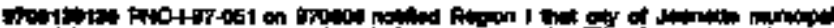

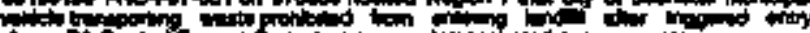

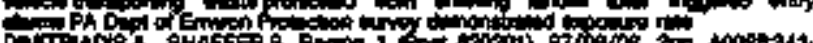

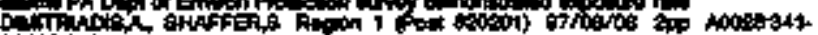
$\operatorname{lom}_{3 \rightarrow \infty} \rightarrow$

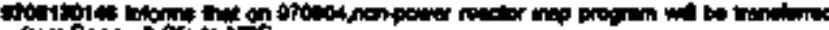

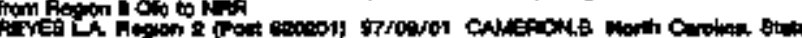

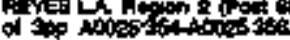

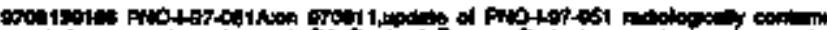

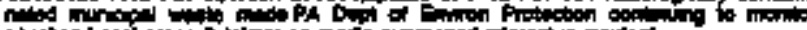

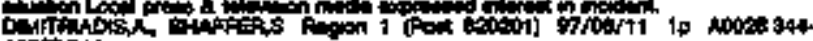
Anotisin

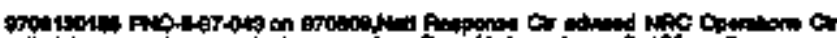

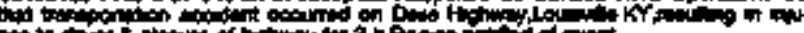

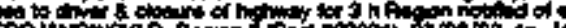

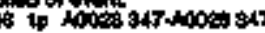

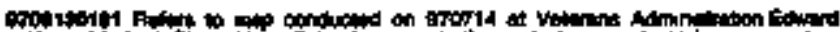

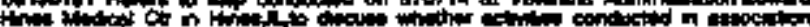

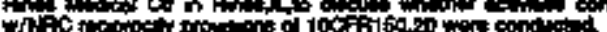

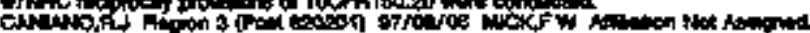
ip 100 o

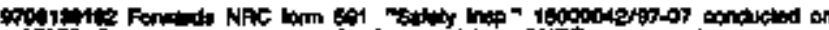

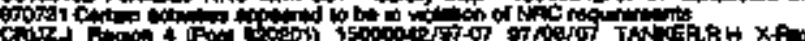

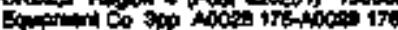

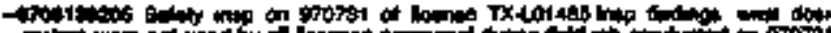

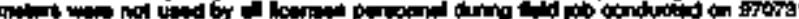

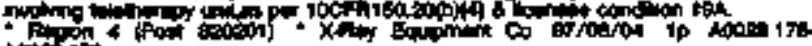
nition. 


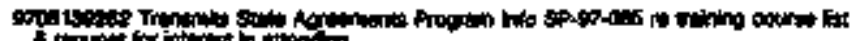

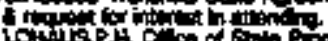

Dow

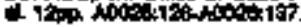

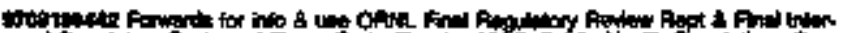

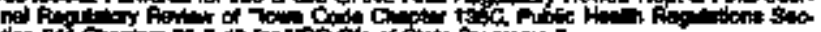

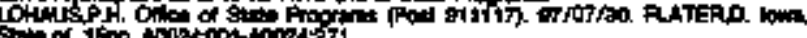

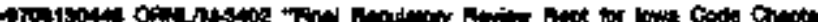

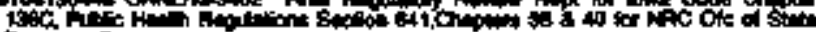
fincting

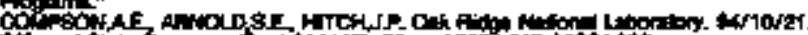

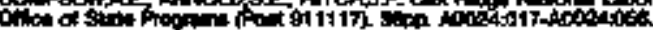

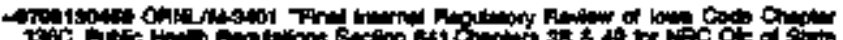
Proprin:

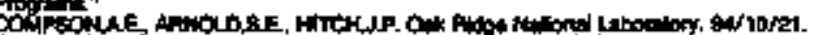

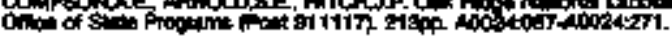

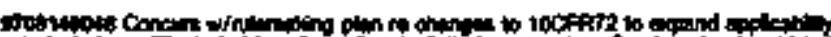

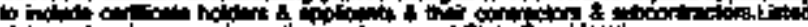

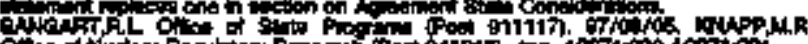

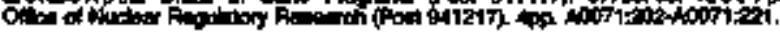

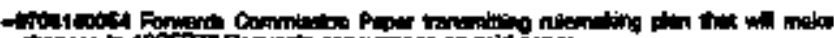

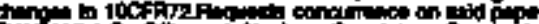

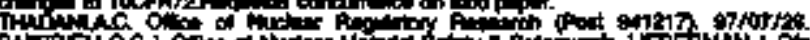
=

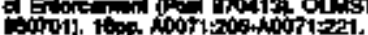

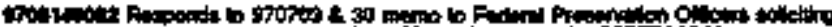

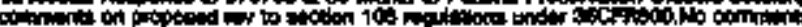

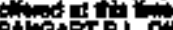

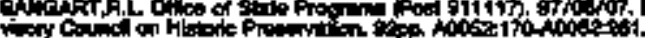

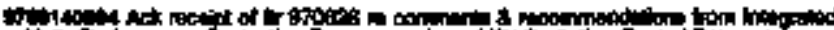

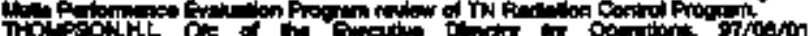

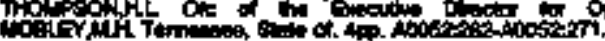

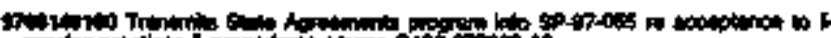

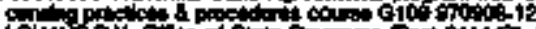

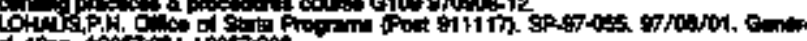
d tson

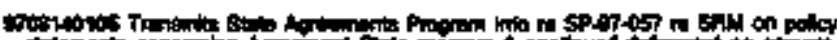

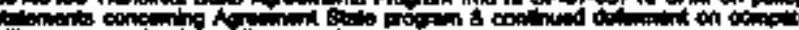

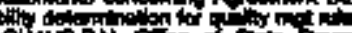

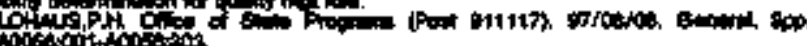

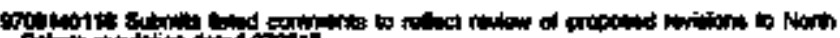

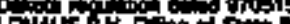

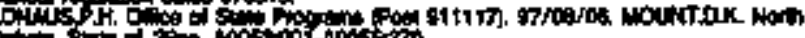

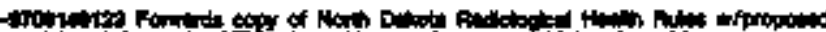

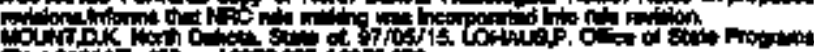

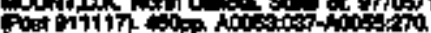

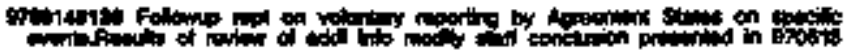

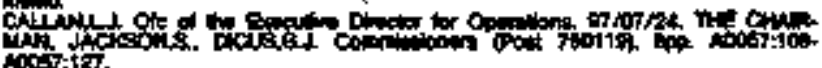

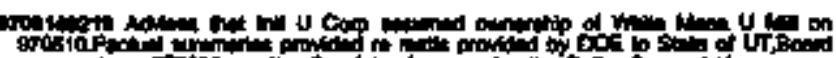

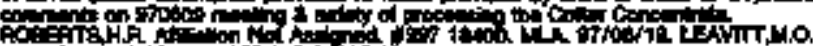

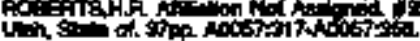

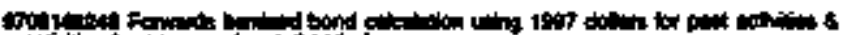

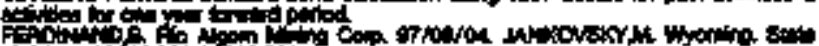

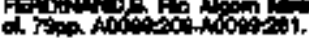

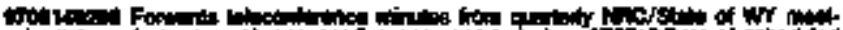

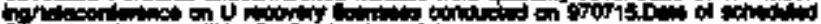

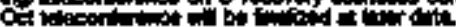

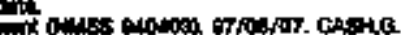

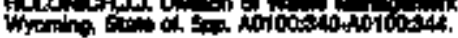

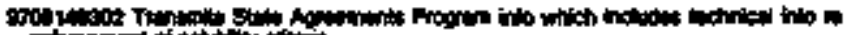

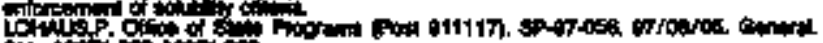

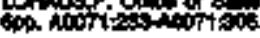

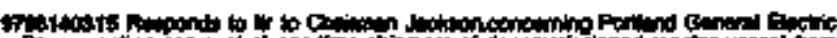
Co rop

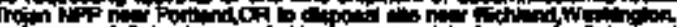

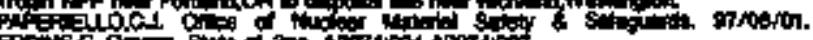

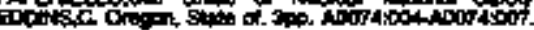

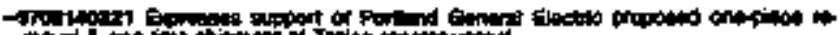

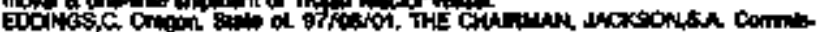

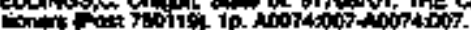

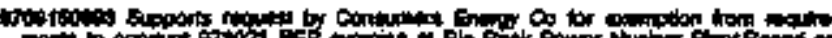

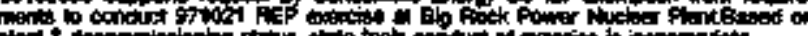

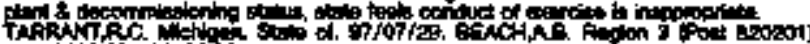

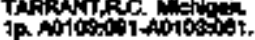

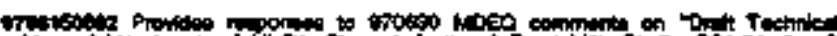

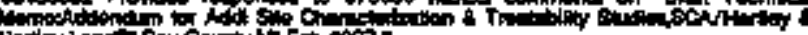

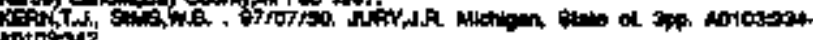

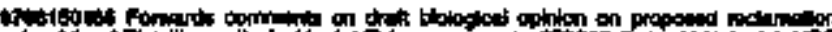

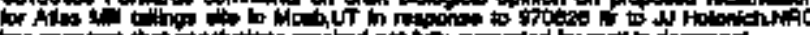

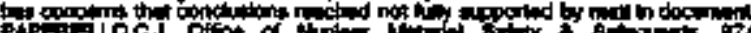

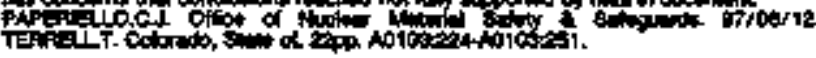

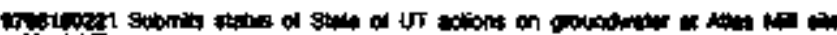
Mater.

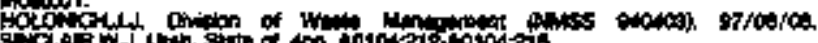

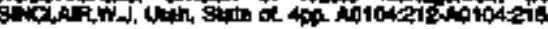

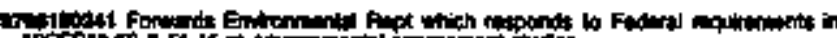
I0

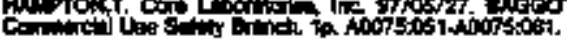

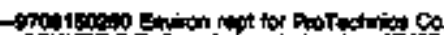

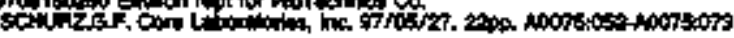

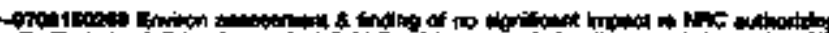

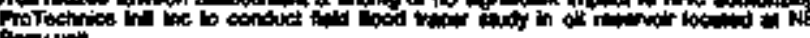

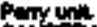

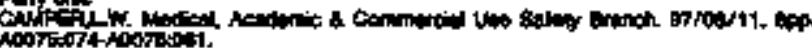

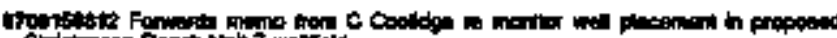

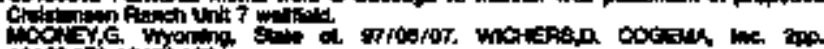

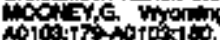

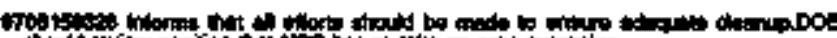

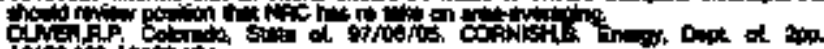
A

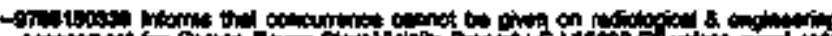

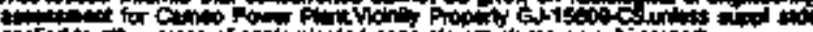

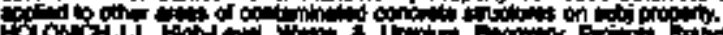

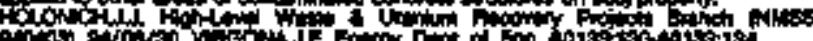

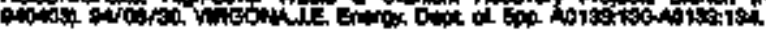

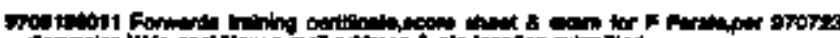

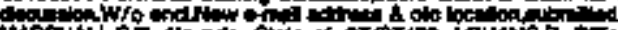

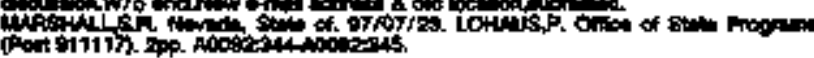

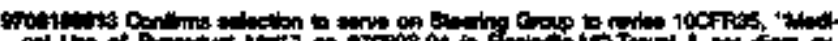

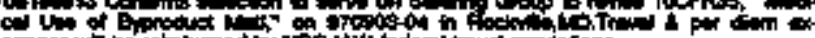

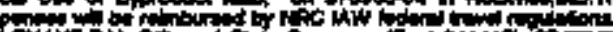

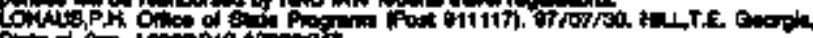

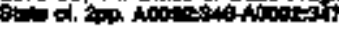

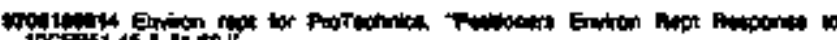

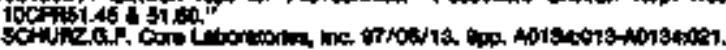

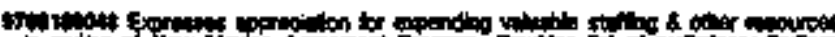

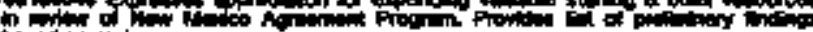
cid

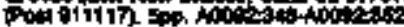

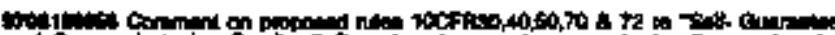

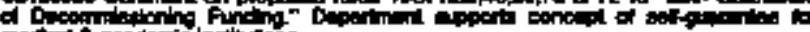

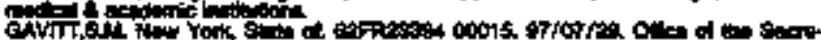

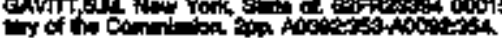

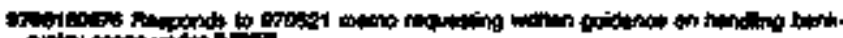
Reto

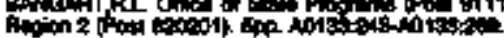

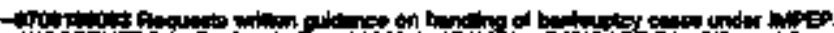

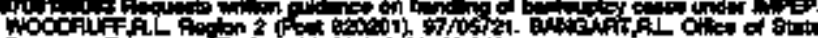

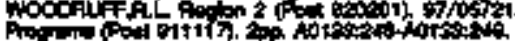

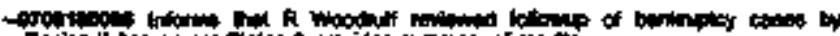

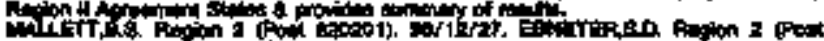

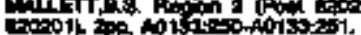

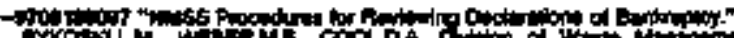

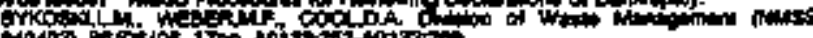

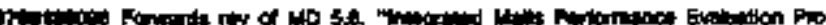

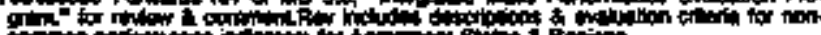

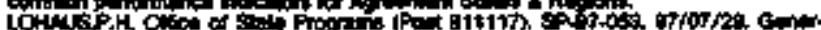

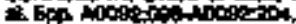




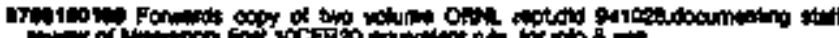

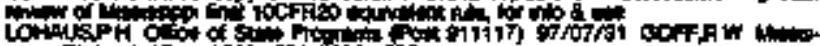

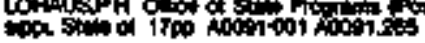

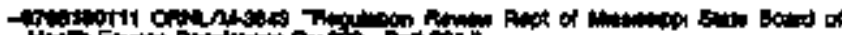

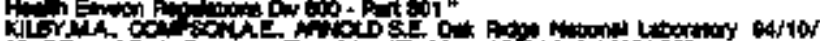

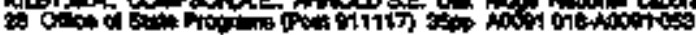

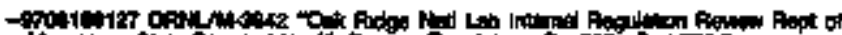

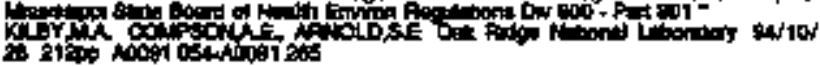

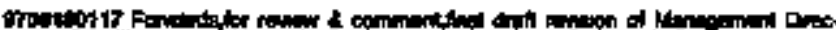

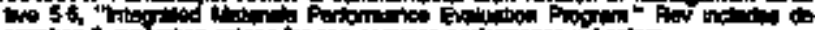

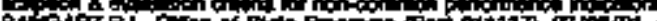

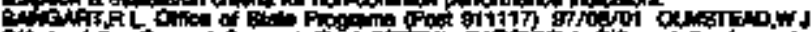
Ont of on a d

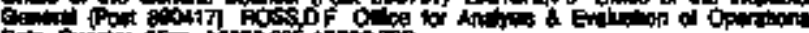

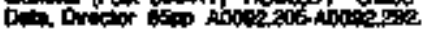

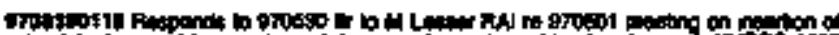

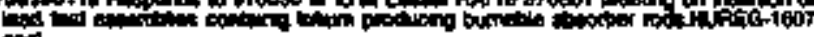

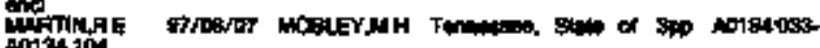

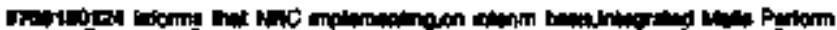

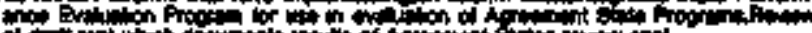

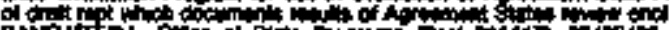

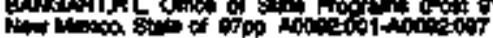

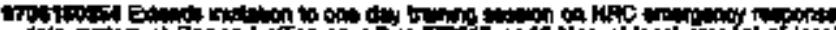

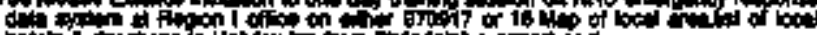

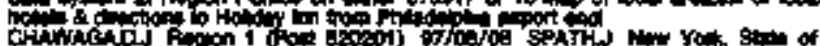

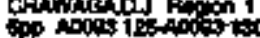

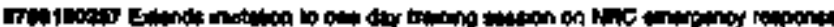

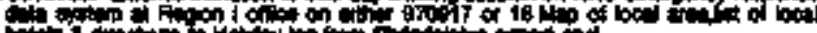

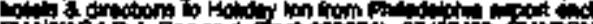

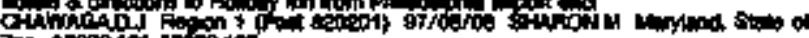

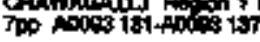

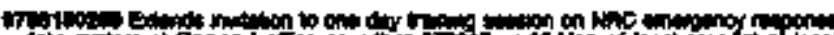

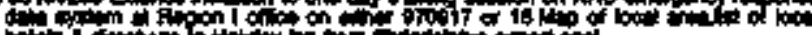
ond s.

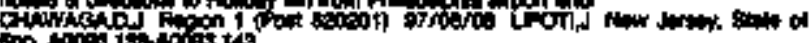
top tom tom

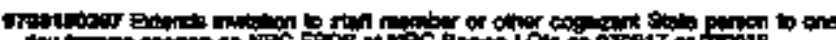

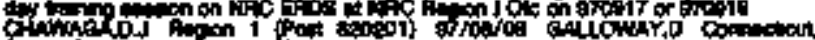

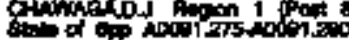

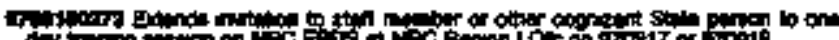

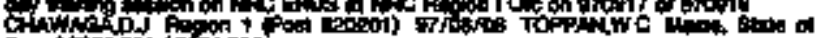

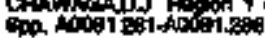

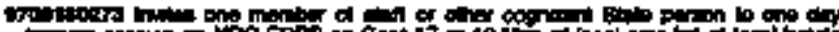

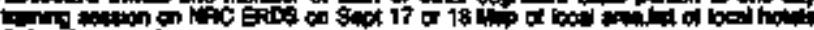

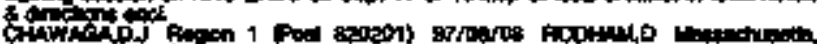

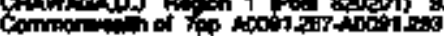

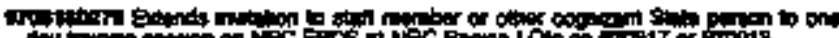

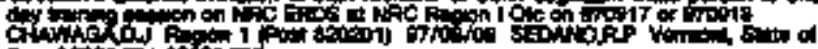

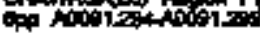

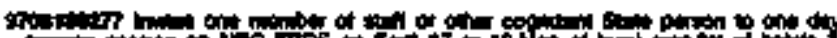

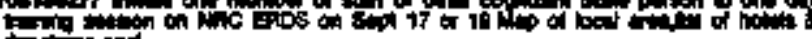

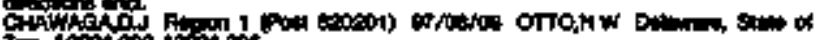

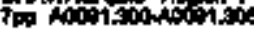

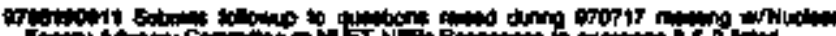

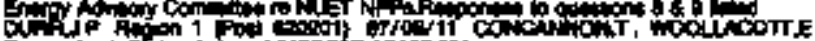
Con

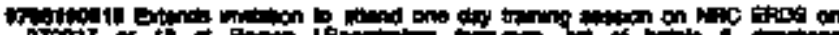

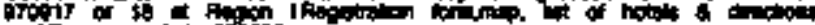

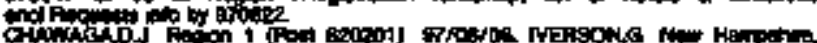

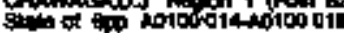

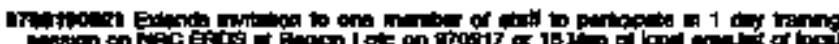

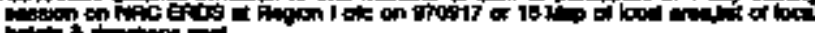

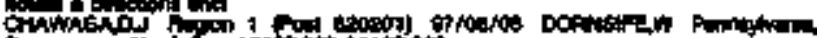

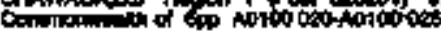

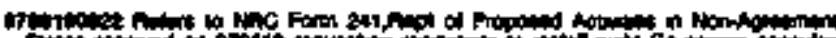

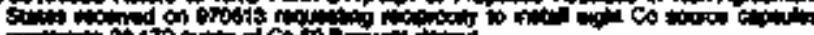

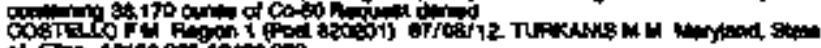
of

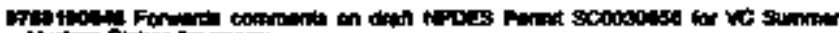

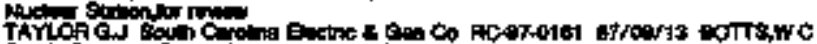

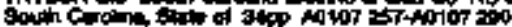

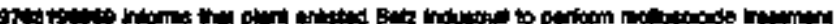

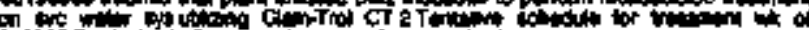
an

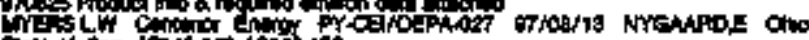

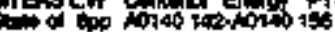

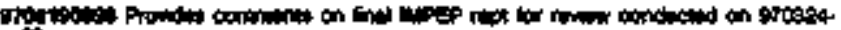

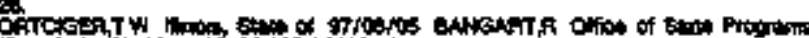

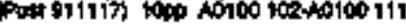

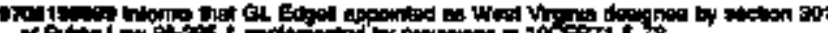
of Pt

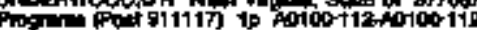

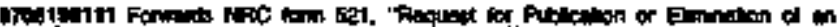

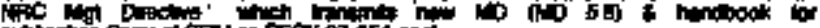

Pow

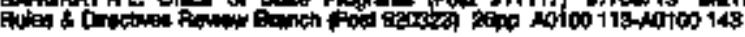

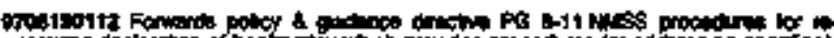

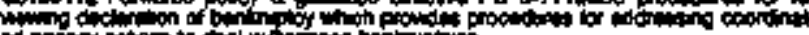

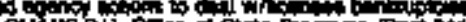

CHAlls Sip A0100 144A0100 $16 x$

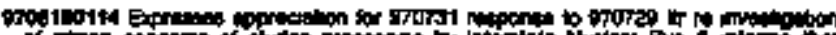

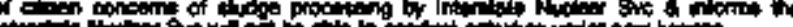

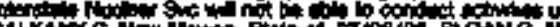

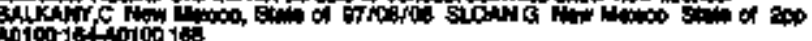

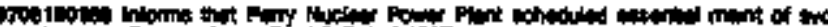

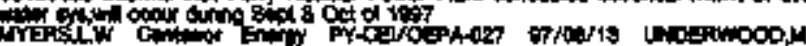

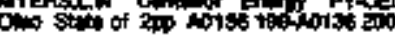

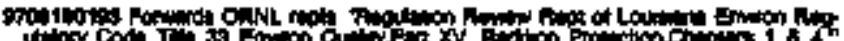

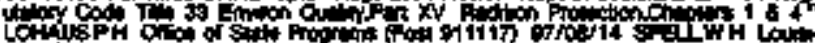

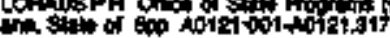

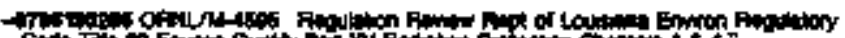

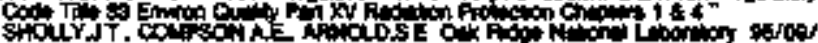

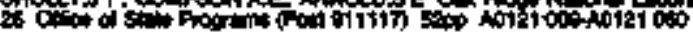

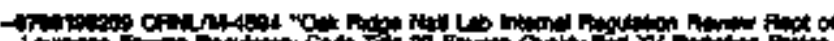

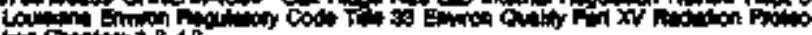

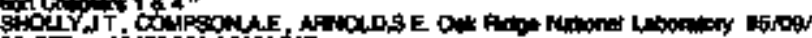

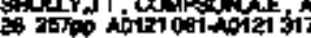

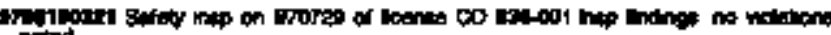

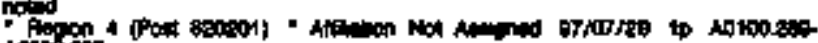
intol 200

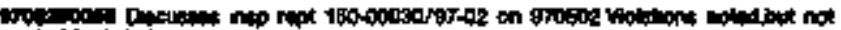

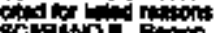

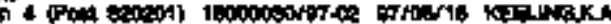

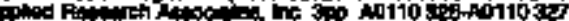

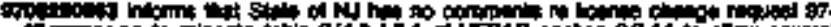

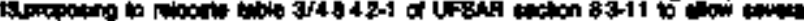

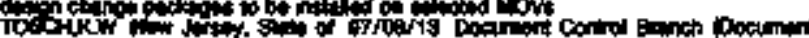

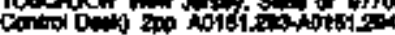

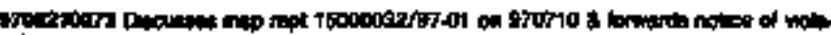

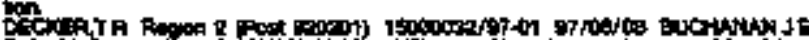

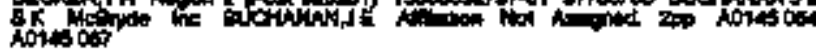

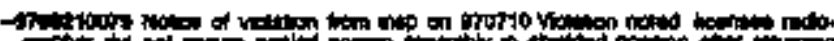

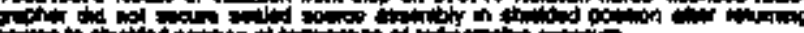

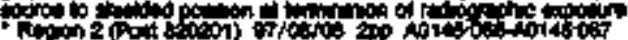

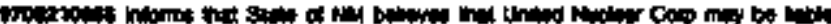

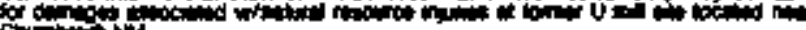

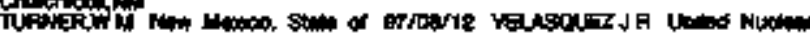

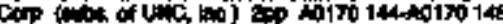

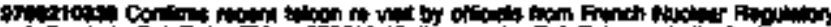

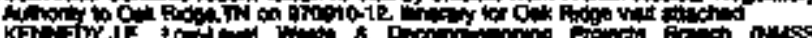

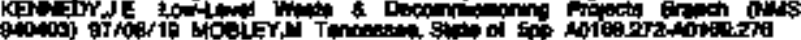

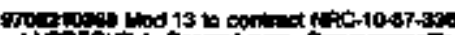

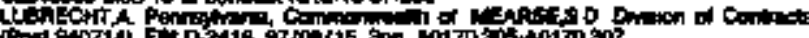

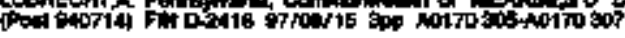

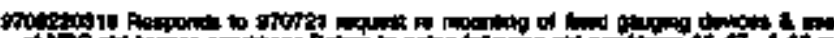

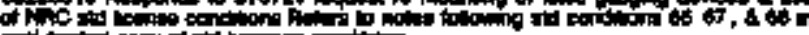

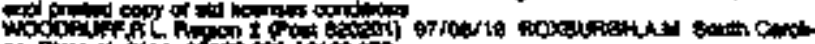

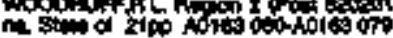

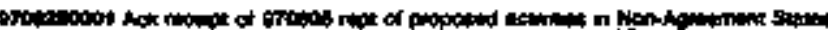

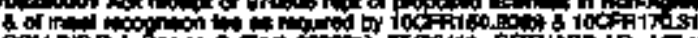
Corlits of J

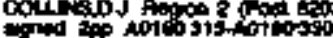




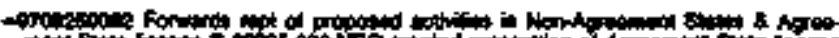

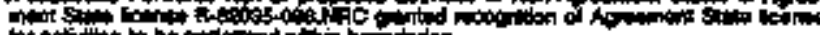

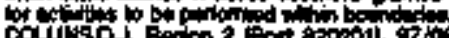

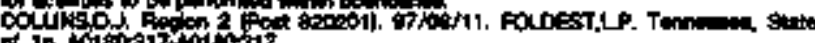

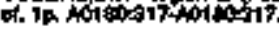

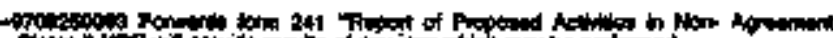

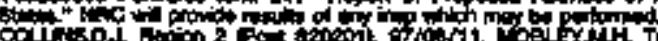

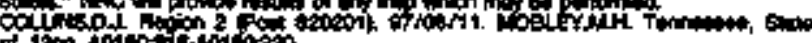

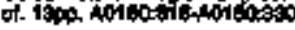

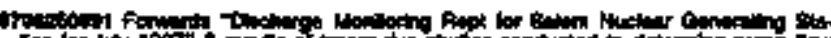

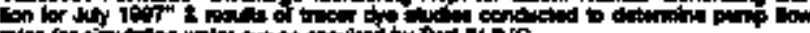

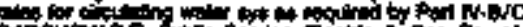

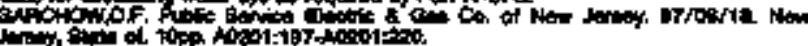

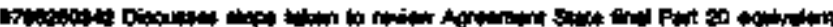

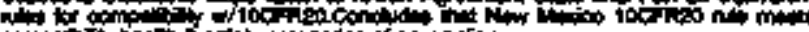

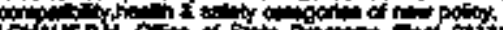

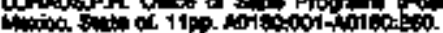

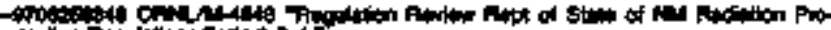

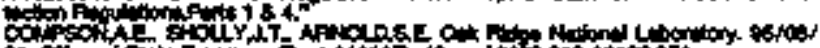

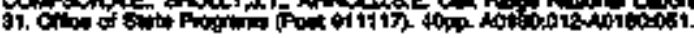

-

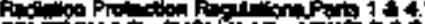

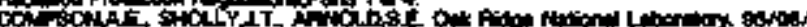

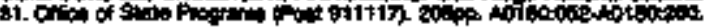

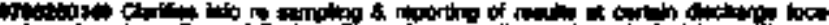

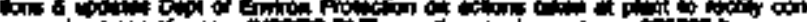
ocices

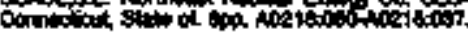

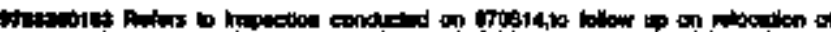

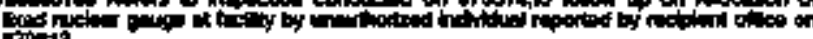

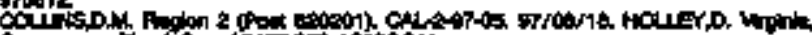

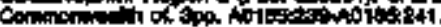

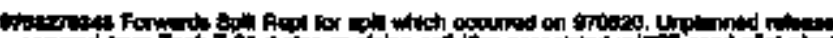

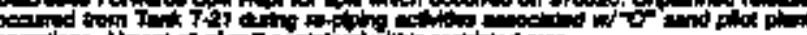

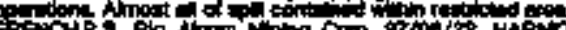

L troatho sont of (1)

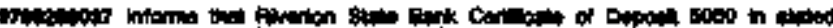

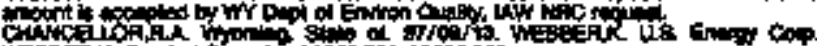

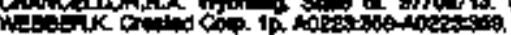

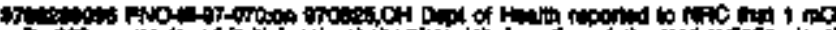

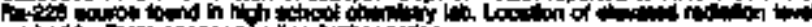

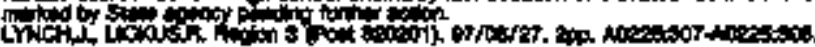

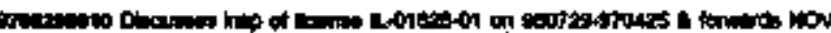

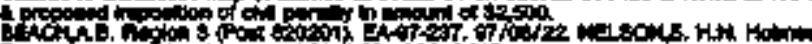

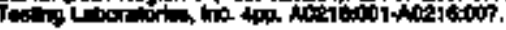

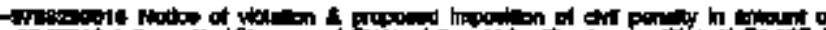

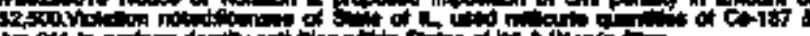

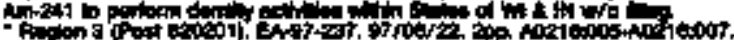

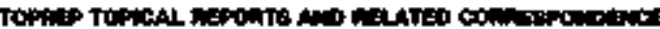

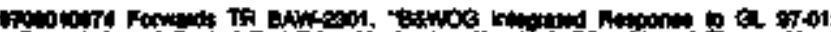

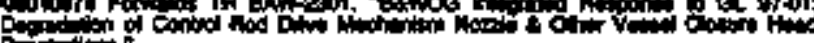

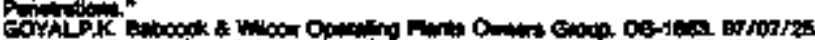

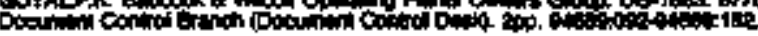

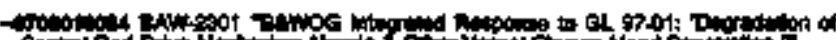

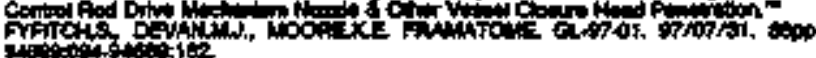

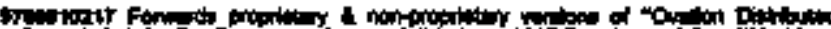

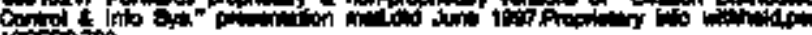

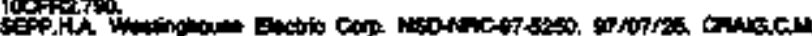

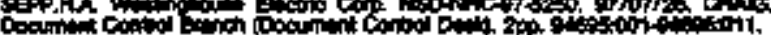

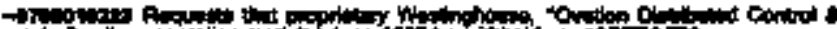

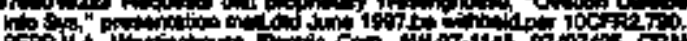

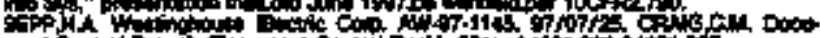

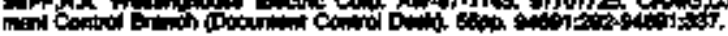

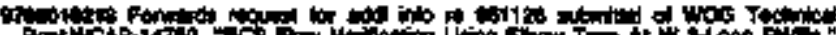

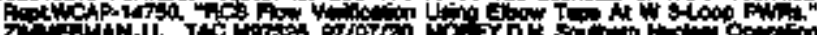

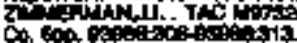

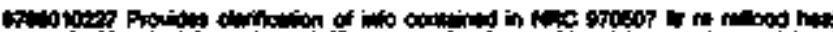

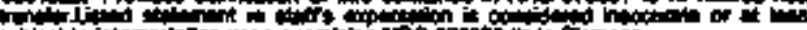

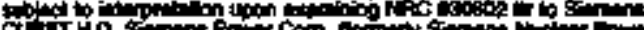

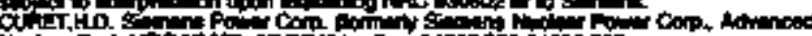

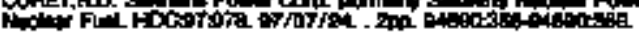

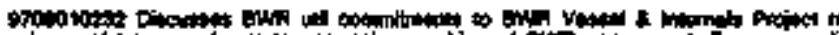

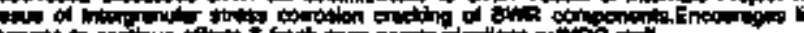
How Ho:

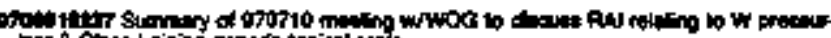

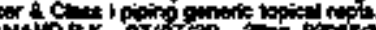
wivith of

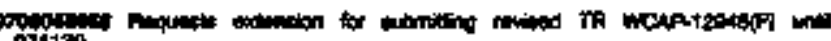
I A

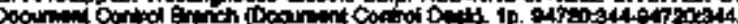

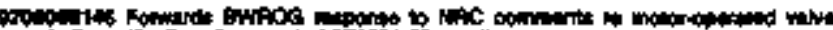

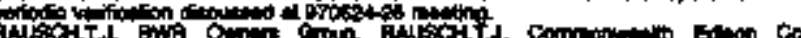

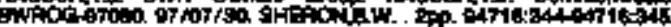

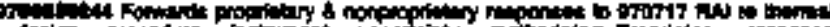

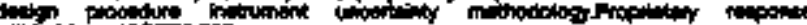

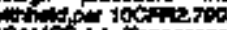

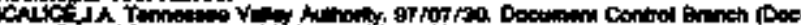

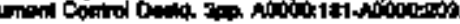

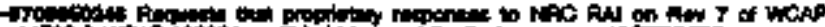

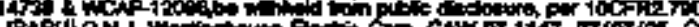

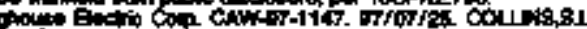

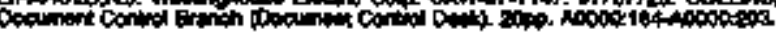

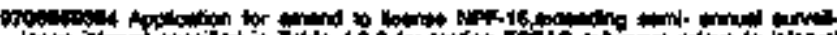

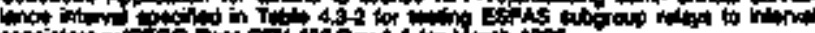

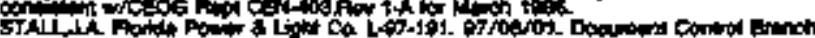

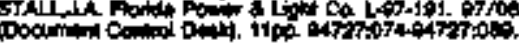

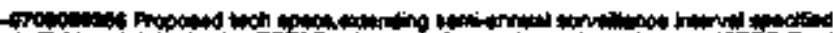

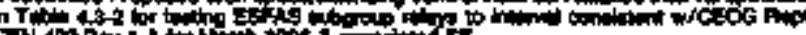

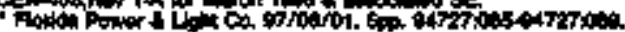

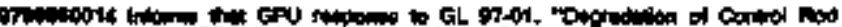

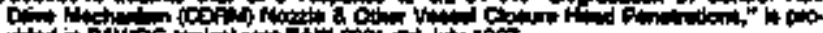

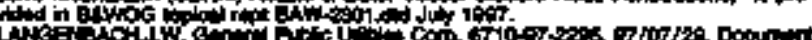

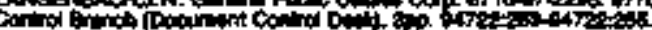

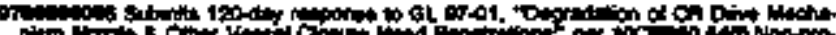

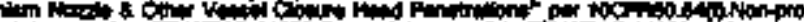

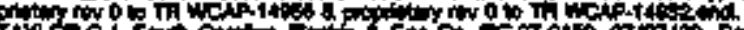

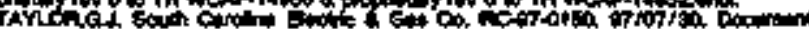

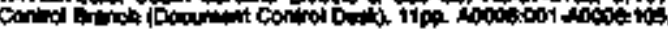

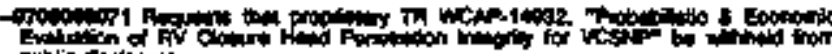

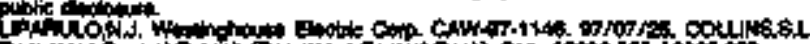

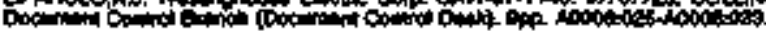

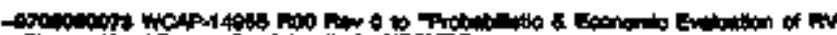

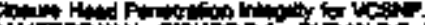

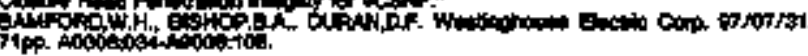

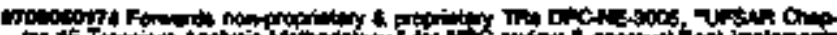

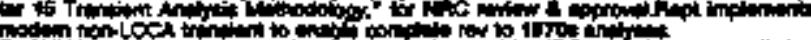

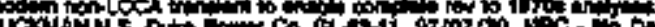

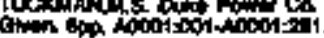

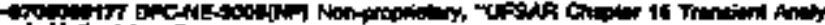

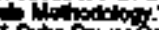

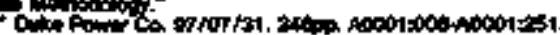

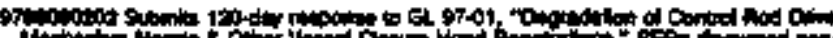

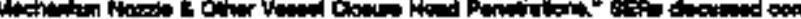

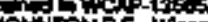

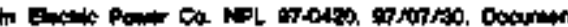

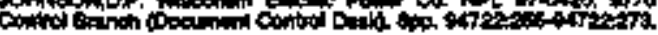

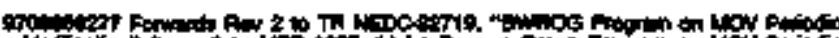

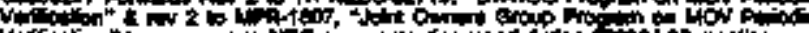

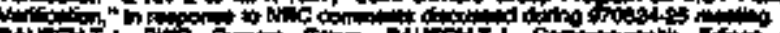

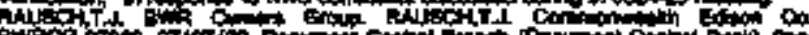

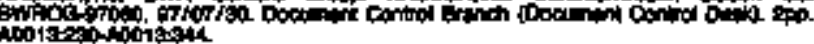

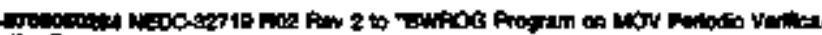

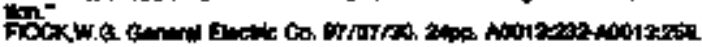

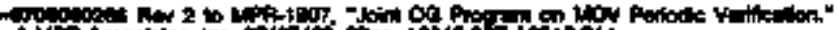

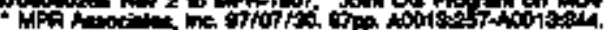

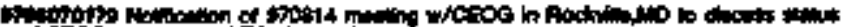

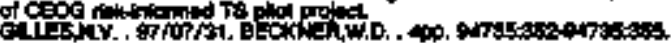

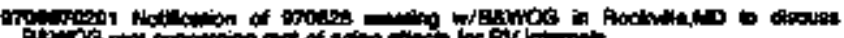

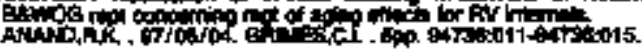

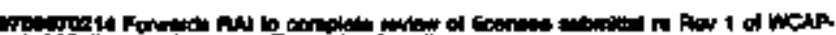

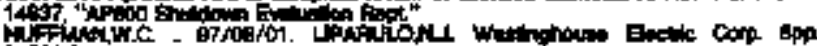

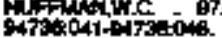




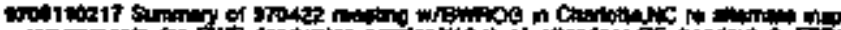

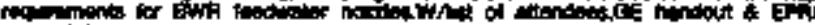

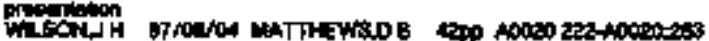

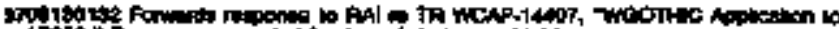

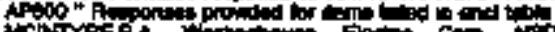

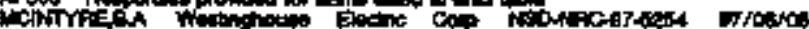

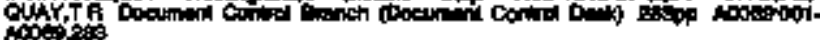

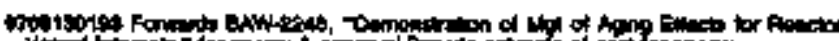

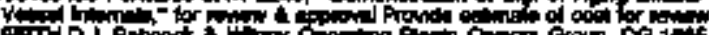

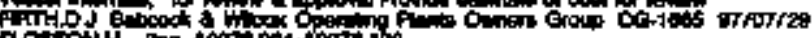

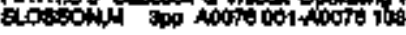

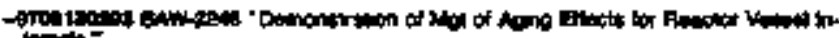

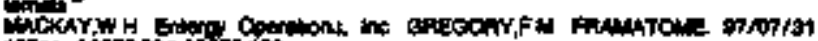

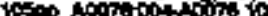

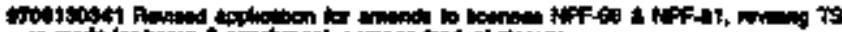

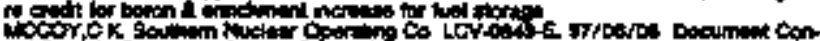

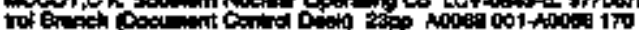

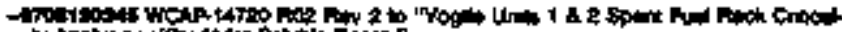

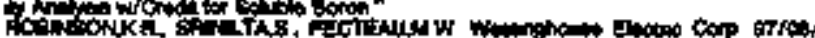

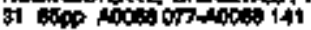

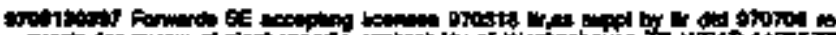

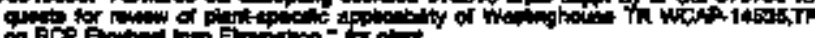

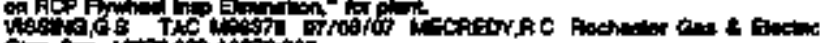
Cap sip notis

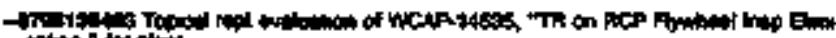

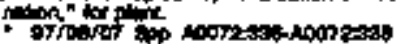

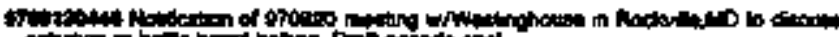

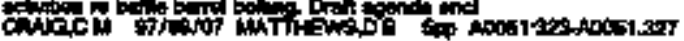

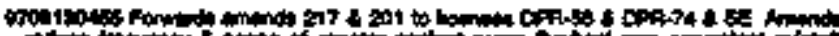

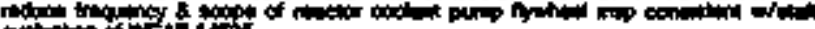

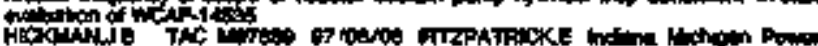

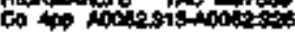

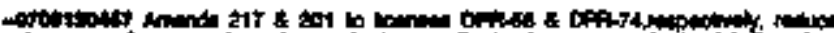

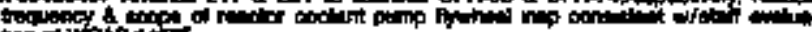

bon

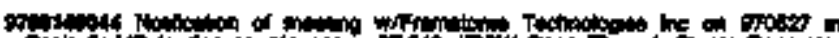

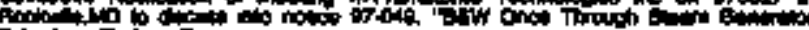

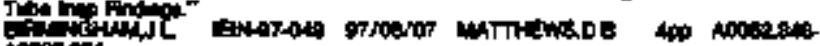
ingersis

Hor OAnF

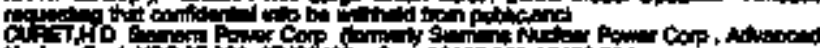

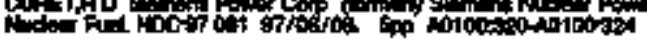

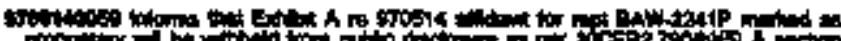

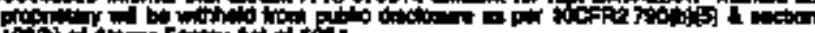

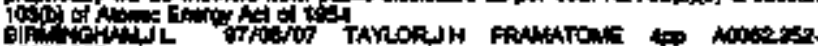
atomes

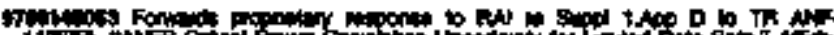

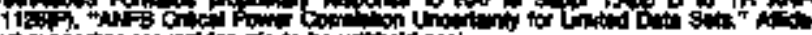

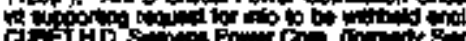

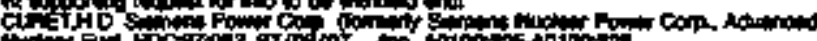

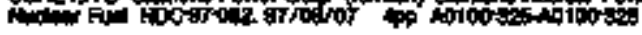

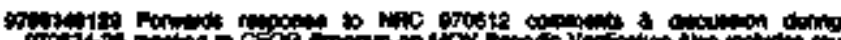

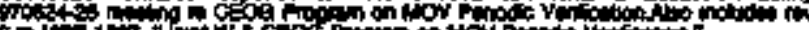

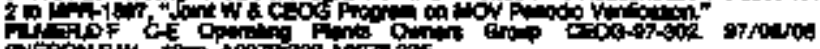

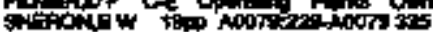

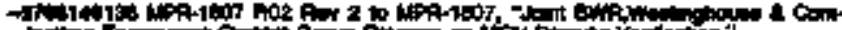

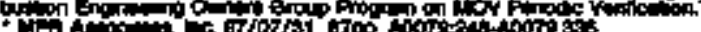

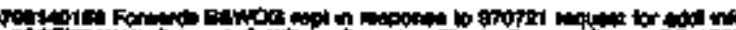
Fos os by

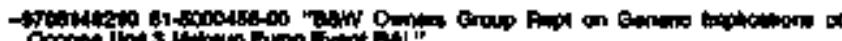

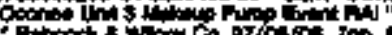

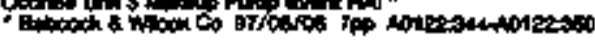

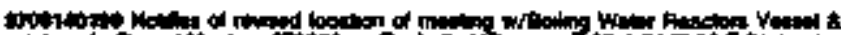
In

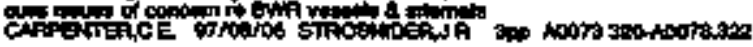

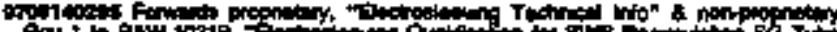

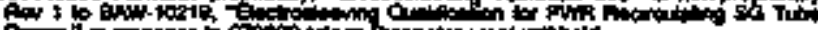

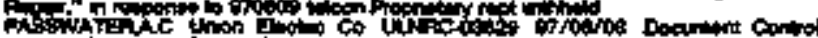

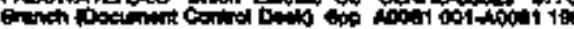

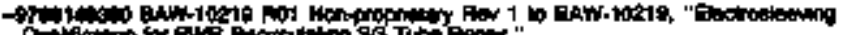

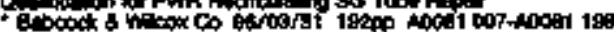

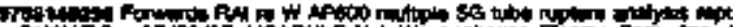

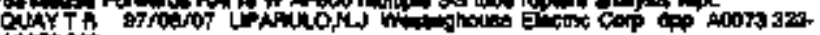
noth

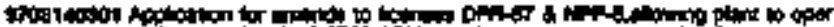

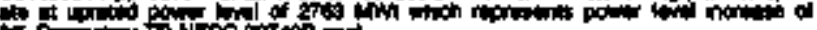
Af Pिy

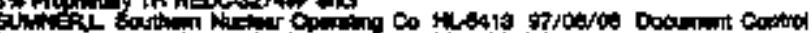

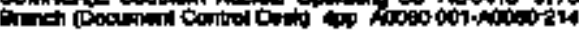

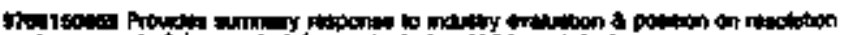
f. .

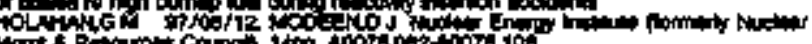

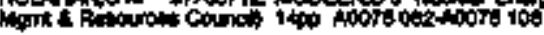

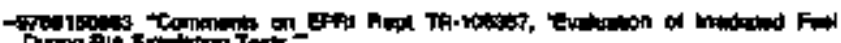

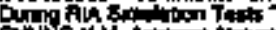

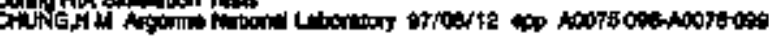

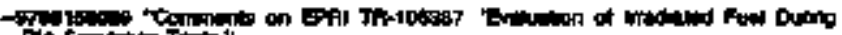

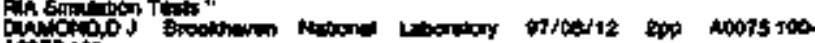
ation

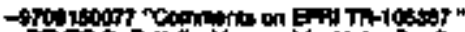
it

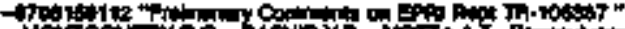

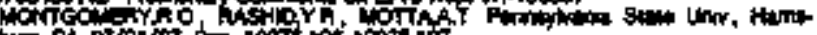

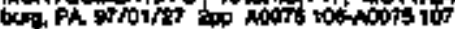

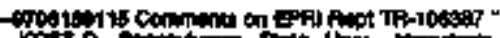

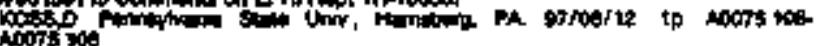

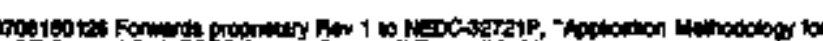

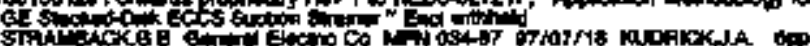

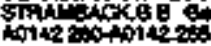

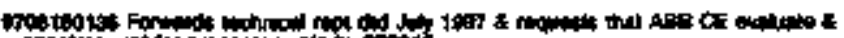

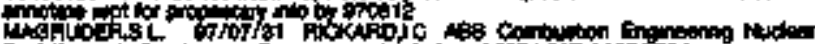

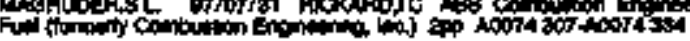

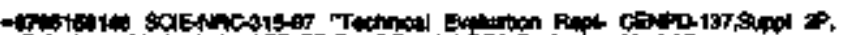

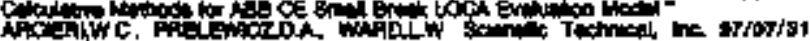

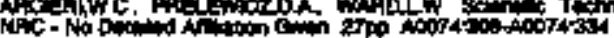

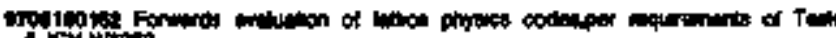

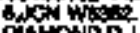
Non

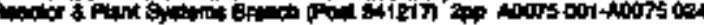

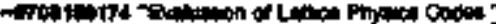

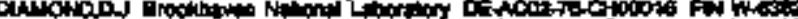

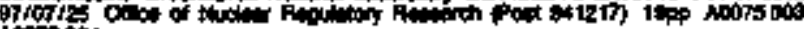

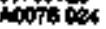

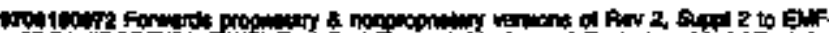

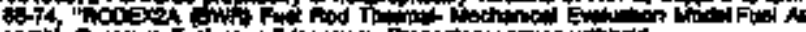

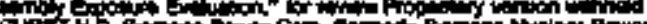
a

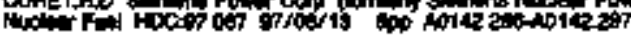

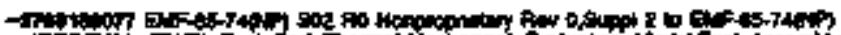

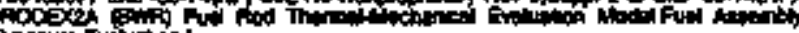

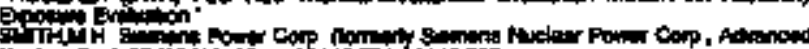

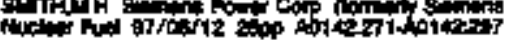

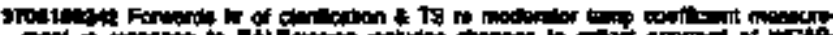

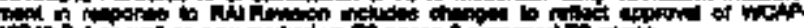

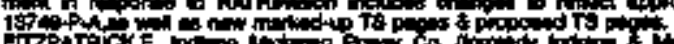

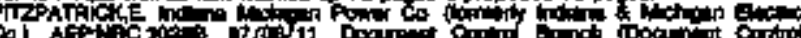

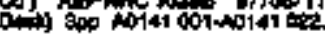

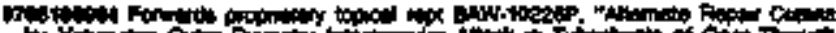

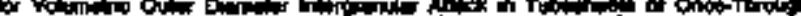

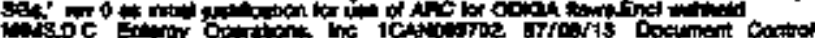

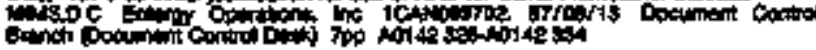

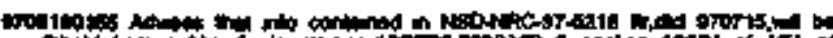

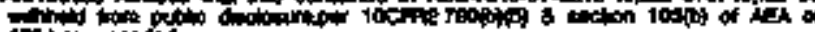

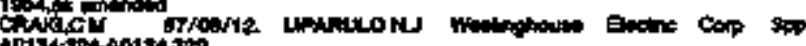

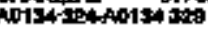

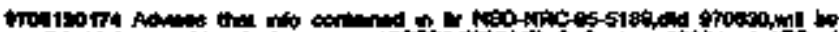

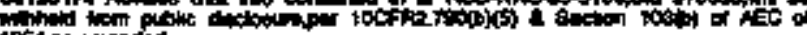

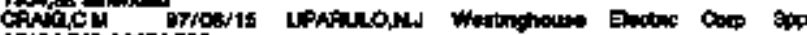

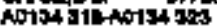

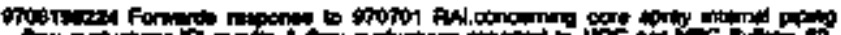

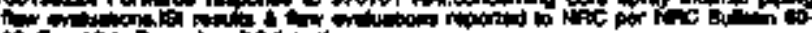

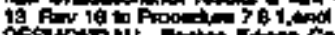

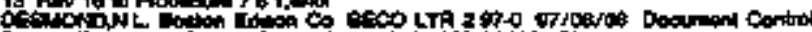

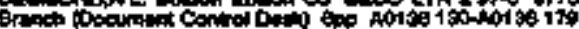




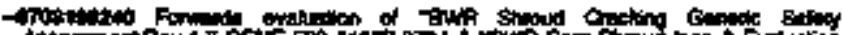

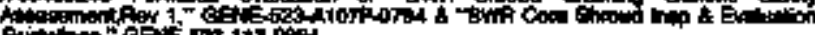

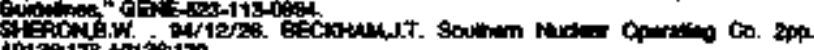

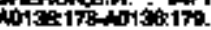

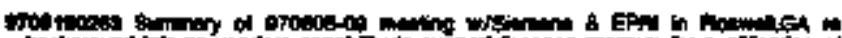

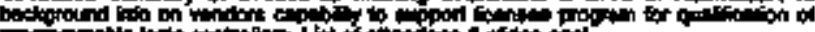

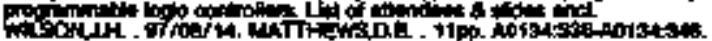

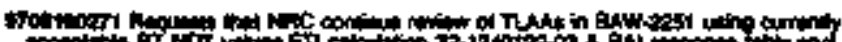

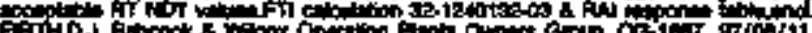

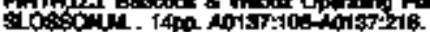

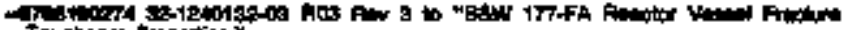

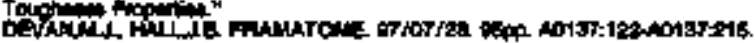

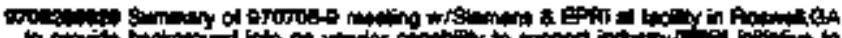

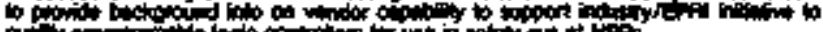

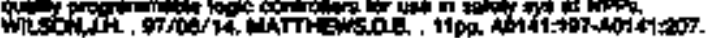

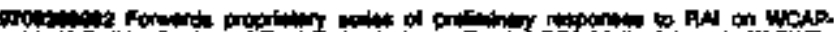

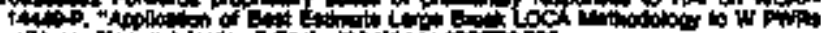

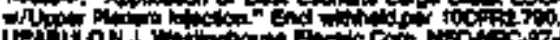

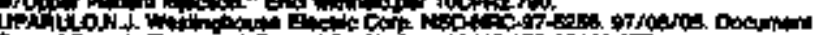

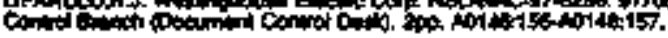

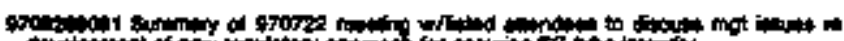

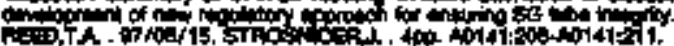

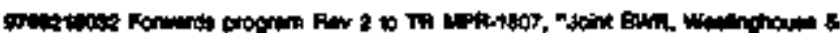

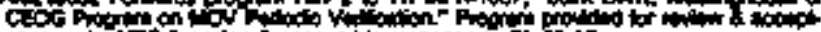

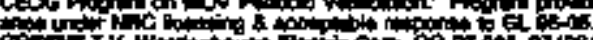

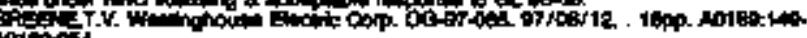

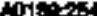

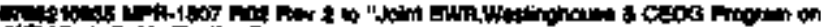

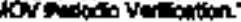

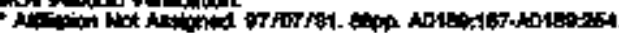

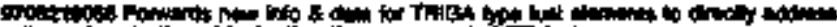

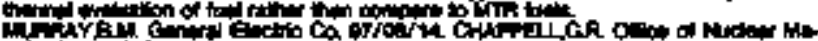

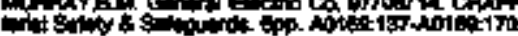

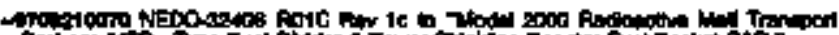

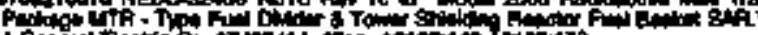

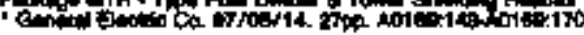

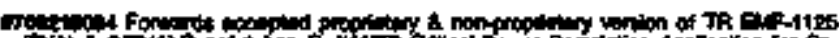

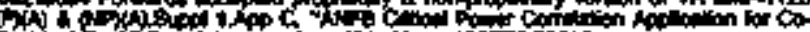

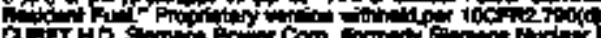

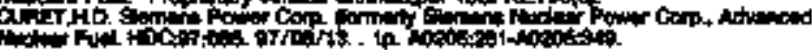

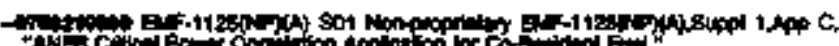

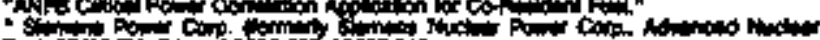

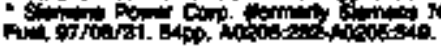

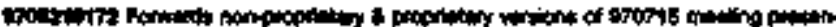

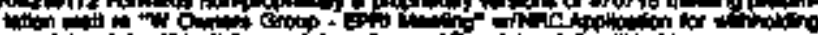

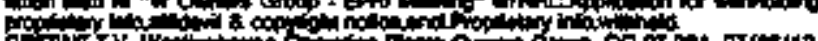

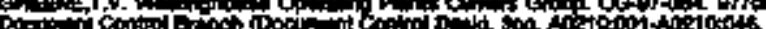

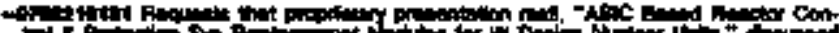

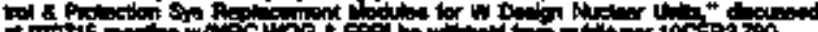

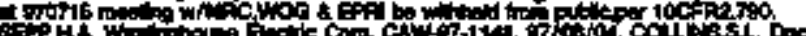

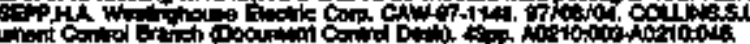

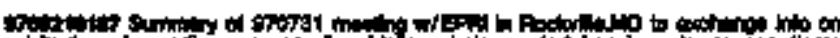

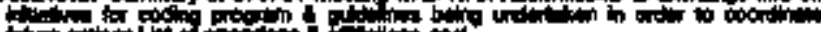

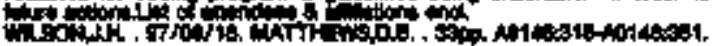

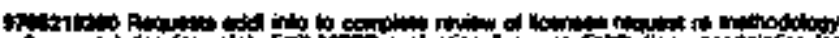

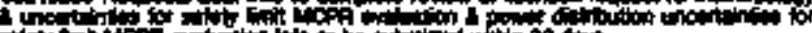

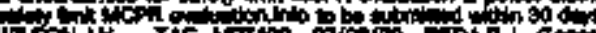

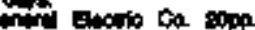

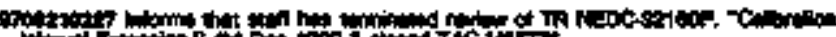

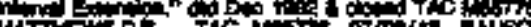
Hor

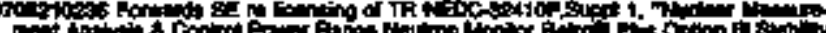

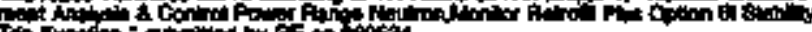

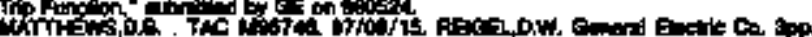
Hotsi:

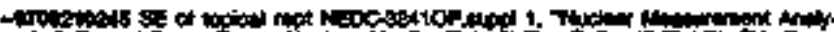

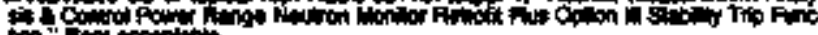

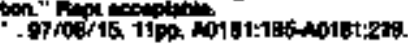

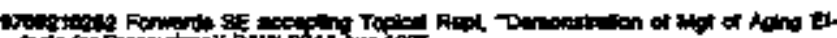

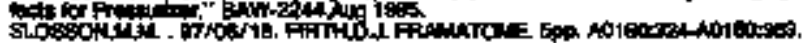

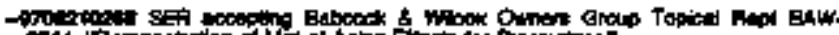

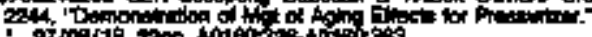

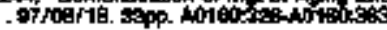

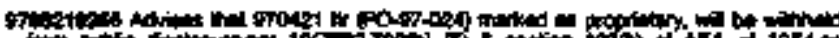

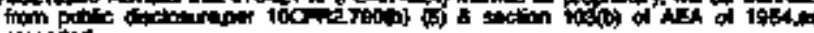

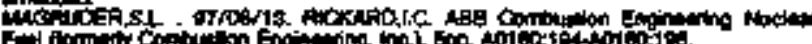

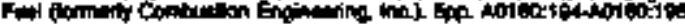

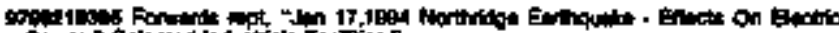

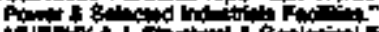

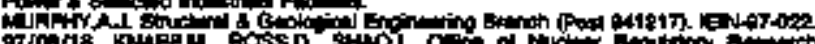

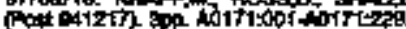

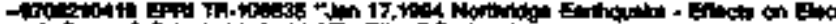

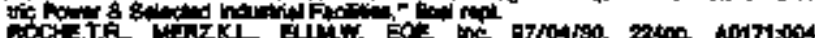
Nortatis?

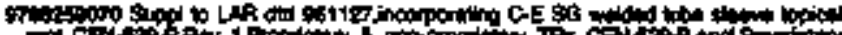

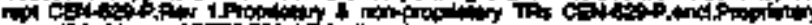

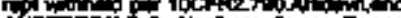

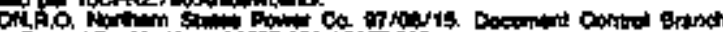

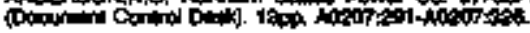

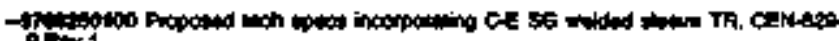

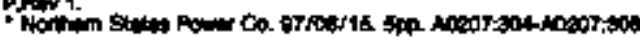

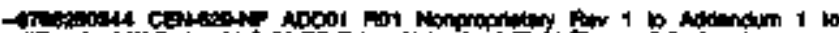

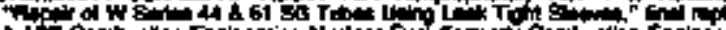

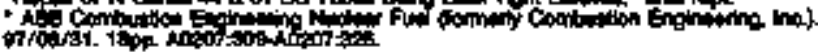

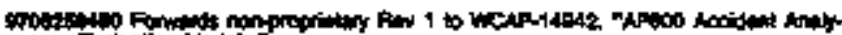

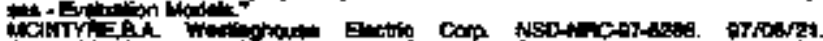

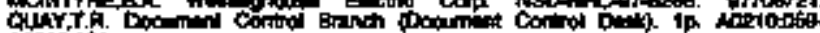

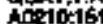

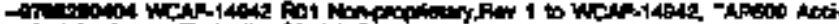

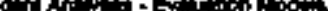

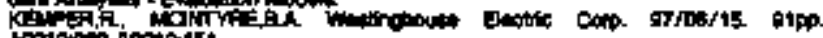
Aretonomineto:151.

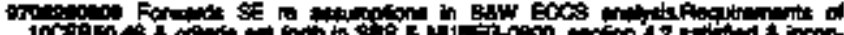

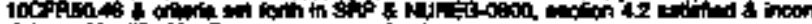

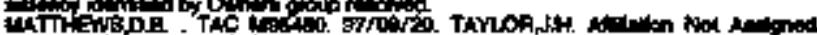

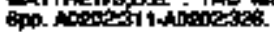

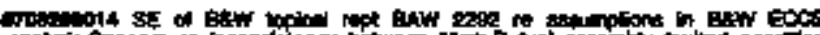

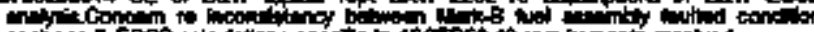

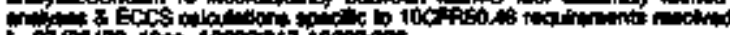

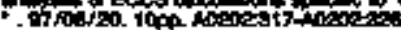

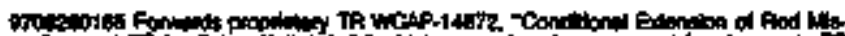

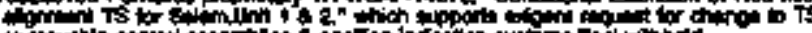
Po

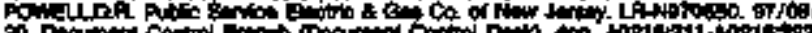

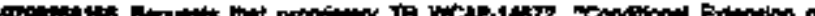

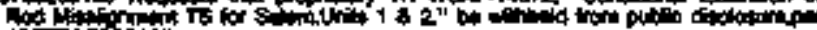

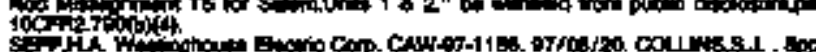

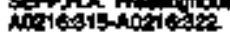

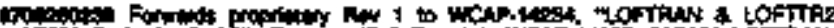

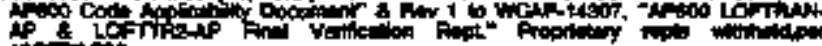
$100=727 \%$

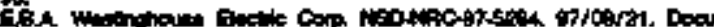

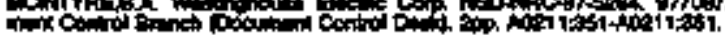

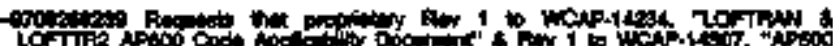

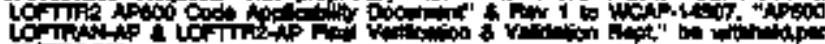

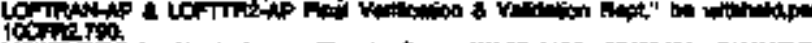
10 inction

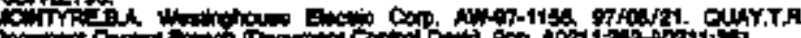

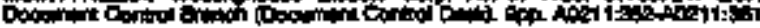

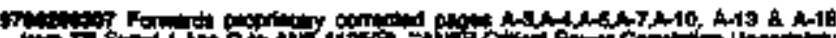

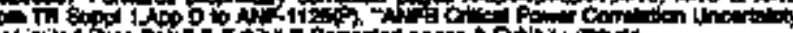

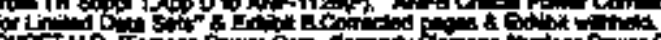

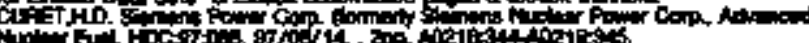

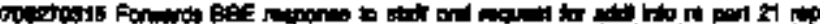

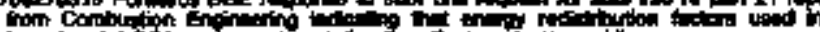

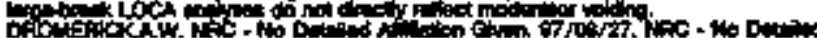

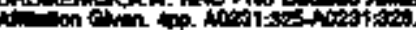

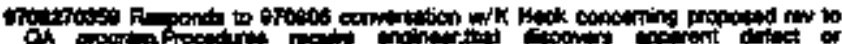

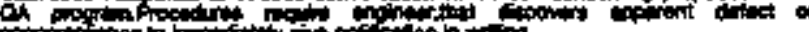

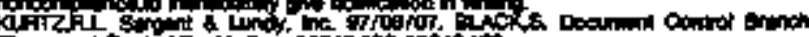

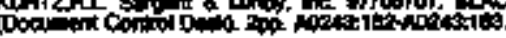

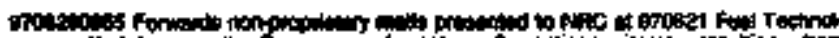

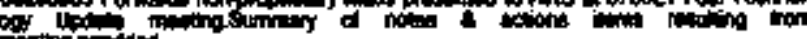

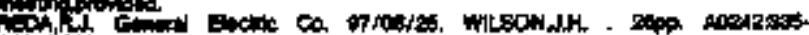
$+0,40$ 


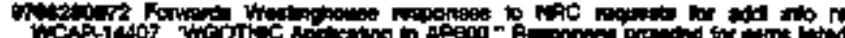

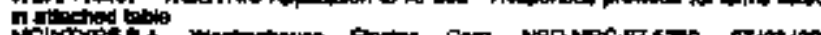

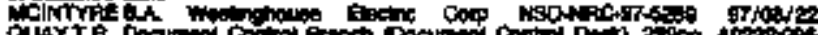
$\operatorname{lom}_{0 \rightarrow 4}$

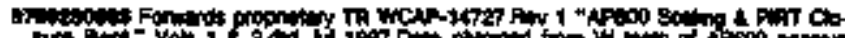

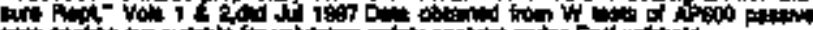

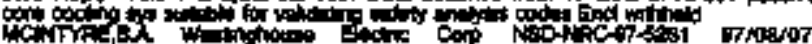

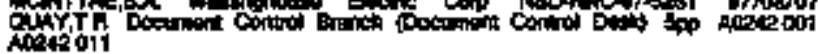

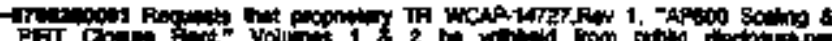

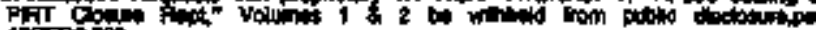
10072780

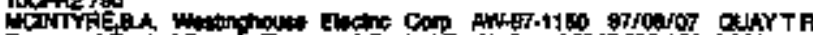

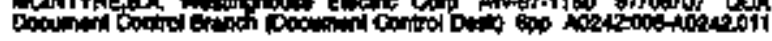

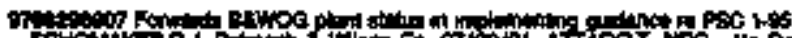

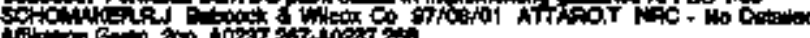

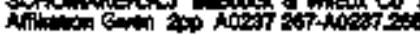

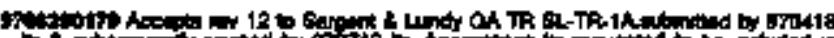

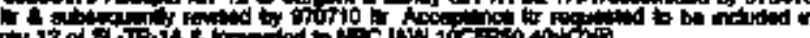

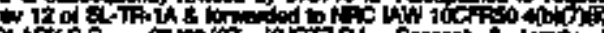

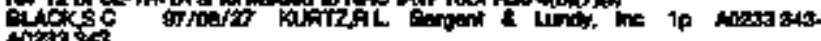

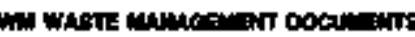

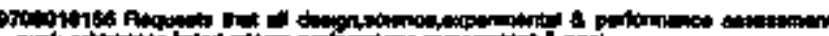

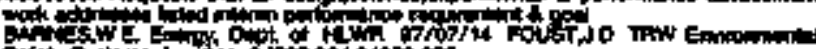

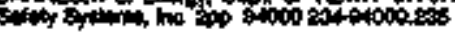

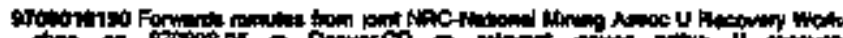

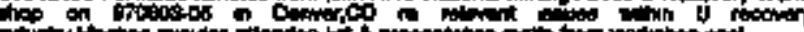

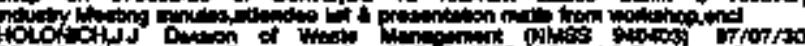

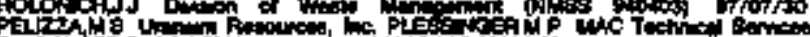

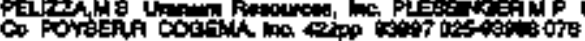

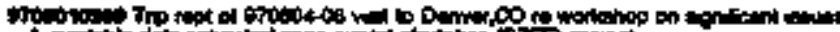

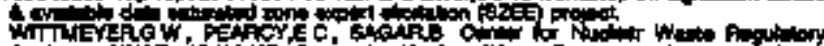

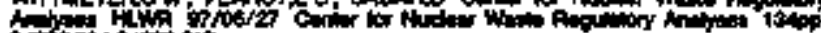

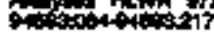

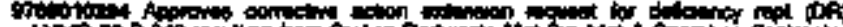
LWho

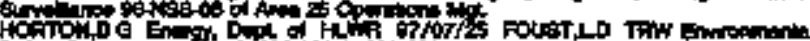

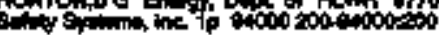

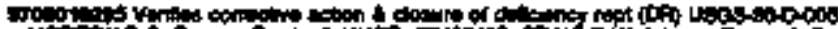

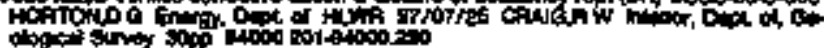

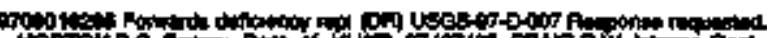

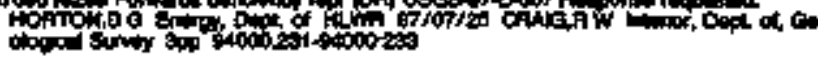

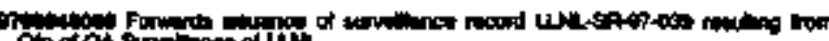

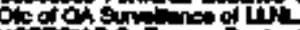

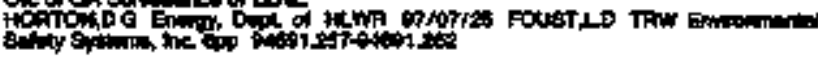

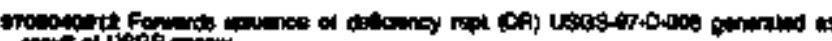

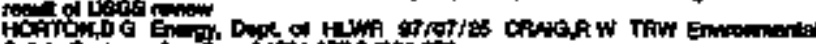

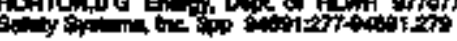

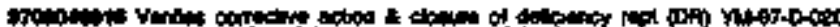

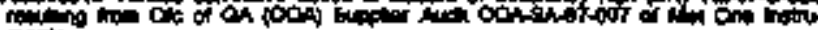

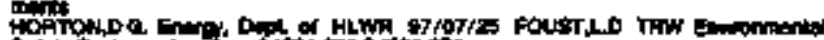

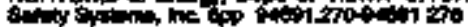

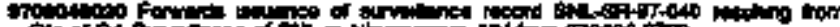

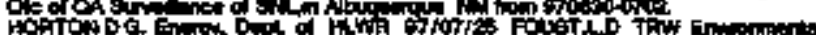
Gidty

If

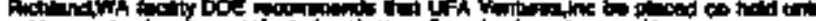

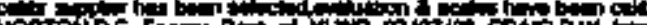

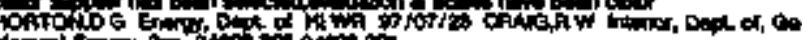

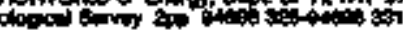

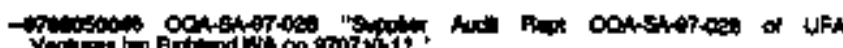

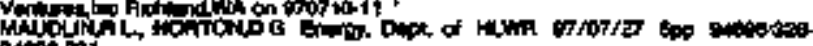
403.

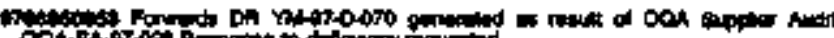

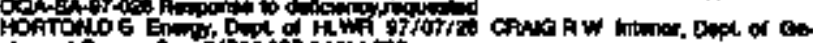

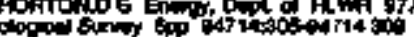

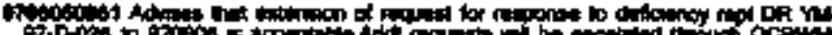

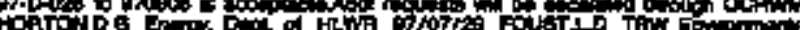

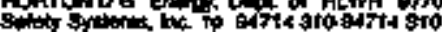

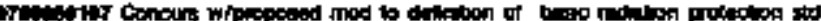

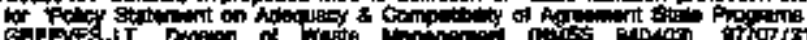

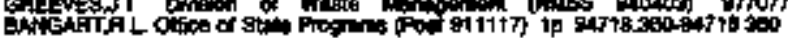

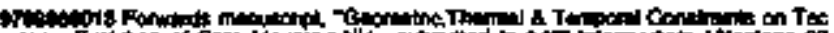

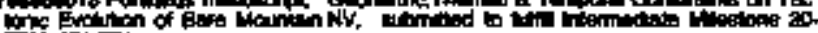

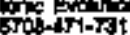

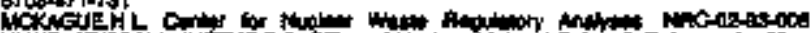

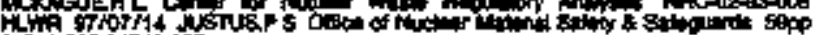
of

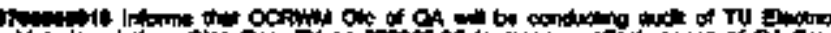

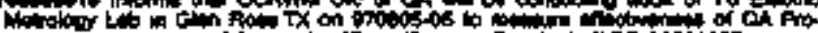

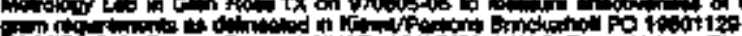

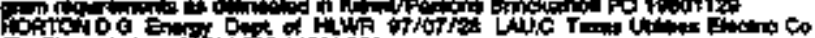
Therind

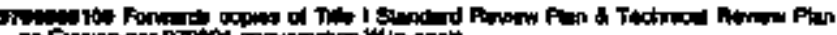

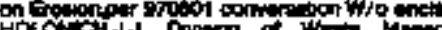

$H$ COCH

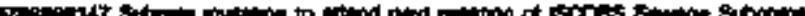

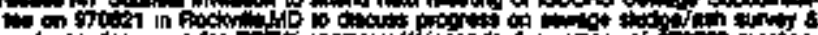

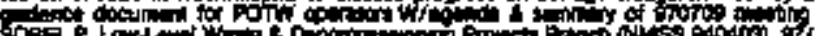

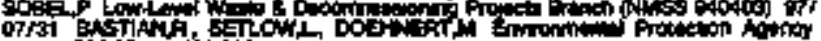
orration

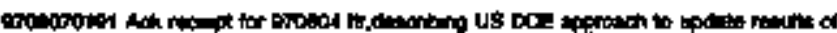

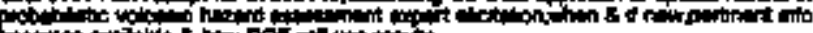

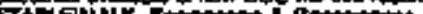

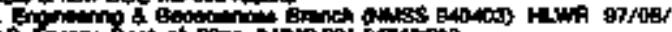
of

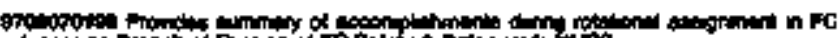

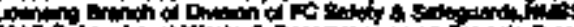

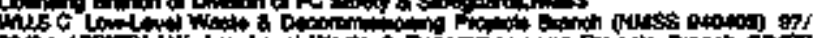

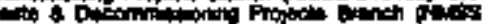

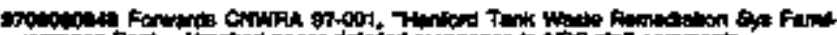

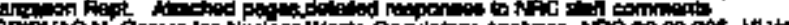

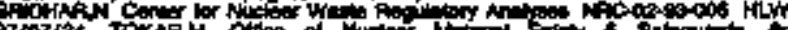

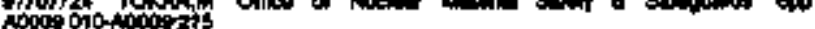

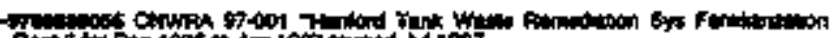

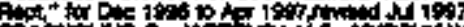

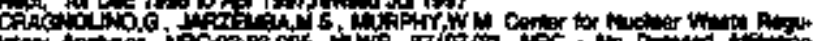

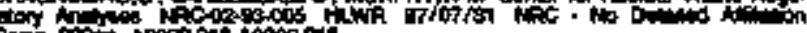

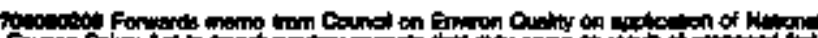

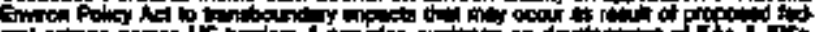

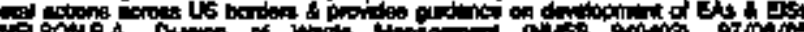

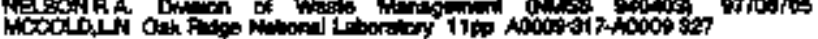

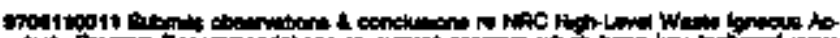

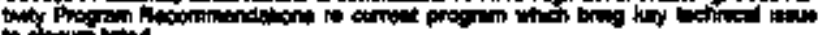

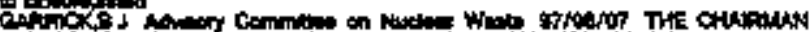

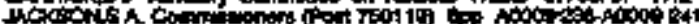

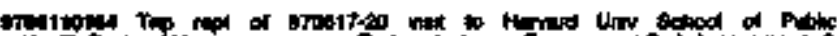

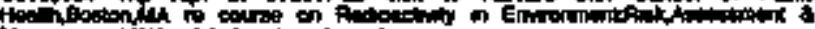

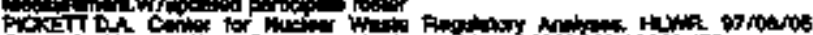

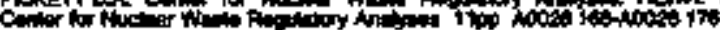

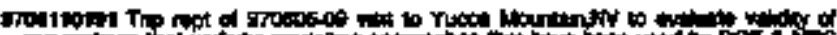

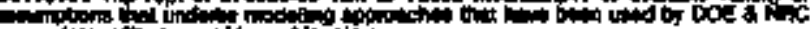
on any

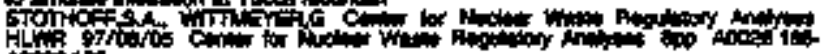
Alote 16

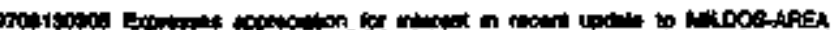

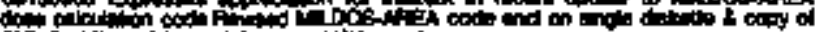

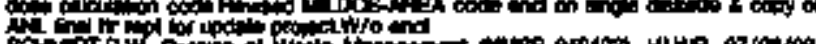

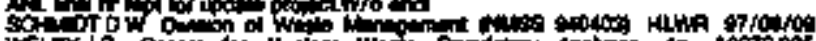

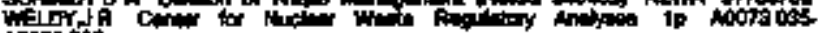
noprost

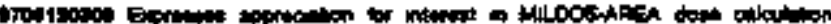

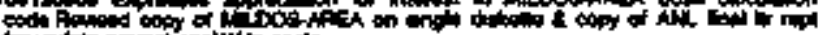

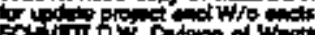

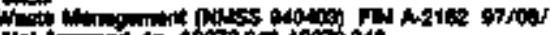

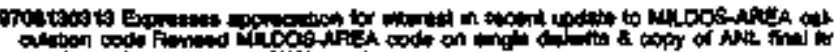

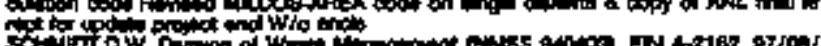

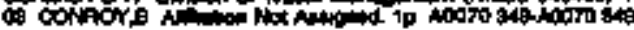

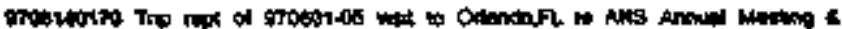

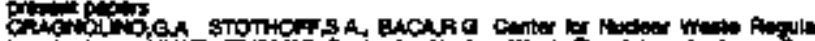

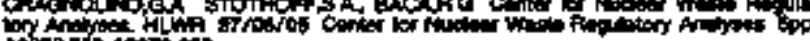

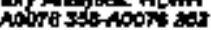




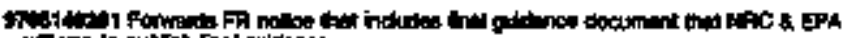

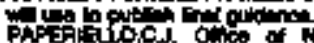

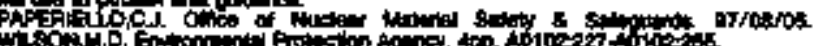

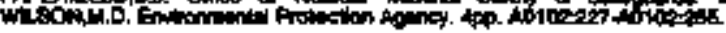

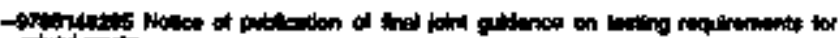

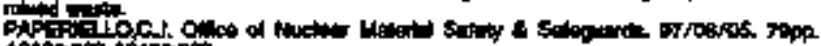

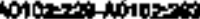

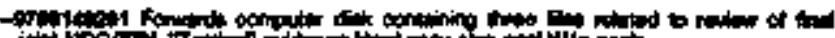

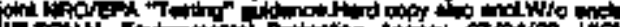

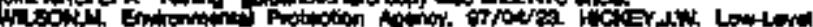

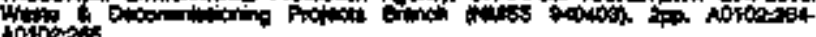

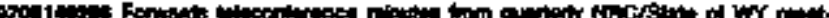

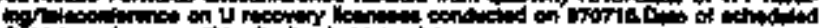

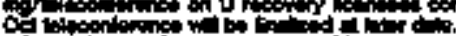

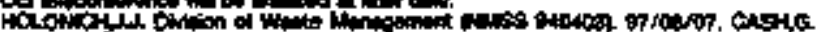

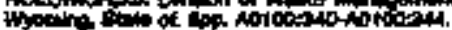

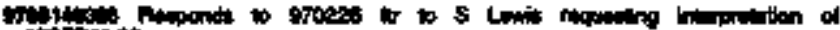

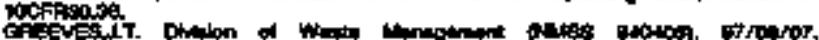

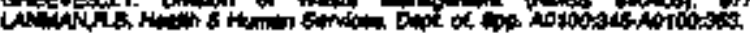

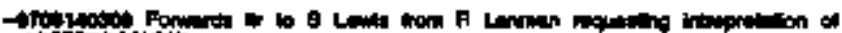
Dofirasidity

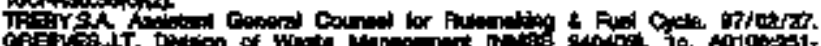
Aotocist.

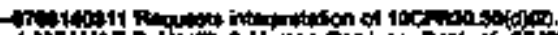

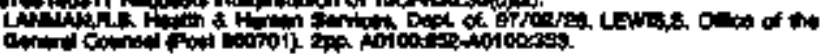

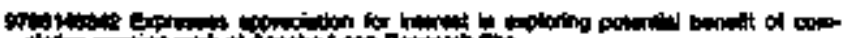

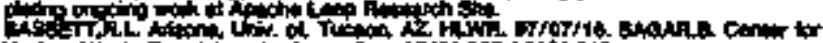

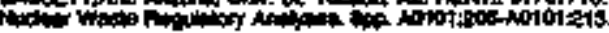

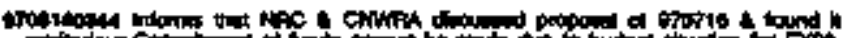

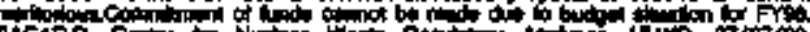

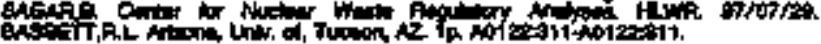

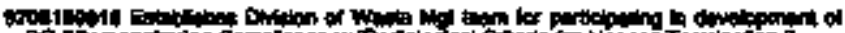

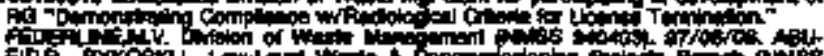

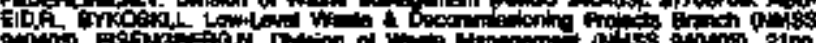

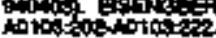

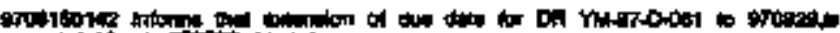

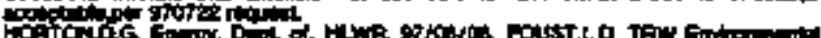

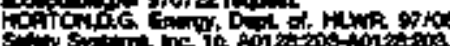

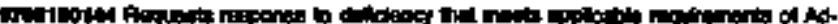

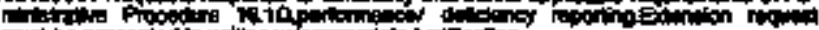

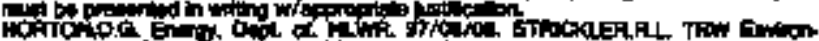

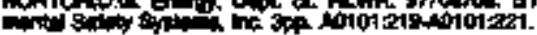

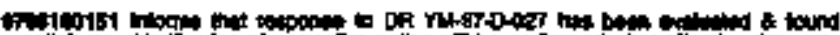

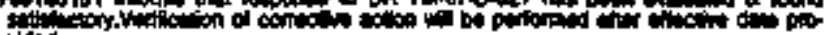

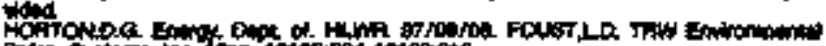

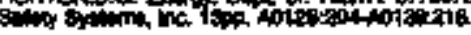

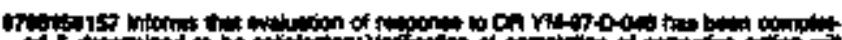

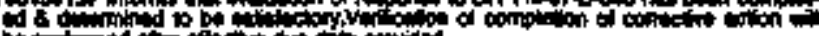

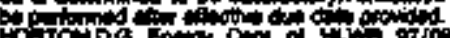

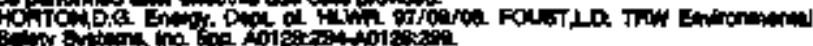

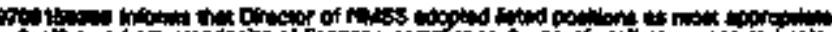

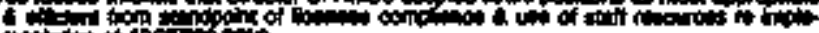

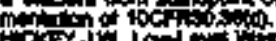

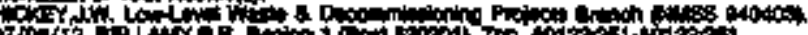

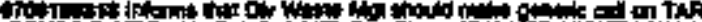

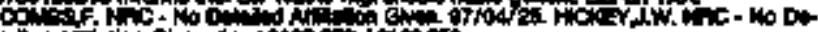

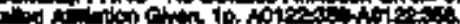

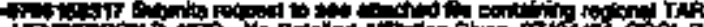

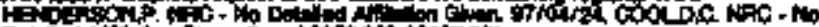

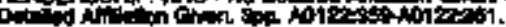

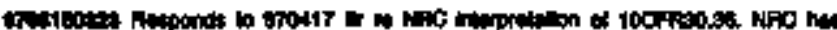

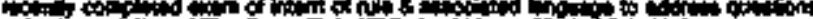

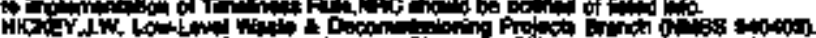
of

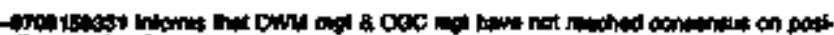

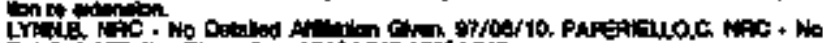

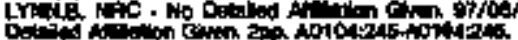

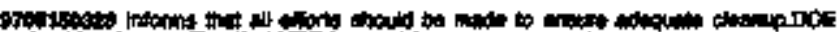
divis

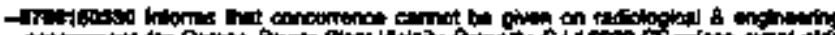

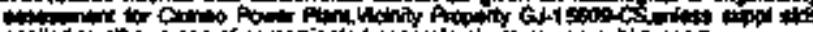

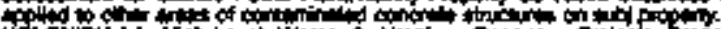

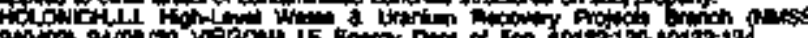

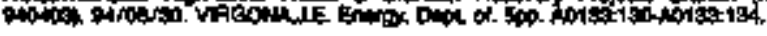

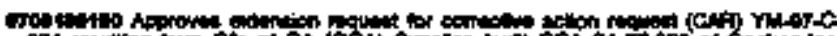

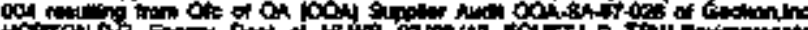

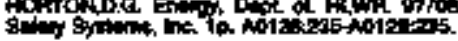

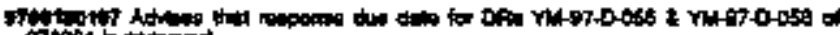

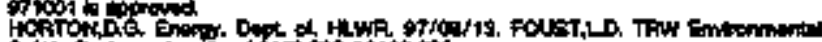

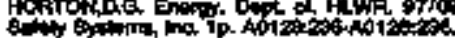

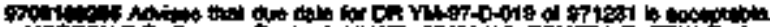

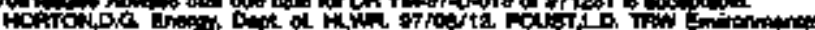

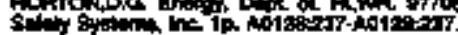

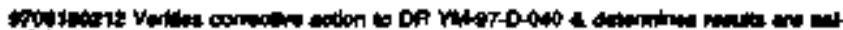

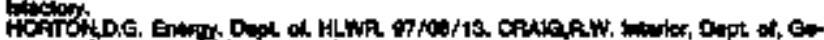

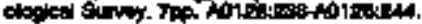

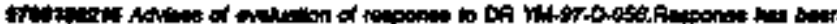

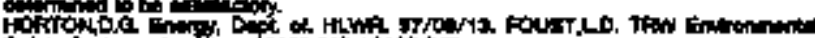

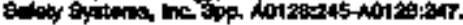

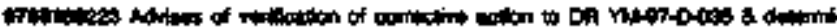

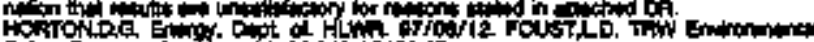

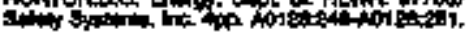

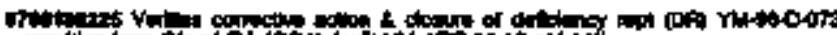

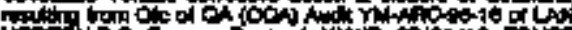

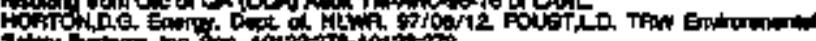

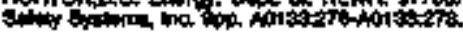

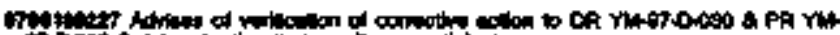

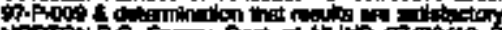

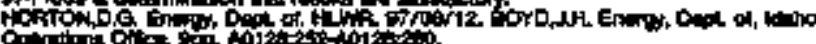

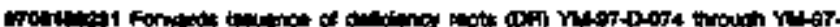

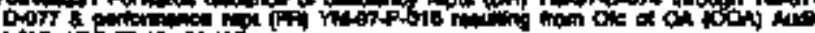

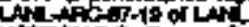

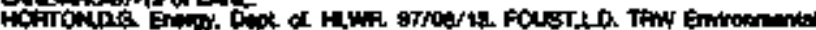

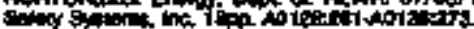

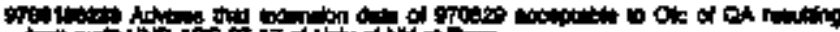

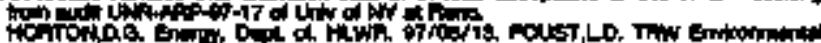

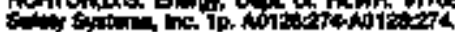

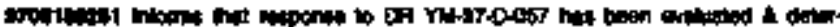

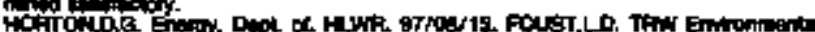

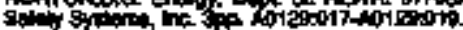

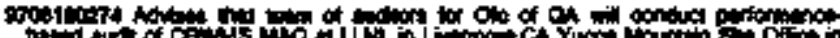

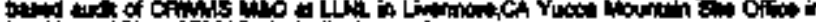

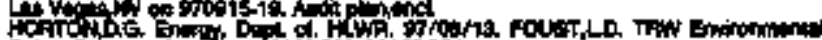

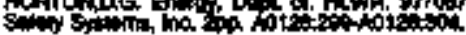

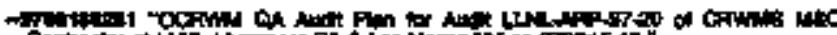

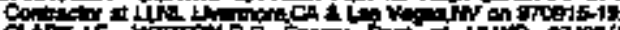

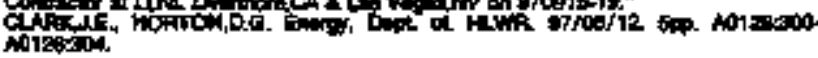

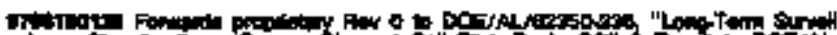

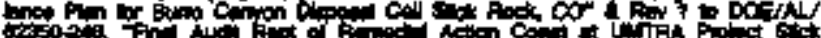

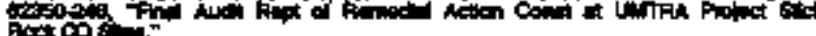

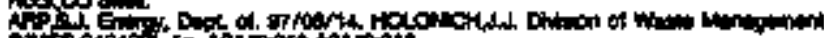

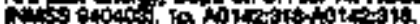

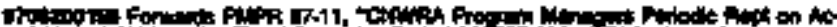

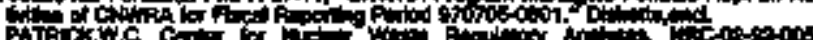

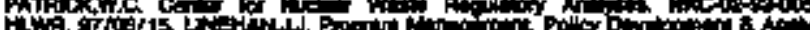

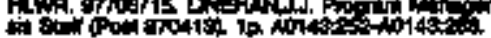

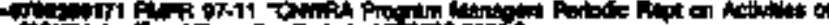

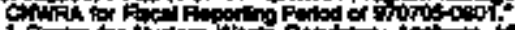

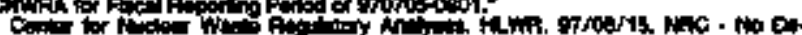

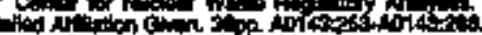

Pine

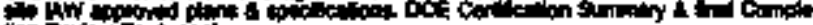

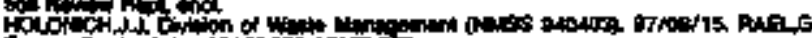

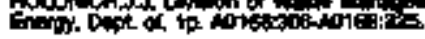

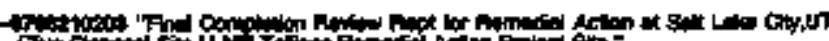

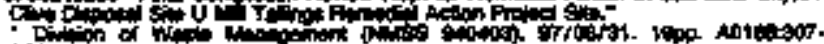
aloing.

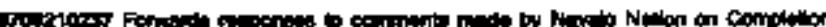

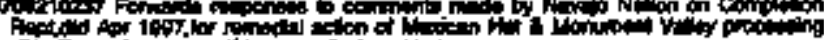

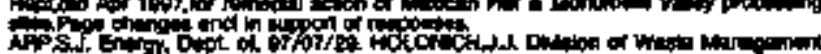
or. 350 o 


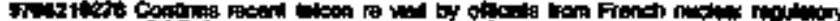

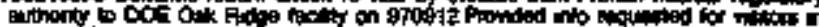

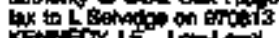

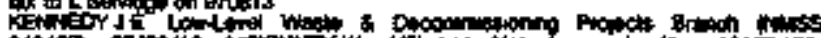

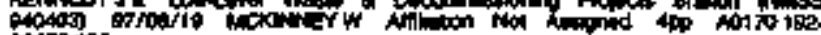
16170 tos

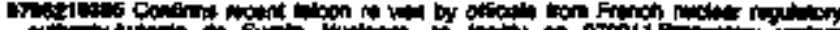

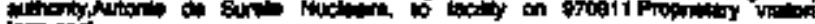
Forand

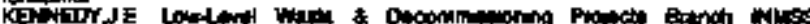

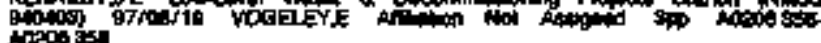

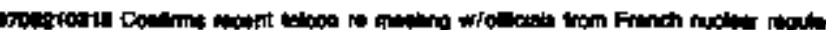

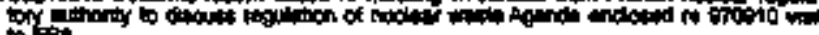

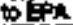

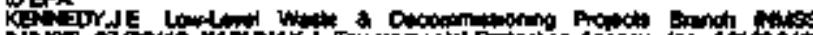

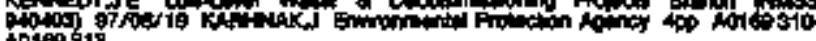
10169518

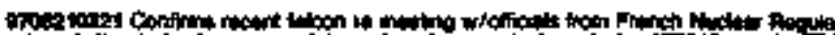

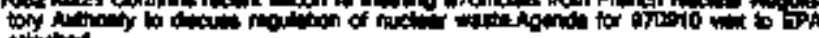
inting

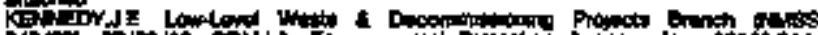

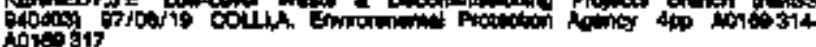

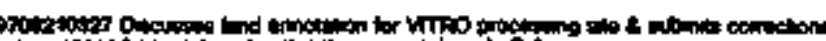

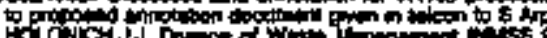

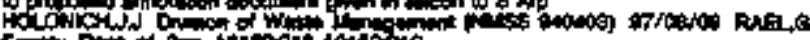

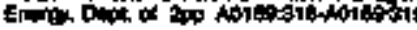

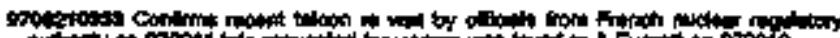

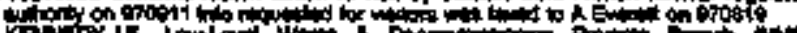

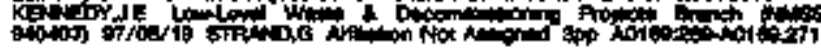

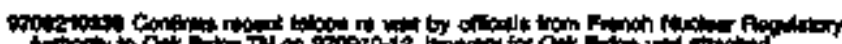

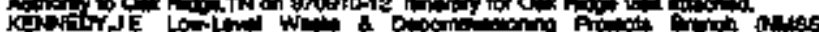

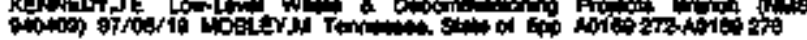

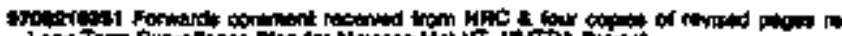

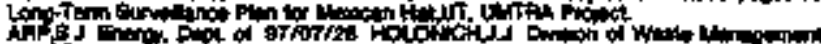

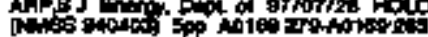

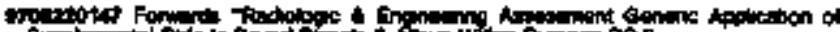

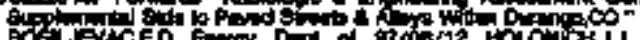

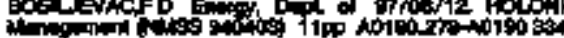

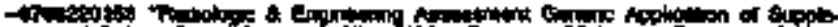

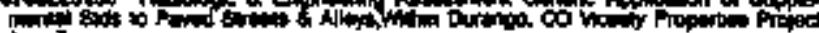

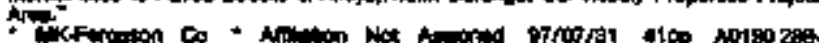
antion s.

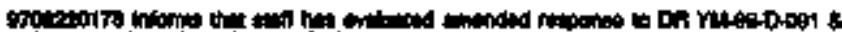

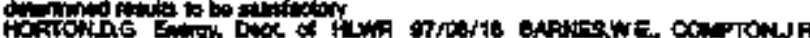

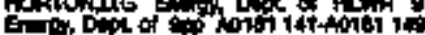

worit:

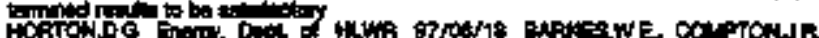

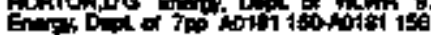

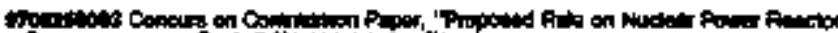

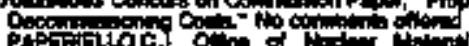

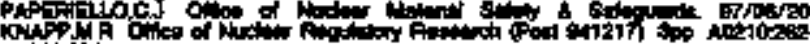
notiosot

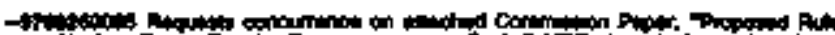

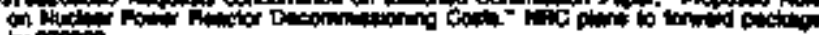

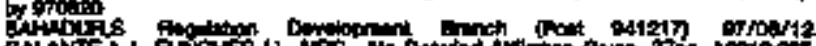

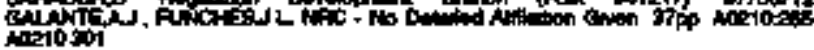

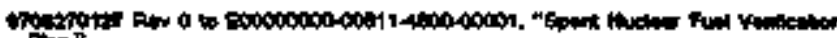

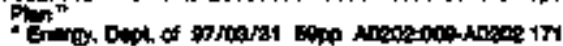

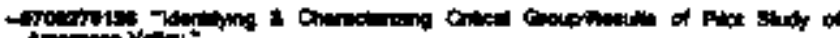

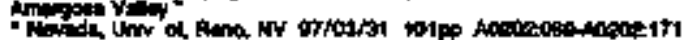

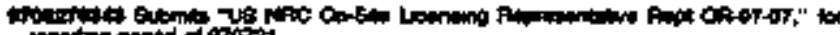

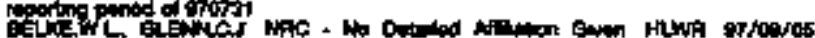
:

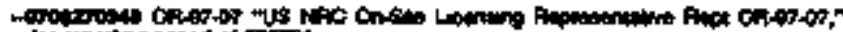

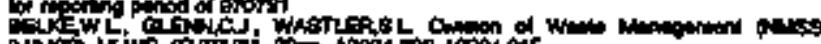

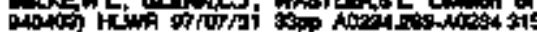

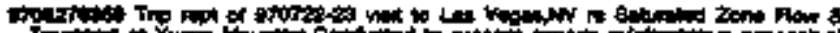

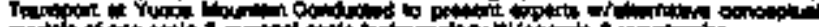

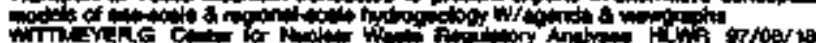

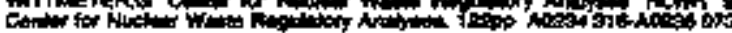

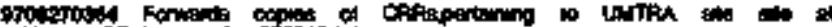

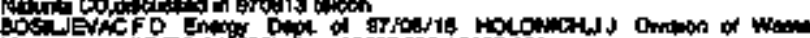
it.

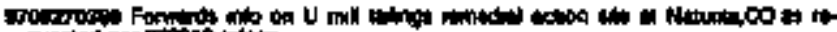

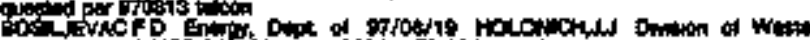

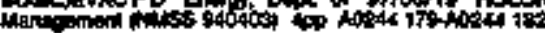

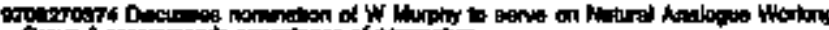

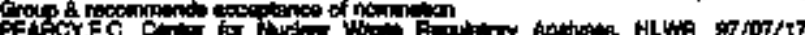

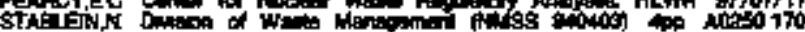
1025173

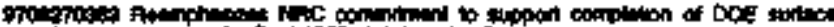

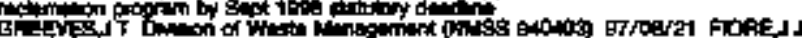

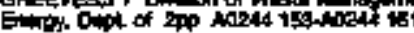

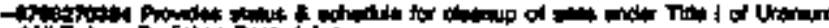

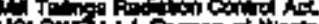
HClow

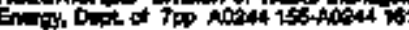

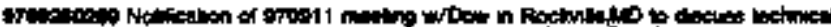

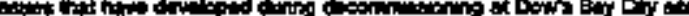

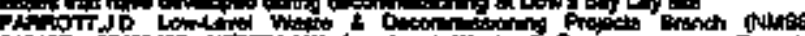

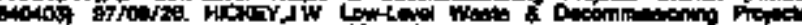

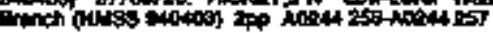

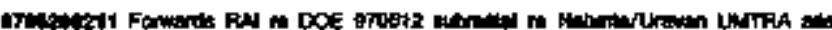

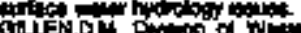

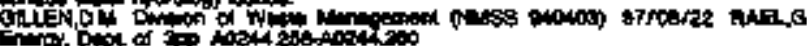

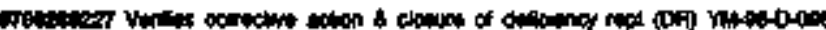

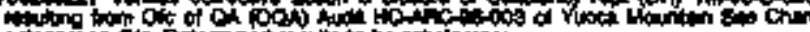

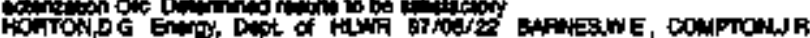

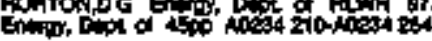

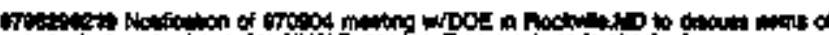

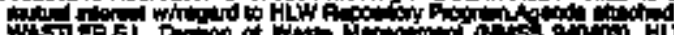

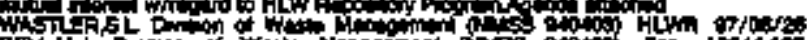
壁 $10 \mathrm{At} 134$

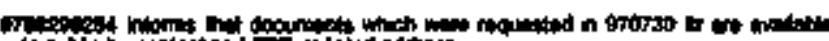

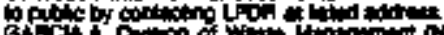

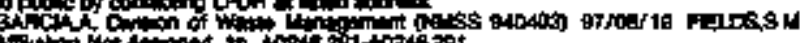

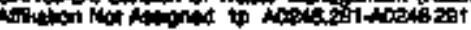

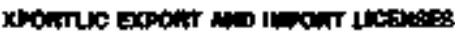

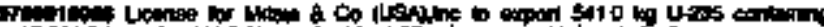

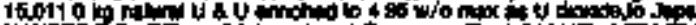
Hulu

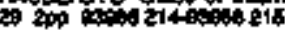

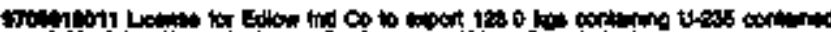

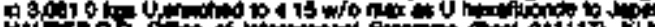

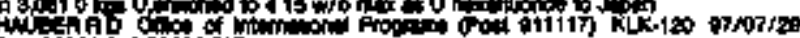

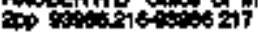

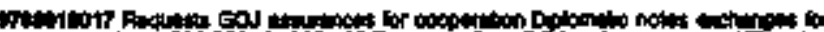

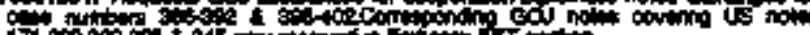

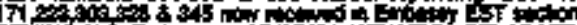

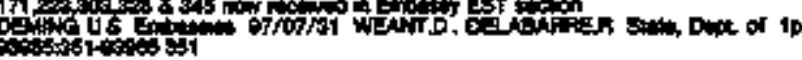

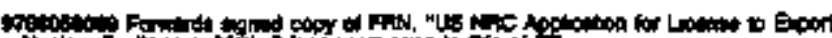

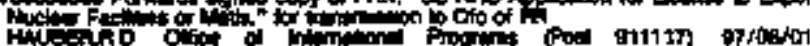

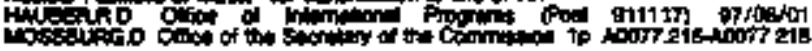

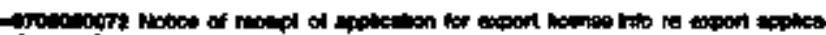
mon the

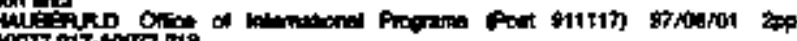

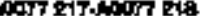

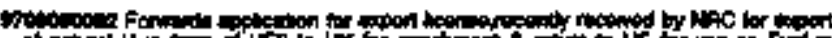

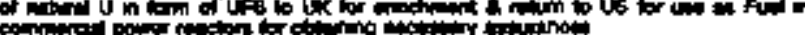

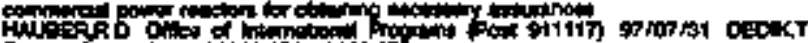

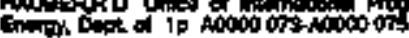

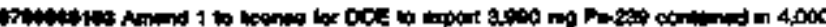

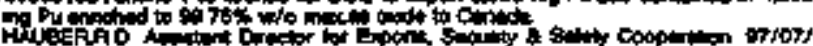

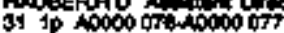

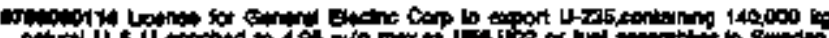

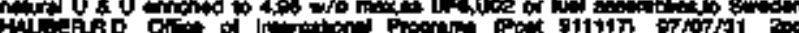

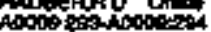

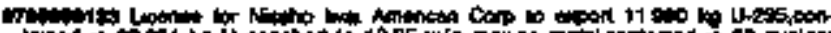

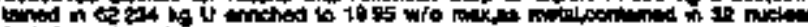

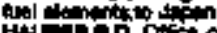

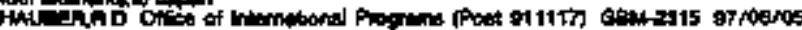

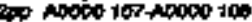




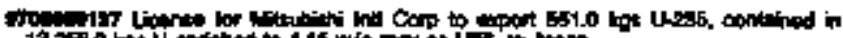

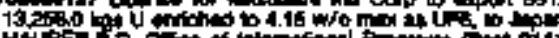

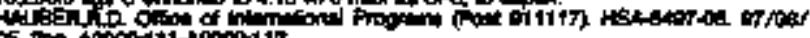

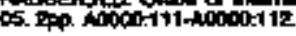

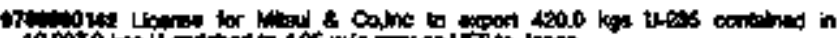

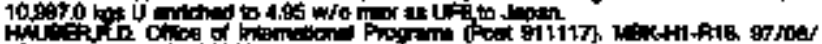
of ape $10000=109+40000+10$.

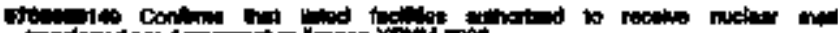

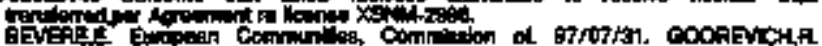

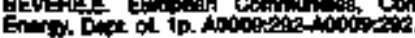

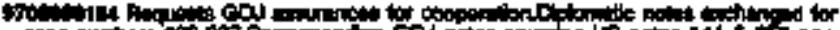

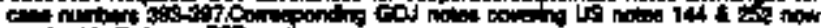

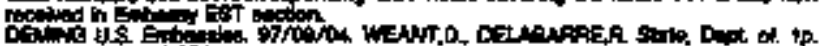

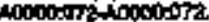

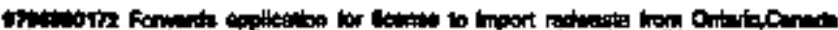

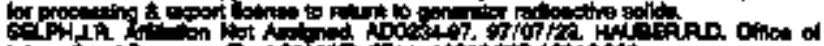

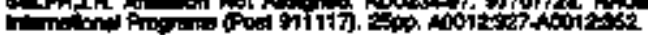

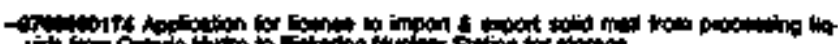

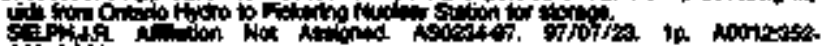
sion

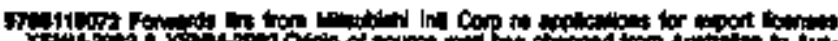
Xoj

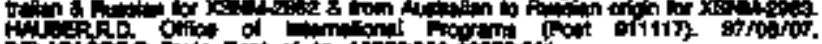

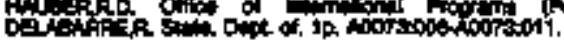

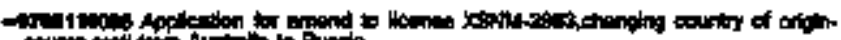

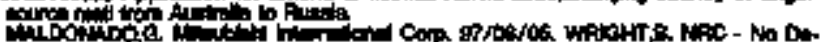

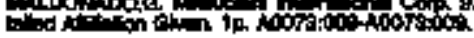

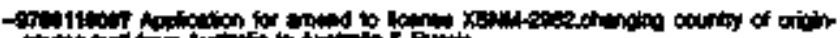

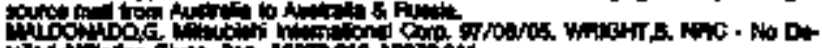

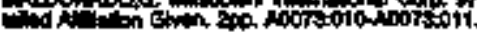

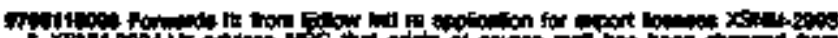

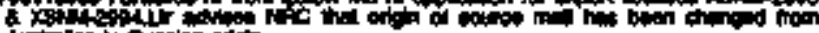

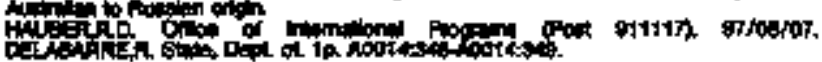

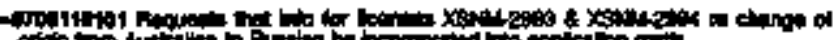

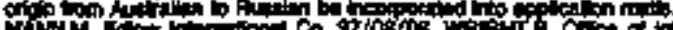

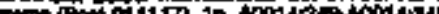

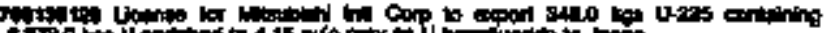

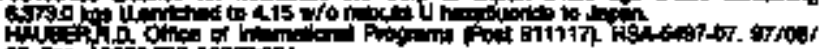

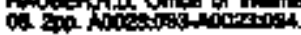

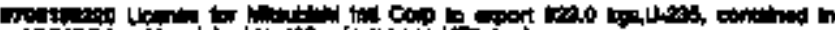

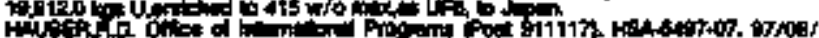

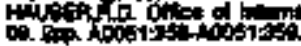

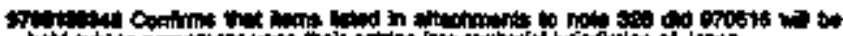

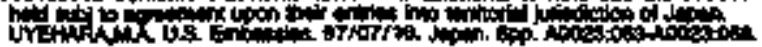

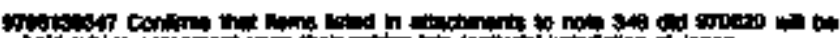

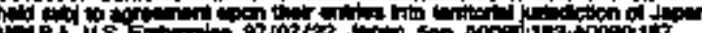

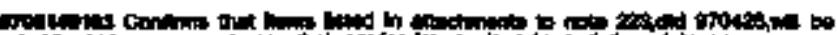

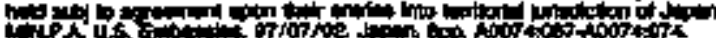

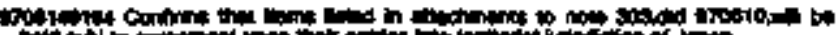

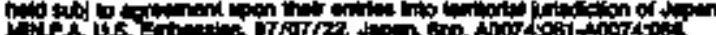

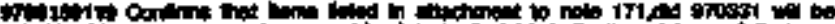

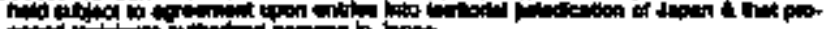

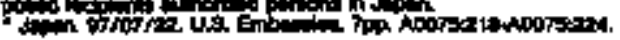

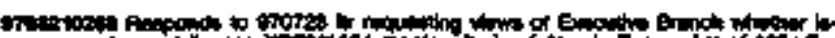

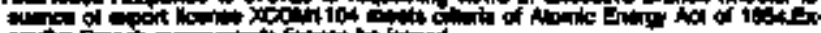

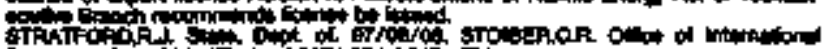

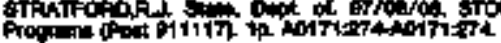

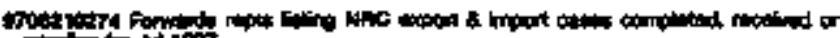

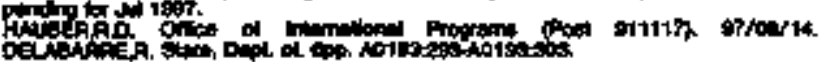

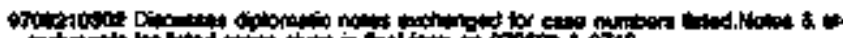

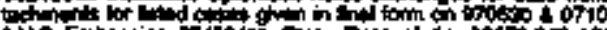

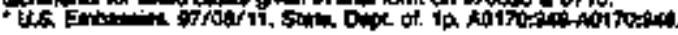

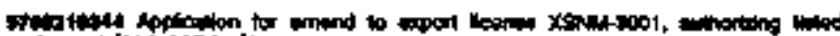

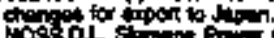

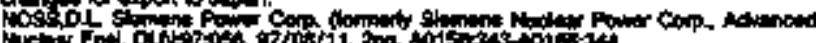

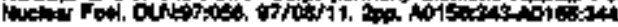

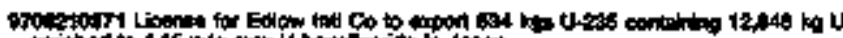

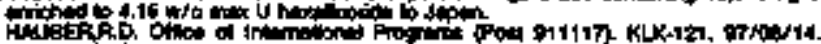

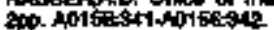

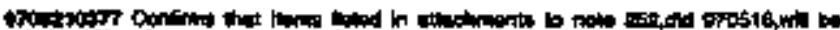

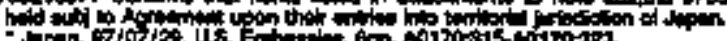

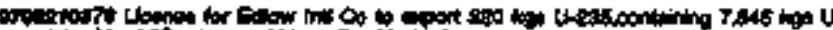

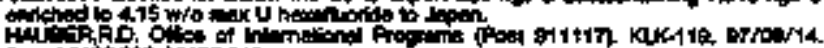

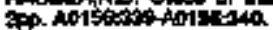

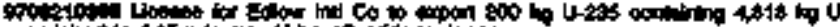

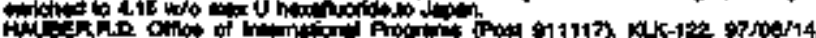

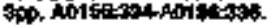

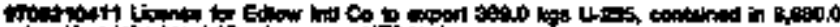

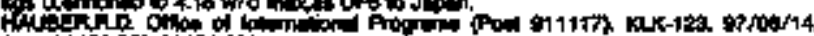

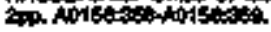

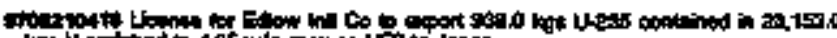

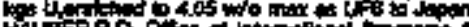

Frot

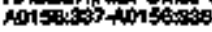

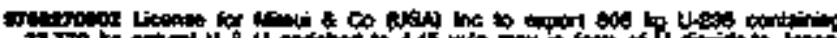

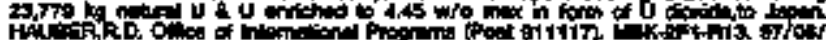

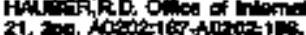

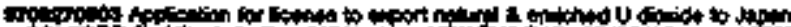

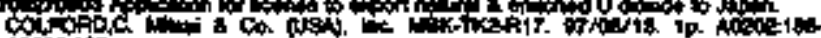
chetich

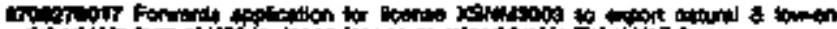

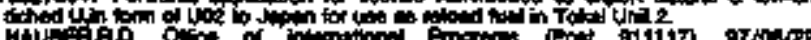

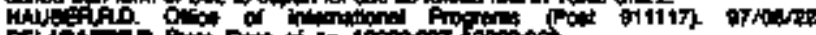

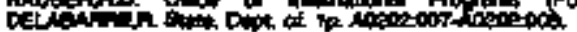

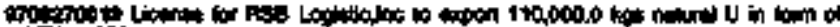
Trout

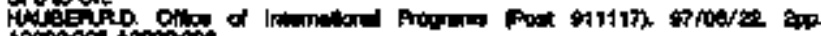

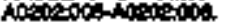

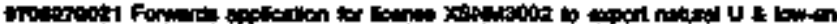

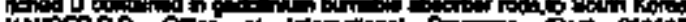
Wul of of on

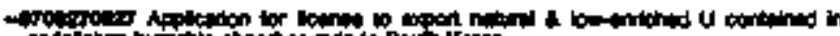

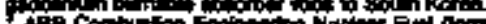

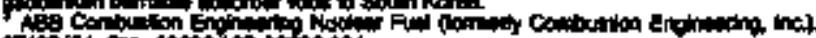

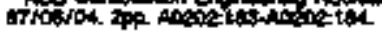

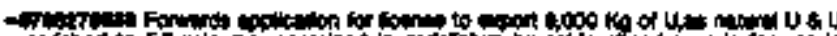

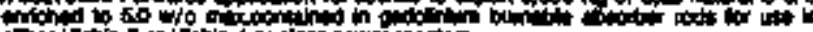

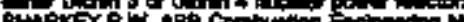

BHare

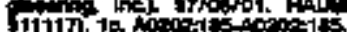

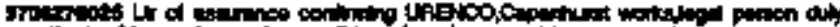

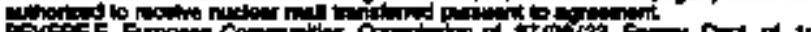
intis Eupow

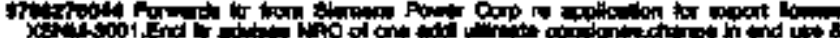

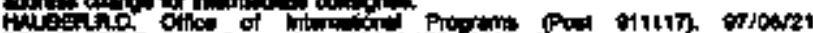

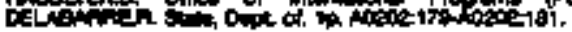

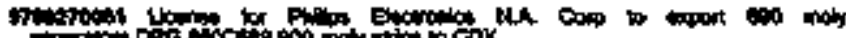

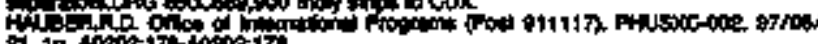

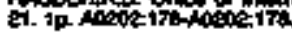

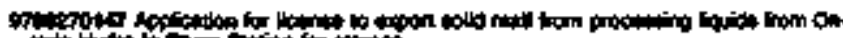

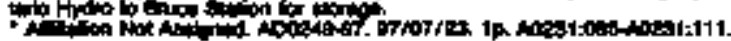

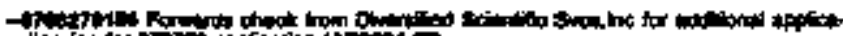

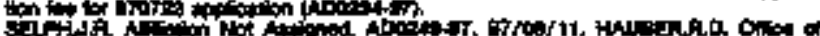

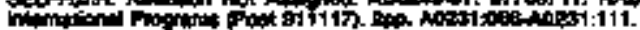




\section{PERSONAL AUTHOR INDEX}

The subject category cohumn in this index gives the docket number and the symbol for the category of information within that docket; for exarmple,50-320 P. The docket subject categories are detined in Appenin A.

Subject categories for nondocketed ftems are also entered in this column by symbol; for example, ACRS. These categories are datined in Appendix B and listed in the contents.
An asterisk following the cocession number in this index indicates that the document is an enclosure to another document. To obtain the accession number of the principal document under which the enclosure is listed, refier to the index entitled "Cross Reference of Enctosires to Principal Documents."

\begin{tabular}{|c|c|c|c|c|}
\hline Mnison & 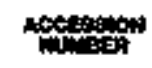 & entistos & & Authos \\
\hline 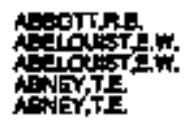 & 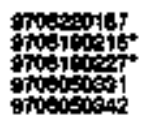 & 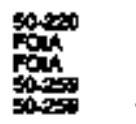 & 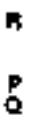 & 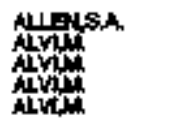 \\
\hline 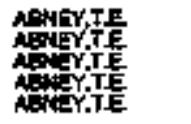 & 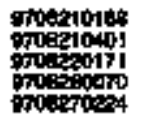 & 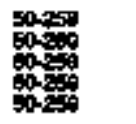 & $\begin{array}{l}P \\
P \\
P \\
P \\
P\end{array}$ & Alyd \\
\hline الم & 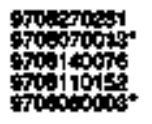 & 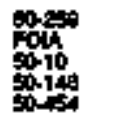 & $\begin{array}{l}\mathbf{F} \\
\mathbf{p} \\
\mathbf{p}\end{array}$ & Alvin \\
\hline 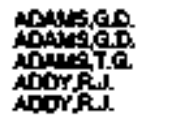 & 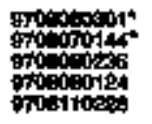 & 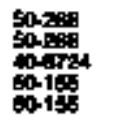 & $\begin{array}{l}3 \\
5 \\
9\end{array}$ & 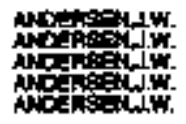 \\
\hline 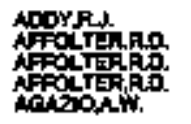 & 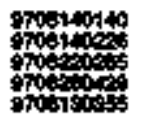 & 我驾 & 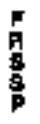 & 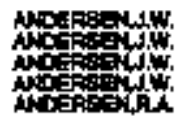 \\
\hline 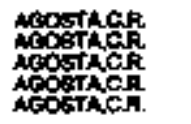 & 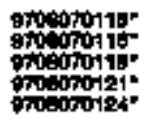 & 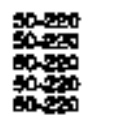 & $\begin{array}{l}p \\
p \\
p \\
p\end{array}$ & 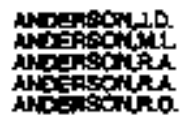 \\
\hline 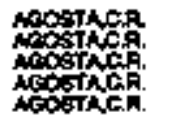 & 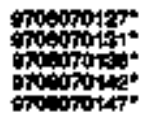 & 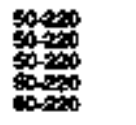 & $\begin{array}{l}p \\
p \\
p \\
p\end{array}$ & 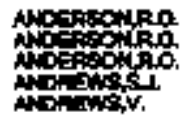 \\
\hline 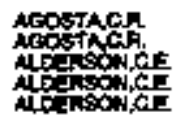 & 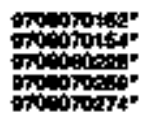 & 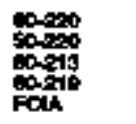 & p & 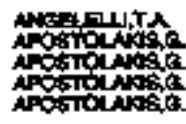 \\
\hline 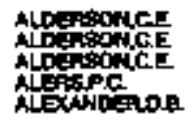 & 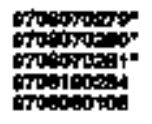 & 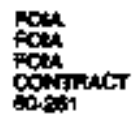 & $P$ & 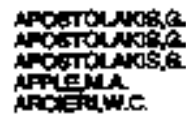 \\
\hline 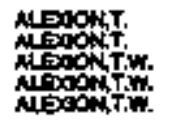 & 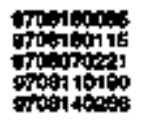 & 舟 & $\begin{array}{l}p \\
p \\
p \\
p\end{array}$ & 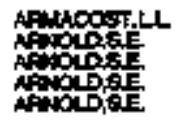 \\
\hline 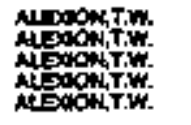 & 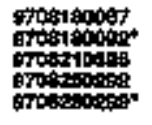 & 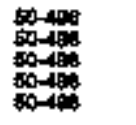 & P & 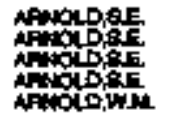 \\
\hline 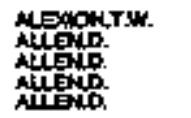 & 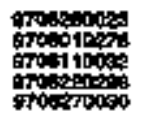 & 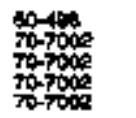 & $\mathbf{P}$ & 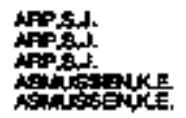 \\
\hline
\end{tabular}

\begin{tabular}{|c|c|c|}
\hline 19tosing & payser & MITHA \\
\hline 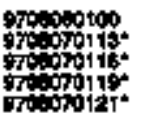 & 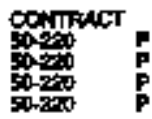 & 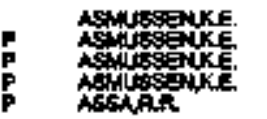 \\
\hline 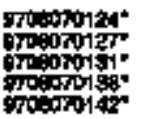 & 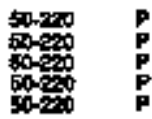 & 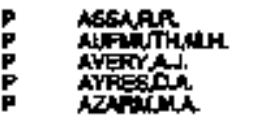 \\
\hline 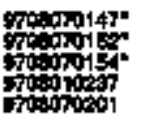 & 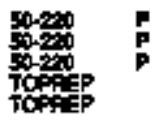 & 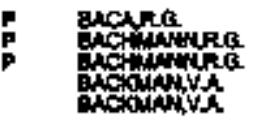 \\
\hline 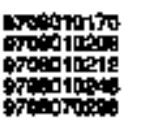 & 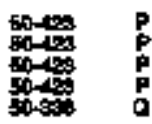 & 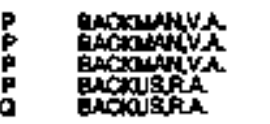 \\
\hline 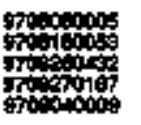 & 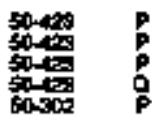 & 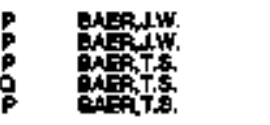 \\
\hline 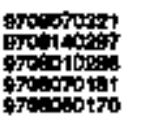 & 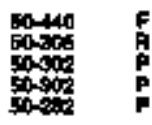 & 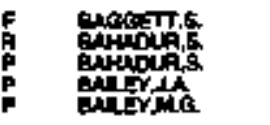 \\
\hline 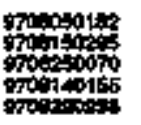 & 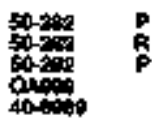 & axpres. \\
\hline 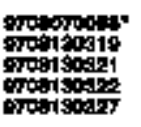 & 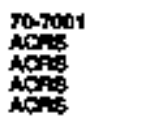 & 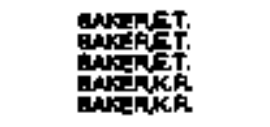 \\
\hline 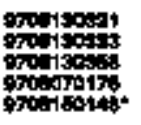 & 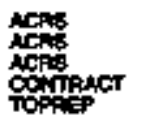 & 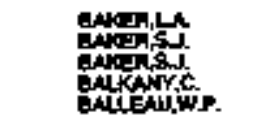 \\
\hline 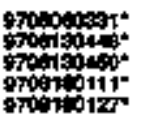 & 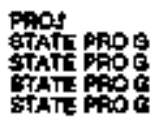 & 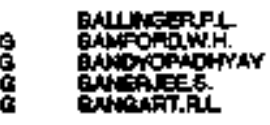 \\
\hline 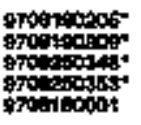 & 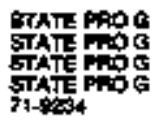 & 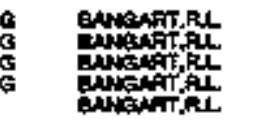 \\
\hline 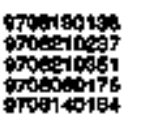 & 我 & 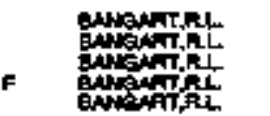 \\
\hline
\end{tabular}

\begin{tabular}{|c|c|}
\hline 1 & ofinger \\
\hline 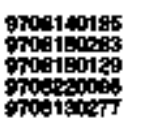 & 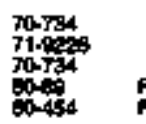 \\
\hline 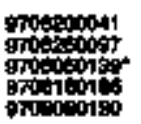 & oper \\
\hline 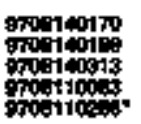 & 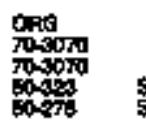 \\
\hline 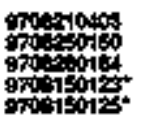 & 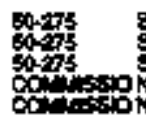 \\
\hline 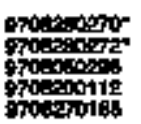 & $\begin{array}{l}0040 \\
50-46 \\
70-14 \\
70-14 \\
70-148\end{array}$ \\
\hline 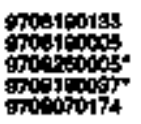 & 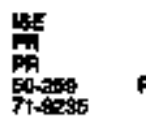 \\
\hline 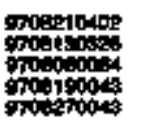 & 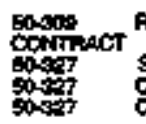 \\
\hline 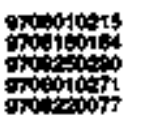 & 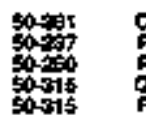 \\
\hline 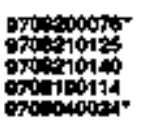 & 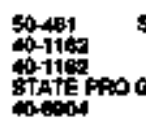 \\
\hline 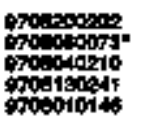 & 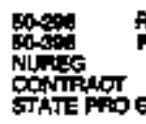 \\
\hline 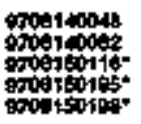 & 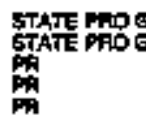 \\
\hline 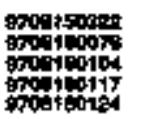 & 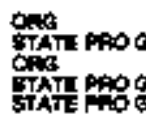 \\
\hline
\end{tabular}




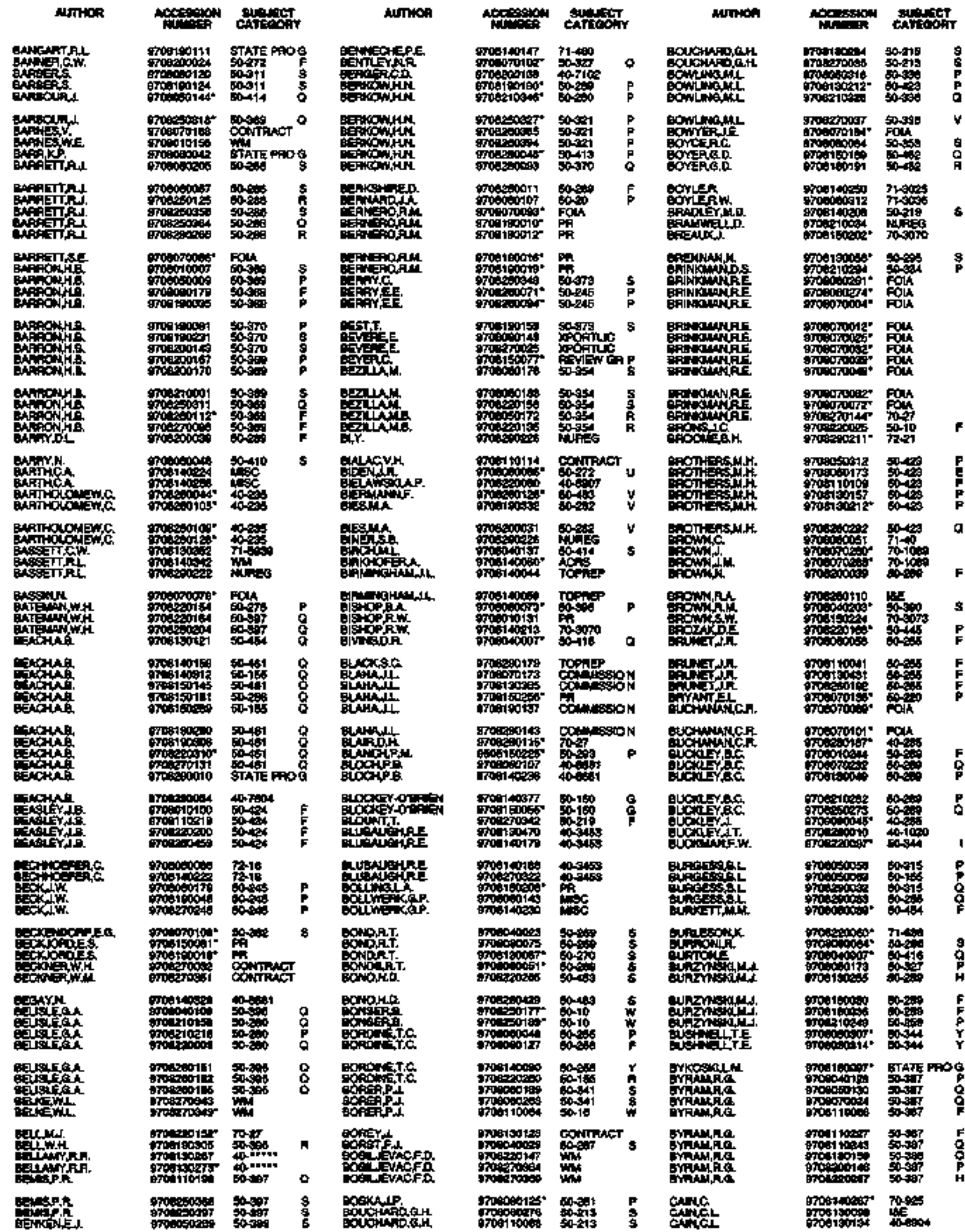




\begin{tabular}{|c|c|c|c|c|c|c|c|c|c|c|c|}
\hline Auntose & 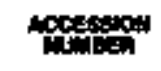 & 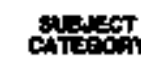 & & 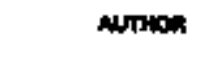 & nofforson & 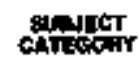 & & Mrthe & 4 & 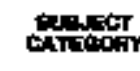 & \\
\hline 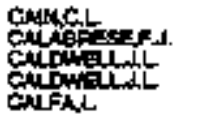 & 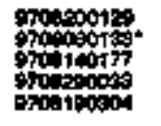 & 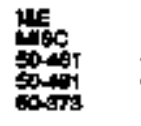 & 8 & 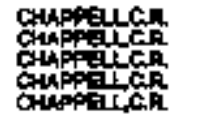 & 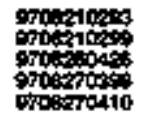 & 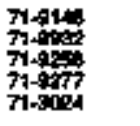 & & 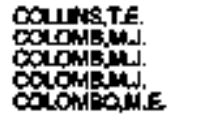 & 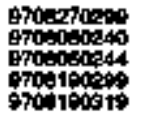 & 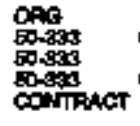 & p \\
\hline 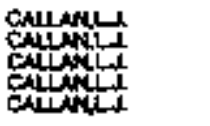 & 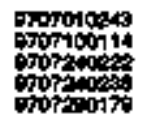 & 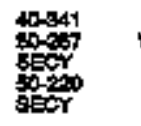 & H & 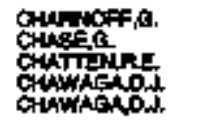 & 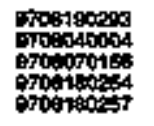 & 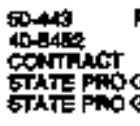 & G & 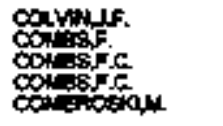 & 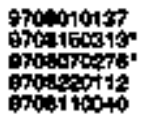 & 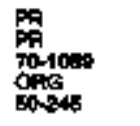 & P \\
\hline 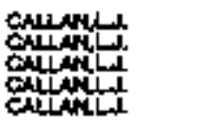 & 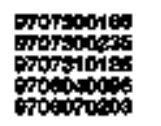 & 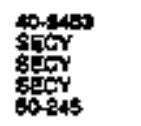 & $p$ & 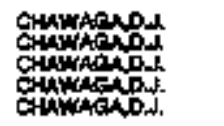 & 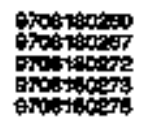 & 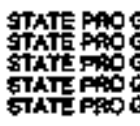 & & 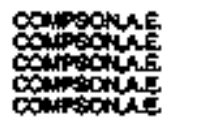 & 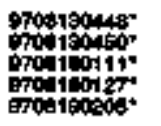 & 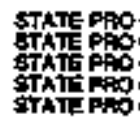 & \\
\hline 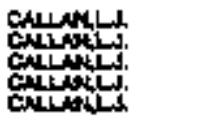 & 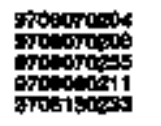 & 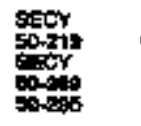 & 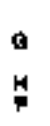 & 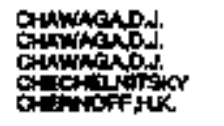 & 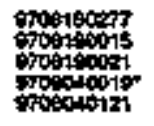 & 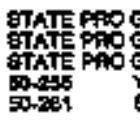 & 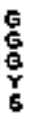 & 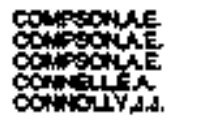 & 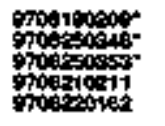 & 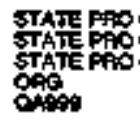 & \\
\hline 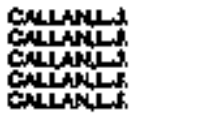 & 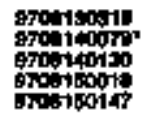 & 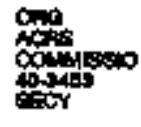 & & 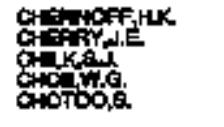 & 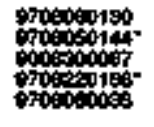 & 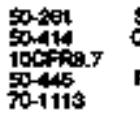 & $\begin{array}{l}5 \\
\text { p }\end{array}$ & 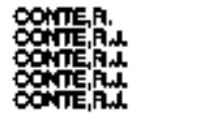 & 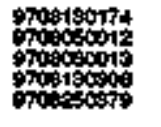 & 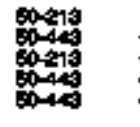 & 8 \\
\hline 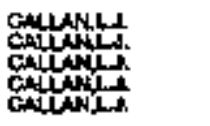 & 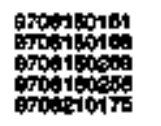 & 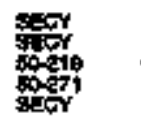 & 9 & 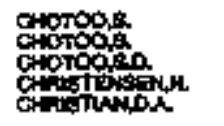 & 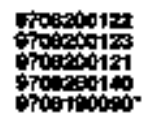 & 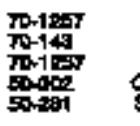 & s & 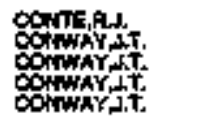 & 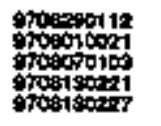 & 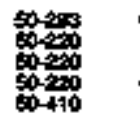 & 0 \\
\hline 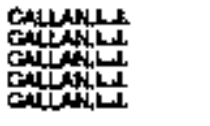 & 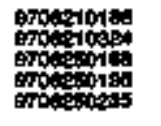 & 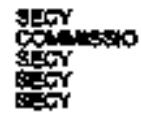 & & 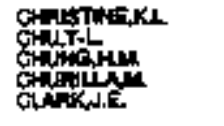 & 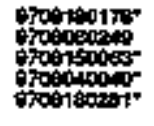 & 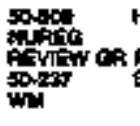 & H & 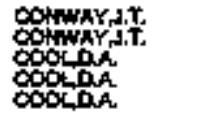 & 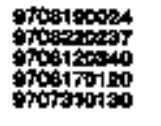 & 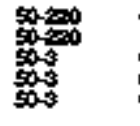 & 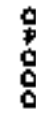 \\
\hline 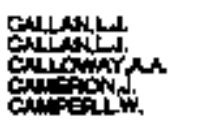 & 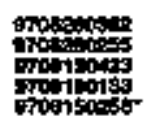 & 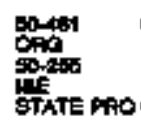 & 0 & 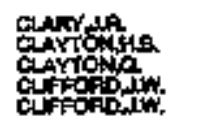 & 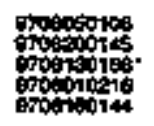 & 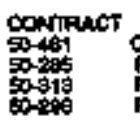 & ? & 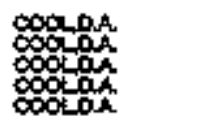 & 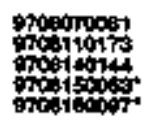 & 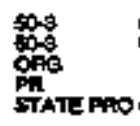 & $\begin{array}{l}8 \\
06\end{array}$ \\
\hline 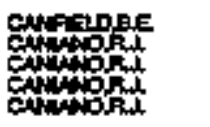 & 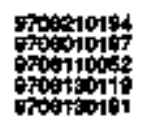 & 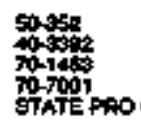 & A & 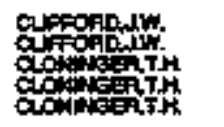 & 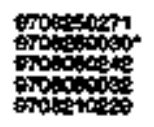 & 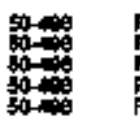 & p & 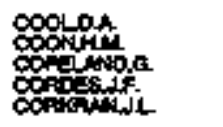 & 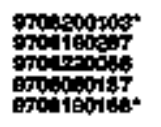 & 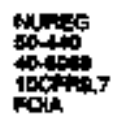 & A \\
\hline 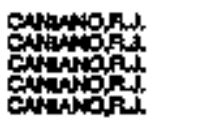 & 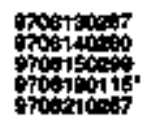 & 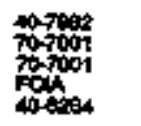 & & 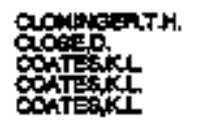 & 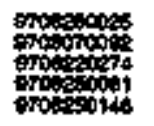 & & $\mathbf{P}$ & 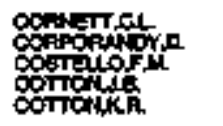 & 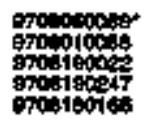 & 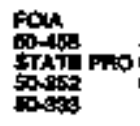 & s: \\
\hline 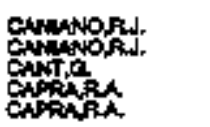 & 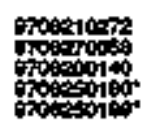 & 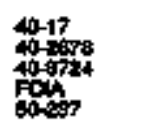 & & 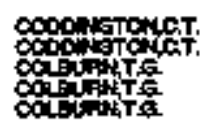 & 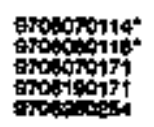 & 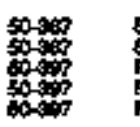 & 5 & 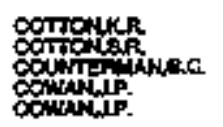 & 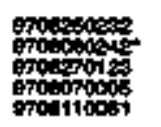 & 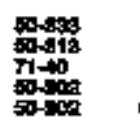 & P \\
\hline 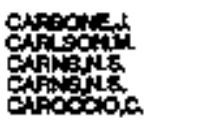 & 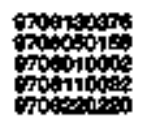 & 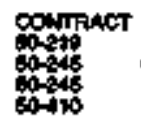 & 8 & 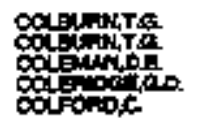 & 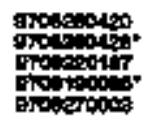 & 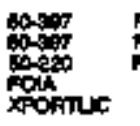 & F & 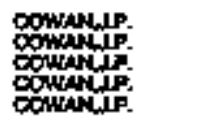 & 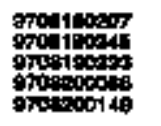 & 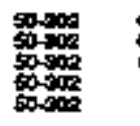 & \\
\hline 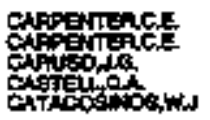 & 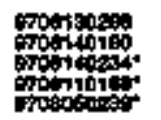 & 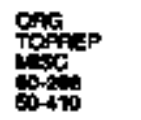 & i & 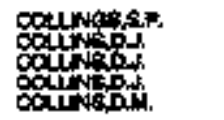 & 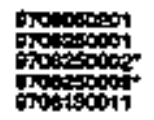 & 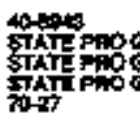 & & 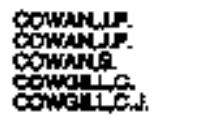 & 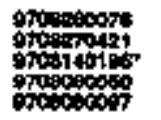 & 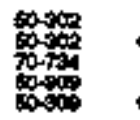 & \\
\hline 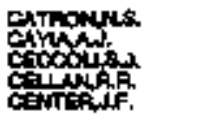 & 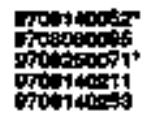 & 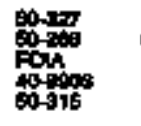 & 0 & 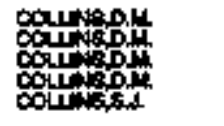 & 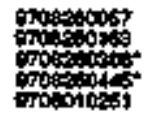 & 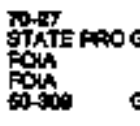 & $\theta$ & 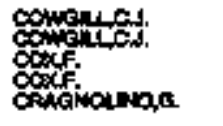 & 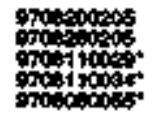 & 禹里 & \\
\hline 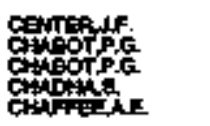 & 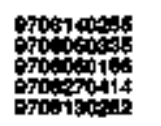 & 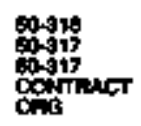 & p & 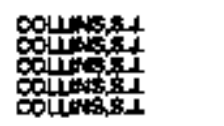 & 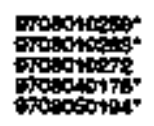 & 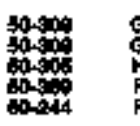 & 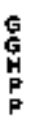 & 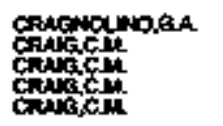 & 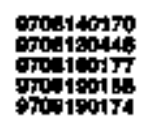 & 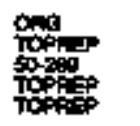 & $p$ \\
\hline 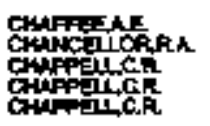 & 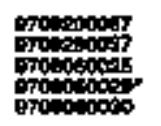 & 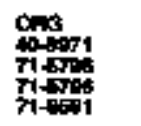 & & 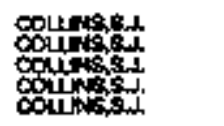 & 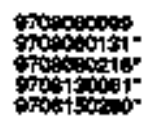 & 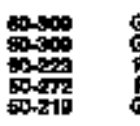 & 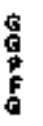 & 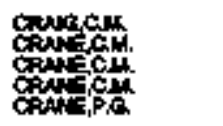 & 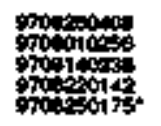 & 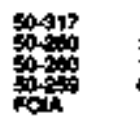 & 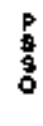 \\
\hline 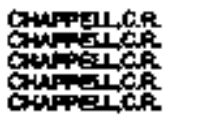 & 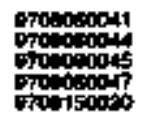 & 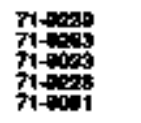 & & 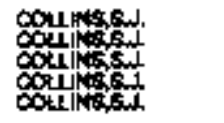 & 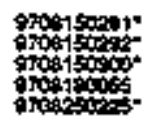 & 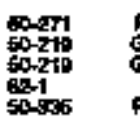 & : & 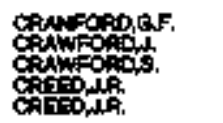 & 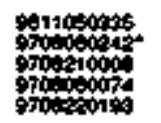 & 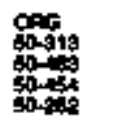 & \\
\hline 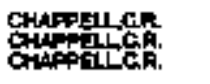 & 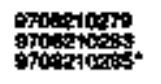 & 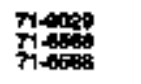 & & 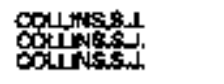 & 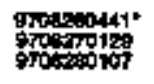 & 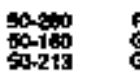 & ge & 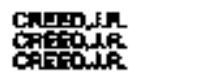 & 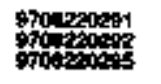 & 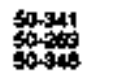 & F \\
\hline
\end{tabular}




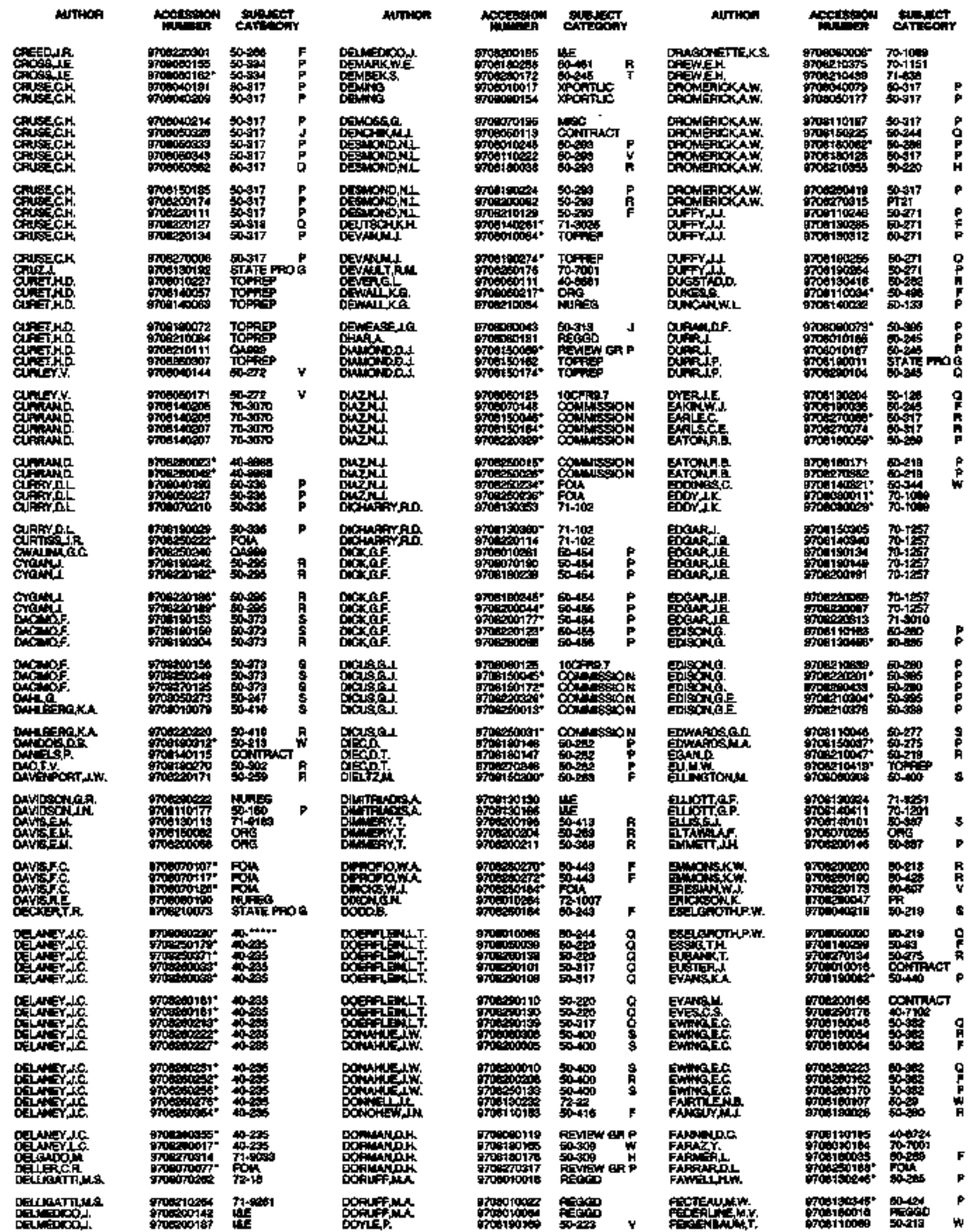




\begin{tabular}{|c|c|c|c|c|c|c|c|c|c|c|c|}
\hline ANTingR & 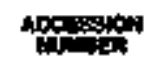 & 엣․ & & 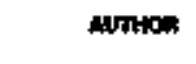 & 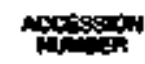 & $\mathrm{m}$ & & ATn:A9 & Mace: & 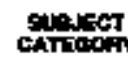 & \\
\hline 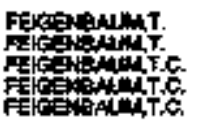 & 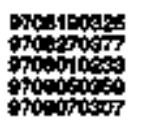 & $\begin{array}{l}5-449 \\
50-213 \\
50-40 \\
50-40 \\
50-40\end{array}$ & $\begin{array}{c}\text { R } \\
\text { 草 } \\
\mathbf{p} \\
\mathbf{p} \\
\mathbf{5}\end{array}$ & 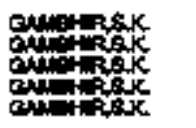 & 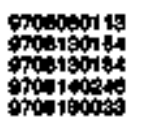 & 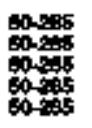 & $\begin{array}{l}\mathbf{p} \\
\mathbf{p} \\
\mathbf{f} \\
0\end{array}$ & 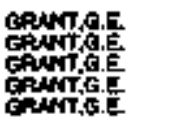 & 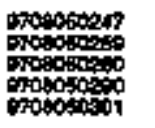 & 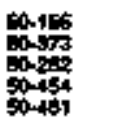 & $\begin{array}{l}0 \\
0 \\
0 \\
0 \\
0\end{array}$ \\
\hline 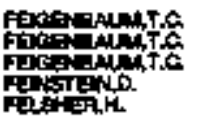 & 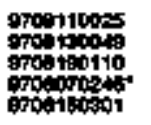 & 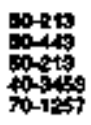 & $\begin{array}{c}\mathbf{H} \\
\mathbf{s} \\
\mathbf{5}\end{array}$ & 年 & 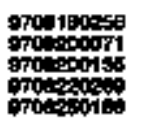 & 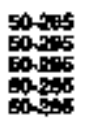 & 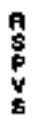 & 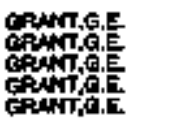 & 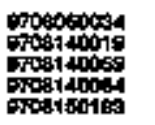 & 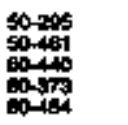 & $\begin{array}{l}8 \\
0 \\
0\end{array}$ \\
\hline 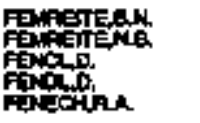 & 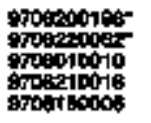 & 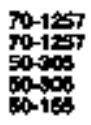 & $\underset{F}{\mathbf{F}}$ & 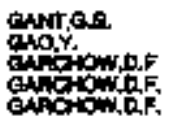 & 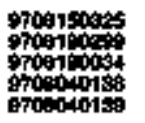 & 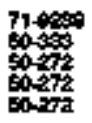 & $\begin{array}{l}\mathbf{A} \\
6 \\
5 \\
5\end{array}$ & 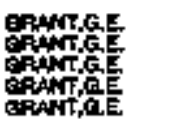 & 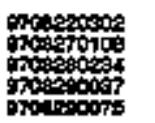 & 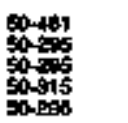 & 0 \\
\hline 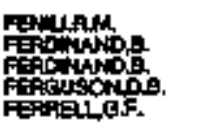 & 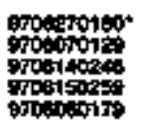 & 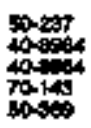 & 9 & 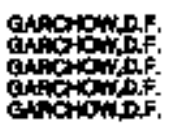 & 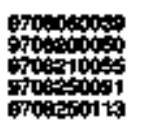 & $\begin{array}{l}50-272 \\
50-511 \\
50-011 \\
50-272 \\
50-272\end{array}$ & $\begin{array}{l}\mathbf{8} \\
\mathbf{5} \\
5 \\
\mathbf{5} \\
0\end{array}$ & 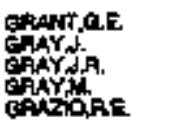 & 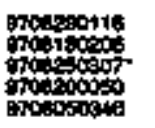 & 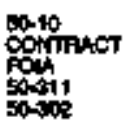 & 8 \\
\hline 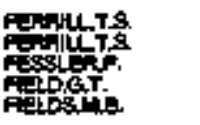 & 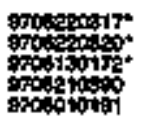 & 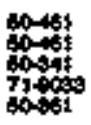 & $\begin{array}{l}9 \\
9 \\
8\end{array}$ & 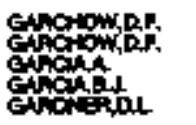 & 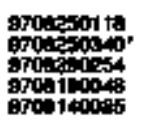 & 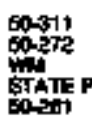 & $\stackrel{A}{A}$ & 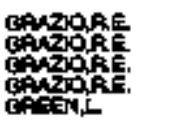 & 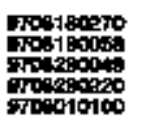 & 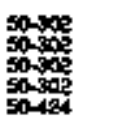 & $\underset{\mathbf{H}}{\mathbf{R}}$ \\
\hline 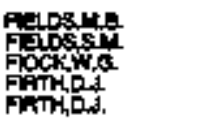 & 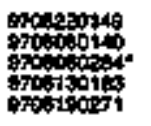 & 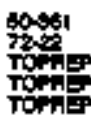 & $P$ & eN & 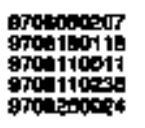 & 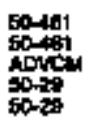 & $\begin{array}{l}5 \\
9 \\
9 \\
9\end{array}$ & 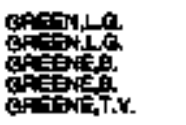 & 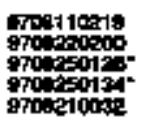 & 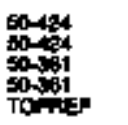 & $\begin{array}{l}\mathbf{F} \\
\mathbf{P} \\
\mathbf{P}\end{array}$ \\
\hline 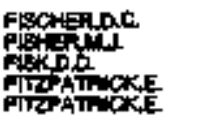 & 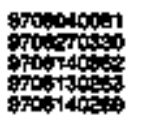 & कi & $\begin{array}{l}p \\
p \\
p\end{array}$ & 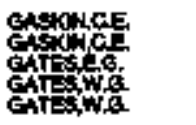 & 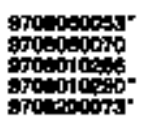 & 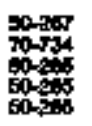 & $\begin{array}{l}r \\
\mathfrak{p} \\
\mathfrak{p}\end{array}$ & 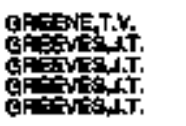 & 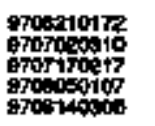 & 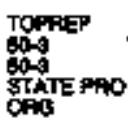 & $\frac{w}{6}$ \\
\hline 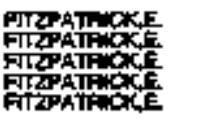 & 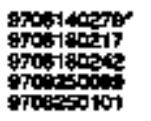 & 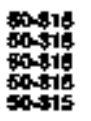 & p & 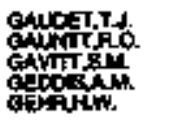 & 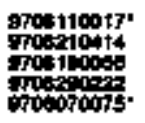 & 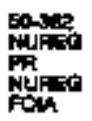 & $\mathbf{s}$ & 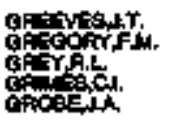 & 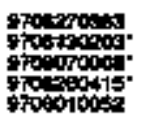 & 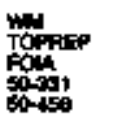 & $\frac{P}{F}$ \\
\hline 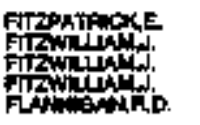 & 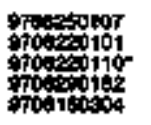 & 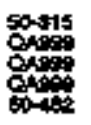 & 8 & 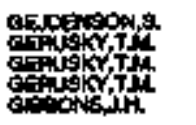 & 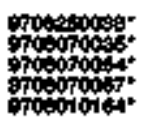 & colns & & $\sum_{k}^{h}$ & orocost & & $\begin{array}{l}\stackrel{g}{0} \\
\frac{g}{g}\end{array}$ \\
\hline 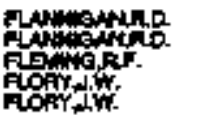 & 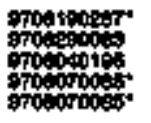 & $\begin{array}{l}\sin _{0} \\
00-2 \\
0+4\end{array}$ & $\frac{8}{5}$ & 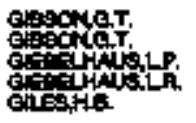 & 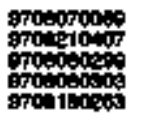 & $\begin{array}{l}00-206 \\
00-17 \\
40-17 \\
00-17 \\
00-311\end{array}$ & $\begin{array}{l}\mathbf{A} \\
\mathbf{n} \\
\mathbf{n}\end{array}$ & h & 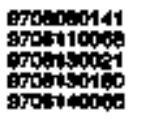 & 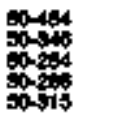 & $\begin{array}{c}g \\
g \\
g \\
q\end{array}$ \\
\hline 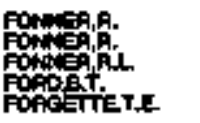 & 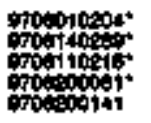 & $\begin{array}{l}40 \\
70 \\
70 \\
0\end{array}$ & 备 & 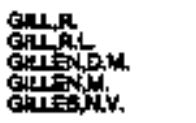 & 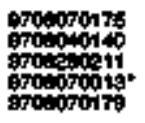 & & $\mathbf{P}$ & A. & 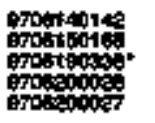 & & $\begin{array}{c}g \\
\mathbf{p} \\
\mathbf{q} \\
\mathbf{p}\end{array}$ \\
\hline 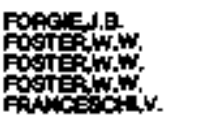 & 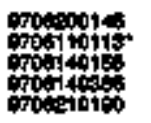 & 然象 & $\begin{array}{l}\mathbf{P} \\
\mathbf{F}\end{array}$ & 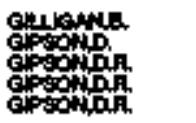 & 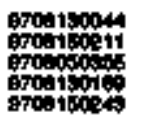 & $\begin{array}{l}72-1000 \\
00-11 \\
00-341 \\
00 ; 11\end{array}$ & $\begin{array}{l}\mathbf{s} \\
\stackrel{0}{\mathbf{p}} \\
\mathbf{p}\end{array}$ & & 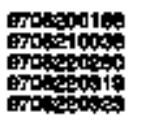 & $\operatorname{lom}_{0 \rightarrow 4}$ & 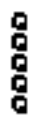 \\
\hline ons. & 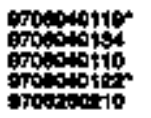 & 50. & $\begin{array}{l}9 \\
0 \\
0 \\
0\end{array}$ & 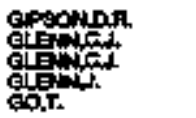 & 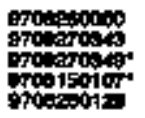 & & P & & 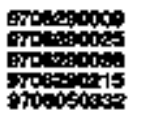 & 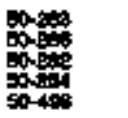 & G \\
\hline$\frac{0}{8}$ & spoloosh & & $\mathbf{n}$ & 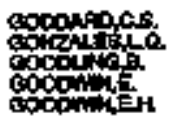 & 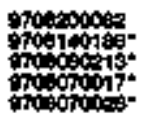 & 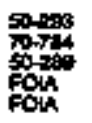 & $\begin{array}{l}\text { P } \\
\text { H }\end{array}$ & Fis. & 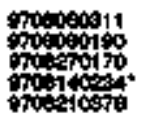 & 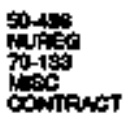 & $p$ \\
\hline 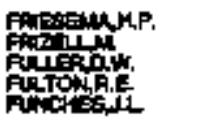 & 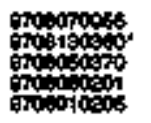 & $\begin{array}{l}40-17 \\
70-100 \\
60-46 \\
70-195\end{array}$ & $\begin{array}{l}\mathbf{F} \\
\mathbf{P}\end{array}$ & 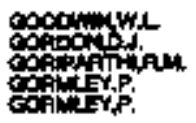 & 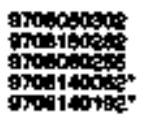 & 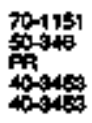 & $f$ & L. & 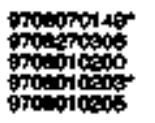 & $\begin{array}{l}\sec _{0} \\
60 \\
00\end{array}$ & $\frac{q}{p}$ \\
\hline 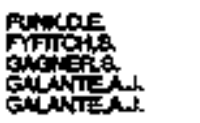 & 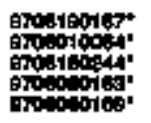 & 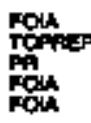 & & 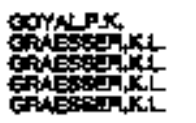 & 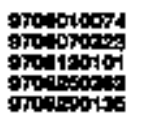 & Torpis & g & Lا & 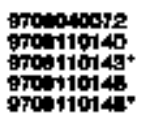 & 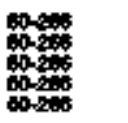 & $\begin{array}{l}P \\
P \\
p \\
p \\
P\end{array}$ \\
\hline 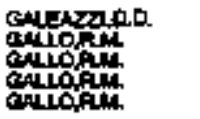 & 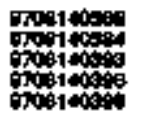 & $\begin{array}{l}50-204 \\
50-500 \\
50-400 \\
50-60\end{array}$ & $\begin{array}{l}\mathbf{s} \\
v \\
v \\
v \\
v\end{array}$ & 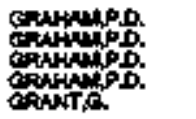 & 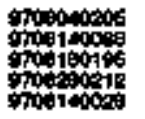 & $\begin{array}{l}60-259 \\
50-200 \\
50-200 \\
50-09 \\
50-16\end{array}$ & لे & 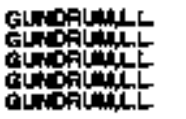 & 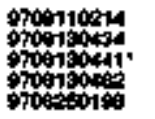 & 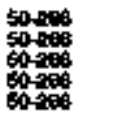 & $\begin{array}{l}p \\
p \\
p \\
p \\
p\end{array}$ \\
\hline 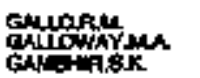 & 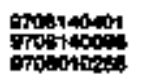 & 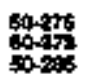 & $\begin{array}{l}\mathrm{W} \\
\mathrm{G}\end{array}$ & 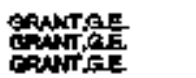 & 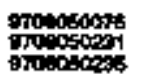 & star-s & 8 & 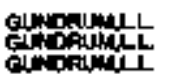 & 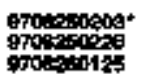 & so-ats & $\begin{array}{l}\mathrm{F} \\
\mathrm{P}\end{array}$ \\
\hline
\end{tabular}




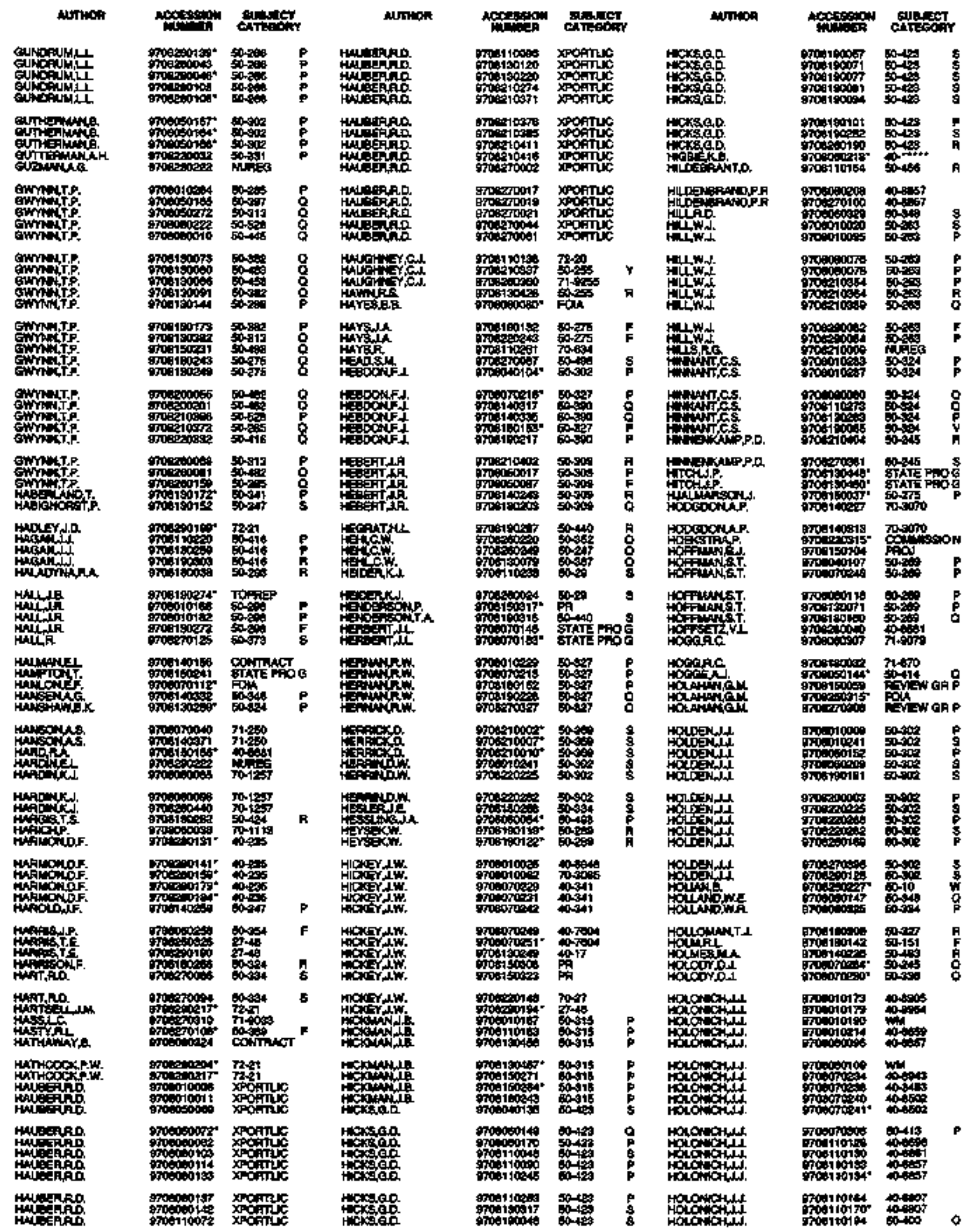


urman

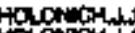

Col

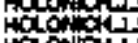

Holowichal.

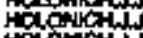

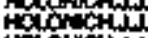

polotits.

HCLONGHd.

Hockint

tol

tcantrits

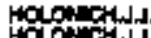

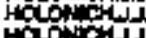

Hon

Acolowhoth

Haphor

Acriyk

loritin

Honth

Harow De

Horiother

Harothor

Hcriotsor.

ratowne

ichontse

asiota

tafowio

wortgkes

Harcitis

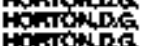

HORTOWDAR

Hojow官

Ho.

tofrotie

Hoctowne

icfiothe

Hotion

prionia

hoptowar

Priphar

tribara

intowa

hortow

AOA

HowAT슨

Hot-50

Hot

10

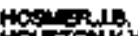

+oustowy,

PNer,

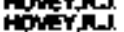

Horer,P.

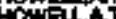

H.

Howitist.

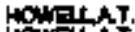

Hit.

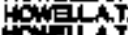

Hot

HONgeAT

Howitr.

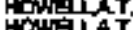

HOWEบAT.

Hortrit

Howit.

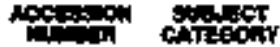

70011004.

rootsos 40 6.

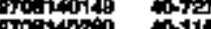

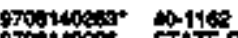

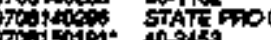

tor

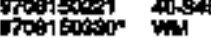

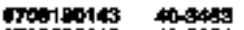

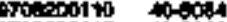

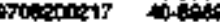

oropicioist

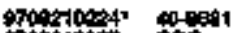

mopioser ofir

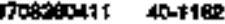

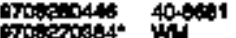

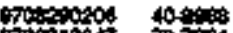

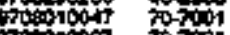

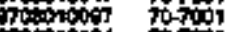

poings thos

ponoses wh

toloses wh

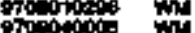

procontot2 wit

rooguche w4

rot wh

grton, wh

cototiot in

0700001 wit

7atsonis

motcondo

meico14t w4

97016015 w4

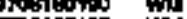

mosteot wh

orosicosos wis

Trostong

fortor wh

(1)

ontitcosi ind

opoces wh

to

1. WM

gitogits in

mepens wh

otyol sol

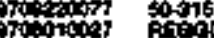

Jitolong

Pondits

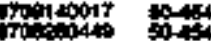

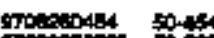

Troateons:

rrodires

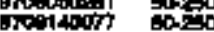

roptor on

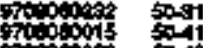

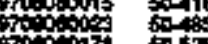

ruidosits

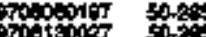

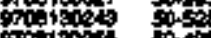

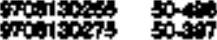

molatasos 50up

molnsiso bo

mostanti

moliteres

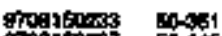

molicis
ArTH:

HOWIILAT

WIII

HOALAT.

Hon: int

TG IIAT.

The

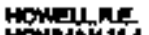

Hownen,

tonstic

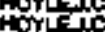

Hone

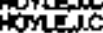

Hor

Hortace

pinctic

tomitas

Honctus

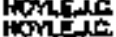

int

kment:

onisto

Hintis

Fon

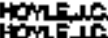

Mintido

Hivence

Homente

on telc

Toming

arimatec

marmence

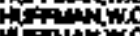

thich

Aluctists

Hucerses

$H(x)-5, T$

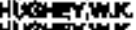

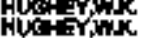

Horeysux

Hetrux

Nuctive

堆严

Aarigrial

Alitis

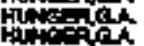

Hucrast

ton

tain

Hidit

HIJTCHAONGR

porgon

anctson

D.

g OEW

品

o

\section{- Kempx}

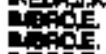

Ninitior.

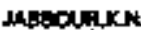

Whacitikn

Actipsion over

Munkan

Mocisingen

ond

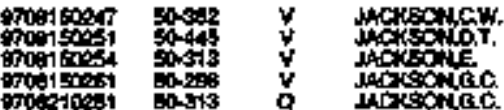

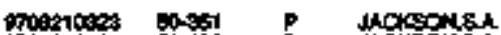

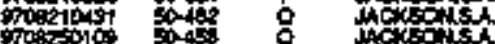

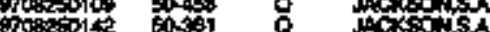

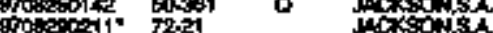

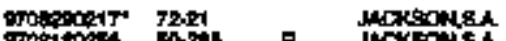

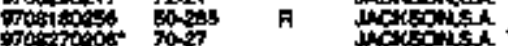

grom

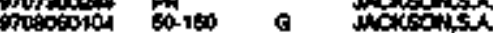

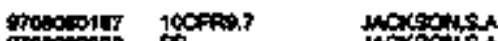

ropopot

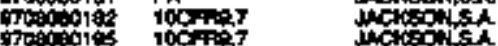

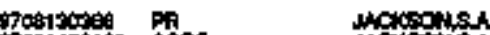

$9708+40015-A C P B$

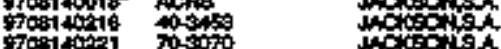

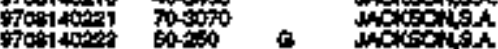

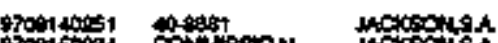

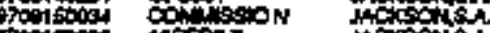

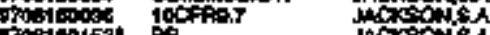

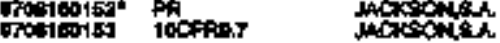

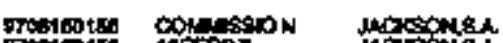

100.

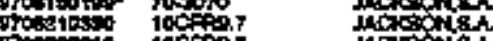

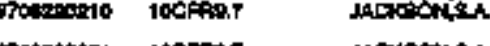

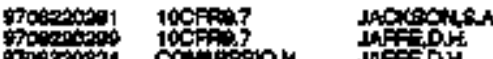

orot

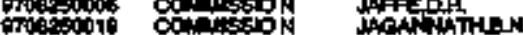

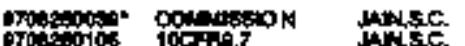

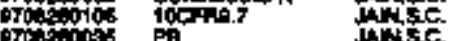

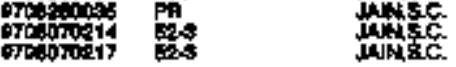

orosicosor 52s JankC.

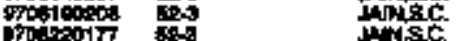

Fon

motolodit

Drotion?

70014012

rondis

gromanon

\%

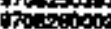

ition

400004

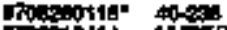

400014

orotions

stropenoto

sinotion

Proton 1

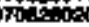

50-50:

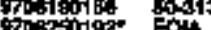

resp

rosicoes bay

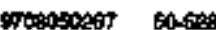

7000000

Tosos

ontos

F

F

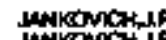

antorip

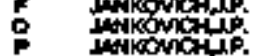

1 invoung

- InkMats.

Perconts

unectorich

Intognchis.

Heromatip

P

streonights.

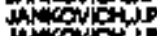

MONG

Atrot.

Mecoura

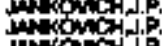

14. OMaja

atomats.

Intconchols

Intondip 


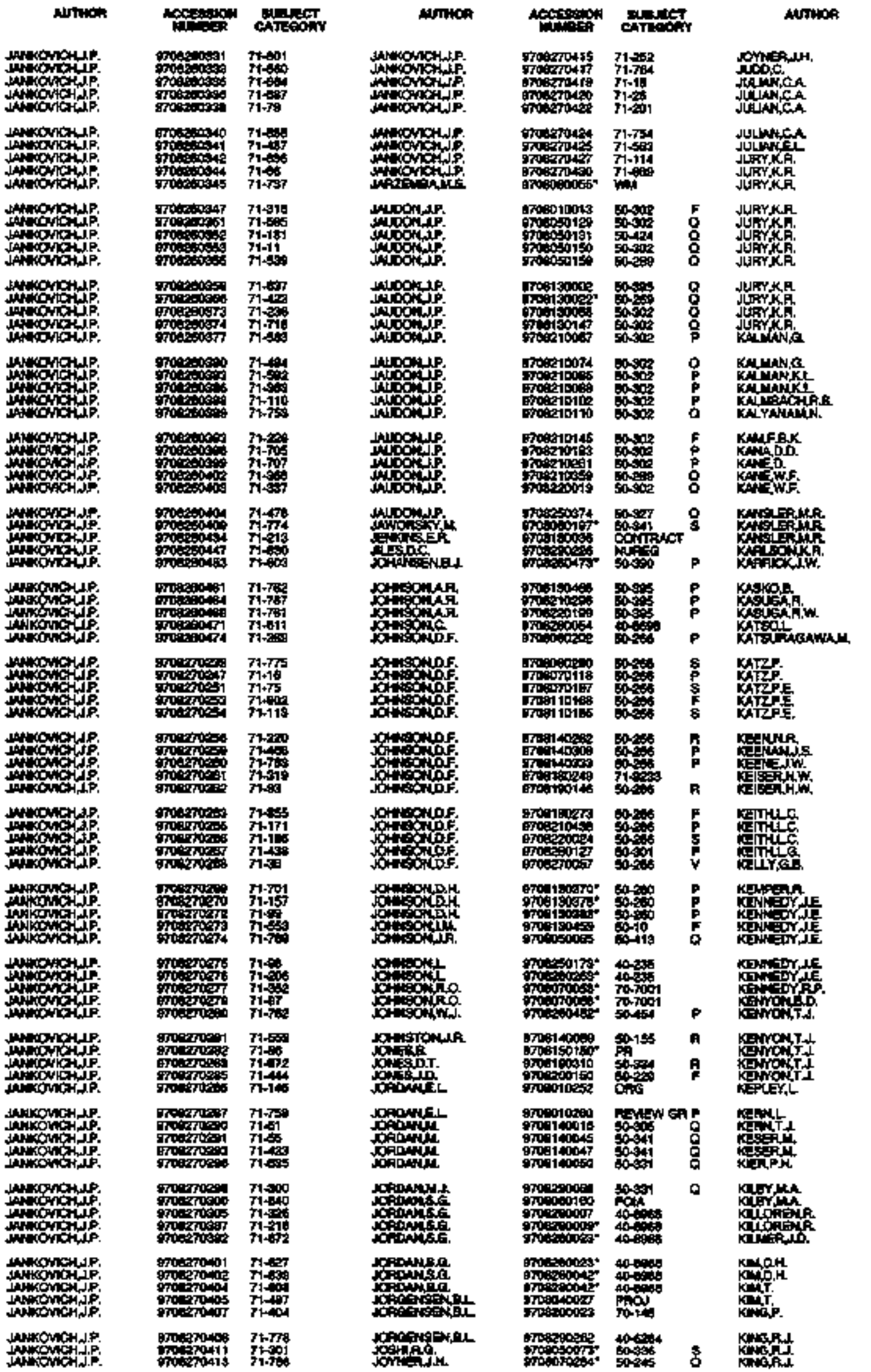

\begin{tabular}{|c|c|c|}
\hline 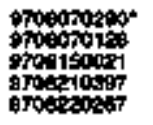 & 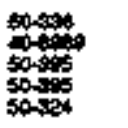 & $\begin{array}{l}0 \\
0 \\
0\end{array}$ \\
\hline 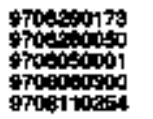 & 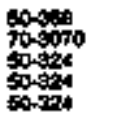 & $\begin{array}{l}\boldsymbol{Q} \\
\mathbf{F} \\
\mathbf{P}\end{array}$ \\
\hline 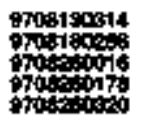 & $\begin{array}{l}50-394 \\
50-304 \\
50-44 \\
00-44\end{array}$ & $\begin{array}{l}\mathbf{p} \\
\mathbf{R} \\
\mathbf{p} \\
\mathbf{f} \\
\mathbf{p}\end{array}$ \\
\hline 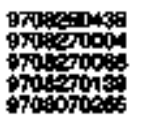 & 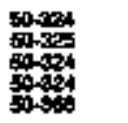 & $\begin{array}{l}q \\
0 \\
p \\
p \\
p\end{array}$ \\
\hline 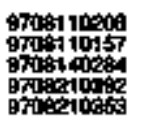 & 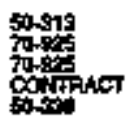 & $\begin{array}{r}F \\
F\end{array}$ \\
\hline 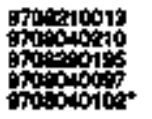 & 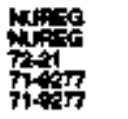 & \\
\hline opest. & & $\begin{array}{l}\text { S } \\
\text { p } \\
\text { f } \\
8\end{array}$ \\
\hline 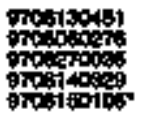 & $\begin{array}{l}17 \\
13 \\
13 \\
1\end{array}$ & $\begin{array}{r}\text { मू } \\
\text { है } \\
\text { ON }\end{array}$ \\
\hline 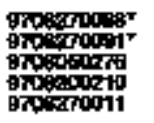 & 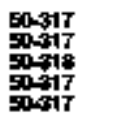 & 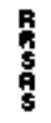 \\
\hline 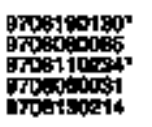 & 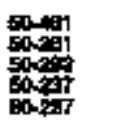 & $\begin{array}{l}\mathbf{s} \\
\frac{9}{5} \\
\mathbf{p}\end{array}$ \\
\hline 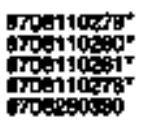 & 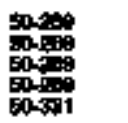 & $\begin{array}{l}\mathbf{0} \\
\frac{0}{0} \\
\frac{0}{p}\end{array}$ \\
\hline 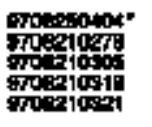 & 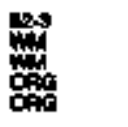 & \\
\hline 10:5 & 为4 & pe \\
\hline 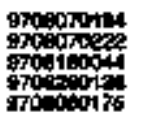 & 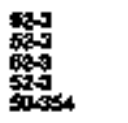 & 8 \\
\hline 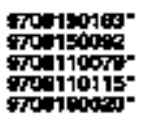 & 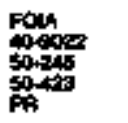 & $\stackrel{\mathbf{n}}{\mathbf{p}}$ \\
\hline 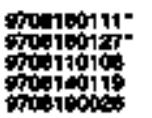 & 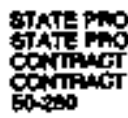 & $\begin{array}{l}08 \\
98 \\
6 \\
6\end{array}$ \\
\hline 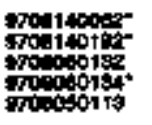 & 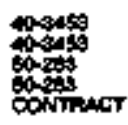 & p \\
\hline tropos & $\begin{array}{l}50-486 \\
50-486 \\
50-488\end{array}$ & 8 \\
\hline
\end{tabular}




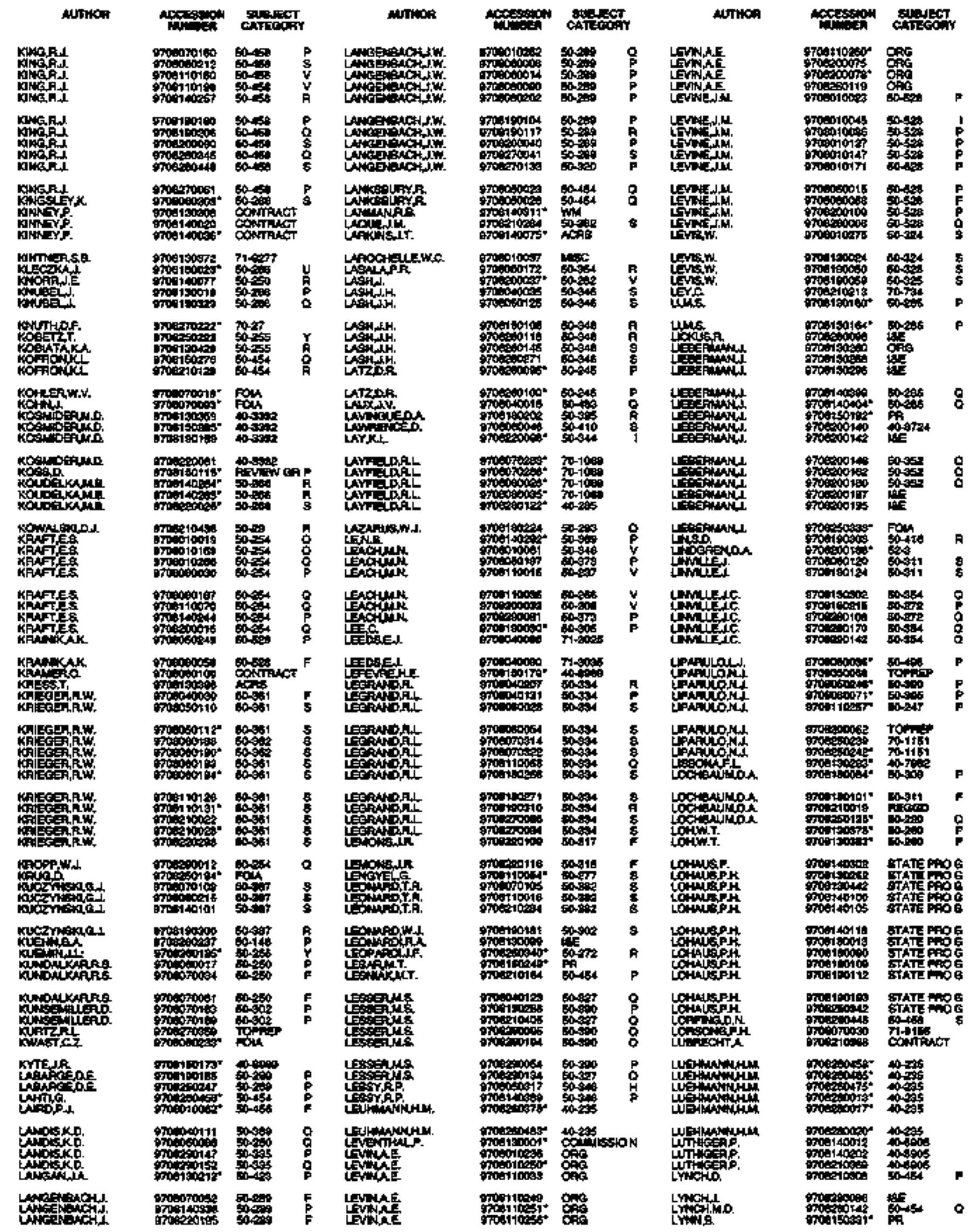




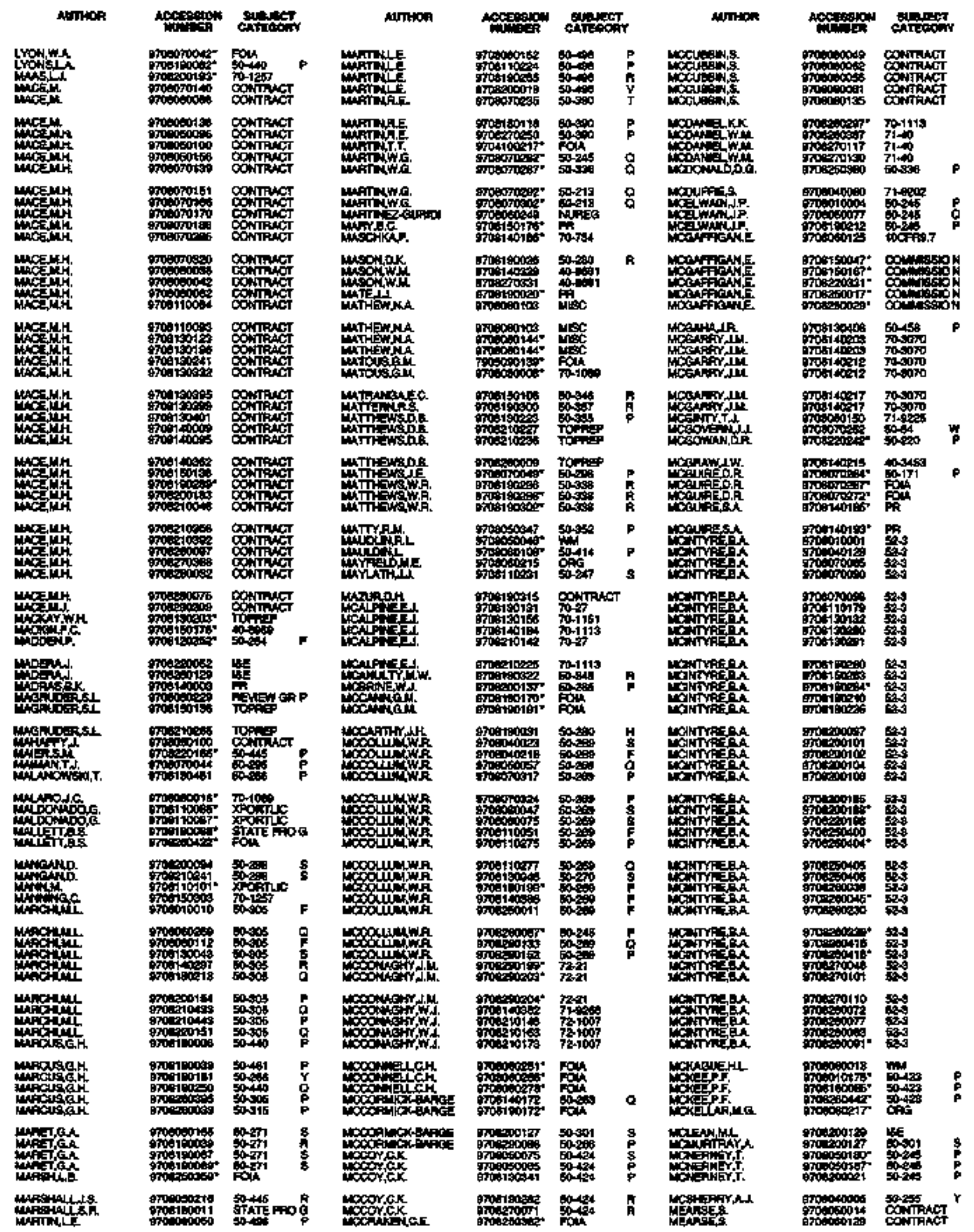




\begin{tabular}{|c|c|c|c|c|}
\hline شתחה & itcisond & Atw & & Amax \\
\hline 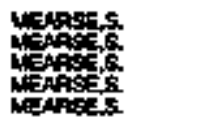 & 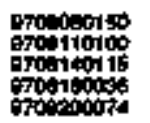 & 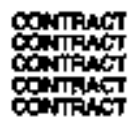 & & 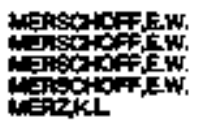 \\
\hline 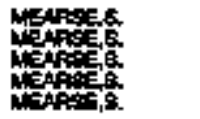 & 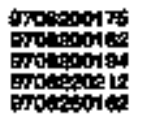 & 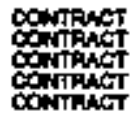 & & 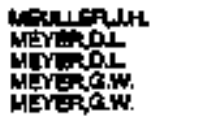 \\
\hline 等 & 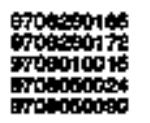 & 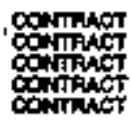 & & 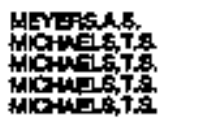 \\
\hline 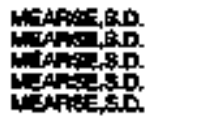 & 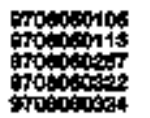 & 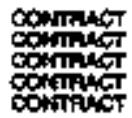 & & 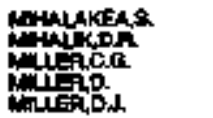 \\
\hline 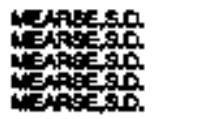 & 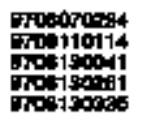 & 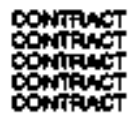 & & 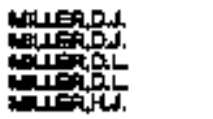 \\
\hline 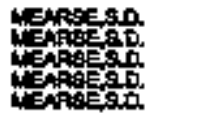 & 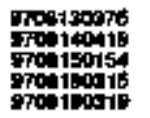 & 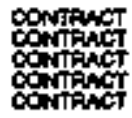 & & 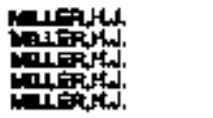 \\
\hline 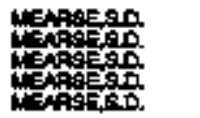 & 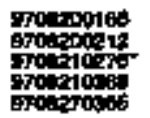 & 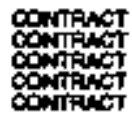 & & 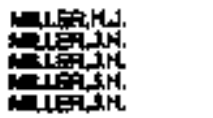 \\
\hline 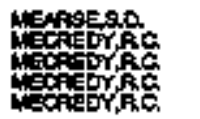 & 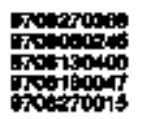 & 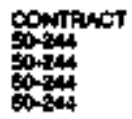 & $\begin{array}{l}p \\
8 \\
8\end{array}$ & tits \\
\hline 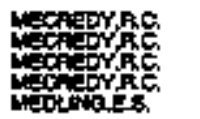 & 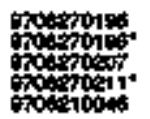 & 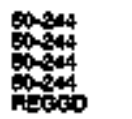 & ? & \\
\hline 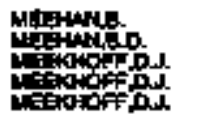 & 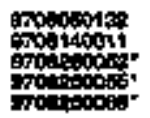 & 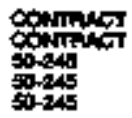 & ? & 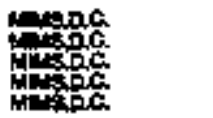 \\
\hline 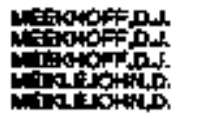 & 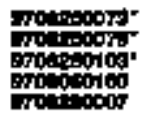 & $\begin{array}{l}\cos 245 \\
50-245 \\
50-240 \\
\text { Foin }\end{array}$ & P & 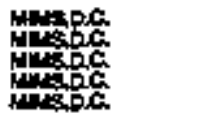 \\
\hline $14 \mathrm{x}$ & 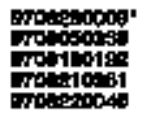 & 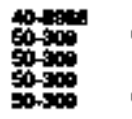 & $\underset{\mathbf{w}}{\mathbf{w}}$ & $\operatorname{tuph}_{\lambda}$ \\
\hline & 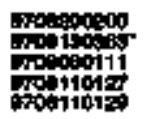 & $\begin{array}{l}50-213 \\
00-20 \\
00-14 \\
0-100 \\
0-6\end{array}$ & 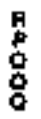 & 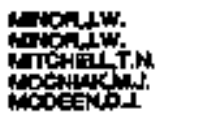 \\
\hline & 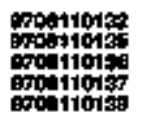 & $\begin{array}{l}\infty 0-190 \\
00-194 \\
50-170 \\
00-90 \\
50-167\end{array}$ & $\begin{array}{l}9 \\
8 \\
8\end{array}$ & 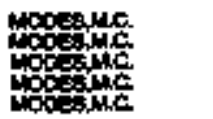 \\
\hline & 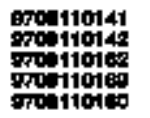 & 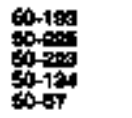 & $\frac{\phi}{\partial}$ & 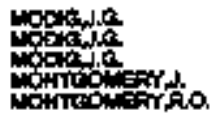 \\
\hline chich & 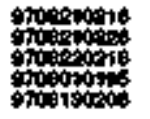 & 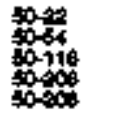 & $\frac{0}{8}$ & 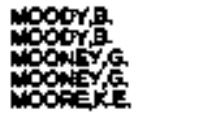 \\
\hline 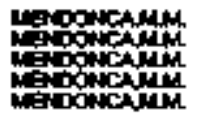 & 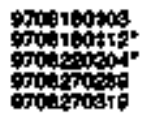 & $\begin{array}{l}00+00 \\
0000 \\
00-2 \\
001\end{array}$ & $\begin{array}{l}9 \\
8 \\
9\end{array}$ & 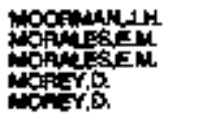 \\
\hline 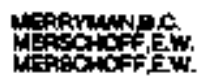 & 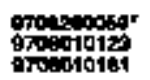 & 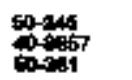 & P & Woro. \\
\hline
\end{tabular}

\begin{tabular}{|c|c|c|c|c|c|c|}
\hline 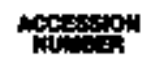 & ave & & Mrnion & Accosions & 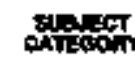 & \\
\hline 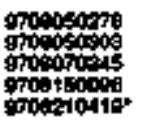 & 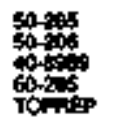 & $\begin{array}{l}0 \\
0\end{array}$ & 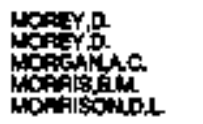 & 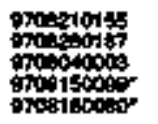 & 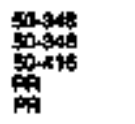 & \\
\hline 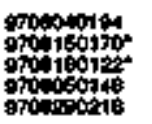 & 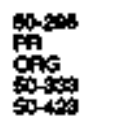 & $\stackrel{\circ}{\forall}$ & 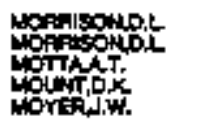 & 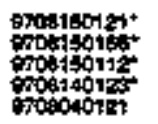 & 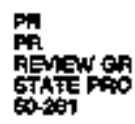 & \\
\hline 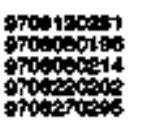 & 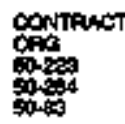 & p & 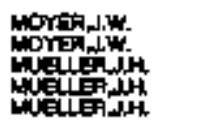 & 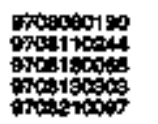 & 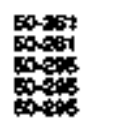 & \\
\hline 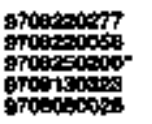 & $\begin{array}{l}50+50 \\
50395 \\
50-10 \\
00-272 \\
000\end{array}$ & $\begin{array}{c}\text { a } \\
\text { w } \\
\text { T }\end{array}$ & 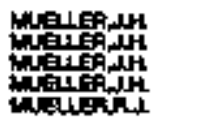 & 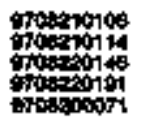 & 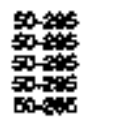 & \\
\hline 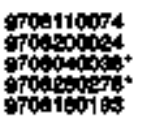 & 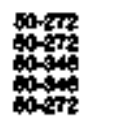 & $\begin{array}{l}5 \\
5 \\
5\end{array}$ & mothen & 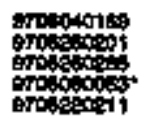 & 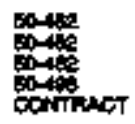 & \\
\hline 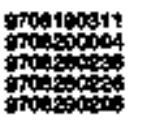 & chent & $\begin{array}{l}9 \\
8 \\
8\end{array}$ & 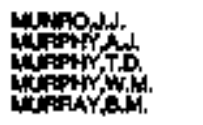 & omosepoy & 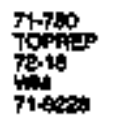 & \\
\hline 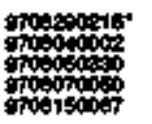 & 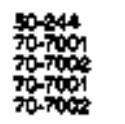 & $a$ & 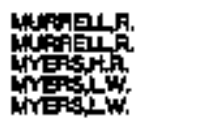 & 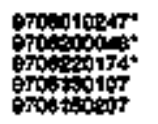 & $\begin{array}{l}\infty 001 \\
0004 \\
0-40 \\
0 \rightarrow 40\end{array}$ & 3 \\
\hline 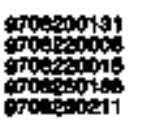 & 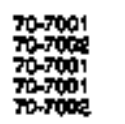 & & 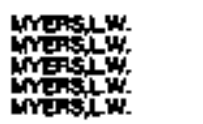 & 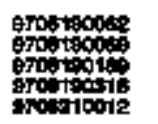 & $\begin{array}{l}00-40 \\
00-40 \\
0-40 \\
0+40 \\
0+40\end{array}$ & P \\
\hline 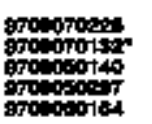 & 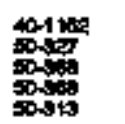 & $\begin{array}{l}\mathbf{5} \\
\mathrm{s} \\
\mathbf{p}\end{array}$ & 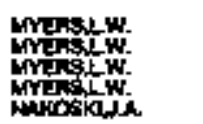 & 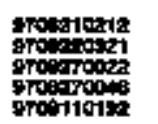 & $\begin{array}{l}50+40 \\
50+40 \\
50+40 \\
50+40\end{array}$ & \\
\hline 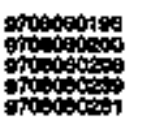 & 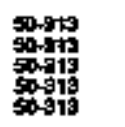 & $\frac{p}{o}$ & 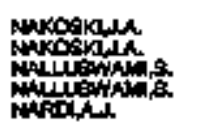 & 等 & 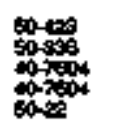 & $p$ \\
\hline 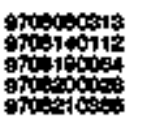 & 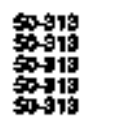 & $\begin{array}{l}p \\
0 \\
0 \\
0 \\
0\end{array}$ & mishing & 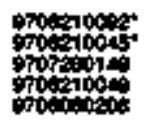 & 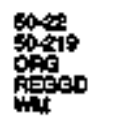 & f \\
\hline 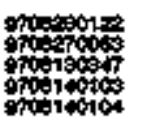 & 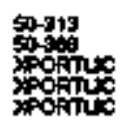 & 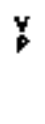 & 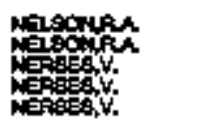 & 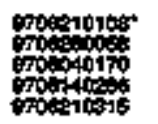 & 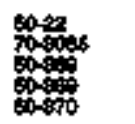 & P \\
\hline 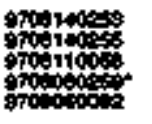 & 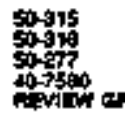 & 角 & 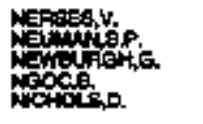 & 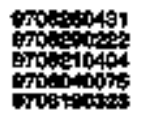 & 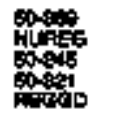 & $\mathbf{p}$ \\
\hline 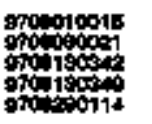 & 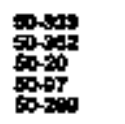 & $\begin{array}{l}\mathbf{F} \\
\mathbf{f} \\
\mathbf{f}\end{array}$ & 等 & 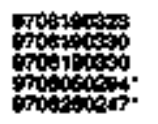 & 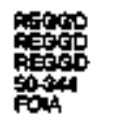 & $Y$ \\
\hline poposis & 然象 & & manar & 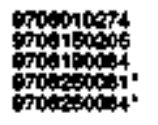 & 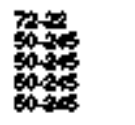 & \\
\hline 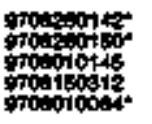 & 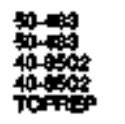 & $\underset{v}{\forall}$ & 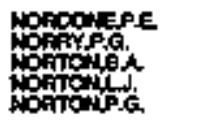 & 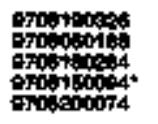 & 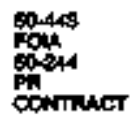 & \\
\hline 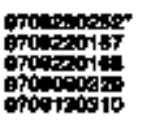 & 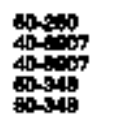 & $\mathrm{Y}$ & 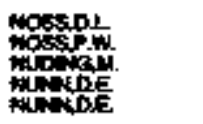 & 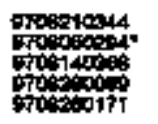 & 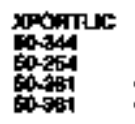 & \\
\hline 1097ons & & $\mathbf{F}$ & A sotath & 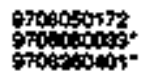 & 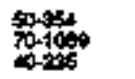 & A \\
\hline
\end{tabular}




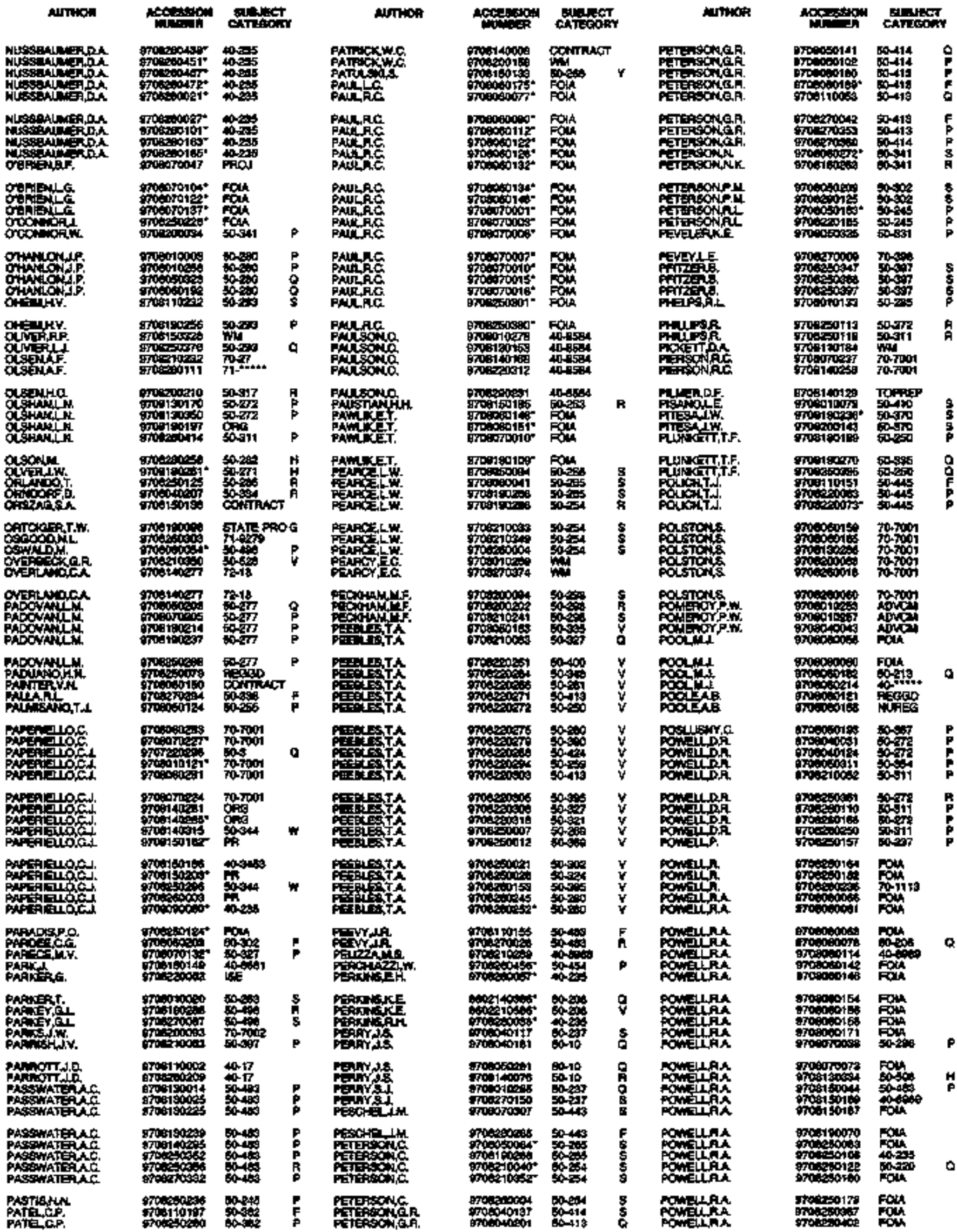




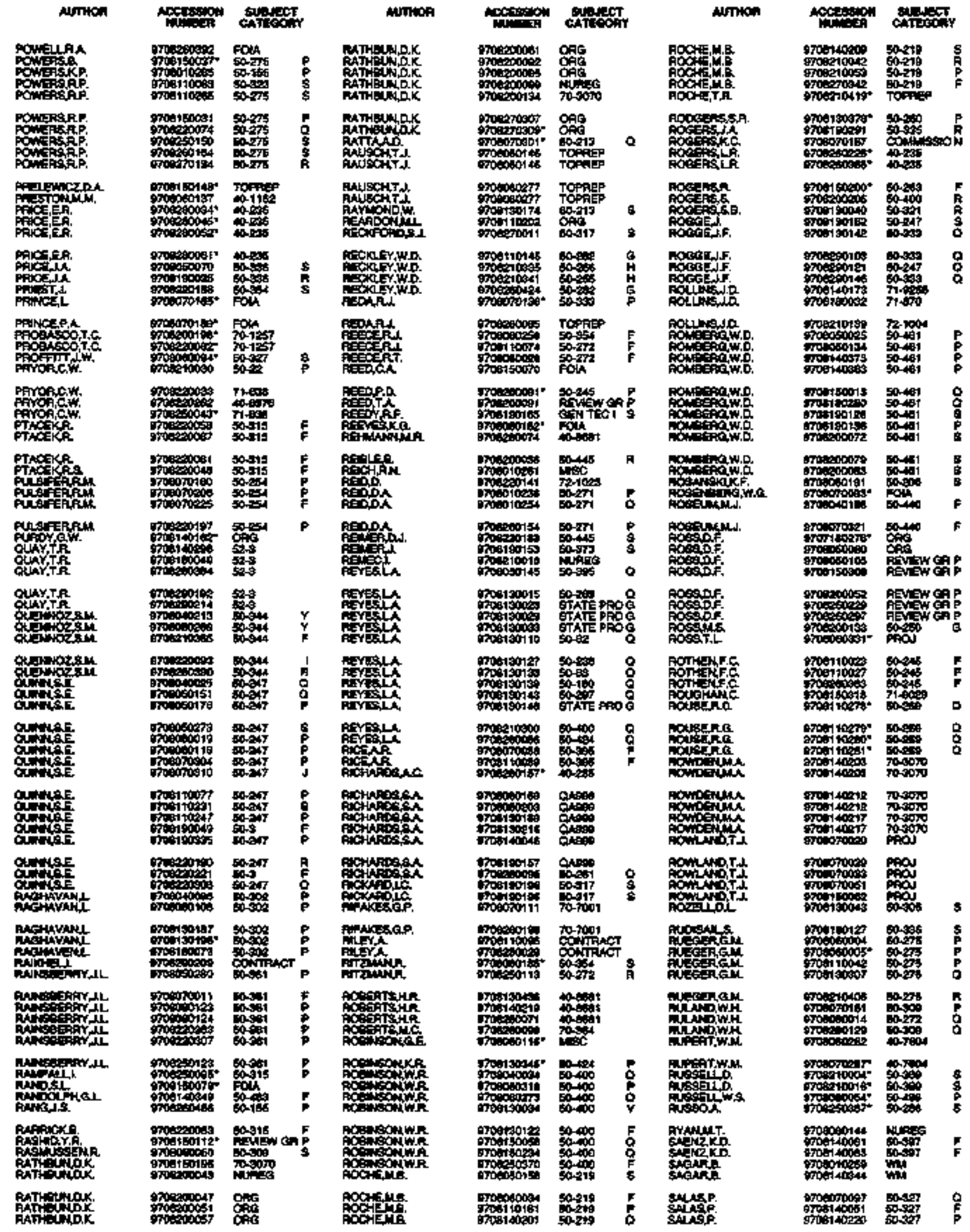




\begin{tabular}{|c|c|c|c|c|c|c|c|c|c|c|c|}
\hline הוnkm & 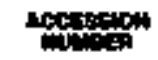 & Find & & AUTKOA & Actionow & aptert & & Mrrap & 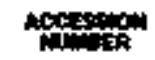 & canter & \\
\hline 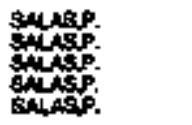 & 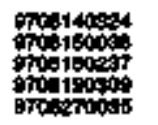 & 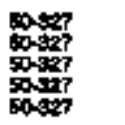 & $\begin{array}{l}\mathbf{p} \\
Q \\
\mathbf{f} \\
\mathbf{R} \\
\mathbf{Q}\end{array}$ & 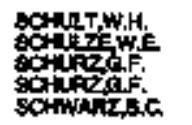 & 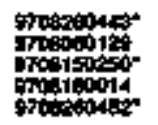 & 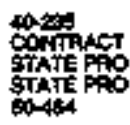 & & 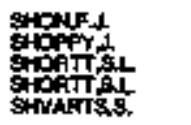 & 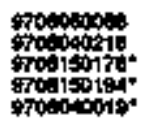 & 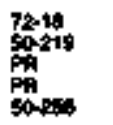 & \\
\hline 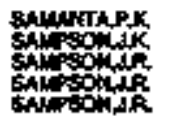 & 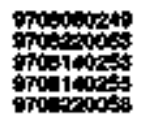 & 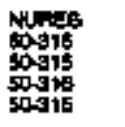 & $\begin{array}{l}\mathbf{F} \\
\mathbf{h} \\
\mathbf{f}\end{array}$ & 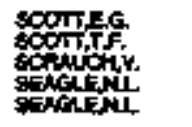 & 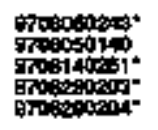 & 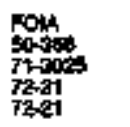 & $\mathbf{s}$ & 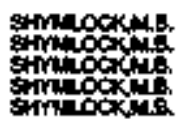 & 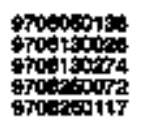 & 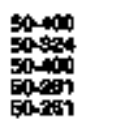 & $\begin{array}{l}0 \\
0 \\
0\end{array}$ \\
\hline 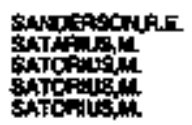 & 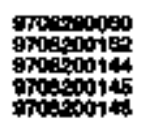 & 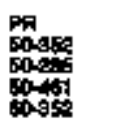 & $\begin{array}{l}\text { o } \\
\text { g } \\
\text { o }\end{array}$ & 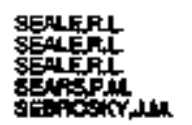 & 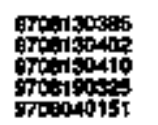 & 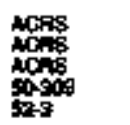 & 3 & 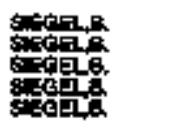 & 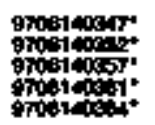 & 窗 & \\
\hline 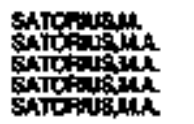 & 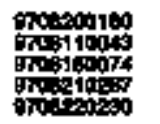 & 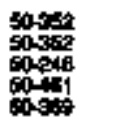 & $\begin{array}{l}a \\
\dot{p} \\
\stackrel{a}{0} \\
0\end{array}$ & 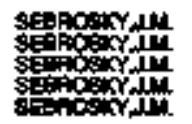 & 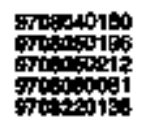 & 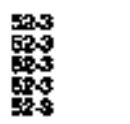 & & 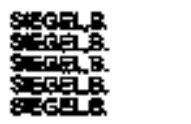 & 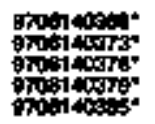 & 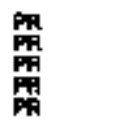 & \\
\hline 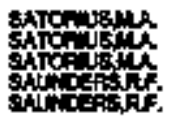 & 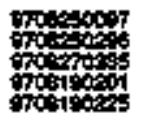 & 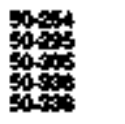 & $\begin{array}{l}\mathrm{D} \\
\mathrm{Q} \\
\frac{0}{\mathrm{P}} \\
\mathrm{P}\end{array}$ & 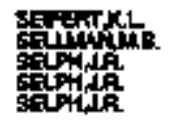 & 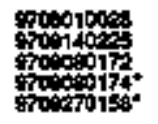 & 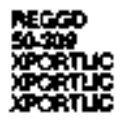 & $w$ & 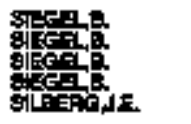 & 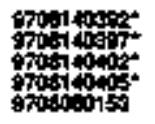 & 举 & \\
\hline 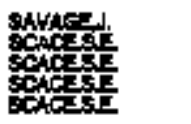 & 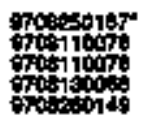 & 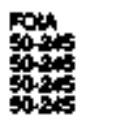 & $\begin{array}{l}\text { A } \\
\text { ĥ } \\
\text { p }\end{array}$ & 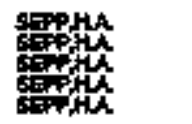 & 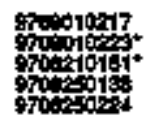 & 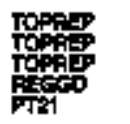 & & 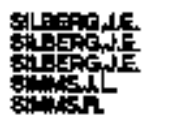 & 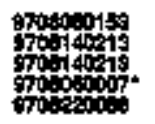 & 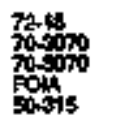 & \\
\hline 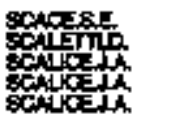 & Tropopds & 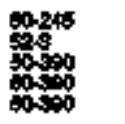 & $\begin{array}{l}\mathbf{F} \\
8 \\
\mathbf{p}\end{array}$ & 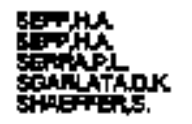 & 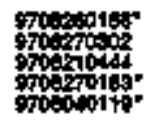 & 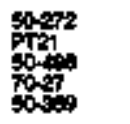 & $\begin{array}{l}\mathbf{p} \\
\mathbf{P} \\
\mathbf{Q}\end{array}$ & 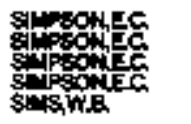 & 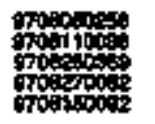 & 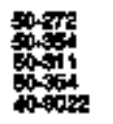 & \\
\hline 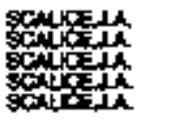 & 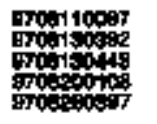 & 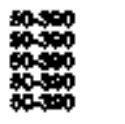 & $\begin{array}{l}\mathbf{q} \\
\mathbf{p} \\
0 \\
\mathbf{p} \\
\mathbf{S}\end{array}$ & 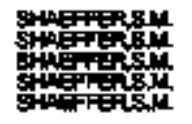 & 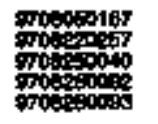 & 最e & $\frac{9}{5}$ & 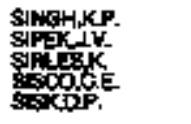 & 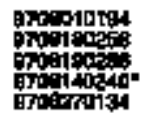 & 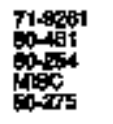 & \\
\hline 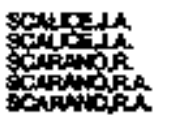 & 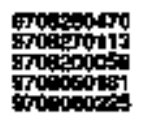 & 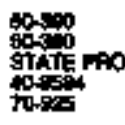 & P & 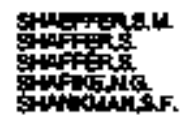 & 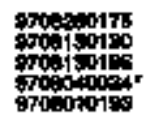 & 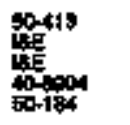 & 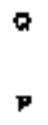 & 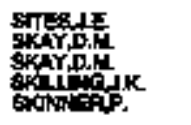 & 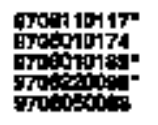 & 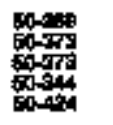 & \\
\hline 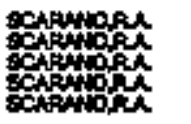 & 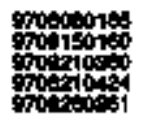 & 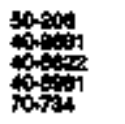 & $\theta$ & 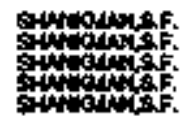 & 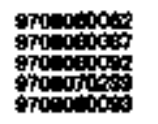 & 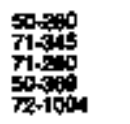 & 0 & 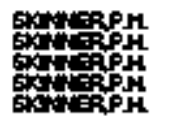 & 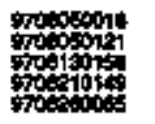 & 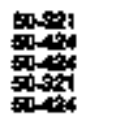 & \\
\hline 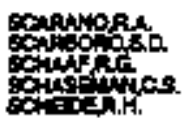 & 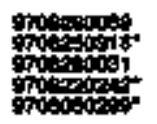 & 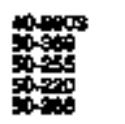 & $\begin{array}{l}0 \\
p \\
p \\
5\end{array}$ & 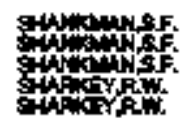 & 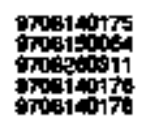 & 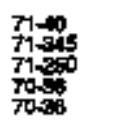 & & 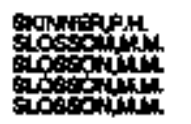 & 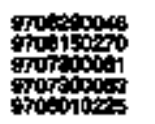 & 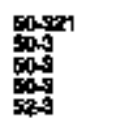 & \\
\hline 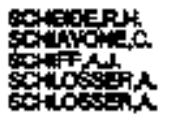 & 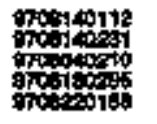 & 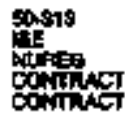 & 5 & 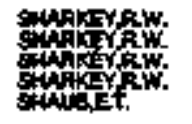 & 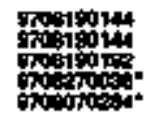 & 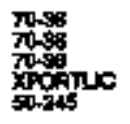 & 0 & 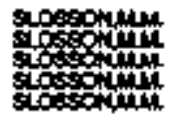 & 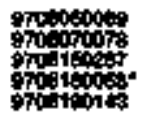 & 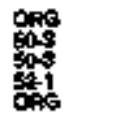 & \\
\hline 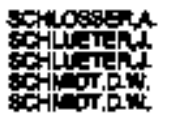 & 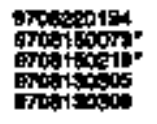 & 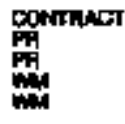 & & 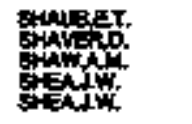 & 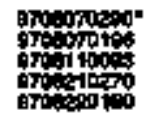 & The & $\ddot{r}$ & 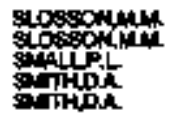 & 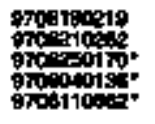 & $\frac{0}{\cos _{0}}$ & \\
\hline 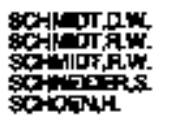 & 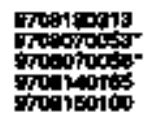 & 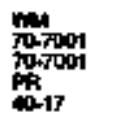 & & 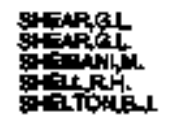 & 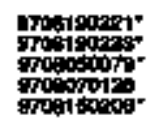 & 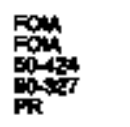 & 8 & tind & 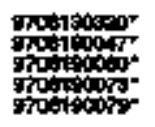 & 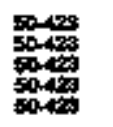 & \\
\hline 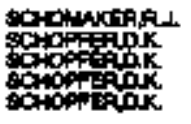 & 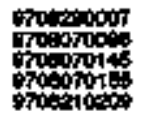 & 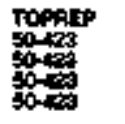 & $\begin{array}{l}\text { p } \\
\mathbf{p} \\
\mathbf{p}\end{array}$ & 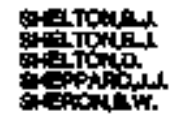 & 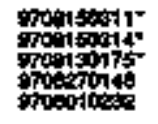 & 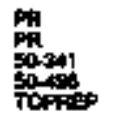 & $\stackrel{p}{F}$ & 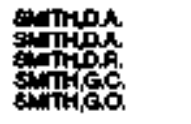 & 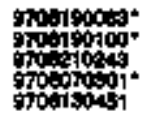 & 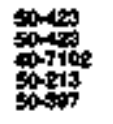 & \\
\hline 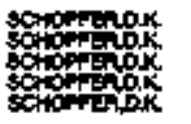 & 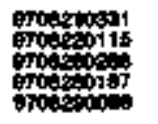 & 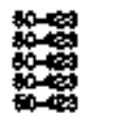 & $\begin{array}{l}q \\
g \\
p \\
p \\
p\end{array}$ & iff. & 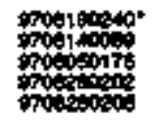 & 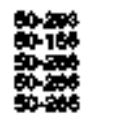 & $\begin{array}{l}\mathrm{p} \\
\mathbf{4} \\
\mathbf{H}\end{array}$ & 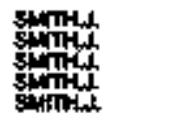 & 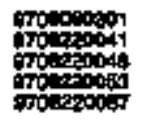 & $\begin{array}{l}0,-315 \\
00-315 \\
00-915 \\
00-315 \\
0,315\end{array}$ & \\
\hline 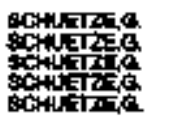 & 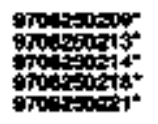 & $\begin{array}{l}\operatorname{moxh}_{\cos } \\
\operatorname{poth}_{\operatorname{coth}}\end{array}$ & & 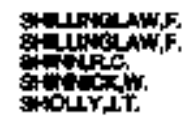 & 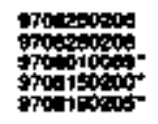 & 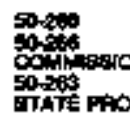 & $\underset{0}{n}$ & 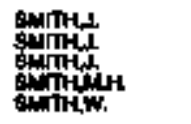 & 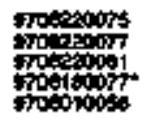 & 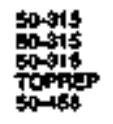 & \\
\hline 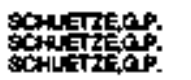 & 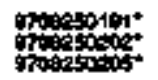 & $\begin{array}{l}\text { rouk } \\
\text { rom }\end{array}$ & & 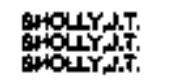 & 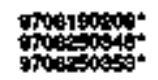 & 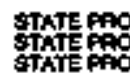 & & 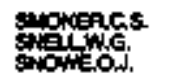 & 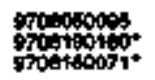 & 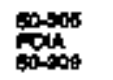 & \\
\hline
\end{tabular}




\begin{tabular}{|c|c|c|c|c|c|c|c|c|c|c|c|}
\hline Antroh & 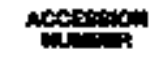 & expyoy & & AUTAOR & Actision & 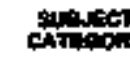 & & Muthos & 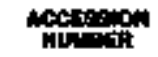 & 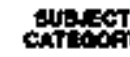 & \\
\hline 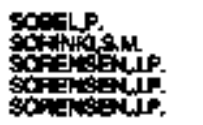 & 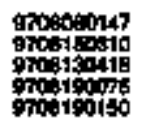 & 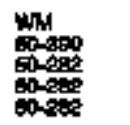 & $\begin{array}{l}\mathbf{p} \\
\text { ค } \\
\text { P }\end{array}$ & 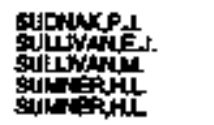 & 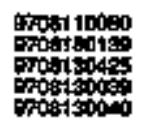 & 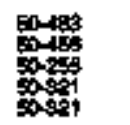 & $\begin{array}{l}\mathbf{E} \\
\mathbf{p} \\
\mathbf{p} \\
\mathbf{p} \\
\mathbf{p}\end{array}$ &  & 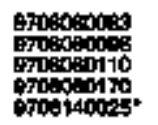 & 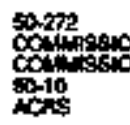 & $\underset{w}{W}$ \\
\hline 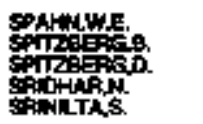 & 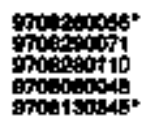 & 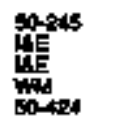 & p & 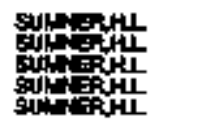 & 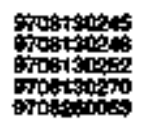 & 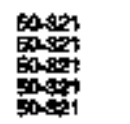 & $\begin{array}{l}f \\
\stackrel{p}{p} \\
\mathbf{p} \\
\mathbf{g} \\
\mathbf{n}\end{array}$ &  & 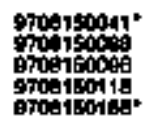 & 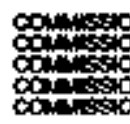 & 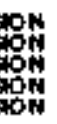 \\
\hline 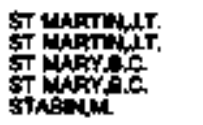 & 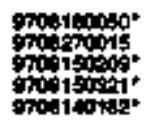 & 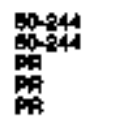 & $\begin{array}{l}\mathbf{8} \\
\mathbf{8}\end{array}$ & 采 & 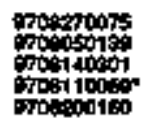 & 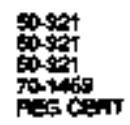 & 号 &  & 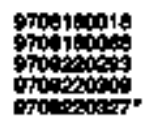 & 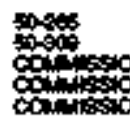 & 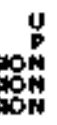 \\
\hline 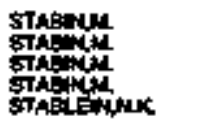 & 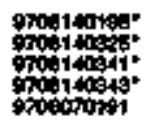 & Pa & & 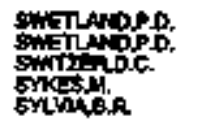 & 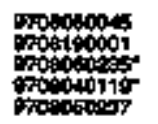 & 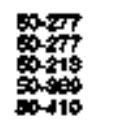 & $\begin{array}{l}0 \\
8 \\
0 \\
1\end{array}$ &  & 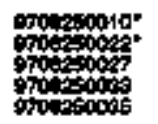 & 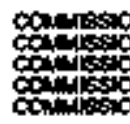 & N \\
\hline 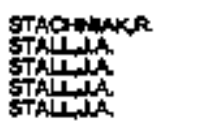 & 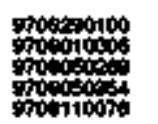 & 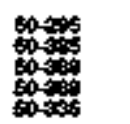 & $\begin{array}{l}\mathbf{5} \\
\mathbf{p} \\
\mathbf{p} \\
\mathbf{p}\end{array}$ & 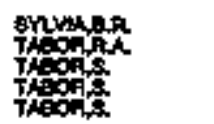 & 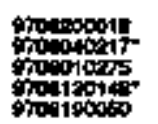 & 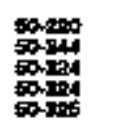 & 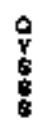 &  & 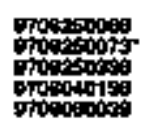 & 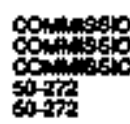 & BN \\
\hline 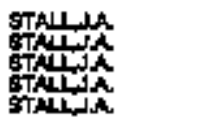 & 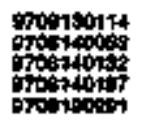 & 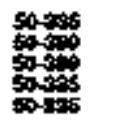 & $\begin{array}{l}\mathrm{R} \\
\frac{6}{5} \\
\mathrm{~s} \\
\mathrm{R}\end{array}$ & 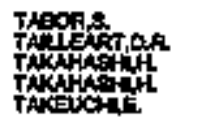 & 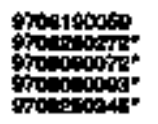 & 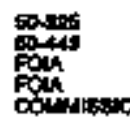 & 8 &  & 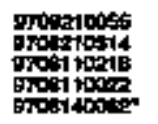 & 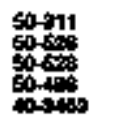 & $\begin{array}{l}8 \\
8 \\
p \\
F\end{array}$ \\
\hline 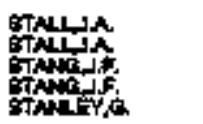 & 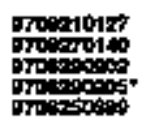 & 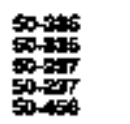 & $\begin{array}{l}\mathbf{Y} \\
\mathbf{p} \\
\mathbf{p}\end{array}$ & 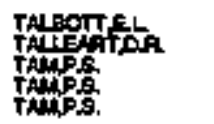 & 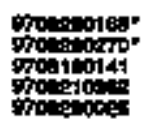 & 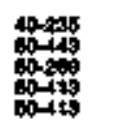 & p &  & 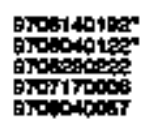 & 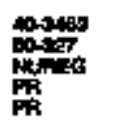 & 0 \\
\hline 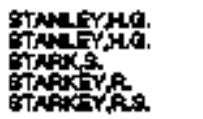 & 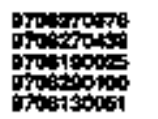 & 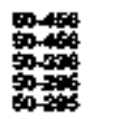 & $\begin{array}{l}\text { p } \\
\text { 昌 } \\
\text { g }\end{array}$ & 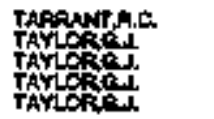 & 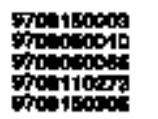 & sones & $\begin{array}{l}\mathbf{p} \\
\mathbf{p} \\
\mathbf{p} \\
\mathbf{p} \\
\mathbf{q}\end{array}$ &  & 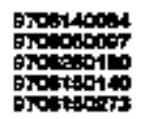 & 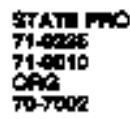 & \\
\hline 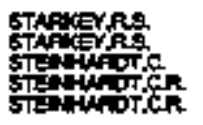 & 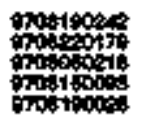 & 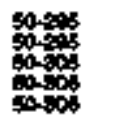 & $\begin{array}{l}R \\
\text { R } \\
\mathbf{p} \\
\mathbf{p} \\
\mathbf{P}\end{array}$ & 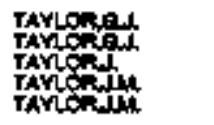 & 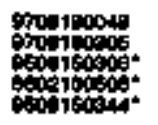 & 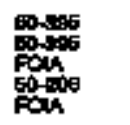 & $\begin{array}{l}\text { P } \\
\mathbf{p}\end{array}$ &  & 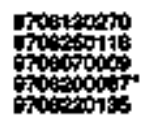 & 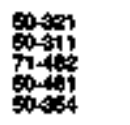 & $\$$ \\
\hline 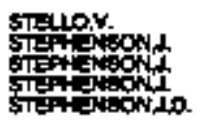 & 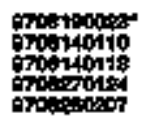 & $\frac{1}{200}$ & $F$ & 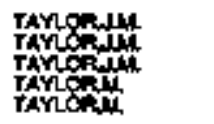 & 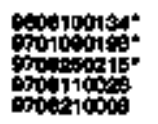 & 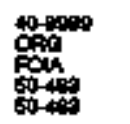 & $\mathbf{F}$ &  & 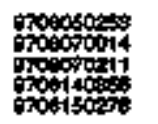 & 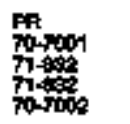 & \\
\hline 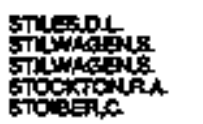 & 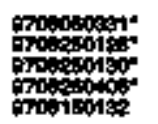 & 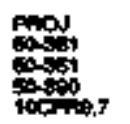 & & 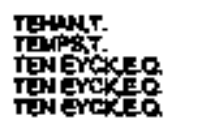 & 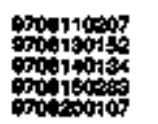 & 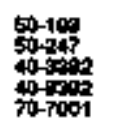 & 8 &  & 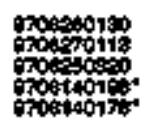 & 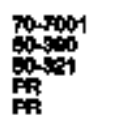 & $\mathbf{A}$ \\
\hline 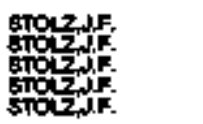 & 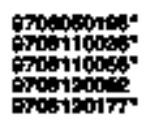 & 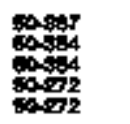 & $\begin{array}{l}\mathbf{p} \\
\mathbf{p} \\
\mathbf{p} \\
\mathbf{p}\end{array}$ & 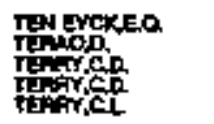 & 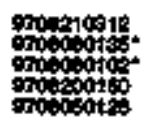 & 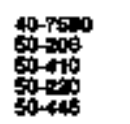 & p &  & 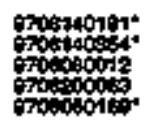 & 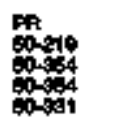 & $\begin{array}{l}5 \\
\mathbf{p} \\
\mathbf{p} \\
\mathbf{8}\end{array}$ \\
\hline 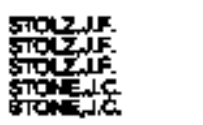 & 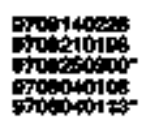 & 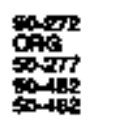 & p & 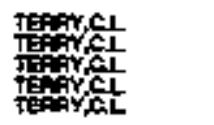 & 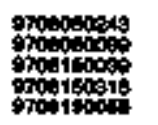 & 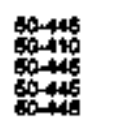 & $\begin{array}{l}\mathbf{p} \\
\mathbf{p} \\
\mathbf{p} \\
\mathbf{p}\end{array}$ &  & 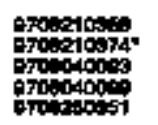 & 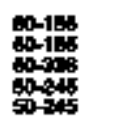 & p \\
\hline 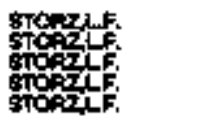 & 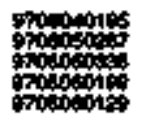 & 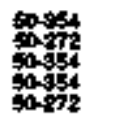 & 0 & 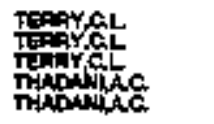 & 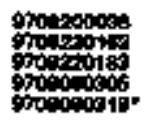 & 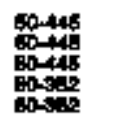 & $\begin{array}{l}\mathbf{R} \\
\mathbf{P} \\
\mathbf{S} \\
\mathbf{H} \\
\mathbf{H}\end{array}$ &  & 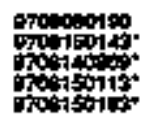 & 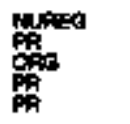 & \\
\hline 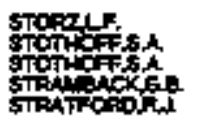 & 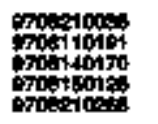 & 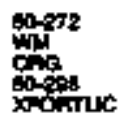 & $\mathbf{F}$ & 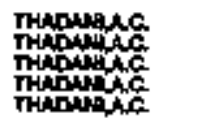 & 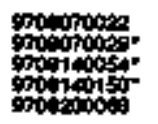 & 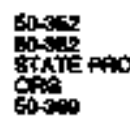 & $\begin{array}{l}\text { d } \\
\text { d } \\
0 \\
0\end{array}$ &  & 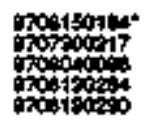 & 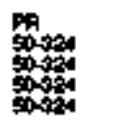 & : \\
\hline 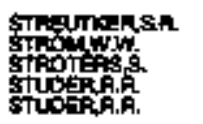 & 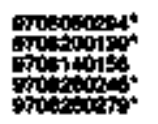 & 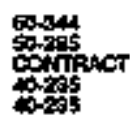 & $Y$ & 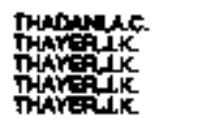 & 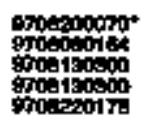 & 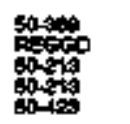 & $\underset{p}{a}$ &  & 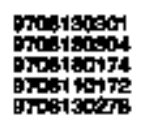 & 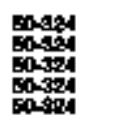 & p \\
\hline 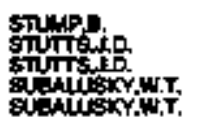 & 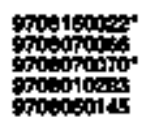 & 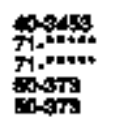 & & 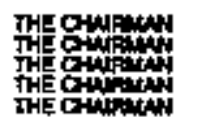 & 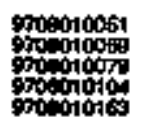 & 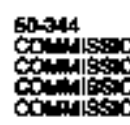 & &  & 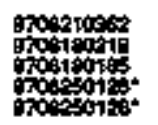 & 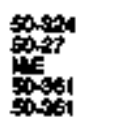 & $p$ \\
\hline 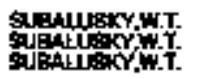 & 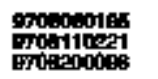 & 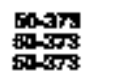 & $\mathbf{P}$ & 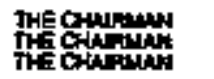 & groposols & rectist & &  & 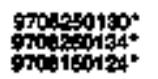 & 然果1 & p \\
\hline
\end{tabular}




\begin{tabular}{|c|c|c|c|c|c|c|c|c|c|c|c|}
\hline urTwa & 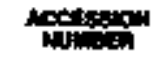 & Pareat & & unnot & 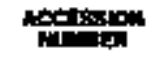 & 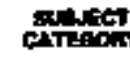 & & autrictit & acatapion & 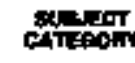 & \\
\hline 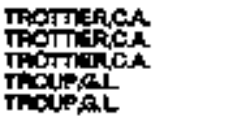 & 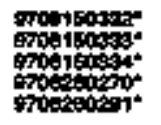 & 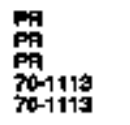 & & 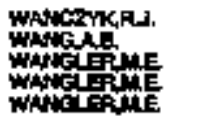 & 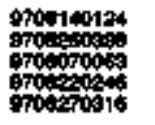 & 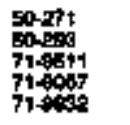 & $\stackrel{6}{\mathbf{p}}$ & 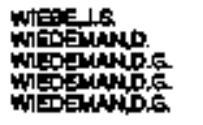 & 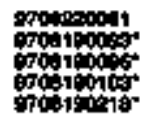 & $\begin{array}{l}\text { onst } \\
\text { Folk } \\
\text { Folk } \\
\text { Fonk }\end{array}$ & $\mathbf{F}$ \\
\hline 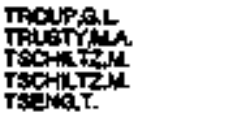 & 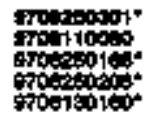 & 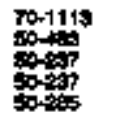 & $\begin{array}{l}\text { p } \\
\text { p } \\
\text { p }\end{array}$ & 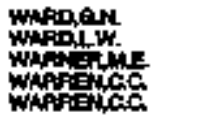 & 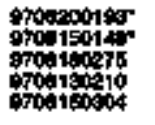 & 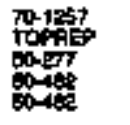 & f & 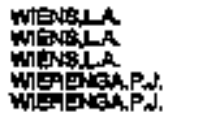 & 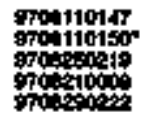 & 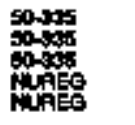 & 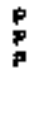 \\
\hline 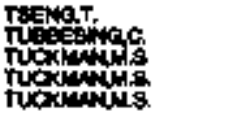 & 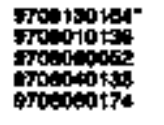 & 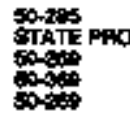 & $\begin{array}{l}p \\
p \\
p\end{array}$ & 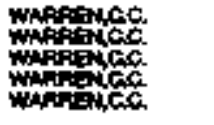 & 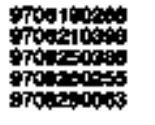 & 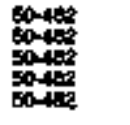 & $\begin{array}{l}\mathbf{g} \\
\mathbf{R} \\
\mathbf{F} \\
\mathbf{E} \\
\mathbf{E}\end{array}$ & 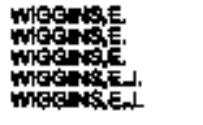 & 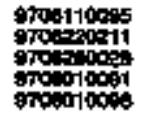 & 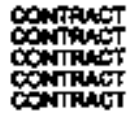 & \\
\hline 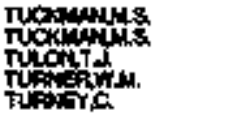 & 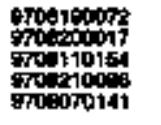 & 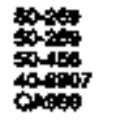 & $\stackrel{p}{p}$ & 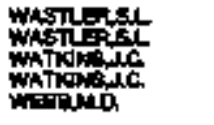 & 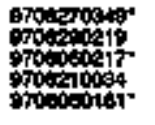 & 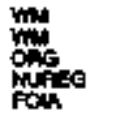 & & 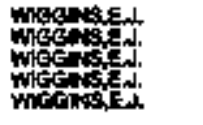 & 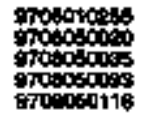 & 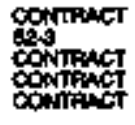 & \\
\hline 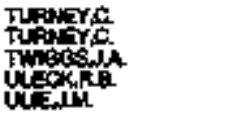 & 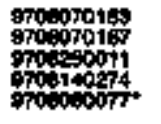 & 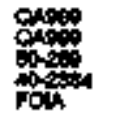 & $F$ & 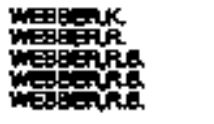 & 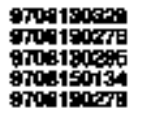 & 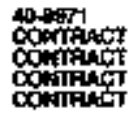 & & 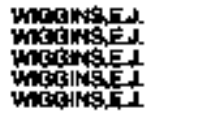 & 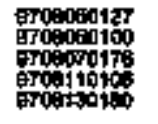 & 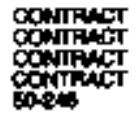 & $r^{2}$ \\
\hline 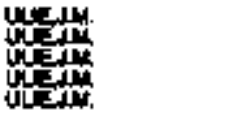 & 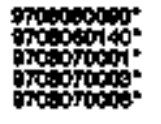 & 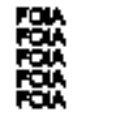 & & 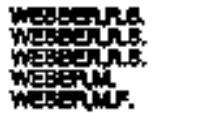 & 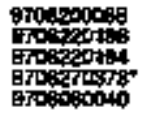 & 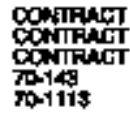 & & 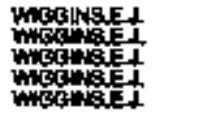 & 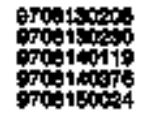 & 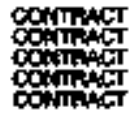 & \\
\hline 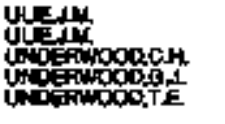 & 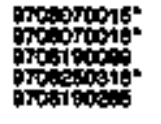 & For & 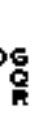 & 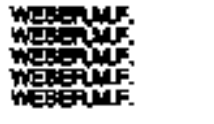 & 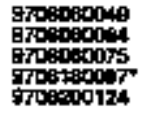 & 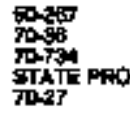 & $\mathbf{r}$ & 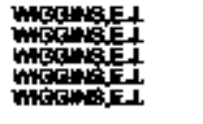 & 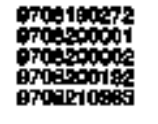 & 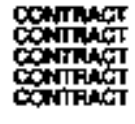 & \\
\hline 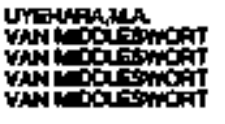 & 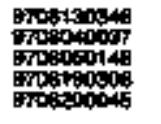 & 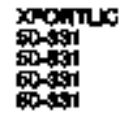 & $\begin{array}{l}\mathbf{S} \\
\mathbf{S} \\
\mathbf{R} \\
\mathbf{5}\end{array}$ & 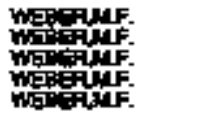 & 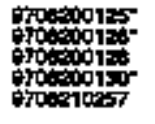 & 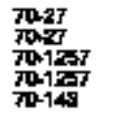 & & 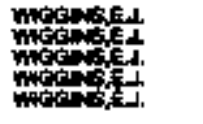 & 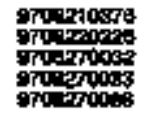 & 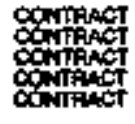 & \\
\hline VAN & 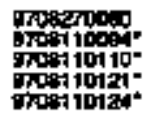 & 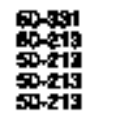 & $\begin{array}{l}8 \\
8 \\
8 \\
8 \\
8 \\
8 \\
8\end{array}$ & 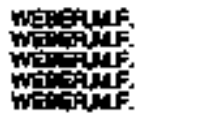 & 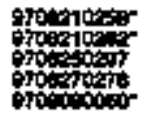 & $\begin{array}{l}70149 \\
70-140 \\
70-7 \\
70-14 \\
40-205\end{array}$ & & 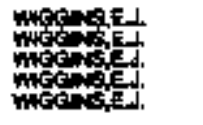 & 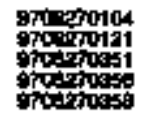 & 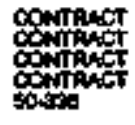 & 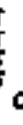 \\
\hline 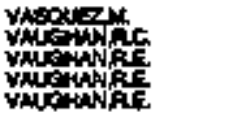 & 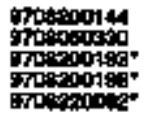 & 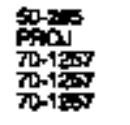 & $\Delta$ & 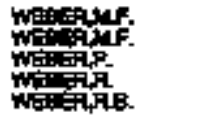 & 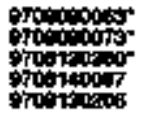 & 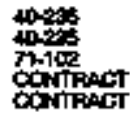 & & 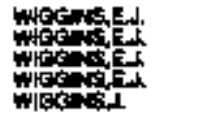 & 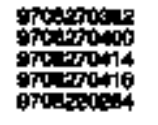 & 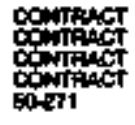 & 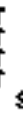 \\
\hline 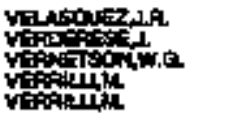 & 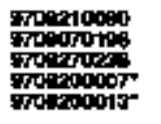 & 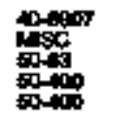 & $\begin{array}{l}\mathbf{P} \\
\mathbf{S} \\
\mathbf{S}\end{array}$ & 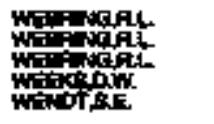 & 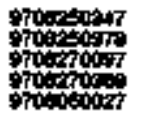 & 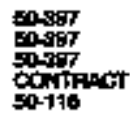 & $\begin{array}{r}\mathbf{s} \\
\mathbf{p} \\
\mathbf{R} \\
\mathbf{R}\end{array}$ & wicesit. & 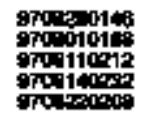 & 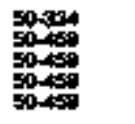 & \\
\hline 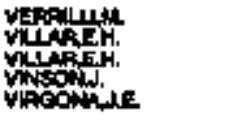 & 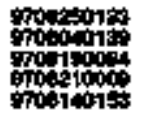 & 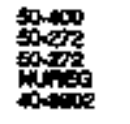 & 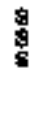 & 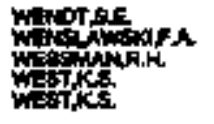 & 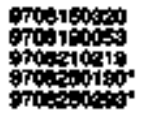 & 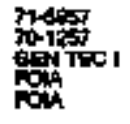 & s & 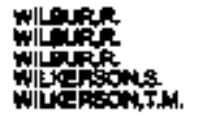 & 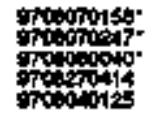 & 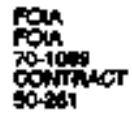 & \\
\hline 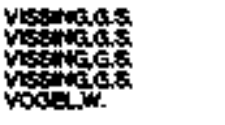 & 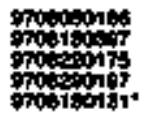 & 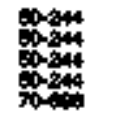 & $\begin{array}{l}p \\
\stackrel{p}{p} \\
p\end{array}$ & 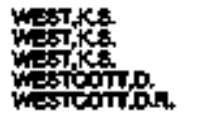 & 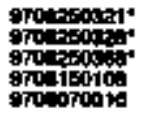 & 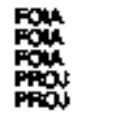 & & 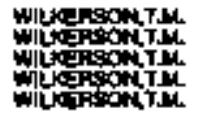 & 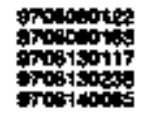 & 我果 & \\
\hline 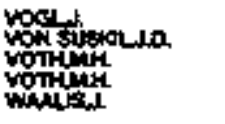 & 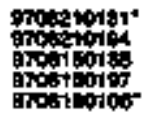 & $\begin{array}{l}0,44 \\
0000 \\
000\end{array}$ & $\begin{array}{l}\text { h } \\
\mathbf{n}\end{array}$ & 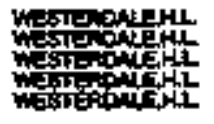 & 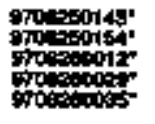 & 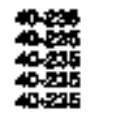 & & 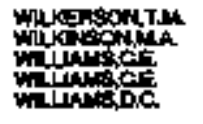 & 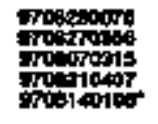 & 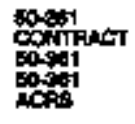 & \\
\hline 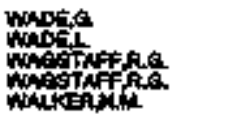 & poporas & 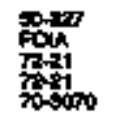 & 0 & 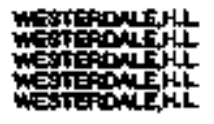 & mostis & 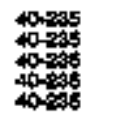 & & 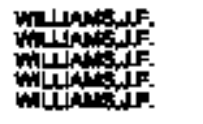 & 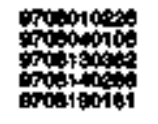 & 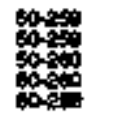 & \\
\hline 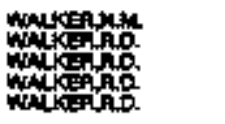 & 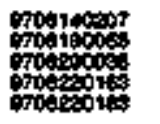 & 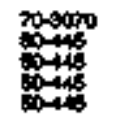 & $\begin{array}{l}\text { p } \\
\text { p } \\
\text { ? }\end{array}$ & 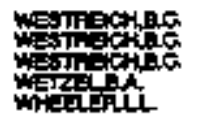 & 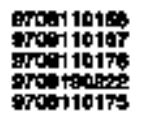 & 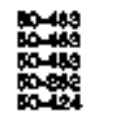 & $\begin{array}{l}\mathbf{p} \\
\mathbf{p} \\
\mathbf{p} \\
\mathbf{p} \\
\mathbf{q}\end{array}$ & 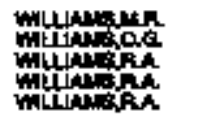 & 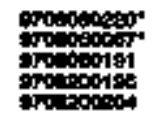 & 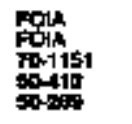 & \\
\hline 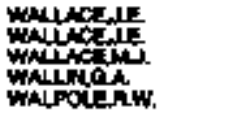 & 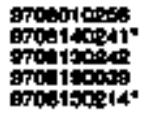 & $\begin{array}{l}000 \\
000 \\
0001 \\
000\end{array}$ & $\begin{array}{l}5 \\
5 \\
5 \\
5\end{array}$ & 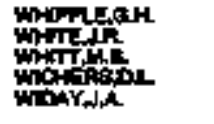 & 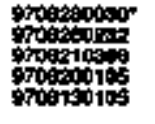 & 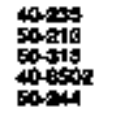 & $\stackrel{a}{\beta}$ & 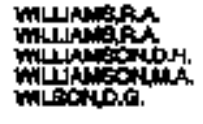 & 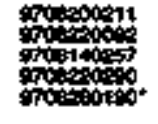 & 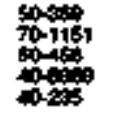 & \\
\hline 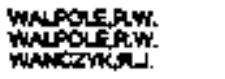 & 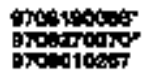 & 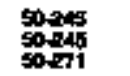 & 8 & 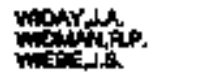 & 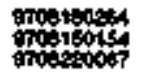 & $\begin{array}{l}5024 \\
000415 \\
50-15\end{array}$ & $\stackrel{n}{F}$ & 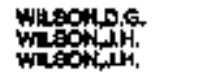 & 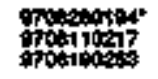 & 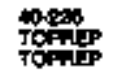 & \\
\hline
\end{tabular}




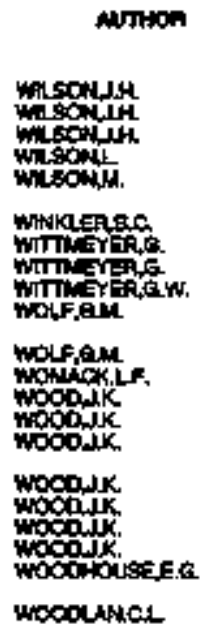

\begin{tabular}{|c|c|c|}
\hline 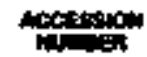 & 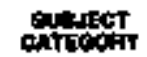 & ANThop \\
\hline 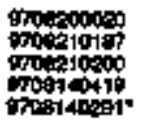 & 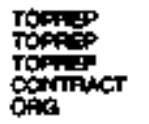 & 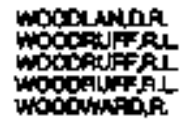 \\
\hline 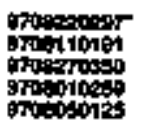 & coninsent & 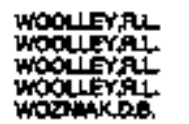 \\
\hline 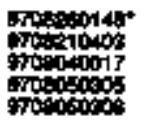 & 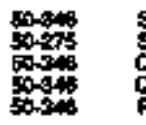 & 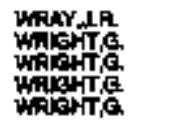 \\
\hline 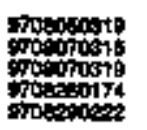 & 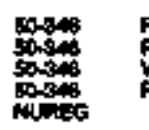 & 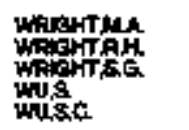 \\
\hline Fugodoero & 90-445 & wurosa. \\
\hline
\end{tabular}

\begin{tabular}{|c|c|c|c|}
\hline $\operatorname{acs} \cos ^{2}$ & cost & & Amion \\
\hline 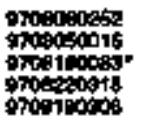 & 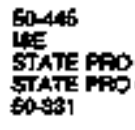 & $\begin{array}{l}P \\
0 \\
0 \\
R\end{array}$ & 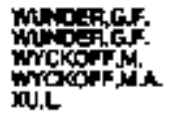 \\
\hline 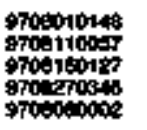 & 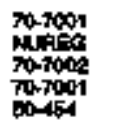 & $\boldsymbol{P}$ & 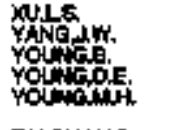 \\
\hline 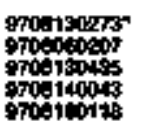 & 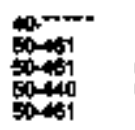 & $\begin{array}{l}\mathbf{s} \\
\stackrel{8}{0} \\
\text { s }\end{array}$ & 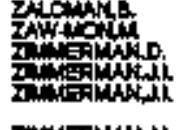 \\
\hline 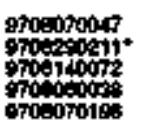 & $\begin{array}{l}\operatorname{mal}_{320} \\
30-11 \\
301113\end{array}$ & 0 & 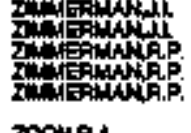 \\
\hline 97001000000 & $50-200$ & 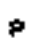 & $2 \times 0$ \\
\hline
\end{tabular}

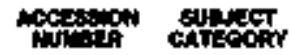

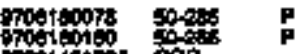

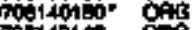

FTol

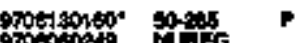

Hownes

ond 1000 N

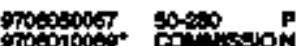

nopioge:

monita

Tot

so-394

croencoes:

notences.

90000010

now.

rotribs:

534

Cor

Sh.

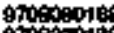

storotio

siogo 


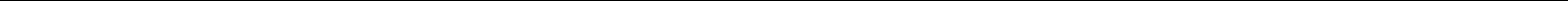




\section{CORPORATE SOURCE INDEX}

The subject category column in this index gives the docket rutriber and the symbol for the category of informatton within thet docket; for example,50-202 ?. The docket subtect categories are defined in Appendix A.

Subject categortes for nondocketed items are also entered in this column by symbol; for exarnple. ACRS.

ANITOR A ATHATION

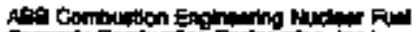

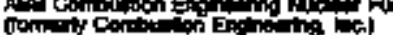

ASEA Bun Bowiti, kio.

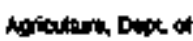

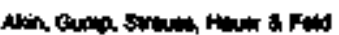

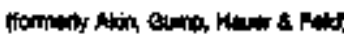

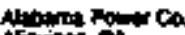

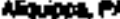
Antid The

Arosiond Cop

Nitan Cop

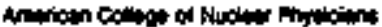

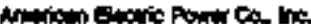

Anemasos Gerp

Am Fury 1 insocotieg

Andere the

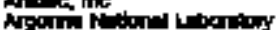

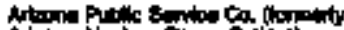

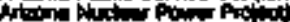

\begin{tabular}{|c|c|}
\hline 970a1 40170 & 70,8 \\
\hline 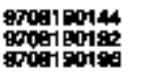 & 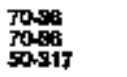 \\
\hline 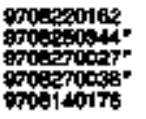 & 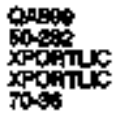 \\
\hline 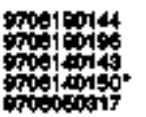 & 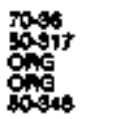 \\
\hline 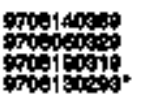 & 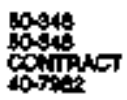 \\
\hline 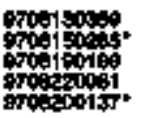 & 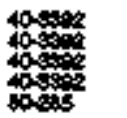 \\
\hline 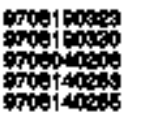 & 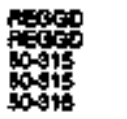 \\
\hline 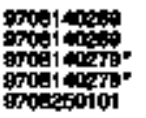 & 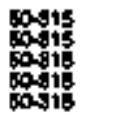 \\
\hline 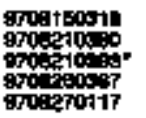 & $\begin{array}{l}71+0009 \\
71+000 \\
71+000 \\
71=0 \\
71=0\end{array}$ \\
\hline 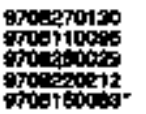 & 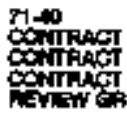 \\
\hline 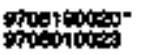 & PA \\
\hline pooploses & 番 \\
\hline 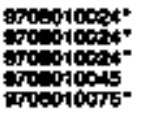 & 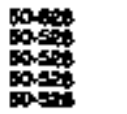 \\
\hline 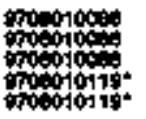 & \\
\hline 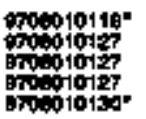 & 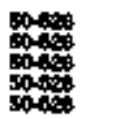 \\
\hline 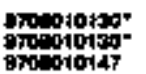 & Doset \\
\hline
\end{tabular}

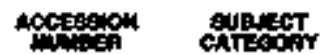

orapost

proporids

oropions:

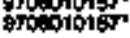

oropolont

7xionon

770.01011.

squon:

grocomont

g800000045

Trosong

Troosols

ratowis

or.ton.

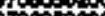

aros

orowononet

Troos

totos

ofos:

oro.0.02\%

9700000

Trosot:

900

Tox

rocosont

ofosos.

Totion

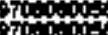

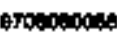

70000

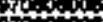

orotion.

moponom.

grotopions:

9rdiparas

Then 100 -

spoosioges

poostod.

(t)

ofteitom

(1)

proticios

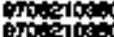

on

roolited

Ntrons Unit. of. Tutaph az

aroectow:

700009

10015. rogr 400ie

rogr 1040

Torotion

Froerposen

citions

trotion

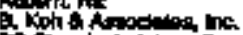

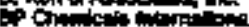

iroporos? mpopis

cosex

60 然

$\$ 060$

so-ge.

so-an

yoge.

sold

0.jo

sorsts

$50=0$

50 s.

50 姲

sors

oses

obta

50.56.

5.5.

50

o.

(2) $P$

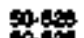

s.t.

sons.

10-6)

tods

Bo-

10-4.

10-6女

06

(1)

\$6;

10-68;

1040\%

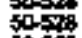

으.

50-50:

50-58

orts.

stots

tots

10-10.

40

04

00

im

tartits

Mpend

40.5.

4001

$40-345$

corterct

of $\rightarrow$ or

10-7004

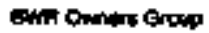




\begin{tabular}{|c|c|c|c|c|}
\hline 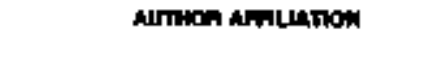 & M & chaper & & Aunkon NFrentiow \\
\hline 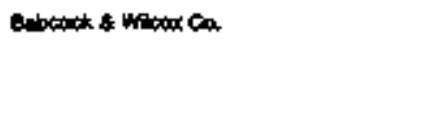 & 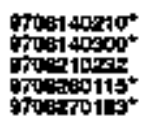 & 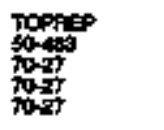 & $\mathbf{P}$ & Eumby Teetnolow the \\
\hline 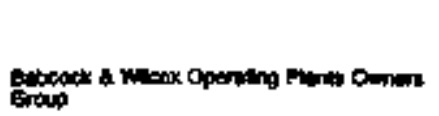 & 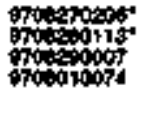 & 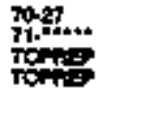 & & 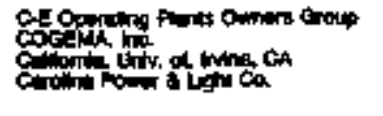 \\
\hline 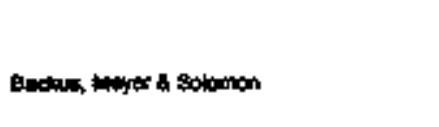 & 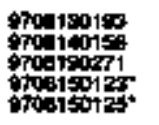 & 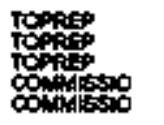 & w & \\
\hline 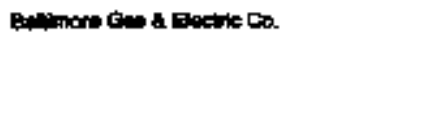 & 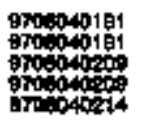 & $\begin{array}{l}50017 \\
50097 \\
50397 \\
50047 \\
50 \% 17\end{array}$ & $\begin{array}{l}p \\
p \\
p \\
p \\
p\end{array}$ & \\
\hline & 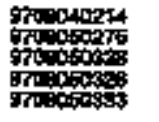 & 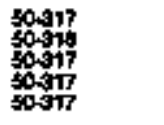 & $\begin{array}{l}p \\
5 \\
j \\
5\end{array}$ & \\
\hline & 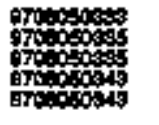 & $\begin{array}{l}60-37 \\
0037 \\
0017 \\
00017\end{array}$ & $\begin{array}{l}p \\
p \\
\mathbf{p} \\
\mathbf{p} \\
\mathbf{p}\end{array}$ & \\
\hline & 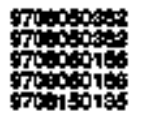 & $\begin{array}{l}0-917 \\
50977 \\
0097 \\
50-97 \\
50-917\end{array}$ & $\begin{array}{l}\frac{q}{0} \\
\frac{0}{p} \\
p \\
p\end{array}$ & \\
\hline & 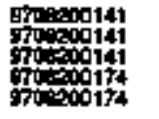 & 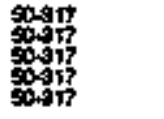 & $\begin{array}{l}\mathbf{F} \\
\mathbf{F} \\
\mathbf{p} \\
\mathbf{p}\end{array}$ & \\
\hline & 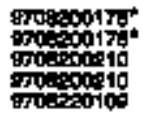 & 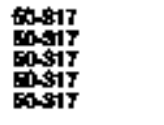 & $\begin{array}{l}\text { P } \\
\text { p } \\
\text { p } \\
\text { p }\end{array}$ & \\
\hline & 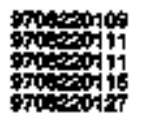 & 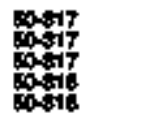 & $\begin{array}{l}\text { p } \\
p \\
p \\
f \\
6\end{array}$ & \\
\hline & 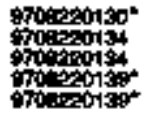 & 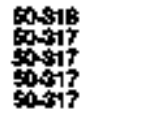 & $\begin{array}{l}0 \\
p \\
p \\
p\end{array}$ & \\
\hline & 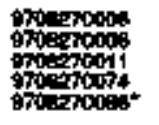 & 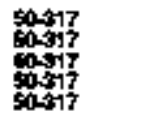 & $\begin{array}{l}8 \\
8 \\
8 \\
\text { h }\end{array}$ & \\
\hline 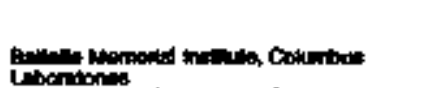 & 9xosecos:" & $\begin{array}{l}50397 \\
50397 \\
71-8037\end{array}$ & 䏴 & \\
\hline 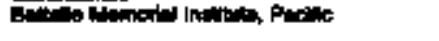 & moroocossi" & PACU & & \\
\hline 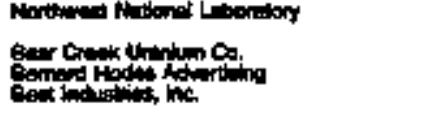 & 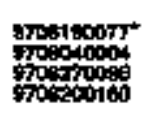 & 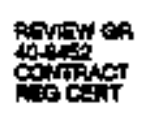 & $\mathbf{D}$ & \\
\hline 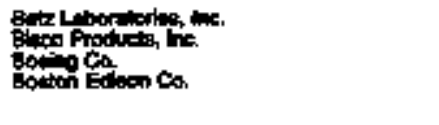 & 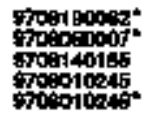 & 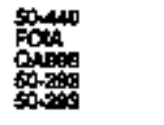 & D & \\
\hline & 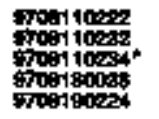 & 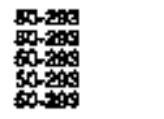 & $\begin{array}{l}\mathbf{Y} \\
\mathbf{5} \\
\mathbf{5} \\
\mathbf{R} \\
\mathbf{D}\end{array}$ & \\
\hline & 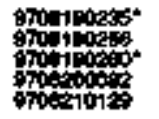 & 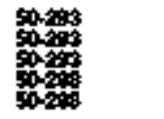 & $\begin{array}{l}p \\
p \\
p \\
\mathbf{p}\end{array}$ & \\
\hline S & 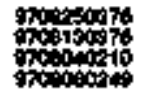 & 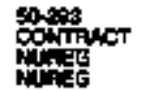 & a & 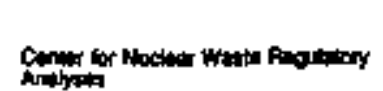 \\
\hline
\end{tabular}

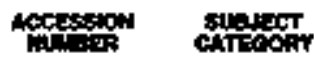

Procosing NAPES

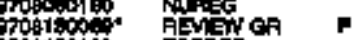

Fos

Fond

on:14015\% Tomep

2000015 40,

ATP010016 CONITACT

mologes

molotos

ixiartas

sorst

to

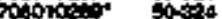

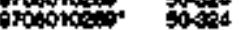

corse

Nongin

97000000 50.93

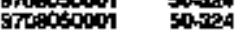

9700000 . $^{*}$

ototosita

$7+00013$

90003

.

gromososon

(1)

50000005

rosoestos

mowionos

porping

T000012

proosonat.

Foroping

Jin

ondives

pitroes

15

t

to

omorrot

moent

mont

9rostos

rot to

rromarar

Totis

$90+400$

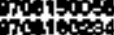

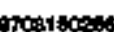

oxosions

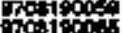

ond 1 to

propocos.

400010

$4+$

totons

970-10139

$700 \mathrm{c}$

Tropisot

97040501 7

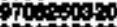

70 ins

7000001

opocrooct

onteroot.

Foroproos

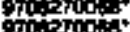

proprots

0404

(1) arosiceots

sores

Dow

$\rightarrow 0$

$54-82$

50 to

tost

50न1

50웅

E0.

c)

极要1

sorst

皮皮

象

soses

50 垈

50

50.424

501:

타면

$50 \times 3 \times 4$

5035

toto

sox

0

身原

Bos

$50=000$

S000

50

Stato

500

sot

s.t.

son

sot

sin

pota

500

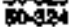

os

$\sin x$

coray

Doss

50

arapocora'

100ipos 7

cons

5 


\begin{tabular}{|c|c|c|c|}
\hline \multirow[t]{3}{*}{ 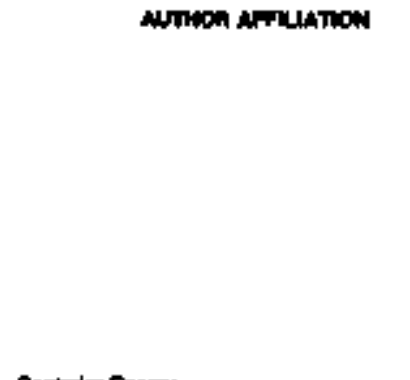 } & Accession & \multicolumn{2}{|c|}{ 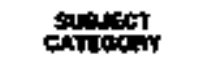 } \\
\hline & 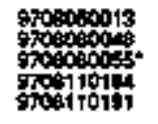 & thy & \\
\hline & 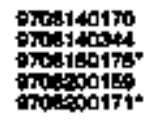 & (4) & \\
\hline \multirow{7}{*}{ 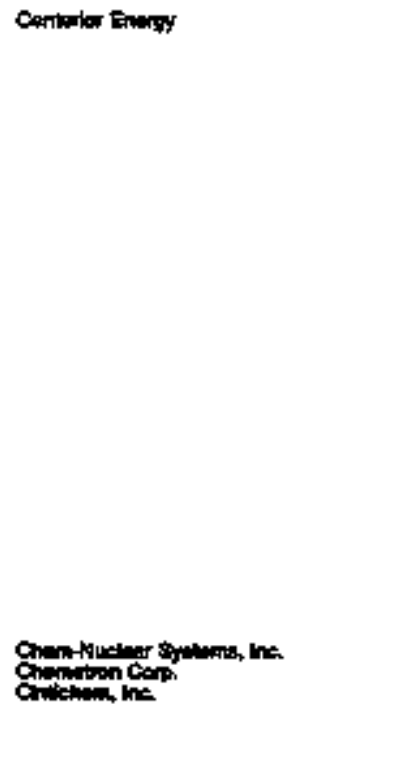 } & 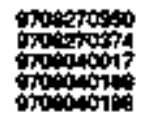 & 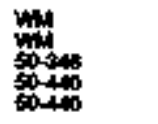 & 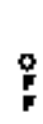 \\
\hline & 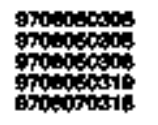 & 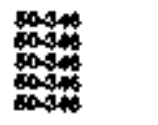 & $\frac{g}{g}$ \\
\hline & 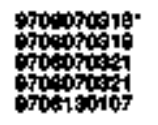 & 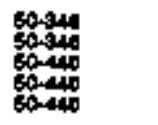 & $\begin{array}{l}p \\
y \\
F \\
p\end{array}$ \\
\hline & 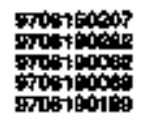 & $\begin{array}{l}5040 \\
50-46 \\
60-410 \\
60-410\end{array}$ & $\begin{array}{l}0 \\
\mathbf{F} \\
\mathbf{p} \\
\mathbf{p}\end{array}$ \\
\hline & 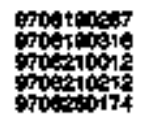 & $\begin{array}{l}s 0-400 \\
60-400 \\
60-440 \\
60-340\end{array}$ & $\begin{array}{l}\text { R } \\
8 \\
\text { p } \\
\text { p }\end{array}$ \\
\hline & 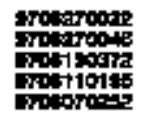 & $\begin{array}{l}0040 \\
50440 \\
71+47 \\
00-54 \\
0.54\end{array}$ & $\begin{array}{l}\mathbf{p} \\
w\end{array}$ \\
\hline & 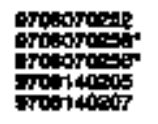 & 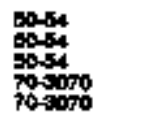 & $w$ \\
\hline \multirow{10}{*}{ 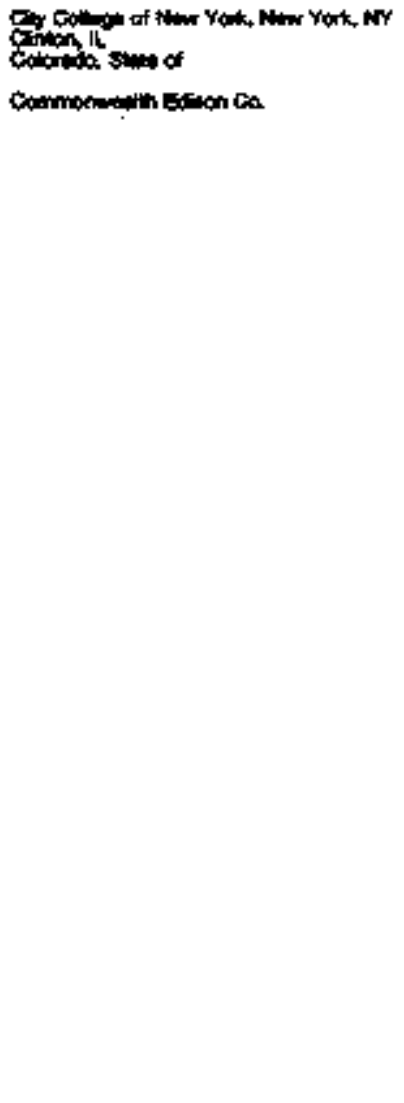 } & 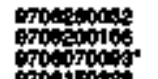 & gontanct & \\
\hline & oropoon & ins & 0 \\
\hline & 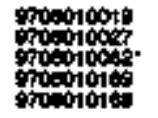 & 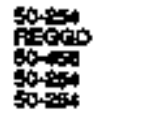 & o \\
\hline & 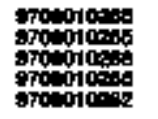 & 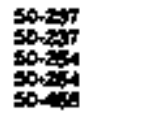 & $\begin{array}{l}Q \\
0 \\
0 \\
0 \\
0\end{array}$ \\
\hline & 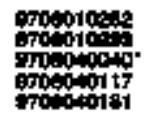 & 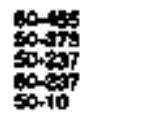 & $\begin{array}{l}\text { p } \\
\text { s } \\
\text { o }\end{array}$ \\
\hline & 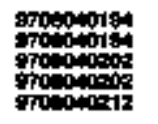 & 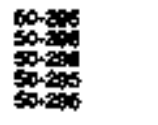 & p \\
\hline & 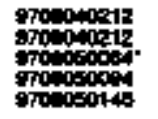 & 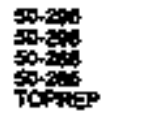 & $\begin{array}{l}\mathbf{p} \\
\mathbf{p} \\
\mathrm{s} \\
\mathrm{g}\end{array}$ \\
\hline & 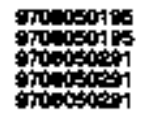 & 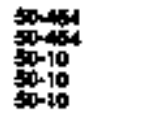 & 官 \\
\hline & 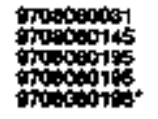 & 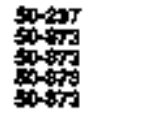 & 吊 \\
\hline & 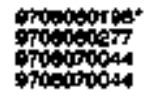 & 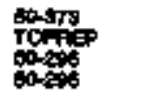 & p \\
\hline
\end{tabular}

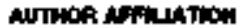
$\operatorname{sectan}$

erceonoteso

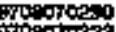
(1) orocoson

oposoco0so 97000041 oroction

F08140164 Crots $\$ 154$ cositer grot 120061

most3006\%

optighes

Toksolol

orisist

tron13011.

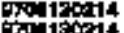

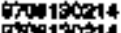

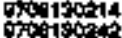

aromixoses

andistes

axd

axpitach

Trescost?

Fiog1 40 it?

$5 \times 140078$

Tid 4000

anderteon

arosinents

(1)

(1)

onditiones

200100068

entorot

emosices

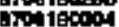

9rosistos

ordexpos

orocos

№p

70:0x000

Not:300600

r.s.

0001000

expertos

orotion

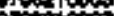

or

posingas

(1)

(1)

(1)

orocoros

000000

srocion

erotion

erocertoros

Fo. 1014

2 licisia

Frosed1913.

o704810131-

Grosiontas

rrove

crosion

srosinter

entertast

orrovers.

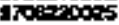

100020005

1003000003

rotinues

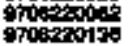

scrars

const

50

$0-15$

0

const

아요

实

60-456

ches

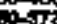

D-4T

a-2

0

(15.

sonst

$50-5$

50-23?

50.

71-

10.205

$00-206$

Bat

$80-4$

50-454

504.

50-10

00-204

04

oro

n-

0006

0

$00-704$

$50-3$

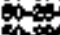

0.

$60-97$

sosm

0037

cost

$50-37$

50-23?

so- 3

50 .

50-3

00 क

s0.tin

(1)

60.

co.

80

s0

$50-296$

so-20

$00-4$

50

$60-6+1$

80-46

$60-4$

$50-454$

$50-45$

50-45.

bot

50-10

so-1

son

皮

50.456 


\begin{tabular}{|c|c|c|c|c|}
\hline \multirow[t]{8}{*}{ 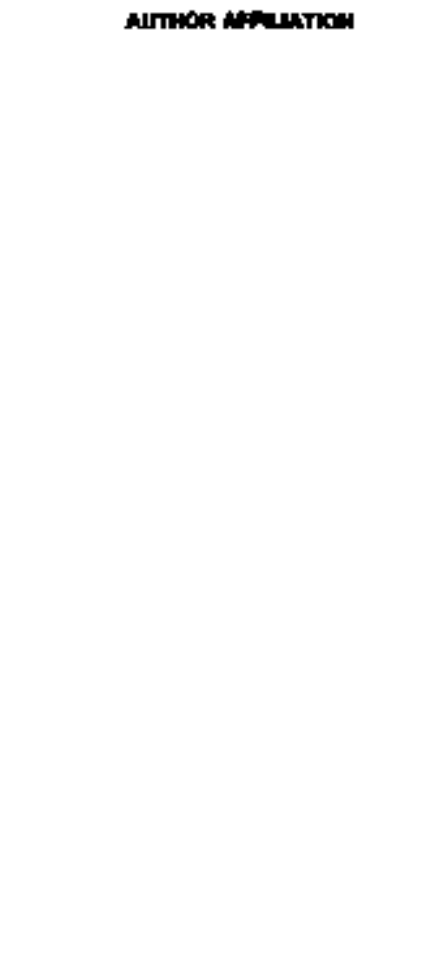 } & m & \multicolumn{2}{|c|}{ 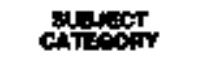 } & \multirow[t]{2}{*}{ 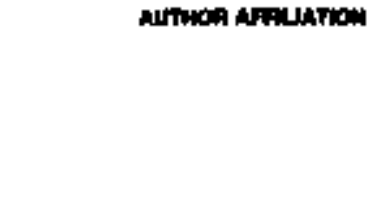 } \\
\hline & 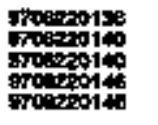 & 50458 & $\begin{array}{l}F \\
8 \\
8\end{array}$ & \\
\hline & 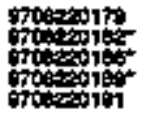 & 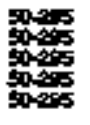 & $\begin{array}{l}\text { R } \\
\text { R } \\
\text { R } \\
\text { F }\end{array}$ & \\
\hline & 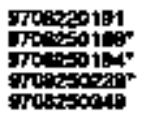 & 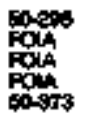 & F & 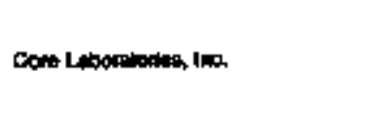 \\
\hline & 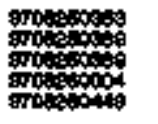 & 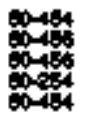 & $\begin{array}{l}q \\
\mathbf{p} \\
\mathbf{p} \\
\mathbf{8} \\
\mathbf{p}\end{array}$ & Deth Gever conp \\
\hline & 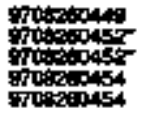 & 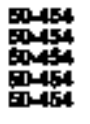 & ? & \\
\hline & 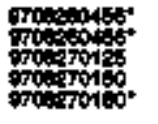 & 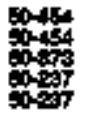 & $\begin{array}{l}\mathrm{P} \\
\mathbf{p} \\
\mathbf{8} \\
\mathbf{8} \\
\mathbf{8}\end{array}$ & \\
\hline & 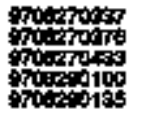 & 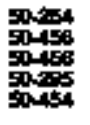 & $\begin{array}{l}p \\
p \\
0 \\
g \\
q\end{array}$ & Dor Chentisal Ca. \\
\hline \multirow[t]{3}{*}{ 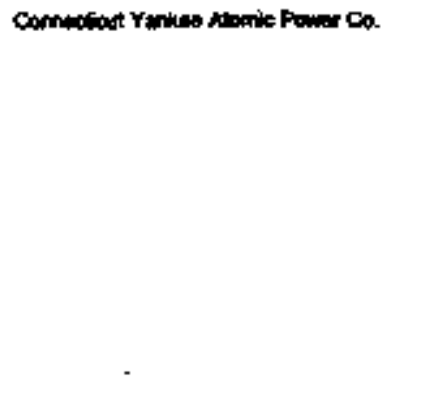 } & 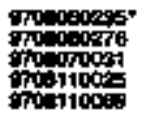 & 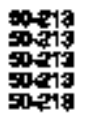 & $\begin{array}{l}0 \\
\text { ह } \\
\text { F } \\
\text { W }\end{array}$ & Duke Poute to \\
\hline & 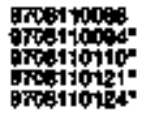 & 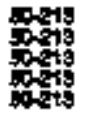 & $\begin{array}{l}9 \\
8 \\
5 \\
5 \\
5\end{array}$ & \\
\hline & 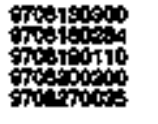 & 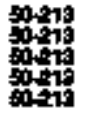 & $\begin{array}{l}\text { W } \\
\text { \& } \\
\text { h } \\
\text { s }\end{array}$ & \\
\hline \multirow[t]{5}{*}{ 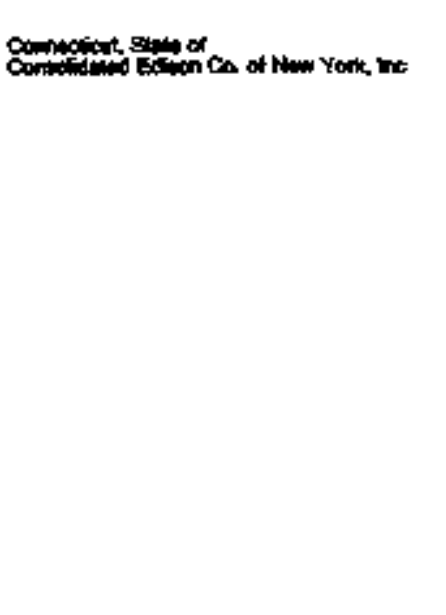 } & 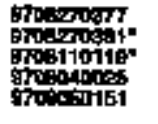 & 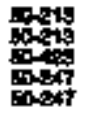 & $\begin{array}{l}\text { w } \\
\text { के }\end{array}$ & \\
\hline & 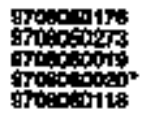 & 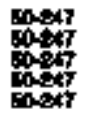 & $\begin{array}{l}\mathrm{p} \\
\mathrm{s} \\
\mathrm{p}\end{array}$ & \\
\hline & 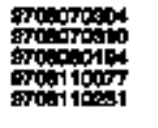 & 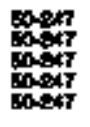 & $\begin{array}{l}P \\
j \\
\frac{5}{8} \\
8 \\
8\end{array}$ & \\
\hline & 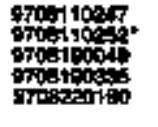 & 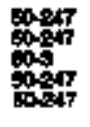 & p & \\
\hline & 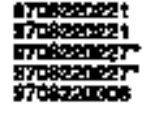 & $\begin{array}{l}00 \\
00 \\
00\end{array}$ & $\begin{array}{l}E \\
F \\
E \\
6\end{array}$ & \\
\hline \multirow{5}{*}{ 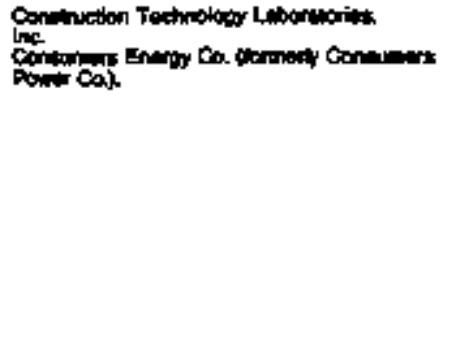 } & co0ts tasez" & roin & & \\
\hline & stosatioes & DO-186 & $p$ & \\
\hline & 97000000124 & cosesc & $p$ & \\
\hline & 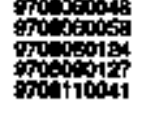 & 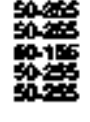 & $\stackrel{p}{q}$ & \\
\hline & 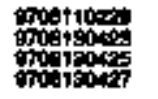 & 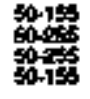 & $\mathbf{F}$ & \\
\hline
\end{tabular}

\begin{tabular}{|c|c|}
\hline Apesen & 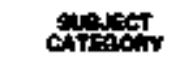 \\
\hline 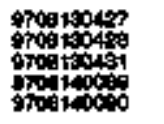 & 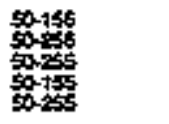 \\
\hline 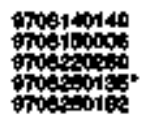 & 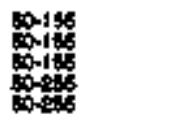 \\
\hline 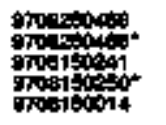 & 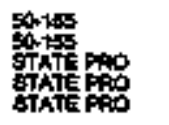 \\
\hline 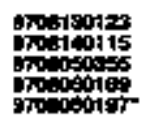 & 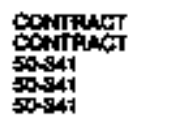 \\
\hline 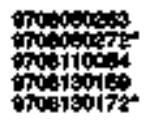 & $\begin{array}{l}30341 \\
00341 \\
40-16 \\
0041 \\
50341\end{array}$ \\
\hline 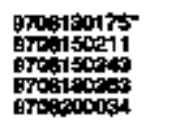 & 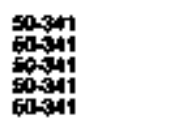 \\
\hline 770000 & 30-31 \\
\hline
\end{tabular}

ondotion:

orocosos rom

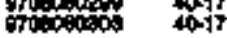

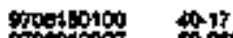

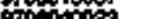

ront

Foofots 0041

groconts soras

900001000

$87000 y$

woth

9rogonest 500\%

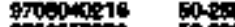

7xoson

row

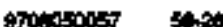

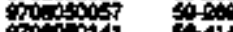

Fon, 5041

of

97000005

intors

700000

STomocos

$0 x=0$

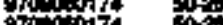

omentor

onctint

rovist

Trodut rot

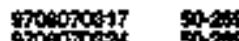

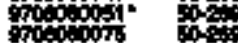

suomin son

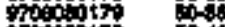

770.010. 5041

Focotion, 0-11

Fno-10061 50.09



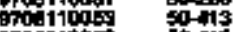

orosituter 304

970011911s" 5020

Proi1919: 5009

porvis: 


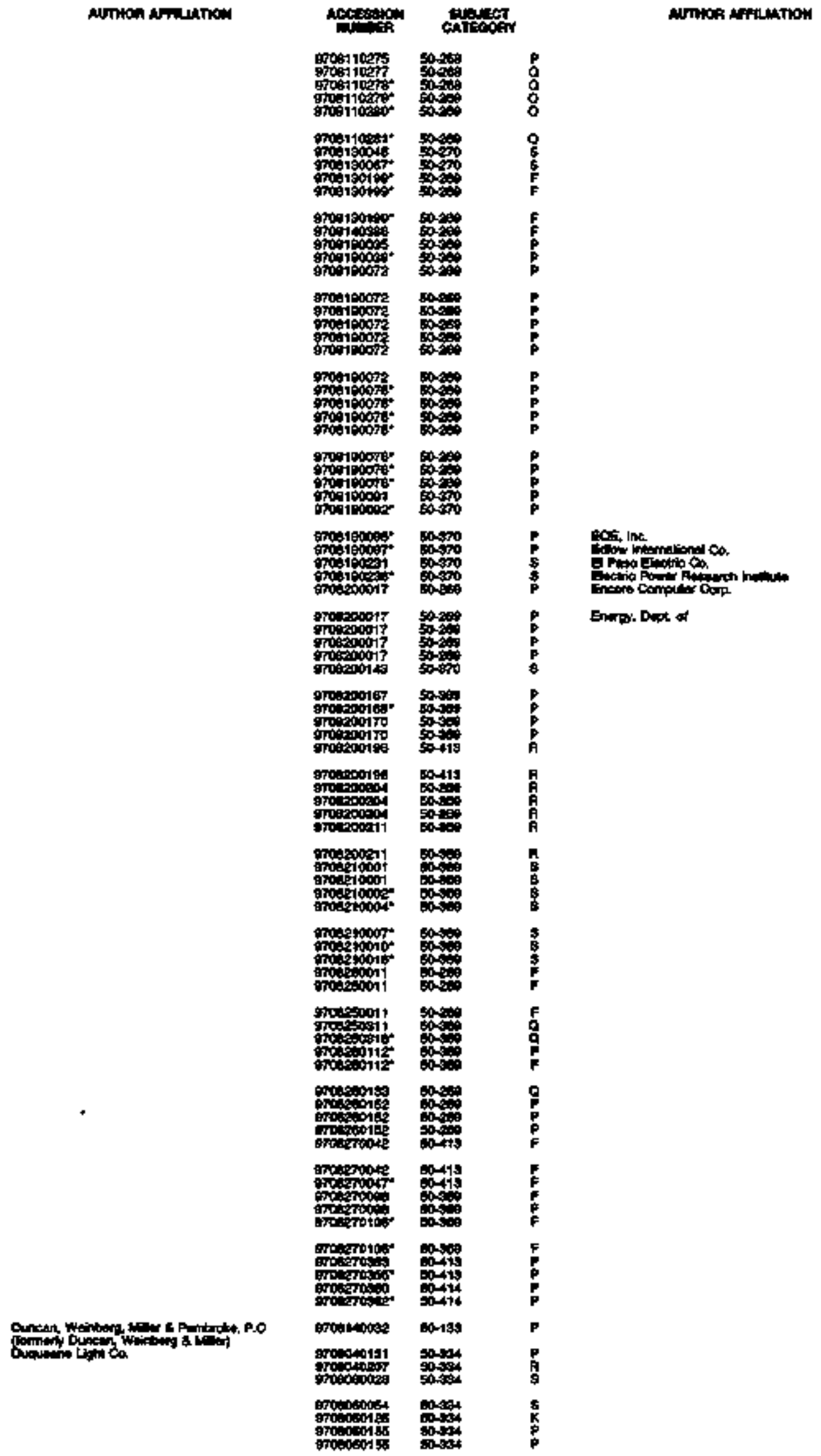




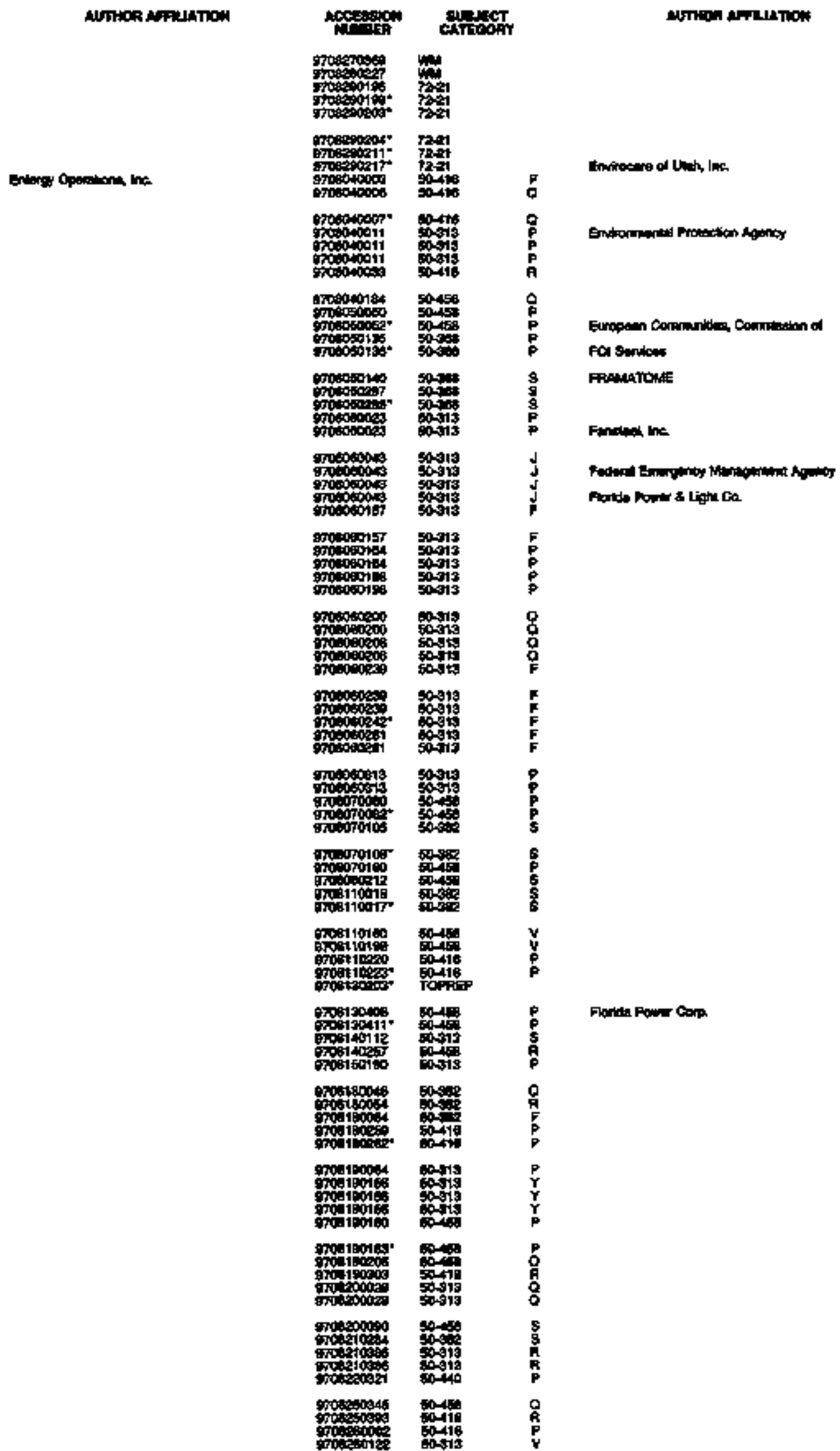

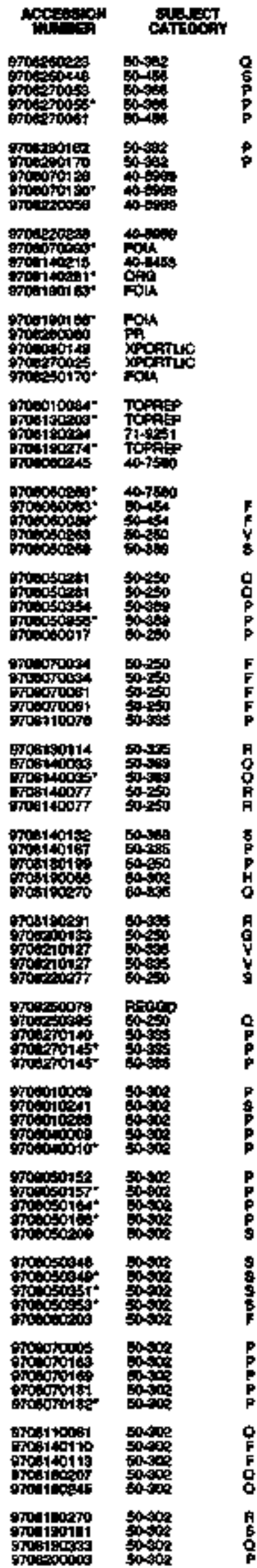


NTTAR AFFU UATOH

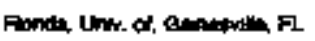

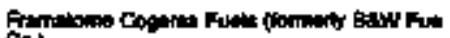
Corl,

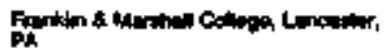

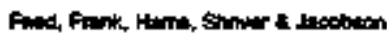

Gin $\operatorname{cop}$

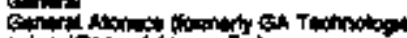

- Inetem

cented Godoto

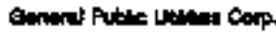

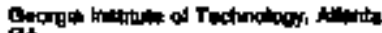

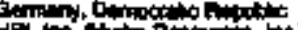

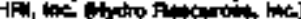

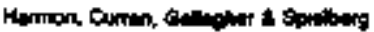

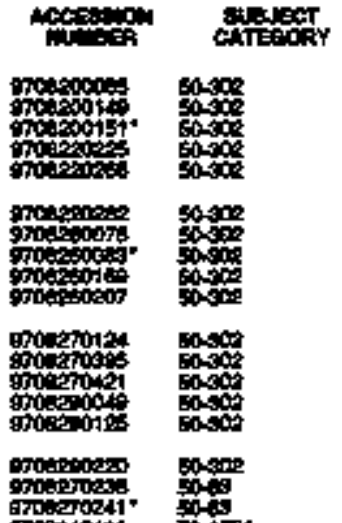

arost40411

broponosis 71-men

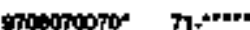

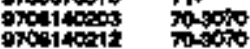

mosticet7 70000

contrict

GTin

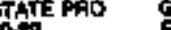

9rod140184.

erolitiss

gropitots

$970+10000$

onotien:25:

9010

Fonent.

goiporis.

popolsos:

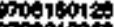

onto

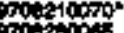

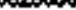

groporome

97000015:

0000001.

grotidid

opownop.

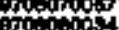

gropios

900 optop

smocouson*

700110161

por row

colitos:

970 ibotion

grotiporot-

970120113

9015015

(1)

97000000

$970+200040$.

7000004

rovistont.

Fot21007

prosion.

Fin.20

orotiont

$70-\pi 4$

70.74

707 년

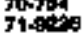

70.74

pose

Q0.6.

Topia

?hespos

solat

7)

roperp

Bones

botis

pos

6006\%

5006

50 .

엄요

0눈:

500\%

60

인성

foses

soyes?

powas

(a)

on.

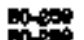

Dor

ortit

E0장

E0-21항

투겅

50<1

60.6\%

tringonts

prop

rant.

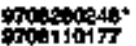

soral

$\infty$

究

(5)

So-14:

97011010"

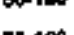

ba-1ed

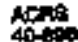

mitese

Norito



$\mathbf{R}$

p

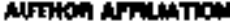

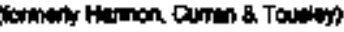

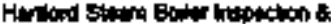

miacte co

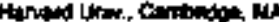

Hider stint of

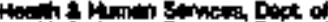

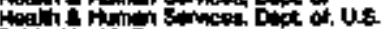

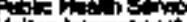

Hotace intemetosts

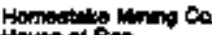

Houte ad Rap.

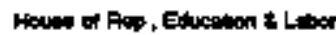

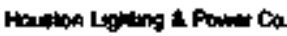

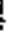

p

$\mathbf{F}$

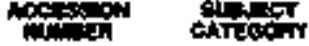

9x:14007 70-30m

oxioges: $10-968$

ar

最asonom:

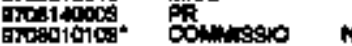

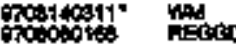

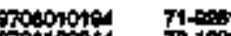

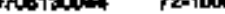

rogeros. sosts

Exosidis.

grontis:

gxosistotion

poptrapas: so-as

grodtooes" 50-20s

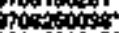

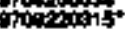

cosinnser

oroutecien

970000

modosom

orototion 1

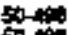

然-

sidis

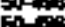

thoctisy

$5+x^{2}$

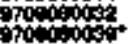

sonite

(1)

s.m.

;on

oronowere.

970000

groodectos.

6rotionsos

s.t.

(ando

spect.

5

propooser

ropopir

Poaticosers

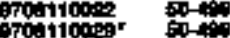

oroen110

Toentopi

Fost10est

सos710e.

80-49:

;on-

6im

50-4ip

orosibaesos

equacis

ofonaros

groveriors

son-

ton

sint

sint

mocaricons'

Thenen

$50+40$

50.4.

30

$\rightarrow \infty$

tontions

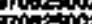

of

$0+001$

sonse

$\sin _{0 \rightarrow \infty}^{\infty}$

inte

sin

$9700=0140$

potosa

po-4a:

tons

sion

solitis

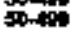

IFF, Im

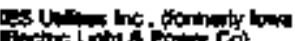

propron

Thiprois

rose

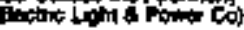

stonows

970 intois

erowosis

protions

orotosoces

$50-31$

crosionoses

groterposs

$970 \mathrm{coc0}$

70 rrot.

9rowooser?

6-木ำ

sis

家1

So-1

10.3

象

(1)

\$ख1

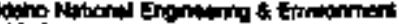

Trogetoos

OAG

mole Pourer co.

popotios

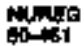




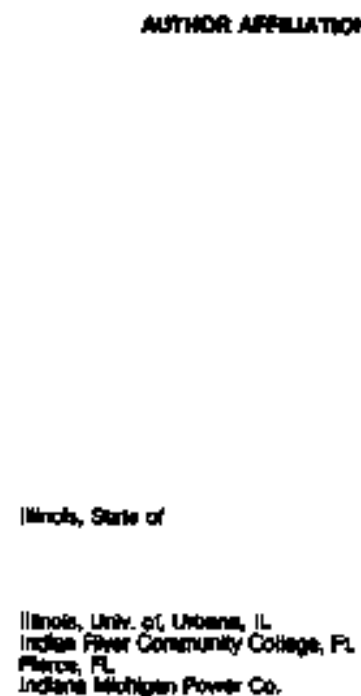

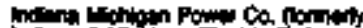

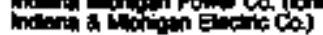

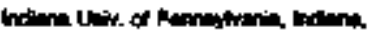
Pan

\begin{tabular}{|c|c|}
\hline 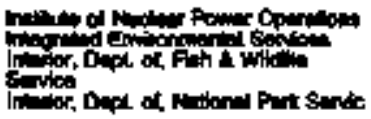 & 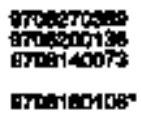 \\
\hline 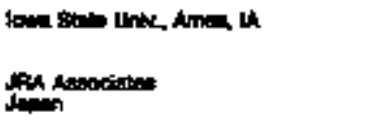 & 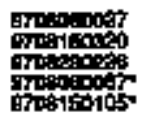 \\
\hline 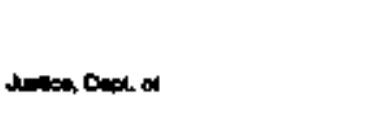 & 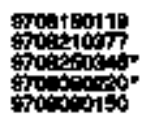 \\
\hline Konneost Corp & 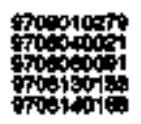 \\
\hline 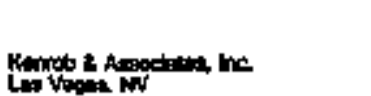 & 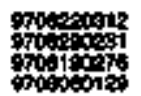 \\
\hline
\end{tabular}

\begin{tabular}{|c|c|c|c|}
\hline 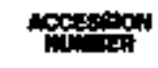 & extes & & AMHOR MFIUATOW \\
\hline 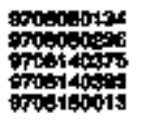 & p-iti & $\begin{array}{l}\mathbf{p} \\
\mathbf{F} \\
\mathbf{p} \\
\mathbf{p} \\
\mathbf{q}\end{array}$ & 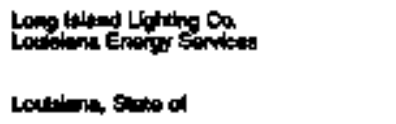 \\
\hline 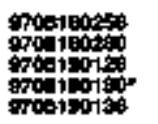 & Somit & $\begin{array}{l}8 \\
0 \\
8 \\
8 \\
8\end{array}$ & 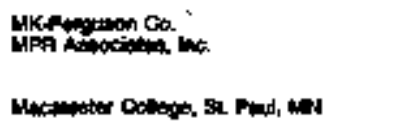 \\
\hline 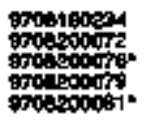 & po-is & $\begin{array}{l}\mathbf{F} \\
5 \\
5 \\
5 \\
5\end{array}$ & 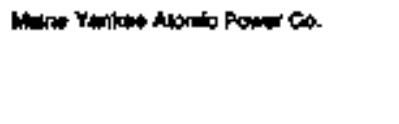 \\
\hline 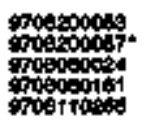 & 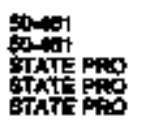 & $\frac{6}{6}$ & \\
\hline 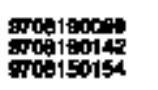 & 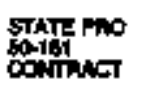 & 早 & \\
\hline proelsoesos & s0315 & $P$ & Ne: indrocth ine \\
\hline 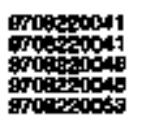 & 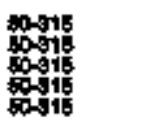 & $\begin{array}{l}F \\
F \\
F \\
F\end{array}$ & 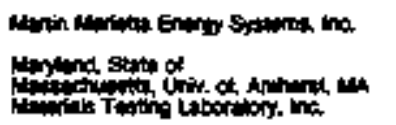 \\
\hline 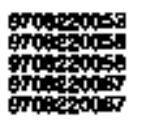 & 40,5 & $\begin{array}{l}\mathbf{F} \\
\mathbf{F} \\
\mathbf{F}\end{array}$ & 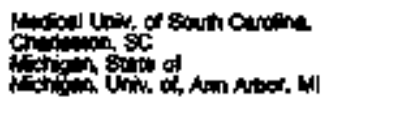 \\
\hline 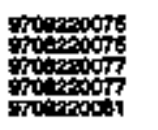 & $\begin{array}{l}\cos 15 \\
\cos 15 \\
\sin 15 \\
60015\end{array}$ & $\begin{array}{l}\mathbf{F} \\
\mathbf{F} \\
\mathbf{F}\end{array}$ & 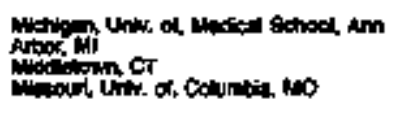 \\
\hline mowases & 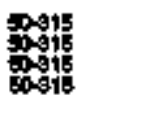 & $\begin{array}{l}\mathbf{F} \\
\mathbf{F} \\
\mathbf{q}\end{array}$ & \\
\hline 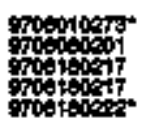 & 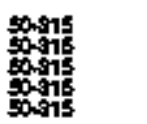 & $\begin{array}{l}q \\
p \\
p \\
p\end{array}$ & 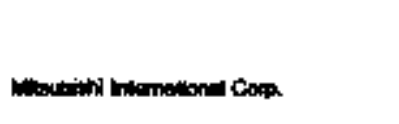 \\
\hline 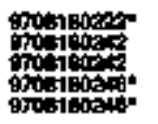 & 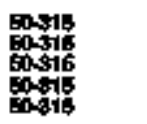 & $\begin{array}{l}p \\
p \\
p \\
p \\
p\end{array}$ & 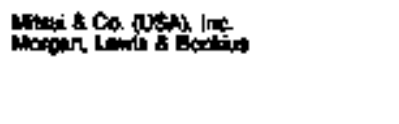 \\
\hline 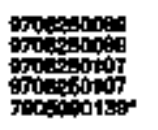 & $\begin{array}{l}\text { costs } \\
\text { Wots } \\
\text { Wois }\end{array}$ & ? & 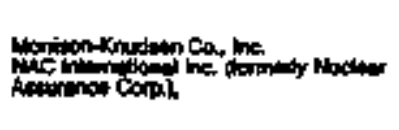 \\
\hline 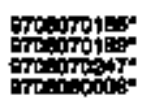 & ron & & 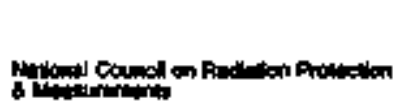 \\
\hline 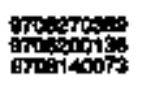 & conpret & & 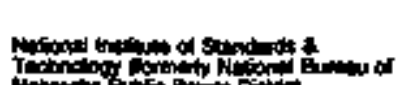 \\
\hline mperemog" & ons & & \\
\hline 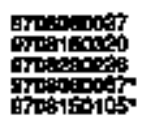 & $\frac{0+116}{20}$ & ค & \\
\hline 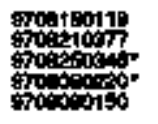 & 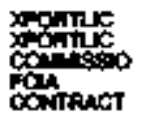 & $\boldsymbol{m}$ & 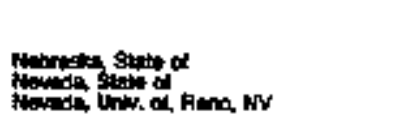 \\
\hline 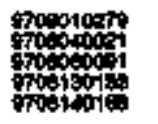 & togen & & 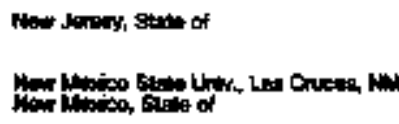 \\
\hline opospis & 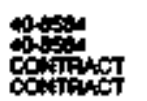 & & 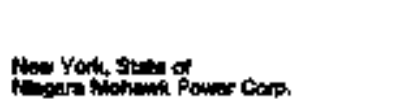 \\
\hline
\end{tabular}

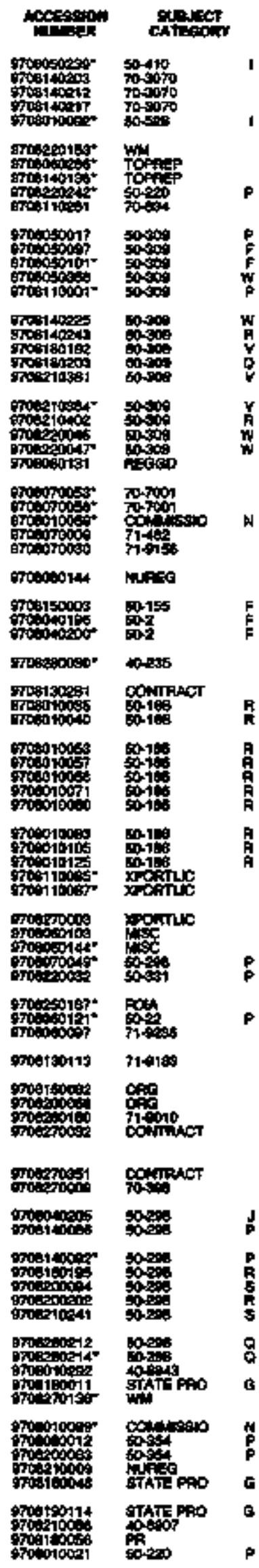




\begin{tabular}{|c|c|c|}
\hline ANTHOA AfFianTion & Actesenow & 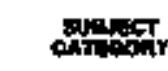 \\
\hline & 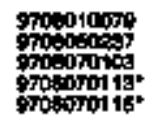 & 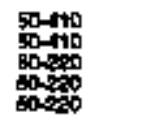 \\
\hline & 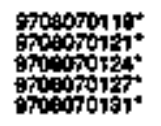 & 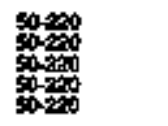 \\
\hline & 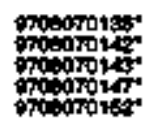 & 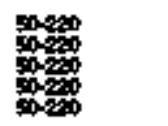 \\
\hline & 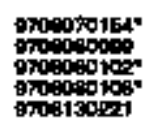 & 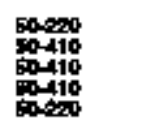 \\
\hline & 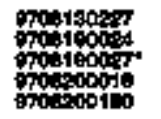 & 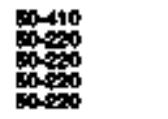 \\
\hline 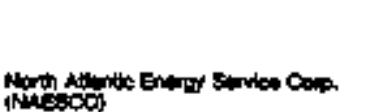 & 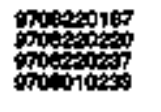 & $\begin{array}{l}50.200 \\
5010 \\
5002020 \\
60.40\end{array}$ \\
\hline & 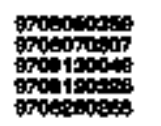 & 舆带 \\
\hline 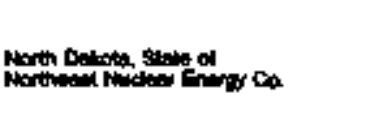 & 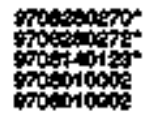 & 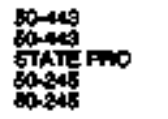 \\
\hline & 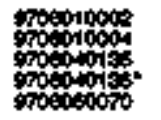 & 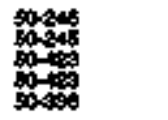 \\
\hline & 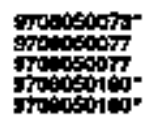 & 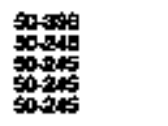 \\
\hline & 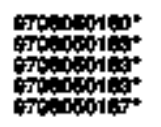 & 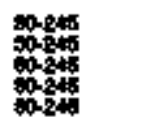 \\
\hline & 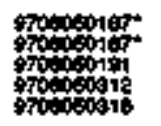 & 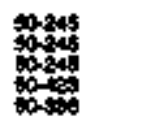 \\
\hline & 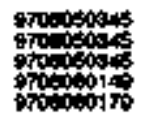 & 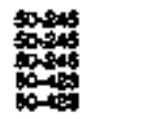 \\
\hline & 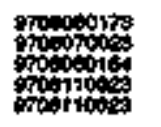 & 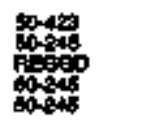 \\
\hline & 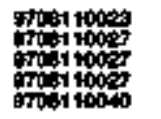 & 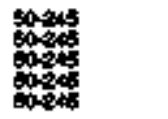 \\
\hline & 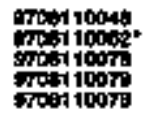 & 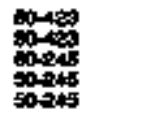 \\
\hline & 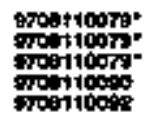 & 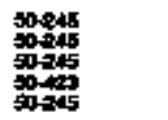 \\
\hline & 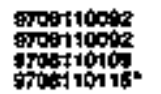 & 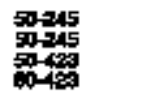 \\
\hline
\end{tabular}

Amka Ariuthon

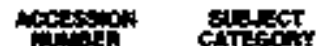

9xiv1034

gxi10

舟,

舟

Brot13007"

grodisotes

onos 30000

orostiosine

grost30000

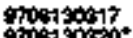

stortsoss

9posisosid

moes boess

proptspes:

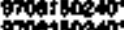

moplote 40

poopoces.

poptoces

opicos

poipos

Trod10004\%"

rot 1000 क

roertoo

9rositopit.

$50-45$

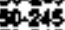

otots

50025

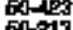

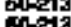

$60-215$

武的

$\rightarrow$ t

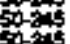

c0-946

Mata

50-64:

然皮

sis

$\rightarrow$ ats

Doetcoort

poptopers.

Don 0001

ipotion:

orotiogor

protrotager

grosisong

ofustiolen

protedpe

970t

oxitot

200100\%

50

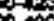

(1)

$+8$

60-200

0

p-ip

4-246

sonsts

tons

otas

(5)

50423

tos

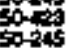

90000000:

potsock

mitotios

ontelost

onesolits

7020

Whis

Fition

ofrosedit

$870=00413$

\%olatis:

monsorst.

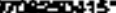

900.6135"

90000004:

970 -

0000 .

gropucos:

9romodoe1-

700000

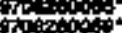

apipaost

900007

Tris

Plonot.

cons

co-45

0 루요

cass

onets

90-4\%

0.2\%

san

0 at:

s0-745

에요.

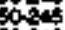

30245

sous

2

tots

totas

soas

trat

50 is

stids

50잔다.

한하영

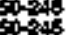

sto-

Dropepos.

.

ontitolis

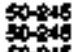

solde

90-46.

F7nctans

int

$7+14$

ofintold

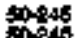

bores

sovis

oropatoso

Grotictite

50-100

cons

EOz) 


\begin{tabular}{|c|c|c|c|c|c|c|}
\hline \multirow[t]{2}{*}{ 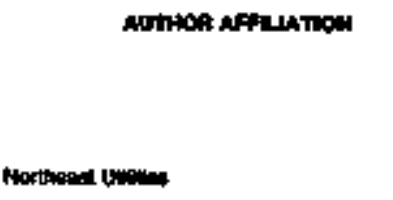 } & \multirow{2}{*}{ 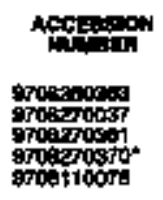 } & \multicolumn{2}{|c|}{$\cos _{x \rightarrow 1}$} & \multirow[t]{2}{*}{ Mrnon AFin MTnON } & \multirow{2}{*}{ 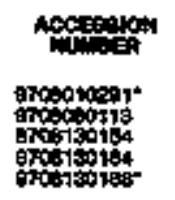 } & 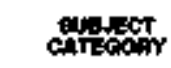 \\
\hline & & 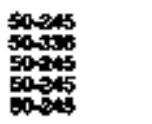 & $\begin{array}{l}\mathbf{F} \\
\mathbf{5} \\
\mathbf{5} \\
\mathbf{n}\end{array}$ & & & 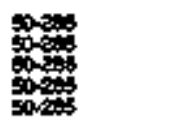 \\
\hline \multirow[t]{9}{*}{ 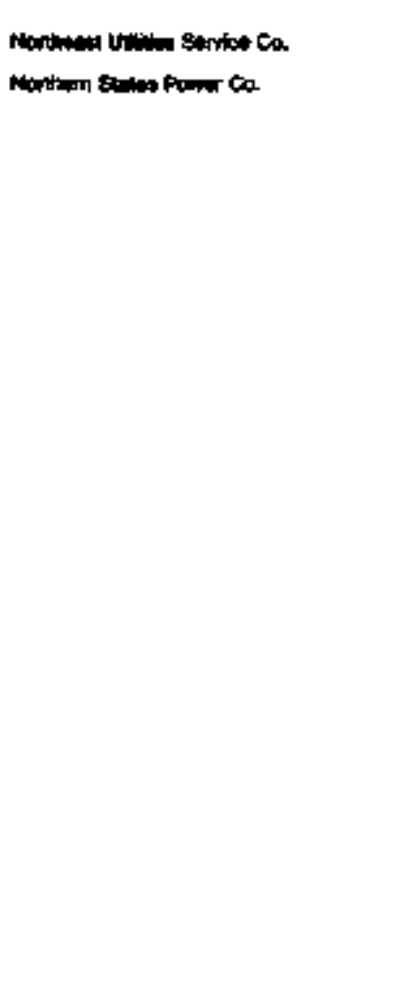 } & 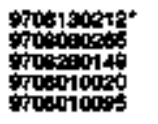 & 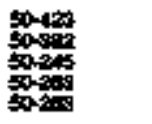 & $\begin{array}{l}\mathbf{p} \\
\mathbf{p} \\
\mathbf{p} \\
\mathbf{8} \\
\mathbf{p}\end{array}$ & & 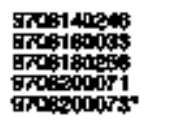 & 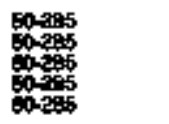 \\
\hline & 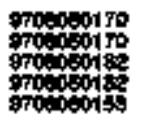 & 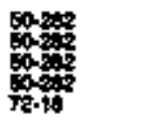 & ? & 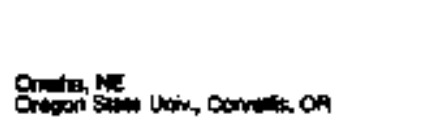 & 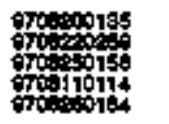 & 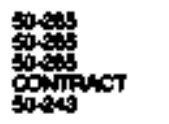 \\
\hline & ocosonstos & 紫然 & $\mathbf{p}$ & 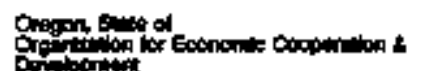 & $\sin 1402011^{\circ}$ & tatien \\
\hline & moninoses & 贺率 & $\begin{array}{l}Y \\
y\end{array}$ & 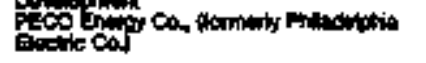 & mopioses & sose \\
\hline & 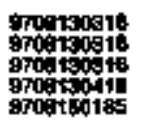 & 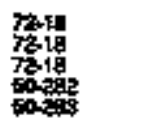 & $\underset{\mathbf{R}}{\mathbf{R}}$ & & 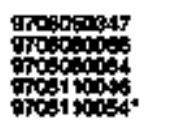 & 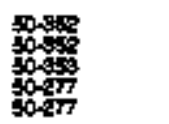 \\
\hline & 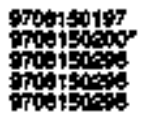 & 象率 & $\begin{array}{l}\mathbf{F} \\
\mathbf{k} \\
\mathbf{R}\end{array}$ & & 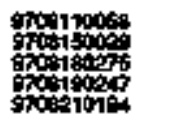 & 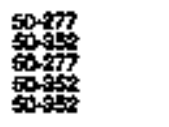 \\
\hline & 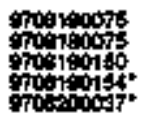 & 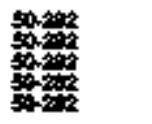 & $\begin{array}{l}\mathrm{g} \\
\mathrm{g} \\
\mathbf{5} \\
\mathbf{8}\end{array}$ & 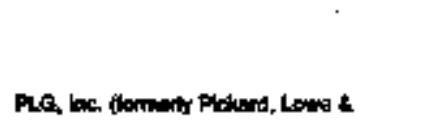 & 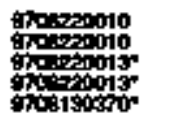 & 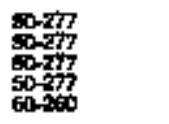 \\
\hline & 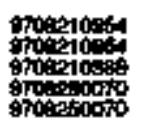 & 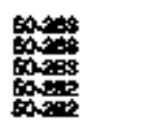 & $\begin{array}{l}\text { p } \\
\stackrel{g}{p} \\
\text { p }\end{array}$ & 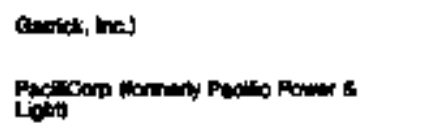 & 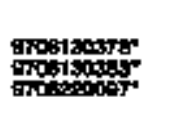 & 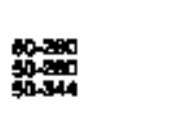 \\
\hline & 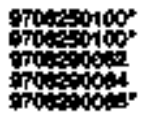 & 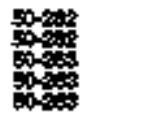 & $\begin{array}{l}p \\
p \\
p\end{array}$ & Puelle Gor a Rocole ca. & 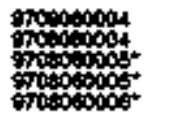 & 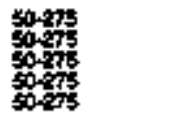 \\
\hline 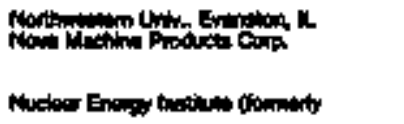 & 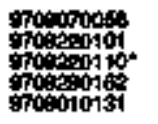 & and & & & 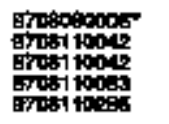 & $\frac{6005}{0005}$ \\
\hline 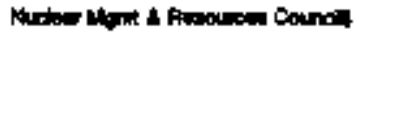 & 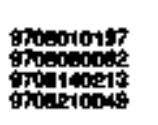 & 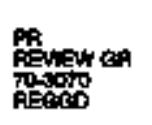 & $\mathbf{P}$ & . & 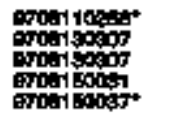 & $\begin{array}{l}\text { pers } \\
00078 \\
00078\end{array}$ \\
\hline 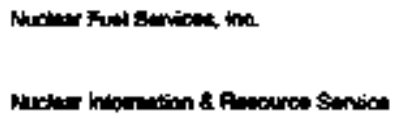 & 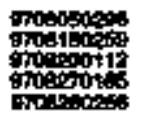 & 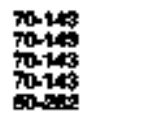 & H & & 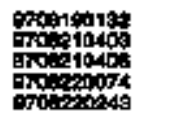 & 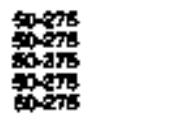 \\
\hline 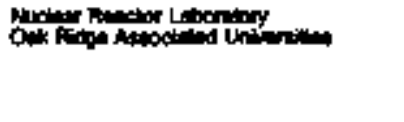 & 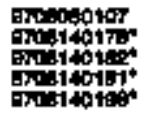 & 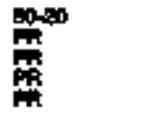 & $\mathbf{P}$ & & 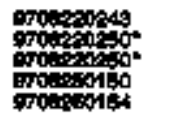 & 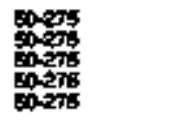 \\
\hline \multirow[t]{3}{*}{ 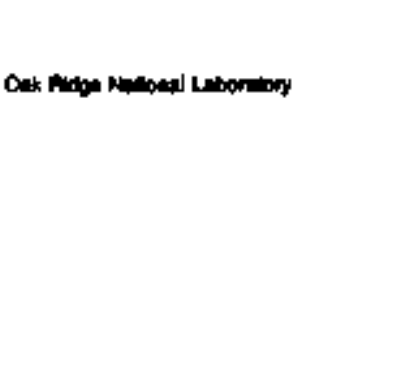 } & 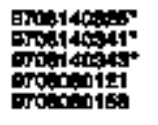 & 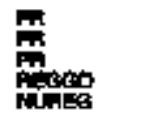 & & 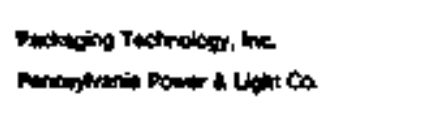 & 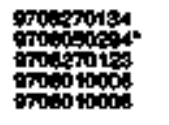 & 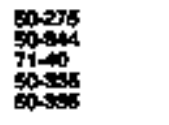 \\
\hline & 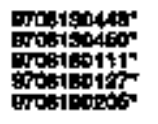 & 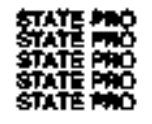 & $\begin{array}{l}\text { 兽 } \\
\text { 高 } \\
0 \\
0\end{array}$ & & 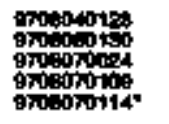 & 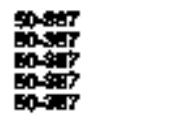 \\
\hline & 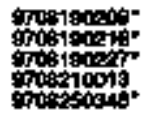 & 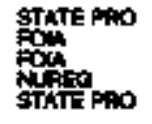 & 0 & & 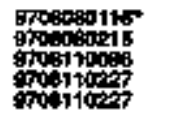 & 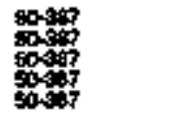 \\
\hline \multirow[t]{2}{*}{ 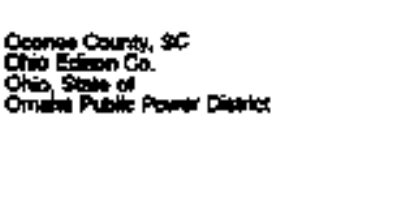 } & 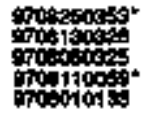 & 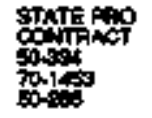 & $\begin{array}{l}p \\
p \\
p\end{array}$ & & 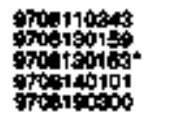 & 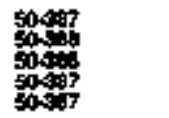 \\
\hline & 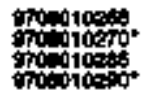 & 悹率 & $\underset{\mathbf{R}}{\mathbf{R}}$ & 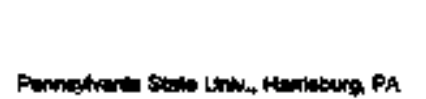 & 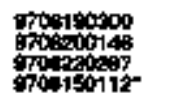 & 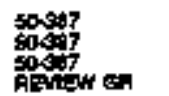 \\
\hline
\end{tabular}




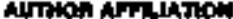

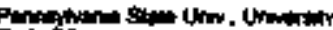
Pantiph

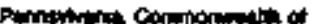

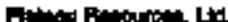

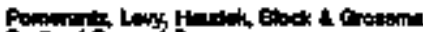

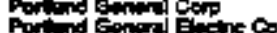

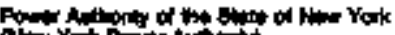

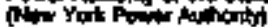

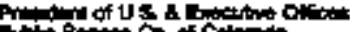

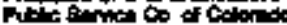

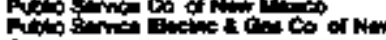
14:

\begin{tabular}{|c|c|c|c|}
\hline 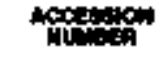 & 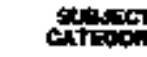 & & 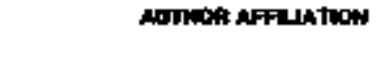 \\
\hline Poptspistos & DAN & $\mathbf{P}$ & \\
\hline Fow & OGNIANT & & \\
\hline 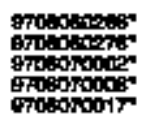 & mon & & \\
\hline 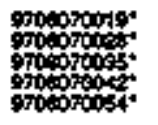 & $\operatorname{son}_{0}$ & & \\
\hline 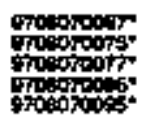 & som & & \\
\hline 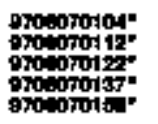 & fouk & & \\
\hline 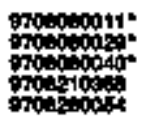 & 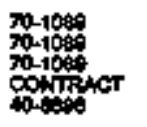 & & \\
\hline 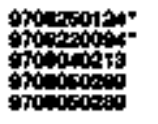 & 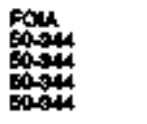 & $\begin{array}{l}y \\
y \\
Y \\
Y\end{array}$ & \\
\hline 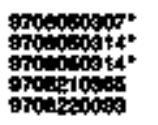 & $\begin{array}{l}00344 \\
00344 \\
000444 \\
00344\end{array}$ & $\begin{array}{l}y \\
y \\
y \\
F \\
y\end{array}$ & \\
\hline mosectos & $\log _{0 \rightarrow 44}$ & Ŕ & \\
\hline monoweoser & $60-288$ & 8 & \\
\hline 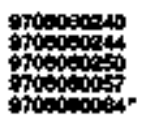 & 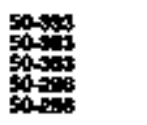 & $\begin{array}{l}8 \\
\mathbf{p} \\
6 \\
8\end{array}$ & 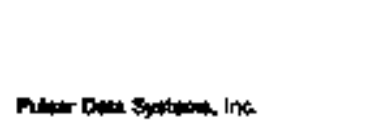 \\
\hline 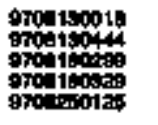 & 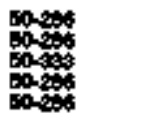 & $\begin{array}{l}\mathbf{P} \\
\mathbf{F} \\
\mathbf{R} \\
\mathbf{R} \\
\mathbf{R}\end{array}$ & 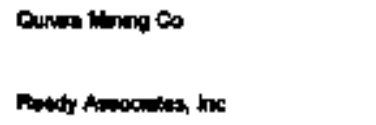 \\
\hline 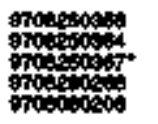 & sons & 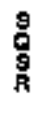 & 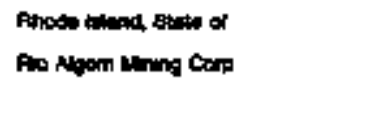 \\
\hline 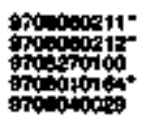 & 10 & s & 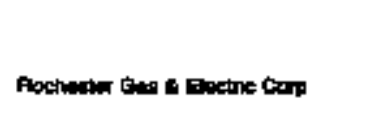 \\
\hline mocinge & $\frac{60 \%}{000}$ & $\begin{array}{l}\text { p } \\
\mathbf{f} \\
\mathbf{p}\end{array}$ & \\
\hline 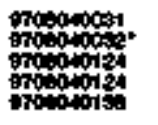 & 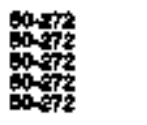 & $\begin{array}{l}\mathbf{p} \\
\mathbf{p} \\
\mathbf{p} \\
\mathbf{p} \\
\mathbf{s}\end{array}$ & \\
\hline 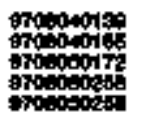 & 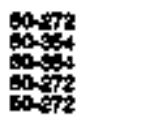 & $\begin{array}{l}\text { g } \\
\text { ด } \\
\text { p } \\
\text { p }\end{array}$ & \\
\hline 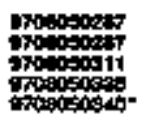 & 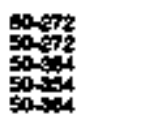 & $\begin{array}{l}0 \\
0 \\
? \\
?\end{array}$ & 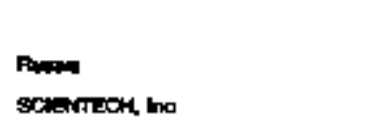 \\
\hline 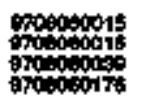 & 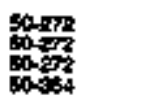 & $\begin{array}{l}\mathbf{F} \\
\mathbf{F} \\
\mathbf{S} \\
\mathbf{S}\end{array}$ & BCA, Ins pate oring \\
\hline
\end{tabular}

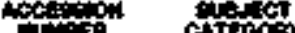

nowores

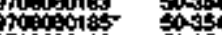

(

(1)

proposones sone

mocost 507

poton 5023

procorar sos

protorso: fom

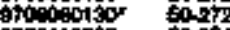

orticit

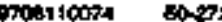

(1)

(1)

ongtist

oroencoses:

Tourpes

$70 \mathrm{esc}$

270 oplour 4

proposest

towo

(4)

산

oroperouss

rot...001

orotion

puespis

(1)

Dito 1

1

Trodson 1

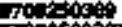

Notosich

witon

grovanoms

molater

corroos

6rotisooul

Oroe140012

gop 1 ten 2

oroet pito

Fin1100

Fon

(1)

70 orton

ofotipdes:

grourases

poustroses

otot?

oropotes

open 19010s

200130115

gos 13019

toon

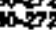

60

sop 72

$\infty 27$

$\infty 2$

027

क-27

sons

$+31$

tos?

con

sond

60.7rs

ons

is

orgex

to-

orit

고11

50.11

-50.472

Donsi1

sos

COMTHCT

40.06.

400

40

WTter।

6

19

40

soment

40

0004

otorat

$\rightarrow 0$

to-ien

8004

ion

70010400, soras

7091904

7001900.

gipop

0004

004

ocan

soezpos

(1)

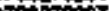

sonest

0

o-

to-ant

orotgroe 11.

soan

ontrosid.

60011004

gonescos

$60-34$

ConTuT contruc

s7oproocst

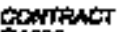

chasos

ato 
Mnitos Nivinow

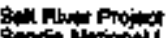

(1)

8monest 5 (und). ine

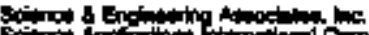

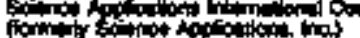

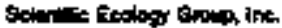

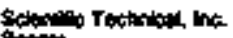

state

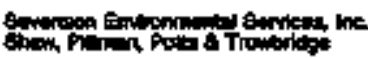

Shaphed iner, ine

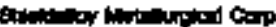

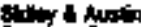

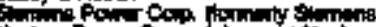
thench

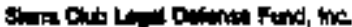

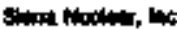

\begin{tabular}{|c|c|}
\hline 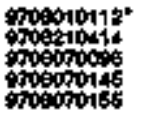 & 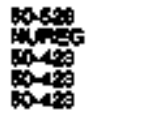 \\
\hline 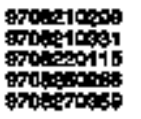 & 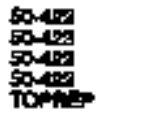 \\
\hline 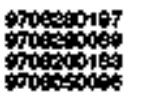 & 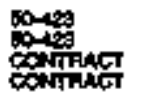 \\
\hline
\end{tabular}

orowos:

groterat 4

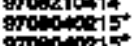

grocomatis

quoputi.

pot cont:

molotict

Hotacts:"

5 thot

riosich

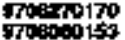

aropototo

97milotip

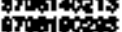

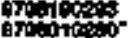

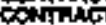

Contract

istis:

sol

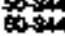

50034

Torate

ofe

언서웅

onto

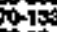

7210

Fon.

10345.

S040

s.4.

97006?

(7)

70.015015.

40-1162

40-1할

419.84

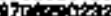

나요

Fropropes

Fin

Fon

ing

topis?

4ropoor

retion

ortolitios

FCRF?

wark

M12.

ofordeonz.

sconson?

oportons

TCPF

Topitis

10120

ofotidis

서영

sponas

powns

opteztos.4

Prit

A6x

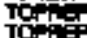

ontion

sropsosut

spoling

minco.

merpos

spontiont

gincides

ofidine?

ofispos

gonanos

thenomitos

c.ing

pototos

growats

protions

pospos.

कTh40010

xomix

satas

7012

Thato

Topipes

reto

colpow

sons

하눙

oft?

60요

pes

orat

past

monchorer 50-25

50.95

monetor

9rotistor 5015

gropenor sors

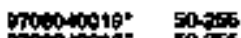

motion:-

pmoposis
ATron AFiLAnOW

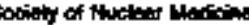

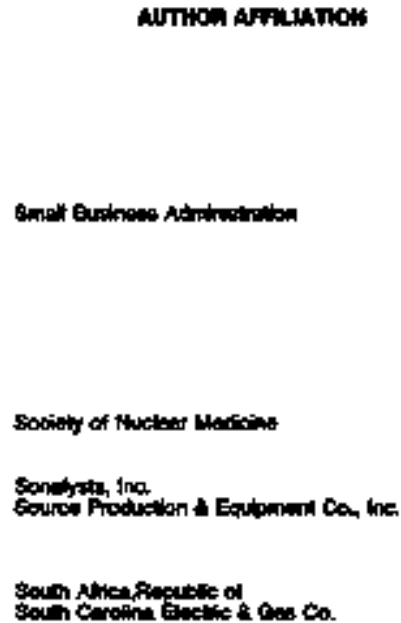

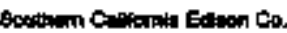

$\mathbf{P}$

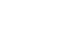

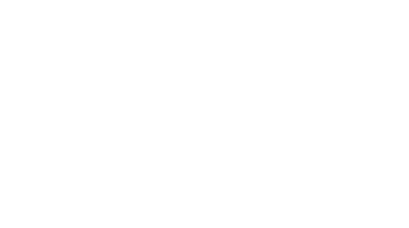

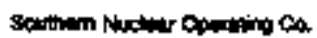

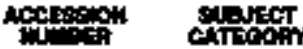

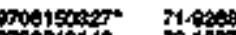

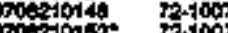

miog?

roes

(1)

incosion

(1)

onction

omotidons?

moegong

Fin

9701000

onosidoso

preserote.

The 1 oros

\$

onters 14

9xisactr

xionosis

1700006

rocosos.

susi 100

721007

ConTanct

ONminc

№n

conitar

Cowinhat

कonirinat

conirint

octipract

Reero

CONTMCT

71-10

11-10;

mosicion*

poertoct

Toentris:0

protionos

toum

soren

rate

Costo

sorstanos

70.010101

rocous.

Fod

50 \%5:5

tot

sols

coses

conson

50031

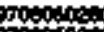

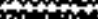

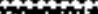

groting.

ondons

nitis.

monom

ond

00000

groeoroot:

970000123

97000123

97000124

10501

10-31

set

tot

14-0.

sost

10.5

solo:

som

thosi

sons

sons

9708410129

Toes1015:

9 oger

motitope

cost

100.1

ordesiono

00 irioto

9rucecos.

vipent?

Notente

onowase

(1)

97100012

(ron

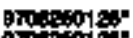

notors.

mitapto

oratopos

sots

0

so.s.

10<01

50.9

osis

$0 \mathrm{sin}$

10.

503

oris

tor

(x)

omanonit

cors:

oning

20.7no

spostopir.

05

$0-44$

$00-4$

970000005

9700000139

Fits rog

growsocs

506

tori

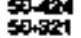

son

s70er 30040

gior

170

\%ol

$50-821$

Hin

然称

970mmoes

970012025

97001300

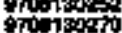

songst

00

窝安 


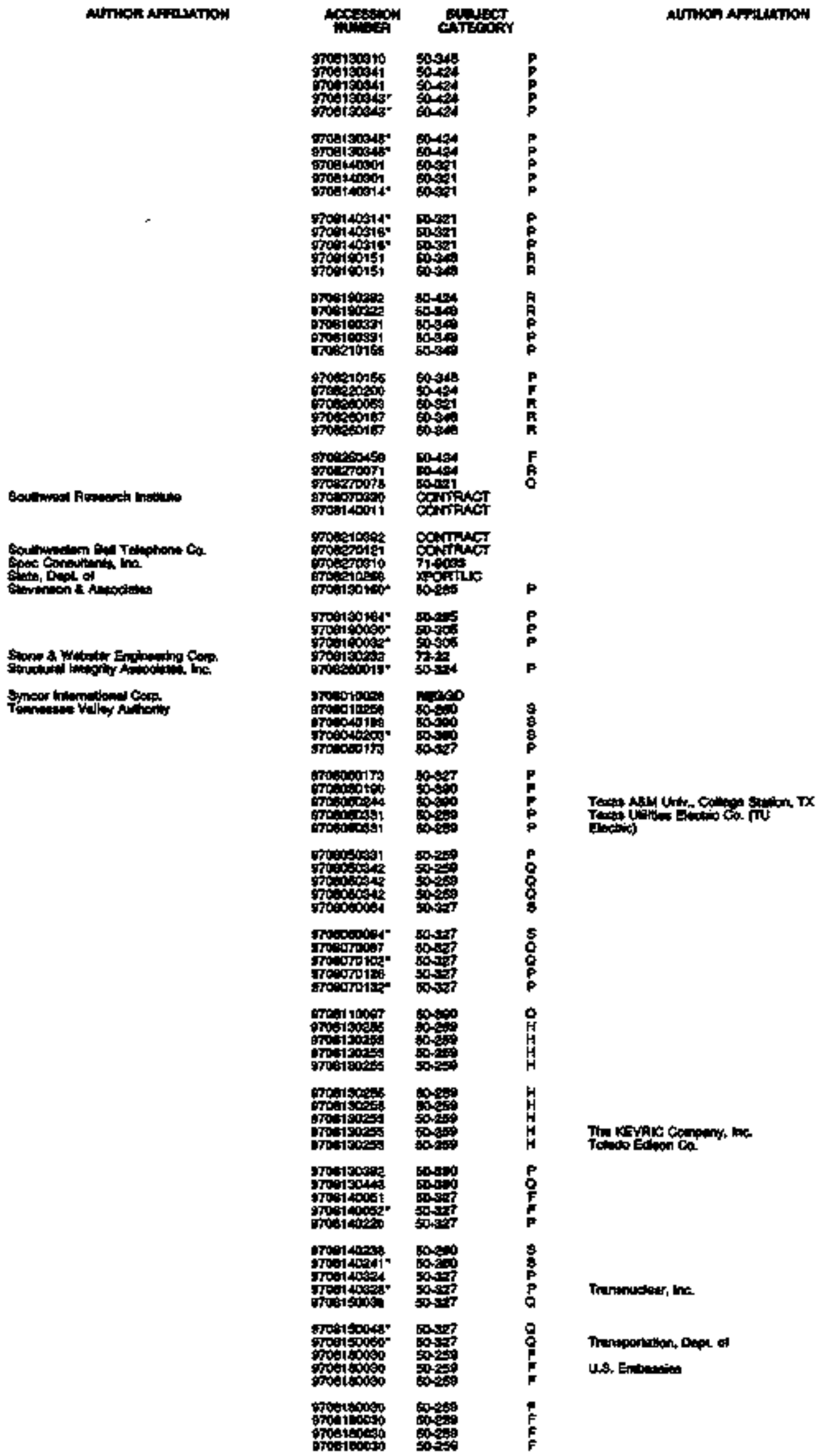




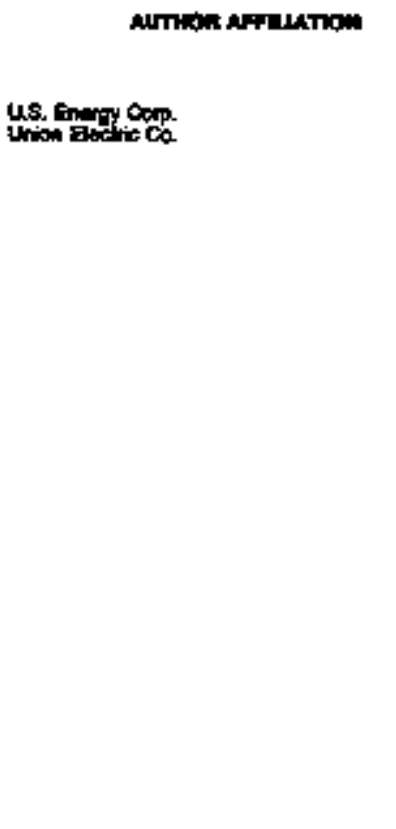

Uniog of Consemid Selomite.

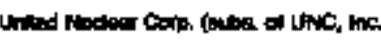

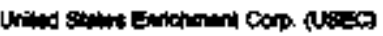

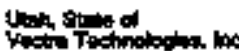

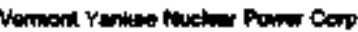

\begin{tabular}{|c|c|c|c|}
\hline 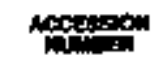 & clivect & & 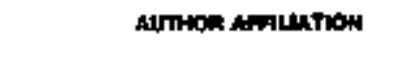 \\
\hline 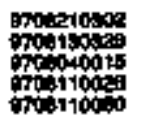 & 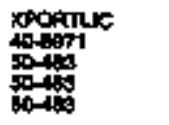 & $\frac{9}{8}$ & 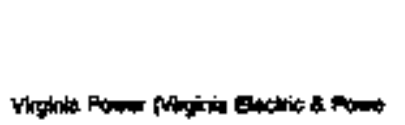 \\
\hline 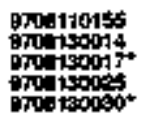 & 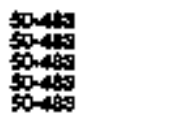 & $\begin{array}{l}\text { F } \\
p \\
p \\
p\end{array}$ & (क) \\
\hline 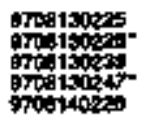 & 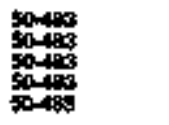 & $\begin{array}{l}p \\
p \\
p \\
p \\
p\end{array}$ & \\
\hline 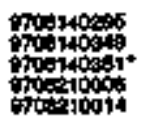 & 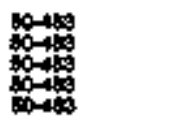 & $\begin{array}{l}p \\
F \\
F\end{array}$ & \\
\hline 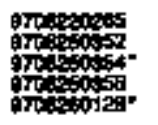 & 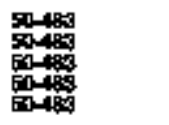 & $\begin{array}{l}\mathbf{s} \\
\mathbf{p} \\
\mathbf{p} \\
\mathbf{p} \\
\mathbf{v}\end{array}$ & \\
\hline 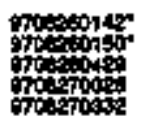 & 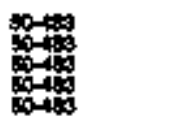 & $\begin{array}{l}v \\
y \\
5 \\
\mathbf{p} \\
p\end{array}$ & \\
\hline 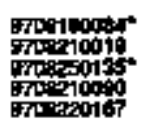 & 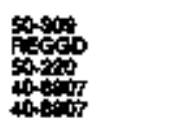 & $\begin{array}{l}p \\
0\end{array}$ & \\
\hline 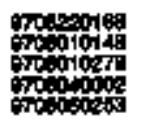 & 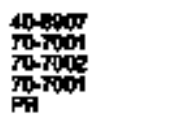 & & 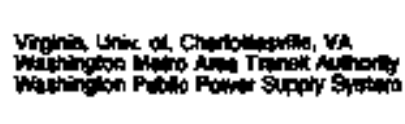 \\
\hline 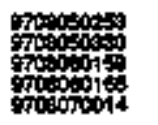 & 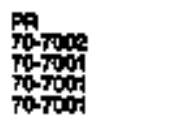 & & \\
\hline 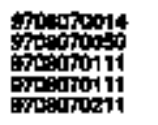 & 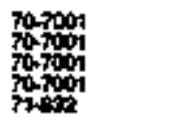 & & \\
\hline 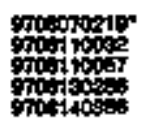 & 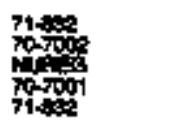 & & 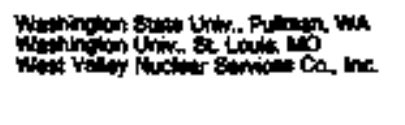 \\
\hline 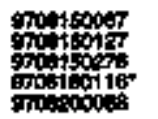 & 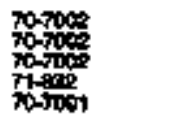 & & 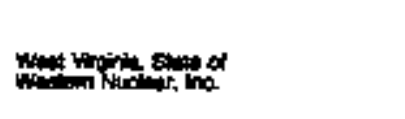 \\
\hline 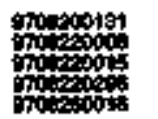 & 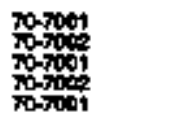 & & Whinthowave Bectic Corp. \\
\hline 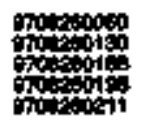 & 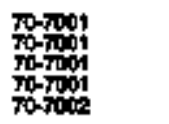 & & \\
\hline 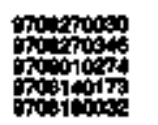 & 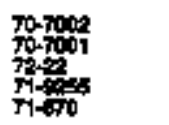 & & \\
\hline 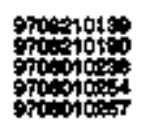 & 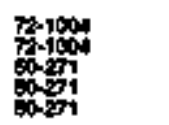 & $\underset{\mathbf{p}}{\mathbf{p}}$ & \\
\hline 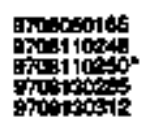 & 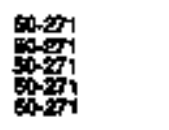 & $\begin{array}{l}\mathbf{s} \\
\mathbf{p} \\
\mathbf{p} \\
\mathbf{p}\end{array}$ & \\
\hline 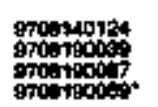 & 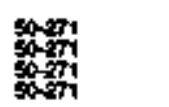 & $\begin{array}{l}\mathbf{s} \\
\mathbf{p} \\
\mathbf{5} \\
\mathbf{5}\end{array}$ & \\
\hline
\end{tabular}

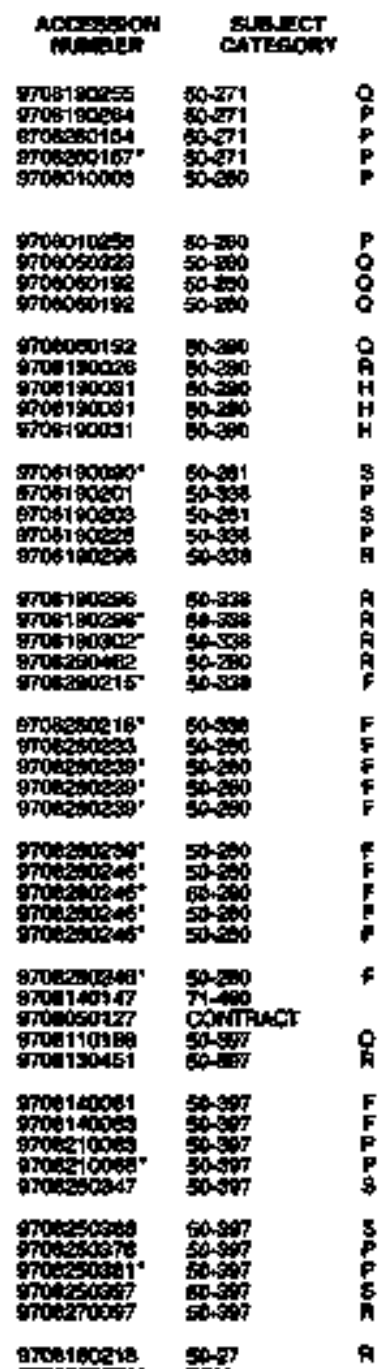

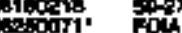

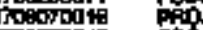

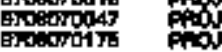

orcotiotod pad

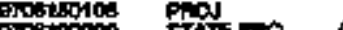

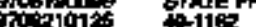

atsolotion

oxpoloom sto

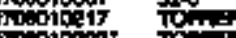

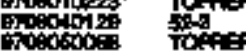

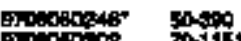

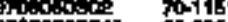

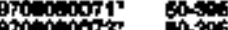

ronotis

monest

proporoc.

oftopooto

grteorosss

grotions

9xicionse

optisote

orostopese

otion

Toin

9 povionat

ortatons:

omolyos.

crotisto

sage

70.1161

6.

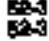

sowas

$\log _{0 \rightarrow 0}$

$\lim _{0 \rightarrow 3}$

53

58

50.4

sat

5

$\sin _{2}$ 


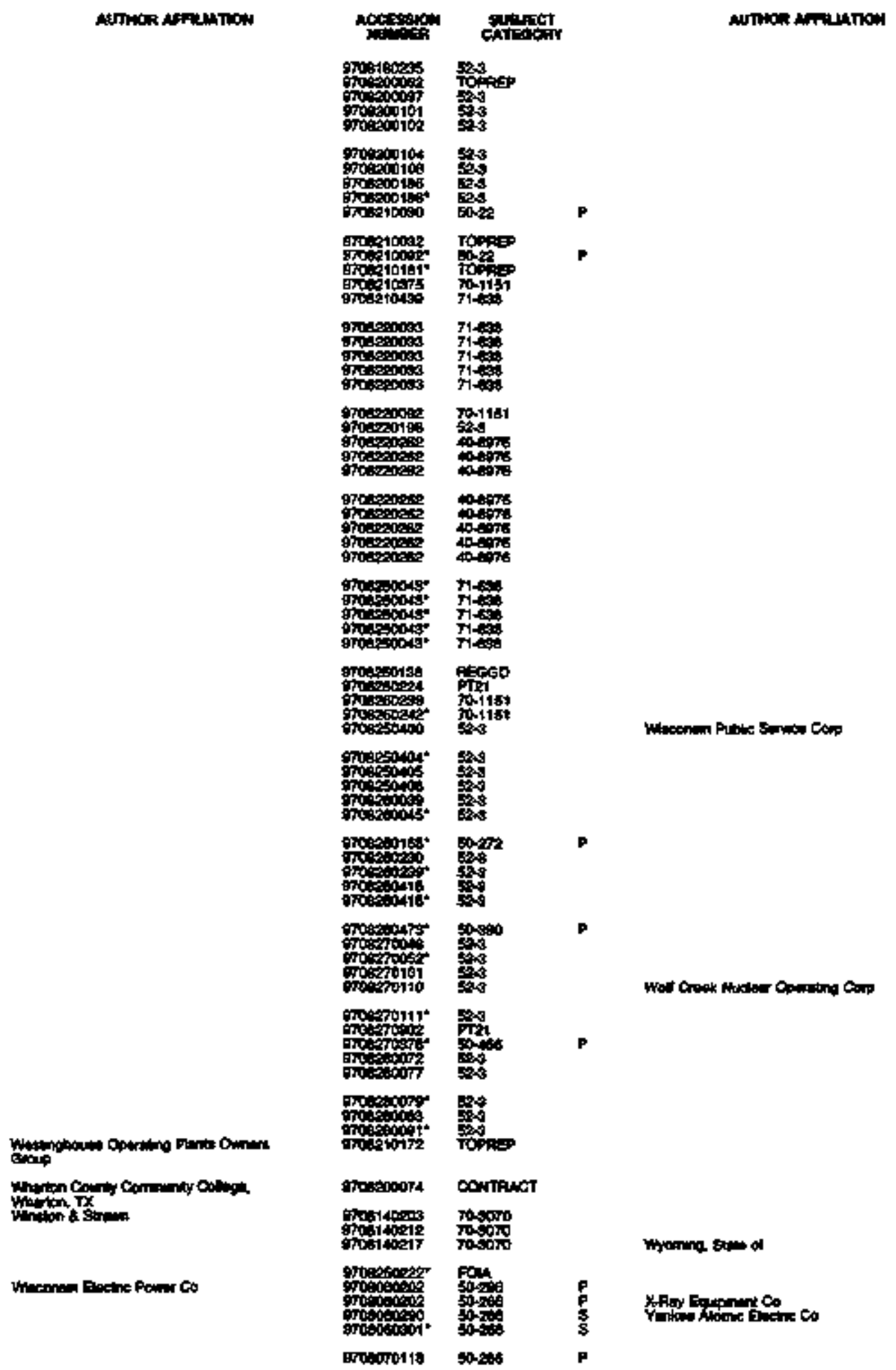

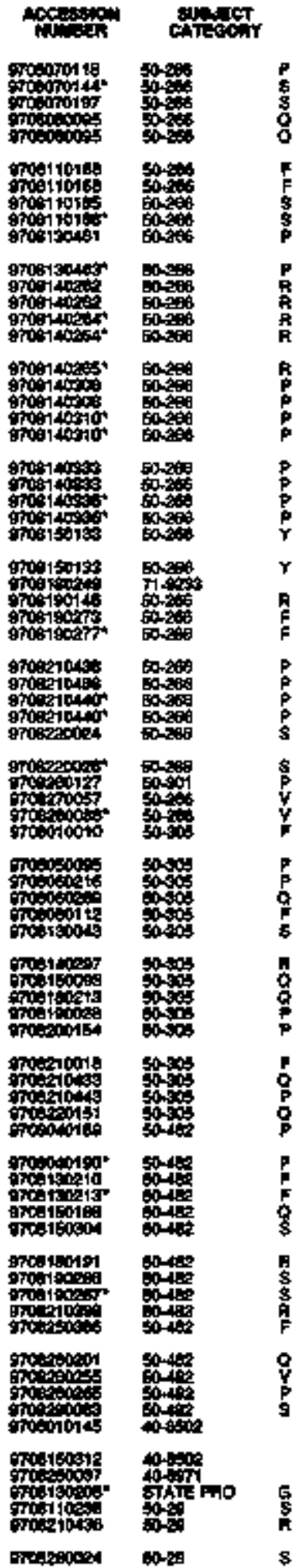




\section{REPORT NUMBER INDEX}

The subject category column in this index gives the docket number and the symbol for the cetegory of information within that docket, for exampla,50-233 P. The docket subiect categories are defined in Apperdix A.

Subject categories for nondocketed iterns are also entered in this column by symbol; for example. ACRS.
These categories are defined in Append|x B and listed in the contents. An asterisk following the accession number in this index indicates that the document is an enclosure to enother document. To obtain the accession number of the principal document undex which the encloesire is listed, fefor to the index entitied "Cross Referenoe of Enclosures to Principal Documents."

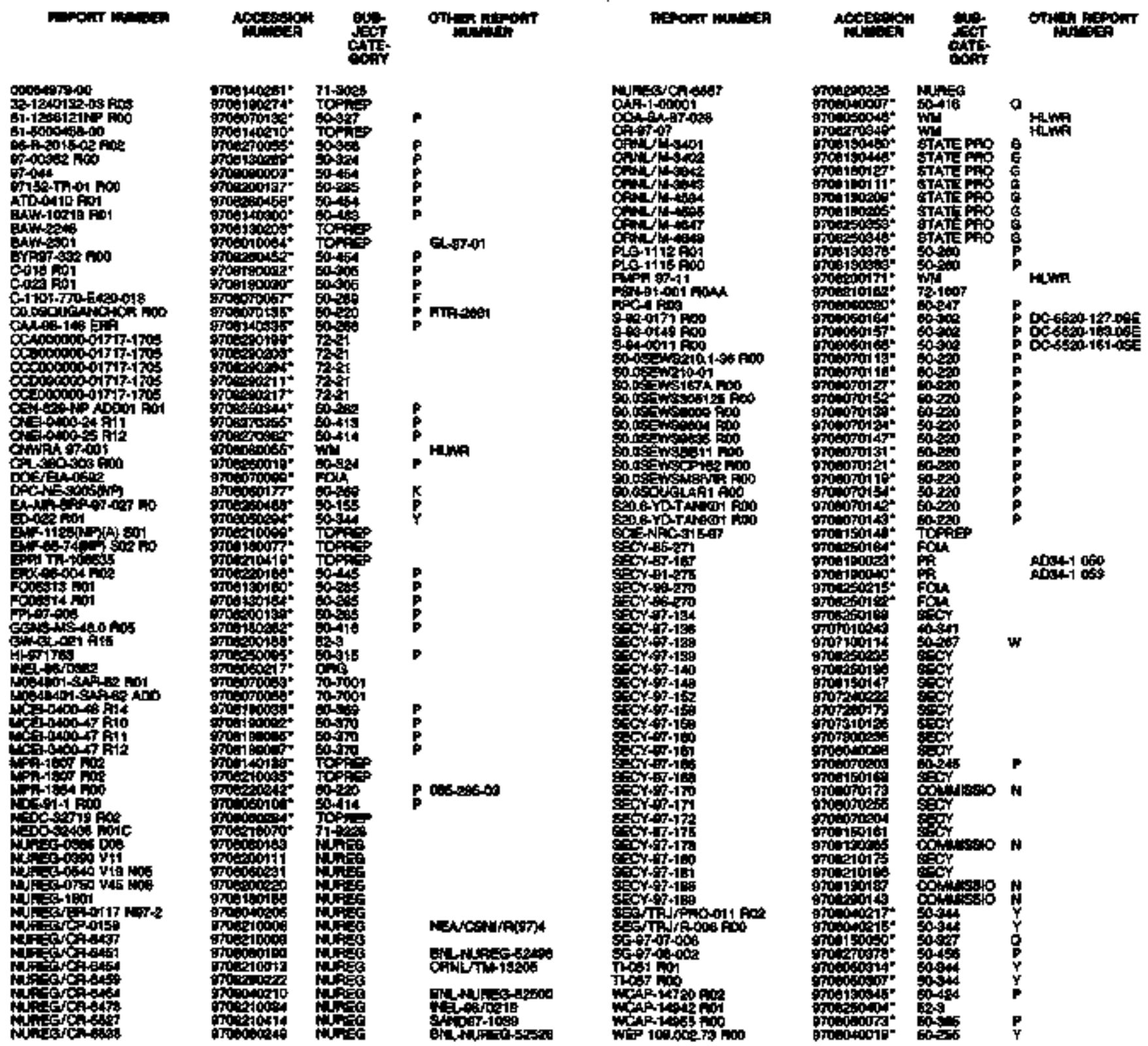




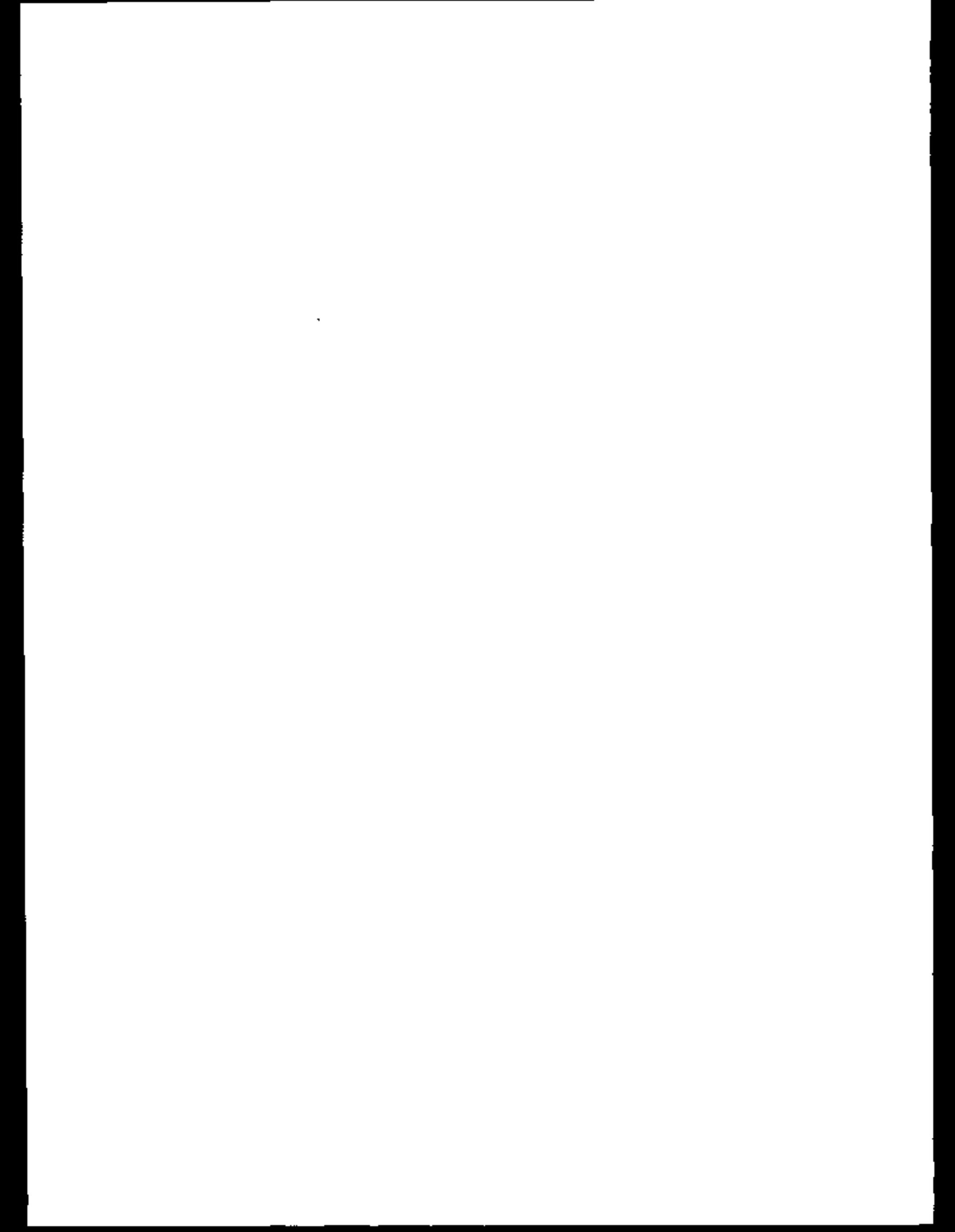




\section{CROSS REFERENCE OF ENCLOSUAES TO PRINCIPAL DOCUMENT}

Item listed as enclosures are those indicated by an asterisk followirg the accession number in the indexes. The second column gives the accession number of the principal document under which the enclosure is listed. Krowing the accession number of the principal document, the date issued,and the calegory enebles locating the tite fisting of the enclosure.

The subject category column in this index gives the docket number and the symbol for the category of information within that docket; for example, 50-320P. The docket subject categories are deflned in Appen. dix A.

Mcotoptis Math

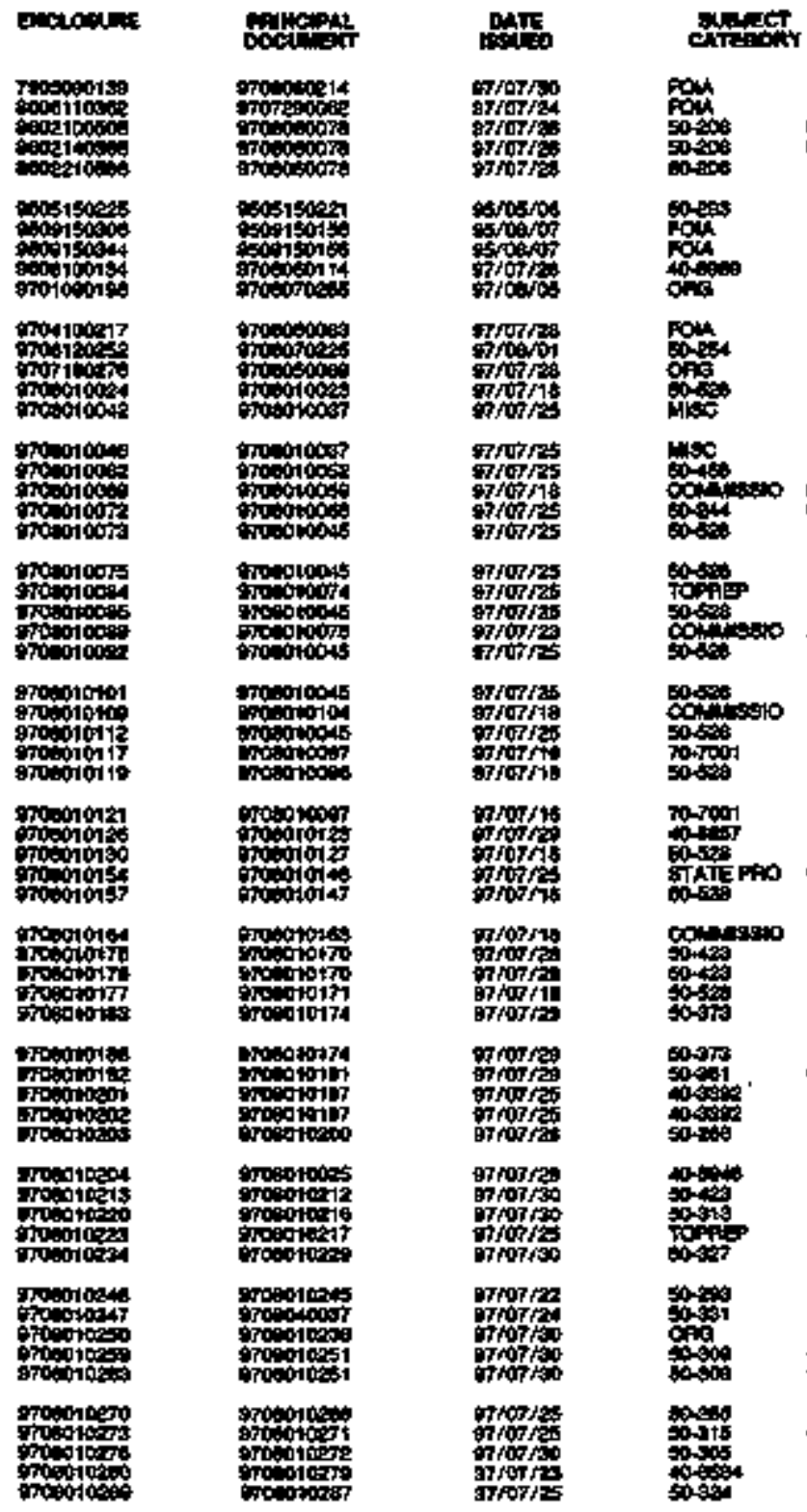

Subject categories for nondocketed items ore also entered In this column by symbol; for example, ACRS. These categories are defined in Appendix B and listed In the Contents.

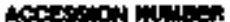

\begin{tabular}{|c|c|c|c|}
\hline 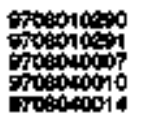 & 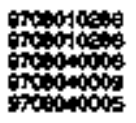 & 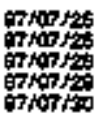 & 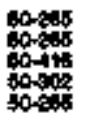 \\
\hline 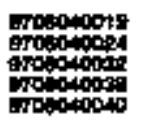 & 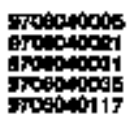 & 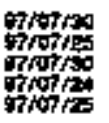 & 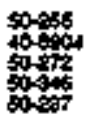 \\
\hline 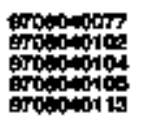 & 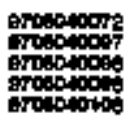 & 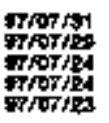 & 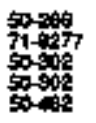 \\
\hline 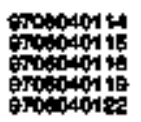 & 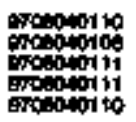 & $\begin{array}{l}\text { morka } \\
\text { mporta } \\
\text { shorkat } \\
\text { mortad }\end{array}$ & 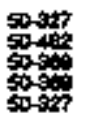 \\
\hline 013 & 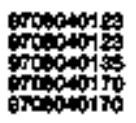 & 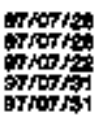 & $\begin{array}{l}\operatorname{sen} \\
\operatorname{son} \\
\text { soted } \\
\text { sosito }\end{array}$ \\
\hline 我里 & 象 & 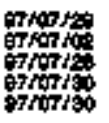 & 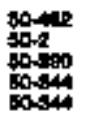 \\
\hline 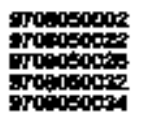 & 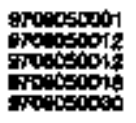 & 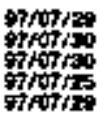 & 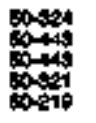 \\
\hline 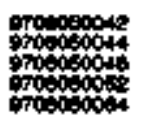 & 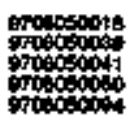 & 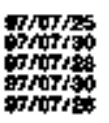 & 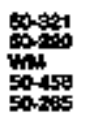 \\
\hline 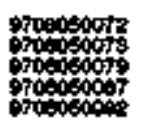 & 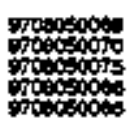 & 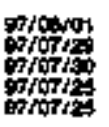 & 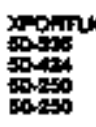 \\
\hline 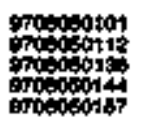 & 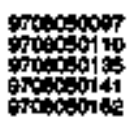 & 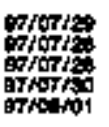 & 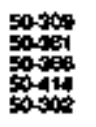 \\
\hline 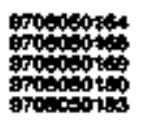 & 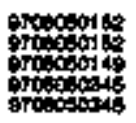 & 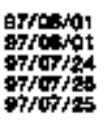 & 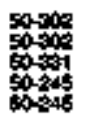 \\
\hline 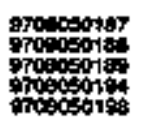 & 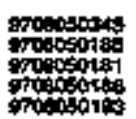 & 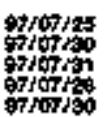 & 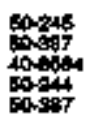 \\
\hline
\end{tabular}


205 CROSS REFEREMCE Of ENCLOSURES TO PRINCIPAL DOCUMENTS

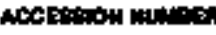

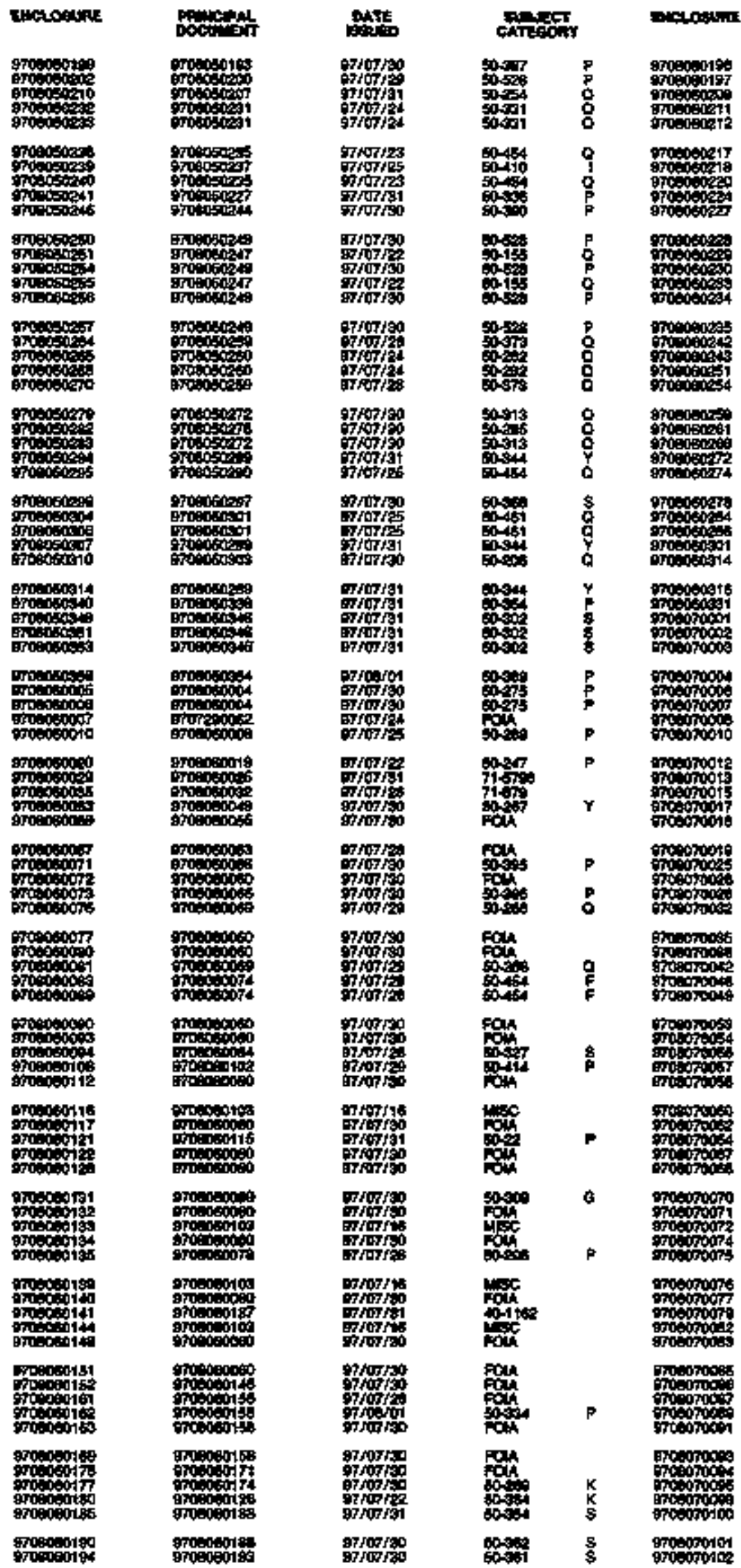

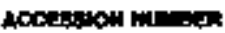

\section{Dopdripint}

매대.

Sroverets

rosogors

9000005

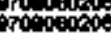

oroposog 15

9 ropepors

popocipt

mocosita

motiont

poitolet

oropeds

000.

octong

sposogocat

20000214

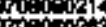

Broboticis

propenets

gropetits

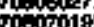

poingorit

Fanecosos

Cur

ofint

omation

$4+000$

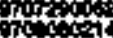

tritation

Omoconte

for

ontonte

orvoricta

10.6.

oratoropers

ofocidid

orcooveres 14

arconent4

$8 x+0,4914$

andorotes

Proorocos

Find

ontorot

gnowosos

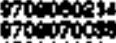

pontos

proprose

oropto

Tointion

wortot

opoopopen

Thiof

rrooneng

gmo.0.007

roopor

roteot

oroeprops

Fontor

orotint

prootrotos

oropocenes
प्रTARण

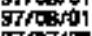

क्षा fort?

कrupto

prrasines

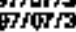

gropra

67107/30

970,13

7 NOF 30

ofrows

grrozso

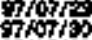

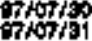

ㅁofrso

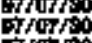

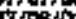

17asig

$9707 / 30$

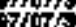

Whophe

orouses

trosio

Fnore

ONTH

ortopiso

0107

Fipra

gropto

optopos

21070

가요

971

oporsso

9 owe

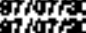

9700

grot

\%

s

oxpho

$\Rightarrow x$

orop/

orkases

or

oroplos

grozras

Iris

Horse

orfaris

orpors

Tho

Hring

蒠打

南10

Haro

orowo

$07 / 0730$

onodo

Frops

rowas

oroyoron axturest

5Pसक

500 .

$50 \%$

40

Cons...

pul...

50

Dora 0

40.t.

Fous

502

rch

xhs

40 .

gow

oind

Fon

T0ma

50.70

sos

PAO

rom

EOra

rom

엇

rou

Eort

rom

gat

corse

Fin

sin

rot

sol.

70001

rot

tot

Notion

socti

co

sot 0

no

74000

rop

son 0

soper 0

roph

sons

Fos

000

Dot

Foy.

sol

Dat.

ROM

$\operatorname{lot}_{0 \rightarrow 7}$ 


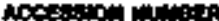

\begin{tabular}{|c|c|c|c|c|c|}
\hline 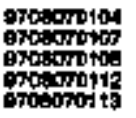 & 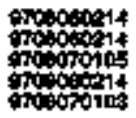 & 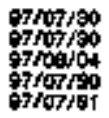 & $\operatorname{low}_{0 \rightarrow 20}$ & $\begin{array}{l}5 \\
\mathrm{P}\end{array}$ & 我电 \\
\hline 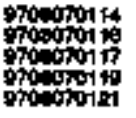 & 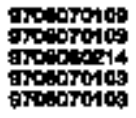 & 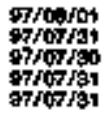 & 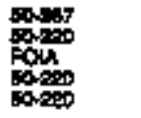 & $\begin{array}{l}\mathbf{s} \\
\mathbf{p}\end{array}$ & 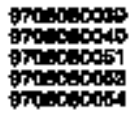 \\
\hline 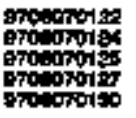 & 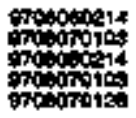 & 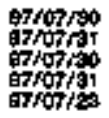 & & $\begin{array}{l}p \\
p\end{array}$ & 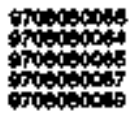 \\
\hline 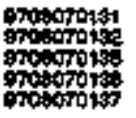 & 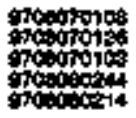 & 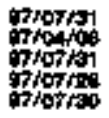 & 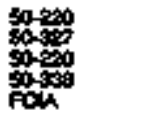 & $\begin{array}{l}\mathbf{p} \\
\mathbf{p} \\
\mathbf{P} \\
\mathbf{p}\end{array}$ & 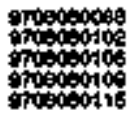 \\
\hline 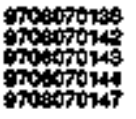 & 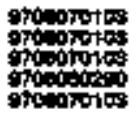 & 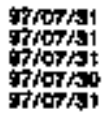 & 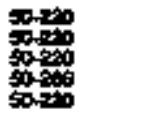 & $\begin{array}{l}p \\
p \\
p \\
p \\
p\end{array}$ & 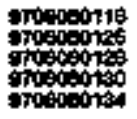 \\
\hline 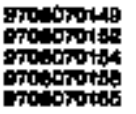 & 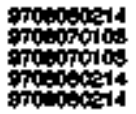 & 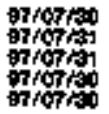 & & : & 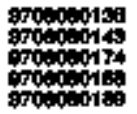 \\
\hline 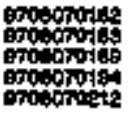 & 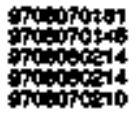 & 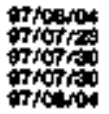 & 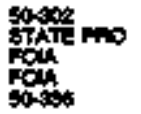 & p & 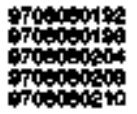 \\
\hline 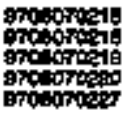 & 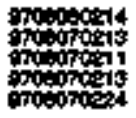 & 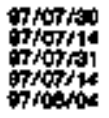 & & $\begin{array}{l}\mathbf{P} \\
\mathbf{P}\end{array}$ & 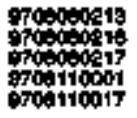 \\
\hline 24 & 象o & 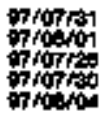 & & $\mathbf{T}$ & 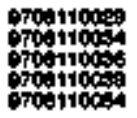 \\
\hline & tep & 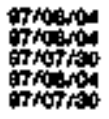 & & $\mathbf{w}$ & 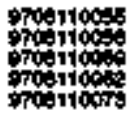 \\
\hline & 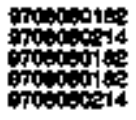 & 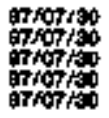 & & $\begin{array}{l}\mathbf{P} \\
\mathbf{P}\end{array}$ & 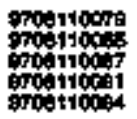 \\
\hline $\begin{array}{l}272 \\
70 \\
70\end{array}$ & 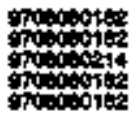 & 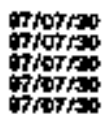 & rou & & 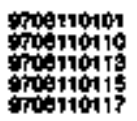 \\
\hline 然 & & 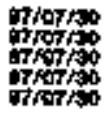 & & 0 & 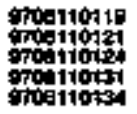 \\
\hline 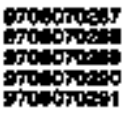 & 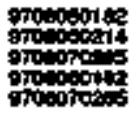 & 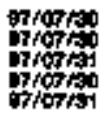 & 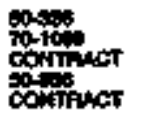 & 0 & 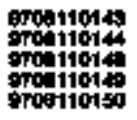 \\
\hline 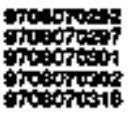 & 象 & 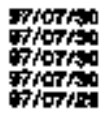 & 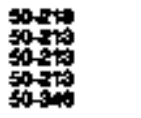 & $\frac{a}{9}$ & 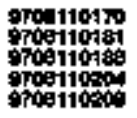 \\
\hline 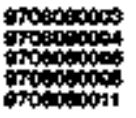 & 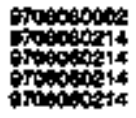 & 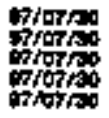 & 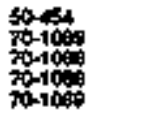 & $\mathbf{P}$ & 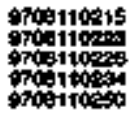 \\
\hline 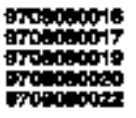 & 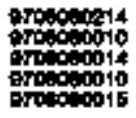 & 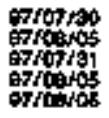 & 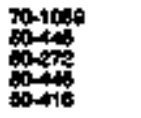 & $\frac{a}{\phi}$ & 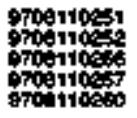 \\
\hline 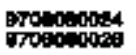 & 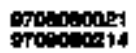 & $\begin{array}{l}\text { erropsa } \\
\text { protas }\end{array}$ & $\frac{00-2.2}{x-1009}$ & $\theta$ & grost10ase \\
\hline
\end{tabular}

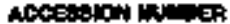

\begin{tabular}{|c|c|c|c|}
\hline 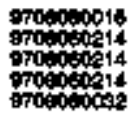 & 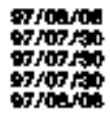 & 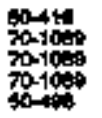 & 0 \\
\hline 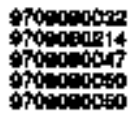 & 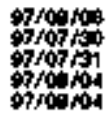 & 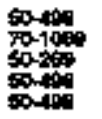 & 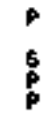 \\
\hline 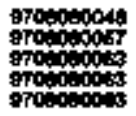 & 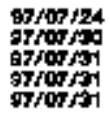 & when & $\begin{array}{l}\mathbf{g} \\
\mathbf{p} \\
\mathbf{p}\end{array}$ \\
\hline 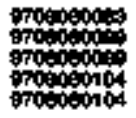 & 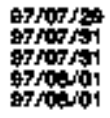 & 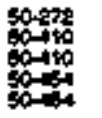 & $\begin{array}{l}\mathbf{y} \\
\mathbf{p} \\
\mathbf{p} \\
\mathbf{q}\end{array}$ \\
\hline 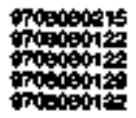 & 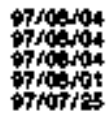 & 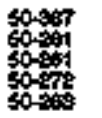 & $\begin{array}{l}\mathrm{s} \\
\mathbf{p} \\
\mathbf{p} \\
\mathbf{p}\end{array}$ \\
\hline 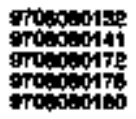 & 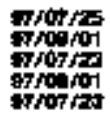 & 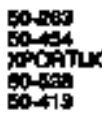 & $\begin{array}{l}\rho \\
0 \\
0\end{array}$ \\
\hline 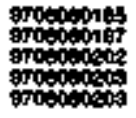 & 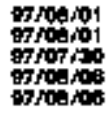 & 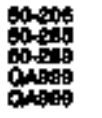 & $\begin{array}{l}\text { Q } \\
\text { 웅 }\end{array}$ \\
\hline 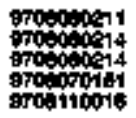 & 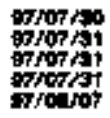 & 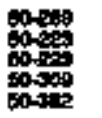 & $\begin{array}{l}\mathbf{H} \\
\mathbf{p} \\
\mathbf{p} \\
\mathbf{8}\end{array}$ \\
\hline 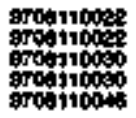 & 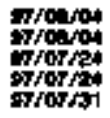 & & $\begin{array}{l}f \\
f \\
\mathbf{p}\end{array}$ \\
\hline 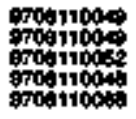 & 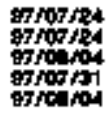 & $\begin{array}{l}00-54 \\
00-54 \\
00-140 \\
00-40 \\
00-40\end{array}$ & $\begin{array}{l}p \\
\dot{p} \\
\dot{s}\end{array}$ \\
\hline 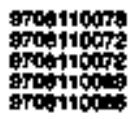 & 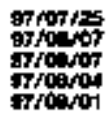 & 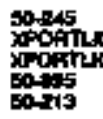 & ค \\
\hline 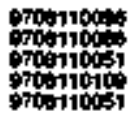 & 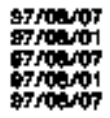 & 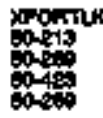 & $\begin{array}{l}\mathbf{s} \\
\mathbf{p} \\
\mathbf{p}\end{array}$ \\
\hline 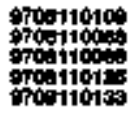 & 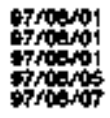 & 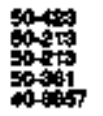 & $\begin{array}{l}\text { P } \\
\text { 皇 } \\
8\end{array}$ \\
\hline 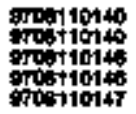 & 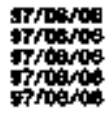 & 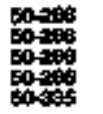 & $\begin{array}{l}p \\
p \\
p \\
p\end{array}$ \\
\hline 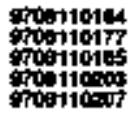 & 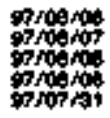 & 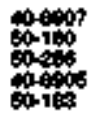 & $\begin{array}{l}\mathbf{P} \\
\mathbf{8} \\
\mathbf{P}\end{array}$ \\
\hline 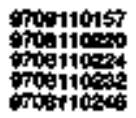 & 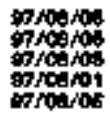 & 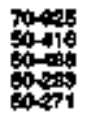 & $\begin{array}{l}\mathbf{p} \\
\mathbf{p} \\
\mathbf{s}\end{array}$ \\
\hline 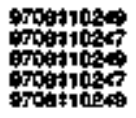 & 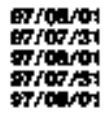 & 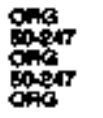 & P \\
\hline $\begin{array}{l}\text { 970d1102s } \\
\text { 9roht1der }\end{array}$ & 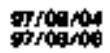 & gons & $s$ \\
\hline
\end{tabular}


268 CROSS REFERENCE OF ENCLOSURES TO PHWCIPAL DOCUMENTS

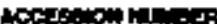

\begin{tabular}{|c|c|}
\hline 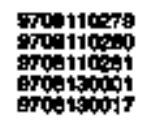 & 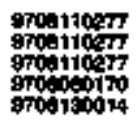 \\
\hline 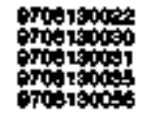 & 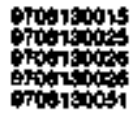 \\
\hline 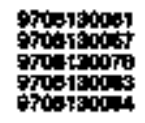 & 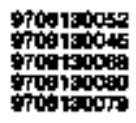 \\
\hline 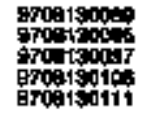 & 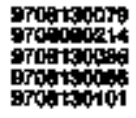 \\
\hline 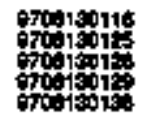 & oponos \\
\hline 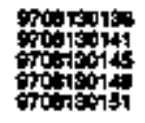 & 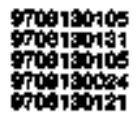 \\
\hline 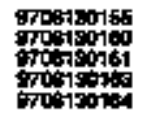 & 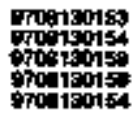 \\
\hline 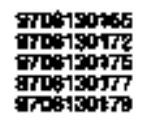 & 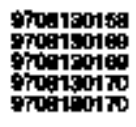 \\
\hline 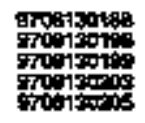 & 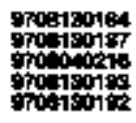 \\
\hline 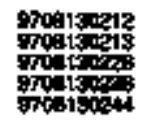 & 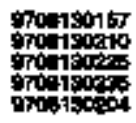 \\
\hline 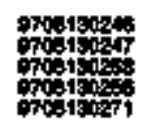 & 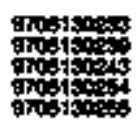 \\
\hline 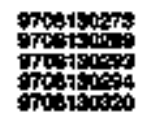 & 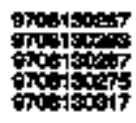 \\
\hline 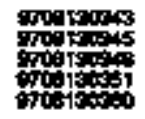 & 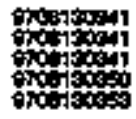 \\
\hline 6 & 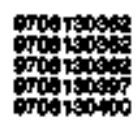 \\
\hline 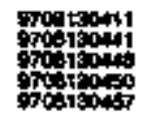 & 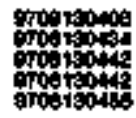 \\
\hline 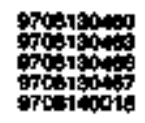 & 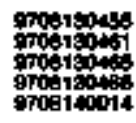 \\
\hline 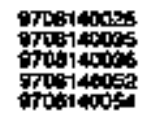 & 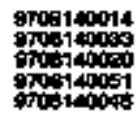 \\
\hline prosto & 90 \\
\hline
\end{tabular}

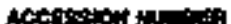

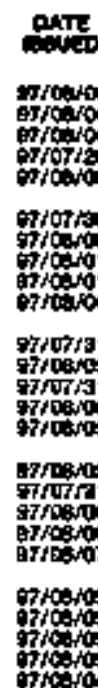

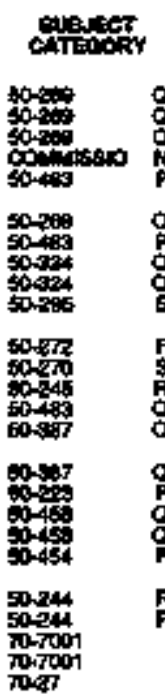

rustarite

\section{Doctiritint}

\section{poivoce}

spoaratis

9rotioling

groatiotos

9r0\%-10123

Oropido1sa

Brog-14150

9roes 1016?

9rotiris

opogitu181

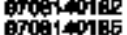

orto하에영

srovforez

ro8140198

EJD8140.74

Erositorio

rositures

ortortos

ofotor

offorta

BTRB:01

groviou

970Bat

prosid

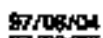

Trosion

miars

Firtzas

sfroska

97005

Wirso

क्रrut

siogsos

Tipsos

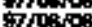

कropti

97somat

provo

oring

orovos

mrowas

$970 \%$

itopo?

ofyor

Troand

S7lowe

710

pitiona

optotios

(ation

tratos

ortor

gatow

ypts

offoris

orjyos

Trots

ritor

omotit

srowit

spoeros

opoor

n7poorod

srow1s

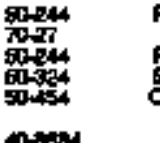

504

propis

pos

orai

0141

(a)

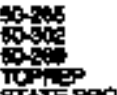

STh

(203

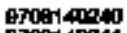

E700140241

67091401

$\sin 400$

orow4024.

Eroplasia

Fropict 4029

emopitiog

कpoprazia

proptriter

groproxion

gropeosts

ropuosid

cropiosing

9rostased

opopers

ompiose

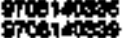

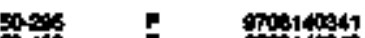

ons.;

$\sin$

Aate.c.

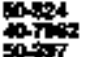

5040

50-4at

9-4a

50201

rike

50

Eve0

ond

gristios

9rictions?

oftolditis

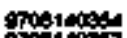

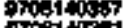

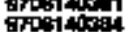

Exatisusa

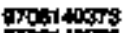

orositusin

of

Tog140

rop

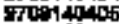

Trostito?

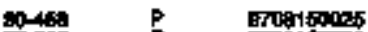

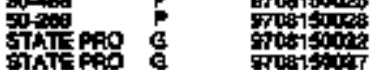

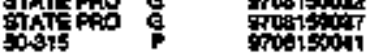

sots

慗家

irsis

spoes sopar

ocis

podisos

orot 1000

NFrs

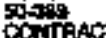

Eximation

0

ixts mosistomo

17.

Tod

grodituog

9roe140118

9709140129

970914014

groelapos

9roe140-14

oroe14unto

Tros14014

9rodicotas

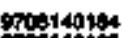

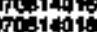

ocipos

(1)

rontinen

930914000

9rodictor:

Toditre?

oresiatares

Protatera

Prod 10:

Thereats?

mol 140.

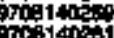

0414001

970140est

moind

90,1400

The 140

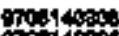

Len 405

rovistor

mo140ils

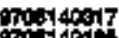

4 is

20

roonostest

roporates

arotyot

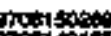

No14\%

mor 0.16

mot

c7atroy

1 -

(7)

pratiples

grog140705

godictor

6upinotes

aropine

mosison

ExDicion

Fos stos

grosisposo

9rosisond

sposisous

wosisops

omateon.

$000140 \mathrm{P}$

9001407

stodisont

6rop15006

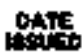

कrotor?

intortit

iroto

srosiot

strosus

97nopos

9707t

STNOF

orrosut

97 stor

97cotos

970stor

errorts

orropos

orropion

riorts

97tosion?

grposua

ofrosion

97 rosos

proser

grouph

optomer

proper

9700

$97 N$ is

grforitos

Fropor

9 pow

9ropo.

and

orfaty

orfora.

orforion

orfaryt

rsivo

proves

errowor

900

然市

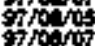

triarso

mpary

Tropror

Troeror

Trogros

Erovo

Proano

orfonow

optostor

Grover

$9707 / 7$

$9700 / 0$

$9700 / 2$

Plote

ortation

\$7

ortorto

optar

9 props

$97 / 040$

970

काNitz

oprarta

groarts

ctarts

40 셔에

Acis:

$50 \%$

STATE PRO $\mathrm{C}$

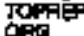

4000 ?

Co: 


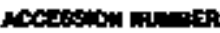

\begin{tabular}{|c|c|}
\hline 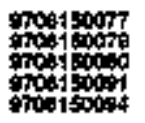 & 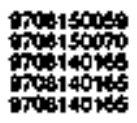 \\
\hline 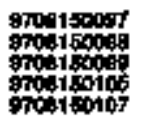 & 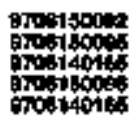 \\
\hline 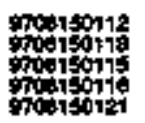 & onsopes \\
\hline 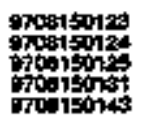 & 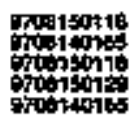 \\
\hline 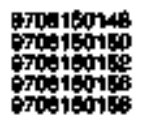 & 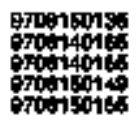 \\
\hline 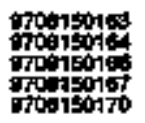 & 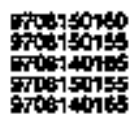 \\
\hline 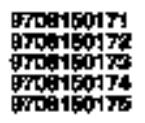 & 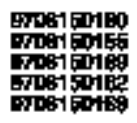 \\
\hline 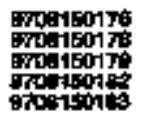 & 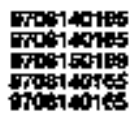 \\
\hline 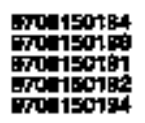 & 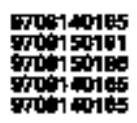 \\
\hline 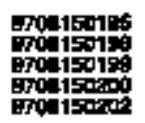 & 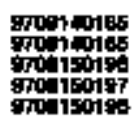 \\
\hline 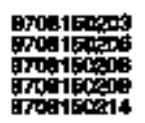 & 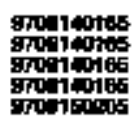 \\
\hline 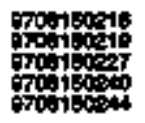 & 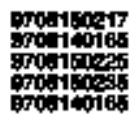 \\
\hline 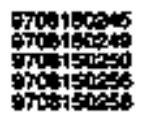 & 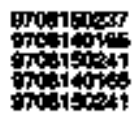 \\
\hline 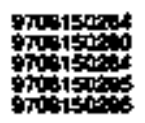 & 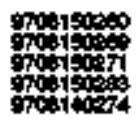 \\
\hline 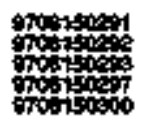 & 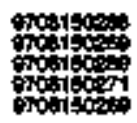 \\
\hline 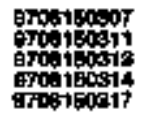 & 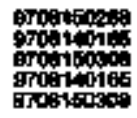 \\
\hline 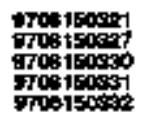 & 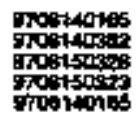 \\
\hline sposiso & sporsors \\
\hline
\end{tabular}

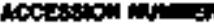

\begin{tabular}{|c|c|c|}
\hline 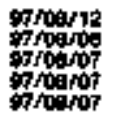 & 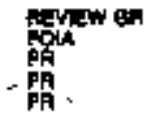 & $\boldsymbol{F}$ \\
\hline 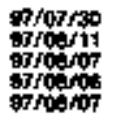 & 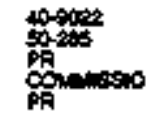 & $\begin{array}{l}\boldsymbol{O} \\
\mathbf{N}\end{array}$ \\
\hline 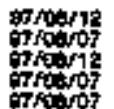 & 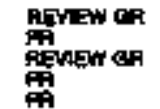 & $\begin{array}{l}\mathbf{P} \\
\mathbf{p}\end{array}$ \\
\hline
\end{tabular}

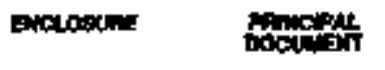

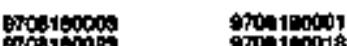

ongersoos

$\cos 2000000$

orisionit

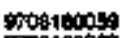

Trosiecos

208100005

Froticoor

arosigocos

$\operatorname{mos} 1000$

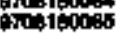

Hon

Froord

Froover

ghod

ghoor

sffor/3

orotor

$9 \operatorname{los}_{10}$

of

$9700 \%$

ororion

97/004a

970 10.

$97 / 0$. 104

जilivis

Proesor

oropis

oflowor

proupr

\%oul2

ondecis

otoento?

970um

ortorio:

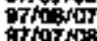

TAR

Thow

othosur

o7fostos

opoeros

orotor

protor

gyopiss

ard

ofowor

OTOSH

Actions

atorio

ition

gyescte

antso

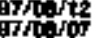

From

proers

9700

770012

Frow

77roerer

Tinos

Fras

gifost

prestor ofd

97000005

orosisocosp

moliopot9

701000

on 1000

stonecost

$\% 7001+006$

stontwors

970a19009

$970 \mathrm{coc0s}$

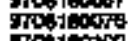

droctock

Troston

opsiento

जo

970015022

prifos

970104

0 ostog 62

oft

orostota

notionso

000

970010000

coobrops

Fotions

artotions

orotions

opepos

500

poorions

Fopopos

oroport

Fosectr

onotion

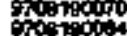

roonespon

irot

97001000

9romiporo

$97001+0001$

groespon

groptipita

970e1s0070

$970 \mathrm{c} 15007$

opoen:

9rode

shispiso

$97 \mathrm{~s} 1900 \mathrm{w}$

or 19015

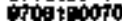

Tronpooso

70.

\%ण 190

grosiporgs
品陵

proyt1

prosion

prots

oftorit

orroes 12

grod

oprotico

now

poape

groas

ofrow

oncey

Trom

जo

(1)

Grospos

othor

Frow

For

How1

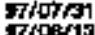

Foeris

Y7toes12

770813

770811

970\%11

7 Toser

ghov1

grows

काrotis

grroort?

9700m

g7ros

9700า

9roart2

9F 000

970075

95000

9700412

$97 \mathrm{Cu}$

$970 / 25$

$97700 / 73$

gropers

(1)

IITS

Grow

g7roarog

grodort

7 o.

ofort

spotis

moto

ondo

gntis

onors

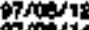

00.1

7 intis

onicy 14

PNow1

mit

07 ovitiz

groed 12

$8 \mathrm{NO} / 12$

ortod

(7)

growis c4ivist

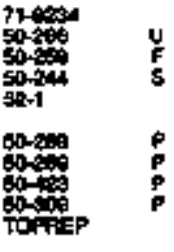

507000

SThTe pho

5049

s0+40

\$0.31

5010 a

OATA

710

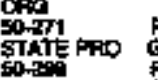

50.72

oncer

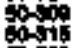

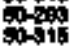

9040
60201

min

䓬

PA

萬

PA

$\mathbf{F r}$

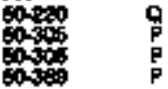

F

6-4a

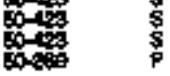

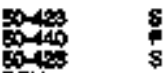

oors is

cotst s

fosin

5000 s

Fon

fork

架高

00

Fon

rop

row

ext

nate

$\vec{p}$ 


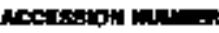

\begin{tabular}{|c|c|}
\hline 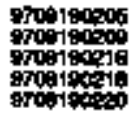 & 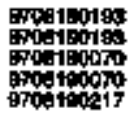 \\
\hline 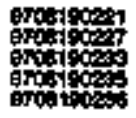 & 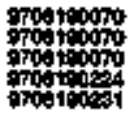 \\
\hline 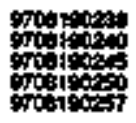 & 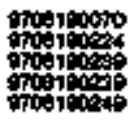 \\
\hline 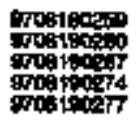 & 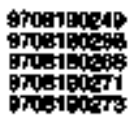 \\
\hline 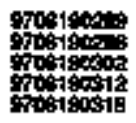 & 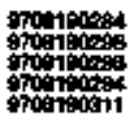 \\
\hline 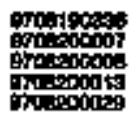 & 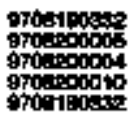 \\
\hline 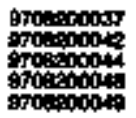 & 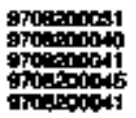 \\
\hline 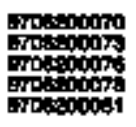 & 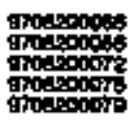 \\
\hline 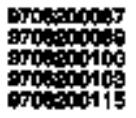 & 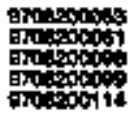 \\
\hline 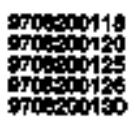 & propis \\
\hline 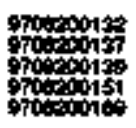 & 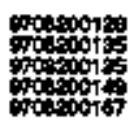 \\
\hline 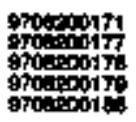 & 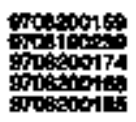 \\
\hline 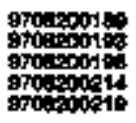 & 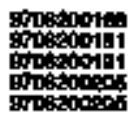 \\
\hline 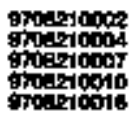 & 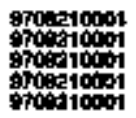 \\
\hline 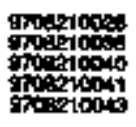 & 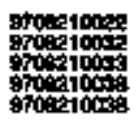 \\
\hline 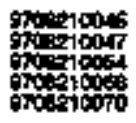 & 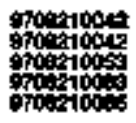 \\
\hline 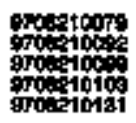 & 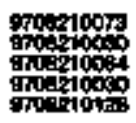 \\
\hline $\begin{array}{l}\text { gropecto434 } \\
\text { 9ropiciotso }\end{array}$ & 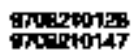 \\
\hline
\end{tabular}

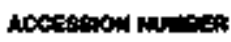

\begin{tabular}{|c|c|c|c|c|}
\hline PATE & triter & & |' & 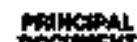 \\
\hline 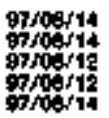 & 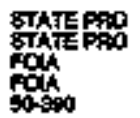 & g & 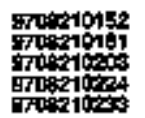 & 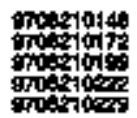 \\
\hline 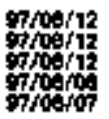 & 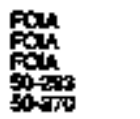 & 8 & 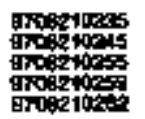 & 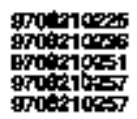 \\
\hline 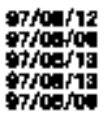 & $\begin{array}{l}\text { Fod } \\
50293 \\
50=44 \\
60=44 \\
50-75\end{array}$ & $\begin{array}{l}p \\
p \\
p\end{array}$ & 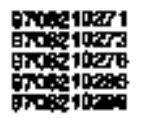 & 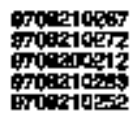 \\
\hline 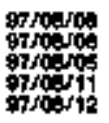 & 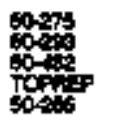 & $\begin{array}{l}\text { q } \\
\text { s } \\
\text { F }\end{array}$ & 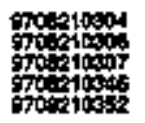 & 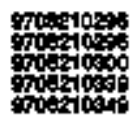 \\
\hline 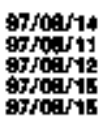 & 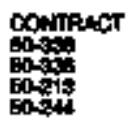 & 曷 & 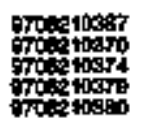 & 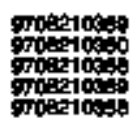 \\
\hline 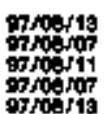 & 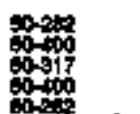 & $\begin{array}{l}v \\
\mathbf{S} \\
\mathbf{d} \\
\mathbf{3} \\
\mathbf{v}\end{array}$ & 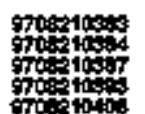 & 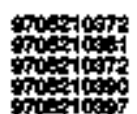 \\
\hline
\end{tabular}

axp:

10

ofrows

groort

errosit

Trasivi

9riogry

ตrosta

g7/para

9708 10

97/03718

97 ropth

9700

mort

ortertis

griosion

mroyt

giroming

orstom

ofoer 15

97 w

Fovi

9roeria

ogrops

groert

9700

proes 10

prow

How

orroesos

ontos

pionto

Fineria

opooris

orions

onto

rifo

The

oftos 12

oproos 15

ond

Freys

ar/oyise

曋

oprortit

Exroarts

Gods

Erow

rroarts

Eropiss

Fropers

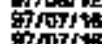

ofrovist

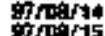

Fnos

Frow

sropos

prowis

Trow.

$9700 \mathrm{~s}$

9rrovita

opes

prostonto

patiplo

Trotipas

onse $G$ oroenons

ont \& 8

c⿻一𠃋十 8 क

so-si \& 97000047

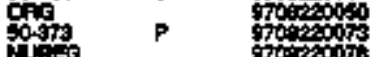

Nin $\rightarrow=0$

71 ose

7 ,

炛国

301.

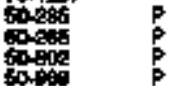

\begin{tabular}{|c|c|c|}
\hline 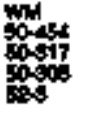 & $\stackrel{p}{p}$ & 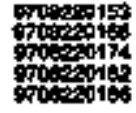 \\
\hline$\frac{x_{0}}{120}$ & $g$ & 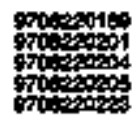 \\
\hline ; & $\begin{array}{l}\mathbf{8} \\
\mathbf{8} \\
\mathbf{8} \\
\mathbf{8} \\
\mathbf{8}\end{array}$ & 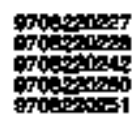 \\
\hline 254 & $\begin{array}{l}8 \\
8 \\
0\end{array}$ & 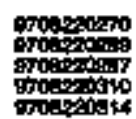 \\
\hline
\end{tabular}

croconose

or

sporation

grogand 10

Foredi23

mocis

morzonis

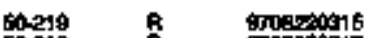

congis

0

tistes

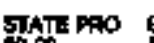

$\operatorname{lot}_{0 \rightarrow 3}$

opos

solest की
800000017

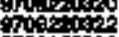

proesosen

grotion

poingosis

oricooss

90000054 sovion

tor

ortion

moeropose

ro.

7rotiog

srosonsons

9riteros

gntoses

grotisoosen

900000

orieriot

oposapion

polerent 27

polponst

opopason 47

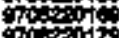

gposin

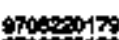

Hos.

pototo

onoment

sotion?

然omet

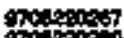

timax.

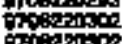

omezinso.

nowats

row

trowi

o7jos/10

Fino

atowi

shit

prow15

Injow

srowt

sistos

ordor

Fort

ore

s) 10

grog/1s

Grog/13

最rowit

Frow1

7 ow

oforid

orfoe/14

Frow

Fow

Irow

ofrous

grov

Exis

etsogio

orsoras

grove

7roino

ornodis

oroes

of 01

770

soets

星地,

9notense

ofoction

ortictast

grosezosa2 oroert

Tho 14

oprou

Troar

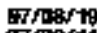

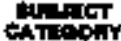

rand

niti

40+6: 1

70-1113

Exis

No.16

400

OONIPUT

10\%?

sow

10.5

离率

sorar 0

60.15

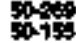

50 s.

5

10

ropes

tot

sor

5

stose

5030

7 然

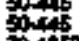

74-125

50 s.t.

50

40

som

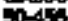

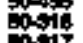

rom

nen

$50+5$

to

sos

oris

at

Bif

not

.010

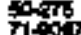

cosed

67 -481

t5it

contsisso

60

sot

孛实

contros

contsosto

Solte

50-110 


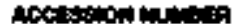

\begin{tabular}{|c|c|c|}
\hline 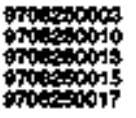 & 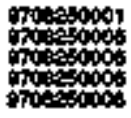 & 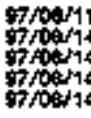 \\
\hline 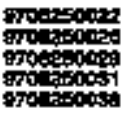 & 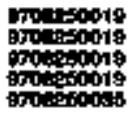 & 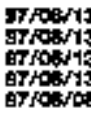 \\
\hline ons & 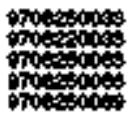 & opoors \\
\hline 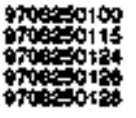 & 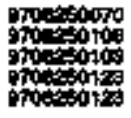 & 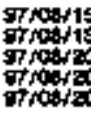 \\
\hline 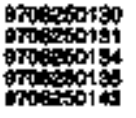 & 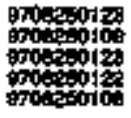 & 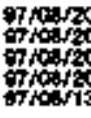 \\
\hline onto & 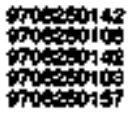 & 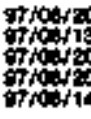 \\
\hline 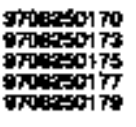 & 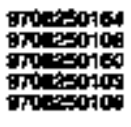 & 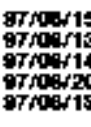 \\
\hline orto & 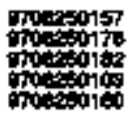 & 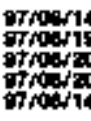 \\
\hline torion & 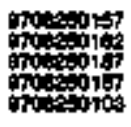 & orionis \\
\hline $\log _{0 \rightarrow 0}$ & 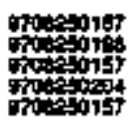 & 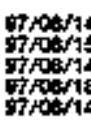 \\
\hline 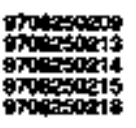 & 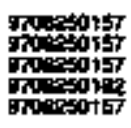 & 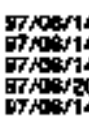 \\
\hline 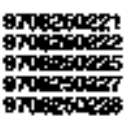 & 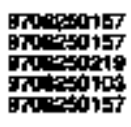 & 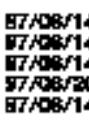 \\
\hline 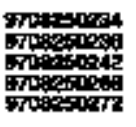 & 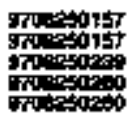 & 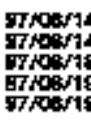 \\
\hline 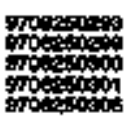 & 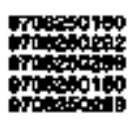 & 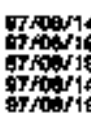 \\
\hline 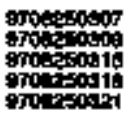 & 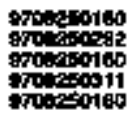 & 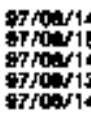 \\
\hline 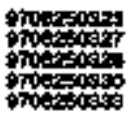 & 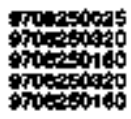 & 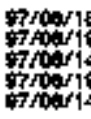 \\
\hline tos & 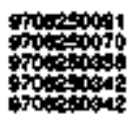 & 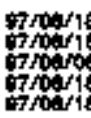 \\
\hline 970100054 & 9rocosose & 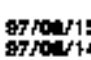 \\
\hline
\end{tabular}

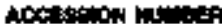

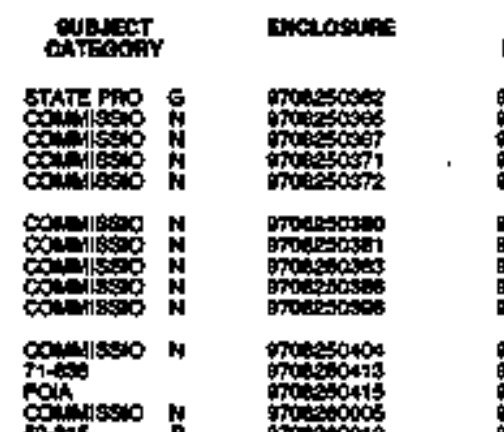

Dictritin

97400100

mosios

protopos

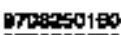

Briagsosta

Brotipose

priagons

ortoptosto

ow

A

pitentort

9700017

Fons

Stoseopos

itesosoto.

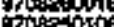

orotoos

कon

phopoos

Dripons

posions

Buthopans

irosionto

epot

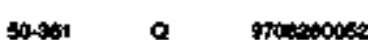

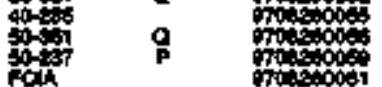

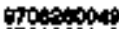

ipotion

orotions

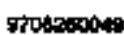

Fon

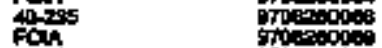

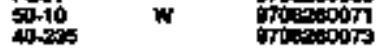

ST:800004:

Troporouts

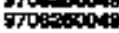

Fon

ron

部格

orionort

पroganous

Fis:

orotion

fit

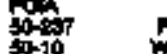

orosonom

700

orotone

oneopis

70.00

potoleste

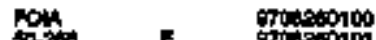

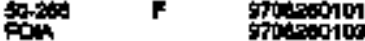

fot

Fin

gan

trigench

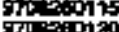

gुopionas

grisentis

$\cos$

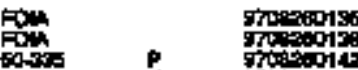

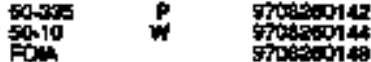

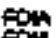

thetoms

Chan

p p gat151

tom

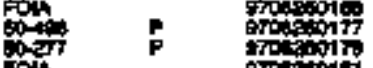

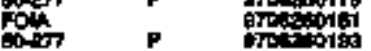

rola sroverists

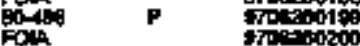

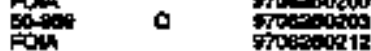

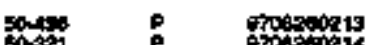

For

gow

ton

prosonoto

Th:00.004:

Tropacos

To0000110

Dopacis

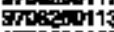

wotgotas

straconers

Tropars

Wuporiz

प्रTe:

Dom:011

Theziditi

(a)

moarsonos

gonoses

ofing

op

prowitos

opocion.

orowerols

orotiotios.

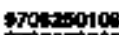

rowasos

7000000

ofostog

orcertorn

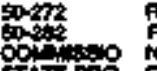

solativg

97005010

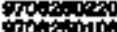

potsing

sotions

bous

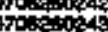

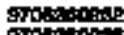

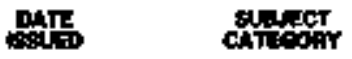

or 14

ordotis

of

GHowis

Trogr14

Trositis

ordogr14

的rowet

of how

grodor

oriowia

Fine

Fon

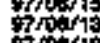

Thor

grow

$8100 / 3$

7roaks

7fous

FNow

grow 19

970019

oroor

grow

grow

Tow

ofow

Fow

구요

grow

类 row

row

spou

frow

Frow

show

grown

$\rightarrow$ ow 2

Froes

कrows

g7ro1/s

Fror/0

Fropto

stion

Prosed

inos

indor/2

That

Fifor

Trovis

90015

groner

orroutis

instit

77 cists

spotoys

How

Fint

Fitow

orow 18

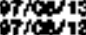

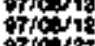

(1)

970014
Fin

sosis s

40.51 $P$

fors.

5044

Four

8

00045

40-2as

TOeq:

co-cis

tors:

40.75

$40-28$

40.5.

0024

0.245

00.245

60245

co-34s

00 ins

50

son-

orio

$0-42$

co-245

$50-20$

$60-245$

Q0.245

64t15

cond

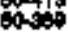

60.311

7

o.

50300

sass

onto

0

40

象

was

pate

50

sols

soms

$+0,5$

50.09

40.65

pros

0

$40-\infty$

40.5

40215

.

$50-213$ 


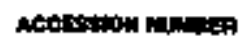

\begin{tabular}{|c|c|}
\hline 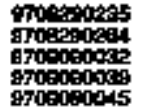 & 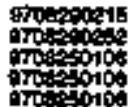 \\
\hline
\end{tabular}

Bing

shrop

Growas

$\rightarrow$ int

ardota

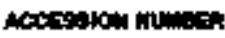

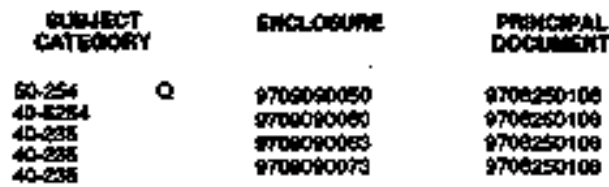

Don

or To6rs

97 intis

4700013

\$TPON13 and

$40-235$

$40-20$

the

$\rightarrow 0-2$ 


\section{APPENDIX A}

\section{DEFINITIONS OF DOCKET CATEGORIES}

\section{DOCKET 27 - Low-Level Radioactive Waste Disposal Sites}

The Docket 27 series contains documentation concerning the licensing of land disposal of low-level radioactive wastes. In the past, waste disposal licensees were assigned unique Docket 27 numbers. Unlike the other docket series, the first two digits of these docket numbers do not correspond to the part of 10 CFR that specifies NRC's authority for issuing these licenses and the regutations that these licensees must follow. The Docket 27 licenses were issued in accordance with Parts 30,40 , and 70 of 10 CFR in the absence of any final regulations governing the licensing of land disposal low-level radjoactive wastes.

In the future, these licensing actions will be governed by 10 CFR Part 61, "Licensing Requirements for Land Disposal of Radioactive Waste." The prefix will then becorme Docket 61 followed by assigned numbers identifying a specific facility. Docunents in these files include:
- Application for land disposal of low-level radioactive waste and supporting documents

- License and amendments

- Correspondence to and from the applicant regarding the application, license, and amendmerts

- Environmental report and supporting documents

- Inspection and enforcement reports and related correspondence

- Adjudicatory documents relating to proceedings before the Licensing Board and the Commission

\section{DOCKET 30 - Byproduct Material Licenses}

The Docket 30 series contains documentation on the possession or use of nuclear byproduct materials for medical, industrial, and academic purposes. These documents are maintained in the PDR on microfiche only.

Documents in this series include:
- Application for a license, license amendments, and license renewals or modifications to the byproduct material license and related correspondence

- Denial, revocation, and suspension of a license and related correspondence 


\section{DEFINITIONS OF DOCKET CATEGORIES}

DOCKET 30 - Byproduct Material Licenses (continued)

- Records related to the NRC inspection program and related correspondence

- Preliminary Notifications of Occurrences (PNOs), enforcement actions, civil penalties, and related correspondence

- Event reports, misadministration repors, and related correspondence

- Correspondence to and from local and State governments
- Adjudicatory documentation rejating to proceedings before the Licensing Board and the Commission

- Other technical, legal, and licensing correspondence between NRC, the licensee, and other interested parties

- Periodic reports from licensees and related conespondence

\section{DOCKET 40 - Nuclear Source Material Facilities}

The Docket 40 series contains documentation concerning the domestic licensing of nuclear byproduct materials rejating to source material licenses. Licenses issued in accordance with 10 CFR Part 40 cover the possession, use, and processing of ores (uranium and thorium) and possession and use of uranium and thorium in a refined form. Each license application is assigned a unique Docket 40 number.

Paper files are maintained in the PDR for only those Docket 40 licensees involvedi in the nuclear fuel cycle; that is, mines, mills, and research and development facilities. Material on other licenses, such as those for possession of material for medical and industrial uses, are available on saicrofiche only. Documents in these files include:

- Application for a license, license amendments and license renewals for source material license
- Source material license and license amendments

- Licensee-jssued Environmental Report (ER) for those license applications that must comply with the National Environmental Policy Act (NEPA)

- NRC-jssued Draft and Final Environmental Statements (DES and FES) for license applications that must comply with NEPA

- Site vísjts and related correspondence

- Inspection reports and related correspondence

- Environmental monitoring reports

- Adjudicatory documents and hearing transcripts relating to proceedings before the Licensing Board and the Commission 


\section{DEFINITIONS OF DOCKET CATEGORIES}

DOCKET 40 - Nuclear Source Material Facilities (continued)

- Other technical, legal, and licensing correspondence between NRC, the licensee, and other interested parties

NOTE: For transcripts of Commission meetings and papers submitted for Commission consideration on a specific docket, see the 10 CFR 9.7 and SECY nondocketed files.

\section{DOCKET 50 - Nuclear Reactors and Fuel Reprocessing Plants}

The Docket 50 series contains documentation concerraing the domestic licensing of production and utilization facilities. Licenses issted in accordance with 10 CFR Part 50 cover commercial nuclear power plants; experimental, research, and test reactors; and fuel fabrication facilities. The NRC staff assigns Docket 50 number when it considers that the application and the Preliminary Safety Analysis Report (PSAR) submitted are complete for staff review. "Pre-docketed" material is assigned a project number and is filed in the applicant's project file.

Because of the large volume of doctumentation accumulated over the lifetine of a facility, each Docket 50 file is stbdivided into the following categories that pertain either to different aspects of the licensing process or to document types.

\section{A. Application/Construction Stage Documents and Related Correspondence}

B. Utility Preliminary Safety Analysis Report (PSAR) and Amendments

C. Utility Environmental Report (ER), Amendments, and Related Correspondence

D. NRC Draft and Final Environmental Statements (DES and FES), Supplements, and Related Correspondence
E. NRC Safety Evaluation Report (SER), Supplements, and Related Correspondence

F. Security, Medical, Emergency, and Fire Protection Plans

G. Non-Antitrust Legal and Adjudicatory Correspondence

H. General Correspondence

I. Financial Information

1. Insurance and Indemnity Information

K. Utility Final and Uptated Safety Analysis Reports (FSAR and USAR) and Amendments

L. Limited Work Authorization (LWA) and Related Correspondence

M. Antitrast Reviews and Related Correspondence

N. Antitrust Transcripts, Testimony, and Briefings

O. Advisory Committee on Reactor Safeguards (ACRS) Reports and Related Correspondence 


\section{DEFINITIONS OF DOCKET CATEGORIES}

DOCKET 50 - Nuclear Reactors and Fuel Reprocessing Plants (continued)

P. Operating License Stage Documents and Related Correspondence

Q. Inspection Reports, Civil Penalties, Responses to Bulletins and Information Notices, and Related Correspondence

R. Periodic Operating Reports and Related Correspondence

S. Reportable Occurrences (ROs), Licensee Event Reports (LERs), Preliminary Notifications of Occurrences (PNOs), Preliminary Notifications of Safeguards Events (PNSs), and Related Correspondence

T. Transcripts, Testimony, and Brietings on Non-Antitrust Matters
U. Executive, Congressional, and State Government Correspondence

V. Licersing of Operators and Related Correspondence

W. Decommissioning Stage Documents and Related Correspondence

X. Onsite Storage of Low-Level Waste and Related Correspondence

Y. Independent Spent Fuel Storage Installatíons (ISFSIs) and Related Correspondence

A detailed description of each category and the documents included in the files for each category follows.

\section{A. APPLICATION/CONSTRUCTION STAGE DOCUMENTS AND RELATED CORRESPONDENCE}

Documents in this category track the application from submittal to the day preceding the issuance of an operating license. It contains all correspondence and other documents with substantive technical content that are not in other specific categories. Included are;

- Application for construction permit

- General information submitted with the construction permit application

- Amendments and changes to the application
- NRC questions, Requests for Additional Information (RAIs), and applicant responses

- Early site reviews

- Correspondenoe and documents relating to unresolved design and construction issues

- Meeting announcements and summaries of the meetings

- Correspondence relating to the utility's Preliminary Safety Analysis Report (PSAR). 


\section{DEFINITIONS OF DOCKET CATEGORIES}

\section{A. APPLICATION/CONSTRUCTION STAGE DOCUMENTS AND RELATED CORRESPONDENCE (CONTINUED)}

- Infornation on laws and changes in regulations or policy that affect the status of the application (National Envíronmental Policy Act (NEPA), Water Quality Improvement Act, Water Pollution Control Act, etc.)

- Construction permit, amendments, related correspondence, and orders issued by the Office of Nuciear Reactor Regulation (NRR) show-caluse and modification orders which do not involve the former Atomic Safety Licensing and Appeal Board

- Scheduting of license application, amendments, and reviews

- Environmental review information (other environmental documents are filed in categories $\mathrm{C}, \mathrm{D}, \mathrm{P}, \mathrm{R}$, or $\mathrm{W}$ )

- Seismic quatification review team (SQRT) reports and related correspondence

- Application for operating license

- General information submitted with the Operating License application

- Amendments and changes to the application for Operating license
- Correspondence related to the utility's Final Safety Analysis Report (FSAR) and Updated Safety Analysis Report (USAR)

- Generic and administrative communications and related documentation that may affect the subject facility and which are not covered by a bulletin or notice

- Safety evaluation reports (SER) issued by the NRC on specific generic issues

- Reports on site visits

- Backup technical documents (e.g., topical reports) submitted in support of the application

- Probabilistic risk assessment (PRA) and related documentation

- Employee allegations and rejated documentation (other documents may be filed in categories $\mathbf{G}, Q$, or $T$ )

- Documents relating to possible equipment failure and emergency instructions

- NRC's changes in internal organizational structure

\section{B. UTILITY PRELIMINARY SAFETY ANALYSIS REPORT (PSAR) AND AMENDMENTS}

This category contains the Preliminary Safety Analysis Report (PSAR) submitted by the utility (fomerly cailed Hazards Summary report or
Safecy Analysis Reports) and the following related material: 


\section{DEFINITIONS OF DOCKET CATEGORIES}

\section{B. UTILITY PRELIMINARY SAFETY ANALYSIS REPORT (PSAR) AND AMENDMENTS (CONTINUED)}

- Amendments and changes to the PSAR

- Transmittal and acknowledgment letters
- Certificates of service to the PSAR

NOTE: All related correspondence is filed in category A.

\section{UTILTY ENVIRONMENTAL REPORT (ER), AMENDMENTS, AND RELATED CORRESPONDENCE}

The category contains the Environmental Report (ER) submitted by the utility and the following related material:

- Amendments and supplements to the ER

- Federal Register notices citing the availability of the $E R$ and amendments

- Transmittal and acknowjedgment letters

- Information on scheduling of submittal or seview of ER
- NRC questions, RAIs and applicant responses

- Correspondence relating to the review of ER

- Meeting announcements and summaries of meetings

- Correspondence relating to certification of pollution control facilities

NOTE: Other environmental documents are filed in categories A, D, P, R, or W.

\section{NRC DRAFT AND FINAL ENVIRONMENTAL STATEMENTS (DES AND FES), SUPPLEMENTS, AND CORRESPONDENCE}

This category contains Draft and Final Environmental Statements (DES and FES) prepared by $\mathrm{NRC}$, and the following related material:

- Supplements to the DES and FES
- Federal Register notices announcing the availability of the DES, FES, and stpplements

- Correspondence relating to the DES and FES 


\section{DEFINITIONS OF DOCKET CATEGORIES}

\section{NRC DRAFT AND FINAL ENVIRONMENTAL STATEMENTS (DES AND FES), SUPPLEMENTS, AND CORRESPONDENCE (CONTINUED)}

- Comments from other Federal, State and local government agencies, organizations, and interested individuals

- Transmittal and acknowiedgment jetters

- Documents rejated to NRC review of environmental issues or review of the ER for purposes of preparing the environumental statements
- Backup technical documents submitted in stupport of the DES and FES

- Reports of site visits to obtain information for the DES or FES

- Meeting announcements and summaries of meeting which the primary subject of discussion is the DES or FES

NOTE: Other environmental documeots and correspondence are filed in categories $A$, C, $\mathrm{P}, \mathbf{R}$, or $\mathbf{W}$

\section{E. NRC SAFETY EVALUATION REPORT (SER), SUPPLEMENTS, AND RELATED CORRESPONDENCE}

This category contains the formal Safety Evaluation Report (SER) prepared by the NRC staff (formerly called NRC-AEC hazard analysis report) in review of PSAR, and the following related material:

- Supplements to the SER

- Federal Register notices announcing the avajlability of the SER and supplements

- Correspondence relating to the SER and supplements

- Information on scheduling of an SER submittal and review
- RAIs for use in preparing the SER; and responses to such requests

- Meeting announcements and meeting summaries at which the primary subject of disctsssion is the SER

- Transmittal and acknowledgment letters

NOTE: Other documents related to specific safety-related istues (that may be referred to as SERs) are filed in categories $A, P$ ot W, depending on the phase of the licensing process. 


\section{DEFINITIONS OF DOCKET CATEGORIES}

\section{F. SECURITY, MEDICAL, EMERGENCY, AND FTRE PROTECTION PLANS}

All plans in this category are individually stbmitted by the applicant or licensee. Emergency and fire protection plans submitted as part of the kicensing application are filed in this category.

- Fire bazard protection plan and surveys

- Plant evacuation correspondence

- Safeguard contingency plan

- Health physics program and its review

- Emergency implementing procedures

- Emergency action levels

- Emergency płan
- Supplements, changes, and revisions to the plans

- Correspondence relating to the aforementioned plans

- Correspondence and đocumentation relating to guard training, emergency response centers, and emergency preparedness

- Documentation on security, non-operator fitness procedures, employee unescorted access and related correspondence

- Transmittal and acknowledgment letters

- Meeting announcements and meeting summaries at which the prinary subject of discussion is related to the aforementioned plans and topics

\section{G. NON-ANTTTRUST LEGAL AND ADJUDICATORY CORRESPONDENCE}

This category contains all non-antitrust legal and adjudicatory documentation, except for transcripts, brieftnos, and testimony, which are fled in category T. Show-cause and moxfification orders issued by the Office of Nucjear Reactor Regulation (NRR), and not before the licensing board, are filed in categorjes $A$ and $P$. Exhibits to the proceedings (hearings) are maintained in the PDR on microfiche only. Paper files of the exhibits are available upon request to the PDR from the Docketing and Services Branch, Office of the Secretary.

- Atomic Safety and Licensing Boaro (ASLB) and the former Atomic Safety and Licensing
Appeal Board (ASLAB) hearing-related documents

- Federal Register notices regarding hearings

- Requests to make appearances at hearings

- Motions, briefs, arguments, statements, and related correspondence filed by participants for hearings

- Transmittal of supporting documents (e.g., SER, FES) to the licensing board

- Correspondence to and from intervenors 


\section{DEFINITIONS OF DOCKET CATEGORIES}

\section{G. NON-ANITTRUST LEGAL AND ADJUDICATORY CORRESPONDENCE (CONTINUED)}

- Board orders and decisions

- Formal petitions for Commission action

- Petitions filed in accordance with 10 CFR 2.206 to the Executive Director for Operations
- Legal filings, director's decisions, Commission orders and decisions

- Orders prohibiting involvement in NRClicensed activities, appeals, and related decisions

\section{H. GENERAL CORRESPONDENCE}

This category contains documents with little or no bearing on the staters of the application or license. Items that have no substantive technical content and administrative doctments are included.

- Change-of-address notifications (extemal only)

- Changes in structure of organization (external only)

- Changes in service lists

- NRC requests to applicants for additional copies of documents

- Correspondence from the public requesting information about the facility, and NRC response

- Correspondence on purely administrative matters
- Letters of support, concem, or opposition to the facility

- Correspondence relating to the Local Public Document Rooms (LPDRs)

- Professional qualifications statements not pertaining to the hearing process

- Constituent, organizational, or utility correspondence forwarded by members of Congress to NRC for response

- Resolutions passed by State and local goveming bodies (e.g, town councils)

NOTE: Letters of concem, support, or opposition for all plants are filed in a General Public Comments tolder and are retained for 6 months from the date of receipt in the PDR.

\section{FINANCIAL INFORMATION}

This category contains all financial inforndation except for that submitted as part of the application or its amendments or that relate to the imposition of civil penalties. 


\section{DEFINITIONS OF DOCKET CATEGORIES}

\section{FINANCLAL INFORMATION (CONTINUED)}

- Company annual reports, auditor statements, and stock prospectuses for all companies holding ownership
- Transcripts and testimony before State commissions pertinent to inancial information requested by NRC

- Separately submitted financial information and related correspondence

- Cecommissioning funding reports

\section{J. INSURANCE AND INDEMNITY INFORMATION}

This category contajns indemnity agreements and amendments as well as:

- Endorsements of insurance policjes
- Other correspondence regarding indemnity and insurance matters

\section{K. UTILITY FINAL AND UPDATED SAFETY ANALYSIS REPORTS (FSAR AND USAR) AND AMENDMENTS}

This category contains the following utility documents:

- Final Safery Analysis Report (FSAR), formerly called Final Hazards Summary Report

- Updated Safety Analysis Report (USAR) periodically updated to incorporate new information or modifications to the facility

- Amendments and changes to the FSAR and USAR
- Federal Register notices announcing the avalability of the FSAR and amendments

- Transmittal and acknowledgement letters

- Certificates of service to the FSAR and USAR

NOTE: All related correspondence is filed in category A or P. Emergency and fire protection plans submitted as part of the licensing application are filed in category $\mathbf{F}$.

\section{LIMITED WORK AUTHORIZATION (LWA) AND RELATED CORRESPONDENCE}

This category contains documents pertaining to the limited work authorization (LWA) whereby applicants can conduct limited site preparation and construction before the NRC grants a construction permit.
- Limited Work Authorization (LWA) and amendments 


\section{DEFINITIONS OF DOCKET CATEGORIES}

\section{LIMTTED WORK AUTHORIZATION (LWA) AND RELATED CORRESPONDENCE (CONTINUED)}

- Correspondence pertaining to work performed under the LWA

\section{ANTITRUST REVIEWS AND RELATED CORRESPONDENCE}

This category contains documents submitted as part of the application for a construction permit andi operating jicense that are entitled

"Information Requested by the Attomey General for Antitrust Review," including:
- Correspondence in which the primary topic of discussion concerns antitrust matters

- All antitrust proceeding documents, except bearing transcripts, testimony, and briefings, which are filed in category $\mathbf{N}$

\section{N. ANTITRUST TRANSCRIPTS, TESTIMONY, AND BRIEFINGS}

This category contains verbatim transcripts of hearings, testimony, and briefings in antitrust matters. Related correspondence is filed in category $\mathrm{M}$.

\section{O. ADVISORY COMMITTEE ON REACTOR SAFEGUARDS (ACRS) REPORTS AND RELATED CORRESPONDENCE}

This category contains plant-specific documents pertaining to ACRS safety reviews. Included are:

- ACRS reports relating to facility license applications
- Announcements of ACRS meetings relating to facility license applications, including descriptions of meeting agenda

- Statements and written testimony by ACRS members or presented by individuals at ACRS proceedings relating to both facility license applications and safecy-related matters 


\section{DEFINITIONS OF DOCKET CATEGORIES}

\section{O. ADVISORY COMMITTEE ON REACTOR SAFEGUARDS (ACRS) REPORTS AND RELATED CORRESPONDENCE (CONTINUED)}

- Technical correspondence and memoranda to and from ACRS, including transmittal and acknowledgment letters relating to facility license applications

NOTE: For generic and document-specific safety-related materiai, transcripts of ACRS subcommittee and fulj committee meetings, minutes, and ACRS reports (consultant, contract, and letter), see ACRS non-docket file.

\section{P. OPERATING LICENSE STAGE DOCUMENTS AND RELATED CORRESPONDENCE}

This category contains general technical doctments dated from the issuance of the operating license until the order for tersmination of the license. Included are:

- Operating licerse, amendments, proposed amendments, changes, and related correspondence

- Show-cause and modification orders issued by NRR (not before the licensing board) for the operating license

- Correspondence regarding the utility's Final and Updated Safery Analysis Report (FSAR and USAR)

- Federal Register notices announcing availability of operating license, amendments, FSAR and USAR

- Technical specifications, proposed changes, revisions and related correspondence

- Backup technical documents (e.g., topical reports) submitted in support of the aforementioned items
- All general technical correspondence, meeting announcements and meeting summaries relating to the technical specifications, the operating license, or technical issues arising after the issuance of the operating license

- Correspondence rejating to codes (c.g., American Society of Mechanical Engineers), regulations, Jaws, and changes in policy that may affect plant operation or the operating license

- NRC questions, RAIs, and licensee responses

- Information on the Standard Review Plan (SRP)

- Results of surveys or tests conducted in accordance with the techrical specifications (not periodic reports, which are filed in category R)

- Cortespondence relating to NRC's System. atic Evaluation Program (SEP)

- Owners' group mexting announcements and reports 


\section{DEFINITIONS OF DOCKET CATEGORIES}

\section{P. OPERATING LICENSE STAGE DOCUMENTS AND RELATED CORRESPONDENCE (CONTINUED)}

- Quality assurance plan and related correspondence

- Environmental matters (technical data)

- Seismic Qualification Review Team (SQRT) reports and related correspondence

- Entergency core cooling system documents

- Radioactive waste information exciuding onsite storage of low-level waste (filed in category $\mathrm{X}$ ) and independent spent fue! installations of high-level waste (filled in category $\mathbf{Y}$ )

- Correspondence relating to expanstion of the spent fuel storage pool

- Startop test reports

- Generic and administrative communications and related documentation that may affect the subject facility but is not covered by a Bulletin or Notice
- SERs on specitic generic issues

- Documents relating to possible equipment failure and emergency instructions

- Documents relating to inservice testing procedures and results (not inservice inspections, which are filled in category $Q$ )

- Specifie plant evaluation tocuments

- Probabilistic Risk Assessment (PRA) reports

- Employee allegations and related documentation (other docurnents may be filed in categories $\mathbf{G}, Q$, or $T$ )

- Reports on site visits

- Application to voluntarily terminate license and to dismantle and dispose of component parts

- Information on changes in NRC's internal organizational structure 


\section{DEFINITIONS OF DOCKET CATEGORIES}

\section{Q. INSPECTION REPORTS, CIVIL PENALTIES, RESPONSES TO BULLETINS AND INFORMATION NOTICES, AND RELATED CORRESPONDENCE}

This category contains the following applicant/icense material:

- Inspection reports

- Responses to Bulletios (formerly called Regulatory Operations, RO Bulletins), Circulars (discontinued in 1981) and Information Notices, and related correspondence

- Applicantlicensec determinations of the proprietary nature of inspection reports

- Letters concerning areas of noncompliance

- Preservice and inservice inspections and procedures

- Notices of violation and proposed impositions of civil penalty and Demands for Information (DFIs)

- Correspondence and orders relating to the imposition and payment of civil penalties. including Enforcement Notifications (ENs) and Significant Enforcement Actions (EAs)

- Notices of deviation and related correspondence

- Notices of enforcement conferences, meetings, and related reports

- Correspondence relating to inspection and enforcement that does not specifically relate to incidents or events as defined in category $\mathbf{S}$

- Health physics inspections and appraisais
- Emergency prepareoness ínspections and appraisals

- Inspections and telated correspondence from the International Atomic Energy Agency (IAEA)

- Risk-based Inspection Gujdes (RIGs)

- Systematic Assessment of Licensee Performance (SALP)

- Incident lnvestigation Team (ITT) reports

- Construction Appraisal Team/Construction Assessment Team (CAT) reports

- Integrated Design Inspection (IDI) reports

- Independent Design Verification Program (IDVP) reports

- Notices of signiticant licensee metings

- Investigation reports and related documentation, and responses to employee allegations (other documents are filed in categories $\mathrm{A}, \mathrm{P}, \mathrm{G}$, or $\mathrm{T}$ )

- Performance Appraișal Team (PAT) reports

- Licensee/vendor interface inspections

- Correspondence relating to the aforementioned items

NOTE: For bulletins and information notices, see non-dacket I\&E file. 


\section{DEFINITIONS OF DOCKET CATEGORIES}

\section{R. PERIODIC OPERATING REPORTS AND RELATED CORRESPONDENCE}

This category contains periodic reports of a routine nature.

- Monthy, semiannual, and annual operating reports (other than financial) for construction, operating, and decommissioning stages

- Periodic environmental monitoring, effluent testing, or similar reports

- Periodic personnel exposure reports

- Periodic fitness for duty performance data

- Periodic facility changes, tests and experiments
- Facility simulation certification four year update reports

- Periodic nonconformance reports (jssued in accordance with licensing board orders)

- Operational logbooks on

- environmental data

- radiological data

- plant systems core

- plant status summary sheets

- Correspondence and other documents relating to the aforementioned items

\section{S. REPORTABLE OCCURRENCES (ROs), LCENSEE EVENT REPORTS (LERs), PRELIMINARY NOTIFICATIONS OF OCCURRENCES (PNOS), PRELIMINARY NOTIFICATIONS OF SAFEGUARDS EVENTS (PNSs), AND RELATED CORRESPONDENCE}

This category contains all materials telating to abnormal, unusual, reportable, and non-routine occurrences or events. These include:

- All 24-hour, 10-day, 30-day, jnterim and final Licensee Event Reports (LERs) and follow-up correspondence (also called incident reports, abnormal accurrences, special reports, and reportable occurrences)

- Potential or possibly reportable Deficiency Reports and related correspondence

- Individual Personnel Exposure Reports
- Construction Deficiency Reports submitted in accordance with 10 CFR 50.55(e)

- Documents related to the issuance of a Preliminary Notification of Occurrence (PNO), Preliminary Notification of Safeguards Events (PNS), and related correspondence

- Environmental technical specifications violation reports and environmental event reports 


\section{DEFINITIONS OF DOCKET CATEGORIES}

\section{S. REPORTABLE OCCURRENCES (ROS), LICENSEE EVENT REPORTS (PRELIMINARY NOTIFICATIONS OF OCCURRENCES (PNOS), PRELIMINARY NOTIFICATIONS OF SAFEGUARDS EVENTS (PNSS), AND RELATED CORRESPONDENCE (CONTINUED)}

- Part 21 reports of defects and noncompliance

- Correspondence and other documents relating to the aforementioned items
NOTE: For PNOs and PNSs, see I\&E. non-docket file.

\section{T. TRANSCRIPTS, TESTIMONY, AND BRIEFINGS ON NON-ANTITRUST MATTERS}

This category contains verbatim transcripts, testimony, and briefings except for congressional matters (filed in category $U$ ) and antitrust matters (filed in category $\mathrm{N}$ ).

- Atomic Safety and Licensing Board (ASLB) and the former Atomic Safety and Licensing Appeal Board (ASLAB) transcripts

- Testimony and statements subaritted to ASLB and the former ASLAB
- Transcripts of Commission meeting

- NRC staff briefings to the Commission

- Statements and written testimony submitted at Commission meetings and hearings

- Transcripts of utility briefings to NRC staff

- Corrections to the aforementioned items

NOTE: Transcripts on specific dockets are also filed in ACRS and 9.7 nondocket files.

\section{U. EXECUTIVE, CONGRESSIONAL, AND STATE GOVERNMENT CORRESPONDENCE}

This category contains documents and related correspondence that have substantive technical and adtrinistrative content, including:

- Statements and testimony before Congress or before bodies/committees appointed by the Executive or Legislative branches
- Technical reports and related information supplied for the record of congressional hearings

- Follow-up questions and answers to the aforementioned 


\section{U. EXECUTIVE, CONGRESSIONAL, AND STATE GOVERNMENT CORRESPONDENCE (CONTINUED)}

- Correspondence to and from Congress and the Executive Branch on other than constituent response matters

- Correspondence to or from State Legislative bodies
NOTE: Routine responses to constituent inquiries are filed in category $\mathbf{H}$.

\section{LICENSING OF OPERATORS AND RELATED CORRESPONDENCE}

This category contains information bearing on licensing of operators and shift engineers, incluting:

- Power plant examination summary sheets

- Correspondence relating to operator examinations
- Documentation on operator Jaboratory tests or reports and related correspondence

- Doctments relating to operator fitness for duty and related correspondence

- Orders prohibiting operators from further nuclear licensed activities, and related correspondence (other documents may be filed in category $\mathbf{G}$ )

\section{W. DECOMMISSIONING STAGE DOCUMENTS AND RELATED CORRESPONDENCE}

This category contains general technical items dated from the order to terminate the operating license.

- Otder granting authority to dismantlef decommission

- Decommissioning Plan, Dismantling Plan, Environmental Report on dismantling and Safety Analysis Report on decommissioning
- Documents relating to the disposal of radioactive material and decontamination of the site

- Other correspondence relating to decommissioning

- All documentation relating to the ongoing operation of the plant which was formerly filed in category $\mathbf{P}$ 


\section{DEFINITIONS OF DOCKET CATEGORIES}

\section{ONSITE STORAGE OF LOW-LEVEL WASTE AND RELATED CORRESPONDENCE}

This category contains material related to onsite storage of Low-Level Waste (LLW).

- Licenste application, license, proposed amendments, and amendments to store LLW at a commercial reactor site
- Technical reports and related correspondence

NOTE: The doctuments in this category will aiso bave a Nuclear Materials Safety and Safeguards (NMSS) Docket 30 license number associated with the Docket 50 number.

\section{Y. INDEPENDENT SPENT FUEL STORAGE INSTALLATIONS (ISFSIS) AND RELATED CORRESPONDENCE}

This category contains material related to license applications, license, amendments and proposed amendments to possess power reactor spent fuel and other radioactive materals associated with spent fuel storage in an independent spent fuel stotage installation. (Such ISFSIs may be on a reactor site or at a separate site.)
- All related correspondence and reports to the aforementioned items

NOTE: The documents in this category will have an NMSS Docket 72 license number. For these installations on a reactor site, the assocjated Docket $\mathbf{5 0}$ number also has been referenced.

\section{Docket 52 - Standard Design Certification, Early Site Permit, and Combined Licenses for Nuclear Power Plants}

The Dacket 52 series contains documentation on the domestic licensing of production facilities. Licenses issued in accordance with 10 CFR Part 52 cover preliminary and final design approval of standard designs, early site pemits, and combined licenses for nuclear power plants. A Docket 52 number is assigned when the NRC considers that the application submitted is complete for staff review. "Predocketed" material is assigned a project number and is filed in the applicant's project file.
Because of the larige volume of documentation accumulated over the lifetime of an application, each Docket 52 file is subdivided into the following categories that pertain either to different aspects of the licensing process or to document types. These categories are similar to those used for the Docket 50 files, but have been modified to retlect the special requirements of Docket 52 applications. 


\section{DEFINITIONS OF DOCKET CATEGORIES}

A. Application for Preliminary Design Approval (PDA), Final Design Approval and Design Certification (FDA/DC), Early Site Permit, Combined Construction' Operating License (COL), and Related Correspondence

B. Vendor Preliminary Standard Safery Anatysis Report (PSSAR-PDA stage), Utility Preliminary Safety Analysis Report (PSAR-COL stage), and Amendments

C. Severe Accident Mitigation Design Altermative Analysis (SAMDA-PDA andFDADC stages), Environmental Reports (ERs-Early Site Permit and $\mathrm{COL}$ stages), Amendments, and Related Correspondence

D. NRC Draft and Final Environmental Statements (DES and FES), Supplements, and Related Correspondence (Eatly Site Permit and COL stages)

E. NRC Safety Evaluation Report (SER), Supplements, and Related Correspondence (Early Site Permit, PDA, FDA/DC, and COL stages)

F Reliability Assurance Program (RAP), Security, Medical, Emergency, and Fire Protection Plans (PDA, FDA/NC, and COL stages)

G. Non-Antitrust Legal and Adjudicatory Correspondence (Early Site Permit, PDA, FDAVC, and COL stages)

H. General Correspondence (Early Site Permit, PDA, FDAVC, and COL stages)

I. Financial Information (COL stage)

J. Ensurance and Indemnity Information (COL stage)
K. Vendor Fưnal Standard Safety Analysis Report (FSSAR-FDA/DC Stage), Utility Final Satety Analysis Report (FSAR-COL Stage), Updated Safety Ajalysis Report (USAR-COL Stage), and Amendments

L. Limited Work Authorization (LWA) and Related Correspondence (COL Stage)

M. Antitrust Reviews and Related Correspondence (COL Stage)

N. Antitrust Hearing Transcripts, Testimony, and Briefings (COL Stage)

O. Advisory Committee on Reactor Safeguards (ACRS) Reports and Related Correspondence (Early Site Permit, PDA, FDA/DC, and COL Stages)

P. Post-Combined Construction/Operating License Stage Doctuments and Related Correspondence (COL Stage)

Q. Inspection Reports, Civit Penalties, Re. sponses to Bulletins and Information Notices, and Rejated Correspondence (COL Stage)

R. Periodic Operating Reports and Related Correspondence (COL Stage)

S. Reportable Occurrences (ROs), Licensee Event Reports (LERs), Preliminary Notifications of Occurrences (PNOs), Preliminary Notifications of Safeguards Events (PNSs), and Related Correspondence (COL Stage)

T. Transcripts, Testimony, and Briefings on Non-Antitrust Matters (Early Site Permit, PDA, FDA/DC, and COL Stages) 


\section{DEFINITIONS OF DOCKET CATEGORIES (CONTINUED)}

U. Executive, Congressional, and State Govemment Correspondence (Early Site Permit, PDA, FDANDC, and COL Stages)

V. Licensing of Operators and Related Correspondence (COL Stage)

W. Decommissioning Stage Documents and Related Correspondence (COL \$tage)
X. Onsite Storage of Low-Level Waste and Related Correspondence (COL and Decommissioning Stages)

Y. Independent Spent Fuel Storage Installations (ISFSIs) and Related Correspondence (COL and Decommissioning Stages)

A detailed description of each category and the documents inciuded in each category follows.

\section{A. APPLICATION FOR PRELIMINARY DESIGN APPROVAL (PDA), FINAL DESIGN APPROVAL AND DESIGN CERTIFICATION (FDA/DC), EARLY SITE PERMIT COMBINED CONSTRUCTION/OPERATING LICENSE (COL), AND RELATED CORRESPONDENCE}

Documents in this category track the application for Preliminary Design Approval (PDA) or Final Design Approval and Design Certification (FDA respectively, or the application of the early site permit until jssuance of the permit. It contains all correspondence and documents with substantive technical content that are not in other specific categories. Included are:

- Application for preliminary design approval

- Application for final design approval and design certification

- Application for early site permit

- General information subraitted with the application

- Amendments and changes submitted by applicants

- NRC questions, Requests for Additional Information (RAIs), and applicant responses
- Meeting announcements and summaries of the meetings

- Correspondence relating to the Preliminary or Final Standard Safety Anabysis Report (SSAR)

- Information on laws and changes in regulations or policy that affect the status of the application (National Environmental Policy Act (NEPA), Water Quality Improvement Act, Water Pollution Control Act, etc.)

- Preliminary Design Approval (PDA), and amendments and correspondence relating to the PDA

- Final Design Approwal (FDA), and amendments and correspondence relating to the FDA

- Early site permit, amendments and related correspondence

- Scheduling documentation

- Backup technical documents (e.g, topical reports) submitted in support of the application 


\section{DEFINITIONS OF DOCKET CATEGORIES}

\section{A. APPLICATION FOR PRELIMINARY DESIGN APPROVAL (PDA), FINAL DESIGN APPROVAL AND DESIGN CERTIFICATION (FDADC), EARLY SITE PERMIT, COMBINED CONSTRUCTION/OPERATING LICENSE (COL), AND RELATED CORRESPONDENCE (CONTINUED)}

- The design-specific Probabilistic Risk Assessment (PRA), and related documentation

- Proposed tests, analyses, and acceptance criteria and related documentation

- Design Acceptance Criteria (DAC) and related correspondence

- Doctments addressing policy issues raised to the Commission via Commission papers
- Documents addressing resolution of issues for the application

- Generic and administrative communications and related documentation which may affect the subject facility and which are nor covered by a Bulletin or Notice

- Employee allegations and related documentation (other documents may be filed in categoties $\mathrm{G}, \mathrm{Q}$, or $\mathrm{T}$ )

- NRC's changes in internal organizational structure

B. VENDOR PRELIMINARY STANDARD SAFETY ANALYSIS REPORT (PSSAR-PDA STAGE), UTILITY PRELIMINARY SAFETY ANALYSIS REPORT (PSAR-COL STAGE), AND AMENDMENTS

This category contains the following documents:

- Preliminary Standard Safety Analysis Report (PSSAR) submitted by vendor

- Amendments and changes to the PSSAR

- Preliminary Safety Analysis Report (PSAR) submitted by the utility
- Amendments and changes to the PSAR

- Transmittal and acknowiedgement letters

NOTE: Al related comespondence is filed in category $\mathbf{A}$

C. SEVERE ACCIDENT MITGATION DESIGN ALTERNATIVE ANAIYSIS (SAMDA-PDA AND FDADC STAGES), ENVIRONMENTAL REPORTS (ERS-EARLY SITE PERMIT AND COL STAGES), AMENDMENTS, AND RELATED CORRESPONDENCE

This category contains the following documents:
- Severe Accident Mitjgation Design Altematives Analysis (SAMDAs) required under the National Environmental Policy Act (NEPA) submitted by vendor 


\section{DEFINITIONS OF DOCKET CATEGORIES}

\section{SEVERE ACCIDENT MITIGATION DESIGN ALTERNATIVE ANALYSIS (SAMDA-PDA AND FDADC STAGES), ENVIRONMENTAL REPORTS (ERs-EARLY SITE PERMIT AND COL STAGES), AMENDMENTS, AND RELATED CORRESPONDENCE (CONTINUED)}

- NRC questions, RAIs, and applicant responses relating to SAMDAS analysis review

- Correspondence relating to the SAMDAS analysís review

- Meeting announcernents and summaries of meetings at which the primary subject of discussion is the SAMDAS analysis

- Environmental Report (ER) submitted by utility

- Amendments and supplements to the ER

- Federal Register notices announcing the availability of the ER and amendments
- Transmittal and acknowledgment letters of ER

- Information on scheduling of submittal or review of ER

- NRC questions, RAIs, and applicant responses relating to review of ER

- Correspondence relating to the review of ER

- Meeting announcements and summaries of meetings at which the primary subject of discussion is the ER

- Correspondence relating to certification of pollution control facilities

NOTE: Other environmental documents are filed in categories A, D, P, R, or W.

D. NRC DRAFT AND FINAL ENVIRONMENTAL STATEMENTS (DES AND FES), SUPPLEMENTS, AND RELATED CORRESPONDENCE (EARLY SITE PERMIT AND COL STAGES)

This category contains the Draft and Final Environfuental Impact Statemepts (DES and FES) prepared by NRC, and supplements to the DES and FES, as well as:

- Federal Register notices announcing the availability of the DES and FES

- Correspondence relating to the DES and FES
- Comments from other Federal, State, and local government agencies, organizations, and interested indiviouals

- Transmittal and acknowledgment letters

- Documents related to NRC review of environmeatal issues or review of the $E R$ obtained for purposes of preparing the environmental statements 


\section{DEFINITIONS OF DOCKET CATEGORIES}

\section{NRC DRAFT AND FINAL ENVIRONMENTAL STATEMENTS (DES AND FES), SUPPLEMENTS, AND RELATED CORRESPONDENCE (EARLY SITE PERMIT AND COL STAGES) (CONTINUED)}

- Backup technical documents submitted in support of the DES and FES

- Reports of site visits to obtain information for the DES or FES
- Meeting announcements and summaries of meetings at which the primary subject of discussion is the DES or FES

NOTE: Other environmental documents and correspondence are filed in categories A, C, P, R, or W.

\section{E. NRC SAFETY EVALUATION REPORT (SER), SUPPLEMENTS, AND RELATED CORRESPONDENCE (EARLY SITE PERMIT, PDA, FDADC, AND COL STACES)}

This category contains the formal Safety Evaluation Report (SER) prepared by NRC in review of the early site permit application, PSSAR

(PDA stage), FSSAR (FDADC stage), PSAR (COL stage), or FSAR (COL stage) and,

Supplements to the SER, as well as:

- Federal Register notices announcing the availability of the SER and suppiements

- Correspondence relating to the SER and supplements

- Information on scheduling of an SER submittal and review
- RAIs for use in preparing the SER and responses to such requests

- Meeting announcements and summaries of meetings at which the primary subject of discussion is the SER

- Transmittal and acknowledgment letters

NOTE: Other documents related to specific safety-rejated issues (that may be referred to as SERs) are fled in categories A, $\mathrm{P}$, or W, depending on the phase of the ficensing process.

\section{F. RELIABILITY ASSURANCE PROGRAM (RAP), SECURITY, MEDICAL, EMERGENCY, AND FIRE PROTECTION PLAN (PDA, FDADC, AND COL STAGES)}

The plans in this category may be individual submissions or may be combined under the Reliability Assurance Program. These plans may be submitted during the review of the PDA, the FDAJDC, and COL applications. Inctuded are:
- Reliability Asstrance Program (RAP)

- Plant evacuation correspondence 


\section{DEFINTIONS OF DOCKET CATEGORIES}

\section{F. RELIABILITY ASSURANCE PROGRAM (RAP), SECURITY, MEDICAL, EMERGENCY, AND FIRE PROTECTION PLAN (PDA, FDADC, AND COL STAGES) (CONTINUED)}

- Safeguard contingency plan

- Heaith physics program and its review

- Emergency implementing procedures

- Emergency action levels

- Emergency plans

- Fire hazard protection plans and surveys

- Supplements, changes, and revisions to the plans
- Correspondence relating to the aforementioned plans

- Correspondence and documentation relating to guard training, emergency response centers, and emergency preparedness

- Documentation on security, non-operator fitness procedures, employee unescorted access and related correspondence

- Transnittal and acknowledgement letters

- Meeting announcements and summaries of meetings at which the primary subject of discussion is related to the aforementioned plans

\section{G. NON-ANTITRUST LEGAL AND ADJUDICATORY CORRESPONDENCE (EARLY SITE PERMIT, PDA, FDA/DC, AND COL STAGES)}

This category contains all non-antitrust legal and adjudicatory documentation, except for transcripts, briefings and testimony, which are filed in category T. Show-cause and modification onders issued by the Office of Nuclear Reactor Regulation (NRR) and not before the Licensing Board are filed in categories $A$ and $P$. Exhibits to the proceeding (hearings) ate maintained in the PDR on microfiche only. Paper files of the exhibjts are available upon request to the PDR from the Docketing and Services Branch, Office of the Secretary.

- Atomic Safety and Licensing Board (ASLB) hearing related documents
- Federal Register notices regarding hearings

- Requests to make appearances at hearings

- Motions, briefs, arguments, statements, and related correspondence filed by participants for hearings

- Transmittal of supporting docuspents (e.g., SER, FES) to the licensing board

- Correspondence to and from intervenors

- Board orders and decistons

- Formal petitions for Commission action 


\section{DEFINITIONS OF DOCKET CATEGORIES}

\section{G. NON-ANTITRUST LEGAL AND ADJUDICATORY CORRESPONDENCE (EARLY SITE PERMTT, PDA, FDADC, AND COL STAGES) (CONTINUED)}

- Petitions filed in accordance with 10 CFR

2.206 to the Executive Director for

Operations

- Legal filings, director's decisions, Com-

mission orders and decisions
- Orders prohibiting involvement in NRC. licensed activities, appeals, and related decisions

\section{H. GENERAL CORRESPONDENCE (EARLY SITE PERMIT, PDA, FDA/DC, AND COL STAGES)}

This category contains documents with little or no bearing on the status of the application or license. Items that have no substantive technical content and administrative documents are included.

- Change of address notifications (external only)

- Changes in structure of organization (external only)

- Changes in service lists

- NRC requests to applicants for additional copies of documents

- Cotrespondence from the public requesting information about the site, design, or facility, and NRC responses

- Correspondence on purley administrative mattters
- Letters of support, concern or opposition to the site, design, or facility

- Correspondence relating to the Local Public Document Rooms (LPDRs)

- Professional qualifications statements not pertaining to the hearing process

- Constituent, organizational or atility correspondence forwarded by members of Congress to NRC for response

- Resolutions passed by state and local governing bodies (e.g., town councils)

NOTE: Letters of concern, support, or opposition for all plants are filed in a General Public Comments folder and are retained for 6 months from the date of receipt in the PDR.

\section{FINANCIAL INFORMATION (COL STAGE)}

This category contains all financial information except for that submitted as part of the applica- tion or its amendments or that relate to the imposition of civil penalties. 


\section{DEFINITIONS OF DOCKET CATEGORIES}

\section{FINANCIAL INFORMATION (COL STAGE) (CONTINUED)}

- Company gnמual reports, auditor statements and stock prospectuses for all comparies holding ownership

- Separately submitted financial information and related correspondence
- Transcripts and testimony before State cortmissions pertinent to financial information requested by NRC

- Decommissioning funding reports

\section{J. INSURANCE AND INDEMNITY INFORMATION (COL STAGE)}

This category contains indernnity agreements and amendments as well as:
- Other correspondence regarding indemnity and insurance matters

- Endorsements of insurance policies
K. VENDOR FINAL STANDARD SAFETY ANALYSIS REPORT (FSSAR-FDADC STAGE), UTILITY FINAL SAFETY ANALYSIS REPORT (FSAR-COL STAGE), UPDATED FINAL SAFETY ANAEYSIS REPORT (USAR-COL STAGE), AND AMENDMENTS

This category contains the following vendor and utility documents:

- Final Standard Safecy Analysis Report (FSSAR) submitted by the vendor

- Final Safety Analysis Report (FSAR) submitted by the utitity

- Updated Safety Analysis Report (USAR) submitted by the utility and periodically updated to incorporate new information or modifications to the facility
- Amendments and changes to the FSSAR, FSAR, and USAR

- Federal Register notices announcing the availability of the FSSAR and FSAR

- Transmittal and acknowledgement letters

- Certificates of service to the FSAR and USAR

NOTE: All related correspondence falls in category $A$ or P. Emergency and Fire Protection Plans submitted as part of the licensing application are filed in category $\mathbf{F}$ 


\section{DEFINITIONS OF DOCKET CATEGORIES}

\section{LIMITED WORK AUTHORIZATION (LWA) AND RELATED CORRESPONDENCE (COL STAGE)}

This category is limited to documents pertaining to Limited work Authorization (LWA) whereby applicants can conduct timited site preparation and construction before NRC grants a combined construction/operating license.
- Limited Work Authorization (LWA) and amendments

- Correspondence pertaining to work performed under LWAs

\section{ANTTTRUST REVIEWS AND RELATED CORRESPONDENCE (COL STAGE)}

This category contains documents submitted as part of the application for a combined construction/operating license entitled, "Information Requested by the Attomey General for Antitrust Review," including:

- Correspondence in which the primary topic of discussion is antitrust matters
- All antitrust proceeding documents, except hearing transcripts, testimony, and briefings, which are filed in category $\mathrm{N}$

\section{N. ANTITRUST HEARING TRANSCRIPTS, TESTIMONY, AND BRIEFINGS (COL STAGE)}

This category contains verbatim transcripts of bearings, testimony and briefings in antitrust matters. Related correspondence is filed in category $\mathbf{M}$.

\section{O. ADVISORY COMMITTEE ON REACTOR SAFEGUARDS (ACRS) REPOKTS AND RELATED CORRESPONDENCE (EARLY SITE PERMIT, PDA, FDADC, AND COL STAGES)}

This category contains project-specific docu* ments pertaining to ACRS safety reviews. Included are:
- ACRS reports relating to a PDA or FDA DC application, early site review, or facility license application 


\section{DEFINITIONS OR DOCKET CATEGORIES}

\section{O. ADVISORY COMMITTEE ON REACTOR SAFEGUARDS (ACRS) REPORTS AND RELATED CORRESPONDENCE (EARLY SITE PERMIT, PDA, FDADC, AND COL STAGES) (CONTINUED)}

- Announcements of ACRS meetings relating to a PDA or FDA/DC application, early site review, or facility license application, inchating descriptions of meeting agenda

- Statements and written testimony by ACRS members or presented by individuals at ACRS proceedings relating to a PDA or FDA/DC application, early site review, facility license application, and safetyrelated matters
- Technical correspondence, and memoranda to and from ACRS, including transmittal and acknowledgement letters relating to a PDA or FDAVDC application, early site review, or facility license application

NOTE: For generic and document-specific safety related material, transcripts of ACRS sulicommittee and fall] committee meetings, minutes, and ACRS reports (consultant, contract and letter), see ACRS non-docket file.

\section{P. POST-COMBINED CONSTRUCTION/OPERATING LICENSE STAGE DOCUMENTS AND RELATED CORRESPONDENCE (COL STAGE)}

This category contains general technical documents dated from the combined construction/operating license until the order for termination of the license. Included are:

- The combined construction/operating license (COL), amendments, proposed amendments, changes, and related correspondence

- Show-cause and modification orders issued by NRR (not before the licensing board) for the COL

- Technical specifications, proposed changes, revisions and related correspondence

- Bactup technical documents (e.g., Topical Reports) submitted in support of the aforementioned items
- Federal Register notices announcing the availability of the COL, FSAR, and amendments

- Correspondence regarding the utility Final Safety Analysis Report (FSAR) and/or the Updated Safety Anaiysis Report (USAR)

- All general technical correspondence, meeting announcements and meeting summaries relating to the technical specifications, the $\mathrm{COL}_{\text {, }}$ or technical issues arising after the issuance of the COL

- Correspondence relating to codes (e.g., American Society of Mechanical Engineers) regulations, laws, and changes in policy that may affect plant operation or bear on the COL

- NRC questions, RAls, and licensee responses 


\section{DEFINITIONS OF DOCKET CATEGORIES}

\section{P. POST-COMBINED CONSTRUCTION/OPERATING LICENSE STAGE DOCUMENTS AND RELATED CORRESPONDENCE (COL STAGE) (CONTINUED)}

- Information an the Standard Review Plan (SRP)

- Results of surveys or tests conducted in accordance with the techenical specifications (not periodic reports, which are filed in category R)

- Owners group meeting announcements and reports

- Quality assurance plan and related correspondence

- Environmental matters (technical data)

- Seismic Qualification Review Team (SQRT) reports and related correspondence

- Radioactive waste information, excluding onsite storage of low-level waste (filed category $X$ ) and independent spent fuel installations of high-level waste (filed in category Y)

- Correspondence relating to expansion of spent fuel storage pool

- Startup test reports

- Generic and administrative communications and related documentation that may affect the subject facility; not covered by a Bulletin or Nofice

- Employee allegations and related documentation (other documents may be filed in categories $\mathrm{G}, \mathrm{Q}$, and $\mathrm{T}$ )

- SERs on specific generic issues

- Documents relating to possible equipment failure and emergency instructions

- Documents relating to inservice testing procedures and results (not inspections which are filed in category $Q$ )

- Specific plant evaluation documents

- Probabilistic Rísk Assessment (PRA) reports

- Employee allegations and related documentation (Other documents may be filed in categories $\mathrm{G}, \mathrm{Q}$, or $\mathrm{T}$ )

- Reports on site visits

- Application to voluntarily terminate license, and to dismantle, and dispose of component parts

- Information on changes in NRC's internal orgarizational structure

Q. INSPECTION REPORTS, CIVIL PENALTIES, RESPONSES TO BULLETINS AND INFORMATION NOTICES, AND RELATED CORRESPONDENCE (COL STAGE)

This category contains the following applicant/ license material:
- Inspection reports 


\section{DEFINITIONS OF DOCKET CATEGORIES}

\section{Q. INSPECTION REPORTS, CIVIL PENALTIES, RESPONSES TO BULIETINS AND INFORMATION NOTICES, AND RELATED CORRESPONDENCE (COL STAGE) (CONTINUED)}

- Responses to Bulletins (formerly called Regulatory Operations, RO Bulletins), Information Notices, and related correspondence

- Applicant licensee determination of the proprietary nature of Inspection Reports

- Letters concerning areas of noncompliance

- Preservice and inservice inspections and procedures

- Notices of violation and proposed impositions of civil penaity and Demand for Information (DFIs)

- Correspondence and orders relating to the opposition and payment of civil penalties, including Enforcement Notification (EN) and Significant Enforcement actions (EA)

- Notices of deviation and related correspondence

- Correspondence relating to inspection and enforcement that does not specifically relate to incidents or events as defined in category $\$$

- Health physics inspections and appraisals

- Emergency preparedness inspections and appraisals
- Inspections and related correspondence from the International Atomic Energy Agency (LAEA)

- Risk-based Inspection Guides (RIGs)

- Systematic Assessment of Licensee Performance (SALP)

- Incident Investigation Team (IIT) reports

- Construction Appraisal Team/Construction Assessment Team (CAT) reports

- Integrated Design Inspection (IDI) reports

- Independent Design Verification program (IDVP) reports

- Notices of significant licensee meetings

- Investigation reports and related documentation, and responses to employee allegations fother documents are filed in categories $A, P, G$, or T)

- Performance Appraisal Team (PAT) reports

- Licensee/vendor interface inspections

- Correspondence relating to the aforementioned items

NOTE* For bulletins and information notices, see nondocket I\&E file. 


\section{DEFINITIONS OF DOCKET CATEGORIES}

\section{R. PERIODIC OPERATING REPORTS AND RELATED CORRESPONDENCE (COL STAGE)}

This category contains periodic reports of a routine nature.

- Monthly, semiannual, and annual operating reports (other than financial) for construction, operating, and decommission stages

- Periodic environmental monitoring, effluent testing, or similar reports

- Periodic fitness for duty performance data

- Periodic personnel exposure reports

- Periodic facility changes, tests and experiments
- Facility simulation certification four year update reports

- Periodic nonconformance reports (issued in accordance with Licensing Board orders)

- Operational Logbooks on

- environmental data

* radiological data

- plant systems core

- plant status summary sheets

- Correspondence or other documents relating to the items listed

\section{S. REPORTABLE OCCURRENCES (ROs), LICENSEE EVENT REPORTS (LERs), PRELIMINARY NOTIFICATIONS OF OCCURRENCES (PNOs), PRELIMINARY NOTIFICATIONS OF SAFEGUARDS EVENTS (PNSS), AND RELATED CORRESPONDENCE (COL STAGE)}

This category contains all materials relating to abnormal, unusual, reportable and non-routine occurrences or events. These include:

- All 24-hour, 10-day, 30-day, interim and final Licensee Event Reports (LERs) and follow-up correspondence (also called incident reports, abnormal occurrences, and reportable occurrences)

- Potential or possibly reportable Deficiency Reports and related correspondence
- Individual Personnel Exposure Reports

- Construction Deficiency Reports submitted in accordance with 10 CFR 50.55 (e)

- Documents related to the issuance of a preliminary Notification of Occurrence (PNOs) and Preliminary Notification of Safeguards Events (PNSs) and related correspondence 


\section{DEFINITIONS OF DOCKET CATEGORJES}

S. REPORTABLE OCCURRENCES (ROS), LICENSEE EVENT REPORTS (LERs), PRELIMINARY NOTIFICATIONS OF OCCURRENCES (PNOS), PRELIMINARY NOTIFICATIONS OF SAFEGUARDS EVENTS (PNSs), AND RELATED CORRESPONDENCE (COL STAGE) (CONTINUED)

- Environmental technical speciffications violation reports and environmental event reports

- Part 21 reports of defects and noncompliance
- Correspondence or other documents relating to the aforementioned items

NOTE: For PNOs and PNSs, see I\&E nondocket files.

\section{T. TRANSCRIPTS, TESTIMONY, AND BRIEFTNGS ON NON-ANTITRUST MATTERS (EARLY SITE PERMIT, PDA, FDA/DC, AND COL STAGES)}

This category contains verbatin transcripts, testimony, and briefings except for congressionad matters (filed in category $U$ ) and antitrust matters (filed in category $\mathbf{N}$ ).

- Atomic Safety and Licensing Board (ASLB) transcripts

- Testimony and statements submitted to ASLB

- Transcripts of Commission meetings
- NRC staff briefings to the Commission

- Statements and written testimony submitted at Commission meetings and hearings

- Transcripts of vendor or utility briefings to NRC staff

- Corrections to the aforementioned items

NOTE: Transcripts on specific dockets are also filed in the ACRS and 10 CFR 9.7 nondocket files.

\section{U. EXECUTIVE, CONGRESSIONAL, AND STATE GOVERNMENT CORRESPONDENCE (EARLY SITE PERMTT, PDA, FDADC, AND COL STAGES)}

This category contains documents and related correspondence that have substantive technical and administrative content, including:
- Statements and testimony before Congress or before bodies/committees appointed by the Executive or Legislative branches 


\section{DEFINITIONS OF DOCKET CATEGORIES}

\section{U. EXECUTIVE, CONGRESSIONAL, AND STATE GOVERNMENT CORRESPONDENCE (EARLY SITE PERMIT, PDA, FDADC, AND COL STAGES) (CONTINUED)}

- Technical reports and related information supplied for the record of congressional hearings

- Follow-up questions and answers to the aforementioned
- Correspondence to and from Congress and the Executive Brazch on other than constituent response matters

- Cotrespondence to or from State Legislative bodies

NOTE: Routine responses to constituent inquiries are filed in category $\mathrm{H}$.

\section{LICENSING OF OPERATORS AND RELATED CORRESPONDENCE (COL STAGE)}

This category contains information bearing on licensing of operators and shift engineers, including:

- Power plant examination summary sheets

- Correspondence relating to operator examinations
- Docurrentation on operator laboratory tests or reports and related correspondence

- Documents relating to operator fitness for duty and related correspondence

- Orders prohibiting operators from further nuclear licensed activities, and related correspondence (other documents may be filed in category $G$ )

\section{W. DECOMMISSIONING STAGE DOCUMENTS AND RELATED CORRESPONDENCE (COL STAGE)}

This category contains general technical items dated from the order for terminating the operating license.

- Order granting authority to dismantle/ decommission

- Decommissioning Plan, Dismantling Plan, Environmental Report on dismantling, and the Safety Analysis Report on decommissioning

- Documents re]ating to the disposal of radioactive material and decontamination of the site

- Other correspondence relating to decommissioning 


\section{DEFINITIONS OF DOCKET CATEGORIES}

\section{W. DECOMMISSIONING STAGE DOCUMENTS AND RELATED CORRESPONDENCE (COL STAGE) (CONIINUED)}

- Afl documentation relating to the ongoing operation of the plant formerly tiled in category $\mathbf{P}$

\section{ONSITE STORAGE OF LOW-LEVEL WASTE AND RELATED CORRESPONDENCE (COL AND DECOMMISSIONING STAGES)}

This category contains material related to onsite storage of Low-Level Waste (LLW).

- License application, license, proposed amendments, and amendments to store low-level waste at a commercial reactor
- Alt related correspondence and reports

NOTE: These documents will also have a Nuclear Materials Safety and Safeguards (NMSS) Docket 30 license number associated with the Docket 52 number.

\section{Y. INDEPENDENT SPENT FUEL STORAGE INSTALLATIONS (ISFSIS) AND RELATED CORRESPONDENCE (COL AND DECOMMISSIONING STAGES)}

This category contains material related to:

- License applications, license, amendments, and proposed amendments to possess power reactor spent fuel and other radioactive materials associated with spent fuel storage in an independent spent fuel storage installation. (Such spent fuel storage installations may be on a reactor site or at a separate site.)
- All related correspondence and reports to the above

NOTE: The documents in this category will have an NMSS Docket 72 number license. For these installations on a reactor site, the associated Docket $\$ 2$ number also has been referenced. 


\section{DEFINITIONS OF DOCKET CATEGORIES}

\section{DOCKET 70 - Special Nuclear Materials}

The Docket 70 files contain documentation concerning the domestic licensing of spectal nuclear material (SNM). Each SNM license application is assigned a unique Docket 70 number. SNM licenses cover the possession and use of radioactive material, including plutoniumenriched uranium. Paper files are maintained in the PDR only for Docket 70 licensees that possess more than 350 grams of uranium-235 or 200 grams of plutonitum. Material on other licensees is available on microfiche only. Documents in these files include:

- Applications for licenses, license amendments, and license renewals for Special Nuclear Material kicenses

- Special Nuclear Materiel licenses and license amendments
- Applicant technical reports concerning the facility, including Safety Analysis Reports (SARs), Environmental Reports (ERs) and amendments

- NRC technical reports concerning the facility

- Emergency and radiological health plans

- Inspection reports and related correspondence

- Adjudicatory documents and bearing transcripts relating to proceedings before the Ijicensing Board

- Other technical, legal, and licensing correspondence between NRC and the licensee or other interested parties

\section{DOCKET 71 - Design of Packages for Transport of Radiogetive Material and Quality Assurance Programs}

The Docket 71 files contain documentation concerning the desizn of radioactive material transport packages and quality assurance (QA) programs for designers, manufacturers, and users of such packages. Each package design and each QA program is individually assigned a unique Docket 71 number.

Al] NRC licensees who design, manufacture, or use zadioactive material packages must comply with NRC-approved package designs and QA programs for these packages. Agreement State licensees who design, manufacture, or use tadioactive material packages do not need a $Q A$ program approval, but the Department of
Transportation requires that NRC approve the package design.

Agreement State licensees who wish to use their packages in States under NRC jurisdiction, or who design and manufacture packages for NRC licensees, must obtain approval for their $Q A$ programs. Documents in these files include:

- Application for QA program approval and amendments to the application

- Application for package design approval, including the Safety Analysis Report (SAR), amendments, and engineering drawings 


\section{DEFINITIONS OF DOCKET CATEGORIES}

DOCKET 71 - Design of Packages for Transport of Radioactive Material and Quality Assurance Programs (continued)

- Certificate of complíance (NRC approval)

for the package design and revisions

- Approval of the QA program

- Correspondence between NRC and the designer and the manufacturer concerning particular package designs

- Requests to be registered as a user of a particular package

- Inspection reports and related correspondence

\section{DOCKET 72 - Independent Spent Fuel Storage Installations}

The Docket 72 series contains documentation concerning certification of storage casks and the licensing of independent spent fuel storage installations. These installations store reactor spent fuel and may be on a reactor site or at a separate site. Each installation is assigned a unique Docket 72 number. Documents in these files include:

- Application for license

- Safety Analysis Reports (SAR) and annual updates

- Certification of completion for vendor design of diry cask storage and related correspondence

- Licensee issued Environmental Report (ER) and amenidments

- Emergency plan and anendments
- Inspection reports and related correspondence

- Reports on accidental criticality or loss of special nucteas material

- QA plan and amendments

- Correspondence relating to the physical security plan

- Decommissioning plan and related correspondence

- Adjudicatory documents and hearing transcripts relating to proceedings between the NRC and the applicant

NOTE: Additional nondocketed information conceming spent fuel storage is available in the Project $M$ nondocket filt. For installations on reactor sites, see category $Y$ in the Docket 50 or 52 series and Docket 70 for NMSS licenses. 


\section{APPENDIX B}

\section{DEFINITIONS OF NONDOCKETED CATEGORIES}

\section{NONDOCKET FILES}

Materials that are not docket-specific are arranged according to the following classification scheme. The capitalized abbreviation is a shorthand description of each topic and corresponds to the way that it appears on the PDR Accession List.

10 CFR 9.7-Commission Meeting

Transcripts and Reports

ACRS-Advisory Committee on Reactor Safeguards Documents

ADVCM-NRC Advisory Committees

COMMISSION-NRC Commistioner Correspondence

CONTRACT-NRC Contracts and Grants

FOIA-Freedom of Information Act

Requests and Appeals

GEN TECH IS-Generic Technical Issues

I\&E-Inspection and Enforcement

Issuances

I\&E Manual-NRC Inspection Manual

I\&E MISC-Draft Generic

Communications
MISC-Public Document Room

Miscellaneous

NUREG-NRC Staff and Contractor

Formal Reports, Conference Proceedings,

Brocbures, International Agreement

Reports, and Related Correspondence

ORG-Organizations

PR-Proposed and Final Rules

PRESS-Press Releases

PRM-Petitions for Rulemaking

PROJ-Projects

PT 21-10 CFR Part 21 Deficiency Reports

QA 999-Vendor Quality Assurance

Inspections

RCSSD-Registration of Certificates for Sealed Sources and Devices

REGGD-Regulatory Guides

RES-Research and Technical Assistance Reports (1974 through 1988)

REVIEW GRP-Review Groups 


\section{DEFINITIONS OF NONDOCKETED CATEGORIES}

\section{NONDOCKET FILES (CONTINUED)}

RM-Rulemakings

SAI - Strategic Assessment Issue Papers

SECY - Staff Position Papers Prepared for the Commission

SPCH-Speeches

STATE PROG-State Programs
TOPREP-Topical Reports and Related Correspondence

WASH-AEC-Issued Formal Reports and Related Correspondence

WM-Waste Manazement Documents

WMRES-Waste Management Contract Research Documents

XPORTLIC_Export and Import Licenses

\section{CFR 9.7 - Commission Meeting Transcripts and Reports}

This file contains documents relating to open and ctosed Commission Meetings.

- Sunshine Federal Register notices

- Full written explanation of closed meetings

- Certification by the General Counsel on closed meetings

- Commission Determination Statements signed by the Secretary regarding the public disclosure of transcripts of Commission meetings
- Verbatim unofficial and official transcripts or portions of transcripts of open and closed Commission meetings made publicly available in accordance with the Sunshine Act and Commission policies

- Enclosures to released transcripts, including applicable viewgraphs, staff papers, and staff correspondence

- Stafí Requirements Memoranda for open meetings, memoranda of record, record of votes of Commissioners on items under consideration at meetings

\section{ACRS - Advisory Committee on Reactor Safeguards Documents}

Documents in this file pertain to safety-related areas reviewed by the ACRS. ACRS serves as an independent advisory committee to the Commission. 


\section{DEFINITIONS OF NONDOCKETED CATEGORIES}

ACRS - Advisory Committee on Reactor Safeguards Documents (continued)

- ACRS reports relating to safety-related matters

- Announcements of ACRS meetings relating to safety-related matters, including meeting agenda

- Transcripts of ACRS subcommittee and full committee meetings relating to both facility license applications and safety-related matters

- Statements and written testimony of ACRS members or presented by individuals at ACRS proceedings related to both vendor or facility license applications and safety-related matters

- Minutes of ACRS subcommittee and full committee meetings relating to both vendor or facility license application and safety॰related matters

- ACRS consultant reports, comments, and related cortespondence relating to both vendor or facility license applications and safety-related matters

- Tecinical correspondence and memoranda to and from ACRS pertaining to safetyrelated matters

- General correspondence and related material about ACRS, but not prepared by ACRS or its consultants

NOTE: For docket-specific technical correspondence, memoranda and announcements of ACRS meetings, see category $O$ of Docket 50 and 52 .

\section{ADVCM - NRC Advisory Committees}

Documents in this file pertain to the responsibilities of all NRC advisory committees other than the Advisory Committee on Reactor Safeguards, including:

- Charters of each advisory committee

- Meeting announcements and meeting agenda
- Minutes and verbation transeripts of committee meetings

- Cormmittee-gentrated reports, memoranda, and correspondence that are publicly available

- Committee-related correspondence 


\section{DEFINITIONS OF NONDOCKETED CATEGORIES}

\section{COMMISSION - NRC Commlssioner Correspondence}

Documents in this file pertain to the daily conduct of Commission business in the Office of the Commission, including:

- Incoming and outgoing Commission-level correspondence relating to official NRC busimess, including reports and other enclosures to the aforementioned correspondence

- Travel vouchers, telephone logs, and official appointment calendars of participating Commissioners. (These are placed in the PDR at the discretion of indjvidual Commissioners.)
- Speeches, interviews and testimony given by Commissioners at meetings, symposia, and congressional hearings

- Vote sheets

- Weekly information reports (numbered as part of the SECY series) prepared for the Commissioners by the Executive Director for Operations (EDO) summarizing key events and items of interest undertaken by individual staff and program offices

NOTE: For transcripts of Commission meetings, see nondocket File 10 CFR 9.7. For ali other staff papers with the SECY-series designation prepared for Commission review, see the SECY nondocket file.

CONTRACT - NRC Contracts and Grants

Documents in this file pertain to AEC and NRC contracts, grants and interagency agreements, including:

- Notification of contracts and grants execution that specifies contract number, project title, contractor name, contract type, funding, and period of performance

- Contractual documents and modifications to contracts 


\section{DEFINITIONS OF NONDOCKETED CATEGORIES}

\section{FOIA - Freediom of Information Act Requests and Appeals}

Documents in this file include requests, denials, and appeals for agency records and information about records released in accordance with the provisions of the Freedom of Information Act (FOIA). Each is assigned a sequential identification number based on the year the request is made, for example, 95-110 refers to the 110th FOIA request made in 1995.

- Requests made under the FOLA for NRC records
- Correspondence between NRC and the requester relevant to the processing, granting, deniat, and appeal of an FOLA request

- Records or portions thereof released in response to FOIA requests or made under FOIA appeals

\section{GEN TECH IS - Generic Technical Issues}

Documents in this file pertain to untesolved safety issues of a generic nature that are being analyzed by the NRC Office of Nuclear Reactor Regulation (NRR), including:

- Draft reports of action plans to accomplish generic tasks in the NRR program
- Correspondence with industry and within NRC discussing methodologies, analyses, the program, and technical aspects of issues

- Meeting notifications and minutes

- Interim findings and status reports on generic tasks addressing related aspects of generic technical issues

\section{I\&E - Inspection and Enforcement Issuances}

Documents in this file include bulletins and information notices that are issued by the NRC Office of Nuclear Reactor Regulation. These issuances describe some event that has occurred at a facility and that may require some follow-up action by the licensee. Preliminary Notifications of Events or Unusual Occurrences (PNOs), Preliminary Notifications of Safeguards
Events or Unusual Occurrences (PNSs),

Enforcement Notifications (ENs) and significant Enforcement Actions (EAs).

- Circulars, that describe an event at a licensed facility and are informational in nature (these were discontinued in 1981) 


\section{DEFINITIONS OF NONDOCKETED CATEGORIES}

\section{1\&E - Inspection and Enforcement Issuances (continued)}

- Information Notices, that are similar to Circulars but are preliminary in nature and are issued shortly after an event has occurted

- Bulletins describing an event at a licensed facility and requesting that afl recipients perform certain actions as a result of that event

- Preliminary Notification of Events or Unusual Oocurrences (PNOs), which provide updated information of an event of safety or public interest
- Preliminary Notifications of Safeguards Events or Unustral Occurrences (PNSs), which provide early notice of events of possible safety significance

- Enforcement Notifications (ENs) and significant Enforcement Actions (EAs), which provide information on a notice of violation and proposed imposition of civil penalties

NOTE: For utility/vendor responses to such issuances and related correspondence, see categories $\mathrm{Q}$ and $\mathrm{S}$ in Dockets 50 and 52 .

IRE MANUAL - NRC Inspection Manual

This fite contains drafts, finats, revisions, Federal Register notices and related correspondence to the NRC Inspection Manual.

\section{I\&E MISC - Draft Generic Communications}

Documents in this file include drafts of Generic Letters, Bulletins, Information Notices and other generic communications issued by the NRC Office of Nuclear Reactor Regulation.
- Draft Bulletins and Information Notices that are being considered for issuance, related comments, and evaluations 


\section{DEFINITIONS OF NONDOCKETED CATEGORIES}

I\&E MISC - Draft Generic Communications (continued)

- Draft Generic Letters and other generic communications that are being considered for issuance, related comments, and correspondence

\section{MISC - Public Document Room Miscellaneous}

This file contains documents about topics not included in the scope of the other nondocketed categories.

\section{NUREG - NRC Stafi and Contractor Formal Reports, Conference Proceedings, Brochures, International Agreement Reports, and Rełated Correspondence}

This file contains formal reports on regulatory, technicai, and administrative issues of interest to staff, industry, other govermental entities, and the public which are published in the NUREG series.

- Results of licensing studies of specific plants or facilities preliminary to licensing actions

- Results of analyses of general or specific problems of a regulatory or technical nature that are of interest to a major segment of the industry

- Action and review plans, as well as guidance, for complying with NRC requirements

- Task force reports on specific topics

- Proceedings of conferences and workshops
- Management and program anajysis reports

- Statistical analyses that are of interest to the staff, the industry, and the public

- Administrative reports that are of interest to the staff, the industry, and the public

- Correspondence from industry, government, and the public commentisg on NUREG series reports

- Internal NRC memoranda summarizing meetings or discussions pertaining to the content of the NUREG reports series

NOTE: NUREG reports on plants or facilities are filed according to each plant's specific docket number. 


\section{DEFINITIONS OF NONDOCKETED CATEGORJES}

ORG - Organizations

This file contains correspondence with professional organizations, govemment agencies, and associations with which NRC is in contact; records pertaining to specific NRC offices; and petitions not assigned Petition for Rulemaking (PRM) numbers that request the Commission to take immediate action on a generic issue.

- Meeting summaries and technical correspondence between the NRC and professional associations

- Correspondence between NRC and other government agencies in which both parties have joint regulatory responsibility, e.g.,
EPA which has authority to control all radioactive politutants emitted into the air

- Correspondence and technical information between NRC and Congress

- Reports, technical information, and correspondence between NRC, foreign governments and international organizations

- lntemal memoranda, trip reports, and scientific papers pertaining to specific NRC offices

\section{PR - Proposed and Final Rules}

This file contains documents of NRC staff proposals to adopt, amend, or repeal a Commission regulation. Proposed Rules (PRs) can be initiated as a result of a Commission directive, a staff proposal, or a Commission determination that sufficient grounds exist for it to proceed with a Petition for change in an NRC regulation (PRM) filed by an outside party.

- Federal Register notice stating the terms or substance of the proposed rute, or a specification of the subject and issues involved

- Text of the Proposed Rule (PR)

- Draft and generic Proposed Rules and Federal Register notices
- Comments, statements, information, opinions, or arguments on the proposed change to a part of the Code of Federal Regulations

- Requests from the nuclear industry, organizations, and other parties for written interpretation of the PR

- Final rules and Federal Register notices

NOTE For staff recommencations to the Commission on whether a PR shoulo be adopted, see the SECY nondocket tille. For Commission review of staff recommendations, see the $10 \mathrm{CFR} 9.7$ nondocket fite. 


\section{DEFINITIONS OF NONDOCKETED CATEGORIES}

\section{PRESS - Press Releases}

This file contains press releases issued by the Office of Public Affairs Headquarters and Regional offices relating to the NRC's regulatory policy and programs, public hearings, and workshops. Press releases are also issued on major licensee programs, changes, proposed fines, and issues effecting the status of licenses.

\section{PRM - Petitions for Rulemaking}

This file contains documents of petitions filed with the Commission by an outside party to issue, amend, or rescind an NRC regulation.

- Petitions filed by any interested person or party to issue, amend, or rescind an NRC regulation

- Federal Register notice of the filing of the petition

- Correspondence between NRC and the petitioner relevant to the status and substance of the petition

- Comments from the nuclear industry, professional associations, public interest groups, and others in favor of or in opposition to the PRM

- Statements, motions, orders, and correspondence dealing with emergency petitions filed by parties on such topics as fire protection and a reanalysis of the capacity of operating U.S. plants to withstand earthquakes

NOTE: For staff recornmendations to the Commissioners on whether the PRM should be denied or issued as a proposed rule, see the SECY nondocket file. For Commission review of staff recommendations, see the 10 CFR 9.7 nondocket file.

\section{PROJ - Projects}

This file contains documents pertaining to four classes of projects: (1) commercial nuclear power plants (Docket 50) for which NRC has full revicw and licensing responsibilities; (2) Governmentowned reactors (also Docket 50) for which NRC proviowes technical expertise and occasional review, (3) design certification and combined licensing (Docket 52) for which NRC has full review and licensing responsibilities; (4) monitored retrieval storage and Topical Reports for storage casks (Project M's) for which NRC has fult review and licensing responsibilities. Each class is identified with a $\mathrm{P}$ prefix, followed by a series of numbers. 


\section{DEFINITIONS OF NONDOCKETED CATEGORIES}

\section{PROJ - Projects (continued)}

These files contain all documentation up to the time NRC considers the application and the preliminary technical reports complete for review. The project is then assigned a docket number and the file is transferred to a docket serjes.

- Applications submitted by the vendors or utility

- Backup technical documents and topical reports submitted in support of the application, and related correspondence

- Preliminary Safety Analysis Report (PSAR), amendments, and related correspondence (Docket 50)

- Pretiminary Standard Safety Analysis Report (PSSAR), amendments, and related correspondence (Docket 52)

- Application for Prelíminary and Final Design Approval and design certification
(Docket 52 and Project M), and related correspondence

- Early site review information

- Meeting notices, summaries, and correspondence

- NRC's or the applicant's changes in intemal arganizational structure

- Reliability Assurance Program and related correspondence (Docket 52)

- Documentation and technical correspondence berween NRC and other government agencies relating to the application

- Adjudicatory correspondence

- Quality Assurance documentation, notices of deviation, and related correspondence

\section{PT 21 - 10 CFR Part 21 Deficiency Reports}

This file contains deficiency reports submitted in compliance with Part 21 of Title 10 of the Code of Federal Regularions.

- Reports by firms involved in the nudear industry reporting noncompliance with NRC regulations or the existence of defects that could create à substantial safety hazard

- Correspondence pertaining to the NRC review of reports to assess the possibility of generic defects

- NRC inspection reports of defects cited 


\section{DEFINITIONS OF NONDOCKETED CATEGORIES}

PT 21 - 10 CFR Part 21 Deficiency Reports (continued)

- Correspondence specifying appropriate

followup actions to be taken by the agency

\section{QA 999 - Vendor Quality Assurance Inspections}

This file contains inspection reports of the vendor/licensee interface from the vendor's view, the vendor's products, and the vendor's programs including equipment environmenta: qualification testing, and followup correspondence. Each vendor is assigned a docket number and is a part of the QA 999 series.

- Vendors are defined as companies, both U.S. and foreign, that are engaged in the design, manufacture, or testing of nuclear components or in supplying services to the nuclear industry. The controlling criteria are found in 10 CFR Part 21, 10 CFR Part 50, the PSAR and FSAR submitted by utilities for specific facilities, and vendor QA topical reports.
- NRC inspection reports of vendor facilities that cover the design, fabrication, and testing of components for use in nuclear facilities and related correspondence

- Civil penalties notices

- Notices of deviation

- Vendor corrective actions and other followup correspondence associated with resolution of inspection findings

NOTE: For vendor/licensee inspections from the licensee's view, see category $Q$ in Docket 50 and 52 .

\section{RCSSD - Registration of Certificates for Sealed Sources and Devices Change}

This file contains doctuments and related corresponderke on radiation sources and device designs. Manufactures and distributors applying for registration of their products must submit for review all safety retated information, design specifications, QA programs and amendments. These documents are maintained in the PDR on microfiche only. 


\section{DEFINITIONS OF NONDOCKETED CATEGORIES}

\section{REGGD - Regulatory Guides}

This file contains new and revised regulatory guides and related correspondence. These guides describe to the industry and to the public methods acceptable to the NRC staff for implementing specific parts of NRC regulations. They also delineate techniques used by the staff in evaluating specific probiems or postulated accidents or provide guidance to applicants.

- Federal Register notices announcing the issuance and availability of a proposed Regulatory Guide and revisions

- Revised or newly issued regulatory guides
- Drafts of proposed regulatory guides or proposed revisions

- NRC value/impact assessment

- Comments submitted by the nuclear industiy and interested members of the public regarding a proposed regulatory guide

- Summaries of meetings held in the NRC to discuss proposed regulatory guides

NOTE: Industry codes and standards cited in regulatory guides are available for reference tise only in the NRC's Technical Library.

\section{RES - Research and Technical Assistance Reports (1974 through 1988)}

This fite contains reports and technical letters stbmitted by NRC contractors providing research and technical assistance to the Com. mission. It also includes research information letters and conference proceedings pertaining to research activities of the Commission.
For final reports of research conducted by contractors, and for conference proceedings, see the NUREG-series nondocket tile. See also the WMRES file for research reports on disposal of high-level waste.

\section{REVIEW GRP - Review Groups}

This file contains documents pertaining to the activities of internal and extemal research review groups. Such groups provide a broad base of technical expertise to aid management in reviewing research in defined technical areas. They review and comment on research plans and pre- dicted results of major experiments, the validity and applicability of research results, possible redirection of research projects, new research projects, and priorities within the technical areas covered by the research review group. 


\section{DEFINITIONS OF NONDOCKETED CATEGORIES}

\section{REVIEW GRP - ReFiew Gronps (continued)}

- Agtnda for quarterly review group meetings

- Summary minutes of review group meetings representing views expressed, including dissenting views

- Research plans and predicted resuits of major experiments and presentations by research contractors
- Comments on meetings from attendees, including representatives from contractors, universities, vendors, and other agencies

- Internal memoranda relating to topics under discussion

- Correspondence related to the aforementioned items

\section{RM - Rulemakings}

This fite contains documents pertaining to public hearings held on proposed charges to the Commission's regulations. Hearings are instituted at the discretion of the Commission, based on the importance and the magnitude of the subject being considered.

- Designation of the presiding officer and any special directions for the conduct of the hearing

- Notifications of Hearing Board meetings
- Petitions, motions, testimony, and statements submitted by parties to the hearings

- Transcripts of the proceedings

- Hearing Board orders and rulings

- Technical reports submitted as evidence in the proceedings

- Reports, if any, of the special Board to the Commission regarding the subject proceeding 


\section{DEFINITIONS OF NONDOCKETED CATEGORIES}

\section{SAI - Strategic Assessment Issue Papers}

This file contains documents pertaining to NRC policy options for assessment and rebaselining for future decisionmaking and regulatory activities.

- Strategic assessment issue papers

- Strategic process and framework papers
- Comments from Congress, other Federal agencies, agreement and nonagreement states, NRC licensees, industry organizations, foreign governments, NRC employees, public interest groups, and the general public (all affected parties are known as stakeholders)

- Transcripts of stakeholder meetings

\section{SECY - Staff Position Papers Prepared for the Commisston}

This file contains staff papers (generally referred to as SECY Papers) prepared for the informa. tion or approval of the Commission. They are assigned a SECY report number.

- Policy issue papers are used to present matters relating to policy formulation

- Rulemaking issue papers are used to present rulemaking activities of the Commission

- Adjudicatory issue papers are used to present matters relating to the granting, suspending, revoking or amending of licenses, or relating to construction permits for nuclear power plants, and activities in which the Commission has an interest

- Information Papers are used to provide information to the Commission. Some (e.g., the weekly report of the Executive Director for Operations) provides general information about agency activities without specific reference to policy formulation, rulemaking, or adjudication

These categories of staff papers may be issued as one of the following: (a) a Commission meeting paper to be discussed at a Commission meeting; (b) an Affimation paper that does not require interactive discussion among the Com* missioners in a Commission meeting but by law must be voted upon by the Commissioners in the presence of each other, (c) a notation vote paper that does not require interactive discussion among all Commissioners or formal vote in a . meeting, thereby lending itself to a written notation process; (d) a negative consent paper that contains issues which the Commission would like brought to its attention before action is taken but which does not require the formality of a Commission vote; and (e) information papers that provide information to the Commission and do not call for any action by the Commission. 


\section{DEFINITIONS OF NONDOCKETED CATEGORUES}

\section{SECY - Staff Position Papers Prepared for the Commission (continued)}

As a general practice, papers that are regularly reieased to the public inciude those that are the subject of ciscussion at a public meeting of the Commission and those that address unclassified matters pertaining to the export and import of nuclear equipment and material.

NOTE: For Weekly Information Reports (WIR) prepared by the NRC Office of the Executive Director for Operations to the Commission providing a summary of key events and items of interest undertaken by staff and program offices, see the Commission Correspondence nondocket tile.

\section{SPCH - Speeches}

This ile contains speeches by the Chairman and Commissioners presented at National and international regulatory information conferences, industrial forums, advisory committees, \$ymposiums, workshops, and energy policy groups.

\section{STATE PROG - State Programs}

This file contains documents that pertain to the Commission's relations with State governments. Areas covered include: (1) the NRC and State Agreement and its amendments, whereby States may assume certain of the NRC's regulatory authority over nuclear materials; and (2) a wide range of liaison and cooperative activities regarding such functions as licensing, inspection procedures, and waste disposal.

- Correspondence between the NRC Office of State Programs and State officials, State liaison officers and radiation control management health officials, regarding:

- Cooperative programs in such technical areas as radiological incidents, water quality, radioactive waste disposal, and transportation surveillance

- NRC reviews of Agrement State radiation control programs

- Technical assistance to Agreement States in reviewing applications, com. pliance actions, developing regulations, and other areas

- Notices prohibiting involvement in NRClicensed activities

- Agenda, reports, and transcripts of regional workshops 


\section{DEFINITIONS OF NONDOCKETED CATEGORIES}

\section{STATE PROG - State Programs (continued)}

- Training programs on current matters of policy and regulations; i.e., decommissioning and radjoactive waste disposal

- State Iegislative reports (discontinued in December 1984)
- Agreement State licensing statistics reports (discontinued in December 1984)

NOTE: For specific uranium milling and mining projects in which the NRC is providing technical assistance to States, see the Project nondocket fíle.

\section{TOPREP - Topical Reports and Related Correspondence}

This file contains Topical Reports, generally of a generic technical nature, submitted by vendors; and correspondence pertaining to the NRC review of these reports. Vendor-related correspondence that is not docket specific and documents generated by vendor-related owners groups are also included in this file.

- Topical reports dealing with such technical matters as specifications, testing, and safety that are submitted by architects, engineers, and other vendors

- Revisions, modifications, and amendments to topical reports
- Correspondence between NRC and vendors pertaining to the review of submittals

- Meeting agenda, summaries, minutes and correspondence pertinent to vendor activities, programs, methodologies, and design criteria

- Vendor-related Owners' Group meeting announcements, ageoda, summaries, reports and correspondence

NOTE: For reports submitted by utilities that are written by vendors on their behalf and that relate to tocketed cases, see the file categories for the docket series.

\section{WASH - AEC-Issued Formal Reports and Related Correspondence}

This file contains formal reports that were issued by the U.S. Atomic Energy Commission (AEC) headquarters staff and related correspondence. The WASH reports, which consisted of administrative, scientific, and technical information, ended in 1975, but correspondence occasionally refers to WASH reports. Post -1975 NRC-issued formal reports carry the NUREG-series designation. 


\section{DEFINITIONS OF NONDOCKETED CATEGORIES}

\section{WM - Waste Management Documents}

This file contains documents related to the activities of the NRC Division of Waste Management. Each topic has been assigned a unique number, prefixed by the letters WM. Documents in these files include:

- NRC and DOE prelicensing activities regarding the disposal of high-level waste in geologic repositories, in accordance with the Nuclear Waste Policy Act of 1982 as follows:

- Technical high-level waste site pre. licensing documentation

- Meeting agenda, minutes and backup documents of DOE/NRC meetings in which DOE presents information on its geologic exploration program to jdentify sites for nuclear waste repositories

- Briefings by DOE on current research on nuclear waste management
- NRC reviews of the site-by-site implementation of DOE's program for uranium mill tailings remedial actions, in accordance with the Uranitm Mill Tailings Radiation Control Act of 1978

- Correspondence and comments between NRC and the Environinental Protection Agency (EPA) pertaining to the bigh-level waste standard

- NRC topical report reviews and associated correspondence

- Corzespondence from the public and other nongovermment organizations for the aforemention items

NOTE: For contractor research on waste management activities, see the WMRES nondocket fife.

\section{WMRES - Waste Management Contract Research Documents}

This file contains interim and final reports and correspondence between NRC and its contractors regarding prelicensing activities in the area of high-level waste disposal in geotogic repositories, including:

- Periodic contractor progress reports
- Tríp reports

- Draft and final reports submitted for NRC review or information 


\section{DEFINITIONS OF NONDOCKETED CATEGORIES}

WMRES - Waste Management Contract Research Documents (continued)

- Correspondence between NRC staff and

contractors related to technical questions or

issues to be resolved

\section{XPORTLIC - Export and Import Licenses}

This file contains records relating to the issuance of licenses to export or import nuclear tacilities and materials, including:

- License applications and amendrnents to export or import special nuclear material, source material, byproduct material, reactors, nuclear facility components, or reactor materials

- Correspondence between NRC and applicant or licensee pertaining to the review or status of a pending application or license amendment

- Commission letters requesting Executive Branch (e.g., U.S. Department of State) views

- Review and findings of the Executive Branch on whether issuance of license would be inimical to the common defense and security of the United States
- Correspondence to and from foreign govemments, international organizations, and members of the public

- Hearing records and decistons

- Memoranda from the Office of the Secretary to the Executive Director for Operations (EDO) regarding the Commission's decision on issuing a proposed license

- Licenses, license amendments, and license renewals

- Periodic report on export actions

NOTE: For transcripts of Commission meetings and papers submitted for Commissioner consideration or information, see the 10 CFR 9.7 and the SECY nondocket file. 